\title{
Al]103 804275
}

NIST

PUELICATIONS

\section{Publications of the}

National Institute of

Standards and Technology

1991 Catalog

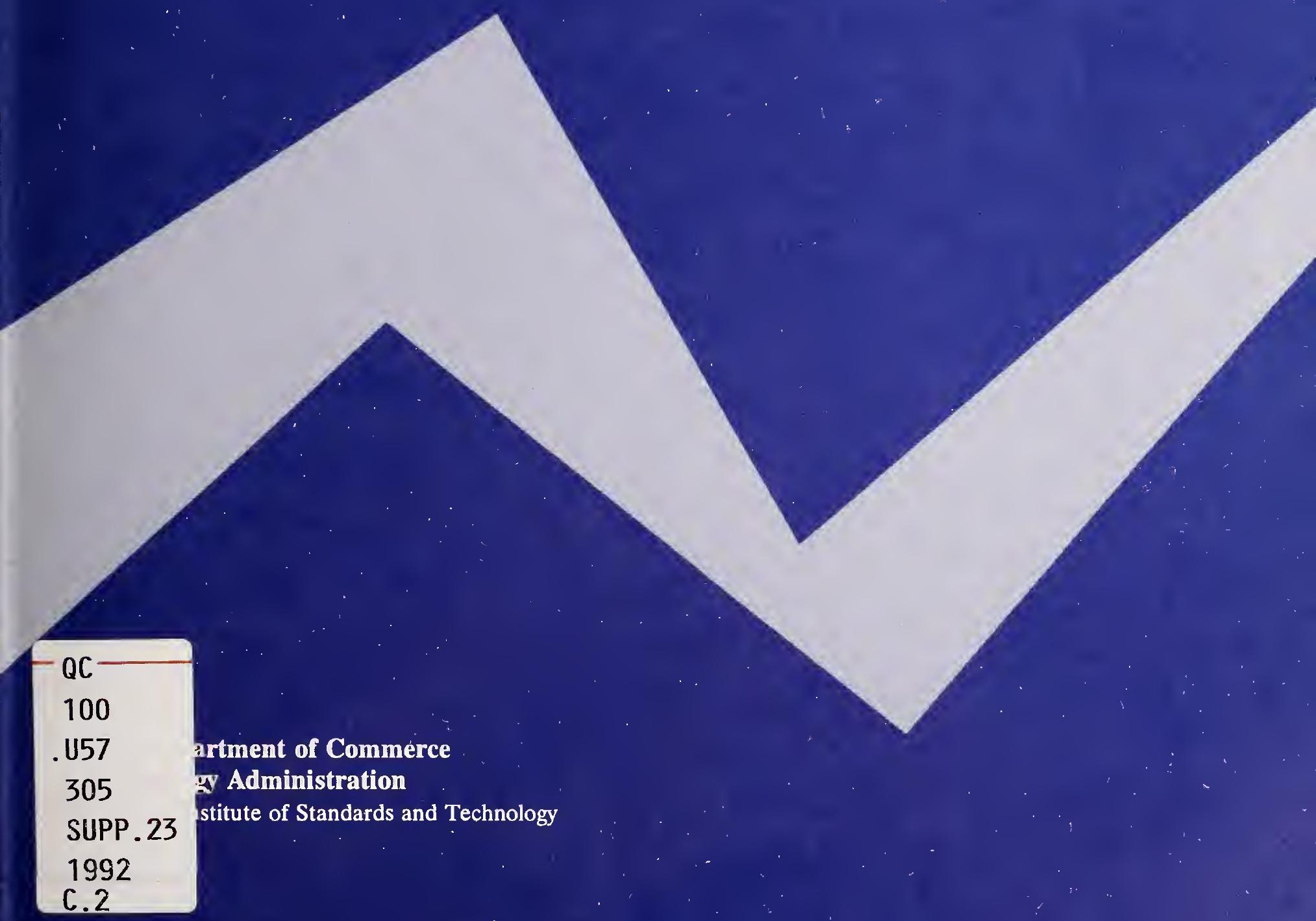


he National Institute of Standards and Technology was established in 1988 by Congress to "assist

industry in the development of technology ... needed to improve product quality, to modernize manufacturing processes, to ensure product reliability . . . and to facilitate rapid commercialization . . . of products based on new scientific discoveries."

NIST, originally founded as the National Bureau of Standards in 1901, works to strengthen U.S. industry's competitiveness; advance science and engineering; and improve public health, safety, and the environment. One of the agency's basic functions is to develop, maintain, and retain custody of the national standards of measurement, and provide the means and methods for comparing standards used in science, engineering, manufacturing, commerce, industry, and education with the standards adopted or recognized by the Federal Government.

As an agency of the U.S. Commerce Department's Technology Administration, NIST conducts basic and applied research in the physical sciences and engineering and performs related services. The Institute does generic and precompetitive work on new and advanced technologies. NIST's research facilities are located at Gaithersburg, MD 20899, and at Boulder, CO 80303. Major technical operating units and their principal activities are listed below. For more information contact the Public Inquiries Desk, 301-975-3058.

\section{Technology Services}

- Manufacturing Technology Centers Program

- Standards Services

- Technology Commercialization

- Measurement Services

- Technology Evaluation and Assessment

- Information Services

\section{Electronics and Electrical Engineering} Laboratory

- Microelectronics

- Law Enforcement Standards

- Electricity

- Semiconductor Electronics

- Electromagnetic Fields'

- Electromagnetic Technology

\section{Chemical Science and Technology} Laboratory

- Biotechnology

- Chemical Engineering ${ }^{1}$

- Chemical Kinetics and Thermodynamics

- Inorganic Analytical Research

- Organic Analytical Research

- Process Measurements

- Surface and Microanalysis Science

- Thermophysics ${ }^{2}$

\section{Physics Laboratory}

- Electron and Optical Physics

- Atomic Physics

- Molecular Physics

- Radiometric Physics

- Quantum Metrology

- Ionizing Radiation

- Time and Frequency

- Quantum Physics'
Manufacturing Engineering Laboratory

- Precision Engineering

- Automated Production Technology

- Robot Systems

- Factory Automation

- Fabrication Technology

Materials Science and Engineering Laboratory

- Intelligent Processing of Materials

- Ceramics

- Materials Reliability

- Polymers

- Metallurgy

- Reactor Radiation

Building and Fire Research Laboratory

- Structures

- Building Materials

- Building Environment

- Fire Science and Engineering

- Fire Measurement and Research

Computer Systems Laboratory

- Information Systems Engineering

- Systems and Software Technology

- Computer Security

- Systems and Network Architecture

- Advanced Systems

Computing and Applied Mathematics Laboratory

- Applied and Computational Mathematics ${ }^{2}$

- Statistical Engineering ${ }^{2}$

- Scientific Computing Environments ${ }^{2}$

- Computer Services ${ }^{2}$

- Computer Systems and Communications ${ }^{2}$

- Information Systems

\footnotetext{
${ }^{1}$ At Boulder, CO 80303.

${ }^{2}$ Some elements at Boulder, CO 80303 .
} 


\section{Publications of the}

National Institute of

Standards and

Technology

1991 Catalog

Ernestine T. Gladden, Editor

Office of Information Services

National Institute of Standards and Technology

Gaithersburg, MD 20899

Issued June 1992

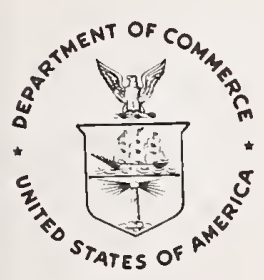

U.S. Department of Commerce

Barbara Hackman Franklin, Secretary

Technology Administration

Robert M. White, Under Secretary for Technology

National Institute of Standards and Technology

John W. Lyons, Director 
National Institute of Standards and Technology Special Publication 305 Supplement 23 to Accompany National Bureau of Standards Special Publication 305 and its Supplements 1 through 22 Natl. Inst. Stand. Technol. Spec. Publ. 305 Suppl. 23, 443 pages (June 1992)

CODEN: NSPUE2

\section{U.S. GOVERNMENT PRINTING OFFICE}

WASHINGTON: 1992

For sale by the Superintendent of Documents, U.S. Government Printing Office, Washington, DC 20402. 


\section{CONTENTS}

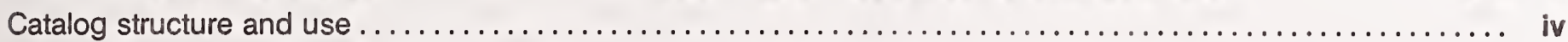

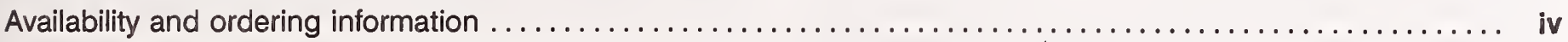

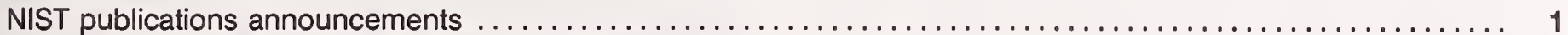

Indexes

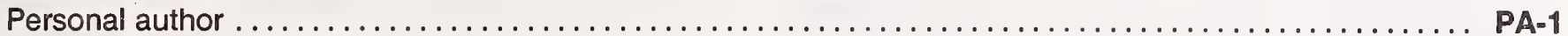

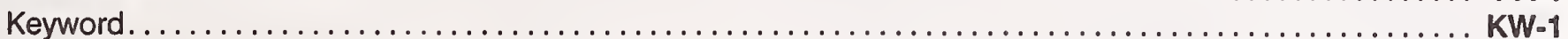

Title ................................................................ Tl

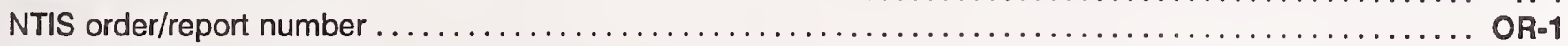

Appendixes

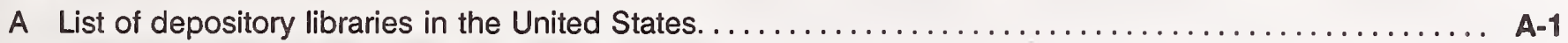

B List of district offices of the U.S. Department of Commerce $\ldots \ldots \ldots \ldots \ldots \ldots \ldots \ldots \ldots \ldots \ldots \ldots$ B 1

Order forms. ..................................................

NIST technical publications program $\ldots \ldots \ldots \ldots \ldots \ldots \ldots \ldots \ldots \ldots \ldots \ldots \ldots \ldots \ldots$ inside back cover

NTIS subject categories $\ldots \ldots \ldots \ldots \ldots \ldots \ldots \ldots \ldots \ldots \ldots \ldots \ldots \ldots \ldots \ldots \ldots \ldots \ldots \ldots \ldots$ back cover 


\section{CATALOG STRUCTURE AND USE}

Full bibliographic citations including keywords and abstracts for National Institute of Standards and Technology (NIST) papers published and entered into the National Technical Information Service (NTIS) collection are cited in the "NIST Publications Announcements" section of this catalog. (Also included are papers published prior to 1991 but not reported in previous supplements of this annual catalog.) Entries are arranged by NTIS subject classifications which consist of 38 broad subject categories (see back cover) and over 350 subcategories. Within a subcategory, entries are listed alphanumerically by NTIS order number.
Four indexes are included to allow the user to identify papers by personal author, keywords, title, and NTIS order/report number. Each entry lists the appropriate title, the NTIS order number, and the abstract number.

Papers may also be identified by searching the NTIS database either online via commercially available systems such as DIALOG, or in the issues of NTIS's Government Reports Announcements and Index and its Government Reports Annual Index.

\section{AVAILABILITY AND ORDERING INFORMATION}

The highest quality and least expensive copies of NIST publications published as Government documents are available from the Superintendent of Documents, U.S. Government Printing Office, Washington, DC 20402. Publications cited with stock numbers (SN) should be ordered by these numbers. GPO will accept payment by check, money order, VISA, MasterCard, or deposit account. For availability and price, write to the GPO or telephone (202) 783-3238. Should an NIST publication be out of print at the GPO, its continued availability is assured at NTIS which sells publications in microfiche or paper copy reproduced from microfiche.

If an entry has a price code, such as PC A04/MF A01, the publication may be ordered from NTIS in paper copy (PC) or microfiche (MF) or both if both codes are given. Order from the National Technical Information Service, 5285 Port Royal Road, Springfield, VA 22161. A copy of the latest price code schedule is available from NTIS. NTIS will accept payment by check, money order, VISA, American Express, MasterCard, or deposit account. NTIS is the sole source of Federal Information Processing Standards (FIPS), Interagency Reports (IRs), and Grant/Contract Reports (GCRs). For more information call (703) 487-4650.

Papers noted "Not Available NTIS" may be obtained directly from the author or from the external publisher cited. Such papers are not for sale by either the GPO or NTIS.

Two other sources for NIST publications are depository libraries (libraries designated to receive Government publications) and Department of Commerce District Offices. The depository libraries listed in Appendix A receive selected NIST publications (see inside back cover for a description of the various NIST publication series). While not every Government publication is sent to all depository libraries, certain depositories designated as Regional Depositories receive and retain one copy of all Government publications made available. Contact the depository library in your area to obtain information on what is available and where.

Department of Commerce District Offices listed in Appendix $B$ provide ready access at the local level to publications, statistical data and summaries, and surveys. Each District Office serves as an official sales agency of the Superintendent of Documents, U.S. Government Printing Office. A wide range of Government publications can be purchased from these offices. In addition, the reference library of each District Office contains review copies of many Government publications. 


\section{SAMPLE ENTRY}

\section{ENVIRONMENTAL \\ POLLUTION \& \\ CONTROL}

\begin{abstract}
Alr Pollution \& Control
100,905

PB91-167353
\end{abstract}

Natlonal Inst. of Standards and Technology (BFRL),

Gaithersburg, MD.

Proposed Standard Practlce for Assessing the

Performance of Gas-Phase Alr Cleaning Equlpment

S. Sllbersteln, Mar 91, 23 p

NISTIR-4523

Contract F-000000

Key words: *Indoor alr pollutlon *Alr cleaners, *Alr pollutlon control equlpment, *Standards, Alr flow, Activated carbon, Servlce life, Alr filters, Performance, Contaminants, Airborne wastes.

The proposed standard practlce provides a general and flexible laboratory method for assessing the performance of equlpment for controlling indoor concentratlons of gas-phase alr contamlnants. Using a canlster fllled with adsorptlon medla, a profile of breakthrough concentratlon over time is obtalned durlng each test conducted at a flxed contaminant challenge concentration. Results of tests performed for different contaminants and different challenge concentratlons can be used for estimating the useful life of alr cleaning equlpment, and for comparing equlpment. The Information will be useful to the engineer for the design and selectlon of such equipment.

\section{NTIS Subject Category}

NTIS Subcategory

Abstract Number

NTIS order number

Corporate or performing organization

Availability Price Codes

\section{Report Title}

Personal authors $\quad$ Report date Page count
Report Number
Contract or grant number

Keywords: * Indlcates keyword index entry

Abstract

\section{ADMINISTRATION \& MANAGEMENT}

\section{Management Practice}

\section{0,001}

PB91-167833

PC A05/MF A01

National Inst. of Standards and Technology, Gaithersburg, MD.

Interim Report to the President and to the Congress on the Malcolm Baldrige National Quality Award.

Interim rept. (Final)

Apr $91,90 \mathrm{p}$

Portions of this document are not fully legible. This publication does not have a series number assigned.

Keywords: *Quality control, "Awards, United States, Productivity, Small business, Manufacturers, Indus- tries, Information transfer, "Quality management, Malcolm Baldrige National Quality Award.

Contents: A Note from the Secretary; Acknowledgments; Executive Summary; The Malcolm Baldrige National Quality Improvement Act of 1987: Background and Purposes; Award Program Design; 1988 Award Program; 1989 Award Program; 1990 Award Program; Program; 1989 Award Program; 1990 Award Program;
Progress in Information Transfer; Benefits of the Award Program; Implications of the Award: Strengthening U.S. Competitiveness: Key Issues; Findings and Conclusions; Appendices.

100,002

PB92-126481

PC A03/MF A01

National Inst. of Standards and Technology, Gaithersburg, MD.

Feasibility of Using a Multiple Award Schedule for Specifying Paints in Government Painting Contracts.

M. E. McKnight. Nov 91,19 p NISTIR-4706

See also PB86-230653. Sponsored by Tri-Services Facilities Coatings Committee.

Keywords: "Government procurement, "Contracts, *Awards, Painting, Coatings, General Services Administration.

The Department of Defense is placing increasing emphasis on the use of commercially available or brand- ed products in lieu of Military or Federal Specification products in building construction and maintenance. However, there are few industry consensus specifications for paints and coatings. Although non-government consensus coating specifications are being developed, a General Services Administration Federal Supply Schedule provides an additional mechanism through which Federal agencies can obtain commercially available coatings. Specifically, the use of a Multiple Award Federal Supply Schedule in a military painting contract is discussed in the report. Procedures for obtaining the lowest cost material that would meet the Government's minimum needs are addressed. A description of need based upon performance criteria for the specific end use, that would be included in a painting contract, is suggested. Advantages and disadvantages of using a Multiple Award Schedule in painting contracts are enumerated. Advantages include providing a mechanism for using new coating technology, taking advantage of manufacturer's technical support and knowledge of regulatory issues, and decreasing the need for revising or preparing new coating specifications. Disadvantages include the additional effort needed to revise guide specifica. tions, train both coating users and suppliers in new procedures and the lack of performance criteria for some end uses. Despite the disadvantages cited, it is concluded that the advantages outweigh the disadvantages and that use of a Multiple Award Schedule in painting contracts is feasible. 


\section{Personnel Management, Labor Relations \& Manpower Studies}

100,003

PB91-231555

PC A05/MF A01

National Inst. of Standards and Technology, Gaithersburg, MD. Office of Personnel and Civil Rights.

NIST Personnel Management Demonstration Project: Design, Implementation and Accomplishments.

A. Cassady. Jul 91, 94p NISTIR-4640

Keywords: "Personnel management, "Payment systems, Government employees, Compensation, Income, Employment, Performance evaluation, Performance standards, Classification, Demonstration projects, Managers, "National Institute of Standards and Technology.

The NIST Authorization Act for Fiscal Year 1987 provided for a 5-year project to demonstrate an alternative personnel management system, which was implemented January 1, 1988. The project system was built on the concepts of total compensation comparability, market sensitivity, pay for performance, administrative simplicity, management flexibility, and governmentwide applicability. Designed to improve hiring and retention of high-quality personnel and to more effectively compensate and retain high performers, the project dramatically changed the way NIST administers pay, position classification, recruitment, qualifications examination, retention, and performance management for former General Schedule employees. Evaluations and feedback from managers and employees have shown that project personnel systems have improved NIST's ability to recruit and retain quality staff; make compensation more competitive; link pay to performance; simplify position classification; streamline processing; improve the staffing process and get new hires aboard faster; and increase the manager's role and accountability in personnel management.

\section{Productivity}

\section{0,004}

PB91-177774

PC A99/MF A04

National Inst. of Standards and Technology, Gaithersburg, MD. Office of Standards Code and Information. Standards Activities of Organizations in the United States.

Special pub. (Final)

R. B. Toth. Feb 91,738 p NIST/SP-806

Supersedes PB85-106151. Also available from Supt. of Docs. See also PB89-221147.

Keywords: "Standardization, "Organizations, "United States, "Directories, Standards, Trade associations, Sources, National government, Specifications, Military organizations, Technical societies, Recommendations.

The directory is a guide to mandatory and voluntary standards activities in the United States at the national level - both governmental and nongovernmental (trade associations, technical and other professional societies). It excludes proprietary (company standards) and state and local levels of government (i.e., county and municipal). It supersedes the 1984 edition (NBS SP 681), 'Standards Activities of Organizations in the United States.' It includes standards distributors, libraries, and information centers, and union lists of standards repositories by regional areas. It also lists organizations that no longer develop standards. Over 750 current descriptive commentaries are formatted, with subject headings to facilitate access to specific information. The main sections cover nongovernment; federal government; sources of standards documents and information; a subject index and related listings covering acronyms and initials, defunct bodies, and those organizations with name changes. Organizations have been included if they develop standards or contribute to the standardization process, whether voluntary or mandatory, or are sources of standards documents or information. An introductory section provides general information on federal (including military) standards activities, a list of 20 major nongovernment standards developers, some historical notes, and an overview of U.S. (national) standardization activities.

\section{Public Administration \& Government}

100,005

PB91-167379

PC A06/MF A01

National Inst of Standards and Technology Gaithersburg, MD. Office of Standards Code and Information. Directory of Federal Government Laboratory ACcreditation/Designation Programs.

Special pub. (Final)

M. Breitenberg. Feb 91, 105p NIST/SP-808

Also avait
201512.

Keywords: "Laboratories, "Certification, "Directories, Specifications, Regulations, Standards, Inspection, Grading, Data bases, "Federal Government certification, Approved products.

The directory is designed to provide updated information on federal government laboratory accreditation and similar type programs conducted by the federal government. The programs designate a set of laboratories or other entities to conduct testing to assist federal agencies in carrying out their responsibilities. The programs include an assessment regarding the capability of the laboratory to conduct the testing. The type and degree of such assessments, however, vary greatly by program. It should be noted that the entries in the directory are based primarily on information provided by the federal agency and reflect the agency's view of its activities. The directory is part of ongoing NIST ef forts to establish and maintain comprehensive databases on standards, regulations, laboratory accreditation and certification programs and related information in accordance with the requirements of the Trade Agreements Act of 1979. The material has been compiled to meet the needs of government, industry, and the public for information on U.S. Government laboratory accreditation and related programs.

\section{0,006}

PB91-222695

PC A05/MF A01

National Inst. of Standards and Technology (BFRL), Gaithersburg, MD

Standards for the Physical Protection of National Resources and Facilities.

Final rept.

R. D. Dikkers. Jul $91,89 p$ NISTIR-4618

Contract IAA-EMW-90-E-3279

Sponsored by Federal Emergency Management Agency, Washington, DC

Keywords: "Federal buildings, "Security, "Standards, Federal agencies, Equipment, Safety engineering, $\mathrm{Na}$ tional government, Facilities, Area security, "Federal Emergency Management Agency.

The specific objectives of the study are: (1) to identify and compile existing standards and guidelines pertaining to the physical protection of facilities and resources; and (2) to prepare a plan and strategies for developing national standards which may be needed to assist Federal departments and agencies in the protection of their facilities and resources.

\section{Research Program Administration \& Technology Transfer}

100,007

PB91-187559 PC A03/MF A01 National Inst. of Standards and Technology, Gaithersburg, MD.

NIST Research Reports, March 1991.

Special pub.

Mar 91, 41p NIST/SP-809

Also available from Supt. of Docs. as SN003-003$03081-3$. See also PB91-112813.

Keywords: "Research, International trade, Federal budgets, High temperature superconductors, Electric contacts, Grants, Technology transfer, Drugs, Chemical analysis, Telecommunication, Polymer matrix composites, Helmets, Integrated circuits, MMIC, US NIST, Advanced Technology Program, Integrated Services Digital Network.

Contents: Research Update; America's Technology Opportunities; 1992 Budget Seeks Increase for NIST; Making Good Contact; Grants to Advance Key Industrial Technologies; Helping Companies Up the Technology Ladder; New Instrument Promises Improved
Tracking of Drugs; Call of the Future; A New Generation of Materials; Manufacturers, Skiers Gain Competitive Edge; Microwaves on a Chip; New Publications; Conference Calendar.

\section{0,008}

PB91-195545

Not available NTIS

National Bureau of Standards (NEL), Gaithersburg MD. Office of Energy-Related Inventions.

Trends in Technological Innovation.

Final rept.

H. Robb. $1988,4 p$

Sponsored by American Society of Mechanical Engineers, New York

Pub. in Proceedings of the Intersociety Energy Conversion Engineering Conference (23rd), Denver, CO., July 31-August 5, 1988, p305-308.

Keywords: "Technology innovation, "Trends, Research and development, Technology utilization, Inventions, Changes, Industries, Manufacturing, Businesses, Reprints.

In the last twelve years, several trends have begun to emerge with respect to the type of inventor and the type and level of technology that have succeeded and will succeed (or fail) in the commercial marketplace. The rate of change taking place in almost all areas of technology is not just accelerating, it is amazing. It goes without saying that the kinds of technologica changes that are taking place are affecting the future of almost all manufacturing industries. In addition, even many institutions in non-technological areas, including some that seem far removed, are being affected by the rate of technological change. For instance, it is interesting to note that in the specific case of banking the rate of change in technological developmen throughout the general scientific community is affecting them in ways that many in the banking industry seem to not be aware of. Yet, these many technological developments are quietly restructuring their industry, from the outside. Similar trends are underway in most areas of business.

100,009

PB91-204032

Not available NTIS

National Bureau of Standards (IMSE), Gaithersburg MD.

Advanced

Final rept.

Final rept.

H. Schwartz. 1988, 8p

Pub. in Proceedings of Israel Materials Engineering 1988, p51-58.

Keywords: "Materials, "Process control, "Research projects, "Data bases, Polymers, Mechanical properties, Cost analysis, Intermetallic compounds, Design criteria, Composite materials, Ceramics, Reprints, Research needs.

Advanced materials include high performance composites, fine ceramics, intermetallic alloys, and advanced polymers. All technologically developed nations have identified these advanced materials along with biotechnology and information technology as the three principal emerging technologies for the next sev eral decades. However, several obstacles must be overcome before the laboratory promise of advanced materials can be converted into practical, economically attrative alternatives to existing materials. Principal among these obstacles are: the demanding process control required to achieve an adequate level of reproducible quality at low cost; the push from international competitors leading to simultaneous rather than sequential development of processing, structure characterization, properties evaluation and performance assessment; the increased variety of materials available for a given application requiring the development of engineering property data bases.

100,010

PB91-206748

PC A03/MF AO1

Itandards and Technology, Gaithersgram.

NVLAP FY 90 Annual Report.

V. R. White. Jun 91,19 p NISTIR-4599 See also PB90-198920.

Keywords: "Laboratories, "Certification, Project management, Research management, Reports, Test facilities, Personnel, Information systems, Research 
projects, Organizational structure, Programs, Program)

The report summarizes activities of the National Voluntary Laboratory Accreditation Program (NVLAP) for the fiscal year which ended September 30,1990. The report covers NVLAP Operations, Technical Activities, Staffing, Publications, Talks and Presentations. The Technical Activities section presents individual descriptions of areas of new activity in FY 90 (Airborne Asbestos Analysis, Secondary Calibration for lonizing Radiation, Plumbing, GOSIP, POSIX, Fasteners, and Calibration), as well as a summary of future program developments.

\section{0,011}

PB91-216531

PC A20/MF A04

National Inst. of Standards and Technology (TS) Gaithersburg, MD. Information Resources and Services Div.

Publications of the National Institute of Standards and Technology, 1990 Catalog.

Rept. for Jan-Dec 90.

E. T. Gladden. Jun 91, 463p NIST/SP-305-SUPPL.

Also available from Supt. of Docs. as $\mathrm{SNOOH}^{-003}$ 03085-6. See also PB90-271818.

Keywords: "Catalogs(Publications), "Bibliographies, Science, Technology Research management, ${ }^{*} \mathrm{Na}-$ tional Institute of Standards and Technology.

Full bibliographic citations including keywords and abstracts for National Institute of Standards and Technology (NIST) (formerly National Bureau of Standards (NBS)) papers published and entered into the Nationa Technical Information Service (NTIS) collection are cited in the 'NIST Publications Announcements' section of the catalog. Entries are arranged by NTIS subject classifications which consist of 38 broad subjec categories and over 350 subcategories. Within a subcategory, entries are listed alphanumerically by NTIS order number. Four indexes are included to allow the user to identify papers by personal author, keywords, title, and NTIS order/report number. Each entry lists the appropriate title, the NTIS order number, and the abstract number.

\section{0,012}

PB92-109172

PC A05/MF A0

National Inst. of Standards and Technology, Gaithers burg, MD. Public Affairs Div.

Research. Services. Facilities. (National Institute of Standards and Technology).

Special pub.

M. A. Bello, G. Porter, and S. A. Shaffer. Aug 91,

$92 \mathrm{p}$ NIST/SP-817

Also available from Supt. of Docs.

Keywords: "Research facilities, *Research programs, US NBS, Cooperation, Government/industry relations, "National Institute of Standards and Technology, US NIST.

Each year more than 1,000 researchers from industry universities, or other government agencies come to NIST to conduct cooperative research projects lasting from a few weeks to several years. The document describes the full spectrum of NIST programs and facilities available for industry participation and use. Each item includes the name and phone number of a NIST program manager or researcher to contact for more information. Contents: Gaining the competitive edge: Serving the customer; Technology services; Electronics and Electrical Engineering Laboratory; Manufacturing Engineering Laboratory; Chemical Science and Technology Laboratory; Physics Laboratory; Materials Science and Engineering Laboratory; Building and Fire Research Laboratory; Computer Systems Laboratory Computing and Applied Mathematics Laboratory; Facilities index; Subject index.

\section{0,013}

\section{PB92-126697}

(Order as PB92-126614, PC A06/MF A02) National Inst. of Standards and Technology, Gaithersburg, MD.

Advanced Technology Program: A New Role for NIST in Accelerating the Development of Commercially Important Technologies.

B. C. Belanger, G. A. Uriano, and R. G. Kammer.

$1991,7 p$

Included in Jnl. of Research of the National Institute of

Standards and Technology, v96 n5 p605-611 Sep/Oct 91.
Keywords: "Technology, Joint ventures, Competition Selection, Awards, "Advanced Technology Program, ATP program, Competitiveness, US NIST.

The Advanced Technology Program (ATP) is a new extra mural program operated by the National Institute of Standards and Technology (NIST) for the Department of Commerce's Technology Administration. The ATP will help enhance U.S. competitiveness by funding the development of pre-competitive, generic technologies in partnership with industry. The paper describes the ATP, the first ATP awards made by NIST, and the prognosis for the success of the ATP.

\section{Exara \\ AERONAUTICS \& AERODYNAMICS}

\section{Aerodynamics}

100,014

PB92-117183

Not available NTIS

National Inst. of Standards and Technology (NEL) Gaithersburg, MD. Fire Measurement and Research Div.

Effects of Global Density Ratio on the Centerline Mixing Behavior of Axisymmetric Turbulent Jets. Final rept.

W. M. Pitts. 1991, 10p

Contract AFOSR-ISSA-85-00012

See also PB87-225413. Sponsored by Air Force Office of Scientific Research, Bolling AFB, DC.

Pub. in Experiments in Fluids 11, p125-134 1991.

Keywords: "Density ration, "Jet mixing flow, "Concentration(Composition), "Turbulent flow, Mixing, Turbulence, Light scattering, Gas flow, Rayleigh scattering, Density(Mass/Volume), Density measurement Reprints.

Measurements, utilizing Rayleigh light scattering, of time-averaged concentration and unmixedness have been made along the centerlines of axisymmetric turbulent jets formed from six pairs of jet and ambien gases. Jet to ambient density ratios range from 0.14 to 5.11. Findings are compared with predictions of an approx similarity analysis and with extensive previous literature measurements. It is shown that virtual origins for plots of inverse time-averaged concentration are strongly dependent on global density ratio. Unmixedness values first grow with increasing distance from the jet source and then achieve an asymptote. The flow distance required to reach this asymptote is a strong function of density ratio.

\section{0,015}

PB92-117209 National Inst. of Standards and Technology (NEL) Gaithersburg, MD. Fire Measurement and Research Div.

Reynolds Number Effects on the Mixing Behavior of Axisymmetric Turbulent Jets.

Final rept.

W. M. Pitts. 1991, 7p

Contract AFOSR-ISSA-85-00012

See also PB87-201836. Sponsored by Air Force Office of Scientific Research, Bolling AFB, DC.

Pub. in Experiments in Fluids 11, p135-141 1991.

Keywords:

${ }^{\star}$ Reynolds

number,

"Concentration (Composition) * Jet mixing flow, *Turbulent flow, Mixing, Turbulence, Light scattering, Gas flow, Rayleigh scattering, Propane, Carbon tetrafluoride, Sulfur hexafluoride, Jets, Reprints.

Measurements of time-averaged jet fluid mass fraction and unmixedness are reported along the centerlines of axisymmetric jets having Reynolds numbers $(\mathrm{Re})$ covering a range of $3,950-11,880$. Jet gases investigated are propane carbon tetrafluoride, and sulfur hexafluoride. The slopes for the fall off of inverse centerline mass fraction with distance are found to be independ ent of Re for moderate downstream distances, but virtual origins for the data are shown to move downstream with increasing Re. Unmixedness measurements show that flows with higher Re require longer flow distances to achieve asymptotic behavior. Results of other investigations reported in the literature are dis cussed which support the conclusions of the work. The relationship between the centerline mixing and entrainment behaviors of these flows is explored.

\section{AGRICULTURE \& FOOD}

\section{Food Technology}

\section{0,016}

PB91-150060

Not available NTIS

National Inst. of Standards and Technology (NML) Gaithersburg, MD. Ionizing Radiation Div.

gamma-Irradiated Seafoods: Identification and Dosimetry by Electron Paramagnetic Resonance Spectroscopy.

Final rept.

M. F. Desrosiers. 1989, 5p

Pub. in Jnl. of Agricultural and Food Chemistry 37, n1 p96-100 Jan/Feb 89.

Keywords: "Seafood, "Food processing, Electron spin resonance, Aquatic animals, Dosimetry, Free radicals Cobalt 60, Acetylglucosamine, Bones, Reprints, "Food irradiation.

Electron paramagnetic resonance (EPR) spectroscopy was used to measure the production of free radicals induced by $(60) \mathrm{Co}$ Gamma-rays in shrimp exoskeleton, mussel shells, and fish bones. The EPR spectrum for irradiated shrimp shell was dose dependent and appeared to be derived from more than one radical. The major component of the radiation-induced spectrum resulted from radical formation in chitin, assigned by comparison with irradiated $\mathrm{N}$-acetyl-D-glucosamine. Other measurements include the total yield of radicals formed as a function of dose and the longevity of the radiation-induced EPR signal. Similar measurements were made for mussel shells and fish bones, and the results are compared and discussed. It was concluded that irradiated shrimp (with shell attached) could definitely be identified by this technique; however, precise determination of absorbed dose was less straightforward. Positive identification of irradiated fish bones was also clearly distinguishable, and dosimetry by EPR appeared to be feasible.

100,017

PB91-194720

Not available NTIS

National Inst. of Standards and Technology (NML) Gaithersburg, MD. lonizing Radiation Div.

Co-Trial on ESR Identification and Estimates of gamma-Ray and Electron Absorbed Doses Given to Meat and Bones.

Final rept.

M. F. Desrosiers, W. L. McLaughlin, L. A. Sheahen, N. J. F. Dodd, J. S. Lea, J. C. Evans, C. C.

Rowlands, J. J. Raffi, and J. P. L. Agnel. 1990, 10p Pub. in International Jnl. of Food Science and Technology 25, p682-691 1990 .

Keywords: "Gamma rays, "Food processing, "Meat "Bones, Electron spin resonance, Poultry, Frogs, Pork Interlaboratory comparisons, Reprints, "Food irradia tion

A multinational co-trial was organized to determine i electron spin resonance (ESR) spectroscopy could be used to monitor foods exposed to ionizing radiation. The bones of chicken legs, frog legs and pork rib bones were prepared and distributed as unknowns to the participating laboratories. In every instance, nonirradiated bones were correctly identified as such Moreover, irradiated bones were not only correctly identified, but relatively good estimates of the absorbed dose were obtained. An intercomparison of the different approaches used by each laboratory is discussed, and recommendations for future trials are presented.

\section{0,018}

PB92-116672

Not available NTIS

National Inst. of Standards and Technology (NML) Gaithersburg, MD. Ionizing Radiation Div. 
Electron Spin Resonance for Monitoring Radiation-Processed Meats Containing Bone.

Final rept.

M. F. Desrosiers. 1991, $2 p$

Pub. in Jnl. of Food Science 56, n4 p1104-1105 1991.

Keywords: "Food processing, "Meat, "Electron spin resonance, "Ionizing radiation, Free radicals, Dose-response relationships, Food industry, Reprints.

Electron spin resonance (ESR) spectroscopy assessed the dose absorbed by radiation-processed meats (containing bone). Additive re-irradiation of the bone generated a dose response curve which could then be used to assess the initial dose from irradiation. An exponential fit to the ESR response to absorbed dose provided a good estimate of initial dose and was a reasonable physical description of the response from absorption of ionizing radiation by bone. These data have implications for regulation of the industry.

\section{ASTRONOMY \& ASTROPHYSICS}

\section{Astrophysics}

\section{0,019}

PB91-148825

Not available NTIS

National Inst. of Standards and Technology (NML), Boulder, CO. Quantum Physics Div.

Modelling the Coronae and Chromospheres of RS CVn Systems by the Analysis of Ultraviolet, $X$-ray and Radio Observations.

Final rept.

J. L. Linsky. 1990, 13p

Contracts NASA-NAG5-82, NASA-H-80531-B

Sponsored by National Aeronautics and Space Administration, Washington, DC

Pub. in Active Close Binaries, p747-759 1990

Keywords: "Stars, Astronomical models, Stellar chromospheres, Stellar coronal, Ultraviolet spectra, Radio spectra, X ray spectra, Binary stars, Reprints.

The author summarizes the present status of chromospheric and coronal models of active stars in RS CVn systems. The first generation models which assumed plane-parallel, one-component layers are now being supplanted by second generation models in which the covering fraction of the plage or flare plasma is estimated by a Doppler-imaging analysis. Third generation models should include the magnetic field geometry and the total energy balance in a self-consistent manner.

\section{0,020}

PB91-148833

Not available NTIS

National Bureau of Standards (NML), Boulder, CO

Quantum Physics Div.

Ultraviolet, Optical, Infrared, and Microwave Observations of HR 5110.

Final rept.

I. R. Little-Marenin, T. Simon, T. R. Ayres, N. L.

Cohen, P. A. Feldman, J. L. Linsky, and S. J. Little. 1986, 11p

Contract NAG5-82

Sponsored by National Aeronautics and Space Administration, Washington, DC.

Pub. in Astrophysical Jnl. 303, n2 p780-790, 15 Apr 86

Keywords: "Binary stars, Ultraviolet spectra, Infrared spectra, Microwave spectra, Optical spectra, Late stars, Reprints, "HR 5110 stars, Algol type stars.

HR 5110 is a close binary system which is viewed nearly pole-on $(i=13 \mathrm{deg})$. A comparison of the characteristics of Algol and RS CVn systems to those of HR 5110 shows that HR 5110 can also be considered an Algol system. Because the primary star is relatively $\mathrm{cool}$ (F2 IV) and there is no apparent emission from an accretion disk, that authors are able to detect in IUE spectra the emission of an active chromosphere and transition region of the cooler (KO IV) secondary. HR 5110 is important because it is the only known Algo system for which the properties of the secondary star can be studied in detail.
100,021

PB91-148957

Not available NTIS

National Inst. of Standards and Technology (NML),

Boulder, CO. Quantum Physics Div.

Studies of $H \mid$ and D I in the Local Interstellar Medium.

Final rept.

J. Murthy, R. C. Henry, H. W. Moos, A. Vidal-Madjar,

J. L. Linsky, and C. Gry. 1990,6p

Pub. in Astrophysical JnI. 356, n1 p223-228, 10 Jun 90.

Keywords: "Interstellar matter, Ultraviolet spectra, Lyman alpha radiation. Emission spectra, Late stars, Hydrogen, Deuterium, Abundance, IUE, Reprints, Capella star.

The authors present high-dispersion IUE spectra of the hydrogen Ly(alpha) chromospheric emission line of two nearby late-type stars, Capella and lambda. Both interstellar $\mathrm{H} 1$ and $\mathrm{D} 1 \mathrm{Ly}$ (alpha) absorption can be seen against the chromospheric line, and the authors have derived the density, velocity dispersion, and bulk velocity of the gas in those lines of sight. They have also placed limits on the $\mathrm{D} / \mathrm{H}$ ratio.

\section{0,022}

PB91-148965

Not available NTIS National Bureau of Standards (NML), Boulder, CO. Quantum Physics Div.

Spatially Resolved Flares in RS CVn Systems.

Final rept.

J. E. Neff, and J. L. Linsky. 1988, 4p

Sponsored by National Aeronautics and Space Administration, Washington, DC.

Pub. in Activity in Cool Star Envelopes, p175-178 1988.

Keywords: "Binary stars, "Stellar flares, Stellar chromospheres, Ultraviolet spectra, Emission spectra, Magnesium, Reprints.

The authors have isolated $\mathrm{Mg} \| \mathrm{k}$ emission line profiles arising solely from the flaring region during flares on AR Lac and $V 711$ Tau. From several high-resolution spectra obtained during the lifetime of the flare, they have determined the size and position of the flaring regions and studied the decay of the emission line width, radial velocity, and integrated line flux.

\section{0,023}

PB91-149021

Not available NTIS

National Inst. of Standards and Technology (NML), Boulder, CO. Quantum Physics Div.

Terminal Velocities for a Large Sample of O Stars, B Supergiants, and Wolf-Rayet Stars.

Final rept.

R. K. Prinja, M. J. Barlow, and I. D. Howarth. 1990 $14 p$

Pub. in Astrophysical Jnl. 361, p607-620, 1 Oct 90

Keywords: "Early stars, "Wolf-Rayet stars, "Supergiant stars, *O stars "Stellar winds, Stellar mass ejection, Terminal velocity, Reprints, Mass loss.

The authors argue that easily measured, reliable estimates of terminal velocities for early-type stars are provided (1) by the central velocity asymptotically approached by narrow absorption features and (2) by the violet limit of zero residual intensity in saturated $P$ Cygni profiles. They use these estimators to determine terminal velocities, nu(infinity) for 1810 stars, 70 early $B$ supergiants, and 35 Wolf-Rayet stars. For OB stars their values are typically $15 \%-20 \%$ smaller than the extreme violet edge velocities, nu(edge), while for WR stars, nu(infinity) .76nu(edge) on average. The authors give new mass-loss rates for WR stars which are thermal radio emitters, taking into account the authors' new terminnal velocities and recent revisions to estimates of distances and to the mean nuclear mass per electron. The authors examine the relationships between nu(infinity) the surface escape velocities, and effective temperatures.

\section{0,024}

PB91-149286

Not available NTIS

National Inst of Standards and Technology (NML), Boulder, CO. Quantum Physics Div.

New Radio Detectors of Early-Type Pre-Main Sequence Stars.

Final rept.

S. L. Skinner, A. Brown, and J. L. Linsky. 1990, 4p

Pub. in Astrophysical Jnl. 357, pL39-L42, 10 Jul 90.

Keywords: "Pre-main sequence stars, "Early stars,

Radio sources(Astronomy). Reprints, Very large array.
The authors present results of VLA radio continuum observations of 13 early-type pre-main-sequence stars selected from the 1984 catalog of Finkenzeller and Mundt. The stars HD 259431 and MWC 1080 were detected at $3.6 \mathrm{~cm}$, while HD 200775 and TY CrA were detected at both 3.6 and $6 \mathrm{~cm}$. The flux density Sv of HD 200775 has a frequency dependence of the form $\mathrm{S}$ (sub nu) varies as nu(sup $0.50+$ or -0.3 ), consisten with the behavior expected for free-free emission originating in a fully ionized wind. However, an observation in A configuration suggests that the source geometry may not be spherically symmetric. In contrast, the spectral index of TY CrA is negative with a flux behavior of the form S(sub nu) varies as nu(sup $-0.15+$ or 0.1 ), implying nonthermal emission. The physical mechanism responsible for the nonthermal emission has not yet been identified, although gyrosynchrotron and synchrotron processes cannot be ruled out.

\section{0,025}

PB91-149849 Not available NTIS National Inst. of Standards and Technology (NML) Boulder, CO. Quantum Physics Div.

Photospheres of Hot Stars. 4. Spectral Type O4. Final rept.

B. Bohannan, S. A. Voels, D. G. Hummer, and D. C. Abbott. 1990,9p

Contract NAGW-766, Grant NSF-AST85-05919

See also PB89-202592. Sponsored by National Aeronautics and Space Administration, Washington, DC. and National Science Foundation, Washington, DC Pub. in Astrophysical Jnl. 365, p729-737, 20 Dec 90

Keywords: "Hot stars, Main sequence stars, Supergiant stars, O stars, Stellar atmospheres, Stellar temperature, Stellar evolution, Reprints.

The basic stellar parameters of a supergiant (zeta Pup) and two main-sequence stars, 9 Sgr and HD 46223, a spectral class $\mathrm{O} 4$ are determined using line profile analysis. The stellar parameters are determined by comparing high signal-to-noise hydrogen and helium line profiles with those from stellar atmosphere models which include the effect of radiation scattered back onto the photosphere from an overlying stellar wind an effect referred to as wind blanketing. At spectral class $\mathrm{O} 4$, the inclusion of wind-blanketing in the mode atmosphere reduces the effective temperature by an average of $10 \%$. The shift in effective temperature is also reflected by shifts in several other stellar parameters relative to previous 04 spectral-type calibrations. It is also shown through the analysis of the two $04 \mathrm{~V}$ stars that scatter in spectral type calibrations is intro duced by assuming that the observed line profile reflects the photospheric stellar parameters.

\section{0,026}

PB91-158956

Not available NTIS

National Inst of Standards and Technology (NML) Gaithersburg, MD. Atomic and Plasma Radiation Div. Working Group 1: Atomic Spectra and Wavelength Standards.

Final rept.

W. C. Martin. $1988,7 p$

Pub in Transactions of the International Astronomical Union, vXXA p111-1171988.

Keywords: "Atomic spectroscopy, Atomic energy levels, Wavelengths, Bibliographies, Reprints.

The bibliographic report describes atomic spectrosco pic research and data of interest for astronomy. It covers the past 3 years.

\section{0,027}

PB91-174664

Not available NTIS

National Inst of Standards and Technology (NML)

Boulder, CO. Quantum Physics Div.

Radio-Continuum Observations of a Variety of Cool Stars.

Final rept.

S. A. Drake, J. L. Linsky, P. G. Judge, and M. Elitzur. $1991,7 p$

Pub. in Astronomical JnI. 101, n1 p230-236 Jan 91.

Keywords: "Radio sources(Astronomy), "Cool stars, Main sequence stars, Supergiant stars, Giant stars, Radio astronomy, Stellar winds, Reprints.

The authors present radio-continuum observations made at 2 and $/$ or $6 \mathrm{~cm}$ of $26 \mathrm{cool}$ stars (FO and later) including $10 \mathrm{~F}-\mathrm{K}$ main-sequence stars and $16 \mathrm{~F}-\mathrm{M}$ giant and supergiant stars. The data were obtained as part of several Very Large Array observing programs. 22 of the observed stars were not detected as sources 
and have typical 3 sigma upper limits of about 0.35 mJy at $2 \mathrm{~cm}$ and about $0.15-0.60 \mathrm{mJy}$ at $6 \mathrm{~cm}$.

\section{0,028}

PB91-174920

Not available NTIS

National Inst. of Standards and Technology (NML),

Boulder, CO. Quantum Physics Div.

Detection of an Expanding H I Shell in the Old Supernova Remnant CTB 80 .

Final rept.

B. C. Koo, W. T. Reach, C. Heiles, R. A. Fesen, and J. M. Shull. 1990, 9p

Sponsored by National Science Foundation, Washington, DC.

Pub. in Astrophysical Jnl. 364, n1 p178-186, 20 Nov 90.

Keywords: "Supernova remnants, "Nebulae, Expansion, Pulars, Reprints.

The authors have detected an expanding $\mathrm{H}$ I shell around the supernova remnant (SNR) CTB 80. The $\mathrm{H}$ shell is clumpy and partially complete, with its SW portion opened. The shell has an expansion velocity of 72 $\mathrm{km} / \mathrm{s}$, a radius of 19 (d sub 2) pc where (d sub 2) is the distance to CTB 80 in $2 \mathrm{kpc}$, and a total H I mass of 1200 (d sup 2, sub 2) solar masses. With its measured kinematic and dynamical properties, CTB 80 provides a 'Rosetta Stone' for studies of old SNRs.

\section{0,029}

\section{PB91-175026}

Not available NTIS

National Inst. of Standards and Technology (NML) Boulder, CO. Quantum Physics Div.

Ground-Truth Observations of Stellar Surface Structure from the Lunar Surface.

Final rept.

J. L. Linsky. 1990, 10p

Grant NAG5-82

Sponsored by National Aeronautics and Space Admin istration, Washington, DC

Pub. in Proceedings of NASA Workshop on Astrophysics from the Moon, Annapolis, MD., February 1990, p168-177.

Keywords: "Stellar activity, "Lunar observatories, Lunar surface, Stellar chromospheres, Starspots, Granulation, Convection, Interferometry, Reprints.

Using increasingly sophisticated observing strategies, astronomers have begun to observe brightness inhomogeneities on the surfaces of stars indicative of starspots, active regions, and chemically-anomalous patches with size scales far smaller than the diffraction limits of the present generation of telescopes. While tantalizing, these first glimpses of stellar surface structures are very crude and not unique. Modest-sized op tical and ultraviolet interferometers located on the lunar surface could resolve these surface structures on nearby, bright stars to provide 'ground truth' to the present crude images and to extend the studies to much smaller and physically interesting scales. An intermediate scale optical/ultraviolet interferometer on the lunar surface with 0.001 to 0.0001 arcsecond angular resolution would provide unique and spectacular results concerning stellar surface structures. The intermediate scale interferometer could be a very useful device for learning how to build larger interferometers that could address more difficult questions.

\section{0,030}

\section{PB91-175034}

Not available NTIS

Boulder, CO. Quantum Physics Div.

Some Concluding Thoughts for Cool Stars VI.

Final rept.

J. L. Linsky. $1989,4 p$

Grant NAG5-82

Sponsored by National Aeronautics and Space Administration, Washington, DC.

Pub. in Proceedings of Cambridge Workshop on Cool Stars, Stellar Systems, and the Sun (6th), Seattle, WA., September 12-14, 1989, p500-503.

Keywords: "Cool stars, Stellar chromospheres, Stellar coronae, Stellar winds, Late stars, Meetings, Reprints.

In the Workshop summary the author highlights some of the major trends in the field of cool stars research and calls attention to some of the important unanswered questions that the author hopes will become the scientific highlights of the next Workshop.
National Inst. of Standards and Technology (NML), Boulder, CO. Quantum Physics Div. Extension of the Class of Magnetic B Star Nonthermal Radio Sources.

Final rept.

J. L. Linsky, S. A. Drake, and T. S. Bastian. 1990, 4p

Grant NAGW-1716

Sponsored by National Aeronautics and Space Administration, Washington, DC

Pub. in Proceedings of Cambridge Workshop on Cool Stars, Stellar Systems, and the Sun (6th), Seattle, WA. September 12-14, 1989, p189-192 1990.

Keywords: "Radio sources(Astronomy), "Magnetic stars, *B stars, Reprints.

The authors have extended the initial Drake et al (1987) survey in 3 subsequent VLA runs, and have no detected a total of 15 sources at $6 \mathrm{~cm}$. Of these stars 3 are also detected at $2 \mathrm{~cm}, 3$ at $3.6 \mathrm{~cm}$, and 5 at $20 \mathrm{~cm}$ The authors have found no additional early- $\mathrm{B} \mathrm{He}$ strong stars, but have detected 10 new stars with spectral types B5-AO that have measured magnetic fields and are generally He-weak and Si-strong. The authors have not yet detected any classical Ap stars despite a number of attempls.

\section{0,032}

PB91-200873

Not available NTIS

National Inst of Standards and Technology (NML)

Boulder, CO. Quantum Physics Div.

Energy Distributions of Symbiotic Novae.

Final rept.

G. L. Bryan, and S. Kwok. 1991, 9p

Pub. in Astrophysical Jnl. 368, p252-260, 10 Feb 91

Keywords: “Symbiotic stars, "Noval, Infrared astronomy satellite, Infrared spectra, Uitraviolet spectra, Stellar envelopes, IUE, Reprints.

The IRAS low-resolution spectra of three recent symbiotic novae (HM Sge. V1016 Cyg, and RR Tel) are fitted with a dust continuum radiative transfer model. It was found that the dust shells are detached from the photosphere and that the sizes of the inner radii are correlated with times since outburst. An analysis of the IUE spectra of HM Sge at different epochs suggests that the strength of the $2200 \mathrm{~A}$ feature is decreasing that the strength of the 2200 A feature is decreasing probably formed in the white dwarf ejecta. A complete accounting of the entire energy budget from radio to $X$ ray shows that most of the energy is emitted by the cool component in the infrared, and a significant fraction of the flux of the hot component is escaping in the far-ultraviolet. The density-bounded nature of the circumstellar gas nebulae could be the result of a bipola geometry of the nebulae. Unlike classical novae, the optical outburst of symbiotic novae is due to the ionization of the preexisting envelope of the cool componen and is not the result of a sudden ejection by the hot component

\section{0,033}

Not available NTIS

National Inst. of Standards and Technology (NML),

Boulder, CO. Quantum Physics Div.

Evolution of Infrared Carbon Stars.

Final rept.

S. J. Chan, and S. Kwok. 1990, 15p

Sponsored by Natural Sciences and Engineering Research Council of Canada, Ottawa (Ontario)

Pub. in Astronomy and Astrophysics 237, p354-368 1990

Keywords: "Carbon stars, *Stellar evolution, Infrared astronomy satellite, Stellar mass ejection, Stellar envelopes, Infrared stars, Late stars, Reprints, Mass loss.

The Infrared Astronomical Satellite (IRAS) survey has shown that carbon stars which were discovered by optical surveys and those identified as such because of their $\mathrm{SiC}$ dust features have very different infrared colors. The former is visually bright and has large excesses in 60 micrometers while the latter (which will be referred to as infrared carbon stars) have blackbodylike energy distributions. In the present paper, the au thors present model calculations on the evolution from visual carbon stars to infrared carbon stars. The au thors have selected a sample of 472 infrared carbon stars from the IRAS Low Resolution Spectra (LRS) Atlas after rejecting mis-classified objects. A new opacity function for the $\mathrm{SiC}$ grain is derived from the LRS of the brightest sources in this sample. The energy distributions of about 150 IRAS infrared carbon stars are fitted with a radiative transfer model. The evolution of infrared carbon stars can be understood by a continuous increase in mass loss rate on the as ymptotic giant branch (AGB).

\section{ATMOSPHERIC SCIENCES}

\section{Physical Meteorology}

\section{0,034}

PB9 1-189340

Not available NTIS National Inst. of Standards and Technology (NML) Gaithersburg, MD. Gas and Particulate Science Div. Reference NO2 Calibration System for GroundBased Intercomparisons during NASA's GTE/CITE II Mission.

Final rept.

A Fried, L Nunnermacker, B Cadoff, R Sams, N. Yates, W. Dorko, R. Dickerson, and E. Winstead. $1990,8 p$

Pub in Jnl. of Geophysical Research - Atmospheres 95, nD7 p139-146 1990

Keywords: "Nitrogen dioxide, Atmospheric composition, Calibration, Reprints, "Reference materials, Global Tropospheric Experiment, Intercomparison.

An NO2 calibration system, based upon a permeation tube and a two stage dynamic dilution system, was de signed, constructed, and characterized at the National Bureau of Standards. The calibration system described was employed as the reference standard in NASA's Global Tropospheric Experiment/CITE-II mission in August of 1986, and was capable of accurately delivering known NO2 concentrations in the 0.4 to 200 ppbv concentration range with a total uncertainty around $10 \%$. Extensive laboratory tests were carried out based upon gravimetry, chemiluminescence de tection, and tunable diode laser absorption spectroscopy, to characterize both the system performance and the permeation emission rate. Upon completion of these tests, the calibration system was mounted on board NASA's research aircraft at both the Wallops Island and Ames research facilities. Known NO2 concentrations in the 0.4 to 1 ppbv range were delivered to four different NO2 detectors in a double blind intercomparison.

\section{0,035}

PB91-236646

Not available NTIS

National Inst. of Standards and Technology (CSTL) Gaithersburg, MD. Organic Analytical Research Div.

Analysis of Wet Deposition (Acid Rain): Determination of the Major Anionic Constituents by Ion Chromatography.

Final rept.

R. A Durst, W. Davison, K. Toth, J. E. Rother, M. E. Peden, and B. Griepink. 1991, 9p

Sponsored by International Union of Pure and Applied Chemistry, Oxford (England)

Pub. in Pure and Applied Chemistry 63, n6 p907-915 1991

Keywords: "Acid rain, "Deposition, "Chemical analysis, "Anions, Air pollution detection, Precipitation(Meteorology), Wet methods, Sampling, Quality assurance, Sample preparation, Comparisons, Reprints, "Ion chromatography.

For the purposes of the document, only the major anionic constituents of wet deposition, i.e., chloride, sulfate and nitrate, will be considered. The objective of the document is to provide a set of recommended procedures for the collection, handling, and analysis of acid rain samples, and the quality assurance of the resulting data. The use of these procedures should result in greater comparability between laboratories and consequently improved reliability in data interpretation. 
educators to reform engineering curricula immediately to improve statistics education, which was judged so inadequate for most engineering colleges as to have resulted in numerous crops of 'statistically illiterate' graduates in the eyes of several industries which employed them. The conference ended with three action items and a resolution to move forward in addressing the critical issues raised.

\section{Education, Law, \& Humanities}

\section{0,036}

PB91-147744

Not available NTIS

National Inst. of Standards and Technology (NCSL), Gaithersburg, MD. Systems and Software Technology Div.

Interactive Courseware Is Leading the Multimedia Movement.

Final rept.

J. Moline. $1990,5 p$

Pub. in UNIX Technology Advisor 2, n7 p14-18 Jul 90.

Keywords: "Computer assisted instruction, *Training devices, Computer systems programs, Operating systems (Computers)

equipment(Computers), Systems engineering, Interactive systems, Reprints, PORTCO(Portable Courseware Project), Multimedia.

The Federal government and other U.S. organizations are developing multimedia training materials for delivery on computer-based interactive training systems. A variety of computers and peripheral devices hosting various operating systems and suites of authoring system software are being used to develop the courseware. The Department of Defense's Portable Courseware Project (PORTCO) is typical of such projects. PORTCO strategy is applicable to all projects that require an open system environment in which to achieve mission objectives.

\section{0,037}

\section{PB91-148114}

Not available NTIS

National Bureau of Standards (NEL), Gaithersburg

MD. Center for Mfg. Engineering.

Agenda for Progress in Technology Education: A Personal View.

Final rept.

D. A. Swyt. 1987, 6

Pub. in Technology Teacher 47, n1 p3-8 Sep/Oct 87

Keywords: "Education, "Technology transfer, Methodology, Training, Reprints, Vocational education.

The U.S. as a productive economy and social system is in the midst of a technological transformation that is fundamentally changing how people live and how they earn their livelihoods. This change is straining our educational system, requiring innovation under duress. With technology as both the source of change and the remedy for the problems which change entails, the matter of teaching about technology has become a strategic issue. As such, the situation calls for a new agenda for progress in technology education. The paper presents a personal view on the situation and an agenda for change by which technology educators might deal with it.

100,038

PB91-158725

Not available NTIS

National Inst of Standards and Technology (NEL) Gaithersburg, MD. Applied and Computational Mathematics Div.

Engineers' Statistical Literacy Is Key to U.S. Competitiveness.

Final rept.

J. T. Fong. $1989,2 p$

Pub. in ASME News $9, n 5 \mathrm{pl}$ and p5 Oct 89

Keywords: "Engineering education, "Statistics, "Probability theory, Curricula, Mathematics, Experimental design, Data analysis, Stochastic processes, Quality control, Reprints, ABET(Accreditation Board for Engineering and Technology).

Highlights of a conference entitled 'Statistics and Probability in Engineering Education' are reported. The conference brought together engineering, mathematics, and statistics educators as well as industry and government leaders to discuss the need for incorporating statistical experimental design, data analysis, stochastic processes, probability, quality management technology, and other related areas into the engineering curricula. Two keynote speakers challenged the

\section{Job Training \& Career Development}

\section{0,039}

PB91-143362 PC A04/MF A01

National Inst. of Standards and Technology (NCTL) Gaithersburg, MD. Office Systems Engineering Group. Multimedia Courseware in an Open Systems Environment: A Federal Strategy.

J. Moline, A. L. Hankinson, and L. A. Welsch. Dec

$90,55 p$ NISTIR-4484

Keywords: *Computer program portability, "Computer assisted instruction, "Training, Interfaces, Standards, Open systems interconnections, *Interactive systems, National government, Strategy, Computer software, Multimedia, National Institute of Standards and Tech nology, Off the shelf computer software, Commercial sector.

The Department of Defense (DoD) Portable Courseware Project (PORTCO) is typical of projects worldwide that require standard software interfaces. The document articulates the strategy whereby PORTCO leverages the open systems movement and PORTCO leverages the open systems movement and al strategy for multimedia courseware is to facilitate the creation of an environment in which high quality portable courseware is available as commercial offthe-shelf products competitively supplied by vendors The Request for Architecture developed cooperatively by DoD and NIST will generate a portable courseware system architecture incorporating standards which meet users' needs. A computer-based interactive training applications profile must be developed which, along with the system architecture, will provide the basis for identifying needed standards. NIST will then accelerate the development of these standards through established standards forums.

BIOMEDICAL
TECHNOLOGY \&
HUMAN FACTORS
ENGINEERING

\section{Biomedical Instrumentation \& Bioengineering}

\section{0,040}

PB91-148668

Not available NTIS

National Inst. of Standards and Technology (MSEL), Gaithersburg, MD. Polymers Div.

Setting Reactions and Compressive Strengths of Calcium Phosphate Cements.

Final rept.

Y. Fukase, E. D. Eanes, S. Takagi, L. C. Chow, and W. E. Brown. 1990, 5p

Sponsored by American Dental Association Health Foundation, Chicago, IL.

Pub. in Jnl. of Dental Research 69, n12 p1852-1856 Dec 90

Keywords: "Calcium phosphates, "Dental materials, *Acid bonded reaction cements; Implantation, Setting time Bones Compressive strength Surface chemistry, Chemical analysis, X-ray diffraction, Time dependence, Reprints

Setting reactions and compressive strengths of a selfhardening calcium phosphate cement (CPC) were investigated. The CPC consists of tetracalcium phos- phate (TTCP) and anhydrous dicalcium phosphate (DCPA) The cement specimens were prepared by mixing $0.7 \mathrm{~g}$ of the powder (TTCP 72.9 wt \% + DCPA $27.1 \mathrm{wt} \%$ ) with $0.175 \mathrm{~mL}$ of the liquid $(25 \mathrm{mmol} / \mathrm{L}$ $\mathrm{H} 3 \mathrm{PO} 4$ and $1.32 \mathrm{mmol} / \mathrm{L}$ sodium fluoride). The specimens were removed from the molds at pre-determined time intervals after being mixed, and their compressive strengths were measured. Immediately afterward, the fractured specimens were rapidly frozen in ethanol ($80 \mathrm{C}$ ), lyophilized, and examined by powder x-ray diffraction and scanning electron microscopy (SEM). The results showed that (1) hydroxyapatite was the only reaction product; (2) the reaction was nearly completed within four $h$, during which both the reaction product and compressive strength increased linearly with time, resulting in a strong correlation between the two; and (3) fully set CPC consisted primarily of small rod-like crystals and some platy crystals.

100,041

PB91-150029

Not available NTIS

National Inst of Standards and Technology (NML), Boulder, CO. Chemical Engineering Science Div.

Low-Cost Low-Volume Carrier (Minilab) for Biotechnology and Fluids Experiments in Low Gravity.

Final rept.

J. M. Cassanto, W. Holemans, T. Moller, P. Todd, R M. Stewart and Z R Korzun 1990, 15p

Sponsored by National Aeronautics and Space Administration, Washington, DC. Commonwealth of Pennsylvania.

Pub. in Space Commercialization: Platforms and Processing, v127 p199-213 1990.

Keywords: "Reduced gravity, "Automatic control, "Space laboratories, "Remote control, Membranes, Interfaces, Interfacial tension, Space flight, Experimental design, Biotechnology, Research projects, Polymeric films, Miniaturization, Reprints.

Research opportunities in biotechnology and fluid echnology under conditions of microgravity can be made available to research workers from developing countries through relevant research projects and lowcost access to microgravity environments. Examples of such projects include polymeric film formation, purification methodology, liquid-liquid diffusion, and growth of organic and protein crystals. An example of a low-cost research device is the Materials Dispersion Apparatus (MDA) which can be used to conduct important low-volume experiments in an automated fashion wherever pairs, or groups of pairs, of liquids must interact. The MDA is designed to be compatible with several carriers: shuttle middeck lockers, Get-Away Special canisters, Hitchhiker, Lifesat, recoverable re-entry vehicles, low-gravity aircraft flights, and sounding rockets. The MDA consists of a pair of sliding blocks, in which wells in one block move into contact with wells in the other block under electronic command. Requirements for power, including temperature control, are minimal. Experiments related to biotechnology have been designed for a single flight of the MDA in which the carrier will be a sounding rocket that provides approximately 6 min of low gravity. Examples of experiments to be conducted include the nucleation of organic crystals in aqueous solution, behavior of immiscible aqueous solutions, solid-liquid surface phenomena at high surface-tension gradients, and the formation of thin polymeric membranes.

\section{0,042}

PB91-158493

Not available NTIS

National Inst. of Standards and Technology (MSEL), Gaithersburg, MD. Polymers Div.

Visco-Elastic Deformation of Dental Porcelain and Porcelain-Metal Compatibility

Final rept.

K. Asaoka, and J. A. Tesk. 1991, 6p

Sponsored by National Inst. of Dental Research, Bethesda, MD.

Pub. in Dental Material 7, n1 p30-35 Jan 91.

Keywords: "Dental materials, Heat treatment, Firing, Dissimilar materials bonding, Metals, Porcelain, Stress analysis, Viscoelasticity, Computerized simulation, Deformation, Thermal expansion, Reprints.

A computer simulation using a visco-elastic stress analysis was conducted to clarify the effect of the heating rate on deformation temperature of dental porcelain during firing. In the simulation, the following temperature-dependent factors were incorporated: elastic modulus, viscosity, and coefficient of thermal expansion. Thermal expansion curves of porcelain with an 
applied load at various heating rates were computed. The results suggest that the temperature where the incompatibility stress develops in the porcelain-fused-tometal strips during cooling can be estimated closely from the deformation point of the heating curve of the porcelain with an applied stress of about 1.2 - 3.1 MPa.

\section{0,043}

PB91-158691 Not available NTIS National Inst. of Standards and Technology (MSEL), Gaithersburg, MD. Polymers Div.

Effect of Catalyst Structure on the Synthesis of a Dental Restorative Monomer.

Final rept.

M. Farahani, A. D. Johnston, and R. L. Bowen. 1991, $6 p$

Sponsored by American Dental Association Health Foundation, Chicago, IL.

Pub. in Jnl. of Dental Research 70, n1 p67-71 Jan 91.

Keywords: *Acid bonded reaction cements, "Dental materials, Molecular isomerism, Synthesis(Chemistry) Catalysts, Amines, Ethylamine, Hexamethylenetetramine, Pyromellitic acid, Reprints, Pyromellitic dianhydride, Diisopropylethyamine.

The addition product of 2-hydroxyethyl methacrylate (HEMA) and pyromellitic dianhydride (PMDA), known as PMDM, is a mixture of two structural isomers. The para PMDM isomer--currently used in mediating adhesive bonding of restorative materials to hard tooth issues-is a crystalline solid. The meta isomer is a liquid. In the synthesis of PMDM, the para isomer which can be purified by crystallization, is usually present to the extent of only $50 \%$ of the product mixture. The effect of the amine catalyst structure was studied relative to its role in increasing the yield of the para isomer, either by a reduction in the amount of the meta isomer or by an increase in the extent of overal reaction. The chemical structure of the amine catalys had an important role in the synthesis of PMDM and influenced the ratio of the isomers. Among aliphatic amines, especially noteworthy as catalysts that gave excellent yields of the para isomer in high purity were $\mathrm{N}, \mathrm{N}$-di-isopropyl-ethylamine and hexametylenetetramine.

\section{0,044}

PB91-159210

Not available NTIS

National Inst. of Standards and Technology (MSEL)

Gaithersburg, MD. Polymers Div.

Dental Biomaterlals and Engineerlng Frontiers for the 90's.

Final rept.

J. A. Tesk. $1990,2 p$

Pub. in Proceedings of the International Kyoto Symposium on Biomedical Engineering (3rd), Kyoto, Japan, November 20-21, 1990, p14-15.

Keywords: *Dental materials, "Biotechnology, Reprints, *Biomedical engineering.

Some areas of dental materials and engineering which should receive increased attention in the 1990's are discussed.

\section{0,045}

PB91-174458

Not available NTIS

National Inst. of Standards and Technology (IMSE) Gaithersburg, MD. Polymers Div.

Glass-Ceramic Inserts Anticipated for 'Megafilled' Composite Restorations.

Final rept.

R. L. Bowen, F. C. Eichmiller, and W. A. Marjenhoff. 1991, 3p

Sponsored by American Dental Association Health Foundation, Chicago, IL.

Pub. in Jnl. of the American Dental Association 122 p71, 73, and 75, Mar 91.

Keywords: "Dental materials, "Composite materials, Ceramics, Glass, Inserts, Color, Dimensional stability, Physical properties, Reprints.

Further improvement in the physical properties of directly placed composites is expected with the introduction of preformed, tooth-colored glass-ceramic inserts for Class I, II and III megafilled restorations. Less microleakage is associated with restorations containing inserts. Inserts are also expected to increase the stiffness, strength and durability of composite restorations, promote dimensional stability of the remaining tooth crown during hardening and function, and offer the esthetic advantage of having inherent tooth shades. It is anticipated that the first commercial inserts kits (perhaps available within the year) will be comprised of three to four standardized shapes in three to four sizes, in the most utilized tooth shades. The additional time required for placement and finishing of inserts by dentists is not expected to be more than two or three minutes. Inserts are expected to add only about a dollar each to the cost of materials used in a composite restoration and should not, therefore, categorically increase current fee structures.

\section{0,046}

\section{PB91-174490}

Not available NTIS

National Bureau of Standards (NEL), Gaithersburg MD. Automated Production Technology Div.

Correction for Converting 2-CM(3)-Coupler Responses to Insertion Responses for Custom in the-Ear Non-Directional Hearing Aids.

Final rept.

E. D. Burnett, and L. B. Beck. 1987,6p

Pub. in Ear Hear 8, p89S-94S Oct 87.

Keywords: "Hearing aids, Fourier analysis, Frequency response, Measurement, Responses, Correction, Comparison, Reprints.

A correction for custom in-the-ear non-directional hearing aids is obtained for converting a frequency response obtained using a 2-cm cubed coupler to an insertion response, approximating that measured using a manikin and ear simulator. The results are compared to those of a previous published study. The methods used for obtaining the responses make use of a signal analyzer with discrete Fourier transform capabilities.

\section{0,047}

PB91-189555

Not available NTIS

National Inst. of Standards and Technology (IMSE) Gaithersburg, MD. Polymers Div.

Protective Coatings for Tooth Crowns.

Final rept.

A. D. Johnston, and R. L. Bowen. 1991, 3p

Sponsored by Americar: Dental Association Health Foundation, Chicago, IL.

Pub. in Jnl. of the American Dental Association 122 p49-51 Apr 91.

Keywords: "Dental materials, "Dental caries, "Polymeric films, "Protective coatings, Surface chemistry, Enamels, Hydrophylic polymers, Reprints.

The application (and occasional renewal) of invisible polymeric films to entire tooth crown surfaces may someday be an effective means of caries prevention for patients of all ages.

\section{0,048}

PB91-194654

Not available NTIS

National Inst. of Standards and Technology (IMSE). Gaithersburg, MD. Polymers Div.

Filler Systems Based on Calcium Metaphosphates. Final rept.

J. M. Antonucci, B. O. Fowler, and S. Venz. 1991, 6p Sponsored by National Inst. of Dental Research, Bethesda, MD

Pub. in Dental Materials 7, p124-129 Apr 91.

Keywords: *Dental materials, *Calcium phosphates, *Dental caries, *Fillers, *Inorganic polymers. Teeth, Chemical radiation effects, Ultraviolet radiation, $\mathrm{Re}$ fractivity, Crack arrest, Composite materials, Microsco py, Reprints.

Calcium metaphosphates (CMP's)--a unique class of phosphate minerals possessing polymeric structures, $(\mathrm{Ca}(\mathrm{PO}) 2) \mathrm{n}$, and having refractive indices of 1.54 1.59 are optically compatible with resins such as BIS GMA. Several types of CMP's were prepared and evaluated for their potential as fillers for visible-light-activated (VLA) dental composites. The vitreous $(V)$ and beta-crystalline forms of CMP were prepared by controlled thermolysis of monocalcium phosphate monohydrate. Hybrid fillers were also prepared by thermal methods. Fillers, characterized by IR spectroscopy and optical microscopy, were prepared in several siz ranges (e.g., 1-100 micrometers). Beta-CMP composites were more moisture-resistant, had higher diametral tensile strengths (from 12 to $33 \mathrm{MPa}$ ), and showed a tendency to arrest brittle fracture. These novel fillers have potential uses in resin-based materials such as dental composites, cements, and adhesives.

\section{0,049}

\section{PB91-202879}

Not available NTIS

National Inst. of Standards and Technology (IMSE), Gaithersburg, MD. Polymers Div.
Self-Setting Calcium Phosphate Cements.

Final rept.

L. C. Chow, S. Takagi, P. D. Costantino, and C. D. Friedman. 1991, 22p

Sponsored by American Dental Association Health Foundation, Chicago, IL.

Pub. in Materials Research Society Symposium Proceedings, v179 p3-24 1991

Keywords: "Calcium phosphates, "Dental materials *Medical supplies, ${ }^{\star}$ Cements, Binary system(Materials), Phosphoric acid, Aqueous solutions, Mixtures, Apatites, Hydroxy compounds, Biocompatibility, Hardness, Reprints, Self-setting.

A mixture of tetracalcium phosphate and dicalcium phosphate, when mixed with a dilute phosphoric acid or other aqueous solutions, will harden like a cementproducing hydroxyapatite as the final product. The latter is the principal mineral component of hard tissue. The chemistry, physical properties, and biocompatibility of the calcium phosphate cement are discussed. The setting property, combined with high biocompatibility, makes calcium phosphate cements useful in many applications in dentistry and medicine.

\section{0,050}

\section{PB91-20408}

Not available NTIS

National Bureau of Standards (IMSE), Gaithersburg MD. Polymers Div.

Microstructure and Elastic Properties of Dental Resin and Resin-Based Glass-Reinforced Composites: XRD, SEM and Ultrasonic Methods.

Final rept.

S. Singh, J. L. Katz, J. Antonucci, R. W. Penn, and J. A. Tesk. $1988,6 p$

Pub. in Materials Research Society Symposium Proceedings, v110 p599-604 1988.

Keywords: "Resin matrix composites, "Fiber compos ites, "Fiberglass, "Dental materials, "Dental caries, Elastic properties, Microstructure, X-ray diffraction, Scanning tunneling microscopy, Mechanical properties, Nondestructive tests, Ultrasonic tests, Amorphous materials, Poisson ratio, Young modulus, Silanes, Velocity, Density, Polymers, Reprints, Energy dispersive spectrometry.

The load-bearing ability of dental restorative materials under cyclic high-stress applications depends upon mechanical properties established by the composition and microstructure. The microstructure and the elastic properties of neat resin and two resin-based glass-re inforced composites have been studied. The microstructure of these materials has been examined using $x$ ray diffractometry (XRD), scanning electron microscopy (SEM) including energy dispersive spectrometry (EDS). The elastic properties, i.e., Young's, shear and bulk moduli and Poisson's ratio were determined from ultrasonic velocities and densities. The ultrasonic velocities were measured using a pulse-through transmission method; density was measured using a buoyant force method. These studies showed that (1) these materials have amorphous structures; (2) these materials have Young's moduli of the order of $20 \mathrm{GPa}$ and (3) the silane coupling agent apparently did no significantly affect the elastic properties of these resinbased composites.

\section{0,051}

PB91-204149

Not available NTIS National Bureau of Standards (IMSE), Gaithersburg, MD. Polymers Div.

Dental Composite Resins: An Update.

Final rept.

S. Venz. $1987,2 p$

Pub. in Modern Dentalab 5, n4 p27-28 Jun/Jul 87.

Keywords: "Dental materials, "Dental caries, *Resin matrix composites, PMMA, Radiation curing, Visible radiation, Methacrylates, Surface treatments, Inorganic compounds, Polymers, Plastics, Reprints, Self-curing.

Since the introduction of self-curing dental restorative resins in the 1940s, numerous modifications have been introduced to enhance the performance of resinbased restorations in the oral cavity. Major developments leading to current dental composite resins are the use of di- or polyfunctional methacrylates instead of the monofunctional methyl methacrylate, the incorporation of surface treated inorganic fillers instead of poly-(methylmethacrylate) powder and the introduction of visible light curing, single component materials instead of two part chemical curing systems. 


\section{Biomedical Instrumentation \& Bioengineering}

\section{0,052}

Not available NTIS

National Inst. of Standards and Technology (MSEL), Gaithersburg, MD. Polymers Div.

Development of an Adhesive System for Bonding to Hard Tooth Tissues.

Final rept.

R. L. Bowen, and W. A. Marjenhoff. 1991, 5p

Sponsored by American Dental Association Health Foundation, Chicago, IL. Pub. in Jnl. of Esthetic Dentistry 3, n3 p86-90 May/Jun
91.

Keywords: "Acid bonded reaction cements, "Adhesives, "Dental materials, Composite materials, Enamels, Dentin, Teeth, Reviews, Inorganic silicates, Resins, Aqueous solutions, Synthesis(Chemistry), Reprints.

The development of an adhesion system for bonding dental composites to dentin and enamel is reviewed. Building on findings concerning adhesion to enamel, $R$. L. Bowen and colleagues at the Paffenbarger Re $R$. L. Bowen and colleagues at the Paffenbarger Re-
search Center, National Institute of Standards and Technology, began addressing and solving problems associated with (1) silicate cements and unfilled resins: (2) bonding in an aqueous environment; and (3) the development of an adhesion system for both dentin and enamel that could withstand various stresses. Although commercial products based on the adhesion system are currently available in the denta materials marketplace, experimentation continues, focusing on the synthesis of potentially better component analogs, the optimization of the individual components, and on improved storage stability and ease of synthesis.

\section{0,053}

PB91-237735

Not available NTIS

National Inst of Standards and Technology (MSEL) Gaithersburg, MD. Polymers Div.

Dentin-Bonding Molar Efficiency Using N-Phenylglycine, N-Phenyl-beta-Alanine, or N-Methyl-NPhenylglycine.

Final rept.

R. E. Webb, and A. D. Johnston. 1991, 4p

Sponsored by American Dental Association Health Foundation, Chicago, IL.

Pub. in Jnl. of Dental Research 70, n3 p211-214 Mar 91

Keywords: *Amino acids, "Bonding strength, "Dentin "Acid bonded reaction cements, *Adhesives, Phenylalanine, Glycine, Methyl compounds, Teeth, Nitrogen organic compounds, Alanines, Oxalates, Iron complexes, Dental materials, Reprints, Molar efficiency.

Three structurally related substituted amino acids ( $\mathrm{N}$ compounds) were studied in a three-step dentin-bonding protocol. In the second step, the amount of the $\mathrm{N}$ compound--either N-phenylglycine (NPG), N-methyl$\mathrm{N}$-phenylglycine (NMNPG), or N-phenyl-beta-alanine (NPBA)-was varied in acetone from $0 \mathrm{~mol} / L$ through $0.5 \mathrm{~mol} / \mathrm{L}$ in $A+0.001 \mathrm{~mol} / \mathrm{L}$ for $N P G$ and $N M N P G$, average bond strength values were $7.4+$ or -2.2 and $10.5+$ or $-2.7 \mathrm{MPa}$. The highest bond strength value for NMNPG was at $0.01 \mathrm{~mol} / \mathrm{L}$, with $13.2+$ or -4.0 $\mathrm{MPa}$. The highest value for NPG was at $0.1 \mathrm{~mol} / \mathrm{L}$, with a value of $11.8+$ or $-2.5 \mathrm{MPa}$. The average bond strength for NPBA did not differ from zero across the entire range of concentrations. Molar efficiency was defined as the bond strength per mole of these applied $\mathrm{N}$-compounds. For the two $\mathrm{N}$-compounds that did provide adhesion to dentin, NPG and NMNPG, the average bond strengths rose, peaked, and fell as the amounts of applied $\mathrm{N}$-compound were increased. The molar efficiency dropped off as the concentration of applied $\mathrm{N}$-compound rose.

\section{Prosthetics \& Mechanical Organs}

\section{0,054}

PB91-194761

Not available NTIS

National Inst. of Standards and Technology (MSEL) Gaithersburg, MD. Polymers Div.

Metal-Polysiloxane Shields for Radiation Therapy of Maxillo-Facial Tumors.

Final rept.

M. Farahani, F. C. Eichmiller, and W. L. McLaughlin. 1991, 6p

Sponsored by American Dental Association Health Foundation, Chicago, IL.
Pub. in Medical Physics 18, n2 p273-278 Mar/Apr 91.

Keywords: "Radiotherapy, "Radiation therapy, "Shielding, "Gamma rays, Tissues, Dosimetry, Particulate composites, Oral diseases, Maxillary neoplasms, Face(Anatomy), Neck(Anatomy), Prosthetic devices, Mouth neoplasms, Molding materials, Cobalt 60, Synthetic elastomers, Resin matrix composites, Siloxanes, Binary alloys, Vinyl compounds, Silver alloys, Copper alloys, Tin alloys, Antimony alloys, Reprints.

In the treatment of some head and neck lesions with high-intensity radiation (teletherapy), an essential procedure is the application of an individually customized shielding appliance, which is designed, modeled, and formed into a working extra- or intraoral stent for the purpose of sparing healthy tissues. The present state of the art is slow and technique intensive, which can add to patient discomfort and inconvenience during molding and fabrication. A new formulation is described, which offers speed and ease of forming a moldable composite stent especially for intraoral use. Tests using collimated gamma-ray beams from a (60) Co teletherapy unit were made in order to measure the dose distribution near interfaces of tissue-simulating polymer and the composite stent material with and without mixtures of metals ( $\mathrm{Ag}-\mathrm{Cu}$ and $\mathrm{Sn}-\mathrm{Sb}$ ). The re sults show that quickly formed composites made of a flexible resin with high concentrations of powdered spherical metal alloys provide effective custom-designed shielding, and, with a thin overlayer of the resin without metal, a dimished back-scattered radiation dose to normal tissues. An example of a successful formulation is a mixture of $90 \%$ by weight $\mathrm{Ag}-\mathrm{Cu}$ alloy powder in a vinyl polysiloxane resin. The material is a moldable putty which, upon polymerization, forms a rigid elastomeric material, providing a half-value layer of approximately 2.5 to $2.8 \mathrm{~cm}$ for a gamma-ray beam from a $(60)$ Co source.

\section{0,055}

PB9 1-194787

Not available NTIS National Inst. of Standards and Technology (MSEL), Gaithersburg, MD. Metallurgy Div.

Corrosion Principles in Dental Implantology.

Final rept.

A. C. Fraker, and F. Eichmiller. 1990, 10p

Pub. in Clark's Clinical Dentistry, Chapter 50, v1 p1-10 1990

Keywords: "Dental materials, "Titanium alloys, "Implants, "Corrosion, "Prosthetic devices, Dissimilar materials bonding, Performance prediction, Apatites, Solid-solid interfaces, Tissues, Surface chemistry, $\mathrm{Hy}$ droxy compounds, Reprints.

Corrosion processes that could occur with dental implants and some guidelines for predicting the corrosion behavior of common prosthetic alloys are given. Interactions between dissimilar materials within the prosthetic device can result in corrosion, and this is discussed. The titanium implant will be covered with a surface oxide or with an applied layer of hydroxyapatite. The implant/tissue interface is described and discussed.

\subsection{6}

PB92-117407

Not available NTIS

National Inst. of Standards and Technology (MSEL), Gaithersburg, MD. Polymers Div.

Comparative Study of Bovine Pericardium Mineralization: A Basic and Practical Approach.

Final rept.

B. B. Tomazic, C. Siew, and W. E. Brown. 1991, 11p Sponsored by American Dental Association Health Foundation, Chicago, IL.

Pub. in Cells and Materials 1, n3 p231-241 1991.

Keywords: "Mineralization, "Pericardium, "Heart valve prosthesis, Cattle, Comparison, In vivo analysis, In vitro analysis, Calcinosis, Histology, Rats, Humans, Reprints.

The biomineralization of bovine pericardium (BP) heart valve bioprostheses was investigated by simulation of the process under in vitro and in vivo conditions. The nature and composition of calcific deposits that formed in human heart valve bioprostheses were compared with the mineral formed on BP. discs immersed in a calcifying medium or subcutaneously implanted into Sprague-Dawley rats. The early stage of experimental biomineralization in vitro took place on the surface only, while in vivo deposition appeared to be intrinsic as documented by histological cross sections. Combined information from in vitro, in vivo and bioprosthe tic mineralization supports the concept that octacal- cium phosphate is a precursor that transforms into bioapatite and is implicated in the calcification of bioprosthetic heart valves.

\section{(20) \\ BUILDING INDUSTRY TECHNOLOGY}

\section{Architectural Design \& Environmental Engineering}

100,057

PB91-147082

Not available NTIS

National Bureau of Standards (NEL), Gaithersburg, MD. Building Physics Div.

Calibration Procedures for Infrared Imaging Systems for Building Diagnostics.

Final rept.

Y. M. Chang, and R. A. Grot. 1986, $8 p$

Sponsored by Department of Energy, Washington, DC.

Pub. in Proceedings of International Symposium on

Temperature Measurement in Industry and Science, Beijing, China, April 16-19, 1986, p277-284.

Keywords: "Calibration, "Infrared equipment, "Temperature measuring instruments, "Heat loss, "Buildings, Temperature measurement, Frequencies, Inspection, Heat transmission, Energy conservation, Standards, Reprints.

The paper describes laboratory measurements for the evaluation of infrared imaging systems commonly used for building inspections. The infrared imaging systems were evaluated at various background temperatures ranging from -20 to $25 \mathrm{C}$. The tests performed on each infrared imaging system were the determination of the minimum resolvable temperature difference (MRTD) at spatial frequencies ranging from 0.03 to $0.25 \mathrm{cy} / \mathrm{mrad}$ and calibration curves of the measured radiosity (isotherm level) versus the equivalent blackbody temperature of the target. The results of the MRTD tests are compared with the theory used in the ASHRAE Standard 101-83. It is shown that the theory accurately predicts the temperature dependence of the MRTD curves only for one infrared imaging system operating in the 2 to 5 micrometer range. For infrared imaging systems in the 8 to 12 micrometer range, the MRTD increases much faster as the object temperature decreases than predicted by the theory. It is also shown that the calibration curves of the two is also shown that the calibration curves of the 8 to 12 micrometer range have a strong temperature dependence.

100,058

PB91-147140

Not available NTIS

National Inst of Standards and Technology (NEL), Gaithersburg, MD. Building Environment Div. Second-Level Post-Occupancy Evaluation Analysis.

Final rept.

B. L. Collins, W. Fisher, G. Gillette, and R. W.

Marans. $1990,24 p$

See also DE89014520

Pub. in Jnl. of the Illuminating Engineering Society, p21-44 1990.

Keywords: "Lighting systems, "Office buildings, "Comfort, Daylighting, Evaluation, Illuminance, Design, Environmental engineering, Illuminating, Interior lighting, Brightness, Reprints.

Post-occupancy evaluations (POE) have been used by a number of researchers as a tool for documenting, evaluating, and improving environmental conditions in offices. In a project sponsored by the US Department of Energy and the New York State Energy Research and Development Authority during 1984-1986 POE data were collected on lighting power densities, photometric levels, and user attitudes for 912 workstations in 13 office buildings that contained lighting systems somewhat typical of current lighting practice. The purpose of the present evaluation is to examine the relationships between individual lighting system type and these data. 
100,059

PB91-14756

Not available NTIS

National Bureau of Standards (NEL), Gaithersburg, MD. Building Physics Div.

Design Heat Loss Factors for Basement and Slab Floors.

Final rept.

T. Kusuda, and J. W. Bean. 1985, 21p

Pub. in Thermal Insulation: Materials and Systems, ASTM ATP 922, p132-152 1985

Keywords: "Heat loss, "Basements, "Floors, Design criteria. Thermal insulation, Thermal analysis, Buildings, Concrete slabs, Walls, Slabs on ground construction, Energy conservation, Reprints.

The heat loss factors for selected types of earth contact surfaces, such as basement walls and floors and slab-on-grade floors, are presented to supplement data in the ASHAAE Handbook of Fundamentals for estimating the building design heat loss. The perimeter heat loss factors are derived from the procedure developed by Mitalas and from monthly normal temperature cycles of many localities in the United States. Except for those for cities in California, the heat loss factors are found to be a relatively well-defined function of degree-days and remain constant for degreedays beyond 3000 (engineering units). The heat loss factors show that the most effective way to reduce the earth contact surface heat loss is to insulate the inside corners of wall/floor joints. The insulation installed below the floor slab is found to be the least effective as long as the wall foundation is left uninsulated.

\section{0,060}

PB91-147876

Not available NTIS

National Bureau of Standards (NEL), Gaithersburg, MD. Building Physics Div.

Ventilation System Performance Evaluation Using Tracer Gas Techniques.

Final rept.

A. K. Persily, and R. A. Grot. 1985, 17p

Sponsored by Department of Energy, Washington, DC. Pub. in Proceedings of AIC Conference (6th) Ventilation Strategies and Measurement Techniques, Netherlands, September 16-19, 1985, p26.1-26.16.

Keywords: "Indoor air pollution, "Ventilation, "Tracers, "Measurement, Performance evaluation, Air quality, Environmental engineering, Leakage, Air flow, Buildings, HVAS systems, Reprints.

Based on current concerns regarding indoor air quality and energy use, there is a need for in situ techniques for evaluating buildings' infiltration and ventilation characteristics. The U.S. National Bureau of Standards has developed and employed equipment and techniques for such evaluation. The measurement of whole building leakage and ventilation rates has been reported on previously. Additional procedures are presented here for a more complete evaluation of the ventilation system operation and the distribution of air within the building. The measurements reveal both the amount of outside air infiltrating through the envelope and the amount of intentional intake through the air handlers. Tracer gas techniques to study the uniformity of air distribution throughout a building are also discussed. These in situ evaluation techniques are described and results from their application are presented.

\section{0,061}

\section{PB91-158535}

Not available NTIS

National Inst. of Standards and Technology (NEL), Gaithersburg, MD. Building Environment Div.

Conference Coverage: Papers Presentations Shine.

Final rept.

B. L. Collins. 1990, 3p

Pub. in Lighting Design and Application, p21-23 Oct 90.

Keywords: "Illuminating, Energy conservation, Lamps, Computer graphics, US NBS, Meetings, Reprints, Baltimore(Maryland).

The technical highlights from the recent IESNA Conference in Baltimore, MD are summarized in some detail. At the session 48 papers on lighting research, technology, design and application were presented. Topics discussed included light sources and conservation issues for displays; efficiency standards for lamps; daylight measurement and modeling; lamp performance including fluorescent, incandescent, meta halide, high pressure sodium, and special sources; tunnel and roadway lighting; measurement and con- trols; modeling and lighting geometry; calculations; visbility and visual performance; computer graphics; and VDT's. Issues relating to energy conservation, such as efficiency standards, daylighting, and new lamp technology generated considerable attention, as did papers on lighting and conservation as well as computer graphics as a design tool.

100,062

PB91-159079

Not available NTIS National Bureau of Standards (NEL), Gaithersburg. MD. Building Equipment Div.

Prototype Expert System for Diagnosing Moisture Problems in Houses.

Final rept.

A. K. Persily. 1986, 10p

Pub. in Proceedinos of BTECC Symposium on Air Infiltration, Ventilation, and Moisture Transfer, Fort Worth, TX., December 1986, 10p.

Keywords: "Expert systems, "Moisture content, "Residential buildings, "HVAC systems, Ventilation, Protoypes, Air flow, Moisture, Indoor air quality, Comfort, Heat loss, Reprints.

A knowledge based expert system is under development to assist in the identification and diagnosis of air leakage problems in residential buildings. The expert system is intended for use by home energy auditors who are familiar with house construction and building performance issues, but do not have the expertise necessary to deal effectively with the wide variety of circumstances encountered in houses. The system development is beginning with a prototype to diagnose moisture-related problems. This prototype is the first step in the development of the more comprehensive expert system that will deal with air leakage problems associated with indoor air quality, thermal comfort, and heat loss and gain. In the paper the moisture-diagnosis prototype is described and discussed. This prototype system requires the user to describe the symptoms of the existing moisture problems and provide information on house characteristics. Based on additional information on the symptoms and the house, this interactive program produces a list of probable causes and recommendations for remedial action. In addition to describing the current prototype system, the paper also discusses the results of an evaluation of the system based on its use by human experts in the field of residential building moisture. This evaluation, along with insights obtained through the efforts of the system's developers, has led to several proposed improvements of the prototype.

100,063

PB91-159293

Not available NTIS MD. Center for Building Technology.

Al: Does It Have a Place in Building Simulation.

Final rept.

R. N. Wright, and J. Hirsch. 1985, 6p

Pub. in Proceedings of Building Energy Simulation Conference, Seattle, WA., August 21, 1985, p169-174.

Keywords: "Artificial intelligence, "Buildings, Designs, Expert systems, Simulation, Decision making, Architecture, Civil engineering, Design criteria, Construction, Reprints.

Artificial intelligence (AI) technologies and simulation technologies can be combined to support excellent decisions throughout the whole building process. A technologies are briefly reviewed. Simulation is generalized as a means to predict prototype performance whether in the building process or in the response of the building in use to its natural and man-made environments. Al can assist the decision maker in selecting the simulation scheme, detailing and conducting the simulation, and assessing the validity and meaning of the results of simulation. Simulation can assist the knowledge engineer in the formulation of knowledge bases to guide in decision making for various building problems. Al and simulation technologies are identified as natural and powerful partners for the guidance of decision makers in all phases of the building process. Substantial research, development and education will be required to realize the potential of these technologies to increase the usefulness, safety and economy of buildings.

\section{0,064}

PB91-167155

PC A03/MF A01

burg, MD.
ZIP: The ZIP-Code Insulation Program, Version 2.0. Economic Insulation Levels for New and Existing Houses by Three-Digit Zip Code. Users' Guide and Reference Manual (Revised Edition).

S. R. Petersen. Jan $91,36 p$ NISTIR-88/3801-1

See also PB89-159446 and PB89-151765. Sponsored by Department of Energy, Washington, DC. Building Systems Div.

Keywords: "Residential buildings, “Thermal insulation, "Economic analysis, Engineering costs, Climate, Prices, Energy conservation, Life cycle costs, Heating, Cooling, Construction, User manuals(Computer programs), Zip codes, ZIP computer program.

ZIP 2.0 is a revised and updated version of ZIP, the Zip-Code Insulation Program. ZIP is a computer program developed to support the DoE Insulation Fact Sheet by providing users with customized estimates of economic levels of residential insulation. These estimates can be made for any location in the United States by entering the first three digits of its ZIP code. The program and supporting files are contained on a single 5-1/4 in. diskette for use with microcomputers having an MS-DOS operating system. The revised ZIP program calculates economic levels of insulation for attics, cathedral ceilings, exterior woodframe and masonry walls, floors over unheated areas, slab floors, basement and crawlspace walls, ducts in unconditioned spaces, and water heaters. Climate parameters are contained in a file on the ZIP diskette and are automatically retrieved by ZIP code. Regional energy prices and insulation costs are also retrieved from the diskette, but these can be overridden to better reflect local conditions.

\section{0,065}

PB91-174607

Not available NTIS

National Inst of Standards and Technology (NEL) Gaithersburg. MD. Building Environment Div.

Refrigerant Charge Effects on Heat Pump Performance.

Final rept

G. S. Damasceno, P. A. Domanski, S. Rooke, and V. Goldschmidt. 1991, 6p

Sponsored by Ranco Controls, Erving, TX

Pub. in ASHRAE (American Society of Heating, Refrigerating and Air-Conditioning Engineers) Transactions, v97 pt $16 \mathrm{p} 1991$

Keywords: "Heat pumps, "Refrigerants, "Performance, Mass transfer, Thermodynamic properties, Air conditioners, Mathematical models, Steady state, Residential buildings, Experimental data, Reprints.

The capability of predicting the effects of refrigerant charge on the steady-state performance of a heat pump is addressed. Rather extensive test data for one particular residential air-to-air heat pump are compared with the predictions of HPSIM a computer model with the capability of tracking refrigerant mass distribution within the various components. The need to properly account for parasitic volumes and all internal volumes of the heat pump is underscored.

\section{0,066}

PB91-174649

National Inst of Standards and Technology (NEL) Gaithersburg, MD. Building Environment Div.

Simulation of an Evaporator with Nonuniform OneDimensional Air Distribution.

Final rept.

P A Domanski. 1991, 10p

Sponsored by Department of Energy, Washington, DC. Pub. in ASHRAE (American Society of Heating, Refrigerating and Air-Conditioning Engineers) Transactions, v97 pt 1 10p 1991 .

Keywords: "Evaporators, "Computerized simulation, "Heat exchangers, "Air conditioning, Heat transfer, Air flow, Mathematical models, Tubes, Mass transfer, Refrigerants, Dehumidification, Reprints.

The paper presents a simulation model of a plate-fin, air-to-refrigerant heat exchanger used as an evaporator in residential air conditioning. The model can account for nonuniform air distribution between coil tubes and simulates refrigerant distribution for coil circuits and individual tubes. The model is based on a tube-by-tube approach. Performance of each tube is analyzed separately by considering the cross-flow heat transfer with the external airstream and the appropriate heat and mass transfer relationships. Each tube is associated with individual refrigerant parameters and mass flow rate, and air mass flow rate, inlet 
temperature, and humidity. A comparison of the model's predictions and laboratory test data is provid ed. Simulation results indicate that air maldistribution may induce maldistribution of a refrigerant, which contributes to the performance degradation of the evaporator

\subsection{7}

PB91-175307

Not available NTIS National Bureau of Standards (NEL), Gaithersburg, MD. Building Physics Div.

Airtightness of Office-Building Envelopes.

Final rept.

A. K. Persily, and R. A. Grot. 1985, 19p

Sponsored by Department of Energy, Washington, DC. Pub. in Proceedings of ASHRAE/DOE/BTECC Conference on Thermal Performance of the Exterior Envelopes of Buildings III, Clearwater Beach, FL., December 1985, p125-143.

Keywords: "Ventilation, "Office buildings, "Leakage, "Infiltration, "Envelopes, Indoor air quality, Computer ized simulation, Air flow, Pressure, Mathematical models, Energy consumption, Reprints.

Although airtightness, infiltration, and ventilation are important considerations in large office buildings, these issues have been studied less in office buildings than in residential buildings. Several features of office buildings make their air exchange characteristics different from homes. These features include curtain wall design and construction, mechanical ventilation systems, specific occupancy patterns, large volumes and building heights, and low surface to volume ratios. The recent development of measuring procedures and computer simulation programs for large buildings has enabled the study of air leakage in large buildings and the effects of air exchange on energy use and indoor air quality. The paper discusses the airtightness andbuilding envelopes in modern office buildings and the relationship between envelope airtightness and air exchange rates. Results of whole building pressurization measurements and tracer gas measurements of air ex change rates in several office buildings are discussed, along with the relationship between these measurement results. A multizone computer simulation program developed at the National Bureau of Standards is applied to two office buildings to examine the relationship further. The results of the simulations reveal the importance of envelope airtightness, floor-to-floor cou pling, and mechanical ventilation to the air change rates in these two buildings.

\section{0,068}

\section{PB91-175471}

Not available NTIS

National Bureau of Standards (NEL), Gaithersburg, MD. Building Physics Div.

Measurement of Thermal Characteristics of Office Buildings.

Final rept

L. K. Norford, A. Rabl, R. H. Socolow, and A. K

Persily. $1985,17 p$

Sponsored by Department of Energy, Washington, DC Pub. in Proceedings of ASHRAE/DOE/BTECC Conference on Thermal Performance of the Exterior Envelopes of Buildings III, Clearwater Beach, FL., December 1985, p272-288.

Keywords: "Office buildings, "Thermal measurements, "Heat loss, Energy consumption, Heat transfer, Spe-
cific heat. Thermal analysis, Solar radiation, Heat transmission, Ventilation, Heating load, Reprints.

Thermal characteristics of two adjacent office buildings have been obtained from measurements of temperature, energy consumption and air exchange. The characteristics include the total heat-loss coefficient, a solar aperture, and building heat capacity. The heatloss coefficient has been determined by steady state and transient analyses; transient analysis is required for the remaining characteristics. The effect of measured infiltration and ventilation rates on the total heatloss coefficient has been identified. Two methods have been used for the transient analysis: the eqquivalent thermal paramter method of Sonderegger and the Fourier method of Subbarao.

\section{0,069}

PB91-187286 Not available NTIS National Inst. of Standards and Technology (NEL), Gaithersburg, MD. Building Environment Div.
BACnet Communication Protocol for Building Automation Systems.

Final rept.

S. T. Bushby, and H. M. Newman. 1991, 6p Pub. in ASHRAE (American Society of Heating, Refrigeration and Air-Conditioning Engineers) Jnl., p14-15 and 18-21 Apr 91.

Keywords: "Energy management systems, "Protocol(Computers), "HVAC systems, Communication networks, Data transmission, Standards, Automatic control, Buildings, Ventilation, Controllers, Reprints,

The building community in the United States and in several other countries has been carefully watching the efforts by ASHRAE to develop a communication protocol for building energy management and control systems. The name of the standard protocol is 'BACnet' which stands for Building Automation and Control network. The article presents an overview of BACnet as it looks today and describes the tasks remaining to be completed. Control devices are modeled as a network-visible collection of objects. The information represented by these objects are accessed and manipulated through the use of a set of application layer services. The standard object-types that are defined and the application services included in the standard are described. Several possibilities for physical media and networking technology are being considered for inclusion in the standard. It is expected that BACnet will be released for public review by the end of January, 1991.

\section{0,070}

\section{PB91-187732}

PC A06/MF A01

Gaithersburg, $M D$

Draft Abstract Test Suite for Determining Conformance to the BACnet Protocol.

S. T. Bushby. Apr 91, 119p NISTIR-4563

Keywords: "Protocol(Computers), "Energy management systems, "Buildings, Communication networks, Data transmission, Tests, Standards, Specifications, Control systems design, "BACNET protocol.

The BACnet communication protocol for building auto mation and control systems is in an advanced state of development and is expected to be released for public review in early 1991. When the review process is completed it will become an ASHRAE standard. One of the important outstanding issues to be resolved is conformance to the standard and how to test devices to determine if they meet the conformance requirements. The report is a draft Abstract Test Suite based on working draft three of the proposed standard. The Abstract Test Suite is a first step in developing the tests which will be used to certify conformance to BACnet. Its purpose is to provide a starting point from which a conformance certification program can be built and to focus discussions on the outstanding conformance issues that need to be resolved before the standard can be considered complete. The role of an abstract test suite in the conformance testing process is described, a proposed BACnet test system architecture is presented and individual test cases are defined. Test cases to determine support for object types and application services defined in working draft three are included. A standard object configuration is also proposed to simplify the testing process.

\section{0,071}

PB91-187757 Gaithersburg, MD.

Intelligent Building Technology in Japan.

A. Rubin. Apr 91, 69p NISTIR-4546

Sponsored by Department of Commerce, Washington, DC. Technology Administration, and Electric Power Research Inst., Palo Alto, CA.

Keywords: "Buildings, "Energy management systems, "Japan, "Technology assessment, Construction, Thermal energy storage systems, Environmental engineering, Ventilation, Air conditioning, Communication networks, "Intelligent buildings, Office automation.

In May 1990, the author of the report visited Japan at the request of the Department of Commerce, to assess the Japanese experiences with 'intelligent building' design, construction and use. The state-ofthe-art was determined by visiting advanced buildings, building complexes, and interviewing architects, engineers, and researchers and academics. Discussions also were conducted with organizations engaged in promoting the use and design of intelligent buildings In general, the Japanese experiences have paralleled those in the United States. In both countries, advanced building technologies have been employed to advance organizational effectiveness and personal productivity. A major problem shared by the two countries has been the lack of standardization of hardware and software (protocols), resulting in major difficulties in integrating equipment from different manufacturers, and in some instances, diverse products from the same manufacturer. Intelligent building design in Japan differs from that in the United States in several ways. They incorporate new systems and products into their buildings as soon as they become available. They stress the need for a high quality environment - amenity - more than we do. The commitment for developing improved intelligent buildings includes active governmental involvement by two major ministries arid other institutions such as banks.

\section{0,072}

PB91-194936 Not available NTIS

National Inst. of Standards and Technology (NEL), Gaithersburg, MD. Building Environment Div.

Rule-Based Diagnostic Method for HVAC Fault Detection.

Final rept.

S. T. Liu, and G. E. Kelly. 1989, 6p

Pub. in Proceedings of Building Simulation ' 89 , Vancouver, British Columbia, Canada, June 23-24, 1989 , p319-324.

Keywords: "HVAC systems, "Energy management systems, "Diagnostic techniques, Ventilation, Heating systems, Cooling systems, Defects, Control systems design, Signal detection, Reprints, "Air handling units.

The paper describes the development of a prototype computerized diagnostic method for the detection of faulty equipment in an HVAC air handling unit (AHU) using sensor data from the building energy management system (BEMS) as input. The method uses a computer model of the AHU to compute a set of optimum performance parameters for the AHU under normal operating conditions and compares the parameters with actual values calculated from the measured sensor data. Deviations between the two sets of parameters are an indication of either an out-of-tune system or malfunction of certain equipment. The program will point out to the building operator the source of the fault and the possible cause in a timely fashion. The modeling part of the program is computation intensive and is written in the FORTRAN language. The diagnostic part is rule-based and is written in the symbolic language Prolog for its inference capability. Example case runs are presented to illustrate the proposed method.

\section{0,073}

PB91-195388

Not available NTIS National Bureau of Standards (NEL), Gaithersburg, MD. Building Environment Div.

Prediction and Fault Detection of Building Energy Consumption Using Multi-Input, Single-Output Dynamical Model.

Final rept.

J. Pakanen. 1988, 11p

Sponsored by Technical Research Center of Finland, Helsinki, and Ministry of Trade and Industry, Helsinki (Finland)

Pub. in Proceedings of International Symposium on Energy Options for the Year 2000 Contemporary Concepts in Technology and Policy, Newark, DE., September 14-17, 1988, v3 p3.57-3.67.

Keywords: "Energy consumption, "Buildings, Environmental engineering, Residential buildings, Space HVAC systems, Computerized simulation, Electrical faults, Degree days, Reprints

For many years degree-days methods have been used to estimate building energy consumption. However, because degree-day method is based only on the outdoor temperature, there may be large differences between the actual and the calculated results. A new method is proposed, based upon on-line measurements of weather and other characteristic data. The method is capable of being used with building automation systems for both failure detection and energy management applications. The method uses a multiinput single-output (MISO) dynamical model to predict the power fluctuation of the building. The model parameters are identified recursively using measurements of the actual power, outdoor temperature, solar radiation, wind velocity and indoor tempeatures. Other 
measurements may also be used as input data. The stochastic variations in power caused by occupants equipment, lights, etc, can be included in the model. Measurements are taken once an hour and are used to update the model parameters by a recursive extended least square algorithm (RELS). The identified mode can be used to predict the energy consumption or to detect the failure of HVAC equipment and systems. Verification of the method has been accomplished using real weather data and the TARP-computer program for the simulation of buildings.

\section{0,074}

PB91-195438

Not available NTIS

National Bureau of Standards (NEL), Gaithersburg, MD. Building Equipment Div.

Diagnostic Techniques for Evaluating Office Building Envelopes.

Final rept.

A. K. Persily, R. A. Grot, J. B. Fang, and Y. M.

Chang. 1988, 20p

Sponsored by Public Buildings Service, Washington, DC.

Pub. in Proceedings of ASHRAE (American Society of Heating, Refrigerating and Air-Conditioning Engineers Winter Meeting, Dallas, TX., January 30, 1988, v94 pt 1 p987-1006.

Keywords: "Office buildings, "Diagnostic techniques, "Thermal analysis, "Energy conservation, Thermal in sulation, Leakage, Heat flux, Transducers, Heat transmission, Thermal resistance, Reprints.

A variety of diagnostic procedures exists for evaluating the thermal performance of office building envelopes. These measurement techniques enable the quantification of various aspects of envelope thermal performance including airtightness, thermal resistance, and insulation system performance. The paper describes several of these diagnostic measurement techniques in terms of instrumentation, test procedures, and data analysis. Representative measurement results are also presented for each technique. The techniques that are described include tracer gas measurements of air exchange rates, pressurization testing of whole building and component airtightness, thermal resistance measurements with calorimeter boxes and heat flux transducers, and infrared thermographic inspection.

\section{0,075}

\section{PB91-206698}

$\mathrm{PC}$ A03/MF A01

National Inst. of

Indoor Ventilation Requirements for Manufactured Housing.

D. M. Burch. May $91,29 p$ NISTIR-4574

Sponsored by Department of Housing and Urban Development, Washington, DC.

Keywords: "Ventilation, "Indoor air pollution, Windows Condensing, Environmental engineering, Formaldehyde, Air flow, Comfort, HVAC systems, Air quality, Houses, "Manufactured housing

In the study, a mathematical analysis is carried out to investigate the mechanical ventilation rates required in manufactured housing. The analysis reveals that the ventilation provided by natural infiltration is inadequate to comply with the ventilation requirements of double-pane window condensation. The study recommends that both single-wide and double-wide mobile homes be equipped with mechanical ventilation equipment having a minimum installed capacity of $0.026 \mathrm{cu}$ $\mathrm{m} / \mathrm{s}$ (55 cu/min). It was found that considerably large ventilation rates are needed to prevent condensation on single-pane windows. Therefore, it is recommended that double-pane windows be required in all heating climates.

\section{0,076}

PC A06/MF A01

National Inst. of Standards and Technology (BFRL), Gaithersburg, MD.

Abstracts of Daylighting Research.

A. I. Rubin, R. Saraiji, R. McCluney, M. Huggins, and C. Emrich. May 91,120 p NISTIR-4578

Prepared in cooperation with Florida Solar Energy Center, Cape Canaveral. Sponsored by Electric Power Research Inst., Palo Alto, CA., and Lighting Research Inst., New York.

Keywords: "Daylighting, "Bibliographies, Research management, Computerized simulation, Mathematical models, Energy conservation, Glare, Abstracts.
The report is an annotated bibliography of Daylight research and practices. It is a compilation of two draft bibliographies, one compiled by the Florida Solar Energy Center, and the other by the National Institute of Standards and Technology. Both were the result of research projects conducted for the Electrical Power Research Institute. The topics covered are as follows: general treatments of daylight, energy saving design, research and design tools, control systems, computer scale, and mathematical models, skylight research and applications, atria, fenestration systems and materials, shading devices, delivery systems--daylight and sunlight, and finally, the responses of people to light.

\section{0,077}

PB91-216655

$\mathrm{PC} \mathrm{A03/MF} \mathrm{A01}$

National Inst. of Standards and Technology (BFRL), Gaithersburg, MD.

Variable Air Volume System Design Guide.

J. Y. Kao. Jun 91, 49p NISTIR-4605

Sponsored by Public Buildings Service, Washington, DC.

Keywords: "Office buildings, "Ventilation, "Air flow, *Design criteria, Air conditioning. Environmental engineering. HVAC systems, Air circulation, Space heating, Control equipment

Variable air volume (VAV) systems have been used extensively in office buildings during recent years. However, there are persistent complaints by building occu pants about the air quality and environmental conditions of these buildings. The guide has been developed to give guidelines on VAV system design to alleviate possible design-caused problems. The guide provides general discussion of VAV systems, air handling system design, system control, and commissioning. The guide has been developed for the General Services Administration to be used by GSA personnel and GSA's contract designers. It includes design check lists as a means of identifying major aspects in VAV system design where new construction and alteration of air systems are involved.

\section{0,078}

PB91-231563

PC A04/MF A01

National Inst. of Standards and Technology (BFRL). Gaithersburg, MD.

Daylight Research Requirements. Workshop Proceedings. Held in Baltimore, Maryland on August $1-2,1990$.

A. Rubin. Jul 91, 65p NISTIR-4639

Sponsored by Electric Power Research Inst., Palo Alto, CA., and Lighting Research Inst., New York, NY.

Keywords: "Buildings, "Daylighting, "Meetings, Research and development, Computer aided design, Architecture, Windows, Energy efficiency, Technology transfer, Electric lighting, Design criteria, Commercia buildings.

The Daylighting Workshop was conducted on August $1-2,1990$ in Baltimore, Maryland. The overall purpose of the meeting was to develop a new research plan for daylighting, including interactions with other systems, such as electrical lighting and HVAC. The major objectives identified were to: determine the needs for new information (i.e. research); and suggest means of encouraging the application of daylighting, based on what is already known.

\section{0,079}

\section{PB91-237800}

Not available NTIS

National Inst. of Standards and Technology (EEEL). Gaithersburg, MD. Electronics and Electrical Engineering Lab. Office.

Effect of Veiling Relections on Vision of Colored Objects.

A. Worthey $1989,6 p$

Pub. in Jnl. of the Illuminating Engineering Society 18 , n2 p10-15 1989.

Keywords: "Illunimating, "Buildings, Research and development, Reflection, Light sources, Color, Reprints.

Familiar light sources vary greatly in the solid angle they subtend. A recent paper examined the effect of light source size, and quantified the obvious facts that a small source makes veiling reflections brighter, but a large source makes them harder to avoid. One conclusion was that veiling reflections are never negligible. The present paper pursues this idea by quantifying the effect of surface reflections on vision of colored ob jects. It is found that speerical lighting reduces the range of colors seen in glossy objects by $37 \%$.

\section{0,080}

PB91-237818

Not available NTIS

National Inst. of Standards and Technology (EEEL),

ing Lab. Office.

Light Source Area, Shading, and Glare.

Final rept

J. A. Worthey. 1991, 8p

Pub. in Jnl. of the Illuminating Engineering Society 20 n2 p29-36 1991.

Keywords: *Light sources, *Illuminating, Glare, Reflection, Luminance, Research management, Reprints.

Familiar light sources vary in the solid angle they subtend by a factor of 100,000 or more. The effects of source area on object shading and on direct illumination of the eye are examined through simplified examples. Previous papers have looked at highlights and veiling reflections in a similar way.

\section{0,081}

PB91-237826

Not available NTIS

National Inst. of Standards and Technology (EEEL) Gaithersburg, MD. Electronics and Electrical Engineering Lab. Office.

Role of Theory in Lighting Research and Design. Final rept.

J. A. Worthey. 1991, 3p

Pub. in Lighting Design and Application 21, n7 p15-17 Jul 91.

Keywords: "Illuminating, "Research management, Spectra, Light sources, Design, Theories, Luminance, Reprints.

Although science in general acknowledges the role of theory the need for theoretical methods is not so well established in the case of lighting research and design. Among the reasons that theory is important to lighting work are these: (1) Theory is what engineers do. (2) it is hard to vary light-source size. (3) It is also hard to vary Spectral Power Distribution. (4) Optics is not controversial. (5) Theory guides experiment. (6) More speculative theories also have a place.

100,082

PB91-240747

PC A04/MF A01

National Inst. of Standards and Technology (BFRL)

Gaithersburg, MD.

Preliminary Results of the Environmental Evalua tion of the Federal Records Center in Overland Missouri.

A. K. Persily, W. S. Dols, S. J. Nabinger, and S.

Kirchner, Jul 91, 52p NISTIR-4634

Sponsored by Public Buildings Service, Washington, DC.

Keywords: "Federal buildings, "Environmental engineering, Indoor air pollution, Air infiltration, Air quality, Carbon dioxide, Carbon monoxide, Formaldehyde, Radon, Ventilation, Missouri.

The National Institute of Standards and Technology (NIST) is studying the thermal and environmental performance of new federal office buildings for the Public Buildings Service of the General Services Administration (GSA). The project involves long-term performance monitoring both before occupancy and during early occupancy in three new office buildings. The performance evaluation includes an assessment of the thermal integrity of the building envelope, long-term monitoring of ventilation system performance, and the measurement of indoor levels of selected pollutants. The report describes the effort being conducted in the second of the three buildings, the Federal Records Center in Overland Missouri, and presents preliminary measurement results from the building. The infrared thermographic inspection of the Overland Building did not reveal any significant thermal defects in the building envelope, though the existence of air leakage and thermal bridging was noted. The whole building pressurization test showed that the building is quite leaky compared to other modern office buildings. The meas ured radon concentrations were $2 \mathrm{pCi} / \mathrm{L}$ or less on the $B 2$ level, and less than or equal to $0.5 \mathrm{pCi} / L$ on the other levels. Formaldehyde concentrations ranged from 0.03 to $0.07 \mathrm{ppm}$, below the $0.1 \mathrm{ppm}$ guideline but above some levels of concern. The measured levels of volatile organic compounds were similar to those observed in other new office buildings, and the impact of building furnishings and construction activities on the VOC levels were noted. The carbon dioxide levels in 
the building have generally been low, as would be expected in a building with low levels of occupancy.

\section{Building Equipment, Furnishings, \& Maintenance}

100,083

PB91-161984

Not available NTIS

National Inst. of Standards and Technology (NEL) Gaithersburg, MD. Building Environment Div

Visibility of Exit Signs and Directional Indicators. Final rept.

B. L. Collins. $1991,17 p$

Sponsored by National Electrical Mfrs. Association, Washington, DC.

Pub. in Jnl. of the Illuminating Engineering Society, p117-1331991.

Keywords: "Sign visibility, "Exits, "Direction signs, Visual perception, Color, Signal visibility, Visual threshold, Signs, Contract, Sign effectiveness, Sign colors, Reprints.

A three-phase experiment assessed the visibility of exit signs and directional indicators. Sign effectiveness was determined in terms of distance to detection, co rect identification, and rated effectiveness, as well as speed through a corridor in one phase. The results indicated that a 2.25 -in. chevron in grey on white with a contrast of about 0.5 (to meet minimum specifications) was identified correctly at the greatest mean distance and received the highest mean ratings of effectiveness, compared to other indicators. The combination of a 2.25-in. chevron with a 6 -in. EXIT sign was identified correctly at a mean distance of about $100 \mathrm{ft}$. Use of color, either red or green increased the identification distance by about 15 to $20 \mathrm{ft}$. Reducing width to heigh ratio reduced identification distance by about $35-40 \mathrm{ft}$ for chevrons of comparable height, although chevrons of 2.6 to 3.75 -in. in height, with a width to height ration of 0.29 to 0.43 , were identified correctly at about 100 $\mathrm{ft}$. These data suggest that chevron width can be reduced if height is increased beyond 2.6-in. to maintain adequate visibility at $100 \mathrm{ft}$. The data indicate the importance of chevron size and configuration as well as sign color and contrast in determining visibility.

\section{0,084}

PB91-187260

Not available NTIS

National Inst. of

Improving the Fire Performance of Building Contents.

Final rept.

R. W. Bukowski. 1991, 5p

Pub. in Construction Specifier 44, n2 p42-46 Feb 91

Keywords: "Building codes, "Fire resistant materials, *Flammability, Furniture, Fire tests, Standards, Meas urement, Risk assessment, Hazards, Upholstery, Reprints.

This is a short article for The Construction Specifier magazine which reviews current activities relative to the regulation of building contents, e.g., upholstered furniture, mattresses, drapes, etc. It reviews the current status of California Technical Bulletin 133, UL, and NFPA standardization activities. The role of computer fire models and measurement methods in providing the data for them in the context of such regulation is discussed.

100,085
PB91-189274

Not available NTIS

National Inst. of Standards and Technology (NEL), Gaithersburg, MD. Building Environment Div.

Measured Performance of Residential Water Heaters Using Existing and Proposed Department of Energy Test Procedures.

Final rept.

A. H. Fanney. $1990,8 p$

Sponsored by Department of Energy, Washington, DC Pub. in ASHRAE (American Society of Heating, Refrig erating and Air-Conditioning Engineers) Transactions, v96, pt1 p288-295 Jan 90.

Keywords: "Water heaters, Residential buildings, Laboratories, Experimental design, Computerized simulation, US DOE, Standards, Reprints.

A computer-based laboratory for testing residential water heaters has been fabricated at the National Insti- tute of Standards and Technology (NIST). The paper describes the automated laboratory, summarizes existing and proposed Department of Energy test procedures for residential water heaters, and presents experimental results for six electric, five gas-fired, and two oil-fired storage-type water heaters obtained from local distributors.

\section{0,086}

PB91-206664

PC A08/MF A01

Dayton Univ., $\mathrm{OH}$. Research Inst.

Technical Reference and User's Guide for FAST/ FFM Version 3

Technical rept. (Final)

M. A. Dietenberger. May 91, 153p NIST/GCR-91/

589

Grant NANB-SD-0557

Sponsored by National Inst. of Standards and Technology (BFRL), Gaithersburg, MD.

Keywords: "Fires, "Furniture, "Burning rate, "Combustion products, "User manuals(Computer programs) Buildings, Smoke, Mathematical models, Compartments, Combustion, Heat flux, FAST/FFM computer program.

The FAST/FFM computer model provides dynamic, quasi-three-dimensional predictions of furniture fire growth and burnout in a room as well as of the spread of both nontoxic and toxic gases, smoke, and fire to other rooms. The reference guide provides a detailed description of the furniture fire model (FFM) equations the program structure of FFM and its insertion into FAST, the FFM data input requirements, and the output produced by version 3 of the model.

\section{0,087}

\section{PB92-108984}

PC A03/MF A01

National Inst. of Standards and Technology (BFRL) Gaithersburg, MD.

Cone Calorimeter Rate of Heat Release Measurements for Upholstered Composites of Polyurethane Foams.

K. M. Villa, and V. Babrauskas. Aug 91, 40p NISTIR4652

Keywords: "Fire tests, "Upholstery, "Polyurethanes Combustion producls, Fire hazards, Furniture, Chairs, Thermal measurements, Calorimeters, Cone calorimeters.

Certain regulatory authorities have recently banned or restricted the use of furniture upholstered with a combination of polyvinyl chloride (PVC) covering and a melamine-treated polyurethane foam padding. Thus, it was endeavored to determine if quantitative measurements would reveal any special hazards associated with this particular combination. The work represents the testing of nine different upholstered composites, made of fabric coverings and polyurethane foam tested at three different irradiance levels in the National Institute of Standards and Technology Cone Calorimeter. Additional combinations using a polyester batting interbarrier were also used. The composite benchscale specimens were tested at $25 \mathrm{~kW} / \mathrm{sq} . \mathrm{m}, 35 \mathrm{~kW} /$ sq. $\mathrm{m}$ and $50 \mathrm{~kW} / \mathrm{sq} . \mathrm{m}$ irradiance levels. For most variables describing fire hazard, the performance of the combination of melamine-treated polyurethane foam and PVC fabric covering was not found to behave in an unusual manner. Only by considering the time period of 15 seconds after ignition was this combination numerically worse than all other combinations tested.

\section{0,088}

PB92-109156

PC A09/MF A02

National Inst. of Standards and Technology (BFRL) Gaithersburg, MD.

International Fire Detection Bibliography, 19751990.

R. W. Bukowski, and N. H. Jason. Sep 91, 185p

NISTIR-4661

See also COM-74-50638. Sponsored by National Fire Protection Research Foundation, Quincy, MA.

Keywords: "Bibliographies, "Fire detection systems *Smoke detectors, "Fire alarm systems, Fire protection, Abstracts, Fire detectors, Sprinkler systems, Gas detectors, Fire safety, Safety devices.

The bibliography was collected from numerous international sources and represents as complete a compilation of publications from the 15 years covered as could be collected. Nearly 1000 references are includ ed, separated into one of 20 topics such as aerosols and smoke, industrial occupancies, ships, smart detectors and systems, and system reliability studies. Each such section begins with a brief summary and cites the more important papers within. The bibliography ends with a commentary on what the overall literature shows, what research is needed to achieve more reliable detection system operation and reduced unwanted alarms. An author index and a key word index are provided.

\section{Building Standards \& Codes}

100,089

PB91-190066

Not available NTIS

National Inst. of Standards and Technology, Gaithersburg, MD. Lab. Accreditation Program. Plumbing Test Lab Accreditation.

Final rept.

J. A. Swaffield, and L. S. Galowin. 1991, 5p Pub. in Heating/Piping/Air Conditioning $63, n 3$ p73-77 Mar 91.

Keywords: "Plumbing, "Building codes, "Test facilities, *Accreditation, Piping systems, Standards, Water conservation, Test methods, Drainage, Performance, Pipes(Tubes), Reprints.

The ability to evaluate plumbing laboratory competency for conducting tests established in standards is assured through the accreditation of testing laboratories. The National Voluntary Laboratory Accreditation Program (NVLAP) of the National Institute of Standards and Technology (NIST) has announced Plumbing ACcreditation as an addition to the Commercial Products Testing Program. NVLAP provides recognition for laboratory competence and support for objectivity in testing and thereby assists industry in interstate commerce. Procurement officials, designers, specifiers and water conservation managers and planners can specify that test data show conformance to specifications in standards in reports for performance of plumbing devices are from NVLAP accredited laboratories. The accreditation process for determining the qualifications for testing laboratories are described. Applications of research for drainage systems and plumbing products are discussed for input to plumbing codes and standards for pipe sizing are shown from experiments and computer model determinations related to pipe pitch and diameter for waste transport.

\section{0,090}

PB91-194811

Not available NTIS

National Inst. of Standards and Technology (BFRL), Gaithersburg. MD.

Meeting the Challenges of a Global Market - Construction Standards, Testing and Certification.

Final rept.

J. G. Gross. 1991, 4p

Pub. in Construction Business Review, p31-34 Mar/ Apr 91.

Keywords: "Standards, "Construction materials, *Europe, "International trade, Test methods, Product development, Certification, Competition, Regulations, Reprints, European Economic Community.

The article provides information on the rapidly changing global market for construction products and services. Particularly, the unification of the European Community and activities in the development of the EC 92 internal market are discussed. Standards development processes in the United States and internationally are reviewed. The importance of standards as the basis for regulation and commerce makes these activities extremely important for the U.S. design profession and building product producers. Related testing, certification and product approval requirements are reviewed. Recommendations for the U.S. construction community are provided.

100,091

PB91-216614

$\mathrm{PC}$ A03/MF A01

National Inst. of Standards and Technology (BFRL), Gaithersburg, MD.

Direct Digital Control Based Building Automation System Design Criteria.

J. Y. Kao. Jun 91, 37p NISTIR-4604

Sponsored by Public Buildings Service, Washington, DC.

Keywords: "Digital command systems, "Automatic control, "Federal buildings, Public buildings, Energy 
management systems, Quality control, HVAC systems, Fire protection, Security, Controllers, Warning sys-
tems, Design criteria, Guidelines, Specifications, "Direct digital control, "Building automation control.

The document serves to provide design guidance for Direct Digital Control (DDC) based Building Automation Systems (BAS). It also provides instructions to design engineers for the BAS design. Explanations of general design philosophy, current unresolved problems confronting the application of DDC in BAS, and considerations for choosing alternative control strategies in specifying application programs are given. The guide is intended for use by GSA and contract designers as a means of identifying major aspects in DDC based BAS design where new construction or major renovations of control systems are included.

\section{0,092}

PB9 1-216697

PC A04/MF A01

National Inst. of Standards and Technology (BFRL), Gaithersburg, MD.

Guide Specification for Direct Digital Control Based Building Automation System.

J. Y. Kao. Jun 91, 72p NISTIR-4606

Sponsored by Public Buildings Service, Washington, DC.

Keywords: "Specifications, "Digital command systems, "Automatic control, "Federal buildings, Energy management systems, Warning systems, Fire protection, Controllers, HVAC systems, Security, Quality assurance, Design criteria, Public buildings, "Building automation systems, "Direct digital control.

The publication is intended for building system designers' use in specifying direct digital control (DDC) based building automation systems (BAS) for construction of Federal buildings. The guide specification conforms with the format of guide specifications used by the General Services Administration (GSA) and its professional services contractors.

\section{Construction Materials, Components, \& Equipment}

100,093

PB91-132134

PC A03/MF A01

National Inst. of Standards and Technology (NEL), Gaithersburg, MD. Center for Fire Research.

Concept of the Calculus of Fire Safety.

R. L. Smith. Nov 90,23 p NISTIR-4459

Keywords: "Fire safety, "Buildings, Artificial intelligence, Statistical analysis, Probability theory, Burns(Injuries), Death, Fire hazards, Computer systems programs, "Risk assessment, Expert systems.

Many people (builders, owners, designers, occupants, etc.) have an interest in appraising the fire safety of existing or proposed buildings. Computers are playing an ever increasing role in fire safety analysis and in the technology transfer of fire science. In the report the concept of the Calculus of Fire Safety is developed as a declarative programming language. This calculus will enable users to specify what fire safety question is to be answered without specifying how the answer is to be obtained. Advances in Artificial Intelligence programming techniques will enable the implementation of the Calculus of Fire Safety on widely available workstations. This will provide a very powerful tool to anyone interested in determining the fire safety of buildings.

\section{0,094}

PB91-143297

PC A04/MF A01

Pennsylvania State Univ., University Park. Dept. of Mechanical Engineering.

Heat Flux, Mass Loss Rate and Upward Flame Spread for Burning Vertical Walls (1990).

Annual rept.

A. K. Kulkarni, C. I. Kim, and C. H. Kuo. Nov 90, 65p NIST/GCR-90/584

Grant NANB8D0849

Sponsored by National Inst. of Standards and Technology (NEL), Gaithersburg, MD. Center for Fire Re. search.

Keywords: "Heat flux, "Walls, "Fire tests, "Flames, Burning rate, Mathematical models, Construction materials, Test facilities, Conduction, Heat transfer, Heat loss.
Progress made during the first year of NIST Grant No 60NANB8D0849 for the period ending August 14 1989 is reported here. The overall objective of the grant is to understand the basic mechanisms of upward flame spread and to develop a methodology to predict the flame spread on practical wall materials, approximately verified by experiments. In the report progress made on the following tasks is described in individual sections, upward flame spread experiments, mathematical model, local mass loss rate apparatus, heat conduction in the interior of burning walls, and Gardon heat flux gage calibration.

\section{0,095}

\section{PB91-144394}

PC A03/MF A01

National Inst. of Standards and Technology (NEL). Gaithersburg, MD. Center for Fire Research

Using the Harvard/NIST Mark VI Fire Simulation.

J. A. Rockett. Nov 90,45 p NISTIR-4464

Keywords: "Fires, "Computerized simulation, "Buildings, Burning rate, Combustion, Plumes, Entrainment, Walls, Algorithms, Flames, "Harvard/NIST VI computer program.

Installation and use of the Harvard/NIST multi-room fire simulation (computer code on the IBM PC (and compatibles) is described. Two separate auxiliary programs, MASBANK and PREPLOT are also described and their use illustrated. Use of the input menus is described and illustrated. Comments are made on the effect of the choice of alternative sub-models on predicted results. Some suggestions for use on other computers are made. The H06.3 disks for use with the report include: Disk No. 1: All files (in packed form) needed to run $\mathrm{H} 06.3$ and the two auxiliary programs MASBANK and PREPLOT. A group of (packed) files including a short data base file and a sample output of H06.3. Disk No 2. Section 1 including 17 out of 25 source code files for H06.3. Disk No. 3: Section 2 including the final 8 source code files of $\mathrm{H} 06.3$ and source code for MASBANK, PREPLOT and the auxiliay source code file DTIME.

\section{0,096}

PB91-144436

PC A06/MF A01

National Inst. of Standards and Technology (NEL) Gaithersburg. MD. Center for Fire Research.

Programmer's Reference Manual for CFAST, the Unified Model of Fire Growth and Smoke Transport.

Technical note (Final).

W. W. Jones, and G. P. Forney. Nov 90, 107p NIST/ $\mathrm{TN}-1283$

Also available from Supt. of Docs. as $\mathrm{SNOO}^{-003}$ 03041-4.

Keywords: "Smoke, "Fires, "Buildings, Mathematica models, Combustion, Transport properties, Combustion products, Computer applications, Plumes, Pyrolysis, Programming manuals, Protocols, Data file, Software engineering, Computer programs, CFAST computer program.

The document describes the unified model of fire growth and smoke spread, CFAST. The paper documents the internal structure of the model and details the method of modifying the model, together with examples. The intent is to provide a framework and methodology for maintenance of the model, together with a method of updating it. The reader is assumed to have a working knowledge of programming, software maintenance and modeling of physical phenomena.

\section{0,097}

PB91-146977

Not available NTIS

National Inst. of Standards and Technology (NEL) Gaithersburg, MD. Fire Measurement and Research Div.

Heat Release Rate: The Single Most Important Variable in Fire Hazard.

Final rept.

V. Babrauskas, and R. D. Peacock. 1990, 14p

Pub. in Proceedings of Fall Meeting Fire Safety Developments and Testing: Toxicity, Heat Release, Product Development, Combustion Corrosivity, Fire Retardant Chemicals Association, Ponte Vedra Beach, FL., October 20-25, 1990, p67-80.

Keywords: "Fire hazards, "Heat of combustion, "Fires, Ignition, Combustion, Fire tests, Burning rate, Thermodynamic properties, Heat transfer, Heat flux, Flammability, Reprints, "Heat release rate.

Heat release rate measurements are sometimes seen by manufacturers and product users as just another piece of data to gather. It is the purpose of the paper to explain why heat release rate is, in fact, the single most important variable in characterizing the 'flammability' of products and their consequent fire hazard. Examples of typical fire histories are given which illustrate that even though fire deaths are primarily caused by oxic gases, the heat release rate is the best predicto of fire hazard. Conversely, the relative toxicity of the combustion gases plays a smaller role. The delays in ignition time, as measured by various Bunsen burner type tests, also have only a minor effect on the development of fire hazard.

\section{0,098}

PB91-147215

National Inst. of Standards and Technology (NEL) Gaithersburg, MD. Fire Science and Engineering Div. Review of Four Compartment Fires with Four Compartment Fire Models.

Final rept.

S. Deal. $1990,20 \mathrm{p}$

Pub. in Proceedings of Fire Retardant Chemicals Association/Fire Safety Developments and Testing Fal Conference, Ponte Vedra Beach, October 21-24, 1990, p1-20.

Keywords: "Fires, "Compartments, "Models, Air flow, Fire tests, Ventilation, Buildings, Interfaces, Fuels, Gas flow, Combustion, Reprints.

A comparison of four compartment zone fire models (FIRST, FAST, CCFM VENTS and FPETOOL) is presented with four experimental compartment fires. The four experiments represent a variation of vent and fue effects upon the fire. The models were compared with regard to their simulated temperature, oxygen concentration, interface height and vent flow. Overall, the models compared well with the experimental data, but some discrepancies existed. These are discussed. The models are qualitatively ranked with regard to the amount of fire science knowledge the user should possess and the detail with which the simulation is pursued.

100,099

PB91-147439

Not available NTIS National Bureau of Standards (NEL), Gaithersburg, MD. Building Materials Div.

Design of High Strength Cement-Based Materials. Part 2. Microstructure.

Final rept.

H. M. Jennings. 1988, $6 p$

See also PB90-152653. Sponsored by Air Force Engineering and Services Center, Tyndall AFB, FL

Pub. in Materials Science and Technology 4, n4 p285290 Apr 88.

Keywords: "High strength concretes, "Cements, "Microstructure, Strength, Mechanical properties, Toughness, Fracture properties, Concrete durability Fractures(Materials), Fracture strength, Design crite ria, Reprints.

The report is Part II of a three part paper that examines relationships between microstructure and strength of cement-based materials. Microstructural fracture that may be related to strength are discussed.

100,100

PB91-147611

Not available NTIS National Bureau of Standards (NEL), Gaithersburg, MD. Building Environment Div.

Temperature Mea

Final rept.

S. T. Liu. $1988,10 p$

Pub. in ASHRAE (American Society of Heating, Refrigerating and Air-Conditioning Engineers) Transactions 94, pt2 p1350-1359 1988.

Keywords: "Windows, "Temperature measurement * Solar radiation, Radiative heat transfer Temperature measuring instruments, Thermal radiation, Thermocouples, Glass coatings, Window glazing, Reflectance, Reprints.

The measurement of the temperature of window glass exposed to direct solar radiation was studied experimentally under clear sky conditions. Thermocouples of different constructions were mounted on the surface of a $3.18 \mathrm{~mm}(1 / 8$-inch) window glass panel and their outputs were monitored simultaneously under both direct solar radiation and shaded conditions. The thermocouples used for the experiment were a pair of thin foil type with butt-bonded bare metal junction, a pair of 
thin foil type encased in thin polymer/glass laminates, and a 40 gauge wire, beaded junction. For the foil types, one from each pair was coated with a highly re-
flective white paint. Shading was applied after stable output was established, and responses from the step change in irradiation level were recorded. It was found that there can be a 0.2 to $0.7 \mathrm{C}$ difference in the measured temperature for the glass surface between a bare metal junction and a junction coated with a high reflectivity (in the shortwave spectrum) paint. The difference in indicated temperature between an unpainted laminated junction and a painted junction can be as high as 1.0C. The radiative properties of the junction's surface, to have equal effect on the surface temperature measurement.

100,101
PB91-147835

Not available NTIS

National Inst. of Standards and Technology (NEL),
Gaithersburg, MD. Fire Measurement and Research

Gaith.

Forced Smolder Propagation and the Transition to Flaming in Cellulosic Insulation.

Final rept.

T. J. Ohlemiller. $1990,12 p$

See also PB86-166657. Sponsored by Department of Energy, Washington, DC

Pub. in Combustion and Flame 81, n3-4 p354-365 Sep 90.

Keywords: "Thermal insulation, "Flame propagation, "Flammability testing, Cellular materials, Construction materials, Combustion, Ignition, Air flow, Reaction kinetics, Fire resistant materials, Flash point, Reprints.

It is well known that a smoldering fuel responds to an increased oxygen supply by becoming faster and hotter until, eventually, flames erupt. The sequence was examined quantitatively for thick horizontal layers of a permeable fuel, i.e., cellulosic insulation. Two configurations are possible, forward and reverse smolder, both were investigated experimentally. Reverse smolder was shown to respond only weakly to an increased air flow and it exhibited no transition to flaming at flow velocities up to $5 \mathrm{~m} / \mathrm{s}$. Forward smolder responded strongly to increased air flow and yielded transition to flaming at about $2 \mathrm{~m} / \mathrm{s}$ for unretarded material. The influence of combustion retardants was also examined; these included boric acid, a smolder retardant, and borax, a flaming retardant. Both prevented the transition to flaming in the absence of adjacent flammable material but were less effective in its presence. The overall response of these various fuel mixtures and configurations suggests that both kinetics (via leading edge heat transfer effects) and oxygen supply rate (not the latter alone) play substantial roles in dictating smolder response to an air flow.

100,102

PB9 1-147843

Not available NTIS

National Inst. of Standards and Technology (NEL), Gaithersburg, MD. Fire Measurement and Research Giv.

Smoldering Combustion Propagation Through a Permeable Horizontal Fuel Layer.

Final rept.

T. J. Ohlemiller. 1990, 13p

Sponsored by Department of Energy, Washington, DC. 90.

Keywords: "Flammability testing, "Thermal insulation, "Flame propagation, Flammability, Fire resistant materials, Combustion, Ignition, Reaction kinetics, Pyrolysis, Combustion products, Air flow, Reprints.

Although the propagation rate of smoldering through porous horizontal fuel layers has been measured for a variety of materials, there has been little work on the structure of the smolder reaction zone and the factors controlling it. These latter aspects are the focus here for the case of thick $(18 \mathrm{~cm})$ layers of wood-based fibers in the form of cellulosic insulation smoldering under natural convection air supply conditions. Twodimensional profiles of temperature, oxygen mole fraction, and residual organic material have been measured both for unretarded insulation and for insulation having $25 \mathrm{wt} \%$ of the smolder retardant, boric acid, added on. It is inferred that the overall wave structure is dominated by oxygen diffusion from above. The heat release chemistry appears to involve both oxidative pyrolysis and char oxidation in a shifting balance depending on depth in the layer. Boric acid is unable to halt the smolder process in these thick fuel layers but it slows its spread by about a factor of 2 by a combination of endothermic and kinetic effects.

\section{0,103}

PB91-147975 Gaithersburg, MD. Building Equipment Div.

Evaluation of Fumed-Silica Insulation for a Thermal Conductivity Standard Reference Material.

Final rept.

B. G. Rennex, T. A. Somers, T. K. Faison, and R. R.

Zarr. 1990, $12 p$

Sponsored by Department of Energy, Washington, DC. Office of Buildings Energy $R$ and $D$.

Pub. in Insulation Materials, Testing, and Applications, ASTM STP 1030, p3-14 1990.

Keywords: "Thermal insulation, "Thermal conductivity, "Silicon dioxide, Thermal resistance, Conductive heat transfer, Heat measurement, Test facilities, Temperature effects, Thermodynamic properties, Heat treatment, Heat transfer, Reprints, "Standard reference materials.

Standard Reference Materials (SRMs) used for thermal conductivity measurements are required by industry, academic, and government laboratories for calibrating heat-flux-meter apparatus or checking the accuracy of guarded-hot-plate apparatus. New thermal conductivity SRMs are sought to improve the accuracy and extend the operational range of these apparatus. An advisory panel recommended the development of a low thermal conductivity SRM having approximately the same thermal conductivity as blown fluorocarbon foams, suitable for a temperature range of -175 to 900 C. The National Bureau of Standards (NBS) examined four lots of fumed-silica insulation and recommended one candidate for further development as a low thermal conductivity SRM. The four lots of fumed-silica insulation were examined for their relative handleability, material uniformity, variability of thermal conductivity measurements, and effect of heat treatment on the measured thermal conductivity of the materials. Thermal conductivity measurements were conducted using the NBS 1-m Guarded Hot Plate for each lot of fumedsilica insulation at a mean specimen temperature of $24 \mathrm{C}$. Analysis of the thermal conductivity data was performed using the NBS statistical analysis program, Dataplot.

\section{0,104}

PB91-148510

Gaithersburg, MD. Center for Fire Research.

Refinement of a Model for FIre Growth and Smoke Transport.

Technical note (Final)

W. W. Jones. Nov 90, 53p NIST/TN-1282

Also available from Supt. of Docs. as SN003-00303043-1.

Keywords: "Fires, "Smoke, "Buildings, "Mathematical models, Fire hazards, Ventilation, Combustion products, Computer applications, Transport properties, Pyrolysis, Mass flow, Computer programs, Data file, Toxicity, Deposition, Hydrogen chloride.

The document describes the changes which have occurred in FAST, FASTplot, FAST in and the distribution disks in the change from 18.3, the release for Hazard I, and the current release 18.5. Included are an errata section for the Technical Note 1262, a revision history that is distributed with the release disks, the addendum to the Technical Note for the new key words, and the description of the implementation of the physical routines.

100,105

PB91-149153

Not available NTIS National Bureau of Standards (NEL), Gaithersburg, MD. Building Materials Div.

Further Investigation of the Effect of Application Parameters on Adheslve-Bonded Seams of SinglePly Roof Membranes.

Final rept.

W. J. Rossiter. 1988, 7p

Sponsored by Corps of Engineers, Washington, DC.

Keywords: "Roofing, "Adhesive bonding, "Ethylene propylene diene polymers, "Peel tests, Synthetic elastomers Process variables, Temperature dependence, Time dependence, Pressure dependence, Surface finishing, Seame(Joints), Quality assurance, Reprints.

A laboratory study was conducted to determine if a Tpeel test was sensitive to differences in initial (7 days)
bond strength for adhesive-bonded seams of EPDM (ethylene propylene diene terpolymer) rubber membranes made under different application conditions. The main categories of application parameters investigated were: surface condition (cleanness, moisture) of the rubber; pressure applied; temperature during seam formation; open time. The T-peel test was found to be sensitive to changes in bond resulting from variation of some of the application parameters. Seams prepared using uncleaned sheets (without removing the talc of other contaminants from their surfaces), or using long open times, had lower average peel strengths than those of the control specimens. Light pressure or high temperature during bond formation produced seams with average peel strengths higher than those of the controls. Wet surfaces and low temperature had no significant effect on the average strength of the seams. The result that uncleaned surfaces had reduced T-peel strength was significant in that lack of proper surface preparation has been cited as a majo factor affecting seam performance in service. This result suggested that the T-peel test offers promise for use in developing a quality assurance methodology to assess whether field-prepared seams are made in accordance with acceptable practice.

\section{0,106}

PB91-157172

PC A03/MF A01

National Inst. of Standards and Technology (NEL) Gaithersburg, MD. Center for Building Technology. Pulse-Echo Ultrasonic Evaluation of the Integrity of Seams of Single-Ply Roofing Membranes: Laboratory Evaluation of a Prototype Test Apparatus. H. Watanabe, and W. J. Rossiter. Dec 90, 47p

NISTIR-4424

Prepared in cooperation with Takenaka Corp., Tokyo (Japan). Technical Research Lab. Sponsored by Civil Engineering Lab. (Navy), Port Hueneme, CA.

Keywords: *Ultrasonic tests, "Test facilities, "Roofing, "Seams (Joints), "Adhesive bonding Transducers, Acoustic measurement, Construction materials, Membranes, Adhesion, Voids, Prototypes, Pressure, Nondestructive tests, "Single-ply roofing membranes.

The feasibility of using NDE (non-destructive evaluation) methods to detect voids in adhesive-bonded seams of single-ply membranes has been under investigation at the National Institute of Standards and Technology (NIST). The report covers the first phase of a two-part study to investigate the applicability of a pulse-echo ultrasonic method for this purpose. A prototype pulse-echo ultrasonic apparatus, called the field scanner and suitable for testing of single-ply seams in the field, was developed. A series of laboratory experiments was conducted using the field scanner to investigate: (1) optimal operating conditions, (2) sensitivity and practical limitations for detecting voids, and (3) variables affecting its response. The equipment was found to be effective in maintaining coupling between the transducer and seam specimens. Two $5-\mathrm{MHz}$ transducers (focusing and non-focusing types) were selected as the most suitable for void detection in the seams. Voids incorporated in laboratory seam specimens were readily detected. The results of the Phase 1 investigation provided quidelines on the optimum conditions for use of the field scanner. Although not without limitations, encouraging evidence was obtained indicating that the field scanner should be applicable to inspections of EPDM seams in service. Consequently, field investigations are being conducted, as planned, in Phase 2 of the study.

100,107

PB91-158543

Not available NTIS

National Bureau of Standards (NEL), Gaithersburg, MD. Fire Safety Technology Div.

Compartment Fire-Generated Environment and Smoke Filling.

Final rept.

L. Y. Cooper. 1988, 23p

Pub. in SFPE Handbook of Fire Protection Engineering, Chapter 7, p2-116-2-138 1988.

Keywords: "Smoke, "Fires, "Mathematical models, "Buildings, "Air flow, Combustion products, Ventilation, Fire safety, Hazards, Fire protection, Reprints.

The Chapter describes some of the key phenomena which occur in compartment fires, and it focuses on Smoke Filling which is one of the simplest quantitative global descriptions of these phenomena. A specific smoke filling model is presented, and solutions to its model equations are discussed along with example applications. 
100,108

PB91-158998

Not available NTIS

National Bureau of Standards (NEL), Gaithersburg,

MD. Fire Safety Technology Div.

Algorithm for the Mass-Loss Rate of a Burning Wall.

Final rept.

H. E. Mitler. $1989,10 p$

Pub. in Proceedings of the International Symposium on p179-188 1989.

Keywords: "Algorithms, *Burning rate, "Fires, "Walls, Pyrolysis, Thermophysical properties, Buildings, Fire safety, Mathematical models, Reprints.

A derivation is given for a simple algorithm which yields the quasi-steady burning rate of a verticle panel of non-charring, non-melting material in an enclosure with stratification of temperature and oxygen concentration. The algorithm requires the solution of a transcendental equation. Among the thermophysical data which are needed, are the mean flame temperature and the height-dependent absorption coefficient. It is found from experiment that the absorption coefficient is well described for PMMA by a two-parameter expression linear in $1 / z$. Comparison with a transient experiment yields good agreemtn for the mass-loss rate, over much of the range.

100,109

PB91-159038

Not available NTIS

National Inst. of Standards and Technology (NEL), Gaithersburg, MD. Fire Measurement and Research Div.

Five Small Flaming Fire Tests in a Simulated Hospital Patient Room Protected by Automatic Fire Sprinklers.

Final rept.

K. A. Notarianni. $1990,23 p$

Pub. in Report of Test FR 3982, p1-23, 31 Oct 90

Keywords: "Fire tests, "Hospitals, "Sprinklers, "Fire protection, Fire damage, Fire safety, Sprinkler systems, Fire fighting, Design criteria, Reprints.

A series of five tests were conducted to measure temperatures, radiation, and carbon dioxide, carbon monoxide, and oxygen concentrations resulting from small flaming wood crib fires within a simulated NIH hospital patient room protected with automatic fire sprinklers. Time to activation of quick and standard response sprinklers and ionization and photoelectric smoke detectors at several locations in the room simulating multiple options for protection of the space were measured. The test series addressed the location of sprinkler heads in the hospital patient room, demonstrated the effect of privacy curtains around the beds, the effect of a shielded fire, and the effect of sidewall sprinklers.

100,110

PB91-159731

PC A03/MF A01

National Inst. of Standards and Technology (NEL), Gaithersburg, MD. Center for Building Technology.

Pulse-Echo Uitrasonic Evaluation of the Integrity of Seams of Single-Ply Roofing Membranes: Results of Field Investigations and Recommendations.

H. Watanabe, and W. J. Rossiter. Nov 90, 42p NISTIR-4425

See also PB91-157172. Prepared in cooperation with Takenaka Corp., Tokyo (Japan). Technical Research Lab. Sponsored by Civil Engineering Lab. (Navy), Port Hueneme, CA.

Keywords: "Ultrasonic tests, *Roofing, "Adhesive bonding, "Seams(Joints), Nondestructive tests, Mechanical properties, Acoustic measurement, Construction materials, Roofs, Membranes, Inspection, Voids, Adhesion, *Single-ply roofing membranes.

The report describes the second and final phase of a study to develop an ultrasonic NDE method for evaluating the integrity of seams of single-ply roofing membranes. The results of the laboratory evaluation of a prototype pulse-echo apparatus (the field scanner) were positive in that voids could be distinguished from well-bonded sections of the specimen using the intensity of the echo from the adhesive layer. In the Phase 2 study, seams of existing EPDM single-ply membranes were examined by roof-top scanning to evaluate the performance of the field scanner in practice. This was followed by laboratory tests to confirm and explain observations made during the field tests of the seams.
The field scanner was sensitive to micro-cavities, which could be generated in adhesive layers. This resulted in a number of 'false positive' readings. The formation of the micro-cavities was attributed to the temperature-induced volatilization of residual solvent remaining in the adhesive layer after its application on the membrane material.

100,111

PB91-162115

Not available NTIS

National Inst. of Standards and Technology (NML)

Boulder, CO. Chemical Engineering Science Div.

Automated High-Temperature Guarded-Hot-Plate Apparatus for Measuring Apparent Thermal Conductivity of Insulations between 300 and $750 \mathrm{~K}$. Final rept.

J. G. Hust, B. J. Filla, J. A. Hurley, and D. R. Smith. $1990,13 p$

Contract ORNL/IA-21428

See also PB88-242060. Sponsored by Oak Ridge National Lab., TN.

Pub. in Insulation Materials, Testing, and Applications, ASTM STP 1030, p710-722 1990.

Keywords: "Thermal insulation, "Thermal conductivity, "Test facilities, "Thermocouples, Temperature measurement, Heat transmission, Algorithms, Heat transfer, Thermodynamic properties, Automatic control, Thermal measurements, Reprints.

An automated guarded-hot-plate apparatus was designed and built to meet the requirements of ASTM standard test method $C 177$ for measuring the thermal transmission properties of thermal insulation. The apparatus is controlled by a scientific desktop computer. Measurements with this apparatus can be performed at temperatures from 300 to $750 \mathrm{~K}$ in environments of different gases at pressures ranging from atmospheric pressure to roughig-pump vacuum. A novel design for a thermocouple device is used; this device more accurately senses the average temperature over the surface of each heater plate. An improved algorithm for the control sequence provides more stable heater powers and specimen temperatures. Initially the algorithm brings the system rapidly to a temperature setpoint with minimal overshoot. It also permits highly sensitive control of the plate temperatures in later phases of the measurement sequence when thermal stability of the specimen boundaries is very important in measuring the thermal conductivity with high precision. Overall uncertainties of thermal conductivities at K.

100,112

PB91-162149

National Bureau of Standards (NEL), Gaithersburg. MD. Center for Building Technology

Computer Simulated Hydration of a Cement Model. Final rept.

S. K. Johnson, and H. M. Jennings. 1986, 10p

Pub. in CIB.86 Proceedings, Washington, DC., September 22-26, 1986, v6 p2086-2095.

Keywords: "Cements, "Hydration, "Microstructure, *Cementing, Silicate cements, Computer simulation, Computer graphics, Bonding, Adhesion, Placing, Reprints.

A computer model is presented, which simulates the development of microstructure in hydrating tricalcium silicate paste. Hydration is programmed to proceed in steps, during which consumption of the anhydrous material occurs in increments and results in the production of calcium silicate hydrate and calcium hydroxide. Information concerning the distribution of the hydration products is processed by the program and is stored in computer files for later production of reports and twodimensional representations of cross-sections of the simulated microstructure. These images can be compared to micrographs of polished samples of hydrated tricalcium silicate. The computer program permits the selection of the initial system parameters prior to the simulation, thus generating the microstructure under a variety of conditions.

100,113

PB91-167189

PC A05/MF A01

National Inst. of Standards and Technology (NCSL), Gaithersburg, MD.

Review of Research Literature on Masonry Shear Walls.

C. W. C. Yancey, S. G. Fattal, and R. D. Dikkers. Feb $91,100 \mathrm{p}$ NISTIR-4512

Portions of this document are illegible.
Keywords: "Building codes, "Masonry, "Walls, Reviews, Design standards, Research, Standards, Loads(Forces), Compressive strength, Shear strength, Costruction.

A review of the technical literature on masonry shear wall tests was conducted to determine the range and depth of research conducted and to identify areas in need of additional research. The review covers documents published from 1976 to 1989 and includes approximately seven hundred masonry wall tests. Both U.S. and foreign research was included in the review. U.S. code and standard requirements for the design of masonry shear walls are discussed and some of the provisions are highlighted in tabular and graphic form. Technical information regarding experimental studies is tabulated for easy reference and a selected number of test programs are examined in greater detail to present the objective and scope, test variables and major findings. Experimental data from comparable research studies are combined and analyzed to determine the influence of key design parameters on the response of shear walls to in-plane lateral loading. Also included is a comparison of two experimentallyderived ultimate shear strength formulae with that in cluded in the 1988 edition of the Uniform Building Code. The findings of the review are summarized and specific research needs are identified.

100,114

PB91-167197

PC A04/MF A01 Harvard Univ., Cambridge, MA. Div. of Applied Sciences.

Ceiling Jet in Fires.

Annual rept.

H. W. Emmons. Dec 90,51p NIST/GCR-90/582

Grant NIST-60NANB8D0845

Sponsored by National Inst. of Standards and Technology (NEL), Gaithersburg, MD. Center for Fire Research.

Keywords: "Fires, "Jet flow, "Air flow, "Ceilings(Architecture), Friction, Heat transfer, Entrainment, Fluid flow, Froude number, Buildings, Mathematical models.

The steady ceiling jet is examined with a simplified 'top heat' theory. Friction causes the jet to change downstream with flow, depth, and/or hydraulic jump adjust. ments to produce Richardson Number $=1$ at the corridor exit, just as in hydraulics. Entrainment has a qualitative effect identical to friction, although there are quantitative differences. Heat transfer has, however, the opposite effect: the Richardson Number moves away from 1 as the flow proceeds. When all effects are included, high friction cases are predictable, while low friction cases are not. New experimental studies are needed to locate the reasons.

100,115

PB91-167304

$\mathrm{PC}$ A03/MF A01 National Inst. of Standards and Technology (BFRL), Gaithersburg, MD

Computational Models Developed for the Corrosion of Prestressing Steel.

S. T. Wu, and J. R. Clifton. Mar 91, 20p NISTIR-4455 Prepared in cooperation with Air Force Office of Scientific Research, Bolling AFB, DC.

Keywords: "Stress corrosion, "Prestressing steels, * Prestressed concrete, Fracturing, Fracture properties, Cracking(Fracturing), Reinforcing steels, Mathematical models, Structural steels, Fractures(Materials), Corrosion.

Modeling for the purpose of estimating the corrosion rates of steel in prestressed concrete are discussed in the paper. For steel in concrete, localized corrosion processes are not well understood. It is premature at present to develop a sophisticated but still incomplete mathematical model. Instead, a simplified approach is proposed for the quantitative evaluation of corrosion rates. A model was developed based upon considerations of diffusion processes and electrochemical reactions. The dominant chemical factors, such as the concentration of oxygen at the electrodes, are treated as primary variables in the differential equations. Two approaches which have been frequently used to treat stress corrosion cracking are briefly described. The first approach is based on a conventional engineering approach by estimating the stress intensity factors under various environments. In the second approach the surface energy at grain boundaries is estimated along the crack path. 
100,116

PC A03/MF A01

National Inst. of Standards and Technology (NEL),

Gaithersburg, MD. Center for Fire Research.

Modern Test Methods for Flammability.

V. Babrauskas. Jun 90, 25p NISTIR-4326

Keywords: "Flammability testing, "Fire tests, Combustion, Flames, Flammability, Calorimeters, Test facilities, Experimental data, Thermal measurements, Fires, Smoke, Ignition, Toxicity, Combustion products, Furniture, Corrosion, Heat transmission.

During the last decade, significant improvements have become available in flammability testing. Rationallybased new methods, derived from fundamental engineering principles are replacing the previously used empirical tests. The major emphasis in the development work has been to provide a basic set of benchscale methods which can be used to predict full-scale product performance. Reference methods for conducting full-scale tests will continue to be needed to handle products or situations where the bench-scale methods are not applicable. The bulk of the testing needs, however, can now be fulfilled by use of benchscale tests which are not only simple to run, but are known to accurately predict the full-scale performance.

\section{0,117
PB91-174573}

Not available NTIS

National Inst. of Standards and Technology (NEL),

Gaithersburg, MD. Fire Science and Engineering Div. Ceiling Jet-Driven Wall Flows in Compartment Fires.

Final rept.

L. Y. Cooper. $1988,12 p$

Pub. in Combustion Science and Technology 62, n4-6 p285-296 1988 .

Keywords: "Fires, "Buildıngs, "Compartments, "Walls, Heat transfer, Plumes, Wall effects, Convection, Air flow, Combustion, Entrainment, Reprints.

Analytic estimates are developed for depth of penetration and lateral entrainment of negatively buoyant, ceiling jet-driven wall flows during early times of compartment fire scenarios. When walls are not 100 far from the fire source of the order of the fire-to-ceiling distance, it is found that the penetration of these downward wall flows is a large fraction of the fire-to-ceiling distance, and that this fraction is relatively independent of the details of fire size and fire-to-wall spacing Also, net rate of entrainment into the wall flow as it is buoyed back upward to the ceiling elevation is found to be several times larger than the flow rate of the driving ceiling jet flow immediately upstream of wall impingement. Data from five studies reported in the literature are reviewed relative to the anlytic results obtained. One of these involved a field model simulation of the flow generated by a buoyant source in an enclosure. Two experimental laboratory studies involved fires in enclosures with characteristic dimension of the order of several meters. Two others involved saltwater plumes in freshwater tanks with characteristic dimension of the order of several tenths of a meter. These data are found to be consistent generally with the analytic results, and, in particular, with the notion that the wall flows in question provide the mechanism for mixing which leads to the deep and rapid stratification typically observed in enclosed compartment fire environments.

100,118
PB91-174789

Not available NTIS

National Bureau of Standards

MD. Fire Safety Technology Div.

Letter to the Editor of Standardization News.

Final rept

D. Gross. $1987,2 p$

Pub. in ASTM (Ámerican Society for Testing and Materials) Standardization News, p7-8 Feb 87.

Keywords: "Fire tests, "Standards, Research facilities, Measurement, Standardization, Metrology, Test facilities, Fires, Combustion, Reprints.

The letter to the editor responds to a short 'In My Opinion' article on the use, for regulatory purposes, of fire test methods designed for research and development (R\&D).

\section{0,119}

PB91-175315

Not available NTIS

National Bureau of Standards (NEL), Gaithersburg, MD. Building Materials Div.
Application of CCRL Data in the Development of Cement Standards.

Final rept.

J. H. Pielert, and C. B. Spring. 1987, 12p

Sponsored by American Society for Testing and Materials, Philadelphia, PA

Pub. in Uniformity of Cement Strength, ASTM STP 961, p30-41 1987.

Keywords: "Cements, "Standards, "Laboratories, Concretes, Quality assurance, Samples, Test facilities, Aggregates, Reinforcing steels, Portiand cements, Construction materials, Reprints.

The Cement and Concrete Reference Laboratory (CCRL) through its laboratory inspection and proficiency sample programs collects data which can be useful in the development of cement and concrete standards. The paper outlines the scope of CCRL activities, discusses the nature of the CCRL data base, discusses specific examples of technical studies which support standard development, and outlines areas of future CCRL support to standards committees. CCRL inspections are conducted in laboratories equipped to perform tests on cement, concrete, concrete aggregate and steel reinforcing bars. The program for the distribution of proficiency samples includes portland cement, portland cement concrete, blended cement and masonry cement. Data from these programs have over the years been made available to the appropriate ASTM committees. CCRL also studies technical problems of interest to ASTM committees C1 and C9. Several CCRL activities will be presented to illustrate the application of CCRL data.

\section{0,120}

PB91-175422

Not available NTIS

National Bureau of Standards (NEL), Gaithersburg, MD. Fire Measurement and Research Div.

Smoke Production and Properties.

Final rept.

G. W. Mulholland. 1988, 10p

Pub. in SFPE (Society of Fire Protection Engineers) Handbook of Fire Protection Engineering, Chapter 25, p368-377 1988.

Keywords: "Smoke, "Fires, "Smoke detectors, Combustion products, Detectors, Transmittance, Visibility, Safety devices, Optical density, Safety engineering, Reprints

The fundamental concepts relevant to smoke are defined including smoke conversion factor, size distribution function, coagulation, light extinction coefficient, optical density, visibility, and smoke detector response function. Tabulated results are presented on smoke emission, average particle size and width of size distribution, specific optical density, and mass optical density for smoke produced by wood and plastics. Illustrative examples are included in regard to analyzing size distribution data, predicting the effect of particle coagulation on number concentration, estimating the visibility of an exit sign for a developing fire, and predicting smoke detector response time.

\section{0,121}

PB91-187278

Not available NTIS

National Inst. of Standards and Technology (NEL), Gaithersburg, MD. Building Environment Div.

Comparison of Two Test Methods for Determining Transfer Function Coefficients for a Wall Using a Calibrated Hot Box.

Final rept.

D. B. Burch, B. A. Licitra, and R. R. Zarr. 1990, 8p

Sponsored by Department of Energy, Washington, DC. Pub. in Jnl. of Heat Transfer 112, n1 p35-42 Feb 90.

Keywords: "Thermal measurements, "Walls, "Heat transfer coefficient, "Temperature measuring instruments. Test facilities, Heat flux. Heat transmission, Heat transfer, Least squares method, Thermal analysis, Heat loss, Reprints.

The paper compares two dynamic test methods for obtaining empirical transfer-function coefficients (TFCs) for a wall specimen using a calibrated hot box (CHB). The wall specimen consisted of an insulated hollow concrete block wall that contained significant thermal bridges and lateral heat flows. The wall specimen was installed between the climatic and metering chambers of a CHB. The climatic chamber generated a dynamic (i.e., time-dependent) excitation function. The metering chamber maintained a typical indoor condition and served as a calorimeter. The transient heat-transfer rate at the inside surface of the wall specimen was determined at hourly time steps from an energy balance of the metering chamber. The first method is called the 'curve fitting method. In the method, a periodic excitation function consisting of a fundamental 24-hour sine wave and several harmonics (e.g.., a sol-air diurnal temperature cycle) was generated in the climatic chamber. Empirical TFCs were obtained by curve fitting the measured specimen heat-transfer rate to a transfer function equation using the method of least squares.

100,122

PB91-187807

PC A04/MF A01

National Inst. of Standards and Technology (BFRL), Gaithersburg, MD.

Building and Fire Research Laboratory Publications, 1990.

N. H. Jason. Apr $91,69 p$ NISTIR-4562

Also available from Supt. of Docs. See also PB90219809.

Keywords: "Buildings, "Fire safety, "Bibliographies, Research management, Fire models, Fire tests Smoke.

Building and Fire Research Laboratory Publications, 1990 is the first edition to reflect the combined publications of the Building and Fire Research Laboratory (BFRL) for calender year 1990. in 1991 the Center for Building Technology (CBT) and the Center for Fire Research (CFR) combined to form BFRL. The publication is a supplement to previous editions of Fire Research Publications and the Building Technology Publications.

100,123

PB91-189324

Not available NTIS

National Inst of Standards and Technology (NEL) Gaithersburg, MD. Automated Production Technology Div.

Acoustic Technique for Evaluation of Thermal In sulation.

Final rept.

D. R. Flynn, D. J. Evans, and T. W. Bartel 1990, 24p Pub. in Insulation, Materials, Testing, and Applications, ASTM STP 1030, p319-342 1990.

Keywords: "Thermal insulation, "Noise(Sound), Frequencies, Fiberglass, Rock wool, Cellulose, Laboratory tests, Nondestructive tests, Sound transmission, Reprints.

A laboratory apparatus has been constructed that en ables rapid measurement of the sound insertion loss of a sample of insulation as a function of frequency. An extensive series of measurements of the sound insertion losses associated with blown samples of fiberglass, rockwool, and cellulose has been completed the results of these acoustic measurements are highly correlated with coverage (mass per unit area) and thermal resistance (R-value). An investigation is planned to extend the acoustic techniques used in the laboratory apparatus to in situ determination of the sound transmission loss through thermal insulation installed in attics. Two possible approaches to such field measurements are described.

100,124

PB91-189993

Not available NTIS

National Inst. of Standards and Technology (NEL). Gaithersburg, MD. Building Materials Div.

Field Testing of Adhesive-Bonded Seams of Rubber Roofing Membranes.

Final rept.

W. J. Rossiter, J. F. Seiler, and P. E. Stutzman.

1989, 10p

Pub. in Proceedings of Conference on Roofing Technology (9th), Gaithersburg, MD., May 4-5, 1989, p7887.

Keywords: "Adhesive bonding, "Seams(Joints), *Roofs, "Rubber adhesives, Adhesion, Bonding, Mechanical properties, Dissimilar materials bonding, Membranes, Bonding strength, Construction materials, Reprints.

Laboratory and field investigations were conducted to obtain data for supporting the development of a methodology to assure the quality of adhesive-bonded seams of vulcanized rubber membranes. The prime factors investigated were surface condition of the rubber, temperature of the rubber at the time of adhesive application and cure time of the adhesive. The laboratory data indicated that the T-peel test is sufficiently sensitive for use in a field methodology for assuring the quality of seams. Based on the laboratory results, it appeared possible to conduct the T-peel 
tests within a day (or perhaps less) of seam formation and to find significant differences in bond strength due to lack of proper surface cleaning of the rubber. Data from field applications are needed to demonstrate whether such differences are found on the job at one day's time or less. Seams sampled from two buildings were found to have low bond strengths, as compared to seams prepared using rubber carefully cleaned in the laboratory.

100,125

PB91-190124

Not available NTIS

National Inst of Standards and Technology (NEL). Gaithersburg, MD. Building Environment Div.

Thermal Conductivity of Fumed Silica Board at Room Temperature.

Final rept.

R. R. Zarr, and T. A. Somers. 1990, 13p

Sponsored by Department of Energy, Washington, DC. Pub. in Thermal Conductivity 21, p247-259 1990.

Keywords: "Thermal insulation, "Silicon dioxide, Thermal conductivity, US NIST, Reprints, Standard Reference Materials.

The thermal conductivity of fumed-silica board insulation was measured using the 1 -metre guarded hot plate at the National Institute of Standards and Technology (NIST). Measurements were conducted for the following ranges: bulk density, 304.5 to $325.4 \mathrm{~kg} / \mathrm{cu} \mathrm{m}$; and air pressure 97.51 to $103.43 \mathrm{kPa}$. The effect of moisture content on room-temperature measurements was minimized by prior conditioning of the specimen at $100 \mathrm{C}$ for 24 hours. A linear equation having two independent parameters, bulk density and air pressure, was fit to thirty-five data points. Certified values of thermal resistance at room temperature were calculated for the following ranges of bulk density and air pressure; 300 to $330 \mathrm{~kg} / \mathrm{cu} \mathrm{m}$ and 97 to $102 \mathrm{kPa}$, respectively. The Office of Standard Reference Materials of NIST in Gaithersburg, Maryland, USA has made available two Standard Reference Materials (SRMS) of fumed-silica board, SRMs-1449 and 1459 .

\section{0,126}

PB91-194696 Not available NTIS National Inst. of Standards and Technology (NEL), Gaithersburg, MD. Building Environment Div.

Evaluation of the Heat Flux Transducer Technique for Measuring the Thermal Performance of Walls. Final rept.

B. M. Burch, and R. R. Zarr. 1991, 11p

Sponsored by Department of Energy, Washington, DC. Pub. in Proceedings of Conference on In-situ Heat Flux Measurements in Buildings Applications and Interpretations of Results, Hanover, NH., May 22-23, 1990 , p111-121 1991.

Keywords: *Heat flux, "Thermal measurements, "Walls, "Energy conservation, Thermal analysis, Temperature distribution. Measuring instruments, Transducers, Heat transmission. Heat transfer, Temperature measurement, Reprints, "Heat flux transducers.

Four wall specimens were instrumented with heat flux transducers and tested in a calibrated hot box to determine the accuracy of the heat flux transducers. The heat flux transducers were installed at the interior surface of the wall specimens and exposed to different steady and dynamic (i.e., time-dependent) temperature differences using a calibrated hox box. Two of the wall specimens were composed of homogeneous and monolithic material layers, which yielded virtually onedimensional heat flux. The other two wall specimens were composed of conventional wall construction with wood structural members, which yielded a two-dimensional heat flux pattern. The heat flux transducers were previously calibrated by exposing them to a uniform heat flux in a guarded hot plate. The steady-state and transient heat flux transducer measurements agreed within $5 \%$ of the calibrated hot box measurements.

100,127
PB91-203521

Not available NTIS

National Bureau of Standards (NEL), Gaithersburg MD. Building Materials Div.

NBS Research on Protective Coatings for Buildings and Structures.

Final rept.

L. W. Masters. 1985, 2p

Pub. in Jnl. of Protective Coatings and Linings 2, n3 p15-16 Mar 85.

Keywords: "Protective coatings, "Structural steels, Research projects, Barrier coatings, Construction ma- terials, US NBS, Performance prediction, Service life, Corrosion prevention, Weathering, Reprints.

The goal of coatings research in the Building Materials Division of the National Bureau of Standards (NBS) is to develop improved technical bases for assuring the quality of and for predicting the service life of protective coatings. The research results lead to test and evaluation methods and criteria to aid in the selection, use, and maintenance of protective coatings.

100,128

PB91-203935

Not available NTIS National Bureau of Standards (NEL), Gaithersburg, MD. Building Materials Div.

Use of Nondestructive Methods for Inspection of Single-Ply Roofing Membranes.

Final rept.

W. J. Rossiter, and J. R. Clifton. 1986, 10p

Sponsored by Naval Civil Engineering Lab., Port Hueneme, CA.

Pub. in Durability of Building Materials 3, p343-352 1986.

Keywords: "Nondestructive tests, "Roofing, "Membranes, Voids, Moisture content, Construction materials, Ultrasonic tests, Infrared thermography, Inspection, Roofs, Seams(Joints), Reprints.

The laboratory study investigated the use of the ultrasonic pulse echo method and the infrared thermography method to detect voids and delaminations nondestructively in seams of single-ply roofing membranes. Voids were intentionally incorporated in seam specimens prepared using a commercially available EPDM (ethylene-propylene-diene terpolymer) roofing membrane material. A number of factors affecting the response of the nondestructive evaluation (NDE) methods was examined, including: pressure applied during seam fabrication, void size, water in the void, temperature, unbonded seam interfaces, and supporting panel under the seam. The results indicated that both NDE methods were successful under certain laboratory conditions in locating hidden voids and delaminations in the seam specimens. Both the ultrasonic pulse echo and the infrared thermography methods need further research before their reliability in locating defects is ascertained and their results can be more fully interpreted.

\section{0,129}

\section{PB9 1-206680}

PC A04/MF A01

Factory Mutual Research Corp., Norwood, MA

Sprinkler/Hot Layer Interaction.

Technical rept. Sept. 1990.

G. Heskestad. May $91,56 p$ NIST/GCR-91/590

Grant NANB-OD1066

Sponsored by National Inst. of Standards and Technology (BFRL), Gaithersburg, MD.

Keywords: *Fires, *Sprinklers, "Cooling, Vaporizing, Spraying, Evaporation, Entrainment, Interfaces, Fire protection. Spray quenching. Mathematical models, Temperature distribution, Boundary layer, Plumes.

A model has been developed for the cooling of a quiescent hot layer by a sprinkler spray in a two-layer zone model (with no direct interaction of the spray with a fire plume), based on existing models of spray-induced flow and heat transfer to evaporating drops. In addition, existing models have been adapted to predict the penetration of the spray-induced flow below the layer interface and associated entrainment of lowerlayer fluid into the upper layer. The cooling model is in good agreement with published results from sprinklered room-fire experiments, for which penetration of spray-induced flow into the lower layer and associated entrainment were calculated to be negligible. No published results have been found to test the validity of the adapted models of penetration and associated entrainment, which do not account for possible effects of the spray below the layer interface.

\section{0,130}

PB91-216648

PC A04/MF A01

National Inst. of Standards and Technology (NEL) Gaithersburg, MD. Robot Systems Div.

Description of a Vibration Compensation System for the Small Scale Model Robot Crane Project. R. Bostelman. Jul 91, 70p NISTIR-4595

Keywords: "Cranes(Hoists), "Robotics, "Vibration damping, Construction equipment, Models, Computer systems hardware, Electronics, Design, Data acquisition, Control systems, Systems engineering, Circuit diagrams, Servo control, SSMRC(Small Scale Model Robot Crane).
The report describes the electronic hardware designed and developed for the Small Scale Model Robot Crane (SSMRC) Project sponsored by the Defense Advanced Research Projects Agency (DARPA). The purpose of the electronic hardware is to provide the necessary servo controls for the three axes of the SSMRC. The report begins with an introduction which explains the overall objectives of the project and describes the SSMRC system configuration. An electronic design section discusses each system component and describes the component from both a hardware and a system point of view. Test procedures and results, which give the troubleshooting methods and data needed for development of the system, are then presented. The report concludes with a brief summary and an appendix which provides electronic design schematics and descriptions of the parts used in the construction of the electronic hardware system.

\section{0,131}

PB91-216689

PC A05/MF A01

National Inst. of Standards and Technology (BFRL), Gaithersburg, MD

Applications of the Generalized Global Equivalence Ratlo Model (GGERM) for Predicting the Generation Rate and Distribution of Products of Combustion in Two-Layer Fire Environments: Methane and Hexanes.

L. Y. Cooper. Jun 91, 78p NISTIR-4590

Keywords: "Combustion, "Fires, "Buildings, "Methan, "Hexane Combustion products, Reaction kinetics, Mathematical models, Fluid flow, Chemical reactions, Steady state.

The Generalized Global Equivalence Ratio Mode (GGERM) was developed to predict the generation rates of oxygen, fuel, and other products of combustion in rooms containing fires. The GGERM extends to general transient conditions the global equivalence ratio model established during times of steady-state in experimental studies involving two-layer compartment fires. The present work uses the GGERM to predict upper layer mass fractions of products of combustion (fuel, oxygen, $\mathrm{CO}$, and others) in these two-layer fire experiments, but during times of transient response. All predicted results are found to be plausible and where transient data are available, predicted and measured results compare favorably. However, available data are limited and additional validation of the GGERM under more varied fire conditions is required before it can be used with confidence in two-layer zone-type compartment fire models.

\section{0,132}

PB91-216788

PC A08/MF A02

National Inst. of Standards and Technology (BFRL), Gaithersburg, MD.

Building and Fire Research Project Summaries, 1991.

N. J. Raufaste. Jun 91, 155p NISTIR-4582

See also PB91-187807.

Keywords: "Research projects, "Fires, "Buildings, Flammability, Fire resistant materials, Combustion, Fire safety, Indoor air quality, Fire hazards, Smoke, Fire protection, "Building and Fire Research Laborato-

In early 1991, as part of an agency-wide reorganization, the National Institute of Standards and Technology (NIST) created the Building and Fire Research Laboratory (BFRL) by merging its Centers for Building Technology and Fire Research. BFRL's mission is to increase the usefulness, safety, and economy of constructed facilities, and reduce the human and economic costs of unwanted fires in buildings. The report summarizes BFRL's research for 1991. The report is arranged by its research programs: structural engineering materials engineering, mechanical and environmental systems, fire science and engineering, and fire measurement and research. Each summary lists the project title, its research, the BFRL point of contact, sponsor, and results.

\section{0,133}

PB91-237479

Not available NTIS

Inst. of Standards and Technology (NEL) Gaithersburg, MD. Building Materials Div. 


\section{Construction Materials, Components, \& Equipment}

Microstructural Aspects of the Fracture of Hardened Cement Paste.

Final rept.

L. Struble, P. Stutzman, and E. Fuller. 1989, 5p

Contract AFOSR-ISSA88-0039

Sponsored by Air Force Office of Scientific Research, Bolling AFB, DC.

Pub. in Jnl. of the American Chemical Society 72, n12 p2295-2299 1989.

Keywords: "Fracture mechanics, "Cements, "Crack propagation, Microstructure, Paste(Consistency) Hardness, Electron microscopy, Microcracks, Construction materials, Reprints.

Microstructures of fractured hardened cement pastes were examined using a scanning electron microscope. Cracks generally appear to pass around unhydrated cement grains and along calcium hydroxide cleavage planes, but to pass through voids, inner hydration product, and outer hydration product with no change in direction. The crack path is often offset, with gaps between offset segments forming bridges. The crack is often branched or forked at the tip, and microcracks are observed just ahead of the tip. The gaps between crack offsets may explain the rising fracture resistance (R-curve) of hardened cement paste, and the microcracking ahead of the crack tip may also provide toughening.

100,134

PB92-108919

National Inst of Standards and Techno3/MF A0 Gaithersburg Of Standards and Technology (NEL) FPETOOL User's Gulde.

H. E. Nelson. Oct 90, 38p NISTIR-4439

Contract GSA/PBS-87-03 Sponsored by Public Buildings Service, Washington,

Keywords: "Fire hazards, "Fire protection, "Computer applications, Computerized simulation, Buildings, Mathematical models, Fire safety, Smoke, Flame propagation, Sprinkler systems, Fire detection systems Ventilation, Flashover, User manuals(Computer programs), "FPETOOL computer program.

FPETOOL is a computerized package of relatively simple engineering equations and models. FPETOOL consists of a package of engineering tools useful in estimating potential fire hazard and the response of the space and fire protection systems to the developing hazard. To a large extent, user instructions are in cluded as screen messages presented at the time of need by FPETOOL. The document covers information useful to the user, but not included as screen messages.

\section{0,135
PBg2-108976}

PC A03/MF A01

National Inst. of Standards and Technology (BFRL) Gaithersburg, MD.

Mathematical Modeling of Enclosure FIres.

H. E. Mitler. May 91, 47p NISTIR-90-4294

See also PB90-129867 and PB81-110520.

Keywords: "Fires, "Mathematical models, "Enclosures, Fire hazards, Fire safety, Fire tests, Combus tion, "Fire models, Field models, Zone models.

After a brief description of the history and physics of enclosure fire modeling, two kinds of deterministic fire models are discussed: field models and zone models. The models consist of sets of coupled equations -. differential, algebraic, or a mixture of the two. Special emphasis is placed on discussing some of the numerica techniques used to solve these equations. Although this is not a comprehensive review article, an attemp has been made to give a sufficiently complete reference section that the interested reader can follow up on any item.

\section{0,136}

\section{PB92-112556}

PC A03/MF A01 California Univ., Berkeley. Dept. of Mechanical Engineering.

Users' Gulde to BREAK1, the Berkeley Algorlthm for Breaklng WIndow Glass In a Compartment FIre. A. A. Joshi, and P. J. Pagni. Oct 91, 29p NIST/GCR$91 / 596$

\section{Grant NANB-80848}

See also PB90-244443 and PB88-111810. Sponsored by National Inst. of Standards and Technology (BFRL), Gaithersburg, MD.

Keywords: "Fires, "Buildings, "Window glass, "Breaking, "Computer applications, Mathematical models
Thermal stresses, Thermal conductivity, Thermal diffusivity. Thermal expansion, Absorption, Modulus of elasticity, Temperature, Heat transfer coefficient, Emissivity, Flames, Radiation, Thermophysical properties, Vents, User manuals(Computer programs).

The report is an instructional manual for the computer program BREAK1, the Berkeley Algorithm for Breaking Window Glass in a Compartment Fire, version 1.0. The thermal response of window glass exposed to a compartment fire and its time to breakage are calculated. The following glass properties, treated here as constant, are required as input: thermal conductivity, thermal diffusivity, absorption length, breaking stress, Young's modulus, coefficient of linear thermal expansion and emissivity. In addition, the ambient temperature, the hot layer temperature heat transfer coefficients and emissivities and the direct flame radiation histories are needed as input. The window geometry is also required. The output describes the evolution of the window temperature field, $T(x, t)$, where $x$ is the direction normal to the glass pane, culminating in the window breakage time, $t$ sub(b). The manual contains a summary of the mathematical model, sample thermophysical and mechanical data tables, some worked examples and advice on using the program.

100,137

PB92-116334

PC A04/MF A01

National Inst. of Standards and Technology, Gaithersburg, MD.

Analysis of Molsture Accumulation in a Wood Frame Wall Subjected to Winter Climate.

D. M. Burch, and W. C. Thomas. Oct $91,57 p$

NISTIR-4674

See also DE90014155 and PB85-199248. Sponsored by Department of Energy, Washington, DC.

Keywords: "Moisture content, "Walls, "Construction materials, "Wood products, Framed structures, Sheathing, Humidity, Heat transfer, Mathematical models, Permeability, Finite difference method, Thermal insulation, Winter.

A transient, one-dimensional, finite-difference model is presented that predicts the coupled transfer of heat
and moisture in a multilayer wall under nonisothermal and moisture in a multilayer wall under nonisotherma the diffusion through the capillary flow regimes. It has a provision to account for convective moisture transfer by including embedded cavities which may be coupled to indoor and outdoor air. The model is subsequently used to predict the time-varying average moisture content in the sheathing and siding of a wood frame wal as a function of time of year. Results are shown for a mild winter climate (Atlanta, GA), an intermediate winter climate (Boston, MA), and a cold winter climate (Madison, WI). The indoor temperature is maintained at $21 \mathrm{C}$, and separate computer runs are carried out for indoor relative humidities of $35 \%$ and $50 \%$. The effect of several construction parameters on the winter moisture accumulation are investigated. The parameters include the interior vapor retarder permeance, sheathing permeance, exterior paint permeance, indoor air leakage, and the amount of insulation.

\section{0,138}

PB92-116342

PC A03/MF A01

National Inst. of Standards and Technology (NCSL), Gaithersburg, MD.

Ultimate Strength of Masonry Shear Walls: Predictions versus Test Results.

S. G. Fattal, and D. R. Todd. Oct 91, 47p NISTIR4633

See also PB91-167189.

Keywords: "Ultimate strength, "Masonry, "Walls, Shear stress, Building codes, Loads(Forces), Mathematical models, Design standards, Earthquake resistant structures, Structural analysis, Earthquake engineering.

The study compares the ability of four different equations to predict the ultimate shear stress in masonry walls failing in shear. Experimental data on full-grouted reinforced shear walls from four different sources are compared with the predictions from the four equations. Wall characteristics from 65 test specimens were used as input to the four predictive equations. The ultimate strength predictions were then compared to the actual measured strength of the 65 test walls. Two of the equations (the existing UBC equation for shear strength of masonry walls and the Architectural Institute of Japan's equation for predicting the shear strength of reinforced concrete shear walls) were found to be inadequate for the prediction of ultimate shear strength of masonry walls. An equation proposed by Shing et al. was found to predict shear strength well for only limited ranges of variables, primarily because excessive weight is given to the contributions of horizontal reinforcement to strength. An equation proposed by Matsumura was found to be the best predictor of the four equations examined, but it lacks the consistency needed to use it as a basis for design

100,139

PB92-116458

Not available NTIS

National Inst. of Standards and Technology (NEL) Gaithersburg, MD. Fire Measurement and Research

Large-Scale Validation of Bench-Scale Fire Toxiclty Tests.

Final rept.

V. Babrauskas, R. H. Harris, E. Braun, B. C. Levin,

M. Paabo, and R. G. Gann. 1991, 24p

Pub. in Jnl. of Fire Sciences 9, n2 p125-148 Mar/Apr 91.

Keywords: "Fire tests, "Standards, "Toxicity, "Construction materials, Test methods, Fire hazards, Test facilities, Chemical properties, Proving. Bench-scale experiments, Buildings, Reprints

A sizable number of bench-scale fire toxicity tests have been proposed over the last two decades. To date, no test method has successfully passed through the standards bodies ISO, ASTM, or BSI. The reasons are varied, but a major concern has been that none of the methods were seen to adequately predict the behavior of real, large-scale building fires. Such validation efforts have been held back both due to a shortage of good quality data, and because agreement had not been reached on the criteria for successful validation. NIST has now completed a pilot project to address both of these issues. In the study, several criteria for validation have been put forth. An initial data set has been compared against these criteria, comprising 2 bench-scale methods, 3 test materials, and a single real-scale fire scenario. The project results indicate that the course being pursued is appropriate, and provide illustrative performance data for the two benchscale methods. The present project was a pilot study; further validation data will have to come from additional test materials and additional real-scale fire scenaros being examined. As a result of the studies, a factorof-3 agreement between bench-scale and real-scale results was established as both useful and practical.

100,140

PB92-116557 Not available NTIS

National Inst. of Standards and Technology (NEL), Gaithersburg, MD. Structures Div.

Impact-Echo: A New Method for Inspecting Construction Materials.

Final rept.

N. J. Carino, and M. Sansalone. $1990,15 p$

Pub. in Nondestructive Testing and Evaluation for Manufacturing and Construction, p209-223 1990.

Keywords: "Construction materials, "Nondestructive tests, "Concretes, Impact tests, Fourier transformation, Stress waves, Measurement, Displacement. Frequency response testing, Surface properties, Reflection, $S$ waves, $P$ waves, Transducers, Frequency, Spectrum analysis, Graphic methods, Cross-sections, Voids, Wave propagation, Delaminating, Defects, $\mathrm{Re}$ prints, "Impact echo technique.

A technique called impact-echo has been developed for flaw detection in heterogenous construction mater als such as concrete. The technique, which is based on a simple concept, uses mechanical impact to generate a short duration stress pulse which travels into the test object as P. and S-waves. The waves are re flected by internal discontinuities or by external boundaries. A displacement transducer located close to the impact point monitors the dynamic surface displacement caused by the arrival of the reflected waves. The stress waves propagate back and forth between the test surface and an internal discontinuity (or external surfaces). Thus, a transient resonance condition is created, and the displacement waveform has a periodicity which is dependent on the P-wave speed and the distance between the reflecting surfaces. The periodicity is determined by using the fast Fourier transform to obtain the dominant frequency in the waveform. Knowing the $\mathrm{P}$-wave speed, the frequency value is used to compute the distance from the surface to the reflecting interface. The paper discusses the theoretical basis of the method and summarizes laboratory 
and field studies, which illustrate the capabilities of the technique. A data analysis technique, called spectral peak plotting, is described.

100,141

PB92-116821

Not available NTIS

National Inst. of Standards and Technology (NEL), Gaithersburg, MD. Building Materials Div.

Integrated Knowledge Systems for Concrete Science and Technology.

Final rept.

G. Frohnsdorff. 1989, 18p

See also PB89-176119.

Pub. in Materials Science of Concrete I, p315-332 1989.

Keywords: "Concretes, "Data bases, "Artificial intelligence, "Expert systems, "Cements, Mathematical models, Software engineering, Knowledge bases(Artificial intelligence), Reprints.

The rapid changes in ability to process, store, and retrieve knowledge brought about by advances in computers and telecommunications have enormous implications for concrete science and technology. Knowledge stored in computers will take on new dimensions as society's collective memory of concrete science and technology grows into a more integrated body of knowledge than has previously been possible. It will make possible powerful decision support systems and will raise problems about the sharing of knowledge. Integrated knowledge systems will consist of text bases, databases, model bases, rule bases, and image bases which may be interfaced through computer networks. It is to be expected that some or all of these forms of knowledge base will become standardized through the voluntary consensus standards process. Development of the integrated knowledge systems will draw attention to the gaps in knowledge and should lead to collaboration between researchers in planning research to fill the gaps. For the systems to develop most effectively, protocols for developing and interlacing the subsystems will need to be developed. The benefits of collaboration will be predictive tools with a power which far exceeds that of anything presently available.

\section{0,142}

\section{PB92-123033}

PC A07/MF A02

National Inst. of Standards and Technology (BFRL), Gaithersburg, MD.

Flammability Characterization of Foam Plastics.

T. G. Cleary, and J. G. Quintiere. Oct 91, 143p

NISTIR-4664

Sponsored by Society of the Plastics Industry, Washington, DC

Keywords: "Flammability, "Polyurethane foam, Ignition, Flammability testing. Flame propagation, Test methods, Test facilities, Standards, Flame calorimeters, Heat measurement, Fire resistance, Construction materials, Polystyrene, Plastics, ASTM E-84 test.

The results of a study to identify an alternative test protocol to the ASTM E-84 (Steiner Tunnel) test as a measure of flammability for foamed plastics are presented. New fire test apparatuses, namely, the Cone Calorimeter and the Lateral Ignition and Flame Spread apparatus were used to more completely characterize foamed plastic flammability. Key flammability properties obtained from these apparatuses describe ignitability, flame spread rate, rate of heat release, and smoke obscuration. An extensive data set of these flammability properties for 10 selected foamed plastics was generated. The tested materials included melting foams (polystyrene foams) and charring foams (polyurethanes, polyisocyanurate and phenolic foams). The effects of melting and dripping were limited by testing the materials in the horizontal orientation. In addition, an integrated approach to material flammability characterization is presented that uses these parameters to predict fire growth potential.

\section{Structural Analyses}

100,143

PB91-134668

Not available NTIS

National Bureau of Standards (NEL), Gaithersburg, MD. Structures Div.
Preliminary Dynamic Analyses of the Ministry of Agriculture Building.

Final rept.

W. C. Stone, and N. R. Cuevas. 1986, 22p

Pub. in Proceedings of International Conference on Mexico Earthquakes - 1985: Factors Involved and Lessons Learned, Mexico City, Mexico, September 19-21, 1986, p233-254

Keywords: "Earthquake resistant structures, "Dynamic structural analysis, "Public buildings, "Concrete structures, Model tests, Mexico City, Concrete slabs, Concrete construction, Reinforced concrete, Seismic waves, Reprints, "Structural vibration, Earthquake engineering. Finite element method.

The Ministry of Agriculture Building on Avenida San Antonio Abad (Mexico City) was originally constructed as a 17-story reinforced concrete waffle slab system with interior reinforced concrete shear walls surrounding the elevator shafts and stairwells. The plan was assymetrical and included an attached laterally braced annex which protruded from the southwest side of the building. During the 1985 Mexico earthquake it suffered significant damage but did not collapse. Rehabilitation plans for the structure are discussed as well as free vibration tests conducted in the post-earthquake configuration. A structural model of the building was prepared as part of a preliminary investigation on its dynamic behavior during the 1985 earthquake. The model was developed using three dimensional finite elements as part of a benchmark study to determine the feasibility of this analytical approach. Observations on the computational burden and difficulty of employing such detailed computer models are discussed.

\section{0,144}

PB91-167239

PC A03/MF A01

Gaithersburg. MD.

Measurement of Structural Response Characteristics of Full-Scale Buildings: Selection of Structures.

R. D. Marshall, L. T. Phan, and M. Celebi. Feb 91,

22p NISTIR-4511

Prepared in cooperation with Geological Survey, Menlo Park, CA. Branch of Engineering Seismology and Geology.

Keywords: "Structural vibration, "Buildings, "Dynamic response, "Measurement, "Earthquake engineering, Structural engineering. Earthquakes, Field tests, Dynamic structural analysis, Measuring instruments, Seismic waves, Loma Prieta Earthquake.

The report describes the selection of existing building structures for subsequent field measurements of lowlevel ambient vibrations. By comparing measurement results with previously recorded high-level responses, it is anticipated that guidance can be developed for improved measurement practice. The buildings selected for the effort represent a cross-section of contemporary structural systems and materials, foundation types, and a range of building heights and aspect ratios. Each building was subjected to strong shaking during the Loma Prieta Earthquake of October 17 . 1989

\section{0,145}

\section{PB91-184846}

PC A14/MF A02

National Inst. of

Gaithersburg, MD. and Retrofit of Structures. U.S.-Japan Panel on Wind and Seismic Effects, UJNR. Held in Gaithersburg, MD., USA, on May 12-14, 1990.

J. O. Jirsa. Apr 91, 305p NISTIR-4515

Prepared in cooperation with Texas Univ. at Austin. Sponsored by National Science Foundation, Washington, DC.

Keywords: "Earthquake engineering, "Meetings, "Structural analysis, "Earthquake resistant structures, Earthquake damage, Retrofitting, Repair, Concrete structures, Steel structures, Reinforced concrete Structural members, Seismic waves, Wind effects.

The report is the Proceedings of an international workshop on Evaluation, Repair, and Retrofit of Structures. The workshop was a joint effort of Task Committees $C$ and D, 'Repair and Retrofit of Existing Structures' and 'Evaluation of Structural Performance' respectively of the U.S.-JAPAN Panel on Wind and Seismic Effects. The workshop was hosted by the National Institute of Standards and Technology during May 12-14 1990. The National Science Foundation provided the finan- cial support. The subjects addressed included: evaluation of structures; performance of existing structures their repair and strengthening; and research on techniques for repairing and retrofitting structures.

\section{0,146}

PB91-204057

Not available NTIS National Bureau of Standards (NEL), Gaithersburg, MD. Structures Div.

Investigation of the Collapse of L'Ambiance Plaza. Final rept.

C. F. Scribner, and C. G. Culver. 1988, 22p

Pub. in Jnl. of Performance of Constructed Facilities 2, n2 p58-79 May 88.

Keywords: "Accident investigations, "Lift slab construction, "Collapse, "Buildings, Failure, Concrete slabs, Loads(Forces), Concrete structures, Concrete construction, Structural members, Structural analysis, Reprints.

Results from an investigation of the collapse of the L'Ambiance Plaza building on April 23, 1987, are presented. The building was being constructed using the lift-slab method; collapse occurred during construction. The investigation included examination of debris at the site of the collapse, review of eyewitness accounts of the collapse, review of project documentation, laboratory and field tests and analyses of the structure. Several potential failure mechanisms were investigated. The most probable cause of the collapse was determined to be loss of support by a lifting jack in the west tower during placement of a group of three floor slabs. The loss of support was likely due to excessive deformation of a shearhead lifting angle which caused a lifting nut to slip off the lifting angle of the shearhead. The failure mechanism was duplicated in laboratory experiments. As loads were redistributed after the initial failure, the remaining jack rods along column line $E$ supporting the package of floor slabs slipped off the lifting angles and the slabs failed in flexure and shear. These slabs fell, causing the slabs below them to fail.

\section{0,147}

PB91-204206

Not available NTIS

National Inst of Standards and Technology (NEL). Gaithersburg, MD. Center for Building Technology. Research and Technology for Structural Engineering Productivity.

Final rept.

R. N. Wright. 1991, 7p

Sponsored by American Society of Civil Engineers, New York.

Pub. in Proceedings of Structures Congress, Indianapolis, IN., April 29-May 1, 1991, p1-7.

Keywords: "Structural engineering, "Productivity, Competition, Research, Structural design, Construction, Artificial intelligence, Automation, Robotics, Safety, Civil engineering, Reprints.

Structural engineers strive to shelter and support human activities with safe, functional, economic and esthetic structures. Engineers' productivity is measured by the value of their results and the efficiency with which their results are obtained. Demands for improved structural engineering services include: reducing vulnerability to natural disasters, conserving and renewing existing structures, building on difficult sites, protecting the environment, conserving scarce resources, and creating structural systems consistent with evolving human aspirations. Structural engineers will be challenged by colleagues abroad to maintain leadership and competitiveness in structural engineering practice in an increasingly international construction marketplace, and by other professions which may take advantage of their growing knowledge and advanced technologies to extend their activities into what is now structural engineering practice. Productivity, value to the customer, should be the deciding factor. Technical leadership is key to the productivity and vitality of structural engineering. Emerging technologies, such as advanced materials, artificial intelligence, automation and robotics, can greatly increase the powers of structural engineers. Strong research, development and educational efforts are needed to adapt and exploit emerging technologies.

\section{0,148}

PB91-206714

$\mathrm{PC}$ A03/MF A01

National Inst. of Standards and Technology (BFRL), Gaithersburg, MD. 


\section{Structural Analyses}

Long Term Performance of Rubber in Seismic and Non-Seismic Bearings: A Literature Review.

J. W. Martin. Jun 91, 30p NISTIR-4613

Keywords: "Vibration insulators, *Rubber, "Bearings, "Earthquake resistant structures, Vibration damping Structural vibration, Seismic waves, Service life, Reviews, Earthquake engineering, Loads(Forces).

The use of seismic isolation bearings to decouple buildings and lifeline structures from strong ground motion has received an increased amount of attention in recent years. While several types of seismic isolator bearings have been developed and proposed for use, the most common type is the laminated rubber (elastomeric) bearing. Because the design lifetime of these bearings is expected to be on the order of 50 to 100 years, the long-term performance of the rubber (elastomer) must be addressed. Therefore, a literature review was conducted to identify potential limits on the long-term performance of rubbers (elastomers) used in bearings. Several issues, including the need for consensus of performance standards and for additional research on the effects of creep, aging, temperature, and high-energy radiation on the properties of rubber.

\section{0,149}

PB91-222570

PC A05/MF A01

National Inst. of Standards and Technology (NEL), Gaithersburg, MD.

Performance of 1/3-Scale Model Precast Concrete Beam-Column Connections Subjected to Cyclic Inelastic Loads. Report No. 2.

G. S. Cheok, and H. S. Lew. Jun 91, 85p NISTIR-

4589

See also PB91-107623.

Keywords: "Cyclic loads, "Earthquake resistant structures, "Beams(Supports), "Precast concrete, "Construction joints, Concrete structures, Concrete construction, Earthquake engineering, Model tests, Ductility, Failure.

Results are presented from the experimental test program on precast concrete beam-column connections subjected to inelastic cyclic loads being conducted at the National Institute of Standards and Technology The report is the second in a series and covers the test results from Phase II of a three phase program. The objective of the test program is to develop an economical moment resistant precast beam-column joint for high seismic zones. Test specimens are 1/3-scale models of a prototype interior concrete beam-column connection. The 1985 UBC design criteria for seismic Zones 2 and 4 were used. Six specimens were tested. The experimental variables include the location of the post-tensioning force and the type of post-tensioning tendons used. Comparisons of the performance among these specimens are made. Comparisons with the monolithic specimens are also presented. These comparisons are made based on the failure mode, energy absorption characteristics, strength and ductility of the connection.

100,150

PB91-236588

Not available NTIS

National Inst. of Standards and Technology (BFRL), Gaithersburg, MD. Structures Div.

Performance of Precast Concrete Beam-toColumn Connections Subject to Cyclic Loading.

Final rept.

G. S. Cheok. 1991, 12p

Pub. in PCl (Prestressed Concrete Institute) Jnl. 36, n3 p56-67 May/Jun 91.

Keywords: "Precast concrete, "Cyclic loads, "Beams(Supports), "Columns(Supports), "Connectors, Earthquake resistant structures, Loads(Forces), Concrete structures, Concrete construction, Joints(Junctions), Structural members, Buildings, Reprints.

An experimental study of the behavior of precast concrete beam-to-column connections subjected to cyclic inelastic loading conducted at the National Institute of Standards and Technology is presented. The study was initiated to provide data for the development of a rational design procedure for such connections in seismically active regions. The objective of the study is to develop a moment resistant precast concrete connection that is economical and can be easily constructed. Four one-third scale monolithic concrete beam-tocolumn connections were tested: two were designed according to the 1985 Uniform Building Code (UBC) Seismic Zone 2 criteria and two to UBC Zone 4 criteria. In addition, two precast, past-tensioned concrete beam-to-column connections similar in design to the monolithic Zone 4 specimens were tested. These tests constitute the first phase of a multi-year test program.

100,151

PB91-237834

Not available NTIS

National Inst. of Standards and Technology (BFRL), Gaithersburg, MD. Building and Fire Research Lab. Office.

Assessment: U.S. Office Building of Moscow.

Final rept.

R. N. Wright, N. J. Carino, J. G. Gross, and M.

Sansalone. $1989,14 p$

Pub. in Jnl. of Performance of Constructed Facilities 3 , n1 p2-15 Feb 89.

Keywords: "Buildings, "Structural analysis, "Damage assessment, Inspection, Construction, Structural members, Masonry, Steels, Concretes, Deterioration Construction materials, Office buildings, "US Embassy(Moscow)

On October 30, 1986, the United States Congress directed the National Bureau of Standards (NBS) to conduct an independent analysis of the new United States Embassy Office Building being constructed in Moscow. The analysis was to include '...an assess. ment of the current structure and recommendations and cost estimates for correcting any structural flaws and construction defect...' The paper describes the history of the building project, the site and building, and the process of investigation, which included field, laboratory and analytical studies, and its findings. The investigation identified important structural defects in the building and defined remedial measures to correct them. While important, these structural defects, in comparison to the total structural system, are modes in scale and fully correctable. Companion papers describe the assessments of: the structural system, the masonry enclosure, and the potential for progressive collapse.

\section{0,152}

PB92-116425

PC A99/MF A06

National Inst. of Standards and Technology (BFRL) Gaithersburg, MD.

Wind and Seismlc Effects. Proceedings of the Joint Meeting of the U.S.-Japan Cooperative Program in Natural Resources Panel on Wind and Seismic Effects (23rd). Held in Tsukuba, Japan, on May 14-17, 1991.

Special pub. (Final).

N. J. Raufaste. Sep $91,639 p$ NIST/SP-820

Also available from Supt. of Docs, as SN003-003 03107-1. See also DE91005427 and PB91-107094.

Keywords: "Wind pressure, "Earthquakes, "Earthquake resistant structures, Highway bridges, Buildings, Dynamic structural analysis, Dynamic loads, Dams, Building codes, Structural design, International coop eration, Wind loads.

The publication is the proceedings of the 23rd Joint Meeting of the U.S.-Japan Panel on Wind and Seismic Effects. The meeting was held at the Public Works Research Institute, Tsukuba, Japan, during May 14-17, 1991. The proceedings include the program, list of members, panel resolutions, task committee reports and 42 technical papers. The papers were presented under seven themes: (1) Wind Engineering; (2) Earthquake Engineering; (3) Storm Surge and Tsunamis; (4) Joint Cooperative Research Program; (5) Performance of Nonstructural Systems; (6) International Decade for Natural Disaster Reduction; and (7) Summaries of Task Committee Workshop Reports.

\section{General}

100,153

PB91-149302

National Bureau of Standards

. Center for Fire Research.

Final rept.

J. E. Snell. 1986, 20p

Pub. in Proceedings of International Meeting of Fire Research and Test Centers, Avila, Spain, October 7-9, 1986, p97-116.

Keywords: Methodology, Technology transfer, Fire tests, Performance tests, Fire safety, Test facilities,
Research management, Reprints, "Center for Fire Research, "Fire research.

The role of the Center for Fire Research is discussed with three examples of recent major projects. It is predicted that the methodology being developed will lead toward performance codes for fire safety.

\section{0,154}

PB91-159764 PC A04/MF A01

National Inst. of Standards and Technology (NEL), Gaithersburg, MD. Center for Computing and Applied Mathematics.

NIST 'Building Life-Cycle Cost' (BLCC) Program (Version 3.0). User's Guide and Reference Manual. S. R. Petersen. Jan 91,54 p NISTIR-4481

Sponsored by Department of Energy, Washington, DC. Federal Energy Management Program Staff.

Keywords: "Buildings, "Economic analysis, "Benefit cost analysis, "Computer applications, Return on investment, Operating costs, Energy conservation, Projects, Savings, Program evaluation, User Cost computer program, National Institute of Standards and Technology.

The NIST Building Life-Cycle Cost (BLCC) computer program provides economic analysis of proposed capital investments that are expected to reduce long-term operating costs of buildings or building systems. It is especially useful for evaluating the costs and benefits of energy conservation projects in buildings. Two or more alternative designs can be evaluated to deter. mine which has the lowest life-cycle cost. Economic measures, including net savings, savings-to-investment ratio, and adjusted internal rate of return, can be calculated for any design alternative relative to the designated base case. BLCC can be used for evaluating both Federal and private sector projects. It complies with ASTM standards related to building economics as well as FEMP and OMB A-94 guidelines for economic analysis of Federal building projects. BLCC is designed to run on an IBM-PC or compatible microcomputer with approximately $640 \mathrm{~K}$ of random access memory, with or without a hard disk.

\section{0,155}

PB91-167254

PC A03/MF A01

National Inst. of Standards and Technology (BFRL), Gaithersburg, MD.

Experimental Study of Top Vented Compartment

K. M. Tu. Feb 91, 34p NISTIR-4499

Keywords: "Fires, Room fires, Ventilation, Tests Ceilings(Architecture), Models, Flow rate, Differential pressure, Vents.

In a top vented compartment fire, both density difference and pressure difference across the horizontal ceiling vent control the vent flow. The research work pursued experimental studies and investigations of exchange flows through a horizontal ceiling vent as related to solely top vented compartment fire situations. A cubic box with inside dimensions of $0.43 \times 0.43 \times 0.43$ $\mathrm{m}$ high $(17 \times 17 \times 17 \mathrm{in})$ and walls of $0.025 \mathrm{~m}(1 \mathrm{in})$ thick Kaowool was employed for the compartment fire tests. For compartment fires of various horizontal ceiling vent sizes: (1) the fuel mass burning rates were measured, and (2) the air and combustion-flue-gas exchange flow rates were estimated based on (i) avail. able theories and (ii) gas concentrations in the fire compartment.

100,156

PB91-167270

PO6/MF A01

National Inst. of Standards and Technology (NEL),

Gaithersburg, MD. Center for Fire Research.

Role of Bench-Scale Test Data In Assessing RealScale Fire Toxicity.

Technical note (Final).

V. Babrauskas, R. H. Harris, E. Braun, B. Levin, M

Paabo, and R. G. Gann. Jan 91, 113p NIST/TN-

1284

Also available from Supt. of Docs.

Keywords: "Fires, "Toxicology, Furniture, Room fires, Lethality, Laboratory animals, Polyurethane resins, Polyvinylchloride, Gases, Bench-scale experiments.

The need was seen for establishing a methodology by which bench-scale fire toxicity methods could be validated against real-scale room fires. The present study is the result of a pilot project in the area. Appropriate 
validation hypotheses have been put forth and examined in the context of some initial data. Three materials - Douglas fir, rigid polyurethane foam, and PVC - were examined in real-scale and bench-scale methods. The real-scale test environment was a post-flashover fire in a three-compartment (room, corridor, room) geometry, with the test specimens comprising wall lining materials. The bench-scale methods examined were the NBS cup furnace method and a new developmental protocol referred to as the 'SwRI/NIST' method. The $\mathrm{N}$-gas Model was applied to the analysis of the data and was found to be consistent with most of the data. The methods were compared for similarity of gas vields, of primary gases, and of types of death. Differences were found in individual cases, but most of those were readily explainable on the basis of an understanding of the test conditions. As a result of these studies, a factor-of-3 agreement between bench-scale and real-scale results was established as both useful and practical.

\section{0,157}

\section{PB91-178848}

PC A03/MF A01 National Inst. of Standards and Technology (CAML), Gaithersburg, MD.

Boussinesq Algorithm for Enclosed Buoyant Convectlon in Two Dimensions.

R. G. Rehm, H. C. Tang, H. R. Baum, J. S. Sims, and D. M. Corley. Mar 91,40 p NISTIR-4540

Keywords: "Convection, Computational fluid dynamics, Finite difference theory, Two-dimensional calculations, Stratified flow, Hydrodynamics, Algorithms, *Building fires, "Boussinesq flow.

Approximate equations for a Boussinesq model with viscous dissipation and thermal conduction describing buoyant convection driven by a heat source in a rectangular enclosure are derived. The finite difference algorithm for computing transient solutions in two dimensions to these equations is presented. The algorithm allows the enclosure fluid to be stratified in a direction parallel to the enclosure walls initially, or for gravity to have an arbitrary direction relative to the enclosure (but with no initial stratification). Computational results of transient, two-dimensional buoyant convection for very high resolution are presented. The hydrodynamics is directly based on the time-dependent Navier Stokes equations; the model is valid in the Boussines approximation. No turbulence model or other empirical parameters are introduced. There is no inflow or outflow at boundaries; this assumption, although rather restrictive, allows the mathematical problem to be properly formulated so that no other empiricism is in troduced by specification of the algorithmic boundary conditions. A finite-difference scheme second-order in space and first-order in time is used to integrate the evolution equations, and an elliptic solver is used to solve the pressure equation.

100,158

PB91-195222

Not available NTIS

National Inst. of Standards and Technology (NEL),

Gaithersburg, MD. Center for Fire Research.

Engineering Analysis of Fire Development in the Hospice of Southern Michigan, December 15, 1985. Final rept.

H. E. Nelson. $1989,12 p$

Pub. in Proceedings of International Symposium on Fire Safety Science (2nd), Tokyo, Japan, June 13-17, 1988, p927-938 1989.

Keywords: "Smoke, "Fires, Investigations, Flashover, Fire safety, Combustion, Reprints.

An analysis of the development of fire and flow of smoke in a multi-fatality fire is presented. The analysis methods used are the least sophisticated available consistent with reasonable accuracy. Fire growth, flashover, pre- and post-flashover spread of products, and impact on victims are addressed. Issues relative to post-flashover combustion chemistry and post-flashover corridor flow are raised.

\section{0,159}

PB91-203620

Not available NTIS

National Bureau of Standards (NEL), Gaithersburg, MD. Fire Science and Engineering Div.

Water Spray Suppression of Fully-Developed Wood Crib Fires In a Compartment.

Final rept.

J. Milke, D. Evans, and W. Hayes. $1985,63 p$

See also PB88-232871. Sponsored by Federal Emergency Management Agency, Washington, DC

Pub. in Report of Test FR 3956, pt1 p1-63 Jan 85.
Keywords: "Fire extinguishing agents, Water, Sprayers, Nozzle flow, Fire fighting, Fire tests, Experimentation, Reprints, Compartment fires.

A series of five experiments examining the effects of a simulated fire fighting water spray introduced into a fully-developed compartment fire were conducted for the Federal Emergency Management Agency by the Center for Fire Research at the National Bureau of Standards per Interagency Agreement (EMW-E-1239 Task Order 4A). Data from these tests were intended to be used as a check of predicted results from the Mission Research Corporation Fire Demand Model. The results illustrate the dynamics of compartment fire suppression using water sprays.

\section{0,160}

\section{PB91-216820}

PC A08/MF A02

National Inst. of Standards and Technology (BFRL),

Gaithersburg, MD.

Building Technology Publlcations 1986-1989.

Final rept.

T. A. Somers. Jun 91, 164p NISTIR-4616

See also PB88-110911.

Keywords: "Buildings, "Research management, "Indexes(Documentation), "Bibliographies, Laboratories, Civil engineering, Construction materials, US NIST

The publication communicates the results of the Center for Building Technology's research during the period 1986 through 1989. Publications constitute a major end product to NIST's efforts. This publication is divided into six chapters: (1) alphabetical listing of publication titles, (2) keyword listing, (3) author listing, (4) title listings classified by NIST Publication Series and by non-NIST publishers such as a professional society. For the former, report titles are listed under six publication designators: Building Science Series, Technical Notes, Special Publications, Journal of Research, Letter Reports, Interagency Reports, (5) technical disciplines - Structures, Materials, Environment, and (6) report reference index used to identify report titles by using the report reference numbers from keywords in Chapter 2 and authors in Chapter 3.

\section{0,161}

PB91-217422

TriData Corp., Arlington, VA

Estimated Impact of the Center for Fire Research Program on the Costs of Fire.

P. Schaenman. Jun 91, 83p NIST/GCR-91/591

Sponsored by National Inst. of Standards and Technology (BFRL), Gaithersburg, MD.

Keywords: "Fire safety, "Cost analysis, Building codes, Economic analysis, Computerized simulation, Smoke detectors, Design standards, Safety engineering, Budgets, Fire detection systems, Flammability testing, CFR(Center for Fire Research).

The Center for Fire Research (CFR) has had a huge impact on reducing casualties and losses from fires. It also has helped stimulate new industries, and saved industry enormous sums by engineering fire safety better, averting business disruption, reducing liability, and in a number of other ways. The dividends of the past continue; CFR's budget essentially has been 'paid' through the Year 2100 by even the most conservative estimates of its impact. This was a first, brief effort to estimate the magnitude of the CFR impact, and how it is distributed across the major components of the total cost of fire. More work is needed on virtually every aspect of the estimation procedures used here CFR's program has made a great contribution to life safety in the United States and has saved business billions of dollars in losses and unnecessary costs of fire. A much greater impact can be achieved with a few millions of dollars more investment in the CFR program each year - the enhanced program.

\section{0,162}

PB91-222604 PC A05/MF A01

National Inst. of Standards and Technology (BFRL), Gaithersburg, MD.

Reduction in Fire Hazard in Corridors and Areas Adjoining Corridors Provided by Sprinklers.

D. Madrzykowski. Jul 91, 90p NISTIR-4631

Sponsored by Public Buildings Service, Washington, DC.

Keywords: "Fire safety, "Sprinklers, "Corridors, Buildings, Graphs(Charts), Oxygen, Carbon dioxide, Carbon monoxide.
Full-scale fire tests were conducted in a noncombustible burn room - corridor - target room test facility using wooden cribs as the fuel load. The facility was instrumented to measure gas temperatures and concentrations of oxygen, carbon dioxide and carbon monoxide. The combustion products were sampled at $1.5 \mathrm{~m} \mathrm{(5 \textrm {ft } )}$ above the floor. The elevation is considered as a characteristic head height. Sprinklers were installed in the burn room and along the corridor. The sprinkler lines were instrumented with pressure switches and clocks to detect the activation time of the sprinklers. Depending on the test configuration, sprinklers were pressurized either with water for suppression ('wet') or air for measurement of activation time ('dry'). The sprinklers were allowed to activate automatically during the suppression tests.

100,163

PB91-231589

PC A03/MF A01

National Inst. of Standards and Technology (BFRL),

Gaithersburg, MD

General Routine for Analysis of Stack Effect.

J. H. Klote. Jul 91,34 p NISTIR-4588

Sponsored by General Services Administration, Washington, DC

Keywords: "Buildings, "Fire tests, "Air infiltration, Stacks, Computerized simulation, Smoke, Ventilation, Air flow.

Stack effect is a major driving force of smoke movement in buildings. The paper presents a general method for evaluation of the location of the neutral plane for a space connected to its surroundings by any number of openings. A computer program, STACK, for analysis of the location of the neutral plane and resulting flows is presented along with example analyses. The examples show that the location of the neutral plane between a space and its surroundings is a weak function of temperature and a strong function of the size of openings. Further, the mass flow rate leaving a space due to stack effect is a strong function of temperature.

100,164

PB91-240770

PC A03/MF A01

National Inst. of Standards and Technology (BFRL), Gaithersburg, MD.

Suppression of Post-Flashover Compartment Fires Using Manually Applied Water Sprays. D. W. Stroup, and D. D. Evans. Jul 91, 41 p NISTIR4625

Sponsored by Swedish Fire Research Board, Stock holm, and Public Buildings Service, Washington, DC.

Keywords: "Fire tests, "Spraying, *Water, Computer ized simulation, Sprinkler systems, Buildings, Fla shover.

A series of four full scale fire tests were conducted to measure the effect of manual fire fighting efforts on post-flashover room fires. One objective of these tests was to generate data for evaluation of computer models of the fire suppression process. The tests were conducted in a room and corridor configuration consisting of a $2.44 \mathrm{~m}$ cube burn room connected to a 12.8 $\mathrm{m}$ long, $2.44 \mathrm{~m}$ wide, and $2.44 \mathrm{~m}$ high corridor. Hose nozzles with different water spray flow rates and medium drop sizes were used in each of the four nominally identical wood crib fires. Gas temperatures, wall surface temperatures and concentrations of oxygen, carbon dioxide, and carbon monoxide were measured in the burn room. Specialized aspirated and shielded thermocouples were used to minimize the effects of the water sprays on gas temperature measurements. The study showed that a water spray flowrate of 36.5 $1 /$ min with volume median drop size of 930 microns was just able to control the post-flashover fire, the flowrate of $16.31 / \mathrm{min}$ with median volume drop size of 800 microns did not control the fire, while the $791 / \mathrm{min}$ flowrate with volume median drop size of $1040 \mathrm{mi}$ crons definitely extinguished the fire.

100,165

PB91-240788

PC A03/MF A01

National Inst. of Standards and Technology (BFRL), Gaithersburg, MD

Framework for Utilizing Fire Property Tests.

T. G. Cleary, and J. G. Quintiere. Aug 91, 37p NISTIR-4619

Keywords: "Fires, "Flammability testing, Buildings, Floors, Walls, Ceilings(Architecture), Flame propogation, Mathematical models. 
A complete approximate set of equations is developed to describe fire spread and its resultant energy release over a surface. Wall, floor, and ceiling orientations are considered. The needed model data are couched in terms of available test method results, e.g., Cone Calorimeter and LIFT apparatuses. Several applications are presented to show how energy release rates can be predicted and how well they represent real data from full-scale and model room lining experiments.

\section{0,166}

\section{PBǵ2-109164}

PC A16/MF A03

National Inst. of Standards and Technology (NEL), Gaithersburg, MD. Center for Fire Research.

Joint Panel Meeting of the UJNR Panel on Fire Research and Safety (11th). Held in Berkeley, CA. on

October 19-24, 1989 .
N. H. Jason, and D. M. Cramer. Oct $90,365 p$

N. H. Jason,
NISTIR-4449

See also PB88-215926.

Keywords: "Meetings, "Fire safety, US NIST, Research management, Evacuating(Transportation), Carbon monoxide, Toxicity, Risk, Fire hazards, Safety engineering, Buildings.

The 11th Joint Meeting of the United States-Japan Panel on Fire Research and Safety was held on the campus of the University of California, Berkeley, October 19-24, 1989. Some disruption of the meeting resulted from the October 17 earthquake. The epicenter was about 60 miles southeast of San Francisco, in the Loma Prieta mountains near Santa Cruz. Thus, some of the papers were not presented, but are included in the volume. The volume comprises a total of 6 progress reports and 30 supporting papers in 3 areas: Risk, Hazard and Evacuation; Fire and Toxicity Chemistry; and Fire and Smoke Physics. The next Panel Meeting will be held in Japan in the autum of 1992 .

\section{0,167}

PB92-110287

(Order as PB92-110261, PC A05/MF A01) National Inst. of Standards and Technology, Gaithersburg, MD

\section{Data for Room Fire Model Comparisons.}

R. D. Peacock, S. Davis, and V. Babrauskas. c1991, $52 p$

Included in Jnl. of Research of the National Institute of Standards and Technology, v96 n4 p411-462 Jul/Aug 91.

Keywords: Data analysis, Fire tests, Buildings, Comparison, "Building fires, "Room fires, Fire models.

With the development of models to predict fire growth and spread in buildings, there has been a concomitant evolution in the measurement and analysis of experimental data in real-scale fires. The report presents the type of analyses that can be used to examine largescale room fire test data to prepare the data for comparison with zone-based fire models. Five sets of experimental data which can be used to test the limits of a typical two-zone fire model are detailed. A standard set of nomenclature describing the geometry of the building and the quantities measured in each experiment is presented. Availability of ancillary data (such as smaller-scale test results) is included.

\section{0,168}

PB92-112218

PC A09/MF A03

National Inst. of Standards and Technology (BFRL),

Gaithersburg, MD

Summaries of BFRL Fire Research In-House Projects and Grants, 1991.

N. H. Jason. Oct $91,199 p$ NISTIR-4686

See also PB91-216788 and report for 1989, PB90127101

Keywords: "Fire prevention, "Fire tests, Research projects, Research management, Buildings, Combustion, Carbon monoxide, Smoke, Furniture, Evacuating(Transportation), Grants.

The report describes the research projects performed in the Building and Fire Research Laboratory (BFRL) Fire Research Program and under its grants program from October 1, 1990 through September 30, 1991. Contents: Carbon Monoxide Prediction; Soot Formation; Turbulent Combustion; Polymer Gasification; Toxic Potency; Furniture Flammability; Wall Fires; Fire Suppression; Fire Hazard Assessment; Engineering Analysis Systems; Large Fires; Fire/Modeling Interactions; Fire Protection Technology.
National Inst. of Standards and Technology, Gaithersburg, MD.

Engineering Analysis of the Fire Development in the Hillhaven Nursing Home Fire, October 5, 1989. H. E. Nelson, and K. M. Tu. Sep 91, 57p NISTIR4665

Sponsored by Health Care Financing Administration, Baltimore, MD.

Keywords: "Fires, "Nursing homes, Smoke, Fire tests, Flashover, Carbon monoxide.

The report presents the methods and results of an analysis of the development and spread of fire and smoke during the October 5, 1989 fire in the Hillhaven Rehabilitation and Convalescent Center, Norfolk, Virginia. The analysis uses data gathered from onsite visits, reports and information for other investigators, fire tests conducted at the National Institute of Stand ards \& Technology, and fire growth models and simila computations. The report details the procedure and data used, the reasons for those selected, and the results obtained. The analysis addresses mass burning rate; rate of heat release; smoke temperature; smoke layer depth; velocity, depth and temperature of the smoke front; oxygen concentration of smoke layer; carbon monoxide concentrations; and other factors. The areas of building analyzed include the room of fire origin, the corridor system exposed by that room, and other patient rooms on that corridor.

\section{0,170}

PB92-112531

PC A05/MF A01

Pennsylvania State Univ., University Park. Dept. of Mechanical Engineering.

Turbulent Upward Flame Spread for Burning Vertical Walls Made of Finite Thickness.

Annual rept. 15 Aug 89-14 Aug 90.

A. K. Kulkarni, C. I. Kim, and C. H. Kuo. Sep 91, 88p NIST/GCR-91/597

Grant NANB-8D0849

See also PB91-143297. Sponsored by National Inst. of Standards and Technology (BFRL), Gaithersburg, MD

Keywords: "Fire tests, "Walls, Mathematical models, Buildings, Turbulent flow, Flame propagation, Mathematical models, Polyurethane, Wood, "Upward flame spread.

The report presents the basic mechanisms of upward flame spread and develops a complete procedure to predict the flame spread on practical wall materials of finite thickness, appropriately verified by experiments. These objectives have been met. Specifically in the past year, several wall materials were tested in the upward flame spread apparatus, mathematical model development for predicting the upward flame spread was completed, and comparisons of data and predictions were made, in addition to completion of many other supporting tasks.

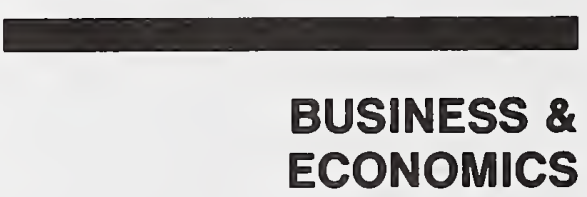

\section{Consumer Affairs}

100,171

PB92-112549

PC A03/MF A01

National Inst. of Standards and Technology (TS), Gaithersburg, MD. Weights and Measures Program.

Checking the Net Contents of Packaged Goods. Third Edition, Supplement 2.

Final rept.

C. S. Brickenkamp, and J. A. Koenig. Oct 91, 17p NIST/HB-133-ED-3-SUPPL 2

Also available from Supt. of Docs. as SN003-003$03111-9$. See also PB91-107144.

Keywords: "Packaging, "Commodities, "Labels, "Handbooks, Requirements, Sampling, Revisions, Tests, Procedures.

Only minor additions and revisions to NIST (formerly NBS) Handbook 133, Third Edition, 'Checking the Net Contents of Packaged Goods,' were adopted by the Conference in 1991. A few editorial changes have also been made. The document consists of change pages to be added to Handbook 133, Third Edition, as amended by the 1990 Supplement.

\section{Domestic Commerce, Marketing, \& \\ Economics}

100,172

\section{PB91-194415}

$\mathrm{PC}$ A03/MF A01

National Inst. of Standards and Technology, Gaithersburg, MD. Office of Standards Services.

Laboratory Accreditation in the United States.

Final rept.

M. A. Breitenberg. May 91, 31p NISTIR-4576

See also PB88-239793.

Keywords: "Test facilities, "Quality assurance, Calibration, Inspection, Tests, United States, USA, "Laboratory accreditation, "Accreditation.

The paper, the third in a series that includes NBSIR $87-$ 3576 , The ABC's of Standards-Related Activities in the United States, and NBSIR 88-3821, The ABC's of Certification Activities in the United States, is designed to provide information on laboratory accreditation to readers not entirely familiar with the topic. The report highlights some important aspects on the topic, furnishes information necessary to make informed decisions on the selection and use of laboratories, and serves as background for using other available documents and services. Readers interested in this area may also wish to review NIST SP 808, Directory of Federal Government Laboratory Accreditation/Designation Programs, which provides updated information on federal government laboratory accreditation and similar type programs conducted by the federal government. Companion volumes on state and local government and private sector laboratory accreditation programs are currently in preparation.

100,173

PB91-195693 Not available NTIS National Bureau of Standards, Gaithersburg, MD. Program Office.

Structural Change and Competitiveness: The U.S. Semiconductor Industry.

Final rept.

G. Tassey. $1990,9 p$

Pub. in Technological Forecasting and Social Change 37, n1 p85-93 Mar 90.

Keywords: Competition, Diversification, Strategy, Japan, USA, Reprints, "Semiconductor industry, Competitiveness, Innovation.

Part of the difficulties being experienced by U.S. semiconductor firms in competing with the Japanese and several European firms can be explained by significant differences in industry structure. Specifically, semiconductors are produced by large electronics firms in Japan which have diversified into many downstream products, while U.S. firms still concentrate on semiconductor components. The diversification smoothes the shocks of individual product cycles and increases the return on investment in semiconductor technology. Although some U.S. semiconductor firms have diversified into several downstream product areas, the degree has not been sufficient and they have diversified hardly at all into related technologies with substantial economic potential such as optoelectronics.

100,174

PB92-108968

PC A05/MF A01

National Inst. of Standards and Technology, Gaithersburg, MD. Office of Standards Code and Information. Directory of State and Local Government Laboratory Accreditation/Designation Programs.

Special pub. (Final).
C. W. Hyer. Jul 91,90 p NIST/SP-815

Contract 43NANBO17411

Also available from Supt. of Docs. as SNO03-003 03093-7. Supersedes PB85-121390. See also PB91167379 and PB91-194415. Sponsored by National Inst. of Standards and Technology, Gaithersburg, MD. Office of Standards Code and Information.

Keywords: "Test facilities, *Quality assurance, "Directories, Calibration, Inspection, Tests, Laboratory accreditation. 
The directory is a guide to laboratory accreditation and similar types of programs conducted by state and local government agencies. These programs accredit or designate laboratories or other entities to conduct testing to assist the agencies in carrying out their responsibilities. The accreditation or designation is based on some type of assessment regarding the capability of the laboratory to conduct the testing. However, the nature of such assessments varies considerably from agency to agency. Entries in the directory are based primarily on information provided by each state and local government agency and reflect the agency's view of its activities.

\section{0,175}

PB92-126465

PC A03/MF A01

National Inst. of Standards and Technology, Gaithersburg, MD. Office of Standards Services.

Questlons and Answers on Quality, the ISO 9000 Standard Series, Quality System Registration, and Related Issues.

M. Breitenberg. Nov 91, 24p NISTIR-4721

See also PB91-194415.

Keywords: "Test facilities, "International trade, *Standards, *Quality assurance, Assessments, Reliability, Inspection, Requirements, Engineering, Specifications, United States, European Community, "Regis tration, Laboratory accreditation, ISO 9000 Series.

The report provides information on the development, content, and application of the ISO 9000 standards to readers who are unfamiliar with these aspects of the standards. It attempts to answer some of the most commonly asked questions on quality; quality systems; the content, application and revision of the ISO 9000 standards; quality system approval/registration; European Community requirements for quality system approval/registration; and sources for additional help.

\section{International Commerce, Marketing, \& Economics}

\section{0,176}

PB91-159061

Not available NTIS

National Bureau of Standards, Gaithersburg, MD.

Office of Standards Code and Information.

Domestic Implementation.

Final rept. 1983-85.

J. Overman. 1986, $18 p$

Pub. in Report to the United States Congress on the Agreement on Technical Barriers to Trade - 'Standards Code', Chapter V, p25-42 Feb 86.

Keywords: "International trade, "Standards, Barriers, United States, Exports, Industries, Regulations, Reprints, Trade Agreements Act.

The report describes the domestic implementation of the GATT Agreement on Technical Barriers to Trade (Standards Code) by the National Bureau of Standards (NBS) and the U.S. Department of Agriculture's Technical Office. During the three year period (1983-1985), NBS reported 59 proposed U.S. regulations to the GATT Secretariat; published 11 issues of tbt news; handled comments on 24 proposed foreign regulations; received 664 notifications of proposed regulations; answered over 6,000 requests for information; and provided standards-related technical assistance to manufacturers and exporters.

\section{0,177}

\section{PB91-187823}

PC A04/MF A01

National Inst. of Standards and Technology, Gaithersburg, MD. Office of Standards Code and Information. GATT Standards Code Activities of the National Instltute of Standards and Technology 1990.

Annual rept.

J. R. Overman. Apr 91, 53p NISTIR-4559

See also PB90-219817.

Keywords: "International trade, "Standards, Technical assistance, Certification, Regulations, Tables(Data), "General Agreement on Tariffs and Trade, Notifications, Trade barriers, US NIST.

The report describes the GATT Standards Code activities conducted by the Standards Code and Information Program, National Institute of Standards and Technology (NIST), for calendar year 1990 . NIST responsibilities include operating the U.S. GATT inquiry point for information on standards and certification activities and the technical office for non-agricultural products; notifying the GATT Secretariat of proposed U.S. Federal Government standards-based rules that might significantly affect trade; assisting U.S. industry with standards-related trade problems; and responding to inquiries about proposed foreign and U.S. regulations.

\section{General}

\section{0,178}

PB91-194498

PC A11/MF A02

burgal Inst. of Standards and Technolo

croup of the Joint U.S.-U.S.S.R. Commercial Commission (1st). Held in Moscow on March 11-13, 1991.

B. G. Simson. May 91,235 p NISTIR-4572

Keywords: "Standardization, "Meetings, International cooperation, Quality control, USSR, USA, Standards Working Group.

In a September 1990 Chairman's meeting of the Joint U.S.-U.S.S.R. Commercial Commission (JCC) in Moscow, senior officials from the U.S. Department of Commerce and the U.S.S.R. Ministry of Foreign Economic Relations established a Standards Working Group for the purpose of developing programs of mutual interest concerning standards development and conformity assessment. During its first meeting in Washington, D.C., and Gaithersburg. Maryland, on March 11-13, 1991, representatives from U.S Government, trade and professional associations, and standard development associations provided the Soviet delegation with insights into U.S. standardization. The visitors described Soviet legislative initiatives now under development.

\section{Analytical Chemistry}

100,179

AD-A227 869/5

PC A02/MF A01

burg, MD.

Scattered Light and Other Corrections In AbsorpScattered Light and Other Corrections In Absorp-
tion Coefficient Measurements in the Vacuum Ultraviolet: A Systems Approach.

R. Klein, W. Braun, A. Fahr, A. Mele, and H. Okabe. Jun 90, 9p ARO-25911.2-CH,

Contract MIPR-119-89

Pub. in Jnl. of Research of the National Institute of Standards and Technology, v95 n3 p337-344 May-Jun 90.

Keywords: "Absorption coefficients, Automation, Auxiliary, Computers, Error analysis, Errors, Gases, Light scattering, Low intensity, Measurement, Reprints, "UItraviolet spectrometers, Systems approach, "Vacuum ultraviolet radiation.

A systems approach in which computer automation is applied to a vacuum ultra-violet spectrometer and auxiliary components is described. The errors associated with the measurement of gaseous absorption coefficients in the vacuum ultraviolet are considered. The presence of scattered light introduces large errors particularly at those wavelengths where the source used is characterized by low intensity. In the case of a D2 light source this occurs in the region 120 to $130 \mathrm{~nm}$. Simple considerations explain the variation of the absorption coefficient determinations in the presence of scattered light and lead to an appropriate treatment of the data to eliminate the error. Experimental results are presented illustrating the efficiency and precision obtainable with the present approach. Keywords: Absorption coefficient; Computer automation; Error analysis; Instrument; Vacuum; Reprints. (jhd)

\section{0,180}

AD-A243 170/8
National Bureau of Standards (NML), Gaithersburg, MD. Center for Chemical Physics.

CID Spectra of Selected Target Molecules.

Final rep Jul 84-Sep 87.

R. I. Martinez. Dec 89,58p AFESC/ESL-TR-88-71, Contracts MIPR-N84-56, MIPR-N84-56-A01 Supported in part by MIPR-N85-22, MIPR-N86-12, MIPR-N87-12.

Keywords: Collisions, Cross sections, Data bases, Dissociation, Dynamics, Energy, Ions, Mass, Molecular properties, Molecules, Spectra, Standardization, Structures, Targets, Mass spectroscopy, "Mass spectra, Quadrumpole moment, Identification, CID(Collisionally Induced Dissociation), Tandem mass spectrometers, Molecular dynamics.

This report describes research into the molecular dynamics of the collisionally induced dissociation (CID) process. This process occurs in tandem mass spectrometers, and is the major process by which primary or parent ions are converted into daughter ions of smaller mass. This work was undertaken in search of a means of standardizing conditions for tandem mass spectrometers so that standard spectral data bases could be collected and used to identify environmental unknowns. This research indicates that CID cross sections for the parent ions can be reproducibly measured as a function of collision energy, and are characteristic of the parent ion structures. Measurement of CID cross sections offers a new means of identifying unknown compounds.

100,181

PB91-147694

Not available NTIS

National Bureau of Standards (NML), Gaithersburg, MD. Chemical Kinetics Div.

Instrument-Independent Tandem Mass Spectrometry Database for XQQ Instruments: The Dynamical Prerequisites.

Final rept.

R. I. Martinez. $1988,6 p$

Pub. in Rapid Commun. Mass Spectrom. 2, n1 p8-13 1988.

Keywords: "Mass spectroscopy, "Data bases, Computer aided design, Reprints

The dynamical prerequisites are discussed in order to focus attention on design considerations for accomplishing an instrument independent MS/MS CAD Database for XQQ instruments.

100,182

PB91-147769

Not available NTIS

National Inst. of Standards and Technology (NML), Gaithersburg, MD. Organic Analytical Research Div Capillary Supercritical Fluid Chromatography of Explosives: Investigations on the Interactions between the Analytes, the Mobile Phase and the Stationary Phase.

Final rept.

A. Munder, S. Chesler, and S. Wise. 1990, 8p

Pub. in Jnl. of Chromatography 521, p63-70 1990

Keywords: "Chromatographic analysis, "Pressure, *Temperature, "Surface properties, Polarity, Siloxanes, Dipole moments, Explosives, Propellants, Solutes, Solid phases, Reprints, "Supercritical fluid chromatography.

The independent effects of pressure and temperature in supercritical fluid chromatography on the capacity ratio on chromatographic resolution of some polar organic model compounds were investigated. Increasing the pressure isothermally leads to a steady decrease in retention and resolution. With changing temperature, a maximum in the capacity ratio as well as the resolution was observed. These observations may be related to a combination of gas chromatographic (GC) and liquid chromatographic (LC) theories of solute interactions with the mobile and stationary phases. However, pure GC or LC-like behavior was not observed either below or above the critical point of the mobile phase. Capacity ratios for various explosives, propellants and related compounds were determined on capillary open tubular columns coated with either a non-polar methyl- or a polar cyanopropyl phenyl-substituted siloxane stationary phase. The mobile phase for all studies was carbon dioxide. On the polar column, many of the solutes exhibited a good correlation between their bulk dipole moment and chromatographic retention. Deviations from the correlation could be explained by means of the physical or steric properties of these solutes. The elution order of the 


\section{Analytical Chemistry}

compounds on the non-polar column was similar to the order achieved using GC rather than LC.

\section{0,183}

PB91-147777

Not available NTIS

National Inst. of Standards and Technology (NML), Gaithersburg, MD. Gas and Particulate Science Div. Use of Single Particle Standards for LAMMA Callbration.

Final rept.

I. H. Musselman, D. S. Simons, J. A. Small, and R.

W. Linton. 1986, 17p

Pub. in Jnl. of Trace and Microprobe Techniques 4, n3 p197-213 1986.

Keywords: "Microanalysis, "Standards, Isotope ratio, Calibration, Reprints, "Laser microprobe mass spectroscopy.

Single particle standards are being used in the development and testing of the relatively new technique of laser microprobe mass analysis (LAMMA). Experiments being conducted at the National Bureau of Standards include the use of particle standards to calibrate isotopic measurements, to investigate detection limits and the accuracy of isotopic analysis in subminickel speciation by LAMMA.

\section{0,184
PB91-148148}

Not available NTIS

National Bureau of Standards (NML), Gaithersburg MD. Organic Analytical Research Div.

Trace Determination of $\mathrm{Cr}$ (VI) by LC/AAS with onLine Preconcentration.

Final rept.

A. Syty, R. G. Christensen, and T. C. Rains. 1986, 4p Pub. in Atomic Spectroscopy 7, n4 p89-92 1986.

Keywords: "Chromium, "Chromates, "Liquid chromatography, "Atomic spectroscopy, Concentrating, Spectrochemical analysis, Surface waters.

A liquid chromatography/atomic absorption spectrometric (LC/AAS) technique for the direct determination of $\mathrm{Cr}(\mathrm{VI})$ at $\mathrm{ng} / \mathrm{mL}$ in the presence of $\mathrm{Cr}(\mathrm{III})$ is described. In this method $\mathrm{Cr}(\mathrm{VI})$ is preconcentrated on a $\mathrm{C}-18$ bonded silica column. The column is connected directly to the aspirating capillary of an atomic absorption spectrometer (AAS). After a preconcentration step the $\operatorname{Cr}(\mathrm{VI})$ is eluted from the column directly into the AAS unit where the chromium absorbance is measured. A preconcentration factor of 300 is attained using a $25-\mathrm{cm}$ long column. The method has been evaluated using samples of natural pond water with 100 percent recovery of spiked $\mathrm{Cr}(\mathrm{VI})$.

\section{0,185}

\section{PB91-148502}

PC A06/MF A01

National Inst. of Standards and Technology (NML) Boulder, CO. Thermophysics Div.

Strategy for Chemical Analysis of Alternative Refrigerants.

Technical note.

T. J. Bruno. Aug 90, 102p NIST/TN-1340

Also available from Supt. of Docs.

Keywords: "Refrigerants, "Substitutes, "Mass spectroscopy, "Gas chromatography, "Infrared spectroscopy, Fluorohydrocarbons, Purity, Contaminants, Water analysis, Adsorbents, Volummetric analysis, Hydrocarbons.

The chemical purity of samples of alternative refrigerants which are used in the determination of the thermophysical properties is of paramount importance. Many properties, such as vapor-liquid equilibria and transport properties such as thermal conductivity, require that measurements be performed on as pure a material as possible. Some common impurities such as water can have an extremely deleterious effect on a measurement, while the effects of other impurities are more subtle. Alternative refrigerants which are not available at purity levels that are normally considered 'research grade' must be characterized in order for the data to be properly interpreted, and for the reported results to be used appropriately. In addition, it is necessary to assess the reactivity of alternative refrigerants in the presence of many of the common construction materials used in experimental apparatus. The National Institute of Standards and Technology has evolved an analytical protocol which is applied to each system. In the paper, the various analytical methods will be discussed, and the data of general usefulness presented. In addition, some of the novel instrumental approaches employed at NIST will be described.
100,186

PB91-148817 Gaith Gaithersburg, MD. Inorganic Analytical Research Div. Characterization of the Mineral Fraction in Botanical Reference Materials and Its Influence on Homogeneity and Analytical Results.

Final rept.

R. M. Lindstrom, A. R. Byrne, D. A. Becker, B.

Smodis, and K. M. Garrity. 1990, 3p

Pub. in Fresenius Jnl. of Analytical Chemistry 338 , p569-571 1990.

Keywords: "Trace elements, "Leaves(Botany), *Standards, "Inorganic materials, Neutron activation analysis, Microscopy, Heterogeneity, Cyclone separators, Reprints, Standard reference materials.

The NIST natural-product leaf Standard Reference Materials have been widely used in developing reliable methods of analysis. A small amount of mineral matter present in these materials was separated by flotation, and characterized qualitatively by microscopy and quantiatively by neutron activation analysis. Several elements are concentrated in the mineral fraction, which can lead to analytical error through incomplete dissolution or sampling statistics. Two new candidate materials prepared by the Office of Standard Reference Materials, SRM 1515 Apple Leaves and SRM 1547 Peach Leaves, have been processed with an airjet mill, resulting in a very finely ground leaf material, with particle size less than 200 mesh. A cyclone classifier in the process discriminates against coarse grit, so that the content of minerals in the ground material is less than in the first-generation materials. Better homogeneity was in fact observed, even down to $100 \mathrm{mg}$ sample size. One must use caution, however, to assure that any inhomogeneity found in real samples are appropriately considered and dealt with.

\section{0,187}

PB91-148858

Not available NTIS

National Bureau of Standards (NML), Gaithersburg, MD. Organic Analytical Research Div.

Reverse-Phase HPLC Separation and Electrochemical Detection of Retinol and Its Isomers.

Final rept.

W. A. MacCrehan, and E. Schonberger. 1987, 14p Pub. in Jnl. of Chromatography - Biomedical Applications 417, n1 p65-78 1987 .

Keywords: "Retinol, "Liquid chromatography, "Vitamin A, Isomers, Blood serum, Electrochemistry, Ultraviolet detectors, Reprints.

Baseline separation of all of the isomers of retinol using reverse-phase liquid chromatography in under 30 minutes is presented. A new approach to the detection of retinol using electrochemical detection is developed. The oxidative electrochemistry of retinol is studied using coulometry and HPLC. The new approach is compared to UV/visible absorbance detection for the determination of retinol in human serum extracts.

\section{0,188}

\section{PB91-148874}

Not available NTIS

National Bureau of Standards (NML), Gaithersburg.

MD. Inorganic Analytical Research Div.

Research at NBS in Direct Potentiometric Measurements in Blood.

Final rept.

G. Marinenko, P. C. Gunaratna, and W. F. Koch.

1987, 11p

Pub. in Proceedings of Meeting of the European Working Group on Ion-Selective Electrodes (8th), Graz, Austria, October 2-4, 1986, p269-279 1987.

Keywords: "Calcium ions, "Potassium ions, "Sodium ions, "Blood, "Activity coefficients, "Potentiometric analysis, Electrolytes, Ion selective electrodes, Electrochemical cells, Mercury amalgams, Reprints.

A research project, sponsored by the National Committee for Clinical Laboratory Standards and funded by several manufacturers' of clinical potentiometric instruments, has been initiated in the Electroanalytical Research Group at the National Bureau of Standards. The goal of the research is to develop reference methods and materials for measuring the activity of free ionized sodium, potassium, and calcium in blood by direct potentiometry with ion selective electrodes. The initial thrust of the research will focus on sodium, wherein it is anticipated that the definitive method for the sodium is anticipated that the definitive method for the sodium
activity measurements will be the sodium analgam cell. A synopsis of the program and the theory supporting the approach will be given. In preparatory work leading to the use of the analgam cell, a new cell with low liquid junction potential has been constructed for use with ion selective electrodes. Dilute ammonium chloride is used as the reference filling solution and agar bridge electrolyte. Preliminary results using the cell for comparing activity and concentration measurements will be discussed. The relative accuracy of the measured activity of sodium using a variety of free sodium ion probes has been evaluated. The sodium analgam cell has been designed and constructed. Preliminary testing of the cell is now underway and appears very promising.

\section{0,189}

\section{PB91-148981}

Not available NTIS National Bureau of Standards (IMSE), Gaithersburg, MD. Polymers Div.

Methods for the Analysis of Organometallic Compounds in Wastes.

Final rept.

G. J. Olson, F. E. Brinckman, and W. R. Blair. 1988 , $16 \mathrm{p}$

Sponsored by Office of Naval Research, Arlington, VA Pub. in Waste Testing and Quality Assurance, ASTM STP 999, p130-145 1988.

Keywords: "Organometallic compounds, "Chemica analysis, "Environmental surveys, Toxic hazards Waste disposal, Water pollution sampling, Chromatography, Separation processes, Trace elements, Reprints, Chemical specification.

Organometallic compounds occur in the environment as a result of anthropogenic and biogenic processes. Since rganiometals are often much more toxic than the corresponding inorganic forms of metals, it is important to be able to speciate the forms of metals in environmental samples at ultratrace levels. Chemical speciation methods, based on the use of chromatographic separations followed by element-selective detection have been developed in the laboratories and elsewhere. These methods, described in part in the paper. are helping the analysis of organometal species at their action levels (down to parts-per-trillion levels) in environmental matrices, leading to a better understanding of environmental occurrence, fate, effects, and transformation. New methods for nondestructive analysis of metal species on surfaces are under development.

\section{0,190}

\section{PB91-149187}

Not available NTIS

National Inst of Standards and Technology (NML) Gaithersburg, MD. Organic Analytical Research Div.

Determination of Bonded Phase Thickness in Liquid Chromatography by Small Angle Neutron Scattering.

Final rept.

L. C. Sander, C. J. Glinka, and S. A. Wise. 1990, 3p Pub. in Analytical Chemistry 62, n10 p1099-11011990.

Keywords: "Bonded coatings, "Silicon dioxide, "Thickness, Porosity, Adsorbents, Liquid chromatography, Neutron scattering, Surface properties, Alkylation, Penetration, Methanol, Reprints.

Preliminary results of research using the technique of small angle neutron scattering (SANS) to characterize phase morphology of alkyl modified silica is reported. The technique of contrast variation was used to reduce scattering interferences from the pore structure. With this procedure, the scattering experiments are carried out on the sample in a liquid environment. By varying the deuterium/hydrogen ratio of the liquid (e.g. $\mathrm{D} 2 \mathrm{O} / \mathrm{H} 2 \mathrm{O}$ ratio), the scattering length density (sld, essentially the index of refraction for neutrons) of the liquid can be varied. This permits the pore structure to be masked by matching the sld of the silica. The thickness of the bonded phase layer was determine for various alkyl modified substrates. In addition, the degree of solvent penetration (volume fraction of methanol associated with the alkyl chains) was determined. The significance of bonded phase thickness to column properties is briefly discussed.

100,191

PB91-158865

Not available NTIS

National Inst. of Standards and Technology (NML) Gaithersburg, MD. Inorganic Analytical Research Div. 
NIST Standards for Sodium and Potassium

Final rept.

W. F. Koch. 1990, 9p

Pub. in Proceedings of International Symposium on Metrology and Clinical Applications of Electrochemical and Fiber Optic Sensors, Rochester, MN., September 22-25, 1989, p3-11 1990.

Keywords: "Potassium, "Sodium, "Blood serum, *Standards, Ion selective electrode analysis, Laboratory tests, Precision, Chemical analysis, Reprints.

The collaborative research project with manufacturers and users of clinical potentiometric instruments, sponsored by the NCCLS and conducted at the National Institute of Standards and Technology (NIST), is nearing attainment of its primary goal to bring conformity to the direct potentiometric measurements of sodium and potassium in human serum through the development of reference materials. Based on the results of the fourth interlaboratory test a candidate Standard Reference Material (SRM) has been produced by the Technicon Corporation, according to the specifications of the steering committee of the NCCLS project. The material was prepared from a single human serum pool, processed, ultra-filtered, and spiked with sodium and potassium resulting in three levels, nominally 120,140 , and $160 \mathrm{mmol} / \mathrm{L}$ sodium, and correspondingly, 6,4 , and $2 \mathrm{mmol} / \mathrm{L}$ potassium. All other components in the sera are at normal levels. The three sera were dispensed into 4-mL glass ampules ( $3.2 \mathrm{~mL}$ per ampoule) and frozen at $-50 \mathrm{C}$. The material is designated as SRM 956, Electrolytes in Human Serum. To test the efficiency of the material, a fifth interlaboratory test was conducted among eight manufacturers and three hospitals, using the candidate SRM and the prototype material from the fourth test. The results of the test indicate a significant improvement in interlaboratory precision when data are subjected to post-calibration. SRM 956 is now in the final stages of certification for sodium and potassium total concentration. These two analytes have been analyzed by the definitive methods (gravimetry and isotope dilution mass spectrometry, respectively) at NIST and the reference flame methods at NIST and two other cooperating laboratories.

\section{2}

PB91-162198

Not available NTIS National Inst. of Standards and Technology (NML), Gaithersburg, MD. Inorganic Analytical Research Div Low-Background Gamma-Ray Assay Laboratory for Activation Analysis.

Final rept.

R. M. Lindstrom, D. J. Lindstrom, L. A. Slaback, and

J. K. Langland. 1990, 5p

Pub. in Nuclear Instruments and Methods in Physics Research A299, p425-429 1990.

Keywords: "Activation analysis, "Gamma counters, Background radiation, Radiation shielding, Cosmic neutrons, Cosmic rays, Comparison, Reprints.

The sources of background in a gamma-ray detector were experimentally determined in underground and surface counting rooms, and an optimized shield was constructed at NIST. The optimum thickness of lead was $10-15 \mathrm{~cm}$, with a greater thickness giving an increased background due to the buildup of tertiary cosmic-ray particles. Neither cadmium, tin, copper nor plastic (hydrocarbon or fluorocarbon) was desirable as a shield liner, since all these increased the background continuum or introduced characteristic peaks into the background spectrum. Two broad peaks in the background result from inelastic scattering of cosmic-ray neutrons $(0.02 / \mathrm{sq} \mathrm{cm} / \mathrm{s})$ in germanium. These neutrons also excite the lower nuclear levels of lead and structural iron to produce additional gamma-ray peaks in the spectrum. The influence of the 20 MW NIST reactor, located $60 \mathrm{~m}$ from the detector, was undetectable. Comparisons among detectors and locations clearly separate cosmic from environmental components of the background.

100,193

PB91-162297

Not available NTIS

National Inst. of Standards and Technology (NML),

Gaithersburg, MD. Electron and Optical Physics Div.

Resonance Ionization Spectroscopy/Resonance Ionization Mass Spectrometry Data Service. II. Data Sheets for $\mathrm{Al}, \mathrm{Ca}, \mathrm{Cs}, \mathrm{Cr}, \mathrm{Co}, \mathrm{Cu}, \mathrm{Kr}, \mathrm{Mg}, \mathrm{Hg}$, and Ni.

Final rept.

E. B. Saloman 1991,60p

Pub. in Spectrochimica Acta 46B, n3 p319-378 1991.

Keywords: "Resonance ionization mass spectroscopy,

*Chemical analysis, Aluminum, Calcium, Cesium,
Chromium, Cobalt, Copper, Krypton, Magnesium, Mer cury, Nickel, Reprints, "Resonance ionization spectroscopy.

A data service has been established at the National Institute of Standards and Technology to provide the necessary information to apply the techniques of Resonance lonization Spectroscopy (RIS) and Resonance Ionization Mass Spectrometry (RIMS) to routine use in analytical chemistry. The service collects and calculates the relevant atomic data, chooses appropriate resonance ionization schemes, and indicates pertinent operating details of successful RIMS studies. The first group of data sheets was published previously covering the elements $\mathrm{As}, \mathrm{B}, \mathrm{Cd}, \mathrm{C}, \mathrm{Ge}, \mathrm{Au}, \mathrm{Fe}, \mathrm{Pb}, \mathrm{Si}$ and $\mathrm{Zn}$. The second group of data sheets is presented here. It covers the elements $\mathrm{Al}, \mathrm{Ca}, \mathrm{Cs}, \mathrm{Cr}, \mathrm{Co}, \mathrm{Cu}, \mathrm{Kr}$, $\mathrm{Mg}, \mathrm{Hg}$ and $\mathrm{Ni}$. Others will be published periodically. Reprints of RIS/RIMS work are solicited so that those efforts may be included in future data sheets.

\section{0,194}

PB91-174235

Not available NTIS

National Inst. of Standards and Technology, Gaithers burg, MD. Standard Reference Materials Program. Appendix: Standard Solutions and Certified Reference Materials.

Final rept.

R. Alvarez. 1990, 9p

Pub. in Official Methods of Analysis, v2 p1214-1222 1990.

Keywords: "Standards, "Solutions, Chemical analysis, Data, Reprints, "Certified reference materials, "Standard reference materials.

The appendix gives the methods to make standard solutions and some standard reference materials.

\section{0,195}

\section{PB91-187641}

(Order as PB91-187617, PC A06/MF A01) National Inst. of Standards and Technology, Gaithersburg, MD.

Multiple Variable-Angle Light Scattering Detector for Gel Permeation Chromatography.

P. H. Verdier. 1991, 13p

Included in Jnl. of Research of the National Institute of Standards and Technology, v96 n2 p177-189 Mar/Apr 91

Keywords: *Gel permeation chromatography, "Light scattering, "Molecular weight, Optical detectors, Polystyrene, Polymers.

A light scattering detector has been designed and constructed for use with gel permeation chromatographs. The detector measures light scattered from the eluting sample simultaneously at nine scattering angles, and is connected to a computer-controlled display which exhibits the angular dependence of the scattering in real time while the sample is eluting. Use of the lightscattering detector in conjunction with the usual concentration-sensitive detector allows the direct determination of molecular weight as a function of elution volume, thereby making the chromatographic system 'self-calibrating.' Tests of the system with a series of linear and branched polystyrenes suggest that it will be a useful tool for the study and characterization of branched polymers.

\section{0,196}

PB91-189282

Not available NTIS National Inst. of Standards and Technology (NML), Gaithersburg, MD. Inorganic Analytical Research Div. Second Dissociation Constant and pH of N-2-hydroxyethylpiperazine-N'-2-ethanesulfonic Acid (HEPES) from 0 to $50 \mathrm{C}$

Final rept.

D. Feng, Y. C. Wu, and W. F. Koch. 1989, 6p

Pub. in Analytical Chemistry 61, n13 p1400-1405 1989.

Keywords: "HEPES, "Buffers(Chemistry), " $\mathrm{pH}$, "Chemical equilibrium, "Dissociation, Sodium chloride, Thermodynamic properties. Electrical potential, Reprints.

HEPES (N-2-Hydroxyethylpiperazine-N'-2-ethanesulfonic acid) has been recommended as a $\mathrm{pH}$ buffer for physiological measurements. The $\mathrm{pH}$ values for thqe buffer system at ionic strengths similar to those in physiological fluids have been determined at temperatures from 0 to $50 \mathrm{C}$ by the emf method. The influence of $\mathrm{NaCl}$ on the buffer and the liqued junction potential associated with the salt have been evaluated. Thus, the practical, operation $\mathrm{pH}$ value can be ascertained.
The second dissociation constant of HEPES has been determined and the thermodynamic properties have been calculated.

\section{0,197}

PB91-189860

Not available NTIS

National Inst. of Standards and Technology (NEL), Gaithersburg, MD. Electricity Div.

Detection of Trace Disulfur Decafluoride in Sulfur Hexafluoride by Gas Chromatography/Mass Spectrometry.

Final rept.

J. K. Olthoff, R. J. Van Brunt, J. T. Heron, and I.

Sauers. 1991, 7p

Sponsored by Department of Energy, Washington, DC Office of Electrical Systems.

Pub. in Analytical Chemistry 63, n7 p726-732, 1 Apr 91.

Keywords: "Sulfur fluorides, *Sulfur hexafluoride, "Mass spectroscopy, "Gas chromatography, Chemical analysis, Pyrolysis, Hydrolysis, Reprints.

A new method is described for detection of $\mathrm{S} 2 \mathrm{~F} 10$ in SF6 in the parts-per-billion (ppb) level. The method utilizes a gas chromatograph/mass spectrometer (GC/ MS) equipped with a heated jet separator. S2F10 is converted to SOF2 on the hot surfaces of the lowpressure portions of the jet separator at temperatures above $150 \mathrm{C}$ by a surface-catalyzed reaction involving $\mathrm{H} 2 \mathrm{O}$. As a consequence of the conversion, peaks corresponding to $\mathrm{S} 2 \mathrm{~F} 10$ appear on single-ion chromatograms at ion masses characteristic of SOF $2(\mathrm{~m} / z=48$, 67 , and 86 ) where there is little or not interterence from features. By this method, a direct analysis of SF6 for S2F10 content can be performed with greater sensitivity than conventional gas chromatographic methods and with a higher degree of reliability and in a time much shorter than required for chromatographic methods that use enrichment procedures. Problems associated with the preparation and stability of reliable S2F10 reference samples are discussed.

\section{0,198}

PB91-189878

Not available NTIS National Bureau of Standards (NML), Gaithersburg, MD. Inorganic Analytical Research Div.

Introduction to Supercritical Fluid Chromatography. 1. Principles and Instrumentation.

Final rept.

M. D. Palmieri. 1988, $1 p$

See also Part 2, PB89-230312.

Pub. in Jnl. of Chemical Education 65, n10 pA254 1988

Keywords: "Supercritical fluid chromatography, Laboratory equipment, Gas chromatography, Liquid chromatography, Reviews, Reprints, ”Supercritical fluids

The basic characteristics of supercritical fluids are described. Supercritical fluids are used as mobile phases in chromatography, and various aspects of supercritical fluid chromatography (SFC), liquid chromatography, and gas chromatography are compared. Finally the basic instrumentation required to perform SFC is described.

\section{0,199}

PB91-190041

Not available NTIS National Bureau of Standards (NML), Gaithersburg, MD. Organic Analytical Research Div.

Evaluation of Column Performance in Liquid Chromatography.

Final rept.

L. C. Sander. $1988,8 p$

Pub. in Jnl. of Chromatographic Science 26, n8 p380 3871988.

Keywords: "Liquid

chromatography, Columns(Process Absorbers(Equipment), Surface properties, Reviews, Selectivity, Performance evaluation, Reprints, Silanol activity, Trace metal activity.

Procedures for the evaluation of chromatographic properties of LC columns are described in the review. General tests for assessing silanol activity, hydrophobicity, trace metal activity, phase type (monomeric vs. polymeric chemistry), and pore size are presented. Also included are specific tests for various solute classes. An emphasis is placed on practical aspects of column testing, and characterization procedures that require bulk packing material (i.e., unpacking the column) have been largely excluded. 
100,200

PB91-195156

Not available NTIS

National Bureau of Standards (NML), Gaithersburg, MD. Inorganic Analytical Research Div.

High Purity Chemicals and Clean Rooms for Trace Metal Analysis.

Final rept.

J. R. Moody. 1988, 4p

Pub. in Proceedings of International Symposium on Trace Analysis in Environmental Samples and Standard Reference Materials, Honolulu, HI., January 6-8, 1988, p97-100.

Keywords: "Trace amounts, "Metals, "Chemical laboratories, Purity, Clean rooms, Reprints, "Trace analysis, "National Bureau of Standards, Environmental samples, Analytical blanks.

The experience of the analytical laboratories at NBS in performing trace metal analysis in environmental samples is analyzed. Specifically, the role of ultra-pure reagents and clean laboratories in reducing the analytical blank is related to significant requirements for environmental samples.

\section{0,201
PB91-195164}

Not available NTIS

National Bureau of Standards (NML), Gaithersburg, MD. Inorganic Analytical Research Div.

Recommended Inorganic Chemicals for Calibration.

Final rept

J. R. Moody, K. W. Pratt, T. C. Rains, and R. R.

Greenberg. 1988, 10

Pub. in Analytical Chemistry 60, n21 p1203 1988.

Keywords: Primary standards, Chemical compounds, Stoichiometry, Purity, Calibration, Chemical analysis, Reprints, "Standard solutions.

Compounds and metals have been critically reviewed in terms of their suitability for use in preparing primary standard solutions for instrumental calibrations. Methods for preparing solutions from these chemicals are discussed. The tables list the available forms and purities for all but the radioactive elements and rare earth gases. A discussion of the relevance of purity, stoichiometry, and assay of chemicals for the analyst is given.

100.202

PB91-195180

Not available NTIS

National Inst. of Standards and Technology (NML),

Gaithersburg, MD. Gas and Particulate Science Div.

Strategies for Background Subtraction in Electron Probe Microanalysis X-ray Compositional Mapping.

Final rept.

R. L. Myklebust, D. E. Newbury, and R. B.

Marinenko. $1989,7 \mathrm{p}$

Pub. in Analytical Chemistry 61, n15 p1612-1618 1989.

Keywords: "Electron probes, "X-ray analysis, "X-ray spectroscopy, "Spectrum analysis, "Background discrimination, Ouantitative analysis, Atomic properties, Reprints, "Compositional mapping.

The background subtraction methods necessary for quantitative compositional mapping with an electron microprobe is discussed. Compositional maps are presented which demonstrate the need for determining a background dependent on average atomic number of the area of the specimen being analyzed. The measurement techniques and requirements for WDS, EDS and combined EDS/WDS compositional maps are discussed. The corrections for EDS analyses are built into the measurement method while for WDS analyses, the average atomic number of each analyzed point must be determined in order to compute the background at that point.

\section{0,203}

PB91-195479

Not available NTIS

National Inst. of Standards and Technology (CSTL). Gaithersburg, MD. Surface and Microanalysis Science Div.

Inner-Shell Ionization Cross Sections.

Final rept.

C. J. Powell. 1990, 8p

Pub. in Microbeam Analysis - 1990, p13-20.

Keywords: "Inner-shell ionization, "Ionization cross sections, "Microanalysis, Auger electron spectroscopy, $X$ ray analysis, Electron impact, Reprints, Electron probe microanalysis, Electron energy loss spectroscopy.
Values of cross sections for ionization of inner-shell electrons by electron impact are required for electron probe microanalysis, Auger-electron spectroscopy, and electron energy-loss spectroscopy. The present author has previously reviewed measurements and calculations of inner-shell ionization cross sections, and the present paper is an update and summary of the earlier reviews with emphasis on data for total inner-shell ionization cross sections as needed for $x$ ray microanalysis. Information is given on the more useful formulae for ionization cross sections and the extent to which they agree with calculations and meas urements for particular elements.

\section{0,204}

B91-195727

Not available NTIS Gaithersburg, MD. Gas and Particulate Science Div.

Accuracy of Transmission Electron Microscopy Analysis

Study.

S. Turner, and E. Steel. 1991, 5p

See also PB87-162111

Pub. in Analytical Chemistry 63, n9 p868-872, 1 May 91.

Keywords: "Asbestos, "Electron microscopy, "Accura cy, "Fibers, Laboratory tests, Filters, Statistical analysis, Interlaboratory comparisons, Reprints.

An interlaboratory study was conducted to determine the accuracy of determinations of the asbestos concentration on filters by use of transmission electron microscopy Replica sections were prepared from single polycarbonate filter that had chrysotile and several types of non-asbestos particles deposited on it surface. Twenty-seven analysts from 15 laboratorie counted a minimum of 3 grid squares and recorded the physical characteristics of the particles and the methods used for identification of the particles. One of the grid squares counted by each analyst was reanalyzed at the National Institute of Standards and Technology (NIST) by using verified counting methods. The mean value of TP/TNS (true positives/total number of struc tures) obtained by the laboratories on the verified grid squares is 0.67 , and the mean value for FP/TNS (false positives/total number of structures) is 0.04 . More than $40 \%$ of the fibers shorter than 1 micrometer were missed, whereas less than $20 \%$ of the fibers longer than 1 micrometer were missed by the analysts. Incor rect translation of the electron microscope stage is likely cause of many false negative values. Other possible reasons for false negatives and false positives are discussed.

\section{0,205}

\section{PB91-203893}

National Inst. of Standards and Technology, Gaithersburg, MD. Office of Measurement Services.

Reference Materials for Analytical Chemistry.

Final rept.

S. D. Rasberry. 1991, 3p

Pub. in Analusis 19, n1 p35-37 Jan 91.

Keywords: "Standards, "Chemical analysis, Accuracy, Reprints.

With every passing year, the role of analytical chemis try seems to increase in prominence. This may be so for several reasons, but perhaps most importantly be cause good measurement is a key to higher productivity. Industrialists are eager to improve quality assurance while reducing wasted energy, manufacturing re jects, and product liability problems. Patients in hosp tals are eager to have accurate clinical analyses to support proper diagnoses and treatment. All mankind is eager for more accurate environmental analyses to help protect the long-term future of our planet.

\section{0,206}

\section{PB91-203901}

Not available NTIS

National Inst. of Standards and Technology, Gaithersburg, MD. Office of Measurement Services.

Role of Reference Materials in Quality Assurance. Final rept.

S. D. Rasberry. $1990,2 p$

Pub. in Analytical Proceedings 27, n11 p296-297 Nov 90.

Keywords: "Chemical analysis, "Ouality assurance, Standards, Decision making, Measurement, Reprints, "Reference materials, National Institute of Standards and Technology

Analytical measurements are increasingly important to industrial quality assurance (OA) and to critical deci- sion making in such fields as clinical, nutritional, and environmental chemistry. Several factors important to attaining analyses that are reliably accurate will be noted. The summary will focus on the role of reference materials in accurate chemical analysis. Fundamenta attributes of reference materials will be described, together with details on how they serve as benchmarks for the measurement process. Some examples will be drawn from materials recently certified at the National Institute of Standards and Technology (NIST)

\section{0,207}

PB91-203968

Not available NTIS

National Inst. of Standards and Technology (NML), Gaithersburg, MD. Radiation Physics Div.

Resonance Ionization Spectroscopy/Resonance lonization Mass Spectrometry Data Service.

Final rept.

E. B. Saloman. $1989,4 p$

See also PB91-162297.

Pub. in Proceedings of the International Symposium on Resonance Ionization Spectroscopy and Its Applications (4th), Gaithersburg, MD., April 10-15, 1989, p255. 258 .

Keywords: "Mass spectroscopy, "Chemical analysis, "Information systems, Cross sections, Data, Oscillator strength, Reprints, "Resonance ionization spectroscopy, "Resonance ionization mass spectrometry.

A data service is being established at the National Bureau of Standards to provide the necessary information to apply the technique of Resonance Ionization Mass Spectrometry (RIMS) to routine use in analytical chemistry. This service will collect and calculate the relevant atomic data, choose appropriate resonance ionization schemes, and indicate pertinent operating details of successful RIMS studies. A sample data sheet is included and others will be available at the Symposium. Suggestions are solicited on how to improve the format and content of the data sheets.

\section{0,208}

PB91-236596

Not available NTIS National Inst. of Standards and Technology (CSTL) Gaithersburg, MD. Surface and Microanalysis Science Div.

Quantitative Analysis of Impurities in SIMOX Samples Using Secondary lon Mass Spectrometry.

Final rept.

P. H. Chi, D. S. Simons, and P. Roitman. 1991, 5p Pub. in Surface and Interface Analysis 17, p57-61 1991.

Keywords: "Gas-solid interfaces, "Silicon, "Ion implantation, "Ouantitative analysis, "Annealing, Mass spectroscopy, Ion microprobe analysis, Microanalysis, Impurities, Process variables, Surface chemistry, Temperature dependence. Time dependence, Chemical analysis, High temperature tests, Diffusion, Nitrogen, Argon, Oxygen ions, Reprints, Simox process.

Silicon films produced by the SIMOX process (separation by implanted oxygen) must be annealed at high temperature to remove the crystal damage introduced during implantation of the high oxygen dose. Different annealing gases, temperatures and times have been investigated. In such processes, various impurities present in the high-temperature ceramic furnace tube, as well as annealing gas species, may be incorporated into the samples. Secondary ion mass spectrometry (SIMS) is used as a quantitative tool to analyze the diffusion of tube components and gases into annealed SIMOX samples. Samples prepared for the investigation were annealed in nitrogen and argon at temperatures ranging from 1250 to $1350 \mathrm{C}$. It was found that most impuritites are present at low levels and are generally trapped in the surface oxide that is grown during the anneal. SIMS analyses of SIMOX samples annealed in nitrogen showed that nitrogen tends to collect in both the surface oxide and buried oxide layers, piling up at the oxide/silicon interfaces.

100,209

PB91-237727

Not available NTIS National Inst. of Standards and Technology (CSTL), Gaithersburg, MD. Inorganic Analytical Research Div. Characteristics of Aerosols Produced by the Spark Discharge.

R. L. Watters, J. A. Small, F. H. Shen, J. R. DeVoe, and R. B. Marinenko. 1989, $8 \mathrm{p}$

Pub. in Analytical Chemistry 61, n17 p1826-1833 1989. 
Keywords: "Electric discharges, "Metals, "Aerosols, Brasses, Copper zinc alloys, Chemical analysis, Spectroscopy, Electron microscopy, Chemical composition, Physical properties, Reprints, Spark erosion craters.

A detailed study of the physical properties and the chemical composition of spark-produced aerosol and corresponding erosion craters has been undertaken. A high repetition rate $(1 \mathrm{kHz})$, electronically controlled waveform spark source was used to generate the aerosol from samples of standard reference materials. The feasibility of using an on-line electrical mobility analyzer to monitor real-time particle concentrations was examined. Consistent bias in the $\mathrm{Zn} / \mathrm{Cu}$ ratios of aerosols from a series of brass SRMs corresponded to a reverse bias in the $\mathrm{Zn} / \mathrm{Cu}$ ratios measured in the spark erosion pits. Collected particles were dissolved and analyzed by inductively coupled plasma spectrometry. A predominance of small particles (100 A) was observed and confirming analyses using the analytical electron microscope was used on individual particles.

100,210

Not available NTIS National Bureau of Standards (NML), Gaithersburg, MD. Inorganic Analytical Research Div.

Laser Enhanced Ionization as a Selectlve Detector for Liquld Chromatographic Determination of Alkyltins in Sediment.

Final rept.

K. S. Epler, T. C. O'Haver, W. A. MacCrehan, and G. C. Turk. $1988,5 p$

Sponsored by Maryland Univ., College Park. Dept. of Chemistry and Biochemistry.

Pub. in Analytical Chemistry 60, n19 p2062-2066 1988.

Keywords: "Tin organic compounds, "Detectors, "Liquid chromatography, "Sediments, Reprints, "Laser enhanced ionization, "Trialkyltins.

Laser-enhanced ionization (LEI), used as an elementspecific detector for liquid chromatography (LC), is applied to trialkyltin determinations in sediment. The high sensitivity of LEI compensates for the degradation in detection limits resulting from chromatographic dispersion. The $L C$ speciates the organotins and resolves spectral interferences from the analytes. A rapid method for extracting $97+$ or $-8 \%$ of the TBT present in sediment into n-butanol is described. The detection limit is $3 \mathrm{ng} / \mathrm{mL}$ tin as TBT or $0.06 \mathrm{ng}$ tin.

100,21

PB92-126499

PC A03/MF A01

National Inst. of Standards and Technology (MSEL)

Gaithersburg, MD. Polymers Div.

Stability of Aqueous Inorganic Lead Solutions in Polycarbonate Containers.

Annual rept. (Oct. 1991).

K. L. Jewett, W. R. Blair, F. E. Brinckman, and F. W.

Wang. Dec $91,27 \mathrm{p}$ NISTIR-4725

See also PB86-189875. Sponsored by Maryland Univ. at Baltimore.

Keywords: "Lead inorganic compounds, "Liquids - Stability "Containers, Concentration(Composition) Water pollution, Monitoring, Acid treatment, Washing, Exposure, pH, Buffers(Cheinistry), Absorption, "Lead acetate, Polycarbonates.

The stability of lead(II) acetate solutions at 50,250 and $1000 \mathrm{mg} / \mathrm{l}$ (ppm as lead) in polycarbonate containers has been studied to assure reliable monitoring. The concentrations of aqueous solutions of lead maintained at $\mathrm{pH} 4.5$ were determined at both short term (hours) and long term (months) exposures. Employing acid washed polycarbonate containers, no significan loss in lead concentration was detected for up to 100 hours after initial exposures to 50,250 and $1000 \mathrm{ppm}$ lead solutions. When containers were pretreated with lead solutions at comparable concentrations that would ultimately be contained within them, no significant loss in concentration was detected over more than 50 days.

100,212

PB92-126614

PC A06/MF A02 National Inst. of Standards and Technology, Gaithersburg, MD.

Journal of Research of the National Instltute of Standards and Technology, September-October 1991. Volume 96, Number 5 .

1991, 65p

Also available from Supt. of Docs. as SN703-027 00042-3. See also PB92-126622 through PB92126721 and PB92-110261. Library of Congress catalog card no. 89-656121.
Keywords: "Research projects, "Metrology, Dimensional measurement, Particle size, Microspheres, Dielectric properties, Permeability, Permittivity, Experimental design, Technology, Block design, Silicon isotopes, Voltametry, Air pollution detection, Dental caries, Chemical analysis, Sulfur dioxide, Carbon dioxide, Force standards Standard reference materials Orthogonal arrays, Advanced Technology Program, Isotope reference materials, Slovak Metrological Society.

Contents: Summary of the Intercomparison of the Force Standard Machines of the National Institute of Standards and Technology, USA, and the Physikalisch-Technische Bundesanstalt Germany; Accurate and Precise Coulometric Determination of Sulfur Dioxide in Compressed Gas Mixtures; Development of a Coulometric Method for Assessing the Concentration of Ambient Levels of $\mathrm{CO} 2 /$ Air in Compressed Gas Mixtures: Certification of NIST SRM 1961--30 micrometers Diameter Polystyrene Spheres; Optimization Techniques for Permittivity and Permeability Determination Taguchi's Orthogonal Arrays Are Classical Designs of Experiments; A Mathematical Model for Dental Caries--A Coupled Dissolution-Diffusion Process; The Advanced Technology Program--A New Role for NIST in Accelerating the Development of Commercially Important Technologies; A List of New Group Divisible Designs; Silicon Reference Materials Certified for Isotope Abundances; Slovak Metrological Society.

100,213

PB92-126713

(Order as PB92-126614, PC A06/MF A02)

National Inst. of Standards and Technology, Gaithersburg, MD.

Sllicon Reference Materlals Certified for Isotope Abundances.

S. Valkiers, P. De Bievre, G. Lenaers, and H. S.

Peiser. 1991, 3p

Prepared in cooperation with Commission of the European Communities, Geel (Belgium). Central Bureau for Nuclear Measurements, and Antwerp Univ., Wilrijk (Belgium). Dept. of Chemistry.

Included in Jnl. of Research of the National Institute of Standards and Technology, v96 n5 p617-619 Sep/Oct 91.

Keywords: "Silicon isotopes, Silicon tetrafluoride, Mass spectroscopy, Atomic weights, "Isotope reference materials, "Reference materials, Isotope abundance.

In a series of gas mass-spectrometric measurements performed near the highest attainable accuracy, samples from two highly homogeneous batches of silicon crystals and silica powder were compared directly with a synthetic mixture of the three stable isotopes of silicon. Thereby, this work not only established the 'absolute' atomic weight of these batches, but also makes portions of these batches available as an Isotopic Reference Material for accurate isotopic abundance measurements in geochemical and other isotopeabundance studies of silicon.

100,214

PB92-127315 Not available NTIS Teknekron Senisor Development Corp., Menlo Park, CA.

Opportunitles for Innovation: Chemical and Blological Sensors.

M. Madou and J P Joseph Oct $91,145 p$ NIST/

GCR-91/593-1

Grant NANB9D0980

See also PB91-107078. Sponsored by National Inst. of Standards and Technology, Gaithersburg, MD.

Paper copy only available from National Institute of Standards and Technology, Gaithersburg, MD.

Keywords: "Chemicals, "Biological detection, "Detectors, Technology transer, Businesses, Markets, Solid state electronics, Measurement Protons, Carbon dioxide, Machining, Semiconductors, Metal oxides, Gas detectors, Acoustic detectors, Improvement, Signal to noise ratio, Monitoring, Metabolin, Microsensors, Amperometric detectors.

Contents:

Application of Micromachining in Solid-State Chemical Sensors:

The Challenges and Opportunities of Sensor Technology;

Metal Oxide Semiconductor Sensors:

Amperometric Gas Sensors:

Opportunities with Acoustic Sensors:

Oxygen, Glucose, Urea, and Cholesterol Sensors for Biomedical Application
Biological Applications of Electrolyte/Insulator/ Silicon Structures:

and Market Introduction Issues for Chemical Microsensors.

\section{Basic \& Synthetic Chemistry}

\section{0,215}

AD-A243 094/0

PC A03/MF $A 01$

National Inst. of Standards and Technology, Gaithersburg, MD.

Determination of the Optical Constants of Thin Chemical-Vapor-Deposited Diamond Windows from 0.5 to $6.5 \mathrm{eV}$.

Technical rept.

L. H. Robins, E. N. Farabaugh, and A. Feldman. 27 Sep $91,19 p$

Contract N00014-90-F-0011

Keywords: Absorption, Absorption spectra, Adsorption, Carbon, Chemicals, Constants, Energy, Etching Fittings, Light scattering, Low energy, Models, Near ultraviolet radiation, "Optical properties, Photons, Reflectance, Refractive index, Regions, Removal, Silicon, Single crystals, Substrates, Surfaces, Thinness, Transmittance, "Chemical vapor deposition, "Diamonds, "Chemical.

The optical constants of thin chemical-vapor-deposited diamond windows were determined as a function of photon energy from 0.5 to $6.5 \mathrm{eV}$ by fitting experimental transmittance and a reflectance data to model that includes the effects of surface optical scatter. Rootmean-squared surface roughness values were also obtained from the analysis. The windows were fabricated by microwave plasma assisted CVD on silicon substrates, followed by partial removal of the substrates by etching. The values of the refractive index were diamonds. Substantial adsorption was found to occur in the visible to near ultraviolet region (2 to $5 \mathrm{eV}$ ) where single-crystal diamond is transparent. The spectrum of the low-energy absorption spectrum is dia mond like amorphous carbon. There is a steep in crease in absorption above the indirect bandgap of diamond $(5.5 \mathrm{eV})$

100,216

PB91-147447

Not available NTIS

National Bureau of Standards (NML), Gaithersburg MD. Ionizing Radiation Div.

Mechanism of OH Radical Reactions with Thymine and Uracil Derivatives.

Final rept

S. V. Jovanovic, and M. G. Simic. 1986, 5p

Pub. in Jnl. of the American Chemical Society $108, n 19$ p5968-5972 1986 .

Keywords: "Uracils, "Thymine, "Oxidation reduction reactions, "Hydroxyl radicals, Chemical radicals, Oxidizers, Nucleotides, Radiolysis, Glycols, Pyrimidines, Reprints.

Yields of reducing, 6-yl, oxidizing, 5-yl, and methyl radicals, generated by $\mathrm{OH}$ radical reaction with uracil, thy mine and 1 -methyluracil, were determined by pulse radiolysis in aqueous solutions at $\mathrm{pH} 7$. The absolute yields and ratio of 5 -yl/6-yl radicals in uracil (20\% 5-yl$U$ and $80 \% 6-y l-U$ ) are altered in methyl substituted derivatives of uracil. For thymine one finds $35 \%$ of $5-y$ l$T$ and $57 \%$ of 6 -yl-T, and for 1 -methyluracil $20 \%$ of 5 $\mathrm{yl}-1-\mathrm{MeU}$ and $65 \%$ of 6 -yl-1-MeU radicals. The yields of pyrimidine glycols in the presence of an oxidizing agent $(G=3.2$ for thymine glycols; 4.3 for uracil gly cols; and 3.7 for 1 -methyluracil glycol), as measured by HPLC, were shown to be equal to the yields of $5-\mathrm{OH}-6$ yl-Py reducing radicals of thymine $(56 \% \mathrm{OH})$, uracil $(80 \% \mathrm{OH})$ and 1 -methyluracil $(65 \% \mathrm{OH})$. It is suggest ed that 5-hydroxy-6-yl radicals are the exclusive precursors of glycols. Mechanisms of glycol formation in the presence and absence of oxidizing agents are proposed and discussed.

100,217

PB91-159012

Not available NTIS National Bureau of Standards (NML), Gaithersburg MD. Chemical Kinetics Div. 


\section{CHEMISTRY}

\section{Basic \& Synthetic Chemistry}

Redox Reactions of Osmium Porphyrins.

Final rept.

S. Mosseri, P. Neta, D. Y. Sabry, P. Hambright, and

A. Harriman. $1988,7 p$

Pub. in Jnl. of the Chemical Society - Dalton Transactions 11, p2705-2711 1988 .

Keywords: "Osmium, "Porphyrins, *Oxidation reduction reactions, Photolysis, Radiolysis, Electrochemistry, Complex compounds, Ligands, Chemical reactions, Reprints.

Cyclic voltammetric, gamma radiolytic, chemical and photochemical studies have shown that the metal centre in osmium porphyrine (OsP) can exist in a wide range of oxidation states, each being stabilized by axial complexation with a particular ligand. Thus, $\mathrm{Os}(\mathrm{II}) \mathrm{P}$ is stabilized by ligation with a CO molecule. Oxidation occurs readily to form the corresponding $\mathrm{Os}$ (III) $\mathrm{P}$ which retains the $\mathrm{CO}$ ligand and can be reduced quantitatively to the original Os(II) complex. Further oxidation can be achieved, at higher oxidant concentration, and the resultant $\mathrm{Os}(\mathrm{IV}) \mathrm{P}$ is stabilized by axial ligation of two alcohol or water molecules. These Os(IV) complexes are stable to both oxidation and reduction. Treating the Os(II)P with an organic peroxide results in formation of an Os(VI)P which is stabilized by two oxo ligands but can be reduced to the Os(VI)P Photolysis of Os(II)P and Os(III)P causes loss of the CO ligand, the naked porphyrin species so formed undergoes oxidative reactions with the solvent, $\mathrm{O} 2$ or trace impurities, in most cases forming an Os(IV)P. Photolysis of Os(II)P in benzene containing small amounts of $\mathrm{O} 2$ generates the di-oxo Os(VI)P.

100,218

PB91-174730 Not available NTIS National Bureau of Standards (NML), Gaithersburg MD. Organic Analytical Research Div.

Oxidation of Organic Compounds by Active Manganese Dioxide.

Final rept.

A. J. Fatiadi. 1986, 142p

Pub. in Organic Syntheses by Oxidation with Metal Compounds, Chapter 3, p119-260 1986.

Keywords: "Manganese oxides, "Oxidation, "Alcohols "Amines, "Hydrazines, Chemical reactivity, Chemical reactions, Reviews, Organic compounds, Reprints.

The recent, synthetic developments in the oxidation of organic compounds by active manganese dioxide has been reviewed. The topics covered are: (1) methods of preparation of active manganese dioxide; (2) aspects of mechanisms of oxidation by active manganese dioxide; an overview also includes (a) oxidation of alcohols and hydroxy compounds, (b) dehydrogenation and oxidative aromatization, (c) oxidation of hydrocarbons, (d) oxidation of amines and hydrazines, and (e) miscellaneous oxidations.

\section{0,219}

B 1 -175125

Not available NTIS

National Inst. of Standards and Technology (IMSE), Gaithersburg, MD. Polymers Div.

Ion Incorporation into Octacalcium Phosphate Hydrolyzates.

Final rept

B. B. Tomazic, I. Mayer, and W. E. Brown. 1991, 13p Sponsored by American Dental Association Health Foundation, Chicago, IL

Pub. in Jnl. of Crystal Growth 108, p670-682 1991.

Keywords: "Calcium phosphates, Chemical analysis, Binary system(Materials), Crystal growth, Nonmetaliferous minerals, Aqueous solutions, Hydrolysis, Ions, Alkali metal compounds, Apatites, Spectroscopy, Solubility, Reprints.

There is considerable evidence indicating that biominerals are formed by precipitation of octacalcium phosphate followed by its hydrolysis. Therefore, the hydrolysis of octacalcium phosphate was studied in aqueous solutions of alkali nitrates/chlorides or carbonates. The hydrolysis was accompanied by incorporation of alkali and carbonate ions into apatite hydrolysis products. These were characterized by chemical analyses, infrared and X-ray diffraction spectroscopy, and solubility measurements. The extent of hydrolysis in carbonate-free media paralleled the extent of ion incorporation: $\mathrm{Li}>\mathrm{Na}>\mathrm{K}$. The hydrolyses in corresponding carbonate media produced less crystalline apatitic products with B-type carbonate substitution.
National Inst. of Standards and Technology, Gaithersburg, MD.

Journal of Research of the National Institute of Standards and Technology. January-February 1991. Volume 96, Number 1.

1991, $150 \mathrm{p}$

Also available from Supt. of Docs as SN703-027-00038-5. See also PB91-184861 and PB91-167411.

Keywords: *Iron complexes, "Iron organic compounds, "Ceramics, Data bases, Calibrating, US NBS, Research projects, Stereo chemistry, Chirality, Carbenes, Biochemistry, Synthesis(Chemistry), Enantiomorphs, Reviews, High-TC superconductors.

Contents: New Chemical and Stereochemical Applications of Organoiron Complexes; International Conference on the Chemistry of Electronic Ceramic Materials; News Briefs; Calibration Services; Standard Reference Data.

\section{0,221 \\ 100,221}

(Order as PB91-184853, PC A07/MF A01)

National Inst. of Standards and Technology, Gaithersburg, MD.

New Chemical and Stereochemical Applications of Organoiron Complexes.

A. J. Fatiadi. 1991, 113p

Included in Jnl. of Research of the National Institute of Standards and Technology, v96 n1 p1-113 Jan-Feb 91.

Keywords: "Iron complexes, "Iron organic compounds, "Chirality, "Biochemistry, Reviews, Synthesis(Chemistry); Enantiomorphs, Carbenes, Stereochemistry, Catalysts. Norbornadiene, Cycloalkanes, Porphyrins, Carbonyls, Ferrocene, Alkaloids.

The objective of the review is to provide a current overview of the rapidly developing chemistry of organometallic complexes and particularly organoiron complexes useful in asymmetric and stereoselective reactions. Also covered are stereoselective reactions of alpha, beta-unsaturated acyl ligands bound to the chiral auxiliary ((eta(sup 5)-C5H5) $\mathrm{Fe}(\mathrm{CO})(\mathrm{PPh} 3)$ ) and new applications of organoiron complexes in the synthesis of natural products. The mechanistic aspects and stabilizing effects of the $\mathrm{Fe}(\mathrm{CO}) 3$ group for alkenes or conjugated dienes are discussed. A brief summary of recent work on the special role of iron in biological reactions is also included.

\section{0,222}

PB91-237347

Not available NTIS

National Inst. of Standards and Technology (NML), Gaithersburg, MD. Ionizing Radiation Div.

Antioxidation Mechanisms of Uric Acid.

Final rept.

M. G. Simic, and S. V. Jovanovic. 1989, 5p

Pub. in Jnl. of the American Chemical Society $111, \mathrm{n} 15$ p5778-5782 1989.

Keywords: "Uric acid, "Oxidation reduction reactions, *Reaction kinetics, Radiolysis, Antioxidants, Free radicals, DNA repair, Reprints.

One-electron oxidation of uric acid generates the urate radical which was studied in aqueous solution by pulse radiolysis and oxygen uptake measurements. Acidbase properties of the radical were determined, i.e. $\mathrm{pKa} 1=3.1+\mathrm{or}-0.1$ and $\mathrm{pKa} 2=9.5+\mathrm{or}-0.1$. The reaction of the radical with oxygen was too slow to be measured, $k<0.01 \mathrm{dm} 2 / \mathrm{mol} / \mathrm{s}$. The one-electron redox potential vs. NHE, E7 $=0.59 \mathrm{~V}$, was derived from the $\mathrm{pH}$ dependence of the redox potential, which was fitted through the values measured at $\mathrm{pH} 7,8.9$ and previously determined at $\mathrm{pH} 13$. Rapid reactions of uric acid with oxidizing species, peroxy radicals and the guanyl radical, were indicative of uric acid as a possible water soluble physiological antioxidant and repair agent of oxidative damage to DNA bases.

\section{0,223}

PB91-237370

Not available NTIS

National Inst of Standards and Technology (NML) Gaithersburg, MD. Organic Analytical Research Div.

Synthesis of 3-Quinuclidinol-(18)O, Benzilic-d5 Acid, and 3-Quinuclidinyl-(18)O, Benzilate-d5.

Final rept.

L. T. Sniegoski, E. White, and G. D. Byrd. 1989, 11p Pub. in Jnl. of Labelled Compounds and Radiopharmaceuticals 27, n9 p983-993 1989 .

Keywords: "Synthesis(Chemistry), "Isotopic labeling, "Benzilic acid, Standards, Mass spectroscopy, Oxygen
18, Purity, Reprints, *Quinuclidinol, *Benzilic acid/ (quinuclidinyl-ester), Bicyclooctanol/aza, Benzene acetic acid/hydroxy-phenyl.

Isotopically labeled analogs of 3-quinuclidinol (Q), benzilic acid (BA), and 3-quinuclidinyl benzilate (BZ), for use as internal standards for isotope dilution measurements and the labeled intermediate 3-quinuclidinone and methyl benzilate used in their production, were synthesized. The isotopic purities of the 1-azabicyclo (2.2.2) octan-3-180-ol, alpha-hydroxy-alpha-phenyld5-benzenacetic acid, and 3-quinuclidinyl-(18)O benzilate-d5 were determined and the electron impact ionization mass spectrum was obtained for each.

\section{Industrial Chemistry \& Chemical Process Engineering}

100,224

PB91-162008

Not available NTIS

National Inst. of Standards and Technology (NML),

Boulder, CO. Chemical Engineering Science Div.

Small Scale Demand Type Neon Liquefaction Plant.

Final rept.

W. P. Dube, A. J. Slifka, R. M. Bitsy, L. L. Sparks,

and K. B. Johnson. 1990, 5p

Pub. in Advances in Cryogenic Engineering, v35 p1797-18011990.

Keywords: "Neon, "Liquefied gases, "Pilot plants, Liquid hydrogen, Cryogenics, Liquefaction, Cost analysis, Circulation, Design, Reprints.

Low temperature measurement of the thermal conductivity of insulating materials is generally made using a boil-off calorimetry technique involving liquid hydrogen (LH2). Liquid neon ( $\mathrm{LNe}$ ) has nearly the same normal boiling point as $\mathrm{LH} 2$, but has a much larger heat of vaporization, allowing extended run times. In the past. the main drawback of using LNe has been its excessive cost; $\$ 170.00$ per liter versus $\$ 1.50$ per liter for LH2 (1989 prices). A neon liquefaction piant has been designed and constructed to capture, purify and refrigerate the neon boil-off from calorimetry experiments. Recycling the neon reduces operating costs to approximately $\$ 20$ per liter. The system consists of a purification section, which removes air and other contaminants from the calorimetry boil-off, a heat exchanger, $\mathrm{LNe}$ and $\mathrm{LH} 2$ storage dewars and a fully automated control system. After purification, neon is liquified in the heat exchanger by $\mathrm{LH} 2$ flowing countercurrently through stainless steel cooling coils. Hydrogen flow is automatically adjusted to keep the neon at its normal saturation temperature, $27 \mathrm{~K}$. The liquid neon is then stored in a dewar placed directly below the heat exchanger. A 5 watt Gifford-McMahon refrigerator provides cooling during extended storage or low-flow applications.

\section{0,225}

PB91-162164 Not available NTIS National Inst. of Standards and Technology (NEL), Gaithersburg, MD. Building Environment Div.

Visualization of Nucleate Flow Boiling for an R22/ R114 Mixture and Its Components.

Final rept.

M. A. Kedzierski, and D. A. Didion. 1990, 17p

Sponsored by Electric Power Research Inst., Palo Alto, CA.

Pub. in Experimental Heat Transfer 3, p447-463 1990.

Keywords: "Nucleate boiling, "Refrigerants, Bubbles, Heat of vaporization, Heat transfer, Thermodynamics, Binary mixtures, Convection, Optical measurement Suppressing, Reprints.

Visualization of bubble nucleation during forced-convective flow inside a horizontal, electrically heated quartz tube was done in order to establish a comparison of this phenomenon between refrigerant mixtures and their pure components. The specific phenomena investigated were the suppression of nucleation due to increased mass flow quality while holding all other conditions fixed and the comparison of the nucleate activity of the binary mixture to the nucleate activity of the pure components. The fluids investigated were a 37.7 mol\% R22/62.3 mol\% R114 binary mixture and the individual components R22 and R114. These fluids were pumped through an abraded, electrically heated quartz tube. A 16-mm high-speed camera was used, at 
$7000 \mathrm{frames} / \mathrm{s}$, to film the boiling process. Detailed measurements of bubble frequency and bubble size were possible at low pressures, allowing direct calculation of the latent heat load required to nucleate a single bubble. Further work is required to develop a method that ensures statistically sound bubble frequency measurements. However, the standard devitions of the bubble diameter measurements were acceptable. The films were used to visually demonstrate the suppression of nucleation with increase in quality or R114, R22, and an R22/R114 mixture. The films suggest that, for a given quality, R114 exhibits much more nucleation than either R22 or the mixture, while the amount of nucleation demonstrated by R22 and the mixture was comparable even though the mixture was mostly R114 by mole. Arguments using the latent heat of vaporization, the vapor density, and the liquid thermal conductivity have been made to explain the visual trends

\section{0,226}

\section{PB91-162222}

Not available NTIS

National Inst. of Standards and Technology (NML), Boulder, CO. Thermophysics Div.

New Wide Range Equation of State for Helium.

Final rept.

R. D. McCarty, and V. D. Arp. 1990, 11p

Pub. in Advances in Cryogenic Engineering 35, p146514751990.

Keywords: "Liquid helium, "Equations of state, Superfluidity, Cryogenics, Accuracy, Reprints.

A new correlation and computer code have been developed for helium. The correlation covers the temperature range of 0.8 to $1500 \mathrm{~K}$ using two equations of state, one for superfluid helium and another for the normal fluid. The equation of state for the normal fluid is valid for temperatures between 2 and $1500 \mathrm{~K}$ and for pressures up to $2000 \mathrm{MPa}$. The new normal fluid equation of state achieves increased accuracy in some regions of pressure and temperature, out not in others. There are unique problems involved in the correlation of helium data which prevent obtaining the accuracies which are more easily obtained for the properties of other fluids. Only the normal fluid equations are given here.

\section{0,227}

PB91-174672

Not available NTIS

National Inst. of Standards and Technology (NML) Boulder, CO. Chemical Engineering Science Div.

Thermal Conductivity of Evacuated Perlite at Low Temperatures as a Function of Load and Load History.

Final rept.

W. P. Dube, L. L. Sparks, and A. J. Slifka. 1991, 4p Sponsored by National Aeronautics and Space Administration, Bay Saint Louis, MS. John C. Stennis Space Center.

Pub. in Cryogenics 31, n3-6 Jan 91

Keywords: "Penlite, "Thermal conductivity, "Evacuating(Vacuum), Thermal cycling tests, Hysteresis, Cryogenics, Insulation, Physical properties, Dewar flasks, Reprints, Mechanical loading.

Perlite, a powdered insulation, is commonly used in large cryogenic storage dewars. When these dewars are thermally cycled, the perlite in the evacuated space between the inner and outer vessels of the dewar experiences a changing mechanical load due to thermal expansion and contraction of the inner vessel. Thermal conductivity data were obtained using a boiloff calorimeter. The apparent thermal conductivity of evacuated perlite increases strongly with applied load. Hysteretic behaviour of the conductivity was observed when perlite was subjected to cyclic mechanical loading.

\section{0,228}

\section{PB91-175463}

Not available NTIS

National Inst. of Standards and Technology (NML), Boulder, CO. Chemical Engineering Science Div.

Overview of Facilitated Transport Membrane Systems.

Final rept.

A. D. Noble, C. A. Koval, and J. J. Pellegrino. 1989, $13 \mathrm{p}$ 89.

Keywords: *Membrane transport, Separation, Reviews, Reprints, "Facilitated transport membrane systems
A review is presented of membrane separation systems which use a reversible chemical reaction to achieve higher selectivities and fluxes that would otherwise be obtainable. Sections describe the system configurations, modeling, theoretical considerations and industrial experience. The authors have also compiled a table of many of the solute and chemical reacting carriers which have been studied for applications in facilitated transport membrane systems.

\section{0,229}

PB91-195487

National Inst of Standards and Technology (NEL). Gaithersburg, MD. Center for Chemical Engineering. Application of Laser Diagnostic Techniques fo the Examination of Liquid Fuel Spray Structure.

Final rept.

C. Presser, A. K. Gupta, H. G. Semerjian, and R. J Santoro. 1990, 28p

Pub. in Chemical Engineering Communications 90 , p75-102 1990.

Keywords: “Drops(Liquids), "Kerosene, "Sprays, Swirling, Air flow, Light scattering, Reprints, *Droplet sizing, Spray combustion, Doppler interferometry.

Results are presented on the spatial distribution of mean droplet size and number density obtained from a hollow cone kerosene spray, introduced into nonswirling and swirling flow fields. An ensemble light scattering technique, based on measurement of the polarization ratio, has been employed to determine local droplet characteristics in dense and dilute regions of the spray. The measurements are complemented with Lorenz-Mie calculations of the scattering characteristics for a polydispersion of droplets of different size and refractive index. The results reveal that the degree of swirl imparted to the surrounding air flow, has a strong influence on spray structure. For all conditions examined the mean droplet size is found to be larger on the spray boundary than towards the centerline. Mean droplet size is also found to increase with distance at all radial positions of the spray; this trend is attributed to the vaporization of smaller droplets and/ or possible coalescence between the droplets. In addition to the ensemble technique, measurements have also been obtained with the phase/Doppler interferometry and light intensity devonolution techniques under identical experimental conditions. The mean droplet sizes obtained with the ensemble approach are in general smaller than those measured with the phase/Doppler technique.

\section{0,230}

PB91-195719

Not available NTIS

National Inst. of Standards and Technology (NML).

Boulder, $\mathrm{CO}$. Chemical Engineering Science Div.

Application of Osmotic Dewatering to the Controlled Crystallization of Biological Macromolecules and Organic Compounds.

Final rept.

P. Todd, S. K. Sikdar, C. Walker, and Z. R. Korszun. 1991, 10p

Pub. in Jnl. of Crystal Growth 110, p283-292 1991.

Keywords: *Lysozyme, *Crystallization, "Dewatering, Crystallography, Proteins, Transport properties, Reprints, "Reverse osmosis.

Several methods of crystallization of biological macromolecules depend upon the transport of water through the vapor phase - a process that is sensitive to ambient conditions (temperature, relative humidity). Other methods depend on the transport of solute by diffusion or through a membrane. By regulating the solute concentration on the outside of a reverse-osmosis membrane it is possible to control the rate at which macromolecules and other solutes are concentrated inside a membrane-bound fluid. The effect of dewatering rate on lysozyme crystal quality and growth rate was as. sessed. A 3-fold increase in concentration over a 9 day period yielded tetragonal crystals $0.5 \mathrm{~mm}$ on a side with sharp edges and with ordering at least to $1.73 \mathrm{~A}$. Transparent crystals of triglycine sulfate were grown by osmotic dewatering; in this case crystal growth could be enhanced or reversed by manipulating the external solution.

\section{0,231}

\section{PB91-237230}

Not available NTIS

National Inst. of Standards and Technology (CSTL), Boulder, CO. Chemical Engineering Div.
Electrokinetic Demixing of Two-Phase Aqueous Polymer Systems. 2. Separation Rates of Polyethylene Glycol-Maltodextrin Mixtures.

Final rept.

K. S. M. S. Raghava Rao, R. M. Stewart, and P. W.

Todd. 1991, 11p

See also Part 1, PB91-159095.

Pub. in Separation Science and Technology 26, n2 p257-267 1991.

Keywords: "Separation processes, "Solvent extraction, "Electrophoresis, "Dextrin, Binary Aqueous solutions Proteins, Electric fields Buffers(Chemistry), Polarity, Temperature dependence, Phosphates, Biochemistry, Reprints.

A method for enhanced demixing of aqueous twophase systems in a thermostated vertical electrophoresis column was therefore studied. The effects of the electric field strength, field polarity, temperature, phase composition, and buffer concentration on demixing rates of a polyethylene glycol-maltodextrin (PEG-MDX) system were quantitatively measured. At normal electrical polarity (anode at the top of the column), using a maximum practicable field strength of $26.4 \mathrm{~V} / \mathrm{cm}$, the demixing rate was twice that in zero electric field at $25+$ or $-2 \mathrm{C}$. With polarity reversed (anode at the bottom, electric field opposing gravitational settling) at a field of $26.4 \mathrm{~V} / \mathrm{cm}$, demixing was 5.5 times as fast as in zero field. Reduction of the temperature from 25 to $14 \mathrm{C}$ caused an increase in demixing rate in the absence of an applied field, while reduced temperature did not change the rate when using electric fields of either normal or reverse polarity. Increased phosphate buffer concentration substantially increased the demixing rate for PEG-MDX mixtures.

100,232

PB91-237297

Not available NTIS

National Inst. of Standards and Technology (CSTL)

Gaithersburg, MD. Thermophysics Div.

Thermodynamics of Solutions Near the Solvent's Critical Point.

Final rept.

J. M. H. Levelt Sengers. 1991, 56p

Pub. in Supercritical Fluid Technology: Reviews in Modern Theory and Applications, Chapter 1, p1-56 1991.

Keywords: "Fluids, "Solutions, "Critical point, Helmholtz free energy, Dilution, Solvents, Thermodynamics, Reprints, Supercritical solubility, Near critical fluids.

This chapter gives a description of dilute-mixture critical behavior that is aimed at the practicing chemical engineer. The basic concepts of critical behavior in pure fluids and fluid mixtures are explained with a maxmum of practical examples and graphical material Many of the peculiarities of dilute near-critical mixtures are described by means of a simple power-series expansion of the classical mixture Helmholtz free energy. Attention is given to the sources of increased error in experiments carried out in near-critical fluids. A number of misleading statements about supercritica solubility that have recently occurred in the literature are reviewed and corrected.

100,233

PB91-237578

Not available NTIS

National Inst. of Standards and Technology (CSTL), Gaithersburg, MD. Thermophysics Div

Thermodynamic Properties of 1,1,1,2,-Tetrafluoroethane (R134a) in the Critical Region.

Final rept.

S. Tang, G. X. Jin, and J. V. Sengers. 1991, 26p Contract DE-FG05-88ER13902

Sponsored by Department of Energy, Washington, DC Pub. in International Jnl. of Thermophysics 12, n3 p515-540 May 91.

Keywords: "Fluorohydrocarbons, "Equations of state, "Critical point, *Thermodynamic properties, Refrigerants, Temperature dependence, Specific heat, Acoustic velocity, Reprints,

A theoretically based simplified crossover model, which is capable of representing the thermodynamic properties of fluids in a large range of temperatures and densities around the critical point, is presented. The model is used to predict the thermodynamic properties of $\mathrm{R} 134 \mathrm{a}$ in the critical region from a limited amount of available experimental information. Values for various thermodynamic properties of R134a at den- 


\section{CHEMISTRY}

\section{Industrial Chemistry \& Chemical Process Engineering}

sities from 2 to $8 \mathrm{~mol} / \mathrm{L}$ and at temperatures from 365 to $450 \mathrm{~K}$ are presented.

\section{Photo \& Radiation Chemistry}

\section{0,234}

AD-A227 868/7 PC A02/MF A01 National Inst. of Standards and Technology, Gaithersburg, MD.

Laser Photolysis of Trimethylgallium at $193 \mathrm{~nm}$ : Quantum Yields for Methyl Radical and Ethane Production

W. Braun, R. Klein, A. Fahr, H. Okabe, and A. Mele. 2 Mar 90, 8p ARO-25911.1-CH,

Contract MIPR-119-89

Pub. in Chemical Physics Letters, v166 n4 p397-403, 2 Mar 90.

Keywords: Chemical reactions, Ethanes, Gallium, Lasers, Methyl radicals. "Photolysis, Production, Quantum efficiency, Reprints, Vapor deposition, Yield, Quantum efficiency, Reprints, Vapor deposition, Yield,
Organic chemistry, " $G a l l i u m$ organic compounds, Isotopic labeling.

Quantum yields for the products from trimethylgallium (TMG) photolysis were determined via a new method involving isotopic analysis using TMG-acetone- $\mathrm{d} 6$ mixtures. The following model-dependent quantum yields were obtained: monomethylgallium (MMG), 0.5; dimethylgallium (DMG), 0.2; gallium, 0.3 ; molecularly eliminated ethane, 0.3 ; free methyl, 1.5 . Two new electronic absorptions at 216 and $220 \mathrm{~nm}$ were found and are tentatively ascribed to DMG. Keywords: Trimethylgallium, Quantum yield, Laser photolysis, Chemical vapor deposition, Reprints. (JS)

\section{0,235}

PB91-147066

Not available NTIS

National Inst. of Standards and Technology (NML), Gaithersburg, MD. Surface Science Div.

Laser Induced Desorption of Molecules from Surfaces.

Final rept.

A. R. Cavanagh, S. A. Buntin, L. J. Richter, and D. S. King. 1990, 12p

Sponsored by Department of Energy, Washington, DC. Pub. in Comments on Atomic and Molecular Physics 24, n6 p365-376 1990.

Keywords: "Surface reactions, Photochemical reactions, Excitation, Heating, Reprints, "Laser induced desorption.

Great insight into the fundamental mechanisms of optically driven surface processes has been obtained through studies of laser induced desorption of species from well characterized surfaces in ultrahigh vacuum. Key fundamental studies are reviewed, with emphasis placed on three distinct processes by which photons can promote surface reactions: heating, resonant adsorbate excitation, and substrate electronic excitation.

\section{0,236}

PB91-147793 Not available NTIS National Inst. of Standards and Technology (NML), Gaithersburg, MD. Chemical Kinetics Div.

Radiation Chemistry of Enones.

Final rept.

P. Neta, and M. Dizdaroglu. 1989, 24p

Sponsored by Department of Energy, Washington, DC. Pub. in Chemistry of Enones, Chapter 16, p757-780 1989.

Keywords: "Aldehydes, "Detones, "Radiation chemistry, Ionizing radiation, Solutions, Chemical radicals, Reviews, Radiolysis, Reprints.

Radiation chemistry deals with the chemical effects of ionizing radiation, such as $\mathrm{X}$-rays, gamma rays, high energy electrons, or other energetic particles. Ionizing radiation is absorbed in organic materials somewhat indiscriminately and causes ionizations and excitations which may result in bond scission. In discussing the radiation chemistry of an organic compound, the authors distinguish between the radiation chemistry of the neat compound, where the energy is absorbed totally by the compound itself, and the radiation chemistry of its solutions, where the energy is absorbed predominantly by the solvent. In the latter case, the solute undergoes chemical changes only via reactions with the primary radicals formed from the solvent. The radi- ation chemistry of enones was studied mainly in solution, as will become clear from the review, and most often it involved aqueous solutions

100,237

PB91-148809

Not available NTIS

National Inst. of Standards and Technology (NML) Boulder, CO. Quantum Physics Div.

Novel Laser Gain and Time-Resolved FTIR Studies of Photochemistry.

Final rept.

S. R. Leone. $1989,13 p$

Contract DAAG-29-85-K-0033, Grant NSF-CHE8408403

Sponsored by Department of Energy, Washington, DC., and National Science Foundation, Washington,

DC.

Pub. in Proceedings of International Conference on Laboratory Research for Planetary Atmospheres (1st), Bowie, MD., October 25-27, 1989, p129-141.

Keywords: "Planetary atmospheres, *Cometary atmospheres, *Photochemical reactions, Infrared spectra, Emission spectra, Acetylene, Tunable lasers, Reprints, Fourier transform infrared spectroscopy.

Several novel techniques are discussed which can be used to explore laboratory photochemical processes and kinetics relevant to planetary atmospheres; these include time-resolved laser gain-versus-absorption spectroscopy and time-resolved Fourier transform in frared (FTIR) emission studies. The laser gain-versusabsorption method employs tunable diode and $\mathrm{F}$. center lasers to determine the yields of excited photofragments and their kinetics. The time-resolved FTIR technique synchronizes the sweep of a commercial FTIR with a pulsed source of light to obtain emission spectra of novel transient species in the infrared. These methods are presently being employed to investigate molecular photodissociation, the yields of excited states of fragments, their subsequent reaction kinetics, Doppler velocity distributions, and velocity changing collisions of translationally fast atoms. Such techniques may be employed in future investi gations of planetary atmospheres, for example to study polycyclic aromatic hydrocarbons related to cometaryemissions, to analyze acetylene decomposition products and reactions, and to determine spectral features in the near infrared and infrared wavelength regions for planetary molecules and clusters.

\section{0,238}

PB91-149252

Not available NTIS

National Inst. of Standards and Technology (NML)

Boulder, CO. Quantum Physics Div.

Periodic Orbits and Diffuse Structures in the Photodissociation of Symmetric Triatomic Molecules. Final rept.

R. Schinke, and V. Engel. 1990, 5p

Pub. in Jnl. of Chemical Physics 93, n5 p3252-3256, 1 Sep 90

Keywords: "Photodissociation, Triatomic molecules, Trajectories, Orbits, Reprints.

The authors reinvestigate the collinear photodissociation of a symmetric triatomic molecule studied a decade ago by Kulander and Light ( $\mathrm{J}$. Chem. Phys 73,4437 (1980)), within the time-dependent formalism. The diffuse structures in the absorption spectrum are uniquely related to three, well separated recurrences which, in turn, are explained by three generic periodic orbits of the corresponding classical Hamiltonian. One of these orbits describes pure symmetric stretch motion while the other two orbits combine symmetric stretch and hyperspherical motion. The applicability of simple one-dimensional, zeroth-order pictures is reviewed in the light of these new results.

\section{0,239}

\section{PB91-149260}

Not available NTIS

National Inst. of Standards and Tech

Photodissociation of C(I)NO in

Photodissociation of $C(I) N O$ in the
Quantum-Mechanical ab initio Study.

Final rept.

R. Schinke, M. Nonella, H. U. Suter, and J. R. Huber.

$1990,9 \mathrm{p}$

Pub. in Jnl. of Chemical Physics 93, n2 p1098-1106, 15 Jul 90.

Keywords: "Photodissociation, Absorption spectra, Vibrational states, Rotational states, Quantum mechanics, Nitric oxide, Potential energy, Reprints, "Nitrosyl chloride.
The authors investigated the photodissociation of CINO (nitrosyl chloride) via the $\mathrm{S}(1)$ electronic state using a three-dimensional (3D) ab initio potentialenergy surface (PES). The dissociation is found to be fast and direct. In the Franck-Condon (FC) region the slope of the potential along the dissociation path is relatively small giving rise to narrow partial absorption peaks. The total absorption spectrum therefore exhibits a broad vibrational structure which is in perfect agreement with recent measurements. The vibrational excitation of the NO fragment is small and can be qualitatively described within the adiabatic approximation. It is found to be very sensitive to the vibrational FC factor in the transition region. The rotational state distribution of NO is highly inverted with a peak around $\mathrm{j}=30$. It is readily explained by the rotational reflection principle. The experimental results are satisfactorily reproduced by the authors' calculations which underlines the overall quality of the calculated PES. Minor adjustments are necessary, however, to quantitatively reproduce the vibrational branching ratio.

\section{0,240}

PB91-203679 Nechnology (NML), Gaithersburg, MD. Chemical Kinetics Div.

of Acetonitrile.

Final rept.

S. Mosseri, P. Neta, and D. Meisel. 1990, 5p

Pub. in Radiation Physics and Chemistry 36, n6 p6836871990.

Keywords: "Acetonitrile, "Cyanides, "Radiolysis, Chemical reactions, Chemical radicals, Peroxy radicals, Solutions, Reprints.

The radiolysis of neat acetonitrile or of its aqueous solutions was found to lead to formation of cyanide ions, but only in the presence of oxygen. The mechanism involved formation of the acetonitrile radical, (dot) $\mathrm{CH} 2 \mathrm{CN}$, which reacts with $\mathrm{O} 2$ to give the peroxyl radical, $\mathrm{NCCH}_{2} \mathrm{O} 2$ (dot). Subsequent decay of the peroxyl radical results in the release of HCN. From the yield of cyanide under various conditions the authors conclude that the peroxyl radicals decay via two main routes, the products of one route from cyanide directly while the product of the second route yield cyanide only after hydrolysis by alkali. The overall yield of $\mathrm{CN}(-)$ in $5 \%$ acetonitrile aqueous solutions saturated with a mixture of $\mathrm{N2O}: \mathrm{O} 2$ (4:1) was $\mathrm{G}$ approximately equal to 6 in alkaline solution and $G$ approximately equal to 3 in neutral solution. The yield of $\mathrm{CH} 2 \mathrm{O}$ in both solutions was $\mathrm{G}$ approximately equal to 1.5 .

\section{0,241}

PB91-236489

Not available NTIS

National Inst. of Standards and Technology (PL), Gaithersburg, MD. Ionizing Radiation Div.

Mechanisms of the Reduction Reactions of $\mathrm{Cr}(\mathrm{VI})$ in the Radiolysis of Acidic Potassium and Silver Dichromate Solutions in the Presence or Absence of Acetic Acid.

Final rept.

M. Al-Sheikhly, and W. L. McLaughlin. 1991, 9p Pub. in Radiation Physics and Chemistry 38, n2 p2032111991

Keywords: "Chromates, "Oxidation reduction reactions, *Reaction kinetics, "Acetic acid, Silver ions, $\mathrm{pH}$, Radiolysis, Chemical dosimeters, Chemical radicals, Computerized simulation, Reprints, Radiation chemical mechanisms.

The mechanisms and the kinetics of reactions in deoxygenated and oxygen-saturated aqueous solutions of potassium dichromate in the range of $\mathrm{pH} 0.4-0.8$, in the presence and absence of acetic acid, were investigated by pulse and steady-state radiolysis. Computer simulation of reaction mechanisms was compared with experimental kinetics data for solutions containing silver dichromate and acetic acid. It was found that carboxymethyl reduces $\operatorname{Cr}(\mathrm{VI})$ to $\mathrm{Cr}(\mathrm{V})$ with a rate constant of $1.8 \times 10$ to the 8 th power $/ \mathrm{M} / \mathrm{s}$, while carboxymethlyperoxyl does not reduce $\mathrm{Cr}(\mathrm{VI})$. Hydroperoxyl reduces $\mathrm{Cr}(\mathrm{VI})$ to $\mathrm{Cr}(\mathrm{V})$, but at a much lower rate constant of $<$ 10 to the 6 th. The oxidation-reduction reaction between radiolytically produced $\mathrm{Cr}(\mathrm{V})$ and $\mathrm{Ag}(\mathrm{II})$ was measured and found to have a rate constant of $1.0 \times$ 10 to the 8 th $p o w e r / M / s$. The mechanisms for the enhanced stability of $\mathrm{Cr}(\mathrm{VI})$ in the presence of $\mathrm{Ag}+$ was studied in the light of the kinetics and the reactivity of the Ag(II) with the intermediate free radicals occurring in this system. The present findings help elucidate the 
mechanisms of the dichromate dosimeter, its doserate independent response and its stability.

\section{Physical \& Theoretical Chemistry}

\section{0,242}

AD-A227 296/1

PC A04/MF A01

Nation

Detection of Excited States by Laser-Induced Fluorescence and Analysis of Energy Transfer. Final rept. Oct $88-$ Sep 89.

J. Borysow, and A. V. Phelps. Sep 90,70p WRDCTR-90-2081,

Contract MIPR-FY-88-N0655

Keywords: Absorption, Collisions, Detection, Diodes Doppler effect, Electric fields, Energy, Energy transfer, Field intensity, Impact, Ionization, Kinetic energy, "Laser induced fluorescence, Lasers, Measurement Nonlinear systems, Profiles, Pulses, Rotation, Time dependence, "Nitrogen ions, "Excited states, Gas ionization.

The laser absorption technique was improved through the use of laser diodes and was applied to absorption measurements of the line profiles for the A2 pi u from $x$ 2 sigmag $(+)$ transition of $N 2(+)$ ions to determine (a) the electric field strength in the positive column of pulsed discharges in N2 from the Doppler shift and broadening, (b) the time dependence of the density of ground state $\mathrm{N} 2(+)$ ions drifting through $\mathrm{N} 2$ resulting from the transfer of kinetic energy of the $\mathrm{N} 2(+)$ to rotational energy. An analysis of the impact of measurements made under this program on models of nonlin ear ionization in N2 leads to the conclusion that ioniza tion by collisions between pairs of metastable $\mathrm{N} 2 \mathrm{~mol}$ ecules in the a' state probably make the largest contribution to the growth of instabilities in high-current discharges. $(\mathrm{RH})$

100,243

AD-A228 082/4

PC A02/MF A01

National Inst. of Standards and Technology, Gaithers burg, MD.

Vibrational Spectra of Molecular Ions Isolated in Solid Neon. III. N4(+)

W. E. Thompson, and M. E. Jacox. 15 Sep 90, 8p ARO-25664.4-CH

Contract MIPR-120-90

Pub. in Jnl. of Chemical Physics, v93 n6 p3856-3862 $15 \operatorname{Sep} 90$.

Keywords: Absorption, Atoms, "Electron spin resonance, Infrared radiation, Infrared spectra, Intensity, Microwaves, "Molecular ions, Neon, Solids, "Vibrational spectra, Nitrogen

When a Ne:N2 $=100$ or 200 mixture is codeposited at $5 \mathrm{~K}$ with a beam of neon atoms excited by a microwave discharge, a weak to moderately intense infrared absorption appears at $2237.6 \mathrm{~cm}-1$ which is assigned to the N4 molecular ion. The analysis of the infrared spectra of the nitrogen-15 substituted species of $\mathrm{N} 4$ supports the conclusion from earlier ab initio calcula tions and electron spin resonance observations that N4 has a linear, centrosymmetric ground-state struc ture. For the N4 species with noncentrosymmetric isotopic substitution, the in-phase end-atom stretching fundamental becomes infrared active and has also been observed. Although the anion responsible for overall charge neutrality of the deposit has not been definitively identified, secondary photolysis studies provide some information regarding its properties. (js)

100,244

AD-A230 $957 / 3$

$\mathrm{PC}$ A03/MF A0

National Inst. of Standards and Technology (NML)

Gaithersburg, MD. Molecular Physics Div.

Vibrational Spectra of Molecular Ions Isolated in Solid Neon. IV.NO(+), NO(-), ONNO $(+)$, and ONNO(-).

M. E. Jacox. 1 Dec 90, 14p ARO-25664.7-CH

Contract ARO-MIPR-120-90

Pub. in Jnl. of Chemical Physics, v93 n11 p7609-7621, 1 Dec 90.

Keywords: Absorption, Atoms, Dimers, Electrons, Infrared radiation, Ionization, Ions, Molecular ions, Neon Photoionization, Solids, Stabilization, Vibrational spectra, Reprints, "Vibrational spectra, "Neon.
When a Ne: NO sample is codeposited at approximately $5 \mathrm{~K}$ with a beam of neon atoms that has been excited in a microwave discharge, photoionization and Penning ionization of the NO leads to the stabilization of both monomer and dimer ions. The most prominent infrared absorption, at $1619.2 \mathrm{~cm}-1$, results from the re action of NO- with No or of an electron with (NO) 2 to form the charge-delocalized trans-ONNO- species. The position of the totally symmetric NO-stretching absorption of trans-ONNO- can be inferred from observation of a combination band. The infrared absorption of $\mathrm{NO}+$ has been detected very close to the gas-phase band center. Other infrared absorptions have been tentatively assigned to NO-, cis-ONNO-, trans-ONNO and the lowest energy isomer of ONNO +. Comparison of the gas-phase vibrational frequencies observed for the weakly bound species cis-ONNO and N2O3 with the corresponding frequencies observed in this neon-matrix study suggests that the matrix shifts for the dimer ion absorptions in the mid-infrared probably amount to less than $1 \%$.

\section{0,245}

\section{AD-A234 043/8}

PC A03/MF A01

National Inst of Standards and Technology (NML) Gaithersburg, MD. Molecular Spectroscopy Div.

Production and Spectroscopy of Small Polyatomic Molecular Ions Isolated in Solid Neon.

M. E. Jacox, and W. E. Thompson. 1990, 11p ARO25664.2-CH,

Contract ARO-MIPR-120-90

Availability: Pub. in High Temperature Science, v28 p225-234 1990. Available only to DTIC users. No copies furnished by NTIS

Keywords: Absorption, Spectroscopy, Neon, "Molecu lar ions, Infrared spectra, Reprints, Molecular clusters, Cluster ions, * Matrix isolation, Solidified gases.

No abstract available.

\section{0,246}

AD-A235 490/0

$\mathrm{PC}$ A03/MF A01

National Inst. of Standards and Technology (IMSE) Gaithersburg, MD. Metallurgy Div.

Thermodynamic and Kinetic Stability of Refractory Materials at Ultra-High Temperatures.

Annual rept. 1 Oct 89-30 Sep 90.

J. W. Hastie, P. K. Schenck, and D. W. Bonnell. Nov 90, 31p AFOSR-TR-91-0447,

Grant AFOSR-ISSA-90-0026

Keywords: Equilibrium(General), Extrapolation, "Hafnium compounds, Heat High temperature Kinetics, Lasers, Mass spectra, Measurement, Oxides, Pulsed lasers, "Refractory materials, "Silicon carbides, Stability, Surface temperature, Thermodynamics, Time Vacuum, Vapor pressure, "Vaporization, LVMS(Laser Vaporization Mass Spectrometry), Mass spectrometry, *Thermal stability, Reaction kinetics, "High temperature, "Ceramic materials, Spectrum analysis, Reaction kinetics.

Using the newly developed measurement technique of Laser Vaporization Mass Spectrometry (LVMS), new vapor pressure data for hafnium oxide and alpha silicon carbide at ultra high temperature have been ob tained. The technique uses pulsed lasers to heat a surface on the timescale of $15 \mathrm{~ns}$, providing an effectively containerless environment. Time-resolved mass spectra establish that the vaporization process is occuring under at least local equilibrium conditions. The vapo pressure data are compared with extrapolations from the literature. The agreement for $\mathrm{HfO} 2$ is excellent, while the comparison for $\mathrm{SiC}$ indicates a significant discrepancy. Both instrumental and physio-chemical in fluences affecting the measurements are discussed and the possibility of a different vaporization mechanism for SiC under vacuum vaporization conditions is suggested. Preliminary optical spectroscopic approaches, to supplement the current indirect methods, to the direct measurement of surface temperatures under short pulse laser heating conditions are presented.

\section{0,247}

AD-A236 485/9

PC A03/MF A01

National Inst. of Standards and Technology, Gaithers burg, MD.
Spatially and Spectrally Resolved Cathodoluminescence of Hot-Filament Chemical-Vapor-Deposited Diamond Particles.

interim rept.

L. H. Robins, E. N. Farabaugh, and A. Feldman. 24 Apr 91, 43p

Contract N00014-90-F-0011

Keywords: Brightness, Broadband, Cathodolumines cence, Deposition, Impurities, Particles, Temperature "Carbon, "Diamond, Crystal growth, Vapor deposition.

Spectrally resolved cathodoluminescence (CL) image and spatially resolved CL spectra were obtained from two specimens grown by hot-filament chemical vapor deposition. Each specimen consisted of a large number of unconnected diamond particles with cubooctahedral and pseudo-five-fold twinned growth habits. The growth temperature was nominally 600 deg $C$ for one specimen and $750 \mathrm{deg} C$ for the other. In the 1.5-3.5 eV range, the spectra are composed of four defect and impurity related bands: there are three bands with zero-phonon lines at $1.68 \mathrm{eV}, 2.156 \mathrm{eV}$ and $2.325 \mathrm{eV}$, and one broad band centered at 2.85 $\mathrm{eV}$. A weak at $5.27 \mathrm{eV}$, due to exciton recombination, was also observed. Spectrally resolved images to the two most intense $\mathrm{CL}$ bands, at $2.126 \mathrm{eV}$ and $2.85 \mathrm{e} \mathrm{V}$, were obtained for several particles. In the low temperature specimen, bright regions in images of the 2.85 $\mathrm{eV}$ band are correlated in some cases with the central regions of (100) facets.

100,248

AD-A237 128/4

$\mathrm{PC}$ A03/MF A01

National Inst. of Standards and Technology (IMSE) Gaithersburg, MD. Ceramics Div.

Spatially and Spectrally Resolved Cathodoluminescence of Hot-Filament Chemical-Vapor-Deposited Diamond Particles.

Interim rept.

L. H. Robins. 24 Apr 91, 40p

Contract N00014-90-F-0011

Keywords: Broadband, "Cathodoluminescence, Growth(General), Impurities, Particles, Temperature, "Diamonds, Crystal growth, "Vapor deosition.

Spectrally resolved cathodoluminescence (CL) image and spatially resolved CL spectra were obtained from two specimens grown by hot-filament chemical vapo deposition. Each specimen consisted of a large number of unconnected diamond particles with cubooctahedral and pseudo-five-fold twinned growth habits. The growth temperature was nominally $600 \mathrm{C}$ for one specimen and $750 \mathrm{C}$ for the other. In the 1.5 $3.5 \mathrm{eV}$ range, the spectra are composed of four defect and impurity related bands: there are three bands with zero-phonon lines at $1.68 \mathrm{eV}, 2.156 \mathrm{eV}$, and $2.325 \mathrm{eV}$ and one broad band centered at $2.85 \mathrm{eV}$. A weak peak at $5.27 \mathrm{eV}$, due to exciton recombination, was also observed. Spectrally resolved images of the two most in tense CL bands, at $2.156 \mathrm{eV}$ and $2.85 \mathrm{eV}$, were obtained for several particles.

\section{0,249}

Not available NTIS

National Inst. of Standards and Technology, Gaithersburg, MD.

Vibrational Spectra of Molecular Ions Isolated in Solid Neon. 6. CO4(-).

M. E. Jacox, and W. E. Thompson. 4 Apr 91, 8p ARO-25664.9-CH

Contract ARO-MIPR-120-90

Pub. in Jnl. of Physical Chemistry, v95 n7 p2781-2787 4 Apr 91 . Available only to DTIC users. No copies fur nished by NTIS.

Keywords: Molecular spectroscopy, "Molecular vibration, Carbonates, Carbon dioxide, Oxygen, Nitrogen, Reprints, "Neon matrices, "Vibrational spectra, "Molecularions.

No abstract available.

100,250

AD-A239 729/7

Not available NTIS

National Inst. of Standards and Technology (NML) Gaithersburg, MD. Molecular Physics Div.

Vibrational Spectra of Molecular Ions Isolated in Solid Neon. 7. $\mathrm{CO}(+), \mathrm{C}_{2} \mathrm{O} 2(+)$, and $\mathrm{C}_{2} \mathrm{O} 2(-)$.

W. E. Thompson, and M. E. Jacox. 15 Jul $91,11 p$ ARO-25664.10-CH

Pub. in Jnl. of Chemical Physics, v95 n2 p735-745, 15 Jul 91. Available only to DTIC users. No copies furnished by NTIS. 


\section{CHEMISTRY}

\section{Physical \& Theoretical Chemistry}

Keywords: "Neon, "Carbon monoxide, "Ion molecule interactions, "Infrared spectra, Photoionization, Ionized gases, Vapor deposition, Absorption, Atomic beams, Electron capture, Reprints, "Molecular ions, Solids, *Vibrational spectra, Penning ionization, Low temperature research.

No abstract available.

\section{0,251}

\section{PB90-244526}

Not available NTIS

American Chemical Society, Washington, DC.

Journal of Physical and Chemical Reference Data, Volume 19, Number 1, 1990.

Quarterly rept.

c1990, 319p

See also PB90-244534 through PB90-244591 and PB90-161241. Prepared in cooperation with American Inst. of Physics, New York. Sponsored by National Inst. of Standards and Technology, Gaithersburg, MD. Available from American Chemical Society, 1155 16th St., NW, Washington, DC 20036.

Keywords: "Magnesium oxides, Thermodynamic properties, Benzenes, Thermal conductivity, Standards, Atomic energy levels, Butanes, Reaction kinetics, Combustion, Periclase, Surface structure, Ion scattering. Aluminum ions, Iron ions, Oxygen atoms, Electronatom collisions, Isobutyl radicals, 2-methylpropane.

Contents: Chemical Kinetic Data Base for Combustion Chemistry. Part 4. Isobutane; Thermodynamic Functions and Properties of $\mathrm{MgO}$ at High Compression and High Temperature; Critical Compilation of Surface Structures Determined by Ion Scattering Methods Benzene--A Further Liquid Thermal Conductivity Standard; Energy Levels of Atomic Aluminum with $\mathrm{Hy}$ perfine Structure; Spectral Data and Grotrian Diagrams for Highly lonized Iron, Fe VIII-XXVI; Updated Excitation and lonization Cross Sections for Electron Impact on Atomic Oxygen.

\section{0,252 \\ PB90-244534}

(Order as PB90-244526)

National Inst. of Standards and Technology (NML) Gaithersburg, MD. Chemical Kinetics Div.

Chemical Kinetic Data Base for Combustion Chem. istry Part 4. Isobutane.

Quarterly rept.

W. Tsang. c1990, 68p

Contract DNA-001-85-C-0022

Sponsored by Defense Nuclear Agency, Washington, DC.

Included in Jnl. of Physical and Chemical Reference Data, v19 n1 p1-68 1990. Available from American Chemical Society, 1155 16th St., NW, Washington, DC 20036.

Keywords: "Butanes, "Reaction kinetics, "Combustion, Pyrolysis, Tables(Data), "Isobutyl radicals, "2 methylpropane, Data bases.

The publication contains evaluated and estimated data on the kinetics of reactions involving isobutane, $t$-buty radical and isobutyl radical and various small inorganic and organic species which are of importance for the proper understanding of isobutane combustion and pyrolysis. It is meant to be used in conjunction with the kinetic data given in earlier publications, which is of direct pertinence to the understanding of methane ethane, methanol and propane pyrolysis and combustion, but which also contains a large volume of data that are applicable to the isobutane system. The temperature range covered is $300-2500 \mathrm{~K}$ and the density range $1 \times 10$ to the 16 power to $1 \times 10$ to the 21 powe molecules/cc. (Copyright (c) 1990 by the U.S. Secretary of Commerce.)

\section{0,253}

PB90-244542

California Univ., Los Angeles.

(Order as PB90-244526) at High Compression and High Temperature.

Quarterly rept.

O. L. Anderson, and K. Zou. c1990, 15p

Grants DOE-ER-13803, NSF-EAR86-17290

Sponsored by Department of Energy, Washington, DC., and National Science Foundation, Washington, DC

Included in Jnl. of Physical and Chemical Reference Data, v19 n1 p69-84 1990. Available from American Chemical Society, 1155 16th St., NW, Washington, DC 20036.
Keywords: "Magnesium oxides, "Thermodynamic properties, Gibbs free energy, Helmholtz free energy, Entropy, " $P$ ericlase, High pressure, High temperature.

The complete thermodynamic functions for $\mathrm{MgO}$ (the mineral periclase) are presented for the temperature range $300-2000 \mathrm{~K}$ and the pressure range $0-150 \mathrm{GPa}$, both as tables and as graphs. Careful attention is given to the temperature and pressure dependence of the coefficient of thermal expansion alpha and the isothermal bulk modulus $\mathrm{K}(\mathrm{T})$, which are the major corrections to the thermodynamic functions in extreme conditions. The Birch-Murnaghan equation of state is used to account for certain terms in the expressions for internal energy and enthalpy. The parameters used in the Birch-Murnaghan equation of state are given as functions of temperature. The parameters which presently limit the calculations of thermodynamic functions to even higher temperatures and pressures and limit the applications of this method to other minerals are given.

100,254

PB90-244559

Oregon State Univ Corvallis. Dept of Chemistry.

Critical Compilation of Surface Structures Determined by lon Scattering Methods.

Quarterly rept.

P. R. Watson. c1990, 27p

Grant NBS-60NANB7D0729

Sponsored by National Inst. of Standards and Technology, Gaithersburg, MD.

Included in Jnl. of Physical and Chemical Reference Data, v19 n1 p85-112 1990. Available from American Chemical Society, 1155 16th St., NW, Washington, DC 20036 .

Keywords: Crystal structure, Reviews, Comparison, * Surface structure, "Ion scattering, Low energy electron diffraction, Semiconductors.

The review critically compiles all surface structures derived by ion scattering techniques reported in the refereed literature prior to January 1988. They are compared with the more extensive low-energy electron diffred Ref. Data 16, 953 (1957)). These investigations cover all types of surfaces including clean and adsorbatecovered metal, semiconductor, and other nonmetallic substrates. The important experimental and theoretical aspects of such investigations have been extracted into easily understood tabular form supplemented by many figures and ancillary tables and complete references. (Copyright (c) 1990 by the U.S. Secretary of Commerce.)

100,255

PB90-244567

Thessaloniki Univ., Salonika (Greece).

Benzene: A Further Liquid Thermal Conductivity Standard.

Quarterly rept.

M. J. Assael, M. L. V. Ramires, C. A. Nieto de

Castro, and W. A. Wakeham. c1990, 3p

Contract DNA-001-85-C-0022

Sponsored by Defense Nuclear Agency, Washington, DC., Lisbon Univ. (Portugal). Dept. de Quimica, and Imperial Coll. of Science and Technology, London (Engand).

Included in Jnl. of Physical and Chemical Reference Data, v19 n1 p113-116 1990. Available from American Chemical Society, 1155 16th St., NW, Washington, DC 20036.

Keywords: *Benzene, "Thermal conductivity, *Standards, Liquids, Literature surveys.

The available experimental liquid-phase thermal conductivity data for benzene have been examined with the intention of establishing a further liquid thermal conductivity standard along the saturation line. The quality of the available data is such that new standard reference values can be proposed with confidence limits better than + or - $1 \%$ for most of the normal liquid range. (Copyright (c) 1990 by the U.S. Secretary of Commerce.)

\section{0,256}

\section{PB90-24459}

(Order as PB90-244526)

$R$ and D Associates, Marina del Rey, CA.
Updated Excitation and Ionization Cross Sections for Electron Impact on Atomic Oxygen.

Quarterly rept.

R. R. Laher, and F. R. Gilmore. c1990, 29p

Contract DNA-001-85-C-0022

Sponsored by Defense Nuclear Agency, Washington,

DC.

Included in Jnl. of Physical and Chemical Reference Data, v19 n1 p277-304 1990. Available from American Chemical Society, 1155 16th St., NW, Washington, DC 20036

Keywords: Upper atmosphere, Excitation Tables(Data), Graphs(Charts), "Electron-atom collisions, "Oxygen atoms, Ionization cross sections, Electron impact.

Cross sections for the excitation and ionization of atomic oxygen by electron impact are presented as the result of a critical review of experimental and theoretical work on this subject. An effort has been made to compile the most accurate and complete set of cross sections available. More than 60 profiles of excitation cross section versus electron-impact energy are presented. These include transitions to the forbidden metastable $O(2 p(4)$ singlet $D)$ and $O((2 p(4)$ singlet $s)$ states, the allowed autoionizing $O(2 p(5)$ triplet $p(0)$ ) state, nine allowed Rydberg series, and twenty-nine forbidden Rydberg series. Recommended ionization cross sections for transitions to the various outer-electron ionization states to the inner-electron ionization state $\mathrm{O}(1+)$ quartet $p$, and to the $\mathrm{O}(2+)$ state are also given. The data presented in the report will be useful in calculations of aeronomical and artificially-induced electron impact on atomic oxygen, an important component of the upper atmosphere. (Copyright (c) 1990 by the U.S. Secretary of Commerce.)

100,257

PB90-244609 Not available NTIS

American Chemical Society, Washington, DC.

Journal of Physical and Chemical Reference Data, Volume 19, Number 2, 1990.

Quarterly rept.

D. R. Lide. c1990, 208p

See also PB90-244617 through PB90-244641 and PB90-244526. Prepared in cooperation with American inst. of Physics, New York. Sponsored by Nationa Inst. of Standards and Technology, Gaithersburg, MD. Available from American Chemical Society, 1155 16th St., NW, Washington, DC 20036.

Keywords: "Physical chemistry, Thermodynamic properties, Chemical properties, Aromatic polycyclic hydro carbons, Physical properties, Dielectric properties, Water, Reaction kinetics, Photochemical reactions, "Reference materials, Debye-Huckel theory, Isomers, Peroxyl radicals, Aqueous solutions.

Contents: Standard Chemical Thermodynamic Properties of Isomer Groups of Monochloroalkanes; Standard Chemical Thermodynamic Properties of Polycyclic Aromatic Hydrocarbons and Their Isomer Groups. III. Naphthocoronene Series, Ovalene Series, and First Members of Some Higher Series; The Dielectric Constant of Water and Debye-Huckel Limiting Law Slopes; Rate Constants for Reactions of Peroxyl Radicals in Fluid Solutions; Cumulative Listing of Reprints and Supplements.

\section{0,258}

PB90-244617

(Order as PB90-244609)

Massachusetts Inst. of Tech., Cambridge. Dept. of Chemistry.

Standard Chemical Thermodynamic Properties of Isomer Groups of Monochloroalkanes.

Quarterly rept.

R. A. Alberty, and M. B. Chung. c1990, 28p

Sponsored by National Inst of Standards and Technology, Gaithersburg, MD.

Included in Jnl of Physical and Chemical Reference Data, v19 n2 p321-348 1990. Available from American Chemical Society, 115516 th St., NW, Washington, DC 20036.

Keywords: "Thermodynamic properties, "Chloroalkanes, Standards, Gibbs free energy, Enthalpy, Entropy, Specific heat, Tables(Data), Chemical properties, Physical properties, Physical chemistry, "Isomers, "Reference materials, Benson method.

The chemical thermodynamic properties of isomer groups of monochloroalkanes from $\mathrm{C} 2 \mathrm{H} 5 \mathrm{Cl}$ to $\mathrm{C} 8 \mathrm{H} 17 \mathrm{Cl}$ in the ideal gas phase have been calculated 
from 298.15 to $1500 \mathrm{~K}$ using new Benson group values from Bozzelli. Increments in isomer group properties per $\mathrm{CH} 2$ have been calculated to show the extent to which thermodynamic properties of higher isomer groups may be obtained by linear extrapolation. Equilibrium mole fractions within isomer groups have been calculated for the ideal gas state. Values of $\mathrm{Cp}, \mathrm{S}$, delta (sub f) $\mathrm{H}$, and delta (sub g) $\mathrm{G}$ are given for all species of monochloroalkanes from $\mathrm{CH} 3 \mathrm{Cl}$ to $\mathrm{C} 8 \mathrm{H} 17 \mathrm{Cl}$ in $\mathrm{SI}$ units for a standard state pressure of 1 bar. The values calculated here are compared with values published by the Thermodynamics Research Center (Texas A and M University) on June 30, 1981. (Copyright (c) 1990 by the U.S. Secretary of Commerce.)

\section{0,259}

\section{PB90-244625}

Massachusetts Inst. of Tech., Cambridge. Dept. of Chemistry.

Standard Chemical Thermodynamic Properties of Polycyclic Aromatic Hydrocarbons and Their Isomer Groups. III. Naphthocoronene Serles, Ovalene Series, and First Members of Some Higher Serles.

Quarterly rept.

R. A. Alberty, M. B. Chung, and A. K. Reif. c1990,

$22 p$

Sponsored by National Inst. of Standards and Technology, Gaithersburg, MD.

ncluded in Jnl. of Physical and Chemical Reference Data, v19 n2 p 349-370 1990. Available from American Chemical Society, 1155 16th St., NW, Washington, DC 20036 .

Keywords: *Thermodynamic properties, *Aromatic polycyclic hydrocarbons, Standards, Numerical analysis, Molecular structure, Gibbs free energy, Tables(Data), Enthalpy, Specific heat, Chemical properties, Naphthalene compounds, Entropy, Physical chemistry, "Isomers, "Reference materials, Benson method, Ovalenes.

The tables in the first two papers on polycyclic aromatic hydrocarbons (J. Phys. Chem. Ref. Data 17,241 (1988) and J. Phys. Chem. Ref. Data (18, 77 (1989)), have been extended by calculating thermodynamic properties for the first two isomer groups in the naphthocoronene series, the first two isomer groups in the ovalene series, and first members of some higher series. Successive isomer groups in each series differ by $\mathrm{C} 4 \mathrm{H} 2$. The properties of individual species have been estimated using Benson group values of Stein and Fahr for temperatures from 298.15 to $3000 \mathrm{~K}$. Values of $\mathrm{C}(\operatorname{sub} p$ ), $S$, delta(sub $f$ ) $H$, and delta(sub $f$ ) $G$ for a standard state pressure of 1 bar are given for isomer groups and for individual species. The isomer group values provide a basis for extrapolating to higher carbon numbers where it is not feasible to consider individual molecular species. (Copyright (c) 1990 by the U.S. Secretary of Commerce.)

\section{0,260}

PB90-244633

National Inst. of Standards an burg, MD.

Dielectric Constant of Water and Debye-Huieckel Limiting Law Slopes.

Quarterly rept.

D. G. Archer, and P. Wang. c1990, 41p

Included in Jnl. of Physical and Chemical Reference Data, v19 n2 p371-412 1990. Available from American Chemical Society, 1155 16th St., NW, Washington, DC 20036

Keywords: "Dielectric properties, "Water, Physical properties, $\quad \begin{aligned} & \text { Thermodynamic } \\ & \text { Graphs(Charts). Numerical analysis, Tables(Data), }\end{aligned}$ Graphs(Charts), Numerical analysis, Tables(Data), Experimental design, Specific heat, Enthalpy, Osmosis, Volume, "Reference materials, "Debye-Huckel theory.

Experimental values of the dielectric constant of water suggest that, for temperatures greater than $400 \mathrm{~K}$, the integral in the Kirkwood dielectric-constant equation, which involves the intermolecular potential function, is a simpler function of temperature and pressure than of temperature and density. An equation has been fitted to values of this integral calculated from experimental values of the dielectric constant for temperatures from $238.15 \mathrm{~K}$ to $823.15 \mathrm{~K}$ and to pressures of approximately $500 \mathrm{MPa}$ for temperatures greater than $273 \mathrm{~K}$. The equation for epsilon thus has explicit variables $\mathrm{T}$, rho, $\mathrm{p}$ and gives a good representation of the available experimental results. The quality of representation of the experimental results has been compared to that of previous correlations of the dielectric constant. The new equation is applicable through wider regions of independent variables than the previous equations and is capable of sufficient accuracy to provide values of Debye-Huckel limiting law slopes which are as accurate as the experimental results allow. Values of Debye-Huckel limiting-law slopes are tabulated. (Copyright (c) 1990 by the U.S. Secretary of Commerce.)

\section{0,261}

PB90-244641

(Order as PB90-244609)

otre Dame Univ, IN Radiation Lab.

Rate Constants for Reactions of Peroxyl Radicals in Fluld Solutions.

Quarterly rept.

P. Neta, R. E. Hue, and A. B. Ross. c1990, 161p

Sponsored by National Inst. of Standards and Technology (NML), Gaithersburg, MD. Chemical Kinetics

Included in Jnl. of Physical and Chemical Reference Data, v19 n2 p413-515 1990. Available from American Chemical Society, 1155 16th St., NW, Washington, DC 20036

Keywords: "Reaction kinetics, Physical chemistry, Chemical reactions, Alkanes, Photolysis, Radiolysis, Chemical properties, Biochemistry, Photochemical reactions, Atmospheric chemistry, Tables(Data), Solvents, "Peroxyl radicals, "Aqueous solutions.

Absolute rate constants for reactions of alkylperoxyl and substituted alkylperoxyl radicals with inorganic and organic compounds in aqueous and non-aqueous fluid solutions have been compiled. The radicals have been generated by radiolysis or photolysis and their rate constants were determined generally by kinetic spectrophotometry or esr. Rate constants are included also for formation of peroxyl radicals by reaction of alkyl radicals with oxygen and for decay of peroxyl radicals by radical-radical reactions. (Copyright (c) 1990 by the U.S. Secretary of Commerce.

\section{0,262}

PB90-244658

Not available NTIS

American Chemical Society, Washington, DC.

Journal of Physical and Chemical Reference Data, Volume 19, Number 3, 1990.

Quarterly rept

D. R. Lide. c1990, 294p

See also PB90-244666 through PB90-244724 and B90-244609. Prepared in cooperation with American Inst. of Physics, New York. Sponsored by National nst. of Standards and Technology, Gaithersburg, MD. Available from American Chemical Society, 1155 16th St., NW, Washington, DC 20036

Keywords: "Research, Collision cross sections, Atomic energy levels, Water vapor, Steam, Specific heat, Alcohol, Carbon dioxide, Photon-atom collisions, Electron-atom collisions, Electron-molecule collisions, Electron-ion collisions, Hydrogen ions, Oxygen atoms, Copper ions, Atom-molecule collisions, Molecule-molecule collisions, Ion-molecule collisions, Refractive index, Alkyl alcohols.

Contents: Energy Levels of Copper, $\mathrm{Cu} I$ through $\mathrm{Cu}$ XXIX; Cross Sections and Related Data for Electron Collisions with Hydrogen Molecules and Molecular Ions; Cross Sections for Collisions of Electrons and Photons with Atomic Oxygen; Cross Sections and Swarm Coefficients for $\mathrm{H}(1+), \mathrm{H}_{2}(+), \mathrm{H}_{3}(+), \mathrm{H}_{1} \mathrm{H}_{2}$ and $\mathrm{H}(1-)$ in $\mathrm{H} 2$ for Energies from $0.1 \mathrm{eV}$ to $10 \mathrm{keV}$. Refractive Index of Water and Steam as Function of Wavelength, Temperature and Density; Heat Capacities of Organic Compounds in the Liquid State I. C1 to 18 1-Alkanols; The Transport Properties of Carbon Dioxide.

\section{0,263}

\section{PB90-244674} Cross Sections and Related Data for Electron Colisions with Hydrogen Molecules and Molecular lons.

H. Tawara, $Y$. Itikawa, $H$. Nishimura, and $M$. Yoshino. c1990, 20p

Prepared in cooperation with Institute of Space and Astronautical Science, Sagamihara (Japan), Niigata Univ. (Japan). Dept. of Physics, and Shibaura Inst. of Tech., Tokyo (Japan).
Included in Jnl of Physical and Chemical Reference Gata, v19 n3 p617-635 1990. Available from American Chemical Society, 1155 16th St., NW, Washington, DC 20036

Keywords: "Collision cross sections, Excitation, Dissociation, Ionization, "Electron-molecule collisions, *Electron-ion collisions, *Hydrogen ions, Molecular ions, Electron impact, Attachment, Rotational states, Vibrational states, Recombination.

Data are compiled and evaluated for collision processes of excitation, dissociation, ionization, attachment, and recombination of hydrogen molecules and molecular ions $(\mathrm{H} 2(1+), \mathrm{H} 3(1+))$ by electron impact as well as for properties of their collision products. (Copyright (c) 1990 by the U.S. Secretary of Commerce.)

100,264

PB90-244682

(Order as PB90-244658)

Institute of Space and Astronautical Science, Sagamihara (Japan).

Cross Sections for Collisions of Electrons and Photons with Atomic Oxygen.

Quarterly rept.

Y. Itikawa, and A. Ichimura. c1990, 13p

Included in Jnl. of Physical and Chemical Reference Data, v19 n3 p637-650 1990. Available from American Chemical Society, 1155 16th St., NW, Washington, DC 20036

Keywords: "Collision cross sections, Atomic energy levels, Scattering cross sections, Excitation, "Electron-atom collisions, *Photon-atom collisions, "Oxygen atoms, Photoionization, Oscillator strengths.

Data have been compiled on the cross sections for the collisions of electrons and photons with atomic oxygen (O). The processes considered are total scattering, elastic scattering, momentum transfer, excitations of electronic states (including fine structure levels of the ground state), ionization, and electron attachment. The cross-section data are presented graphically. Energy levels, transition probabilities and some other properties of atomic oxygen are summarized to aid understanding of the collision processes. The literature was surveyed through December 1988 , but more recent data, if they are available to the authors, are included also. (Copyright (c) 1990 by the U.S. Secretary of Commerce.)

100,265

PB90-244716

Order as PB90-244658) (Czechoslovakia)

Heat Capacities of Organic Compounds in the Liquid State I. C1 to C18 1-Alkanols.

Quarterly rept.

M. Zabransky, V. Ruzicka, and V. Majer. c1990, 44p Grant NBS-60NANB9DO912

Prepared in cooperation with Delaware Univ., Newark. Dept. of Chemistry. Sponsored by National Inst. of Standards and Technology, Gaithersburg, MD.

Included in Jnl. of Physical and Chemical Reference Data, v19 n3 p719-760 1990. Available from American Chemical Society, 1155 16th St., NW, Washington, DC 20036

Keywords: "Specific heat, "Alcohols, Liquids, Polynomials, Tables(Data), Spline functions, Calorimetry Temperature dependence, Methanol, Ethanol, Alkyl alcohols, Propanols, Butanols.

Heat capacities of liquid $\mathrm{C} 1$ to $\mathrm{C} 18$ 1-alkanols measured by calorimetric methods were compiled and evaluated. The selected experimental data were fitted as a unction of temperature with cubic splines using weighted least squares minimization. The parameters of the cubic spline polynomials and the recommended values for heat capacities are presented. A new quasipolynomial equation which permits extrapolation of heat capacities outside the temperature range of experimental values was derived and its parameters for C1 to C10 1-alkanols are presented. (Copyright (c) 1990 by the U.S. Secretary of Commerce.)

100,266

PB90-244724 (England). Dept. of Chemical Engineering and Chemical Technology. 


\section{Transport Properties of Carbon Dioxide.}

Quarterly rept.

V. Vesovic, W. A. Wakeham, G. A. Olchowy, J. V.

Sengers, J. T. R. Watson, and J. Millat. c1990, 46p

Prepared in cooperation with Maryland Univ., College Park. Inst. for Physical Science and Technology, National Engineering Lab., East Kilbride (Scotland), and Rostock Univ. (German D.R.).

Included in Jnl. of Physical and Chemical Reference Data, v19 n3 p763-808 1990. Available from American Chemical Society, 115516 th St., NW, Washington, DC 20036.

Keywords: "Carbon dioxide, "Thermal conductivity, "Viscosity, Thermodynamic properties, Transport properties.

The paper contains new, representative equations for the viscosity and thermal conductivity of carbon dioxide. The equations are based in part upon a body of experimental data that have been critically assessed for internal consistency and for agreement with theory whenever possible. In the case of the low-density thermal conductivity at high temperatures, all available data are shown to be inconsistent with theoretical expectation and have therefore been abandoned in favor of a theoretical prediction. Similarly, the liquid-phase thermal conductivity has been predicted owing to the small extent and poor quality of the experimental information. The complete correlations cover the temperature range $200 \mathrm{~K}=$ or $<T<1500 \mathrm{~K}$ for viscosity and $200 \mathrm{~K}=$ or $<\mathrm{T}=$ or $<1000 \mathrm{~K}$ for thermal conductivity, and pressures up to $100 \mathrm{MPa}$. Tables of the viscosity and thermal conductivity generated by the representative equations are provided to assist with the confirmation of computer implementations of the calculation procedure.

\section{0,267}

PB91-134494 Not available NTIS National Inst. of Standards and Technology (NML) Boulder, CO. Thermophysics Div.

Critical Locus, (Vapor + Liquid) Equilibria, and Coexisting Densities of (Carbon Dioxide + Propane) existing Densities of (Carbon Dioxide
at Temperatures from $311 \mathrm{~K}$ to $361 \mathrm{~K}$.

Final rept.

V. G. Niesen, and J. C. Rainwater. 1990, 19p

Sponsored by Department of Energy, Washington, DC. Office of Basic Energy Sciences.

Pub. in Jnl. of Chemical Thermodynamics 22, p7777951990.

Kaywords: "Thermodynamic equilibrium, "Carbon dioxide, "Propane, Measurement, Vapors, Liquids, Density(Mass/volume), Critical point, Critical pressure, Correlation, Comparison, Mathematical models, Dew point, Wet bulb temperature, Reprints, Temperature dependence.

Isothermal (vapor + liquid) equilibria and coexisting densities for (carbon dioxide + propane) have been measured at $(311.05,327.75,344.43$, and 361.15$) \mathrm{K}$ from the vapor pressure of propane to the critical pressure of the mixture, and the critical locus has thus been determined. Away from the critical locus, the measurements agree within combined experimental uncertainties with those of Reamer, Sage, and Lacey. The measurements confirm the observation of Roo and Baron that the critical pressures reported by Poettmann and Katz and by Reamer et al. are substantially in error. The critical pressures from the study are lower than those of Reamer et al. by as much as $0.785 \mathrm{MPa}$ and of Roof and Baron by as much as $0.034 \mathrm{MPa}$. The dew- and bubble-points are correlated by three methods, the Soave-Redlich-Kwong (SRK) equation, the DDMIX program of Ely, and the Leung Griffiths model as modified by Moldover and Rainwater. Except for poor predictions of liquid densities by the SRK equation, all three models provide good correlations of the measurements, but the modified Leung-Griffiths model is superior very near the critical locus.

\section{0,268}

\section{PB91-134676}

Not available NTIS

National Inst. of Standards and Technology (NML)

Boulder, CO. Thermophysics Div.

Neutron Diffraction from Sheared D-Glycerol: Preliminary Studies.

Final rept.

G. C. Straty, and H. J. M. Hanley. 1988, 8p

Sponsored by Department of Energy, Washington, DC Office of Basic Energy Sciences.

Pub. in Proceedings of Symposium on Energy Engineering Sciences (6th), May 4-6, 1988, p136-143.

Keywords: Neutron diffraction, Supercooling, Shear properties, Reprints, "Glycerols, Structure factors.
The paper reports on the experimental segment of a wide range investigation of the nature of complexity in liquids, as manifested by their behavior under mechanical stress. An apparatus constructed for a feasibility study of such behavior is described. Preliminary neutron diffraction measurements of the static structure factor of supercooled liquid glycerol under shear are reported. Data were taken on the SANS facility at NBS in the range $0.02=$ or $\angle Q=$ or $\angle 0.39 / \mathrm{A}, \mathrm{Q}$ equal to $0.8,1.35$, and $3.5 / \mathrm{A}$. Comparisons between the sheared data and data for the system at equilibrium are given. The tentative conclusion is that the influence of shear on the structure factor may be strong, but the data are too qualitative for a significant interpretation.

100,269

PB91-134957

Not available NTIS

National Inst. of Standards and Technology (NML), Boulder, CO. Thermophysics Div.

Structure of a Stage-3 Cs - Graphite Intercalate. Final rept.

P. A. Wielopolski, J. W. White, and H. J. M. Hanley.

1990, $11 p$

Pub. in Molecular Physics 69, n6 p947-957 1990.

Keywords: "Graphite, "Cesium, Computerized simulation, Structural analysis, Reprints, "Intercalation, Motion, Structural analysis, Reprints, "Intercalation, Mo-
lecular dynamics, Temperature dependence, Multilayers.

The influence of interlayer coupling on the in-plane structural properties of the stage-3 cesium-graphite system is investigated using a multilayer molecular-dynamics simulation. It is shown that the in-plane structure, represented by the circularly averaged pair distribution function, is insensitive to the interlayer coupling over a wide range of temperatures. The structure of the layer is investigated as a function of the param eters of the Cs-Cs potential and of the barrier height for free diffusion created by the graphite periodic potential

\section{0,270}

PB91-146985

Not available NTIS

National Bureau of Standards (NML), Gaithersburg, MD. Surface Science Div.

Adsorption of Water and Oxygen on $\mathrm{Ag}(110)$ : $\mathrm{A}$ Study of the Interactions among Water Molecules, Hydroxyl Groups, and Oxygen Atoms.

Final rept.

K. Bange, T. E. Madey, J. K. Sass, and E. M. Stuve. $1987,29 p$

Pub. in Surface Science 183, n3 p334-362 1987

Keywords: "Water, "Oxygen, "Chemisorption, "Surface chemistry, Silver, Electron beams, Desorption, Chemical bonds, Hydroxyl radicals.

The adsorption of water on clean and oxygen covered $\mathrm{Ag}(110)$ in ultra-high vacuum was studied with thermal desorption spectroscopy (TDS), electron stimulated desorption ion angular distribution (ESDIAD), low energy electron diffraction (LEED), work function measurements, and Auger electron spectroscopy. Water adsorbs on the clean surface at $80 \mathrm{~K}$ with no long-range order and only weak short-range order as determined by LEED and ESDIAD, respectively. Electron bombardment of water adlayers on clean Ag(110) results in dissociation to form $\mathrm{OH}$ groups at $80 \mathrm{~K}$. Water also reacts with preadsorbed oxygen, either in atomic or molecular form, to form $\mathrm{OH}$ groups at $80 \mathrm{~K}$. The $\mathrm{O}-\mathrm{H}$ bond axis of the $\mathrm{OH}$ groups is tilted with respect to the surface normal and its thermodynamically stable, azimuthal orientation is along the (001) direc. tion. In the absence of excess, molecular $\mathrm{H} 2 \mathrm{O}$, the $\mathrm{OH}$ groups exhibit no hydrogen bonding. The $\mathrm{OH}$ groups form $(\mathrm{lxm})(m=2,3)$ LEED compression patterns upon annealing to $200 \mathrm{~K}$. These LEED patterns require the OH groups to diffuse across the ridges of the surface. The gamma adsorption state, which evolves water in a desorption peak at $225 \mathrm{~K}$, was carefully studied. This state consists of coadsorbed $\mathrm{H} 2 \mathrm{O}$ and $\mathrm{OH}$ in a $1: 2$ ratio. The results of the work are combined with earlier TDS and vibrational measurements for this system to propose an adsorption model for the gamma state, in which the $\mathrm{H} 2 \mathrm{O}$ molecules are hydrogen bonded to the $\mathrm{OH}$ groups and the $\mathrm{OH}$ groups occupy a binding site different from the case where no excess water exists.

100,271

PB91-147009

Not available NTIS

National Inst. of Standards and Technology (NML) Gaithersburg, MD. Thermophysics Div.
Critical Exponent for Viscosity.

Final rept.

R. F. Berg, and M. R. Moldover. 1990, 4p Sponsored by National Aeronautics and Space Administration, Washington, DC.

Pub. in Physical Review A 42, n12 p7183-7186, 15 Dec 90.

Keywords: "Viscosity, "Carbon dioxide, "Xenon, Liquefied gases, Critical point, Reduced gravity, Exponents, Reprints.

The authors have measured the critical exponent $y$ characterizing the divergence of the viscosity eta varies as absolute value of (T-T(c))(sup(-y)) for carbon dioxide and xenon. The values of $y$ for both fluids fall within the range $y=0.041+$ or -0.001 and are consistent with the range $y=0.042+$ or -0.002 spanned by the earlier data for four binary liquid mixtures. The agreement is the strongest evidence that pure fluids and binary liquids are in the same dynamic universality class; however, the results for $y$ are inconsistent with the recent theoretical value of 0.032 .

100,272

PB91-147041

Not available NTIS National Inst. of Standards and Technology (NML) Boulder, CO. Chemical Engineering Science Div.

Heat Capacity and Thermodynamic Properties of Deuterated Ammonium Hexafluorophosphate ND4PF6 from $5.8 \mathrm{~K}$ to $347 \mathrm{~K}$

Final rept.

J. E. Callanan, R. D. Weir, and E. F. Westrum. 1990 , $12 p$

Sponsored by Department of National Defence, Ottawa (Ontario)

Pub. in Jnl. of Chemical Thermodynamics 22, p9799901990.

Keywords: "Specific heat, "Calorimetry, "Ammonium compounds, "Inorganic phosphates, Isotopic labeling, Temperature dependence, Thermodynamic properties, Deuteration, Tables(Data), Order disorder transformations, Reprints.

The heat capacity of deuterated ammonium hexafluorophosphate ND4PF6 was measured from $5.8 \mathrm{~K}$ to 347 $\mathrm{K}$ by adiabatic calorimetry. Two lambda-shaped anomalies were found in the curve for heat capacity against temperature. The upper anomaly reached a peak heat capacity (at constant pressure) of approx 600R at $(194.4+$ or -0.05$) \mathrm{K}$ with Delta(sub trs)S(sup 0) $(1.013+$ or -0.016$) R(R=8.3145 \mathrm{~J} / \mathrm{K} / \mathrm{mol})$. The lower anomaly is also lambda-shaped but is gradual, continuous, and reaches its maximum heat capacity = $19.2 \mathrm{R}$ at $(137.5+$ or -1.0$) \mathrm{K}$ with Delta(sub trs)S(sup $0)=(0.562+$ or -0.011$)$ R. Both anomalies are char acteristic of order-disorder transitions. Smoothed values of the standard thermodynamic quantities are tabulated up to $350 \mathrm{~K}$

\section{0,273}

PB91-147058

Not available NTIS National Inst. of Standards and Technology (NML) Boulder, CO. Chemical Engineering Science Div. Heat Capacity of Deuterated Ammonium Tetrafluoroborate ND4BF 4 from 7 to $348 \mathrm{~K}$.

Final rept.

J. E. Callanan, R. D. Weir, and E. F. Westrum. 1990 $12 p$

Sponsored by Department of National Defence. Ottawa (Ontario)

Pub. in Jnl. of Chemical Thermodynamics 22, p9579681990.

Keywords: "Calorimetry, "Ammonium compounds, "Fluoborates, "Specific heat, Isotopic labeling, Deuteration. Temperature dependence, Tables(Data), Thermodynamic properties, Order disorder transformations, Reprints, "Foreign technology.

The heat capacity of deuterated ammonium tetrafluoroborate ND4BF 4 was measured at constant pressure from $7 \mathrm{~K}$ to $348 \mathrm{~K}$ by adiabatic calorimetry. The curve for heat capacity against temperature is smooth with broad maximum between $120 \mathrm{~K}$ and $165 \mathrm{~K}$, and the peak value of approximately $14.9 \mathrm{R}$ occurs between $145 \mathrm{~K}$ to $155 \mathrm{~K}$. The shape of the broad maximum is similar to that found in other anisotropic salts and is believed to be a result of contributions both from the rotation of the ND4(sup +) ion and from a larger than usual dilation of the unit cell. Values of the standard thermodynamic quantities are tabulated up to $350 \mathrm{~K}$. 
100,274

PB91-147157

Not available NTIS

National Bureau of Standards (NML), Gaithersburg,

MD. Organic Analytical Research Div.

Cryogenic-Temperature Fluorescence Spectra of Polynuclear Aromatic Hydrocarbons of Molecular Weight 302

Final rept.

A. L. Colmsjo, and S. A. Wise. 1986, 9p

Pub. in Analytica Chimica Acta 187, p129-137 1986.

Keywords: "Aromatic polycyclic hydrocarbons, "Fluorescence spectroscopy, "Pyrenes, Cryogenics, Sensitivity, Selectivity, Molecular weight, Reprints, "Fluoranthenes, "Perylenes, *Shpol'skii effect.

Shpol'skii cryogenic temperature fluorescence spectra of 19 of the possible 33 polynuclear aromatic hydrocarbons with a molecular weight of 302 are reported. The group consists of the benzo- and naphtho-pyrenes and fluoranthenes and also the benzoperylenes. There is a large variation in the degree of selectivity and sensitivity achieved by the Shpol'skii technique within this group of compounds.

100,275

PB91-147199

Not available NTIS

National Inst. of Standards and Technology (NML),

Boulder, CO. Chemical Engineering Science Div.

Hydrogen Slush Production with a Large Auger.

Final rept.

D. E. Daney, V. D. Arp, and R. O. Voth. 1990, 10p

Sponsored by National Aeronautics and Space Admin-

stration, Cleveland, $\mathrm{OH}$. Lewis Research Center.

Pub. in Advances in Cryogenic Engineering 35, p1767-

17761990

Keywords: "Cryogenics, "Solid cryogens, "Solidified gases, *Augers, Refrigerants, Mathematical models, Generators, Slush, Reprints, "Solid hydrogen.

The design and construction of a $178 \mathrm{~mm}$ (7 in) diameer auger type hydrogen slush generator is described. A supercritical helium flow loop, which simulates the performance of a helium refrigerator, cools the generaor. The coolant temperature varies down to $5 \mathrm{~K}$ and the flow varies about the $1.4 \mathrm{~L} / \mathrm{s}(3 \mathrm{cfm})$ design point. The computer model of the auger type generator shows that coolant temperature and auger speed have the greatest influence on slush production rate, although coolant flow rate and auger radial clearance are also important. All these effects will be evaluated in the tests of the system.

100,276

B 91-147298

Not available NTIS

National Bureau of Standards (NML), Gaithersburg,

MD. Surface Science Div.

Soluble Models in Surface Collision Dynamics.

Final rept.

J. W. Gadzuk. 1988, $7 p$

Pub. in Solvay Conference on Surface Science, v14 p310-316 1988.

Keywords: "Molecular collisions, "Surface reactions, Molecular excitation, Dynamics, Phonons, Reprints.

Aspects of electron-hole pair, phonon, and intra-moecular vibrational excitation, as illustrated in some soluble models in surface collision dynamics, are considered. Emphasis is placed on energy redistribution and sticking out metal surfaces.

100,277

PB91-147363 Not available NTIS National Inst. of Standards and Technology (NML),

Gaithersburg, MD. Thermophysics Div.

Limiting versus Apparant Critical Behavior of Henry's Constants and K Factors.

Final rept.

A. H. Harvey, R. Crovetto, and J. M. H. Levelt

Sengers. $1990,4 p$

Pub. in AlChE Jnl. 36, n12 p1901-1904 Dec 90.

Keywords: Asymptotic properties, Critical point, Thermodynamics, Solubility, Mixtures, Reprints, *Henry constant.

In previous work, an asymptotic relationship, linear with the appropriate choice of variables, was derived for the variation of Henry's constant for a solute in the neighborhood of the solvent's critical point. When experimental Henry's-constant data in several solvents were plotted in a manner suggested by the asymptotic relationship, linearity was observed over a wide range of conditions. Since these lines remained straight at the highest (closet-to-critical) temperatures at which data existed, it was assumed that they represented the asymptotic behavior. Further study, however, has shown that this assumption is mistaken and that the Henry's-constant plot attains its asymptotic behavior only in a small region closer to the critical point than the extent of the data. A related asymptotic linear relationship involving the vapor-liquid partition coefficient K-factor) appears to be a more reliable indicator of the true asymptotic behavior, in part because the limiting value at the critical point is known a priori. The work explains the discrepancy noted in previous work between slopes of Henry's-constant plots and the initial slopes of mixture critical lines.

100,278

PB91-147405

Not available NTIS

National Inst. of Standards and Technology (NML), Boulder, CO. Thermophysics Div.

Extension of an Improved One-Fluid Conformal Solution Theory to Real Fluid Mixtures with Large Size Differences.

Final rept.

M. L. Huber, and J. F. Ely. 1990, 10p

Sponsored by Department of Energy, Washington, DC.

Pub. in International Jnl. of Thermophysics $11, \mathrm{n} 1 \mathrm{p} 87$ 96 Jan 90.

Keywords: "Fluids, Lennard-Jones potential, Perturbation theory, Statistical mechanics, Helmholtz free energy, Hydrocarbons, Mixtures, Reprints.

Conformal solution theories have been shown to be inadequate as the size ratio of the molecules in a mixture increases. The authors present an improved van der Waals-1 fluid conformal solution theory which incorporates a correction term developed using statistical mechanical perturbation theory. The correction addresses the effect of different size molecules on the Helmholtz free energy of the mixture. Results of the new model are compared with other conformal solution theories for model Lennard-Jones systems. They lso show how to extend the model to perform computations on real fluid mixtures. Results for selected hydrocarbon mixtures are given

100,279

PB91-147454

Not available NTIS

National Bureau of Standards (NML), Gaithersburg,

MD. Molecular Spectroscopy Div.

Structure of Carbon Dioxide Dimer from Near Infrared Spectroscopy.

Final rept.

K. W. Jucks, Z. S. Huang, D. Dayton, R. E. Miller,

and W. J. Lafferty. 1987,6p

Pub. in Jnl. Chemical Physics 86, n8 p4341-4346 1987.

Keywords: "Molecular structure, Near infrared radiation, Infrared spectra, Dimers, Reprints, "Carbon dioxide dimers.

An F-center laser-molecular beam spectrometer has been used to obtain a sub-Doppler resolution spectrum of the CO2 dimer. The vibrational mode investigated in corresponds to the nu(1) + nu(3) combination band of the monomer located at $3716 / \mathrm{cm}$. A qualitative assignment of the spectrum shows that the equilibrium structure of the molecule has $\mathrm{C}(2 \mathrm{~h})$ symmetry, as, due to zero spin nuclei, half of the expected transitions are missing in the observed spectrum. This implies that the molecule has a slipped parallel structure rather than a T-shaped geometry.

100,280

PB91-147470

Not available NTIS

National Inst. of Standards and Technology (NML), Gaithersburg, MD. Chemical Kinetics Div.

Alkyl Transfer Reactions between Protonated Alcohols and Ethers. The Gas-Phase Alkylation of Formaldehyde.

Final rept.

Z. Karpas, and M. Mautner, $1989,5 p$

Pub. in Jnl. of Physical Chemistry 93, n5 p1859-1863 1989.

Keywords: "Organic ions, "Alcohols, "Ethers, "Reaction kinetics, Stability, Chemical reactivity, Activation energy, Molecular structure, Reprints, “Alkyl transfer reactions.

Alkyl transfer reactions between protonated ethers and alcohols are of the general type R2OR(prime) $(+)$ $+\quad \mathrm{R}$ (double prime)2O 20 (double alkyl radical. The reactions are classified according to the degree of alkylation, with up to $n=5$ alkyl groups in the system. For $n=2$ the reaction is alkyl transfer between alcohols $\mathrm{ROH} 2(+)$ and $\mathrm{ROH}$ to yield protonated ethers $\mathrm{R} 2 \mathrm{OH}(+)$. The rate constants for these reactions increase with increasing stability of the transferred $R(+)$ group, possibly due to lowered activation barrier of the initial partial $\mathrm{R}(+)-\mathrm{OH}_{2}$ dissociation. However, the reaction with $\mathrm{R}(+)=\mathrm{CH} 3(+)$ is faster and with $R(+)=t-C 4 H 9(+)$ is slower than expected, possibly due to steric effects. For $n=3$, ie., reactions between the ethers $\mathrm{R} 2 \mathrm{OH}(+)$ and the alcohols $\mathrm{R}$ (prime) $\mathrm{OH}$, both the formation of $\mathrm{R}$ (prime) $\mathrm{ORH}(+)$ and of the tertiary oxonium ions R2OR(prime) $(+)$ are observed. For $n=4$, the reaction between the ethers $\mathrm{R} 2 \mathrm{OH}(+)$ and $\mathrm{R} 2 \mathrm{O}$ to give $\mathrm{R} 2 \mathrm{OR}(+)$ is observed. For $\mathrm{n}=5$, a completely alkylated system, an example is methyl exchange between $\left(\mathrm{CH}_{3}\right) 2 \mathrm{OCD} 3(+)$ and (CH3)2O, which is a very slow thermoneutral reaction with a large activation energy of $13 \mathrm{kcal} / \mathrm{mol}$.

100,281

PB91-147538

Not available NTIS National Bureau of Standards (NEL), Gaithersburg, MD. Chemical Process Metrology Div.

Instability in pH Measurements of Sputtered IrO2 Films.

Final rept.

K. G. Kreider, S. Semancik, and J. E. Erickson. 1987,

Pub. in Proceedings of International Conference on Sensors and Actuators (4th) - Transducers '87, Tokyo, Japan, June 2-5, 1987, p734-737.

Keywords: "Iridium oxides, ${ }^{*} \mathrm{pH}$ meters, $\mathrm{pH}$ value, Instability, Sensors, Thin films, Photoelectron spectroscopy, Crystallinity, Amorphous materials, Reprints.

Sputter deposition of iridium oxide can produce thin films that show significant promise for use as the active coating in $\mathrm{pH}$ sensing devices. Among the desirable characteristics of sputtered iridium oxide films are their superior corrosion resistance, high temperature capabilities, and their ability to be fabricated in forms suitable for miniaturized probes. The results of testing of SIROF $\mathrm{pH}$ sensors produced in the study indicate that both anhydrous, crystalline films and amorphous films yield similar responses of approximately 56-59 $\mathrm{mv} / \mathrm{pH}$. The open circuit potential of these films did vary with various fabrication conditions and environmental exposures. Deliberate attempts to modify the open circuit potential of the SIROFs included air baking at $523 \mathrm{~K}$, which significantly increased the output voltage and autoclave heating at $523 \mathrm{~K}$, which significantly decreased the voltage. XPS measurements on SIROF films showed that iridium is present in $\mathrm{a}+4$ oxidation for a variety of treatments. Oxygen in core level data suggest that certain test treatments considered here may cause surface hydroxylation.

\section{0,282}

PB91-147645

Not available NTIS National Bureau of Standards (NML), Gaithersburg, MD. Chemical Kinetics Div.

Resonance Enhanced Multiphoton Ionization Spectroscopy of $\mathrm{CHCl} 2$ and $\mathrm{CDCl} 2$.

Final rept.

G. R. Long, and J. W. Hudgens. 1987, 3p

Pub. in Jnl. of Physical Chemistry 91, p5870-5872 1987.

Keywords: Deuterium compounds, Rydberg states, Multi-photon processes, Ultraviolet spectra, Vibrational states, Reprints, "Dichloromethane radicals, Multiphoton ionization.

Resonance enhanced multiphoton ionization spectra between 355 and $375 \mathrm{~nm}$ of $\mathrm{CHCl} 2$ and $\mathrm{CDCl} 2$ radicals are reported. The origin of the observed spectra was at $370.1 \mathrm{~nm}$ for $\mathrm{CHCl} 2$ and $370.4 \mathrm{~nm}$ for $\mathrm{CDCl} 2$. The REMPI mechanism is determined to be a $2+1$ photon absorption, with the resonant intermediate state tentatively assigned as a $3 d$ Rydberg state. Vibrational structure originated from activity in the $\mathrm{C}-\mathrm{Cl}$ symmetric stretching and the out-of-plane bending modes. The $\mathrm{C}$ $\mathrm{Cl}$ symmetric stretch frequencies are $845 / \mathrm{cm}$ in $\mathrm{CHCl} 2$ and $814 / \mathrm{cm}$ in $\mathrm{CDCl} 2$.

100,283

PB91-147710

Not available NTIS

National Bureau of Standards (NML), Gaithersburg. MD. Chemical Kinetics Div. 


\section{Physical \& Theoretical Chemistry}

Multicomponent Cluster Ions. 2. Comparative Stabilities of Cationic and Anionic Hydrogen Bonded Networks: Mixed Clusters of Water and Hydrogen Cyanide.

Final rept.

M. Mautner, and C. V. Speller. 1989, 4p

See also AD-A167 880.

Pub. in Jnl. of Physical Chemistry 93, n9 p3663-3666 1989.

Keywords: "Protons, "Hydroxyl radicals, Hydration, Enthalpy, Acidity, Alkalinity, Stability, Hydrogen bonds, Reprints, "Cyanide ions, "Cluster ions.

The thermochemistry of cluster ions containing $\mathrm{H} 2 \mathrm{O}$ and $\mathrm{HCN}$ was obtained from clustering equilibria. The clustering of $\mathrm{H} 2 \mathrm{O}$ about $\mathrm{H} 3 \mathrm{O}(+)$ and $\mathrm{OH}(-)$, and the clustering of $\mathrm{HCN}$ about $\mathrm{HCNH}(+)$ show distinct shell filling effects, but the clustering of $\mathrm{H} 2 \mathrm{O}$ and $\mathrm{HCN}$ about $\mathrm{CN}(-)$ does not. Hydration by one water molecule eliminates the difference of $5 \mathrm{kcal} / \mathrm{mol}$ between the proton affinities of $\mathrm{HCN}$ and $\mathrm{H} 2 \mathrm{O}$. Hydration also decreases the difference between the intrinsic deprotonation energies, ie., delta $\mathrm{H}(0)$ acid' of $\mathrm{H} 2 \mathrm{O}$ and $\mathrm{HCN}$ from 37.7 $\mathrm{kcal} / \mathrm{mol}$, to $19.8 \mathrm{kcal} / \mathrm{mol}$ in the 4 -fold hydrated species. In the protonated clusters, exchange of $\mathrm{H} 2 \mathrm{O}$ by $\mathrm{HCN}$ does not significantly affect the total stability of the cluster.

\section{0,284}

PB91-147785 Not available NTIS National Inst. of Standards and Technology (NML), Gaithersburg, MD. Chemical Kinetics Div.

Slow Electron Transfer Reactions between Alkylhydrazines.

Final rept.

S. F. Nelsen, D. T. Rumack, and M. Mautner. 1987,

$7 p$

Pub. in Jnl. of American Chemical Society 109, n5 p1373-1379 1987

Keywords: "Hydrazines, "Reaction kinetics, "Electron transfer, Organic ions, Gibbs free energy, Reprints, Energy barriers.

Unlike other gas-phase exoergic electron transfer reactions, reactions between alkylhydrazines, which in volve large geometry changes, proceed with unusually low efficiencies, i.e., $\mathrm{k} / \mathrm{k}$ (collision) $=0.01-0.0001$. The temperature coefficient of Me2NNMe2 + EtMeNNMeEt --. EtMeNNMeEt $(+)+$ Me2NNMe2 $\mathrm{E}(\mathrm{sub} \mathrm{a})=+2.7 \mathrm{kcal} / \mathrm{mol}$ indicates an energy barrier of $16 \mathrm{kcal} / \mathrm{mol}$ for electron transfer within the complex. The energy barrier results from the large geometry change required for relaxation of the product R2NNR2 $(+)$ ions from a perpendicular to a planar geometry. Nevertheless, the energy barrier is substantially smaller than would result from vertical electron transfer in the complex $(44 \mathrm{kcal} / \mathrm{mol})$, indicating geometry relaxation in the transition state. The major trends observed include: slowing of reactions of acyclic vs. cyclic reactants; and small positive or negative temperature coefficients. In these trends, electron transfer is similar to heavy particle transfer.

\section{0,285}

PB91-147801

Not available NTIS

National Bureau of Standards (NML), Gaithersburg,

MD. Chemical Kinetics Div.

Pulse Radiolysis Studies of Organic Electron Transfer Reactions.

Final rept.

P. Neta, and A. Harriman. 1988, 53p

Pub. in Photoinduced Electron Transfer, Chapter 2.3, p110-162 1988.

Keywords: "Porphyrins, "Electron transfer, Oxidation reduction reactions, Organic ions, Radiolysis, Catalysts, Reprints

Pulse radiolysis studies on organic electron transfer reactions are reviewed, with special emphasis on porphyrins and redox catalysts.

\section{0,286}

PB9 1.147884

Not available NTIS

National Bureau of Standards (IMSE), Gaithersburg MD. Ceramics Div.

Effects of Pressure on the Thermal Decomposition Kinetics and Chemical Reactivity of Nitromethane. Final rept.

G. J. Piermarini, S. Block, and P. J. Miller. 1989,6p Pub. in Jnl. of Physical Chemistry 93, n1 p457-462 1989.

Keywords: "Nitromethane, "High pressure, "Thermal degradation, "Reaction kinetics, Chemical reactivity,
Isotope effect, Melting points, Ammonium formate, Water, Reprints.

The effects of pressure and temperature on the melt ing point, thermal decomposition rate, and chemical reactivity of nitromethane have been studied. A diamond anvil high pressure cell in conunction with (1) an optical polarizing microscope for melting point and chemical reactivity observations, and (2) an automated Fourier transform infrared instrument for thermal decomposition kinetic measurements, were used. Both pressure and temperature were found to increase the rate of thermal decomposition indicating an overall bimolecular reaction mechanism. However, the mechanism is complex and appears to vary over large changes in pressure. Nevertheless, a chemical mech anism is proposed which explains the bimolecularity and also accounts for the observed decomposition products, ammonium formate and water. A dynamic stress-induced catastrophic reaction in nitromethane, which appears to be crystal orientation dependent with respect to the applied Stress, was found. Under the same conditions, deuterated nitromethane did not exhibit this catastrophic reaction.

\section{0,287}

\section{PB91-148015}

National Inst. of Standards and Technology (NML) Gaithersburg, MD. Surface Science Div.

Resonant Photoemission and PSD of Rare Earth Oxides.

Final rept.

J. Schmidt-May, F. Senf, J. Voss, C. Kunz, and A

Flodstrom. 1985, 4p

Pub. in Desorption Induced by Electronic Transitions DIET II, Springer Series in Surface Sciences, v4 p94 971985.

Keywords: "Europium oxides, "Samarium oxides, "Ytterbium oxides, "Photoemission, Photoelectron spectroscopy, Reprints, Photon stimulated desorption.

The resonant enhancement in photoelectron spectra at the $4 \mathrm{~d}$ edges of rare earth atoms and metals is also found in photoyield spectra of desorbed ions from the surfaces of the oxides of $\mathrm{Sm}, \mathrm{Eu}$ and $\mathrm{Yb}$ following the photon excitation. The analysis of the $4 \mathrm{~d} 4 \mathrm{f}$ resonance leads to a picture of an indirect mechanism of ion desorption which is caused by the flux of energetic 4 bulk photoelectrons. Photoemission and ion desorption measurements of $\mathrm{Sm}$ metal with oxidized $\mathrm{Yb}$ overlayer strongly support this idea. The dominant desorption through secondary processes limits the use of the photon-stimulated desorption (PSD) in the sense that it is not known to which type of atom the desorbing species was attached.

\section{0,288}

PB91-148080

Not available NTIS

National Bureau of Standards (NML), Gaithersburg, MD. Molecular Spectroscopy Div.

Fluoromethanol: Synthesis, Microwave Spectrum, and Dipole Moment.

Final rept

R. D. Suenram, F. J. Lovas, and H. M. Pickett. 1986, $10 p$

Pub. in Jnl. of Molecular Spectroscopy 119, n2 p4464551986

Keywords: "Carbinols, "Fluorine organic compounds "Microwave spectra, "Dipole moments, Synthesis(Chemistry), Rotational spectra, Electric moments, Conformational changes.

The microwave spectrum of the new molecular species $\mathrm{CH}$ FOH has been observed and analyzed. The molecule exhibits a gauche conformation which is in accord with previous ab initio calculations. This is also the conformation that is predicted based on anomeric effect arguments. The rotational transitions are split by the tunneling of the molecule between equivalent gauche conformations. A simultaneous fit of both tunneling states allows the determination of perturbation free rotational parameters as well as the tunneling frequency and a tunneling distortion parameter. A dipole moment $(\mathrm{dm})$ analysis yields values of all three dipole moment components and the $\mathrm{dm}(\mathrm{b}) \times \mathrm{dm}(\mathrm{c})$ term which indicates that $\mathrm{dm}(\mathrm{b})$ and $\mathrm{dm}(\mathrm{c})$ have opposite signs.

\section{0,289}

PB91-148171

Not available NTIS

National Inst. of Standards and Technology (NML), Gaithersburg, MD. Thermophysics Div.
Ergodic Convergence Properties of Supercooled Liquids and Glasses.

Final rept.

D. Thirumalai, and R. D. Mountain. 1990, 14p

See also PB90-254814. Sponsored by National Science Foundation, Washington, DC.

Pub. in Physical Review A 42, n8 p4574-4587, 15 Oct 90.

Keywords: "Supercooling, Amorphous materials, Ergodic processes, Binary alloys, Glass, Convergence, Reprints, Molecular dynamics.

The dynamical properties of two measures to probe ergodic behavior in Hamiltonian systems with a large number of degrees of freedom are investigated. The measures, namely, the energy metric $d(t)$ and the fluctuation metric Omega(t) are both based on the timeaveraged energies of the individual particles comprising the system. These ideas are used to study the behavior of supercooled and glassy states of soft-sphere binary alloys using microcanonical molecular dynamics.

\section{0,290}

PB91-148650

National Inst. of Standards and Technology (NML), Gaithersburg, MD. Molecular Physics Div.

Microwave Spectrum of Ar-H2O: Dipole Moment, Isotopic Studies, and (17)O Quadrupole Coupling Constants.

Final rept.

G. T. Fraser, F. J. Lovas, R. D. Suenram, and K Matsumura. 1990, 16p

Pub. in Jnl. of Molecular Spectroscopy 144, p97-112 1990.

Keywords: "Argon complexes, Microwave spectra, Deuterium compounds, Isotope effects, Oxygen 17, Oxygen 18, Dipole moments, Intermolecular forces, Van der Waals forces, Dimers, Water, Reprints.

Microwave spectra for the $\mathrm{K}=0$ states of $\mathrm{Ar}-\mathrm{H} 2 \mathrm{O}, \mathrm{Ar}$ $\mathrm{D} 2 \mathrm{O}, \mathrm{Ar}-\mathrm{HOD}, \mathrm{Ar}-\mathrm{H} 2(18) \mathrm{O}$, and $\mathrm{Ar}-\mathrm{H} 2(17) \mathrm{O}$ were obtained using a pulsed-nozzle Fourier-transform microwave spectrometer. For the Ar-H2O species, the results are in excellent agreement with the far-infrared data of Cohen et al. ( $\mathrm{J}$. Chem. Phys. 89, 4494-4504 (1988) and 92, 169-177 (1990)). The present data are also compared with predictions from the three aniso. tropic potentials proposed by Hutson (J. Chem. Phys. $92,157-168(1990))$ to interpret the far-infrared spectrum of the complex.

\section{0,291}

PB91-148726

Not available NTIS National Inst. of Standards and Technology (NML) Boulder, CO. Quantum Physics Div.

Photodissociation Dynamics of Water in the Second Absorption Band: Vibrational Excitation of OH (A (2)Sigma).

Final rept.

B. Heumann, K. Kuhl, K. Weide, R. Duren, B. Hess, U. Meier, S. D. Peyerimhoff, and R. Schinke. 1990 , $6 p$

Pub. in Chemical Physics Letters 166, n4 p385-390, 2 Mar 90.

Keywords: "Water, "Photodissociation, Excited states, Potential energy, Vibrational states, Excitation, Reprints.

A three-dimensional potential energy surface for the second excited state of water is caluclated by thsecond absorption band. The authors find in accordance with experimental data, vibrational excitation of $\mathrm{OH}($ (Sup 2) Sigma) to be generally weak. This result can be readily explained in terms of the calculated potential surface.

\section{0,292}

PB9 1-148783

Not available NTIS National Inst of Standards and Technology (NEL) Boulder, CO. Chemical Engineering Science Div.

Kinetics of Copper Extraction Using (anti)-2-Hydroxy-5-Nonlybenzophenone Oxime.

Final rept.

M. F. Jin, F. C. Michel, and R. D. Noble. 1989, 6p $\mathrm{Pub}$. in Industrial and Engineering Chemistry Research 28, n2 p193-198 Feb 89

Keywords: "Copper complexes, "Chelating agents, "Oximes, "Reaction kinetics, Transport properties, Liquid-liquid interfaces, Benzophenone, Decanes, Rotation, Diffusion, Reprints. 
For the reaction $(\mathrm{Cu}(2+))$ (aq) +2 (HR)(org) (CuR2)(org) $+2(\mathrm{H}(1+))$ (aq) using the chelating agen anti-2-hydroxy-5-nonylbenzophenone oxime, the reaction orders for $(\mathrm{Cu}++)$, the active isomer of the oxime, and $(\mathrm{H}+)$ were measured experimentally using a rotating diffusion cell. The reaction orders were firs order in $(\mathrm{Cu}++)$, first order in the active isomer of oxime, and negative first order in $(\mathrm{H}+)$. Extrapolation of the diffusion cell data for the kinetically limited case was an important advancement of the technique. The forward and reverse reaction rate constants were calculated. The results were obtained using a resistance model of copper transport.

\section{0,293}

PB91-148841

Not available NTIS

National Inst. of Standards and Technology (NML) Boulder, CO. Quantum Physics Div.

Mode Specific Internal and Direct Rotational Predissociation in HeHF, HeDF, and HeHCL: van der Waals Complexes in the Weak Binding Limit.

Final rept.

C. M. Lovejoy, and D. J. Nesbitt. 1990, 21p

Grant NSF-CHE86-05970

Sponsored by National Science Foundation, Washington, DC.

Pub. in Jnl. of Chemical Physics 93, n8 p5387-5407, 15 Oct 90.

Keywords: *Helium complexes, Hydrogen chloride, Hydrogen fluoride, Deuterium compounds, Van der Waals forces, Intermolecular forces, Infrared spectra Predissociation, Reprints, Feshbach resonances, Supersonic expansion.

The near-infrared vibration-rotation spectra of the weakly bound complexes $\mathrm{HeHF}$, $\mathrm{HeDF}$ and $\mathrm{HeHCl}$ are observed in a slit supersonic expansion. The spectra correspond to simultaneous excitation of vibration and internal rotation of the $H(D) X$ subunit within the complex. The HeHF and HeDF P/R branch transitions show J-dependent excess linewidths, which are attributed to rapid predissociation of the excited states from intramolecular rotation-translation energy transfer The corresponding P/R branch transitions in $\mathrm{HeHCl}$ are not observed despite good $S / N$ on the $Q$ branch suggesting even more rapid predissociation for the upper state of the complex. In addition to isotropic intermolecular potentials, the HeHF/HeDF data yield considerable information on the potential anisotropy in the region sampled by the bound and quasibound states. The information so obtained is complementary to results from scattering studies and provides sensitive tests for refining trial potential energy surfaces.

\section{0,294}

PB91-148882

Not available NTIS

National Inst. of Standards and Technology (NML), Gaithersburg, MD. Molecular Physics Div.

Structures of the NH3-HCCCCH and $\mathrm{H} 2 \mathrm{O}-\mathrm{HCCCCH}$ Complexes by Fourier-Transform Microwave Spectroscopy.

Final rept.

K. Matsumura, F. J. Lovas, and R. D. Suenram

$1990,16 \mathrm{p}$

Pub. in Jnl. of Molecular Spectrscopy 144, p123-138 1990.

Keywords: *Complexes, *Molecular structure, Acetylene, Ammonia, Water, Microwave spectra, Hydrogen bonds, Rotational spectra, Electric dipole moments, Dimers, Reprints, *Ammonia complexes, *Water complexes, Diacetylene, Fourier transform microwave spectroscopy.

The microwave spectra of the ammonia-diacetylene and water-diacetylene complexes have been observed with a pulsed-beam, Fabry-Perot cavity, Fourier-transform microwave spectrometer. In addition to the normal isotopic forms, the authors have also ob served the spectra of ND3-HCCCCH, (15) NH3 $\mathrm{HCCCCH}, \mathrm{HDO}-\mathrm{HCCCCH}$ and D2O-HCCCCH. Present results derived for the diacetylene complexes are compared with those of the acetylene complexes. It is noteworthy that the induced dipole moment of the ammonia-diacetylene complex is about $50 \%$ large than that of the ammonia-acetylene complex. The ef fects of the conjugation of the carbon-carbon triple bonds are discussed.

\section{0,295}

PB91-148924

Not available NTIS

National Inst. of Standards and Technology (NML), Boulder, CO. Quantum Physics Div.
Multireference-Configuration Interaction (MR-Cl) Calculations on $\mathrm{HS}(2+)$ and Experimental Observation via Electron Impact Ionization of H2S. Final rept.

P. J. Miller, S. A. Rogers, J. Senekowitsch, S. V. ONeil, and S. R. Leone. 1990, 15p

Contract AFOSR89-0073

Sponsored by Air Force Office of Scientific Research Bolling AFB, Washington, DC

Pub. in International Jnl. of Mass Spectrometry and Ion Processes 100, p505-519 1990.

Keywords: "Hydrogen sulfides, Metastable state, Electron impact, Sulfur ions, Ionization, Reprints, Dictation

The stability of the $\mathrm{HS}(2+)$ dictation is investigated both theoretically and experimentally. The theoretica analysis involves complete active-space self-consistent field (CASSCF) and multi-configuration referenceconfiguration interaction (MR-CI) theory. A metastable (sup 2) Pi ground state is found with a calculated barrie to dissociation of $1.52 \mathrm{eV}$. No other metastable states for energies up to $10 \mathrm{eV}$ above the ground state are found. In conjunction with the theoretical results, electron impact ionization of $\mathrm{H} 2 \mathrm{~S}$ is able to generate the $\mathrm{HS}(2+)$ dication, demonstrating that the species exists on a time scale longer than 10 microseconds. The appearance potential for $\mathrm{HS}(2+)$ from $\mathrm{H}, \mathrm{S}$ is 42.3 + or $-1.0 \mathrm{eV}$. Evidence is also presented for the electron impact production of $\mathrm{S}(2+), \mathrm{H} 2 \mathrm{~S}(2+)$ and $\mathrm{H} 3 \mathrm{~S}(2+)$.

\section{0,296}

PB91-148940 Not available NTIS National Bureau of Standards (NEL), Gaithersburg, MD. Fire Measurement and Research Div.

Applicability of Smoluchowski's Equation with a Constant Kernel to Coalescence.

Final rept.

G. W. Mulholland, and R. D. Mountain. 1986, 2p Pub. in Jnl. of Chemical Physics 84, n7 p4109-4110, 1 Apr 86.

Keywords: "Coalescence, Computerized simulation, Reprints, *Smoluchowski equation.

A computer simulation of particle size independent coagulation is carried out to test the applicability of Smo luchowski's coagulation theory for coalescence. A small time enhancement in the coagulation rate is observed for the simulation. A straightforward generalization of the coagulation theory to include a transien term does not yield results in quantitative agreement with the simulation.

\section{0,297}

PB91-148973

Not available NTIS

National Inst of Standards and Technology (NML)

Boulder, CO. Quantum Physics Div.

$\mathrm{H}+\mathrm{O} 3$ Fourier-Transform Infrared Emission and Laser Absorption Studies of $\mathrm{OH}(\mathrm{X}(2) \mathrm{Pi})$ Radica: An Experimental Dipole Moment Function and State-to-State Einstein A Coefficients.

Final rept.

D. D. Nelson, A. Schiffman, D. J. Nesbitt, J. J.

Orlando, and J. B. Burkholder. 1990, 17p

Contract AFOSR-F 49620-86-C-0056

Sponsored by Air Force Office of Scientific Research Bolling AFB, DC.

Pub. in Jnl. of Chemical Physics 93, n10 p7003-7019, 15 Nov 90.

Keywords: *Hydroxyl radicals, Vibrational spectra, Dipole moments, Einstein coefficients, Atmospheric radiation, Reprints, Fourier transform infrared spectroscopy.

The relative intensities of 88 pairs of rovibrational transitions of $\mathrm{OH}(X$ (Sup 2) Pi) distributed over 16 vibrational bands ( $n u$ prime $<9$, delta nu $=-1,-2$ ) have been measured using Fourier transform infrared (FTIR) emission/absorption spectroscopy. Each pair of transitions originates from a common vibrational, rotational, and spin-orbit state, so that the measured relative intensities are independent of the $\mathrm{OH}$ number density and quantum state distribution. These data are combined with previous nu $=1<-0$ relative intesntiy absorption measurements and nu $=0,1$, and 2 permanent dipole moments to determine the $\mathrm{OH}$ dipole moment function as a cubic polynomial expanded about the equilibrium bond length. The relative intensities provide detailed information about the shape of the $\mathrm{OH}$ dipole moment function mu(r) and hence the absolute Einstein A coefficients. Absolute Einstein A coefficients from the present mu(r) are therefore presented in order to provide the most reliable experimen- tal numbers for modeling of near IR atmosphere $\mathrm{OH}$ emission phenomena.

\section{0,298}

PB91-149005

Not available NTIS

National Inst. of Standards and Technology (NML), Boulder, CO. Quantum Physics Div.

Laser-Induced Fluorescence Measurements of Drift-Velocity Distributions for $\mathrm{Ba}(+)$ in $\mathrm{Ar}$ : Moment Analysis and a Direct Measure of Skewness.

Final rept

S. M. Penn, J. P. M. Beijers, R. A. Dressler, V. M

Bierbaum, and S. R. Leone. 1990, 10p

Contract AFOSR84-02

Sponsored by Air Force Office of Scientific Research, Bolling AFB, DC

Pub. in Jnl. of Chemical Physics 93, n7 p5118-5127, Oct 90 .

Keywords: "Barium ions, Laser induced fluorescence, Ion mobility, Ion temperature, Velocity distribution, Doppler effect, Skewness, Argon, Drift, Reprints.

Velocity distributions for $\mathrm{Ba}(1+)$ ions drifting in argon under the influence of an electric field are measured using single-frequency, laser-induced fluorescence techniques. A moment analysis of these data yields values for the ion mobilities, temperatures, and, for the first time, skewness parameters of the ion velocity distributions. Doppler profiles of the drifting ions are obtained with the laser beam propagating both paralle and perpendicular to the electric field direction as a function of $\mathrm{E} / \mathrm{N}$ up to $148 \mathrm{Td}$.

\section{0,299}

PB91-149161

Not available NTIS

National Inst. of Standards and Technology (NML)

Boulder, CO. Chemical Engineering Science Div.

Applied Electric Fields for Downstream Processing.

Final rept

S. R. Rudge, and P. Todd. 1990, 27p

Pub. in Protein Purification from Molecular Mechanisms to Large-Scale Processes, ACS Symposium Series 427, Chapter 16, p244-270 1990.

Keywords: "Ions, "Interfaces, "Electric fields, Thermodynamics, Electrolytes, Electrophonesis, Reviews, Proteins, Reprints, "lonic partitioning.

Electric potentials at interfaces are governed by the distribution of ions between the phases. The fundamental thermodynamics of ionic partitioning are reviewed, and the implications of the resulting double layer for transport processes in electric fields are discussed. The special considerations of electric field applications, concerning heat and mass transport, are presented. Electric fields have been applied to aqueous biphasic demixing and to cell separations by density gradient electrophoresis and free flow electrophoresis, for the purpose of producing gram quantities of protein. Analytical applications of electric fields, for the purpose of monitoring downstream processes, have also been successful. These applications include capillary electrophoresis, electrospray mass spectrometry, and pulsed field electrophoresis.

\section{0,300}

PB91-149369

Not available NTIS

National Bureau of Standards (NML), Gaithersburg MD. Surface Science Div.

Influence of Sulfur on Methanation over Tungsten(110).

P. D. Szuromi, R. D. Kelley, and T. E. Madey. 1986

$9 p$

Pub. in Jnl. of Physical Chemistry 90, n24 p6499-6507 1986

Keywords: "Sulfur, *Tungsten, "Reaction kinetics, "Surface chemistry, "Catalysts, Carbon monoxide, Hydrogen, Oxygen, Auger electron spectroscopy, Clumps, Activation energy, Methane, Reprints, *Methanation.

The effect of adsorbed sulfur on the rate of methanation over W(110) is reported for a range of sulfur coverages (0.03-0.55 mL) and temperatures (600-750K) fo 1 Torr $\mathrm{CO}$ and 100 Torr hydrogen. The rate drop sharply for low sulfur coverages (by $40 \%$ at $0.05 \mathrm{~mL}$ ) but decreases less dramatically for higher coverages. Postreaction Auger spectra reveal that the sulfur-free surface for the sample is covered with both carbidic carbon and oxygen, and is oxygen rich. Increasing 


\section{Physical \& Theoretical Chemistry}

sulfur coverages leads to little change in the postreaction carbon level but does cause the oxygen coverage to fall substantially, to zero by $0.40 \mathrm{ml} \mathrm{S}$. The activation energy for methanation is unaffected by the presence of sulfur, suggesting that the decrease in rate is caused by sulfur limiting the saturation coverages that can be attained by the reactants. This possibility motivated a thermal desorption study of $\mathrm{CO}$ and hydrogen adsorption on sulfur precovered surfaces. Good agreement is obtained between the changes in these saturation coverages and the decrease in methanation rate due to the presence of sulfur when small coverages of coadsorbed oxygen are present. These results taken as a whole suggest that sulfur adatoms inhibit methanation through long-range effects, which appear to be moderated by the formation of clusters. Such cluster formation is itself inhibited at low sulfur coverages by the presence of oxygen, which causes sulfur to poison the reaction more effectively at such low coverages.

\section{0,301}

Not available NTIS National Bureau of Standards (NML), Gaithersburg, MD. Surface Science Div.

Summary Abstract: The Influence of Sulfur on Methanation over $W(110)$.

Final rept.

P. D. Szuromi, R. D. Kelley, and T. E. Madey. 1987,

Pub. in Jnl. of Vacuum Science and Technology A 5 n4 p867-868 1987.

Keywords: "Sulfur, "Tungsten, "Reaction kinetics, "Surface chemistry, "Catalysts, Carbon monoxide, Hy drogen, Oxygen, Auger electron spectroscopy, Clumps, Activation energy, Methane, Reprints, "Methanation.

The effect of adsorbed sulfur on the rate of methanation over $W(110)$ is reported for a range of sulfur coverages $(0.03-0.55 \mathrm{~mL})$ and temperatures $(600-750 \mathrm{~K})$ for 1 Torr $\mathrm{CO}$ and 100 Torr hydrogen. The rate drops sharply for low sulfur coverages (by $40 \%$ at $0.05 \mathrm{~mL}$ ) but decreases less dramatically for higher coverages. Postreaction Auger spectra reveal that the sulfur-free surface for the sample is covered with both carbidic carbon and oxygen, and is oxygen rich. Increasing sulfur coverages leads to little change in the postreaction carbon level but does cause the oxygen coverage to fall substantially, to zero by $0.40 \mathrm{~mL}$ of $\mathrm{S}$. The activation energy for methanation is unaffected by the presence of sulfur, suggesting that the decrease in rate is caused by sulfur limiting the saturation coverages that can be attained by the reactants. This possibility motivated a thermal desorption study of $\mathrm{CO}$ and hydrogen adsorption on sulfur precovered surfaces. Good agreement is obtained between the changes in these saturation coverages and the decrease in methanation rate due to the presence of sulfur when small coverages of coadsorbed oxygen are present. These results taken as a whole suggest that sulfur adatoms inhibit methanation through long-range effects, which appear to be moderated by the formation of clusters. Such cluster formation is itself inhibited at low sulfur coverages by the presence of oxygen, which causes sulfur to poison the reaction more effectively at such low coverages.

\section{0,302}

PB91-149385

Not available NTIS

National Inst. of Standards and Technology (NML),

Boulder, CO. Quantum Physics Div.

General Method for Doppler Determination of Cylindrically Symmetric Velocity Distributions: An Application of Fourier Transform Doppler Spectroscopy.

Final rept.

C. A. Taatjes, J. I. Cline, and S. R. Leone. 1990, 6p Grant NSF-PHY86-04504

Sponsored by National Science Foundation, Washington, DC., and Air Force Weapons Lab., Kirtland AFB, NM.

Pub. in Jnl. of Chemical Physics 93, n9 p6554-6559, 1 Nov 90.

Keywords: Velocity distribution, Doppler effect, Hermite polynomials, Anisotropy, Reprints, "lodine atoms, Fourier transform Doppler spectroscopy.

A general method is developed for determination of cylindrically symmetric velocity distributions from Doppler profile measurements. The method applies Kinsey's Fourier transform Doppler spectroscopy (J. L. Kinsey, J. Chem. Phys. 66,2560 (1977)) to distributions arising from photodissociation and uses an orthogonal polynomial expansion to perform the integral transforms analytically. The method is shown to offer an improvement in stability over direct numerical solution of the integral equation and to have applicability to distributions which are not 'separable,' that is, which canno be separated into a product of speed- and angle-dependent factors. The method is applied to experimental measurements of the collisional relaxation of a fast anisotropic distribution of 1 (doublet $P(1 / 2)$ ) atoms in thermal bath. It is shown that the nascent distribution is separable, but the distribution does not remain separable throughout the relaxation process.

\section{0,303}

PB91-149393

Not available NTIS

National Inst. of Standards and Technology (MSEL), Gaithersburg, MD. Ceramics Div.

Relation between the Surface Energy and the Debye Temperature for Cubic Solids.

Final rept.

V. K. Tewary, and E. R. Fuller. 1990, 5p

Pub. in Jnl. of Materials Research 5, n5 p1118-1122 May 90

Keywords: "Specific heat, "Surface energy, "Simple cubic lattices, "Solids, Atomic mass, Reprints.

It is shown that a phenomenological relation exists be tween the Debye temperature theta (in degree Kelvin) and the surface energy Gamma (in ergs/sq $\mathrm{cm}$ ) of cubic solids: theta $=(71.9)(\mathrm{Gamma} / \mathrm{M})(\sup 1 / 2)$ where $M$ is the atomic weight. This relation is derived theoretically in the Debye isotropic approximation by assuming that the interatomic potential is central. No restrictions are imposed on the range of the potential. The relation is obeyed very well by the observed values of theta and gamma in the case of many solids.

\section{0,304}

PB91-149831

Not available NTIS

National Bureau of Standards (NEL), Boulder, CO Chemical Engineering Science Div.

Thermodynamics of Ammonium Indates. 2. The Molar Heat Capacity of the Ammonium Pentabromoindate Monohydrate Salt (NH4)2 InBr5. $22 \mathrm{O}$ from 7.8 to $348 \mathrm{~K}$

Final rept.

S. M. Acosta, E. F. Westrum, R. J. C. Brown, J. E.

Callanan, and R. D. Weir. 1988, 6p

See also PB89-123905.

Pub. in Jnl. of Chemical Thermodynamics 20, p1321 13261988.

Keywords: "Indium inorganic compounds, "Specific heat, Calorimetry, Adiabatic conditions, Reprints, "Ammonium pentabromoindate monohydrate.

The molar heat capacity of ammonium pentabromoindate monohydrate $(\mathrm{NH} 4) 2 \ln \mathrm{Br} 5$ - $\mathrm{H} 2 \mathrm{O}$ was measure from 7.8 to $348 \mathrm{~K}$ using adiabatic calorimetry. The curve of heat capacity as a function of temperature is continuous. There is no sign of the phase transition predicted by Yamada and Weiss (Ber. Bunsenges Phys Chem. 1983, 87, 932). Values of the standard molar thermodynamic quantities for $(\mathrm{NH} 4) 21 n \mathrm{Br} 5$ $\mathrm{H} 2 \mathrm{O}$ are presented to $345 \mathrm{~K}$

\section{0,305}

\section{PB91-149864}

Not available NTIS

National Inst. of Standards and Technology (NML) Boulder, CO. Chemical Engineering Science Div.

Thermodynamics of the Divalent Metal Fluorides. 3. Heat Capacity of the Fast lon Conductor $\mathrm{Sr} \mathrm{SnF}$ from 6 to $344 \mathrm{~K}$

Final rept.

J. E. Callanan, R. Shaviv, E. F. Westrum, and R. D.

Weir. 1989, 7p

See also PB91-133850.

Pub. in Jnl. of Solid State Chemistry 81, p51-57 1989.

Keywords: "Specific heat, Ionic conductivity, Temperature dependence, Reprints, "Strontium tin fluorides *Strontium tetrafluorostannates.

The heat capacity of the fast ion conductor $\mathrm{SrSnF} 4$ was measured by adiabatic calorimetry from $6<\mathrm{T} / \mathrm{K}$ $<344$. A phase transition was not detected, but an anomalous rise in the molar heat capacity was found above $280 \mathrm{~K}$ that showed no sign of lessening at 344 $K$. The rise is coincident with the temperature range where a rapid drop in the $(19) \mathrm{F}$ spin-lattice relaxation time $T(1)$ occurs. Standard molar thermodynamic functions are given at selected temperatures from 5 to 345
100,306

PB91-149906

National Inst. of Standards and Technology (NML), Gaithersburg, MD. Molecular Physics Div.

Vibrational Spectra of Molecular Ions Isolated in Solid Neon. 5. N2O(1+) and NNO2(1-).

Final rept.

M. E. Jacox. $1990,10 p$

See also PB91-149914 and PB91-112714. Sponsored by Army Research Office, Research Triangle Park, NC. Pub. in Jnl. of Chemical Physics 93, n11 p7622-7631, 1 Dec 90.

Keywords: "Molecular ions, "Nitrous oxide, "Vibrational spectra, Matrix isolation, Infrared spectra, Solidified gases, Penning effect, Absorption spectra, Photoionization, Photolysis, Cryogenics, Reprints, Solid neon.

When a Ne:N2O $=200$ or 800 sample is codeposited at approximately $5 \mathrm{~K}$ with a beam of neon atoms that has been excited in a microwave discharge, the infrared spectrum of the products includes absorptions contributed by the two stretching fundamentals of ground-state $\mathrm{N} 2 \mathrm{O}(\mathrm{H})$. An abosrption near $1200 / \mathrm{cm}$ also results from the stabilization of $\mathrm{NNO} 2(1-)$ on reaction of $\mathrm{O}(1-)$ with $\mathrm{N} 2 \mathrm{O}$. The two other stretching fundamentals of NNO2 have also been identified. Detailed isotopic substitution studies support these identifications and permit a normal coordinate analysis for both $\mathrm{N} 2 \mathrm{O}(1+)$ and NNO2. The nitrogen-nitrogen bond of NNO2(1-) has approximately single bond character, and the nitrogen-oxygen bonds are relatively weak. The photodestruction threshold for NNO2 in this experimental system is near $420 \mathrm{~nm}$. Photolysis of NNO2(1-) is accompanied by growth in the absorptions of cis ONNO. Several other infrared absorptions in the initial sample are tentatively assigned to vibrations of $\mathrm{N} 2 \mathrm{O}$ complexed to various ions

100,307

PB91-149914 Not available NTIS National Inst. of Standards and Technology (NML) Gaithersburg, MD. Molecular Physics Div.

Vibrational Spectra of Molecular Ions Isolated in Solid Neon. 4. NO(1+), NO(1-), ONNO(1+), and ONNO(1-).

Final rept.

M. E. Jacox, and W. E. Thompson. 1990, 13p See also PB91-149906 and PB91-112714. Sponsored by Army Research Office, Research Triangle Park, NC. Pub. in Jnl. of Chemical Physics 93, n11 p7609-7621, 1 Dec 90 .

Keywords: "Molecular ions, "Nitric oxide, "Vibrational spectra, Matrix isolation, Solidified gases, Photoionization, Photolysis, Infrared spectra, Penning effect, Cryogenics, Reprints, Solid neon.

When a Ne:NO sample is codeposited at approximately $5 \mathrm{~K}$ with a beam of neon atoms that has been excited in a microwave discharge, photoionization and Penning ionization of the NO lead to the stabilization of both monomer and dimer ions. The most prominent infrared absorption, at $1619.2 / \mathrm{cm}$, results from the reaction of NO with NO or of an electron with (NO)2 to form the charge-delocalized trans-ONNO(1-) species. The position of the totally symmetric NO-stretching absorption of trans ONNO(1-) can be inferred from observa. tion of a combination band. The infrared absorption of $\mathrm{NO}(1+)$ has been detected very close to the gas phase band center. Other infrared absorptions have been tentatively assigned to $N O$, cis ONNO $(1-)$, trans ONNO, and the lowest energy isomer of ONNO $(1+)$. Comparison of the gas-phase vibrational frequencies observed for the weakly bound species cis ONNO and $\mathrm{N} 2 \mathrm{O} 3$ with the corresponding frequencies observed in this neon-matrix study suggests that the matrix shifts for the dimer ion absorptions in the mid-infrared probably amount to less than $1 \%$.

100,308

PB91-149955

Not available NTIS

National Inst of Standards and Technology (NML) Gaithersburg, MD. Molecular Physics Div.

Application of Uitrafast Broadband Infrared Spectroscopy to Measurement of Metal-Carbonyl Dynamics.

Final rept

S. A. Angel, P. A. Hansen, E. J. Heilweil, and J. C. Stephenson. 1990, 3p

Pub. in Springer Series in Chemical Physics, v53 p4804821990. 
Keywords: "Metal carbonyls, Carbon monoxide, Nitric xide, Infrared spectra, Picosecond pulses, Liquid phases, Vapor phases, Vibrational spectra, Reprints. Population lifetimes and energy transfer dynamics obtained from transient multichannel infrared spectra of $\mathrm{CO}$ and $\mathrm{NO}(\mathrm{v} \doteq 1)$ vibrations for gas and liquid phase metal-carbonyl systems are presented.

100,309

PB91-150003

Not available NTIS

National Inst. of Standards and Technology (NEL),

Boulder, $\mathrm{CO}$. Chemical Engineering Science Div.

Fusion of Mercury. A New Certified Standard for Differential Scanning Calorimetry.

Final rept.

J. E. Callanan, K. M. McDermott, and E. F. Westrum $1990,6 p$

Pub. in Jnl. of Chemical Thermodynamics 22, p2252301990.

Keywords: "Mercury, "Heat of fusion, "Melting points, Standards, "Differential thermal analysis, Calorimetry, Adiabatic conditions, Reprints.

From the results of adiabatic-calorimetric measurements, mercury has been certified as a standard reference material for temperature and enthalpy of fusion for differential scanning calorimetry. The fusion temperature is $(234.30+$ or -0.03$) \mathrm{K}$ and the molar enthalpy of fusion is $(2.301+$ or -0.001$) \mathrm{kJ}$ per mole. Adiabatic-calorimetric measurements made by heating continuously at $0.00017 \mathrm{~K}$ per second through the transition showed a fusion temperature of $(234.32+$ or - 0.03)K. Differential-scanning-calorimetric measurements gave a fusion temperature of $(234.34+$ or $0.48) \mathrm{K}$

100,310

B91-150052

Not available NTIS Boulder, CO. Quantum Physics Div.

Diode Laser Probing of I*((2)P1/2) Doppler Profiles: Time Evolution of a Fast, Anisotropic Velocity Distribution in a Thermal Bath.

Final rept.

J. I. Cline, C. A. Taatjes, and S. R. Leone. 1990, 11p Sponsored by Weapons Lab., Kirtland AFB, NM.

Pub. in Jnl. of Chemical Physics 93, n9 p6543-6553, 1 Nov 90.

Keywords: Velocity distribution, Doppler effect, Infrared spectra, Anisotropy, Reprints, "lodine atoms.

The relaxation of a nonthermal translational population distribution of fast $I^{*}$ (doublet $P(1 / 2)$ ) atoms dilutely dispersed in a gaseous bath at thermal equilibrium is studied by time-resolved Doppler spectroscopy. The ast, anisotropic velocity distribution of * $^{*}$ atoms is produced by pulsed laser photolysis of $n$-perfluoropropyl odide (n-C3F7l) at $266 \mathrm{~nm}$. A frequency-narrowed, GaAsinP diod e laser is tuned across the iodine transition at $1315 \mathrm{~nm}$ to measure the Doppler gain profile of the $I^{*}$ photofragments. The velocity distribution is expressed as a separable product of a radial speed function and an angular function describing the anisotropy. The collision-induced time evolution of both the speed and anisotropy components of the nascent velocity population distribution relaxing to form a $300 \mathrm{~K}$ Maxwellian equilibrium distribution is determined.

100,311

B $91-158717$

Not available NTIS

National Inst. of Standards and Technology (IMSE),

Gaithersburg, MD. Reactor Radiation Div.

New Different Forms of Ammonium Loaded and Partly Deammoniated Zeolite Rho Studied by Neutron Powder.

Final rept.

R. Fischer, W. Baur, R. Shannon, J. Parise, and J.

Faber. $1989,7 p$

Pub. in Acta Cryst. C45, p983-989 1989

Keywords: "Zeolites, "Ion exchange resins, Crystal structure, Neutron diffraction, Ammonia, Powder(Particles), Simple cubic latties, Deuteration, Calcines, Roasting, Cesium compounds, Aluminum compounds, Inorganic silicates, Ions, Reprints, Zeolite RHO, Rietveld method.

Zeolite ND4-Rho, prepared by shallow-bed calcination of ammonium-exchanged Rho under flowing dry nitrogen at $873 \mathrm{~K}$, subsequently reloaded with ammonium, deuterium exchanged and dehydrated: D2.5(ND4)7.4Cs0.7Al10.9Si37.1096, M(sub r) = 3073.45, cubic, $143 \mathrm{~m}, \mathrm{a}=14.5265(7) \mathrm{A}, \mathrm{V}=3065.37 \mathrm{cu}$
A, $Z=1, D x=1.685 \mathrm{~g} / \mathrm{cc}$, Reitveld refined refinement A collected at room temperature. Zeolite ND4-Rho, prepared by deep-bed calcination of ammonium-exchanged Rho in air at $633 \mathrm{~K}$, yielding a partly deammoniated sample, subsequently deuterium exchanged and dehydrated: Dx(ND4)4.6Cs0.55Al10.1Si37.9-096, $M($ sub $r)=3047.28$, cubic, $143 \mathrm{~m}, \mathrm{a}=14.4247(5) \mathrm{A}, \mathrm{V}$ $=3001.38 \mathrm{cuA}, Z=1, D x=1.685 \mathrm{~g} / \mathrm{cc}$, Rietveld refinement based on fixed-wavelength neutron powder diffraction data collected at room temperature, lambda $=1.5423(6) \mathrm{A}$

100,312

PB91-158790

Not available NTIS

National Inst of Standards and Technology (NML), Gaithersburg, MD. Chemical Kinetics Div.

Zonal Model for Corona Discharge-Induced Oxidation of SF6 in SF6/O2/H2O Gas Mixtures.

Final rept.

J. T. Herron, and R. J. Van Brunt. 1989,6p

Sponsored by Department of Energy, Washington, DC Pub. in Proceedings of International Symposium on Plasma Chemistry (9th), Pugnochiuso, Italy, September 4-8, 1989, v1 p257-262.

Keywords: "Sulfur hexafluoride, "Reaction kinetics, "Oxidation, "Corona discharges, "Mathematical models, Oxygen, Oxygen fluorides, Mixtures, Water vapor, Reprints.

A chemical kinetics model for oxidation of SF6 in negative glow-type corona discharges is proposed. The model is applied to highly localized dc, point-plane negative corona discharges in SF6/O2 mixtures containing water vapor, and resulting predictions for oxidation by-product yields are compared with recent data on rates for SOF2, SOF4, SO2F2, SO2, and S2F10 formation from corona discharges in SF6 and SF6/O2 mixtures.

\section{0,313}

PB9 1-158857

Not available NTIS

National Bureau of Standards (NEL), Gaithersburg, MD. Thermophysics Div

Wetting Layers on Solid Substrates.

Final rept.

R. F. Kayser. $1987,19 p$

Pub. in KINAM, v8 ptA p87-105 1987.

Keywords: "Wetting, Wettability, Substrates, Glass, Dispersion, Reprints, lonic forces.

When two fluids coexist near a substrate, a thin layer of one fluid often intrudes between the other fluid and the substrate. The thickness of such an intruding layer s often determined by a competition between gravitatonal forces, which tend to thin the layer, and longrange dispersion forces, which tend to thicken the ayer. The paper reviews the theory of wetting layers stabilized by dispersion forces and delimits the applicability of the theory. It also presents a new derivation of the wetting layer thickness for wetting layers stabilized by ionic forces. The mechanism differs from that of dispersion forces and arises when a solid surface can become electrically charged. Elements of both theories are needed to explain recent measurements of wetting layers on glass.

100,314

B9 1-161927

Not available NTIS

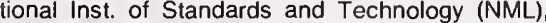

Gaithersburg, MD. Surface Science Div.

of Co/Pt(111)

Final rept.

J. D. Beckerle, M. P. Casassa, E. J. Heilweil, R. R Cavanagh, and J. C. Stephenson. 1990, 10p

Sponsored by Air Force Office of Scientific Research, Bolling AFB, DC

Pub. in Jnl. of Electron Spectroscopy and Related Phenomena 54/55, p17-26 1990.

Keywords: "Carbon monoxide, "Vibrational states, Vibrational spectra, Infrared spectroscopy, Picosecond pulses, Chemisorption, Platinum, Surfaces, Reprints.

The vibrational dynamics of chemisorbed $\mathrm{CO}$ on $\mathrm{Pt}(111)$ are investigated by picosecond IR transient saturation spectroscopy. An intense ps pump pulse excites the $\mathrm{CO}$ stretch vibration for $\mathrm{CO} / \mathrm{Pt}(111)$ and the transient differential IR spectrum of the vibrationally excited adlayer is monitored with a second IR probe pulse. The transient spectrum exhibits a dispersive bandshape, with both bleach and absorption features. The transient spectrum appears prior to the pump pulse and decays within a few ps following the pump pulse. The results are interpreted by comparison with analogous measurements on metal carbonyl molecules, and model calculations including the effects of optical coherence.

100,315

PB91-161968

Not available NTIS

National Bureau of Standards (NEL), Gaithersburg, MD. Thermodynamics Div.

Research at NBS on Dynamic Measurements of Thermophysical Properties at High Temperature (Abstract)

Final rept.

A. Cezairliyan. 1988, $1 \mathrm{p}$

Pub. in Thermal Conductivity, p3 1988

Keywords: "Thermophysical properties, Electric ccnductors, High temperature, Reduced gravity, Measurement, US NBS, Reprints.

Techniques will be described for the dynamic measurements of selected thermophysical properties of electrically conducting substances at temperatures above $1500 \mathrm{~K}$ and up to about $10,000 \mathrm{~K}$. The techniques are based on rapid resistive self-heating of the specimen from room temperature to any desired high temperature in less than one second by the passage of an electrical current pulse through it and on measuring the pertinent quantities with appropriate time resolution. Both millisecond and microsecond resolution techniques developed at NBS will be described, and examples of measured properties will be given. The potential applications of the technique, including property measurements in a microgravity environment, will be discussed.

100,316

PB91-162057

Not available NTIS

National Inst. of Standards and Technology (NML)

Gaithersburg, MD. Molecular Physics Div.

H202: Spectroscopy, Structure and Dynamics.

Final rept.

G. T. Fraser. $1991,18 p$

Pub. in International Review in Physical Chemistry 10 ,

n2 p189-206 1991

Keywords: "Water, "Dimerization, "Hydrogen bonds, Electron tunneling. Molecular structure, Molecular spectroscopy, Reprints.

Tunnelling in water dimer allows each of the hydrogen atoms to participate in the hydrogen bonding, giving rise to a complicated energy-level scheme and rotational spectrum. As a result of new theoretical and experimental developments, great progress has been made in the analysis and interpretation of the spectrum, yielding detailed information about this hydrogen-bonded interaction. The paper reviews the spectroscopy of the highly non-rigid dimer, which is becoming a prototype system for the study of multidimensional tunnelling problems in weakly bound complexes.

100,317

PB91-162065

Not available NTIS

National Inst. of Standards and Technology ( $\mathrm{NML}$ ), Gaithersburg, MD. Surface Science Div.

Resonant Tunneling with Electron-Phonon Interactions: An Exactly Solvable Model Applied to Desorption.

Final rept

J. W. Gadzuk, 1990, 10p

Pub. in Jnl. of Electron Spectroscopy and Related Phenomena 54/55, p201-2101990.

Keywords: "Resonant tunneling, "Desorption, Electron phonon interactions, Electron tunneling, Hot electrons, Surface reactions, Molecules, Atoms, Reprints, Laser induced desorption, Quantum wells.

The problem of hot electron and/or laser induced desorption of atoms or molecules on surfaces is considered. The equivalence between a previous wavepacket model for this process and the problem of inelastic resonant electron tunneling through a quantum well with electron-phonon coupling is established. Numerical consequences of some exactly solved quantum well models are explored as they relate to desorption.

100,318

PB91-162073

Not available NTIS 
National Inst. of Standards and Technology (NML), Boulder, CO. Thermophysics Div.

Phase Transitions.

Final rept.

M. S. Green, and J. M. H. Levelt Sengers. 1990, 5p

Pub. in Encyclopedia of Physics, p897-901 1990.

Keywords: "Phase transformations, Order disorder transformations, Phase diagrams, Phase rule, Liquid crystals, Ising model, Order parameters, Reprints, Lattice gas.

The report is an update of the original Article on Phase Transitions written by M. S. Green. Several phase transitions have been added to the ones covered by M. S. Green. The presentations of the concepts of order-disorder phenomena, order parameter and critical-point universality have been expanded and updated. Illustrations have been improved and new ones added.

\section{0,319}

PB91-162081

Not available NTIS

National Inst. of Standards and Technology (NML). Gaithersburg, MD. Thermophysics Div.

Unified Description of Infinite-Dilution Thermodynamic Properties for Aqueous Solutes.

Final rept.

A. H. Harvey, J. M. H. Levelt Sengers, and J. C.

Tanger. 1991, $6 p$

Pub. in Jnl. of Physical Chemistry 95, n2 p932-937 1991.

Keywords: "Solubility, "Henrys law, "Argon, "Xenon, "Ethylene, "Specific heat, "Aqueous solutions, Critical point, Density(Mass/volume), Thermodynamics, Analysis(Mathematics), Reprints, "Infinite dilution, *Molar volume.

A linear expression, asymptotically correct near a solvent's critical point, is derived relating Henry's constant of a solute to the density of the pure solvent. Since the expression is general (i.e. not restricted to the solvent's saturation curve), it may be differentiated to yield other infinite-dilution thermodynamic properties such as partial molar volumes. This enables these properties for a solute to be predicted from a single fit of the Henry's constant expression to solubility data. Such predictions are compared with published measurements of apparent molar volumes and a apparent
molar heat capacities for aqueous argon, xenon, and molar heat capacities for aqueous argon, xenon, and range of conditions including the region near water's critical point, where large effects are observed. Agreement with heat capacity data is only qualitative; the authors show that this may be in part a consequence of a higher order term in the asymptotic expression that becomes important after two differentiations. The divergent behavior of the infinite-dilution properties near the critical point is determined by divergences in pure-solvent properties; the only solute dependence is in the amplitude of the divergence, which is proportional to the slope of the Henry's constant expression. While the experimental Henry's constant data yield a very large linear region, it now appears that the slope in the region is not the true asymptotic slope. Nevertheless, it appears that predictions of infinite-dilution thermodynamic properties based on Henry's constants are adequate over a wide range of conditions.

\section{0,320}

PB91-162289

Not available NTIS

National Inst. of Standards and Technology (NML), Gaithersburg, MD. Surface Science Div.

Surface-State-Mediated Photochemistry: Laser-Induced Desorption of NO from Si (111).

Final rept.

L. J. Richter, S. A. Buntin, D. S. King, and R. R.

Cavanagh. 1990, $4 \mathrm{p}$

Contract DE-Al05-84ER 13150

Sponsored by Department of Energy, Washington, DC. Pub. in Physical Review Letters 65, n15 p 1957-1960, 8 Oct 90.

Keywords: "Nitric oxide, "Desorption, Chemisorption, Surface chemistry, Silicon, Lasers, Reprints.

Laser-induced desorption of NO from Si(111) has been investigated using state-specific detection techniques. Characterization of the internal-state population distributions with desorption-laser wavelengths in the range $1907-355 \mathrm{~nm}$ indicate that, at low initial NO coverages, desorption is driven by a common, nonthermal mechanism. The wavelength dependence of the yield and the effects of coadsorbates on the yield establish that photogenerated holes in a Si intrinsic surface state mediate the laser-adsorbate interaction.
100,321

PB91-167213

PC A05/MF A01

National Inst. of Standards and Technology, Gaithersburg, MD.

Technical Activities 1990, Standard Reference Data Program.

M. W. Chase. Jan 91, 83p NISTIR-4505

See also PB90-185109.

Keywords: Physical properties, Chemical properties, Thermodynamics, Materials, Data bases, "Chemical information systems, "Standard reference data, US NIST, NSRDS system.

Standard Reference Data is a program office within the Office of Measurement Services in Technology Services, National Institute of Standards and Technology. Standard Reference Data develops and disseminates publications and databases of critically evaluated physical, chemical, and materials properties of substances. These publications and databases are available through NIST and private publishers, on magnetic tape and PC diskettes, and from online retrieval systems. Standard Reference Data is responsible for management and coordination of the program. Work is carried out through a decentralized network of data centers and projects referred to as the National Standard Reference Data System (NSRDS). The volume summarizes the activities of the program for the fiscal year 1990

\section{0,322}

PB9 1-174763

Not available NTIS

National Inst. of Standards and Technology (NEL), Boulder, CO. Thermophysics Div.

Viscosity Surface for Mixtures of Methane and Ethane.

Final rept.

D. G. Friend. $1990,8 p$

Pub. in Cryogenics 30, p105-112 Feb 90

Keywords: "Ethane, "Methane, "Viscosity, "Binary mixtures, Fluids, Physical properties, Experimental data, Statistical analysis, Reprints

The viscosity surface for methane-ethane mixtures has been correlated as a function of composition, density and temperature. The correlation is based on measurements, made at the National Institute of Standards and Technology, using a torsionally oscillating quartz crystal viscometer, which have been reported previously. The viscosity data cover the composition range including both pure fluids and three of their binary mixtures and include temperatures from 100 to $320 \mathrm{~K}$ with pressures to $30 \mathrm{MPa}$. The correlation comprises contributions from the dilute gas state, based entirely on Chapman-Enskog mixture theory and an empirical excess function. The excess function exploits corresponding states arguments and, with suitable reductions of the viscosity, density and temperature, the data are seen to conform closely to a single function of the reduced quantities. The very steep rise of viscosity at high densities is described by a simple rational polynomial and this is added to another polynomial in reduced density and temperature to completely represent the reduced excess viscosity. For the 624 experimental points which were considered, the average absolute deviation from the correlation is less than $1.5 \%$

\section{0,323}

PB91-174805

Not available NTIS

National Inst. of Standards and Technology (NML) Boulder, CO. Chemical Engineering Science Div

Increased Facilitated Transport Related to Microstructural Changes in Heat-Treated lon-Exchange Membranes.

Final rept.

M. D. Heaney, and J. J. Pellegrino. 1989, 19p

Sponsored by Department of Energy, Morgantown, WV. Morgantown Energy Technology Center.

Pub. in Jnl. of Membrane Science 47, p143-161 1989

Keywords: "Ion exchange materials, "Membrane transport, "Membranes, Microstructure, Heating, Temperature dependence, Hydrophylic polymers, Perfluoro compounds, Polymeric films, Swelling, Corrosive gases, Permeability, Small angle scattering Carbon dioxide, Separation processes, Reprints, Ionomers.

Poly(perfluorosulfonic acid) ionomer films were subjected to a heat treatment, with a low volatility polar solvent, which swells the film. These swollen films were used as supports for liquid membranes using water as a solvent. The diffusive and facilitated transport of acid gases was measured and found to be greatly increased in these membranes versus untreated ones. Microstructural analysis of the films, using small angle $X$-ray scattering, indicates that there is an increase in the size of the ionic (hydrophilic) clusters. The increase in ionic cluster size correlates with the observed increase in permeability of the acid gases. The effective diffusion coefficient of the carbon dioxide carrier complex (determined from a model for facilitated transport) has increased by 1-2 orders of magnitude, while that of the carbon dioxide alone has increased only 4-fold over the same range of heat treatment conditions. The microstructural changes persist over extended periods of time, and therefore the heat treatment is possibly a practical means of increasing permeability for this polymer in commercial applications. In limited tests it has been previously confirmed that the selectivity of the treated membranes for carbon dioxide versus carbon monoxide remains high, with separation factors of 126 to 70 .

\section{0,324}

PB91-174813

Not available NTIS National Bureau of Standards (NML), Gaithersburg MD. Molecular Spectroscopy Div.

Population Lifetimes of $\mathrm{OH}(v=1)$ and $O D(v=1) \mathrm{Vi}$ brations in Alcohols, Silanols and Crystalline Micas.

Final rept.

E. J. Heilweil, M. P. Cassassa, R. R. Cavanagh, and J. C. Stephenson. 1986, 4p

Sponsored by Air Force Office of Scientific Research, Bolling AFB, DC

Pub. in Springer Series in Chemical Physics 'Ultrafast Phenomena $V^{\prime}$, v46 p465-468 1986.

Keywords: "Vibrational states, "Alcohols, "Mica, Silanes, Deuterium compounds, Chemical bonds, Electronic states, Lifetime, Reprints, Hydroxy group, Silanols.

Vibrational energy dynamics of excited fundamenta modes of ground electronic state condensed-phase molecules is suspected of playing a role in many bondbreaking physical and chemical processes. As a first step in elucidating this role, it is necessary to compare the vibrational $v=1$ lifetimes ( $T$ sub 1 ) of a specific functional group in a variety of molecules and chemical environments. It should then be possible to determine whether structural or other vibrational properties of a molecular family influence that particular group's chemical reactivity.

\section{0,325}

\section{PB91-174821}

National Bureau of Standards (NML), Gaithersburg MD. Chemical Kinetics Div.

Thermochemistry of Sulfoxides and Sulfones.

Final rept.

J. T. Herron. 1988, 12p

Pub. in Chem. Sulphones Sulphoxides, p95-106 1988.

Keywords: "Sulfoxides, "Sulfones, "Sulfates, "Sulfites, "Thermochemical properties, Experimental data, Reviews, Reprints, Chemical structure.

The report is a review of the experimental data on the thermochemistry of sulfoxides, sulfones, sulfites, and sulfates, and the use of such data in developing estimation methods, deriving bond strengths, and interpreting data on the thermal stability and chemical kinetics of these species. Group values are presented for the estimation of thermochemical properties using the method of group additivity.

\section{0,326}

PB91-174839

Not available NTIS National Bureau of Standards (NML), Gaithersburg, MD. Chemical Kinetics Div.

Formation and Stability of SF5 and S2F 10.

Final rept.

J. T. Herron, and W. Tsang. 1987, 6p

Pub. in Proceedings of International Symposium on Gaseous Dielectrics (5th), p199-204 1987.

Keywords: "Sulfur fluorides, "Sulfur hexafluoride, "Dielectric properties, "Thermal stability, Thermodynamic properties, Reaction kinetics, Water vapor, Computerized simulation, Reprints.

The simulation of the dielectric breakdown of SF6 through the use of computer modeling offers many advantages over more costly direct testing methods for determining the conditions under which potentially 
toxic by-products may be generated. A complete model calculation requires a knowledge of the rate constants or cross sections for all relevant processes. A complete data base of that nature has not yet been assembled. However enough data exist to begin to answer some of the pressing questions regarding the formation and stability of various proposed reaction intermediates. Computer modeling studies have been carried out for very simple systems in order to deter mine the conditions under which S2F10 is formed and the role of water vapor in that process. Many other recombination products of undetermined toxicity such as S2F4, S2F6, and S2F8 can be formed following the breakdown of SF6. The thermal stability of SF5 and S2F 10 is discussed and the lifetimes of these species given as a function of temperature.

\section{0,327}

\section{PB91-174847}

Not available NTIS National Bureau of Standards (NML), Gaithersburg, MD. Chemical Kinetics Div.

Letter to Editor of Chemical and Engineering News.

Final rept.

J. T. Herron, and W. Tsang. 1987, 1p

Pub. in Chemical and Engineering News $65, n 44$ p3 1987.

Keywords: "Combustion, Chemical reactions, Reaction kinetics, Reprints

This is a one-page letter to the editor of Chemical and Engineering News, on the subject of combustion chemistry.

\section{0,328}

PB91-174870

Not available NTIS

Nationa Bureau of Standards (NML), Gaithersburg. MD. Chemical Kinetics Div.

Resonance Enhanced Multiphoton Ionization Spectroscopy of $\mathrm{CHCl} 2$ and $\mathrm{CDCl} 2$.

Final rept.

J. W. Hudgens, and G. R. Long. 1988, 3p

Pub. in AlP Conference Proceedings, n172 p337-339 1988.

Keywords: "Electronic spectra, *Ionization, Spectrum analysis, Spectral lines, Free radicals, Isotope effect, Molecular vibration, Reprints, *Chloromethyl radicals, Rydberg states.

Resonance enhanced multiphoton ionization spectra of $\mathrm{CHCl} 2$ and $\mathrm{CDCl} 2$ were observed between $355-375$ nanometers $(\mathrm{nm})$ via a $2+1$ excitation process. Electronic origins tentatively assigned to $3 \mathrm{~d}$ Rydberg states were observed at $370.1 \mathrm{~nm}$ (frequency $=54,024$ per $\mathrm{cm}$ ) for $\mathrm{CHCl} 2$ and at $370.4 \mathrm{~nm}$ (frequency $=53,980$ per $\mathrm{cm}$ ) for $\mathrm{CDCl} 2$. The $\mathrm{C}-\mathrm{Cl}$ symmetric stretch frequencies are 845 per $\mathrm{cm}$ in $\mathrm{CHCl} 2$ and 814 per $\mathrm{cm}$ in $\mathrm{CDCl} 2$.

\section{0,329}

PB91-174995

Not available NTIS

National Inst. of Standards and Technology (NML) Gaithersburg, MD. Quantum Metrology Div.

Polarized X-ray Emission Studies of Methyl Chloride and the Chlorofluoromethanes.

Final rept.

D. W. Lindle, P. L. Cowan, T. Jach, R. E. LaVilla, R

D. Deslattes, and R. C. C. Perera. 1991, 14p

Sponsored by Department of Energy, Washington, DC Pub. in Physical Review A 43, n5 p2353-2366, 1 Mar 91.

Keywords: "Chloromethanes, "Methyl chloride, "Dichlorodifluoromethane, Polarization(Waves), Synchrotron radiation, $X$ ray fluorescence, Molecular orbitals, $X$ rays, Reprints, "Chlorotrifluoromethane, " Trichlorofluoromethane.

A new technique sensitive to molecular orientation and geometry, and based on measuring the polarization of $\mathrm{x}$-ray emission, has been applied to the $\mathrm{Cl}$-containing molecules methyl chloride $(\mathrm{CH} 3 \mathrm{Cl})$ and the chlorofluoromethanes (CF3Cl, $\mathrm{CF} 2 \mathrm{Cl} 2$, and $\mathrm{CFCl}$ ) in the gas phase. Upon selective excitation using monochromatic synchrotron radiation in the $\mathrm{Cl}$ K-edge $(\mathrm{Cl} 1 \mathrm{~s})$ near-threshold region, polarization-selective $x$-ray emission studies reveal highly polarized molecular valence $x$-ray fluorescence for all four molecules. It is shown that the polarized $x$-ray emission technique can be used to infer, directly from experiment, symmetries of occupied and unoccupied valence molecular orbitals, anisotropies in absorption and emission, and orientational and geometrical information. It is suggested that the $x$-ray polarized-fluorescence phenomenon, re- ported here for simple molecules, can be used as a new approach to study more complicated systems in a variety of environments.

\section{0,330}

Not available NTIS National Inst. of Standards and Technology (NML) Gaithersburg, MD. Chemical Kinetics Div.

Group Reactivities in the Gas Phase Reactions of Hydroxyl Radicals with Ethers.

Final rept.

R. Liu, T. J. Wallington, P. Dagaut, and M. J. Kuryio $1989,4 p$

Pub. in Wuli Huaxue Xuebao 5, n2 p210-213 1989.

Keywords: "Hydroxy radicals, "Ethers, "Reaction kinetics, Chemical reactivity, Photolysis, Reprints Chemical functional groups, Gas phase.

The absolute rate constant was determined for the gas phase reaction of $\mathrm{OH}$ radicals with diethoxy methane using the flash photolysis resonance fluorescence technique. $\mathrm{OH}+\mathrm{CH} 3 \mathrm{CH} 2 \mathrm{OCH} 2 \mathrm{OCH} 2 \mathrm{CH} 3->$ prod ucts (1) Experiments were performed at total pres sures (using Ar diluent gas) between 25-50 torr. The authors' earlier kinetic measurements for normal aliphatic mono-ethers are used to calculate reactivity values for $\mathrm{CH} 3$ and $\mathrm{CH} 2$ which are significantly enhanced over those in normal alkanes. These values predioct the rate constant for reaction (1) quite accurately, indicating that there is no further reactivity en hancement due to the second functional group.

100,331

PB91-175133

Not available NTIS

National Bureau of Standards (NML), Gaithersburg MD. Chemical Kinetics Div.

Single Pulse Shock Tube Study of the Reactions o Hydrogen Atoms with Aromatics. 4. Chloroto luenes.

Final rept.

W. Tsang, Y. Z. He, W. G. Mallard, and J. P. Cui.

$1988,8 p$

Pub. in Shock Tubes and Waves, p467-474 1988

Keywords: *Hydrogen, "Atoms, *Reaction kinetics, Displacement reactions, Thermodynamics, Shock tubes, Reprints, "Chlorotoluenes, Hydrogen atom abstraction.

The hydrogen atom induced decomposition of para and ortho chlorotoluene was studied in single pulse shock tube experiments. The hydrogen atoms are generated through the decomposition of trace quantities of hexamethylethane in large excesses of the chlorotoluene. The displacement products, chlorobenzene and toluene, are directly detected. The importance of the abstraction channel is determined by the differ ence between the total amount of isobutene, produced in equal molar quantities with the $\mathrm{H}$-atoms, and the displacement products. With rate constants for hydrogen atom attack on methane as internal standards, the rate expressions for the three parachlorotoluene decomposition channels have been found to be, $\mathrm{k}(\mathrm{H}+\mathrm{p}$ $\left.\mathrm{ClC} 6 \mathrm{H}_{4} \mathrm{CH} 3-\mathrm{H} 2+\mathrm{ClC} 6 \mathrm{H}_{4} \mathrm{CH} 2\right)=9.6 \times 10$ to the 10 th power Exp(-4265/T) $\mathrm{l} / \mathrm{mol}-\mathrm{s} \mathrm{k}(\mathrm{H}+\mathrm{p}-\mathrm{ClC} 6 \mathrm{H} 4 \mathrm{CH} 3$ $\mathrm{CH}+\mathrm{ClC} 6 \mathrm{H} 5)=3.5 \times 10$ to the 10th power Exp($3710 / \mathrm{T}) \mathrm{l} / \mathrm{mos} \mathrm{k}(\mathrm{H}+\mathrm{p}-\mathrm{ClC} 6 \mathrm{H} 4 \mathrm{CH} 3-\mathrm{Cl} 3+\mathrm{C} 6 \mathrm{H} 4 \mathrm{CH} 3)$ $=4.8 \times 10$ to the tenth power $\operatorname{Exp}(-4795 / \mathrm{T}) \mathrm{l} / \mathrm{mol}-\mathrm{s}$ at 1010 - 1130 . The measurments for orthoclorotoluene are more difficult due to the presence of toluene and chlorotoluene impurities. The rate constants for the comparable processes are close to that for the parachloro compound and have been found to be factors of $1.2,1.3$, and 1.1 of the values given above. The au thors results are compared with previously published numbers of toluene and phenol.

\section{0,332}

PB91-175174

Not available NTIS

National Bureau of Standards (NML), Gaithersburg, MD. Molecular Spectroscopy Div.

Molecular Spectroscopy Division of the National Bureau of Standards.

Final rept.

A. Weber. 1987, 2

Pub. in Applied Spectroscopy 41, n6 p1085-1086 1987

Keywords: *Molecular spectroscopy, US NBS, Research, Reprints.

The report is a brief description of the activities of the Molecular Spectroscopy Division at the National Bureau of Standards.
100,333

PB91-175257

Not available NTIS

National Bureau of Standards (NEL), Boulder, CO. Chemical Engineering Science Div

Book Review: Partitioning in Aqueous Two-Phase Systems: Theory, Methods, Uses, and Applications to Biotechnology.

Final rept.

G. F. Slaff. 1987, 1p

Pub in Jnl of American Chemical Society 109, n5 p1606 1987.

Keywords: "Phase, "Partition, Solvent extraction, Sep aration, Reviews, Biotechnology, Reprints, "Aqueous systems.

This is a review of the book, 'Partitioning in Aqueous Two-Phase Systems

100,334

PB91-175505

Not available NTIS

National Inst. of Standards and Technology (NML) Boulder, CO. Quantum Physics Div.

Rotational Predissociation, Vibrational Mixing, and van der Waals Intermolecular Potentials of NeDF. Final rept.

C. M. Lovejoy, and D. J. Nesbitt. 1991, 16p

Grant NSF-CHE86-05970

Sponsored by National Science Foundation, Washing ton, DC.

Pub. in Jnl. of Chemical Physics 94, n1 p208-223, 1 Jan 91.

Keywords: "Neon complexes, Van der Waals forces, Hydrogen fluoride, Deuterium compounds, Supersonic jet flow, Infrared spectroscopy, Laser spectroscopy, Binding energy, Near infrared radiation, Predissociation, Neon 20, Neon 22, Potentials, Reprints.

The near-infrared spectrum of NeDF formed in a slit free jet expansion is recorded with a high resolution tunable laser spectrometer. Four bands, consisting of the DF stretching fundamental and three internal rotation and van der Waals stretch combination bands, are observed and analyzed for both the $20 \mathrm{Ne}$ and $22 \mathrm{Ne}$ isotopomers. All three combination bands reveal a sudden onset of rotational predissociation at modest $\mathrm{J}$ which is modeled with effective one-dimensional potentials to determine the binding energy. The experimental results are compared with predictions of a re cently published ab initio anisotropic potential surface, and an improved potential is developed and tested.

100,335

PB91-175562

Not available NTIS

National Inst. of Standards and Technology (NML). Gaithersburg, MD. Quantum Metrology Div.

Direct Determination of Molecular Orbital Symmetry of H2S Using Polarized X-ray Emission.

Final rept.

R. Mayer, D. W. Lindle, S. H. Southworth, and P. L. Cowan. 1991, 7p

Pub. in Physical Review A 43, n1 p235-241, 1 Jan 91

Keywords: "Hydrogen sulfide, "Molecular orbitals, Polarization(Waves), Emission spectra, $X$ rays, Symmetry, Reprints.

$\mathrm{X}$-ray emission from the molecule $\mathrm{H} 2 \mathrm{~S}$ is strongly polarized following excitation of a sulfur K-shell electron to an unoccupied subthreshold molecular orbital with a polarized $x$-ray beam. Changes in the polarization of the emission spectrum are observed as the incident beam's energy is swept across the subthreshold ab sorption resonance. The previously unresolved absorption resonance is shown experimentally to be primarily associated with a molecular orbital of b2 symmetry, but with a high-excitation-energy component due to an orbital with a1 symmetry. Satellite emission intensity is shown to depend on the primary photon energy, and is therefore associated with multivacancy effects and not with contamination, as previously suggested.

100,336

PB91-187138

Not available NTIS

National Inst. of Standards and Technology (NML) Gaithersburg, MD. Center for Chemical Physics. 


\section{CHEMISTRY}

Physical \& Theoretical Chemistry

Concerning the Formation and the Kinetics of Phenylium lons.

Final rept.

P. Ausloos, S. G. Lias, T. J. Buckley, and E. E.

Rogers. 1989, 13p

Pub. in International JnI. Mass Spectrom. Ion Process es 92 , p65-77 1989

Keywords: "Reaction kinetics, Organic ions, Isomerization, Hydrocarbons, Molecular structure, Potential energy, Reprints, "Phenylium ions, Ion molecule reactions, Halobenzenes, Pulsed ion cyclotron spectrometry.

Kinetic measurements carried out in a pulsed ion cyclotron spectrometer show that $\mathrm{C} 6 \mathrm{H} 5(+)$ ions formed in unimolecular and bimolecular processes can exhibi two populations of different reactivities. As expected, ground-state singlet phenylium ions are predominantly formed in low energy processes, while higher energy isomeric (mainly acyclic) ions appear in the high energy electron impact fragmentation of $\mathrm{C} 6 \mathrm{H} 6$ and halobenzenes, as well as in highly exothermic ion $/ \mathrm{mole}$ cule reactions. The energy barrier for ring opening was estimated to be $2.0+$ or $-0.3 \mathrm{eV}$, in reasonably good agreement with theory. Phenylium ions react readily with hydrocarbons and polar molecules. By means of isotopic labelling it was shown that alkanes transfer $\mathrm{H} 2$ via a two-stage process consisting of the transfer of $H(-)$ to the phenylium ion followed by a transfer in the complex of $\mathrm{H}(+)$ to the resulting benzene moiety. Cyclopropane was found to transfer $\mathrm{CH}_{2}$ to the phenylium ion in a direct concerted process. In contrast, reaction with unsaturated hydrocarbons involves extensive hydrogen and carbon atom scrambling. Contrary to earlier findings phenylium ions were found to react at least ten times faster with acetylene than the other $\mathrm{C} 6 \mathrm{H} 5(+)$ isomer(s)

\section{0,337}

PB91-187195

Not available NTIS

National Bureau of Standards (NEL), Gaithersburg. MD. Thermodynamics Div.

Critical Exponent for the Viscosity of Four Binary Liquids.

Final rept.

R. F. Berg, and M. R. Moldover. 1988, 11p

Contract NASA-C-86129D

Sponsored by National Aeronautics and Space Administration, Cleveland, $\mathrm{OH}$. Lewis Research Center.

Pub. in Jnl. of Chemical Physics 89, n6 p3694-3704 1988.

Keywords: "Viscosity, "Binary mixtures, "Liquids, Vis cometry, Viscoelasticity, Critical temperature, Reprints.

We have measured the viscosity of four binary mix tures near their consolute points: (1) methanol $+\mathrm{cy}$ clohexane, (2) isobutyric acid + water, (3) nitroethane +3 -methylpentane, and (4) 2-butoxyethanol + water The viscosity data are consistent with the power-law divergence: eta approximately equal to (T-TC) $(-y)$, with an apparent viscosity exponent in the range $0.0404<$ $y<0.0444$. Recent theoretical estimates for $y$ are near 0.032 , which is outside the experimental range. The value of $y$ is independent of whether the critical point is an upper or a lower consolute point and of whether the approach toward Tc is at constant pres sure or at constant volume. Our torsion oscillator viscometer is unique in its simultaneous low frequency ( $\mathrm{Hz}$ ) and low shear rate $(0.1 / \mathrm{s})$, allowing its use close to the critical point before encountering non-Newtonian fluid behavior associated with critical slowing down Nevertheless, we find quantitative evidence for viscoelasticity near the critical point.

100,338

PB91-187435

Not available NTIS

National Bureau of Standards (NML), Gaithersburg.

MD. Radiometric Physics Div.

Shape Resonances in Molecular Fields.

Final rept.

J. L. Dehmer, D. Dill, and A. C. Parr. 1988, 23p

See also DE84009206.

Pub. in Fundamental Processes of Atomic Dynamics p541-563 1988.

Keywords: Electron-molecule collisions, Electron scattering, Photoionization, Molecular models, Spectroscopy, Nitrogen, Reprints, "Shape resonances.

A shape resonance is a quasibound state in which a particle is temporarily trapped by a potential barrie (i.e., the shape of the potential), through which it may eventually tunnel and escape. This simple mechanism plays a prominent role in a variety of excitation processes in molecules, ranging from vibrational excitation by slow electrons to ionization of deep core levels by $x$ rays. Moreover, their localized nature makes shape resonances a unifying link between otherwise dissimilar circumstances. One example is the close connection between shape resonances in electron-molecule scattering and in molecular photoionization. Another is the frequent persistence of tree-molecule shape resonant behavior upon adsorption on a surface or condensa. tion into a molecular solid. The main focus of the article is a discussion of the basic properties of shape resonances in molecular fields, illustrated by the sigma(sub u) shape resonance in N2. Other aspects to be discussed are vibrational effects of shape resonances, connections between shape resonances in different physical settings, and examples of shape resonant behavior in more complex cases, which form current challenges in the field.

\section{0,339}

\section{1-187658}

(Order as PB91-187617, PC A06/MF A01) National Inst. of Standards and Technology, Gaithersburg, MD.

Proposed New Electrolytic Conductivity Primary Standards for $\mathrm{KCl}$ Solutions.

Y. C. Wu, W. F. Koch, and K. W. Pratt. 1991, 11p

Included in Jnl. of Research of the National Institute of Standards and Technology, v96 n2 p191-201 Mar/Apr 91

Keywords: "Primary standards, "Aqueous electrolytes, "Potassium chloride, "Standards, Electrical conductivity, "Electrolytic conductivity, Cell constants, Demal.

An absolute determination of aqueous electrolytic conductivity has been made for 0.01 molal $(\mathrm{m})$ and $0.1 \mathrm{~m}$ potassium chloride solutions, over the temperature range of 0 to $50 \mathrm{C}$ in 5 degree intervals. A cell with a removable center section of accurately known length and area was used for the measurements. Values were adjusted to be in conformity with the ITS- 90 temwere adjusted to be in conformity with the ITS-90 tem-
perature scale. The overall uncertainty over the entire temperature range is estimated to be $0.03 \%$. Values at $25 \mathrm{C}$ for 0.01 and $0.1 \mathrm{~m}$ are 0.00140823 and $0.0128246 \mathrm{~S} / \mathrm{cm}$, respectively. It is proposed that these values be adopted as primary standards for aqueous electrolytic conductivity, replacing the demal scale.

\section{0,340}

B91-189233

Not available NTIS

National Inst. of Standards and Technology (NML), Gaithersburg, MD. Chemical Thermodynamics Div. Combustion Calorimeters.

Final rept.

E. S. Domalski. $1990,61 p$

Pub. in Advances in Calorimetry and Thermochemistry, v1 p51-111 1990.

Keywords: "Heat measurement, "Combustion, "Calorimetry, Physical chemistry, Thermodynamics, Reviews, Thermochemistry, US'NBS, Reprints.

The paper provides a survey of ongoing activities in combustion calorimetry in the Chemical Thermodynamics Division at the National Bureau of Standards (NBS), USA. For some time, many physical chemists have felt that the frontiers of combustion calorimetry have disappeared and that little novelty or innovation is required for the application of the technique. Moreover, they also felt that the impact of new developments in the area of physical chemistry would not be very significant. The paper seeks to demonstrate that significant innovation in combustion calorimetric methods is occurring, and that significant accomplishments have been made and should be expected for the future in the area of research.

\section{0,341}

PB9 1-189241

Not available NTIS

National Inst. of Standards and Technology (IMSE), Gaithersburg, MD. Polymers Div.

How Does Surface Roughness Affect PolymerSurface Interactions.

Final rept.

J. F. Douglas. $1989,10 p$

Pub. in Macromolecules 22, n9 p3707-3716 Sep 89.

Keywords: "Surface roughness, "Fractals, "Adsorption, "Fluid-solid interactions, "Polymers, Liquid-solid interfaces, Entropy, Mathematical models, Monte Carlo Method, Invariance, Reprints.

A simple model is introduced to qualitatively describe the effect of surface roughness on the propertiies of surface interacting polymers and comparison is made with recent Monte Carlo data and renormalization group calculations. The model indicates that increasing the surface irregularity (i.e. the fractal dimension) has the effect of enhancing polymer-surface interactions relative to the idealized planar interface. This is a consequence of a greater probability of polymer-surface intersection with increasing roughness. It is also argued that adsorption occurs more readily on fractal surfaces since then adsorption requires a smaller 'entropic price'. A rough surface (fractal dimension = 3) may adsorb a polymer when a corresponding smooth surface (fractal dimension $=2$ ) of the same material will not adsorb. Roughness can thus alter the effective polymer-surface interaction in a fundamental way.

100,342

PB91-189381

Not available NTIS National Bureau of Standards (NEL), Gaithersburg, MD. Thermophysics Div.

Modeling the Thermodynamic Properties of Sodium Chloride in Steam Through Extended Corresponding States.

Final rept.

J. S. Gallagher, and J. M. H. Levelt Sengers. 1988

$13 p$

Pub. in International Jnl. of Thermophysics 9, n5 p6496611988.

Keywords: "Sodium chloride, "Solutions, "Thermodynamic properties, "Phase transformations, Aqueous electrolytes, Steam, Helmboltz free energy, Critical pressure, Critical temperature, Mathematical models, Reprints, "Apparent molar specific heat, "Apparent molar volume, Corresponding states.

Recent precise data on anomalous behavior of apparent molar properties of electrolyte solutions in nearcritical steam have raised important questions as to how the thermodynamic properties of these systems should be described. Current Gibbs tree energy models fail for highly compressible solutions. Here, Helmholtz free energy formulation is presented as a first step in modelling compressible dilute aqueous electrolyte solutions. Comparisons are made with the known critical line, coexistence curves, apparent molar volumes and specific heats of $\mathrm{NaCl}$ in steam, and conclusions presented on improving the model.

100,343

PB91-189415 Not available NTIS National Bureau of Standards (NML), Gaithersburg, MD. Molecular Spectroscopy Div.

Spectroscopic Constants for the nug Infrared Band of $\mathrm{HNO}_{3}$.

Final rept.

A. Goldman, A. G. Maki, C. J. Howard, J. B.

Burkholder, and R. Escribano. 1988, 6p

Pub. in JnI. of Molecular Spectroscopy 131, n1 p195 2001988.

Keywords: "Nitric acid, "Spectrum analysis, "Infrared spectroscopy, Vibrational spectra, Rotational spectra Atmospheric chemistry, Air pollution, Reprints.

High resolution infrared measurements have been made on the nu 9 band of $\mathrm{HNO} 3$ from 414 to $500 / \mathrm{cm}$. Over 2300 transitions have been measured, assigned, and fit to obtain 14 ro-vibrational constants for the nu9 $=1$ state that reproduce the observed spectrum with a RMS deviation of $0.0004 / \mathrm{cm}$. The band center or nu 9 is at $458.2887+$ or $-0.0005 / \mathrm{cm}$.

\section{0,344}

PB91-189431

Not available NTIS

National Inst. of Standards and Technology (NEL) Gaithersburg, MD. Thermophysics Div.

Effect of Long Range Interfacial Forces on the Dynamics of Wetting Layers at a Liquid Vapor Interface.

Final rept.

J. L. Harden, and R. F. Kayser. 1989, 10p

Pub. in Jnl. of Colloid and Interface Science 127, n2 p548-557 1989.

Keywords: "Liquid-vapor interfaces, Van der Waals forces, Light scattering, Capillary waves, Thickness, Wetting, Reprints.

For certain binary liquid solutions, the denser fluid wil form a thin wetting layer between the less dense fluid and the vapor. The authors consider the possible effect of interfacial van der Waals forces on the capillary waves residing on such wetting layers. In the limit where wetting layer thickness, $h$, is much smaller than 
the capillary wavelength, they find a damped peristaltic mode that behaves as (omega squared) varies as ( squared)/(h cubed) for typical wave numbers. Such a mode, if observable, could provide an alternate means of measuring wetting layer thicknesses and could be used to check the dependence of wetting layer thickness on long range interfacial forces.

\section{0,345}

\section{PB91-189514}

Not available NTIS

National Bureau of Standards (NEL), Gaithersburg, MD. Thermophysics Div.

Mobility Fluctuations and Electrophoretic Light Scattering from Macromolecular Solutions.

Final rept.

J. B. Hubbard, and D. A. McQuarrie. 1988, 15p

Pub. in Jnl. of Statistical Physics 52, n5-6 p1247-1261 1988.

Keywords: "Macromolecules, Light scattering, Stochastic processes, Electrophoresis, Solutions, Mobility, Reprints.

The authors discuss the origins and the effects of mobility fluctuations of rigid, globular macromolecules on a solution's electrophoretic light scattering spectrum. Assuming a dilute solution, a modified van Hove selfcorrelation function is calculated via van Kampen's time-ordered cumulant method, and the results are compared with less rigorous approaches. The consequences of generalizing to dynamic external fields are briefly considered.

\section{0,346}

\section{PB91-189548}

Not available NTIS National Bureau of Standards (NML), Gaithersburg, MD. Chemical Kinetics Div.

Electronic Spectrum of the GeH3 Radical.

Final rept.

R. D. Johnson, J. W. Hudgens, and B. P. Tsai. 1988, $6 p$

Pub. in Jnl. of Chemical Physics 89, n8 p4558-4563 1988.

Keywords: Free radicals, Rydberg states, Vibrationa states, Chemical reactions, Chlorine, Fluorine, $R e$ prints, "Germyl radicals, Multiphoton ionization.

The germyl ( $\mathrm{GeH} 3$ ) radical has been observed by resonance enhanced multiphoton ionization (REPI) spectroscopy in the region of $370-430 \mathrm{~nm}$. The spectrum arises from two-photon resonances with the $5 p$ doublet $A$ double prime, sub 2 (D sub 3 h) Rydberg state that possesses an origin at $419.1 \mathrm{~nm}(v(0-0)=47,705$ $\mathrm{cm})$. A vibrational progression of $758 / \mathrm{cm}$ was as signed to the 'umbrella' mode, $v$ ' sub 2. The observed $X$ doublet $A$ sub 1 (C sub $3 V$ ) ( $V$ double prime, sub 2) $=2$ to $(V$ double prime, sub 2$)=0$ vibrational interva is $663 / \mathrm{cm}$ which leads to an estimated barrier to inversion of $1530 / \mathrm{cm}$.

\section{0,347}

PB91-189613

Not available NTIS

National Inst. of Standards and Technology (NML) Gaithersburg, MD. Chemical Thermodynamics Div.

Energy of Combustion of Triphenylphosphate. Final rept

D. R. Kirklin, and E. S. Domalski. 1989, 8

Pub. in Jnl. of Chemical Thermodynamics $21, n 5$ p 449 4561989

Keywords: "Heat of combustion, "Heat of formation, Phosphorus organic acid esters, Heat measurement Heatof fusion, Heat of vaporization, Reprints, "Triphonylphosphate.

The enthalpy of combustion of crystalline triphenylphosphate was measured in the NBS aneroid adiabatic rotating calorimeter. The standard molar enthalpy of combustion at $298.15 \mathrm{~K}$ and 1 bar $\mathrm{P}(0)=100,000 \mathrm{~Pa}$ for the reaction: $\mathrm{C} 18 \mathrm{H} 15 \mathrm{PO} 4(\mathrm{cr})+21 \mathrm{O} 2(\mathrm{~g})=18$ $\mathrm{CO} 2(\mathrm{~g})+(\mathrm{H} 3 \mathrm{PO} 4+6 \mathrm{H} 2 \mathrm{O})(\mathrm{aq})$ is-(9259.31 + or 4.96) $\mathrm{kJ} / \mathrm{mol}$. The derived enthalpy of formation for crystalline triphenylphosphate is $-(829.26+$ or -5.72$)$ $\mathrm{kJ} / \mathrm{mol}$. Enthalpies of formation of the liquid and gas phases were calculated using literature values of the enthalpies of fusion and vaporization. Group contribution values were derived for estimating enthalpies of formation of aromatic phosphates in the gas, liquid, and solid phases.

100,348

PB91-189639

Not available NTIS

National Bureau of Standards (NML), Gaithersburg

MD. Molecular Spectroscopy Div.
Hydrogen Bonding in Pt Ammine Complexes. Final rept.

M. Krauss, K. J. Miller, and H. Basch. 1988, $4 p$ Pub. in Chemical Physics Letters 148, n6 p577-580 1988.

Keywords: "Platinum complexes, "Ammino com pounds, "Bonding strength, "Hydrogen bonds, Oligonucleotides, Mathematical models, Calculation methods, Guanines, Formaldehyde, Carbonyl compounds, Ligands, Reprints.

Intra- and inter-ligand hydrogen bonding stabilize the binding of $\mathrm{Pt}$ ammine complexes to oligonucleotides. Model calculations explore the energetics of intraligand binding of the ammine to $G(06)$ in PtA3G $(\mathrm{A}=$ ammine, $\mathrm{G}=$ guanine) and the inter-ligand binding of a carbonyl oxygen to the ammine in a complex of PtA4 and formaldehyde. Hydrogen bonding interaction to the $\mathrm{G}(06)$ site yields a weak bonding interaction but permits the close approach of the guanine(06) and ammine $(\mathrm{H})$ atoms. Inter-ligand hydrogen bonding is strong as expected for an optimal ionic hydrogen bond.

\section{0,349}

PB9 1-189837

Not available NTIS

National Bureau of Standards (NEL), Gaithersburg. MD. Thermophysics Div.

Modelling Aqueous Solutions Near the Critical Point of Water.

Final rept

G. Morrison. 1988, 21p

Pub. in Jnl. of Solution Chemistry 17, n9 p887-907 1988

Keywords: "Aqueous electrolytes, "Solutions, "Thermodynamic properties, "Partial molar volume, Water Dilution, Critical temperature, Critical pressure, Helm holtz free energy, Mathematical models, Reprints "Nonelectrolytes, "Partial molar isobaric thermal expansion.

The thermodynamic properties of a dilute solution near the critical point of the solvent are discussed. Two ex amples are discussed, a solution of a nonelectrolyte and a solution of an electrolyte. The limiting behavio of the electrolyte solutions is modelled with a Debye Huckel term in the Helmholtz free energy. The partia molar properties, in particular the volume and isobaric thermal expansion are examined in detail. The derivation of these properties is introduced by considering the geometry of the thermodynamic surfaces near to and far from the critical point of the solvent. The authors conclude that the properties of solutions near the solvent critical point are dominated by that feature; so lution properties cannot be adequately modelled with out including the functional forms associated with the critical point.

\section{0,350}

PB91-189845

Not available NTIS

National Inst. of Standards and Technology (NEL) Gaithersburg, MD. Thermophysics Div.

Ergodic Measures for the Simulation of Dielectric Properties of Water.

Final rept.

R. D. Mountain, and D. Thirumalai. 1991, 8p

Sponsored by National Science Foundation, Washing ton, DC.

Pub. in Computer Physics Communications 62, p352 3591991.

Keywords: "Water, Dielectric properties, Time intervals, Dipole moments, Computerized simulation, Hydrogen bonds, Reprints, Molecular dynamics.

The authors determine the time interval t(obs), required for effective ergodic convergence of the fluctuations in dipole moment which are needed for the computation of the dielectric properties of water. It is established that the value of $t$ (obs) can be computed from the scaling relations obeyed by the fluctuation metric Omega sub mu (t), associated with the time-averaged values of the dipole moment of the individual wate molecules. The theoretical ideas behind Omega sub mu (t) are used to compute t(obs) at several tempera tures for the simple point-charge model of water. It is found that $t$ (obs) increases exponentially with decreas ing temperature, the barrier being associated with the difficulty in making collective orientational rearrangements. For the simple point-charge model considered in the paper, the barrier for these collective motions is estimated to be $3.5 \mathrm{kcal} / \mathrm{mole}$. The study provides a firm basis for obtaining reliable values of the dielectric properties of dipolar fluids using molecular dynamics simulations.
100,351

PB91-189852

Not available NTIS National Bureau of Standards (NML), Gaithersburg MD. Chemical Kinetics Div.

Polyelectrolyte Stabilized Metal Oxide Hydrosols as Catalysts for the Photooxidation of Water by Zinc Porphyrins.

Final rept.

G. S. Nahor, S. Mosseri, P. Neta, and A. Harriman. $1988,6 p$

Pub. in Jnl. of Physical Chemistry 92, n15 p4499-4504 1988.

Keywords: "Iridium oxides, "Ruthenium oxides, "Catalysts, "Water, "Hydrosols, "Photooxidation, Porphyrins, Quantum efficiency, Oxygen, Solar energy, Reaction kinetics, Reprints.

Colloids of ruthenium dioxide and iridium oxide have been prepared and characterized. These colloids, which are inherently negatively-charged in neutral water, have been stabilized with a surface layer of polyelectrolyte. Electrostatic binding occurs between the stabilized colloids and water-soluble zinc porphyrins of the opposite electronic charge. Such electrostatic forces affect the rate constant for interfacial electron transfer between the colloids and radical cations derived from the zinc porphyrins. The products of these reactions depend upon the relative charges of the reactants. For oppositely charged reactants, the rate of interaction was very high but $\mathrm{O} 2$ generation was no observed. In cases where the porphyrin and colloid possess the same charge, the system can be used to oxidize water to $\mathrm{O} 2$ under photochemical conditions. The yield of $\mathrm{O} 2$ depends upon the solution $\mathrm{pH}$ and the nature of both reactants. With negatively charged reactants in alkaline solution, the authors have reported quantum efficiencies for $\mathrm{O} 2$ generation in the range of $50-60 \%$. With positively charged reactants, oxygen formation could be observed in acidic solution, al though the quantum efficiencies were less than $10 \%$

100,352

PB91-189902

Not available NTIS

National Inst. of Standards and Technology (NML)

Gaithersburg, MD. Chemical Kinetics Div.

Proton Affinity of Cyanogen and Ion-Molecule Reactions of $\mathrm{C} 2 \mathrm{~N} 2(+)$

Final rept

S. A. H. Petrie, M. Mautner, M. J. McEwan, and C. G Freeman. 1989, 10p

Pub. in International Jnl. of Mass Spectrometry and Ion Processes 90, n3 p241-250 1989.

Keywords: "Cyanogen, Nitriles, Reaction kinetics, Thermochemistry, Thermodynamics, Organic ions, Hydrocarbons, Carbon monoxide, Carbon dioxide, Re prints, "Proton affinity, "Ion molecule reactions.

The proton affinity (PA) of C2N2 has been determined by proton transfer equilibria using forward and reverse rate coefficients from SIFT measurements. The results yield a PA for $\mathrm{CH} 3 \mathrm{Cl}$ of $673+$ or $-4 \mathrm{~kJ} / \mathrm{mol}$ and a PA for C2N2 of $674+$ or $-4 \mathrm{~kJ} / \mathrm{mol}$ relative to a PA for $\mathrm{C} 2 \mathrm{H} 4$ of 680 + or $-2 \mathrm{~kJ} / \mathrm{mol}$. $\mathrm{C} 2 \mathrm{~N} 2 \mathrm{H}+$ is also formed by the reactions of $\mathrm{C}_{2} \mathrm{~N}_{2}(+)$ with $\mathrm{H}_{2}$ and $\mathrm{H}_{2} \mathrm{O}$. In the latter, charge transfer competes with $\mathrm{H}$ atom transfer while in the reaction of $\mathrm{C}_{2} \mathrm{~N} 2(+)$ with $\mathrm{C}_{2} \mathrm{H}_{2}$ and $\mathrm{C} 2 \mathrm{H} 4$ only charge transfer occurs. In each instance for these reactions of $\mathrm{C} 2 \mathrm{~N} 2(+)$, the most exothermic charnel is dominant and occurs at or near the collision rate. Rate coefficients are also reported for association (condensation) of $\mathrm{C}_{2} \mathrm{~N}_{2}(+)$ with $\mathrm{CO}, \mathrm{CO} 2, \mathrm{C}_{2} \mathrm{H}_{2}$ and $\mathrm{C}_{2} \mathrm{H}_{4}$

100,353

PB91-189936

Not available NTIS

National Inst. of Standards and Technology (NML) Gaithersburg, MD. Surface Science Div.

Recent Developments in Applied Surface Science. Final rept.

C. J. Powell. 1989, 2p

Pub. in Vide, Couches Minces 44, n248 p427-428 1989.

Keywords: "Surfaces, Reprints, Overviews.

An overview is given of the recent growth of applied surface science and of the recent proposal to form an Applied Surface Science Division within the International Union of Vacuum Science, Technique and Applications 


\section{Physical \& Theoretical Chemistry}

American Chemical Society, Washington, DC

Journal of Physical and Chemical Reference Data, Volume 19, Number 4, July-August 1990.

Bimonthly rept.

D. R. Lide. c1990, 270 p

See also PB91-192443 through PB91-192468 and PB90-244658. Errata sheet inserted. Prepared in cooperation with American Inst. of Physics, New York. Sponsored by National Inst. of Standards and Technology, Gaithersburg, MD.

Available from American Chemical Society, 1155 16th St., NW, Washington, DC. 20036-9976.

Keywords: "Sulfur, "Atomic energy levels, "Atomic spectra, "Specific heat, "Entropy, "Solids, Phase transformations, Sulfur ions, Thermodynamic properties, Enzymes, "Liquids, "Krebs Cycle, "Organic acids, ties, Enzymes, "Liquids, "Krebs Cycle, "Organic acids,
"Amino acids, "Organic compounds, Oxidation reduction reactions.

Contents:

Energy Levels of Sulfur, S(I) Through S(XVI);

Heat Capacities and Entropies of Organic Compounds in the Condensed Phase, Volume II;

The Thermodynamics of the Krebs Cycle and Related Compounds.

\section{0,355}

\section{PB91-192443}

Not available NTIS

National Inst. of Standards and Technology (NML), Gaithersburg, MD. Center for Atomic, Molecular and Optical Physics.

Energy Levels of Sulfur, SI Through S XVI.

Bimonthly rept.

W. C. Martin, R. Zalubas, and A. Musgrove. c1990,

$60 p$

Included in Jnl. of Physical and Chemical Reference Data, v19 n4 p821-880 Jul/Aug 90. Available from American Chemical Society, 1155 16th St., NW, Washington, DC. 20036-9976

Keywords: "Sulfur, "Atomic energy levels, "Atomic spectra, Sulfur ions, Ionization potentials, Electron configurations.

Energy level data are given for the atom and all positive ions of sulfur $(Z=16)$. These data have been critically compiled, mainly from published and unpublished material on measurements and analyses of the optical spectra. The authors have derived or recalculated the levels for a number of the ions. In addition to the level values in reciprocal $\mathrm{cm}$ and the parity, the $\mathrm{J}$ value and the configuration and term assignments are listed if known. Leading percentages from the calculated eigenvectors are tabulated or quoted wherever available. lonization energies are given for all spectra.

100,356

PB91-192450 Not available NTIS National Inst. of Standards and Technology (NML), Gaithersburg. MD. Chemical Thermodynamics Div. Heat Capacities and Entropies of Organic Compounds in the Condensed Phase. Volume 2. Bimonthly rept.

E. S. Domalski, and E. D. Hearing. c1990, 168p Included in Jnl. of Physical and Chemical Reference Data, v19 n4 p881-1048 Jul/Aug 90. Available from American Chemical Society, 115516 th St., NW, Washington, DC. 20036-9976.

Keywords: "Specific heat, "Entropy, "Liquids, "Solids, "Organic compounds, Tables(Data), Reviews, Thermodynamic properties, Phase transformations.

The compilation of data on the heat capacities, entropies, and phase transitions of organic compounds in the condensed phase supplements the document published earlier on the subject, namely 'Heat Capacities and Entropies of Organic Compounds in the Con densed Phase' by E. S. Domalski, W. H. Evans, and E. D. Hearing, J. Phys. Chem. Ref. Data, 13, Suppl. 1, (1984). It provides data on approximately 1300 organic compounds. About half of the articles examined contain data published prior to 1982. A total of 565 articles have been examined, evaluated, and referenced. In addition to values for the heat capacity and entropy at $298.15 \mathrm{~K}$, phase transitions for solid/solid, solid/liquid, and in some instances, solid/gas and liquid/gas are tabulated as encountered from the articles examined and evaluated.

100,357

PB91-192468

Not available NTIS

California Univ., San Diego, La Jolla. Dept. of Chemistry.
Thermodynamics of the Krebs Cycle and Related Compounds.

Bimonthly rept.

S. L. Miller, and D. Smith-Magowan. c1990, 25p Grant NANB6-D0656

Prepared in cooperation with National Inst. of Standards and Technology (NML), Gaithersburg, MD. Chemical Thermodynamics Div.

Included in Jnl. of Physical and Chemical Reference Data, v19 n4 p1049-1074 Jul/Aug 90. Available from American Chemical Society, 115516 th St., NW, Washington, DC. 20036-9976.

Keywords: "Krebs Cycle, "Entropy, "Heat of forma tion, "Gibbs free energy, "Aspartic acid, "Citric acid, "Acetic acid, "Fumaric acid, "Formic acid, "Glutamic acid, "Succinic acid, *Organic acids, "Alanines, "Glycine, Chemical equilibrium, Metabolism, Enzymes, Catalysis, Oxidation reduction reactions.

A survey is made of the enthalpies of formation, third law entropies and Gibbs energies available for Krebs cycle and related compounds. These include formate, acetate, succinate, fumarate, glycine, alanine, aspartate and glutamate. The potential of the NAD + /NADH couple is recalculated based on the ethanol/acetaldehyde and isopropanol/acetone equilibria. The reported enzyme catalyzed equilibrium constants of the Krebs cycle reactions are evaluated with estimated errors These 28 equilibria form a network of reactions that is solved by a least squares regression procedure giving Gibbs energies of formation for 21 Krebs cycle and related compounds. They appear to be accurate to + or $-0.4 \mathrm{~kJ} / \mathrm{mol}$ for some compounds but + or $-1 \mathrm{~kJ} / \mathrm{mo}$ in less favorable cases. This procedure indicates which third law delta(sub f) and enzyme equilibria are inaccurate, and allows very accurate delta(sub f) to be determined for compounds related to the Krebs cycle by measuring enzyme equilibrium constants.

\section{0,358}

\section{PB9 1-192476}

Not available NTIS

American Chemical Society, Washington, DC.

Journal of Physical and Chemical Reference Data, Volume 19, Number 5, September-October 1990.

Bimonthly rept.

D. R. Lide. c1990, 202p

See also PB91-192484 through PB91-192534. Prepared in cooperation with American Inst. of Physics, New York. Sponsored by National Inst. of Standards and Technology, Gaithersburg, MD.

Available from American Chemical Society, 1155 16th St., NW, Washington, DC. 20036-9976.

Keywords: "Thermodynamic properties, Liquid oxygen, Polyatomic gases, Thermal conductivity, Carbon tetrafluoride, Methane, Molten salts, Phase diagrams, Rare gases, Water.

\section{Contents:}

Transport Properties of Fluid Oxygen

Thermal Conductivity of Nine Polyatomic Gases at Low Density:

The Thermal Conductivity of Methane and Tetrafluoromethane in the Limit of Zero Density;

Coupled Phase Diagram-Thermodynamic Analysis of the 24 Binary Systems, A2CO3-AX and A2SO4-AX Where $A=L i, N a, K$ and $X=C l, F$, $\mathrm{NO} 3, \mathrm{OH}_{i}$

Equilibrium and Transport Properties of Gas Mixtures at Low Density--Eleven Polyatomic Gases and Five Noble Gases;

A Unified Fundamental Equation of State for the Thermodynamic Properties of $\mathrm{H} 2 \mathrm{O}$.

\section{0,359}

\section{PB91-192484} Boulder, CO. Thermophysics Div.

\section{Transport Properties of Fluid Oxygen.}

Bimonthly rept.

A. Laesecke, R. Krauss, K. Stephan, and W. Wagner. c1990, 34p

Prepared in cooperation with Stuttgart Univ. (Germany, F.R.). Inst. fuer Technische Thermodynamik und Thermische Verfahrenstechnik, and Ruhr Univ., Bochum (Germany, F.R.). Inst. fuer Thermo- und Fluiddynamik. Included in Jnl. of Physical and Chemical Reference Data, v19 n5 p1089-1122 Sep/Oct 90. Available from American Chemical Society, 115516 th St., NW, Washington, DC. 20036-9976.

Keywords: "Liquid oxygen, "Oxygen, Transport properties, Thermal conductivity, Viscosity, Equations of state, Graphs(Charts)
Supplementing the recently completed IUPAC tables for the thermodynamic properties of oxygen, the paper presents a data evaluation of the transport properties, viscosity, and thermal conductivity. From a comprehensive literature survey the available data have been compiled, and their quality was assessed. Selected measurements were correlated to generate skeleton tables of the most reliable data along the vapor-liquid coexistence curve and for the fluid region at pressures from 0.1 to $100 \mathrm{MPa}$ and at temperatures from 70 to $1400 \mathrm{~K}$. The set of correlations which was developed includes residual concept formulations as well as transport equations of state. These allow the direct calculation of viscosities and thermal conductivities from pressure and temperature as input variables. The simplified crossover model was employed to represent the enhancement of the thermal conductivity in the critical region.

100,360

PB91-192492

Not available NTIS Universidad Autonoma Metropolitana, Mexico City. Dept. of Fisica.

Thermal Conductivity of Nine Polyatomic Gases at Low Density.

Bimonthly rept.

F. J. Uribe, E. A. Mason, and J. Kestin. c1990, 14p Grants NSF-CHE85-09416, NSF-CHE88-19370

Prepared in cooperation with Brown Univ., Providence RI. Sponsored by National Science Foundation, Washington, DC., and National Inst. of Standards and Technology, Gaithersburg, MD.

Included in Jnl. of Physical and Chemical Reference Data, v19 n5 p1123-1136 Sep/Oct 90. Available from American Chemical Society, 115516 th St., NW, Washington, DC. 20036-9976.

Keywords: "Polyatomic gases, "Thermal conductivity, Nitrogen, Oxygen, Nitrogen oxide(NO), Nitrogen oxide(N2O), Carbon monoxide, Carbon dioxide, Carbon tetrafluoride, Sulfur hexafluoride, Methane, Tables(Data)

The authors present a complete set of easily programmable computer algorithms, and a set of numerical tables, for the thermal conductivities of the nine gases: $\mathrm{N} 2, \mathrm{O} 2, \mathrm{NO}, \mathrm{CO}, \mathrm{CO} 2, \mathrm{~N} 2 \mathrm{O}, \mathrm{CH}_{4}, \mathrm{CF}_{4}$, and $\mathrm{SF} 6$. This complements their earlier corresponding-states work on the equilibrium and transport properties of these gases (J. Phys. Chem. Ref. Data 16, 445 (1987); 17 $255(1988))$. The results embrace the temperature range from $\mathrm{T}$ star $=\mathrm{kT} / \mathrm{epsilon}=1$ up to a nominal upper limit of $3000 \mathrm{~K}$. The accuracy achieved is specified, and the correlation can be used in a predictive mode.

100,361

PB91-192500

Not available NTIS

Imperial Coll. of Science and Technology, London (England). Dept. of Chemical Engineering and Chemical Technology.

Thermal Conductivity of Methane and Tetrafluoromethane in the Limit of Zero Density.

Bimonthly rept.

M. J. Assael, J. Millat, V. Vesovic, and W. A.

Wakeham. c1990, 11p

Sponsored by National Inst. of Standards and Technology, Gaithersburg, MD., Department of Industry, London (England), and Rostock Univ. (German D.R.) Included in Jnl. of Physical and Chemical Reference Data, v19 n5 p1137-1148 Sep/Oct 90. Available from American Chemical Society, 1155 16th St., NW, Washington, DC. 20036-9976.

Keywords: "Carbon tetrafluoride, "Methane, *Therma conductivity, Polyatomic gases, Transport properties, Tables(Data)

The paper presents accurate representations of the thermal conductivity of methane and tetrafluoromethane in the limit of zero density. The theoretically-based correlations provided are valid for the temperature range $120-1000 \mathrm{~K}$ and $280-750 \mathrm{~K}$ for methane and tetrafluoromethane respectively. The methane correlation has associated uncertainties of + or - $2 \%$ be tween 300 and $500 \mathrm{~K}$, rising to + or $-2.5 \%$ at the low, and + or $-4 \%$ at the high, temperature extremes. The tetrafluoromethane correlation has uncertainties of $1 \%$ between $280 \mathrm{~K}$ and $450 \mathrm{~K}$, rising to + or $-5 \%$ at the highest temperature. A comparison with some earlier correlations is given. The paper also includes an improved correlation for the temperature dependence of the zero-density viscosity of tetrafluoromethane. 
100,362

PB91-192518

Not available NTIS

Ecole Polytechnique, Montreal (Quebec).

Coupled Phase Diagram-Thermodynamic Analysis of the 24 Binary Systems, A2CO3-AX and A2SO4AX Where $A=\mathrm{Li}, \mathrm{Na}, \mathrm{K}$ and $\mathrm{X}=\mathrm{Cl}, \mathrm{F}, \mathrm{NO}, \mathrm{OH}$.

Bimonthly rept.

Y. Dessureault, J. Sangster, and A. D. Pelton. c1990, 30p

Sponsored by American Ceramic Society, Columbus, $\mathrm{OH}$., and National Inst. of Standards and Technology, Gaithersburg, MD.

Included in Jnl. of Physical and Chemical Reference Data, v19 n5 p1149-1178 Sep/Oct 90. Available from American Chemical Society, 1155 16th St., NW, Washington, DC. 20036-9976.

Keywords: "Molten salts, "Phase diagrams, Thermodynamic properties, Lithium halides, Sodium halides, Potassium halides, Chlorides, Carbonates, Fluorides Nitrates, Sulfates, Hydroxides.

A complete bibliographic search for all thermodynamic and phase diagram data on the 24 binary systems A2CO3-AX and A2SO4-AX (where $\mathrm{A}=\mathrm{Li}, \mathrm{Na}, \mathrm{K}$ and $\mathrm{X}=\mathrm{F}, \mathrm{Cl}, \mathrm{OH}, \mathrm{NO}$ ) was carried out. A computer-assisted simultaneous evaluation of all data was performed in order to obtain optimized equations for the thermodynamic properties of the phases. A re-evaluation of the thermodynamic data for several of the pure salts was also carried out. The optimized thermodynamic parameters are reported as well as the phase diagrams calculated from these equations. These are considered to be the best evaluated phase diagrams which can be deduced from the data currently available. Estimated error limits of all binary assessments are given.

100,363

PB9 1-192526

Not available NTIS

Brown Univ, Providence, Rl. Div of Engineering

Equilibrium and Transport Properties of Gas Mixtures at Low Density: Eleven Polyatomic Gases and Five Noble Gases.

Bimonthly rept.

J. Bzowski, J. Kestin, E. A. Mason, and F. J. Uribe.

c1990, 44p

Grant NANB7-D0703

Sponsored by National Inst. of Standards and Technology, Gaithersburg, MD.

Included in Jnl. of Physical and Chemical Reference Data, v19 n5 p1179-1232 Sep/Oct 90. Available from American Chemical Society, 1155 16th St., NW, Washington, DC. 20036-9976.

Keywords: *Polyatomic gases, *Rare gases, Transport properties, Chemical equilibrium, Mixtures, Algorithms.

The paper uses results from statistical-mechanical theory, applied through a combination of an extended principle of corresponding states with some knowledge of intermolecular potentials, to the calculation of the transport and equilibrium properties of gas mixtures at low density. The gases involved are: N2, O2, $\mathrm{NO}, \mathrm{CO}, \mathrm{CO} 2, \mathrm{~N} 2 \mathrm{O}, \mathrm{CH} 4, \mathrm{CF} 4, \mathrm{SF} 6, \mathrm{C} 2 \mathrm{H} 4, \mathrm{C} 2 \mathrm{H} 6$, and $\mathrm{He}, \mathrm{Ar}, \mathrm{Ne}, \mathrm{Kr}, \mathrm{Xe}$. The properties included are: second virial coefficient, viscosity, diffusion, and thermal diffusion, but not thermal conductivity. The calculations are internally, thermodynamically consistent and the resulting algorithms, which are fully programmable, operate in an entirely predictive mode by means of validated combination rules. The paper is a sequel to one on the five noble gases and all their possible mixtures and a second on the above eleven polyatomic gases. The paper contains ten tables (mainly intended for the checking of computer codes) and 201 graphs of deviation and comparison plots.

100,364

PB91-192534

Not available NTIS

cal Engineering.

Unified Fundamental Equation for the Thermodynamic Properties of $\mathrm{H} 2 \mathrm{O}$.

Bimonthly rept.

P. G. Hill. c1990, 42p

Sponsored by National Inst. of Standards and Technology, Gaithersburg, MD.

Included in Jnl. of Physical and Chemical Reference

Data, v19 n5 p1233-1274 Sep/Oct 90. Available from American Chemical Society, 1155 16th St., NW, Washington, DC. 20036-9976.

Keywords: *Water, "Thermodynamic properties, Equations of state, Compressive properties, Acoustic velocity, Specific heat, Graphs(Charts).
A new unified equation of state for $\mathrm{H} 2 \mathrm{O}$ is presented, which includes the revised and extended scaling equation of Levelt Sengers, Kamgar-Parsi, Balfour and Sengers, is continuous over all single phase states of $\mathrm{H} 2 \mathrm{O}$ from triple point pressure and temperature to $1000 \mathrm{MPa}$ (or the melting line) and $1000 \mathrm{C}$ and provides accurate representation of existing thermodynamic data in that range. In addition it provides a smooth transition from singular critical region functions to the nonsingular far-field functions. This is demonstrated by the variations of isochoric specific heat, isothermal compressibility, speed of sound, specific heat ratio and coexistence line properties in the critical region

100,365

B91-192542

Not available NTIS

American Chemical Society, Washington, DC

Journal of Physical and Chemical Reference Data, Volume 19, Number 6, November-December 1990. Bimonthly rept.

D. R. Lide. c1990, 389p

See also PB91-192559 through PB91-192583. Errata sheet inserted. Prepared in cooperation with American Inst. of Physics, New York. Sponsored by National Inst. of Standards and Technology, Gaithersburg, MD. Available from American Chemical Society, 1155 16th St., NW, Washington, DC. 20036-9976.

Keywords: * Research, Thermal conductivity, Viscosity, Rare gases, Line spectra, Stark effect, Polyatomic molecules, Molecular energy levels, Vibrational states, Organic nitrogen compounds, Amines, Thermodynamic properties, Thermophysical properties, Transient molecules.

\section{Contents:}

The Viscosity and Thermal Conductivity of Pure Monatomic Gases from Their Normal Boiling Point up to $5000 \mathrm{~K}$ in the Limit of Zero Density and at $0.101325 \mathrm{MPa}$ :

Experimental Stark Widths and Shifts for Spectral Lines of Neutral and Ionized Atoms (A Critical Review of Selected Data for the Period 1983 through 1988)

Vibrational and Electronic Energy Levels of Polyatomic Transient Molecules Supplement 1;

Thermodynamic and Thermophysical Properties of Organic Nitrogen Compounds. Part 1. Methanamine, Ethanamine, 1- and 2 . Propanamine, Benzenamine, 2-, 3-, and 4Methylbenzenamine.

\section{0,366}

PB91-192559

Not available NTIS

Rostock Univ. (German D.R.). Fachbereich Chemie.

Viscosity and Thermal Conductivity of Pure Monatomic Gases from Their Normal Boiling Point Up to $5000 \mathrm{~K}$ in the Limit of Zero Density and at 0.101325 $\mathrm{MPa}$.

Bimonthly rept

E. Bich, J. Millat, and E. Vogel. c1990, 17p

Sponsored by National Inst. of Standards and Technology, Gaithersburg, MD.

Included in Jnl. of Physical and Chemical Reference Data, v19 n6 p1289-1306 Nov/Dec 90. Available from American Chemical Society, 1155 16th St., NW, Washington, DC. 20036-9976.

Keywords: "Thermal conductivity, "Viscosity, "Helium, "Neon, "Argon, "Krypton, "Xenon, Transport properties, Kinetic theory, Tables(Data).

The kinetic theory of gases in the limit of zero density and that of moderately dense gases is used to generate accurate tables of the viscosity and thermal conductivity of the pure monatomic gases for zero density and for a pressure of $0.101325 \mathrm{MPa}$. The theoreticallybased tables cover the temperature range from the normal boiling point of the relevant gas up to $5000 \mathrm{~K}$. The associated uncertainties of the proposed data are detailed in the paper. A comparison of the correlated data with experimental results and some other recent correlations is given.

\section{0,367}

PB91-192575

Not available NTIS

National Inst. of Standards and Technology (NML)

Gaithersburg, MD. Molecular Physics Div.

Vibrational and Electronic Energy Levels of Polyatomic Transient Molecules. Supplement 1. Bimonthly rept.

M. E. Jacox. c1990, 160p

Sponsored by National Inst. of Standards and Technology, Gaithersburg, MD.
Included in Jnl of Physical and Chemical Reference D19 6 . 387-1546 Nov/Dec 90 . Available from American Chemical Society, 1155 16th St., NW, Washington, DC. 20036-9976.

Keywords: "Polyatomic molecules, "Molecular energy levels, "Vibrational states, Laser induced fluorescence, Photoelectron spectroscopy Emission spectra Free radicals, Infrared spectra, Hydrides, Matrix isolation, Molecular ions, Radiative lifetime, Raman spectra, Ultraviolet absorption, Tables(Data), "Transient molecules.

New information on the experimentally determined vibrational and electronic energy levels of approximately 500 neutral and ionic transient molecules possessing from 3 to 16 atoms has been evaluated and added to the previously established database for these species. There has been selective extension of the compilation to somewhat less reactive species such as $\mathrm{HNCO}$ $\mathrm{HCNO}, \mathrm{H} 2 \mathrm{O} 2$, and cis- and trans-HONO, as well as to many transient molecules which include atoms beyond the third row of the Perjodic Table. Electronic spectral data are also given for a number of transient molecules which possess more than six atoms. Radiative life-times and the principal rotational constants are included. Observations in the gas phase, in molecular beams, and in rare-gas and nitrogen matrices are evaluated. The types of measurement surveyed include conventional and laser-based absorption and emission techniques, laser absorption with mass analysis, and photoelectron spectroscopy.

\section{0,368}

PB91-192583

Not available NTIS

Texas $A$ and $M$ Univ., College Station. Thermodynamics Research Center.

Thermodynamic and Thermophysical Properties of Organic Nitrogen Compounds. Part 1. Methana. mine, Ethanamine, 1- and 2-Propanamine, Benzenamine, 2-, 3-, and 4-Methylbenzenamine.

Bimonthly rept

J. Chao, N. A. M. Gadalla, B. E. Gammon, K. N. Marsh, A. S. Rodgers, G. R. Somayajulu, and R. C. Wilhoit. c1990, 70p

Sponsored by National Inst. of Standards and Technology, Gaithersburg, MD.

Included in Jnl. of Physical and Chemical Reference Data, v19 n6 p1547-1616 Nov/Dec 90. Available from American Chemical Society, 1155 16th St., NW, Washington, DC. 20036-9976.

Keywords: *Organic nitrogen compounds, "Amines, "Thermodynamic properties, "Thermophysical properties, Boiling points, Freezing, Critical point, Specific efficients, Density, Combustion, Thermochemistry.

The thermodynamic and thermophysical properties of eight primary amines, methanamine, ethanamine, 1 and 2-propanamine, benzenamine, and 2-, 3-, and 4 methylbenzenamine have been evaluated. Recommended values are given for the following properties: normal boiling, freezing, and triple-point temperatures, critical constants, thermodynamic properties in the solid and liquid phases, vapor pressure, enthalpy of vaporization, density, second virial coefficients, and enthalpy of combustion. Ideal gas thermodynamic properties have been calculated by statistical mechanical methods.

\section{0,369}

PB91-194472

PC A04/MF A01

National Inst. of Standards and Technology (CSTL) Gaithersburg, MD.

Experimental Thermal Conductivity, Thermal Diffusivity, and Specific Heat Values for Mixtures of Nitrogen, Oxygen, and Argon.

Final rept.

R. A. Perkins, and M. T. Cieszkiewicz. Mar 91, 65p NISTIR-3961

See also PB89-148407. Sponsored by Wright Research and Development Center, Wright-Patterson AFB, OH., and National Aeronautics and Space Administration, Cleveland, $\mathrm{OH}$. Lewis Research Center.

Keywords: "Thermal conductivity, "Thermal diffusivity, "Specific heat, "Ternary systems, "Nitrogen, "Oxygen, "Argon, Binary system(Materials), Hot wire anemometers, Tables(Data), Temperature dependence, Pressure dependence, Standards. Thermodynamic properties, Measurement, Supercritical state, Mixtures, Equations of state, Correlations, Liquefied gases, Air. 
Experimental measurements of thermal conductivity and thermal diffusivity obtained with a transient hotwire apparatus are reported for three mixtures of nitro. gen, oxygen, and argon. Values of the specific heat $\mathrm{Cp}$, are calculated from these measured values and the density calculated with an equation of state. The measurements were made at temperatures between 65 and $303 \mathrm{~K}$ with pressures between 0.1 and $70 \mathrm{MPa}$. The data cover the vapor, liquid, and supercritical gas phases for the three mixtures. The total reported points are 1066 for the air mixture $(78.11 \%$ nitrogen $20.97 \%$ oxygen $-0.92 \%$ argon), 1058 for the $50 \%$ ni trogen - $50 \%$ oxygen mixture, and 864 for the $25 \%$ nitrogen - $75 \%$ oxygen mixture. Empirical thermal conductivity correlations are provided for the three mixtures.

\section{0,370}

PB91-194837

Not available NTIS National Inst. of Standards and Technology (NML) Gaithersburg, MD. Molecular Spectroscopy Div.

Theoretical Model of Metal Binding Sites in Proteins.

Final rept.

M. Krauss, and W. J. Stevens. 1989, 13p

Pub. in Progress in Clinical and Biological Research 289, p95-107 1989 .

Keywords: "Subtilisins, "Molecular orbitals, "Binding energy, "Calcium ions, "Magnesium ions, "Sodium ions, Proteins, Cations, Metals, Reprints, "Binding sites.

$\mathrm{Ab}$ initio molecular orbital calculations of the binding energy of metal cations to clusters of water, formamide, and formate ligands are used to analyze $\mathrm{Ca}$ bind ing sites in proteins. The ab initio energetics of the first coordination shell provide a basis for evaluating the conformation behavior and the selectivity of cation binding. The enthalpies of binding are modeled by estimating the Born polarization energy relative to the model cluster of the first shell. The data is applied to an analysis of binding sites in the protein, Subtilisin BPN. In the study the $\mathrm{Ca}, \mathrm{Mg}$, and $\mathrm{Na}$ cation energetic selectivity is compared for binding to two of the sites.

\section{0,371}

1-194944

Not available NTIS

National Inst. of Standards and Technology (IMSE) Gaithersburg, MD. Ceramics Div.

Reflection-Extended-X-ray-Absorption-Fine-

Structure Spectroscopy at the Carbon K-Edge.

Final rept.

G. G. Long, D. R. Black, and D. K. Tanaka. 1989, 6p Pub. in Materials Research Society Symposia Pro ceedings, v143 p157-1621989.

Keywords: *Glassy carbon, Graphite, Chemical bonds Vitreous state, Backscattering, Reprints, Extended Xray absorption fine structure spectroscopy, K-edge energy.

The carbon K-edge Reflection-Extended X-Ray Absorption Fine Structure (refl-EXAFS) spectra from graphite, diamond and glassy carbon have been investigated. There is good phase shift transferability be tween the two well-known bonding types in diamond and graphite, provided that appropriate inner potential corrections to the K-edge ( $E$ (sub o) are made. The model spectra from diamond and graphite were used to investigate the nature of glassy carbon. It was found that for the particular form of glassy carbon used in the study, the bonding more closely resembled $\mathrm{sp} 3$ than sap2. The result is preliminary pending the authors' evaluation of the influence of surface oxygen.

\section{0,372}

PB91-194951

Not available NTIS

National Inst. of Standards and Technology (IMSE), Gaithersburg, MD. Ceramics Div.

Surface-Extended X-ray-Absorption Fine-Structure Experiments at Atmospheric Pressure by Means of a Photocathode Proportional Counter with Monolayer Sensitivity.

Final rept.

G. G. Long, D. K. Tanaka, J. Kruger, G. A. Danko,

and D. A. Fischer. 1989, 7p

Pub. in Physical Review B 39, n15 p651-657 1989.

Keywords: "Iron oxides, X-ray absorption, Proportional counters, Photocathodes, Thin films, Substrates, Surfaces, Reprints.

Surface-EXAFS (extended x-ray absorption fine structure) measurements at atmospheric pressure were made by means of a new photocathode proportional counter on the oxide films that form on bulk iron substrates. Both thermally-formed and chemically-formed films were studied. Near-edge spectra with effective monolayer sensitivity were used to investigate the ionicity of cations in ultra-thin films

\section{0,373}

PB91-194985

Not available NTIS

National Inst. of Standards and Technology (NML).

Gaithersburg, MD. Center for Chemical Technology.

PLOTMD: An Interactive Program to Modify Molecular Plots on a Graphics Terminal.

Final rept.

J. C. Luo, G. L. Gilliland, and H. L. Ammon. 1989, 1p Pub. in Jnl. of Applied Crystallography 22, p186 Apr 89

Keywords: * "Crystallography, "Stereochemistry, "Graphic methods, "Crystal structure, "Computer programs, Molecular structure, Reprints, PLOTMD program.

A program was written to display an HP 7550 A plot file on a MicroVax II workstation. Some of the modifica tions of the picture that can be performed are: (1) change between $8.5 \times 11$ and $11 \times 8.5$ inch formats; ( 2 ) rotate about Z-axis; (3) move picture up-down and right-left; (4) increase or decrease picture size; (5) insert dashed lines between atoms, perhaps to indicate a weak interaction; (6) manipulate labels. Labeltext information can be deleted, inserted, moved, al tered and increased or decreased in size. Stereo-labeling is carried out automatically from manipulations on the left-eye image. The program is written in VAX FORTRAN(V4.0) on the MicroVMS operating system(V4.5). The graphics procedures are based on the MicroVMS workstation graphics software(V3.0) The program is implemented on a standard MicroVAX II workstation with a 20 inch monochrome monitor and a mouse.

\section{0,374}

PB91-195008

Not available NTIS

National Inst. of Standards and Technology (IMSE), Gaithersburg, MD. Metallurgy Div.

Atomistic Diffusion Equations and Kinetic Forces. Final rept.

J. R. Manning. 1989, 15p

Sponsored by Office of Naval Research, Arlington, VA Pub. in Diffusion Analysis and Applications, p3-17 1989.

Keywords: "Diffusion, "Reaction kinetics, Thermodynamic equilibrium, Metals, Theories, Equations, Vacancies, Crystal structure, Reprints.

There are two major approaches to diffusion theory (1) the atomistic-kinetic approach in which the motions of individual atoms are followed and (2) the continuumthermodynamic approach in which thermodynamic forces are described which move the system toward an equilibrium state by inducing atom fluxes. In the present paper, atomistic-kinetic diffusion equations are developed from a consistent point of view based on calculations of the energy change resulting from individual atom jumps. The approach, which is generally applicable, is employed here to give for the first time a fully atomistic calculation of kinetic driving forces from coherency strains, the energy of mixing and the gradient energy. Effects on the kinetic equations from vacancy concentration gradients, vacancy fluxes and dependencies of vacancy formation energy on composition are also calculated; and a general atomistic-kinetic expression for the atom flux between neighboring planes is developed. The equation is shown to provide the same type of forces and terms as those in the general thermodynamic diffusion equations. Thus, the two approaches yield identical results. They can be usefully combined to provide practical applications of diffu sion theory that would not be obtained from either ap proach alone.

\section{0,375}

\section{PB91-195065}

Not available NTIS

National Bureau of Standards (NML), Gaithersburg MD. Chemical Kinetics Div.

Carbon Acidities of Aromatic Compounds. 2. Ionic Probes of Aromaticity in Annelated Rings.

Final rept.

M. Mautner, J. F. Liebman, and S. A. Kafafi. 1988,

$5 p$

Pub. in Jnl. of the American Chemical Society 110, n18 p5937-5941 1988 .

Keywords: "Aromatic compounds, "Chemical bonds "Aromatic polycyclic hydrocarbons, "Hydrogen ions 1 plus, Acidity, Carbon, Atomic models, Resonance charge exchange, Benzene, Pyridines, Ionization, Polarizability, Napthalene, Indene, Semiempirical equations, Stability, Reprints, Protonation, Annelation, Charge stabilization.

lonization by the deprotonation of benzene and pyridine, and by the protonation of pyridine, involve lone pairs in the sigma plane without significant pi effects. In these cases annelation by a benzene ring increases the acidity or proton affinity by a constant $6+$ or -1 $\mathrm{kcal} / \mathrm{mol}$, ascribed to increased polarizability. In comparison, protonation of benzene and deprotonation of cyclopentadiene disrupts or creates a 6-electron aromatic system, respectively, and in their annelated derivatives, naphthalene and indene, a secondary 4-electron conjugated pi system. These cases involving pi electrons show annelation effects that are substantially larger $(13.4 \mathrm{kcal} / \mathrm{mol})$ or smaller $(1.0 \mathrm{kcal} / \mathrm{mol}) \mathrm{re}$ spectively, than just the electrostatic effect. Analysis of these data suggests that the stability of secondary 4electron systems in the annelated rings is smaller by 6 + or $-\mathrm{kcal} / \mathrm{mol}$ than the aromatic 6-electron systems. In fair agreement with Herndon's structure resonance values for these species. Annelation effects are reproduced well by Dewar's AM1 semiempirical method.

100,376

PB91-195073

Not available NTIS

National Inst. of Standards and Technology (NML),

Gaithersburg, MD. Chemical Kinetics Div.

Thermal Decomposition of lons. 3. Protonated Ethanol and Diethyl Ether.

Final rept.

M. Mautner, and L. W. Sieck. 1989, 11p

See also PB87-162301.

Pub. in International Jnl. of Mass Spectrometry and lon Processes 92, p123-1331989.

Keywords: "Organic ions, "Ethanol, "Ethyl ether, "Pyrolysis, Reaction kinetics, Activation energy, Protons, Reprints.

Above $550 \mathrm{~K}$, the $\mathrm{C}_{2} \mathrm{H} 5 \mathrm{OH} 2+$ ion decomposes to yield $\mathrm{H} 3 \mathrm{O}+$ with the loss of $\mathrm{C} 2 \mathrm{H} 4$. An alternative decomposition channel is the formation of $\mathrm{CH} 3 \mathrm{CHOH}+$ where in the $\mathrm{H} 2$ lost, deuteration studies suggest that one hydrogen atom originates from hydroxyl and another from the ethyl group. The decomposition products regenerate $\mathrm{C} 2 \mathrm{H} 5 \mathrm{OH} 2+$ by proton transfer to $\mathrm{C} 2 \mathrm{H} 5 \mathrm{OH}$, forming reaction cycles that reach steadystates. At higher concentrations of $\mathrm{C} 2 \mathrm{H} 5 \mathrm{OH}$, condensation with $\mathrm{C}_{2} \mathrm{H} 5 \mathrm{OH} 2+$ forms $(\mathrm{C} 2 \mathrm{H} 5) 2 \mathrm{OH}+$, which in turn decomposes with the loss of $\mathrm{C} 2 \mathrm{H} 4$ to regenerate $\mathrm{C} 2 \mathrm{H}_{5} \mathrm{OH} 2+$, resulting in another steady-state cycle. Decomposition rate constants are calculated from steady-state ion ratios, and $150-670 \mathrm{~K}$ are in the range of 1 to $100000 / \mathrm{sec}$ for all the reactions. The decompositions of $\mathrm{C} 2 \mathrm{H} 5 \mathrm{OH} 2+$ are at the low-pressure limit and of $(\mathrm{C} 2 \mathrm{H} 5) 2 \mathrm{OH}+$ are at the high-pressure limit at $2-8$ torr. The decompositions show Arrhenius activation energies of 10 to $30 \mathrm{~K} \mathrm{cal} / \mathrm{mol}$.

\section{0,377}

PB91-195131 Not available NTIS

National Inst. of Standards and Technology (NEL), Gaithersburg, MD. Thermophysics Div.

Dipole Moments of Seven Partially Halogenated Ethane Refrigerants.

Final rept.

C. W. Meyer, and G. Morrison. 1991, 7p

Pub. in Jnl. of Physical Chemistry 95, n9 p3860-3866, 2 May 91.

Keywords: "Refrigerants, "Fluorinated alphatic hydrocarbons, "Ethane, "Dipole moments, "Gases, Temperature dependence, Refractivity, Measurement, Dielectric properties, Polarizability, Reprints.

Dielectric constant measurements have been performed on seven partially halogenated ethanes, currently being considered as alternative refrigerants, in the gaseous state over the range $305-415 \mathrm{~K}$ in order to determine their dipole moments. Argon was also studied to test the apparatus. Five of the refrigerants have temperature-independent moments: R125 (CF3CHF2) $\mathrm{mu}=1.563+$ or $-0.005 \mathrm{D} ; \mathrm{R} 134 \mathrm{a}$ (CF3CH2F) $\mathrm{mu}=2.058+$ or $-0.010 \mathrm{D}$ : R152a (CHF2CH3), $\mathrm{mu}=2.262+$ or $-0.008 \mathrm{D}$; and R124 (CF3CHCIF), mu $=1.469+$ or $-0.012 \mathrm{D}$. Two refrigerants have conformer-dependent moments, making their time-averaged moments temperature dependent: R134 (CHF2CHF2), with a moment ranging from 0.991 + or $-0.004 \mathrm{D}$ at $309 \mathrm{~K}$ to $1.250+$ or $-0.004 \mathrm{D}$ at 413 $\mathrm{K}$; and R143 (CHF2CH2F), where the moment ranges 
from $1.680+$ or $-0.001 \mathrm{D}$ at $309 \mathrm{~K}$ to $1.745+$ or $0.001 \mathrm{D}$ at $410 \mathrm{~K}$. Accurate estimation of a molecule's temperature-dependent dipole moment requires an in dependent determination of its polarizability; this determination has been made from index of refraction measurements. Where possible, the present results are compared with earlier determinations of dipole mo ments. In all but one instance, the agreement is good

\section{0,378}

PB91-195214

Not available NTIS

National Bureau of Standards (NML), Gaithersburg, MD. Chemical Kinetics Div.

Proton Affinities and pKa Values of Tetraalkylhydrazines.

Final rept

S. F. Nelsen, M. Mautner, D. T. Rumack, and L. W.

Sieck. 1988,6p

Pub. in Jnl. of the American Chemical Society $110, n 19$ p6303-6308 1988.

Keywords: "Hydrazine, "Proton affinity, "Pyridines, "Dissociation, "Tertiary amines, Alkyl compounds, Amines, Thermodynamic quilibrium, Solvation, Hydrogen ions 1 plus, Reprints, Proton affinity, Protonation. Proton affinities for 16 saturated tetraalkylhydrazines were determined by equilibration with triethylamine, 2,6-dimethyl pyridine, and 2,6-diethylpyridine using high pressure mass spectrometry, and pKa measurements in water were made for the 14 compounds which proved soluble enough. Proton transfer equilibria for these compounds are compared with electron transfer equilibria previously measured.

\section{0,379}

PB91-195230

Not available NTIS

National Inst. of Standards and Technology (NML) Gaithersburg, MD. Chemical Kinetics Div.

Rate Constants for Reduction of Substituted Methylperoxyl Radicals by Ascorbate lons and N,N,N',N'-Tetramethyl-Para-Phenylenediamine.

Final rep

P. Neta, S. Steenken, L. V. Shastri, R. E. Huie, S

Mosseri, J. P. Mittal, and P. Maruthamuthu. 1989, 6p

Pub. in Jnl. of Physical Chemistry 93, n10 p4099-4104 1989.

Keywords: *Reaction kinetics, "Reduction(Chemistry) "Free radicals, Ascorbic acid, lons, Peroxy radicals Radiolysis, Thermodynamic equilibrium, Pulse techniques, Alkyl compounds, Reprints, Tetramethyl-pphenylenediamine, Taft constants.

Absolute rate constants $(k)$ for reduction of substituted methylperoxyl radicals by ascorbate ions and by TMPD (N,N,N',N'-tetramethyl-p-phenylenediamine) in aqueous solutions have been determined by pulse radiolysis. The rate constants vary from 10 to the 6 th powe to 10 to the 9 th power $/ \mathrm{M} / \mathrm{s}$, increasing as the electron withdrawing capacity of the substituent on the peroxy group increases. Linear correlations are observed between log $k$ and the Taft substituent constants sigma for a wide variety of substituents, but not all substituents fit the same line. In the case of ascorbate as reductant, the points for peroxyl radicals that contain halogens on the alpha-carbon lie on a different line (rho* $=0.41$ ) than that for the other substituents (rho* $=1.25$ ). In the case of TMPD there are also two families of peroxyl radicals: those comprising the electron donating groups Me through $\mathrm{t}$-Bu (rho* $=5.6$ ) and those containing electron-withdrawing substituents $\left(\mathrm{rho}^{*}=0.64\right)$

100,380

PB91-195255

Not available NTIS

National Bureau of Standards (IMSE), Gaithersburg, MD. Reactor Radiation Div.

Dynamics of Molecular Hydrogen Adsorbed in CoNa-A Zeolite.

Final rept.

J. M. Nicol, J. Howard, and J. Eckert. 1988, 5p

Pub. in Jnl. of Physical Chemistry 92, n25 p7117-7121 1988.

Keywords: "Hydrogen, *Adsorption, "Zeolites, Cobal ions, Sodium ions, Neutron scattering. Chemical bonds, Reprints, "Molecular dynamics, Vibrational spectra.

The dynamics of molecular hydrogen adsorbed in the cavities of partially cobalt exchanged type $A$ zeolite (C04.1 Na3.8-A) has been investigated in the energy range $0.40 \mathrm{meV}$ by incoherent inelastic neutron scattering. Both rotational and vibrational excitations are identified in the spectra. The rotational tunnel splitting of the vibrational ground state of the coordinated molecular hydrogen is observed at $3.8 \mathrm{meV}$. Analysis of the data in terms of a twofold cosine potential with two degrees of rotational freedom suggests that the hydrogen molecules are bound end-on to the Co cations and perform $180 \mathrm{deg}$. reorientations with a barrier of 55-68 $\mathrm{meV}(1.3-1.5 \mathrm{Kcal} / \mathrm{mole})$. A mode at $15.3 \mathrm{meV}$ is identified as a vibration of the bound hydrogen. Evidence for the torsional mode of Al(OH) 4 complexes, formed in the beta-cages during ion-exchange, is found in the vibrational spectra at $21 \mathrm{meV}$.

100,381

PB91-195297

Not available NTIS National Bureau of Standards (NML), Gaithersburg, MD. Quantum Metrology Group.

Anomalous Th M3O1 X-ray Emission Spectrum of ThO2

Final rept.

M. Ohno, and A. E. LaVilla. 1988, 4p

Pub. in Physical Review B 37, n18 p915-918 1988.

Keywords: "Thorium oxides, "X ray spectra, Emission spectra, Greens function, Coster-Kronig transitions, Reprints.

The Th M301 (triplet $P(3 / 2)$ (sup -1) -> 5s(sup -1)) $x$ ray emission spectrum (XES) of ThO2 was measured with a high resolution vacuum double crystal spectrometer. The spectrum is the first evidence of the splitting of a $5 \mathrm{~s}$ hole level into two main structures which are due to a strong configuration interaction 5 s(sup -1) <--> 5p(sup -1)5d(sup -1)5(n, epsilon) super Coster-Kronig process. The $\mathrm{O}(1 \mathrm{a})$ and $\mathrm{O}(1 \mathrm{~b})$ hole energies of ThO2 determined by the presen measurements are respectively, 293.9 and $276.2 \mathrm{eV}$. A large energy shift (order of about $15 \mathrm{eV}$ ) to lower binding energy from the theoretically predicted atomic energies is observed even when one takes into accoun the atom-oxide energy shift. The cause of discrepancy is suggested to be due to a strong configuration interaction of the final $5 \mathrm{p} 5 \mathrm{~d}$ double hole.

100,382

PB91-195305

Not available NTIS

National Inst. of Standards and Technology (NML). Gaithersburg, MD. Quantum Metrology Div.

Many Electron Effects in Lgamma2,3 X-ray Emission Spectroscopy Spectrum of $\mathrm{BaO}$.

Final rept.

M. Ohno, and R. E. LaVilla. 1989, 7p

Pub. in Physical Review B 39, n13 p8845-8851 1989.

Keywords: "Barium oxides, "X ray spectra, CosterKronig transition, Emission spectra, Greens function, Reprints.

The L(gamma 2,3)(2s(sup -1) $\rightarrow>4 p($ sup -1) x-ray emission spectrum (XES) of $\mathrm{BaO}$ was measured in fluorescence with a high resolution vacuum double crystal spectrometer. The spectrum was also calculated by the Green's function method. It is shown that the spectrum can be interpreted essentially in terms of a two level system in the final state, e.g., the $4 p$ hole leve interacting with the product of the $4 p($ sup -1$)<-->$ $4 \mathrm{~d}$ (sup -2) $4 \mathrm{f}$ super Coster-Kronig process. It is also shown that it is necessary to take into account the $4 d$ (sup -2) 4 f multiplet.

\section{0,383}

PB91-195313

National Inst. of Standards and Technology (NML) Gaithersburg, MD. Quantum Metrology Div.

Many-Electron Effects in Lgamma2,3 XES Spectra from Rare Earth Elements.

Final rept.

M. Ohno, and R. E. LaVilla. 1989, 6p

Pub. in Physical Review B 39, n13 p8852-8857 1989.

Keywords: "Neodymium oxides, "Samarium oxides, "X ray spectra, Coster-Kronig transitions, Emission spectra, Greens function, Reprints.

The L(gamma 2,3)(2s(sup -1)-> 4p(sup -1)) $x$-ray emission spectra of $\mathrm{Nd} 2 \mathrm{O} 3$ and $\mathrm{Sm} 2 \mathrm{O} 3$ were measured in order to study the strong configuration interaction between a $4 \mathrm{p}$ hole level and the double $4 \mathrm{~d}$ hole $4 \mathrm{f}$ (sup $n+1$ ) electron levels. The spectra are calculated also using the Green's function method. The agreement between theory and experiment is reasonably good. The spectrum can be interpreted in terms of the spectral function of the final $4 \mathrm{p}$ hole where it is shown that the one-electron picture of the $4 \mathrm{p}$ hole breaks down due to the strong $4 p($ sup -1$)<-->4 d($ sup -2$) 4 f($ sup $n+1)$ super Coster-Kronig process.

\section{0,384}

PB9 1-195370

Not available NTIS (NML). Gaithersburg, MD. Chemical Kinetics Div.

Reactions of $\mathrm{C}_{5} \mathrm{H} 3(+)$ and $\mathrm{C} 5 \mathrm{H} 5(+)$ lons with Acetylene and Diacetylene.

Final rept.

F. Ozturk, S. G. Lias, M. Moini, F. W. Brill, and T. J.

Buckley. 1989, 7p

See also AD-A197 087

Pub. in Jnl. of Physical Chemistry 93, n10 p4038-4044 1989

Keywords: "Acetylene, "Alkene compounds, "Alkyne compounds, lon cyclotron-resonance, Reaction kinetics, Reprints, "Organic ions, "Diacetylene, "Butyne, Fourier transform mass spectroscopy, lon molecule reactions.

The reactions of $\mathrm{C} 5 \mathrm{H} 3(1+)$ and $\mathrm{C} 5 \mathrm{H} 5(1+)$ ions with acetylene and diacetylene were investigated using a Fourier transform ion cyclotron resonance (FTICR) mass spectrometer. The ejection capabilities of FTICR were used to determine the reaction mechanisms and rate constants for the reactions of ions produced from a number of different precursors. While different structures could be attributed to $\mathrm{C} 5 \mathrm{H} 3(1+)$ ions produced from different precursors on the basis of reactivity, no significant differences in the rate constants of C5H5(1+) ions were observed. Different percentages of reactive $\mathrm{C} 5 \mathrm{H} 5(1+)$ structure(s) were produced from different precursors using different charge transfe gases. Formation of $\mathrm{C} 5 \mathrm{H} 5(1+)$ from norbornadiene and cycloheptatriene was studied in more detail and existence of a second formation mechanism was shown to be possible.

\section{0,385}

PB91-195735

Not available NTIS National Bureau of Standards (IMSE), Gaithersburg. MD. Ceramics Div.

High-Temperature

from Liquid Water.

Final rept.

G. E. Walrafen, W. H. Yang, M. S. Hokmabadi, and G. J. Piermarini. 1988, 3p

Pub. in Jnl. of Physical Chemistry 92, n15 p4540-4542 1988.

Keywords: "Raman spectra, "Water, Hydrogen bonds, Pressure dependence, High pressure, High temperature, Reprints, Diamond anvils.

Raman spectra were obtained from liquid water at 33 kbar and at 170 and $200 \mathrm{C}$ using a diamond anvil cell. Pressures were determined from the ice VII-wate melting temperatures. Comparison of the present data, with data from Lindner and Franck, indicates that the $\mathrm{OH}$-stretching peak frequency, in $\mathrm{cm}($ sup -1), de creases with increasing pressure at constant temperature. Further analysis of the data suggests that the double-to-single, hydrogen-bond potential-well transformation, which is known to occur for linear hydrogen bonds, also occurs for severely bent, nonhydrogenbonded, $\mathrm{O}-\mathrm{H}-\mathrm{O}$ configurations.

100,386

PB91-200782

Not available NTIS

National Inst. of Standards and Technolo

Reactivities of Chlorine Atoms and Peroxyl Radicals Formed in the Radiolysis of Dichloromethane. Final rept.

Z. B. Alfassi, S. Mosseri, and P. Neta. 1989, 6p Sponsored by Department of Energy, Washington, DC Pub. in Physical Chemistry 93, n4 p1380-1385 1989

Keywords: "Methylene chloride, "Peroxy radicals, "Chlorine, "Atoms, "Reactivity, "Reaction kinetics, Radiolysis, Anilines, Chemical radicals, Phenols, Hy drogen transfer, Ethers, Reprints.

Radiolysis of dichloromethane (DCM) leads to formation of primary oxidizing radicals and carbon-centered radicals. The latter react with oxygen to yield peroxy radicals. The yields and chemical behavior of these in termediates were studied by pulse radiolysis of DCM solutions containing various solutes: phenols, anilines, dimethoxybenzene, hexamethylbenzene, cyclohex ene, dimethyl sulfoxide, and zinc tetratolylporphyrin. At low concentrations, some of these solutes were found to be oxidized by two peroxyl radicals, $\mathrm{CHClO}$ ? (dot) and $\mathrm{CHCl} 2 \mathrm{O} 2$ (dot), with different rate constants higher concentrations, the solutes react also with the 


\section{Physical \& Theoretical Chemistry}

primary radicals: $\mathrm{Cl}$ atoms and the radical cations $\mathrm{CH} 2 \mathrm{Cl} 2(+$ dot) with diffusion-controlled rate constants. The rates of these reactions were determined by competition kinetics because of the very short lifetimes of the species. $\mathrm{Cl}$ atoms were found to have a half-life of about $5 \mathrm{~ns}$ in DCM, reacting predominantly with the solvent by hydrogen abstraction. The radica cations decay within a fraction of a nanosecond. The total yield of these primary radicals was determined to be $\mathrm{G}=3.6$ and appears to be divided about equally between $\mathrm{Cl}$ and the radical cations. The total yield of oxidation, by the primary and the peroxyl radicals, was found to be $\mathrm{G}=7.5 \mathrm{Cl}$ atoms were found to be very reactive in electron transfer as well as addition and hydrogen abstraction reactions.

\section{0,387}

PB91-202846

Not available NTIS

National Bureau of Standards (NML), Gaithersburg

MD. Molecular Spectroscopy Div.

Time- and State-Resolved Measurements of Nitric Oxide Dimer Infrared Photodissociation.

Final rept.

M. P. Casassa, J. C. Stephenson, and D. S. King

$1988,11 \mathrm{p}$ Pub. in Jnl. of Chemical Physics 89, n4 p1966-1976

Keywords: "Nitric oxide, "Photodissociation, Laser in duced fluorescence, Van der Waals forces, Vibrationa states, Picosecond pulses, Infrared spectra, Predissociation, Lifetime, Dimers, Reprints.

Picosecond and nanosecond lasers and pulsed mo lecular beam techniques have been used to measure the infrared photodissociation spectra, the product state distributions, and the predissociation lifetimes of vibrationally excited nitric oxide dimer, (NO)2. Results for the nu(1) $(v=1)$ symmetric NO stretching mode and the $n u(4)(v=1)$ antisymmetric NO stretching mode are presented. Predissociation lifetimes are determined by time-resolved laser induced fluorescence probing of the NO monomer product appearance rate. The observations are discussed in terms of various vibrational predissociation mechanisms, including vibrational potential coupling and electronically nonadiabatic predissociation

\section{0,388}

PB91-202853

Not available NTIS National Bureau of Standards (IMSE), Gaithersburg, MD.

Grain Boundaries in a 2-D Superlattice.

Final rept.

P. Cenedese, R. Kikuchi, and J. W. Cahn. 1988, 6p Pub. in Materials Research. Society Symposium Pro ceedings 122, p231-236 1988.

Keywords: "Crystal structure, "Grain boundaries, "Superlattices, "Bicrystals, Cluster analysis, Predictions, Two-dimensional calculations, Microstructure, Thermodynamic properties, Mathematical models, Gases, Dihedral angle, Wetting, Transition temperature, Critical temperature, Temperature dependence, Adsorption, Reprints.

In bicrystals with CSL orientation relationships two kinds of coincidence site lattices form, resulting in two kinds of grain boundaries. The thermodynamic properties are examined, using a lattice gas model, and make microstructural predictions about the dihedral angles at junctions between these boundaries. The behavio of these boundaries is examined for low temperature and through the critical disordering temperature Some aspects of nonstoichiometry are presented. A wetting transition is found at low temperatures, in which one kind of grain boundary is converted into the other by expelling antiphase domain boundary. No wetting tran sition is found at the critical temperature because an unusually high critical exponent of 3 is found for the difference in grain boundary energies between the symmetric and antisymmetric cases.

\section{0,389}

PB91-202986 Not available NTIS

National Inst. of Standards and Technology (NML)

Boulder, CO. Quantum Physics Div.

Causes of $\mathrm{SiH} 4$ Dissociation in Silane dc Discharges.

D. A. Doughty, and A. Gallagher. 1990, 5p

Contract SERI-XB-2-02189

Sponsored by Solar Energy Research Inst., Golden,

CO.

Pub. in Physical Review A 42, n10 p6166-6170, 15 Nov 90.
Keywords: "Silane, "Gas discharges, "Chemical dissociation, lon-molecule collisions, Silicon hydrides, Amorphous silicon, Semiconducting films, Substrates, Glass, Reprints.

Hydrogenated amorphous-silicon (alpha-Si:H) film growth on glass fibers strung between discharge electrodes is used to measure the distribution of film-producing radicals in a silane dc discharge. The measured distribution, as well as film deposition rates on the electrodes, show that typically $>80 \%$ of the depositing radicals are produced in the cathode sheath. Discharge models, confirmed by the spatial distribution of optical emission, rule out the possibility that this dissociation in the sheath is due to electron impact. Collisions of energetic ions and neutrals with silane are clearly implicated as the cause of the sheath dissociation. In contrast, due to much lower ion kinetic energies, almost all dissociation is due to electron collisions in the low-power if discharges most commonly used for film production. In addition, the ratio of the number of Si atoms deposited on all surfaces to the total number of ions collected at the dc-discharge cathode is measured to be 30 , demonstrating the dominance of neutral radical deposition in these discharges.

\section{0,390}

PB91-202994

Not available NTIS

National Inst. of Standards and Technology (NML), Boulder, CO. Quantum Physics Div.

Silane Dissociation Products in Deposition Discharges.

Final rept

J. R. Doyle, D. A. Doughty, and A. Gallagher. 1990 , $10 \mathrm{p}$

Contract SERI-XG-1-1216

Sponsored by Solar Energy Research Inst., Golden, $\mathrm{CO}$.

Pub. in Jnl. of Applied Physics 68, n9 p4375-4384, 1 Nov 90.

Keywords: "Silane, "Gas discharges, "Chemical dissociation, Surface reactions, Amorphous silicon, Semiconducting films, Reprints.

Time-dependent production of higher-silane gases and alpha-Si:H film are measured relative to decomposed silane in if and dc, hot and cold cathode, static gas discharges. From the absence of higher-silane production in very low silane partial-pressure discharges, it is inferred that most higher silanes are procharges, it is inferred that most higher silanes are prohigher silanes are thus tracers of $\mathrm{SiH} 2$, while the film production traces the fraction of $\mathrm{H}, \mathrm{SiH}$, and $\mathrm{SiH} 3$ in the initial decomposition. From the measured stable product yields, the authors deduce that $\mathrm{SiH}_{4}->\mathrm{SiH} 2$ $+2 \mathrm{H}$ is the dominant electron-collisional dissociation channel.

\section{0,391}

\section{PB91-203018}

Not available NTIS

National Bureau of Standards (NML), Gaithersburg

MD. Chemical Kinetics Div.

Ultraviolet Absorption of the Vinyl Radical and Reaction with Oxygen.

Final rept.

A. Fahr, and A. H. Laufer. 1988, 4p

Pub. in Jnl. of Physical Chemistry 92, n26 p7229-7232 1988.

Keywords: *Vinyl radicals, "Ultraviolet spectra, Photolysis, Reaction kinetics, Fan ultraviolet radiation, Absorptivity, Oxygen, Reprints

Two absorption features at 1647.1A and 1683.3A have been detected from the vacuum ultraviolet flash photolysis of either $\mathrm{Sn}\left(\mathrm{C}_{2} \mathrm{H}_{3}\right) 4$ or $\mathrm{Hg}\left(\mathrm{C}_{2} \mathrm{H} 3\right)_{2}$. The features are assigned to the vinyl radical. Extinction coefficients of $1150+$ or $-500 / \mathrm{cm} / \mathrm{atm}$ and $1120+$ or $500 / \mathrm{cm} / \mathrm{atm}$ (base e) for the two bands, respectively have been measured and a rate constant of $(6.7+$ or $2.7 \times 10$ to the -12$) \mathrm{cc} / \mathrm{molec} / \mathrm{sec}$ for the reaction of $\mathrm{C} 2 \mathrm{H} 3+02$ has been determined

\section{0,392}

PB91-203034 Not available NTIS National Inst. of Standards and Technology (IMSE) Gaithersburg, MD. Ceramics Div.

Lanthanide Coordination Chemistry: Spectroscopic Properties of Terbium and Europium Borate.

Final rept.

R. A. Faltynek. $1989,8 p$

Pub. in Jnl. of Coordination Chemistry 20, n1 p73-80 1989.
Keywords: "Lanthanum complexes, "Spectrum analysis, "Borates, "Terbium complexes, "Europium complexes, Charge transfer, Infrared spectra, Ultraviolet spectra, Imidazoles, Pyrazoles, Luminescence, Ligands, Electron spectroscopy, Reprints.

Complexes formed between poly(pyrazol-1-yl) - or poly(imidazol-1-yl) borate anions and tripositive cations of terbium or europium were examined by infrared, UV absorption, and emission spectroscopy. Trisand tetrakis(heterocycle)borates yielded isostructural compounds with both lanthanides, having a different molecular geometry than the bis(heterocycle)borate complexes. Electronic spectra indicate that the poly(pyrazol-1-yl)borate complexes emit from a ligandto-metal (LMCT) charge transfer state. The free ligands are UV transparent however, suggesting that he sensitizing chromophore responsible for emission is created only upon complexation. The poly(imidazol 1-yl)borate compounds, exhibit a more complex excited state profile, with emission apparently originating from both LMCT and intraligand states.

100,393

PB91-203075

Not available NTIS National Inst. of Standards and Technology (NEL) Gaithersburg, MD. Chemical Process Metrology Div. Chemical and Electronic Properties of Pd/ SnO2(110) Model Gas Sensors.

Final rept.

T. B. Fryberger, J. W. Erickson, and S. Semancik. 1989,7p

Pub. in Surface and Interface Analysis 14, n1-2 p83-89 Jan/Feb 89

Keywords: "Catalysts, "Palladium, "Tin oxides, "Vapor deposited coatings, "Ceramics, *'Gas detectors, Metal films, Solid-solid interfaces, Monomolecular films, Annealing, Surface chemistry, Surface resistivity, Metallography, Hydrogen, Time dependence, Pressure dependence, Chemical properties, Electronic structure, Scattering, Reprints.

The $\mathrm{Pd} / \mathrm{SnO} 2(110)$ interface has been investigated as a model gas sensor system. Techniques including XPS, UPS, ISS, and 4-point conductivity measurements were used to study the growth mode and chemical interaction of $\mathrm{Pd}$ with this $\mathrm{SnO} 2$ surface for coverages between 0.1 and 10.0 monolayer-equivalents (ML-EQ). It was found that the Pd overlayer grows by clustering at $300 \mathrm{~K}$ and that the deposited Pd is primarily metallic by 3 ML-EQ. Heating Pd-covered surfaces up to $800 \mathrm{~K}$ causes a decrease in the XPS $\mathrm{Pd} / \mathrm{Sn} 3 \mathrm{~d}$ intensity ratio, possibly due to further clustering of the overlayer above room temperature. Annealing did not, however, change the metallic nature of the deposited $\mathrm{Pd}$. The gas sensing behavior of these model surfaces was tested by measuring the surface conductivity vs. time at several hydrogen pressures and the conductivity response for a $3 \mathrm{ML}-\mathrm{EQ} \mathrm{Pd} / \mathrm{SnO} 2(110)$ surface was found to be 16 times greater than that for the Pd- free surface.

100,394

PB91-203232 Not available NTIS National Inst. of Standards and Technology (NML). Gaithersburg, MD. Chemical Kinetics Div.

Rate Constants for Hydrogen Abstraction Reactions of the Sulfate Radical, SO4- Alkanes and Ethers.

Final rept.

A. E. Huie, and C. L. Clifton. 1989, 9p

Pub. in International Jnl. of Chemical Kinetics 21, n8 p611-619 Aug 89.

Keywords: *Sulfates, "Radicals, "Hydrogen trasfer, "Reaction kinetics, "Alkanes, "Ethers, Photolysis, Persulfate, Chemical reactions, Reprints.

Rate constants have been determined for the reactions of SO4(-) with a series of alkanes and ethers. The $\mathrm{SO} 4(-)$ radical was produced by the laser-flash photolysis of persulfate, $S 208(-2)$. For methane, only an upper limit of $1 \times 10$ to the 6 th power $\mathrm{l} / \mathrm{mol} / \mathrm{sec}$ could be determined. For ethane, propane, and 2-methylpropane, rate constants of $0.44,4.0$, and $10.5 \times 10$ to the 7th power were found. For ethyl and n-propyl ether, rate constants of $1.3 \times 10$ to the 8 th power and $2.2 \times 10$ to the 8 th power $\mathrm{I} / \mathrm{mol} / \mathrm{sec}$ were found and for 1,4 dioxane and tetrahydrofuran, rate constants of $7.2 \times 10$ to the 7 th power and $2.8 \times 10$ to the 8 th power were obtained. The reaction of SO4(-) with allyl alcohol was also studied and found to have a rate constant of $1.4 \times$ 10 to the 9 th power $\mathrm{I} / \mathrm{mol} / \mathrm{sec}$. 
100,395

PB91-203273

Not available NTIS

National Inst. of Standards and Technology (NML), Gaithersburg, MD. Molecular Physics Div.

Production and Spectroscopy of Small Polyatomic Molecular Ions Isolated in Solid Neon.

Final rept.

M. E. Jacox, and W. E. Thompson. 1990, 10p

Sponsored by Army Research Office, Arlington, VA

Pub. in High Temperature Science 28, p225-234 1990.

Keywords: *Oxygen ions, "Molecular ions, "Carbon dioxide, "Infrared spectra, Molecular structure, Neon, Vibrational states, Reprints, Cluster ions.

The use of a newly developed discharge sampling configuration has led to the stabilization of several small polyatomic molecular ions in solid neon in sufficient concentration for detection of their infrared absorptions. The results obtained in these experiments will be illustrated by observations of the ions which appear when carbon dioxide or oxygen is present in the system. In the experiments on carbon dioxide, the antisymmetric stretching absorptions of both $\mathrm{CO} 2(+)$ and $\mathrm{CO} 2(-)$ have been identified. An infrared absorption of O4(-) had previously been observed in systems in which an alkali metal atom, $M$, was codeposited with an Ar:O2 mixture. The appearance of this absorption in the discharge sampling experiments on oxygen definitively excludes an alternate assignment of the absorption to an MO4 structure in which two $\mathrm{O} 2$ units are coordinated to a central alkali metal atom. Detailed isotopic substitution experiments support the assignment of four infrared absorptions to a vibrational fundamental and combination bands of $\mathrm{O} 4(+)$ for which no spectroscopic data have previously been reported. Analysis of the spectrum provides information regarding the molecular structure of $\mathrm{O} 4(+)$.

\section{0,396}

PB91-203281

Not available NTIS

National Bureau of Standards (NML), Gaithersburg, MD. Chemical Thermodynamics Div.

Resonance Enhanced Multiphoton Ionization Spectra of the GeF and GeCl Radicals from 400$500 \mathrm{~nm}$.

Final rept.

R. D. Johnson, J. W. Hudgens, and B. P. Tsai. 1988, $5 p$

Pub. in Jnl. of Chemical Physics 89, n10 p6064-6068 1988.

Keywords: "Electron transitions, "Rydberg states, *Germanium, "Electron spectra, Electron structure, Energy levels, Excited states, Reprints, "Germanium fluoride radicals, "Germanium chloride radicals, "Multiphoton ionization.

The resonance enhanced multiphoton ionization spectra from 400 to $500 \mathrm{~nm}$ of the Germanium chloride and Germanium fluoride radicals are reported.

\section{0,397}

PB91-203331

Not available NTIS

National Inst of Standards and Technology (NML),

Gaithersburg, MD. Molecular Spectroscopy Div.

Molecular Desorption from Solid Surfaces: Laser Diagnostics and Chemical Dynamics.

Final rept.

D. S. King, and R. R. Cavanagh. 1989, 45p

Pub. in Advances in Chemical Physics 76, p45-89 1989.

Keywords: "Surface chemistry, "Desorption, "Solids, Spectroscopy, Reviews, Heating, Laser radiation, Nitric oxide, Reprints, Molecular dynamics.

Review of recent state-resolved experiments exploring thermal and laser-driven desorption processes at metal surfaces is presented.

\section{0,398}

PB91-203448

Not available NTIS

National Inst of Standards and Technology (NML) Gaithersburg, MD. Molecular Spectroscopy Div.

Microwave Spectrum and Molecular Structure of the N2-H2O Complex.

Final rept.

H. O. Leung, M. D. Marshall, R. D. Suenram, and F. J. Lovas. $1989,13 p$

Pub. in Jnl. of Chemical Physics 90, n2 p700-712, 15 Jan 89.

Keywords: "Complex compounds, "Water, "Nitrogen, *Microwave spectra, *Molecular structure, Molecular rotation, Hydrogen bonds, Dipole moments, Nuclear quadrupole resonance, Electron tunneling, Reprints.

The a-type, $\mathrm{K}=0$ microwave spectrum of the $\mathrm{N} 2-\mathrm{H} 2 \mathrm{O}$ complex has been observed using a pulsed molecular beam Fabry-Perot cavity microwave spectrometer. Seven isotopic species have been studied in the range of $5-23 \mathrm{GHz}$. The $\mathrm{N} 2-\mathrm{H} 2 \mathrm{O}$ complex exhibits tunnelng motions similar to the $1->2$ tunnelling motion of the $\mathrm{H} 2 \mathrm{O}-\mathrm{DOD}$ complex which gives rise to four components for each rotational transition.

\section{0,399}

PB91-203471

Not available NTIS

National Inst. of Standards and Technology (NML), Gaithersburg, MD. Surface Science Div.

Chemical Effects of $\mathrm{Ne}+$ Bombardment on the MoS2(0001) Surface Studied by High-Resolution Photoelectron Spectroscopy.

Final rept.

J. R. Lince, T. B. Stewart, M. M. Hills, P. D.

Fleischauer, J. A. Yarmoff, and A. Taleb-Ibrahimi 1989, 19p

Pub. in Surface Science 210, n3 p387-405 1989

Keywords: "Molybdenum disulfide, "Ion bombardment, Photoelectron spectroscopy, High resolution, Neon ions, Surfaces, Reprints.

The effect of $1 \mathrm{KeV} \mathrm{Ne}(1+)$ ion bombardment on the clean, ordered MoS2(0001) surface was studied using high-resolution photoelectron spectroscopy excited with synchrotron radiation. A qualitative depth distribution of the chemical species present after $\mathrm{Ne}(1+)$ bombardment was determined by varying the photon energies employed for core-level spectroscopy. The results indicate that the preferential sputtering of sulfur over molybdenum occurs predominately through a mechanism involving chemical bonding effects, specifically through the preferential emission of polysulfide ions over other species in the bombarded region.

100,400

PB91-203547

Not available NTIS

National Bureau of Standards (NML), Gaithersburg, MD. Chemical Kinetics Div.

Ionic Hydrogen Bonds. Part 1. Thermochemistry, Structural Implications, and Role in Ion Solvation. Final rept.

M. Mautner 1987, 34p

See also AD-A178 839.

Pub. in Molecular Structure and Energetics, Chapter 3, v4 p71-104 1987.

Keywords: "Hydrogen bonds, "Clustering, "Solvation, *Ions, Water, Heat of solution, Thermochemistry, Reviews, Molecular structure, Reprints.

The energetics and structural implications of ionic hydrogen bonds are reviewed. Gas-phase clustering properties are compared with solvation in liquid water.

100,401

PB91-203562

Not available NTIS

National Inst. of Standards and Technology (NML),

Boulder, CO. Quantum Physics Div.

Structural Dependence of HF Vibrational Red Shifts in ArnHF, $n=1-4$, via High-Resolution Slit Jet Infrared Spectroscopy.

Final rept.

A. Mcllroy, R. Lascola, C. M. Lovejoy, and D. J.

Nesbitt. 1991, 9p

Grant NSF-CHE86-05970

Sponsored by National Science Foundation, Washington, DC.

Pub. in Jnl. of Physical Chemistry 95, n7 p2636-2644 1991.

Keywords: "Argon complexes, Van der Waals forces, Supersonic jet flow, Vibrational spectra, Infrared spectroscopy, Hydrogen fluoride, High resolution, Red shift, Reprints.

The rotationally resolved nu $=1<-0 \mathrm{HF}$ stretching spectra of $\operatorname{Ar}(n) \mathrm{Hf}, \mathrm{n}=1-4$, have been observed by using a slit jet, difference frequency infrared laser spectrometer. The red shift of the HF vibrational frequency is seen to be sensitively dependent on the placement of the Ar with respect to the projection of the HF dipole moment; the largest incremental red shift is observed for the ArHF linear geometry. The $n$ $=1-3$ red shifts account for almost half (9.65-19.26/ $\mathrm{cm}$ ) of the shift observed for HF in an Ar matrix (42.4/ $\mathrm{cm}$ ), suggesting that only the nearest neighbors contribute significantly to the perturbation of the HF vibrational frequency. The geometry of the experimentally observed isomer of Ar4HF places the fourth Ar in what would be the second coordination layer from the HF where it has little effect $(<2 \%)$ on the observed red shift, supporting the proposed dominance of the nearest neighbors in determining the HF vibrational frequency. In all spectra, no evidence for vibrational predissociation is observed, indicating an extremely long excited state lifetime (approx $=$ or $>16 \mathrm{~ns}$ ).

\section{0,402}

PB91-203661

Not available NTIS

National Inst. of Standards and Technology (NML), Gaithersburg, MD. Chemical Kinetics Div.

Radiolytic Studies of the Redun neactions of Ruthenium Porphyrins.

Final rept.

S. Mosseri, P. Neta, and P. Hambright. 1989, 5p

Pub. in Jnl. of Physical Chemistry 93, n6 p2358-2362 1989.

Keywords: "Ruthenium compounds, "Porphyrins, "Oxidation reduction reactions, Radiolysis, Reaction kinetics, Ligands, Cyanides, Reprints.

Oxidation of Ru(II) porphyrins to the Ru(III) and Ru(IV) states and ligand exchange reactions of the various states have been studied by radiolytic methods. RU(II) porphyrins, stabilized with a CO ligand, undergo one electron oxidation on the porphyrin ring to form the piradical cation. When Ru(II)(P)(CO) (P-octaethylporphyrin (OEP) or tetraphenylporphyrin (TPP)) is oxidized by irradiation in $\mathrm{CH} 2 \mathrm{Cl} 2$, the initial radical cation combines with $\mathrm{Cl}-$, formed by radiolysis of the solvent or added beforehand, with a rate constant of about $100000 \mathrm{l} / \mathrm{mol} / \mathrm{s}$ to yield $\mathrm{Ru}(\mathrm{II})(\mathrm{P}+)(\mathrm{CO})(\mathrm{Cl}-)$. Irradiation of the same porphyrins in acetonitrile does not result in oxidation but rather in uptake of the $\mathrm{CN}$ - produced by the radiolysis to form $\mathrm{Ru}(\mathrm{III}) \mathrm{P}(\mathrm{CN}-) 2$. When this product is oxidized by irradiation in acetonitrile/ $\mathrm{CCl}(4)$ solutions, oxidation occurs on the metal center to give $\mathrm{Ru}(\mathrm{III}) \mathrm{P}\left(\mathrm{CN}-\mathrm{C}_{2}\right.$ as the final product. Chemical oxidations and reductions were carried out to complement the radiolysis results.

\section{0,403}

PB91-203695

Not available NTIS

National Inst. of Standards and Technology (NML), Boulder, CO. Quantum Physics Div.

Progress on the Determination of Intermolecular Potential Energy Surfaces from High Resolution Spectroscopy.

Final rept.

D. J. Nesbitt. $1990,10 p$

Grants NSF-CHE86-05970, NSF-PHY86-04504

Sponsored by National Science Foundation, Washington, DC.

Pub. in Dynamics of Polyatomic Van der Waals Complexes, p461-470 1990.

Keywords: *Complexes, Van der Waals forces, Vibrational states, Infrared spectroscopy, Intermolecular forces, Hydrogen bonds, High resolution, Predissociation, Rare gases, Water, Reprints, "Potential energy surfaces.

The determination of intermolecular potentials between chemical species has been pursued via a varie ty of methods including scattering experiments, line broadening, virial coefficients and transport phenomena. A powerful alternative approach is to orient the collisional intermediate in a weakly bound complex, and sample the bound region of the potential via high resolution IR spectroscopy on excited intermolecular vibrational states. The author illustrates recent theoretical and experimental progress toward this goal. Molecular systems are discussed that illustrate large amplitude motion characteristic of a (1) 'ball + ball', (2) 'ball + stick', and (3) 'ball+top' intermolecular potential energy surface. The examples are drawn from high resolution near IR measurements on rare gas-hydrogen halide and rare gas-water complexes.

100,404

PB91-203703

Not available NTIS

National Inst. of Standards and Technology (NML)

Boulder, CO. Quantum Physics Div.

Multiple Intermolecular Bend Vibrational Excitation of a Hydrogen Bond: An Extended Infrared Study of OCOHF.

Final rept

D. J. Nesbitt, and C. M. Lovejoy. 1990, 15p

Grant NSF-CHE86-05970

Sponsored by National Science Foundation, Washing. ton, DC. 
Pub. in Jnl. of Chemical Physics 93, n11 p7716-7730, 1 Dec 90.

Keywords: "Hydrogen bonds, "Complexes, Near infrared radiation, Supersonic jet flow, Vibration spectra Carbon dioxide, Hydrogen fluoride, Laser radiation, Predissociation, Excitation, Bending, Reprints.

The authors report the use of near infrared tunable difference frequency laser absorption methods to investigate low-frequency bending of the intermolecular hydrogen bond in OCOHF complexes. By deliberate thermal warming of the slit jet expansion to $16 \mathrm{~K}$, they observe bending 'hot band' transitions built on the fundamental nu(HF) $=<-0 \mathrm{HF}$ stretch from the lowest five internally excited bending states which correspond to low-frequency, skeletal bending of the intermolecular hydrogen bond. In addition, much weaker paralle (delta $\mathrm{I}=0$ ) combination band transitions are observed.

\section{0,405}

PB91-203760

Not available NTIS

National Bureau of Standards (NML), Gaithersburg

MD. Chemical Kinetics Div.

Dissociation Energetics of Simple Dlazirlnes by Photon and Electron Impact.

Final rept.

H. Okabe. $1987,14 p$

1987.

Keywords: "Dissociation energy, Halohydrocarbons Absorption spectra, Photolysis, Mass spectra, Heat of formation, Reviews, Thermodynamic properties, Re prints, "Diazirine, Methyl compounds, Difluorodiazirine.

Dissociation energetics by photon and electron impact for six simple diazirines has been reviewed. The data on absorption spectra, UV photolysis, the heats of for mation, mass spectra and appearance potentials are presented.

\section{0,406}

PB91-203844

Not available NTIS

National Bureau of Standards (NML), Gaithersburg, MD. Molecular Spectroscopy Div.

Vibrational, Rotational, and Tunnellng Dependence of Vibrational Predissoclation in the HF Dimer.

Final rept.

A. S. Pine, and G. T. Fraser. $1988,8 p$

Pub. in Jnl. of Chemical Physics 89, n11 p6636-6643 1988

Keywords: "Hydrogen fluoride, "Predissociation, Vibrational spectra, Laser spectroscopy, Line width, Dimers, Reprints.

Vibrational predissociation linewidths have been resolved in the two H-F stretching bands of the HF dimer using an optothermal (bolometer-detected) molecularbeam color-center laser spectrometer. In addition to the strong vibrational mode dependence reported earlier by several groups, one observes a substantial Krotational and tunneling dependence to the longerlived mode, nu(1), which is associated with the 'free- $\mathrm{H}$ ' stretch. The predissociation linewidths (FWHM in $\mathrm{MHz}$ ) for this vibration are $6.4(5)$ for $\mathrm{K}=0(+), 9.5(5)$ for $K=0(-), 10.2(5)$ for $K=1(+)$ and $11.8(5)$ for $K=(-)$, where the +1 - superscripts refer to the symmetric/ antisymmetric tunneling states. The $K=0$ levels of the 'bound- $\mathrm{H}$ ' stretch have tunneling-independent widths of $330(30) \mathrm{MHz}$. Extraneous broadening due to saturation effects was observed and corrected for in these measurements.

\section{0,407}

Not available NTIS Gaithersburg, MD. Biotechnology Div.

Frequency Dependence of Catalyzed Reactions in a Weak Oscillating Fleld.

Final rept.

B. Robertson, and R. D. Astumian. 1991, 6p

Pub. in Jnl. of Chemical Physics 94, n11 p7414-7419, 1 Jun 91 .

Keywords: "Reaction kinetics, *Catalysis, "Frequency response, "Mathematical models, Ion mobility, Phosphatases, Erythrocytes, Reprints, "Lorentz curves, "Oscillating fields, Sodium/potassium adenosine triphosphatase.

The frequency dependence of the average rates of reactions catalyzed by one or more catalysts in a weak oscillating field is derived. The average rates are sums of Lorentzian curves whose characteristic frequencies are the inverse relaxation times of the normal modes of the kinetic system and whose amplitudes are quadratic in the field. The signs of the Lorentz amplitudes can be either positive or negative, so the rates versus frequency can have a variety of shapes, including frequency windows. One can get relaxation times and amplitudes by measuring steady-state rates as a function of the frequency of the field. The theory is applied to determine the Lorentz amplitudes and characteristic frequencies of ion transport rates catalyzed by $\mathrm{Na}(+)$ $\mathrm{K}(+) \mathrm{ATP}$ ase in erythrocytes.

\section{0,408}

PB91-204008

Not available NTIS National Inst. of Standards and Technology (NML), Boulder, CO. Quantum Physics Div.

HIgh-Resolution Infrared Flash Kinetic Spectroscopy of $\mathrm{OH}$ Radicals.

Final rept.

A. Schifiman, D. D. Nelson, M. S. Robinson, and D.

J. Nesbitt. 1991, 8p

Contract AFOSR-F49620-86-C-0056

Sponsored by Air Force Office of Scientific Research, Bolling AFB, DC.

Pub. in Jnl. of Physical Chemistry 95, n7 p2629-2636 1991.

Keywords: "Hydroxyl radicals, "Reaction kinetics, "Ethane, "Butanes, "Propane, Infrared lasers, Infrared spectra, Photolysis, Atmospheric circulation, Reprints, Flash kinetic spectroscopy.

A high-resolution infrared flash kinetic spectrometer is used for time- and frequency-resolved studies of the $\mathrm{OH}$ radical. $\mathrm{OH}$ is produced by $193-\mathrm{nm}$ excimer lase photolysis of $\mathrm{HNO} 3 /$ buffer gas mixtures in a $100-\mathrm{cm}$ flow tube and is probed via weak fractional absorption of light from a widely tunable (2.35-3.59 micrometers) single-mode (frequency spread $<$ or $=2 \mathrm{MHz}$ ) color center laser. The IR absorption technique allows fas ( $<$ or $=0,000001 \mathrm{~s}$ ), sensitive $(<1$ billion radicals $/ \mathrm{cc}$ per quantum state) detection of $\mathrm{OH}$ and is designed to permit determination of absolute $\mathrm{OH}$ number densities. The spectrometer is used to measure rate constants for the reactions of $\mathrm{OH}$ with ethane (k1), propane (k2) $\mathrm{n}$-butane $(\mathrm{k} 3)$, and isobutane $(\mathrm{k} 4)$. The reliability of these measurements is tested on a variety of rotational, spin-orbit, and lambda-doublet states, with several buffer gases, and over more than an order of magnitude of alkane concentrations. The resulting rate constants are, in units of 10 to the -12 th power $\mathrm{cc} / \mathrm{mole}$ cule/sec, k1 $=0.243+$ or $-0.012, \mathrm{k} 2=1.02+$ or $0.05, \mathrm{k} 3=2.35+$ or -0.08 , and $\mathrm{k} 4=2.11+$ or 0.09 . The rate constants for the ethane, $n$-butane, and isobutane reactions agree with some previous determinations but are found to be between $10 \%$ and $25 \%$ lower than values currently used in atmospheric modeling; it is recommended that these values be revised to reflect the lower rates from the study. Curren models of atmospheric air flow based on these rate constants, as well as those of previously accepted values, are found to be inconsistent with daily changes in observed atmospheric alkane concentrations.

\section{0,409}

\section{PB91-204024}

Not available NTIS

National Inst. of Standards and Technology (NML) Boulder, CO. Quantum Physics Div.

Investigation of Internal Rotor Dynamics of NeDC and ArDCl via Infrared Absorption Spectroscopy Final rept.

M. Schuder, D. Nelson, and D. J. Nesbitt. 1991, 16p Grants NSF.CHE86-05970, NSF-PHY86-04504

Sponsored by National Science Foundation, Washington, DC.

Pub. in Jnl. of Chemical Physics 94, n9 p5796-5811, 1 May 91.

Keywords: "Argon complexes, "Neon complexes, Van der Waals forces, Supersonic jet flow, Intermediate in frared radiation, Laser radiation, Vibrational spectra, Deuterium compounds, Hydrogen chloride, Infrared spectroscopy, Predissociation, Reprints, Potential energy surfaces.

The van der Waals complexes, $\mathrm{NeDCl}$ and $\mathrm{ArDCl}$, are produced in a slit jet supersonic expansion and observed via direct absorption of tunable mid-infrared $\mathrm{Pb}$ salt diode laser radiation. The relative fundamental vs bend combination band intensity distributions are very different for the two complexes. The ArDCl fundamental to $\mathrm{Pi}$ bend combination band intensity ratio is $4: 1$, whereas for $\mathrm{NeDCl}$ the corresponding ratio is $1: 8$. This anomalous intensity pattern for $\mathrm{NeDCl}$ and the proxim- ity of the bend combination bands to the $\mathrm{DCl} R(0)$ line indicate that the $\mathrm{DCl}$ diatomic is exhibiting nearly free rotation within the complex compared to more restricted librational motion of $\mathrm{DCl}$ in ArDCl. Experimentally determined rotational constants, vibrational frequencies, and relative intensities are compared to predictions based on existing empirical potential surfaces.

\section{0,410}

PB9 1-204065

Not available NTIS

National Inst. of Standards and Technology (NEL), Gaithersburg, MD. Thermophysics Div.

Thermodynamic Properties of Aqueous Solutions at High Temperatures; Needs, Methods, and Challenges.

Final rept.

J. M. H. Levelt Sengers. 1990, 17p

Pub. in International Jnl. of Thermophysics 11, n2 p399-415 1990.

Keywords: "Aqueous solutions, "Thermodynamic properties, "Thermophysical properties, "Hydrothermal systems, Autoclaves, Debye-Huckel theory, High temperature tests, High pressure tests, Data bases, Methodology, Reviews, Hot-water systems, BWR type reactors, Hydroelectric power, Reprints, Research needs.

Needs exist for thermophysical data on aqueous solutions at high temperatures and pressures in many different areas of science and engineering. These needs are reviewed and references are given to recent relevant conference proceedings, reviews and papers. Aspects and drawbacks are discussed of current methods, which are most often extensions to high temperatures of methods developed for liquid water. The challenges posed by engineering needs and by new phenomena resulting from experimental breakthroughs are discussed. Some examples are given of alternative approaches more suitable for highly compressible media.

100,411

\section{PB91-216713}

(Order as PB91-216705, PC A07/MF A01) National Inst. of Standards and Technology, Boulder, $\mathrm{CO}$

High-Temperature Transient Hot-wire Thermal Conductivity Apparatus for Fluids.

R. A. Perkins, H. M. Roder, and C. A. Nieto de Castro. 1991, 23p

Prepared in cooperation with Lisbon Univ. (Portugal). Dept. de Quimica.

Included in Jnl. of Research of the National Institute of Standards and Technology, v96 n3 p247-269 May/Jun 91.

Keywords: "Thermal conductivity, "Thermal diffusivity, Measuring instruments, Hot-wire gages, Specific heat, High temperature, Toluene, Nitrogen, Argon, Fluids.

A new apparatus for measuring both the thermal conductivity and thermal diffusivity of fluids at temperatures from 220 to $775 \mathrm{~K}$ at pressures to $70 \mathrm{MPa}$ is described. The instrument is based on the step-powerforced transient hot-wire technique. Performance of the instrument was verified with measurements on liquid toluene as well as argon and nitrogen gas. In particular, new data for the thermal conductivity of liquid toluene near the saturation line, between 298 and 550 $K$, are presented. These new data can be used to illus trate the importance of radiative heat transfer in transient hot-wire measurements. Thermal conductivity data for liquid toluene, which are corrected for radiation, are reported. From the measured thermal conductivity and thermal diffusivity, the authors can calculate the specific heat, $\mathrm{Cp}$, of the fluid, provided that the density is measured, or available through an equation of state.

\section{0,412}

PB91-23652

Not available NTIS

National Inst. of Standards and Technology (MSEL), Gaithersburg, MD. Office of Intelligent Processing of Materials.

Problem of Detailed Balance and Model Lineshapes in Collision-Induced Rotovibrational Bands: $\mathrm{H} 2-\mathrm{H} 2$ and $\mathrm{H}_{2}-\mathrm{He}$.

Final rept.

G. Birnbaum, and A. Borysow. 1991, 12p

Pub. in Molecular Physics 73, n1 p57-68 1991

Keywords: "Molecular collisions, Vibrational spectra, Hydrogen, Helium, Line shape, Reprints. 
When account is taken of the vibrational dependence of the potential function, the usual condition of detailed balance taken with respect to the vibrational-rotational frequencies does not apply, and model spectral functions satisfying the condition are inaccurate. A model correlation function is derived that deals with the case of vibration-dependent potentials. The parameters of the model are computed from relations involving the spectral moments. The model is shown to give spectral shapes, for a number of examples involving the fundamental and first overtone bands of $\mathrm{H} 2-\mathrm{He}$ and $\mathrm{H} 2-\mathrm{H} 2$ at room temperature and $1000 \mathrm{~K}$, in excellent agreement with spectra calculated quantum mechanically from first principles. The model is useful for predicting the collision-induced vibrational spectra for these and other systems for which the vibration-dependent induced dipole and potential are known.

\section{0,413}

PB91-236547

Not available NTIS National Inst. of Standards and Technology (CSTL), Gaithersburg, MD. Surface and Microanalysis Science Div.

Photodissociation Dynamics of Mo(CO)6 at $266 \mathrm{~nm}$ and $355 \mathrm{~nm}$ : CO Photofragment Kinetic-Energy and Internal-State Distributions.

Final rept.

S. A. Buntin, R. R. Cavanagh, L. J. Richter, and D. S. King. 1991, 14p

Sponsored by Department of Energy, Washington, DC Pub. in Jnl. of Chemical Physics 94, n12 p7937-7950, 15 Jun 91.

Keywords: "Carbon monoxide, "Photodissociation, Near ultraviolet radiation, Photofragment spectroscopy, Laser induced fluorescence, Kinetic energy, Photolysis, Reprints, "Hexacarbonylmolybdenum.

The internal-state and kinetic-energy distributions of the CO photofragments from the 266 and $355 \mathrm{~nm}$ photolysis of $\mathrm{Mo}(\mathrm{CO}) 6$ have been measured under collision-free conditions using vacuum-ultraviolet laser-induced fluorescence. The Doppler-broadened CO photofragment line shapes indicate that the translational energy distributions are isotropic and Maxwellian. There is no photolysis-laser wavelength or internalstate dependence to the extracted translational "temperatures.' The observed energy partitioning and kinetic-energy distributions are inconsistent with an impulsive ejection of a single $\mathrm{CO}$ ligand. The results also suggest that the photodissociation of $\mathrm{Mo}(\mathrm{CO}) 6$ at 266 and $355 \mathrm{~nm}$ involves a common initial 'state' and that similar exit channel effects are operative.

\section{0,414}

PB91-236570

Not available NTIS

National Inst. of Standards and Technology (MSEL), Gaithersburg, MD. Polymers Div.

Temperature Gradient in Differential Scanning CaIorimetry.

Final rept.

S. S. Chang. 1991, 7p

Pub. in Thermochimica Acta 178, p195-201 1991.

Keywords: "Calorimeters, "Heat measurement, "Thermal analysis, "Temperature gradients, Reaction heat, Heat flow, Dynamic tests, Measurement, Epoxy resins, Mathematical models, Curing, Reprints.

A temperature gradient is required for any type of heat flow process. In a dynamic calorimeter, temperature gradients exist in the entire calorimetric system during the measurement of temperature differences or heat flow. Most organic materials are of relatively low thermal conductivity in comparison to metallic and inorganic materials. Therefore large temperature gradients are expected to exist in organic materials during the measurement. The magnitude of the temperature gradient can be estimated through a simple modeling procedure. The results of experimentally determined temperature gradients during the cure of an epoxy resin in a differential scanning calorimeter are presented to confirm the expected magnitude of temperature nonuniformity.
Rotational Spectrum and Structure of the Complex Ar-Ch3CN.

Final rept.

R. S. Ford, R. D. Suenram, G. T. Fraser, F. J. Lovas, and K. R. Leopold. 1991, 7p

Grant NSF-CHE88-07895

Sponsored by National Science Foundation, Washington, DC.

Pub. in Jnl. of Chemical Physics 94, n8 p5306-5312, 15 Apr 91.

Keywords: "Argon complexes, "Acetonitrile, Van der Waals forces, Microwave spectra, Molecular structure, Reprints.

The microwave spectrum of the weakly bound complex Ar-CH3CN has been observed using a pulsednozzle Fourier-transform microwave spectrometer The spectrum is characteristic of an asymmetric rotor with nearly free internal rotation of the methyl group. Spectroscopic constants for the ground internal rotor state, in megaHertz, are given. The complex has a Tshaped geometry in which the acetonitrile axis is nearly perpendicular to the line joining the centers of mass of the monomers, and the methyl group undergoes nearly free internal rotation. The center-of-mass separation is 3.6505 (7) A and the dipole moment component along the $b$ inertial axis of the complex is $3.802(5) \mathrm{D}$. The latter is quantitatively accounted for by the sum of the monomer permanent moment and the dipole-induced dipole in the argon.

100,416

PB91-236679

Not available NTIS

National Inst of Standards and Technology (PL),

Gaithersburg, MD. Molecular Physics Div.

Optothermal-Detected Microwave-Sideband $\mathrm{CO} 2$ Laser Spectroscopy of Ar-NH3.

Final rept.

G. T. Fraser, A. S. Pine, and W. A. Kreiner. 1991, 7p Pub. in Jnl. of Chemical Physics 94, n11 p7061-7067, 1 Jun 91

Keywords: "Argon complexes, "Ammonia, Intermolecular forces, Van der Waals forces, Laser spectroscopy, Carbon dioxide lasers, Infrared spectra, Reprints.

A microwave-sideband CO2-laser optothermal spectrometer with a resolution better than $1 \mathrm{MHz}$ has been used to record the infrared spectrum of $\mathrm{Ar}-\mathrm{NH} 3$ in the vicinity of the $a R(O, D)$ line of the nu(2) vibration of free $\mathrm{NH}$. A Pi <- Sigma type band is observed, giving a positive l-type doubling constant q, of $90.9 \mathrm{MHz}$ for the upper state. The nu(2) vibrationally excited complex is found to predissociate in less than the $0.9 \mathrm{~ms}$ transit time between the bolometer detector and laser-excitation region. A lower limit to the upper-state lifetime can be obtained from the observed linewidths, which range from 1.5 to $3 \mathrm{MHz}$ (FWHM). The present results agree with and extend the previous free-jet diode-laser absorption measurements on this band.

\section{0,417}

\section{PB91-236737}

Not available NTIS

National Inst. of Standards and Technology (PL), Gaithersburg, MD. Molecular Physics Div.

Van der Waals Complexes in 1,3-Dipolar Cycloaddition Reactions: Ozone-Ethylene.

Final rept.

C. W. Gillies, J. Z. Gillies, R. D. Suenram, F. J.

Lovas, E. Kraka, and D. Cremer. 1991, 10p

Pub. in Jnl. of the American Chemical Society 113, n7 p2412-2421 1991

Keywords: *Oxygen complexes, *Ozone, *Ethylene, Van der Waals forces, Rotational spectra, Microwave spectra, Electric dipole moments, Deuterium compounds, Molecular structure, Cyclization, Reprints.

Microwave spectra of $\mathrm{O} 3-\mathrm{CH}_{2}=\mathrm{CH} 2, \mathrm{O} 3-\mathrm{CD} 2=\mathrm{CH} 2$, O3-trans-CHD $=\mathrm{CHD}$, and $\mathrm{O} 3$-cis- $\mathrm{CHD}=\mathrm{CHD}$ have been observed with a pulsed-beam Fabry-Perot cavity, Fourier transform microwave spectrometer. Internal motion in the van der Waals complex give two states for the normal, 1,1-dideuterated and trans-1,2-dideuterated isotopic forms. The c-type transitions of the two states for the isotopic species above, as well as the one observed isotopic form of O3-cis-CHD $=\mathrm{CHD}$, independently fit to an asymmetric top Watson Hamiltonian. Stark effect measurements were performed.

\section{0,418}

PB91-236778

Not available NTIS

National Inst. of Standards and Technology (CSTL), Gaithersburg, MD. Thermophysics Div.
Phase Equilibrla and Critical Lines in Model Water/ Salt Mixtures.

Final rept.

A. H. Harvey. 1991, 6p

Pub. in Jnl. of Chemical Physics 95, n1 p479-484, 1 Jul 91

Keywords: *Solutions, "Aqueous electrolytes, "Mathematical models, *Phase diagrams, *Thermodynamic equilibrium, "Critical point, Thermodynamics, Dipoles, Spheres, Binary mixtures, Reprints, ${ }^{*}$ Critical lines, Mean spherical approximation.

The mean spherical approximation solution for the thermodynamics of a mixture of equal-sized dipolar hard spheres and charged hard spheres is used to calculate phase equilibria and critical lines. The binary system is characterized by the dimensionless ratio of the dipolar strength to the strength of the charges. At high values of this ratio, the critical curve is interrupted by a liquid-liquid equilibrium and the phase diagram (under the usual classification scheme) is type III. As the ratio is lowered, the critical curve becomes continuous; this is type I or II behavior. The continuous critcal line is maintained until the critical temperature of the ionic component exceeds that of the dipolar component by a factor of approximately 3.56 ; such a lengthy unbroken critical line is unusual in ordinary fluid systems but is observed in many mixtures of water with strong electrolytes. At sufficiently low values of the dimensionless ratio, the critical line is interrupted very near the critical point of the dipolar component; these systems exhibit type IV or $V$ behavior. The simple model used here reproduces the qualitative features of the phase diagrams of real water/salt systems. Insights from the model suggest at least partial explanations for the unusually long unbroken critical lines observed in many of these systems.

\section{0,419}

PB91-236836

Not available NTIS

National Inst. of Standards and Technology (PL), Gaithersburg, MD. Molecular Physics Div.

Vibrational Spectra of Molecular Ions Isolated in Solid Neon. 6. CO4(1-)

Final rept.

M. E. Jacox, and W. E. Thompson. 1991, 7p

See also PB91-149906.

Pub. in Jnl. of Physical Chemistry 95, n7 p2781-2787, 4 Apr 91

Keywords: "Neon, "Matrix isolation, *Molecular ions, *Vibrational spectra, Infrared spectra, Cryogenics, Carbon dioxide, Oxygen, Molecular structure, Reprints, *Percarbonate ion, Force constants.

When a Ne:CO2:O2 =200:1:1 mixture is codeposited at approximately $5 \mathrm{~K}$ with a beam of neon atoms that have been excited in a microwave discharge, several prominent infrared absorptions appear in the resulting deposit. In addition to the absorptions of unreacted $\mathrm{CO} 2$, absorptions previously assigned to $\mathrm{O} 4(1+)$ and O4(1-) are present, and new absorptions appear at $697,1256,1865$, and $1895 \mathrm{~cm}-1$. Detailed isotopic substitution experiments demonstrate that these new absorptions are contributed by a product of formula $\mathrm{CO} 4$ in which the $\mathrm{CO} 2$ moiety is joined to $\mathrm{O} 2$ in such a way that the two $\mathrm{O}$ atoms of each of these groups are nonequivalent. Arguments are presented indicating that this product is the $\mathrm{CO4}(1-)$ anion. The infrared frequencies of the isotopically substituted species have been used for a least-squares force constant adjustment calculation, assuming a planar O2CdashO2(1-) $\mathrm{C}(\mathrm{s})$ structure in which most of the negative charge remains on the $\mathrm{O} 2$ moiety. The photodestruction threshold of CO4(-) lies near $260 \mathrm{~nm}$. Evidence has been obtained for the formation of $\mathrm{CO} 3(1-)$ or of $\mathrm{CO}(3)$ in an excited state with 3-fold symmetry, for which randomization of the oxygen isotopic substitution occurs.

\section{0,420}

PB91-236851

Not available NTIS National Inst. of Standards and Technology, Gaithersburg, MD.

Temperature Measurements in Flames Using Thermally Assisted Laser-Induced Fluorescence of Ga. Final rept.

R. Joklik, J. Horvath, and H. Semerjian. 1991, 8p Contract N0001989IPB7387

Sponsored by Naval Air Systems Command, Washington, DC.

Pub. in Applied Optics 30, n12 p1497-1504, 20 Apr 91

Keywords: *Temperature measurement, "Flames, Laser induced fluorescence, Gallium, Reprints. 


\section{CHEMISTRY}

\section{Physical \& Theoretical Chemistry}

The use of thermally assisted fluorescence (THAF) for temperature measurements has been investigated in a laminar, premixed $\mathrm{C} 2 \mathrm{H} 2 / \mathrm{O}_{2} / \mathrm{Ar}$ flame seeded with $\mathrm{Ga}$ atoms. Average temperature measurements were made with an uncertainty of less than + or $-100 \mathrm{~K}$ in flames $>2150 \mathrm{~K}$ and were found to be in agreement with sodium line reversal temperature measurements and equilibrium calculations. In both fuel rich and lean flames spanning equivalence ratios from 0.75 to 2.0 , it was found that composition influenced the measured temperatures, resulting in an accuracy of + or $-100 \mathrm{~K}$ over the range of flame conditions. Results indicate that THAF with gallium as the thermometric species is limited to cases in which an inefficient quencher, such as a rare gas, is the primary diluent.

\section{0,421}

PB9 1-236869

Not available NTIS

National Inst. of Standards and Technology (PL), Gaithersburg, MD. Molecular Physics Div.

Molecular-Beam Optothermal Spectroscopy of the 9.6 mum nu14.

Final rept.

M. L. Junttila, J. L. Domenech, G. T. Fraser, and A

S. Pine. $1991,8 p$

Sponsored by National Aeronautics and Space Administration, Washington, DC

Pub. in Jnl. of Molecular Spectroscopy 147, p513-520 1991.

Keywords: "Benzene, Intermediate infrared radiation, Carbon dioxide lasers, Infrared spectra, Ground state, Molecular beams, Reprints.

The nu(sub 14) e(sub 1u)-symmetry fundamental band of $\mathrm{C}(6) \mathrm{H}(6)$ has been recorded with sub-Doppler resolution under collisionless low-temperature molecularbeam conditions using bolometric detection of molecules excited by tunable microwave sidebands of a Lamb-dip-stabilized $\mathrm{CO} 2$ laser. The sidebands cover about $40 \%$ of the spectrum between laser lines in which 117 transitions for $\mathrm{J}, \mathrm{K}=$ or $<11$ have been observed and fit to a precision of + or $-24 \mathrm{kHz}$, yielding improved ground and excited state rotational constants with nearly microwave accuracy. The authors' measured ground state rotational constant, B(sub $0)=0.18977389(3) / \mathrm{cm}$, is compared with several disparate values reported previously.

\section{0,422}

PB91-236835

Not available NTIS

National Inst. of Standards and Technology (MSEL), Gaithersburg, MD. Polymers Div.

Temperature Dependence of Probe Diffusion in Bulk Polymer Matrices.

Final rept.

H. Kim, D. Waldow, C. Han, Q. Tran-Cong, and M.

Yamamoto. 1991, 5p

Pub. in Polymer Communications 32 , n4 p108-112 1991.

Keywords: "Polymerization, *Diffusion, *Rayleigh scattering *Probes, Chemical radiation effects, Pre dictions, Matrix materials, Temperature dependence, Photochemical reactions, Melts, Measurement Dimers, Reprints, Cyclophane.

The temperature dependence of the diffusion coefficients for a photobleachable molecular probe in various polymer matrices has been studied using forced Rayleigh scattering (FRS). The probe molecule, a cyclophane derivative, is demonstrated to be a suitable probe for the FRS experiment due to the characteristics of the photodimerization reaction. The dynamic range of the FRS experiment enabled the measurement of the self diffusion coefficients over a range of nearly eight orders of magnitude. The temperature dependence of the self diffusion coefficients is well described by the Williams, Landel, Ferry (WLF) equation for all the polymer matrices studied. In addition, the data from the individual polymer matrices can be shifted to construct a universal curve which is also well de scribed by the WLF equation.

\section{0,423}

\section{PB91-236984}

Not available NTIS

National Inst. of Standards and Technology (NML)

Boulder, CO. Time and Frequency Div.

Heterodyne Frequency Measurements of $\mathrm{CO}$ and OCS Beyond $2100 \mathrm{~cm}(-1)$

Final rept.

A. G. Maki, J. S. Wells, and D. A. Jennings. 1990, 6p Sponsored by National Aeronautics and Space Admin istration, Washington, DC. Office of Atmospheric Research.
Pub. in Jnl. of Molecular Spectroscopy 144, p224-229 1990

Keywords: *Carbon dioxide, "Molecular spectra, *Infrared spectra, "Carbon oxysulfide, Frequencies, Tables(Data), Band spectra, Lasers, Reprints.

Infrared heterodyne frequency measurements are given for absorption lines of the 04(0)0-00(0)0 band of OCS and the fundamental band of CO. Transition frequencies of several isotopomers of $\mathrm{CO}$ are given. The frequency measurements were based on doubled CO2 laser radiation used as a local oscillator and as a fre quency standard. Tables of wavenumber standards based on both OCS and CO absorption lines are given.

\section{0,424}

PB91-237016 Standards and Technology (PL) Gaithersburg, MD. Molecular Physics Div.

3.5mum Region.

Final rept.

D. W. Merdes, J. Pliva, and A. S. Pine. 1991, 17p Pub. in Jnl. of Molecular Spectroscopy 147, p431-447 1991

Keywords: "Cyclopropane, *Infrared spectra, Intermediate infrared radiation, Laser spectroscopy, Band spectra, High resolution, Reprints.

The spectrum of the cyclopropane molecule was measured in the region 2898.5 to $3157.3 / \mathrm{cm}$ with Doppler-limited resolution using a difference frequency laser spectrometer. Deconvolution was used to further enhance the effective resolution in the main part of the highly perturbed spectrum. The region below about $3060 / \mathrm{cm}$ contains perpendicular bands. The nu(2) + nu(9)E' state is also perturbed by anharmonic interac tions with additional $E^{\prime}$ states. A comprehensive analy sis of this complex system of perpendicular bands based on a Hamiltonian model of the main interactions yielded much improved spectroscopic constants, as well as information on seven tentatively identified perturbing states.

\section{0,425}

PB91-237032 National Inst. of Standards and Technology (CSTL) Gaithersburg, MD. Surface and Microanalysis Science Div.

Direct Detection of Atom Vaporization by Laser Resonance Ionization as a Probe of Gas-Surface Chemisorption Mechanisms.

Final rept.

M. H. Mintz, P. Shuker, and J. Fine. 1990, 5p

Pub. in Surface Science Letters 238, pL473-L477 1990.

Keywords: "Chemisorption, *Surface chemistry, *Metals, "Laser spectroscopy, Mechanism, Low temperature Ambient temperature Oxygen Magnesium Polycrystals, Measurement, Laser heating, Vaporizing, Reprints, Laser resonance ionization.

A new method for monitoring the development of chemisorption processes on metallic surfaces (either single crystals or polycrystalline), is demonstrated. The method involves direct detection of metal vaporization by laser resonance ionization. It has a remarkably high sensitivity and selectivity for detecting vapor densities (of the order of a few atoms $\mathrm{cm} / \mathrm{cc}$ ) enabling vaporization measurements even at relatively low temperatures (about room temperature). The method is demonstrated for the case of room-temperature chemisorp'ion of oxygen on polycrystalline magnesium. A random initial chemisorption stage followed by an island growth stage is seen to dominate the chemisorption process.

\section{0,426}

PB91-237149

Not available NTIS

National Bureau of Standards (NEL), Boulder, CO. Chemical Engineering Science Div.

Mass Transfer Coefficients in Two-Phase Aqueous Extraction.

Final rept.

T. A. Patil, S. K. Sikdar, S. B. Sawant, and J. B.

Joshi. 1988, $6 \mathrm{p}$

Pub. in Chemical Engineering Jnl. and Biochemical Engineering Jnl. 39, n1 pB1-B6 1988.

Keywords: "Mass transfer, Bovine serum albumin, $\mathrm{pH}$, Temperature, Molecular weight, Polyoxyethylene, Separation, Extraction, Reprints.
Mass transfer coefficients of bovine serum albumin in two-phase aqueous systems were measured in a 51 $\mathrm{mm}$ i.d. stirred cell with a plane interface. Two systems, sodium sulfate-PEG and dextran-PEG were employed. The effects of stirring speed, $\mathrm{pH}$, temperature and the PEG molecular mass on mass transfer and partition coefficients were investigated. The authors strong dependence of mass transfer and partition on $\mathrm{pH}$. Using the variation of the overall mass transfer coefficients with $\mathrm{pH}$, the authors were able to determine the individual-phase mass transfer coefficients.

\section{0,427}

PB91-237164

National Inst. of Standards and Technology (PL) Gaithersburg, MD. lonizing Radiation Div

Molecular-Orbital Studies via Satellite-Free $X$-ray Fluorescence: $\mathrm{Cl}$ K Absorption and K-ValenceLevel Emission Spectra of Chlorofluoromethanes. Final rept.

R. C. C. Perera, P. L. Cowan, D. W. Lindle, R. E. LaVilla, T. Jach, and R. D. Deslattes. 1991, 10p Sponsored by Department of Energy, Washington, DC. Pub. in Physical Review A 43, p3609-3618 1991.

Keywords: "Dichlorodifluoromethane, ${ }^{*}$ Chloromethanes, $X$ ray fluorescence, $X$ ray absorption, Synchrotron radiation, Molecular orbitals, Reprints, "Chlorotrifluoromethane.

$X$-ray absorption and emission measurements in the vicinity of the chlorine K edge of the three chlorofluoromethanes have been made using monochromatic synchrotron radiation as the source of excitation. By selectively tuning the incident radiation to just above the $\mathrm{Cl}$ is single-electron ionization threshold for each molecule, less complex $x$-ray-emission spectra are obtained. This reduction in complexity is attributed to the elimination of multielectron transitions in the CI K shell, which commonly produce satellite features in $x$-ray emission. The resulting 'satellite-free' $x$-ray-emission spectra exhibit peaks due only to electrons in valence molecular orbitals filling a single $\mathrm{Cl}$ is vacancy. These simplified emission spectra and the associated $x$-ray absorption spectra are modeled using straightforward procedures and compared with semiempirical groundstate molecular-orbital calculations. Good agreement is observed between the present experimental and theoretical results for valence-orbital energies and those obtained from ultraviolet photoemission and between relative radiative yields determined both experimentally and theoretically in the work.

100,428

PB91-237172

Not available NTIS

National Inst. of Standards and Technology (PL), Gaithersburg, MD. Molecular Physics Div.

Hydration of Carbon Dioxide: The Structure of H2O-H2O-CO2 from Microwave Spectroscopy.

Final rept.

K. I. Peterson, R. D. Suenram, and F. J. Lovas. 1991, $12 p$

Pub. in Jnl. of Chemical Physics 94, n1 p106-117, 1 Jan 91.

Keywords: "Carbon dioxide, *Hydration, Electric dipole moments, Molecular structure, Molecular spectra, Rotational spectra, Hydrogen bonds, Complexes, Trimers, Water, Reprints.

The structure of the gas-phase trimeric complex $\mathrm{H} 2 \mathrm{O}$ $\mathrm{H} 2 \mathrm{O}-\mathrm{CO} 2$ is determined through an analysis of the rotational spectia of ten isotopically substituted species. These spectra were measured in the region between 7.5 and $18 \mathrm{GHz}$ using a pulsed-molecular-beam Fourier-transform microwave spectrometer. The structure is found to be cyclical with the dimer-type bond lengths within the trimer being approximately the same as those found in the free heterodimers. One water molecule is oxygen bound to the carbon atom of the $\mathrm{CO} 2$ and is also hydrogen bonded to the oxygen of the second water molecule. The second water molecule is in turn hydrogen bonded to one of the oxygens of the $\mathrm{CO} 2$ molecule. The observed splittings are most likely due to a hydrogen-exchanging internal rotation of the second water molecule.

100,429

PB91-237487

Not available NTIS

National Inst. of Standards and Technology (PL), Gaithersburg, MD. Molecular Physics Div. 
Microwave Spectra and Electric Dipole Moments of $X(4)$ Sigma(1-)1/2 VO and $\mathrm{NbO}$.

Final rept.

R. D. Suenram, G. T. Fraser, F. J. Lovas, and C. W. Gillies. 1991, $9 p$

Pub. in Jnl. of Molecular Spectroscopy 148, p114-122 1991.

Keywords: *Niobium oxides, "Vanadium oxides, "Electric dipole moments, Microwave spectra, Rotationa spectra, Fourier transform spectrometers, Reprints.

Microwave spectra and electric dipole moments have been measured for the X(sup 4) Sigma(sub 1/2, sup -) states of $\mathrm{VO}$ and $\mathrm{NbO}$ using a pulsed-nozzle Fouriertransform microwave spectrometer with a laser-vaporization source. An analysis of the Stark effect of hyperfine components of the $\mathrm{J}=3 / 2<-1 / 2$ transition yields dipole moments of $3.355(14)$ and $3.498(7) \mathrm{D}$ for $\mathrm{VO}$ and $\mathrm{NbO}$, respectively. The dipole-moment measurements are compared with recent ab initio calculations.

100,430

PB91-237529

Not available NTIS

National Inst. of Standards and Technology (CSTL) Gaithersburg, MD. Chemical Kinetics and Thermodynamics Div.

Stabilities of Substituted Benzyl Radicals: Dissociation Rates of Aminoethylbenzenes, Hydroxyethylbenzenes, and Cyanoethylbenzenes.

Final rept.

M. M. Suryan, and S. E. Stein. 1989, 4p

Pub. in Jnl. of Physical Chemistry 93, n21 p7362-7365 1989.

Keywords: "Reaction kinetics, "Dissociation, "Chemical stability, Decomposition reactions, Pyrolysis, Low pressure research, Free radicals, Chemical bonds. $R e$ prints, "Benzyl radicals, Benzene/aminoethyl, Benzene/hydroxyethyl, Benzene/cyanoethyl.

Rates of unimolecular thermal decomposition of amino, hydroxy, and cyano ethylbenzenes have been determined by the very low pressure pyrolysis technique. These rates relative to the rate of dissociation of ethylbenzene, yield the following substituent effects on benzylic $\mathrm{C}-\mathrm{C}$ bond strengths: NH2 (ortho)-2.7, (meta)benzylic C-C bond strengths: $\mathrm{NH} 2$ (ortho)-2.7, (meta)(para)-1.1; CN (ortho)-0.3, (meta)0.4, and (para)-1.5. These effects are generally smaller than those for substituted anisoles and follow different trends. Along with results of ESR studies of Nicholas and Arnold, the present substituent effects indicate that a $1 \mathrm{G}$ change in the benzylic hyperfine coupling constant corresponds to a $1.8 \mathrm{kcal} / \mathrm{mol}$ change in bond strengths.

\section{0,431}

NB91-237537 Not available NTIS National Inst. of Standards and Technology (CSTL), Gaithersburg, MD. Chemical Kinetics and Thermodynamics Div.

Dissoclation of Substituted Anisoles: Substituent Effects on Bond Strengths.

Final rept.

M. M. Suryan, S. E. Stein, and S. A. Kafafi. 1989, 7p Pub. in Jnl. of the American Chemical Society 111, n13 p4594-4600 1989.

Keywords: *Anisoles, "Dissociation, "Molecular structure, Chemical bonds, Aryl ethers, Free radicals, Reaction kinetics, Reprints, "Bond strength, *Substituent effects.

Rates of O-methyl homolysis of 24 substituted anisoles were determined by the very-low-pressure pyrolysis technique. All ortho substituents weakened the 0 methyl bond, with effects ranging from -0.2 to $-7.4 \mathrm{kcal}$. Effects of para substituents varied over the narrowerrange 1.2 to $-2.9 \mathrm{kcal}$, while in the meta position effects were even smaller $(1.1$ to $-1.0 \mathrm{kcal}$, including substituents previously studied). There was some correlation between the measured differences in substituent effects on bond strengths (DeltaBDE) and literature rates of $\mathrm{H}$-abstraction from substituted phenols, but differences were also clear, presumably reflecting polar effects in the abstraction reactions. Overall, DeltaBDE showed a fair correlation with the substituent constant (sc) (cor. coeff. $=0.91$ ). Deviations were attributed to contributions to $\mathrm{sc}$ from substituent bond dipole-charge interaction. A good correlation was also found between DeltaBDE and ESR hyperfine coupling constants (hfcc) for $\mathrm{O}$ - and $\mathrm{p}$-substituted phenoxy radicals. However, substituents affected the degree of apparent spin density alternation in the ring rather than absolute values, obscuring the physical connection between DeltaBDE and hfcc.
100,432

PB91-237636

Not available NTIS

National Bureau of Standards (IMSE), Gaithersburg. MD. Reactor Radiation Div.

Neutron Spectroscopic Evidence for Adsorbed Hydroxyl Species on Platinum Black. Final rept.

T. J. Udovic, J. J. Rush, and R. R. Cavanagh. 1988 $2 \mathrm{p}$

Pub. in Jnl. of the American Chemical Society $110, \mathrm{n} 16$ p5590-5591 1988 .

Keywords: "Platinum black, "Catalysts, "Adsorption, "Surface chemistry, "Hydroxyl compounds, Neutron scattering, Monomolecular films, Hydrogen, Vibrational spectra, Spectrum analysis, Volumetric analysis, Chemical reactions, Synthesis(Chemistry), Oxygen, Reprints, Electron energy loss spectroscopy.

The scarcity of vibrational spectroscopic studies confirming the stability of hydroxyl species on high-surface-area metallic materials has led to the use of incoherent inelastic neutron scattering (IINS) to investigate the possible extent of surface hydroxyl formation from the reaction of oxygen with hydrogen on platinum black. The titration of 0.4 monolayers residual $H$ on the reduced Pt black surface with $02(\mathrm{O}: \mathrm{H}=1)$ at $80 \mathrm{~K}$ followed by equilibration at $300 \mathrm{~K}$ and recooling to $80 \mathrm{~K}$ led to the disappearance of residual hydrogen vibra tional peaks at 104 and $152 \mathrm{meV}$ and the formation of a vibrational peak maximized at $128 \mathrm{meV}$. The peak became more intense upon the further addition of equimolar amounts of $\mathrm{H} 2$ and $\mathrm{O} 2$ (ca. 0.5 more monolayers each of $\mathrm{O}$ and $\mathrm{H}$ ) to the $\mathrm{Pt}$ surface at $80 \mathrm{~K}$ followed by similar $300 \mathrm{~K}$ equilibration and $80 \mathrm{~K}$ recooling steps. Comparison of the IINS spectra with electron energy loss results on $\mathrm{Pt}(111)$ suggest that the 128 meV peak is indeed due to the $\mathrm{Pt}-\mathrm{O}-\mathrm{H}$ bending mode of adsorbed $\mathrm{OH}$ species present as the dominant hydrogenous species on the Pt black surface for $\mathrm{O}: \mathrm{H}$ ratios of unity.

\section{0,433}

PB91-237651

Not available NTIS

National Inst. of Standards and Technology (CSTL) Boulder, CO. Thermophysics Div.

Extended Phase Rule for Nonreactive, Multiphase, Multicomponent Chemical Systems.

Final rept.

L. J. Van Poolen. 1990,13p

Pub. in Fluid Phase Equilibria 58, n1-2 p133-145, 1 Jun 90.

Keywords: "Phase rule, "Mixtures, Phase diagrams, Phase transformations, Degrees of freedom, Thermodynamics, Reprints, Total density, Total composition, Phase volume fractions.

A comprehensive phase rule for nonreacting, multicomponent, multiphase systems is developed which considers the system intensive variables, overall or total density, overall composition and phase volume fractions. This extended phase rule, especially as it incorporates overall density and composition, may be of use to engineers who design chemical processing equipment and to theoreticians concerned with the nature of thermodynamic equilibrium. The degrees of freedom are given as $(C+1)$ where $(C)$ is the number of components. The Gibbs phase rule is incorporated into this formulation. For a single phase the system intensive variables are the same as phase intensive variables and the rule given here and that of Gibbs are coincident. When the maximum possible number of phase is present, e.g., for a pure substance at its triple point, only system intensive variables such as total density or phase volume fractions can be specified. For cases other than the single phase or maximum number of possible phases it is shown that no phase intensive variables need be specified to fix the state of thermodynamic equilibrium. However, in the case of vapor-liquid equilibria it is useful to fix the phase intensive variable temperature along with the system intensive variables total density and composition. Along such thermodynamic paths mixtures are seen to behave similarly to pure fluids in that the mixture can be represented by pure-fluid-like vapor pressure and coexistence density curves.

100,434

PB91-237693

Not available NTIS

National Inst. of Standards and Technology (MSEL), Gaithersburg, MD. Ceramics Div.
Database and Retrieval System for the NBS Tables of Chemical Thermodynamic Properties.

Final rept.

P. Wang, and D. B. Neumann. 1989, 8p

Pub. in Jnl. of Chemical Information and Computer Sciences $29, n 1$ p $31-381989$

Keywords: "Thermodynamic properties, "Chemical properties, "Information systems, Data base manage ment, Inorganic compounds, Organic compounds, Information retrieval, Reprints.

The organization and structure of a database for the 'NBS tables of chemical thermodynamic properties Selected values for inorganic and $\mathrm{C} 1$ and $\mathrm{C} 2$ organic substances in SI units' is explained. The use of a commercial database management system for the storage and retrieval system is discussed. The file constructs necessary to provide rapid retrieval by elemental composition in the type of database management system are shown. Both implementations on a mini-computer and on personal computers are discussed.

100,435

PB91-237701

Not available NTIS

National Inst. of Standards and Technology (MSEL) Gaithersburg, MD. Ceramics Div.

Surface Oxidation Kinetics of $\mathrm{Si}_{3} \mathrm{~N}_{4}-4 \% \mathrm{Y}_{2} \mathrm{O} 3$ Powders Studied by Bremsstrahlung-Excited Auger Spectroscopy.

Final rept.

P. S. Wang, S. M. Hsu, S. G. Malghan, and T. N Wittberg. 1991, 4p

Pub. in Jnl. of Materials Science 26, p3249-3252 1991

Keywords: "Oxidation, "Reaction kinetics, "Silicon nitrides, "Ceramics, "Auger electron spectroscopy, Powders, Surface chemistry, Yttrium oxides, Bremsstrahlung, Time dependence, Silicon oxides, Activation energy, Solid-solid interfaces, Sintering, Reprints.

Samples of silicon nitride powder containing $4.0 \%$ $\mathrm{Y} 2 \mathrm{O} 3$ in weight were heated in air at temperatures between 900 and $1000 \mathrm{C}$. The average $\mathrm{SiO} 2$ layer thickness on the Si3N4 powder particles, as a function of time at a particular temperature, was measured by Bremsstrahlung-excited Auger electron spectroscopy. Oxidation was found to follow a linear rate law with an activation energy of $56+$ or $-1.5 \mathrm{kcal} / \mathrm{mol}$. The yttrium level measured by $X$-ray photoelectron spectroscopy was also found to decrease as a function of the oxide layer thickness. This suggests that there is a reaction between the Si3N4 and $\mathrm{Y}_{2} \mathrm{O} 3$ particles which results in the formation of an yttrium-rich phase at the interface between the surface $\mathrm{SiO} 2$ layer and the underlying Si3N4 particle.

100,436

PB91-237719

Not available NTIS

National Inst. of Standards and Technology (MSEL), Gaithersburg, MD. Ceramics Div.

Oxidation Kinetics of Silicon Carbide Whiskers Studied by X-ray Photoelectron Spectroscopy. Final rept.

P. S. Wang, S. M. Hsu, and T. N. Wittberg. 1991, $4 p$ Pub. in Jnl. of Materials Science 26, p1655-1658 1991

Keywords: "Oxidation, "Reaction kinetics, "Silicon carbides, "Ceramics, Whiskers, Photoelectron spectroscopy, $X$ ray analysis, Surface chemistry, Thickness, Silicon oxides, Equations, Calculation methods, Activation energy, Measurement, Reprints.

Silicon carbide whisker surfaces were characterized by $X$-ray photoelectron spectroscopy (XPS) to determine changes in the surface oxide film which occurred as a result of heating in air at temperatures from 600 to 800 C. Equations were derived for the calculation of surface oxide film thickness from the $\mathrm{SiC}$ to $\mathrm{SiO} 22 \mathrm{p}$ intensity ratios. Oxidation was found to follow a linear rate law in the temperature range for the first $10 \mathrm{~nm}$ of oxide growth. An activation energy of $17.2+$ or -2.8 $\mathrm{kcal} / \mathrm{mol}(72+$ or $-12 \mathrm{~kJ} / \mathrm{mol})$ was measured.

100,437

PB91-237784

Not available NTIS

National Inst. of Standards and Technology (NML),

Gaithersburg, MD. Center for Chemical Physics. 
Rovibrational Analysis of the nu7(1) Intermolecular Hydrogen Bond Bending Vibration in HCN-HF Using Far Infrared Fourier Transform Spectroscopy.

Final rept.

B. A. Wofford, W. J. Lafferty, W. B. Olsen, R. S.

Ram, A. Quinonez, and J. W. Bevan. 1988, 6p

Pub. in Chemical Physics Letters 152, n4-5 p299-304 1988.

Keywords: "Hydrogen bonds, "Hydrogen cyanide, "Hydrogen fluoride, "Molecular vibration, "Vibrational spectra, Infrared spectroscopy, Molecular structure, Reprints, "Hydrogen bonded complexes, Rotational constants.

The nu7(1) fundamental of the hydrogen bonded complex. HCN--HF has been observed in the gas phase in the far infrared using a cooled static long path gas cell. The frequency of this intermolecular vibration is $73.5835(6) / \mathrm{cm}$. Rotational constants have been determined.

100,438

\section{PB91-237859}

Not available NTIS

National Inst. of Standards and Technology (IMSE),

Gaithersburg, MD. Polymers Div.

Evaluation of Liquid Junction Potentials and Determination of pH Values of Strong Acids at Moderate lonic Strengths.

Final rept.

Y. C. Wu, W. F. Koch, and D. Feng. 1989, 9p

Pub. in Jnl. of Solution Chemistry 18, n7 p641-649 1989.

Keywords: "Electric potential, "pH, Electrochemistry, Inorganic acids, Solutions, lonic strength, Reprints, *Junction potential, Strong acids.

A new method is proposed for the evaluation of the liquid junction potential and the single ion activity. Neither of these quantities can be determined independently. However, the ratio of the activity coefficients may be evaluated by following the method suggested by Frank, from which the liquid junction potential may be computed, so also the $\mathrm{pH}$ values at higher ionic strengths $(<1 \mathrm{~m})$. Experimental results are presented and uncertainty is discussed.

\section{0,439}

PB91-237867

Not available NTIS

National Inst. of Standards and Technology (CSTL)

Gaithersburg, MD. Biotechnology Div.

Electrochemical Behavior of Ferrocene in Polyethylene Oxide -400 .

Final rept.

W. T. Yap, E. A. Blubaugh, R. A. Durst, and C. K

Chiang. $1989,4 p$

Pub. in Jnl. of the Electrochemical Society 136, n9 p2588-2591 1989.

Keywords: "Polyethylene glycols, *Solid electrolytes, ${ }^{\star}$ Conductive polymers, "Ferrocene, "Electrochemistry, Photovoltaic cells, Battery charging, Solutes, Electron transfer, Volt-ampere characteristics, Chemical reactions, Diffusion, Reprints.

There have been many studies on and applications of polymer-based (polyethylene oxides) solid electrolytes. These polymeric electrolytes are utilized in areas, such as, solid state batteries and photovoltaic cells. The high boiling point of these polymer electrolytes makes them potentially useful as solvents for electrochemical reactions at elevated temperatures. Additionally, the study of the physical properties for dissolved analyte such as, diffusion, chemical reactivities are of more fundamental but important, in nature. Presented in the work are studies involving the electroactivity of ferrocene in polyethylene glycol-400. Discussions are included involving the relative contributions of electron transfer rates and uncompensated re sistance to the current potential curves obtained in the studies.

\section{0,440}

PB92-110113

Not available NTIS

American Chemical Society, Washington, DC

Journal of Physical and Chemical Reference Data, Volume 20, Number 1, 1991.

Bimonthly rept.

D. R. Lide. c1991, 202p

See also PB92-110121 through PB92-110154, PB92 110162, PB92-110212, and PB91-192542. Errata sheet inserted. Prepared in cooperation with America Inst. of Physics. New York. Sponsored by Nationa Inst. of Standards and Technology, Gaithersburg, MD.
Available from American Chemical Society, 115 16th St., NW, Washington, DC. 20036-9976.

Keywords: "Physical properties, Spectrum analysis, Tables(Data), Copper ions, Atomic energy levels, Magnesium, Alkyl compounds, Alkoxy compounds, Wavelengths, Enthalpy, Liquid crystals, Ionization, Molecular structure, Hydrides, Diatomic molecules, Transition temperature, "Reference materials, Benzoic acid/ (phenyl-ester), Grotrian diagrams.

Contents:

Spectral Data and Grotrian Diagrams for Highly Ionized Copper, Cu X-Cu XXIX;

Wavelengths and Energy Level Classifications of Magnesium Spectra for All Stages of Ionization (Mg I thru XII);

Spectroscopy and Structure of the Alkali Hydride Diatomic Molecules and Their Ions:

Critical Evaluation of Liquid Crystal Transition Temperatures I:

4,4'-Alkyl/alkylphenylbenzoates;

Cumulative listing of Reprints and Supplements.

\section{0,441}

PB92-110121

(Order as PB92-110113)

Japan Atomic Energy Research Inst., Tokai.

Spectral Data and Grotrian Diagrams for Highly Ionized Copper, Cu X-Cu XXIX.

T. Shirai, T. Nakagaki, Y. Nakai, J. Sugar, and K. Ishii. c1991, $81 p$

Prepared in cooperation with National Inst. of Standards and Technology, Gaithersburg, MD., Kyoto Univ (Japan), and Hiroshima-Denki Inst. of Tech. (Japan) Included in Jnl. of Physical and Chemical Reference Data, v20 n1 p1-81 1991. Available from American Chemical Society, 1155 16th St., NW, Washington, DC. 20036-9976.

Keywords: “Spectrum analysis, *Copper ions, Wavelengths, Tables(Data), Atomic energy levels, Graphs(Charts), lonization, "Reference materials, *Grotrian diagrams.

Wavelengths, energy levels, level classifications, intensities, and transition probabilities for the coppe spectra $\mathrm{Cu} X$ to $\mathrm{Cu} X \mathrm{XIX}$ are compiled. The data are critically evaluated and the best results, in the authors' judgement are quoted. A short review of the work on each stage of ionization is included. Grotrian diagrams are also presented to provide graphical overviews. The literature has been surveyed to March 1990. (Copyright (c) 1991 by the U.S. Secretary of Commerce.)

\section{0,442}

PB92-110139

(Order as PB92-110113)

National Inst. of Standards and Technology, Gaithersburg, MD.

Wavelengths and Energy Level Classifications of Magnesium Spectra for All Stages of Ionization (Mg I through Mg XII).

V. Kaufman, and W. C. Martin. c1991, 70p

Included in Jnl. of Physical and Chemical Reference Data, v20 n1 p83-152 1991. Available from American Chemical Society, 1155 16th St., NW, Washington, DC. 20036-9976.

Keywords: "Wavelengths, "Spectrum analysis, "Atomic energy levels, "Magnesium, Tables(Data) Listings, Ionization, Critical point, "Reference materials.

Wavelengths and their classifications are compiled for the spectra of magnesium. Selections of data are based on the critical evaluations in the compilation of energy levels by Martin and Zalubas with some updating from the more recent literature. All classifications have been verified with predictions made by differencing the energy levels. In addition to the spectra ordered by ionization stage, two finding lists are included, one containing $\mathrm{Mg} I$ to $\mathrm{Mg}$ III and the other $\mathrm{Mg}$ IV to Mg XII. (Copyright (c) 1991 by the U.S. Secretary of Commerce.)

\section{0,443}

PB92-110147

lowa Univ., lowa City. (Order as PB92-110113)

Spectroscopy and Structure of the Alkali Hydride Diatomic Molecules and Their lons.

W. C. Stwalley, W. T. Zemke, and S. C. Yang. c1991,

$35 \mathrm{p}$
Prepared in cooperation with Wartburg Coll., Waverley, IA. Dept. of Chemistry, and Rhode Island Univ., Kingston. Dept. of Chemistry.
Included in Jnl. of Physical and Chemical Reference Data, v20 n1 p153-187 1991. Available from American Chemical Society, 1155 16th St., NW, Washington, DC. 20036-9976.

Keywords: Spectrum analysis, "Molecular structure, *Metal hydrides, *Diatomic molecules, *Ions, Sodium hydrides, Potassium hydrides, Cesium hydrides, Atomic energy levels, Rubidium hydrides, Alkali metals, Hydrides, Deuterium compounds, Laser spectroscopy, Excitation, *Reference materials.

All significant experimental measurements and theoretical calculations of the spectroscopy and structure of the alkali hydrides $\mathrm{NaH}, \mathrm{KH}, \mathrm{RbH}$ and $\mathrm{CsH}$, and the corresponding alkali deuterides, are identified and reviewed. Published molecular constant determinations from conventional and laser spectroscopy are evaluated; recommended spectroscopic constants for Chi(1)Sigma(+) and Alpha(1)Sigma(+) states are tabulated. RKR and hybarid potential energy curves are evaluated: recommended RKR curves for CHI(1)Sigma( + ) and Alpha(1)Sigma( + ) states are $\mathrm{CHI}(1)$ Sigma( $(+)$ and Alpha(1)Sigma( + ) states are
tabulated. Ground state dissociation energy (De) estimates are evaluated; recommended Chi(1)Sigma(+) and Alpha(1)Sigma(+) state De and D6 values are tabulated. Accurate electronic structure calculations (Hartree Fock or better) are listed and described briefly; all excited electronic states considered are included. Experimental and theoretical radiative and dipole properties are noted and discussed. Calculations on the positive and negative ions of the four diatomic alkali hydrides are also listed and described briefly. (Copyraight (c) 1991 by the U.S. Secretary of Commerce.)

100,444

PB92-110154

Kent State Univ., $\mathrm{OH}$.

(Order as PB92-110113)

Critical Evaluation of Liquid Crystal Transition Temperatures I: 4,4'-Alkyl/alkoxyphenylbenzoates.

T. T. Blair, M. E. Neubert, M. Tsai, and C. Tsai.

c1991, 16p

Included in Jnl. of Physical and Chemical Reference Data, v20 n1 p189-204 1991. Available from American Chemical Society, 1155 16th St., NW, Washington, DC. 20036-9976.

Keywords: "Liquid crystals, "Transition temperatures, *Alkyl compounds, "Alkoxy compounds, Melting points, Enthalpy, Alkoxy compounds, Melting Tables(Data), Graphs(Charts), Error analysis, Molecular structure, "Benzoic acid/(phenyl-ester).

Transition temperataures for 366 straight chain 4,4 'alkyl/alkoxyphenylbenzoates (through Nov. 1990) have been compiled and critically evaluated. Information was obtained wherever possible, with the exception of the patent literature, and examined for editorial coherency. Line graphs of the melting and clearing temperatures were plotted as a function of the chain length of either side, while holding constant the other side, and evaluated for consistency, particularly for the persistence of the odd-even alternation in the clearing curve. It became apparent that major breaks in the curve indicated errors in the data which, when corrected, restored the alternating curve. Mesophase identification was reviewed by evaluating bar graphs and block diagrams. Usually, a given phase appeared first in a monotropic form, then in an enantiotropic form, and finally disappeared without reappearing in the series. Further evaluation of the data suggested that a number of phases have been missed. Evaluated transition temperatures are available on 38 dialkyl, 91 alkyl/alkoxy, 100 alkoxy/alkyl, and 137 dialkoxy compounds. Enthalpies for 93 of the esters are also included. (Copyright (c) 1991 by the U.S. Secretary of Commerce.)

100,445

Not available NTIS American Chemical Society, Washington, DC.

Journal of Physical and Chemical Reference Data, Volume 20, Number 2, March-April 1991.

Bimonthly rept.

D. R. Lide. c1991, 234p

See also PB92-110170 through PB92-110204, PB92110212 . PB92-110113, and PB91-192542. Prepared in cooperation with American Inst. of Physics, New York Sponsored by National Inst. of Standards and Technology, Gaithersburg, MD

Available from American Chemical Society, 1155 16th St., NW, Washington, DC. 20036-9976. 
Keywords: "Physical chemistry, Thermochemistry, Combustion, Propenes, Thermodynamic properties, Thermophysical properties, Ethane, Information systems, Specific heat, Polymers, Organic compounds, Liquid phases, Alkanes, "Reference materials, ATHAS system.

\section{Contents:}

Chemical Kinetic Data Base for Combustion Chemistry Part V. Propene

Thermophysical Properties of Ethane;

Heat Capacity and Other Thermodynamic Properties of Linear Macromolecules. X. Update of the ATHAS 1980 Data Bank;

Heat Capacities of Organic Compounds in Liquid State. II. C1 to C18 n-Alkanes;

Cumulative Listing of Reprints and Supplements.

100,446

PB92-110170

(Order as PB92-110162) National Inst. of Standards and Technology (NML), Gaithersburg, MD. Chemical Kinetics Div.

Chemical Kinetic Data Base for Combustion Chemistry. Part 5. Propene.

W. Tsang. c1991, 53p

Included in Jnl. of Physical and Chemical Reference Data, v20 n2 p221-273 Mar/Apr 91. Available from American Chemical Society, 1155 16th St., NW, Washington, DC. 20036-9976.

Keywords: "Reaction kinetics, "Combustion, "Propenes, "Thermochemistry, Inorganic compounds, Pyrolysis, Alkanes, Transport properties, Thermodynamic properties, Vapor phases, Tables(Data), "Reference materials.

The publication contains evaluated and estimated data on the kinetics of reactions involving propene and allyl radical and various small inorganic and organic species which are of importance for the proper understanding of the early stages of propene and the intermediate stages of propane and isobutane combustion and pyrolysis. It is meant to be used on conjunction with the kinetic data given in earlier publications, which is of direct pertinence to the understanding of methane, ethane, methanol, propane and isobutane pyrolysis and combustion, but which also contains a large volume of data that are applicable to the propene system. The temperature range covered is $300-2500 \mathrm{~K}$ and the density range 10 to the 16 th power to 10 to the 21st power molecules/cu cm. (Copyright (c) 1991 by the U.S. Secretary of Commerce.)

100,447

PB92-110188

(Order as PB92-110162)

National Inst. of Standards and Technology (NML), Boulder, CO. Thermophysics Div.

Thermophysical Properties of Ethane.

D. G. Friend, H. Ingham, and J. F. Ely. c1991, 73p

Included in Jnl. of Physical and Chemical Reference

Data, v20 n2 p275-347 Mar/Apr 91. Available from American Chemical Society, 1155 16th St., NW, Washington, DC. 20036-9976.

Keywords: "Thermophysical properties, "Ethane, Liquid phases, Thermodynamic properties, Equations of state, Transpoort properties, Specific heat, Tables(Data), Boundary layer transition, Phase rule, Viscosity, Ideal gas, Thermal conductivity, "Reference materials.

New correlations for the thermophysical properties of fluid ethane are presented. The correlations are based on a critical evaluation of the available experimental data and have been developed to represent these data over a broad range of the state variables. Estimates for the accuracy of the equations and comparisons with measured properties are given. The reasons for the new study of ethane include significant new and accurate data and improvements in the correlating functions which allow increased accuracy of the correlations--especially in the extended critical region. Short tables of the thermophysical properties of ethane are included. The study complements an earlier study of methane and uses the same correlating equations and format. (Copyright (c) 1991 by the U.S. Secretary of Commerce.)

\section{0,448}

PB92-110204

Vysoka Skola Chemicko-Torder as PB92-110162) (Czechoslovakia).
Heat Capacities of Organic Compounds in Liquld State. 2. C1 to $\mathrm{C} 18 \mathrm{n}$-Alkanes.

V. Rizicka, M. Zabransky, and V. Majer. c1991, 52p Prepared in cooperation with Clermont-Ferrand-2 Univ., Aubiere (France).

Included in Jnl. of Physical and Chemical Reference Data, v20 n2 p405-444 Mar/Apr 91. Available from American Chemical Society, 1155 16th St., NW, Washington, DC. 20036-9976.

Keywords: "Specific heat, "Organic compounds, "Alkanes, "Liquid phases. Thermodynamic properties, Tables(Data), Experimental design, Least square methods, Temperature, "Reference materials.

Heat capacities of liquid $\mathrm{C} 1$ to $\mathrm{C} 18$ n-alkanes measured by calorimetric methods have been compiled and evaluated. The selected experimental data were fitted as a function if temperature with cubic splines using weighted least squares minimization. The parameters of the cubic spline polynomials and the recommended values for heat capacities are presented. Heat capacities were also fitted by a quasipolynomial equation permitting extrapolation of heat capacities outside the temperature range of experimental values. (Copyright (c) 1991 by the U.S. Secretary of Commerce.)

\section{0,449}

PB92-110212

Not available NTIS American Chemical Society, Washington, DC

Journal of Physical and Chemical Reference Data, Volume 20, Number 3, May-June 1991.

Bimonthly rept.

D. R. Lide. c1991, 128p

See also PB92-110220 through PB92-110253, PB92. 110113 and PB92-110162. Prepared in cooperation with American Inst. of Physics, New York. Sponsored by National Inst. of Standards and Technology, Gaithersburg, MD.

Available from American Chemical Society, 1155 16th St., NW, Washington, DC 20036-9976.

Keywords: "Physical chemistry, Argon ions, Nitrogen, Reaction kinetics, Vapor phases. Thermodynamic properties, Nitroxyl radicals, Organic compounds, Sodium bromides, Argon, Nitrogen ions, Atomic energy levels, Ion-molecule collisions, Molecule-molecule collisions, Solubility, Carbon dioxide, Critical point, Water, Cross sections, "Reference materials, Chemical reaction mechanisms.

Contents:

Kinetics and Mechanisms of the Gas-Phase Reactions of the NO3 Radical with Organic Compounds:

Thermodynamic Properties of the $\mathrm{NaBr}+\mathrm{H} 2 \mathrm{O}$ System

Cross Sections and Swarm Coefficients for Nitrogen lons and Neutrals in N2 and Argon lons and Neutrals in Ar for Energies from 0.1 $\mathrm{eV}$ to $10 \mathrm{keV}$

Evaluation of Solubility Data of the System CO2. $\mathrm{H} 2 \mathrm{O}$ from $273 \mathrm{~K}$ to the Critical Point of Water;

Cumulative Listing of Reprints and Supplements.

100,450

\section{PB92-110220}

California Univ., Riverside. Statewide Air Pollution Re search Center.

Kinetics and Mechanisms of the Gas-Phase Reactions of the NO3 Radical with Organic Compounds. R. Atkinson. c1991, 49p

Included in Jnl. of Physical and Chemical Reference Data, v20 n3 p459-507 May/Jun 91. Available from American Chemical Society, 1155 16th St., NW, Washington, DC 20036-9976.

Keywords: "Reaction kinetics, "Nitroxyl radicals, "Organic compounds, Vapor phases, Reviews, Chemical reactions, Free radicals, Nitrates, "Chemical reaction mechanisms, "Reference materials.

A substantial data base concerning the rate constants for the gas-phase reactions of the nitrate (NO3) radical with organic compounds is now available, with rate constants having been determined using both absolute and relative rate methods. To date, the majority of these kinetic data have been obtained at room temperature using relative rate techniques utilizing both the reactions of the NO3 radical with other organic compounds and the equilibrium constants for the $\mathrm{NO} 3+\mathrm{NO} 2$ in equilibrium with N2O5 reactions as the reference reaction. In the article, the literature kinetic and mechanistic data for the gas-phase reactions of the NO3 radical with organic compounds (through late
1990) have been tabulated, reviewed and evaluated. While the available data base exhibits generally good agreement and self-consistency, further absolute rate data are needed, preferably as a function of temperature. Most importantly, mechanistic and product data for the reactions of the NO3 radical with organic compounds need to be obtained. (Copyright (c) 1991 by the U.S. Secretary of Commerce.)

100,451

PB92-110238

(Order as PB92-110212)

National Inst. of Standards and Technology, Gaithersburg, MD

Thermodynamic Propertles of the $\mathrm{NaBr}+\mathrm{H} 2 \mathrm{O}$ System.

D. G. Archer. c1991, 47p

Included in Jnl. of Physical and Chemical Reference Data, v20 n3 p509-555 May/Jun 91. Available from American Chemical Society, 115516 th St., NW, Washington, DC 20036-9976.

Keywords: "Thermodynamic properties, "Sodium bromides, "Water, Chemical reactions, Experimental design, Thermodynamic activity, Reaction kinetics, Gibbs iree energy, Enthalpy, Entropy, "Reference materials.

Equations that described the thermodynamic properties of the $\mathrm{NaBr}+\mathrm{H} 2 \mathrm{O}$ system were obtained from a fit to experimental results for the system. The experimental results included in the fit spanned the range of temperature of approximately 260 to $623 \mathrm{~K}$ and the range of pressure from the vapor pressure of the solution to $150 \mathrm{MPa}$. New equations and/or values for the following properties are given in the present work: (1) the change in chemical potential with respect to temperature and pressure for $\mathrm{NaBr}(\mathrm{cr})$, valid from 200 to $900 \mathrm{~K}$. (2) delta(sub f) G(sub $\mathrm{m}$ ) and delta(sub f)H(sub $\mathrm{m}$ ) from the elements, as well as $\mathrm{S}($ sub $\mathrm{m}$ ) and $\mathrm{C}$ (sub $\mathrm{p}, \mathrm{m}$ ), all for $298.15 \mathrm{~K}, 0.1 \mathrm{MPa}$ for $\mathrm{NaBr}: 2 \mathrm{H} 2 \mathrm{O}(\mathrm{cr}),(4)$ the change in chemical potential for both $\mathrm{NaBr}$ and $\mathrm{H} 2 \mathrm{O}$ in $\mathrm{NaBr}(\mathrm{aq})$ as a function of temperature, pressure, and molality, valid from 260 to $600 \mathrm{~K}$ and from the vapor pressure of the solution to $150 \mathrm{MPa}$. (Copyright (c) 1991 by the U.S. Secretary of Commerce.)

100,452

PB92-110246

(Order as PB92-110212)

Joint Inst. for Lab. Astrophysics, Boulder, CO.

Cross Sections and Swarm Coefficients for Nitrogen lons and Neutrals in N2 and Argon lons and Neutrals In N2 and Argon lons and Neutrals in Ar for Energles from $0.1 \mathrm{eV} 10 \mathrm{keV}$.

A. V. Phelps. c1991,17p

Included in Jnl. of Physical and Chemical Reference Data, v20 n3 p557-573 May/Jun 91. Available from American Chemical Society, 115516 th St., NW, Washington, DC 20036-9976.

Keywords: "Ion-molecule collisions, "Molecule-molecule collisions, "Cross sections, Nitrogen ions, Nitrogen, Argon ions, Argon, Atomic energy levels, Charged-particle transfer, Reaction kinetics, Excition, Tables(Data), Graphs(Charts).

Graphical and tabulated data and the associated bibliography are presented for cross sections for elastic, excitation, and ionization collisions of $\mathrm{N}(+), \mathrm{N} 2(+), \mathrm{N}$, and N2 with N2 and for $\mathrm{Ar}(+)$ and $\mathrm{Ar}$ with Ar for laboratory energies from $0.1 \mathrm{eV}$ to $10 \mathrm{keV}$. Where appropriate, drift velocities and reaction or excitation coefficients are calculated from the cross sections and recommended for use in analyses of swarm experiments and electrical discharges. In the case of $N(+)$ in N2, cross sections for momentum transfer, charge transfer, electronic excitation, and electron production are recommended. In the case of N in N2, only cross sections for momentum transfer are recommended. For N2 in N2, cross sections for momentum transfer, electronic excitation, and electron production are recommended. Collisions of electronically excited states with $\mathrm{N} 2$ are not included. For $\mathrm{Ar}(+)$ in Ar, cross sections for charge transfer, electronic excitation, and electron production are recommended. For $\mathrm{Ar}$ in $\mathrm{Ar}$, cross sections for momentum transfer, electronic excitation, and electron production are recommended.

100,453

PB92-110253

(Order as PB92-110212)

National Inst. of Standards and Technology (NML) Gaithersburg, MD. Thermophysics Div. 
Evaluation of Solubility Data of the System CO$\mathrm{H} 2 \mathrm{O}$ from $273 \mathrm{~K}$ to the Critical Point of Water.

R. Crovetto. c1991, 15p

Included in Jnl. of Physical and Chemical Reference Data, v20 n3 p575-589 May/Jun 91. Available from American Chemical Society, 1155 16th St., NW, Washington, DC 20036-9976.

Keywords: "Solubility, "Carbon dioxide, "Water, High temperature tests, Critical point, Pressure, Henry's law, Equations of state, Vapor phases.

The critical review covers the existing literature on the solubility of $\mathrm{CO} 2$ in water from $273 \mathrm{~K}$ to the critical temperature of the solvent $(647 \mathrm{~K})$. Results of the evaluation are expressed in the form of fitting equations for the infinite dilution Henry's constant, $k$, as a function of the density of the solvent, and also as an explicit function of the temperature. The pressure effect on the solubility is considered in the formulation. Different equations of state were used for the description of the $\mathrm{CO} 2$ $\mathrm{H} 2 \mathrm{O}$ vapor phase and the effect on the calculated Henry's constant values are analyzed. The 'best' solubility estimates are presented in smoothed tabular form. (Copyright (c) 1991 by the U.S. Secretary of Commerce.)

\section{0,454}

PB92-116326

PC A03/MF A01

National Inst. of Standards and Technology (CSTL), Boulder, CO. Chemical Engineering Div.

Transient Hydrogen Heat Transfer. Final Report for April 1986-April 1989.

B. Louie, and W. G. Steward. Oct 91, 42p NISTIR-

3951

Contract WPAFB MIPR FY1455-89-N0604

See also PB90-254764. Prepared in cooperation with

FluidTherm Engineering, Boulder, CO.

Keywords: "Liquid hydrogen, "Heat transfer, Cryogenics, Transients, Thin films, Carbon, Platinum, Heat of vaporization, Nucleate boiling, Heat measurement Substrates, Ceramics, Aluminum oxide, Quartz, Glass expoxy composites, Electric conductivity, Power generation.

Transient heat transfer to liquid hydrogen was investigated. Thin carbon films and $\mathrm{Pt}$ foils submerged in liquid hydrogen received stepped inputs of power ranging from 1 to $42 \mathrm{~W} / \mathrm{sq} \mathrm{cm}$, and the onset of nucle ate or film boiling was obtained for each power level The critical heat flux was found to be approximately 8 $\mathrm{W} / \mathrm{sq} \mathrm{cm}$, with the transition to film boiling occurring in times less than $0.0 \mathrm{C}$. s. Premature film boiling can be related to the positive temperature coefficient of resistance and the narrowness of the heaters. Thermometric devices and power generation equipment selection are discussed.

\section{0,455}

PB92-116508

Not available NTIS

National Inst. of Standards and Technology (NML)

Boulder, CO. Thermophysics Div.

Hydrogen Component Fugacity Coefficients in Binary Mixtures with Etane: Pressure Dependence. Final rept.

T. J. Bruno, J. A. Schroeder, and S. L. Outcalt. 1990, $8 p$

See also PB89-124853. Sponsored by Gas Research Inst., Chicago, IL

Pub. in International Jnl. of Thermophysics $11, \mathrm{n} 5$ p889-896 1990.

Keywords: "Fugacity, "Binary mixtures, "Hydrogen, "Ethane, Test chambers, Comparison, Reprints.

The fugacity coefficients of hydrogen in binary mixtures with ethane were measured. Data were taken using an experimental chamber which is divided into two regions by a semipermeable membrane through which hydrogen, but not ethane can penetrate. The measurement of the gas pressures inside and outside the membrane gives the hydrogen component fugacity at a given temperature, binary mixture mole fraction and mixture pressure. In the paper, results are reported at mixture pressures of $5.25,6.97,10.21$, and 13.47 $\mathrm{MPa}$. In each case, the temperature of the mixture was maintained at an average value of $130 \mathrm{C}(403.15 \mathrm{~K})$ The general qualitative features of the data are discussed, and comparisons are made with predictions obtained from the Redlich-Kwong and Peng-Robinson equations of state.

\section{0,456}

PB92-116524

Not available NTIS

National Inst. of Standards and Technology (CSTL) Boulder, CO. Chemical Engineering Div.
Thermodynamics of the Divalent-Metal Fluorides. 4. Heat Capacity of MSnF4, M $=\mathrm{Pb}, \mathrm{Ba}$, or $\mathrm{Sr}$, at Temperatures from $300 \mathrm{~K}$ to $660 \mathrm{~K}$.

Final rept.

J. E. Callanan, and R. D. Weir. 1991, 10p

See also PB91-149864. Sponsored by Department of National Defence, Ottawa (Ontario).

Pub. in Jnl. of Chemical Thermodynamics 23, p4114201991

Keywords: "Ionic conductivity, "Calorimetry, "Specific heat, "Stannates, Thermodynamic properties, Temperature dependence, Strontium compounds, Barium compounds, Lead compounds, Phase transformations, Monoclinic lattices, Tetragonal lattices, Cubic lattices, Fluorine 19, Enthalpy, Entropy, Transition temperature, Reprints, "Fast ion conductors, Tetrafluorostannates.

The low-temperature adiabatic calorimetric measurements on three fast-ion conductors have been ex tended to $660 \mathrm{~K}$ by differential scanning calorimetry (d.s.c.). For strontium tetrafluorostannate $\mathrm{SrSnF} 4$ the heat capacities from the d.s.c. merged smoothly with those from the adiabatic measurements and no phase transition was observed up to $660 \mathrm{~K}$. The anomaly between $280 \mathrm{~K}$ and $350 \mathrm{~K}$ was confirmed and a second anomaly was found near $475 \mathrm{~K}$ coincident with changes in the spin-lattice relaxation time of (19)F The heat capacity of BaSnF 4 rose smoothly from the anomaly between $210 \mathrm{~K}$ and $320 \mathrm{~K}$ noted previously in the adiabatic measurements. No evidence of a phase transition was observed up to $660 \mathrm{~K}$, although the slope of the heat capacity against temperature curve increased above $510 \mathrm{~K}$. In lead tetrafluorostannate $\mathrm{PbSnF} 4$ the transition from the monoclinic alphaphase to tetragonal beta-phase was complete at $365 \mathrm{~K}$ with an associated enthalpy $=(306.0+$ or -0.2$) \mathrm{J} /$ mol and entropy $=(0.97+$ or -0.02$) \mathrm{J} / \mathrm{K} / \mathrm{mol}$. The heat capacity then rose smoothly to $500 \mathrm{~K}$ where increased sharply leading up to the tetragonal betaphase to cubic gamma-phase transition at $600 \mathrm{~K}$ with enthalpy $=(2.53+$ or -0.02$) \mathrm{kJ} / \mathrm{mol}$ and entropy $=$ $(4.03+$ or -0.05$) \mathrm{J} / \mathrm{K} / \mathrm{mol}$. Thermodynamic functions are tabulated to $600 \mathrm{~K}$ for $\mathrm{SrSnF} 4$ and to $650 \mathrm{~K}$ for $\mathrm{BaSnF} 4$ and $\mathrm{PbSnF} 4$.

\section{0,457}

PB92-116649 National Inst. of Standards and Technology (NML), Gaithersburg, MD. Molecular Physics Div.

Rotational-Tunneling Spectrum of the Ar-SO2 van der Waals Complex.

Final rept.

L. H. Coudert, K. Matsumura, and F. J. Lovas. 1991 , $15 p$ Pub. in Jnl. of Molecular Spectroscopy 147, p46-60 1991

Keywords: "Microwave spectra, "Argon compounds, "Sulfur dioxide, Gases, Molecular structure, Spectrum analysis, Fourier transformation, Fabry-perot interferometer, Sulfur 32 reactions, Sulfur 34 reactions, Rotaometer, Sulfur 32 reactions, Sulfur 34
tional states, Coriolis force, Reprints.

The microwave spectrum of Ar-So2 has been observed with a pulsed beam, Fabry-Perot cavity, Fourier transform microwave spectrometer. This complex dis plays a- and c-type spectra, the latter of which is shifted by internal motion of the $\mathrm{SO} 2$ unit. In addition to the spectrum of the normal isotopic form, the spectrum of $\mathrm{Ar}$ - (34) SO2 was observed. Rotational analysis of each spectrum provides the constants $A=$ $9151.235(5) \mathrm{MHz}, 1 / 2(B+C)=1402.0444$ (3) $\mathrm{MHz}$, and $\mathrm{B}-\mathrm{C}=167.808$ (2) $\mathrm{MHz}$ for the normal species and $A=9112.734(6) \mathrm{MHz}, 1 / 2(\mathrm{~B}+\mathrm{C})=1385.149$ (6) $\mathrm{MHz}$, and $\mathrm{B}-\mathrm{C}=162.838$ (2) $\mathrm{MHz}$ for the $\mathrm{Ar}-$ (34) SO2 species. Constants relevant to the Coriolis coupling between the tunneling motion and the overall rotation of the molecule are also determined and, along with the rotational constants, make possible an unambiguous determination of the angle between the a axis of the complex and the $\mathrm{C} 2$ axis of the $\mathrm{SO} 2$ molecule.

\section{0,458}

PB92-116839

Not available NTIS

National Inst. of Standards and Technology (NML), Gaithersburg, MD. Thermophysiçs Div.

Apparatus for Acoustic Measurements of Gases with Applications for High Temperatures.

Final rept.

K. A. Gillis, and M. R. Moldover. 1991, $8 p$

Contracts DE-Al05-88ER13823, N0002490MP33493

Sponsored by Department of Energy, Washington, DC., and Department of the Navy, Washington, DC.
Pub. in Proceedings of Symposium on Energy Engineering Sciences Fluid and Dynamical Systems (9th), Argonne, IL., May 13-15, 1991, p310-317.

Keywords: "Sound waves, "Gases, "Sound transducers, Specific heat, Virial equation, Thermodynamic properties, High temperature tests, Refrigerants, Reprints, Bid(difluoromethyl) ether.

Accurate measurements of the speed of sound in gases are conventionally made using metal resonators with small transducers embedded in their walls. The authors have extended the method to include gases at high temperatures and gases that are corrosive or incompatible with elastomers and thermoplastics. The authors use remote transducers coupled to a resonator through acoustic waveguides. Thin metal diaphragms separate the waveguides from the resonator and confine the test gas within an all-metal environment. In the present apparatus, any gas compatible with gold and stainless steel can be studied. The apparatus was tested by determining the ideal-gas heat capacity and second acoustic virial coefficient of bis(difluoromethyl) ether. The ether is an environmentally benign compound; however, its solubility in polymer o-rings made it incompatible with conventional acoustic apparatus containing elastomers.

100,459

PB92-116854

Not available NTIS

National Inst. of Standards and Technology (CSTL)

Gaithersburg, MD. Biotechnology Div.

Thermodynamics of the Disproportionation of Adenosine 5'-diphosphate to Adenosine 5'-triphosphate and Adenosine 5'-monophosphate. 1. Equilibrium Model.

Final rept.

R. N. Goldberg, and Y. B. Tewari. 1991, 21p

Pub. in Biophysical Chemistry 40, p241-261 1991.

Keywords: "Disproportionation, "Enzymes, "Adenosine phosphates, "ADP, "Magnesium ions, "ATP Activity coefficients, Thermodynamic equilibrium, Mathematical models, Specific heat, AMP, Enthalpy, Blood cells, Nucleotides, Protons, Gibbs free energy, Hemoglobin, Catalysts, Biochemistry, Entropy, Reprints, Adenylate kinase, lonic strength.

The thermodynamic treatment of the disproportionation reaction of adenosine 5'-diphosphate to adenosine 5'-triphosphate and adenosine 5'-monophosphate is discussed in terms of an equilibrium model which includes the effects of the multiplicity of ionic and metal bound species and the presence of long range electrostatic and short range repulsive interactions. Calculated quantities include equilibrium constants, enthalpies, heat capacities, entropies, and the stoichiometry of the overall reaction. The matter of how these calculations can be made self-consistent with respect to both calculated values of the ionic strength and the motality of the free magnesium ion is discussed. The thermodynamic data involving proton and magnesium-ion binding data for the nucleotides involved in this reaction have been evaluated.

100,460

PB92-117035

Not available NTIS

National Inst. of Standards and Technology (MSEL),

Gaithersburg, MD. Ceramics Div.

Surface X-ray Absorption Spectroscopy, EXAFS and NEXAFS, for the In situ and Ex situ Study of Electrodes.

Final rept.

G. G. Long, and J. Kruger. 1991, 43p

Sponsored by National Science Foundation, Washington, DC., and Air Force Office of Scientific Research, Bolling AFB, DC

Pub. in Techniques for Characterization of Electrodes and Electrochemical Processes, Chapter 4, p167-209 1991.

Keywords: "Electrodes, "Surface chemistry, "Electrochemistry, Corrosion inhibitors, $X$ ray analysis, $R e-$ views, On-line measurement systems, Characterization, Passivity, Electrolytes, Reprints, Extended x-ray absorption fine structure.

The report is a review of extended $x$-ray absorption fine structure (EXAFS) and NEXAFS spectroscopy as it applies to important issues in electrochemistry. The theory and the resolution of some of the problems encountered are given. Techniques and instrumentation are reviewed. And finally, examples are given of applications from passivity, corrosion inhibitors and batteries. 
100,461

PB92-117050

Not available NTIS

National Inst. of Standards and Technology (NML) Gaithersburg, MD. Thermophysics Div.

Semiclassical Calculation of Self-Broadening in O2, N2, and CO Raman Spectra.

Final rept

J. P. Looney, and G. J. Rosasco. 1991, 12p

Sponsored by Army Research Office, Research Triangle Park, NC.

Pub. in Jnl. of Chemical Physics 95, n4 p2379-2390, 15 Aug 91.

Keywords: "Raman spectra, "Oxygen, "Nitrogen, Carbon monoxide, Semiclassical approximation, Calculation methods. Spectrum analysis, Temperature dependence, Gases, Line broadening, Reprints

Semiclassical calculations of the $\mathrm{J}$ and temperature dependence of the Raman Q1 and So self-broadened linewidths of $\mathrm{O} 2, \mathrm{~N} 2$, and $\mathrm{CO}$ have been performed within the framework of Robert and Bonamy with $\mathrm{J}=$ $0-30$ and $T=300-1500 \mathrm{~K}$. The calculation of the $\mathrm{CO}$ self-broadened $\mathrm{R} 1$ linewidths are also reported for $\mathrm{T}$ $=100-300 \mathrm{~K}$ and $\mathrm{J}=0-20$. Comparison of the results of these calculations with recent experiments shows good overall agreement.

100,462

PB92-117092

Not available NTIS

National Inst. of Standards and Technology (NEL)

Gaithersburg, MD. Semiconductor Electronics Div.

XANES of Transition Metal Zinc-Blende Semiconductors.

Final rept.

D. A. Mckeown. 1991, 3p

Pub. in X-ray Absorption Fine Structure, Chapter 85 p346-348 1991

Keywords: "X ray analysis, "Electron transitions, Semiconductors(Materials), "Transition metals, Sulfides, Iron compounds, Copper compounds, Zinc compounds, Chalcopyrite, Sphalerite, Atomic structure, Reprints, $X$ ray absorption near-edge structure, Zinc blende.

XANES (X-ray absorption near-edge structure) is known to be sensitive to both the arrangement of atoms around, as well as the atomic states of the absorbing atom. Therefore, it is not surprising that XANES data, collected on compounds having different arrangements of atoms around the absorbing atom, can have very different features. In the study, XANES data were gathered for three transition metals: $F e$ and $\mathrm{Cu}$ in chalcopyrite (CuFeS2), and $\mathrm{Zn}$ in sphalerite $(\mathrm{ZnS})$, where all three cations are in nearly identical (ZnS), where all three cations are in nearly identical structures in III-V semiconductors). Since the environments are similar, any change in the XANES should, to first approximation, be due entirely to atomic effects of the absorbing atom. The rationale behind the study is to see if any changes in the near-edge data can be assigned to electronic transitions of the absorbing atom; this may be useful for interpreting XANES for III$V$ semiconductors. Previously, $\mathrm{Zn}, \mathrm{Cu}$, and $\mathrm{Fe}$ edges were presented separately, but no comparisons or calculations have been made for all three edges.

100,463

PB92-117175

Not available NTIS

National Inst. of Standards and Technology (NML) Gaithersburg, MD. Molecular Physics Div

High-Resolution Infrared Spectrum of the nut Band of OC10.

Final rept.

J. Ortigoso, R. Escribano, J. B. Burkholder, C. J

Howard, and W. J. Lafferty. 1991, 25p

Pub. in Jnl. of Molecular Spectroscopy 148, p346-370 1991.

Keywords: "Chlorine oxides, "Infrared spectra, High resolution, Absorption spectra, Band spectra, Reprints, Fourier transform infrared spectroscopy.

The infrared absorption spectrum of the nu(sub 1) band of $\mathrm{OClO}$ (care must be taken to distinguish it from $\mathrm{CIOO}$, which also exists) has been recorded in the $950 / \mathrm{cm}$ region, with a Fourier transform infrared spectrometer with an instrumental resolution of about $0.004 / \mathrm{cm}$. Most lines appear as doublets owing to the spin-rotation interaction present in this molecule. Around 2800 lines have been assigned for the (35) ClO2 species and about 800 for the $(37) \mathrm{ClO} 2 \mathrm{spe}$ cies. In addition, a number of lines of the 'hot band' nu(sub 1) + nu(sub 2) - nu(sub 2) have been assigned for the (35) $\mathrm{ClO} 2$ species. Effective rotational and spinrotational spectroscopic constants have been obtained for the ground and the nu(sub 1) - 1 vibrational states of (35) ClO2 and (37) ClO2 and the correspond ing band origins have also been determined. Ferm resonance between the 2nu(sub 2) and nu(sub 1) bands has been found to be negligible; however, a weak resonance between the $\mathrm{K}$ (sub alpha) $=7$ levels of nu(sub 1) $=1$ and the K(sub alpha) =9 levels of nu(sub 2) $=2$ has been observed. The measured integrated nu(sub 1) band strength is $87+$ or $-6 / \mathrm{sq} \mathrm{cm} /$ atm at $300 \mathrm{~K}$.

\section{0,464}

PB92-117290

Not available NTIS

National Inst of Standards and Technology (MSEL) Gaithersburg, MD. Metallurgy Div.

Laser Vaporization Mass Spectrometry of Refractory Materials: Graphite and YBa2Cu3Ox.

Final rept.

P. K. Schenck, D. W. Bonnell, and J. W. Hastie

$1989,5 p$

Pub. in Jnl. of Vacuum Science and Technology A 7 n3 p1745-1749 1989.

Keywords: "Graphite, Laser target interactions, High temperature superconductors, Mass spectroscopy, Reaction kinetics, Thermochemistry, Vaporization, Targets, Plumes, Reprints, "Yttrium barium cuprates.

A very high pressure sampling mass spectrometer has been used to study the thermochemistry and kinetics of laser induced plumes from graphite and $\mathrm{YBa} \mathrm{Cu} 3 \mathrm{O}(\mathrm{x})$ targets. Time resolved mass spectra of $C($ sub $n)(n=1-9)$ neutral vapor species were obtained from graphite targets under conditions of varying lasersurface interaction geometry and vapor plume-sampling geometry lonic species $\mathrm{C} 1(1+), \mathrm{C} 2(1+)$ $\mathrm{C} 3(1+)$, and impurities $\mathrm{Na}(1+)$ and $\mathrm{K}(1+)$ were also observed in the laser induced plume. Mass spectra obtained from superconducting $\mathrm{YBa} 2 \mathrm{Cu} 3 \mathrm{O}(\mathrm{x})$ targets showed an abundance of species in the laser induced plumes including both neutral and ionic $\mathrm{Y}, \mathrm{Ba}$, and $\mathrm{Cu}$ In addition, species such as $\mathrm{BaO}, \mathrm{CuO}(1+) \mathrm{YO}$ and bimetallics ( $\mathrm{BaCu}, \mathrm{YCu}$ ) were observed. Similar neutra and ionic metal atom species were observed using time resolved optical spectroscopy of the plume.

\section{0,465}

PB92-123157

PC A04/MF A01

National Inst. of Standards and Technology, Gaithersburg, MD.

NIST X-Ray Photoelectron Spectroscopy (XPS) Database.

Technical note (Final)

C. D. Wagner. Oct 91, 62p NIST/TN-1289

Also available from Supt. of Docs. as SN003-003 03104-6. Prepared in cooperation with Surfex Co. Oakland, CA.

Keywords: "X ray photoelectron spectroscopy, "Photoelectron spectroscopy, "Data bases, "Bibliogra phies, Chemical shift, Binding energy, Auger electrons, Tables(Data)

The technique known as XPS (X-Ray Photoelectron Spectroscopy) involves $x$-ray irradiation of surface samples under high vacuum. Electrons escaping from the samples are sorted and arranged to form a spectrum. A compilation of data for binding energy and kinetic energy of sample electrons from all elements has been collected. Depending on the nature of the chemi$\mathrm{cal}$ bond the chemical shift can be as much as $10 \mathrm{eV}$. Over the past 6 years the author has indexed articles related to the subject area. The data bank contains a total of 13,200 records, from a total of 800 papers.

100,466

PB92-148063

Not available NTIS

American Chemical Society, Washington, DC.

Journal of Physical and Chemical Reference Data, Volume 20, Number 4, July/August 1991.

Bimonthly rept.

D. R: Lide. c1991, 168 p

See also PB92-148071 through PB92-148105 and PB92-110212. Prepared in cooperation with American Inst. of Physics, New York. Sponsored by National Inst. of Standards and Technology, Gaithersburg, MD. Available from American Chemical Society, 115 16th St., NW, Washington, DC. 20036-9976.

Keywords: "Physical chemistry, Supercritical fluids Reaction kinetics, Combustion, Propellants, Molecula orbitals, Thermodynamic properties, Cyclopentadiene, Solutes, Liquids, Solids, Solubility, Nitrogen, Oxygen,
Molecular vibration, Molecular rotation, Constants, Carbon dioxide, Ab-initio calculations.

\section{Contents:}

Chemical Kinetic Data Base for Propellant Combustion. I. Reactions Involving NO, NO2, $\mathrm{HNO}, \mathrm{HNO} 2, \mathrm{HCN}$, and N2O:

Ab-Initio Calculations and Ideal Gas Thermodynamic Functions of Cyclopentadiene and Cyclopentadiene Derivatives;

Improved Fits for the Vibrational and Rotational Constants of Many States of Nitrogen and Oxygen:

Solubilities of Solids and Liquids of Low Volatility in Supercritical Carbon Dioxide.

100,467

PB92-148071

(Order as PB92-148063)

National Inst. of Standards and Technology (NML) Gaithersburg, MD. Chemical Kinetics Div.

Chemical Kinetic Data Base for Propellant, Combustion. 1. Reactions Involving NO, NO2, HNO, $\mathrm{HNO} 2, \mathrm{HCN}$ and $\mathrm{N} 2 \mathrm{O}$

W. Tsang, and J. T. Herron. c1991, 55p

ncluded in Jnl. of Physical and Chemical Reference Data, v20 n4 p609-663 Jul/Aug 91. Available from American Chemical Society, 1155 16th St. NW. Wash ington, DC. 20036-9976.

Keywords: "Reaction Kinetics, "Combustion, "Propellants, Transport properties, Nitrogen oxides, Chemical equilibrium, Chemical reactions, Hydrogen, Oxygen, Oxygen compounds, Carbon monoxide, RDX, Explosives, Cyanides, Tables(Data)

The publication contains evaluated chemical kinetic data on a number of single elementary reactions involving small polyatomic molecules which are of importance in propellant combustion. The work involves the collection and evaluation of mechanistic and rate information and the use of various methods for the extrapolation and estimation of rate data where information does not exits. The conditions covered range from $500-2500 \mathrm{~K}$ and 10 to the 17 power -10 to the 22 power particles/cu cm. The results of the first years effort lead to coverage of all pertinent reactions of the following species; $\mathrm{H}, \mathrm{H} 2, \mathrm{H} 2 \mathrm{O}, \mathrm{O}, \mathrm{OH}, \mathrm{HCHO}, \mathrm{CHO}$ $\mathrm{CO}, \mathrm{NO}, \mathrm{NO} 2, \mathrm{HNO} \mathrm{HNO} 2, \mathrm{HCN}$, and $\mathrm{N} 2 \mathrm{O}$. (Copyright (c) 1991 by the U.S. Secretary of Commerce.)

100,468

PB92-148089

Technion - Israel Inst. of Tech. Haifa.

Ab-Initio Calculations and Ideal Gas Thermody namic Functions of Cyclopentadiene and Cyclopentadiene Derivatives.

M. Karni, I. Oref, and A. Burcat. c1991, 19p

Included in Jnl of Physical and Chemical Reference Data, v20 n4 p665-683 Jul/Aug 91. Available from American Chemical Society, 1155 16th St., NW, Washington, DC. 20036-9976.

Keywords: "Thermodynamic properties, "Molecular orbitals, "Cyclopentadiene, Cycloalkenes, Combus tion, Fuels, Ideal gas, Molecular structure, Enthalpy, Thermochemistry, Entropy, Free radicals Tables(Data), Moments of inertia, Molecular vibrations, Specific heat, "Ab-initio calculations.

Analysis of recent experiments in combustion kinetics of cyclopentene (6), requires knowledge of the thermodynamic parameters of cyclopentadienyl radicals. Structures, frequencies and energies, ideal gas thermodynamic properties and values, have been calculated for cyclopentadiene, cyclopentadienols, and a number of radicals derived from them. The necessan molecular information for these calculations was found by ab-initio molecular orbital calculations. The geometries, vibrational frequencies and moments of inertia of 8 species are reported. In order to estimate the accu racy of the computations the molecular parameters were compared with known values reported in the literature whenever those were available. (Copyright (c) 1991 by the U.S. Secretary of Commerce.)

100,469

PB92-148097

$\mathrm{A}$ and D Associates, Los Angeles, CA. 
Improved Fits for the Vibrational and Rotational Constants of Many States of Nitrogen and Oxygen. R. R. Laher, and F. R. Gilmore. c1991, 28p Contract DNA-001-88-C-0046

Sponsored by Defense Nuclear Agency, Washington, DC

Included in Jnl. of Physical and Chemical Reference Data, v20 n4 p685-712 Jul/Aug 91. Available from American Chemical Society, 1155 16th St., NW, Washington, DC. 20036-9976.

Keywords: "Nitrogen, "Oxygen, "Molecular vibration, "Molecular vibration, "Molecular rotation, "Constants. Molecular energy levels, Excitations, lons, Graphs(Charts), Tables(Data), Spectrum analysis.

All pertinent measurements of the vibrational intervals delta $G$ (upsilon $+1 / 2$ ) and rotational constants $b$ (sub upsilon) for 11 states of $\mathrm{N} 2$, four states of $\mathrm{N} 2(+)$, the ground state of $\mathrm{O} 2$, and four states of $\mathrm{O} 2(+)$ that could be found in published papers have been assembled and plotted against upsilon. (These are the states important in modeling the fluorescence produced when air is bombarded by fast electrons.) These values of delta $G$ and $B$ (sub upsilon) are compared with values calculated from the standard polynomials in powers of upsilon $+1 / 2$, using the coefficients tabin powers of upsilon $+1 / 2$, using the coefficients tab-
ulated by Huber and Herzberg (1979), as well as coefficients derived by later analysts, when available. In about 25 percent of the states considered, the coefficients of Huber and Herzberg are found to still yield good fits to the latest available spectroscopic data. In another 25 percent, good fits are obtained from more recently published coefficients. For the remaining 50 percent of the states, new improved coefficients have been derived by least-squares fitting. The results are tabulated and plotted. (Copyright (c) 1991 by the U.S. Secretary of Commerce.)

100,470

PB92-148105

Leeds Univ. (England).

(Order as PB92-148063) in Supercritical Carbon Dioxide.

K. D. Bartle, A. A. Clifford, S. A. Jafar, and G. F.

Shilstone. c1991, 42p

Included in Jnl. of Physical and Chemical Reference Data, v20 n4 p713-756 Jul/Aug 91. Available from American Chemical Society, 1155 16th St., NW, Washington, DC. 20036-9976.

Keywords: "Carbon dioxide, "Solubility, "Solids, "Liquids, "Solutes, Supercritical fluids, Temperature, Pressure, Tables(Data), Gravimetric analysis, Chromatographic analysis, Phase studies, Phase stability, Equations of state, Graphs(Charts), Spectrum analysis.

A table is given of the compounds of low volatility, whose experimental solubilities in supercritical carbon dioxide have been published up to the end of 1989 , with the temperature and pressure ranges of the experimental measurements, the experimental method, and references to the source of data. The data for pure compounds, which were presented in tabular form in the original publications, are shown in a series of figures along with correlation lines for each isotherm. The method of correlation was to fit the experimental data for each isotherm, in the form of the natural logarithm of the product of mole fraction and pressure, to a linear function of density above a pressure of 100 bars. The constants obtained from the fitting procedures are given in a table. Procedures for estimating, from these constants, the solubilities of the compounds at temperatures and pressures different from those of the experimental data are suggested. (Copyright (c) 1991 by the U.S. Secretary of Commerce.)

100,471

PB92-148113

Not available NTIS

American Chemical Society, Washington, DC.

Journal of Physical and Chemical Reference Data, Volume 20, Number 5, September/October 1991.

Bimonthly rept.

D. R. Lide. c1991, 289p

See also PB92-148121 through PB92-148154 and PB92-148063. Prepared in cooperation with American Inst. of Physics, New York. Sponsored by National Inst. of Standards and Technology, Gaithersburg, MD Available from American Chemical Society, 1155 16th St., NW, Washington, DC. 20036-9976.

Keywords: "Thermodynamic properties, "Energy levels, Aluminum ions, Krypton ions, Oxygen, Water, Steam, Multicharged ions, Wavelengths, Tables(Data).
Wavelengths and Energy Level Classifications for the Spectra of Aluminum (Al 1 through Al XIII); Energy Levels of Krypton, $\mathrm{Kr}_{\mathrm{r}} \mathrm{I}$ through $\mathrm{Kr}$ XXXV|;

Thermodynamic Properties of Oxygen from the Triple Point to $300 \mathrm{~K}$ with Pressures to 80 $\mathrm{MPa}$;

Sixteen Thousand Evaluated Experimental

Thermodynamic Property Data for Water and Steam

Cumulative Listing of Reprints and Supplements.

\section{0,472
PB92-148121}

(Order as PB92-148113)
National Inst. of Standards and Technology, Gaithers burg, MD.

Wavelengths and Energy Level Classifications for the Spectra of Aluminum (All through AI XIII).

$\checkmark$. Kaufman, and W. C. Martin. c1991, 82p

Included in Jnl. of Physical and Chemical Reference Data, v20 n5 p775-858 Sep/Oct 91. Available from American Chemical Society, 115516 th St., NW, Washington, DC. 20036-9976.

Keywords: "Aluminum ions, "Aluminum, "Energy levels, "Wavelengths, Atomic energy levels, Atomic spectra, Multicharged ions, Positive jons, Tables(Data)

Wavelengths and their classifications have been compiled for the spectra of the atom and all positive ions of aluminum $(Z=13)$. The selections of data are based on the compilations of energy levels by Martin and $\mathrm{Za}$ lubas $(1970, \mathrm{~J}$. Phys. Chem. Ref. Data 8, 817-864), with some updating from the more recent literature. Wavelengths (or wavenumbers) calculated from the differences of the energy levels are given, along with the observed values for all classified lines; these calculated wavelengths should in general be more accurate than the observed values wherever the two values differ significantly. Calculated wavelengths are also given for a number of lines that have not yet been observed including some important forbidden transitions. The most complete data are given in separate tables for the different spectra. No limitation has been imposed on the wavelength range of the classified lines, except for the omission of $x$-ray transitions in the neutral atom. Two finding lists are also included, one for Al I through AI III and the other AI IV through AI XIII. (Copyright (c) 1991 by the U.S. Secretary of Commerce.)

\section{0,473}

PB92-148139

National Inst. of Standards (Order as PB92-148113) Gaithersburg. MD. Atomic Physics Div.

Energy Levels of Krypton, $\mathrm{Kr} I$ through $\mathrm{Kr}$ XXXVI. J. Sugar, and A. Musgrove. c1991, 57p

Sponsored by Department of Energy, Washington, DC. Office of Magnetic Fusion Energy.

Included in Jnl. of Physical and Chemical Reference Data, v20 n5 p859-915 Sep/Oct 91. Available from American Chemical Society, 115516 th St., NW, Washington, DC. 20036-9976.

Keywords: "Krypton ions, "Krypton, "Energy levels, Atomic energy levels, Multicharged ions, lonized gases, Spectra, Tables(Data).

The energy levels of the krypton atom, in all stages of ionization for which experimental data are available, have been compiled. No data has yet been published for $\mathrm{Kr}$ XI through $\mathrm{Kr}$ XVII. For $\mathrm{H}$-like krypton very accurate calculated level values are compiled. In all, data for 29 spectra are given. Experimental g-factors are included for $\mathrm{Kr} \mathrm{I}$ and $\mathrm{Kr}$ II. Calculated percentage compositions of levels are given for 12 ions. A value for the ionization energy of each ion, either experimental or theoretical, is included. (Copyright (c) 1991 by the U.S. Secretary of Commerce.)

\section{0,474}

PB92-148147

Idaho Univ., Moscow.

(Order as PB92-148113)

Thermodynamic Properties of Oxygen from the

Triple Point to $300 \mathrm{~K}$ with Pressures to $80 \mathrm{MPa}$.

c1991, 105p

Prepared in cooperation with Ruhr Univ., Bochum (Germany, F.R.). Inst. fuer Thermo- und Fluiddynamik. Included in Jnl. of Physical and Chemical Reference Data, v20 n5 p917-1021 Sep/Oct 91. Available from American Chemical Society, 115516 th St., NW, Washington, DC. 20036-9976.
Keywords: "Thermodynamic properties, "Oxygen Equations of state, Acoustic velocity, Specific heat, Critical point, Triple point, Tables(Data).

A joint project by the authors has resulted in two new thermodynamic property formulations for oxygen. The fundamental equation explicit in Helmholtz energy by Schmidt and Wagner has been used for the calculation of the property tables presented here, and for comparisons of calculated properties to the experimental data. The formulation by Stewart and Jacobsen is used in the paper in comparisons of properties calculated by the two formulations. These comparisons provide the basis for independent assessment of the accuracy of the available data and calculated properties. The fundamental equation is valid for thermodynamic properties of oxygen from the freezing line to $300 \mathrm{~K}$ at pressures to $80 \mathrm{MPa}$. A separate vapor pressure equation and equations for the saturated liquid and saturated vapor densities and the ideal gas heat capacity are included. Functions for calculating internal energy, enthalpy, entropy, isochoric heat capacity (Cv), isobaric heat capacity $(\mathrm{Cp})$ and velocity of sound are also included. Tables of thermodynamic properties of oxygen are given within the range of validity of the fundamental equation. The fundamental equation reported here may be used to calculate densities with an uncertainty of 0.10 percent, heat capacities within 2.0 percent, and velocity of sound values within 1.0 percent. These uncertainty values are valid for the range outside of the critical region. (Copyright (c) 1991 by the U.S. Secretary of Commerce.)

100,475

PB92-148154

Order as PB92-148113)

Keio Univ., Yokohama (Japan). Dept. of Mechanica Engineering.

Sixteen Thousand Evaluated Experimental Ther modynamic Property Data for Water and Steam.

H. Sato, K. Watanabe, J. M. H. L. Sengers, J. S.

Gallagher, and P. G. Hill. c1991, 22p

Prepared in cooperation with National Inst. of Stand ards and Technology (NML), Gaithersburg, MD. Thermophysics Div., British Columbia Univ., Vancouver. Dept. of Mechanical Engineering, Technische Univ. Muenchen (Germany, F.R.), and Ruhr Univ., Bochum (Germany, F.R.). Inst. fuer Thermo- und Fluiddynamik. Included in Jnl. of Physical and Chemical Reference Data, v20 n5 p1023-1044 Sep/Oct 91. Available from American Chemical Society, 115516 th St., NW, Washington, DC. 20036-9976.

Keywords: *Thermodynamic properties, *Steam tables(Thermodynamics), "Water, Equation of state, Acoustic velocity, Vapor pressure, Metastable state, Pressure dependence, Density, Enthalpy, Virial coefficients, Temperature dependence, Tables(Data).

As part of the activities of the International Association for the Properties of Water and Steam, all reliable sources of experimental data on the thermodynamic properties of ordinary (light) water and steam have been collected and converted to common temperature, pressure, volume, mass and heat scales. The data are grouped by state or phase: ideal-gas properties; sublimation and melting curves; saturation properties; properties of liquid water at ambient pressure; thermodynamic properties of the single-phase state; and those of metastable states. In each category, a subdivision is made by property. Properties include the volume, enthalpy, heat capacities, sound velocity, internal energy and Joule-Thomson and related coefficients. The total data collection contains approximately 16,000 data points and covers a century of experimental work at temperatures from 253 to $1273 \mathrm{~K}$ and pressures up to $1 \mathrm{GPa}$. The report characterizes the data and gives the literature references. The actual data collection is available in computerized form. (Copyright (c) 1991 by the U.S. Secretary of Commerce.)

100,476

PB92-148162

Not available NTIS

American Chemical Society, Washington, DC.

Journal of Physical and Chemical Reference Data, Volume 20, Num

Bimonthly rept.

D. L. Lide. c1991, 346p

See also PB92-148170 through PB92-148220 and PB92-148113. Errata sheet inserted. Prepared in cooperation with American Inst. of Physics, New York. 
Sponsored by National Inst. of Standards and Technology, Gaithersburg, MD.

Available from American Chemical Society, 1155 16th St., NW, Washington, DC. 20036-9976.

Keywords: "Physical chemistry, Atomic weights, Standards, Equations of State. Thermodynamic prop. erties, Methane, Propulsion, Sulfuric acid, Carbon dioxide, Solubility, Reaction kinetics, High temperature tests, Combustion, Atmospheric chemistry, "Foreign technology, Isotopic composition.

Contents:

A New Equation of State and Tables of Thermodynamic Properties for Methane Covering the Range from the Melting Line to $625 \mathrm{~K}$ at Pressures up to $1000 \mathrm{MPa}$ :

Thermodynamic Properties of the Aqueous Sulfuric Acid System to $350 \mathrm{~K}$;

The Solubility of Carbon Dioxide in Water at Low Pressure;

Chemical Kinetic Data Sheets for HighTemperature Reactions. Part II:

Atomic Weights of the Elements 1989;

Isotopic Compositions of the Elements 1989.

\section{0,477}

PB92-148170

(Order as PB92-148162) Ruhr Univ., Bochum (Germany, F.A.). Inst. fuer Thermo- und Fluiddynamik.

New Equation of State and Tables of Thermodynamic Properties for Methane Covering the Range from the Melting Line to $625 \mathrm{~K}$ at Pressures up to $1000 \mathrm{MPa}$.

U. Setzmann, and W. Wagner. c1991, 95p

Included in Jnl. of Physical and Chemical Reference Data, v20 n6 p1061-1 155 Nov/Dec 91. Available from American Chemical Society, 1155 16th St., NW, Washigton, DC 20036-9976.

Keywords: "Equations of State, "Thermodynamic properties, "Methane, Tables(Data), Pressures, Specific heat, Helmholtz free energy, Enthalpy, Phase transformation, Ideal gas, Melting, Liquid-vapor interfaces, Saturation, Virial coefficients.

The work reviews the data on thermodynamic properties of methane which were available up to the middle of 1991 and presents a new equation of state in the form of a fundamental equation explicit in the Helmholtz free energy. A new strategy for optimizing the structure of empirical thermodynamic correlation equations was used to determine the functional form of the equation. The Helmholtz function containing 40 fitted coefficients was fitted to selected experimental data of the following properties: (1) thermal properties of the single phase (P rho T) and (2) of the liquid-vapor saturation curve ( $($ (sub s)rho' rho') including the Maxwell criterion, (3) speed of sound $w$, (4) isochoric heat capacity $c$ upsilon, (5) isobaric heat capacity c(sub p). (6) difference of enthalpy delta $h$, and (7) second virial coefficient $B$. Independent equations are also included for the vapor pressure, the saturated liquid and vapor densities, the isobaric ideal gas heat capacity and the melting pressure as functions of temperature. Tables for the thermodynamic properties of methane from 90 $\mathrm{K}$ to $620 \mathrm{~K}$ for pressures up to $1000 \mathrm{MPa}$ are presented. To verify the accuracy of the new formulation, the calculated property values are compared with selected experimental results and existing equations of state for methane. (Copyright (c) 1991 by the U.S. Secretary of Commerce.)

\section{0,478}

\section{PB92-148188}

National Aeronautics and (Order as PB92-148162) Cleveland, $\mathrm{OH}$. Lewis Research Center.

Thermodynamic Properties of the Aqueous Sulfuric Acid System to $350 \mathrm{~K}$.

F. J. Zeleznik. c1991, 44p

Included in Jnl. of Physical and Chemical Reference

Data, v20 n6 p1157-1200 Nov/Dec 91. Available from American Chemical Society, 1155 16th St., NW, Washington, DC. 20036-9976.

Keywords: "Thermodynamic properties, "Sulfuric acid, Electrolytes, Liquid phases, Specific heat, Enthalpy, Melting points, Electromotive force, Tables(Data), Osmosis, Activity coefficients, Heat of fusion, Solid phases, Gibbs free energy, Freezing, Phase transformations, Entropy.

Experimental measurements for aqueous sulfuric acid and its related pure, solid phases have been thermo- dynamically analyzed and correlated as a function of temperature and composition from pure water to pure acid. The pure phases included anhydrous sulfuric acid, five of its hydrates and ice. Experimental data which were used in the correlation included measurements of the enthalpy of dilution, both solution and pure phase heat capacities, electromotive force and solution freezing points. The correlation yielded mutually consistent expressions for the Gibbs energy of each phase and these functions generally reproduce the experimental data to + or -0.75 percent. The Gibbs energy functions of the pure solid phases were used to generate tables of their thermodynamic properties from $\mathrm{OK}$ to the melting points. The Gibbs energy function for aqueous sulfuric acid was used to produce tables of both integral and partial molar solution properties as a sunction of sulfuric acid mole fraction every 50 degrees from 200 to $350 \mathrm{~K}$. (Copyright (c) 1991 by the U.S. Secretary of Commerce.)

\section{0,479}

PB92-148196

(Order as PB92-148162)

Alberta Univ., Edmonton. Dept of Chemical EngineerSolubility of Carbon Dioxlde in Water at Low Pressure.

J. J. Carrol, J. D. Slupsky, and A. E. Mather. c1991, $9 \mathrm{p}$

Included in Jnl. of Physical and Chemical Reference Data, v20 n6 p1201-1209 Nov/Dec 91. Available from American Chemical Society, 1155 16th St., NW, Washington, DC. 20036-9976.

Keywords: "Carbon dioxide, "Solubility, Henrys law, Low pressure tests, Water, Enthalpy, Vapor phases, Chemical composition.

The system carbon dioxide-water is of great scientific and technological importance. Thus, it has been studied often. The literature for the solubility of carbon dioxide in water is vast and interdisciplinary. An exhaustive survey was conducted and approximately $100 \mathrm{ex}$ perimental investigations were found that reported equilibrium data at pressures below $1 \mathrm{MPa}$. A model based on Henry's law was used to correlate the low pressure data (those up to $1 \mathrm{MPa}$ ). The following correlation of the Henry's constants (expressed on a mole fraction basis) was developed. $\operatorname{In}(\mathrm{H} 21 / \mathrm{MPa})=-6.8346$ $+12817 / \mathrm{T}-3766800 / \mathrm{sq} \mathrm{T}+2.997 \times 10$ to the 8 th power/cu T. The correlation is valid for $273<\mathrm{T}<433$ $\mathrm{K}(0<\mathrm{t}<160 \mathrm{C})$ where $\mathrm{T}$ is in $\mathrm{K}$. Any experimental data that deviated significantly from this model were duly noted. (Copyright (c) 1991 by the U.S. Secretary of Commerce.)

\section{0,480
PB92-148204}

Aerospace Corp., Los Angeles, CA.

(Order as PB92-148162)

Chemical Kinetic Data Sheets for High-Temperature Reactions. Part 2.

c1991, 100p

Included in Jnl. of Physical and Chemical Reference Data, v20 n6 p1211-1311 Nov/Dec 91. Available from American Chemical Society, 1155 16th St., NW, Washington, DC. 20036-9976.

Keywords: "Reaction kinetics, "High temperature tests, "Combustion, "Atmospheric chemistry, "Propulsion, Vapor phases, Graphs(Charts). Thermochemistry, Oxygen atoms, Alkanes, Hydroxyl radicals, Halogen organic compounds, Oxygen, Ammonia.

Rate coefficient measurements for over fifty gasphase bimolecular reactions were critically evaluated and compared to theoretical calculations. The results of the work are summarized here in forty-nine Data Sheets, one sheet for each reaction or set of reactions of a single pair of reagents. The reactions chosen are of interest in propulsion, combustion, and atmospheric chemistry. Each Data Sheet consists of two pages that include a brief resume of the important experimental measurements and theoretical calculations, a graphi$\mathrm{cal}$ presentation of the data, a recommended rate coefficient expressed as a function of temperature, $k(T)$ $=A T(\sup n) \exp (-B / T)$, with probable uncertainty limits, a discussion of the basis for the recommendation, an equilibrium constant and a rate coefficient for the reverse reaction where applicable, and pertinent references. (Copyright (c) 1991 by the U.S. Secretary of Commerce.)

\section{0,481}

PB92-148212
(Order as PB92-148162)

Curtin Univ. of Technology, Bentley (Australia)

Atomic Weights of the Elements 1989.

J. R. De Laeter, and K. G. Heumann. c1991, 13p

Prepared in cooperation with Regensburg Univ. (Germany, F.R.).

Included in Jnl. of Physical and Chemical Reference Data, v20 n6 p1313-1325 Nov/Dec 91. Available from American Chemical Society, 1155 16th St., NW, Washington, DC. 20036-9976.

Keywords: "Atomic weights, "Standards, Chemical elements, Radioisotopes, Half life, Extraterrestrial matter, Tables(Data), Isotopic composition.

The biennial review of atomic weight, $A($ sub $r)(E)$, determinations, and other cognate data has resulted in changes for nickel from $58.69+$ or -0.01 to 58.6934 tor -0.0002 and for antimony from 121.75 + or 0.03 to $121.757+$ or -0.003 due to new calibrated measurements. Because the measurement of the isotopic composition of mercury has also been improved during the last two years, the Commission was able to reduce the uncertainty of the atomic weight of this element from $200.59+$ or -0.03 to $200.59+$ or -0.02 . Due to the nearly constant isotopic composition of protactinium in nature, where (231) $\mathrm{Pa}$ is the predominant isotope, the atomic weight of this element was fixed to $231.03588+$ or -0.00002 . The Table of Isotopic Compositions of the Elements 1989 will be published as a companion paper to that on Atomic Weights of the Elements 1989. The Table of Standard Atomic Weights Abridged to Five Significant Figures and current data on isotopic compositions of nonterrestrial material are included to benefit users who are more concerned with the length of time during which a given table has full validity to the precision limit of their interest. The Table of Atomic Weights to Four Significant Figures was prepared and has been published separately. (Copyright (c) 1991 by the U.S. Secretary of Commerce)

100,482

PB92-148220

(Order as PB92-148162)

Curtin Univ. of Technology, Bentley (Australia)

Isotopic Compositions of the Elements 1989

J. R. De Laeter, K. G. Heumann, and K. J. R.

Rosman. c1991, 11p

Prepared in cooperation with Regensburg Univ. (Germany, F.R.).

Included in Jnl of Physical and Chemical Reference Data, v20 n6 p1327-1337 Nov/Dec 91. Available from American Chemical Society, 1155 16th St., NW, Washington, DC. 20036-9976.

Keywords: Atomic weights, Chemical elements, Mass spectroscopy, Tables(Data), "Isotopic composition.

The Subcommittee for Isotopic Abundance Measurements (SIAM) of the IUPAC Commission on Atomic Weights and Isotopic Abundances has carried out its biennial review of isotopic compositions, as determined by mass spectrometry and other relevant methods. The Subcommittee's critical evaluation of the published literature element by element forms the basis of the Table of Isotopic Compositions of the Elements as Determined by Mass Spectrometry 1989 . which is presented in the Report. Atomic Weights calculated from the tabulated isotopic abundances are consistent with $A($ sub $r)(E)$ values listed in the Table of Standard Atomic Weights 1989. (Copyright (c) 1991 by the U.S. Secretary of Commerce).

\section{Polymer Chemistry}

100,483

PB91-144402

PC A05/MF A01

National Inst. of Standards and Technology (NML) Boulder, CO. Chemical Engineering Science Div.

Thermal Conductivity of a Polymide Film between 4.2 and $300 \mathrm{~K}$, with and without Alumina Particles as Filler.

D. L. Rule, D. R. Smith, and L. L. Sparks. Aug 90 77p NISTIR-3948

Sponsored by Ship Structure Committee, Washington DC.

Keywords: "Polymeric films, "Thermal conductivity, "Composite materials, "Polyimide resins, Ceramics, Polymer matrix composites, Aluminum oxide, Particles, 


\section{CHEMISTRY}

\section{Polymer Chemistry}

Fillers, Cryogenics, Temperature dependence, Tables(Data), Graphs(Charts), Pressure dependence. The thermal conductivity of several types of a commercial polyimide (specifically, polypyromellitimide: PPMI) film was measured over a range of temperatures from 4.2 to $300 \mathrm{~K}$ using an unguarded steadystate parallel-plate apparatus. Specimens were made by stacking multiple layers of film together. Conductive grease was used between layers of film to reduce thermal contact resistance. Two specimens were made from two different types of neat (unadmixed) film with a thickness of 76 micrometers, and three specimens were made from films containing two different amounts of admixed alumina filler and having thick. nesses of 25 micrometers or 76 micrometers. The conductivity of PPMI film increases with the amount of alumina filler present. The thermal conductivity of specimens made from film of the same type but of different thickness is independent of film thickness, within the limits of experimental uncertainty. The thermal conductivity of a specimen subjected to a simulated curing process by being held at a temperature of $150 \mathrm{C}$ for ninety minutes was indistinguishable from that of a similar, control specimen not subjected to such treatment.

100,484

Not available NTIS National Bureau of Standards (IMSE), Gaithersburg, MD. Polymers Div.

Incorporation of Molecular Orientation into Systems of Lamellar Morphology. 1. Effects of Packing Entropy on the Lamellar Thickness of Block Copolymers.

Final rept.

E. A. DiMarzio. $1988,8 p$

Pub. in Macromolecules 21, n7 p2262-2269 Jul 88.

Keywords: "Block copolymers, "Entropy, "Lamellar structure, Membranes, Orientation, Packing, Miceller systems, Molecular models, Thickness, Equations, Reprints.

Equations for the dimensions of the $A$ and $B$ regions of monodisperse diblock copolymers with lameliar morphology are obtained. An important feature of the treatment is the incorporation of the orientation-dependent packing entropy into the formalism. The equations are believed to be accurate over the whole range of orientation of bonds, from random orientation to perfect alignment. Copolymer thicknesses vary as the $2 / 3$ power of molecular weight to the first power depending on the amount of orientation induced by the packing entropy and the energetics. The amount of bond orientation in stretched molecules in bulk material is twice what the molecules would have if they were stretched (by the same stretch ratio) in solvent. It is observed that the effect is important to those systems of micelles, vesicles, membranes, soaps, liquid crystals, and block copolymers that experimentally are known to display significant orientation, and it is suggested that extant theories can be modified to include the effect.

\section{0,485}

\section{PB91-148890}

Not available NTIS

National Inst. of Standards and Technology (MSEL).

Gaithersburg, MD. Polymers Div.

Chain Conformation of a Block Polymer in a Microphase-Separated Structure.

Final rept.

Y. Matsushita, K. Mori, Y. Mogi, R. Saguchi, I. Noda, M. Nagasawa, T. Chang, C. Glinka, and C. Han.

$1990,5 \mathrm{p}$

Pub. in Macromolecules 23, n19 p4317-4321, 17 Sep 90.

Keywords: "Block copolymers, "Polystyrene, "Viny copolymers, "Radius of gyration, Neutron scattering Lamallar structure, Molecular weight, Pyridines, Measurement, Reprints.

The radii of gyration of a polystyrene block along the directions parallel and perpendicular to the lamellae in the lamellar structure of poly(styrene-b-2-vinylpyridine) were measured by small-angle neutron scattering. It was found that the block polymer is extended along the perpendicular direction, while it is contracted along the parallel direction. The extension is in accord with the theories of microphase-separated structure, but no theory predicts the contraction. From the molecular weight dependences of the radius of gyration along the directions parallel and perpendicular to the lamellae, it is concluded that the block polymer is contracted along the parallel direction to keep the volume occu- pied by the block chain constant when the microphase separation occurs.

\section{0,486}

PB91-158626

Not available NTIS

National Bureau of Standards (IMSE), Gaithersburg, MD. Polymers Div.

Measurement of Space Charge Fields in Polymers. Final rept.

A. S. DeReggi, M. G. Broadhurst, G. T. Davis, and F. I. Mopsik. 1987, $6 p$

Pub. in Proceedings of Annual Report Conference on Electrical Insulation and Dielectric Phenomena, Gaithersburg, MD., October 18-22, 1987, p307-312.

Keywords: "Polymeric films, "Electrical transients, "Dielectric films, Pulses, Surface energy, Imbeddings(Mathematics), Electric fields, Electrodes, Laser heating, Polyethylene, Calibrating, Measurement, Laser heating, Space charge, Reprints, Ionomers.

Progress in analyzing and interpreting electrical transients induced by imbedded space charge when the surface of a dielectric polymer film is heated with short thermal pulses is reported. The thermal pulse currently comes from the energy of a dye laser absorbed by the electrodes. Measurement of the transient with and without a potential difference applied to the film allows (1) scaling and combining the transients obtained by heating each side of the film and (2) quantifying the resulting Fourier coefficients of the electric field distribution due to the imbedded space charge. The calibra. tion procedure is all electrical, and does not require a knowledge of the energy in the pulse, the electrode absorbency, or thermal properties of the polymer film.

100,487

PB91-159095

Not available NTIS National Inst. of Standards and Technology (NML) Boulder, CO. Chemical Engineering Science Div.

Electrokinetic Demixing of Two-Phase Aqueous Polymer Systems. 1. Separation Rates of Polyethylene Glycol-Dextran Mixtures.

Final rept.

K. S. M. S. Raghava Rao, R. M. Stewart, and P.

Todd. 1990, 12p

Pub. in Separation Science and Technology 25, n9-10 p985-996 1990.

Keywords: "Separation processes, "Solvent extraction, "Electrophoresis, "Polymers, Phase separation(Materials), Aqueous solutions, Electric fields, Buffers(Chemistry), Polyoxyethylene, Dextran, Polarity. Convection, Phosphates, Electrokinetics, Rates, Binary system(Materials), Reprints.

The separation of biomolecules and cells using aqueous two-phase systems provides a mild, nontoxic extraction medium, in contrast with conventional organicaqueous phase extraction. However, due to their similar physical properties, immiscible aqueous phases do not separate rapidly. Because a net surface potential occurs on phase droplets due to the unequal partitioning of certain dissolved ions, a study was undertaken in which the resulting motion in an electric field (electrophoresis) was explored as a possible method for rapid demixing of aqueous two-phase systems in a vertical electrophoresis column. The effects of electric field strength, buffer concentration, and field polarity on the demixing rate of mixtures of polyethylene glycol and dextran in phosphate buffer were measured. It was
found that an optimum field strength of around $29.2 \mathrm{~V} /$ $\mathrm{cm}$ exists at which demixing is most rapid and occurs at about twice the rate in zero field at $25+$ or $-2 C$ using normal polarity (anode at the top of the column). With reverse polarity (anode at the bottom; electric field opposing gravitational settling) at a field strength of $14.6 \mathrm{~V} / \mathrm{cm}$ the rate was 3 times as fast as in zero field. Strong convection was observed at high field strengths. Increasing the phosphate concentration increased the demixing rate.

\section{0,488}

PB91-159715

PC A07/MF A01 National Inst of Standards and Technology (MSEL). Gaithersburg, MD. Polymers Div.

Polymers: Technical Activities 1990.

L. E. Smith, and B. M. Fanconi. Jan 91, 134p NISTIR-4396

See also PB86-165024.

Keywords: "Polymers, "US NBS, "Research projects, Resins, Polymer matrix composites, Inks, Optical materials, Dental materials, Medical supplies, Biodeterior- ation, Injection molding, Process control, Mathematical models, Fluid flow, Mechanical properties, Polymer blends, Test equipment.

Technical Activities of the Polymers Division for FY 90 are reviewed. Included are descriptions of the 6 Tasks of the Division, project reports, publications, and other technical activities.

\section{0,489}

\section{PB91-162099}

Not available NTIS

National Inst. of Standards and Technology (MSEL), Gaithersburg, MD. Polymers Div.

Spinodal Decomposition in a Hydrogen-Bonded Polymer Blend.

Final rept.

M. He, Y. Liu, Y. Feng, M. Jiang, and C. C. Han.

Pub. in Macromolecules 24, n2 p464-473 1991.

Keywords: "Polymer blends, Hydrogen bonds, Binary system(Materials), Polystyrne, Polyacrylates, Fluoropolymers, Opacity, Coarseness, Temperature measurement, Phase diagrams, Light scattering, Optical properties, Reprints, "Spinodal decomposition.

The spinodal decomposition of a hydrogen-bonded binary blend, which consists of a polystyrene with 1.5 $\mathrm{mol} \%$ of $\mathrm{p}-(1,1,1,3,3,3$-hexafluoro-2-hydroxyisopropyl)-alpha-methylstyrene comonomer and a poly-(butyl methacrylate), has been studied. The system has a LCST with a very asymmetrical phase diagram. Also the critical point does not coincide with the temperature minimum of either the spinodal or the cloud-point curve. The kinetics follows the Cahn-Hilliard-Cook model for early spinodal decomposition and a selfsimilar mechanism for late-stage coarsening for most cases. For shallow quench cases and for close to glass transition temperature cases, double peaks have been observed during spinodal decomposition, which suggests that a second or an alternative relaxation mechanism may be dominating the coarsening process. A special procedure has been developed to measure the cloud point for the system, which should be applicable to any polymer blend system that has a cloud temperature close to the glass transition temperature.

100,490

PB91-162131

Not available NTIS

National Inst. of Standards and Technology (MSEL), Gaithersburg, MD. Polymers Div.

Time-Resolved Small-Angle Neutron Scattering in Intermediate- and Late-Stage Spinodal Decomposition of (DPB/HPI) Blends.

Final rept.

H. Jinnai, H. Hasegawa, T. Hashimoto, and C. C. Han. 1991, 8p

Pub. in Macromolecules 24, nl p282-289 1991.

Keywords: "Polymer blends, "Spinodal decomposition, Optical properties, Polyisoprene, Polybutadiene, Synthetic elastomers, Light scattering, Phase diagrams, Time resolution, Deuteration, Small angle scattering, Reprints.

Time-resolved small-angle neutron scattering (SANS) experiments were performed to study the later stage (i.e., the intermediate stage and the late stage) of spinodal decomposition (SD) of a critical mixture of perdeuterated polybutadiene (DPB) and protonated polyisoprene (HPI) with an LCST-type phase diagram and a critical temperature Te $=36.1 \mathrm{C}$ Time-sliced SANS intensity, $\mathrm{S}(\mathrm{q}, \mathrm{t})$, is generally composed of the scattering due to growing domains, I (sub d) $(q, t)$, and that due to the thermally induced local composition fluctuations inside the domain I(sub tau)(q,t) where $q$ and $t$ are the scattering vector and time, respectively. $S(q, t)$ as a function of g shows the 'spatial crossover' such that at $q$ much below and above a time-dependent wavenumber, $q($ sub s)(t), it depends only on I(sub d) $(q, t)$ and I(sub tau) $(q, t)$, respectively. The time evolution of the composition difference between the two coexisting domains, delta phi( $(t)$, was determined from I(sub tau) $(q, t)$ which was found to be consistent with the prediction of the scattering theory based upon the random-phase approximation. Delta phi(t) was found to increase with and reach a constant equilibrium value delta phi(sub 0 ) at $t>+(\mathrm{cr})$, where $\mathrm{t}(\mathrm{cr})$ is the crossover time from the intermediate to the late-stage SD. The crossover wavenumber, $\mathrm{g}(\mathrm{sub} \mathrm{s})(\mathrm{t})$, also underwent a characteristic change at $\mathrm{t}(\mathrm{cr})$ 
100,491

PB91-162255

Not available NTIS

National Bureau of Standards (NML), Gaithersburg MD. Inorganic Analytical Research Div.

High Purity Fluoropolymer Materials: Trace Element Content and Leaching.

Final rept.

J. R. Moody, E. S. Beary, D. S. Bushee, and P. J

Paulsen. 1988, 6p

Pub. in Jnl. of Crystal Growth 89, p43-48 1988

Keywords: "Fluoropolymers, Solvent extraction, Trace elements, Mass spectroscopy, Purification, Leaching, Crystal growth, Chemical analysis, Resins, Impurities, Reprints.

Many problems in analytical chemistry are shared by the high purity crystal growth community since both are greatly concerned with the effects of impurities either upon an analysis or upon a device. Fluoropolymers are widely used today in applications where inertness or high purity or both are needed. The behavior of these polymers in high purity solvent systems has been investigated by several analytical techniques. Both the resin from which finished parts are made as well as blow molded bottles have been evaluated to determine cationic impurities in both the resin and in leachates.

\section{0,492}

PB91-174482

Not available NTIS

National Bureau of Standards (IMSE), Gaithersburg, MD. Polymers Div.

Fluorescence Monitoring of Polymer Processing: Mixing and Zero Shear Viscosity.

Final rept.

A. Bur, F. Wang, and R. Lowry. 1988, 4p

Pub. in Proceedings of Society of Plastics Engineers ANTEC '88, Atlanta, GA., April 18-22, 1988, p11071110.

Keywords: "Propellants, "Polymers, "Fluorescence spectroscopy, Process variables, Monitors, Temperature dependence, Doped materials, Mixing, Binders(Materials), Rheological properties, Reprints.

Fluorescence spectroscopy is being employed to monitor processing parameters such as non-Newtonian viscosity, molecular orientation, intersegmental penetration, and the degree of mixing of product ingre dients. In order to do this, the processing ingredients are doped at a low concentration with fluorescent chromophores which are added as separate entities or chemically bound to the polymer binder. The chromophores are chosen in accordance with their sensitivity to a particular processing parameter. Information about the process being examined is obtained with the aid of known correlations between fluorescence spectra, rheological data, and other processing parameters. Two experiments determined: (1) the measure ment of the change in fluorescence intensity as a func tion mixing time for a two component specimen, an (2) the measurement, at zero shear, of the depend ence of the intensity of the fluorescence spectra from a doped polymer binder as a function of temperature. The feasibility of the fluorescence monitoring tech nique is demonstrated in each case.

\section{0,493}

\section{PB91-174508}

Not available NTIS

National Inst. of Standards and Technology (NML)

Boulder, CO. Chemical Engineering Science Div.

Statistical Mechanical Model of Aqueous Two-

Phase Systems.

Final rept.

H. Cabezas, J. D. Evans, and D. C. Szlag. 1989, 10p

Pub. in Fluid Phase Equilibria 53, p453-462 1989.

Keywords: "Binary system(Materials), "Polymer blends, Phase diagrams, Aqueous solutions, Statistica mechanics, Mathematical models, Forecasting, Interactions, Molecular weight, Polyoxyethylene, Dextran, Methyl cellulose, Virial coefficients, Reprints.

A predictive model for the phase diagrams of two-poly mer aqueous two-phase systems has been developed from the solution theory of Hill. This theory gives chemical potentials in terms of isobaric isothermal osmotic virial coefficients representing polymer pair interactions. Predictive expressions for the dependence of these coefficients on molecular weight and polydispersity from Renormalization Group theory were developed. For a two-polymer system the model contain three monomer interaction coefficients, one for each polymer pair, and two scaling exponents, one for each polymer. Phase diagrams for four different systems have been calculated from the model at ambient conditions and shown to agree with experiment within 1 $2 \%$ in polymer weight fraction. Two of the systems containpolyethylene glycol and dextran with molecula weights from 4000 to 280,000 . The other two systems contain methylcellulose and dextran with molecular weights from 30,000 to 280,000 .

100,494

PB91-187153

Not available NTIS

National Inst. of Standards and Technology (IMSE) Gaithersburg, MD. Polymers Div.

Small-Angle Neutron Scattering Studies of Compatible Blends of Linear Poly(Vinyl Methyl Ether) and Cross-Linked Deuterated Polystyrene.

Final rept.

B. J. Bauer, R. M. Briber, and C. C. Han. 1989, 9p Pub. in Macromolecules 22, n2 p940-948 Feb 89

Keywords: "Polymer blends, "Small angle scattering "Binary system(Materials), "Vinyl ether resins, Phase separation(Materials), Networks, Temperature de pendence, Crosslinking, Deuteration, Labeled sub stances, Graphs(Charts), Swelling, Reprints.

Small angle neutron scattering (SANS) from single phase polymer blends of crosslinked deuterated polystyrene (PSD) and linear polyvinylmethylether (PVME) has been studied as a function of temperature. Phase separation during the polymerization resulted if the PVME present is greater than the amount which would swell the PSD network at equilibrium. SANS from the single phase linear blend and the lowest crosslink density sample exhibited linear behavior for inverse zero angle scattering, $1 / \mathrm{S}(\mathrm{O})$ and inverse correlation length squared, $1 /(\mathrm{xi})$ sup 2 , versus $1 / \mathrm{T}$ while the highe crosslink density sample showed pronounced curva ture in the same plot, eventually crossing over the curves for the other two samples. Linear plots for $\mathrm{S}(\mathrm{O})$ and $\mathrm{xi}$ versus $1 / \mathrm{T}$ for the highest crosslink density single phase sample could be obtained with the expo nents $1 / \mathrm{S}(\mathrm{O})$ sup 57 and $1 /$ (xi)sup 1.44 The highes crosslink density single phase sample was then deformed to $L / L=2.5$ and the SANS examined as a function of temperature. The scattering obtained was anisotropic with different values of $S(O)$ and $x i$ in the directions parallel and perpendicular to the deformation. Plots of $1 / S(O)$ and $1 /(x i)$ sup 2 versus $1 / T$ indicated that the sample phase separated in the direction parallel to the deformation at a lower temperature than in the perpendicular direction.

\section{0,495}

PB91-187229 National Inst. of Standards and Technology (NEL) Gaithersburg, MD. Fire Measurement and Research Div.

Molecular Dynamics Study of the Depolymerization Reaction in Simple Polymers.

Final rept.

E. Blaisten-Barojas, and M. R. Nyden. 1990, 7p

Sponsored by National Science Foundation, Washing ton, DC

Pub. in Chemical Physics Letters 171, n5-6 p499-505, 17 Aug 90.

Keywords: "Depolymerization, "Monomers, "Synthesis(Chemistry), "Combustion, Computerized simulation, Reaction kinetics, Thermal degradation Oxidation, Agglomerates, Infrared spectra, Temperature dependence, Reprints, "Molecular dynamics.

A molecular dynamics experiment was designed to follow the sequence of depolymerization reactions oc curring when a polymer degrades into its constituent monomers. The simulation addresses interna changes that certain materials undergo at the momen of burning. Polymer fragments were thermally generated from the random scission of longer polymer chains containing 50-950 units. Subsequently, these therma fragments depolymerized and coiled dramatically, forming incipient 'agglomerates'. These agglomerates cooled while depolymerizing; the cooling mechanism remarkably inhibits the depolymerization reaction and eventually terminates the degradation process leading behind a sample of cold stable agglomerates. The size distribution of the polymer fragments is given as well as the IR spectrum of a typical sample at $2000 \mathrm{~K}$

\section{0,496}

\section{PB91-187385}

Not available NTIS

National Inst. of Standards and Technology (IMSE), Gaithersburg, MD. Polymers Div.
Phenomenological Theory of the Influence of Strain History on the Rate of Isothermal Stress Relaxation.

Final rept.

B. D. Coleman, and L. J. Zapas. $1989,16 p$

Pub. in Jnl. of Rheology 33, n3 p501-516 1989.

Keywords: "Stress relaxation, "Polymers, "Strain tests, "Isotherms, Constitutive equations, Viscoelasticity, Theories, Time dependence, Rheological properties, Incompressible flow, Reprints, Strain history, BKZ.

Constitutive relations are formulated for a class of in compressible viscoelastic fluids for which internal structural changes occur at a rate that is influenced by the history of the strain. For the materials considered, the contribution to the stress at time $t$ made by the strain at an earlier time $T$ is a function of that strain, the true elapsed time $\mathrm{t}$ - tau, and a quantity sigma ( $\mathrm{t}$, tau) that can be interpreted as the elapsed time measured by a clock whose rate of advance, because it is tied to the rate of structural change, is affected by the history of the strain. The functional relating delta sigma ( tau)/delta $t$ to the history of the strain up to time $t$ is assumed to have the same domain and a structure similar to that relating stress to strain history. The present theory reduces to the theory of $B K Z$ fluids in the (extreme) special case in which sigma $(t, t a u)=t$ tau, i.e., in which delta sigma ( $t$, tau)/delta $t=1$. It is shown that there is a sense in which constitutive relations recently found to account well for observed discrepancies between experimental observations and predictions of the BKZ theory can be considered firstorder approximations to the relations formulated here.

100,497

PB91-187427

Not available NTIS

National Inst of Standards and Technology (MSEL)

Gaithersburg, MD. Polymers Div.

Multistep Stress-Relaxation Study of a Single Crystal of n-Eicosane $\left(\mathrm{C}_{2} \mathrm{OH} 42\right)$ in Torsion.

Final rept

J. M. Crissman. $1989,7 p$

89

Keywords: "Single crystals, "Stress relaxation, "Polymers, Hydrocarbons, Viscoelasticity Constitutive equations, Torsion tests, Time dependence, Reprints, Eicosane.

Multistep stress-relaxation experiments in torsion have been done on a single crystal of the long chain hydrocarbon compound n-eicosane. It was found that at very small strains the behavior approaches that of a linear viscoelastic material whereas at larger strains the behavior becomes increasingly nonlinear and time dependent. In the region where the stress-strain behavior becomes nonlinear it is shown that the second step response, in an experiment in which the magnitude of the second step is one half that of the first step, can be described very well using a recent constitutive equation derived by Zapas.

100,498

PB91-187450

Not available NTIS

National Inst of Standards and Technology (IMSE)

Gaithersburg, MD. Polymers Div.

Statistics of a Polymer Molecule in the Presence of Asymmetric Obstacles.

Final rept.

E. A. Di Marzio. 1991, 10p

Pub. in Macromolecules 24, n7 p1595-1604 1991.

Keywords: "Polymers, "Stereochemistry, "Statistical analysis, "Statistical mechanics, Configuration, Molecular weight, Barriers, Orientation, Chemical bonds, Probability, Reprints.

The number of configurations $W$ of a polymer in a field of fixed obstacles is obtained. The cubic lattice of $N$ sites has a coordination number $z$ and is of $d=z / 2$ dimensions. The obstacles are modeled as rigid rods or rigid but bent polymers. A fraction of the obstacle bonds are oriented in orientation $i$. The flexible polymer which is placed into the field of rigid obstacles is of length $\mathrm{M}$ and has beta(sub i) of its bonds lying in orientation $\mathrm{i}$. The formula for $\mathrm{W}$ results in expectation values only a few percent different from the exact expressions for the known special cases. The beta(sub i) are not fixed numbers but rather occur with a probability given by $W$. With the maximum term method the dimensions of the polymer are calculated. The polymer is elongated in the direction of alignment of the obsta- 


\section{CHEMISTRY}

\section{Polymer Chemistry}

cles. The volume of the polymer is found to increase with volume fraction of obstacles for isotropically ordered obstacles, but it decreases for high concentrations of obstacles if the obstacles are strongly aligned. The scaling law exponent describing molecular weight dependence of linear polymer dimension is 0.6 for each of the three dimensions but deviates from the value for large elongation. Seven possible applications of the formula are discussed.

100,499

PB91-194456

PC A03/MF A01

National Inst. of Standards and Technology (MSEL),

Gaithersburg, MD. Polymers Div.

Synthesis of Non-Ionic Water-Dispersible Resins for Use in Intaglio Inks Curing by Electron Beam Radiation.

Annual rept.

B. Dickens, B. J. Bauer, and W'. J. Pummer. May 91 , 27p NISTIR-4564

Sponsored by Bureau of Engraving and Printing, Washington, DC.

Keywords: "Inks, Aqueous solutions, Resins, Polymeric films, Radiation curing, Electron beams, Polymerization, Engraving, Printing. Chemical radiation effects, Polyethylene glycols, Methacrylates, Siloxanes, Phosphazene, PMMA, Polyurethanes, Isocyanates, Viscosity, Synthesis(Chemistry), "Intaglio.

Several types of non-ionic resins which cure under the action of radiation such as UV or electron beam have been synthesized. The resins disperse in neutral water when uncured. The water dispersibility is conferred by incorporating polyethylene oxide sequences in the resin molecules. The polymerizing action is conferred by methacrylate groups. Typically, one end of each polyethylene sequence is attached to a methacrylate group, and the other end is chemically linked in various ways to produce resins varying from 2 to 4 methacrylate functionality. The linking moieties used are (1) a phosphazene ring, (2) a siloxane center, (3) a poly (methyl methacrylate backbone, and (4) a mixture of difunctional and trifunctional isocyantes. When the resins containing the urethane linkages were used with low viscosity crosslinkers, the mixtures were of appropriate viscosity for use in intaglio inks and cured with less than two megarads exposure to give reasonably flexible scuff-free films. The other resins were not viscous enough for use in intaglio inks and in some cases cured too slowly.

100,500

B 1-194688

Not available NTIS

National Inst. of Standards and Technology (MSEL), Gaithersburg, MD. Polymers Div.

Small-Angle Neutron Scattering of Blends of Cross-Linked and Linear Polystyrene.

Final rept.

R. M. Briber, and B. J. Bauer. 1991, 6p

Pub. in Macromolecules 24, n8 p1899-1904, 15 Apr 91.

Keywords: "Small angle scattering, "Neutron, "Polymer blends, "Polystyrene, Labeled substances, Crosslinking, Networks, Thermodynamic properties, Deuteration, Isotope exchange, Synthesis(Chemistry), Divinyl benzene, Elastic properties, Free energy, Phase separation(Materials), Binary system(Materials), separation(Materials), Binary system(Materials),
Density(Mass/volume), Mixing, Rubber, Reprints.

Small-angle neutron scattering (SANS) has been used to study the scattering function and thermodynamics of blends of linear protonated polystyrene (PSH) and cross-linked deuterated polystyrene (PSD). Two series of samples were synthesized. In both cases the samples were made by dissolving the linear PSH in deuterated (d8) styrene monomer containing a small amount of divinylbenzene, which was then polymerized to form the PSD network around the linear PSH chains. The samples were all made at a concentration of $50 / 50$ by weight PSD/PSH. Series 1 is a set of samples with the same cross-link density varying the length of the linear chain. Series 2 is a set of samples containing the same length linear chain varying the cross-link density systematically. By extrapolation of $S(q)$ obtained from SANS using an Ornstien-Zernike plot $(1 / S(q)$ versus $q$ squared) to $q=0$, the zero-angle scattering, $S(0)$, was obtained. $S(0)$ is inversely proportional to the second derivative of the free energy with respect to composition. For the series 1 and 2 samples it was found that the samples could be driven to phase separation by increasing either the length of the linear chain or the network density. In the series 1 samples the zeroangle scattering scaled as $1 / S(0)$ approximately $=1 /$ $\mathrm{N}$ (sub b). In the series 2 samples the zero-angle scat- tering scaled as $1 / \mathrm{S}(0)$ approximately $=1 / \mathrm{N}$ (sub $\mathrm{c}$ ). In addition, the scattering was found to increase as $N$ (sub c) decreased for the series 2 samples, a behavior opposite to that predicted by combining classical rubber elasticity and mixing theories.

\section{0,501}

PB91-195099

Not available NTIS National Bureau of Standards (IMSE), Gaithersburg, MD. Polymers Div.

Relaxation of Crosslinked Networks: Theoretical Models and Apparent Power Law Behavior. Final rept.

G. B. McKenna, and R. J. Gaylord. 1988, $6 p$ Pub. in Polymer 29, n11 p2027-2032 Nov 88

Keywords: "Viscoelasticity, "Relaxation, "Synthetic elastomers, "Crosslinking, Networks, Theories, Mathematical models, Density(Mass/Volume) Superposition(Mathematics), Time dependence, Reprints.

The viscoelastic behavior of crosslinked polymer networks is discussed. Models of the long term relaxation based on the retracing of dangling chains are described. It is pointed out that the theories all predict power law time relation but differ in predictions of the crosslink density dependence of the power law exponent. The experimental data of Chasset and Thirion is examined. It is shown that the power law relation works only over a limited time span with deviations occurring at long times and more markedly for more highly crosslinked systems. Furthermore, the validity of time-crosslink density superposition for networks is confirmed, thereby precluding a crosslink density de pendent exponent in any power law representation of the data.

\section{0,502}

PB91-195107

Not available NTIS

National Inst. of Standards and Technology (IMSE), Gaithersburg, MD. Polymers Div.

Study of the Linear Viscoelastic Properties of Cyclic Polystyrenes Using Creep and Recovery Measurements.

Final rept.

G. B. McKenna, B. J. Hostetter, N. Hadjichristidis, L. J. Fetters, and D. J. Plazek. 1989, 19p

Pub. in Macromolecules 22, n4 p1834-1852 Apr 89.

Keywords: "Polystyrene, "Viscoelasticity, "Molecular weight, "Rheological properties, "Fractionation, Creep properties, Dilution, Measurement, Contamination, Cyclic compounds, Polymer blends, Chromatography, Reprints, Size exclusion chromatography, Zero shear viscosity, Recoverable compliance, Plateau compliance, Linear molecules.

The viscoelastic behavior of macrocyclic polystyrene fractions having molecular weights ranging from 19400 to 390000 was studied. Measurements of zero shea viscosity and recoverable compliance were made on the so-called Strasbourg fractions, Akron fractions and two of the former which were refractionated. Also, studies of the effects of blending of up to $15.75 \%$ linear chains with the refractionated Strasbourg fractions were carried out. The studies show that the presence of linear chain contaminants in the macrocyclic fractions has the effect of decreasing the plateau com pliance and increasing the steady state value of the recoverable compliance. Also, the presence of small amounts of linear contaminant increases dramatically the viscosity of the cyclic fractions. The results of analysis of the good and moderate fractions gives a picture of the cycles as showing 'classical' viscoelastic behavior, i.e., the steady state recoverable compliance in creases with increasing molecular weight and attains a constant value at high molecular weights (MW greater than 180,000), as do linear polymers. The zero shea viscosity-molecular weight relation is well described by an equation of the form viscosity $=A M+B M$ (sup a) where $\mathrm{a}=3.9$, which is somewhat higher than the 3.4 power obtained for entangled linear chains.

100,503

B91-195271

Not available NTIS National Inst. of Standards and Technology (NEL) Gaithersburg, MD. Fire Measurement and Research Div.

Molecular Dynamics of Inltial Events In the Thermal Degradation of Polymers.

Final rept.

M. Nyden, and D. W. Noid. 1991, 6p

Pub. in Jnl. of Physical Chemistry 95, n2 p940-945 1991.
Keywords: "Combustion, "Polyethylene, "Thermal degradation, Polymers, Computerized simulation, $\mathrm{Re}$ action kinetics, Mechanisms, Chemical bonds, Hydrogen bonds, Mathematical models, Reprints, "Molecular dynamics.

Computer simulations, based on molecular dynamics, have been used to reveal kinetic and mechanistic as pects of initial events in the thermal degradation of polyethylene and related polymers. Simulations were performed on a series of model polymers and the rate constants for random scission of the carbon-carbon bonds were computed. The results are consistent with the predictions of statistical theories of unimolecular reactivity. Observations of the detailed motions of the model polymers have also revealed pronounced coiling actions in the vicinity of dissociating bonds. The behavior is examined in the light of proposed mechanisms for intramolecular hydrogen transfer.

\section{0,504}

PB91-195685 Gaithersbur. of Standards and Technology (IMSE) Difunctional MD. Polymers Div.

Monomers Capable of Cyclopolymerization.

Final rept

J. W. Stansbury. 1991, 7p

Sponsored by National Inst. of Dental Research, Bethesda, MD.

Pub. in Macromolecules 24, n8 p2029-2035 1991

Keywords: "Acrylates, "Esters, "Cyclic compounds, "Monomers, "Polymerization, Synthesis(Chemistry) Aldehyde polymers, Dental materials, Ethers, Reaction kinetics, Steric hindrance, Solubility, Oligomers, Reprints, Diazabicyclooctane.

The reaction of an acrylate ester with paraformaldehyde in the presence of diazabicyclo(2.2.2)octane has been shown to provide access to novel ether-fused dimethacrylate-like monomers that can undergo cyclopolymerization. The study examined the influence of the pendant ester functionality on the synthesis and polymerization of these monomers. While bulky ester groups were generally found to reduce the rate of reaction in monomer synthesis, the more hindered monomers appear to polymerize through the available in tramolecular cyclization pathway with greater efficiency than monomers without significant steric constraints. Polymerizations in solution lead to mainly cyclized, soluble polymers up to relatively high monomer concentrations. Bulk polymerizations provided brittle, cross-linked polymers with high degrees of conversion. The work was extended to include the synthesis of a multifunctional oligomer based on the same 1,6diene substructure. The polymerization of this oligomer produced a tough, highly cross-linked polymer.

\section{0,505}

PB91-202887

Not available NTIS

National Inst. of Standards and Technology (IMSE), Gaithersburg, MD. Polymers Div.

Examination of Mechanisms Responsible for Incoherent Scattering of Subthermal Neutrons by Polymers.

Final rept.

L. D. Coyne, and W. L. Wu. 1989, 3p

Pub. in Polymer Communications $30, n 10$ p312-314 1989.

Keywords: "Small angle scattering, "Thermal neutrons, "PMMA, "Polymers, Incoherent scattering, ( Methylene radicals, Hydroxy analysis, Methyl radicals, tion temperature. Protons, Labelled compounds, Reprints, Methyne compounds.

Proper account for the large incoherent contribution is vital for quantitative evaluation of small-angle neutron scattering data of polymers. Knowledge of the relative contributions of hydrogen atoms in differing chemical substituents will aid in a priori estimates of incoherent scattering cross-sections for polymers of complex structure. From subthermal neutron transmission measurements on poly(methyl methacrylate) and hydrogenous and partially deuterated poly(vinyl alcohol) it is established that contributions to levels of incoherent scattering by protons in polymers are ordered quantitatively as $-\mathrm{CH} 3>-\mathrm{OH}>-\mathrm{CH} 2-,=\mathrm{CH}$. . No apparent effect of the glass transition on incoherent scattering of subthermal neutrons by polymers was discerned. 
100,506

PB91-203224

Not available NTIS

National Inst. of Standards and Technology (MSEL) Gaithersburg, MD. Polymers Div.

Morphology of Polymer Films and Single Molecules.

Final rept.

B. Howell, and D. H. Reneker. 1990, 20p

Pub. in Jnl. of Applied Polymer Science 40, n9-10 p1663-1682 1990 .

Keywords: "Monomolecular films, "Polymeric films, *Plastics, "Polystyrene, Labelled compounds, Electron microscopy, Thin films, Molecular weight, Peek Polyethylene, Elastomers, Deuteration, PMMA, Block copolymers, Ruthenium oxide, Polyacetylenes, Polybutadiene, Glutamates, Reprints, Poly4BCMU, Polybenzyl-I-glutamate.

Seven polymeric substances were examined by high resolution electron microscopy. Features on the scale of the diameter of single molecular chains were observed. Polymers examined include: (1) linear polyeth ylene (Mw $=52,000)$, (2) polyethylene (Mw approximately $5,000,000)$, (3) poly-(cis-1,4-butadiene), (4) poly-gamma-benzyl-L-glutamate,

poly(etheretherketone) (PEEK), (6) deuterated polystyrene - poly(methylmethacrylate) (PMMA) block co polymer, and (7) a polydiacetylene, poly $(1,12$ di(butoxy-carbonylmethylurethanyl)-5,8-dodecadiyne), also called poly-4BCMU. A variety of methods were used to prepare dispersed single molecules, and very thin films, some of which had regions with strands con taining only a few molecules. Staining with RuO4 re vealed structures near the surface of the films that were reproducible and characteristic of each polymer.

100,507

PB91-203364

Not available NTIS

National Inst. of Standards and Technology (MSEL) Gaithersburg, MD. Polymers Div.

Simulation Studies of Excluded Volume Effects on Polymer Chaln Dynamics in Several Nonlattice Models.

Final rept

D. KranbuehI, D. Eichinger, and P. H. Verdier. 1991 , $9 p$

Pub. in Macromolecules 24, n9 p2419-2427 1991.

Keywords: *Polymers, "Monte Carlo method, "Statistical mechanics, Stereochemistry, Computerized simulation, Mathematical models, Scale(Ratio), Relaxation time, Diffusion, Molecular chains, Reprints, Markov chains, Chain length, Excluded volume, Off lattice.

Computer simulations of off-lattice bead-stick models of polymer chains with a variety of move rules have been carried out to gauge the effect of these move rules on the dynamical behavior of the chains. Long relaxation times, translational diffusion constants, and mean-square end-to-end length for chains of from 9 to 99 beads, both with and without excluded volume, are reported. For several move rules the excluded volume constraints increase the chain-length dependence of the long relaxation times by about the square root of chain length, roughly twice the exponent expected from simple scaling arguments based on the expansion of equilibrium dimensions by excluded volume. Move rules analogous to some of those used in the present study have been employed by others in previous lattice-model studies. Those earlier lattice-mode results appeared to suggest that relaxation times scale like mean-square chain dimensions. Present results suggest, however, that the earlier result is an artifact resulting from the combination of lattice constraints with the use of move rules explicitly dependent upon local chain conformation.

\section{0,508}

PB91-203570

Not available NTIS

Natonal Inst. of Standards and Technology (IMSE)

Gaithersburg, MD. Polymers Div.

Tests of the Flory-Rehner Hypothesis: Comparison of the Elastic Free Energy Function for Crosslinked Rubber in the Dry and Swollen States.

Final rept.

G. B. McKenna, K. M. Flynn, and Y. Chen. 1989, 5p

Pub. in Molecular Basis of Polymer Networks, p127 1311989.

Keywords: "Polymers, "Solvents, "Elastomers, "Rubber, "Free energy, "Swelling, Networks, Elastic properties, Conformational changes, Compression tests, Torsion tests, Thermodynamic properties, Reprints, Frenkel-Flory-Rehner hypothesis.
One of the presumptions of the Frenkel-Flory-Rehner (FFR) hypothesis is that the form of the elastic component of the free energy function is not affected by the presence of solvent molecules. The change in elastic properties of the swollen rubber are then attributed to changes in chain conformation alone. Experiments in which the derivative of the elastic free energy with respect to the deformation for both dry and swollen rubber was measured were used to test the aspect of the FFR hypothesis. Torsional and compression ex periments were carried out on samples of dicumyl peroxide crosslinked natural rubber. Results show that the elastic free energy function of a rubber network is unaffected, at least to first order, by the presence of solvent. This appears to be true over the entire range of experimental parameters investigated, i.e., crosslink density, solvent quality, and degree of swelling.

\section{0,509}

Not available NTIS National Bureau of Standards (NEL), Gaithersburg MD. Building Materials Div.

Reflection/Absorption FTIR Spectral Characteristics of Thin and Thick Crosslinked Epoxy Films on Steel Substrates.

Final rept.

T. Nguyen, D. Bentz, and W. Byrd. 1988, $5 p$

Pub. in Proceedings of ACS Division of Polymeric Materials Science and Engineering, Los Angeles, CA. September 26-30, 1988, v59 p459-463.

Keywords: "Polymeric films, "Epoxy resins, "Steels, "Coatings(Materials), Thickness, Crosslinking, Thin films, Spectrum analysis, Amines, Infrared spectra Calculation methods, Fourier transformation, Theories, Transmission, Substrates, Absorptivity, Reflection Comparative evaluation, Reprints.

The study examined, both theoretically and experimentally, band shapes of the reflection/absorption FTIR spectra of thin and thick crosslinked epoxy films on steel substrates. Amine-cured epoxy films of between $10 \mathrm{~nm}$ and $25 \mathrm{um}$ were spin coated on mechanically polished cold rolled steel samples, and reflec tion/absorption FTIR (RA-FTIR) spectra were taken at 84 degree angle of incidence using single reflection and parallel polarized light. The theoretical curves were calculated using Greenler's boundary-value equations for films on metals. For thin films, except for the broad and strong bands at 1510 and $12471 / \mathrm{cm}$ which shifted to higher frequency, other bands were similar to that obtained by the transmission technique. For micrometer films, numerous bands shifted to higher frequency and the bands at 1510 and 12471 $\mathrm{cm}$ distorted and split. The theory agreed well with ex perimental results for thin films. However, for microme ter thick films, although the theory correctly predicted distortion and splittings of strong bands, it underestimated the intensity of the splitted bands. Comparison between experimental data and theoretical calcula tions on the relationship between RA-FTIR band intensity and film thickness will also be discussed.

\section{0,510}

PC A07/MF A02 National Inst. of Standards and Technology (MSEL) Gaithersburg, MD. Polymers Div.

Assessment of the State-of-the-Art for Process Monitoring Sensors for Polymer Composites. D. Hunston, W. McDonough, B. Fanconi, F. Mopsik F. Wang, F. Phelan, and M. Chang. 1 Jun $91,145 p$ NISTIR-4514

Keywords: "Polymers, "Ultrasonics, "Dielectrics, "De tectors, "Spectroscopy, "Optical scanners, Proces control, Sensor characteristics, Composite materials, "Process monitoring, Research and development.

A variety of techniques have been applied to monitoring the changes that occur in the resin during composite processing. The report identifies and analyzes those techniques that have the most promise for online application, namely: ultrasonic, dielectric, spectroscopic, and optical techniques. All these method have advantages and disadvantages, and the best technique for a given application will depend strongly on the materials and processes involved. The report discusses these on-line monitoring techniques and compares them in the areas of measurement speed sampling volume, sensitivity to different resins, effects of fiber type, resistance to the manufacturing environment, interpretation of the data, adaptability for other uses, and temperature capabilities. The most important comparison, however, concerns the research and development work that is needed to achieve the ful potential of each technique in applications on the fac- tory floor. Dielectric and ultrasonic methods have an (n) connection because commercial equipment designed for process monitoring is already available. The various spectroscopic and optical methods are generally in an earlier stage of development. The great potential inherent in these latter methods, however, makes it highly desirable to actively pursue their developments as well.

100,511

PB91-236638

Not available NTIS

National Inst. of Standards and Technology (MSEL), Gaithersburg, MD. Polymers Div.

Semiempirical Theory of Relaxation: Concentrated Polymer Solution Dynamics.

Final rept.

J. F. Douglas, and J. B. Hubbard. 1991, 15p 91.

Keywords: *Polymers, "Viscoelasticity, "Stress relax ation, "Glass, "Melts, Diffusion, Molecular weight, Cluster model, Transport theory, Homogeneity, Viscosity, Reprints, Memory effects.

Classical models of viscoelasticity neglect memory ef fects arising from material inhomogeneity and cooperative molecular motion. These memory effects are modeled by using an integral equation approach in which physical arguments are employed to estimate the memory kernel. The model memory kernel de pends on two parameters: beta, which is interpreted as a measure of material inhomogeneity, and phi, which is interpreted as a measure of cooperativity of molecula motion. Here the authors apply a relaxation model to polymer melt dynamics. An idealized 'cluster' model of stress relaxation in glasses is introduced to estimate beta. This model rationalizes well the observed stress relaxation of polymer glasses. A cluster model is also introduced to estimate the molecular weight dependence of the zero-shear rate viscosity and the diffusion coefficient of entangled polymers. For flexible polymers in three dimensions the viscosity molecular weight exponent ranges in the interval 3.3 to 3.7 , depending on the strength of the excluded-volume inter action. The diffusion coefficient molecular weight ex ponent in three dimensions is predicted to lie in the interval -2.3 to -2.5 . Results similar to the reptation model are obtained in four dimensions, and the Rouse theory is recovered in the limit of infinite dimensiona lity. Estimates of the concentration dependence of entangled polymer solution transport properties are also given.

100,512

PB91-236653

Not available NTIS National Inst of Standards and Technology (NML) Boulder, CO. Chemical Engineering Science Div.

Thin and Composite High-Flux Membranes of Perfluorosulfonated Ion-Exchange Polymer.

Final rept.

B. K. Dutta, D. Randolph, and S. K. Sikdar. 1990,

$11 \mathrm{p}$

Pub. in Jnl. of Membrane Science 54, p51-61 1990

Keywords: "Perfluoro compounds, "Sulfonic acids, "Membrane transport, "Polymeric films, "Castings, Composite structures, Ion exchange materials, Curing, Amino acids, Permeability, Thin films, Electron micros copy, Mixtures, Glycine, Alanines, Separation processes, Reprints.

The techniques of casting thin and composite mem branes of perfluorosulfonic acid polymer (PFSA) are reviewed. The thin PFSA membrane was prepared by a hot-casting method using an aqueous methanol solution with nonsolvents cyclohexanol and dimethylformamide. The composite PFSA membrane was cold cast and subsequently cured at a higher temperature. Scanning electron micrographs showed the thin membrane to be asymmetric, whereas the composite membrane had a thin homogeneous polymer layer on the porous support. Permeation through the membranes was tested using several amino acids and their mixtures as model permeants. It was found that membranes of both types provided higher fluxes than the commercial membrane of the same polymeric mater al. Permeation studies on amino acid mixtures using the composite membrane revealed that glycine permeated faster than either L-alanine or L-phenylalanine, and that glycine separation factors were higher for the composite PFSA membrane than for other polymeric membranes. 


\section{0,513}

PB91-236786

Not available NTIS

National Inst. of Standards and Technology (CSTL) Gaithersburg, MD. Process Measurements Div.

Small-Angle Neutron Scattering and Light Scattering Studies on the Miscibility of Protonated Polyisoprene/Deuterated Polybutadiene Blends.

Final rept.

H. Hasegawa, S. Sakurai, M. Takenaka, T.

Hashimoto, and C. C. Han. 1991, 7p

Pub. in Macromolecules 24, n8 p1813-1819 1991.

Keywords: "Synthetic elastomers, "Polyisoprene, "Polybutadiene, Polymer blends, Neutron scattering, Light scattering, Solubility, Isotope exchange, Protons, Deuterium, Hydrogen, Binary system(Materials), Smal angle scattering, Mixtures, Phase diagrams, Fluid-fluid interactions, Reprints.

Binary mixtures of protonated polyisoprene and deuterated polybutadiene with particular microstructures exhibit an LCST-type phase behavior. Small-angle neutron scattering (SANS) and time-resolved smallangle light scattering (SALS) techniques were employed to study the static and dynamic phase behavio of the mixtures. From the static study by SANS, binary interaction parameters and the phase diagram were obtained. The spinodal temperature at the critical blend composition obtained by the analysis of SANS profiles from the single-phase state was in good agreement with that obtained by the time-resolved SALS measurements. The component polymers are prepared by living anionic polymerization and hence have well-defined molecular characteristics. Therefore, this system is considered to be an excellent polymer mixture system for the study of the relationships between static and dynamic phase behaviors of the polymer mixture and the molecular parameters such as molecular weight, polydispersity, microstructure, etc.

\section{0,514}

PB91-237842

Not available NTIS

National Inst. of Standards and Technology (IMSE) Gaithersburg, MD. Polymers Div.

Network Structure in Epoxies. 6. The Growth Process Investigated by Neutron Scattering.

Final rept.

W. L. Wu, W. Su, and B. J. Bauer. 1989, 5p

See also PB88-195094.

Pub. in Polymer 30, n8 p1384-1388 1989.

Keywords: "Epoxy resins, Curing, Molecular structure, Molecular weight, Neutron scattering, Percolation, Reprints.

The curing process of epoxies in bulk prior to the gelation threshold was investigated using small angle neutron scattering. Both the radius of gyration and the molecular weight of the partially cured molecular network were measured at various extent of cure. Two curing agents with a difference in their functionality were in cluded to elucidate the curing mechanism. The experimental results were interpreted in terms of the classical Flory-Stockmayer theory in one case and the percolation theory for the other case.

\section{0,515}

\section{PB92-110196}

(Order as PB92-110162)

Tennessee Univ., Knoxville. Dept. of Chemistry. Heat Capacity and Other Thermodynamic Properties of Linear Macromolecules. 10. Update of the ATHAS 1980 Data Bank.

M. Varma-Nair, and B. Wunderlich. c1991, 56p

Prepared in cooperation with Oak Ridge National Lab. TN. Chemistry Div.

Included in Jnl. of Physical and Chemical Reference Data, v20 n2 p349-404 Mar/Apr 91. Available from American Chemical Society, 1155 16th St., NW, Washington, DC. 20036-9976.

Keywords: "Specific heat, "Thermodynamic properties, "Polymers, Linear systems, Information systems, Gibbs free energy. Experimental design, Aromatic compounds, Tables(Data), "Reference materials, ATHAS system.

The prior published ATHAS 1980 Data Bank of experimental heat capacities of linear macromolecules that included critically reviewed material on almost 100 polymers is updated. In addition, the data bank has been computerized so that future updates can be made continuously, and new print-outs or computer files will be available from the authors from 1990 on (Copyright (c) 1991 by the U.S. Secretary of Commerce.)
100,516

PB92-116284

PC A07/MF A02

National Inst. of Standards and Technology (MSEL), Gaithersburg, MD.

Polymers: Technical Activities 1991. (NAS-NRC Assessment Panel, February 13-14, 1992).

L. E. Smith, and B. M. Fanconi. Oct 91, 131p

NISTIR-4696

See also PB91-159715.

Keywords: "Polymers, "Polymer matrix composites, * Polymer blends, Medical supplies, Dental materials, Standards, On-line measurement systems, Technology transfer, Shear, Phase separation(Materials), Dissimilar materials bonding, Corona discharges, Vinyl polymers, Rheological properties, Automobiles, Thin films, Computerized simulation, Neutron scattering Biodegradation, Porcelain, US NIST, Standard Reference Materials.

All of the Division's programs are described in the report, organized according to these six Groups. These Groups have made a number of significant accomplishments during the last year. Some of these are summarized below: A computer simulation was performed to model resin flow in liquid molding of the front end structure of a Ford Escort; A NIST/industry consortium has evolved for the purpose of developing inline measurement technology based on optical and fluorescence methods; A combined light scattering photometer/rheometer has been constructed to examine phase separation behavior of polymer blends and solutions under the influence of a simple shear field; $A$ unique new tool has been developed to study the physics involved in the adhesion between a polymer and a solid surface like glass; The distribution of polarization in poly(vinylidene fluoride) poled by corona discharge rather than by contacting electrodes indicated positive charge injection near the surface; Proton spin diffusion results indicated domain sizes in poly(butylmethacrylate)/hydroxy modified polystyrene blends with minimum dimensions in the $6-10 \mathrm{~nm}$ range and stoichiometries of the order of 75/25; Novel, siloxane-containing difunctional and multifunctional viny monomers were prepared; The apparent 'shift' in polymer blend phase separation temperature, Tc, under shear flow has been interpreted with the mode-coupling theory for mean-field polymer blend systems The radial flow behavior was successfully predicted from measurements in unidirectional flow; An exposure technique that rapidly determines whether or not a polymer is biodegradable has been developed; Instrumentation has been set up and calibrated to measure the dielectric constant of thin polymer films at high electric fields; A viscoelastic model of stresses in porcelain-metal strips has been developed.

\subsection{7}

PB92-116698 Not available NTIS National Inst. of Standards and Technology (MSEL), Gaithersburg, MD. Polymers Div.

Flexible Polymer with Excluded Volume at an Interacting Penetrable Surface of Variable Dimension: A Multiple epsilon Perturbation Theory.

Final rept.

J. F. Douglas, and M. K. Kosmas. 1989, 8p

Pub. in Macromolecules 22, n5 p2412-2419 May 89

Keywords: "Polymers, "Surface properties, Perturbation theory, Calculation methods, Interactions, Renormalization, Reprints, "Excluded volume.

Application of the renormalization group method to the perturbative treatment of surface ineracting polymers with excluded volume currently requires the surface dimension to be fixed. A multiple epsilon perturbation expansion method is proposed to circumvent the technical restriction. There are numerous potential applications of multiple epsilon perturbation theory (e.g. combined treatment of binary and ternary excluded volume interactions) which can be pursued once the internal consistency of the method is demonstrated in higher order calculations. The surface interaction model provides a good starting point for studying the multiple method because of the relative simplicity of the perturbative calculations which provide the input into the multiple epsilon renormalization group calculations. Another convenient aspect of the model is that the consistency of the multiple epsilon method can be checked against accurate results for the limits of each interaction (surface and excluded volume) alone and the combined interactions for the special case of a two dimensional surface.

\section{0,518}

PB92-117266

Not available NTIS
National Inst. of Standards and Technology (MSEL). Gaithersburg, MD. Polymers Div.

Microstructure Effects on the Lower Critical Solution Temperature Phase Behavior of Deuterated Polybutadiene and Protonated Polyisoprene Blends Studied by Small-Angle Neutron Scattering.

Final rept.

S. Sakurai, H. Jinnai, H. Hasegawa, T. Hashimoto, and C. C. Han. 1991, 5p

Pub. in Macromolecules 24, n17 p4839-4843 1991.

Keywords: "Synthetic elastomers, "Polymer blends, "Neutron scattering, "Polybutadiene, "Polyisoprene, Binary system(Materials), Microstructure, Small angle scattering, Deuteration, Isotope exchange, Labelled compounds, Solubility, Solutions, Critical temperature, Phase diagrams, Vinyl polymers, Phase separation(Materials), Randomness, Reprints.

The miscibility of a blend of protonated polyisoprene (HPI) with the 3,4-linkage microstructure in the range $7-15 \%$ and of deuterated polybutadiene (DPB) with the 1,2 -linkage microstructure in the range $12-28 \%$ was studied by small-angle neutron scattering (SANS). It was found that all blends studied here show lower critical solution temperature (LCST) type phase behaviors; i.e., the phase separation occurs by raising the temperature. It was also found that the miscibility is quite sensitive to the microstructures of the polydienes used. The effective thermodynamic interaction parameter (chi(eff)) per segment between two polymers was determined by fitting SANS data in the single-phase state with a theoretical scattering curve obtained on the basis of the random-phase approximation. The temperature dependence of (chi(eff)) showed a systematic change with the microstructure. For a given $\mathrm{HPI}$, the (chi(eff)) values decreased, and therefore, the blends became more miscible, with an increase in the vinyl content (i.e., 1,2-linkage content) in DPB. On the contrary, for a given DPB, the values increased, and therefore, the blends became more immiscible, with an increase in the vinyl content (i.e., 3,4-linkage content) in $\mathrm{HPI}$. An alternative explanation for the LCST phase behavior is proposed based on treatment for the random copolymer blends.

\section{0,519}

PB92-117274

Not available NTIS

National Inst. of Standards and Technology (MSEL), Gaithersburg, MD. Polymers Div.

Small-Angle Neutron Scattering and Light Scattering Study on the Miscibility of Poly(styrene-ran-butadiene)/Polybutadiene Blends.

Final rept.

S. Sakurai, T. Izumitani, H. Hasegawa, T. Hashimoto, and C. Han. 1991, 8p

See also PB91-236786.

Pub. in Macromolecules 24, n17 p4844-4851 1991.

Keywords: "Solubility, "Polymer blends, "Synthetic elastomers, "Styrene butadine resins, "Polybutadiene, Binary system(Materials), Copolymers, Small angle scattering, Randomness, Light scattering, Neutron scattering, X-ray scattering, Predictions, Reprints.

The miscibility of binary blends of polybutadiene (PB) and poly(styrene-ran-butadiene)(SBR) was studied on the basis of segmental interaction parameters. In the analysis PB was assumed to be a random copolymer comprising 1,2-linkages (V) and 1,4-linkages (B) and SBR to be the one comprising styrene (S) and butadiene with $V$ and $B$. The segmental interaction parameters between $V$ and $S$ (chi vs), $B$ and $S$ (chi bs), and $V$ and $B$ (chi vb) were determined on the basis of the copolymer blend theory from the effective interaction parameters (chi eff's) for the blends of PB and SBR, and those for poly(styrene-b-butadiene-b-styrene) triblock copolymer (SBS), with different copolymer compositions and microstructures of polybutadienes. The values chi eff wer determined from either the smallangle neutron or $X$-ray scattering of the mixtures in the single-phase state. By use of these segmental interaction parameters, chi vs, chi bs, and chi vb, the miscibility of the SBR/PB blends was predicted for a given set of $V, B$, and $S$ contents in PB and SBR. The predictions of the miscibility for several SBR/PB blends agreed well with the results of the light scattering experiments. 


\section{General}

\section{0,520}

\section{PB92-112499}

National Inst of Standards and Ter Gaithersburg, MD.

NIST Calibration Services for Humidity Measurement.

A. Wexler, R. W. Hyland, S. Hasegawa, and P. H. Huang. Oct $91,65 p$ NISTIR-4677

See also PB270 049.

Keywords: *Humidity measurement, "Calibration, "Hygrometers, Water vapor, Moisture content, Calibration standards, Gas generating systems, Vapor pressure, Error analysis, Tables(Data), US NIST.

The National Institute of Standards and Technology (NIST), formerly National Bureau of Standards (NBS) provides a service to Government agencies and the public for the calibration of humidity measuring instruments. Calibrations are performed by subjecting the instrument under test to atmospheres of known moisture content produced by the NBS two-pressure humidity generator. The most accurate calibrations are made with the NBS standard hygrometer, a device based on the gravimetric method. The internal report documents the original work for the NBS standard hygrometer, and for the NBS two-pressure humidity generator. The operations, tests, calibrations of component parts, and sources of errors of the standard hygrometer and the humidity generator are summarized. The random and systematic errors affecting the overall accuracy of the standard hygrometer and the humidity generator are analyzed and shown by tables.

\section{CIVIL ENGINEERING}

\section{Construction Equipment, Materials, \& \\ Supplies}

100,521

PB91-134189

Not available NTIS National Bureau of Standards (NEL), Gaithersburg, MD. Building Materials Div.

Techniques to Observe the Fracture Zone in Mortar and Concrete.

Final rept.

L. I. Knab, H. Jennings, H. N. Walker, J. R. Clifton,

and J. W. Grimes. 1986, 7p

Pub. in Fracture Toughness and Fracture Energy of Concrete, p241-2471986.

Keywords: "Crack propagation, "Concretes, "Microscopy, Mortars(Material), Fractures(Materials), Electron microscopy, Fluorescence, Cracking(Fracturing), Fracture tests, Fracture zones, Building materials, Reprints.

There have been relatively few studies of the microstructural details of the fracture zone of propagating cracks in mortar and concrete. This lack of detailed information has hindered the development and validation of tracture mechanics theories based on the micromechanisms of crack propagation. The paper presents the application of two techniques to observe the microstructural details of the fracture zone: fluorescent light microscopy using thin sections, and scanning electron microscopy using backscattered electrons. Preliminary findings indicate that both techniques have the potential to provide new information on the fracture zone in mortar and concrete, both near the exterio and in the interior of specimens.

\section{0,522}

\section{PB91-143321}

PC A04/MF A01

National Inst of Standards and Technology (NEL) Gaithersburg, MD. Center for Building Technology. Outline of a National Plan on High-Performance
Concrete: Report on the NIST/ACI Workshop. Held in Gaithersburg, MD on May 16-18, 1990.

N. J. Carino, and J. R. Clifton. Dec 90, 63p NISTIR4465

Keywords: *High strength concretes, "Meetings, Alkali aggregate reactions, Mechanical properties, Service life, Specifications, Concrete durability, Standards, Tests, Curing, Mixing, Recommendations.
A workshop on high-performance concrete (HPC) was held in Gaithersburg, MD on May 16, 17, and 18, 1990. The workshop was co-sponsored by the American Concrete Institute. High-performance concrete was defined as concrete having desired properties and unjformity which cannot be obtained routinely using only conventional constituents and normal mixing, placing, and curing practices. Eight working groups were organized to address different topics. The report summarizes the discussions and conclusions of the working groups. Each chapter begins with a brief introduction providing background on the nature of the problems addressed by the working group. Specific research topics are identified, and discussions are provided to explain the rationale for the needed research. The recommended research is proposed as the basis for a national program to exploit the potential of high-performance concrete and ensure U.S. competitiveness in concrete technology. Recommendations for implementing the plan are provided.

\section{0,523}

PB91-148072

Not available NTIS

National Bureau of Standards (NEL), Gaithersburg. MD. Building Materials Div.

Microstructure and Fracture at the Cement PasteAggregate Interface.

Final rept.

L. Struble. $1988,10 p$

Sponsored by Air Force Office of Scientific Research Bolling AFB, DC

Pub. in Materials Research Society Symposium Proceedings, v114 p11-201988.

Keywords: "Microstructure, "Bonding strength, " $\mathrm{Ce}$ ments, "Aggregates, "Fractures(Materials), Cementing, Interfaces, Cement aggregate reactions, Concretes, Bonding, Cracks, Reprints.

The mechanical behavior of concrete in thought to be affected by the microstructure of the paste-aggregate interface, which differs in several respects from the microstructure of bulk paste. General aspects of interfacial microstructure, which appear to be fairly well understood, are reviewed. Recent microstructural studies using back-scattered electron imaging show that there is a zone along the interface (approximately $50 \mathrm{mi}$ crometers wide) within which there are generally few large unhydrated clinker grains, many large voids (greater than 5 micrometers), a generally porous $\mathrm{mi}-$ crostructure, many hollow-shell hydration grains, and in some cases little calcium hydroxide. The tendency for cracks to develop or grow along the paste-aggregate interface is discussed in relation to specific features of the interfacial microstructure.

\section{0,524}

PB91-148528

PC A03/MF A01

National Inst. of Standards and Technology (NEL). Gaithersburg, MD. Center for Building Technology

Guide to the Use of the Cement and Concrete Research Remote Bulletin Board System (RBBS) Computer.

L. J. Kaetzel, and J. R. Clifton. Dec 90, 49p NISTIR4473

Keywords: "Cements, "Concretes, "Research projects, "Data bases, Computer systems hardware Information systems, Computer systems programs, Data transmission, Data retrieval, "Remote bulletin board system, National Institute of Standards and Technology.

A computer system has been installed at the Nationa Institute of Standards and Technology, Building Materials Division, for exchanging and disseminating information related to cement and concrete research. The computer is accessible, remotely, through telephone lines. Messages, and computer stored files in many formats can be exchanged among cement researchers. Also, information can be retrieved and/or viewed that describes: scheduled symposia, archived computer based models, project summaries, and published papers. The document describes how to use the computer system.

\section{0,525}

PB91-149922

Not available NTIS

National Bureau of Standards (NEL), Gaithersburg, MD. Building Materials Div.

Pozzolan Programs of the Cement and Concrete Reference Laboratory.

Final rept.

J. H. Pielert, and R. M. Kolos. 1987,13p

See also PB80-160047. Sponsored by American Society for Testing and Materials, Philadelphia, PA.
Pub. in Proceedings of the International Ash Utilization Symposium (8th), p19-1-19-13 1987

Keywords: "Laboratories, "Concretes, "Cements, "Pozzolans, Aggregates, Inspection, Fly ash, Admix tures, Concrete durability, Standards, Quality assurance, Fieprints.

The Cement and Concrete Reference Laboratory (CCAL) located in the Center for Building Technology of the National Bureau of Standards has as its goal the improvement in the quality of testing of construction materials. This is accomplished through the inspection of laboratories who test construction materials, distribution of proficiency samples, studies of testing problems, and participation on technical committees. Until recently, the inspection and proficiency sample programs considered portland, blended and masonry cements; portland cement concrete; aggregates; and reinforcing steel. In response to requests of the Join ASTM C1/C9 Subcommittee on the CCRL, two new programs related to pozzolans have been added; pozzolan laboratory inspection program was begun in January 1987 and the distribution of a fly ash reference sample is planned for September 1987. The paper will provide a short overview of CCRL programs for cement, concrete, aggregates and reinforcing steel and give specifics on the pozzolan programs including scope of coverage, schedules, costs, anticipated benefits, and participation information.

100,526

PB91-174524

Not available NTIS

National Inst. of Standards and Technology (NEL) Gaithersburg, MD. Structures Div.

Introduction to $\mathrm{ACl}$ 306.1-87: Specification for Cold Weather Concreting.

Final rept.

N. J. Carino $1988,8 p$

Pub. in Concrete International 10, p50-57 Oct 88

Keywords: "Cold weather construction, "Concrete construction, "Specifications, Concrete durability, Structural forms, Concrete structures, Concretes, Freezing, Construction, Strength, Peprints.

The historical developments leading to the new $\mathrm{ACl}$ specification on cold weather concreting are reviewed. Attention is focused on the evolution of the curren definition of 'cold weather' and on changes in the protection requirements to prevent damage from freezing at an early age. The evolution of the current criteria for form removal is also reviewed, and the paper concludes with a presentation of key aspects of the standard specification.

100,527

PB91-175117

Not available NTIS National Inst. of Standards and Technology (NEL) Gaithersburg, MD. Structures Div.

Rate Constant Functions for Strength Development of Concrete.

Final rept.

R. C. Tank, and N. J. Carino. 1991, 10p

Pub. in ACI Materials Jnl. 88, n1 p74-83 Jan/Feb 91

Keywords: "Concretes, *Curing, "Strength, Concrete construction, Temperature effects, Concrete durability, Mechanical properties, Aging tests(Materials), Construction materials, Concrete structures, Life(Durability), Reprints.

The rate constant for strength development of a particular concrete mixture is the initial slope of the relative strength-versus-age curve at constant temperature curing. The form of the rate constant versus temperature function is needed to describe the combined effects of time and temperature on strength development. The study investigates the relationship between the rate constant and curing temperature. Based on strength gain data for concrete and mortar specimens made with Type I cement and cured at 10,23, and 40 $\mathrm{C}(50,73$, and $104 \mathrm{~F})$, the following conclusions are drawn: (1) strength gain can be represented by a three-parameter hyperbolic function; (2) the rate constant is a nonlinear function of curing temperature and a simple exponential function describes the relationship; (3) tests of appropriate mortar specimens provide the information needed to predict relative strength development of the corresponding concrete; and (4) the proposed rate constant model accurately describes the development of relative strength as a function of the equivalent age. 


\section{0,528}

\section{PB91-178863}

PC A03/MF A01

Gaithersburg.

Characterization of Field Concrete.

P. E. Stutzman. Jan 91, 35p NISTIR-4516

Keywords: "Concretes, "Field tests, Construction materials, Performance prediction, Service life, Chemical analysis, Characterization, Crack propagation, Microstructure, Compressive strength, Sampling, Tests.

Field inspection and laboratory analysis of concrete and concrete structures are necessary for condition evaluation, determination of the need and extent of repairs, and for the prediction of concrete service life. A detailed evaluation includes field inspection, field testing, sampling, and laboratory analyses and also involves the description of the structure, concrete, and aggregates. Field inspection for overall structure condition includes the identification and description of surface defects, cracks, and cracking patterns. Field testing determines relative concrete quality and location of degraded concrete, and provides guidance in developing a sampling plan. Laboratory analyses provide detailed information on the microstructure, phase composition, chemical composition, and strength of the concrete.

\section{0,529}

\section{PB91-187179}

Not available NTIS

National Inst. of Standards and Technology (NEL), Gaithersburg, MD. Building Materials Div.

Percolation of Phases in a Three-Dimensional Cement Paste Microstructural Model.

Final rept.

D. P. Bentz, and E. J. Garboczi. 1991, 20p

Sponsored by National Science Foundation, Washington, DC.

Pub. in Cement and Concrete Research 21, p325-344 1991.

Keywords: "Cements, "Microstructure, "Hydration, *Percolation, Water cement ratio, Admixtures, PorosiA three-dimensional digital-image-based simulation model of cement hydration is used to study the percolation or connectivity of phases as a function of hydration. Results from an investigation of the effects of water-to-cement ratio, degree of hydration, and the substitution of inert and pozzolanic mineral admixtures for cement, on the connectivity of the capillary porosity are presented. For all scenarios studied, plotting pore connectivity vs. total porosity results in a single universal curve. Based on the curve, the degree of hydration required to achieve pore discontinuity as a function of water-to-cement ratio and pozzolanic mineral admixture concentration has been determined. Similar universal curves have been obtained for the connectivity of the calcium silicate hydrate and calcium hydroxide phases in hydrated neat cement when plotted against the appropriate phase fraction. Simulation results are analyzed using percolation theory, and are applied to interpreting observed experimental results concerning cement properties as a function of hydration.

\section{0,530}

PB91-189621

Not available NTIS

National Inst. of Standards and Technology (NEL), Gaithersburg, MD. Building Materials Div.

Evaluation of Test Methods for Measuring the Bond Strength of Portland Cement-Based Repair Materials to Concrete.

Final rept

L. I. Knab, and C. Spring. $1989,12 p$

See also PB88-215488. Sponsored by Tri-Service Building Materials Committee, Washington, DC Pub. in Cement, Concrete and Aggregates 11, n1 p3-
141989.

Keywords: "Portland cements, "Bonding strength, Tension tests, Tensile stress, Repair, Bonding, Mechanical properties, Dissimilar materials bonding, Concretes, Tensile strength, Concrete construction, Reprints.

Three bond test methods were evaluated for screening and selecting repair materials used in overlaying and patching portland cement concrete. The bond strengths of three repair materials to base concrete were investigated using two uniaxial tensile bond test methods and a slant shear bond test method. The differing strength characteristics of the repair materials as compared to the base concrete resulted in different failure patterns, which had to be taken into account in the analyses of the failure stresses. Substantial differences in the failure stresses of the two test types (slant shear and uniaxial tension) were attributed to their completely different geometries and loading conditions. Differences in the failure stress of the two tes types emphasized the importance of selecting a test method that simulates, in so far as possible, the anticipated in-service conditions of repair material. It was concluded that both the slant shear test method and the pipe nipple grips uniaxial tensile test method can be used to screen and select repair materials of the type investigated (portland cement concrete or latex modified concrete) for overlaying or patching portland cement concrete.

\section{0,531}

\section{PB91-203836}

Not available NTIS

National Inst. of Standards and Technology (NEL), Gaithersburg, MD. Building Materials Div.

Activities of the Construction Materials Reference Laboratories Related to Laboratory Accreditation. Final rept.

J. H. Pielert. $1989,7 \mathrm{p}$

Sponsored by American Association of State Highway and Transportation Officials, Washington, DC.

Pub. in Accreditation Practices for Inspections, Tests, and Laboratories, ASTM STP 1057, p30-36 1989

Keywords: "Construction materials, Test facilities, Aggregates, Concretes, Standards, Reprints, “Laboratory accreditation.

A conference was held at the National Institute of Standards and Technology (formerly The Nationa Bureau of Standards) in May 1986 to consider the status of existing evaluation and accreditation programs for construction materials testing laboratories and to determine the need for a coordinated national system. The conference attendees concluded that there is a need for a coordinated national system for the accreditation of construction materials testing laboratories and its development should be initiated.' The paper will discuss activities since the conference, including the work of a planning committee formed to define the goals, scope, format, and procedures of such a system. There will be particular emphasis on activities of CCRL and AMRL, which make up the Con struction Materials Reference Laboratories. They are research associate programs at NIST sponsored by ASTM and AASHTO, respectively, and provide laboratory assessment and proficiency sample services, but do not accredit laboratories. However, the AASHTO Executive and Policy Committee approved the AASHTO Accreditation Program utilizing AMRL technical programs for implementation in mid-1988. The Ex ecutive Group of the Joint ASTM C1/C9 Subcommittee on the CCRL is changing CCRL programs for concrete and concrete aggregates to comply with new ASTM laboratory evaluation standards.

\section{0,532}

\section{PB91-240804}

PC A03/MF AO

National Inst. of Standards and Technology (BFRL) Gaithersburg, MD.

Guidelines for the Development of Computer Based Models in a Cementitious Materials Modeling Laboratory.

L. J. Kaetzel, J. R. Clifton, and L. J. Struble. Aug 91, 35 p NISTIR-4650

Prepared in cooperation with Illinois Univ. at UrbanaChampaign. Dept. of Civil Engineering.

Keywords: "Cements, "Materials tests, "Mathematica models, "Computerized simulation, Construction ma terials, US NBS, Software engineering, Recommendations, Standards, National science Foundation, Data bases, Equipment interfaces, Computer networks.

The paper presents guidelines and considerations for the development, archiving, and distribution of computer models for a centralized cementitious materials modeling laboratory. An analysis of the approach used by cement researchers to develop large complex computer models reveals the need for guidelines in selecting computer platforms, software languages and tools, software engineering and documentation. A modeling laboratory established at NIST associated with the NSF Center for Science and Technology of Advanced Cement-Based Materials is discussed and the important of such a facility in promoting the exchange of information (i.e. ideas, models, data). The computer models currently archived in the modeling laboratory are used as test cases to describe the facility.

\section{0,533}

PB92-116300

$\mathrm{PC} \mathrm{A03/MF} \mathrm{A01}$

National Inst. of Standards and Technology (BFRL), Gaithersburg, MD

Uniaxial Tensile Tests to Measure the Bond of In situ Concrete Overlays.

R. G. Mathey, and L. I. Knab. Oct 91, 37p NISTIR-

4648

See also PB90-204520. Sponsored by U.S. Army Corps of Engineers, Washington, DC, Naval Facilities Engineering Command, Alexandria, VA, Air Force Engineering and Services, AFB, Washington, DC.

Keywords: "Pavement overlays, "Concrete pavements, "Bonding strength, "Tension tests, Tensile properties, Concrete slabs, Dissimilar materials bonding, Concretes, Mechanical properties, Tensile strength, Concrete durability, Test facilities, Mechanical tests.

The feasibility of two in situ tensile test methods for use in the field to measure the bond of concrete overlays was investigated in the laboratory. The two test methods used pneumatic and hydraulic loading apparatuses. The uniaxial tensile tests were conducted using partial-depth in situ cores drilled through overlay concrete and into previously cast slabs. Comparisons of the magnitude and repeatability of the tensile strength results for the two test methods indicated that they were comparable. The study demonstrated that both the pneumatic and hydraulic test methods are applicable for field use for measuring the tensile strength of the bond between a relatively thick overlay and its base concrete.

\section{Highway Engineering}

\section{0,534}

PB91-187849

PC A04/MF A01

National Inst. of Standards and Technology (NEL), Gaithersburg, MD. Center for Building Technology. Proposed Design Criteria for Shallow Bridge Foundations.

F. Y. Yokel. Feb 90, 57p NISTIR-90/4248

Sponsored by Federal Highway Administration, McLean, VA. Office of Research, Development, and Technology.

Keywords: "Bridge foundations, "Design criteria, "Settlement(Structural), Loads(Forces), Structural engineering, Highway bridges, Displacement, Bearing capacity, Ultimate strength, Failure, Stresses.

Criteria for the design of spread footings for highway bridges are proposed. The criteria address working load as well as load and resistance factor design (LRFD) procedures. Importance factors to be used in conjunction with the LRFD design format are proposed. The importance factors increase the design loads as the span length increases and also otherwise account for the severity of the consequences of a structural or foundation failure. Further data on LRFD design will be available from studies presently in progress. Available information on tolerances of highway bridges and other structures to foundation displacements are reviewed. On the basis of the information, allowable foundation-displacement limits are proposed. Unconditionally allowable foundation displacements will not affect the strength and serviceability of bridges and therefore do not require structural design modifications. 


\section{COMBUSTION, ENGINES, \& PROPELLANTS}

\section{Combustion \& Ignition}

\section{0,535}

\section{PB91-134510}

Not available NTIS National Bureau of Standards (NEL), Gaithersburg, MD. Fire Measurement and Research Div. Aspects of Smoldering Combustion.

Final rept.

T. J. Ohlemiller. 1987, 9p

Pub. in Proceedings of International Conference on the Physical and Chemical Processes Occurring in a Burning Cigarette, Winston-Salem, NC., April 26-29, 1987, p346-354.

Keywords: "Combustion, Reprints, "Cigarettes, Fire models.

Several application areas of smoldering combustion phenomena are reviewed and inter-compared to demonstrate the range of length and time scales possible, as well as the dependence of controlling factors on the configuration of the smolder wave. Existing models for these same application areas are briefly examined.

\section{0,536}

PB91-144428

PC A04/MF A01

National Inst. of Standards and Technology (NML) Boulder, CO. Chemical Engineering Science Div.

Ignition Characteristics of the Nickel-Based Alloy UNS N07001 in Pressurlzed Oxygen.

J. W. Bransford, and P. A. Billiard. Sep 90, 53p NISTIR-3947

Prepared in cooperation with Boeing Co., Seattle, WA Sponsored by National Aeronautics and Space Administration, Huntsville, AL. George C. Marshall Space Flight Center. Keywords: "Ignition, "Laser heating, "Waspaloy, lasers, Nickel alloys, Graphs(Charts), Oxygen supply equipment, Pressurized cabins.

The development of ignition and combustion in pressurized oxygen atmospheres was studied for the nickel-based alloy UNS N07001. Ignition of the alloy was achieved by heating the top surface of a cylindrical specimen with a continuous-wave $\mathrm{CO} 2$ laser. Two heating procedures were used. In the first, laser power was adjusted to maintain an approximately linear increase in surface temperature. In the second, lase power was periodically increased until autoheating (self-heating) was established. It was found that the alloy would autoheat to combustion from temperatures below the solidus temperature. In addition, the alloy had a tendency to develop combustion zones (hot spots) at high oxygen pressures when the incremental (step) heating test mode was used. Unique points on the temperature-time curves that describe certain events are defined and the temperatures at which these events occur are given for the oxygen pressure range of 1.72 to $13.8 \mathrm{MPa}$ (250 to $2000 \mathrm{psia}$ ).

100,537

PB91-147280

Not available NTIS National Bureau of Standards (NML), Gaithersburg. MD. Chemical Kinetics Div.

Mechanlsm of Soot Formation in AcetyleneOxygen Mixtures.

Final rept.

M. Frenklach, D. W. Clary, W. C. Gardiner, S. E.

Stein, and T. Yuan. 1986, 37p

Pub. in Combustion Science and Technology $50, n 1-3$ p79-115 1986.

Keywords: "Acetylene, "Soot, "Oxygen, "Reaction kinetics, Computation, Decomposition reactions, Pyrolysis, Chemical reactions, Chemical radicals, Shock tubes, Reprints.

A computational study of the chemical kinetic effects of oxygen addition on the process of soot formation from hot acetylene is reported. The results, which are supported by trends observed in shock-tube experiments, reveal that the reaction pathway to soot identified previously for acetylene pyrolysis remains essentially unchanged in a oxidative environment. The main effects of oxygen are: (1) promotion of fuel decomposition, which slightly alters the initiation route to soot; (2) supplementary rapid production of hydrogen atoms in the initial, small-molecule reactions, which drives the concentration of hydrogen above the equilibrium value with respect to $H(2)$ and thus enhances polymeric growth of polycyclic aromatics; (3) oxidation of aromatic radicals by molecular oxygen, which removes them from the polymeric growth. The computational results indicate a crucial need for kinetic studies of high-temperature reactions between molecular oxygen and hydrocarbon radicals.

100,538

PB91-147306

Not available NTIS

National Bureau of Standards (NEL), Gaithersburg $M D$. Fire Science and Engineering Div.

Radiant Heat Fluxes from 100-200 MW Natura Gas/Air Diffusion Flames.

Final rept.

J. P. Gore, G. M. Faeth, D. Evans, and D. B.

Pienning. 1987, 4p

Pub. in Proceedings of Fall Technical Meeting Chemical and Physical Processes in Combustion, San Juan, PR., December 15-17, 1986, p21.1-21.4 1987.

Keywords: "Diffusion flames, "Thermal radiation, "Radiative heat transfer, "Natural gas, "Heat flux, Heat transfer, Combustion, Flame propagation, Flames, Flame temperature, Radiant flux density, Reprints.

Extended abstract on the calculation of radiation from 100-200 MW natural gas/air diffusion jet flames.

100,539

PB91-147934

Not available NTIS

National Bureau of Standards (NEL), Gaithersburg MD. Chemical Process Metrology Div.

Velocity and Droplet Size Measurments in a Fue Spray.

Final rep

C. Presser, A. K. Gupta, R. J. Santoro, and H. G.

Semeriian. $1986,11 p$

Sponsored by Department of Energy, Washington, DC Pub. in Proceedings of AIAA Aerospace Sciences Meeting (24th), Reno, NV., January 6-9, 1986, p1-11.

Keywords: "Kerosene, "Flame spraying "Drops(Liquids) "Particle size distribution, Air flow, Mass transfer, Light scattering, Swirling, Velocity, Reprints.

Experimental results are presented for size, number density and velocity of droplets in a pressure atomized hollow cone kerosene spray introduced into nonswirling and swirling air flow fields. Laser Doppler velocimetry has been employed to measure the axial, radial and tangential velocity components of the spray dropets, introduced at the exit plane of a moveable-vane swirl-stabilized burner. Spatial distributions of droplet size and number density have been obtained by utilizing an ensemble light scattering technique, based on the measurement of the polarization ratio. Lorenz-Mie calculations of the scattering characteristics of a polydispersion of droplets have been carried out to complement the light scattering measurements. High speed cinematography was also used to observe the overall qualitative features of the spray and flame. The results reveal that swirl has a significant influence on the spray jet spread downstream of the nozzle where fuel/air mixing occurs. Swirl modifies both the drople size and number density, in addition to the expected modification of the velocity distribution. A large number of droplets are captured by the centrally located recirculation region and returned back upstream. These droplets are then transported radially outwards into the surrounding combustion air. The correlation between fuel droplet size and velocity due to its interaction with the swirled combustion air is of major importance toward understanding the phenomenological trends associated with spray flames.

\section{0,540}

PB91-148056

Not available NTIS

National Inst. of Standards and Technology (NEL) Gaithersburg, MD. Fire Measurement and Research Div.
Signal Detection Efficiency in Multiphoton Ionization Flame Measurements.

Final rept.

K. C. Smyth and P. J. H. Tjossem. 1990, 8p

Pub. in Applied Optics 29, n33 p4891-4898, 20 Nov 90.

Keywords: "Diffusion flames, Multi-photon processes, Signal detection, Carbon monoxide, Argon, Methane Fluorescence, Reprints, "Multiphoton ionization.

Multiphoton ionization is often the most sensitive method available for detecting radical species in flame environments. To make accurate relative concentration measurements, however, the electron (or ion) detection efficiency as a function of flame position must be known. Two methods are presented for determining this quantity in a laminar $\mathrm{CH}$ 4/air diffusion flame burning at atmospheric pressure. The results show significant variation of the electron detection efficiency in the lean, stoichiometric, and rich flame regions, with the greatest detection sensitivity observed in the high-temperature, primary reaction zones (i.e., near stoichiometric conditions). Corrections to multiphoton ionization data obtained for $\mathrm{H}$ atoms are discussed in terms of determining relative concentration profiles across the methane/air diffusion flame.

100,541

PB91-149013

Not available NTIS National Bureau of Standards (NEL), Gaithersburg. MD. Thermophysics Div.

Laser Diagnostics for Characterization of Fuel Sprays.

Final rep

C. Presser, A. K. Gupta, R. J. Santoro, and H. G. Semeriian. 1987, 8p

Sponsored by Department of Energy, Washington, DC Pub. in Proceedings of International Congress on Applications of Lasers and Electro-Optics (5th), ICALEO '86, Arlington, VA., November 10-13, 1986, p160-167 1987 .

Keywords: "Fuel sprays, Drop size, Density(Number/ volume), Light scattering, Laser applications, Combustion, Reprints, Phase Doppler velocimetry, Deconvelution.

Experimental measurements are presented for a pres sure-atomized hollow cone spray introduced into nons wirling and swirling air flow fields. An ensemble light scattering technique, based on measurement of the polarization ratio, is used to obtain detailed information on mean droplet size and number density in dense re gions of the spray. The results reveal that combustion air swirl has a significant influence on the spray struc ture. Mean droplet size on the spray boundary is found to be larger than that on the centerline for both the nonswirling and swirling cases.

\section{0,542}

PB91-157180

PC A12/MF A02

California Inst. of Tech., Pasadena. Guggenheim Je Propulsion Center.

Species Produced in Fires Burning in Two-Layered and Homogeneous Vitiated Environments.

Rept. for 1 Aug 89-31 Jul 90.

J. H. Morehart, E. E. Zukoski, and T. Kubota. Dec $90,275 p$ NIST/GCR-90/585

Grant NANB900958

Sponsored by National Inst. of Standards and Technology (NEL), Gaithersburg, MD. Center for Fire Research.

Keywords: "Combustion products, "Diffusion flames, "Stoichiometry, Natural gas, Plumes, Fires, Combustion, Ethylene, Chemical reactions, Air, Pyrolysis.

The chemical species produced in a buoyant, turbulen diffusion flame exposed initially to a supply of fresh air and extending into a reduced-oxygen environment containing products of combustion are investigated. The stably stratified, vitiated region is formed by placing a hood above a burner so that it accumulates the gases of the fire plume, while the direct injection of air into the upper portion of the hood allows conditions to be studied where the stoichiometry of the collected gases is different than that of the plume flow crossing the interface between these two regions. Measurements of the composition show that the species produced in the flame depend primarily on the stoichiometry of the gases in the vitiated region, but are independent of the fuel-air ratio of the mass transported across the interface by the plume. Experiments were conducted with natural gas, ethylene, and propylene fuels. For natural gas fires, a weak dependence of species con- 


\section{Combustion \& Ignition}

centrations on the temperature of the product gas layer was observed over the range 500 to $900 \mathrm{~K}$.

\section{0,543}

\section{PB91-157206} neering.

Fire Propagation in Concurrent Flows.
Final progress rept. 1 Aug $89-31$ Jul 90.

A. C. Fernandez-Pello. Dec 90, 133p NIST/GCR-90/

586

Grant NANB7D0737

See also PB90-151754. Sponsored by National Inst. of Standards and Technology (NEL), Gaithersburg, MD. Center for Fire Research.

Keywords: "Flame propagation, "Fire tests, "Air flow, Flames, Mathematical models, Experimental data Combustion, Ignition, Burning rate, Turbulent flow, Velocity measurement.

A research program is being conducted to study the mechanisms controlling the spread of fire in turbulent forced gas flows moving in the direction of flame propagation. The research tasks completed during the reporting period include experimental studies of the effect of flow turbulence on the rate of concurrent flame spread, and of mass burning when the combustible material is in a floor configuration. The results of the experiments with thick PMMA sheets show that flow turbulence affects significantly the flame spread and mass burning processes. As the turbulence intensity is increased, the flame spread rate decreases because the flame length sharply decreases, and the mass burning rate increases because the surface heat flux increases. Currently underway is a complementary study with the combustible material placed in a ceiling geometry to observe the effect of buoyancy on the flame spread and mass burning processes. An additional task also completed during the period is a review of the processes of ignition and flame spread of solid combustibles. The review presents a novel approach to the analysis and prediction of both processes based in their close interrelationship.

\section{0,544}

\section{PB91-174474}

Not available NTIS

National Bureau of Standards (NEL), Boulder, CO. Chemical Engineering Science Div.

Ignition Characteristics of Selected SSME Alloys. Final rept.

J. W. Bransford, P. A. Billiard, J. A. Hurley, and I.

Vasquez. 1986, 21p

(ndics and Space Administration, Huntsville, AL. George C. Marshall Space Flight Center.

Pub. in Proceedings of NASA Conference on Advanced Earth-to-Orbit Propulsion Technology, Huntsville, AL., May 13-15, 1986, p366-386.

Keywords: "Heat resistant alloys, "Metals, "Combustion, "Ignition temperature, Laser heating, Differential thermal analysis, Phase transformations, Oxygen supply systems, Hyperbaric oxygenation, Oxides, Reprints.

Data from the measurement of the surface and interior temperatures of laser heated cylindrical specimens and from Differential Thermal Analysis (DTA) thermograms have shown that the ignition of alloys is a complex process. The complexity of the process arises from the numerous complex oxides that are formed during the heating process prior to alloy ignition. The ignition process can involve one or more phase transitions and can also be dependent upon the experimental procedures. The ignition temperature as a function of oxygen pressure has been determined for a series of alloys used in or proposed for use in high pressure oxygen systems. The results for five alloys - UNS presented and discussed.

\section{0,545
PB9 $1-174797$}

Not available NTIS

National Bureau of Standards (NEL), Gaithersburg, MD. Center for Fire Research.

Letter to the Editor, Fire Technology, Comments on T.Z. Harmathy Viewpoint, May 1985.

D. Gross. $1985,3 p$

Pub. in Fire Technology 21, n4 p324-326 Nov 85.

Keywords: "Heat transfer, "Absorptivity, "Inertia, "Fires, Heat flux, Heat transmission, Transport properties, Thermodynamic properties, Absorption, Reprints.
Comments on Viewpoint by T.Z. Harmathy, Fire Technology, May 1985 (rho) or the square root of K(rho)c-Thermal Inertia or Thermal Absorptivity.

\section{0,546}

PB91-175331

Not available NTIS National Bureau of Standards (NEL), Gaithersburg, MD. Fire Safety Technology Div.

Surface Flame Spread.

Final rept.

J. G. Quintiere. $1988,8 p$

Pub. in SFPE (Society of Fire Protection Engineers) Handbook of Fire Protection Engineering, Chapter 24 p360-367 1988 .

Keywords: "Flame propagation, "Fires, Flames, Combustion, Combustible flow, Combustion physics, Liquid fuels, Forest fires, Mathematical models, Burning rate, Reprints.

The contribution is intended for a proposed handbook currently being organized by a committee of the Society of Fire Protection Engineers. It attempts to address the student or practicing fire protection engineer by its tutorial format. It covers both opposed flow and windaided flame spread over solids in some detail. Approximate formulas are developed using simple assumptions in order to illustrate the role of the relevant physi$\mathrm{cal}$ and chemical variables. The relationship between these approximate formulas and more exact results and data are discussed. Although an extensive review is not presented, an attempt is made to illustrate the extent of knowledge and opportunities for application. Flame spread over liquid fuels and in the forest are briefly discussed.

\section{0,547}

\section{PB91-178830}

PC A03/MF A01

National Inst. of Standards and Technology (BFRL), Gaithersburg, MD.

FIREDOC Users Manual, 2nd Edition.

N. H. Jason. Mar 91, 36p NISTIR-4526

See also PB90-271800.

Keywords: "Fire research, "Information dissemination, "Information retrieval, "User manuals(Computer programs), Data bases, Bibliographies, US NIST, On line systems, "FIREDOC systems, "Fire Research Information Services.

FIREDOC is the on-line bibliographic database which reflects the holdings (published reports, articles, books, and audiovisual items) of the Fire Research Information Services (FRIS), at the Building and Fire Research Laboratory, National Institute of Standards and Technology. The manual provides a step-by-step technique for entering and exiting the database via telecommunication lines, as well as a number of techniques for searching the database and processing the results of the searches. The Second Edition incorporates changes from a recent STAR sof tware upgrade, a section of Advanced Techniques and procedures for using FIREDOC with an IBM PC, XT, AT and compatible computers and with a Macintosh computer.

100,548
PB9 1-203794

Not available NTIS

National Inst. of Standards and Technology (NEL) Gaithersburg, MD. Fire Measurement and Research Div.

Kinetic Properties of the Components of DouglasFir and the Heat of Combustion of Their Volatile Pyrolysis Products.

Final rept.

W. J. Parker, and S. L. LeVan. 1989, 17p

Pub. in Wood and Fiber Science 21, n3 p289-305 1989.

Keywords: "Douglas fir wood, "Pyrolysis, "Combustion products. "Heat of combustion, Cellulose, Lignin, Xylans, Nitrogen, Activation energy, Mass balance, Reprints.

Specimens of the chemical components of Douglas-fir which include cellulose, lignin, mannan, and xylan were pyrolyzed in nitrogen in the temperature range between 290 and $370 \mathrm{C}$, which is characteristic of the pyrolysis temperature range of wood in a fire environment. From the data the effective activation energy, preexponential factor, and the net heat of combustion of the volatiles were calculated as a function of mass retention fraction of the specimen. The kinetic parameters were used to calculate the mass loss rate of Douglas-fir and a prepared mixture of the components in a pyrolyzer based on their measured temperature history. The calculated mass loss rates were compared with the measured values.
100,549

PB91-203851

Not available NTIS

National Inst. of Standards and Technology (NEL), Gaithersburg, MD. Fire Measurement and Research Div.

Wind Effects on Fires.

Final rept.

W. M. Pitts. 1991, 52p

Sponsored by Defense Nuclear Agency, Washington, DC.

Pub. in Progress in Energy and Combustion Science 17. $\mathrm{p} 83-1341991$.

Keywords: "Fires, "Urban areas, "Nuclear explosion effects, Firestorms, Wind(Meteorology), Models, Reprints.

In the review characteristics of the two types of mass fire--fire storm and conflagration--are discussed. Brief histories of urban mass fire and research efforts on the topic are given. Models which have been developed to predict the initiation, development, spread, and behavior of mass fires following the detonation of a nuclear device in an urban environment are summarized. The current understanding of the fire processes which are believed to control mass fire behavior are reviewed. Particular emphasis is placed on the wind-fire interactions mentioned in the last paragraph. The discussion forms the basis for an analysis of the effectiveness of existing models for mass fire growth and behavior. It is concluded that the understanding of the important physical processes is incomplete and that models for mass fire development and behavior are likely to be subject to large and uncharacterized errors. The possiblity of improving our understanding of the underlying physical and chemical processes utilizing reducedscale experiments is assessed.

100,550

PB91-203869

Not available NTIS

National Inst. of Standards and Technology (NEL), Gaithersburg, MD. Chemical Process Metrology Div. Effect of Atomization Air on Droplet Dynamics of Spray Flames.

Final rept.

C. Presser, A. K. Gupta, and H. G. Semerjian. 1988, $4 p$

Sponsored by Department of Energy, Washington, DC. Pub. in Proceedings of Fall Technical Meeting on Chemical and Physical Processes in Combustion, Clearwater Beach, FL., December 5-7, 1988, p105-1. $105-4$

Keywords: "Atomization, "Sprays, "Flames, "Droplets, "Dynamics, Combustion, Spatial distribution, Aerodynamic characteristics, Flow visualization, Reprints, Atomization air.

Fuel spray combustion is an important part of a wide variety of propulsion and power systems such as furnaces and gas turbine combustors, afterburners, fuelinjection internal combustion engines, liquid rocket engines, etc. Recent studies using air-assist nozzles have shown that the design and fabrication of these nozzles can directly influence spray circumferential uniformity, i.e., the presence of asymmetrical fuel flux profiles in combustors. The practical implications of these fuel flux nonuniformities are that they seriously alter the spray structure, which subsequently affects droplet/air interactions, local fuel/air mixing, overall flame characteristics and combustor performance, and pollutant emission levels. In addition, the effect of aerodynamic factors (i.e., combustion air swirl and atomization air flow rate) on spray characteristics has been investigated. The paper discusses the effect of atomization air on the droplet dynamics of spray flames formed by an air-assist nozzel. Presented are spatial distributions of mean droplet velocity and their probability distributions, which provide quantitative information for examination of the observed spray flame features.

100,551

PB91-216804

PC A03/MF A01

National Inst. of Standards and Technology (BFRL), Gaithersburg, MD.

Interaction of an Isolated Sprinkler Spray and a Two-Layer Compartment Fire Environment.

L. Y. Cooper. May 91, 49p NISTIR-4587

Sponsored by American Architectural Mirs. Association, Des Plaines, IL 
Keywords: "Fire tests, "Sprinkler systems, Spraying, Mathematical models, Nozzles, Computerized simulation, Water, Combustion, Buildings.

A mathematical model is developed to simulate the interaction of an isolated operating sprinkler and a two-layer fire environments under arbitrary conditions of sprinkler-nozzle elevation, and upper-and lowerlayer thickness and temperature. The sprinkler is characterized by water flow rate, nozzle diameter, and three other device parameters. The model takes account of all effects of the sprinkler spray as it entrains drives downward (by aerodynamic drag on the spray drops), humidifies, and cools (by drop evaporation) gases from both the high temperature upper layer and the relatively cooler lower layer. The model provides a means of predicting the rates of flow of mass, enthalpy, products of combustion, and evaporated water to each of the two layers as a result of sprinkler operation. An algorithm for such predictions is presented in a manner that is suitable for general use in two-layer zone-type compartment fire models. The model is ex ercised in example calculations which simulate the interaction between the spray of a real sprinkler device and both fire and non-fire environments. The calcula tions revealed an important generic interaction phenomenon, namely, an abrupt and large change in the growth rate of an upper layer that would accompany an increase in upper layer thickness beyond a critica thickness (for a given upper layer temperature) or an increase in upper layer temperature beyond a critical temperature (for a given upper layer thickness). Exceeding critical values would lead to very large rate of growth of upper layer thickness, a growth that would ikely lead to rapid and complete smoke filling of even the largest compartments of fire origin.

\section{0,552}

\section{PB91-216838}

PC A99/MF E08

National Inst. of Standards and Technology (CSTL), Gaithersburg, MD

ICLASS-91 Proceedings of the International Conference on Liquid Atomization and Spray Systems (5th). Held in Gaithersburg, MD. on July 15-18, 1991.

Special pub. (Final)

H. G. Semeriian. Jul 91, 874p NIST/SP-813

Also available from Supt. of Docs. as SN003-00303086-4.

Keywords: "Meetings, "Atomization, "Sprays, "Liq uids, Atomizers, Diesel engines, Spraying, Atomizing, Fuel systems, Fuel sprays, Metal powder, Combustion, Size determination, Drops(Liquids).

The NIST Special Publication contains the Proceed ings of the Fifth International Conference on Liquid Atomization and Spray Systems, sponsored and organized by the Institute for Liquid Atomization and Spray Systems (ILASS-Americas) and the National Institute of Standards and Technology (NIST), held at NIST, in Gaithersburg, Maryland on July 15-18, 1991. One hundred papers were presented at the Conference, including four keynote lectures on Physics of Atomization, Experimental and Theoretical Studies on the Structure of Fuel Sprays in Diesel Engines, Atomization of Liquid Metals for the Manufacture of Metal Powder and for Spray Forming, and Twin Fluid Atomization: Factors Influencing Mean Drop Size. Technical sessions were organized on Evaporation and Mass Transfer in Sprays, Spray Coating, Basic Atomization Processes Pressure Atomizers, Diesel Sprays, Drop Size Distribu tion of Sprays, Novel Atomizers, Diagnostics, Atomization of Special Liquids, Spray Combustion, Twin Fluid Atomization, Spray Modeling, Airblast and Electrostatic Atomization, and Airbreathing and Rocket Engines.

\section{0,553}

PB91-237024

Not available NTIS

National Inst. of Standards and Technology (NEL) Gaithersburg, MD. Fire Measurement and Research Div.

Partial Equilibrium in Laminar Hydrocarbon Diffusion Flames.

Final rept.

J. H. Miller, and K. C. Smyth. 1988, $4 p$

Pub. in Chemical Physics Process Combustion, p16/1 $16 / 41988$.

Keywords: "Diffusion flames, "Hydrogen, "Oxygen, "Atoms, Hydroxyl radicals, Hydrocarbons, Equilibrium, Chemical radicals, Reprints, Flame structure, Combustion chemistry.

Possible methods of estimating $\mathrm{H}$-atom and $\mathrm{O}$-atom concentrations from $\mathrm{OH}$ - and major species measure- ments are discussed. Assumptions of full and partial equilibrium are examined.

\section{0,554}

PB9 1-237099

Not available NTIS

National Inst. of Standards and Technology (BFRL) Gaithersburg, MD. Fire Measurement and Research

Laser-Induced Fluorescence of CH. in a Laminar CH4/Air Diffusion Flame: Implications for Diagnos tic Measurements and Analysis of Chemical Rates. Final rept.

T. S. Norton, and K. C. Smyth. 1991, 20p

Pub. in Combustion Science and Technology 76, p1. 201991

Keywords: *Methane, "Diffusion flames, "Combustion kinetics, Laser applications, Free radicals, Aromatic polycyclic hydrocarbons, Concentration(Composition) Hydrogen atoms, Reprints, "Laser induced fluorescence, "Methyl radicals.

Relative $\mathrm{CH}$ radical concentration profiles have been measured in a laminar, flowing methane/air diffusion flame using laser-induced fluorescence from the $(0,0)$ band of the A(sup 2)delta-.- $>$ X(sup 2) pi transition. $\mathrm{CH}$ has the narrowest profile of any species yet measured in the flame. The $\mathrm{CH}$ peak does not coincide with the position of the $\mathrm{OH}$ and temperature maxima, but ap pears on the fuel-rich side, between the peak concentrations of hydrogen atoms and methyl radicals. If $\mathrm{CH}$ fluorescence is detected with a narrow bandpass dielectric filter, rather than using a monochromator, severe interference attributed to fluorescence from polycyclic aromatic hydrocarbons (PAH) is observed. The $\mathrm{CH}$ concentration decreases, while $\mathrm{PAH}$ fluorescence interference increases rapidly with height above the burner Implications for $\mathrm{CH}$ imaging experiments in turbulent diffusion flames are discussed. The net chemi$\mathrm{cal}$ production/destruction rate of $\mathrm{CH}$ is determined as a function of flame position at a height $\mathrm{H}$ of $9 \mathrm{~mm}$ above the burner. The $\mathrm{CH}$ production rate profile is used to derive a relative mole fraction profile for triplet methylene $3 \mathrm{CH}_{2}$. The concentration ratio (3) $\mathrm{CH} 2: \max /(\mathrm{CH}) \max$ is found to be $13-300$, where the major source of uncertainty is the reaction rate of (3) $\mathrm{CH}_{2}:+\mathrm{H}-\rightarrow \mathrm{CH}+\mathrm{H}_{2}$. Maximum concentrations of the $\mathrm{CH}$, radicals are not well established in the flame. However, if the derived $((3) \mathrm{CH} 2:) \max /(\mathrm{CH}$.) max ratio is combined with the calculated value of $25 \mathrm{ppm}$ for $((3) \mathrm{CH} 2$ ) max from Puri et al. (1987), one estimates that the peak $\mathrm{CH}$ mole fraction lies between 0.08 and 2 ppm at $\mathrm{H}-9 \mathrm{~mm}$.

\section{0,555}

PB91-237412

Not available NTIS

ND. Fire Sureau of Standards (NEL),

Experimental Study of the Pyrolysis Rate of a Poly(methyl methacrylate) (PMMA) Wall Panel in a Reduced-Scale Enclosure.

Final rept.

K. D. Steckler, and H. E. Mitler. 1988, 4p

Pub. in Chem. Phys. Processes Combust., p73/1-73/4 1988.

Keywords: "PMMA, "Plastics, "Construction materials, *Pyrolysis, Combustion chambers, Reaction kinetics, Wallboard, Panels, Fire tests, Flammability, Mathematical models, Comparison, Ventilation, Linings, Reprints.

A series of experiments was conducted to study the pyrolysis rate of a fully-ignited fixed-area wall panel in a reduced-scale enclosure for a wide range of ventilation conditions. The wall panel was PMMA, a non-charring homogeneous material which is amenable to theoretical analysis. Experimental results are compared with theoretical results obtained from a steady-state algebraic model for non-charring, non-melting, homogeneous materials.

\section{0,556}

PB92-112432 PC A11/MF A03

Michigan State Univ., East Lansing. Dept. of Mechanical Engineering.

Theoretical Investigation of Piloted Ignition of Wood.

L. S. Tzeng, and A. Atreya. Aug 91, 230p NIST/

GCR-91-595

Grant NANB8D0861

Sponsored by National Inst. of Standards and Technology (BFRL), Gaithersburg, MD.

Keywords: "Wood fuels, "Combustion, "Pyrolysis, Solids, Flame propagation, Gases, Equations, Diffu- sion flames, Mathematical models, Activation energy, Ignition, Heat transfer, Predictions, Pilot lights.

A theoretical model for piloted ignition of a flame in the gas phase above a vaporizing or pyrolyzing solid has been developed. Using the model it has been found that (1) The postulated simplified governing equations adequately explain the pre-ignition flashes that are often observed experimentally; (2) A rational criterion for positioning the pilot flame exists: (3) The hea losses to the surface play an important role, indicating that the fuel evolution rate by itself is insufficient for predicting the onset of piloted ignition. In the investigation, a numerical integration scheme is developed tha accounts for the often vastly different rates between chemical reaction and convection or diffusion processes in the equations of combustion theory. The new numerical scheme is found to be very efficient for the piloted ignition problem, which involves both pre-mixed and diffusion flames. Finally, a numerical model for pi loted ignition of wood which includes transient solid phase decomposition has been developed. It has been found that the activation energy for the combustion of the evolved fuel is $49 \mathrm{Kcal} / \mathrm{mol}$.

100,557

PB92-112473

PC A04/MF A01

National Inst. of Standards and Technology (BFRL)

Gaithersburg, MD

Data for Fire Hazard Assessment of Selected NonHalogenated and Halogenated Fire Retardants: Report of Test FR 3983

R. H. Harris, V. Babrauskas, B. C. Levin, and M

Paabo. Oct $91,56 \mathrm{p}$ NISTIR-4649

Sponsored by Huber (J.M.) Corp., Norcross, GA Solem Div. and Aluminum Co of America, Alcoa Center, PA. Alcoa Technical Center.

Keywords: "Fire resistant materials, "Plastics, "Fire hazards, "Flammability testing, Halogens, Additives, Comparison, Toxicity, Calorimetry, Combustion prod ucts, Thermal degradation, Hydrogen chloride, Hydro gen bromide, Smoking, Ignition, Gases, Cone calorimeters.

Five plastic materials, with and without fire retardants, were studied to compare the fire hazards of non-halogenated fire retardant additives with halogenated flame retardants. The plastic materials were identified by the sponsors as unsaturated polyesters, thermoplastic high density, low density and cross-linked low density polyethylenes, polypropylene, flexible and rigid poly(vinyl chlorides), and cross-linked and thermoplas tic ethylene-vinyl acetate copolymers. The non-halo genated fire retardants tested were aluminum hydroxide $(\mathrm{Al}(\mathrm{OH}) 3)$ also known as alumina trihydrate (ATH) sodium alumino-carbonate, and magnesium hydroxide. The halogenated flame retardants were chlorine or bromine/antimony oxides. The plastics were stud red using the Cone Calorimeter and the cup furnace smoke toxicity method (high density polyethylene only). The Cone Calorimeter provided data on mass consumed, time to ignition, peak rate and peak time of heat release, total heat released, effective heat of combustion, average yields of $\mathrm{CO}, \mathrm{CO} 2, \mathrm{HCl}$, and $\mathrm{HBr}$, and average smoke obscuration. The concentrations of toxic gases generated in the cup furnace smoke toxicity method were used to predict the toxic potency of the mixed thermal decomposition products. The data from the Cone Calorimeter indicate that the non-halogenated fire retardants were, in most of the tested plastic formulations, more effective than the halogenated flame retardants in increasing the time to ignition. The non-halogenated fire retardants were also more effective in reducing the mass consumed, peak rate of heat release, total heat released, and effective heat of combustion, and in reducing the amount of smoke produced. The use of halogenated flame retardants increased smoke production and $\mathrm{CO}$ yields and additionally, produced the known acid gases and toxic irritants, $\mathrm{HCl}$ and $\mathrm{HBr}$, in measureable quantities.

\section{0,558}

PB92-117191

Not available NTIS

National Inst. of Standards and Technology (NEL) Gaithersburg, MD. Fire Measurement and Research Div.

Large-Scale Turbulent Structures and the Stabilization of Lifted Turbulent Jet Diffusion Flames. Final rept.

W. M. Pitts. $1990,8 p$

Pub. in Proceedings of International Symposium on Combustion/The Combustion Institute (23rd), Orleans, France, July 22-27, 1990, p661-668. 
Keywords: "Diffusion flames, "Vortices, "Jet flow, Turbulence, Rayleigh scattering, Turbulent flow, Light scattering, Velocity measurement, Flame photometry, Hot wire anemometers, Combustion stability, Reprints.

The physical mechanisms responsible for the stabilization of lifted axisymmetric turbulent jet diffusion flames remain uncharacterized. Flame stabilization has been shown to take place at radial locations in the corresponding isothermal jets where the flow has an intermittent character. Recently developed flow diagnostics capable of simultaneous multi-point concentration and velocity measurements are used to investigate the large-scale turbulent structures in the intermitten region of an isothermal jet of propane. The findings, in conjunction with earlier literature results, show that large-scale structures form as the result of organized turbulent motion during which strong outward ejections of fluid form central regions of the jet flow into the ambient surroundings. Once formed, the large-scale structures are convected downstream for long distances. The downstream edges of the structures are regions of significantly higher concentration gradients and shear than regions further upstream. Occasionally, the jet fluid loses its turbulent energy and is no longer transported by the flow. The fluid can be reentrained by the passage of later large-scale structures It is concluded that the processes responsible fo flame stabilization must occur in these large-scale turbulent structures.

100,559

PB92-117365

Not available NTIS

National Inst. of Standards and Technology (NEL) Gaithersburg, MD. Fire Measurement and Research Div.

Relative $\mathrm{H}$-Atom and O-Atom Concentration Meas urement in a Laminar, Methane/Air Diffusion Flame.

Final rept.

K. C. Smyth, and P. J. H. Tjossem. 1990, 9p

Pub. in Proceedings of Symposium on Combustion The Combustion Institute (23rd), France, July 22-27, 1990, p1829-1837.

Keywords: "Ionization, "Diffusion flames, "Concentration(Composition), "Fluorescence, "Laser
applications, Quantitative analysis, Chemical analysis Electrophotometry, Laser beams, Methane, Hydrogen, Flame photometry, Laser spectroscopy, Laminar flow, Oxygen, Reprints.

Relative $\mathrm{H}$-atom and $\mathrm{O}$-atom concentration profiles have been measured in a laminar, co-flowing methane/air diffusion flame burning at atmospheric pressure. Multiphoton ionization $(2+1$ process at $243 \mathrm{~nm})$ has been used to detect $\mathrm{H}$ atoms, while laser-induced fluorescence at $845 \mathrm{~nm}$ excited by two-photon absorption at $226 \mathrm{~nm}$ was employed to observe $\mathrm{O}$ atoms. In both cases it was found that low photon intensities were required in order to avoid the photolytic production of the species of interest. Doppler-free measure ments were carried out using a retroreflected-beam geometry in order to provide sufficient signal levels. Under these experimental conditions it is necessary to correct the raw profile data for variations in collisional quenching and electron detection sensitivity as a function of flame position. Establishing absolute radical concentrations is discussed in terms of partial equilibrium considerations and detailed flame structure calculations

\section{Fuel \& Propellant Tanks}

\section{0,560}

PB9 1-175570

Not available NTIS

National Inst. of Standards and Technology, Boulder CO. Chemical Engineering Science Div.

Reusable Cryogenic Foam Insulation for Advanced Aerospace Vehicles.

Final rept.

P. S. McAuliffe, A. H. Taylor, L. L. Sparks, and W. P. Dube. 1991, 12 p

Pub. in Proceedings of AIAA Aerospace Sciences Meeting (29th), Reno, NV., January 7-10, 1991, p1-12.

Keywords: "Propellant tanks, "Thermal insulation, "Cryogenic fluid storage, Cryogenic rocket propellants, Rocket propellants, Storage tanks, Fuel tanks, Thermal conductivity, Foams, Aerospace vehicles, Reprints.
Future high-speed aircraft and aerospace vehicles using cryogenic propellants will require an advanced reusable insulation system for the propellant tank structure. The cryogenic insulation system must be lightweight, structurally and thermally efficient, and capable of multiple reuse without cracking or degraded performance. The paper presents recent progress in the development of a reusable cryogenic foam insulation system having a maximum service temperature of $400 \mathrm{~F}$. The system consists of pre-shaped, pre-cut blocks of rigid polymethacrylimide foam insulation, wrapped with a high-temperature Kapton and aluminum foil vapor barrier which is adhesively bonded to the propellant tank well.

\section{Reciprocation \& Rotating Combustion Engines}

100,561

PB91-189498

Not available NTIS

National Inst. of Standards and Technology (IMSE) Gaithersburg, MD. Ceramics Div.

Ceramic Wear Maps.

Final rept.

S. M. Hsu, D. S. Lim, and R. G. Munro. 1988, 10p

Pub. in Proceedings of the International Symposium on Ceramic Materials and Components for Engines (3rd) Las Vegas, NV., November 27-30, 1988, p1236-1245

Keywords: "Wear, "Ceramics, *Graphic methods, *Tribology, "Heat engines, Friction, Mapping, Tests, Methodology, Reprints.

Friction and wear characteristics of ceramics are crucial properties for their successful application to new engine designs. Tests for friction and wear performance usually are based on empirical field trials. These tests are very expensive. Laboratory measurements of tribological properties would be much less expensive. However, laboratory tests are specific to a particular wear tester and the specific operating conditions. Translation of laboratory results to industrial application and design is often difficult and fraught with uncertainty. The paper describes a new systematic effort to measure and represent the wear characteristics of ceramics in a uniform and unitied methodology. For any material, the methodology leads to a set of wear maps which collectively provide a comprehensive representation of the wear properties of the materials. The maps provide critical guidance to design engineers for materials selection.

\section{COMMUNICATION}

\section{Common Carrier \& Satellite}

\section{0,562}

FIPS PUB 159

National Inst. of Standards and Technology (CSL), Gaithersburg, MD.

Detail Specification for 62.5-mum Core Diameter/ 125-mum Cladding Diameter Class la Multimode, Graded-Index Optical Waveguide Fibers.

Federal information processing standards (Final).

S. M. Radack, and A. G. Hanson. 27 Dec 90, 24p

Also pub. as American National Standards Inst., New York rept. no. ANSI/EIA/TIA-492AAAA-1989. Pre pared in cooperation with American National Standards Inst., New York, and National Communications System, Arlington, VA.

Three ring vinyl binder also available: North American Continent price \$7.00; all others write for quote.

Keywords: *Standards, "Optical waveguides, *Glass fibers, "Telecommunications, "Data processing systems, Cladding, Cores, Acceptability, Industries, Protective coatings, Performance tests, Measurement, Attenuation coefficients, Bandwidth, Length, Federal Information Processing Standards.

The standard, by adoption of American National Standard/EIA/TIA-492AAAA-1989, defines the opti- cal, geometrical, environmental, and mechanical specifications for glass (EIA/TIA-458-A-1984 Class la) multimode optical waveguide fibers. Minimum acceptable values for all characteristics are given, and applicable industry standards for their measurement are referenced. The standard supersedes former draft Federal Standard (FED-STD) 1070 in its entirety.

100,563

PB91-134825

Not available NTIS

National Inst. of Standards and Technology (NCSL), Gaithersburg, MD. Systems and Network Architecture Div.

Performance of OSI Transport over ACCUNET and IBERPAC.

Final rept.

E. Vazquez, R. Colella, J. Vinyes, J. Fox, and J.

Berrocal. 1988, 10p

Sponsored by Universidad Politecnica de Madrid (Spain). Escuela Tecnica Superior de Ingenieros de Telecomunicacion.

Pub. in Proceedings of Annual Joint Conference IEEE (Institute of Electrical and Electronics Engineers) Computer and Communications Societies - IEEE INFOCOM (7th), New Orleans, LA., March 27-31, 1988, p651-660.

Keywords: *Computer networks, Simulation, Specifications, Reprints, "Computer communications, "Protocols, Throughput.

The paper presents the results of a research project intended to assess the effect of several transport mechanisms on throughput observed between two transport users communicating over concatenated X.25 networks. Two main areas of effort are covered. First, the protocol design and development process is addressed, including the formal specification of class 4 transport and the tools used to support protocol implementation and testing. The main advantages of ESTELLE, the formal specification language used, and the development method are outlined. The second area covered is a set of simulated and live experiments investigating transport performance over X.25 networks. The transport mechanisms of potential interest (splitting, concaternation and acknowledgement withholding) are described and the main simulation results are presented. Finally, the simulation results are validated with live experiments over two concatenated X.25 networks. As indicated in the conclusion, use of the three mechanisms considered in the paper result in significant transport user throughput improvements.

100,564

PB91-148734

Not available NTIS

National Inst. of Standards and Technology (NEL), Boulder, CO. Electromagnetic Fields Div.

Quasi-Static Analysis of a Two-Wire Transmission Line Located at an Interface.

Final rept

D. A. Hill. $1990,6 p$

Pub. in Radio Science 25, n4 p435-440 Jul/Aug 90.

Keywords: "Transmission lines, Characteristic impedance, Permeability, Permittivity, Interfaces, Reprints.

Simple quasi-static expressions have been derived for the propagation constant, the characteristic impedance, and the field distribution of a two-wire transmission line located at the air-Earth interface. Both the complex permittivity and the complex permeability of the Earth are allowed to differ from the free-space values, and a numerical solution of the mode equation shows that quasi-static approximation is valid when the wire separation is much less than a free-space wavelength. The quasi-static approximation can be used to determine both the complex permittivity and the complex permeability of the Earth from measurements of the propagation constant and the characteristic impedance of the transmission line.

100,565

PB91-158584

Not available NTIS National Bureau of Standards (NEL), Gaithersburg, MD. Building Equipment Div.

Toward Modular Semantic Application Layer Protocols (MS-ALPs): Communicatlon between Heterogeneous Information Systems Using Artificial In. telligence (AI) Programming Techniques.

Final rept.

W. F. Danner. 1986, 10p

Pub. in Proceedings of Annual Technical Symposium (25th) Distributed Information Systems: Emerging 
Uses and Technology, Gaithersburg, MD., June 12 , 1986, p63-72

Keywords: "Computer communications, "Protocols, *Artificial intelligence, Information systems, Computer aided design, Data base management systems, Distributed computer systems, Computer programming, Knowledge representation, LISP programming language, Reprints, Object-oriented programming.

A communication capability between incompatible information systems is presented. Formats for Modular Semantic Application Layer Protocols (MS-ALPs) are described. A Meta-Information (MI) Format supports the exchange of knowledge necessary for each system to understand the other. Subsequently an Information (I) Format provides for the exchange of information in the context of that knowledge while maintaining system semantics. The MS-ALP Formats make use of two artificial intelligence (AI) programming techniques: frame-based knowledge representation; and object-oriented programming capabilities as an integral part of the frame-based representation. These 'self-descriptive' formats provide for a virtual extension of an information management system (IMS) which does not require a detailed understanding of IMS operations for access to information. An implementation is described which operates within LISP programming environments. However, the concepts developed during the course of this research are believed to have wider applicability.

\section{0,566}

PB91-175018 Not available NTIS National Bureau of Standards (ICST), Gaithersburg, MD. Systems and Network Architecture Div. Appllcation of Formal Description Techniques to the Specification of Distributed Test Systems. Final rept.

R. J. Linn, and J. P. Favreau. 1988, 14p

Pub. in Proceedings of IEEE INFOCOM Conference on Computer Communications, New Orleans, LA., March 27-31, 1988, p96-109.

Keywords: "Distributed processing, "Tests, "Electronic mail, Specifications, Protocols, Distributed computer systems, Translators, Computer networks, Computer communications, Reprints, Department of Defense, OSI(Open Systems Interconnection).

As part of a planned transition by the Department of Defense (DoD) from military standard to Open Sys tems Interconnection (OSI) protocols, the National Bureau of Standards (NBS) is implementing and testing two application-layer gateways. NBS is applying description techniques developed within the International Organization for Standardization (ISO) and the Consultative Committee for International Telephone and Telegraph (CCITT) to the specification of distributed test systems for the gateways between OSI and DoD networks. Translators, developed at NBS, for Estelle and ASN.I aid in automating the implementation of the test systems. An overview of the test system for an electronic mail gateway and the methods employed to realize it are presented. Measures of productivity of the methods employed are also presented.

\section{0,567}

\section{PB91-187682}

Order as PB91-187617, PC A06/MF A01) National Inst. of Standards and Technology, Boulder, CO.

Limited International Intercomparison of Responsivity Scales at Fiber Optic Wavelengths.

R. L. Gallawa, J. L. Gardner, D. H. Nettleton, K. D.

Stock, T. H. Ward, and X. Li. 1991, 6p

Prepared in cooperation with National Measurement Lab., Lindfield (Australia), National Physical Lab., Teddington (England), and Physikalisch-Technische Bundesanstalt, Brunswick (Germany, F.R.)

Included in Jnl. of Research of the National Institute of Standards and Technology, v96 n2 p225-245 Mar/Apr 91.

Keywords: "Optical communication, Near infrared radiation, $\mathrm{Ge}$ semiconductor detectors, Interlaboratory comparisons, Optical detectors, Optical fibers, Fiber optics, Standards, Optical power, Intercomparison.

The authors report here on a recent limited international intercomparison of responsivity scales at wavelengths of interest to the optical communications community. Participants in the comparison were the national laboratories in the United States, the United Kingdom, Germany, and Australia. The wavelengths tested were 1300 and $1550 \mathrm{~nm}$. Data taken at $850 \mathrm{~nm}$ are only briefly discussed. The disagreement between the national laboratories' responsivity scale is comfortably within the uncertainty claimed by each laboratory.

\section{0,568}

B9 1-187724

PC A03/MF A01

National Inst. of Standards and Technology (CSL) Gaithersburg, MD.

Validation of an OSI Transport Class 4 Simulator. $\mathrm{O}$. Kim, S. Heatley, and B. Bishop. May 91, 36p NISTIR-4530

Keywords: "Computer communications, "Computer networks, "Protocols, Scheduling, Algorithms, Simulation, Models, Computer systems hardware, Computer software, OSI transport.

Most work on scheduling in communications protocols has considered only the MAC layer. Token bus, token ring, and FDDI all have priority mechanisms. But mechanisms for scheduling must be extended into the upper OSI layers to be effective. The National Institute of Standards and Technology (NIST) has chosen to study possible scheduling mechanisms for Transport Class 4 by first developing a detailed Transport Class 4 simular. Simscript II.5 and simulates the Intel iNA960 implementation of Transport Class 4 running on a $186 / 51$ ront-end board with a 286/10 host. In the paper, the model is described, the processing times which serve as inputs into the model are specified and the validation experiments are documented.

\section{0,569}

PC A05/MF A01 National Inst. of Standards and Technology (CSL) Gaithersburg, MD.

Issues in Transparent File Access.

Special pub. (Final).

K. Olsen, and J. Barkley. Apr 91, 90p NIST/SP-500/

Also available from Supt. of Docs. as SN003-00303079-1.

Keywords: "Communication networks, "File management systems, "Access methods, Operating systems(Computers), Standards, UNIX(Operating system), Semantics, File structures, Access control Applications programs(Computers), Protocols, Interfaces, Remote systems, Computer programs, NES(Network File System), TFA(Transparent File Access), POSIX

For a computer system attached to a network, the network provides connectivity to many other systems whose file systems may be very different from the local file system. However, there is no standard way for an application to 'transparently' access files on severa file systems whose access characteristics may differ from the access characteristics of the local file system. Transparent file access means that remote files are accessed as though they were local. The Institute of Electrical and Electronics Engineers (IEEE) 1003.8 Transparent File Access (TFA) Working Group of the POSIX Standards Committee (IEEE P1003) has undertaken the development of an application programming interface specification based on the IEEE 1003.1-1990 Standard. The objective of IEEE 1003.8 is to permit access to the widest possible range of file system which can resemble the file system of IEEE 1003.11990. The report presents the major issues and probems whose resolution form the basis of the IEEE 1003.8 TFA Standard. Some issues are illustrated with examples and demonstrations using the Network File System (NFS), the most widely used implementation for accessing remote files on a network.

\section{0,570}

PB91-203091

Not available NTIS

National Inst of Standards and Technology (NEL) Boulder, CO. Electromagnetic Technology Div.

Frequency Stabilization of an Erbium-Doped Fiber Laser: A Potential Wavelength Standard for Optical Communications.

Final rept.

S. L. Gilbert. 1991, 1p

Pub. in Proceedings of Optical Fiber Communication Conference, San Diego, CA., February 18-22, 1991, p85.

Keywords: *Optical communication, Near infrared radiation, Infrared lasers, Tunable lasers, Frequency standards, Frequency stability, Acetylene, Reprints, "Erbium lasers, Fiber lasers, Wavelength standards.

A single frequency, $=$ or $<1.6 \mathrm{MHz}$ linewidth, tunable $\mathrm{Er}(+)$-doped fiber laser has been constructed. Stabili- zation to an absorption line of acetylene near $1.53 \mathrm{mi}-$ crometers reduced the laser frequency noise to less than $500 \mathrm{kHz}$ rms.

100,571

PB91-216622

PC A03/MF A01

National Inst. of Standards and Technology (CSL), Gaithersburg, MD

Electronic Data Interchange in Message Handling Systems.

P Markovitz Jun 91, 39p NISTIR-4608

Sponsored by Internal Revenue Service, Washington, DC.

Keywords: "Message processing, "Electronic mail, "Data transmission, Standardization, Computer communications, Protocols, Data structures, Data processing security, Models, Dictionaries, EDl(Electronic Data Interchange). MHS(Message Handling System), IPMS(Interpersonal Messaging Service).

Electronic Data Interchange (EDI) identifies a family of standards used for the electronic transmission of business oriented data (e.g., invoices and purchase orders). EDI standards specify data formats, but are designed independent of a communications protocol. In June, 1990, the Consultative Committee on International Telephony and Telegraphy (CCITT) drafted two Recommendations (F.435:EDI Messaging Service, X.435: EDI Messaging System) which define a stand ardized service and protocol for transmitting EDI data via the Message Handling System (MHS). Using the MHS, EDI data can be transferred between compatible EDI applications implemented on heterogeneous com puter systems. The paper introduces the MHS, the car rier service for EDI data; and the Interpersonal Messaging Service, the only MHS application currently standardized and the model for the EDI Messaging Service. Following the introductory material is a detailed review of the EDI Messaging draft Recommen dations. The transmission of EDI data via the MHS is described as well as the relationships between EDI Messaging and directory, security, and physical deliv ery services. Three appendices are also included in the paper. Appendix A contains a list of abbreviations. Appendix B provides a glossary of MHS terms, and Appendix $\mathrm{C}$ briefly describes EDI messaging elements of service.

\section{0,572}

PB92-102201

PC A05/MF A01

National Inst. of Standards and Technology (NCSL) Gaithersburg, MD. Advanced Systems Div.

ISDN Conformance Testing: Layer 1. Physical Layer. Part 1. Basic Rate S/T Interface, User Side. Special pub. (Final).

S. A. Wakid, and K. M. Roberts. Sep 91, 91p NIST SP-500/194

Also available from Supt. of Docs. as SN003-003 03109-7.

Keywords: Telecommunication, Test methods, Interfaces, Communication networks, Communications management, "Integrated Services Digital Network Test specifications, Conformance tests.

The American National Standard for Telecommunications T1.605-1989 specifies the Layer 1 requirements to provide for satisfactory transmission between a Terminal Equipment (TE) and Network Termination (NT) It describes both the physical interface and the electrical characteristics of the signals appearing at the $S$ and $T$ reference points. Equipment designed to operate on the Integrated Services Digital Network (ISDN) Basic Rate $S$ or $T$ interiace must conform with this set of requirements. The document describes a set of test specifications which test conformance of TEs and NTs to the ISDN Physical Layer at the S/T reference point as defined in ANS T1.605-1989. These tests were de veloped and approved by members of North American ISDN Users' Forum (NIU-Forum)

\section{0,573}

PB92-102219

PC A08/MF A02

National Inst of Standards and Technology (CSL) Gaithersburg, MD. Advanced Systems Div.

North American ISDN Users' Forum Agreements on Integrated Services Digital Network.

Special pub. (Final).

S. A. Wakid, and K. M. Roberts. Sep 91, 152p NIST/ SP-500/195

Also available from Supt. of Docs. as SN003-003-

03110-1. See also PB91-171967, PB91-197004, PB92-112200 and PB92-102201. 


\section{Common Carrier \& Satellite}

Keywords: "Standards, Communication networks Computer networks, Computer software, Specifications, User needs, Tests, "ISDN(Integrated Services Digital Network), Basic rate interface, Primary rate interface.

The document compiles the existing North American ISDN Users' Forum (NIU-Forum) agreements for an In tegrated Services Digital Network (ISDN) developed and approved in the NIU-Forum as of November 1990 These agreements cover: Layer 1 Basic Rate Interfac (BRI) at the $U$, and $S / T$ reference points; Layer 1 Primary Rate Interface (PRI) at the $U$ reference point; Layer 2 BRI and PRI; Layer 3 BRI Basic Call Control for Class I equipment; Layer 3 PRI Basic Call Control for Class II equipment; and Generic Control procedure for Class I BRI Supplementary Services. In addition the document references the Conformance tests which have been completed by the NIU-Forum. These include: Layer 1 BRI S/T interface; and Layer 2 BRI Link Access Procedure, D-channel (LAPD). Finally, the document contains the Application Profile for four of the Incoming Call Management applications which have been submitted to the NIU-Forum.

\section{Graphics}

\section{0,574}

PB91-187773

$\mathrm{PC}$ A03/MF $\mathrm{AO}$

National Inst. of Standards and Technology (NCTL) Gaithersburg, MD. Office Systems Engineering Group. Government Document Processing Requirements Report.

R. F. Sies. Apr 91, 16p NISTIR-4560

Keywords: "Standards, Documents, User needs, *Electronic publishing, Electronic Information Ex change Standards, ODA(Office Document Architecture), SGML(Standard Generalized Markup Language), SPDL(Standard Page Description Language) ODL(Office Document Language).

The report describes several activities of the Office Systems Engineering Group in the area of electronic publishing standards. It gives an account of the July 30, 1990 workshop on Electronic Information Exchange Standards Used in Document Processing Applications and the list of User Requirements that came
out of that workshop. The report also talks about other efforts the Office Systems Engineering Group has made to help bring about the harmonization of elec tronic publishing standards.

\section{Policies, Regulations, \& Studies}

100,575

\section{N91-25760/0}

(Order as N91-25755/0, PC A99/MF A04) National Inst. of Standards and Technology (NML) Boulder, CO. Time and Frequency Div.

Trapped-Ion Frequency Standards.

D. J. Wineland, W. M. Itano, J. C. Bergquist, J. J.

Bollinger, and D. J. Heinzen. May $90,8 p$

Pub. in Proceedings of Annual Precise Time and Time Interval (PTTI) Applications and Planning Meeting (22nd) p53-60 (See N91-25755 17-70). Sponsored by AFOSR and Onr

Keywords: Atoms, "Frequency standards, Ions *Trapped particles, Clocks, Microwaves, Optical equipment, Radio frequencies, Time measurement.

Frequency standards based on stored atomic ions are briefly reviewed. Specific examples are chosen to illustrate what is currently possible. Both $\mathrm{rf} / \mathrm{microwave}$ and optical devices are discussed. The present limitations to existing experiments and possibilities for future im provement are outlined.

\section{0,576}

\section{N91-25766/7}

(Order as N91-25755/0, PC A99/MF A04) National Inst. of Standards and Technology, Boulder, CO.
GPS Time Transfer with Implementation of Selective Availability.

D. W. Allan, M. P. Granveaud, W. J. Klepczynski, and W. W. Lewandowski. May 90, 11p

Pub. in Proceedings of Annual Precise Time and Time Interval (PTTI) Applications and Planning Meeting (22nd) p145-155

Keywords: "Global positioning system, "Metrology, "Navigation satellites, "Time measurement, Atomic clocks, Navigation, Observatories.

The international community of time metrology is facing a major challenge with the Selective Availability (SA) degradation of GPS satellite signals. At present there are 6 Block 1 satellites and 8 Block 2 satellites operating. According to the policy of the U.S. Department of Defence the Block 1 satellite signals will not be degraded, but these satellites are old with a finite life. The Block 2 satellites, which have all been launched since 1988, were subject to Selective Availability from March 25, 1990 . The effect of SA should be to limit precision to about 100 meters for navigation and $167 \mathrm{~ns}$ for timing. A study was conducted in order to understand the nature of the actual introduced degradation, and to elaborate the means of removing the effects of this degradation on time transfer. This study concerns the time extraction from GPS satellites at NIST, USNO and Paris Observatory, and the comparison of atomic clocks between these laboratories by common view approach. The results show that when using the data taken over several days the time extraction can be achieved with uncertainty of a few tens of nanoseconds, while strict common-view has removed entirely the effects of SA during the periods under study

\section{0,577}

PB91-134593

Not available NTIS

National Bureau of Standards (NML), Boulder, CO. Time and Frequency Div.

Israel's New Synchronized Time Scale, UTC(INPL) Final rept.

A. Shenhar, W. Litman, A. Lepek, A. Citrinovitch, and D. W. Allan. $1988,5 p$

Pub. in Proceedings of Annual Symposium on Frequency Control 1988 (42nd), Baltimore, MD., June 1-3, 1988, p485-489.

Keywords: "Time measurement, Cesium frequency standards, Frequency stability, Synchronism, Israel, Standards, Frequency sta
Reprints, Software clocks.

The National Physical Laboratory of Israel (INPL) together with Time and Frequency Limited (TFL) and
NBS is building a software clock to be used as the Israeli national time base, UTC(INPL). The software clock is based on several commercial Cs clocks (HP and TFL) whose outputs are routed sequentially through a TFL programmed switch into a time interval counter. The paper presents the principles of generating the software clock, its performance and the method to compare it to UTC and UTC(NBS). The paper also presents the optimization procedures for synchronizing and syntonizing UTC(INPL) with UTC as a coordinated time scale.

\section{0,578}

PB91-162388

Not available NTIS

National Inst. of Standards and Technology (NML), Boulder, CO. Time and Frequency Div.

Calculated from the Frequency Domain: An Update.

Final rept

F. L. Walls, J. Gary, A. O'Gallagher, L. Sweet, and R. Sweet. 1990, 8p

Pub. in Proceedings of the European Frequency and Time Forum (4th), Neuchatel, Switzerland, March 13 $15,1990, \mathrm{p} 197-204$

Keywords: "Frequency stability, Time domain, Reprints, Allan variance, Phase noise.

The authors investigate the dependence of the fractional frequency stability measures and mode on the parameter for common power-law noise types. The authors have implemented the calculations using numerical techniques which make it possible to perform the calculations for virtually any value of the parameter for common power-law noise types and a wide variety of high-frequency filters.

\section{0,579}

PB91-192989

PC A06/MF A02

National Inst. of Standards and Technology, Gaithersburg, MD. Office of Standards Services.
Conformity Assessment Workshop on Electromagnetic Compatibility.

B. G. Simson. Jun 91,111 p NISTIR-4611

See also PB91-192997. Sponsored by American Society of Mechanical Engineers, Washington, DC., and American Council of Independent Labs., Washington, DC.

Keywords: *Electromagnetic compatibility, "Meetings, Government policies, Recommendations, Competition, European Economic Community, Conformity assessment.

On April 4, 1991, the National Institute of Standards and Technology (NIST) cosponsored a conformity assessment workshop on electromagnetic compatibility (EMC) with the American Council of Independent Laboratories (ACIL) and the American Electronics Association (AEA). The purpose of the workshop, which focused on information technology equipment (ITE), was to explore how the U.S. Government can assist EMC aboratories in gaining acceptance of their test results in such other markets as the European Community (EC). The following consensus recommendations were reached by the private sector panelists: (1) establishing an EMC advisory committee; (2) harmonizing the National Voluntary Laboratory Accreditation Program (NVLAP) and Federal Communications Commission (FCC) programs; (3) negotiating an agreement with the EC to permit establishing notified bodies in the United States; (4) establishing a formal U.S. Government role in accrediting conformity assessment programs; and (5) establishing a proactive role for NIST in disseminating information in to affected interests in the EMC area.

\section{0,580}

PB91-200790

Not available NTIS

National Inst. of Standards and Technology (NML), Boulder, CO. Time and Frequency Div.

New Inexpensive Frequency Calibration Service from NIST.

Final rept

D. W. Allan, D. D. Davis, J. Levine, M. A. Weiss, and N. Hironaka. $1990,10 \mathrm{p}$

Pub. in Proceedings of Annual Symposium on Frequency Ccontrol (44th), Baltimore, MD., May 23-25, 1990, p107-116.

Keywords: Frequency stability, Accuracy, Reprints, * Frequency calibration. Automated computer time system, ACTS system, US NIST.

A new inexpensive frequency calibration service from NIST is now available. The service takes advantage of the operation of the NIST Automated Computer Time System (ACTS), which was begun in 1988. Software to access the service from several types of computers was released at the same time. Time and frequency dissemination by the modest-accuracy service depends on the reciprocity of the telephone system. The round-trip delay is measured by the NIST equipment. The advance of an on-time marker is adjusted so as to arrive at the user's site on time. A frequency calibration method taking advantage of the service has been designed and preliminary tests conducted. A computer is not required to access the service. All that is required is a telephone modem, a simple peripheral circuit to generate an on time marker and standard time and frequency measurement and data processing equipment

100,581

PB91-200808

Not available NTIS

National Inst. of Standards and Technology (NML) Boulder, CO. Time and Frequency Div.

Rubidium Frequency Standard and a GPS Receiv er: A Remotely Steered Clock System with Good Short-Term and Long-Term Stability.

Final rept.

D. W. Allan, and J. Levine. 1990, 10p

Pub. in Proceedings of Annual Symposium on Frequency Control, Baltimore, MD., May 23-25, 1990 p151-160.

Keywords: "Rubidium frequency standards, "Global positioning system, *Frequency stability, Cesium frequency standards, Frequency synchronization, Reprints, Time transfer.

The short-term stability of a rubidium gas-cell frequency standard is usually better than that of commercial cesium-beam frequency standards. Cesium almost always has better stability in the long-term because cesium has less sensitivity to environmental perturbations. For example, cesium has little or no frequency 
drift, whereas rubidium usually does. Improving a clock's environment invariably improves the long-term performance, especially in the case of rubidium. Satellite time transfer shows a day-to-day stability of about a nanosecond. If a rubidium standard, in a good environment with the above performance in the short-term, were married to a satellite time-transfer system, then the combined performance of the system could have better short-term and better long-term stability than a stand-alone, free-running, commercial cesium standard. The authors have taken some data to test the idea. The conclusions confirm the hypothesis. The authors have also replaced the rubidium oscillator with a quartz oscillator and with a high-performance commercial cesium standard. In both cases the system had significantly improved long-term stability over what otherwise would be obtainable from either oscillator by itself.

\section{0,582}

PB9 1-202937

Not available NTIS

National Inst. of Standards and Technology (NML),

Boulder, CO. Time and Frequency Div.

Frequency and Time Stability of GPS and GLONASS Clocks.

Final rept.

P. Daly, I. D. Kitching, D. W. Allan, and T. K. Peppler. 1990, 13p

Pub. in Proceedings of Annual Symposium on Frequency Control (44th), Baltimore, MD., May 23-25, 1990, p127-139.

Keywords: "Global positioning system, "Frequency stability, Frequency standards, Time standards Atomic clocks, Comparison, Reprints, "GLONASS system.

The frequency stability and reliability of the clocks are critical to the success of the GPS and GLONASS programs. The authors show some of the similarities and differences between the clocks involved in these two systems. On-board clocks and the stability of the master control clocks for these systems are analyzed. The authors discuss the attributes of these two systems as time and frequency references. Their relationship to UTC will also be illustrated. More data over a longer period of time was available for the authors from GPS than from GLONASS. Even so it is obvious that both systems have matured. Though the GLON. ASS system was developed later, its overall clock performance has improved more rapidly. Some of the more recent GLONASS clock performance is at about the same level as that of the GPS clocks. The analysis has yielded some very interesting contrasts, comparisons, and changes in these systems that should be of great interest for time and frequency users, as well as for clock vendors and receiver vendors.

\section{0,583}

PB91-203174

Not available NTIS Nation

Advances in Science, Emerging Technologies and Global Competition: Opportunities and Challenge. Final rept.

H. Hellwig. 1988, 9p

Pub. in Proceedings European Frequency and Time Forum (2nd), Neuchatel, Switzerland, March 16-18, 1988, p13-21 1988.

Keywords: "Frequency standards, "Time standards, Competition, Technology, Oscillators, Clocks, Reprints.

For the remainder of the century, the time and frequency community is faced with significant new opportunities and challenges. The roots of current time and frequency technology date back half a century; scientific advances have been largely evolutionary and have gradually expanded the boundaries of these known foundations. In stark contrast to this, the technological world is undergoing nearly revolutionary changes which are driven by 3 interrelated forces: Global Competition, Emerging Technologies and Advances in Science. In the following, specific examples of these three areas are described as they relate to time and frequency. The opportunities which can be derived from emerging technologies and scientific advances are quite large, but seemingly require more efficient methods of moving an idea from the laboratory to market readiness and a more sophisticated pooling of resources. Thus, winning ideas and organizations probably will be based on significant levels of cooperation both vertically (research, engineers, marketeers) as well as horizontally (between organizations nationally and internationally).
100,584

PB9 1-203182

Not available NTIS

National Bureau of Standards, Gaithersburg. MD. Office of the Associate Director for Programs, Budget and Finance.

Established Microwave Frequency Standards.

Final rept.

H. Hellwig. $1989,2 p$

Pub. in Frequency Standards and Metrology, p44-45 1989.

Keywords: "Frequency standards, Historical aspects, Atomic clocks, lon storage, Microwaves, Rubidium, Cesium, Hydrogen, Masers, Reprints.

The paper is the introduction to a series of papers by other authors on 'established microwave frequency standards'. A historical background is given tracing the development of microwave frequency standards since the experiments by Stern-Gerlach in 1921. A brief summary of their salient technical features is given, followed by a discussion of performance and applications. Finally, the advent of a new contender, the mercury ion storage principle, is briefly described.

\section{0,585}

PB91-231605

PC A03/MF A01

National Inst. of Standards and Technology (NML),

Boulder, CO. Time and Frequency Div.

NIST Time and Frequency Services.

Special pub. (Final).

R. E. Beehler, and M. A. Lombardi. Jun $91,32 p$

NIST/SP-432

Also available from Supt. of Docs.

Keywords: *Radio broadcasting, "Frequency standards, "Time signals, High frequency, Low frequency, Navigational aids, Computer networks, Voice communication, Air traffic control, GOES satellites, Calibration, Uses, US NIST.

NIST Time and Frequency Services (Special Publication 432 (Revised 1990)) is a revision of SP 432, last published in 1979. It describes services available, as of December 1990, from NIST radio stations WWV, WWVH, and WWVB; from GOES satellites; from Loran-C; by telephone (voice and modem); and from the NIST Frequency Measurement Service.

\section{6}

\section{PB91-236950}

Not available NTIS

National Inst. of Standards and Technology (PL), Boulder, CO. Time and Frequency Div.

Precise Ephemerides for GPS Time Transfer.

Final rept.

W. Lewandowski, and M. A. Weiss. 1988, 11p

Pub. in Proceedings of Annual Precise Time and Time Interval (PTTI) Applications and Planning Meeting (21st), Redondo Beach, CA., November 28-30, 1990, p95-105 1988

Keywords: "Ephemeris time, Global positioning system, Precision, Comparison, Reprints, "Broadcast ephemerides, "Time transfer.

The present technology of atomic clocks motivates time transfer techniques with nanosecond accuracy. Global Positioning System (GPS), the most common means for international time comparisons could achieve such accuracy over short distances (up to $1000 \mathrm{~km}$ ). Over intercontinental distances, the accuracy of the GPS time transfer ranges between 20 and 30 ns. Some of the principal error sources are the broadcast ephemerides, the broadcast ionospheric model, and the local antenna coordinates. The study investigates the quality of broadcast ephemerides by comparing them with precise ephemerides and by using precise ephemerides for time transfer. Another aspect of the work is to suggest a strategy to overcome the planned degradation of GPS satellite messages via Selective Availability (SA)

\section{0,587}

\section{PB92-123132}

PC A03/MF A01

National Inst. of Standards and Technology, Boulder, CO.

Time Domain Frequency Stability Calculated from the Frequency Domain Description: Use of the SIGINT Software Package to Calculate Time Domain Frequency Stability from the Frequency Domain.

F. L. Walls, J. Gary, A. O'Gallagher, R. Sweet, and L. Sweet. Sep 91, 39p NISTIR-89/3916-REV Supersedes PB90-257684.
Keywords: "Frequency stability, Time domain, SIGINT computer code, Fortran 77 programming language, Frequency domain, Allan variance.

The authors describe the use of SIGINT, an interactive software package developed by the National Institute of Standards and Technology, which facilitates the calculation of time domain frequency stability from frequency domain data as a function of measuring time in terms of either the Allan variance (sigma squared(sub y))(tau); the modified Allan variance, mod(sigma squared(sub y))(tau); or (sigma squared(sub y)) $(\mathrm{tau})=(\mathrm{tau} /(\mathrm{square}$ root of 3)) mod(sigma squared(sub y))(tau). Except for the graphic output, the 77 and runs on AT compatible computers that have a math co-processor. It also runs on many other systems; however, calls to an available graphics library will need to be substituted for those that are included in this version. The program uses either a user defined function for the input noise or default functions that describe the noise types commonly found in oscillators, amplifiers, frequency multipliers, frequency dividers, and general signal processing equipment including up to four coherent bright lines in the noise spectra. These default functions make it simple to analyze the time domain frequency stability as a function of measuring bandwidth using realistic first- second- or third-order lowpass filters or the simplified infinitely sharp cutoff parameter. The default functions are also set up to examine the effect of various servo parameters on the performance of a frequency source locked to a frequency reference.

\section{Radio \& Television Equipment}

100,588

PB91-134874

Not available NTIS

National Inst. of Standards and Technology (NML), Boulder, CO. Time and Frequency Div.

Extending the Range and Accuracy of Phase Noise Measurements.

Final rept.

F. L. Walls, A. J. D. Clements, C. M. Felton, M. A. Lombardi, and M. D. Vanek. 1988, $10 p$

Pub. in Proceedings of Symposium on Frequency Control (42nd), Baltimore, MD., June 1-3, 1988, p432-441.

Keywords: Electrical measurement, Quartz resonators, Frequency stability, Oscillators, Accuracy, Reprints, "Phase noise, Calibration.

The paper describes recent progress in extending high accuracy measurements of phase noise in oscillatoors and other devices for carrier frequencies from the of to the millimeter region and Fourier frequencies up to $10 \%$ of the carrier (or a maximum of about $1 \mathrm{GHz}$ ). A brief survey of traditional precision techniques for measuring phase noise is included as a basis for comparing their relative performance and limitations.

100,589

PB91-134882

Not available NTIS

National Inst. of Standards and Technology (NML),

Boulder, CO. Time and Frequency Div.

Accuracy Model for Phase Noise Measurements.

Final rept.

F. L. Walls, C. M. Felton, A. J. D. Clements, and T.

D. Martin. $1989,16 p$

Pub. in Proceedings of Annual Precise Time and Time Interval (PTTI) Applications and Planning Meeting (21st), Redondo Beach, CA., November 28-30, 1989 , p295-310.

Keywords: Microwave amplifiers, Microwave oscilla tors, Frequency synthesizers, Spectrum analyzers Frequency stability, Electrical measurement, Accuracy, Reprints, "Phase noise, Time domain, Calibration.

The authors have recently completed a new modular system for accurate measurements of phase noise in oscillators, amplifiers, frequency synthesizers, and passive components. This new system is capable of measuring the phase noise at carrier frequencies from $5 \mathrm{MHz}$ to $1.5 \mathrm{GHz}, 1.5$ to $26 \mathrm{GHz}$, and 33 to $50 \mathrm{GHz}$. Other frequency ranges can be measured using external mixers to convert the signals into one of the above frequency ranges. The analysis bandwidths vary from $0.1 \mathrm{~Hz}$ to $10 \%$ of the carrier frequency up to a maximum of about $1 \mathrm{GHz}$. Extensive internal calibration of the system is used to correct for all gain variations with 


\section{Radio \& Television Equipment}

analysis frequency including phase-locked-loop and cable-loss effects. Detailed descriptions of the new calibration procedures, including a sample table of uncertainties, are given. High accuracy determinations of phase noise can be used to compute accurate values of short-term, time-domain frequency stability.

\section{0,590}

PB91-162040

Not available NTIS

National Inst. of Standards and Technology (NEL). Boulder, CO. Electromagnetic Fields Div.

Evaluation of Dual-Port Circularly Polarized Probes for Planar Near-Field Measurements.

Final rept.

M. H. Francis, and K. MacReynolds. 1990, 6p

Pub. in Proceedings of Annual Meeting and Symposium on Antenna Measurement Techniques Association (12th), Philadelphia, PA., October 7-16, 1990, p13-313-8.

Keywords: "Probes(Electromagnetic), Extremely high frequency, Millimeter waves, Near field, Circular polarization, Cross polarization, Gain, Reprints, *Antenna measurements.

Accurate near-field cross-polarization measurements on circularly polarized (CP) antennas at millimeterwave frequencies require well-characterized probes with low axial ratios. The authors have recently obtained and calibrated dual-port CP horns for use as near-field probes at frequencies of $40-50 \mathrm{GHz}$. The authors present some gain, axial ratio, and pattern measurements for these probes and show that they give accurate cross-polarization measurements.

\section{0,591}

PB91-162156

Not available NTIS

National Inst of Standards and Technology (NEL) Boulder, CO. Electromagnetic Fields Div.

Microstrip Patch Antenna as a Standard Transmitting and Receiving Antenna.

Final rept.

M. Kanda. $1989,3 p$

See also PB90-206038

Pub. in Proceedings of International Symposium on Electromagnetic Compatibility, Nagoya, Japan, September 8-10, 1989, p460-462.

Keywords: "Microstrip antennas, Antenna radiation patterns, Electrical impedance, Standards, Reprints, Patch antennas.

The paper discusses the possibility of employing a microstrip patch antenna as a standard transmitting and receiving antenna. The intrinsic properties of the substrate used for the antenna are determined by careful impedance measurements. The experimental results indicate that the transmitting characteristics of a microstrip antenna can be theoretically determined from its geometry. The microstrip patch antenna discussed here is physically small $(20 \mathrm{~cm}$ square for $450 \mathrm{MHz}$ ) and can be well matched to a power delivery system $(S W R=1.17)$.

\section{Verbal}

100,592

PB91-189910

Not available NTIS

National inst of Standards and Technology (NCSL) Gaithersburg, MD. Advanced Systems Div.

Phone-Mediated Word Alignment for Speech Recognition Evaluation.

Final rept.

J. Picone, G. R. Doddington, and D. S. Pallett. 1990,

$4 \mathrm{p}$

Pub. in IEEE (Institute of Electrical and Electronics Engineers) Transactions on Acoustics, Speech and Signal Processing 38, n3 p559-562 Mar 90.

Keywords: "Speech recognition, "Algorithms, Automation, Performance evaluation, Speech analysis, Reprints.

The paper presents an algorithm that produces a word-based alignment of two text strings by post-processing the output of a phone-based alignment procedure. The alignment is performed using an unconstrained endpoint dynamic optimization framework and minimizes the readjustment of word boundaries as determined by the phone-level alignment.
100,593

PB91-195396

Not available NTIS

National Bureau of Standards (ICST), Gaithersburg, MD. Advanced Systems Div.

Scoring Continuous Speech in the DARPA Speech Recognition Program: Part 2.

Final rept.

D. S. Pallett. $1988,5 p$

Pub. in Proceedings of AVIOS ' 88 Voice I/O System Applications Conference, San Francisco, CA., October $4-6,1988, p^{1-5}$

Keywords: "Speech recognition, "Computer applications, "Performance evaluation, Automation, Tests, Protocol, Computer software, Reprints.

The paper outlines considerations taken in developing and implementing Benchmark Test Procedures for evaluating the performance of large vocabulary continuous speech recognition systems using the DARPA Resource Management Speech Database. These tests were implemented in March and October 1987 and in June of 1988 , with future tests planned using the same test protocol. The emphasis of the discussion in the paper is on scoring the results of the tests and includes a brief discussion of results obtained using scoring software.

\section{0,594}

PB9 $1-505370$

CD-ROM $\$ 1200.00$ National Inst. of Standards and Technology (CSL), Gaithersburg, MD. Automated Speech Recognition Group.

DARPA Air Travel Information System (ATISO) Speaker-Dependent Training Data (on CD-ROM).

Data file.

Nov 90, CD-ROM NIST/DF/VD-91/003

Microsoft Extension version 2.1. User instructions on disc 'readme.doc'. See also PB91-505362, PB91505354, PB90-501776, PB89-226666, PB90-500547, PB91-505065, PB91-506592, and PB90-500539.

The datafile is on four, 4.72 inch discs.

Keywords: "Data file, "Speech recognition, Training, Air traffic, Information systems, Acoustics, Models, Microphones, CD-ROM.

The set of discs is the third release in a series of CDAOMs containing recordings of speech in the DARPA Air Travel Information System (ATIS) domain. The set contains 'read' speech intended to permit acoustic modelling for 10 of the speakers of an earlier release (CD5-1.1) in the series, and can be used in training speaker-dependent speech recognition systems. Discs 5-3.1 and 5-4.1 contain speech data for the close-talking (Sennheiser) microphone, and 5-5.1 and 5-6.1 contain data for the desk-top (Crown PCC-160) microphone. The set of CD-ROMs augments material on two earlier releases, PB91-505354 and PB91505362. The first release contains spontaneous utterances elicited in a 'Wizard-of-Oz' simulation of a spoken language system capable of providing air travel information derived from a simplified version of the Official Airline Guide, along with the relational database containing the travel information. The second release contains 'read' versions of the spontaneous utterances for 20 of the speakers, along with 40 adaptaterances for 20 of the speakers, along with
tion utterances for each of these speakers.

100,595

PB91-509802

CD-ROM $\$ 500.00$

National Inst of Standards and Technology (CSL)

Gaithersburg, MD. Automated Speech Recognition Group

Road Rally Conversational Speech Corpora (on CD-ROM)

Data file.

Sep 91, CD-ROM NIST/DF/VD-91/015

System: Sun Microsystems $3 / 180$; OS 4.1 operating system. Microsoft Extension Version 2.1. No color requirement or hard disk required. 'Readme.doc' on Disc. Software is written in 'C' Source Code. See also PB89-226666, PB90-501776, PB90-500539, PB91505362, PB91-505354, PB90-500547, PB91-505065 and PB91-506592.

The datafile is on one, 4.72 inch disc. Data format: ISO 9660.

Keywords: "Data file, "Speech recognition, Words(Language), Planning, CD-ROM, Conversation, Road rallys.

The 'Road Rally' corpora were designed for the development and testing of word-spotting systems and were collected in a conversational domain using a road rally planning task as the topic. The corpora actually consists of two sub-corpora 'Stonehenge' and 'Waterloo'. The Stonehenge corpus contains road rally planning conversations as well as some read speech collected using high quality microphones and a telephone-simulating filter. The Waterloo corpus contains read speech in a road rally planning domain collected using actua telephone lines.

\section{COMPUTERS, CONTROL \& INFORMATION THEORY}

\section{Computer Hardware}

100,596

N91-25793/1

(Order as N91-25755/0, PC A99/MF A04)

National Inst. of Standards and Technology (NML) Boulder, CO. Time and Frequency Div.

Synchronizing Computer Clocks Using a Local Area Network

J. Levine. May $90,9 p$

Pub. in Proceedings of Annual Precise Time and Time Interval (PTTI) Applications and Planning Meeting (22nd) p409-417 (See N91-25755 17-70).

Keywords: Calibrating, "Clocks, "Local area networks, "Time measurement, Protocol (Computers). Telephones, "Computer networks, "Synchronism.

Researchers completed the first tests of a method to synchronize the clocks of networked computers to the National Institute of Standards and Technology (NIST) time scale. The method uses a server computer to disseminate the time to other clients on the same local area network. The server is synchronized to NIST using the ACTS protocol over a dial-up telephone line. The software in both the server and the parameters of this model are used to adjust the time of the local clock and the interval between calibration requests in a statistically optimum way. The algorithm maximizes the time between calibrations while at the same time keep ing the time of the local clock correct within a specific tolerance. The method can be extended to synchronize computers linked over wide-area networks, and an experiment to test the performance of the algorithms over such networks is being planned.

100,597

PB91-147652

Not available NTIS

National Inst of Standards and Technology (NEL), Gaithersburg, MD. Mathematical Analysis Div.

Closure and Precision in Level-Index Arithmetic. Final rept.

D. W. Lozier, and F. W. J. Olver. 1990, 10p Pub. in SIAM (Society for Industrial and Applied Mathematics) Jnl. of Numerical Analysis 27, n5 p1295-1304 Oct 90 .

Keywords: "Arithmetic units, "Computer systems hardware, Numerical precision, Error analysis, Computer calculations, Floating point arithmetic, Reprints.

First it is proved that two recently introduced systems of computer arithmetic, the level-index (ii) and symmetric level-index (sli) systems are closed under the four basic arithmetic operations, provided that division by zero is excluded and the operations are executed in finite precision. In consequence, the li and sli systems are free from the defects of overflow and underflow. Second, measures of precision are discussed and compared. Third, the ranges and local precisions of numbers stored in the li and sli systems are compared with corresponding ranges and local precisions of numbers stored in the floating-point system.

100,598

PB91-147660

Not available NTIS

National Inst. of Standards and Technology (ICST) Gaithersburg, MD. Advanced Systems Div. 
Workloads, Observables, Benchmarks and Instrumentation.

Final rept.

G. Lyon, and R. D. Snelick. 1990,12p

See also PB90-207770.

Pub. in Proceedings of Joint International Conference on Vector and Parallel Processing, Zurich, Switzerland, September 10-13,1990, p86-97.

Keywords: "Computer systems performance, "Performance evaluation, Benchmarks, Comparison, Trees(Mathematics), Observation, Work measurement, Reprints, Workloads.

A compact user-level summary, a use-tree, explains system variabilities across benchmarks. The tree provides a clear, static declaration of the relationships among a limited number of major system resources that determine most performance variance. The new approach is especially effective with hardware instrumentation that decouples workload from observation (otherwise, a compact set of observables may be unavailable). A tree supports simple predictions and promotes more meaningful comparisons of workloads. Accounting for the sources of performance variation shown in the tree can inspire new methods of assessment; a 'time dilation' technique illustrates this for loosely-coupled systems with local clocks.

100,599

PB91-158766

Not available NTIS

National Bureau of Standards (ICST), Gaithersburg, MD. Systems and Software Technology Div.

1987: The Year of the 386.

Final rept.

A. L. Hankinson. 1988, 2p

Pub. in IEEE (Institute of Electrical and Electronics Engineers) Spectrum 25, n1 p32-33 Jan 88

Keywords: "Personal computers, "Chips(Electronics), Workstations, Microcomputers, Computer software, Reprints, Intel 80386 computers.

Users of personal computers will likely remember 1987 as the year of the Intel 80386 . This chip had a major influence on the three themes that characterized the significant PC related milestones during 1987 . The first theme is the convergence of PC's and engineering workstations. The second theme is the blurring of the distinction between software for PC's minicomputers and mainframes. The final theme is the end of the era of the 'safe buy'. Each of these themes is presented in the context of the significant PC related milestones that occurred during 1987.

\section{0,600}

PB91-158899

Not available NTIS

National Inst. of Standards and Technology (NEL),

Gaithersburg, MD. Factory Automation Systems Div.

Choosing a Name for Your Computer.

Final rept.

D. Libes. $1990,8 p$

Pub. as Choosing a Name for Your Computer, p1-8 Aug 90.

Keywords: “Computers, Guidelines, Reprints, "Domain name system, Hostname, Computer name.

In order to easily distinguish between multiple computers, the authors give them names. Experience has taught that it is as easy to choose bad names as it is to choose good ones. The essay presents guidelines for deciding what makes a name good or bad.

\section{0,601}

\section{PB91-159319}

Not available NTIS

National Inst. of Standards and Technology (ICST),

Gaithersburg, MD. Advanced Systems Div.

Hardware Instrumentation Approach for Performance Measurement of a Shared-Memory Multiprocessor.

Final rept.

G. Nacht, and A. Mink. 1989, 16p

See also PB89-186852. Sponsored by Defense Advanced Research Projects Agency, Arlington, VA

Pub. in Proceedings of International Conference on Modeling Techniques and Tools for Computer Performance Evaluation (4th), Palma, Spain, September $14-16,1988, p 249-2641989$.

Keywords: "Multiprocessors, "Computer performance evaluation, Parallel processors, Measuring instruments, Computer systems hardware, Reprints.

Two approaches for the design of performance measurement instrumentation for a shared memory, tightly coupled, MIMD multiprocessor are presented. The TRAce Measurement System (TRAMS) is a hybrid measurement tool used to obtain trace measurement information. The Resource Measurement System (REMS) is a non-intrusive hardware measurement tool used to obtain both trace measurement and resource utilization information. The TRAMS approach provides a hardware assist to the more traditional software approach of obtaining timestamps from the operating system at each event to be measured. The hardware assist reduces the artifact that is introduced in a test program and is a feasible and economical approach to providing measurement capabilities to a wide range of multiprocessors. Manufacturers could offer this type of measurement tool as a plug-in option. The REMS approach provides more detailed and extensive measurement information than does the TRAMS approach and introduces no artifact to the test program, but it does this at a significantly higher cost. When access to pertinent signals is restricted the applicability of such a hardware tool is limited.

\section{0,602}

\section{PB91-189894}

Not available NTIS

National Inst of Standards and Technology (NCSL).

Gaithersburg, MD. Advanced Systems Div.

Conformance Tester for X.25 DTE Implementations.

Final rept.

H. Peng, D. H. Su, and S. A. Wakid. 1989, 4p

Pub. in IEEE (Institute of Electrical and Electronics Engineers) Network Magazine 3, n4 p27-30, 39, Jul 89.

Keywords: Standards, Tests, Reprints, "Data terminal equipment, Defense Data Network, C programming language, Protocols.

A validation suite is presented to test the conformance of Data Terminal Equipment (DTE) to the ISO International Standard of the $1984 \times .25$ protocol (ISO 7776 and 8208 ) as well as to the CCITT recommendations of the 1980 version of the protocol. The test cases are based on ISO 8882 and the testing methodology itself complies with ISO 9646 . The paper describes the design, instrumentation, and execution of this tester which is written in ' $C$ ' and available in the public domain.

\section{0,603}

\section{PB91-201822}

PC A05/MF A01

Gaithersburg.

National Computer Systems Laboratory Annual Report, 1990.

E. Lennon, S. Radack, and R. Roach. Dec 90, 76p NISTIR-4492

Also available from Supt. of Docs.

Keywords: *Computers, "Computer software, Information systems, Systems engineering, Computer security, Computer networks, Computer architecture, Telecommunication, Technology transfer, Federal information processing standards, "National Computer Systion processing
tems Laboratory.

The report describes the annual computer and related telecommunications activities and accomplishments of the National Computer Systems Laboratory (NCSL). Following the Director's Foreword, an overview of the Laboratory is presented, including a current NCSL Organization Chart and selected staff accomplishments. Overviews of NCSL's five technical divisions are featured, followed by a section on Technology Transfer which details the vehicles NCSL uses to disseminate research and information to the public and technical communities. A list of Federal Information Processing Standards (FIPS) and FIPS order information conclude the annual report.

\section{0,604}

PB92-108950 Gaithersburg, MD. Electricity Div.

Video Processing with the Princeton Engine at NIST.

Technical note (Final)

B. F. Field, and C. Fenimore. Aug 91, 54p NIST/TN1288

Also available from Supt. of Docs. as SN003-00303091-1.

Keywords: "Image processing, "Supercomputers, Computer aided design, Computer architecture, Parallel computers, Real time operations, Data compression, Modules, Fortran, "Princeton Engine, Video processing, SIMD(Computers).
The document describes the NIST program in digital processing, including a newly created Image Processing Laboratory at NIST that is available to governmental, industrial, and academic researchers working on digital image processing. The centerpiece of the laboratory is a video supercomputer, the Princeton Engine, designed and constructed by the David Sarnoff Re. search Center. The engine provides real-time video and image-processing capability, accepting a variety of video formats over multiple wideband input channels and outputting real-time video for immediate viewing. Because the Engine is programmable, it is possible to use it to evaluate prototypes of image processing components rapidly and efficiently. The hardware capabilities of the Princeton Engine are described as well as the available supporting video equipment in the Laboratory. Two programming examples are included to demonstrate the unusual programming environment and 'language' used to program the Engine. Appendices list the available predefined library modules, and the processor assembly language instructions.

100,605

PB92-116409

PC A05/MF A01

National Inst. of Standards and Technology (NCSL), Gaithersburg, MD. Advanced Systems Div.

Test Methods for Optical Disk Media Characteristics (for $356 \mathrm{~mm}$ Ruggedized Magneto-optic Media).

Special pub.

F. L. Podio. Sep 91, 79p NIST/SP-500/191

Also available from Supt. of Docs. as SN003-00303103-8. Sponsored by National Aeronautics and Space Administration, Hampton, VA. Langley Research Center

Keywords: "Tests, "Computer storage devices, "Optical disks, Optical data storage materials, Environmental tests, Radiation tests, Substrates, Standards, Magneto-optic media.

Standard test methods for computer storage media characteristics are essential and allow for conformance verification to media interchange standards. Tests methods are also needed to develop procedures which would allow for repeatability of results among different industry and U.S. Government sites. The test procedures documented in the publication reflect the work done by the NIST/NASA Working Group for the Development of Test Methods and Specifications for $356 \mathrm{~mm}$ Ruggedized Rewritable Media. The test methods were developed for $356 \mathrm{~mm}$ two-sided laminated glass substrate with a magneto-optic active layer media technology. These test methods may be used for testing other media types, but in each case their applicability must be evaluated. Test methods are included for a series of different media characteristics including: operational, non-operational, and storage environments, mechanical and physical characteristics, and substrate, recording layer, and preformat characteristics. Tests for environmental qualification and media lifetimes are also included. The test methods include testing conditions, testing procedures, a description of the testing setup, and the required calibration procedures.

100,606

PB92-123124

PC A05/MF A01

National Inst. of Standards and Technology (CSL), Gaithersburg, MD. Advanced Systems Div.

Monitoring and Reporting Techniques for Error Rate and Error Distribution in Optical Disk Systems.

Final rept.

F. L. Podio. Oct 91, 92p NIST/SP-500/198

Also available from Supt. of Docs. as SN003-00303125-9. Proceedings of a workshop held in Colorado Springs, Colorado on August 5, 1991.

Keywords: "Optical disks, "Meetings, State of the art, Mechanical drives, Error analysis, Recommendations, Error detection, Error correction.

The report constitutes the proceedings of the workshop on Monitoring and Reporting Techniques for Error Rate and Error Distribution in Optical Disk Systems held on August 5, 1991, in Colorado Springs, Colorado. The objectives of the workshop were to identify the state of the art on error rate monitoring and reporting techniques in optical disk systems and to promote discussions on possible future implementations. The workshop presentations included the description of a Computer Systems Laboratory of the National Institute of Standards and Technology (CSL/NIST) program for investigating error reporting capabilities of optical disk 


\section{Computer Hardware}

drives, Federal Government needs on error detection, correction and reporting, and the state of the art on error reporting capabilities in current generation drives. Presentations also included the description of the capabilities of error correction and detection current chips and discussions on error management strategies. During a discussion panel, the participants identified a preliminary set of user requirements and it was suggested that a Government/industry working group be organized in order to document a consensus position between the Federal Government and industry in error reporting capabilities for future generation drives.

\section{Computer Software}

100,607

AD-A233 961/2

PC A04/MF A01

National Inst. of Standards and Technology, Gaithers burg, MD.

Ada Compiler Validation Summary Report. Certificate Number: 901109\$1.11053, Digital Equipment Corporation VAX Ada, Version 2.2 VAX $8800=>$ VAX 8800 .

Final rept.

12 Dec 90, 59p Rept no. AVF-NIST90DEC505-1-1.11 Keywords: "Ada programming language, "Compilers, "Validation summary reports, Standards, Tests.

The Ada implementation described above was tested according to the Ada Validation Procedure (Pro90) against the Ada Standard (Ada83) using the current Ada Compiler Validation Capability (ACVC). This Validation Summary Report (VSR) give an account of the testing of this Ada implementation.

\section{0,608}

- A234 083/4 burg, MD.

Ada Compiler Validation Summary Report: DDC International A/S, DACS VAX/VMS to 80186 Bare Ada Cross Compiler System, Version 4.6, VAX 8530 (Host) to Bare Board iSBC 186/93A (Host), 90112951.11079

Final rept.

30 Nov 90, 98p Rept no. NIST-90DDC500-8-1.11

Keywords: "Ada programming language, "Compilers, "Validation summary reports, Standards, Tests

The Ada implementation described above was tested according to the Ada Validation Procedures Pro90 against the Ada Standard Ada83 using the current Ada Compiler Validation Capability (ACVC). This Validation Summary Report (VSR) gives an account of the testing of this Ada implementation.

\section{0,609}

\section{AD-A234 118/8}

PC A06/MF A01

National Inst. of Standards and Technology, Gaithersburg, MD.

Ada Compiler Validation Summary Report: Certificate Number: 901129S1.11112 DDC International A/S DACS 80386 DMS/OS Ada Compiler System, Version 4.6 IBM PS/2 Model $80-311=>$ IBM PS $/ 2$ Model 80-311.

Final rept.

30 Nov 90, 117p Rept no. NIST90DDC500-9-1.11

Keywords: "Ada programming language, ${ }^{*}$ Compilers, Validation summary reports, Standards, Tests.

The Ada implementation described above was tested according to the Ada Validation Procedures (Pro90) against the Ada Standard (Ada83) using the current Ada Compiler Validation Capability (ACVC). This Validation Summary Report (VSR) gives an account of the testing of this Ada implementation.

\section{0,610}

AD-A234 323/4

PC A04/MF A01

National Inst. of Standards and Technology, Gaithersburg, MD.

Ada Compiler Validation Summary Report: Certificate Number: 901129S1.11050 DDC International A/S DACS VAX/VMS Native Ada Compiler System, Verslon 4.6 VAX $8530 \Rightarrow$ VAX 8530

30 Nov $90,53 p$

Keywords: "Ada programming language, "Compilers, "Validation summary reports, Standards, Tests.
The Ada implementation described above was tested according to the Ada Validation Procedures Pro90 against the Ada Standard Ada83 using the current Ada Compiler Validation Capability (ACVC). This Validation Summary Report (VSR) gives an account of the testing of this Ada implementation.

100,611

AD-A234 350/7

PC A04/MF A01

National Inst. of Standards and Technology, Gaithersburg, MD.

Ada Compiler Validation Summary Report: Digital Equipment Corporation, VAX Ada, Version 2.2, BAX 8800 (Host) I VAX MicroVAX II Running VAXELN Version 4.1 (Target), 901109S1.11054. Fina! rept.

12 Dec 90, 59p Rept no. NIST-90DEC505-2-1.11

Keywords: "Ada programming language, "Compilers, *Validation summary reports, Standards, Tests.

The Ada implementation described above was tested according to the Ada Validation Procedures Pro90 against the Ada Standard Ada83 using the current Ada Compiler Validation Capability (ACVC). This Validation Summary Report (VSR) gives an account of the testing of this Ada implementation.

100,612

AD-A234 380/4

PC A03/MF A01

National Inst. of Standards and Technology, Gaithersburg, MD.

Ada Compiler Validation Summary Report: Certificate Number: 901129S1.11051, DDC International A/S, DACS VAX/VMS to 68020 Bare Cross Compiler System, Version 4.6 MicroVAX 3100 Greater Than or Equal to Motorola MVME133.

Final rept. 17 Dec 90-1 Mar 91

1 Mar $91,45 p$

Keywords: "Ada programming language, "Compilers, "Validation summary reports, Standards, Tests.

The Ada implementation described above was tested according to the Ada Validation Procedures Pro 90 against the Ada Standard Ada 83 using the current Ada Compiler Validation Capability (ACVC). This Validation Summary Reports (VCR) gives an account of the testing of this data implementation.

100,613

AD-A234 438/0

PC A06/MF A01

National Inst. of Standards and Technology, Gaithersburg, MD.

Ada Compiler Validation Summary Report: Certificate Number: 901129S1.11077 DDC International A/S DACS VAX/VMS to 80186 Bare Ada Cross Compiler System with Rate Monotonic Scheduling, Version 4.6 VAX $8530=>$ Bare Board iSBC/03A. Final rept.

30 Nov $90,101 p$

Keywords: "Ada programming language, "Compilers, "Validation summary reports, Standards, Tests.

The Ada implementation described above was tested according to the Ada Validation Procedures (Pro 90) against the Ada Standard (Ada 83) using the current Ada Compiler Validation Capability (ACVC). This Validation Summary Report (VSR) gives an account of the testing of this Ada implementation.

\section{0,614}

AD-A234 439/8

PC A04/MF A01

National Inst of Standards and Technology, Gaithersburg, MD

Ada Compiler Validation Summary Report: Certificate Number: 901129S1.11075 DDC International A/S DACS 80386 UNIX V Ada Compiler System Version 4.6 ICL DRS $300=>$ ICL DRS 300 .

Final rept.

30 Nov $90,72 p$

Keywords: "Ada programming language, "Compilers, "Validation summary reports, Standards, Tests.

The Ada implementation described above was tested according to the Ada Validation Procedures Pro90 against the Ada Standard Ada83 using the current Ada Compiler Validation Capability (ACVC). This Validation Summary Report (VSR) gives account of the testing of this Ada implementation.
National Inst. of Standards and Technology, Gaithersburg, MD.

Ada Compiler Validation Summary Report: Certifi cate Number: 901129S1.11074 DDC International A/S DACS VAX/VMS 6080386 PM Bare Ada Cros Compiler System, Version 4.6 VAX $8530=>$ Bare Board iSBC 386/21.

Final rept.

30 Nov $90,101 p$

Keywords: "Ada programming language, "Compilers, "Validation summary reports, Standards, Tests.

The Ada implementation described above was tested according to the Ada Validation Procedures Pro90 against the Ada Standard Ada83 using the current Ada Compiler Validation Capability (ACVC). This Validation Summary Report (VSR) gives an account of the testing of this Ada implementation.

\section{0,616}

AD-A239 715/6

PC A06/MF A02

National Inst. of Standards and Technology (NCSL), Gaithersburg, MD. Software Standards Validation Group.

Ada Compiler Validation Summary Report. Certificate Number: 910510S1.11161 UNISYS Corporation, UCS Ada, Version 1R1, 2200/600 (Host and Target). Revision.

Final rept.

24 Jul 91, 123p

Revision of report dated 9 Apr-10 May 91

Keywords: "Ada programming language, "Compilers Standardization, Test and evaluation Computers, Computer program verification, "Validation summary reports.

This Validation Summary Report describes the exten to which a specific Ada compiler conforms to the Ada Standard, ANSI/MIL-STD-1815A. This report explains all technical terms used within it and thoroughly reports the results of testing this compiler using the Ada Compiler Validation Capability. An Ada compiler must be implemented according to the Ada Standard, and any implementation-dependent features must conform to the requirements of the Ada Standard. The Ada Standard must be implemented in its entirety, and nothing can be implemented that is not in the Standard. Even though all validated Ada compilers conform to the Ada Standard, it must be understood that some differences do exist between implementations. The Ada Standard permits some implementation depend encies--for example, the maximum length of identifiers or the maximum values of integer types. Other differences between compilers result from the characteristics of particular operating systems, hardware, or implementation strategies. All the dependencies observed during the process of testing this compiler are given in this report. The information in this report is derived from the test results produced during validation testing. The validation process includes submitting a suite of standardized tests, the ACVC, as inputs to an Ada compiler and evaluating the results.

100,617

AD-A240 511/6

PC A05/MF A0

National Inst. of Standards and Technology, Gaithers burg, MD.

Ada Compiler Validation Summary Report: Certificate Number: 910626S1.11179 US. Navy Ada/M, Version 4.0 (OPTIMIZE) VAX $11 / 785=>$ AN/AYK14 (Bare Board)

Final rept.

30 Jul $91,89 p$

Keywords: "Ada programming language, "Compilers, Standardization, Test and evaluation, "Validation summary reports.

This Validation Summary Report describes the extent to which a specific Ada compiler conforms to the Ada Standard, ANSI/MIL-STD-1815A. This report explains all technical terms used within it and thoroughly reports the results of testing this compiler using the Ada Compiler Validation Capability. An Ada compiler must be implemented according to the Ada Standard, and any implementation-dependent features must conform to the requirements of the Ada Standard. The Ada Standard must be implemented in its entirety, and nothing can be implemented that is not in the Standard. Even though all validated Ada compilers conform to the Ada Standard, it must be understood that some differences do exist between implementations. The Ada Standard permits some implementation depend- 
encies--for example, the maximum length of identifiers or the maximum values of integer types. Other differences between compilers result from the characteristics of particular operating systems, hardware, or implementation strategies. All the dependencies observed during the process of testing this compiler are given in this report. The information in this report is derived from the test results produced during validation testing. The validation process includes submitting suite of standardized tests, the ACVC, as inputs to an Ada compiler and evaluating the results.

\section{0,618}

PC A04/MF A01 National Inst. of Standards and Technology, Gaithersburg, MD

Ada Compiler Validation Summary Report: Certlficate Number 910705S1.11192 InterAct Corporatlon InterAct Ada Mips Cross-Compiler System, Release 2.0 MicroVAX 3100 Cluster $\Rightarrow$ Lockheed Sanders STAR MVP R3000/R3010 board (Bare Machine).

Final rep

31 Jul 91,63p

Keywords: "Ada programming language, "Compilers, Standardization, Test and evaluation, "Validation summary reports.

This Validation Summary Report describes the extent to which a specific Ada compiler conforms to the Ada Standard, ANSI/MIL-STD-1815A. This report explains all technical terms used within it and thoroughly reports the results of testing this compiler using the Ada Compiler Validation Capability. An Ada compiler must be implemented according to the Ada Standard, and any implementation-dependent features must conform to the requirements of the Ada Standard. The Ada Standard must be implemented in its entirety, and nothing can be implemented that is not in the Standard. Even though all validated Ada compilers conform to the Ada Standard, it must be understood that some differences do exist between implementations. The Ada Standard permits some implementation dependencies--for example, the maximum length of identifiers or the maximum values of integer types. Other differences between compilers result from the characteristics of particular operating systems, hardware, or implementation strategies. All the dependencies observed during the process of testing this compiler are given in this report. The information in this report is derived from the test results produced during validation testing. The validation process includes submitting a suite of standardized tests, the ACVC, as inputs to an Ada compiler and evaluating the results.

\section{0,619}

AD.A240 610/6 A03/MF A01

National Inst. of Standards and Technology, Gaithersburg, MD.

Ada Compiler Validation Summary Report: Certificate Number: 910502S1.11158 DDC International A/S, DACS VAX/VMS to 80860 Bare Ada Cross Compiler System, Version 4.6.1, VAX 8530 (Host) to Tadpole Technology PLC TP860M (Bare Board) (Target).

24 Jul $91,36 p$

Keywords: "Ada programming language, "Compilers, Standardization, Test and evaluation, "Validation summary reports.

This Validation Summary Report describes the exten to which a specific Ada compiler conforms to the Ada Standard, ANSI/MIL-STD-1815A. This report explains all technical terms used within it and thoroughly reports the results of testing this compiler using the Ada Compiler Validation Capability. An Ada compiler mus be implemented according to the Ada Standard, and any implementation-dependent features must conform to the requirements of the Ada Standard. The Ada Standard must be implemented in its entirety, and nothing can be implemented that is not in the Standard. Even though all validated Ada compilers conform to the Ada Standard, it must be understood that some differences do exist between implementations. The Ada Standard permits some implementation dependencies--for example, the maximum length of identifiers or the maximum values of integer types. Other differences between compilers result from the characteristics of particular operating systems, hardware, or implementation strategies. All the dependencies obgiven in this report. The information in this report is derived from the test results produced during valida- tion testing. The validation process includes submitting a suite of standardized tests, the ACVC, as inputs to an Ada compiler and evaluating the results.

100,620

AD-A240 611/4

PC A05/MF A01

National Inst. of Standards and Technology, Gaithers burg, MD.

Ada Compiler Validation Summary Report: Certificate Number: 910626S1.11176 U.S. Navy, Ada/L Version 4.0 (/Optimize), VAX 11/785 (Host) to AN/ UYJ-43 (Single CPU) (Bare Board) (Target).

Final rept.

30 Jul $91,79 p$

Keywords: "Ada programming language, "Compilers, Standardization, Test and evaluation, "Validation summary reports.

This Validation Summary Report describes the extent to which a specific Ada compiler conforms to the Ada Standard, ANSI/MIL-STD-1815A. This report explains all technical terms used within it and thoroughly reports the results of testing this compiler using the Ada Compiler Validation Capability. An Ada compiler must be implemented according to the Ada Standard, and any implementation-dependent features must conform to the requirements of the Ada Standard. The Ada Standard must be implemented in its entirety, and nothing can be implemented that is not in the Stand ard. Even though all validated Ada compilers conform to the Ada Standard, it must be understood that some differences do exist between implementations. The Ada Standard permits some implementation dependencies--for example, the maximum length of identifiers or the maximum values of integer types. Other differences between compilers result from the characteris tics of particular operating systems, hardware, or im plementation strategies. All the dependencies observed during the process of testing this compiler are given in this report. The information in this report is derived from the test results produced during validation testing. The validation process includes submitting a suite of standardized tests, the ACVC, as inputs to an Ada compiler and evaluating the results.

\section{0,621}

AD-A240 612/2

PC A05/MF A01

National Inst. of Standards and Technology, Gaithers burg, MD

Ada Compiler Validation Summary Report: Certificate Number: 910626\$1.11174 U.S. Navy, Ada/M, Verslon 4.0 (/Optimlze), VAX 8550, Running VAX VMS Version 5.3 (Host) to AN/UYK-44 (EMR) (Bare Board) (Target)

Final rept.

$30 \mathrm{Jul} 91,89 \mathrm{p}$

Keywords: "Ada programming language, "Compilers, Standardization, Test and evaluation, "Validation summary reports.

This Validation Summary Report describes the exten to which a specific Ada compiler conforms to the Ada Standard, ANSI/MIL-STD-1815A. This report explains all technical terms used within it and thoroughly reports the results of testing this compiler using the Ada Compiler Validation Capability. An Ada compiler must be implemented according to the Ada Standard, and any implementation-dependent features must conform to the requirements of the Ada Standard. The Ada Standard must be implemented in its entirety, and nothing can be implemented that is not in the Standard. Even though all validated Ada compilers conform to the Ada Standard, it must be understood that some differences do exist between implementations. Th Ada Standard permits some implementation depend encies--for example, the maximum length of identifiers or the maximum values of integer types. Other differences between compilers result from the characteristics of particular operating systems, hardware, or im plementation strategies. All the dependencies observed during the process of testing this compiler are given in this report. The information in this report is derived from the test results produced during valida tion testing. The validation process includes submitting a suite of standardized tests, the ACVC, as inputs to an Ada compiler and evaluating the results.

\section{0,622}

AD-A240 613/0

PC A05/MF A01

National Inst. of Standards and Technology, Gaithersburg, MD.
Ada Compiler Validation Summary Report: Certifi cate Number: 910626S1.11175 U.S. Navy Ada/M, V

Final rept.

30 Jul $91,87 p$

Keywords: "Ada programming language, "Compilers Standardization, Test and evaluation, "Validation summary reports.

This Validation Summary Report describes the extent to which a specific Ada compiler conforms to the Ada Standard, ANSI/MIL-STD-1815A. This report explains all technical terms used within it and thoroughly re ports the results of testing this compiler using the Ada Compiler Validation Capability. An Ada compiler must be implemented according to the Ada Standard, and any implementation-dependent features must conform to the requirements of the Ada Standard. The Ada Standard must be implemented in its entirety, and nothing can be implemented that is not in the Stand ard. Even though all validated Ada compilers conform to the Ada Standard, it must be understood that some differences do exist between implementations. The Ada Standard permits some implementation dependencies--for example, the maximum length of identifiers or the maximum values of integer types. Other differences between compilers result from the characteristics of particular operating systems, hardware, or implementation strategies. All the dependencies observed during the process of testing this compiler are given in this report. The information in this report is derived from the test results produced during valida tion testing. The validation process includes submitting a suite of standardized tests, the ACVC, as inputs to an Ada compiler and evaluating the results.

100,623

AD-A240 762/5

PC A04/MF A01

National Inst. of Standards and Technology, Gaithersburg, MD.

Ada Compiler Validation Summary Report. Certiflcate Number: 910705 S1.11191, InterACT Corporation InterACT Ada 1750A Compiler System, Release 3.5 , MicroVAX 3100 cluster $=>$ InterACT MIL-STD-1750A Instruction Set Architecture Simulator, Release 2.3 (Bare Machine).

Final rept

31 Jul $91,75 p$

Keywords: "Ada programming language, "Compilers, Computer program verification, "Validation summary reports, Standardization, Test and evaluation.

The Ada implementation described above was tested according to the Ada Validation Procedures (Pro90) against the Ada Standard (Ada83) using the current Ada Compiler Validation Capability (ACVC). This Validation Summary Report (VSR) gives an account of the testing of this Ada implementation. For any technical terms used in this report, the reader is referred to Pro90. A detailed description of the ACVC may be found in the current ACVC User's Guide (UG89).

100,624

AD-A240 763/3

PC A05/MF A01

National Inst. of Standards and Technology, Gaithers burg, MD.

Ada Compiler Validation Summary Report. Certificate Number: 910626S1.11178, U.S. Navy $\mathrm{Ada} / \mathrm{M}$ Version 4.0 (/OPTIMIZE) VAX $11 / 785 \Rightarrow$ AN/ UYK-44 (EMR) (Bare Board).

Final rept.

30 Jul $91,89 p$

Keywords: "Ada programming language, "Compilers, Computer program verification, "Validation summary reports, Standardization, Test and evaluation.

The Ada implementation described above was tested according to the Ada Validation Procedures (Pro90) against the Ada Standard (Ada83) using the current Ada Compiler Validation Capability (ACVC). This Validation Summary Report (VSR) gives an account of the testing of this Ada implementation. For any technical terms used in this report, the reader is referred to Pro90. A detailed description of the ACVC may be found in the current ACVC User's Guide (UG89).

\section{0,625}

PC A05/MF A01

National Inst. of Standards and Technology, Gaithers burg, MD. 
Ada Compiler Validation Summary Report: Certificate Number 910626S1.11173 U.S. Navy Ada/L, Version 4.0 (/Optimize) VAX $855=$ AN/UYK-43 (EMR) (Bare Board).

Final rept.

30 Jul $91,80 p$

Keywords: "Ada programming language, "Compilers, Computer program verification, "Validation summary reports, Standardization, Test and evaluation.

The Ada implementation described above was tested according to the Ada Validation Procedures (Pro90) against the Ada Standard (Ada83) using the current Ada Compiler Validation Capability (ACVC). This Validation Summary Report (VSR) gives an account of the testing of this Ada implementation. For any technical terms used in this report, the reader is referred to Pro90. A detailed description of the ACVC may be found in the current ACVC User's Guide (UG89).

\section{0,626}

\section{AD-A240 784/9} burg, MD.

Ada Compiler Validation Summary Report: Certiflcate Number Number 910626\$1.11172 U.S. Navy UYK-43 (Single CPU) (Bare Board).

Final rept.

30 Jul $91,80 p$

Keywords: "Ada programming language, "Compilers, Computer program verification, "Validation summary reports, Standardization, Test and evaluation.

The Ada implementation described above was tested according to the Ada Validation Procedures (Pro90) against the Ada Standard (Ada83) using the current Ada Compiler Validation Capability (ACVC). This Validation Summary Report (VSR) gives an account of the testing of this Ada implementation. For any technical terms used in this report, the reader is referred to Pro90. A detailed description of the ACVC may be found in the current ACVC User's Guide (UG89).

\section{0,627}

AD-A240 785/6

PC A03/MF A01

National Inst. of Standards and Technology, Gaithersburg, MD.

Ada Compiler Validation Summary Report: Certificate Number: 910502S1.11159 DDC International A/S DACS Sun-3/Sunos to 68030 Bare Ada Cross Compiler System, Version 4.6.4, MRI IEEE 695 (BASIC-MODE) Sun-3/50 = Motorola MVME 143 Board (68030/68882).

Final rept.

24 Jul $91,50 p$

Keywords: "Ada programming language, "Compilers, Computer program verification, "Validation summary reports, Standardization, Test and evaluation.

The Ada implementation described above was tested according to the Ada Validation Procedures (Pro90) against the Ada Standard (Ada83) using the current Ada Compiler Validation Capability (ACVC). This Validation Summary Report (VSR) gives an account of the testing of this Ada implementation. For any technical terms used in this report, the reader is referred to Pro90. A detailed description of the ACVC may be found in the current ACVC User's Guide (UG89).

\section{0,628}

AD-A240 849/0

PC A03/MF A01

National Inst. of Standards and Technology, Gaithersburg, MD.

Ada Compiler Validation Summary Report. Certificate Number: 910502S1.11160 DDC International A/S DACS Sun-3/SunOS to 68030 Bare Ada Cross Compiler System, Version 4.6.4, MRI IEEE 695 MVME143 Board (68030/68882).

Final rept

24 Jul $91,50 p$

Keywords: "Ada programming language, "Compilers, Security, Standardization, Test and evaluation, "Validation summary reports, Computer program verification.

This Validation Summary Report describes the extent to which a specific Ada compiler conforms to the Ada Standard, ANSI/MIL-STD-1815A. This report explains all technical terms used within it and thoroughly reports the results of testing this compiler using the Ada
Compiler Validation Capability. An Ada compiler must be implemented according to the Ada Standard, and any implementation-dependent features must conform to the requirements of the Ada Standard. The Ada Standard must be implemented in its entirety, and nothing can be implemented that is not in the Standard. Even though all validated Ada compilers conform to the Ada Standard, it must be understood that some differences do exist between implementations. The Ada Standard permits some implementation dependencies--for example, the maximum length of identifiers or the maximum values of integer types. Other differences between compilers result from the characteristics of particular operating systems, hardware, or implementation strategies. All the dependencies observed during the process of testing this compiler are given in this report. The information in this report is derived from the test results produced during validation testing. The validation process includes submitting a suite of standardized tests, the ACVC, as inputs to an Ada compiler and evaluating the results.

100,629
AD-A240 850/8

PC A05/MF A01

National Inst. of Standards and Technology, Gaithersburg, MD.

Ada Compller Validation Summary Report. Certlficate Number: 910626S1.11177 U.S. NAVY Ada/L, UYK-43 (EMR) (Bare Board).

Final rept

26 Jun 91, 80p

Keywords: "Ada programming language, *Compilers, Test and evaluation, "Validation summary reports, Computer program verification, Standards.

This Validation Summary Report describes the extent to which a specific Ada compiler conforms to the Ada Standard, ANSI/MIL-STD-1815A. This report explains all technical terms used within it and thoroughly reports the results of testing this compiler using the Ada Compiler Validation Capability. An Ada compiler must be implemented according to the Ada Standard, and any implementation-dependent features must conform to the requirements of the Ada Standard. The Ada Standard must be implemented in its entirety, and nothing can be implemented that is not in the Standard. Even though all validated Ada compilers conform to the Ada Standard, it must be understood that some differences do exist between implementations. The Ada Standard permits some implementation dependencies--for example, the maximum length of identifiers or the maximum values of integer types. Other differences between compilers result from the characteristics of particular operating systems, hardware, or implementation strategies. All the dependencies observed during the process of testing this compiler are given in this report. The information in this report is derived from the test results produced during validation testing. The validation process includes submitting a suite of standardized tests, the ACVC, as inputs to an Ada compiler and evaluating the results.

100,630
AD-A242 268/1

PC A08/MF A02

National Inst. of Standards and Technology, Gaithersburg, MD.

Ada Compller Validatlon Summary Report: Certificate Number: 910517 S1.11163 U.S. Navy, AdaVAX, Version 5.0, (/NO OPTIMIZE) VAX 8350 (Host and Target).

Final rept.

17 May $91,175 p$

No software available for distribution.

Keywords: "Ada programming language, "Compilers, Standardization, Test and evaluation, "Validation summary reports, Computer program verification.

This Validation Summary Report describes the extent to which a specific Ada compiler conforms to the Ada Standard, ANSI/MIL-STD-1815A. This report explains all technical terms used within it and thoroughly reports the results of testing this compiler using the Ada Compiler Validation Capability. An Ada compiler must be implemented according to the Ada Standard, and any implementation-dependent features must conform to the requirements of the Ada Standard. The Ada Standard must be implemented in its entirety, and nothing can be implemented that is not in the Standard. Even though all validated Ada compilers conform to the Ada Standard, it must be understood that some differences do exist between implementations. The Ada Standard permits some implementation dependencies--for example, the maximum length of identifiers or the maximum values of integer types. Other differences between compilers result from the characteristics of particular operating systems, hardware, or implementation strategies. All the dependencies observed during the process of testing this compiler are given in this report. The information in this report is derived from the test results produced during validation testing. The validation process includes submitting a suite of standardized tests, the ACVC, as inputs to an Ada compiler and evaluating the results.

100,631

AD-A242 269/9

PC A05/MF A01 burg, MD.

Ada Compiler Validation Summary Report: Certificate Number: 910517 S1.11162 U.S. Navy, AdaVAX version 5.0, (/OPTIMIZE) VAX 8600 (Host and Target).

Final rept.

17 May 91, 76p

No software available for distribution.

Keywords: "Ada programming language, "Compilers, Standardization, Test and evaluation, "Validation summary reports, Computer program verification.

This Validation Summary Report describes the extent to which a specific Ada compiler conforms to the Ada Standard, ANSI/MIL-STD-1815A. This reports the results of testing this compiler using the ADa Compile Validation Capability. An Ada compiler must be implemented according to the Ada standard, and any implementation-dependent features must conform to the requirements of the Ada standard. the Ada Standard must be implemented in its entirety, and nothing can be implemented that is not in the standard. Even though all validated Ada compilers conform to the Ada Standard, it must be understood that some differences do exist between implementations. The Ada Standard permits some implementation dependencies-for example, the maximum length of identifiers or the maximum values of integer types. Other differences between compilers result from the characteristics of particular operating systems, hardward, or implementation strategies. All the dependencies observed during the process of testing this compiler are given in this report. The information in this report is derived from the test results produced during validation testing. The validation process includes submitting a suite of standardized tests, the ACVC, as inputs to an Ada compiler and evaluating the results.

\section{0,632}

AD-A242 270/7 PC A04/MF A01

National Inst. of Standards and Technology, Gaithersburg, MD.

Ada Compiler Validation Summary Report: Certificate Number: 910517 S1.11165 U.S. Navy, Ada VAX, Version 5.0 (/NO-OPTIMIZE), VAX 11/785 (HOSt and Target).

17 May $91,72 p$

No software available for distribution.

Keywords: "Ada programming language, "Compilers, Standardization, Test and evaluation, *Validation summary reports, Computer program verification.

This Validation Summary Report describes the extent to which a specific Ada compiler conforms to the Ada Standard, ANSI/MIL-STD-1815A. This report explains all technical terms used within it and thoroughly reports the results of testing this compiler using the Ada Compiler Validation Capability. An Ada compiler must be implemented according to the Ada Standard, and any implementation-dependent features must conform to the requirements of the Ada Standard. The Ada Standard must be implemented in its entirety, and nothing can be implemented that is not in the Standard. Even though all validation Ada compilers conform to the Ada Standard, it must be understood that some differences do exist between implementations. The Ada Standard permits some implementation dependencies--for example, the maximum length of identifiers or the maximum values of integer types. Other differences between compilers result from the characteristics of particular operating systems, hardware, or implementation strategies. All the dependencies observed during the process of testing this compiler are given in this report. The information in this report is derived from the test results produced during validation testing. The validation process includes submitting a suite of standardized tests, the ACVC, as inputs to an Ada compiler and evaluating the results. 
100,633

AD-A242 273/1

PC A04/MF A0

National Inst. of Standards and Technology, Gaithersburg, MD.

Ada Compiler Validation Summary Report: Certificate Number: $910517 \$ 1.11164$ U.S. Navy, AdaVAX, Version 5.0, (/OPTIMIZE), VAX11/785 (Host and Target).

Final rept.

17 May $91,72 p$

No software available for distribution.

Key...rds: "Ada programming language, "Compilers, Standardization, Test and evaluation, "Validation summary reports, Computer program verification.

This Validation Summary Report describes the extent to which a specific Ada compiler conforms to the Ada Standard, ANSI/MIL-STD-1815A. This report explains all technical terms used within it and thoroughly reports the results of testing this compiler using the Ada Compiler Validation Capability. An Ada compiler mus be implemented according to the Ada Standard, and any implementation-dependent features must conform to the requirements of the Ada Standard. The Ada Standard must be implemented in its entirety, and nothing can be implemented that is not in the Standard. Even though all validation Ada compilers conform to the Ada Standard, it must be understood that some differences do exist between implementations. The Ada Standard permits some implementation dependencies--for example, the maximum length of identifiers or the maximum values of integer types. Other differences between compilers result from the characteris. tics of particular operating systems, hardware, or im plementation strategies. All the dependencies observed during the process of testing this compiler are given in this report. The information in this report is derived from the test results produced during validation testing. The validation process includes submitting a suite of standardized tests, the ACVC, as inputs to an Ada compiler and evaluating the results.

100,634

AD-A242 609/6

PC A05/MF A01

National Inst. of Standards and Technology, Gaithersburg, MD.

Ada Compller Validation Summary Report: Certificate Number 910918S1.11216, NEC Corporation, NEC Ada Compller System for EWS-UX/V (Rel 4.0), Version Release 2.1(4.6) EWS4800/220 EW $4800 / 220$.

Final rept.

18 Sep $91,77 \mathrm{p}$

No software available with this document

Keywords: Standardization, Test and evaluation, "Compilers, "Ada programming language, "Validation summary reports, Computer program verification.

This Validation Summary Report describes the extent o which a specific Ada compiler conforms to the Ada Standard, ANSI/MIL-STD-1815A. This report explains all technical terms used within it and thoroughly reports the results of testing this compiler using the Ada Compiler Validation Capability. An Ada compiler must be implemented according to the Ada Standard, and any implementation-dependent features must conform to the requirements of the Ada Standard. The Ada Standard must be implemented in its entirety, and nothing can be implemented that is not in the Standard. Even though all validated Ada compilers conform to the Ada Standard, it must be understood that some differences do exist between implementations. The Ada Standard permits some implementation dependencies--for example, the maximum length of identifiers or the maximum values of integer types. Other differences between compilers result from the characteris tics of particular operating systems, hardware, or implementation strategies. All the dependencies observed during the process of testing this compiler are given in this report. The information in this report is derived from the test results produced during validation testing. The validation process includes submitting a suite of standardized tests, the ACVC, as inputs to an Ada compiler and evaluating the results.

\section{0,635}

AD-A242 896/9

PC A04/MF A01

National Inst. of Standards and Technology, Gaithersburg, MD.
Ada Compiler Validation Summary Report. Certificate Number: 911025S1.11226, Digital Equipment Corporation DEC Ada, Version 1.0 DECstation 5000 Model $200=>$ DECstation 5000 Model 200 Final rept.

28 Oct $91,71 p$

Keywords: Standardization, Test and evaluation "Compilers, "Ada programming language, "Validation summary reports, Computer program verification.

This Validation Summary Report describes the exten to which a specific Ada compiler conforms to the Ada Standard, ANSI/MIL-STD-1815A. This report explains all technical terms used within it and thcroughly ? ports the results of testing this compiler using the Ada Compiler Validation Capability. An Ada compiler must be implemented according to the Ada Standard, and any implementation-dependent features must conform to the requirements of the Ada Standard. The Ada Standard must be implemented in its entirety, and nothing can be implemented that is not in the Stand ard. Even through all validated Ada compilers conform to the Ada Standard, it must be understood that some differences do exist between implementations. The Ada Standard permits some implementation dependencies--for example, the maximum length of identifiers or the maximum values of integer types. Other differences between compilers result from the characteristics of particular operating systems, hardware, or implementation strategies. All the dependencies observed during the process of testing this compiler are given in this report. The information in this report is derived from the test results produced during validation testing. The validation process includes submitting suite of standardized tests, the ACVC, as inputs to an Ada compiler and evaluating the results.

100,636

FIPS PUB 120-1A

C E09

National Inst. of Standards and Technology (CSL) Gaithersburg, MD

Graphical Kernel System (GKS). Pascal Binding; Category: Software Standard; Subcategory: Graphics.

Federal information processing standards (Final).

D. R. Benigni. c1991, 179p

Supersedes FIPS PUB 120. See also FIPS PUB 120-1 and FIPS PUB 120-1B. Also pub. as American National Standards Inst., New York rept. no. ANSI-X3.124.2 1988. Prepared in cooperation with American National Standards Inst., New York.

Three ring vinyl binder also available; North American Continent price $\$ 7.00$; all others write for quote.

Keywords: "Computer graphics, "Interactive graphics, Software, Requirements, Subroutines, Specifications, Federal Information Processing Standards Publications 120-1A, FIPS 120-1A, *Graphical Kernel System, American National Standard Graphical Kernel System (ANSGKS) ANSI X3.124-1985, Language binding, Graphics software standard.

The revision supersedes FIPS PUB 120 and modifies the standard by adding a requirement for validation of GKS implementations that are acquired by Federal agencies. FIPS 120-1 adopts American National Standard Graphical Kernel System (ANS GKS), ANS X3.124-1985, Functional Description, which consists for four parts (X3.124.1-1985 FORTRAN Binding $\times 3.124 .2-1988$ Pascal Binding X3.124.3-1989 Ada Binding), as a FIPS. ANS GKS specifies a library (or toolbox package) of subroutines for an application pro. grammer to incorporate within a program in order to produce and manipulate two-dimensional pictures. The purpose of the standard is to promote portability of graphics application programs between different installations. The standard is for use by implementors as the reference authority in developing graphics software systems; and by other computer professionals who need to know the precise syntactic and semantic rules of the standard.

\section{0,637}

PB91-134536

Not available NTIS

National Bureau of Standards (ICST), Gaithersburg, MD. Systems and Software Technology Div.

Developing Federal Software Standards: A New Direction.

Final rept.

W. M. Osborne, and A. L. Hankinson. 1986, 2p

Pub. in Proceedings of Computer Standards Conference Striking a Balance between Technology, Economics, Politics, and Reality - for Substance, Not Form, San Francisco, CA., May 13-15, 1986, p1-2.
Keywords: Reprints, "Federal information processing "Computer software, "Software engineering Software tools, Computer software maintenance, Computer program reliability, Computer software management.

The software community has made great strides in the application of engineering methods and tools to improve software quality and to enhance the productivity of the software development process. The discussion describes efforts underway at the National Institute of Standards and Technology to use software standards as a framework for developing, acquiring, and main taining software during its operational life.

\section{0,638}

PB91-143289

PC A03/MF A01

National Inst. of Standards and Technology (NEL) Gaithersburg, MD. Robot Systems Div.

Assembly Code to Compute Sine and Cosine Using the Cordic Algorithm.

J. A. Horst. Dec 90, 20p NISTIR-4480

Keywords: "Computer calculations, Assembly languages, Algorithms, Computation, Computer programs, "CORDIC algorithm, Sine, Cosine, Intel 8051 microcontrollers.

The CORDIC algorithm is commonly used to approximate certain elementary functions. Many microproces sor and microcontroller chips without the availability of math coprocessor chips could benefit from the efficient implementation of this algorithm. The focus of the work is to report on a specific implementation in assembly code (for an 8051 microcontroller) that computes the sine and cosine to eleven bits of accuracy.

100,639

PB9 1-149179

Not available NTIS

National Bureau of Standards (ICST), Gaithersburg. MD. Center for Computer Systems Engineering.

UNIX Expert: A Prototype Knowledge-Based Software Development Workstation.

Final rept.

S. B. Salazar. $1985,6 \mathrm{p}$

Pub. in Intelligent Systems: Their Development and Application, p103-108 1985

Keywords: *UNIX(Operating system), "Expert sys tems, Knowledge bases(Artificial intelligence), Software engineering, Workstations, Prototypes, Reprints.

$\mathrm{U}$-Expert is a prototype expert system that assists in resolving UNIX needs. Implementing this system has raised issues regarding the construction of complex knowledge-based systems, among them problems of organizing large amounts of knowledge and providing an acceptable interface for users of varying experience levels. These observations suggest that a programmer's assistant should combine artificial intelligence methods, such as rules, frames, and natural language processing, with more traditional approaches, such as graphics.

\section{0,640}

PB91-149336

Not available NTIS National Bureau of Standards (NEL), Gaithersburg, MD.

Algorithm Design for Large-Scale Computations.

Final rept.

F. Sullivan. 1987, $7 p$

tions $1, n 1$ International Jn

Keywords: "Computer programming, Ising model, Su percomputers, Computation, Algorithms, Reprints, "Algorithm design, Fractal dimensions, Molecular dynamics.

Success in large scale scientific computations often depends on algorithm design. Even the fastest ma chine may prove to be inadequate unless sufficient attention is paid to the way in which the computation is organized. In the paper, the authors use several problems from computational physics to illustrate the importance of good algorithms, and they offer some very general principles for designing algorithms. Two subthemes are: first, the strong connection between the algorithm and the architecture of the target machine; and second, the importance of non-numerical meth ods in scientific computations. 


\section{Computer Software}

National Inst. of Standards and Technology (NCSL), Gaithersburg, MD

Proceedings of the Object-Oriented Database Task Group Workshop, Tuesday, October 23, 1990,

Chateau Laurier Hotel, Ottawa, Canada.
E. N. Fong. Jan 91,150 p NISTIR-4488

Keywords: "Meetings, "Data bases, "Standardization, Data base management systems, Programming languages, Standards, Models, Data processing security, Query languages, "Object-oriented programming.

The report constitutes the proceedings of a one-day workshop on standardization of object database systems. The workshop was the second attempt to solicit public input to identify what aspects of object database systems may be candidates for consensus that can lead to standards. The workshop goals focused on concrete proposals for languages or module interfaces, exchange mechanisms, abstract specifications, common libraries, or benchmarks. The workshop an nouncement also solicited papers on relationships of object database systems capabilities to existing standards, including assertions that question the wisdom of standardization. The proceedings consist of 13 position papers covering various aspects where standardization on object database systems may be possible.

\section{0,642}

PB91-159160

Not available NTIS National Bureau of Standards, Gaithersburg, MD. Program Office.

Agency Exploits Flexibility of Software in Moving Info.

Final rept.

A. H. Sher, and P. B. Saunders. 1986, 1p

Pub. in Government Computer News 5 , n9 p35(2), 9 May 86.

Keywords: "Microcomputers, "Computer software, Data bases, Word processing, Data conversion, Data base management systems, Text processing, Data dictionaries, Reprints.

The article examines problems encountered when data is not in a form immediately suitable for a particular software package. It describes two applications. In the first, global word processor commands are used to convert text files created on a variety of hardware and software systems to a format that can be imported directly into a database file; in the second, the use of database management software enhances the utility of a directory by permitting queries on any information field or part thereof without restricting searches to a rigid list of prespecified keywords.

\section{0,643}

PB91-159723

PC A14/MF A02

National Inst. of Standards and Technology (NCSL), Gaithersburg, MD

Proceedings of the Object-Oriented Database Task Group Workshop. Held in Atlantic City, New Jersey on May 22, 1990.

E. N. Fong, and C. W. Thompson. Feb 91, 308p

NISTIR-4503

See also PB91-157198. Prepared in cooperation with Texas Instruments, Inc., Dallas.

Keywords: "Meetings, "Data bases, "Standardization, Data base management systems, Programming languages, Standards, Models, Information systems,
Query languages, "Object-oriented programming.

The report constitutes the proceedings of a one-day workshop on standardization of object database systems. The workshop was the first of two workshops held to solicit public input to identify what aspects of object database systems may be candidates for consensus that can lead to standards. The workshop attempted to focus on concrete proposals for language or module interlaces, exchange mechanisms, abstract specifications, common libraries, or benchmarks. The workshop announcement also solicited papers on the relationship of object database system capabilities to existing standards, including assertions that question the wisdom of standardization. The proceedings consists of 22 position papers covering various aspects where standardization on object database systems may be possible.

100,644

PB91-167387

PC A04/MF A01

National Inst. of Standards and Technology (NCSL), Gaithersburg, MD.
Functional Benchmarks for Fourth Generation Languages.

Special pub. (Final).

M. M. Gray, and G. E. Fisher. Mar $91,61 p$ NIST/SP. $500 / 184$

Also available from Supt. of Docs. as $\mathrm{SNOO3}^{-003-}$ 03071-6. See also PB87-210332.

Keywords: “Tests, Data base management, High level languages, Methodology, Man computer interface, Computer graphics, Data processing security, Software engineering, Application programs(Computers) ware engineering, Application programs(Computers), marks.

In recent years, fourth generation language (4GL) usage has expanded in data processing organizations especially where end-users have assumed increased programming responsibilities. Since there are no $4 \mathrm{GL}$ standards, managers selecting a $4 \mathrm{GL}$ need some method of determining how well a particular $4 \mathrm{GL}$ will meet organizational, application, and user requirements. The report provides a methodology to assist in that determination. It contains 'functional benchmarks' (as opposed to performance benchmarks) consisting of a testing methodology and descriptions of tests to evaluate the capabilities of a particular $4 \mathrm{GL}$ in relation to organizational requirements. Test results are evalu ated at two levels: the 4GL's ability to perform a task and the ease of performing it. These evaluations are combined with user-defined weighted requirements to produce an overall rating for each 4GL tested.

\section{0,645}

PB91-174441

Not available NTIS

National Inst of Standards and Technology (NEL) Gaithersburg, MD. Applied and Computational Mathematics Div.

DEQSOL and ELLPACK: Problem-Solving Environments for Partial Differential Equations.

Final rept.

R. F. Boisvert, and D. K. Kahaner. 1991, 13p

Pub. in Office of Naval Research Asian Óffice Scientific Information Bulletin 16, n1 p7-19 Jan/Mar 91.

Keywords: "Partial differential equations, "Computer programming, Elliptic differential equations, Boundary value problems, Numerical integration, Supercomputers, Fortran, Reprints, DEQSOL system, ELLPACK system.

A number of integrated problem-solving environments for the solution of partial differential equations have been developed in recent years. Such systems permit very-high-level descriptions of the mathematical problem and its solution algorithm. These descriptions are translated into efficient Fortran programs that produce solutions in both tabular and graphical forms. In the paper the authors compare and contrast two such systems: DEQSOL, a Japanese project, and ELLPACK an American project. For each system, the authors present a brief history, describe its capabilities, and present several examples of its use.

100,646

PB91-175455

Not available NTIS

National Bureau of Standards (ICST), Gaithersburg MD. Information Systems Engineering Div.

Designing Data Entity Naming Conventions.

Final rept.

J. Newton. 1988, $11 p$

Pub. in Proceedings of International Conference on Entity-Relationship Approach (6th), New York, NY. November $9-11,1987$, p439-449 1988

Keywords: "Data bases, "Data management, Data base administrators, Data dictionaries, Standards, Data structures, Reprints, Information Resource Dictionary System.

A coherent set of naming conventions for data entities is crucial to the central management of data. Name content and format must be designed to maximize the information content and relationship to the logical structure of the data. Information Resource Dictionary System (IRDS) metaname structure provides a framework for name development; a methodology is described for mapping between an entity's data dictionary names.

100,647

PB9 1-185108

$\mathrm{PC}$ A04/MF AO

National Inst. of Standards and Technology (NEL) Gaithersburg, MD. Applied and Computational Mathematics Div.
VolksGrapher: a FORTRAN Plotting Package User's Guide, Version 3.0.

D. K. Kahaner, and W. E. Anderson. Feb 90, 55p NISTIR- $90 / 4238$

Keywords: "Plotting, "Subroutines, Fortran programming language, Two dimensional, Interactive systems, Printers(Data processing), User manuals(Computer programs), VolksGrapher computer program.

VolksGrapher(VG) is a collection of FORTRAN callable subroutines for plotting two dimensional data. The main features of VG are: Portability; Ease of use; Realistic flexibility; Built-in interactivity; Support for several printers; Works in the window environment of the Sun; $V G$ is in the public domain.

100,648

PB91-187567

PC A04/MF A01

National Inst. of Standards and Technology (CSL),

Gaithersburg, MD.

Guide to Design, Implementation and Management of Distributed Databases.

Special pub.

E. N. Fong, C. L. Sheppard, and K. A. Harvill. Feb

$91,60 \mathrm{p}$ NIST/SP-500/185

Also available from Supt. of Docs. as SNO03-00303076-7.

Keywords: "Data base management systems, "Distributed computer systems, Data bases, Life cycles, Computer systems hardware, Computer systems programs, Systems engineering, Computer networks, Computer communications, Computer software, Data dictionaries, Protocols.

For an organization to operate in a distributed database environment, there are two related but distinct tasks that must be accomplished. First, the distributed database environment must be established. Then, a distributed database application can be designed and installed within the environment. The guide describes both of these activities based on a development lifecycle phase framework. The guide provides practical information and identifies skills needed for systems designers, application developers, database and data administrators who are interested in the effective planning design installation, and support for a distributed database environment. In addition, the guide instructs system analysts and application developers with a step-by-step procedure for the design, implementation and management of a distributed data base management system (DBMS) application. The guide also notes that truly heterogeneous distributed database technology is still a research consideration.

\section{0,649}

PB91-187716

PC A04/MF A01

National Inst. of Standards and Technology (NCSL), Gaithersburg. MD. Software Standards Validation Group. Programming Languages and Database Language
SQL. Validated Processor List Including GoSIP Conformance Testing Registers.

J. B. Kailey. Apr 91, 59p NISTIR-4557

Keywords: “Compilers, *Operating systems(Computers), "Validity, Federal information processing standards, PASCAL programming language, Query languages, Tests, Data bases, Ada programming language, $\mathrm{COBOL}$ programming language, Fortran programming language, SQL programming Fortran programming language, SQL programming
language, Federal Information Resources Management Regulation.

The Validated Processor List identifies those COBOL, Fortran, Ada, and Pascal programming language processors that have a current validation certificate and those SQL language processors that have a registered test report, referencing the applicable Federal Information Processing Standard (FIPS) as of the date of the publication. The testing of language processors to determine the degree to which they conform to the Federal Standards is required by Government agencies as specified by the FIPS, Federal Information Resources Management Regulation (FIRMR) Parts 201.13 and 201.39 , and the associated Federal ADP and Telecommunications Standards Index. The List is updated and published quarterly.

100,650

PB91-189456

Not available NTIS National Inst. of Standards and Technology (NCSL), Gaithersburg, MD. Systems and Software Technology Div. 
Difficulties in Parsing SGML.

Final rept.

J. Heath, and L. Welsch. 1988, 7p

Pub. in Proceedings of ACM Conference on Document Processing Systems, Santa Fe, NM., December 5-9, 1988, p 71-77.

Keywords: "Parsers, Documents, Parsing algorithms, Programming languages, Translators, Reprints, "SGML(Standard Generalized Markup Language).

A frequently cited problem with the Standard Generalized Markup Language (SGML) is that applications using the standard have been slow in arriving. Part of this delay is because of the instability of the standard and part because of constructs of the language that are functionally redundant and/or add unnecessary complexity to both machine and human processing. The paper examines the implementation of an SGML parser using commonly available tools for building programming language translators. It describes the problems encountered and suggests modifications to SGML to eliminate those problems. The modified language can be implemented using well tested tools and will be more stable and more amenable to both computer and human processing while maintaining all of the fundamental strengths of SGML.

\section{0,651}

\section{PB91-201772}

PC A03/MF A01

National Inst. of Standards and Technology (NCSL), Gaithersburg, MD. Software Standards Validation Group.

FORTRAN Temporary Program Fixes, FCVS78 Version 2.0, Level 26.

1 Feb $87,22 p$

See also PB85-226736 and PB82-250903.

Keywords: "Compilers, "Error correction codes, Errors, Tests, Computer program verification, Computer software maintenance, Debugging(Computers), *Fortran programming language.

The report is a supplement to the 1978 FORTRAN Compiler Validation System (FCVS78) User's Guide which documents the errors that are contained in Version 2.0 of the FCVS78. An update to the FCVS78 is referred to as Temporany Program Fix (TPF). These TPF's must be applied to the FCVS78 population tape to correct the identified errors and will be used in conducting an official validation. An explanation of each error is provided in the 'description of problem' paragraph together with the name of the test(s), if any, affected by the error.

\section{0,652}

\section{PB91-216598}

PC A03/MF A01

National Inst. of Standards and Technology (CAML), Gaithersburg, MD.

Portable Vectorized Software for Bessel Function Evaluation.

R. F. Boisvert, and B. V. Saunders. Jun $91,18 p$

NISTIR-4615

Keywords: "Bessel functions, "Computer programming, Vector processing, CRAY computers, Computation, Algorithms, Fortran 77 programming language, Chebyshev series, Portability, VFNLIB system.

A suite of computer programs for the evaluation of Bessel functions and modified Bessel functions of orders zero and one for a vector of real arguments is described. Distinguishing characteristics of these programs are that (1) they are portable across a wide range of machines, and (2) they are vectorized in the case when multiple function evaluations are to be performed. The performance of the new programs are compared with software from the FNLIB collection of Fullerton on which the new software is based.

\section{0,653}

PB9 1-222588

PC A03/MF A01

National Inst. of Standards and Technology (CSL), Gaithersburg, MD. Advanced Systems Div.

Performance Evaluation of Hypercube Applications: Using a Global Clock and Time Dilation.

R. D. Snelick. Jul 91, 27p NISTIR-4630

Sponsored by Defense Advanced Research Projects Agency, Arlington, VA., and Department of Energy, Washington, DC.

Keywords: *Hypercube multiprocessors, Parallel processing, Performance evaluation, Time dilation.

Time dilation provides an accurate method for investigating the performance of an application using a varie- ty of physical transport speeds. Measuring sources of communication delay in a global domain reveals performance data that are important in the analysis of loosely-coupled machine applications. Together, time dilation and communication delay measurement provide an environment that offers insight for the development and analysis of concurrent algorithms and architectures. Logical and physical transport latencies indicate whether a poorly performing application needs a new algorithm (less logical delay) or a faster interconnection hardware (less physical delay). Other algorithmic and architectural characteristics are revealed with the performance evaluation measurements. The runtime statistics should be available to programmers so that necessary steps can be taken to improve their programs.

\section{0,654}

PB91-231571

PC A03/MF A01 National Inst. of Standards and Technology (CSL), Gaithersburg, MD. Database and Graphics Group. Computer Implementation of a Discrete Set Alge-

L. Gallagher. Jul 91, 31p NISTIR-4637

Keywords: "Data management, "Set theory, Trees(Mathematics), Data structures, Boolean algebra, Algorithms, Binary trees.

Large finite sets occur naturally in computer data management. Discrete elements such as numbers, pointers, icons, and object identifiers all have fixed-length bit sequence representations that may be viewed as base-two integers. The efficiencet storage and manipulation of large collections of such items is a longstanding problem in computer science. In particular, many data management algorithms may be specified in terms of set operations (e.g. union, intersection, crossproduct) on these collections. Often a large set is stored in reduced form for storage efficiency. A specific problem is then to find efficient algorithms for performing set operations over the reduced representations. The paper presents a binary tree storage mechanism for efficient representation of arbitrary sets of discrete elements taken from a fixed universe. It then develops high-level algorithms for mapping these sets to and from their tree representations and for executing set operations directly on the storage representation itself. This approach provides a method for direct computer implementation of mathematical models based on Boolean algebras defined over finite sets.

\section{0,655}

B 1-236687

Not available NTIS National Inst. of Standards and Technology (CSL), Gaithersburg, MD. Systems and Software Technology Div.

Functional Meta-Structure for Hypertext Models and Systems.

Final rept.

R. Furuta, and P. D. Stotts. $1990,27 p$

Pub. in Electronic Publishing 3, n4 p179-205 Nov 90.

Keywords: "Models, Systems analysis, Publishing, Information retrieval, Reprints, "Hypertext, Meta-structure.

The authors describe a hypertext 'meta-structure'-one that provides an organization for the architecture of hypertext models and systems. The meta-structure was initially developed to help us understand the architecture of a specific hypertext model (the Trellis hypertext model). However, its organization seems generally applicable to a wide range of other models and systems as well. As such, the meta-structure is a good candidate for a high-level hypertext reference model, and so they refer to it as the Trellis hypertext reference model, or the r-model. The r-model represents a hypertext at five levels of abstraction-two abstract levels, two concrete levels, and one visible level. In the paper, they present the r-model, use it to classify four different hypertext (and hypertext-like) systems, and then discuss its relationship to various hypertext-defined concepts.

100,656
PB9 $1-237040$

Not available NTIS

(CSL), Gaither.

Book Review: Hypertext Hands-On.

Final rept.

J. Moline. $1990,2 p$

Pub. in Electronic Publishing 3, n3 p173-174 Aug 90.

Keywords: "Books, *Reviews, Information retrieval, Publishing, Computer software, Systems engineering, Reprints, "Hypertext.
Hypertext Hands-On, by Ben Shneiderman and Greg Kearsley, is an excellent introduction to hypertext whether in book or electronic form. The serious user of the material should start with the software. It provides quick introduction to this new way of organizing and accessing information through examples of potential hypertext applications that could not be represented in the book. These applications provide a context for the concepts of hypertext. The authors have included material on how hypertext works, why one might use it, things of concern to system designers, and help on authoring and implementing a hypertext. Further, they have included a discussion of many of the commercial hypertext systems and also articles on the major personalities in the development of the hypertext concept. The book, whether as a hyperbook or a hardback, is an excellent introduction to this new approach of organizing and accessing information.

100,657

PB91-237057

Not available NTIS

National Inst. of Standards and Technology (CSL). Gaithersburg, MD. Systems and Software Technology Div.

Linking Information to Objects: A Hypertext Prototype for Numismatists.

Final rept.

J. Moline. 1991, 17p

Pub. in Visual Resources VII, p361-377 1991

Keywords: "Museums, "Information systems, Computer applications, Prototypes, Data bases, Man computer interface, Parallel processing, Reprints, "Numismatics, *Hypertext.

The researcher discusses a hypertext prototype developed to provide for the information needs of numismatists, particularly those working with early Arab coins. The structure of the prototype is discussed showing the organization of the information bases, the links among information bases, and the links to tools which facilitate the work of the researcher. The information bases include image bases, object bases, and document bases. Hypertext provides a user interface with simple links to allow non-sequential browsing of the material, as well as complex links which integrate other components into the prototype. The purpose of the paper to present a system of limited scope as a basis for discussing how it deals with some of the issues which may be important in museum settings. It is expected that this research could serve as a model for the development of parallel systems for other user groups. Further, it is expected that this prototype will provide a better understanding of the technologies available and their role in facilitating information use by a particular user community.

\section{0,658}

PB91-237685

Not available NTIS

National Inst of Standards and Technology (CSL), Gaithersburg, MD. Systems and Software Technology Div.

Software Verification and Validation: An Overview. Final rept.

D. R. Wallace, and R. U. Fujii. 1989, 8p

See also PB90-111691.

Pub. in IEEE (Institute of Electrical and Electronics Engineers) Software 6, n3 p10-17 May 89.

Keywords: "Computer program verification, "Software engineering, "Computer software management, Standards, Guidelines, Computer software maintenance, Tests, Reprints.

The application of software verification and validation (V\&V) to the development and maintenance of computer software is governed by three types of software engineering standards and guidelines: those identifying requirements for planning and performing sof tware $V \& V$, those requiring software $V \& V$, and those includ. ing software V\&V activities as part of their domain. Specific definitions, requirements, and approaches vary among them but together they provide strong direction for planning and implementing a software verification and validation effort which includes software testing.

100,659

PB91-240754

PC A03/MF A01

National Inst. of Standards and Technology (MEL),

Gaithersburg, MD. Robot Systems Div. 
Depot: A Framework for Sharing Software Installation Across Organizational and Unix Platform Boundaries.

K. L. Manheimer, S. N. Clark, B. A. Warsaw, and W.

K. L. Manheimer, S. N. Clark, B. A. W
P. Rowe. Sep 90, 14p NISTIR-4436

Prepared in cooperation with Century Computing, Inc. Laurel, MD.

Keywords: "Distributed computer systems, "Computer software, "Installing, UNIX(Operating system), File management systems, Distributed processing, USA local.

The depot provides a coherent and accomodating framework for distributing and administering nonstandard UNIX applications across extensibly numerous and diverse computer platforms. It is designed to promote sharing of expertise and disk resources necessary to maintain elaborate third-party applications, supplementing the utility of ad hoc /usr/local arrangement in a formal scheme that allows extension across non-homogeneous clusters of hosts. In particular, it facilitates software installation, release, and accomodation across multiple platforms and diverse host configura. tions. The authors have implemented the Depot using conventional UNIX subsystems and resources combined with policies for coordinating them. The paper presents the specific aims, structure, and rationale of the depot framework in sufficient detail to facilitate its implementation elsewhere.

\section{0,660}

\section{PB9 1-507699}

MUMPS System Lab., Nagoya (Japan)

Backup), 1991.

Backup),

1 Mar 91 .

System: DEC PDP-11/34; Vax/VMS Version V5.3 op erating system. Language: MUMPS. File format: Vax/ VMS Backup. Other formats available as PB91. 507707 (for Microcomputers), PB91-507715 (ANSI) and PB91-507723 (TAR). Sponsored by National Inst. of Standards and Technology, Gaithersburg, MD. Available in 9-track, ASCII character set, 1600 or 6250 bpi.

Keywords: "Software, "Compilers, "Tests, Federal information processing standards, Precompilers, Validation summary reports, Computer systems programs, Computer program verification, Interpreters, Magnetic tapes, "MUMPS programming language.

Version 7.6 of the ANSI/MDC X11.1-1984 MUMPS Validation Suite (the MVS) is the revision of Version 7.51 (August, 1990) designed to meet the requirements in FIPS PUB 125 for a conformance test method. The revision was made with U.S. reviewers' comments and suggestions in order to incorporate into the validation suite such features as computer detection of integrity of the validation suite, pass/fail conditions of individual tests, checkpointing test runs to enable interrupted test process to restart from the most suitable position, instructions and warnings at adequate points, and fully automated tabulation of the Validation Summary Report (the VSR). Provision of such features will facilitate evaluation of the validation process by relegating these time-consuming tasks to the computer, assisted by a few experienced personnel.

\section{0,661}

PB91-507707

MUMPS System Lab., Nagoya (Japan).

MUMPS Vaiidation Suite, Version 7.6, 1991 (for Microcomputers).

Software.

1 Mar 91, 2 diskettes NIST/SW/DK-91/012

System: IBM PC/XT; MS DOS 3.0 or higher operating system. Language: MUMPS. Supersedes PB90500125. Other formats available as PB91-507699 (Vax/VMS), PB91-507715 (ANSI), and PB91-507723 (TAR). Sponsored by National Inst. of Standards and Technology, Gaithersburg, MD.

The software is on two $1.2 \mathrm{M}, 51 / 4$ inch diskettes, high density. The diskettes are in ASCII format.

Keywords: "Software, "Compilers, "Tests, Microcomputers, Federal information processing standards, Precompilers, Validation summary reports, Computer systems programs, Computer program verification, Interpreters, Diskettes, "MUMPS programming language.

Version 7.6 of the ANSI/MDC $\times 11.1-1984$ MUMPS Validation Suite (the MVS) is the revision of Version 7.51 (August, 1990) designed to meet the require- ments in FIPS PUB 125 for a conformance test method. The revision was made with U.S. reviewers comments and suggestions in order to incorporate into the validation suite such features as computer detection of integrity of the validation suite, pass/fail conditions of individual tests, checkpointing test runs to enable interrupted test process to restart from the most suitable position, instructions and warnings at adequate points, and fully automated tabulation of the Validation Summary Report (the VSR). Provision of such features will facilitate evaluation of the validation process by relegating these time-consuming tasks to the computer, assisted by a few experienced personnel.

\section{0,662}

PB91-507715

MUMPS System Lab., Nagoya (Japan).

CP T99

MUMPS Validation Suite, Version 7.6 (ANSI), 1991.

Software.

1 Mar 91, mag tape NIST/SW/MT-91/013

System: DEC PDP-11/34. Language: MUMPS. Supersedes PB90-500117. Other formats available as PB91 507699 (Vax/VMS Backup), PB91-507707 (for Microcomputers), and PB91.507723 (TAR). Sponsored by National Inst. of Standards and Technology, Gaithers. burg, MD.

Available in 9-track, ASCII character set, 1600 or 6250 bpi.

Keywords: "Software, "Compilers, "Tests, Federal information processing standards, Precompilers, Valida-
tion summary reports, Computer systems programs, Computer program verification, Interpreters, Magnetic tapes, "MUMPS programming language.

Version 7.6 of the ANSI/MDC $\times 11.1-1984$ MUMPS Validation Suite (the MVS) is the revision of Version 7.51 (August, 1990) designed to meet the requirements in FIPS PUB 125 for a conformance test method. The revision was made with U.S. reviewers comments and suggestions in order to incorporate into the validation suite such features as computer detec tion of integrity of the validation suite, pass/fail conditions of individual tests, checkpointing test runs to enable interrupted test process to restart from the most suitable position, instructions and warnings at adequate points, and fully automated tabulation of the Validation Summary Report (the VSR). Provision of such features will facilitate evaluation of the validation process by relegating the time-consuming tasks to the computer, assisted by a few experienced personnel.

\section{0,663}

PB91-507723

MUMPS System Lab. Nagoya (Japan)

MUMPS Validation Suite, Version 7.6 (TAR), 1991.

Software.

1 Mar 91, mag tape NIST/SW/MT-91/014

System: DEC PDP-11/34; VAX/VMS Version V5.3 operating system. Language: MUMPS. Other formats available as PB91-507699 (Vax/VMS Backup), PB91 507707 (for Microcomputers), and PB91-507715 (ANSI). Sponsored by National Inst. of Standards and Technology, Gaithersburg, MD.

Available in 9-track, ASCll character set, 1600 or 6250 bpi.

Keywords: "Software, "Compilers, "Tests, Federal in formation processing standards, Precompilers, Valida tion summary reports, Computer systems programs Computer program verification, Interpreters, Magnetic tapes, "MUMPS programming language.

Version 7.6 of the ANSI/MDC X11.1-1984 MUMPS Validation Suite (the MVS) is the revision of Version 7.51 (August, 1990) designed to meet the requirements in FIPS PUB 125 for a conformance tes method. The revision was made with U.S reviewers comments and suggestions in order to incorporate into the validation suite such features as computer detec tion of integrity of the validation suite, pass/fail conditions of individual tests, checkpointing test runs to enable interrupted test process to restart from the most suitable position, instructions and warnings at adequate points, and fully automated tabulation of the Validation Summary Report (the VSR). Provision of such features will facilitate evaluation of the validation process by relegating these time-consuming tasks to the computer, assisted by a few experienced personnel.

\section{0,664}

PB91-937301

Not available NTIS

National Inst. of Standards and Technology, Gaithers. burg, MD.
Validated Processor List (Cobol, Fortran, ADA, Pascai, SQL). July 1991. Jul 91,65 p NISTIR/4623

Keywords: Cobol Programming Language, Fortran Programming Language, Language Programming.

Validated Processor List identifies those COBOL, Fortran, ADA and Pascal programming language processors that have a current validation certificate and those SQL language processors that have a registered test report, referencing the applicable Federal Information Processing Standard (FIPS) as of the date of this publication. This list also includes GOSIP Conformance Testing Registers. The testing of language processors to determine the degree to which they contorm to the Federal Standards is required by Government agencies in accordance with Federal Information $\mathrm{Re}$ sources Management Regulation (FIRMR) Parts 201.13 and 201.39, and the associated Federal ADP and Telecommunications Standards Index. The list is updated and published quarterly.

100,665

PB91-937302

Not available NTIS

National Inst. of Standards and Technology, Gaithersburg, MD.

Validated Processor List (Cobol, Fortran, ADA, Pascal, SQL). October 1991.

Oct $91,94 \mathrm{p}$ NISTIR/4690

Supersedes PB91-937301.

Keywords: Cobol Programming Language, Fortran Programming Language, Language Programming.

Validated Processor List identifies those COBOL, Fortran, ADA and Pascal programming language processors that have a current validation certificate and those SOL language processors that have a registered test report, referencing the applicable Federal Information Processing Standard (FIPS) as of the date of this publication. This list also includes GOSIP Conformance Testing Registers. The testing of language processors to determine the degree to which they conform to the Federal Standards is required by Government agencies in accordance with Federal Information Re. sources Management Regulation (FIRMR) Parts 201.13 and 201.39, and the associated Federal ADP and Telecommunications Standards Index. The list is updated and published quarterly.

\section{0,666}

PB92-108943

PC A07/MF A02

National Inst. of Standards and Technology (CSL), Gaithersburg, MD.

Development of an Optical Disk System for the Automated Retrieval of EASEAR Records.

Final rept.

N. Willman. Aug 91, 139p NISTIR-4654

Sponsored by Social Security Administration, Baltimore, MD.

Keywords: "Information retrieval, "Optical disks, Programming manuals, Computer programs, Magnetooptics, Automation, Feasibility, Pilot studies, "Social Security Administration, EASEAR pilot system, EASEAR records.

The Social Security Administration (SSA) maintains records of the yearly wages earned by every person in the United States. Each year, approximately 2.5 gigabytes of data are collected on self-employed wage earners, and 47 gigabytes of data are collected on other wage earners. The records are currently stored on over 110,000 rolls of microfilm. It takes over 400 scouts to retrieve information needed by the professional staff. Often, the needed roll of film is missing from the file due to being currently in use, misfiled, or misplaced. In addition, the information obtained from the microfilm is not always the most current, and decisions are sometimes based on obsolete data. Advances in peripheral mass storage technology during the 1980 s (e.g. magneto-optic recording) now allow for alternate approaches to data storage and retrieval. It is believed that an automated retrieval system would provide a more accurate, timely, and cost effective means of retrieving information. The pilot application detailed in the document is intended to demonstrate the feasibility of a full scale automated records retrieval system, to study the impact of an automated system on the users, and to gain insight into the factors which would impact the design of a full scale system. 
National Inst. of Standards and Technology (CSL), Gaithersburg, MD.

Proceedings of the Workshop on High Integrity Software. Held in Gaithersburg, MD. on January 22-23, 1991.

Special pub. (Final).

D. R. Wallace, D. R. Kuhn, and J. C. Cherniavsky.

Aug $91,11 \mathrm{p} \mathrm{NIST/SP-500/190}$

Also available from Supt. of Docs. as $\mathrm{SNOOH}^{-003}$ 03108-9. Prepared in cooperation with National Science Foundation, Washington, DC

Keywords: "Meetings, "Computer program reliability, "Computer software, "Standards, Standardization, Computer security, Benefit cost analysis, Safety, Data integrity, Computer software management, Hazards, Criticality, "NIST.

The paper provides information related to the National Institute of Standards and Technology (NIST) effort to coordinate an effort to produce a comprehensive set of standards and guidelines for the assurance of high integrity software. The effort may include adapting or adopting existing standards as appropriate. In particular, the paper presents the results of a Workshop on the Assurance of High Integrity Software held at NIST on January 22-23, 1991. Workshop participants addressed techniques, costs and benefits of assurance, controlled and encouraged practices, and hazard analysis. A preliminary set of recommendations was prepared and future directions for NIST activities in this area were proposed.

100,668

PB92-112267

PC A04/MF A01

National Inst. of Standards and Technology (CSL) Gaithersburg, MD.

Proceedings of the Forum on Standards for High Integrity Software (DOD, Government, Industry). Held in Gaithersburg, Maryland on June 28, 1991. D. R. Wallace, M. Brown, and A. McKinlay. Sep 91 , $64 p$ NISTIR-4656

See also PB92-109040. Prepared in cooperation with Naval Surface Warfare Center, Dahlgren, VA., and McDonnell Douglas Corp., St. Louis, MO.

Keywords: "Meetings, *Computer software, "Computer security, "Standards, Quality assurance, Safety, Software engineering, Hazards, Computer program reliability, Computer program verification.

The report provides information related to the Forum on Standards for High Integrity Software (Department of Defense, Government, and Industry) held at the $\mathrm{Na}$ tional Institute of Standards and Technology (NIST) on June 28,1991 . At the forum, software engineering ex. perts presented their perspectives on the role of software engineers in software safety, a comparison fo safety and computer security issues in standards, hazard analysis, assurance standards, and software certification. Future directions for NIST activities for assurance of high integrity software were proposed.

\section{0,669}

PB92-116417

PC A03/MF A01

National Inst. of Standards and Technology (CSL), Gaithersburg, MD.

Software Reengineering: A Case Study and Lessons Learned.

Special pub. (Final)

M. K. Ruhl, and M. T. Gunn. Sep 91, 42p NIST/SP$500 / 193$

Also available from Supt. of Docs. as SN003-00303100-3. See also N91-24047.

Keywords: *Software engineering, Computer software maintenance, Case studies, Cost effectiveness, Recommendations, "Software reuse, CASE(Computer Aided Software Engineering).

The report is aimed at managers and technical personnel (both Federal Government and industry) who need to understand: the concepts and issues of software reengineering; the use of Computer Aided Software Engineering (CASE) tools in the reengineering process; and the application of the technology to organizational problems. Software reengineering involves the use of existing software and documentation to specify requirements, design, documentation, and to produce software for a target platform. CASE tools are expected to play an important role in automating parts of the reengineering process. In the report software reengineering and other related terms are defined and possible benefits that relate to the technology are described. The use of CASE tools for reengineering are examined. A case study that examines the feasibility and cost-effectiveness of software reengineering is described. Study results are addressed along with recommendations for organizations that are considering the use of reengineering.

\section{0,670}

PB92-117308

Not available NTIS National Inst. of Standards and Technology (NCSL), Gaithersburg, MD. Systems and Software Technology

Interactive Video Software Portability: Migrating a DOS Application to POSIX.

Final rept.

R. D. Schneeman. 1991, 9p

Pub. in Proceedings of the Annual Technical Symposium (29th) Advanced Information Interfaces: Making Data Accessible, Washington, DC., June 19-20, 1991, p1-9.

Keywords: "Computer program portability, *Disk operating system(DOS), Video disks, Interactive systems, Computer program transferability, Computer software, Interruption, Computer graphics, Reprints, "POSIX(Portable Operating System Interface for Computer Environments).

Research on the migration of an MS-DOS based Interactive Video (IV) application to the Portable Operating System Interface for Computer Environments (POSIX) has revealed several key portability concerns. Namely, when (1) mapping the MS-DOS software interrupt application interface into a suitable POSIX paradigm; (2) implementing the necessary subset of IV commands from the Interactive Mulitmedia Association's (IMA) Recommended Practices for Multimedia Portability document; and (3) supplanting the original graphics capabilities with an Open Systems windowing solution. The paper addresses the above concerns in the context of migrating an MS-DOS application into a POSIX environment. The computing environment used in the research study and the target application are described fully.

\section{0,671}

\section{PB92-937300}

Slanding Order

burg, MD.

Validated Products List (Cobol, Fortran, ADA, Pascal, MUMPS, SQL).

1992, 1p

Supersedes PB91-937300

Paper copy available on Standing Order, deposit account required (U.S., Canada, and Mexico \$100; all others \$200). Single copies also available in paper copy only.

Keywords: "Cobol programming language, "Fortran programming language, Language programming. *Federal Information Processing Standards, "Validation summary reports, " $\mathrm{Pascal}$ programming language, "Ada programming language, SQL programming language.

The Validated Processor List identifies those COBOL, Fortran, Ada and Pascal programming language processors that have a current validation certificate and those SQL language processors that have a registered test report, referencing the applicable Federal Information processing Standard (FIPS) as of the date of the publication. The list also includes GOSIP Conformance Testing Registers. The testing of language processors to determine the degree to which they conform to the Federal Standards is required by Government agencies in accordance with Federal Information Resources Management regulation (FIRMR) Parts 201.13 and 201.39, and the associated Federal ADP and Telecommunications Standards Index. The list is updated and published quarterly.

\section{Information Processing Standards}

\section{0,672}

FIPS PUB 100-1

$\mathrm{PC} \mathrm{A03}$

National Inst. of Standards and Technology (NCSL), Gaithersburg, MD.
Interface between Data Terminal Equipment (DTE) and Data Circuit-Terminating Equipment (DCE) for Operation with Packet-Switched Data Networks (PSDN), or between Two DTEs, by Dedicated Circuit. Category: Hardware Standard. Subcategory: Data Transmission.

Final rept.

c1989, 24p

Also pub. as American National Standards Inst., New York rept. no. ANSI-X3.100-1989. Supersedes FIPS PUB 100. Prepared in cooperation with American National Standards Inst., New York.

Three ring vinyl binder also available; North American Continent price \$7.00; all others write for quote.

Keywords: "Computer networks, "Data processing terminals, Telecommunication, "Interfaces, "Federal Information Processing Standards, " ${ }^{*}$ 'ommunication networks, Communication terminals, X-25 protocol, Packet switching.

The Federal Information Processing Standard (FIPS) specifies the interface between data terminating equipment (DTE) such as automated data processing (ADP) equipment and telecommunication system terminal equipment, and data circuit-terminating equipment (DCE) for operation in the packet mode on packet-switched data networks (PSDN), or between two DTEs, by dedicated circuit. The revised standard adopts American National Standard ANSI X3.1001989 which in turn adopts CCITT Recommendation X25-1988 developed by the Consultative Committee on International Telephone and Telegraph, and ISO 7776 1986 and ISO 8208-1987 developed by the International Organization for Standardization. The revision supersedes FIPS 100/FED-STD 1041. More detailed specifications on the applicable interface points for the standard in an Integrated Services Digital Network (ISDN) environment will be developed for a future FIPS for ISDN.

100,673

FIPS PUB 120-1

PC E15

National Inst. of Standards and Technology (CSL), Gaithersburg, MD.

Graphical Kernel System (GKS); Category: Software Standard; Subcategory: Graphics.

Federal information processing standards (Final)

D. F. Benigni. c1991, 401p

Supersedes FIPS PUB 120 . See also FIPS PUB $120-$ $1 \mathrm{~A}$ and FIPS PUB 120-1B. Also pub. as American National Standards Inst. New York rept. no. ANSIX3.124-1985. Prepared in cooperation with American National Standards Inst., New York.

Three ring vinyl binder also available; North American Continent price \$7.00; all others write for quote.

Keywords: *Computer software, "Federal information processing standards, Computer graphics, Fortran, Pascal programming language, Validation, Ada programming language, "Graphic Kernel System, Language binding.

The report's revision supersedes FIPS PUB 120 and modifies the standard by adding a requirement for validation of GKS implementations that are acquired by Federal agencies. FIPS $120-1$ adopts American $\mathrm{Na}$ tional Standard Graphical Kernel System (ANS GKS), ANSI X3.124-1985, Functional Description, which consists of four parts (X3.124.1-1985 FORTRAN Binding, X3.124.2-1988 Pascal Binding, X3.124.3-1989 Ada Binding), as a FIPS. ANS GKS specifies a library (or toolbox package) of subroutines for an application programmer to incorporate within a program in order to produce and manipulate two-dimensional pictures. The purpose of the standard is to promote portability of graphics application programs between different installations. The standard is for use by implementors as the reference authority in developing graphics software systems; and by other computer professionals who need to know the precise syntactic and semantic rules of the standard.

\section{0,674}

FIPS PUB 120-1B

PC E12

National Inst. of Standards and Technology (CSL), Gaithersburg, MD.

Graphlcal Kernel System (GKS). Ada Binding; Category: Software Standard; Subcategory: Graphics. Federal information processing standards (Final).

D. R. Benigni. c1991, $218 \mathrm{p}$

Supersedes FIPS PUB 120. See also FIPS PUB 120-1 and FIPS PUB 120-1A. Also pub. as American National Standards Inst., New York rept. no. ANSI-X3.124.3- 


\section{Information Processing Standards}

1989. Prepared in cooperation with American National Standards Inst., New York.

Three ring vinyl binder also available; North American Continent price \$7.00; all others write for quote.

Keywords: "Computer software, "Federal information processing standard, Computer graphics, Fortran, Pascal programming language, Validation, Ada programming language, "Graphic Kernel System, "Language binding.

The report revision supersedes FIPS PUB 120 and modifies the standard by adding a requirement for validation of GKS implementations that are acquired by Federal agencies. FIPS $120-1$ adopts American National Standard Graphical Kernel System (ANS GKS) ANSI X3.124-1985, Functional Description, which consists of four parts (X3.124.1-1985 FORTRAN Binding X3.124.2-1988 Pascal Binding, X3.124.3-1989 Ada Binding), as a FIPS. ANS GKS specifies a library (or toolbox package) of subroutines for an application programmer to incorporate within a program in order to produce and manipulate two-dimensional pictures. The purpose of the standard is to promote portability of graphics application programs between different installations. The standard is for use by implementors as the reference authority in developing graphics software systems; and by other computer professionals who need to know the precise syntactic and semantic rules of the standard.

\section{0,675}

PC A05/MF A01

National Inst. of

Gaithersburg, MD.

Government Open Systems Interconnection Profile (GOSIP); Category: Hardware and Software Standards; Subcategory: Computer Network Protocols.

3 Apr 91,810

Supersedes FIPS PUB 146

Three ring vinyl binder also available: North American Continent price \$7.00; all others write for quote.

Keywords: "Computer networks, "Standards, Protocols, Specifications, "Open systems interconnections, "Federal information processing standards.

The publication is a revision of Federal Information Processing Standards (FIPS) 146 and supersedes FIPS 146 in its entirety. FIPS 146 adopted the Govern ment Open Systems Interconnection Profile (GOSIP) which defines a common set of data communication protocols that enable systems developed by different vendors to interoperate and the users of different applications on those systems to exchange information. The revision contains all of the protocols in FIPS 146 plus additional protocols which provide new services useful to Federal agencies and increase the interoperability achievable among end systems of different manufacture. The revision also includes minor technical changes to the protocols in FIPS 146.

\section{0,676}

National Inst. of Standards and Technology (NCSL), Gaithersburg, MD.

American National Standard for C. Category: Software Standard. Subcategory: Programming Language.

K. A. Miles. c1990, 361p

Also pub. as American National Standards Inst., New York rept. no. ANSI-X3.159-1989. Prepared in cooperation with American National Standards Inst., New York

Three-ring vinyl binder also available; North American Continent price \$7.00; all others write for quote.

Keywords: "Programming languages, Standards, Data processing, ${ }^{\star}$ Federal Information Processing Stand ard, Information processing, Computer software, ${ }^{*} \mathrm{C}$ programming language.

The publication announces the adoption of American National Standard for C, ANSI X3.159-1989, as a Fed eral Information Processing Standard (FIPS). The American National Standard for $\mathrm{C}$ specifies the form and establishes the interpretation of programs written in the $\mathrm{C}$ programming language. The purpose of the standard is to promote portability of $C$ programs for use on a variety of data processing systems. The standard is for use by implementors as the reference authority in developing compilers, interpreters, or othe forms of high level language processors; and by othe computer professionals who need to know the precise syntactic and semantic rules adopted by ANSI.
100,677

FIPS PUB 161

National Inst. of Standards and Technology (NCSL) Gaithersburg, MD

Electronic Data Interchange (EDI). Category: Software Standard. Subcategory: Electronic Data Interchange.

Final rept.

R. G. Saltman. 29 Mar $91,10 p$

Three ring vinyl binder also available; North American Continent price \$7.00; all others write for quote.

Keywords: "Data transmission, Electronics, Telecommunication, Businesses, "Federal Information Processing Standard, "Electronic data interchange.

The publication announces the adoption, as a Federal Information Processing Standard, of recognized national and international standards for EDI In EDI, data that would be traditionally conveyed on paper documents are transmitted or communicated electronically according to established rules and formats. The data that are associated with each type of functional document, such as a purchase order or invoice, are transmitted together as an electronic message. The formatted data may be transmitted from originator to recipient via telecommunications or physically transported on electronic storage media.

\section{0,678}

FIPS PUB 54-1

PC E 18

National Inst. of Standards and Technology (CSL), Gaithersburg, MD

Computer Output Microform (COM) Formats and Reduction Ratios, 16MM and 105MM. Category: Hardware Standard; Subcategory: Media.

Federal information processing standards (Final).

T. C. Bagg. c15 Jan 91, 24p

Supersedes FIPS PUB 54. Also pub. as American National Standards Inst. New York rept. no. ANSI/AlIMMS14-1988. Prepared in cooperation with American National Standards Inst., New York, and Association for Information and Image Management, Silver Spring MD.

Three ring vinyl binder also available: North American price \$7.00; all others write for quote.

Keywords: "Microfilm, "Standards, Data processing Reduction, "Federal information processing standards, "Microform, "Microfiche, Computer output microards,

The FIPS PUB specifies the image arrangement, size, and reduction ratios for $16 \mathrm{~mm}$ and $105 \mathrm{~mm}$ microforms generated by Computer Output Microfilmers. It is limited to systems using business-oriented fonts similar to line printer output. The revised FIPS adopts American National Standard for Information and Image Management -- Microfiche ANSI/AIIM MS5-1991, and American National Standard for Information and Image Mancan National Standard for Information and Image ManMicrofilm, ANSI/AIIM MS14-1988.

\section{0,679}

FIPS PUB 9-1 PC A02/MF A01 National Inst. of Standards and Technology (NCSL), Gaithersburg, MD.

Congressional Districts of the United States. Category: Federal General Data Standard, Representations and Codes.

Federal information processing standards (Final).

H. Tom. 30 Nov $90,10 p$

Supersedes FIPS PUB 9

Three ring vinyl binder also available, North American Continent price \$7.00; all others write for quote.

Keywords: "United States, "Congress, *Federal Information Processing Standards, * Specifications, Representation, Codes, Guidelines, "Congressional Districts.

The standard provides the structure of numeric codes for representing congressional districts and similar areas defined for the various congresses of the United States. Congressional districts are legislatively defined subdivisions of a State for the purpose of electing representatives or delegates to the House of Representaresentatives or delegates to the House of Representalent entity may comprise a single congressional district or similar representational area.

\section{0,680}

FIPS SET $1991 \quad$ PC\$2300.00 National Inst. of Standards and Technology, Gaithersburg, MD.
Federal Information Processing Standards Publication.

Federal Information Processing Standards.

1991, 2895p-in 162v

Set includes FIPS PUB 0 through FIPS PUB 161 and 13 ring vinyl binders. Supersedes FIPS SET 1990.

Keywords: "Computer Software, "Federal Information Processing Standards.

No abstract available.

100,681

PB91-132126

PC A03/MF A01

National Inst. of Standards and Technology, Gaithersburg, MD.

Fed-X: The NIS S. N. Clark. Aug 90, 18p NISTIR-4371-REV

See also PB90-269507.

Keywords: "Translators, Parsers, Standards, "Product data exchange, "PDES(Product Data Exchange Specification), "EXPRESS programming language, Software tools, Computer aided manufacturing.

The Product Data Exchange Specification (PDES) is an emerging standard for the exchange of product information among various manufacturing applications. PDES includes an information model written in the Express language; other PDES-related information models are also written in Express. The National PDES Testbed at NIST has developed software to manipulate and translate Express models. This software consists of an in-memory working form and an associated Express language parser, FED-X. The design and capabilities of FED-X and the Express Working Form are discussed.

100,682

PB91-132159

PC A03/MF A01

National Inst. of Standards and Technology, Gaithersburg, MD.

NIST PDES Toolkit: Technical Fundamentals. National PDES Testbed Report Series (Revised).

S. N. Clark. Nov 90, 34p NISTIR-4335/REV See also PB90-250093.

Keywords: Standards, Translators, "Product data exchange, "PDES(Product Data Exchange Specification), "STEP(Standard for the Exchange of Product Model Data), "Software tools, Computer aided manufacturing, Software libraries, Application programs(Computers), EXPRESS programming language.

The Product Data Exchange Specification (PDES) is an emerging standard for the exchange of product information among various manufacturing applications. A software toolkit for manipulating PDES data has been developed at the National PDES Testbed at NIST. A technical overview of this PDES Toolkit is provided. Fundamental software libraries are described, and techniques for creating applications based on the Toolkit are discussed.

\section{0,683}

PB91-132183

PC A03/MF A01

National Inst. of Standards and Technology (NCSL) Gaithersburg, MD.

NIST Working Form for STEP: National PDES Testbed Report Series (Revised)

S. N. Clark. 19 Nov 90, 11 p NISTIR-4351/REV See also PB90-250044.

Keywords: "Standards, "Parsers, "Product data exchange, "PDES(Product Data Exchange Specification), *STEP(Standard for the Exchange of Product Model Data), "Computer aided manufacturing, Software tools.

The Product Data Exchange Specification (PDES) is an emerging standard for the exchange of product information among various manufacturing applications. The neutral exchange medium for PDES product models is the STEP physical file format. The National PDES Testbed at NIST has developed software to manipulate and translate STEP models. This software consists of an in-memory working form and an associated physical file parser, STEPparse. The design and capabilities of STEPparse and of the STEP Working Form are discussed.

\section{0,684}

PB91-132209

$\mathrm{PC}$ A03/MF A0 
National Inst. of Standards and Technology (NCSL), Gaithersburg, MD

Use of the IRDS Standard in CALS (Revised)

D. K. Jefferson, and C. M. Furlani. Sep 89, 16p

NISTIR-89-4169-REV

See also PB90-112467.

Keywords: "Information systems, "Standards, "Data base management systems, "CALS(Computer aided Acquisition and Logistics Support), "IRDS(Information Resource Dictionary System), Data dictionaries, Distributed data bases.

The objective of the point paper is to show how the Information Resource Dictionary System (IRDS) can fulfill critical design and operational requirements for CALS Phase II. First, a series of assumptions are made about the data management services which are needed by CALS Phase II. Next, these assumptions are used to develop a series of requirements for a dictionary system. The structure of the IRDS family of tandards is then described. Examples are provided to illustrate how the IRDS could meet the requirements. A schedule is presented to show that the IRDS and other data management standards will be available when needed to meet the immediate requirements of CALS. An architecture is presented to illustrate additional standards required to achieve longer-range goals of distributed database. Finally, development tasks are recommended.

\section{0,685}

PB91-134635

Not available NTIS

National Bureau of Standards (ICST), Gaithersburg, MD. Information Systems Engineering Div.

Information Systems Engineering Program.

Final rept.

F. E. Spielman. 1987, 3p

Pub. in Proceedings of International Conference on Interactive Information and Processing Systems for Meteorology, Oceanography, and Hydrology (3rd) New Orleans, LA., January 12-16, 1987, p58-60.

Keywords: "Standards, "Information systems, Programming languages, Computer graphics, Systems engineering, Reprints, "Data bases, "Data management, Data dictionaries, Data base management systems, Computer applications, National Institute of Standards and Technology.

Because of the increased use of packaged software for data management and applications development, there is a renewed interest in Federal, national, and international standards for database languages, data dictionary systems, computer graphics, data interchange, and interfaces to programming languages. Along with such specific standards, there is a need for guidance documents on data administration, logical database design, use of standard codes and representations, selection of DBMS and graphics systems, and applications development. The presentation discusses the Information Systems Engineering program within he Institute for Computer Sciences and Technology (ICST) at the National Institute of Standards and Technology (NIST) and identifies standards, research activities, and guidance projects in these areas.

\section{0,686}

PB91-144444 National Inst. of Standards and Technology (NCSL), Gaithersburg, MD.

Working Implementation Agreements for Open Systems Interconnection Protocols.

See also PB90-259763. Presented at the NIST Work shop for Implementors of OSI Plenary Assembly, Gaithersburg, MD., September 14, 1990.

Keywords: "Protocols, Standards, Computer networks, Message processing. File management systems, Computer security, Access, Data base manage ment, Local area networks, ${ }^{*}$ OSI(Open Systems Interconnection), National Institute of Standards and Technology.

The document records Working Agreements on Implementation details of Open Systems Interconnection (OSI) Protocols among the organizations participating in the National Institute of Standards and Technology (NIST)/OSI Workshop Series for Implementors of OS। Protocols. These decisions are documented to facilitate organizaions in their understanding of the status of agreements. It is a standing document that is updated after each workshop (about 4 tımes a year).
National Bureau of Standards (ICST), Gaithersburg, MD. Information Systems Engineering Div. Specifications for a Federal Information Process. ing Standard Data Dictionary System.

Final rept.

A. H. Goldfine. 1984, 13p

Pub. in Proceedings of NASA Administrative Data Base Management Systems Conference, Greenbelt MD., May 25-26, 1983, p121-1331984.

Keywords: "Federal information processing standards Data dictionaries, Data base management systems, Computer software, Specifications, Reprints.

The paper describes the data dictionary software specifications being developed by the Institute for Computer Sciences and Technology. The functionality and user interfaces in the final specifications will be incorporated into a planned Federal Information Processing Standard (FIPS) Data Dictionary System. The paper also discusses the focus and status of the effort to develop the planned FIPS DDS.

\section{0,688}

B91-158915

Not available NTIS

(ICST), Gaithersburg MD. Systems and Network Architecture Div.

Features and Facilities of Estelle.

Final rept.

R. J. Linn. 1986, 26p

Pub. in Protocol Specification, Testing and Verification, p271-296 1986 .

Keywords: "Standards, Protocols, Pascal programming language, Specifications, Finite state machines, ISO(International Organization for Standardization).

Estelle is a formal description technique developed by the International Organization for Standardization (ISO) in collaboration with the International Telegraph and Telephone Consultative Committee (CCITT) It is a draft proposed standard within ISO. Estelle was developed to be used by standards committees for the specification of data communications protocols which are destined to become international standards. A specification in Estelle is comprised of a set of modules which communicate with each other. Modules are specified as extended finite state machines using facilities in Estelle which consist of a set of extensions to ISO Pascal.

\section{0,689}

PB91-171967

PC A99/MF E06

National Inst. of Standards and Technology (BFRL), Gaithersburg, MD.

Stable Implementation Agreements for Open Systems Interconnection Protocols. Version 4, Edition 1, December 1990.

Special pub. (Final)

T. Boland. Mar $91,803 p$ NIST/SP-500/183

Also available from Supt. of Docs. as SN903-01500000-4. Supersedes PB90-212192.

Based on the Proceedings of the NIST Workshop for Implementors of OSI. Held in Gaithersburg, Maryland.

Keywords: "Protocols, "Computer communications *Computer networks, Standards, Agreements, Local area networks, Access methods, Message processing, Computer security, File management systems, Direc tories, Data base management systems, "OSI(Open Systems Interconnection)

The document records current Stable Agreements for Open Systems Interconnection (OSI) Protocols among the organizations participating in the National Institute of Standards and Technology/OSI Workshop Series for Implementors of OSI Protocols.

100,690

PB91-187542

PC A03/MF A01

National Inst. of Standards and Technology (NCSL) Gaithersburg, MD. Advanced Systems Div.

Standard Reference Materials: Calibration of NIST Standard Reference Material 3201 for 0.5 Inch (12.65 mm) Serial Serpentine Magnetic Tape Cartridge.

tridge.

M. P. Williamson, N. E. Willman, and D. S. Grubb.

Feb 91, 39p NIST/SP-260-115

Also available from Supt. of Docs. as SN003-00303068-6.

Keywords: "Magnetic tapes, "Standards, Calibration, Test methods, *Standard reference materials.

The publication describes the test system design and operation for the calibration of the NIST secondary standard reference tapes SRM 3201 for 0.5 inch (12.65mm) 22 and 48 track serial serpentine magnetic tape cartridges. The importance of producing a Stand ard Reference Material for the magnetic tape cartridge is to promote the ability to interchange data both within and among various computer installations. Reliable interchange is assured when the media is designed and manufactured on the basis of a comparison to a known and accepted standard reference media.

\section{0,691}

PB91-197004

PC A11/MF A02

National Inst of Standards and Technology, Gaithersburg, MD.

Stable Implementation Agreements for Open Systems Interconnection Protocols. Version 4, Edition 1, March 1991. Change Pages (Supplement).

Special pub. (Final)

T. Boland. Jun $91,236 p$ NIST/SP-500/183SUPPL1 Also available from Supt. of Docs. as SN903-015 00000-4. See also PB91-171967.

Keywords: "Protocols, Changes, Computer networks Message processing, File management systems, Computer security, Data processing security, Algorithms, "OSI(Open Systems Interconnection), National Institute of Standards and Technology.

The document records current Stable Agreements for Open Systems Interconnection (OSI) Protocols among the organizations participating in the National Institute of Standards and Technology (NIST)/OSI Workshop Series for Implementors of OSI Protocols.

\section{0,692}

PB91-201004

PC A05/MF A01

National Inst. of Standards and Technology (CSL) Gaithersburg, MD

Application Portability Profile (APP): The U.S. Government's Open System Environment Profile OSE/ 1 Version 1.0

Special pub. (Final)

G. Fisher. Apr 91,78p NIST/SP-500/187

Also available from Supt. of Docs.

Keywords: "Computer applications, "Computer program portability, "Specifications, Computer program transferability, Protocols, Data structures, Nationa government, United States, Information systems, Federal agencies, "Open System Environment, *APP(Application Portability Profile), National Institute of Standards and Technology.

An Open System Environment (OSE) encompasses the functionality needed to provide interoperability portability, and scalability of computerized applications across networks of heterogeneous hardware/software platforms. The OSE forms an extensible framework that allows interfaces, services, protocols, and supporting data formats to be defined in terms of nonproprietary specifications that evolve through open (public), consensus-based forums. A selected suite of specifications that define these interfaces, services, protocols, and data formats for a particular class or domain of applications is called a profile. The Application Portability Profile (APP) is the U.S. Government's OSE profile. It was developed to provide functionality across a broad range of Federal applications. The report describes the service areas and components included in the APP and provides evaluations of recommended specifications for the majority of the service area components. Organizations should use the report to assist in determining which specifications may be applicable to their particular environments.

\section{0,693}

PB91-203133

Not available NTIS

National Inst of Standards and Technology (NCSL) Gaithersburg, MD. Systems and Software Technology Div.

How the Government Shapes UNIX Standards.

Final rept.

J. A. Hall. $1989,1 p$

Pub. in UNIX World 6, n4 p87(2) Apr 89

Keywords: "UNIX(Operating system), "Federal information processing standards, Reprints APP(Applications Portability Profile), POSIX(Portable Operating System Interiace for Computer Environments), National Institute of Standards and Technology.

The National Institute of Standards and Technology (NIST) is actively supporting standards developing ac tivities relating to the Applications Portability Profile 


\section{Information Processing Standards}

(APP). A major component of the APP is POSIX (the Portable Operating System Interface for Computer Environments), and its relationship to the UNIX operating system.

\section{0,694}

\section{PB92-110105}

PC A05/MF A01

National Inst. of Standards and Technology (CSL), Gaithersburg, MD.

GOSIP Conformance and Interoperation Testing and Registration.

J. S. Nightingale. Mar 91, 94p NISTIR-4594

See also FIPS PUB 146-1 and PB91-187716.

Keywords: "Federal information processing standards "Government procurement, "Computer networks, Protocols, Acquisition, Computer communications, Computer program verification, Tests, Message process ing, "GOSIP(Government Open Systems Interconnection Profile).

The development of Federal Information Processing Standard (FIPS) 146 which specifies the Government Open Systems Interconnection Profile (GOSIP) resulted in the need to establish policy and procedures aimed at ensuring that Federally procured data communications products adhere to the technical documents referenced by GOSIP and that they interoperate. The goal of the report is to aid a Federal Acquisition Authority in procurement of GOSIP products by employing publicly accessible registers verifying supplier claims of conformance and documenting instances of interoperability of GOSIP conformant products.

\section{0,695}

PB92-112200

PC A04/MF A01

National Inst. of Standards and Technology (CSL), Gaithersburg, MD.

Stable Implementation Agreements for Open Systems Interconnection Protocols. Version 4, Edition 1, June 1991 Change Pages (Supplement).

Final rept.

T. Boland, and B. Gray. Sep 91, 68p NIST/SP5001832

Also available from Supt. of Docs. as SN903-015 00000-4. See also PB91-171967 and PB91-197004.

Keywords: "Protocols, "Computer communications, "Computer networks, Standards, Changes, Agreements, Local area networks, Message processing. File management systems, Directories, "OSI(Open Systems Interconnection), NIST.

The document records current Stable Agreements for Open Systems Interconnection (OSI) Protocols among the organizations participating in the National Institute of Standards and Technology (NIST)/OSI Workshop Series for Implementors of OSI Protocols.

\section{0,696}

\section{PB92-119676}

PC A08/MF A02

National Inst. of Standards and Technology (CSL), Gaithersburg, MD.

Government Open Systems Interconnection Profile Users' Guide, Version 2.

Final rept.

T. Boland. Oct 91, 167p NIST/SP-500/192

Also available from Supt. of Docs. Supersedes PB90-

111212. See also PB91-171967. PB91-197004, PB92-

112200 and FIPS PUB 146-1.

Keywords: "Protocols, "Computer communications, "Computer networks, Standards, Message processing, OSI(Open Systems Interconnection), "GOSIP(Government Open System Interconnection), Federal agencies.

The document assists Federal agencies in planning for and procuring OSI. It provides tutorial information on OSI Protocols as well as information on OSI Registration, GOSIP Technical Evaluation, and GOSIP Transition Strategies.
Stable Implementation Agreements for Open Systems Interconnection Protocols. Version 4, Edition 1, Change Pages September 1991. Output from the September 1991 OSI Implementors Workshop. Held in Gaithersburg, Maryland.

Final rept.

T. Boland. Nov 91, 242p NIST/SP500-183 3

Also available from Supt. of Docs. as SN903-015 $00000-4$. See also PB91-171967, PB91-197004 and PB92-112200.

Keywords: "Protocols, Computer networks, Local area networks, "OSI(Open System Interconnection), National Institute of Standards and Technology.

The document records current Stable Agreements for Open Systems Interconnection Protocols among the organizations participating in the NIST/OSI Implementors Workshop series.

\section{0,698}

PB92-126523

PC A17/MF A04

National Inst. of Standards and Technology (NCSL)

Gaithersburg, MD.

Working Implementation Agreements for Open

Systems Interconnection Protocols.

T. Boland. Mar 91, 394p NISTIR-4507

Proceedings of the NIST workshop held in Gaithers burg, MD., December 14, 1990. See also PB91 144444.

Keywords: "Protocols, Standards, Computer networks, Message processing. File management systems, Local area networks, "OSI(Open Systems Interconnection), National Institute of Standards and Technology.

The document records Working Implementation Specification Agreements of Open Systems Interconnection Protocols among the organizations participating in the NIST/OSI Workshop Series for Implementors of OSI Protocols. These decisions are documented to assist organizations in their understanding of the status of agreements. This is a standing document that is updated after each workshop (about 4 times a year).

\section{0,699}

PB92-126580 Gaithersburg, MD.

Guidelines for the Evaluation of File Transfer, Access and Management Implementations.

Special pub.

P. Markovitz, S. Trus, and C. Royster. Oct 91, 85p NIST/SP-500/196

Also available from Supt. of Docs. See also PB90 111212, PB91-171967, PB91-107565 and PB92110105. Sponsored by Army Information Systems Command, Fort Huachuca, AZ., Air Force Communications Command, Scott AFB, IL., and Internal Revenue Service, Washington, DC.

Keywords: *Computer network, *Computer communications, File management systems, Access methods, "OSI(Open Systems Interconnection).

The document advances the goals of the Government Open Systems Interconnection Profile (GOSIP) by providing guidelines for evaluating File Transfer, Access and Management (FTAM) implementations. These guidelines can assist a user in determining which implementation, among several candidates, will best meet the functional and performance requirements of that user. Specifically, the document contains: (1) guidelines for evaluating the functional specifications of FTAM implementations, (2) guidelines for measuring the performance of FTAM implementations, and (3) guidelines for matching the functional and performance specifications of an FTAM implementation to the functional and performance requirements of the user.

\section{Pattern Recognition \& Image Processing}

100,700

PC A03/MF A01

National Inst. of Standards and Technology (NEL) Gaithersburg, MD. Robot Systems Div. Real-Time Model-Based Tracking Combining Spa-
tial and Temporal Features.

K. Chaconas, and M. Nashman. Jun 91, 24 p NISTIR4610

Keywords: "Tracking(Position), Moving targets, Real time, Correlation, Algorithms, "Robot vision.

The paper describes a method for tracking moving image features by combining spatial and temporal edge information with model based feature information. The algorithm updates the two-dimensional position of object features by correlating predicted model features with current image data. The results of the correlation process are used to compute an updated model. The algorithm makes use of a high temporal sampling rate with respect to spatial changes of the image features and operates in a real-time multi-processing environment. Preliminary results demonstrate successful tracking for image feature velocities between 1.1 and 4.5 pixels every image frame.

100,701

PB92-112275

PC A03/MF A01

National Inst. of Standards and Technology (NEL), Gaithersburg, MD. Robot Systems Div.

Integration-Based Method for Depth Estimation.

D. Raviv. Sep 91, 24p NISTIR-4669

Prepared in cooperation with Florida Atlantic Univ., Boca Raton.

Keywords: *Image reconstruction, *Depth finding, Computer vision, Three dimensional, Edge detection, Motion, Algorithms.

A closed-form, integration-based, and massively-parallel algorithm for determining depth of points in 3-D using one moving camera is presented. It is based on analyzing a sequence of images that result from a known rectilinear motion of a camera (with no rotation) in a stationary environment. A traceable point in an image sequence is reconstructed using an integration operation (no differentiation operator is involved). The method arose from two simple observations: (1) Stationary points in the 3-D scene appear to move away from the focus of expansion (FOE); (2) The distance of a point in 3-D space from the camera motion-axis is the same at all instants of time. Any visible moving point in the image can be processed independently of, and concurrently with, any other point. Laboratory results for the case where the optical axis is parallel to the motion axis show an error of less than $0.6 \%$ in absolute distance.

\section{0,702}

PB92-112507

PC A03/MF A01

National Inst. of Standards and Technology, Gaithersburg, MD.

Three Dimensional Reconstruction from Optical Flow Using Temporal Integration.

R. Rangachar, T. H. Hong, M. Herman, and R. Luck. Apr 91,14 p NISTIR-4570

Prepared in cooperation with American Univ., Washington, DC., Maryland Univ., College Park. Dept. of Mechanical Engineering, and Äspex, Inc., New York.

Keywords: "Image reconstruction, Three dimensional, Image segmentation, Optical flow. Temporal integration, PIPE(Pipelined Image Processing Engine).

Image flow, the apparent motion of brightness patterns on the image plane can provide important visual information such as distance, shape surface orientation, and boundaries. It can be determined by either feature tracking or spatio-temporal analysis. The optical flow thus determined can be used to reconstruct the 3-D scene by determining the depth from camera of every point in the scene. However, the optical flow determined by either of the methods mentioned above will be noisy. As a result, the depth information obtained from optical flow can not be successfully used in practical applications such as image segmentation, 3-D reconstruction, path planning, etc. By using temporal integration, the accuracy of both the optical flow and the depth determined from optical flow can be increased. In the work, the authors describe an incremental integration scheme called the running average method to temporally integrate the image flow. They integrate the depth from camera obtained using optical flow determined from gradient based methods, and show that the results of temporal integration are much more useful in practical applications than the results from local edge operators. Finally, they consider an image segmentation example and show the advantages of temporal integration. 
100,703

PB92-112564

$\mathrm{PC} \mathrm{A03/MF} \mathrm{A0} 1$

National Inst. of Standards and Technology, Gaithersburg, MD.

Analysis of Optical Flow Estimation Using Epipolar Plane Images.

R. Rangachar, T. H. Hong, M. Herman, and R. Luck. Apr $91,14 \mathrm{p}$ NISTIR-4569

Prepared in cooperation with American Univ., Washington, DC., Maryland Univ., College Park. Dept. of Mechanical Engineering, and Äspex, Inc., New York.

Keywords: "Image analysis, Image reconstruction, Edge detection, Distance, Motion, "Optical flow, PIPE(Pipelined Image Processing Engine).

Image flow, the apparent motion of brightness patterns on the image plane, can provide important visual information such as distance, shape, surface orientation, and boundaries. It can be determined by either feature tracking or spatio-temporal analysis. The authors consider spatio-temporal methods, and show how differential range can be estimated from time-space imagery. The authors generate a time-space image by considering only one scan line of the image obtained from a camera moving in the horizontal direction at each time interval. At the next instant of time, the authors shift the previous line up by one pixel, and obtain another line from the image. The authors continue the procedure to obtain a time-space image, where each horizontal line represents the spatial relationship of the pixels, and each vertical line the temporal relationship. Each feature along the horizontal scan line generates an edge in the time-space image, the slope of which depends upon the distance of the feature from the camera. The authors apply two mutually perpendicular edge operators to the time-space image, and determine the slope of each edge. The authors show that this corresponds to optical flow. The authors use the result to obtain the differential range, and show how this can be implemented on the Pipelined Image Processing Engine (PIPE). The authors use a simple technique to calibrate the camera and show how the depth can be obtained from optical flow. The authors provide a statistical analysis of the results of $3-D$ reconstruction of the scenes using optical flow determined from $3 \times 3,5 \times 5$, and $7 \times 7$ edge operators.

\section{General}

100,704

PB91-148486

PC A09/MF A02

National Inst. of Standards and Technology (NCSL), Gaithersburg, MD

Bibliography of Selected Computer Security Publications, January 1980-October 1989.

Special pub (January 1980-October 1989).

R. Turn, and L. E. Bassham. Dec 90, 200p NIST/SP$800 / 1$

Contract 43NANB922203

Also available from Supt. of Docs. as SN003-00303060-1. Prepared in cooperation with Turn (Rein) Associates, Pacific Palisades, CA.

Keywords: "Computer security, "Bibliographies, Access control, Data processing security, Computer privacy, Data bases, Computer networks, Cryptography, Secure communications, Auditing, Data integrity.

The bibliography cites selected books and articles on computer security published from January 1980 through October 1989. To have been selected, an article had to be substantial in content and have been published in professional or technical journals, magazines, or conference proceedings. Only very substantial articles from the popular or trade press were in cluded. English language articles from foreign journals were included as available. The citations are listed under nine categories. A tenth category of pre-1980 publications is also provided, as well as an appendix containing addresses of all journals and magazines referenced in the bibliography.

100,705

PB91-159228

Not available NTIS

National Bureau of Standards (ICST), Gaithersburg

MD. Systems and Software Technology Div.

Thwarting the Hackers.

Final rept.

E. F. Troy. $1984,6 p$

Pub. in Datamation, 6p, 1 Jul 84
Keywords: "Computer security, "Access control, Computer communications, Operating systems(Computers), Modems, Reprints.

The article provides general information about a new class of device designed to enhance communications security for computer systems. The "port protection device' (PPD) is fitted on a computer's dial-up telephone line between the telephone set and the computer port, and operates independently of the computer. The PPD in its simplest form requires the dial-up user to supply a special password before gaining access to the computer line. Additional features described in the article include: camouflage of the port by initially hiding the characteristic modem tone; call-back to the authorized user telephone number for independent verification before connection; and logging of all activity on the line. The article includes a general table of characteristics for devices from the eleven known vendors without performing any qualitative evaluation of them.

\section{0,706}

PB9 1-187740

PC A03/MF A01

National Inst. of Standards and Technology, Gaithersburg, MD.

Computer Security: Selected Articles.

Internal rept.

M. Swanson, and E. Lennon. cApr 91, 46p NISTIR4545

Keywords: "Computer security, Local area networks, Risk, Computer viruses, Computer privacy, Data integ rity, Data processing security, Computer software, Personal computers, Data encryption, Computer networks, Internet.

Contents: Is Your System Safe; Proper assignment of responsibility for data security; Assessing Security; NIST Group Explores Risk-Assessment Packages Crackdown on software pirates; Memo: Computer Viruses and Personal Computers; Reflections on Trusting Trust; The Science of Computing: The Internet Worm; and Secret Codes.

\section{0,707}

PB91-187781

PC A06/MF A01 National Inst. of Standards and Technology, Gaithersburg, MD.

National Aeronautics and Space Administration's (NASA) Automated Information Security Handbook.

E. Roback. Mar 91, 107p NISTIR-4518, NHB-2410.9 Sponsored by National Aeronautics and Space Administration, Washington, DC. Information Resources Management Office.

Keywords: "Data processing security, "Handbooks, Risk assessment, Telecommunication, Microcomputers, NASA * Computer security, *Information security, Automated Information Security programs, Accreditation, Contingency plans.

The National Aeronautics and Space Administration's (NASA) Automated Information Security Handbook provides NASA's overall approach to automated information systems security including discussions of such aspects as: program goals and objectives, assignment of responsibilities, risk assessment, foreign national access, contingency planning and disaster recovery, awareness training, procurement, certification, planning, and special considerations for microcomputers.

\section{0,708}

PB91-187864

PC A08/MF A01

National Inst. of Standards and Technology (CSL), Gaithersburg, MD.

Public-Key Cryptography. Computer Security,

Special pub. (Final).

J. Nechvatal. Apr 91, 172p NIST/SP-800/2

Also available from Supt. of Docs. as SN003-00303078-3.

Keywords: *Cryptography, "Computer security, Cryptology, Data encryption, Data processing security, Computer information security, Data integrity, Access control, Secure communications, Authentication, Pro tocols, Local area networks, Algorithms, Computer architecture, "Public-key systems, Digital signatures, Hash functions.

The paper surveys public-key cryptography. It discusses the theory of public-key cryptography and examines several examples of public-key cryptosystems. It also treats the related topics of digital signatures, hash functions, and, more briefly, zero-knowledge protocols. Modes of implementation of public-key crypto- systems are discussed, including implementation in networks. Examples of existing or proposed implementations are summarized. Comparisons to secretkey cryptography are made. Relevant mathematics is covered in appendices. An extensive bibliography is included.

100,709

PB91-189571

Not available NTIS National Inst. of Standards and Technology (NEL), Gaithersburg, MD. Semiconductor Electronics Div.

Knowledge Verification of Machine-Learning Procedures Based on Test Structure Measurements. Final rept.

D. Khera, L. W. Linholm, R. A. Allen, M. W.

Cresswell, V. C. Tyree, W. Hansford, and C. Pina.

1991, 5p

Pub. in Proceedings of International Conference on Microelectronic Test Structures, Kyoto, Japan, March 18-20, 1991, p145-149.

Keywords: "Machine learning, "Knowledge bases(Artificial intelligence), Export systems, Systems engineering, Tests, Heuristic methods, Comparison, Reprints.

The paper describes an approach for evaluating and refining the rules, based on test structure measurements, to be entered into the knowledge base of an expert system that characterizes device performance. The objective is to qualify the performance of rules determined by a machine-learning classification application with the best knowledge available from the human experts. The technique combines a machine-learning approach with the traditional heuristic based development of an expert system. Strengths and weaknesses of the individual techniques are compared.

\section{0,710}

PB91-203380

Not available NTIS

National Bureau of Standards (ICST), Gaithersburg, MD. Systems and Software Technology Div.

Static Analysis Tools for Software Security Certification.

Final rept

D. R. Kuhn. 1988, 9p

Pub. in Proceedings of the National Computer Security Conference (11th), October 17-20, 1988, p290-298.

Keywords: "Computer software, "Computer security, * Software tools, Cryptography, Electronic funds transfer, Data encryption, Data processing security, Computer program verification, Reprints, Static analysis.

The paper describes a suite of tools used in evaluating software for security certification. The tools are currently being used on cryptographic software for secure Electronic Funds Transfer, but could be applied to other applications as well.

100,711

PB91-204107

Not available NTIS

National Inst. of Standards and Technology (NCSL), Gaithersburg, MD. Computer Security Div.

Data' Encryp tion Standard: Past and Future.

Final rept.

M. E. Smid, and D. K. Branstad. 1988, 10p

Pub. in Proceedings of the IEEE (Institute of Electrical and Electronics Engineers) 76, n5 p550-559 1988.

Keywords: "Data encryption, *Standards, Cryptography, Algorithms, Data processing security, Electronic funds transfer, Reprints.

The Data Encryption Standard (DES) is the first, and to the present date, only, publicly available cryptographic algorithm that has been endorsed by the U.S. Government. The paper deals with the past and future of the DES. It discusses the forces leading to the development of the standard during the early 1970's, the controversy regarding the proposed standard during the mid 1970's, the growing acceptance and use of the standard in the 1980's, and some recent developments that could affect the future of the standard.

100,712

PB91-216671

PC A07/MF A02

National Inst. of Standards and Technology (CSL), Gaithersburg, MD. 
Standard Security Label for GOSIP: An Invitational Workshop. Held on April 9-10, 1991.

N. Nazario. Jun 91, 133 p NISTIR-4614

Keywords: "Meetings, "Computer security, "Standards, Protocols, United States government, Computer networks, Federal information processing standards, Data processing security, Government policies, Cryptography, Data integrity, "GOSIP(Government Open Systems Interconnection Profile), National Institute of Standards and Technology, Security labels.

On April 9 and 10, 1991 the Protocol Security Group at the National Institute of Standards and Technology (NIST) held its Second Workshop on Security Labels. Forty representatives from the U.S. Government, Industry, and the Canadian Government gathered for two days to discuss a NIST proposed Standard Security Label for the U.S. Government Open Systems Interconnection Profile (GOSIP). Issues on security policy and security object registration were also discussed in reference to the proposed label. The information shared during the two days of discussion and the recommendations of the group are documented in the proceedings.

\section{0,713}

PB91-222638 National Inst. of Standards and Technology, Gaithersburg, MD.

Automated Information Systems Security Program Handbook.

E. Roback. Jul 91, 156p NISTIR-4636

Reprinted with Permission by U.S. Department of Human Services.

Keywords: "Computer information security, "Handbooks, Data processing security, Risk assessment, Personal computers, Word processing, Telecommunication, "Computer security, Contingency plans, US HHS

The Interagency Report presents the U.S. Department of Health and Human Services' (HHS) Automated Information Systems Security Program Handbook which provides a comprehensive description of the program elements which comprise HHS's approach to computer security. Among the varied items included are: security policy and responsibilities, security level designators, security level requirements, security administration, risk management, contingency planning, personnel security, facility security, application systems and data security, personal computers, data communications, and acquisitions and contracts.

\section{0,714}

PB91-237677

Not available NTIS

National Inst. of Standards and Technology (MEL), Gaithersburg, MD. Robot Systems Div.

Geometric Reasoning for Constructing 3D Scene Descriptions for Images.

Final rept.

E. L. Walker, and M. Herman. 1988, 16p

Pub. in Artificial Intelligence 37, n1-3 p275-290 Dec 88.

Keywords: "Computer vision, "Three dimensional models, "Image processing, Reasoning, Expert systems, Knowledge bases(Artificial intelligence), Robotics, Knowledge representation, Reprints.

There are many applications for a vision system which derives a three-dimensional model of a scene from one or more images and stores the model for easy retrieval and matching. The derivation of a 3D model of a scene involves transformations between four levels of representation: images, 2D features, 3D structures, and $3 \mathrm{D}$ geometric models. Geometric reasoning is used to perform these transformations, as well as for the eventual model matching. Since the image formation process is many-to-one, the problem of deriving 3D features from 2D features is ill-constrained. Additional constraints may be derived from knowledge of the domain from which the images were taken. The 3D Mosaic system has successfully used domain specific knowledge to drive the geometric reasoning necessary to acquire 3D models for complex real-world urban scenes. To generalize this approach, a framework for the representation and use of domain knowledge for geometric reasoning for vision is proposed.
Guide to Expert System Building Tools for Microcomputers.

Special pub. (Final).

C. E. Dabrowski, and E. N. Fong. Jul 91, 151p NIST/ SP-500/188

Also available from Supt. of Docs. as SNO03-00303088-1.

Keywords: "Expert systems, "Microcomputers, "Systems engineering, "Software tools, Knowledge bases(Artificial intelligence), Knowledge representation, Man computer interface, Computer software, Object-oriented programming.

Microcomputer-based expert system building tools (microcomputer-based ESBTs), sometimes known as expert system shells, are software packages for development of expert systems that run on microcomputers. The report provides system managers, planners, and potential expert system developers with a readable description of ESBTs for microcomputers including a detailed description of specific tool features and the capabilities they support. The technical content of the report is based on analysis of commercially available ESBTs. However, individual commercial products are not described, compared, or ranked.

\section{0,716}

PB92-112259

PC A09/MF A02

National Inst

Glossary of Computer Security Terminology

Glossary of Computer Security Termin
E. Roback. Sep 91, 177p NISTIR-4659

Keywords: "Computer information security, "Data processing security, "Dictionairies, Computer security, Computer privacy.

The Glossary of Computer Security Terminology provides a summary of frequently encountered computer and communications security terms and various definitions used by federal agencies for those terms. The glossary does not provide a single definition per term rather it reflects the variations in use of these terms among federal organizations.

\section{0,717}

PB92-112390

PC A03/MF AO

National Inst. of Standards and Technology (CSL).

Gaithersburg, MD. Computer Security Div.

Computer Security Bulletin Board System. User's Guide.

M. Skandera, and M. Swanson. Sep 91, 40p NISTIR4667

Keywords: "Computer information security, Use manuals, Bulletin Board System, BBS system, Computer security, Computer privacy, US NIST.

The Computer Security Act of 1987 assigned to the National Institute of Standards and Technology (NIST) the responsibility for providing federal agencies with advice and assistance in the area of computer security. To accomplish a portion of the task, the NIST Com puter Security Division maintains an electronic bulletin board system (BBS) which focuses on computer security issues. The NIST Computer Security BBS makes a wide variety of computer security information available to federal agencies and the public and encourages the sharing of information which can help users and managers protect their data and systems. The document describes the BBS and provides detailed instructions on how to use the many functions.

\section{0,718}

PB92-116391

PC A05/MF A01

National Inst. of Standards and Technology (CSL), Gaithersburg, MD

Security in ISDN.

Special pub. (Final)

W. E. Burr. Sep 91, 77p NIST/SP-500/189

Also available from Supt. of Docs. as SN003-003 $03112-7$. See also PB90-198946 and N89-25634.

Keywords: "Computer security, ."Computer networks *Communication networks, Standards, Access control, Data encryption, Data processing security, Computer privacy, Data integrity, Protocols, *ISDN(Integrated Services Digital Network), OSI(Open Systems Interconnection).

The Integrated Services Digital Network (ISDN) standards will provide worldwide digital communication service and will play a key role in the transition to electronic documents and business transactions. ISDN has been developed with little thought to security.
ISDN security will become a pressing concern for both government and business. ISDN's digital nature facilitates adding security, but the deployment of ISDN in the public network is well under way and the present investment in ISDN equipment, as well as the commercial necessity to deploy ISDN in a timely manner, constrains how security features may be added. ISDN security standards should take advantage of, and be compatible with, emerging standards for Open Systems Interconnection (OSI) security. International Standard 7498-2 defines five security services for OSI: Confidentiality, Access Control, Authentication, Data Integrity and Non-repudiation. The challenge of ISDN security is to extend these concepts to all ISDN applications, including voice use of the public network. Terminal-to-terminal link encryption provides a powerful ISDN security mechanism, because of ISDN's ability to provide circuit switched connections throughout the world. A standard for the reliable authentication of human users is badly needed for ISDN security.

\section{0,719}

PB92-117068

Not available NTIS

National Inst. of Standards and Technology (NCSL), Gaithersburg, MD.

Book Review: 'The Art of Computer Systems Performance Analysis' by R. Jain.

Final rept.

G. Lyon. 1991, 2p

Sponsored by Defense Advanced Research Projects Agency, Arlington, VA.

Pub in IEEE (Institute for Electrical and Electronics Engineers) Design and Test of Computers, p90-91 Sep 91.

Keywords: "Computer systems performance, "Computer performance evaluation, Reviews, Experimental design, Probability theory, Computerized simulation, Mathematical models, Statistical analysis, Queueing theory, Reprints.

The book covers a wide spectrum of performance topics. Its review is organized as follows: Overview. Professional sources, projects, mistakes, techniques, metrics; Measurement. Workloads (type, selection, characterization), monitors, accounting logs, capacity planning, benchmarking, data presentation, honesty in comparisons; Probability and Statistics. Summarizing measured data, comparing systems, regressions (linear and other); Design of Experiments. Screening: to the $k$ power, 2 to the $k$ power $r$ factorials, 2 to the $(k-$ p) power fractional factorials; Comparing: One-factor experiments, two-factor full factorials (without and with replication, $r$ ), full factorials with $\mathrm{k}$ factors; Simulation. Background, mistakes, verification, random numbers and their tests, random-variates, 20 common distributions; Queueing Models. Single queues, networks, operational laws, mean-value analysis, convolution, hierarchical decomposition.

\section{0,720}

\section{PB92-123140}

PC A03/MF A01

National Inst. of Standards and Technology (CSL)

Gaithersburg, MD.

Establishing a Computer Security Incident Response Capability (CSIRC)

Special pub. (Final)

J. P. Wack. Nov $91,47 p$ NIST/SP-800/3

Also available from Supt. of Docs as SN003-00303121-6. See also PB90-115601.

Keywords: "Computer information security, Data processing security, Computer viruses, Risk analysis, Threat evaluation, Vulnerability, "CSIRC, Computer Security Incident Response Capabilities.

Government agencies and other organizations have begun to augment their computer security efforts because of increased threats to computer security. Incidents involving these threats, including computer viruses, malicious user activity, and vulnerabilities associated with high technology, require a skilled and rapid response before they can cause significant damage. These increased computer security efforts, described here as Computer Security Incident Response Capabilities (CSIRCs), have as a primary focus the goal of reacting quickly and efficiently to computer security incidents. CSIRC efforts provide agencies with a centralized and cost-effective approach to handling computer security incidents so that future problems can be efficiently resolved and prevented. While the risks to computer security have increased, agencies have also become more dependent on computers. Many systems in widespread use today do not contain safeguards to guarantee protection from these threats. Ad- 
ditionally, as systems become more complex, they are more prone to vulnerabilities that can increase the risk of malicious exploitation. Due to greater availability of computers, users are often de facto system managers, however many have neither the requisite skills nor time to manage their systems effectively. These factors make it clear that agencies need to augment their computer security capabilities before they suffer from serious computer security problems that can harm their missions, result in significant expense, and tarnish their images. A CSIRC can help agencies resolve computer security problems in a way that is both efficient and cost-effective. Combined with policies for centralized reporting, a CSIRC can reduce waste and duplication while providing a better posture against potentially devastating threats. A CSIRC is a proactive approach to computer security, one that combines reactive capabilities with active steps to prevent future incidents from occurring.

\section{DETECTION \& COUNTERMEASURES}

\section{Magnetic Detection}

100,721

AD-A230 438/4

PC A02/MF A01

National Inst. of Standards and Technology (NEL) Boulder, CO. Electromagnetic Fields Div. Summary of Experiments with the Separated Aperture Technique of Dielectric Anomaly Detection. Final rept.

F. R. Clague. Dec 89, 8p Rept no. SR-723-29-89

Keywords: Anomalies, Antennas, "Antipersonnel mines, "Countermeasures, Cross polarization, "Detection, Dielectrics, Holography, Horn antennas, Images Intrusion, Measurement, "Mine detectors, Narrowband, Plates, Time domain.

This report summarizes experiments conducted for the Mine Detection Division, Counter-Mine, Counter-Intrusion Department. The objective of this report is to provide a condensed overview of the major things tried with an indication of how well they worked, and a reference to the original report that will allow finding the more detailed description if desired. The objective of the experiments was to study and improve what has generally been called the separated aperture system of dielectric anomaly detection. The detection portion of the system is a 'head' made of a transmit antenna and a receive antenna separated by a flat plate called the 'septum'. While the items tested were primarily the different versions of the separated aperture heads, a small number of other antennas was also evaluated. These included broadband horns used for time domain measurements, a narrow band horn used for holographic imaging, and cross polarized antennas that were used in an antipersonnel mine detector.

\section{Radiofrequency Detection}

100,722

PB91-143347

PC A03/MF A01

National Inst. of Standards and Technology (NEL), Boulder, CO. Electromagnetic Fields Div.

Near-Field and Far-Field Excitation of a Long Conductor in a Lossy Medium.

D. A. Hill. Sep 90, 38p NISTIR-3954

Sponsored by Army Belvoir Research Development and Engineering Center, Fort Belvoir, VA.

Keywords: "Magnetic fields, "Electric conductors, Electric fields, Field strength, Far fields, Near fields, Electric dipoles, Line current, Plane waves, Excitation, Tunnels, Grounded conductors, Insulated conductors, Underground conductors, Electromagnetic detection.

Excitation of currents on an infinitely long conductor is analyzed for horizontal electric dipole or line sources and for a plane-wave, far-field source. Any of these sources can excite strong currents which produce strong scattered fields for detection. Numerical results for these sources indicate that long conductors produce a strong anomaly over a broad frequency range. The conductor can be either insulated or bare to model ungrounded or grounded conductors.

\section{ELECTROTECHNOLOGY}

\section{Antennas}

100,723

PB91-132274

PC A07/MF A01 National Inst. of Standards and Technology (NEL) Boulder, CO. Electromagnetic Fields Div.

Spherical Near-Field Scanning: Experimental and Theoretical Studies.

R. C. Wittmann, and C. F. Stubenrauch. Jul 90, 127p NISTIR-3955

Sponsored by Rome Air Development Center, Hanscom AFB, MA. Electromagnetics Directorate.

Keywords: Spherical harmonics, Spherical waves, Far field, Algorithms, "Antenna measurements, Microstrip antennas, Probes(Electromagnetic), Near field.

The report documents the evaluation of spherical near-field scanning algorithms and computer code de veloped at the National Institute of Standards and Technology. The experimental work is primarily a com parison of probe-compensated spherical and planar near-field measurement results for a common test antenna. Theoretical work is largely supportive of the ex perimental effort, but some peripheral topics are developed: For example, (1) application of spherical nearfield measurements to the determination of inciden fields in compact ranges; and, (2) spherical-wave expansions for the fields of a uniformly excited circular aperture (to facilitate the creation of analytic test data)

100,724

PB91-134239 Not available NTIS National Inst. of Standards and Technology (NEL) Boulder, CO. Electromagnetic Fields Div.

Calibration of Antenna Factor at a Ground Screen Field Site Using an Automatic Network Analyzer. Final rept.

E. B. Larsen, R. L. Ehret, D. G. Camell, and G. H.

Koepke. $1989,6 \mathrm{p}$

Sponsored by Naval Air Systems Command, Washington, DC

Pub. in Proceedings of IEEE (Institute of Electrical and Electronics Engineers) National Symposium on Electromagnetic Compatibility, Denver, CO., May 23-25, 1989, p19-24.

Keywords: "Antennas, "Electromagnetic fields, "Network analyzers, "Test facilities, Electromagnetic radiation, Field strength, Antenna radiation patterns, Amplification, Dipoles, Reprints, "Calibration.

The technique now employed at the National Institute of Standards and Technology (NIST) for calibrating antenna factor at frequencies from 25 to $1000 \mathrm{MHz}$ use a standard open-circuit half-wave receiving dipole to measure the electric field strength. Unfortunately, the dipole responds to ambient fields over a large frequency range. This approach is compared with a three-an tenna method which uses an accurate automatic network analyzer with $120 \mathrm{~dB}$ dynamic range to measure insertion loss between the transmitting and receiving antennas. A field site having a $20 \mathrm{~m} \times 60 \mathrm{~m}$ ground screen which acts as a good reflector is used.

100,725

PB91-148239

Not available NTIS

National Inst. of Standards and Technology (NEL)

Boulder, CO. Electromagnetic Fields Div.

Spherical Near-Field Scanning: Determining the Incident Field Near a Rotatable Probe.

Final rept.

R. C. Wittman. 1990, 4p

Pub. in Proceedings of Antennas and Propagation Symposium Digest, Dallas, TX., May 7-11, 1990, p224227.
Keywords: Probes(Electromagnetic), Electromagnetic compatibility, Electromagnetic interference, Near field Scanning, Algorithms, Reprints, "Antenna measurements.

Many RCS, EMI/EMC, and antenna measurements require a known incident field within a test volume. To evaluate systems designed to produce a specific incident field (compact ranges, for example), one must measure the actual illumination for comparison with design specifications. Beyond its diagnostic value, this incident field data can also be used for error estimation and for calculating first order corrections. In the paper the author develops a spherical near-field scanning algorithm for determining incident fields inside a probe's 'minimum sphere.' This differs from the well-known spherical near-field scanning formulation which determines fields outside the source's minimum sphere The scanner size depends on the extent of the region of interest and not on the extent of the (possibly much larger) source. The data may be collected using a standard roll-over-azimuth positioner.

100,726

PB91-185124

$\mathrm{PC}$ A03/MF A01

National Inst. of Standards and Technology (NEL) Boulder, CO. Electromagnetic Fields Div.

NIST Calibration Procedure for Vertically Polarized Monopole Antennas $30 \mathrm{kHz}$ to $300 \mathrm{MHz}$.

Technical note.

D. G. Camell, E. B. Larsen, J. E. Cruz, and D. A. Hill. Jan $91,26 p$ NIST/TN-1347

Also available from Supt. of Docs. as $\mathrm{SNOOH}_{-003}$ 03077-5.

Keywords: "Monopole antennas, "Calibration, Low frequency, Medium frequency, High frequency, Very high frequency, Polarization, Antenna factors.

The report describes the theoretical basis and test procedure for vertically polarized monopole antenna calibrations at the National Institute of Standards and Technology (NIST). The standard field method applies the theoretical equations of a vertical monopole antenna to calculate the vertical electric field. The method is used at the NIST open field site in the frequency range of $30 \mathrm{kHz}$ to $300 \mathrm{MHz}$. The uncertainty in the antenna factor of the antenna under test (AUT) is now + or - 1 dB.

\section{0,727}

PB91-236919

Not available NTIS

National Inst. of Standards and Technology (NEL) Boulder, CO. Electromagnetic Fields Div.

Alignment Fixture for Millimeter Waveguide.

Final rept.

D. P. Kremer, and A. C. Newell. 1990, 4p

Pub in IEEE (Institute of Electrical and Electronics En gineers) Antennas and Propagation Society Magazine $32, n 3$ p 45-48 Jun 90

Keywords: "Antennas, Millimeter waves, Alignment Fixtures, Reprints, "Waveguide flanges.

Millimeter wave measurements require that care be exercised in the connection and handling of the waveguide flanges and their contact surfaces. When properly connected these flanges can provide many years of reliable and repeatable measurements. Improper use will limit the flange use to just a few connections and result in large measurement errors. These errors are especially acute in situations requiring repeated connecting and disconnecting of these flanges, such as in antenna or insertion loss measurements. Severa factors contribute to these errors, but the largest are: improperly installed waveguide flanges, misalignmen in flange connections, and excess strain on the waveguide or the flange. The National Institute of Standards and Technology has addressed these problems by developing a mechanical alignment fixture for millimeter band waveguide. Two fixtures were developed; one for small devices such as standard gain horns which can be supported by the fixture and another for larger devices. These systems along with a properly installed flange can reduce the uncertainty of the connection from greater than $1 \mathrm{~dB}$ to a few hundredths of a decibel.

100,728

PB91-237073

Not available NTIS

National Inst. of Standards and Technology (EEEL). Boulder, CO. Electromagnetic Fields Div. 


\section{Antennas}

General Technique to Correct Probe Position Errors in Planar Near-Field Measurements to Arbitrary Accuracy.

Final rept.

L. A. Muth, and R. L. Lewis. $1990,8 \mathrm{p}$

Pub. in IEEE (Institute of Electrical and Electronics Engineers) Transactions on Antennas and Propagation 38, n12 p1925-1932 Dec 90.

Keywords: "Antennas, Probes(Electromagnetic), Taylors series, Error analysis, Near field, Far field, Reprints.

A general theoretical procedure is presented to remove known probe position errors when planar nearfield data are transformed to the far field. The authors represented the measured data as a Taylor series whose terms contain the error function and the idea spectrum of the antenna. This representation is then assumed to be an actual near field existing on an error free regularly spaced two dimensional scan plane. Then by inverting the Taylor series, they obtain the ideal spectrum in terms of the measured data and the position errors. The solution is given by an infinite series of an error operator acting on data containing errors of measurement. The error operator is the Taylor series without the zeroth-order term. The nth order approximation to the ideal near field of the antenna can be explicitly constructed by inspection of the structure of the error operator.

100,729

PB92-110279

(Order as PB92-110261, PC A05/MF A01)

National Inst. of Standards and Technology, Boulder. CO.

General Analytic Correction for Probe-Position Errors in Spherical Near-Field Measurements.

L. A. Muth. c1991, 20p

Included in Jnl. of Research of the National Institute of Standards and Technology, v96 n4 p391-410 Jul/Aug 91.

Keywords: Antenna radiation patterns, Probes(Electromagnetic), Computerized simulation, Spherical configuration, Near field, Correction, Graphs(Charts).

A general theoretical procedure is presented to remove known probe-position errors in spherical nearfield data to obtain highly accurate far fields. The measured data is represented as a Taylor series in terms of the displacement errors and the ideal spectrum of the antenna. The representation is then assumed to be an actual near field on a regularly spaced error-free spherical grid. The ideal spectrum is given by an infinite series of an error operator acting on data containing errors of measurement. This error operato is the Taylor series without the zeroth-order term. The nth-order approximation to the ideal near field of the antenna can be explicitly constructed by inspection of the error operator. Computer simulations using periodic error functions show that the authors are dealing with a convergent series, and the error-correction technique is highly successful.

\section{0,730}

PB92-112283

PC A04/MF A0 1

National Inst. of Standards and Technology (EEEL) Boulder, CO. Electromagnetic Fields Div.

Personal Computer Codes for Analysis of Planar Near Fields.

L. A. Muth, and R. L. Lewis. Jun 91, 64p NISTIR-

3970

Sponsored by Air Force Guidance and Metrology Center, Newark AFS, OH

Keywords: *Antenna radiation patterns, Data management, Personnel computers, Computer applications, Near field, Far field, Metrology, Computation, Subroutines, Fortran, PNFC codes.

Fortran codes were developed for analysis of planar near-field data. The report describes some of the inner workings of the codes, the data management schemes, and the structure of the input/output sections to enable scientists and programmers to use these codes effectively as a research tool in antenna metrology. The open structure of the codes allows a user to incorporate into the package new applications for future use with relative ease. The subroutines currently in existence are briefly described, and a table showing the interdependence among these subroutines is constructed. Some basic research problems, such as transformation of a near field to the far field and correction of probe position errors, are carried out from start to finish to illustrate use and effectiveness of these codes. Sample outputs are shown. The advantage of a high degree of modularization is demonstrated by the use of DOS batch files to execute FORTRAN modules in a desired sequence.

\section{Circuits}

100,731

PB91-148700

Not available NTIS

National Bureau of Standards (NEL), Boulder, CO. Electromagnetic Technology Div.

High Speed Superconducting A/D Converter. Final rept.

C. A. Hamilton, F. L. Lloyd, and R. L. Kautz. 1984, 4p Contract N00014-83-F-0012

Sponsored by Office of Naval Research, Arlington, VA Pub. in Proceedings of Government Microcircuit Applications Conference, Las Vegas, NV., November 6-8, 1984, p140-143.

Keywords: *Analog to digital converters, *Superconducting devices, Signal processing, Reprints.

Superconducting electronics has demonstrated impressive performance capabilities for small digital systems and analog signal processing applications. One of the areas where superconductivity offers a unique advantage is in ultra-high speed A/D conversion. The paper describes the operation and current status of a 6 -bit, 4 gigasample/second A/D converter which has been developed at NBS.

100,732

PB91-149310

Not available NTIS National Inst. of Standards and Technology (NEL), Gaithersburg, MD. Electricity Div.

Comprehensive Approach for Modeling and Testing Analog and Mixed-Signal Devices.

Final rept.

T. M. Souders, and G. N. Stenbakken. 1990, 8p

Pub. in Proceedings of International Test Conference, Washington, DC., September 10-14, 1990, p169-176 1990.

Keywords: "Models, "Tests, Analog to digital converters, Optimization, Computer software, Reprints, "Analog devices, "Mixed-signal devices.

An approach is presented for optimizing the testing of analog and mixed-signal devices. The entire process is performed with algebraic operations on an appropriate model. The paper demonstrates how this is accomplished using simple calls with public-domain software. Examples of test results achieved using this approach are included.

\section{0,733}

PB91-149401

Not available NTIS National Inst. of Standards and Technology (NEL). Gaithersburg, MD. Electricity Div.

Device for Audio-Frequency Power Measurement. Final rept

G. Q. Tong, Z. T. Qian, X. Y. Xu, and L. X. Liu. 1990, $5 p$

Pub. in IEEE (Institute of Electrical and Electronics Engineers) Transactions on Instrumentation and Measurement 39, n3 p540-544 Jun 90.

Keywords: "Power measurement, Electric current meters, Voltage measuring instruments, Audio frequencies, Reprints.

A new device for the measurement of audio-frequency power is introduced. The device can also be used to measure audio-frequency voltage and current. The full range of power factors are accomodated (cos theta $=$ 0 to 1). Voltage and current measuring ranges are 15 $600 \mathrm{~V}$ and $0.1-10 \mathrm{~A}$, respectively. When cos theta $=1$, the permissible error of the power measurement is from 50 to $150 \mathrm{ppm}$ over the frequency range of $40 \mathrm{~Hz}$ to $10 \mathrm{kHz}$ (including the line power frequency).

100,734

PB91-236554

Not available NTIS

National Inst of Standards and Technology (EEEL) Gaithersburg, MD. Electricity Div.
Investigating the Use of Multimeters to Measure Quantized Hall Resistance Standards.

Final rept.

M. E. Cage, D. Y. Yu, B. M. Jeckelmann, R. L.

Steiner, and R. V. Duncan. 1991, 5p

See also PB91-101097.

Pub. in IEEE (Institute of Electrical and Electronics Engineers) Transactions on Instrumentation and Measurement 40, n2 p262-266 Apr 91.

Keywords: "Electrical resistance, "Multimeters, *Standards, Josephson junctions, Calibration, Resistors, Accuracy, Reprints, "Quantum Hall effect, "Resistance standards, Josephson arrays.

A new generation of digital multimeters was used to compare directly the ratios of the resistances of several wire-wound resistors and a quantized Hall resistor. The accuracies are better than $0.1 \mathrm{ppm}$ for ratios as large as $4: 1$ if the multimeters are calibrated with a Josephson array.

100,735

PB91-236802

Not available NTIS

National Inst. of Standards and Technology (EEEL), Gaithersburg, MD. Electricity Div.

RF-DC Differences of Thermal Voltage Converters Arising from Input Connectors.

Final rept.

D. X. Huang, J. R. Kinard, and G. Rebuldela. 1991

$6 p$

See also PB91-101295.

Pub. in IEEE (Institute of Electrical and Electronics Engineers) Transactions on Instrumentation and Measurement 40, n2 p360-365 Apr 91.

Keywords: Electric connectors, Transmission lines, Skin effect, Reprints, "Thermal voltage converters, Thermal converters.

The RF-dc differences of thermal voltage converters (TVC's) caused by skin effect and transmission-line effects of different length input structures have been studied. Some discrepancies do exist between simple mathematical models and measured results for commonly used input connectors. The paper reports a study of these discrepancies and some worst-case results of changes in RF-dc difference due to connection and disconnection of TVC's.

\section{0,736}

PB91-236810

Not available NTIS

National Inst. of Standards and Technology (NEL),

Boulder, CO. Electromagnetic Technology Div.

Excess Low-Frequency Flux Noise in dc Squids Incorporating $\mathrm{Nb} / \mathrm{Al}-\mathrm{Oxide} / \mathrm{Nb}$ Josephson Junctions.

Final rept.

M. E. Huber, and M. W. Cromar. 1990, 2p

Pub. in Physica B 165-166, p77-78 1990.

Keywords: "SQUID devices, Electromagnetic noise, Josephson junctions, Direct current, Low frequency, Aluminum oxide, Niobium, Reprints, SIS(Superconductors).

The authors have fabricated thin-film dc SQUIDs (Superconducting QUantum Interference Devices) incorporating $\mathrm{Nb} / \mathrm{Al}-\mathrm{Oxide} / \mathrm{Nb}$ tunnel junctions. The spectral density of the voltage noise, $S(v)$, of stripline SQUIDs is characterized between $1 \mathrm{~Hz}$ and $2000 \mathrm{~Hz}$. In this frequency range, $S(v)$ is proportional to the square of the responsivity over a significant range of bias conditions with an unusual frequency dependence. In a $7 \mathrm{pH}$ SQUID, the spectral density of the flux noise, $\mathrm{S}$ (phi) at $1 \mathrm{~Hz}$ is less than 10 to the -11 power (Phi(0)) squared $/ \mathrm{Hz}$ where Phi(0) is identically $=\mathrm{h} / 2 \mathrm{e}$. The observed noise does not appear to be environmental; also, it is independent of the value of the junction shunt resistance and whether the stripline material is a Pbin alloy or $\mathrm{Nb}$. Subject to the constraint of a constant product of the junction area, critical current density, and SQUID self-inductance in the SQUIDS studied, S(phi) is inversely proportional to the junction area.

100,737

PB91-236893

Not available NTIS

National Inst. of Standards and Technology (EEEL), Gaithersburg, MD. Electricity Div.

AC-DC Difference Relationships for Current Shunt and Thermal Converter Comblnations.

Final rept.

J. R. Kinard, T. E. Lipe, and C. B. Childers. 1991, 4p See also PB91-101378. 
Pub. in IEEE (Institute of Electrical and Electronics Engineers) Transactions on Instrumentation and Measurement 40, n2 p352-355 Apr 91.

Keywords: Alternating current, Direct current, Mutua inductance, Electrical measurement, Electric converters, Reprints, *Thermal converters, Current shunts, Thermal voltage converters, Thermal current converters.

The paper describes the relationship between the overall ac-dc difference of a current-shunt/thermalconverter combination and the individual characteristics of the current shunt and thermal converter (TC) considered separately. Equations governing these characteristics are derived for the general case where the characteristics include mutual inductance between the shunt and the TC circuits, and for the simplified case such as the replacement of just a thermoelement (TE) within the TC, where the change in mutual inductance is negligible. Predicted and measured results are given for shunts used with different TC's.

\section{0,738}

PB91-237107

Not available NTIS

National Inst. of Standards and Technology (EEEL),

Gaithersburg, MD. Electricity Div.

New Low-Voltage Standards in the DC to $1-\mathrm{MHz}$ Frequency Range.

Final rept.

N. M. Oldham, and R. M. Henderson. 1991, 5p

See also PB91-101493.

Pub. in IEEE (Institute of Electrical and Electronics Engineers) Transactions on Instrumentation and Measurement 40, n2 p368-372 Apr 91.

Keywords: "Standards, Electrical measurement, Audio frequencies, Radio frequencies, Low voltage, Potentiometers(Resistors), Electric potential, Dividers, Reprints, "Voltage standards. Thermal voltage converters, Thermal converters.

Two new instruments for generating and measuring 1 $200-\mathrm{mV}$ signlas have been developed and used to test a number of different low-voltage measurement techniques. Differences between the various measurement miques. Differences between the various measurement in the $1-20 \mathrm{kHz}$ range and within + or $-300 \mathrm{ppm}$ out to $1 \mathrm{MHz}$.

\section{0,739}

PB91-237446

Not available NTIS

National Inst of Standards and Technology (EEEL) Gaithersburg, MD. Electricity Div.

Improvements for Automating Voltage Calibrations Using a 10-V Josephson Array.

Final rept

R. L. Steiner, and R. J. Astalos. 1991, 5p

See also PB91-101592.

Pub. in IEEE (Institute of Electrical and Electronics Engineers) Transactions on Instrumentation and Meas. urement 40, n2 p321-325 Apr 91.

Keywords: *Calibration, *Standards, Josephson junc tions, Direct current, Digital systems, Automation, Voltmeters, Reprints, "Voltage standards, Josephson arrays.

A voltage standard system based on a $10-\mathrm{V}$ Josephson array have been completely automated with three novel developments. First, a unique way of connecting Zener voltage standards, a digital voltmeter (DVM) and the array to a commercial standard cell scanner has provided necessary switching flexibility. Second using a programmable millimeter wave attenuator has greatly simplified the selection of voltage steps. Third and last, programmed error checking, which verifies array steps by comparing measurement scatter to previously characterized system noise levels, has proven more reliable than visual observation. The operation o this new system is simplified enough for an inexper user while the calibration uncertainty ( 1 sigma) is still a few parts in 100 million.

\section{0,740}

\section{PB91-237586}

Not available NTIS

National Inst. of Standards and Technology (PL)

Gaithersburg, MD. Physics Lab. Office.

New International Electrical Reference Standards

Based on the Josephson and Quantum Hall Ef fects.

B. N. Taylor, and T. J. Witt. 1989, 16p

Sponsored by Bureau International des Poids et Mesures, Sevres (France).

Pub. in Metrologia 26, n1 p47-62 1989.
Keywords: "Electrical resistance, "Voltage, "Standards, Josephson effect, International, Reprints, "Resistance standards, *Voltage standards, Quantum Hall effect, Ohm, Volt.

The authors discuss the background and basis for the new international electrical reference standards of voltage and resistance that are to come into effect worldwide, starting on 1st January 1990. Founded on the Josephson and Quantum Hall effects, respectively, these new reference standards will improve significantly the international uniformity of electrical meas urements and their consistency with the SI.

\section{0,741}

PB92-117076

Not available NTIS

National Inst. of Standards and Technology (NEL),

Gaithersburg, MD. Electricity Div.

Need for Power Quality Testlng Standards.

Final rept.

F. D. Martzloff. 1990, 2p

Pub. in Proceedings of National Conference Powe Quality for End-Use Applications (2nd), San Francisco CA., March 21-23, 1990, p171-172.

Keywords: *Power supply circuits, "Standards, "Quality, "Surges, Voltage regulation, Power measurement, Electronic equipment, Power, Voltage regulators, Overvoltage, Overcurrent, Reprints.

The quality of the power supplied to sensitive electronic equipment is an important issue. Quantifying this quality, however, is difficult under the present state of nonexistent or uncoordinated standards. Improve ments in the situation described as poor power quality can be achieved by reducing the sensitivity of equipment to power line disturbances, or by limiting the injection of disturbances. While these remedies might seem obvious in principle, their implementation ap pears more difficult. Voluntary standards provide a guide for such an impleınentation. The formation by IEEE of a Standards Coordinating Committee on Power Quality responds to the need.

\section{0,742}

PB92-117431

Not available NTIS

National Inst of Standards and Technology (EEEL) Gaithersburg, MD. Electricity Div.

Performance Evaluation of a New Audio-Frequency Power Brldge.

Final rept.

B. C. Waltrip, and N. M. Oldham. 1991, 4p

See also PB91-101634.

Pub. in IEEE (Institute for Electrical and Electronics Engineers) Transactions on Instrumentation and Measurement 40, n2 p380-383 Apr 91.

Keywords: "Electric bridges, Electrical measurement, Alternating current, Audio frequencies, Power factor, Electrical impedance, Performance evaluation, Wattmeters, Reprints.

Several techniques for measuring active and reactive power in the $50 \mathrm{~Hz}$ to $20 \mathrm{kHz}$ frequency range are described. The approaches include: (1) the development of a high-precision sampling wattmeter using a resistive attenuator, a shunt, and two commercially available sampling voltmeters configured as a dual-channe equivalent-time sampler; (2) the development of another high-precision sampling wattmeter using the same shunt and attenuator, a high-impedance, wideband differential amplifier, and a commercially avail able, dual-channel, direct-sampling waveform analyzer; (3) for zero power factor measurements, the use of a digital generator to produce precise phase shifts from $+\mathrm{pi} / 2$ to $\mathrm{pi} / 2$; and (4) the use of simultaneous thermal voltage and current measurements for unity power factor measurements. These approaches were developed to evaluate a new high-accuracy, audio-frequency power bridge that is based on ac voltage and impedance measurements.

\section{Electromechanical Devices}

\section{0,743}

PB91-236620

Not available NTIS

National Inst. of Standards and Technology (EEEL), Boulder, CO. Electromagnetic Fields Div.
Determining Adapter Efficiency by Envelope Averaglng Swept Frequency Peflection Data.

Final rept.

W. C. Daywitt. 1990, 5p

Pub. in IEEE (Institute of Electrical and Electronics En gineers) Transactions on Microwave Theory and Tech. niques $38, \mathrm{n} 11 \mathrm{p} 1748-1752$ Nov 90.

Keywords: *Adapters, Electric connectors, Microwave equipment, Millimeter waves, Metrology, Efficiency, Reprints, Automated network analyzers.

A simple automated network analyzer swept frequency technique for measuring adapter efficiency across its entire frequency band is described. Envelope averag ing is used to avoid the model assumptions usually found in regression averaging techniques. Calcula tions show that errors arising from the theoretical as sumptions leading to the technique are around 0.004 B for a common WR 42 waveguide-to-coaxial adapt-

\section{Optoelectronic Devices \& Systems}

100,744

AD-A227 836/4

PC A01/MF A01

National Inst. of Standards and Technology, Gaithers burg, MD.

Photomultiplier Housing for Vacuum Operation of Side-on 1P28-Type Tubes.

W. Braun, R. Klein, A. Fahr, and A. Mele. 1990, 4p ARO-25911.3-CH,

Contract MIPR-ARO-119-89

Pub. in Review of Scientific Instruments, v61 n5 p1558-1560 May 90.

Keywords: *Housings, Operation, Photometry, *Photo multiplier tubes, Reprints, Sides, Tubes, Vacuum, Vacuum ultraviolet radiation.

A design for the mounting of a side-on tube such as the 1P28 type which has proven simple to construct reliable, and evacuatable is described. Keywords: Re prints; Housing; Photomultiplier; Vacuum Ultraviolet photometry; design. (jhd)

100,745

PB91-134734

Not available NTIS

National Bureau of Standards (NEL), Boulder, CO Electromagnetic Technology Div.

Progress in the Development of Miniature Optical Fiber Current Sensors.

Final rept.

D. Tang, and G. W. Day. 1988,1 p

Sponsored by Defense Nuclear Agency, Washington, DC., and Los Alamos National Lab., NM.

Pub. in Proceedings of IEEE (Institute of Electrical and Electronics Engineers) Lasers and Electro-Optics Society 1988 LEOS Annual Meeting, Santa Clara, CA. November 2-4, 1988, p306.

Keywords: *Electric current meters, "Fiber optics, Far aday effect, Birefringence, Optical measuring instru ments, Reprints, *Optoelectronic devices, "Optical fibers, Sensors.

Recent improvements in fiber annealing technology have allowed the authors to substantially reduce the size and increase the number of turns of fiber curren sensing coils. Coils as small as $7 \mathrm{~mm}$ diameter have been successfully annealed. Coils with more than 100 turns and diameters of 1 or $3 \mathrm{~cm}$ are routinely produced. The linear birefringence of such coils is small enough that Faraday rotation is not measurably dimin ished. Increased loss as a result of annealing is minimal.

\section{0,746}

PB91-134742

Not available NTIS

National Inst of Standards and Technology (NEL) Boulder, CO. Electromagnetic Technology Div. Practical Considerations in the Design of Optical Fiber Current Sensors.

Final rept.

D. Tang, A. H. Rose, and G. W. Day. 1990, 6p Sponsored by National Aeronautics and Space Administration, Washington, DC., Department of Defense Washington, DC., and Department of Energy, Washington, DC. 


\section{Optoelectronic Devices \& Systems}

Pub. in Proceedings of International Congress on Optical Science and Engineering. The Hague, Netherlands, March 12-15, 1990,6p.

Keywords: "Electric current meters, "Fiber optics, Optical measuring instruments, Birefringence, Reprints, "Optoelectronic devices, "Optical fibers, Sensors.

While current sensors based on the Faraday effect in bulk materials have shown good success in field tests the use of single mode fiber as the sensing element has both technical and economic advantages. In the paper the authors describe some of the practical problems that have inhibited the development of fiber cur rent sensors. Recent research suggests that most of these problems, including especially the problem of linear birefringence in the sensing coils, can be solved. Instruments providing a measurement quality approaching that set by fundamental material parameters can now be achieved.

\section{0,747}

PB91-148254

Not available NTIS National Bureau of Standards (ICST), Gaithersburg, MD. Advanced Systems Div.

Conformance Verification of FDDI Stations.

Final rept.

L. Zuqiu, and W. E. Burr. 1988, 15p

Pub. in Fiber and Integrated Optics 7, n3 p181-195 1988.

Keywords: "Local area networks, Fiber optics, Verification, Standards, Tests, Reprints, "FDDI(Fiber Distributed Data Interface), "Fiber distributed data interface, "Token ring networks.

The Fiber Distributed Data Interface (FDDI) is an emerging standard for a $100 \mathrm{Mbit} / \mathrm{s}$. fiber optic token ring Local Area Network. The paper outlines an approach to testing FDDI stations to verify their conformance to the FDDI standards.

\section{0,748}

PB91-148999

Not available NTIS

National Inst. of Standards and Technology (NEL)

Gaithersburg, MD. Semiconductor Electronics Div.

Micromachined Thermal Radiation Emitter from a Commercial CMOS Process.

Final rept.

M. Parameswaran, A. M. Robinson, D. L. Blackburn, M. Gaitan, and J. Geist. 1991, 3p

Pub. in IEEE (Institute of Electrical and Electronics Engineers) Electron Device Letters 12, n2 p57-59 Feb 91

Keywords: "Infrared sources, "Thermal radiation CMOS, Reprints, Thermal scene simulation.

Fabrication of thermally isolated micromechanical structures capable of generating thermal radiation for dynamic thermal scene simulation (DTSS) is described. Complete compatibility with a commercial CMOS process is achieved through design of a novel, but acceptable, layout for implementation by the CMOS foundry using its regular process sequence. Following commercial production and delivery of the CMOS chips, a single maskless etch in an aqueous ethylenediamine-pyrocatechol mixture (EDP) is performed to realize the micromechanical structures. The resulting structures are suspended plates consisting of polysilicon resistors encapsulated in the field and CVD oxides available in the CMOS process. The plates are suspended by aluminum heater leads that are also encapsulated in the field and CVD oxides. Studies of the suitability of these structures for DTSS have been initiated, and early favorable results are reported.

\section{0,749}

\section{PB91-149146}

Not available NTIS

National Inst of Standards and Technology (NEL). Boulder, CO. Electromagnetic Technology Div.

Performance and Limitations of Faraday Effect Sensors.

Final rept.

A. H. Rose, M. N. Deeter, D. Tang, and G. W. Day

\section{0, 1p}

Sponsored by Department of Energy, Washington, DC., and Department of Defense, Washington, DC.

Pub. in Proceedings of Electrochemical Society Fall Meeting, Seattle, WA., October 14-19, 1990, p1093.

Keywords: "Optical measuring instruments, "Electric current meters, "Magnetic fields, "Faraday effect, Measurement, Sensors, Reprints.

Sensors that use the Faraday effect to measure electric current and magnetic fields are becoming more prevalent because of their sensitivity, bandwidth, stability, and ability to operate in the presence of high voltage or EMI. They are now routinely used to measure large current pulses and are becoming available to the power industry. They can measure currents from milliamperes to megamperes and magnetic fields in the nanotesla range. Their size has been reduced and stability increased. Their speed is limited by material ef fects or transit time, but often extends to hundreds of megahertz.

\section{0,750}

PB91-162206

Not available NTIS

National Inst. of Standards and Technology (NEL), Boulder, CO. Electromagnetic Fields Div.

Photonic Probes for the Measurement of Electromagnetic Fields Over Broad Bandwidths.

Final rept.

K. D. Masterson, L. D. Driver, and M. Kanda. 1989,

$6 p$

Pub. in Proceedings of IEEE (Institute of Electrical and Electronics Engineers) National Symposium on Electromagnetic Compatibility, Denver, CO., May 23-25, 1989, p1-6.

Keywords: "Photonics, Electromagnetic fields, Directional couplers, Pockels cell, Fiber optics, Sensors, Reprints, "Electric field meters, Optical modulators.

The characteristics of photonic systems which make them especially well suited for use as broadband electromagnetic field sensors are discussed. Transfe functions are given for the individual components of such a measurement system, with special emphasis given to those of Pockels-cell and modified-directional-coupler optical modulators. An isotropic electricfield meter having $15 \mathrm{~cm}$ resistively tapered dipole elements combined with bulk crystal, Pockels-cell modu lators is described. The meter's frequency response is flat between $30 \mathrm{kHz}$ and $100 \mathrm{MHz}$, except for resonances in the modulator crystals that occur between 1 and $10 \mathrm{MHz}$. A photonic probe that uses a modified directional-coupler modulator is also briefly described.

\section{0,751}

PB91-174615 Boulder, CO. Electromagnetic Technology Div. Iron-Garnet Magnetic Field Sensors with 100 pT/ per square root $\mathrm{Hz}$ Noise-Equivalent Field.

Final rept.

M. N. Deeter, A. H. Rose, and G. W. Day. 1990, 4p

Pub. in Proceedings of Optical Fibre Sensors Conference, Sydney, New South Wales, Australia, Decembe 2-6, 1990, p341-344.

Keywords: "Magnetic detection, "Fiber optics, Yttrium iron garnets, Faraday effect, Magnetic fields, Magnetooptics, Substitutes, Gallium, Sensors, Reprints.

The sensitivity of Faraday-effect sensors incorporating diamagnetically substituted yttrium iron garnet $(Y \mid G)$ is potentially much higher than of sensors employing pure YIG. Results of Faraday rotation linearity and sensitivity measurements are presented for gallium-substituted YIG. At $500 \mathrm{~Hz}$, the noise-equivalent magnetic field is approximately $100 \mathrm{pT} /$ square root of $\mathrm{Hz}$.

\section{0,752}

\section{PB91-174706}

Not available NTIS

National Bureau of Standards

Electronic and Radiometric Characteristics of Near Infrared Photodiodes.

Final rept.

G. Eppeldauer, M. Tsudagawa, E. Zalewski, and J.

Houston. 1987, 10p

Pub. in Proceedings of International Symposium on Tech. Comm. Photon Detect., Int. Meas. Confed., v13 p215-224 1987

Keywords: *Photodiodes, *Radiometry, Near infrared radiation, Quantum efficiency, Indium phosphides, $R e$ prints, Gallium indium arsenides.

Three different types of infrared photodiodes from three manufacturers have been examined for their electronic and radiometric performance. Measurements were made of the shunt resistance, absolute response, reflectance, uniformity and linearity. The results of these measurements are presented in a manner that selects the best device for radiometric applications. From the limited sampling of devices available at the time of the study, InGaAs/InP photodiodes appear to be the most suitable. They have a very high internal quantum efficiency, are linear and reasonably uniform, and may be expected to perform well in high gain applications. Their high internal quantum efficiency can be interpreted as indicating that they may be suitable for self-calibration in a manner resembling silicon photodiodes.

\section{0,753}

\section{PB91-175109}

Not available NTIS

National Inst. of Standards and Technology (NEL), Boulder, CO. Electromagnetic Fields Div.

Optical Fiber Current Sensors with Temperature Stabilities Near the Material Limit.

Final rept.

D. Tang, A. H. Rose, and G. W. Day. 1990, 4p Pub. in Proceedings of Optical Fibre Sensors Conference (7th), Sydney, New South Wales, Australia, December 2-6, 1990, p77-80.

Keywords: "Electric current meters, "Optical fibers, Thermal stability, Faraday effect, Sensors, Reprints.

The authors describe an optical fiber current sensor with a normalized temperature coefficient of with a normalized temperature coefficient is within $20 \%$ of the limit set by the temperature dependence of the Verdet constant measured in bulk silica. Packaging of the sensor coil degrades its stability, but a fully packaged coil with a stability of $+0.00017 / \mathrm{K}$ over the range from -30 to $+125 \mathrm{C}$ has also been demonstrated.

\section{0,754}

PB91-175158 National Inst. of Standards and Technology (NEL), Boulder, CO. Electromagnetic Technology Div. Faraday Effect Current Sensing Using a Sagnac Interferometer with a $3 \times 3$ Coupler.

Final rept.

L. R. Veeser, and G. W. Day. 1990, 189p

Pub. in Proceedings of Optical Fibre Sensors Conference (7th), Sydney, New South Wales, Australia, December 2-6, 1990, p325-328.

Keywords: "Optical measuring instruments, "Electric current meters, "Fiber optics, Optical interferometers, Sagnac effect, Faraday effect, Sensors, Reprints, Optical couplers.

The authors demonstrate a fiber optic current sensor based on a Sagnac interferometer with a $3 \times 3$ fiber coupler. Compared to the more common Sagnac interferometer with a $2 \times 2$ coupler, the design offers the additional benefits of a greater response for small signals and the unambiguous interpretation of signals that exceed the period of the response function.

\section{0,755}

\section{PB91-187625}

(Order as PB91-187617, PC A06/MF A01) National Inst. of Standards and Technology, Gaithersburg, MD.

Linearity of a Silicon Photodiode at $30 \mathrm{MHz}$ and Its Effect on Heterodyne Measurements.

A. L. Migdall, and C. Winnewissser. 1991, 4p Included in Jnl. of Research of the National Institute of Standards and Technology, v96 n2 p143-146 Mar/Apr 91.

Keywords: *Photodiodes, Si semiconductor detectors, Silicon diodes, Light modulation, Laser radiation, High frequency, Heterodyning, Irradiance, Linearity.

The effect of optical irradiance on the linearity of a Si photodiode was studied. These results are compared for light modulated at $30 \mathrm{MHz}$ and at dc as the optical irradiance was varied over a 9 decade range. The authors discuss how these results affect the use of the detector as a heterodyne receiver. As the optical irradiance varied from 0.01 to $.1000 \mathrm{~mW} / \mathrm{sq} \mathrm{cm}$, while maintaining constant total power, the photocurrent was constant to about $1 \%$, but as the power density increased further, the photocurrent increased about $13 \%$. At the highest densities that the authors could achieve, about 60 million $\mathrm{mW} / \mathrm{sq} \mathrm{cm}$, there was only slight evidence of the onset of saturation. These results are of importance in the work to use optical heterodyne detection to measure filter transmittances over a wide dynamic range. The results provide guidelines for achieving maximum accuracy when using this particular diode as an optical heterodyne receiver. 
National Inst. of Standards and Technology (NEL), Boulder, CO. Electromagnetic Technology Div. Hydrogenated Amorphous Germanium Detectors Deposited onto Channel Waveguides.

Final rept.

D. R. Larson, and R. J. Phelan. 1990, 3p

Pub. in Optics Letters 15, n10 p544-546, 15 May 90.

Keywords: *Ge semiconductor detectors, "Photodeectors, Amorphous materials, Integrated optics, Lithium niobates, Picosecond pulses, Subtrates, Glass, Doped materials, Phosphorus, Reprints.

The authors have fabricated hydrogenated amorphous germanium photodetectors coupled to channel waveguides in glass and lithium niobate substrates. They measured a pulse response duration of 140 psec (FWHM), which is shorter than that of any previously reported photodetectors deposited onto dielectric waveguides. The optical gap, which determines the spectral response characteristics, is approximately $1.2 \mathrm{eV}$. A photoconductive gain of 18 was measured in phosphorus-doped detectors.

100,757

B91-203349

Not available NTIS

National Bureau of Standards (NML), Gaithersburg, MD. Radiation Physics Div.

Stable, High Quantum Efficiency Silicon Photodiodes for Vacuum-UV Applications.

Final rept.

R. Korde, L. R. Canfield, and B. Wallis. 1988, 8 p Pub. in Proceedings of SPIE (Society of Photo-Optical Instrumentation Engineers) Ultraviolet Technology II, Orlando, FL., April 4-5, 1988, p153-160.

Keywords: "Ultraviolet detectors, "Photodiodes, Vacuum ultraviolet radiation, Silicon diodes, Quantum efficiency, Radiometry, Standards, Reprints.

Silicon photodiodes have been developed by defectree phosphorus diffusion having practically no carrier recombination at the Si-SiO2 interface or in the front diffused region. The quantum efficiency of these phoodiodes was found to be around $120 \%$ at $100 \mathrm{~nm}$. Unlike the previously tested silicon photodiodes, the developed photodiodes exhibit extremely stable quantum efficiency over extended periods of time. Current$y$, the authors are investigating the possibility of using these photodiodes as vacuum ultraviolet detector standards.

\section{0,758}

PB91-203976

Not available NTIS

National Inst. of Standards and Technology (NEL),

Boulder, CO. Electromagnetic Technology Div.

Integrated-Optic Waveguide Glass Lasers.

Final rept.

N. A. Sanford, K. J. Malone, and D. R. Larson. 1991, $1 \mathrm{p}$

Pub. in Proceedings of Optical Fiber Communication Conference, San Diego, CA., February 18-22, 1991 , p27.

Keywords: "Integrated optics, "Waveguide lasers, Mode locked lasers, $Q$ switched lasers, Glass lasers, Rare earth elements, Doped materials, Chemical vapor deposition, Reprints.

Rare-earth-doped integrated-optic waveguide devices offer new miniaturized $\mathrm{cW}$ and pulsed lasers, amplifiers, and other active elements. Fabrication methods which use bulk glasses as well as chemical-vapor deposition techniques are being explored.

100,759

PB91-204131

Not available NTIS

National Inst of Standards and Technology (NEL)

Boulder, CO. Electromagnetic Technology Div.

Analyzing Integrated Optical Waveguides: A Comparison of Two New Methods.

Final rept.

Y. Tu, I. C. Goyal, and R. L. Gallawa. 1990, 3p

Pub. in Applied Optics 29, n36, p5313-5315, 20 Dec 90.

Keywords: "Optical waveguides, "Integrated optics, WKB approximation, Fourier series, Numerical analysis, Comparison, Reprints.

The authors present a comparison of two recently developed methods of analyzing optical waveguides. One of them is numerical, and the other is an approximate analytical method.
National Inst. of Standards and Technology (EEEL), Gaithersburg, MD. Semiconductor Electronics Div. Generalized Photodiode Self-Calibration Formula. Final rept.

R. Kohler, J. Geist, and J. Bonhoure. 1991, 3p

Pub. in Applied Optics 30, n7 p884-886, 1 Mar 91.

Keywords: "Photodiodes, "Calibration, Quantum efficiency, Silicon diodes, Reprints, Self calibration, Oxide bias experiment, Reverse bias experiment.

The authors have derived the photodiode self-calibration formula for calculating the internal quantum efficiency of silicon photodiodes from the results of one, two, or three independent self-calibration experiments and from the results of ozide bias and reverse bias experiments in conjunction with a calculation of the effect of Auger recombination. They show that the formula published elsewhere for these three effects is not correct.

100,761

B91-237792

Not available NTIS

National Inst. of Standards and Technology (EEEL), Gaithersburg, MD. Electronics and Electrical Engineering Lab. Office.

Dealing with Obvious Issues in Lighting.

Final rept.

J. A. Worthey. 1991, 4p

Pub. in Lighting Design and Application 21, n8 p15, 17 19, Aug 91

Keywords: *Illuminating, "Daylighting, Research management, Design criteria, Luminance, Light sources, Lighting systems, Color, Reprints.

An early 'controlled study' of lighting effects is reviewed. Although the authors did not present it in that way, the study controlled for most obvious differences between two light sources, then looked for possible effects of more subtle physical differences. The outcome was null -- no difference in the light sources. The suggestion is made that while subtle differences may be easier to study in a simple experiment, obvious differences may be more important. To deal with obvious facts, an approach involving more theory and calculation is appropriate.

100,762

PB92-110261

PC A05/MF A02

National Inst. of Standards and Technology, Gaithersburg, MD.

Journal of Research of the National Institute of Standards and Technology. July-August 1991. Volume 96, Number 4.

c1991, 100p

Also available from Supt. of Docs as SN703-027-0041. 5. See also PB92-110279 through PB92-110311 and P891-216705. Library of Congress catalog card no. 89-656121.

Keywords: "Photodiodes, "Computerized simulation, Antenna radiation patterns, Building fires, Room fires. Contents:

General Analytic Correction for Probe-Position Errors in Spherical Near-Field Measurements: Data for Room Fire Model Comparisons;

Numerical Modeling of Silicon Photodiodes for High-Accuracy Applications--Part I. Simulation Programs:

Numerical Modeling of Silicon Photodiodes for High-Accuracy Applications--Part II. Interpreting Oxide-Bias Experiments:

Numerical Modeling of Silicon Photodiodes for High-Accuracy Applications--Part III. Interpolating and Extrapolating Interna Quantum-Efficiency Calibrations.

\section{0,763}

PB92-110295

(Order as PB92-110261, PC A05/MF A01) National Inst. of Standards and Technology, Gaithersburg, MD.

Numerical Modeling of Silicon Photodiodes for High-Accuracy Applications. Part 1. Simulation Programs.

J. Geist, D. Chandler-Horowitz, A. M. Robinson, and C. R. James. c1991, 7p

Prepared in cooperation with Alberta Univ., Edmonton. Included in Jnl. of Research of the National Institute of Standards and Technology, v96 n4 p463-469 Jul/Aug 91.

Keywords: "Photodiodes, "Computerized simulation, Mathematical models, Silicon diodes, Quantum effi- ciency, One dimensional, Accuracy, PC-1D computer program.

The suitability of the semiconductor-device modeling program PC-1D for high-accuracy simulation of silicon photodiodes is discussed. A set of user interface programs optimized to support high-accuracy batch-mode operation of PC-1D for modeling the internal quantum efficiency of photodiodes is also described. The optimization includes correction for the dark current under reverse- and forward-bias conditions before calculating the quantum efficiency, and easy access to the highest numerical accuracy available from PC-1D, neither of which is conveniently available with PC-1D's standard user interface.

100,764

PB92-110303

(Order as PB92-110261, PC A05/MF 01) National Inst. of Standards and Technology, Gaithersburg, MD.

Numerical Modeling of Silicon Photodiodes for High-Accuracy Applications. Part 2. Interpreting Oxide-bias Experiments.

J. Geist, R. Koehler, R. Goebel, A. M. Robinson, and C. R. James. c1991, 9p

Prepared in cooperation with Bureau International des Poids et Mesures, Sevres (France), and Alberta Univ. Edmonton.

Included in Jnl. of Research of the National Institute of Standards and Technology, v96 n4 p471-479 Jul/Aug 91

Keywords: "Photodiodes, "Computerized simulation Silicon diodes, Quantum efficiency, Mathematical models, Numerical solution, Visible radiation, PC-1D computer program, Oxide bias experiments.

The semiconductor device modeling program PC-1D and the programs that support its use in high-accuracy modeling of photodiodes, all of which were described in Part I of the series of papers, are used to simulate oxide-bias self-calibration experiments on three different types of silicon photodiodes. It is shown that these simulations can be used to determine photodiode characteristics, including the internal quantum efficiency for the different types of photodiodes. In the latter case, the simulations provide more accurate values than can be determined by using the conventional data reduction procedure, and an uncertainty estimate can be derived. Finally, it is shown that $0.9997+$ or - is a nominal value for the internal quantum efficiency of one type of photodiode over the 440 to $460 \mathrm{~nm}$ spectral region.

\section{0,765}

PB92-110311

(Order as PB92-110261, PC A05/MF A01) National Inst. of Standards and Technology, Gaithersburg, MD.

Numerical Modeling of Silicon Photodiodes for High-Accuracy Applications. Part 3. Interpolating and Extrapolating Internal Quantum-Efficiency Calibrations.

J. Geist, A. M. Robinson, and C. R. James. c1991, 12p

Prepared in cooperation with Alberta Univ., Edmonton. Included in Jnl. of Research of the National Institute of Standards and Technology, v96 n4 p481-492 Jul/Aug 91.

Keywords: "Photodiodes, "Computerized simulation, Silicon diodes, Mathematical models, Quantum efficiency, Visible radiation, Near infrared radiation, Interpolation, Extrapolation, PC-1D computer program.

The semiconductor device modeling program PC-1D and the programs that support its use in high-accuracy modeling of photodiodes, all of which were described in Part I of the series of papers, are used to simulate the interpolation of high-accuracy calibrations in the spectral region between $450 \mathrm{~nm}$ and $850 \mathrm{~nm}$. Convenient interpolation formulae that depend only upon wavelength are derived. Uncertainty spectra for a number of sources of error are also derived. The formulae are normalized to experimental internal-quantum efficiency calibrations in the 440 to $470 \mathrm{~nm}$ spectral region and at $860 \mathrm{~nm}$ and are used to interpolate the calibration values between these wavelengths. The results of the interpolations are compared with experimental calibration data that are available at a few wavelengths between 440 and $860 \mathrm{~nm}$. 
100,766

PB92-112382 PC A05/MF A02 National Inst. of Standards and Technology, Gaithersburg, MD.

HgCdTe Detector Reliability Study for the GOES Program.

D. G. Seiler, G. G. Harman, J. R. Lowney, S. Mayo, and W. S. Liggett. Sep $91,100 p$ NISTIR-4687

Sponsored by National Oceanic and Atmospheric Administration, Rockville, MD.

Keywords: "GOES satellites, "Infrared detectors, Intermediate infrared radiation, Mercury cadmium tellurides, Satellite-borne

Reliability(Electronics), Degradation

The report summarizes the results of a special assessment concerning the reliability of certain infrared detectors for the Geostationary Operational Environmental Satellite (GOES) system. The data made available by ITT on detector resistances and signals support the conclusion that degradation of some detector responses has occurred, even when the estimated measurement uncertainty is included. Statistical analysis of the 11-micrometer detectors confirmed that detector 11-105 decreased in signal with time. The existing data available to NIST is not sufficient to identify uniquely the cause of degradation or unstable behav ior present in a number of detectors. NIST's physical examination of several detectors by optical and SEM microscopy methods and an examination and analysis of the Detector Measurement Database has yielded several plausible possible mechanisms for the observed degradation. The possible mechanisms are related to the detector fabrication or processing steps and include: Incomplete or poor passivation procedures, excess mercury diffusion resulting from the ionbeam milling fabrication step, poor indium electrical contacts produced by the indium-plated fabrication step, and delamination of the $\mathrm{ZnS}$ antireflection optical coating. Other observed problems are also discussed.

100,767

PB92-116763

Not available NTIS

National Inst of Standards and Technology (NML), Gaithersburg, MD. Radiometric Physics Div.

Temperature Monitored/Controlled Silicon Photodiodes for Standardization.

Final rept.

G. Eppeldauer. 1991, 7p

Pub. in Proceedings of SPIE (Society of Photo-Optical Instrumentation Engineers) - Surveillance Technologies, v1479 p71-77 1991.

Keywords: "Photometers, "Radiometers, Silicon diodes, Temperature measurement, Temperature control, Standardization, Photodiodes, Correction, Reprints.

Two alternative approaches to reducing errors in radio meters and photometers caused by temperature variations involve temperature monitoring and temperature control. In the first method, the measurement results are interpreted using the temperature of the detector at the time of measurement. The other method is to control the temperature of the detector to a constant value. Design considerations and examples of both approaches are discussed.

\section{0,768}

PB92-116771

Not available NTIS

National Inst. of Siandards and Technology (NML).

Gaithersburg, MD. Radiometric Physics Div.

Fourteen-Decade Photocurrent Measurements with Large-Area Silicon Photodiodes at Room Temperature.

Final rept

G. Eppeldauer, and J. E. Hardis. 1991, 9p

Pub. in Applied Optics 30, n22 p3091-3099, 1 Aug 91.

Keywords: "Optical detectors, "Photodiodes, "Photocurrents, Electrical measurement, Silicon diodes, Room temperature, Radiometry, Bandwidth, Sensitivity, Reprints.

Recent improvements in commercial silicon photodiodes and operational amplifiers permit electrical noise to be reduced to an equivalent of $0.1 \mathrm{fA}$ of photocurrent when a measurement time of $400 \mathrm{~s}$ is used. This is equivalent to a photocurrent resulting from fewer than 800 photons/s, and it implies a dynamic range of 14 orders of magnitude for a detector circuit. The authors explain the circuit theory, paying particular attention to the measurement bandwidth, the causes of noise and drift, and the proper selection of circuit components. These optical radiation detectors complement the primary radiometric standards. These detectors may replace photomultiplier tubes that have been used traditionally and or that were too costly to be used.

\section{Power \& Signal Transmission Devices}

\section{0,769}

PB91-133892 Not available NTIS

National Inst. of Standards and Technology (NEL), Boulder, CO. Electromagnetic Technology Div.

AC Loss Measurements of Two Multifilamentary NbTi Composite Strands.

Final rept

E. W. Collings, K. R. Marken, M. D. Sumption, R. B. Goldfarb, and R. J. Loughran. 1990, 8p

See also DE90005388. Sponsored by Department of Energy, Washington, DC. Div. of High Energy Physics. 36, p169-176 1990 .

Keywords: Filament winding, Magnetization, Hysteresis, Reprints, "Superconducting wires, "AC losses, Niobium titanium.

As part of an interlaboratory comparative testing program conducted in support of the Versailles Agreement on Advanced Materials and Standards (VAMAS) transversefield DC hysteresis loss measurements were made at liquid-helium temperatures at fields of up to $3 \mathrm{~T}(30 \mathrm{kG}$ ) on two samples of multifilamentary $\mathrm{NbT}$ composite. The strands differed widely in filament number were comparable in filament diameter, and one of them was provided with a Cu-Nibarrier between the filaments. The results have been analyzed, and magnetically deduced critical current density values obtained (for comparison with directly measured transport data) using various standard techniques. Based on these studies, a figure-of-merit for AC loss is recommended. The Cu-matrix strand, with its interfilamentary spacing of less than 1 micrometer, exhibited pronounced proximity-effect-induced coupling losses; this was not observed in the mixed-matrix strand which possessed not only a Cu-Ni barrier but also an interfilamentary spacing of typically 4 micrometers.

\section{0,770}

PB91-134114

Not available NTIS

National Inst. of Standards and Technology (NEL)

Boulder, CO. Electromagnetic Fields Div.

Determination of Scattering Parameters with Respect to the Characteristic Impedance of Precision Coaxial Air-Line Standards.

Final rept.

D. R. Holt. $1990,2 p$

Pub. in Proceedings of Conference on Precision Electromagnetic Measurements (CPEM 90), Ottawa Canada, June 11-14, 1990, p282-283.

Keywords: "Coaxial cables, Characteristic impedance, Skin effect, Surface roughness, Scattering, Standards, Reprints.

Scattering parameter expressions with respect to the characteristic impedances in correspondence to the principal mode are developed for the coaxial air-line standard. Dimensional variations of the inner and outer conductors and skin effect loss are included in the model. The local characteristic impedance, which is found from the stored energy principle, is derived from the forward and backward voltage and current waves of the principal mode. Four sources of error for absolute value of $S$ (sub 11) are discussed.

100,771

Not available NTIS National Inst. of Standards and Technology (NEL), Gaithersburg, MD. Electricity Div.

Partial Discharges in Low Voltage Cables.

Final rept.

J.P. Steiner, and F. D. Martzloff. 1990, 4p

Pub. in Proceedings of IEEE (Institute of Electrical and Electronics Engineers) International Symposium on Electrical Insulation, Toronto, Canada, June 3-6, 1990 p149-152.

Keywords: "Electrical insulation, "Electric discharges, Signal processing, Reprints, "Electric cables, Low voltage, Test methods.

Testing of high voltage apparatus for partial discharges has long been recognized as an important part of quality control for these devices. Recently, interest has been focused on methods for testing low voltage cables to determine their integrity under adverse operating conditions such as a loss of coolant accident. A new method using partial discharges, is presented which has the potential for locating breaches in the insulation of in situ, low voltage, multiconductor cables.

100,772

PB91-134759

Not available NTIS

National Inst of Standards and Technology (NEL). Boulder, CO. Electromagnetic Technology Div.

Magnetoresistance of Multifilament Al/Al-Alloy Conductors.

Final rept.

C. A. Thompson, and F. R. Fickett. 1990, 7p

Pub. in Advances in Cryogenic Engineering Materials, v36 p663-669 1990.

Keywords: "Electric conductors, Aluminum alloys, Reprints, "Magnetoresistance, Multifilaments.

Previously the authors have shown that composite monofilament conductors consisting of very pure aluminum confined in an Al-Fe-Ce alloy sheath show an anomalously high magnetoresistance compared to pure aluminum. Some monofilament conductors showed values of Delta R/A in excess of 50 at $4 \mathrm{~K}$ in fields of $10 \mathrm{~T}$, whereas pure aluminum values are usually an order of magnitude smaller. Concerns that similar anomalous behavior might occur in multifilament wires of the same materials prompted this study. Multifilamentary conductors with pure aluminum filaments contained in an $\mathrm{Al}-\mathrm{Fe}$-Ce matrix have been investigated.

\section{0,773}

PB91-161943

Not available NTIS National Inst of Standards and Technology (MSEL), Gaithersburg, MD. Polymers Div.

Proceedings of Cable ' 89 Workshop.

Final rept.

M. G. Broadhurst, and E. Kelly. 1990, 119p

Pub. in Proceedings of JICABLE/EPRI/CEA Workshop 'Cable 89' The Aging of Extruded Dielectric Cables, St. Petersburg, FL., November 2-3, 1990, $119 p$

Keywords: "Meetings, "Polymers, "Electric cables, "Dielectric materials, Power lines, Extrusion, Aging tests Maintenance, Performance prediction, Accelerated tests, Electrical insulators, Reprints.

The report presents the proceedings of the workshop 'Cable 89' on The Aging of Extruded Dielectric Cables. The workshop was held in St. Petersburg. FL on November $2-3,1989$. The sponsoring organizations are The Electric Power Research Institute (US), the Canadian Electric Association and JICABLE (France). Attendance at the workshop was by invitation only, and attendees included 60 representatives of universities, research organizations, electric utilities, and manufacturers of insulating polymers and power cables, from eight countries. The workshop was created as a forum for world experts to relate and compare their service experiences, theoretical, fundamental and experimental studies, and test results on the topic of aging of solid dielectric cables. The overall topic included materials aging, cable and accessories aging, diagnostic tests accelerated aging and service experiences and conditions. The first half day of the workshop featured six overview presentations by international experts. The presentations and the brief discussions that followed each presentation are included in the report. The second half day was devoted to discussions within three separate working groups dealing with the three topic areas: materials, cables, and accelerated tests. Reports from the three working groups were given on the third half day, and are included in the report with the general discussions that followed each one.

\section{0,774}

PB91-236752

Not available NTIS National Inst. of Standards and Technology (EEEL), Boulder, CO. Electromagnetic Technology Div.

Methods of Analyzing Planar Optlcal Waveguldes. Final rept.

I. C. Goyal, R. L. Gallawa, and A. K. Ghatak. 1991,

Pub. in Optic Letters 16, n1 p30-32, 1 Jan 91. 
Keywords: "Optical waveguides, "Optical fibers, "Fiber optics, Wave equations, Integrated optics, WKB approximation, Reprints.

The authors present a new approximate solution of the scalar-wave equation for planar optical waveguides with arbitrary refractive-index profiles. Test calculations are done for an index profile with a known solution. The comparison demonstrates the accuracy of their method. The method may also be applied to circularly symmetric optical fibers.

100,775

PB91-236992

Not available NTIS

National Inst. of Standards and Technology (EEEL), Boulder, CO. Electromagnetic Fields Div.

Characteristic Impedance Determination Using Propagation Constant Measurement.

Final rept.

R. Marks, and D. Williams. 1991, 3p

Pub. in IEEE (Institute of Electrical and Electronics Engineers) Microwave and Guided Wave Letters 1, n6 p141-143 Jun 91

Keywords: "Transmission lines, * Characteristic impedance, Electrical conductivity, Electrical measurement Reprints, Microwave monolithic integrated circuits, $\mathrm{Co}$ planar waveguides.

A method is demonstrated where by the characteristic impedance of transmission lines may be easily determined from a measurement of the propagation constant. The method is based on a rigorous analysis using realistic approximations to account for the effects of imperfect conductors. Numerical studies indicate that high accuracy is possible, and experiments using coplanar waveguide demonstrate the advantage of the method in the interpretation of S-parameters.

100,776

PB92-116656

Not available NTIS

National Inst. of Standards and Technology (EEEL). Boulder, CO. Electromagnetic Fields Div.

Mode-Stirred Chamber for Measuring Shielding Effectiveness of Cables and Connectors: Assessing MIL-STD-1344A Method 3008.

Final rept.

M. L. Crawtord, and J. M. Ladbury. 1989, 7p See also PB89-149264

Pub. in Connection Technology 5, n6 p45-51 Jun 89.

Keywords: "Electromagnetic shielding, "Electric connectors, "Electric cables, Communication cables, UItrahigh frequency, Superhigh frequency, Electrical measurement, Recommendations, Reprints, Mode stirred method.

The mode-stirred method for measuring the shielding effectiveness (SE) of cables and connectors as specified in MIL-STD-1344A Method 3008 is examined. Problems encountered in applying the method are identified and recommendations to improve the measurement results are provided. These include chamber design, type and placement of transmitting and reference receiving antenna, determination and correction for VSWR of the reference antenna and equipment under test (EUT), and the measurement approach to use at specified test frequencies. Design and measurement setups for a small mode-stirred chamber suitable for performing SE measurements in the frequency range $(1-18) \mathrm{GHz}$ with dynamic ranges up to $130 \mathrm{~dB}$ are given along with SE measurement results of some sample EUTs.

\section{0,777}

PB92-117324

Not available NTIS National Inst. of Standards and Technology (EEEL), Boulder, CO. Electromagnetic Fields Div.

Air Gage Size Measurement of Microwave Stand-

ards.

Final rept.

G. V. Sherwood. 1991, 10p

Pub. in Proceedings of Measurement Science Conference, Anaheim, CA., January 31-February 1, 1991, p110.

Keywords: "Transmission lines, "Coaxial cables, *Waveguides, "Standards, Dimensional measurement Microwave equipment, Surface roughness, Mechanical measurement, Metrology, Calibration, Reprints.

Size measurement of wave guides and coaxial transmission line standards has been performed at NIST for many years. Recently, the air gaging systems used to perform these measurements have been enhanced using digital data acquisition methods to facilitate com- puter analysis. Initial experiments were performed using air gaging probes on a coordinate measuring machine to correlate linear position with size attributes. Related geometric and dimensional characteristics that contribute to the uncertainty of measuremen were also studied. Alternative assessment methods have been used to provide a more complete characterization of these features and complement air gage size measurements using a systems approach. The article is a report of these developments at NIST.

\section{Resistive, Capacitive, \& Inductive Components}

100,778

N91-25797/2

(Order as N91-25755/0, PC A99/MF A04) National Inst. of Standards and Technology (NML). Boulder, CO. Time and Frequency Div.

Environmental Sensitivities of Quartz Crystal Oscillators.

F. L. Walls. May $90,22 p$

Pub. in Proceedings of Annual Precise Time and Time Interval (PTTI) Applications and Planning Meeting (22nd) p465-486 (See N91-25755 17-70).

Keywords: "Crystal oscillators, Frequencies, Quartz crystals, Sensitivity, Electric fields, Humidity, Loads (Forces), Magnetic fields, Precision, Pressure distribution, Vibration, *Quartz resonators.

The frequency, amplitude, and noise of the output signal of a quartz crystal controlled oscillator is affected by a large number of environmental effects. The physical basis for the sensitivity of precision oscillators to temperature, humidity, pressure, vibration, magnetic field, electric field, load and radiation is discussed. The sensitivity of crystal oscillators to radiation is a very complex topic and poorly understood. Therefore only a few general results are mentioned. The sensitivity to most external influences often varies significantly from one oscillator type to another and from one unit of given type to another. For a given unit, the sensitivity to one parameter often depends on the value of other parameters and history.

100,779

PB91-134320

Not available NTIS

(NML), Gaithersburg.

MD. Temperature and Pressure Div.

Stability of Thermistors.

Final rept.

B. W. Mangum. 1987, $6 \mathrm{p}$

Pub. in Proceedings of International Symposium on Temperature Measurement in Industry and Science, p170-175 1987.

Keywords: "Thermistors, "Thermal stability, Aging tests(Materials), Reprints.

Thirty-two bead-in-glass probe-type and 67 disc-type thermistors from six manufacturers have been investigated for stability upon thermal cycling from 0 to 300 or $150 \mathrm{C}$ for the beads and 0 to $100 \mathrm{C}$ for the discs. Also 12 bead-in-glass probes and 11 discs were aged for about 4000 hours at $100 \mathrm{C}$. After the thermistors were heat treated at the elevated temperatures, their resistances were measured over the range from 0 to $100 \mathrm{C}$ The disc-type thermistors were much less stable than the bead-in-glass probe types. The bead thermistors were not very stable when subjected to $300 \mathrm{C}$ environments nor were the discs when subjected to $100 \mathrm{C}$ environments.

\section{0,780}

PB91-134338

Not available NTIS

National Bureau of Standards (NML), Gaithersburg MD. Temperature and Pressure Div.

SRM 1970 - Succinonitrile Triple-Point Standard . And Its Use in Calibration of Thermistors.

Final rept.

B. W. Mangum. 1987, $6 \mathrm{p}$

Pub. in Proceedings of International Symposium on Temperature Measurement in Industry and Science. p60-65 1987

Keywords: *Thermistors, Reprints, *Standard reference materials, "Succinonitrile, *Calibration.

The triple-point temperature of succinonitrile (SCN) has been found to be a good reference point at about
$58.08 \mathrm{C}$, a value in a region devoid of fixed points. Cells with reentrant wells and containing approximate ly 60 grams of zone-refined SCN have been investigated for use as a Standard Reference Material (SRM) SRM 1970. They were designed to accept thermistor thermometers primarily, but will accept any thermometer that is less than $4.5 \mathrm{~mm}$ in diameter. Through a three-point calibration obtained by the use of SRM 1970, SRM 1968 (the Gallium Melting-Point Standard), and the triple point of water, thermistors can be calibrated with an uncertainty as small as + or -2 or $3 \mathrm{mK}$ over the range from 0 to $70 \mathrm{C}$

100,781

PB91-134866

Not available NTIS

National Bureau of Standards (NML), Boulder, $\mathrm{CO}$. Time and Frequency Div.

Environmental Effects on the Medium and Long Term Frequency Stability of Quartz Oscillators.

Final rept.

F. L. Walls. $1988,9 p$

Pub. in Proceedings of European Frequency and Time Forum (2nd), Neuchatel, Switzerland, March 16-18 1988, p719-727. Keywords: "Quartz resonators, "Frequency stability,
Environmental tests, Atomic clocks, Pressure, Humidjty, Frequency standards, Reprints.

The medium and Iongterm frequency stability of most quartz oscillators is degraded by various environmental effects, the most important of which appear to be acceleration, temperature, load change, pressure, and possibly humidity. In the paper, preliminary data are shown which indicate that the medium and long term frequency stability of some ocillators can be improved by controlling the pressure and humidity around the oscillator. These data were obtained on only one oscillator of each type and may or may not be representative. If these improvements can be obtained in other precision crystal oscillators, then crystal oscillators may become usable in some applications generally thought to require atomic standards.

100,782

PB9 1-174912

Not available NTIS

National Inst. of Standards and Technology (NEL) Boulder, CO. Electromagnetic Technology Div.

Noise-Affected I-V Curves in Small Hysteretic Josephson Junctions.

Final rept.

R. L. Kautz, and J. M. Martinis. 1990, 35p

Sponsored by Office of Naval Research, Arlington, VA Pub. in Physical Review B 42, n16 p9903-9937, 1 Dec 90.

Keywords: "Josephson junctions, Electromagnetic noise, Quasi particles, Hysteresis, Reprints.

The authors investigate the noise-affected I-V curves of small-area Josephson junctions through experiment, simulation, and theory. In particular, the authors consider I-V curves in which two different states of finite voltage coexist at the same de bias: a high-voltage state that corresponds to the usual quasiparticle branch and a low-voltage state that is characterized by thermally activated phase diffusion. In addition, the authors develop analytic expressions for three key parameters of the I-V curve of junctions displaying hysteresis between the phase-diffusion and quasiparticle branches: the initial slope of the phase-diffusion branch, the bias level at which the junction switches from the phase-diffusion branch to the quasiparticle branch, and the bias level at which it returns to the phase-diffusion branch.

100,783

PB91-190116

Not available NTIS

National Inst. of Standards and Technology (NEL)

Gaithersburg, MD. Chemical Process Metrology Div.

Transparent Thin Film Thermocouple.

Final rept.

M. Yust, and K. G. Kreider. 1989, 6p

See also PB91-110635

Pub. in Thin Solid Films 176, n1 p73-78 Sep 89

Keywords: "Thin films, "Thermocouples, "Indium oxides, "Tin oxides, Sputtering, Ceramics, Transparence, Platinum, Calibrating, Temperature measuring instruments, Reprints.

A transparent thin film thermocouple has been produced by reactive sputtering which has a Seebeck coefficient of $0.14 \mathrm{mv} / \mathrm{C}$. The positive leg of the thermocouple is indium tin oxide (ITO) and the negative leg is 


\section{ELECTROTECHNOLOGY}

\section{Resistive, Capacitive, \& Inductive Components}

indium oxide ( $\ln 203)$. The $\ln 203$ and ITO thin films were also tested with sputtered thin film platinum to obtain their individual thermoelectric potentials. Stability of the transparent thermocouple at $575 \mathrm{~K}$ and calibration data of sputtered ITO and platinum are also reported.

\section{0,784}

PB91-195446

Not available NTIS

National Inst. of Standards and Technology (NEL), Boulder, CO. Electromagnetic Technology Div.

Sidelobe Suppression in Small Josephson Junctions.

Final rept.

A. L. Peterson. 1991, 4p

Pub. in Cryogenics 31, p132-135 Feb 91.

Keywords: "Josephson junctions, "Critical current, Diffraction patterns, Sidelobes, Reprints.

Many applications of Josephson junctions require that the sidelobes of the critical-current patterns as a function of magnetic field be as small as possible. The sidelobes can be significantly suppressed by appropriate choice of junction geometry. The paper emphasizes that suppression is not just a long-junction effect. For a wide variety of shaped small junctions, the diffraction patterns are given by simple expressions, in spection of which quickly shows the overall features of the patterns. For some shapes the highest sidelobes can be kept well under $1 \%$ of the central lobe. For applications requiring long junctions, the geometry of small junctions giving the desired pattern is a practical starting point for a long-junction analysis.

\section{Semiconductor Devices}

\section{0,785}

DE91002169

$\mathrm{PC} A 03 / \mathrm{MF} \mathrm{A01}$

Solar Energy Research Inst., Golden, CO.

Scanning tunneling microscopy studies of the surfaces of a-Si:H and a-SiGe:H films. Annual report, December 1989-31 January 1991.

Progress rept.

A. Gallagher, R. Ostrom, and D. Tannenbaum. Jun

91, 35p SERI/TP-214-4409

Contract ACO2-83CH10093

Sponsored by Department of Energy, Washington, DC.

Keywords: "Germanium Compounds, "Silicon Compounds, Amorphous State, Chemical Reactions, Chemical Vapor Deposition, Electric Discharges, Etch ing, Hydrogen Compounds, Microstructure, Progress Report, Scanning Electron Microscopy, Silanes, Sola Cells, Stoichiometry, Thin Films, EDB/360602, EDB/ $140501, \mathrm{EDB} / 400102$.

The report contains a detailed description of the experimental complexities encountered in developing scanning tunneling microscope (STM) probing of atomic structure on the surface of freshly-grown hydrogenated-amorphous semiconductors. It also contains a speculative microscopic film-growth model that explains differences between the disorder in CVD grown a-Ge: $\mathrm{H}$ versus a-Si:H films. This model is derived from prior results obtained in the chemical analysis of GeH(sub 4) plasmas, combined with surface reaction and thermodynamic considerations. The neutral radical fragments of silane, disilane and germane dissociation in discharges, which dominate the vapor and filmgrowth reactions, have been deduced from detailed analysis of prior data and are reported. 4 refs., 7 figs.

\section{0,786}

\section{PB91-134692}

Not available NTIS

National Inst. of Standards and Technology (NEL) Gaithersburg, MD. Semiconductor Electronics Div.

Current Density Dependence of Electromigration t50 Enhancement Due to Pulsed Operation.

Final rept.

J. S. Suehle, and H. A. Schafft. 1990, 5p

Pub. in Proceedings of Annual Conference on Reliability Physics 1990 (28th), New Orleans, LA., March $27-$ 29, 1990, p106-110.

Keywords: "Integrated circuits, Reliability(Electronics), Current density, Metallizing, Reprints, "Electromigration, Electric pulses.

Two effects that complicate the electromigration characterization of metallization for pulsed stress have been observed. One is the dependence of the $t(50)$ enhancement (due to pulsed operation) on current density and the other is a decrease of this enhancement over a range of frequencies $(0.2$ to $2 \mathrm{MHz})$ that is connected with the joule heating. These effects are discussed in terms of changes in the buildup and relaxation response times of the excess vacancy concen. tration.

100,787

PB91-14718

Not available NTIS

National Inst. of Standards and Technology (NEL), Boulder, CO. Electromagnetic Technology Div.

Low-Temperature, Electrically Calibrated SOS Bolometer for Power and Energy Measurements. Final rept.

R. M. Craig, and R. J. Phelan. 1990, 11p

Sponsored by Aerospace Guidance and Metrology Center, Newark AFS, OH

Pub. in Proceedings of Measurement Science Conference, Anaheim, CA., February 8-9, 1990, p3A-1-3A-11 1990.

Keywords: "Bolometers, Visible radiation, Infrared radiation, Power measurement, Radiometry, Cryogenics, Reprints, SOS(Semiconductors), Silicon on sapphire.

A system has been constructed to allow absolute, electrically calibrated radiometry of low power $(\mathrm{pW} / \mathrm{Hz}$ to the $1 / 2$ pawer) and tector is based on an adaptation cf silicon-on-sapphire tector is based on an adaptation of silicon-on-sapphire
(SOS) technology with an integral absorber/heater for real-time electrical substitution. Cooling is provided by a custom closed-cycle helium liquefaction system built inside an optical access Dewar. Convenient control and data analysis are provided by use of the GPIB bus and a computer. This arrangement will allow routine laser measurements and detector calibrations at $1 \%$ $5 \%$ accuracy across the 0.4 micrometer to 15 micrometers wavelength range.

100,788

PB91-147520

Not available NTIS

National Inst of Standards and Technology (NEL) Gaithersburg, MD. Semiconductor Electronics Div. Verification of the Relation between Two-Probe and Four-Probe Resistances as Measured on Silicon Wafers.

Final rept.

J. J. Kopanski, J. Albers, G. P. Carver, and J. R.

Ehrstein. 1990, 7p

Pub. in Jnl. of the Electrochemical Society $137, \mathrm{n} 12$ p3935-3941 Dec 90

Keywords: "Electrical resistance, "Silicon, Electrical measurement, Probes(Electromagnetic), Laplace equation, Wafers, Reprints, Spreading resistance, Multilayers.

The predicted relation between the two-probe resistance (spreading resistance) and the four-probe resistance, and the dependence of the four-probe resistance on the ratio of layer thickness to probe spacing have been experimentally verified. The verified behavior is predicted from calculations, based upon the solution of Laplace's equation, of the two- and four-probe resistance for arbitrary, vertical resistivity profiles. Arrays of lithographically fabricated, geometrically well-defined contacts on silicon wafers were utilized to make the necessary precise, reproducible resistance measurements. Additional measurements using point pressure contacts were also made. The dependence of the four-probe resistance on the ratio of layer thickness to probe spacing was verified for both the in-line and square probe configurations.

100,789

PB91-147819

Not available NTIS

National Bureau of Standards (NEL), Gaithersburg, MD Semiconductor Electronics Div.

Thermal Characterization of Integrated Circuits - A Tutorial.

Final rept

F. F. Oettinger. 1986, $4 p$

Pub. in Proceedings of Symposium SEMI-THERM 3 ,

Scottsdale, AZ., December 10, 1986, 4p.

Keywords: "Integrated circuits, Thermal analysis, Computation, Experimentation, Reprints.

The tutorial deals with the practicalities of the thermal characterization of integration of integrated circuit devices and packages, using both computational and experimental techniques.
100,790

B9 1-147827

Not available NTIS

National Bureau of Standards (NEL), Gaithersburg, MD. Semiconductor Electronics Div.

Thermal Measurements of VLSI Packages: A Critical Review.

Final rept.

F. F. Oettinger. 1985, 4p

Pub. in Program and Extended Abstracts VLSI Packag ing Workshop, Gaithersburg. MD., September 9-11. 1985, p26-29.

Keywords: "Very large scale integration, "Integrated circuits, "Thermal measurements, Computerized simu lation, Chips(Electronics), Reviews, Reprints.

Techniques to thermally characterize ceramic and plastic VLSI packages are discussed. Computer simulations and both direct and indirect thermal evaluation techniques are highlighted. Limitations and strengths of the various techniques are identified.

100,791

PB91-147918

Not available NTIS

National Bureau of Standards (NEL), Gaithersburg. MD. Semiconductor Electronics Div.

Investigation of Photoconductive Picosecond Microstripline Switches on Self-Implanted Silicon on Sapphire (SOS).

Final rept.

P. Pollack-Dingels, G. Burdge, C. H. Lee, A. C.

Seabaugh, R. T. Brundage, M. I. Bell, and J. Albers.

1987, 5p

See also PB90-218124.

Pub. in Proceedings of OSA-IEEE (LEOS) Meeting (2nd) Picosecond Electronics and Optoelectronics II, Incline Village, NV., January 14-16, 1987, p232-236

Keywords: ^Semiconductor switches, Amorphous silicon, Electrical resistivity, Microstrip devices, Ion implantation, Silicon ions, Raman effect. Photoconduc tivity, Radiation damage, Lifetime, Reprints SOS(Semiconductors), Picosecond pulses.

Silicon on sapphire (SOS) switches, damaged by Si im plantation at fluence levels of 10 to the 13 power to 10 to the $15 \mathrm{power} / \mathrm{sq} \mathrm{cm}$, were characterized by picosecond cross-correlation, Raman, and resistivity measurements.

\section{0,792}

PB91.148197

Not available NTIS

National Inst. of Standards and Technology (NEL) Gaithersburg, MD. Semiconductor Electronics Div. Comparison of High-Resistivity Measurements of Silicon by AC Impedance, DC Resistance, van der Pauw, and Four-Probe Methods.

Final rept.

W. R. Thurber, J. R. Ehrstein, and J. R. Lowney. $1990,2 p$

Pub. in Extended Abstracts, Electrochemical Society 1990 Fall Meeting, Seattle, WA., October 14-19, 1990, v90-2 p581-582.

Keywords: "Electrical resistivity, "Silicon, Electrical impedance, Electrical measurement, Probes(Electromagnetic), Comparison, Reprints.

Resistivity measurements by different techniques are compared for high-resistivity silicon. The ac impedance method is emphasized as it is seldom used for silicon but has certain advantages for high-resistivity material. Slices with implanted and annealed surface were measured by the impedance method and two terminal dc resistance. For material with lapped surfaces, results were obtained by ac impedance, van der Pauw, and four-probe methods. The agreement was within $5 \%$ for slices and ingot sections greater than 0.1 $\mathrm{cm}$ in length and resistivity above $5 \mathrm{~K}(\mathrm{ohm})-\mathrm{cm}$.

100,793

PB91-149062

Not available NTIS

National Bureau of Standards (NEL), Gaithersburg, MD. Semiconductor Electronics Div.

Characteristics of the Breakdown Voltage of Power MOSFETs After Total Dose Irradiation. Final rept.

R. D. Pugh, A. H. Johnston, and K. F. Galloway. $1986,5 p$

Sponsored by Air Force Inst. of Tech., Wright-Patterson AFB, OH.

Pub. in IEEE (Institute of Electrical and Electronics Engineers) Transactions on Nuclear Science NS-33, n6 1460-1464 Dec 86 
Keywords: "Field effect transistors, "MOSFET, "Radiation effects, "Breakdown(Electronic threshold), Gamma irradiation, Radiation hardening, Silicon, Reprints.

The effects of total dose irradiation on the breakdown voltage of p-channel power MOSFETs are examined. Although breakdown voltage for $\mathrm{p}$-channel devices increased at higher dose levels, as expected, some devices exhibited an initial decrease in breakdown at very low levels of total dose. The interaction of ionizing radiation effects with the junction termination methods designed to increase the voltage at which breakdown occurs is analyzed.

100,794
PBg 1-149070

Not available NTIS

National Inst. of Standards and Technology (NEL), Boulder, CO. Electromagnetic Fields Div.

MMIC Related Metrology at the National Institute of Standards and Technology.

Final rept.

G. R. Reeve, R. Marks, and D. L. Blackburn. 1990

Pub. in Proceedings of IEEE (Institute of Electrical and Electronics Engineers) Instrumentation and Measurement Technology Conference, San Jose, CA., February 13-15, 1990, p196-199.

Keywords: "Integrated circuits, "Metrology, Gallium arsenides, Probes(Electromagnetic), Wafers, Reprints, "MMIC, US NIST.

Last year a program was instituted at the National Institute of Standards and Technology (NIST) specifically directed at developing improved metrology methods and standards to support microwave monolithic integrated circuit (MMIC) technology. The paper describes how the program was developed, the modes of interaction with the industrial community and the DARPA MIMIC initiative, and the particular projects being undertaken which will result in a more consistent measurement base for those engaged in the design and manufacture of MMIC devices.

\section{0,795}

B91-149120

Not available NTIS

National Bureau of Standards (NEL), Gaithersburg, MD. Semiconductor Electronics Div. Measurement of Capacitance on Wafers.

Final rept.

P. Roitman, J. S. Suehle, T. J. Russell, and M.

Gaitan. 1986, 9p

Pub. in Proceedings of IEEE (Institute of Electrical and Electronics Engineers) VLSI (Very Large Scale Integration) Workshop on Test Structures, Long Beach, CA., February 17-18, 1986, p96-104.

Keywords: "Very large scale integration, "Integrated circuits, "Capacitance, Electrical measurement, Probes(Electromagnetic), Wafers, Reprints.

Capacitance measurements of both capacitor and transistor structures can provide critical parameters for process monitoring, process modeling, device modeling and circuit modeling. However, accurate measurements of capacitance on small devices located on large silicon wafers are very difficult. The problem is simply that very low level analog measurements must be made at the end of a necessary system of cables and probes Several authors have proposed building and probes. Several authors have proposed building relatively high level outputs to the external test system. relatively high level outputs to the external test system. measure capacitance on wafers directly. This improvement involved three parts of the experiment: the design of the capacitors, the design of the probe fixturing, and the instrumentation. These are discussed in turn.

\section{0,796}

PB91-149880

Not available NTIS National Inst. of Standards and Technology (NEL) Gaithersburg, MD. Precision Engineering Div.

Selective-Area Epitaxial Growth of Gallium Arsenide on Silicon Substrates Patterned Using a Scanning Tunneling Microscope Operating in Air.

Final rept.

J. A. Dagata, W. Tseng, J. Bennett, C. J. Evans, J.

Schneir, and H. H. Harary. 1990, 3p

See also AD-A209 079.

Pub. in Applied Physics Letters 57, n23 p2437-2439, 3

Dec 90.

Keywords: "Gallium arsenides, "Epitaxial growth Scanning tunneling microscopy, Substrates, Silicon, Reprints, Nanotechnology.
Selective-area epitaxial growth of gallium arsenide on n-Si(100) substrates is reported, where the oxide (SiOx) mask consists of 1-2 monolayer-thick features patterned onto a silicon substrate using a scanning tunneling microscope (STM) operating in air. The technique for generating the STM patterns on hydrogenpassivated silicon was reported recently $(1990)$. The GaAs epilayer was grown by migration-enhanced epitaxy at $580 \mathrm{C}$ and its morphology was investigated by scanning electron microscopy. The chemical selectivity of the STM-patterned regions was verified by imaging time-of-flight secondary-ion mass spectrometry. The implications of these results for the development of a unique, STM-based nanostructure fabrication technology are discussed.

100,797

PB91-149989

Not available NTIS

National Inst. of Standards and Technology (NEL) Gaithersburg, MD. Applied and Computational Mathematics Div

Modelling Gallium Arsenide Transistors.

Final rept.

J. L. Blue, and C. L. Wilson. 1990, 9p

Pub. in Lectures in Applied Mathematics 25, p89-97 1990.

Keywords: "Transistors, "Gallium arsenides, "Mathematical models, Partial differential equations, Monte Carlo method, Quantum mechanics, Thermodynamics, Reprints

The usual equations used in modelling semiconductor devices are a set of three coupled partial differential equations first formulated forty years ago. These PDEs are adequate to model present-day silicon transistors, but are inadequate for modelling GaAs transistors. Suitable PDEs can be formulated using quantum me. chanics and statistical thermodynamics models obtained by Monte-Carlo calculations.

\section{0,798}

PB91-158873

Not available NTIS

National Bureau of Standards (NEL), Gaithersburg, MD. Precision Engineering Div.

NBS Submicron Particle Standards for Microcontamination Measurement.

Final rept

T. R. Lettieri. 1989, 7p

Pub. in Semiconductor Fabrication: Technology and Metrology, ASTM STP 990, p215-221 1989.

Keywords: "Semiconductor devices, "Standards, Contamination Microspheres Substrates, Silicon Surfaces, Wafers, Reprints, "Particle standards, US NIST.

There is a recognized need for standard artifacts with which to calibrate the laser-scanning instruments which detect and monitor microcontamination on semiconductor wafers. Although commercial calibration wafers are available for this purpose, the present paper proposes the use of National Bureau of Standards (NBS) particle-sizing standard microspheres, deposited on polished silicon substrates, as an alternative working standard until such time as a true national calibration artifact is developed. To this end, several techniques for depositing the microspheres on semiconductor surfaces are presented in the paper. In addition, the technques used to certify the NBS particle standards and the measurement results from each technique are summarized.

\section{0,799}

B91-159087

Not available NTIS

National Inst. of Standards and Technology (NEL).

Gaithersburg, MD. Precision Engineering Div.

Submicrometer SEM Magnification Standard.

Final rept.

M. T. Postek, and R. C. Tiberio. 1989, $4 \mathrm{p}$

Pub. in National Nanofabrication Facility Research Accomplishments 1988-89, 4p 1989.

Keywords: "Dimensional measurements, "Scanning electron microscopy, "Standards, Line widths, Lithography, Prototypes, Reprints, "Magnification standards, Nanofabrication, Standard reference materials.

The National Institute of Standards and Technology (NIST) in conjunction with the National Nanofabrication Facility (NNF) has had a continuing effort for the development of a submicrometer scanning electron microscope (SEM) magnification standard prototype. The program is one portion of a feature-size measurement program specifically aimed at the development and certification of SEM magnification and linewidth standards and the associated techniques for their cali- bration and use. The program has several distinct lasks that have undergone simultaneous development at NIST (Postek et al., 1987, Postek et al., 1989, Postek, 1989). One of these tasks has been the development and fabrication of a lithographically produced submicrometer SEM magnification standard useful at low accelerating voltages. With the assistance of the NNF, a prototype magnification standard has been fabricated and has undergone further refinements in materials selection and etching techniques during the year.

\section{0,800}

PB91-159244

Not available NTIS National Inst. of Standards and Technology (NEL), Gaithersburg, MD. Semiconductor Electronics Div. Permittivity Measurements on Molecular-Sized Samples.

Final rept.

A. van Roggen, L. Yuwono, H. Zhou, P. H. E. Meijer, and J. J. Kopanski. 1990, $6 \mathrm{p}$

Sponsored by National Science Foundation, Washington, DC.

Pub. in Proceedings of Conference on Electrical Insulation and Dielectric Phenomena, Pocono Manor, PA. October 29-31, 1990, p385-390.

Keywords: "Molecular electronics, "Organic semiconductors, "Permittivity, Measurement, Polyethylene, Reprints.

A new laboratory on Molecular Electronics has been started at the Physics Department of the Catholic University of America. In their efforts to make organic bistable devices, one of the research functions of the laboratory is to measure the electrical properties of materials and active devices made with molecular (mainly organic) materials. The size of material samples, and the specimens used for measurement, is exceedingly small, typically layers with a thickness of the order of $100 \mathrm{~nm}$. Consequently, the setups used for normal dielectric and conductivity measurements $=$ or $>10$ $\mathrm{mm}$ electrode size) cannot be used, and special cells and instrumentation have to be developed.

\section{0,801}

PB91-159749

PC A03/MF A01

National Inst. of Standards and Technology (NEL) Gaithersburg, MD. Semiconductor Electronics Div. Center for Electronics and Electrical Engineering Technical Progress Bulletin Covering Center Programs, July to September 1990, with 1991 CEEE Events Calendar.

J. A. Gonzalez. Jan 91, 48p NISTIR-4496 See also PB91-107201.

Keywords: "Semiconductor devices, "Metrology, Electromagnetic interference, Optical fibers, Integrated circuits, Antennas, Silicon, Electrooptics, Superconduc tors, Signal processing, Waveforms, Progress report

The report is the thirty-second issue of a quarterly pubication providing information on the technical work of the National Institute of Standards and Technology (formerly the National Bureau of Standards) Center for Electronics and Electrical Engineering. The issue of the CEEE Technical Progress Bulletin covers the third quarter of calendar year 1990. Abstracts are provided by technical area for both published papers and papers approved by NIST for publication. Genera topics discussed include the following: Semiconductor Technology Program; Signals and Systems Metrology Program; Additional Information; 1991 CEEE Calendar; Sponsor List; and Key Contacts in Center, Center Organization.

100,802

PB91-167163

$\mathrm{PC} \mathrm{A03/MF} \mathrm{A01}$

National Inst of Standards and Technology (NEL)

Gaithersburg, MD. Center for Mfg. Engineering

Standard Reference Materials: Bright-Chromium Linewidth Standard, SRM 476, for Calibration of Optical Microscope Linewidth Measuring Systems. Special pub (Final)

C. F. Vezzetti, R. N. Varner, and J. E. Potzick. Jan 91, 47p NIST/SP-260/114

Also available from Supt. of Docs. as SN003-00303047-3.

Keywords: "Integrated circuits, "Dimensional measurement, "Optical microscopes, "Calibration, "Photomasking, "Line width, Process control, Precision,

Chromium, "Standard reference materials. 


\section{ELECTROTECHNOLOGY}

\section{Semiconductor Devices}

Standard Reference Material, SRM 476, was developed for use in calibrating optical microscopes used to measure linewidths in the range of 0.9 to 10.8 micrometers on bright chromium photomasks, such as those used in the production of integrated circuits. The SRM, the measurement system, and the procedures used to calibrate the SRM are described. The algorithm for determining the line edge location uses a threshold criterion derived from analysis of microscope image profiles. The profiles are predicted by computer modeling based on the theory of partial coherence. The performance of the system is monitored by measuring line features on a control photomask before and after calibrating each SRM. Precautions concerning care and handling and instruction for the use of SRM 476 to calibrate optical microscopes for photomask linewidth measurement are given.

\section{0,803}

PB91-174581

Not available NTIS

National Bureau of Standards (NEL), Gaithersburg,

MD. Semiconductor Devices and Circuits Div.

Design, Fabrication, and Testing of an Interconnect Test Structure for Evaluating VLSI Processes. Final rept.

M. W. Cresswell, E. S. Coleman, W. D. Partlow, and

L. W. Linholm. 1985, 2p

Pub. in Proceedings of Government Microcircuit Applications Conference, Orlando, FL., November 5-7, 1985, p359-360.

Keywords: "Very large scale integration, "Integrated circuits, Test equipment, Test methods, Lithography, Fabrication, Aluminum, Silicon, Design, Reprints.

The paper describes a systematic approach to the comparative experimental evaluation of alternative sub-micron lithographic methods using microelectronic test structures. Measurements are presented for both polysilicon and aluminum lines with design geometries of 0.6 to 4.0 micrometers. These structures provide unambiguous results which can be used as a tool to improve the control and performance of VLSI devices.

100,804

B'1-175000

Not available NTIS

National Bureau of Standards (NEL), Gaithersburg, MD. Semiconductor Devices and Circuits Div.

Characterizing VLSI Processes Using Test Struc-

tures.

Final rept.

L. W. Linholm, J. A. Mazer, and K. F. Galloway

1985, 3p

Pub. in Proceedings of Digest of the DOD/NBS Conference on Microelectronic Electromagnetic Susceptibility, Gaithersburg, MD., March 12-13, 1985, p16-18.

Keywords: "Very large scale integration, "Integrated circuits, Test equipment, Test methods, Electromagnetic interference, Microelectronics, Wafers, Reprints.

Functional tests alone cannot fully evaluate complex integrated circuits (ICs). Comprehensive test programs are needed to assure, with acceptable confidence, that desired performance has been built into these circuits. Results from measurements obtained using specially designed microelectronic test structures can be an important element in a comprehensive test program to assure circuit performance and reliability. In the paper, six categories of test structures are identified and the step at which they impact VLSI circuit production is indicated. An example of the use of test structures is given. Finally, a brief discussion considering the use of test structures for measurements importan to understanding effects such as electromagnetic interference $(E M I)$ is given.

\section{0,805}

PB91-175489

Not available NTIS

National Bureau of Standards (NEL), Gaithersburg, MD. Semiconductor Electronics Div.

Evaluation and Improvement of E-Beam Exposure Routines by Use of Microelectronic Test Structures.

Final rept.

T. W. O'Keeffe, M. W. Cresswell, L. W. Linholm, and D. J. Radack. 1986, 13p

Pub. in Proceedings of IEEE VLSI Workshop on Test Structures, Long Beach, CA., February 17-18, 1986 , p82-94.

Keywords: "Very large scale integration, "Integrated circuits, Test equipment, Test methods, Chips(Electronics), Electron beams, Microelectronics Line width, Reprints.
The paper describes the use of the cross-bridge test structure in conjunction with a series of interconnect test structures to assess and improve the exposure routines and procedures in the replication of submicron features. The interconnect test structures used in the experiment are resistors which include both serpentine and comb-like interconnect patterns and can be used to assess line continuity and line-to-line isolation. Results obtained during the evaluation of line continuity, resolution, linewidth, and proximity exposure effects are presented.

\section{0,806}

PB91-189399

Not available NTIS

National Inst. of Standards and Technology (NEL) Gaithersburg, MD. Semiconductor Electronics Div.

Uniform Junction Temperature AIGaAs/GaAs Power Heterojunction Bipolar Transistors on Silicon Substrates.

Final rept.

G. Gao, Z. Fan, D. L. Blackburn, M. S. Unlu, J. Chen K. Adomi, and H. Morkoc. 1991, 3p

Sponsored by Air Force Office of Scientific Research Bolling AFB, DC

Pub. in Applied Physics Letter 58, n10 p1068-1070, 11 Mar 91.

Keywords: "Bipolar transistors, Aluminum gallium arsenides, Thermal stability, Heterojunctions, Substrates, Silicon, Reprints, Power transistors.

AlGaAs/GaAs power heterojunction bipolar transistors on Si substrates exhibiting uniform junction temperature distribution are reported. Owing to a unique device design, the temperature spread across the entire device area is about 1 deg C. The device exhibits a common emitter current gain of 20 , a maximum collector current of $0.6 \mathrm{~A}$, and a collector base junction breakdown voltage of $25 \mathrm{~V}$.

\section{0,807}

PB91-189779

Not available NTIS

National Inst. of Standards and Technology (NML) Gaithersburg, MD. Inorganic Analytical Research Div. Neutron Activation Analysis in Electronic Technology.

Final rept.

A. M. Lindstrom. $1988,1 \mathrm{p}$

Pub. in Jnl. of the Electrochemical Society 135, n8 pC372 1988

Keywords: "Neutron activation analysis, Chemical analysis, Trace amounts, Semiconductors, Silicon, Reprints.

The procedure of neutron activation analysis is reviewed, with recent examples of the application of this and other nuclear analytical methods in semiconductor technology. These methods in many situations offe highly specific and sensitive elemental analysis capability, often wholly instrumental. The sample physics involved in neutron activation in many cases make systematic errors different in kind and less important in quantity than in analytical methods relying on atomic phenomena.

\section{0,808}

B9 1-194712

Not available NTIS National Inst. of Standards and Technology (NEL) Gaithersburg, MD. Precision Engineering Div.

Pattern Generation on Semiconductor Surfaces by a Scanning Tunneling Microscope Operating in Air. Final rept.

J. A. Dagata, J. Schneir, H. H. Harary, J. Bennett, and $W$. Tseng. 1991,5p

Pub. in Jnl. of Vacuum Science and Technology $B$, n2 p1384-1388 Mar/Apr 91.

Keywords: "Semiconductor devices, "Pattern making, *Scanning tunneling microscopy, Gallium arsenides, Silicon, Epitaxy, Masking, Surfaces, Scanning electron microscopy, Reprints, Nanotechnology.

Recent results employing scanning tunneling microscope-based techniques for the generation of nanometer-scale patterns on passivated semiconductor surfaces are presented. Preparation and characterization of hydrogen-passivated silicon and sulfur-passivated gallium arsenide surfaces are described, and the determination of the chemical and morphological properties of the patterned regions by scanning electron microscopy and time-of-flight secondary ion mass spectrometry are discussed. The authors' demonstration that ultrashallow, oxide features written by scanning tunneling microscope (STM) can serve as an effective mask for selective-area GaAs heteroepitaxy on silicon is used to illustrate key requirements necessary for the realization of a unique, STM-based nanotechnology.

100,809

PB91-194829

Not available NTIS

National Inst. of Standards and Technology (NEL), Gaithersburg, MD. Semiconductor Electronics Div.

High Spatial Resolution Mapping of Semiconductor Resistivity.

Final rept.

J. J. Kopanski, G. P. Carver, J. R. Lowney, D. S.

Miles, and D. B. Novotny. 1991, 2p

Pub. in Extended Abstracts, v91-1 p698-699 1991.

Keywords: "Mercury cadmium tellurides, "Gallium arsenides, "Silicon, "Electrical resistivity, High resolution, Laplace equation, Semiconductors, Lithography, Reprints, "Resistivity mapping, Spreading resistance.

A new approach to the resistivity mapping of semiconductors uses an array of lithographically defined contacts (at a density of 60,000 sites per sq $\mathrm{cm}$ and an automated probe station for data acquisition. Resistivity growth striations in silicon as narrow as 45 micrometers in width and with + or - $5 \%$ variation from the background resistivity have been resolved. Solution of the Laplace equation for the measurement geometry and measurements on ion implanted test structures are described. Anticipated applications include resistivity mapping of liquid encapsulated Czochralski GaAs, $\mathrm{Hg}(1-x) \mathrm{Cd}(\mathrm{x}) \mathrm{Te}$, and fine scale resistivity variations in processed silicon.

\section{0,810}

PB91.203141

Not available NTIS

National Inst. of Standards and Technology (NEL), Gaithersburg, MD. Semiconductor Electronics Div. Cratering.

G. G. Harman. 1989, 13p

Pub. in Reliability and Yield Problems of Wire Bonding in Microelectronics, Chapter 5, p141-163 1989.

Keywords: "Electric contacts, "Microelectronics, Integrated circuits, Reliability(Electronics), Fracture mechanics, Gallium arsenides, Test methods, Silicon, Failure, Reprints, "Wire bonds.

The monograph describes the several methods of testing the quality of wire bonds, including the pull test, the shear test, and the high-temperature stress test. Essentially all metallurgical, chemical, and mechanical bond failure modes are described, including cratering, 'plaque,' the effect of various contaminants, cleaning and cleaning methods, poor bonding machine control and the effect of external environments. Methods of preventing or detecting these failures are given. In conclusion how 'new technology' processing and packaging have changed classical wire bond failures is discussed. Discussion of proposed 'new' wire and pad metallizations and general guidelines for anticipating the reliability of such new materials is also given.

100,811

PB91-203752

Not available NTIS

National Bureau of Standards (NEL), Gaithersburg MD. Precision Engineering Div.

New Approach to Image Modeling and Edge Detection in the SEM.

Final rept.

D. Nyyssonen. $1988,9 p$

Sponsored by CD Metrology, Inc., Germantown, MD. Pub. in Proceedings of SPIE (Society of Photo-Optical Instrumentation Engineers) - Integrated Circuit Metrology, Inspection, and Process Control II, v921 p48-56 1988.

Keywords: *Scanning electron microscopy, "Edge detection, "Imaging techniques, "Very large scale integration. Probability density functions, Monte Carlo method, Electron scattering, Integrated circuits, Process control, Line width, Reprints, Secondary electrons, Micrometrology.

The need for feature-size measurements on microchips for VLSI and other developing technologies with micrometer and submicrometer dimensions has resulted in the use of scanning electron microscopes (SEMs) for process control measurements. However good measurement practice requires the ability to accurately predict the observed signal output for any given structure of the feature. The model forms the basis for measurement algorithms, error analysis, and proper calibration techniques. The SEM, especially for 
secondary electron imaging and low beam voltages, has lacked the ability to quantitatively predict image structure at the 0.01 micrometer level needed for submicrometer dimensional control. A new approach to secondary-electron image modeling has been developed consisting of a surface integral (over the line structure) of a probability density function which describes the likelihood of a secondary electron being generated by the primary beam and emitted at a given point in space, if that point coincides with the surface of a line structure. This probability density function can be determined by using either a state-of-the-art Monte Carlo technique or by using a modified diffusion model. The calculation of the image from this probability density function takes into account edge geometry and shadowing due to nearby edges as well as field effects due to any bias voltage on the electron-detector grid.

\section{0,812}

PB91-203992

Not available NTIS National Inst. of Standards and Technology (NEL) Gaithersburg, MD. Semiconductor Electronics Div. Building-in Reliability: Making it Work.

Final rept.

H. A. Schafft, D. A. Baglee, and P. E. Kennedy.

1991, 7p

Contract ARPA-3882

Sponsored by Defense Advanced Research Projects Agency, Arlington, VA.

Pub. in Proceedings of International Reliability Physics Symposium, Las Vegas, NV., April 9-11, 1991, p1-7.

Keywords: "Reliability(Electronics), Microelectronics, Semiconductor devices, Process control, Quality control, Packaging, Measurement, Reprints.

Aggressive reliability and market-entry demands will require the use of a building-in approach to reliability To adopt this approach and make it work requires that very significant breaks be made from the traditional ways of improving and appraising reliability. The nature of these breaks are discussed in the context of describing the basic elements of the approach of buildscribing the basic elements of the approach of build-
ing-in reliability and the obstacles that hinder its adoption. To help visualize how the approach can be implemented, initial steps to make the transition and some specific examples of its use are described.

\section{0,813
PBg $1-216739$}

(Order as PB91-216705, PC A07/MF A01) National Inst. of Standards and Technology, Gaithersburg, MD.

Automated Reverse-Bias Second-Breakdown Transistor Tester.

D. Berning. 1991, 14p

Included in Jnl. of Research of the National Institute of Standards and Technology, v96 n3 p291-304 May/Jun 91.

Keywords: "Nondestructive tests, "Transistors, *Second breakdown, Power transistors, Reverse bias. An automated instrument is described for generating curves for the reverse-bias, safe-operating area of transistors nondestructively. A new technique for detecting second breakdown that makes automation possible is highlighted. Methods to reduce stress to the device under test are discussed, as are several other innovations that enhance automation. Measurements using the tester are described, and limitations on nondestructive testability are discussed.

\section{0,814}

PB91-236513

Not available NTIS

National Inst. of Standards and Technology (EEEL)

Gaithersburg, MD. Semiconductor Electronics Div.

Automated Reverse-Bias Second-Breakdown

Transistor Tester.

Final rept.

D. W. Berning. 1991, 8p

Pub. in Proceedings of Applied Power Electronics Conference and Exposition, Dallas, TX., March 10-15, 1991, p339-346.

Keywords: *Transistors, Electromagnetic testing, Nondestructive tests, Automation, Reprints, "Second breakdown, Reverse bias.

An automated instrument is described for generating curves for the reverse-bias, safe-operating area of transistors nondestructively. A new technique for detecting second breakdown that makes automation possible is highlighted. Methods to reduce stress to the device under test are discussed, as are several other innovations that enhance automation. Measure- ments using the tester are described, and limitations on nondestructive testability are discussed.

\section{0,815}

PB91-236703

Not available NTIS

National Inst. of Standards and Technology (CSL) Gaithersburg, MD. Systems and Software Technology Div.

Emitter Ballasting Resistor Design for, and Current Handling Capability of AIGaAs/GaAs Power Heterojunction Bipolar Transistors.

Final rept.

G. Gao, M. S. Unlu, H. Morkoc, and D. L. Blackburn. $1991,12 p$

Pub. in IEEE (Institute of Electrical and Electronics Engineers) Transactions on Electron Device 38, n2 p185 196 Feb 91.

Keywords: "Bipolar transistors, Aluminum gallium arsenides, Thermal analysis. Heterojunctions, Resistors, Reprints, Power transistors, Emitter ballasting.

A systematic investigation of the emitter ballasting resistor for power Heterojunction Bipolar Transistors (HBTs) is presented. The current-handling capability of power HBTs is found to improve with ballasting resistpower HBTs is found to improve with ballasting resistance. An equation for the optimal ballasting resistance is presented, where the effects of thermal conductivity of the substrate material and the temperature coefficient of the ballasting resistor are taken into account. Current levels of 400 to $800 \mathrm{~mA} / \mathrm{mm}$ of emitter periphery at case temperatures of 25 to $-80 \mathrm{C}$ for powe AlGaAs/GaAs HBTs have been obtained using an on chip lightly-doped GaAs emitter ballasting resistor. Device temperature has been measured using both an infrared micro-radiometer and temperature-sensitive electrical parameters. Steady-state and transient thermal modeling were also performed. Although the measured temperature is spatially nonuniform, the modeling results show that such nonuniformities occur for a uniform current distribution, as is to be expected for an HBT with emitter ballasting resistors.

\section{0,816}

PB91-237255

Not available NTIS National Inst. of Standards and Technology (NEL) Boulder, CO. Electromagnetic Fields Div.

Microwave Monolithic Integrated Circuit-Related Metrology at the National Institute of Standards and Technology.

Final rept.

G. R. Reeve, R. Marks, and D. L. Blackburn. 1990,

$4 \mathrm{p}$

Pub. in IEEE (Institute of Electrical and Electronics Engineers) Transactions on Instrumentation and Measurement 39, n6 p958-961 Dec 90.

Keywords: "Integrated circuits, "Metrology, Microwave circuits, Gallium arsenides, Probes(Electromagnetic), Wafers, Reprints, US NIST.

The paper describes how the National Institute of Standards and Technology (NIST) interacts with the GaAs community and the Defense Advanced Research Projects Agency (DARPA) MIMIC initiative. The organization of a joint industry and government laboratory consortium, for MIMIC related metrology research is described, along with some of the initial technica developments at NIST done in support of the consortium.

\section{0,817}

B91-240739

$\mathrm{PC}$ A03/MF A01

National Inst. of Standards and Technology (EEEL), Gaithersburg, MD.

etrology for the Semiconductor Industry.

R. I. Scace. Sep 91, 47p NISTIR-4653

Keywords: "Integrated circuits, "Metrology, Semiconductor devices, Semiconductor materials, Microwave equipment, Process control, Optoelectronics, Manufacturing, Automation, Market surveys, "Semiconductor industry.

The metrological needs of the semiconductor industry and its supporting materials and manufacturing equipment industries for the 1990's are set forth. The information in the report derives from technological workshops sponsored by the American Society for Testing and Materials, NIST, SEMATECH, Semiconductor Equipment and Materials International, and the Semiconductor Research Corporation; from industry information sources such as Dataquest and VLSI Research; from NIST colleagues; and from personal discussions with many others in the field. The data from these sources have been analyzed for their implica- tions for measurement science and technology, on which the report focuses. It contains a brief introduction to the economic significance of the semiconducto industry, an overview of the relevant technologies, their development trends and the metrological needs thus implied, and evidence of needs expressed by sources outside of NIST. The report deals with the needs for measurements of all kinds, but intentionally does not discuss the means by which these may be met. The text is also to be published as one chapter in a more comprehensive assessment covering the electronics industry as a whole.

\section{0,818}

PB92-116466

Not available NTIS

National Inst. of Standards and Technology (NEL) Gaithersburg, MD. Scientific Computing Div.

Three-Dimensional Simulations of High-Resolution Photoresist Processing.

Final rept.

E. Barouch, B. Bradie, and H. A. Fowler. 1991, 12p Sponsored by Defense Advanced Research Projects Agency, Arlington, VA., and Air Force Office of Scientific Research, Bolling AFB, DC.

Pub. in Jnl. of Imaging Science 35, n4 p240-251 Jul/ Aug 91.

Keywords: "Integrated circuits, "Computerized simulation, Computer graphics, Three dimensional, Dissolving, Reprints, "Microlithography, Photoresists.

A computer simulation package for optical microlithography contains modules for the processing of positive photoresists in the three stages of aerial-image transfer, exposure-bleaching, and dissolution. Evolving and final resist profiles are displayed in three dimensions a several dissolution times, thus simulating real systems in their natural form. Results presented for a variety of examples demonstrate the robustness and versatility of the package.

\section{0,819}

PB92-116599

Not available NTIS

National Inst. of Standards and Technology (NEL) Gaithersburg, MD. Semiconductor Electronics Div.

Ellipsometry SRM's for Use in Thin Film Measurements.

Final rept.

D. Chandler-Horowitz, J. F. Marchiando, and B. J.

Belzer. 1990, 11p

Pub. in Proceedings of Measurement Science Conference, Anaheim, CA., February 8-9, 1990, p5A-11-5A21.

Keywords: "Silicon dioxide, "Film thickness, "Dimensional measurement, "Ellipsometry, Helium neon lasers, Refractive index, Thin films, Polarimetry, Semiconductors, Reprints, *Standard reference materials.

A Standard Reference Material (SRM) consisting of a film of silicon dioxide on a silicon substrate has been designed, fabricated, measured, and certified at NIST for the ellipsometric angles, Delta and psi, and for the derived film thickness and refractive index. The SRM can be used as an aid in the evaluation of the performance of optical and mechanical thickness-monitoring instruments as well as ellipsometers. The optical instruments are based on the theory describing reflection of light from a sample. The film thickness is determined by using a model having one or two uniform isotropic films atop a substrate. The calculated thick nesses rely on accurate values of the indices of refraction of the substrate and/or film at the necessary frequencies of light. The measurement procedure used here to certify the ellipsometric angles uses an accurate rotating-analyzer ellipsometer and $\mathrm{HeNe}$ laser source operating near the principal angle of incidence. The measurement data from several samples are analyzed collectively to determine the certified film thicknesses and refractive index. At the present time, three different film thicknesses, 50, 100, and $200 \mathrm{~nm}$, are being certified. Future work may involve certifying thinner layers of oxide.

\section{0,820}

\section{PB92-116607}

Not available NTIS

National Inst. of Standards and Technology (NEL)

Gaithersburg, MD. Semiconductor Electronics Div. 


\section{ELECTROTECHNOLOGY}

\section{Semiconductor Devices}

Sensitivity of Ellipsometric Modeling to the 'Islands' of Silicon Precipitates at the Bottom of the Buried Oxide Layer in Annealed SIMOX.

Final rept.

D. Chandler-Horowitz, J. F. Marchiando, M. Doss, S. Krause, and S. Visitserngtrakul. 1990, $2 p$ Pub. in Proceedings of IEEE SOS/SOI Technology Conference, Key West, FL., October 2-4, 1990, p73-
74 .

Keywords: Transmission electron microscopy, Precipitates, Silicon, Sensitivity, Reprints, *SIMOX, Spectroscopic ellipsometry, Multilayers.

Spectroscopic ellipsometry is a nondestructive probe which can be highly sensitive to the multilayer structure of materials such as SIMOX (Separation by IMplanted OXygen). Recent TEM micrographs of highflux single-implant SIMOX annealed at $1300 \mathrm{C}$ for 6 hours, show 'islands' of silicon precipitates near the bottom of the buried oxide layer. Spectroscopic ellipsometric measurements were performed on these samples at various implant doses and beam current densities to observe how the measured data fit the data theoretically predicted for various models of SIMOX that lead to the presence of these 'islands'.

\section{0,821}

PB92-116664

Not available NTIS

National Inst. of Standards and Technology (NEL) Gaithersburg, MD. Semiconductor Electronics Div. Modified Sliding Wire Potentiometer Test Structure for Mapping Nanometer-Level Distances. Final rept.

M. W. Cresswell, M. Gaitan, R. A. Allen, and L. W. Linholm. 1991, 6p

Pub. in Proceedings of IEEE (Institute for Electrical and Electronics Engineering) International Conference on Microelectronic Test Structures, Kyoto, Japan, March 18-20, 1991, v4 n1 p129-134.

Keywords: "Potentiometers(Instruments), "Dimensional measurement, Wafers, Reprints, $X$ ray lithography, Nanometer measurement.

The paper presents a modified voltage-dividing potentiometer test structure which overcomes a problem typical in scaling electrical test structures; it provides a correction for electrical length shortening of a resistor strip caused by the attachment of voltage taps of non negligible width. The test structure was implemented in chrome on quartz and measurements of displace ments between 10 and $500 \mathrm{~nm}$ with + or - 12-nm random error were made using available test equipment. Applications for the test structure may include: monitoring the self-registration accuracy and precision of primary pattern generator systems and monitoring level-to-level overlay in advanced wafer fabrication.

\section{0,822}

PB92-116862

Not available NTIS

National Inst. of Standards and Technology (NEL)

Gaithersburg, MD. Semiconductor Electronics Div.

Experimentally Verified IGBT Model Implemented in the Saber Circuit Simulator.

Final rept.

A. R. Hefner, and D. M. Diebolt. 1991, 10p

Pub. in Proceedings of Annual IEEE (Institute for Electrical and Electronics Engineers) Power Electronics Specialists Conference (22nd), Cambridge, MA., June 24-27, 1991, p10-19.

Keywords: *Bipolar transistors, Reprints, "Circuit simulators, Saber model, Power transistors.

A physics-based IGBT (Insulated Gate Bipolar Transistors) model is implemented into the general purpose circuit simulator Saber (TM). The IGBT model includes all of the physical effects that have been shown to be important for describing IGBTs, and the model is valid for general external circuit conditions. The Saber IGBT model is evaluated for the range of static and dynamic conditions in which the device is intended to be operat ed, and the simulations compare well with experimental results for all of the conditions studied.

\section{0,823}

Test Chip for the Evaluation of Surface-Diffusion Phenomena in Sputtered Aluminum Planarization Processes.

Final rept.

M. A. Jones, J. A. Roberts, C. H. Ellenwood, M. W. Cresswell, and R. A. Allen. 1991, 6p

Pub. in Proceedings of IEEE (Institute for Electrical and Electronics Engineers) International Conference on Microelectronic Test Structures, Kyoto, Japan, March 18-20, 1991, v4 n1 p35-40.

Keywords: Very large scale integration, Scanning electron microscopy, Circuit interconnections, Chips(Electronics), Metallizing, Integrated circuits, Aluminum, Reprints, "Test chips, Planarization.

A test chip has been designed and fabricated for the evaluation of surface-diffusion phenomena in sputtered aluminum planarization processes. It allows for confirmation of the correct sample cross-section bevel angle and orientation for scanning electron microscope (SEM) inspection of step coverage. These features eliminate ambiguities that may otherwise arise in the interpretation of the SEM images. The chip design provides arrays of vias with multiple combinations of provides arrays of vias with multiple combinations of
size and spacing to enable characterization and modsize and spacing to enable characterization and modeling of the aluminum planarization phenomena for a full range of deposition parameters. It also incorporates electrically readable test structures that allow relating the SEM images of step coverage to corresponding electrical properties, such as electromigra-
tion, of the deposited metal. These are extracted from standardized electrical test structures designed and characterized for that purpose. The overall objective is to enable the selection of deposition parameters that simultaneously produce visually acceptable step-coverage images as well as optimized electrical properties of the film.

\section{0,824}

B92-116979 National Inst. of Standards and Technology (NEL) Gaithersburg, MD. Semiconductor Electronics Div. Knowledge Extraction Techniques for Expert System Assisted Wafer Screening.

Final rept.

D. Khera, M. W. Cresswell, L. W. Linholm, G.

Ramanathan, J. Buzzeo, and A. Nagarajan. 1990, 6p Pub. in Proceedings of International Semiconducto Manufacturing Science Symposium, Burlingame, CA. May 21-23, 1990, p44-49.

Keywords: *Integrated circuits, Expert systems, Screening, Yield, Wafers, Reprints.

The paper describes a procedure for using inductionbased classification techniques for identifying relationships between work-in-process (WIP) test structure data and future IC yield at wafer test on a wafer-bywafer or lot-by-lot basis. The relationships are extracted from databases of previously processed WIP wafer test structure measurements and final wafer yield. They are presented in the form of rules relating WIP data to final yield. It is further shown that these rules, when incorporated into expert systems, can advise the
human operator responsible for screening wafers which are likely to produce sub-marginal yield if processed to completion. These rules also identify the WIP test structure parameters and values which have historically provided the highest and lowest final wafer yields.

\section{0,825}

PB92-116987

Not available NTIS

National Inst. of Standards and Technology (NEL), Gaithersburg, MD. Semiconductor Electronics Div.

Assessment of Reliability Concerns for Wide-Temperature Operation of Semiconductor Devices and

Circuits.

J. J. Kopanski, D. L. Blackburn, G. G. Harman, and

D. W. Berning. 1991, 6p

Sponsored by National Aeronautics and Space Administration, Cleveland, $\mathrm{OH}$. Lewis Research Center.

Pub. in Proceedings of International High Temperature Electronics Conference (1st), Albuquerque, NM., June 16-20, 1991, p137-142 1991.

Keywords: "Semiconductor devices, *Reliability(Electronics), Temperature effects, Arrhenius equation, High temperature, Accelerated tests, Circuits, Reprints.

Factors that may affect the long-term ( 30 yrs = approx 1 million h) reliability of electronic systems that can withstand temperature extremes $(-150$ to $+300 \mathrm{C})$ are discussed. There is ample evidence that a straightforward application of the Arrhenius equation, with activation energies determined from high-temperature accelerated stress testing, is not strictly valid for predicting real device lifetime. The relevance and validity of this traditional reliability assurance methodology, especially near the high-temperature operating limit, is critiqued. Some of the barriers to long-term reliable operation of devices and circuits subject to extreme temperatures are identified in the broad areas of: (1) semiconductor materials, (2) components and circuit design, and (3) packaging and assembly. Finally, alternative approaches to reliability assurance, not dependent on elevated temperature testing, which may be ap plicable to high-temperature electronics, are discussed.

\section{0,826}

PB92-117084

Not available NTIS

National Inst. of Standards and Technology (NEL) Gaithersburg, MD. Semiconductor Electrónics Div. Persistent Photoconductivity in SIMOX Films. Final rept.

S. Mayo, J. R. Lowney, P. Roitman, and D. B.

Novotny. 1990, 2p

Contract DNA-IACRO-88-800

See also PB91-112409. Sponsored by Defense Nuclear Agency, Washington, DC

Pub. in Proceedings of IEEE SOS/SOI Technology Conference, Key West, FL., October 2-4, 1990, p156157.

Keywords: "Photoconductivity, Film resistors, Time de pendence, Interfaces, Wafers, Reprints, "SIMOX SIMOX(Separation by IMplanted OXygen), Photoinduced transient spectroscopy.

Photoinduced transient spectroscopy (PITS) was used to measure the persistent photoconductive (PPC) response in film resistors fabricated on two different commercial n-type SIMOX (Separation by IMplanted Oxygen) wafers. A broadband, single-shot, flashlamppumped dye laser pulse was used to photoexcite interband electrons in the film, and the decay in the induced excess carrier population was measured at temperatures in the 60- to $220-\mathrm{K}$ Range. The post-illumination conductivity transients observed show PPC signals exhibiting nonexponential character. They were recorded for periods of time up to $30 \mathrm{~s}$ at constant temperature. The photoconductive data from these film resistors are analyzed by using the Queisser and Theodorou potential barrier model, and a logarithmic time decay dependence is confirmed for the first time in SIMOX material. The sensitivity of PITS is demonstrated to be appropriate for characterization of the SIMOX interface structure and for material qualification.

100,827

PB92-117159

Not available NTIS

National Inst of Standards and Technology (NEL) Gaithersburg, MD. Semiconductor Electronics Div. Band-Gap Narrowing and III-V Heterostructure FETs.

Final rept.

D. R. Myers, J. A. Lott, J. R. Lowney, J. F. Klem, and C. P. Tigges. $1990,4 p$

See also DE91000401.

Pub. in Proceedings of International Electron Devices Meeting, San Francisco, CA., December 9-12, 1990 , p31.1.1-31.1.4.

Keywords: *Field effect transistors, "Energy gap, Aluminum gallium arsenides, Quantum wells, Valence bands, Reprints, Gallium indium arsenides, Density of states, Heterostructures.

The authors calculate the magnitude of band-gap narrowing for GaAs-based alloys, and have included these results into one-dimensional heterojunction device models for strained In(0.15)Ga(0.85)As quantum-well MODulation-doped Field-Effect Transistors (MODFETs). Equivalent rigid shifts of as much as 102 $\mathrm{meV}$ are obtained for the valence band of depleted $p$ type $\mathrm{Al}(0.15) \mathrm{Ga}(0.85)$ as doped as $5 \times 10$ to the 18 th power/cc. Their simulations suggest that band-gap narrowing is most significant for $p$-channel MODFETs. The predicted effect of band-gap narrowing in $p$-channel MODFETs is the formation of parasitic conduction in the low-mobility parent dopant region. The parasitic conduction would reduce the intrinsic gain. 
National Inst. of Standards and Technology (NEL), Gaithersburg, MD. Precision Engineering Div

Application of Transmission Electron Detection to $X$-ray Mask Calibrations and Inspection. Final rept.

M. T. Postek, R. D. Larrabee, W. J. Keery, and E.

Marx. 1991, 13p

Pub. in Proceedings of SPIE (Society of Photo-Optical Instrumentation Engineers) - Integrated Circuit Metrology, Inspection, and Process Control V, v1464 p35-47 1991.

Keywords: "Integrated circuits, "Calibration, Transmission electron microscopy, Scanning electron microscopy, Electron detection, Dimensional measurement, Metrology, Inspection, Reprints, " $X$ ray masks, $X$ ray lithography.

Masks used for the manufacture of integrated circuits by $x$-ray lithography can be calibrated and inspected in a scanning electron microscope by using the transmitted electron detection mode. By their nature, these masks present a measurement subject unique from most (if not all) other objects used in semiconductor processing because the support membrane is, by design, $x$-ray transparent. The characteristic can be used as an advantage in electron beam-based mask metrology since, depending upon the incident electron beam voltages, substrate composition and substrate thickness, the membrane can also be essentially electron transparent. The areas of the mask where the absorber structures are located are essentially $x$-ray opaque as well as electron opaque. Viewing the sample from a perspective below an $x$-ray mask can provide excellent electron signal contrast (depending upon the instrument conditions) between the absorber structure and the membrane. Thus, the mask can be viewed in the transmitted electron detection mode of the scanning electron microscope, and precise and potentially accurate dimensional measurements can be made.

\section{0,829}

PBó2-117233

Not available NTIS

National Inst. of Standards and Technology (NEL), Gaithersburg, MD. Semiconductor Electronics Div.

Resldual Defects in SIMOX: Threading Dislocations and Pipes.

Final rept.

P. Roitman, M. Edelstein, S. Krause, and S

Visitserngtrukul. 1990, 2p

Contract DNA-IACRO-88-800

Sponsored by Defense Nuclear Agency, Washington, DC.

Pub. in Proceedings of IEEE SOS/SOI Technology Conference, Key West, FL., October 2-4, 1990, p154155.

Keywords: "Crystal defects, Crystal dislocations, Very large scale integration, Bipolar transistors, Stacking faults, Integrated circuits, Electron microscopy, Silicon, Reprints, "SIMOX

In the past few years, due to improved control of the ion implantation process and improved annealing sequences, a qualitative improvement has been realized in the structural quality of SIMOX films. The dense network of oxide precipitates and threading dislocations in the top silicon can be annealed out, reducing the dislocation density from about 10 to the 10 th power/sq $\mathrm{cm}$ to about $100,000 / \mathrm{sq} \mathrm{cm}$ or less. CMOS transistors and circuits have been successfully fabricated in this material. However, bipolar devices are sensitive to defect densities in this range, as is VLSI yield. Therefore, the defect density must be monitored and reduced. The authors discuss below some techniques for monitoring dislocations and stacking faults in SIMOX films. Also, a different type of defect, a silicon 'pipe' running through the buried oxide has been observed. The origin of these defects, and a technique for detecting them, will be described.

\section{0,830}

PB92-117241

Not available NTIS

National Inst. of Standards and Technology (NEL).

Gaithersburg, MD. Semiconductor Electronics Div.

Round-Robin Study of Implants in Si and $\mathrm{SiO}_{2}$ by SIMS, RBS, and NAA.

Final rept.

P. Roitman, D. S. Simons, P. H. Chi, R. M. Lindstrom, G. E. Lux, S. Baumann, S. W. Novak, R. G. Wilson,

D. Farrington, J. Keenan, F. A. Stevie, J. L. Moore, R. B. Irwin, A. J. Filo, C. W. Magee, R. Alcorn, and D. File. $1990,3 p$

Pub. in Proceedings of International Conference on Secondary lon Mass Spectrometry (SIMS VII) (7th), Monterey, CA., September 3-8, 1989, p115-117 1990.
Keywords: "Ion implantation, "Silicon dioxide, "Silicon, Carbon, Sodium, Aluminum, Chromium, Iron, Copper, Reprints, Secondary ion mass spectroscopy.

A round-robin study of implants of $\mathrm{C}, \mathrm{Na}, \mathrm{Al}, \mathrm{Cr}, \mathrm{Fe}$, and $\mathrm{Cu}$ into $\mathrm{Si}$ and $\mathrm{SiO} 2$ has been conducted. The results are reported.

\section{0,831}

PB92-117282

Not available NTIS National Inst. of Standards and Technology (NEL), Gaithersburg, MD. Semiconductor Electronics Div. Measurement, Use, and Interpretation of TCR.

Final rept.

H. A. Schafft. 1991, 7p

Pub. in Proceedings of International Wafer Level Reliability Workshop, Lake Tahoe, CA., October 7-10, 1990, p211-2171991.

Keywords: "Integrated circuits, Stress testing, Electromigration, Metallizing Measurement, Reprints, *Temperature coefficient of resistance.

A few comments are made about the measurement, use, and interpretation of the temperature coefficient of resistance (TCR). TCR is important when one wants to determine the temperature of a metallization line in an electromigraion stress test.

\section{General}

100,832

PB91-132241

PC A06/MF A01

National Inst of Standards and Technology (NEL)

Boulder, CO. Electromagnetic Fields Div.

Bibliography of the NIST Electromagnetic Fields

Division Publications.

R. M. Lyons, and K. A. Gibson. Aug 90, 104p

NISTIR-3945

Supersedes PB90-163635

Keywords: "Electromagnetic fields, Electrical measurement, Dielectric properties, Electromagnetic interference, Electromagnetic noise, Remote sensing, Radiation hazards, Antennas, Metrology, Waveforms, Standards, Time domain, Near field, US NIST.

The bibliography lists the publications by the staff of the Electromagnetic Fields Division of the National Institute of Standards and Technology for the period January 1970 through July 1990 . It supersedes PB90163635 which listed the publications of the Electromagnetic Fields Division from January 1970 through August 1989. Topics covered include antennas, dielectric measurements, electromagnetic interference, microwave metrology, noise, remote sensing, time domain, and waveform metrology. Selected earlier publications from the Division's predecessor organizations are included.

\section{0,833}

PB91-132266

A04/MF A01

National Inst. of Standards and Technology (NEL),

Boulder, CO. Electromagnetic Technology Div.

Metrology for Electromagnetic Technology: A Bibliography of NIST Publications.

M. E. DeWeese. Aug 90,66p NISTIR-3946

Supersedes PB90-161670.

Keywords: "Metrology, "Bibliographies, Optical communication, Superconductors, Electrooptics, Cryogenics, Lasers, "Electromagnetic metrology, Cryoelectronics, Optical fibers, Superconducting devices, US NIST.

The bibliography lists the publications of the personnel of the Electromagnetic Technology Division of NIST during the period from January 1970 through publication of this report. Topics include cryoelectronics, electromagnetic metrology, lasers, magnetics, optical fibers, and superconducting materials. A few earlier references that are directly related to the present work of the Division are also included.

\section{0,834}

PB91-133868

Not available NTIS

National Inst. of Standards and Technology (NEL), Boulder, CO. Electromagnetic Technology Div.
Eddy Current Probe Characterization Using an Impedance Plane Display Instrument.

Final rept.

T. E. Capobianco. $1989,9 p$

Sponsored by Army Materials Technology Lab., Watertown, MA.

Pub. in Proceedings of Defense Conference on Nondestructive Testing (38th), San Antonio, TX., October 31-November 2, 1989, p193-201.

Keywords: "Eddy current tests, "Electrical impedance, "Standards, Display devices, Nondestructive tests, Electromagnetic testing, Calibrating, Conductivity, Aluminum alloys, Probes, Reprints, "Calibration.

The U.S. Army is sponsoring work at the National Institute of Standards and Technology to develop a military standard for characterizing eddy current probe performance. Presently, the test method of this draft standard is the measurement of the probe impedance change when the probe is applied to test blocks of two different conductivities. The authors hoped that this impedance measurement would be easy to perform in the field, but they discovered that field and depot level operations lack the equipment for measuring impedance. This has become a serious obstacle to the implementation of the standard. However, depot operations often have an eddy current instrument which displays flaw signals in the impedance plane. These instruments do not display the actual impedance values of the flaw signals presented but could possibly be calibrated for this purpose. Results of an experiment where a calibration technique was tried and eddy current probe impedances measured are presented.

\section{0,835}

PB91-134213 Not available NTIS

National Inst. of Standards and Technology (NEL), Gaithersburg, MD. Electricity Div

Transition from Trichel-Pulse Corona to Dielectric Barrier Discharge.

Final rept.

S. V. Kulkarni, R. J. Van Brunt, and V. K. Lakdawala. $1990,8 \mathrm{p}$

Sponsored by Department of Energy, Washington, DC. Div. of Electric Energy Systems.

Pub. in Proceedings of Annual Report Conference on Electrical Insulation and Dielectric Phenomena, Pocono Manor, PA., October 28-31, 1990, p267-274.

Keywords: Electrical insulation, Electric discharges, Dielectrics, Reprints, "Corona discharges, Trichel pulses.

Experiments are conducted to investigate the conditions under which the transition from negative corona to dielectric barrier controlled discharge occurs. A negative point-plane electrode (covered with PTFE dielectric) geometry is studied using a newly developed partial discharge detection technique. At a critical gapdistance, an abrupt transition from a rapid pulsating behavior to a widely distributed random pulse behavior is observed. The critical distance increases with increasing diameter of the solid dielectric and decreases with increase in applied voltage. The influence of dielectric surface charging on the Trichel pulse behavior is manifested by the measured pulse height and time separation distributions. As the influence of dielectric charging increases, the pulse separation distribution begins to broaden significantly and the corresponding pulse height distribution becomes narrower.

100,836

PB91-134528

Not available NTIS National Inst. of Standards and Technology (NEL), Gaithersburg, MD. Electricity Div.

Collisional Electron-Detachment and Ion-Conversion Processes In SF6.

Final rept.

J. K. Olthoff, R. J. Van Brunt, Y. Wang, L. D Doverspike, and R. L. Champion. 1990, 16p

Sponsored by Department of Energy, Washington, DC. Pub. in Nonequilibrium Etfe

Keywords: "Sulfur hexafluoride, Electric discharges, Cross sections, Reprints, "Electron detachment, "Ionmolecule collisions, Fluorine ions, Sulfur fluorides.

Results are summarized from the first direct measurements of absolute cross sections for electron-detachment and ion-conversion processes involving interactions of SF6(1-), SF5(1-) and F(1-), with SF6. These cross sections are used to calculate electron-detachment and ion-conversion reaction coefficients as func- 


\section{General}

tions of electric field-to-gas density ratios $(E / N)$ for various reactions. The relevance of these results is discussed to the interpretation of data from uniformfield drift-tube measurements and measurements of electrical-discharge initiation processes.

\section{0,837}

PB91-134684

Not available NTIS

National Inst. of Standards and Technology (NEL), Gaithersburg, MD. Electricity Div.

Electrical Breakdown and Streamer Statistics in $\mathrm{N}$ hexane under Uniform Field Conditions.

Final rept

K. L. Stricklett, E. F. Kelley, H. Yamashita, H. Kawai, and C. Fenimore 1990, 4p

Sponsored by Department of Energy, Washington, DC. Office of Energy Storage and Distribution.

Pub. in Proceedings of IEEE (Institute of Electrical and Electronics Engineers) International Symposium on Electrical Insulation, Toronto, Canada, June 3-6, 1990, p61-64.

Keywords: "Hexanes, "Dielectric breakdown, "Electric discharges, High speed photography, Liquids, Reprints, "Breakdown(Electronic threshold), Streamer prints, "Briation.

Under uniform field conditions, two modes of electrical breakdown are observed in liquid $n$-hexane: breakdown may be initiated by either cathode or anode streamers. This observation suggests that two unique sets of phenomena lead to electrical breakdown. In the work described, high-speed photography is employed to obtain a record of each breakdown event thereby providing statistical information regarding the relative frequencies of anode and cathode processes. The degree to which the relative probability for either process is influenced by experimental conditions is discussed.

\section{0,838}

PB91-134916

Not available NTIS

National Inst. of Standards and Technology (NEL) Boulder, CO. Electromagnetic Fields Div.

Dual Six-Port Reflectometer Systems Using Waveguide in the Frequency Range 18-50 GHz.

Final rept.

C. M. Weil, F. E. Marler, J. R. Major, M. F. Weidman, and D. H. Russell. 1989, 13p

Pub. in ARFTG Conference Digest (33rd), Long Beach CA., June 15-16, 1989, p76-88.

Keywords: "Microwave reflectometers, Extremely high frequencies, Superhigh frequencies, Millimeter waves, Waveguides, Scattering, Bolometers, Reprints, Six port, Uncertainty.

The development and evaluation of three dual six-port reflectometer systems, that use WR-42, WR-28 and WR-22 waveguides are discussed; these cover the frequency range $18-50 \mathrm{GHz}$. The systems are capable of automated or semiautomated operation and will pro vide complex scattering parameter data for customer waveguide components, as well as effective efficiency data for power sensors. Some representative measurement data are presented that demonstrate that these systems yield results that do not differ significantly from those obtained using older measurement systems. Some discussion of measurement uncertainties is also included.

\section{0,839}

PB91-135020

Not available NTIS

National Inst. of Standards and Technology (NEL) Gaithersburg, MD. Electricity Div.

Effect of High Pressure on Prebreakdown Phenomena in n-Hexane.

Final rept.

H. Yamashita, H. Kawai, K. L. Stricklett, and E. F.

Kelley. 1990, 6p

Sponsored by Department of Energy, Washington, DC Pub. in Proceedings of International Conference on Conduction and Breakdown in Dielectric Liquids (10th), Grenoble, France, September 10-14, 1990, p404-409.

Keywords: "Dielectric breakdown, "Electric discharges, Liquids, Electric fields, Electric potential, Shadowgraph photography, Reprints, "Pressure effects, *Hexane, Nonuniform fields, Streamer initiation.

The effect of pressure on the initiation of prebreakdown streamers in a-hexane in a non-uniform field is investigated. Using a high-magnification (100x) highresolution (1 micrometers) optical system and a highspeed camera, the initial growth of low-density stream. ers propagating from a needle cathode is examined at pressures ranging from 0.1 to $1.1 \mathrm{MPa}$. The initial streamer is a single filament which grows to approximately 4 micrometers in diameter and 8 micrometers in length before the appearance of a dense bushy structure at its tip. Oscillatory behavior of the initial streamer is observed in which the diameter of the intitial streamer expands and contracts as the streamer grows. The pressure dependence of the streamer initiation voltage is shown.

\section{0,840}

PB91-147223

National Inst of Standards and Technology (NEL). Boulder, CO. Electromagnetic Technology Div.

High Speed Magnetic Field Sensors Based on Iron Garnets.

Final rept

M. N. Deeter, A. H. Rose, and G. W. Day. 1990, 2p

Pub. in Proceedings of AFCEA Department of Defense Fiber Optics Conference, McLean, VA., March 20-23, 1990, p423-424.

Keywords: "Yttrium iron garnets, "Magnetic measurement, "Magnetic fields, "Magnetooptics, Faraday effect, Optical fibers, Fiber optics, Sensors, Reprints.

The authors characterize magnetic field sensors based on the Faraday effect in ferrimagnetic iron garnets in terms of their sensitivity and frequency response. Signal-to-noise measurements at $80 \mathrm{~Hz}$ on a sample of yttrium iron garnet yield noise equivalent magnetic fields of $10 \mathrm{nT} / \mathrm{Hz}$. Frequency response measurements exhibit virtually flat response to approximately $700 \mathrm{MHz}$.

100,841

$1-147702$

Not available NTIS National Bureau of Standards (NEL), Gaithersburg, MD. Precision Engineering Div.

Determination of Fields Near a Silver Strip on a Glass Substrate.

Final rept.

E. Marx, and E. C. Teague. $1988,9 p$

Pub. in Proceedings of SPIE (Society of Photo-Optical Instrumentation Engineers) - Scanning Microscopy Technologies and Applications, v897 p176-184 1988.

Keywords: "Dimensional measurement, "Light scattering, Electromagnetic scattering, Optical scanning, Integral equations, Resonance scattering, Near field, Reprints, "Silver strips.

The theory and numerical considerations that are used in the computation of the scattered electromagnetic fields near the surface of a silver strip on a glass substrate are presented. These calculations provide theoretical guidance for the measurement of the width of the strip by means of near-field optical scanning. The dimensions of the strip cross section, e.g. $300 \mathrm{~nm}$ by $100 \mathrm{~nm}$ can be a fraction of the wavelength of the incident light $6328 \mathrm{~nm}$. The flux $1 \mathrm{~nm}$ above the surface shows sharp spikes at the edges of the strip. The features of the fields near such a surface could be used for accurate determination of the width of the strip by measurements up to about $30 \mathrm{~nm}$ above the strip. The effects of other variables are also shown in the figures.

\section{0,842}

PB91-148676 Not available NTIS National Inst. of Standards and Technology (NEL). Boulder, CO. Electromagnetic Technology Div. Integrity Tests for High-(T sub c) and Conventional Critical-Current Measurement Systems.

Final rept.

L. F. Goodrich, and S. L. Bray. 1990, $8 p$

Sponsored by Department of Energy, Washington, DC Pub. in Advances in Cryogenic Engineering (Materials), v36 ptA p43-50 1990

Keywords: "Critical current, High temperature superconductors, Electrical measurement, Power supplies, Test methods, Voltmeters, Reprints, Ground loops.

Critical-current measurement systems must be extremely sensitive to the small differential voltage that is present across the test specimen as it changes from the zero resistance state to the flux-flow resistance state. Consequently, these measurement systems are also sensitive to interfering voltages. Such voltages can be caused by ground loops and by common mode voltages. Specific methods for testing the sensitivity of critical-current measurement systems and for detecting the presence of interfering voltages are discussed. These include a simple procedure that simulates the zero resistance state and the use of an electronic circuit that simulates the flux-flow resistance state.

\section{0,843}

PB91-158816

Not available NTIS

National Bureau of Standards (NEL), Gaithersburg, MD. Chemical Process Metrology Div.

Surface Acoustical Wave Spectroscopy for High Humidity Sensing

Final rept.

P. H. Huang. $1987,5 p$

Pub. in Proceedings of International Conference on Solid-State Sensors and Actuators (4th). Tokyo, Japan, June 2-5, 1987, p462-466

Keywords: "Surface acoustic wave devices, "Humidity measurement, High humidity, Lithium niobates, Electric fields, Acoustic fields, Sensors, Reprints, "SAW devices.

Polar water molecules in ambient moist air can be made to interact with the electric and acoustic fields of surface acoustical waves(SAW) in plezoelectric crysta surface. The interaction causes a change in the amplitude of the received surface wave which is proportional to the energy density of the wave. An amplitudemodulated SAW technique, which does not use ad sorptive coatings, has been used to study this electroacoustic interaction for high humidity sensing in the range 90 to $98 \% \mathrm{RH}$. The sensor system consists of two identical high frequency, wide band SAW delay lines. One is used as a reference channel with shorted electric fields at the surface. The reference delay line is maintained in an environment of nearly zero percen relative humidity. The ratio of voltage output from the sensing channel to that of the reference channel has been measured. An exponential relationship exists between the measured voltage ratio and the relative hu midity in the range from 89 to $98 \%$ for SAW frequencies at $210,220,230,240$, and $250 \mathrm{MHz}$, respectively Measurements of spectral group delay reveal the limitation on the transmission bandwidths of the sensor system for high humidity sensing.

\section{0,844}

PB91-161919 Not available NTIS National Inst. of Standards and Technology (NEL) Boulder, CO. Electromagnetic Fields Div.

Improved Technique for Determining Complex Permittivity with the Transmission/Reflection Method.

Final rept.

J. Baker-Jarvis, E. J. Vanzura, and W. A. Kissick.

$1990,8 p$

Pub. in IEEE (Institute of Electrical and Electronics En gineers) Transactions on Microwave Theory and Techniques 38, n8 p1096-1103 Aug 90.

Keywords: "Permittivity, Dielectric properties, Permeability, Transmission, Reflection, Reprints.

The transmission/reflection method for complex permittivity and permeability determination is studied. The special case of permittivity measurement is examined in detail. New robust algorithms for permittivity determination that eliminate the ill-behaved nature of the commonly used procedures at frequencies corresponding to integer multiples of one-half wavelength in the sample are presented. An error analysis is presented which yields estimates of the errors incurred due to the uncertainty in scattering parameters, length measurement, and reference plane position. In addition, new equations are derived for determining complex permittivity independent of reference plane position and sample length.

\section{0,845}

PB91-161992

National Inst. of Standards and Technology (NEL)

Boulder, CO. Electromagnetic Fields Div.

TEM/Reverberating Chamber Design/Concept Study: A Single Facility for Large System Radiated EMC Testing, $10 \mathrm{kHz}=40 \mathrm{Ghz}$.

Final rept.

M. L. Crawford. 1989, 15p

Pub. in Proceedings of EMC EXPO Conference Record, Washington, DC., August 1-3, 1989, 15p

Keywords: "Reverberation chambers, "Electromagnetic compatibility, Transmission lines, Scale models Performance, Design, Reprints, US NIST.

The paper describes work in progress at NIST to develop a single, integrated facility using a large shielded enclosure configured as a TEM transmission line 
driven reverberating chamber. TEM test fields are generated at frequencies below multimode cutoff, and mode-stirred test fields are generated at frequencies above multimode cutoff. The paper discusses a proposed facility design, advantages and limitations, the theoretical basis for the work, and the proposed experimental approach for evaluating a $1 / 10$ scale model of a large enclosure having a test volume of $8 \mathrm{~m} \times 16 \mathrm{~m} \times$ $30 \mathrm{~m}$.

\section{0,846}

PB91-162362 Not available NTIS National Inst. of Standards and Technology (NEL), Gaithersburg, MD. Electricity Div. Cutting the High Cost of Testing.

Final rept.

T. M. Souders, and G. N. Stenbakken. 1991, 4p Pub. in IEEE (Institute of Electrical and Electronics Engineers) Spectrum, p48-51 Mar 91.

Keywords: "Test methods, Computer applications, Analog circuits, Linear equations, Mathematical models, Reprints.

An approach is presented for optimizing the testing of analog and mixed-signal devices. The entire process is performed with algebraic operations on an appropriate model. The paper demonstrates how this is accomplished, using simple calls with public-domain software. Examples of test results achieved using this approach are included.

100,847

PB91-162370

Not available NTIS

National Bureau of Standards (NEL), Boulder, CO. Electromagnetic Fields Div.

Intercomparison of NBS Noise Calibration Serv-

ices.

Final rept.

D. F. Wait, W. C. Daywitt, and G. Counas. 1988, 2p Pub. in Proceedings of Conference on Precision Electromagnetic Measurements 'CPEM ' 88 Digest', Tsukuba, lbaraki, Japan, June 7-10, 1988, p209-210.

Keywords: "Thermal noise, "Standards, Microwave radiometers, Noise temperature, Reflectometers, Calibration, Reprints, Intercomparison.

New, less restrictive thermal noise calibration services recently established in the frequency range of $2-12.4$ $\mathrm{GHz}$ overlap prior NBS services and provide an opportunity for intercomparison. The agreement between old and new calibration systems is better than $0.4 \%$.

100,848

PB91-171959

PC A08/MF A0

National Inst. of Standards and Technology (NEL).

Boulder, CO. Electromagnetic Fields Div.

Transmission/Reflection and Short-Circuit Line Permittivity Measurements.

Technical note.

J. Baker-Jarvis. Jul 90, 156p NIST/TN-1341

Also available from Supt. of Docs. as SN003-00303058-9.

Keywords: "Dielectric properties, Microwaves, Electrical measurement, Calibration, Permeability, Reflection, Uncertainty, Coaxial cables, Computer programs, "Permittivity measurement, Loss factors.

The transmission/reflection and short-circuit line methods for measuring complex permittivity are examined. Equations for permittivity are developed from first principles. New robust algorithms that eliminate the illbehaved nature of the commonly used transmission/ reflection method at frequencies corresponding to integral multiples of one-half wavelength in the sample are presented. These allow measurements to be made on samples of any length. An uncertainty analysis is presented which yields estimates of the errors incurred due to the uncertainty in scattering parameters, length measurement and reference plane position. The equations derived here indicate that the minimum uncertainty for transmission/reflection measurements of nonmagnetic materials occurs at integral multiples of one-half wavelength in the material. In addition, new equations for determining complex permittivity independent of reference plane position and sample length are derived. New equations are derived for permittivity determination using the short-circuit line allow positioning the sample arbitrarily in the sample holder.

100,849

PB91-175240 Not available NTIS National Inst. of Standards and Technology (NEL), Gaithersburg, MD. Electrosystems Div.
Recent Developments in Digital Oscilloscopes.

Final rept.

H. K. Schoenwetter $1989,2 \mathrm{p}$

Pub. in Proceedings of IEEE (Institute of Electrical and Electronics Engineers) Instrumentation and Measurement Technology Conference, Washington, DC., April 25-27, 1989, p154-155.

Keywords: "Oscilloscopes, Digitizers, Reprints, "Digital oscilloscopes, Waveform recorders.

The paper reviews the latest developments in digital storage oscilloscopes (DSOs) as reported in the open literature. DSOs are used to digitize and store waveforms which may be compared, analyzed, and manipulated. DSO capabilities usually include programmability, automatic waveform parameter measurement, the display of pre-trigger signal activity, and waveform averaging to reduce noise and ripple.

\section{0,850}

PB91-175356

Not available NTIS

National Bureau of Standards (NEL), Boulder, CO. Electromagnetic Fields Div.

Lattice Approach to Environments Irradiated by Unknown Sources.

Final rept.

J. Randa, and M. Kanda. 1987, 5p

Pub. in Proceedings of the International Zurich Symposium and Technical Exhibition on Electromagnetic Compatibility (7th), Zurich, March 3-5, 1987, p191-195.

Keywords: "Electromagnetic environments, Maxwells equations, Numerical solution, Reprints, Successive overrelaxation method, III posed problems.

An approach is suggested to the characterization of electromagnetic environments irradiated by unknown sources. The approach is based on the numerical solution of Maxwell's equations subject to the constraints imposed by the measured values of the field at a small number of measurement points and by boundary conditions. The idea is demonstrated using a particular method for the numerical solution. Examples which demonstrate the approach but reveal deficiencies in the numerical method are given. Possible future directions are suggested.

100,851

PB91-175398

Not available NTIS

National Inst. of Standards and Technology (NML),

Gaithersburg, MD. Electricity Div.

Measurements of Power Frequency Magnetic Fields Away from Power Lines.

Final rept.

M. Misakian, J. M. Silva, and R. S. Baishiki. 1991,

$11 p$

Pub. in IEEE (Institute of Electrical and Electronics Engineers) Transactions on Power Delivery 6, n2 p901911 Apr 91

Keywords: "Magnetic fields, Residential buildings, Industrial buildings, Environments, Measurement, Calibration, Reprints.

Recent epidemiological studies have focused attention on the measurement of ambient level power frequency magnetic fields in residential and industrial settings. These fields can be as much as two orders of magnitude smaller than power line magnetic fields and can also contain significant levels of harmonic content. Because the existing IEEE standard for characterizing power frequency magnetic fields is intended for measurements near power lines, it has a number of inadequacies if used alone for guidance during the measurement of residential fields. The paper describes the instrumentation, calibration procedures, and outlines measurement strategies which can overcome some of the shortcomings of the existing standard. Examples of ambient level magnetic field measurements are also provided.

\section{0,852}

\section{PB91-184754}

PC A03/MF A01

National Inst of Standards and Technology (NEL) Gaithersburg, MD. Center for Electronics and Electrical Engineering.

Center for Electronics and Electrical Engineering Technical Publication Announcements Covering Center Programs, July to September 1990, with 1991 CEEE Events Calendar.

J. A. Gonzalez. Apr 91, 31p NISTIR-4537

See also PB90-206491.

Keywords: "Semiconductor devices, "Metrology, Electrical engineering, Electronics, Electromagnetic inter- ference, Integrated circuits, Antennas, Optical fibers, Signal processing, Sensors, Waveforms, Electrooptics. Photodetectors, High temperature superconductors, Progress report.

This is the twenty-sixth issue of a quarterly publication providing information on the technical work of the $\mathrm{Na}$ tional Institute of Standards and Technology (formerly the National Bureau of Standards) Center for Electronics and Electrical Engineering. This issue of the CEEE Technical Publication Announcement covers the third quarter of calendar year 1990. Topics discussed include the following: Semiconductor Technology Program; Signals \& Systems Metrology Program; Fast Signal Acquisition, Processing, \& Transmission; Electrical Systems; Electromagnetic Interference; 1991 CEEE Calendar.

100,853

PB91-189290

Not available NTIS

National Inst. of Standards and Technology (NML), Gaithersburg, MD. Electricity Div.

Measurement Reliability: The Detection of Nonlinearities.

Final rept.

C. P. Fenimore, and G. J. FitzPatrick. 1991, 6p Sponsored by Defense Nuclear Agency, Washington, DC.

Pub. in Proceedings of Space Nuclear Power Systems Symposium (8th), Albuquerque, NM., January 7-10, 1991, p1113-1118.

Keywords: "Electrical measurement, Spacecraft power supplies, Reliability(Electronics), Failure(Electronics), Photomultiplier tubes, Voltage dividers, Kerr cells, Error analysis, Reprints.

The detection of a single measurement failure in a compound measurement system consisting of a voltage divider and a Kerr cell is demonstrated. The comparison of measurement devices based on distinct technologies is inherently robust; they may be expected to have distinct failure characteristics. The Kerr comparison is based on model fitting applied to numerically-generated data and experimental, digitallyrecorded waveforms. The characteristic signatures of two measurement errors are found: for a quadratic nonlinearity in the detector and for an overdriven photodetector. The length of the data records permits the detection of nonlinearities which are comparable to the noise in magnitude. Detection of such errors is a prerequisite to recalibration in software which enables error correction in remote applications, such as space power systems.

100,854

PB91-189308

Not available NTIS

National Inst of Standards and Technology (NML) Gaithersburg, MD. Electricity Div.

Incompatibility between the 100/1300 Surge Test and Varistor Failure Rates.

Final rept.

C. P. Fenimore, and F. D. Martzloff. 1991, 6p

Pub. in Proceedings of International Zurich Symposium (9th), and Technical Exhibition on Electromagnetic Compatibility, Zurich, Switzerland, March 12-14, 1991 , p525-530.

Keywords: "Surges, "Varistors, Mathematical models, Nonlinear systems, Circuits, Tests, Reprints.

A proposed high-energy surge test featuring a $100 /$ 1300 microsec waveform and a peak voltage of 2.3 times the peak voltage of the low-frequency mains is under consideration by the IEC. The energy storage capacitor suggested for the surge generator, originally specified as high as 25000 microfared, has been scaled down but is still at a level of several thousand microfarads. To determine the energy dissipated in various surge tests, numerical integration is applied to a simple but realistic mathematical model of a test circuit. The energy that would be deposited into a varistor of the voltage rating commonly used in protecting load equipment, if subjected to this test, far exceeds the capability of the varistor, but reported varistor failure rates do not reflect such a situation. Thus, a re-examination of the premises that led to the $100 / 1300$ microsec test specifications appears necessary.

100,855

PB91-189811

Not available NTIS

National Inst. of Standards and Technology (NML), Gaithersburg, MD. Electricity Div. 


\section{ELECTROTECHNOLOGY}

\section{General}

Real, Realistic Ring Waves for Surge Testing. Final rept

F. D. Martzloff, and G. Pellegrini. 1991,6p

Pub. in Proceedings of the International Zurich Symposium and Technical Exhibition on Electromagnetic Compatibility (9th), Zurich, Switzerland, March 12-14 1991, p499-504.

Keywords: "Surges, Data transmission systems, Transmission lines, Power lines, Lightning, Transients, Test methods, Reprints, Ring waves.

Five independent investigations on the coupling of surges into low-voltage circuits (data or power lines) and of their effects, show that a damped oscillatory transient is a real, realistic stress for equipment connected to these lines.

\section{0,856}

PB91-202895

Not available NTIS

National Bureau of Standards (NEL), Boulder, CO.

Electromagnetic Fields Div.

TEM Driven Reverberating Chamber, a Single Facility for Radiated EMS/V Testing, $10 \mathrm{kHz}-18 \mathrm{GHz}$ Final rept.

M. L. Crawiord. 1987, $11 \mathrm{p}$

Pub. in Proceedings of International Conference on Electromagnetic Compatibility EMC EXPO 87, San Diego, CA., May 19-21, 1987, pT11.18-T11.28.

Keywords: "Reverberation chambers, "Electromagnetic testing, Electromagnetic susceptibility, Vulnerability, Test facilities, Performance evaluation, Oper ation, Design, Reprints.

The paper discusses the design, operation, and evaluation of a reverberating chamber, excited by a transverse electromagnetic (TEM) transmission line, for use in establishing radiated electromagnetic fields for susceptibility/vulnerability (EMS/V) testing of electronic equipment. The potential range of application is from $10 \mathrm{kHz}$ to $18 \mathrm{GHz}$. Included are brief descriptions of the facility, the operation procedures, the methodfor determining the test field amplitude inside the chamber, and the evaluation of the chambers electrical parameters such as VSWR and E-field strength as a function of input power. Also the E-field spatial uniformity and a summary of measurement uncertainties and conclusions derived from the test results are pre sented.

\section{0,857}

PB91-203885

Not available NTIS

National Inst. of Standards and Technology (NEL)

Boulder, CO. Electromagnetic Fields Div.

Simultaneous versus Independent Injection Testing of Nonlinear Multiport Systems.

Final rept.

J. Randa. 1991, 4p

Pub. in Proceedings of International Zurich Symposium and Technical Exhibition on Electromagnetic Compatibility (9th), Zurich, Switzerland, March 12-14, 1991,

p71.74

Keywords: "Electromagnetic susceptibility, Electromagnetic interference, Nonlinear systems, Reprints *Injection testing.

The paper is a theoretical investigation of the question whether, in injection testing of a multiport system, all ports must be injected and tested simultaneously. A general, nonlinear, three-port system is analyzed. Con ditions under which the ports can be tested separately are derived, and problems with the practical applica tion of these conditions are pointed out. Systems with memory are also treated, and the extension to general multiports is given. The relevance to bulk injection testing is discussed.

\section{0,858}

$1-236844$

Not available NTIS

National Inst. of Standards and Technology (EEEL) Boulder, CO. Electromagnetic Fields Div.

Improved Technique for Measuring Permittivity of Thin Dielectrics with a Cylindrical Resonant Cavity. Final rept.

M. D. Janezic, and J. H. Grosvenor. 1991, 5p

Sponsored by Department of the Navy, Washington, DC.

Pub. in Proceedings of IEEE (Institute of Electrical and Electronics Engineers) Instrumentation and Measure ment Technology Conference Enhancing Productivity with Instrumentation and Measurement Technologies, Atlanta, GA., May 14-16, 1991, p580-584.

Keywords: "Dielectrics, "Permittivity, Electrical measurement, Dielectric properties, Cavity resonators, Alu- minum oxide, Magnesium titanates, Polystyrene, Microwaves, Reprints.

A new technique for measuring the relative permittivity of thin, low-loss dielectric materials in a cylindrical resonant cavity has been developed. A thin dielectric sample, of unknown characteristics, is placed upon a thicker dielectric sample whose permittivity is well characterized. Both samples are then placed on the endplate in the cylindrical resonant cavity. In this way, the thin sample is placed in a region of the cavity where interaction with the electromagnetic fields is greater. From knowledge of the cavity's resonant frequency, dimensions of the cavity and both dielectric samples, and the permittivity of the thicker sample, the authors are able to use iterative techniques to accu-
rately determine the permittivity of the thin dielectric sample. A derivation and discussion of the theory used in this layered-dielectric permittivity measurement technique is provided. Also, measurement results, at frequencies between 9 and $10 \mathrm{GHz}$, are given.

\section{0,859}

\section{PB91-237214}

Not available NTIS

National Inst. of Standards and Technology (EEEL), Gaithersburg, MD. Electricity Div.

Precision Qualification of Watthour Meters.

Final rept.

J. D. Ramboz, C. Fenimore, and S. B. Schiller. 1991,

Pub. in IEEE (Institute of Electrical and Electronics Engineers) Transactions on Instruments and Measurement 40, n2 p406-409 Apr 91

Keywords: "Watt hour meters, Electric energy meters, Experimental design, Electrical measurement, Precision, Standards, Reprints.

One of the NIST Measurement Assurance Programs transfers the unit of the watthour using transpor meters. For the application, the response of these meters to variations in environmental conditions must be well-characterized. A statistically planned experiment is employed to determine corrections for the response of each meter to varying conditions of voltage, current, temperature, and power factor. This qualification procedure is designed to be efficient with the number of test points and to yeild estimates of the model parameters describing the corrections.

\section{0,860}

PB91-237222

Not available NTIS

National Inst of Standards and Technology (NEL) Boulder, CO. Electromagnetic Fields Div.

Theoretical Considerations for a Thermo-Optic Microwave Electric-Field-Strength Probe.

Final rept.

J. Randa. $1990,8 p$

Sponsored by Naval Ocean Systems Center, San Diego, CA.

Pub. in Jnl. of Microwave Power and Electromagnetic Energy 25, n3 p133-140 1990.

Keywords: "Electric fields, "Probes(Electromagnetic), Microwave equipment, Electrical measurement, Ultrahigh frequency, Superhigh frequency, Millimeter waves, Reprints.

Theoretical background for the design of a microwave electric-field probe is presented. The design uses a fiberoptic thermometer to measure the temperature rise of a resistive sphere or spherical shell in an electromagnetic field. Design parameters are chosen to optimize sensitivity and frequency response for the $1 \mathrm{GHz}$ to $10 \mathrm{GHz}$ range. These parameters also result in good frequency response well into the millimeter-wave range. Advantages of the design are that it is small nompertubing, and can be used in high electromagnet ic fields.

\section{0,861}

\section{PB91-237644}

Not available NTIS National Inst. of Standards and Technology (EEEL) Gaithersburg, MD. Electricity Div.

Influence of Memory Propagation on Phase-Resolved Stochastic Behavior of AC-Generated Partial Discharges.

Final rept

R. J. Van Brunt, and E. W. Cernyar. 1991, 3p

Sponsored by Department of Energy, Washington, DC Office of Energy Storage and Distribution.

Pub. in Applied Physics Letters 58, n23 p2628-2630, 10 Jun 91.

Keywords: "Electric discharges, Stochastic processes, Static electricity, Alternating current, Dielectrics, Reprints.
Using measurements of phase-restricted conditional partial-discharge pulse-amplitude and phase distributions, it is shown for the first time that the stochastic properties of a dielectric-barrier type of partial discharge generated by an ac voltage are significantly in fluenced by memory associated with charge deposited on the dielectric surface by preceding discharge events. The memory effect must be considered in any attempt to interpret results of phase-resolved partialdischarge measurements.

\section{0,862}

PB91-237776

Not available NTIS

National Inst. of Standards and Technology (EEEL) Boulder, CO. Electromagnetic Fields Div.

Translate LRL and LRM Calibrations.

Final rept.

D. Williams, R. Marks, and K. Phillips. 1991, 5p

Pub. in Microwaves and RF $30, \mathrm{n} 2$ p78, $80-82$ and 84 , Feb 91.

Keywords: "Calibration, Probes(Electromagnetic), Microwaves, Wafers, Reprints, LRM method, LRL method, Line reflect match method, Line reflect line method, Automatic network analyzers.

The Line-Reflect-Match calibration technique (LRM) is reviewed. Unless the match standard is perfect, calibration using the most common form of LRM differs from that of the Line-Reflect-Line technique (LRL). The authors present an explicit transformation which relates the two calibrations.

\section{0,863}

PB9 1-240838

PC A03/MF A01

National Inst. of Standards and Technology (NEL). Gaithersburg, MD. Center for Electronics and Electrical Engineering.

Center for Electronics and Electrical Engineering Technical Publication Announcements Covering Center Programs, October to December 1990, with 1991 CEEE Events Calendar.

J. A. Gonzales. Aug 91, 32p NISTIR-4645 See also PB90-265232 and PB91-184754.

Keywords: "Electrical engineering, "Electronics, Semiconductors(Materials), Metrology, Signal processing, Electromagnetic interference, Antennas, Lasers, Magnetic materials, Microwaves, Fiber optics, Superconductors, Electrooptics, Abstracts, Technology innovation, Semiconductor devices, NIST(National Institute of Standards and Technology), CEEE(Center for Electronics and Electrical Engineering)

This is the twenty-seventh issue of a quarterly publication providing information on the technical work of the National Institute of Standards and Technology (formerly the National Bureau of Standards) Center for Electronics and Electrical Engineering. The issue of the Center for Electronics and Electrical Engineering Technical Publication Announcements covers the fourth quarter of calendar year 1990. Abstracts are provided by technical area for papers published this quarter.

\section{0,864}

PB92-112309

PC A03/MF A01

National Inst. of Standards and Technology (NEL), Gaithersburg, MD. Semiconductor Electronics Div.

Electronics and Electrical Engineering Laboratory Technical Publication Announcements Covering Laboratory Programs, January to March 1991, with 1991 EEEL Events Calendar.

J. A. Gonzalez. Sep 91, 20p NISTIR-4675 See also PB91-240838.

Keywords: "Electromagnetic interference, "Microelectronics, "Electrooptics, "Metrology, Integrated circuits, Bipolar transistors, High temperature superconductors, Signal processing, Millimeter waves, Antennas, Lasers, Microwaves, Optical fibers, Magnetic materials, Sensors, Fiber optics, Progress report, Abstracts.

The report is the twenty-eighth issue of a quarterly publication providing information on the technical work of the National Institute of Standards and Technology, Electronics and Electrical Engineering Laboratory. The issue of the EEEL Technical Publication Announcements covers the first quarter of calendar year 1991. Abstracts are provided by technical area for papers published this quarter. Major topic areas include the following: Semiconductor microelectronics; Signal acquisition, processing, and transmission; Electrical systems; Electromagnetic interference. 
100,865

PB92-112325 PC A03/MF A01

National Inst. of Standards and Technology, Boulder, $\mathrm{CO}$

Optimization Techniques for Permittivity and Permeability Determination.

P. D. Domich, J. Baker-Jarvis, and R. G. Geyer. Jun 91, 23p NISTIR-4571

Keywords: "Permeability, "Permittivity, Regression analysis, Complex variables $S$ matrix, Optimization, Microwaves, Transmission lines, Glass, Yttrium iron garnets, Barium titanates, Nickel ferrite, Polystyrene, Polymers.

The paper discusses optimization techniques for the determination of complex permittivity and permeability in transmission lines. The traditional theoretical mode using scattering parameters is extended into a mathematical regression model that can be solved with widely accepted numerical techniques. The new model produces accurate primary mode results for the samples tested including nonmagnetic materials with high dielectric constants. An extension of the model includes responses due to higher order modes. The general model determines parameters to specify the spectral functional form of complex permittivity and permeability and is capable of small corrections to independent variable data including angular frequency, sample length, sample position, and cutoff wavelength. The method provides reliable determination for both low and high permittivity materials.

\section{0,866}

PB92-116367 PC A06/MF A02

National Inst. of Standards and Technology (EEEL) Boulder, CO. Electromagnetic Technology Div. Bibliography of the NIST Electromagnetic Fields Division Publications.

R. M. Lyons, and K. A. Gibson. Aug 91, 108p

NISTIR-3973

Supersedes PB91-132241.

Keywords: "Electromagnetic fields, "Bibliographies, Electrical measurement, Dielectric properties, Electromagnetic interference, Electromagnetic noise, Remote sensing, Time domain, Radiation hazards Near field, Antennas, Metrology, Waveforms, Standards, Microwaves, Attenuation, Dielectric measurements, Microwave metrology, Microwave power, Impedance, Near-field antenna measurements, Noise, Nonionizing radiation, Waveform metrology.

The bibliography lists the publications by the staff of the Electromagnetic Fields Division of the National Institute of Standards and Techology for the period January 1970 through July 1991. It supersedes NISTIR 3945 , which listed the publications of the Electromagnetic Fields Division from January 1970 through July 1990. Selected earlier publications from the Division's predecessor organizations are included.

\section{0,867}

PB92-116375

PC A04/MF A01

National Inst. of Standards and Technology (EEEL).

Boulder, CO. Electromagnetic Technology Div.

Metrology for Electromagnetic Technology: A Bibliography of NIST Publications.

M. E. DeWeese. Aug 91, 69p NISTIR-3972

Supersedes PB91-132266.

Keywords: "Metrology, "Bibliographies, Optical communication, Optical fibers, Magnetic measurement, Superconducting devices, Superconductors, Optoe lectronic devices, Electrooptics, Lasers, Cryoelectron ics, Electromagnetic metrology, Superconducting materials, "Electromagnetic metrology, Cryoelectronics.

The bibliography lists the publications of the personne of the Electromagnetic Technology Division of NIST during the period from January 1970 through publication of the report. A few earlier references that are directly related to the present work of the Division are also included.

\section{0,868}

PB92-116516

Not available NTIS

National inst of Standards and Technology (NEL)

Boulder, CO. Electromagnetic Technology Div.

Voltage Calibration Systems Using Josephson Junction Arrays.

Final rept.

C. J. Burroughs, and C. A. Hamilton. 1990, 4p

Pub. in IEEE (Institute for Electrical and Electronics

Engineers) Transactions on Instrumentation and

Measurement 39, n6 p972-975 Dec 90.
Keywords: "Josephson junctions, "Standards, "Calibration, Computerized control systems, Arrays, Uses, Reprints, "Voltage standards.

The recent development of large arrays of Josephson junctions is allowing an ever increasing number of laboratories to maintain intrinsic Josephson voltage standards at an accuracy near $0.05 \mathrm{ppm}$. The paper reviews the fundamentals of Josephson voltage standards and how computer control makes these standards simple to use in a variety of applications.

\section{0,869}

PB92-117167

Not available NTIS

National Inst. of Standards and Technology (EEEL). Gaithersburg, MD. Electricity Div

Influence of Nonsinusoidal Waveforms on Voltmeters, Ammeters, and Phase Meters.

Final rept.

N. M. Oldham, and T. L. Nelson. 1991, $6 p$

Pub. in Proceedings of IEEE (Institute for Electrical and Electronics Engineers) Winter Power Meeting. New York City, NY., February 3-7, 1991, p7-12.

Keywords: "Phase meters, "Voltmeters, "Ammeters, Waveforms, Tests, Reprints.

The operating principles of various voltmeters, ammeters, and phase meters are described. The results of tests on these instruments at different levels of distortion indicate that phase meters are subject to large often unpredictable errors while most voltmeters and ammeters respond to the runs value, independent of waveshape.

\section{0,870}

PB92-123082

National Inst. of Standards and Technology (EEEL). Gaithersburg, MD.

Electronics and Electrical Engineering Laboratory. 1991 Strategic Plan. Supporting Technology for U.S. Competitiveness in Electronics.

R. M. Powell. Nov $91,38 p$ NISTIR-4714

See also PB92-112309.

Keywords: "Electrical measurement, Electronics in dustry, Optical communication, Semiconductor devices, Microwave equipment, Video equipment, Integrated circuits, Microelectronics, Signal processing, Optical fibers, Strategy, Trends, Facilities, Sensors, Metrology, Electronics and Electrical Engineering Laboratory, Competitiveness, US NIST.

Among U.S. manufacturing industries, the electronics industry is the largest employer (1.94 million) and is a close second in shipments (\$266 billion) to the chemical industry (1990). U.S. competitiveness in many fields of electronics has been declining. Improved competitiveness will require outstanding performance from manufacturers in every step required to put product into service to a buyer: research and development, manufacturing, marketplace exchange, and after-sales support. All of these steps are highly measurement intensive and have outstripped available measurement support. Improved measurement support is an essential part of any successful strategy for improving U.S. competitiveness. The Electronics and Electrical Engineering Laboratory (EEEL), within the National Institute of Standards and Technology, as part of a continuing study of the electronics industry, is identifying measurement needs in important fields of electronics: semiconductors, superconductors, magnetics, microwaves, lightwaves (optoelectronics) power networks, video, electromagnetic compatibility, and complex-system description and testing. The document describes EEEL's strategic plan for a response in terms of both general strategic directions across all of these fields and specific strategic directions for fou fields: semiconductors, microwaves, optical-fiber com munications, and video. The plan relates these directions to important goals for improved U.S. competitiveness.

\section{0,871}

\section{PB92-126663}

(Order as PB92-126614, PC A06/MF A02)

National Inst. of Standards and Technology, Boulder $\mathrm{CO}$

Optimization Techniques for Permittivity and Permeability Determination.

P. D. Domich, J. Baker-Jarvis, and R. G. Geyer.

1991, 11p

Included in Jnl. of Research of the National Institute of

Standards and Technology, v96 n5 p565-575 Sep/Oct 91.
Keywords: "Dielectric properties, "Permeability, "Permittivity, Mathematical models, Superhigh frequency, Nonlinear analysis, Microwave frequencies, $\mathrm{S}$ matrix, Optimization, Yttrium iron garnets, Barium titanates, Nickel ferrite, Polystyrene, Polymers, Glass.

The paper discusses optimization techniques for the determination of complex permittivity and permeability in transmission lines. The traditional theoretical mode using scattering parameters is extended into a mathematical regression model that can be solved with widely accepted numerical techniques. This new model produces accurate primary mode results for the samples tested including nonmagnetic and magnetic materials with high dielectric constants. An extension of the model includes responses due to higher orde modes. The general model determines parameters to specify the spectral functional form of complex permittivity and permeability and is capable of small corrections to independent variable data including angula frequency, sample length, sample position, and cutof wavelength. The method provides reliable determination for both low and high permittivity materials.

ENERGY

\section{Electric Power Transmission}

\section{0,872}

PB91-162248

Not available NTIS

National Inst. of Standards and Technology (NEL) Gaithersburg, MD. Electricity Div.

Evaluation of Instrumentation Used to Measure AC Power System Magnetic Fields.

Final rept.

M. Misakian. 1991, 11p

Pub. in IEEE (Institute of Electrical and Electronics Engineers) Transactions on Power Delivery 6, n1 p1-11 Jan 91

Keywords: "Power systems, "Magnetic fields, "Magnetic measurement, AC systems, Instruments, Exposure meters, Performance, Meetings, Tests, Reprints.

A workshop was organized by the AC Fields Working Group for the purpose of evaluating instrumentation designed for measuring power system magnetic fields. The instruments tested varied from simple single axis survey meters to microcontroller based instruments designed for long term data collection and analysis. The working group designed a series of tests which were used to evaluate each instrument. These included calibration and harmonic response tests, tests of susceptibility to high $60 \mathrm{~Hz}$ electric fields and electromagnetic interference and the measurement of fields typical of transmission line, appliance, substation and office/shop environments. Results for each of these tests are presented and discussed. With some mino exceptions, the peformance of all instruments was satisfactory.

100,873

PB91-216770

PC A04/MF A01

National Inst. of Standards and Technology (EEEL), Gaithersburg, MD.

NIST Measurement Services: Calibration Service for Current Transformers.

Special pub. (Final).

J. D. Ramboz, and O. Petersons. Jun 91, 62p NIST/ SP-250/36

Also available from Supt. of Docs. as SN003-003 03087-2.

Keywords: "Current transformers, "Calibration, Electrical measurement, Electric power distribution, Stand ards, Uncertainty, US NIST.

A calibration service at the National Institute of Stand ards and Technology (NIST) for laboratory-quality current transformers is described. The service provides measurements of the current ratio and the phase angle between the secondary and primary currents. In the Report of Calibration or Test, the measured ratio is reported as the product of the marked (nominal) ratio and the ratio correction factor. The measured phase angle is reported directly in milliradians (mrad) and is 
positive if the secondary current leads the primary. The range of primary-to-secondary current ratios that can be measured with the equipment at NIST extends from $0.25 \mathrm{~A}: 5 \mathrm{~A}$ to $12000 \mathrm{~A}: 5 \mathrm{~A}$. The maximum current at the present time is about $20000 \mathrm{~A}$. Estimates of calibration uncertainties, including their sources, are given and quality control procedures are described. For routine calibrations, uncertainties of + or $-0.01 \%$ for the ratio and + or $-0.1 \mathrm{mrad}$ for the phase angle are quoted. However, lower uncertainties--to + or - 0.005 mrad or 5 microrads for phase angle--are possible under the provisions of Special Tests.

\section{0,874}

PB92-112341

PC A06/MF A02

National Inst. of Standards and Technology (EEEL),

Gaithersburg, MD. Electricity Div.

Research for Electric Energy Systems: An Annual Report.

Annual rept. 1990

W. E. Anderson. Jun 91, $105 p$ NISTIR-4691

See also report for 1989 , PB90-228032. Sponsored by Department of Energy, Washington, DC. Div. of Electric Energy Systems

Keywords: "Surges, "Power transmission lines, "Electric fields, "Dielectrics, Kerr cells, Power lines, Chemical detection, Mathematical models, Graphs(Charts).

The report documents the technical progress in four investigations which make up the project 'Support of Research Projects for Electrical Energy Systems,' funded by the U.S. Department of Energy and performed by the Electricity Division of the National Institute of Standards and Technology (NIST). Specifically these investigations include: (1) Measurements of magnetic fields in support of epidemiological and in vitro studies of biological field effects; (2) development of a technique to measure trace amounts of S2F10 in the presence of SF6 and the development of an improved stochastic analyzer for pulsating phenomena; (3) optical and electrical measurements of negative streamers preceding electric breakdown in liquid dielectrics; and (4) the development of a reference resistive divider for high voltage impulse measurements. The work discussed in the report is part of an ongoing research activity at NIST.

\section{Fuel Conversion Processes}

\section{0,875}

\section{PB91-134940} Gaithersburg, MD. Ceramics Div.

Effect of Slag Penetration on the Performance of Magnesia Chrome Refractories in Slagging Gasifi-

ers.

S. M. Wiederhorn, R. F. Krause, J. Sun, and G. V

Sklizkov. 1987, 8p

Sponsored by Department of Energy, Oak Ridge, TN. Fossil Energy Program.

Pub. in Proceedings of Symposium on Materials for Coal Gasification, Cincinnati, OH., October 10-15, 1987, p121-128.

Keywords: "Coal gasification, "Magnesite refractories "Refractory materials, "Creep rupture tests, Slags, Magnesium oxides, Microstructure, Creep rupture $\begin{array}{lll}\text { Magnesium oxides, Microstructure, Creep rupture } & \text { Brength, } \\ \text { streepsicks, }\end{array}$ Fractures(Materials), Reprints.

The purpose of the paper is to present a summary of a study on the morphological changes that occur when refractory bricks are penetrated by slag. The study consisted primarily of a microstructural analysis of specimens from a brick that had been removed from a coal gasifier. Laboratory studies were also conducted on newly manufactured brick to clarify the nature of the reactions that occur in practice. Data suggests a strong correlation between the structural stability of the refractory and the viscosity of the intergranular vitreous phase that forms as a result of slag penetration. In the course of the paper, the effect of viscosity on grain growth and on the creep and creep rupture behavior of this material is discussed.

\section{0,876}

PB91-158592

Not available NTIS

National Bureau of Standards (IMSE), Gaithersburg. MD. Ceramics Div.
Rapporteur's Summary Materials Development for Coal Gasification.

Final rept.

S. J. Dapkunas. $1987,3 p$

Pub. in Proceedings of ASM Conterence on Materials for Coal Gasification, Cincinnati, OH., October 10-15, 1987, p183-185.

Keywords: "Coal gasification, "Corrosion, "Refractories, Steels, Hydrogen sulfide, Chromium additions, Erosion, Sulfidization, Heat resistant materials, Linings, Aluminum oxide, Ceramics, Slags, Concrete, Reviews, Reprints.

The operating conditions found in coal gasification systems have long been recognized as placing severe demands on materials of construction. An assessment of the performance of German wartime synfuels plants specifically cited hydrogen sulfide attack of alloy steels in high pressure Lurgi gasifiers as a problem and noted that 23 percent chromium steels were required for adequate corrosion resistance. The energy crisis of the 1970's raised renewed concern for the behavior of materials in coal gasifiers, and several assessments of the performance of materials, particularly for internal environments of dry ash gasifiers, were conducted. Inplant tests and extensive laboratory testing programs identified sulfidation attack of alloys and, in some applications, erosion to be primary modes of alloy deterioration.

\section{Fuels}

100,877

AD-A242 816/7

$\mathrm{PC} \mathrm{A03} / \mathrm{MF} \mathrm{A01}$

National Inst. of Standards and Technology, Gaithersburg, MD.

Short-Duration Autoignition Temperature Measurements for Hydrocarbon Fuels.

Final rept. May 89-Sep 90.

K. C. Smyth, and N. P. Bryner. Jan 91, 49p AFESC/ ESL-TR-90-43,

Keywords: Air, "Autoignition, Control, Decomposition, Determination, Fuels, Heat, High temperature, Hydrocarbons, Ignition, Measurement, Metals, Mixtures, Nickel, Reliability, Short range(Time), Stainless steel, Surfaces, "Temperature, Test methods, Titanium, "Hydrocarbon fuels.

This study develops an experimental method for the reliable determination of auto ignition temperatures reliable determination of auto ignition temperatures tion exposures of controlled fuel/air mixtures on three tion exposures of controlled fuel/air mixtures on three determinations have been made for the ignition of 15 hydrocarbons on heated nickel, stainless steel, and titanium surfaces for three different fuel/air mixtures. The measured autoignition temperatures generally decrease for the larger hydrocarbons and for richer mixtures, with the $\mathrm{C} 2$ hydrocarbons having particularly low values. The highest autoignition temperature are observed for nickel surfaces and the lowest for the stainless steel, with titanium being an intermediate case. Relationships between autoignition, radical-based chemical mechanisms and end-gas autoignition (engine knock) are discussed. Most of the previous autoignition data are unsatisfactory since measurements are typically carried out using ASTM E659 test. This test method attempts to measure the autoignition temperature of hydrocarbons fuels in the gas-phase under experimental conditions where it is more likely that ignition temperatures for a complex mixture of the products of fuel decomposition are being determined rather than those of the original fuel.

100,878

PB91-133942

Not available NTIS

MD. Chemical Thermodynamics Div.

MSW Calorimetry.

Final rept.

E. S. Domalski, K. L. Churney, A. E. Ledford, and M. L. Reilly. 1986, $4 p$

Pub. in Mechanical Engineering, p32-35 Aug 86.

Keywords: "Colorimeters, "Calorific value, "Fuels, "Garbage, Thermodynamic properties, Solid waste disposal, Heat measurement, Combustion, Sampling, Test facilities, Reprints, "Municipal wastes, Solid wastes.
For many years, it has been the opinion of combustion engineers that one cannot sample a multi-ton quantity of municipal solid waste (MSW) and remove a representative gram-size sample for bomb calorimetric measurement. As a response to this concern, a multikilogram capacity combustion flow calorimeter has been designed, constructed, and placed into operation to assess the credibility of the bomb calorimetric determination using gram-size samples. Calorific values derived from both gram-size samples in a bomb calorimeter and multi-kilogram-size samples in a large flow calorimeter have been compared and found to be in good agreement. The study has shown that the determination of the calorific value of MSW using gram-size samples is credible provided proper attention is paid to sampling, sample size-reduction, and sample subdivision.

100,879

PB91-134403 National Bureau of Standards (NEL), Gaithersburg, MD. Chemical Process Metrology Div.

Gas Flow Measurement: Calibration Facilities and Fluid Metering Traceability at the National Bureau of Standards.

Final rept.

G. E. Mattingly. 1987, 24p

Pub. in Natural Gas Energy Measurement, p219-242 1987

Keywords: "Flowmeters, "Flow measurement, "Natural gas, Fluid flow, Gas flow, Standards, Test facilities Calibrating, Accuracy, Reprints, "Calibration.

As the value of scarce fluid resources increases in today's domestic and international market places and process industries so does the need for improved fluid measurement and for improved traceability to primary standards. Both buyers and sellers of fluid products are increasingly concerned about accurate custody transfer. Designers and operators of industrial processes are increasingly concerned about the precision of their fluid measurement to optimize the performance of their continuous production technologies. To satisfy these expressed needs for improved fluid measurements and traceability in the wide range of fluids and conditiions required, is a considerable task. The calibration facilities which flow gas and which are currently in use at the National Bureau of Standards (NBS) are described. The performance characteristics of these facilities are given together with corresponding levels of uncertainties.

\section{0,880}

PB91-147025

Not available NTIS

National Inst. of Standards and Technology (NML) Boulder, CO. Chemical Engineering Science Div. Experimental Evaluation of Selected Orifice Flow meter Upstream Installations.

Final rept.

J. A. Brennan, C. F. Sindt, and M. A. Lewis. 1990, 9p Sponsored by Gas Research Inst., Chicago, IL Pub. in Proceedings of American Gas Association Distribution/Transmission Conference, Los Angeles, CA. May 7-9, 1990, p594-602.

Keywords: "Orifice meters, "Gas meters, "Standards Roughness, Flowmeters, Orifices, Specifications, Flow measurement, Gas flow, Pipes(Tubes), Gas pipes, Reprints.

There are two standards for orifice flow measuremen that are the primary references used in the gas industries around the world. These standards differ significantly on some installation specifications. The differences can profoundly affect the design, cost, and measurement accuracy of meter stations. Revisions to the standards are currently being completed. A new flow equation has been developed, and serious consideration given to changing some of the installation specifications. New experimental test results are the basis for changing the installation specifications on pipe roughness and flow conditioner location. These two parameters are being studied both individually and collectively. Results from tests on orifice flowmeters ranging from 3 to 24 in are presented.

100,881

PB91-148791

Not available NTIS

National Inst. of Standards and Technology (NML), Boulder, CO. Chemical Engineering Science Div. 
Effect of Tube Bundle Flow Conditioners on Orifice Meter Discharge Coefficients.

Final rept.

K. M. Kothari, J. A. Brennan, and J. Gorter. 1990, 9p Sponsored by Gas Research Inst., Chicago, IL

Pub. in Proceedings of International Gas Research Conference, Tokyo, Japan, November 6-9, 1989, p541-549 1990 .

Keywords: "Flow regulators, "Flow measurement, Flowmeters, Natural gas, Nitrogen, Tubes, Reynolds number, Reprints, "Tube discharge flow conditioners, "Discharge coefficients.

Two experimental test programs on the use of tube bundle flow conditioners upstream of an orifice flowmeter have been completed. One program used a flange tapped $100 \mathrm{~mm}$ orifice flowmeter in nitrogen gas at pressures of approximately $4 \mathrm{MPa}$ and the other used a $600 \mathrm{~mm}$ orifice flowmeter in natural gas at pressures of approximately $6 \mathrm{MPa}$. The $100 \mathrm{~mm}$ flowmeter ests were run at a Reynolds number near 1,000,000 and the $600 \mathrm{~mm}$ flowmeter test covered the Reynolds number range of $1,000,000$ to $55,000,000$ and included D-D/2, flange, and corner tappings. Both programs included several beta ratio orifice plates. Test results clearly show that placement of the flow conditioner 7 pipe diameters upstream of the orifice plate can cause bias measurement errors compared to long pipe data. For some beta ratios, these bias errors exceeded $1 \%$.

100,882

PB91-162214

Not available NTIS

National Bureau of Standards (NEL), Boulder, CO. Thermophysics Div.

Speed of Sound of Natural Gas Mixtures.

Final rept.

R. D. McCarty. 1986, 7p

Pub. in Proceedings of International Symposium on

Fluid Flow Measurement, Arlington, VA., November $16-19,1986,7 \mathrm{p}$

Keywords: *Natural gas, "Acoustic velocity, Equations of state, Mathematical prediction, State of the art, Mixtures, Density, Reprints.

Accurate values for the speed of sound in natural gas mixtures are important in the application of sonic melering devices and in many design applications. In the case of mixtures, it is not possible to obtain experimentally determined speed of sound data for all possible compositions of the pure components found in natural gases. The alternative is a mathematical model of acceptable accuracy which allows the prediction of the speed of sound at an arbitrary state point composition. The paper describes the 'state of the art' for the prediction of the speed of sound for natural gases.

\section{0,883}

PB91-162271

Not available NTIS

National Inst. of Standards and Technology (MSEL), Gaithersburg, MD. Polymers Div.

Bioprocessing of Fossil Fuels Using Hyperthermophilic Archaebacteria.

Final rept

G. J. Olson, T. L. Peeples, I. I. Blumentals, R. N.

Schicho, and S. H. Brown. 1989, 16p

Pub. in Proceedings of Symposium on Biological Processing of Coal and Coal-Derived Substances, Palo Alto, CA., May 15-19, 1989, p2-53-2-68.

Keywords: "Coal, "Metabolism, "Sulfur, "Desulfurization, Bacteria, Thermophiles, Hydrogen sulfide, Cystine, Thiols, Sulfides, Reprints, "Pyrococcus furiosus, "Bioprocessing.

Studies on the metabolism of sulfur in model compounds and in coals at 98-100 C were conducted using the hyperthermophilic archaebacterium, Pyrococcus furiosus (P. furiosus). The bacterium produced hydro gen sulfide from elemental sulfur, cystine, methyl trisulfide and thiocystine. In addition, mercaptans were detected when the organism was grown in the presence of disulfides. Instability of several of the model compounds at the high incubation temperatures may have accounted for some of the observed transformations. P. furiosus produced small amounts of hydrogen sulfide when grown in the presence of coals given oxidative or perchloroethylene treatments. A continuous feed process at $98 \mathrm{C}$ was demonstrated with refuse coal containing over $1 \%$ by weight elemental sulfur. At a dilution rate of 0.25 per hour, coal sulfur decreased from 6.4 to $5.2 \%$.
National Bureau of Standards (NEL), Gaithersburg, MD. Applied and Computational Mathematics Div. Gas Analysis Modeling System. Final rept

R. P. O'Neill, W. G. Kurator, B. Mariner-Volpe, W. A Trapmann, and J. Heinkel. 1984, 19p

Pub. in Analytic Techniques for Energy Planning, p2652831984.

Keywords: "Natural gas, " $G a s$ pipelines, " $G$ as supply, "Energy models, Gas distribution, Reserves, Fuel consumption, Regulations, Mathematical models, Reprints, *Gas Analysis Modeling System.

The Gas Analysis Modeling System (GAMS) has been developed to provide EIA with the ability to study issues associated with natural gas supply and pipeline regulatory options. GAMS represents aggregates of the major United States pipelines, domestic supply sources and end uses as well as the corresponding regulations.

\section{0,885}

PB91-195339

Not available NTIS

National Bureau of Standards (IMSE), Gaithersburg, MD. Polymers Div.

Recent Progress in Coal Bioprocessing Research in the United States: Scope and Areas Needing Further Research.

Final rept.

G. J. Olson. $1988,7 p$

Pub. in Conservation and Recycling 1, n3-4 p319-325 Aug 88.

Keywords: *Coal preparation, Desulfurization, Microorganisms, Research management, Solubility, Reprints.

The paper summarizes the scope of recent coal bioprocessing research in the United States including desulfurization, solubilization, and other aspects of coal cleaning. Measurement and standards needs are discussed, areas of controversy or neglect which require additional research are also described.

\section{0,886}

PB91-203323

Not available NTIS

National Inst. of Standards and Technology (IMSE), Gaithersburg, MD. Polymers Div.

Biological Sulfur Oxidation and Reduction for Coal Sulfur Speciation and Desulfurization.

Final rept

R. M. Kelly, R. N. Schicho, S. H. Brown, J. P.

Soisson, I. I. Blumentals, G. J. Olson, and E. J.

Parks. 1989, 15p

Pub. in Proceedings of the Annual EPRI (Electric Power Research Institute) Conference (13th) on Fue Science and Conversion, Santa Clara, CA., May 18-19, 1988, p7-3-7-17 1989

Keywords: "Qualitative analysis, "Sulfur ions, "Coal, Desulfurization, "Bioassay, Biochemistry, Oxidation Reduction(Chemistry), Bacteria, Thiobacillus oxidans, Carbon sulfides, Microbiology, Reprints, Pyrococcus furiosus, Pyrodictium brockii, Speciation.

Sulfur speciation in coal is limited by uncertainties in the analytical methods used. Assays based on biological activity may eliminate some of these limitations. Using bacteria capable of elemental sulfur oxidation(Thiobacillus thiooxidans) or elemental sulfur reduction (Pyrococcus furiosus and Pyrodictium brockii), it was possible to determine the approximate levels of the sulfur species in four different coal samples. It was found that in three of the coal samples (lllinois No. 6, Indiana No. 5, and an Australian coal), only trace amounts of elemental sulfur could be detected by bioassay. However, an Indiana bog coal was found to have in excess of $1 \%$ (by weight) elemental sulfur content. Existing analytical methodology for coal sulfur speciation was shown to characterize the sulfur fraction as organic. In all cases, the results obtained by bioassay were reinforced through chemical analysis following CS2 extraction of the coals. Elemental sulfur levels in the Indiana bog coal could be determined by either oxidative or reductive microbial processing. The results obtained here suggest that bioassay can be used to screen coals for elemental sulfur content and may provide an alternate and complementary probe for sulfur speciation.

100,887

PB92-108927

PC A03/MF A01

National Inst. of Standards and Technology (NEL). Gaithersburg, MD. Center for Fire Research.
Short-Duration Autoignition Temperature Measurements for Hydrocarbon Fuels.

K. C. Smyth, and N. P. Bryner. Dec 90, 48p NISTIR4469

Contract JON-2104-3039

See also AD-778 998. Sponsored by Air Force Engineering and Services Center, Tyndall AFB, FL.

Keywords: "Autoignition, "Hydrocarbons, "Fuels Temperature measurement, Tests, Laboratory equip ment.

The autoignition of hydrocarbon fuels on hot surfaces is a process of practical significance which occurs when self-sustained combustion of a gas mixture is initiated. Possible prevention can be accomplished by choosing fuels of the proper molecular structure, by choosing the composition, coatings, or treatment of the hot surface, or by other intervention strategies. The goal of the present investigation is to devise a reliable method for measuring the autoignition behavior of hydrocarbon fuels in order to formulate appropriate strategies to reduce autoignition tendencies.

\section{Heating \& Cooling Systems}

100,888

PB91-13422

Not available NTIS

National Bureau of Standards (NEL), Gaithersburg, MD. Building Physics Div.

Method for Measuring Heat Loss from Underground Heat Distribution Systems.

Final rept.

T. Kusuda, J. B. Fang, and W. M. Ellis. 1987, 17p Pub. in Thermal Insulation: Materials and Systems, ASTM STP 922, p52.68 1987.

Keywords: "Heat loss, "Heat measurement, "Therma measurements, Pipes(Tubes), Distribution systems Heat transmission, Field tests, Heat transfer, Model tests, Reprints, "Heat distribution systems.

A simple procedure to obtain the in situ determination of heat loss from an underground heat distribution system is described. The proposed procedure for estimating the heat loss from underground heat distribution systems consists of making a series of temperature and thermal conductivity measurements on the ground grid in the vicinity of the pipe system and deducing the heat loss by statistically determining the parameters of a mathematical model using the gathered data. Verification of the procedure was tested in a simulated scale model of an underground system using an insulated sandbox. The scale model is a two-pipe system buried $0.3045 \mathrm{~m}$ (12 in.) below the top surface of a $0.66-\mathrm{m}(26$-in.)-wide, 0.71 -m(28-in.)-high, and 1.22 m(48-in.)-long sandbox. The test pipes are two electrically heated steel pipes having an outside diameter of $3.37 \mathrm{~cm}(1.325 \mathrm{in}$ ). The walls of the sandbox are insulated by $5.08-\mathrm{cm}(2-\mathrm{in}$.)thick expanded polystyrene board. The heat loss measurements from this simulated underground heat distribution system were obtained for pipe surface temperatures of $66 \mathrm{C}(150.9 \mathrm{~F})$ $93.8 \mathrm{C}(200.8 \mathrm{~F})$, and $149.2 \mathrm{C}(300.7 \mathrm{~F})$ for three different sand moisture contents (dry, $10 \%$, and $13 \%$ ). Relatively good agreement was obtained-between the measured electrical power input to the test pipes and the system heat loss estimated by the sand temperaure profile around the pipes and sand thermal conductivity measured by the thermal needle technique.

\section{0,889}

PB91-144386

PC A05/MF A01

National Inst. of Standards and Technology (NEL), Gaithersburg, MD. Center for Building Technology. Computer Program for Simulation of HVAC/Lighting Interactions: Initial Report.

G. N Walton. Dec 90, 99p NISTIR-4472

Sponsored by Department of Energy, Washington, DC., and Electric Power Research Inst., Palo Alto, CA.

Keywords: "HVAC systems, "Lighting systems, "Computerized simulation, "Buildings, Algorithms, Interac tions, Thermal measurements, Energy audits, HLITE computer program, National Institute of Standards and Technology.

The report describes the initial release of the HUITE program, which simulates the thermal interaction of lighting and HVAC systems. The program was developed to extend the results of an experimental study in 
HVAC/lighting interaction being conducted at the $\mathrm{Na}$ tional Institute of Standards and Technology (NIST). It will serve in planning future experimental test cases and in the development of algorithms that can be incorporated into larger building energy analysis programs. The interim report covers the first phase of the development of HLITE which simulates the NIST HVAC/lighting test facility. Future planned developments will expand its capabilities to larger facilities with more complete thermal interactions. The computer program is based on a simple combined explicit and implicit time integration scheme for a finite volume model which may be applicable to a much broader range of building simulations.

\section{0,890}

PB91-144410

PC A03/MF A01

National Inst. of Standards and Technology (NML), Boulder, CO. Chemical Engineering Science Div.

Effect of Noble Gas Mixtures on the Performance of Regenerative-Type Cryocoolers Analytical Estimate.

D. E. Daney. Sep 90, 38p NISTIR-90/3936

Sponsored by Air Force Space Technology Center, Kirtland AFB, NM.

Keywords: "Regenerative cooling, "Helium, "Krypton, *Argon, "Binary mixtures, "Heat transfer, Cryogenics, Prandtl number, Viscosity, Thermal conductivity, Performance evaluation, Graphs(Charts).

The authors compare the performance of regenerators that use noble gas mixtures to the performance of those that use pure helium gas. Both helium-argon and helium-krypton mixtures are investigated. For some heat transfer surfaces, a modest gain in heat transfer can be achieved with these mixtures. The concomitant increase in pressure drop, however, more than offsets the heat transfer gain so the net regenerator loss increases for all cases they evaluated. The dependence of heat transfer on Prandtl number $(\mathrm{Pr})$ has not been measured for the range associated with noble gas mix tures, $0.2<\operatorname{Pr}<0.5$, and they estimate that the un certainty from the source can exceed 20 percent. The authors give the estimates for the transport properties (Prandtl number, viscosity, and thermal conductivity) of helium-argon and helium-krypton mixtures because of the absence of experimental data at low temperature.

\section{0,891}

PB91-174516

Not available NTIS

National Bureau of Standards (NEL), Gaithersburg MD. Building Environment Div.

Research and Development of Heat Pumps Using Nonazeotropic Mixture Refrigerants.

Final rept.

J. M. Calm, and D. A. Didion. 1985, 8p

Sponsored by Electric Power Research Inst., Palo Alto, CA.

Pub. in ASHRAE (American Society of Heating, Refrigerating and Air-Conditioning Engineers) Technical Data Bulletin, v1 n9 p132-139 Jun 85.

Keywords: "Refrigerants, "Heat pumps, Air conditioning equipment, Cooling systems, Air conditioners, Research projects, Thermodynamic properties, Coolants Heating systems, Working fluids, Reprints, "Nonazeotropic mixtures.

Although nearly a century has passed since the use of multicomponent refrigerants was first proposed, such refrigerants have achieved only extremely limited application. Further research and development will be needed before widespread use of nonazeotropic mixture refrigerants will be commercially attractive. The paper summarizes a workshop conducted to identify these research and development needs.

\section{0,892}

\section{PB91-206706}

PC A04/MF A01

National Inst. of Standards and Technology (BFRL),

Gaithersburg, MD.

Measurement and Evaluation of Lighting/HVAC Interaction.

S. J. Treado, and J. W. Bean. May 91, 55p NISTIR4429

Keywords: "Lighting systems, "HVAC systems, "Cooling load, "Energy consumption, Temperature measurement, Energy conservation, Heat transfer, Heat transmission, Walls, Ventilation, Test facilities.

The interaction of building lighting and HVAC systems and the effects on cooling load and lighting system performance are being evaluated using a full-scale test facility at the National Institute of Standards and Tech- nology (NIST). The test facility and measurement methodology are described, along with sample test data and performance evaluation results. The implications of measurement uncertainty on results are discussed.

\subsection{3}

PB92-126432

PC A07/MF A02

National Inst. of Standards and Technology, Gaithersburg, MD.

Evaporator Performance Investigation for Residential Air-Conditioning Application Using Mixed Refrigerants.

M. Chwalowski. Nov 91, 148p NISTIR-4723

See also PB87-152286. Sponsored by Electric Power Research Inst., Palo Alto, CA. Nuclear Power Div.

Keywords: "Evaporators, "Air conditioning, "Reliability, Residential buildings, Heat exchangers, Refrigerants, Mixtures, Performance evaluation, Design criteria, Effectiveness, Profiles, Nonazeotropic.

The design of the heat exchanger utilizing nonazeotropic refrigerant in an air conditioning application presents unique problems due to the phase change of the moist air and the variable specific heat of the evaporating refrigerant mixture. The study discusses the performance analysis and the design procedure of a cross counterflow heat exchanger working as an evaporator in an experimental system which simulated a residential air conditioning application. The effect of the change of the mixture composition on heat exchanger performance was evaluated. The focus of the theoretical study was the development of the effectiveness/ Number of Transfer Units (NTU) (Exit/NTU) relationships with the use of the experimentally derived quantities for moist air flowing across the heat exchanger

\section{0,894}

PB92-126440

PC A04/MF A01

National Inst. of Standards and Technology, Gaithersburg, MD.

Testing and Rating of an Atmospheric, Gas-Fired Furnace Equipped with a Burner Air Inlet Damper. S. T. Liu, G. E. Kelly, and C. P. Terlizzi. Nov 91, 61p NISTIR-4717

See also PB-289 484. Sponsored by Department of Energy, Washington, DC. Office of Conservation and Renewable Energy.

Keywords: "Gas furnaces, Air flow, Heat exchanger Draft control systems, Energy consumption, Trace techniques, Tests, Flue gases, Gas burners, "Burner box inlet dampers, Atmospheric gas furnaces, Flue dampers, Draft diverters.

An atmospheric furnace with an integral draft diverter and an electro-mechanical burner box inlet damper was tested by the tracer gas method for the development of a test procedure. Tracer gas tests were conducted under two conditions: with the diverter open and with the diverter sealed and the stack restricted. Test results indicated that the flue gas flow pattern inside the heat exchanger were different for the two conditions. There was reverse flow in one of the clam shells when the diverter was open, but no flow reversa when the diverter was sealed. The off-cycle sensible loss which was a measure of the effectiveness of the burner box inlet damper gave similar value for both conditions. Because of the change in flow pattern and the fact that the furnace normally operated in the field with the diverter open, a recommended test procedure was developed which requires that the tracer gas test should be conducted with the diverter open. A calculation procedure was developed to compute the annual fuel utilization efficiency for the type of furnaces that employ a burner box inlet damper or flue damper for off-cycle loss reduction.

\section{Policies, Regulations \& Studies}

100,895

PB91-147728

Not available NTIS

National Bureau of Standards (NEL), Gaithersburg, MD. Office of Energy-Related Inventions,

Feasibility of Using Knowledge-Based Systems for Aiding Inventors.

Final rept.

J. L. Merchant. 1986, 14p

Pub. in Proceedings of the Annual Meeting of the Technology Transfer Society (11th), Indianapolis, IN., June 23-26, 1986, p1-12.
Keywords: "Research management, "Expert systems, Research projects, Knowledge bases(Artificial intelligence), Inventions, Computers, Computer systems programs, Computer applications, Reprints, "ERIP(Energy-Related Inventions Program), Department of Energy.

The Energy-Related Inventions Program (ERIP) is a small program whose purpose is to provide support to independent inventors and small businesses with promising energy-related inventions and to move these inventions closer to the marketplace. The $\mathrm{Na}$ tional Bureau of Standards (NBS) provides, at no cost to the inventor, evaluations of energy-related inventions and recommends those it considers promising to the Department of Energy (DOE). In turn, DOE provides financial support and/or help in marketing an inventor's idea. The objective of the task is to estimate the potential for use of computers to assist inventors in the development of their ideas or to provide synergism for the development of new ideas. The paper will explore the current state of knowledge-based systems and other computer software as they could relate to invention, and recommend ERIP actions that would lead to realization of their potential.

\section{0,896}

PB91-158659

Not available NTIS

National Bureau of Standards (NEL), Gaithersburg, MD. Office of Energy-Related Inventions.

Evaluation of Building Systems Inventions.

Final rept.

J. S. Dhillon, and T. A. Coultas. 1986, 4p

Pub. in Proceedings of Conference on Energy: An Integrated Approach, Chattanooga, TN., April 30-May 2, 1986, p97-100.

Keywords: "Grants, "Inventions, "Buildings, Meetings, Energy conservation, Research management, US NBS, US DOE, Reprints.

One part of the United States Federal Nonnuclear Energy Research and Development Act of 1974 directs the National Bureau of Standards (NBS) to evaluate all promising nonnuclear energy-related inventions for the purpose of obtaining direct grants for their development from the Department of Energy (DOE). The program provides an opportunity for independent inventors and small businesses to obtain Federal assistance in developing and commercializing their inventions.

100,897

PB91-167288

PC A03/MF A01

National Inst. of Standards and Technology (CAML), Gaithersburg, MD.

DISCOUNT-A Program for Discounting Computations in Life-Cycle Cost Analyses. User's Guide and Reference Manual.

Final rept.

S. R. Petersen. Jan 91, 22p NISTIR-4513

Prepared in cooperation with Federal Energy Management Program, Washington, DC

Keywords: "Energy conservation, "Energy sources, "Life cycle costs, "Cost analysis, Economic analysis, Projects, Buildings, Value, Discounted cash flow, Interest rate, Present worth, Return on investment, User manuals(Computer programs), DISCOUNT computer program.

The DISCOUNT computer program has been prepared as an aid to implementing life-cycle cost evaluations of potential energy conservation and renewable energy projects in new and existing Federal buildings. It can also be used for evaluating similar projects in the private sector and at the state and local government levels. The DISCOUNT program computes discount factors and related present values, future values, and periodic payment values of cash flows occurring at known points in time. DISCOUNT computations are especially useful in life-cycle cost analysis, financial analysis, and engineering-economics problems not requiring comprehensive summation and reporting. DISCOUNT performs all of the functions of standard discounting tables, computing present values of future amounts, future values of present amounts, present and future values of periodic payments, and periodic payments corresponding to present and future amounts. In addition. DISCOUNT computes the present value of periodic payments which increase at known rates over time, and the present value of energy costs which increase at rates projected by the U.S. Department of Energy for use in Federal life-cycle cost analyses. DISCOUNT provides the added flexibil- 
ity of accepting non-integer discount rates, time periods, and escalation rates in its computations. DIS COUNT runs on most IBM PC and compatible micro computers with no special equipment requirements.

100,898

PB9 1-507970

CP D02

National Inst. of Standards and Technology (CAML) Gaithersburg, MD. Office of Applied Economics.

Buildlng Life Cycle Cost Computer Program, Version 3.2 (for Microcomputers).

Software.

Jul 91, 2 diskettes NIST/SW/DK-91/013

Supersedes PB90-501206 and PB90-501198.

The software is on two $360 \mathrm{~K}, 51 / 4$ inch diskettes, double density. Documentation included; may be or dered separately as PB91-159764.

Keywords: "Software, "Buildings, "Life cycle costs "Economic analysis, Operating costs, Benefit cos analysis, Energy conservation, Savings, Return on in vestment, Cash flow, Project management, Diskettes, Savings-to-investment ratio.

Building Life Cycle Cost (BLCC) Computer Program 3.0 is an economic analysis software package for evaluating investments in buildings and building systems which reduce their long-term operating costs. It is especially useful for evaluating the costs and savings of energy conservation projects in buildings. BLCC computes life-cycle costs, savings-to-investment ratio, net savings, internal rate of return, and cash flow analysis for project alternatives. It is appropriate for Federal and private sector use, as well as for state and local governments. BLCC runs on most IBM-PC and com patible microcomputers, with or without a hard disk. No special computer hardware or graphics capabilities are needed.

\section{0,899}

PB92-112515

PC A04/MF A01

National Inst. of Standards and Technology (NEL) Gaithersburg, MD. Center for Computing and Applied Mathematics.

Energy Prices and Discount Factors for Llfe-Cycle Cost Analysis 1992. Annual Supplement to NIST Handbook 135 and NBS Special Publication 709.

B. C. Lippiatt. Oct 91, 63p NISTIR-85/3273-6

See also report for 1991, PB91-113613. Sponsored by Department of Energy, Washington, DC. Federa Energy Management Program Staff.

Keywords: "Cost analysis, "Prices, "Life cycle costs, Fuels, Residential buildings, National government, Electric appliances, Economic analysis, Energy conservation, Energy supplies, Tables(Data), "Federal Energy Management Program.

The report is the 1992 annual edition of energy prices and discount factors for performing life-cycle cost analyses of energy conservation and renewable energy projects. It supports the Federal life-cycle costing methodology by updating the energy price projections and descount factors that are described, ex plained, and illustrated in NIST Handbook 135 (HB 135). It supports private-sector life-cycle cost analysis by updating the energy price indices that are de scribed, explained, and illustrated in NBS Special Publication 709 (SP 709). It also supports the Energy Conservation Mandatory Performance Standards for New Federal Residential Buildings (10 CFR 435) by providing a table of factors for updating appliance labe values.

\section{Reserves}

100,900

N91-20219/2

(Order as N91-20207/7, PC A05/MF A01) National Bureau of Standards (IMSE), Gaithersburg, MD.

Kelvin Water-Drop Experiment.

R. D. Shull. Jan $90,3 p$

In NASA, Langley Research Center, National Educators' Workshop: Update 1988. Standard Experiments in Engineering Materials Science and Technology $P$ $51-53$.

Keywords: "Electric potential, Experimentation, "Procedures, "Water, Copper, Electrons, Metal plates, Students.
This experiment was originally designed and performed by Lord Kelvin (William Thomson) in the late 1800 's to demonstrate the creation of an electric potential simply by means of dividing up a body of flowing water. The objective is to demonstrate the power of electrical forces in a material as common as water and to help teach the student that even simple, well understood phenomena sometimes present unexpected results that, at first thought, defeat explanation. The experimental equipment and procedure are explained.

\section{Solar Energy}

100,901

PB91-159152

Not available NTIS

National Bureau of Standards (NEL), Gaithersburg MD. Building Equipment Div.

Method for Characterizing the Thermal Performance of a Solar Storage Wall from Measured Data. Final rept.

J. Seem, and E. Hancock. 1985, 12p

Sponsored by Department of Energy, Washington, DC. Passive and Hybrid Solar Energy Div.

Pub in Proceedings of ASHRAE/DOE/BTECC Conference on Thermal Performance of the Exterior Envelopes of Buildings III, Clearwater Beach, FL., December 2-5, 1985, p1304-1315.

Keywords: "Heat transfer, "Thermal energy storage systems, "Solar energy, "Walls, Regression analysis, Least squares method, Calorimeters, Heat flux, Sola architecture, Heat transmission, Construction materials, Reprints.

A technique is presented for characterizing the dynamic performance of a thermal-storage wall based on the data obtained from a series of temperature and heat flux measurements. It is shown that the coefficients of a transfer function model can be estimated directly from data using linear least squares regression. Data from the National Bureau of Standards (NBS) Passive Solar Component Calorimeter are used to demon strate that the technique can be successfully applied. The transfer function techniques are potentially applicable to linear systems with time-invariant properties. It is also shown that a very simple set of parameters can be derived from the transfer function coefficients to characterize the steady-state performance. Only one parameter for each system input is required to predict long-term average thermal performance of the component.

\section{General}

\section{2}

PB91-178871

PC A09/MF A01

National Inst. of Standards and Technology, Gaithersburg, MD. Office of Energy-Related Inventions.

Energy Related Inventions Program: A Joint Program of the Department of Energy and the National Institute of Standards and Technology. Status Report for Recommendations 1 through 250.

Mar $91,179 \mathrm{p}$ NISTIR-4533

Supersedes PB90-225988.

Keywords: "Inventions, "US NIST, "US DOE, Recommendations, Indexes(Documentation).

A brief description of the Energy-Related Inventions Program and all inventions recommended by the $\mathrm{Na}$ tional Institute of Standards and Technology to the Department of Energy since the inception of the program, including a brief summary of the current status of each.

\section{0,903}

PB91-184770

National Inst. of Standards and Technology, Gaithersburg, MD. Office of Energy-Related Inventions.

Energy Related Inventions Program. A Joint Program of the Department of Energy and the National Institute of Standards and Technology. Status Report for Recommendations 251 through 523. Mar 91, 298p NISTIR-4534 Supersedes PB90-221813.

Keywords: *Inventions, Technology innovation, Product development, Recommendations, Evaluation,
Grants, US DOE, "Energy related inventions program, US NIST.

The Energy-Related Inventions Program was estab lished in 1975. Since its inception over 27,000 inventions have been evaluated. As of the printing of the report, 523 have been recommended to the Department of Energy. The report supercedes NISTIR 4313 and summarizes the status of Inventions 251 through 523. A companion report (NISTIR 4533) summarizes recommended inventions 1 through 250 .

ENVIRONMENTAL POLLUTION \& CONTROL

\section{Air Pollution \& Control}

\section{0,904}

PB91-158741

Not available NTIS

National Bureau of Standards (NML), Gaithersburg. MD. Chemical Thermodynamics Div.

Thermodynamic Data for Modeling of Flue Gas Cleanup Systems (Abstract for Poster Session). Interim rept. Sep 86-Jun 87

D. Garvin, M. W. Chase, and S. Abramowitz. 1987,

3p

-Al22-83PC60425

Sponsored by Department of Energy, Pittsburgh, PA Pittsburgh Energy Technology Center.

Pub. in Proceedings of Coal Utilization and Environmental Control Contractors' Review Meeting, Gaithersburg, MD., July 7-9, 1987, 3p.

Keywords: "Sulfur dioxide, "Nitrogen oxides, "Flue gases, Thermochemistry, Air pollution control, Stationary sources, Abstracts, Data, Reprints.

A thermodynamic data system has been designed for use with flue gas control systems. Values of thermodynamic properties have been selected for the major species in SOx-NOx systems. For each selected value a reliability is given together with documentation of the source of the value. Data are given for chemical proc esses in the gas, solid and solution phases. Both tabular and graphical presentations are used. A new analysis has been made of the solubility of $\mathrm{SO} 2$ and its ionization in aqueous solution.

100,905

PB91-167353

PC A03/MF A01

National Inst of Standards and Technology (BFRL) Gaithersburg, MD.

Proposed Standard Practice for Assessing the Performance of Gas-Phase Air Cleaning Equipment.

S. Silberstein. Mar 91, 23p NISTIR-4523

Keywords: "Indoor air pollution, "Air cleaners, "Air pollution control equipment, "Standards, Air flow, Activated carbon, Service life, Air filters, Performance, Contaminants, Airborne wastes.

The proposed standard practice provides a genera and flexible laboratory method for assessing the performance of equipment for controlling indoor concentrations of gas-phase air contaminants. Using a canis ter filled with adsorption media, a profile of breakthrough concentration over time is obtained during each test conducted at a fixed contaminant challenge concentration. Results of tests performed for different contaminants and different challenge concentrations can be used for estimating the useful life of air cleaning equipment, and for comparing equipment. The information will be useful to the engineer for the design and selection of such equipment.

100,906

PB91-175539

Not available NTIS

National Bureau of Standards (NML), Gaithersburg MD. Molecular Spectroscopy Div. 


\section{Air Pollution \& Control}

Infrared Spectroscopy Applied to Atmospheric Chemistry.

Final rept.

A. G. Maki. $1985,7 p$

Pub. in Proceedings of CMA/NBS Workshop on Atmospheric Spectra, Gaithersburg, MD., November 3-4, 1983, p1-7 1985.

Keywords: "Atmospheric composition, "Infrared spectroscopy, Absorption spectra, Trace amounts, High resolution, Monitoring, Detection, Reprints.

The requirements of laboratory infrared spectroscopy for applications to detection and monitoring of molecular species present in trace amounts in the earth's atmosphere are reviewed. A critical evaluation is presented of the problems encountered in accurate determination of the frequencies, intensities, and line shapes of the absorption features. Problems encountered with transient, unstable species and the effect of perturbations on the intensities are discussed.

\section{0,907}

PB91-187146

Not available NTIS National Inst. of Standards and Technology (NEL), Gaithersburg, MD. Building Environment Div.

Multi-Zone Dispersal Analysis by Element Assem. bly.

Final rept

J. W. Axley. 1989, 18p

Sponsored by Department of Energy, Washington, DC., Environmental Protection Agency, Washington, DC., and Consumer Product Safety Commission, Washington, DC.

Pub. in Building and Environment 24, n2 p113-130 1989.

Keywords: "Indoor air quality, "Transport properties, "Flow models, Particulates, Mass flow, Air pollution, Mathematical models, Computerized simulation, Buildings, Air flow, Reprints.

An element-assembly formulation of multi-zone contaminant dispersal theory that is not limited to the wellmixed zone idealization is described. In the approach flow systems are idealized as assemblages of elements that model specific instances on contaminant mass transport within a system. A general form and specific examples of element equations are presented. The process of assembling the element equations to form system equations and the qualitative character of the resulting system equations is discussed. Solutions options are outlined, examples of application are presented, and one implementation of the theory, the CONTAM family of programs developed at NBS, is briefly described.

\section{0,908}

PB91-195347

Not available NTIS

National Bureau of Standards (NML), Gaithersburg,

MD. Inorganic Analytical Research Div.

Development of a New Tracer Technology Using Enriched Rare-Earth Isotopes.

Final rept.

J. M. Ondov, and W. R. Kelly. 1988, $1 p$

Pub. in Abstracts of Papers of the American Chemical Society 195, p84 Jun 88.

Keywords: "Isotopic labeling, "Rare earth elements, "Fly ash, "Flue gases, "Electric power plants, "Neodymium 148, Technology assessment, Field tests, Coal combustion, Design, Stationary sources, Deposition, Tracer techniques, Reprints.

Under funding from the Electric Power Research Institute, the authors are developing a new particulate tracer technology suitable for use in power plant studies using enriched rare-earth (RE) isotopes and advanced tracer delivery techniques. Highly enriched $R E$ isotopes are nontoxic, chemically and radiologically stable, and are available at modest cost. The relative abundances of most RE isotopes are invariant in nature and can be measured with great precision by thermal ionization mass spectrometry. Since there are no industrial sources of enriched RE isotopes, they can be used as definitive tracers. Design calculations suggest that signal-to-noise (S:N) ratios > 500:1 could be achieved in ambient aerosols 100-km downwind of a $425-M W(e)$ power plant with a release rate of only $126 \mathrm{mg}(148) \mathrm{Nd} / \mathrm{hr}$. Error analyses suggest that the technique offers $a>40,000$-fold advantage over previously proposed whole-element tracer schemes. Results of limited field tests suggest that $\mathrm{S}: \mathrm{N}$ ratios adequate for detection at $100 \mathrm{~km}$ could be achieved in ambient aerosol particles less than or equal to $10 \mathrm{mi}-$ crometers from a $100 \mathrm{MW}(\mathrm{e})$ coalfired power plant with a release rate as little as $13 \mathrm{mg} / \mathrm{hr}$. The $S: N$ ratios of submicrometer particles are enormous and should permit detection over $1000-\mathrm{km}$ distances. The tests further suggest that fly ash particles can be effectively 'tagged' by coagulation with fine residue particles made with simple two-fluid atomizers operated at elevated pressure.

\section{0,909}

PB91-195420

Not available NTIS National Bureau of Standards (NEL), Gaithersburg, MD. Building Environment Div.

Air Exchange and Pollutant Concentration Measurements in Two Office Buildings.

Final rept.

A. Persily, W. S. Dols, and R. Grot. $1988,10 p$

Sponsored by Geological Survey, Reston, VA., and

General Services Administration, Washington, DC.

Pub. in Proceedings of CIB Conference Health Buildings ' 88 , Stockholm, Sweden, September 1988, p591-
600 .

Keywords: "Air pollution monitoring, "Commercial buildings, "Carbon monoxide, "Carbon dioxide, "Formaldehyde, "Radon, "Indoor air pollution, "Air circulation, Air quality, Air flow, Ventilation, Volatile organic compounds, Garages, Tracer techniques, Office buildings, Reprints, Tracer gas analysis.

Long term measurements of air exchange rates and indoor pollutant concentrations were made in two, mechanically ventilated office buildings. The measurements employed automatic tracer gas measurement systems for the continuous monitoring of air exchange rates. Automated measurements of carbon monoxide and dioxide were made concurrently. Passive monitors were employed to measure concentrations of radon and formaldehyde. Measurements were also made of particulate concentrations and for the identification of volatile organic compounds. The first building had a history of indoor air quality and thermal comfort complaints, but the air exchange rates were very high and no excessive pollutant levels were identified. Problems of intake air quality did occur periodically. The second building is recently constructed and has had few indoor air quality problems. Airflow from the underground parking garage into the office space has led to elevated carbon monoxide levels at times.

100,910

PB91-206722

PC A07/MF A01

National Inst. of Standards and Technology (BFRL), Gaithersburg, MD.

User Manual NBSAVIS CONTAM88. A User Interface for Air Movement and Contaminant Dispersal Analysis in Multizone Buildings.

R. A. Grot. Jun 91,150 p NISTIR-4585

Sponsored by Department of Energy, Washington, DC. Building Services Div., Consumer Product Safety Commission, Washington, DC. Directorate of Engineering Science, and Environmental Protection Agency, Research Triangle Park, NC. Atmospheric Research and Exposure Assessment Lab.

Keywords: User manuals(Computer programs), "Indoor air quality, "Air flow, Buildings, Indoor air pollution, Ventilation, Steady state, Air quality, Contaminants, Leakage, Environmental quality, CONTAM 88 computer program.

The manual describes the usage of three computer programs for analyzing the air movement and indoor air quality in multizone buildings. The first program NBSAVIS creates and edits a building description and generates the leakage, fan and contaminant data necessary to predict the air infiltration and internal air movement in a building and perform an indoor air quality analysis. These data are used by the program CONTAM88 which calculates the air flows and both dynamic and steady state levels of indoor contaminants. CONTAMEZ also produces an output file which can serve as the input for the NIST programs CONTAM86 and CONTAM87 developed by Dr. James Axley.

\section{1}

PB92-116805

Not available NTIS

National Inst. of Standards and Technology (NML), Gaithersburg, MD. Gas and Particulate Science Div. New Way to Mount Particulate Material for Laser Microprobe Mass Analysls.

Final rept.

R. A. Fletcher. $1989,4 p$

Pub. in Analytical Chemistry 61, n8 p914-917 1989.
Keywords: "Particulates, "Carbon, "Sample preparation, Quartz, Fibers, Aerosols, Air pollution, Mass spectroscopy, Reprints, Laser microprobe.

A new particle mounting sample preparation technique for the Laser Microprobe Mass Analyzer is presented. The substrate is high purity quartz fiber material conventionally used to collect airborne particulate matter. Advantages to using quartz fiber are low contamination, ease of sample preparation, and low background due to the transparency and high melting point of the quartz.

\section{0,912}

\section{PB92-126630}

(Order as PB92-126614, PC A06/MF A02) National Inst. of Standards and Technology, Gaithers burg, MD.

Accurate and Precise Coulometric Determination of Sulfur Dioxide in Compressed Gas Mixtures.

G. D. Mitchell, and A. A. Bell. 1991, 6p

Included in Jnl. of Research of the National Institute of Standards and Technology, v96 n5 p541-546 Sep/Oct 91.

Keywords: "Voltametry, "Air pollution detection "Chemical analysis, "Sulfur dioxide, Acid rain, Gas cylinders, Compressed air, Binary mixtures, Performance evaluation, Standards, Electrochemistry, Comparison, Concentration(Composition), "Standard reference materials.

Sulfur dioxide (SO2) in the atmosphere is a common pollutant and is a major contributor to the formation of acid rain. Accurate and precise determinations of SO2 in the atmosphere are essential to determine the mag nitude of the problem. Reference gas mixtures such as NIST SRMs are an important part of the measuremen procedure. Coulometry has been established as an im portant and reliable method for the determination of acidic compounds. The analytical method and simple apparatus described here are applied to the precise and accurate determination of sulfur dioxide in nitrogen, specifically in compressed gas cylinders at nominal concentrations of 50 and $100 \mathrm{micromol} / \mathrm{mol}(\mathrm{ppm})$ The method is constant current coulometry where the magnitude of the current is set by the balance between the electrochemical generation of $\mathrm{OH}(-)$, the flow of $\mathrm{SO} 2$, and the chemical reaction of the solution. The method is direct, rapid, and can be refined further to provide analysis at the nanomol/mol level.

\section{0,913}

\section{PB92-126648}

(Order as PB92-126614, PC A06/MF A02) National Inst. of Standards and Technology, Gaithersburg, MD.

Development of a Coulometric Method for Assessing the Concentration of Ambient Levels of $\mathrm{CO} 2 /$ Air in Compressed Gas Mixtures.

G. D. Mitchell, and A. A. Bell. 1991, 4p

Included in Jnl. of Research of the National Institute of Standards and Technology, v96 n5 p547-550 Sep/Oct 91.

Keywords: "Air pollution detection, "Chemical analy sis, "Carbon dioxide, "Voltametry, Concentration(Composition), Greenhouse effect, Comparison, Standards, Performance evaluation Nondispersive infrared spectroscopy, Experimental design, Binary mixtures, Compressed air, Gas cylinders, "Standard reference materials.

The understanding of global 'greenhouse' issues as they relate to $\mathrm{CO} 2$ in the atmosphere is a current environmental concern. At the National Institute of Standards and Technology there is a continuous search fo methods of analysis that yield results that are traceable to fundamental quantities. The coulometric method presented here is a reliable method for the direct analysis of $\mathrm{CO} 2 /$ air cylinder gas mixtures. It is based on Faraday's laws of electrolysis and therefore no external standardization is required. A series of $\mathrm{CO} 2 /$ air cylinder gas mixtures ranging in concentration from 300 to $375 \mathrm{micromol} / \mathrm{mol}(\mathrm{ppm})$ were analyzed and the results compared to those results obtained by non-dispersive infrared (NDIR) analysis with traceability to gravimetric standards. The coulometric method is rapid, sensitive, precise, and with the proper experimental controls, will yield accurate results. 


\section{Environmental Health \& Safety}

\section{0,914}

PB92-116359

PC A07/MF A02

National Inst of Standards and Technology (MEL) Gaithersburg, MD.

Response of Personal Noise Dosimeters to Continuous and Impulse-Like Signals.

Technical note (Final).

D. J. Evans, D. R. Flynn, V. Nedzelnitsky, and E. D.

Burnett. Jun $91,148 \mathrm{p}$ NIST/TN-1286

Also available from Supt. of Docs. as $\mathrm{SNO03}^{-003}$

03116-0. See also PB80-176084 and PB86-109139.

Sponsored by Occupational Safety and Health Administration, Washington, DC.

Keywords: "Personnel dosimetry, "Dosimeters, "Noise meters, "Occupational safety and health "Sound level meters, Occupational exposure, Regulations, Law enforcement, Design criteria, Performance evaluation, Continuous noise, Pulses, Signal-to-noise ratio, Standards, Calibrating, Acoustic detection, Sound intensity.

A study of the capabilities of noise dosimeters to measure personal exposure to time-varying and im pulse-like noises was carried out. Ten commercial noise dosimeters were obtained. A laboratory reference noise dosimeter was constructed to provide a demonstrably accurate basis with which to compare the commercial noise dosimeters. Each commercial dosimeter, when ordered from the manufacturer, was specified to have: (1) a threshold A-weighted sound level of $80 \mathrm{~dB}$, (2) a criterion sound level of $90 \mathrm{~dB}$, and (3) an exchange rate of $5 \mathrm{~dB}$ and/or $3 \mathrm{~dB}$. The performance of the commercial dosimeters was compared with theory and with results obtained from the reference dosimeter. Except in a few isolated cases, the commercial dosimeters were in general agreemen with the performance specification of the appropriate American National Standard and with OSHA regulations.

\section{Solid Wastes Pollution \& Control}

100,915

\section{DE91018580}

PC A09/MF A02

Institute of Paper Science and Technology, Atlanta,

GA. Chemical Recovery Group.

Fundamental studies of black liquor combustion. Report No. 4, Phases, 2, 3, and 4: Final report, December 1987--December 1989.

Progress rept.

D. T. Clay, S. J. Lien, T. M. Grace, C. A. Brown, and

H. L. Empie. Mar 90, 190p DOE/CE/40637-T9

Contract AC02-83CE 40637

Sponsored by Department of Energy, Washington, DC.

Keywords: "Black Liquids, "Paper Industry, Boilers, Cogeneration, Combustion Properties, Energy Conservation, Energy Recovery, Furnaces, Mathematical Models, Progress Report, Waste Product Utilization $\mathrm{EDB} / 320303, \mathrm{EDB} / 320304$

The fundamentals of black liquor combustion are being studied in a project being carried out for the US Department of Energy by the Institute of Paper Science \& Technology (IPST, formerly the Institute of Paper Chemistry) and the National Institute of Science \& Technology (NIST, formerly the National Bureau of Standards). The project was divided into four phases. This report covers the completion of Phase 1 (in-flight processes), the results of all of the work on Phase 2 (char bed processes), Phase 3 (fume processes), and Phase 4 (furnace simulation). 41 refs., 62 figs., 30 tabs.

100,916

PB91-147264 Not available NTIS National Bureau of Standards (NML), Gaithersburg, MD. Chemical Thermodynamics Div.

NBS Research Program in Municipal Solid Waste Combustion.

Final rept. 1 Oct 85-30 Jun 86.

E. S. Domalski. $1986,28 p$

Sponsored by Department of Energy, Washington, DC. Biofuels and Municipal Waste Technology Div.

Pub. in Proceedings of Biofuels and Municipal Waste

Technology Division Management Review Meeting.

Washington, DC., July 29-30, 1986, 28p.
Keywords: "Solid wastes, "Combustion, "Research projects, Free radicals, Calorimeters, Reaction kinetics, Thermodynamics, Solid waste disposal.

Research activities conducted at NBS for DOE are summarized covering the period 1 October 1985 to 30 June 1986. Major accomplishments consist of: (1) reporting of research results (8 publications), (2) describing modifications to the calorimeter's combustor, (3) studying reaction kinetics using $\mathrm{H}$ atoms and $\mathrm{OH}$ radicals as reactants, and (4) conducting combustion measurement which more closely simulate field conditions of real-world incinerators.

100,917

PB91-174631

Not available NTIS

National Bureau of Standards (NML), Gaithersburg. MD. Chemical Thermodynamics Div.

Monitoring the Fate of Chlorine from MSW Sampling through Combustion.

Final rept.

E. S. Domalski, K. L. Churney, A. E. Ledford, S. S. Bruce, T. J. Buckley, R. M. Parris, and S. N. Chesler. 1986, $14 p$

Pub. in Proceedings of Municipal Solid Waste as a Utiity Fuel, Madison, WI., November 20-22, 1985, p16-1. 16-14 1986.

Keywords: "Chlorine, "Solid wastes, "Municipal wastes, Chemical analysis, Combustion, Field tests, Monitoring, Reprints, Baltimore County(Maryland), Brooklyn(New York).

The total chlorine and water soluble chlorine contents of the components of municipal solid waste (MSW) have been determined from sampling studies carried out at two sites, Baltimore County, MD, and Brooklyn. $\mathrm{NY}$, for a five-day period. The total chlorine contents of the MSW samples from Baltimore County, MD, and Brooklyn, NY, are 0.45 and 0.89 mass \%, respectively.

\section{Water Pollution \& Control}

100,918

\section{PB91-195040}

Not available NTIS

National Inst. of Standards and Technology (IMSE), Gaithersburg, MD. Polymers Div.

Simultaneous Butyltin Determinations in the Microlayer, Water Column and Sediment of a Northern Chesapeake Bay Marina and Receiving System.

Final rept.

C. L. Matthias S. J. Bushong, L. W. Hall, J. M.

Bellama, and F. E. Brinckman. 1988, 6p

Pub. in Appl. Organomet. Chem. 2, n6 p547-552 1988.

Keywords: "Chesapeake Bay, "Water pollution, "Metal alkyl compounds, Sediments, Estuaries, Marinas, Antifouling coatings, Toxic substances, Biodeterioration, Reprints, "Tributyltins, Microlayers.

Butyltins were determined in the microlayer, water column and sediment of a northern Chesapeake Bay marina and its receiving system. Concentrations of the toxicant species tributyltin (TBT) ranged from 60 to $4130 \mathrm{ng} / \mathrm{L}$ in the microlayer from 34 to $367 \mathrm{ng} / \mathrm{L}$ in the water column and from 0.05 to 1.4 microgram $/ \mathrm{g}$ (dry weight) in sediment. TBT concentrations in all three environmental compartments were higher in the marinas than in the receiving system. Concentrations of TBT in the micro-layer and water column of the study area were potentially toxic to sensitive aquatic biota. The microlayer appears to be depleted in dibutyltin relative to both water column and sediment, suggesting that the rate of stepwise dealkylation of dibutyltin is faster in the microlayer than in the other compartments studied.

\section{General}

100,919

\section{PB91-189761}

Not available NTIS

National Inst. of Standards and Technology (NEL). Gaithersburg, MD. Statistical Engineering Div.
Replicate Measurements in the Interpretation of Environmental Monitoring.

Final rept.

W. S. Liggett. 1990, 7p

Pub. in Environmental Monitoring, Restoration, and Assessment (What Have We Learned), p249-255 1990

Keywords: "Environmental monitoring, "Replicating, "Measurement, Errors, Probability density functions, Estimating, Analysis of variance, Sampling, Reprints.

In simultaneous monitoring at several sites, replicate samples and measurements, when chosen properly, provide a useful and unambiguous basis for data interpretation. Simultaneous monitoring permits calculation of indices of environmental change that are not influenced by weather changes. When the small changes measured by such an index are of interest, comparison of the index with the sampling and measurement erro is appropriate. This comparison is made possible by the replicates. The familiar two-way table obtained by sampling a set of locations at several times is also considered. The indices considered are ones that perform well when large errors are more frequent than predicted by the normal distribution. Through the use of replicates, the variation in such indices caused by erro alone can be assessed and thus environmental change is determined.

INDUSTRIAL \& MECHANICAL. ENGINEERING

\section{Hydraulic \& Pneumatic Equipment}

\section{0,920}

PB91-134411

Not available NTIS

National Bureau of Standards (NEL), Gaithersburg. MD. Chemical Process Metrology Div.

Flowmeter Installation Effects: A New Approach to an Old but Prevalent Problem.

Final rept.

G. E. Mattingly, and T. T. Yeh. 1986, 22p

See also PB89-132856.

Pub. in Proceedings of International Conference on Flow Measurement in the Mid 80's, East Kilbride, Glasgow, June 9-12, 1986, p1-22.

Keywords: "Pipe flow, "Flow measurement, "Flow visualization. Flowmeters Velocity measurement, Fluid flow, Turbulent flow, Pipes(Tubes), Reprints, Laser doppler velocimeters, Velocity distribution.

For a selected piping configuration, elbows-out-ofplane, preliminary results are presented for laser Doppler velocimetry (LDV) profiles taken in the downstream piping near tis configuration. These results quantify pipeflow profiles for both time-averaged and turbulent-velocity components as well as swirl-angle and power-spectral density distributions. It is planned that these experiments will describe the pipeflows downstream of this configuration. These results are also intended to provide validation data for computer modelling studies of this flow. It is also planned that future phases of this research include pipeflows from other prevalently encountered piping configurations. Additionally, these phases can also focus on the benefit-cost aspects (capital and pressure loss) of flow-conditioning elements.

\section{0,921}

PB91-134445

Not available NTIS

National Inst. of Standards and Technology (NML) Boulder, CO. Chemical Engineering Science Div.

Effect of the Location of an In-Line Tube Bundle on Orifice Flowmeter Performance.

Final rept.

S. E. MCFaddin, C. F. Sindi, and J. A. Brennan. $1989,6 p$

Contract GRI-5081-353-0422

Sponsored by Gas Research Inst., Chicago, IL.

Pub. in Flow Meas. Instrum. 1, p9-14 Oct 89. 
Keywords: "Orifice meters, "Flowmeters, "Orifice flow, Finishes, Flow measurement, Fluid flow, Surface properties, Reprints, "Rod bundles, Discharge coefficient.

The Gas Research Institute has sponsored many significant flow measurement studies at the National Institute of Standards and Technology. The main thrust of the 1988 research project was to determine the effect of the location of an in-line tube bundle flow conditioner on the orifice discharge coefficient. An in-line tube bundle was placed at four locations-7, 12, 17, and 27 pipe diameters upstream of the orifice plate in a $100 \mathrm{~mm}$ (4 inch) orifice meter. The research has determined that if the in-line tube bundle is placed at 7 pipe diameters, the minimum distance specified in the flow measurement standards for a 0.75 beta ratio plate, as much as $1 \%$ error in the discharge coefficient occurs. At 17 pipe diameters, the discharge coefficients of all the beta ratios are statistically the same as would be determined in 'ideal' conditions. These new experimental data will help to improve the current flow measurement standards and suggest the direction of future research.

\section{0,922}

\section{PB91-147108}

Not available NTIS National Inst. of Standards and Technology (NML), Gaithersburg, MD. Center for Chemical Technology. Stabilization Techniques for Spinning Rotor Gage Residual Drag.

Final rept.

S. H. Choi, S. Dittmann, and C. R. Tilford. 1990, 7p Sponsored by Department of Energy, Washington, DC. Office of Fusion Energy.

Pub. in Jnl. of Vacuum Science and Technology A 8 n6 p4079-4085 Nov/Dec 90

Keywords: "Vacuum gages, Rotor dynamics, Stabiliza tion, Measurement, Instability, Reprints, Residual drag.

The pressure-independent residual drag on a spinning ball requires an offset correction for the accurate use of a spinning rotor vacuum gage (SRG) at low pressures. Instabilities in the residual drag (RD) can cause significant errors in low-pressure SRG measurements. The instabilities usually occur as discontinuous shifts when a ball is levitated by the magnetic suspension, or as continuous changes as the ball slows down. The discontinuous shifts in residual drag have been found to be caused by changes in the orientation of the ball's magnetic moment, induced by the suspension field of the inductive drive circuit. Premagnetizing the ball in a strong field has stabilized the orientation of the magnetic moment and the RD value. The continuous changes are caused by competition between magnetic and inertial forces, which result in a frequency-dependent orientation of the rotational axis of the ball. Etching a spot on the ball alters its shape and defines an inertial axis. Magnetizing the ball in a preferred direction with respect to the axis minimizes both the residual drag and its frequency dependence.

\section{0,923}

\section{PB91-147488}

Not available NTIS

National Inst. of Standards and Technology (NML) Boulder, CO. Chemical Engineering Science Div. SHOOT Flowmeter and Pressure Transducers. Final rept.

A. Kashani, R. A. Wilcox, A. L. Spivak, D. E. Daney, and C. E. Woodhouse. 1990, $6 p$

Pub. in Cryogenics 30, p286-291 Mar 90

Keywords: "Pressure transducers, "Flowmeters, Spaceborne experiments, Liquid helium, Superfluidity, Reprints, SHOOT experiment.

A venturi flowmeter has been designed and constructed for the Superfluid Helium On-Orbit Transfer (SHOOT) experiment. The calibration results obtained from the SHOOT venturi demonstrate the ability of the flowmeter to meet the requirements of the SHOOT experiment. Flow rates as low as $20 \mathrm{cu} \mathrm{dm} / \mathrm{h}$ and as high as $800 \mathrm{cu} \mathrm{dm} / \mathrm{h}$ have been measured with the flowmeter. The pressure drop in the flowmeter is measured by two Validyne differential pressure transducers. The ranges of the two transducers are $0.86 \mathrm{kPa}(0.125$ p.s.i.d.) and $8.62 \mathrm{kPa}(1.25$ p.s.i.d.). The low range transducer measures flow rates less than $200 \mathrm{cu} \mathrm{dm} /$ $\mathrm{h}$, whereas the high range transducer measures flow rates greater than $200 \mathrm{cu} \mathrm{dm} / \mathrm{h}$. The repeatability of the flowmeter at the nominal flow rate of $500 \mathrm{cu} \mathrm{dm} / \mathrm{h}$ is within + or $-1 \%$ of the flow rate. Performances of the SHOOT differential and absolute pressure transducers, which have undergone calibration and vibra tion tests, are also included. Throughout the tests, the responses of the transducers remained linear and re- peatable to within + or $-1 \%$ of the full scales of the transducers.

\section{Laboratory \& Test Facility Design \& Operation}

\section{0,924}

\section{N91-21361/1}

(Order as N91-21331/4, PC A24/MF A03)

National Inst. of Standards and Technology (NML) Gaithersburg, MD. Thermophysics Div.

High-Speed Spatial (Linear) Scanning Pyrometer: A Tool for Diagnostics, Temperature Mapping, and Property Determinations at High Temperatures. A. Cezairliyan, R. F. Chang, and G. M. Foley. 1 May $90,5 p$

In JPL, Proceedings of the First Workshop on Containerless Experimentation in Microgravity p 352-356.

Keywords: High temperature environments, "Pyrometers, Scanners, Temperature distribution, "Temperature measurement, Linear arrays, Photodiodes, Radiance, Reduced gravity, Silicon, Spectra.

Development of a fast spatial scanning pyrometer for temperature measurements above $1500 \mathrm{~K}$ is described. The salient features of the pyrometer are: (1) it measures spectral radiance temperature (at 0.65 micron) at 1024 points along a straight line $(25 \mathrm{~mm}$ long) on the target; (2) it has no moving parts and uses a self-scanning linear array of silicon photodiodes as the detector; (3) its output is recorded digitally every 1 microsec with a full-scale resolution of about 1 part in 4000 , permitting performance of a complete cycle of measurements (1024 points) in about $1 \mathrm{~ms}$. Operational characteristics of the pyrometer are given. Examples of measurements of the temperature along rapidly heated (resistive self-heating) specimens (rod, tube strip) are presented. Potential use of the pyrometer in the experiments, both ground-based and in microgra vity, requiring temperature mapping and property distribution of the specimen at high temperatures is discussed.

\section{0,925}

\section{PB91-132282}

PC A04/MF A01

National Inst. of Standards and Technology (MSEL). Boulder, CO. Materials Reliability Div.

Data-Reduction and Analysis Procedures Used in NIST's Thermomechanical Processing Research. Y. W. Cheng, and C. L. Sargent. Aug 90, 68p

NISTIR-3950

Keywords: *Computer systems programs, "Data processing, Data reduction, Phase transformations, Test equipment, Deformation, Strain rate, Loads(Forces), Cooling rate, Time dependence, "Thermomechanical treatments, Temperature dependence.

The report described the data-reduction procedures and computer programs used to reduce and analyze the data obtained with a hot-deformation apparatus. The measured raw data with the apparatus include temperature vs. time, specimen's relative length (dilation) vs. time, actuator movement (stroke) vs. time, and load vs. time. Four computer programs were written for data reduction and analysis to determine the cooling rates, the true stress-true strain curves, the true strain rates, and the phase-transformation temperatures. Local averaging techniques were used to smooth the data of temperature, dilation, stroke, and load. Source codes for the computer programs are included. Example results of the analyses are presented and an example of the program execution is given.

\section{0,926}

PB91-147272

Not available NTIS

National Inst. of Standards and Technology (NML), Gaithersburg. MD. Thermophysics Div.

Study of the Linearity of Transfer Leaks and a Helium Leak Detector.

Final rept.

C. D. Ehrlich, S. A. Tison, H. Y. Hsiao, and D. B.

Ward. 1990,6 p

Pub. in Jnl. of Vacuum Science and Technology A 8 , n6 p4086-4091 Nov/Dec 90.

Keywords: "Leak detectors, "Linearity, Helium, Leakage, Gas detectors. Flow measurement, Accuracy, Errors, Drift(Instrumentation), Mass spectrometers, Random variables, Reprints.
A study has been performed of the linearity of two types of variable-reservoir-pressure leaks and a commercial tuned magnetic sector mass-spectrometer helium leak detector. While the leaks exhibit predict able (but not always linear) behavior over a broad range, the linearity of the leak detector depends strongly on properly correcting for observed drift and random fluctuations in the measured leak rate.

\section{0,927}

\section{PB91-158519}

Not available NTIS National Bureau of Standards (NML), Gaithersburg, MD. Inorganic Materials Div.

Levitation Calorimetry.

Final rept.

D. W. Bonnell, R. L. Montgomery, B. Stephenson, P C. Sundareswaran, and J. L. Margrave. 1988, 34p Sponsored by Rice Univ., Houston, TX. Dept. of Chemistry.

Pub. in Specific Heat of Solids, p265-298 1988.

Keywords: "Calorimetry, "Leviation melting, "Liquid metals, Specific heat, Thermodynamic properties, High temperature tests, Heat measurement, Reviews, Reprints.

The determination of high temperature thermodynamic properties is of fundamental importance in chemistry, physics, and engineering. The temperature region above $2000 \mathrm{~K}$ has been generally inaccessable to direct measurement, with basic properties even of elements often being obtained by extrapolation. The development of levitation calorimetry has provided the technique for precision measurements of conducting materials in the previously difficult to study regime. The chapter presents a review of the development of the field, from the first successful calorimeter system in the late 1960's, to the reviewed, and the various considerations in coil development are discussed. Actual calorimeter systems are discussed, with a detailed development of the data analysis being presented. A comprehensive review of the literature is presented, with examples of results obtained and comparisons of the current state of liquid properties.

\section{0,928}

PB91-159004

Not available NTIS

National Inst. of Standards and Technology (MSEL),

Gaithersburg, MD. Metallurgy Div.

Mechanical Testing Revitalized.

Final rept.

L. Mordfin. 1990, 1p

Pub. in Experimental Techniques 14, n5 p20 Sep/Oct 90.

Keywords: "Mechanical tests, "Strain gages, Test facilities, Stress analysis, Strain measurement, Mechanical properties, Nondestructive tests, Experimentation, Materials testing, Reprints.

The fiftieth anniversary of the invention of the bonded resistance strain gage was celebrated last year. Over a period of about twenty years, which encompassed World War II and the postwar years, this device completely revolutionized the field of experimental stress analysis. Today, this field is considered relatively mature; although strain gages continue to be used in ever-increasing quantities, significant new advancements in the way they are used have become rare. The field of mechanical testing, which is closely intertwined with that of experimental stress analysis, grew in much the same way. .. until the 1980s. The last ten years have witnessed a revival of interest in mechanical testing, with major efforts devoted to the development of new mechanical test methods and techniques to satisfy needs for new kinds of measurements on new kinds of materials. A new group on Mechanical Properties and Performance was established at NIST to address these needs. It is suggested that the Society for Experimental Mechanics could fulfill an important role as a central forum for the revitalized field of mechanical testing.

100,929

PB91-175067

Not available NTIS

National Inst. of Standards and Technology (IMSE), Gaithersburg, MD. Ceramics Div.

High-Resolution Small-Angle $X$-ray Scattering Camera for Anomalous Scattering.

Final rept.

G. G. Long, P. R. Jemian, J. R. Weertman, D. R.

Black, H. E. Burdette, and R. Spal. 1991, 8p

Sponsored by Department of Energy, Washington, DC. 
Pub, in Jnl. of Applied Crystallography 24, p30-37 1991.

Keywords: "Small angle scattering, "Cameras, "X-ray analysis, "Photodetectors, Test equipment, Measuring instruments, Steels, Microstructure, Temperature dependence, Photodiodes, Precipitates, Calibrating, AC generators, Correlators, Reprints.

The design and operation of a new small-angle $\mathrm{X}$-ray scattering instrument, optimized for high throughput at a synchrotron source, high angular and wave-length resolution, large sample cross-sectional area, accurate energy tuning, excellent signal-to-noise ratio and harmonic rejection are presented. The principles of design and implementation are given, as are the details of primary calibration of absolute intensity and experimental desmearing. The instrument has been tested for application to anomalous-scattering measurements near the chromium $\mathrm{K}$ edge. Preliminary results on samples of a heat-treated steel are presented as a demonstration of the capability of this experiment to separate the microstructure evolution as a function of temperature of a chromium-rich precipitate from the thermal behavior of other precipitates in the steel.

\section{0,930}

PB91-175216

Not available NTIS

National Inst. of Standards and Technology (MSEL),

Boulder, CO. Fracture and Deformation Div.

Jlc Data Analysis with a 'Negative Crack Growth' Correction Procedure.

Final rept.

Y. A. Rosenthal, R. L. Tobler, and P. T. Purtscher.

1990, 4p

Pub. in Jnl. of Testing and Evaluation 18, n4 p301-304 Jul 90 .

Keywords: "Crack propagation, " $\mathrm{J}$ integral, "Mechanical tests, Crack initiation, Fracture mechanics, Austenitic steels, Measurement, Standards, Fracturing, Fracture properties, Toughness, Reprints.

It often occurs in J-R testing that some of the initial crack extension data points have anomalous negative values. ASTM Methods 813-87 and 813-81, however, do not specify any procedures for treating such data. The authors propose a procedure for data analysis which utilizes the negative data point values and is consistent with ASIM E 813-87. The proposed correction procedure is illustrated using a data set for austenitic steel; also, J values obtained using ASIM E 813-87 are compared to those obtained from its predecessor E 813-81.

\section{0,931}

PB91-175406

Not available NTIS National Bureau of Standards (NEL), Gaithersburg, MD. Mechanical Production Metrology Div.

Eccentric Load Sensitivity of Force Sensors.

Final rept.

R. A. Mitchell, R. L. Seifarth, and C. P. Reeve. 1986, 7p

Pub. in Mechanical Problems in Measuring Force and Mass, p275-281 1986.

Keywords: "Force, "Sensors, "Eccentricity, Loads(Forces), Measurement, Statistical analysis, Mathematical models, Tension, Compressive properties, Bending moments, Sensitivity, Reprints.

A test has been developed to measure the eccentric load sensitivity of universal (tension and compression) force sensors. The test can also be used to calibrate the transverse bending moment measuring bridges of multi-axis force-moment sensors. Results of tests of five sensors are given. The results are analyzed in terms of a sinusoidal statistical model.

100,932

100,932
PB91-187633

(Order as PB91-187617, PC A06/MF A01) National Inst. of Standards and Technology, Gaithersburg, MD.

Use of the Electrostatic Classification Method to Size 0.1 micrometer SRM Particles: A Feasibility Study.

P. D. Kinney, D. Y. H. Pui, G. W. Mulholland, and N.

P. Bryner. 1991, 30p

Sponsored by Minnesota Univ., Minneapolis.

Included in Jnl. of Research of the National Institute of Standards and Technology, v96 n2 p147-176 Mar/Apr 91.

Keywords: *Dimensional measurement, "Particle size, "Microspheres, Aerosol generators, Atomizers, Con- densation nuclei, Polystyrene, Electrostatics, Mobility, Feasibility, "Standard reference materials.

The use of the electrostatic classification method for sizing monodisperse 0.1 micrometer polystyrene latex (PSL) spheres has been investigated experimentally. The objective was to determine the feasibility of using electrostatic classification as a standard method of particle sizing in the development of a 0.1 micrometer particle diameter Standard Reference Material (SRM). The mean particle diameter was calculated from a measurement of the mean electrical mobility of the PSL spheres as an aerosol using an electrostatic classifier. The performance of the classifier was investigated by measuring its transfer function, conducting a sensitivity analysis to verify the governing theoretical relationships, measuring the repeatability of particle sizing, and sizing NIST SRM 1691, 0.269 micrometer and NIST SRM 1690, 0.895 micrometer particles.

\section{0,933}

PB91-206730

PC A03/MF A01

National Inst. of Standards and Technology (BFRL), Gaithersburg, MD

Calibration at $24 \mathrm{C}$ of a Heat-Flow-Meter Apparatus Having $610 \mathrm{~mm}$ Square Plates.

R. R. Zarr, and B. A. Licitra. May $91,36 p$ NISTIR-

4539

Sponsored by Department of Energy, Washington, DC. Office of Buildings Energy $R$ and $D$.

Keywords: "Heat flow meters, "Calibration, Heat measurement, Heat flux, Heat transmission, Test facilities, Plates, Data acquisition, Thermal insulation, Thermal measurements, Thermal conductivity.

Results are summarized for 38 individual calibration measurements conducted at $24 \mathrm{C}$ and atmospheric conditions for a heat-flow-meter apparatus having 610 $\mathrm{mm}$ square plates. The apparatus was calibrated using a 26.2-mm-thick specimen of fibrous-glass board having a density of $139 \mathrm{~kg} / \mathrm{cu} \mathrm{m}$. The specimen was selected from an internal lot (Lot 1970) of Standard Reference Material (SRM) similar to SRMs 1450 $1450 \mathrm{a}$, and $1450 \mathrm{~b}$. Values of apparent thermal conductivity were predicted using a regression equation developed for the lot of fibrous-glass insulation. Calibration measurements varied + or $-0.4 \%$ with small drift of $0.4 \%$ over 250 days. The apparent thermal conductivity of the calibration specimen was also measured using the National Institute of Standards and Technology's one-meter Line-Heat-Source Guarded Hot Plate. Agreement between measurements of thermal conductivity of the guarded hot plate and predicted values were within +0.2 to $-0.3 \%$ at $24 \mathrm{C}$. The report describes the heat-flow-meter apparatus and the computer data-acquisition-system used to collect data from the apparatus.

100,934

PB91-222646

PC A08/MF A02

National Inst. of Standards and Technology (TS),

Gaithersburg, MD.

Directory of Accredited Laboratories, 1991. National Voluntary Laboratory Accreditation Program (NVLAP).

Final rept.

N. M. Trahey, V. R. White, and J. Horlick. Apr 91 ,

170 p NIST/SP. 810

Also available from Supt. of Docs. as SN003-00303082-1. See also PB90-198920.

Keywords: "Laboratories, "Directories, Acoustics, Asbestos, Carpets, Computer applications, Construction materials, Dosimetry, Electromagnetic compatibility, Paints, Papers, Plastics, Sealers, Seals(Stoppers), Stoves, Telecommunication, Thermal insulation, "National Voluntary Laboratory Accreditation program, NVLAP program.

The annual Directory provides a listing of laboratories accredited as of March 1, 1991, by the National Institute of Standards and Technology, National Voluntary Laboratory Accreditation Program (NVLAP). The names of approximately 900 laboratories in 15 fields of testing are included. A brief description of the NVLAP program is given, and a summary of laboratory participation is provided. To aid the user, indexes cross reference the laboratories by company name, NVLAP Lab Code Number, field of testing, and geographic location (state or country). A listing of the test methods (scope of accreditation) is provided for each laboratory.
National Inst. of Standards and Technology (CSTL), Gaithersburg, MD. Process Measurements Div. Procedure for the Effective Recalibration of Liquid-in-Glass Thermometers.

Special pub. (Final).

J. A. Wise. Aug 91, 19p NIST/SP-819

Also available from Supt. of Docs. as SN003-003. 03102-0.

Keywords: "Temperature measuring instruments, "Thermometers, "Calibration, Volume, Liquid in glass thermometers, Ice baths, ITS-90.

High quality liquid-in-glass thermometers require only one complete calibration in their lifetime and it is possible to avoid the usual requirement for complete recalibration of the instrument by the recalibration of a single previously calibrated temperature. The need for recalibration of properly manufactured liquid-in-glass thermometers is due to the gradual relaxation of residual mechanical strains in the glass that have a significant effect on the volume of the bulb. The recalibration of a single point provides a reliable indication of the effect of this change in volume and provides a means for the accurate adjustment of the remainder of the scale. The paper describes a procedure for the single temperature recalibration of liquid-in-glass thermometers that can be performed in the user's laboratory and the subsequent adjustment of the entire scale. The adjustment of the scale that is required by the recent introduction of the new International Temperature Scale (ITS-90) is also described.

100,936

PB92-126416

PC A10/MF A03

National Inst of Standards and Technology (TS)

Gaithersburg, MD. Physical Measurement Services Program.

NIST Calibration Services Users Guide, 1991

Special pub.

J. D. Simmons. Oct 91, 221p NIST/SP-250 91ED

Also available from Supt. of Docs, as SN003-003-

03115-2. Supersedes PB89-200216. See also PB90127820.

Keywords: "Fees, "Metrology, Manual, Measuring instruments, Test facilities, Standards, Dimensions, Mechanical tests, Acoustics, Ultrasonics, Microwaves, Thermodynamics, Ionizing radiation, Electromagnetic properties, Prices, "National Institute of Standards and Technology, "Calibration.

The National Institute of Standards and Technology (NIST) Calibration Services Users Guide provides detailed descriptions of currently available NIST calibration services, measurement assurance programs, and special test services. The following measurement areas are covered: (1) dimensional; (2) mechanical, including flow, acoustic, and ultrasonic; (3) thermodynamic; (4) optical radiation; (5) ionizing radiation; and (6) electromagnetic, including $\mathrm{dc}$, ac, $\mathrm{ff}$, and microwave. A separate Fee Schedule is issued annually, providing current prices for the services offered, updates on points of contact, and information on measurement seminars.

\section{0,937}

PB92-126655

(Order as PB92-126614, PC A06/MF A02) National Inst. of Standards and Technology, Gaithersburg, MD.

Certification of NIST SRM 1961: 30 micrometers Diameter Polystyrene Spheres.

A. W. Hartman, T. D. Doiron, and G. G. Hembree.

1991, 13p

Included in Jnl. of Research of the National Institute of Standards and Technology, v96 n5 p551-563 Sep/Oct 91.

Keywords: "Dimensional measurement, "Particle size, * Microspheres, Electron microscopy, Optical microscopy, Polystyrene, "Standard reference materials.

The report describes the certification of SRM 1961, an NIST Standard Reference Material for particle diameter. It consists of an aqueous suspension of monosize 30 micrometers diameter polystyrene spheres. The primary technique used optical microscopy; it gave a mean diameter value $\mathrm{D}(\mathrm{bar})=29.62+$ or $-0.04 \mathrm{mi}$ crometer and a standard deviation of the size distribution of 0.21 micrometer. Over 2000 spheres were measured. The supporting technique used electron microscopy, which yielded $\mathrm{D}($ bas $)=29.68+$ or -0.11 micrometer. Ninety spheres were measured. 


\section{Laboratory \& Test Facility Design \& Operation}

\section{0,938
PB92-126721}

(Order as PB92-126614, PC A06/MF A02) Slovak Metrological Society, Bratislava (Czechoslovakia).

\section{Slovak Metrologicai Society.}

J. Mandak. 1991, 2p

Included in Jnl. of Research of the National Institute of Standards and Technology, v96 n5 p621-622 Sep/Oct 91.

Keywords: "Metrology, Czechoslovak organizations, Historical aspects, Meetings, "Slovak Metrological Society.

The brief communication describes the establishmen of the Slovak Metrological Society (SMS). SMS came into being on October 16,1990, as the result of a special founding Congress held in DT Zilina with 137 dele gates from the whole of Slovakia in attendance. I brings together individuals involved in both the technical and legal aspects of metrology and its aim is to help in developing metrology and measurement in Slovakia. SMS functions under the direction of the $\mathrm{Czech}$ oslovak Metrological Institute (CSMU), the center of Czechoslovak metrology. Together with the Federa Weights and Measures Office (FUNM), state metrolo gical centers, centers of the calibration service, and production plant metrological centers, it establishes the principal tasks of present-day metrology in the Czechoslovak Federal Republic (CSFR).

\section{0,939}

PB92-163864 Gathechnology (TS) Program.

Modernized Metrlc System (Chart).

Final rept

Aug 91 , 1p NIST/SP-304-91-ED

Keywords: "Metric system, Units of measurement, Training aids, Metric system, SI, Units of measurement.

The chart presents a popularized yet technically accurate guide to SI base units, supplementary units, multiples and prefixes, and common conversions. The intended audience is (mainly) school children and the general public.

\section{0,940}

PB92-163872

Not available NTIS

National Inst. of Standards and Technology (TS) Gaithersbur

Brlef History of Measurement Systems with Chart of the Modernized Metric System.

Aug 91, 1 p NIST/SP-304A-91-ED

Keywords: *Metric system, International system of units, Measurement, History.

The Modernized Metric System (also called the International System of Units) is made up of seven base units, two supplementary units, and many derived units. The chart describes this entire system, including details about the standards for each base unit and information on how the system is used. The reverse side of the chart contains a brief history of measurement systems. The need for measurement began with primative man, who used parts of his body and his natura surroundings for measurement standards and measuring instruments. As societies evolved, weights and measures became more complex. Two systems became predominant: the English system, rooted in the history and tradition of England; and the metric system, a scientifically based system using decima notation. The metric system, with its inherent decima advantages, gained widespread acceptance and is now the official measurement system in nearly al countries of the world.

\section{0,941}

PB92-163880

Not available NTIS

National Inst. of Standards and Technology (TS)

Gaithersburg, MD. Physical Measurement Services

Program.

Metric Conversion Card.

Final rept.

J. D. Simmons. Aug 91, 1p NIST/SP-365-91-ED

Keywords: Metric system, "Metrication, Units of meas urement.

NIST SP365 is a pocket size plastic Metric Conversion Card with approximate conversions to and from metric for the most commonly used units of measurement on the opposite sides of the card.

\section{0,942}

PB92-163898

National Inst. of Standards and Technology (TS), Gaithersburg, MD. Physical Measurement Services Program.

Metric Measures Up (Ruler).

Final rept.

Aug 91, 1p NIST/SP-376-91-ED

Keywords: "Units of measurement, Metric system.

This ruler provides metric linear measure (17.75) and customary-unit linear equivalents (approximately 7 inches).

\section{Manufacturing Processes \& Materials Handling}

100,943

PC A05/MF A01 National Inst. of Standards and Technology (MSEL), Gaithersburg, MD. Polymers Div.

Development of Test Methods to Determine the Compatibility of Llquid Hazardous Materials with Polyethylene Packagings.

Final rept. 11 Jun $90-31$ Dec 91

J. M. Crissman, and M. A. Schen. Dec 91, 77p

NISTIR-4729

Contract DTRS-56-90-X-70006

See also PB-280 215 and PB90-235417. Sponsored by Department of Transportation, Washington, DC Office of Hazardous Materials Regulations.

Keywords: "Hazardous materials, "Polyethelene, *Shipping containers, Compatibility, Test methods, Packaging, Oxidation, Permeability, Cracks, Degradation, Peroxides, Transportation safety, Regulations, Hazardous materials transportation, Organic peroxides, Hazardous liquids.

The report describes work done for the Department of Transportation, Office of Hazardous Materials Transportation, to develop test methods which can be used to determine whether a liquid hazardous material may be shipped in a specific type of polyethylene container. Current federal regulations require that each prospective lading be tested individually in proposed polyethylene packagings. The central task of the project is to ene packagings. The central task of the project is to determine the feasibility of substituting a set of standard liquids for the purpose of compatibility testing with ardous liquids with polyethylene there are three major areas of concern, permeation and/or swelling, environmental stress cracking, and oxidative degradation. ronmental stress cracking, and oxidative degradation. Specific recommendations are made for a set of
standard liquids which address all three aspects of compatibility. Special attention is given the class of organic peroxides which may be unstable at the test temperatures currently in use. In the area of permeation, an empirical method known as the 'Permachor' scheme is proposed as a means of estimating the permeability of many liquid hazardous materials, and for ranking individual members within a given class of liquids.

\section{Nondestructive Testing}

\section{0,944}

$-187120$

Not available NTIS National Inst. of Standards and Technology (IMSE), Boulder, CO. Fracture and Deformation Div. Eddy Current Reflection Probe: Theory and ExperIment.

Final rept.

B. A. Auld, J. C. Moulder, S. Jefferies, P. J. Shull, and S. Ayter. 1989, 11p

Pub. in Research in Nondestructive Evaluation 1, n1 p1-11 1989.

Keywords: "Eddy currents, "Nondestructive tests, Signal processing, Measuring instruments, Electric coils, Defects, Probes, Finite difference method, Reflection, Experimental data, Aluminum, Reprints.

Eddy current reflection probes are two-port devices consisting of a drive coil encircling a differential pair of pickup coils. In operation, the drive coil is excited with an alternating current and the signal detected is the emf induced in the pickup coils. When no flaw is present there is no output signal, but when a flaw is present, the perturbed field from the drive coil induces a signal in the pickup coils. In this respect, reflection probes are fundamentally different from absolute eddy current probes, which are generally operated as passive elements in a bridge circuit. With an absolute probe, the impedance of the probe is monitored and a flaw is detected by the change in probe impedance it causes. A general delta $Z$ theory of eddy current reflection probes was previously developed for application to new types of robotic sensors. In the paper the theory is applied to the characterization of surfaceconnected flaws. Flaw signals were calculated using a finite-difference implementation of the delta $Z$ theory developed at Stanford University. A special air-core eddy-current reflection probe was fabricated at NBS and used to obtain flaw signals for a number of rectangular-shaped slots in aluminum. An automatic impedance analyzer was used to measure flaw signals as the probe was scanned down the length of a flaw. The paper compares the experimental results with predictions of the theory.

100,945

PB91-236562

Not available NTIS

National Inst of Standards and Technology (EEEL). Boulder, CO. Electromagnetic Technology Div. Eddy Current Probe Sensitivity as a Function of Coil Construction Parameters.

Final rept.

T. E. Capobianco, J. Splett, and H. Iyer. 1990, 18p Pub. in Res. Nondestr. Eval. 2, p169-186 1990.

Keywords: "Nondestructive tests, "Eddy current tests, *Electric coils, Notch tests, Eddy currents, Defects, Permeability, Measuring instruments, Wire, Sensitivity, Inspection, Reprints.

The authors report the results of the first phase of a study designed to quantify the relationship between eddy current coil construction and the performance of these coils used in nondestructive evaluation (NDE) inspections. The ferrite core coils wound for the study are small but typical of the sizes commonly used in commercially manufactured eddy current probes. The data reported here were produced from a set of 27 probes scanned over a single defect. The defect was an electrical-discharge-machined (EDM) notch in a 19 $\mathrm{mm}$ thick 7075-T6 aluminum alloy specimen. The partcircular EDM notch was $9 \mathrm{~mm}$ long and $3 \mathrm{~mm}$ deep and $0.1 \mathrm{~mm}$ wide. Analysis of the data shows that the number of turns, the winding distance, the coil aspect ratio, and the backside ferrite length all affect the coil sensitivity. Winding inhomogeneity is significant for coils having many winding layers and can be considerably larger than the contribution made by variations in some of the construction factors. Wire gauge, ferrite diameter, and permeability showed no significant ef fects on their measure of sensitivity in the study.

\section{0,946}

PB91-237305

Not available NTIS

National Inst of Standards and Technology (IMSE), Boulder, CO. Fracture and Deformation Div.

Characterization of Capacltive Array for NDE Applications.

Final rept.

P. J. Shull, A. V. Clark, P. R. Heyliger, J. C. Moulder, and B. A. Auld. 1990, 17p

Pub. in Research in Nondestructive Evaluation 2, p11. 271990

Keywords: "Nondestructive tests, "Capacitance, "Dielectrics, "Arrays, Detectors, Ferroelectric materials, Capacitors, Measuring instruments, Dielectric properties, Reprints.

The authors have developed a capacitive array sensor which responds to the complex dielectric constant of an interrogated material. The sensor requires only single-sided access and operates in a differential mode for detection of discontinuities in the relative dielectric constant, or in the absolute mode where the interest is in absolute quantities. The device in genera is noncontacting but can be fabricated as an embedded sensor. Various proof-of-concept studies have been performed to explore possible applications of the device. In the differential mode, small surface features (notches) were detected in a conductor; in dielectrics, both surface and subsurface features were detécted. The probe was sensitive to change in impedance caused by a curing epoxy, viewed through a graphite- 
epoxy composite panel. A study was performed to assess the use of the probe for noncontact characterzation of sintering of ceramics. In this (absolute) mode, the effects of liftoff and dielectric constant must be separated. They propose a scheme based on the ability to multiplex the capacitive array probe to accomplish this. Preliminary investigation shows that the effect of parasitic capacitance between the probe and ground points in the environment must be suppressed.

100,947

PB91-237313

Not available NTIS

National Bureau of Standards (IMSE), Boulder, CO. Fracture and Deformation Div.

Capacitive Array Sensors for Nondestructive Evaluation.

Final rept.

P. J. Shull, P. Heyliger, B. A. Auld, and A. V. Clark.

1988,10

Pub. in Jnl. of Metals 40, n11 p112 1988.

Keywords: "Capacitance, "Detectors, "Nondestructive tests, "Arrays, "Ceramics, Dielectric materials, Sintering, Surface properties, Epoxy resins, Coatings(Materials), Defects, Insulators, Electric conductors, Reprints.

Capacitive array sensors can be used to characterize: (1) surface features on conductors; (2) surface and subsurface features on dielectrics. The versatility is a decided advantage over inductive probes. The study characterized the sensor on both conductors and dielectrics for a variety of both real and simulated surface and subsurface (dielectrics only) flaws. This was done with the probe elements configured for both absolute and differential modes. In the absolute mode there are two electrodes - a source and a receiver. A voltage is impressed across these elements. In the differential mode there are three electrodes: one source and two receiver electrodes. These receiver electrodes are then connected to a differential amplifier. The probe's potential was examined for the following applications: monitoring of the cure of epoxy resins, thickness and porosity of thermal barrier coatings, flaws in ceramics and subsurface flaws in insulators.

\section{0,948 \\ PB91-237610}

Not available NTIS

National Inst. of Standards and Technology (MSEL), Gaithersburg, MD. Materials Reliability Div.

Theory of Capacitive Probe Method for Noncontact Characterization of Dielectric Properties of Materials.

Final rept.

V. K. Tewary, P. R. Heyliger, and A. V. Clark. 1991,

10p

Pub. in Jnl. of Materials Research 6, n3 p629-638 Mar 91.

Keywords: "Dielectric materials, "Capacitance, Finite element analysis, Hilbert transformation, Reliability, Characterization, Nondestructive tests, Capacitance, Measurement, Monitors, Theories, Surface energy, Reprints, Capacitive probe.

The capacitive probe method for noncontact characterization and monitoring of dielectric materials is analyzed theoretically. An analytical method based upon the Hilbert transform technique and a numerical method using the finite element technique for calculating the potential distribution and change in admittance of the probe caused by presence of the dielectric material as a function of liftoff (distance between the probe plane and the surface of the dielectric material) are described. The two methods are compared with each other and their relative advantages discussed. The possibility of extracting useful information about the dielectric constant of the material from experimental data is also discussed in the light of the proposed theory.

100,949

PB92-117423

Not available NTIS

National Inst. of Standards and Technology (MSEL). Gaithersburg, MD. Metallurgy Div.

Advanced Sensing of Materials Processing.

Final rept.

H. N. G. Wadley, A. H. Kahn, and W. L. Johnson.

1988, 10p

Pub. in Materials Research Society Symposium Proceedings, v117 p109-118 1988 .

Keywords: "Nondestructive tests, "Process control, "Metals, "Detectors, Artificial intelligence, Reviews, Microstructure, Measurement, Ultrasonic tests, Acoustic emission, Eddy currents, Reprints.
To implement new process control strategies including Intelligent Processing of Materials, advanced sensors are required to nonintrusively evaluate process and microstructure variables. Researchers increasingly are looking to innovative extensions of traditional nondestructive evaluation technologies, such as ultrasonics, acoustic emission, and eddy current methodologies for this. Here, the nature and characteristics of emerging sensors based upon these new measurement methods are described and examples of their application discussed.

\section{LIBRARY \& INFORMATION SCIENCES}

\section{Information Systems}

\section{0,950}

PB91-157123

PC A03/MF A01

National Inst. of Standards and Technology, Gaithersburg, MD.

STEP On-Line Information Service User's Guide. National PDES Testbed Report Series.

S. Katz. 2 Jan 91,25 p NISTIR-4491

Supersedes PB91-143354.

Keywords: "Information services, "On line systems, In formation systems, Access methods, User manuals, Documents, Computer software Computer networks, STEP(Standard for the Exchange of Product Model Data), PDES(Product Data Exchange using STEP), National Institute of Standards and Technology

The document describes how to access the on-line STEP (Standard for the Exchange of Product Model Data) information service provided by the National PDES (Product Data Exchange using STEP) Testbed at the National Institute of Standards and Technology (NIST). All items available through the STEP information service are public domain materials. The Testbed will maintain the on-line system; however, the contributing organizations are responsible for the content and mation service contains both documents and software modules.

\section{0,951}

PB91-159756

PC A02/MF A01 National Inst. of Standards and Technology, Gaithers burg, MD.

National PDES Testbed Mail Server User's Guide. National PDES Testbed Report Series.

S. Ressler. 31 Jan 91,10 p NISTIR-4508

Keywords: "Electronic mail, Document circulation, Archives, Documents, Access methods, User manuals, *PDES(Product Data Exchange using STEP) STEP(Standard for the Exchange of Product Model Data).

As part of the Nation PDES Testbed Project, the Information Services Center has established a facility to electronically distribute documents. The facility is based on software known as an 'archive server' or 'mail server'. Electronic documents may be requested and in turn received via electronic mail (e-mail). The report is intended to function both as a user's guide and as a general explanation of the system and its capabilities.

100,952

PB91-167312

PC A04/MF A01

National Inst. of Standards and Technology (TS), Gaithersburg, MD.

NIST Standard Reference Data Products 1991 Catalog.

Special pub.

M. W. Chase, and J. C. Sauerwein. Feb 91, 53p

NIST/SP-782

Also available from Supt. of Docs. as SNO03-00303063-5. Supersedes PB90-219841.

Keywords:

"Catalogs(Publications),

Indexes(Documentation), Chemical analysis, Atomic physics, Reaction kinetics, Properties, Molecular structure, Molecular spectroscopy, Thermochemistry, Ther mophysical properties, "National Institute of Standards and Technology, "Data bases, Materials science.

The National Institute of Standards and Technology's Standard Reference Data Program provides reliable well-documented data to scientists and engineers for use in technical problem-solving, research, and development. The catalog lists classic data compilations in hard-copy form and current databases in the Standard Reference Database Series. The edition of the catalog updates many new databases. These data compilations have been subdivided into seven categories. Prices and ordering information are located at the back of the document.

100,953

PB9 1-189795

Not available NTIS

National Bureau of Standards, Gaithersburg, MD.

Office of Standards Code and Information.

Standardizing Standards.

Final rept.

D. R. Mackay. $1988,2 p$

Pub. in Visions of the Future, p45-46 1988.

Keywords: "Standards, Guidelines, Reprints, Standards Engineering Society, Keywords, Titles.

The paper describes the activities of the Standards Engineering Society relating to the development of a draft standard for standards incorporating guidelines for designating standards, selecting titles and keywords for standards, providing abstracts of standards, and establishing formats for different types of standards. A second standard establishing guidelines for the production of catalogs and indexes is described as under development.

100,954

PB92-109016

PC A07/MF A02

National Inst. of Standards and Technology (TS), Gaithersburg, MD. Information Resources and Services Div.

Data Bases Available in the Research Information Center of the National Institute of Standards and Technology.

Special pub. (Final).

D. Cunningham. Sep 91,131 NIST/SP-818

Supersedes PB91-107284. Also available from Supt of Docs. as SN003-003-03101-1.

Keywords: *Data bases, "Information services, Direc tories, Indexes(Documentation), Subject indexing. Vendors, Tables(Data), "NIST.

The publication supersedes and revises NIST SP 799 which is dated September 1990. Data bases are listed by acronyms and by full titles. Citations also include dates covered, brief descriptions, kinds of information each contains, producers, the titles of corresponding hard copy, and vendors. A list of vendors with address es and telephone numbers precedes the list. The General Subject Index is arranged alphabetically by subject categories and the Cross Reference Index lists variant forms of the names of the data bases in the left column with cross references to the name of the data base used in the publication on the right.

100,955

PB92-126556

PC A03/MF A01

National Inst. of Standards and Technology (CSL),

Gaithersburg, MD

Guide to Schema and Schema Extensibility.

Final rept.

B. K. Rosen, and I. des Fontaines. Nov $91,35 p$

NIST/SP-500/197

Also available from Supt. of Docs. See also PB90147919 and PB90-219866. Prepared in cooperation with Geological Survey, Reston, VA. Water Resources Div.

Keywords: "Information systems, "Data management Dictionaries, Data base management, Computer aided software engineering, Information resources management.

The guide was developed to assist both the casual user of Information Systems (IS) as well as ADP fessionals in understanding the concepts behind databases and data dictionary schemas and schema extensibility. It was developed in the context of its application and pertinence to the ANSI standard X3.138 1988, Information Resource Dictionary Systems (IRDS). The guide begins with a set of definitions that 
provide an understanding of data dictionary and repository terminology. It then follows with a discussion of the IRDS. After establishing these basic definitions, the discussion of schema is initiated. The document to the exchange of information between Computer Aided Software Engineering (CASE) tools. Included is a discussion of the CASE tool to IRDS data exchange prototype that was developed at NIST. The document concludes with a discussion of possible future impacts of schema extensibility on the development of standards in areas related to data dictionaries and repositor ies.

\section{Reference Materials}

100,956

\section{FIPS PUB 11-3}

PC E14

National Inst. of Standards and Technology (CSL), Gaithersburg, MD

Guideline: American National Dictionary for Information Systems. Category: Software; Subcategory: Documentation.

Federal information processing standards (Final).

S. M. Radack. c1991, 150p

Supersedes FIPS PUB 11-2. Also pub. as American Standards Committee, New York rept. no. ANSIX3.172-1990. Prepared in cooperation with American National Standards Committee, New York

Three ring vinyl binder also available: North American Continent price $\$ 7.00$; all others write for quote.

Keywords: "Dictionaries, "Information systems, National government, Data processing, Computers, "Federal information processing standards.

The publication adopts ANSI X3.172-1990, AMERICAN NATIONAL DICTIONARY FOR INFORMATION SYSTEMS (ANSDIS), as a FIPS Guideline. The FIPS PUB provides a common reference within the Federal Government for terms and definitions used in the field of information systems including computers, data communications, data processing, text processing, and related fields. The DICTIONARY consists of an alphabetic listing of terms and their definitions. The DICTIONARY includes terms and definitions from the ISO Vocabulary -- Information Systems developed by ISO/ IEC JTC 1/TC 97/SC1: Vocabulary. The revision supersedes FIPS PUB 11-2 (ANSI X3/TR-1-82) in its entirety.

\section{0,957}

PB91-136507

PC A99/MF A99

National Inst of Standards and Technology, Gaithers burg, MD.

National Bureau of Standards Publications 1977 1987. Volume 1. Citations, Key Words, and Abstracts.

Special pub (Final rept. 1977-87)

R. Pardee, E. Gladden, and D. Harris. Aug 90, 1675p NIST/SP-790-VOL-1

Also available from Supt. of Docs. See also PB91136515

Keywords: "US NBS, "Bibliographies, Subject index terms, Abstracts, "National Bureau of Standards, US NIST.

Volume 1 contains full bibliographic citations, key words, and abstracts for 15,746 National Bureau of Standards (NBS) (as of August 23, 1988 the National Institute of Standards and Technology (NIST)) papers published and entered into the National Technical Information Service (NTIS) collection between 1977 and 1987. Also included are several papers published prior to 1977 but not entered into the NTIS collection unti 1977. Volume 2 (PB91-136515) contains four indexes to allow the user to identify papers by personal author, key words, title, and NTIS order/report number.

\section{0,958}

PB91-136515

PC A99/MF A99

EnviroSystems Supply, Inc., Fort Myers, FL.

Natlonal Bureau of Standards Publlcations 1977 -

1987. Volume 2 . Indexes.

Special pub (Final rept. 1977-87).

R. Pardee, E. Gladden, and D. Harris. Aug 90, 1442p

NIST/SP-790-VOL-2

Also available from Supt. of Docs. See also PB91136507 .
Keywords: "US NBS, "Bibliographies, Subject index terms, Indexes(Documentation), Authors, "National Bureau of Standards, US NIST.

Volume 1 contains full bibliographic citations, key words, and abstracts for 15,746 National Bureau of Standards (NBS) (as of August 23, 1988 the National Institute of Standards and Technology (NIST)) papers published and entered into the National Technical Information Service (NTIS) collection between 1977 and 1987. Also included are several papers published prior to 1977 but not entered into the NTIS collection until 1977. The volume, Volume 2 , contains four indexes to allow the user to identify papers by personal author, key words, title, and NTIS order/report number.

\section{0,959}

PB9 1-148494

PC A03/MF A01

National Inst. of Standards and Technology, Gaithersburg, MD. Information Resources and Services Div. Abstract and Index Collection in the Research Information Center of the National Institute of Standards and Technology (Fourth Edition). Special pub. (Final).

D. Cunningham. Dec 90, 36p NIST/SP.803

Also available from Supt. of Docs. as SN003-00303056-2. Supersedes PB89-103790.

Keywords: "Indexes(Documentation), "Abstracts, Technical reports, Information centers, Descriptive cataloging, Subject indexing, "National Institute of Centers, Research Information Center(NIST).

An alphabetical arrangement of abstracts and indexes available in the Research Information Center (RIC) of the National Institute of Standards and Technology (NIST) is listed by most current title of the publication. Other information includes description of the abstract or index, RIC holdings, principal sources, publisher or association, corresponding RIC data base and the classification number. A general subject and former title/data base name index follow the main text of the report.

\section{0,960}

PB91-171330

PC A12/MF A02

National Inst of Standards and Technology, Gaithersburg, MD. Office of Information Services.

NIST Serial Holdings, 1991.

Special pub. (Final).

M. L. Kingston. Feb 91, 268p NIST/SP-777

Also available from Supt. of Docs. as SN003-00303075-9. Supersedes PB90-183245.

Keywords: "Periodicals, "Catalogs(Documentation), "Collection, "Information centers, Standards, Libraries, Metrology * US National Institute of Standards and Technology, "NIST.

The publication contains holdings information for approximately 5,000 titles held in the National Institute of Standards and Technology (NIST) Research Information Center, representing current and noncurrent journals, periodicals, annuals, memoirs, proceedings, and transactions.

\section{General}

\section{0,961}

\section{PB91-189522}

Not available NTIS

National Inst of Standards and Technology (NCSL), Gaithersburg, MD. Systems and Software Technology

Div.

Final rept.

R. Hunter, P. Kaijser, and F. Nielsen. 1989, 11p Pub. in Computer Communications 12, n2 p69-79 Apr 89.

Keywords: "Documents, "Standards, Data processing, Information transfer, Format, Reprints, *ODA(Office Document Architecture), "Document processing.

The document provides a tutorial overview to the Office Document Architecture (ODA) standard--ISO 8613. The document presents historical information about the development of the standard and summarizes the features of ODA. The prime objective of ODA is to provide for the representation and encoding of documents so that they can be transferred between different document processing systems. ODA docu- ments may be transferred in three forms: formatted form, processable form, and formatted processable form. These forms are described as well as other concepts of ODA including: logical and layout structures, document constituents, content architectures, and so on. The ODA standard is briefly, yet thoroughly, de scribed.

\section{0,962}

PB92-116383

PC A03/MF A01

National Inst. of Standards and Technology, Gaithersburg, MD.

Guide for the Use of the International System of Units: The Modernized Metric System.

Special pub. (Final).

A. O. McCoubrey. Sep 91, 39p NIST/SP-811

Also available from Supt of Docs. as SNO03-00303113-5. See also PB92-109032 and N73-26969.

Keywords: *International system of units, *Metric system, "Conversion tables, Units of measurement.

The Guide for the Use of the Modernized Metric System is a practical tool for the use of authors in the preparation of technical manuscripts in conformance with the NIST policy that requires the use of the International System of Units (SI) for all publications. For this purpose the Guide replaces Exhibit 2-D of Chapter 2 in the NBS Communications Manual for Scientific Technical, and Public Information; it has also been prepared to support the broad objectives of the NIST pro gram for metric conversion. The Guide is further in tended to replace NBS Letter Circular LC 1120 (1979) as a document suitable for distribution to other Government agencies and the public.

\section{MANUFACTURING TECHNOLOGY}

\section{Computer Aided Design (CAD)}

100,963

PB91-147579 Not available NTIS National Bureau of Standards (NEL), Gaithersburg, MD. Factory Automation Systems Div.

UNIX in the Government's Automated Manufacturing Research Facility (AMRF).

Final rept.

D. Libes. $1986,6 p$

Pub. in Government Computer News $5, n 6$ p1-6, 14 Feb 86.

Keywords: "UNIX(Operating system), "Research projects, Computer aided manufacturing, Automation, Computer aided design, Robotics, Computer systems hardware, Programming languages, Operating systems(Computers), Reprints, *Automated Manufacturing Research Facility.

The report discusses all projects in the Automated Manufacturing Research Facility (AMRF) using UNIX Brief descriptions of each project include machine, language and version of UNIX used.

100,964

PB91-157164

PC A04/MF A01

National Inst. of Standards and Technology, Gaithers burg, MD.

NIST Express Working Form Programmer's Reference (Revised November 1990). Natlonal PDES Testbed Report Serles.

S. N. Clark. Dec 90, 52p NISTIR-4407

See also PB90-269531.

Keywords: *Computer aided design, "Computer aided manufacturing "Standards, "Software tools, Computer programming, Mathematical models, Programming manuals, Parsers, Translators, *PDES(Product Data Exchange Specifications), National Institute of Stand ards and Technology.

The Product Data Exchange Specification (PDES) is an emerging standard for the exchange of product information among various manufacturing applications. PDES includes an information model written in the Ex- 
press language; other PDES-related information models are also written in Express. The National PDES Testbed at the National Institute of Standards and Technology (NIST) has developed software to manipulate and translate Express models. The software consists of an in-memory working form and an associated Express language parser, Fed- $X$. The internal operation of the Fed-X parser is described. The implementation of the data abstractions which make up the Express Working Form is discussed, and specifications are given for the Working Form access functions. The creation of Express language translators using Fed- $X$ is discussed.

\section{0,965}

PB91-167262

PC A03/MF A01

National Inst. of Standards and Technology (NCSL) Gaithersburg, MD. Information Systems Engineering Div.

\section{SQL3 Support for CALS Applications.}

L. Gallagher. Feb 91, 39p NISTIR-4494

Keywords: "Computer aided manufacturing, "Data base management systems, Computer aided design Federal information processing standards, Standardization, Data processing security, Recursive functions, "CALS, "SQL3 database language, ANSI(American National Standards Institute), ISO(International Organization for Standardization).

Previous reports to CALS have identified the importance of Database Language SOL in CALS Phase II requirements. In particular, a July 1989 point paper on $S Q L$ and RDA identified features in the existing SQL standard and its near-term SQL2 replacement that are most appropriate to CALS data management concerns. The report focuses on SQL3, a follow-on standardization project for major new SQL enhancements that is expected to be adopted by American National Standards Institute (ANSI), International Organization for Standardization (ISO), and as a Federal Information Processing Standard (FIPS) in the mid 1990's. Many of the proposed SQL3 features are of particular importance to the Standard for the Exchange of Product model data (STEP) because of that standard's unique data modeling and data access requirements. Existing and planned features in SQL3 may not satisfy all STEP requirements, but they should provide an appropriate base from which many requirements can be suitably addressed. Since features in SQL3 are just now being specified, they are open to modification and improvement to best suit CALS needs. The report identifies the major enhancements under consideration by the ANSI and ISO SQL standardization committees and relates them to known manufacturing and product management requirements. It also discusses the status of these features in the SQL3 specification and indicates opportunities available to CALS to influence further development.

\section{0,966}

\section{PB91-18478}

PC A03/MF A01

National Inst. of

Gaithersburg, MD. for the Exchange of Product STEP: Standard for the Exchange of Product Syntactic Rules.

W. F. Danner, D. T. Sanford, and Y. Yang. Mar 91 , 21p NISTIR-4528

Keywords: "Standards, "Models, Computer aided design, Semantics, Syntax, "STEP(Standard for the Exchange of Product Model Data), PDES(Product Data Exchange using STEP), National Institute of Standards and Technology.

The report documents the rules that are used in the integration of Standard for the Exchange of Product Model Data (STEP) draft resource models. The rules are applied in the development of the STEP Integrated Resource that satisfies application requirements for STEP.

\section{0,967}

\section{PB91-216663}

PC A03/MF A0

of Standards (NEL), Gaithersburg.

D. Machine Intelligence Group.

Apparel STEP Translator.

H. T. Moncarz, and Y. T. Lee. Jun 91, 19p NISTIR4612

Sponsored by Defense Logistics Agency, Alexandria, VA.

Keywords: "Clothing industry, "Computer aided design, Computer aided manufacturing, Prototypes,
Data structures, File structures, Format, Translators, Man computer interface, Models, Standardization "STEP(Standard for the Exchange of Product Model Data), National Institute of Standards and Technology.

The paper describes the implementation of a prototype computer program for translating apparel pattern data between different file storage formats. The program demonstrates the feasibility of using a single, standard format as a mechanism for exchanging data between dissimilar pattern design systems. The software was developed as part of an ongoing project to incorporate apparel applications into the Standard for the Exchange of Product Model Data (STEP), an emerging international standard. In the short term, the project goal is to develop a neutral data format for exchanging two-dimensional pattern data between apparel computer aided design (CAD) systems. In the longer term, the goal is to develop an information model that can be used to encompass the entire apparel life cycle. This achievement would allow all of the processes involved in the apparel life cycle to share the same data.

\section{0,968}

PB91-222620

PC A03/MF A01 National Inst. of Standards and Technology, Gaithersburg, MD.

Configuration Management of the STEP Documents: Procedures and System Requlrements. $\mathrm{Na}$ tlonal PDES Testbed Report Series.

S. B. Katz. 10 Jul 91, 20p NISTIR-4629

Sponsored by Assistant Secretary of Defense (Production and Logistics), Washington, DC. Computer-Aided Acquisition and Logistic Support Program.

Keywords: "Computer aided manufacturing, "Standards, "Product development, Computer aided design "STEP(Standard for the Exchange of Product Mode Data), ISO(International Organization for Standardization), PDES(Product Data Exchange using STEP), National Institute of Standards and Technology, Configuration management.

The paper proposes configuration management procedures that support the International Organization for Standardization's (ISO'S) approval process for the Standard for the Exchange of Product Model Data (STEP), and introduces requirements for incorporating those procedures into a configuration management system.

\section{0,969}

PB92-112374

PC A04/MF A01

National Inst. of Standards and Technology, Gaithersburg, MD.

Proposed Testing Methodology for STEP Application Protocol Validation. National PDES Testbed Report Series.

M. J. Mitchell. 26 Sep 91, 51p NISTIR-4684

Sponsored by Assistant Secretary of Defense (Production and Logistics), Washington, DC. Computer-Aided Acquisition and Logistic Support Program.

Keywords: "Protocols, *Tests, "Product development "Standards, Computer aided design, Computer aided manufacturing, Standardization, Specifications, Models, Methodology, "STEP(Standard for the Ex change of Product Model Data), PDES(Product Data Exchange Using STEP).

An Application Protocol (AP) is a specification for a subset of the data described by the Standard for the Exchange of Product Model Data (STEP). Application Protocols are designed to permit practical implementations of STEP. Validation is needed to ensure that the technical solutions provided by the AP will work in a practical sense. The document proposes that the STEP development policy be strengthened to require that Application Protocols be validated prior to being submitted for standardization. Justification for the additional requirement on Application Protocols is provided. The body of the paper describes a series of validation techniques that are apropriate for the development methods used by STEP. A process is proposed under which these validation techniques should be applied. In addition, the paper describes the contribution that AP validation could make to conformance testing.

\section{0,970}

PB92-112523

PC A04/MF A01

National Inst. of Standards and Technology, Gaithersburg, MD.
Test Plan for Validating a Context Driven Integrated Model (CDIM) for Sheet Metal Die Design. National PDES Testbed Report Series.

K. K. Jurrens. Aug $91,55 p$ NISTIR-4699

Sponsored by Assistant Secretary of Defense (Production and Logistics), Washington, DC. Computer-Aided Acquisition and Logistic Support Program.

Keywords: "Computer aided design, "Computer aided manufacturing, "Models "Metal sheets, *Dies, Product development, Tests, Requirements, Protocols, Software tools, Facilities, Training, Scheduling CDIM(Context Driven Integrated Model), PDES(Product Data Exchange Using STEP) STEP(Standard for the Exchange of Product Model Data).

The document defines the Test Plan that will govern testing of the first Context Driven Integrated Model (CDIM) developed by the PDES, Inc. Sheet Metal Project (CDIM SM1). The Test Plan specifies the test objectives, methodology, constraints, requirements, evaluation criteria, resources, deliverables, and issue procedures for the testing activity. In addition, the document provides a brief introductory overview of CDIM SM1.

100,971

PB92-123090

PC A06/MF A02

National Inst. of Standards and Technology, Gaithersburg, MD.

Validation Testing Laboratory User's Guide. NatIonal PDES Testbed Report Series.

J. N. Breese, M. McLay, and G. Silvernale. Sep 91 105p NISTIR-4683

See also PB91-107581. Sponsored by Assistant Secretary of Defense (Production and Logistics), Washington, DC. Computer-Aided Acquisition and Logistic Sup port Program.

Keywords: *Computer aided design, *Computer aided manufacturing, "Standardization, "Product development, Standards, Data processing, UNIX(Operating system), Utility routines, Computer vision, Translato routines, Compilers, Parsers, Editing routines, Data base management systems, User manuals(Compute programs), Computer systems hardware, Computer systems programs, PDES(Product Data Exchange using STEP), STEP(Standard for The Exchange of Product model data), CALS, US NIST.

Product Data Exchange Using STEP (PDES) is the United States activity supporting the Standard for the Exchange of Product Model Data (STEP), a proposed international standard for product data representation and exchange. The document is a quick reference for those using the Validation Testing Laboratory of the National PDES Testbed. It is not intended for those wishing to learn about the tools in the Testbed but is rather for those who already have a working knowl edge of the programs and terminology. The Testbed was initiated in 1988 under the sponsorship of the U.S. Department of Defense Computer-aided Acquisition and Logistic Support (CALS) program. A major goal of the Testbed is to provide technical leadership in a national effort to implement a complete and useful specification for the exchange of product data. The specification must be designed to meet the needs of U.S. industry and the CALS program. The National PDES Testbed supports and actively participates in the international effort to develop (STEP).

\section{Computer Aided Manufacturing (CAM)}

\section{0,972}

PB91-132142

$\mathrm{PC}$ A03/MF A0

National Inst of Standards and Technology (NEL) Gaithersburg, MD. Factory Automation Systems Div. Software and Computer Integrated Manufacturing H. M. Bloom. Nov 90,11 p NISTIR-4468

Keywords: "Computer aided manufacturing, "Computer software, Systems engineering, Production management, Data management, Communications management, Standards, Data bases.

The paper outlines present and future directions for software in a Computer Integrated Manufacturing (CIM) environment. The three major CIM components production management, data management, and communications management -- are described in 
terms of software available today and future software based on emerging international standards. Key standards efforts such as product data exchange, open systems interfaces, database systems frameworks, and CIM frameworks are described.

\section{0,973}

PB91-134718

Not available NTIS National Bureau of Standards (NEL), Gaithersburg, MD. Center for Mfg. Engineering.

$A l$ in Manufacturing: The NBS AMRF as an Intelllgent Machine.

Final rept.

D. A. Swyt. $1988,16 p$ Expert Systems and the Leading Edge in Production Planning and Control, Intelligent Manufacturing (1st), p357-372 1988 .

Keywords: *Artificial intelligence, Robots, Standards, Automation, Reprints, "Automated Manufacturing Research Facility, "Computer aided manufacturing, National Institute of Standards and Technology, Control systems, Knowledge representation.

Consisting of robots, machine tools and computers, the Automated Manufacturing Research Facility (AMRF) is being integrated using a real-time, sensoryfeedback, data-driven hierarchical control architecture. As such, the AMRF is a research tool for investigating the manufacturing enterprise as a system of in telligent machines. The report describes the AMRF in terms of its real-time control system architecture; notes the role of symbolic languages, knowledge-representation, sensory-processing and other aspects of artificial intelligence (Al) in its development; and speculates on further application of $\mathrm{Al}$ in future intelligen manufacturing systems similar in form to the AMRF.

100,974

PB91-147595

Not available NTIS

National Bureau of Standards (ICST), Gaithersburg, MD. Systems and Network Architecture Div.

Application of OSI Protocols for Plant Information Networks.

Final rept.

R. J. Linn. 1987, 21p

R. J. Linn. 1987, 21p
Pub. in Proceedings of International Conference on Foundations of Computer Aided Process Operations, Park City, UT., July 5-10, 1987, p453-473.

Keywords: "Computer aided manufacturing, "Communication networks, "Protocols, Standards, Computer networks, Information systems, Computer architecture, Reprints, OSI(Open Systems Interconnection), MAP(Manufacturing Automation Protocol), GOSIP(Government Open Systems Interconnection Profile), TOP(Technical and Office Protocols).

The International Organization for Standardization (ISO) has developed a set of standards defining data communications protocols for heterogeneous computer systems. Collectively, these protocols are called the Open Systems Interconnection (OSI) protocols. Computer and communications equipment manufacturers are beginning to provide implementations of these protocols as product offerings which can be applied to manufacturing plant information networks. Summaries are provided of the functional aspects of selected international standards, and their relationships to U.S. Government and industrial profiles of the same stand ards. These profiles are the Government Open Systems Interconnection Profile (GOSIP), Manufacturing Automation Protocol (MAP) and Technical and Office Protocols (TOP). The MAP profile was developed under the leadership of General Motors Corporation with the objective of applying emerging international standards to plant information networks in highly automated factories. The TOP and GOSIP profiles extend the application of the same kernel of protocols to scientific, engineering, and office environments which may be geographically or locally distributed. Application of OSI protocols for plant information networks is illustrated by example.

100,975

(148122

Not available NTIS

National Bureau of Standards (NEL), Gaithersburg, MD. Center for Mfg. Engineering.

CIM, Data and Standardization withln the NBS AMRF.

Final rept.

D. A. Swyt. $1988,7 p$

Pub. in Robotics 4, n2 p193-199 Jun 88

Keywords: "Computer aided manufacturing, "Data management, "Standards, Automation, Robotics,
Computer aided design, Reprints, Automated Manufacturing Research Facility, National Institute of Standards and Technology.

Computer-Integrated-Manufacturing (CIM) is key to the factory of the future, shared data is key to integration, and interface standards are key to data-sharing. Within its Automated Manufacturing Research Facility (AMRF), the U.S. National Bureau of Standards is addressing issues of standardized interfaces in the four principal data activities of the automated factory: data preparation, data administration, data communication, and data-driven control. By means of such interface standards, the flexibly-automated robotics-based, computer-integrated factory of the future can be realized in a modular, easily-integratable, multi-vendor form.

\section{0,976}

B91-148130

Not available NTIS

MD. Center for Mfg. Engineering

Innovation in Manufacturing Technology: A View from NBS.

Final rept.

D. A. Swyt. $1985,16 p$

Pub. in Proceedings of Conference on Manufacturing Research: Organizational and Institutional Issues, Stuttgart, West Germany, August 19-23, 1985, p1-16.

Keywords: "Computer aided manufacturing, "Manufacturing, "Technology assessment, Automation, Process control, Robotics, Automatic control, Man machine systems, Standardization, Industrial plants, Industries, Reprints.

Based on work in measurements and standards for the automated manufacturing of discrete parts, the view of manufacturing technologies from the National Bureau of Standards is one of substantial change and growth following the current seminal stage of development. Economics and the desire for advanced capabilities drive the change. Relative to technological ideas being developed in laboratories such as NBS, current industrial robots are crude and the most automated processes not under adequate control. Simple extrapolation of current work will result in profoundly more advanced robotic systems and effective automated control of entire factories. This impending change provides substantial challenges for coordinated research in three key areas: the basics of unit processes, control systems for intelligent machines, and the integration of unit processes and intelligent machines into whole factory systems.

100,977

PC A03/MF A01 National Inst. of Standards and Technology (NEL), Gaithersburg, MD. Factory Automation Systems Div. Engineering Design Laboratory Guide.

A. B. Feeney. 19 Feb 91,19 p NISTIR-4519

Keywords: "Computer aided manufacturing, "Computer aided design, Structural analysis, Finite element analysis, Knowledge base, Computerized simulation, Numerical control, Clothing, US NIST, "Engineering Design Laboratory, "Factory automation, Geometric modeling.

The document provides a brief description of the systems available for use in the Engineering Design Laboratory at the National Institute of Standards and Technology, Gaithersburg, Maryland. The Engineering Design Laboratory was established to study the process of design, how design information can be represented, and how to make design information available to necessary systems throughout a product's life cycle. The document also discusses opportunities for collaborative or independant research in the Engineer: ing Design Lab.

\section{0,978}

PB91-167700

PC A04/MF A01

National inst of Standards and Technology (CAML), Gaithersburg, MD. Office of Applied Economics.

Automan 2.0: Decision Support Software for Automated Manufacturing Investments. User Manual. S. F. Weber. Mar 91, 75p NISTIR-4543, NIST/SW/ DK-91/007A , NIST/SW/DK-91/009A

For system on diskette, see PB91-506568 and PB91. 507194.

Keywords: "Computer aided manufacturing, "Investments, "Decision support sysems, Automation, Life cycle costs, Present worth, Evaluation, Comparison, Ranking, Ratings, User manuals(Computer programs), Documentation, Automan computer program.
The manual documents Automan, a microcomputer program designed to support multi-criteria decisions about automated manufacturing investments. The program permits users to combine quantitative and qualitative criteria in evaluating investment alternatives. Quantitative criteria could include such traditional financial measures as Life-Cycle Cost and Net Presen Value as well as such engineering performance measures as throughput and setup time Qualitative criteria could include flexibility and product quality. First, the user specifies the evaluation criteria and the investment alternatives to be evaluated. Second, the use makes pairwise comparisons between criteria to establish their weights. Third, the user rates each investment alternative with respect to the criteria. The pairwise comparison process helps the user rate the qualitative criteria, while measured performance data, such as cost, setup time, or throughput, can be entered for quantitative criteria. Automan combines ratings with criteria weights into an overall rating for each investment alternative and then ranks alternatives. Finally, graphical sensitivity analysis can be conducted to visualize the overall ratings of every alternative for every possible weight that could be assigned to any criterion. Automan comes with sample decision models and a manual that includes a detailed tutorial, a glossary of evaluation criteria, a bibliography, and an index.

100,979

PB91-174623

Not available NTIS

National Inst of Standards and Technology (NEL) Gaithersburg, MD. Precision Engineering Div.

High Precision Gaging with Computer Vision Systems.

Final rept.

T. D. Doiron. 1990, 12p

Contract DE-A105-85OR21584

Sponsored by Department of Energy, Oak Ridge, TN Pub. in Industrial Metrology 1, p43-54 1990.

Keywords: "Computer vision, Edge detection, Video equipment, Interfaces, Cameras, Precision, Accuracy Reprints, "Gaging, Robot vision.

In order to use a computer vision system for high accuracy gaging, the intensity array reported to the computer from the camera must correspond closely to the geometry of the part to be measured. To verify this correspondence for two different vision systems, a number of tests are reported and discussed. A number of effects due to the camera sensor geometry, the type of edge finder employed, the thermal properties of the camera, and the interface method used between the camera and computer are explored.

\section{0,980}

PB91-174938

Not available NTIS

National Bureau of Standards (NEL), Gaithersburg MD. Factory Automation Systems Div.

Error Prevention and Detection in Data Prepara tion for a Numerically Controlled Milling Machine. Final rept.

T. R. Kramer, and W. T. Strayer. 1987, 19p

Grant 70NANB4H0006

Sponsored by Catholic Univ. of America, Washington DC.

Pub. in Proceedings of Symposium on Intelligent and Integrated Manufacturing Analysis and Synthesis, Boston, MA., December 13-18, 1987, p195-213.

Keywords: "Computer aided manufacturing, "Error detection codes, *Milling machines, Numerical control, Workstations, Detection, Software(Computers), Errors, Checking(Proving), Milling(Machining), Quality control, Reprints.

In the Vertical Workstation (VWS) of the NBS Automated Manufacturing Research Facility, metal parts are machined automatically from a feature-based design A simple two-and-a-half dimensional part may be designed and machined within an hour, allowing half the time for design input. Workstation activity may be divid ed into design, process planning, data execution, and physical execution stages. In order to make VWS operation safe and accurate, extensive error prevention and detection (verification) procedures have been incorporated in the data preparation stages, particularly design and data execution. The software for these stages is about 700 pages of LISP code and runs on a Sun computer. About a quarter of the code is solely for error prevention. Automatic verification includes design editor dialogs, design enhancement, design verification (subdivided into parameter type checks, feature verification, and reference feature fit checking), process plan verification, workpiece verification, 
part model checking, and other items. Interactive verification includes design drawing, workpiece mode drawing, and tool path drawing. The feature verifiers are prepared by a rule-based automatic programming subsystem.

100,981

PB91-175091

Not available NTIS

National Bureau of Standards (NEL), Gaithersburg,

MD. Center for Mfg. Engineering.

U.S. Manufacturing Systems: Factories Past, Present, and Future.

Final rept.

D. A. Swyt. $1987,13 p$

Pub. in Proceedings of U.S./Japan Conference on Manufacturing Research Perspectives, Tokyo, Japan, October 27-29, 1986, p38-50 1987

Keywords: "Computer aided manufacturing, "Robots Automation, United States, Competition, Industria plants, Robotics, Reprints, Automated Manufacturing Research Facility.

U.S. manufacturing is in the throes of a fundamental changeover in its production technologies and systems. Under pressures of rapid globalization of its own markets, fierce competition from technologically-capable trading partners, and an aging industrial base, the United States is beginning to move from the labor-intensive mass production factory of the past to the in formation-intensive, custom-quantity factory of the future. The principle technology of the factory of the future is robotic-based, computer-integrated, flexible automation. In machine-tool-based factory production the computer and robot have been joined with the machine tool to form the flexible manufacturing cell, the first realization on the shop floor of the central principles of the factory of the future. To allow achievement of the total factory of the future, manufacturing research in the United States, represented by work at the Automated Manufacturing Research Facility, is directed at bringing the entire factory under computerintegrated control.

100,982

PB9 1-193367

PC A05/MF A01

National Inst. of Standards and Technology (NEL) Gaithersburg, MD. Factory Automation Systems Div. Concurrent Engineering through Product Data Standards.

G. P. Carver, and H. M. Bloom. May 91, 77p NISTIR4573

Keywords: "Computer aided manufacturing, "Standards, Concurrent processing, Automation, Information processing, Data bases, Computer aided design, "Concurrent engineering, STEP(Standard for the Exchange of Product Model Data), PDES(Product Data Exchange using STEP), National Institute of Standards and Technology.

Concurrent engineering involves the integration of people, systems and information into a responsive, efficient system. Integration of computerized systems allows additional benefits: automatic knowledge capture during development and lifetime management of a product, and automatic exchange of that knowledge among different computer systems. Critical enabler are product data standards and enterprise integration frameworks. A pioneering assault on the complex technical challenges is associated with the emerging international Standard for the Exchange of Product Model Data (STEP). Surpassing in scope previous standards efforts, the goal is a complete, unambiguous, computer-readable definition of the physical and functional characteristics of a product throughout its life cycle. U.S. government agencies, industrial firms, and standards organizations are cooperating in a program, Product Data Exchange using STEP (PDES), to develop and implement STEP in a shared-database environment. PDES will lead to higher, integrated levels of automation based upon information standards and frameworks. U.S. manufacturers will benefit from concurrent engineering without sacrificing the historical strengths and traditions of individuality, initiative, and intellectual property rights. Concurrent engineering, through information technology and stand ards, represents the power of a new industrial revolution. The role of the National Institute of Standards and Technology (NIST) National PDES Testbed, technical leadership and a testing-based foundation for the development of a STEP, is described.
National Inst. of Standards and Technology, Gaithersburg, MD.

Extracting STEP Geometry and Topology from a Solid Modeier: Parasolid-to-STEP.

Solid Modeier: Parasolid-to-STEP.

Prepared in cooperation with Catholic Univ. of America, Washington, DC. Sponsored by Assistant Secretary of Defense (Production and Logistics), Washing ton, DC. Computer-Aided Acquisition and Logistic Support Program.

Keywords: "Solids, "Three dimensional models, *Translators, Mathematical models, Computer software, Translator routines, Topology, Geometry *STEP(Standard for the Exchange of Product Model Data), "Parasolid software system.

Parasolid is a commercial solid modeling software system. It uses a manifold boundary representation. STEP is an emerging international Standard for the EXchange of Product Model Data. Included in STEP are proposed standards for Topology and Geometry which provide the definitions of data types required for building boundary representations) and for physical files representing products. Parasolid-to-STEP is a software system written in the $C$ programming language for translating a Parasolid format boundary rep resentation file giving the shape of an object into a STEP format file describing the same shape. The system handles elementary curves (lines, circle, ellipse) and surfaces (plane, sphere, cylinder, cone, torus).

100,984

PB91-194480

PC A03/MF A01

National Inst. of Standards and Technology, Gaithersburg, MD.

Configuration Management Concepts Document. National PDES Testbed Report Series.

S. B. Katz. 26 Apr 91, 33p NISTIR-4538

Keywords: "File management systems, "Access, Computer aided manufacturing, Documents, Software engineering, Quality assurance, "CMS(Configuration Management System), "STEP(Standard for the Exchange of Product Model Data), ISO(International Organization for Standardization).

The purpose of the document is to establish Configuration Management (CM) concepts to be applied in support of the development of the Standard for the Exchange of Product Model Data (STEP). Configuration management is the management of change. It is a formal discipline which provides methods and tools to: identify components, versions and baselines of selected items; and control changes to those items. CM provides a method for logically grouping related components throughout the various stages of product development. It also provides visibility and traceability for the evolving status of each item. An effective CM system thus identifies, controls, records, and reports on any functional, physical or status changes to the controlled items.

100,985

PB91-195172

Not available NTIS National Bureau of Standards (NEL), Gaithersburg, MD. Robot Systems Div.

CAD Directed Robotic Deburring

Final rept.

K. N. Murphy, R. J. Norcross, and F. M. Proctor

1988, 7p

Pub. in Proceedings of International Symposium on Robotics and Manufacturing (2nd): Research, Education, and Applications, Albuquerque, NM., November 16-18, 1988, p959-965.

Keywords: "Robots, "Deburring, "Computer aided manufacturing, "Computer aided design, Robotics, Real time systems, Off line systems, Programming, Control systems, Paths, Automation, Interactive graphics, Algorithms, Data structures, Reprints, Automated Manufacturing Research Facility.

At the Automated Manufacturing Research Facility, research is being conducted on techniques to automate robot programming. A technique has been developed and demonstrated in the Cleaning and Deburring Workstation which uses Computer Aided Design (CAD) geometry data to automatically generate robo deburring paths. Using a graphics interface, an operator specifies the edges on a part to be deburred, the deburring tools to be used, and the speeds, feed rates, and contact forces desired. Deburring paths are generated and sent to a PUMA 760 robot controlled by the Real-Time Control System. The robot uses a two-pass technique for deburring. On the first pass, the robot uses force feedback to correct the deburring path points to account for robot kinematic errors, tool wear, and minor part misplacement. On the second pass, the robot follows the corrected path, deburring the part The paper describes the techniques, algorithms and data formats used in the robotic deburring system.

\section{0,986}

PB91-195495

Not available NTIS

National Inst. of Standards and Technology (NEL) Gaithersburg, MD. Robot Systems Div.

Automating Robot Programming in the Cleaning and Deburring Workstation of the AMRF.

Final rept.

F. M. Proctor, K. N. Murphy, and R. J. Norcross

$1989,11 \mathrm{p}$

Pub. in Proceedings of SME Conference Deburring and Surface Conditioning ' 89 , San Diego, CA., February 13-16, 1989, p1-11.

Keywords: "Robots, "Automation, "Workstations, "Computer aided manufacturing, Robotics, Scheduling, Materials handling, Deburring, Cleaning, Precision finishing, Buffing, Process control, Reprints, Automated Manufacturing Research Facility.

In the cleaning and deburring workstation, two robots cooperate to accomplish deburring, buffing, cleaning, and handling of machined parts. A technique has been developed which uses part geometry data to automatically generate robot paths. Using a graphics interface, an operator specifies how a part is to be gripped, fixtured, deburred, buffed, and cleaned. A path planner combines the process plan with geometry data to compute robot paths. A workstation controller coordinates the actions of both robots, allowing various steps in the finishing process to be performed simultaneously. The paper describes the methods used to automate the finishing process.

100,987

PB91-203299

Not available NTIS

National Inst. of Standards and Technology (NEL) Gaithersburg, MD. Center for Mfg. Engineering.

Issues in the Design and Implementation of a System Architecture for Computer Integrated Manufacturing.

Final rept.

A. Jones, E. Barkmeyer, and W. Davis. 1989, 12p Pub. in International Jnl. of Computer Integrated Manu facturing 2, n2 p65-76 Mar/Apr 89.

Keywords: "Computer aided manufacturing, "Systems engineering, "Computer architecture, Production planning, Production control, Data management, Automation, Computer systems hardware, Computer systems programs, Reprints.

The advent of sophisticated automation equipment and computer hardware and software is changing the way manufacturing is carried out. To be competitive, manufacturing companies must integrate these new technologies into their existing and future factories. In addition, they must integrate the planning, control, and data management methodologies needed to make effective use of those technologies. The paper discusses several issues related to the design and implementation of a system architecture which can serve as the basis for those integration efforts. That architecture includes separate architecture for production planning and control, data management, and data communications.

\section{0,988}

PB91-203356

National Inst. of Standards and Technology (NEL) Gaithersburg, MD. Factory Automation Systems Div. Parser That Converts a Boundary Representation into a Features Representation.

Final rept.

T. R. Kramer. $1989,10 \mathrm{p}$

Contract NANB-7-H0716

See also PB89-160634. Spons
of America, Washington, DC.

Pub. in International Jnl. of Computer Integrated Manufacturing 2, n3 p154-163 May/Jun 89.

Keywords: "Computer aided manufacturing, "Machining, "Parsers, Workstations, File structures, Feature extraction, Computer aided design, Pattern recognition, Boundaries, Reprints, VWS2 computer program, Automated Manufacturing Research Facility, 


\section{MANUFACTURING TECHNOLOGY}

\section{Computer Aided Manufacturing (CAM)}

PDES(Product Data Exchange Specification), STEP(Standard for The Exchange of Product Data).

The VWS2 B-rep Parser is a computer program written in LISP that takes a file giving the boundary representation of a part as input and produces a file giving a feature-based representation of the part as output. The format of the input file is a Product Data Exchange Specification (PDES)/Standard for The Exchange of Product Data (STEP) boundary representation, and the format of the output file is that required by the VWS2 system of the National Institute of Standards and Technology (NIST) Automated Manufacturing Research Facility (AMRF). The parser deals with a limited range of two-and-a-half dimensional parts. The general approach to parsing is to expect that the part is parsable and look for arrangements of faces which are the signatures of features. The initial implementation of the approach recognizes five feature types. The approach is extendible to a wider range of feature and subfeature types, and to parts which have features made from several sides. Parts having features which intersect in a complex manner are likely to test the limits of the approach, or be beyond the limits. With the addition of the parser, the AMRF Vertical Workstation is capable of making a part from a PDES/STEP file without human intervention.

\section{0,989}

PB91-203737

Not available NTIS

National Bureau of Standards (NEL), Gaithersburg D. Robot Systems Div.

Control Structure for Multi-Tasking Workstations. Final rept.

R. J. Norcross. $1988,3 p$

Pub. in Proceedings of IEEE (Institute of Electrical and Electronics Engineers) International Conference on Robotics and Automation, Philadelphia, PA., April 2429, 1988, p1133-1135.

Keywords: "Workstations, "Control systems, "Manufacturing, Operating systems(Computers), Concurrent processing, Resource allocation, Multiprocessing, Reprints.

Manufacturing control modules, which are based on hierarchical control theory, decompose commands from a supervisory controller into elementary tasks to be performed by subordinate systems. The ability to simultaneously manage coordinated and independent functions of subordinates, while also processing new commands from the supervisory controller, is beneficial in advanced implementations of the controllers. The paper describes a control structure, based on computer operating system principles, which provides the desired capabilities. Utilizing concurrent processing and coordinating tasks via resource allocation provides extensive modularity which simplifies integration, gives a multi-processing environment, and produces the aforementioned capabilities.

\section{0}

PB91-222596

PC A03/MF A01

National Inst. of Standards and Technology, Gaithers burg, MD.

Development Plan: Application Protocols for Mechanical Parts Production. National PDES Testbed Report Series.

C. Stark, and M. Mitchell. 2 Jul 91, 36p NISTIR-4628 Sponsored by Assistant Secretary of Defense (Production and Logistics), Washington, DC. Computer-Aided Acquisition and Logistic Support Program.

Keywords: "Computer aided manufacturing, "Mechanical devices, "Protocols, Computer applications, Production planning, Standards, "STEP(Standard for the Exchange of Product Model Data), PDES(Product Data Exchange using STEP), National Institute of Standards and Technology.

The Standard for the Exchange of Product Model Data (STEP) is being developed as an international standard. The National Product Data Exchange using STEP (PDES) Testbed was established at the National Institute of Standards and Technology (NIST) specifically to address the development and testing of STEP, and to serve U.S. industry in its use of the standard. An Application Protocol (AP) is a specification for a subset of STEP data that can be implemented in an application system. The APs for Mechanical Parts Production effort will produce an early example of APs being used together in an operational prototype. This effort will develop, fully test, validate, and deliver a set of APs to the STEP Production Cell application systems which support the following: detailed design, process planning, and numerical control programming. The docu- ment describes the plan for developing and validating a series of three APs required as input to the STEP Production Cell. The resultant candidate APS, that will be submitted to the International Organization for Standardization (ISO) for inclusion in the standard, will have been validated and thoroughly tested prior to their submission.

\section{0,991}

\section{PB91-240796}

$\mathrm{PC}$ A03/MF A01

National Inst. of Standards and Technology (MEL), Gaithersburg, MD.

Manufacturing Systems Integration Control Entity Interface Document.

M. K. Senehi, S. Wallace, E. Barkmeyer, S. Ray, and E. K. Wallace. Jun 91, 47p NISTIR-4626

Keywords: "Computer aided manufacturing, "Control systems, "Interfaces, Automation, Hierarchies, Integrated systems. Production planning. Production control, Systems engineering, Models, NIST(National Institute of Standards and Technology), MSI(Manufacturing Systems Integration).

The document defines a set of interfaces for controllers to be incorporated into an integrated manufacturing production planning and control environment that conforms to the National Institute of Standards and Technology (NIST) Manufacturing Systems Integration (MSI) architectural model.

100,992

PB92-108885

PC A03/MF A01

National Inst. of Standards and Technology (MEL), Gaithersburg, MD.

Clock for the Manufacturing Systems Integration Testbed.

D. Libes. 5 Sep 91, 19p NISTIR-4666

Keywords: "Computer aided manufacturing, "Clocks, "Computer systems programs, Timing devices, UNIX(Operating system), Scheduling, Automation, Man computer interface, Time, MSi(Manufacturing Systems Integration).

The paper describes a software module that provides timing services to the Manufacturing Systems Integration (MSI) Testbed in the automated factory. The software 'alarm clock' provides services to other MSI software including: synchrony; real-time, or nonreal-time adjusted in a variety of ways; and alarms at relative or absolute intervals. By providing a central time service, these services are provided more reliably, efficiently, and flexibly than could any client on its own. The paper describes the implementation, interfaces, and how to design and write programs that use it.

\section{0,993}

PB92-112234

$\mathrm{PC} \mathrm{A03/MF} \mathrm{A01}$

National Inst. of Standards and Technology, Gaithersburg, MD.

Conceptual Architecture for a Mechanical Parts Production System Based on STEP. National PDES Testbed Rept. Series.

E. J. Barkmeyer, J. E. Fowler, and S. P. Magleby. 27

E. J. Barkmeyer, J. E. Fow $91,39 p$ NISTIR-4685

Prepared in cooperation with Brigham Young Univ., Provo, UT

Keywords: "Computer aided manufacturing, "Mechancal devices, "Standards, "Data processing, Computer aided design, File management systems, Protocols, Production models, Systems design, Data bases, Computer systems programs, Product development, *STEP(Standard for the Exchange of Product Model Data), PDES(Product Data Exchange Using STEP).

The Standard for the Exchange of Product Model Data (STEP) is an emerging standard addressing the problems of data exchange and representation of produced goods in a variety of manufacturing enterprises. Given that the initial STEP specifications largely pertain to mechanical parts production, this domain is an appropriate context for intial STEP implementations. The document describes an architecture for systems realizing a mechanical parts production capability using STEP data exchange. The functions of the major systems and relationships between systems is discussed. Software components which could be used to implement major systems are identified. Major emphasis is given as to how STEP is implemented and used in the context of the architeture.

100,994

PB92-112242
National Inst. of Standards and Technology (MEL), Gaithersburg, MD. Manufacturing Systems Integration: Initial Architecture Document.

M. K. Senehi, E. Barkmeyer, M. Luce, S. Ray, E. K Wallace, and S. Wallace. Sep 91, 70p NISTIR-4682 See also PB91-240796. Sponsored by Naval Research Lab., Washington, DC. Navy Manufacturing Technology Program.

Keywords: "Computer aided manufacturing, "Systems engineering, "Production planning, "Production control, Computer architecture, Systems design, Automation, Industrial plants, Hierarchies, Models, Error correction codes, Process control, Control systems, Data processing, Man computer interface, Materials handling, Data management, Resource allocation, Production management.

The goal of the Manufacturing Systems Integration (MSI) project at the National Institute of Standards and Technology (NIST) is to develop an approach to solving the problem of incompatible data and control processes within a manufacturing enterprise. A major activty of the MSI project is the development of a system architecture that incorporates an integrated production planning and control environment. A particular difficulty in developing such an architecture is the sharing of information between engineering, production management and control systems; there are no standards specifying the interactions among such systems. The MSI team is developing a testbed environment which allows experimentation with integrated production management and control systems. A critical feature of the testbed is the specification of an architecture and interfaces which allow the incorporation of commercial products and university-built prototype systems that support production engineering and control. The validation of the architecture will be via a demonstration of the production of selected parts, using either actual or emulated shop floor equipment or any combination of both. The document describes the first draft of that architecture and serves as an interim report to record progress in the architecture design.

\section{0,995}

PB92-112572

PC A05/MF A01

National Inst. of Standards and Technology (MSEL) Gaithersburg, MD.

Intelligent Processing of Materials, Technical ACtivities 1991. (NAS-NRC Assessment Panel, November $14-15,1991)$

Annual rept.

H. T. Yolken. 1991, 96p NISTIR-4693

See also PB89-151823 and PB91-143339.

Keywords: "Artificial intelligence, "Process control, "Materials, *Nondestructive tests, Magnetic properties, Steels, Feasibility analysis, Expert systems, Thermomechanical treatments, Metals, Powder metallurgy, Aluminum, Ceramics, Polymers, Detectors, On-line systems, Standards, US NIST.

Contents: Intelligent Processing of Rapidly solidified metal powders by inert gas atomization; Progress To wards Development of an Intelligent Processing System for On-line Control of Steel Sheet Formability Thermomechanical Processing of Steels; Magnetic Methods for Evaluation of Mechanical Properties of Steels; Ultrasonic Sensing of Liquid/Solid Interfaces in Metals; Physical and Mechanical Metallurgy and Sensor Technology Development for Intelligent Processing of Soldered Assemblies; Temperature Measurement in Aluminum Processing; Fluorescence and Optical Monitoring of Polymer Processing; Coating Technology Consortium; Measurement of Dispersion of Ceramic Powders in Dense Slurries Using Electroacoustics; Application of NMR Imaging Technique for Intelligent Processing of Materials; Eddy Current Sens ing of Carbon-Carbon Composites During Processing Development of Electromagnetic Probes for Intelligen Processing of Dielectric Materials; Transient Elastic Waves in Laminates; X-Ray Radioscopy Standards: Advancement of Documentary Standards for Nondestructive Evaluation; Eddy Current Standards and Methods; Ultrasonics and Acoustic Emission Standards and Methods; Nondestructive Evaluation Using Magnetic Particles.

\section{0,996}

PB92-117027

Not available NTIS

National Bureau of Standards (NEL), Gaithersburg MD. Automated Production Technology Div. 
Mare Island Flexible Manufacturing Workstation. Final rept.

K. Lee, and C. Lee. 1988, 10p

Sponsored by Naval Research Lab., Washington, DC. Navy Manufacturing Technology Program.

Pub. in Proceedings of International Conference on Computer Integrated Manufacturing, Troy, NY., May 23-25, 1988, p9-18.

Keywords: "Computer aided manufacturing, "Workstations, Automation, Robotics, Robots, Quality control, Reprints, NIST, Flexible manufacturing

The Mare Island Flexible Manufacturing Workstation is being developed at the National Institute of Standards and Technology (NIST). Advanced features include hierarchical control architecture, combined machining and turning operations in one machine, adaptable workholding, flexible robot end-effector, robotic changing of tools, chuck jaws, and gripper fingers, robotic workpiece loading and unloading, and automated buffer storage of supplies. Quality control is achieved by using in-process gaging, tool setting, and tool condition monitoring. When completed, the Mare Island Flexible Manufacturing Workstation will be the first small-batch flexible manufacturing system in the U.S. capable of continuous unattended production of RISIC. parts.

\section{0,997}

PB92-123058

PC A03/MF A01

National Inst. of Standards and Technology (NEL), Gaithersburg, MD. Factory Automation Systems Div. Multi-Enterprise Concurrent Engineering through International Standards.

G. P. Carver, and H. M. Bloom. Oct 91, 27p NISTIR4708

See also PB91-193367.

Keywords: "Computer aided manufacturing, "Product development, "Standards, Automation, Standardization, Data bases Concurrent processing "Concurrent engineering, PDES(Product Data Exchange Using STEP), STEP(Standard for The Exchange of Product model data)

Concurrent engineering involves the integration of people, systems and information into a responsive, ef ficient system. Integration of computerized systems allows additional benefits: automatic knowledge capture during development and lifetime management of a product, and automatic exchange of that knowledge among different computer systems. Critical enablers are product data standards and enterprise integration frameworks. A pioneering assault on the complex technical challenges is associated with the emerging international Standard for the Exchange of Product international Standard for the Exchange of Product
Model Data (STEP). Surpassing in scope previous standards efforts, the goal is a complete, unambiguous, computer-readable definition of the physical and functional characteristics of a product throughout its life cycle. The use of STEP will lead to higher, integrated levels of automation based upon information stand-
ards and frameworks. Concurrent engineering, through information technology and standards, represents the power of a new industrial revolution.

\section{Computer Software}

\section{0,998}

PB91-144378 PC A03/MF A01

National Inst. of Standards and Technology, Gaithersburg, MD

NIST STEP Working Form Programmer's Reference. National PDES Testbed Report Series (Revised).

S. N. Clark. 29 Nov 90,27 p NISTIR-4353/REV See also PB90-250077.

Keywords: "Standards, "Computer aided design "Computer aided manufacturing, Mathematical models, Computer software, Parsers, File structures, Translators, Computer programming, *PDES(Product Data Exchange Specification), "STEP(Standard for the Exchange of Product Model Data), National Institute of Standards and Technology.

The Product Data Exchange Specification (PDES) is an emerging standard for the exchange of product information among various manufacturing applications. The neutral exchange medium for PDES product models is the Standard for the Exchange of Product
Model Data (STEP) physical file format. The National PDES Testbed at the National Institute of Standards and Technology (NIST) has developed software to manipulate and translate STEP models. The software consists of an in-memory working form and an associated physical file parser, STEPparse. The internal operation of the STEPparse parser is described. The implementation of the data abstractions which make up the STEP Working Form is discussed, and specifications are given for the Working Form access functions. The creation of STEP translators using STEPparse is discussed.

\section{Job Environment}

\section{0,999}

PB91-167403

PC A06/MF A01

National Inst of Standards and Technology (NEL) Gaithersburg, MD. Center for Fire Research.

Users Guide for RAPID, Version 2.3.

Special pub. (Final).

R. D. Peacock, J. N. Breese, and C. L. Forney. Jan

91, 113 p NIST/SP-798

Supersedes PB87-100996. Also available from Supt. of Docs. as SN003-003-03046-5.

Keywords: "Fire tests, "Data reduction, "Computer applications, Data acquisition, Data processing, Data file, Input output processing, Utility routines, Digital command systems, User manuals(Computer programs), RAPID computer program.

The voluminous amount of data that can be collected by automatic data acquisition systems during large scale fire tests requires the use of a digital computer for the reduction of data. RAPID is a stand-alone program specifically designed to convert raw instrument voltages collected during such tests into a meaningful form. The reduced data can also be used alone or in combinations to obtain derived quantities. The program is written with the ability to accept data from a user defined data acquisition system and to check the correctness of data being analyzed. The data can be converted into meaningful scientific units and then presented in tabular or printer plot form, or stored for further processing. The guide provides detailed instructions for the use of the program.

\section{1,000}

\section{PB91-184762}

PC A03/MF A01

National Inst. of Standards and Technology (BFRL), Gaithersburg, MD.

Visibility of Exit Directional Indicators.

B. L. Collins, and P. J. Goodin. Mar 91, 44p NISTIR4532

See also PB91-161984. Sponsored by National Electrical Mfrs. Association, Washington, DC

Keywords: *Emergency planning, "Human factors engineering, Display devices, Indicator lights, Safety, Visibility, Human behavior, Luminance, Egress, Exit signs.

A three-phase experiment assessed the effectiveness of different configurations for exit signs and directional indicators. Two phases involved visibility assessments, while a third phase was a behavioral assessment. In the experiment, sign effectiveness was determined in terms of distance to detection, correct identification, and rated effectiveness, as well as speed through a corridor. The results indicated that a chevron in grey on white with a contrast of about 0.4 to 0.5 (to meet minimum specifications) was identified correctly at the greatest mean distance and received the highest mean ratings of effectiveness, as compared to other directional indicators. The combination of a 2.25 in chevron with a 6 -in EXIT sign was identified correctly at a mean distance of about $100 \mathrm{ft}$. Use of color, either red or green, increased the distance by about 15 to 20 ft. Visibility is best predicted by total chevron area, with chevrons with larger total areas seen at greater distances. Analysis of the movement data from the behavioral phase indicated that chevrons of 225 -in provided adequate visibility at about $100 \mathrm{ft}$, but that speed of movement is not a sensitive indicator for sign visibility. Finally, the data from all three phases indicate the importance of chevron size and configuration as well as sign color and contrast in determining visibility.

\section{Joining}

101,001

PB91-134619

Not available NTIS

National Inst. of Standards and Technology (IMSE)

Boulder, CO. Fracture and Deformation Div.

Report on 1989 Actions by International Institute of Welding.

Final rept.

T. A. Siewert, and M. J. Houle. 1990, 3p

Pub. in Materials Evaluation 48, p408, 411-412 Mar 90

Keywords: "Welding, "Nondestructive tests, Standards, Reprints, "Foreign technology, International In stitute of Welding, International organizations.

The summary describes the activities at the 1989 meeting of the International Institute of Welding Commission V - Testing, Measurement, and Control of Welds. The summary makes U.S. industry aware of European research and standard development in this area, and solicits U.S. input for international documents.

101,002

PB91-149278

Not available NTIS

National Inst. of Standards and Technology (IMSE) Boulder, CO. Fracture and Deformation Div.

Dynamic Arc-Power Source Response in GMA Welding.

T. A Siewert and G. Kohn 1989, 4p

Pub. in Proceedings of American Welding Society Annual Meeting (68th) and International American Welding Society Brazing Conference (18th), Chicago, IL., March 22-27, 1987, p43-46 1989.

Keywords: "Gas metal arc welding, "Dynamic response, Weld metal, Welding current, Base metal, Electric current, Filler metal, Weldments, Welding, Reprints.

Previous studies of droplet transfer frequency in gas metal arc (GMA) welding have been reported primarily as a function of the welding current. The study investigates both current and voltage effects in the short circuiting mode and reports the additional effects of the power supply characteristics and other welding procedure parameters on the droplet transfer. A compute simulation of short-circuiting transfer has been developed. The effect of changes in the welding parameters in the model are compared to actual welds. The simulated weld data match the actual weld data to within $10 \%$.

\section{1,003}

PB91-162339

Not available NTIS

National Inst of Standards and Technology (MSEL) Boulder, CO. Fracture and Deformation Div.

Analysis and Characterization of Commercial Welding Fluxes.

Final rept.

T. A. Siewert, and G. L. Franke. 1990, 9p

Sponsored by David Taylor Research Center, Bethes da, MD.

Pub. in Welding Research Supplement, p247s-255s Jul 90

Keywords: "Welding, "Composition(Property), "Spectroscopic analysis, $X$ ray fluorescence, $X$ ray diffraction, Fluxes, Welding rods, Welding electrodes, Chemical properties, Quality control, Reprints.

Various quantitative and qualitative flux analysis tech niques were evaluated using a series of highly basic submerged arc welding fluxes. The test matrix included some replicate testing so the precision of the primary measurement techniques could be compared. The results indicate that optical emission spectroscopy can match the precision of $x$-ray fluorescence, while providing data on many more elements, some of which could be important monitors of the quality of the flux. $X$-ray diffraction and particle size analysis provide additional information that complement the compositional data provided by the first two techniques. The techniques were able to identify compositional variations that explained the difference in the color of two flux samples and revealed a composition change with particle size for one flux sample. For applications where a tighter control of flux composition is warrant$e d$, these techniques can provide quality control information beyond that provided by typical quality control procedures. 
101,004

PB91-174250

National Inst. of Standards and Technology (MSEL)

Boulder, CO. Fracture and Deformation Div.

Sensing of GMAW Droplet Transfer Modes Using an ER 100S-1 Electrode.

Final rept.

G. Adam, and T. A. Siewert. 1990,6p

Pub. in Welding Research Supplement, p103-s-108-s

Mar 90.

Keywords: "Gas metal ore welding, "Control systems design, "Computer applications, Welding current, Welding electrodes, Weld metal, Microcomputers Computer aided manufacturing, Weldments, Statistical analysis, Sensors, Reprints.

The authors evaluated the capability of a $16-\mathrm{MHz}$ microcomputer with a fast analog-to-digital conversio board to capture welding data and to develop throughthe-arc sensing and control parameters for the gas metal arc welding process. After software was customized for the application, current and voltage data at sampling rates up to $50 \mathrm{kHz}$ could be recorded for relatively long times, limited only by available disk space. The analysis software enabled them to extract numerical data and manipulate it using statistical analysis. Fourier transforms, amplitude frequency histograms, peak-searching algorithms and smoothing procedures. To determine the capabilities of the system, it was applied to the study of welding with an ER100S-1 elec trode. The current and voltage were recorded for 197 welds which formed a matrix of welding conditions en compassing short-circuiting, globular and spray transter modes, as well as the transition regions between these modes.

\section{Manufacturing, Planning, Processing \& Control}

\section{1,005}

PB91-162230

Not available NTIS

National Bureau of Standards (NEL), Gaithersburg.

MD. Factory Automation Systems Div.

Research Tackles Automation Issues.

Final rept.

C. R. McLean. $1986,5 p$

Pub. in Design News 7, p94-98 Jul 86

Keywords: "Automation, "Manufacturing, "Technology utilization, Industrial plants, Research projects, Computer aided manufacturing. Fabrication, Assembly lines, Production management, Industries, Production engineering, Reprints, "Automated Manufacturing Research Facility.

Major technical obstacles have inhibited the spread of automation within key sectors of American industry. Factory automation researchers at the National Bureau of Standards in Gaithersburg, Md. are working on overcoming some of those obstacles. A small batch manufacturing system testbed, the Automated Manufacturing Research Facility (AMRF), currently under construction at the National Bureau of Standards (NBS), is designed to support research in factory automation standards. The article briefly describes the technical obstacles inhibiting factory automation, the goals of the project, key participants, system concepts, and the architecture of the AMRF testbed.

\section{1,006}

PB91-240812

PC A04/MF A01

National Inst. of Standards and Technology (MEL), Gaithersburg, MD

New Concepts of Precision Dimensional Measurement for Modern Manufacturing.

D. A. Swyt. Aug 91, 59p NISTIR-4644

Keywords: "Manufacturing, "Dimensional measurement, Error analysis, Accuracy, Precision, Machine tools, Length, Distance measuring equipment.

A new scheme for the analysis of the origin and propogation of errors in length-based dimensions is presented, one which is applicable to the characterization of measuring machines, machine tools, and the dimensioned parts which they characterize or form. The scheme involves a matrix of four dimensional-typespecific errors (associated with the length-based quantities of displacement, position, distance and ex tension) and three measurement-axiom-specific errors (associated with rules governing the zero, the unit and the scale of measurement). Application of the scheme is illustrated by a propogation of error analysis of the use of a laser-interferometer-based coordinate measuring machine for the determination of the length of a simple part. Also indicated is how an identical analysis applies to the characterization of the performance of machine tools which shape such parts.

\section{Optics \& Lasers}

\section{1,007}

PB91-162107 Not available NTIS National Inst. of Standards and Technology (MSEL), Gaithersburg, MD. Ceramics Div.

Analytic Solution for the Three-Layer Multiple Beam Interferometer.

Final rept.

R. G. Horn, and D. T. Smith. 1991, 7p

Contract ONR-N0014-8-F-0034

Sponsored by Office of Naval Research, Arlington, VA. Pub. in Applied Optics 30, n1 p59-65, 1 Jan 91.

Keywords: "Interferometers, Diffraction patterns, Coatings(Materials), Silver, Silica, Sapphire, Mica, Thin films, Measurement, Analytical solution, Metal films, Reprints.

A simple analytic solution for the condition of constructive interference for light transmitted through an interferometer incorporating three ideally transparent layers of arbitrary thickness and refractive index is presented. Also considered are the effect of adding two metallic coatings to the outer surfaces of the interferometer and empirical expressions for the associated phase changes for silver coatings on silica, sapphire, and mica substrates. A particular application to fringes of equal chromatic order can be utilized to obtain precise measurements of the thickness of extremely thin films sandwiched between two substrates.

\section{Quality Control \& Reliability}

\section{1,008}

PB91-143339

PC A05/MF A01

National Inst. of Standards and Technology (MSEL), Gaithersburg, MD.

Materials Reliability. Technical Activities, 1990. (NAS-NRC Assessment Panel, January 31-February 1, 1991).

H. I. McHenry. Dec 90, 82p NISTIR-90/4395

Keywords: "Research projects, "Reliability, Aircraft construction materials, Ultrasonic tests, Nondestruc tive tests, Polymer-matrix composites, Greens function, US NBS, Cryogenics, Aluminum alloys, Thermomechanical treatment, Lithium alloys, Packaging, Soldered joints, Stress analysis, Steels, Charpy impact test, Standards, Arc welding, Super conductors.

Selected Highlights of the Materials Reliability Division are as follows: Composites NDE: A high resolution ultrasonic system has been developed for inspecting thick polymer-matrix composites; NDE Instruments: Field trials were conducted on two prototype ultrasonic NDE instruments. A formability sensor system was delivered to the Ford Motor Company for evaluation at their Dearborn stamping plant. An ultrasonic system for roll-by inspection of railroad wheels is being evaluated at the American Association of Railroads test track in Pueblo, Colorado; Elastic Waves in Composites: A powerful technique using a time-dependent Green's function method has been developed for studying propagation of elastic waves and their scattering from discontinuities in anisotropic solids; Electronic Packaging: Computer programs have been developed to convert coordinate points on solder joint surfaces obtained by x-ray laminography and optical inspection into finite element meshes for stress analysis; Thermomechanical Processing: The continuous cooling transformation (CCT) characteristics and the high-temperature, high strain-rate flow properties were measured for microalloyed SAE 1141 forging steel Charpy Standards: Over 1000 industrial customers were supplied with Charpy V-notch reference specimens and calibration services for certification of Charpy impact test machines to ASTM Standard E23;
Cryogenic Testing: A 5 MN (1 million pound-force) servohydraulic testing machine was refurbished and equipped with a cryostat and dewar capable of testing specimens $2 \mathrm{~m}$ long and $50 \mathrm{~cm}$ in diameter in liquid helium; Aluminum-Lithium Alloys: A cooperative program with NASA indicated that aluminum-lithium alloys have sufficient oxygen compatibility for use in cryogenic tankage for the Advanced Launch System; Automated Welding: An intelligent welding program was initiated for the U.S. Navy in conjunction with Babcock and Wilcox and INEL; and Superconductors: Using an ionic-crystal model and ultra sound-velocity measurements, some physical properties of the Y1Ba2Cu3OX superconductor were studied as a function of oxygen content $(x=6-7)$.

\section{1,009}

PB91-147132

Not available NTIS

National Bureau of Standards (NML), Gaithersburg, MD. Radiometric Physics Div.

Fundamentals and Applications of Infrared Thermography for Nondestructive Testing.

Final rept.

J. Cohen. $1987,43 p$

Pub. in International Advances in Nondestructive Testing, v13 p39-81 1987.

Keywords: "Nondestructive tests, "Infrared thermography, "Thermography, Temperature measurement, Thermal radiation, Imaging techniques, Reprints.

The recent availability of highly sophisticated thermal imaging systems, in particular, digitized scanners with storage and computational capabilities, has engendered a rash of new NDT applications for infrared thermography which pervades industry. The paper treats two areas: fundamental physical concepts of infrared thermography, which provides the rationale for experimentation, and applications of thermography to NDT. Topics included in the former area are thermal radiation; thermal imaging systems, their operation, performance characteristics, and external influences; targets; and radiation thermometry. The latter area is a critical review of applications literature, with emphasis on the 'new thermography' and with examples selected to illustrate new trends and techniques.

101,010

PB91-147900

Not available NTIS National Inst of Standards and Technology (NML), Gaithersburg, MD. Ionizing Radiation Div.

Real-Time Radiology Standards: Results of a Workshop.

Final rept.

R. C. Placious, and T. A. Siewert. 1987, 2p

Pub. in Materials Evaluation 45, p1270-1271 Nov 87.

Keywords: "Radiology, "Radiography, "Standards, Real time, US NBS, Meetings, Reprints.

A two day workshop was held recently to review $\mathrm{Na}$ tional Bureau of Standards (NBS) activities in real-time radiology, to obtain the opinion of experts on standards needs in the rapidly developing technology, and to guide NBS in identifying, in order of priority, those activities that would most benefit the industry.

101,011

PB91-148163

Not available NTIS

National Bureau of Standards, Gaithersburg, MD. Program Office.

Industry and Government Strategies for Product Quality.

Final rept.

G. Tassey. $1989,15 p$

Pub. in International Jnl. of Technology Management 4, n2 p 189-203 1989.

Keywords: "Quality, "Product development, Government policies, Industries, Technology innovation, Competition, Manufacturing, Reliability, Marketing, Reprints.

Quality has become an increasingly important part of competitiveness strategies in the United States and other nations. Yet, the concept of quality and the approaches to implementing strategies to improve quality are not well developed. In particular, the term quality connotes to many a highly subjective image in which qualitative reactions by customers is the primary measuring stick. Views on the barriers to achieving higher levels of quality suffer from a singular focus on technical or organization factors. Quality in manufactured products is a combination of performance levels, performance stability, reliability, and longevity. Ad- 
vancing any or all of these elements of quality requires a range of technological, organizational, and marketing changes. Specific technical solutions cannot be effectively conceived and implemented in isolation from geted markets. This implies a trend toward integration of research and development, production, and marketing. Moreover, an increasing fraction of technical solutions will have to come from outside the corporate entity as will information on the broader technological and market trends.

\section{1,012}

PB91-149088

Not available NTIS National Bureau of Standards (NML), Gaithersburg, MD.

NBS Quality Assurance Support: Current and Planned Services.

Final rept.

C. W. Reimann. 1986, $9 p$

Pub. in Proceedings of American Society for Quality Control, Annual Quality Congress Transactions (40th), Anaheim, CA., May 19-21, 1986, p53-61.

Keywords: "Quality assurance, "US NBS, Measurement, Calibration, Uses, Reprints, "National Bureau of Standards, Reference materials, US NIST.

The National Bureau of Standards (NBS) is the central reference laboratory for the U.S. that provides measurement services - calibrations, standard reference materials, and standard reference data - for process and quality control applications, adaptable to a wide range of technologies and quality assurance management systems. Measurement provides a quantitative foundation for quality assurance. Chemical and physical tests are used increasingly throughout the cycle of production and plant operations, and measuremen now represents a significant manufacturing cost element. While measurement science has made enormous gains, the state of measurement practice lags in many areas. Despite the growing need for reliable measurement, there are a number of barriers to wider use of standards and related quality assurance services. Cooperative projects and broader community input to NBS priorities offer avenues to improve use of NBS quality assurance services and to stimulate the development of needed services. The paper summarizes growing application areas, measurement trends, NBS' current services and plans, and outlines mechanisms for effective interaction with NBS.

\section{1,013}

PB91-149930 Not available NTIS National Bureau of Standards (NML), Gaithersburg, $\mathrm{MD}$

Measurement Quality Assurance.

Final rept.

C. W. Reimann. 1987, 10p

See also PB89-147508.

Pub. in Proceedings of American Society for Quality Control Annual Quality Congress Transactions (41st), Minneapolis, MN., May 4-6, 1987, p389-398.

Keywords: "Quality assurance, "US NBS, Measurement, Calibration, Uses, Reprints, "National Bureau of Standards, Standards laboratories, US NIST, Traceability.

Measurements in the U.S. economy now cost more than $\$ 200 \mathrm{~B}$ annually. The principal uses of measurements are as appraisal tools in quality assurance applications affecting products, processes, environmental quality, and health care. High technology areas such as advanced materials, aerospace and defense systems, opto-electronics, and biotechnology incur measurement costs three to five times higher than such costs in traditional technologies. In addition, high technology areas demand greater accuracy, in many cases challenging state-of-the-art capabilities. The paper summarizes the rationale for measurement quality, key elements contained in such a system, linkages between standards laboratories and plant operations, and the role of the National Bureau of Standards (NBS). The paper will include an update of services available from NBS to support corporate quality assurance.

\section{1,014}

PB91-159103

Not available NTIS National Inst of Standards and Technology, Gaithersburg, MD. Standard Reference Materials Program.
Reference Materials: Their Role in Measurement Accuracy.

Final rept

W. P. Reed, and S. D. Rasberry. 1990, 9p

Pub. in Impact of Science on Society, n157 p71-79 1990.

Keywords: Materials tests, Measurement, Accuracy, Reprints, "Reference materials, US NIST.

Measurement compatibility and the establishment of production standards are very important aspects of manufacturing, material processing, and environmental monitoring. Organizations such as the US National Institute of Standards and Technology play a key role in providing the wide variety of reference materials re quired.

\section{1,015}

PB91-162354

Not available NTIS

National Inst. of Standards and Technology (NEL) Gaithersburg, MD. Precision Engineering Div.

Stylus Profiling at High Resolution and Low Force. Final rept.

J. F. Song, and T. V. Vorburger. 1991, 9p

Pub. in Applied Optics 30, n1 p42-50, 1 Jan 91.

Keywords: Gratings(Spectra), Calibration, Surface roughness, High resolution, Diamonds, Pens, Reprints, "Atomic force microscopy, "Profilometry, Styluses.

The paper describes experimental work to improve the lateral resolution of stylus instruments. The efforts in volve (1) use of a fine stylus, (2) low stylus load, (3) high magnification in the lateral direction, and (4) specimens with fine surface structure by which the lateral resolution of stylus instruments could be detected. By using styli with tip widths between 0.05 and 0.15 . micrometer a stylus load of $0.6-1.2 \times 10$ to the -6 power $\mathrm{N}$ (0.06-0.12-mgf), and a piezostage for lateral displacement, the authors detected 0.05-0.15 micromete lateral resolution on the surfaces of different kinds of specimens. To get a high lateral resolution, the most important consideration is a fine stylus with small tip size.

101,016
PB91-167221

PC A03/MF A01

National Bureau of Standards (NEL), Gaithersburg MD. Machine Intelligence Group.

Review of Current Geometric Tolerancing Theorles and Inspection Data Analysis Algorithms. S. C. Feng, and T. H. Hopp. Feb 91, 24p NISTIR4509

Sponsored by Naval Research Lab., Washington, DC. Navy Manufacturing Technology Program.

Keywords: "Dimensional measurement, "Tolerances(Mechanics), State of the art Computer aided design, Computer aided manufacturing, Inspection, Algorithms, Reviews, "Coordinate measuring machines.

The report provides an overview of the state of the art in mechanical dimensioning and tolerancing theories and CMM inspection data analysis technology. It is expected that the information included in this review will benefit CMM software developers, CMM users, and researchers of new CMM technology. The document is the result of a survey of published geometric dimensioning and tolerancing theories and post-inspection data analysis algorithms. Both traditional and modern theories have been reviewed. Principles on which current national standards and international standards based have been stated. These geometric dimensioning and tolerancing principles are commonly used in mechanical design and part inspection. Post-inspection data analysis algorithms, used for extracting features and evaluating tolerances, have also been reviewed. The effects of using different fitting criteria are discussed. From the theory and algorithm review, the authors recommend directions for future developmen in these areas. The bibliography covers activities and accomplishments of the research in advancing inspection technology

\section{1,017}

PB91-187765

PC A03/MF A01

National Inst. of Standards and Technology (NEL) Gaithersburg, MD. Robot Systems Div.

AMRF Composites Fabrication Workstation: A Test Methodology to Measure the Quality of Thermoplastic Composite Parts.

J. A. Falco. Apr 91, 23p NISTIR-4552

Keywords: "Composite fabrication, "Nondestructive tests, Composite materials, Workstations, Robotics,
Manipulators, Consolidation, Reinforced plastics, Quality control, Inspection, Methodology, Automated Manufacturing Research Facility, Thermoplastic composites.

The Robot Systems Division of the National Institute of Standards and Technology (NIST) requires a test methodology to measure the quality of the composite parts produced by an advanced manufacturing workstation being assembled in the Automated Manufac turing Research Facility (AMRF). The workstation will be used to study methods of fabricating complex shaped, continuous carbon fiber reinforced, thermoplastic composite parts using pre-impregnated tow. The paper discusses the mechanisms of thermoplastic consolidation and the various defects associated with poor consolidation. Several testing techniques are dis cussed concerning their ability to locate, identify and or quantify these defects. The discussion is followed by a trade off analysis of all considered testing techniques in an attempt to determine the most effective test methodology to measure the quality of thermoplastic composite parts.

101,018

PB91-190058

Not available NTIS

National Inst of Standards and Technology (MSEL) Boulder, CO. Fracture and Deformation Div.

Image Quality Indicator Design for Radioscopy and Tomography.

Final rept.

T. A. Siewert, D. W. Fitting, D. A. Shepherd, M. W. Austin, and C. N. McCowan. 1990, 8p

Pub. in Review of Progress in Quantitative Nondestructive Evaluation, v9 p383-390 1990.

Keywords: "Tomography, Monte Carlo method, X-ray magery, Radiation transport, Image analysis, Resolu tion, Reprints, "Image quality indicators, "Radioscopy.

A new image quality indicator design is proposed for real-time radioscopy systems. The design has spherical symmetry, so it provides the same image quality information independent of rotation. The resolution of several prototype indicators was measured by image analysis of a fine-grained radiograph. The resulting mage intensity profile was compared to a radiation transport model.

\section{1,019}

PB91-194993

Not available NTIS

National Inst. of Standards and Technology (NEL) Gaithersburg, MD.

Process Quality Enhancement for Machine Tools: Measurements and Standards.

Final rept.

J. W. Lyons. 1989,11

Pub. in Proceedings of U.S.-China Science, Technology, and Economic Development Program - Technica Transformation of Traditional Industries, May 1988 p70-80 1989

Keywords: "Quality assurance, "Process control Process computers, Machine tools, Measurement Standards, Mathematical models, Error correcting devices, Error detection codes, Reprints.

A careful study of three-dimensional coordinate meas uring machines (CMM) has been made to ascertain the errors inherent in the machines and to learn how to provide measurement services for such devices. The errors in such machines are largely repeatable, systematic errors; the random errors are generally much smaller. Improved performance can be achieved with these machines by measuring the systematic errors and then removing them by adding corrections to the control computer. Next, the similar but more difficult problem of error determination and correction for cutting machines was considered. Besides the quasistatic errors found in the CMMs there are additiona dynamic errors, principal among which are from thermal gradients arising in spindle bearings and othe points of friction. Ways were devised to compute these errors from real-time sensory information combined with stored data on machine behavior and, also in real time, to add correction information to the control signals. Ultimately, quality assurance for dimensions of parts will be attained in the factory of the future by an extension of the above approach rather than by postproduction gaging. The computer is making it possible to understand the behavior of the process machines and to use mathematical models of this behavior in the process control computers so that the machines are self-correcting and must perform within the specified control limits. 


\author{
101,020 \\ PB91-203463 \\ Not available NTIS \\ National Bureau of Standards (NEL), Gaithersburg \\ MD. Precision Engineering Div. \\ Capacitance versus Stylus Measurements of Sur- \\ face Roughness. \\ Final rept. \\ A. G. Lieberman, T. V. Vorburger, C. H. W. Giauque, \\ D. G. Risko, R. Resnick, and J. Rose. 1988, 16p \\ Pub. in Surface Topography 1, p315-330 1988.
}

Keywords: *Surface roughness, Mathematical models, Capacitance, Profiles, Probes, Metals, Pens, Reprints, Styluses.

Roughness measurements were performed upon a large variety of mechanical metal surfaces using capacitance roughness gauges and high-quality stylus instruments. Profile measurements obtained from the stylus instruments served as the basis for modeling the response of the capacitance gauge to the surfaces. The probing element of the capacitance gauges consisted of a $2.0 \times 16.8 \mathrm{~mm}$ flexible platen, which, togeth er with the rough metal surface, formed an electrical capacitor. Modeling the probe involved a detailed understanding of (1) how each element of the rough surface affects the sensed capacitance and (2) the way the flexible platen rests on top of the highest peaks of the surface and sags between these peaks. The model was realized as a FORTRAN computer program that uses a digitized stylus-generated profile as input data and computes the capacitive-roughness paramete $R$ (c) as output. The model was validated by comparing the computed value of $\mathrm{R}(\mathrm{c})$ to that measured using the capacitance gauge for each of 41 different surfaces. The correlation of $R$ (c) with more traditional definitions of roughness is also addressed for these same surfaces.

\section{1,021}

\section{PB91-216721}

(Order as PB91-216705, PC A07/MF A01)

National Inst. of Standards and Technology, Gaithersburg, MD.

Standard Reference Specimens in Quality Control of Engineering Surfaces.

J. F. Song, and T. V. Vorburger. 1991, 19p

Included in Jnl. of Research of the National Institute of Standards and Technology, v96 n3 p271-289 May/Jun 91.

Keywords: "Surface roughness, *Quality control, Calibration, Standard reference materials.

In the quality control of engineering surfaces, the authors aim to understand and maintain a good relationship between the manufacturing process and surface function. This is achieved by controlling the surface texture. In the paper, the characteristics, utilizations, and limitations of different classes of precision roughness calibration specimens are described. A measuring procedure of engineering surfaces, based on the calibration procedure of roughness specimens at NIST, is proposed. This procedure involves utilization of check specimens with waveform, wavelength, and other roughness parameters similar to functioning engineering surfaces. These check specimens would be certified under standardized reference measuring conditions, or by a reference instrument, and could be used for overall checking of the measuring procedure and for maintaining accuracy and agreement in engineering surface measurement.

\section{1,022}

\section{PB92-112416}

PC A08/MF A02

National Inst. of Standards and Technology (TS) Gaithersburg, MD. Weights and Measures Program.

Uniforms Laws and Regulations as Adopted by the National Conference on Weights and Measures (76th), 1991.

Handbook.

C. S. Brickenkamp, and J. A. Koenig. Oct 91, 173p

NIST/HB-130-1992 ED

Also available from Supt. of Docs. as SN003-003 03105-4. Supersedes PB91-107102.

Keywords: "Weight measurement, "Law(Jurisprudence), "Regulations, "Handbooks, Automotive fuels, Consumer affairs, Packaging, Standardization, Commodities, Prices, Labels, Food, Sales ${ }^{*}$ Weights and measures, Weightmaster law, Open dating, Unit pricing

The handbook, which is revised annually, compiles the uniform laws and regulations developed by the Com- mittee on Laws and Regulations of the National Conference on Weights and Measures (NCWM). The compilation itself was approved by the NCWM in 1979, and the edition includes amendments adopted by the Conference at its annual meeting in 1991. The edition also contains an updated reprint of Section 2 from NCWM Publication 3, 'Policy, Interpretations, and Guidelines.' The NCWM recommends adoption and promulgation by the States of these uniform laws and regulations as updated in the handbook.

\section{1,023}

PB92-112424

PC A10/MF A03

National Inst. of Standards and Technology (TS), Gaithersburg, MD. Weights and Measures Program.

Specifications, Tolerances, and Other Technical Requirements for Weighing and Measuring Devices as Adopted by the 76 th National Conference on Welghts and Measures, 1991.

Handbook.

H. V. Oppermann. Oct 91, 214p NIST/HB-44-1992

$\mathrm{ED}$

Also available from Supt. of Docs. as SNOO3-00303114-3. Supersedes PB91-107136.

Keywords: "Measuring instruments, "Weight indicators, "Handbooks, Tolerances(Mechanics), Volume unit meters, Dimensional measurement, Specifications, Length, Odometers, Liquids, Vapors.(25I)Grain moisture, Taximeters.

Handbook 44 was first published in 1949, having been preceded by similar handbooks of various designations and in several forms beginning in 1918. The 1992 edition was developed by the Committee on Specifications and Tolerances of the National Conference on Weights and Measures with the assistance of the Office of Weights and Measures of the National Institute of Standards and Technology. It includes amendments adopted by the 76th annual meeting of the $\mathrm{Na}$ tional Conference on Weights and Measures in 1991. Handbook 44 is published in its entirety each year following the annual meeting of the National Conference on Weights and Measures.

\section{1,024}

PB92-112465

PC A 16/MF A03

National Inst. of Standards and Technology (TS), Gaithersburg, MD. Weights and Measures Program.

Report of the National Conference on Weights and Measures (76th). Held in Philadelphia, PA. on July 14-19, 1991.

Special pub. (Final).

C. S. Brickenkamp, and A. H. Turner. Sep 91, 374p

NIST/SP-816

Also available from Supt. of Docs. as SNO03-00303115-1. See also report for 1990, PB91-112763. Library of Congress catalog card no. 26-27766.

Keywords: "Weight measurement, *Regulations, *Meetings, Automotive fuels, Consumer affairs Tolerances(Mechanics), Law(Jurisprudence), Railroad tracks, Food packaging, Measuring instruments, Standardization, Specifications, Commodities, Metrology, Training, Safety, "Weights and measures, National Type Evaluation Program, NTEP program.

The theme of the 76th Annual Meeting of the National Conference on Weights and Measures (NCWM) was 'Weights and Measures for the Twenty-first Century.' In the address to the delegates. Chairman David Smith of Nospection Service and NCWM joint program; the National Type Evaluation Program; and the needs for State metrology. Special meetings included those of the Metroligists, the Associate Membership Committee, the Retired Officials Committee the Scale Manufacturers' Association, the American Petroleum Institute, the Industry Committee on Packaging and Labeling, the State regional weights and measures associations, and the National Association of State Departments of Agriculture Weights and Measures Division, and the National Council on State Metrication. Reports by the standing and annual committees of the Conference comprise the major portion of the publication along with the addresses delivered by Conference officials and other authorities from government and industry.

\section{Research Program Administration \& Technology Transfer}

101,025

PB91-14935

Not available NTIS

National Bureau of Standards (NEL), Gaithersburg,

MD. Center for Mfg. Engineering.

Transferring NBS Technology to Small Manufacturers Through State and Local Centers.

Final rept.

D. A. Swyt. $1988,7 p$

Pub. in Jnl. of Technology Transfer 13, n1 p7-13 1988.

Keywords: "Technology transfer, "Small businesses, Manufacturers, Government/industry relations, Robotics, Reprints, Automated Manufacturing Research Facility.

The paper provides a model of a technology-transfer system in which a federal laboratory -- the National Bureau of Standards's Automated Manufacturing Research Facility -- is linked to small manufacturers through state- and community-based technology centers, where the various institutions in the system are matched along dimensions of: level of government: stage in cycle of innovation; education of prototypical worker; and nature of capital-revenue base.

\section{Robotics/Robots}

101,026

PB91-157214

PC A03/MF A01

National Inst. of Standards and Technology (NEL), Gaithersburg, MD. Intelligent Controls Group.

TROI User's Guide.

B. Warsaw, and J. Michaloski. Jan 91, 46p NISTIR4471

Keywords: "Robots, "Control systems, "Man machine systems, Space stations, Man computer interface, User manuals(Computer programs), Real time systems, Interactive systems, *TROI(TeleRobotic Operator Interface), Object-oriented programming.

The document provides an introduction to the TeleRobotic Operator Interface (TROI) system and a user guide to TROI programming and operation. TROI provides a flexible, extensible, object-oriented interface to the NASREM robot control system (RCS). It consists of two major portions, the X-window system Graphical User Interface (GUI), and the RCS's data-server interface modules. TROI provides a highly dynamic environment for interacting with the RCS. The user is able to view and modify state variables of a running control system, and to edit, save, and load graphical interface configurations while connected to a running control system. In this way, the user can interactively perform diagnostics, switch diagnostic contexts by creating and destroying interactive objects, and reconfigure data flow networks, allowing control of RCS operations without the costs of switching operating modes. TROI merges a user-initiated window system event model and an independent data driven event model into a single event stream.

101,027

PB91-158568

Not available NTIS

National Bureau of Standards (NEL), Gaithersburg, MD. Robot Systems Div.

Self-Adjustment of a Robot Joint Controller.

Final rept.

N. Dagalakis, and D. R. Myers. 1986, $8 p$

Pub. in Proceedings of Annual IEEE (Institute of Electrical and Electronics Engineers) Industrial Electronics Society Conference (12th), Milwaukee, WI., September 1986, p530-537.

Keywords: "Robots, *Joints(Anatomy), "Self adaptive control systems, Man machine systems, Optimization, Reprints.

A self-adjustment technique based on performance scheduling is applied to a robot joint controller. The technique allows a user to specify the desired time response of a joint to a step input. Using an optimization search routine the robot controller modifies its gain settings until its joint response matches the reference response. The adjustment technique has been tested on the wrist rotation joint of an industrial robot using a joint controller excitation and examination procedure 
previously developed at the National Bureau of Standards. The results indicate that the technique does permit the robot controller to modify joint response to match a user-specified desired performance.

101,028

PB91-158576

Not available NTIS MD. Robot Systems Div.

Application of the Joint Excitation Gear Adjustment Technique to Low Frequencies and Heavy Robot Joints.

Final rept.

N. G. Dagalakis, and D. R. Myers. 1987, 11p

Contract N00014-83-K-0236

Sponsored by Office of Naval Research, Arlington, VA Pub. in Proceedings of SME Conference on Robots, Chicago, IL., April 27-30-, 1987, p17-43-17-54.

Keywords: "Robots, "Gears, Performance evaluation, Adjusting, Accelerometers, Low frequencies, Reprints

Previous work has demonstrated the use of the robot oint excitation technique and coherence analysis to adjust robot joint gears of light links for maximum precision. Band limited random joint motions are induced and the response of the link is monitored by one or more accelerometers. The gears are adjusted to minimize the effect of nonlinearities caused by backlash or mize the effect of nonlinearities caused by backlash or tight meshing. The application of the technique to low perimental results are given from several robot adjustment tests as one recommendation for the easiest way to use this technique.

\section{1,029}

PB91-159194 Not available NTIS National Bureau of Standards (NEL), Gaithersburg, MD. Automated Production Technology Div

Design and Implementation of a Five-Axis Robotic Micromanipulator for Inserting Parts into Precision Collets.

Final rept.

A. H. Slocum, L. Greenspan, and J. P. Peris. 1988 ,

Pub. in International Jnl. of Machine Tools and Manufacture 28, n2 p131-1391988.

Keywords: "Manipulators, "Robotics, Servomechanisms, Hydraulic cylinders, Hydraulic motors, Degrees of freedom, Machine tools, Reprints.

The design, construction, and testing of a five-axis micromanipulator is described. It is designed to be attached to the end of a machine tool tending robot and to insert a part into a collet which is only $0.1-0.2 \mathrm{~mm}$ $(.004-.008$ in) larger in diameter than the part; thus compensating for robot positioning errors. The microcompensating for robot positioning errors. The micro-
manipulator weighs $22 \mathrm{~N}$ ( $5 \mathrm{lbs})$ and is $64 \mathrm{~mm}(2.5 \mathrm{in})$ hick and $127 \mathrm{~mm}(5 \mathrm{in})$ square with two linear axes with $+/ .3 \mathrm{~mm}(0.13 \mathrm{in})$ of travel, two angular axes with $+/-4$ angular degrees of motion, and one linear degree of freedom with $+/-25 \mathrm{~mm}(1.0$ in) of travel. Hydraulic actuators, with $6.9 \mathrm{MPa}(1000 \mathrm{psi})$ fluid, provide
$2700 \mathrm{~N}$ (600lbs) of force along the linear axes, and 68 $2700 \mathrm{~N}(600 \mathrm{lbs})$ of force along the linear axes, and 68
$\mathrm{~N}-\mathrm{m}(600 \mathrm{in}-\mathrm{lbs})$ of torque about angular axes. Repeatability of the device is $.013 \mathrm{~mm}$ (.0005 in)

\section{1,030}

PB9́1-16736

PC A03/MF A01

National Inst. of Standards and Technology (NEL) Gaithersburg, MD. Robot Systems Div.

Robot Characterization Testing.

N. Dagalakis. Jan 91, 19p NISTIR-4510

Keywords: "Robots "Characteristics, "Performance evaluation, Tests, Manpower, Equipment, Capabilities, Standards, Parameter identification, Man machine systems.

The document describes the field of robot characterzation which is broken into the areas of performance, parameter identification, and environmental interaction. Each area is explored by considering the tests, equipment, and manpower required to characterize the capabilities and performance of robots.

101, 031
PB91-174961

Not available NTIS

National Bureau of Standards (NEL), Gaithersburg, MD. Robot Systems Div.

Robot Sensor Language.

Final rept.

S. Leake. $1987,8 p$

Pub in Proceedings of Goddard Conference on Space Applications of Artificial Intelligence (Al) and Robotics, Greenbelt, MD., May 13-14, 1987, p1-8.
Keywords: "Robot sensors, "Programming languages, Control systems, Syntax, Interactive systems, Algorithms, Debugging(Computers), Reprints, RSL(Robot Sensor Language).

Robot Sensor Language (RSL) is a data-driven, semiinterpreted, hierarchical, user extensible, robot task description language. It provides four levels of task decomposition, with structures and syntax specialized for each level. The user can add commands for new sensors appropriate to the task at hand. The language is highly interactive, easing debugging and algorithm development. It may also be used as an interface to a task planning system.

101,032

PB91-174979

Not available NTIS

National Bureau of Standards (NEL), Gaithersburg, MD. Robot Systems Div.

Control of Flexible Robot Arm.

Final rept.

J. D. Lee, L. S. Haynes, B. L. Wang, and K. H. Tsai. 1987, 11p

Pub. in Proceedings of Conference on Modeling and Control of Robotic Manipulators and Manufacturing Processes, Boston, MA., December 13-18, 1987 , p241-251.

Keywords: "Robot arms, "Control systems, Computerized simulation, Sampling, Degrees of freedom, Damping, Reprints.

The work is a computer simulation of the control of the flexible robot arm. The dynamic equations for a singlelink flexible robot arm have been derived rigorously. The arm has two degrees of freedom in rotation and one in translation so that the workspace is three-dimensional. The payload is simulated by attaching additional mass to the arm at a specified location. The gov erning equations of the plant and the measurements are nonlinear. The process of control is divided into two stages: coarse control and fine control. Based on the pole-placement method, a linear observer is constructed for fine control. The numerical results of several cases are presented here. The effects of damping and sampling rate are also discussed.

\section{1,033}

PB91-194969

Not available NTIS

National Inst of Standards and Technology (NEL) Gaithersburg, MD. Robot Systems Div.

NASREM Robot Control System and Testbed.

Final rept.

R. Lumia, J. Fiala, and A. Wavering. 1990, 7p

Pub. in International Jnl. of Robotics and Automation 5, n1 p20-26 1990.

Keywords: "Robots, "Control systems, Hierarchies, Robotics, Manipulators, Remote sensing, Mathematical models, Algorithms, Test equipment, Reprints "NASREM, National Institute of Standards and Technology, National Aeronautics and Space Administration.

The National Aeronautics and Space Administration (NASA) National Institute of Standards and Technology (NIST) Standard Reference Model for Telerobot Control System Architecture (NASREM) provides the framework for a complete manipulator control system It is composed of three hierarchies: task decomposition, world modeling, and sensory processing. In the process of building NASREM, a great deal of effort has been spent in the definition of the interfaces between levels of the hierarchy so that the majority of robot control and sensory processing algorithms in the literature can be implemented. This allows the realization of the NASREM architecture to serve the dual purpose of robot controller and testbed for robot control algorithms. The paper describes the purpose and overall organization of NASREM. Then, two examples of NASREM task decomposition modules are discussed in terms of function as well as interface requirements.

\section{1,034}

PB91-203190

Not available NTIS

National Bureau of Standards (NEL), Gaithersburg MD. Robot Systems Div.

Application of the PIPE Image Processing Machine to Scanning Microscopy.

Final rept.

M. Herman. $1988,5 p$

Pub. in Proceedings of SPIE (Society of Photo-Optical Instrumentation Engineers) - Scanning Microscopy Technologies and Applications, Los Angeles, CA. January $13-15,1988$, v897 p169-173.
Keywords: "Robots, "Computer vision, "Image processing, Pipelining(Computers), Robotics, Microscopy, Scanners, Stereoscopy, Motion, Reprints.

PIPE is a pipelined image processing device that was designed for real-time robot vision applications. It accepts image from a video camera 60 times per second and contains hardware for digitizing, displaying and performing operations on these images at video rates. Each stage of the pipeline contains arithmetic and logic units, convolvers, image buffers, and look-up tables. The purpose of the paper is to introduce PIPE and some of its applications to the scanning microscopy community. Three kinds of applications are de scribed. The first is stereo analysis, whose purpose is to automatically extract range from two cameras mounted side by side. The second application is motion analysis and tracking. The application involves detecting motion, measuring its velocity, using it to obtain three-dimensional information, and tracking it through time. The final application is inspection of two dimensional patterns.

\section{1,035}

PB91-203208

Not available NTIS

National Inst. of Standards and Technology (NEL) Gaithersburg, MD. Robot Systems Div.

Video Compression for Remote Vehicle Driving. Final rept.

M. Herman, K. Chaconas, M. Nashman, and T. H.

Hong. $1989,8 p$

Pub. in Proceedings of SPIE (Society of Photo-Optical Instrumentation Engineers) - Mobile Robots III, Cambridge, MA., November 10-11, 1988, v1007 p136-143 1989.

Keywords: "Robotics, "Ground vehicles, "Teleopera tors, Image processing, Data compression, Real time Remote control, Algorithms, Pipelining(Computers) Reprints.

In order to effectively drive a remote ground vehicle using video images, the operator must be provided with a natural, real-time video sequence and the imagery must be accurate and detailed enough so that the operator can make mobility and survivability deci sions. Unfortunately, high data rate communication channels are often not feasible for the task. To accom plish remote driving using a low data rate channel video compression techniques must be incorporated. The paper discusses the remote vehicle driving problem and describes several video compression algo rithms that have been implemented on PIPE, a real time pipelined image processing machine. The pape then discusses how the algorithms are evaluated on real-world remote driving tests. Finally, advanced tech niques for providing video compression are proposed.

101,036

PB91-217414

PC A03/MF A01

National Inst. of Standards and Technology (NEL), Gaithersburg, MD. Robot Systems Div.

Generation of Smooth Trajectories without Planning.

J. C. Fiala. Jun 91, 19p NISTIR-4622

Keywords: "Manipulators, "Control systems, "Trajectory control, Dynamical systems, Control theory, Robotics, Smoothing. Trajectory optimization, Robot dynamics, Simulation.

A technique for generating smooth trajectories of a system is presented. The approach is to find a dynamical equation with the desired transient behavior and use it as the basis of the control algorithm. It is shown that an appropriate dynamical equation can be obtained by varying the position and velocity gains of a proportional-derivative control loop over the duration of the movement. The dynamical equation can be used to generate trajectories online with minimal planning. The resulting manipulator control system responds to path errors in a more reasonable fashion than traditional approaches to trajectory control since explicit replanning is not required.

101,037

PB92-117019

Not available NTIS

National Bureau of Standards (NEL), Gaithersburg. MD. Robot Systems Div 
Performance Measures of a Robotic Micropositioner.

Final rept.

J. D. Lee, J. S. Albus, N. G. Dagalakis, and T. M.

Tsai. $1988,10 \mathrm{p}$

Pub. in Proceedings of International Symposium on Robotics and Manufacturing: Research, Education, and Applications (2nd), Albuquerque, NM., November 16-18, 1988, p1047-1056.

Keywords: "Robotics, "Robot dynamics, "Control systems, Computerized simulation, Robot arms, Manipulators, Algorithms, Positioning devices(Machinery), Reprints.

The work describes a computer simulation of the dynamics and control of a robotic micropositioner. The robotic micropositioner is a parallel link manipulator which has six actuators, each controlled independently by a hydraulic system. The dynamic equations of the micropositioner are derived. The control algorithm for path tracing is formulated and tested. In the work, the performance of the micropositioner is investigated as a function of damping, speed, payload, and location of target.

\section{Tooling, Machinery, \& Tools}

\section{1,038}

PB91-146993

Not available NTIS

National Inst. of Standards and Technology (NEL),

Gaithersburg, MD. Chemical Process Metrology Div.

State-of-the-Art in Pressure Metrology.

Final rept.

V. E. Bean. $1990,19 p$

Pub. in Proceedings of Symposium Semana de la Calidad y la Metrologia en El Politecnico, Mexico City, Mexico, November 26-30, 1990, p231-249 1990.

Keywords: "Pressure measurement, "Metrology, "Standards, State of the art, Manometers, Reviews, Reprints, Piston gages.

The state-of-the-art in pressure primary standards is reviewed. Among the areas that require continued research are: (1) The dependence of the effective area of a gas-operated piston gage on the gas species; (2) The dependence of the effective area of a gas-operated piston gage upon whether the gage is operated in the gage or absolute mode; (3) Methods for obtaining improved distortion coefficients for piston gages.

\section{1,039}

PB91-147751

Not available NTIS

National Bureau of Standards (IMSE), Boulder, CO. Fracture and Deformation Div.

Progress in Uniform Field Eddy Current Methods. Final rept

J. D. Moulder, N. Nakagawa, and P. J. Shull. 1987,

9p

\section{Contract W-7405-ENG-82}

Sponsored by Air Force Wright Aeronautical Labs. Wright-Patterson AFB, OH. Materials Lab.

Pub. in Review of Progress in Quantitative Nondestructive Evaluation, v7A p147-155 1987.

Keywords: "Eddy current tests, "Nondestructive tests, Electric current, Hysteresis, Magnetic fields, Eddy currents, Magnetic properties, Electrical properties, Field rents, Magnetic properties, Electrical properties, Field
strength, Electromagnetic testing, Measuring instruments, Reprints.

The use of eddy current probes with a spatially uniform magnetic field distribution is especially advantageous for quantitative NDE, since the theoretical analysis of field-flaw interactions is much simpler for a uniform field. The authors reported here recent progress in the design and fabrication of high frequency uniform field eddy current probes. The goal has been to achieve higher frequency operation and greater bandwidth than previous designs so that smaller flaws may be studied over a greater range of skin depths. Measurements on artificial flaws designed to simulate crack 'bridging' phenomena are also described.

Using Surface Roughness as a Wear Criterion in High-Speed Steel End-Milling.

Final rept.

L. Evans. $1986,6 p$

Pub. in Proceedings of North American Manufacturing Research Conference (14th), Minneapolis, MN., May 28-30, 1986, p177-182.

Keywords: "Surface roughness, "Machine tools, "Wear, "Steels, Machining, Surface properties, Finishes, Wear tests, Metal working, Reprints.

At the present time, there is no good quantitative wearout criterion that relates wear on the tool to the fitness of the tool for use in machining and therefore no goo one for tool wear testing. A criterion based on flank wear is commonly used but not universally accepted because it provides no indication of other kinds of wear occurring on the tool, and little information about the surface finish or dimensions of the machined part. It is important to choose a criterion related to the workpiece, since it is the quality of the part that matters. In the study, a wear-out criterion based on surface roughness is chosen and high-speed steel end-milling tests performed in order to judge the usefulness of the criterion. These tests do not monitor surface roughness inprocess; two indirect methods, feed force and cutting power, are studied as potential techniques to detect when a tool can no longer produce parts with a quality surface finish.

101,041

PB91-159186

Not available NTIS

National Bureau of Standards (NEL), Gaithersburg, MD. Automated Production Technology Div.

Kinematic Couplings for Precision Fixturing. Part 2. Experimental Determination of Repeatability and Stiffness.

Final rept.

A. H. Slocum, and A. Donmez. 1988, 8 p

Sponsored by Oak Ridge National Lab., TN.

Pub. in Precision Engineering 10, n3 p115-122 Jul 88.

Keywords: *Couplings, Fasteners, Stiffness, Precision, Test facilities, Ball bearings, Couplers, Fittings, Lubrication, Reproducibility, Reprints.

The paper describes results of tests performed to determine the repeatability of a large heavily preloaded kinematic coupling. The coupling was constructed from two $355.6 \mathrm{~mm}$ (14 in) diameter $101.6 \mathrm{~mm}$ (4 in) thick cast iron disks with hardened steel gothic arch inserts and $28.6 \mathrm{~mm}$ (1.125 in) diameter balls. A special load frame was constructed for performing cyclic
tests of kinematic coupling while applying a $5800 \mathrm{~N}$ $(1300 \mathrm{lbf})$ preload. Different types of balls and lubrication at the interface were tested. When using lubricated silicon nitride balls, axial and radial repeatability was on the order of 0.25 micrometers ( + or $-10 \mathrm{mi}-$ croinches). Stiffness was on the order of $180 \mathrm{kN} / \mathrm{mm}$ (10 (exp 6) lbf/in)

\section{1,042}

PB91-189225 National Inst. of Standards and Technology (NEL), Gaithersburg, MD. Precision Engineering Div.

Statistical Process Control Tactics for Coordinate Measuring Machines.

Final rept.

T. Doiron. 1991, 12p

Pub. in Proceedings of Measurement Science Conference, Anaheim, CA., January 31-February 1, 1991, $12 \mathrm{p}$.

Keywords: "Dimensional measurement, "Process control, Standards, Artifacts, Metrology, Accuracy, Reprints, "Coordinate measuring machines, Gage blocks, Ball bars, Hole plates.

As coordinate measuring machines (CMMs) become the primary instruments for dimensional measurement in industry, the problem of process control for CMM accuracy is receiving increasing attention. While there is no obvious 'best' method for CMM statistical Process Control (SPC), there are.a number of strategies based on the measurement of artifacts which are currently in use. A number of these methods are presented, and their strengths and weaknesses are discussed with respect to accuracy, ease of use, reproducibility, flexibility, and response to common CMM problems.

101,043

PB9 1-202978

Not available NTIS

Nechnology (NEL) Gaithersburg, MD. Precision Engineering Div.
Computer Vision Based Station for Tool Setting and Tool Form Measurement.

Final rept.

T. D. Doiron. $1989,8 p$

Contract DE-A105-85OR21584

Sponsored by Department of Energy, Oak Ridge, TN. Pub. in Precision Engineering 11, n4 p231-238 Oct 89.

Keywords: "Computer vision, "Tools, "Metrology, Algorithms, Measuring instruments, Shape, Position(Location), Accuracy, Workstations, Reprints.

The paper describes a computer vision based system which measures the shape of a turning tool edge and determines its position. The system consists of three subsystems: a computer vision system with a solid state camera and microscope as the sensor, a metrology frame to provide scale and position information and a set of algorithms to extract the needed information from the video output from the camera. The accuracy limitations of each subsystem are discussed and test data are compared with the accuracy predictions. The tool form measurements exhibited an accuracy which varied from 0.3 to 1.5 micrometers depending on the edge orientation. The tool setting accuracy was found to be near 2 micrometers. Suggestions for im provement are discussed.

\section{1,044}

PB92-123066

PC A03/MF A01

National Inst. of Standards and Technology (NEL) Gaithersburg, MD. Factory Automation Systems Div. Use of Solid Modeling in the Design of M3 Compo nents.

A. B. Feeney, and P. F. Brown. Sep 91, 35p NISTIR4710

See also PB90-136938 and PB90-242207.

Keywords: Computer aided design, Vibration isolators, Dynamic control, Microelectronics, Metrology, Mass, * Molecular measuring machines, Coordinate measuring machines, Solid modeling, Nanotechnology, M3 project.

The paper documents the work done for the Molecula Measuring Machine (M3) Project by the Engineering Design Laboratory (EDL) at NIST. The M3 Project is developing and fabricating a device capable of making repeatable measurements of atomic scale features within a volume of $50 \mathrm{~mm} \times 100$ micrometers. Extraordinary measures are being taken to isolate the device from mechanical vibrations, thermal loading and acoustic noise. The finest level of control over mechanical vibrations is achieved through an active vibration isolation system. The EDL created solid models of the components and assemblies related to the active vibration isolation system in order to obtain the mass properties calculations required by the dynamic control system. The paper provides an overview of the M3 Project, describes the modeling work that was done within the EDL, and gives the mass property calculation results for the M3 parts and assemblies. Additionally, the paper discusses the advantages of using solid models as an integrated shape representation and a number of ways solid models are being used in product lifecycle applications.

\section{Tribology}

\section{1,045}

PB91-203943

Not available NTIS

National Inst. of Standards and Technology (MSEL), Gaithersburg, MD. Ceramics Div.

Numeric Database for Tribology: Format and Application Issues.

Final rept.

A. W. Ruff, J. Rumble, and S. Jahanmir. 1991, 17p

Sponsored by Department of Energy, Washington, DC., National Science Foundation, Washington, DC. and Department of the Army, Washington, DC.

Pub. in Computerization and Networking of Materials Databases: Second Volume, ASTM STP 1106, p180. 1961991.

Keywords: "Data bases, "Tribology, Personal computers, Meetings, Friction, Wear, Mathematical models, Design criteria, Stress tests, Temperature dependence, Software engineering, Reprints, ACTIS data base. 
A computerized tribology information system designed for use with personal computers and including a numeric database is presently under development. The status of the system (named ACTIS) is summarized. Two important issues associated with the numeric database component were the data format and the organization of numeric data for user applications. Based on information developed at a National Institute of Standards and Technology (NIST) workshop on tribology information, a set of data fields has been identified for use in a database on friction and wear of materials, and on material properties. Evaluated data in these categories have been assembled and loaded into that format using commercial database management software. Over 360 records are presently contained in the database covering a broad range of materials of tribological significance. The data are organized for direct application by the user in material selection, wear model calculations, tribological component design, and with other software codes such as stress and temperature calculations.

\section{General}

101,046

BB91-147942

Not available NTIS

National Inst. of Standards and Technology (NML)
Boulder, $\mathrm{CO}$. Chemical Engineering Science Div.

Review of Pulse Tube Refrigeration.

Final rept.

R. Radebaugh. $1990,15 p$

Sponsored by National Aeronautics and Space Administration, Moffett Field, CA. Ames Research Center Pub. in Advances in Cryogenic Engineering 35, p1191. 12051990.

Keywords: "Refrigerators, "Cryogenic cooling, Cryogenics, Stirling cycle, Coolers, Heat transfer, Refrigerating, Thermodynamic cycles, Efficiency, Mass flow, Pressure, Reprints, "Pulse tube refrigerators.

The paper reviews the development of the three types of pulse tube refrigerators: basic, resonant, and orifice types. The principles of operation are given. It is shown that the pulse tube refrigerator is a variation of the Stirling-cycle refrigerator, where the moving displacer is substituted by a heat transfer mechanism or by an orifice to bring about the proper phase shifts between pressure and mass flow rate. A harmonic analysis with phasors is described which gives reasonable results for the refrigeration power yet is simple enough to make clear the processes which give rise to the refrigeration. The efficiency and refrigeration power are compared with those of other refrigeration cycles. A brief review is given of the research being done at various laboratories on both one and two-stage pulse tubes. A preliminary assessment of the role of pulse tube refrigerators is discussed.

101,047
PB91-147967

Not available NTIS

National inst of Standards and Technology (NML) Boulder, $\mathrm{CO}$. Chemical Engineering Science Div.

Apparatus for the Measurement of Regenerator Performance in Pulse Tube Refrigerators.

Final rept.

W. Rawlins, and R. Radebaugh. 1990, 9p

Sponsored by National Aeronautics and Space Admin istration, Moffett Field, CA. Ames Research Center. Pub. in Advances in Cryogenic Engineering 35, p121312211990.

Keywords: "Refrigerators, "Cryogenic cooling, "Regenerators, "Measuring instruments, "Effectiveness, Coolers, Cryogenics, Mass flow, Heat loss, Heat ex changers, Cooling systems, Reprints, "Pulse tube refrigerators.

The paper discusses the design and construction of an apparatus to measure the ineffectiveness of regenerators used for pulse tube refrigerators. Because of the fairly large mass flow rates which occur in pulse tube refrigerators, the regenerator ineffectiveness must be made quite small. The apparatus described here allows for the measurement of the regenerator's heat loss under actual operating conditions in a pulse tube refrigerator. A low temperature heat sink of liquid nitrogen is used since it approximates the temperatures normally achieved in a one-stage pulse tube.
National Inst. of Standards and Technology (NEL), Boulder, CO. Chemical Engineering Science Div.

Analytical Model for the Refrigeration Power of the Orifice Pulse Tube Refrigerator.

Technical note.

P. J. Storch, R. Radebaugh, and J. E. Zimmerman

Dec $90,95 \mathrm{p}$ NIST/TN-1343

Also available from Supt. of Docs. Sponsored by $\mathrm{Na}$ tional Aeronautics and Space Administration, Moffett Field, CA. Ames Research Center.

Keywords: "Refrigeration, "Mathematical models, *Cryogenic cooling, Refrigerating, Refrigerating machinery, Coolers, Mass flow, Refrigerators, Cryogenics, Orifices, Pulses, "Pulse tube refrigerators.

The pulse tube refrigerator is a cryocooler with potential for high reliability. The regenerative refrigerator operates with low average pressures, small compression ratios, and only one moving part. In research conducted at the National Institute of Standards and Technology, a pulse tube refrigerator with an orifice located at the closed, hot end of the system has been used to reach $60 \mathrm{~K}$ in one stage. A gross refrigeration power of $12 \mathrm{~W}$ has been measured at $80 \mathrm{~K}$ with a pulse tube volume of $30 \mathrm{cu} \mathrm{m}$. The paper presents the development of an analytical model to predict the refrigeration power produced in an orifice pulse tube. Three versions of the model are presented: the first calculates quantities which are independent of dead volume; the second neglects dead volume; and the third describes a system with dead volume. Experimental results confirm predictions by the model that refrigeration power is proportional to the average pressure, the pulse frequency, the mass flow ratio, and the square of the dynamic pressure ratio. Results are presented that are useful for approximate design of orifice pulse tube systems. The model assumes adiabatic conditions and an energy balance is performed in terms of net enthalpy flow. Phasor analysis was found to be very useful for analyzing the oscillations of pressure, temperature and mass flow rate.

\section{1,049}

PB91-192997

PC A06/MF A01

National Inst of Standards and Technology, Gaithersburg. MD. Office of Standards Services.

Conformity Assessment Workshop on Pressure Vessels.

B. G. Simson. Apr 91, 103p NISTIR-4542

Sponsored by American Society of Mechanical Engineers, New York.

Keywords: "Pressure vessels, "Boilers, "Meetings, Federal assistance programs, Government policies, European communities, Common market, Recommendations, Standards, International trade, Conformity assessment.

On January 31, 1991, The American Society of Mechanical Engineers (ASME) and the National Institute of Standards and Technology (NIST) cosponsored a workshop on pressure vessels at the Department of Commerce auditorium in Washington D.C. The purpose of the workshop, attended by one hundred and thirty two persons, was to explore how the U.S. Gov ernment can assist the pressure vessel industry in conformity assessment activities aimed at gaining acceptance of their products in such other markets as the European Community. The following recommendations were reached by the private sector panelists: (1) U.S. Government promotion of national consensus standards: (2) enhancing NIST's standard information capability; (3) U.S. Government negotiation with the European Commission for establishing notified bodies for pressure vessels in the United States; and (4) U.S. Government establishment of a sectoral technical advisory organization.

\section{1,050}

PB91-194464 National Inst. of Standards and Technology (CSTL) Boulder, CO. Chemical Engineering Div.

Heat Transfer and Pressure Drop in a Compact Pin-Fin Heat Exchanger with Pin Orientation at 18 deg to the Flow Direction.

\section{A. Olson. Feb 91, 78p NISTIR-3965}

Sponsored by National Aeronautics and Space Administration, Hampton, VA. Langley Research Center.

Keywords: "Heat transfer, "Pressure gradients, "Friction factor, Turbulent flow, Reynolds number, Hea flux, Nusselt number, Heat exchangers, Temperature distribution, Experimental data, Mathematical models, "Compact heat exchangers.
The authors have measured the heat transfer and pressure drop characteristics of a novel, compact heat exchanger in helium gas at $3.5 \mathrm{MPa}$ and Reynolds numbers of 450 to 12,000 . The 'pin-fin' specimen consisted of pins, $0.51 \mathrm{~mm}$ high and spaced $2.03 \mathrm{~mm}$ on centers, spanning a channel through which the helium flows; the angle of the row of pins to the flow direction was 18 degrees. The specimen was radiatively heated on the top side at heat fluxes up to $74 \mathrm{~W} / \mathrm{sq} \mathrm{cm}$ and insulated on the back side. Correlations were developed for the friction factor and Nusselt number. The Nusselt number compares favorably to those of past studies of staggered pin-fins, when the measured temperatures are extrapolated to the temperature of the wall-fluid interface.

\section{1,051}

PB91-203877

Not available NTIS

National Bureau of Standards (NEL), Boulder, CO

Chemical Engineering Science Div.

Optimization of a Pulse Tube Refrigerator for a Fixed Compressor Swept Volume.

Final rept.

R. Radebaugh, K. Chowdhury, and J. Zimmerman

$1988,13 p$

Sponsored by National Aeronautics and Space Admin istration, Moffett Field, CA. Ames Research Center.

Pub. in Proceedings of the International Cryocoole Conference (5th), Monterey, CA., August 18-19, 1988 , p113-125.

Keywords: "Cryogenic cooling, Refrigerators, Optimization, Cryogenic equipment, Refrigerating equipment, Cryogenics, Cooling, Ultralow temperatures, Refriger ating, Compressors, Cooling systems, Reprints, "Pulse tube refrigerators.

The paper discusses experiments which were done to determine the minimum temperature and the maximum refrigeration power available with an orifice pulse tube refrigerator driven by a compressor with a fixed swept volume of $25 \mathrm{cu} \mathrm{cm}$. The frequency of the compressor motion could be varied between 10 and $25 \mathrm{~Hz}$ Only screen regenerators were studied but several mesh sizes and regenerator volumes were used. The screen materials tested were phosphor bronze, stainless steel, and nylon. Three different pulse tube volumes were investigated for most regenerators. The lowest temperatures achieved were about $67 \mathrm{~K}$ with the following conditions: pulse tube volume of $7.9 \mathrm{cu}$ $\mathrm{cm}$, regenerator gas volume of $9.2 \mathrm{cu} \mathrm{cm}$, regenerato screen of 250 mesh stainless steel, frequency of 10-15 $\mathrm{Hz}$, and average pressures of $1500-2000 \mathrm{kPa}$. These optimum volumes resulted in a pressure ratio of about 1.3

101,052

PB92-116482

Not available NTIS

National Inst of Standards and Technology (NML) Boulder, CO. Chemical Engineering Science Div.

Measurement of Regenerator Performance in a Vuilleumier Refrigerator.

Final rept.

P. E. Bradley, and R. Radebaugh. 1991, 10p

Pub. in Proceedings of Interagency Meeting on Cryo coolers (4th), Plymouth, MA., October 24, 1990, p195 2041991.

Keywords: "Refrigerators, "Performance evaluation, "Regenerators, Cooling systems, Heat exchangers, Thermal efficiency, Mathematical models, Cryogenics, Low temperature tests, Refrigerating, Refrigerating machinery, Reprints, "Vuilleumier refrigerator.

A 3-stage Vuilleumier refrigerator developed for Wright-Patterson Air Force Base has been used to measure the performance of third-stage regenerators. The refrigerator operates between 2.5 to $5.0 \mathrm{~Hz}$ and achieves temperatures of 10 to $20 \mathrm{~K}$ at the third-stage cold end. The purpose of the paper is to present a comparison of regenerator performance for two regenerator materials and to compare experimental results with a third order numerical model and a first order approximation for calculating the regenerator performance. Measurements of two regenerator materials are presented. Even though Brass has a volumetric heat capacity approximately 15 times lower than that of $\mathrm{Pb}+5 \% \mathrm{Sb}$, the regenerator loss with brass is only $60 \%$ higher than that of $\mathrm{Pb}+5 \% \mathrm{Sb}$ because of the large heat capacity contribution of the helium gas in the regenerator void volume. These results are in fair agreement with a third order numerical model. 
101,053

PB92-123041

PC A05/MF A01

National Inst. of Standards and Technology (MEL),

Gaithersburg, MD.

Publications of the Manufacturing Engineering Laboratory Covering the Period January 1989September 1991.

P. Nanzetta, C. F. Hutchins, and L. L. Wood. Nov 91 , 95p NISTIR-4713

See also PB90-130568.

Keywords: "Bibliographies, Dimensional measurement, Artificial intelligence, Production engineering, Automation, Metrology, Precision, Robotics, "Manufacturing Engineering Laboratory.

The document provides a list of publications by staff of the Manufacturing Engineering Laboratory for the period of January 1989 through September 1991. Publications cover research done by the Laboratory in the areas of the precision dimensional measurement and precision engineering; robotics and intelligent machines; manufacturing data description, data administration, and information processing; and, sensors for manufacturing processes.

\section{MATERIALS SCIENCES}

\section{Carbon \& Graphite}

\section{1,054}

PB91-158683 Gaithersburg, MD. Ceramics Div.

Effects of Different $\mathrm{CH} 4-\mathrm{H} 2$ Gas Compositions on the Morphology and Growth of Diamond Grown by Hot Filament CVD.

Final rept.

E. N. Farabaugh, and A. Feldman. 1990,6p

Pub. in Materials Research Society Symposium Proceedings, v162 p127-1321990.

Keywords: "Diamonds, "Thin films, Chemical vapor deposition, Crystal growth, Single crystals, Substrates, Methane, Hydrogen, Electron microscopy, Surface roughness, Process variables, $X$ ray diffraction, Silicon, Mullite, Reprints.

Diamond films have been grown on single crystal $\mathrm{Si}$ and polycrystalline mullite substrates by hot filament chemical vapor deposition. Both substrates offer a good thermal expansion match with diamond. Gas mixture ratios of $\mathrm{CH} 4: \mathrm{H} 2$ ranged from $0.1-1.0 \%$. The remaining deposition parameters were: substrate temperature, $750 \mathrm{C}$; filament temperature, $1800 \mathrm{C}$; gas pressure, $5000 \mathrm{~Pa}$; gas flow rate, $52 \mathrm{sccm}$ except for the $0.1 \% \mathrm{CH} 4: \mathrm{H} 2$ ratio deposition in which the flow the $0.1 \% \mathrm{CH} 4: \mathrm{H} 2$ ratio deposition in which the flow
rate was $120 \mathrm{sccm}$. Film thicknesses were determined from cross sectional SEM micrographs. The average growth rates on the mullite increased nearly linearly with increasing $\mathrm{CH} 4$ gas fraction, ranging from $0.05 \mathrm{mi}$ crometers/hr to 0.21 micrometers/hr. Growth rates on the Si substrates were slightly lower for identical the Si substrates were slightly lower for identical that the rougness of the films decreased with increasing $\mathrm{CH} 4$ gas fraction during deposition. X-ray diffraction patterns showed that crystalline grain size in the films decreased with increasing $\mathrm{CH}_{4}$ gas fraction. No preferred crystallographic orientation was seen in the diffraction patterns.

\section{1,055}

PB92-116540

Not available NTIS

National Inst. of Standards and Technology (MSEL), Gaithersburg, MD. Reactor Radiation Div.

Neutron Measurements of Intramolecular Vibrational Modes in C60.

Final rept.

R. L. Cappelletti, J. R. D. Copley, W. A.

Kamitakahara, F. Li, J. S. Lannin, and D. Ramage.

1991, 4p

Pub. in Physical Review Letters 66, n25 p3261-3264, 24 Jun 91

Keywords: "Vibrational spectra, "Carbon, Spectrum analysis, Neutron scattering, Measurement, Intramole- cular structures, Reprints, "Buckeyballs, "Buckminsterfullerence.

Neutron-inelastic-scattering measurements have been made on a sample of highly purified solid C60. New features in the spectrum of intramolecular modes no obtainable by optical spectroscopy have been identified. Available theoretical calculations show trends in accord with the experimental results but disagree in important details, and improved calculations are desirable.

\section{Ceramics, Refractories, \& Glass}

\section{1,056}

AD-A243 049/4

PC A08/MF A02

National Inst. of Standards and Technology (IMSE),

Gaithersburg, MD. Ceramics Div.

Strength and Microstructure of Ceramics.

Final technical rept. 1 Oct $89-30$ Sep 91.

B. R. Lawn. Oct 91, 170p AFOSR-TR-91-0892

Grants AFOSR-ISSA-90-0003, AFOSR-ISSA-91-0002

Keywords: Aluminum oxides, Ceramic materials, Composite materials, Cracks, Cycles, Degradation, Documents, Fatigue, Friction, Grain boundaries, Interfaces, Mechanical properties, "Microstructure, Models, Predictions, Residual stress, Routing, Scanning electron microscopes, Strength(General), Test and evaluation, Theory, "Ceramics, "Strength(Mechanics), Grain size, Crystallization, "Fracture(Mechanics), Toughness, Advanced composites, Cracking(Fracturing), Scanning electron microscopy, Bridging, Tensile tests, Processing, Micromechanics.

Results of a program on the toughness properties of monophase and two phase ceramics that toughen by bridging are presented. Fracture mechanics models describing this behavior, in the particular context of strength, are developed. Results of strengths tests confirming the essential predictions of the theory are presented. Innovative processing routes suggested by the models are shown to lead to two phase composites with impressive flaw insensitivity. A partial list of publications included in this report are: (1) The role of crystallization of an intergranular glassy phase in determining grain boundary residual stresses in debased aluminas; (2) In situ measurements of bridged crack interfaces in scanning electron microscopes; (3) Cyclic fatigue from frictional degradation at bridging grains in alumina; (4) Microstructure, toughness curves and mechanical properties of alumina ceramics; (5) Fabrication of flaw tolerant aluminum titanate reinforced alumina; and (6) Influence of grain size and degree of crystallization of intergranular glassy phase on the mechanical behaviour of a debased alumina.

\section{1,057}

PB9 1-132233

PC A08/MF A01

National Inst of Standards and Technology (MSEL). Gaithersburg, MD. Ceramics Div.

Institute for Materials Science and Engineering, Ceramics: Technical Activities 1990.

S. M. Hsu. Nov 90, 170p NISTIR-4394

See also report for 1989 , PB90-163981.

Keywords: "Ceramics, Silicon nitrides, Phase diagrams, Data retrieval, Standards, Superconductors, Thin films, Optical materials, Construction materials, Powder(Particles), Microstructure, Creep properties, Crack propagation, Surface energy, Glass, Data bases, Lubricants, Diamonds, Tribology, Characterization, Ceramic matrix composites, Fracture mechanics, Solid-solid interfaces.

Current programs of the Ceramics Division are reviewed. In 1990, technology transfer was accom. plished by the preparation and publication of 180 papers, the presentation of 150 talks and the submission of 6 invention disclosures. Leadership of national and international standards activities continued. The Structural Ceramics Database (SCD) Version 1.0 which contains evaluated thermomechanical data for industrial designers has been offered for public distribution by the Office of Standard Reference Data. Other database activities continued with the distribution of A Computerized Tribology Information System (ACTIS) and the completion of Volumes 7 and 8 of the NIST/American Ceramic Society Phase Diagrams for Ceramists. A new activity, an assessment of the New Diamond Technology in Japan was undertaken by division personnel in 1990. In the structural materials area, research on the chemistry of ceramic slurries and suspensions has led to identification of mechanisms for coating silicon nitride powders with discrete sintering aids. Significant advances in the ability to measure surface forces between dissimilar materials were achieved. Basic research on bridging effects in fracture have been extended to microstructural design and studies of cyclic fatigue. Phase diagram determinations and studies of the role of processing environments on microstructural features of high temperature superconductors continued. The role of rare earth substitutions on YBaCO properties were evaluated and provided insight into the effect of atomic size of substitutional atoms.

101,058

PB91-147330

Not available NTIS National Inst. of Standards and Technology (MSEL). Gaithersburg, MD. Metallurgy Div.

Dihedral Angles in Magnesia and Alumina: Distributions from Surface Thermal Grooves.

Final rept.

C. A. Handwerker, J. M. Dynys, R. M. Cannon, and R. L. Coble. 1990, 7p

Pub. in Jnl. of the American Ceramic Society $73, n 5$ p1371-1377 May 90.

Keywords: "Aluminum oxide, "Magnesium oxides, "Dihedral angle, Grain boundaries, Polycrystalline, Surfaces, Reprints.

Dihedral angles, psi, from surface thermal grooves were measured using a metal reference line technique for polycrystalline $\mathrm{MgO}$, undoped $\mathrm{Al} 2 \mathrm{O} 3$, and $\mathrm{MgO}$. doped $\mathrm{Al} 2 \mathrm{O} 3$. The values of psi, span the following ranges: $89 \mathrm{deg}$ to $116 \mathrm{deg}$ for $\mathrm{MgO}$ at $1520 \mathrm{~K}, 76 \mathrm{deg}$ to $166 \mathrm{deg}$ for undoped $\mathrm{Al} 2 \mathrm{O} 3$ at $1870 \mathrm{~K}$, and $90 \mathrm{deg}$ to $139 \mathrm{deg}$ for $\mathrm{MgO}$-doped $\mathrm{Al} 2 \mathrm{O} 3$ at $1870 \mathrm{~K}$. The range of inclination angles of the grain boundary to the surface was a function of psi, with the maximum inclination angle of about $13 \mathrm{deg}$, in quantitative agreement with theory.

\section{1,059}

PB91-147348

Not available NTIS

National Inst. of Standards and Technology (MSEL), Gaithersburg, MD. Metallurgy Div.

Metal Reference Line Technique for Obtaining Dihedral Angles from Surface Thermal Grooves.

Final rept.

C. A. Handwerker, J. M. Dynys, R. M. Cannon, and

R. L. Coble. 1990, $6 p$

Pub. in Jnl. of the American Ceramic Society $73, \mathrm{n} 5$ p1365-1370 May 90.

Keywords: *Grain boundaries, "Dihedral angle, Scanning electron microscopy, Aluminum oxide, Magnesium oxides, Grain growth, Surface energy, Reprints

A metal reference line (MRL) technique is described for the measurement of surface-grain-boundary dihedral angles, psi, from thermal grooves at a sample surface using scanning electron microscopy (SEM). Meta lines deposited onto a thermally grooved surface using photolithography conform to the contours of the grainboundary groove and provide a high-contrast reference line for measuring psi, by SEM. Measurements of psi from optical interferometry and calculated from groove dimensions using surface diffusion models of thermal grooving are compared with the metal reference line measurements from the same thermally grooved surface of $\mathrm{MgO}$-doped $\mathrm{Al} 2 \mathrm{O} 3$. Distributions of psi are found to shift to lower angles and approach the true psi value as the resolution of the technique increases, with the MRL technique having the highest resolution.

101,060

PB91-147389

Not available NTIS National Bureau of Standards (IMSE), Gaithersburg, MD. Ceramics Div.

Application of Analytical Electron Microscopy to Glass-Bonded Aluminas.

Final rept.

B. J. Hockey. 1986, 4p

Proceedings of Annual Meeting Electron Microscopy Society of America (44th), Albuquerque, NM., August 10-15, 1986, p436-439.

Keywords: *Ceramics, "Aluminum oxide, Electron microscopy, Electron diffraction, $\mathrm{X}$ ray analysis, Crystallization, Glass, Reprints.

The application of analytical electron microscopy to multi-phase ceramic materials is illustrated by studies 
MATERIALS SCIENCES

Ceramics, Refractories, \& Glass

on glass-bonded aluminas. Particular emphasis is placed on describing not only the morphology of the glassy intergranular phase, but its composition using energy dispersive $X$-ray analyses (EDX). Similarly, $E D X$, in conjunction with electron diffraction, is also used to determine the specific nature and distribution of intergranular crystalline phases that form as a result of devitrification during heat treatment of these aluminas.

101,061

PB91-147553

Not available NTIS National Bureau of Standards (IMSE), Gaithersburg, MD. Ceramics Div.

Quantitative Determination of Amorphous Content in Ceramic Materials Using X-ray Powder Diffraction.

Final rept.

M. A. Kuchinski, C. R. Hubbard, L. A. Johnson, and

V. A. Greenhut. $1988,9 p$

Pub. in Proceedings of International Conference on Ceramic Powder Processing Science (1st), Orlando, FL., November 1-4, 1987, p321-329 1988.

Keywords: "Ceramics, "Amorphous materials, $X$ ray diffraction, Cristobalite, Reprints, Powder diffraction.

A quantitative technique which employs a modified method of additions approach to analyze for low amorphous content in crystalline matrices was developed and tested. Known amounts of amorphous material are added to the starting powder ('spiking'). The method uses the ratio of a measure of the intensity of the amorphous phase corrected for background to the background corrected intensity of a reference line from a crystalline phase. The amorphous spiking phase must be close in composition to the amorphous phase existing in the analyte. A critical step of the method is to correctly establish the background intensity. A completely crystalline material of similar scattering power was used to establish background intensity.

101,062

PB9'1-147983

Not available NTIS

National Bureau of Standards (IMSE), Gaithersburg, MD. Ceramics Div.

Hermetically Sealed Inert Atmosphere Cell for Xray Powder Diffraction.

Final rept.

J.J. Ritter. 1988, 2p

Pub. in Powder Diffraction 3, n1 p30-31 Mar 88.

Keywords: "X ray diffraction, "Inert atmospheres, Hermetic seals, Barium oxides, Titanium boride, Reprints, Powder diffraction.

The construction and use of a captive inert atmosphere cell for the preservation of atmospherically sensitive samples during $\mathrm{x}$-ray diffraction is described.

101,063

PB91-147991

Not available NTIS

National Bureau of Standards (IMSE), Gaithersburg,

MD. Ceramics Div.

Graphics Program for Binary and Ternary Ceramic Phase Diagrams.

Final rept.

P. K. Schenck, and J. R. Dennis. 1987, 7p

Pub. in Proceedings of International Conference on User Applications of Alloy Phase Diagrams, ASM Materials Week '86, Lake Buena Vista, FL., October 1986, p255-261 1987.

Keywords: "Ceramics, "Phase diagrams, "Computer graphics, Computer applications, Data bases, Reprints.

The paper describes the computer graphics program developed for the National Bureau of Standards (NBS) - American Ceramic Society (ACerS) Phase Diagrams for Ceramists Data Center for computer capture and reproduction of ceramic phase diagrams. The graphics program allows diagrams to be digitized, or entered by program allows diagrams to be digitized, or entered by the keyboard, and provides a number of powerful editdiagrams for future volumes of 'Phase Diagrams for Ceramists'. The database of graphics files generated using the program will eventually be integrated with ther databases under development in the Data Center, as described in a companion paper in the Center,

101,064

PB91-14822

Not available NTIS

National Bureau of Standards (IMSE), Gaithersburg,

MD. Ceramics Div.
Effects of Crystal Bonding on Brittle Fracture.

Final rept.

G. S. White, E. A. Fuller, S. W. Freiman, and T. L.

Baker. 1988, 7p

Pub. in Jnl. of Materials Research 3, n3 p491-497 1988

Keywords: "Ceramics, "Semiconductors(Materials), "Fracture mechanics, "Brittle materials, Crack propagation, Indentation, Crystal structure, Environmental tests, Reprints.

Indentation techniques have been used both to obtain fracture energies as a function of board ionicity in various IV, III-V, and II-VI compounds and to determine the ability of environments to enhance crack growth in some of these materials. The fracture energies are compared to those obtained using a simplistic interplaner stress model and are found to agree within approximately $50 \%$. Limitations of the indentation technique for evaluating $n$-values are discussed.

101,065

PB91-148767

Not available NTIS

National Bureau of Standards (IMSE), Gaithersburg, MD. Ceramics Div.

Physical and Thermo-Mechanical Properties of Monoclinic Single Crystals.

Final rept.

R. P. Ingel, P. A. Willging, B. A. Bender, and T. W.

Coyle. 1988, 11p

Sponsored by Naval Research Lab., Washington, DC Pub. in Advances in Ceramics 24A, p459-469 1988.

Keywords: "Zirconium oxides, "Ceramics, "Single crystals, "Monoclinic lattices, Electron microscopy, Temperature dependence, Mechanical hysteresis, Tetragonal lattices, Reprints, Thermomechanical properties.

Single crystals of unstabilized pure $\mathrm{ZrO} 2$ were successfully gorwn by the skull melting technique. TEM analysis revealed that the material was a monoclinic single crystal but heavily twinned with dislocations clearly visible. Physical property measurements (density, x-ray, etc.) were typical of monoclinic $\mathrm{ZrO} 2$. Strength and fracture toughness measurements as a function of temperature showed significant increases at $1200 \mathrm{C}$, just above the $\mathrm{A}$ (sub f) temperature of 1190 $C$. The strength and toughness increased from 174 $\mathrm{MPa}$ and 2.4 MPam(Sup 1/2) at room temperature to $350 \mathrm{MPa}$ and $5 \mathrm{MPam}($ Sup $1 / 2)$ at $1200 \mathrm{C}$. Testing at $1100 \mathrm{C}$ showed large differences in behavior depend. ing on whether the material was heated to the test emperature or cooled to the test temperature from the tetragonal phase field. These results indicate that there is a mechanical hysteresis which parallels the thermal expansion hysteresis. These effects are expected based on the stress-driven nature of the Monoclinic $<->$ Tetragonal transformation.

\section{1,066}

PB9 1-149096

Not available NTIS

National Bureau of Standards (IMSE), Gaithersburg, MD. Ceramics Div.

Low Temperature Chemical Process for Precursors to Boride and Carbide Ceramics Powders.

Final rept.

J. Ritter. 1987, 21p

Pub. in Proceedings of Conference on Ceramic Powder Science and Technology, Boston, MA., August $6,1986,21 \mathrm{p} 1987$.

Keywords: "Ceramics, "Precursors, "Silicon carbides, "Titanium borides, "Boron carbides, Synthesis(Chemistry), Powder(Particules), Low tempersture, Reprints.

The controlled reductive dehalogenation of elemental halides has been studied as a low temperature approach to boride and carbide precursors. It was shown that $\mathrm{TiCl} 4$ and $\mathrm{BCl} 3$ react with metallic sodium at $130 \mathrm{C}$ in a nonpolar solvent to give the precursor to TiB2. Similar type reactions with $\mathrm{SiCl} 4$ and $\mathrm{CCl} 4$ or with $\mathrm{BCl} 3$ and $\mathrm{CCl} 4$ have produced the precursors to $\mathrm{SiC}$ and $\mathrm{B} 4 \mathrm{C}$ respectively. The chemistry is generally applicable to any combination of elemental halides which can be reduced by alkali metals or alkali metals alloys. The reactions in these systems are facilitated by highspeed, high-shear stirring and reaction temperatures of $110-130 \mathrm{C}$. Product $\mathrm{NaCl}$ is separated from the amorphous precursors by vacuum sublimation at 900 C. The TiB2 precursor undergoes initial crystallization at this temperature while the $\mathrm{SiC}$ precursor requires heating in excess of $1500 \mathrm{C}$ to achieve crystallinity. The procedure has been successfully applied to the synthesis of precursors to the particulate composites $\mathrm{SiC} / \mathrm{TiC}$ and $\mathrm{SiC} / \mathrm{TiN}$.

101.067

PB91-149104

Not available NTIS National Bureau of Standards (IMSE), Gaithersburg, MD. Ceramics Div.

Low Temperature Synthesis of Ceramic Powders for Structural and Electronic Applications.

Final rept.

J. J. Ritter, and K. G. Frase. 1986, 497p

Pub. in Proceedings of International Conference on UItrastructure Processing of Ceramics, Glasses and Composites (2nd), Palm Coast, FL., March 1985, 497p 1986.

Keywords: "Ceramics, "Precursors, "Titanium borides, "Silicon carbides, "Boron carbides, Barium titanates, Aluminum oxide, Tantalum compounds, Alcoholates, Synthesis(Chemistry), Low temperature, Yttrium compounds, Metals, Reprints.

Low temperature chemical routes to ceramic powders have received considerable attention within recent years. A novel, low temperature approach to TiB2, SiC and $\mathrm{B} 4 \mathrm{C}$ ceramics is described as well as studies on alkoxide routes for barium polytitanates, beta/beta' aluminas and yttrium tantalate. The concept of controlling powder crystalline phases, phase stability and particle size through manipulation of synthesis parameters is discussed.

101,068

PB91-149112

Not available NTIS

National Inst. of Standards and Technology (MSEL), Gaithersburg, MD. Ceramics Div.

In situ Measurements of Bridged Crack Interfaces in the Scanning Electron Microscope.

Final rept.

J. Rodel, J. F. Kelly, and B. R. Lawn. 1990, 6p

Sponsored by Air Force Office of Scientific Research, Bolling AFB, DC.

Pub. in Jnl. of the American Ceramic Society $73, \mathrm{n} 11$ p3313-3318 Nov 90

Keywords: "Ceramics, "Crack propagation, "Fracture mechanics, Electron microscopy, Aluminum oxide, Solid-solid interfaces, Test equipment, Crack opening displacement, Reprints, In-situ tests.

A device for in situ SEM examination of crack propagation during loading of compact tension specimens is described with a specific demonstration on an alumina ceramic. The device facilitates direct qualitative observations of the inception and subsequent frictional pullout of grain-localized bridges at the crack interface. Quantitative data on the bridging mechanism are obained from measurements of the crack-opening displacements behind the crack tip. The crack profile is found to be closer to linear than parabolic at the bridged interface. Deconvolution of these crack-opening data allow for an evaluation of the closure tractions operative at the crack walls within the bridging zone, and thence the R-curve.

101,069

PB91-149419

Not available NTIS

National Inst. of Standards and Technology (MSEL), Gaithersburg, MD. Metallurgy Div.

Comparison of Flux Dynamics in Two Samples of YBa2Cu307 with Different Pinning.

Final rept.

M. Turchinskaya, L. H. Bennett, L. J.

Swartzendruber, A. Roitburd, C. K. Chiang, M. Hill, J. E. Blendell, and K. Sawano. 1990, 4p Pub. in Materials Research Society Symposium Proceedings, v169 p931-934 1990.

Keywords: "Ceramics, "Pinning, Kinetics, Magnetization, Time dependence, Temperature, Superconductors, Reprints, " Yttrium barium cuprates.

The kinetics of magnetization in two samples of YBa2Cu3O7 prepared by different methods, are studed. Magnetization vs. time data were obtained at various temperatures for a number of applied fields. The data display (after some short period of time) logarithmic behavior and (after a sufficiently long time) exponential behavior. The pinning parameter 'activation volume' was determined from the experimental data for both samples. 
National Inst. of Standards and Technology (MSEL), Boulder, CO. Fracture and Deformation Div. Mechanical Loss in a Glass-Epoxy Composite. Final rept.

M. Weller, and H. Ledbetter. 1990, 3p

Sponsored by Department of Energy, Washington, DC. Office of Fusion Energy.

Pub. in Jnl. of Materials Research 5, n5 p913-915 May 90.

Keywords: *Fiber composites, "Epoxy matrix composites, "Mechanical measurement, "Glass fiber reinforced plastics, Elastic properties, Torsional stress, Reprints.

Using a computer-controlled inverted torsion pendulum at frequencies near $1 \mathrm{~Hz}$, the mechanical losses in a uniaxially fiber-reinforced composite were determined. The composite comprised glass fibers in an epoxy-resin matrix. Three fiber contents: 0,41 , and 49 vol. $\%$ were studied. Three mechanical-loss peaks appeared: above $300 \mathrm{~K}$, near $200 \mathrm{~K}$, and near $130 \mathrm{~K}$. They correspond closely to alpha, beta, and gamma peaks found previously in many polymers. A mechanical-loss peak for either the glass or the glass-resin interface was not seen. Between 300 and $4 \mathrm{~K}$, the torsion modulus increased in the resin by a factor of 3.30 and in the 0.49 glass-epoxy by a factor of 2.37 .

\section{1,071}

PB91-150037

Not available NTIS

National Inst. of Standards and Technology (MSEL), Gaithersburg, MD. Ceramics Div.

Improved Analysis for Flexural Creep with Application to Sialon Ceramics.

Final rept.

C. F. Chen, and T. J. Chuang. 1990, $8 p$

Contract DE-A105-850421569

Pub. in Jnl. of American Ceramic Society 73, n8 p23662373 Aug 90.

Keywords: "Ceramics, "Sialon, "Creep strength, Performance prediction, Indentation, Least squares method, Compression tests, Engines, Tension tests, Bend tests, Comparison, Reprints.

By using a statistical least-squares method to minimize the differences between predicted and measured load-point displacement rates from four-point bend specimens, power-law creep parameters for tension and compression were estimated. An alternative but simpler method of estimating power-law creep parameters from flexural creep data is also proposed. The method entails the direct measurements of steadystate creep strain rates at two stress levels by an indentation technique. Based on a closed-form solution, the power-law creep parameters could then be estimated from both the measured neutral axis locations and curvature rates. The results from these two methods compare favorably with one another, and with the simple compressive creep data. Both methods yield a high stress exponent of about 14 for tension and a stress exponent of about unity for compression. Cavitation-enhanced creep in tension and diffusional creep in compression are responsible for the asymmetric behavior.

101,072

PB91-158600

Not available NTIS

National Bureau of Standards (IMSE), Gaithersburg, MD. Ceramics Div.

Near Net Shape Forming of Ceramics.

Final rept.
S. J. Dapkunas, and A. L. Dragoo. 1987, 13p

Pub. in Proceedings of ASME Winter Annual Meeting Interdisciplinary Issues in Materials Processing and Manufacturing, Boston, MA., December 13-18, 1987 , p19-31.

Keywords: *Ceramics, Processing, Manufacturing, Intelligence, Process control, Reprints, "Net shaping.

The widespread use of advanced ceramics requires the development of reliable, economic, reproducible manufacturing. Near net shape forming is required to achieve these manufacturing goals and the many types of these forming processing offer a variety of benefits for specific applications. Ultimately the development of intelligent processing systems is required.

101,073

PB91-158774

Not available NTIS

National Bureau of Standards (IMSE), Gaithersburg,

MD. Ceramics Div.
National Bureau of Standards-American Ceramic Society Phase Diagram Data Program.

Final rept.

J. W. Hastie. $1987,5 p$

Pub. in Proceedings of International CODATA Conference (10th), Computer Handling and Dissemination of Data, Ottawa, Canada, July 14-17, 1986, p179-183 1987

Keywords: *Ceramics, "Data bases, Phase diagrams, Computer graphics, Mathematical models, US NBS Thermodynamic equilibrium, Reprints.

In recent years the National Bureau of StandardsAmerican Ceramic Society phase diagram data program has made increased use of computers in handling and disseminating the data. Computers play a key role in many areas, such as: liter ature retrieval, bibliographic file development, phase diagram editing, coupled thermo-chemical-phase diagram evaluation, isothermal sectioning, multi-dimensional representation, and model predictions. Preparation of hard-copy compilations of phase diagrams, together with bibliographic and critical evaluation information, is also facilitated using the computer stored data. A discussion is given of the current status and future directions of the recently expanded program.

101,074

B9 1-158840

Not available NTIS

National Bureau of Standards (NEL), Gaithersburg, MD. Building Materials Div.

Towards Computer-Based Microstructure Models for Cement-Based Systems.

Final rept.

H. M. Jennings. 1987, 10p

Pub. in Materials Research Society Symposium Proceedings, v85 p291-300 1987.

Keywords: *Cements, Mathematical models, Microstructure, Software engineering, Computerized simulation, Reprints.

Aspects of a computer model for cement-based sysems is described. It can incorporate separately many assumptions in the form of sub-models. Two different types of sub-model are described, one that represents average characteristics of the system and one that sums individual contributions to particular characteristics of the system. The core of the overall model is a file of numbers representing the microstructure. The file is operated on to simulate hydration and the development of bulk properties.

\section{1,075}

PB91-159277

Not available NTIS

National Bureau of Standards (IMSE), Gaithersburg MD. Ceramics Div

Tensile Creep Behavior of Structural Ceramics.

Final rept.

S. M. Wiederhorn, L. Chuck, and T. J. Chuang. 1986, $20 p$

Pub. in Proceedings of Automotice Technology Development Contractors' Coordination Meeting (24th), Dearborn, MI., October 27, 1986, 20p.

Keywords: "Ceramics, Heat engines, Tension tests, Creep properties, Heat resistant materials, Silicon carbides, Fracture mechanics, Performanc prediction Sampling, Reprints.

The paper describes an inexpensive method of studying tensile creep of ceramic materials up to temperatures of $1500 \mathrm{C}$. The technique used self aligning test fixtures, and simple grinding procedures for specimen preparation. Results suggest that tensile testing can be carried out with almost the same ease and cost as flexural testing. Since the tensile test configuration re sults in a simple state of stress, data from tensile testing can be used to understand creep behavior in more complex states of mechanical loading. Following the line of reasoning, data presented in the paper are being used to predict the creep behavior and state of stress in flexural bars of siliconized, silicon carbide subjected to creep at elevated temperatures.

\section{1,076}

B9 1-162321

Not available NTIS

National Inst. of Standards and Technology (MSEL) Gaithersburg, MD. Metallurgy Div.

Superconducting Properties of Bi2-xyPbxSnySr2Ca2Cu3Oz.

Final rept.

H. M. Seyoum, J. M. Habib, L. H. Bennett, W. Wong$\mathrm{Ng}$, and A. J. Shapiro. 1990,6p

Pub. in Supercond. Sci. Technol. 3, p616-621 1990
Keywords: "High-TC superconductors, "Ceramics, Stannates, Transition temperature, Strontium compounds, Bismuth oxides, Lead oxides, Tin oxides, Strontium oxides, Calcium oxides, Copper oxides, $X$ ray diffraction, Electron microscopy, Magnetization, Tin additions, Lead additions, Reprints.

Two systematically processed series, $\mathrm{Bi} 1.6 \mathrm{~Pb} \times \mathrm{Sn}(0.4$ x) $\mathrm{Sr} 2 \mathrm{Ca} 2 \mathrm{Cu} 3 \mathrm{Oz}$ and $\mathrm{Bi}(1.9-\mathrm{x}) \mathrm{PbxSn0}$. $1 \mathrm{Sr} 2 \mathrm{Ca} 2 \mathrm{Cu} 3 \mathrm{Oz}$ (where $X$ ranges from 0 to 0.4 ) have been examined via magnetization measurements, $x$-ray diffraction and scanning electron microprobe. In addition to observing the often reported high Tc superconducting transition temperatures at $80 \mathrm{~K}(2212)$ and $110 \mathrm{~K}(2223)$, a range of well resolved transition temperatures have been found, implying the possible existence of new phases. The presence of $S n$ in these materials appeared to suppress the formation of the low Tc Raveau 2201 phase. Also observed was an ordered variation in the amount of flux pinning as the $\mathrm{Sn}$ and $\mathrm{Pb}$ concentrations were changed. It is suggested that $\mathrm{Sn}$ increases flux pinning by forming the compound $\mathrm{Sr} 3 \mathrm{Sn} 2 \mathrm{O} 7$.

\section{1,077}

PB91-175075

Not available NTIS National Inst. of Standards and Technology (IMSE) Gaithersburg, MD. Ceramics Div.

Small-Angle-Scattering Determination of the Microstructure of Porous Silica Precursor Bodies.

Final rept.

G. G. Long, S. Krueger, P. R. Jemian, D. R. Black, H. E. Burdette, J. P. Cline, and R. A. Gerhardt. 1990,

$10 p$

Pub. in Jnl. of Applied Crystallography 23, p535-544 1990

Keywords: *Small angle scattering, "Silica, "Ceramics, $X$-ray analysis, Neutron scattering, Sintering, Inorganic silicates, Potassium compounds, Particle size distribution, Colloids, Microstructure, Reprints, "Ceramic precursors.

Small-angle $X$-ray and small-angle neutron scattering measurements were carried out on a series of porous silica precursor (unsintered) bodies with different starting chemistries. The samples were prepared from mixtures containing 10 to 30 wt $\%$ colloidal silica sol and 90 to 70 wt \% potassium silicate. Particle-size distributions were derived from the data using a maximumentropy technique. Scattering data from the porous silica samples are especially suitable for such an analysis because the colloidal particles and clusters and aggregates of these particles are verified in detail to be spherical, and the scattering instrument use for the study covered the entire range of sizes in the material and was very well calibrated. It was found that the lower the amount of colloidal silica, the broader the size distribution of the silica aggregates.

\section{1,078}

PB91-175190

Not available NTIS

National Bureau of Standards (IMSE), Gaithersburg, MD. Ceramics Div.

Low Temperature Chemical Route to Precursors of Boride and Carbide Ceramic Powders.

Final rept.

J. J. Ritter. 1986, 6p

Pub. in Materials Research Society Symposia Proceedings, v73 p367-372 1986.

Keywords: "Ceramics, Borides, Carbides, Low temperature, Synthesis(Chemistry), Dehalogenation, Silicon chlorides, Silanes, Boron chlorides, Binary system(Materials), Titanium compounds, Reduction(Chemistry), Particulate composites, Sintering, Reprints, *Ceramic precursors.

The controlled reductive dehalogenation of elemental halides has been studied as a low temperature approach to boride and carbide precursors. It is shown that $\mathrm{SiCl} 4$ and $\mathrm{CCl} 4$ can be reacted with metallic sodium at 130 degrees in a non-polar solvent to give the precursor to SiC. Similar type reactions with $\mathrm{TiCl} 4$ and $\mathrm{BCl} 3$ or with $\mathrm{BCl} 3$ and $\mathrm{CCl} 4$ have produced the precursors to TiB2 and $\mathrm{B} 4 \mathrm{C}$ respectively. This procedure has also been used to generate the precursors to the two-phase composites $\mathrm{SiC} / \mathrm{TiC}$ and $\mathrm{SiC} / \mathrm{TiN}$. The method is generally applicable to any combination of elemental halides which can be reduced by alkali metals or alkali metal alloys. 
National Inst. of Standards and Technology (NEL), Gaithersburg, MD. Building Environment Div. Summary of Low-Density Glass-Fiber Reference Materials at NIST: 1980-1989.

Final rept.

R. R. Zarr. 1991, 10p

Pub. in Jnl. of Thermal Insulation 14, p211-220 Jan 91.

Keywords: "Thermal insulation, "Glass fibers, "Standards, Samples, Manmade fibers, Calibrating, Metrology, Measurement, Reprints, *Standard reference materials, National Institute of Standards and Technology.

The paper reviews three programs at the National Institute of Standards and Technology (NIST) that provide specimens of low-density glass-fiber insulation as reference materials. The National Voluntary Laboratory Accreditation Program (NVLAP) provides specimens as Proficiency Test Materials; the Standard Reference Materials Program (SRMP), as Standard Reference Materials; and the Center for Building Technology (CBT), as Calibrated Transfer Specimens. The programs of SRMP and CBT are summarized for the years 1980 to 1989 . The paper includes a catalogue of NIST publications covering the technical development of the low-density glass-fiber insulation used in the programs of NVLAP, SRMP and CBT.

101,080

PB91-187237

Not available NTIS

National Inst. of Standards and Techno'ogy (IMSE), Gaithersburg, MD. Ceramics Div.

Isothermal Phase Behavior of Ag3SbS3, ZnGeP2, and $\mathrm{ZnS}$.

Final rept.

$S$. Block, G. J. Piermarini, R. G. Munro, and E. Fuller.

$1989,12 p$

Pub. in Physica A 156, n1 p341-352, 15 Mar 89.

Keywords: "Isothermal treatment, "Phase transformations(Materials), "Silver inorganic compounds, "Antimonides, "Germanides, "Single crystals, Pressure dependence, Bulk modulus, Sulfides, Phosphides, Ceramics, Wurtzite, Sphalerite, Compressibility, Equations of state, Young modulus, Reprints.

The isothermal phase behavior as a function of pressure and the bulk moduli of Ag3SbS3, ZnGeP2, and $\mathrm{ZnS}$ have been determined. The respective transformation pressures observed optically with single crystals are $4.9+$ or $-0.15 \mathrm{GPa}, 20$ + or $-5 \mathrm{GPa}$, and 15.0 + or $-0.2 \mathrm{GPa}$. The latter transformation pressure is for both the wurtzite and sphalerite forms of $\mathrm{ZnS}$. ZnGeP2 also transforms to a second high pressure phase at approximately $32 \mathrm{GPa}$. Under hydrostatic pressure zinc sulfide shows no transformation beween the wurtzite and sphalerite forms. The bulk modulus was determined using the Birch, Birch-Murnaghan, Murnaghan, and Tait equations of state with both the entire sets of compressibility data and only the linear range. The full sets of data are not as self consistent as the linear range and are useful only for interpolation. The linear sets give more reliable values for determining physical parameters such as Young's modulus. The linear sets of compressibility values yield bulk moduli and pressure derivatives: wurtizite 80 and $3.7 \mathrm{GPa}$, sphalerite 67 and $4.8 \mathrm{GPa}$ Ag3SbS3 28 and $5.6 \mathrm{GPa}$ and $\mathrm{ZnGeP2} 79$ and $3.2 \mathrm{GPa}$

\section{1,081}

PB91-189449

Not available NTIS

National Inst. of Standards and Technology (MSEL). Gaithersburg, MD. Reactor Radiation Div.

Structures and Properties of New Zeolite X-Type Zincophosphate and Beryllophosphate Molecular Sieves.

Final rept.

W. T. A. Harrison, T. E. Gier, K. L. Moran, J. M.

Nicol, H. Eckert, and G. D. Stucky, 1991, 3p

Pub. in Chemical Materials 3, n1 p27-29 1991.

Keywords: "Zeolites, "Molecular sieves, "Faujasite, "Zinc phosphates, "Beryllium phosphates, Neutron scattering, $X$ ray scattering, Crystal structure, Phase diagrams, Aluminum silicates, FCC lattices, Reprints.

The crystal structures and properties of two new faujasite analogues containing zinc and phosphorus (ZnPO-X) and beryllium and phosphorus (BePO- $\mathrm{X}$ ) as the only framework tetrahedral atoms, are reported. These phases were prepared at ambient conditions and characterized by Rietveld refinement of powder neutron data $(\mathrm{BePO}-\mathrm{X}$ ) and powder $\mathrm{X}$-ray data (ZnPO$X)$. Both crystallize in the face-centered cubic space group Fo bar 3 and display 1:1 $\mathrm{Zn} / \mathrm{Be}: \mathrm{P}$ tetrahedral- atom order. Extra-framework cation positions have been established and these are compared with known aluminosilicate analogues. BePO-X is stable to calcination to at least $500 \mathrm{C}$, whilst the $\mathrm{ZnPO}-\mathrm{X}$ framework transfers to a new phases at $300 \mathrm{C}$. The BePO-X displays a typical zeolitic ion-exchange chemistry. These novel phases are members of a large new family of non-aluminosilicate ( $\mathrm{Zn} / \mathrm{Be}, \mathrm{P} / \mathrm{AsO})$ molecular sieves.

101,082

B91-189704

Not available NTIS

National Inst. of Standards and Technology (IMSE) Gaithersburg, MD. Ceramics Div.

Fundamental Condition for Existence of Microcrack Clouds in Monophase Ceramics.

Final rept.

B. R. Lawn. 1991, 4p

Sponsored by Air Force Office of Scientific Research, Bolling AFB, DC

Pub. in Jril. of the European Ceramic Society 7, p17-20 1991.

Keywords: "Ceramics, "Fracture mechanics, "Polycrystals, Microcracks, Crack propagation, Grain size, Defects, Residual stress, Reprints, Microcrack clouds.

Conditions for the existence of a microcrack cloud about a primary crack front in monophase polycrystalline ceramics are examined. With the assumption that microcracks initiate from sub-facet flaws, and that these flaws scale with the grain size, an expression is derived for the cloud radius. The cloud radius dimin ishes rapidly with grain size, from unlimited dimensions at the critical size for spontaneous, general microcracking to sub-grain level at some fraction of the critical size. The corresponding grain-size 'window' is dependent on the flaw size but is restrictively small for typical monophase ceramics.

\section{1,083}

PB91-190082

Not available NTIS

National Inst. of Standards and Technology (IMSE), Gaithersburg, MD. Ceramics Div.

Indentation Determination of Crack Growth $\mathrm{Pa}$ rameters in Gallium Arsenide.

Final rept.

G. S. White, S. W. Freiman, and A. M. Wilson. 1991 , $3 p$

Contract N00014-87-F-0007

Sponsored by Office of Naval Research, Arlington, VA Pub. in Jnl. of the American Ceramic Society 74, n2 p419-421 Feb 91

Keywords: "Ceramics, "Indentation, "Crack growth, "Fracture mechanics, "Gallium arsenides, Measurement, Environmental tests, Water, Acetonitrile, Mechanical properties, Reprints.

Indentation measurements of the environmentally enhanced crack growth parameter, $n$, in GaAs have been made in water, acetonitrile, and methanol. Reasonable agreement with $n$ values obtained from double-cantilever-beam tests was obtained. It is concluded that the range of $n$ values which can be measured by the indentation technique is much wider than previously observed, and is strongly dependent upon the experimental configuration employed.

101,084

PB91-194670 Not available NTIS National Inst. of Standards and Technology (MSEL). Gaithersburg, MD. Ceramics Div.

Effect of Thermochemical Treatments on the Strength and Microstructure of SiC Fibers.

Final rept.

B. A. Bender, J. S. Wallace, and D. J. Schrodt. 1991, $7 p$

Sponsored by Naval Air Systems Command, Washington, DC

Pub. in Jnl. of Materials Science 26, p970-976 1991

Keywords: "Ceramic fibers, "Ceramic matrix composites, *Tensile strength, "Silicon carbides *Thermochemical properties, Thermal cycling tests, Microstructure, Thermal degradation, Fabrication, High temperature, Carbon monoxide, Annealing, Electron microscopy, Polymers, Optical microscopy, Auger electron spectroscopy, Reprints.

The room-temperature strength of commercially available polymer-derived $\mathrm{SiC}$ fibers degrades during the typical high-temperature thermal cycle used in ceramic matrix composite fabrication. Substantial improvements in retained room-temperature strength for two different commercially available fibers were observed after annealing in carbon powder at temperatures up to $1600 \mathrm{C}$. Further improvements in strength were observed for both fibers when heat treated in $\mathrm{CO}$ atmospheres. X-ray diffraction, TEM, SEM, auger electron spectroscopy, and optical microscopy were used to characterize the microstructure and chemistry of the heat-treated fibers in order to understand better the degradation mechanisms of the fibers as well as the improved strength retention.

101,085

PB91-195628

Not available NTIS

National Inst. of Standards and Technology (IMSE) Gaithersburg. MD. Ceramics Div.

Phase Equilibria of the System Strontium OxideCalcium Oxide-Cupric Oxide.

Final rept.

R. S. Roth, C. J. Rawn, J. J. Ritter, and B. Burton. $1989,5 p$

Pub. in Jnl. of the American Ceramic Society $72, n 8$ p1545-1549 1989

Keywords: "Ceramics, "Phase diagrams, "Crystal structure, "Calcium oxides, "Strontium oxides, "Copper oxides, X-ray diffraction, Orthorhombic lat tices, Solid solutions, Tetragonal lattices, Reprints.

A new phase, probably $\mathrm{CaCuO} 2$, stable only to $740 \mathrm{C}$ was found to have an orthorhombic subcell. Cmca a $=10.588(1) \mathrm{b}=2.8122(4), \mathrm{c}=6.3245(6) \times 0.1 \mathrm{~nm}$ with weak diffraction spots of two superimposed larger cells, one requiring 10 times the subcell $b$ axis, the other belonging to a monoclinic lattice (interchanging $b$ and c) with the short axis multiplied by 6 times and beta approximately $=105.5$ degrees. The ternary system at $950 \mathrm{C}$ shows large regions of solid solution. Complete solid solution is observed for the series $(\mathrm{S} r$, CA)2Cu03. The compound $\mathrm{SrCuO} 2$ accepts $\mathrm{Ca}$ up to Sro.25Ca0.75O2 and $\mathrm{Sr} 14 \mathrm{Cu} 24 \mathrm{O} 41$ extends to at least Sr7Ca7Cu24O41. A new ternary phase exists with a small homogeneity region (Ca1-xSrx) CuO2 with $x$ approximately $=0.15$. The composition (Ca0.84Sr0.16)CuO2 has a primitive tetragonal unit cell with $\mathrm{a}-3.867(2)$ and $\mathrm{c}=3.2195(2) \times 0.1 \mathrm{~nm}$.

\section{1,086}

PB91-195669

Not available NTIS

National Bureau of Standards (IMSE), Gaithersburg.

MD.

Standardization of Advanced Ceramics.

Final rept.

S. J. Schneider, and D. R. Bradley. 1988, 7p

Pub. in Advanced Ceramic Materials 3, n5 p442-448 1988

Keywords: "Standardization, *Ceramics, Tests, Methodology, Terminology, Mechanical properties, Data bases, Classifications, Reliability, Nomenclature, Reprints

Advanced Ceramics, because of the superior and multifaceted properties, are being extensively developed and brought to market as rapidly as possible. A wide spectrum of commercial standards (test methods, classification system, reference materials and data) however, are lacking, and the lack may impede furthe marketplace diffusion and industrial acceptance. The paper elaborates upon general Advanced Ceramics standards needs and the mechanisms and organizations by which the needs could be addressed and met. Currently, a unified classification system, along with standard terminology/nomeclature are needed to set the basis for unanimity in information transfer between designers, manufacturers and product users. Standard mechanical property behavior tests, particularly high temperature procedures, have been identified as one additional singular need as mechanical integrity and reliability are pervasive requirements for all Advanced Ceramics.

\section{1,087}

PB91-202820

Not available NTIS

National Bureau of Standards (IMSE), Gaithersburg MD Ceramics Div.

Comparison of Creep Rupture Behavior in Tension and Bending.

Final rept.

$1988,7 p$

Pub. in Ceramic Engineering and Science Proceedings 9, n7-8 p635-641 1988.

Keywords: *Ceramics, "Creep properties, "Silicon carbides, Bend tests, Tension tests, Comparison, Rupture, Compression tests, Cavities, Failure, Service life, 
Performance prediction, Stress strain relations, Reprints.

Creep-rupture data in flexure are analyzed in terms of creep-rupture data in tension, and creep data obtain in both tension and compression. Based upon a critical strain criterion for cavity coalescence, and the assumption that the failure modes in tension and flexure are the same, accurate predictions of component lifetime in flexure were made.

\section{1,088}

PB91-202838

Not available NTIS

National Bureau of Standards (IMSE), Gaithersburg MD. Ceramics Div.

High Temperature Creep Testing of Ceramics.

Final rept.

D. F. Carroll, and S. M. Wiederhorn. 1988, 15p

Pub. in International Jnl. of High Technology Ceramics 4, n2-4 p227-241 1988.

Keywords: "Silicon carbides, "Ceramics, "High temperature tests, Creep properties, Heat resistant mater als, Construction materials, Tension tests, Compression tests, Loads(Forces), US NBS, Methodology, Characterization, Binary system(Materials), Failure, Characteriz
Reprints.

In structural applications, materials are often subjected to complex modes of loading, simple tensile or compressive stresses being uncommon. During creep deformation, these complex modes of loading result in time dependent stress and strain distributions because ceramics tend to creep faster in tension than in compression. Consequently, creep of ceramic materials must be characterized in both tension and in compres sion before any predictions can be made on how component will behave under complex stress states. Experimental techniques currently being used at the National Bureau of Standards to characterize the creep behavior of two phase ceramic materials at high temperatures are described. Techniques for creep studies in both tension and compression are usable to temperatures of $1500 \mathrm{C}$. The reliability of the test techniques with regard to linearity of loading and stability during testing is discussed.

\section{1,089}

PB9́1-203489

Not available NTIS

National Bureau of Standards (IMSE), Gaithersburg MD. Ceramics Div.

Small Angle Neutron Scattering Characterization of Microporous Silica.

Final rept.

G. G. Long, S. T. Krueger, and R. A. Gerhardt. 1988 ,

Pub. in Ceramic Transactions, v5 p171-179 1988.

Keywords: "Small angle scattering, "Ceramics, "Porosity, "Silica, On-line measurement systems, Neutron scattering, Characterization, Microstructure, Densification, Heat treatment, Nondestructive tests, Reprints.

The new technique of multiple small angle neutron scattering has been used to study the densification of microporous silica as a function of thermal processing One of the advantages of small angle neutron scattering is that all of the scatterers, which in this case are pores within ceramic bodies, are measured whether they are open or closed, to yield a statistically significant determination of microstructure morphology. Fur thermore, the neutron scattering techniques are nondestructive, and the time scale of data collection has been reduced, offering future possibilities for in situ measurements of microstructure evolution during processing.

\section{1,090}

PB9 1-203786

Not available NTIS National Bureau of Standards (IMSE), Gaithersburg, MD. Reactor Radiation Div.

Refinement of the Structure of Beta'-MoO3.

Final rept.

J. B. Parise, E. M. McCarron, A. W. Sleight, and E.

Prince. $1988,4 \mathrm{p}$

Pub. in Materials Science Forum 27-28, p85-88 1988.

Keywords: "Crystal structure, "Molybdenum oxides, Rhenium oxides, Neutron diffraction, Monoclinic lat tices, Tungsten oxides, Deuteration, Labelled compounds, Oxidation, Instability, Reprints

The structure of beta'-MoO3, a new modification of $\mathrm{MoO} 3$, is closely related to the structure of $\mathrm{ReO} 3$ and has been refined using high resolution neutron powder diffraction data and the Rietveld technique. The struc- ture is monoclinic ( $P 2$ (sub 1) $/ \mathrm{n}, \mathrm{a}=7.415(1), \mathrm{b}=$ 7.433(1) c 7.654(1)A Beta MoO3 is isostructural with the room temperature monoclinic modification of WO3 and may be derived from the deuterated intercalate (DxMoO3), also with $\mathrm{ReO} 3$ type structure, by heating in oxygen at $200 \mathrm{C}$. The modification is structurally intermediate between the WO3-type and the thermodynamically stable alphaMoO3.

\section{1,091}

PB9́1-204172

Not available NTIS

National Bureau of Standards (IMSE), Gaithersburg, MD. Ceramics Div,

Creep and Reliability of Ceramic Materials at Elevated Temperatures.

Final rept.

S. M. Wiederhorn, T. J. Chuang, and D. F. Carroll.

$1988,14 p$

Contract DE-AI05-85OR21569

Sponsored by Department of Energy, Oak Ridge, TN

Pub. in Proceedings of International Symposium on Fine Ceramics Arita '87, Japan, November 17-19, 1987, p117-130 1988

Keywords: "Ceramics, "Heat engines, "Heat resistant materials, "Creep properties, "Silicon carbides, Constitutive equations, Failure, Reliability, Fracture mechanics, Compression tests, Tension tests, Stress strain relations, Defects, Holes(Mechanics), Cavities, Bend tests, Surface properties, Methodology, Rates(Per time), Performance prediction, Reprints.

Failure at elevated temperatures in structural ceramics often occurs as a consequence of creep deformation, which results in the generation of cavities within the ceramic. With time, cavities grow and then coalesce to form the cracks that eventually are the cause of structural failure. The generation of cavities not only leads to failure of structural ceramics, but also changes the constitutive relation between creep rate and applied stress, so that, as a consequence of cavitation, creep in tension is more sensitive to stress than creep in compression. The net effect of this asymmetric behavior is a time dependence in the stress distribution when components are subjected to bending moments. Methods of measuring the creep rate in ceramic materials are discussed. Creep in tension is shown to be at least 20 times that in compression for a commercial grade of reaction bonded silicon carbide. Also, bimodal creep rate curves are observed in both tension and compression. As an example of the application of the data to more complex configurations, the constitutive equations developed for this material are used to predict the stress and strain distribution and the zone of cavitation in four point-flexure bars. Experimental data on cavitation and strain are in satisfactory agreement with predictions.

\section{1,092}

PB91-236927

Not available NTIS

National Inst. of Standards and Technology (MSEL), Gaithersburg, MD. Ceramics Div.

Cyclic Fatigue from Frictional Degradation at Bridging Grains in Alumina.

S. Lathabai, J. Rodel, and B. R. Lawn. 1991, 9p

Pub. in Jnl. of the American Ceramic Society $74, n 6$ p1340-1348 Jun 91

Keywords: "Ceramics, "Aluminum oxide, "Fatigue life, "Tension tests, "Grain size, "Fracture mechanics, Cyclic loads, Friction, Microstructure, Fracture properties, Wear, Electron microscopy, Polycrystals, Reprints, Grain bridging

Tension-tension cyclic loading tests have been conducted on a coarse-grained alumina ceramic that exhibits toughness-curve behavior by grain-interlock bridging. Fatigue effects are observed in the regions of both short cracks, using indentation flaws, and long cracks, using compact-tension specimens. A true mechanical fatigue effect is demonstrated by running the tests below the static fatigue limit. A custom-made device for in situ observation of crack propagation in the scanning electron microscope enables us to identify bridge degradation as a cause of the fatigue process. 'Wear' debris cumulates at the sliding intergranular frictional contact points, indicating a loss of traction at the junction. The basis of a fracture mechanics model describing the effect of the frictional degradation in reducing crack-tip shielding is outlined and fitted to the data.

101,093
PB91-236935
National Inst. of Standards and Technology (MSEL), Gaithersburg, MD. Ceramics Div.

Fracture Mechanics Model for Subthreshold Indentation Flaws. Part 1. Equilibrium Fracture.

Final rept.

S. Lathabai, J. Rodel, T. Dabbs, and B. R. Lawn.

1991, 12p

Sponsored by Office of Naval Research, Arlington, VA. Pub. in Jnl. of Materials Science 26, p2157-2168 1991.

Keywords: "Crack propagation, "Models, "Silica glass, * Fractures(Materials), Residual stress, Defects, Equilibrium, Deformation, Cracking(Fracturing), Fracture properties, Stress intensity factors, Reprints.

A fracture mechanics model for subthreshold indentation flaws is described. The model describes the initiation and extension of a microcrack from a discrete deformation-induced shear fault (shear crack) within the contact zone. A stress-intensity factor analysis for the microcrack extension in residual-contact and applied-stress fields is used in conjunction with appropriate fracture conditions, equilibrium in Part 1 and nonequilibrium in Part II, to determine critical instability configurations. In Part I, the K-field relations are used in conjunction with the Griffith requirements for crack equilibrium in essentially inert environments to determine: (I) the critical indentation size (or load) for spontaneous radial crack pop-in from a critical shear fault under the action of residual stresses alone; (II) the inert strengths of surfaces with subthreshold or postthreshold flaws. The theory is fitted to literature data for silicate glasses. These fits are used to 'calibrate' dimensionless parameters in the fracture mechanics expressions, for later use in Part II. The universality of the analysis in its facility to predict the main features of crack initiation and propagation in residual and applied fields will be demonstrated. Special emphasis is placed on the capacity to account for the significant increase in strength (and associated scatter) observed on passing from the postthreshold to the subthreshold domain.

101,094

PB91-23713

Not available NTIS

National Inst. of Standards and Technology (MSEL), Gaithersburg, MD. Ceramics Div.

Cathodoluminescence Measurement of Strained Alumina Single Crystals.

Final rept.

Pub. in JnI. of the European Ceramic Society 7, p109. 1161991.

Keywords: "Cathodoluminescence, "Aluminum oxide, "Single crystals, "Vickers hardness, "Residual stress, Emission spectroscopy, Measurement, Electron microscopy, Spectrum analysis, Stress strain relations, Annealing, Indentation, Reprints.

Alumina single crystals indented with a Vickers hardness indenter were investigated by cathodoluminescence (CL) imaging and spectroscopy in a scanning electron microscope. The spatial resolution of the $\mathrm{CL}$ images was approximately 0.4 micrometers. CL spectra were measured with wavelength resolution of $1.0 \mathrm{~nm}$ in the wavelength range of 200 to $900 \mathrm{~nm}$; in the immediate region of the ruby lines $(693 \mathrm{~nm})$, spectra were measured with a resolution of $0.15 \mathrm{~nm}$. Stressinduced frequency shifts of the ruby lines were utilized to measure the residual stresses in the region surrounding the indentation.

101,095

PB91-237339

Not available NTIS

National Bureau of Standards (IMSE), Gaithersburg, MD. Ceramics Div.

New Layered Cuprate Structure-Type, (A1XA'x) 14Cu24041.

Final rept.

T. Siegrist, L. F. Schneemeyer, R. S. Roth, J. V.

Waszczak, and S. A. Sunshine. 1988, 10p

Pub. in Materials Research Bulletin 23, n10 p142914381988.

Keywords: "Ceramics, "Semiconductors(Materials), *Crystal structure. "Alkaline earth metal compounds, *Cuprates, Calcium compounds, Lanthanum compounds, Melts, Oxides, Orthorhombic lattices, Strontium compounds, Bismuth compounds, Barium compounds, Reprints.

Phases crystallizing in a new structure-type with general formula $\left(A(1-x) A^{\prime} x\right) 14$ Cu24 O41 $(A=$ alkaline earth 
metal, $\mathrm{A}^{\prime}=$ trivalent metal) and symmetry $\mathrm{Cccm}$ with an extended stoichiometry range have been found in studies of the Sr-Y-Cu-O, Ba-Sr-Cu-O, Ca-Sr-Bi-Cu-O and $\mathrm{Ca}-\mathrm{La}$-Cu-O systems. Single crystal $\mathrm{X}$-ray studies on several crystals grown from different alkaline earth/ metal oxide-cuprate melts reveal a common orthorhombic F-centered subcell of $a=11.3 \mathrm{~A}, \mathrm{~b}=13.0 \mathrm{~A}$ and $\mathrm{c}=3.9 \mathrm{~A}$. Super-structure is observed in crystals, leading to a 7 -fold increase of the $c$-axis and a change in symmetry to space group $\mathrm{Cccm}$. As in Ba2YCu3O7. the $\mathrm{Cu}$ atoms are found as $\mathrm{Cu}-\mathrm{O}$ planes and linear $\mathrm{Cu}$ $\mathrm{O}$ chains. Due to shear in the planes, half of the $\mathrm{Cu}-\mathrm{O}$ squares share edges, producing $\mathrm{Cu}$-Cu zigzag chains, similar to the planes observed in $\mathrm{CaCu} 2 \mathrm{O} 3$. In the linear $\mathrm{Cu}-\mathrm{O}$ chains, the $\mathrm{Cu}-\mathrm{O}$ squares share edges as well, leading to a short $\mathrm{Cu}$-Cu contact of 2.75A. Experiments on ceramic samples indicate that the oxygen content is fixed and that the samples are semiconducting.

\section{1,096}

101,096
PB91-237628

Not available NTIS

National Inst. of Standards and Technology (IMSE), Gaithersburg, MD. Polymers Div.

Fourier Transform Infrared Analysis of Ceramic Powders: Quantitative Determination of Alpha, Beta, and Amorphous Phases of Silicon Nitride. Final rept.

T. K. Trout, R. A. Faltynek, F. E. Brinckman, and J. M. Bellama. $1989,5 p$

Pub. in Jnl. of Materials Research 4, n2 p399-403 1989.

Keywords: "Fourier transformation, "Infrared spectroscopy, "Ceramics, "Quantitative analysis, "Silicon nitrides, Compositions, Powders, Spectrum analysis Amorphous materials, Crystal structure, Ternary systems, Phase diagrams, Reflectance, Reprints.

Fourier transform infrared spectroscopy (FT-IR) forms the basis for determining the morphological composition of mixtures containing alpha, beta, and amorphous phases of silicon nitride. The analytical technique involving multiple linear regression treatment of Kubelka-Munk absorbance values from diffuse reflectance measurements, yields specific percent composition data for the amorphous phase as well as the crysalline phases in ternary mixtures of $0-1 \%$ by weight Si3N4 in potassium bromide.

\section{1,097}

\section{PB91-237743} Gaithersburg, MD. Ceramics Div.

Environmentally Enhanced Fracture of Gallium Arsenide.

Final rept

G. S. White, S. W. Freiman, and A. M. Wilson. 1989 3p

Contract N00014-87-F-0007

Sponsored by Office of Naval Research, Arlington, VA. Pub. in Jnl. of the American Ceramic Society 72, n11 p2193-2195 Nov 89.

Keywords: "Gallium arsenides, "Ceramics, "Fracture mechanics, Stress corrosion cracking Environmental tests, Single crystals, Nitrogen, Crack propagation, Ammonia, Water, Methanol, Acetonitrile, Humidity, Reprints.

GaAs has been found to undergo environmentally enhanced fracture in water, acetonitrile, formanide, and methanol. In contrast, no effect on crack growth has been observed in ammonia gas or N2 gas at $50 \%$ relative humidity. These results are consistent with the model proposed by Michelske, et al. of electrostatically enhanced bond rupture. The lack of water controlled regions in ammonia or N2 gas is presently not understood.

\section{1,098}

PB92-116797

Not available NTIS

National Inst. of Standards and Technology (NML) Boulder, CO. Chemical Engineering Science Div.

Design and Fabrication of a Miniature High-Temperature Guarded-Hot-Plate Apparatus.

Final rept.

B. J. Filla. $1990,8 p$

Pub. in Proceedings of International Thermal Conductivity Conference (21st), Lexington, KY., October 15. 18, 1989, p67-74 1990.

Keywords: "Test equipment, Ceramics, Thermal conductivity, Measurement, Ceramic matrix composites, Oxidation, Corrosion, High temperature tests, Design

criteria, Fabrication, Thickness, Platinum, Rhodium, Reprints, "Hot plates, Miniaturization.

A miniature guarded-hot-plate apparatus has been built to measure thermal conductivity of ceramics and ceramic composites at temperatures between $700 \mathrm{~K}$ and $1500 \mathrm{~K}$ in a neutral or mildly oxidizing atmosphere. The system is composed of a set of $7.94 \mathrm{~cm}$ diameter boron nitride disks supported by high-purity alumina insulation board. Thermocouple-grade platinum and platinum-rhodium wiring are used for both the heater and thermometry elements. A typical specimen examined in this apparatus is $7 \mathrm{~cm}$ in diameter with a thickness between 1 and $8 \mathrm{~mm}$. Optimal specimen thermal conductivities fall in the range of 0.05 to $5 \mathrm{~W} /(\mathrm{m})(\mathrm{K})$ Design features and choice of materials used in this apparatus are discussed.

\section{1,099}

PB92-116995

Not available NTIS

National inst. of Standards and Technology (MSEL),

Gaithersburg, MD. Ceramics Div.

Test Method for Tensile Creep of Structural Ceramics Using Flexure Beams.

Final rept.

R. F. Krause, and T. J. Chuang. 1991, 10p

Pub. in Ceramics Today - Tomorrow's Ceramics, p1865-1874 1991.

Keywords: "Ceramics, "Construction materials, "Flexural strength, Creep tests, Silicon carbides, Tension tests High temperature tests, Displacement, tests, High temperature tests, Displacement,
Loads(Forces), Compression tests, Bend tests, SinLoads(Forces), Compression tests, Bend tests, Sin-
tered materials, Flexing, Beams(Supports), Reprints, Power law.

A novel method of analysis is described for evaluating the power-law parameters for tensile creep of structural ceramics at elevated temperatures. Displacement rate, invariant with time in the secondary mode of creep, is measured versus load on both compression and flexure specimens of a material. The analysis takes into account that the material behaves differently in tensile and compressive creep. The power-law parameters for compressive creep are evaluated directly from strain rate versus stress in the compression tests. Using these values for compressive creep, those for tensile creep are evaluated from displacement rate versus bending moment in the flexure tests. The compressive and tensile creep properties of the material determine the curvature and neutral-axis position in the flexure specimen under a given bending moment. The power-law parameters for creep of sintered alpha silicon carbide are evaluated to demonstrate the usefulness of the method.

\section{1,100}

PB92-117001

Not available NTIS

Pational Inst. of Standards and Technology (MSEL) Gaithersburg, MD. Reactor Radiation Div.

Characterization of the Densification of Alumina by Multiple Small-Angle Neutron Scattering.

Final rept.

S. Krueger, G. G. Long, and R. A. Page. 1991, 9p
Contracts MIPR-ARO-102-90, DE-FG05-84ER45063

Sponsored by Army Research Office, Research Triangle Park, NC., and Department of Energy, Washington,

Pub. in Acta Crystollagraphica A47, p282-290 1991

Keywords: "Aluminum oxides, "Porosity, Neutron scattering, Ceramics, Small angle scattering, Sintering, Microstructure, Reprints, Pore size.

Multiple small-angle neutron scattering was used to follow the evolution of the pore-size distribution in alpha-Al2O3 through the intermediate and final stages of sintering. The new technique enables the study of microstructure in the 0.08-10 micrometers size regime which is the size range of importance for many materials systems, without needing to increase the resolution of currently available small-angle scattering instruments. The microstructure evolution results indicate a nearly constant effective pore radius for the alumina throughout the intermediate sintering stage, ranging from 0.19 micrometers at $54 \%$ of theoretical density to 0.17 micrometers at $79 \%$ dense. As the alumina densifies further, there is a transition region after which the effective pore radius grows rapidly to greater than or equal to 0.6 micrometers at $97.5 \%$ dense.

101,101

B92-117043

National Inst. of Standards and Technology (MSEL)

Gaithersburg, MD. Ceramics Div.
Effect of Green Density and the Role of Magnesium Oxide Additive on the Densification of Alumina Measured by Small-Angle Neutron Scattering. Final rept.

G. G. Long, S. Krueger, and R. A. Page. 1991, 7p Pub. in Jnl. of the American Ceramic Society $74, n 7$ p1578-1584 Jul 91.

Keywords: "Aluminum oxides, "Densification, "Ceram ics, Neutron scattering, Small angle scattering, Density, Green strength, Sintering, Magnesium oxides, Measurement, Additives, Porosity, Microstructure, Characterization, Reprints.

Small-angle neutron scattering measurements were used to examine the effect of green density and the role of $\mathrm{MgO}$ additive on the evolution of the porous microstructure of alumina during intermediate- and final-stage sintering. It was found that the initial connectivity in the green state plays a dominant role in establishing the channel diameters during the intermediate stage of sintering, and contributes also to determining the onset density at which the final stage of sin tering begins. The role of $\mathrm{MgO}$ as a sintering aid lies, a least in part, in prolonging the stability of intermediatestage sintering such that the body achieves greate density before the transition to final-stage sintering after which isolated pores are formed.

101,102

PB92-117357

Not available NTIS

National Inst. of Standards and Technology (NML) Boulder, CO. Chemical Engineering Science Div.

Apparent Thermal Conductivity of Fumed-Silica Standard Reference Materials.

Final rept.

D. A. Smith. $1990,16 p$

Contract ORNL/IA-21428

Sponsored by Oak Ridge National Lab. TN.

Pub. in Proceedings of International Thermal Conduc tivity Conference (21st), Lexington, KY., October 15 18,1989, p261-276 1990

Keywords: "Vapor deposition, "Thermal conductivity * Silica, "Insulating bosition, "Thermes croporosity, Temperature dependence, Atmospheric pressure, Pressure dependence, Density, Statistica analysis, Moisture content, Reprints, "Fumed silica "Standard reference materials, Hot plates.

Measurements of apparent thermal conductivity of microporous fumed-silica insulation board (MFS) are re ported here for mean specimen temperatures ranging from 318 to $733 \mathrm{~K}(45$ to $460 \mathrm{C}$ ) and environmental ai pressures from $83.5 \mathrm{kPa}$ (626 Torr) down to $27 \mathrm{kPa}$ (200 Torr). Apparent conductivity is correlated with temperature, pressure, and density; the correlation obtained represents the data within a standard deviation of 0.68 percent. MFS with a density of $300 \mathrm{~kg} / \mathrm{cu} \mathrm{m}$ and at an ambient pressure of $83 \mathrm{kPa}$ has an apparen thermal conductivity of $19.8 \mathrm{~mW} /(\mathrm{m} \mathrm{K})$ at $300 \mathrm{~K}$. It is suitable for use as a standard reference material of very low conductivity from 297 to $733 \mathrm{~K}$ ( 24 to $460 \mathrm{C}$ ). Adsorbed moisture within this material must be driven off by prolonged heating at $110 \mathrm{C}$ before its conductivity is measured. Great care in handling MFS is necessary because of its fragility.

101,103

PB92-117449

Not available NTIS

National Inst. of Standards and Technology (MSEL) Gaithersburg, MD. Ceramics Div.

Effect of Surface Forces on Subcritical Crack Growth in Glass.

Final rept.

S. M. Wiederhorn, and E. R. Fuller. $1989,4 p$

Pub. in Jnl. of the American Ceramic Society $72, n 2$ p248-251 Feb 89

Keywords: "Surface forces, "Crack propagation Silica glass, Fracture mechanics, Aqueous solutions, Stress intensity factors, $\mathrm{pH}$, Surface chemistry, Reprints.

Data on the growth of cracks tested in aqueous soluions were interpreted in terms of surface force theory. For applied stress intensity factors greater than 0.25 MPa (m(sup 0.5)), the position and the slope of the curves and their dependence on $\mathrm{pH}$ and ion concentration can be explained in terms of surface force theory, provided these forces are of a magnitude and range that are representative of those involved in the cohesive bonding of solids. Weaker forces, such as structural, double-layer, or dispersion forces, have little 


\section{Ceramics, Refractories, \& Glass}

effect on crack growth in silica glass for $K($ sub 1$)>$ $0.25 \mathrm{MPa}$ (m(sup 0.5))

\section{1,104}

\section{PB92-126507}

PC A09/MF A02

National Inst. of Standards and Technology (MSEL), Gaithersburg, MD. Ceramics Div.

Ceramics: Technical Activities 1991. (NAS-NRC Assessment Panel, February 13-14, 1992).

Annual rept.

S. W. Freiman. Feb $92,176 p$ NISTIR-4694

S. W. Freiman. Feb 92
See also PB91-132233

Keywords: "Research projects, "Ceramics, Standards, Materials, Mechanical properties, Processing, Superconductors, Microstructure, Synchrotron radiation, Tribology, Patents, Vapor deposition, Databases, Photonic materials, Optoelectronic materials, Advanced ceramics, Ferroelectric oxide films, High temperature superconductors

In 1991, the Ceramics Division continued its program of standards development, data generation and evaluation, and research focused on the characterization and understanding of material behavior. The research program focuses on the critical issues which control the implementation of advanced ceramics and is developed through internal assessment and consultation with representatives from ceramic industries and academia. Some areas of research were: ferroelectric oxide films, glass compositions, advanced ceramics powder processing, modelling and superconductivity.

\section{1,105}

PB92-126564

PC A05/MF A01

National Inst. of Standards and Technology (EEEL)

Boulder, CO. Electromagnetic Technology Div.

High-Temperature Superconductivity: Abstracts of NIST Publications, 1987-1991.

Final rept.

M. E. DeWeese. Nov 91, 93p NIST/SP-826

Also available from Supt. of Docs. Supersedes PB89 148340.

Keywords: "Ceramics, "Superconductivity, "Abstracts, Bismuth, Standards, Thallium, Yttrium, High temperature tests, Thin films, $X$ ray diffraction, Crystal defects, Phase transformations.

The report is a collection of abstracts from 243 papers published between March 1987 and August $1991 \mathrm{cov}$ ering various aspects of superconductivity research. The work of nine divisions of the National Institute of Standards and Technology (formerly the Nationa Bureau of Standards) in both Boulder, Colorado, and Gaithersburg, Maryland, is represented.

\section{Coatings, Colorants, \& Finishes}

\section{1,106}

AD-A243 095/7 PC A03/MF A01

National Inst. of Standards and Technology, Gaithersburg, MD.

Chemical Vapor Deposited Diamond.

Technical rept.

A. Feldman, E. N. Farabaugh, and L. H. Robins. 27 Sep $91,50 p$

Contract N00014-90-F-0011

Keywords: Chemical reactions, Chemicals, Coefficients, Deposition, "Diamonds, Diffusion, Electrical resistance, Far infrared radiation, Frequency, Friction, Measurement, Modulus of elasticity, Optics, Permeability, Room temperature, Single crystals, Therma conductivity, Transparencies, "Vapor deposition, "Chemical vapor deposition.

There is considerable interest in using diamond for nu merous applications because of the development of methods that allow for the deposition of diamond by chemical vapor deposition (CVD) techniques over large areas. The interest in new applications for diamond rests on combinations of superior properties that diamond possesses. These extreme properties, which are based on measurements in bulk single crystal diamond, include greatest hardness, highest elastic moduli, and highest thermal conductivity at room temperature of any material. Other important properties include optical transparency over an extensive wave length range from the ultraviolet through the far infrared, high electrical resistivity, dopability to be a semi-

conductor, low permeability to diffusion, chemical inertness, and low coefficient of friction.

101,107

PC A05/MF A01

National Inst. of Standards and Technology (MSEL), Gaithersburg, MD.

U.S. Assessment of the New Diamond Technology in Japan.

Special pub. (Final)

A. Feldman, and L. H. Schwartz. Jan 91, 100p NIST/ SP.807

Also available from Supt. of Docs. as $\mathrm{SNOOH}^{-003}$. 03067-8.

Keywords: "Diamonds, "Vapor deposited coatings, "Chemical vapor deposition "Japan, Etching. Abra sives, Polishing, Reviews, Epitaxy, Ion implantation, Laser cutting, Cutting tools, Heat sinks, Electro-optics, Optical materials, Research projects, Lithography, International cooperation, Boron nitrides, Ceramics, Industrial engineering

The assessment of Japanese diamond technology was undertaken at the request of the joint committees for implementation of the 1988 United States-Japan Agreement on Cooperation in Research and Development in Science and Technology under the auspices of the Committee on Materials, Office of Science and Technology Policy. The purposes of the study were to assess the level of scientific activity, areas of intended commercialization, progress in commercialization, the role of the Japanese government in assisting industry to exploit the new diamond technology, and the rela-
tive positions of Japan and the U.S. in all aspects of synthesized diamond technology. Japanese efforts were chosen for assessment because Japan is the apparent world leader in the drive for commercialization of the technology. The principal focus of the study was diamond produced by chemical vapor deposition, with a peripheral investigation of cubic boron nitride and diamond-like carbon. Based on the assessment, a set of conclusions and recommendations is presented. The assessment is based on site visits in Japan between May 28 and June 5, 1990

101, 108
PB9 1-195248

Not available NTIS National Bureau of Standards (NEL), Gaithersburg MD. Building Materials Div.

Fourier Transform Infrared Spectroscopic Studies of the Degradation of One-Component Polyether Polyurethane Protective Coatings on Steel.

Final rept.

T. Nguyen, and W. E. Byrd. 1988, 20p

Pub. in Proceedings of FATIPEC Congress (19th), v3 p255-274 1988 .

Keywords: "Protective coatings, "Steels, "Polyurethanes, "Polyethers, "Corrosion mechanisms, Polymers, Construction materials, Fourier transformation, Infrared spectra, Degradation, Time dependence, Brittle materials, Dissociation, Reprints.

The degradation of one-component polyether polyurethane coatings on steel in corrosive environments was investigated using reflection/absorption Fourier transform infrared spectroscopy (FTIR-RA). Coatings of about 2 micrometers were applied uniformly to the mechanically polished ( 0.25 micrometer) cold-rolled steel substrates using a spin coater. After curing at room conditions, the coated specimens were exposed to an aerated $40 \mathrm{C}$ and $80 \% \mathrm{RH}$ environment. The studies showed that considerable chemical changes occurred in the structure of the coating after 8 months exposure to the exposure environment. Besides changes in the intensities of the amino, carbonyl and isocyanate
groups, some of the hydrogen bonded amino groups also become dissociated. Exposure in the environmen also results in extensive brittleness and crazing in the polyurethane coatings. The competitive formation and degradation reactions of various chemical groups in polyurethane during exposure will also be discussed.

\section{1,109}

PB91-203729 Not available NTIS National Bureau of Standards (NEL), Gaithersburg, MD. Building Materials Div.

Characterization of Epoxide Coatings on Steel by Reflection/Absorption Fourier Transform Infrared Spectroscopy: Quantitative Study.

Final rept.

T. Nguyen, W. E. Byrd, and D. P. Bentz. 1988, 18p

See also PB88-175518.

Pub. in Proceedings of FATIPEC Congress (19th), v2 p229-246 1988 .
Keywords: "Polymeric films, "Epoxy resins, "Steels, "Coatings(Materials), Thickness, Characterization, Thin films, Spectrum analysis, Infrared spectra, Calcu lation methods, Fourier transformation, Theories, Substrates, Absorptivity, Reflection, Transmission, Comparative evaluation, Reprints.

The study examines, both theoretically and experimentally, the effects of film thickness and angle of incidence on band shape intensities of an epoxy coating on a steel substrate. Crosslinked epoxy films between $10 \mathrm{~nm}$ and 25 micrometers were uniformly spin coated on $25 \times 25 \mathrm{~mm}$, mechanically polished $(0.25$ micrometers), cold-rolled steel samples. The most intense band of an epoxy coating, the $15101 / \mathrm{cm}$ band, was used for the study. There is a good linearity between band intensity and film thickness up to about $200 \mathrm{~nm}$ above that it deviates form Beer-Lambert law. Above $250 \mathrm{~nm}$, the theory underestimates the intensity and in this range a power law expression is more useful to estimate film thickness from the peak height of a FTIRRA spectrum. For thin films $(100 \mathrm{~nm})$, the theory agrees very well with experimental results on the relationship between band intensity and angle of incidence. How ever, for thick films the theory underestimates the intensity at low angles of incidence and overestimates at high angles of incidence, particularly in the micromete thickness range. There are certainly some differences between the spectrum of an epoxy on steel substrate obtained by the FTIR-RA technique and that of a film on $\mathrm{NaCl}$ obtained by the transmission technique. These differences become quite pronounced in thick films

\section{1,110}

PB91-204073

Not available NTIS

National Inst of Standards and Technology (IMSE). Gaithersburg, MD. Metallurgy Div.

Magnetic Properties of Iron/Silica Gel Nanocomposites.

Final rept.

R. D. Shull, J. J. Ritter, A. J. Shapiro, L. J.

Swartzendruber, and L. H. Bennett. 1989, 6p

Pub. in Materials Research Society Symposium Proceedings, v132 p179-184 1989.

Keywords: "Silica gel, "Iron, "Particulate composites, "Magnetic properties, "Paramagnetic materials, Matrix materials, Spin glass, Homogeneous mixtures, X-ray diffraction, Porosity, Particle size, Mossbauer effect, Spectrum analysis, Heating, Temperature dependence, Hydrogen, Ammonia, Superparamagnetism, Reprints, "Nanocomposites.

Homogeneous gelled composites of iron and silica containing 5-30 wt. \% Fe have been prepared by low temperature polymerization of aqueous solutions of ferric nitrate, tetraethoxysilane, and ethanol (with an HF catalyst). X-ray diffraction data indicated that these bulk materials are comprised of nanometer-sized regions of iron compounds embedded in a silica gel matrix. Scanning electron microscopy observations show that this matrix is characterized by the presence of many interconnected pores and that the size of these pores is related to the particle size of the Fecontaining regions. The paramagnetic nature of these materials at room temperature, as well as small size of the iron-containing regions, is indicated by the appearance in many of the sample of only a high intensity central doublet in the (57) Fe Mossbauer spectra. The Mossbauer effect data indicated that the form of the iron can be changed by a subsequent treatment in an atmosphere of ammonia or hydrogen at elevated temperatures. In addition, magnetic susceptibility measurements indicated that the hydrogen treated material becomes a spin glass at low temperatures.

101,111

PB91-237669

Not available NTIS National inst. of Standards and Technology (MSEL), Gaithersburg, MD. Ceramics Div.

Variations in the Practice of Ceramic Technology in Different Cultures: A Comparison of Korean and Chinese Celadon Glazes.

Final rept.

P. B. Vandiver, L. A. Cort, and C. A. Handwerker.

$1989,42 p$

Pub. in Ceram. Civiliz. 4, p347-388 1989.

Keywords: "Ceramic glazing, "Archaeological specimens, "Glazed ware, "Porcelain, Korea, China, Tech nology transfer, Comparison, Reprints, "Celadon.

Korean celadon glazes remained quite constant in visual appearance and technology during the Koryo 
MATERIALS SCIENCES

Coatings, Colorants, \& Finishes

dynasty (A.D. 918-1392), the result of a conservative craft workshop tradition which supplied green-glazed wares for the court, and they changed very little during the early Choson dynasty (fifteenth century) when they became a popular ware. The technological feat is contrasted with the diversity in visual appearance and ceramic technology found in Chinese celadons. The potential interactions of these traditions are explored.

\section{1,112}

\section{PB92-116813}

Not available NTIS

National Bureau of Standards (IMSE), Gaithersburg, MD. Ceramics Div.

Heat Conductivity of Oxide Coatings by Photothermal Radiometry between 293 and $1173 \mathrm{~K}$.

Final rept.

H. P. R. Frederikse, and X. T. Ying. 1988, $4 p$

Pub. in Applied Optics 27, n22 p4672-4675 1988.

Keywords: "Thermal conductivity, "Coatings(Materials), "Metal oxides, "Ceramics, Photothermal conversion, Nondestructive tests, Substrates, Radiometers, Reprints, Thermal waves.

Optical generation and optical detection of thermal waves are being used to determine the thermal properties of oxide coatings on metal substrates.

\section{Composite Materials}

101,113

AD-A231 778/2

PC A03/MF A01

National Inst. of Standards and Technology (IMSE),

Gaithersburg, MD. Metallurgy Div.

Influence of Water on the Mechanical Properties of a Glass-Epoxy Matrix Composite.

Technical rept. 1 Feb 89-1 Feb 91.

M. R. Stoudt, E. Escalante, and R. E. Ricker. 12 Feb $91,14 p$

Contract N00014-89-F-0072

Keywords: Absorption, Degradation, Desorption, Epoxy compounds, Glass, High pressure, Intervals, Matrix materials, Mechanical properties, Orientation(Direction), Pressure, Sampling, Water, Weight, "Composite materials.

The influence of exposure to water at ambient pressure and at an elevated pressure on the mechanical properties of a glass fiber epoxy matrix composite was investigated. The mechanical properties of three orientations of the composite were determined in the dry condition, after exposure to water at ambient pressure and after exposure to water at 5.9 MPa. Then, to determine the mechanism of the observed degradation, the mechanical properties of samples exposed at the two pressures were determined after the absorbed water was removed. The rate and extent of water absorption and desorption was evaluated by measuring the weight change at periodic intervals.

\section{1,114}

\section{AD-A239 509/3}

PC A03/MF A01

National Inst. of Standards and Technology (MSEL), Gaithersburg, MD. Corrosion Group.

Influence of Moisture and Pressure on the Mechanical Properties of a Glass-Epoxy Matrix Composite and a Graphite-Epoxy Mat rix Composite. Technical rept. no. 2, 1 Feb 89-1 Feb 91. M. R. Stoudt, E. Escalante, and R. E. Ricker. Jul 91, $30 \mathrm{p}$

Contract N00014-89-F-0072

Keywords: Absorption, "Composite materials, Degradation, Desorption, Dry materials, Epoxy compounds, Exposure(General), Fibers, Graphited materials, High pressure, Intervals, Matrix materials, Mechanical properties, Moisture, Orientation(Direction), Pressure, Sampling, Strength(Mechanics), Water, Weight, Wet strength, Yield strength, "Glass, “Graphite epoxy composites, "Epoxy composites, Hydrostatic pressure, Water absorption, Moisture content, Fiber reinforced composites, Polymers, Plasticity, Glass fibers.

The influence of exposure to water at ambient pressure and at elevated pressure was evaluated on the mechanical properties of a glass fiber-epoxy matrix composite and a graphite fiber-epoxy matrix composite. The mechanical properties of three different fiber orientations for each material were measured in the dry condition, after exposure to water at ambient pres- sure and after exposure to water at $5.9 \mathrm{MPa}$. In order to determine if the observed degradation was permanent, the mechanical properties were also measured after the absorbed water was removed. During exposure, the rate and extent of water absorption and desorption was evaluated by measuring the weight change at periodic intervals. Substantial reductions in the yield strength and ultimate strength were observed in the wet condition for the glass epoxy samples at both pressures, while the strength of the graphite epoxy composite was found to be insensitive to the water exposure. The wet strength of the glass epoxy composite was found to be independent of water pres sure during absorption and upon desorption, complete recovery of the dry strength was demonstrated. Evidence of compression-induced failure was observed for the graphite-epoxy composite in the wet condition.

\section{1,115}

32191

PC A05/MF A01

National Inst. of Standards and Technology (MSEL)

Gaithersburg, MD.

Polymer Composite Processing. Industry Workshop (2nd). Held at Gaithersburg, Maryland on May 18, 1990.

C. Johnson, S. S. Chang, and D. Hunston. Dec 90 , 87p NISTIR-446 See also PB88-179882.

Keywords: "Thermosetting resins, "Composite materials, "Fiber composites, "Meetings, Processing, Molding techniques, Pressing(Forming), Filament winding, Preimpregnating, Standards, Heat transfer, Delaminating, Creep properties, Fatigue life, Environmental tests, Microstructure, "Polymer matrix composites, "Resin matrix composites, Prepregs, Solid-Solid interfaces.

Industry representatives identified the most important processing methods, and scientific and technical barriers to improved polymer composite processing. Two processing methods were selected as most important: pressure molding and liquid molding. Seven scientific and technical barriers to the full exploitation of these processing methods were identified. The three highest priority items are the need to understand and control resin flow and fiber orientation, to develop process monitoring sensors for on-line control, and to understand and control the fiber-matrix interface. The remaining areas where there is a need for improvement are data validation and testing standards, determination and control of morphology, surface quality and dimensional stability, and understanding of heat flow. The Workshop also identified and prioritized eight technologies that complement processing and are important for the future. The three highest ranked items were fiber placement, new methods to prepare prepreg, and joining. The remaining items are preform preparation, recycling, environmental safety, tooling, and alternate sources of energy. The majority of people at the Workshop felt that thermosets were still the dominant resin system in most applications. The Workshop also selected seven performance issues that they felt were critical for the future: impact, environmental attack, delamination, dimensional changes, thermal stability, fatigue, and creep.

\section{1,116}

\section{PB91-147090}

Not available NTIS National Inst. of Standards and Technology (MSEL), Gaithersburg, MD. Polymers Div.

Effect of Logarithmic Singularity on the Free Edge Stress Intensity Factor of Composite Laminates.

Final rept.

M. Y. M. Chiang, and H. K. Stolarsky. 1990, 14p Pub. in Proceedings of Technical Conference on Composite Materials (5th), East Lansing, Ml., June 11-14, 1990, p336-349.

Keywords: "Laminates, "Stress intensity factors, Composite materials, Singularity, Reprints.

The study explains the relative importance of two types of stress singularities (r(sup delta) and log r), which may occur at the free edge of laminates. The $r$ (sup delta) singularity and $\log r$ singularity are merged to describe the edge effects of composite laminates. A combination of asymptotic analysis, which gives the strength of the singularity, and enriched finite element method (global/local technique) is used to solve a complete boundary value problem. The numerical results have been obtained for three different composite laminates: (45 deg/-45 deg) laminate, $(90 \mathrm{deg} / 15 \mathrm{deg})$ laminate and $(-15 \mathrm{deg} / 75 \mathrm{deg}$ laminate. It is concluded that the inclusion of the additional weaker singularity $(\log r)$ may significantly change the value of the stress intensity factor based on consideration of the strong singularity (r(sup delta)) alone.

\section{1,117}

PB91-159178

Not available NTIS

National Inst. of Standards and Technology (MSEL), Gaithersburg, MD. Metallurgy Div.

Spin Glass Magnetic Behavior of Iron/Silica Gel Nanocomposites.

Final rept.

R. D. Shull, and J. J. Ritter. 1990, 6p

Pub. in Materials Research Society Symposium Proceedings, v195 p435-440 1990.

Keywords: "Composite materials, "Iron compounds, "Silica gel, "Spin glass, "Polymers, Magnetic properties, Paramagnetic materials, Magnetic permeability, Measurement, Cryogenics, Anisotropy, Ferromagnetic materials, X-ray diffraction, Mossbauer effect, Electron microscopy, Reprints, "Nanocomposites.

Homogeneous gelled composites of iron and silica containing 5.40 wt. \% Fe prepared by low temperature polymerization of aqueous solutions of ferric nitrate, tetraethoxysilane, and ethanol (with an HF catalyst) were heated to $380 \mathrm{C}$ in the presence of hydrogen gas. $X$-ray diffraction and Mossbauer effect measurements, and transmission electron microscope (TEM) observations show these materials are comprised of nanometer-sized regions of iron compounds embedded in a silica gel matrix. Magnetic susceptibility data indicate the materials became either superparamagnetic or ferromagnetic at room temperature. On cooling, the mag netization data furthermore show that the hydrogenat ed materials containing $11-30 \% \mathrm{Fe}$ become magnetic spin glasses at temperatures less than $30 \mathrm{~K}$. Magnetic history effects are observed in addition to displaced hysteresis loops below their spin freezing temperatures (Tf). For field-cooled materials at $10 \mathrm{~K}$, the displacement of the hysteresis loops along the field axis indicates the presence of a unidirectional anisotropy which decreases with the cooling field. Both superparamagnetic-to-spin glass and ferromagnetic-to-spin glass transitions are observed in these nanocomposites. Tf varies with the Fe content from approximately $30 \mathrm{~K}$ for the $11 \% \mathrm{Fe}$ nanocomposite to approximately $10 \mathrm{~K}$ for a content near $33 \% \mathrm{Fe}$.

\section{1,118}

PB91-162180

Not available NTIS

National Inst. of Standards and Technology (IMSE), Boulder, CO. Fracture and Deformation Div.

Mechanical Properties of Alumina-Peek Unidirectional Composite: Compression, Shear, and Tension.

Final rept.

R. D. Kriz, and J. D. McColskey. 1990, 7p

Sponsored by National Aeronautics and Space Administration, Moffett Field, CA. Ames Research Center. Pub. in Advances in Cryogenic Engineering (Materials) 36, p921-927 1990.

Keywords: "Aluminum oxide, "Fiber composites, "Ceramic matrix composites, Cryogenics, Compression tests, Low temperature, Thermoplastics, Fracture strength, Fatigue, Tension tests, Shear tests, Peek, Reprints.

A new $\mathrm{Al} 2 \mathrm{O} 3$ (alumina) fiber composite with high strain to failure was fabricated with a thermal plastic PEEK (poly-ether-ether-ketone). The Al2O3-PEEK composite shows a marked improvement over thermally setting composite in that it absorbs 150 percent more elastic strain energy at $76 \mathrm{~K}$ than at room temperature. This increase in fracture toughness at low temperatures can provide improved fatigue performance for thermal isolation straps at low temperature. Other me. chanical property results suggest improvements for applications where graphite-epoxy materials are presently being used at low temperatures and where light weight is not a critical issue.

101,119

PB91-174656

Not available NTIS

National Inst. of Standards and Technology (NML), Gaithersburg, MD. Ionizing Radiation Div.

Emissivity of Aluminized Mylar.

Final rept.

S. R. Domen. 1991, 3p

Pub. in Radiation Physics and Chemistry $37, \mathrm{n} 2$ p1992011991.

Keywords: "Thermal insulation, "Polyethylene terephthalate, "Aluminum coatings, Calorimeters, Com- 


\section{Composite Materials}

posite materials, Plastics, Graphite, Thermal emission, Polymeric films, Metallizing, Reprints.

Commercially-available aluminized Mylar, 6 micrometers thick, is a useful material for reducing heat losses by thermal radiation in some calorimeters, such as those constructed of graphite or A-150 plastic. The present experiment shows that clean aluminized layers have a thermal emissivity near 0.044 .

101,120

PB91-174946

Not available NTIS

National Inst. of Standards and Technology (MSEL),

Boulder, CO. Fracture and Deformation Div.

Numerical Simulation and Visualization Models of Stress Wave Propagation Graphite/Epoxy Composites.

Final rept.

R. D. Kriz, and J. M. Gary. 1990, 8p

Pub. in Review of Progress in Quantitative Nondestructive Evaluation, v9 p125-132 1990

Keywords: "Stress waves, "Wave propagation, "Computerized simulation, "Finite difference method, "Composite materials, Nondestructive tests, Supercomputers, Finite element method, Elastic waves, Wave phases, Reflection, Reprints.

Based on previous results by finite elements the authors have extended their studies to include a fourth order splitting finite difference scheme of MacCormack. The finite difference method has allowed the authors to solve much larger problems. With a finite element method a mesh with 16458 degrees of freedom was solved in 2.2 hours on the NIST Cyber 205. With the finite difference scheme a mesh with 221502 degrees of freedom was solved in 70 seconds without using the full memory capacity of the Cyber 205. With this improved numerical method more accurate stress wave propagation simulations were possible. The authors studied some interesting phenomena observed in previous studies where the mesh was too coarse to conclude meaningful results. They studied the reflection of a single pulse in an off-axis unidirectional graphite/epoxy composite where the large deviation angle of the propagating wave resulted in a short lived surface wave shift of the reflected wave. A colored movie of this phenomena shows $\mathrm{L}, \mathrm{qL}$, qt and $\mathrm{t}$ modes and other physical phenomena not predicted by simple plane-wave theory.

101,121

PB91-185116

PC A09/MF A01

National Inst of Standards and Technology (MSEL),

Boulder, CO. Materials Reliability Div.

Effect of a Free Surface on Stress Distribution in a Bimaterial Composite.

Special pub. (Final).

V. K. Tewary, and R. D. Kriz. Mar 91, 186p NIST/SP. 802

Also available from Supt. of Docs.

Keywords: "Stress analysis, "Free surfaces(Crystallography), "Composite materials, Binary system(Materials), Greens function, Elastic properties, Loads(Forces), Grain boundaries, Equaeffect.

The paper is in two parts. In Part I, the SI Green's function is calculated and applied to plane-strain problems in cubic solids. In Part II, an extension of the Green's function method to the generalized-plane-strain problem is described, which enables the calculation of the elastic response of a solid to a prescribed out-of-plane strain. It is applied to calculate the stress distribution in a composite solid subjected to an out-of-plane load. The final results are in the form of an analytical closed integral representation which can be calculated numerically as well as analytically. The analytical evaluation of the integral by using the contour integration method gives the result in the form of a series. The series contains singular as well as non-singular terms. In general, it is difficult to ascertain the convergence of the series representation. The analytical series representation can be used to identify precisely the nature and the weight of each singularity in the stress distribution.

101,122

PB91-187336

Not available NTIS National Inst. of Standards and Technology (IMSE), Gaithersburg, MD. Polymers Div.
Interlaminar Shear Fracture of Laminated Composites.

Final rept.

H. Chai. 1990, 15p

Pub. in International Jnl. of Fracture 43, p117-131 1990.

Keywords: "Resin matrix composites, "Composite ma terials, "Fracture mechanics, Fracture strength, Electron microscopy, Surface energy, Adhesion, Plasticity, Deformation, Performance prediction, Reprints, Interlaminar shear.

The interlaminar fracture toughness in mode II and mode III of a number of advanced composites was studied using beam type test specimens and scanning electron microscopy. Special emphasis was placed on elucidating the material aspects of the fracture process and on quantifying the effect of matrix on fracture energy. The fracture energy in mode II was independent of crack extension while that for mode III exhibite a rather probabilistic 'resistance' behavior that was at tributed to the effect of fiber bridging. The initiation fracture energy, considered here the true measure of $\mathrm{G}(\mathrm{III})$, coincided with $\mathrm{G}(\mathrm{IIc})$. For either mode, the in erlaminar region ahead of the crack tip exhibited considerable plastic deformations, the severity that is believed to control the laminate toughness. The interlaminar fracture energy in shear, hereby denoted as $\mathrm{G}$ (sc) (= $\mathrm{G}(\mathrm{Ilc})=\mathrm{G}$ (III)) , was accurately predicted rom a straightforward adhesive joint fracture test provided the adhesive thickness coincide with the thick ness of the interlaminar resin layer.

\section{1,123}

PB91-194878

Not available NTIS

. Boulder, CO. Fracture and Deformation Div

Elastic Constants of Fiber-Reinforced Composites: A Fresh Measurement Approach.

Final rept.

H. Ledbetter, and M. Lei. 1990, 3p

ub. in Proceedings of Ultrasonics Symposium, Honolulu, HI., December 4-7, 1990, p1267-1269.

Keywords: "Fiber composites, "Elastic properties "Metal matrix composites, "Graphite composites, "UI trasonic tests, Measurement, Methodology, Magnesium, Reprints.

Composites reinforced with uniaxial fibers often possess transverse-isotropic symmetry and, therefore, five independent elastic constants: $\mathrm{C} 11, \mathrm{C} 33, \mathrm{C} 44$ C66, C13. ( $X 3$ is the fiber direction, $X 1$ the platenormal direction.) Measurements on a single specimen along principal axes give all the Cij except $\mathrm{C}_{13}$. To get $\mathrm{C} 13$, the usual approach is to propagate waves in nonprincipal directions such as (110). A fresh ap proach is proposed that requires measurements only along principal directions: $\langle 100\rangle$. The approach also avoids the thin-plate problem, where measuring C22 and C33 may be difficult. In the new approach, a pulseecho (megahertz-frequency) method is used to measure $\mathrm{C} 11, \mathrm{C} 44, \mathrm{C} 66$. (All three involve waves propagating along $X_{1}$, through the plate.) $A$ resonance (kilohertz-frequency) method is used to measure two elas ic compliances: $\mathrm{S} 11$ and $\mathrm{S} 33, \mathrm{~S} 44$, and $\mathrm{S} 12$ com from simple formulas containing $\mathrm{C} 44$ and $\mathrm{C} 66$. S13 comes from a relatively simple formula containing $S 11$, $\mathrm{S} 12, \mathrm{S33}$, and C11. The new approach is applied to a graphite-magnesium composite.

\section{1,124}

PB91-195362

Not available NTIS

National Inst. of Standards and Technology (IMSE) Gaithersburg, MD. Ceramics Div.

Reduction in Sintering Damage of Fiber Reinorced Composites.

Final rept.

C. P. Ostertag. $1990,8 p$

Pub. in Ceramic Transactions 7, p745-752 1990.

Keywords: "Sintering, "Fiber composites, "Ceramic matrix composites, "Fracture mechanics, "Residual stress, Stress tests, Crack propagation, Loads(Forces), Compression tests, Processing, Re prints.

Sintering of fiber reinforced composites results in the creation of residual stresses. Previous experiments revealed that these stresses initiate in the early stage of sintering usually during the heating cycle, and may be responsible for the sintering damage observed in fiberreinforced composites. Processing techniques were developed to reduce stress generation during the heating cycle by applying uniaxial compressive stresses in the range of $0.5 \mathrm{MPa}$ to $2.3 \mathrm{MPa}$ to the green composites and compare the results with composites under no load. The application of a compressive load increases the matrix density and hence, after the load is removed, the critical stress necessary for crack formation is increased. No sintering damage such as crack formation was observed after the composites were subjected to a compressive stress of 1.5 $\mathrm{MPa}$.

\section{1,125}

\section{PB91-202952}

Not available NTIS National Bureau of Standards (IMSE), Gaithersburg, MD. Ceramics Div.

Effect of Thermal Expansion Mismatch on Fiber Pull-Out in Glass Matrix Composites.

Final rept

U. V. Deshmukh, A. Kanei, S. W. Freiman, and D. C. Cranmer. 1988, 6p

Pub. in Materials Research Society Symposium Proceedings, v120 p253-258 1988 .

Keywords: "Interfacial tension, "Thermal expansion, *Fiber composites, "Ceramic fibers, "Glass, "Matrix materials, Carbon fibers, Silicon carbides, Sliding friction, Borosilicate glass, Silica glass, Alkali glass, Moisture content, Shear strength, Stress tests, Wetting, Reprints, Fiber pull-out.

Single fiber pull-out tests can be used to directly measure the fiber-matrix interfacial shear stress in glass matrix composites. The system under investigation consisted of a soda-lime-silica glass matrix containing $\mathrm{SiC}$ monofilaments with a carbon-rich surface. The presence of the carbon-rich layer on the surface of the fibers makes them non-wetting to most glasses; hence the fibers are held in the matrix only by frictional forces acting at the interface. The mechanical gripping responsible for the force can be changed by manipulating the glass matrix/fiber thermal expansion coefficient mismatch. Frictional stresses (tau) and friction coefficients (mu) obtained for $\mathrm{SiC}$ monofilaments in a soda-lime-silica glass matrix were compared with previously obtained data on a borosilicate glass matrix (tau $=2-3 \mathrm{MPa}, \mathrm{mu}=0.72+$ or -0.36 ). For the sodaor - 0.03 were obtained. Tau in the soda-lime-silica system is higher due to the larger difference in thermal expansion mismatch between the fiber and matrix. The differences in mu may be due to lubrication effects caused by water at the fiber-matrix interface.

\section{1,126}

PB91-203059

Not available NTIS

National Inst of Standards and Technology (IMSE) Boulder, CO. Fracture and Deformation Div.

Monitoring of Anisotropic Material Elastic Properties Using Ultrasonic Receiving Rays.

Final rept.

D. W. Fitting, and A. V. Clark. 1989, 8p

Sponsored by National Research Council of Canada, Ottawa (Ontario)

Pub. in Proceedings of International Symposium on Nondestructive Characterization of Materials (3rd), Saarbruchen, FRG, October 3-6, 1988, p91-98 1989.

Keywords: "Anisotropy, "Elastic properties, "Ultrasonic tests, "Piezoelectric gages, "Materials tests, On-line measurement systems, Monitors, Measurement Arrays, Graphite-epoxy composites, Phase velocity, Nondestructive tests, Receivers, Reprints.

A robust technique has been developed for determining the elastic constants of an anitstropic material and for on-line monitoring of changes in the elastic properties. Use of an array of small piezoelectric elements as the receiver permits gathering information on the angle-of-arrival of the ultrasonic beam as well as the arrival time. On-line monitoring of changes in the elastic properties of a material is carried out by directing an ultrasound beam from a stationary transmitting transducer into the sample in a specified direction. Alterations in the stiffness constants of the material (as a result of such things as moisture absorption) will cause the direction of the energy flux to deviate. These deviations are readily monitored with the array of receivers. The arrays fabricated for monitoring elastic properties and the electronics required to process their signals are described herein. Use of the arrays for measuring the quasilongitudinal and quasitransverse wave fields in an anisotropic material (graphite-epoxy composite) is described and the importance of the measurement in correctly determining group and phase velocities is discussed. 
MATERIALS SCIENCES

Composite Materials

101,127

PB91-203687

Not available NTIS

National Bureau of Standards (IMSE), Gaithersburg MD. Ceramics Div.

Role of Glassy Interfaces in High Temperature Crack Growth in SiC Fiber Reinforced Alumina.

Final rept.

S. V. Nair, K. Jakus, and C. Ostertag. $1988,6 \mathrm{p}$

Pub. in Proceedings of Annual Conference on Com posites and Advanced Ceramic Materials (12th), p681. 6861988

Keywords: "Composite materials, "Ceramic matrix composites, "Ceramic fibers, "Fracture mechanics, Aluminum oxide, Silicon carbides, Crack arrest, High temperature tests, Fiber composites, Interfacial tension, Time dependence, Bridging, Temperature dependence, Loads(Forces), Borosilicate glass Coatings(Materials), Crack propagation, Reprints, Fiber pull-out

Glassy alumina reinforced with continuous $\mathrm{SiC}$ fibers and pure alumina reinforced with SiC fibers with and without a coating of a borosilicate glass were studied in this investigation. The experimental component in volved conducting constant displacement rate and constant load tests at approximately $1000 \mathrm{C}$. These tests were interrupted at various times to examine crack morphology changes with temperature and time dependent effects on fiber bridging of cracks. A significant result was that cracks were found to open in a time dependent fashion by fiber pull out prior to eventual failure. It is shown that such a time dependent in terfacial slip can be modeled based on viscous slip in a glassy layer at the fiber/matrix interface.

101,128

PB91-237123

Not available NTIS

National Inst. of Standards and Technology (MSEL) Gaithersburg, MD. Ceramics Div.

Differential Sintering.

Final rept.

C. Ostertag. 1991,7p

Pub. in Science of Sintering, p453-459 1991.

Keywords: "Ceramic matrix composites, "Fiber composites, "Sintered materials, Stress relaxation, Stress tests, Crack initiation, Fracture mechanics, Ceramic coatings, Aluminum oxide, Reprints.

The paper discusses an extreme example of differential sintering, namely sintering of fiber-reinforced composites. The stress that develops during sintering of composites initiate during the heating cycle, where the matrix has a low density and, hence, a low strength. Crack formation occurs mainly during the stage of sintering. Stress relaxation methods by coating the fibers with coarse-grained alumina particles is being dis cussed.

101,129

PB91-237321

Not available NTIS

National Inst. of Standards and Technology (IMSE) Gaithersburg, MD. Fracture and Deformation Div.

Change in Magnetic State of Fe + Silica Gel Nanocomposites Due to Low Temperature Treatment in Ammonia.

Final rept.

R. D. Shull, J. J. Ritter, and L. J. Swartzendruber.

$1991,3 p$

Pub. in Jnl. of Applied Physics 69, n8 p5144-5146, 15 Apr 91.

Keywords: "Magnetic materials, "Iron compounds "Silica gel, "Composite materials, "Nanostructures, Ferromagnetic materials, Polymerization, Ambient temperature, Aqueous solutions, Superparamagnetism, Mossbauer effect, Hydrogen, Ammonia, Annealing, Spin glass, Reprints.

Homogeneous gelled composites of iron and silica containing 11-40 wt. \% Fe were prepared by room temperature polymerization of aqueous solutions of ferric nitrate, tetraethoxysilane, and ethanol (with an hydrogen fluoride catalyst). The effect on the magnetic state of these nanocomposites following a low temperature $(T<400 \mathrm{C}$ ) treatment in $1 \mathrm{~atm}$ of ammonia (after a prior anneal in $1 \mathrm{~atm}$ of hydrogen) is presented along with the dependence on the $\mathrm{H} 2$ pretreatment. In all cases the room temperature Mossbauer spectra for the material in the $\mathrm{NH} 3$-treated and $\mathrm{H} 2$-pretreated conditions were similar. However, when treated in $\mathrm{H} 2$ at $770 \mathrm{C}(2 \mathrm{~h})$ the Mossbauer spectra also contained a significant component having a large isomer shift (1.3 $\mathrm{mm} / \mathrm{s})$ and quadrupole splitting $(3.2 \mathrm{~mm} / \mathrm{s})$. The mate- rial was also slightly ferromagnetic at all temperatures below $300 \mathrm{~K}$ For materials pretreated in hydrogen below $400 \mathrm{C}$, a threefold enhancement in the magnetic susceptibility was measured following treatment in ammonia. In addition, both the field and temperature dependence of the susceptibility indicated the presence of spin-glass behavior at $10 \mathrm{~K}$ for $\mathrm{NH} 3$-treated samples containing up to $40 \% \mathrm{Fe}$. At room temperature, these latter ammonia-treated nanocomposites were either superparamagnetic (Fe contents, $\mathrm{C}(\mathrm{sub} \mathrm{Fe}$ ) up to $25 \%$ ) or ferromagnetic (C(sub $\mathrm{Fe})<25 \%$ ).

\section{1,130}

PB92-117258

Not available NTIS

National Inst. of Standards and Technology (CSTL) Boulder, CO. Chemical Engineering Div.

Thermal Conductivity of Alumina Fiber/Epoxy and Alumina Fiber/PEEK from 4.2 to $310 \mathrm{~K}$

Final rept.

D. L. Rule, and L. L. Sparks. $1990,15 p$

See also PB89-218358.

Pub. in Proceedings of International Thermal Conduc tivity Conference (21st), Lexington, KY., October 15 18,1989, p671-685 1990.

Keywords: "Thermal conductivity, "Peek, "Epoxy matrix composites, "Polymer matrix composites, "Resin matrix composites, "Fiber composites, Temperature dependence, Aluminum oxides, Ceramic fibers, Thermal cycling tests, Orientation, Measurement, Reprints.

The thermal conductivities of poly-ether-ketone (PEEK), of alumina fiber in a matrix of PEEK, and of alumina fiber in a matrix of epoxy were determined along with the effects of fiber orientation and thermal cycling. Thermal conductivity was measured over the temperature range 4.2 to $310 \mathrm{~K}$ using a steady-state apparatus. These data are presented and discussed relative to specimen characteristics. After differen fiber fractions in the specimens were accounted for the thermal conductivity of the PEEK composite material is less than that of the epoxy composite material in particular temperature ranges for the heat flow parallel to the fiber direction.

101,131

PB92-117332

Not available NTIS

National Inst. of Standards and Technology (MSEL) Gaithersburg, MD. Metallurgy Div.

Magnetic Behavior of Nanocomposites Prepared in a Vitreous Alumina Gel.

Final rept.

R. D. Shull, J. J. Ritter, A. J. Shapiro, L. J.

Swartzendruber, and L. H. Bennett. 1991, 6p

Pub. in Materials Research Society Stmposia Proceedings, v206 p455-460 1991.

Keywords: "Ceramic matrix composites, "Gels, "Alu minum oxides, "Polymerization, "Iron compounds, Magnetic materials, Iron nitrates, Aqueous solutions, Vitreous state, Alcoholates, Ferromagnetism, Nitric acid, Electron microscopy, $X$-ray diffraction, Moss bauer effect, Embedding, Magnetic permeability, Temperature dependence, Hydrogen, Reduction(Chemistry), Paramagnetism, Reprints, Nanocomposites.

Homogeneous gelled composites of iron and vitreous alumina containing $10-40 \%$ Fe have been prepared by room temperature polymerization of aqueous aluminum alkoxide solutions containing ferric nitrate and nitric acid at low $\mathrm{pH}$. Scanning electron microscopy, $x$ ray diffraction, and Mossbauer spectroscopy demonstrated that this bulk material is comprised of nanometer-sized regions of iron compounds embedded in a vitreous alumina gel matrix. Magnetization data showed that in the as-cured condition these nanocomposites areparamagnetic at room temperature and become either superparamagnetic or ferromagnetic on cooling to $10 \mathrm{~K}$. The magnetic susceptibility increased with the Fe content and with decreasing temperature. Analysis of the temperature dependence of the magnetic susceptibility indicated the magnetic moment per Fe atom was 1.87 for the $10 \%$ Fe nanocomposite and that it increased linearly with composition to 1.96 fo the $40 \% \mathrm{Fe}$ material. Mossbauer effect data showed that subsequent treatment of these materials in a gaseous environment of hydrogen at elevated temperatures ( $T<400 \mathrm{C}$ ) changed the form of the iron in the magnetic regions. These results are compared to that observed for similar nanocomposites prepared using a silica gel matrix.

\section{Corrosion \& Corrosion Inhibition}

101,132

PB9 1-134700

Not available NTIS

National Bureau of Standards (IMSE), Gaithersburg. MD. Metallurgy Div.

Corrosion, Repassivation and Corrosion Fatigue Behavior of the Surgical Implant Alloy, Co-Cr-Mo. Final rept.

P. Sung, and A. C. Fraker. 1987, 10p

Pub. in Proceedings of Conference on Environmenta Degradation of Engineering Materials III, University Park, PA., April 13-15, 1987, p471-480.

Keywords: "Cobalt alloys, "Chromium alloys, "Molybdenum alloys, "Corrosion alloys, Surgical transplanta tion, Implantation, Orthopedics, Passivity, Bones Joints(Anatomy), Reaction kinetics, Surface chemistry, Protective coatings, Fatigue life, Reprints.

The cobolt-chromium-molybdenum (Co-Cr-Mo) alloy is used widely as an orthopedic surgical implant material. In-vivo degradation of the alloy due to corrosion or corrosion fatigue can lead to metal ion release into the body or fracture of the device. The continual destruc tion of the protective film can promote crack formation and thereby cause fracture of the implant. Scully, and Ambrose and Kruger were among the earliest workers to emphasize the importance of passive film and re passivation results in the metal ions adjacent to the ruptured films being dissolved before another protec tive film can be formed. However, if the repassivation rate is much faster than the rate of film rupture, meta ons may not be dissolved, and corrosion may not occur. By monitoring the current transient after a bare metal surface is formed by abrading, while maintaining a constant potential with a potentiostat, the repassivation kinetics can be measured.

101,133

PB91-149047

Not available NTIS

National Bureau of Standards (IMSE), Gaithersburg, MD. Metallurgy Div.

Cleavage Step Formation and Resistance to Trans granular Stress Corrosion Cracking.

Final rept.

E. N. Pugh, and M. J. Kaufman. 1986, 11p

Pub. in Proceedings of Materials Technology Congress, Adelaide, Australia, May 19-21, 1986, v2 p1-11

Keywords: "Stress corrosion, "Metals, "Fracture me chanics, "Transgranular corrosion, Crack propagation, hardening, Reprints.

A new approach to improving the resistance of alloys to transgranular SCC is described. It is based on the view that this important service failure occurs by discontinuous cleavage and that resistance to this form of cracking can be achieved by impeding the formation of steps between parallel but displaced cleavage facets. It is suggested that the steps in the case of susceptible alloys are produced by plastic shearing on slip planes and that this low energy process can be restricted either by ensuring that ready cross-slip can occur (by avoiding low stacking fault energy and short range order) or by increasing the shear strength by precipitation hardening. This approach does not affect the intrinsic susceptibility of the material but is designed to impart resistance by inhibiting crack propagation. The approach also provides new insight into the mechanism of the embrittlement process.

101,134

PB91-150078

Not available NTIS

National Inst of Standards and Technology (IMSE)

Gaithersburg, MD. Metallurgy Div.

Concepts of Underground Corrosion.

Final rept.

E. Escalante. 1989, 14p

AST. in Effects of Soil Charact

Keywords: "Metals, "Electrochemical corrosion, "Soi properties, "Steels, Soil microbiology, Electrical resistivity, Aeration, Oxygenation, Reprints.

Corrosion in soil is a complex phenomenon, but there are some basic concepts that are useful in understanding the process. Underground corrosion is electrochemical in character, and this fact is used to de scribe the corrosion process in terms of an ordinary dry cell. The differences between corrosion in disturbed and undisturbed soil are discussed, and data 
are presented to emphasize these differences. The results reveal that soil composition is less important than soil resistivity, but both are subordinate in importance to oxygen availability. Thus, corrosion is negligible in undisturbed soils where oxygen concentration is low.

\section{1, 135
PB9 $1-158667$}

Not available NTIS

National Bureau of Standards (IMSE), Gaithersburg, MD. Metallurgy Div.

Corrosion Testing in Soil.

\section{Final rept.}

E. Escalante. 1987, 4p

Pub. in Metals Handbook, v13 p208-211 1987.

Keywords: *Corrosion tests, "Soils, "Metals, Measurement, Underground corrosion, Sampling, Oxygen, Design criteria, Reprints.

An approach to evaluating the durability of a metal in soil will be described. Specimen design, preparation, burial, and retrieval techniques will be discussed. The type of information sought during soil-induced corrosion evaluation controls the design configuration and he nature of the corrosion measurements. Consideration of these factors during the planning stage will help the corrosio engineer obtain a maximum amount of information with a minimum number of future problems in the program. The corrosion of metals underground can be divided into two broad categories: corrosion in undisturbed soils and corrosion in disturbed soils. Corrosion in undisturbed soil is always low, regardless of soil conditions, and is limited only by the availability of the oxygen necessary for the cathodic reaction. Steel piles driven into soil fall under this category and therefore undergo limited corrosive attack.

101, 136
PB91-174714

Not available NTIS

National Inst. of Standards and Technology (MSEL)

Gaithersburg, MD. Metallurgy Div.

Measuring the Corrosion of Metals in Soil.

Final rept.

E. Escalante. 1990, 13p

Pub. in Corrosion Testing and Evaluation: Silver Anniversary Volume, ASTM STP 1000, p112-125 1990.

Keywords: "Soils, "Underground corrosion, "Copper, Corrosion tests, Measurement, Polarization, Galvanic corrosion, Alternating current, Pile structures, Reprints.

Polarization resistance and galvanic current measurements, used for underground corrosion studies, are described, and problems encountered in making these measurements in the field, along with solutions, are discussed. Polarization measurements, supported by physical measurements, have shown that induced alternating current increases the corrosion rate of copper concentric neutrals compared to copper neutrals without ac. These measurements also revealed that driven piles undergo less corrosion attack than similar piles in backfilled trenches. Galvanic current measurements provide valuable information on the behavior of galvanically coupled materials and can be used to compare the corrosivity of soil environments.

\section{1,137}

PB91-175182

Not available NTIS

National Inst. of Standards and Technology (MSEL), Gaithersburg, MD. Metallurgy Div.

Stress Corrosion Cracking of $\mathrm{Al}-\mathrm{Li}$ Alloys: The Role of Grain Boundary Precipitates.

Final rept.

R. E. Ricker, J. L. Fink, and A. K. Vasudevan. 1991,

Pub. in Metallurgical Transactions A 22A, p264-267 Jan 91.

Keywords: "Aluminum alloys, "Lithium alloys, "Stress corrosion cracking, Grain boundaries, Precipitates, Precipitation hardening, Microstructure, Heat treatment, Copper alloys, Binary alloys, Ternary alloys, Yield strength, Fracture mechanics, Reprints.

The stress corrosion cracking (SCC) behavior of precipitation hardened alloys may depend on a large number of microstructural parameters that vary during fabrication and heat treatment such as grain size, grain boundary (gb) solute segregation, precipitate size, precipit free zone, and matrix slip character. Since all of these factors vary simultaneously during normal heat treatments, it is difficult to assess independently the contribution of each microstructural factor to the SCC behavior of the alloy In particular, a series of experiments were designed which would allow the evaluation of the role of gb precipitates in the SCC behavior of Al$\mathrm{Li}$ and $\mathrm{Al}-\mathrm{Li}-\mathrm{Cu}$ alloys independent of the other factors (such as matrix precipitate microstructure) that normaly vary during aging treatments. For these experiments, the matrix precipitates of a binary $\mathrm{Al}-\mathrm{Li}$ alloy were held constant, keeping the yield strength constant, while the gb precipitate size and volume percent were systematically varied. In contrast, to keep the yield strength of the ternary Al-Li-Cu alloy constant at the same level as the binary alloy, the matrix precipitate size and distribution were varied with the gb precipitate size.

101,138

PB9 1-187690

PC A03/MF A01

National Inst. of Standards and Technology (BFRL) Gaithersburg, MD.

Chloride Ion Diffusion in Low Water-to-Solid Cement Pastes.

J. R. Clifton, L. I. Knab, E. J. Garboczi, and L. X.

Xiong. Apr 91, 31p NISTIR-4549

Prepared in cooperation with Shanghai Research Inst. (China). Sponsored by Nuclear Regulatory Commission, Washington, DC.

Keywords: "Corrosion tests, "Reinforced concrete, "Composite materials, "Reinforcing steels, "Chlorine ions, Diffusion, Portland cements, Moisture conten Mathematical models, Service life, Performance prediction, Radioactive waste management, Low-level radioactive wastes.

Diffusion coefficients of 0.3 water to solids ratio $(\mathrm{w} / \mathrm{s})$ hydrated portland cement paste specimens were measured using a conventional diffusion cell. Specimens were made from both ASTM Type I and Type II portland cements and blends containing mineral admixtures (fly ash, granulated blastfurnace slag, or silica fume). The average diffusion coefficient for the portland cement paste specimens was $14 \times 10$ (sup -13)sq $\mathrm{m} / \mathrm{s}$. The effects of the depth of concrete cover ove reinforcing steel and of the chloride ion diffusion coefficient on the service life of reinforced concrete exposed to chloride ions were predicted based on a diffusion model. Based on the model, the effect of the cover was shown to be proportional to the square of the cover depth. A 10-fold decrease in the diffusion coefficient of concrete was predicted to result in a 10 fold increase in the predicted service life.

101,139

PB91-203588

Not available NTIS

National Inst. of Standards and Technology (NEL), Gaithersburg, MD. Building Materials Div.

Detection and Quantitative Characterization of Blistering and Corrosion of Coatings on Stee Using Infrared Thermography.

Final rept.

M. E. McKnight, and J. W. Martin. 1989, 6p

Contract NCEL-86WR60188

Sponsored by Naval Civil Engineering Lab., Port Hueneme, CA.

Pub. in Jnl. of Coatings Technology $61, n 775$ p57-62 Aug 89.

Keywords: "Protective coatings, "Structural steels, *Barrier materials, "Corrosion prevention, Corrosion products, Characterization, Weathering, Quantitative analysis, Thermography, Blisters, Infrared thermal detectors, Process variables, Air flow, Organic compounds, Thickness, Image analysis, Time dependence, Panels, Construction materials, Interfaces, Reprints.

Results of studies to assess the effect of several key experimental variables on the detection of blisters and corrosion spots at the interface of organic coating and steel using infrared thermography are presented. Coating defects, such as blisters and corrosion spots, are detected as a result of differences in the therma properties of degraded and non-degraded areas of a coated panel when a temperature gradient is induced through the thickness of the coated panel. The resulting thermographic image is then analyzed using computer image processing to determine size, location and extent of degradation. Therefore, the method provides a quantitative, nondestructive procedure for determining the extent of deterioration on coated metal panels. The results of the studies show that experimental variables such as the method of heating test panels, the panel temperature, the flow rate of air, the time between initiation of heating and acquisition of the thermographic image and the thickness of the organic coatings can affect the results.

\section{Elastomers}

101,140

PB91-132167

$\mathrm{PC}$ A03/MF A01

National Inst of Standards and Technology (NEL) Gaithersburg, MD. Center for Fire Research.

Reduction of Hydrogen Cyanide Concentrations and Acute Inhalation Toxicity from Flexible Poly urethane Foam Combustion Products by the Addition of Copper Compounds. Part 3. The Effect of Copper Additives on the Flammability Characteristics of Flexible Polyurethane Foam.

B. C. Levin, E. Braun, J. R. Shields, and D. Lowe. Oct $90,39 p$ NISTIR-4441

Sponsored by International Copper Research Association, Inc., New York, and Society of the Plastics Industry, Inc., New York.

Keywords: "Hydrogen cyanide, "Combustion products, "Copper compounds, *Foam rubber, "Polyurethane resins, Exhaust gases, Flammability testing, Toxicity, Thermal degradation, Respiration, Statistical analysis, Heat measurement, Ignition, Oxidation.

The report addresses the issue of whether the addition of a copper compound to a flexible polyurethane foam would affect the flammability characteristics of the foam. The following properties were examined: (1) ignitability in three systems (the NBS Toxicity Test Method, the Cone Calorimeter, and Lateral Ignition and Flame Spread Test (LIFT)), (2) heat release rate under small-scale (Cone Calorimeter) and mediumscale (furniture calorimeter), (3) smoke obscuration (Cone Calorimeter), and (4) rate of flame spread (LIFT). In all cases, no differences in flammability characteristics between the treated and untreated foam were observed.

101,141

PB91-195081

Not available NTIS

National Bureau of Standards (IMSE), Gaithersburg, MD. Polymers Div.

Mechanical and Swelling Behavior of Crosslinked Natural Rubber: Consequences of the FloryRehner Hypothesis.

Final rept.

G. B. McKenna, K. M. Flynn, and Y. Chen. 1988, 4p Pub. in Polymer Communications 29, n9 p272-275 Sep 88.

Keywords: "Natural rubber, "Swelling, "Crosslinking, Networks, Benzene, Methyl ethyl ketone, Ethyl acetate, Acetone, Density(Mass/Volume), Elastic properties, Heat of mixing, Reprints, "Flory-Rehner hypothesis, "Flory-Huggins parameter, Dicumyl peroxide.

The Frenkel-Flory-Rehner (FFR) hypothesis that the elastic free energy of a swollen network is balanced by the free energy of mixing has been examined using experiments on dicumyl peroxide crosslinked natural rubber. Swelling was performed in good solvent (benzene) and poor solvent (Methyl ethyl ketone, Ethyl acetate, acetone) conditions crosslink density varied from $2.3 \times 10$ (sup -5) to $3.26 \times 10$ (sup -4) moles/cu m. Validity of the FFR hypothesis demands that the FloryHuggins interaction parameter, chi, in the expression for the free energy of mixing be dependent upon the crosslink density, upsilon. Then it is found that there is an apparently universal relationship between the volume fraction of rubber, $v 2$, and the effective interaction parameter chi (effective), viz, v2 $=$ v2 (chi (effective)) = upsilon2 (chi, upsilon). The value of chi (effective) depends upon crosslink density as (chi (effective) - chi(0)) = (a)upsilon where for the natural rubber and solvents studied appears to be independent of solvent.

\section{Fibers \& Textiles}

\section{1,142}

AD-A236 708/4

PC A02/MF A01

National Inst. of Standards and Technology (IMSE), Gaithersburg, MD. Ceramics Div. 
Effects of Multiple Filament Geometry in the Hot Filament Deposition of Diamond Films.

Interim rept.

E. N. Farabaugh, A. Feldman, and L. H. Robins. 12 Apr $91,9 p$

Contract N00014-90-F-0011

Keywords: Chambers, Deposition, "Diamonds, Feeding, "Filaments, Films, Flow rate, Gas flow, Gases, Geometry, Growth(General), High rate, Hot wire, Morphology, Parameters, Pressure, Rates, Surface properties, Volume, Methane, Pyrolysis.

Scaleup of hot filament chemical vapor deposition of diamond films requires knowledge of how filament geometry affects the deposition process. The effect of multiple filament geometry on the growth rate and surmultiple filament geometry on the growth rate and suractors were varied: the number of helical turns in a filament $-5,10$ or 15; the number of filaments present -1 or 2; and the $\mathrm{CH} 4$ fraction in the $\mathrm{CH} 4-\mathrm{H} 2$ feed gas mixture $-0.25 \%, 0.5 \%, 0.75 \%$ or $1.0 \%$. Other depositions parameters were $750 \mathrm{C}$ substrate temperature, 52 standard $\mathrm{cm} / \mathrm{m}$ total feed gas flow rate, and 5300 $\mathrm{Pa}$ deposition pressure. The deposition chamber volume was 1.9 liters. Increasing the $\mathrm{CH} 4$ concentration in the feed gas results in higher growth rates. However, increasing the number of filament turns in dual filament systems resulted in lower than expected growth rates for dual 10 and 15 turn filaments.

101,143

PB91-149948

Not available NTIS

National Inst. of Standards and Technology (NML),

Boulder, CO. Chemical Engineering Science Div.

Round Robin on Apparent Thermal Conductivity of Several Loose-Fill Insulations.

Final rept

R. D. Adams, and J. G. Hust. 1990, 27p

Sponsored by Oak Ridge National Lab. TN

Pub. in Insulation Materials, Testing, and Applications, ASTM STP 1030, p263-289 1990

Keywords: "Thermal insulation, "Thermal conductivity, Interlaboratory comparisons, Cellulose, Rock wool Glass fibers, Fabrics, Measurement, Precision, Bias, Fillers, Reprints

The primary means of establishing the thermal performance of loose-fill insulation is ASTM Standard Practice C 687. To provide information for the development of a statement of precision and bias for the Standard Practice, a round robin was carried out by eleven laboratories measuring apparent thermal conductivity of four common loose-fill insulation products cellulose, rock/slag wool, and bonded and unbonded glass fiber) and a glass fiber blanket. The test results on the glass fiber blanket indicate that the measurement capability of the participants can be characterized by an imprecision of $3.0 \%$, as measured by two ized by an imprecision of $3.0 \%$, as measured by two standard deviations, and a negligible bias of the mean. acterized by an imprecision ranging from $21 \%$ for cellulose to $10 \%$ for the rock/slag wool. The increase in imprecision for the loose-fill materials is attributed primarily to inadequately defined procedures for preparmarily to inadequately defined proced
ing and conditioning the specimens.

\section{Iron \& Iron Alloys}

101,144

N91-20218/4

(Order as N91-20207/7, PC A05/MF A01) National Bureau of Standards (IMSE), Gaithersburg MD.

Low Carbon Steel: Metallurgical Structure vs. Mechanical Properties.

R. D. Shull. Jan $90,4 p$

In NASA, Langley Research Center, National Educators' Workshop: Update 1988. Standard Experiments in Engineering Materials Science and Technology $p$ $47-50$.

Keywords: Experimentation, "Low carbon steels, "Mechanical properties, "Metallurgy, Heat treatment, Low cost, Phase diagrams, Pins, Procedures, Students, Temperature effects, "Thermomechanical treatment.

The objective is to provide a low cost, simple experiment for either demonstration purposes or as a laboratory experiment that will teach the student the importance of the thermal-mechanical history of a metallic alloy in determining that material's mechanical behavor. Hairpins are subjected to various treatments. The experimental equipment and procedures are discussed.

101,145

PB91-134627

Not available NTIS

National Inst of Standards and Technology (IMSE).

Boulder, CO. Fracture and Deformation Div.

Cryogenic Material Properties of Stainless Steel Tube-to-Flange Welds.

Final rept.

T. A. Siewert, C. N. McCowan, and D. P. Vigliotti.

$1990,9 \mathrm{p}$

Pub. in Cryogenics 30, p356-364 Apr 90

Keywords: "Chromium nickel alloys, "Weldments, "Manganese alloys, "Cryogenics, Pipes(Tubes), Mechanical properties, Superconductors, Flanges, Tension tests, Tensile strength, Notch strength, Fatigue life, Failure, Thermal cycling tests, Loads(Forces), Reprints, "Stainless steel-304L, "Stainless steel-316L, "Superconducting super collider.

The mechanical properties of stainless steel tube-toflange welds for a cryogenic piping application were measured. A planar specimen was developed to duplicate the constraint, loading and heat-sink properties of the circular joint, while reducing preparation time and cost. Specimens were evaluated containing welds between the tube material (21 $\mathrm{Cr}-6 \mathrm{Ni}-9 \mathrm{Mn})$ and the three stainless steels being considered for the flange materials: type $304 \mathrm{~L}$, type $316 \mathrm{~L}$ and $21 \mathrm{Cr}-6 \mathrm{Ni}-9 \mathrm{Mn}$. The mechanical property tests consisted of three phases: simple tensile testing to failure, tensile testing of notched specimens (where the notch simulated fabrication flaws) and fatigue testing of notched specimens for the $4 \times 10$ (sup 4) cycle design life of the structure. The type $316 \mathrm{~L}$ stainless steel flange produced welds with the best combination of strength and ductility at 295 and $4 \mathrm{~K}$ in all three phases of testing.

101,146

PB91-147686

Not available NTIS

National Bureau of Standards (NEL), Gaithersburg, MD. Building Materials Div.

Roughness Measures of Blasted Steel Surfaces Remotely Imaged with a Thermographic Camera. Final rept.

J. W. Martin, and D. P. Bentz. 1987, 18p

Pub in Proceedings of Symposium on Corrosion Protection by Organic Coatings, v87-2 p179-196 1987.

Keywords: "Surface roughness, "Steels, "Panels, "Thermography, "Abrasive blasting, Abrasion, Surface properties, Roughness, Profilometers, Finishes, Blasting, Reprints.

A standard series of blasted steel panels were imaged with a thermographic camera and characterized with respect to surface roughness using fractal and depth mapping techniques. It was shown that the fractal dimensions of these blasted steel panels correlate very well with their perceived roughness and that a computer rendering of a fractal surface looks like a blasted surface. At present, depth mapping on blasted stee substrates is too complicated to perform; instead, a series of precision drilled and milled holes were used. For milled holes, a good relationship exists between thermographically and physically measured diameter and depth measurements. For drilled holes, a good relationship exists for diameter, but not depth, since the emission effects of hole depth and drill angle have not been separated out.

101,147

PB91-148106

Not available NTIS

National Bureau of Standards (IMSE), Gaithersburg, MD. Metallurgy Div.

Study of the Galling of Two Steels Using Two Test Methods.

Final rept.

P. A. Swanson, L. K. Ives, E. P. Whitenton, and M. B. Peterson 1988, 17p

Pub. in Wear 122, n2 p207-223, 1 Mar 88.

Keywords: "Tribology, "Steels, "Galling, Wear tests, Measurement, Carburizing Quenching(Cooling), Comparison, Methodology, Reprints, -Steel-AIS|-1541, *Steel-AISI-8620.

The galling behavior of two heat treated steels, AISI 1541 and 8620 , was investigated. Two laboratory tests were used. The first utilized a sphere-on-flat contact geometry and the second a flat-on-flat geometry. The galling damage that was generated was measured by using a stylus profilometer to collect a series of parallel profiles through the damage zone. The maximum peak-to-valley distance for each profile was calculated and the average of this parameter was then used to quantify the amount of galling that was produced. The measurement technique enabled the authors to not only assess the galling behavior of the test materials but it also allowed the authors to determine the variability inherent in each test method. Significantly less damage was obtained for carburized 8620 on 'asquenched' 1541 steel than was observed when the quenched 1541 steel was tested under self-mated conditions. The greatest amount of damage was generated when hot-rolled 1541 steel was run against itself. Possible reasons for these results will be reviewed.

101,148

PB91-158980

Not available NTIS

National Inst of Standards and Technology (MSEL), Boulder, CO. Fracture and Deformation Div.

Fracture Toughness of $316 \mathrm{~L}$ Stainless Steel Welds with Varying Inclusion Contents at $\mathbf{4} \mathrm{K}$.

Final rept.

C. N. McCowan, and T. A. Siewert. 1990, 8p

Sponsored by Department of Energy, Washington, DC. Pub in Advances in Cryogenic Engineering (Materials), v36 p1331-1338 1990 .

Keywords: "Fracture strength, *Stainless Steel-316L, "Weld defects, "Inclusions, Hardness, Welding, Stainless steels, Loads(Forces), Voids, Cracks, "Cryogenics, Reprints.

The $4 \mathrm{~K}$ fracture toughness of a type $316 \mathrm{~L}$ stainless steel weld composition increased significanlty when the inclusion contents of the GMA welds were decreased. For the three welds tested (Ni-13 wt. $4 \mathrm{~K}$ yields strngth approx. $740 \mathrm{MPa}$ ), the fracture toughness increased $35 \%$ as the inclusion contents were decreased by $65 \%$. Expressing the effect of inclusions on the toughness in terms of the inclusion spacing (inverse square root of the inclusion density), the fracture toughness of the $316 \mathrm{~L}$ welds increased $18 \mathrm{MPa}$.m(exp 1/2) per micron increase in the average inclusion spacing. Fractographic studies were conducted to characterize differences in the initiation of fracture as a function of the inclusion content. Fracture specimens that were examined after loading into the blunting range had stable crack growth lengths of 0.1 to 0.25 $\mathrm{mm}$. Crack growth occurs as voids which formed at inclusions link by microcracking, rather than coalescence.

101,149

PB91-167171

$\mathrm{PC}$ A03/MF A01

National Inst. of Standards and Technology (MSEL), Gaithersburg, MD. Metallurgy Div.

Examination of the Excessive Retained Austenite on the Surface of a Section of 17-7 Precipitation Hardening Stainless Steel.

G. E. Hicho, W. J. Boettinger, L. Swartzendruber, and T. R. Shives. Jan $91,32 p$ NISTIR-4502

Keywords: "Austenite, "Stainless steels, "Chromiumnickel steels, Surface properties, Precipitation hardening, Heat treatment, Microstructure, Aluminum additions.

A 'mishap' section, and other selected sections made from 17-7 precipitation hardening stainless steel were metallurgically examined, and it was concluded that the unusually large amount of retained austenite, greater than $7 \%$, measured on the surface appears to have been produced during both the solution annealing and austenite conditioning steps of the heat treating process, but was revealed only after the austenite conditioning step. It is suspected that the excessive retained austenite occurred during the heat treating process where these sections may have been exposed to a furnace atmosphere that contained too much carbon or nitrogen. The exposure could have led to an increase in the amount of retained austenite both on the surface and internally.

101,150

PB91-167346

PC A03/MF A01

National Inst of Standards and Technology (MSEL) Gaithersburg, MD. Metallurgy Div. 


\section{Iron \& Iron Alloys}

Crack Arrest Fracture Toughness Measurements of Normalized and Inclusion Shape Controlled AAR TC128 Grade B Steel, and Micro-Alloyed, Control-Rolled, and Inclusion Shape Controlled A $8 \times X$ Grade B Steel.

G. E. Hicho. Feb 91, 18p NISTIR-4501, REPT-21

Keywords: "Fracture mechanics, "Carbon steels, "Railroad cars, Tank cars, Fracture strength, Crack arrest, Measurement, Inclusions, Process variables, Process control.

The crack arrest fracture toughness was determined for normalized AAR TC 128 grade $B$ steel and control rolled A $8 \times X$ grade $B$ steel. Both steels were made using inclusion shape control practice. The crack arrest fracture toughness of the AAR TC 128 steel was slightly better than that for the A $8 \times X$ steel.

101,151

PB9 1-175372

Not available NTIS

National Inst. of Standards and Technology (IMSE),

Boulder, CO. Fracture and Deformation Div

Effect of Nitrogen and Carbon on FCC-HCP Stability in Austenitic Steels.

Final rept.

R. P. Reed, and M. W. Austin. 1989, 4p

Pub. in Scripta Metallurgica 23, n8 p1359-1362 1989.

Keywords: "Austenitic steels, "Nitrogen additions, Austenite, Ferrite, Martensitic transformation, FCC lattices, HCP lattices, "Carbon additions, Reprints.

High-nitrogen austenitic steels have recently been developed for low-temperature use. For a long time it has been recognized that both nitrogen and carbon increase austenite stability with respect to formation of delta ferrite at high temperatures and body-centered cubic (bcc) martensite at low temperatures. Yet, the effects of nitrogen and carbon on austenite (fcc) stability relative to hexagonal close-packed (hcp) martensite and the stacking-fault energy have not been adequately clarified.

\section{1,152}

\section{PB91-175448}

Not available NTIS

National Inst. of Standards and Technology (NML), Gaithersburg, MD. Surface Science Div.

Mechanical Sputtering of Structural Stainless Steels.

Final rept.

T. Nenadovic, N. Popovic, and J. Fine. 1989, 8p

Pub. in Jnl. of Materials Science 24, p3699-3706 1989.

Keywords: "Sputtering, "Austenitic stainless steels, "Erosion, Structural steels, Gas turbine blades, Pressure vessels, Deterioration, Wear, Surfaces, Pitting, Reprints.

In the paper the results of an investigation of the beam modification (erosion) of stainless steel are presented. The possible similarity of the martensitic stainless steel degradation for turbine engine blades and austenitic stainless steel used as a wall of the vacuum vessel for fusion reactor application has been pointed out. Changes appearing during the interaction have been investigated on the rotating turbine blade material. Simultaneously morphological changes of the 'target' and the change of shape of bombarding species have been analyzed.

\section{1, 153}

PB9 1-184747

PC A03/MF A01

National Inst. of Standards and Technology (MSEL). Boulder, CO. Materials Reliability Div.

Microstructure, Composition, and Hardness of Rockwell C Hardness Blocks.

T. A. Siewert, and A. Tomer. Jan 91, 26p NISTIR3960

Keywords: "Rockwell hardness, "Standards, "Steels, Compositions, Microstructure, Hardness, Purity.

The microstructure, composition, and hardness of hardness blocks (Rockwell C scale) that are commonly available in the country were examined. Blocks near HRC levels of 25,45 , and 65 were obtained from each of six sources to represent the HRC measurement range. It was found that the steels used in the blocks had surprisingly high levels of impurity elements, which are one source of scatter in the hardness values. Other than the concern for the steel purity, it is concluded that most of these hardness block producers possess the technology necessary to manufacture a reference hardness block, or series of blocks, if it is found desirable for NIST to distribute a national hardness standard.
101,154

PB9 1-184838

PC A03/MF A01

National Inst. of Standards and Technology (NEL) Gaithersburg, MD. Center for Fire Research. Prediction of Elevated Temperature Deformation of Structural Steel under Anisothermal Conditions. B. A. Fields, and R. J. Fields. Jan 91, 46p NISTIR4497

Sponsored by American Iron and Steel Inst., Washington, DC.

Keywords: "Structural steels, Performance prediction High temperature tests, Deformation, Strain tests, Creep properties, Computer programs, Heating, Elastic properties, Equations, Tables(Data), Strain hardening, Steel-ASTM-A36, Anisothermal.

Using a previously formulated equation which calculates the elastic, plastic and creep strains during loading at a constant elevated temperature, a method and a computer program have been developed that will predict the strain due to creep during anisothermal tests at constant load. Comparisons were made with results from anisothermal tests for AS A149, an Australian steel close to the specification for ASTM A36. Agreement is excellent for several linear heating rates and one nonlinear heating rate.

101, 155

PB91-187856

PC A03/MF A01

National Inst of Standards and Technology (MSEL),

Boulder, CO. Materials Reliability Div.

Continuous-Cooling Transformation Characteristics and High-Temperature Flow Behavior of a Microalloyed SAE 1141 Steel.

Y. W. Cheng, and A. Tomer. Feb 91, 39p NISTIR-

3964

Prepared in cooperation with Israel Atomic Energy Commission, Beersheba. Nuclear Research CenterNegev.

Keywords: *Low carbon steels, "Heat resistant alloys, "Thermomechanical treatment, Temperature dependence, Phase transformations, Stress tests, Deformation, Fluid flow, Viscosity, Flow stress, Austenite, Ferrite, Pearlite, Microstructure, Crystallization, Automobiles, "Steel-SAE-1141, "Microalloyed steels, Continuous cooling.

The report presents the results of a thermomechanical processing (TMP) study on a microalloyed SAE 1141 forging steel. The primary objective of the study is to investigate the effects of deformation temperature on the phase-transformation kinetics and to determine the high-temperature flow characteristics of the steel. One-hit compression tests at a constant true strain rate of $10 / \mathrm{s}$ were performed with a TMP simulator. Tests were performed at 900,1000 , and $1100 \mathrm{C}$. The results show that flow stress increased with decreasing temperature. In the strain range 0.35 to 0.6 , the effect of temperature on the flow stress can be described by the equation, sigma (MPa) $=2.93 \exp$ $(4944 / T$ (K)). Continuous-cooling transformation (CCT) diagrams determined following deformation at 1000 and $1100 \mathrm{C}$ were similar. However, deformation at $900 \mathrm{C}$ shifted the ferrite-plus-pearlite nose to a at $900 \mathrm{C}$ shifted the ferrite-plus-pearlite nose to a pearlite microstructure. This is because the steel does not recrystallize at $900 \mathrm{C}$ after deformation imposed in the study. The usefulness of the CCT diagram and the relationship between deformation and austenite recrystallization are discussed.

\section{1,156}

PB 1-189464

Not available NTIS National Bureau of Standards (IMSE), Gaithersburg, MD. Metallurgy Div.

Effects of Varying Austenitizing Temperature and Cooling Rate on the Ability of HSLA-80 Steel to Achieve a Yield Strength Comparable to HSLA100.

Final rept.

G. E. Hicho, and R. J. Fields. 1988, 9p

Sponsored by David Taylor Research Center, Bethesda, MD.

Pub. in Jnl. of Heat Treating 6, n2 p77-85 1988

Keywords: "Austenitic steels, "Yield strength, "Heat treatment, Tensile strength, Quenching(Cooling) Temperature dependence, Metal plates, Optimization, Rates(Per time), Reaction kinetics, Thickness, High strength steels, Low alloy steels, Reprints, "Steel HSLA-80, "Steel HSLA-100.

Heat treating experiments were conducted on HSLA80 in order to maximize the yield strength. The paper concentrates on the optimization of the austenitizing treatment and the cooling rate. The optimum austenitization temperature was found to vary with plate thickness. In all cases, the time-at-temperature was $60 \mathrm{~min}$ utes followed by an immersion in water. Double austenitization and other cooling rates did not significantly improve the yield strength. A heat treatment was found for the $19 \mathrm{~mm}$ and $32 \mathrm{~mm}$ thick plates that improved the yield strength beyond those specified for HSLA100.

\section{1,157}

\section{PB91-189944}

Not available NTIS

National Inst. of Standards and Technology (MSEL) Boulder, CO. Fracture and Deformation Div.

Influence of Interstitial Content on Fracture Toughness.

Final rept.

P. T. Purtscher, and R. P. Reed. 1989, 5p

Pub. in Proceedings of International Conference on High Nitrogen Steels, Lille, France, May 18-20, 1988, p189-1931989.

Keywords: "Interstitials, "Crystal defects, "Cryogenics, *Austenitic steels "Fracture mechanics, Martensitic transformation, Fracture strength, Tension tests, Ductility, Nitrogen additions, Inclusions, Metallography, Nucleation, Yield strength, Mathematical models Voids, Stress concentration, Reprints.

The effects of interstitial content on tensile properties, martensitic transformation, and inclusion content are examined in relationship to the measured fracture toughness of austenitic stainless steels at cryogenic temperatures. Metallographic sections show that void nucleation occurs ahead of a crack only after the applied $\mathrm{J}$ exceeds a large fraction of the measured toughness, $\mathrm{J}$ (sub Ic). A stress intensity factor is calculated for void nucleation from a simple model that considers the yield strength and inclusion spacing.

\section{1,158}

PB91-189951

Not available NTIS

National Inst. of Standards and Technology (MSEL), Boulder, CO. Fracture and Deformation Div.

Metallographic Study of the Crack-Tip Region from Fracture Mechanics Specimens of Austenitic and Ferritic Steels.

Final rept.

P. T. Purtscher, R. P. Reed, and D. K. Matlock. 1989 , $6 p$

Sponsored by Department of Energy, Washington, DC Pub. in Proceedings of MRS International Meeting on Advanced Materials, Tokyo, Japan, May 31-June 4 1988, p391-396 1989.

Keywords: "Fracture mechanics, "Crack opening displacement, "Austenitic steels, "Ferritic stainless steels, "Strain hardening, Fracture strength, Electron microscopy, Etching, Metallography, Microhardness Ductility, Reprints.

Metallographic sections of blunted and growing cracks in steels with different strain-hardening capacities are observed in the light and scanning electron microscope. The strain distribution around the crack-tip region is inferred from the etching behavior and microhardness readings. The direction of initial growth is approximately straight ahead of the fatigue precrack in the high strain-hardening austenitic steels and at an angle of about 45 degrees in the low strain-hardening ferritic steel. These observations are discussed in terms of different criteria for ductile fracture in fracture mechanics specimens.

101,159

PB91-189977

Not available NTIS National Inst. of Standards and Technology (MSEL), Boulder, CO. Fracture and Deformation Div.

Discontinuous Yielding during Tensile Tests at Low Temperatures.

Final rept.
R. P. Reed, and N. J. Simon. 1990, 10p

Sponsored by Department of Energy, Washington, DC Pub. in Advances in Cryogenic Engineering, v36 p1077-1086 1990.

Keywords: "Tensile tests, "Austenitic steels, "Low temperature tests, Yield strength, Temperature effects, Stresses, Strains, Mechanical properties, Yield point, Tensile properties, Compressive properties, Reprints.

The effects of temperature and coolant on the initiation of discontinuous yielding were studied. As the 
temperature is increased above $4 \mathrm{~K}$, discontinuous yielding begins at higher stresses and strains; discontinuous yielding is not present at temperatures above $30 \mathrm{~K}$. Results are interpreted in terms of macroscopic heat balances and localized generation of heat from moving dislocations.

\section{1,160}

PB9́1-194795

Not available NTIS

National Inst. of Standards and Technology (NML),

Boulder, $\mathrm{CO}$. Chemical Engineering Science Div.

High-Temperature Thermal Properties of UNS

\$44004 Using Multivariant Analysis.

Final rept.

L. J. Freiberger, and J. W. Bransford. 1990, 10p

Sponsored by National Aeronautics and Space Administration, Huntsville, AL. George C. Marshall Space Flight Center.

Pub. in Advanced Earth-to-Orbit Propulsion Technology 1990, vl p113-122.

Keywords: *Stainless steel-440, "Liquid metals, "Combustion, Thermophysical properties, Mathematical models, Melting points, Thermal conductivity, Extrapolation. Thermodynamic properties, Spacecraft construction materials, Multivariate analysis, High temperature tests, Reprints.

In order to model the thermal behavior of steel alloys in the temperature range at which ignition and combustion may occur, their thermophysical properties at temperatures up to their melting points, approximately 1600 to $1800 \mathrm{~K}$ for many iron nickel and cobalt-based alloys, must be known. Published values of thermal conductivity, specific heat, and emissivity are either given for much lower temperatures or are absent for alloys of special interest. Therefore, the development of a simple experimental method coupled with a numerical technique that can be used to extrapolate existing data or to estimate property values where no data exists was undertaken. The paper describes the experiment and numerical technique. The application of the procedures to estimate the thermophysical properties of UNS S44004 (AISI 440-C) are discussed.

101,161

PB91-195321

Not available NTIS

National Inst. of Standards and Technology (IMSE), Gaithersburg, MD. Polymers Div.

Rate of Pyrite Bioleaching by 'Thiobacillus ferrooxidans': Results of an Interlaboratory Comparison.

Final rept.

G. J. Olson. 1991, 3p

Pub. in Applied and Environmental Microbiology 57, n3 p642-644 Mar 91

Keywords: "Pyrite, "Beneficiation, "Leaching, "Biotechnology, Standards, Iron, Extractive metallurgy, Interlaboratory comparisons, Rates(Per time), Variability, Thiobacillus ferroxidans, Variability, Reprints.

Ten laboratories participated in an interlaboratory comparison of determination of bioleaching rates of a pyrite reference material. A standardized procedure and a single strain of Thiobacillus ferrooxidans were used in the study. The mean rate of bioleaching of the pyrite reference material was $12.4 \mathrm{mg}$ of $\mathrm{Fe}$ per liter per $h$, with a coefficient of variation (percent relative standard deviation) of $32 \%$ as determined by eight laboratories. These results show the precision among laboratories of the determination of rates of pyrite bioleaching when a standard test procedure and reference material are used.

\section{1,162}

PB9́1-195404

Not available NTIS

National Inst. of Standards and Technology (IMSE), Gaithersburg, MD. Polymers Div.

Characterization by High Performance Liquid Chromatography (HPLC) of the Solubilization of Phosphorus in Iron Ore by a Fungus.

Final rept.

E. J. Parks, G. J. Olson, F. E. Brinckman, and F

Baldi. 1990, 7p

Pub. in Jnl. of Industrial Microbiology 5, n2-3 p183-189 1990.

Keywords: "Biotechnology, "Fungi, "Liquid column chromatography, "Iron, "Phosphorus, "Beneficiation, Contamination, Characterization, Leaching, Metalliferous ores, Apatites, Hydroxy compounds, Solvation, Oxalic acid, Metabolites, Solubilization, Hydrochloric acid, Separation processes, Reprints.

The value of iron ore is adversely affected by phosphorus in concentrations over 0.03 percent by weight. The present research concerns using metabolic products of fungi to leach insoluble phosphates (hydroxyapatite) from ores. Ion chromatography was used to measure metabolization of glucose into acidic fragments. The rate and products of glucose degradation depend on both the chemical composition of the growth medium (buffered or not) and incubation conditions (shaken or quiescent). The principal products are identified as oxalic acid and tautomers of propylene dicarboxylic acid, mainly itaconic acid. Slow metabolization of the latter generates more oxalic acid. Aliphatic acids were not detected. Both iron ore phosphate and calcium phosphate are partially solubilized by either the spent broth or aqueous oxalic acid. Solubilization of ore phosphorus is greatly assisted by hydrochloric acid added to the spent broth in small increments. The data suggest biological and chemical leaching procedures for iron ore dephosphorization.

\section{1,163}

PB911-195529

Not available NTIS

National Inst. of Standards and Technology (MSEL), Boulder, CO. Fracture and Deformation Div. Nitrogen Strengthening of Austenitic Stainless Steels at Low Temperatures.

Final rept.

R. P. Reed, and N. J. Simon 1989, 9p

Sponsored by Department of Energy, Washington, DC Pub. in Proceedings of International Conference on High Nitrogen Steels, Lille, France, May 18-20, 1988 , p180-188 1989.

Keywords: "Austenitic stainless steels, "Nitrogen, Yield strength, "Stress relaxation, "Low temperature Hardening(Materials), Tensile properties, Mechanical properties, Stresses, Strains, Elastic properties, Reprints.

Additions of nitrogen to austenitic steels significantly increase their flow strengths at low temperatures. Tensile stress-strain, stress relaxation, and strain-ratechange measurements on $\mathrm{Fe}-\mathrm{Cr}-\mathrm{Ni}$ alloys have been completed. For these same alloys, elastic constants and lattice parameters were measured from 295 to 4 K. Regression analyses of NBS data was used to assess the dependence of yield strength on carbon and nitrogen content. These data were interpreted in terms of strengthening mechanisms.

\section{1,164}

B9́1-236505

Not available NTIS National Inst. of Standards and Technology (MSEL) Boulder, CO. Materials Reliability Div.

Spatially Overdetermined Analysis for Propagation Toughness Using Strain Gages.

Final rept.

J. R. Berger, and J. W. Dally. 1990, 7p

Pub. in Mechanics Research Communications 17, n2 p93-99 1990.

Keywords: "Strain gages, "Toughness, "Stainless steels, "Fracture properties, Fracture tests, Fractures(Materials), Strain measurement, Mechanica properties, Crack propagation, Cracking(Fracturing), Spatial distribution, Reprints.

Recent work by the authors has shown the applicability of strain measurements to static and dynamic fracture characterizations. Here they develop a methodology to analyze the spatial variations in strain sensed by a series of strain gages as a crack propagates beneath them. They analyze the dynamic fracture of a 4340 steel compact specimen using the developed methodology. The propagation toughness was determined to be $119 \mathrm{MPa}$ square root of $\mathrm{m}$ at a crack speed of 644 $\mathrm{m} / \mathrm{s}$

\section{1,165}

\section{PB9 1-236943}

Not available NTIS

Nechnology (MSEL)

Boulder, CO. Materials Reliability Div.

at $4 \mathrm{~K}$.

Final rept.

H. M. Lee, R. P. Reed, and J. K. Han. 1990, 10p

Sponsored by Department of Energy, Washington, DC Pub. in Advances in Cryogenic Engineering (Materials), v36 p1273-1282 1990 .

Keywords: "Tension tests, "Low temperature tests, "Loads(Forces), "Austenitic stainless steels, "High strength steels, Tensile properties, Ultimate strength, Yield point, Fracture properties, Mechanical properties, Yield strength, Reprints.

Load-controlled tensile tests were conducted at $4 \mathrm{~K}$ on high-strength austenitic steels. The rate of loading was varied from 5 to $5000 \mathrm{~N} / \mathrm{s}$. This change of loading rate affected the onset of discontinuous yielding and, in turn, the fracture characteristics of the steels. Ultimate strength decreased at higher loading rates. The role of discontinuous yielding in affecting the dependence of these properties on loading rate is discussed. If conventional pressure vessel codes are used for the designation of structural design stresses, these data suggest that the ultimate strength, not the yield strength. controls the assignment.

101,166

PB1-237081

Not available NTIS

National Inst. of Standards and Technology (MSEL), Boulder, CO. Materials Reliability Div.

Interlaboratory Tension and Fracture Toughness Test Results for CSUS-JN1 (Fe-25Cr-15Ni-0.3N) Austenitic Stainless Steel at $4 \mathrm{~K}$.

Final rept.

H. Nakajima, K. Yoshida, S. Shimamoto, R. L. Tobler, R. P. Reed, R. P. Walsh, and P. T. Purtscher. 1990, $8 \mathrm{p}$

Sponsored by Department of Energy, Washington, DC. Office of Fusion Energy.

Pub. in Advances in Cryogenic Engineering (Materials), v36 p261-268 1990

Keywords: *Austenitic stainless steels, "Low temperature tests, "Fracture properties, "Toughness, Fractures(Materials), Standards, Experimental data, Fracture tests, Mechanical properties, Cryogenics, Tension tests, Reprints.

Interlaboratory tests are part of the U.S.-Japan cooperative program in fusion energy to establish cryogenic test standards for structural alloys. The second round of $4 . \mathrm{K}$ tension and fracture toughness tests for CSUSJN1 (Fe-25Cr-15Ni-0.35N) austenitic stainless steel are described in the paper. The scatter of interlaboratory measurements is acceptable if some fracture toughness data are excluded as outliers.

101,167

PB9 1-237206

Not available NTIS

National Inst. of Standards and Technology (MSEL) Boulder, CO. Materials Reliability Div.

Effect of Processing on 4-K Mechanical Properties of a Microalloyed Austenitic Stainless Steel.

Final rept.

P. T. Purtscher, M. C. Mataya, L. M. Ma, and R. P.

Reed. 1990, 8p

Sponsored by Department of Energy, Washington, DC Office of Fusion Energy.

Pub. in Advances in Cryogenic Engineering (Materials), v36 p79-86 1990

Keywords: "Austenitic stainless steels, "Toughness *Fracture properties, Tensile properties, Hot rolling Alloy steels, Mechanical properties, Hot working, Annealing, Cryogenics, Low temperature tests, Reprints.

Tensile and fracture toughness tests were performed at $4 \mathrm{~K}$ as a function of hot-rolling temperature and compared with similar data for the steel in the annealed condition. Results show that the properties in hot-rolled conditions were comparable to annealed steel so there was no advantage to annealing afte processing. Microalloy precipitates were effective a controlling grain size during processing at temperatures below $1150 \mathrm{C}$, and did influence the flow stress at $4 \mathrm{~K}$

101,168

PB91-237248

Not available NTIS . Boulder, CO. Materials Reliability Div.

Strain Rate Effect on Tensile Properties at $4 \mathrm{~K}$ of a VAMAS Round-Robin Austenitic Steel.

Final rept.

R. P. Reed, R. P. Walsh, and R. L. Tobler. 1990, 8p Pub. in Advances in Cryogenic Engineering (Materials), v36 p1061-1068 1990.

Keywords: "Tensile properties, "Strain rate, "High strength steels, "Austenitic stainless steels, "Low temperature tests, Ultimate strength, Mechanical tests, Mechanical properties, Tension tests, Yield point, Displacement, Reprints.

A high-strength austenitic steel with nominal composition $\mathrm{Fe}-25 \mathrm{Cr}-14 \mathrm{Ni}-0.37 \mathrm{~N}$ was included in an international round-robin measurement program. Tensile and fracture toughness tests were conducted at $4 \mathrm{~K}$ by leading low temperature test laboratories in Japan Europe, and the U.S. The paper reports on the effect 


\section{MATERIALS SCIENCES}

\section{Iron \& Iron Alloys}

of strain rate on displacement-controlled tensile tests at $4 \mathrm{~K}$. Similar to other recent results on austenitic steels, a transition in ultimate strength was observed as a function of strain rate: at higher strain rates the strength decreased about $10 \%$. The onset of discontinuous yielding was also strain-rate dependent. The reduction of ultimate tensile strength is associated with the change from the nucleate to film heat transfe mechanism from the specimen surface to liquid helium.

\section{1,169}

PB92-10890

PC A03/MF A01

National Inst. of Standards and Technology (MSEL), Gaithersburg, MD. Metallurgy Div.

Mechanical Properties and Fracture Toughness of AAR TC128 Grade B Steel in the Normalized, and Normalized and Stress Relieved Conditions.

G. E. Hicho, and D. E. Harne. Sep 91, 46p NISTIR4660, REPT-24

See also PB90-207796

Keywords: "Fracture mechanics, "Toughness, "Carbon steels, "Fracture strength, Tensile strength, Normalizing(Statistics), Stress relaxation. Temperature dependence, Crack opening displacement, Manganese alloys, Tank cars, J integral.

Traditional mechanical property tests, ultimate and yield strength, reduction in area, elongation, impact, and the nil-ductility temperature were performed on AAR TC128 grade B steel in the normalized, and the normalized and stress relieved conditions from $-51 \mathrm{C}$ to room temperature In addition the crack initiation fracture toughness, as function of temperature, was determined using crack tip opening displacement (CTOD) and $J$ integral test procedures. The crack arrest fracture toughness, as a function of temperature were also determined. It was found that the normalized and stress relieved steel had overall better mechanica and fracture toughness properties than the normalized steel.

\section{1,170}

PB92-116615

Not available NTIS

National Boulder, CO. Fracture and Deformation Div.

Ultrasonic Measurement of Sheet Steel Texture and Formability: Comparison with Neutron Diffraction and Mechanical Measurements.

Final rept

A. V. Clark, R. B. Thompson, Y. Li, R. C. Reno, G. V. Blessing, D. V. Mitrakovic, R. E. Schramm, and D. Matlock. 1990, 19p

Pub. in Research in Nondestructive Evaluation 2 p239-257 1990

Keywords: "Steels, "Metal sheets, "Ultrasonic tests, Rolling, Nondestructive tests, On-line measuremen systems, Plastic deformation, Electroacoustic transducers, Predictions, Measurement, Orientation, Comparison, Neutron diffraction. Formability, Texture, Lamb waves, Reprints, Orientation distribution coefficients, R-value.

Ultrasonic measurements were made on a set of thin steel sheets, using the lowest-order shear horizontal mode (SH(sub 0)-mode) and lowest-order symmetric Lamb-wave mode (S(sub 0)-mode). The velocities of these modes were measured as a function of angle relative to the sheet rolling direction. From the data reduction it is, in theory, possible to (1) partially characterize the texture of the sheet, and (2) predict the plastic strain ratio (r-value). The plate texture can be completely characterized by quantities known as orientation distribution coefficients (ODCs). The lowest-order ODCs can be obtained from the measurements; these were compared with ODCs measured by neutron diffraction, with good agreement for the dominant ODC. The r-value is a commonly used measure of sheet formability. It is typically measured mechanically with uniaxial tension specimens subjected to large plastic strain. Therefore, the $r$-value test is destructive and time consuming. A good correlation was found between S(sub 0)0mode velocity measurements and the average in-plane r-value. Consequently, the use of noncontacting electromagnetic-acoustic transducers (EMATs) may offer an online nondestructive measurement of sheet formability.

\section{1,171}

PB92-126515

PC A07/MF A02

National Inst. of Standards and Technology (MSEL), Gaithersburg, MD. Metallurgy Div.

Metallurgy Division, Technical Activities 1991. (NAS-NRC Assessment Panel February 13-14, 1992).

Annual rept. 1 Oct $90-30$ Sep 91

E. N. Pugh, and S. C. Hardy. Dec $91,136 p$ NISTIR4697

See also PB91-132225.

Keywords: "Metallurgy, "Research projects, Meta matrix composites, Alloys, Superconductors, Corrosion, Electrodeposition, Magnetic materials, Mechanical properties, Process control, Intermetallic alloys, Metals processing, Process sensors.

The report summarizes the FY 1991 activities of the Metallurgy Division of the National Institute of Standards and Technology (NIST). These activities center upon the structure-processing-properties relations of metals and alloys and on methods of measurement; and also include the generation and evaluation of critical materials data. Efforts comprise studies of metals processing and process sensors; advanced materials, including metal matrix composites, intermetallic alloys and superconductors; corrosion and electrodeposition; mechanical properties; magnetic materials; and high temperature reactions.

\section{Lubricants \& Hydraulic Fluids}

\section{1,172}

PB91-158808

Not available NTIS

National Bureau of Standards (IMSE), Gaithersburg, MD. Ceramics Div.

Mechanisms of Additive Effectiveness

Final rept.

S. M. Hsu, P. Pei, C. S. Ku, R. S. Lin, and S. T. Hsu.

1986, $10 p$

Sponsored by Department of Energy, Washington, DC. Pub. in Proceedings of International Colloquim Additives for Lubricants and Operational Fluids (5th), January $14-16,1986, \mathrm{p} 3.14-1-3.14-10$

Keywords: "Lubricant additives, "Wear, "Tribology Oxidation, Corrosion, Fluid-solid interactions, Reaction kinetics, Polar compounds, Reprints.

The interactions among naturally occurring polar molecular species with additives in wear and oxidation are studied. Base oil hetroatoms such as sulfur, nitrogen, and oxygen have pronounced effects on additive performance. Interaction studies in wear and oxidation tests suggest a complex chemical reaction mechanism that has heretofore been little understood but is beginning to be defined now by critical experiments.

\section{1,173}

PB9 1-158923 (IMSE), Gaithersburg, MD. Ceramics Div.

Heats of Immersion, Friction, and Wear of Base Oil Fractions.

Final rept.

F. E. Lockwood, K. Bridger, and S. M. Hsu. 1989 ,

Pub. in Tribology Transactions 32, n4 p506-516 1989.

Keywords: "Lubricating oils, "Mixed base crudes, Friction, Adsorption, Surface chemistry, Thermodynamic properties, Fluid flow, Wear, Interfacial tension, Reprints, * Heat of immersion.

Heats of immersion were determined for three base oils, their fractions as separated by polarity, and model compounds on a 52,100 steel surface. The method consists of calculating the heat of immersion based on surface tension and contact angle measurements made at temperatures from $25 \mathrm{C}$ to $175 \mathrm{C}$. Friction and wear tests were performed on the same oils using micro-sample four-ball wear tests. The heat of immersion data were found to be inversely proportional to friction and wear. Model compounds with different functional groups similarly tested, however, suggest molecular structures have a significant role in influencing friction and wear when chemical reactions become the dominant lubrication mechanism rather than adsorption.

101,174

PB91-174862

National available NTIS Gaithersburg, MD. Ceramics Div.
Mechanisms of Additive Effectiveness.

Final rept.

S. M. Hsu, P. Pei, C. S. Ku, R. S. Lin, and S. T. Hsu $1989,20 p$

Sponsored by Department of Energy, Washington, DC Pub. in Lubrication Science 1, n2 p165-184 Jan 89.

Keywords: "Lubricant additives, *Tribology, Interactions, Lubricating oils, Wear, Oxidation, Quantity ratio, Calorimetry, Mechanism, Reprints, FRT, ZDP, Fourball test.

The article discusses the nature and extent of base oiladditive and additive-additive interactions. The firs interaction is in the bulk oil phase, where solution chemistry is involved. The second interaction occurs a the wear surfaces. Various experimental techniques (DSC, thin film oxygen uptake. FRT, four ball) were used to measure interactions in an examination of base oil-additive-interactions on oxidation stability, various species of base oils exert a strong influence on the performance of antioxidants. In additive-additive interactions, the data suggest a strong concentration of dependent interaction patterns among the various additives and the base oils. Mechanistic studies are recommended to elucidate some of the observed phenonema.

101,175

PB91-174953

Not available NTIS

National Bureau of Standards (IMSE), Gaithersburg MD. Ceramics Div.

Study of Additive Response in a Series of Rerefined Base Oils Typical of Current Commercial Practice.

Final rept.

C. S. Ku, E. E. Klaus, and S. M. Hsu. 1985, 13p

ub. in Proceedings of European Tribology Congress (4th), Ecully, France, September 9-12, 1985, v1 p1-13.

Keywords: "Automobiles, "Lubricating oils, "Tribology, Lubricant additives, Compositions, Refining, Oxidation, Comparison, Recycled materials, Thin films, Reprints, Oxygen uptake.

A group of 14 rerefined base oils representing 6 refining processes and 10 geographic areas of the U.S. and Canada have been evaluated extensively to determine compositional detail. The additive responses of these base oils to six commercial automotive additive packages were studied. The NBS thin film oxygen uptake test (TFOUT) was used in the study with test conditions which showed good correlation with IIID engine sequence test results. A group of 43 virgin base oils with a common additive package was also tested for comparison with the rerefined base oils. The oxidation induction times from the TFOUT with six commercial additive packages were correlated with basestock compositional parameters. The study shows that satisfactory quality commercial motor oils can be formulated with rerefined base oils and a number of commercial additive packages. Correlational studies show that the major compositional parameters that are important with rerefined oils are sulfur, nitrogen, and polars, all of which have a negative effect on additive response.

101,176

PB91-189407

Not available NTIS

National Inst. of Standards and Technology (IMSE) Gaithersburg, MD. Ceramics Div.

Study on the Nature of Boundary Lubricating Film: Analytical Method Development.

Final rept.

R. S. Gates, S. M. Hsu, and K. L. Jewett. 1989, 8p Sponsored by Department of Energy, Washington, DC Energy Conversion and Utilization Technologies Div. Pub. in Tribology Transactions 32, n4 p423-430 1989.

Keywords: "Tribology, "Lubrication, "Polymeric films, *Chemical analysis, "Fluid-solid interactions, Refractivity, Organometallic compounds, Ultraviolet spectra, Absorption spectroscopy, Molecular weight, Surface chemistry, Interfaces, Oxidation, Wear, Reprints, Size exclusion chromatography.

The nature of the boundary lubricating film and its formation mechanism has been the subject of research in the last several decades. The study describes the development of a novel analytical technique designed specifically for the analysis of small amounts of lubricating film using an integrated size exclusion chromatography-refractive index-ultraviolet-graphite furnace atomic absorption (SEC-RI-UV-GFAA) system. Experiments under static simulation conditions as well as under dynamic rubbing conditions were conducted. 
Analysis of the reaction products in each case indicates the formation of high molecular weight organometallic compounds with molecular weights range from 1,000 to 100,000 . The paper describes the methodology, equipment, and the procedures developed to measure nanogram quantities of the organometallic compounds generated by the tribochemical reactions in the boundary contact of a four ball wear tester.

101,177

PB91-189480

Not available NTIS

National Inst. of Standards and Technology (IMSE), Gaithersburg, MD. Ceramics Div.

Mechano-Chemical Descriptive Model for Wear Under Mixed Lubrication Conditions.

Final rept.

S. M. Hsu, E. E. Klaus, and H. S. Cheng. 1988, 17p Sponsored by Department of Energy, Washington, DC. Office of Basic Energy Sciences.

Pub. in Wear 128, n3 p307-323, 15 Dec 88.

Keywords: "Tribology, "Lubrication, "Wear, "Surface chemistry, Probabilistic estimation, Performance prediction, Process control, Reprints.

The processes of lubrication are systematically analyzed and explained with the aid of recent experimental discoveries. Effective lubrication often involves surface chemical reactions which are dependent on contact geometry, load, speed, and environmental influences. Materials properties such as hardness, elasticity and others also affect the wear outcome. Fluid mechanics under the influence of interfacial pressures and temperatures control a significant portion of the wearing processes. A conceptual model is proposed to link all these factors in a line of defense framework, and to discuss how wear can be predicted based on a probabilistic model. The model proposes a series of criteria for various combinations of chemically controlled, and materials properties controlled situations.

101,178

PB9́1-195412

Not available NTIS

National Inst. of Standards and Technology (IMSE), Gaithersburg, MD. Ceramics Div.

Diesel Deposit Forming Tendencies - Microanalysis Methods.

Final rept.

J. M. Perez, P. Pei, Y. Zhang, and S. M. Hsu. 1991,

$10 \mathrm{p}$

Sponsored by Department of Energy, Washington, DC. Pub in SAE Technical Paper Series 910750 International Congress and Exposition, Detroit, MI., February 25-March 1, 1991, p1-10.

Keywords: "Lubricants, "Liquids, "Diesel engines, "Deposits, "Tribology, Thermal degradation, Oxidation, Automotive engineering, Chemical analysis, Wear tests, Microanalysis, Reprints.

Liquid lubricants are cruicial to the successful development of advanced engines for the next decade. Engines are being optimized to meet emission standards as well as improved durability and fuel economy. Lubricant research is focused on the severe environment and temperature requirements of advanced engines with a top ring reversal temperature of over $400 \mathrm{C}$. The paper describes key lubricant considerations including oxidation and thermal stability, volatility, deposit formation, friction and wear control. Cooperative research efforts between industry and NIST resulted in several candidate high temperature liquid lubricants.

\section{1,179}

PB91-203810

Not available NTIS

National Inst. of Standards and Technology (MSEL), Gaithersburg, MD. Ceramics Div.

Characterization of -Tricresylphosphate Lubricating Films by Micro-Fourier Transform Infrared Spectroscopy.

Final rept.

J. M. Perez, C. S. Ku, P. Pei, B. E. Hegemann, and S. M. Hsu. $1990,9 p$

Pub. in Tribology Transactions 33, n1 p131-139 1990

Keywords: "Lubricant additives, "Films, "Tribology, *TCP, Infrared spectra, Fourier transformation, Characterization, Microanalysis, Wear tests, Calorimetry, Oxidation, Temperature dependence, Corrosion products, Chemical analysis, Methodology, Reprints.

The characterization of tricresylphosphate lubricating films by use of micro-Fourier Transform Infrared Spectroscopy is reported. The study uses a combination of test methods to establish the sequence of chemical reactions that result in the formation of anti-wear films observed. A four-ball wear test sequence, using a 6 microliter sample to increase test severity, generated the tribological films. A pressurized differential scanning calorimeter (DSC) was used to obtain oxidized samples at various temperatures. The wear contact temperatures are estimated by comparison of the spectra analyses of the oxidation products to the wear film analyses. The combination of test methods is rapid and reliable. DSC oxidation and spectra analyses coupled with the sequential wear test method enabled characterization of the films formed. The approach is useful in evaluating additive effectiveness.

\section{1,180}

\section{PB91-236711}

Not available NTIS

National Inst. of Standards and Technology (MSEL). Gaithersburg, MD. Ceramics Div.

Effect of Selected Chemical Compounds on the Lubrication of Silicon Nitride.

Final rept.

R. S. Gates, and S. M. Hsu. 1991, 9p

Sponsored by Department of Energy, Washington, DC. Energy Conversion and Utilization Technologies Program.

Pub. in Tribology Transactions 34, n3 p417-425 1991.

Keywords: "Tribology, "Lubricant additives, "Ceramics, "Friction, "Wear tests, "Paraffin, "Silicon nitrides, Sulfonic acids, Monomolecular films, Oleic acid, Salicylic acid, Imidazoles, Phenol, Lubricating oils, Fluidsolid interactions, Phosphorus compounds, Glycols, Reprints.

Information on the chemical interactions of ceramics is scarce. This is especially true for chemical interactions with regard to lubrication of these materials. The paper investigates the influence of selected chemical compounds on the friction and wear of silicon nitride under boundary lubrication conditions. A ball-on-three-flat modification of the four-ball wear tester was utilized to evaluate the tribological characteristics of a ho pressed silicon nitride lubricated with a paraffinic base oil containing 1 weight percent additives. Friction, wear, and film formation tendencies were observed for a range of oil soluble chemical compounds containing oxygen, sulfur, nitrogen, chlorine, and phosphorus. A wide range of additive response was observed. Friction coefficient varied from increases of 30 percent to decreases of 47 percent below that of the base case of paraffin oil without additive. Wear was measured as the wear scar diameter increase above the Hertz diameter. Relative to the base case, wear additive response ranged from an increase in diameter of 61 percent to a decrease of 96 percent. Successful wear reduction was obtained by all phosphorus containing compounds, several glycol compounds, oleic acid, sulfonates, a salicylate, an imidazoline, and a phenol, and was generally associated with the formation of a film in the contact region.

\section{Materials Degradation \& Fouling}

\section{1,181}

PB91-143313

$\mathrm{PC} \mathrm{A03/MF} \mathrm{A01}$

National Inst. of Standards and Technology (IMSE), Gaithersburg, MD. Polymers Div.

Studies on the Degradation Products of Paper with and without Pollutants in a Closed Environment. 1. Preliminary Results.

E. J. Parks, C. M. Guttman, K. L. Jewett, and F. E.

Brinckman. Dec 90, 47p NISTIR-4456

Sponsored by National Archives and Records A.dministration, Washington, DC.

Keywords: "Newsprint, "Papers, "Degradation, "Sulfur dioxide, "Acetic acid, Aging tests(Materials), Organic acids, Gas chromatography, Mass spectroscopy, Liquid chromatography, Graphs(Charts).

The authors have developed methods using Mass Spectroscopy, Liquid Chromatography and Gas Chromatography to detect degradation products of rag paper and newsprint in the presence of some common air pollutants. They have searched for products that might themselves be autocatalytic to encourage degradation of these materials. In particular, they have looked at gaseous degradation products and those degradation products which are mobile and which may be transferred one paper to another by surface or gas phase diffusion. Six organic acids have been tentatively identified as degradation products which are surface mobile on newsprint and rag paper. Acetic acid is a major organic acid gas phase component. The results of these studies were correlated with more traditional bulk paper properties tests.

\section{1,182}

PB91-144345

$\mathrm{PC}$ A03/MF A01

National Inst. of Standards and Technology (MSEL), Gaithersburg, MD. Polymers Div

Synthesis of Prototype Resins for Use as BEP Intaglio Ink Vehicles Curing by Electron Beam Radiation.

Annual rept.

B. J. Bauer, and B. Dickens. Dec 90, 45p NISTIR

4474

Sponsored by Bureau of Engraving and Printing, Washington, DC

Keywords: "Radiation curing, "Resins, "Electron beams, "Inks, $X$ ray analysis, Neutron scattering, Chemical radiation effects, Polymeric films, Transition temperature, Polymethyl methacrylate, Copolymers, Polyoxyethylene, "Intaglio ink.

Macromonomers have been investigated as components in electron beam curing resins for use in intaglio inks. Thermal studies of cured samples of macromonomer-conventional monomer networks show a single transition for macromonomer containing samples and multiple transitions for equivalent samples made with unfunctionalized polymer. Small angle neutron and $x$ ray scattering studies show that samples made with macromonomers are much more uniform than those made with unfunctionalized polymers. Cure studies show that macromonomers with different polymerizable end groups have very different rates of cure, most of the groups inhibiting the cure.

101,183

PB91-159269

Not available NTIS National Bureau of Standards (IMSE), Gaithersburg, MD. Metallurgy Div.

Method for Quantitative Measurement of Galling Damage.

E. P. Whitenton, M. B. Peterson, and L. K. Ives.

1987, 16p

Pub. in Proceedings of Metal Transfer and Galling in Metallic Systems Symposium, Orlando, FL., October 8-9, 1986, p155-1701987.

Keywords: *Galling, *Tribology, *Metals, Quantitative analysis, Measurement, Damage assessment, Profilometers, Sensor mapping, Surface roughness, Wear, Computerized simulation, Reprints.

Galling damage at sliding contacts is recognized by the occurrence of a significant increase in surface roughness, characterized by gouges, grooves, prows, transferred lumps of material, and sometimes cracks. In the past, little effort has been made to measure the severity or nature of the damage quantitatively. Rather, a description based on visual observation was usually the only means employed to distinguish between the damage produced by different tribological contact conditions or exhibited by different types of materials. The method described employs an automated profilometer system to map a relatively large area of the damaged surface. From the topographic data obtained it was possible to evaluate parameters that are descriptive of the area as a whole rather than along a single trace as is normally done in profilometer based roughness measurements. Three rather simple parameters were found to give a good account of observed galling damage. The parameters were the average maximum peak to valley roughness, Rt ave., the displaced volume, DV, and a measure of the shape or aspect ratio of damage feature, AR. Results using these and other parameters are given. In addition, different galled surfaces are compared using computer generated damage maps.

\section{1,184}

PB91-174904

Not available NTIS National Inst. of Standards and Technology (IMSE) Gaithersburg, MD. Ceramics Div.

Base.

Final rept.

S. Jahanmir, S. M. Hsu, and R. G. Munro. 1989, 9p Pub. in Computerization and Networking of Materials Data Bases, ASTM STP 1017, p340-348 1989. 
Keywords: "Data bases, "Tribology, International cooperation, Technology transfer, Friction, Lubrication, Wear, Design criteria, Reprints, "Actis code.

Tribology is being increasingly recognized as a critical discipline that can play a key role in raising the level of U.S. competitiveness. The transfer of tribology research information into general engineering practice can be made quickly and efficiently by the computerizing of tribology data. The tribology community has been participating in an international effort to centralize and computerize tribology data that goes beyond the establishment of bibliographic data bases. The community is developing a computerized tribology information system as a self-sustaining activity, with government providing the initial funding for research and prototype construction of a PC-based system that contains six data-base components: numeric, design, newsletter, research-in-progress, bibliography, and product and services directory. The numeric data base, which contains 'best judgment' values compiled by experts, and the design data base, which contains validated design programs, are being developed in the first two years of a six-year program. These two data bases present a formidable technical challenge, since not only must they be self-contained, but they must also interact.

\section{1,185
PB9 $1-187328$}

Not available NTIS

National Bureau of Standards (NML), Gaithersburg, MD. Inorganic Analytical Research Div.

Method to Enhance Porosity of Micro-particles.

Final rept.

B. S. Carpenter, L. C. Sander, C. Horvath, and W. R. Brown. 1988, 9p

Pub. in Jnl. of Radioanalytical and Nuclear Chemistry 124, n2 p523-531 1988

Keywords: "Silica, "Fission products, "Uranium, "Porosity, "Ceramics, Coatings(Materials), Trace elements, Fission tracks, Microparticles, Reprints.

The Nuclear Track Technique (NTT) is used to enhance the porosity of silica micro-particles. The enhanced porosity is a result of the formation of surface and interior pores or tracks in the silica by the action of external and internal fission fragments. The fission tracks produced at the surface and within the interior of the micro-particles are a result of coating the particles with trace quantities of uranium, instead of having trace quantities of uranium incorporated within the silicon dioxide matrix.

\section{1,186}

\section{PB9́1-189530}

Not available NTIS

National Inst. of Standards and Technology (IMSE) Gaithersburg, MD. Ceramics Div.

Tribological Characteristics of Synthesized Diamond Films on Silicon Carbide.

Final rept.

S. Jahanmir, L. K. Ives, A. Feldman, E. Farabaugh,

and D. E. Deckman. 1989, 9p

Pub. in Wear 133, n1 p73-81 1989.

Keywords: "Tribology, "Vapor deposited coatings, "Diamonds, "Silicon carbides, "Ceramics, "Wear, $X$ ray analysis, Friction, Surface chemistry, Thin films, Graphite, Reprints, Carbon films.

The purpose of the research is to explore the possible use of synthesized diamond films as wear resistant, low friction materials for tribological applications. Silicon carbide specimens, in the form of small disks, were coated with diamond films using the hot filament CVD process. A ball-on-three-flat contact geometry was used in the wear experiments. The experimental results confirmed that wear rate of the disk specimens can be reduced by a factor of 200 , when they are deposited with a diamond film. The fraction coefficient was reduced by almost one order of magnitude. Although it was not explicitly shown the wear resistance may be related to the hardness of the diamond film. EDAN analysis of the worn surface of the diamond indicated that the SiC counterface undergoes tribochemical reactions with the air atmosphere producing hymical reactions with the air atmosphere producing hytion product cannot be responsible for low friction coefficients, since the same material is formed in $\mathrm{SiC}$ / $\mathrm{SiC}$ tests. It is, therefore, hypothesized that the low friction coefficient of diamond may be related to formation of a thin film of graphite at the real area of contact. Removal of these graphitic regions by wear would then produce a smooth wear surface.
National Inst. of Standards and Technology (IMSE), Gaithersburg, MD. Ceramics Div.

Comparison of Standard Test Methods for NonLubricated Sliding Wear.

Final rept.

A. W. Ruff. $1989,9 p$

Pub. in Wear 134, n1 p49-57 1989

Keywords: "Standards, "Interlaboratory comparisons, "Wear tests, "Tribology, Sliding friction, Methodology, Variability, Metals, Solid-solid interfaces, Reprints.

The paper analyzes non-lubricated sliding wear data obtained using three standard test methods and geometries: block-on-ring crossed-on-cylinder and pinon-disk. The data discussed here were obtained in interlaboratory testing programs where the conditions were carefully specified, and where the test materials were provided to the different laboratories. Such an approach should provide the most consistent data and should allow direct comparison of the intrinsic characteristics of the different test methods. However, results showed that when the above mentioned three tests were carried out under similar load and speed conditions, the typical within-laboratory variation in reproducing wear volume measurements in metal-on-metal sliding ranged from $21 \%$ to $57 \%$. By comparison, using standard test methods one finds the variation to be about $5 \%$ for abrasive wear, and about $5 \%$ for erosive wear.

101,188

PB91-190025

Not available NTIS

National Inst. of Standards and Technology (MSEL), Gaithersburg, MD. Ceramics Div.

Sliding Wear Studies of Nickel-Copper Composition-Modulated Coatings on Steel.

Final rept

A. W. Ruff, and Z. X. Wang. 1989, 14p

Pub. in Wear 131, n2 p259-272 1989.

Keywords: "Tribology, "Wear tests, "Steels, "Coatings(Materials), "Nickel alloys, "Copper alloys, Friction alloys, Sliding friction, Binary alloys, Compositions, Laminates, Loads(Forces), Reprints.

Sliding wear measurements have been conducted on $\mathrm{Ni}-\mathrm{Cu}$ composition-modulated coatings on steel. The coatings consisted of alternate layers of nickel and copper with layer spacings of $10 \mathrm{~nm}$ and $100 \mathrm{~nm}$. Pure nickel and pure copper coatings were also prepared in the same way and studied. The coated steel cylinders were slid against 52100 steel in a crossed-cylinder test under clean conditions without lubrication at various loads and sliding distances. It was found that the most wear-resistant coating was the one having the smallest layer spacing. This effect was most pronounced at low loads. Both composition-modulated coatings showed less wear than coatings of pure $\mathrm{Cu}$ and $\mathrm{Ni}$ from the same solution. The layer microstructure in these coatings is believed to provide internal barriers to wear damage that lead to the improvements in wear resistance. Results from examination of the worn specimens and collections of wear debris are also described.

\section{Miscellaneous Materials}

\section{1,189}

PB91-147074

Not available NTIS

National Inst. of Standards and Technology (NML) Gaithersburg, MD. Thermophysics Div.

Alternative Refrigerants R123a, R134, R141b, R142b, and R152a: Critical Temperature, Refractive Index, Surface Tension, and Estimates of Liquid, Vapor, and Critical Densities.

Final rept.

H. B. Chae, J. W. Schmidt, and M. R. Moldover. $1990,6 p$

Sponsored by Department of Energy, Washington, DC. Pub. in Jnl. of Physical Chemistry 94, n25 p8840-8845 13 Dec 90.

Keywords: "Refrigerants, Critical temperature, Critical density, Refractive index, Interfacial tension, Surface tension, Reprints, Chlorofluorocarbons.

Differential capillary rise and refractive index data are reported for five alternative refrigerants: $\mathrm{R} 141 \mathrm{~b}(\mathrm{CCl} 2 \mathrm{~F}-\mathrm{CH} 2), \mathrm{R} 142 \mathrm{~b}(\mathrm{CClF} 2-\mathrm{CH} 3)$ and R152a(CHF2-CH3). The data extend from about $25 \mathrm{C}$ to the critical point of each fluid and directly yield the critical temperature Tc and the temperature-dependent capillary length. The present data were combined with liquid density data (near ambient temperature) to determine the Lorentz-Lorenz constant. The LorentzLorenz relation is used to estimate the liquid, vapor, and critical densities, and the surface tension.

101,190

PB91-149344

Not available NTIS

National Bureau of Standards (IMSE), Gaithersburg, MD. Ceramics Div.

Tensile-Fracture Resistance Mechanisms in Brittle Polycrystals: An Ultrasonics and In-situ Microscopy Investigation.

Final rept.

P. L. Swanson. 1987, 22p

Contract USGS-414274

Sponsored by Geological Survey, Menlo Park, CA.

Pub. in Jnl. of Geophysical Research-Solid Earth and Planets 92, nNB8 p8015-8036 1987.

Keywords: "Brittle materials, "Polycrystals, "Fracture mechanics, Ceramics, Microscopy, Ultrasonic tests, Granite, Aluminum oxide, Rocks, Reprints, In-situ tests.

The material-breakdown processes associated with mode-I fracture propagation in brittle polycrystals is in vestigated using ultrasonic-wave probing and in-situ optical microscopy. Emphasis is placed on delineation of the size and shape of the 'fracture process zone' and identification of its constitutive micromechanisms. Two materials were investigated: imperfectly-elastic multi-phase Westerly granite and near perfectly-elastic single-phase polycrystalline alumina (Al2O3). In both materials a zone of 'tractions' was observed to develop along the macrocrack flanks behind the visually identified primary fracture tip. The tractions, or restraining forces, were provided by both frictional interlocking of the fracture surfaces and 'bridging' by intact material left behind the advancing fracture front. Localized microcracking (friction-induced microcracking and material-bridge rupture) was found to be associated with the traction sites. Traction-zone lengths in the granite were as long as $15-40 \mathrm{~mm}(0.75 \mathrm{~mm}$ grain size) and $2 \mathrm{~mm}$ in the alumina (20-100 micrometer grain size). The crack-interface restraining mechanisms are incorporated into a simple fracture mechanics formulation which is used to describe several commonly observed features (e.g. R-curves) of macroscopic fracture behavior.

101,191

PB91-159137 Not available NTIS National Bureau of Standards (IMSE), Gaithersburg, MD.

International Co-operation: The Versailles Project on Advanced Materials and Standards.

Final rept.

L. H. Schwartz, and B. W. Steiner. 1988, 2p

Pub. in ATAS Bulletin 5, p135-136 May 88.

Keywords: "International cooperation, "Materials, *Standards, Reprints, "VAMAS Project.

The formation and activity of the Versailles Project on Advanced Materials and Standards (VAMAS) is described.

101,192

PB91-159145

Not available NTIS

National Bureau of Standards (IMSE), Gaithersburg, MD.

VAMAS Bulletin.

Final rept.

L. H. Schwartz, and B. W. Steiner. Jul 87, 16p

Pub. as VAMAS Bulletin, n6 p1-16 Jul 87.

Keywords: "Materials, "Standards, "International cooperation, Reprints, "VAMAS project.

Recent work of the Versailles Project on Advanced Materials and Standards (VAMAS) is described.

101,193

PB91-162024

Not available NTIS

National Inst of Standards and Technology (MSEL) Gaithersburg, MD. Polymers Div. 
Effect of Pyrophosphate Concentrations on Calcium Phosphate Growth on Well-Crystallized Octacalcium Phosphate and Hydroxyapatite Seed Crystals.

N. Eidelman, W. E. Brown, and J. L. Meyer. 1991, 9p Sponsored by American Dental Association Health Foundation, Chicago, IL.

Pub. in Jnl. of Crystal Growth 108, p385-393 1991.

Keywords: *Calcium phosphates, "Pyrophosphates, Crystal growth, Concentration(Composition), Apatites, Hydroxy compounds, Inhibitors, Comparison, Dental materials, Reprints.

The comparative effects of $0.3,1$ and 3 micromolar P2O7(sup 4-) concentrations on the growth of calcium phosphate on octacalcium phosphate (OCP) and on 'well-crystallized' hydroxyapatite (OHAp) seeds were studied. The control growth rates (without P2O7(sup 4) were adjusted by weight or surface area of the seed crystals. The induction periods were longer with higher P2O7(sup 4-) concentrations and longer on OCP seeds than on the OHAp seeds. However, the final growth rates were about the same on both kinds of seeds when equal concentrations of P2O7(sup 4-) were used, suggesting that the same phase was growing on both kinds of seeds. The growth rates were faster when higher concentrations of OHAp seeds were used. The crystal growth on both OCP and OHAP seeds was accelerated when most of the labeled P2O7(sup 4-) disappeared from the solution. The composition of the initial phase that grew on either OCP or OHAp seed crystals appears to be OCP or partially hydrolyzed OCP. These results lead to the conclusion that apparently OCP, but not OHAp, grew on OCP and OHAp seeds in the presence of P2O7(sup 4-).

101,194

PB91-174243

Not available NTIS

National Inst. of Standards and Technology (NEL). Gaithersburg, MD. Building Environment Div.

Experimentation, Analysis, and Correlation of Refrigerant-22 Flow Through Short Tube Restrictors. Final rept.

D. A. Aaron, and P. A. Domanski. 1990, 13p

Sponsored by Department of Energy, Washington, DC. Pub. in ASHRAE (American Society of Heating, Refrigerating and Air-Conditioning Engineers) Transactions, v96 pt1 13p 1990.

Keywords: "Refrigerants, "Pipe flow, *Heat pumps, Tubes, Orifices, Air conditioners, Pressure reduction, Expansion, Diameters, Fluid flow, Mass flow, Reprints.

Refrigerant-22 flow through short tube restrictors was investigated. The analysis pertained to initially subcooled refrigerant flowing through short tubes with $5<$ $L / D<20$. The flow conditions studied were those typically found in heat pumps. Flow dependencies upon upstream subcooling, upstream pressure, downstream pressure, tube length, tube diameter, entrance chamfering, and exit chamfering were examined. A correlation and flow charts for mass flow rate prediction were developed from a large experimental data base.

\section{1,195}

PB91-175414 Not available NTIS National Inst. of Standards and Technology (NML), Gaithersburg, MD. Thermophysics Div. Thermodynamic Properties of Two Alternative Refrigerants: 1,1-dichloro-2,2,2-trifluoroethane (R123) and 1,1,1,2-tetrafluoroethane (R134a).

Final rept.

G. Morrison, and D. K. Ward. 1991, 22p

Sponsored by Department of Energy, Washington, DC. Pub. in Fluid Phase Equilibria 62, p65-86 1991.

Keywords: *Refrigerants, "Thermodynamic properties, Temperature effects, Vapor pressure, Working fluids, Critical point, Thermophysical properties Density(Mass/volume), Measurement, Pressure, Reprints.

The paper describes two different sets of measurements of the properties of two proposed alternative refrigerants, 1,1,1,2-tetrafluoroethane (R134a) and 1,1 dichloro-2,2,2-trifluoroethane (R123). The first are vapor-liquid equilibrium measurements made in a simple variable-volume sapphire cell. The vapor pressures and saturation liquid densities were determined for both materials, between $268 \mathrm{~K}$ and the critical temperature for $\mathrm{R} 134 \mathrm{a}$ and between $303 \mathrm{~K}$ and $373 \mathrm{~K}$ for R123. The saturation vapor density was also determined for R134a from $298 \mathrm{~K}$ to the critical point, where the critical conditions were measured. The second set

of measurements were made with a vibrating tube densimeter from the saturation pressure to $5500 \mathrm{kPa}$ for $\mathrm{R} 134 \mathrm{a}$ and to $3500 \mathrm{kPa}$ for $\mathrm{R} 123$ in the temperature range $278 \mathrm{~K}-368 \mathrm{~K}$. These data are then compared with other determinations of similar properties.

\section{1,196}

PB91-189563

Not available NTIS

National Inst. of Standards and Technology (NEL) Gaithersburg, MD. Building Environment Div.

Study of Flow Boiling Heat Transfer with Refrigerant Mixtures.

Final rept

D. S. Jung, M. O. McLinden, R. Radermacher, and D. A. Didion. 1989, 14p

Sponsored by Electric Power Research Inst., Palo Alto, CA.

Pub. in International Jnl. of Heat Mass Transfer. 32, n9 p1751-1764 1989

Keywords: *Heat pumps, "Refrigerants, Heat transfer coefficient, Mixtures, Nucleate boiling, Reprints.

Mixture effects are studied on horizontal flow boiling heat transfer with both azeotropic and non-azeotropic refrigerant mixtures. More than 2000 local heat trans fer coefficients are obtained with the azeotropic R12/ R152a mixture and compared against the previously measured data with the non-azeotropic R22/R114 mixture In a convective evaporation region, small mass transfer resistance is found for mixtures. The variation of physical properties due to mixing is responsible for almost all of the heat transfer degradation. In a partial boiling region, however, severe degradation of heat transfer with mixtures, similar to that in nucleate pool boiling heat transfer with mixtures, is found. A suppression of nucleate boiling at lower qualities due to loss of wall superheat with mixtures is responsible for the degradation. An analysis is developed to predict a transition quality by using Hsu's onset of nucleate boiling theory. The prediction agreed well with observed transition qualities for both pure and mixed refrigerants. Correlations, based on the supposition of Chen and using only phase equilibrium data to consider mixture effects, are developed with mean deviations of 7.2 and $9.6 \%$ for pure and mixed refrigerants.

\section{1,197}

PB91-236695

Not available NTIS

National Bureau of Standards (NEL), Gaithersburg, MD. Thermophysics Div.

Thermodynamic Diagrams for Refrigerant Mixtures.

Final rept.

J. S. Gallagher, M. O. McLinden, and G. Morrison

$1988,18 p$

Sponsored by Electric Power Research Inst., Palo

Alto, CA.

Pub. in ASHRAE (American Society of Heating, Refrigerating and Air-Conditioning Engineers) Transactions v94 pt2 p2119-21361988.

Keywords: "Refrigerants, "Equations of state, "Thermodynamic properties, Thermochemical diagrams, Fluorohydrocarbons, Entropy, Thermodynamics, Temperature, Pressure, Thermophysical properties, Thermochemical properties, Reprints, "Thermodynamic diagrams.

Several highly detailed diagrams of the thermodynamc properties of refrigerant mixtures have been generated. These diagrams are based on the CarnahanStarling-DeSantis equation of state and are useful in the analysis and design of refrigeration equipment using refrigerant mixtures. Presented are pressure-enthalpy diagrams for $R 13 / 12, R 22 / 114$ and $R 22 / 11$ mixtures at compositions of $60 / 40$ and $40 / 60$ weigh percent for each mixture. Temperature-entropy diagrams for the R22/114 mixture as well as for pure R22 and R114 are also presented. The CSD equation of state is briefly reviewed along with a description of the routines used to produce the diagrams.

101,198

PB92-112366

A05/MF A01

National Inst. of Standards and Technology (CSTL),

Boulder, CO. Thermophysics Div.

Survey of Current Worldwide Research on the Thermophysical Properties of Alternative Refrigerants.

M. O. McLinden, W. M. Haynes, J. T. R. Watson, and K. Watanabe. Jun $91,82 p$ NISTIR-3969

Prepared in cooperation with National Engineering Lab., East Kilbride (Scotland), and Keio Univ., Yokohama (Japan). Dept. of Mechanical Engineering. Spon- sored by Department of Energy, Washington, DC Office of Buildings and Community Systems.

Keywords: "Refrigerants, "Thermophysical properties, Transport properties, Surveys, Research, Thermody namic properties, Tables(Data), Fluorohydrocarbons Permittivity, Refractivity, Foreign technology, Global, Hydrochlorofluorocarbons, Hydrofluorocarbons.

The survey represents an exhaustive compilation of the research activities throughout the world concerned with either measurements or correlations of the thermophysical properties of alternative refrigerants. The properties covered in the study include thermodynamic, transport, phase equilibria, and other properties such as dielectric constant and refractive index. The survey has included a wide range of fluids (including R23, R32, R125, R143a, R22, R134a, R152a, R134 R124, R142b, R123, R123a, R141b) along with mix tures containing at least one of these fluids. The report presents in tabular form summary information about each research activity; the survey does not present raw data or correlating equations.

\section{1,199}

PB92-116748

Not available NTIS

National Inst. of Standards and Technology (NML) Boulder, CO. Thermophysics Div.

Predictive Extended Corresponding States Model for Pure and Mixed Refrigerants.

Final rept.

J. F. Ely, and M. L. Huber. 1990, 10p

Pub. in Proceedings of ASHRAE-Purdue CFC and IIRPurdue Refrigeration Conference, West Lafayette, IN. July 17-20, 1990, 10p.

Keywords: "Thermophysical properties, "Refrigerants Enthalpy, Equations of state, Mixtures, Entropy, Thermal conductivity, Computer programs, Thermodynamic properties, Forecasting, Viscosity, Models, Density(Mass/volume), Thermodynamic equilibrium, Reprints, PROZPER computer program.

The authors have developed a predictive correspond ing states model for the thermophysical properties of pure refrigerants and refrigerant mixtures. The bulk phase properties such as the density, enthalpy and entropy are predicted using the principle of extended corresponding states. The theoretically based model uses shape factors to insure conformality among the various components and R134a is used as the reference fluid. The shape factors are found by mapping saturation boundaries of the fluids of interest onto the reference fluid. In the case where no saturation data are available, the shape factors are predicted. In addition to equilibrium properties, a one-fluid corresponding states model is used to predict the viscosity and thermal conductivity. Phase equilibria is predicted using a Peng-Robinson equation of state. The corresponding states model is contained in an interactive microcomputer program, PROZPER (PRoperties of OZone Protecting Environmentally acceptable Refrigerants). In addition, the program can 'learn' new components using only a minimum amount of information--the molecular weight, the normal boiling point and the critical parameters of the chemical species. Additional information such as vapor pressures, saturated liquid densities, saturated liquid thermal conductivities and viscosities may be input to improve the predictive capability of the model.

\section{1,200}

PB92-116946

Not available NTIS

National Inst. of Standards and Technology (NEL) Gaithersburg, MD. Building Equipment Div.

Horizontal Flow Boiling Heat Transfer Experiments with a Mixture of R22/R114.

Final rept.

D. Jung, M. O. McLinden, R. Radermacher, and D. A Didion. $1989,15 p$

Sponsored by Electric Power Research Inst., Palo Alto, CA

Pub. in International Jnl. of Heat and Mass Transfer 32, n1 p131-145 1989.

Keywords: "Refrigerants, "Thermodynamic properties, *Heat transfer, "Boiling, Heat transfer coefficient, Hea flux, Mixtures, Concentration(Composition), Liquid phases, Fluid flow, Transport properties, Reprints.

An experimental study on horizontal flow boiling heat transfer for pure R22 and R114 and their mixtures under uniform heat flux condition is reported. More than 1200 local heat transfer coefficients are obtained for annualar flow at a reduced pressure of 0.08 and 


\section{MATERIALS SCIENCES}

\section{Miscellaneous Materials}

qualities up to $85 \%$ for various heat fluxes and mass fluxes. The results show that local heat transfer coefficients for mixtures are as much as $44 \%$ lower than a linear mole fraction weighting of the pure component values for the same flow condition. A composition variation of up to 0.07 mole fraction in the annular liquid film was measured between the top and bottom of the tube. The effects of the composition variation and nonideal variations in physical properties account for the most of the heat transfer degradation seen with mixtures.

\section{Nonferrous Metals \& Alloys}

\section{1,201}

AD-A229 229/0 PC A10/MF A02 National Inst. of Standards and Technology, Boulder, CO.

Aluminum Alloys for Cryogenic Tanks: Oxygen Compatibility. Volume 1.

Interim rept. Nov 88-Jun 90

R. P. Reed, N. J. Simon, J. D. McColskey, and J. R.

Berger. Sep 90, 207p AL-TR-90-063-VOL-1,

Keywords: Alloys, "Aluminum alloys, Compatibility, Contamination, "Cryogenics, Documents, Experimen tal data, Laboratories, *Liquid oxygen, Oxygen, Porta ble equipment, "Tanks(Containers), Test and evaluation, Test facilities, Test methods, 'Lithium alloys, Compatibility, Lox(liquid oxygen), Aluminum allo 8090 , Aluminum alloy 2090 , Aluminum alloy 2219 , Ad vanced Launch System.

In Part I of this program, Al-Li alloys $8090-\mathrm{T} 3$ and 2090-T81 and Al alloy 2219 (tempers T851, T37) were ested for compatibility with liquid oxygen using pres surized mechanical-impact apparatuses at two NASA laboratories, Marshall Space Flight Center (MSFC) and White Sands Test Facility (WSTF). Specimens and data from tests at Santa Susana Field Laboratory (SSFL), Rocketdyne, on alloy 2090-T81 were supplied by ALCOA. Pressurized mechanical-impact data on alloy WL049-T351 were produced by WSTF. In addition, WSTF conducted open-cup mechanical-impact tions occurred in some specimens of all alloys during pressurized mechanical-impact tests at MSFC. There were no reactions during similar tests at WSTF and SSFL. The reactions at MSFC are not attributed to specimen contamination. This interim report analyze the ignitions found in the MSFG pressurized mechanical-impact tests, compares the results and test methodologies of both MSFC and WSTF facilities (since each laboratory is producing test data that are apparently divergent), and addresses the current test standard document NASA NHB 8060.1B as it pertains to this study. (TTL)

\section{1,202}

AD-A229 231/6 PC A09/MF A02 National Inst. of Standards and Technology, Boulder CO.

Review of Cryogenic Mechanlcal and Thermal Properties of Al-Li Alloys and Alloy 2219.

Final rpt. Nov 88-Jun 90 .

N. J. Simon, E. S. Drexler, and R. P. Reed. Sep 90 197p AL-TR-90-064,

Keywords: Air force facilities, Air force systems command, "Aluminum alloys, Astronautics, Constants, Cryogenic storage devices, Cryogenics, Elastic prop erties, Elongation, Fracture(Mechanics), High strength alloys, Laboratories, "Launching, "Lithium alloys, "Mechanical properties, Specific heat, Supervisors,
"Tanks(Containers), Tensile strength, Thermal con"Tanks(Containers), Tensile strength, Thermal con-
ductivity, Thermal expansion, "Thermal properties, Tcı.ıhness, Validation, Yield strength, Launchers, Alumirulin alloy 2219, Cryogenic tankage, Advanced Launch System.

The review of cryogenic mechanical and thermal properties presented here is part of a broader NIST program to assess new high-strength Al-Li alloys for use in the cryogenic tankage of the Advanced Launch in the cryogenic tankage of the Advanced Launch
System. This program is sponsored by the Air Force Systems Command, Astronautics Laboratory, Edwards Air Force Base, with Bao Nguyen, Task Manag er. It is part of the Materials and Process Validation (3101) of the Structures, Materials, and Manufacturing $(3000)$ portion of the ALS Advanced Development Pro gram. Since the purpose of the NIST program has been to assess the relative suitability of high-strength $\mathrm{Al}-\mathrm{Li}$ alloys and alloy 2219 for us in ALS cryogenic tanks, data on Al-Li alloys 8090,2090 , WeldaliteTM 049 , and $\mathrm{Al}$ alloy 2219 have been included in the survey. Properties covered in this survey are tensile strength, yield strength, elongation, fracture toughness, elastic constants, specific heat, thermal conductivity, and thermal expansion. (ttl)

\section{1,203}

AD-A231 830/1

PC A07/MF A01

National Inst. of Standards and Technology, Boulder, CO.

Aluminum Alloys for ALS Cryogenic Tanks: Oxygen Compatibility. Volume 2.

Final rept. Nov 88-Jun 90

R. P. Reed, N. J. Simon, J. D. McColskey, C. N. MicCowan, and E. S. Drexler. Sep 90, 128p AL-TR-

90-0630-VOL-2

See also Volume 1, AD-A229 229

Keywords: Alloys, Compatibility, Cryogenics, Drop tests, Electron microscopes, Electronic scanners, Eye Gases, Height, Liquid oxygen, Lithium alloys, Magnification, Microscopy, Optical analysis, Oxygen, Potential energy, Power, Tanks(Combat vehicles), Test facilities. Aluminum alloys, Aluminum-lithium alloys, Weldalite.

In part II of this program, selected tempers of alloys 8090,2090 WL 049 and 2219 were tested for compatibility with liquid oxygen in modified open-cup and pressurized mechanical-impact equipment at White Sands Test Facility. Drop height (potential energy), pressure, and environment (liquid oxygen, LOX, and gaseous oxygen, GOX were varied in these tests. Additional promoted-combustion tests were also conducted a WSTF. Reactions, ranging in size from those that could be observed with the naked eye to those that required use of the scanning electron microscope (SEM), were identified in all alloys. Those that require little or no magnifying pewer to view are labeled macroreactions; those that require the use of optical microscopy or SEM are called microreactions. AI-L alloys had more macroreactions than 2219; 2219 had more, or an equivalent number of microreactions than each of the Al-Li alloys.

\section{1,204}

AD-A242 956/1

PC A07/MF A02

CO.

Aluminum Alloys for ALS Cryogenic Tanks: Comparative Measurements of Cryogenic Mechanical Properties of Al-Li Alloys and Alloy 2219.

Final rept. Aug 89-Mar 90.

R. P. Reed, P. T. Purtscher, N. J. Simon, J. D.

McColskey, and R. P. Walsh. Oct 91, 146p

Keywords: "Aluminum alloys, "Lithium alloys, "Cryogenics, Mechanical properties, Tensile properties, Fracture(Mechanics), Toughness, Delamination, Cracking(Fracturing), Low temperature alloys, Grain size, Aluminum - Lithium Alloys, Cryogenic Mechanica Properties, Alloy 2219, Surface Flaw.

Tensile and plane-strain fracture toughness properties were obtained at cryogenic temperatures to compare the Al-Li alloys 8090, 2090, and WLO49 and alloy 2219 in various tempers and specimen orientations. The strongest alloy at very low temperatures is WLO49 T851, which is about $10 \%$ stronger than $2090-$ T81. Both alloys are considerably stronger than 2219-T87. Alloy 2090-T81 is tougher in the in-plane orientations (about 50\%) than WL049-T851 at low temperatures; the higher in-plane toughness is attributed to the presence of less constituent particles and the tendency to crack out-of-plane or delaminate at low temperatures. This delamination tends to divide the moving crack, thus separating it into smaller regions where plan stress (rather than plane strain) conditions are conducive to increased toughness. Thus, a dichotomy: re duced toughness in the through-thickness or out-ofplane orientations leads to increased toughness in the in-plane orientations. In service, a leak in the tank is considered failure, and a leak will be caused by a crack in the panels of the tankage growing through the pane thickness. To measure the resistance to crack growth under these conditions, surface-flawed panel tests are recommended.

\section{1,205}

PC A03/MF A01

National Inst. of Standards and Technology, Boulder. CO.
Guideline for Nb(sub 3)Sn critical current measurements using fiberglass-epoxy composite sample mandrels.

L. F. Goodrich. 1989, 18p CONF-890701-31

Contract Al01-86ER52132

International cryogenic materials conference, Los Angeles, CA (United States), 24-28 Jul 1989. Sponsored by Department of Energy, Washington, DC.

Keywords: "Critical Current, "Niobium Base Alloys, Tin Alloys, Bonding, Composite Materials, Fiberglass, Intermetallic Compounds, Measuring Methods, Meetings, EDB/360104, "Cryogenics, "Superconductors.

This guideline was prompted, in part, by presentations given at the 6th Japan-US Workshop on High Field SUperconductors (February 22--24, 1989, Boulder, CO) and subsequent discussions. The results of the recent Versailles Project on Advanced Materials and Standards (VAMAS) Nb(sub 3) Sn critical-current round robin indicate that increased consistency in interlaboratory measurements might be achieved by a more detailed specification of the critical-current (I(sub c)) measurement technique. However, a competition exists between the benefit of a rigidly specified measurement technique, which ensure measurement reproducibility, and a less restrictive technique that would be practical for a greater number of laboratories. Ideally, the measurement technique should have the least number of restrictions that is consistent with measurement reproducibility. This measurement guideline is intended to be one of a few different measurement techniques in a second $\mathrm{Nb}$ (sub 3 ) Sn I(sub c) round robin that is envisioned to determine the preferred measurement method for $\mathrm{Nb}$ (sub 3 ) Sn. At this time, there is no consensus regarding the materials used for the reaction mandrel, measurement mandrel, or sample bonding. There are advantages and disadvantages for all known materials. This guideline was written for one combination of materials. This measurement guideline assumes that the reader has the basic knowledge and experience with I(sub c) measurements of $\mathrm{NbTi}$ and $\mathrm{Nb}$ (sub 3)Sn conductors.

101,206

N91-21347/0

(Order as N91-21331/4, PC A24/MF A03) National Inst. of Standards and Technology (NML), Gaithersburg, MD. Thermophysics Div.

Dynamic Technique for Measuring Surface Tension at High Temperatures in Microgravity Environment.

A. P. Miiller, and A. Cezairliyan. 1 May 90, 5p In JPL, Proceedings of the First Workshop on Containerless Experimentation in Microgravity p 243-247.

Keywords: Heating, High temperature, "Interfacial tension, "Liquid metals, Melting, Microgravity applications, Pipes (Tubes), Reduced gravity, C-135 aircraft, Copper, Electric current, Melting points, Refractory metals, Simulation, Tantalum, "Surface tension.

The feasibility of a dynamic technique for measuring surface tension of liquid metals at high temperatures in a microgravity environment was demonstrated. The basic method involves heating a tubular specimen resistively from ambient temperature through its melting point in about $1 \mathrm{sec}$ by passing an electrical current pulse through it, while simultaneously recording the pertinent experimental quantities. Static equilibrium for the molten specimen is achieved in a microgravity environment by splitting the current after it passes through the specimen tube and returning a fraction along the tube axis, and the remaining fraction outside the specimen. Adjustments to the current split enable a balance between the magnetic and surface tension forces acting on the specimen. Values for surface tension are determined from measurements of the equilibrium dimensions of the molten specimen tube, and the magnitudes of the currents. Rapid melting experiments, performed during microgravity simulations with the NASA KC-135 aircraft, yield a value for the surface tension of copper at its melting point which is in agreement with literature data. Measurements of surface tension of a refractory metal (tantalum) are underway.

101,207

PB91-132225

PC A07/MF A01

National Inst. of Standards and Technology (MSEL), Gaithersburg, MD. Metallurgy Div. 
Institute for Materials Science and Engineering: Metallurgy Divlsion, Technical Activities 1990. Annual rept. 1 Oct $89-30$ Sep 90 . E. N. Pugh, and J. H. Smith. Dec 90, 142p NISTIR4397 See also report for 1989, PB90-161159.

Keywords: "Metallurgy, Magnetic materials, Electrodeposited coatings, Corrosion, Processing, Process control, Superconductors, Intermetallic compounds, High temperature tests. Heat resistant alloys, Steels, Microstructure, Weldments, Phase diagrams, Construction materials, Detectors, Metal matrix composites, International cooperation.

The report summarizes the FY 1990 activities of the Metallurgy Division of the National Institute of Stand ards and Technology (NIST). These activities center upon the structure-processing-properties relations of metals and alloys and on methods of measurement and also include the generation and evaluation of criti$\mathrm{cal}$ materials data. Efforts comprise studies of metals processing and process sensors; advanced materials, including metal matrix composites, intermetallic alloys and superconductors; corrosion and electrodeposition; mechanical properties; magnetic materials; and high temperature reactions. The work described also includes two cooperative programs with professiona societies (the Alloy Phase Diagram Program with ASM International, and the Corrosion Data Program with the National Association of Corrosion Engineers); two with trade associations (the Temperature Sensor Program with the Aluminum Association, and the Steel Sensor Program with the American Iron and Steel Institute) and several with industry including the Powder Atomization Consortium with three companies.

\section{1,208}

\section{PB91-134551}

Not available NTIS

National Bureau of Standards (IMSE), Gaithersburg, MD. Metallurgy Div.

Literature Review of the Galling Process.

Final rept.

M. B. Peterson, L. K. Ives, and K. J. Bhansali. 1987. $25 \mathrm{p}$

Pub. in Proceedings of Symposium on Metal Transfer and Galling in Metallic Systems, Orlando, FL., October 8-9, 1986, p1-25 1987.

Keywords: "Galling, *Reviews, "Metals, Plastic deformation, Surface roughness, Microstructure. Crystal structure, Loads(Forces), Strain hardening, Reprints, Preferred orientation, Temperature dependence, Asperity.

A review was conducted of the technical literature relating to the process of galling. Approximately 250 references pertinent to galling were reviewed and key references selected. A principal focus of the review concerned the relationship between galling and plastic deformation. Attention was directed to two deformation models; a soft asperity model and a hard asperity model. From these models a postulate on frictional behavior has been developed. The severity of galling is determined not only by contact conditions such as load and contact geometry but also by factors affecting deformation characteristics such as crystal structure, preferred orientation, work hardening rate, and microstructure. The severity of galling is found to increase with increasing load, reduced surface roughness, higher adhesion, reduced strain hardening rate and higher temperature. These and other factors relating to galling severity are discussed.

\section{1,209}

PB91-147314 Not available NTIS National Inst. of Standards and Technology (MSEL), Gaithersburg, MD. Metallurgy Div.

Phase Formation in Electrodeposited and Thermally Annealed Al-Mn Alloys.

Final rept.

B. Grushko, and G. R. Stafford. 1990, 11p

Sponsored by Office of Naval Research, Arlington, VA. Pub. in Metallurgical Transactions A 21A, p2869-2879 Nov 90.

Keywords: "Aluminum manganese alloys, Amorphous materials, Electrodeposition, Aluminum intermetallics, Annealing, Icosahedrons, BCC lattices, FCC lattices, Reprints, Icosahedral phase, Quasicrystals, Manganese intermetallics.

Aluminum-manganese alloys with compositions ranging from 0 to 50 wt pct $\mathrm{Mn}$ were electrodeposited onto copper substrates from a chloroaluminate molten salt to $325 \mathrm{C}$. The structures of these electrodeposits were then compared to those observed when metastable electrodeposits were thermally annealed at $200 \mathrm{C}$ to $610 \mathrm{C}$. The alloys were characterized by scanning electron microscopy, transmission electron microscopy (TEM), energy dispersive spectroscopy, and $\mathrm{X}$-ray diffraction. At deposition temperatures of $150 \mathrm{C}$ to 250 $C$, no stable structure other than the strongly supersaturated and highly dislocated Al-face-centered cubic (fcc) solid solution is observed. An amorphous phase and body-centered cubic (bcc) Al8Mn5 are observed at higher manganese compositions.

\section{1,210}

PB91-147322 Not available NTIS National Bureau of Standards (IMSE), Gaithersburg, MD. Metallurgy Div.

Effect of Coherency Strain on Alloy Formation: Migration of Liquid Films.

Final rept.

C. A. Handwerker, J. W. Cahn, D. N. Yoon, and J. E. Blendell. 1985, $18 \mathrm{p}$

Sponsored by Army Research Office, Research Triangle Park, NC.

Pub in Proceedings of TMS-AIME Fall Meeting Diffusion in Solids: Recent Developments, Detroit, MI., September 17, 1984, p275-292 1985.

Keywords: "Alloys, Mathematical models, Thermodynamics, Diffusion, Reprints, "Liquid films.

When solid solution grains are not in chemical equilibrium with the liquid in which they are imbedded, equilibration frequently occurs by dissolution and reprecipitation of saturated solid solution, rather than by solid state diffusion. At small volume fractions of liquid, this mechanism has the appearance of liquid film migration and is modelled by diffusion of the components of the solid through the liquid film, driven by differences in solubility in the liquid between the dissolving grain and the growing grain. A thermodynamic analysis is given for the solubilities of the grains, which includes local interfacial curvature and local coherency stresses due to lattice parameter variations in the diffusion zones. An expression for the migration velocity derived from the model contains no unmeasurable parameters. Except for curvature and liquid film thickness, the parameters are all thermodynamic, elastic, crystallographic or diffusive and can be measured independent of the liquid film migration process.

\section{1,211}

PB91-147512

Not available NTIS $\mathrm{MD}$

Phase Diagrams of Hexagonal Binary Ordering Alloys with Anisotropic Interactions.

Final rept.

R. Kikuchi, and J. W. Cahn. 1987,6p

Pub. in Proceedings of International Conference on User Applications of Alloy Phase Diagrams, Lake Buena Vista, FL., October 4-9, 1986, p19-24 1987.

Keywords: "Binary alloys, "Phase diagrams, Hexagonal close packed lattices, Order disorder transformations, Interactions, Reprints, Cluster variation method.

Phase diagrams between the disordered and the $D O(19)$ ordered (A(3)B phases are calculated for the hexagonal lattice using the tetrahedron approximation of the cluster-variation method. Nearest-neighbor pairwise interactions are assumed, but the bonds within the basal plane are allowed to differ from the bonds between basal planes, $r$ being the ratio of interaction energies. When $r=1$, the disorder-A(3)B phase boundary agrees exactly with that calculated for fcc, although the entropy expressions for the hcp and fcc cases are different. For $0.8<r<1.2$, the boundary Shape is qualitatively the same as the $r=1$ case.
When $0<r<0.7$, the present calculations for the $B$ rich side of the $A(3) B$ phase becomes unstable.

\section{1,212}

PB91-147637

Not available NTIS

National Bureau of Standards (IMSE), Gaithersburg, MD. Ceramics Div.

Structure of Surface Films on Magnesium and on Magnesium Alloys.

Final rept.

G. G. Long, J. Kruger, D. K. Tanaka, and Z. Zhang.

$1988,1 \mathrm{p}$

Pub. in Jnl. of the Electrochemical Society 135, n3 pC144 1988 .

Keywords: "Magnesium oxides, "Magnesium, "Surfaces, $X$ ray spectroscopy, Crystal structure, Oxygen, Films, Reprints, "Magnesium alloy AZ61.
Surface reflection x-ray spectroscopy measurements at the oxygen K-edge are reported for surface films on high purity magnesium and on AZ61 magnesium alloy. Surface-EXAFS data was extracted for the films and for a single crystal MgO standard sample. The experimental curves were fitted by theoretical curves to obtain oxygen nearest neighbor distances, coordination numbers, and a measure of the disorder in the surface films.

101,213

PB91-148866

Not available NTIS National Bureau of Standards (IMSE), Gaithersburg. MD. Reactor Radiation Div.

Neutron Powder Diffraction and Inelastic Scattering Study of the Structures of Zr2Pd, Zr2PdD1.70, and $Z$ r2PdD1.96.

Final rept.

A. J. Maeland, E. Lukacevic, J. J. Rush, and A.

Santoro. 1987, 15p

Pub. in Jnl. of the Less Common Metals 129, p77-91 Feb 87.

Keywords: "Zirconium hydrides, "Palladium, "Alloys, Crystal structure, Deuteration, Isotope effects, Neutron diffraction, Intermetallic compounds, Tetragonal lattices, Reprints.

The compounds $\mathrm{Zr} 2 \mathrm{Pd}$ Zr2PdD 1.70 , and $\mathrm{Zr} 2 \mathrm{PdD}$ 1.96 have been studied with the neutron powder diffraction technique and the Rietveld method of profile analysis. All three materials crystallize with the symmetry of space group $14 / \mathrm{mmm}, Z=2$. The lattice parameters are $a=3.3085(9)$ and $c=10.8907(6)$ lambda for $\mathrm{Zr} 2 \mathrm{PdD} 1.70$, and $\mathrm{a}=3.3715(3)$ and $\mathrm{c}=$ 11.438 (2) lambda for $\mathrm{Zr2PdD} 1.96$. The $\mathrm{D}$ atoms are located at the centers of slightly distorted tetrahedra of $\mathrm{Zr}$ atoms with a $\mathrm{Zr}$-D distance of 2.071(1) lambda in $\mathrm{Zr} 2 \mathrm{PdD} 1.70$ and 2.092(2) in Zr2PdD 1.96 and with $\mathrm{Zr}$ $\mathrm{D}-\mathrm{Zr}$ angles ranging from $106.6 \mathrm{deg}$ to $110.9 \mathrm{deg}$. The hydrogen location in the structure has been confirmed by neutron inelastic scattering measurements. These show a broad hydrogen vibration peak at $135 \mathrm{meV}$ analogous to that observed for hydrogen bound in relatively undistorted tetrahedral sites in other hydrides. As a consequence of the insertion of deuterium in the structure of the intermetallic compound, the $\mathrm{Zr}$ - $\mathrm{Zr}$ distances in the deuterides are considerably different from the corresponding ones intermetallic compound, the $\mathrm{Zr}$ - $\mathrm{Zr}$ distances in the deuterides are considerably different from the corresponding ones in $\mathrm{Zr} 2 \mathrm{Pd}$. How ever the $\mathrm{Zr}$-Pd separation remains practically identical in the three materials.

101,214

PB91-149039

Not available NTIS

National Bureau of Standards (IMSE), Gaithersburg,

MD. Metallurgy Div.

Materials Research at the National Bureau of Standards.

Final rept.

E. N. Pugh. 1986, 3p

Pub. in Proceedings of Materials Technology Congress, Adelaide, Australia, May 19-21, 1986, v1 p1-3.

Keywords: "Research projects, "Metals, "Nondestructive tests, "US NBS, International cooperation, Australia, Processing, Reprints.

Materials research at NBS is centered in the Institute for Materials Science and Engineering, one of four major organizational units at the Bureau. The Institute is comprised of five Divisions, namely Ceramics, Fracture and Deformation, Metallurgy, Polymers, and Reactor Radiation; it also manages a Bureau wide interdisciplinary program in nondestructive evaluation. As an agency of the Department of Commerce, NBS has traditionally provided the measurement foundation to support our industrial economy, and the Institute continues to generate measurement methods, critical materials data and standards for the industrial and scientific community. The Institute's research programs support the measurement activities and, in addition, are moving increasingly to address the science base underlying both traditional and new materials technologies. The lecture outlines these activities, focusing mainly on those of the Metallurgy Division and the NDE program.

101,215

PB91-149427

Not available NTIS

National Inst. of Standards and Technology (IMSE), Gaithersburg, MD. Polymers Div. 


\section{MATERIALS SCIENCES}

\section{Nonferrous Metals \& Alloys}

Structural Instabilities and Superconductivity in Quasi-Binary Mn5Si3-Type Compounds.

Final rept.

R. M. Waterstrat, R. Kuentzler, and J. Muller. 1990,

$10 p$

Sponsored by American Dental Association Health Foundation, Chicago, IL.

Pub. in Jnl. of the Less-Common Metals 167, p169 1781990.

Keywords: "Binary alloy systems, "Superconductivity, "Zirconium alloys, "Platinum alloys, Iridium alloys Osmium alloys, Specific heat, Magnetic permeability Gold additions, Transition temperature, Low temperature, Reprints, Manganese silicides.

Measurements have been made of the low temperature specific heat, magnetic susceptibility, lattice parameters and superconducting behavior of quasibinary compounds based on the Mn5Si3-type struc ture and having the general formula Zr5Ir(1-v) Tv where $T$ is either platinum or osmium. The atomic displace ments previously reported for the Zr51r3 structure occur only near this binary composition: other alloys in the quasi-binary systems have an undistorted Mn5Si3 type structure. Superconductivity occurs in these quasi-binary systems, and Tc is a continuous function of the electron concentration reaching a maximum $\mathrm{Tc}=7.2 \mathrm{~K}$ for the binary compound Zr5Pt3. Substitution of small amounts of gold for platinum in Zr5Pt3 apparently destabilizes the Mn5Si3 type structure and produces alloys of unknown structure with lower Tc values (about $4 \mathrm{~K}$ ).

\section{1,216}

PB9 1-149450

Not available NTIS

National Bureau of Standards (NEL), Gaithersburg,

MD. Automated Production Technology Div.

Material Dependency of Chip-Form Detection

Using Acoustic Emission.

Final rept.

K. W Yee. 1987,5 p

Pub. in Proceedings of NAMRC (North American Manufacturing Research) Conference (15th), Bethlehem, PA., May 27-29, 1987, p458-462.

Keywords: "Chips, "Machining, "Acoustic detectors Metal spinning, Microcomputers, Monitoring, Aluminum, Steels, Metal products, Metal scrap, Metal working, Reprints.

An acoustic-emission-based microcomputer chip-form monitor has been designed and built at the Nationa Bureau of Standards. The monitor implements algorithms based on research by the University of California, Berkeley. The ability to identify chip form in turning of three types of metal (aluminum, low-carbon steel, and an alloy steel) has been previously reported. The monitor performs best for the easy-to-machine metals where chip breaking is most difficult and disastrous tangles are most likely. Subsequent experiments have shown that the successful identification of chip form is dependent on the work-place metal. Additional results from the machining of two-nonmagnetic stainless steels and a copper alloy are contrasted with the previous results.

\section{1,217}

PB91-158485

Not available NTIS

National Inst of Standards and Technology (MSEL), Gaithersburg, MD. Reactor Radiation Div.

Rapid Low-Temperature Hopping of Hydrogen in a Pure Metal: The ScHx System.

Final rept.

I. S. Anderson, N. F. Berk, J. J. Rush, T. J. Udovic,

R. G. Barnes, A. Magerl, and D. Richter. 1990 4p

Pub. in Physical Review Letters 65, n12 p1439-1442,

17 Sep 90.

Keywords: "Scandium, "Hydrogen, Low temperature research, Neutron scattering, Structure factors, Diffusion, Reprints.

The localized motion of hydrogen in scandium has been studied by quasielastic neutron scattering. The results reveal that the quasielastic linewidth has a pronounced minimum near $100 \mathrm{~K}$ and rises at lower temperature with approximate 1/T dependence, indicative of nonadiabatic behavior that can be associated with weak coupling of hydrogen to the metal conduction electrons. This is the first such observation in a pureelectrons. This is the first such observation in a pure-
metal-hydrogen system. The simultaneous determinametal-hydrogen system. The simultaneous determinapreted in terms of labile and nonlabile hydrogen configurations in a manner consistent with recent estimates of pairing and activation energies in the rareearth-hydrogen systems.
101,218

PB91-158527

Not available NTIS National Bureau of Standards (IMSE), Gaithersburg. MD. Metallurgy Div.

Acoustic Emission Monitoring of Laser Drilling. Final rep

R. B. Clough, R. J. Schaefer, and H. N. G. Wadley. 1984, 10p

Pub. in Proceedings of ASM Metals Congress Metals/ Materials Technology Series, Detroit, ML., September $15-10,1984, \mathrm{p} 1-10$

Keywords: "Metals, "Laser drilling, "Acoustic emission, Manufacturing, Nondestructive tests, Process control, Laser radiation, Aluminum, Reprints.

Laser drilling, because of its speed, controllability, and ability to penetrate tough or exceptionally hard materials, is a rapidly growing manufacturing method. However, beam power spikes and interventing disintegration products can disrupt or diminish energy transmission to the hole site, resulting in variable hole depth. To improve the situation, a combination of acoustic emission monitoring and laser settings has been inves tigated to improve hole depth prediction. The emission is suggested to be generated principally by liquid expulsion; intergranular cracking appears to be a secondary source. Acoustic emission shows promise as an experimental method for study of directed energy beam-material interactions.

\section{1,219}

\section{PB9 1-158550}

Not available NTIS

National Inst. of Standards and Technology (MSEL), Gaithersburg, MD. Metallurgy Div.

Convective and Morphological Instabilities during Directional Solidification.

Final rept.

S. R. Coriell, and G. B. McFadden 1990, 15p

Sponsored by National Aeronautics and Space Administration, Washington, DC

Pub. in Principles of Solidification and Materials Processing, Chapter 16, v1 p365-379 1990.

Keywords: "Directional solidification(Crystals), "Binary alloys, Liquid-solid interfaces, Convection, Rayleigh scattering, Crystal growth, Separation, Morphology, Finite difference theory, Liquid metals, Stability, Mathematical models, Time dependence, Reprints.

During the directional solidification of a binary alloy at constant velocity, solute segregation may arise due to either interface instability or fluid flow in the melt. Recent calculations of cellular shapes in the absence of fluid flow and thermosolutal convection caused by the rejection of a lighter solute during growth vertically upwards are presented. Three-dimensional steadystate solutions for nonplanar interface morphologies are computed numerically by finite differences. A linear temperature field is assumed, and the solute field in the melt and the unknown crystal-melt interface position are obtained self-consistently. For a model of an alumimun-chromium alloy with a distribution coefficient greater than unity, the calculations predict hexagonal nodes near the onset of instability. Numerical results for the solute segreation caused by thermosolutal convection are obtained using finite differences in a twodimensional, time-dependent model that assumes a planar-crystal melt interface. The system is assumed periodic in the horizontal direction, and the possibility of multiple flow states sharing the same period is examined. As the solutal Rayleigh number is varied, multiple steady states, time-periodic states, and quasiperiodic states occur.

\section{1,220}

1-158949

Not available NTIS National Bureau of Standards (IMSE), Gaithersburg, MD. Metallurgy Div.

Rapid Solidification Alloys: Crystals, Quasicrystals, and Metallic Glass.

Final rept.

J. R. Manning. $1986,6 p$

Pub. in ASTM (American Society for Testing and Materials) Standardization News 14, n9 p26-31 Oct 86.

Keywords: "Metals, "Alloys, Reviews, Phase tranformations, Metallic glasses, Microstructure, Reprints "Quasicrystals, "Rapid solidification process.

An overview is given of rapid solidification processes and the advantages rapid solidification can provide to alloys. The advantages include finer microstructures, decreased segregation, extended solid solubility, and formation of new types of phases, including the recently discovered quasicrystal phases.
101,221

PB91-158964 Not available NTIS National Bureau of Standards (IMSE), Gaithersburg MD. Metallurgy Div.

Transformation of the Icosahedral Phase in Rapidly Quenched Al-rich Al-Mn Alloys.

Final rept.

A. J. McAlister, L. A. Bendersky, R. J. Schaefer, and F. S. Biancaniello. 1987, 4p

Pub. in Scripta Metallurgica 21, n2 p103-106 1987

Keywords: "Aluminum manganese alloys, Phase transformations, Crystallization, Icosahedrons, Alum num intermetallics, Thermal analysis, $X$ ray diffraction Electron microscopy, Activation energy, Reaction kinetics, Heat of crystallization, Reprints, *Quasicrystals, Icosahedral phase, Manganese intermetallics.

Crystallization of the quasicrystalline icosahedral phase formed in Al-rich Al-Mn alloys has been studied by constant heat rate DTA, XRD, and TEM. Heats and activation energies of transformation were obtained, and evidence presented which suggests that the transformation is diffusion controlled.

101,222

PB91-159111

Not available NTIS

National Bureau of Standards (IMSE), Boulder, CO. Fracture and Deformation Div.

Use of Neutron Pole Figures to Calibrate Ultrasonic Techniques for On-Line Texture Control of Aluminum Plates.

Final rept.

R. C. Reno, A. V. Clark, G. V. Blessing, R. J. Fields, A. Govada, R. B. Thompson, P. P. Del Santo, R. B. Mignogna, and J. F. Smith. 1987, 7p

Pub. in Proceedings of ASM Symposium on Intelligent Processing of Materials and Advanced Sensors, Orlando, FL., 1986, p77-83 1987.

Keywords: "Metal plates, "Aluminum, "Neutron diffraction, "Process control, Intelligence, Calibrating, UItrasonic tests, Texture, Metal rolling, Crystallography, On-line systems, Monitors, Detectors, Comparison, Nondestructive tests, Reprints.

Neutron pole figures were used to determine the crys tallographic texture in rolled aluminum plates that were previously characterized with ultrasonic techniques. The orientation distribution function coefficients (ODC's) determined by neutron diffraction are in excelent agreement with those derived from ultrasound measurements, thus confirming the efficacy of ultrasound as an on-line monitoring technique.

101,223

PB91-159202

Not available NTIS National Bureau of Standards (IMSE), Gaithersburg, MD. Metallurgy Div.

Electrodeposition of Aluminum from Molten Salts. Final rept.

G. Stafford, and C. Turner. 1987, 13p 1987.

Keywords: "Electrodeposition, "Aluminum manganese alloys, "Fused salts, Amorphous materials, Aluminum intermetallics, Electrolytes, Manganese additions, Metallic glasses, Heat treatment, $X$ ray diffraction, $R e$ prints, Manganese intermetallics.

The effect of additions of Mn to molten salt electroIytes will be discussed. It was found that aluminum manganese alloys exhibit x-ray diffraction patterns consistent with a metallic glass structure which upon heat treatment transforms to the crystalline intermetallic Al6Mn. Some electrochemical phenomena operative in these melts will be discussed.

\section{1,224}

PB91-167338

PC A04/MF A01

National Inst. of Standards and Technology, Gaithers burg, MD.

Intercomparison Study of Rockwell Hardness Test Blocks.

T. R. Shives, and J. H. Smith. Feb 91, 74p NISTIR4531

Keywords: "Standards, "Rockwell hardness, "Metals, Comparison, Test equipment, Diamond pyramid hardness tests, Aging tests(Materials), Calibrating.

The National Institute of Standards and Technology undertook an intercomparison study of Rockwell hard- 
ness test blocks marketed in the United States. Test blocks from six different manufacturers were included in the study. Measurements were made generally on seven sets of test blocks at three hardness levels for each of the HRC, HRB, HR3ON and HR3OT hardness scales. Even for the nearly ideal conditions of the study, it was found that there are significant differences among hardness test blocks of different manufacturers for some hardness levels. This is especially true for the high hardness part of the HRC scale, the lower and middle parts of the HRB scale, and parts of both the HR3ON and HR30T scales. In these regions, the ranges of test results for blocks of different manufacturers that have similar assigned values exceed the tolerance limits for standardized test blocks according to ASTM Standard E 18-89a. Reevaluation of HRB and HR30T test blocks four to five years after initial evaluation indicated that many of these are unstable. There were significant changes in hardness in a number of the blocks.

101,225

PB91-174466

Not available NTIS National Bureau of Standards (IMSE), Gaithersburg, MD. Reactor Radiation Div.

Properties of Amorphous Zirconlum Rhodium Hydride (Zr3RhHx) Prepared from Glassy and Crystalline Alloys.

Final rept.

R. C. Bowman, J. S. Cantrell, K. Samwer, J. Tebbe, E. L. Venturini, and J. J. Rush. 1988, 13p

Pub. in Physics Review B: Condensed Matter 37, n15 p8575-8587 1988.

Keywords: "Amorphous materials, "Zirconium alloys, "Rhodium alloys, "Metal hydrides, Metallic glasses, Hydrogenation, Neutron scattering, Nuclear magnetic resonance, Electronic structure, Thermal degradation, Magnetic permeability, $X$ ray diffraction, Calorimetry, Reprints.

The a-Zr3RhHx samples with $x<5.5$ were studied by $x$-ray diffraction, proton nuclear magnetic resonance, magnetic susceptibility, low temperature heat capacity, differential scanning calorimetry, and inelastic neutron scattering. All hydride samples are amorphous with similar properties which are shown to be independent of the structure for the initial alloy. Namely, the solidstate reaction of hydrogen with crystalline c-Zr3Rh appeared to produce an equivalent amorphous phase to hydrogenated glassy alloys. The changes in the electronic and magnetic properties upon hydrogenation imply a systematic decrease in the Fermi level density of states in a-Zr3RhHx as the hydrogen content in creases. The thermal stabilities of the amorphous hydrides (with respective to the irreversible formation of crystalline $\mathrm{ZrHx}$ phases) also decrease with increasing hydrogen stoichiometry. Whereas the hydrogen atoms predominantly occupy tetrahedral interstitial sites coordinated with zirconium atoms there is strong circum stantial evidence for occupancies of different and less stable sites at the higher compositions.

\section{1,226}

PB91-174771

Not available NTIS Nation

N-Dimensional Crystallographic Description of the Icosahedral Phases; the Example of the Al73Mn21Si6 Quasiperiodic Structure.

Final rept.

D. Gratias, J. W. Cahn, M. Bessiere, Y. Calvayrac, S. Lefebvre, A. Quivy, and B. Mozer. 1987, 12p

Pub. in Proceedings of NATO Advanced Research Workshop, Italy, October 4-8, 1987, $12 p$.

Keywords: "Aluminum alloys, "Icosahedrons, "Phase diagrams, $X$ ray diffraction, Neutron diffraction, Electron diffraction, Crystallography, Reprints, Quasiperiodic.

Icosahedral phases in aluminium based alloys have been extensively studied by means of x-ray, neutron and electron diffraction. Many spectra have been collected, but no fully satisfying crystallographic model that matches properly the observed diffracted intensities has been proposed. The structural characterization of quasiperiodic phases is a formidable task due to the numerous parameters which have to be determined in addition to those of standard 3D crystallography.

\section{1,227}

PB91-187161

Not available NTIS

National Inst. of Standards and Technology (MSEL),

Gaithersburg, MD. Metallurgy Div.
Role of Elastic Energy in the Morphological Development of a Ni-Ti-Al Alloy.

Final rept.

L. A. Bendersky, P. W. Voorhees, W. J. Boettinger, and W. C. Johnson. $1988,6 p$

Pub. in Scripta Metallurgica 22, n7 p1029-1034 Jul 88. Keywords: "Strain energy methods, "Morphology, *Nickel alloys, "Titanium alloys, "Aluminum alloys, Elastic properties, Microstructure, Quenching(Cooling), Electron microscopy, Annealing, Phase transformations(Materials), Transmission electron microscopy, Heusler alloys, Precipitates, Coherence, Reprints, "Rapid solidification process.

The late-stage microstructural development of a rapidly solidified Ni50Ti37.5 alloy, studied by TEM is de. scribed. During $800 \mathrm{C}$ annealing, the following transformations occur: Heusler single phase to modulated precipitation of $\mathrm{B} 2$ phase to cuboidal precipitates of Heusler phase in B2 matrix to interconnected Heusler/ B2 structure. The morphological evolution of the coherent system is controlled primarily by elastic strain energy

\section{1,228}

\section{PB91-187187}

Not available NTIS

National Inst. of Standards and Technology (NEL),

Gaithersburg, MD. Building Materials Div.

Characterization of Cylindrical Holes in Metallic Substrates via Their Infrared Emission Patterns. Final rept.

D. P. Bentz, J. W. Martin, and M. E. Batts. 1991, 12p Pub. in Wear 143, p255-266 1991.

Keywords: "Thermal emission, "Holes(Mechanics), "Aluminum, "Steels, "Metals, "Infrared mapping, Infrared thermal detectors, Metal plates, Depth measurement, Temperature measuring instruments, Comparison, Theories, Milling(Machining), Geometry, Substrates, Ambient temperature, Calibrating, Surface energy, Reprints

IR emission patterns for a series of cylindrical holes milled into aluminum and steel plates were compared with those predicted from existing theory. The emission patterns were found to be influenced by the hole geometry, the substrate material and the IR camera system itself. For holes milled into an aluminum plate, the results were adequately explained by existing theory for diffuse materials. For holes milled into a steel plate, however, the theory was found not to apply and empirical calibration was required. It is concluded that the IR technique may have promise in characterizing the diameter and depth of milled holes at temperatures near ambient.

\section{1,229}

\section{PB91-187203}

Not available NTIS

Gaithersburg. Of Standards and

Particle Size Measurement of Inert Gas Atomized Powder.

Final rept.

F. S. Biancaniello, J. J. Conway, P. I. Espina, G. E Mattingly, and S. D. Ridder. 1990, 6p

Pub. in Materials Science and Engineering A A124, n1 p9-14, 10 Apr 90.

Keywords: "Atomizers, "Powder metallurgy, "Particle size distribution, "Supersonic flow, Measurement, Metal powder, Reliability, Reproducibility, Graphic methods, Reprints, "Supersonic inert gas metal atomizer.

Metal powder produced by a Supersonic Inert Gas Metal Atomizer (SiGMA) has been analyzed by using several diagnostic methods. These analyses have brought several interesting results. Some of these unexpected results are the reliability of the various techniques, the procedures for proper (reproducible) particle size analysis, and the graphical representation of the data that best shows the powder characteristics. The study has shown that gas atomized powder produced in the SiGMA facility has distinct size distribution characteristics that do not follow the log-normal pattern. The fragmentation mechanisms leading to droplet formation are examined which explain the SiGMA powder size distribution data. These powder analysis procedures are applicable to all inert gas atomized powder and could lead to a better understanding of the atomizing system's operative liquid disruption mechanisms.
National Inst. of Standards and Technology (IMSE), Gaithersburg, MD. Reactor Radiation Div.

Relationship of Hydrogen Site Occupancy to Diffusion Behavior in Crystalline and Amorphous Zr2PdHx.

Final rept.

R. C. Bowman, D. R. Torgeson, R. G. Barnes, A. J.

Maeland, and J. J. Rush. 1989, 6p

Pub. in Z. Phys. Chem. 163, n2 p425-430 1989.

Keywords: "Amorphous materials, "Zirconium alloys, "Palladium alloys, "Zirconium hydrides, "Diffusion, Neutron scattering, Neutron diffraction, Nuclear magnetic resonance, Relaxation time, Crystal structure, Surface chemistry, Protons, Reprints.

New measurements of the proton relaxation times have resulted in more thorough analyses of the diffusion properties in crystalline and amorphous $\mathrm{Zr} 2 \mathrm{PdH}$. The influences of host metal and hydrogen site occupancies were better recognized when additional information from the proton second moments, inelastic neutron scattering spectra, and published neutron diffraction data was included. Complex motion is indicated when multiple sites become occupied at the higher stoichiometries of the crystalline phases or in the glassy hydride.

\section{1,231}

PB91-189423

Not available NTIS

National Inst of Standards and Technology (IMSE), Gaithersburg, MD. Metallurgy Div.

Structural Study of Electrodeposited AluminumManganese Alloys.

Final rept.

B. Grushko, and G. Stafford. 1989, 9p

Pub. in Meta

Keywords: "Aluminum alloys, "Manganese additions, Electrodeposited transformations(Materials), Liquid metals, Compositions, FCC lattices, Substrates, Copper, Amorphous materials, Binary alloys, $\mathrm{X}$-ray diffraction, Electron microscopy, Aluminum chlorides, Solutes, Reprints, "Rapid solidification process, Icosahedral phase.

Al-Mn alloys with compositions ranging between 0-27 wt $\% \mathrm{Mn}$ were electrodeposited at $150 \mathrm{C}$ onto copper substrates from a chloroaluminate melt with controlled addition of $\mathrm{MnCl} 2$. The specimens were studied by SEM, TEM, EDAX and $x$-ray diffraction. The addition of small amounts of $\mathrm{Mn}$ results in the formation of a supersaturated $\mathrm{fcc}$ solid solution of $\mathrm{Mn}$ in Al. At the higher $\mathrm{Mn}$ content an amorphous phase is established. The highly facted crystalline surface of pure Al and Al$\mathrm{Mn}$ solid solution becomes a smooth nearly specular surface when the amorphous phase is present. There is a concentration discontinuity between the above limit and the higher $\mathrm{Mn}$ concentration limit of the fcc phase (about $9 w \%$ ). Appearance of the amorphous phase in the alloy results in a decrease in the Mn concentration in solid solution to about $2 \mathrm{wt} \%$. The crystallization of the amorphous phase starts at the fcc-amorphous phase interface at $230 \mathrm{C}$. As a result of treatment at 230-340C, the amorphous phase completely transforms into Al6Mn, while the fcc phase is unaffected. Prior to crystallization, the amorphous phase shows a modification that could be interpreted as the formation of fine-grain icosahedral phase. The formation of phases by electrodeposition and rapid solidification is discussed.

101,232

PB91-189696

Not available NTIS

National Inst. of Standards and Technology (IMSE)

Gaithersburg, MD. Metallurgy Div.

Surface Properties and Coatings of Materials Microtailored Alloys.

Final rept.

D. Lashmore. 1988, $7 p$

Pub. in Israel Jnl. of Technology 24, n3-4 p469-475 1988

Keywords: "Electrochemical coating, "Electrodeposition, "Process control, "Alloys, Compositions, Surface properties, Microstructure, X-ray diffraction, Coppe alloys, Cobalt alloys, Nickel alloys, Interfaces, Reprints.

Electrochemical deposition is becoming recognized as an important processing technique to produce alloys whose properties can be controlled on a near atomic level. In the paper alloy deposition is reviewed with particular emphasis on a deposition process incorpo- 


\title{
MATERIALS SCIENCES
}

\author{
Nonferrous Metals \& Alloys
}

rating a feedback system allowing a high degree of control over composition. Such a process can be used to produce unique microstructures which may be tailored on a near atomic level resulting in materials whose properties may be very finely tailored. Such a control has implication for the design of new alloys whose interfacial properties must be precisely controlled.

\section{1,233}

PB91-189985

Not available NTIS

National Inst. of Standards and Technology (MSEL), Boulder, CO. Fracture and Deformation Div.

Creep of Copper: 4 to $295 \mathrm{~K}$

Final rept.

R. P. Reed, N. J. Simon, and R. P. Walsh. 1990, 9p Sponsored by Department of Energy, Washington, DC Pub. in Advances in Cryogenic Engineering, v36 p1175-11831990.

Keywords: "Copper, "Creep properties, "Creep tests, Least squares method, Strains, Shear properties, Tensile properties, Steady state, Mechanical properties, Shear flow, Experimental data, Reprints.

Creep measurements at 295,76 and $4 \mathrm{~K}$ have been conducted on C10400 copper. Specimens were held under constant tensile load (dead weight) for periods of time sometimes exceeding one month. Creep data have been fitted to a series of expressions relating creep strain to time by a nonlinear least-squares procedure. The coefficients of the terms in the equations were related to applied stress levels. Primary, logarithmic, and steady-state creep ranges are discussed.

\section{1,234}

PB9 1-194852

Not available NTIS

National Inst. of Standards and Technology (MSEL), Boulder, CO. Fracture and Deformation Div.

Elastic Constants of a Tungsten-Particle CopperMatrix Composite.

Final rept.

H. Ledbetter, and S. Datta. 1991, 4p

Pub. in JSME International Jnl., Series I, v34 n2 p1941971991.

Keywords: "Elastic properties, "Metal matrix composites, "Particulate composites, "Copper, *Tungsten, UItrasonic tests, Young modulus, Shear strength, Bulk modulus, Mathematical models, Poisson ratio, Anisotropy, Reprints.

Using a megahertz-frequency ultrasonic method, a measurement of the elastic constants of a composite consisting of seventy-volume-percent tungsten particles in a copper matrix was done. The Young, shear, and bulk moduli and the Poisson ratio are reported Tungsten is much stiffer than copper: the Young-modulus ratio equals $410 / 129=3.2$. Thus, from a linear rule-of-mixture, large departures were found. (The Poisson ratio comes close to a linear rule-of-mixture.) Unexpectedly, a substantial elastic anisotropy was found: eighteen percent in the shear modulus. To model the system, a scattered-plane-wave ensembleaverage approach was used. For the four elastic constants listed above, reasonable model-measuremen agreement was found, one-to-seven percent for the averaged-over-direction measurements. Assuming oblatespheriodal $(\mathrm{c} / \mathrm{a}=0.5)$ particles improves the agreement: two percent or less for the four cases.

\section{1,235}

PB91-195537

Not available NTIS

MD. Metallurgy Div.

Coherency Strain Induced Instability of SolidLiquld Interfaces in the Mo-NI System.

Final rept.

W. H. Rhee, C. A. Handwerker, and D. N. Yoon

$1988,7 p$

Pub. in Proceedings of MRS Symposium on Interfacial Structure, Properties and Design, v122 p205-211 1988

Keywords: "Instability, "Liquid-solid interfaces, "Molybdenum alloys, "Nickel alloys, "Binary alloys, Time dependence, Equations, Microstructure, Diffusion, Grain boundaries, Migration length, Films, Grain growth, Liquid metals, Liquids, Reprints, Coherency strain.

An instability at solid-liquid interfaces induced by coherency strain has been studied in the Mo-Ni system. The coherency strain is developed in the diffusion zone by solute diffusion into the solid when the lattice parameter varies with composition. As a result of the strain, the originally planar solid-liquid interface becomes unstable by dissolution of the initial solid and reprecipitation of the new solid, forming a sinusoidal interface shape. In a previous study, the coherency strains have been shown to be the driving force for the solution and reprecipitation process of the instability. The paper analyzes the evolution of the instability with time, from initiation to healing of the instability. Using the equations developed for liquid film migration (LFM) the change of microstructure is explained qualitatively. In addition, the relationships between the instability and diffusion induced grain boundary migration (DIGM) and LFM are discussed.

101,236

$-195651$

Not available NTIS

National Inst. of Standards and Technology (IMSE), Gaithersburg, MD. Metallurgy Div.

Phase Selection in Non-Equilibrium Processing.

Final rept.

R. J. Schaefer. $1989,14 p$

Pub. in Proceedings of Indo-US Workshops on Solidification Principles and Materials Processing, Hyderabad, India, January 15-21, 1988, p381-394 1989.

Keywords: "Quenching(Cooling), "Phase diagrams, "Solidification, "Aluminum alloys, "Manganese alloys, Microstruction, Nucleation, Crystallization, Rates(Per time), Binary alloys, Reprints, *Rapid solidification process.

When an alloy is solidified rapidly, it is often thermodynamically possible for several different phases to form. Which of the phases dominates the final microstructure depends on the nucleation and growth kinetics of the several possible phases and also on the detailed thermal conditions of the specific solidification process. The effects are discussed with reference to the aluminum-manganese system, where the results of several types of experiments can be understood in terms of the strikingly different nucleation and growth behavior of several stable and metastable phases.

101,237

PB91-202945 Not available NTIS National Inst. of Standards and Technology, Gaithersburg, MD.

Uitrasonic Texture and Stress Measurements in Anisotropic Polycrystalline Aggregates.

Final rept.

P. P. Delsanto, A. B. Mignogna, and A. V. Clark.

$1990,10 p$

Sponsored by Office of Naval Research, Arlington, VA. Pub. in Jnl. of the Acoustical Society of America 87, n1 p215-224 Jan 90.

Keywords: "Acoustics, "Ultrasonic tests, "Metal plates, *Anisotropy, Residual stress, Texture, Reliability, Comparative evaluation, Methodology, Neutron diffraction, Strain gages, Polycrystals, Aggregates, Rayleigh waves, Time-of-flight method, Deformation, Homogeneity, Reprints.

A perturbative treatment of ultrasonic Rayleigh wave propagation is applied to investigate the case of an initally deformed metal plate, assumed to be homogeneous and to consist of an orthotropic distribution of cubic crystallites. Time-of-flight measurement techniques are proposed for the determination of both stress and texture. An analysis of these experimental results and a comparison with results of both ultrasonic bulk waves and neutron diffraction techniques confirm the reliability of this method. The special case of residual stresses is discussed in detail. Measurements of residual stresses with this technique agree with the results of strain gage measurements.

\section{1,238}

PB91-204156

Not available NTIS

National Inst. of Standards and Technology (IMSE), Gaithersburg, MD. Metallurgy Div.

Crystalline and Glassy Phases of Transition-Metal Metalloid Systems.

Final rept.

R. E. Watson, and L. H. Bennett. 1991, 11p

Sponsored by Department of Energy, Washington, DC. Pub. in Physical Review B 43, n14 p11 642-11 652, 15 May 91.

Keywords: "Metallic glasses, "Nickel alloys, "Iron alloys, "Metalloid, "Polymorphism, Phosphorus additions, Boron additions, Silicon additions, Carbon additions, Nitrogen additions, Crystal structure, Reprints.

Glasses formed in the Ni-P and Fe-B systems are known to be polymorphic. The crystalline phases in these systems display markedly different metalloid environments and a one-to-one relationship can be made between those environments and the glassy phase for Ni-P. It is the purpose of the present paper to explore the prospects for polymorphism in other transition-metal-rich metalloid glassy systems in which the metalloids are $\mathrm{P}, \mathrm{Si}, \mathrm{B}, \mathrm{C}$, and $\mathrm{N}$. The known crysta structures of the phosphides, silicides, carbides, $\mathrm{ni}$ trides, and borides have been inspected using radical Voronoi (Wigner-Seitz) -cell constructs to deduce metalloid-site nearest-neighbor arrangements. The occurence of differences, such as those obtained in Ni-P and $F e-B$, is taken as indicative of possible polymorphism in the glasses. A list of such glasses has been constructed. It is seen that polymorphism is most likely to occur in the phosphides and silicides, and very un likely in the nitrides.

\section{1,239}

PB91-236760

Not available NTIS National Bureau of Standards (IMSE), Gaithersburg MD. Metallurgy Div.

Microstructural Control Through Diffusion-Induced Grain Boundary Migration.

Final rept.

C. A. Handwerker, and J. W. Cahn. 1988, 7p

Pub. in Materials Research Society Symposium Proceedings, v106 p127-133 1988

Keywords: "Microstructure, *Grain boundaries, *Diffusion, "Metals, "Ceramics, Migration length, Vapor deposition, Low temperature, Silicon, Process control, Polycrystals, Compositions, Reprints.

Diffusion-induced grain boundary migration (DIGM) is a common, but only recently discovered low tempera ture phenomenon that results in high rates of both chemical mixing (or unmixing) and grain boundary migration. DIGM is found in many situations where chemical heterogeneities lead to diffusion. For example DIGM is observed during diffusion and compound formation in polycrystalline multilayer contact systems produced by low temperature deposition techniques. The diffusional mixing along the moving grain bounda$r y$ is high, localized, and results in a distinctive composition profile behind the moving interface. Theory has indicated, and experiments have confirmed, which conditions lead to DIGM and which conditions suppress it. The microstructural changes can result in either a grain refinement as seen in many metallic systems or in enhanced grain growth as seen in polysilicon. In either case these microstructural and compositional changes are controllable in a way that may allow fabrication of unique devices.

101,240

PB92-108992

PC A04/MF AO

National Inst. of Standards and Technology (CAML), Gaithersburg, MD.

Phase-Field Model for Isothermal Phase Transitions in Binary Alloys.

A. A. Wheeler, W. J. Boettinger, and G. B.

McFadden. Aug 91, 52p NISTIR-4662

Keywords: "Binary alloys, "Phase transformations, Copper nickel alloys, One-dimensional calculations, Two-dimensional calculations, Asymptotic methods, Interfaces, Separation.

The authors describe a new phase-field model, which models isothermal phase transitions between ideal binary alloy solution phases. Equations are developed for the temporal and spatial variation of the phase field, which describes the identity of the phase, and for the composition. The authors conduct an asymptotic analysis as the gradient energy coefficient of the phase field becomes small. From their analysis they show that their model recovers classical sharp inter face models of this situation when the interfacial layers are thin, and they show how to relate the parameters appearing in the phase-field model to material and growth parameters in real systems. Further, the authors identify three stages of temporal evolution; the first corresponding to interfacial genesis which occurs very rapidly, the second to interfacial motion controlled by the local energy difference across the interface and diffusion; the last taking place on a long time scale in which curvature effects are important, and corresponds to Ostwald ripening. The authors also present results of numerical calculations. 
National Inst. of Standards and Technology (MSEL), Gaithersburg, MD. Metallurgy Div. MIcrostructural Studies of TI-AI Alloys In the VIcinlty of the 'Eutectoid' Reaction.

Final rept.

S. A. Jones, R. D. Shull, A. J. McAlister, and M. J. Kaufman. $1988,6 p$ Pub. in Scripta Metallurgica 22, n8 p1235-1240 Aug
88.

Keywords: "Binary alloys, "Titanium alloys, "Aluminum alloys, Phase transformations, Phase diagrams, Intermetallic compounds. Thermal analysis, Electron microscopy, High temperature tests, Eutectoids, Compositions, Reprints.

The Ti-Al phase diagram in the composition range between 35 and 50 atomic percent Al has been studied by means of optical and transmission electron microscopy and differential thermal analysis. The observations indicate inconsistencies with the alpha to alpha(2) + gamma eutectoid interpretation of the phase equilibria near $1100 \mathrm{C}$ at 45 at. \% Al and suggest the low temperature 'eutectoid-like' structure may be produced by the decomposition of the high temperature alpha phase via a cellular reaction: alpha (solid solution) to alpha + gamma.

\section{Plastics}

\section{1,242}

PB91-148247

Not available NTIS National Inst. of Standards and Technology (MSEL), Gaithersburg, MD. Polymers Div.

Multistep Stress-Relaxation Behavior in Uniaxial Extension of an Ethylene-Hexene Copolymer. Final rept.

L. J. Zapas, and J. M. Crissman. 1990, 13p

Pub. in Jnl. of Rheology 34, n1 p1-13 Jan 90

Keywords: "Polyethylene, "Stress relaxation, "Ethylene copolymers, "Hexenes, Viscoelasticity, Performance prediction, Constitutive equations, Reprints.

For crystallizable polymers such as polyethylene it is found that the mechanical response in a stress relaxation experiment is nonlinear, even at quite small deformations, and the behavior for some multistep stress relaxation strain histories cannot be described quantitatively by the BKZ theory. A better description of the behavior is obtained using a new constitutive equation proposed by Zapas. The predictions of both the BKZ theory and the new description are shown for a series of single and multistep stress-relaxation experiments done in uniaxial extension on an ethylene-hexene copolymer. The results of the calculations using the new description are in good agreement with experiment.

\section{1,243}

\section{PB91-150086}

Not available NTIS

National Inst. of Standards and Technology (NML), Boulder, $\mathrm{CO}$. Chemical Engineering Science Div.

Biodegradable Plastics: An Idea Whose Time Has Come.

Final rept.

J. D. Evans, and S. K. Sikdar. 1990, $5 p$

Pub. in CHEMTECH 20, p38-42 Jan 90.

Keywords: "Packaging materials, "Plastics, "Biodeterioration, "Waste disposal, Enzymes, Photochemical reactions, Valeric acid, Polymers, Earth fills, Butyric acid, Reprints.

Because of severe environmental impacts of the use of plastics in recent times, regulatory and government authorities in many countries are beginning to outlaw certain plastic products, especially in packaging. Biodegradable plastics have emerged lately to redress the problems. In the article the progress made in this endeavor is reviewed and a few commercial successes are highlighted.

101,244

PB91-189712

Not available NTIS

National Bureau of Standards (IMSE), Gaithersburg, MD. Polymers Div.

Effect of Crosslink Density on Physical Aging of Epoxy Networks.

Final rept.

A. Lee, and G. B. McKenna. 1988, $6 p$

Pub. in Polymer 29, n10 p1812-1817 Oct 88
Keywords: "Networks, "Crosslinking, "Aging tests(Materials), "Epoxy resins, Temperature dependence, Density(Mass/volume), Polyoxypropylene, Stress relaxation, Glass, Transition temperature, Specific heat, Calorimetry, Superposition(Mathematics), Thermodynamic equilibrium, Quenching(Cooling), Reprints, DGEBA.

Physical aging of different crosslink density polypropylene oxide/DGEBA networks were investigated using the small-strain stress relaxation technique in simple extension. The effects of crosslink density on the glass transition temperature, $\mathrm{Tg}$, and the change in specific heat at $\mathrm{Tg}$ were measured using a scanning calorimeter in heating. Although an increase in $\mathrm{Tg}$ was observed as crosslink density increased, contrary to other studies of crosslinked polymers, the specific heat did not change as the crosslink density changed. Aging was studied at several values of temperature below $\mathrm{Tg}$ after quenching from above $\mathrm{Tg}$. The stress relaxation curves at different aging times, temperatures and crosslink densities were able to superimposed to form a single master curve, demonstrating the applicability of a time-aging time-temperaturecrosslink density superposition principle to this type of network. Under all aging conditions, the double logarithmic shift rate was found to decrease with increasing crosslink density while being independent of temperature for a given network. Furthermore, at temperatures of $10 \mathrm{C}$ and $5 \mathrm{C}$ below $\mathrm{Tg}$, the network glasses were aged into structural equilibrium.

\section{1,245}

PB91-189720

Not available NTIS

National Inst of Standards and Technology (IMSE), Gaithersburg, MD. Polymers Div.

Viscoelastic Response of Epoxy Glasses Subjected to Different Thermal Treatments.

Final rept.

A. Lee, and G. B. McKenna. 1989, 4p

Pub. in Proceedings of ANTEC 89 Annual Technical Conference of Society of Plastics Engineers (47th), New York, NY., May 1-4, 1989, p1170-1173.

Keywords: "Epoxy resins, "Crosslinking, "Networks, "Viscoelasticity, Thermodynamic equilibrium, Heat treatment, Glass, Transition temperature, Polymers, Aging tests(Materials), Quenching(Cooling), Stress relaxation, Time dependence, Temperature dependence.
Density(Mass/volume) Superposition(Mathematics), Deformation, Reprints.
Sensity(Mass/volume

Physical aging studies were made using model epoxy network glasses. Linear and nonlinear viscoelastic responses were measured after quenching the glass from above $\mathrm{Tg}$ to the temperature of interest. In the linear viscoelastic regime, the authors studied the effects of temperature and crosslink density on physical aging. The stress relaxation curves at different aging times, temperatures and crosslink densities were able to be superimposed to form a single master curve, demonstrating the validity of a time-aging time - temperature - crosslink density superposition principle for this type of epoxy network. Furthermore, at temperature of $10 \mathrm{C}$ and $5 \mathrm{C}$ below $\mathrm{Tg}$, the network glasses were aged into structural equilibrium, thus obtaining t star, the time required to reach structural equilibrium. Results in the nonlinear viscoelastic regime showed that $t$ star did not change with the applied stress; therefore, aging is not 'erased' by large mechanical stimuli. These results support the argument that the volume recovery which occurs during aging impact the small deformation response differently than it does the large deformation response.

\section{1,246}

PB91-189829

Not available NTIS

National Bureau of Standards (IMSE), Gaithersburg, MD. Polymers Div.

Physical Aging and the Viscoelastic Response of Network Glasses.

Final rept.

G. B. McKenna, and A. Lee. $1988,4 p$

Pub. in Proceedings of the International Conference on Deformation, Yield and Fracture of Polymers ( 7 th) Cambridge, UK., April 11-14, 1988, p2/1-2/4.

Keywords: "Epoxy resins, "Networks, "Aging tests(Materials), Superposition(Mathematics), Deformation, Viscoelasticity, Glass, Transition temperature, Stress tests, Time dependence, Quenching(Cooling), Crosslinking, Reprints.

Physical aging studies were made using a model epoxy network. Linear and nonlinear viscoelastic responses were measured after quenching the glass from above $\mathrm{Tg}$ to temperatures near $\mathrm{Tg}$. Results are presented which show that aging is not 'erased' by large mechanical deformations rather the time required for the glass to age into equilibrium is independent of the applied stress.

\section{1,247}

PB91-236612

Not available NTIS

National Inst. of Standards and Technology (MSEL), Gaithersburg, MD. Polymers Div.

Long Time Creep and LlfetIme Behavior in Uniaxia Extension of a Linear Low Density Polyethylene. Final rept.

J. M. Crissman. 1991, 7p

Pub. in Polymer Engineering and Science 31, n8 p541. 547 Apr 91.

Keywords: "Polymers, "Plastics, "Polyethylene, Time dependence, "Creep tests, Density, Predictions, Services life, Stress tests. Temperature dependence, Graphic methods, Reprints.

The paper describes uniaxial creep measurements done on a linear low density polyethylene over a wide that the creep behavior overall is quite complex and that the one quantity which can best be used to predict useful lifetime of this material is the time at which necking is observed. The time to neck data has been used to generate a composite curve which can serve as an upper bound to lifetime prediction.

\section{Wood \& Paper Products}

101,248

DE91007688

PC A05/MF A01

National Inst. of Standards and Technology (NEL) Gaithersburg, MD. Center for Chemical Engineering. Advanced sensor development program for the pulp and paper Industry. Final report.

Progress rept.

J. D. Allen, S. R. Charagundla, A. Macek, H. G Semeriian, and J. R. Whetstone. Oct 90,100p DOE/ CE/40748-T1

Contract Al01-85CE40748

Sponsored by Department of Energy, Washington, DC.

Keywords: Emission Spectra, Flames, "Paper Industry, "Sodium, Absorption, Boilers, "Combustion Control, "Emission Spectroscopy, Hydroxyl Radicals, Mathematical Models, Measuring Instruments, Potassium, Probes, Progress Report, Radicals, Remote Sensing, Research Programs, Spent Liquors, Temperature Measurement, Windows, EDB/320300, EDB/440500, "Air pollution control equipment.

This report describes experimental and theoretical studies toward development of a remote sensing technique for non-intrusive temperature measurement based on optical spectroscopic analysis of recovery boiler. The overall objectives were (a) construction of a fiber-optic system for measurement of spectroscopic emission intensities at several wavelengths and (b) development of a computer program relating these intensities to temperatures of the emitting species. The emitting species for temperature measurements in flames can be either naturally occurring free radicals $(\mathrm{OH}, \mathrm{CH}, \mathrm{C}($ sub 2)) or atoms which, in turn, can be either naturally occurring or seeded into flames. Sodium atoms, the obvious emitters in recovery boilers, are not promising as thermometric species because of their high concentration. At high concentrations, strong self-absorption results cause optical depths to be much smaller than the sampling depths desired for recovery boilers. An experimental program was, therefore, undertaken with the objective of identification and spectroscopic detection and measurement of other naturally occurring thermometric species. The program consisted of several laboratory studies and four field trips to different recovery boilers. 19 refs., 43 figs., 8 tabs.

\section{General}




\section{General}

National Bureau of Standards (NML), Gaithersburg, MD. Office of Standard Reference Data.

Factual Materials Databanks - The Need for Standards.

Final rept.

H. Krockel, K. Reynard, and J. Rumble. 1987, 60p

Pub. in VAMAS (Versailles Project on Advanced Materials and Standards) Report, pi-51 Jul 87.

Keywords: "Materials, "Data bases, "Standards, Information systems, Data analysis, Access, Reprints, $\mathrm{Na}$ tional Institute of Standards and Technology.

The VAMAS Task Group on Factual Materials Databanks has identified the areas where standards development would significantly and positively impact the building and dissemination of materials databanks. Materials databanks are becoming substantial elements of the computerized flow of information on materials properties from the generation of the information to its use. Their features and operational conditions will therefore be influenced by all standards related to any phase of the information flow. Accordingly, the review includes the aspects of data generation data anlysis, data presentation, access to data, and use of data; and the report presents sets of recommendations under four headings: Basic considerations for handling data; Material data generation and reporting; Materials databanks; and Access to materials data.

\section{1,250}

PB91-148049

Not available NTIS

National Inst. of Standards and Technology (NML), Boulder, $\mathrm{CO}$. Chemical Engineering Science Div. Apparatus for Measurement of Coefficient of Friction.

A. J. Slifka, J. D. Siegwarth, L. L. Sparks, and D. K. Chaudhuri. $1990,7 p$

Sponsored by National Aeronautics and Space Administration, Huntsville, AL. George C. Marshall Space Flight Center.

Pub. in Advances in Cryogenic Engineering (Materials) 36, p1119-1125 1990 .

Keywords: "Coefficient of friction, "Friction measurement, Controlled atmospheres, Cryogenics, Steels, Reprints.

An apparatus has been built at the National Institute of Standards and Technology in Boulder, Colorado to measure the coefficient of friction in certain controlled atmospheres. The machine uses either of two configurations of specimens. A cone-on-cone configuration can be loaded to produce a contact stress of $137 \mathrm{MPa}$ (20 ksi) and surface velocities from $0.03 \mathrm{~m} / \mathrm{s}$ to $1.2 \mathrm{~m} /$ s. A ball-on-flat configuration can be loaded to produce a Hertzian contact stress in excess of $1830 \mathrm{MPa}$ (267 $\mathrm{ksi}$ ) and surface velocities from $0.06 \mathrm{~m} / \mathrm{s}$ to $2.0 \mathrm{~m} / \mathrm{s}$. The designed temperature-range of operation is 80 to $1030 \mathrm{~K}$. The machine is described and some test results are presented.

\section{1,251}

PB91-204040

Not available NTIS

National Inst. of Standards and Technology (IMSE), Gaithersburg, MD.

International Collaboration in Prestandards Research on Advanced Materials within the Context of VAMAS.

Final rept.

L.H. Schwartz and B. W. Steiner. $19896 \mathrm{p}$ Pub. in ASTM (American Society for Testing and Materials) Standardization News 17, n2 p28-33 Feb 89.

Keywords: "Materials tests, "Data bases, "Standards, "Research projects, "International cooperation, Surface chemistry. Chemical analysis, Characterization, Ceramics, Polymer matrix composites, Methodology, Cryogenics, Superconductors, Wear, Corrosion, Weldments, Creep tests, Fracture mechanics, Fatigue, Reprints, "Vamas(Versailles Project on Advanced Materials and Standards).

VAMAS, the Versailles Project on Advanced Materials and Standards, now encompasses international collaboration in thirteen areas of prestandards research on advanced materials. One of the most successful projects has stimulated the development of a consensus on high priority action essential for the development of compatible computerized materials databanks. Another has established a framework for a coherent international research effort laying the foundation for standards in surface chemical analysis. Six projects address critical problem areas in the characterization of particular classes of advanced materials: ceramics, polymer blends, polymer composites, efficient test procedures for polymer properties, superconducting and cryogenic structural materials, and bioengineering materials. The other five projects dea with characterization of the response of advanced materials to particularly challenging performance situations: wear, hot salt corrosion resistance, weld characteristics, creep crack growth, and low cycle fatigue Recent results of VAMAS activity in these various areas are surveyed and directions for the future are indicated.

\section{1,252}

PB92-126424

PC A05/MF AO

National Inst. of Standards and Technology (MSEL), Gaithersburg, MD.

Materials Reliability. Technical Activities, 1991. (NAS-NRC Assessment Panel, February 13-14, 1992).

H. I. McHenry. 1992, 95p NISTIR-4695

See also PB91-143339.

Keywords: "Materials, "Research projects, Quality, Reliability, Safety, Process control, Nondestructive tests, Standards, Cryogenics, Automation, Fracture mechanics, Metals, Microstructure, Physical properties, Ceramics, Superconductors, Advanced materials, Intelligent welding, Acoustic microscopy.

The report describes the 1991 fiscal-year programs of the Materials Reliability Division of the Materials Sci ence and Engineering Laboratory. It summarizes the principal accomplishments in three general research areas: materials performance, properties, and processing. The Fracture Mechanics, Fracture Physics, Nondestructive Evaluation, and Composite Materials Groups work together to detect damage in metals and composite materials and to assess the significance of the damage with respect to service performance. The Cryogenic Materials and Physical Properties Groupsin vestigate the behavior of materials at low temperature and measure and model the physical properties of advanced materials, including composites, ceramics, and the new high-critical-temperature superconductors. The Welding and Thermomechanical Processing Groups investigate the nonequilibrium metallurgical changes that occur during processing and affect the quality, microstructure, properties, and performance of metals.

\section{1,253}

\section{PB92-126606}

PC A15/MF A03

National Inst of Standards and Technology (MSEL) Gaithersburg, MD.

National Educators Workshop: Update '90. Standard Experiments in Engineering Materials Science and Technology.

Final rept.

J. S. Harris, and J. A. Jacobs. Nov 91, 341p NIST/ SP-822

Also available from Supt. of Docs as SN003-003 03124-1. See also N90-24350 and N91-20207. Pro ceedings of a Workshop held in Gaithersburg, MD. November $13-15,1990$. Prepared in cooperation with Norfolk State Univ., VA., and National Aeronautics and Space Administration, Washington, DC. Sponsored by ASM International, Detroit, MI., and Battelle Pacific Northwest Labs., Richland, WA.

Keywords: "Education, "Materials tests, "Fracture(Mechanics), "Structural properties, Standards, Microstructure, Steels, Metals, Strain rate, Fiber reinforced composites, Ceramics, Tensile properties Metal working, Thermoplastics, Biodegradation, Universities, Experimentation.

The report includes proceedings of a workshop sponsored jointly by NIST and the Sichools of Technolog and Science, Norfolk State University, Norfolk, Virginia, and held in Gaithersburg, Maryland, November 13 15, 1990. The workshop theme was strengthening materials education. Material in the publication can serve as a valuable guide to faculty who are interested in useful experiments for their students. There was blend of experiments on new materials and traditional materials. Uses of computers in MS\&E, experuimental design and an approach to systematic materials are among the topics presented.
MATHEMATICAL SCIENCES

\section{Algebra, Analysis, Geometry, \& Mathematical Logic}

\section{1,254}

PB91-147850 Nationdards and Technology (NEL), Gaithersburg, MD. Scientific Computing Div.

Computing the Eigenvalues and Eigenvectors of Symmetric Arrowhead Matrices.

Final rept.

D. P. O'Leary, and G. W. Stewart. 1990, 9p

Pub. in Jnl. of Computational Physics 90, n2 p497-505 Oct 90 .

Keywords: "Sparse matrix, "Matrices, Fermi liauids, Eigenvectors, Eigenvalues, Radiationless decay, Computation, Algorithms, Reprints.

The paper treats the eigenvalue problem for a symmetric matrix which is zero except for its main diagonal and one row and column. Such problems arise in the description of radiationless transitions in isolated molecules and of oscillators vibrationally coupled with a Fermi liquid. In these applications, the order of the matrix A can be in the thousands. The purpose of the paper is to present formulas and efficient algorithms for computing eigenvalues and eigenvectors of such matrices.

\section{1,255}

PB91-148742

Not available NTIS

National Inst. of Standards and Technology (NML), Boulder, CO. Quantum Physics Div.

Asymptotic Shooting Method for the Solution of Differential Equations.

Final rept.

A. Holubec, A. D. Stauffer, P. Acacia, and J. A.

Stauffer. 1990, 15p

Pub. in Jnl. of Physics A: Mathematical and General 23, p4081-4095 1990 .

Keywords: "Differential equations, Numerical integration, Analytic continuation, Asymptotic methods, Series(Mathematics), Polynomials, Eigenvalues, Reprints.

The authors present a method for the solution of ordinary differential equations over a semi-infinite interval including the determination of eigenvalues, which is in principle capable of arbitrary accuracy. The solution is expressed in terms of finite polynomials, rendering the integration or determination of zeros of such solutions a straightforward matter. The authors have applied the method to a number of examples including the determination of the eigenvalues of the screened Coulomb potential and the three-dimensional quartic oscillator.

\section{1,256}

PB91-148759 Not available NTIS National Inst. of Standards and Technology (NML), Gaithersburg, MD. Ionizing Radiation Div.

Certain Theorems on Bilateral Generating Functions Involving Hermite, Laguerre, and Gegenbauer Polynomials.

Final rept.

J. H. Hubbell, and H. M. Srivastava. 1990, 11p

Pub. in Jnl. of Mathematical Analysis and Applications 152, p343-353 1990.

Keywords: *Generating functions, Hermite polynomials, Laguerre polynomials, Bessel functions, Gamma transport theory, Theorems, Reprints, Gegenbauer polynomials, Konhauser polynomials.

The object of the paper is to present simpler proofs of the various generalizations of some interesting results on bilateral generating functions which were derived recently by group-theoretic methods. It is also shown how one of our main theorems on generating functions would apply not only to the Bessel function but indeed also to the Konhauser biorthogonal polynomials whose special case when $s=2$ was encountered in 
certain analytical calculations involving the penetration of gamma rays through matter.

101,257

PB9́1-159046

Not available NTIS

National Bureau of Standards (NEL), Gaithersburg,

MD. Mathematical Analysis Div.

SImplified Error Bounds for Newton's Rule.

Final rept.

F. W. J. Olver. 1986, 7p

Pub. in IMA Jnl. of Numerical Analysis 6, n3 p373-379 1986.

Keywords: "Iterative methods, "Newton method, Roots of equations, Error analysis, Analytic functions, Approximation, Precision, Reprints, Interval analysis.

A posteriori error bounds are given for approximations to zeros of a function f obtained by Newton's rule. Here $f$ is a differentiable function of a real variable, or an analytic function of a complex variable. The bounds are somewhat simpler and sharper than existing results. Numerical examples are included.

101,258

PB91-175281

Not available NTIS

National Bureau of Standards (NEL), Gaithersburg,

MD. Mathematical Analysis Div.

Some Algorithms for Approximating Convolutions. Final rept.

D. P. O'Leary. $1988,13 p$

Pub. in Computer Vision, Graphics, and Image Processing 41, n3 p333-345 Mar 88.

Keywords: "Image processing, "Convolution, Parallel processing, Factorization, Polynomials, Algorithms, Reprints, Singular value decomposition

The paper presents some algorithms for approximating two-dimensional convolution operators of size $n \times$ $n$, $n$ odd, by a product, or sum of products, of $3 \times 3$ convolutions. Inaccuracies resulting from the approximation as well as from fixed point computation are discussed, and examples are given.

\section{1,259}

\section{PB91-187245}

Not available NTIS

National Inst. of Standards and Technology (NEL), Gaithersburg.

Algorithms for Special Tridiagonal Systems.

Final rept.

R. F. Boisvert. 1991, 20p

Pub. in SIAM (Society for Industrial and Applied Mathematics) Jnl. on Scientific and Statistical Computing 12, n2 p423-442 Mar 91

Keywords: "Matrices, Elliptic differential equations, Poisson equation, Fourier analysis, Factorization, Algorithms, Reprints, "Tridiagonal matrices.

Algorithms for the solution of symmetric diagonally dominant tridiagonal systems of linear equations with constant diagonals are considered. Such systems occur, for example, when solving certain constant-coefficient elliptic partial differential equations by the Fourier method. In particular, the specialized LU factorization method of Malcolm and Palmer (SpLU), the cyclic reduction method of Hockney (CR), and the reversed triangular factorization method of Evans (RTF) are considered. An interesting property of the first two algorithms is that they may be terminated early for highly diagonally dominant systems. A new implementation of RTF that also has the property is presented significantly reducing its operation count in many cases. The slightly perturbed systems that arise from problems with Neumann or periodic boundary conditions are also considered, with extensions given for SpLU to the periodic case and RTF to the Neumann case. Floating-point operation counts are given for each method, and the results of experiments on a single scalar processor are reported.

101,260

PB91-187799

PC A03/MF A01

National Inst. of Standards and Technology (NEL), Gaithersburg, MD. Applied and Computational Mathematics Div.

Convergence Properties of a Class of Rank-Two Updates.

P. T. Boggs, and J. W. Tolle. Apr $91,49 p$ NISTIR4554

Contracts AFOSR-ISSA-90-0004, AFOSR-88-0267

Prepared in cooperation with North Carolina Univ. at

Chapel Hill. Sponsored by Air Force Office of Scientific

Research, Bolling AFB, DC.
Keywords: "Numerical analysis, Mathematical programming, Sequences(Mathematics), Newton method, Approximation, Iteration, Convergence, Matrices, Theorems, "Foreign technology, Unconstrained optimization, Constrained optimization.

Many optimization algorithms generate, at each iteration, a pair $x$ sub $\mathrm{k}, \mathrm{H}$ sub $\mathrm{k}$ consisting of an approximation to the solution, $x$ sub $k$ and a Hessian matrix approximation, $\mathrm{H}$ sub $\mathrm{k}$, which contains local secondorder information about the problem. Much is known about the convergence of the $x$ sub $k$ to the solution of the problem but relatively little about the behavior of the sequence of matrix approximations. The authors analyze the sequence $H$ sub $k$ generated by the extended Broyden class of updating schemes independ ently of the optimization setting in which they are used, deriving various conditions under which convergence is assured and delineating the structure of the limits. Rates of convergence are also obtained. The results extend and clarify those already in the literature.

101,261

PB91-203497

Not available NTIS

National Inst. of Standards and Technology (NML), Gaithersburg, MD.

Nature of Collinearity.

Final rept.

J. Mandel. 1989, 9p

Pub. in Jnl. of Quality Technology 21, n4 p268-276 989.

Keywords: "Collinearity, Regression analysis, Quality control, Reprints, Multiple linear regression, Effective prediction domain, Singular value decomposition.

Collinearity is treated as an indication that use of the regression equation for prediction is limited to a specific portion of the sample space regressors. A method is presented for determination of this portion, called the Effective Prediction Domain (EPD). The same basic technique is also used for the detection of collinearity. Numerical examples are discussed in detail.

101,262

PB91-216754

(Order as PB91-216705, PC A07/MF A01) National Inst. of Standards and Technology, Gaithersburg, MD.

Root Projection of One-Sided Time Series

J. A. Simmons. $1991,11 \mathrm{p}$

Included in Jnl. of Research of the National Institute of Standards and Technology, v96 n3 p333-343 May/Jun 91

Keywords: "Time series analysis, "Signal processing, Least squares method, Laplace transformation, Complex variables, Prony method, Gram-Schmidt algorithm, Root projection, Deconvolution.

Until recently it has been impossible to accurately determine the roots of polynomials of high degree, even for polynomials derived from the $Z$ transform of time series where the dynamic range of the coefficients is generally less than $100 \mathrm{~dB}$. In a companion paper, two new programs for solving such polynomials were discussed and applied to signature analysis of one-sided time series. The author presents here another technique, that of root projection (RP), together with a Gram-Schmidt method for implementing it on vectors of large dimension. This technique uses the roots of the $Z$ transform of a one-sided time series to construct a weighted least squares modification of the time series whose $Z$ transform has an appropriately modifed root distribution. Such a modification can be employed in a manner which is very useful for filtering and deconvolution applications. Examples given here include the use of boundary root projection for front end noise reduction, and a generalization of Prony's method.

\section{1,263
PB91-216762}

(Order as PB91-216705, PC A07/MF A01)

National Inst. of Standards and Technology, Gaithersburg, MD.

Deconvolution of Acoustic Emission and Other Causal Time Series.

J. A. Simmons. 1991, 25p

Included in Jnl. of Research of the National Institute of Standards and Technology, v96 n3 p345-369 May/Jun 91.

Keywords: "Time series analysis, "Signal processing, *Acoustic emissions, Fast Fourier transforms, One dimensional, Transducers, Calibration, Comparison, Algorithms, Graphs(Charts).
A new technique, root projection (RP), is given for quantitative deconvolution of causal time series in the presence of moderate amounts of noise. Deconvolu. tion is treated as a well-conditioned but underdetermined problem and a priori information is employed to obtain comparable noise reduction to that achieved by singular value decomposition (SVD) techniques while providing more accurate frequency information about the inverse. Two detailed examples are given. The first gives noise analysis for alternate methods for deconvolution with a Gaussian kernel. The second example presents a model acoustic emission transducer calibration problem with typical noisy and incomplete output data. This example is treated by the use of a robust cross-cutting algorithm combining both the RP and SVD methods.

\section{1,264}

PB92-112408

PC A09/MF A02

National Inst of Standards and Technology (CAML), Gaithersburg, MD.

NBS-INA. The Institute for Numerical Analysis. UCLA 1947-1954.

Special pub. (Final).

M. R. Hestenes, and J. Todd. Aug 91, 185p NIST/ SP-730

Also available from Supt. of Docs. as SN003-00303094-5. Prepared in cooperation with California Univ. Los Angeles. Dept. of Mathematics, and California Inst. of Tech., Pasadena. Dept. of Mathematics. Sponsored by Mathematical Association of America, Washington, DC.

Keywords: Differential equations, Numerical integration, Numerical analysis, Digital computers, Computer applications, Linear programming. Historical aspects, Matrices, US NBS, "Institute for Numerical Analysis, UCLA.

The report is a history of the Institute for Numerical Analysis (INA) with special emphasis on its research program during the period 1947 to 1956 . The Institute for Numerical Analysis was located on the campus of the University of California, Los Angeles. It was a section of the National Applied Mathematics Laboratories, which formed the Applied Mathematics Division of the National Bureau of Standards (now the National Institute of Standards and Technology), under the U.S. Department of Commerce. The history of the program at INA is concerned primarily with the development of mathematics pertinent to solving numerical computations. The development could happen only if some mathematicians were proficient in handling the electronic digital computers. To insure that there would be some, INA was constituted. It was well funded, and could attract first class mathematicians to take a year off for research at INA. They were in the midst of people solving problems of considerable difficulty using digital computers. They were thus enticed into using them. When this happened, many important developments emerged. The history is centered around these people and discusses who they were, what their interests were, and what they did.

\section{1,265}

PB92-123108

PC A03/MF A01

National Inst. of Standards and Technology (CAML), Gaithersburg, MD. Applied and Computational Mathematics Div.

Computing Delaunay Triangulations for CometShaped Polygons.

J. Bernal. Nov $91,19 p$ NISTIR-4716

See also PB89-129480.

Keywords: "Computational geometry, Computation, Polygons, Algorithms, "Delauney triangulations, Computational complexity, Voronoi diagrams.

The author presents two triangulation algorithms which combined produce an algorithm for computing Delaunay triangulations for comet-shaped polygons. The first algorithm constructs in linear time a triangulation for a comet-shaped polygon. The second algorithm constructs a Delaunay triangulation for a polygon from any triangulation for the polygon. The algorithms can be used for deleting vertices in a Delaunay triangulation and for computing constrained Delaunay triangulations. 


\section{Operations Research}

101,266

PB91-148775

Not available NTIS

National Bureau of Standards (NEL), Gaithersburg,

Seconter for Applied Mathematics.

Second Order Sensitivity Analysis in Factorable Programming: Theory and Applications.

Final rept.

R. H. F. Jackson, and G. P. McCormick. 1988, 273p Pub. in Mathematical Programming 41, n1 p1-273 May 88.

Keywords: "Mathematical programming, "Optimization, Nonlinear programming, Tensors, Reprints, Second-order sensitivity analysis.

Second-order sensitivity analysis methods are developed for analyzing the behavior of a local solution to a constrained nonlinear optimization problem when the problem functions are perturbed slightly. Specifically, formulas involving third-order tensors are given to compute second derivatives of components of the local solution with respect to the problem parameters. When in addition, the problem functions are factorable it is shown that the resulting tensors are polyadic in nature.

\section{1,267}

PB91-174540

Not available NTIS

National Bureau of Standards (NEL), Gaithersburg, MD. Mathematical Analysis Div.

Applications of Operations Research Techniques to System Design and Evaluation.

Final rept.

R. E. Chapman. 1986, 2p

Pub. in Proceedings of Symposium on ASME (American Society of Mechanical Engineers) Codes and Recent Advances in PVP and Valve Technology, Chicago, IL., July 20-24, 1986, p139-140.

Keywords: "Systems design, "Systems analysis, *Operations research, Systems engineering, Economic analysis, Mathematical programming, Monte Carlo methods, Simulation, Mathematical models, Reprints.

The paper illustrates how several operations research techniques can be used to facilitate the system design/evaluation process. Two classes of techniques, mathematical programming and Monte Carlo simulation, are used to demonstrate how cooperative efforts between engineering professionals and operations research analysts can promote more informed decision making. The paper concludes with a discus sion of the role of standardized practices for performing economic evaluations of competing system designs.

\section{1,268}

PB91-174896

Not available NTIS National Bureau of Standards (NEL), Gaithersburg, MD. Operations Research Div.

State-of-the-Art of Computational Testing of Mathematical Programming Algorithms.

Final rept.

R. H. F. Jackson. $1985,6 p$

Pub. in Mathematical Programming Society Committee on Algorithms Newsletter, n12 p8-13 May 85

Keywords: "Mathematical programming, Algorithms, Tests, Computer program verification, Reprints, "Computational testing.

The article summarizes some of the philosophical aspects on computational testing, including some historical background.

\section{1,269}

PB91-187815 PC A03/MF A01

National Inst. of Standards and Technology (NEL), Gaithersburg, MD. Applied and Computational Mathematics Div.

Interior-Point Method for Linear and Quadratic Programming Problems.

P. T. Boggs, P. D. Domich, J. E. Rogers, and C. Witzgall. Apr 91, 13p NISTIR-4556

Keywords: "Linear programming, "Ouadratic programming, Mathematical programming, Algorithms, Interior point methods.

The authors have been working on a particular class of interior point methods for solving linear programming problems for several years. The methods combine several search directions that are readily computed at each iteration. The final step is then calculated by computing the step that solves the original problem re stricted to the subspace spanned by these several directions. In the paper, they propose an extension of these ideas to the case of convex quadratic programming

\section{1,270}

\section{PB91-204198}

Not available NTIS

National Inst. of Standards and Technology (NEL) Gaithersburg, MD. Applied and Computational Mathematics Div.

Convergence Behavior of Trajectories for Linear Programming.

Final rept.

C. Witzgall, P. T. Boggs, and P. D. Domich. 1990 $27 p$

Contract ONA-N-0014-87-F0053

Sponsored by Office of Naval Research, Arlington, VA Pub. in Contemporary Mathematics 114, p161-187 1990.

Keywords: "Linear programming, *Trajectories, "Convergence, Linear inequalities, Logarithm functions, Constraints, Reprints, Analytic centers, Center manifolds.

The convergence behavior of center trajectories aris ing from the use of the logarithmic barrier function in linear programming is examined based on the concep of analytic center of a system of linear constraints. Main results are the convergence of A-trajectories to g-centers of the optimal face and the convergence of their tangential directions. Both results hold in the presence of primal and dual degeneracies. g-center varieties are introduced extending the concept of ana lytic g-centers.

\section{1,271}

PB92-116318

PC A03/MF A01

National Inst. of Standards and Technology (CAML) Gaithersburg, MD.

Merit Function for Inequality Constrained Nonlinear Programming Problems.

P. T. Boggs, J. W. Tolle, and A. J. Kearsley. Oct 91 , $34 \mathrm{p}$ NISTIR-4702

See also PB90-123944 and PB86-105830. Prepared in cooperation with North Carolina Univ, at Chapel Hill. Dept. of Mathematics, and Rice Univ., Houston, TX Dept. of Mathematical Sciences.

Keywords: "Nonlinear programming, Optimization, Ouadratic programming, Constraints, Convergence, Algorithms, Tables(Data), "Merit functions.

The authors consider the use of the sequential quadratic programming (SOP) technique for solving the in equality constrained minimization problem min sub $x$ $f(x)$ subject to: $g($ sub $i)(x)<$ or $=0, i=1, \ldots, m$. SOP methods require the use of an auxiliary function, called a merit function or line-search function, for assessing the steps that are generated. The authors derive a merit function by adding slack variables to create an equality constrained problem and then using the merit function developed earlier by the authors for the equality constrained case. They stress that they do not solve the slack variable problem, but only use it to construct the merit function. The resulting function is simplified in a certain way that leads to an effective procedure for updating the squares of the slack variables. The final form of the merit function has many desirable properties, and is demonstrated to be effective in practice.

\section{Statistical Analysis}

\section{1,272}

PB91-147017 Not available NTIS

National Inst. of Standards and Technology (NEL) Gaithersburg, MD. Applied and Computational Mathematics Div

Orthogonal Distance Regression.

Final rept.

P T Boggs, and J. R. Donaldson. 1989, 12p

See also PB90-151747.

Pub. in Proceedings of Summer Research Conference on Contemporary Mathematics, Arcata, CA., June 1016, 1989, v112 p183-194 1990

Keywords: „Error analysis, Maximum likelihood estimation, Confidence limits, Computation, Computer ap- plications, Reprints, “Orthogonal distance regression, Parameter estimation, Covariance matrices.

Orthogonal distance regression (ODR) is the name given to the computational problem associated with finding the maximum likelihood estimators of parameters in measurement error models in the case of normally distributed errors. The authors examine the stable and efficient algorithm of Boggs, Byrd, and Schnabel (1987) for finding the solution to the problem when the underlying model is assumed to be nonlinear in both the independent variable and the parameters. The authors also describe the associated publicdomain software package, ODRPACK. The authors then review the results of a simulation study that compares ODR with ordinary least squares (OLS). The authors also present the new results of an extension to the study. Finally the authors discuss the use of the asymptotic covariance matrix for computing confidence regions and intervals for the estimated parameters. Their conclusions are that ODR is better than OLS for the criteria considered, and that ODRPACK can provide effective solutions and useful statistical information for nonlinear ODR problems.

101,273

PB91-149443

Not available NTIS

National Bureau of Standards (NEL), Boulder, CO. Statistical Engineering Div.

Linear Calibration When the Coefficient of Variation is Constant

Final rept.

Y. C. Yao, and D. F. Vecchia. 1988, 13p

Pub. in Probability and Statistics, p297-309 1988.

Keywords: "Calibrating, "Coefficient of variation, $\mathrm{Pa}$ rameter identification, Maximum likelihood estimates, Reprints, Pitman closeness.

The report considers point estimation of the unknowns in the calibration problem when the calibration curve is a straight line through the origin and the responses are normal with variances proportional to the square of their mean. It presents four different estimators and compares their performances using the criterion of Pitman closeness. For all the practical ranges of parameter values, one of these estimators is found to dominate the others under the normality assumption. Non-normal cases are not considered.

101,274

PB91-158824

Not available NTIS

National Bureau of Standards (NEL), Gaithersburg,

MD. Statistical Engineering Div.

Design and Analysis of Experiments.

Final rept.

J. S. Hunter, M. G. Natrella, E. H. Barnett, W. G.

Hunter, and T. L. Koehler. 1988, 81p

Pub. in Juran's Quality Control Handbook, Section 26 , p26.1-26.81 1988 .

Keywords: "Experimental design, Factorial design, Block design, Laboratory tests, Mixtures, Statistics, Reprints.

The chapter outlines some general considerations in planning experiments and some tools for sound experimentation. Various statistical experiment designs are classified according to their structure, their purpose, and the kind of information obtained. Detailed explanations of the design structure and analysis are given for completely randomized designs, various kinds of block designs, factorial and fractional fractorial designs, and mixture designs. Some methods for planning the size of the experiment, and for planning interlaboratory tests are also given.

101,275

PB91-158907

Not available NTIS

National Inst. of Standards and Technology (NEL), Gaithersburg, MD. Statistical Engineering Div.

New Yorker in Japan.

Final rept.

W. Liggett. 1990, 2p

Pub. in Industrial Science and Technology 31, p46-47 1990.

Keywords: Culture(Social sciences), United States, Japan, Travel, Statistics, Reprints, "National Research Laboratory of Metrology, US NIST.

A personal note is provided on experiences at NALM (National Research Laboratory of Metrology) in Japan, which relate NIST to NRLM, and U.S. culture to that of Japan. 
101,276

PB91-158931

Not available NTIS

National Bureau of Standards (NEL), Gaithersburg,

MD. Statistical Engineering Div.

Calibration with Working Standards.

Final rept.

T. Lwin, and C. H. Spiegelman. 1986, 6p

Contract ONR-00014-83-005

Sponsored by Office of Naval Research, Arlington, VA

Pub. in Applied Statistics 35, n3 p256-261 1986.

Keywords: "Calibration, Error analysis, Regression analysis, Nonparametric statistics, Reprints, Working standards.

Certified values of working standards used for calibrations are rarely exact, and calibration curve procedures should take into account all sources of error, including errors in the working standards. When the errors in working standards have a known finite bound the authors give an easily implementable accurate calibration curve procedure. It produces conservative confidence intervals based on an expansion.

101,277

PB91-203414

Not available NTIS

National Inst. of Standards and Technology (NEL), Gaithersburg, MD. Statistical Engineering Div. Estimators for Censored (Log)Normal Samples.

Final rept.

J. A. Lechner. $1989,7 p$

Pub. in Proceedings of Annual Reliability and Maintainability Symposium, Atlanta, GA., January 24-26, 1989 , p262-268

Keywords: Life tests, Reliability, Reprints, “Lognormal distribution, Parameter estimation.

The paper presents an evaluation of the properties of several estimators of the parameters of the (two-parameter) logNormal distribution, for sample sizes from 10 to 60 and for right-censoring of 0 to $80 \%$ of the sample. Correction factors to reduce bias and recommendations are included. The estimators are also applicable to right-censored Normal samples.

\section{1,278}

PB91-507954

CP T06

National Inst. of Standards and Technology (NEL) Gaithersburg, MD. Statistical Engineering Div.

OMNITAB 80 (ASCII): An Interactive System for Statistical and Numerical Data Analysis (Version 7.0).

Software.

Mar 91, mag tape NIST/SW/MT-91/011

System: SUN/UNIX; VMS V4.7 operating system. Language: TAR UNIX command FO. Other formats available as PB91-507962 (UNIX)

Available in 9-track, ASCII character set, 1600 or 6250 bpi. For $6250 \mathrm{bpi}$, the price is T06. Documentation in cluded; may be ordered separately as PB87-172235.

Keywords: *Software, "Statistical analysis, "Numerical analysis, "Data analysis, Computer calculations, Computation, Tabulation processes, Integrated systems, Interactive systems, Batch processing, Regression analysis, Correlation, Magnetic tapes.

OMNITAB 80 is a high-level integrated general purpose programming language and statistical software computing system. The system enables the user to use a digital computer to perform statistical and numerical data analysis without having any prior knowledge of computers or programming language. It permits one to perform arithmetic, complex arithmetic, trigonometric calculations as well as data manipulation, special function calculations, statistical analysis, and matrix and array operations. The system responds to simple instructions to obtain accurate results since reliable, varied, and sophisticated algorithms for data analysis and manipulation are referenced. It may be used either interactively or in batch mode. Some of the major statistical capabilities are regression, correlation analysis and oneway/twoway analysis. OMNITAB 80 has been installed nationally and internationally. The source language of the system is in FORTRAN for implementation on the VAX computer. Input/output is done in ASCII format. A load and run module is also included as well as a module of test problems.

National Inst. of Standards and Technology (NEL), Gaithersburg, MD. Statistical Engineering Div.
OMNITAB 80 (UNIX): An Interactive System for Statlstical and Numerical Data Analysis. Version 7.0.

Software.

May 91, mag tape NIST/SW/MT-91/012

System: SUN/UNIX; VMS V4.7 operating system. Language: TAR UNIX command FO. Other formats available as PB91-507954 (ASCII).

Available in 9-track, ASCII character set, 1600 or 6250 bpi. For 6250 bpi, the price is T06. Documentation in cluded; may be ordered separately as PB87-172235.

Keywords: "Software, *Statistical analysis, "Numerical analysis, "Data analysis, Computer calculations, Computation, Tabulation processes, Integrated systems, Interactive systems, Batch processing, Regression analysis, Correlation, Magnetic tapes.

OMNITAB 80 is a high-level integrated general purpose programming language and statistical software computing system. The system enables the user to use a digital computer to perform statistical and numerical data analysis without having any prior knowledge of computers or programming language. It peredge of computers or programming language. It pertrigonometric calculations as well as data manipulation, special function calculations, statistical analysis, and matrix and array operations. The system responds to simple instructions to obtain accurate results since reliable, varied, and sophisticated algorithms for data analysis and manipulation are referenced. It may be used either interactively or in batch mode. Some of the major statistical capabilities are regression, correlation analysis and oneway/twoway analysis. OMNITAB 80 has been installed nationally and internationally. The source language of the system is in FORTRAN for implementation on the VAX computer. Input/output is done in UNIX format. A load and run module is also included as well as a module of test problems.

\section{1,280}

\section{PB9́2-12667}

(Order as PB92-126614, PC A06/MF A02) National Inst. of Standards and Technology, Gaithers burg, MD.

Taguchi's Orthogonal Arrays Are Classical Designs of Experiments.

R. N. Kacker, E. S. Lagergren, and J. J. Filliben.

1991, 15p

Included in Jnl. of Research of the National Institute of Standards and Technology, v96 n5 p577-591 Sep/Oct 91

Keywords: "Experimental design, Factorial design, Matrices, "Orthogonal arrays, Taguchi arrays.

Taguchi's catalog of orthogonal arrays is based on the mathematical theory of factorial designs and difference sets developed by R. C. Bose and his associates. These arrays evolved as extensions of factorial designs and latin squares. The paper (1) describes the structure and constructions of Taguchi's orthogonal arrays, (2) illustrates their fractional factorial nature, and (3) points out that Taguchi's catalog can be expanded to include orthogonal arrays developed since 1960.

\section{1,281
PB92-126705}

(Order as PB92-126614, PC A06/MF A02)

Birsa Agricultural Univ., Ranchi (India). Dept. of Statistics.

\section{List of New Group Divisible Designs.}

K. Sinha 1991, 3p

Included in Jnl. of Research of the National Institute of Standards and Technology, v96 n5 p613-615 Sep/Oct 91.

Keywords: "Experimental design, "Block design, Tables(Data).

Group divisible designs are the most important class of partially balanced incomplete block (PBIB) designs. A list of new group divisible designs with $r, k$ or $<10$ is provided.

\section{General}

101,282

PB91-132175 PC A03/MF A01

National Inst. of Standards and Technology (NEL) Gaithersburg, MD. Applied and Computational Mathe. matics Div.
Guide to Available Mathematical Software Problem Classification System.

A. F. Boisvert, S. E. Howe, and D. K. Kahaner. Nov $90,33 p$ NISTIR-4475

See also PB90-216508.

Keywords: *Applications of mathematics, "Classifica tions, Subject indexing, Cataloging, Mathematica models, "Computer software, Computer calculations.

A vast collection of reusable mathematical and statistical software is now available for use by scientists and engineers in their modeling efforts. This software represents a significant source of mathematical expertise, created and maintained at considerable expense. Unfortunately, the collection is so heterogeneous that it is a tedious and error-prone task simply to determine what software is available to solve a given problem. In mathematical problem-solving environments of the future such questions will be fielded by expert software advisory systems. One way for such systems to systematically associate available software with the probems they solve is to use a problem classification system. The paper describes a detailed tree-structured problem-oriented classification system appropriate for such use.

\section{MEDICINE \& BIOLOGY}

\section{Biochemistry}

101,283

PB 1-134544

Not available NTIS

National Inst. of Standards and Technology (NML) Boulder, CO. Chemical Engineering Science Div.

Chitin-Chitosan Membranes: Separations of Amino Acids and Polypeptides.

Final rept.

J. J. Pellegrino, S. Geer, K. Maegley, R. Rivera, and D. Steward. $1990,16 p$

Pub. in Annals of the New York Academy of Sciences $589, \mathrm{p} 229-244,20$ May 90

Keywords: "Adsorption, "Amino acids, "Peptides, *Chitins, "Membranes, Measurement, Yeasts, Alcohol oxidoreductases, Dehydrogenase, Mixtures, Permeability, Molecular weight, Diffusion theory, Crosslink ing, Aldehydes, Gels, Reprints, "Separation processes, Chitosan, Glutaraldehyde.

The permeation of several amino acids and yeast alco hol dehydrogenase (YADH) has been measured for membranes made from poly(N-acetyl-D-glucosamine)/poly(D-glucosamine)(chitin/chitosan) membranes. Sorption data for a number of amino acids has also been measured and indicates that those with polar aromatic residues are preferentially sorbed. Prefrential sorption combines with the molecular size effects on diffusion, to cause permeation to not be monotonic with molecular weight. Other interaction effects due to $\mathrm{pH}$ and the presence of co-ions have been observed. Limited mixture studies indicate separation factors slightly higher than those predicted by pure component permeation are obtained. It was also found that films which are initially impermeable to YADH may be made gel-like and thus YADH-permeable by controlled treatment with glutaraldehyde crosslinking.

101,284

PB9 1-174565

Not available NTIS

National Inst. of Standards and Technology (NML)

Gaithersburg, MD. Organic Analytical Research Div.

Planar Waveguide Optical Immunosensors.

Final rept.

S. J. Choquette, L. Locascio-Brown, and R. A. Durst. 1990, 6p

Pub. in Proceedings of SPIE (Society of Photo-Optical Instrumentation Engineers) Chemical, Biochemical and Environmental Fiber Sensors II, San Jose, CA. September 19-21, 1990, v1368 p258-263.

Keywords: "Theophylline, Monoclonal antibodies, Fluorescence, Liposomes, Reprints, "Planar waveguides, *Immunosensors.

Monoclonal antibodies were covalently bonded to the surfaces of planar waveguides to confer immunoreac- 


\section{Biochemistry}

tivity. Silver-ion diffused waveguides were used to measure theophylline concentrations in a fluorescence immunoassay, and silicon nitride waveguides were used to detect theophylline in an absorbancebased immunoassay. Liposomes were employed in both assays as the optically detectable label in a competitive reaction to monitor antigen-antibody complexation. Regeneration of the active antibody site will be discussed.

\section{1,285}

PB91-175349

Not available NTIS

National Bureau of Standards (NML), Gaithersburg, MD. Chemical Thermodynamics Div.

Neutron Crystallography of Proteins.

Final rept.

N. V. Raghavan, and A. Wlodawer. 1987, 31p

Pub. in Methods of Experimental Physics, v23 ptC p335-365 1987

Keywords: "Single crystals, "Neutron diffraction, "Proteins, "Biochemistry, Crystal structure, Solvents, Solvolysis, Hydrogen isotopes, Labeled reactions, Isotope exchange, Reprints.

Single crystal neutron diffraction has reached the stage where the efforts of the last fifteen years are beginning to provide results. Three facilities are now available for data collection. Although large crystals are still desirable, their sizes no longer limit successful neutron analyses. The results of hydrogen exchange experiments and of methyl rotor analyses provide unique methods for correlating the dynamic properties of proteins with their three-dimensional structures. Such motions in proteins are thought to play an important role in their function and may affect processes such as ligand binding, enzyme catalysis, and electron transfer. Finally, the improved description of the bound solvent that is obtained by neutron diffraction and the differentiation of solvent components is also gaining in importance.

\section{1,286}

PB91-189472

Not available NTIS

National Inst. of Standards and Technology (NML), Gaithersburg, MD. Ionizing Radiation Div.

8-Hydroxyguanine Content of Isolated Mitochondria Increases with Lipid Peroxidation.

Final rept.

A. M. Hruszkewycz, and D. S. Bergtold. $1990,6 p$

Pub. in Mutation Research 244, n2 p123-128 1990.

Keywords: "Lipid peroxidation, "Mitochondria, Alphatocopherol, Deoxyribonucleic acids, DNA damage, Agar gel electrophoresis, Reprints, "8-hydroxyguanine.

When lipid peroxidation was induced in isolated mitochondria there was a marked increase in the 8-hydroxchondria there was a marked increase in the 8-hydrox-
yguanine content of the nucleic acids extracted from these mitochondria. The elevation of 8-hydroxyguanine levels was associated with an extensive alteration of normal electrophoretic mobility of mitochondrial DNA. However, suppression of lipid peroxidation with alpha-tocopherol proportionally attenuated 8-hydroxyguanine production and limited the electrophoretic mobility change of mitochondrial DNA.

101,287

PB91-189886

Not available NTIS

National Inst. of Standards and Technology (NML), Boulder, CO. Chemical Engineering Science Div.

Enhanced Transport and Liquid Membranes in Bioseparations.

Final rept.

J. Pellegrino, and R. Noble. $1990,9 p$

Pub. in Trends in Biotechnology 8, p216-224 Aug 90.

Keywords: "Biological transport, "Chemical reactions, "Artificial membranes, Reprints, "Bioseparations.

Membranes that use a reversible chemical reaction or sequestration to achieve high selectivities and productivities show great potential for use in bioseparations was studied. The concept of liquid membranes, with and without a complexing agent (carrier), and the types of system configurations and carriers that may be used with these membranes, are discussed.

\section{1,288}

PB91-194803

National Inst. of Standards and Technology (CSTL)

Gaithersburg, MD. Biotechnology Div.
Thermodynamics of Hydrolysis of Oligosaccharides.

Final rept.

R. N. Goldberg, D. Bell, Y. B. Tewari, and M. A.

McLaughlin. 1991, 8p

Pub. in Biophysical Chemistry 40, p69-76 1991.

Keywords: "Thermodynamics, *Oligosaccharides, Hydrolysis, Enthalpy, High pressure liquid chromatography, Chemical reactions, 1,4-alpha-glucosidase, Reprints, Beta-fructofuranosidase.

Microcalorimetry has been used to determine enthalpy changes for the hydrolysis of a series of oligosaccarides. High-pressure liquid chromatography was used to determine the extents of reaction and to check for any possible side reactions. The enzyme glucan $1,4-$ alpha-glucosidase was used to bring about the following hydrolysis reactions: (A) maltose (aq) $+\mathrm{H} 2 \mathrm{O}$ (liq) $=$ 2D-glucose (aq); (B) maltotriose (aq) $+2 \mathrm{H} 2 \mathrm{O}$ (liq) $=3 \mathrm{D}$ glucose(aq); (C) maltotetraose(aq) $+3 \mathrm{H} 2 \mathrm{O}$ (liq) $=4 \mathrm{D}$ glucose(aq); (D) maltopentaose $(a q)+4 \mathrm{H} 2 \mathrm{O}($ liq) $=5 \mathrm{D}$ glucose (aq); (E) maltohexaose $(a q)+5 \mathrm{H} 2 \mathrm{O}$ (liq) $=6 \mathrm{D}$ glucose $(\mathrm{aq}) ;(\mathrm{E})$ maltohexaose $(\mathrm{aq})+5 \mathrm{H} 2 \mathrm{O}(\mathrm{liq})=6 \mathrm{D}-$
glucose $(\mathrm{aq}) ;(\mathrm{F})$ maltoheptaose $(\mathrm{aq})+6 \mathrm{H} 2 \mathrm{O}(\mathrm{liq})=7 \mathrm{D}-$ glucose $(\mathrm{aq}) ;(\mathrm{F})$ maltoheptaose $(\mathrm{aq})+6 \mathrm{H} 2 \mathrm{O}$ (liq) $=7 \mathrm{D}$ -
glucose $(\mathrm{aq}) ;(\mathrm{G})$ amylose $(\mathrm{aq})+\mathrm{nH} 2 \mathrm{O}($ liq) $=(n+1) \mathrm{D}$ glucose $(\mathrm{aq})$; and $(\mathrm{H})$ panose $(\mathrm{aq})+2 \mathrm{H} 2 \mathrm{O}$ (liq) $=3 \mathrm{D}$ glucose (aq); (J) isomaltotriose $(\mathrm{aq})+2 \mathrm{H} 2 \mathrm{O}$ (liq) $=3 \mathrm{D}$ glucose(aq). The enzyme beta-fructofuranosidase was used for the reactions; (K) raffinose $(\mathrm{aq})+\mathrm{H} 2 \mathrm{O}$ (liq) $=$ alpha-D-melibiose(aq) + D-fructose(aq); and (L) stachyose $(\mathrm{aq})+\mathrm{H} 2 \mathrm{O}(\mathrm{liq})=0$-alpha-D-galactopyranosyl- $(1->>6)$-alpha-o-D-galactopyranosyl- $(1->6)$ alpha-D-glucopyranose +D-fructose(aq). These processes correspond to the hydrolysis of the following linkages: glucose-glucose (alpha, $1->4$ ) glucose-glucose (alpha $1->6$ ), and glucose-fructose $(1->6)$. The respective enthalpy changes accompanying the hydrolysis of these linkages are $-453,5.8$ and -15.0 $\mathrm{kJ} / \mathrm{mol}$. Both these results and available thermodynamic data in the literature demonstrate that additivity works well in predicting the thermodynamics of hydrolysis reactions involving oligosaccharides.

101,289

PB91-195701

Not available NTIS

National Inst. of Standards and Technology (CSTL), Gaithersburg, MD. Biotechnology Div.

Thermodynamics of Hydrolysis of Disaccharides: Lactulose, alpha-D - Melibiose, Palatinose, D - Trehalose, D - Turanose and 3-0-beta-D-Galactopyranosyl-D-arabinose.

Final rept.

Y. B. Tewari, and R. N. Goldberg. 1991, 9p

See also PB89-186761.

Pub. in Biophysical Chemistry 40, p59-67 1991.

Keywords: "Thermodynamics, "Disaccharides, Hydrolysis, High pressure liquid chromatography, Enthalpy, Entropy, Beta-galactosidases, Fructose, Galactose, Sucrose, Glucose, Reprints.

High-pressure liquid chromatography and microcalorimetry have been used to study the thermodynamics of the hydrolysis reactions of a series of dissaccharides. beta-galactosidase for lactulose and 3-o-beta-D-galactopyranosyl-D-arabinose; beta-glucosidase for alpha$\mathrm{D}$-melibiose; beta-amylase for D-trehalose; isomaltase for platinose; and alpha-glucosidase for D-turanose. The buffer used was sodium acetate $(0.02-0.10 \mathrm{M}$ and $\mathrm{pH}$ 4.44-5.65). These six processes correspond, respectively, to the hydrolysis of the following linkages: galactose-fructose $(1->4)$, galactose-glucose $(1->$ 6), glucose-fructose $(1->6)$, glucose-glucose $(1->$ $\left.1^{\prime}\right)$, glucose-fructose $(1->3)$, and galactose-ambinose $(1->3)$. Using available data in the literature, the heat capacity changes for these processes are found to be in the range -36 to $-69 \mathrm{~J} / \mathrm{mol} / \mathrm{K}$. The available thermodynamic data on the hydrolysis of disaccharides is summarized. It is found that the entropy changes for the hydrolysis of disaccharides are in the range $31-56 \mathrm{~J} / \mathrm{mol} / \mathrm{K}$ and are well represented by an average value of $40+$ or $-7 \mathrm{~J} / \mathrm{mol} / \mathrm{K}$. The abnormally high enthalpy of hydrolysis of aqueous sucrose $(-14.96$ $\mathrm{kJ} / \mathrm{mol}$ ) can be explained by consideration of the enthalpy of conversion of fructopyranose to fructofuranose.

101,290

PB92-116953

Not available NTIS National Inst. of Standards and Technology (NML), Gaithersburg, MD. Ionizing Radiation Div.
Formation of ortho-Tyrosine by Radiation and Organic Solvents in Chicken Tissue.

Final rept.

L. R. Karam, and M. G. Simic. 1990, 5p

Pub. in Jnl. of Biological Chemistry 265 , n20 p11581 11585,15 Jul 90

Keywords: "Poultry, "Tyrosine, *Organic solvents, *Radiation, Tissues(Biology), Mass fragmentography, Dose-response relationships, Reprints.

Fresh chicken breast and beef incubated in water were found to contain no o-Tyr at the current levels of detection $(0.1 \mathrm{ppm})$ by capillary gas chromatography/mass spectrometry and selective ion monitoring. In contrast, samples incubated at $37 \mathrm{C}$ in the presence of ethanol, benzene, or carbon tetrachloride (used in fat extrac tion) contained large quantities (2.5-5.1 ppm) of o-Tyr No o-Tyr was detected in the water-insoluble fraction of meat treated with carbon tetrachloride after triple extraction by water. However, reaction of radiation generated $\mathrm{OH}$ in gamma-irradiated fresh chicken tissue with endogenous phenylalanine yields 0 -Tyr with a linear yield-dose response in both water-soluble and -insoluble tissue fractions.

\section{Clinical Chemistry}

101,291

PB91-147629

Not available NTIS

National Inst. of Standards and Technology (NML) Gaithersburg, MD. Organic Analytical Research Div.

Liposome Flow Injection Immunoassay: Implications for Sensitivity, Dynamic Range, and Antibody Regeneration.

Final rept.

L. Locascio-Brown, A. L. Plant, V. Horvath, and R. A. Durst. 1990, 7p

Pub. in Analytical Chemistry 62, n23 p2587-2593, 1 Dec 90 .

Keywords: "Liposomes, "Immunoassay, "Theophylline, Antibodies, "Flow injection analysis.

The report describes the use of a liposome-based flow injection immunoassay (FIIA) system for quantitation of a clinical analyte, theophylline. With very minor changes in assay format, the procedure can also be used for the quantitation of anti-theophylline. Automated sequential analyses were performed at room temperature with picomole sensitivity and a day-to-day coefficient of variation of less than $5 \%$ for aqueous solutions. The system components include liposomes that contain fluorophores in their aqueous centers and an immobilized-antibody reactor column. The immunoreactor was regenerated hundreds of times over 3 months of continuous use with no measurable loss of antibody activity. The two assay formats studied produced distinct dynamic ranges for their respective analytes. The special advantages of using flow injection analysis for immunoassays and of using liposomes in FIIA are discussed.

\section{1,292}

PB91-189266

Not available NTIS

National Bureau of Standards (NML), Gaithersburg MD. Organic Analytical Research Div.

Stability of Uric Acid in Ammonium Hydroxide.

Final rept.

P. Ellerbe, A. Cónen, M. J. Welch, and E. White V.

$1988,3 p$

Pub. in Clinical Chemistry 34, n11 p2280-2282 1988

Keywords: "Uric acid, "Stability, "Ammonia, Decomposition reactions, Chemical analysis, Mass spectroscopy, Allantoin, Urea, Reprints.

The stability of uric acid in dilute aqueous ammonium hydroxide solution was examined by mass spectrometry. Uric acid decomposes in ammonium hydroxide even at the low concentration of $0.015 \mathrm{M}$ at a mole ratio of ammonium hydroxide to uric acid of $50: 1$. The slope of the decomposition curve indicates that uric acid was being destroyed at an initial rate of severa percent per hour. There are at least four products of the decomposition two of which have been identified as allantoin and urea. Uric acid does not detectably decompose in ammonium hydroxide at a concentration of $0.001 \mathrm{M}$ and mole ratio of ammonium hydroxide to uric acid of 3.4 or less. The results have certain implications. Firstly, any method for the determination of 
uric acid that involves treating the analyte with ammonium hydroxide before analysis may destroy it. Secondly, a published 'definitive' method for uric acid could produce incorrect results because the uric acid is stored in $0.015 \mathrm{M}$ ammonium hydroxide at a mole ratio oif over 120:1 of ammonium hydroxide to uric acid.

\section{Clinical Medicine}

101,293

B9 1-195198

Not available NTIS

National Inst. of Standards and Technology (NML), Gaithersburg, MD. Ionizing Radiation Div.

Intensifying Effect of Metallic Screens on the Sensitivity of X-ray Films for $400-k V$ Bremsstrahlung Photons.

Final rept.

E. Navon, C. E. Dick, and G. Barnea. 1991,6p Pub. in Medical Physics 18, n2 p299-304 Mar/Apr 91.

Keywords: "Radiographic films, "X ray film, "Radiation effects, "Electron bombardment, Bremsstrahlung, Lead(Metal), Aluminum, Copper, Foils(Materials), Sensitivity, Reprints, Metal screens, Intensification factors.

Measurements are carried out to determine the relative photographic effect produced by electrons emanating from metallic screens on a typical radiographic film. The electrons are produced in metal foils of aluminum, copper, and lead by the interaction of photons in $400-\mathrm{kV}$ bremsstrahlung beam. Intensification factors of up to $1.65,2.05$, and 5.90 for aluminum, copper, and of up to $1.65,2.05$, and 5.90 for aluminum, copper, and lead screens, respectively, are determined as a funccreens are determined to be $20 \mathrm{mg} / \mathrm{sq} \mathrm{cm}$ for aluminum and $30 \mathrm{mg} / \mathrm{sq} \mathrm{cm}$ for copper and lead. These results are compared with the absorbed dose in the film emulsions, calculated by Monte Carlo methods. The emulsions, calculated by Monte Carlo methods. The
results of the present work are compared with those results of the present work are compared with those for (60)Co and (137)Cs photon beams. The dependence of the equilibrium thicknes at the screen material is summarized and explained.

101,294

PB91-203745

Not available NTIS National Bureau of Standards (IMSE), Gaithersburg, MD. Metallurgy Div.

Mossbauer Imaging.

Final rept.

S. J. Norton 1988, 1p

See also PB89-176895

Pub. in Physics Bulletin 39, n5 p182 1988.

Keywords: "Mossbauer effect, "Imaging techniques, "Computerized tomography, *Spectroscopy, Metallography, Diagnostic techniques, Tests, Reprints.

Imaging techniques are playing an increasingly imporant role in many areas of research. Although NMR has long been employed as an important spectroscopic tool in chemistry, biology and materials science, a significant recent advance is nuclear-magnetic-resonance (NMR) imaging. Now the Mossbauer effect, an other well-established spectroscopic technique, has also been proposed as the basis of a new imaging method (S. J. Norton, Nature, 12 Nov., 1987).

\section{1,295}

PB92-116623

Not available NTIS

National Inst. of Standards and Technology (NEL), Gaithersburg, MD. Statistical Engineering Div.

Use of Cross-Validation as a Stopping Rule in Emission Tomography Image Reconstruction.

Final rept.

K. J. Coakley. 1991, $8 p$

Pub. in Proceedings of SPIE (Society of Photo-Optical Instrumentation Engineers) - Medical Imaging $V$ : Image Physics, v1443 p226-233 1991

Keywords: "Image reconstruction, "Tomography, Stopping rules(Mathematics), Reprints, "Positron emission tomography, EM algorithm, Cross validation.

Simulated and real Positron Emission Tomography (PET) images are reconstructed using the iterative EM algorithm. The data is split up into independent parts. The EM algorithm is applied to each part and stopped according to a cross-validation procedure. For a varie ty of simulated and real data sets, stopping points

were reached. For simulated data, the average of the econstructions from a four-way split of the data was visually superior to the reconstruction obtained by applying the EM algorithm to the full data and stopping at a subjectively chosen iteration. For real data, such an mprovement was not observed. To remove point artifacts, the reconstructions were filtered.

101,296

Not available NTIS National Inst. of Standards and Technology (NML), Gaithersburg, MD. lonizing Radiation Div.

Photon Energy Dependence of the Sensitivity of Radiochromic Film and Comparison with Silver Halide Film and LiF TLDs Used for Brachytherapy Dosimetry.

Final rept.

P. J. Muench, A. S. Meigooni, R. Nath, and W. L. McLaughlin. 1991, 7p

Sponsored by Public Health Service, Rockville, MD. Pub. in Medical Physics 18, n4 p769-775 Jul/Aug 91.

Keywords: "Brachytherapy, "Film dosimetry, "Thermoluminescent dosimetry, "Photons, Comparison, Iridium, Silver halides, lonizing radiation, Polymerization, Dyes, Reprints, "Radiochromic film.

There is a new radiochromic film, a highly uniform, thin (100 micrometers) etector whose sinsitive layer ( $6 \mathrm{mi}$ crometers thick) changes from colorless to blue by dye polymerization without processing, upon exposure to ionizing radiation. Because the dose gradients around brachytherapy sources are steep, the high spatial resolution offered by film dosimetry is an advantage over other detectors such as thermoluminescent dosimeters (TLDs). This compares the photon energy dependence of the sensitivities of GafChromic film, silver halide verification film (Kodak X-Omat V Film), and lithum fluoride TLDs (Harshaw), over the photon energy range $28 \mathrm{keV}$ to $1.7 \mathrm{MeV}$, which is of interest in brachytherapy. Sensitivity of the radiochromic film is observed to decrease by about $30 \%$ as effective photon energy decreases from $1710 \mathrm{keV}$ (4-MV $x$ rays) to 28 $\mathrm{keV}$ (60-kV x rays, 2-mm Al filter). In contrast, the sensitivity of verification film increases by $980 \%$ and that of LiF TLDs increases by $41 \%$. Radiochromic film, like LiF TLDs, does not exhibit the drastic sensitivity changes below $127 \mathrm{keV}$ that silver halide film exhibits. Dose distribution in the immediate vicinity of a high activity $(370 \mathrm{GBq}$ ) brachytherapy (192) Ir source has been mapped using radiochromic film and is presented to illustrate the applicability of this new technology to brachytherapy dosimetry.

\section{Cytology, Genetics, \& Molecular Biology}

101,297

PB91-134767 Not available NTIS

National Inst. of Standards and Technology (NML) Boulder, CO. Chemical Engineering Science Div. Gravity-Dependent Phenomena at the Scale of the Single Cell.

Final rept.

P. Todd. 1989, 19p

Pub. in American Society for Gravitational and Space Biology Bulletin 2, p95-113 Aug 89.

Keywords: *Cells(Biology), Reduced gravity, Sedimentation, Diffusion, Solutes, Gravity, Reprints, Gravitational effects, Intracellular transport, Convective flow.

Progress in gravitational cell biology research will depend on the continuing evaluation of a wide variety of physical phenomena affected by gravity and their roles in extracellular, intercellular, and intracellular processes. The paper examines those responses of organisms to gravity which depend on functions at the single cell level. Single cell functions are affected by perturbations in their internal and external environment by a variety of factors, one of which is the effect of gravity. In a microgravity environment extracellular solutes must be transported by diffusion or active circulatory processes in the absence of density gradientdriven convection, and flocculation and coalescence are reduced by the lack of motion of aggregates.

101,298

B9 1-148098

National Bureau of Standards (NML), Gaithersburg MD. Chemical Thermodynamics Div.
Multiple Conformations of Amino Acid Residues in Ribonuclease A.

Final rept.

A. Svensson, L. Sjolin, G. L. Gilliland, B. C. Finzel, and A. Wlodawer. 1986, 6p

Pub. in Proteins: Struct., Funct., Genet. 1, n4 p370-375 1986.

Keywords: "Amino acids, "Pancreatic ribonuclease, "Protein conformation, Structure-activity relationship, Protein engineering, Reprints.

Highly refined $1.26 \mathrm{~A}$ structure $(\mathrm{R}=0.15)$ of phosphate-free bovine pancreatic ribonuclease A was modeled with 13 residues having discrete multiple conformations of side chains. These residues are widely distributed over the protein surface, but only one of them Lys 61 , is involved in crystal packing interactions. The discrete conformers have no unusual torsion angles and their interactions with the solvent and with other atoms of the protein are similar to those residues modeled with a single conformation. For three of the residues, Val 43, Asp 83, and Arg 85, two correlated conformations are found. The observed plasticity in the protein surface will be of significance in analyzing structure - function relationships and in performing protein engineering

101,299

PB91-148213

Not available NTIS

National Bureau of Standards (NML), Gaithersburg, MD. Chemical Thermodynamics Div.

Crystallizing Catabolite Gene Activator Protein with CAMP for Structural Analysis.

Final rept.

I. T. Weber. $1988,8 p$

Pub. in Methods in Enzymology 159, p278-285 1988.

Keywords: "Adenosine cyclic monophosphate, "Catabolite gene activator proteins, Binding sites, Protein kinases, Protein conformation, DNA-binding proteins, Regulator genes, Reprints.

The crystal structure of the CAP dimer with CAMP has provided many insights into the action of this gene regulatory protein. The CAP subunit is divided into two domains that are connected by a hinge region. The carboxy-terminal domains bind to DNA and show both sequence and structural homologies with many other gene regulatory proteins from bacteria and viruses. The amino-terminal domain forms a binding site for CAMP and has been used to model the CAMP-binding domains of the regulatory subunits of mammalian cAMP-dependent protein kinase.

101,300

PB91-149229

Not available NTIS

National Inst. of Standards and Technology (NML), Boulder, CO. Chemical Engineering Science Div.

Hydrodynamics and Mass Transfer in Two-Phase Aqueous Extraction Using Spray Columns.

Final rept.

S. B. Sawant, S. K. Sikdar, and J. B. Joshi. 1990, 7p Pub. in Biotechnology and Bioengineering 36, p109. 1151990.

Keywords: "Hydrodynamics, "Mass transfer, "Sprays, "Albumins, "Separation, Polyoxyethylene, Dextran Phase velocity, Reprints, "Two phase aqueous extraction.

Spray columns can be used to isolate and purify proeins using the two-phase aqueous extraction technique based on polyethylene glycol (PEG) and dextran. The fractional dispersed phase (PEG) holdup and overall mass transfer coefficients were measured in a $9.7 \mathrm{~mm}$ i.d. spray column. The authors found that the dispersed phase holdup increased with increasing PEG phase velocity. The overall mass transfer coefficients for bovine serum albumin, normalized for the PEG holdup, were found to be independent of the PEG phase velocity. The result was expected, since true mass transfer coefficients do not vary with phase velocity.

101,301

PB91-175604

Not available NTIS

National Bureau of Standards (NML), Gaithersburg MD. Chemical Thermodynamics Div. 


\section{Cytology, Genetics, \& Molecular Biology}

Crystal Structure of Phosphate-Free Ribonuclease.

Final rept.

A. Wlodawer, G. I. Gilliland, L. Sjolin, and L. A

Svensson. 1987, 10p

Pub. in UCLA (Úniversity of California, Los Angeles)

Symposium on Molecular and Cellular Biology 69, n2

p109-118 1987.

Keywords: "Ribonucleases, "Crystal structure, "Protein conformation, Pancreas, Amino acids, Phosphates, Binding sites, Butanols, Molecular models, Reprints.

The crystal structure of phosphate-free bovine pancreatic ribonuclease has been refined with $1.26 \mathrm{~A}$ resolution $X$-ray diffraction data. The final $R$ factor was $15 \%$ with the rms deviation of bond lengths from ideality of $0.024 \mathrm{~A}$. Even in the absence of the anion bound in the active site, only minor movements of the side chains were noticed in that area. Thirteen amino acids, all but one of them on the protein surface, were modeled with two different conformations of the side chains. The bound solvent of the refined model includes a t-butanol and 188 fully or partially occupied water sites. The structure was compared in detail with three other highly refined models of ribonuclease.

\section{1,302}

PB91-187476 Not available NTIS National Inst. of Standards and Technology (CSTL), Gaithersburg, MD. Biotechnology Div.

46Gas Chromatography-Mass Spectrometry of Free Radical-Induced Products of Pyrimidines and Purines in DNA.

Final rept.

M. Dizdaroglu. 1990, 16p

M. Dizdaroglu. 1990, 16p
Pub. in Methods of Enzymology 193, p842-857 1990.

Keywords: "Deoxyribonucleic acids, "Pyrimidines, *Purines, "DNA damage, "Free radicals, Mass fragmentography, Hydrolysis, $\mathrm{pH}$, Reprints.

A description is provided of the application of the gas chromatography-mass spectrometry (GC-MS) technique to measurement of products of pyrimidines and purines in DNA, which are formed by reactions of free radicals with DNA. Acidic or enzymatic hydrolyses are used to free base products as bases or nucleosides prior to their analysis by GC-MS. Acidic hydrolysis releases DNA base-amino acid crosslinks from nucleoprotein. The trimethylsilyl derivatives of all these products provide characteristic mass spectra, which contain a molecular ion and other typical ions of this class of compounds. The tbutyldimethylsilyl derivatives are also used for measurement of modified bases. The use of the selected-ion monitoring technique permits the characterization and quantitative measurement of the products at low concentrations. Examples of mass spectra are presented and the fragmentation patterns of the molecules are discussed.

\section{1,303}

PB9́1-187484

Not available NTIS

National Inst of Standards and Technology (CSTL). Gaithersburg, MD. Biotechnology Div.

Measurement of DNA Base Damage and DNA-Protein Cross-Links in Mammalian Chromatin.

Final rept.

M. Dizdaroglu. 1990, 14p

Sponsored by Department of Energy, Washington, DC. Pub. in lonizing Radiation Damage to DNA: Molecular Aspects, p17-301990.

Keywords: "DNA damage, "DNA-binding proteins, "Chromatin, lonizing radiation, Cross-linking reagents, Mass fragmentography, Mammals, Free radicals, Reprints.

Methodologies incorporating the technique of gas chromatography/mass spectrometry (GC/MS) have been developed in recent years for measurement of DNA damage produced by ionizing radiation or by other free radical-generating systems. GC/MS with selected-ion monitoring (SIM) facilitates unequivocal identification and quantitation of a plethora of products of all four bases in DNA. In addition, DNA-protein cross-links in mammalian chromatin are unequivocally identified and quantitated. The sensitivity and selectivity of the GC/MS-SIM technique also enables the measurement of DNA base products in isolated mammalian chromatin without the necessity of first isolating DNA. Recent results demonstrate the usefulness of the GC/MS technique for measurement of DNA damage in DNA as well as in mammalian chromatin under a vast variety of conditions of free radical production.

\section{1,304}

PB91-189365

Not available NTIS

National Inst. of Standards and Technology (CSTL), Gaithersburg, MD. Biotechnology Div.

Hydroxyl Radical Induced Cross-Linking of Cytosine and Tyrosine in Nucleohistone.

Final rept.

E. Gajewski, and M. Dizdaroglu. 1990, 4p

Pub. in Biochemistry 29, n4 p977-980 1990.

Keywords: "Cytosine, "Tyrosine, "Hydroxyl radicals, Cross-linking reagents, Mass fragmentography, Gamma rays, Hydrolysis, Reprints, "Nucleohistones.

Hydroxyl radical induced formation of a DNA-protein cross-link involving cytosine and tyrosine in nucleohistone in buffered aqueous solution is reported. The technique of gas chromatography-mass spectrometry was used for the investigation. A gamma-irradiated aqueous mixture of cytosine and tyrosine was first investigated in order to obtain gas chromatographic mass spectrometric properties of possible cytosine-tyrosine cross-links. One cross-link was observed, and its structure was identified as the product from the formation of a covalent bond between carbon 6 of cytosine and carbon 3 of tyrosine. With the use of gas chro matography-mass spectrometry with selected-ion monitoring, this cytosine-tyrosine cross-link was identified in acidic hydrolysates of calf thymus nucleohistone gamma-irradiated in N2O-saturated aqueous solution. The yield of the DNA-protein cross-link in nucleohistone was found to be a linear function of the radiation dose in the range of $100-500 \mathrm{~Gy}(\mathrm{~J} / \mathrm{kg})$. The yield amounted to $0.05 \mathrm{nmol} / \mathrm{J}$. Mechanisms uderlying the formation of the cytosine-tyrosine cross-link in nucleohistone were proposed to involve radical-radical and or radical addition reactions of hydroxyl adduct radicals of cytosine and tyrosine moieties, forming a covalent bond between carbon 6 of cytosine and carbon 3 of tyrosine. When oxygen was present in irradiated solutions, no cytosine-tyrosine cross-links were observed.

101,305

PB91-190074

Not available NTIS

National Inst. of Standards and Technology (NML) Gaithersburg, MD. Chemical Thermodynamics Div.

Crystal Structure of a CAMP-Independent Form of Catabolite Gene Activator Protein with Adenosine Substituted in One of 2 cAMP-Binding Sites. Final rept.

M. C. Vaney, G. L. Gilliland, J. G. Harman, A Peterkofsky, and I. T. Weber. 1989, 7p

Pub. in Biochemistry 28, n11 p4568-4574 1989

Keywords: "Catabolite gene activator proteins, "Adenosine cyclic monophosphate, "Crystallization, "Protein conformation, "Adenosine, Binding sites, Genetic transcription, Operon, Escherichia coli, Mutation, X-ray diffraction, Reprints.

Catabolite gene activator protein (CAP) in the presence of CAMP stimulated transcription from severa operons in E. coli. A cAMP-independent variant, in which Ala144 is replaced by Thr (CAP91), has been crystallized as the CAMP complex. CAP91, unlike the wild-type CAP, is activated by analogs of CAMP, including adenosine. In order to test the effect of adenosine on the structure, a crystal of CAP91 was soaked in a solution of $10 \mathrm{mM}$ adenosine, and $X$-ray diffraction data were measured to $3.5 \mathrm{~A}$ resolution. The difference Fourier map calculated with phases from the CAP91 structure showed significant negative density at the position of the phosphate of CAMP bound in the 'closed' subunit of the CAP91 dimer. This was consistent with the replacement of adenosine for CAMP in the 'closed' subunit. The cAMP-binding site of the 'open' subunit was apparently still occupied by cAMP. The coordinates were refined by restrained least-square to an $\mathrm{A}$ factor of $20.2 \%$. Adenosine is bound in the cAMP-binding pocket in almost the same position as CAMP, however, the new position of $5^{\prime} \mathrm{OH}$ of adenosine allowed formation of two new hydrogen bonds with Ser 83 , instead of the interactions of the phosphate of CAMP with Arg 82 and Ser 83.

\section{1,306}

PB91-200824

National Inst. of Standards and Technology (NML) Gaithersburg, MD. Chemical Kinetics Div.
Iron Ion-Dependent Modification of Bases in DNA by the Superoxide Radical-Generating System Hypoxanthine/Xanthine Oxidase.

Final rept.

O. I. Aruoma, B. Halliwell, and M. Dizdaroglu. 1989 ,

Pub. in Jnl. of Biological Chemistry 264, n22 p1302413028, 5 Aug 89.

Keywords: "Deoxyribonucleic acids, "Superoxide, *Hypoxanthines, "Xanthine oxidase, "Iron, Mass fragmentography, Hydroxyl radicals, DNA damage, Hydrolysis, lons, Reprints.

Damage to the bases in DNA produced by the hypoxanthine/xanthine oxidase system in the presence of iron ions was studied. The base products in DNA were measured using gas chromatography-mass spectrometry with selected ion monitoring after acidic hydrolysis of DNA and trimethylsilylation. Products identified were cytosine glycol, thymine glycol, 5,6-dihydroxycytosine, 4,6-diamino-5-formamidopyrimidine, 8-hydroxyadenine, 2,6-diamino-4-hydroxy-5-formamidopyrimiyadenine, 2,6-diamino-4-hydroxy-5-formamidopyrimi-
dine, and 8-hydroxyguanine. These are typical hydroxyl radical-induced products of the bases in DNA. 2,6-Diamino-4-hydroxy-5-formamidopyrimidine, 5,6-dihydroxycytosine, 4,6-diamino-5-formamidopyrimidine, and 8-hydroxyguanine were proposed as the products in DNA to measure if one aims to measure DNA products as indices of oxidative DNA damage involving hydroxyl radicals in vivo.

\section{1,307}

PB91-202960

Not available NTIS

National Inst. of Standards and Technology (NML), Gaithersburg, MD. Chemical Kinetics Div.

Selected-Ion Mass Spectrometry: Assays of Oxidative DNA Damage.

Final rept.

M. Dizdaroglu, and E. Gajewski. 1990, 15p

Pub. in Methods in Enzymology 186, p530-544 1990.

Keywords: "Mass spectrometry, "Oxidation, "DNA damage, lons, Mass fragmentrography, Hydrolysis, Hydroxyl radicals, Cross-linking reagents, Nucleoproteins, DNA-binding proteins, Reprints.

Chemical characterization and quantitative measurement of oxidative damage to DNA caused by free radicals is essential for an understanding of the biological consequences of such damage. The present article describes the technical details of the application of the gas chromatography-mass spectrometry (GC-MS) technique to measurement of DNA damage caused by hydroxyl radicals. Reactions of hydroxyl radicals form a variety of base products in DNA and DNA-protein crosslinks in nucleoprotein. Acidic or enzymatic hydrolyses are used to free base products as bases or nucleosides prior to their analysis by GC-MS. Acidic hydrolysis releases DNA base-amino acid crosslinks from nucleoprotein. The trimethylsilyl derivatives of all these products provide characteristic mass spectra, which contain a molecular ion and other typical ions of this class of compounds. The use of the selected-ion monitoring technique permits the characterization and quantitative measurement of the products at low concentrations.

\section{1,308}

PB91-237198

Not available NTIS

National Inst. of Standards and Technology (CSTL), Gaithersburg, MD. Biotechnology Div.

Immobilization of Binding Proteins on Nonporous Supports Comparison of Protein Loading, Activity, and Stability.

Final rept.

A. L. Plant, L. Locascio-Brown, W. Haller, and R. A. Durst. 1991, 16p

Pub. in Applied Biochemistry and Biotechnology 30 , p83-98 1991

Keywords: "Proteins, "Binding sites, "Glass, "Nylon, "Polystyrene, Monoclonal antibodies, Theophylline, Biotin, Ligands, Radioactivity, Reprints.

Four different nonporous particulate materials, nylon, polystyrene, soda-lime silicate glass, and fused silica glass, have been evaluated for their appropriateness as immobilization supports for immunoglobulins. A method of protein quantitation that is usually applied to solutions, the bicinchoninic acid (BCA) assay, was used successfully to directly measure ng amounts of protein immobilized on the supports. Two proteins, a monoclonal antibody to theophylline and the biotin binding protein avidin, were studied. Radioactive theo- 
phylline and radioactive biotin were used to measure the activity of the immobilized protein. Ligand binding capacity per sqmm of support was measured as a function of amount of protein immobilized. The binding activity of biotinyl-antibody conjugate immobilized on avidin-adsorbed polystyrene is stable, even when stored for over 22 wk. Antibody covalently immobilized on soda-lime silicate glass beads retains its binding.

\section{1,309}

PB9́1-237362

Not available NTIS

National Inst. of Standards and Technology (MSEL)

Gaithersburg, MD. Reactor Radiation Div.

$\mathrm{Ab}$ Initio Phase Determination for X-ray Diffraction Data from Crystals of a Native Protein.

Final rept.

L. Sjolin, E. Prince, L. Anders Svensson, and G. L.

Gilliland. 1991, $8 p$

Pub. in Acta Crystallographica A47, p216-223 1991

Keywords: "Proteins, "Crystal structure, "Phase diagrams, Algorithms, $X$ ray diffraction, Fourier transformation, Electron density, Molecular structure, Cattle, Reflection, Entropy, Reprints, *Bovine chymosin.

An efficient algorithm for the determination of an everywhere positive electron-density distribution that agrees with observed structure amplitudes has been used to determine the phases of X-ray diffraction data from recombinant bovine chymosin, a protein with 323 amino-acid residues in the molecular chain whose structure was recently determined using molecular replacement methods. A systematic procedure for testing the signs of centric reflections, using the total entropy of the map as a figure of merit, was used to produce a low-resolution map. The phases of acentric and additional centric reflections were then chosen by adding them to the map with various possible phases and computing the total entropy of the resulting map. The molecule is many times larger than any whose structures have previously been determined without the use of isomorphous replacement, molecular re placement or anomalous dispersion, and the map demonstrates the potential of maximum-entropy methods in macromolecular structure determination.

\section{1,310}

PB91-237560

Not available NTIS

National Bureau of Standards (NEL), Boulder, CO Chemical Engineering Science Div.

Low-Cost Aqueous Two-Phase System for Enzyme Extraction.

Final rept.

D. C. Szlag, and K. A. Giuliano, $1988,6 \mathrm{p}$

Pub. in Biotechnol. Tech. 2, n4 p277-282 1988

Keywords: "Enzymes, "Extraction, Purification, Alcohol dehydrogenase, Polyoxyethylene, Dextran, Separation, Viscosity, Yeasts, Reprints, Maltodextrins.

Several low-cost maltodextrins formed two liquid phases with polyethylene glycol (PEG) in aqueous solution. The physical characteristics of these maltodextrin/PEG systems are similar in many respects to dextran/PEG systems except for the cost. Maltodextrins are currently available for $\$ 0.56 / \mathrm{kg}$ as compared to $\$ 150 / \mathrm{kg}$ for fractionated dextran (industrial grade). The remarkable cost savings makes the large scale application of polymer-polymer aqueous two-phase extractions commercially viable. The authors have measured some of the physical properties of the maltodextrin/PEG two-phase systems and found them to exhibit low viscosities and excellent processibility. Extraction of alcohol dehydrogenase from homogenized yeast cells and yeast enzyme concentrate was performed in both PEG/dextran and PEG/maltodextrin systems using PEG bound Cibacron blue. Greater purification factors were measured for the PEG/maltodextrin system for similar recoveries.

\section{1,311
PB91-237602}

Not available NTIS National Inst. of Standards and Technology (CSTL), Gaithersburg, MD. Biotechnology Div. Thermodynamics of Industrially Important Enzyme Catalyzed Reactions.

Final rept.

Y. B. Tewari. $1990,17 p$

Pub. in Appl. Biochem. Biotechnol. 23, n3 p187-203 1990.

Keywords: "Thermodynamics, "Enzymes, "Catalysis, Penicillins, Chemical reactions, Amino acids, Saccharides, Enthalpy, Reprints.

The paper presents an examination of the thermodynamics of ten industrially important enzyme catalyzed reactions. The reactions discussed are: the conversions of penicillin $\mathrm{G}$ to 6 -amino-penicillinic acid using the enzyme penicillin acylase; starch to glucose using amylases; glucose to fructose using glucose (xylose) isomerase; cellulose to glucose using cellulase; fumaric acid and ammonia to $L$-aspartic acid using $L$-aspartase; transcinnamic acid and ammonia to L-phenylalanine using L-phenylalanine ammonia-lyase; L-histidine to urocanic acid and ammonia using L-histidine ammonia-lyase; lactose to glucose and galactose using lactase; and the reactions catalyzed by amino acylases and proteases. The selection of these processes was based on the economic value of the products and their intrinsic industrial importance. The available thermodynamic properties, such as equilibrium constants, Gibbs energies (Delta G), enthalpies (Delta $H$ ), and heat capacity changes (Delta C(sub p)) of these enzyme catalyzed reactions are reviewed and summarized. Recommendations are made for future research in this area.

\section{1,312}

PB92-116912

Not available NTIS

National Inst. of Standards and Technology (NML)

Gaithersburg, MD. Chemical Thermodynamics Div.

Damage to the Bases in DNA Induced by Stimulated Human Neutrophils.

Final rept.

J. H. Jackson, P. A. Hyslop, I. U. Schraufistatter, C.

G. Cochrane, M. Dizdaroglu, A. F. Fuciarelli, and E.

Gajewski. 1989, 6p

Pub. in Jnl. of Clinical Investigation 84, n5 p1644-1649 1989

Keywords: "DNA damage, "Neutrophils, Free radicals, Mass fragmentography, Carcinogens, Base pairs, Thymus gland, Reprints.

Inflammatory leukocytes (neutrophils) have been associated with carcinogenesis in chronic inflammation and malignant transformation of cells. Stimulated neutrophils have been known to cause DNA damage. Here, the authors report on the chemical characterization of base damage in DNA induced by a tumor promotor-stimulated human neutrophils. Calf thymus DNA was treated with stimulated neutrophils in the presence and absence of iron ions, and then analyzed by gas chromatography-mass spectrometry with selected-ion monitoring. Cytosine glycol, thymine glycol, 4,6diamino-5-formamidopyrimidine, 8-hydroxyadenine, 2,6-diamino-4-hydroxy-5-formamidopyrimidine and 8 hydroxyguanine were identified as base products in DNA, and their yields were measured. These products are the typical hydroxyl radical-induced products of DNA bases, which are well known from the radiation chemistry of DNA in aqueous solution. This fact suggests that they were likely due to hydroxyl radicals produced in the neutrophil system.

\section{1,313}

PB92-117399

Not available NTIS

National Inst. of Standards and Technology (NML) Gaithersburg, MD. Chemical Thermodynamics Div.

Thermodynamics of the Disproportionation of Adenosine 5'-diphosphate to Adenosine 5'-triphosphate and Adenosine 5'-monophosphate. 2. Experimental Data.

Final rept.

Y. B. Tewari, R. N. Goldberg, and J. V. Advani. 1991, $14 p$

See also PB92-116854

Pub. in Biophysical Chemistry 40, p163-176 1991.

Keywords: "Thermodynamic equilibrium, "Blood cells, "Disproportionation, "Magnesium ions, "Adenosine phosphates, Liquid column chromatography, Calorimetry, Enthalpy, Entropy, ADP, Enzymes, Catalysts, Biochemistry, ATP, Temperature dependence, AMP, Measurement, $\mathrm{pH}$, Hydrolysis, Specific heat, Activity coefficients, Reprints.

High-pressure liquid-chromatography and microcalorimetry have been used to determine equilibrium constants and enthalpies of reaction for the disproportion reaction of adenosine 5'-diphosphate (ADP) to adenosine 5'-triphosphate (ATP) and adenosine 5'-monophosphate (AMP). Adenylate kinase was used to catalyze the reaction. The measurements were carried out over the temperature range 286 to $311 \mathrm{~K}$, at ionic strengths varying from 0.06 to $0.33 \mathrm{~mol} / \mathrm{kg}$, over the $\mathrm{pH}$ range 6.04 to 8.87 , and over the $\mathrm{pMg}$ range 2.22 to 7.16 , where $\mathrm{pMg}=-\log \mathrm{a}(\mathrm{Mg}(\sup 2+))$. The equilibrium model developed by Goldberg and Tawari was used for the analysis of the measurements. For the reference reaction: 2 ADP (3-)(a o) to AMP (2-)(ao) + ATP(4-)(ao), $K=0.225+$ or -0.010 , Delta $G=3.70+$ or $-0.11 \mathrm{~kJ} / \mathrm{mol}$, Delta $H=-1.5+$ or $-1.5 \mathrm{~kJ} / \mathrm{mol}$, Delta $5=-17+$ or $-5 \mathrm{~J} / \mathrm{mol} / \mathrm{K}$ and Delta $\mathrm{C}(\mathrm{sub} \mathrm{p})=$ $46 \mathrm{~J} / \mathrm{mol} / \mathrm{K}$ at $298.15 \mathrm{~K}$ and $0.1 \mathrm{MPa}$. These results and the thermodynamic parameters for the auxiliary equilibria in solution have been used to model the thermodynamics of the disproportionation reaction over a wide range of temperature, $\mathrm{pH}$, ionic strength, and magnesium ion molality. Under approximately physiological conditions $(311.15 \mathrm{~K} . \mathrm{pH} 6.94,(\mathrm{Mg}(2+))=1.35$ $\times 10$ (sup 3-) $\mathrm{mol} / \mathrm{kg}$, and I $=0.23 \mathrm{~mol} / \mathrm{kg}$ ) the apparent equilibrium constant for the overall disproportionation reaction is equal to $0.93+$ or -0.02 . Thermody namic data on the disproportionation reaction and literature values for this apparent equilibrium constant in human red blood cells are used to calculate a molality of $1.94 \times 10$ (sup -4) mol/ $\mathrm{kg}$ for free magnesium ion in human red blood cells. The results are also discussed in relation to thermochemical cycles and compared with data on the hydrolysis of the guanosine phosphates.

\section{Dentistry}

101,314

PB91-187666

(Order as PB91-187617, PC A06/MF A01)

National Inst. of Standards and Technology, Gaithersburg, MD.

Digital Image Analysis Assisted Microradiography Measurement of Mineral Content of Caries Lesions in Teeth.

L. C. Chow, S. Takagi, W. Tung, and T. H. Jordan. 1991, 12p

Prepared in cooperation with American Dental Association Health Foundation, Chicago, IL.

Included in Jnl. of Research of the National Institute of Standards and Technology, v96 n2 p203-214 Mar/Apr 91.

Keywords: "Dental caries, "Microradiography, "Image analysis, Digital data, Lesions, Measurement, Thickness, Mineral content, Remineralization.

The study investigated the feasibility of using a digital image analysis system to process the information contained in microradiographs of tooth sections that included dental caries lesions. The results show that by using an aluminum step wedge to provide a range of thickness standards and a sound area of the sample as an internal reference, data on tooth mineral content as a function of the location can be obtained with an estimated error of less than $5 \%$ relative to the mineral content of sound area. The microradiographic technique allows the response of tooth samples to a remineralization treatment to be quantitatively measured and statistically analyzed.

101,315

PB91-194746

Not available NTIS

National Inst. of Standards and Technology (IMSE) Gaithersburg, MD. Polymers Div.

Effects of $\mathrm{pH}$ and Calcium on Hydrolysis of Na2SiF6 and Na2SnF6: A Quasi-Constant Composition Titration Study.

Final rept.

N. Eidelman, and L. C. Chow. 1991, 7p

Sponsored by American Dental Association Health Foundation, Chicago, IL

Pub. in Caries Research 25, p101-107 1991.

Keywords: "Dental caries, "Calcium ions, "Reaction kinetics, "Hydrolysis, "Acid resistance, $\mathrm{pH}$, Calcium phosphates, Titration, Surface chemistry, Inhibitors, Tin compounds, Fluoridation, Reprints, Sodium hexafluorostannate, Sodium hexafluorosilicate.

Hydrolysis reactions of sodium hexafluorosilicate (Na2SiF6) and sodium hexaflurostannate (Na2SnF6) were studied at various combinations of $\mathrm{pH}$ and calcium ion $(\mathrm{Ca}(2+))$ concentrations using a quasi-constant composition titration method. Under high concentrations of hydrogen ion and $\mathrm{Ca}(2+)$ the promoting effect of $\mathrm{Ca}(2+)$ on the hydrolysis of Na2SiF6 was stronger than the inhibition effect of hydrogen ion. However, the inhibition effect of hydrogen ion on the hydrolysis of $\mathrm{Na2SnF6}$ was stronger than the promoting effect of $\mathrm{Ca}(2+)$. Na2SiF6 and Na2SnF6 were found to have hydrolysis properties that may make them suitable for use with an acidic calcium phosphate solution in a topical fluoride treatment which forms dicalcium phosphate dihydrate as an intermediate. 


\section{Dentistry}

101,316

PB92-126689

(Order as PB92-126614, PC A06/MF A02) National Inst. of Standards and Technology, Gaithersburg, MD.

Mathematical Model for Dental Caries: A Coupled Dissolution-Diffusion Process.

T. M. Gregory, L. C. Chow, and C. M. Carey. 1991,

$12 p$

Included in Jnl. of Research of the National Institute of Standards and Technology, v96 n5 p593-604 Sep/Oct 91.

Keywords: *Dental caries, "Teeth, Diffusion, Hydroxy compounds, Apatites, Computerized simulation, Demineralizing, Dissolution, Mathematical models, Thermodynamic equilibrium, Calcium ions, Phosphates, pH, Plaque formation, Membranes.

Demineralization of tooth mineral in the caries process was studied using a computer model that simulates a diffusion controlled dissolution process. The solution in the inner ('lesion') compartment was in equilibrium with the tooth mineral, but its composition changed in response to diffusion of ions between the two solutions through an infinitely thin barrier. A steady state condition is eventually reached under which the ratio of flux of $\mathrm{Ca}$ to that of $\mathrm{P}$ becomes $5 / 3$. The results of the simulation show that for a given 'plaque' $\mathrm{pH}$, the rate of demineralization at steady state was the highest for cation and the lowest for anion permselective membranes.

\section{Nutrition}

101,317

PB91-147413

Not available NTIS

National Bureau of Standards (NML), Gaithersburg, MD. Inorganic Analytical Research Div.

Dietary Collection Procedures.

Final rept.

G. V. lyengar. 1987, 7p

Pub. in Clinical Nutrition 6, n4 p147-153 Jul/Aug 87.

Keywords: "Food analysis, "Standards, Food consumption, Chemical analysis, Nutritive value, Diets, Nutrition.

There is no single method that may be regarded as entirely satisfactory for the purpose of estimating the human dietary intake of nutrients and toxic substances in foods, since each approach has some limitation or the other. Yet, many decisions of public health concern need to be made with analytical information available from ongoing biomedical trace element research studies. Therefore, every effort should be made to generate reliable analytical data from these investigations. More efforts are urgently needed to develop several kinds of dietary reference materials and to promote their use in standardizing analytical procedures in the food analysis laboratories if the results generated from field studies are to be used for the development of newer concepts for improving the currently used dietary intake models and to provide new guidelines.

\section{1,318}

PB91-147421

Not available NTIS

National Bureau of Standards (NML), Gaithersburg, $M D$. Inorganic Analytical Research Div.

Practical Constraints in Monitoring Micronutrient Intake.

Final rept.

G. V. lyengar. $1987,5 p$

Pub. in Clinical Nutrition 6, n4 p154-158 Jul/Aug 87.

Keywords: *Trace elements, "Monitoring, Chemical analysis, Nutrition, Nutritive value, Diets, Food consumption, "Biomonitoring.

Monitoring of human subjects for the daily intake of essential and toxic trace elements poses certain inherent difficulties. The reasons include, among others (1) there are only a few situations in which a direct association between specific elements, target specimens, and dietary intake have been established, and (2) in many other situations, meaningful information can be derived only when simultaneous analyses of several different specimens from the same subject has been carried out. It is important to recognize that solutions to many of the biomonitoring type of problems require a multidisciplinary approach to the problem as a whole, a sound understanding of the metabolic roles played by each element, as well as the gastrointestinal absorpion properties as observed from different kinds of foods.

\section{Pharmacology \& Pharmacological Chemistry}

101,319

PB91-195149

Not available NTIS

National Inst. of Standards and Technology (IMSE), Gaithersburg, MD. Polymers Div.

Adsorption and Orientation of Tetracycline on Hydroxyapatite.

Final rept.

D. N. Misra. 1991, 6p

Sponsored by American Dental Association Health Foundation, Chicago, IL.

Pub. in Calcified Tissue International 48, p362-367 1991.

Keywords: "Hydroxy compounds, "Apatites, "Tetracyclines, "Separation processes, Adsorbents, Calcification, Bone, Teeth, Polymers, Orientation, Tissues, Eth anols, Chloromethanes, Surface chemistry, Hydrogen bonds, Reprints.

Adsorption of tetracycline from separate solutions of ethanol, p-dioxane, and chloroform onto synthetic hydroxyapatite (containing about 1.5 monolayer of physisorbed water) was studied in order to understand its interaction with bone and teeth. The adsorption isoth erms of tetracycline are reversible and Langmuirian from ethanol and p-dioxane and are almost identical The isotherm is irreversible from chloroform, and a constant amount of adsorbate is removed from the solutions above a certain concentration. An analysis of the reversible isotherms showed that at maximum cov erage the ring or polycyclic structure of the molecule stands perpendicular to the surface with appropriate hydroxyl groups and ketooxygens hydrogen bonded to the surface. The process of adsorption does not affect the chemical integrity of tetracycline.

\section{Public Health \& Industrial Medicine}

\section{1,320}

PB91-204099

Not available NTIS

National Bureau of Standards (NML), Gaithersburg MD. Gas and Particulate Science Div.

Visibility of Chrysotile Asbestos in the Scanning Electron Microscope.

Final rept.

J. A. Small. 1985, 18p

Sponsored by Ontario Research Foundation, Mississauga.

Pub. in Proceedings of Workshop on Asbestos Fibre Measurements in Building Atmospheres, Toronto, Canada, March 1985, p69-86.

Keywords: "Asbestos, "Scanning electron microscopy, "Visibility, Contrast, Chemical analysis, Fibers, Resolution, Reprints, "Chrysotile.

Most manufacturers of conventional scanning electron microscopes (SEM)s claim resolution of the order of 3 $10 \mathrm{~nm}$ for their instruments which is substantially less than the diameters of $20-30 \mathrm{~nm}$ reported for single fibril chrysotile. With this level of resolution, it appears that these instruments should be able to image single fibrils of chrysotile asbestos under normal operating conditions in a real-time imaging mode appropriate to rapid sample searching. The limiting resolution performance of an SEM however can only be realized with an ideal high contrast sample such as a thin edge or a strongly scattering particle viewed against a weakly scattering background. In anlayzing a material such as chrysotile asbestos with a low electron scattering coefficient, the visibility of chrysotile asbestos fibers is limited by the contrast of the sample rather than the ultimate resolution of the SEM used for the analysis.

\section{Radiobiology}

101,321

PB91-190108

Not available NTIS

National Inst of Standards and Technology (CSTL) Gaithersburg, MD. Biotechnology Div.

Mechanistic Studies of Ionizing Radiation and Oxidative Mutagenesis: Genetic Effects of a Single 8Hydroxyguanine (7-Hydro-8-oxoguanine) Residue Inserted at a Unique Site in a Viral Genome.

Final rept.

M. L. Wood, M. Dizdaroglu, E. Gajewski, and J. M.

Essigmann. 1990, 9p

Pub. in Biochemistry 29, n30 p7024-7032 1990.

Keywords: "Ionizing radiation, *Mutagenesis, "Vira genes, DNA adducts, Escherichia coli, High pressure liquid chromatography, Mass fragmentography, Ultraviolet spectroscopy, $\mathrm{T}$ phages, Phosphorylation, Codon, Polyribonucleotide synthetase, Mutation, Reprints, *8-hydroxyguanine.

T4 RNA ligase was used to construct a deoxypentanucleotide containing a single 8-hydroxyguanine (7hydro-8-oxoguanine; $\mathrm{G}$ (sup $8-\mathrm{OH}$ ) residue, which is one of the putatively mutagenic DNA adducts produced by oxidants and ionizing radiation. The pentamer d(GCTAG(sup 8-OH)p was prepared by the ligation of a chemically synthesized acceptor molecule, d(GCTA), to an adducted donor, 8-hydroxy-2'-deoxyguanosine 5',3'-bisphosphate. The acceptor was efficiently converted to the reaction product $(>95 \%)$, and the final product yield was $50 \%$. Following 3 '-dephosphorylation, the pentamer was characterized by UV spectroscopy, by high-pressure liquid chromatography, and by gas chromatography-mass spectrometry of the nucleosides released by enzymatic hydrolysis. Both d(GCTAG(sup 8-OH) and an unmodified control were 5'-phosphorylated by using (gamma-32P) ATP and incorporated covalently by DNA ligase into a fivebase gap at a unique Nhel restriction site in the other duplex genome of an M13mp19 derivative. The ligation product contained $\mathrm{G}$ (sup $8-\mathrm{OH}$ ) at the 3 ' residue of an in-frame amber codon (5'-TAG-3') (genome position 6276) of the phage lacZa gene. The adduct was part of a nonsense codon in a unique restriction site in order to facilitate the identification and selection of mutants generated by the replication of the modified genome in Escherichia coli.

\section{1,322}

PB91-195057

Not available NTIS

National Inst. of Standards and Technology (NML), Gaithersburg, MD. Ionizing Radiation Div.

Absorbed Dose to Water: Comparison of Several Methods Using a Liquid Ionization Chamber. Final rept.

O. Mattsson, H. Svensson, G. Wickman, S. R.

Domen, J. S. Pruitt, and R. Loevinger. 1990, 6p Sponsored by Sahlgren Hospital, Gothenburg (Sweden), and Umea Univ. (Sweden).

Pub. in Acta Oncologica 29, n2 p235-240 1990.

Keywords: "Radiation doses, Liquid ionization chambers, Iron sulfates, Gamma rays, Calorimeters, Dosimetry, Comparison, Water, Reprints.

A liquid ionization chamber has been used as a transfer instrument for the quantity absorbed dose to water in a cobalt -60 gamma ray beam. The characteristics of the liquid ionization chamber are described. The trans. ferred dosimetric information has been compared with absorbed dose determinations using air ionization chamber dosimetry, water calorimetry and ferrous sulphate dosimetry. The agreement between the different measured absorbed dose values is very good, i.e., within $0.2 \%$. This is an indication that the consistency in the methods to determine absorbed dose to water is good. The impact of the new standard for air-kerma, introduced in 1986 by the BIPM, on the air ionization chamber is investigated. It is shown that any differences in the dosimetry when using the old or the new set of data cancel out for the cobalt- 60 beam. The investigation also shows that the value of (epsilon sub $\mathrm{m}) \mathrm{G}$ for the ferrous sulphate dosimeter recommended in ICRU 35 for electrons can be used also in the cobalt -60 beams.

101,323

PB91-203265

Not available NTIS

National Inst. of Standards and Technology (NML), Gaithersburg, MD. Ionizing Radiation Div. 
Interlaboratory Comparlson of Actinides in Human Tissue (239) Pu + (240)Pu.

Final rept.

K. G. W. Inn, W. S. Liggett, H. L. Volchok, M. S.

Feiner, J. F. Mclnroy, D. S. Popplewell, D. R

Percival, R. A. Wessman, V. T. Bowen, H. D.

Livington, R. L. Kathren, and H. Kawamura. 1990,

$11 p$

Pub. in Jnl. of Radioanalytical and Nuclear Chemistry 138, n2 p219-229 1990.

Keywords: "Actinides, "Tissues(Biology), "Plutonium 39. *Plutonium 240, "Interlaboratory comparisons, Humans, Reprints, Standard Reference Materials.

An international laboratory intercomparison of actindes in human tissues was organized by the United States Transuranic Registry and the National Bureau Standards. Five laboratories from the United States, United Kingdom, and Japan participated in the intercomparison. The laboratories were requested to anayze Standard Reference Materials 4351 (Human Lung) and 4352 (Human Liver) for (239)Pu + (240)Pu concentration. In general, approximately equivalent measurement capabilities were found among the participants. The results of the intercomparison were staistically comparable to those used for the original certification of the SRMs, and were combined for a reevaluation of the certified values. The sum of the data sets resulted in notable improvements in the certification for the plutonium content in these SRMs.

101,324

PB91-236497

Not available NTIS

Nechnology (PL), Gaithersburg, MD. Ionizing Radiation Div.

Use of Storage Phosphor Imaging Plates in Portal Imaging and High-Energy Radlography: The Intensifying Effect of Metallic Screens on the Sensitivity.

Final rept.

G. Barnea, E. Navon, A. Ginzburg, J. Politch, H.

Roehrig, C. E. Dick, and R. C. Placious. 1991, 7p

Pub. in Medical Physics 18, n3 p432-438 May/Jun 91.

Keywords: "Biomedical radiography, "Dosimetry, *Phosphors *Nuclear medicine, Sensitivity, Metals, Images, Radiation transport, Photon beams, Cobalt 60 , Spectrum analysis, Radiation doses, Monte Carlo method, Reprints.

The sensitivity of storage phosphor imaging plates (SPIP) at megavolt photon energies (Cobalt $60,6-10$ and $18-\mathrm{MV}$ radiotherapy beams) is studied both experimentally and by Monte Carlo radiation transport calcuations. In addition, the same techniques are used to investigate the intensifying effect of metal screens on the sensitivity of the SPIP. The results provide evidence that the sensitivity of the SPIPs is proportional to the absorbed energy in the phosphor layer per cGy. The spectral sensitivity is calculated for photon energies between $10 \mathrm{keV}$ and $20 \mathrm{MeV}$ for various SPIP. screen combinations.

\section{1,325}

PB91-236877

Not available NTIS

National Inst. of Standards and Technology (PL), Gaithersburg, MD. Ionizing Radiation Div.

Biomarkers of OH Radical Damage In vivo.

Final rept.

L. R. Karam, D. S. Bergtold, and M. G. Simic. 1991, $7 p$

Sponsored by Defense Nuclear Agency, Washington, DC.

Pub. in Free Rad. Res. Comms. 12-13, p11-16 1991.

Keywords: *Chemical radiation effects, "Free radicals, *Hydroxyl radicals, "In vivo, "Biological effects, Mechanism, Synthesis(Chemistry), Tyrosine, Thymine, Glycols, Urine, Biological markers, Reprints.

Mechanisms of formation of o-tyrosine (o-Tyr) and thy mine glycol (TG), the two possible markers of $\mathrm{OH}$ radical generation in biosystems and in vivo are described. The o-Tyr measurements require invasive approaches, while TG detection may be accomplished by noninvasive analysis in the urine.

101,326

PB91-237008

Not available NTIS

National Inst. of Standards and Technology (PL) Gaithersburg, MD. Ionizing Radiation Div.
Sensitometry of the Response of a New Radiochromic Film Dosimeter to Gamma Radiation and Electron Beams.

Final rept.

W. L. McLaughlin, C. Yun-Dong, C. G. Soares, A.

Miller, G. Van Dyk, and D. F. Lewis. 1991, 12p

Pub. in Nuclear Instruments and Methods in Physics Research A302, p165-176 1991.

Keywords: "Dosemeters, "Film dosimetry, "Gamma rays, "Electron beams, Biomedical radiography, Industrial radiography, Images, Irradiation, Nondestructive tests, Sensitivity, Spectrum analysis, Temperature dependence, Reprints.

A new radiation-sensitive imaging material, called GafChromic (TM) Dosimetry Media, offers advances in high-dose radiation dosimetry and high-resolution radiography for gamma radiation and electrons. The potential uses in radiation processing, radiation sterilization of medical devices, population control of insects by irradiation, food irradiation, blood irradiation for rgan-transplant immuno-suppression, clinical radiography, and industrial radiography have led to the present sensitometric study over the breadth of the wide dynamic range of the new routine detector and maging material, namely, absorbed doses from $10 \mathrm{~Gy}$ to $5 \times 10$ to the 4 th power Gy. The thin-coated film is colorless before irradiation, and registers a deep-blue image upon irradiation, with two absorption bands at about $650 \mathrm{~nm}$ (major band) and $600 \mathrm{~nm}$ (minor band). The response to electrons, in terms of increase in absorbance per unit absorbed dose, is the same as that to gamma radiation within the estimated uncertainty of the measurements ( + or - $5 \%, 95 \%$ confidence level). The spatial resolving power is $>1200$ lines $/ \mathrm{mm}$. After the first 24 hours, the image is stable over many months (within + or - $5 \%$ in absorbance), however, the system should be irradiated and analyzed at approximately the temperatures used during calibration, because of temperature dependence during irradiation and readout, and temperatures greater than $55 \mathrm{C}$ should be avoided.

101,327

PB91-237388

Not available NTIS

National Inst. of Standards and Technology (PL), Gaithersburg, MD. Ionizing Radiation Div.

Calibration of Ophthalmic Applicators at NIST: A Revised Approach.

Final rept.

C. G. Soares. 1991, 7p

Pub. in Medical Physics 18, n4 p787-793 Jul/Aug 91.

Keywords: "Radiation doses, "Calibrating, "Film dosimetry, "Opthalmology, "Beta dosimetry, Electric currents, Extrapolation chambers, Irradiation, Spectrum rents, Extrapolation chambers, lrradiation, Spectrum mic applicators.

A revised approach to the problem of measuring a surface-absorbed dose from beta-particle-emitting ophthalmic applicators is presented. The technique chosen employs an extrapolation chamber equipped with a 4-mm-diam collecting electrode to make current measurements at air gaps from 0.08 to $0.20 \mathrm{~mm}$ at $0.02-\mathrm{mm}$ intervals. These data yield a linear relationship between current and air gap, the slope of which is used to determine average surface-absorbed-dose rate over the central area of the source. For additional information about the distribution of the activity over the source surface, autoradiographs using calibrated radiochromic dye films are analyzed to map the doserate profile across the surface of the applicator. Experiments varying several parameters of the extrapolation chamber measurement, including collecting electrode area, voltage gradient, range of air gaps used, and entrance foil material, are described. Also treated are calibrations of, and a description of the use of, radiochromic dye films for source profiling. Experiments for determining correction factors for the extrapolation chamber measurements are described, and an assessment of the uncertainties associated with these measurements is given.

\section{Toxicology}

101,328

PB91-162032

Not available NTIS

National Inst. of Standards and Technology (IMSE), Gaithersburg, MD. Polymers Div.
Newest Approaches to Quantitative Assessment of Bioactive Organotins.

Final rept.

I. C. Felkner, B. Worthy, G. J. Olson, and F. E.

Brinckman. 1989, 14p

Pub. in Chemical Speciation and Bioavailability 1, n3 p79-92 Oct 89

Keywords: "Organotin compounds, "Bacillus subtilis, *Toxicity, Bioassay, Lasers, Genetic engineering, Reprints, "Toxic substances.

New approaches for assessing the toxicity of organotin chemical species have been developed. These approaches are based upon the selective responses of sensitive biodetectors whose chemical and molecular interactions with a wide variety of toxicants have been previously determined and reported. Bioassays capable of quantitating and chemically speciating toxicants for impacts on diverse bacterial sensors are discussed herein. The principal new development is a laser/bacterial bioassay which is capable of differentiating between various toxic chemicals and specifically distinguishing between the different organotin species based on their mechanism of toxic action. The system uses a battery of isogenic Bacillus subtilis strains genetically engineered to respond differentially to specific toxicants. The response is monitored by differential light scattering of a laser which is integrated with a computerized system that collects and analyzes the data. The system routinely generates fully analyzed data within $66 \mathrm{~min}$ for most samples. It is capable of making 1,200 measurements on each sample within 2 to 4 seconds, and shows promise as a rapid and inexpensive system to monitor organotins and various other toxicants on site.

101,329

PB91-187070

Not available NTIS

National Inst. of Standards and Technology (NML), Gaithersburg, MD. Chemical Kinetics Div.

Base Modifications in Plasmid DNA Caused by Potassium Permanganate.

Final rept.

S. A. Akman, J. Doroshow, and M. Dizdaroglu. 1990

Pub. in Archives of Biochemistry and Biophysics 282 n1 p202-205 Oct 90.

Keywords: "Plasmids, "Deoxyribonucleic acids, "Potassium permanganate, "DNA damage, "Toxicity, Mass fragmentography, Mutagenicity tests, Cell survival, lons, Reprints.

$\mathrm{KMnO} 4$ is a powerful oxidizing agent which has been used to modify DNA bases. In previous studies, mild $\mathrm{KMnO} 4$ treatment has been shown to preferentially modify Thy; Cyt and Gua are modified only under harsher conditions to as yet unidentified products. In the present study, denatured plasmid pCMV beta gal DNA was exposed to 0.015-1.5 mM KMnO4, pH 8.6, at $4 \mathrm{C}$ for $5 \mathrm{~min}$, after which the DNA was hydrolyzed in formic acid, trimethylsilylated, and analyzed for modified base content by gas chromatography-mass spectrometry/selected ion monitoring. The study data suggest that previous studies which have used treatment with $\mathrm{KMnO} 4$ to study the mutagenicity of Thy glycol specifically or as a Thy-specific probe in DNA structure should be interpreted with caution.

101,330

PB91-187104

Not available NTIS

National Inst. of Standards and Technology (CSTL), Gaithersburg, MD. Biotechnology Div.

Copper-Ion-Dependent Damage to the Bases in DNA in the Presence of Hydrogen Peroxide.

Final rept.

O. I. Aruoma, B. Halliwell, E. Gajewski, and M Dizdaroglu. 1991, 4p

Pub. in Biochemistry JnI. 273, p601-604 1991

Keywords: "DNA damage, "Hydrogen peroxide, *Toxicity, "Copper, "Iron, lons, Hydroxyl radicals, Catalase, Superoxide dismutase, Ascorbic acid, Reprints, Hypoxanthine-xanthine oxidase system.

Mixtures of $\mathrm{Cu}($ sup $2+$ ) and $\mathrm{H} 2 \mathrm{O} 2$ at $\mathrm{pH} 7.4$ caused damage to the bases in DNA greater than that caused by mixtures of $\mathrm{Fe}\left(\mathrm{Sup} 3+\right.$ ) and $\mathrm{H}_{2} \mathrm{O} 2$. Addition of ascorbic acid to the Cu(Sup $2+$ )/ $/ \mathrm{H} 2 \mathrm{O} 2$ system caused a very large increase in base damage, much greater than that produced by the $\mathrm{Fe}(\sup 3+) / \mathrm{H}_{2} \mathrm{O} 2 /$ ascorbic acid system. The products of base damage in the presence of Cu(sup $2+$ ) were typical products that have been shown to result from attack of hydroxyl radi- 
cals upon the DNA bases. Cytosine glycol, thymine glycol, 8-hydroxyadenine and especially 8-hydroxyguanine were the major products in both the Cu(sup $2+) / \mathrm{H}_{2} \mathrm{O} 2$ and the $\mathrm{Cu}(\sup 2+$ )/H2O2/ascorbic acid systems. Base damage in DNA by these systems was inhibited by the chelating agents EDTA and nitrilotriacetic acid and by catalase, but not by superoxide dismutase, nor by the hydroxyl-radical scavenger mannitol. It is proposed that Cu(sup $2+$ ) ions bound to the DNA react with $\mathrm{H} 2 \mathrm{O} 2$ and ascorbic acid to generate hydroxyl radicals, which then immediately attack the DNA bases in a site-specific manner. A hypoxanthine/ xanthine oxidase system also caused damage to the DNA bases in the presence of $\mathrm{Cu}(\mathrm{sup} 2+$ ) ions. This was inhibited by superoxide dismutase and catalase. The high activity of $\mathrm{Cu}(\sup 2+$ ) ions, when compared with Fe(sup $3+$ ) ions, in causing hydroxyl-radical-dependent damage to DNA and to other biomolecules. means that the availability of Cu(sup $2+$ ) ions in vivo must be carefully controlled.

\section{1,331}

PB91-187112

National Inst. of Standards and Technology (NML) Gaithersburg, MD. Chemical Kinetics Div.

Damage to the Bases in DNA Induced by Hydrogen Peroxide and Ferric Ion Chelates.

Final rept.

O. I. Aruoma, B. Halliwell, E. Gajewski, and M.

Dizdaroglu. 1989, $4 \mathrm{p}$

Pub. in Jnl. of Biological Chemistry 264, n34 p2050920512, 5 Dec 89.

Keywords: “DNA damage, *Hydrogen peroxide, *Iron, "Chelating agents, Toxicity, Hydroxyl radicals, Enzyme inhibitors, Superoxide dismutase, Carcinogens, Kidney, Reprints, Hypoxanthine-xanthine oxidase system.

Incubation of a number of ferric ion chelates with $\mathrm{H} 2 \mathrm{O} 2$ at $\mathrm{pH} 7.4$ generated a reactive species able to produce chemical modifications of the bases in DNA that are very similar to those produced in DNA by the hypoxanthine/xanthine oxidase system. Products were identified and quantitated by the use of gas chromatography-mass spectrometry with selected-ion monitoring. Compared with other complexes used, ferric ion-nitrilotriacetic acid produced by far the largest amount of the base products. Typical hydroxyl radi$\mathrm{cal}$ scavengers and superoxide dismutase provided significant decreases in the yields of the products. On this basis, it is proposed that ferric ion complexes react with $\mathrm{H} 2 \mathrm{O} 2$ to produce hydroxyl radical; this was also shown using the deoxyribose assay.

\section{1,332}

PB91-187468 Not available NTIS National Inst. of Standards and Technology (CSTL), Gaithersburg, MD. Biotechnology Div.

Chemical Determination of Free Padical-Induced Damage to DNA.

Final rept.

M. Dizdaroglu. 1991, 18p

Sponsored by Department of Energy, Washington, DC. Pub. in Free Radical Biology and Medicine 10, p2252421991

Keywords: "Free radicals, "DNA damage, "Toxicity, Mass fragmentography, Hydroxyl radicals, Cross-linking reagents, Histones, Chromatin, Mammals, Reprints.

Free radical-induced damage to DNA in vivo can result in deleterious biological consequences such as the initiation and promotion of cancer. Chemical characterization and quantitation of such DNA damage is essential for an understanding of its biological consequences and cellular repair. Methodologies incorporating the technique of gas chromatography/mass spectrometry (GC/MS) have been developed in recent years for measurement of free radical-induced DNA damage. The use of GC/MS with selected-ion monitoring (SIM) facilitates unequivocal identification and quantitation of a large number of products of all four DNA bases produced in DNA by reactions with hydroxyl radical, hydrated electron, and $\mathrm{H}$ atom. Hydroxyl radical-induced DNA-protein cross-links in mammalian chromatin, and products of the sugar moiety in DNA are also unequivocally identified and quantified. The sensitivity and selectivity of the GC/MS-SIM technique enables the measurement of DNA base products even in isolated mammalian chromatin without the necessity of first isolating DNA, and despite the presence of histones. Recent results demonstrate the usefulness of the GC/MS technique for chemical determination of free radical-induced DNA damage in DNA as well as in mammalian chromatin under a vast variety of conditions of free radical production.

\section{1,333}

PB91-187492

Not available NTIS

National Inst. of Standards and Technology (CSTL), Gaithersburg, MD. Biotechnology Div.

Modification of Bases in DNA by Copper Ion-1,10Phenanthroline Complexes.

Final rept

M. Dizdaroglu, O. I. Aruoma, and B. Halliwell. 1990, $5 p$

Pub. in Biochemistry 29, n36 p8447-8451 1990.

Keywords: "DNA damage, "Toxicity, Superoxide, Pyrimidines, Purines, Hydroxyl radicals, Catalase, Dimethyl sulfoxide, Reprints, Copper ion-phenanthroline complexes.

Damage to the bases in DNA by the cupric ion-1,10phenanthroline complex was investigated. Ten base products in DNA were identified and quantitated by the use of gas chromatography/mass spectrometry with selected-ion monitoring. DNA damage by the cupric ion-1,10-phenanthroline complex required the presence of a reducing agent such as ascorbic acid or mercaptoethanol. Products identified were typical hydroxyl radical induced products from the pyrimidines and purines in DNA, well-known from previous studies using various hydroxyl radical producing systems such as ionizing radiation, hypo-xanthine/xanthine oxidase, or hydrogen peroxide in the presence of transition metal ions. Product formation was not significantly inhibited by typical scavengers of hydroxyl radical such as mannitol and sodium formate, but there was partial inhibition by dimethyl sulfoxide. Catalase substantially decreased formation of base products, and added hydrogen peroxide stimulated it, indicating the hydrogen peroxide dependency of DNA base damage. Superoxide dismutase afforded only a partial reduction in product yields in systems containing ascorbic acid. On the basis of the types of base products formed, the hydrogen peroxide dependency of product formation, and a previous report suggesting that DNA damage is due to a diffusible species the study suggests that DNA base damage is caused by hydroxyl radical.

101,334

PB91-187518

Not available NTIS National Inst. of Standards and Technology (CSTL), Gaithersburg, MD. Biotechnology Div.

Structure and Mechanism of Hydroxyl Radical-Induced Formation of a DNA-Protein Cross-Link Involving Thymine and Lysine in Nucleohistone. Final rept.

M. Dizdaroglu, and E. Gajewski. 1989, 5p

Pub. in Cancer Research 49, p3463-3467, 1 Jul 89

Keywords: "DNA damage, "DNA-binding proteins, *Thymine, "Lysine, "Hydroxyl radicals, *Toxicity, Mass fragmentography, Cross-linking reagents, Amino acids, Reprints, "Nucleohistones.

Hydroxyl radical-induced formation of a DNA-protein cross-link involving thymine and lysine in calf thymus nucleohistone in vitro is reported. Basic amino acids such as lysine constitute a very high proportion of the amino acids of histones, and help histones to bind to DNA in chromatin. For this reason, basic amino acids are likely to participate in DNA-protein cross-linking. For identification of the thymine-lysine cross-link in nucleohistone, hydroxyl radical-induced cross-linking of thymine to lysine was investigated first using a mode system, i.e., an aqueous mixture of thymine and lysine. Hydroxyl radicals were generated by exposure of the mixture to ionizing radiation after $\mathrm{N} 2 \mathrm{O}$ saturation. The technique of gas chromatography-mass spectrometry was used to analyze the samples for possible crosslinks. One thymine-lysine cross-link was found and its links. One thymine-lysine cross-link was found and its
structure was elucidated. Using gas chromatographymass spectrometry with selected-ion monitoring, this thymine-lysine cross-link was identified in acidic hydroysates of calf thymus nucleohistone gamma-irradiated in $\mathrm{N} 2 \mathrm{O}$-saturated aqueous solution.

\section{1,335}

PB91-187526

Not available NTIS

National Inst. of Standards and Technology (CSTL) Gaithersburg, MD. Biotechnology Div.
Chemical Nature of In vivo DNA Base Damage in Hydrogen Peroxide-Treated Mammalian Cells. Final rept.

M. Dizdaroglu, Z. Nackerdien, B. C. Chao, E.

Gajewski, and G. Rao. 1991, 3p

Sponsored by Department of Energy, Washington DC., and National Science Foundation, Washington,

Pub. in Archives of Biochemistry and Biophysics 285, n2 p388-390 Mar 91.

Keywords: "DNA damage, "Hydrogen peroxide, "Toxicity, Mammals, Cells(Biology), In vivo analysis, Mass fragmentography, Hydroxyl radicals, Chromatin, Reprints.

Hydrogen peroxide is generated in mammalian cells by normal metabolism or by treatment with external agents. Treatment of mammalian cells with this oxidiz ing agent results in DNA damage. Little is known about the chemical nature of hydrogen peroxide-mediated DNA damage in mammalian cells. The study reports on the chemical characterization of in vivo base damage to nuclear DNA in mammalian cells caused by exposure to $\mathrm{H} 2 \mathrm{O} 2$ Chromatin was isolated from cells and analyzed by gas chromatography/mass spectrometry with selected-ion monitoring. Ten DNA base products were identified and quantitated. Modified bases identified were typical hydroxyl radical-induced products of DNA bases. Results indicate involvement of hydroxyl radicals in the mechanism of nuclear DNA damage in mammalian cells caused by $\mathrm{H}_{2} \mathrm{O} 2$.

101,336

PB91-189217

Not available NTIS

National Inst. of Standards and Technology (CSTL) Gaithersburg, MD. Biotechnology Div.

Damage to the DNA Bases in Mammalian Chromatin by Hydrogen Peroxide in the Presence of Ferric and Cupric lons.

Final rept.

M. Dizdaroglu, G. Rao, B. Halliwell, and E. Gajewski. 1991, 8p

Sponsored by Department of Energy, Washington, DC 2 in Archives of

Keywords: "DNA damage, "Chromatin, "Hydrogen peroxide, "Iron, "Copper, "Toxicity, Cations, Mammals, Superoxide dismutase, Hydroxyl radicals, Reprints.

Modification of DNA bases in mammalian chromatin upon treatment with hydrogen peroxide in the presence of ferric and cupric ions was studied. Ten DNA base products in mammalian chromatin were identified and quantitated by the use of gas chromatographymass spectrometry with selected-ion monitoring after hydrolysis of chromatin and trimethylsilylation of hydrolysates. Hydrogen peroxide in the presence of cupric ions caused more DNA damage than in the presence of ferric ions. Chelation of cupric ions caused a marked inhibition in product formation. By contrast, DNA was damaged more extensively in the presence of chelated ferric ions than in the presence of unchelated ferric ions. The presence of ascorbic acid generally increased the yields of the products, indicating increased production of hydroxyl radical by reduction of metal ions by ascorbic acid.

101,337

PB91-189357 Not available NTIS National Inst. of Standards and Technology (CSTL), Gaithersburg, MD. Biotechnology Div.

Bleomycin-Dependent Damage to the Bases in DNA Is a Minor Side Reaction.

Final rept.

E. Gajewski, O. I. Aruoma, M. Dizdaroglu, and B.

Halliwell. 1991, 5p

Pub. in Biochemistry 30, n9 p2444-2448 1991.

Keywords: "Bleomycin, "Toxicity, "DNA damage, Antineoplastic agents, Gas fragmentography, Catalase Hydroxyl radicals, Iron, Hydrogen peroxide, Superoxide dismutase, Thiobarbituric acid, Reprints.

The antitumor antibiotic bleomycin degrades DNA in the presence of ferric ions and $\mathrm{H} 2 \mathrm{O} 2$ or in the presence of ferric ions, oxygen, and ascorbic acid. When DNA degradation is measured as formation of base propenals by the thiobarbituric acid assay, it is not in hibited by superoxide dismutase and scavengers of the hydroxyl radical or by catalase (except that catalase inhibits in the bleomycin/ferric ion/H2O2 system by removing $\mathrm{H} 2 \mathrm{O} 2$ ). Using the technique of gas chro- 
matography/mass spectrometry with selected-ion monitoring, the study shows that DNA degradation is accompanied by formation of small amounts of modified DNA bases. The products formed are identical with those generated when hydroxyl radicals react with DNA bases. Base modification is significantly inhibited by catalase and partially inhibited by scavengers of the hydroxyl radical and by superoxide dismutase. The study suggests that the bleomycin-oxo-iron ion complex that cleaves the DNA to form base propenals can decompose in a minor side reaction to generate hydroxyl radical, which accounts for the base modification in DNA. However, hydroxyl radical makes no detectable contribution to the base propenal formation.

101,338

PB9 1-189373

Not available NTIS

National Inst. of Standards and Technology (CSTL) Gaithersburg, MD. Biotechnology Div.

Modificatlon of DNA Bases in Mammalian Chroma tin by Radiatlon-Generated Free Radicals.

Final rept.

E. Gajewski, G. Rau, Z. Nackerdien, and M.

Dizdaroglu. 1990, 7p

Pub. in Biochemistry 29, n34 p7876-7882 1990.

Keywords: "DNA damage, "Chromatin, "Free radicals, "Toxicity, "Ionizing radiation, Mammals, Hydrolysis, Superoxide, Mass fragmentography, Reprints.

Modification of DNA bases in mammalian chromatin in aqueous suspension by ionizing radiation generated free radicals was investigated. Argon, air, N2O and $\mathrm{N} 2 \mathrm{O} / \mathrm{O} 2$ were used for saturation of the aqueous $\mathrm{N} 2 \mathrm{O} / \mathrm{O} 2$ were used for saturation of the aqueous system in order to provide different radical environments. Radiation doses ranging from 20 to $200 \mathrm{~Gy}$ ( $\mathrm{J} /$ interactions with pyrimidines and purines in chromatin were identified and quantitated by using the technique of gas chromatography/mass spectrometry with selected-ion monitoring after acidic hydrolysis and trilected-ion monitoring after acidic hydrolysis and trimethylsilylation of chromatin. The methodology used permitted analysis of the modified bases directly in chromatin without the necessity of isolation of DNA from chromatin first. The results indicate that the radical environment provided by the presence of different gases in the system had a substantial effect on the
types of products and their quantities. Some products were produced only in the presence of oxygen, whereas other products were detected only in the absence of oxygen. Products produced under all four gaseous conditions were also observed Generally, the pres ence of oxygen in the system increased the yields of ence of oxygen in the system increased the yields of the products with the exception of formamidopyrimiair, and to a lesser extent in the presence of $\mathrm{N} 2 \mathrm{O} / \mathrm{O} 2$ had no effect on product formation.

\section{MILITARY SCIENCES}

\section{Logistics, Military Facilities, \& Supplies}

101,339

PB91-158832

Not available NTIS

National Inst. of Standards and Technology (NCSL) Gaithersburg, MD. Information Systems Engineering Div.

Data Management Standards in Computer-Aided Acquisition and Logistic Support (CALS)

Final rept.

D. K. Jefferson. 1990, 30p

Pub. in Proceedings of Technology for Space Station Evolution Workshop, Dallas, TX., January 16-19, 1990 v2 p197-226 1990.

Keywords: "Logistics support, "Computer applications, "Data management, "Standards, Federal information processing standards, Data bases, Query languages, Quality control, Reprints, IRDS(Information Resource Dictionary System), RDA(Remote Database Access), SQL

The Computer-aided Acquisition and Logistic Support (CALS) initiative intends to replace the present flow of paper between islands of automation with, initially, digital data interchange, and, ultimately, an integrated

shared database. These objectives can be attained in an open systems (non-proprietary) environment only through the use of national and international standards for data interchange and database. The presentation describes the current status and future expectations for the key database standards: the SQL family for database definition and manipulation; the IRDS family for metadata management; and the RDA family for data. base distribution. Together these standards will provide a robust, flexible foundation for data management solutions to important national problems of efficiency and quality control.

\section{1,340}

PB91-167247

PC A05/MF A01

National Inst. of Standards and Technology (NEL) Gaithersburg, MD. Center for Building Technology.

Field Study of the Performance of EPDM Roofing at Air Force Facilities.

W. J. Rossiter, J. F. Seiler, W. P. Spencer, and P. E. Stutzman. Jan 91,78 p NISTIR-4504

Sponsored by Air Force Engineering and Services Center, Tyndall AFB, FL.

Keywords: "Military facilities, "Roofs, "Inspection, Membranes, Performance, Mechanical tests, Field tests, Surveys, Defects, Adhesion, Seams(Joints).

A study was conducted at the request of the Air Force Engineering and Services Center to obtain and analyze information on the in-service performance of lowsloped EPDM roofing systems at Air Force installations. Because of the benefits to be gained in having available alternative materials for fabricating membranes for low-sloped roofing systems, the Air Force has proposed developing a guide specification for EPDM roofing. Technical data are needed to support the development of the guide specification. The information obtained in the study contributes to the data base. Fifteen USAF installations in 11 states were visited, and 61 EPDM roofs were inspected. This represented about 50 percent of the number of Air Force installations and buildings with EPDM roofing. The age of the roof systems ranged from 3 to 156 months, although 40 percent were only 30 months old or less. The inspections were performed by walking over the roofs during which notes were recorded and photos were taken. During the field visits, discussions were held with base engineering personnel to determine their views of the performance of EPDM roofing under their responsibility. Considering the relatively young age of the roofs inspected, their overall performance was found to be satisfactory. About half were visually seen to be in fine condition, while another third displayed only minor defects which were limited in scope and were considered to be readily reparable with routine maintenance. On a less positive note, in the latter case the observed defects had gone without repair. This illustrated a key concern expressed by field personnel that they lacked ability to perform routine maintenance.

101,341

PB91-167296 Gaithersburg, MD.

Raster Graphics Conformance Testing.

Keywords: "Computer graphics, "Sweep generators, "Laboratories, "Tests, Conformity, Standards, Evaluation, Programs, Management, "Computer-aided Acquisition and Logistic Support, Department of Defense.

The report evaluates the alternatives for identifying and selecting a conformance testing laboratory for raster graphics in support of the Department of Defense (DoD) Computer-aided Acquisition and Logistic Support (CALS) Program. It discusses and analyzes four different approaches to selecting a conformance testing laboratory. After discussing the approaches, it recommends an alternative for DoD to pursue in selecting a laboratory for conducting conformance testing of raster graphics implementations. The annexes to the report describe the requirements, procedures, forms, and criteria necessary for establishing and managing a raster graphics conformance testing program.

101,342

\section{PB91-178889}

PC A12/MF A02

National Inst. of Standards and Technology (NCSL), Gaithersburg, MD.
Collection of Technical Studies Completed for the Computer-Alded Acquisition and Logistic Support (CALS) Program. Fiscal Year 1988. Volume 1 of 3. Text, Security and Data Management.

Rept. for Oct 87-Sep 88

R. S. Morgan. Apr $90,253 p$ NISTIR-4315

See also PB91-178897 and PB91-178905. Sponsored by Assistant Secretary of Defense (Production and Lo gistics), Washington, DC. Computer-Aided Acquisition and Logistic Support Program.

Keywords: "Data management, "Text processing, "Computer security, Publishing. Federal information processing standards, Computer graphics, Tests, ${ }^{\text {} C A L S(C o m p u t e r-a i d e d ~ A c q u i s i t i o n ~ a n d ~ L o g i s t i c ~ S u p-~}$ port), National Institute of Standards and Technology, PDES(Product Data Exchange Specification).

Computer-aided Acquisition and Logistic Support (CALS) Program is a Department of Defense (DoD) and Industry strategy to transition from paper-intensive acquisition and logistic processes to a highly automated and integrated mode of operation for the weapon systems of the 1990s. The three volumes of the report document and accomplishments of the National Institute of Standards and Technology (NIST) to advance the development of technology and standards in support of CALS. In Volume 1, work on text and graphics standards in the CALS publishing environment is described, including technology assessments, application guidance, conformance test plans and a draft Federal Information Processing Standard (FIPS) for ODA ODIF. Additionally, a technology assessment and proposed conformance testing strategy for page description management tools is presented with a discussion of computer security issues. The use of the Informa tion Resource Dictionary System, (IRDS, ANSI Stand ard $\mathrm{X} 3.138-1988$ ) is proposed as an integration and configuration management mechanism for the Product Data Exchange Specification (PDES)

101,343

PB91-178897

PC A21/MF A03

National Inst. of Standards and Technology (NCSL), Gaithersburg, MD.

Collection of Technical Studies Completed for the Computer-Aided Acquisition and Logistic Support (CALS) Program, Fiscal Year 1988. Volume 2 of 3. Graphics, CGM MIL-SPEC

Rept. for Oct 87-Sep 88

R. S. Morgan. Mar 91, $482 p$ NISTIR-4316

See also PB91-178889 and PB91-178905. Sponsored by Assistant Secretary of Defense (Production and Logistics), Washington, DC. Computer-Aided Acquisition and Logistic Support Program.

Keywords: "Computer graphics, "Standards, Tests, Information systems, Data processing, Text processing, Protocols, "CALS(Computer-aided Acquisition and Logistic Support), National Institute of Standards and Technology, CGM(Computer Graphics Metafile), Department of Defense.

Computer-aided Acquisition and Logistic Suppor (CALS) Program is a Department of Defense (DoD) Industry strategy to transition from paper-intensive acquisition and logistic processes to a highly automated and integrated mode of operation for the weapon systems of the 1990s. The three volumes of the repor document the accomplishments of the National Institute of Standards and Technology (NIST) to advance the development of technology and standards in support of CALS. In Volume 2, progress in the Compute Graphics Metafile (CGM) standard is described, including work in the graphics standards committees and the expansion and updating of the CALS CGM application profile. A draft Military Specifications for CGM is included. A plan for Extended CGM is presented, including documentation of relevant standards committee work.

101,344

PB91-178905

PC A08/MF A01

National Inst. of Standards and Technology (NCSL), Gaithersburg, MD.

Collection of Technical Studies Completed for the Computer-Aided Acquisition and Logistic Support (CALS) Program, Fiscal Year 1988. Volume 3 of 3. CGM Registration.

Rept. for Oct 87 -Sep 88

R. S. Morgan. Apr 90, 173p NISTIR-4317

See also PB91-178897 and PB91-178889. Sponsored by Assistant Secretary of Defense (Production and Logistics), Washington, DC. Computer-Aided Acquisition and Logistic Support Program. 


\title{
MILITARY SCIENCES
}

\author{
Logistics, Military Facilities, \& Supplies
}

Keywords: Registration, Programming languages, Standardization, "CALS(Computer-aided Acquisition and Logistic Support), "CGM(Computer Graphics Metafile), National Institute of Standards and Technology. Computer-aided Acquisition and Logistic Support (CALS) Program is a Department of Defense (DOD) and Industry strategy to transition from paper-intensive acquisition and logistic processes to a highly automated and integrated mode of operation for the weapon systems of the 1990s. The three volumes of the report document the accomplishments of the National Institute of Standards and Technology (NIST) to advance the development of technology and standards in support of CALS. Volume 3 documents work accomplished to meet CALS needs for Computer Graphics Metafile (CGM) functionality through the registration of graphical elements within the standardization process.

\section{1,345}

PB91-184812

PC A03/MF A01

National Inst. of Standards and Technology (NCSL), Gaithersburg, MD.

Standard Generalized Markup Language Encoding of the Office Document Architecture Document Application Profile.

R. B. Wilson. Apr 91, 34p NISTIR-4547

Keywords: "Information processing, "Documents, Standards, Text processing, Logistics support, Computer applications, "Computer-aided Acquisition and Logistic Support, "SGML(Standard Generalized Markup Language), ODA(Office Document Architecture), DAP(Document Application Profile), Abstract Syntax Notation One.

The Office Systems Engineering Group (OSE) at the National Institute of Standards and Technology (NIST) was tasked by the Computer-aided Acquisition and Logistic Support (CALS') Project Office to bring CALS' requirements to the Open Systems Interconnection (OSI) Implementor's Workshop sponsored by NIST. CALS tasked the OSE Group to assist in the SGML encoding of the Office Document Architecture (ODA) Document Application Profile (DAP). NIST offered to encode the ODA DAP in the Standard Generalized Markup Language (SGML) to illustrate the similarities between the two standards and to provide a common SGML/ASN.1 profile. The report describes in various levels of detail the two international standards. It then discusses, by offering a simple example, the methodology involved in performing an SGML encoding. Subsequently, it examines the SGML encoding of the ODA DAP. The report provides two accompanying tables with the SGML encoding and finishes with a brief summary of the two standards.

\section{1,346
PB91-187708}

PC A06/MF A01

National Inst. of Standards and Technology (CSL),

Gaithersburg, MD.

Tiled Raster Graphics and MIL-R-28002A: A Tutorial and Implementation Guide.

F. E. Spielman, and L. H. Sharpe. Apr 91, 106p NISTIR-4567

Sponsored by Assistant Secretary of Defense (Production and Logistics), Washington, DC. Computer-Aided Acquisition and Logistic Support Program.

Keywords: "Computer graphics, "Sweep generators, Standards, Documents, Standardization, Image processing. Data compression. Coding. Software tools, "Military specification MIL-R-28002A, Abstract Syntax "Military specification MIL-R-28002A, Abstract Syntax tic Support.

The report examines the technical issues facing an implementor of the raster data interchange format de fined in military specification MIL-R-28002A. Information previously scattered throughout several standards is incorporated into the report for ease of reference. The National Institute of Standards and Technology Office Document Architecture Raster Document Application Profile (NIST ODA Raster DAP) is analyzed with regard to both notation and intent.

\section{1,347}

PB91-193821

PC A03/MF A01

National Inst. of

NIST Support of the CALS Program: 1990 Synop-

sis. J. Kemmerer. Jun 91, 22p NISTIR-4609

Keywords: "Project management, Data transmission, Computer graphics, Documents, Standards, Adminis- tration, Data management, Computer security, Telecommunication, Bibliographies, "CALS(Computeraided Acquisition and Logistics Support), *NIST(National Institute of Standards and Technology).

The report summarizes overall Computer-aided Acquisition and Logistics Support (CALS) program management, technical support, and administration provided by National Institute of Standards and Technology (NIST). A brief summary of some of the 1990 activities is offered in each of the general technical support areas: electronic data interchange, graphics, document standards, raster compression, data management, security, and data communication. Most of the NIST deliverables given to the CALS Office have since been published for easier access by the CALS community. The report offers the titles and brief abstracts of such published deliverables, as well as titles and abstracts for those NIST CALS deliverables published in previous years.

101,348

PB91-194506

PC A14/MF A02

National Inst. of Standards and Technology (CSL), Gaithersburg, MD.

NIST Support for the Computer-Aided Acquisition and Logistic Support (CALS) Program in the Area of Graphics Standards, Calendar Year 1990.

Rept. for Oct 89-Dec 90

D. R. Benigni. May 91,311 p NISTIR-4579

See also PB90-228016 and PB90-257759. Sponsored by Office of the Secretary of Defense, Washington,

Keywords: "Logistics support, "Computer applications, "Computer graphics, "Standards, Requirements, Federal information processing standards, Specifications, Software tools, CALS(Computer-aided Acquisition and Logistic Support), NIST(National Institute of Standards and Technology), Department of Defense.

Computer-aided Acquisition and Logistic Support (CALS) is a program of the Office of the Secretary of Defense. Its objective is to establish an integrated set of standards and specifications for the creation, management, and exchange of logistics data and product development data--including graphical data--by computer. Since FY86, the National Institute of Standards and Technology (NIST) has been funded to recommend the standards to satisfy CALS requirements for system integration and digital data transfer, and to accelerate standards implementation. The report comprises the continuing work of the NIST Graphics Software Group in support of the CALS Program for 1990. The format of the report combines the separate task deliverables assigned to the NIST Graphics Software Group for 1990 which were as follows: (1) update Computer Graphics Metafile (CGM) Application Profile; (2) inject CALS requirements into the standards committees' work on Amendments to the CGM standard; (3) produce a software tool to determine conformance of a metafile to the CGM standard and to MIL-D28003; and (4) explore potential sources of generator and interpreter conformance test capabilities.

\section{1,349}

PB92-112440

National Inst. of Standards and Technology (CAML), Gaithersburg, MD. Office of Applied Economics.

Economic Analysis for Military Construction (MILCON) Design. Concepts, Techniques, and Applications for the Analyst. Instructor's Manual. R. T. Ruegg, and S. K. Fuller. Sep $91,637 \mathrm{p}$ NISTIR$90 / 4256$

See also Student's manual, PB92-112457. Sponsored by Corps of Engineers, Huntsville, AL. Huntsville Training Div.

Keywords: "Military training, "Construction management, "Economic analysis, "Instructors, Benefit cost analysis, Energy conservation, Life cycle costs, Structural design, Manuals, Requirements, Buildings, Military facilities, Case studies.

The Instructor's Manual is a detailed guidebook for teaching the five-day course, 'Economic Analysis for Military Construction Design: Concepts, Techniques, and Applications for the Analyst'. It provides numerous aids for teaching the course, including pre-course check-lists, descriptions of instructional techniques, tips for making the course more effective, and step-bystep lesson plans. Copies of all slides and vugraphs step lesson plans. Copies of all slides and vugraphs entation text, exercises, and solutions. Through spe- cial fonts and a page numbering system geared to the Student Manual, the instructor always knows exactly what the student sees without the necessity of manipulating both manuals at once.

\section{1,350}

PB92-112457

PC A21/MF A04

National Inst. of Standards and Technology (CAML) Gaithersburg, MD. Office of Applied Economics.

Economic Analysis for Military Construction (MILCON) Design. Concepts, Techniques, and Applications for the Analyst. Student's Manual.

R. T. Ruegg, and S. K. Fuller. Sep $91,476 p$ NISTIR$90 / 4255$

See also Instructor's manual, PB92-112440. Sponsored by Corps of Engineers, Huntsville, AL. Huntsville Training Div.

Keywords: "Military training, "Construction management, "Economic analysis, "Students, Benefit cost analysis, Energy conservation, Life cycle costs, Structural design, Requirements, Military facilities, Buildings, Case studies, Manuals.

The manual is the class workbook for a five-day course, 'Economic Analysis for Military Construction Design: Concepts, Techniques, and Applications for the Analyst'. The course equips design professionals to conduct, document, and review economic studies of building and facility design alternatives in accordance with Army and Air Force requirements. It demonstrates a variety of applications through realistic examples and case studies. The workbook covers 16 training modules; including orientation, pre and post tests, aids to learning, time value of money, mathematical operations, general economic studies, energy conservation studies, data, computer software, and uncertainty and risk analysis. Each of the technical modules lists learning objectives and summarizes key points. The manual is designed not as a stand-alone tutorial, but as a working document for a course taught by an instructor who provides additional information.

\section{NATURAL RESOURCES \& EARTH SCIENCES}

\section{Cartography}

101,351

\section{PB91-147678}

Not available NTIS National Bureau of Standards (NEL), Gaithersburg MD. Mathematical Analysis Div.

Non-Linear Contour-to-Grid Digital Interpolation. Final rept.

B. A. Mandel. 1986, 9p

See also AD-A180 632 .

Pub. in Proceedings of DOD Mapping, Charting, and Geodesy (MC\&G) Conference, Alexandria, VA., May 8 1986, 9p.

Keywords: "Contours, "Grids(Coordinates), "Maps *Terrain models, Triangulation, Mapping, Conversion, Elevation, Interpolation, Methodology, Nonlinear systems, Digital systems, Sampling, Reprints.

A technical approach to the task of performing a contour-to-grid conversion using non-linear interpolation has been studied. The approach consists of reconstructing a terrain surface from the digitized contour lines on a map. The approximating surface is defined in terms of a partition of the region into irregular triangles obtained by a Voronoi method. The vertices of the triangles are selected by sampling a large set of digitized contour data with a tolerance band technique. The result is a smooth synthetic surface with which the elevation of any given point can be calculated. Therefore, grids of any given dimensions can be generated. 


\section{Forestry}

101,352

PB91-143305

PC A04/MF A01

National Inst. of Standards and Technology (NEL) Gaithersburg, MD. Center for Fire Research.

Canadian Mass Fire Experiment, 1989.

Final rept.

J. G. Quintiere. Nov 90, 64p NISTIR-4444

Sponsored by Defense Nuclear Agency, Washington, DC.

Keywords: "Fire tests, "Forest fires, "Environmental impacts, "Atmospheric motion, Combustion, Smoke, impacts, "Atmospheric motion, Combustion, Smoke, Clouds, Prescribed burning.

Working with Forestry Canada and the Ontario Ministry of Natural Resources, the Defense Nuclear agency carried out an extensively instrumented experiment of a prescribed burn in forest debris to simulate conditions of a mass fire. In addition to the Canadian team, a multi-institutional US team made both ground and airborne measurements of the fire and smoke conditions. The fire reported on was in Hill Township, Ontario and covered nearly 480 ha in its overall burning area. Both flaming and smoldering modes contributed to the energy and combustion products of the fire. Significant quantities measured and determined included estimations of energy release rate, emission factors for smoke particulates and species, ground level wind and temperatures, and aspects of cloud dynamics and cloud particles. The fire caused a capping cloud to form and reach a level of $6.5 \mathrm{~km}$. Rain, snow, hail and lightning were reported along with ground level fire whirls and water spouts on the adjoining lakes. Fire spread rates reached $1 \mathrm{~m} / \mathrm{s}$ and fire induced winds reached $12 \mathrm{~m} / \mathrm{s}$.

\section{Geology \& Geophysics}

101,353

PB91-203026

Not available NTIS

National Inst. of Standards and Technology (NML), Boulder, CO. Quantum Physics Div.

Lunar Laser Ranging.

Final rept.

J. E. Faller, and J. O. Dickey. 1990, 2p

Pub. in EOS 71, n21 p725-726, 22 May 90.

Keywords: "Lunar rangefinding, "Geodesy, Apollo 11 flight, Laser range finders, Earth rotation, Lunar landing, Gravitation, Reprints, Lunar libration, Nordved effect.

At the 1990 AGU Spring Meeting in Baltimore, Md., on Tuesday morning, May 29, there will be the special joint Geodesy-Planetology session Apollo Lunar Laser Ranging and Our Lunar Return. The session of invited papers will focus on and, yes, celebrate lunar laser ranging, which has been made possible by simple laser reflectors of the type first left by Buzz Aldrin on the powdery surface of the Sea of Tranquility. The session has been arranged to recall that on July 20 of this past year we celebrated the twentieth anniversary of the first human landing on the Moon.

\section{1,354}

PB91-237396

Not available NTIS

National Bureau of Standards (NML), Gaithersburg, MD. Gas and Particulate Science Div.

Accelerator (14)C Dating of Late Pleistocene Megafauna.

T. Stafford. $1988,3 p$

Pub. in Current Research in the Pleistocene, v5 p41-43 1988. Keywords: "Radiocarbon dating, "Age determination,
"Fossils, "Geologic ages, Mass spectroscopy, Pleistocene epoch, Bones, Wildlife, Stratigraphy, Reprints.

A significant application of tandem accelerator mass spectrometry $14 \mathrm{C}$ dating has been attempting to resolve two, decades-old controversies---the timing of human migrations into the New World and the dating of Late Pleistocene megafaunal extinctions, which are nearly coincident with the Clovis-period hunters. The precise dating of the Clovis culture (Haunes 1984) has not been accomplished for the extinct, terminal Pleistocene faunas, which are dated to $10,000-12,000 \mathrm{yr}$
B.P. (Martin 1984) and into the Holocene (Kurten and Anderson 1980). Two questions remain to be resolved---are post 10,500 yr B.P. $14 \mathrm{C}$ dates accurate and what is the time range within which the genera became extinct. Direct dating of fossils is mandatory because the approach eliminates uncertainty from stratigraphic interpretation, bioturbation and sediment recycling. Although the problems with bone dating are ongstanding (Taylor 1982; Ptorsch 1986; Stafford et al 1987), fossil bone can now be dated accurately if a rigorous biochemical and geochemical approach is taken (Stafford, Brendel and Duhamel 1988).

\section{1,355}

\section{PB9 1-237404}

Not available NTIS National Inst of Standards and Technology (NML) Gaithersburg, MD. Gas and Particulate Science Div. Accelerator Radiocarbon Dates on Charcoal, Shell, and Human Bone from the Del Mar Site, California. Final rept.

T. W. Stafford, and R. A. Tyson. 1989, 7p

Pub. in American Antiquity 54, n2 p389-395 1989

Keywords: "Radiocarbon dating, "Age determination, *Fossils, "Site surveys, "Geologic ages, California, Mass spectroscopy, Amino acids, Bones, Charcoal Hemispherical shells, Reprints.

Accelerator radiocarbon dates on shell, charcoal, and human bone samples are an indication that human activity at the Del Mar site dates to 5000 yr B.P. and pos sibly from 7000 yr B.P. The shell and charcoal fragments were stratigraphically dislocated by extensive bioturbation and are evidence that accelerator dates on extremely small samples should be used with cau tion. Certain depositional environments will require multiple-dating within a site because single-sample dating will not yield definitive ages. Dating of individua amino acids from the Del Mar sphenoid yielded an av erage age of 4900 ( + or -$) 40 \mathrm{yr}$ and conclusively es tablishes the age for the Del Mar skull. The previously dated tibia was found to be contaminated with exogenous amino acids, thereby making its age uncertain

\section{Mineral Industries}

\section{1,356}

PB91-144337

PC A05/MF A01

Maryland Univ., College Park. Dept. of Mechanical Engineering.

Investigation of Simulated Oil-Well Blowout Fires. Annual rept.

J. P. Gore, S. M. Skinner, and U. S. Ip. Nov $90,82 p$ NIST/GCR-90/581

\section{Grant NANB8D0834}

Sponsored by National Inst. of Standards and Technology (NEL), Gaithersburg, MD. Center for Fire Research.

Keywords: "Blowouts, "Oil wells, "Fires, "Fire protection, Heat flux. Temperature measurement, Flames, Fire extinguishers, Fire tests, Reaction kinetics, Burning rate.

A study of simulated oil well blowout fires aimed at improving predictive capabilities needed for the develop ment of radiation and fire suppression technology is described. Measurements of temperature distributions and radiative heat flux to representative locations are used to evaluate the analysis. Methane/air flames with suppression and heptane + methane/air flames without suppression are considered. The analysis consists of (i) construction of state relationships for fuel with water addition and two phase fuel mixtures using species concentration data for single fuels with the help of mixing rules and (ii) application of an existing flow solver under the locally homogeneous flow approximasolver under the locally homogeneous flow approxima-
tion. The predictions and measurements are in reasontion. The predictions and measurements are in reasonrule for state relationships and treatment of two phase flow effects is necessary for further improvement.

\section{1,357}

PB91-231597

PC A03/MF A01

National Inst. of Standards and Technology (NEL) Gaithersburg, MD. Unmanned Systems Group.

Task Decomposition and Algorithm Development for Real-Time Motion Control of a Continuous Mining Machine.

H. M. Huang, J. A. Horst, and R. Quintero. May 91 46p NISTIR-459

Sponsored by Bureau of Mines, Pittsburgh, PA
Keywords: "Mining equipment, "Algorithms, "Underground mining, "Coal mining, "Control systems design, Cutting, Real time operations, Systems engineering, Mining engineering, Error analysis, "Continuous mining machines.

The drive toward increased safety for coal miners has led to the development of computer-assisted methods of underground coal mining. The development of con trol architectures and accompanying code for the con trol of the movement of continuous mining machines (tramming control) is an important part of this overall effort The tramming control algorithm design de scribed is in concert with hierarchical architecture design principles developed at National Institute of Standards and Technology (NIST), referred to as the Real-Time Control Systems (RCS) methodology. The algorithm design with accompanying code allows for the control of both cutting and free-space movement by a continuous mining machine (CM) and allows for a high degree of human operator interaction.

\section{Natural Resource Management}

101,358

PB92-123116

PC A05/MF A01

Maryland Univ., College Park. Dept. of Fire Protection Engineering.

Development of a Technique to Assess the Adequacy of the Municipal Water Supply for a Residential Sprinkler System.

J. A. Milke, and J. L. Bryan. Nov 91, 83p NBS/GCR$91 / 600$

Grant NANB8D0814

See also PB88-155825. Sponsored by National Inst. of Standards and Technology (NEL), Gaithersburg, MD Center for Fire Research, and Fire Administration, Emmitsburg, MD.

Keywords: "Assessments, "Water supply, "Municipalities, Residential buildings, Cost effectiveness, Water storage, Storage tanks, Plumbing, Prototypes, Water flow, Sprinklers, Piping systems, Evaluation.

The research effort developed a technique to assess the adequacy of the municipal water supply for residential sprinkler systems installed in one- and twofamily dwellings. The effort is a continuation of a recently completed project which investigated cost-effective techniques for alleviation of deficiencies in the municipal water supply. In that effort, a need was identified to develop a technique to evaluate the adequacy of the municipal water supply. The report includes characterizing typical plumbing flow fixtures in residences to permit an analysis of the domestic water supply within a residence. Having characterized the residential flow devices, techniques to evaluate the domestic water supply are investigated. The investigation considers the feasibility of developing an inexpensive prototype apparatus with which to conduct the water supply evaluations.

\section{Soil Sciences}

101,359

PB91-174680 Not available NTIS National Inst. of Standards and Technology (NML) Gaithersburg, MD. Gas and Particulate Science Div. Wawayandaite, a New Calcium Manganese Beryllium Boron Silicate from Franklin, New Jersey. Final rept.

P. J. Dunn, D. R. Peacor, J. D. Grice, F. J. Wicks, and P. H. Chi. 1990, 4p Pub. in American Mineralogist 75, p405-408 1990.

Keywords: "Nonmetalliferous minerals, "Inorganic silicates, New Jersey, Crystal structure, Calcium compounds, Manganese compounds, Beryllium compounds, Boron compounds, Reprints, "Wawayandaite.

Wawayandaite, a new mineral from Franklin, New Jersey, is found associated with willemite, friedelite, and numerous other minerals. It is monoclinic, space group $\mathrm{P} 2 / \mathrm{c}$ or $\mathrm{Pc}$, with $\mathrm{a}=15.59(2), \mathrm{b}=4.78(1), \mathrm{c}=$ 18.69 (4) $\mathrm{A}$, beta $=101.84(15)$ degrees, $Z=1$. Chemi $\mathrm{cal}$ analyses yielded $\mathrm{BeO}$ 17.6. $\mathrm{MgO} 1.9 \mathrm{CaO} 24.8$ $\mathrm{MnO} 9.8, \mathrm{ZnO} 1.1, \mathrm{~B} 2 \mathrm{O}, 3.8, \mathrm{SiO} 2$ 28.2, $\mathrm{H} 2 \mathrm{O} 9.6, \mathrm{C}$ 


\section{Soil Sciences}

3.0, less $\mathrm{O}=\mathrm{Cl} 0.7$, total $=99.1 \mathrm{wt} \%$. The idealized chemical formula

is Ca12Mn4B2Be18Si12O46(OH,Cl)30. Wawayandaite many are strongly curved crystals with a pearly luster, many are strongly curved. Cleavage is perfect on
$(010), \mathrm{D}$ (sub measured) is approximateLy 3.0 , D (sub calculated) $=2.98 \mathrm{~g} / \mathrm{cc}$. It is biaxial, negative, with $2 \mathrm{~V}$ $=85$ degrees alpha $=1.619$, beta $=1.631$, and gamma $=1.641$

\section{NAVIGATION, GUIDANCE, \& CONTROL}

\section{Navigation \& Guidance System \\ Components}

\section{1,360}

PB91-195354 Not available NTIS National Inst. of Standards and Technology (NEL), Gaithersburg, MD. Robot Systems Div.

Real-Time Algorithms and Data Structures for Underwater Mapping.

Final rept.

D. N. Oskard, T. H. Hong, and C. A. Shafer. 1990, 7p Pub. in IEEE (Institute of Electrical and Electronics Engineers) Transactions on Systems Man and Cybernetics 20, n6 p 1469-1475 1990 .

Keywords: "Autonomous navigation, "Mapping, Models, Underwater navigation, Data bases, Algorithms, Data structures, Real time systems, Hierarchies, Sonar detection, "MAUV(Multiple Autonomous Underwater Vehicles).

As part of the Multiple Autonomous Underwater Vehicle (MAUV) project at the National Institute of Standards and Technology, a spatial mapping system has been developed to provide a model of the underwater environment suitable for autonomous navigation. The system is comprised of multi-resolution depth maps designed to integrate sensor data with a priori model, an object/attribute database for storing information known about detected objects, and a set of flags to monitor abnormal or emergency conditions in the environment. The paper describes the organization and structure of the mapping system and the algorithms used to map terrain and obstacles detected by acoustic sonar.

\section{NUCLEAR SCIENCE \& TECHNOLOGY}

\section{Isotopes}

\footnotetext{
101,361
PB92-116789

Not available NTIS

National Bureau of Standards (NML), Gaithersburg, MD. Ionizing Radiation Div.

Observation of Anomalous Isotope Ratios in SIRIS Measurements of Molydenum.

Final rept.

W. M. Fairbank, M. T. Spaar, J. E. Parks, and J. M.

R. Hutchinson. 1988, 4p

Pub. in Proceedings of International Symposium on Resonance Ionization Spectroscopy and Its Applications (4th), Gaithersburg, MD., April 10-15, 1988, p293296.

Keywords: "Molybdenum isotopes, "Tin isotopes, "Isotope ratio, "Resonance ionization mass spectroscopy, Laser radiation, Measurement, Hyperfine structure, Sputter-ion pumps, Reprints, Sputter-initiated resonance ionization spectroscopy.
}

Isotope ratio measurements in Mo and Sn by SputterInitiated Resonance Ionization Spectroscopy show hat the response for odd isotopes and even isotopes can be dramatically different in some cases, even if the laser linewidth is larger than the span of the hyperfine structure and isotope shifts. Power broadening on the resonance transition has not removed the odd/even effect in the cases studied.

\section{Nuclear Instrumentation}

\section{1,362}

\section{PB91-178855}

PC A08/MF A01

National Inst. of Standards and Technology (NML) Gaithersburg, MD. Center for Radiation Research. Center for Radiation Research. 1990 Technical Activities.

C. E. Kuyatt. Feb 91,158 p NISTIR-4506

See also PB90-130279.

Keywords: "Research projects, "Radiation measuring instruments, "Radiometric analysis, Methodology, Calibrating, US NIST, Tests, Radiation sources, Radiation dosage, Neutron fluence, "NIST Center for Radiation Research.

The report summarizes research projects, measurement method development, calibration and testing and data evaluation activities that were carried out during Fiscal Year 1990 in the NIST Center for Radiation Research. These activities fall in the areas of radiometric physics, radiation sources and instrumentation, and ionizing radiation.

\section{1,363}

PB92-116441

Not available NTIS

National Inst. of Standards and Technology (NML)

Gaithersburg, MD. lonizing Radiation Div.

Effects of Absorbed Dose Rate, Irradiation Temperature and Post-Irradiation Temperature on the Gamma Ray Response of Red Perspex Dosimeters.

Final rept.

M. Al-Sheikhly, W. J. Chappas, W. L. McLaughlin,

and J. C. Humphreys. 1991, 16p

Pub. in Proceedings of International Symposium on High Dose Dosimetry for Radiation Processing Vienna, Austria, November 5-9, 1990, p419-434 1991.

Keywords: "Radiation curing, "Dosimeters, Gamma rays, Irradiation, Plastics, Calibrating, Temperature dependence, PMMA, Dyes, Color centers, Measurement Reprints, Adsorbed dose, Radiochromic dosimeters.

The influences of irradiation temperature and gamma radiation dose rate on the response of red $4034 \mathrm{Per}$ spex dosimeters have been reported in the literature to be of relatively minor consequence, particularly for irradiations at up to a temperature of $40 \mathrm{C}$ and at absorbed dose rates of the order of $7 \mathrm{kGy} / \mathrm{h}$ (about $2 \mathrm{~Gy}$ / s). The present study shows this to be true; however, if there are marked differences in the temperature $(22 \mathrm{C}$ to $50 \mathrm{C}$ ) during extended storage periods (up to 16 hours) after irradiation, there is a tendency to overestimate an unknown dose administered at the elevated temperature, as a result of using a calibration made at an ambient lower temperature (e.g. $22 \mathrm{C}$ ). The presen study of dose rate effects also shows that there is a pronounced tendency to overestimate an unknown gamma ray dose administered at a relatively low dose rate ( 3 and $7 \mathrm{kGy} / \mathrm{h}$ ) as a result of using a calibration made at a considerably higher dose rate (e.g. 31 kGy/ h), even when the temperature of irradiation and stor age is controlled at $22 \mathrm{C}$.

\section{1,364}

PB92-116961

Not available NTIS National Inst. of Standards and Technology (MSEL) Gaithersburg, MD. Polymers Div.

Radiochromic Film Dosimeter for Gamma Radiation in the Absorbed-Dose Range 0.1 - $10 \mathrm{kGY}$. Final rept.

H. M. Khan, M. Farahani, and W. L. McLaughlin. 1991, 4p

Pub. in Radiation Physics and Chemistry 38, n4 p395 3981991.

Keywords: "Film dosimetry, "Gamma dosimetry, Food irradiation, Gamma rays, Dose-response relationships, Spectrophotometry, Reprints, "Radiochromic film.
A commercially available leuco-dye film (FWT-63-02), having a thickness of $0.55 \mathrm{~mm}$, has been investigated spectrophotometrically for its characteristics as a radiochromic dosimeter and for its potential use in food irradiation applications. The gamma-ray irradiation of the nearly colorless, transparent film induces blue color with an absorption maximum at $600 \mathrm{~nm}$. The increase in absorbance at $600 \mathrm{~nm}$ per unit thickness of film is linear with dose in the dose range up to $8 \mathrm{kGy}$, with a slope of $0.91 / \mathrm{mm} / \mathrm{kGy}$. After a modest additional increase during the first day following irradiation, the radiation-induced color is stable when stored at room temperature at least for 5 weeks. The response slope is $16 \%$ higher when stored at $60 \mathrm{C}$, however, after the initial 1-day increase it is stable for several weeks when stored at that temperature. The response of the dosimeter is independent of dose rate in the range 0.5 $170 \mathrm{~Gy} / \mathrm{min}$.

101,365

PB92- 117100

Not available NTIS National Inst. of Standards and Technology (NML) Gaithersburg, MD. Ionizing Radiation Div.

Dosimetry: New Approaches for New Challenges. Final rept.

W. L. McLaughlin. 1991, $31 p$

Pub. in Proceedings of Nordion Gamma Processing Seminar (4th), Ottawa, Ontario, Canada, May 26-31, 1991, p1-31.

Keywords: "Dosimetry, "Radiation curing, "Chemical radiation effects, "Plastics, Polymeric films, Gamma rays, PMMA, Polymers, Quality control, Mapping, Dosimeters, Dichromates, Photographic film dosimeters, Reprints, Adsorbed dose, Radiochromic dosimeters.

During the past few years, since the time of the 3rd Gamma-Processing Seminar in 1983, new and improved dosimetry systems and applications have been introduced for radiation processing. These include better reference and transfer standards; more accurate calorimeters; alanine (ESR analysis) with greate precision and wider dose ranges; several liquid dosimeters (UV and visible spectrophotometry), and radiochromic dye organic solutions; several solid polymeric dosimeters, including new polymethylmethacrylate (Perspex) and Nylon and other plastic films. The latter systems are commercially available in large quantities, and are relatively inexpensive and easy to use over the absorbed dose ranges encountered in radiation processing (10-10 to the 5 th power Gy). Some of these are especially useful for dose mapping in radiation processed products, as a means of determining the locations and mean values of minimum and maximum doses and the dose uniformity ratios, and others are used for day-to-day quality control measurements.

\section{1,366}

PB92-117126

Not available NTIS

National Inst. of Standards and Technology (NML), Gaithersburg, MD. lonizing Radiation Div.

2-Deoxy-D-Ribose Aqueous Solution as a GammaRay Dosimeter.

Final rept.

W. L. McLaughlin, M. Farahani, and J. H. Liang 1991, 13p

Pub. in Proceedings of International Symposium on High Dose Dosimetry for Radiation Processing Vienna, Austria, November 5-9, 1990, p159-171 1991.

Keywords: "Dosimeters, "Gamma rays, "Deoxyribose, "Arabinose, Optical activity, Aqueous solutions, Irradiation, Chemical radiation effects, Radiation curing Polymers, Maltose, Storage life, Time dependence, Temperature dependence, Saccharides, Sugars, Reprints.

The change in the angle of optical rotation (delta alpha) of buffered aqueous solution $(\mathrm{pH} 7)$ of 2-deoxyD-ribose due to irradiation with gamma radiation gives a convenient means of dosimetry. The method is shown to be suitable with solutions stored and irradiated in sealed glass ampoules. The dosimeter solution is not sensitive to light and is not affected by contamination by trace organic or inorganic impurities. The radiation induced value of (delta alpha) is reproducible to within + or - $3 \%(95 \%$ confidence level) at dose levels of 50-500 kGy. The useful radiation response range of the dosimeter is 10 to $1000 \mathrm{kGy}$, and this represents a more sensitive and reproducible response than the conventional optical rotation of glucose or sucrose solution. Comparisons were also made with solutions of $\mathrm{D}-(-)$ arabinose and D-(+)-maltose. The value of (delta alpha) of 2-deoxy-D-ribose does not change during storage and reading, over at least several weeks stor- 
age period. There is no appreciable variation of response with dose rate, but the irradiation temperature coefficient over the temperature range 0 to $17 \mathrm{C}$ is approximately $+1.5 \% \mathrm{C}$, and at higher temperatures up to $33 \mathrm{C}$ is $+3.0 \% \mathrm{C}$.

\section{Radioactive Wastes \& Radioactivity}

101,367

NUREG/CR-5711

PC A06/MF A02

National Inst. of Standards and Technology, Gaithersburg, MD.

Assessment of Uncertainties in Measurement of $\mathrm{pH}$ in Hostile Environments Characteristic of Nuclear Repositories.

Technical rept. 30 Jun 87-28 Feb 91.

K. G. Kreider, M. J. Tarlov, and P. H. Huang. Oct 91 , $102 p$

Also available from Supt. of Docs. See also NUREG/ CR-5166 and NUREG/CR-5484. Sponsored by Nuclear Regulatory Commission, Washington, DC. Div. of Engineering.

Keywords: "Electrodes, "Radioactive wastes, "pH, "Thin films, Iridium oxides, Data covariances, Tuff, Measurement, Corrosion resistance, Aqueous solutions, Ceramics, Sputtering, Spectrum analysis, Electrochemistry, High temperature tests.

The report focuses on evaluation and characteristics of sputtered thin film pH electrodes which can be used to assess the corrosivity of hot (100 C) aqueous solutions present in nuclear repositories. Sputtered thin films have the advantages of high temperature capability, ruggedness, and low cost. The sputtered iridium oxide films (SIROF) were found to have a linear, 58 $\mathrm{mV} / \mathrm{pH}$, response to changes in $\mathrm{pH}$. They had little hysteresis but drifted approximately $0.2 \mathrm{~V}$ over a period of two days exposure to $\mathrm{pH} 2-12$ solutions. The films were found to be insensitive to interference from most ions such as alkali ions but had redox sensitivity to ferri-/ferrocyanide ions. Although special surface treatments were needed for the films for good adherence at $200 \mathrm{C}$ the films were not degraded after 20 hours exposure at $\mathrm{pH} 4,7$, and 10 at $200 \mathrm{C}$. Ruthenium oxide sputtered films performed equally well to the iridium oxide films in parallel tests. The report also contains information on electrochemistry and testing of thin film electrodes and the characterization of the thin films by $x$-ray photoemission spectroscopy, ultraviolet photoemission spectroscopy, and ion scattering spectroscopy.

101,368

NUREG/CR-5716

PC A05/MF A01

Arizona Univ., Tucson. Dept. of Soil and Water Science.

Model Validation at the Las Cruces Trench Site.

Technical rept. 1 Nov 90-20 May 91.

R. G. Hills, and P. J. Wierenga. Jun $91,94 p$

Also available from Supt. of Docs. Prepared in cooperation with New Mexico State Univ., Las Cruces. Dept. of Mechanical Engineering. Sponsored by Nuclear Regulatory Commission, Washington, DC. Div. of Engineering.

Keywords: "Radioactive waste facilities, "Low level radioactive wastes, Field tests, Water flow, Radionuclide migration, Soils, Ground disposal, Las Cruces Trench Site.

A series of dynamic field experiments have been performed at the Las Cruces Trench Site to provide data to test deterministic and stochastic models for water flow and solute transport in spatially variable unsaturated soils. Two experiments were performed to provide support for model validation efforts during Phase I of INTRAVAL (an international effort towards validation of geosphere models for transport of radionuclides) and a third experiment is currently underway to support the INTRAVAL Phase II efforts. The third experiment utilized different boundary and initial conditions and additional chemical tracers. The report sumtions and additional chemical tracers. The report sumefforts and presents the INTRAVAL Phase II validation plans. The Phase II validation strategy is discussed in detail.

\section{Reactor Engineering \& Nuclear Power Plants}

\section{1,369}

PB91-222687

PC A10/MF A03

National Inst. of Standards and Technology (EEEL), Gaithersburg, MD. Electricity Div.

Annotated Bibliography: Diagnostic Methods and Measurement Approaches to Detect Incipient Defects Due to Aging of Cables.

F. D. Martzloff, and A. G. Perrey. Jul 91, 224p

NISTIR-4485

Sponsored by Nuclear Regulatory Commission, Washington, DC

Keywords: "Bibliographies, "Defects, "Nuclear power plants, "Cables, "Nondestructive tests, Surveys, Cable insulation, Wire, Detection, Aging, Damage assessment.

Open-literature papers and some limited-distribution documents were reviewed in a search to identify promising approaches to the in-situ detection of incipient defects in nuclear power-plant cables. The search was extended to the topics of detection of any defect, to radiation effects, and to basic considerations on partial discharges. The report presents a review of 150 papers that appeared significant on the basis of their title, but many of which were found not applicable upon close review. A compilation of 850 references cited in the reviewed papers is included in the report.

101,370

PB92-123074

PC A04/MF A01

National Inst. of Standards and Technology, Gaithersburg, MD.

Fire-Plume-Generated Ceiling Jet Characteristics and Convective Heat Transfer to Ceiling and Wall Surfaces in a Two-Layer Zone-Type Fire Environment: Uniform Temperature Ceiling and Walls.

L. Y. Cooper. Nov 91, 58p NISTIR-4705

Sponsored by Nuclear Regulatory Commission, Washington, DC.

Keywords: "Fires, "Heat transfer, "Reactor safety, "Walls, "Ceilings(Architecture), Subroutines, Mathematical models, Algorithms, Computer programs, Temperature distribution, Jet flow, Heat flux, Plumes, Velocity, CEILHT Subroutine.

The work presents a model to predict the instantaneous rate of convective heat transfer from fire plume gases to the overhead ceiling surface in a room of fire origin. The room is assumed to be a rectangular parallelopiped and, at times of interest, ceiling temperatures are simulated as being uniform. Also presented is an estimate of the convective heat-transfer, due to ceiling-jet-driven wall flows, to both the upper and lower portions of the walls. The effect on the heat transfer of the location of the fire within the room is taken into account. Finally presented is a model of the velocity and temperature distributions in the ceiling jet. The model equations were used to develop an algorithm and associated modular computer subroutine to carry out the indicated heat transfer calculations. The subroutine is written in FORTRAN 77 and called CEILHT. The algorithm and subroutine are suitable for use in two-layer zone-type compartment fire model computer codes.

101,371

PB92-126598

PC A05/MF A01

National Inst. of Standards and Technology (BFRL), Gaithersburg, MD.

Predicting the Remaining Service Life of Concrete. J. R. Clifton. Nov $91,84 \mathrm{p}$ NISTIR-4712

See also PB89-215362 and PB91-107219. Prepared in cooperation with Oak Ridge National Lab., TN., and Nuclear Regulatory Commission, Washington, DC.

Keywords: *Nuclear power plants, "Service life, "Concretes, Performance, Estimates, Comparisons, Accelerated tests, Statistical models, Mathematical models, Degradation, Corrosion, Sulfates, Alkali-aggregate reactions, Frost, Leaching, Radiation.

The report examines the basis for predicting the remaining service lives of concrete materials of nuclear power facilities. Methods for predicting the service life of new and in-service concrete materials are analyzed. These methods include (1) estimates based on experience, (2) comparison of performance, (3) accelerated testing, (4) stochastic methods, and (5) mathematical modeling. New approaches for predicting the remaining service lives of concrete materials are proposed and recommendations for their further development are given. Degradation processes are discussed based on considerations of their mechanisms, likelihood of occurrence, manifestations, and detection. They include corrosion, sulfate attack, alkali-aggregate reactions, frost attack, leaching, radiation, salt crystallization, and microbiological attack.

\section{Reactor Fuels \& Fuel Processing}

\section{1,372}

PB91-158733

Not available NTIS National Inst of Standards and Technology (MSEL). Gaithersburg, MD. Metallurgy Div.

Corrosion Behavior of Zirconium Alloy Nuclear Fuel Cladding.

Final rept.

A. C. Fraker, and J. Harris. 1990, $8 \mathrm{p}$

Sponsored by Nuclear Regulatory Commission, Washington, DC. Office of Nuclear Material Safety and Safeguards.

Pub. in Materials Research Society Symposium Proceedings, v176 p549-556 1990.

Keywords: "Zircaloys, "Corrosion tests, "Nuclear fuel claddings, Passivity, Polarization, Crevice corrosion, Surface finishing. Electrical faults, Chemical radiation effects, Alkalinity, Temperature dependence, Reprints.

Zircaloy-2 and -4 are used as nuclear fuel cladding Both alloys are more than ninety-eight percent zirconium and are corrosion resistant to various media. Electrochemical measurements using polarization techniques have been made on these alloys in aqueous media with a pH of 8.5 and varying ionic concentration (1X and $10 \mathrm{X}$ ) at temperatures of $22 \mathrm{C}$ and $95 \mathrm{C}$. Results showed that under the test conditions of the study these alloys passivated and had negligible corrosion rates, but there were some variations in passivation due to surface preparation and some crevice corrosion was observed. Data are presented and discussed in terms of passivity, breakdown potential and susceptibility to localized corrosion.

\section{Reactor Materials}

101,373

PB91-189316

Not available NTIS

National Inst of Standards and Technology (MSEL), Gaithersburg, MD. Metallurgy Div.

Plastic Zone Formation Around an Arresting Crack.

Final rept.

R. J. Fields, and R. deWit. $1990,8 p$

Sponsored by Nuclear Regulatory Commission, Washington, DC.

Pub. in International Jnl. of Fracture 42, n3 p231-238 1990.

Keywords: "Crack arrest, "Pressure vessels, "Reactor materials, "Steels, "Fracture mechanics, "Strain tests, "Plasticity, Strain gages, Reactor components, Velocity, Reprints.

The evolution of strain near the tip of an arresting cleavage crack was followed using a strain gage technique. The results were obtained in a body so large that reflected elastic waves did not contribute significantly to the strain records during the time interval of interest. The measurements were made using a linear array of strain gages located along the intended crack path and $0.65 \mathrm{~B}(\mathrm{~B}=$ specimen thickness) above this plane. The gages were read using instrumentation capable of resolving strain in time intervals of less than 10 microseconds. To within the resolution of the technicque, the results did not suggest plasticity near the rapidly propagating, cleavage crack tip. At the instant of arrest, however, the strain gages detected a plastic zone which increased in intensity over a period of several milliseconds. This increasing intensity was interpreted as an elastic-plastic boundary emanating from the arresting crack tip and eventually reaching an equilibrium size, i.e., that predicted from the applied stress intensity factor and the static plastic properties. A simple analysis is presented to estimate the velocity of 
the elastic-plastic boundary from the strain data and results are given for several experiments.

\section{OCEAN TECHNOLOGY \& ENGINEERING}

\section{Biological Oceanography}

101,374

PB9 1-184796

PC A03/MF A01

National Inst. of Standards and Technology (CSTL)

Gaithersburg, MD

Alaska Marine Mammal Tissue Archival Project:

Revised Collection Protocol.

P. R. Becker, S. A. Wise, B. J. Koster, and A

Zeisler. Mar 91, 40p NISTIR-4529

Prepared in cooperation with National Ocean Service Anchorage, AK. Arctic Environmental Assessment Anchorage, AK. Alaska Outer Continental Shelf Office. Keywords: "Alaska, "Aquatic animals, "Mammals "Tissues(Biology), Archives, Sampling, Procedures, Field tests.

In 1987, the Outer Continental Shelf Studies Program of the Minerals Management Service (MMS) provided funds to the Ocean Assessments Division (OAD) Alaska Office, National Oceanic and Atmospheric Administration (NOAA), to establish and conduct a program of collecting tissues from Alaska marine mammals and storing them under conditions which would allow for future analyses for substances indicative of contamination from offshore oil and gas, and mining activities. Cataloging and archiving of samples are conducted at the Alaska Marine Mammal Tissue $\mathrm{Ar}$ chive, which is maintained by NIST in its National Biomonitoring Specimen Bank (NBSB), Gaithersburg. Maryland. The report presents the protocol used in the collection and archival process.

\section{Marine Engineering}

101,375

PB91-158782

Not available NTIS

National Bureau of Standards (NEL), Gaithersburg, MD. Robot Systems Div.

Real-Time Hierarchical Planning for Multiple Mobile Robots.

Final rept.

M. Herman, and J. S. Albus. 1987, 10p

Pub. in Proceedings of DARPA Knowledge-Based Planning Workshop, Austin, TX., December 8-10, 1987, p22-1-22-10.

Keywords: "Underwater vehicles, "Robotics, Control systems, Real time, Knowledge bases(Artificial intelligence), Reprints, MAUV(Multiple Autonomous Underwater Vehicles), Hierarchical control.

The Multipule Autonomous Underwater Vehicles (MAUV) project is described. The goal of the project is to have multiple underwater vehicles exhibiting intelligent, autonomous, cooperative behavior. The MAUV control system is hierarchically structured and incorporates sensing, world modeling, planning and execution. The levels in the hierarchy include a mission level a group level, a vehicle task level, and an elemental action level. Issues of realtime planning and dynamic replanning in unstructured environments are discussed.

\section{1,376}

PB92-108935 PC A05/MF A01

National Inst. of Standards and Technology (BFRL), Gaithersburg, MD

Navy Safety Center Data on the Effects of Fire Protection Systems on Electrical Equipment.

R. S. Levine. Apr 91, 86p NISTIR-4620

Portions of this document are not fully legible. Sponsored by Nuclear Regulatory Commission, Washington, DC. Center. Sponsored by Minerals Management Service

Keywords: "Fire protection, "Electrical equipment, "Fire damage, "Fire extinguishing agents, "Shipboard fire control, Fire extinguishers, Sprinkler systems, Safety engineering. Fire hazards, Accident records, Tables(Data). Fire detection systems, Naval ships, Submarines, Halon, Carbon dioxide, Military facilities, Accident statistics.

Records of the Navy Safety Center, Norfolk, VA were reviewed to find data relevant to inadvertant operation of installed Fire Extinguishing Systems in civilian Nuclear power plants. Navy data show the incidence of collateral fire or other damage by fresh water on operating electrical equipment in submarines, and in shore facilities is about the same as the civilian experience, about $30 \%$. Aboard surface ships, however, the collateral damage incidence is much lower, about $15 \%$. With sea water, the collateral damage incidence is at least $75 \%$. It is concluded that the fire extinguisher water has to be contaminated, as by rust in sprinkler systems or deposited salt spray, for most collateral damage to occur. Reasons for inadvertant operation (or advertant operation) of firex systems at shore facilities, submarines, and surface ships resemble those for nuclear power plants. Mechanical or electrical failures lead the list, followed by mishaps during maintenance. Detector and alarm system failures are significant problems at navy shore facilities, and significant at nuclear power plants. Fixed halon and $\mathrm{CO} 2$ systems in shore facilities cause no collateral damage. Lists of individual Navy incidents with water and with halon and carbon dioxide are included as appendices to the report.

\section{Physical \& Chemical Oceanography}

\section{1,377}

PB91-149237 Not available NTIS

National Inst. of Standards and Technology (NML)

Gaithersburg, MD. Organic Analytical Research Div.

Preparation and Analysis of a Marine Sediment Reference Material for the Determination of Trace Organic Constituents.

Final rept

M. M. Schantz, B. A. Benner, S. N. Chesler, B. J.

Koster, and K. E. Hehn. 1990, 14p

Pub. in Fresenius Jnl. of Analytical Chemistry 338 , p501-514 1990

Keywords: "Sediments, *Marine environments, "Standards, "Aromatic polycyclic hydrocarbons, "Polychlorobiphenyl compounds, Liquid chromatography, Gas chromatography, Mass spectroscopy, Trace elements, Sulfur, Contaminants, Reprints, Standard reference materials.

A new marine sediment Standard Reference Material (SRM) has been prepared and analyzed for the deter mination of trace organic constituents. SRM 1941. Organics in Marine Sediment, has been certified for concentrations of 11 PAHs using results obtained from gas chromatography (GC) with flame ionization detection, gas chromatography-mass spectrometry, and liquid chromatography with fluorescence detection. Non-certified values for 24 additional PAHs are also reported. GC with electron capture detection was used to provide non-certified concentrations for 15 PCB cogeners and 7 chlorinated pesticides. In addition to the geners and 7 chlorinated pesticides. In addition to the trace elements were determined using neutron activation analysis, and the sulfur content was also determined using isotope dilution thermal ionization mass spectrometry.

\section{ORDNANCE}

\section{Ammunition, Explosives, \& Pyrotechnics}

101,378

PC A09/MF A02

National Inst. of Standards and Technology (EEEL). Boulder, CO. Electromagnetic Fields Div.
PB92-116292
Quantifying Standard Performance of Electromagnetic-Based Mine Detectors.

W. L. Gans, R. G. Geyer, and W. K. Klemperer. Oct 91,180 p NISTIR-3982

See also AD-A226 626. Sponsored by Army Belvoir Research Development and Engineering Center, Fort Belvoir, VA:

Keywords: "Mine detectors, Buried objects, Dielectric properties, Portable equipment, Electromagnetic properties, Soil properties, Remote sensing, Basic programming language, Computer programs, Permeability, Permittivity, Sensitivity, Algorithms.

This is a final report to sponsor on work performed by National Institute of Standards and Technology (NIST) personnel from January 1, 1985 to December 31, 1990. An overview of the theory of the electromagnetic properties of soils is presented along with a brief review of existing technologies for the detection of buried objects using electromagnetics. The critical electromagnetic performance factors for portable EM mine detectors that NIST has identified are presented, along with a discussion of measurement systems for measuring the constitutive properties of soil and minelike materials. Recommendations are then presented for a measurement system configuration that should meet most of the Army's requirements. A recommended mine detector testing strategy is then presented along with a set of instructions for specific tests and an algorithm for comparatively scoring the performance of detectors. The tests and the scoring algorithm are as specific and as detailed as is possible at this stage of development. Last, a section is included that contains NIST's recommendations for the test data that should be archived.

\section{Combat Vehicles}

101,379

PB91-134726

Not available NTIS

National Inst. of Standards and Technology (NEL), Gaithersburg, MD. Robot Systems Div.

Control System Architecture for Unmanned Ground Vehicles.

Final rept.

S. Szabo, H. Scott, and R. Kilmer, 1990, 9p

Pub. in Proceedings of AUVS-90 Technical Symposium, Dayton, OH., July 30-August 1, 1990, p258-266.

Keywords: *Ground vehicles, "Military vehicles, Remote control, Artificial intelligence, Systems engineering, Reprints, "Antonomous control, Teleoperation, Robotics, Control systems, Real time systems, National Institute of Standards and Technology.

The U.S. Army Laboratory Command is spearheading a program designed to demonstrate cooperative realtime control of multiple unmanned ground vehicles. The program, titled TEAM, involves the teleoperated control of two vehicles from remotely located operator control stations. The function of the system is to provide remote operation of military vehicles equipped with any of a variety of reconnaissance or related mission packages. The National Institute of Standards and Technology's (NIST's) role in the program is to apply their hierarchically-structured real-time sensorbased control architecture, originally developed for the control of industrial robots, to the design of the supervisory control systems in the two vehicles and the operator control stations. The paper presents a description of the NIST Real-time Control System (RCS) architecture for the TEAM program. A general description of the TEAM operational scenarios and functional requirements relevant to these control systems is provided. The overall control architecture developed on the basis of these requirements is presented. 


\section{PHOTOGRAPHY \& RECORDING DEVICES}

\section{PHYSICS}

\section{Acoustics}

\section{Recording Devices}

\section{1,380}

PB91-132258

PC A03/MF A01

National Inst. of Standards and Technology, Gaithersburg, MD.

Overview of the Product Data HyperStandard CDROM Prototype.

S. Ressler. Nov 90, 16p NISTIR-4470

Keywords: *Information systems, *Information retrieval, "Standardization, Specifications, Prototypes, Data storage devices, Cost effectiveness, Computer systems hardware, " $\mathrm{CD}$ ROM, "Product data exchange. "Product Data HyperStandard CD ROM STEP(Standard for the Exchange of Product Mode Data), PDES(Product Data Exchange using STEP) CALS(Computer aided Acquisition and Logistics Support), Computer aided design.

The paper describes the contents of the Product Data HyperStandard CD-ROM Prototype containing information related to product data standards. HyperStandard is a generic term describing the application of hypertext and multimedia technologies to standards in general. The CD-ROM contains both browsers for perusing the documents, and a number of other items of interest to the product data community. These include: IGES Version 5; the CALS specifications; preliminary STEP drafts, PDES, Inc. technical documents; the NIST PDES Toolkit; plus miscellaneous other pieces of information. (The sources of data for the documen browsers on the CD-ROM prototype were the original electronic documents used to print those documents.) All of these data combined should serve as a usefu repository of information for the community of technical experts developing product data standards. This variety of information has been assembled to demonstrate that CD-ROM's are a convenient and cost-effective medium for use by the people involved in produc data standardization efforts.

101,381

PB92-126549

PC A04/MF A01

National Inst. of Standards and Technology (CSL), Gaithersburg, MD.

3480 Type Tape Cartridge: Potential Data Storage Risks, and Care and Handling Procedures to Minimize Risks.

Final rept.

M. P. Williamson. Nov $91,60 p$ NIST/SP-500/199

Also available from Supt. of Docs. See also PB83 237271 and PB88-233135. Sponsored by National Environmental Satellite, Data, and Information Service, Washington, DC.

Keywords: "Data storage devices, "Computer storage devices, "Magnetic tapes, CLASS 3480 cartridge tape system, Magnetic tape cartridges.

The 3480 type media was introduced to the data storage industry in 1984. Early problems were reported due to premature degradation of the polyester polyurethane binder. In addition, the lack of guidelines for proper care and handling increased the risks associated with the storage of data on the 3480 type media. After approximately seven years experience, media manufacturers have significantly improved their binde formulations. Furthermore, 3480 type media users from government and industry have experienced the dependability and, conversely, the problems associated with the storage of data on the 3480 type media. The National Institute of Standards and Technology (NIST), under the sponsorship of the National Oceanic and Atmospheric Administration (NOAA), has undertaken an appraisal of the potential risks associated with the storage of data on the 3480 type media. In addition, the study summarizes reasonable procedures for the care and handling of the 3480 type media in order to minimize potential risks. The conclusions of the NIST study are based on information gathered by NIST from pertinent scientific literature, and interviews with 3480 type technology users and manufacturers.

\section{PB92-117456}

\section{1,382}

PB92-116847

National Inst of Standards and Not available NTIS

Gaithersburg, MD. Thermophysics Div.

Accurate Acoustic Measurements in Gases Under Difficult Conditions.

Final rept.

K. A. Gillis, M. R. Moldover, and A. R. H. Goodwin. $1991,5 p$

Sponsored by Department of Energy, Washington DC., and Department of the Navy, Washington, DC. Pub. in Review of Scientific Instruments 62, n9 p22132217 Sep 91.

Keywords: "Test equipment, "Waveguides, "Sound waves, "Gases, "Acoustics, Measurement, Diaphragms(Mechanics), Transducers, Resonators, Reprints.

Accurate measurements of the speed of sound in gases are often made using metal resonators with small transducers that perturb the resonance frequencies in minor and predictable ways. The method is extended to gases that may be corrosive and to high temperatures by using remote transducers coupled to a resonator by acoustic waveguides. Thin metal diaphragms separate the waveguides from the resonator. Thus, only metal parts come into contact with the test gas. In the present apparatus, any gas compatible with gold and stainless steel can be studied.

\section{1,383}

Not available NTIS

(EEEL) Boulder, CO. Electromagnetic Fields Div.

Probe-Corrected Spherical Near-Field Scanning Theory in Acoustics.

Final rept.

R. C. Wittmann. 1991, 4p

Pub. in Proceedings of IEEE (Institute for Electrical and Electronics Engineers) Instrumentation and Measurement Technology Conference Enhancing Productivity with Instrumentation and Measurement Technologies, Atlanta, GA., May 14-16, 1991, p283-286.

Keywords: "Acoustic fields, "Sound transducers *Transducers, Acoustic measurement, Spherical waves, Near field, Scanning, Probes, Reprints.

Spherical near-field scanning is well known in electromagnetics. The acoustical analog is outlined here Data are taken, with an arbitrary probe, on a spherical surface surrounding an unknown transducer. The algorithms uses these data to characterize the fields of the transducer everywhere outside the measuremen sphere. The results can be corrected for probe effects if the probe's receiving pattern is known.

\section{Fluid Mechanics}

\section{1,384}

PC A03/MF A01

National Inst. of Standards and Technology, Boulder, $\mathrm{CO}$. Thermodynamics Div.

Integrated theoretical and experimental study of the thermophysical properties of fluid mixtures. Progress report (February 1990-February 1991).

$J$. F. Ely. Nov 90, 24p DOE/ER/13992-T1

Contract A105-89ER13992

Sponsored by Department of Energy, Washington, DC.

Keywords: "Suspensions, Colloids, Computerized Simulation, "Fluids, Microstructure, Mixtures, Neutrons, Polystyrene, Progress Report, Scattering, EDB/360603, "Thermophysical properties.

In this report we highlight the progress made during the period February 1990 through February 1991. The objective of this research is twofold: 1 . Development of predictive and correlative procedures for the thermophysical properties of complex fluids and fluid mixtures; and 2. Furthering our basic understanding of fluid behavior with advances in microscopic theory, computer simulation and selected experimentation. Substantial progress has been made in both of these areas. 39 refs., 4 figs.

101,385

PB91-134585 $\begin{aligned} & \text { Not available NTIS } \\ & \text { National Inst. of Standards and Technology (NEL) }\end{aligned}$

Not available NTIS Boulder, CO. Thermophysics Div.

Phase Equilibria from the One-Fluid Model. Final rept.

K. D. Romig, and H. J. M. Hanley. 1989, 3p

Sponsored by Department of Energy, Washington, DC. Pub. in Cryogenics 29, n1 p65-67 Jan 89.

Keywords: Mathematical models, Predictions, Reprints, "Binary fluids, Binary mixtures, Phase equilibrium, Lennard-Jones potential.

The prediction of possible phase behavior in binary fluid mixtures is discussed in the context of the Scott and van Konynenberg scheme. It is shown that the corresponding states one-fluid model for LennardJones mixtures gives qualitative agreement with ex periment.

\section{1,386}

PB91-147892

Not available NTIS National Bureau of Standards (NEL), Gaithersburg $M D$. Fire Measurement and Research Div.

Development of a Line Camera for Real-Time Measurements of Concentration in Turbulent Flow Fields.

Final rept

W. M. Pitts. 1987,9 p

Contract AFOSR-ISSA86-0008

Sponsored by Air Force Office of Scientific Research, Bolling AFB, Washington, DC

Pub. in Proceedings of International Congress on Aplications of Lasers and Electrooptics (5th). Arlington, VA., November 10-13, 1986, p7-15 1987.

Keywords: "Flow visualization, *Turbulent flow, "Cameras, Rayleigh scattering, Light scattering, Real time operations, Concentration(Composition), Image intensifiers, Laser applications, Gas flow, Propane, Reprints.

The development of an intensified digital line camera for laser-induced Rayleigh light scattering measurements of real-time concentration fluctuations in turbulent flow fields is described. Particular attention is given to the need for and choice of an image intensifier. Preliminary results for a turbulent jet of propane flowing into air are presented.

101,387

PB91-148031

Not available NTIS National Inst of Standards and Technology (NML) Boulder, $\mathrm{CO}$. Chemical Engineering Science Div.

Effect of Pipe Surface Finish on Gas Flow Measurement with an Orifice Meter.

Final rept.

C. F. Sindt, J. A. Brennan, S. E. McFaddin, and K. M. Kothari. $1990,8 \mathrm{p}$

Sponsored by Gas Research Inst., Chicago, IL.

Pub. in Proceedings of International Gas Research Conference, Tokyo, Japan, November 6-9, 1989, p1-8 1990.

Keywords: "Flow measurement, "Orifice meters, *Pipes(Tubes), Surface properties, Roughness, Gas flow, Reynolds number, Reprints.

The National Institute of Standards and Technology (NIST) under the sponsorship of the Gas Research Institute (GRI) has completed an investigation of the change in the orifice discharge coefficient caused by the surface finish in the pipe upstream of the orifice plate. Three sizes of pipes of three roughnesses were tested. The results from this investigation show that the orifice discharge coefficient is affected by pipe surface finish and the effect depends upon the Reynolds number and the orifice beta ratio.

\section{1,388}

PB91-162263

Not available NTIS

National Inst. of Standards and Technology (NML). Gaithersburg, MD. Thermophysics Div. 


\section{PHYSICS}

\section{Fluid Mechanics}

Computer Simulation of Fluid-Fluid Phase Coexistence in Mixtures of Nonadditive Soft Disks.

Final rept.

R. D. Mountain, and A. H. Harvey. 1991, 6p

Pub. in Jnl. of Chemical Physics 94, n3 p2238-2243, 1 Feb 91

Keywords: "Equilibrium, "Binary mixtures, "Liquids, Gibbs equations, Thermodynamic properties, Monte lecular flow, Mixing, Mixtures, Reprints.

Fluid-fluid phase equilibrium in binary mixtures of positively nonadditive soft disks is studied using both molecular dynamics and Gibbs ensemble Monte Carlo simulations. The molecular dynamics simulations are unable to provide quantitative results for the phase transition, but they do indicate that some sort of ordering takes place as the temperature is lowered. In contrast, the Gibbs ensemble simulations demonstrate unambiguously the presence of a first-order transition and give quantitative results for the coexistence curve however, convergence is very slow for mixtures that are not symmetric. Comparison with the Gibbs ensemble results indicates that a simple first-order perturbation theory provides good results far from the consolute point. Closer to the consolute point, the theory does not reproduce the flatness of the coexistence curve; the limitation is intrinsic in any mean-field theory. Comments are made regarding the relative merits of molecular dynamics and Gibbs ensemble simulations for studying fluid-fluid phase coexistence.

\section{1,389}

PB91-162347

Not available NTIS

National Inst. of Standards and Technology (NML)

Boulder, CO. Chemical Engineering Science Div.

Effect of Pipe Surface Finish on the Orifice Discharge Coefficient.

Final rept.

C. F. Sindt, J. A. Brennan, S. E. McFaddin, and R.

Wilson. $1989,8 p$

Sponsored by Gas Research Inst., Chicago, IL

Pub. in VDI Berichte 768, p49-56 1989

Keywords: "Pipe flow, "Surface roughness, "Orifice meters, "Orifice flow, Finishes, Surface properties, Pipes(Tubes), Orifices, Flow measurement, Reynold number, Gas flow, Reprints.

The U.S. National Institute of Standards and Technolo gy (NIST), under the sponsorship of the Gas Research Institute (GRI), investigated the change in the orifice discharge coefficient caused by the interior surface finish in the pipe upstream of the orifice plate. Three sizes of meters and three surface finishes were tested over a range of pipe Reynolds numbers from $4.3 \times 10$ (to the power of 5) to $1.2 \times 10$ (to the power of 7). The results of the investigation show that the orifice discharge coefficient changed more than one percent at a Reynolds number of $7 \times 10$ (to the power of 6 ) between a smooth pipe, 2.8 micrometer $\mathrm{Ra}$, and a rough pipe, 8.9 micrometer $\mathrm{Ra}$, for a beta ratio of 0.74 . For beta ratios less than 0.5 the change in the orifice discharge coefficient was not measurable for the same change in pipe roughness.

\section{1,390}

PB91-195263

Not available NTIS

National Inst. of Standards and Technology (NEL) Boulder, CO. Scientific Computing Div.

Use of Computer Algebra to Locate Critical Loci in Fluid Mixtures.

Final rept.

G. C. Nielson, M. O. McLinden, and G. Morrison.

$1990,10 p$

Pub. in Jnl. of Symbolic Computation 10, n5 p499-508 Nov 90.

Keywords: "Fluids, "Mixtures, "Loci, "Computer calculations, Algebra, Rational functions, Linear algebraic equations, Symbolic codes, Phase diagrams, Refrigerants, Reprints.

The authors have explored the use of the symbolic manipulator MACSYMA to generate FORTRAN code for finding critical loci in 2-component fluid mixtures. For a given equation of state and mixing rules, MAC SYMA can generate the equations satisfied at a critical point. MACSYMA also produces FORTRAN code that can be used to develop codes for finding critical loci. The results for test cases where traditional methods have been applied are identical to earlier work. They describe the application of the technique to the Carnahan-Starling-De Santix equation, a non-cubic function consisting of a hard-sphere kernal and a Redlich-
Kwong-like attractive term. Examples of its application to refrigerant mixtures are described.

101,391

PB91-195636

Not available NTIS

National Inst. of Standards and Technology, Boulder, CO. Thermodynamics Div.

Non-Equilibrium Molecular Dynamics Simulations of Structured Molecules. Part 1. Isomeric Effects on the Viscosity of Butanes.

Final rept.

R. L. Rowley, and J. F. Ely. 1991, 16p

Pub. in Molecular Physics 72, n4 p831-846 1991.

Keywords: "Viscosity, "Butane, "lennard-Jones potential, "Nonequilibrium flow, Rheology, Simulation, Reprints, Molecular dynamics.

Corresponding-states theories fail to predict the large difference observed between n-butane and isobutane viscosities at similar reduced conditions. To investigate the molecular cause of the structural effects upon gate the molecular cause of the structural effects upon viscosity, non-equilibrium molecular dynamics simulan-butane and isobutane are performed over much of the density range for which experimental data are available. Simulated viscosities at zero shear agree very well with experimental data over the entire density range. Site size, non-equilibrium molecular alignmen and molecular geometry are the primary factors causing both the similarities and differences between the isomers' viscosity and rheology.

\section{1,392}

PB91-200832

Not available NTIS

National Bureau of Standards (NEL), Boulder, CO

Thermophysics Div.

Taking the Measure of Fluid Properties Data Bases.

Final rept.

M. E. Baltatu, H. J. M. Hanley, and N. A. Olien. 1987, $1 \mathrm{p}$

Pub. in Mechanical Engineering, p64 Dec 87.

Keywords: "Fluids, "Thermophysical properties, "Data bases, Critical point, Melting points, Thermochemical properties, Thermodynamic properties, Pressure, Temperature, Reprints.

The status of thermophysical properties data bases today is discussed with comments on needs for the future. Recommendations for obtaining the best possible bases are discussed. Future work will require close cooperation between the scientist and the engineer. Examples of such cooperation are given.

\section{1,393}

PB91-203000

Not available NTIS National Inst. of Standards and Technology (NML), Boulder, CO. Thermophysics Div.

Analysis of Laminar Vortex Shedding Behind a Circular Cylinder by Computer-Aided Flow Visualization.

Final rept.

B. E. Eaton. 1987, 29p

Sponsored by National Research Council of Canada, Ottawa (Ontario)

Pub. in Jnl. of Fluid Mechanics 180, p117-145 1987.

Keywords: "Flow visualization, "Computational fluid dynamics, "Vortex shedding, "Cylinders, Finite element method, Numerical analysis, Navier-Stokes equations, Reynolds number, Two dimensional flow, Wakes, Critical point, Vortex flow, Vortices, Reprints

Streamline, streakline, and material-line flow-visualization techniques have been numerically simulated in the vortex-shedding flow field from a finite-element simulation of the two-dimensional Navier-Stokes equations a a Reynolds number of 110 . The results have been used (i) to characterize the wake in terms of its criticalpoint trajectories, and (ii) to verify that the two-dimensional Navier-Stokes model predicts the mechanism of vortex shedding experimentally observed by Gerrard (1978). A technique for determining vorticity balances in the flow field is also presented.

\section{1,394}

PB91-204123

Not available NTIS

National Inst. of Standards and Technology (NEL), Gaithersburg, MD. Thermophysics Div.
Thermodynamic Behavior of Fluids in the Supercritical Region.

Final rept.

S. Tang, and J. V. Sengers. 1991, $4 p$

Contract DE-FG05-88ER13902

Sponsored by Department of Energy, Washington, DC. Pub. in Proceedings of International Symposium on Supercritical Fluids (2nd), Boston, MA., May 20-22, 1991, p254-257.

Keywords: "Thermodynamic properties, "Fluids, "Supercritical state, Free energy, Temperature, Critical temperature, Critical point, Thermodynamics, Pressure, Equations of state, Reprints.

A procedure for constructing a thermodynamic free energy of fluids is discussed which incorporates a crossover from singular thermodynamic behavior at the critical point to regular thermodynamic behavior far away from the critical point. The procedure is based on an approximate solution of the renormalization-group theory of critical phenomena and yields an accurate representation of the thermodynamic properties of fluids in a large range of temperatures and densities around the critical point.

101,395

PB91-222679

PC A03/MF A01

National Inst. of Standards and Technology (CSTL), Boulder, CO. Chemical Engineering Div.

Unsteady Laminar Flow in a Circular Tube: A Test of the HERCOL (Hermitian Collocation) Computer Code.

J. F. Welch, J. A. Hurley, M. P. Glover, R. D. Nassimbene, and M. R. Yetzbacher. May $91,27 p$ NISTIR-3963

Keywords: "Laminar flow, "Computational fluid dynamics, "Pipe flow, "Velocity distribution, Fluid flow, Pressure gradients, Unsteady flow, Computer programs, Partial differential equations, Numerical analysis, Tubes, HERCOL computer program.

HERCOL, a computer code for the integration of second-order differential equations in one space dimension by Hermitian collocation was used to calculate the unsteady velocity profiles for laminar flow in a circular tube. The code was tested for stability and accuracy on the problem for which an analytical solution exists prior to application to a like problem in which the initial and boundary conditions preclude the existence of analytical solutions. The test problem is one in which a pressure gradient is imposed on a fluid initially at rest in a circular tube; the fluid accelerates and at steady state has a parabolic velocity profile. A second example was constructed from the first; a pressure gradient equal but opposite in sign is imposed on the fluid with a fully developed parabolic velocity profile. At steady state, the velocity is again parabolic but in the opposite direction to that at the initial conditions. Excellent agreement with the analytical solution was obtained in the first problem; in the second, the behavior was as expected. This example is suitable for first-time users of the code.

101,396

PB92-112291

PC A03/MF A01

National Inst. of Standards and Technology (NEL), Gaithersburg, MD. Applied and Computational Mathematics Div.

Effect of Gravity Modulation on Thermosolutal Convection in an Infinite Layer of Fluid.

B. V. Saunders, B. T. Murray, G. B. McFadden, S. R Coriell, and A. A. Wheeler. Oct $91,42 p$ NISTIR-4679 See also PB90-265281.

Keywords: *Convection, Binary alloys, Reduced gravity, Instability, "Thermosolutal convection, Thermohaline convection, Buoyant convection, Gravity modulation, Directional solidification.

The effect of time-periodic vertical gravity modulation on the onset of thermosolutal convection in an infinite horizontal layer with stress-free boundaries is investigated using Floquet theory for the linear stability analysis. The authors consider situations for which the fluid layer is stably stratified in either the fingering or diffusive regimes of double-diffusive convection. Results are presented both with and without steady background acceleration. Modulation may stabilize an unstable base solution or destabilize a stable base solution. In addition to synchronous and subharmonic response to the modulation frequency, instability in the double-diffusive system can occur via a complex conjugate mode. In the diffusive regime, where oscillatory 
onset occurs in the unmodulated system, regions of resonant instability occur and exhibit strong coupling with the unmodulated oscillatory frequency.

101,397

PB92-116490

Not available NTIS

National Inst. of Standards and Technology (CSTL), Boulder, CO. Chemical Engineering Div.

Choosing Flow Conditioners and Their Location for Orifice Flow Measurement.

Final rept.

J. A. Brennan, C. F. Sindt, M. A. Lewis, and J. L.

Scott. 1991, 5 p

Contract GRI-5088-271-1680

Sponsored by Gas Research Inst., Chicago, IL.

Pub. in Flow Meas. Instrum. 2, p40-44 Jan 91.

Keywords: "Orifice meters, "Orifice flow, "Rod bundles, Fluid flow, Flowmeters, Orifices, Flow measure ment, Flow control, Turbulence, Reprints, "Flow conditioners.

Three different tube bundles, a zanker, and an etoile flow conditioner have been tested in a $100 \mathrm{~mm}$ orifice flowmeter. The tube bundles included a seven- and a 19-tube configuration. Tests were designed to determine a location for the flow conditioner that would remove the effects of flow disturbances and keep the length of the meter tube as short as possible. The disturbances included an inlet tee two out-of-plane elbows, and a single elbow. All disturbances were preceded by another long radius elbow located 12 pipe diameters upstream. Results indicate that it may be possible to move the conditioner closer to the disturbance than current standards permit.

\section{Optics \& Lasers}

\section{1,398}

AD-A207 806/1

PC A06/MF A01

National Inst. of Standards and Technology, Gaithersburg, MD.

Annual Report to the Strategic Defense Initiative Organization on the Free-Electron Laser Driven by the NIST CW Microtron.

Annual rept. 1 Apr 88-31 Mar 89.

A. G. Johnson. 5 May 86, $101 \mathrm{p}$

Contract N00014-87-F-0066

Keywords: Acceleration, Damage, Electric current, Engineering, "Free electron lasers, Mirrors, Peak power, Short pulses, Test and evaluation, "Antimissile defense systems, "Strategic Defense Initiative.

Excellent progress has been made during the past year on all areas critical to the NIST-NRL FEL project. A contract for the construction of a wiggler was signed early in this reporting period. The contractor has completed the engineering design of the wiggler and is well along in construction. Several methods to increase the peak current in the RTM were studied. The conceptual design of the injector for the method selected was completed. A study on the problem of mirror damage has been completed, and commercial suppliers of mirrors that can withstand the high intracavity power of the FEL have been identified. The design of the room in which the FEL is located has been improved, an the design of the users area has been completed. Calculations of FEL performance have been extended to include short-pulse effects and the effects of wiggle magnetic field errors. A major activity in this period has been preparation of the RTM for one-pass acceleration to $17 \mathrm{MeV}$. One-pass tests were started, and preliminary measurements of beam quality were better than design goals by a factor of two.

\section{1,399}

PC A03/MF A01

National Inst. of Standards and Technology, Gaithersburg, MD

NIST-NRL Free-Electron Laser.

Status rept.

P. H. Debenham, R. L. Ayres, W. A. Cassatt, B. C.

Johnson, and R. G. Johnson. 1990, 12p

Contract N00014-87-F-0066

Keywords: Accuracy, Cavities, Electron beams, Errors, Facilities "Free electron lasers, Frequency, Length, Losses, Mirrors, Models, Oscillators, Pulses, Simulation, Three dimensional, User needs, White light, Racetrack microtrons, Picosecond pulses, Tunable lasers.
A free-electron laser (FEL) user facility is being constructed. The FEL, which will be operated as an oscillator, will be driven by the $17 \mathrm{MeV}$ to $185 \mathrm{MeV}$ to 185 $\mathrm{MeV}$ electron beam of the NIST continuous-wave racetrack microtron. Anticipated performance of the FEL includes: waveleng th tuneable from $200 \mathrm{~nm}$ to 10 micrometers; a continuous train of 3-ps pulses at either 16.5 or $66.1 \mathrm{MHz}$; and average power of $10 \mathrm{~W}$ to 200 $W$. Construction of the RTM will be completed in January 1991 . The measured $\mathrm{rms}$ field error is $0.6 \%$, which is sufficiently small for good gain. With a full-scale model of the 9-m-long optical cavity, we have developed a method of aligning the cavity end mirrors to the required accuracy using white light and an autocollimator/telescope. We have performed three-dimensiona simulations of performance including the effects of the electron beam (emittance, pulse length and shape, and timing jitter), undulator field errors, and cavity losses. These calculations predict adequate gain fo lasing across the full wavelength range. $(\mathrm{RH})$

101,400

AD-A236 746/4

PC A02/MF A01

(1)

britical Assessment of Optical Properties of CVD Diamond Films.

Interim rept.

A. Feldman, and L. H. Robins. 12 Apr 91, 10p

Contract N00014-90-F-0011

Keywords: Blue(Color), Coatings, Deposits, Diamonds, Energy bands, Infrared windows, Laser materials, Luminescence, Optical materials, Optical properties, Phonons, Quality, Quantum efficiency, Surface roughness, Vapor deposition, $X$ rays, Thin films, "Diamond films, Chemical vapor deposition, Progress report.

The basic phenomena that determine the optical properties of diamond, which include the electronic band structure, the phonon structure, and defects, are discussed. Experiments on chemical vapor deposited diamond relevant to its applications as an optical material are reviewed. The most immediate application is the diamond $x$-ray window. Other applications, such as infrared transmissive elements and coatings, will require considerable improvement in material quality. Surface roughness is a major impediment. The ability to depos it smooth surfaces would make diamond considerably more attractive as optical material. Diamond also has promise as a blue luminescent or laser material. Identifying and controlling the relevant luminescent centers will be needed to improve the quantum efficiencies of such devices.

\section{1,401}

PC A03/MF A01

National Inst of Standards and Technology, Gaithersburg, MD.

Use of Diamond as an Optical Material.

Technical rept.

A. Feldman. 27 Sep $91,17 p$

Contract N00014-90-F-0011

Keywords: Absorption, Adhesion, Carbon, Chemical reactions, Coatings, Films, Impurities, Lithography, Materials, Methodology, "Optical materials, "Optical properties, Optics, Phase, Polishing, Scattering, Single crystals, Substrates, Surface roughness, Vapor deposition, $X$ rays, "Diamonds, Chemical vapor deposition.

Chemical vapor deposition (CVD) processes promise inexpensive diamond optics and coatings with large dimensions. Near term optical applications include $x$-ray windows, membranes for $x$ ray lithography, and infrared windows and domes. CVD diamond, which is mainly polycrystalline, shows materials problems that include scattering due to large surface roughness, absorption due to defects, non diamond carbon phases, and impurities, and poor diamond/substrate adhesion. Free carrier absorption in CVD diamond has also been reported. While diamond films less than 3 um thick can be made transmissive in the visible and in the ultraviolet, at these wavelengths, thicker components scatter excessively and show absorption due to defects. Continuing research is improving the optical quality of CVD diamond. New polishing methods have yielded smooth surfaces in reasonable polishing times. Recent research holds promise for large optics made from single crystal diamond.

\section{1,402
PBg $0-244708$}

Order as PB90-244658)

Technische Univ. Muenchen (Germany, F.R.). Lehrstuhl fuer Thermodynamik.
Refractive Index of Water and Steam as Function of Wavelength, Temperature and Density

Quarterly rept.

P. Schiebener, J. Straub, J. M. H. Levelt Sengers, and J. S. Gallagher. c1990, 41p

Prepared in cooperation with National Inst. of Standards and Technology (NEL), Gaithersburg, MD. Thermophysics Div.

Included in Jnl, of Physical and Chemical Reference Data, v19 n3 p677-717 1990. Available from American Chemical Society, 115516 th St., NW, Washington, DC 20036.

Keywords: "Water, "Water vapor, "Steam, Near infrared radiation. Near ultraviolet radiation, Tables(Data) "Refractive index, Visible radiation, Temperature de pendence.

Based on a comprehensive collection of data previously obtained by Thormahlen et al. on the experimental refractive index of water and steam from the 1870 s to the present, a new formulation is presented for the range of 0.2 to 2.5 micrometers in wavelength, -10 to $+500 \mathrm{deg} C$ in temperature and 0 to $1045 \mathrm{~kg} / \mathrm{cu} \mathrm{m}$ in density. The Lorentz-Lorenz function or molar refraction, a strong function of wavelength but only weakly dependent on density and temperature, is fitted to a selected set of accurate refractive index data. The NBS/NAC equation of state for water and steam, the new international standard, is used to convert the experimental pressures to density. The deviations of all experimental data from the formulation are shown. A detailed assessment of the accuracy of the formulation is presented. It is demonstrated that several recent formulations of optical properties of liquid wate over large ranges of wavelength need improvement in the range covered here. The new formulation is used to generate tables of the refractive index of water and steam at six wavelengths in the visible, near-infrared and near-ultraviolet, from 0 to $500 \mathrm{deg} C$ and up to 100 $\mathrm{MPa}$ in pressure.

101,403

PB91-132308

PC A10/MF A02

National Inst of Standards and Technology (NEL)

Boulder, CO. Electromagnetic Technology Div.

Technical Digest-Symposium on Optical Fiber Measurements, 1990

G. W. Day, and D. L. Franzen. Sep 90, 211p NIST/ SP-792

Also available from Supt. of Docs. See also PB89129555. Prepared in cooperation with Optical Society of America, Washington, DC.

Keywords: "Fiber optics, "Optical measurement "Meetings, Optical communication, Electrooptics, $\mathrm{Re}$ flectometers, "Optical fibers, Fiber optics transmission lines, Sensors.

The digest contains summaries of 45 papers presented at the Symposium on Optical Fiber Measurements, held September 11-12, 1990, at the National Institute of Standards and Technology, Boulder, Colorado.

101,404

PB91-134106

Not available NTIS

National Bureau of Standards (NML), Boulder, CO Time and Frequency Div.

Modulatable Narrow-Linewidth Semiconductor Lasers.

Final rept.

L. Hollberg, and M. Ohtsu. 1988, 3p

See also PB88-239470.

Pub. in Applied Physics Letters 53, n11 p944-946 Sep 88

Keywords: "Semiconductor lasers, Frequency control, Frequency stability, Line width, Optical communication, Reprints, Frequency division multiplexing, Line narrowing.

The authors found that using the technique of optical feedback locking, to narrow semiconductor linewidths, does not sacrifice the ability to modulate the laser's frequency via the injection current. The frequency of a laser is stabilized to a separate Fabry-Perot reference cavity using resonant optical feedback and can be modulated efficiently at frequencies related by rational fractions to the free-spectral range of the reference cavity. This system can provide an array of narrowlinewidth, frequency-stable laser lines and shows promise for applications in frequency-division-multiplexed coherent communications, as well as laser frequency control and precision measurement systems. 


\section{1,405}

PB91-134130

National Inst. of Standards and Tot available NTIS

Boulder CO Time Standards and Technology (NML),

Accurate Frequency of the $119 \mathrm{mu} \mathrm{m}$ Methanol

Laser from Tunable Far-Infrared Absorption Spec-

troscopy.

M. Inguscio, L. R. Zink, K. M. Evenson, and D. A

Jennings. $1990,5 p$

Pub. in IEEE (Institute of Electrical and Electronics Engineers) Jnl. of Quantum Electronics 26, n3 p575-579 Mar 90.

Keywords: "Frequency measurement, Far infrared radiation, Absortion spectra, Infrared lasers, Reprints "Methanol lasers, "Laser frequencies.

The authors report on high accuracy absorption spectroscopy of $\mathrm{CH} 3 \mathrm{OH}$ in the far infrared. In addition to 22 transitions in the ground state, the authors have meas ured the frequency of the $(n, t a u, J, K),(0,1,16,8)->$ $(0,2,15,7)$ transition in the nu sub 5 excited vibrational level, which is responsible for the laser emission at 119 micrometers. An accurate remeasurement of the lase emission frequency has also been performed, and the results are in good agreement.

\section{1,406}

PB91-147207

Not available NTIS

National Inst. of Standards and Technology (NEL) Boulder, CO. Electromagnetic Technology Div.

Standard Reference Fibers for Calibration of the Optical Time Domain Reflectometer.

Final rept.

B. L. Danielson, C. D. Whittenberg, and T. Drapela. $1989,4 \mathrm{p}$

Pub. in Proceedings of International Instrumentation Symposium (35th), Orlando, FL., May 1-4, 1989, p895898.

Keywords: "Reflectometers, "Calibration, Optical fibers, Reprints, "Standard reference fibers, "Reference materials, "Optical time domain reflectometers. OTDR(Optical time domain reflectometers).

Calibration of optical time domain reflectometers by military and industrial users can be achieved by a number of published test procedures. For some performance parameters, a particularly convenient way for establishing measurement verification and traceability to national standards is through the use of a standard reference fiber. At NIST the authors have begun a program to evaluate such test lightguides. Prototype standard reference fibers have been characterized for spectral attenuation, group delay, group index, and length. The authors describe measurement methods and tolerances for these devices.

\section{1,407}

PB91-147868

Not available NTIS

National Inst. of Standards and Technology (NML), Boulder, CO. Chemical Engineering Science Div.

Fiber-Optic Fluorescence Array to Study Free Convection in Porous Media.

Final rept.

R. A. Perkins, and M. C. Jones. 1989 , 6p

Sponsored by Department of Energy, Washington, DC. Office of Basic Energy Sciences.

Pub. in Review of Scientific Instruments $60, \mathrm{n} 11$ p3492-3497 Nov 89.

Keywords: "Convection, "Fiber optics, "Fluorescence, Fluorescent dyes, Labelled substances, Porous materials, Design, Fluid mechanics, Reprints.

A 64-point fiber-optic fluorescence array is integrated into a heated-packed bed to study free convection in porous media. The array consists of 64 individual fluorescence coupler-detector modules. The array modules are designed for rhodamine $6 G$ dye and allow real-time measurement of tracer concentrations to about $40 \mathrm{ppb}$. The coupler-detectors are inexpensive and allow a single fiber to carry both the excitation ligh and the emitted fluorescence light between the detectors and the remote probe terminations at known locations within the bed. Dilute pulses of tracer dye are injected into the packed bed and the dye concentration at each of the 64 fiber-optic probe terminations is monitored as a function of elapsed time. The tracer concentration as a function of time and position allows the fluid movement to be followed within the porous medium.
National Inst. of Standards and Technology (NEL), Boulder, CO. Electromagnetic Technology Div.

Subpicosecond Pulse Compression and Raman Generation Using a Mode-Locked Erbium-Doped Fiber Laser-Amplifier.

Final rept.

J. B. Schlager, P. D. Hale, and D. L. Franzen. 1990 , 3p

Pub. in IEEE (Institute of Electrical and Electronics Engineers) Photonics Technology Letters 2, n8 p562-564 Aug 90.

Keywords: "Pulse compression, Near infrared radiation, Mode locked lasers, Picosecond pulses, Doped materials, Raman effect, Raman lasers, Solitons, Reprints, Femtosecond pulses, Erbium fibers.

Pulses of $20 \mathrm{ps}$ duration from a $1536 \mathrm{~nm}$ erbium-doped fiber laser mode-locked at $10 \mathrm{MHz}$ are soliton-compressed in a $1 \mathrm{~km}$ fiber to $1.5 \mathrm{ps}$. The pulses are then amplified in an erbium-doped fiber amplifier and compressed in a second fiber to less than 300 fs. Pulses give rise to stimulated Raman scattering in fibers; the direct output from the erbium fiber laser-amplifier has sufficient peak power to pump fiber Raman lasers.

\section{1,409}

PB91-148478

PC A99/MF A04

CO.

Laser Induced Damage in Optical Materials: 1989. Special pub.

H. E. Bennett, L. L. Chase, A. H. Guenther, B. E. Newnam, and M. J. Soileau. Oct $90,674 \mathrm{p}$ NIST/SP. 801

Also available from Supt. of Docs. as SNO03-003 03061-9. See also report for 1988, PB90-185570. Errata sheet inserted. Proceedings of Annual Symposium on Optical Materials for High-Power Lasers (21st), Boulder, CO., Nov. 1-3, 1989. Prepared in coop. with American Society for Testing and Materials, Phila delphia, PA., SPIE-The International Society for Optical Engineering, Bellingham, WA.

Keywords: "Optical materials, "Radiation damage, "Laser damage, "Meetings, Physical radiation effects, Laser materials, Thin films, Optical coatings, Surfaces, Mirrors.

The Twenty-First Annual Symposium on Optical Materials for High-Power Lasers was divided into sessions concerning Materials and Measurements, Mirrors and Surfaces, Thin Films, and, finally, Fundamental Mechanisms. As in previous years, the emphasis of the papers presented at the Symposium was directed toward new frontiers and new developments. Particular emphasis was given to materials for high power apparatus. The wavelength range of the prime interest was from 10.6 micrometers to the uv region. Highlights included surface characterization, thin film substrate boundaries, and advances in fundamental laser-matte threshold interactions and mechanisms. The scalling of damage thresholds with pulse duration, focal area, and wavelength was discussed in detail.

\section{1,410}

PB91-148908

Not available NTIS

National Inst. of Standards and Technology (NML), Gaithersburg, MD. Radiometric Physics Div.

Use of Heterodyne Detection to Measure Optical Transmittance over a Wide Range.

Final rept.

A. L. Migdall, B. Roop, Y. C. Zheng, J. E. Hardis, and G. J. Xia. $1990,9 p$

Pub. in Applied Optics 29, n34 p5136-5144, 1 Dec 90.

Keywords: "Transmittance, Optical filters, MachZehnder interferometers, Demodulation, Heterodyning. Acoustooptics, Optical measurement, Reprints, Densitometry.

The authors are developing a heterodyne detection technique to measure optical transmittance with high accuracy over an unprecedented dynamic range. They have measured filters spanning a wide range of transmittances (12 orders of magnitude) and have evaluated the absolute uncertainties and discuss the ultimate accuracies that may be achieved. Their setup uses a two-beam Mach-Zehnder interferometer with acoustooptic frequency shifting to produce a frequency difference between the two light beams. The authors determine the optical transmittance of a filter by inserting it into one of the interferometer arms and measuring the change in amplitude of the signal at the difference frequency on the interferometer output beam. The method allows direct comparisons between optical and if attenuators, ultimately tying optical transmittance measurements to if attenuation standards in an absolute way.

101,411

PB91-149138

Not available NTIS National Bureau of Standards (NML), Gaithersburg, MD. Temperature and Pressure Div.

Dispersion of the Electronic Contribution to the Third-Order Nonlinear Susceptibility of $\mathrm{H2}$

Final rept.

G. J. Rosasco, and W. S. Hurst. 1986, $6 p$

Pub. in Jnl. of the Optical Society of America B 3, n10 p1251-1256 Oct 86.

Keywords: "Hydrogen, Raman spectra, Kerr effect, Reprints, Nonlinear susceptibility, Second harmonic generation, Third harmonic generation.

A new value is reported for the electronic contribution (the electronic hyperpolarizability) to the third-order nonlinear susceptibility of $\mathrm{H}_{2}$. The result is derived from a measurement of the ac (optical)-Kerr constant referenced to the resonant Q-branch susceptibility of D2. A long-known dispersion formula is applied to compare the authors' value of the electronic hyperpolarizability with values determined by other nonlinear optical techniques. The dispersion formula is shown to account systematically for the results of other nonlinear techniques.

101,412

PB91-149195

Not available NTIS

National Bureau of Standards (NEL), Boulder, CO. Electromagnetic Technology Div.

Laser Measurements.

Final rept.

A. A. Sanders. 1983, 3p

Pub. in Proceedings of Measurement Science Conference, Palo Alto, CA., January 20-21, 1986 p198-200 1983.

Keywords: "Laser radiation, "Lasers, Power measurement, Beam profiles, Calorimeters, Metrology, US NBS, Reprints.

The report will review some of the national standards and measurement services for lasers available at NBS, highlight some of the current research, and indicate some of the future needs and direction of NBS laser metrology research.

101,413

PB91-158709

Not available NTIS

National Bureau of Standards (IMSE), Gaithersburg, MD. Ceramics Div.

Stoichiometry of Reactively Evaporated Films.

Final rept.

A. Feldman, E. N. Farabaugh, and Y. N. Sun. 1988, $4 p$

Pub. in Proceedings of Optical Interference Coatings Topical Meeting, Tucson, AZ., April 12-15, 1988, p1417.

Keywords: "Optical films, "Silicon oxides, "OXidation, Photoelectron spectroscopy, Dielectric films, Thin films, Stoichiometry, Reprints

If an optical film is not fully oxidized, its refractive index will be different from expected, and significant optical absorption may result. In order to ensure full oxidation of oxide films, evaporations are frequently carried out in reactive atmospheres containing significant partial pressures of oxygen. $\mathrm{SiO}(\mathrm{x})$ films provide a convenient system for studying the oxidation state of reactively evaporated films, not only because they are important coating materials, but because structural models have been developed for the system. In the paper, the degree of oxidation of $\mathrm{SiO}(\mathrm{x})$ films, prepared by reactive electron-beam evaporation of $\mathrm{Si}$ under different partial pressures of oxygen, has been examined by $X$ ray photoelectron spectroscopy (XPS). The analysis suggests tha the silicon in the films is not as fully oxidized as might be expected on the basis of the measured oxygen content.

101,414

PB91-158972

Not available NTIS National Inst. of Standards and Technology (NEL), Gaithersburg, MD. Applied and Computational Mathematics Div. 
Algorithm and Computer Program for the Calculation of Envelope Curves.

Final rept.

M. McClain, A. Feldman, D. Kahaner, and X. T. Ying

1991, 4p

See also PB90-155409.

Pub. in Computers in Physics, p45-48 Jan/Feb 91

Keywords: "Curve fitting, "Light transmission, Refractive index, Absorptivity, Transmittance, Oscillations, Interpolation, Algorithms, Reprints, Envelope curves.

A procedure has been developed to calculate numerically the envelope functions of an oscillatory curve. The method has been shown to be applicable to optical transmission data, but it is general enough to be able on request.

101,415

PB9 1-174854

Not available NTIS

National Bureau of Standards (NML), Gaithersburg, MD. Atomic and Plasma Radiation Div.

Laser-Driven Ionization and Photoabsorption Spectroscopy of Atomic lons.

Final rept.

W. T. Hill, and C. L. Cromer 1987,12p

Pub. in Springer Ser. Opt. Sci. 54, p183-194 1987.

Keywords: "Atomic ions, "Photoabsorption, Barium ions, Cesium ions, Xenon ions, Isoelectronic sequence, Laser radiation, Ionization, R matrix, Reprints.

The application of laser-driven ionization techniques to photoabsorption spectroscopy of atomic ions will be discussed. A summary of the experimental results which confirm that a collisional mechanism is responsible for the nearly complete ionization following laser irradiation is given, along with a bibliography of the photoabsorption measurements reported to date. The importance of these investigations, demonstrated in two studies involving the $\mathrm{Ba}$ nuclear sequence (i.e. $\mathrm{Ba}$, $\mathrm{Ba}(1+)$, and $\mathrm{Ba}(2+))$ and the $\mathrm{Xe}$ isoelectronic sequence (i.e. $\mathrm{Xe}_{\mathrm{e}} \mathrm{Cs}(1+)$ and $\left.\mathrm{Ba}(2+)\right)$, will be discussed. Finally, a tabulation of quantum defect parameters for $\mathrm{Xe}, \mathrm{Cs}(1+)$, and $\mathrm{Ba}(2+)$ based on a re-analysis of the Xe-sequence spectra via a shifted R-matrix quantum defect approach will be presented.

101,416

PB91-175141

Not available NTIS

National Inst. of Standards and Technology (NEL) Boulder, CO. Electromagnetic Technology Div.

Semiconductor Claddings on Glass Waveguides for Polarizers and Detectors.

Final rept.

D. L. Veasey, D. R. Larson, R. J. Phelan, and T. E.

Batchman. 1990, 1p

Pub. in Proceedings of Optical Society of America Annual Meeting Technical Digest, Boston, MA., November 4-9, 1990, pMA5.

Keywords: "Polarizers, Amorphous silicon, Waveguides, Detectors, Semiconductors, Reprints, Claddings.

$T E$ and $T M$ polarizers and polarization sensitive detectors were fabricated by cladding glass channel waveguides with hydrogenated amorphous silicon. Extinction ratios as high as $48 \mathrm{~dB}$ have been observed for the TE pass device and up to $34 \mathrm{~dB}$ for the TM pass device.

\section{1,417
PB91-175596}

Not available NTIS

National Inst. of Standards and Technology (NML), Boulder, CO. Quantum Physics Div.

Correlated Spontaneous Emission in a Zeeman Laser.

Final rept.

M. P. Winters, and J. L. Hall. $1990,4 \mathrm{p}$

Contract N000 14-89-J-1227, Grant NSF-PHY86-

04504

Sponsored by Office of Naval Research, Arlington, VA., and National Science Foundation, Washington, DC.

Pub. in Physical Review Letters 65, n25 p3116-3119, 17 Dec 90.

Keywords: "Helium neon lasers, Spontaneous emission, Laser interferometry, Sensitivity, Reprints, "Zeeman lasers.

The authors have observed phase-diffusion noise $40 \%$ below the Schawlow-Townce limit in the relative phase of a two-mode HeNe Zeeman laser due to the correlated-emission-laser effect.
101,418

PB91-187096 Gaithersburg, MD. Radiometric Physics Div. Temperature Dependence of High Accuracy-Photometer Heads.

Final rept.

G. Andor. 1989, 2p

Pub. in Applied Optics 28, n22 p4733-4734, 15 Nov 89.

Keywords: "Photometers, "Photodiodes, Silicon diodes, Temperature dependence, Transmittance, Reprints.

The redefinition of the Candela in 1979 permits the use of upsilon(lambda) response photodetectors as primary photometric standards. Their long-term stability and temperature dependence is of prime importance in this application. Modern high-accuracy photometer heads consist of a silicon photodiode, a upsilon(lambda) matched glass filter and a current to voltage converter. The purpose of the letter is to report an investigation of the temperature characteristics for three types of high-quality silicon photodiodes and their matching filters. The temperature dependence of a properly designed current to voltage converter in the practical illuminance range of 0.0001 to 10,000 lux is negligible.

101,419

PB91-187419

Not available NTIS

National Inst of Standards and Technology (MSEL) Gaithersburg, MD. Ceramics Div.

Design Diagrams for Heavy Metal Fluoride Glass Windows.

Final rept.

D. Cranmer, T. Chuang, S. Freiman, and A. Raynes. 1990, 17p

Sponsored by Naval Research Lab., Washington, DC. and Center for Naval Analyses, Alexandria, VA. Naval Warfare Operations Div.

Pub. in Proceedings of High Power Laser Optical Components Topical Meeting, China Lake, CA., October 30-31, 1989, p98-114 1990 .

Keywords: "Design criteria, "Glass, "Window glass, "Fluorides, "Optical materials, Lasers, Service life, Finite difference theory, Fatigue limit, Weibull density functions, Reliability, Stress analysis, Mathematical models, Reprints, Heavy metals.

In order to design structures with materials such as heavy metal fluoride glasses, it is necessary that the stresses under which the structure will operate and the probability that the structure will survive these stresses be known. An experimental and theoretical methodology is described that combines stress determination using a finite element method with experimental determinations of the requisite fracture parameters, leading to the prediction of structural lifetimes. The stresses for an assumed geometry will be presented and combined with the fracture parameters needed to describe the behavior of the glass. These parameters include measures of the strength and strength distribution as well as the material's sensitivity to the service environment. A preliminary stress-lifetime diagram for the material has been created and compared to the results of the finite element model.

101,420

PB91-187617

PC A06/MF A01

National Inst. of Standards and Technology, Gaithersburg, MD.

Journal of Research of the National Institute of Standards and Technology. March-April 1991. Volume 96 , Number 2

1991, 109p

Also available from Supt. of Docs. as SN703-027$00039-3$. See also PB91-187625 through PB91187682 and PB91-184853. Library of Congress catalog card no. 89-656121.

Keywords: "Photodiodes, Dimensional measurement, Particle size, Microsphere, Gel permeation chromatography, Light scattering, Molecular weight, Potassium chloride, Standards, Dental caries, Optical communication, Standard reference materials, Electrolytic conductivity, Bidirectional scattering distribution function.

Contents:

Linearity of a Silicon Photodiode at $30 \mathrm{MHz}$ and Its Effect on Heterodyne Measurements;

Use of the Electrostatic Classification Method to Size 0.1 micrometer SAM Particles--A Feasibility Study;
A Multiple Variable-Angle Light Scattering Detector for Gel Permeation Chromatography; Proposed New Electrolytic Conductivity Primary Standards for $\mathrm{KCl}$ Solutions

Digital Image Analysis Assisted

Microradiography--Measurement of Mineral Content of Caries Lesions in Teeth;

Bidirectional Scattering Distribution Function (BSDF)--A Systematized Bibliography;

A Limited International Intercomparison of Responsivity Scales at Fiber Optic Wavelengths.

101,421

PB91-187674

(Order as PB91-187617, PC A06/MF A01) National Inst. of Standards and Technology, Gaithersburg, MD

Bidirectional Scattering Distribution Function (BSDF): A Systematized Bibliography.

C. Asmail. 1991, 9p

Included in Jnl. of Research of the National Institute of Standards and Technology, v96 n2 p215-223 Mar/Apr 91

Keywords: "Light scattering, "Bibliographies, Distribution functions, Inverse scattering, Specular reflection, Diffuse reflection, Surface properties, Polarization, *BSDF(Bidirectional Scattering Distribution Function), *Bidirectional scattering distribution function.

In conjunction with the development of a bidirectional scattering metrology project, a large number of papers pertaining to the theory and measurement of bidirectional scattering from optical surfaces were collected and categorized. The collection includes papers that deal with various aspects of the bidirectional scattering distribution function (BSDF), its measurement, interpretation, use, and implications. Each paper is classified in one or more subject categories on the basis of its technical content. The subject categories are included just to serve as a key to the most salient characteristics of each paper cited. Because of the interest in the field, the bibliography is being published as a service to the public.

101,422

PB91-189332

Not available NTIS

National Inst. of Standards and Technology (IMSE). Gaithersburg, MD. Ceramics Div.

Refractive Index Gratings in Rare-Earth-Doped Alkaline Earth Glasses.

Final rept

V. A. French, R. C. Powell, D. H. Blackburn, and D.

C. Cranmer. $1991,5 \mathrm{p}$

Sponsored by Army Research Office, Research Triangle Park, NC.

Pub. in Jnl. of Applied Physics 69, n2 p913-917, 15 Jan 91.

Keywords: "Gratings, Laser applications, Refractive index, Doped materials, Europium ions, Silicates, Glass, Reprints.

Four-wave-mixing techniques were used to produce permanent laser-induced refractive index gratings in Eu( $3+)$-doped silicate glasses. The gratings are associated with a thermally induced change in the local glass structure at the site of the $\mathrm{Eu}(3+)$ ions, leading to a double-minimum potential well for the electronic energy levels of the $\mathrm{Eu}(3+)$ ions. The effects on the characteristics of the permanent laser-induced gratings produced by changing the divalent modifier ions of the glass host are reported, and a theoretical model is presented to explain the physical origin of the change in the refractive index of the material.

\section{1,423}

PB91-189738

Not available NTIS

National Inst. of Standards and Technology (NML), Gaithersburg, MD. Electricity Div.

Effects of Colored Pump Noise on Intensity Correlations of a Single Mode Dye Laser.

Final rept.

P. D. Lett, and E. C. Gage. 1989, 7p

Pub. in Physical Review A 39, n3 p1193-1199, 1 Feb

Keywords: "Dye lasers, Optical pumping, Quantum optics, Langevin equation, White noise, Intensity, Reprints.

Recent theoretical analyses of the single mode laser with colored noise pump fluctuations have allowed the detailed prediction of the short-time behavior of the in- 
tensity autocorrelations of such a laser. In particular, a rounding-off of the intensity correlation function on the time scale of the correlation time of the pump fluctuations has been predicted. The authors present results from experiments performed on a single mode dye laser system that clearly confirm these predictions. Numerical simulations of the laser Langevin equations including the colored noise pump fluctuations are used to fit the experimental results.

101,424

PB911-189803

Not available NTIS

National Inst. of Standards and Technology (NML),

Gaithersburg, MD. Molecular Spectroscopy Div.

Fourier Transform Infrared Spectroscopy at the

NIST: High Temperature Molecules and Atmospheric Molecules.

Final rept.

A. G. Maki. 1989, 4p

Pub. in Proceedings of High Resolution Fourier Transform Spectroscopy Topical Meeting, Santa Fe, NM., February 13-15, 1989, p98-101.

Keywords: "Fourier spectroscopy, "Molecular spectroscopy, "Infrared spectroscopy, Atmospheric chemistry, Air pollution, High temperature, Infrared instruments, Calibration, Reprints, US NIST.

The paper gives some of the interesting results from three projects that involve Fourier Transform Spectroscopy (FTS) measurements, studies of molecules involved in atmospheric chemistry, high temperature studies, and the compilation of data for wavenumber calibration of infrared instrumentation. Earlier work on these projects was conducted with a tunable diode laser, now many of the measurements are made with a FTS spectrometer. The FTS instrument is not as sensitive as the diode laser spectrometer, nor does it give as high resolution, but it has the advantage of conveniently and quickly giving broad band spectra with thousands of absorption lines.

\section{1,425}

PB9́1-195644

Not available NTIS

National Inst of Standards and Technology (NML), Gaithersburg, MD. Radiometric Physics Div.

Roughened Quartz Surfaces and Teflon as SmallAngle Diffusers and Depolarizers between 200 and $400 \mathrm{~nm}$

Final rept

R. D. Saunders, and H. J. Kostkowski. 1989, 4p Pub. in Applied Optics 28, n15 p3242-3245 1989.

Keywords: "Spectroradiometers, "Diffusers, Rocketborne instruments, Near ultraviolet radiation, Small angle scattering, Depolarization, Quartz, Teflon, Reprints, "Depolarizers.

Laboratory tests of transmission diffusers are reported for quariz and teflon. These tests were conducted in order to select transmission diffusers for the NOAA rocket-flight UV spectroradiometers.

101,426

PB9́1-203042

Not available NTIS

National Bureau of Standards (IMSE), Gaithersburg,

MD. Ceramics Div.

Alternate Representation of Prism Refraction for Light Incident at the Brewster Angle.

Final rept.

A. Feldman. 1988, 2p

Pub. in Applied Optics 27, n22 p4608-4609 1988

Keywords: "Optics, "Optical materials, "Prismatic bodies, "Brewster angle, Graphs(Charts), Visible radiation, Reprints.

An alternate representation of prism refraction provides a convenient context for solving the problem of a beam incident on a wedged plate at the Brewste angle and allows for a graphical solution based on a simple geometric construction.

101,427

PB91-203109

Not available NTIS

National Inst of Standards and Technology (NEL),

Boulder, CO. Electromagnetic Technology Div.

Frequency Stabilization of a Tunable ErbiumDoped Fiber Laser.

Final rept

S. L. Gilbert. 1991, 3p

Sponsored by Naval Sea Systems Command, Washington, DC

Pub in Optics Letters 16, n3 p150-152, 1 Feb 91.

Keywords: Near infrared radiation, Optical communication, Frequency standards, Frequency stability, In- frared lasers, Tunable lasers, Acetylene, Reprints Erbium lasers, Fiber lasers, Wavelength standards.

A single-frequency $\mathrm{Er}$-doped fiber laser that is tunable rom 1.52 to $\mathbf{1 . 5 8}$ micrometers has been constructed. The laser linewidth was determined to be less than 1.6 $\mathrm{MHz}$ FWHM by observing the spectrum of the beat beween the fiber laser and a 1523 micrometer $\mathrm{He}-\mathrm{Ne}$ laser. The frequency of the fiber laser was locked to several absorption lines of acetylene near $1.53 \mathrm{mi}$ crometers. The research demonstrates the inherent tability of fiber lasers and their potential for use in a wavelength standard for optical communications.

\section{1,428}

\section{PB91-203158}

Not available NTIS

National Bureau of Standards (NEL), Gaithersburg MD. Precision Engineering Div.

Note on the Precision Cementing of Small Optical Components.

Final rept.

A. W. Hartman. $1988,2 p$

Pub. in Review of Scientific Instruments 59, n12 p2617-2618 1988 .

Keywords: "Optical equipment, "Cementing, Alignment, Substrates, Precision, Shrinkage, Reprints.

When optical or mechanical components are cemented to a substrate that exhibits errors in surface figure or tilt angle, the possibility exists that these errors will propagate to the work piece. If the cement layer thickness shrinks by a given fraction $p$ during setting of the cement, the error propagation will occur at the same fraction causing losses in flatness, tilt angle, etc. These losses can be reduced to near harmless levels by adopting a two-stage cementing process, involving an intermediate substrate.

\section{1,429}

\section{PB91-203802}

Not available NTIS

National Inst. of Standards and Technology (NEL). Boulder, CO. Electromagnetic Fields Div.

New Triple Correlator Design for the Measurement of Ultrashort Laser Pulses.

Final rept.

N. G. Paulter, and A. K. Majumdar. 1991, 6p

Pub. in Optics Communications 81, n1-2 p95-100, 1 Feb 91

Keywords: "Optical correlators, "Correlators, Optica measurement, Laser radiation, Light pulses, Nonlinear optics, Design, Reprints.

A measurement device, a triple correlator, is proposed for the characterization of ultrashort optical pulses. The proposed triple correlator uses two consecutive nonlinear optical interactions, a sum frequency generation followed by a difference frequency generation, to produce a triple correlation output at the same optical frequencies as the input pulse. Phase matching for the triple correlator is greatly simplified by using the given design. The operation of the triple correlator is
described, and examples of expected output signals described, and examples of expected output signals sensitive to noise, as shown by computer simulations.

\section{1,430}

\section{PB91-203984}

Not available NTIS

National Inst. of Standards and Technology (NML),

Gaithersburg, MD. Atomic and Plasma Radiation Div.

Precise Laser Wavelength Measurements: What Can We Learn from Classical Spectroscopy.

Final rept.

C. J. Sansonetti. 1989, 6p

Pub. in Proceedings of International Laser Science Conference (4th), Advances in Laser Science IV, Atlanta, GA., 1988, p548-553 1989.

Keywords: "Dye lasers, "Laser radiation, "Wavemeters, Fabry Perot interferometers, Continuous wave lasers, Fourier spectrometers, Reprints, "Wavelength measurement.

The wavelengths of $\mathrm{cw}$ dye lasers are typically meas ured using Michelson wavemeters, but the absolute accuracy of such instruments is limited by their sensitivity to alignment of the laser beams. This systematic problem can be largely eliminated by diffusely scattering the beams and using classical spectroscopic methods to measure the wavelength. We have developed a laser wavemeter, based on a static Fabry-Perot interferometer, with an accuracy of a few parts in a billion and an update rate of about $2 \mathrm{~Hz}$. The system is insensitive to the alignment and collimation of the incoming laser beams. The potential for a more precise wave- meter based on techniques of Fourier transform spectroscopy is also considered.

101,431

PB91-204016

Not available NTIS

National Inst. of Standards and Technology (NML) Boulder, CO. Quantum Physics Div.

Production and Diagnosis of a Highly Spin-Polarized $\mathrm{Na}$ Beam.

Final rept.

G. W. Schinn, X. L. Han, and A. Gallagher. 1991, 5p Contract DE-FG02-87ER13720

Sponsored by Department of Energy, Washington, DC. Office of Basic Energy Sciences.

Pub. in Jnl. of the Optical Society of America B 8, n1 p169-173 Jan 91

Keywords: "Sodium, "Optical pumping, Laser induced fluorescence, Polarization(Spin alignment), Atomic beams, Reprints.

The authors describe optically pumping a beam of sodium atoms to $>96 \% \mathrm{~m}(\mathrm{~s})$ and $>92 \% \mathrm{~m}(\mathrm{~s}), \mathrm{m}(\mathrm{l})$ state selection. For the optical pumping both ground hyperfine states are pumped, using single-mode $\mathrm{cw}$ dye-laser radiation tuned to the triplet $S(1 / 2)$-triplet $\mathrm{P}(1 / 2)$ transition that is phase modulated in a LiTaO3 crystal to produce first-order sidebands at approximately the $1772-\mathrm{MHz}$ hyperfine splitting of the ground state. The z-directed optical pumping is performed in a $z$-directed magnetic field of (about) $5 \mathrm{G}$. The state-selected atoms then move, in (about) $1 \mathrm{~cm}$ into an (about) $200 \mathrm{G}$, z-directed field. The downstream probe laser beam is scanned through the triplet $S(1 / 2)(\mathrm{m}(\mathrm{s})$ $m(I)->$ triplet $P(3 / 2)\left(m^{\prime}(s)\right.$, $\left.m(I)\right)$ transitions, which are spectroscopically resolved at $200 \mathrm{G}$, and the fluorescence intensities portray the residual populations in each of the eight triplet $S(1 / 2)$ states.

101,432

PB91-204180

Not available NTIS

National Inst. of Standards and Technology (NML), Boulder, CO. Quantum Physics Div.

Correlated Spontaneous Emission in a Zeeman Laser.

Final rept.

M. P. Winters, and J. L. Hall. 1990, 9p

Contract N00014-89-J-1227, Grant NSF-PHY86

04504

See also PB91-175596. Sponsored by Office of Naval Research, Arlington, VA., and National Science Foundation, Washington, DC

Pub. in New Frontiers in Quantum Electrodynamics and Quantum Optics, p193-2011990.

Keywords: Gravitational wave detectors, Spontaneous emission, High sensitivity, Reprints, "Zeeman lasers, Correlated emission lasers, Laser interferometers, Squeezed states.

In recent years there has been great interest in the development of high sensitivity laser interferometers for use in the detection of gravitational waves. Ordinarily, the sensitivity of these devices is limited by quantum fluctuations that take the form of shot noise in passive interferometers and spontaneous emission noise in active interferometers. Proposals for increasing the sensitivity of these interferometers include squeezed states for passive devices and the correlated emission aser (CEL) for active devices. Generation of squeezed states has now been demonstrated by several groups and it is the purpose of the article to describe an experimental investigation of the CEL

101,433

PB91-237065

Not available NTIS

National Inst. of Standards and Technology (NML), Gaithersburg, MD. Atomic and Plasma Radiation Div. Exploding Foil Photoionization X-ray Laser.

Final rept.

P. D. Morley, and J. Sugar. 1988, 4p

Pub. in Physical Review A 38, n6 p3139-3142 1988.

Keywords: "X ray lasers, Multicharged ions, lodine ions, Soft $x$ rays, Photoionization, Inner-shell ionization, Reprints, "Copper-like ions, Exploding foils.

By combining the techniques of exploding foils and inner-shell photoionization, it is predicted that lasing can occur for $x$-rays less than $50 \mathrm{~A}$. As an example, the authors work out the case for Cu-like iodine $(1(24+))$ which should lase on the $3 d(10) 4 p-3 d(9) 4 s(2)$ doublet calculated at $24.565 \mathrm{~A}$ and $26.026 \mathrm{~A}$ with integrated line intensities comparable to the selenium $\mathrm{x}$-ray laser. 
101,434

PB91-237156

Not available NTIS

National Inst. of Standards and Technology (EEEL)

Boulder, CO. Electromagnetic Fields Div.

New Triple Correlation Technique for Measuring

Final rept.

N. G. Paulter, and A. Majumdar. 1991, 12p

See also PB91-203802.

Pub. in Review of Scientific Instruments 62, n3 p567. 578 Mar 91.

Keywords: "Laser radiation, "Light pulses, Picosecond pulses, Nonlinear optics, Optical measurement, Reprints, Triple correlation method.

A new triple correlation technique for measuring the complete intensity profile of ultrashort optical pulses is described. The triple correlation preserves the phase information of the input pulse so that a reconstruction of the triply correlated signal will provide a unique reconstruction of the input. The new technique de scribed here uses two second-order, nonlinear optical interactions for the generation of a triply correlated signal. A derivation of the measured triple-correlation signal and the pulse reconstruction is given. The effects of noise on the measured signal are also exam. ined.

\section{1,435}

\section{PB92-102185}

Not available NTIS

National Inst of Standards and Technology (NML),

Gaithersburg, MD. Radiometric Physics Div.

LBIR Facility User Handbook.

Final rept.

R. U. Datla, S. C. Ebner, J. Proctor, and A. C. Parr

Jun 91,31 p NIST/HB-147

Sponsored by Army Strategic Defense Command, Huntsville, AL.

Keywords: "Research facilities, "Handbooks, Blackbody radiation, Clean rooms, Standards, "Low Background Infrared Calibration Facility(LBIR), *LBIR facility, Absolute cryogenic radiometer, US NIST.

A handbook has been prepared to aid prospective users of NIST's Low Background Infrared Calibration Facility(LBIR). A detailed overview of the facility is given, including directions to the NIST site and places to stay. The sponsorship of the facility by the Strategic Defense Command to serve the community using blackbody sources in a cryogenic environment is stated in the introduction. The vacuum and cryogenic hardware associated with the facility is discussed in detail, as well as procedures the user must follow regarding clean room practice, vacuum compatibility, and electrical hookup. Much discussion is also given to Absolute Cryogenic Radiometer (ACR) which serves as the standard detector for the system. An electrical substitution radiometer, the ACR measures the total flux emitted from a blackbody.

\section{1,436}

\section{PB92-116532}

Not available NTIS

National Inst. of Standards and Technology (NEL). Gaithersburg, MD. Precision Engineering Div.

Light-Scattering Measurement of the RMS SIopes of Rough Surfaces.

Final rept.

L. X. Gao, T. V. Vorburger, A. G. Lieberman, and T.

R. Lettieri. 1991, 7p

Pub. in Applied Optics 30, n22 p3221-3227, 1 Aug 91.

Keywords: "Surface roughness, *Optical measurement, Laser applications, Light scattering, Metrology, Texture, Slope, Reprints.

Angle-resolved light scattering (ARLS) is used to estimate the root-mean-square (rms) slopes of rough surfaces having a well-defined lay, and the effect on slope measurements caused by changing the angles of incidence and scattering is investigated. The ARLS patterns are taken with the Detector Array for Laser Light Angular Scattering (Dallas) research instrument, and the rms slopes are obtained from the angular widths of these patterns. In general, it was found that the angular width, and thus the estimated rms slope, is surprisingly insensitive to relatively large changes in both the incident and scattering angles of light. These results are independent of surface material and are valid for both sinusoidal and random rough surface with lay. The principles, experiments, analyses, and conclusions involved in using ARLS to estimate rms surface slopes are described.
101,437

-116888

Not available NTIS

National Inst. of Standards and Technology (EEEL),

Boulder, CO. Electromagnetic Fields Div.

Diffraction by a Half-Plane in a Lossy Medium.

Final rept.

D. A. Hill. 1991, 3p

Sponsored by Army Belvoir Research and Develop ment Center, Fort Belvoir, VA.

Pub. in Jnl. of Applied Physics 69, n12 p8405-8407, 15 Jun 91.

Keywords: "Wave diffraction, "Diffraction, Plane waves, Fresnel integrals, Fresnel zones, Polarization Remote sensing, Reprints.

The classical problem of plane-wave diffraction by half-plane is extended to allow for loss in the surrounding medium. The loss causes the arguments of the integral functions to become complex. Numerical results show that the relative importance of the edge-diffracted field decreases as the loss is increased. This effect is important in interpreting the effects of off-path scatterers in remote sensing of lossy media. The results for both electric and magnetic polarizations are in qualitative agreement with previous results based on the Kirchhoff approximation.

\section{1,438}

PB92-116896

Not available NTIS

National Inst. of Standards and Technology (NML), Boulder, CO. Time and Frequency Div.

CW Dye Lasers.

Final rept.

L. Hollberg. 1990, 54p

Pub. in Dye Laser Principles with Applications, Chapter 5, p185-238 1990.

Keywords: "Continuous wave lasers, *Dye lasers, Historical aspects, Frequency control, Performance, Design, Theory, Reprints

The chapter describes the principles, construction, performance, and some of the theory of Cw dye lasers. It briefly reviews the history of $\mathrm{Cw}$ dye laser development and also the modern frequency control technologies

101,439

PB92-116904

Not available NTIS

National Inst. of Standards and Technology (NML), Boulder, CO. Time and Frequency Div.

Diode Lasers and Their Application to Spectrosco-

py.

Final rept.

L. Hollberg. 1990, 9p

Pub. in Applied Laser Spectroscopy, p117-125 1990.

Keywords: "Semiconductor lasers, "Spectroscopy, Semiconductor diodes, Reviews, Uses, Reprints.

The paper is a brief review of lasers and their application to spectroscopy.

101,440

PB92-126531

PC A08/MF A02

National Inst of Standards and Technology (EEEL),

Boulder, CO. Electromagnetic Technology Div.

Modified Airy Function and WKB Solutions to the Wave Equation.

A. K. Ghatak, R. L. Gallawa, and I. C. Goyal. Nov 91 , $175 p$ NIST/MONO-176

Also available from Supt of Docs. See also PB91236752. Prepared in cooperation with Indian Inst. of Tech., New Delhi. Dept. of Physics.

Keywords: *Optical waveguides, Optical fibers, Wave equations, Integrated optics, WKB approximation, Reprints, Airy Functions.

The WKB method has served in problems of quantum mechanics and nonuniform optical waveguides, the discipline treated in the monograph. The monograph discusses the WKB and the Modified Airy Function (MAF) methods in considerable detail, to the end that the reader gains an appreciation of the strengths and weaknesses of each. The authors treat eigenvalue problems as well as initial value problems. The two methods could be illustrated via any of several disciplines, but they chose to use problems that are of current interest to the optical waveguide community. They have also used Schrodinger's equation for typical potential well problems to further illustrate the method. Both the wave functions and the eigenvalues are found. They have also included several exercises, to be solved by the reader. They give several examples to illustrate the method for problems of interest in optical telecommunications devices.

\section{Plasma Physics}

\section{1,441}

AD-A227 656/6

PC A02/MF A01

National Inst of Standards and Technology, Boulder,

CO.

Liquid and Solid Ion Plasmas.

Annual rept. 1 Oct 89-31 Sep 90

D. J. Wineland, and J. J. Bollinger. $15 \operatorname{Sep} 90,10 p$

Contract N00014-89-F-0020

Keywords: Angular momentum, Cooling Coupling(Interaction), Radiative transfer, Distribution functions, Electromagnetic fields, Images, Ions, Laser applications, "Plasmas(Physics), Spatial distribution, Spectroscopy, Temperature, Velocity, Penning Traps, Laser Cooling.

Atomic ions which are stored in electromagnetic fields are an example of nonneutral plasmas. Laser techniques allow control of plasma angular momentum and provide plasma cooling to temperatures much less than $1 \mathrm{~K}$. Using imaging techniques, plasma spatial information is achieved. Laser spectroscopic techniques allow measurement of plasma velocity distribution functions. Liquid and solid behavior of ion plasmas is studied. Keywords: Nonneutral plasma; One component plasma; Strongly coupled plasma; Liquid and solid plasma, Plasma distribution functions; Penning trap; Laser cooling. (jhd)

\section{1,442}

PB91-147504

Not available NTIS

National Inst. of Standards and Technology (NML), Gaithersburg, MD. Atomic and Plasma Radiation Div. Overview of the Ion Dynamic Effect in Line Broadening, and a Generalization of the Unified Theory. Final rept.

D. E. Kelleher, D. H. Oza, J. Cooper, and R. L.

Greene. 1990, 7p

Pub. in Jnl. of Quantitative Spectroscopy and Radiative Transfer 44, n1 p101-107 1990.

Keywords: "Thermal plasmas, "Line broadening, Computation, Reprints, Ion dynamics.

The authors present a brief overview of the collisional broadening of hydrogenic radiators in thermal plasmas. Their focus is on lines with unshifted central components, particularly the alpha lines (Delta $=1$ ), for which ion motion effects can be very large. The calculations span a wide range of electron densities, temperatures, and radiator - ion perturber reduced masses mu. The computations were made using a numerical simulation approach. Numerical methods have proven necessary to deal with the overlapping strong collisions of different ion perturbers. Most current analytical methods, such as the unified theory, are not valid when strong collisions overlap, as is often the case with ion perturbers.

\section{1,443}

PB91-158618

Not available NTIS

National Inst. of Standards and Technology (NML), Gaithersburg, MD. Radiometric Physics Div.

Relative Populations of Excited Levels within the Ground Configuration of Si-Like $\mathrm{Cu}, \mathrm{Zn}, \mathrm{Ge}$, and Se lons.

Final rept.

R. U. Dalta, J. R. Roberts, and A. K. Bhatia. 1991, 4p Contract DE-EA-77-A-01-6010

Sponsored by Department of Energy, Washington, DC. Pub. in Physical Review A 43, n2 p1110-1113, 15 Jan 91.

Keywords: "Copper ions, "Zinc ions, "Germanium ions, "Selenium ions, Multicharged ions, Excited states, TEXT devices, M1-transitions, Electron collisions, Reprints, Proton collisions.

Populations of $3 p(2)$ singlet $D(2)$, triplet $P(1)$, triplet $\mathrm{P}(2)$ levels in Si-like $\mathrm{Cu}, \mathrm{Zn}, \mathrm{Ge}$, and Se ions have been deduced from the measurements of absolute intensities of magnetic dipole transitions within the $3 s(2)$ $3 p(2)$ ground configuration. Observations have been made in the Texas Experimental Tokamak (TEXT). The measured population ratios are compared with 


\section{PHYSICS}

\section{Plasma Physics}

theoretical calculations based on the distorted-wave approximation for the electron collisions and a semiclassical approximation for the proton collisions. The observed deviation from the statistical distribution for the excited-level populations within the ground configuration along the silicon isoelectronic sequence is in agreement with theoretical prediction.

\section{1,444}

PB92-117225

Not available NTIS National Inst. of Standards and Technology (EEEL), Gaithersburg, MD. Electricity Div.

Off-Axis Measurements of Ion Kinetic Energies in RF Plasmas.

Final rept.

S. B. Radovanov, J. K. Olthoff, and R. J. Van Brunt

1991, 2p

Sponsored by SEMATECH, Austin, TX.

Pub. in Proceedings of International Conference on Phenomena in lonized Gases (20th), Barga, Italy, July $8-12,1991, \mathrm{p} 835-836$

Keywords: "Argon plasma, Argon ions, Kinetic energy Gas discharges, RF systems, Reprints, Reference cells.

Ion kinetic energy distributions are presented for argon plasmas in the GEC RF reference cell and are compared with other previous measurements.

\section{1,445}

\section{PB92-126473}

PC A04/MF A01

National Inst. of Standards and Technology (NML), Gaithersburg, MD. Ionizing Radiation Div.

Mass Energy-Transfer and Mass Energy-Absorption Coefficients, Including In-Flight Positron Annihilation for Photon Energies $1 \mathrm{keV}$ to $100 \mathrm{MeV}$ P. D. Higgins, F. H. Attix, J. H. Hubbell, S. M. Seltzer, M. J. Berger, and C. H. Sibata. Nov 91, 70p NISTIR4680

See also PB87-116141, PB88-109830, UCRL-50400V6-REV-2 and UCRL-50174-SEC-2-R1. Prepared in cooperation with Cleveland Clinic Foundation, $\mathrm{OH}$. Dept. of Radiation Therapy, and Wisconsin Univ.-Madison. Dept. of Medical Physics.

Keywords: "Energy transfer, "Photon cross sections, "Energy absorption, Scattering, Computation, Pair production, $X$ rays, Gamma rays, Bremmstrahlung, Photoelectric emission, Irradiation, Corrections, Radiography, Positrons.

Mass energy-transfer (Mu(sub u)/Rho) and mass energy-absorption coefficients (Mu(sub en)/Rho) are tabulated in units of $(\mathrm{sqcm} / \mathrm{g}$ ) for photon energies between $1 \mathrm{keV}$ and $100 \mathrm{MeV}$ for 29 elements $(Z=1-92)$, and 14 mixtures and compounds of general dosimetric interest. Cross sections for photo-effect, incoherent scattering, pair and triplet production are those com piled or generated by the National Institute of Standards and Technology (NIST) (formerly the National Bureau of Standards). Corrections are included for inflight positron annihilation, previously not applied in NIST calculations for energies above $10 \mathrm{MeV}$.

\section{Radiofrequency Waves}

101,446

\section{P891-203505}

Not available NTIS

National Inst. of Standards and Technology (NEL), Gaithersburg, MD. Precision Engineering Div.

Computed Fields Near the Edge of a Dielectric Wedge.

Final rep

E. Marx. 1990, 5p

Pub. in IEEE (Institute of Electrical and Electronics Engineers) Transactions on Antennas and Propagation 38 , n9 p1438-1442 Sep 90.

Keywords: "Electromagnetic scattering, Electromagnetic fields, Integral equations, Edges, Reprints, Dielectric wedges.

The behavior of the electromagnetic field near the edge of an infinite sharp dielectric wedge has not been unequivocally established, and a numerical experiment is performed to learn about this behavior. The fields scattered by a finite wedge are determined by solving an integral equation. The fields near the edge of the wedge are computed from a boundary function by integration. The theory of the fields near the edge of the dielectric wedge appears to imply that some of the components diverge, but the analysis is based on a power series expansion that has not been shown to exist. The numerical experiment shows that the behavior of the fields near the edge does not follow the expected behavior. The problem of the perfectly conducting wedge is well understood and is used here to verify the numerical methods.

101,447

891-203513

Not available NTIS (NEL), Gaithersburg, MD. Precision Engineering Div. Interface. Final rept.

E. Marx. 1989, 10p

Pub. in IEEE (Institute of Electrical and Electronics Engineers) Transactions on Antennas and Propagation 37, n5 p619-628 1989.

Keywords: "Electromagnetic scattering, Radar cross sections, Maxwells equations, Integral equations, Plane waves, Interfaces, Cylinders, Dielectrics, Reprints.

The problem of the determination of the fields scattered by an infinite cylinder of arbitrary cross section located at the interface between two semi-infinite media is reduced to the solution of integral equations for unknown functions defined on the boundaries. These boundary functions are chosen so as to minimize their number. The incident field is that of a plane monochromatic wave. The derivation of the integral equations is given for the TE mode for a dielectric cylinder and for a perfectly conducting cylinder. The exact electromagnetic fields are obtained from the solutions of the integral equations by integration, and the radar cross section can be computed from the far-field approximation. Sample outputs of the computer programs that implement the solution are shown.

\section{Solid State Physics}

\section{1,448}

AD-A231 818/6

PC A02/MF A01 National Inst. of Standards and Technology (IMSE), Gaithersburg, MD. Ceramics Div.

Influence of Filament Geometry on Hot Filament Growth of Diamond Films.

Technical rept.

E. N. Farabaugh, A. Felfman, and L. Robins. 5 Feb 91, 8p Rept no. TR-1

Contract N00014-90-F-0011

Keywords: Filaments, Silicon, Single crystals, Substrates, "Diamond films.

The influence of filament geometry on growth rate and morphology has been observed on diamond films deposited on single crystal silicon substrates in a hot fila ment CVD reactor. Single and dual helical $W$ filaments having 5,10 , or 15 turns and $\mathrm{CH}_{4}: \mathrm{H} 2$ ratios of $0.25 \%$ $1.00 \%$ were used. With single filaments the deposition rate was approximately proportional to the number of turns in the filaments produced lower deposition rates, compared to single filaments for depositions carried out at the same $\mathrm{CH}_{4}: \mathrm{H} 2$ of $0.5 \%$. Employing dual filaments doubled the area of uniform growth. Faceting o our films changed from (111) to (100), as the $\mathrm{CH} 4: \mathrm{H} 2$ ratio was increased.

\section{1,449}

PC A03/MF A01

National Inst. of Standards and Technology, Gaithersburg, MD.

High Resolution Electron Microscopy of Diamond Film Growth Defects and their Interactions.

Technical rept.

D. Shechtman, E. N. Farabaugh, L. H. Robins, and

A. Feldman. 25 Sep 91, 25p

Contract N00014-90-F-0011

Keywords: Angles, Crystal growth, Crystals, Defects(Materials), Density, Diamonds, Electron microscopy, Grain size, High rate, High resolution, Locus Microstructure, Nucleation, Sites, Twinning(Crystallography), "Diamond films, Chemica vapor deposition.

High resolution electron microscopy of plasma-assist ed chemical vapor deposition (CVD) diamond films was performed. The film was fine grained with a grain size of the order of 0.1 micrometer. Several features of the microstructure were studied and their importance to the understanding of the diamond film growth was evaluated. The observation include: 1 . Twinning density rises as a function of the distance from the center of the crystal. 2. The twins have an important role in the rapid growth of this kind of film. The reentrant angle between intersecting twins serves as a nucleation site for the growth of new (111) planes. 3 . The center point of a twin quintuplet has five reentrant angles and thus serves as a preferred nucleation site for new planes as the crystal grows. 4 . Misfit boundaries, being the locus of intersection points of the growing planes on two ad jacent twins can serve as an indicator for the loca crystal growth direction. The central nucleation site serve as an indicator for the local crystal growth direction. The central nucleation site for the growing planes can thus be traced back in many cases to a quintuplet twin point.

\section{1,450}

AD-A243 096/5

PC A03/MF A01 burg, MD.

Transmission Electron Microscopy of the CVD Diamond Film/Substrate Interface.

Technical rept.

D. Shechtman. 27 Sep 91, 14p

Contract N00014-90-F-0011

Keywords: Diamonds, Electrical properties, Germanium, Grain boundaries, Interfaces, Mechanical properties, Migration, Semiconductors, Silicon, Substrates, "Diamond films, Transmission electron microscopy Chemical vapor deposition.

The structure of grain boundaries in the diamond lattice, and twin boundaries as a special case, received considerable attention in over thirty years. This is mainly due to the importance of defects in silicon and germanium to their usefulness as efficient semiconductors. Twin boundaries have equal importance to various properties of diamond films. Electrical and mechanical properties as well as migration mechanisms are all affected by the twin boundaries and their structure. The ever presence of twins in CVD diamond films makes their study even more important compared to semiconductors which can be grown without bound aries. The purpose of the study reported here is, therefore, to investigate the crystallography of twin boundaries which form in chemical vapor deposited (CVD) diamond films, and to compare them to the ones which were found in silicon and in germanium.

\section{1,451}

AD-A243 220/1

PC A03/MF A01

National Inst. of Standards and Technology, Gaithers burg, MD.

Spatially and Spectrally Resolved Cathodoluminescence Measurements of CVD-Grown Diamond Particles and Films.

Technical rept

L. H. Robins, E. N. Farabaugh, and A. Feldman. 27 Sep 91, 15p Rept no. NIST-TR-6

Contract N00014-90-F-0011

Keywords:

Cathodoluminescence

Concentration(Composition), Cross sections, Depth Gradients, Impurities, Interfaces, Low temperature, Luminescence, Measurement, Particles, Polycrystalline, Recombination reactions, Silicon, Spatial distribution, Substrates, "Diamonds, Color centers, Crystal structure, Chemical vapor deposition, "Diamond films.

Spatially and spectrally resolved cathodoluminescence (CL) was used to investigate the spatial distribution of luminescence centers in CVD-grown diamond particles and polycrystalline films. For single particles grown at a low substrate temperature (nominally 650 C), one of the two most intense CL bands, the 2.156 $\mathrm{eV}$ band, was found to be associated with (111) facets. The CL image of the other intense band, the $2.85 \mathrm{eV}$ band, showed considerable particle-to-particle variation among the same particles. The images of the $2.156 \mathrm{eV}$ and $2.85 \mathrm{eV} \mathrm{CL}$ bands were found to have a complementary relationship for some particles. A model of competing recombination centers is proposed to help explain these results. Cross-sectional measurements of the $\mathrm{CL}$ spectra of polycrystalline films on silicon showed that the intensity of a siliconimpurity-related CL band decreases with increasing distance from the film-substrate interface. This depth variation is interpreted as due to a silicon impurity concentration gradient. 
101,452

DE91016275

$\mathrm{PC}$ A03/MF A01

National Inst. of Standards and Technology, Boulder, CO.

Integrity tests for high-(Tc) and conventional critical-current measurements systems.

L. F. Goodrich, and S. L. Bray. 1989, 15p CONF-

890701-30

Contract Al01-86ER52132

International cryogenic materials conference, Los Angeles, CA (United States), 24-28 Jul 1989. Sponsored by Department of Energy, Washington, DC.

Keywords: "Superconductors, Electric Impedance, Electrical Testing, High-Tc Superconductors, Measuring Methods, Simulation, Meetings, EDB/420500, $\mathrm{EDB} / 360204$.

A set of simple procedures that will test the integrity of measurement systems used for critical-current determinations on high-(TC) and conventional superconductors has been developed. These tests include a finite resistance, a zero resistance, and a superconductor voltage-current simulator. In the measurement of the critical current, voltage sensitivity is a key factor. The zero resistance test is the most effective test to detect the presence of interfering voltages such as ground loop or common-mode voltages and will determine the voltage sensitivity limit of a measurement system. 5 refs., 3 figs.

\section{1,453}

\section{N91-20208/5}

(Order as N91-20207/7, PC A05/MF A01) National Bureau of Standards (IMSE), Gaithersburg, MD.

High T(sub C) Superconductors: Are They Magnetic.

R. D. Shull. Jan $90,5 p$

In NASA, Langley Research Center, National Educators' Workshop: Update 1988. Standard Experiments in Engineering Materials Science and Technology $p$ 15.

Keywords: Experimentation, "High temperature superconductors, Magnetic properties, Oxides, Procedures, Transition temperature, "Y ttrium barium cuprates.

The objective is to demonstrate the magnetic characteristics of the new superconducting oxides with transition temperatures above liquid nitrogen temperature $(77 \mathrm{~K})$. The first task was to find out whether $\mathrm{YBa} 2 \mathrm{Cu} 307-\mathrm{x}$ is magnetic at liquid nitrogen temperature, and if so, what the sign of its magnetic susceptibility is. Details of the experimental procedure are given.

\section{1,454}

PATENT-4 954481

Not available NTIS Department of Commerce, Washington, DC. Superconductor-Polymer Composites.

A. S. DeReggi, C. K. Chiang, and G. T. Davis. Filed 29 Dec 88, patented 4 Sep 90, 2p PB91-176032, 29 Dec 88, patented

PAT-APPL-7-292 601
This Government-owned invention available for U.S. licensing and, possibly, for foreign licensing. Copy of patent available Commissioner of Patents, Washington, DC $20231 \$ 1.50$.

Keywords: "Superconducting films, "Polymer matrix composites, *Particulate composites, *Patents, Rare earth alloys, Cuprates, Barium oxides, Thermoplastic resins, Polymeric films, Thin films, PAT-CL-505-1, Vinylidene fluoride resins.

Superconductor-polymer composite materials comprise a matrix formed of a thermoplastic polymer and a superconductor powder dispersed in the matrix. The superconductor powder preferably has a composition $\mathrm{RBa2Cu} 3 \mathrm{O}(7-\mathrm{x})$ wherein $\mathrm{A}$ is a rare earth metal and $\mathrm{x}$ is less than or equal to 1 . The thermoplastic polymer matrix comprises a vinylidene fluoride homopolymer or copolymer. The composite materials may be formed as shaped products, sheets or films.

101,455

PB91-133827

Not available NTIS

National Inst of Standards and Technology (NEL)

Boulder, CO. Electromagnetic Technology Div.

Current Supply for High-T(sub c) Superconductor Testing.

Final rept.

S. L. Bray, and L. F. Goodrich. 1990, 4p

Sponsored by Department of Energy, Washington, DC.

\section{Pub. in Meas. Sci. Technol. 1, p491-494 1990}

Keywords: "Superconductors, Electrical measurement, Direct current, Power supplies, Design, Reprints, "High temperature superconductors, "Critical current, *Current sources.

Precise and accurate measurements of the DC critical current of high-T(c) superconductors often require a current supply that has high input-to-output isolation, high isolation from ground and low output ripple. Also, to ensure precise current control, the supply should have low current drift and its input-output characteristic should be linear. A design for a simple and inexpensive current supply that has these characteristics is presented. The primary power source is a $12 \mathrm{~V}$ wet-cell battery and the typical operating range is from $10 \mathrm{~mA}$ to $10 \mathrm{~A}$. The supply's current ripple is $<0.4 \mathrm{~mA}$ RMS over this operating range. At an output current of $5 \mathrm{~A}$ the current drift is $<2 \% / h$ without a warm-up period. The maximum variation of the supply's output from linearity, over its full operating range is $<5 \%$.

\section{1,456}

B 9́1-133983

Not available NTIS

National Inst. of Standards and Technology (NEL). Boulder, CO. Electromagnetic Technology Div.

Transport Critical Current of Aligned Polycrystalline Y1Ba2Cu307-delta and Evidence for a Nonweak-Linked Component of Intergranular Current Conduction.

Final rept.

J. W. Ekin, H. R. Hart, and A. R. Gaddipati. 1990 ,

$11 p$

Sponsored by Department of Energy, Washington, DC., and Office of Naval Research, Arlington, VA

Pub. in Jnl. of Applied Physics 68, n5 p2285-2295, 1 Sep 90.

Keywords: "Superconductors, "Grain structure, Magnetic fields, Direct current, Polycrystalline, Alignment, Reprints, "High temperature superconductors, *Yttrium barium cuprates, " Critical current, Weak links.

A study of grain alignment and its effect on the dc transport critical current in fine-grained bulk $\mathrm{Y} 1 \mathrm{Ba} 2 \mathrm{Cu} 3 \mathrm{O}$ (7-delta) is reported in magnetic fields from $0.0001 \mathrm{~T}$ to $26 \mathrm{~T}$. The data clearly demonstrate that alignment alone significantly reduces the weaklink problem in fine-grained polycrystalline samples with low-aspect-ratio (4:1) grains (unlike melt-grown samples where there has been some ambiguity as to the relative importance of alignment versus large grain growth). Furthermore, the results provide strong evidence that there are two parallel components of intergranular current conduction, one consisting of weaklinked material, the other behaving like intrinsic intragranular material that is not weak-linked. Field-cooled and force-free $\mathrm{J}(\mathrm{c})$ data are also presented, along with detailed measurements of the shapes of the voltagecurrent characteristics.

\section{1,457}

PB9́1-134056

Not available NTIS

National Inst. of Standards and Technology (NEL), Boulder, CO. Electromagnetic Technology Div.

High T(sub c) Superconductors and the Critical Current Measurement.

Final rept.

L. F. Goodrich, and S. L. Bray. 1990, 11 p

Pub. in Cryogenics 30, p667-677 Aug 90.

Keywords: "Superconductors, Electrical measurement, Recommendations, Reprints, "High temperature superconductors, "Critical current, Interlaboratory comparisons.

With the introduction of high $\mathrm{T}(\mathrm{c})$ superconductors, a number of problems associated with critical current $I$ (c) measurement have arisen. The existing I(c) measurement practices were developed and proved for low T(c) superconductors. There are substantial differences between the two classes of materials. When the I(c) concept was casually extended to the high T(c) conductors, measurement inconsistency, ambiguity and, in some cases, invalidity followed. A discussion of the underlying philosophy of I(c) measurement is presented, and a number of measurement variables that can influence the measured I(c) are discussed. Many of the problems stem from inadequate reporting practices, and recommendations are given for improving measurement reports.

\section{1,458}

PB9́1-134148

Not available NTIS Boulder, CO. Electromagnetic Technology Div.
Fundamental and Harmonic Susceptibilities of YBa2Cu307-delta.

Final rept.

T. Ishida, and R. B. Goldfarb. 1990, 12p

Sponsored by Ministry of Education, Science and Culture, Tokyo (Japan).

Pub. in Physical Review B 41, n13 p8937-8948, 1 May 90.

Keywords: "Superconductors, Magnetic measurement, Magnetic fields, Magnetization, Reprints, "High temperature superconductors, "Magnetic susceptibility, "Yttrium barium cuprates, Temperature dependence.

The authors have examined the complex harmonic magnetic susceptibilities chi(sub $n$ ) of the sintered high-critical-temperature superconductor YBa2Cu3O(7-delta). The experimental variables for the measurements of chi(sub n) were the sample temperature, the ac magnetic field amplitude, frequency, and the magnitude of a superimposed dc field $\mathrm{H}(\mathrm{dc})$. Results are given.

101,459

PB91-134254

Not available NTIS

National Inst of Standards and Technology (IMSE)

Boulder, CO. Fracture and Deformation Div.

Madelung Potentials and Valences in the Y1Ba2Cu307 Superconductor.

Final rept.

$H$. Ledbetter, and M. Lei. 1990, 3p

Pub. in Physica C 166, p483-485 1990.

Keywords: "Superconductors, Valence, Reprints, "High temperature superconductors, "Yttrium barium cuprates, Ewald method.

Using Ewald's method, the authors calculated the ionsite potentials and Madelung energy of Y1Ba2Cu307(orthorhombic Pmmm, No 47) They considered the effects of copper-ion and oxygen-ion valences. Among seven suggested copper-oxygen ion-charge configurations, only two give a low electrostatic bonding energy to agree with oxygen vacancies on $\mathrm{O}(1)$ sites. Only one configuration gives a realistic bulk modulus. The authors perturbed this configuration by equalizing the $\mathrm{Cu}(1)-\mathrm{Cu}(2)$ valences and introducing a hole into the $\mathrm{CuO} 2$ plane at the oxygen sites. This configuration also agrees well with observation.

101,460

PB91-134262

Not available NTIS

National Inst of Standards and Technology (IMSE) Boulder, CO. Fracture and Deformation Div

Elastic Constants, Debye Temperatures, and Electron-Phonon Parameters of Superconducting $\mathrm{Cu}$ prates and Related Oxides.

Final rept.

H. Ledbetter, M. Lei, and S. Kim. 1990, 10p

Pub. in Phase Transitions 23, p61-70 1990.

Keywords: *Superconductors, Strontium titanates, Barium titanates, Elastic properties, Electron phonon interactions, Bulk modulus, Reprints, "High temperature superconductors, Lanthanum strontium cuprates, Yttrium barium cuprates, Lanthanum cuprates, Debye temperature, Kresin model, Born model.

Using both measurements and modeling, the authors studied the cohesive and related properties of several oxides. Superconducting oxides include $\mathrm{La}(1.85) \mathrm{Sr}(0.15) \mathrm{CuO} 4, \mathrm{Y} 1 \mathrm{Ba} 2 \mathrm{Cu} 3) 7$, and $(\mathrm{Bi}-$ $\mathrm{Pb} 2 \mathrm{Sr} 2 \mathrm{Ca} 2 \mathrm{Cu} 3 \mathrm{O} 10$. Related nonsuperconducting oxides include $\mathrm{SrTiO}_{3}, \mathrm{BaTiO}_{3}$, and $\mathrm{La} \mathrm{CuO} 4$. For these materials, the authors give the complete quasiisotropic elastic constants corrected to the void-free state. From elastic constants and atomic volume, the authors caluclate Debye characteristic temperatures. The authors support their elastic-constant measurements with Born-model calculations of the bulk modulus.

101,461

PB91-134270

Not available NTIS National Inst. of Standards and Technology (IMSE) Boulder, CO. Fracture and Deformation Div.

Elastic Constants of Polycrystalline Bi-Pb-Sr-CaCu-O Superconductor.

Final rept.

H. M. Ledbetter, S. A. Kim, R. B. Goldfarb, and K Togano. $1989,4 p$

89. 


\section{Solid State Physics}

Keywords: "Superconductors, Electron phonon interactions, Shear modulus, Bulk modulus, Modulus of elasticity, Poisson ratio, Critical temperature, Bismuth oxides, Lead oxides, Strontium oxides, Calcium oxides, Polycrystalline, Reprints, "High temperature superconductors, Young modulus, Debye temperature, Kresin model, Temperature dependence, Cuprates.

For polycrystalline (Bi-Pb)2Sr2Ca2Cu3010, the authors report the 5-295-K elastic constants: shear modulus $(G)$, Young modulus (E), bulk modulus $(B)$, and Poisson ratio (nu). Both $\mathrm{G}$ and $\mathrm{E}$ show nearly normal temperature variation. During cooling, $B$ softens be tween 215 and $65 \mathrm{~K}$. The Poisson ratio decreases abruptly at $215 \mathrm{~K}$ and decreases almost five percent in the 295-5-K region. In its temperature dependence, this compound resembles YBa2Cu307. However, it shows much lower elastic stiffness. Corrected to the void-free state, the authors calculate a Debye temperature of $312 \mathrm{~K}$, versus $436 \mathrm{~K}$ for $\mathrm{YBa} 2 \mathrm{Cu} 307$. The Poisson ration, 0.20 , agrees well with that of YBa2Cu3O7, 0.21. The authors use Kresin's model to interrelate Debye temperature, critical temperature, and electron-phonon interaction parameter for fou classes of copper-oxide superconductors.

\section{1,462}

PB91-134478

Not available NTIS

National Inst. of Standards and Technology (NEL) Boulder, CO. Electromagnetic Technology Div. Break Junction Tunneling Spectroscopy of SingleCrystal Bismuth-Based High-Temperature Superconductors.

Final rept.

J. Moreland, C. K. Chiang, and L. J. Swartzendruber. $1990,7 p$

Pub. in Advances in Cryogenic Engineering (Materials) 36, p619-625 1990 .

Keywords: Electron tunneling, Energy gap, Single crystals, Reprints, "High temperature superconductors *Superconducting junctions, Bismuth strontium calcium cuprates, Break junctions.

The authors have measured the tunneling spectra of some high temperature superconducting crystal break junctions at $4 \mathrm{~K}$. The samples were thin plates of $\mathrm{Bi} 2 \mathrm{SrCa} 2 \mathrm{Cu} 2 \mathrm{O} 5$ compound. The tunneling spectra (conductance versus voltage) were not typical of BCS superconductor tunneling electrodes. The spectra of higher-resistance break-junction settings $(R>1 \mathrm{M}$ $\mathrm{hm}$ ) show a tunneling gap on top of a linearly increas ing conductance background signal. 'Harmonic' dip features in the spectra of lower resistance break junction settings ( $R<1 \mathrm{M}$ ohm) indicated tunneling between multiple particles in the vicinity of the primary (highest resistance) contact of the junction. The dips occurred at about the same current but shifted in voltage when the resistance of the break junction was continuously adjusted to new settings.

\section{1,463}

PB91-134569

Not available NTIS

National Inst of Standards and Technology (NEL) Boulder, CO. Electromagnetic Technology Div.

Magnetization of Anisotropic Superconducting Gralns.

Final rept.

R. L. Peterson. 1990, 4p

Pub. in Jnl. of Applied Physics 67, n11 p6930-6933, 1 Jun 90 .

Keywords: "Type 2 superconductors, "Superconductors, Magnetic anisotropy, Magnetic hysteresis, Mag netization, Grain size, Single crystals, Reprints, "High temperature superconductors, Critical current, Bean model.

A critical-state calculation of the magnetization of hard Aye-II superconducting grains having anisotropic critical-current densities is given. The analysis shows how the critical-current densities should be deduced from the critical-current densities should be deduced from phologies. A universal curve of the magnetization hysteresis versus one of the grain dimensions is presented, showing that the hysteresis does not change linearly with grain size. Applications to single crystals and to bulk materials are made.
Calculation of Energy of Low-Angle Grain Boundaries.

Final rept

A. C. Shi, Y. He, and C. Rottman. 1987, 13p

Pub. in Philosophical Magazine A-Defects and Mechanical Properties 55, n4 p499-511 1987.

Keywords: "Grain boundaries, Reprints, Crystal dislocations, Elasticity

Various techniques for calculating the linear elastic energy of low-angle grain boundaries are reviewed. The dependence of both elastic and inelastic contributions on boundary-plane orientation (n) and axis of rotation $(u)$ is considered in detail. Particular attention is devoted to the treatment of the elastic contribution near the dislocation cores. The differences in elastic energy between the recent calculations of Rey and Saada (1977) and Bonnet (1981) with the classic calculation of Read and Shockley (1950) are explained by the differing treatments used by these authors for the region near the cores. The elastic energy for low-angle grain boundaries composed of three sets of parallel, equally spaced dislocations is calculated, within an easily understood convention for handling the region near the cores, for the first time. The dependence of the total energy, elastic plus core, on (n) and (u) is given.

\section{1,465}

\section{PB91-147165}

Not available NTIS

National Inst. of Standards and Technology (MSEL) Gaithersburg, MD. Ceramics Div.

Phase Equilibria in the System TI-Ca-Ba-Cu-O. 1 Stability of the 2122 Phase under Conditions of Oxygen Annealing.

Final rept.

L. P. Cook, C. K. Chiang, W. Wong-Ng, L. J.

Swartzendruber, and L. H. Bennett. 1990, 4p

Pub. in Materials Research Society Symposia Proceedings, v169 p137-140 1990.

Keywords: "High temperature superconductors, "Superconductors, X-ray diffraction, Magnetic susceptibiliperconductors, X-ray diffraction, Magnetic susceptibili-
ty, Reprints, "Thallium barium calcium cuprates, Phase equilibrium.

Results are presented for $850 \mathrm{C}$ oxygen annealing experiments (up to $62 \mathrm{hr}$ ) on the 2122 (T1:Ca:Ba:Cu) oxide phase, with and without intermediate grinding Samples were characterized by powder X-ray diffraction and a.c. magnetic susceptibility. The 2122 phase appears to be thermodynamically stable in oxygen under conditions of both extensive grinding, and, annealing of long duration. However, onset T(c)'s vary from $71 \mathrm{~K}$ to $109 \mathrm{~K}$ for apparently single-phase 2122 samples with nearly identical X-ray powder patterns, depending upon the heat treatment.

101,466

PB91-147397

Not available NTIS

National Bureau of Standards (IMSE), Gaithersburg MD. Ceramics Div.

RIR - Measurement and Use in Quantitative XRD. Final rept.

C. R. Hubbard, and R. L. Snyder. 1988, 4p

Pub. in Powder Diffraction 3, n2 p74-77 Jun 88

Keywords: * $X$ ray diffraction, Quantitative analysis, $X$ ray analysis, Reprints, "Reference intensity ratio.

The Reference Intensity Ratio (RIR) is a general, instrument-independent constant for use in quantitative phase analysis by the $\mathrm{X}$-ray powder diffraction internal standard method. When the reference standard is corundum, RIR is known as I/I(c); These constants are collected in the Powder Diffraction File (1987), can be calculated, and can be measured. Recommended methods for accurate measurement of RIR constants are presented, and methods of using these constants for quantitative analysis are discussed. The numerous, complex constants in Copeland and Bragg's method introduced to account for superimposed lines can be simply expressed in terms of RIR constants and relative intensities. This formalism also permits introduction of constraints and supplemental equations based on elemental analysis.

\section{1,467}

PB91-147603

National Inst. of Standards and Technology (NEL) Gaithersburg, MD. Semiconductor Electronics Div.
Phonon-Assisted Magneto-Donor Optical Transitions In $\mathbf{n}-\mathbf{I n S b}$

Final rept

C. L. Littler, W. Zawadzki, M. R. Loloee, X. N. Song, and D. G. Seiler. 1990, 3p

Grant NSF-DMR86-17823

Sponsored by National Science Foundation, Washington, DC.

Pub. in Semicond. Sci. Technol. 5, n3S p169-171 Mar 90.

Keywords: "Indium antimonides, "Optical transition, Excited states, Phonons, Reprints.

The authors have observed and described new optical transitions between magneto-donor states in InSb assisted by optic phonon emission. The phonon-assisted transitions provide a unique opportunity to investigate high excited states of the magneto-Coulomb system. High-resolution data reveal the presence of excited magneto-donor states belonging to the same Landau sub-band.

101,468

PB91-147736

Not available NTIS

National Inst. of Standards and Technology (NEL). Gaithersburg, MD. Semiconductor Electronics Div.

Morphology and Barrler-Height Development of $\mathrm{Bi} /$ InP(110) Interfaces.

Final rept.

K. E. Miyano, T. Kendelewicz, R. Cao, C. J. Spindt,

and I. Lindau. 1990, 7p

Sponsored by Defense Advanced Research Projects Agency, Arlington, VA., and Office of Naval Research, Arlington, VA

Pub. in Physical Review B 42, n5 p3017-3023, 15 Aug 90.

Keywords: *Indium phosphides, "Bismuth, *Interfaces, Band theory, Substrates, Photoelectron spectroscopy, Reprints.

The development of the interface between cleaved $n$ and p-type $\ln \mathrm{P}(110)$ substrates and overlayers of $\mathrm{Bi}$ has been studied in the coverage range of 0.01 to 10 monolayers with use of soft-x-ray photoemission spectroscopy. The attenuation and narrowing of the substrate in $4 d$ and $P 2 p$ core-level spectra, as well as the line-shape development of the adatom Bi $5 d$ signal, indicate that the morphology is of the Stranski-Krastanov type, as has been verified previously for Sb and Bi over-layers on GaAs(110). Specifically, the Bi grows in ordered two-dimensional patches that merge at 1 monolayer coverage, and beyond the coverage the deposited adatoms form three-dimensional clusters. The band bending as measured from energy shifts of the In $4 d$ and $P$ 2p spectra approaches midgap near 0.3 monolayer coverage, but between 0.3 and 1.0 monolayer the band bending for both doping types exhibits a reversal.

\section{1,469}

PB91-147926 Not available NTIS National Inst. of Standards and Technology (NEL), Gaithersburg, MD. Precision Engineering Div.

Low Accelerating Voltage SEM Imaging and Metrology Using Backscattered Electrons.

Final rept.

M. T. Postek. $1990,5 p$

Pub. in Review of Scientific Instruments $61, \mathrm{n} 12$ p3750-3754 Dec 90.

Keywords: *Scanning electron microscopy, "Semiconductors, "Metrology, Microchannel electron multipliers, Electron scattering, Line width, Backscattering, Reprints.

An approach to measure semiconductor structures for nondestructive submicrometer metrology in the scanning electron microscope (SEM) at low accelerating voltage is described, using the collection and measurement of only the backscattered electron signal rather than the more commonly used secondary electron signal. In this technique, the backscattered electron signal is collected using a high-efficiency microchannel-plate electron detector system with the front face of the detector biased negatively to reject the lowenergy secondary electrons thus collecting only the backscattered electrons. The advantage of using the backscattered electron signal is discussed, as well as a comparison to measurements using the secondary electron signal. The potential of this technique for application to accurate SEM metrology and standards development is also discussed. 
101,470

PB91-148023

Not available NTIS

National Inst. of Standards and Technology (MSEL),

Gaithersburg, MD. Reactor Radiation Div.

Crystal and Spin Structures of Nd2CuO4.

Final rept.

S. Skanthakumar, and J. W. Lynn. 1990, 3p

Pub. in Physica C 170, p175-177 1990.

Keywords: "Crystal structure, Single crystals, Tetragonal lattices, Neutron diffraction, $X$-ray diffraction, Electron spin, Reprints, "Neodymium cuprates, Magnetic ordering.

$X$-ray and neutron diffraction have been used to study the magnetic and structural properties of single crystal $\mathrm{Nd} 2 \mathrm{CuO} 4$. The authors previously observed a small distortion of the basic tetragonal structure, whereby peaks were observed with both $X$-ray and neutron diffraction. The authors explicity show that these are not due to lambda/2 wavelength contamination, as recently suggested. The authors also clarify the nature of the magnetic structures observed in the material. In particular, with the present neutron diffraction data on multidomain samples, it is not possible to distinguish between the collinear and noncollinear spin configurations which have been proposed for this system.

101,471

PB91-148064

Not available NTIS

National Inst. of Standards and Technology (NML), Gaithersburg, MD. Gas and Particulate Science Div. Secondary Ion Yield Changes in Silicon and Gallium Arsenide Due to Topography Changes during Oxygen or Cesium Ion Bombardment.

Final rept.

F. Stevie, P. Kahora, D. Simons, and P. Chi. 1988 , $5 p$

Pub. in Jnl. of Vacuum Science and Technology A 6, n1 p76-80 1988.

Keywords: "Gallium arsenides, "Silicon, "Ion bombardment Scanning electron microscopy, Oxygen ions, Cesium ions, Topography, Reprints, Secondary ion mass spectroscopy, Depth profiles.

Changes in secondary ion yields of matrix and dopant species have been correlated with changes in surface topography during $\mathrm{O} 2(1+)$ bombardment of $\mathrm{Si}$ and GaAs. It is concluded that changes in surface topography under $\mathrm{O} 2$ ion bombardment can affect secondary ion yields of matrix and dopant species in Si and GaAs. In light of these observations, the practice of normalizing SIMS dopant profiles to a matrix signal must be reexamined.

101,472

PB91-148155

Not available NTIS

National Inst. of Standards and Technology (NEL), Gaithersburg, MD. Semiconductor Electronics Div.

Strontium-Induced Oxygen Defect Structure and Hole Doping in La2-xSrxCuO4.

Final rept.

Z. Tan, M. E. Filipkowski, J. I. Budnick, E. K. Heller, D. L. Brewe, B. L. Chamberland, C. E. Bouldin, J. C. Woicik, and D. Shi. $1990,4 p$

Sponsored by Department of Energy, Washington, DC. Pub. in Physical Review Letters 64, n22 p2715-2718, 28 May 90

Keywords: "High temperature superconductors, "Superconductors, Strontium additions, Crystal defects, Doped materials, Polycrystalline, Reprints, "Lanthanum strontium cuprates, Lanthanum cuprates.

The authors have discovered that the apical oxygen with a 2.35-A La-O bond length is removed when La is substituted by $\mathrm{Sr}$ in polycrystalline $\mathrm{La}(2-x) \mathrm{Sr}(\mathrm{x}) \mathrm{CuO} 4$ under normal preparation conditions. This apical oxygen can be partially filled by oxygen annealing. It is reasoned that a defect oxygen is trapped at an interstitial site near the $\mathrm{Sr}$ atom. The authors present evidence that this defect oxygen is intrinsic to $\mathrm{Sr}$ doping, independent of processing conditions. The authors propose that this defect oxygen serves as a mechanism for hole doping similar to that in the superconducting oxygen-rich $\mathrm{La} 2 \mathrm{CuO}(4+\mathrm{y})$.

101,473

PB91-148189 Not available NTIS National Inst. of Standards and Technology (NEL), Boulder, CO. Electromagnetic Technology Div.
Cryogenic Properties of Copper (Wall Chart). Final rept.

C. A. Thompson, W. M. Manganaro, and F. R.

Fickett. 1990, 1p

Keywords: "Copper, "Copper alloys, "Graphs(Charts), "Magnetic properties, "Electrical resistivity, "Thermodynamic properties, Cryogenics, Magnetoresistivity.

The document is a wall chart prepared using data selected from the extensive literature on the low temperature properties of copper and from the author's measurements made over the past two decades. It is a successor to, and an update of, an earlier chart with the same title.

\section{1,474}

PB91-148205

Not available NTIS

National Inst. of Standards and Technology (IMSE),

Gaithersburg, MD. Ceramics Div.

Strength and Fracture Behavior of Ba-Y-Cu-O Superconducting Ceramics.

Final rept.

J. S. Wallace, E. R. Fuller, A. S. Raynes, S. W

Freiman, J. E. Blendell, M. L. Balmer, and M. Hill.

$1989,13 p$

Pub. in Proceedings of International Symposium on High Temperature Superconducting Compounds: Processing and Related Properties, Las Vegas, NV., February 27-28, 1989, p597-609.

Keywords: "High temperature superconductors, "Superconductors, Fracture strength, Phase transformations, Microcracks, Toughness, Strains, Reprints, "Yttrium barium cuprates.

Mechanical reliability is an important aspect of the technological application of high-temperature ceramic superconductors. The paper discusses the strength and fracture behavior of $\mathrm{Ba}-\mathrm{Y}-\mathrm{Cu}-\mathrm{O}$ superconducting ceramics, and, more importantly, the influence that the microstructures of these materials impart to the mechanical properties. The sintering conditions are seen to have a profound influence on the microstructure development in these materials. In addition, large internal strains can develop on cooling from the sintering temperature. These strains result either from the phase transformation that occurs during oxygenation or from anisotropy in the thermal expansion. Since strain-relief by plastic deformation or diffusion is generally not active below the sintering temperature in these materials, these strains are most easily accommodated by microcracking. Such microcracking was seen to have a profound influence on the strength behavior.

\section{1,475}

PB91-148916

Not available NTIS

National Bureau of Standards (NEL), Gaithersburg, MD. Thermophysics Div.

Theory of Specific Heat of Solids.

Final rept.

A. P. Miiller. $1988,89 p$

Pub. in Specific Heat of Solids, Chapter 1, p1-89 1988.

Keywords: "Specific heat, Lattice vibrations, Band theory, Superconductors, Spin waves, Spin glass state, Kondo effect, Reviews, Reprints.

A review of the theory describing the contributions of the lattice vibrations, conduction electrons and various magnetic excitations to the specific heat of solids is presented. The lattice contribution is described in terms of a number of models based on the harmonic approximation, namely, the simple Einstein and Debye models and the more realistic Born-von Karman model. The effect of anharmonicity of the lattice vibrations and of the formation of lattice vacancies on the specific heat at elevated temperatures is also considered. The contribution of the conduction of the conduction electrons in their normal state is described in terms of the free electron model and deviations from free electron behavior due to band structure and many-body enhancement effects are also discussed. The results for the specific heat of superconductingstate electrons as predicted by classical thermodynamics and BCS theory are described. Discussions of theoretical results describing magnetic contributions include the topics: spin-wave specific heat, specific heat in the critical temperature region, Schottky anomalies and, the Kondo and spin-glass effects. An extensive list of references is included.
Possible 'Proximity Matrix' Route to High Current Conductors.

Final rept.

J. Moreland, Y. Li, J. W. Ekin, and L. F. Goodrich.

1990, 9p

Sponsored by Department of the Navy, Washington,

Pub. in Advances in Cryogenic Engineering (Materials), v36 ptA p413-421 1990

Keywords: "High temperature superconductors, "Superconducting films, "Yttrium barium cuprates, "Silver coatings, Electrical conductivity, Vacuum deposition, Proximity effect, Powder(Particles), Reprints.

The conductance of point contacts between the surfaces of superconducting $\mathrm{YBa} 2 \mathrm{Cu} 3 \mathrm{O}$ (7-delta) thin films is very low. This is probably due to a native insulating surface layer. The conductance of these point contacts can be markedly increased by vacuum depositing and subsequently annealing a thin layer of $\mathrm{Ag}$ into the surface of the films. The authors believe that what might be described as a normal-metal superconducting 'proximity matrix', is formed at the surface of the Ag coated YBa2Cu3O(7-delta) films. In the paper, they describe their efforts to adapt this method to YBa2Cu3O(7-delta) powder. In particular, they have developed a procedure for vacuum deposition of very thin $\mathrm{Ag}$ coatings onto the surface of $\mathrm{YBa} 2 \mathrm{Cu} 3 \mathrm{O}(\mathrm{X})$ powder grains. The Ag-treated powder is then pelletized, sintered, annealed, and cut to form small conducting bars for electrical transport testing.

\section{1,477}

PB91-149294

Not available NTIS National Inst. of Standards and Technology (NML), Boulder, CO. Quantum Physics Div.

Study of the GaAs-Si(100) Interface Using Laser Probing of Thermal Desorption Kinetics. Final rept. R. V. Smilgys, D. J. Oostra, and S. R. Leone. 1990 , $7 p$

Contract AFOSR87-0119

Sponsored by Air Force Office of Scientific Research, Bolling AFB, DC

Pub. in Jnl. of Vacuum Science and Technology B 8, n5 p1102-1108 Sep/Oct 90.

Keywords: "Gallium arsenides, "Interfaces, "Desorption, Laser induced fluorescence, Surface reactions, Silicon, Reprints, Thermal desorption.

The thermal desorption kinetics of Ga from initially Asterminated $\mathrm{Si}(100)$ is investigated using laser-induced fluorescence spectroscopy. During the Ga desorption process the surface does not remain fully As terminated because a significant fraction of the initial As coverage simultaneously desorbs with the $\mathrm{Ga}$. The results on the GaAs system are compared with those previously reported on the InAs system, which show that In interacts with As on Si(100) less strongly than does $\mathrm{Ga}$.

\section{1,478}

PB91-149328

Not available NTIS National Bureau of Standards (NML), Gaithersburg, MD. Surface Science Div.

Epitaxial fcc Fe Films on Cu(100).

Final rept.

D. A. Steigerwald, and W. F. Egelhoff. 1988, 1p Pub. in Physical Review Letters 60, n24 p2558, 13 Jun 88

Keywords: "Iron, "Epitaxial growth, Substrates, Metal films, Magnetic films, FCC lattices, Magnetism, Diffusion, Copper, Reprints.

A comment is given of a recent report on the magnetic properties of ultra-thin films of fcc Fe on Cu(001). The criticism is based on investigations of the epitaxial growth processes in terms of diffusion and segregation.

101,479

PB91-149898

Not available NTIS

National Bureau of Standards (NML), Gaithersburg, MD. Surface Science Div.

\section{Hall Effect.}

Final rept.

S. M. Girvin. 1987, 2p

See also N87-28431.

Pub. in McGraw-Hill Encyclopedia of Science and Technology, p296-297 1987. 


\section{Solid State Physics}

Keywords: "Hall effect, Transport properties, Magnetic fields, Electrical conductivity, Reprints, "Quantum Hall effect.

The classical and quantum Hall effects are briefly defined and discussed.

\section{1,480}

PB91-149963

Not available NTIS

National Inst. of Standards and Technology (NEL), Boulder, CO. Electromagnetic Technology Div.

Analysis of the YBa2Cu307/SrTiO3 Interface as a Function of Post-Deposition Annealing Temperature.

Final rept.

S. E. Asher, A. J. Nelson, A. R. Mason, A. B

Swartzlander, R. Dhere, L. Kazmerski, J. Halbritter T. E. Harvey, J. A. Beall, and R. H. Ono. 1990, 7p Pub. in Proceedings of American Vacuum Society Fall Meeting, Boston, Ma., October 10-15, 1990, p205-211.

Keywords: "High temperature superconductors, "Superconducting films, "Yttrium barium cuprates, Strontium titanates, Annealing, Interfaces, Thin films, Reprints.

A multiple technique approach is used to study $\mathrm{YBa} 2 \mathrm{Cu} 3 \mathrm{O} 7$ grown on $\mathrm{SrTiO} 3$ as a function of postdeposition annealing temperature. X-ray diffraction data are used to determine the relative amounts of aaxis and c-axis oriented growth. These results are compared to the surface morphology of the films observed by SEM. Secondary ion mass spectrometry (SIMS) is used to study the diffusion of substrate ele(SIMS) is used to study the diffusion of substrate elements into the YBCO films as a function of post-deposition annealing temperature. The data obtained from all these techniques are correlated to determine an optimized temperature for post-deposition annealing. The results of the study show that the desired C-axis of substrate elements into the film at annealing temperatures of $750 \mathrm{C}$.

\section{1,481}

PB91-149997

Not available NTIS National Bureau of Standards (IMSE), Gaithersburg, MD.

Emergence of Modern Nucleation Theory.

Final rept.

J. W. Cahn. 1987, 15p

Pub. in Materials Research Society Symposia Proceedings, v57 p41-55 1987.

Keywords: "Nucleation, "Crystal growth, Theories, Reprints.

A series of important papers by David Turnbull and his collaborators in the late 1940's and early 1950's laid the experimental and theoretical foundation of modern nucleation theory. The elegance, versatility, and genwith brilliant and insightful experimental confirmation, with brilliant and insightful experimental confirmation, sparked widespread application which continues other subjects grew directly or indirectly from that work.

\section{1,482}

\section{PB91-150045}

Not available NTIS

National Bureau of Standards (IMSE), Gaithersburg, MD. Ceramics Div.

Amorphization and Conductivity of Silicon and Germanium Induced by Indentation.

Final rept.

D. R. Clarke, C. M. Kroll, P. D. Kirchner, R. F. Cook, and B. J. Hockey. 1988, 4p

Pub. in Physical Review Letters 60, n21 p2156-2159, 23 May 88

Keywords: *Germanium, "Silicon, Transmission electron microscopy, Electrical conductivity, Indentation, Reprints, "Amorphization.

The authors report the observation, by transmission electron microscopy, that single crystal silicon and germanium is converted to an amorphous state at room temperature directly under both Vickers and Knoop indentations. The effect is seen for crystal orientations of (brackets: 001), (brackets: 011) and (brackets: 111), and with applied loads between $0.1 \mathrm{~N}$ and $0.5 \mathrm{~N}$. They also observe that the materials become electrically conducting under load and that the process is reversible on subsequent unloading and reloading. Furthermore, the transformed phase is found to make ohmic contact to the surrounding, untransformed, semiconductor

\section{1,483}

B91-158501

National Inst. of Standards and Technology (NEL) Gaithersburg, MD. Applied and Computational Mathematics Div.

Micromagnetic Calculations of $180 \mathrm{deg}$ Surface Domain-Wall Magnetization Profiles with Comparison to Measurements.

Final rept.

J. L. Blue, and M. R. Scheinfein. 1990, 3p

See also PB91-107557. Sponsored by Office of Naval Research, Arlington, VA

Pub. in Jni. of Applied Physics 68, n12 p6504-6506, 15 Dec 90.

Keywords: "Domain walls, Scanning electron microscopy, Mathematical models, Ferromagnetism, Magnetization, Permalloys, Comparison, Reprints, Landau Lifshitz-Gilbert equation, Surface magnetism, Micromagnetics.

The authors compare measurements of magnetization profiles across 180 deg surface domain walls in permalloy ferromagnet with calculations from micromagnetic models. The models were solved both by re laxation and by a time-evolution calculation. Th measurements were made using scanning electron microscopy with polarization analysis. The authors obtain good agreement without postulating any surface anisotropy effect. This is the first successful comparison between experiment and a time-evolution calculation of domain walls.

\section{1,484}

$-158642$

Not available NTIS National Bureau of Standards (NML), Gaithersburg, MD. Quantum Metrology Group.

$X-r a y$ Interferometry and gamma-ray Wavelengths.

Final rept.

R. D. Deslattes, $1988,16 p$

Pub. in Art of Measurement: Metrology in Fundamental and Applied Physics, p193-208 1988.

Keywords: *Interferometry, "X-rays, X-ray diffraction, Crystal structure, Silicon, Reprints.

The role of $x$-ray interferometry in linking the visible, $x$ ray and gamma-ray domains is briefly reviewed, and several applications are considered. Resolution of the conflict between two optical determinations of Si lattice periods is reported.

\section{1,485}

PB91-159020

Not available NTIS

National Inst. of Standards and Technology (NML) Gaithersburg, MD. Gas and Particulate Science Div. Modeling Electron Beam Interactions in Semiconductors.

Final rept.

D. Newbury. 1989, 40p

Pub. in SEM Microcharacterization of Semiconductors, Chapter 2, p29-68 1989.

Keywords: "Electron beams, "Semiconductors, Scanning electron microscopy, Beam interactions, Elastic scattering, Monte Carlo methods, Electron trajector ies, Mathematical models, Analytic functions, Inelastic scattering, Reprints.

A quantitative description of electron beam - specimen interactions with solids forms the basis for developing models by which scanning electron microscope images of semiconductor materials and devices can be calculated. Elastic and inelastic scattering processes are considered, including both discrete and continuous energy loss descriptions. These processes can be described by analytic functions, and by considering both elastic and inelastic scattering, simulation of individual electron trajectories by Monte Carlo calcula tions is possible. Applications to specific problems of interest to semiconductor science and technology are described, including spatial resolution at interfaces, details of contrast in charge collection microscopy (electron beam induced conductivity), and energy deposition in solids.

\section{1,486}

PB91-159053

Not available NTIS

National Inst. of Standards and Technology (IMSE)

Gaithersburg, MD. Ceramics Div.

Chemical Modification of the Orthorhombic Superconductor Ba2YCu307-delta.

Final rept.

C. P. Ostertag, F. Beech, and E. R. Fuller. 1988, 10p

Pub. in Ceramic Transactions 1, n2 p501-510 1988.
Keywords: *High temperature superconductors, *Superconductors, Orthorhombic lattices, Chemical reactions, Modification, Silicon carbides, Acetone, Mullite Reinforcing materials, Whiskers, Milling, Reprints, *Yttrium barium cuprates.

The superconducting orthorhombic phase of Ba2Y Cu3O(7-delta) has been observed to undergo low-temperature chemical reaction with acetone when used as a milling medium. The chemically modified powder is non-superconducting. The orthorhombic structure and superconductivity can be fully regenerated by annealing in oxygen at $950 \mathrm{C}$. The modified powder is unstable against and reactive with potentia reinforcing materials, such as silicon carbide and mullite whiskers.

\section{1,487}

PB91-159129 Not available NTIS National Bureau of Standards (IMSE), Gaithersburg, MD. Reactor Radiation Div.

Phonon Density of States of Superconducting YBa2Cu3O7 and the Nonsuperconducting Analog YBa2Cu3O6.

Final rept.

J. J. Rhyne, D. A. Neumann, J. A. Gotaas, F. Beech, L. Toth, S. Lawrence, S. Wolf, M. Osofsky, and D. U. Gubser. 1987, 4p

Pub. in Physical Review B 36, n4 p2294-2297, 1 Aug 87.

Keywords: *High temperature superconductors, *Superconductors, *Phonons, Orthorhombic lattices, Neutron scattering, Atomic structure, Reprints, *Yttrium barium cuprates, Density of states.

Neutron scattering has been used to study the vibrational density of states and the atomic structure of the high temperature superconductor $\mathrm{YBa} 2 \mathrm{Cu} 3 \mathrm{O} 7$. The oxygen atoms were found to occupy four sites and to form chains along the $b$ axis direction of the orthorhombic Pmmm cell. The density of states shows a strong double peak at about $20 \mathrm{meV}$ and a second major maximum near $70 \mathrm{meV}$ with additional less-intense features present at intermediate energies.

\section{1,488}

PB91-159772

PC A05/MF A01

National Inst of Standards and Technology (MSEL), Gaithersburg, MD. Reactor Radiation Div.

NIST Reactor: Summary of Activities July 1989 through June 1990.

Technical note.

C. O'Connor. Dec $90,90 p$ NIST/TN-1285

Also available from Supt. of Docs. as SN003-00303057-1. See also PB90-169996.

Keywords: "NBSR reactor, Research reactors, Activation analysis, Cold neutrons, Crystal structure, Neutron diffraction, Neutron radiography, Nondestructive tests, High temperature superconductors, Polymeric films, Isotopes, Molecular dynamics, Magnetic semiconductors.

The report summarizes all those programs which use the NIST reactor. It covers the period for July 1989 through June 1990 . The programs range from the use of neutron beams to study the structure and dynamics of materials, through nuclear physics and neutron standards to sample irradiations for activation analysis, isotope production, neutron radiography, and nondestructive evaluation.

101,489

PB91-161935

Not available NTIS

National Inst of Standards and Technology (MSEL) Gaithersburg, MD. Reactor Radiation Div.

Structural and Magnetic Properties of Er Thin Films and Er/Y Superlattices: Magnetoelastic Effects.

Final rept.

J. A. Borchers, M. B. Salamon, R. W. Erwin, J. J.

Rhyne, and R. R. Du. 1991, 14p

Pub. in Physical Review B 43, n4 p3123-3136, 1 Feb 91.

Keywords: "Erbium, *Yttrium, Thin films, Superlattices, Molecular beam epitaxy, Magnetic properties, $X$ ray scattering, Magnetization, Neutron diffraction, Comparison, Interfacial tension, Ferromagnetic materials, Magnetoelastic waves, Strain tests, Reprints.

Crystalline erbium thin films and $\mathrm{Er} / \mathrm{Y}$ superlattices with varying Er-layer thicknesses have been grown by molecular-beam epitaxy. The magnetic and structural 
properties of these samples have been analyzed by $\mathrm{x}$ ray-scattering, bulk magnetization, and neutron-diffraction techniques. From a comparison of the data for the two systems, the importance of interfacial strain relative to artificial modulation in shaping the magnetic behavior has been determined. Though the basic nature of the erbium magnetic order is not qualitatively altered in either the thin films or superlattices, the conical ferromagnetic phase is suppressed in all of the samples considered. The enhanced critical fields exhibit a systematic dependence on Er-layer thickness. These effects appear to follow directly from the epitaxial basal-plane strain which is measurable in films over $14,000 \mathrm{~A}$ thick. The strain, along with a 'clamping' of the Er thermal expansion to the $Y$ lattice, leads to a reduction of the magnitude of the magnetoelastic energy that drives the ferromagnetic transition. The dependence of the magnetoelastic energy on the epitaxial strain is described by a model which accounts for the elastic coupling of the erbium lattice to the yttrium.

\section{1,490}

Not available NTIS Technology (NML), Gaithersburg, MD. Electron and Optical Physics Div. Uses of Spin-Polarised Electrons in Fundamental Electron-Atom Collision Processes and the Analysls of Magnetic Microstructures.

Final rept.

M. H. Kelley. $1990,18 p$

Sponsored by Department of Energy, Washington, DC. Pub. in Australian Jnl. of Physics 43, p565-582 1990.

Keywords: "Electron-atom collisions, Electron scattering, Ferromagnetic materials, Magnetic storage, Microstructure, Reprints, "Electron spin polarization, Secondary electrons, Sodium atoms.

Two experimental programs are discussed which exploit the use of polarized electrons for studies of fundamental processes and physical properties. In one program, collisions between spin-polarized electrons and optically pumped sodium atoms provide a very detailed characterization of the spin-dependent interactions important in low-energy electron-atom collisions. The results of these measurements provide a critical test for the reliability of state-of-the-art electron scattering calculations. In the second program, the spin polarization of secondary electrons ejected by high-energy electron impact is used to determine the magnetic structure of ferromagnetic materials with very high spatial resolution (about $60 \mathrm{~nm}$ ). The ability to perform such studies with high resolution has been exploited both in studies of the basic magnetic properties of ferromagnetic materials and in studies of how these basic properties affect the magnetic structure and performance of devices used for magnetic information storage.

\section{1,491}

PB91-162305 Not available NTIS National Inst. of Standards and Technology (NML), Gaithersburg, MD. Electron and Optical Physics Div. Micromagnetics of Domain Walls at Surfaces.

Final rept.

M. R. Scheinfein, J. Unguris, J. L. Blue, K. J.

Coakley, and D. T. Pierce. 1991, 28p

Sponsored by Office of Naval Research, Arlington, VA. Pub. in Physical Review B 43, n4 p3395-3422, 1 Feb 91.

Keywords: ^Domain walls, Scanning electron microscopy, Bloch wall, High resolution, Nickel rich permalloy, Magnetic films, Single crystals, Iron, Reprints, Surface magnetism, Neel wall.

High-spatial-resolution magnetization maps of ferromagnetic surfaces are generated with use of scanning electron microscopy with polarization analysis (SEMPA). The structure of surface Neel walls is measured by SEMPA and compared directly to the results of micromagnetics simulations. The authors find that the surface magnetic properties observed with SEMPA can be modeled using standard micromagnetic theory using only bulk parameters. Surface-domain-wall magnetization profiles were measured, using two different probe diameters in each case, for an Fe(100) single crystal and for Permalloy films with thicknesses of $0.12,0.16,0.20$, and 0.24 micrometer. The micromagnetic simulations show the 180 deg wall of the bulk turning over into a Neel wall at the surface with the magnetization in the plane of the surface. The Neel wall extends from the surface into the bulk over a depth approximately equal to a Bloch-wall width.
National Inst. of Standards and Technology (NEL), Gaithersburg, MD. Semiconductor Electronics Div. Narrow-Gap Semiconductors and Related Materials.

Final rept.

D. G. Seiler, and C. L. Littler. 1990, 345p

See also AD-A223 534.

Pub. in Jnl. of Semiconductor Science and Technology $5, \mathrm{n} 5 \mathrm{pS} 1-\mathrm{S} 345$ May 90.

Keywords: "Narrow gap semiconductors, "Semiconductors, *Meetings, Reprints.

An introduction to the Conference on Narrow Gap Semiconductors and Related Materials held in Gaithesburg, MD, June 12-15, 1989 is given

\section{1,493}

174532

Not available NTIS

National Bureau of Standards (NML), Gaithersburg, MD. Radiation Physics Div.

Book Review: Polarized Electrons at Surfaces by J. Kirschner.

Final rept.

R. J. Celotta. 1986, 1p

Pub. in Applied Optics 25, n12 p1860, 15 Jun 86

Keywords: "Surfaces, Polarization(Spin alignment), Reprints, "Electron spin polarization, Surface magnetism, Book reviews.

The article reviews the book entitled 'Polarized Electrons at Surfaces' by J. Kirschner.

101,494

PB91-174557

Not available NTIS

National Inst. of Standards and Technology (NEL)

Gaithersburg, MD. Robot Systems Div.

Development of a Three-Dimensional Finite Element Program for Large Strain Elastic-Plastic Solids.

Final rept.

J. H. Chiou, J. D. Lee, and A. G. Erdman. 1990, 15p Pub. in Computers and Structures $36, n 4$ p631-645 1990.

Keywords: "Plastic properties, "Three dimensional models, "Computer calculations, Mathematical models, Elastic properties, Plastic deformation, Strain rate, Finite element method, Numerical solution, Reprints.

In recent years the progress in computational mechan cs has made it possible to adopt more sophisticated constitutive models for solving complicated plasticity problems. For the formulation of elastic-plastic theory at finite strain, E. H. Lee decomposed the deformation gradient into a product of the elastic and plastic parts instead of assuming that the strain rate is the combina tion of the elastic and plastic strain rates. A finite element model has been formulated based on E. H. Lee's theory. Accordingly, a three-dimensional general purpose finite element software package has been developed. The simple tension problem and the simple shear problem are solved as examples so that the numerical solutions and the exact analytical solutions can be compared. Finally, a tensile test of a thin-walled aluminum alloy tube is solved and compared with the experimental data. The good agreement between the finite element solution and the experimental data demonstrates the validity and applicability of the proposed numerical model.

\section{1,495}

PB91-174698

Not available NTIS

National Bureau of Standards (NML). Gaithersburg, MD. Surface Science Div.

$X P S$ and Auger Forward Scattering in Epitaxial Films.

Final rept.

W. F. Egelhoff. 1987, 1p

Pub. in Physics Today, pS-69 Jan 87.

Keywords: "X ray photoelectron spectroscopy, "Interfaces, "Epitaxy, Forward scattering, Auger electrons, Thin films, Copper, Nickel, Reprints.

Very recently, a phenomenon long known in angle-resolved $x$-ray solved $x$-ray photoelectron spectroscopy (XPS) has peak intensities are enhanced at angles correspondpeak intensities are enhanced at angles correspondmmediate neighboring atoms. These enhanced intensities thus identify the bond axes present near the surface. In the paper, examples are presented of the great power of the 'XPS searchlight' effect as a new tool for studying epitaxy and interfaces.

\section{1,496}

PB91-175232

Not available NTIS National Inst. of Standards and Technology (MSEL), Gaithersburg, MD. Reactor Radiation Div

Models for Oxygen Ordering and Diffusion in Ba2YCu3Ox and Ba2YCu3-yMyOx (M = Fe, Co, Al, Ga).

Final rept.

A. Santoro. $1990,4 p$

Pub. in Materials Research Society Symposia Proceedings, v169 p185-188 1990.

Keywords: "High temperature superconductors, Vacancies(Crystal defects), Twinning, Substitutes, "Superconductors, Reprints, "Yttrium barium cuprates.

One of the most important aspects of the structure of $\mathrm{Ba} 2 \mathrm{YCu} 3 \mathrm{O}(\mathrm{x})$ is the local arrangement of the oxygen atoms on the basal plane of the unit cell for compositions comprised between $x=6.0$ and $x=7.0$ (the 'basal plane' is defined by the copper atoms forming the Cu-O2 chains). The paper discusses, from a geometric point of view, models of vacancy formation and ordering in the pure compound, and possible configurations of the oxygen atoms in metal doped materials. These models take into account the existence of twin ning in samples of composition $6.5<$ or $=x<$ or $=$ 7.0 , are consistent with the observed movement of the twin walls occurring when the oxygen stoichiometry is varied and explain the symmetry and the composition of the metal doped compounds. As the oxygen atoms relevant in the study are those located on the basal plane of the structure, in what follows one needs to consider only the atomic configuration on the plane and one may ignore all the other atoms in the unit cell.

101,497

PB91-175323

Not available NTIS

National Bureau of Standards (NML), Gaithersburg, MD. Radiation Physics Div.

Book Review: 'Polarized Electrons at Surfaces' by J. Kirschner.

Final rept.

D. T. Pierce. $1986,1 p$

Pub. in Jnl. of the Optical Society of America B 3, n7 p1040 Jul 86.

Keywords: "Surfaces, Magnetic materials Polarization(Spin alignment), Polarized beams, $\mathrm{Re}$ prints, "Electron spin polarization, Book reviews, Surface magnetism.

The application of electron spin polarization to the study of surfaces is a highly exciting and rapidly growing research area. The monograph gives the author's incisive and up-to-date (as of 1984) view of the field. The book has four main parts: Basic Concepts, Experimental Considerations, Results from Non-Magnetic Crystals, and Results from Magnetic Materials. The book is sufficiently self-contained to provide an introduction to the field, for students at the graduate level, for example, as well as to serve as a useful reference work.

101,498

PB91-175554

Not available NTIS National Inst. of Standards and Technology (NML) Gaithersburg, MD. Surface Science Div.

lon-Induced Radiation-Enhanced Diffusion of Silver in Nickel.

Final rept.

D. Marton, J. Fine, and G. P. Chambers. 1989, 5p Pub. in Materials Science and Engineering A115 p223-227 1989 .

Keywords: *Silver, Auger electron spectroscopy, Meta films, Thin films, Nickel, Reprints, "Radiation enhanced diffusion, Multilayers.

Radiation-enhanced diffusion (RED) was observed during the Auger electron spectroscopy sputter depth profiling of multilayered $\mathrm{Ag} / \mathrm{Ni}$ thin films. Broadening of the thin $(4 \mathrm{~nm})$ silver layers occurred during sputter profiling and resulted in silver Auger intensity profiles for each of these five layers which were asymmetric exhibiting a steep leading edge followed by a more slowly decreasing tail. These findings can be interpreted in terms of interface broadening due to two main factors: (1) surface roughening--symmetric broadening: (2) RED of silver--asymmetric broadening. With the model, it has been possible to separate these factors and to determine the rate of RED. 


\section{PHYSICS}

\section{Solid State Physics}

\section{1,499}

PB91-175612

Not available NTIS

National Inst of Standards and Technology (NML)

Gaithersburg, MD. Surface Science Div.

Electron Attenuation Lengths at SiO2/Si Interfaces.

Final rept.

J. A. Yarmoff, S. A. Joyce, E. Cartier, and F. R

McFeely. 1990, 7p

Pub. in Jnl. of Electron Spectroscopy and Related Phenomena 52, p221-227 1990.

Keywords: "Silicon dioxide, "Electron transport, Electron phonon interactions, Kinetic energy, EV range 1 10, EV range 10-100, Photoemission, Interfaces, Reprints, Attenuation length.

The attenuation length, lambda, was measured as a function of kinetic energy for low energy electrons (8$70 \mathrm{eV}$ ) traveling through $\mathrm{SiO} 2$ with the use of core level soft $x$-ray photoemission. Two oxides of differen thickness were compared, and the extra attenuation resulting from the additional oxide on the thicker sample was used to determine the attenuation length The curve resulting from an analysis which employs an exponential attenuation model displays a minima at an electron kinetic energy of about $35 \mathrm{eV}$, which is result of scattering from the bulk plasmon. Below 35 $\mathrm{eV}$, lambda increases and reaches a local maxima about $20 \mathrm{eV}$, at which point lambda begins to decrease again. The decrease in lambda at low electron energies arises in part from electron-phonon scattering This is confirmed by the shape of the bulk Si 2 p photoemission peak observed on the thicker oxide sample. which is distorted by the phonon-induced loss processes.

\section{1,500}

\section{PB9 1-178822}

PC A23/MF A03

National Inst of Standards and Technology, Gaithers

burg, MD.

Chemistry of Electronic Ceramic Materials.

Final rept.

P. K. Davies, and R. S. Roth. Jan 91, 535p NIST/SP. 804

Also available from Supt. of Docs. Prepared in cooperation with Pennsylvania Univ., Philadelphia. Sponsored by National Aeronautics and Space Administration, Washington, DC., Office of Naval Research, Arlington, VA., and Idaho National Engineering Lab., Idaho Falls.

Proceedings of the International Conference on the Chemistry of Electronic Ceramic Materials. Held in Jackson, Wyoming on August 17-22, 1990.

Keywords: "Meetings, "Ceramics, "Superconductors, "Dielectric materials, Dielectric properties, Surface chemistry, Synthesis(Chemistry), Characterization, Crystal growth, Perovskites, Oxides, Computerized simulation.

Partial Contents: Chemical reaction controlled microstructures and properties of ferroelectric ceramics; Chemistry and properties of temperature compensated microwave dielectrics; Molecular chemistry and the synthesis of precursors to electronic ceramic materials; Polymeric synthesis of perovskite powders and films: Electrodeposition of nanomodulated electronic ceramic thin films; Properties of some mixed uranium oxides; The kinetics and mechanism of the crystallization of Mg2Al4Si5O18 from MgAl2O4 and SiO2 in the presence of a bismuth oxide flux; Alkaline earth nitrides and hydrides; Oxygen diffusion in Y2O3-containing tetragonal zirconia polycrystals (Y-TZP); Microstructures in high temperature superconductors; Calorimetric studies of ceramics; Surface energy barrier formed by adsorbed oxygen in porous $\mathrm{ZnO}$; A scanning tunneling microscopy study of single crystal ZnO and $\mathrm{TiO} 2$ surfaces; Computer simulation studies of electronic ceramics; Molecular dynamics simulations of ion motion in divalent and mixed monovalent-divalent beta'-alumina.

\section{1,501}

PB91-187294

Not available NTIS National Bureau of Standards (IMSE), Gaithersburg, MD.

6-D Structural Model for the Icosahedral (AI, SI)-Mn Quasicrystal.

Final rept.

J. W. Cahn, D. Gratias, and B. Mozer. 1988, 9p

Pub. in Jnl. of Physics 49, n7 p1225-12331988.

Keywords: "Crystal models, Aluminum manganese alloys, Silicon containing alloys, X-ray diffraction, $\mathrm{Re}$ prints, "Quasicrystals, Icosahedral phase.
A 6-dimensional (6-D) periodic model is proposed for the Al-Mn-Si icosahedral quasiperiodic crystal. The model results from an embedding of the periodic cubic alpha structure in 6-D. In the Janner-Janssen-Bak description, it consists of three concentric spherica shells of respectively $\mathrm{Mn}, \mathrm{Al}$ and $\mathrm{Al}$ aligned in perpendicular space around the lattice nodes and two additional shells of $\mathrm{Al}$ around the body centers. The mode is shown to match the $X$-ray powder diffraction data with a satisfactory residual R-factor of 0.128 .

\section{1,502}

\section{PB91-187344} Gaithersburg, MD. Ceramics Div.

Lead Zirconate-Titanate Thin Films Prepared by the Laser Ablation Technique.

Final rept.

C. K. Chiang, L. P. Cook, P. K. Schenck, P. S. Brody, and J. M. Benedetto. 1990, 7p

Pub. in Proceedings of Materials Research Society Symposium Ferroelectric Thin Films, San Francisco, CA., April 16-20, 1990, v200 p133-139.

Keywords: "Lead zirconate titanates, "PZT, Ferroelectric materials, Dielectric properties, Electrical resistivity, Thin films, Reprints, Laser ablation.

Lead zirconate-titanate (PZT) thin films were prepared by the laser ablation technique. The PZT $(\mathrm{Zr} / \mathrm{Ti}=53$ / 47) target was irradiated using a focused q-switched $\mathrm{Nd}$ YAG laser (15 $\mathrm{ns}, 100 \mathrm{~mJ}$ at 1.064 micrometers). The as-deposited films were amorphous as indicated by $X$-ray powder patterns, but crystallized readily with brief annealing above $650 \mathrm{C}$. The dielectric constan and the resistivity of the crystallized films were studied using a parallel-plate type capacitor structure.

\section{1,503}

PB91-187351

Not available NTIS

National Inst of Standards and Technology (MSEL), Gaithersburg, MD. Ceramics Div.

Levitation of Superconducting Composites.

Final rept.

C. K. Chiang, M. Turchinskaya, L. J. Swartzendruber, R. D. Shull, and L. H. Bennett. 1990, 5p

Pub. in Proceedings of Advances in Materials Science and Applications of High Temperature Superconductors, Greenbelt, MD., April 2-6, 1990, p181-185

Keywords: "High temperature superconductors, "Superconducting composites, Electrical resistivity, Magnetic susceptibility, Magnetic hysteresis, Flux pinning Levitation, Reprints, "Yttrium barium cuprates, *Cyanoacrylate.

The inverse levitation of a high temperature superconductor-polymer composite consisting of powdered quench-melt-growth $\mathrm{Ba} 2 \mathrm{YCu} 3 \mathrm{O}$ (7-delta) and cyanoacrylate is reported. Magnetic hysteresis loop measurements for the composite are compared to those measured for the bulk material prior to powdering. Differ ences in the flux pining capibility bewteen the two ma terial forms are small but significant.

\section{1,504}

\section{PB91-187369}

Not available NTIS

National Inst. of Standards and Technology (MSEL)

Gaithersburg, MD. Ceramics Div.

Processing $\mathrm{Bi}-\mathrm{Pb}-\mathrm{Sr}-\mathrm{Ca}-\mathrm{Cu}-\mathrm{O}$ Superconductors from Amorphous State.

Final rept.

C. K. Chiang, W. Wong-Ng, L. P. Cook, S. W

Freiman, N. M. Hwang, M. Vaudin, M. D. Hill, R. D.

Shull, A. J. Shapiro, L. J. Swartzendruber, and L. H.

Bennett. 1990, 10p

Pub in Proceedings of Advances in Materials Science and Applications of High Temperature Superconductors, Greenbelt, MD., April 2-6, 1990, p127-136.

Keywords: "High temperature superconductors, "Superconductors, Magnetic susceptibility, Electrical resistivity, Amorphous state, Crystallization, X-rays, Reprints, "Bismuth strontium calcium cuprates.

The bismuth-based high-T(c) superconductors can be processed via an amorphous $\mathrm{Bi}-\mathrm{Pb}-\mathrm{Sr}-\mathrm{Ca}-\mathrm{Cu}$ oxide. The amorphous oxides were prepared by melting the constituent powders in an alumina crucible at $1200 \mathrm{C}$ in air, followed by pouring the liquid onto an aluminum plate and rapidly pressing with a second plate. In the amorphous state, no crystalline phase was identified in the powder X-ray diffraction pattern of the quenched materials. After heat treatment at high temperature, the amorphous materials crystallized into a glass-ceramic containing a large fraction of the
$\mathrm{Bi} 2 \mathrm{Sr} 2 \mathrm{Ca} 2 \mathrm{Cu} 3 \mathrm{O}(\mathrm{x})$ phase $(\mathrm{T}(\mathrm{C})=110 \mathrm{~K})$. The process ing method, crystallization, and results of dc electrical resistivity and ac magnetic susceptibility measurements are discussed.

\section{1,505}

PB91-187377 available NTIS National Inst. of Standards and Technology (IMSE), Gaithersburg, MD. Reactor Radiation Div.

Magnetic Ordering of Er in Powder and Single Crystals of ErBa2Cu307.

Final rept.

T. W. Clinton, and J. W. Lynn. 1991, 4p

Pub. in Physica C 174, p487-490 1991.

Keywords: "High temperature superconductors, "Superconductors, Temperature measurement, Powder(Particles), Single crystals, Reprints, "Erbium barium cuprates, "Magnetic ordering.

A comment is made on the article ' $2 D$ and $3 D$ Magnetic Ordering of Er in $\mathrm{ErBa2Cu} 3 \mathrm{O}(\mathrm{x})(6=$ or $<\mathrm{x}=$ or $<$ 7)', by Maletta et al. We point out that our originally published Meissner Effect data conclusively shows that our sample is fully superconducting, contrary to their assertion. Secondly, we note that our temperature measurements are done via a standard four-probe resistance measurement, which gives a correct value for the resistance of the calibrated thermometer, while their resistance measurements yield values which are systematically high, thus providing an incorrect temperature scale. Hence their own thermometry is in error rather than ours, contrary to their assertion.

\section{1,506}

PB91-189258

Not available NTIS

National Inst. of Standards and Technology (NML), Gaithersburg, MD. Surface Science Div.

X-ray Photoelectron Forward Scattering Studies of Surface Segregation in Epitaxial $\mathrm{Cu}-\mathrm{Ni}-\mathrm{Ni}(100)$ Sandwich Structures.

Final rept

W. F. Egelhoff. 1989, 5p

Pub. in Jnl. of Vacuum Science and Technology A 7. n3 p2060-2064 1989.

Keywords: "Surfaces, Electron scattering, Metal films, Photoelectrons, Separation, Epitaxy, Copper, Nickel, $X$-rays, Reprints, Multilayers.

The forward scattering of the $\mathrm{Cu} 2 \mathrm{p}(3 / 2)$ core level photoelectrons by overlying $\mathrm{Ni}$ lattice atoms which occurs in the photoemission process has been used to follow the segregation of $\mathrm{Cu}$ to the surface in epitaxial $\mathrm{Ni}$-Cu-Ni(100) sandwich structures. When 1-monolayer of $\mathrm{Cu}$ is covered by 1-2 monolayers (ML) of Ni, surface diffusion processes constitute the mechanism by which the segregation occurs, whereas if $1 \mathrm{ML} \mathrm{Cu}$ is covered by $10 \mathrm{ML} \mathrm{Ni}$ or more, the segregation involves the usual bulk (vacancy) diffusion mechanism.

\section{1,507}

PB91-189506 Not available NTIS National Bureau of Standards (IMSE), Gaithersburg, MD. Metallurgy Div.

Magnetic Hysteresis of High-Temperature YBa2Cu3OX-AgO Superconductors: Explanation of Magnetic Suspension.

Final rept.

C. Y. Huang, Y. Shapira, E. J. McNiff, P. N. Peters,

B. B. Schwartz, M. K. Wu, R. D. Shull, and C. K

Chiang. $1988,6 p$

Pub. in Modern Physics Letters B 2, n7 p869-874 Aug 88.

Keywords: "High temperature superconductors, "Superconducting composites, Magnetic hysteresis, Flux pinning, Magnetization, Levitation, Silver oxides, Reprints, "Yttrium barium cuprates.

The authors have measured the magnetization $M$ of superconducting $\mathrm{YBa} 2 \mathrm{Cu} 3 \mathrm{O}(\mathrm{x})-\mathrm{AgO}$ composites with $\mathrm{T}$ (c) approximately equal to $92 \mathrm{~K}$ as a function of an applied magnetic field $\mathrm{H}$ at 77 and $87 \mathrm{~K}$. A very pronounced $\mathrm{M}-\mathrm{H}$ hysteresis loop occurs even at $87 \mathrm{~K}$, indicating the presence of extremely strong pinning centers. The results of these measurements, together with a simple model, explain quantitatively why these superconductors could be suspended below a magnet.

\section{1,508}

PB91-189589

Not available NTIS

MD. Metallurgy Div. 
Superconducting Thin Fllms of BI-Sr-Ca-Cu-O Obtained by Laser Ablation Processing.

Final rept.

B. F. Kim, J. S. Wallace, L. J. Swartzendruber, J. Bohandy, L. H. Bennett, F. J. Adrian, E. Agostinelli, W. J. Green, K. Moojani, R. D. Shull, and T. E Phillips. 1988, 3p

Pub. in Applied Physics Letters 53, n4 p321-323 1988.

Keywords: "High temperature superconductors, "Superconducting films, Electrical resistivity, Thin films, Reprints, "Bismuth strontium calcium cuprates, Laser ablation, Microwave absorption.

Thin films of Bi-Sr-Ca-Cu-O, deposited on (100) cubic zirconia by laser ablation from a bulk superconducting target of nominal composition $\mathrm{BiSrCaCu} 2 \mathrm{O}(\mathrm{x})$, have been investigated by dc resistance and magnetically modulated microwave absorption measurements. The latter technique reveals important features regarding the phase purity of superconducting samples that are masked in the $\mathrm{dc}$ resistance measurements. The superconducting behavior of the films, as a function of the substrate temperature during deposition and the post-deposition annealing conditions, is discussed.

\section{1,509}

PB91-189647

Not available NTIS

National Inst. of Standards and Technology (NEL) Gaithersburg, MD. Chemical Process Metrology Div. Sputtered Thin Film YBa2Cu3On.

Final rept.

K. G. Kreider, J. P. Cline, A. Shapiro, J. L. Pena, A.

Rojas, J. A. Azamar, L. Maldonado, and L. Del

Castillo. $1988,8 p$

Pub. in Proceedings of Topical Conference on High Tc Superconducting Thin Films, Devices and Applications of the American Vacuum Society, Atlanta, GA., September 1988 , p53-60.

Keywords: "High temperature superconductors, "Superconducting films, Temperature dependence, Partial pressure, Stoichiometry, Substrates, Oxygen, Sputtering, Thin films, Reprints, “Yttrium barium cuprates.

The study was carried out to determine the effect substrate temperature, target to substrate angle, and the partial pressure of oxygen in the sputtering atmosphere on the stoichiometry. Films were deposited by planar magnetic sputtering of stoichiometric 1:2:3 pressed and sintered targets on $\mathrm{MgO}, \mathrm{ZrO2}(\mathrm{Y})$ and ZrO2 coated Al2O3 substrates. DEX and WDS were used to determine the chemical composition of the thin films. X-ray diffraction was used to identify the structure after film crystallization at $1175 \mathrm{~K}$ and oxidation a $800 \mathrm{~K}$. The partial pressure of oxygen appears to have the most profound effect on the stoichiometry by lowering the barium content. Negative ion resputtering of the growing film apparently also slows film growth rate. Films deposited on alumina circuitboard with a $\mathrm{ZrO} 2$ barrier layer have demonstrated transitions to superconducting behavior at $95 \mathrm{~K}$ when the stoichiometric ratio is preserved.

\section{1,510}

\section{PB91-189654}

Not available NTIS

National Inst. of Standards and Technology (IMSE), Gaithersburg, MD. Ceramics Div.

Dynamical Diffraction Imaging (Topography) with $X$-ray Synchrotron Radiation.

Final rept.

M. Kuriyama, R. C. Dobbyn, and B. W. Steiner. 1989 , $15 \mathrm{p}$

Pub. in Annual Review of Materials Science 19, p1832071989.

Keywords: Synchrotron radiation, X-rays, Topography, Electrooptics, Reprints, "Diffraction imaging.

New materials with enormous practical interest are now being produced in industry by far more sophisticated processing methods than those used a decade ago. The structure of these advanced materials is not in a 'stable' state normally found in nature. Even traditional structural materials used for familiar industrial components are virtually all frozen configurationally from states that are metastable or unstable before freezing. Moreover, some materials contain non-equilibrium atomic level dispersions of impurities and topologically metastable structures. In the areas of electronics and electrooptics, materials have already been made with structural control at the atomic level. The wide use of single crystals and single crystal layers as industrial materials requires precise information on atomic behavior during modern processing, such as Czochralski growth and chemical vapor deposition.
Success with such 'high-tech' materials requires the knowledge of new ways to arrange atoms in order to achieve the desired properties.

101,511

PB91-189662

Not available NTIS

National Bureau of Standards (IMSE), Gaithersburg. MD. Ceramics Div.

Streaking Images That Appear Only in the Plane of Diffraction in Undoped GaAs Single Crystals: Diffraction Imaging (Topography) by Monochromatic Synchrotron Radiation.

Final rept.

M. Kuriyama, U. Laor, D. Larson, R. C. Dobbyn, M.

Brown, and B. Steiner. 1988, $7 p$

See also N88-30437.

Pub. in Physical Review B 38, n17 p2421-2427 1988

Keywords: Monochromatic radiation, Synchrotron radiation, Gallium arsenides, Single crystals, Topography, Reprints, "Diffraction imaging.

Streaking images restricted to the direction of the diffraction (scattering) vector have been observed on transmission through undoped GaAs. These disruption images (caused by the reduction of diffraction in the direction of observation) appear both in the forward and in Bragg diffracted directions in monochromatic synchrotron radiation diffraction imaging. This previously unobserved phenomenon can be explained in terms of the effective volume of planar defects or platelets which affects the absorption coefficient in anomalous transmission. Such regions of the crystal look perfect despite the presence of imperfections when the scattering vector is not perpendicular to the normal of the platelets.

\section{1,512}

PB91-189670

Not available NTIS National Bureau of Standards (NML), Gaithersburg MD. Surface Science Div.

Initial Stages of Degradation of Superconductor Surfaces: $\mathrm{O} 2, \mathrm{H} 2 \mathrm{O}, \mathrm{CO} 2$ and $\mathrm{CO}$ Chemisorption on $\mathrm{La2}-\mathrm{xSrxCuO} 4$.

Final rept.

R. L. Kurtz, T. E. Madey, L. Toth, R. Stockbauer, A. Shih, and D. Mueller. 1988, 4p

Pub. in Physical Review B 37, n13 p7936-7939 1988

Keywords: "High temperature superconductors, "Superconductors, "Chemisorption, Photoelectron spectroscopy, Ultraviolet spectroscopy, Water vapor Carbon dioxide, Carbon monoxide, Oxygen, Degradation, Surfaces, Reprints, "Lanthanum strontium cuprates.

The initial stages of degradation of high-T(c) superconductor surfaces by interaction with atmospheric gases
have been studied using ultraviolet photoelectron spectroscopy. $\mathrm{O} 2$ has little effect, while $\mathrm{H} 2 \mathrm{O}$ adsorbs dissociatively with an initial sticking coefficient $S(0) 1$ and forms a surface hydroxide. $\mathrm{CO} 2$ adsorbs forming a carbonate-like surface species with an initial sticking coefficient of $0.3 \mathrm{CO}$ is observed to result in similar adsorbed surface species with a much lower sticking coefficient of 0.0003 .

\section{1,513}

PB91-189688

Not available NTIS

National Inst. of Standards and Technology (NML)

Gaithersburg. MD. Surface Science Div.

Electronic Structure of High-Tc Superconductors Studied Using Photoelectron Spectroscopy.

Final rept.

R. L. Kurtz, S. W. Robey, R. L. Stockbauer, D.

Mueller, A. Shih, L. Toth, A. K. Singh, and M.

Osofsky. 1989, 5p

Pub. in Vacuum 39, n7-8 p611-615 1989.

Keywords: "High temperature superconductors, "Electronic structure "Superconductors, Photoelectron spectroscopy, Synchrotron radiation, Valence bands, Photoemission, Stoichiometry, Carbon dioxide, Carbon monoxide, Water vapor, Oxygen, Surfaces, Reprints, Lanthanum strontium cuprates, Yttrium barium $\mathrm{cu}$ prates, Bismuth strontium calcium cuprates, Thallium barium calcium cuprates.

Fundamental information about the structure of the valence band and the chemical valence states of the various constituents of the $\mathrm{La}-\mathrm{Sr}-\mathrm{Cu}, \mathrm{Y}-\mathrm{Ba}-\mathrm{Cu}, \mathrm{Bi}-\mathrm{Sr}-\mathrm{Ca}$ $\mathrm{Cu}$ and TI-Ca-Ba-Cu oxides have been obtained using photoelectron spectroscopy. These results show that the one-electron theories do not adequately describe the electronic structure of these superconductors. The atomic origins of the features observed in the valence bands have been investigated by studying photoemission resonances and changes in excitation cross-sections with photon energy. Results to date suggest that these materials have varying densities of states at the Fermi level, valence bands composed of $\mathrm{O} 2 \mathrm{p}$ and $\mathrm{Cu}$ $3 d$ states, and display no significant changes in the band structure associated with the superconducting behavior when the temperature is lowered below $T(c)$. In addition, the complex surface chemistry of these oxides make it essential to study the surface stoichiometry and the interaction of simple molecules. $\mathrm{O} 2$ and $\mathrm{CO}$ are found to interact only weakly with the surfaces of the materials studied to date, while $\mathrm{H} 2 \mathrm{O}$ and $\mathrm{CO} 2$ react strongly, forming hydroxides and carbonates.

101,514

\section{PB91-189787}

Not available NTIS

National Inst of Standards and Technology (IMSE) Gaithersburg, MD. Reactor Radiation Div.

Phonon Density of States and Superconductivity in $\mathrm{Nd} 1.85 \mathrm{Ce} 0.15 \mathrm{CuO} 4$.

Final rept.

J. W. Lynn, I. W. Sumarlin, D. A. Neumann, J. J.

Rush, J. L. Peng, and Z. Y. Li. 1991, 4p

Pub. in Physical Review Letters 66, n7 p919-922, 18 Feb 91.

Keywords: "High temperature superconductors, "Superconductors, Neutron scattering, Inelastic scattering, Electron tunneling, Phonons, Reprints, "Neodymium cerium cuprates, Density of states.

Inelastic-neutron-scattering techniques have been used to measure the generalized phonon density of states in superconducting $\mathrm{Nd}(1.85) \mathrm{Ce}(0.15) \mathrm{CuO} 4$ There is reasonable agreement at low energies between the authors' measurements and recent pointcontact-tunneling density-of-states measurements reported by Huang et al, which suggests that phonons play a major role in pairing in the electron-superconductor systems. The correspondence at higher energies, however, is not particularly good, but probably reflects the difficulty in obtaining quantitative tunneling data.

\section{1,515}

PB91-189928 Not available NTIS National Inst. of Standards and Technology (NEL) Gaithersburg, MD. Precision Engineering Div

Microchannel-Plate Detection Systems for Low Accelerating Voltage SEM.

Final rept.

M. T. Postek. 1991, 8p

Pub. in Hitachi Instrument News, p3-10 Feb 91

Keywords: "Scanning electron microscopy, "Microchannel electron multipliers, Secondary emission, Electron scattering, Line width, Reprints.

Microchannel-plate electron detector systems provide a high gain, low noise capability for low-voltage scanning electron microscopy for the collection of both secondary and backscatteed electrons. Coupled with field emission scanning electron microscopes, such as the Hitachi S-800 and the Hitachi S-4000, microchannel-plate detector systems enable the investigation of secondary electron induced contrast mechanisms and backscattered electron detection at extremely low accelerating voltages (i.e., below $1.0 \mathrm{keV}$ for an array of new applications in scanning electron microscopy).

101,516

PB91-189969

Not available NTIS National Inst. of Standards and Technology (NML) Gaithersburg, MD. Gas and Particulate Science Div. Fe Implantation in In0.53Ga0.47 As/InP.

Final rept.

M. V. Rao, D. S. Simons, P. E. Thompson, N. R Keshavarznia, J. M. Kuo, P. M. Amirtharaj, and T. Y. Chang. 1989, 5p

Pub. in Jnl. of Applied Physics 65, n2 p481-485 1989

Keywords: "Ion implantation, "Iron, Indium phosphides, Annealing, Reprints, "Gallium indium arsenides, Secodary ion mass spectroscopy, Depth profiles Photoreflectance.

Single and multiple energy $\mathrm{Fe}$ ion implants are performed in n-type InGaAs. Rapid thermal and furnace anneals are used to activate the implanted material Surface Fe accumulation, multiple Fe peaks and deep in-diffusion of $\mathrm{Fe}$ are observed in the secondary ion mass spectrometry depth profiles of the implanted material. The crystal lattice perfection of the annealed 


\section{PHYSICS}

\section{Solid State Physics}

material is evaluated qualitatively by photoreflectance measurements.

\section{1,517}

PB91-190090

National Inst. Not available NTIS Gaithersburg. Of Standards and Technology (NML), Geometric and Electronic Properties of Cs Structures on III-V (110) Surfaces: From 1D and 2D Insulators to 3D Metals.

Final rept.

L. J. Whitman, J. A. Stroscio, R. A. Dragoset, and R. J. Celotta. 1991, 4p

Sponsored by Office of Naval Research, Arlington, VA. Pub. in Physical Review Letters 66, n10 p1338-1341, 11 Mar 91.

Keywords: "Chemisorption, "Cesium, Scanning tunneling microscopy, Indium antimonides, Gallium arsenides, Superlattices, Adsorption, Surfaces, Reprints.

The authors report the structural and electronic properties of $\mathrm{Cs}$ adsorbed on room-temperature GaAs and InSb (110) surfaces as observed with scanning t unneling microscopy. Cs initially forms long one-dimensional (1D) zigzag chains on both surfaces. Additional Cs adsorption on GaAs(110) results in the formation of a 2D overlayer consisting of five-atom Cs polygons arranged in a $c(4 \times 4)$ superlattice. The tunneling gap measured over these insulating structures narrows with the transition from $1 \mathrm{D}$ to $2 \mathrm{D}$, with metallic characteristics observed following saturation with a second Cs overlayer.

\section{1,518}

PB91-194662 Not available NTIS

National Inst. of Standards and Technology (NEL),

Boulder, CO. Electromagnetic Technology Div.
YBa2Cu307-delta/Insulator Multi-Layers for

YBa2Cu307-delta/Insulat
Crossover Fabrication.

Final rept.

J. A. Beall, M. W. Cromar, T. E. Harvey, M. E.

Johansson, and R. H. Ono. 1991, 4p

Sponsored by Defense Advanced Research Projects Agency, Arlington, VA

Pub. in IEEE (Institute of Electrical and Electronics Engineers) Transactions on Magnetics 27, n2 p15961599 Mar 91.

Keywords: "High temperature superconducters, "Superconducting films, Strontium titanates, Thin films, Substrates, Dielectrics, Crossovers, Reprints, "Yttrium barium cuprates, Laser ablation, Multilayers.

The development of thin-film dielectrics compatible with the epitaxial growth of YBa2Cu3O(7delta)(YBCO) is crucial to the fabrication of multi-layer device and circuit structures. The authors have investigated the YBCO/SrTiO3(STO) system by fabricating YBCO/STO bilayers and simple YBCO/STO/YBCO crossover structures. The thin films were deposited in situ by pulsed laser deposition and analyzed using $x$ ray diffraction and scanning electron microscopy. The film interfaces were characterized by secondary ion mass spectrometry (SIMS) depth profiling. They have developed photolithographic and wet-etching processes for patterning the crossovers whidh are compatible with these materials. The crossover structures were characterized by resistance and insulator pinhole density as well as the superconducting properties of the patterned top and bottom YBCO electrodes (critical temperature, $T(c)$ and critical current density, $J(c))$. Using $\mathrm{SrTiO} 3$ as the insulating layer,they have made crossovers with good isolation between layers $(>100$ Megohm) and high $J(c)$ even in the top electrode $(\mathrm{J}(\mathrm{C})(76 \mathrm{~K})>100,000 \mathrm{~A} / \mathrm{cm}$ squared.

\section{1,519}

PB91-194704

Not available NTIS

National Inst. of Standards and Technology (NEL), Boulder, CO. Electromagnetic Technology Div.

Anomalous Low-Frequency Butterfly Curves for Subsidiary and Ferromagnetic Resonance Overlap

at $3 \mathrm{GHz}$.
Final rept

R. W. Cross, C. E. Patton, G. Srinivasan, J. G. Booth, and $M$. Chen. 1991, $5 p$

Contract USAF-F19628-85-0002

Sponsored by Rome Air Development Center, Griffiss AFB, NY.

Pub. in Jnl. of Applied Physics 69, n3 p1569-1573, 1 Feb 91

Keywords: "Yttrium iron garnets, Ferromagnetic resonance, Ultrahigh frequency, Spin waves, Line width, Thin films, Damping, Reprints.
Subsidiary absorption butterfly curves of spin-wave instability threshold versus static in-plane field have been obtained for yttrium iron garnet (YIG) thin films a $3 \mathrm{GHz}$. The butterfly curves have been found to be rather anomalous, typically displaying a pronounced dip and a very low minimum threshold. These anomalous features are attributed to the overlap of the subsidiary absorption field region with ferromagnetic resonance (FMR). First-order instability theory was extended to include the uniform mode response near FMR. The extended theory yields good fits to the data for reasonable values of the YIG FMR linewidths. The theoretical analysis also shows a predicted flip in the azimuthal propagation angle phi(sub $k$ ) for the unstable spin waves in the region of FMR overlap. With in creasing field, there are predicted discontinuous changes in phi(sub k) from $90 \mathrm{deg}$. to $0 \mathrm{deg}$. and back to $90 \mathrm{deg}$. in the region of FMR.

\section{1,520}

\section{PB91-194738}

Not available NTIS

National Bureau of Standards (NML), Gaithersburg MD. Radiation Physics Div.

Soft X-ray Absorption and Emission Spectra and the Electronic Structure of Some Exotic Materials. Final rept.

D. L. Ederer, L. R. Canfield, T. A. Callcott, K. L.

Tsang, C. H. Zhang, and E. T. Arakawa. 1988, 5 p See also DE89001207.

Pub. in Proceedings of SPIE (Society of Photo-Optical Instrumentation Engineers) - X-ray Vac. Ultraviole Interact. Data Bases, Calc., Meas., v911 p75-79 1988.

Keywords: "Electronic structure, High temperature superconductors, Aluminum manganese alloys, $X$-ray fluorescence, $\mathrm{X}$-ray spectroscopy, Soft $\mathrm{X}$-rays, Synchrotron radiation, Absorption spectra, Emission spectra Band theory, Reprints, Lithium aluminides, Quasicrystals.

The technique of soft $x$-ray fluorescence spectroscopy (SXE) is complimentary to that of photoemission spectroscopy (PES). SXE probes the local partial density of states (PDOS), selects dipole allowed symmetries, and is not necessarily surface sensitive. PES on the othe hand, averages over tHe DOS and can be used to measure the dispersion of the energy bands. PES is also very surface sensitive. The authors present meas urements on the high T(c) superconductors, the quas crystalline phase of AIMn, and the LiAl intermetallic alloy. These measurements provide insight for theoretical modeling. In the case of the high T(c) compound and the intermetallic compound, the measurements are in good agreement with the theory. However, for the quasicrystals, the measurements provide new in sights to challenge theory.

\section{1,521}

PB91-194753

Not available NTIS

National Inst of Standards and Technology (NEL)

Boulder, CO. Electromagnetic Technology Div.

Current Record in Superconductors.

Final rept.

J. W. Ekin, K. Salama, and V. Selvamanickam. 1991 $1 \mathrm{p}$

Dy Office of Naval Research, Arlington, VA. Pub. in Nature 350, p26, 7 Mar 91.

Keywords: "High temperature superconductors, *Critical current, "Superconductors, Magnetic fields, Reprints, "Yttrium barium cuprates.

The authors report the first direct demonstration that high transport $J$ (c) can be achieved in bulk melt-grown YBa2Cu3O7 at magnetic fields up to $30 \mathrm{~T}$ at liquid nitrogen temperature.

\section{1,522}

PB91-194779

Not available NTIS

National Inst. of Standards and Technology (NML) Gaithersburg, MD. Electron and Optical Physics Div. System for the Study of Magnetic Materials and Magnetic Imaging with the Scanning Tunneling Microscope.

Final rept.

P. N. First, J. A. Stroscio, D. T. Pierce, R. A.

Dragoset, and R. J. Celotta. 1991, 6p

Sponsored by Office of Naval Research, Arlington, VA Pub. in Jnl. of Vacuum Science and Technology B 9 , n2 p531-536 Mar/Apr 91.

Keywords: "Scanning tunneling microscopy, "Ferromagnetic materials, Magnetic films, Magnetization, Feasibility, Reprints, Electron spin polarization, Secondary electrons.
A report of work in progress to determine the feasibility of imaging the magnetization of ferromagnetic samples with the scanning tunneling microscope (STM) is presented. A vacuum system was designed to test several different proposals as well as to prepare and characterize thin films of magnetic materials by conventional means, including STM. This was begun with an attempt to detect spatially resolved spin-polarization of secondary electrons emitted from the sample when operating the STM in the scanning field emission mode. Results are currently inconclusive, but encouraging. A weak spin-polarization signal has been observed corresponding to one of the in-plane components of magnetization. However, topographic feedthrough has not been fully eliminated as a possible spurious source of contrast.

101,523

PB91-194860

Not available NTIS

National Inst. of Standards and Technology (MSEL), Boulder, CO. Fracture and Deformation Div.

Group and Phase Sound Velocities in an Eu1Ba2Cu307 Superconductor and Related Perovskite Oxides.

Final rept.

H. Ledbetter, C. M. Fortunko, and S. Lin. 1990, 5p

Pub. in Proceedings of Ultrasonics Symposium, HonoIulu, HI., December 4-7, 1990, p1215-1219.

Keywords: "Acoustic velocity, "Superconductors, Barium titanates, Strontium titanates, Phase velocity, Group velocity, Ultrasonic radiation, Reprints, "Europium barium cuprates.

A material's longitudinal sound-wave velocity relates strongly to the electron-lattice interaction, which affects both conductivity and superconductivity. Measuring phase velocity versus frequency, v(omega), reveals dispersion, which may arise from various sources. To measure v(omega) accurately over a large frequency range, the authors used a modified amplitude-spectrum, pulse-echo method. Specifically, they used a buffer rod whose elastic impedance differed substantially from that of the specimen. Also, they used very broadband ultrasonic transducers and a broadband receiver with large dynamic range. They established the validity of their measurement method by measuring $\mathrm{BaTiO}_{3}$ and $\mathrm{SrTiO} 3$ polycrystals. They then used the method to measure the longitudinalwave velocities in an Eu1Ba2Cu307 superconductor both at 295 and $76 \mathrm{~K}$. Against expectation, they found no remarkable dispersion.

\section{1,524}

\section{PB91-194894}

Not available NTIS

National Bureau of Standards (IMSE), Gaithersburg, MD. Reactor Radiation Div.

Long-Range Antiferromagnetic Order of the $\mathrm{Cu}$ in Oxygen Deficient RBa2Cu3O6 $+x$.

Final rept.

W. H. Li, J. W. Lynn, H. A. Mook, B. C. Sales, and Z. Fisk. $1988,4 p$

Pub. in Physical Review B 37, n16 p9844-9847 1988.

Keywords: "High temperature superconductors, *Antiferromagnetism, "Superconductors, Tetragonal lattices, Neutron diffraction, Single crystals, Polarized beams, Copper ions, Reprints, "Yttrium barium cuprates, "Neodymium barium cuprates, "Magnetic ordering.

The authors have employed polarized and unpolarized neutron diffraction techniques on both powders and single crystals to establish the nature of the magnetic order of the $\mathrm{Cu}$ ions in oxygen deficient RBa2Cu3O $(6+x)(R=Y, N d)$. Magnetic Bragg peaks of the type $(\mathrm{h} / 2, \mathrm{k} / 2, \mathrm{l})$ are observed, which yield a magnetic structure in which the Cu spins are coupled antiferromagnetically both in the Cu-O planes as well as along the tetragonal $\mathrm{C}$-axis, with the spin direction in the tetragonal plane. The Neel temperature is very sensitive to the oxygen content, with $T(N)(X$ approx $=0$ ) approx $=500 \mathrm{~K}$, while the spin configuration is independent of $x$.

\section{1,525}

PB91-194902

Not available NTIS

National Inst. of Standards and Technology (IMSE), Gaithersburg, MD. Reactor Radiation Div. 
Crystal-Field Splittings in the Cubic Heusler Alloys HoPd2Sn and ErPd2Sn.

Final rept.

W. H. Li, J. W. Lynn, H. B. Stanley, T. J. Udovic, R. N. Shelton, and P. Klavins. 1989, 8 . Pub. in Physical Review B 39, n7 p4119-4126, 1 Mar 89.

Keywords: *Crystal field, Time-of-flight method, Neutron scattering, Inelastic scattering, Magnetic moments, Heusler alloys, Reprints, ${ }^{*}$ Holmium palladium stannides, "Erbium palladium stannides, Magnetic superconductors.

Neutron inelastic scattering measurements have been taken with triple-axis and time-of-flight techniques to determine the crystal field levels of the rare-earth ions in the cubic Heusler alloys HoPd2Sn and ErPd2Sn. The observed excitations have been identified as crystal field in origin by their dispersionless character as well as by the temperature and wave-vector dependence of the intensities. Analysis of the data shows that the energies and intensities can be understood on the basis of a crystalline electric field with cubic point symmetry, with Lea, Leask, and Wolf parameters of $W=$ $0.0287(4) \mathrm{meV}, x=0.3248(8)$ for HoPd2Sn, and $W=$ -0.0450 (4) meV, $x=0.3022(6)$ for ErPd2Sn. The crystal field parameters for the other rare-earth elements have also been calculated by scaling to the values determined for HoPd2Sn.

\section{1,526}

\section{PB91-194977}

Not available NTIS

National Inst. of Standards and Technology (IMSE), Gaithersburg, MD. Ceramics Div.

Studies of Magnetic Flux Penetration in a Chemically Synthesized $\mathrm{Bi}(\mathrm{Pb}) \mathrm{SrCaCuO}$ Superconductor.

Final rept.

D. Lundy, J. Ritter, L. Swartzendruber, R. Shull, and L. Bennett. 1989, 7p

Pub. in Jnl. of Superconductivity 2, n2 p273-279 Jun 89.

Keywords: *High temperature superconductors, "Superconductors, Magnetic measurement, Magnetic hysteresis, Magnetic flux, Flux pinning, Reprints, "Bismuth lead strontium calcium cuprates.

Magnetic measurements were conducted on a sample of chemically synthesized $\mathrm{Bi}(1.5) \mathrm{Pb}(0.5) \mathrm{Sr}(1.5) \mathrm{Ca}(1.75) \mathrm{Cu} 2 \mathrm{O}(\mathrm{x})$ using an ac susceptometer and a vibrating sample magnetometer. The sample showed a large fraction of the phase with a superconducting onset temperature of $110 \mathrm{~K}$. This high temperature superconducting phase appears to have an extremely narrow hysteresis loop showing a small number of flux pinning sites.

\section{1,527}

PB91-195024

Not available NTIS

National Inst. of Standards and Technology (CSTL), Gaithersburg, MD. Surface and Microanalysis Science Div.

Ion Bombardment Induced Diffusion: A Case Study on a Sputtered Ag/Ni Layered System.

Final rept.

D. Marton, and J. Fine. 1990, 19p

Pub. in Periodica Polytechnica 34, n1-3 p37-55 1990.

Keywords: "Diffusion, lon bombardment, Metal films, Separation, Surfaces, Nickel, Silver, Reprints, Multilayers.

The layered $\mathrm{Ag} / \mathrm{Ni}$ system has been shown to exhibit surface segregation of $\mathrm{Ag}$ when $\mathrm{Ag}$ layer buried between two Ni layers is sputter profiled at room temperature (J. Fine et al., 1983). While the study was concerned with the analysis of radiation induced segregation (RIS), it also demonstrated the role of bombardment enhanced diffusion in the transport of $\mathrm{Ag}$ atoms to the surface. The segregation rate was shown to obey first order kinetic equations, and segregation rates for I keV and $4 \mathrm{keV}$ sputtering were determined.

\section{1,528}

PB91-195115

Not available NTIS

National Bureau of Standards (IMSE), Gaithersburg, MD. Metallurgy Div.

Wigner-Seitz Local-Environment Study of the High Tc Superconductors.

Final rept.

M. Melamud, L. H. Bennett, and R. E. Watson. 1988, $8 p$

Pub. in Physical Review B 38, n7 p4624-4631 1988

Keywords: "High temperature superconductors, "Chemical bonds, Wigner-Seitz method, Anisotropy, Reprints, Yttrium barium cuprates, Lanthanum cuprates.

The neighborhood and bonding of atoms in the high TC superconductors are studied using a Wigner-Seitz-cell construction. Assuming metallic radii for the atoms, it is shown that the $\mathrm{Ba}, \mathrm{Y}$., and $\mathrm{La}$ atoms have large coordination numbers. A three-dimensional bonding scheme is suggested. The same bonding characteristics result for both the La-Cu-O type (about 40K) and the Y-BaCu-O type (about $90 \mathrm{~K}$ ) superconductors.

\section{1,529}

PB91-195461 Gaithersburg, MD. Precision Engineering Div.

SEM Imaging and Metrology at Low Accelerating Voltages Using Backscattered Electrons.

Final rept.

M. T. Postek. 1990, 1p

Pub. in Proceedings of Scanning ' 91 , Atlantic City, NJ., April 8-12, 1991, pl-21 1990.

Keywords: *Scanning electron microscopy, "Semiconductors, Microchannel electron multipliers, Electron scattering, Backscattering, Metrology, Reprints, Secondary electrons.

An approach to measure semiconductor structures for nondestructive submicrometer metrology in the scanninng electron microscope (SEM) at low accelerating voltage is described, using the collection and measurement of only the backscattered electron signal rather than the more commonly used secondary electron signal. In the technique, the backscattered electron signal is collected using a high-efficiency microchannel-plate electron detector system with the front face of the detector biased negatively to reject the lowenergy secondary electrons thus collecting only the backscattered electrons. The advantage of using the backscattered electron signal is discussed, as well as a comparison to measurements using the secondary electron signal. The potential of the technique for application to accurate SEM metrology and standards development is also discussed.

101,530

Not available NTIS National Bureau of Standards (NEL), Gaithersburg, MD. Chemical Process Metrology Div.

Comparison of Amorphous and Quasicrystalline Films of Sputtered AI0.72Mn0.22Si0.06.

Final rept.

J. L. Robertson, S. C. Moss, and K. G. Kreider. 1988, $4 \mathrm{p}$

Pub. in Physical Review Letters 60, n20 p2062-2065 1988.

Keywords: *Aluminum manganese alloys, Amorphous materials, Metallic glasses, Sputtering, Thin films, Silicides, Comparison, Reprints, "Quasicrystals.

X-ray data were collected on films of Films showed a typical metallic glass structure fa ctor $\mathrm{S}(\mathrm{Q})$, while at $230 \mathrm{C}$ the structure was quasicrystalline plus Al. A combined broadening function was applied to the normalized and reduced $S(Q)$ for the $230 \mathrm{C}$ film to bring it essentially into coincidence with the amorphous $(45 C) S(Q)$. This involved both a Lorentzian broadening for particle size and a broadening proportional to $Q$ squared associated with phason strain, and leads the authors to conclude that the glass, or amorphous, phase is a defect limit of the quasicrystal.

\section{1,531}

PB9 1-195560

Not available NTIS

National Inst. of Standards and Technology (IMSE)

Gaithersburg, MD. Ceramics Div.

Inverse Correlation between the Intensity of Luminescence Excited by Electrons and by Visible Light in Chemical-Vapor-Deposited Diamond Films.

Final rept.

L. H. Robins, E. N. Farabaugh, A. Feldman, and L. P. Cook. 1991, 6p

Pub. in Physical Review B 43, n11 p9102-9107, 15 Apr 91.

Keywords: "Cathodoluminescence, "Photoluminescence, Chemical vapor deposition, Raman spectra, Thin films, Reprints, "Diamond films.

Diamond films grown by filament-assisted or microwave-plasma-assisted chemical-vapor deposition
(CVD) were characterized by photoluminescence (PL), cathodoluminescence (CL), and Raman spectroscopies. The laser-excited PL spectra of these films in and near the carbon Raman region $(1100-1800 / \mathrm{cm}$ from the 514.5-nm laser line, or 2.18-2.28 eV) are broad and featureless; $C L$ spectra measured within a wider spectral range (1.5-3.5 eV) shows several distinct components. Because of its correlation with the Raman band of sp2-bonded carbon, the visible-laser-excited PL in the carbon Raman region is attributed to sp2-bonded carbon clusters. The spectrally integrated CL intensity is found to vary from specimen to specimen approximately inversely with the intensity of the laser-excited $\mathrm{PL}$. The inverse correlation is especially strong for one component of the $\mathrm{CL}$, a broad band at $2.85 \mathrm{eV}$. To explain these results, it is proposed that the luminescence centers in these CVD diamond films can be classified into two types with differing excitation and recombination properties. Rate equations are derived for the luminescence kinetics of such a system of two types of recombination centers.

101,532

PB91-195578

Not available NTIS

National Bureau of Standards (IMSE), Gaithersburg, MD. Ceramics Div.

Optically Detected Magnetic Resonance Study of Antisite-to-Acceptor and Related Recombination Processes in as-Grown in P-Zn.

Final rept.

L. H. Robins, T. A. Kennedy, and P. C. Taylor. 1988, 10p

Pub. in Physical Review B 38, n18 p3227-3236 1988.

Keywords: "Indium phosphides, Electron spin resonance, Magnetic resonance, Point defects, Doped materials, Photoluminescence, Zinc, Reprints, Antisites.

The paramagnetic state of the phosphorus-on-indium antisite has been observed by optically detected magnetic resonance (ODMR) in as-grown zinc-doped indium phosphide. The antisite resonance is seen both as an enhancing ODMR signal on the antisite-acceptor photoluminescence (PL) at $0.8 \mathrm{eV}$ and as a quenching ODMR signal on the shallow-donor-acceptor $\mathrm{PL}$ at $1.37 \mathrm{eV}$. The dependence of the ODMR on microwave power, microwave modulation frequency, and photoexcitation intensity is examined, and a rate equation model is developed for the important recombination processes. The experimental results suggest that the antisite-acceptor recombination rate is approximately $40,000 / \mathrm{s}$; the antisite electron spins are unthermalized, but recombine with spin-thermalized holes; and the antisite concentration may be greater than $2 \times 10$ to the 15th power/cc. Two other resonances are also observed, the shallow donor resonance at $g=1.217$ and an unidentified broad resonance at $\mathrm{g}=2.0$.

101533

PB91-195586

Not available NTIS National Inst. of Standards and Technology (IMSE) Gaithersburg, MD. Ceramics Div.

Photoluminescence Excitation by Band-Gap Optical Absorption in Chemical Vapor Deposition Diamond Films.

Final rept.

L. H. Robins, P. J. H. Tjossem, K. C. Smyth, P. Y Barnes, E. N. Farabaugh, and A. Feldman. 1991, 7p Pub. in Jnl. of Applied Physics 69, n6 p3702-3708, 15 Mar 91

Keywords: *Photoluminescence, Chemical vapor deposition, Laser spectroscopy, Thin films, Excitation, Reprints, "Diamond films.

Photoluminescence excitation (PLE) spectra at photon energies near the indirect band gap of diamond have been obtained for diamond films grown by the filament-assisted chemical vapor deposition (CVD) method. The PLF intensity was observed to increase abruptly with photon energy above $5.5 \mathrm{eV}$. This in. crease coincides with the onset of phonon-emissionassisted interband absorption, which was observed in dependently by diffuse transmittance measurements. A lower-energy PLE threshold at about $5.25 \mathrm{eV}$, which coincides approximately with the onset of phonon-absorption-assisted interband absorption, was observed in the spectrum of a gem-quality natural diamond, but not in the spectra of the CVD-grown films. Emissio spectra of the luminescence excited by above-bandgap photons have features similar to luminescence spectra of the same specimens excited by $20-\mathrm{keV}$ electrons. The spectrally integrated intensities of the luminescence excited by above-band-gap photons and by electrons were found to vary from specimen to 


\section{Solid State Physics}

specimen in a linearly related manner, suggesting that similar recombination processes occur in both cases.

101,534

PB91-195594

Not available NTIS

National Inst. of Standards and Technology (NEL)

Boulder, CO. Electromagnetic Technology Div.

Morphology of Silver on YBa2Cu307-delta Thin

Films.

Final rept.

A. Roshko, R. H. Ono, J. A. Beall, J. Moreland, A. J.

Nelson, and S. Asher. 1991, 3p

Pub. in IEEE (Institute of Electrical and Electronics Engineers) Transactions on Magnetics 27, n2 p16161618 Mar 91.

Keywords: "High temperature superconductors, "Superconducting films, Strontium titanates, Magnesium oxides, Thin films, Microstructure, Morphology, Annealing, Substrates, Silver, Reprints, "Yttrium barium cuprates, Lanthanum aluminates, Laser ablation.

The morphology of silver layers deposited and annealed on laser ablated $\mathrm{YBa}$ 2Cu3O(7-delta) films has been examined. Silver was found to dewet the YBa2Cu3O(7-delta) (001) surface on annealing in oxygen and nitrogen. This dewetting behavior may be kineticly inhibited by using either thick silver layers or short anneals and low temperatures. Silver layers 70 $\mathrm{nm}$ to 2.5 micrometers thick were studied on films de posited on $\mathrm{SrTiO} 3, \mathrm{LaAlO} 3$ and $\mathrm{MgO}$ substrates. Anneal times were varied from $6.5 \mathrm{~min}$ to $15 \mathrm{hrs}$ at temperatures from 200 to $700 \mathrm{C}$.

\section{1,535}

\section{PB9 1-195677}

Not available NTIS

National Bureau of Standards (IMSE), Gaithersburg MD. Ceramics Div.

Parent Structure of the Layered High-Temperature Superconductors.

Final rept.

T. Siegrist, S. M. Zahurak, D. W. Murphy, and R. S. Roth. 1988, 2p

Pub. in Nature 334, n6179 p231-232 1988.

Keywords: "High temperature superconductors, "Crystal structure, *Superconductors, Tetragonal lattices, $X$ ray diffraction, Reprints, "Calcium strontium cuprates.

Crystals of composition ( $\mathrm{Ca}(0.86) \mathrm{Sr}(0.14)) \mathrm{CuO} 2$ have been grown and characterized by single crystal X-ray diffraction. The crystals are tetragonal with space group $\mathrm{P} 4 / \mathrm{mmm}$ (No. 123), $\mathrm{a}=3.8611$ (2)A, c $=$ $3.1995(2) \mathrm{A}, \mathrm{c} / \mathrm{a}=0.829, \mathrm{Z}=1$. The structure con tains planar $\mathrm{CuO} 2$ layers separated by $\mathrm{Ca}(\mathrm{Sr})$ atoms. The structure is a simple defect perovskite with ordered oxygen vacancies and can be regarded as the $n$ $=$ infinity parent of the $A 2 B 2 C a(n-1) C u(n) O(4+2 n)(A$ $=\mathrm{Bi}, \mathrm{Tl} ; \mathrm{B}=\mathrm{Sr}, \mathrm{Ba}$ ) superconductors.

\section{1,536}

PB91-195743

Not available NTIS

National Inst of Standards and Technology (NEL). Gaithersburg, MD. Mathematical Analysis Div.

Effect of an Electric Field on the Morphological Stability of the Crystal-Melt Interface of a Binary Alloy. 3. Weakly Nonlinear Theory

Final rept

A. A. Wheeler, G. B. McFadden, S. R. Coriell, and D. T. J. Hurle. 1990, 11p

See also PB90-193541.

Pub. in Jnl. of Crystal Growth 100, n1-2 p78-88 Feb 90.

Keywords: Binary alloys, Crystal growth, Electric fields, Electromigration, Electroepitaxy, Stability, Reprints, "Crystal-melt interface.

The effect of a constant electric current on the crystalmelt interface morphology during directional solidification at constant velocity of a binary alloy is considered. A linear temperature field is assumed, and thermoelectric effects and Joule heating are neglected; electromigration and differing electrical conductivities of crysta and melt are taken into account. A two-dimensional weakly nonlinear analysis is carried out to third order in the interface amplitude, resulting in a cubic amplitude equation that describes whether the bifurcation from the planar state is supercritical or subcritical. For wavelengths corresponding to the most dangerous mode of linear theory, the authors calculate the demarcation between supercritical behavior as a function of processing conditions and material parameters. The bifurcation behavior is a sensitive function of the magnitude and direction of the electric current and of the electrical conductivity ratio.
101,537

B9 $1-195750$

Not available NTIS National Inst. of Standards and Technology (NML), Gaithersburg, MD. Electron and Optical Physics Div. Scanning Tunneling Microscopy Study of Clean and Cs-Covered InSb(110).

Final rept.

L. J. Whitman, J. A. Stroscio, R. A. Dragoset, and R. J. Celotta. 1991, $5 p$

Sponsored by Office of Naval Research, Arlington, VA Pub. in Jnl. of Vacuum Science and Technology $B$, n2 p770-774 Mar/Apr 91

Keywords: "Indium antimonides, Scanning tunneling microscopy, Crystal defects, Surfaces, Coatings, Cesium, Reprints.

Scanning tunneling microscopy has been employed to study clean and Cs-covered InSb(110) surfaces. Atomic-resolution images of both the filled and empty electronic state densities have been obtained. The surface relaxation determined from these images is in good agreement with that predicted by structure calculations. A variety of surface defects have been observed, with the most common appearing to be simple $\mathrm{Sb}$ vacancies. Adjacent In and Sb vacancies (Schottky defects) have also been observed. The perturbation of the surface surrounding these defects is asymmetrical along (in brackets: 001), as might be expected due to the asymmetry in the surface. Surprisingly, the perturbation is also asymmetrical along (in brackets: $1,-1,0$ ) where symmetry is expected. Cs adsorbed on roomtemperature InSb(110) forms one-dimensional zig-zag chains along (in brackets: $1,-1,0$ ), similar to those previously observed on GaAs(110).

\section{1,538}

PB91-200865

Not available NTIS National Inst. of Standards and Technology (NEL) Gaithersburg, MD. Mathematical Analysis Div.

Numerical Study of Two-Dimensional Crysta Growth Forms in the Presence of Anisotropic Growth Kinetics.

Final rept.

L. N. Brush, and R. F. Sekerka. 1989, 23p

Sponsored by Carnegie-Mellon Univ., Pittsburgh, PA Pub. in Jnl. of Crystal Growth, p419-441 1989.

Keywords: "Crystal growth, "Two-dimensional calculations, "Anisotropy, "Kinetics, Single crystals Rates(Per time), Morphology, Mathematical models Liquid-solid interfaces, Fourier transformation, Hea flow, Cooling, Surface tension, Stability, Computation, Quasi-steady states.

A model of the free growth of a two-dimensional single crystal of pure material from an undercooled melt is presented. Quasi-steady-state heat flow and anisotropic interfacial properties couple in the model, gov erning the morphology of the evolving crystal-melt interface. The evolution of an initially slightly perturbed circular crystal as it grows from the linear into the nonlinear regime both in the absence and in the presence of anisotropic interfacial growth kinetics is studied. The calculations show that in the absence of kinetic anisotropy, splitting of the tip of an initial perturbation occurs For an initial shape consisting of two Fourier components, a resonant growth effect of the high frequency Fourier component occurs for special initial configurations. Furthermore, the overall rate of crystal growth is shown to be only of the heat extracted from the system and is virtually independent of the interface shape; this is a consequence of the quasi-steady state approxima tion to heat flow. In the presence of anisotropic growth kinetics, tip stabilization may be observed for certain initial conditions. However, a resonant growth effect can still act to destabilize the tip even in the presence of anisotropic interfacial growth kinetics.

\section{1,539}

PB91-203117

Not available NTIS

National Inst. of Standards and Technology (NEL) Boulder, CO. Electromagnetic Technology Div.

Switching in High-Tc Superconductor Current Transport Measurements.

Final rept.

L. F. Goodrich, J. Moreland, and A. Roshko. 1991,

Pub. in IEEE (Institute of Electrical and Electronics Engineers) Transactions on Magnetics 27, n2 p11941197 Mar 91.

Keywords: "High temperature superconductors, Electrical measurement, Critical current, Niobium stannides, Reprints, Switching voltages, Yttrium barium cuprates, Niobium titanium, Weak links.
Switching voltages can occur in four-wire current transport measurements of sintered high-T(c) superconductors. These switching voltages are irreversible shifts in the voltage-current characteristic of the superconductor that result in multiple branches. The voltage along these branches can be very nonlinear as a function of current and can be positive or negative in polarity relative to the current direction. These voltages can interface with the correct determination of resistivity and critical current density. Experimental data on unaligned sintered $\mathrm{Y} 1 \mathrm{Ba} 2 \mathrm{Cu} 3 \mathrm{O}$ (7-delta) which illustrate the complex nature of the voltages and the confusion they can create are presented. Models based on weak links and $H(c 1)$ and on other effects, are discussed as are observations on $\mathrm{NbTi}$ and $\mathrm{Nb} 3 \mathrm{Sn}$ based superconductors.

\section{1,540}

PB91-203539

Not available NTIS

National Inst. of Standards and Technology (NML), Gaithersburg, MD. Surface Science Div.

Core Hole Screening for Intermediate Size Metal Particles.

Final rept

J. A. D. Matthew. 1990, 5p

Pub. in Solid State Communication 73, n2 p179-183 1990.

Keywords: "Dielectric properties, "Molecular relaxation, "Metal particles, "Solid state physics, Electrical properties, Binding, Dielectrics, Metals, Particles, Molecular structure, Reprints.

A hierarchy of dielectric sphere models is presented to account for the shift in binding energy with particle size for a core hole at the center of a jellium sphere of radius $R$. It is shown that the classical final state interaction between the screening charge deficit at the cluster surface and the localized core hole is indeed very important as recently suggested in the literature, so that for a wide range of $R$ the relaxation energy shift is only weakly dependent on metal dielectric properties. The breakdown of the simple classical rule observed below $R 20$ au requires further study both theoretically and under more controlled experimental conditions.

\section{1,541}

PB91-203554

Not available NTIS

National Bureau of Standards (NEL), Gaithersburg, MD. Semiconductor Electronics Div.

Lattice Relaxation in Silicon Doped with $4 d-$ and 5d-Transition Metals.

Final rept.

S. Mayo, and J. R. Lowney. 1988, 6p

Pub. in Jnl. of Applied Physics 64, n9 p4538-4543 1988.

Keywords: "Silicon, Ionization cross sections, Electron transitions, Conduction bands, Valence bands, Doped materials, Photoionization, Silver, Platinum, Gold, Phonons, Reprints, Lattice relaxation.

Photoionization cross-section spectra from deep centers in silicon doped with technologically important $4 \mathrm{~d}$ and $5 \mathrm{~d}$-transition elements were analyzed by the Ridley and Amato lattice coupling model to determine threshold energy and lattice relaxation parameters corresponding to optically induced transitions involving either band. The average optic phonon energy is 50 meV. Electron transitions to the conduction band from the silver, platinum, and gold acceptor centers have, respectively, threshold energies (in meV) $E(T o)=550$, 226 , and 570 . For silver and gold, the Huang-Rhys parameter $S$ could not be determined because of a mixture of both allowed and forbidden transitions; for platinum, $S=0.3$. Electron transition data from the donor centers of these elements to the conduction band are not available or insufficient to allow analysis of the threshold region.

101,542

PB91-203596

Not available NTIS

National Bureau of Standards (NML), Gaithersburg, MD. Surface Science Div.

Method of FIM-FEEM Specimen Preparation of Superconducting and Other Oxides.

Final rept.

A. J. Melmed. 1988,5

Pub. in Jnl. of Physics C6, p67-71 1988.

Keywords: "Field ion microscopy, "Ion microscopy, "Superconductors, Cuprates, Oxides, Reprints, "Field electron microscopy. 
A new method is described for preparation of oxide specimens for field-ion and field-electron emission microscopy.

\section{1,543}

PB9́1-203604

Not available NTIS

National Bureau of Standards (NML), Gaithersburg, MD. Surface Science Div.

Progress in Fleld lon Microscopy Imaglng of HighTc Superconducting Oxides.

Final rept.

A. J. Melmed, R. D. Shull, and C. K. Chiang. 1988,

Pub. in Jnl. of Physics C6, p459-464 1988.

Keywords: "High temperature superconductors, "Field ion microscopy, "Superconductors, Image analysis, Reprints, Yttrium barium cuprates, Lanthanum strontium cuprates.

Field ion Microscopy (FIM) of many of the new hightransition-temperature superconducting oxides has become routine. The older $\mathrm{La}-\mathrm{Sr}-\mathrm{Cu}-\mathrm{O}$ type and the newer $\mathrm{R}-\mathrm{Ba}-\mathrm{Cu}-\mathrm{O}\left({ }^{\prime} 1,2,3^{\prime}\right)$ type, with $\mathrm{R}=\mathrm{Y}, \mathrm{Yb}, \mathrm{Sm}$ $\mathrm{Gd}, \mathrm{Dy}, \mathrm{Ho}, \mathrm{Er}, \mathrm{Pr}, \mathrm{Eu}$ or La, consistently image well at low temperature in hydrogen FIM and argon FIM at higher temperatures, and less consistently in neon $\mathrm{FIM}$. The newest Bi-Sr-Ca-Cu-O type and TI-Ba-Ca-Cu$O$ type can be imaged by hydrogen FIM, but the Bibased specimens thus far have been mechanically weak and phase identification in both types poses a significant problem. Some details of FIM image interpretation for the ' $1,2,3$ ' superconductors are addressed in particular, the various degrees of order in the images and the question of identity of the imaging species.

\section{1,544
PB91-203612}

Not available NTIS National Bureau of Standards (NML), Gaithersburg, MD. Surface Science Div.

Atomic Fingerprint of YBa2Cu3O7-x-Type HighTemperature Superconductors Observed by Field Ion Microscopy.

Final rept.

A. J. Melmed, R. D. Shull, C. K. Chiang, and H. A

Fowler. 1988, 6p

Pub. in Materials Science and Engineering 100, pL27. L32 Apr 88.

Keywords: *High temperature superconductors, "Crystal defects, "Superconductors, Field ion microscopy, Grain boundaries, Crystal dislocations, Twinning, Reprints, Yttrium barium cuprates, Ytterbium barium cuprates, Samarium barium cuprates, Gadolinium cuprates, Samarium cuprates.

The high-transition-temperature superconducting oxides YBa2Cu3O(7-x), YbBa2Cu3O(7-x), $\mathrm{SmBa} 2 \mathrm{Cu} 3 \mathrm{O}(7-\mathrm{x})$ and $\mathrm{GdBa} 2(\mathrm{Cu}(0.96) \mathrm{Fe}(0.04)) 3 \mathrm{O}(7-$ $x)(0<X<0.05)$ have been found to exhibit a common, unusual but characteristic field ion microscope image at low temperatures, due to inhomogeneous field evaporation, field ionization or both, resulting in preferentially imaged atoms. The characteristically striated images enable identification of various lattice defects such as dislocations, twin boundaries and grain boundaries.

\section{PB9́1-203646}

Not available NTIS

National Inst. of Standards and Technology (NEL) Boulder, CO. Electromagnetic Technology Div. Prospects for High Temperature Superconductor Semiconductor Contacts.

Final rept.

J. Moreland, J. W. Ekin, and T. Larson. 1991, 7p

Contract DE-AI05-89ER14044

Sponsored by Department of Energy, Washington, DC Pub. in Proceedings of ASM International Electronic Materials and Processing Congress (3rd), San Francis Co, CA., August 20-23, 1990, p195-201 1991.

Keywords: "High temperature superconductors, *Semiconductor devices, "Circuit interconnections, "Electric contacts, "Superconductors, Gallium arsenides, Silicon, Reprints, Yttrium barium cuprates, Ohmic contacts.

Development of high temperature superconductor semiconductor contacts with very low contact resistivities is required for microelectronic applications such as circuit interconnects or superconductor - semiconduc tor - superconductor (S/Semi/S) proximity effect devices. Several thin-film depositon methods have been developed for forming very low resistance contacts to high temperature superconductors. Such a contact could potentially be part of a superconductor - semi conductor contact that relies on an intervening norma metal, spanning superconductor and semiconductor interfaces. Presently the authors are surveying materials commonly used to make ohmic contacts to $\mathrm{Si}$ and GaAs for their compatibility with high-temperature superconductors. Prime material characteristics necessary for potential contact structures are (1) low chemical reactivity of the intervening materials with hightemperature superconductors, (2) limited interdiffusion of multilayer constituents, and (3) stable, low contact resistivity, ohmic $\mathrm{I}-\mathrm{V}$ characteristics.

\section{1,546}

Not available NTIS

National Inst. of Standards and Technology (NEL) Boulder, CO. Electromagnetic Technology Div.

Tunnellng Stabilized Magnetlc Force Mlcroscopy Prospects for Low Temperature Applicatlons to Superconductors.

Final rept.

J. Moreland, and P. Rice. 1991, 4p

Pub. in IEEE (Institute for Electrical and Electronics Engineers) Transactions on Magnetics 27, n2 p11981201 Mar 91.

Keywords: "Images, "Tunnel effect, Cryogenics, Superconductors, Magnetic forces, Resolution, Superconducting junctions, Superconducting films, Reprints, "Tunneling stabilized magnetic force microscopy Magnetic force microscopes, Flux lattice, Bitter patterns, Yttrium barium cuprates.

The authors have recently demonstrated an imaging technique referred to as tunneling stabilized magnetic force microscopy or TSMFM. TSMFM is performed using a scanning tunneling microscope (STM) with a flexible, magnetic, tunneling tip in place of the usual rigid tunneling tip. TSMFM images are therefore comrigid tunneling tip. TSMFM images are therefore com-
binations of topography and the magnetic forces between the tip and the sample. Room temperature TSMFM images of magnetic bit tracks on a hard disk have $100 \mathrm{~nm}$ resolution and are comparable to Bitter patterns made using a ferrofluid. They have built a low temperature STM with the hope of getting TSMFM images of the flux lattice in superconductors. Preliminary TSMFM images of a $\mathrm{YBa}(2) \mathrm{Cu}(3) \mathrm{O}(\mathrm{x})$ (YBCO) film (T(c) $-88 \mathrm{~K}$ ) in a $50 \mathrm{mT}$ field show that relatively large magnetic forces are acting on the flexible tip while scanning at $48 \mathrm{~K}$.

\section{1,547}

PB91-203950

Not available NTIS

National Inst. of Standards and Technology (NEL) Boulder, CO. Electromagnetic Technology Div.

Properties of YBa2Cu307-delta Thin Films Grown on Off-Axis-Cut MgO Substrates.

Final rept.

S. E. Russek, B. Jeanneret, D. A. Rudman, and J. W. Ekin. 1991, 4p

Pub. in IEEE (Institute of Electrical and Electronics Engineers) Transactions on Magnetics 27, n2 p931-934 Mar 91

Keywords: *High temperature superconductors, *Superconducting films, Transition temperature, Magnesium oxides, Crystal growth, Thin films, Substrates,

A series of $\mathrm{YBa} 2 \mathrm{Cu} 3 \mathrm{O}$ (7-delta) films has been reactively sputtered on off-axis-cut $\mathrm{MgO}$ substrates. All the films were oriented with the $c$ axis normal to the substrate regardless of substrate orientation, indicating that growth dynamics is a major factor influencing film orientation on non-lattice-matched substrates. As the substrate orientation is moved off the $(100)$ direction the films showed a decrease in transition temperature and showed properties indicative of an increased density of weak links. The films grown on high-angle substrates showed better properties than the films grown on low-angle substrates. Films grown on (110) $\mathrm{MgO}$ were as good as films grown on (100) $\mathrm{MgO}$

\section{1,548
PB91-216747}

(Order as PB91-216705, PC A07/MF A01)

National Inst. of Standards and Technology, Gaithersburg, MD.

High Resolution Synchrotron X-Radiation Diffractlon Imaging of Crystals Grown in Microgravity and Closely Related Terrestrlal Crystals.

B. Steiner, R. C. Dobbyn, D. Black, H. Burdette, and

M. Kuriyama. 1991, 27p

Prepared in cooperation with EG and G Energy Measurements, Inc., Goleta, CA., National Aeronautics and
Space Administration, Hampton, VA. Langley Research Center, Alabama A and M Univ., Huntsville, and GTE Labs., Inc., Waltham, MA.

Included in Jnl. of Research of the National Institute of Standards and Technology, v96 n3 p305-331 May/Jun 91.

Keywords: "Crystal growth, "Crystal defects, $\mathrm{X}$ ray diffraction, Synchrotron radiation, Czochralski method Bridgman method, Mercury iodides, Gallium arsenides Reduced gravity, High resolution, Electrooptics, Lead tin tellurides, Triglycine sulfates.

Irregularities in three crystals (useful in electrooptic detector research) grown in space and in four terrestrial crystals grown under other wise comparable condi tions have been observed in high resolution diffraction imaging. The images provide important new clues to the nature and origins of irregularities in each crystal. For two of the materials, mercuric iodide and lead tin telluride, more than one phase (an array of non diffracting inclusions) was observed in terrestrial samples; but the formation of these multiple phases appears to have been suppressed in directly comparable crystals grown in microgravity. The terrestrial seed crystal of triglycine sulfate displayed an unexpected layered structure, which propagated during directly comparable space growth. Terrestrial Bridgman re growth of gallium arsenide revealed a mesoscopic structure substantially different from that of the origina Czochralski material. A directly comparable crystal is to be grown shortly in space.

101,549

PB91-236729

Not available NTIS

National Inst. of Standards and Technology (EEEL) Gaithersburg, MD. Semiconductor Electronics Div.

Testing the Accuracy of Calculated Equilibrium Carrier Concentrations in the Presence of Surface Flelds.

Final rept

J. Geist, J. R. Lowney, C. R. James, and A. M.

Robinson. 1991, 7p

91.

Keywords: "Carrier density, One-dimensional calculations, Charge carriers, Mathematical models, Diffusion theory, Iterative methods, Semiconductors, Tests, Reprints.

Simple analytic expressions for the one-dimensional majority-carrier concentration in a uniformly doped semi-infinite semiconductor with a charge-accumulated front surface are derived. These expressions are based on the assumption that the effective intrinsic carrier concentration depends only upon the concentration of the majority dopant. Within the framework of this assumption, the expressions derived here are rigorously accurate for intrinsic material, and are accurate to well within 100 parts per million (ppm) for doping concentrations above one trillion/cc. The results of calculations of the majority-carrier concentration carried out using these expressions and using a widely available one-dimensional semiconductor device modeling program are compared to illustrate how these expressions are used for testing the accuracy of iterative solutions of the drift-diffusion equations in the presence of surface fields.

\section{1,550}

PB91-236968

Not available NTIS

National Inst of Standards and Technology (EEEL) Gaithersburg, MD. Semiconductor Electronics Div. Majority and Minority Electron and Hole Mobilities in Heavily Doped GaAs.

Final rept.

J. R. Lowney, and H. S. Bennett. 1991, 9p

Pub. in Jnl. of Applied Physics 69, n10 p7102-7110, 15 May 91

Keywords: *Gallium arsenides, "Electron mobility, "Hole mobility, Minority carriers, Semiconducto doping, Doped materials, Reprints.

The majority electron and minority hole mobilities have been calculated in GaAs for donor densities between 5 $x 10$ to the 16 th power and $1 \times 10$ to the 19 th power $\mathrm{cc}$ Similarly, the majority hole and minority electron mobilities have been calculated for acceptor densities between $5 \times 10$ to the 16 th power and $1 \times 10$ to the 20 th power cc. All the important scattering mechanism have been included. The ionized impurity and carriercarrier scattering processes have been treated with a phase-shift analysis. These calculations are the first to 


\section{Solid State Physics}

use a phase-shift analysis for minority carriers scattering from majority carriers. The results are in good agreement with experiment, but predict that at high dopant densities minority mobilities should increase with increasing dopant density for a short range of densities. The results are important for device modeling ecause of the need to have values for minority mobilities.

\section{1,551}

\section{PB9́1-237180}

Not available NTIS

National Inst. of Standards and Technology (NEL) Boulder, CO. Electromagnetic Technology Div.

Critical-Current Diffraction Patterns of GrainBoundary Josephson Weak Links.

Final rept.

R. L. Peterson, and J. W. Ekin. 1990, 5p

Pub. in Physical Review B 42, n13 p8014-8018, 1 Nov 90.

Keywords: "Josephson junctions, "Critical current, High temperature superconductors, Superconducting junctions, Diffraction patterns, Grain boundaries, Magnetic fields, Reprints, Weak links.

The authors discuss the diffraction patterns and other characteristics of the critical current as a function of magnetic field in grain-boundary Josephson barriers. Diffraction patterns occur not just for SIS junctions but for all types of Josephson links, including SNS junctions, which may be present at grain boundaries in high-T(c) superconductors. The authors discuss the generality of the Airy diffraction pattern, which is expected to characterize grain-boundary barriers in bulk material more accurately than the Fraunhofer pattern. The transport critical-current density in many bulk, granular high-T(c) superconductors has a power-law dependence on very low magnetic fields, characteristic of averaged diffraction patterns, and cannot be fitted by an exponential magnetic-field dependence, which may result from the material properties of the barriers.

\section{1,552}

\section{PB91-237263}

Not available NTIS

National Inst. of Standards and Technology (MSEL), Gaithersburg, MD. Ceramics Div.

Surface Extended-X-ray-Absorption Fine Structure and Scanning Tunneling Microscopy of $\mathrm{Si}(001) 2 \times 1-\mathrm{Sb}$.

Final rept.

M. Richter, J. C. Woicik, J. Nogami, P. Pianetta, K. E. Miyano, A. A. Baski, T. Kendelewicz, C. E. Bouldin

W. E. Spicer, C. F. Quate, and I. Lindau. 1990, 4p

W. E. Spicer, C. F. Quate, a

Sponsored by Office of Naval Research, Arlington, VA Pub. in Physical Review Letters 65, n27 p3417-3420 31 Dec 90.

Keywords: * Silicon, "Antimony, "Interfaces, Scanning tunneling microscopy, $X$ ray absorption, Chemica bonds, Surfaces, Dimers, Reprints, Low energy electron diffraction.

Surface extended-x-ray-absorption fine structure (SEXAFS) has been combined with scanning tunneling microscopy (STM) to determine both the local and long-range bonding properties of the Si(001) $2 \times 1-\mathrm{Sb}$ interface. Sb $L(3)$ edge SEXAFS shows that Sb dimers occupy a modified bridge site on the Si(001) surface with a Sb-Sb near-neighbor distance of $2.88+$ or $0.03 \mathrm{~A}$. Each Sb atom of the dimer is bonded to two $\mathrm{S}$ atoms with a Sb-Si bond length of $2.63+$ or $-0.04 \mathrm{~A}$ STM resolves the dimer structure and provides the long-range periodicity of the surface. Low-energy-electron diffraction of vicinal $\mathrm{Si}(001)$ shows that the $\mathrm{Sb}$ dimer chains run perpendicular to the original Si dimer chains.

\section{1,553}

National Inst. of Standards and Technology (CAML) Gaithersburg, MD. Applied and Computational Mathematics Div.

Micromagnetic Calculations of 180 deg Surface Domain Walls.

Final rept.

M. R. Scheinfein, and J. L. Blue. 1991, 12p

See also PB91-158501.

Pub. in Jnl. of Applied Physics 69, n11 p7740-7751, 1 Jun 91.

Keywords: "Domain walls, Mathematical models, Microstructure, Permalloys, Iron, Reprints, Landau-Lifshitz-Gilbert equation, Surface magnetism, Micromagnetics.
The authors compare quantitative results from two methods of solving the micromagnetics equations for 180 deg domain walls. The first method solves the Landau-Lifshitz-Gilbert equation by time integration of the stiff differential equations. The second method uses a relaxation scheme to determine the equilibrium domain wall magnetization configuration. They compare results from these calculations for various damping parameters and discretization mesh densities, and also compare their results with domain wall measurements made using scanning electron microscopy with polarization analysis. They conclude that equilibrium domain wall microstructure is insensitive to the damping parameter used in the time method of solution for domain walls in magnetically soft iron and Permalloy. For large damping parameters, the approach to equilibrium is the same for the time method and the relaxation method. Further, they can obtain metastable equilibrium states with both methods.

\section{1,554}

PB91-237289

Not available NTIS

National Inst. of Standards and Technology (PL), Gaithersburg. MD. Electron and Optical Physics Div. Scanning Electron Microscopy with Polarization Analysis (SEMPA)-Studies of Domains, Domain Walls and Magnetic Singularities at Surfaces and in Thin Films.

Final rept.

M. R. Scheinfein, J. Unguris, M. Aeschlimann, D. T. Pierce, and R. J. Celotta. 1991, 7p

See also PB91-112672. Sponsored by Office of Naval Research, Arlington, VA.

Pub. in Jnl. of Magnetism and Magnetic Materials 93, p109-1151991.

Keywords: "Magnetic domains, "Domain walls, Scanning electron microscopy, Magnetic recording, Magnetic films, Thin films, Magnetooptics, Microstructure, Terbium alloys, Cobalt alloys, Iron alloys, Permalloys, Reprints, "Surface magnetism, Neel walls, SEMPA system, Electron spin polarization, Micromagnetics.

Scanning Electron Microscopy with Polarization Analysis (SEMPA) is used to investigate the surface magnetic microstructure of domain walls in thin permalloy films and the domain structure of magneto-optic TbFeCo alloys. Domain wall measurements confirm the results of micromagnetic theory.

\section{1,555}

PB91-237420

Not available NTIS National Bureau of Standards (NML), Gaithersburg. MD. Surface Science Div.

Structural Study of the Epitaxial Growth of fcc-Fe Films, Sandwiches, and Superlattices on $\mathrm{Cu}(100)$. Final rept.

D. A. Steigerwald, I. Jacob, and W. F. Egelhoff.

$1988,21 \mathrm{p}$

Pub. in Surface Science 202, n3 p472-492 1988.

Keywords: "Iron, "Epitaxial growth, Metal films, Thin films, Superlattices, Agglomeration, FCC lattices, Magnetism, Substrates, Copper, Reprints.

The epitaxial growth of ultrathin films of pseudomorphic fcc-Fe on Cu(100) has been studied with several structure sensitive techniques. It has been found that the growth is not via a Frank-van der Merwe (layer-bylayer) mechanism, as proposed by several others, but rather exhibits $\mathrm{Fe}$ agglomeration, and at or slightly above $300 \mathrm{~K}$, Cu surface segregation. This manner of growth is shown to be consistent with the generally recognized parameters which affect thin film growth Several adsorbed gas species are found to strongly affect the growth process and the resulting film morphology. The conditions for growth of the best quality Fe films, sandwiches, and superlattices on $\mathrm{Cu}(100)$ are presented and are contrasted with the conditions which have been used in previous studies.

\section{1,556}

\section{PB9 1-237438}

Not available NTIS

National Inst. of Standards and Technology (IMSE),

Gaithersburg, MD. Ceramics Div.

Structural Anomalies in Undoped Gallium Arsenide Observed in High Resolution Diffraction Imaging with Monochromatic Synchrotron Radiation.

Final rept.

B. Steiner, M. Brown, R. C. Dobbyn, M. Kuriyama, U. Laor, and Larson. 1989, 10p

See also N89-11595.

Pub. in Jnl. of Applied Physics 66, n2 p559-568 1989.

Keywords: *Gallium arsenides, *Crystal defects, $X$ ray diffraction, Synchrotron radiation, Monochromatic radi- ation, Grain boundaries, High resolution, Anomalies, Reprints.

Novel streak-like disruption features restricted to the plane of diffraction have recently been observed in synchrotron radiation diffraction from undoped, semiinsulating gallium arsenide crystals. These features were identified as ensembles of very thin platelets (or interfaces) normal to the various 110 directions, and a structural model consisting of antiphase domain boundaries was proposed. The other principal characteristics observed in high resolution monochromatic synchrotron radiation diffraction images are: (quasi)cellular structure, linear low angle subgrain boundaries in 110 directions, and stripes in a $110 \mathrm{di}-$ rection, as well as systematic differences in the acceptance angle for various diffractions. When these detailed structures are considered together, a unifying picture of these features emerges. That is, the presence of ensembles of thin 110 antiphase platelet regions or boundaries is generally consistent not only with the streak-like diffraction features but with all other observations of defects as well. For the formation of such regions, the authors propose two mechanisms, operating in parallel, that appear to be consistent with the various defect features observed by a variety of techniques.

\section{1,557}

PB91-237453

Not available NTIS National Inst. of Standards and Technology (CSTL) Gaithersburg, MD. Surface and Microanalysis Science Div.

Theory of Ballistic-Electron-Emission Spectroscopy of $\mathrm{NiSi} / \mathrm{Si}(111)$ Interfaces.

Final rept.

M. D. Stiles, and D. R. Hamann. 1991, 4p

Pub. in Physical Review Letters 66, n24 p3179-3182, 17 Jun 91.

Keywords: *Nickel silicides, "Silicon, Scanning tunneling microscopy, Electronic structure, Electron transport, Interfaces, Epitaxy, Reprints, Ballistic electron emission microscopy.

The authors discuss theoretical calculations of ballistic-electron-emission-microscopy spectra based in part on a first-principles computation of the transmission across the interiaces. They propose a way of presenting experimental data that highlights the transmission process with respect to contributions from the tunneling distribution. They also present a specific ap plication to $\mathrm{A}$ - and B-type $\mathrm{NiSi2} / \mathrm{Si}(\mathrm{III})$ interfaces, shwing a factor three difference between them at low voltages.

101,558

PB91-237545

Not available NTIS National Bureau of Standards (IMSE), Gaithersburg MD. Metallurgy Div.

Mossbauer Study of the Effect of Oxygen Stoichiometry on the High

Final rept.

L. J. Swartzendruber, L. H. Bennett, M. Z. Harford, and M. Rubinstein. 1988, 12p

Pub. in Jnl. of Superconductivity 1, n2 p219-230 1988

Keywords: "High temperature superconductors, "Mossbauer effect, "Superconductors, Room temperature, Doped materials, Stoichiometry, Iron 57 Oxygen, Reprints, "Yttrium barium cuprates, "Praseodymium barium cuprates, Magnetic ordering.

The Mossbauer effect of oxygen-depleted YBa2Cu3O(7-x) and $\mathrm{PrBa} 2 \mathrm{Cu} 3 \mathrm{O}(7-\mathrm{x})$ doped with $3 \%$ (57)Fe has been investigated at room temperature. $A$ manifold of quadrupole-split spectra has been found wjose parameters are in general agreement with those found by other workers. In addition, the Mossbaue spectra show that a fraction of the $\mathrm{Fe}$ sites develop magnetic order at room temperature when the oxygen content is reduced. It is demonstrated that the observed asymmetries in the Mossbauer spectra can be the result of a preferential alignment of the platelike crystallites that arises during the normal sample preparation process. The tendency to bond with the oxygen atoms is presumed to be responsible for the discreteness of the Mossbauer spectra as a function of oxygen depletion. 
National Inst. of Standards and Technology (MSEL), Gaithersburg, MD. Metallurgy Div.

Low-Field Flux Pinning in Twinned and Detwinned Single Crystals of $\mathrm{YBa} 2 \mathrm{Cu} 3 \mathrm{O} 7-\mathrm{x}$.

Linal rept. Bennett, and A. Roytburd. 1991, 3p

Pub. in Applied Physics Letters 58, n14 p1566-1568, 8 Apr 91.

Keywords: "High temperature superconductors, "Flux pinning, "Superconductors, Single crystals, Twinning, Reprints, "Yttrium barium cuprates.

Flux trapping in single crystals of $\mathrm{YBa} 2 \mathrm{Cu} 3 \mathrm{O}(7-\mathrm{x})$ before and after twin removal has been measured for vortices parallel and perpendicular to the $c$ axis. The results show clearly that, for low applied magnetic fields, twins contribute significantly to flux pinning over the temperature range 60 to $85 \mathrm{~K}$ when the vortices are parallel to the twin boundaries (i.e., parallel to the $c$ axis).

\section{1,560}

\section{PB91-240820}

PC A04/MF A01

National Inst. of Standards and Technology (NML), Gaithersburg, MD. Surface Science Div.

Technical Activities 1990 Surface Science Division. C. J. Powell. May $91,57 p$ NISTIR-4643

See also PB90-161985.

Keywords: Surface chemistry, Surface reactions, Superconducting films, High temperature superconductors, Magnetic recording. Magnetic alloys, Ion irradiation, Synchrotron radiation, Standards, Electron transport, Thin films, Magnetooptics, Semiconductors, Adsorption, Interfaces, Bibliographies, "Surface science, US NIST.

The report summarizes technical activities and accomplishments of the NIST Surface Science Division during Fiscal Year 1990. Overviews are presented of the Division and of its three constituent groups: Surface Dynamical Processes, Thin Films and Interfaces, and Surface Spectroscopies and Standards. These overviews are followed by reports of selected technical accomplishments during the year. A summary is given of Division outputs and interactions that includes lists of publications, talks, committee assignments, seminars (including both Division seminars and Interface Science seminars arranged through the Division), conferences organized, and a standard reference material certified. Finally, lists are given of Division staft and of guest scientists who have worked in the Division during the past year.

\section{1,561}

\section{PB92-108877}

PC A03/MF A01

National Inst. of Standards and Technology (MSEL). Gaithersburg, MD. Reactor Radiation Div.

NIST*LATTICE: A Program to Analyze Lattice Relationships. Spring 1991 Version.

Technical note (Final).

V. L. Karen, and A. D. Mighell. Sep 91, 41p NIST/ $\mathrm{TN}-1290$

Also available from Supt. of Docs. as $\mathrm{SNOOH}_{-003}$ 03106-2. See also PB86-166774.

Keywords: "Crystal lattices, *Computer programming, Programming manuals, Crystal symmetry, Fortran, NIST star LATTICE computer program, Matrix inversion.

The NIST * LATTICE program is written in standard FORTRAN and is designed to be used in any analytical laboratory. The software is multifunctional and can be used to analyze various types of lattice relationships. The present version of the program performs several functions including: (1) the determination of symmetry, and the evaluation of experimental error, through Converse-Transformation analysis; (2) the generation of transformation matrices relating any two unit cells; (3) the calculation of the reduced cell of the lattice, and the calculation and reduction of specified derivative supercells and/or subcells; (4) unit cell transformations; and (5) matrix inversions. It is planned to incorporate additional features in forthcoming versions of the program.

\section{1,562}

PB92-109008

PC A08/MF A02

National Inst. of Standards and Technology (MSEL), Gaithersburg, MD.
High Resolution Diffraction Imaging of Crystals Grown in Microgravity and Closely Related Terrestrial Crystals.

Technical note (Final).

B. Steiner, R. Dobbyn, D. Black, H. Burdette, M Kuriyama, R. Spal, L. van den Berg, A. Fripp, R. Simchick, R. Lal, A. Batra, D. Mattiesen, and B.

Ditchek. Aug 91, 165p NIST/TN-1287

Also available from Supt. of Docs. as SN003-00303092-9. Sponsored by National Aeronautics and Space Administration, Washington, DC. Microgravity Science and Applications Div.

Keywords: "Mercury iodides, "Gallium arsenides, *Crystal growth, "Crystal structure, "Reduced gravity, $X$ ray diffraction, Microgravity applications, $\mathrm{Hg} / 2 \mathrm{semi}$ conductor detectors, Synchrotron radiation, Monochromatic radiation, High resolution, "Lead tin tellurides, "Triglycine sulfates.

The paper reports on irregularities found in three crystals grown in space, in four crystals grown entirely on the ground, and compares the two sets. Irregularities have been observed in mercuric iodide, lead tin telluride, triglycine sulfate, and gallium arsenide by high resolution synchrotron $x$-radiation diffraction imaging Radiation detectors made from mercuric iodide crystals grown in microgravity have been reported to perform far better than conventional detectors grown from the same material under full gravity. Effort is now underway to reproduce these 'space' crystals, optimize their properties, and extend comparable superiority to other types of material.

\section{1,563}

PB92-112333

PC A05/MF A02

National Inst. of Standards and Technology (MSEL), Boulder, CO. Materials Reliability Div.

Oxides and Oxide Superconductors: Elastic and Related Properties.

M. Lei, and H. Ledbetter. Aug 91, 100p NISTIR-3980

Keywords: "High temperature superconductors, "Superconductivity, Electron phonon interactions, Elastic properties, Debye temperature, Bulk modulus, Barium titanates, Strontium titanates, "Yttrium barium cuprates, Kresin model, Born model.

Using both measurements and modeling, the elastic and related properties of some oxides and oxide superconductors were studied. The polycrystal elastic constants were measured using a $\mathrm{MHz}$-frequency pulse-echo method between 295 and $4 \mathrm{~K}$ and correct ed to the void-free state by using a model for a composite material containing spherical particles. The elastic moduli of the high-T(c) superconductor $\mathrm{Y} 1 \mathrm{Ba} 2 \mathrm{Cu} 3 \mathrm{O} 7$ (YBCO) were compared with that of oxides, especially the perovskites $\mathrm{BaTiO} 3$ and $\mathrm{SrTiO}$, which are crystal-structure building blocks for the YBCO superconductor. The bulk moduli were also calculated using a Born ionic model with two energy terms: electrostatic (Madelung) and ion-core-repulsion. The calculated bulk modulus of $Y B C O, 98 \mathrm{GPa}$, agrees well with measurement, $101 \mathrm{GPa}$. Based on monocrystal measurements combined with analysistheory, elastic stiffnesses C(ij) for orthorhombic YBCO were estimated. The bulk modulus obtained from the estimated $\mathrm{C}(\mathrm{ij})$ by the Voigt-Reuss-Hill averaging method agrees with the monocrystal measurement. From the measured polycrystalline elastic constants, the Debye characteristic temperatures were calculated.

\section{1,564}

PB92-116565

Not available NTIS

National Inst. of Standards and Technology (MSEL) Gaithersburg, MD. Reactor Radiation Div.

Theoretical Model for the Tunneling-Gap Anisotropy Observed in Layered Copper-Oxide High-Temperature Superconductors.

Final rept.

R. C. Casella. 1991, 3p

Pub. in Solid State Communications 78, n5 p377-379 1991.

Keywords: "High temperature superconductors, "Superconductors, Band theory, Single crystals, Reprints, "Bismuth strontium calcium cuprates, Tunneling gap anisotropy.

A two-band model, in which the pair constituents in one band are linked via double intermediate boson (IB) exchange with a third fermion in the other band, is invoked to understand the tunneling-gap anisotropy observed in $\mathrm{Bi} 2 \mathrm{Sr} 2 \mathrm{CaCu} 2 \mathrm{O} 8$ single crystals. The coordinates of the two IBs and of the third fermion are inte- grated out, leading to effective pair correlations. The theory is presently in agreement with the data and subject to further experimental test via more precise measurements. Within the model, present data imply that the IB cut-off omega $>0.3 \mathrm{eV}$. The observed broad i.r. excitation centered at approximately $3000 /$ $\mathrm{cm}$ is taken as a candidate for the (possibly composite) IB. Experimental implications are discussed in a general context.

101,565

PB92-116631

Not available NTIS

National Inst. of Standards and Technology (CSTL), Gaithersburg, MD. Surface and Microanalysis Science Div.

Magnetic Anisotropies in Ultrathin fcc Fe(001) Films Grown on Cu(001) Substrates.

Final rept.

J. F. Cochran, W. B. Muir, J. M. Rudd, B. Heinrich, Z. Celinski, T. T. Le-Tran, W. Schwarzacher, W.

Bennett, and W. F. Egelhoff. 1991, 3p

Sponsored by Natural Sciences and Engineering Research Council of Canada, Ottawa (Ontario).

Pub. in Jnl. of Applied Physics 69, n8 p5206-5208, 15 Apr 91.

Keywords: "Thin films, "Magnetic fields, "Molecular beam epitaxy, "Magnons, Monomolecular films, FCC lattices, Copper, Substrates, Anisotropy, Polishing, Surface finishing, Etching, Smoothing, Brillouin effect, Light scattering, Measurement, Ferromagnetic resonance, Reprints, "Ultrathin films.

Brillouin light scattering and ferromagnetic resonance have been used to measure the magnetic field dependence of the magnon frequency at room temperature for 3 monolayer thick films of fcc Fe(001) grown by means of molecular beam epitaxy on $\mathrm{Cu}(001)$ substrates. These films exhibit a strong uniaxial magnetic anisotropy which causes the magnetization to be oriented perpendicular to the film plane in zero applied field. Four specimens were grown on copper substrates prepared using a chemical polishing technique which avoided the use of abrasives; this treatmen greatly reduced the density of etch pits on the copper surface. The uniaxial anisotropies found for these specimens were very consistent and comparable to that previously measured for the best film grown on a mechanically polished copper substrate. There is evidence that substrate smoothness is improved, and hence that the uniaxial anisotropy is increased, when the substrate is covered with a few monolayers of epitaxial copper before the iron films are grown.

\section{1,566}

PB92-116680

Not available NTIS

National Bureau of Standards (NEL), Boulder, CO.

Electromagnetic Technology Div.

Single-Target Magnetron Sputter-Deposition of High-Tc Superconducting Bi-Sr-Ca-Cu-O Thin Films.

Final rept.

N. G. Dhere, J. P. Goral, A. R. Mason, R. G. Dhere, and R. H. Ono. $1988,3 p$

Pub. in Jnl. of Applied Physics 64, n10 p5259-5261, 15 Nov 88.

Keywords: "High temperature superconductors, "Superconducting films, Tetragonal lattices, $X$ ray diffraction, Sputtering, Thin films, Reprints, "Bismuth strontium calcium cuprates.

Single-target RF magnetron sputtering was used to de posit superconducting thin films of $\mathrm{Bi}-\mathrm{Sr}-\mathrm{Ca}-\mathrm{Cu}-\mathrm{O}$ with a $\mathrm{T}(\mathrm{co})$ above $80 \mathrm{~K}$. Varying $\mathrm{P}(\mathrm{O} 2)$ modified the concentrations of $\mathrm{Bi}, \mathrm{Cu}$, and $\mathrm{O}$ in the films by $10-20 \%$. Higher annealing temperatures, especially with brief melting, favored the formation of the higher $T(c)$ phases. Tetragonal phases $(6-\mathrm{K}$ and $75-\mathrm{K} \mathrm{T}(\mathrm{c}))$, with a 3.8097 A, C - 24.607 A, and Bi2Sr2CuO6 composition, and $\mathrm{a}=3.812 \mathrm{~A}, \mathrm{c}=30.66 \mathrm{~A}$, and $\mathrm{Bi} 2 \mathrm{Sr}(2-$ x) $\mathrm{Ca}(1+\mathrm{x}) \mathrm{Cu} 2 \mathrm{O} 8$ composition, were identified; 70-84 $K$ films contained large proportions of a new tetragonal phase, with $\mathrm{a}=3.81 \mathrm{~A}$, and $\mathrm{c}=55.23 \mathrm{~A}$.

101,567

PB92-116730

Not available NTIS

National Bureau of Standards (NML), Gaithersburg MD. Surface Science Div. 


\section{PHYSICS}

\section{Solid State Physics}

Interpretation of Field Ion Microscopy (FIM) images of Asymmetrical Specimens of 1,2,3-Type High-Tc Superconductors.

Final rept.

H. B. Elswijk, H. A. Fowler, and A. J. Melmed. 1988, $5 p$

Pub. in Jnl. de Physique 49, nC-6 p489-493 1988

Keywords: "High temperature superconductors, *Field ion microscopy, "Superconductors, Image analysis, Asymmetry, Reprints, Yttrium barium cuprates.

Field Ion Microscopy (FIM) specimens of '1,2,3'-type high-Tc superconducting materials, typically have an asymmetrical shape. This results from the anisotropic properties, which are involved in the preparation techniques. As a consequence of this shape, the magnification and resolution depend on the direction in the FIM image. Assuming that the elliptical poles in published micrographs are due to tip shape, the authors calculate an asymmetry in the magnification as large as a factor of four. Therefore, the current image interpretation is revisited quantitatively, keeping in mind the magnification asymmetry. The strong effect enhances the striped appearance of the images, and has not been fully recognized so far.

\section{1,568}

\section{PB92-117316}

Not available NTIS

National Inst. of Standards and Technology (NEL) Gaithersburg, MD. Semiconductor Electronics Div. Intrinsic Carrier Concentrations in Long Wave length $\mathrm{HgCdTe} B$ ased on the New, Noniinear Temperature Dependence of $\mathrm{Eg}(x, \mathrm{~T})$.

Final rept.

D. G. Seiler, J. R. Lowney, C. L. Littler, and I. T.

Yoon. 1991, 5p

Pub. in Materials Research Society Symposia Proceedings, v216 p59-63 1991.

Keywords: "Mercury cadmium tellurides, Temperature dependence, Energy gap, Carrier density, Nonlinear analysis, Reprints, Magnetoabsorption.

Intrinsic carrier concentrations of narrow-gap $\mathrm{Hg}(1$ $x) \mathrm{Cd}(\mathrm{x}) \mathrm{Te}$ alloys $(0.17=$ or $<\mathrm{x}=$ or $<0.30$ ) have been calculated as a function of temperature between 0 and $300 \mathrm{~K}$ by using the new nonlinear temperature dependence of the energy gap obtained previously by two-photon magneto-absorption measurements for samples with $0.24=$ or $<x=$ or $<0.26$. The authors report here experimental values for $E(s u b g)(x, T)$ for samples with $x=0.20$ and 0.23 obtained by one photon magneto-absorption measurements. These data confirm the validity of the new $E(\operatorname{sub} g)(x, T)$ relationship for these $x$ values. In this range of composition and temperature, the energy gap of mercury cadmium telluride is small, and very accurate values are needed for the gap to obtain reliable values for the intrinsic carrier density. Large percentage differences exist be tween their new calculations and previous values for $\mathrm{n}(\mathrm{sub} \mathrm{i})$ at low temperatures. Even at $77 \mathrm{~K}$, differences approaching 10 percent exist, confirming the importance of using the new n(sub i) results for materials and device characterization and a proper understand ing of device operation in long-wavelength materials.

\section{1,569}

\section{PB92-117340}

Not available NTIS

National Inst. of Standards and Technology (MSEL) Gaithersburg, MD. Reactor Radiation Div.

Antiferromagnetic Order of $\mathrm{Cu}$ in $\mathrm{Sm} 2 \mathrm{CuO} 4$.

Final rept.

S. Skanthakumar, J. W. Lynn, J. L. Peng, and Z. Y.

Li. 1991, 3p

Pub. in Jnl. of Applied Physics 69, n8 p4866-4868, 15 Apr 91.

Keywords: "Samarium compounds, "Cuprates, "Copper ions, Antiferromagnetism, Neutron diffraction Single crystals, Ceramics, Comparison, Praseodymium compounds, Neodymium compounds, Tetragonal lattices, Neel temperature, Spin waves, Reprints, Samarium cuprate.

Neutron diffraction techniques have been used to study the magnetic order of the $\mathrm{Cu}$ ions in a single crystal of Sm2CuO4. The measurements revealed the development of long-range magnetic order of the $\mathrm{Cu}$ moments at $T($ sub $N)=280+$ or $-1 \mathrm{~K}$, with a relative ly simple antiferromagnetic configuration of spins as found in $\mathrm{Nd}_{2} \mathrm{CuO} 4$ and Pr2CuO4. However, the spin directions in $\mathrm{Sm} 2 \mathrm{CuO} 4$ are rotated by 90 degrees from the spin directions in Nd2CuO4 and $\mathrm{Pr} 2 \mathrm{CuO} 4$. The detailed spin structure can be either collinear or noncollinear, and in these tetragonal systems it is not possi- ble to distinguish between them with the present neutron diffraction data on multidomain samples. Measurements demonstrate that there are no spin reorientations below the Neel temperature, in contrast to the behavior found for $\mathrm{Nd}_{2} \mathrm{CuO} 4$.

\section{1,570}

PB92-117373

Not available NTIS

National Inst. of Standards and Technology (NML), Gaithersburg, MD. Electron and Optical Physics Div. Kinematic Theory of Ballistic Electron Emission Spectroscopy of Silicon-Silicide Interfaces.

Final rept.

M. D. Stiles, and D. R. Hamann. 1991, 5p

Pub. in Jnl. of Vacuum Science and Technology B 9 , n4 p2394-2398 Jul/Aug 91

Keywords: "Cobalt silicides, "Nickel silicides, "Silicon, "Interfaces, Electronic structure, Electron transport, Band theory, Epitaxy, Reprints, Ballistic electron emission spectroscopy.

The electronic structure of the materials being measured has a strong effect on the spectroscopy of the interface between them measured by ballistic electron emission microscopy (BEEM). Specific calculations fo $\mathrm{CoSi} / \mathrm{Si}(111)$ and $\mathrm{NiSi} / \mathrm{Si}(111)$ based on the calculated band structures of the materials illustrate some of the observable effects due to band structures, particularly of the overlayer. The BEEM spectra for $\mathrm{CoSi} / \mathrm{Si}(111)$ show a delayed onset due to a mismatch of the states near the conduction band minimum in the Si. The spectra for $\mathrm{NiSi} / \mathrm{Si}(111)$ show structure due to a decrease in the density of states in the $\mathrm{NiSi} 2$ at about $1.8 \mathrm{eV}$ above the Fermi level.

\section{1,571}

PB92-117381

Not available NTIS National Inst. of Standards and Technology (IMSE), Gaithersburg, MD.

Theory of Orientation Textures Due to Surface Energy Anisotropies.

Final rept.

J. E. Taylor, and J. W. Cahn. 1988, 3p

Pub. in Jnl. of Electronic Materials 17, n5 p443-445 1988.

Keywords: "Crystallization, "Nucleation, "Surface energy, Epitaxy, Anisotropy, Substrates, Orientation, Liquid-solid interfaces, Free energy, Surfactants, Wetting, Electronics, Texture, Reprints, Wulff construction

The effect of surface free energy on the orientation o nuclei on a substrate is examined. In general, the orientation of the nuclei is not determined by minimizing the nucleus-substrate interfacial free energy alone: all interfacial anisotropies must be considered. That orientation preferences should result from anisotropies in the substrate-nucleus interfacial free energies is obvious, but it was found that strong orientation effects are present even if the energy is assumed to be independent of the nucleus orientation, provided that the nutrient-nucleus interfacial free energies are anisotropic Furthermore, varying the magnitude of even an isotropic nucleus-substrate energy will change the orientation of the nuclei. The theory points to some simple rules for prediction of nucleation orientations.

\section{1,572}

PB92-117415

Not available NTIS

National Inst. of Standards and Technology (NML), Gaithersburg, MD. Electron and Optical Physics Div. Observation of Two Different Oscillation Periods in the Exchange Coupling of $\mathrm{Fe} / \mathrm{Cr} / \mathrm{Fe}(100)$.

Final rept.

J. Unguris, R. J. Celotta, and D. T. Pierce. 1991, 4p Pub. in Physical Review Letters 67, n1 p140-143, 1 Jul 91.

Keywords: "Magnetization, Scanning electron microscopy, Antiferromagnetism, Ferromagnetism, Chromium, Iron, Oscillations, Magnetoresistivity, Reprints.

Oscillations of ferromagnetic to antiferromagnetic exchange coupling between two Fe layers separated by a $\mathrm{Cr}$ spacer of linearly increasing thickness were investigated by imaging the magnetic domains with scan ning electron microscopy with polarization analysis. Up to six oscillations in the coupling with a period of 10-12 $\mathrm{Cr}$ layers were observed, and, in the case of an extremely well ordered $\mathrm{Cr}$ interlayer, additional oscillations with a period of $2 \mathrm{Cr}$ layers were observed.

101,573

PB92-117464

Not available NTIS
National Inst. of Standards and Technology (MSEL), Gaithersburg, MD. Reactor Radiation Div.

Low Temperature Magnetization and Magnetic Excitations in Amorphous Fe78B 13 Si9.

Final rept.

S. C. Yu, J. W. Lynn, J. J. Rhyne, and G. E. Fish. 1991, 5p

Pub. in Jnl. of Magnetism and Magnetic Materials 97, p286-290 1991.

Keywords: "Iron alloys, "Magnetization, Boron containing alloys, Silicon containing alloys, Amorphous tions, Neutron scattering, Spin waves, Reprints.

The authors have carried out a study of the low-temperature magnetization and spin-wave dispersion in the amorphous metallic ferromagnet $\mathrm{Fe}(78) \mathrm{B}(13) \mathrm{Si}(9)$. The temperature dependence of the magnetization was measured with a vibrating sample magnetometer and the spin-wave measurements were carried out using inelastic neutron scattering techniques. The magnetization is found to obey the usual law for M(sub s)(T), while the spin waves follow the conventionai ferromagnetic dispersion relation for $E(s u b q)$. The spin wave stiffness constant $D(T)$ is found to be substantially larger than the value inferred from the magnetization data, similar to the behavior observed in other Invartype materials.

\section{Structural Mechanics}

\section{1,574}

PB91-132217

PC A09/MF A01

National Inst. of Standards and Technology (MSEL), Boulder, CO. Materials Reliability Div.

Study of Static and Dynamic Fracture Using Strain Measurements.

J. R. Berger, and J. W. Dally. Sep 90, 188p NISTIR3952

Keywords: "Strain "Crack measurement, "Fractures(Materials), 'Strain "Crack propagation, instruments, instruments, Pressure vessels,

The analysis of strain fields surrounding both stationary and propagating cracks is presented. Series expansions of the static and dynamic strain fields are developed. Gage orientation angles are then studied to optimize the strain response. The orientation angles are found to be dependent on gage type and material. Algorithms are developed which use the temporal or spatial strain variations to extract fracture parameters. The accuracy of the parameter determinations is shown to be excellent, and limits are placed on the validity of the developed methods. The methods are then applied to the analysis of a large scale crack arrest test conducted in a pressure vessel steel. The behavior of the crack-tip position with time and the propagation toughness with time, temperature and position are determined. From this information, details of the conditions at crack arrest are extracted. The propagation toughness-crack-velocity relation is then constructed.

\section{1,575}

PB91-147033

Not available NTIS National Inst. of Standards and Technology (NEL), Gaithersburg, MD. Applied and Computational Mathematics Div.

Mechanism for High Strain-Rate Shear Band Formation.

Final rept.

T. J. Burns. $1990,4 p$

Pub. in Shock Compression of Condensed Matter 1989, p345-348 1990.

Keywords: "Torsion tests, Mathematical models, Viscoplasticity, Shear properties, Strains, Reprints.

Two numerical simulations of a model of the highstrain-rate torsion test are discussed, one with isothermal temperature boundary conditions, the other with adiabatic boundary conditions. It is shown that a moving boundary of rigid unloading, starting from isothermal ends of the thin-walled tube, caused by heat conduction, is a plausible mechanism for shear band formation during the test. 
101,576

PB91-147256

Not available NTIS

National Inst. of Standards and Technology (MSEL),

Boulder, CO. Fracture and Deformation Div.

Experimental and Numerical Studles of the J-Integral for a Surface Flaw.

Final rept.

R. H. Dodds, and D. T. Read. 1990, 21p

Sponsored by David Taylor Research Center, Bethesda, MD.

Pub. in International Jnl. of Fracture 43, p47-67 1990.

Keywords: *Fractures(Materials), "Fracture properties, *J integral, "Crack propagation, Displacement, Deformation. Fracture tests, Tensile stress, Strains, Cracking(Fracturing), Surface properties, Reprints.

Applied J-integral values for a surface cracked tensile panel are experimentally evaluated by measuring strain and displacement quantities along an instrumented contour located on the longitudinal symmetry plane. Nonlinear, 3-D, finite-element analyses are employed to obtain corresponding estimates of the contour and area integral contributions to a 3-D J-integral. Finite element results indicate that the area integral contribution is negligibly small on the symmetry plane; the fracture driving force is thus adequately characterized by the experimental contour values. Detailed comparisons of the experimental and numerical results (crack mouth opening displacement, J-values, and strains along the contour) reveal that the one-quarter symmetric, finite element model accurately predicts the panel response for overall (gauge length) strains approaching 1.6 times the material yield strain, beyond which the observed deformation patterns exhibited globally asymmetric shear bands.

\section{1,577}

PB91-147371

Not available NTIS

National Inst. of Standards and Technology (IMSE), Boulder, CO. Fracture and Deformation Div.

Stress Intensity Factors by Enriched Mixed Finite Elements.

Final rept.

P. R. Heyliger, and R. D. Kriz 1989, 13p

Pub. in International Jnl. for Numerical Methods in Engineering 28, p1461-1473 1989.

Keywords: *Fracture mechanics, "Stress intensity factors, Finite element method, Crack initiation, Fracturing, Stresses, Strains, Elastic properties, Linear systems, Approximation, Displacement, Reprints.

An enriched finite element model for linear elastic fracture mechanics is developed for a mixed variational statement. The independent approximations for the displacement and stress components are enriched by adding the near-field analytic expressions for a cracked body to the polynomial approximations of a conventional element. This allows for an accurate representation of the stress and displacement fields near the crack tip and also results in the direct calculation of the appropriate stress intensity factors. The accuracy of the formulation is demonstrated through several numerical examples.

101,578

PB91-149211 Not available NTIS National Inst. of Standards and Technology (NML), Boulder, CO. Quantum Physics Div.

Thermal Noise in Mechanical Experiments.

Final rept.

P. P. Saulson. $1990,9 p$

Pub. in Physical Review D 42, n8 p2437-2445, 15 Oct 90.

Keywords: "Mechanical oscillators, "Thermal noise, Gravitational wave detectors, Suspension devices, Pendulums, Anelasticity, Reprints.

The fluctuation-dissipation theorem is applied to the case of low-dissipation mechanical oscillators, wose losses are dominated by processes occurring inside the material of which the oscillators are made. In the common case of losses described by a complex spring constant with a constant imaginary part, the thermal noise displacement power spectrum is steeper by one power of omega than is predicted by a velocity-damping model. The author constructs models for the thermal noise spectra of systems with more than one mode of vibration, and evaluate a model of a specific design of pendulum suspension for the test masses in a gravitational-wave interferometer.
National Inst. of Standards and Technology (NEL), Gaithersburg, MD. Applied and Computational Mathematics Div.

Mechanism for Shear Band Formation in the High Strain-Rate Torsion Test.

Final rept.

T. J. Burns. 1990, 9p

See also PB89-215370.

Pub. in Transactions of the ASME 57, p836-844 Dec 90.

Keywords: "Viscoelasticity, "Torsion tests, "Shear properties, Thermoelasticity, Mechanical properties, Rheological properties, Elastic properties, Strains, Stresses, Heat transfer, Plastic flow, Mathematical models, Reprints.

A numerical study of a one-dimensional model of the high strain-rate torsion test shows that a moving boundary of rigid unloading, starting from the ends of the thin-walled tubular specimen, is a plausible mechanism for adiabatic shear band formation during the test. Even though the dimensionless thermal diffusivity parameter is very small, the moving boundary is due to heat transfer from the specimen through its ends, which are assumed to be isothermal heat sinks. The mathematical model is based on a physical model of thermoelastic-plastic flow and a phenomenological Arrhenius model for the plastic flow surface. The numerical technique used is the semi-discretization method of lines.

\section{1,580}

PB91-174268

Not available NTIS

National Inst. of Standards and Technology (IMSE) Boulder, CO. Fracture and Deformation Div.

Determining the Dynamic Stress Intensity Factor with Strain Gages Using a Crack Tip Locating Algorithm.

Final rept.

J. R. Berger, J. W. Dally, and R. J. Sanford. 1990

$12 \mathrm{p}$

Sponsored by National Science Foundation, Washington, DC.

Pub. in Engineering Fracture Mechanics 36, n1 p1451561990.

Keywords: *Strain measurement, "Stress intensity factors, ${ }^{*}$ Crack initiation, Strain gages, Stress analysis, Algorithms, Strains, High alloy steels, Fracture mechanics, Crack propagation, Stress concentration, Reprints.

Recent experimental studies have shown that strain gages can be employed to determine either static or dynamic stress intensity factors, with relatively simple experiments. The paper describes an analysis method for determining stress intensity factors associated with a propagating crack. The analysis is based on a three parameter representation of the strain field near the crack tip. An algorithm is developed where triangulation is used to precisely locate the crack tip position. The algorithm also includes an iteration scheme which permits an accurate determination of two key parameters in the series representation of the strain field. The new method of analysis is demonstrated in a dynamic experiment with a high strength 4340 alloy steel.

\section{1,581}

PB91-174433

Not available NTIS

National Inst of Standards and Technology (IMSE). Boulder, CO. Fracture and Deformation Div.

Improved Optical Diffraction Strain Measurement System.

Final rept.

J. R. Berger, and R. D. Kriz. 1989, 7p

Pub. in Proceedings of SEM Spring Conference on Experimental Mechanics, Cambridge, MA., May 29-June 1, 1989, p572-578

Keywords: "Strain measurement, "Diffraction, "Laser applications, "Fracture mechanics, Gratings(Spectra) Optical equipment, Image processing, Strains, Diffraction patterns, Optical measuring instruments, Crack propagation, Reprints.

Improvements on an automated optical post-processor previously developed at NIST Boulder are described. All three components of in-plane strain as well as rigid body rotations are determined at a maternal point by observing changes in the diffraction pattern from a high frequency grating. The procedure is automated for full field analysis by using digital image processing and a computer controlled positioning device. Spatial resolution and strain sensitivity were improved by decreasing the laser beam diameter, using a sepa- rate camera for each diffraction peak, and using frame averaging techniques.

101,582

PB91-174599

Not available NTIS

National Inst of Standards and Technology (IMSE),

Boulder, CO. Fracture and Deformation Div.

Use of Birefringent Coatings in Fracture Mechanics.

Final rept.

J. W. Dally, J. R. Berger, and K. C. Ham. 1989, 8p Grant NSF-MSM85-13037

Sponsored by National Science Foundation, Washington, DC.

Pub. in Proceedings of SEM Spring Conference on Experimental Mechanics, Cambridge, MA., May 29-June $1,1989, \mathrm{p} 513-520$

Keywords: "Strain measurement, "Stress intensity factors, "Fracture mechanics, "Birefringent coatings, Birefringence, Optical equipment, Refractivity, J integral, Plastic deformation, Strains, Elastic properties, Reprints.

The equations which describe the response of birefringent strips, patches and arrays of strips to the strains developed in a plane body with a through thickness crack subjected to opening mode loading are described. The strain-optic equations are shown in terms of $\mathrm{K}(\mathrm{I})$ for small scale yielding where an elastic analysis is sufficient. The optical response is also shown in terms of $\mathrm{J}$ for a power law hardening material where a plastic region has developed and small scale yielding s not valid. Design concepts for very simple optical gages to measure both $\mathrm{K}(\mathrm{I})$ and $\mathrm{J}$ are introduced.

101,583

PB91-175208

Not available NTIS

National Inst. of Standards and Technology (IMSE), Boulder, CO. Fracture and Deformation Div.

Computerization of a Thermomechanical Processing Research System.

Final rept.

Y. Rosenthal, and Y. W. Cheng. 1989, 31p

Pub. in Intelligent Instruments and Computers, p58-88 Mar/Apr 89

Keywords: "Data acquisition, "Mechanical properties, *Computerized simulation, Mechanical tests, Software(Computers), Stress strain diagrams, Data analysis, Personal computers, High temperature tests, Processing, Reprints.

Software programs for data acquisition and analysis were developed for a personal computer that operates a thermomechanical processing research system. The programs enable data acquisition during mechanical testing at high temperatures, and at constant true strain rates, as well as enabling post-test data analysis. The system is described and results (mechanical properties, engineering and true stress-strain curves, continuous cooling--transformation diagrams) are presented.

101,584

PB91-175224

Not available NTIS

National Bureau of Standards (NEL), Gaithersburg, MD. Structures Div.

Extended Abstract: A Finite Element Study of the Stress and Displacement Fields Produced by Point Impact.

Final rept

M. Sansalone, N. J. Carino, and N. N. Hsu. 1986, 4p Pub. in Jnl. of Acoustic Emission 5, n1 pS24-S27 JanMar 86.

Keywords: "Stress waves, "Wave propagation, "Finite element method, "Displacement, Nondestructive tests, Impact tests, Defects, Greens function, Concretes, Plates, Stresses, Reprints, "Impact-echo tests.

The National Bureau of Standards is working to develop a nondestructive test method for finding flaws within heterogenous materials, such as concrete. The method, which is referred to as the impact-echo method, involves introducing transient stress waves into a test object by mechanical impact (point source) and monitoring reflections of the waves from internal defects and external boundaries using a displacement transducer (point receiver) which is located close to the impact point. The impact-echo test is a simple procedure; however, successful interpretation of recorded surface displacement waveforms requires an understanding of the interaction of transient stress waves with internal defects. To develop a basis for the 


\section{PHYSICS}

\section{Structural Mechanics}

method, Green's function solutions, finite element so lutions, and experimental studies of transient stres wave propagation in solid plates and in plates containing flaws have been carried out. The abstract will discuss some significant findings of the finite element studies.

\section{1,585}

\section{PB91-202812}

Not available NTIS National Inst. of Standards and Technology (NEL), Gaithersburg, MD. Mathematical Analysis Div. Identification of Dynamic Green's Functions in Structural Networks.

Final rept.

A. S. Carasso, and E. Simiu. 1989, 8p

Sponsored by Air Force Office of Scientific Research Bolling AFB, DC., and Directorate of Aerospace Studies, Kirtland AFB, NM.

Pub. in AIAA (American Institute of Aeronautics and Astronautics) Jnl. 27, n4 p492-499 Apr 89.

Keywords: "Dynamic structural analysis, "Greens function, Large space structures, Timoshenko beams, Integral equations, Reprints, III posed problems, Deconvolution.

The paper presents the mathematical and computational basis of a method for experimentally identifying the dynamic behavior of linear structural systems. The method consists of exciting the structure with specific pulses and reconstructing the dynamic Green's functions by deconvolution of the measured response. The reconstruction procedure involves the solution of an illposed integral equation in the presence of noise. Inverse Gaussian functions, which have mathematical properties that render the deconvolution problem tractable, are used as probing pulses. Two examples are presented for which dynamic Green's functions are reconstructed from synthetic response data: (1) a nondispersive medium consisting of a mechanical slider with free body motion, and (2), a network that may be representative of a large orbiting structure, with rigidly connected torsional members and Timoshenko beams. It is shown that residual effects of noise that may be detectable in the reconstructed Green's functions, are effectively smoothed out when these functions are used to predict the response to forces such as may be induced by control systems. Finally, an example is presented of deconvolution implemented in slow motion.

\section{1,586}

\section{PB91-236794}

Not available NTIS

National Inst. of Standards and Technology (IMSE), Gaithersburg, MD. Fracture and Deformation Div. Resonating-Orthotropic-Cube Method for Elastic Constants.

Final rept.

P. Heyliger, H. Ledbetter, and M. Austin. 1990, 10p

Pub. in Dynamic Elastic Modulus Measurements in Materials, ASTM STP 1045, p100-109 1990.

Keywords: "Elastic constants, "Vibration tests, Piezoelectric transducers, Anisotropy, Mechanical properties, Rayleigh-Ritz method, Reprints.

Following studies by Demarest (1969) and by Ohno (1976), the authors describe measurements and analysis that yield, from a single cube-shape specimen, in a single measurement, the complete set of anisotropic elastic-stiffness constants, the $\mathrm{C}(\mathrm{ij})$. Experimentally, they place a cubic specimen between two piezoelectric transducers, which excite and detect the cube's macroscopic free-vibration (fundamental-mode) frequencies, up to $10 \mathrm{MHz}$. From the specimen's shape, size, and mass, and from the measured resonance-frequency spectrum, they analyze for the $C(i j)$ within a given tolerance.

\section{1,587}

PB91-237354

Not available NTIS

National Inst. of Standards and Technology (NEL), Gaithersburg, MD. Structures Div.

Impulse Response Functions for Elastic Structures with Rigid Body Degrees of Freedom.

Final rept.

E. Simiu, and G. Cook. 1989, 14p

Sponsored by Air Force Office of Scientific Research, Bolling AFB, DC

Pub. in Jnl. of Sound and Vibration 135, p275-288, 8 Dec 89.

Keywords: "Dynamic response, "Greens function, "Degrees of freedom, "Elastic analysis, Manipulators, Structural analysis, Functions, Fourier transformation,
Many body problem, Robotics, Structural engineering, Reprints.

A procedure based on the theory of generalized functions is developed which allows the calculation of dynamic Green's functions for discrete and continuous elastic systems with rigid body degrees of freedom. For any given degree of freedom the procedure divides the Fourier transform of the dynamic Green's function into two parts. The first part contains the singularity due to the rigid body degree of freedom. Its time domain counterpart is obtained in closed form. The second part exhibits no singularities, and its time domain counterpart is obtained by numerical integration using a Fast Fourier Transform routine. The procedure is applied to two systems for which closed form solutions are also obtained: a system consisting of two point masses connected by an elastic spring which slide without friction on a horizontal plane, and a nondispersive mechanical slider with elastic deformations due to shear strain. The agreement between the results yielded by the proposed procedure and the closed form solutions is shown to be excellent. The procedure is then used to caluclate the dynamic Green's function for the tip displacement of a dispersive medium consisting of a flexible robot manipulator with the mechanical properties of a Timoshenko beam.

\section{1,588}

\section{PB92-116573}

Not available NTIS

National Inst. of Standards and Technology (MSEL) Gaithersburg, MD. Polymers Div.

Buckling and Post-Buckling Behavior of Elliptical Plates: Part 1. Analysis.

Final rept.

H. Chai. $1990,8 \mathrm{p}$

See also Part 2. PB92-116581.

Pub. in Jnl. of Applied Mechanics 57, p981-988 Dec 90.

Keywords: "Structural analysis, "Composite structures, "Plates, "Elliptical configuration, "Buckling, Calculation methods, Polynomials, Ritz method, Delaminating, Stress analysis, Displacement, Deformation, Loads(Forces), Substrates, Reprints.

A polynomial series expansion for displacements is used in conjunction with the Rayleigh-Ritz energy method to produce buckling and post-buckling stress solutions for an elliptically-shaped surface layer that has been delaminated from the main load-bearing body. Plate deformations are induced by a combined in-plane displacement field applied to the plate boundary and normal pressure. Convergence of the plate solution is assessed by systematically increasing the numbre The convergence of membrane and bending stresses at the plate boundary was generally slow and nonuniform. The degrees-of-freedom necessary for a satisfactory solution typically increase with increasing complexity or magnitude of the plate deformations. By employing as many as 77 displacement terms, practically exact stress solutions are obtained for a wide variety of basic delamination plate problems. The proposed solution procedure is highly efficient and economical and it may be easily extended to other plate geome tries of loading conditions.

\section{1,589}

PB92-116581

Not available NTIS

National Inst of Standards and Technology (MSEL) Gaithersburg, MD. Polymers Div.

Buckling and Post-Buckling Behavior of Elliptical Plates. Part 2. Results.

Final rept.

H. Chai. 1990, 6p

See also Part 1, PB92-116573.

Pub. in Jnl. of Applied Mechanics 112, p989-994 Dec 90.

Keywords: "Composite structures, "Delaminating "Buckling, Elliptical configuration, Stress analysis, Structural analysis, Graphic methods, Substrates, Membrane structures, Bend tests, Loads(Forces), Displacement, Plates, Reprints.

The large-deflection plate solution developed in Part 1 is used here to study the buckling and post-buckling deformation and stress characteristics of an elliptically-shaped surface layer that has been delaminated from a large material body. The economical, yet accu rate nature of the solution, together with available graphic routines, has made it possible to present, figu ratively, a comprehensive description of the plate behavior. The conditions for a layer-substrate overlap and the variations of membrane and bending stresses along the plate boundary are emphasized. Deformations were induced either by a normal pressure or a biaxial displacement field applied to the plate boundary. The problem variables are plate size and shape, details of load biaxiality, and load level.

\section{1,590}

\section{PB92-126622}

(Order as PB92-126614, PC A06/MF A02) National Inst. of Standards and Technology, Gaithersburg, MD.

Summary of the Intercomparison of the Force Standard Machines of the National Institute of Standards and Technology, USA, and the Physikalisch-Technische Bundesanstalt, Germany.

S. L. Yaniv, A. Sawla, and M. Peters. 1991, 12p

Prepared in cooperation with Physikalisch-Technische Bundesanstalt, Brunswick (Germany, F.R.).

Included in Jnl. of Research of the National Institute of Standards and Technology, v96 n5 p529-540 Sep/Oct 91.

Keywords: "Standards, Force(Mechanics), Interlaboratory comparisons, Static loads, Transducers, "Force measurement, "Force standards, Deadweight machines, US NIST.

A comparison of force measurements performed at the National Institute of Standards and Technology, USA, and at the Physikalisch-Technische Bundesanstalt, Germany is reported. The focus of the study was the intercomparison of the forces realized by the two Institutes rather than the measurement process. The transfer standards used in the comparison consisted of force transducers and associated readout instrumentation. The results of the intercomparison reveal that over a range of $50 \mathrm{kN}$ to $4.5 \mathrm{MN}$, the forces realized at NIST and at PTB compare favorably. For forces up to $900 \mathrm{kN}$ the agreement is within + or - $40 \mathrm{ppm}$; above $900 \mathrm{kN}$ the agreement is within + or $-100 \mathrm{ppm}$.

\section{General}

\section{1,591}

\section{AD-A227 806/7}

PC A03/MF A01

(N)

Boulder, CO. Time and Frequency Div.

Cooled Ion Frequency Standard.

Annual rept. 1 Oct $89-31$ Sep 90.

D. J. Wineland. 15 Sep $90,17 p$

Contract N00014-90-F-0004

Keywords: Atomic spectroscopy, Cooling, Doppler effect, Electromagnetism, "Frequency standards, High resolution, lons, Laser applications, Radiation pressure, Residuals, Radiative transfer, Storage, Transitions, Traps, Penning Traps, Laser Cooling, Ion traps, Penning traps.

The purpose of this work is to develop techniques to overcome the fundamental limits of present frequency standards--the second order and residual first-order Doppler shifts. To this end suitable frequency reference transitions are studied for ions which are stored on electromagnetic traps and cooled by radiation pressure to less than 1K. Keywords: Atomic spectroscopy; Doppler narrowing; Doppler shifts; Frequency standards; High resolution spectroscopy; Ion storage; Laser spectroscopy; Penning trap; Laser cooling. (jhd)

\section{1,592}

AD-A242 029/7 PC A02/MF A01 Na.

\section{Liquid and Solid Ion Plasmas.}

\section{Annual rept. 1 Oct $90-31$ Sep 91}

D. J. Wineland, and J. J. Bollinger. 15 Sep 91, 8p Contract N00014-89-F-0020

Keywords: Angular momentum, Cooling, Distribution functions, Electromagnetic fields, Images, Ions, Lasers, Plasmas(Physics), Solids, Spatial distribution, Spectroscopy, Temperature, Velocity, Laser cooling, Penning traps, "Ion plasmas.

Atomic ions which are stored in electromagnetic fields are an example of nonneutral plasmas. Laser techniques allow control of plasma angular momentum and provide plasma cooling to temperatures much less than $1 \mathrm{~K}$. Using imaging techniques, plasma spatial information is achieved. Laser spectroscopic techniques 
allow measurement of plasma velocity distribution functions. Liquid and solid behavior of ion plasmas is studied.

\section{1,593}

\section{AD-A242 030/5}

PC A02/MF A01

National Inst. of Standards and Technology, Boulder. $\mathrm{CO}$.

Cooled Ion Frequency Standard.

Annual rept. 1 Oct $90-31$ Sep 91

D. J. Wineland. 15 Sep $91,8 p$

Contract N00014-90-F-0004

Keywords: Doppler effect, "Frequency standards, lons, Radiation pressure, Residuals, Transitions, "Laser cooling, Penning traps, "Atomic spectroscopy.

The purpose of this work is to develop techniques to overcome the fundamental limits of present frequency standards--the second order and residual first-order Doppler shifts. To this end we study suitable frequency reference transitions in ions which are stored on electromagnetic traps and cooled by radiation pressure to less than $1 \mathrm{~K}$

\section{1,594}

\section{PB90-244575}

(Order as PB90-244526)

Massachusetts Univ., Amherst. Dept. of Physics and Astronomy.

Energy Levels of Atomic AlumInum with Hyperfine Structure.

Quarterly rept.

E. S. Chang. c1990, 7p

Contract DNA-001-85-C-0022

Sponsored by Defense Nuclear Agency, Washington,

DC

Included in Jnl. of Physical and Chemical Reference Data, v19 n1 p119-126 1990. Available from American Chemical Society, 115516 th St., NW, Washington, DC 20036.

Keywords: "Atomic energy levels, Hyperfine structure, Electron transition, Ionization potentials, Spectra, *Aluminum ions.

A new energy level table for Al I has been constructed to include hyperfine structure from observations within the last decade. Improvement in accuracy over olde tables is about an order of magnitude. The analysis of high-I Rydberg levels using the polarization formula results in a new value for the ionization potential which is $0.110 / \mathrm{cm}$ or five standard deviations above the old value. (Copyright (c) 1990 by the U.S. Secretary of Commerce.)

\section{1,595}

\section{PB90-244583}

Japan Atomic Energy Research Inst., Tokai.

(Order as PB90-244526) Spectral Data and Grotrian Diagrams for Highly Ionized Iron, Fe VIII-XXVI.

Quarterly rept.

T. Shirai, Y. Funatake, K. Mori, J. Sugar, W. L.

Wiese, and Y. Nakai. c1990, 149p

Sponsored by Department of Energy, Washington, DC. Prepared in cooperation with Hiroshima-Denki Inst. of Tech. (Japan), and National Inst. of Standards and Technology, Gaithersburg, MD.

Included in Jnl. of Physical and Chemical Reference Data, v19 n1 p127-276 1990. Available from American Chemical Society, 115 16th St., NW, Washington, DC 20036

Keywords: Transition probabilities, Atomic energy levels, Spectra, Tables(Data), Graphs(Charts), "Iron ions, Multicharged ions, Grotrian diagrams, Oscillator strengths.

Wavelengths, energy levels, level classifications, oscillator strengths, and atomic transition probabilities for the iron ions Fe VIII to Fe XXVI are critically reviewed and tabulated. Grotrian diagrams are also presented to provide graphical overviews. The literature has been surveyed to March 1988 . (Copyright (c) 1990 by the U.S. Secretary of Commerce.)

\section{1,596}

\section{PB90-244666}

(Order as PB90-244658)

National Inst. of Standards and Technology (NML), Gaithersburg, MD. Center for Atomic, Molecular and Optical Physics.
Energy Levels of Copper, $\mathrm{Cu} I$ through $\mathrm{Cu} X X \mid X$. Quarterly rept.

J. Sugar, and A. Musgrove. c1990, 90p

Included in Jnl of Physical and Chemical Reference

Data, v19 n3 p527-616 1990. Available from American

Chemical Society, 115516 th St., NW, Washington, DC 20036.

Keywords: "Atomic energy levels, Spectra,

Tables(Data), "Copper ions, Multicharged ions.

The energy levels of the copper atom, in all stages of ionization for which experimental data are available have been compiled. Ionization energies, either experimental or theoretical, and experimental g-factors are given. Leading components of calculated eigenvectors are listed. (Copyright (c) 1990 by the U.S. Secretary of Commerce.)

\section{1,597}

\section{PB90-244690}

(Order as PB90-244658)

Joint Inst. for Lab. Astrophysics, Boulder, CO.

Cross Sections and Swarm Coefficients for $\mathrm{H}+$ $\mathrm{H} 2+, \mathrm{H} 3+, \mathrm{H}, \mathrm{H} 2$, and $\mathrm{H}$-in $\mathrm{H} 2$ for Energies from $0.1 \mathrm{eV}$ to $10 \mathrm{keV}$

Quarterly rept.

A. V. Phelps. c1990, 23p

Included in Jnl. of Physical and Chemical Reference

Data, v19 n3 p653-673 1990. Available from American

Chemical Society, 1155 16th St., NW, Washington, DC 20036 .

Keywords: "Collision cross sections, Momentum transfer, Excitation, Dissociation, "Atom-molecule collisions, "Molecule-molecule collisions, "Ion-molecule collisions, "Hydrogen ions 1 minus, "Hydrogen ions 1 plus, "Hydrogen ions 2 plus, "Hydrogen ions 3 plus, Charge exchange, Rotational states, Vibrational states, Swarm coefficients.

Graphical and tabulated data and the associated bibliography are presented for cross sections for elastic excitation and ionization collisions of $\mathrm{H}(1+), \mathrm{H} 2(1+)$ $\mathrm{H}_{3}(1+), \mathrm{H}_{2} \mathrm{H}_{2}$, and $\mathrm{H}(1-)$ with $\mathrm{H}_{2}$ at laboratory energies from 0.1 to $10 \mathrm{keV}$. Where appropriate, drift ve locities and reaction or excitation coefficients are cal culated from the cross sections and recommended for use in analyses of swarm experiments and electrica discharges. Collisions of electronically excited states with $\mathrm{H} 2$ are not included. (Copyright (c) 1990 by the U.S. Secretary of Commerce.)

101,598

PB91-132290

PC A04/MF A01

National Inst. of Standards and Technology (NML)

Gaithersburg, MD. Center for Radiation Research.

Absorbed-Dose Calibration of Ionization Chambers in a(60)Co Gamma-Ray Beam.

Special pub. (Final)

J. S. Pruitt. Oct 90,56 p NIST/SP-250/40

Also available from Supt. of Docs. as SN003-00303034-1.

Keywords: "Ionization chambers, Radiation dosage, Cobalt 60 Calorimeters, Gamma rays, Water, "Radiation doses, "Calibration, Interlaboratory comparisons, Gamma dosimetry.

Measurement Service C. 1 from the National Institute of Standards and Technology (NIST) publication SP 250 provides calibrations for customer-owned ionization chambers so that they may be used to determine absorbed dose to water in (60)Co gamma-ray beams The calibrations are based on calorimetric measurement of absorbed dose to graphite in a graphite phantom. Transformation of the calibrations to a water phantom is made with a specially-designed graphite ionization chamber, and requires knowledge of photon mass attenuation coefficients and the perturbation of the graphite chamber in the water medium. The determination of these quantities is described in detail along with the operational techniques normally used to transfer the calibration to customer-owned chambers. Appendix A lists experimental data used to test the photon-fluence scaling theorem. Appendices B C and $D$ describe international comparisons of the chamber calibrations, and Appendix $\mathrm{E}$ shows a sample calibration report.

\section{1,599}

PB91-133900

Not available NTIS

National Inst of Standards and Technology (NML), Gaithersburg, MD. Quantum Metrology Div.
Parity-Unfavored Transitions in Resonant Photoemission from $\mathrm{Ar}, \mathrm{Kr}$, and $\mathrm{Xe}$ : Experimental and Theoretical Results.

Final rept.

J. W. Cooper, D. W. Lindle, T. A. Carlson, D. A

Mullins, C. E. Beall, B. W. Yates, J. W. Taylor, and F. A. Grimm. 1990, 10p

Pub. in Jnl. of Electron Spectroscopy and Related Phenomena 51, p397-4061990.

Keywords: "Argon, "Krypton, "Xenon, Synchrotron radiation, Electron transitions, Auger electrons, Angular distribution, Reprints, "Photoemission, Electron spectroscopy, High resolution.

High-resolution angle-resolved electron spectroscopy with synchrotron radiation has been used to study the angular distributions of resonant-Auger processes near the core-level thresholds $\mathrm{Ar} 2 \mathrm{p}, \mathrm{Kr} 3 \mathrm{~d}$ and $\mathrm{Xe} 4 \mathrm{~d}$ Angular-distribution parameters (beta) were measured for all resolved peaks in the electron spectra, and large negative values of beta were found for some peaks. A quantitative method for calculating the angular-distribution parameters is described and results of calculations for the $\operatorname{Ar} 2 \mathrm{p}(3 / 2)->4 s$ resonant-Auger spectrum are compared with the experimental results.

101,600

PB91-134486

Not available NTIS

National Inst. of Standards and Technology (NEL)

Boulder, CO. Electromagnetic Technology Div.

High-Resolution, Tunneling-Stabilized Magnetic Imaging and Recording.

Final rept

J. Moreland, and P. Rice. 1990, 3p

90.

Keywords: "Magnetic recording, Metal films, Iron, $\mathrm{Re}$ prints, Surface magnetism, Imaging techniques, High resolution, Scanning tunneling microscopy, Magnetic films.

The authors have used a scanning tunneling microscope (STM) to record and image magnetic regions on the surface of a hard disk. The usual rigid STM tip was replaced by a compliant magnetized Fe film tip. As a result, tunneling images were combinations of the sur face topography and variations in the magnetic force between the Fe film tip and the disk surface. The authors believe that the recording process relied on maintaining the proximity of the magnetized Fe film tip near the disk surface. Apparently, the magnetic field was focused near the Fe film tip with sufficient intensity to change the surface magnetization of the disk. The authors have recorded spots on the disk within a 500 $\mathrm{nm} \times 500 \mathrm{~nm}$ area. These spots were subsequently imaged with the same STM tip. Their best magnetic image resolution was $20 \mathrm{~nm}$. The compliance of the Fe film tips was such that image contrast due to variation of the magnetic force on the tip corresponded to $\mathrm{z}$ motions of the piezoelectric translator as large as $50 \mathrm{~nm}$.

101,601

PB91-134502

Not available NTIS

National Inst. of Standards and Technology (NML), Boulder, CO. Thermophysics Div.

Thermal Conductivity of a Moderately Dense Gas. Final rept.

C. A. Nieto de Castro, D. G. Friend, R. A. Perkins, and J. C. Rainwater. $1990,8 p$

Pub. in Chemical Physics 145, p19-26 1990.

Keywords: "Thermal conductivity, "Gases, Nitrogen, Argon, Reprints, Lennard-Jones potential, Virial coefficients, Density dependence.

Recent extensive measurements of the thermal conductivity of argon and nitrogen have enabled us to evaluate the first density correction as a function of temperature and to compare it with a theoretical prediction. The prediction follows from a previously published microscopically based theory, which includes effects due to collisional transfer, collisions among three molecules, and monomer-dimer collisions. The first density correction was evaluated for a Lennard-Jones interaction potential. An ad hoc modification of the theory accounts for contributions to the thermal conductivity from internal degrees of freedom. The comparison shows good agreement over a wide range of temperatures. 


\section{PHYSICS}

\section{General}

National Inst. of Standards and Technology (NML), Boulder, $\mathrm{CO}$. Thermophysics Div.

Thermal Conductivity and Heat Capacity of Gaseous Argon.

Final rept.

H. M. Roder, R. A. Perkins, and C. A. Nieto de

Castro. 1989, 24p

Pub. in International Jnl. of Thermophysics 10, n6 p1141-1164 Nov 89

Keywords: "Argon, "Thermal conductivity, "Thermal diffusivity, "Specific heat, Gases, Reprints, Transient hot wire technique.

The paper presents new absolute measurements of the thermal conductivity and of the thermal diffusivity of gaseous argon obtained with a transient hot-wire instrument. The authors measured seven isotherms in the supercritical dense gas at temperatures between 157 and $324 \mathrm{~K}$ with pressures up to $70 \mathrm{MPa}$ and densities up to $32 \mathrm{~mol} / \mathrm{L}$ and five isotherms in the vapor at temperatures between 103 and $142 \mathrm{~K}$ with pressures up to the saturation vapor pressure. The instrument is capable of measuring the thermal conductivity with an accuracy better than $1 \%$ and thermal diffusivity with an accuracy better than $5 \%$. Heat capacity results were determined from the simultaneously measured values of thermal conductivity and thermal diffusivity and from the density calculated from measured values of pressure and temperature from an equation of state. The heat capacities presented in the paper, with a nominal accuracy of $5 \%$, prove that heat capacity data can be obtained successfully with the transient hot wire technique over a wide range of fluid states. The technique will be invaluable when applied to fluids which lack specific heat data or an adequate equation of state.

101,603

PB91-134643

Not available NTIS

National Inst. of Standards and Technology (NML), Boulder, CO. Quantum Physics Div.

Lunar Gravitational Wave Antenna Using a Laser Interferometer.

Final rept.

R. T. Stebbins, and P. L. Bender. 1990, 14p

Pub. in AIP Conference Proceedings 202, Physics and Astrophysics from a Lunar Base, NASA Workshop (1st), Stanford, CA., May 19-20, 1989, p188-201 1990.

Keywords: Angular resolution, Sensitivity, Reprints, * Gravitational wave antennas, Laser interferometers, Lunar bases, Lunar surface.

A moon-based laser interferometer for detecting gravitational radiation could detect signals in the band 0.1 to $10,000 \mathrm{~Hz}$. A preliminary evaluation of the noise budget for an optimistic antenna design is reported here and compared to that for other planned gravitational wave interferometers. Over most of the frequency range, the sensitivity is controlled by the thermal noise in the test mass suspensions. As pointed out by others, the most important role of a lunar antenna would be the improved angular resolution made possible by the long baseline to terrestrial antennas.

101,604

PB9 1-144360

PC A03/MF A01

National Inst. of Standards and Technology (NEL), Gaithersburg, MD. Center for Computing and Applied Mathematics.

Space Marching Difference Schemes in the Nonlinear Inverse Heat Conduction Problem.

A. S. Carasso. 21 Nov 90, 41 p NISTIR-4482

Keywords: *Conduction, "Gun barrels, Nonlinear differential equations, Finite difference theory, One-dimensional calculations, Mathematical models, Heat transfer, Fourier analysis, Error analysis, III posed problems, Inverse problems.

The Lax-Richtmyer theory is used to study the error amplification properties of 18 space marching finite difference schemes, for the 1-D nonlinear inverse heat conduction problem. A non-dimensional parameter Omega, involving the time step Delta $t$, the effective thermal diffusivity alpha, and the distance I from the sensor to the active surface, provides a measure of the numerical difficulty of the inverse calculation. All 18 schemes are unstable and blow-up like 10(sup lambda Omega), where the constant lambda depends on the particular numerical method. However, there are substantial differences in the lambda's, and some newly constructed algorithms, employing forward time differences at nonadjacent mesh points, are shown to produce relatively low values of lambda. Using synproduce relatively low values of lambda. Using syn- considered for which Omega $=25$. This problem simulates heat transfer in gun barrels when a shell is fired. It is shown that while most of the 18 schemes cannot recover the thermal pulses at the gun tube wall, two of the new methods provide reasonably accurate results. A tendency to underestimate peak values in fast, narrow thermal pulses, is also noted.

101,605

PB9 1-147116

Not available NTIS

National Bureau of Standards (NML), Gaithersburg,

MD. Radiation Physics Div.

Giant Resonances in the Transitlon Regions of the Periodic Table.

Final rept.

C. W. Clark, and T. B. Lucatorto. 1986, 15p

Sponsored by Air Force Office of Scientific Research, Bolling AFB, DC.

Pub. in Giant Resonances in Atoms, Molecules, and Solids, p137-151 1986.

Keywords: "Transition metals, "Rare earth elements, Atomic orbitals, Giant resonance, Atomic structure, Reprints, Orbital collapse.

The behavior of the transition elements and the lanthanides is described in terms of an independent electron model. The properties of the transition elements and the lanthanides which are associated with orbital collapse are described in terms of an independent electron model. Special attention is focused on elements at the beginning of the transition period, such as $\mathrm{Cs}, \mathrm{Ba}$, and $\mathrm{La}$, to show that the dramatic changes in such properties as the photoabsorbtion can be explained in terms of orbital collapse, provided one carefully treats exchange and correlation effects. Theoretical models are compared with experimental results in isoelectronic and isonuclear systems.

101,606

B9 1-147124

Not available NTIS

National Bureau of Standards (NML), Gaithersburg,

MD. Electricity Div.

Adjustment of the Fundamental Physical Constants: A Report of the CODATA Task Group on Fundamental Constants, 1986.

Final rept.

E. R. Cohen, and B. N. Taylor. 1986, 39p

Sponsored by Rockwell International, Thousand Oaks, CA. Science Center.

Pub. in CODATA Bulletin, n63 39p 1986.

Keywords: "Fundamental constants, Least squares method, Adjusting, Correction, Reprints.

The paper summarizes in some detail the 1986 leastsquares adjustment of the fundamental physical constants carried out by the authors under the auspices tand mental constants, and gives tables of recommended values of the constants for international use resulting from the 1986 adjustment. The 1986 CODATA set of values entirely replaces its immediate predecessor, that recommended for international use by CODATA in 1973. The latter was based on the 1973 least-squares adjustment of the fundamental constants which was also carried out by the authors under the auspices and guidance of the Task Group.

101,607

PB91-147173

Not available NTIS

National Inst. of Standards and Technology (NML), Gaithersburg, MD. Quantum Metrology Div. Selective Excitation of X-ray Emission Spectra. Final rept.

P. L. Cowan. 1990, 7p

Pub. in Physica Scripta T31, p112-118 1990.

Keywords: " $X$ ray spectra, Synchrotron radiation, $X$ ray absorption, $X$ ray fluorescence, Emission spectra, Excitation, Reprints.

While $X$-ray emission spectroscopy is a relatively old technique, it takes on important new characteristics when samples are selectively excited by a carefully prepared beam of X-rays. The method is made practical by the availability of synchrotron radiation sources and it extends the capabilities of the traditional technique. Both the energy and the polarization of the excitation $X$-rays can have significant effects on the emission spectra. Selective control of these parameters can provide specific information on both the electronic structure and the atomic structure of molecules or solids. Examples of the new types of studies include: satellite free $X$-ray emission, multi-vacancy excitation, polarized $X$-ray excitation and emission, and state selective excitation of neutral atoms and molecules.
101,608

PB91-147249

Not available NTIS National Bureau of Standards (NML), Gaithersburg MD. Ionizing Radiation Div.

Preparation and Properties of Monomolecular Films for Use as Radioactive Source Mounts.

Final rept.

L. J. Dobrilovic, M. Simovic, D. J. Bek-Uzarov, J. M.

R. Hutchinson, and W. B. Mann. 1988, 7p

Pub. in Applied Radiation and Isotopes 39, n9 p999. 10051988.

Keywords: "Monomolecular films, "Radioactivity, *Mountings, Chemisorption, Adsorption, Reprints, Calcium stearate, Multilayers.

The physical properties of the calcium-stearate monomolecular layer have been examined using an automatic torsion balance. These layers were obtained by adsorption of $\mathrm{Ca}(2+)$ ions from $0.0001 \mathrm{M} \mathrm{CaCl} 2$ onto the stearic-acid monomolecular layer. The introduction also briefly reviews previous work on the preparation of monomolecular layers and their use, for some 50 years, for radioactivity measurements.

\section{1,609}

PB91-147355

Not available NTIS

National Inst. of Standards and Technology (NML) Gaithersburg, MD. Center for Radiation Research.

Electrofission in the Quasifree and Delta Regions. Final rept.

K. Hansen, J. O. Adler, K. I. Blomqvist, D. Nilsson, A Sandell, B. Schroder, W. R. Dodge, J. W. Lightbody,

J. S. Connell, J. R. Calarco, J. P. Connelly, F. W.

Hersman, W. Kim, and M. Leuschner. 1990, $6 p$

Pub. in Physical Review C 41, n4 p1619-1624 Apr 90

Keywords: "Uranium 238, "Uranium 233, "Fission cross sections, "Electrofission, MeV range 100-1000, Reprints

The coincident electrofission (e,e'f) cross sections of $(233,238) \mathrm{U}$ were measured in the excitation range $150-550 \mathrm{MeV}$. The probability of fission was found to be near unity in the delta resonance region (250-550 $\mathrm{MeV}$ ), but less than unity in the quasifree scattering region $(150-250 \mathrm{MeV})$. The data are analyzed with a model based on the quasifree and delta reaction mechanisms. An excitation energy of $25 \mathrm{MeV}$ is found for the residual nucleus in the quasifree region and over $40 \mathrm{MeV}$ in the delta region.

101,610

PB91-147462

Not available NTIS

National Inst. of Standards and Technology (NML) Gaithersburg, MD. Atomic and Plasma Radiation Div. Transitions and Energy Levels for Cu-Like $\mathrm{Yb}(41+), \mathrm{Ta}(44+)$, and $\mathrm{U}(63+)$.

Final rept.

D. R. Kania, B. J. MacGowan, C. J. Keane, C. M.

Brown, and J. O. Ekberg. 1990, 4p

Sponsored by Department of Energy, Washington, DC. Pub. in Jnl. of the Optical Society of America B 7, n10 p1993-1996 Oct 90.

Keywords: *Ytterbium ions, "Tantalum ions, "Uranium ions, "Atomic energy levels, "Electron transitions, Plasma spectra, Multicharged ions, Soft $x$ rays, Laserproduced plasma, Reprints.

Transitions from the $n=4,5$, and 6 excited levels of the $\mathrm{Cu}$-like ions $\mathrm{Yb}(41+), \mathrm{Ta}(44+)$, and $\mathrm{U}(63+)$ were observed in the spectra from plasmas that were produced by one or two beams of the Nova laser. Wave lengths in the range 9-140 A were measured with an uncertainty of + or $-0.015 \mathrm{~A}$ and were compared with previously measured and predicted wavelengths. The energy levels were derived from the observed wavelengths.

101,611

PB91-147496

Not available NTIS

National Inst of Standards and Technology (NEL)

Boulder, CO. Electromagnetic Technology Div.

Global Stability of the Chaotic State Near an Interi-

or Crisis.

Final rept.

R. L. Kautz. $1989,20 p$

Contract N00014-85-F-0085

Sponsored by Office of Naval Research, Arlington, VA

Pub. in Structure, Coherence and Chaos in Dynamical

Systems, p207-226 1989. 
Keywords: *Chaos, Josephson junctions, Thermal noise, Attractors, Stability, Reprints.

In dissipative systems, chaotic trajectories are locally unstable in that they show a sensitive dependence on initial conditions but globally stable in that they are represented by attractors. The global stability of the chaotic state is examined in the neighborhood of an interior crisis using the rf-biased Josephson junction as an example. A measure of global stability is developed by considering the response of the system to therma noise. In the presence of noise, the system occasionally escapes from the region of state space containing the attractor and the temperature dependence of the average escape time is characterized by an activation energy. The activation energy is a useful measure of the global stability of the chaotic state, being analo gous to the barrier height for a particle in a potentia well.

101,612

PB91-147587

Not available NTIS

National Bureau of Standards (NML), Gaithersburg,

MD. Center for Radiation Research.

Possible (e,e'2N) Studies at CEBAF.

Final rept.

J. W. Lightbody. 1988, 9p

Pub. in Proceedings of Workshop on Perspectives in Nuclear Physics at Intermediate Energies (3rd), Trieste, Italy, May 18-22, 1987, p455-473 1988.

Keywords: "Knock-out reactions, "Electron scattering, CEBAF accelerator, High energy particles, Spectrometers, Hadrons, Reprints.

Multi-hadron knockout by high energy electron scattering will be one of the high priority experimental programs at CEBAF. The motivation for such reaction studies has both an experimental and theoretical side and is discussed herein. The equipment required for such studies, the kinematics employed, estimates of counting rates, and related issues are also discussed.

101,613

PB91-147959

Not available NTIS

National Inst. of Standards and Technology (NML),

Gaithersburg, MD. Center for Radiation Research.

Muon Stlcking in Catalysed d-t Fusion: Nuclear Resonance Effect.

Final rept.

J. Rafelski, B. Muller, M. Danos, G. Velarde, and E.

Minguez. 1987, 6p

Pub. in Proceedings of International Conference on Emerging Nuclear Energy Systems (4th). Madrid Spain, June 30-July 4, 1986, p383-388 1987.

Keywords: "Muon-catalyzed fusion, Deuteron reactions, Triton reactions, Reprints, Nuclear resonance.

The kinetic energy of a muon attached to the recoiling particle in $d$-t fusion reduces the energy available in the nuclear fusion channel by about $(90+$ or -50 deV.) The highly resonant $d$-t reaction amplitude is deuned and therefore the reaction channel is further suppressed in comparision to the nonsticking reaction.

\section{1,614}

PB91-148684 National Inst. of Standards and Technology (NML), Boulder, CO. Quantum Physics Div.

Close-Coupling Calculation for the (2)(Sigma sub u) $(+)$ State of ( $\mathrm{H}$ sub 2$)(-)$

Final rept.

T. Gorczyca, and D. W. Norcross. 1990, 7p

Grant NSF-PHY86-04504

Sponsored by National Science Foundation, Washing ton, DC

Pub. in Physical Review A 42, n9 p5132-5138, 1 Nov 90.

Keywords: *Hydrogen, Molecular structure, Negative ions, Bound state, Reprints.

The authors demonstrate the utility of the close-coupling approximation, usually applied to scattering problems, for calculations of molecular bound states. Their primary test case is the doublet Sigma (sub u)(sup +) state of $\mathrm{H} 2(1-)$. While limited to Hartree-Fock accuracy, their analysis of this resonant state within the bound region parallels closely the results from more precise quantum-chemical calculations. Specifically, the crossing between the neutral and negative-ion curves occurs at an internuclear separation of 3.025 a.u. in their calculation, in good agreement with a multireference configuration-interaction approach of previous authors. The shape and total energy of the anion curve, when corrected for by the correlation energy of the target at the crossing, is also in good agreement with earlier results. The method is then extended into the unbound or resonant-state region by studying the shape-resonance behavior of scattering eigenphase sums.

101,615
PB91-148692

Not available NTIS Ptandards (NML), Gaithersburg, MD. Quantum Metrology Group.

Determination of the Neutron Mass and Implications for Fundamental Constants.

G. L. Greene, E. G. Kessler, M. S. Dewey, and R. D. Deslattes. 1987, 5p

Pub. in Proceedings of International Conference on Neutron Physics, Kiev, Ukraine, USSR, September 21 25, 1987, p76-80.

Keywords: Quantum electrodynamics, Fundamental constants, Rest mass, Reviews, Tests, Reprints, Neutron mass.

The neutron mass, while not well understood theoretically, is known to quite high accuracy by a combination of absolute gamma ray spectroscopy and high accuracy mass spectroscopy. A review of the current experimental status of the neutron mass is given, along with a discussion of the prospects for improvement in the near future. The implication of these results for the fundamental constants as well as for tests of QED is briefly reviewed.

\section{1,616}

91-148718

Not avallabIe NTIS

National Inst. of Standards and Technology (NEL).

Boulder, CO. Thermophysics Div.

Anisotropic Structure of a Simple Liquid.

Final rept.

H. J. M. Hanley. $1988,16 p$

Sponsored by Department of Energy, Washington, DC Office of Basic Energy Sciences.

Pub. in Proceedings of the Winter Meeting on Statistical Physics Lectures on Thermodynamics and Statistical Mechanics (17th), Oaxtepec, Mexico, January 5-8, 1988 , p109-124.

Keywords: "Non-Newtonian fluids, Couette flow, Structure factors, Reprints, Molecular dynamics.

Non-Newtonian behavior in a sheared simple fluid is reviewed briefly. The author points out that such behavior is more often associated with fluids of very complex structure, such as polymers. The concept of the Maxwell relaxation time is introduced and it is shown that the product of the time and a shear rate is a key parameter that indicates if a fluid will display non-Newtonian characteristics, regardless of molecular structure. The author discusses the distorted structure factor of a dense two-dimensional soft disk liquid un dergoing Couette flow. The structure factor is determined experimentally by light scattering from the output of a nonequilibrium molecular dynamic simula tion. The distorted pair correlation function is also discussed.

\section{1,617}

Not available NTIS National Inst. of Standards and Technology (NML) Gaithersburg, MD. Atomic and Plasma Radiation Div. Precise Experimental Test of Calculated Two-Electron Lamb Shifts in Helium.

Final rept.

C. J. Sansonetti, J. D. Gillaspy, and C. L. Gromer.

$1990,4 p$

Pub. in Physical Review Letters 65, n20 p2539-2542, 12 Nov 90.

Keywords: "Helium, "Lamb shift, Quantum electrodynamics, Electron transitions, Metastable state, Laser spectroscopy, Atomic energy levels, Tests, Reprints.

The authors have measured the wave number of the (4) He 2 singlet $S->3$ singlet $P$ transition by Dopplerfree spectroscopy in a metastable helium beam. The result, $19931.924794(45) / \mathrm{cm}$, represents an order of magnitude improvement over previous measurements and provides a sensitive test of two-electron QED effects. The result shows an unexpectedly large deviation from theoretical prediction. The authors derive improved term values for a number of low-lying helium levels. All levels with $n=2$ show significant deviations from theory that are correlated with the size of calcu lated two-electron contributions to the Lamb shifts.

\section{1,618}

PB91-149245
National Inst. of Standards and Technology (NML) Boulder, CO. Quantum Physics Div.

Nonresonant Charge Transfer in the Threshold Region for $(3) \mathrm{He}(+)+(4) \mathrm{He}($ sub $<-$ sup- $>$ ) (3) He $+(4) \mathrm{He}(+)$.

Final rept

M. M. Schauer, S. R. Jefferts, and G. H. Dunn. 1990 6p

Grant NSF-PHY86-0450

Sponsored by National Science Foundation, Washing ton, DC.

Pub. in Physical Review A 42, n9 p5332-5337, 1 Nov 90.

Keywords: "Helium, *Charge transfer, Isotope effects, Helium 3, Helium 4, Cryogenics, Reprints

Measurements of the ratio of the rate coefficient for charge transfer between (3) $\mathrm{He}(1+)$ and (4) $\mathrm{He}$ to that for the reverse process have been made in the temperature range $8 \mathrm{~K}$ approx $=$ or $<\mathrm{T}$ approx $=$ or $<$ $80 \mathrm{~K}$. A 1.1-meV difference in the ionization potentia of these species leads to an endothermicity in the for ward direction of the reaction, and these experiments constitute the only measurements of a threshold re sulting from such a small endothermicity. In addition, a limited number of direct measurements of the rate coefficients for these processes have been made and are included here. The measurements were done in a cooled Penning trap using Fourier transform spectroscopy of the axial trapping frequency. The measurements are compared to theoretical estimates.

101,619

PB91-149971

Not available NTIS

National Inst. of Standards and Technology (NML) Boulder, CO. Quantum Physics Div.

Optical Potential Approach to Electron and Positron Scattering from Noble Gases. 2. Neon.

Final rept.

K. Bartschat, R. P. McEachran, and A. D. Stauffer. 1990, 7p

Pub. in Jnl. of Physics B: Atomic and Molecular Physics 23, p2349-2355 1990 .

Keywords: "Electron-atom collisions, "Positron-atom collisions, *Neon, Electron scattering Differential cross sections, Total cross sections, Optical models, Reprints.

The authors have extended their previous work on an optical potential method to electron and positron scattering from neon. In addition, they have modified the approach to include the diagonal potentials previously neglected. This required the use of a numerical form of the Green function. Comparison with recent experimental measurements with positrons indicates the necessity of including absorption effects at higher energies, although these effects appear to be smaller for neon than they were for argon. At energies just above the inelastic excitation thresholds, however, the picture is not so clear at the moment.

101,620

PB91-150011

Not available NTIS

National Inst. of Standards and Technology (MSEL), Gaithersburg, MD. Reactor Radiation Div.

Nonlocal Phase Shifts Induced by Static Electric Fields in Neutron Interferometers When the Path Enclosed Charge Vanishes.

Final rept.

R. C. Casella. $1990,4 p$

Pub. in Physical Review Letters 65, n18 p2217-2220, 29 Oct 90

Keywords: Electric fields, Phase shift, Reprints, "Neutron interferometry.

An experiment, analogous to that of Cimmino et al., is proposed to measure the phase shift induced by static electric fields on the passage of neutrons through an interferometer when the path-enclosed linear charge density is zero. A finite shift is predicted and discussed in light of the Aharonov-Casher result.

101,621

PB91-158634

Not available NTIS

National Bureau of Standards (NML), Gaithersburg, MD. Center for Basic Standards.

Accurate Spectroscopy In the X-ray Region. Final rept.

R. D. Deslattes. $1988,8 p$

Pub. in Nuclear Instruments and Methods in Physics

Research B31, n1-2 p51-58 Apr 88 . 


\section{PHYSICS}

\section{General}

Keywords: "X ray spectroscopy, Multicharged ions, Heavy ions, Precision, Accuracy, Reprints.

There is currently not only keen interest in accurate spectroscopy of one- and two-electron ions but also the by now well-founded hope that these spectra can be produced under conditions where Doppler and spectator electron problems are under adequate control. These developments will require that one confront problems of wavelength normalization at a more refined level than has been necessary in the past. The report briefly outlines the present-day situation in the case of using normal $x$-ray lines as references, and the steps which might be taken to transcend limitations inherent in the approach.

\section{1,622}

PB91-158881

Not available NTIS

National Bureau of Standards (NML), Gaithersburg, MD. Molecular Spectroscopy Div.

Explicit Mapping of the Set of Dynamical Symmetries onto the Set of Equivalent Lagrangians for Systems with One Degree of Freedom.

Final rept.

C. Leubner, and M. A. M. Marte. 1986, 6p

Pub. in Physics Letters A 117, n2 p67-72, 28 Jul 86.

Keywords: "Mechanics, Lagrangian functions, Dynamics, Reprints, One degree of freedom.

For newtonian systems with one degree of freedom, for which the existence of a relationship between the dynamical symmetries of the equation of motion and its equivalent lagrangian descriptions is well known, an explicit method is given for establishing the lagrangian associated with any particular dynamical symmetry.

101,623

PB91-159236

Not available NTIS

National Inst. of Standards and Technology (NEL)

Gaithersburg, MD. Electricity Div.

Preface to Book Entitled 'Nonequilibrium Effects in Ion and Electron Transport'.

Final rept

R. J. Van Brunt. 1990, 2p

Pub. in Nonequilibrium Effects in Ion and Electron Transport, pv-vi 1990

Keywords: "Gas discharges, "Electron transport Breakdown(Electronic threshold), Electric discharges, Collision cross sections, Electron collisions, Atomic collisions, Reprints, "Ion transport, Swarm seminar.

The volume presents the contributions of the participants in the Sixth International Swarm Seminar, held August 2-5, 1989, at the Webb Institute in Glen Cove, New York. The Swarm Seminars are traditionally held as relatively small satellite conferences of the International Conference on the Physics of Electronic and Atomic Collisions (ICPEAC) which occurs every two years. The 1989 ICPEAC took place in New York City prior to the Swarm Seminar. The focus of the Swarm Seminars has been on basic research relevant to understanding the transport of charged particles, mainly electrons and ions, in weakly ionized gases. This is a field that tends to bridge the gap between studies of fundamental binary atomic and molecular collision processes and studies of electrical breakdown or discharge phenomena in gases. Topics included in the 1989 seminar ranged the gamut from direct determinations of charged-particle collision cross sections to use of cross sections and swarm parameters to model the behavior of electrical gas discharges.

101,624

PB91-15925

Not available NTIS

National Bureau of Standards (NML), Gaithersburg.

MD. Office of Standard Reference Data.

Steam Tables.

Final rept.

H. J. White. $1987,10 p$

Pub. in Encyclopedia of Physical Science and Technology, v13 p328-337 1987.

Keywords: "Steam tables(Thermodynamics), Physical properties, Encylopedias, Reprints.

Steam tables are defined and a brief history of the development is given. Emphasis is based on steam tables receiving international acceptance, i.e., those of the International Association for the Properties of Steam (IAPS) and its predecessors. A listing of current releases from IAPS, including automated versions and archival documents supporting those releases, are available.
101,625

PB91-159285

Progress a. Time and Frequency Div.

, NIST on Absolute Frequency Standards Using Stored lons.

Final rept.

D. J. Wineland, J. C. Berquist, J. J. Bollinger, W. M. Itano, D. J. Heinzen, S. L. Gilbert, C. H. Manney, M. G Raizen, and C. S. Weimer. 1990, 6p

See also PB90-188616. Sponsored by Office of Naval Research, Arlington, VA., and Air Force Office of Scientific Research, Bolling AFB, DC .

Pub. in Proceedings of European Frequency and Time Forum (4th), Neuchatel, Switzerland, March 13-15, 1990, p267-272.

Keywords: "Atomic clocks, *Frequency standards, "Standards, "Ion storage, Atomic spectroscopy, High resolution, Laser spectroscopy, Beryllium 9, Mercury 199, Reprints, Ion storage, Penning traps, Paul traps, Laser cooling.

Experiments at NIST, whose goal is to realize írequency standards of high accuracy using stored ions, are briefly summarized. In one experiment, an rf oscillator is locked to a nuclear spin-flip hyperfine transition in (9) $\mathrm{Be}(1+)$ ions which are stored in a Penning trap and sympathetically laser-cooled. Uncertainty in Doppler shifts is estimated to be less than $5 \times 10$ (sup -15). In a second experiment, a stable laser is used to probe an electric quadrupole transition (frequency $=1.07 \times$ 10 (sup 15) $\mathrm{Hz}$ ) in a single laser-cooled $(199) \mathrm{Hg}(1+)$ ion stored in a Paul trap. The measured $Q$ value of the transition is approximately 10 (sup 13).

101,626

PB91-159301

Not available NTIS

National Bureau of Standards (NML), Gaithersburg, MD. Radiation Physics Div.

Photoionization and Collisional Ionization of Excited Atoms Using Synchrotron and Laser Radiations.

Final rept.

F. J. Wuilleumier, D. L. Ederer, and J. L. Picque.

1987, 90p

Pub. in Advances in Atomic and Molecular Physics 23, p197-286 1987.

Keywords: "Excited states, "Photoionization, Laser radiation, Synchrotron radiation, Autoionization, Atoms, Reprints, Collisional ionization.

The article is devoted to a description of various ionization processes which may be studied in excited atoms. Some of these processes are: photoionization, autoionization, and collisional ionization. The development of the field has been accelerated by combining tunable lasers, which excite the atomic species, with synchrotron radiation, which probes the excited atoms. The authors concentrate on the two-step photoionization in free atoms where one electron is promoted to an outer orbital and another is promoted either to a highly excited state which subsequently autoionizes, or to a continum state.

101,627

PB91-161976

National Inst. of Standards and Technology (NEL),

Gaithersburg, MD. Statistical Engineering Div.

Cross-Validation Procedure for Stopping the EM Algorithm and Deconvolution of Neutron Depth Profiling Spectra.

Final rept.

K. Coakley. 1991, 7

Pub. in IEEE (Institute of Electrical and Electronics Engineers) Transactions on Nuclear Science 38, n1 p9. 15 Feb 91.

Keywords: "Neutron spectra, "Image reconstruction, Stopping rules(Mathematics), Iterations, Reprints, Emission tomography, EM algorithm, Deconvolution, Depth profiles.

The iterative EM algorithm is used to deconvolve neutron depth profiling spectra. Because of statistical noise in the data, artifacts in the estimated particle emission rate profile appear after too many iterations of the EM algorithm. To avoid artifacts, the EM algorithm is stopped using a cross-validation procedure. The data are split into two independent halves. The EM algorithm is applied to one half of the data to get an estimate of the emission rates. The algorithm is stopped when the conditional likelihood of the other half of the data passes through its maximum. The roles of the two halves of the data are then switched to get a second estimate of the emission rates. The two estimates are then averaged.

\section{1,628}

PB91-162123

Not available NTIS

National Inst. of Standards and Technology (NML) Boulder, CO. Time and Frequency Div.

Quantum Optics of Single, Trapped lons.

Final rept.

W. M. Itano, J. C. Bergquist, F. Diedrich, and D. J.

Wineland. 1990,5 p

Sponsored by Air Force Office of Scientific Research Bolling AFB, DC., and Office of Naval Research, Arlington, VA

Pub. in Coherence and Quantum Optics VI, p539-543 1990.

Keywords: "Mercury ions, "Ion storage, "Quantum optics, Trapping(Charged particles), Photons, Reprints, Laser cooling, Ion traps.

Quantum jumps in laser-cooled, trapped, mercury ions were observed. These quantum jumps were used to infer some quantum properties of the electromagnetic field, such as photon antibunching and sub-Poissonian photon statistics. A single mercury ion was cooled to the ground (zero-point) energy in the harmonic effective potential of the trap.

\section{1,629}

\section{PB91-167205}

PC A03/MF A01

National Inst of Standards and Technology (NEL) Gaithersburg, MD. Center for Computing and Applied Mathematics.

Uncertainty and Accuracy in Physical Measurements.

Special pub. (Final).

H. H. Ku. Dec 90,14p NIST/SP-805

Also available from Supt. of Docs.

Keywords: "Uncertainty, "Accuracy, Measurement Recommendations, Standards, Calibration.

The formulation of the 'uncertainty' of a reported value always involves a certain degree of arbitrariness, de pending primarily on how the value is going to be used. Currently there are at least two schools of thought on the subject: physicists interested in detecting differences among result are in favor of the recommendations of the International Bureau of Weights and Meas ures (BIPM/CIPM 1981), whereas those involved in calibration work and routine measurements follow the orthodox method (see e. NBS Special Publication 644). In the note, the suggestion is made to use the word 'accuracy/inaccuracy' for standards that follow BIPM's recommendation, and the word 'uncertainty' for standards that follow the orthodox method. It is believed that the use of different terms for distinct purposes will resolve some of the basic difficulties facing international groups who are attempting to write their own standards.

101,630

PB91-174722

Not available NTIS

National Inst. of Standards and Technology (NML), Boulder, CO. Quantum Physics Div.

Gyroscope-Weighing Experiment Revisited -- with a Null Result.

Final rept.

J. E. Faller, W. J. Hollander, P. G. Nelson, and M. P. McHugh. 1990, 5p

Pub. in Proceedings of Recontre de Moriond New and Exotic Phenomena (25th), Les Arcs, Savoie, France, January 20-27, 1990, p197-201.

Keywords: "Weight reduction, "Gyroscopes, Spinning(Motion), Experimentation, Gravity, Reprints.

A recent experiment that reported finding an anomalous weight reduction for a spinning gyroscope weighed on a pan balance has been repeated during six days in the laboratory. No anomalous weight changes of the magnitude reported, were found that depended on rotor speed and/or its direction of rotation about the vertical axis.

101,631

PB91-174748

National Inst. of Standards and Technology (NEL)

Boulder, CO. Electromagnetic Technology Div. 
Anomalous Magnetoresistance in Al/Al-Alloy Composite Conductors.

Final rept.

F. R. Fickett, and C. A. Thompson. 1990, $8 p$

Sponsored by Wright Research and Development Center, Wright-Patterson AFB, OH

Pub. in Advances in Cryogenic Engineering (Materials), v36 p671-678 1990.

Keywords: "Magnetoresistance, Electric conductors, Composite materials, Aluminum, Cryogenics, Anomalies, Reprints.

The transverse magnetoresistance of several composite conductors containing a large single filament of pure aluminum in a matrix of $\mathrm{Al}-\mathrm{Fe}-\mathrm{Ce}$ has been measured at $4 \mathrm{~K}$ to fields of $10 \mathrm{~T}$. The magnetoresistance (delta $R / R$ ) of the composite is very large, rising to 55 in the 'worst' case. Previous measurements on pure polycrystalline aluminum have always shown a rapidly saturating behavior with a very small linear component; delta A/R rarely exceeds a value of 5-6. In addition, the magnetoresistance of the composite samples shows a structure as the field is rotated around the current axis.

\section{1,632}

PB91-174755

Not available NTIS

National Inst of Standards and Technology (NML) Gaithersburg, MD. Ionizing Radiation Div.

New Test of Quantum Mechanics: Is Planck's Constant Unlque.

Final rept.

E. Fischbach, G. L. Greene, and R. J. Hughes. 1991, $4 \mathrm{p}$

Sponsored by Department of Energy, Washington, DC Pub. in Physical Review Letters 66, n3 p256-259, 21 Jan 91.

Keywords: *Quantum mechanics, "Conservation laws, "Plancks constant, Fundamental constants, Invariance, Tests, Reprints

The possibility that different realms of physics are described by distinct quantization constants is considered. From the consistency of existing data, the authors infer limits on the differences between hypothetically distinct quantization constants for different elementary particles. Since the existence of multiple Planck constants implies violations of space-time symmetries, these limits may be viewed as precise tests of fundamental conservation laws, including the conservation of linear momentum and energy.

101,633

PB91-174888

Not available NTIS

National Inst. of Standards and Technology (NML) Gaithersburg, MD. Surface Science Div.

Instrumentation of X-ray Beam Lines with PIN Diode Detectors.

Final rept.

T. J. Jach. 1990,40

Pub. in Nuclear Instruments and Methods in Physics Research A299, p76-79 1990.

Keywords: "Synchrotron radiation sources, " $X$-ray detection, "PIN diodes, Silicon diodes, Photodiodes, Performance, Reprints, Beamlines.

Much of the $X$-ray work on synchrotron-radiation beam lines is still done using ion chambers as detectors. Silicon PIN photodiodes offer considerable advantages over ion chambers for many applications. In addition to greater efficiency over a wide energy range (1-20 keV). they possess a flat configuration, large areas, an $a b$ sence of bias requirements, high dynamic range, and compatability with ultrahigh vacuum. The researchers have characterized the properties of several commercial PIN photodiodes at X-ray energies, have had diodes commercially produced which were specialized for use in synchrotron beam lines, and have produced new photodiode devices for synchrotron-radiation applications. The performance of these devices was reviewed over extended periods of time in beam-line control and as detectors in experiments.

101,634

PB91-174987

Not available NTIS

National Bureau of Standards (NEL), Gaithersburg. MD. Precision Engineering Div.

Optical Levitation of SIngle Particles.

Final rept.

T. R. Lettieri. 1988, 10p

Pub. in Proceedings of Scientific Conference on Obscuration and Aerosol Research, Edgewood, MD. June 26-30, 1987, p305-314 1988.
Keywords: *Radiation pressure, "Levitation, Laser radiation, Drops(Liquids), Microspheres, Reprints, "Optical levitation.

Experimental techniques are presented for optically levitating single particles using laser radiation pressure.

\section{1,635}

PB91-175083

Not available NTIS

National Inst. of Standards and Technology (NML), Boulder, CO. Quantum Physics Div.

Gravitational Radiation Observations on the Moon. Final rept.

R. T. Stebbins, J. W. Armstrong, P. L. Bender, R. W P. Drever, R. W. Hellings, and P. R. Saulson. 1990 $10 \mathrm{p}$

Pub. in Proceedings of NASA Workshop on Astrophys ics from the Moon, Annapolis, MD., February 5-7, 1990, p637-646.

Keywords: "Gravitational wave antennas, "Lunar observatories, Laser interferometry, Lunar bases, Reprints.

A Laser-Interferometer Gravitational-Wave Observato ry (LIGO) is planned for operation in the United States, with two antennas separated by several thousand kilometers. Each antenna would incorporate laser interferometers with $4 \mathrm{~km}$ arm lengths, operating in vacuum. The frequency range covered initially would be from a few tens of $\mathrm{Hz}$ to a few $\mathrm{kHz}$, with possible extension to lower frequencies later. Similar systems are likely to be constructed in Europe, and there is a possibility of at least one system in Asia or Australia. It will be possible to determine the direction to a gravitational wave source by measuring the difference in the arrival times at the various antennas for burst signals or the phase difference for short duration nearly periodic signals The addition of an antenna on the Moon, operating in support of the Earth-based antennas, would improve the angular resolution for burst signals by about a factor 50 in the plane containing the source, the Moon, and the Earth.

\section{1,636}

PB91-175265

Not available NTIS

National Bureau of Standards (NML), Gaithersburg, MD. Ionizing Radiation Div.

Status of Beta-Partlcie Calibration Studies at NBS. Final rept.

C. G. Soares, and J. S. Pruitt. 1987, 3p

Pub. in Proceedings of Department of Energy Work shop on Beta Measurements, Albuquerque, NM., January 23-24, 1986, p169-171 1987

Keywords: "Ionization chambers, "Beta detection, "Beta sources, "Test facilities, "Calibration, Radiation protection, Electron beams, US NBS, Reprints.

A calibration facility for beta-particle instruments and sources used in radiation protection has been established at the National Bureau of Standards (NBS). The facility consists of standardized beta-particle sources and extrapolation ionization chambers for the accurate measurement of beta-particle absorbed dose. The facility also includes a series of nearly monoenergetic electron beams for measurement of instrument response functions. A service which has been established for the calibration of beta-particle sources and transfer-quality ionization chambers is described. The latest improvements to the monoenergetic electron facility are also discussed.

\section{1,637}

PB91-175273

Not available NTIS

National Inst. of Standards and Technology (NML) Boulder, CO. Quantum Physics Div.

Test of Newton's Inverse Square Law of Gravity Using the $300 \mathrm{~m}$ Tower at Erie, Colorado: Newton Vindicated on the Plains of Colorado.

Final rept.

C. C. Speake, T. M. Niebauer, M. P. McHugh, P. T.

Keyser, J. E. Faller, J. Y. Curz, J. C. Harrison, J.

Makinen, and R. B. Beruff. 1990, 8p

Pub. in Proceedings of Rencontre de Moriond New and Exotic Phenomena (25th), Les Arcs, Savoie, France, January 20-27, 1990, p255-262.

Keywords: "Gravitation, “Gravity, Tests, Reprints, Meteorological towers, Inverse square laws, Newton laws.

A 300-m meteorological tower was used to measure gravity at eight different heights above the surface of the earth. The observed gravity values were corrected for tides and gravimeter screw error. Also, tests were performed to look for systematic effects due to tower motion. The resulting values are compared to values predicted by Newton's inverse-square law from upward continuation of surface gravity. The result places new constraints on any non-Newtonian interaction.

101,638

PB91-175364

Not available NTIS

National Inst of Standards and Technology (PL) Gaithersburg, MD. Atomic Physics Div.

Spectrum and Energy Levels of the Xenonlike Ion ce V.

Final rept

A. Redfors, and J. Reader. 1991, 7p

Sponsored by Department of Energy, Washington, DC.

Pub. in Physical Review A 43, n5 p2367-2373, 1 Mar

Keywords: "Cerium ions, "Energy levels, "Ultraviolet spectra, Vacuum ultraviolet radiation, Multicharged ions, Hartree-Fock approximation, Reprints.

The spectrum of four-times ionized cerium $\mathrm{Ce} v$ has been observed with a sliding-spark discharge and a 10.7-m normal-incidence vacuum spectrograph. A total of 107 lines have been classified as transitions between 51 energy levels. All levels of the $5 p(6), 5 p$ quintet $4 f, 5 p$ quintet $5 d, 5 p$ quintet $6 s, 5 p$ quintet $6 p$ and $5 p$ quintet $6 d$ configurations have been determined. Because of collapse of the $4 f$ shell, the $5 p$ quintet $4 \mathrm{f}$ configuration is situated well below $5 p$ quintet $5 d$. $\mathrm{Ab}$ initio calculations and least-squares fits of the energy parameters to the experimental energy levels are reported. The ionization energy is revised to $527100+$ or $-2000 / \mathrm{cm}(65.35+$ or $-0.25 \mathrm{eV})$.

101,639

PB91-175380

Not available NTIS

National Inst. of Standards and Technology (NML) Boulder, CO. Quantum Physics Div.

Search for a Composition Dependent Force Using a Liquid-Supported Torsion Balance.

Final rept.

M. P. McHugh, P. T. Keyser, and J. E. Faller. 1990

Pub. in Proceedings of the Recontre de Moriond New and Exotic Phenomena (25th), Les Arcs, Savoie, France, January 20-27, 1990, p233-236

Keywords: "Basic interactions, Torsion balances Lead(Metal), Copper, Reprints, "Fifth force, Eotvos ex periment.

Current efforts are discussed to search for an interme diate range composition dependent interaction, using a liquid-supported torsion balance containing a $\mathrm{Pb}-\mathrm{Cu}$ composition dipole. The mass inhomogeneity of the basement laboratory serves as the source for the interaction. The expected sensitivity and preliminary results are presented.

101,640

B91-175430

Not available NTIS

National Bureau of Standards (NEL), Boulder, CO.

Chemical Engineering Science Div.

Saran Carbon Cryogenic Regenerator for Liquid Helium.

Final rept.

L. V. Munukutla, J. A. Jones, and R. Radebaugh. $1988,10 p$

Pub. in Proceedings of Interagency Meeting on Cryocoolers, Monterey, CA., August 17, 1988, p109-118.

Keywords: "Carbon, *Regenerators, "Liquid helium, Cryogenics, Adsorption, Heat exchangers, Therma conductivity, Reprints.

A promising solution to the problems of regenerator neffectiveness in the $4 \mathrm{~K}$ to $15 \mathrm{~K}$ region is the use of saran carbon with adsorbed helium as the regenerato material. Preliminary measurements indicate that this material has a higher volumetric specific heat than any other known material in the $4 \mathrm{~K}$ to $15 \mathrm{~K}$ temperature range. Furthermore, the estimated thermal conductivity of saran carbon is low enough to permit its use as a continuous matrix between the hot and cold ends of the regenerator, but it does not adversely affect the longitudinal conduction heat loss along the regenerator length. An NBS computer regenerator program has been used to show that a saran carbon/ adsorbed helium, parallel-plate geometry regenerator is likely to be superior to any other known regenerator for cooling in the $4 \mathrm{~K}$ to $15 \mathrm{~K}$ temperature region. 


\section{PHYSICS}

\section{General}

\section{1,641}

PB91-175497

Not available NTIS

National Inst. of Standards and Technology (NML)

Boulder, CO. Quantum Physics Div.

Spin-Dependent Electron-Impact Excitation of Sodium.

Final rept

H. L. Zhou, B. L. Whitten, G. Snitchler, D. W

Norcross, and J. Mitroy. 1990, 6p

Contract DE-AI05-86ER53237

Sponsored by Department of Energy, Washington, DC Office of Basic Energy Sciences.

Pub. in Physical Review A 42, n7 p3907-3912, 1 Oct 90.

Keywords: "Electron scattering, "Sodium, Atomic energy levels, Electron spin, Excitation, EV range 01 10, R matrix, Reprints.

The spin-dependent cross sections for electron excitation of the $3 p$ level of sodium at energies just above threshold are studied using the R-matrix approach. In addition to the total cross section for excitation, the authors present results for the excitation cross sections as a function of the change in the spin and orbital angular-momentum projection of the target. Calculations were made in the four- $(3 s, 3 p, 4 s, 3 d)$ five$(+4 p, 4 d)$ approximations, for electron energies ranging from threshold to $4.0 \mathrm{eV}$. The results are compared with other calculations and with the recent experimental results of Han, Schinn, and Gallagher (Phys. Rev. A $38,535(1988)$ )

\section{1,642}

PB91-175513

Not available NTIS

National Bureau of Standards (NML), Gaithersburg. MD. Radiation Physics Div.

Of What Use Is Basic Research.

Final rept.

T. B. Lucatorto. $1987,1 p$

Pub. in Candela Wavelength, p5 1987.

Keywords: "Racetrack microtrons, "Beam steering, Linear accelerators, Electron accelerators, Electron beams, MeV range 10-100, Design, Reprints.

In the NBS-Los Alamos racetrack microtron (RTM) the $17 \mathrm{MeV}$ electron beam, which has made one pass through the RTM linac, is deflected $180 \mathrm{deg}$ in one end magnet and is returned to the same end of the (stand ing wave) linac for a second pass. A pair of dipole magnets on the linac axis compensate for the beam displacement caused by the end magnet so that the beam enters the linac on axis. These two magnets are designed to have equal field integrals in order to produce a pure displacement. Matching the field integrals was complicated by the quite different widths of the two magnets which have different beam clearance requirements. In addition, the wider magnet contains a quadrupole coil for beam steering. Design considerations are presented. Magnetic field measurements show that critical design goals have been achieved.

101,643

PB91-175521

Not available NTIS

National Bureau of Standards (IMSE), Gaithersburg.

MD. Reactor Radiation Div.

Fe-W Supermirrors for Polarizing Neutrons.

Final rept.

C. F. Majkrzak, D. A. Neumann, J. R. D. Copley, and

R. P. DiNardo. $1988,6 p$

Pub. in Proceedings of 1987 Fall MRS Meeting, v103 p115-120 1988 .

Keywords: "Polarizers, Polarization(Spin alignment) Metal films, Thin films, Substrates, Tungsten, Glass, Iron, Sputtering, Reprints, "Neutron polarizers, "Supermirrors, Multilayers.

Thin film bilayers of ferromagnetic $\mathrm{Fe}$ and nonmagnetic $W$ have been deposited by sputtering on flat glass substrates according to a sequence of gradually vary ing bilayer thicknesses which in effect extends the critical angle for external mirror reflection for neutrons of one spin state when the $\mathrm{Fe}$ is magnetized in the plane of the film. The measured reflectivity of the Fe-W multilayer system is compared with that of other supermirror polarizers consisting of different materials and layer sequences.

\section{1,644}

PB91-175547

Not available NTIS

National Bureau of Standards (NML), Gaithersburg,

MD. lonizing Radiation Div.
Calculation of Beta-Particle Counting Efficiency for Liquid ScIntillation Systems with Three Phototubes.

Final rept.

A. G. Malonda, and B. M. Coursey. 1988, 6p

Pub. in Applied Radiation and Isotopes - International Jnl. of Radiation Applications and Instrumentation 39 n12 p1191-1196 1988.

Keywords: "Scintillation counters, "Liquid scintillators, "Beta detection, Phototubes, Counting, Efficiency, Computation, Reprints.

Formulae are derived for the computation of beta-particle counting efficiency for three-phototube liquidscintillation counters. It is shown that the counting efficiency for triple coincidences may be computed by considering either the pulse-production probabilities or the non-detection probabilities. Effects due to asymmetry of the phototubes are also evaluated.

\section{1,645}

PB91-175588

Not available NTIS National Bureau of Standards (NML), Gaithersburg, MD. Atomic and Plasma Radiation Div.

Working Group 2: Atomic Transition Probabilities. Final rept.

W. L. Wiese, $1988,7 p$

Pub. in Reports of Astronomy, vXXA p117-123 1988

Keywords: "Electron transitions, "Transition probabilities, Oscillator strengths, Literature surveys, Reprints.

Some new activities on the determination of atomic transition probabilities are briefly described and an exhaustive list of new literature references is given which covers all transition probability data for the three year period August, 1984-August, 1987.

\section{1,646}

B91-187088

Not available NTIS

(NML), Gaithersburg MD. Atomic and Plasma Radiation Div.

Electric Ouadrupole and Magnetic Dipole Transition Probabilities In Potassium Isoelectronic Sequence.

Final rept.

M. A. Ali, and Y. K. Kim. 1988, 6p

Sponsored by Department of Energy, Washington, DC Office of Fusion Energy.

Pub. in Physical Review A 38, n8 p3992-3997 1988.

Keywords: "E2-transitions, "M1-transitions, Forbidden transitions, Transition probabilities, Multicharged ions, Molybdenum ions, Potassium, Reprints.

Electric quadrupole and magnetic dipole transition probabilities between 3p(6)4s doublet $S(1 / 2)$ and probabilities between $3 p(6) 4$ s doublet $S(1 / 2)$ and $3 p(6) 3 d$ doublet $D(3 / 2)$, doublet $D(5 / 2)$ levels for $K$
through $M o(23+)$ have been calculated in the relativistic Dirac-Fock single configuration approximation. The positions of the (excited) $4 s$ doublet $S(1 / 2)$ level for $\mathrm{Mn}(7+)-\mathrm{Mo}(23+)$ are predicted along with the fine structure splitting in the ground configuration $3 p(6) 3 d$.

\section{1,647}

\section{PB91-187211}

Not available NTIS

National Bureau of Standards (NML), Gaithersburg MD. Temperature and Pressure Div.

$\mathrm{H} 2 \mathrm{O}$ (I)-H2O(III)-H2O(L) Triple Point of Water as a Fixed Pressure Point.

Final rept

N. Bignell, and V. E. Bean. 1988, $5 p$

Pub. in Metrologia 25, n4 p205-209 1988.

Keywords: "Pressure measurement, "Water, Phase diagrams, Triple point, Reprints.

An apparatus to use the $\mathrm{H} 2 \mathrm{O}(\mathrm{I})-\mathrm{H} 2 \mathrm{O}$ (III)- $\mathrm{H} 2 \mathrm{O}(\mathrm{L})$ triplepoint as a fixed pressure point is described. A technique to establish the three phases simultaneously in the cell is given. The value of the pressure was meas ured with a piston gage and was found to be 208.829 + or $-0.025 \mathrm{MPa}$ at the 99.7 percent confidence level.

\section{1,648}

PB91-187302

Inst. of Standards and Technology (NML),

Gaithersburg, MD. Quantum Metrology Div.

Angular Distribution of Ejected Electrons in Resonant Auger Processes of $\mathrm{Ar}, \mathrm{Kr}$, and $\mathrm{Xe}$.

Final rept

T. A. Carlson, D. R. Mullins, C. E. Beall, B. W. Yates J. W. Taylor, D. W. Lindle, and F. A. Grimm. 1989

$16 p$

Pub, in Physical Review A: General Physics 39, n3 p1170-11851989.
Keywords: "Argon, "Krypton, "Xenon, "Auger effect, Photoelectron spectroscopy, Autoionization, Angula distribution, Reprints.

Angle-resolved electron spectroscopy with the help of synchrotron radiation has been used to study resonate Auger processes near the core shells of Ar $2 p, \mathrm{Kr} 30$ and $\mathrm{Xe} 4 \mathrm{~d}$. Results for the lowest energy resonances have received special attention: argon (Ar2p(3/2) $>4 \mathrm{~s}$ ) at a photon energy of $244.4 \mathrm{eV}$, krypton (Kr3d(5) 2) $->6 p$ ) at $91.2 \mathrm{eV}$, and xenon (Xe4d(5/2)->6p) a $65.1 \mathrm{eV}$. The angular distribution parameters, beta, are evaluated for each of the resolved Auger peaks. Most striking is the occurrence of large negative beta values for some of the higher kinetic energy peaks. The results are most apparent under high electron resolution. The beta values near -1 are justified in terms of the angular-momentum transfer theory, which predicts beta $=-1$ for parity unfavored transitions. Results on the Auger spectra are also given at lower kinetic energies, for shake-up states, and for higher energy resonances, especially those involving vacancies in the core shells of the lower spin states.

\section{1,649}

PB91-187310

Not available NTIS

National Bureau of Standards (NML), Gaithersburg MD. Quantum Metrology Group.

Unusual Degree of Angular Anisotropy in the Res onant Auger Spectrum of $\mathrm{Kr}$.

Final rept.

T. A. Carlson, D. R. Mullins, C. E. Beall, B. W. Yates J. W. Taylor, D. W. Lindle, B. P. Pullen, and F. A

Grimm. 1988, 4p

Pub. in Physical Review Letters 60, n14 p1382-1385 1988.

Keywords: "Krypton, "Auger effect, Photoemission, Anisotropy, Excitation, Reprints.

The angular distribution parameter, beta, has been measured for the resonant Auger spectra in $\mathrm{Kr}$ following $3 d->5 p$ excitation. Unusually low values, close to 1 , were seen for two bands. From optical spectroscopy data these bands are assigned to the final states $4 p(4)$ (triplet $P) 5 p(q u a r t e t P(5 / 2)$, quartet $P(3 / 2)$. Accordintet $\mathrm{D}(5 / 2)$

\section{1,650}

\section{PB91-187393}

Not available NTIS

( Inst. of Standards and Technology (NML), Gaithersburg, MD. Quantum Metrology Div.

Angular Distributions of Electrons in Resonant Auger Spectra.

Final rept.

J. W. Cooper. 1989, 3p

Pub. in Physical Review A 39, n7 p3714-3716, 1 Apr 89.

Keywords: "Auger electrons, Angular distribution, Auger effect, Argon, Reprints.

Recent measurements have shown that the angular distribution of Auger electrons observed in near threshold resonant excitation with energy resolution adequate to partially resolve the multiplet structure corresponding to final core states exhibited a marked change in the angular distributions as a function of final electron energy. A method of calculating these angular distributions is proposed which extends the formalism developed for treating angular distributions in photoeffect via a separation into parity favored and unfavored components. As an example of the use of the method, calculations are presented for the $2 p-4 s$ resonant Auger process in Argon and compared with recent experiments.

\section{1,651}

Not available NTIS National Inst. of Standards and Technology (NML) Gaithersburg, MD. Quantum Metrology Div. Polarization and Anisotropy of X-ray Emission from Molecules.

Final rept.

P. L. Cowan. $1990,14 \mathrm{p}$

Pub in Proceedings of International Conference on $X$ Ray and Inner-Shell Processes (15th), Knoxville, TN. July 9-13, 1990, p696-709.

Keywords: "Molecular gases, "X-rays, Angular distribution, Polarization, Anisotropy, Reprints.

$X$-ray emission from selectively excited core hole states in molecular gases can exhibit strong polarization and anisotropic angular distributions. These ef- 
fects are attributable to the creation of an aligned ensemble of excited molecules within the otherwise randomly oriented molecular gas. This alignment can be created because the resonant excitation probability of core hole states can be strongly dependent upon the instantaneous molecular orientation. This effect presents a novel opportunity for studying molecular symmetries and the structure of matter in disordered phases.

\section{1,652}

\section{PB91-187443}

Not available NTIS National Inst. of Standards and Technology (NML), Gaithersburg, MD. Quantum Metrology Div. Some Historical Remarks Concerning These Conferences: With Emphasis on the X-ray Component Final rept.

R. D. Deslattes. $1990,10 \mathrm{p}$

Pub. in Proceedings of International Conference on $X$ ray and Inner-Shell Processes (15th), Knoxville, TN July $9-13,1990$, p3-12

Keywords: "X-rays, Inner-shell excitation, Inner-shell ionization, Synchrotron radiation, Historical aspects, Meetings, Reprints

The text is an essay dealing with historical aspects of the series o International Conference on X-ray and Inner Shell Physics. It is intended as part of the preface to the Proceedings volume of the year's Conference, $X-90$. It emphasizes not only the earliest meetings but also one that took place earlier and, though not gener ally considered part of the present sequence, anticipated much of what has transpired subsequently.

\section{1,653}

PB91-189597

Not available NTIS

of Standards (NML), Gaithersburg.

MD. Atomic and Plasma Radiation Div.

Quantum Electrodynamic Contributions to Spin Orbit Splitting in the Ground State of AluminumLlke lons.

Final rept.

Y. K. Kim, V. Kaufman, J. Sugar, and M. A. Ali. 1988 $5 p$

Sponsored by Department of Energy, Washington, DC Office of Fusion Energy, and Howard Univ., Washing ton, DC

Pub. in Jnl. of the Optical Society of America B 5, n10 p2225-2229 1988.

Keywords: Multicharged ions, Quantum electrodynamics, L-S coupling, Ground state, Lamb shift, Cobalt ions, Xenon ions, $M$ shell, Reprints, *Aluminum-like ions.

It is shown that the inclusion of QED corrections for the $\mathrm{M}$-shell electrons substantially improves the agreement between multiconfiguration Dirac-Fock calculations and experimental values on the spin-orbit splitting in the ground state $(3 s(2) 3 p(2 P))$ of Al-like ions. Recommended values of the spin-orbit splitting are presented for $\mathrm{Co}(14+)$ through $\mathrm{Xe}(41+)$.

\section{1,654}

\section{PB91-189605}

Not available NTIS

National Bureau of Standards (NML), Gaithersburg, MD. Atomic and Plasma.Radiation Div.

Relativistic and Correlation Effects in the 2s3p Configuration of Beryllium-Like Ions.

Final rept.

Y. K. Kim, A. W. Weiss, and W. C. Martin. 1988, 10p Sponsored by Department of Energy, Washington, DC

Office of Fusion Energy.

Pub. in Jnl. of the Optical Society of America B 5, n10 p2215-2224 1988 .

Keywords: Multicharged ions, Oscillator strengths, Excited states, Relativistic effects, Energy levels, Correlation, Reprints, "Beryllium-like ions.

Relativistic and correlation effects cause several inversions of the 'triplet' and 'singlet' $J=1$ levels in the $2 s 3 p$ configuration of Be-like ions. The authors have studied these effects in the range of atomic number $Z=6-54$ using both relativistic and nonrelativistic multiconfiguration wave functions. Theoretical predictions of energies of these levels with respect to the $2 \mathrm{~s}(2)$ single $S(0)$ ground level are compared with experimental data, and the authors conclude that overall, the predic tions for the ions having $Z=$ or $>12$ are probably more accurate than the available measurements. $\mathrm{Ca}$ culated oscillator strengths for these resonance transitions are also reported. Near cadmium $(Z=48)$, the authors confirm still another inversion due to the complete dominance of relativistic effects, which has been reported earlier in the literature.
101,655

\section{PB91-189746}

Not available NTIS National Bureau of Standards (NML), Gaithersburg MD. Electricity Div

Observation of Atoms Laser-Cooled Below the Doppler Limit.

Final rept.

P. D. Lett, R. N. Watts, C. I. Westbrook, W. D.

Phillips, and P. L. Gould. 1988, 4p

Sponsored by Office of Naval Research, Arlington, VA Pub. in Physical Review Letters 61, n2 p169-172, 11 Jul 88.

Keywords: Temperature measurement, Cryogenics, Reprints, "Ultracold atoms, Sodium atoms, Laser cooling.

The authors have measured the temperature of a gas of sodium atoms in 'optical molasses' to be as low as $43+$ or -20 (micro)K. Surprisingly, this strongly violates the generally accepted theory of Doppler cooling which predicts a limit of 240 (micro)K. Several complementary measurements of the ballistic motion of atoms released from the molasses were used to determine the temperature.

\section{1,656}

PB91-189753

Not available NTIS

National Inst. of Standards and Technology (NML) Gaithersburg, MD. Quantum Metrology Div.

Doppler Shift Attenuation Llfetime Measurements in (54) Cr Following Thermal Neutron Capture.

Final rept.

K. P. Lieb, H. G. Borner, M. S. Dewey, J. Jolie, S. J. Robinson, S. Ulbig, and C. Winter. 1988, 5p Pub. in Physics Letters B 215, n1 p50-54, 8 Dec 88

Keywords: "Chromium 54, Interacting boson model Neutron capture, Thermal neutrons, Doppler broadening, Lifetime, Reprints.

The double crystal spectrometer GAMS4 in combination with the ILL high flux reactor has been used to determine the lifetimes of the $3074 \mathrm{keV}$ ( 2 sub 3 , sup $+)$ and $3720 \mathrm{keV}(1,2)+$ states in $(54) \mathrm{Cr}$. The initia recoil energy of about $0.5 \mathrm{keV}$ imparted by the primary gamma-radiation after thermal neutron capture in (53) $\mathrm{Cr}$ produces Doppler broadened line shapes of the secondary transitions. The large (2 sub 3 , sup +$)(2$ sub 1 , sup +$) M 1$ strength of $B(M 1)=0.39(6)(\mathrm{mu}$ sub n) squared suggests the $(2$ sub 3 , sup +$)$ state to be of mixed symmetry within the Interacting Boson mode IBM-2.

101,657

PB91-190009

Not available NTIS

National Inst of Standards and Technology (NML)

Gaithersburg, MD. Atomic and Plasma Radiation Div. Dielectronic Recombination Rate Coefficients of $\mathrm{O}(5+)$ and $\mathrm{O}(2+)$

Final rept.

L. J. Roszman. 1989, 3p

Pub. in Physica Scripta T28, p36-38 1989.

Keywords: *Oxygen ions, Multicharged ions, L-S cou pling, Autoionization, Reprints, Dielectronic recombination.

The dielectronic recombination rate coefficients for $\mathrm{O}(5+)$ and $\mathrm{O}(2+)$ in a single-configuration and LScoupled atomic model are presented. A model calculation for the effects of thermal equilibration of the final states with the plasma electrons is presented and examined.

\section{1,658}

PB91-190033

Not available NTIS

National Inst. of Standards and Technology (NML), Gaithersburg, MD. Radiation Physics Div.

Energy Levels and Transition Probabilities in the Ground State Configuration of Sulfur-Like Ions.

Final rept.

E. B. Saloman, and Y. K. Kim. 1989, 18p

Pub. in Atomic Data and Nuclear Data Tables 41, n2 p339-356 1989 .

Keywords: "Energy levels, Forbidden transitions, Multicharged ions, Transition probabilities, Ground state, Reprints, "Sulfur-like ions.

Theoretical energy levels have been calculated for the terms of the ground state configuration of all the sulfurlike ions of atomic number $Z=16-92$. Transition probabilities have been obtained for the $M 1$ and $E 2$ transitions within the configuration. The calculations were by means of a multiconfiguration-Dirac-Fock technique which included perturbatively the Breit interaction and the Lamb shift for the K- L-, and M-shell electrons. Results are presented which use experimental and semi-empirical fits to the term energies for ions with atomic numbers up to $Z=42$. Above $Z=42$, purely theoretical results are given. It is shown that the agreement between the authors' theoretical results and the semi-empirical values of the term energies improves rapidly as $Z$ increases, with all values in excellent agreement by $Z=42$.

\section{1,659}

PB91-192567

Not available NTIS Institute of Physics, Belgrade (Yugoslavia)

Experimental Stark Widths and Shifts for Spectral Lines of Neutral and Ionized Atoms: A Critical Review of Selected Data for the Period 1983 through 1988

Bimonthly rept.

N. Konjevic, and W. L. Wiese. c1990, 79p

Prepared in cooperation with National Inst. of Standards and Technology, Gaithersburg MD.

Included in Jnl. of Physical and Chemical Reference Data, v19 n6 p1307-1386 Nov/Dec 90. Available from American Chemical Society, 1155 16th St., NW, Washington, DC. 20036-9976.

Keywords: "Line spectra, "Stark effect, Neutral atoms Positive ions, Line width, Line broadening, Spectra shift, Reviews, Tables(Data)

A critical review of the available experimental data on Stark widths and shifts for spectral lines of non-hydrogenic neutral atoms and positive ions has been carried out. The review covers the period from 1983 throug the end of 1988 and represents a continuation of earlier critical reviews up to 1982. Data tables containing the selected experimental Stark broadening param eters are presented with estimated accuracies. Guidelines for the accuracy estimates have been developed during the previous reviews and are summarized. The data are arranged according to elements and spectra, and these are presented in alphabetical and numerica order, respectively Comparisons with comprehensive calculations based either on the semiclassical theory, or--for multiply ionized atoms--on the modified se miempirical approximation, are made whenever possible, since the comparison with theory has often been a principal motivation for the experiments.

\section{1,660}

\section{PB91-194399}

PC A04/MF A01

National Inst of Standards and Technology, Gaithersburg, MD.

Stopping Power of Fast Charged Particles in Heavy Elements.

H. Bichsel. Apr 91, 74p NISTIR-4550

Prepared in cooperation with Bichsel (Hans), Seattle, WA.

Keywords: "Stopping power, Proton bombardment Charged particles, Excitation, Bethe-Bloch theory.

The stopping power formula from Bethe's theory contains terms which are known only approximately and must be estimated with the use of experimental data. These terms include a material constant, the mean ex citation energy of the medium, and the shell-, Blochand Barkas-corrections. In an analysis of measured proton and alpha-particle stopping powers and ranges, modifying parameters have been introduced into these corrections, and the mean excitation energy was simultaneously adjusted, so as to get the closest possible agreement with experimental results. Such an analysis is reported here for elements with atomic numbers $Z=$ or $>57$. The modification parameters introduced for the shell corrections have a simple relation to atomic energy levels. The Bethe theory with the adopted mean excitation energies and proposed ad justments of the shell- and Burkas- corrections predicts stopping powers that are in close agreement with experimental values, within the experimental uncertainties. This agreement was obtained for protons with kinetic energies above about $0.5 \mathrm{MeV}$, and for heavie ions of charge $z$ at energies above $(z-1.5) \mathrm{MeV} / \mathrm{u}$

\section{1,661}

PB91-194407

PC A03/MF A01

National Inst. of Standards and Technology, Gaithers burg, MD. 


\section{PHYSICS}

\section{General}

Model for the Non-Perturbative QCD (Quantum Chromodynamics) Vacuum.

M. Danos, D. Gogny, and D. Iracane. Apr 91, 34p NISTIR-4555

Prepared in cooperation with CEA Centre d'Etudes de Bruyeres-le-Chatel, Montrouge (France).

Keywords: "Quantum chromodynamics, "Vacuum states, Meissner-Ochsenfeld effect, Bose-Einstein condensation, Lagrangian functions, Quarks, Gluons. By treating the high-momentum gluon and the quark sector as an in principle calculabe effective Lagrangian, the authors obtain a non-perturbative vacuum state for QCD as an infrared quark-gluon condensate. This vacuum is removed from the perturbative vacuum by an energy gap and supports a Meissner-Ochsenfeld effect. It is unstable below a minimum size and it also suggests the existence of a universal hadronization time. This vacuum thus exhibits all the properties required for color confinement.

\section{1,662}

PB91-194423

PC A04/MF A01

National Inst of Standards and Technology (NML). Gaithersburg, MD. Electron and Optical Physics Div. Technical Activities 1990, Electron and Optical Physics Division.

C. W. Clark. May $91,72 p$ NISTIR-4553

See also report for 1989, PB90-207267.

Keywords: "Research, Far ultraviolet radiation, Soft $x$ rays, Synchrotron radiation, SURF II storage ring, Multi-photon processes, Scanning tunneling microscopy, Electron-atom collisions, Atomic physics, Radiometry, Calibration, Magnetic films.

The report summarizes technical activities of the NIST Electron and Optical Physics Division during Fiscal Year 1990. These fall into five general areas: soft x-ray radiometry, operation of the SURF-II synchrotron storage ring; electron microscopy and basic surface physics; soft $x$-ray emission studies; and multiphoton processes. A listing is given of calibration services, publications, talks, and other relevant activities of the Division's staff.

\section{1,663}

PB91-194886

Not available NTIS

National Bureau of Standards (NML), Gaithersburg, MD. Electricity Div.

Prospects for Electromagnetic Manipulation and Trapping of Antihydrogen.

Final rept.

P. D. Lett, P. L. Gould, and W. D. Phillips. 1988, 14p

Pub. in Hyperfine Interactions 44, n1-4 p335-348 1988.

Keywords: "Antimatter, Storage, Reprints, "Antihydrogen, "Atom traps, Laser cooling.

The storage and handling of atomic antimatter presents special problems because contact with the walls of any material container is prohibited. Laser cooling and trapping and magnetic trapping techniques for neutral atoms dre therefore especially appealing. Here the authors review the basic principles of electromagnetic trapping and laser cooling for neutral atoms, and briefly describe a number of already-demonstrated laser and magnetic traps. They discuss limits on the temperatures achievable by laser cooling, as well as some special problems in the laser cooling of hydrogen/antihydrogen. In particular, they present some new results for the case of pulsed cooling.

\section{1,664}

PB91-194910

Not available NTIS

National Inst. of Standards and Technology (NML) Gaithersburg, MD. Quantum Metrology Div.

Photoionization Studies of Atoms and Molecules Using Synchrotron Radiation.

Final rept.

D. W. Lindle. $1989,6 p$

Pub. in Nuclear Instruments and Methods in Physics Research 280, n2-3 p161-166, 10 Aug 89.

Keywords: "Photoionization, $X$ ray fluorescence, Synchrotron radiation, Reprints.

Photoionization studies of free atoms and molecules have undergone considerable development in the past decade, in large part due to the use of synchrotron radiation. The tunability of synchrotron radiation has permitted the study of photoionization processes near valence- and core-level ionization thresholds for atoms and molecules throughout the Periodic Table. A general illustration of these types of study will be presented, with emphasis on a few of the more promising new directions in atomic and molecular physics being pursued with synchrotron radiation.

\section{1,665}

PB91-194928

Not available NTIS National Bureau of Standards (NML), Gaithersburg, MD. Quantum Metrology Group.

Angle-Resolved Photoemission from the Ar 2p Subshell.

Final rept.

D. W. Lindle, L. J. Medhurst, T. A. Ferrett, P A

Heimann, M. N. Piancastelli, S. H. Liu, D. A. Shirley,

T. A. Carlson, P. C. Deshmukh, G. Nasreen, and S.

T. Manson. 1988, 4p

Pub. in Physical Review A 38, n5 p2371-2374, 1 Sep

Keywords: "Inner-shell ionization, "Photionization, *Argon, Synchrotron radiation, Photoemission, $X$ rays, Reprints

The angular distribution for $\mathrm{Ar} 2 \mathrm{p}$ photoionization has been measured from just above threshold to $400 \mathrm{eV}$ photon energy, and calculated in the same energy range using the relativistic random-phase approximation. The experimental and theoretical results exhibit good agreement, but the present results disagree somewhat with earlier Hartree-Fock (HF) calculations, with the HF values found to be significantly higher in the near-threshold region. Possible reasons for this discrepancy are discussed with relevance to the general understanding of inner-shell photoionization phenomena.

101,666

PB91-195016

Not available NTIS

National Inst of Standards and Technology (NML), Gaithersburg, MD. Radiometric Physics Div.

Low-Pressure Symmetric Discharge Heat Pipe and DROGS of Eu.

Final rept.

H. Maoqi, L. Ronggen, and C. L. Cromer. $1990,5 p$ Pub. in Acta Optica Sinica 10, n5 p385-389 May 90

Keywords: "Heat pipes, "Europium, Atomic spectra, Excited states, Metal vapors, Reprints, Double resonance optogalvanic spectroscopy.

The authors describe a new low pressure symmetric discharge heat pipe (LPDP) which may be used to generate high $Z$ low pressure metal vapor sample. The authors have studied the double resonance opto-galvanic spectroscopy (DROGS) of Eu atom in different pressure with the LPDP. Four new lines of Eu from 17341/ $\mathrm{cm}$ excited level and three from $16612 / \mathrm{cm}$ are observed for the first time. It is verified that such a new LPDP is suitable for studying highly excited state spectroscopy of high Z metal elements.

\section{1,667}

\section{PB91-195032}

Not available NTIS

National Inst. of Standards and Technology (NEL).

Gaithersburg, MD. Precision Engineering Div.

Relativistic Probability Amplitudes and State Preparation.

Final rept.

E. Marx. 1989, 3p

Pub. in Bell's Theorem, Quantum Theory and Conceptions of the Universe, p127-129 1989.

Keywords: "Quantum electrodynamics, Klein-Gordon equation, Relativistic effects, Quantum mechanics, Wave functions, Charged particles, Antiparticles, Reprints, Probability amplitudes.

The wave function for a spinless charged particle in an electromagnetic field is decomposed into two probability amplitudes, one for a particle and one for an antiparticle. The particle amplitude is specified at the initial time and the antiparticle amplitude at the final time. Time reflection invariance indicates that the preparation of the antiparticle state at the final time should be carried out by observers composed of antiparticles. A model of elementary particles shows how relativistic quantum mechanics can be used to describe all reactions.

\section{1,668}

PB91-195123

Not available NTIS

National Bureau of Standards (NML), Gaithersburg MD. Center for Radiation Research.
Glueballs and Hadron Spectroscopy.

Final rept.

S. Meshkov. $1988,9 p$

Pub in Proceedings of the Conference on the Intersections between Particle and Nuclear Physics (3rd) Rockport, ME., May 14-19, 1988, p796-804 1988.

Keywords: "Glueballs, Pseudoscaler mesons, Spectroscopy, Tensors, Hadrons, Reprints.

Identifying glueballs is intimately intertwined with the study of meson spectroscopy. In the brief review, recent experimental results are compared with QCD inspired predictions for the masses of the $0(-+), 1(+$ ), $1(--), 1(++)$ mesons. They agree remarkably well. For the $0(-+)$ and $1(++)$ systems, these agreements lead to the posibility of identifying additional states as candidates for exotica. A major mystery is why the $(2(t+)$ sub gT) states are not seen in $J / p s i->$ gamma phi phi, in view of the fact that the OZI-violating mechanism should be a good way to produce glue balls.

\section{1,669}

PB91-195206 Not available NTIS National Inst. of Standards and Technology (NML) Gaithersburg, MD. lonizing Radiation Div.

Intensifying Effect of Metallic Screens on the Sensitivity of X-ray Films at $662 \mathrm{keV}$.

Final rept.

E. Navon, C. E. Dick, and G. Barnea. 1989, 4p

Pub. in Jnl. of Applied Physics 65, n7 p2852-2855 1989.

Keywords: * $X$ ray film, "Radiation effects, "Electron bombardment, KeV range 100-1000, Foils(Materials) Lead(Metal), Aluminum, Copper, Radiation doses, Comparison, Sensitivity, Reprints, Intensification factors, Metal screens.

The relative photographic effect of electrons originating from $662 \mathrm{keV}$ photons interacting in aluminum copper and lead foils was measured as a function of the foil thickness. Intensification factors on the sensitivity of KODAK AA x-ray films of up to $5.5,4.6$, and 8.7 were determined for aluminum, copper and lead respectively. The equilibrium thicknesses for back screens were found to be 40,65 and $110 \mathrm{mg} / \mathrm{sq} \mathrm{cm}$ respectively. For front screens, the equilibrium thicknesses is $110 \mathrm{mg} / \mathrm{sq} \mathrm{cm}$ in all cases. The results were compared with the absorbed dose in the film's emulsions, calculated by Monte Carlo methods and found to agree within 10 percent.

101,670

\section{PB91-195289}

Not available NTIS

National Bureau of Standards (NML), Gaithersburg

MD. Quantum Metrology Group.

Anomalous Lgamma2,3 X-ray Emission Spectrum of Xe.

Final rept.

M. Ohno, and R. E. LaVilla. 1988, 5p

Pub. in Physical Review A 38, n7 p3479-3483 1988.

Keywords: * Xenon, " $X$ ray spectra, Emission spectra, Greens function, Fluorescence; Reprints.

The L(gamma 2,3)(2s(sup -1)-> $4 p($ sup -1)) x-ray emis sion spectrum (XES) of gaseous xenon was measured in fluorescence with a high resolution vacuum double crystal spectrometer. The spectrum was also calculated by the Green's function method. The agreement is excellent. It is shown that the spectrum can be interpreted essentially in terms of the spectral function of the $4 p$ hole. The effects of the initial $2 s$ hole state on the final $4 p$ hole state are small. The spectrum shows that the quasi-particle picture of a $4 p$ hole in xenon breaks down due to strong $4 p($ sup -1$)<-->4 d$ (sup -2) $4(n$, epsilon)f super Coster-Kronig processes.

101,671

PB91-195453

Not available NTIS

National Inst. of Standards and Technology (NML) Gaithersburg, MD. Electricity Div.

Observation of Atoms Laser Cooled Below the Doppler Limit.

Final rept.

W. D. Phillips, C. I. Westbrook, P. D. Lett, R. N.

Watts, P. L. Gould, and H. J. Metcalf. 1989, 3p

Pub. in At. Phy. 11, p633-635 1989.

Keywords: Reprints, "Ultracold atoms, Sodium atoms, Laser cooling. 
The authors have measured the temperature of a three-dimensional gas of laser cooled $\mathrm{Na}$ atoms to be as low as 40 + or - 20 microK. This is in strong dis agreement with the expected Doppler-cooling limit of 240 microK.

\section{1,672}

PB91-195503

Not available NTIS National Inst. of Standards and Technology (NML), Gaithersburg, MD. Atomic and Plasma Radiation Div. Relativistic Distorted-Wave Cross Sections for Electron-Impact Excitations of Berylliumllke Ions. Final rept

W. J. Qian, J. P. Desclaux, and Y. K. Kim. 1989, 9p Pub. in Physical Review A 39, n9 p4509-4517 1989 Keywords: "Electron-ion collisions, Electron impact, Relativistic effects, Wave functions, Cross sections, Excitation, Reprints, "Beryllium-like ions.

Relativistic distorted-wave Born (RDWB) cross sections of Be-like ions for excitations from the ground state, $2 s(2)$ singlet $S(0)$, to the $2 s 2 p$ triplet $P(1)$ and singlet $P(1)$ state by electron impact are reported for $\mathrm{Ne}(6+)$ through $\mathrm{U}(88+)$. Multiconfiguration DiracFock (MCDF) wave functions were used to describe the target ion states, and the relativistic continuum wave functions were calculated in the field of (frozen) target ion charge distribution with the configuration-average exchange potential. Cross sections are presented in compact fitting formulas that allow interpolations to determine cross sections with high precision for arbitrary Be-like ions $(10=$ or $<Z=$ or $<92)$ and arbitrary incident energies ( $\mathrm{T}=$ or $<20 \mathrm{keV}$ ).

\section{1,673}

PB91-195511

Not available NTIS

National Inst. of Standards and Technology (PL) Gaithersburg, MD. Atomic Physics Div.

Spectrum and Energy Levels of Seven-Times-Ionized Krypton (Kr VIII) and Resonance Lines of Eight-Times-Ionized Krypton (Kr IX).

Final rept.

J. Reader, N. Acquista, and V. Kaufman. 1991, 10p

Sponsored by Department of Energy, Washington, DC Pub. in Jnl. of the Optical Society of America B 8, n3 p538-547 Mar 91

Keywords: "Krypton ions, "Line spectra, "Energy levels, Extreme ultraviolet radiation, Reprints, Copperlike ions.

The spectrum of the copperlike ion $\mathrm{Kr}$ VIII was observed with a low-inductance spark and 10.7-m normal- and grazing-incidence spectrographs. A total of 46 new lines with wavelengths ranging from 115 to 696 A were identified: 18 of the type $3 \mathrm{~d}(10) \mathrm{nl}$ $3 d(10) n l ', 10$ of the type $3 d(10) 4 s-3 d(9) 4 s 4 p$, and 18 of the type $3 d(10) 4 p-3 d(9) 4 p(2)$. From these lines 37 new energy levels were determined: 14 of the type $3 \mathrm{~d}(10) \mathrm{nl}, 10$ belonging to $3 \mathrm{~d}(9) 4 \mathrm{~s} 4 \mathrm{p}$, and 13 to $3 d(9) 4 p(2)$. New values for all known levels were derived by combining the present results with measurements at longer wavelength by Gallardo et al. (Appl. Opt. 28, 5088 (1989)). The ionization energy was found to be $1014665+$ or $-25 / \mathrm{cm}(125.802+$ or $0.003 \mathrm{eV}$ ). New wavelength measurements were made for the $3 d(10)-3 d(9) 4 p$, $4 f$ resonance lines of the nickellike ion $\mathrm{Kr}$ IX.

\section{1,674}

PB91-195602

Not available NTIS

National Inst. of Standards and Technology (NML), Gaithersburg, MD. Atomic and Plasma Radiation Div. Dielectronic Recombination Rate Coefficients for Ions of the Fluorine Isoelectronic Sequence.

Final rept.

L. J. Roszman. 1989, 2p

Sponsored by Department of Energy, Washington, DC. Office of Fusion Energy.

Pub. in Physical Review A 39, n2 p913-914 1989.

Keywords: Isoelectronic sequence, Krypton ions, Molybdenum ions, Selenium ions, Reprints, "Fluorine-like ions, "Dielectronic recombination.

The dielectronic-recombination rate coefficients for the fluorine isoelectronic sequence have been revised due to an error for the rate coefficients of $\operatorname{Kr}(27+)$ and $\mathrm{Mo}(33+)$. Additionally, the calculations of the dielec tronic-recombination rate coefficients associated with the delta $n=0$ radiative transitions have been extended to include orbital angular momentum values for the Rydberg and continuum electrons as high as 15 and 17 respectively. A separate calculation has been made for the rate coefficient of $\mathrm{Se}(24+)$ in order that comparisons can be made with recently completed relativistic calculations.

\section{1,675}

PB91.195610

Not available NTIS

National Inst. of Standards and Technology (NML)

Gaithersburg MD. Atomic and Plasma Radiation Div.

and Thermal Equilibration upon Dielectronic Recombination in Low Density to

L. J. Roszman. 1989, 5p

Sponsored by Department of Energy, Washington, DC. Office of Fusion Energy.

Pub. in Physical Review A 39, n4 p2073-2077 1989.

Keywords: Isoelectronic sequence, Metastable state Excited states, Reprints, ${ }^{*}$ Oxygen-like ions, Dielectronic recombination.

The decrease of the dielectronic recombination rate coefficient by the equilibration of the highly excited final states of the process with the plasma continuum is examined for the oxygen isoelectronic sequence. The enhancement of the effective rate of dielectronic recombination by the presence of metastable states in the ground configuration of the initial ion is analyzed for $\mathrm{Fe}(18+)$.

\section{1,676}

$-200816$ Gaithersburg, MD. Atomic Physics Div.

$5 s(2) 5 p(2)-(5 s(2) 5 p 5 d+5 s 5 p(3)+5 s(2) 5 p 6 s+$ $5 s(2) 5 p 7 s$ ) Transitions in $s b \quad I I$ and $5 s(2) 5 p$ $(5 s 5 p(2)+5 s(2) n l)$ Transitions in Sb III.

Final rept.

B. Arcimowicz, Y. N. Joshi, and V. Kaufman. 1989,

7p

Pub. in Canadian Jnl. of Physics 67, n6 p572-578 1989

Keywords: "Antimony ions, "Ultraviolet spectra, Electron transitions, Ground state, Reprints.

The spectrum of antimony was photographed in the 575-2300 A region using a hollow cathode and a triggered spark source. The analysis of the $5 \mathrm{~s}(2) 5 \mathrm{p}(2)$ $(5 s(2) 5 p 5 d+5 s 5 p(3)+5 s(2) 5 p 6 s+5 s(2) 5 p 6 s+$ $5(2) 5 p 7 s$ ) transitions in Sb II spectrum was revised and interpreted on the basis of multiconfiguration interaction calculations. Accurate wavelength measurements of Sb III lines lead to a revised ground-state $5 s(2) 5 p$ doublet $P$ interval value of $6574.5 / \mathrm{cm}$.

\section{1,677}

Not available NTIS National Inst. of Standards and Technology (NML) Boulder, CO. Time and Frequency Div.

Clocks, Atomic and Molecular.

Final rept.

J. A. Barnes, and J. J. Bollinger. 1991, 2p

See also PB81-236606.

Pub. in Encyclopedia of Physics, p154-155 1991.

Keywords: *Frequency standards, "Atomic clocks, lon traps(Instrumentation), Cesium, Time, Reprints, Laser cooling.

Atomic and molecular clocks are described in simple terms using the cesium atomic clock as an example. Some applications of atomic clocks are listed. Curren research on frequency metrology including ion traps, laser cooling, and optical frequency standards are discussed.

\section{1,678}

Not available NTIS National Inst. of Standards and Technology (NML) Boulder, CO. Time and Frequency Div.

Observation of Correlations in Finite, Strongly Coupled Ion Plasmas.

Final rept.

J. J. Bollinger, S. L. Gilbert, D. J. Heinzen, W. M. Itano, and D. J. Wineland. 1990, 11p

Sponsored by Air Force Office of Scientific Research, Bolling AFB, DC., and Office of Naval Research, Arlington, VA

Pub. in Proceedings of Yamada Conference (24th) on Strongly Coupled Plasma Physics, Lake Yamanaka, Japan, August 29-September 2, 1989, p177-187 1990.

Keywords: Laser induced fluorescence, Beryllium ions Reprints, "Ion plasmas, Penning traps, Laser cooling

The authors have observed spatial correlations with up to $15,000 \mathrm{Be}(1+)$ ions in a Penning trap with Gamma
100. These correlations are strongly affected by the boundary conditions and take the form of concentric shells as predicted by computer simulations. In the paper the authors briefly describe the experimental confinement geometry and the laser cooling of the ions. Experimental techniques for observing the shell structure are described. The relatively large spacings between the ions permit the shells to be directly viewed by imaging the $\mathrm{Be}(1+)$ laser-induced fluorescence onto a photon-counting camera. Diagnostic techniques capable of measuring the ion diffusion are then discussed. Qualitative observations of the ion diffusion are compared with theoretical predictions.

101,679

PB91-200881

Not available NTIS

National Inst of Standards and Technology (NML) Boulder, CO. Quantum Physics Div.

Near-Threshold Vibrational Excitation of $\mathrm{H} 2$ by Electron Impact: Resolution of Discrepancies between Experiment and Theory.

Final rept.

S. J. Buckman, M. J. Brunger, D. S. Newman, G.

Snitchler, S. Alston, D. W. Norcross, M. A. Morrison,

B. C. Saha, G. Danby, and W. K. Trail. 1990, 4p See also PB91-101584

Pub. in Physical Review Letters 65, n26 p3253-3256, 24 Dec 90.

Keywords: "Electron-molecule collisions, "Hydrogen, Vibrational states, Excitation, Reprints.

New measurements and calculations of near-threshold $(0.5-5.0 \mathrm{eV})$ e- $\mathrm{H} 2$ vibrational-excitation cross sections challenge previous determinations based on transport analysis. Elastic and $m u(0)=0->m u=1$ cross sections measured in a crossed electron-molec ular-beam apparatus agree well with values calculated using a vibrational close-coupling theory with a separable representation of exchange, particularly at energies below $2.0 \mathrm{eV}$, but are incompatible with cross sections derived via analysis of electron-swarm transport data.

\section{1,680}

PB91-202903

Not available NTIS

National Inst of Standards and Technology (NEL) Boulder, CO. Electromagnetic Technology Div.

Enhanced Flux Creep in Nb-Ti Superconductors After an Increase in Temperature.

Final rept.

R. W. Cross, and R. B. Goldfarb. 1991, 2p

Sponsored by Department of Energy, Washington, DC Pub. in Applied Physics Letters 58, n4 p415-416, 28 Jan 91.

Keywords: "Superconducting super collider, "Superconducting cables, Superconducting magnets, Tem perature dependence, Magnetization, Relaxation, Reprints, Flux creep, Niobium titanium.

The magnetic fields of Superconducting Super Collider (SSC) dipole magnets change with time when the magnets are operated at constant current. The decay of the field is thought to be a consequence of flux creep in the Nb-Ti filaments in the superconducting cables. However, measured magnetic relaxation of small samples of SSC cable as a function of time is unlike the large decays that are observed in the fields of the actual magnets. The authors have made relaxation measurements on sample SSC conductors at 3.5 and $4.0 \mathrm{~K}$ after field cycling. The decay at both temperatures was $2.8 \%$ in 50 min. However, the relaxation measured after a temperature increase from 3.5 to 4.0 $\mathrm{K}$ was $4.8 \%$ in 50 min. A likely reason for the greater magnetization decay is that, after an increase in temperature, the $\mathrm{Nb}-\mathrm{Ti}$ is in a supercritical state, with shielding currents flowing at a density greater than the new critical current density. This causes enhanced flux creep. The authors suggest that a small temperature rise during the operation of SSC magnets may contribute to the unexpectedly large magnetic field decay

101,681

PB91-202911

Not available NTIS

National Inst. of Standards and Technology (NEL) Boulder, CO. Electromagnetic Technology Div

Hall Probe Magnetometer for SSC Magnet Cables: Effect of Transport Current on Magnetization and Flux Creep.

Final rept.

R. W. Cross, and R. B. Goldfarb. 1991, 3p

Sponsored by Department of Energy, Washington, DC. 


\section{PHYSICS}

\section{General}

Pub. in IEEE (Institute of Electrical and Electronics Engineers) Transactions on Magnetics 27, n2 p1796 1798 Mar 91

Keywords: "Superconducting super collider, "Superconducting cables, "Magnetometers, Superconducting magnets, Magnetic hysteresis, Magnetization, Hall effect, AC losses, Reprints, Flux creep.

The authors constructed a Hall probe magnetometer to measure the magnetization hysteresis loops of Superconducting Super Collider magnet cables. The instrument uses two Hall-effect field sensors to measure the applied field $\mathrm{H}$ and the magnetic induction $\mathrm{B}$. Magnetization $M$ is calculated from the difference of the two quantities. The Hall probes are centered coaxially in the bore of a superconducting solenoid with the probe against the sample's broad surface. An alternative probe arrangement, in which $M$ is measured directly, aligns the sample probe parallel to the field. The authors measured $\mathrm{M}$ as a function of $\mathrm{H}$ and field cycle rate both with and without a dc transport current. Flux creep as a function of current was measured from the dependence of ac loss on the cycling rate and from the decay of magnetization with time. Transport currents up to $20 \%$ of the critical current have minimal effect on magnetization and flux creep.

\section{1,682}

\section{PB9 1-202929}

Boulder Inst. Of Standards and Technology (NEL)

Boulder, CO. Electromagnetic Technology Div.

Flux States Generated by Optical Switching of a Superconducting Circuit.

Final rept.

C. E. Cunningham, G. S. Park, B. Cabrera, and M. E. Huber. $1990,2 p$

Sponsored by Office of Naval Research, Arlington, VA. Pub. in Physica B 165 and 166, p113-114 1990.

Keywords: Optical switching, Magnetic flux, Light pulses, Reprints, "Superconducting switches.

The authors pulse a superconducting microbridge with light changing the quantum flux state of a superconducting circuit. Long sequences of pulses are used to measure the degree of correlation between successive flux states. In a series of runs, the pulse length was changed over six decades from $6 \mathrm{~ns}$ to $10 \mathrm{~ms}$. The correlations fit a simple Fokker-Planck conditional probability model.

\section{1,683}

PB91-203067

Not available NTIS

National Inst. of Standards and Technology (NML), Boulder, CO. Quantum Physics Div.

Probe for Real-Time Images of Particle Beams and Their Analyses in a Merged-Beams Apparatus.

\section{Final rept.}

J. L. Forand, C. Timmer, E. Wahlin, B. D. DePaola G. H. Dunn, D. R. Swenson, and K. Rinn. 1990, 6p Contract DE-Al05-86ER53237

Sponsored by Department of Energy, Washington, DC Pub. in Review of Scientific Instruments 61, n11 p3372-3377 Nov 90 .

Keywords: "Probes(Electromagnetic), "Particle beams, Charged particles, Real time, Density, Reprints.

The authors have developed a real-time charged particle beam probe which can be used both as a beam diagnostic (beam tuning) and to quantitatively determine the three-dimensional density distribution of a beam. The probe consists of a microchannel plate, an aluminized phosphor screen deposited on a fiber-optic faceplate, a flexible fiber-optic bundle, and a charge injection device (CID) in tandem. Digital or analog video output of the CID is fed into a computer or oscilloscope, respectively, for analysis or observation. The probe can be moved continuously along the beams' paths to obtain 'pictures' of the beam at any desired interval thus giving three-dimensional form factors. Either beam singly or both beams simultaneously may be observed on the monitor facilitating the task of beam adjustment for optimum overlap. The digital output is used for quantitative computation of the beam overlaps. The device is linear with beam intensity, but the performance degrades with time due to beam damage of the microchannel plate.

\section{1,684}

PB91-203083

Not available NTIS

National Inst. of Standards and Technology (NML), Boulder, CO. Quantum Physics Div.
Measurements of Velocity-Changing Collision Kernels.

Final rept.

K. E. Gibble, and A. C. Gallagher. 1991, 15p

Grant NSF-PHY86-04504

Sponsored by National Science Foundation, Washington, DC.

Pub. in Physical Review A 43, n3 p1366-1380, 1 Feb 91.

Keywords: "Atomic collisions, Line broadening, Ground state, Rare gases, Rubidium, Reprints, "Collision kernals.

Measurements of velocity-changing collision (VCC) kernels are obtained using velocity-selective optica pumping. This is the first measurement of the shape of an elastic VCC kernel over the full thermal range of velocity changes. Since ground-state collisions are studied (quintet $5(1 / 2)$ ) $\mathrm{Rb}$ and $\mathrm{He}, \mathrm{Ne}, \mathrm{Ar}, \mathrm{Kr}$, or $X e)$,very low buffer-gas pressures can be used, and therefore pressure broadening (dephasing collisions) does not obscure the effects of VCC's. The data are deconvolved to eliminate the effects of the natural linewidth and are then extrapolated with respect to buffer-gas pressure to determine the single-collision limit. For small initial velocities, the measured kernels are consistent with a hard-sphere model for large ve locity changes, but differ significantly for small velocity changes. The measured VCC kernels for large initia velocities and large velocity changes do not exhibit the agreement with the hard-sphere kernel. This implies that information about the inter-atomic potential can be obtained from a measurement of VCC kernels. The authors also show the inadequacy of the much-used Keilson-Storer kernel (J. Appl. Math. 10, 243 (1952)).

\section{1,685}

PB91-203125

Not available NTIS

National Inst of Standards and Technology (NML)

Boulder, CO. Quantum Physics Div.

Squeezed Quantum States of Relic Gravitons and Primordial Density Fluctuations.

Final rept.

L. P. Grishchuk, and Y. V. Sidorov. $1990,9 p$

Pub. in Physical Review D 42, n10 p3413-3421, 15 Nov 90 .

Keywords: "Gravitons, Gravitational waves, Particle production, Quantum mechanics, Black holes, Cosmology, Reprints.

The close relationship between the theory of particle creation in external fields and the theory of quantum mechanical squeezed states is clarified. It is shown that relic gravitons (and other primordial perturbations), created from zero-point quantum fluctuations in the course of cosmological evolution, should now be in strongly squeezed states. The statistical properties of the stochastic collection of relic gravitational waves are investigated. Some other examples of particle creation, and in particular Hawking's process of black hole evaporation, are considered in the context of the theory of squeezed states.

\section{1,686}

PB91-203166

Not available NTIS

National Inst. of Standards and Technology (NML) Gaithersburg, MD. Center for Radiation Research.

Polarizability of the Photon.

Final rept.

E. Hayward. 1989, 3p

Pub. in Physics Letters B 223, n2 p133-135, 8 Jun 89

Keywords: *Protons, Scattering cross sections, Dispersion relations, Polarization, Reprints.

The relationship between the static polarizability of the proton and the electric and magnetic polarizabilities obtained from low-energy photon scattering is dis cussed. It is shown that a consistent picture emerges if one associates the static polarizability with the imaginary part of the forward scattering amplitude, and the photon scattering cross section with the real part.

\section{1,687}

PB91-203216 Not available NTIS

National Inst. of Standards and Technology (NEL), Boulder, CO. Electromagnetic Fields Div.

Generalization of the Cornu Spiral for Lossy Media.

Final rept.

D. A. Hill. 1991, 3p

Pub. in Jnl. of Applied Physics 69, n3 p1772-1774, 1 Feb 91.
Keywords: "Fresnel diffraction, Fresnel integrals, Fresnel zones, Complex variables, Lossy media, Half planes, Reprints, Cornu spiral.

The classical problem of Fresnel diffraction by a straightedge is extended to allow for loss in the medium. The loss modifies the usual Cornu spiral solution because the argument of the Fresnel integral becomes complex. For high loss, the contributions of the outer Fresnel zones are highly attenuated.

\section{1,688}

PB91-203240

Not available NTIS

National Inst. of Standards and Technology (NML), Boulder, CO. Quantum Physics Div.

Asymptotic Expansions of the Kernel Functions for Line Formation with Continuous Absorption.

Final rept.

D. G. Hummer. 1991,6p

Contract NAGW-766, Grant NSF-AST88-02937

Sponsored by National Aeronautics and Space Administration, Washington, DC., and National Science Foundation, Washington, DC

Pub. in Jnl. of Quantitative Spectroscopy and Radiative Transfer 45, n4 p211-216 1991.

Keywords: "Line spectra, Radiative transfer, Asymptotic series, Analytic functions, Kernel functions, Line broadening, Reprints.

Asymptotic expressions are obtained for the kernel functions Mn2(tau, alpha, beta) and K2n(tau, alpha, beta) appearing in the theory of line formation with complete redistribution over a Voigt profile with damping parameter alpha in the presence of a source of continuous opacity parameterized by beta. For alpha $>0$, each coefficient in the asymptotic series is expressed as the product of analytic functions of alpha and eta identically = beta(tau) separately. For Doppler broadening, only the leading term can be evaluated analytically.

\section{1,689}

PB91-203257

Not available NTIS

National Inst. of Standards and Technology (NML), Gaithersburg, MD. lonizing Radiation Div.

NIST High-Dose Calibration Services.

Final rept.

J. C. Humphreys. $1989,5 p$

Pub. in Nuclear Instruments and Methods in Physics Research B40-41, pt2 p1173-1177 Apr 89.

Keywords: "Radiation doses, "Dosimeters, "Calibration, Gamma rays, Measurement, Reprints, Traceability, US NIST.

There is a need for standardization of high-dose measurements used in the radiation processing industry in order to provide measurement assurance and traceability to national standards. NIST provides dosimetry calibration services to this industry. One of these services involves the administering of known absorbed doses of gamma rays to customer-supplied dosimeters. The dosimeters are packaged to provide appropriate electron equilibrium conditions and are irradiated in a standard (60)Co calibration facility; this provides a calibration of the response of that batch of dosimeters. Another service consists of supplying to a customer calibrated transfer dosimeters for irradiation with the customer's in-house radiation source. The irradiated transfer dosimeters are then returned to NIST for analysis and the results reported to the customer, thereby providing a calibration of the dose rate of the customer's source.

\section{1,690}

PB91-203307 Not available NTIS National Inst. of Standards and Technology (NML), Gaithersburg, MD. Molecular Spectroscopy Div.

Notes and Comments on Roundtable Discussion on Laser-Assisted Collisions and Collision-Induced Spectra.

Final rept.

P. S. Julienne, and L. Frommhold. 1989, 7p

Pub. in Spectral Line Shapes, p800-806 1989.

Keywords: Collision broadening, Line broadening, Line spectra, Dipole moments, Reprints, "Laser assisted collisions, "Collision induced spectra.

It is especially fitting at the conference, which for the first time brings together the communities on line broadening and collision-induced absorption, that the roundtable sessions on laser-assisted collisions and collision-induced spectra be combined into one dis- 
cussion. This enables one to point out the very strong similarities between these two fields, both in the types of phenomena treated and in the methodology used to calculate the spectra. Therefore, as a major theme of the discussion, the authors would like to point out the similarities and differences between the two fields.

\section{1,691}

PB91-203315

Not available NTIS

National Inst. of Standards and Technology (NEL),

Boulder, CO. Electromagnetic Fields Div.

Estimation of Electromagnetic Flelds In Complex Environments.

Final rept.

M. Kanda, and J. Randa. 1991, 6p

Pub. in Proceedings of the International Zurich Symposium and Technical Exhibition on Electromagnetic Compatibility (9th), Zurich, Switzerland, March 12-14 1991, p337-342.

Keywords: "Electromagnetic fields, "Field strength, "Estimating, Statistical analysis, Finite element method, Directional measurement, Reprints, Nationa Institute of Standards and Technology.

The problem of measuring and characterizing complicated multiple-source, multiple-frequency electromagnetic environments is becoming more important and more difficult as electrical devices proliferate. The paper reviews three general approaches to the problem which have been investigated at the National Institute of Standards and Technology (NIST). The three approaches are: a statistical treatment of the spatial distribution of electromagnetic field intensities; a nu merical computation using a finite-element (or lattice) form of the electromagnetic action functional; and use of a directional probe to scan a volume. All three methods are still in the development stage, but each appears promising.

101,692

PB9́1-203372

Not available NTIS National Bureau of Standards (NEL), Gaithersburg MD. Statistical Engineering Div.

Experimentation and Measurement.

Final rept.

H. H. Ku. $1988,5 p$

See also PB84-233659.

Pub. in Basic Questions in Fatigue: ASTM STP 924, v1 p9-13 1988.

Keywords: "Experimental design, Statistical analysis, Variability, Planning, Reprints.

The paper reviews a number of statistical concepts and methods that could be helpful to experiments in the planning stage. These include: (1) sharpening the objective of the experiment, (2) avoiding confounding of experimental factors, and (3) providing realistic estimates of variabilities. Two examples are given.

\section{1,693}

\section{PB91-203398}

Not available NTIS

National Bureau of Standards (NML), Gaithersburg, MD. Ionizing Radiation Div.

Cross-Section Measurements in the IntermediateEnergy Standard Neutron Field.

Final rept.

G. P. Lamaze, C. M. Eisenhauer, J. A. Grundl, E. D.

McGarry, and F. J. Schima. 1988, 4p

Pub. in Proceedings of International Conference on Nuclear Data for Science and Technology, Mito Japan, May 30, 1988, p1033-1036.

Keywords: "Neutron cross sections, Cobalt 59 target Gold 197 target, Indium 115 target, Scandium 45 target, Silver 109 target, Sodium 23 target, Neutron reactions, Fast neutrons, Reprints.

Measured and calculated results are reported for the spectrum-averaged cross sections of the $(115) \ln \left(n, n^{\prime}\right)(115 \mathrm{~m}) \ln , \quad$ (197)Au(n,gamma)(198)Au, (23) Na(n,gamma)(24) Na, (59)Co(n,gamma) (60)Co and (45)Sc(n,gamma)(46)Sc reactions in the Interme diate Energy Standard Neutron Field (ISNF) at the National Bureau of Standards (NBS). The ISNF spectrum resembles a fission spectrum at energies above 3.7 $\mathrm{MeV}$; between 0.18 and $3.7 \mathrm{MeV}$, the spectrum is dominated by neutrons returning from the graphite after several collisions; and at lower energies the shape is determined by the $1 / \mathrm{v}$ absorption in the (10)B shell. The median energy is about $0.58 \mathrm{MeV}$, with $98 \%$ of the neutron fluence rate occuring between $1,2 \mathrm{keV}$ and $5.6 \mathrm{MeV}$. The fluence was determined by a fluence transfer reaction $((235) \mathrm{U}(\mathrm{n}, \mathrm{f})(\mathbf{1 4 0}) \mathrm{Ba})$ from a well-calibrated (252) Cf fission source.
101,694

PB91-203406

Not available NTIS National Bureau of Standards (NML), Gaithersburg, MD. lonizing Radiation Div.

Spectrum-Averaged Cross-Section Measurement of $(103) R h(n, n)(103 m) R h$ in the $(252) C f$ Fission Neutron Spectrum.

Final rept.

G. P. Lamaze, F. J. Schima, C. M. Eisenhauer, and

V. Spiegel. 1988, $5 p$

Pub. in Nuclear Science and Engineering 100, n1 p4347 Sep 88.

Keywords: "Rhodium 103 target, "Neutron cross sections, Neutron scattering, Fission neutrons, Inelastic scattering, Dosimetry, Califomium 252, Reprints.

Because of the close correspondence of the (103)Rh $\left(n, n^{\prime}\right)$ differential cross section and the kerma muscle response function for neutrons, rhodium is an attractive neutron kerma monitor. In support of its use as a neutron monitor, the spectrum averaged cross section, sigma(bar), has been measured for a (252)Cf fission neutron spectrum. Pairs of rhodium foils were irradiated on opposite sides of a thinly-encapsulated (252)Cf neutron source. The neutron fluence incident on the foils is determined by the source strength, average foil distance, and length of time of the irradiation. Corrections are made for neutron scattering, saturation of activity, and attenuation of the $x$-rays in the foil during counting. The $x$-rays were detected with an intrinsic germanium $x$-ray detector. The results of five separate irradiations yield a value of
sigma(bar)(star) $P(K x)=62.3+$ or $-19 \mathrm{mb}$. A dscussions of systematic uncertainties is given.

\section{1,695}

\section{PB91-203422}

National Inst. of Standards and Technology (NEL), Gaithersburg, MD. Precision Engineering Div.

Calibration of the NBS 10 mum Space Beads.

Final rept.

T. R. Lettieri, A. W. Hartman, G. G. Hembree, and E. Marx. 1989, 11p

Pub. in Jnl. of Colloid Interface Science 131, n2 p5505601989

Keywords: "Microspheres, "Dimensional measurement, Electron microscopy, Light scattering, Particle size, Diameters, Calibration, Certification, Reprints, "Standard reference materials, Space beads, Micrometrology.

A summary is presented of the procedures used to certify NBS Standard Reference Material 1960, nominal 10 micrometer 'space beads'. Three independent micrometrology techniques were used, with the results agreeing to about $0.1 \%$. Center distance finding, a method based on optical microscopy, gave a value of $9.89+$ or -0.04 micrometers which was chosen as the certified mean diameter. Two supporting measurements, made using metrology electron microscopy and resonance light scattering, yielded mean diameters of $9.886+$ or -0.029 micrometers and $9.898+$ or size distribution for this material is nearly Gaussian with a certified standard deviation of 0.09 micrometrs, excluding outlying particles. The SRM 1960 microspheres were grown in microgravity aboard the NASA space shuttle 'Challenger', making the material the first commercial product to be made in space.

\section{1,696}

\section{PB91-203430}

Not available NTIS

National Inst. of Standards and Technology (NEL) Gaithersburg, MD. Precision Engineering Div.

Dimensional Calibration of an NBS 0.3-mum-Diameter Particle-Sizing Standard.

Final rept.

T. R. Lettieri, and G. G. Hembree. $1989,7 p$

Pub. in Jnl. of Colloid Interface Science 127, n2 p566 5721989

Keywords: "Microspheres, "Dimensional measurement, Transmission electron microscopy, Light scattering, Particle size, Calibration, Certification, Diame ters, Reprints, "Standard reference materials, Micrometrology.

The article summarizes the procedures used at the $\mathrm{Na}$ tional Bureau of Standards (NBS) in the certification of a new submicrometer particle-sizing Standard Reference Material (SRM 1691). The mean diameter of the polystyrene microspheres was accurately measured using two independent micrometrology techniques.
One technique, transmission electron microscopy, measured the particles dried and in a vacuum, and gave a result of $0.269+$ or -0.007 micrometer for the certified mean diameter. The supporting method, quasi-elastic light scattering, yielded a value of 0.276 + or - 0.007 micrometer for the diameter of the microspheres in liquid suspension. Descriptions of the experimental techniques, data analyses, and sources of error are discussed in the paper. The calibrated $\mathrm{mi}$ crospheres are now available from NBS for use as a primary length standard in the submicrometer size range. Applications include microcontamination measurement in semiconductor processing, atmospheric sampling, and electron-microscope calibration.

\section{1,697}

PB91-203455

Not available NTIS

National Bureau of Standards (NML), Gaithersburg. MD. Office of Standard Reference Data.

Critical Data in Physics and Chemistry.

Final rept.

D. R. Lide, and B. B, Molino. 1987, 12p

Pub. in Encyclopedia of Physical Science and Technology, v3 p786-797 1987

Keywords: "Physical properties, "Chemical properties, Atomic weights, Fundamental constants, Thermody namics, Fluids, Historical aspects, Reprints, Critical data.

The history and current status of activities related to critical evaluations of physical and chemical data are summarized. A general description of methods for evaluating data is given. Representative tables of important physical and chemical constants are included.

\section{1,698}

PB91-203638

Not available NTIS

National Inst. of Standards and Technology (NML)

Boulder, CO. Quantum Physics Div.

Observation of the Cesium Clock Transition Using

Final rept.

C. Monroe, H. Robinson, and C. Wieman. 1991, 3p

Pub. in Optics Letters 16, n1 p50-52, 1 Jan 91.

Keywords: "Atomic clocks, Electron transitions Cesium, Reprints, Atom traps, Laser cooling.

Cesium atoms in a vapor cell have been trapped and cooled by using light from laser diodes. The $6 S \mathrm{~F}=4$ $\mathrm{m}=0->6 \mathrm{SF}=3, \mathrm{~m}=0$ hyperfine clock transition was excited as these atoms then fell $2.5 \mathrm{~cm}$ in darkness. The authors observed a linewidth of $8 \mathrm{~Hz}$ with good signal-to-noise ratio. This gave a short-term frac tional frequency resolution of $6.5 \times 10$ to the -12 power/sec (sup 1/2), and there is potential for substantial improvement. The apparatus is extremely simple and compact, consisting of a small cesium vapor cell and two diode lasers.

\section{1,699}

\section{PB91-203778}

Not available NTIS

National Inst. of Standards and Technology (NML) Gaithersburg, MD. Radiation Physics Div.

High Harmonic Generation in Hydrogenic Ions. Final rept.

L. Pan, K. T. Taylor, and C. W. Clark. 1989, 4p 89.

Keywords: "Harmonic generation, Multi-photon processes, Nonlinear optics, Reprints, "Hydrogen-like ions "Hydrogenic ions.

The authors have calculated the frequency-dependent nonlinear susceptibilities of hydrogenic ions up to very high order of nonlinearity. The critical driving intensity at which the radiated harmonic intensity of the $(q+2)$ th order, produced by the direct process, exceeds that of the qth order, is found to decrease rapidly with $q$. The computed values of critical intensity are comparable to those at which 'plateau' behavior is observed in recent experiments.

\section{1,700}

PB91-203828

Not available NTIS

National Inst. of Standards and Technology (NEL)

Boulder, CO. Electromagnetic Technology Div. 


\section{PHYSICS}

\section{General}

Effect of Mechanical Deformation on Nb-Ti Filament Proximity-Effect Coupling at the Edges of SSC Cables.

Final rept.

T. W. Petersen, and R. B. Goldfarb. 1991 2p

Sponsored by Department of Energy, Washington, DC Div. of High Energy Physics.

Pub. in IEEE (Institute of Electrical and Electronics Engineers) Transactions on Magnetics 27, n2 p1809 1810 Mar 91.

Keywords: "Superconducting super collider, "Superconducting cables, Superconducting magnets, Magnetic fields, Proximity effect, Time dependence, Mag netization, Deformation, Reprints, Niobium titanium.

Magnetization as a function of transverse magnetic field and time was measured for short strands extracted from the centers and edges of five $\mathrm{Nb}$-Ti Rutherford cables designed for use in Superconducting Supe Collider dipole magnets. The multifilamentary strands all had 6-micrometer diameter filaments. Edge samples, which had severe mechanical deformation, showed small magnetic coupling losses at low fields compared to no coupling losses for undeformed center strands. Sharp strand bends at cable edges decreases the interfilament spacing to the order of the coherence length in the normal matrix material which increases the effective filament diameter and hystere sis loss at low fields. Microscopic studies of the cables cross sections confirmed smaller interfilament separa tions in these samples. Flux creep measurements, rep resented by the time dependence of magnetization, showed little difference between edge and center samples. This indicates that the proximity-coupled matrix in edge samples is not a significant source of flux creep.

101,701

PB91-203919

Not available NTIS

National Inst. of Standards and Technology (NEL)

Boulder, CO. Electromagnetic Technology Div.

New Look at the Bitter Method of Magnetic Imag-

ing.

Final rept.

P. Rice, and J. Moreland. 1991, 2p

Pub. in Review of Scientific Instruments $62, n 3$ p844 845 Mar 91.

Keywords: Scanning tunneling microscopy, Magnetic storage, Magnetic disks, High resolution, Imaging techniques, Ferrofluids, Reprints, "Bitter method, *Magnetic imaging.

A scanning tunneling microscope (STM) was used in place of an optical microscope in the Bitter method to image the magnetic ferrofluid particles on the surface of a hard disk. The resolution obtainable with the usua Bitter method is limited by the optical viewing of the magnetic particles. Using the scanning tunneling microscope, the authors have obtained image resolution limited only by the ferrofluid particle size.

\section{1,702}

PB91-204115

Not available NTIS

National Inst. of Standards and Technology (NML) Boulder, CO. Quantum Physics Div.

Test of the Inverse-Square Law of Gravitation Using the $300-m$ Tower at Erie, Colorado.

Final rept.

C. C. Speake, T. M. Niebauer, M. P. McHugh, P. T. Keyser, J. E. Faller, J. Y. Cruz, J. C. Harrison, J. Makinen, and R. B. Beruff. 1990, 5p

Pub. in Physical Review Letters 65, n16 p1967-1971 15 Oct 90.

Keywords: "Gravitation, Basic interactions, Towers Tests, Reprints, Inverse square laws, Fifth force.

Gravity was measured at eight different heights on a $300-m$ meteorological tower using LaCoste and Romberg gravimeters. The observed values were adjusted for tides, drift, and gravimeter screw errors, and tested for systematic effects due to tower motion. These re sults are compared with values predicted using Newton's inverse-square law from surface gravity. The differences exhibit no systematic trends and their rms value is well within the estimated errors of the experiment. This result places new constraints on the possible strength and range of any non-Newtonian force.

101,703

PB91-204164

Not available NTIS

National Inst. of Standards and Technology (NML),

Boulder, CO. Quantum Physics Div.
Collisional Energy Transfer between Excited $\mathrm{Sr}$ Atoms.

Final rept.

H. G. C. Werij, M. Harris, J. Cooper, A. Gallagher,

and J. F. Kelly. 1991, 13p

Contract NSF-PHY86- 35

Sponsored by National Science Foundation, Washington, DC

Pub. in Physical Review A 43, n5 p2237-2249, 1 Mar 91.

Keywords: "Atomic collisions, "Strontium, Excited states, Reprints, Collisional energy transfer.

The following energy-transfer reactions have been observed in a Sr vapor following pulsed excitation of the 5 singlet $P(1)$ state. The $r$ singlet $D(2)$ state was populated by a nonlinear stimulated process. By measuring the rate coefficients for these reactions for over 40 final states $n(\sup (2 s+1)) L$, one looks for insight into how these reactions depend on energy defect, spin, and angular momentum. It is found that there is not a very strong dependence on the energy defect, and that singlet and triplet final states are produced in comparable amounts. The final states tend to be populated more effectively as their angular momentum increases. Total rate coefficients are of the order of gaskinetic rates.

\section{1,704}

PB91-216705

PC A07/MF A02

National Inst. of Standards and Technology, Gaithersburg, MD.

Journal of Research of the National Institute of Standards and Technology. May-June 1991. Volume 96, Number 3.

1991, 150p

Also available from Supt. of Docs. as SN703-02700040-7. See also PB91-216713 through PB91216762 and PB91-187617. Library of Congress catalog card no. 89-656121.

Keywords: *Research, Thermal conductivity, Thermal diffusity, Fluids, Surface roughness, Quality control Nondestructive tests, Transistors, Crystal growth, Crystal defects, Time series analysis, Signal processing, Acoustic emissions, Second breakdown.

Contents:

A High-Temperature Transient Hot-Wire Thermal Conductivity Apparatus for Fluids:

Standard Reference Specimens in Quality Control of Engineering Surfaces;

An Automated Reverse-Bias Second-Breakdown Transistor Tester;

High Resolution Synchrotron X-Radiation Diffraction Imaging of Crystals Grown in Microgravity and Closely Related Terrestrial Crystals;

Root Projection of One-Sided Time Series;

Deconvolution of Acoustic Emission and Other Causal Time Series.

\section{1,705}

PB9́1-216812

PC A04/MF A01

National Inst. of Standards and Technology (PL),

Gaithersburg. MD.

Evidence for Surface alpha Particle Clusters in (nat)AG(197)Au from the (e,alpha) Reaction.

W. R. Dodge. Jun 91, 57p NISTIR-4495

Keywords: "Alpha particles, "Cluster model, "Gold 197 target, "Silver, *Electron reactions, MeV range 100-1000, MeV range 10-100, Electroproduction, Protons, Graphs(Charts)

The (nat) $\mathrm{Ag}$ and (197) $\mathrm{Au}(\mathrm{e}, \mathrm{p})$ and (e, alpha) energy and angular distributions were measured at 6 electron bombarding energies between 50 and $115 \mathrm{MeV}$ at the National Institute of Standards and Technology. The (nat) $\mathrm{Ag}$ and (197) $\mathrm{Au}(\mathrm{e}, \mathrm{p})$ angular distributions exhibit an asymmetric component which increases from 0 to $50 \%$ as the proton energy increases from the proton Coulomb barrier to $26 \mathrm{MeV}$. The (nat) $\mathrm{Ag}$ and (197) Au (e, alpha) angular distributions exhibit an asymmetric component which increases from 0 to $30 \%$ as the alpha energy increases from the alpha particle Coulomb barrier to $26 \mathrm{MeV}$. The authors conclude that the asymmetric component of the proton and alpha particle yields are the result of direct or semidirect processes rather than resonance processes and hence, because of the short mean free paths of alpha particles in nuclear matter, give evidence for the existence of alpha particle clusters in the nuclear surface.
National Inst. of Standards and Technology (MSEL), Gaithersburg, MD. Reactor Radiation Div. Transmission Properties of a Counter-Rotating Pair of Disk Choppers.

Final rept.

J. R. D. Copley. 1991, 10p

Pub. in Nuclear Instruments and Methods in Physics Research A303, p332-341 1991

Keywords: "Neutron choppers, Time-of-flight spectrometers, High resolution, Crosstalk, Design, Reprints.

Counter-rotating pairs of disk choppers are used to provide pulsed neutron beams for thermal neutron scattering experiments. An important advantage of this type of device, compared with the single disk chopper, is that each disk can be equipped with several slots. A choice of slot widths is then made possible, simply by changing the relative phasing of the two disks. The resolution of an instrument with counter-rotating chopper pairs can therefore be altered without having to modify the incident wavelength or the speed of the choppers. A potential problem, when there are several slots in each disk, is that there will be 'cross talk', i.e. neutrons will be transmitted by a pair of slots other than the intended combination. The author examines the transmission properties of a counter-rotating pair of choppers, and describes methods to determine whether or not cross talk can occur. An important conclusion is that the axial separation between the two choppers must be included in the analysis; this separation, which is generally small (of order several $\mathrm{cm}$ ), significantly increases the potential for unwanted cross talk. The author briefly discusses the implications of cross talk in the design of a high resolution time-offlight spectrometer.

101,707

PB91-236745

National Inst of Standards and Technology (PL), Gaithersburg, MD. Electron and Optical Physics Div. Associative Ionization of Ultra-Cold Laser Trapped Sodium Atoms.

Final rept.

P. L. Gould, P. D. Lett, R. N. Watts, C. I. Westbrook, P. S. Julienne, W. D. Phillips, H. R. Thorsheim, and J. Weiner. 1989, 14p

Pub. in At. Phys. 11, p215-228 1989

Keywords: "Sodium atoms, Reprints, "Associative ionization, Ultracold atoms, Laser cooling, Laser trapping.

The authors describe their recent investigations of associative ionization in laser cooled sodium confined in a new type of laser trap. Their measured cross section at a temperature $<1 \mathrm{mK}$ is three orders of magnitude larger than that measured in previous experiments at higher temperatures. The authors have also seen a dramatic influence of laser intensity on the collision dynamics.

\section{1,708}

PB91-236828

Not available NTIS

National Inst. of Standards and Technology (PL), Boulder, CO. Time and Frequency Div.

Reply to Comment on 'Quantum Zeno Effect'.

Final rept.

W. M. Itano, D. J. Heinzen, J. J. Bollinger, and D. J.

Wineland. 1991, 2p

See also PB90-254715

Pub. in Physical Review A 43, n9 p5168-5169, 1 May 91.

Keywords: Quantum mechanics, lon storage, Reprints, *Quantum Zeno effect, "Zeno effect, Laser cooling, Penning traps.

Various interpretations of quantum mechanics are valid insofar as they predict the same experimental results. Some invoke 'wave-function collapse' and some do not. An interpretation based on the collapse postulate provides a simple explanation for a recent experimental demonstration of the quantum Zeno effect (Itano et al., Phys. Rev. A-41, 2295 (1990)), but other interpretations are also valid.

101,709 
Cryogenic Fatigue of High-Strength Aluminum Alloys and Correlations with Tensile Properties. Final rept.

L. Ma, J. K. Han, R. L. Tobler, R. P. Walsh, and R. P. Reed. $1990,8 p$

Sponsored by Department of Energy, Washington, DC. Office of Fusion Energy, and Universities Pesearch Association, Berkeley, CA. Superconducting Super Collider Central Design Group.

Pub. in Advances in Cryogenic Engineering (Materials), v36 p375-382 1990.

Keywords: "Superconducting super collider, "Aluminum alloys, "Stress tests, *Cryogenic storage devices, Tensile strength, Fatigue life, Metal sheets, Reprints Aluminum alloy 7075 , Aluminum alloy 7475 , Aluminum alloy 2219, Aluminum alloy 2090 .

Notched and unnotched sheet specimens of four aluminum alloys were fatigue tested to evaluate potential use in the Superconducting Super Collider. Alloys 7075-T6, 7475-T761, 2219-T87, and 2090-T8E41 were tested in axial fatigue at a stress ratio of 0.1 . The unnotched specimens were tested at 295,76 , and $4 \mathrm{~K}$ whereas notched specimens were tested at $76 \mathrm{~K}$ only. The fatigue strengths of these alloys were compared with a practical interest in a life of 100,000 cycles. Fatigue strengths with static tensile strengths for notched and unnotched specimens.

101,710

PB91-237115

Not available NTIS

National Inst. of Standards and Technology (EEEL)

Gaithersburg, MD. Electricity Div.

Monltoring the Mass Standard via the Comparison of Mechanical to Electrical Power.

Final rept.

P. T. Olsen, W. L. Tew, E. R. Williams, R. E.

Elmquist, and $H$. Sasaki. 1991, $6 p$

See also PB91-101501.

Pub. in IEEE (Institute of Electrical and Electronics Engineers) Transactions on Instrumentation and Measurement 40, n2 p115-120 Apr 91 .

Keywords: *Mass, "Standards, Superconducting mag. nets, Rest mass, Hysteresis, Monitoring, Balances, Comparison, Reprints, "Kilogram, Watt.

The paper presents the current status of the NIST SI watt experiment. Included are goals for the near future as well as projections regarding the viability of monitoring and/or replacing the kilogram mass standard. Although several significant systematic errors have ye to be evaluated, the standard deviation of the mean of the authors' present measurement distributions is 0.05 ppm.

\section{1,711}

PB91-237461 Not available NTIS National Inst. of Standards and Technology (NML), Gaithersburg, MD. Gas and Particulate Science Div. Structure of Odd-Odd (132)Sb.

Final rept.

C. A. Stone, W. B. Walters, and S. H. Faller. 1989 ,

Pub. in Physical Review C 39, n5 p1963-1971 1989. Keywords: *Antimony 132, Nuclear energy levels, Nuclear structure, Fission products, Lifetime, Reprints.

New information is presented on the decay of 40-s (132) Sb. A second beta-fed $1+$ level was identified in $\mathrm{Sb}$; it lies at an energy of $2268 \mathrm{keV}$ and is fed by a relatively strong $0.83 \%$ beta-decay branch. Four gamma rays were identified that form a weakly populated cascade from a level at $483 \mathrm{keV}$. Coincidence data demonstrate that the 254-keV level in this cascade is the 102-ns isomer identified previously by Clark et al. A 96-keV, isomeric gamma ray has also been observed that is placed in the decay of the 4.1 - min, 8isomer in (132) Sb. It may also be the 96-keV transition that is associated with the 1.8 microsec isomer identified in the $A=132$ chain by Clark et al. The splitting of the levels in the proton-neutron multiplets is discussed and compared with the results of several recent shell model calculations.

\section{1,712}

\section{PB91-237495}

Not available NTIS

National Inst. of Standards and Technology (PL), Gaithersburg, MD. Atomic Physics Div.

Extreme-Ultravlolet Spectra of $\mathrm{Pd}, \mathrm{Ag}, \mathrm{Cd}$, and $\mathrm{Sn}$ lons Observed In a 1.3-keV Tokamak Plasma. Final rept.

J. Sugar, V. Kaufman, and W. L. Rowan. 1991, 4p

Sponsored by Department of Energy, Washington, DC.
Pub. in Jnl. of the Optical Society of America B 8, n5 p913-916 May 91.

Keywords: *Plasma spectra, Isoelectronic sequence, Extreme ultraviolet radiation, UItraviolet spectra, Palladium, Silver, Cadmium, Reprints, Cadmium-like ions, Chlorine-like ions, Potassium-like ions, Sulfur-like ions.

The elements $\mathrm{Pd}, \mathrm{Ag}, \mathrm{Cd}$, and $\mathrm{Sn}$ were each injected by laser ablation into a tokamak plasma of $1.3-\mathrm{keV}$ electron temperature. Spectra in the range of 50-400 A were observed photographically with a 2.2-m grazingincidence spectrograph. The authors identified lines in the isoelectronic sequences of $\mathrm{S}, \mathrm{Cl}, \mathrm{Ar}$ and $\mathrm{K}$ that were not previously classified. They fall in the range of 51-78 $\mathrm{A}$ and were measured with an uncertainty of or $-0.010 \mathrm{~A}$. Graphs of the differences between the measured and the calculated transition energies vary smoothly and confirm the identifications. Leastsquares fits to these differences permit accurate predictions for elements $\mathrm{Tc}, \mathrm{Ru}, \mathrm{Rh}$, and In that have not been investigated. Similar fits to ground-state double splittings in the $\mathrm{Cl}$ and $\mathrm{K}$ sequences provide accurate values of magnetic dipole transition energies for these ions.

\section{1,713}

PB91-237503

Not available NTIS

National Inst. of Standards and Technology (PL), Gaithersburg, MD. Atomic Physics Div.

Spectra of the P I Isoelectronic Sequence from Co XIII to Mo XXVIII.

Final rept.

J. Sugar, V. Kaufman, and W. L. Rowan. 1991, 5p

Contract DE-AC05-78ET-53043

Sponsored by Department of Energy, Washington, DC Pub. in Jnl. of the Optical Society of America B 8, n1 p22-26 Jan 91.

Keywords: "Plasma spectra, Isoelectronic sequence, M1-transitions, Soft $x$ rays, Reprints, *Phosphorus-like ions.

Phosphorus like spectra $\mathrm{Cu} X V$ to Mo XXVIII have been identified in tokamak- and laser-produced plasmas in the wavelength range of 83 to $163 \mathrm{~A}$. These were obtained with the TEXT tokamak at the University of Texas and the 1-GW Nd:glass laser at the National Institute of Standards and Technology, using 2.2- and 10.7-m grazing-incidence spectrographs, respectively. Wavelength measurements were made with an uncertainty of + or $-0.005 \mathrm{~A}$. All the classified lines arise from the $3 s(2) 3 p(3)-3 s(2) 3 p(2) 3 d$ array. They were identified through detailed comparisons with HartreeFock calculations of wavelengths and relative intensities along the isoelectronic sequence. All spectra from $\mathrm{Cu}$ to Mo were observed, except for $\mathrm{Rb}$ and $\mathrm{Sr}$, whose wavelengths are predicted by interpolation with an uncertainty of + or $-0.02 \mathrm{~A}$. By means of previously observed magnetic-dipole (M1) lines within the $3 s(2) 3 p(3)$ ground configuration and comparisons with published Dirac-Fock calculations, all the energy levels of the configuration were determined with an uncertainty of + or -10 to $/ 100 \mathrm{~cm}$ for Co to Mo. These levels were used to predict all the $M 1$ wavelengths. A correction to the Co XIV ground configuration is made by means of a previously published list of $\mathrm{M} 1$ lines.

\section{1,714}

PB9́1-237511

Not available NTIS

National Inst. of Standards and Technology (PL), Gaithersburg, MD. Atomic Physics Div.

Beam-Foil Study of Two-Electron Transitions in Cu-Llke lons.

Final rept.

J. Sugar, E. Trabert, G. Moller, P. H. Heckmann, J.

$H$. Blanke, and I. Martinson. 1991, 6p

Pub. in Physica Scripta 43, p484-489 1991

Keywords: Extreme ultraviolet radiation, Ultraviolet lasers, Electron transitions, Niobium ions, Molybdenum ions, Silver ions, Reprints, "Copper-like ions.

The transition $3 d(10) 4 p$ doubletP(0)-3d(9)4s(2) doublet $\mathrm{D}$ in $\mathrm{Cu}$-like ions, the XUV laser transition proposed by Morley and Sugar, has now been identified in delayed spectra obtained with foil-excited ion beams of $\mathrm{Nb}, \mathrm{Mo}$ and $\mathrm{Ag}$ Calculations of the wavelengths agree closely with the observations, but the observed decay times are significantly larger than the theoretically expected lifetimes. It is shown that the lifetime is probably obscured by long-lived cascades from the lowest-lying quartet levels.
National Inst. of Standards and Technology (PL), Gaithersburg, MD. Physics Lab. Office.

Basic Standards and Fundamental Constants.

Final rept.

B. N. Taylor. 1989, 3p

Pub. in IEEE (Institute of Electrical and Electronics Engineers) Transactions on Instrumentation and Measurement 38, n2 p164-166 1989 .

Keywords: "Fundamental constants, "Electrical measurement, *Standards, Josephson effect, Least squares method, Reprints, Quantum Hall effect.

These introductory remarks to the panel discussion on Basic Standards and Fundamental Constants at the 1988 Conference on Precision Electromagnetic Measurements (CPEM) touch upon two topics: the importance of precision electromagnetic measurements to the fundamental constants and vice versa; and the idea that recent advances in the determination of a number of constants may have made future leastsquares adjustments of the constants unnecessary.

\section{1,716}

PB91-237750

Not available NTIS

National Inst. of Standards and Technology (NML), Gaithersburg, MD. Atomic and Plasma Radiation Div. Spectroscopic Data Base for Carbon and Oxygen. Final rept.

W. L. Wiese. $1989,2 p$

Pub. in Physica Scripta T28, p10-11 1989.

Keywords: "Carbon, *Oxygen, "Atomic energy levels, *Transition probabilities, "lonization, Spectrum analysis, Wavelengths, Reprints, "Spectroscopic data.

Comprehensive compilations of critically evaluated spectroscopic data for all stages of ionization of carbon and oxygen are briefly reviewed, especially with respect to their scope and the accuracy of the listed data. The spectroscopic quantities covered are wavelengths, energy levels, and transition probabilities.

101,717

PB91-237768

Not available NTIS

National Inst of Standards and Technology (NML), Gaithersburg, MD. Atomic and Plasma Radiation Div. Unified Set of Atomlc Transltion Probabilities for Neutral Argon.

Final rept.

W. L. Wiese, J. W. Brault, K. Danzmann, M. Kock, and $V$. Helbig. 1989, 11p

Pub. in Physical Review A 39, n5 p2461-2471 1989

Keywords: "Argon, "Atomic energy levels, "Transition probabilities, Atomic spectra, Electron transitions, Reprints, "Atomic transition probabilities, "Atomic lifetimes.

While the atomic transition probabilities and radiative lifetimes of neutral argon have been the subject of numerous experiments and calculations, the results exhibit numerous discrepancies and inconsistencies. The authors present for the first time a unified set of atomic transition probabilities, which is consistent with essentially all recent results, sometimes after critical re-analysis. The data consistency and scale confirmation has been achieved in two ways: (1) The authors have carried out some lifetime/branching ratio measurements for a principal $5 p$ level and the associated $4 s-5 p$ transitions. These measurements have very closely confirmed the accuracy of the results of recent independent emission experiments. (2) The authors have critically re-analyzed and revised the literature data for the $4 \mathrm{~s}-4 \mathrm{p}$ transitions, as well as utilized the results of a similar critical analysis for the $4 s-5 p$ transition array, to establish complete sets of absolute data for these arrays. They have found these data to be mutually consistent from cross-correlation checks between the two arrays using recent literature data. $\mathrm{Fi}$ nally, they have proposed renormalization factors for other argon transitions based on the analysis.

101,718

PB91-240762

PC A04/MF A01

Inst. of Standards and Technology (MSEL), Gaithersburg, MD. Polymers Div.

HIgh Magnetlc Fleld Facilities In Japan Reiated to Superconductivity Research (Japan Technology Program)

R. D. Shull. Aug 91, 62p NISTIR-4593

Keywords: "Magnetic fields, "Superconductors, Research facilities, Superconducting magnets, High tem- 


\section{PHYSICS}

\section{General}

perature superconductors, Critical current, Critical field, Magnetic resonance, Japan, "Foreign technology, "High magnetic fields.

The report describes the high magnetic field facilities presently available in Japan and how those facilities are being used for the study of superconducting materials. Particular attention is devoted to Japanese technological advances in the development of superconducting materials which may have an impact on the construction of future high magnetic field facilities Both pulsed magnetic field facilities and constant field generation are evaluated. In addition, future facilities to become available in Japan are listed.

\section{1,719}

PB92-109032 PC A04/MF A01

National Inst. of Standards and Technology (PL)

Gaithersburg, MD

International System of Units (SI).

Special pub. (Final).

B. N. Taylor. Aug 91, 64p NIST/SP-330

Also available from Supt. of Docs. as SN003-003

03099-6. Supersedes PB86-244159.

Keywords: "International system of units, "Metric system, "Units of measurement, Primary standards Translations, "Foreign technology, International Bureau of Weights and Measures.

The booklet is the United States of America edition of the English-language translation of the sixth edition of Le Systeme International d'Unites (SI), the definitive reference on the SI published in 1991 by the International Bureau of Weights and Measures (BIPM) in the French language. The USA edition conforms in substance with the English-language translation that follows the French-language text in the BIPM publication. That translation was a joint effort of the BIPM, the $\mathrm{Na}$ tional Institute of Standards and Technology (NIST) in the United States, and the National Physical Laboratory (NPL) in the United Kingdom. However, to make the booklet helpful to the broadest community of users in the USA, it was necessary to follow current Federa policy, to recognize present USA practices as they are found in the literature of the authors domestic voluntary standards organizations such as ASTM and IEEE and to use American spelling of certain words. Thus, the USA edition differs from the English-language ver sion in the BIPM publication in the following details: (1) the dot is used instead of the comma as the decimal marker; (2) the American spellings 'meter', 'liter', and 'deka' are used instead of 'metre', 'litre', and 'deca'; (3) a small number of footnotes are added for explanatory purposes and to identify USA practices that differ from those suggested in the BIPM publication; (4) in a few instances, American rather than British spelling or usage is followed for a few common words; and (5) the index has been moderately expanded.

\section{1,720}

PB92-112358

PC A04/MF A01

National Inst. of Standards and Technology

Boulder, CO. Electromagnetic Technology Div. Magnetic Measurements for High Energy Physics Applications.

Final rept. Jun 1588 -Jun 1491

R. B. Goldfarb. Aug $91,69 p$ NISTIR-3975

Contract DE-AI05-85-ER40240

Sponsored by Department of Energy, Washington, DC. Div. of High Energy Physics.

Keywords: "Superconducting magnets, "Superconducting cables, Superconducting wires, Superconducting super collider. High energy physics, Time depend ence, Magnetic fields, Critical current, Eddy currents, Magnetization, AC losses, Niobium titanium, Flux creep.

The report is a collection of papers describing magnetic measurements on multifilamentary $\mathrm{Nb}$ - $\mathrm{Ti}$ supercon ductor wires and cables as a function of magnetic field and time at liquid-helium temperatures. The papers deal with ac losses and interfilament coupling by proximity effect and eddy currents. Flux creep was investigated under different experimental conditions. A Hallprobe magnetometer, which was used to measure magnetization and flux creep in the presence of a transport current, is described. A method for increasing the critical current of superconducting cable by controlling twist pitch is demonstrated. A critical-state model for the magnetization of superconductors was developed for samples with field-dependent critical current density and rectangular cross section.
National Inst. of Standards and Technology (TS), Gaithersb

Criteria for the Operation of Federally-Owned Secondary Calibration Laboratories (lonlzing Radiation).

Special pub. (Final)

E. H. Eisenhower. Aug $91,66 p$ NIST/SP-812

Also available from Supt. of Docs. as SN003-00303095-3.

Keywords: "Ionizing radiation, "Survey monitors, "Dosimeters, "Calibration, "Laboratories, Performance standards, Gamma rays, X rays, Beta particles, Alpha particles, Neutrons, Criteria, Secondary laboratories, Accreditation.

The document contains standards of performance for laboratories that calibrate instrumentation used to measure ionizing radiation. Such standards are useful for the development of a secondary level of calibration aboratories that can provide a high-quality link be tween the National Institute of Standards and Technology and those who make routine measurements at the field level. The standards may also be used as criteria on which a decision is based regarding accreditation of a particular laboratory. They were developed by representatives of federally-owned laboratories that perform calibrations of the type addressed by the docu ment. The first major part contains general criteria that must be satisfied by all laboratories seeking accreditation. It includes requirements relating to management and staff, physical aspects of the laboratory, calibrations facilities and equipment, operational procedures, accuracy and quality assurance, and records and reports. Five subsequent major parts establish criteria for calibration of survey instruments, irradiation of personnel dosimeters, calibration of sources, calibration of instruments for diagnostic levels, and calibration of reference-class instruments. The types of radiation covered include gamma rays, $x$ rays, beta particles, neutrons, and alpha particles. An appendix describes the proficiency tests administered by NIST to secondary laboratories as a prerequisite for their accreditation.

\section{1,722}

\section{PB92-116474}

Not available NTIS Boulder, CO. Time and Frequency Div.

303-MHz Frequency Standard Based on Trapped $\mathrm{Be}(+)$ lons.

Final rept.

J. J. Bollinger, D. J. Heinzen, W. M. Itano, S. L.

Gilbert, and D. J. Wineland. 1991, 3p

Sponsored by Office of Naval Research, Arlington, VA., and Air Force Office of Scientific Research, Bolling, and Air AFB, DC.
ing

Pub. in IEEE (Institute of Electrical and Electronics Engineers) Transactions on Instrumentation and Measurement 40, n2 p126-128 Apr 91

Keywords: "Frequency standards, "Atomic clocks, Hyperfine structure, Beryllium ions, Beryllium 9, Ground state, Ultrahigh frequency, Doppler effect, Reprints, Laser cooling, Penning traps, Ion traps.

A 303-MHz hyperfine transition in the ground state of (9) Be(1+) was used as a basis of a frequency standard. The ions were stored in a Penning ion trap. Linewidths as narrow as 900 micro $\mathrm{Hz}$ were obtained. The frequency stability was measured to be better than $3 \times 10$ to the -12 power/square root of tau. The inaccuracy in the second-order Doppler shift was reduced to 5 parts in 10 to the 15th power by laser cooling. An apparent pressure shift with an unexpectedly large value was discovered which limits the accuracy of the current experiment to approximately 1 part in 10 to the 13 power.

\section{1,723}

PB92-116706

Not available NTIS National Bureau of Standards (NML), Gaithersburg, MD. lonizing Radiation Div.

Dosimetry of a Nearly Monoenergetic 6- to 7-MeV Photon Source by Nal(TI) Scintillation Spectrometry.

Final rept.

K. C. Duvall, S. M. Seltzer, C. G. Soares, and B. W. Rust. $1988,5 p$

Pub. in Nuclear Instruments and Methods in Physics Research 272, n3 p866-870 Nov 88.

Keywords: "Gamma dosimetry, "Nal detectors, Scintillation counters, MeV range 01-10, Radiation protection, Fluorine 19 target, Proton reactions, Spectra unfolding, Calibration, Kerma, Reprints.
The dosimetry of a nearly-monoenergetic 6- to $7-\mathrm{MeV}$ photon source developed at the National Bureau of Standards (NBS) for radiation protection instrument calibration has been carried out by Nal(TI) scintillation spectrometry. The approach uses calculated 3 -inch $x$ 3 -inch Nal(TI) detector-response functions that have been shown to be reasonably accurate up to $20 \mathrm{MeV}$. A least-squares fit of the appropriate response functions to a selected region of the pulse-height distribution determines the primary 6- to 7-MeV photon fluence. The uncertainty in the fluence determination is based on the chi squared of the fit, the statistics of the data, and the uncertainty in the response functions. The air kerma delivery due to the primary photons at a reference point in the photon field is calculated from the primary photon fluence.

\section{1,724}

PB92-116714 Not available NTIS National Bureau of Standards (NML), Gaithersburg, MD. Atomic and Plasma Radiation Div.

Atomic Spectroscopy in the 20th Century - A Tribute to Sitterly, Charlotte Moore, on the Occasion of Her 90 th Birthday.

Final rept.

B. Edlen, and W. C. Martin. 1988, 4p

Pub. in Jnl. of the Optical Society of America B 5, n10 p2039-2042 1988 .

Keywords: "Atomic spectroscopy, Reprints, Sitterly Charlotte Moore.

The introduction describes several areas of atomic spectroscopy represented by 24 papers to be published in a feature issue of J. Opt. Soc. Am. B. This feature issue i a tribute to Charlotte Moore Sitterly on the ocassion of her 90 th birthday. The introductory material includes an appreciation of Dr. Sitterly's career at NBS.

\section{1,725}

PB92-116722

Not available NTIS

National Inst. of Standards and Technology (NML), Gaithersburg, MD. Temperature and Pressure Div. Differences between Thermodynamic Temperature and $t$ (IPTS-68) in the Range $230 \mathrm{C}$ to $660 \mathrm{C}$.

Final rept.

R. E. Edsinger, and J. F. Schooley. 1989, 12p

Pub. in Metrologia 26, p95-106 1989.

Keywords: "Temperature scales, Temperature measurement, Melting points, Aluminum, Uncertainty, Comparison, Reprints, International Practical Temperature parison, Reprints, International Practical Temperature
Scale of 1968 , Kelvin Thermodynamic Temperature Scale of 1968, Kelvin Thermodyna
Scale, Gas thermometry, IPTS-68.

Determination of the differences between the Kelvin Thermodynamic Temperature Scale (KTTS) and the International Practical Temperature Scale of 1968 (IPTS-68) in the range from $230 \mathrm{C}$ to $660 \mathrm{C}$ has been accomplished by gas thermometry. The estimated random uncertainty of the results ranges from + or $0.005 \mathrm{C}$ to + or $-0.008 \mathrm{C}$; the major uncertainties appear to lie in the determination of the volume of the gas bulb and the determination of its IPTS-68 temperature. The present results differ noticeably from earlier gas-thermometric determinations in the range of temperature overlap. The value found for the thermodynamic temperature of the freezing point of $\mathrm{Al}$ is $(660.342+$ or -0.015$) \mathrm{C}$.

101,726

PB92-116870

Not available NTIS

National Inst. of Standards and Technology (NML), Boulder, CO. Time and Frequency Div.

Test of the Linearity of Quantum Mechanics by of Spectroscopy of the $(9) \mathrm{Be}(+)$ Ground State.

Final rept.

D. J. Heinzen, J. J. Bollinger, W. M. Itano, S. L.

Gilbert, and D. J. Wineland. 1990, 3p

See also PB90-205899. Sponsored by Air Force Office of Scientific Research, Bolling AFB, DC., and Office of Naval Research, Arlington, VA.

Pub. in Proceedings of Rochester Conference on Coherence and Quantum Optics (6th), Rochester, NY., June 26-28, 1989, p479-481 1990.

Keywords: "Quantum mechanics, "Beryllium 9, Nuclear magnetic resonance, Radiofrequency spectroscopy, Frequency standards, Ground state, Beryllium ions, Linearity, Tests, Reprints, Ion storage, Penning traps

A frequency standard based on an $r$ hyperfine resonance in the ground state of $(9) \mathrm{Be}(1+)$ ions is used to set limits on possible nonlinearities in quantum me- 
chanics. A limit of $2.4 \times 10$ to the -20 power $\mathrm{eV}$ is placed on the size of a possible nonlinear correction to the $(9) \mathrm{Be}(1+)$ nuclear Hamiltonian.

101,727

PB92-117118

Not available NTIS

National Inst. of Standards and Technology (NML), Gaithersburg, MD. lonizing Radiation Div.

Novel Radiation Dosimetry Systems.

Final rept.

W. L. McLaughlin. 1991, 25p

Pub. in Proceedings of International Symposium on High Dose Dosimetry for Radiation Processing Vienna, Austria, November 5-9, 1990, p3-27 1991.

Keywords: "Dosimeters, Electron spin resonance, Free radicals, Luminescence, Polymeric films, Optical activity, Alanines, Reprints, Dye dosimeters, Quartz dosimeters, Telemetering dosimetry, Telemetering dosimetry, Radiochromic dyes, Radiation processing.

New developments of high dose dosimetry systems have led to a number of improved adaptations of existing dosimeters and several promising innovations. These systems include the following: thin film and polymeric 'radiochromic' systems; liquid and gel phase radiochromic systems; free radical systems analyzed by either electron spin resonance (ESR), color complex spectrophotometry, selective electrode potentiometry, or luminescence analysis; saccharide solutions analyzed by ESR or optical rotation measurement; bleachable aqueous dye solutions or dye films analyzed by visible spectrophotometry; inorganic solid state sensors, such as $\mathrm{SiO} 2$, by potentiometric methods or ESR analysis; telemetering dosimetry systems based on laser beam analysis of radiochromic sensors.

101,728

PB92-117134 Not available NTIS National Inst. of Standards and Technology (NML), Gaithersburg, MD. Ionizing Radiation Div.

Temperature Dependence of Radiochromic Film Dosimeters.

Final rept.

W. L. McLaughlin, J. C. Humphreys, W. Z. Ba, H. M. Khan, M. Al-Sheikhly, and W. J. Chappas. 1991, 12p Pub. in Proceedings of International Symposium on High Dose Dosimetry for Radiation Processing, Vienna, Austria, November 5-9, 1990, p305-316 1991.

Keywords: "Photographic film dosemeters, "Film dosimetry *Dosimeters, Temperature dependence, Gamma radiation, Polymeric films, Reprints, Radiochromic dyes, Radiation processing.

Three types of transparent radiochromic films, two forming a blue image and one a red image, are now widely used in high dose dosimetry. These are FWT60-00, a free standing nylon base film (nominal absorbed dose range: 1-50 kGy) which is usually measured at 605 and $510 \mathrm{~nm}$ wavelengths, GafChromic dosimetry media, a coated polyester film (nominal dose range: $0.05-30 \mathrm{kGy}$ ), which is measured at a variety of visible wavelengths from 675 down to $400 \mathrm{~nm}$, and Riso B3, a free standing polyvinylbutyral base film (nominal absorbed dose range 1-200 kGy) which is measured at a single wavelength, $554 \mathrm{~nm}$. Each of these film types is also supplied in other forms for special applications and different useful dose ranges, so that the systems are now able to measure doses and make radiographic images (without development) over a wide dynamic range, namely 10 to 200,000 Gy, with reasonable precision, nominally + or $-3 \%$ at $95 \%$ confidence limits, without appreciable dose rate dependence. The high resolution radiographic images can be used for archival dose distribution records.

\section{1,729}

\section{PB92-126572}

$\mathrm{PC}$ A03/MF A01

Gaithersburg. of

Interpretation of the SI for the United States and Metrlc Conversion Policy for Federal Agencies.

Final rept.

B. N. Taylor. Oct $91,20 p$ NIST/SP-814

Also available from Supt. of Docs. See also PB92109032.

Keywords: "International system of units, "Metric system, Units of measurement, Policies, Federal agencies.

The National Institute of Standards and Technology (NIST) Special Publication replaces National Bureau of Standards (NBS) Letter Circular LC1132 published April 19, 1982. It reprints the Department of Com- merce, NIST, Federal Register notice of December 20 , 1990 titled 'Metric System of Measurement; Interpretation of the International System of Units for the United States' (the International System of Units, abbreviated $\mathrm{SI}$, is the modernized metric system); the Department of Commerce, Office of the Secretary. Federal Register notice of January 2, 1991 titled 'Metric Conversion Policy for Federal Agencies'; and Executive Order 12770 issued by the President of the United States on July 25, 1991 titled 'Metric Usage in Federal Government Programs.' The first Federal Register notice restates the Department of Commerce's interpretation of the International System of Units for the United States that was last published by the Department in 1982; the second revises the Code of Federal Regulations (CFR) to remove the voluntary aspect of the conversion to the metric system of measurement for Federal agencies; and the Executive Order provides Presidential authority and direction for the use of the metric system of measurement by Federal departments and agencies in their programs. Also included is a diagram that shows graphically how the 19 SI derived units with special names are derived in a coherent manner from the SI base and supplementary units.

\section{PROBLEM-SOLVING INFORMATION FOR STATE \& LOCAL GOVERNMENTS}

\section{Police, Fire, \& Emergency Services}

101,730

1-216796

Herndom Group, Inc., Chapel Hill, NC

PC A04/MF A01

First Pass at Computing the Cost of Fire Safety in a Modern Society.

W. P. Meade Jun 91,51p NIST/GCR-91/592

Contract NIST-525BNB1C6678

Sponsored by National Inst. of Standards and Technology (BFRL), Gaithersburg, MD.

Keywords: "Fire safety, "Cost estimates, US NIST, Residential buildings, Commercial buildings, Research management, Economic analysis, Insurance, Construction.

The report provides an estimate of the total annual dollar costs of establishing and maintaining fire safety in 20th-Century United States. The cost of fire safety estimate is needed to serve as a basis for evaluating the benefits and appropriateness of current and pro. posed fire research programs of NIST. This was a relatively modest effort to obtain a first-order estimate of these costs and much of what is reported in these two reports is anecdotal. Nonetheless, the central conclusions one comes to in reading them are that the burden of fire on our society is substantially greater than previously realized and that significant reductions in this burden may be readily achievable through the continued development of fire science and the rapid transfer of fire research results. These findings are particularly relevant as the U.S. economy and its manufacturing infrastructure adjust to the competitive challenges of the global marketplace.

\section{SPACE TECHNOLOGY}

\section{Astronautics}

\section{1,731}

PB91-144352

PC A04/MF A01

National Inst. of Standards and Technology (NEL), Gaithersburg, MD. Robot Systems Div.
Short-Term Evolution for the Flight Telerobotic Servicer.

R. Lumia. Dec 90, 61p NISTIR-4463

Keywords: "Robotics, "Teleoperators, "Remote manipulator system, Space maintenance, Automatic control, Control systems design, Computer vision, Auto mation, Space station servicing, Space telerobotics, Flight telerobotic servicer.

The document identifies near term technology developments which would have significant impact on the evolution of the FTS toward autonomous operation. Analysis of anticipated FTS tasks is used to identify operations that might be performed autonomously rather than in a purely teleoperated fashion. Alternative techniques for automating these operations are then described. A discussion of FTS long term evolution is included as an appendix.

101,732

PB91-185090

PC A06/MF A01

National Inst. of Standards and Technology (NEL),

Gaithersburg, MD. Robot Systems Div.

Recommended Fine Positioning Test for the Development Test Fllght (DTF-1) of the NASA Flight Telerobotic Servicer (FTS)

N. Dagalakis, A. J. Wavering, and P. Spidaliere. Feb $91,124 p$ NISTIR-4478

Prepared in cooperation with National Aeronautics and Space Administration, Greenbelt, MD. Goddard Space Flight Center.

Keywords: "Spacecraft maintenance, "Telerobotics, "Positioning, "Flight tests, Robots, Robot sensors, Robot arms, Standards, Off line systems, Computer programming, DTF-1(Development Test Flight), tion), FTS(Flight Telerobotic Servicer).

The purpose of the report is to propose test procedures for the NASA DTF (Development Test Flight)-1 positioning tests of the FTS (Flight Telerobotic Servicer). The unique problems associated with the DTF-1 mission are discussed, standard robot performance tests and terminology are reviewed and a very detailed description of flight-like testing and analysis is presented. The major technical problem associated with DTF1 is that only one position sensor can be used, which will be fixed at one location, with a working volume which is probably smaller than some of the robot errors to be measured. Radiation heating of the arm and the sensor could also cause distortions that would interfere with the test. Two robot performance testing committees have established standard testing procedures relevant to the DTF-1. Due to the technical problems associated with DTF-1 these procedures cannot be applied directly. These standard tests call for the use of several test positions at specific locations. Only one position, that of the position sensor, can be used by DTF-1. Off-line programming accuracy might be impossible to measure and in that case it will have to be replaced by forward kinematics accuracy.

\section{Extraterrestial Exploration}

\section{1,733}

PB91-237875 Not available NTIS National Inst. of Standards and Technology (NML), Gaithersburg, MD. lonizing Radiation Div.

Possible Use of Pattern Recognition for the Analysis of Mars-Rover X-ray-Fluorescence Spectra. Final rept.

L. I. Yin, J. I. Trombka, S. M. Seltzer, R. G. Johnson, and J. A. Philpotts. $1989,8 \mathrm{p}$

Pub. in Jnl. of Geophysical Research 94, nNB10 p3611-3618 1989

Keywords: "Mars sample return missions, "Mars surface samples, "X-ray fluorescense analysis, "Pattern recognition, "Soils, Chemical analysis, Reprints.

In the Mars Sample Return Mission, a rover vehicle may be used to collect and select samples from different locations on the Martian surface to be brought back to earth for laboratory studies. It is anticipated that an in-situ energy-dispersive $x$-ray fluorescence (XRF) spectrometer will be on-board the rover. In such a mission sample selection is of higher priority than insitu quantitative chemical analysis. With this in mind, a simple, direct and speedy pattern-recognition method 


\section{Extraterrestial Exploration}

has been developed as an alternative to detailed chemical analysis of the XRF spectra. The authors present and discuss laboratory demonstrations of the efficacy of this pattern-recognition technique in the analysis of many XRF spectra obtained from a series of geological samples, including a simulated Martian soil.

\section{Manned Spacecraft}

101,734

PB91-184804

PC A05/MF A01

National Inst. of Standards and Technology (NML)

Boulder, $\mathrm{CO}$. Chemical Engineering Science Div.

Heat Transfer in a Compact Heat Exchanger Containing Rectangular Channels and Using Helium Gas.

D. A. Olson. Jan 91, 99p NISTIR-3959

See also PB91-107573. Sponsored by National Aeronautics and Space Administration, Hampton, VA Langley Research Center.

Keywords: "Heat exchangers, "Heat transfer, Friction factor, Channel flow, Heat transfer coefficient, Reynolds number, Turbulent flow, Experimental data, Heat flux, Temperature distribution, Nusselt number. Helium, Mass flow, National Aerospace Plane.

Development of a National Aerospace Plane (NASP) which will fly at hypersonic speeds, requires nove cooling techniques to manage the anticipated high heat fluxes on various components. The author has constructed a compact heat exchanger consisting of 12 parallel, rectangular channels in a flat piece of commercially pure nickel. The channel specimen was radiatively heated on the top side at heat fluxes of up to $77 \mathrm{~W} / \mathrm{sq} \mathrm{cm}$ insulated on the back side, and cooled with helium gas flowing in the channels at 3.5 to 7.0 $\mathrm{MPa}$ and Reynolds numbers of 1400 to 28,000 . The measured friction factor was lower than that of the accepted correlation for fully developed turbulent flow, although our uncertainty was high due to uncertainty in the channel height and a high ratio of dynamic pres sure to pressure drop. The measured Nusselt number when modified to account for differences in fluid properties between the wall and the cooling fluid, agreed with past correlations for fully developed turbulent flow in channels. Flow nonuniformity from channel-to-channel was as high as $12 \%$ above and $19 \%$ below the mean flow.

\section{1,735}

PB9 1-216606

PC A05/MF A01

National Inst. of Standards and Technology (BFRL), Gaithersburg, MD.

Material Flammability Test Assessment for Space Station Freedom.

T. J. Ohlemiller, and K. M. Villa. Jun 91, 79p NISTIR4591, NASA-CR-187115

Sponsored by National Aeronautics and Space Administration, Cleveland, $\mathrm{OH}$. Lewis Research Center.

Keywords: "Spacecraft construction materials, "Space stations, "Flammability testing, Reduced gravity, Test methods, Fire tests, Ignition, Comparison, Space hazards, NASA, Graphs(Charts), US NIST.

The NASA Upward Flame Propagation test, which measures response to a well-defined laminar flame a the bottom of a test sample, is currently used to screen for flammability all materials intended for use in the interior of manned spacecraft. The response of a series of materials was compared in the test and in the stand ard NIST flammability tests (Cone Calorimeter for rate of heat release and LIFT tests for ignitability and latera flame spread). The goal was to see if these differing flammability assessment approaches provide comparable information on the potential hazards of a material. At the present a firm relation between the behavior in the NASA test and in the NIST tests has not been established. Recommendations are given for future testing.

\section{Spacecraft Trajectories \& Flight Mechanics}

101,736

$91 \cdot 175166$
National Inst. of Standards and Technology (NML) Boulder, CO. Quantum Physics Div.

Orbit Determination and Gravitational Field Accuracy for a Mercury Transponder Satellite.

Final rept.

M. A. Vincent, and P. L. Bender. 1990, 5p

Grant NAGW-822

Sponsored by National Aeronautics and Space Administration, Washington, DC.

Pub. in Jril. of Geophysical Research 95, nB13 p21,357-21,361, 10 Dec 90.

Keywords: *Gravitational fields, *Orbit calculation, *Mercury(Planet), Mercury spacecraft, Range finding, Circular orbits, Polar orbits, Transponders, Librations, Reprints.

Covariance studies were performed to investigate the orbit determination problem for a small transponder satellite in a nearly circular polar orbit with 4-hour period around Mercury. With $\mathrm{X}$ band and $\mathrm{Ka}$ band Doppler and range measurements, the analysis indicates that the gravitational field through degree and order 10 can be solved for from as few as 40 separate 8-hour arcs of tracking data. In addition, the Earth-Mercury distance can be determined during each ranging period with about $6-\mathrm{cm}$ accuracy. The expected geoid accuracy is $10 \mathrm{~cm}$ up through degree 5 , and $1 \mathrm{~m}$ through degree 8 . The main error sources were the geocentric range measurement error, the uncertainties in higher degree gravity field terms, which were not solved for, and the solar radiation pressure uncertainty.

\section{Unmanned Spacecraft}

101,737

PB91-161950

National Inst. of Standards and Technology (NEL), Gaithersburg, MD. Scientific Computing Div.

Probe Waveforms and the Reconstruction of Structural Dynamic Green's Functions.

Final rept.

A. S. Carasso. 1991, 5p

Contracts AFOSR-ISSA-88-0036, ONR-N00014-89-F0013

Sponsored by Air Force Office of Scientific Research, Bolling AFB, DC., and Office of Naval Research, Arlington, $V A$.

Pub. in AIAA JnI. 29, n1 p114-118 Jan 91.

Keywords: "Large space structures, Dynamic structural analysis, Greens function, Waveforms, Pulses, Reprints, Deconvolution.

Experimental identification of dynamic behavior in linear structural systems can be achieved by exciting the structure with a specifically synthesized pulse, and reconstructing the relevant dynamic Green's function by deconvolution of the measured response. The re construction procedure involves the solution of an illposed integral equation in the presence of noise. The paper underlines the rich variety of infinitely divisible pulse shapes that may be used while still retaining a tractable deconvolution problem. Flexibility in pulse shape is necessary to allow for possible perturbations caused by interfacing devices that convert electrical voltages into mechanical forces. A numerical experiment, using synthetic noisy data, shows that the reconstruction procedure remains effective even when the probe waveform deviates strongly from the preferred unimodal shape. A dispersive structural network that may be representative of a large space structure is used as an illustrative example.

\section{General}

101,738

PB91-162016

National Inst. of Standards and Technology (NML)

Boulder, CO. Chemical Engineering Science Div.
Apparatus for Measurement of Thermal Conductivity of Insulation Systems Subjected to Extreme Temperature Differences.

Final rept.

W. P. Dube, L. L. Sparks, A. J. Slifka, and R. M. Bitsy. 1990, 7p

Sponsored by National Aeronautics and Space Administration, Hampton, VA. Langley Research Center. Pub. in Advances in Cryogenic Engineering (Materials), v36 p853-859 1990.

Keywords: "Thermal insulation, "Thermal conductivity, *Test facilities, "Spacecraft design, "Thermal measurements, Thermal analysis, Thermal protection, Cryogenics, Temperature measurement, Heat transmission, Heat transfer, Reprints.

Advanced aerospace designs require thermal insulation systems which are consistent with cryogenic fluids, high thermal loads, and design restrictions such as weight and volume. To evaluate the thermal performance of these insulating systems, an apparatus capable of measuring thermal conductivity using extreme temperature differences $(27$ to $1100 \mathrm{~K})$ is being developed. The system is described along with estimates of precision and accuracy in selected operating conditions. Preliminary data are presented.

\section{TRANSPORTATION}

\section{Air Transportation}

101,739

PB92-112226

PC A05/MF A01

National Inst. of Standards and Technology (MSEL), Gaithersburg, MD. Metallurgy Div.

Static and Dynamic Strength Tests on Electrical Conductor Cables Specified for Airport Landing Structures.

Final rept.

R. J. Fields, S. R. Low, and D. E. Harne. 27 Oct 88 , 82p NISTIR-88-3884

Sponsored by Federal Aviation Administration, Washington, DC. Navigation and Landing Div.

Keywords: "Static tests, "Strength, "Electric wire, "Landing aids, Electric conductors, Dynamic tests, Experimental data, Test facilities, Displacement, Strains, Mechanical properties, Fatigue(Mechanical), Wire, Impact tests, Loads(Forces), Mechanical tests.

The report covers a series of static and dynamic tests on electrical conductors specified for use in landing aids on airport runways carried out by NIST for the Federal Aviation Administration. The structures are intended to be frangible so that they will break up readily if impacted, thus minimizing damage to the impacting aircraft. While the structures are frangible, they contain electrical cables which, due to the requirement of electrical conduction, are not frangible. In an actual impact, these cables do not break readily and tend to wrap around the aircraft. The tests authorized by the FAA were carried out to assess the force required to break through various types of FAA specified cables by a simulated aircraft impact. Furthermore, the effectiveness of using break-away connectors was evaluated to determine if they would reduce the total load on an impacting aircraft. In order to correctly design the dynamic test apparatus, it was necessary to know the approximate, expected load levels and cable elongations at fracture. Therefore a series of quasi-static tests were performed on the cables and break-away connectors. The report describes these quasi-static tests as well as the construction and application of the dynamic test apparatus.

101,740

PB92-112580

PC A06/MF A02

National Inst of Standards and Technology (NCSL). Gaithersburg, MD

Prototyping the IRDS: An Airport Application. A. P. Sani. Oct $91,119 p$ NISTIR-4688

See also PB89-136329. Sponsored by Univ. of Toronto, Mississauga (Ontario). Inst. for Land Information Management. 
Keywords: "Geographic information systems, "Data management, "Airports, Software tools, Data bases, *Information Resource Dictionary System, "IRDS system, GIRDS system, Federal information processing standards.

The management of geographic information resources (GIRS) continues to be plagued by problems of monitoring, locating and controlling the array of geographic information in complex organizations. Software tools to support these functions are fundamental to any effort to maintain the integrity of geographic information as it changes. In addition, such tools are desirable when formalizing, then managing the integration of geographic information resources within an organization. One of the major approaches to the problem in the area of database systems has been the development of the information resource dictionary (IRD) which contains meta-data. An Information Resource Dictionary System (IRDS) is a database of meta-data along with software and procedures for the creation and maintenance of the IRD. In 1989 the American National Standards Institute (ANSI) X3.138-1988 IRDS (ANSIIRDS) was adopted as Federal Information Processing Standard 156 by the United States Government. ANSIIRDS is intended to support the definition, management and control of mete-data. The study presents the first known attempt to actually apply ANSI-IRDS in the geographic information management domain.

\section{Railroad Transportation}

\section{1,741}

PB91-222653

PC A03/MF A01 Boulder, CO. Materials Reliability Div. Ultrasonic System Using EMATs (ElectromagneticAcoustic Transducers). Report No. 23.

Final rept. Sep 85-Dec 90.

R. E. Schramm, A. V. Clark, D. V. Mitrakovic, S. R Schaps, and T. J. McGuire. May 91, 47p NISTIR3968

\section{Contract DTFR-53-89-X-00018}

See also PB91-222661. Sponsored by Federal Railroad Administration, Washington, DC. Office of Research and Development.

Keywords: "Ultrasonic tests, "Railroad cars, "Wheels, *Residual stress, Detection, Inspection, Accident prevention, Nondestructive tests, Signal processing, Transducers, Surface waves.
The report covers research performed by the National Institute of Standards and Technology for the Federal Railroad Administration. The report covers a project by the Materials Reliability Division (formerly the Fracture and Deformation Division) to develop and build a system to detect and measure residual stress in the rims of railroad wheels. Acoustic birefringence is the underlying principle of operation. This is a measure of the relative difference in the propagation times of two shear waves polarized in radial and circumferential directions. Measurements employ an EMAT (electromagnetic-acoustic transducer) as an ultrasonic probe. This type of transducer requires little or no surface preparation and no acoustic couplant. The system operates in a pulse-echo mode. A short burst of shear The rotation of the EMAT determines the orientation of the polarization vector, radial or circumferential. Precise timing of echoes in both directions reveals the degree of birefringence. Changes are due to both stress state and metallurgical texture. Initial tests indicate it may be possible to separate these two.

\section{1,742}

PB91-222661

PC A04/MF A01

National Inst. of Standards and Technology (MSEL), Boulder, CO. Materials Reliability Div.

Tread Crack Detection in Railroad Wheels: An UItrasonic System Using EMATs (ElectromagneticAcoustic Transducers). Report No. 22.

Final rept. Sep 85-Dec 90.

R. E. Schramm, A. V. Clark, D. V. Mitrakovic, Y.

Cohen, P. J. Schull, and S. R. Schaps. May 91, 69p NISTIR-3967

Contract DTFR-53-89-X-00018

See also PB91-222653. Sponsored by Department of Transportation, Federal Railroad Administration Washington, DC. Office of Research and Development.

Keywords: *Ultrasonic tests, "Railroad cars, "Wheels, "Cracks, Fractures(Materials), Detection, Treads, Nondestructive tests, Accident prevention, Inspection, Signal processing.

The report covers research performed by the National Institute of Standards and Technology (NIST) for the Federal Railroad Administration (FRA). The report covers a project by the Materials Reliability Division (formerly the Fracture and Deformation Division) to develop and build an ultrasonic system to detect cracktype flaws in the tread of railroad wheels. To achieve fully automatic operation, the sensor is built into the rail so testing occurs as the train rolls past. Signal analysis takes place in real time. The ultrasonic probe is an EMAT (electromagnetic-acoustic transducer). As con- figured here, the EMAT has a small footprint, and, due to its principle of operation, it does not require any acoustic couplant. The system operates in a pitchcatch mode. A short burst of Rayleigh (surface) waves travels around the wheel tread, and an echo indicates a flaw's presence and size. Testing was performed on both a short track in the NIST laboratory and the fullscale facilities of the Transportation Test Center (TTC) in Pueblo, Colorado. The report documents the design, construction, and testing of the system. It is also to serve as an operational guide for the equipment being delivered to the FRA.

\section{Road Transportation}

101,743

PB91-157156

PC A03/MF A01

National Inst. of Standards and Technology (NEL) Gaithersburg, MD. Robot Systems Div.

New Approach to Vision and Control for Road Following.

D. Raviv, and M. Herman. Jan 91, 46p NISTIR-4476

Prepared in cooperation with Florida Atlantic Univ., Boca Raton.

Keywords: "Robot dynamics, "Control systems "Computer vision, *Ground vehicles, Robots, Optical processing, Automous navigation, Image processing.

The paper deals with a new quantitative, vision-based approach to road following. It is based on the theoretical framework of the recently developed optical flowbased visual field theory. By building on this theory, the authors suggest that motion commands can be generated directly from a visual feature, or cue, consisting of the projection into the image of the tangent point to the edge of the road, along with the optical flow of this point. Using this cue, they suggest several different vision-based control approaches. There are several advantages to using this visual cue: it is extracted directly from the image, i.e., there is no need to reconstruct the scene; for many road following situations this is the only necessary visual cue; only the horizontal component of the optical flow of the tangent point needs to be extracted; it has a scientific basis, i.e., the described techniques are not ad-hoc; and the related computations are relatively simple and thus suitable for real-time applications. For each control approach, they derive the value of the related steering commands. 


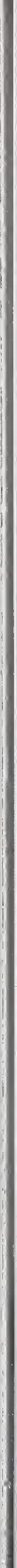




\section{SAMPLE ENTRY}

S. Silbersteln

Proposed Standard Practice for Assessing the Performance of Gas-Phase Air Cleaning Equipment

PB91-167353
100,905
Author name(s)

Title

NTIS order number
AARON, D. A.

Experimentation, Analysis, and Correlation of Refrigerant-22 Flow Through Short Tube Restrictors.

PB91-174243

101,194

ABBOTT, D. C. Photospheres of Hot Stars. 4. Spectral Type O4.
PB91-149849

ABRAMOWITZ, S.
Thermodynamic Data for Modeling of Flue Gas Cleanup Systems (Abstract for Poster Session).
PB91-158741
100,904

Asymptotic Shooting Method for the Solution of Differential Equations.
PBg1-14874

101,255

ACOSTA, S. M.

Thermodynamics of Ammonium Indates. 2. The Molar Heat Capacity of the Ammonium Pentabromoindate Monohydrate Salt (NH4)2 InBr5. H2O from 7.8 to $348 \mathrm{~K}$

PB91-14983

100,304

ACOUISTA, $\mathbf{N}$.

Spectrum and Energy Levels of Seven-Times-Ionized KrypIon ( $\mathrm{Kr}$ VIII) and Resonance Lines of Eight-Times-Ionized Krypton $(\mathrm{Kr}$ IX)

PB91-195511

101,673

ADAM, G.

Sensing of GMAW Droplet Transter Modes Using an ER 100S-1 Electrode.

PB91-174250

101,004

ADAMS, R. D.

Round Robin on Apparent Thermal Conductivity of Several Loose-Fill Insulations.

PB91-149948

101,143

ADLER, J. 0.

Electrofission in the Quasifree and Delta Regions. PB91-147355

101,609

ADOMI, $K$.

Uniform Junction Temperature AIGaAs/GaAs Power Heterojunction Bipolar Transistors on Silicon Substrates. PB91-189399
ADRIAN, F. J.

Superconducting Thin Films of Bi-Sr-Ca-Cu-O Obtained by Laser Ablation Processing.

101,508

ADVANI, J. V.

Thermodynamics of the Disproportionation of Adenosine 5'Thermodynamics of the Disproportionation of Adenosine 5 5'-monophosphate. 2. Experimental Data.
PB92-117399

101,313

AESCHLIMANN, $M$.

Scanning Electron Microscopy with Polarization Analysis (SEMPA)-Studies of Domains, Domain Walls and Magnetic Singularities at Surfaces and in Thin Films.

PB91-237289

101,554

AGNEL, J. P. L

Co-Trial on ESR Identification and Estimates of gamma-Ray and Electron Absorbed Doses Given to Meat and Bones. PB91-194720

AGOSTINELLI, E.

Superconducting Thin Films of Bi-Sr-Ca-Cu-O Obtained by Laser Ablation Processing.

PB91-189589

101,508

AKMAN, S. A.

Base Modifications in Plasmid DNA Caused by Potassium Permanganate.
PB91-187070

101,329

AL-SHEIKHLY, $M$.

Effects of Absorbed Dose Rate, Irradiation Temperature and Post-Irradiation Temperature on the Gamma Ray Re-

sponse of Red Perspex Dosimeters.
PB92-116441 PB92-116441

Mechanisms of the Reduction Reactions of $\mathrm{Cr}(\mathrm{VI})$ in the Radiolysis of Acidic Potassium and Silver Dichromate Solutions in the Presence or Absence of Acetic Acid.

PB91-236489

100,241

Temperature Dependence of Radiochromic Film Dosi-

meters.

101,728

ALBERS, J.

Investigation of Photoconductive Picosecond Microstripline Switches on Self-Implanted Silicon on Sapphire (SOS) PB91-147918
Verification of the Relation between Two-Probe and FourProbe Resistances as Measured on Silicon Wafers. PB91-147520

ALBERTY, R. A.

Standard Chemical Thermodynamic Properties of Isomer Groups of Monochloroalkanes.

PB90-244617

100,258

Standard Chemical Thermodynamic Properties of Polycyclic Aromatic Hydrocarbons and Their Isomer Groups. III. Naphthocoronene Series, Ovalene Series, and First Members of Some Higher Series.

PB90-244625

100,259

ALBUS, J. S. Performance Measures of a Robotic Micropositioner.
PB92-117019

101,037

Real-Time Hierarchical Planning for Multiple Mobile Robots.
PB91-158782

ALCORN, $R$.

Round-Robin Study of Implants in $\mathrm{Si}$ and $\mathrm{SiO} 2$ by SIMS RBS, and NAA.

PB92-117241

ALFASSI, Z. B.

Reactivities of Chlorine Atoms and Peroxyl Radicals Formed in the Radiolysis of Dichloromethane.

PB91-200782

100,386

ALI, M. A.

Electric Quadrupole and Magnetic Dipole Transition Probabilities in Potassium Isoelectronic Sequence.

PB91-187088

101,646

Quantum Electrodynamic Contributions to Spin Orbit Splitting in the Ground State of Aluminum-Like lons.

PB91-189597

101,653

ALLAN, D. W.

Frequency and Time Stability of GPS and GLONASS Clocks.

PB91-202937

100,582

GPS Time Transfer with Implementation of Selective Availability.

N91-25766/7

100,576

Israel's New Synchronized Time Scale, UTC(INPL) PB91-134593 


\section{PERSONAL AUTHOR INDEX}

New Inexpensive Frequency Calibration Service from NIST PB91-200790 Rubidium Frequency Standard and a GPS Receiver: A Remotely Steered Clock System with Good Short-Term and Long-Term Stability.

PB91-200808

100,581

ALLEN, J. D.

Advanced sensor development program for the pulp and paper industry. Final repor

101,248

ALLEN, R. A.

Knowledge Verification of Machine-Learning Procedures Based on Test Structure Measurements.

100,709

Modified Sliding Wire Potentiometer Test Structure for Mapping Nanometer-Level Distances. 100,821

Test Chip for the Evaluation of Surface-Diffusion Phenomena in Sputtered Aluminum Planarization Processes.
PB92-116920

\section{ALSTON, S.}

Near-Threshold Vibrational Excitation of $\mathrm{H} 2$ by Electron Impact: Resolution of Discrepancies between Experiment and Theory.

ALVAREZ, $\boldsymbol{R}$.

101,679

Appendix: Standard Solutions and Certified Reference Ma-

terials.

100,194

AMIRTHARAJ, P. M.

Fe Implantation in In0.53Ga0.47As/InP.

PB91-189969

101,516

AMMON, H. L.

PLOTMD: An Interactive Program to Modify Molecular Plots on a Graphics Termina.

100,373

ANDERS SVENSSON, L.

Ab Initio Phase Determination for $\mathrm{X}$-ray Diffraction Data from Crystals of a Native Protein.

ANDERSON, $\mathrm{S}$.

101,309

Rapid Low-Temperature Hopping of Hydrogen in a Pure Metal: The ScHx System.

PB91-158485

101,217

ANDERSON, O. L.

Thermodynamic Functions and Properties of $\mathrm{MgO}$ at High Compression and High Temperature.

PB90-244542

100,253

ANDERSON, W. E.

Research for Electric Energy Systems: An Annual Report.
PB92-112341

VolksGrapher: a FORTRAN Plotting Package User's Guide, Version 30.

PBersion 3.0 .

ANDOR, G.

100,647

Temperature Dependence of High Accuracy-Photometer

PB91-187096

101,418

ANGEL, S. A.

Application of Ultrafast Broadband Infrared Spectroscopy to Measurement of Metal-Carbonyl Dynamics.

ANTONUCCI, J.

Microstructure and Elastic Properties of Dental Resin and Resin-Based Glass-Reinforced Composites: XRD, SEM and Ultrasonic Methods.

PB91-204081

100,050

ANTONUCCI, J. M.

Filler Systems Based on Calcium Metaphosphates. PB91-194654

ARAKAWA, E. T.

Soft $X$-ray Absorption and Emission Spectra and the Electronic Structure of Some Exotic Materials.

PB91-194738

ARCHER, D. G.

Dielectric Constant of Water and Debye-Huieckel Limiting Law Slopes.

100,260

Thermodynamic Properties of the $\mathrm{NaBr}+\mathrm{H} 2 \mathrm{O}$ System.

ARCIMOWICZ, $B$.

$5 s(2) 5 p(2)-(5 s(2) 5 p 5 d+5 s 5 p(3)+5 s(2) 5 p 6 s+$ $5 \mathrm{~s}(2) 5 \mathrm{p} 7 \mathrm{~s})$ Transitions in $\mathrm{Sb} I 1$ and $5 \mathrm{~s}(2) 5 \mathrm{p} \cdot(5 \mathrm{~s} 5 \mathrm{p}(2)+$ $5 \mathrm{~s}(2) \mathrm{nl)}$ Transitions in Sb III.

PB91-200816

101,676

ARMSTRONG, J. $w$

Gravitational Radiation Observations on the Moon PB91-175083

101,635

Hydrogen Slush Production with a Large Auger

New Wide Range Equation of State for Helium. PB91-162222

100

ARUOMA, O. I. Bleomycin-Dependent Damage to the Bases in DNA is a
Minor Side Reaction.
PB91-189357

101,337

Copper-Ion-Dependent Damage to the Bases in DNA in the Presence of Hydrogen Peroxide.

101,330

Damage to the Bases in DNA Induced by Hydrogen Perox-

ide and Ferric lon Chelates. PB91-187112

101,331

Iron Ion-Dependent Modification of Bases in DNA by the Superoxide Radical-Generating System Hypoxanthine/Xanthine Oxidase.

101,306

Modification of Bases in DNA by Copper Ion-1,10-Phenanthroline Complexes.

PB91-187492

101,333

ASAOKA, $K$

Visco-Elastic Deformation of Dental Porcelain and Porcelain-Metal Compatibility.

PB91-158493

100,042

ASHER, $\mathbf{S}$.

Morphology of Silver on YBa2Cu3O7-delta Thin Films.

101,534

\section{ASHER, S. E.}

Analysis of the $\mathrm{YBa} 2 \mathrm{Cu} 3 \mathrm{O} 7 / \mathrm{SrTiO} 3$ Interface as a Function of Post-Deposition Annealing Temperature.

101,480

ASMAIL, $C$.

Bidirectional Scattering Distribution Function (BSDF): A Systematized Bibliography.

101,421

ASSAEL, M. J.

Benzene: A Further Liquid Thermal Conductivity Standard.

PB90-244567 100,255

Thermal Conductivity of Methane and Tetrafluoromethane in the Limit of Zero Density

PB91-192500

100,361

ASTALOS, R. J.

Improvements for Automating Voltage Calibrations Using a $10-V$ Josephson Array

100,739

STUMIAN, R. D.

Frequency Dependence of Catalyzed Reactions in a Weak Oscillating Field.
PB91.203927

ATKINSON, $R$

Kinetics and Mechanisms of the Gas-Phase Reactions of the NO3 Radical with Organic Compounds.
PB92-110220 PB92-110220

Theoretical Investigation of Piloted Ignition of Wood. PB92.112432

ATTIX, F. H

Mass Energy-Transfer and Mass Energy-Absorption Coeffi-

cients, Including In-Flight Positron Annihilation for Photon

Energies $1 \mathrm{keV}$ to $100 \mathrm{MeV}$.

PB92-126473

101,445

AULD, B. A.

Capacitive Array Sensors for Nondestructive Evaluation.

100,947

Characterization of Capacitive Array for NDE Applications.

PB91-237305 100,946

Eddy Current Reflection Probe: Theory and Experiment.

PB91-187120

100,944

AUSLOOS, $P$.

Concerning the Formation and the Kinetics of Phenylium

lons.

100,336

AUSTIN, $M$.

Resonating-Orthotropic-Cube Method for Elastic Constants.

AUSTIN, M. W.

Effect of Nitrogen and Carbon on FCC-HCP Stability in Aus-

PB91-175372

101,151

Image Quality Indicator Design for Radioscopy and Tomography.

PB91-190058

101,018

AXLEY, J. W.

Multi-Zone Dispersal Analysis by Element Assembly. PB91-187146

100,907

AYRES, R. L.

NIST-NRL Free-Electron Laser
AD-A227 $310 / 0$

101,399

AYRES, T. R.

Ultraviolet, Optical, Infrared, and Microwave Observations of HR 5110.
PB91-148833

100,020

A YTER, $\mathbf{S}$.

Eddy Current Reflection Probe: Theory and Experiment.

PB91-187120

AZAMAR, J. A.

Sputtered Thin Film YBa2Cu3On

PB91-18964

101,509

BA, w. $\mathbf{z}$.

Temperature Dependence of Radiochromic Film Dosimeters.

PB92-117134

101,728

BABRAUSKAS, $v$.

Cone Calorimeter Rate of Heat Release Measurements for Upholstered Composites of Polyurethane Foams.
PB92-108984

Data for Fire Hazard Assessment of Selected Non-Haloge-

PB92-112473

100,557

Data for Room Fire Model Comparisons.

PB92-110287
Heat Release Rate: The Single Most Important Variable in Fire Hazard.

PB91-146977

100,097

Large-Scale Validation of Bench-Scale Fire Toxicity Tests.

PB92-116458

Modern Test Methods for Flammability.
PB91-167676

100,139

100,116

Role of Bench-Scale Test Data in Assessing Real-Scale Fire Toxicity.

100,156

BAGG, T. C.

Computer Output Microform (COM) Formats and Reduction Ratios, $16 \mathrm{MM}$ and $105 \mathrm{MM}$. Category: Hardware Standard: Subcategory: Media.

FIPS PUB 54-1

100,678

BAGLEE, D. A.

Building-in Reliability: Making It Work.

PB91-203992

100,812

BAISHIKI, R. S.

Measurements of Power Frequency Magnetic Fields Away from Power Lines

BAKER-JARVIS, $\mathbf{J}$.

100,851

Improved Technique for Determining Complex Permittivity with the Transmission/Reflection Method.

PB91-161919

100,844

Optimization Techniques for Permittivity and Permeability Determination.

100,865

Optimization Techniques for Permittivity and Permeability Determination.
PB92-126663

100,871

Transmission/Reflection and Short-Circuit Line Permittivity Measurements
PB91-171959

100,848

BAKER, T. L.

Effects of Crystal Bonding on Brittle Fracture.

PB91-14822

101,064

BALDI, F.

Characterization by High Performance Liquid Chromatography (HPLC) of the Solubilization of Phosphorus in Iron Ore by a Fungus.

101,162

BALMER, M. L

Strength and Fracture Behavior of Ba-Y-Cu-O Superconducting Ceramics.

PB91-148205

101,474

BALTATU, M. E.

Taking the Measure of Fluid Properties Data Bases.

PB91-200832

101,392

BANGE, $K$

Adsorption of Water and Oxygen on $\mathrm{Ag}(110)$ : A Study of the Interactions among Water Molecules, Hydroxyl Groups, and Oxygen Atoms. 
Intensifying Effect of Metallic Screens on the Sensitivity of X-ray Films for 400-kV Bremsstrahlung Photons.

101,293

Use of Storage Phosphor Imaging Plates in Portal Imaging and High-Energy Radiography: The Intensitying Effect of

PB91-236497

101,324

BARNES, J. A

Clocks, Atomic and Molecular.

PB91-200840

101,677

BARNES, P. $Y$

Photoluminescence Excitation by Band-Gap Optical $\mathrm{Ab}$ sorption in Chemical Vapor Deposition Diamond Films.
PB91-195586

101,533

BARNES, R. G.

Rapid Low-Temperature Hopping of Hydrogen in a Pure Metal: The ScHx System.

PB91-158485

101,217

Relationship of Hydrogen Site Occupancy to Diffusion Behavior in Crystalline and Amorphous $\mathrm{Zr} 2 \mathrm{PdHx}$.

PB91-187252

101,230

BARNETT, E. H.

Design and Analysis of Experiments.

PB91-158824

101,274

BAROUCH, E.

Three-Dimensional Simulations of High-Resolution Photoresist Processing.

PB92.116466

100,818

BARTEL, T. W.

Acoustic Technique for Evaluation of Thermal Insulation.

PB91-189324

100,123

BARTLE, K. D.

Solubilities of Solids and Liquids of Low Volatility in Supercritical Carbon Dioxide.

PB92-148105

100,470

BARTSCHAT, $K$.

Optical Potential Approach to Electron and Positron Scattering from Noble Gases. 2. Neon.

PB91-149971

101,619

BASCH, $\mathrm{H}$.

Hydrogen Bonding in Pt Ammine Complexes. PB91-189639

100,348

BASKI, A. A.

Surface Extended-X-ray-Absorption Fine Structure and

Scanning Tunneling Microscopy of Si(001)2x1-Sb.
PB91-237263

101,552

BASSHAM, L. E.

Bibliography of Selected Computer Security Publications, January 1980 -October 1989

PB91-14848

100,704

Extension of the Class of Magnetic B Star Nonthermal Extension of the Class of Magnetic B Star Nontherma
Radio Sources.
PB91.175042
100,031

BATCHMAN, T. E.

Semiconductor Claddings on Glass Waveguides for Polarizers and Detectors.

PB91-175141

101,416

BATRA, A.

High Resolution Diffraction Imaging of Crystals Grown in Microgravity and Closely Related Terrestrial Crystals. PB92-109008

101,562

BATTS, M. E.

Characterization of Cylindrical Holes in Metallic Substrates via Their Infrared Emission Patterns.

PB91-187187

101,228

BAUER, B. J.

Network Structure in Epoxies. 6. The Growth Process Investigated by Neutron Scattering

100,514

Small-Angle Neutron Scattering of Blends of Cross-Linked and Linear Polystyrene.

100,500

Small-Angle Neutron Scattering Studies of Compatible Small-Angle Neutron Scattering Studies of Compatible
Blends of Linear Poly(Vinyl Methyl Ether) and Cross-Linked Blends of Linear Poly(Vinyl Methyl Ether) and Cross-Linked
Deuterated Polystyrene.
100,494 PB91-187153

100,494

Synthesis of Non-lonic Water-Dispersible Resins for Use in Intaglio Inks Curing by Electron Beam Radiation.

100,499

Synthesis of Prototype Resins for Use as BEP Intaglio Ink Vehicles Curing by Electron Beam Radiation. PB91-144345

101,182

BAUM, H. R.

Boussinesq Algorithm for Enclosed Buoyant Convection in Two Dimensions.

BAUMANN, $\mathbf{S}$.

100,157

Round-Robin Study of Implants in Si and SiO2 by SIMS, RBS, and NAA.

100,830

BAUR, $W$.

New Different Forms of Ammonium Loaded and Partly Deammoniated Zeolite Rho Studied by Neutron Powder.
PB91-158717

100,311

C. E.

Angular Distribution of Ejected Electrons in Resonant Auger Processes of $\mathrm{Ar}, \mathrm{Kr}$, and $\mathrm{Xe}$

101,648

Parity-Unfavored Transitions in Resonant Photoemission from $\mathrm{Ar}, \mathrm{Kr}$, and $\mathrm{Xe}$ : Experimental and Theoretical Results. PB91-133900

101,599

Unusual Degree of Angular Anisotropy in the Resonant Auger Spectrum of $\mathrm{Kr}$.

101,649

BEALL, J. A.

Analysis of the $\mathrm{YBa} 2 \mathrm{Cu} 307 / \mathrm{SrTiO} 3$ Interface as a Function f Post-Deposition Annealing Temperature.

PB91-149963

101,480

Morphology of Silver on YBa2Cu3O7-delta Thin Films.

PB91-195594 101,534

YBa2Cu3O7-delta/Insulator Multi-Layers for Crossover Fabrication.

PB91-194662

101,518

BEAN, J. W.

Design Heat Loss Factors for Basement and Slab Floors.

PB91-147561

100,059

Measurement and Evaluation of Lighting/HVAC Interaction.

PBN $V$

$\mathrm{H} 2 \mathrm{O}(\mathrm{I})-\mathrm{H} 2 \mathrm{O}(\mathrm{III})-\mathrm{H} 2 \mathrm{O}$ (L) Triple Point of Water as a Fixed Pressure Point.

PB91-187211

State-of-the-Art in Pressure Metrology.

PB91-146993

101,647

BEARY, E. S

High Purity Fluoropolymer Materials: Trace Element Content and Leaching.

PB91-16225

100,491

BECK, L. B.

Correction for Converting 2-CM(3)-Coupler Responses to Insertion Responses for Custom in-the-Ear Non-Directiona Hearing Aids.

PB91-174490

100,046

BECKER, D. A

Characterization of the Mineral Fraction in Botanical Reference Materials and Its Influence on Homogeneity and Anaytical Results.

BECKER, P. $\mathbf{R}$

100,186

Alaska Marine Mammal Tissue Archival Project: Revised Collection Protocol.

PB91-184796

101,374

BECKERLE, J. D.

Picosecond IR Studies of the Vibrational Dynamics of $\mathrm{CO}$ $P t(111)$.

PB91-161927

100,31

BEECH, $\mathbf{F}$.

Chemical Modification of the Orthorhombic Superconductor Ba2YCu3O7-delt

$1-159053$

101,486

Phonon Density of States of Superconducting YBa2Cu3O7 and the Nonsuperconducting Analog YBa2Cu3O6. PB91-159129

BEEHLER, R. E.

NIST Time and Frequency Services.

PB91-231605

BEIJERS, J. P. M.

Laser-Induced Fluorescence Measurements of Drift-Velocity Distributions for $\mathrm{Ba}(+)$ in $\mathrm{Ar}$ : Moment Analysis and Distributions for $\mathrm{Ba}(+)$, in Direct Measure

100,298

\section{BEK-UZAROV, D. J.}

Preparation and Properties of Monomolecular Films for Use as Radioactive Source Mounts.

PB91-147249

BELANGER, B. C.

101,608

Advanced Technology Program: A New Role for NIST in Accelerating the Development of Commercially Important Technologies
PB92-126697

BELL, A. A.

100,013

Accurate and Precise Coulometric Determination of Sulfur Dioxide in Compressed Gas Mixtures. PBe2-1260?

100,912

Development of a Coulometric Method for Assessing the Concentration of Ambient Levels of CO2/Air in Compressed Gas Mixtures.

PB92-126648

100,913

BELL, D.

Thermodynamics of Hydrolysis of Oligosaccharides. PB91-194803

101,288

BELL, M. I.

Investigation of Photoconductive Picosecond Microstripline Switches on Self-Implanted Silicon on Sapphire (SOS)

PB91-147918

BELLAMA, J. $M$.

Fourier Transform Infrared Analysis of Ceramic Powders: Quantitative Determination of Alpha, Beta, and Amorphous Phases of Silicon Nitride.

PB91-237628

101,096

Simultaneous Butyltin Determinations in the Microlayer ( Wyarina and Receiving System.

PB91-195040

100,918

\section{BELLO, M. A.}

Research. Services. Facilities. (National Institute of Standards and Technology)

PB92-109172

100,012

BELZER, B. J.

Ellipsometry SRM's for Use in Thin Film Measurements. PB92-116599

100,819

BENDER, B. A.

Effect of Thermochemical Treatments on the Strength and Microstructure of SiC Fibers.

PB91-194670

101,084

Physical and Thermo-Mechanical Properties of Monoclinic Single Crystals

BENDER, P. L.

101,065

Gravitational Radiation Observations on the Moon

PB91-175083

101,635

Lunar Gravitational Wave Antenna Using a Laser Interfer ometer.

PB91-134643

101,603

Orbit Determination and Gravitational Field Accuracy for a Mercury Transponder Satellite.

PB91-175166

101,736

BENDERSKY, L. A.

Role of Elastic Energy in the Morphological Development of a Ni-Ti-Al Alloy.

101,227

Transformation of the Icosahedral Phase in Rapidly Quenched Al-rich Al-Mn Alloys.

PB91-158964

101,221

BENEDETTO, J. M.

Lead Zirconate-Titanate Thin Films Prepared by the Laser Ablation Technique

PB91-187344

101,502

Graphical Kernel System (GKS). Ada Binding; Category: Software Standard; Subcategory: Graphics. Toftware StandGraphical Kernel System (GKS); Category: Software Stand-
ard; Subcategory: Graphics.
FIPS PUB 120-1 Graphical Kernel System (GKS). Pascal Binding; Category: Software Standard; Subcategory: Graphics.
FIPS PUB 120-1A NIST Support for the Computer-Aided Acquisition and Logistic Support (CALS) Program in the Area of Graphics Standards, Calendar Year 1990.

PB91-194506

101,348

BENNER, B. A.

Preparation and Analysis of a Marine Sediment Reference Material for the Determination of Trace Organic Constituents.

PB91-149237

101,377 


\section{PERSONAL AUTHOR INDEX}

Mossbauer Study of the Effect of Oxygen Stoichiometry on the High Tc Superconductor Y1Ba2(CuO.97Fe0.03)3O7-x. PB91-237545

101,558

Phase Equilibria in the System TI-Ca-Ba-Cu-O. 1. Stability of the 2122 Phase under Conditions of Oxygen Annealing. PB91-147165 101,465

Processing $\mathrm{Bi}-\mathrm{Pb}-\mathrm{Sr}-\mathrm{Ca}-\mathrm{Cu}-\mathrm{O}$ Superconductors from Amorphous State.

101,504 Superconducting Properties of $\mathrm{Bi2}-\mathrm{x}-\mathrm{yPb} \mathrm{P}$ SnySr2Ca2Cu $3 \mathrm{Oz}$. PB91-162321 101,076 Superconducting Thin Films of Bi-Sr-Ca-Cu-O Obtained by Laser Ablation Processing.

101,508

Wigner-Seitz Local-Environment Sludy of the High Tc Superconductors.

PB91-195115.

101,528

Magnetic Anisotropies in Ultrathin fcc Fe(001) Films Grown on Cu(001) Substrates.

PB92-116631

101,565

\section{BENTZ, D.}

Reflection/Absorption FTIR Spectral Characteristics of Thin and Thick Crosslinked Epoxy Films on Steel Substrates.
PB91-203711

100,509

\section{BENTZ, D. P.}

Characterization of Cylindrical Holes in Metallic Substrates via Their Infrared Emission Patterns.

PB91-187187

101,228

Characterization of Epoxide Coatings on Steel by Reflection/Absorption Fourier Transform Infrared Spectroscopy: Ouantitative Study.

PB91-203729

101,109

Percolation of Phases in a Three-Dimensional Cement Paste Microstructural Model.

100,529

Roughness Measures of Blasted Steel Surfaces Remotely Imaged with a Thermographic Camera.

101.146

PB91-1476

Critical Exponent for the Viscosity of Four Binary Liquids.
PB91-187195

Critical Exponent for Viscosity

PB91-147009

100,271

BERGER, J. R.

Aluminum Alloys for Cryogenic Tanks: Oxygen Compatibility. Volume 1 .

101,201

Determining the Dynamic Stress Intensity Factor with Strain Gages Using a Crack Tip Locating Algorithm.

101,580

Improved Optical Diffraction Strain Measurement System. PB91-174433

101,581

Spatially Overdetermined Analysis for Propagation Toughness Using Strain Gages.

101,164

Study of Static and Dynamic Fracture Using Strain Meas-

PB91-132217

101,574

Use of Birefringent Coatings in Fracture Mechanics.

PB91-174599

101,582

\section{BERGER, M. J.}

Mass Energy-Transfer and Mass Energy-Absorption Coefficients, Including In-Flight Positron Annihilation for Photon Energies $1 \mathrm{keV}$ to $100 \mathrm{MeV}$

PB92-126473

101,445

BERGQUIST, J. C.

Ouantum Optics of Single, Trapped lons

PB91-162123

101,628

Trapped-Ion Frequency Standards.

N91-25760/O

100,575

BERGTOLD, D. $S$.

8-Hydroxyguanine Content of Isolated Mitochondria Increases with Lipid Peroxidation.

PB91-189472

101,286

Biomarkers of $\mathrm{OH}$ Radical Damage In vivo.

PB91-236877

101,325

BERK, N. F.

Rapid Low-Temperature Hopping of Hydrogen in a Pure Metal: The $\mathrm{ScH}$ S System.

PB91-158485

101,217

BERNAL, J.

Computing Delaunay Triangulations for Comet-Shaped Polygons.

101,265

BERNING, $D$.

Automated Reverse-Bias Second-Breakdown Transistor Tester.

PB91-216739

100,813

BERNING, D. $W$.

Assessment of Reliability Concerns for Wide-Temperature Operation of Semiconductor Devices and Circuits.
PB92-116987

Automated Reverse-Bias Second-Breakdown Transistor Tester.
PB91-236513

100,814

ERQUIST, J. C.

Progress at NIST on Absolute Frequency Standards Using Stored lons.

BERROCAL, J.

101,625

Performance of OSI Transport over ACCUNET and IBER

PB91-134825

100,563

BERUFF, $\mathrm{R}, \mathrm{B}$.

Test of Newton's Inverse Square Law of Gravity Using the $300 \mathrm{~m}$ Tower at Erie, Colorado: Newton Vindicated on the Plains of Colorado.

PB91-175273

101,637

Test of the Inverse-Square Law of Gravitation Using the 300-m Tower at Erie, Colorado.

101,702

PBS1:

N-Dimensional Crystallographic Description of the Icosahedral Phases; the Example of the Al73Mn21Si6 Ouasiperiodic Structure.

PB91-17477

101,226

AN, J. W

Rovibrational Analysis of the nu7(1) Intermolecular Hydrogen Bond Bending Vibration in HCN-HF Using Far Infrared Fourier Transform Spectroscopy.

PB91-237784

100,437

BHANSALI, K. J.

Literature Review of the Galling Process.

PB91-134551

101,208

BHATIA, A. K

Relative Populations of Excited Levels within the Ground Configuration of Si-Like $\mathrm{Cu}, \mathrm{Zn}, \mathrm{Ge}$, and Se lons.

PB91-158618

101,443

BIANCANIELLO, F. S.

Particle Size Measurement of Inert Gas Atomized Powder. PB91-187203

Transformation of the Icosahedral Phase in Rapidly Ouenched Al-rich Al-Mn Alloys.

P891-158964

101,221

$\mathrm{BICH}, \mathrm{E}$.

Viscosity and Thermal Conductivity of Pure Monatomic Gases from Their Normal Boiling Point Up to $5000 \mathrm{~K}$ in the Limit of Zero Density and at $0.101325 \mathrm{MPa}$

P891-192559

100,366

BICHSEL, $\mathrm{H}$.

Stopping Power of Fast Charged Particles in Heavy Elements.
PB91-194399

101,660

\section{BIERBAUM, V. $M$.}

Laser-Induced Fluorescence Measurements of Drift-Velocity Distributions for $\mathrm{Ba}(+)$ in $\mathrm{Ar}$ : Moment Analysis and Direct Measure of Skewness.

PB91-149005

100,298

BIGNELL, $N$.

$\mathrm{H} 2 \mathrm{O}(\mathrm{l})-\mathrm{H} 2 \mathrm{O}(\mathrm{III})-\mathrm{H} 2 \mathrm{O}(\mathrm{L})$ Triple Point of Water as a Fixed Pressure Point.

PB91-187211

101,647

BILLIARD, P. A.

Ignition Characteristics of Selected SSME Alloys. $\quad 100,544$
PB91-174474

100,544
PB91-174474 N07001 in Pressurized Oxygen.

PB91-144428

100,536

BIRNBAUM, G.

Problem of Detailed Balance and Model Lineshapes in Collision-Induced Rotovibrational Bands: $\mathrm{H} 2-\mathrm{H} 2$ and $\mathrm{H} 2-\mathrm{He}$. PB91-236521

100,412

BISHOP, $B$.

Validation of an OSI Transport Class 4 Simulator.

100,568

\section{BITSY, R. M.}

Apparatus for Measurement of Thermal Conductivity of Insulation Systems Subjected to Extreme Temperature Differ-

Small Scale Demand Type Neon Liquefaction Plant.
PB91-162008

101,738

BLACK, D.

High Resolution Diffraction Imaging of Crystals Grown in Microgravity and Closely Related Terrestrial Crystals. High Resolution Synchrotron X-Radiation Diffraction Imaging of Crystals Grown in Microgravity and Closely Related Terrestrial Crystals.

PB91-216747

101,548

High-Resolution Small-Angle $X$-ray Scattering Camera for Anomalous Scattering.

100,929

Reflection-Extended-X-ray-Absorption-Fine-Structure Spectroscopy at the Carbon K-Edge.

100,371

Small-Angle-Scattering Determination of the Microstructure of Porous Silica Precursor Bodies.

P891-175075

101,077

BLACKBURN, D. H.

Refractive Index Gratings in Rare-Earth-Doped Alkaline Earth Glasses.

BLACKBURN, D. L.

Assessment of Reliability Concerns for Wide-Temperature Operation of Semiconductor Devices and Circuits.

PB92-116987

100,825

Emitter Ballasting Resistor Design for, and Current Handling Capability of AlGaAs/GaAs Power Heterojunction Bipolar Pransistors.

100,815

Micromachined Thermal Radiation Emitter from a Commercial CMOS Process.

PB91-148999

100,748

Microwave Monolithic Integrated Circuit-Related Metrology at the National Institute of Standards and Technology. MMIC Related Metrology at the National Institute of Standards and Technology.

P891-149070

100,794

Uniform Junction Temperature AIGaAs/GaAs Power Heterojunction Bipolar Transistors on Silicon Substrates.

P891-189399

100,806

BLAIR, T. T.

Critical Evaluation of Liquid Crystal Transition Temperatures I: 4,4'-Alkyl/alkoxyphenylbenzoates.

P892-110154

100,444

BLAIR, W. R.

Methods for the Analysis of Organometallic Compounds in

WBa1-148981

100,189

Stability of Aqueous Inorganic Lead Solutions in Polycarbonate Containers.

PB92-126499

100,211

BLAISTEN-BAROJAS, E.

Molecular Dynamics Study of the Depolymerization Reaction in Simple Polymers.

100,495

BLANKE, J. H.

Beam-Foil Study of Two-Electron Transitions in Cu-Like lons.

PB91-237511

101,714

BLENDELL, J. E.

Comparison of Flux Dynamics in Two Samples of $\mathrm{YBa} 2 \mathrm{Cu} 3 \mathrm{O} 7$ with

101,069

Effect of Coherency Strain on Alloy Formation: Migration of Liquid Films.

101,210

Strength and Fracture Behavior of Ba-Y-Cu-O Superconducting Ceramics.

PB91.148205

101,474

Ultrasonic Measurement of Sheet Steel Texture and Formability: Comparison with Neutron Diffraction and Mechanical Measurements.

PB92-116615

101,170 
PB91-149989

100,797

BLUMENTALS, I. I.

Biological Sulfur Oxidation and Reduction for Coal Sulfur Speciation and Desulfurization.

100,886

Bioprocessing of Fossil Fuels Using Hyperthermophilic ArChaebacteria

100,883

BOETTINGER, W. J.

Examination of the Excessive Retained Austenite on the Surface of a Section of 17.7 Precipitation Hardening Stain less Steel.

101,149

Phase-Field Model for Isothermal Phase Transitions in Binary Alloys.

101,240

Role of Elastic Energy in the Morphological Development of Ni-Ti-Al Alloy.

PB91-18716

101,227

BOGGS, P. T.

Convergence Behavior of Trajectories for Linear Programming.

101,270

Convergence Properties of a Class of Rank-Two Updates.

PB91-187799 101,260

Interior-Point Method for Linear and Quadratic Programming

101,269

PB91-187815

Merit Function for Inequality Constrained Nonlinear Pro-
gramming Problems.

PB92-116318

101,272

Orthogonal Distance Regression

PB91-147017

Superconducting Thin Films of Bi-Sr-Ca-Cu-O Obtained by

Laser Ablation Processing.

PB91-189589

101,508

BOHANNAN, $B$.

Photospheres of Hot Stars. 4. Spectral Type O4.

PB91-149849

BOISVERT, R. F.

Algorithms for Special Tridiagonal Systems.

DEQSOL and ELLPACK: Problem-Solving Environments for

Partial Differential Equations.
PB91-174441 100,645

Guide to Available Mathematical Software Problem Classification System.

PB91-132175

101,282

Portable Vectorized Software for Bessel Function Evalua-

PB91-216598

100,652

BOLAND, T.

Government Open Systems Interconnection Profile Users' Guide, Version 2.

PB92-119676

100,696

Stable Implementation Agreements for Open Systems Interconnection Protocols. Version 4, Edition 1, Change Page September 1991. Output from the September 1991 OSI Implementors Workshop. Held in Gaithersburg, Maryland.

PB92-126408

100,697

Stable Implementation Agreements for Open Systems Interconnection Protocols. Version 4, Edition 1, December 1990.
PB91-171967 Stable Implementation Agreements for Open Systems Interconnection Protocols. Version 4, Edition 1, June 199 Change Pages (Supplement)

PB92-112200

100,695

Stable Implementation Agreements for Open Systems Interconnection Protocols. Version 4, Edition 1, March 1991. Change Pages (Supplement).

100,691

Working Implementation Agreements for Open Systems Interconnection Protocols.

100,686

Working Implementation Agreements for Open Systems Interconnection Protocols.

100,698

303-MHz Frequency Standard Based on Trapped $\mathrm{Be}(+)$

101,722

Clocks, Atomic and Molecular

Clocks, Atomic and Molecular.
PB91-200840

Liquid and Solid Ion Plasmas.
AD-A227 656/6

101,677

Liquid and Solid Ion Plasmas.

101,441

AD-A242 029/7

101,592

Observation of Correlations in Finite, Strongly Coupled Ion Plasmas.

PB91-200857

101,678

Progress at NIST on Absolute Frequency Standards Using

Stored lons.

101,625

Reply to Comment on 'Quantum Zeno Effect'.
PB91-236828

101,708

Test of the Linearity of Quantum Mechanics by if Spectroscopy of the $(9) \mathrm{Be}(+)$ Ground State.

PB92-116870

101,726

Trapped-Ion Frequency Standards.

N $91-25760 / 0$

100,575

RE,

Generalized Photodiode Self-Calibration Formula.

PB91-236901

100,760

BONNELL, D. W.

Laser Vaporization Mass Spectrometry of Refractory Materials: Graphite and $\mathrm{YBa} 2 \mathrm{Cu} 3 \mathrm{OX}$

PB92-117290

100,464

Levitation Calorimetry

PB91-158519

100,927

Thermodynamic and Kinetic Stability of Refractory Materials at Uitra-High Temperatures.

AD-A235 490/0

100,246

BOOTH, J. G.

Anomalous Low-Frequency Butterfly Curves for Subsidiary and Ferromagnetic Resonance Overlap at $3 \mathrm{GHz}$.

ORCHERS, J. A

Structural and Magnetic Properties of Er Thin Films and Er/ Y Superlattices: Magnetoelastic Effects

PB91-161935

BORNER, H. G.

Doppler Shift Attenuation Lifetime Measurements in (54) $\mathrm{Cr}$ Following Thermal Neutron Capture.

101656

BORYSOW, A.

Problem of Detailed Balance and Model Lineshapes in Collision-Induced Rotovibrational Bands: $\mathrm{H} 2-\mathrm{H} 2$ and $\mathrm{H} 2-\mathrm{He}$

PB91-236521

100,412

BORYSOW, J.

Detection of Excited States by Laser-Induced Fluorescence and Analysis of Energy Transfer.

AD-A227 296/1

100,242

BOSTELMAN, $R$.

Description of a Vibration Compensation System for the Small Scale Model Robot Crane Project.

PB91-216648

Strontium-Induced Oxygen Defect Structure and Hole Doping in $\mathrm{La2}-\mathrm{xSr} \times \mathrm{CuO} 4$

101,472

Surface Extended-X-ray-Absorption Fine Structure and Scanning Tunneling Microscopy of Si(001)2x1-Sb.

PB91-237263

101,552

BOWEN, R. L.

Development of an Adhesive System for Bonding to Hard Tooth Tissues.

PB91-236539

100,052

Effect of Catalyst Structure on the Synthesis of a Dental Restorative Monomer.

PB91-15869

00,043

Glass-Ceramic Inserts Anticipated for 'Megafilled' Compos ite Restorations.

PB91-174458

Protective Coatings for Tooth Crowns.

PB91-189555

100,045

BOWEN, V. T.

100,047

nterlaboratory Comparison of Actinides in Human Tissue (239) Pu + (240)Pu.

PB91-203265

101,323

BOWMAN, R. C.

Properties of Amorphous Zirconium Rhodium Hydride (Zr3RhHx) Prepared from Glassy and Crystalline Alloys

101,225

Relationship of Hydrogen Site Occupancy to Diffusion Behavior in Crystalline and Amorphous $\mathrm{Zr} 2 \mathrm{PdH}$.

101,230

BRADIE, $B$.

Three-Dimensional Simulations of High-Resolution Photore sist Processing
PB92-116466

BRADLEY, D. $R$.

Standardization of Advanced Ceramics.

PB91-195669

100,818

BRADLEY, P. E.

101,086

Measurement of Regenerator Performance in a Vuilleumier Refrigerator.

BRANSFORD, J. W.

101,052

High-Temperature Thermal Properties of UNS $\$ 44004$ Using Multivariant Analysis.

Ignition Characteristics of Selected SSME Alloys PB91-174474

100,544

Ignition Characteristics of the Nickel-Based Alloy UNS No7001 in Pressurized Oxygen

PB91-144428

100,536

BRANSTAD, D. $K$

Data Encryption Standard: Past and Future.

PB91-204107

100,711

BRAULT, J. W.

Unified Set of Atomic Transition Probabilities for Neutral Argon.

PB91-237768

101,717

BRAUN, E.

Large-Scale Validation of Bench-Scale Fire Toxicity Tests.

B92-116458

00,139

Reduction of Hydrogen Cyanide Concentrations and Acute Inhalation Toxicity from Flexible Polyurethane Foam Combustion Products by the Addition of Copper Compounds. Part 3. The Effect of Copper Additives on the Flammability Characteristics of Flexible Polyurethane Foam.

PB91-132167

101,140

Role of Bench-Scale Test Data in Assessing Real-Scale Fire Toxicity.
PB91-167270

100,156

\section{BRAUN, $W$.}

Laser Photolysis of Trimethylgallium at $193 \mathrm{~nm}$ : Ouantum Yields for Methyl Radical and Ethane Production.

AD-A227 868/

100,234

Photomultiplier Housing for Vacuum Operation of Side-on 1P28-Type Tubes

100,744

Scattered Light and Other Corrections in Absorption Coefficient Measurements in the Vacuum Ultraviolet: A Systems

AD-A227 869/5

100,179

BRAY, S. L.

Current Supply for High-T(sub c) Superconductor Testing.
PB91-133827

101,455

High T(sub c) Superconductors and the Critical Current PB91-134056

101,457

Integrity Tests for High-(T sub $\mathrm{c})$ and Conventional CriticalCurrent Measurement Systems.

PB91-148676

100,842

Integrity tests for high-(Tc) and conventional critical-current measurements systems.

DE91016275

101,452

BREESE, J. N.

Users Guide for RAPID, Version 2.3

PB91-167403

100,999

Validation Testing Laboratory User's Guide. National PDES Testbed Report Series.

PB92-123090

100,971

BREITENBERG, $M$.

Directory of Federal Government Laboratory Accreditation/

Designation Programs

Ouestions and Answers on Ouality, the ISO 9000 Standard Series, Quality System Registration, and Related Issues.

PB92-126465

BREITENBERG, M. A 


\section{PERSONAL AUTHOR INDEX}

Uniforms Laws and Regulations as Adopted by the National Conference on Weights and Measures (76th), 1991. PB92-112416

101,022

BRIDGER, $K$.

Heats of Immersion, Friction, and Wear of Base Oil Fractions.

PB91-158923

101,173

BRILL, F. W.

Reactions of $\mathrm{C}_{5} \mathrm{H} 3(+)$ ) and $\mathrm{C} 5 \mathrm{H} 5(+)$ lons with Acetylene and Diacetylen

BRINCKMAN, F. E.

100,384

Characterization by High Performance Liquid Chromatography (HPLC) of the Solubilization of Phosphorus in Iron Ore by a Fungus.

101,162

Fourier Transform Infrared Analysis of Ceramic Powders: Ouantitative Determination of Alpha, Beta, and Amorphous Phases of Silicon Nitride.

PB91-237628

101,096

Methods for the Analysis of Organometallic Compounds in Wastes.

100,189

Newest Approaches to Ouantitative Assessment of Bioac Newest Approaches to Ouantitative Assessment of Bioac-
tive Organotins.
PB91-162032
101,328

Simultaneous Butyltin Determinations in the Microlayer Water Column and Sediment of a Northern Chesapeake Bay Marina and Receiving System.

100,918

Stability of Aqueous Inorganic Lead Solutions in Polycarbonate Containe

100,211

Studies on the Degradation Products of Paper with and without Pollutants in a Closed Environment. 1. Preliminary Results.

PB91.143313

101,181

BROADHURST, M. G.

Measurement of Space Charge Fields in Polymers.

PB91-158626

Proceedings of Cable ' 89 Workshop.

PB91-161943

100,486

BRODY, P. S.

Lead Zirconate-Titanate Thin Films Prepared by the Lase Ablation Technique.

PB91-187344

101,502

BROWN, A.

New Padio Detectors of Early-Type Pre-Main Sequence

Stars.

100,024

BROWN, C. A.

Fundamental studies of black liquor combustion. Report No. 4, Phases, 2, 3, and 4: Final report, December 1987December 19

BROWN, C. M

100915

Transitions and Energy Levels for Cu-Like $\mathrm{Yb}(41+)$ $\mathrm{Ta}(44+)$, and $\mathrm{U}(63+)$

PB91-147462

101,610

BROWN, M.

Proceedings of the Forum on Standards for High Integrity Software (DOD, Government, Industry). Held in Gaithersburg, Maryland on June 28, 1991.

PB92-112267

100,668

Streaking Images That Appear Only in the Plane of Diffraction in Unoped GaAs Single Crystals: Diffraction Imaging (Topography) by Monochromatic Synchrotron Radiation. 101,511 Structural Anomalies in Undoped Gallium Arsenide Observed in High Resolution Diffraction Imaging with Monochromatic Synchrotron Radiation.

PB91-237438

101,556

BROWN, P. F.

Use of Solid Modeling in the Design of M3 Components. PB92-123066

101,044

BROWN, R. J. C.

Thermodynamics of Ammonium Indates. 2. The Molar Heat Capacity of the Ammonium Pentabromoindate Monohydrate Salt (NH4) 2 InBr5. $\mathrm{H} 2 \mathrm{O}$ from 7.8 to $348 \mathrm{~K}$

PB91-149831

100,304

BROWN, S. H.

Biological Sulfur Oxidation and Reduction for Coal Sulfur Speciation and Desulfurization.

Bioprocessing of Fossil Fuels Using Hyperthermophilic Archaebacteria.
PB91-162271

BROWN, W. E

100,883

Comparative Study of Bovine Pericardium Mineralization: A Basic and Practical Approach.

Basic and 117407

100,056

Effect of Pyrophosphate Concentrations on Calcium Phos phate Growth on Well-Crystallized Octacalcium Phosphate and Hydroxyapatite Seed Crystals.

PB91-162024

101,193

Ion Incorporation into Octacalcium Phosphate Hydrolyzates.
PB91-175125

100,219

Setting Reactions and Compressive Strengths of Calcium Phosphate Cements.

148668

100,040

WN, W. R.

Method to Enhance Porosity of Micro-particles. PB91.187328

BRUCE, S. S.

Monitoring the Fate of Chlorine from MSW Sampling hrough Combustion.

PB91-174631

100,917

BRUNDAGE, R. T.

Investigation of Photoconductive Picosecond Microstripline

Switches on Self-Implanted Silicon on Sapphire (SOS).

PB91-147918

100,791

RUNGER, M. J.

Near-Threshold Vibrational Excitation of $\mathrm{H} 2$ by Electron Impact: Resolution of Discrepancies between Experiment and Theory.

101,679

BRUNO, T. J.

Hydrogen Component Fugacity Coefficients in Binary Mixures with Etane: Pressure Dependence.

100,455

-116508

100,185

PB91-148502

BRUSH, L. N.

Numerical Study of Two-Dimensional Crystal Growth Forms in the Presence of Anisotropic Growth Kinetics.

101,538

BRYAN, G. L.

Energy Distributions of Symbiotic Novae.

PB91-200873

100,032

\section{BRYAN, J. L.}

Development of a Technique to Assess the Adequacy of the Municipal Water Supply for a Residential Sprinkler System

101,358

YNER, N. P.

Short-Duration Autoignition Temperature Measurements for Hydrocarbon Fuels.

AD-A242 816/7

100,877

Short-Duration Autoignition Temperature Measurements for Hydrocarbon Fuels.

Use of the Electrostatic Classification Method to Size 0.1 micrometer SRM Particles: A Feasibility Study PB91-187633

100,932

CKLEY, T.

Concerning the Formation and the Kinetics of Phenylium

B91-187138

100,336

Monitoring the Fate of Chlorine from MSW Sampling through Combustion.

PB91-174631

100,917

Reactions of $\mathrm{C}_{5} \mathrm{H} 3(+)$ ) and $\mathrm{C} 5 \mathrm{H} 5(+)$ lons with Acetylene and Diacetylene.
PB91-195370

BUCKMAN, S. J.

100,384

Near-Threshold Vibrational Excitation of $\mathrm{H} 2$ by Electron Impact: Resolution of Discrepancies between Experiment and Theory.

BUDNICK, J. I.

Strontium-Induced Oxygen Defect Structure and Hole Doping in La2-xSrxCuO4.

PB91-148155

101,472

BUKOWSKI, R. W.

Improving the Fire Performance of Building Contents.
PB91-187260 PB91-187260 PB92-109156

\section{BUNTIN, S. A.}

Laser Induced Desorption of Molecules from Surfaces. PB91-147066

100,235

Photodissociation Dynamics of Mo(CO) 6 at $266 \mathrm{~nm}$ and $355 \mathrm{~nm}$ : CO Photofragment Kinetic-Energy and Internal$355 \mathrm{~nm}$ : CO Pho

State Distribution
PB91-236547

100,413

Surface-State-Mediated Photochemistry: Laser-Induced Desorption of NO from Si (111).

PB91-162289

100,320

BUR, A.

Fluorescence Monitoring of Polymer Processing: Mixing and Zero Shear Viscosity.

PB91-17448

100,492

Ab-Initio Calculations and Ideal Gas Thermodynamic Func-
tions of Cyclopentadiene and Cyclopentadiene Derivatives.

tions of Cyclopentadiene and Cyclopentadiene Derivatives.
PB92-148089

BURCH, B. M.

Evaluation of the Heat Flux Transducer Technique for Measuring the Thermal Performance of Walls.

100,126

BURCH, D. B.

Comparison of Two Test Methods for Determining Transfer Function Coefficients for a Wall Using a Calibrated Hot

B91-187278

100,121

BURCH, D. M.

Analysis of Moisture Accumulation in a Wood Frame Wall

Aubjected to Winter Climate.

PB92-116334

100,137

Indoor Ventilation Requirements for Manufactured Housing.
PB91-206698

BURDETTE, $H$.

High Resolution Diffraction Imaging of Crystals Grown in Microgravity and Closely Related Terrestrial Crystals PB92-109008

101,562

High Resolution Synchrotron X-Radiation Diffraction ImagHicrogravity and Closely Related Terrestrial Crystals.

PB91-216747

101,548

\section{BURDETTE, H. E.}

High-Pesolution Small-Angle X-ray Scattering Camera for PB1-175067

Small-Angle-Scattering Determination of the Microstructure of Porous Silica Precursor Bodies.

101,077

\section{BURDGE, G.}

Investigation of Photoconductive Picosecond Microstripline Switches on Self-Implanted Silicon on Sapphire (SOS)

PB91.147918

100,791

BURKHOLDER, J. B.

$\mathrm{H}+\mathrm{O} 3$ Fourier-Transform Infrared Emission and Lase Absorption Studies of $\mathrm{OH}(X(2)$ Pi) Radical: An Experimental Dipole Moment Function and State-to-State Einstein A Coefficients.

High-Resolution Infrared Spectrum of the nu1 Band of OC10.

PB92-117175

100,463

Spectroscopic Constants for the nu9 Infrared Band of

B91-189415

100,343

BURNETT, E. D.

Correction for Converting 2.CM(3)-Coupler Responses to

Hearing Aids.

100,046

Response of Personal Noise Dosimeters to Continuous and mpulse-Like Signals.

PB92-11635

100,914

BURNS, T. J.

Mechanism for High Strain-Rate Shear Band Formation.

PB91-147033 101,575

Mechanism for Shear Band Formation in the High StrainRate Torsion Test.

PB91-149872

101,579

BURR, W. E.

Conformance Verification of FDDI Stations.

PB91-148254 
BYRD, W.

Reflection/Absorption FTIR Spectral Characteristics of Thin and Thick Crosslinked Epoxy Films on Steel Substrates. PB91-203711

BYRD, W. E.

Characterization of Epoxide Coatings on Steel by Reflection/Absorption Fourier Transiorm Infrared Spectroscopy: Ouantitative Study

PB91-203729

101,109

Fourier Transform Infrared Spectroscopic Studies of the Degradation of One-Component Polyether Polyurethane Protective Coatings on Steel.

PB91-195248

101,108

\section{BYRNE, A. $R$.}

Characterization of the Mineral Fraction in Botanical Reference Materials and Its Influence on Homogeneity and Analytical Results.

PB91-148817

100,186

BZOWSKI, J.

Equilibrium and Transport Properties of Gas Mixtures at Low Density: Eleven Polyatomic Gases and Five Noble

PB91-192526

100,363

CABEZAS, $\mathrm{H}$.

Statistical Mechanical Model of Aqueous Two-Phase Systems.

PB91-174508

100,493

CABRERA, $B$.

Correlation of Flux States Generated by Optical Switching of a Superconducting Circuit.

PB91-202929

101,682

CADOFF, $B$.

Reference NO2 Calibration System for Ground-Based Intercomparisons during NASA's GTE/CITE II Mission.

comparisons

100,034

CAGE, M. E.

Investigating the Use of Multimeters to Measure Quantized Hall Resistance Standards.

PB91-236554

100,734

CAHN, J. W.

6-D Structural Model for the Icosahedral (Al, Si)-Mn Ouasicrystal.

101,501

Effect of Coherency Strain on Alloy Formation: Migration of

Liquid Films. 147322

101,210

Emergence of Modern Nucleation Theory.

101,481

Grain Boundaries in a 2-D Superlattice.

PB91-202

100,388

Microstructural Control Through Diffusion-Induced Grain Boundary Migration.

PB91-236760

101,239

N-Dimensional Crystallographic Description of the Icosahedral Phases; the Example of the Al73Mn21Si6 Ouasiperiodic Structure.

101,226

Phase Diagrams of Hexagonal Binary Ordering Alloys with Anisotropic Interactions.

101,211

Theory of Orientation Textures Due to Surface Energy Ani$\begin{array}{ll}\text { sotropies. } & 101,571\end{array}$

CALARCO, J. R.

Electrofission in the Quasifree and Delta Regions PB91-147355

CALLANAN, J. E.

Fusion of Mercury. A New Certified Standard for Differential Scanning Calorimetry.

PB91-150003

100,309

Heat Capacity and Thermodynamic Properties of Deutrated Ammonium Hexafluorophosphate ND4PF6 from $5.8 \mathrm{~K}$ to

$347 \mathrm{~K}$.
PB91-147041

100,272

Heat Capacity of Deuterated Ammonium Tetrafluoroborate ND4BF4 from 7 to $348 \mathrm{~K}$

PB9 1.147058

100,273

Thermodynamics of Ammonium Indates. 2. The Molar Heat Capacity of the Ammonium Pentabromoindate Monohydrate

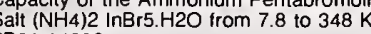

100,304

PB91-149831

100,304

Thermodynamics of the Divalent Metal Fluorides. 3. Heat Capacity of the Fast Ion Conductor Sr SnF4 from 6 to 344 PB91-149864 100,305

Thermodynamics of the Divalent-Metal Fluorides. 4. Heat Capacity of MSnF4, M $=\mathrm{Pb}, \mathrm{Ba}$, or $\mathrm{Sr}$, at Temperatures $300 \mathrm{~K}$ to $660 \mathrm{~K}$

PB92-116524

100,456

CALLCOTT, T. A.

Soft X-ray Absorption and Emission Spectra and the Electronic Structure of Some Exotic Materials.

PB91-194738

101,520

CALM, J. M.

Research and Development of Heat Pumps Using Nonazeotropic Mixture Refrigerants.
PB91-174516

100,891

ALVAYRAC, $Y$

N-Dimensional Crystallographic Description of the Icosahedral Phases; the Example of the A173Mn21Si6 Quasiperiodic Structure.

CAMELL, D. G.

101,226

Calibration of Antenna Factor at a Ground Screen Field Site Using an Automatic Network Analyzer.

PB91-134239

100,724

NIST Calibration Procedure for Vertically Polarized Monopole Antennas $30 \mathrm{kHz}$ to $300 \mathrm{MHz}$.

PB91-185124

100,726

\section{ANFIELD, L. R.}

Soft $X$-ray Absorption and Emission Spectra and the Electronic Structure of Some Exotic Materials.

101,520

Stable, High Ouantum Efficiency Silicon Photodiodes for Vacuum-UV Applications.

PB91-203349

100,757

CANNON, R. M.

Dihedral Angles in Magnesia and Alumina: Distributions from Surface Thermal Grooves.

101,058

PB91-147330

Dihedral

Metal Reference Line Technique for Obtaining
Angles from Surface Thermal Grooves.

Angles from Sur
PB91-147348

101,059

CANTRELL, J. S.

Properties of Amorphous Zirconium Rhodium Hydride (Zr3RhHx) Prepared from Glassy and Crystalline Alloys.

101,225

CAO, L. $X$.

Light-Scattering Measurement of the RMS Slopes of Rough Surfaces.

PB92-116532

101,436

CAO, R.

Morphology and Barrier-Height Development of $\mathrm{Bi} / \mathrm{InP}(110)$ Interfaces.

PB91-147736

101,468

CAPOBIANCO, T. E.

Eddy Current Probe Characterization Using an Impedance Plane Display Instrument.

100,834

PB91-133868

Coil Con-

struction Parameters.

PB91-236562

100,945

CAPPELLETTI, R. L.

Neutron Measurements of Intramolecular Vibrational Modes

PB92-116540

101,055

CARASSO, A. S.

Identification of Dynamic Green's Functions in Structural

Networks.
PB91-202812

101,585

Probe Waveforms and the Reconstruction of Structural Dynamic Green's Functions.

PB91-161950

101,737

Space Marching Difference Schemes in the Nonlinear Inverse Heat Conduction Problem.

101,604

Mathematical Model for Dental Caries: A Coupled Dissolution-Diffusion Process.

PB92-126689

101,316

ARINO, N. J.

Assessment: U.S. Office Building of Moscow.

100,151

Extended Abstract: A Finite Element Study of the Stress and Displacement Fields Produced by Point Impact.

PB91-175224 101,584

Impact-Echo: A New Method for Inspecting Construction

PB92-116557 100,140

Introduction to $\mathrm{ACl} 306.1-87$ : Specification for Cold Weather Concreting.

100,526

Outline of a National Plan on High-Performance Concrete: Report on the NIST/ACI Workshop. Held in Gaithersburg, MD on May 16-18, 1990.

PB91-143321

100,522

Rate Constant Functions for Strength Development of Con-

PB91-175117

100,527

CARLSON, T. A.

Angle-Resolved Photoemission from the Ar $2 p$ Subshell.

PB91-194928 101,665

Angular Distribution of Ejected Electrons in Resonant Auger Processes of $\mathrm{Ar}, \mathrm{Kr}$, and $\mathrm{Xe}$.

101,648

Parity-Unfavored Transitions in Resonant Photoemission from $\mathrm{Ar}, \mathrm{Kr}$, and $\mathrm{Xe}$ : Experimental and Theoretical Results.

PB91-133900

Resonant

Unusual Degree of Angular Anisotropy in the Resonant Auger Spectrum of $\mathrm{Kr}$.

101,649

CARPENTER, B. S.

Method to Enhance Porosity of Micro-particles. PB91.187328

101,185

CARROL, J. J.

Solubility of Carbon Dioxide in Water at Low Pressure.

PB92-148196

CARROLL, D. F.

Comparison of Creep Rupture Behavior in Tension and

Bending.

PB91-202820

101,087

Creep and Reliability of Ceramic Materials at Elevated Temperatures.

PB91-204172

101,091

High Temperature Creep Testing of Ceramics.

101,088

CARTIER, E.

Electron Attenuation Lengths at $\mathrm{SiO} 2 / \mathrm{Si}$ Interfaces. PB91-175612

101,499

\section{CARVER, G. P.}

Concurrent Engineering through Product Data Standards

PB9 1-19336

100,982

High Spatial Resolution Mapping of Semiconductor Resis-

PBo

100,809

Multi-Enterprise Concurrent Engineering through International Standards.

PB92-123058

100,997

Verification of the Relation between Two-Probe and FourPB91-147520

100,788

CASASSA, M. P.

Picosecond IR Studies of the Vibrational Dynamics of $\mathrm{CO} /$ Pt(111)

Time- and State-Resolved Measurements of Nitric Oxide

Dimer Infrared Photodissociation.
PB91-202846

100,387

CASELLA, R. C.

Nonlocal Phase Shifts Induced by Static Electric Fields in Neutron Interferometers When the Path-Enclosed Charge

PB91-150011

101,620

Theoretical Model for the Tunneling-Gap Anisotropy Observed in Layered Copper-Oxide High-Temperature Superconductors.

101,564

CASSADY, A.

NIST Personnel Management Demonstration Project: Design, Implementation and Accomplishments.

100,003

CASSANTO, J. M 


\section{PERSONAL AUTHOR INDEX}

CELOTTA, R. J.

Book Review: Polarized Electrons at Surfaces by J. KirschPB91-174532

101,493

Geometric and Electronic Properties of Cs Structures on III$\checkmark$ (110) Surfaces: From $1 D$ and $2 D$ Insulators to $3 D$ Metals. PB91-190090 101,517

Observation of Two Different Oscillation Periods in the Exchange Coupling of $\mathrm{Fe} / \mathrm{Cr} / \mathrm{Fe}(100)$.

101,572 Scanning Electron Microscopy with Polarization Analysis
(SEMPA)-Studies of Domains, Domain Walls and Magnetic Singularities at Surfaces and in Thin Films.

101,554 PB91-237289

and CsCovered InSb(110)

PB91-195750

101,537

System for the Study of Magnetic Materials and Magnetic Imaging with the Scanning Tunneling Microscope P891-194779

101,522

\section{CENEDESE, $P$.}

Grain Boundaries in a 2-D Superlattice.

PB91-202853

100,388

\section{CERNYAR, E. W.}

Influence of Memory Propagation on Phase-Resolved Stochastic Behavior of AC-Generated Partial Discharges.

P891-237644

100,861

CEZAIRLIYAN, A

Dynamic Technique for Measuring Surface Tension at High Temperatures in Microgravity Environment

101,206 N91-21347/0 High-Speed Spatial (Linear) Scanning Pyrometer: A Tool for
Diagnostics, Temperature Mapping, and Property DetermiDiagnostics, Temperature Mapr

nations at High Temperatures.

Research at NBS on Dynamic Measurements of Thermophysical Properties at High Temperature (Abstract). PB91-161968

100,315

CHACONAS, $K$.

Real-Time Model-Based Tracking Combining Spatial and Temporal Features.

100,700

Video Compression for Remote Vehicle Driving

PB91-203208

101,035

\section{CHAE, H. B.}

Alternative Refrigerants R123a, R134, R141b, R142b, and R152a: Critical Temperature, Refractive Index, Surface Tension, and Estimates of Liquid, Vapor, and Critical Densities.
P891-147074 CHAI, $\mathrm{H}$.

Buckling and Post-Buckling Behavior of Elliptical Plates: Part 1. Analysis.

101,588

Buckling and Post-Buckling Behavior of Elliptical Plates. Part 2. Results

101,589

Interlaminar Shear Fracture of Laminated Composites.

PB91-187336 101,122

CHAMBERLAND, B. L.

Strontium-Induced Oxygen Defect Structure and Hole Doping in La2-xSrxCuO4.

PB91-148155

101,472

CHAMBERS, G. P.

Ion-Induced Radiation-Enhanced Diffusion of Silver in

Nickel.
PB91-175554

101,498

CHAMPION, R. L.

Collisional Electron-Detachment and Ion-Conversion Processes in SF6.

CHAN, S. J.

100,836

Evolution of Infrared Carbon Stars.

PB91-202861

100,033

CHANDLER-HOROWITZ, D.

Ellipsometry SRM's for Use in Thin Film Measurements PB92-116599

100,819

Numerical Modeling of Silicon Photodiodes for High-Accuracy Applications. Part 1. Simulation Programs.

100,763

Sensitivity of Ellipsometric Modeling to the 'Islands' of Silicon Precipitates at the Bottom of the Buried Oxide Layer in Annealed SIMOX

PB92-116607

100,820

CHANG, E. S.

Energy Levels of Atomic Alumınum with Hyperfine Structure.

101,594

CHANG, $M$.

Assessment of the State-of-the-Art for Process Monitoring Sensors for Polymer Composites.

PB91-222612

100,510

CHANG, R. F.

High-Speed Spatial (Linear) Scanning Pyrometer: A Tool for Diagnostics. Temperature Mapping, and Property Determinations at High Temperatures.
N91-21361/1

100,924

CHANG, S. S.

Polymer Composite Processing. Industry Workshop (2nd). Held at Gaithersburg, Maryland on May 18, 1990.

B91-132191

101,115

Temperature Gradient in Differential Scanning Calorimetry.

PB91-236570

100,414

CHANG, $T$.

Chain Conformation of a Block Polymer in a Microphase Separated Structure.

PB91-148890

100,485

CHANG, T. Y.

Fe Implantation in In0.53Ga0.47As/InP.

PB91-189969

101,516

CHANG, Y. M.

Calibration Procedures for Infrared Imaging Systems for Building Diagnostics.

100,057

Diagnostic Techniques for Evaluating Office Building EnvePB91-195438

100,074

CHAO, B. C.

Chemical Nature of In vivo DNA Base Damage in Hydrogen Peroxide-Treated Mammalian Cells.

PB91.187526

101,335

CHAO, J.

Thermodynamic and Thermophysical Properties of Organic Nitrogen Compounds. Part 1. Methanamine, Ethanamine, Methylben PB91-192583

100,368

CHAPMAN, R. E.

Applications of Operations Research Techniques to System Design and Evaluation.

PB91-174540

101,267

CHAPPAS, W. J.

Effects of Absorbed Dose Rate, Irradiation Temperature and Post-Irradiation Temperature on the Gamma Ray Response of Red Perspex Dosimeters.

101,363

Temperature Dependence of Radiochromic Film DosiTemperature Dependence of Radiochromic Film Dosi-
meters.
PB92-117134

01,728

CHARAGUNDLA, S. $R$.

Advanced sensor development program for the pulp and

paper industry. Final report.
DE91007688

\section{CHASE, L. L.}

Laser Induced Damage in Optical Materials: 1989. PB91-148478

101,409

CHASE, M. W.

NIST Standard Reference Data Products 1991 Catalog.
PB91-167312

Technical Activities 1990, Standard Reference Data Pro-

gram.

100,321

Thermodynamic Data for Modeling of Flue Gas Cleanup Systems (Abstract for Poster Session).

100,904

CHAUDHURI, D. $K$. Apparatus for Measurement of Coefficient of Friction.
PB91-148049 101,250

101,250

Improved Analysis for Flexural Creep with Application to

Sialon Ceramics.

101,071

CHEN, J.

Uniform Junction Temperature AIGaAs/GaAs Power Heterojunction Bipolar Transistors on Silicon Substrates. PB91-189399

100,806

CHEN, M.

Anomalous Low-Frequency Butterfly Curves for Subsidiary

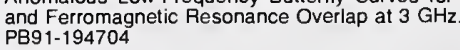

101,519

CHEN, $Y$

Mechanical and Swelling Behavior of Crosslinked Natural Rubber: Consequences of the Flory-Rehner Hypothesis.

101,141

Tests of the Flory-Rehner Hypothesis: Comparison of the Elastic Free Energy Function for Crosslinked Rubber in the

PB91-203570

100,508

CHENG, H. S.

Mechano-Chemical Descriptive Model for Wear Under Mixed Lubrication Conditions.

PB91-189480

101,177

CHENG, Y. $W$.

Computerization of a Thermomechanical Processing Research System
P891-175208

101,583

Continuous-Cooling Transformation Characteristics and High-Temperature Flow Behavior of a Microalloyed SAE 1141 Steel.

PB91.187856

101,155

Data-Reduction and Analysis Procedures Used in NIST's Thermomechanical Processing Research.

100,925

Performance of Precast Concrete 8eam-to-Column Connections Subject to Cyclic Loading.

PB91-236588

100,150

Performance of 1/3-Scale Model Precast Concrete BeamColumn Con

Report No. 2
PB91-222570

CHERNIAVSKY, J. C.

100,149

Proceedings of the Workshop on High Integrity Software Held in Gaithersburg, MD. on January 22-23, 1991.

P892-109040

100,667

CHESLER, S

Capillary Supercritical Fluid Chromatography of Explosives: Cavestigations on the Interactions between the Analytes, the Mobile Phase and the Stationary Phase.

PB91-147769

100,182

CHESLER, S. N.

Monitoring the Fate of Chlorine from MSW Sampling through Combustion.

PB91-174631

100,917

Preparation and Analysis of a Marine Sediment Reference Material for the Determination of Trace Organic Constitu-

PB91.149237

101,377

CHI, P.

Secondary lon Yield Changes in Silicon and Gallium Arsenide Due to Topography Changes during Oxygen or Cesium

PB91.148064

101,471

CHI, P. H.

Quantitative Analysis of Impurities in SIMOX Samples Using Secondary lon Mass Spectrometry.

PB91.236596 100,208

Round-Robin Study of Implants in Si and SiO2 by SIMS,

RBS, and NAA.
PB92-117241 100,830

Wawayandaite, a New Calcium Manganese Beryllium Boron Silicate from Franklin, New Jersey.

PB91.174680

101,359

CHIANG, C. $K$

Atomic Fingerprint of YBa2Cu3O7-x-Type High-Temperature Supercond

Break Junction Tunneling Spectroscopy of Single-Crystal Bismuth-Based High-Temperature Superconductors.
PB91-134478 462 Flux Dynamics in Two Samples of Comparison of Flux Dynamics in Two Samples of
YBa2Cu3O7 with Different Pinning.
PB91-149419

Electrochemical Behavior of Ferrocene in Polyethylene Oxide-400.

100,439

Lead Zirconate-Titanate Thin Films Prepared by the Laser Ablation Technique.

PB91-187344

101,502

Levitation of Superconductıng Composites.

101,503 
CHOW, L. C.

Digital Image Analysis Assisted Microradiography Measurement of Mineral Content of Caries Lesions in Teeth.
PB91-187666 Effects of $\mathrm{pH}$ and Calcium on Hydrolysis of Na2SiF6 and Na2SnF6: A Quasi-Constant Composition Titration Study.
PB91-194746 Mathematical Model for Dental Caries: A Coupled Dissolution-Diffusion Process.

tion-Diffusion
PB92-126689

101,316

Self-Setting Calcium Phosphate Cements.

100,049

Setting Reactions and Compressive Strengths of Calcium Phosphate Cements.

PB91-148668

CHOWDHURY, $K$.

Optimization of a Pulse Tube Refrigerator for a Fixed Compressor Swept Volume.

PB91-203877

101,051

CHRISTENSEN, R. G.

Trace Determination of $\mathrm{Cr}(\mathrm{VI})$ by LC/AAS with on-Line Preconcentration.

100,184

CHUANG, T.

Design Diagrams for Heavy Metal Fluoride Glass Windows.

PBU 18749

Comparison of Creep Rupture Behavior in Tension and Bending.

101,087

Creep and Reliability of Ceramic Materials at Elevated Tem-

peratures.

101,091

Improved Analysis for Flexural Creep with Application to Sialon Ceramics.

101,071

Tensile Creep Behavior of Structural Ceramics.

101,075

Test Method for Tensile Creep of Structural Ceramics Using Flexure Beams.

101,099

CHUCK, L.

Tensile Creep Behavior of Structural Ceramics.

PB91-159277

101,075

CHUNG, M. B.

Standard Chemical Thermodynamic Properties of Isomer Groups of Monochloroalkanes.

100,258

Standard Chemical Thermodynamic Properties of Polycyclic Aromatic Hydrocarbons and Their Isomer Groups. III. Naphthocoronene Series, Ovalene Series, and First Members of Some Higher Series.

PB90-244625

100,259

CHURNEY, K. L.

Monitoring the Fate of Chlorine from MSW Sampling through Combustion.

PB91-174631

MSW Calorimetry.

PB91-133942

100,917

CHWALOWSKI, $M$.

Evaporator Performance Investigation for Residential Air-

Conditioning Application Using Mixed Refrigerants.
PB92-126432

CIESZKIEWICZ, M. T.

Experimental Thermal Conductivity, Thermal Diffusivity, and Specific Heat Values for Mixtures of Nitrogen, Oxygen, and Argon.
PB91-194472

CITRINOVITCH, A.

100,369

Israel's New Synchronized Time Scale, UTC(INPL)

AGUE, $F$.

Summary of Experiments with the Separated Aperture Technique of Dielectric Anomaly Detection.

100,721

CLARK, A. V.

Capacitive Array Sensors for Nondestructive Evaluation

PB91-237313 100,947 Characterization of Capacitive Array for NDE Applications.
PB91-237305

Monitoring of Anisotropic Material Elastic Properties Using Ultrasonic Receiving Rays

101,126

Residual Stress Detection in Railroad Wheels: An Uitrasonic System Using EMATs (Electromagnetic-Acoustic Transducers). Report No. 23.

101,741

Theory of Capacitive Probe Method for Noncontact Characterization of Dielectric Properties of Materials.

100,948

Tread Crack Detection in Railroad Wheels: An Ultrasonic System Using EMATs (Electromagnetic-Acoustic Transduc ers). Report No. 22.

PB91-222661

101,742

Ultrasonic Measurement of Sheet Steel Texture and Formability: Comparison with Neutron Diffraction and Mechanical Measurements.
PB92-116615

101,170

Ultrasonic Texture and Stress Measurements in Anisotropic Polycrystalline Aggregates.

101,237

Use of Neutron Pole Figures to Calibrate Ultrasonic Techniques for On-Line Texture Control of Aluminum Plates.
PB91-159111 CLARK, C. W.

Giant Resonances in the Transition Regions of the Periodic Table.

PB91-147116

101,605

PB91-203778

101,699

Technical Activities 1990, Electron and Optical Physics Division.

101,662

CLARK, S. N.

Depot: A Framework for Sharing Software Installation Across Organizational and Unix Platform Boundaries.

PB91-240754 100,659

Fed-X: The NIST Express Translator. Revised, November

1990.
PB91-132126 100,681

NIST Express Working Form Programmer's Reference (Revised November 1990). National PDES Testbed Report

PB91-157164

100,964

NIST PDES Tookit: Technical Fundamentals. National PDES Testbed Report Series (Revised)
PB91-132159

100,682

NIST STEP Working Form Programmer's Reference. National PDES Testbed Report Series (Revised).

PB91-144378

100,998

NIST Working Form for STEP: National PDES Testbed Report Series (Revised)

100,683

CLARKE, D. $R$

Amorphization and Conductivity of Silicon and Germanium Induced by Indentation.

CLARY, D. W.

101,482

Mechanism of Soot Formation in Acetylene-Oxygen Mixtures.

PB91-147280

100,537

CLAY, D. T.

Fundamental studies of black liquor combustion. Report No. 4, Phases, 2, 3, and 4: Final report, December 1987 December 1989 .

018580

100,915

CLEARY, T. G.

Flammability Characterization of Foam Plastics.

Framework for Utilizing Fire Property Tests

PB91-240788

100,142

CLEMENTS, A. J. D.

Accuracy Model for Phase Noise Measurements.

Extending the Range and Accuracy of Phase Noise Measurements.

CLIFFORD, A. A.

100,588

Solubilities of Solids and Liquids of Low Volatility in Supercritical Carbon Dioxide.

PB92-148105

100,470

CLIFTON, C. L.

Rate Constants for Hydrogen Abstraction Reactions of the Sulfate Radical, SO4- Alkanes and Ethers.

PB91-203232

100,394

LIFTON, J. R.

Chloride Ion Diffusion in Low Water-to-Solid Cement PBa1-187690

101,138

Computational Models Developed for the Corrosion of Prestressing Steel.
PB91.167304

100,115

Guide to the Use of the Cement and Concrete Research Remote Bulletin Board System (RBBS) Computer. 100,524

Guidelines for the Development of Computer Based Models
in a Cementitious Materials Modeling Laboratory.
PB91-240804 PB91-240804

Outline of a National Plan on High-Performance Concrete: Report on the NIST/ACI Workshop. Held in Gaithersburg MD on May 16-18, 1990

100,522

Predicting the Remaining Service Life of Concrete.

PB92-126598

101,371

Techniques to Observe the Fracture Zone in Mortar and Concrete.

100,521

Use of Nondestructive Methods for Inspection of Single-Ply Roofing Membranes.

PB91-203935

100,128

CLINE, J. I.

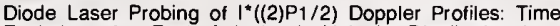
Evolution of a Fast, Anisotropic Velocity Distribution in a Thermal Bath.

PB91-150052

100,310

General Method for Doppler Determination of Cylindrically Symmetric Velocity Distributions: An Application of Fourie Transform Doppler Spectroscopy.

PB91-14938

100,302

NE, J.P.

Small-Angle-Scattering Determination of the Microstructure of Porous Silica Precursor Bodies.

101,077

Sputtered Thin Film YBa2Cu3On

PB91-189647

101,509

CLINTON, T. W.

Magnetic Ordering of $\mathrm{Er}$ in Powder and Single Crystals of $\mathrm{ErBa2Cu} 3 \mathrm{O} 7$

PB91-187377

101,505

LOUGH, R. B.

Acoustic Emission Monitoring of Laser Drilling.

PB91-158527

101,218

AKLEY, $K$.

Cross-Validation Procedure for Stopping the EM Algorithm and Deconvolution of Neutron Depth Profiling Spectra.

PB91-161976

OAKLEY, K. J.

Micromagnetics of Domain Walls at Surfaces.

101,49

Use of Cross-Validation as a Stopping Rule in Emission Tomography Image Reconstruction.

101,295

COBLE, R. L.

Dihedral Angles in Magnesia and Alumina: Distributions from Surface Thermal Grooves

PB91-147330

101,058

Metal Reference Line Technique for Obtaining Dihedral Angles from Surface Thermal Grooves.

101,059

COCHRAN, J. F.

Magnetic Anisotropies in Ultrathin fcc $\mathrm{Fe}(001)$ Films Grown on Cu(001) Substrates.

PB92-116631

101,565

COCHRANE, C. G.

Damage to the Bases in DNA Induced by Stimulated Human Neutrophils.

PB92-116912

101,312

COHEN, A.

Stability of Uric Acid in Ammonium Hydroxide

PB91-189266

101,292

OHEN, E. R.

Adjustment of the Fundamental Physical Constants: A Report of the CODATA Task Group on Fundamental Constants, 1986.

101,606

COHEN, J.

Fundamentals and Applications of Infrared Themography for Nondestructive Testing

101,009

COHEN, N. L.

Ultraviolet, Optical, Infrared, and Microwave Observations of HR 5110.

100,020

COHEN, Y.

Tread Crack Detection in Railroad Wheels: An Ultrasonic System Using EMATs (Electromagnetic-Acoustic Transducers). Report No. 22

PB91-222661

101,742

COLELLA, $R$

Performance of OSI Transport over ACCUNET and IBERPAC

PB91-134825

100,563

COLEMAN, B. D.

Phenomenological Theory of the Influence of Strain History 


\section{PERSONAL AUTHOR INDEX}

CONNELL, J. S.

Electrofission in the Quasifree and Delta Regions PB91-147355

101,609

CONNELLY, J. P.

Electrofission in the Quasifree and Delta Regions.

PB91-147355

101,609

CONWAY, J. J.

Particle Size Measurement of Inert Gas Atomized Powder. PB91-187203

101,229

COOK, G.

Impulse Response Functions for Elastic Structures with Rigid Body Degrees of Freedom.

PB91-237354

101,587

COOK, L. P.

Cathodoluminescence Measurement of Strained Alumina Single Crystals.

101,094

Inverse Correlation between the Intensity of Luminescence Excited by Electrons and by Visible Light in ChemicalVapor-Deposited Diamond Films.

PB91-195560

101,531

Lead Zirconate-Titanate Thin Films Prepared by the Laser Ablation Technique.

101,502

Phase Equilibria in the System TI-Ca-Ba-Cu-O. 1. Stability 2122 Phase under Conditions of Oxygen Annealing PB91-147165 101,465

Processing Bi-Pb-Sr-Ca-Cu-O Superconductors from Amorphous State.

101,504

COOK, R. F.

Amorphization and Conductivity of Silicon and Germanium Induced by Indentation.

PB91-150045

101,482

COOPER, J.

Collisional Energy Transfer between Excited Sr Atoms. PB91-204164 101,703

Overview of the Ion Dynamic Effect in Line Broadening, and a Generalization of the Unified Theory.

101,442

COOPER, J.W.

Angular Distributions of Electrons in Resonant Auger Spec-

Pra. 187393

101,650

Parity-Unfavored Transitions in Resonant Photoemission from $\mathrm{Ar}, \mathrm{Kr}$, and $\mathrm{Xe}$ : Experimental and Theoretical Results. PB91-133900

101,599

COOPER, L. Y.

Applications of the Generalized Global Equivalence Ratio Model (GGERM) for Predicting the Generation Rate and Distribution of Products of Combustion in Two-Layer Fire Environments: Methane and Hexanes.

100,131

Ceiling Jet-Driven Wall Flows in Compartment Fires
PB91-174573

100,117

Compartment Fire-Generated Environment and Smoke Fill-

ing. 158543

100,107

Fire-Plume-Generated Ceiling Jet Characteristics and Convective Heat Transfer to Ceiling and Wall Surfaces in a Two-Layer Zone-Type Fire Environment: Uniform Temperature Ceiling and Walls.

PB92-123074

101,370

Interaction of an Isolated Sprinkler Spray and a Two-Layer Compartment Fire Environment.

PB91-216804

100,551

COPLEY, J. R. D.

Fe-W Supermirrors for Polarizing Neutrons

101,643

Neutron Measurements of Intramolecular Vibrational Modes

in C60.
PB92-116540

Transmission Properties of a Counter-Rotating Pair of Disk Choppers.

101,706

CORIELL, S. R.

Convective and Morphological Instabilities during Directional Solidification

PB91-158550 $\quad 101,219$

Effect of an Electric Field on the Morphological Stability of the Crystal-Melt Interface of a Binary Alloy. 3 . Weakly Nonlinear Theory.

101,536

Effect of Gravity Modulation on Thermosolutal Convection in an Infinite Layer of Fluid.

PB92-11229

101,396

CORLEY, D. M.

Boussinesq Algorithm for Enclosed Buoyant Convection in Two Dimensions.

CORT, L. A.

100,157

Variations in the Practice of Ceramic Technology in Different Cultures: A Comparison of Korean and Chinese Celadon Glazes.

101,111
COSTANTINO, P. D.

Self-Setting Calcium Phosphate Cements

COUDERT, L. H.

Rotational-Tunneling Spectrum of the Ar-SO2 van der Waals Complex

COULTAS, T. A

Evaluation of Building Systems Inventions.
PB91-158659

\section{COUNAS, $\mathrm{G}$.}

Intercomparison of NBS Noise Calibration Services. PB91-162370

COURSEY, B. $M$.

Calculation of Beta-Particle Counting Efficiency for Liquid Scintillation Systems with Three Phototubes.

PB91-175547

101,644

\section{COWAN, P. L}

Direct Determination of Molecular Orbital Symmetry of H2S Using Polarized X-ray Emission.

100,335

Molecular-Orbital Studies via Satellite-Free $X$-ray Fluorescence: $\mathrm{Cl} \mathrm{K}$ Absorption and $\mathrm{K}$-Valence-Level Emission Spectra of Chlorofluoromethanes

100,427

Polarization and Anisotropy of X-ray Emission from Mole-

PB91-187401

101,651

Polarized X-ray Emission Studies of Methyl Chloride and the Chlorofluoromethanes.

174995

100,329

Selective Excitation of X-ray Emission Spectra.

PB91-147173

107,607

COYLE, T. $W$

Physical and Thermo-Mechanical Properties of Monoclinic Single Crystals.
PB91-148767

101,065

COYNE, L. D.

Examination of Mechanisms Responsible for Incoherent Scattering of Subthermal Neutrons by Polymers.

100,505

CRAIG, R. $M$.

Low-Temperature, Electrically Calibrated SOS Bolometer for Power and Energy Measurements.

CRAMER, D. M

Joint Panel Meeting of the UJNR Panel on Fire Research and Safety (11th). Held in Berkeley, CA. on October 19-24,

PB92-109164

100,166

CRANMER, D.

Design Diagrams for Heavy Metal Fluoride Glass Windows. PB91-187419

101.419

CRANMER, D. C.

Effect of Thermal Expansion Mismatch on Fiber Pull-Out in Glass Matrix Composites.

101,125

91-202952

Alkaline

Earth Glasses.

P891-189332

CRAWFORD, $M$. L.

Mode-Stirred Chamber for Measuring Shielding Effectiveness of Cables and Connectors: Assessing MIL-STD-1344A Method 3008 .

100,776

TEM Driven Reverberating Chamber, a Single Facility for Radiated EMS $/ V$ Testing, $10 \mathrm{kHz}-18 \mathrm{GHz}$

100,856

TEM/Reverberating Chamber Design/Concept Study: A Single Facility for Large System Radiated EMC Testing, 10 Single Facility for Large System Radiated EMC Testing, 10
$\mathrm{kHz}-40 \mathrm{Ghz}$. $\mathrm{kHz}-40 \mathrm{Ghz}$.

\section{CREMER, D.}

100,845

Van der Waals Complexes in 1,3-Dipolar Cycloaddition Reactions: Ozone-Ethylene

CRESSWELL, M. W.

100,417

Design, Fabrication, and Testing of an Interconnect Test Structure for Evaluating VLSI Processes PB91-174581

100,803

Evaluation and Improvement of E-Beam Exposure Routines by Use of Microelectronic Test Structures.

100,805

Knowledge Extraction Techniques for Expert System Assisted Wafer Screening

100,824

Knowledge Verification of Machine-Learning Procedures Based on Test Structure Measurements.

PB91-189571

100,709

Modified Sliding Wire Potentiometer Test Structure for Mapping Nanometer-Level Distances.

100,821

Test Chip for the Evaluation of Surtace-Diffusion Phenomena in Sputtered Aluminum Planarization Processes

PB92-116920

CRISSMAN, J. M.

Development of Test Methods to Determine the Compatibility of Liquid Hazardous Materials with Polyethylene Packagings. 20457

100,943

Long Time Creep and Lifetime Behavior in Uniaxial Extension of a Linear Low Density Polyethylene.

101,247

Multistep Stress-Relaxation Behavior in Uniaxial Extension

of an Ethylene-Hexene Copolymer. 101,242

Multistep Stress-Relaxation Study of a Single Crystal of nEicosane (C2OH42) in Torsion.

PB91-187427

100,497

CROMAR, M. W.

Excess Low-Frequency Flux Noise in dc Squids Incorporating Nb/Al-Oxide/Nb Josephson Junctions.

100,736

YBa2Cu3O7-delta/Insulator Multi-Layers for Crossover Fab-

rication.

PB91-194662

101,518

CROMER, C. L.

Laser-Driven Ionization and Photoabsorption Spectroscopy of Atomic lons.

101,415

Low-Pressure Symmetric Discharge Heat Pipe and DROGS

PB91.195016

101,666

Precise Experimental Test of Calculated Two-Electron Lamb Shifts in
PB91-149203

101,617

CROSS, R. W.

Anomalous Low-Frequency Butterfly Curves for Subsidiary and Ferromagnetic Resonance Overlap at $3 \mathrm{GHz}$.

101,519

Enhanced Flux Creep in Nb-Ti Superconductors After an Increase in Temperature.

PB91-202903

101,680

Hall Probe Magnetometer for SSC Magnet Cables: Effect of

Transport Current on Magnetization and Flux Creep

101,681

CROVETTO, R.

Evaluation of Solubility Data of the System CO-H2O from $273 \mathrm{~K}$ to the Critical Point of Water.

100,453

Limiting versus Apparant Critical Behavior of Henry's ConStants and K Factors.
PB91-147363

100,277

CRUZ, J. E.

NIST Calibration Procedure for Vertically Polarized Monopole Antennas $30 \mathrm{kHz}$ to $300 \mathrm{MHz}$

100,726

CRUZ, J. Y.

Test of the Inverse-Square Law of Gravitation Using the 300-m Tower at Erie, Colorado.

101,702

CUEVAS, N. R.

Preliminary Dynamic Analyses of the Ministry of Agriculture Building.

PB91-134668

100,143

CUI, J. P.

Single Pulse Shock Tube Study of the Reactions of Hydrogen Atoms with Aromatics. 4. Chlorotoluenes.

100,331

CULVER, C. G.

Investigation of the Collapse of L'Ambiance Plaza.

PB91-204057

100,146

CUNNINGHAM, C. E. 
DAGALAKIS, $N$.

Recommended Fine Positioning Test for the Development Test Flight (DTF-1) of the NASA Flight Telerobotic Servicer (FTS)

PB91-185090

101,732

Robot Characterization Testing

PB91-167361

Self-Adjustment of a Robot Joint Controller. PB91-158568

101,030

AGALAKIS, N. G.

101,027

Application of the Joint Excitation Gear Adjustment Technique to Low Frequencies and Heavy Robot Joints.

PB91-158576 101,028

Performance Measures of a Robotic Micropositioner. PB92-117019

101,037

DAGATA, J. A.

Pattern Generation on Semiconductor Surfaces by a Scanning Tunneling Microscope Operating in Air.

100,808

PB91-194712

Selective-Area Epitaxial Growth of Gallium Arsenide on Sili-
con Substrates Patterned Using a Scanning Tunneling Mi\begin{tabular}{l} 
con Substrates Patterned Using a Scanning Tunneling Mi- \\
croscope Operating in Air. \\
PB91-149880 \\
\hline
\end{tabular}

Group Reactivities in the Gas Phase Reactions of Hydroxyl Radicals with Ethers

PB91-175059

100,330

Determining the Dynamic Stress Intensity Factor with Strain Gages Using a Crack Tip Locating Algorithm.

101,580

Spatially Overdetermined Analysis for Propagation Toughness Using Strain Gages

101,164

Study of Static and Dynamic Fracture Using Strain Measurements.

Use of Birefringent Coatings in Fracture Mechanics. PB91-174599

101,574

DALTA, R. U.

Relative Populations of Excited Levels within the Ground Configuration of Si-Like Cu, Zn, Ge, and Se Ions.

PB91-158618

101,443

DALY, P.

Frequency and Time Stability of GPS and GLONASS Clocks.
PB91-202937

100,582

DAMASCENO, G. S.

Refrigerant Charge Effects on Heat Pump Performance. PB91-174607

DANBY, G.

Near-Threshold Vibrational Excitation of $\mathrm{H}_{2}$ by Electron Impact: Resolution of Discrepancies between Experiment and Theory.

and Theory.
PB91-200881

101,679

DANEY, D. E.

Effect of Noble Gas Mixtures on the Performance of Regenerative-Type Cryocoolers Analytical Estimate.

PB91-144410

100,890

Hydrogen Slush Production with a Large Auger.

PB91-147199

SHOOT Flowmeter and Pressure Transducers. PB91-147488

100,275

DANIELSON, B. L.

100,923

Standard Reference Fibers for Calibration of the Optical

Time Domain Reflectometer.
PB91-147207

101,406

DANKO, G. A.

Surface-Extended X-ray-Absorption Fine-Structure Experiments at Atmospheric Pressure by Means of a Photocathode Proportional Counter with Monolayer Sensitivity

PB91-194951

100,372

DANNER, $\boldsymbol{W}$. $\mathbf{F}$.

STEP: Standard for the Exchange of Product Model Data Resource Integration: Semantic and Syntactic Rules. PB91-184788

100,966
is (MS-

Toward Modular Semantic Application Layer Protocols (MSALPS): Communication between Heterogeneous Information

$\begin{array}{lr}\text { Systems Using Artificial Intelligence (AI) Programming } \\ \text { Techniques. } \\ \text { PB91-158584 } & 100,565\end{array}$ PB91-158584

DANOS, $M$.

Model for the Non-Perturbative QCD (Quantum Chromodyn-

amics) Vacuum
PB91-194407

101,661

esonance

Muon

PB91-147959

101,613

DANZMANN, $K$.

Unified Set of Atomic Transition Probabilities for Neutral

Argon.
PB91-237768

DAPKUNAS, S. J.

Near Net Shape Forming of Ceramics.
PB91-158600

101,072

Rapporteur's Summary Materials Development for Coal Gasification.

DATLA, R. U.

LBIR Facility User Handbook.

PB92-102185

100,876

DATTA, $\mathbf{S}$.

Elastic Constants of a Tungsten-Particle Copper-Matrix Composite.

PB91-194852

101,234

AVIES, P. K.

Chemistry of Electronic Ceramic Materials.

PB91-17882

101,500

DAVIS, D. D.

New Inexpensive Frequency Calibration Service from NIST. PB91-200790

100,580

DAVIS, G. T.

Measurement of Space Charge Fields in Polymers.

B91-158626

Superconductor-Polymer Composites

PATENT-4 95448

100,486

AVIS, $S$.

Data for Room Fire Model Comparisons

PB92-110287

101,454

DAVIS, $w$.

Issues in the Design and Implementation of a System Architecture for Computer Integrated Manufacturing.

PB91-203299

DAVISON, $W$.

Analysis of Wet Deposition (Acid Rain): Determination of the Major Anionic Constituents by lon Chromatography.

AY, G. W.

100,035

G. W.

Faraday Effect Current Sensing Using a Sagnac Interferometer with a $3 \times 3$ Coupler

PB91-175158

100,754

High Speed Magnetic Field Sensors Based on Iron Gar-

nets.

100,840

Iron-Garnet Magnetic Field Sensors with 100 pT/per square root $\mathrm{Hz}$ Noise-Equivalent Field.

100,751

Optical Fiber Current Sensors with Temperature Stabilities Near the Material Limit.

PB91.175109

100,753

Performance and Limitations of Faraday Effect Sensors.

PB91-149146 100,749

Practical Considerations in the Design of Optical Fiber Current Sensors.

100,746

Progress in the Development of Miniature Optical Fiber Cur-

rent Sensors.
PB91-134734

100,745

Technical Digest-Symposium on Optical Fiber Measurements, 1990 .

101,403

\section{DAYTON, D.}

Structure of Carbon Dioxide Dimer from Near Infrared PB91-147454

DAYWITT, W. C.

Determining Adapter Efficiency by Envelope Averaging Swept Frequency Reflection Data.

PB91-236620

100,743

Intercomparison of NBS Noise Calibration Services. 100,847

DE BIEVRE, $P$.

Silicon Reference Materials Certified for Isotope Abun-

dances.

100,213

DE LAETER, J. $R$.

Atomic Weights of the Elements 1989

PB92-148212

100,481

sotopic Compositions of the Elements 1989 PB92-148220

100,482

DEAL, $\mathbf{S}$.

Review of Four Compartment Fires with Four Compartment Fire Models.

DEBENHAM, P. $\mathrm{H}$

100,098

NIST-NRL Free-Electron Laser

AD-A227 310/0

101,399

DECKMAN, D. E.

Tribological Characteristics of Synthesized Diamond Films on Silicon Carbide.

PB91-189530

101,186

High Speed Magnetic Field Sensors Based on Iron Garnets. 147223

100,840

Iron-Garnet Magnetic Field Sensors with $100 \mathrm{pT} /$ per square root $\mathrm{Hz}$ Noise-Equivalent Field.

PB91.174615

100,751

Performance and Limitations of Faraday Effect Sensors.

PB91-149146

100,749

DEHMER, J. L.

Shape Resonances in Molecular Fields.

PB91-187435

100,338

DEL CASTILLO, L.

Sputtered Thin Film YBa2Cu3On.

PB91-189647

101509

DEL SANTO, P. P.

Use of Neutron Pole Figures to Calibrate Ultrasonic Tech.

niques for On-Line Texture Control of Aluminum Plates.

PB91-159111

101,222

DELSANTO, P. P.

Ultrasonic Texture and Stress Measurements in Anisotropic Polycrystalline Aggregates.

PB91-202945

101,237

DENNIS, J. $R$

Graphics Program for Binary and Ternary Ceramic Phase Diagrams.

PB91-147991

101,063

DEPAOLA, B. D.

Probe for Real-Time Images of Particle Beams and Their Analyses in a Merged-Beams Apparatus.

PB91-203067

101,683

DEREGGI, A. S.

Measurement of Space Charge Fields in Polymers.

PB91-158626

Superconductor-Polymer Composites.

PATENT-4 954481

100,486

DES FONTAINES,

Guide to Schema and Schema Extensibility

PB92-126556

101,454

DESCLAUX, J. P.

Relativistic Distorted-Wave Cross Sections for ElectronImpact Excitations of Berylliumlike Ions.

PB91-195503

101,672

DESHMUKH, P. C

Angle-Resolved Photoemission from the Ar $2 p$ Subshell. PB91-194928

101,665

DESHMUKH, U. $V$.

Effect of Thermal Expansion Mismatch on Fiber Pull-Out in Glass Matrix Composites.

PB91-202952

101,125

DESLATTES, $R$. $D$.

Accurate Spectroscopy in the $\mathrm{X}$-ray Region.

PB91-158634

101,621

Determination of the Neutron Mass and Implications for Fundamental Constants.

PB91-148692

101,615

Molecular-Orbital Studies via Satellite-Free X-ray Fluorescence: $\mathrm{Cl} \mathrm{K}$ Absorption and $\mathrm{K}$-Valence-Level Emission Spectra of Chlorofluoromethanes.

PB91-237164

100,427

Polarized X-ray Emission Studies of Methyl Chloride and the Chlorofluoromethanes. 


\section{PERSONAL AUTHOR INDEX}

DEWEY, M. S.

Determination of the Neutron Mass and Implications for Fundamental Constants.

101,615

Doppler Shift Attenuation Lifetime Measurements in (54)Cr Following Thermal Neutron Capture.

PB91-189753

101,656

DEWIT, $R$.

Plastic Zone Formation Around an Arresting Crack. PB91-189316

101,373

DHERE, N. G.

Single-Target Magnetron Sputter-Deposition of High-Tc Superconducting $\mathrm{Bi}-\mathrm{Sr}-\mathrm{Ca}-\mathrm{Cu}-\mathrm{O}$ Thin Films.

101,566

DHERE, $\mathbf{R}$.

Analysis of the $\mathrm{YBa} 2 \mathrm{Cu} 3 \mathrm{O} 7 / \mathrm{SrTiO} 3$ Interface as a Function of Post-Deposition Annealing Temperature.

101,480

49963

Single-Target Magnetron Sputter-Deposition of High-Tc Superconducting Bi-Sr-Ca-Cu-O Thin Films.

PB92-116680

101,566

DHILLON, J. S.

Evaluation of Building Systems Inventions.

PB91-158659

100,896

DI MARZIO, E. A.

Statistics of a Polymer Molecule in the Presence of Asymmetric Obstacles.

\section{DICK, C. E.}

100,498

Intensifying Effect of Metallic Screens on the Sensitivity of $X$-ray Films at $662 \mathrm{keV}$.

101,669

Intensifying Effect of Metallic Screens on the Sensitivity of Intensifying Effect of Metallic Screens on the Sensitivity of
X-ray Films for 400-kV Bremsstrahlung Photons.
PB91-195198

101,293

Use of Storage Phosphor Imaging Plates in Portal Imaging and High-Energy Radiography: The Intensifying Effect of Metallic Screens on the Sensitivity.

101,324

$-236497$

Synthesis of Non-Ionic Water-Dispersible Resins for Use in Intaglio Inks Curing by Electron Beam Radiation.
PB91-194456

Synthesis of Prototype Resins for Use as BEP Intaglio ink Vehicles Curing by Electron Beam Radiation

PB91-144345

101,182

DICKERSON, $\mathrm{A}$

Reference NO2 Calibration System for Ground-Based Intercomparisons during NASA's GTE/CITE II Mission PB91-189340

100,034

DICKEY, J. 0.

Lunar Laser Ranging.

PB91-203026

101,353

DIDION, D. A

Horizontal Flow Boiling Heat Transfer Experiments with a Mixture of R22/R114.

101,200

Research and Development of Heat Pumps Using Nonazeotropic Mixture Refrigerants.

100,891

Study of Flow Boiling Heat Transfer with Refrigerant Mix-

tures.
PB91-189563,196

Visualization of Nucleate Flow Boiling for an R22/R114 Mixture and Its Components

PB91-162164

100,225

DIEBOLT, D. M.

Experimentally Verified IGBT Model Implemented in the Saber Circuit Simulato

PB92-1168

100,822

DIEDRICH, F.

Quantum Optics of Single, Trapped lons.

101,628

DIETENBERGER, M. A.

Technical Reference and User's Guide for FAST/FFM Version 3 .

PB91-206664

100,086

DIKKERS, R. D.

Review of Research Literature on Masonry Shear Walls.

PB91-167189 100,113

Standards for the Physical Protection of National Re-

sources and Facilities.

PB91-222695

100,006

DILL, D.

Shape Resonances in Molecular Fields

PB91-187435

100,338

DIMARZIO, E. A.

Incorporation of Molecular Orientation into Systems of Lamellar Morphology. 1. Effects of Packing Entropy on the Lamellar Thickness of Block Copolymers.

100,484

DINARDO, R. P.

Fe-W Supermirrors for Polarizing Neutrons.
PB91-175521

101,643

TTCHEK, $B$.

High Resolution Diffraction Imaging of Crystals Grown in Microgravity and Closely Related Terrestrial Crystals.

PB92-109008

DITTMANN, $\mathbf{S}$.

Stabilization Techniques for Spinning Rotor Gage Residual Drag. 147108

DIZDAROGLU, $M$.

46Gas Chromatography-Mass Spectrometry of Free Radical-Induced Products of Pyrimidines and Purines in DNA

PB91-187476

Base Modifications in Plasmid DNA Caused by Potassium Permanganate

101,329

Bleomycin-Dependent Damage to the Bases in DNA is a Minor Side Reaction.

PB91.189357

101,337

Chemical

to DNA.

101,332

Chemical Nature of In vivo DNA Base Damage in Hydrogen Peroxide-Treated Mammalian Cells.

101,335

Copper-lon-Dependent Damage to the Bases in DNA in the Presence of Hydrogen Peroxide.

101,330

Damage to the Bases in DNA Induced by Hydrogen Peroxide and Ferric lon Chelates.

101,331

Damage to the Bases in DNA Induced by Stimulated Human Neutrophils.

101,312

Damage to the DNA Bases in Mammalian Chromatin by $\mathrm{Hy}$ drogen Peroxide in the Presence of Ferric and Cupric lons.
PB91-189217 Hydroxyl Radical Induced Cross-Linking of Cytosine and Tyosine in Nucleohiston

B91-189365

101,304

Iron Ion-Dependent Modification of Bases in DNA by the Superoxide Radical-Generating System Hypoxanthine/Xanthine Oxidase.

PB91-200824

101,306

Measurement of DNA Base Damage and DNA-Protein

Cross-Links in Mammalian Chromatin.

101,303

Mechanistic Studies of lonizing Radiation and Oxidative Mutagenesis: Genetic Effects of a Single 8-Hydroxyguanine (7Hydro-8-oxoguanine) Residue Inserted at a Unique Site in a Viral Genome.

101,321

PB91-190108

Modification of Bases in DNA by Copper lon-1,10-Phenan-
throline Complexes. throline Complexes.

101,333

Modification of DNA Bases in Mammalian Chromatin by Radiation-Generated Free Radicals.

PB91-189373

101,338

Radiation Chemi

100,236

Selected-Ion Mass Spectrometry: Assays of Oxidative DNA Damage.

B91-202960

101,307

Structure and Mechanism of Hydroxyl Radical-Induced Formation of a DNA-Protein Cross-Link Involving Thymine and Lysine in Nucleohistone.

101,334

\section{DOBBYN, $R$.}

High Resolution Diffraction Imaging of Crystals Grown in Microgravity and Closely Related Terrestrial Crystals.
PB92-109008

DOBBYN, R. C.

Dynamical Diffraction Imaging (Topography) with X-ray SynDhrotron Radiation.

101,510

High Resolution Synchrotron X-Radiation Diffraction Imaging of Crystals Grown in Microgravity and Closely Related Terrestrial Crystals.

PB91-216747

101,548

Streaking Images That Appear Only in the Plane of Diffraction in Unoped GaAs Single Crystals: Diffraction Imaging (Topography) by Monochromatic Synchrotron Radiation. 101,511 Structural Anomalies in Undoped Gallium Arsenide Ob-
served in High Resolution Diffraction Imaging with Monochserved in High Resolution Diffrac

romatic Synch

101,556

DOBRILOVIC, L. J.

Preparation and Properties of Monomolecular Films for Use as Radioactive Source Mounts.

PB91-147249

101,608

DODD, N. J. F.

Co-Trial on ESR Identification and Estimates of gamma-Ray and Electron Absorbed Doses Given to Meat and Bones.

100,017

\section{DODDINGTON, G. R.}

Phone-Mediated Word Alignment for Speech Recognition Evaluation.

PB91-189910

100,592

DODDS, R. $\mathrm{H}$.

Experimental and Numerical Studies of the J-Integral for a Surface Flaw.

DODGE, $W . R$.

Electrofission in the Ouasifree and Delta Regions.

PB91-147355

101,609

Evidence for Surface alpha Particle

PB91-216812

Clusters in

101,705

DOIRON, T.

Statistical Process Control Tactics for Coordinate Measuring Machines.

101,042

DOIRON, T. D.

Certification of NIST SRM 1961: 30 micrometers Diameter

Polystyrene Spheres.
PB92-126655 100,937

Computer Vision Based Station for Tool Setting and Tool Form Measurement

High Precision Gaging with Computer Vision Systems.

PB91-174623

100,979

DLS, W. S.

Air Exchange and Pollutant Concentration Measurements in

Two Office Buildings.
PB91-195420 100,909

Preliminary Results of the Environmental Evaluation of the Federal Records Center in Overland Missouri.

PB91-240747

100,082

DOMALSKI, E. S

Combustion Calorimeters.

PB91-189233

100,340

Energy of Combustion of Triphenylphosphate.

100,347

Heat Capacities and Entropies of Organic Compounds in the Condensed Phase. Volume 2

100,356

Monitoring the Fate of Chlorine from MSW Sampling through Combustion.

PB91-174631

100,917

MSW Calorimetry.

PB91-133942

100,878

NBS Research Program in Municipal Solid Waste Combus-

tion. 147264

100,916

DOMANSKI, P. A.

Experimentation, Analysis, and Correlation of Refrigerant-22 Flow Through Short Tube Restrictors.

PB91-174243

101,194

Refrigerant Charge Effects on Heat Pump Performance.

100,065
PBg1-174607 sional Air Distribution.

PB91-174649

100,066

DOMEN, S. $R$.

Absorbed Dose to Water: Comparison of Several Methods Using a Liquid lonization Chamber. 
PB91-187070

101,329

DosS, $M$.

Sensitivity of Ellipsometric Modeling to the 'Islands' of Silicon Precipitates at the Bottom of the Buried Oxide Layer in Annealed SIMOX

PB92-116607

100,820

DOUGHTY, D. A.

Causes of SiH4 Dissociation in Silane dc discharges.
PB91.202986

Silane Dissociation Products in Deposition Discharges.

PB91-202994

s.

DOUGLAS, J. F.

Flexible Polymer with Excluded Volume at an Interacting Penetrable Surface of Variable Dimension: A Multiple epsiIon Perturbation Theory.

PB92-116698

100,517

How Does Surface Roughness Affect Polymer-Surface Interactions.

PB91-18924

100,341

Semiempirical Theory of Relaxation: Concentrated Polymer Solution Dynamics.

Solution Dynami
PB91-236638

100,511

DOVERSPIKE, L. D.

Collisional Electron-Detachment and Ion-Conversion Processes in SF6.

100,836

DOYLE, J. $R$.

Silane Dissociation Products in Deposition Discharges.
PB91-202994

DRAGOO, A. L.

Near Net Shape Forming of Ceramics.

PB91-158600

101,072

DRAGOSET, R. A.

Geometric and Electronic Properties of Cs Structures on III$\mathrm{V}(110)$ Surfaces: From $1 \mathrm{D}$ and $2 \mathrm{D}$ Insulators to $3 \mathrm{D}$ Metals.
PB91-190090

Scanning Tunneling Microscopy Study of Clean and Cs. Covered InSb(110).

PB91-195750

101,537

System for the Study of Magnetic Materials and Magnetic Imaging with the Scanning Tunneling Microscope.

PB91-194779

101,522

DRAKE, S. A.

Extension of the Class of Magnetic B Star Nontherma Radio Sources.
PB91-175042

100,031

Radio-Continuum Observations of a Variety of Cool Stars.

PB91-174664

100,027

DRAPELA, T.

Standard Reference Fibers for Calibration of the Optical Time Domain Reflectometer

PB91-147207

101,406

DRESSLER, R. A.

Laser-Induced Fluorescence Measurements of Drift-Velocity

Distributions for $\mathrm{Ba}(+)$ in $\mathrm{Ar}$ : Moment Analysis and a Direct Measure of Skewness.

PB91-149005

100,298

DREVER, R. W. P.

Gravitational Radiation Observations on the Moon.
PB91-175083 PB91-175083
DREXLER, E. S.

Aluminum Alloys for ALS Cryogenic Tanks: Oxygen Compatibility. Volume 2

101,203

AD-A231 830/1

Review of Cryogenic Mechanical and Thermal Properties of
Al-Li Alloys and Alloy 2219 .
AD-A229 231/6 101,202

AD-A229 231/6

Photonic Probes for the Measurement of Electromagnetic Fields Over Broad Bandwidths.

PB91-162206

100,750

DU, R. R.

Structural and Magnetic Properties of Er Thin Films and Er/ $Y$ Superlattices: Magnetoelastic Elfects.

PB91-161935

101,489

DUBE, $W . P$.

Apparatus for Measurement of Thermal Conductivity of Insulation Systems Subjected to Extreme Temperature Differ-

ences.
PB91-162016

101,738

Reusable Cryogenic Foam Insulation for Advanced Aerospace Vehicles.

Small Scale Demand Type Neon Liquefaction Plant
PB91-162008

100,560

Thermal Conductivity of Evacuated Perlite at Low Temperatures as a Function of Load and Load History.

100,227

DUNCAN, R. V.

Investigating the Use of Multimeters to Measure Quantized Hall Resistance Standards.

PB91-236554

100,734

DUNN, G. H.

Nonresonant Charge Transfer in the Threshold Region for

$(3) \mathrm{He}(+)+(4) \mathrm{He}($ sub $<-$ sup- $>)$ (3) $\mathrm{He}+(4) \mathrm{He}(+)$.
PB91-149245

101,618

Probe for Real-Time Images of Particle Beams and Their Analyses in a Merged-Beams Apparatus.

PB91-203067

101,683

\section{DUNN, P. J.}

Wawayandaite, a New Calcium Manganese Beryllium Boron Silicate from Franklin, New Jersey.

PB91-174680

101,359

DUREN, $\mathrm{B}$

Photodissociation Dynamics of Water in the Second ADsorption Band: Vibrational Exitation of $\mathrm{OH}$ (A (2)Sigma).

PB91-148726 100,291

\section{JRST, R. A.}

Analysis of Wet Deposition (Acid Rain): Determination of the Major Anionic Constituents by Ion Chromatography.
PB91-236646 $\begin{array}{ll}100,035 & \\ \text { PB91-236646 } & \end{array}$ Electrochemical Behavior of Ferrocene in Polyethylene
Oxide-400. PB91-237867

Immobilization of Binding Proteins on Nonporous Supports Comparison of Protein Loading, Activity, and Stability
PB91-237198

101,308

Liposome Flow Injection Immunoassay: Implications for Sensitivity, Dynamic Range, and Antibody Regeneration.
PB91,291

Planar Waveguide Optical Immunosensors.

PB91-17456

101,284

DUTTA, B. K.

Thin and Composite High-Flux Membranes of PerfluorosulPB91.236653

DUVALL, K. C.

Dosimetry of a Nearly Monoenergetic 6- to $7-\mathrm{MeV}$ Photon Source by $\mathrm{Nal}(\mathrm{TI})$ Scintillation Spectrometry.

PB92-116706

101,723

DYNYS, J. M.

Dihedral Angles in Magnesia and Alumina: Distributions from Surface Thermal Grooves.

PB91-147330

101,058

Metal Reference Line Technique for Obtaining Dihedral Angles from Surface Thermal Grooves. PB91-147348

101,059

EANES, E. D.

Setting Reactions and Compressive Strengths of Calcium Phosphate Cements.

PB91-148668

100,040

\section{EATON, B. E.}

Analysis of Laminar Vortex Shedding Behind a Circular Cylinder by Computer-Aided Flow Visualization.

PB91.203000

101,393

EBNER, S. C.

LBIR Facility User Handbook

PB92-102185

101,435

ECKERT, $H$.

Structures and Properties of New Zeolite X-Type Zinco phosphate and Beryllophosphate Molecular Sieves
PB91-189449

101,081

ECKERT, J.

Dynamics of Molecular Hydrogen Adsorbed in CoNa-A Zeolite.

100,380

EDELSTEIN, $M$.

Residual Defects in SIMOX: Threading Dislocations and Pipes.

PB92-117233

100,829

EDERER, D. L

Photoionization and Collisional Ionization of Excited Atoms Using Synchrotron and Laser Radiations.

PB91-159301

101,626

Soft X-ray Absorption and Emission Spectra and the Electronic Structure of Some Exotic Materials.

101,520

EDLEN, $B$.

Atomic Spectroscopy in the 20th Century - A Tribute to Sitterly, Charlotte Moore, on the Occasion of Her 90th Birth day.

101,724

EDSINGER, R. E.

Differences between Thermodynamic Temperature and t(IPTS-68) in the Range $230 \mathrm{C}$ to $660 \mathrm{C}$.

PB92-116722

101,725

EGELHOFF, W. F.

Epitaxial fcc Fe Films on Cu(100)

PB91-149328

101,478

Magnetic Anisotropies in Ultrathin fcc Fe(001) Films Grown on Cu(001) Substrates.

Structural Study of the Epitaxial Growth of fcc-Fe Films Sandwiches, and Superlattices on $\mathrm{Cu}(100)$.

101,555

$X$-ray Photoelectron Forward Scattering Studies of Surface Segregation in Epitaxial Cu-Ni-Ni(100) Sandwich Structures. PB91-189258

XPS and Auger Forward Scattering in Epitaxial Films.

PB91-174698

101,495

EHRET, R. L.

Calibration of Antenna Factor at a Ground Screen Field Site Using an Automatic Network Analyzer.

PB91-134239

100,724

EHRLICH, C. D.

Study of the Linearity of Transfer Leaks and a Helium Leak Detector

PB91-147272

100,926

EHRSTEIN, J. R.

Comparison of High-Resistivity Measurements of Silicon by AC Impedance, DC Resistance, van der Pauw, and FourProbe Methods.

PB91-148197

100,792

Verification of the Relation between Two-Probe and FourProbe Resistances as Measured on Silicon Wafers.

PB91-147520

100,788

EICHINGER, D.

Simulation Studies of Excluded Volume Effects on Polymer Chain Dynamics in Several Nonlattice Models.

100,507

203364

EICHMILLER, $\boldsymbol{F}$.

Corrosion Principles in Dental Implantology.

PB91-194787

100,055

EICHMILLER, F. C.

Glass-Ceramic Inserts Anticipated for 'Megafilled' Composite Restorations

100,045

Metal-Polysiloxane Shields for Radiatıon Therapy of Maxillo-Facial Tumors.

PB91-19476

100,054

EIDELMAN, $\mathbf{N}$.

Effect of Pyrophosphate Concentrations on Calcium Phosphate Growth on Well-Crystallized Octacalcium Phosphate

and Hydroxyapatite Seed Crystals.
PB91-162024

101,193

Effects of $\mathrm{pH}$ and Calcium on Hydrolysis of Na2SiF 6 and Na2SnF6: A

EISENHAUER, C. M.

Cross-Section Measurements in the Intermediate-Energy Standard Neutron Field

PB91-203398

101,693

Spectrum-Averaged Cross-Section Measurement of $(103) R h(n, n)(103 \mathrm{~m}) \mathrm{Rh}$ in the $(252) \mathrm{Cf}$ Fission Neutron Spectrum. 203406

101,694

EISENHOWER, E. H

Criteria for the Operation of Federally-Owned Secondary Calibration Laboratories (lonizing Radiation)

PB92-11248

101,721

EKBERG, J. 0.

Transitions and Energy Levels for Cu-Like $\mathrm{Yb}(41+)$ ), $\mathrm{Ta}(44+)$, and $\mathrm{U}(63+)$

101,610

EKIN, J. W.

Critical-Current Diffraction Patterns of Grain-Boundary Josephson Weak Links.

B91-237180

101,551

Current Record in Superconductors 
ELSWIJK, H. B.

Interpretation of Field Ion Microscopy (FIM) Images of Asymmetrical Specimens of 1,2,3-Type High-Tc SuperconPB92-116730

101,567

ELY, J. F.

Extension of an Improved One-Fluid Conformal Solution Theory to Real Fluid Mixtures with Large Size Differences. Integrated theoretical and experimental study of the thermophysical properties of fluid mixtures. Progress report (February 1990-February 1991)

DE91012660

101,384

Non-Equilibrium Molecular Dynamics Simulations of Structured Molecules. Part 1. Isomeric Effects on the Viscosity of

101,391

Predictive Extended Corresponding States Model for Pure and Mixed Refrigerants.

PB92-116748

101,199

Thermophysical Properties of Ethane

PB92-110188

100,447

EMMONS, H. W.

Ceiling Jet in Fires.

PB91-167197

100,114

EMPIE, H. L

Fundamental studies of black liquor combustion. Report No. 4, Phases, 2, 3, and 4: Final report, December 1987.December 1989.
DE91018580

EMRICH, C.

Abstracts of Daylighting Research

PB91-206755

100,915

ENGEL, $V$.

100,076

Periodic Orbits and Diffuse Structures in the Photodissocia-

tion of Symmetric Triatomic Molecules.

100,238

EPLER, K. S.

Laser Enhanced Ionization as a Selective Detector for Liquid Chromalographic Determination of Alkyltins in Sedi-

PB92-116755

100,210

EPPELDAUER, G

Electronic and Radiometric Characteristics of Near Infrared Photodiodes.

100,752

Fourteen-Decade Photocurrent Measurements with LargeArea Silicon Photodiodes at Room Temperature.
PB92-116771

Temperature Monitored/Controlled Silicon Photodiodes for
Standardization. PB92-116763

ERDMAN, A. G.

Development of a Three-Dimensional Finite Element Program for Large Strain Elastic-Plastic Solids.
PB91.174557

101,494

ERICKSON, J. E.

Instability in pH Measurements of Sputtered IrO2 Films.
PB91-147538

100,281

ERICKSON, J. W.

Chemical and Electronic Properties of Pd/SnO2(110) Model Gas Sensors

100,393

ERWIN, R. W.

Structural and Magnetic Properties of Er Thin Films and $\mathrm{Er} /$ $Y$ Superlattices: Magnetoelastic Elfects.

PB91-161935

101,489

ESCALANTE, E.

Concepts of Underground Corrosion.

PB91-150078

101,134

Corrosion Testing in Soil.

101,135

Influence of Moisture and Pressure on the Mechanical Properties of a Glass-Epoxy Matrix Composite and a Graphite-Epoxy Matrix Composite.

AD-A239 509/3

101,114

Influence of Water on the Mechanical Properties of a Glass-Epoxy Matrix Composite.

101,113

Measuring the Corrosion of Metals in Soil.

101,136

ESCRIBANO, R

High-Resolution Infrared Spectrum of the nu1 Band of

100,463

Spectroscopic Constants for the nug Infrared Band of HNO3.

PB91-189415

100,343

ESPINA, P. I.

Particle Size Measurement of Inert Gas Atomized Powder. PB91-187203

101,229

ESSIGMANN, J. M.

Mechanistic Studies of Ionizing Radiation and OxIdative Mutagenesis: Genetic Effects of a Single 8-Hydroxyguanine (7-
Hydro-8-oxoguanine) Residue Inserted at a Unique Site in a Viral Genome.

101,321

EVANS, $\mathrm{C}$. J.

Selective-Area Epitaxial Growth of Gallium Arsenide on Silicon Substrates Patterned Using a Scanning Tunneling Microscope Operating in Air.

PB91.149880

100,796

EVANS, D.

Radiant Heat Fluxes from 100-200 MW Natural Gas/Air Diffusion Flames

100,538

PB91-147306

Water Spray Suppression of Fully-Developed Wood Crib Fires in a Compartment.

B $91-203620$

100,159

EVANS, D. D.

Suppression of Post-Flashover Compartment Fires Using Manually Applied Water Sprays.

EVANS, D. J.

100,164

Acoustic Technique for Evaluation of Thermal Insulation

PB91-189324 100,123

Response of Personal Noise Dosimeters to Continuous and Impulse-Like Signals

PB92-116359

100,914

EVANS, J. C.

Co-Trial on ESR Identification and Estimates of gamma-Ray and Electron Absorbed Doses Given to Meat and Bones. PB91-194720

EVANS, J. D.

Biodegradable Plastics: An Idea Whose Time Has Come. 101,243 Statistical Mechanical Model of Aqueous Two-Phase Sys-

PB91-174508

100,493

EVANS, L.

Using Surface Roughness as a Wear Criterion in HighSpeed Steel End-Milling

\section{EVENSON, K. M.}

Accurate Frequency of the $119 \mathrm{mu} \mathrm{m}$ Methanol Laser from Tunable Far-Infrared Absorption Spectroscopy.

101,040 PB91-134130

101,405

FABER, J.

New Different Forms of Ammonium Loaded and Partly Deammoniated Zeolite Rho Studied by Neutron Powder.
PB91-158717

FAETH, G. M.

Radiant Heat Fluxes from 100-200 MW Natural Gas/Air Diffusion Flames.

FAHR, $A$.

Laser Photolysis of Trimethylgallium at $193 \mathrm{~nm}$ : Quantum Yields for Methyl Radical and Ethane Production. AD-A227 868/7

ADotomulliplier Housing for Vacuum Operation of 1P28-Type Tubes
AD-A227 836/4

Side-on

Scattered Light and Other Corrections in Absorption Coefficient Measurements in the Vacuum Ultraviolet: A Systems

AD-A227 869/5

100,179

Ultraviolet Absorption of the Vinyl Radical and Reaction with Oxygen.

FAIRBANK, W. M.

Observation of Anomalous Isotope Ratios in SIRIS Measurements of Molydenum. PB92-116789

101,361

FAISON, T. K.

Evaluation of Fumed-Silica Insulation for a Thermal Conductivity Standard Reference Material.

100,103

FALCO, J. A.

AMRF Composites Fabrication Workstation: A Test Meth. odology to Measure the Quality of Thermoplastic Composite Parts. PB91-187765

101,017

FALLER, J. E.

Gyroscope-Weighing Experiment Revisited .- with a Null

101,630

Lunar Laser Ranging.

101,353

Search for a Composition Dependent Force Using a Liquid. Supported Torsion Balance.

101,639

Test of Newton's Inverse Square Law of Gravity Using the
$300 \mathrm{~m}$ Tower at Erie, Colorado: Newton Vindicated on the Plains of Colorado.

PB91-175273

101,637

Test of the Inverse-Square Law of Gravitation Using the 300-m Tower at Erie, Colorado.

101,702

FALLER, S. H.

Structure of Odd-Odd (132)Sb.

PB91-23746

101,711

FALTYNEK, R. A.

Fourier Transform Infrared Analysis of Ceramic Powders: Quantitative Determination of Alpha, Beta, and Amorphous Phases of Silicon Nitride.

PB91-237628

101,096

Lanthanide Coordination Chemistry: Spectroscopic Properties of Terbium and Europium Poly(Pyrazol-1-YI) Borate and Poly(Imidazol-1-YI) Borate.

PB91-203034

100,392

AN, $z$.

Uniform Junction Temperature AIGaAs/GaAs Power Heter-

ojunction Bipolar Transistors on Silicon Substrates.
PB91-189399

FANCONI, B.

Assessment of the State-of-the-Art for Process Monitoring Sensors for Polymer Composites.

FANCONI, B. M.

Polymers: Technical Activities 1990

PB91-159715

100,510

Polymers: Technical Activities 1991. (NAS-NRC Assessment Panel, February 13-14, 1992)

FANG, J. B.

100,516

Diagnostic Techniques for Evaluating Office Building Envelopes.

100,074

Method for Measuring Heat Loss from Underground Hea Distribution Systems.

PB91-134221

100,888

FANNEY, A, H

Measured Performance of Residential Water Heaters Using Existing and Proposed Department of Energy Test Procedures.

PB91-189274

100,085

FARABAUGH, E.

Tribological Characteristics of Synthesized Diamond Films on Silicon Carbide.

FARABAUGH, E. N.

101,186

Chemical Vapor Deposited Diamond.

AD-A243 095/7

101,106

Determination of the Optical Constants of Thin ChemicalVapor-Deposited Diamond Windows from 0.5 to $6.5 \mathrm{eV}$

AD-A243 094/0 100,215
10

Effects of Different CH4-H2 Gas Compositions on the Morphology and Growth of Diamond Grown by Hot Filament PB91-158683

101,054

Effects of Multiple Filament Geometry in the Hot Filament Deposition of Diamond Films

AD-A236 708/4

101,142

High Resolution Electron Microscopy of Diamond Film Growth Defects and their Interactions.

101,449

Influence of Filament Geometry on Hot Filament Growth of

Diamond Films.
AD-A231 818/6
101,448

Inverse Correlation between the Intensity of Luminescence Excited by Electrons and by Visible Light in ChemicalVapor-Deposited Diamond Films.

PB91-195560

101,531

Photoluminescence Excitation by Band-Gap Optical $A b-$ sorption in Chemical Vapor Deposition Diamond Films. PB91-195586

101,533

Spatially and Spectrally Resolved Cathodoluminescence Measurements of CVD-Grown Diamond Particles and Films.
AD-A243 220/1 
Oxidation of Organic Compounds by Active Manganese Dioxide.

100,218

FATTAL, S. G.

Review of Research Literature on Masonry Shear Walls.

PB91-167189 100,113

Ultimate Strength of Masonry Shear Walls: Predictions versus Test Results.

PB92-116342

100,138

FAVREAU, J. P.

Application of Formal Description Techniques to the Specification of Distributed Test Systems.

100,566

FEENEY, A. B.

Engineering Design Laboratory Guide.

100,977

Use of Solid Modeling in the Design of M3 Components. PB92-123066

101,044

FEINER, M. S.

Interlaboratory Comparison of Actinides in Human Tissue (239)Pu $+(240) \mathrm{Pu}$.

PB91-203265

101,323

FELDMAN, A.

Algorithm and Computer Program for the Calculation of En-

$\begin{array}{ll}\text { velope Curves. } & 101,414 \\ \text { PB91-158972 } & \end{array}$

Alternate Representation of Prism Refraction for Light Incident at the Brewster Angle.

PB91-203042

101,426

Chemical Vapor Deposited Diamond.

AD-A243 095/7

101,106

Critical Assessment of Optical Properties of CVD Diamond Films.

AD-A236 746/4

101,400

Determination of the Optical Constants of Thin ChemicalVapor-Deposited Diamond Windows from 0.5 to $6.5 \mathrm{eV}$.

AD-A243 094/0

100,215

Effects of Different $\mathrm{CH} 4-\mathrm{H} 2$ Gas Compositions on the Morphology and Growth of Diamond Grown by Hot Filament CVD.

101,054

Effects of Multiple Filament Geometry in the Hot Filament Deposition of Diamond Films.

101,142

High Resolution Electron Microscopy of Diamond Film Growth Defects and their Interactions.

101,449

Inverse Correlation between the Intensity of Luminescence Inverse Correlation between the Intensity of Luminescence
Excited by Electrons and by Visible Light in ChemicalVapor-Deposited Diamond Films.

PB91-195560

101,531

Photoluminescence Excitation by Band-Gap Optical Absorption in Chemical Vapor Deposition Diamond Films.

PB91-195586

101,533

Spatially and Spectrally Resolved Cathodoluminescence Measurements of CVD-Grown Diamond Particles and Films. AD-A243 220/1 101,451

Spatially and Spectrally Resolved Cathodoluminescence of Hot-Filament Chemical-Vapor-Deposited Diamond Particles.

Stoichiometry of Reactively Evaporated Films.

100,247

101,413

Tribological Characteristics of Synthesized Diamond Films on Silicon Carbide.

101,186

U.S. Assessment of the New Diamond Technology in Japan.

PB91-167395

101,107

Use of Diamond as an Optical Material.

101,401

FELDMAN, P. A.

Ultraviolet, Optical, Infrared, and Microwave Observations of $\mathrm{HR} 5110$.

PB91-148833

FELFMAN, A.

Influence of Filament Geometry on Hot Filament Growth of Diamond Films.
AD-A231 $818 / 6$

FELKNER, I. C.

Newest Approaches to Ouantitative Assessment of Bioactive Organotin

FELTON, C. M.

Accuracy Model for Phase Noise Measurements.

PB91-134882 100,589 Extending the Range and Accuracy of Phase Noise Measurements.

PB91-134874

100,588

ENG,

Evaluation of Liquid Junction Potentials and Determination of pH Values of Strong Acids at Moderate lonic Strengths.
PB91-237859 Second Dissociation Constant and $\mathrm{pH}$ of $\mathrm{N}$-2-hydroxyethylpiperazine-N'-2-ethanesulfonic Acid (HEPES) from 0 to $50 \mathrm{C}$.
PB91-189282

100,196

ENG, S. C.

Review of Current Geometric Tolerancing Theories and Inspection Data Analysis Algorithms.

FENG, $Y$.

101,016

Spinodal Decomposition in a Hydrogen-Bonded Polymer

PB91-162099

100,489

FENIMORE, C.

Electrical Breakdown and Streamer Statistics in N-hexane under Uniform Field Conditions.

PB91-134684

100,837

Precision Qualification of Watthour Meters.

PB91-237214

100,859

PB92-108950

100,604

FENIMORE, C. P.

Incompatibility between the $100 / 1300$ Surge Test and Varistor Failure Rates.

PB91.189308

100,854

Measurement Reliability: The Detection of Nonlinearities.

100,853

FERNANDEZ-PELLO, A. C.

Fire Propagation in Concurrent Flows.

PB91-157206

100,543

FERRETT, T. A.

Angle-Resolved Photoemission from the Ar $2 p$ Subshell. PB91-194928

101,665

FESEN, R. A.

Detection of an Expanding $\mathrm{H}$ I Shell in the Old Supernova Remnant CTB 80.

PB91-174920

100,028

ETTERS, L. J.

Study of the Linear Viscoelastic Properties of Cyclic Polys. tyrenes Using Creep and Recovery Measurements.
PB91-195107

FIALA, J.

NASREM Robot Control System and Testbed. PB91-194969

101,033

FIALA, J. C.

Generation of Smooth Trajectories without Planning PB91-217414

\section{FICKETT, F. R.}

Anomalous Magnetoresistance in Al/Al-Alloy Composite PB91-174748
PBnductors.

101,631

Cryogenic Properties of Copper (Wall Chart).

101,473

Magnetoresistance of Multifilament Al/Al-Alloy Conductors. PB91-134759

100,772

FIELD, B. F.

Video Processing with the Princeton Engine at NIST.

PB92-108950

100,604

FIELDS, B. A.

Prediction of Elevated Temperature Deformation of Structural Steel under Anisothermal Conditions.

PB91-184838

101,154

FIELDS, R. J.

Effects of Varying Austenitizing Temperature and Cooling Rate on the Ability of HSLA-80 Steel to Achieve a Yield Strength Comparable to HSLA-100.

PB91-189464

101,156

Plastic Zone Formation Around an Arresting Crack.

PB91-189316

101,373

Prediction of Elevated Temperature Deformation of Structural Steel under

101,154

Static and Dynamic Strength Tests on Electrical Conductor Cables Specified for Airport Landing Structures.
PB92-112226

101,739

Use of Neutron Pole Figures to Calibrate Ultrasonic Techniques for On-Line Texture Control of Aluminum Plates.

FILE, $D$.

Round-Robin Study of Implants in Si and $\mathrm{SiO} 2$ by SIMS,

RBS, and NAA
PB92-117241

100,830

FILIPKOWSKI, M. E.

Strontium-Induced Oxygen Defect Structure and Hole Doping in $\mathrm{La} 2-x \mathrm{SrxCuO} 4$

FILLA, B. J.

101,472

Automated High-Temperature Guarded-Hot-Plate Apparatus for Measuring Apparent Thermal Conductivity of Insulations between 300 and $750 \mathrm{~K}$.

100,111

Design and Fabrication of a Miniature High-Temperature Guarded-Hot-Plate Apparatus.

PB92-116797

101,098

FILLIBEN, J. J.

Taguchi's Orthogonal Arrays Are Classical Designs of Experiments.

PB92-126671

101,280

FILO, A. J.

Round-Robin Study of Implants in Si and $\mathrm{SiO} 2$ by SIMS, RBS, and NAA

FINE, $J$.

Direct Detection of Atom Vaporization by Laser Resonance Ionization as a Probe of Gas-Surface Chemisorption Mech-

100,425

Ion Bombardment Induced Diffusion: A Case Study on a Sputtered Ag/Ni Layered System.

101,527

Ion-Induced Radiation-Enhanced Diffusion of Silver in Nickel. Nickel.

391-175554 Mechanical Sputtering of Structural Stainless Steels
PB91-175448

101,152

FINK, J. L.

Stress Corrosion Cracking of Al-Li Alloys: The Role of Grain Boundary Precipitates.

PB91-175182

101,137

FINZEL, B. C.

Multiple Conformations of Amino Acid Residues in Ribonuclease A.

PB91-148098

101,298

FIRST, P. N.

System for the Study of Magnetic Materials and Magnetic

Imaging with the Scanning Tunneling Microscope.

101,522

FISCHBACH, E.

New Test of Ouantum Mechanics: Is Planck's Constant Unique.

101,632

FISCHER, D. A.

Surface-Extended X-ray-Absorption Fine-Structure Experiments at Atmospheric Pressure by Means of a Photocathode Proportional Counter with Monolayer Sensitivity.

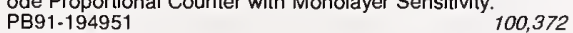

FISCHER, $R$.

New Different Forms of Ammonium Loaded and Partly Deammoniated Zeolite Rho Studied by Neutron Powder PB91-158717

100,311

FISH, G. E.

Low Temperature Magnetization and Magnetic Excitations in Amorphous Fe78B13Si9.

PB92-117464

101,573

FISHER, G.

Application Portability Profile (APP): The U.S. Government's Open System Environment Profile OSE/1 Version 1.0 PB91-201004

FISHER, G. E.

Functional Benchmarks for Fourth Generation Languages. PB91-167387

100,644

SHER, W. Second-Level Post-Occupancy Evaluation Analysis.

100,058

FISK, $\mathbf{Z}$.

Long-Range Antiferromagnetic Order of the $\mathrm{Cu}$ in Oxygen PB91-194894

101,524

FITTING, D. W.

Image Ouality Indicator Design for Radioscopy and Tomog-

raphy.

101,018

Monitoring of Anisotropic Material Elastic Properties Using Ultrasonic Receiving Rays.

PB91-203059 
Tests of the Flory-Rehner Hypothesis: Comparison of the Elastic Free Energy Function for Crosslinked Rubber in the

Dry and Swollen States.

100,508

FOLEY, G. M.

High-Speed Spatial (Linear) Scanning Pyrometer: A Tool for Diagnostics, Temperature Mapping, and Property Determinations at High Temperatures.

N91-21361/1

100,924

\section{FONG, E, N.}

Guide to Design, Implementation and Management of Distributed Databases.

PB91-187567 100,648

Guide to Expert System Building Tools for Microcomputers. Proceedings of the Object-Oriented Database Task Group
Workshop. Held in Atlantic City, New Jersey on May 22,

PB91-159723

100,643

Proceedings of the Object-Oriented Database Task Group Workshop, Tuesday, October 23, 1990, Chateau Laurier Hotel, Ottawa, Canada.

PB91-157198

100,641

FONG, J. T.

Engineers' Statistical Literacy is Key to U.S. Competitiveness.

FORAND, J. L.

100,038

Probe for Real-Time Images of Particle Beams and Their Analyses in a Merged-Beams Apparatus.

PB91-203067

101,683

FORD, R. S.

Rotational Spectrum and Structure of the Complex ArCh3CN

PB91-236661

100,415

FORNEY, C. L

Users Guide for RAPID, Version 2.3

PB91.167403

FORNEY, G. P.

100,999

Programmer's Reference Manual for CFAST, the Unified Model of Fire Growth and Smoke Transport

PB91-144436

100,096

FORTUNKO, C. M.

Group and Phase Sound Velocities in an Eu1Ba2Cu3O7 Superconductor and Related Perovskite Oxides.

PB91-194860

101,523

FOWLER, B. 0.

Filler Systems Based on Calcium Metaphosphates.

PB91-194654

FOWLER, H. A.

100,048

Atomic Fingerprint of $\mathrm{YBa} 2 \mathrm{Cu} 3 \mathrm{O} 7-\mathrm{x}-\mathrm{T}$ ype High-Temperature Superconductors Observed by Field Ion Microscopy.

Interpretation of Field Ion Microscopy (FIM) Images of Asymmetrical Specimens of 1,2,3-Type High-Tc Supercon-

PB92-116730

101,567

Three-Dimensional Simulations of High-Resolution Photoresist Processing

FOWLER, J. E

100,818

Conceptual Architecture for a Mechanical Parts Production System Based on STEP. National PDES Testbed Rept.

PB92-112234

100,993

FOX, J.

Performance of OSI Transport over ACCUNET and IBER PAC. 134825

100,563

FRAKER, A. C.

Corrosion Behavior of Zirconium Alloy Nuclear Fuel CladPBing. 158733

101,372

Corrosion Principles in Dental Implantology.

100,055

Corrosion, Repassivation and Corrosion Fatigue Behavior of the Surgical Implant Alloy, Co-Cr-Mo.

the Surgical Impt

101,132

FRANCIS, M. $\mathbf{H}$.

Evaluation of Dual-Port Circularly Polarized Probes to Planar Near-Field Measurements.

PB91-162040

100,590

RANKE, G. L

Analysis and Characterization of Commercial Welding Fluxes.

101,003

FRANZEN, D. L.

Subpicosecond Pulse Compression and Raman Generation Using a Mode-Locked Erbium-Doped Fiber Laser-Amplifier.

PB91-148007 101,408

Technical Digest-Symposium on Optical Fiber Measurements, 1990.

FRASE, K. G.

101,403

Low Temperature Synthesis of Ceramic Powders for Structural and Electronic Applications.
PB91-149104

\section{FRASER, G. T.}

$\mathrm{H} 2 \mathrm{O} 2$ : Spectroscopy, Structure and Dynamics. PB91-162057

Microwave Spectra and Electic Dipole $X(4)$ Sigma(1-1) $1 / 2$ VO and $\mathrm{NbO}$. $\mathrm{X}(4)$ Sigma(1-)1/2 VO and $\mathrm{NbO}$.
PB91-237487

Microwave Spectrum of Ar-H2O: Dipole Moment, Isotopic Studies, and (17)O Quadrupole Coupling Constants.

PB91-148650

100,290

Molecular-Beam Optothermal Spectroscopy of the 9.6 mum PB14.

100,421

Optothermal-Detected Microwave-Sideband CO2-Laser Spectroscopy of $\mathrm{Ar}-\mathrm{NH} 3$

100,416

Rotational Spectrum and Structure of the Complex ArCh3CN.
100,415

Vibrational, Rotational, and Tunneling Dependence of Vibrational Predissociation in the HF Dimer.

PB91-203844

100,406

FREDERIKSE, $\mathrm{H}$, P. R.

Heat Conductivity of Oxide Coatings by Photothermal Radiometry between 293 and $1173 \mathrm{~K}$

PB92-116813

FREEMAN, C. G.

Proton Affinity of Cyanogen and Ion-Molecule Reactions of $\mathrm{C} 2 \mathrm{~N} 2(+)$.

FREIBERGER, L. J.

High-Temperature Thermal Properties of UNS $\$ 44004$ Using Multivariant Analysis.

PB91-194795

101,160

FREIMAN, S. Design Diagrams for Heavy Metal Fluoride Glass Windows.
PB91-187419

FRETMAN, S. W.

Ceramics: Technical Activities 1991. (NAS-NRC Assessment Panel, February 13-14, 1992).

ment Panel, 7

101,104

Effect of Thermal Expansion Mismatch on Fiber Pull-Out in Glass Matrix Composites.

PB91-202952

101,125

Effects of Crystal Bonding on Brittle Fracture.

$\quad 101,064$

Environmentally Enhanced Fracture of Gallium Arsenide.

PB91-237743 101,097

Indentation Determination of Crack Growth Parameters in Gallium Arsenide.

PB91-190082

101,083

Processing Bi-Pb-Sr-Ca-Cu-O Superconductors from Amor-

phous State.
PB91-187369 101,504

Strength and Fracture Behavior of Ba-Y.Cu-O Superconducting Ceramics.

FRENCH, V. A.

Refractive Index Gratings in Rare-Earth-Doped Alkaline Earth Glasses.

FRENKLACH, $M$.

Mechanism of Soot Formation in Acetylene-Oxygen Mixtures.

100,537

FRIED, A.

Reference NO2 Calibration System for Ground-Based Intercomparisons during NASA's GTE/CITE II Mission. PB91-189340

FRIEDMAN, C. D.

Self-Setting Calcium Phosphate Cements.

PB91-202879

\section{FRIEND, D. G.}

Thermal Conductivity of a Moderately Dense Gas.

PB91-134502

Thermophysical Properties of Ethane.

PB92-110188

Viscosity Suriace for Mixtures of Methane and Ethan PB91-174763

FRIPP, A.

High Resolution Diffraction Imaging of Crystals Grown in Microgravity and Closely Related Terrestrial Crystals.

PB92-109008

FROHNSDORFF, G

Integrated Knowledge Systems for Concrete Science and Technology.

FROMMHOLD, L

Notes and Comments on Roundtable Discussion on LaserAssisted Collisions and Collision-Induced Spectr

PB91-203307

FRYBERGER, T. B.

Chemical and Electronic Properties of Pd/SnO2(110) Model Gas Sensors.

PB91-203075

100,393

FUCIARELLI, A. F.

Damage to the Bases in DNA Induced by Stimulated uman Neutrophils

PB92-116912

101,312

FUJII, R. U.

Software Verification and Validation: An Overview. B91-237685

100,658

FUKASE, $Y$.

Setting Reactions and Compressive Strengths of Calcium Phosphate Cements.

$-148668$

100,040

FULLER, E.

Isothermal Phase Behavior of Ag3SbS3, ZnGeP2, and ZnS.
PB91-187237

Microstructural Aspects of the Fracture of Hardened Cement Paste.

FULLE, E. R.

100,133

Chemical Modification of the Orthorhombic Superconductor Ba2YCu3O7-delta.

PB91-159053

101,486

Effect of Surface Forces on Subcritical Crack Growth in Glass.

PB92-117449

101,103

Effects of Crysta

101,064

Relation between the Surface Energy and the Debye Temperature for Cubic Solids.

PB91-149393

100,303

Strength and Fracture Behavior of Ba-Y-Cu-O Superconducting Ceramics.

FULLER, S. $K$.

Economic Analysis for Military Construction (MILCON) Design. Concepts, Techniques, and Applications for the Analyst. Instructor's Manual.

PB92-112440

101,349

Economic Analysis for Military Construction (MILCON) Design. Concepts. Techniques, and Applications for the Analyst. Student's Manual.

2-112457

101,350

FUNATAKE, $Y$.

Spectral Data and Grotrian Diagrams for Highly lonized ron, Fe VIII-XXVI.

PB90-244583

101,595

FURLANI, C. M.

Use of the IRDS Standard in CALS (Revised) B91-132209

100,684

FURUTA, R.

Functional Meta-Structure for Hypertext Models and SysPB91-236687

100,655

GADALLA, N. A. M.

Thermodynamic and Thermophysical Properties of Organic Nitrogen Compounds. Part 1. Methanamine, Ethanamine, 1and 2-Propanamine, Benzenamine, 2-, 3-, and 4-Methylben-

zBami-192583

100,368

GADDIPATI, A. R.

Transport Critical Current of Aligned Polycrystalline $\checkmark 1 \mathrm{Ba} 2 \mathrm{Cu} 307-$ delta and Evidence for a Nonweak-Linked Component of Intergranular Current Conduction B91-133983

101,456

GADZUK, J. W.

Resonant Tunneling with Electron.Phonon Interactions: An Exactly Solvable Model Applied to Desorption. PB91-162065

100,317

Soluble Models in Surface Collision Dynamics. PB91-147298

100,276

GAGE, E. C.

Effects of Colored Pump Noise on Intensity Correlations of a Single Mode Dye Laser

PB91-189738 
Damage to the Bases in DNA Induced by Hydrogen Peroxide and Ferric Ion Chelates.

101,331

Damage to the Bases in DNA Induced by Stimulated Human Neutrophils.

101,312

PB92-116912

Damage to the DNA Bases in Mammalian Chromatin by $\mathrm{Hy}$ drogen Peroxide in the Presence of Ferric and Cupric lons.
PB91-189217

Hydroxyl Radical Induced Cross-Linking of Cytosine and Tyrosine in Nucleohistone.

101,304

Mechanistic Studies of Ionizing Radiation and Oxidative Mutagenesis: Genetic Effects of a Single 8-Hydroxyguanine (7Hydro-8-oxoguanine) Residue Inserted at a Unique Site in a Viral Genome.

PB91-190108 101,321

Modification of DNA Bases in Mammalian Chromatin by Radiation-Generated Free Radicals.

101,338

Selected-Ion Mass Spectrometry: Assays of Oxidative DNA Damage.

101,307

Structure and Mechanism of Hydroxyl Radical-Induced Formation of a DNA-Protein Cross-Link Involving Thymine and Lysine in Nucleohistone.

PB91-187518

101,334

GALLAGHER, A.

Causes of $\mathrm{SiH} 4$ Dissociation in Silane dc discharges.
PB91-202986 PB91-202986
Collisional Energy Transfer between Excited Sr Atoms.

Collisional Energy Transfer between Excited Sr Atoms.
PB91-204164

Production and Diagnosis of a Highly Spin-Polarized $\mathrm{Na}$ Beam

101,431

Scanning tunneling microscopy studies of the surfaces of a$\mathrm{Si} \mathrm{H}$ and $\mathrm{a}-\mathrm{SiGe}: \mathrm{H}$ films. Annual report, 1 December 1989 31 January 1991

100,785

Silane Dissociation Products in Deposition Discharges.

Silane Dissociation Products in Deposition Discharges.
100,390

GALLAGHER, A. C.

Measurements of Velocity-Changing Collision Kernels.
PB91-203083

GALLAGHER, $J$,

Modeling the Thermodynamic Properties of Sodium Chloride in Steam Through Extended Corresponding States.
PB91-189381 100,342
PB91-189381

101,402 length, Temperat
PB90-244708

Sixteen Thousand Evaluated Experimental Thermodynamic Property Data for Water and Steam.

100,475

Thermodynamic Diagrams for Refrigerant Mixtures.

PB91-236695

GALLAGHER, L.

Computer Implementation of a Discrete Set Algebra.
PB91-231571

SOL3 Support for CALS Applications.

PB91-167262

100,965

GALLAWA, R. L.

Analyzing Integrated Optical Waveguides: A Comparison of

$\begin{array}{ll}\text { Two New Methods. } & \text { 100,759 } \\ \text { PB91-204131 }\end{array}$

Limited International Intercomparison of Responsivity Scales at Fiber Optic Wavelengths.

100,567

Methods of Analyzing Planar Optical Waveguides.
PB91-236752

100,774
PB91-236752

Modified Airy Function and WKB Solutions to the Wave
Equation.
PB92-126531
101,440

GALLOWAY, K. F.

Characteristics of the Breakdown Voltage of Power MOSFETs After Total Dose Irradiation.

PB91-149062

Characterizing VLSI Processes Using Test Structures.

PB91-175000

100,793

GALOWIN, L. S.

Plumbing Test Lab Accreditation.

PB91-190066

GAMMON, B. E.

Thermodynamic and Thermophysical Properties of Organic Nitrogen Compounds. Part 1. Methanamine, Ethanamine, 1 and 2-Propanamine, Benzenamine, 2-, 3-, and 4-Methylbenzenamine.

100,368

GANN, R. G.

Large-Scale Validation of Bench-Scale Fire Toxicity Tests.
PB92-116458

Role of Bench-Scale Test Data in Assessing Real-Scale Fire Toxicity.

GANS, $W$. L.

Quantifying Standard Performance of ElectromagneticBased Mine Detectors.
PB92-116292

101,378

GAO, G.

Emitter Ballasting Resistor Design for, and Current Handling Capability of AIGaAs/GaAs Power Heterojunction Bipolar

$\begin{array}{ll}\text { Transistors. } & 100,815\end{array}$

Uniform Junction Temperature AIGaAs/GaAs Power Heterojunction Bipolar Transistors on Silicon Substrates.

PB91-189399

GARBOCZI, E. J.

Chloride Ion Diffusion in Low Water-to-Solid Cement Pastes.

PB91-187690

101,138

Percolation of Phases in a Three-Dimensional Cement Paste Microstructural Model.

100,529

GARDINER, W. C

Mechanism of Soot Formation in Acetylene-Oxygen Mixtures.

$1-147280$

100,537

GARDNER, J.

Limited International Intercomparison of Responsivity Scales at Fiber Optic Wavelengths.
PB91-187682

PB91-187682

100,567

GARRITY, K. $M$

Characterization of the Mineral Fraction in Botanical Reference Materials and Its Influence on Homogeneity and Analytical Results.
PB91-148817

100,186

GARVIN, D.

Thermodynamic Data for Modeling of Flue Gas Cleanup Systems (Abstract for Poster Session).

100,904

GARY, J.

Calculated from the Frequency Domain: An Update.

PB91-162388

Time Domain Frequency Stability Calculated from the Frequency Domain Description: Use of the SIGINT Software Package to Calculate Time Domain Frequency Stability rom the Frequency Domain

PB92-123132

100,587

GARY, J. M.

Numerical Simulation and Visualization Models of Stress Wave Propagation Graphite/Epoxy Composites.

PB91-174946

101,120

GATES, R. S.

Effect of Selected Chemical Compounds on the Lubrication of Silicon Nitride.

PB91-236711

101,180

Study on the Nature of Boundary Lubricating Film: Analytical Method Development.

101,176

GAYLE, F. W.

Low-Field Flux Pinning in Twinned and Detwinned Single Crystals of YBa2Cu3O7-x

PB91-237552

101,559

GAYLORD, R. J.

Relaxation of Crosslinked Networks: Theoretical Models and Apparent Power Law Behavior.

PB91-195099

100,501

GEER, $\mathbf{S}$.

Chitin-Chitosan Membranes: Separations of Amino Acids and Polypeptides.

PB91-134544

101,283

GEIST, J.

Generalized Photodiode Self-Calibration Formula. $\quad 100,760$
PB91-236901

Micromachined Thermal Radiation Emitter from a Commercial CMOS Process.

PB91-148999

100,748

Numerical Modeling of Silicon Photodiodes for High-Accuracy Applications. Part 1. Simulation Programs.

cy Applications.
PB92-110295

100,763

Numerical Modeling of Silicon Photodiodes for High-Accuracy Applications. Part 2. Interpreting Oxide-bias Experiments. PB92-110303

100,764

Numerical Modeling of Silicon Photodiodes for High-Accuracy Applications. Part 3. Interpolating and Extrapolating Internal Ouantum-Efficiency Calibrations.

100,765

Testing the Accuracy of Calculated Equilibrium Carrier Concentrations in the Presence of Surface Fields.

PB91-236729

101,549

GERHARDT, R. A

Small Angle Neutron Scattering Characterization of Microporous Silica.

101,089

Small-Angle-Scattering Determination of the Microstructure of Porous Silica Precursor Bodies.

PB91-175075

101,077

GEYER, R. G.

Optimization Techniques for Permittivity and Permeability

Determination.
PB92-112325

Optimization Techniques for Permittivity and Permeability

Determination.

PB92-126663

100,871

Ouantifying Standard Performance of ElectromagneticBased Mine Detectors

GHATAK, A. K

101,378

Methods of Analyzing Planar Optical Waveguides.

100,774

Modified Airy Function and WKB Solutions to the Wave Equation.

PB92-126531

101,440

GIAUQUE, C. H. W.

Capacitance versus Stylus Measurements of Surface Roughness.

PB91-203463

101,020

GIBBLE, K. E.

Measurements of Velocity-Changing Collision Kernels. PB91-203083

101,684

GIBSON, K. A.

Bibliography of the NIST Electromagnetic Fields Division

Publications.
PB91-132241 100,832

Bibliography of the NIST Electromagnetic Fields Division PB92-116367

GIER, T. E.

100,866

Structures and Properties of New Zeolite X-Type Zincophosphate and Beryllophosphate Molecular Sieves.

PB91-189449

101,081

GILBERT, S. L.

303-MHz Frequency Standard Based on Trapped Be $(+)$

PB92-116474

101,722

Frequency Stabilization of a Tunable Erbium-Doped Fiber PB91-203109

101,427

Frequency Stabilization of an Erbium-Doped Fiber Laser: A Potential Wavelength Standard for Optical Communications. PB91-203091

100,570

Observation of Correlations in Finite, Strongly Coupled Ion

Plasmas.
PB91-200857 101,678

Progress at NIST on Absolute Frequency Standards Using PBor-159285

101,625

Test of the Linearity of Quantum Mechanics by if Spectroscopy of the $(9) \mathrm{Be}(+)$ Ground State.

101,726

GILLASPY, J. D.

Precise Experimental Test of Calculated Two-Electron Lamb Shifts in Helium

101,617

GILLETTE, G.

Second-Level Post-Occupancy Evaluation Analysis

100,058

GILLIES, C. W.

Microwave Spectra and Electric Dipole Moments of $X(4)$ Sigma(1-) $1 / 2$ VO and $\mathrm{NbO}$

Van der Waals Complexes in 1,3-Dipolar Cycloaddition Reactions: Ozone-Ethylene.

PB91-236737

100,417

GILLIES, J. Z

Van der Waals Complexes in 1,3-Dipolar Cycloaddition Reactions: Ozone-Ethylene.

PB91-236737

100,417

GILLILAND, G. I.

Crystal Structure of Phosphate-Free Ribonuclease. PB91-175604

101,301

GILLILAND, G. L.

Ab Initio Phase Determination for $X$-ray Diffraction Data 
Updated Excitation and Ionization Cross Sections for Electron Impact on Atomic Oxygen

GINZBURG, A.

Use of Storage Phosphor Imaging Plates in Portal Imaging and High-Energy Radiography: The Intensifying Effect of Metallic Screens on the Sensitivity.

PB91-236497

GIRVIN, S. M.

Hall Effect.

GIULIANO, K. A.

Low-Cost Aqueous Two-Phase System for Enzyme Extraction.

101,324

101,479

GLADDEN, E.

National Bureau of Standards Publications 1977-1987. Volume 1. Citations, Key Words, and Abstracts.

PB91-136507

National Bureau of Standards Publications 1977-1987. Volume 2. Indexes.

PB91-136515

GLADDEN, E. T.

Publications of the National Institute of Standards and Technology, 1990 Catalog.

GLINKA, C.

Chain Conformation of a Block Polymer in a MicrophaseSeparated Structure.

PB91.148890

100,485

GLINKA, C. J.

Determination of Bonded Phase Thickness in Liquid Chromatography by Small Angle Neutron Scattering.

100,190

GLOVER, M. P.

Unsteady Laminar Flow in a Circular Tube: A Test of the HERCOL (Hermitian Collocation) Computer Code.

PB91-22267

101,395

GOEBEL, $\mathbf{R}$

Numerical Modeling of Silicon Photodiodes for High-Accuracy Applications. Part 2. Interpreting Oxide-bias Experiments.
PB92-110303

GOGNY, D.

Model for the Non-Perturbative OCD (Ouantum Chromodynamics) Vacuum.
PB91-194407

GOLDBERG, R. N.

101,661

Thermodynamics of Hydrolysis of Disaccharides: Lactulose, alpha-D - Melibiose, Palatinose, D - Trehalose, D - Turanose and 3-o-beta-D-Galactopyranosyl-D-arabinose.

Thermodynamics of Hydrolysis of Oligosaccharides.
PB91-194803

Thermodynamics of the Disproportionation of Adenosine 5' diphosphate to Adenosine $5^{\prime}$-triphosphate and Adenosine 5 '-monophosphate. 1. Equilibrium Model.

100,459

Thermodynamics of the Disproportionation of Adenosine $5^{\circ}$. diphosphate to Adenosine 5 'triphosphate and Adenosine $5^{\prime}$-monophosphate. 2. Experimental Data.

101,313

GOLDFARB, R. B.

AC Loss Measurements of Two Multifilamentary NbTi Com posite Strands
PB91-133892

100,769

Effect of Mechanical Deformation on Nb-Ti Filament Proximity-Effect Coupling at the Edges of SSC Cables. 101,700 Elastic Constants of Polycrystalline Bi-Pb-Sr-Ca-Cu-O Superconductor.

101,461

Enhanced Flux Creep in Nb-Ti Superconductors After an Increase in Temperature.

101,680

Fundamental and Harmonic Susceptibilities of $\mathrm{YBa} 2 \mathrm{Cu} 3 \mathrm{O} 7$. delta.

101,458

Hall Probe Magnetometer for SSC Magnet Cables: Eifect of Transport Current on Magnetization and Flux Creep. PB91-202911 101,681 Magnetic Measurements for High Energy Physics Applications.

101,720

Specifications for a Federal Information Processing Standard Data Dictionary System

PB91-158758

100,687

GOLDMAN, A.

Spectroscopic Constants for the nu9 Infrared Band of PB91-189415

GOLDSCHMIDT, $v$.

Refrigerant Charge Effects on Heat Pump Performance.
PB91-174607

GONZALES, J. A.

Center for Electronics and Electrical Engineering Technical Publication Announcements Covering Center Programs, Oc-
tober to December 1990, with 1991 CEEE Events Calendar.
PB91-240838

100,863

\section{GONZALEZ, J. A}

Center for Electronics and Electrical Engineering Technical Progress Bulletin Covering Center Programs, July to September 1990 , with 1991 CEEE Events Calendar. PB91-159749

100,801

Center for Electronics and Electrical Engineering Technical Publication Announcements Covering Center Programs, July to September 1990, with 1991 CEEE Events Calendar.
PB91-184754

100,852

Electronics and Electrical Engineering Laboratory Technical Publication Announcements Covering Laboratory Programs, January to March 1991, with 1991 EEEL Events Calendar. POODIN,

Visibility of Exit Directional Indicators.

PB91-184762

100,864

\section{OODRICH, L. F.}

Current Supply for High-T(sub c) Superconductor Testing. PB91-133827

Guideline for $\mathrm{Nb}$ (sub 3)Sn critical current measurements using fiberglass-epoxy composite sample mandrels. DE91016274

High T(sub c) Superconductors and the Critical Current Measurement

101,457

Integrity Tests for High-(T sub c) and Conventional CriticalCurrent Measurement Systems.

PB91-148676

100,842

Integrity tests for high-(Tc) and conventional critical-current measurements systems.

101,452

Possible 'Proximity Matrix' Route to High Current ConducPB91-148932

101,476

Switching in High-Tc Superconductor Current Transport Measurements.

GOODWIN, A. R. H

101,539

Accurate Acoustic Measurements in Gases Under Difficult Conditions.

PB92-116847

101,382

GORAL, J, P.

Single-Target Magnetron Sputter-Deposition of High-Tc Superconducting Bi-Sr-Ca-Cu-O Thin Films.

PB92-116680

101,566

GORCZYCA, T.

Close-Coupling Calculation for the (2)(Sigma sub $\mathrm{u})(+)$ State of $(\mathrm{H}$ sub 2)(-)

GORE, J. P.

Investigation of Simulated Oil-Well Blowout Fires.

101,356

Radiant Heat Fluxes from 100-200 MW Natural Gas/Air Diffusion Flames.
PB91-147306

GORTER, J.

Effect of Tube Bundle Flow Conditioners on Orifice Meter Discharge Coefficients.

GOTAAS, J. A.

Phonon Density of States of Superconducting YBa2Cu3O7 and the Nonsuperconducting Analog YBa2Cu3O6.

PB91-159129

101,487

GOULD, P. L.

Associative Ionization of Ultra-Cold Laser Trapped Sodium

PB91-236745

101,707

Observation of Atoms Laser-Cooled Below the Doppler

Limit. 189746

101,655

Observation of Atoms Laser Cooled Below the Dopple

PB91-195453

101,671

Prospects for Electromagnetic Manipulation and Trapping of Antihydrogen.

101,663

GOVADA, A.

Use of Neutron Pole Figures to Calibrate UItrasonic Techniques for On-Line Texture Control of Aluminum Plates.

PB91-159111

101,22

GOYAL, I. C.

Analyzing Integrated Optical Waveguides: A Comparison of Two New Methods.

PB91-204131

100,759

Methods of Anal

100,774

Modified Airy Function and WKB Solutions to the Wave Equation.

PB92-12653

101,440

GRACE, T. M.

Fundamental studies of black liquor combustion. Report No. 4, Phases, 2, 3, and 4: Final report, December 1987. December 198

100,915

GRANVEAUD, M. P.

GPS Time Transfer with Implementation of Selective Availability.

N91-25766/7

100,576

GRATIAS, D.

6-D Structural Model for the Icosahedral (Al, Si)-Mn Quasicrystal.

101,501

N-Dimensional Crystallographic Description of the Icosahedral Phases; the Example of the Al73Mn21Si6 Quasiperiodic Structure.

101,226

GRAY, B.

Stable Implementation Agreements for Open Systems Interconnection Protocols. Version 4, Edition 1, June 1991 Change Pages (Supplement).

100,695

GRAY, M. M.

Functional Benchmarks for Fourth Generation Languages.
PB91-167387 GREEN, M. $S$.

Phase Transitions.

PB91-162073

100,318

GREEN, W. J.

Superconducting Thin Films of Bi-Sr-Ca-Cu-O Obtained by Laser Ablation Processing.

PB91-189589

101,508

GREENBERG, $R$. $R$

Recommended Inorganic Chemicals for Calibration. PB91-195164

100,201

GREENE, G. L.

Determination of the Neutron Mass and Implications for Fundamental Constants.

PB91-148692

101,615

New Test of Quantum Mechanics: Is Planck's Constant Unique.

101,632

GREENE, R. L.

Overview of the Ion Dynamic Effect in Line Broadening, and a Generalization of the Unified Theory.

PB91-147504

101,442

GREENHUT, V. A.

Ouantitative Determination of Amorphous Content in $\mathrm{Ce}$ ramic Materials Using X-ray Powder Diffraction.

PB91-147553

101,061

GREENSPAN, L

Design and Implementation of a Five-Axis Robotic Micromanipulator for Inserting Parts into Precision Collets. PB91-159194

101,029

GREGORY, T. M.

Mathematical Model for Dental Caries: A Coupled Dissolution-Diffusion Process

101,316

GRICE, J. D.

Wawayandaite, a New Calcium Manganese Beryllium Boron Silicate from Franklin, New Jersey.

PB91-174680

101,359

GRIEPINK, B.

Analysis of Wet Deposition (Acid Rain): Determination of the Major Anionic Constituents by lon Chromatography.
PB91-236646

GRIMES, J. $W$.

Techniques to Observe the Fracture Zone in Mortar and Concrete.

PB91-134189

100,521

GRIMM, F. A.

Angular Distribution of Ejected Electrons in Resonant Auger Processes of $\mathrm{Ar}, \mathrm{Kr}$, and $\mathrm{Xe}$.

PB91-187302

101,648

Parity-Unfavored Transitions in Resonant Photoemission from $\mathrm{Ar}, \mathrm{Kr}$, and $\mathrm{Xe}$ : Experimental and Theoretical Results.

Unusual Degree of Angular Anisotropy in the Resonant
Auger Spectrum of $\mathrm{Kr}$. Auger Spectrum

101,649

GRISHCHUK, L. P.

Squeezed Ouantum States of Relic Gravitons and Primordial Density Fluctuations.

PB91-203125

101,685 
GROSVENOR, J. $\mathbf{H}$

Improved Technique for Measuring Permittivity of Thin Dielectrics with a Cylindrical Resonant Cavity. $\quad 100,858$ GROT, $\mathbf{R}$.

Air Exchange and Pollutant Concentration Measurements in Two Office Buildings.

PB91-195420

100,909

GROT, R. A.

Airtightness of Office-Building Envelopes.

100,067

Calibration Procedures for Infrared Imaging Systems for Building Diagnostics.

100,057

$\begin{array}{ll}\text { PB91-147082 100,057 } & \end{array}$

Diagnostic Techniques for Evaluating Office Building Enve-
lopes.
PB91-195438

100,074 User Manual NBSAVIS CONTAM88. A User Interface for
Air Movement and Contaminant Dispersal Analysis in Multizone Buildings

100,910

Ventilation System Performance Evaluation Using Tracer Gas Techniques.

PRUBB, D. $\mathbf{S}$.

100,060

GRUBB, D. S.

Standard Reference Materials: Calibration of NIST Standard Reference Material 3201 for 0.5 Inch $(12.65 \mathrm{~mm})$ Serial Serpentine Magnetic Tape Cartridge.

PB91-187542

100,690

GRUNDL, J. A

Cross-Section Measurements in the Intermediate-Energy Standard Neutron Field

PB91-203398

101,693

GRUSHKO, B.

Phase Formation in Electrodeposited and Thermally An. nealed Al-Mn Alloys.

101,209

Structural Study of Electrodeposited Aluminum-Manganese Alloys.

101,231

GRY, C.

Studies of $\mathrm{HI}$ and $\mathrm{DI}$ in the Local Interstellar Medium.

PB91-148957

100,021

GUBSER, D. U.

Phonon Density of States of Superconducting $\mathrm{YBa} 2 \mathrm{Cu} 3 \mathrm{O} 7$ and the Nonsuperconducting Analog YBa2Cu3O6.
PB91-159129
101,487

Laser Induced Damage in Optical Materials: 1989. PB91-148478

101,409

GUNARATNA, P. C

Research at NBS in Direct Potentiometric Measurements in

Blood.
PB91-148874

100,188

GUNN, M. T.

Soltware Reengineering: A Case Study and Lessons Learned.

PB92-116417

100,669

GUPTA, A. $K$

Application of Laser Diagnostic Techniques for the Examination of Liquid Fuel Spray Structure.

100,229

Effect of Atomization Air on Droplet Dynamics of Spray Flames.

PB91-203869

100,550

Laser Diagnostics for Characterization of Fuel Sprays.
PB91-149013

Velocity and Droplet Size Measurments in a Fuel Spray.

PB91-147934

100,539

GUTTMAN, C. M.

Studies on the Degradation Products of Paper with and without Pollutants in a Closed Environment. 1. Preliminary

PB91-143313

101,181

HABIB, J. M.

Superconducting Properties of $\mathrm{Bi} 2-x-y \mathrm{PbxSnySr} 2 \mathrm{Ca} 2 \mathrm{Cu} 3 \mathrm{O} z$ PB91-162321 101,076

HADJICHRISTIDIS, $\mathrm{N}$.

Study of the Linear Viscoelastic Properties of Cyclic Polystyrenes Using Creep and Recovery Measurements. HALBRITTER, J.

Analysis of the $\mathrm{YBa} 2 \mathrm{Cu} 3 \mathrm{O} 7 / \mathrm{SrTiO} 3$ Interface as a Function of Post-Deposition Annealing Temperature.

101,480

PB91-149963

Subpicosecond Pulse Compression and Raman Generation Using a Mode-Locked Erbium-Doped Fiber Laser-Amplifier. PB91-148007

HALL, J. A.

How the Government Shapes UNIX Standards PB91.203133

100,693

HALL, J. L.

Correlated Spontaneous Emission in a Zeeman Laser.
PB91-175596

101,417

Correlated Spontaneous Emission in a Zeeman Laser PB91-204180

101,432

HALL, L. W.

Simultaneous Butyltin Determinations in the Microlayer Water Column and Sediment of a Northern Chesapeake Bay Marina and Receiving System.

PB91-195040

100,918

HALLER, $W$.

Immobilization of Binding Proteins on Nonporous Supports Comparison of Protein Loading. Activity, and Stability.

PB91-237198

HALLIWELL, $B$.

Bleomycin-Dependent Damage to the Bases in DNA is a PB91-189357

101,337

Copper-Ion-Dependent Damage to the Bases in DNA in the Presence of Hydrogen Peroxide.

101,330

Damage to the Bases in DNA Induced by Hydrogen Peroxide and Ferric Ion Chelates.

PB91-187112

101,331

Damage to the DNA Bases in Mammalian Chromatin by Hydrogen Peroxide in the Presence of Ferric and Cupric lons.
PB91-189217

101,336

Iron Ion-Dependent Modification of Bases in DNA by the Superoxide Radical-Generating System Hypoxanthine/Xan-
thine Oxidase.
PB91-200824

01,306

Modification of Bases in DNA by Copper Ion-1,10-Phenanthroline Complexes.

HAM, K. C.

101,333

Use of Birefringent Coatings in Fracture Mechanics.

PB91-174599

101,582

HAMANN, D. R.

Kinematic Theory of Ballistic Electron Emission Spectroscopy of Silicon-Silicide Interfaces.

PB92-117373

101,570

Theory of Ballistic-Electron-Emission Spectroscopy of NiSi2/Si(111) Interfaces.

HAMBRIGHT, P.

101,557

Radiolytic Studies of the Redox Reactions of Ruthenium Porphyrins.

PB91-203661

Redox Reactions of Osmium Porphyrins
PB91-159012

HAMILTON, C. A.

High Speed Superconducting A/D Converter

PB91-148700

100,402

Arrays.

PBrays. 116516

100,217

HAN, C.

Chain Conformation of a Block Polymer in a Microphase Separated Structure.

PB91-148890

100,485

Small-Angle Neutron Scattering and Light Scattering Study on the Miscibility of Poly(styrene-ran-butadiene)/Polybutadiene Blends

100,519

Temperature Dependence of Probe Diffusion in Bulk Poly mer Matrices.
PB91-236885

100,422

\section{HAN, C. C.}

Microstructure Effects on the Lower Critical Solution Temperature Phase Behavior of Deuterated Polybutadiene an Protonated Polyisoprene Blends Studied by Small-Angle

Neutron Scattering.
PB92-117266

100,518

Small-Angle Neutron Scattering and Light Scattering Studies on the Miscibility of Protonated Polyisoprene/Deuterated Polybutadiene Blends.

PB91-236786

100,513

Small-Angle Neutron Scattering Studies of Compatible Blends of Linear Poly(Vinyl Methyl Ether) and Cross-Linked

PB91-187153

100,494

Spinodal Decomposition in a Hydrogen-Bonded Polymer Blend.

PB91-162099

100,489

Time-Resolved Small-Angle Neutron Scattering in Intermediate- and Late-Stage Spinodal Decomposition of (DPB/ HPI) Blends.

HAN, J. K.

100,490

Cryogenic Fatigue of High-Strength Aluminum Alloys and Correlations with Tensile Properties.

PB91-236976

101,709

Load-Controlled Tensile Tests of Austenitic Steels at $4 \mathrm{~K}$.

PB91-236943

101,165

HAN, $X$. L.

Production and Diagnosis of a Highly Spin-Polarized $\mathrm{Na}$ Beam.

101,431

\section{HANCOCK, E.}

Method for Characterizing the Thermal Performance of a Solar Storage Wall from Measured Data.

PB91-159152

100,901

\section{DWERKER, C. A.}

Coherency Strain Induced Instability of Solid-Liquid Inter faces in the Mo-Ni System

PB91-195537

101,235

Dihedral Angles in Magnesia and Alumina: Distributions rom Surface Thermal Grooves.

101,058

Effect of Coherency Strain on Alloy Formation: Migration of iquid Films.

PB91-147322

101,210

Metal Reference Line Technique for Obtaining Dihedral Angles from Surface Thermal Grooves.

101,059

Microstructural Control Through Diffusion-Induced Grain

Boundary Migration.
PB91-236760

101,239

Variations in the Practice of Ceramic Technology in Different Cultures: A Comparison of Korean and Chinese Celadon Glazes.

PB91-237669

101,111

HANKINSON, A. L

1987: The Year of the 386

PB91-158766

100,599

Developing Federal Software Standards: A New Direction.

PB91-134536 100,637

Multimedia Courseware in an Open Systems Environment: A Federal Strategy.

PB91-143362

100,039

HANLEY, H. J. M.

Anisotropic Structure of a Simple Liquid.

PB91-148718

101,616

Neutron Diffraction from Sheared D-Glycerol: Preliminary Studies.

PB91-134676

100,268

Phase Equilibria from the One-Fluid Model.

PB91-134585

101,385

Structure of a Stage-3 Cs - Graphite Intercalate. PB91-134957

100,269

Taking the Measure of Fluid Properties Data Bases. PB91-200832

101,392

HANSEN, $K$.

Electrofission in the Quasifree and Delta Regions

PB91-147355

101,609

HANSEN, P. A.

Application of Ultrafast Broadband Infrared Spectroscopy to Measurement of Metal-Carbonyl Dynamics.

PB91-149955

100,308

HANSFORD, W.

Knowledge Verification of Machine-Learning Procedures

Based on Test Structure Measurements
PB91-189571

100,709

HANSON, A. G.

Detail Specification for 62.5-mum Core Diameter/125-mum 
$\mathrm{HgCdTe}$ Detector Reliability Study for the GOES Program. PB92-112382

100,766

\section{HARMAN, J.G.}

Crystal Structure of a cAMP-Independent Form of Catabolite Gene Activator Protein with Adenosine Substituted in One of 2 CAMP-Binding Sites.

PB91-190074

101,305

HARNE, D. E.

Mechanical Properties and Fracture Toughness of AAR TC128 Grade B Steel in the Normalized, and Normalized and Stress Relieved Conditions.

PB92-108901

101,169

Static and Dynamic Strength Tests on Electrical Conducto Cables Specified for Airport Landing Structures.

PB92-112226

101,739

\section{HARRIMAN, A.}

Polyelectrolyte Stabilized Metal Oxide Hydrosols as Catalysts for the Photooxidation of Water by Zinc Porphyrins.
PB91-189852

Pulse Radiolysis Studies of Organic Electron Transfer Reactions.

PB91-147801

100,285

Redox Reactions of Osmium Porphyrins

PB91-159012

100,217

HARRIS, D.

National Bureau of Standards Publications 1977-1987. Volume 1. Citations, Key Words, and Abstracts.

PB91-136507 100957

National Bureau of Standards Publications 1977-1987. Volume 2. Indexes

PB91-136515

100,958

HARRIS, J.

Corrosion Behavior of Zirconium Alloy Nuclear Fuel Clad ding.

101,372

HARRIS, J. S.

National Educators Workshop: Update '90. Standard Experiments in Engineering Materials Science and Technology. $202-126606$

101,253

HARRIS, M.

Collisional Energy Transfer between Excited Sr Atoms. PB91-204164

101,703

HARRIS, R. $\mathrm{H}$.

Data for Fire Hazard Assessment of Selected Non-Halogenated and Halogenated Fire Retardants; Report of Test FR

PB92-112473

100,557

Large-Scale Validation of Bench-Scale Fire Toxicity Tests.

PBg2-116458 100,139

Role of Bench-Scale Test Data in Assessing Real-Scale Fire Toxicity.

PB91-167270

100,156

AARRISON, J. C.

Test of Newton's Inverse Square Law of Gravity Using the $300 \mathrm{~m}$ Tower at Erie, Colorado: Newton Vindicated on the Plains of Colorado.

$\begin{array}{ll}\text { PB91-175273 } & 101,637\end{array}$

Test of the Inverse-Square Law of Gravitation Using the 300-m Tower at Erie, Colorado.

PB91-204115

101,702

HARRISON, W. T. A.

Structures and Properties of New Zeolite X-Type Zincophosphate and Beryllophosphate Molecular Sieves.
PB91-189449
101,081

HART, H. R.

Transport Critical Current of Aligned Polycrystalline 1Ba2Cu3O7-delta and Evidence for a Nonweak-Linked Component of Intergranular Current Conduction.

101,456

HARTMAN, A. W.

Calibration of the NBS 10 mum Space Beads.

101,695

Certification of NIST SRM 1961: 30 micrometers Diameter Polystyrene Spheres

100,937

Note on the Precision Cementing of Small Optical Compo-

nents.

101,428

HARVEY, A. H.

Computer Simulation of Fluid-Fluid Phase Coexistence in Mixtures of Nonadditive Soft Disks.

PB91-162263

101,388

Limiting versus Apparant Critical Behavior of Henry's Constants and K Factors.

PB91-147363

100,277

Phase Equilibria and Critical Lines in Model Water/Salt Mix-

tures.

100,418

Unified Description of Infinite-Dilution Thermodynamic Propenties for Aqueous Solutes.

PB91-162081

100,319

HARVEY, T. E.

Analysis of the $\mathrm{YBa} 2 \mathrm{Cu} 3 \mathrm{O} 7 / \mathrm{SrTiO} 3$ Interface as a Function of Post-Deposition Annealing Temperature.
PB91-149963

101,480

YBa2Cu3O7-delta/Insulator Multi-Layers for Crossover Fab rication.

101,518

HARVILL, K. A.

Guide to Design, Implementation and Management of Distributed Databases

PB91-187567

100,648

HASEGAWA, $\mathrm{H}$.

Microstructure Effects on the Lower Critical Solution Temperature Phase Behavior of Deuterated Polybutadiene and Protonated Polyisoprene Blends Studied by Small-Angle Neutron Scattering.

PB92-117266

100,518

Small-Angle Neutron Scattering and Light Scattering Studies on the Miscibility of Protonated Polyisoprene/Deuterat

PB91-236786

100,513

Small-Angle Neutron Scattering and Light Scattering Study on the Miscibility of Poly(styrene-ran-butadiene)/Polybutadiene Blends.

PB92-117274

100,519

Time-Resolved Small-Angle Neutron Scattering in Intermediate- and Late-Stage Spinodal Decomposition of (DPB)

PB91-162131

100,490

HASEGAWA, S.

NIST Calibration Services for Humidity Measurement PB92-112499

100,520

HASHIMOTO, T.

Microstructure Effects on the Lower Critical Solution Temperature Phase Behavior of Deuterated Polybutadiene and Protonated Polyisoprene Blends Studied by Small-Angle Neutron Scattering

PB92-117266

100.518

Small-Angle Neutron Scattering and Light Scattering Studies on the Miscibility of Protonated Polyisoprene/Deuterated Polybutadiene Blends.

PB91-236786

100513

Small-Angle Neutron Scattering and Light Scattering Study on the Miscibility of Poly(styrene-ran-butadiene)/Polybutadiene Blends

PB92-117274 100,519

Time-Resolved Small-Angle Neutron Scattering in Intermediate- and Late-Stage Spinodal Decomposition of (DPB) HPI) Blends.

$1-16213$

100,490

HASTIE, J. W.

Laser Vaporization Mass Spectrometry of Refractory Materials: Graphite and $\mathrm{YBa} 2 \mathrm{Cu} 3 \mathrm{OX}$

100,464

National Bureau of Standards-American Ceramic Society Phase Diagram Data Program.

PBa1-158774

101,073

Thermodynamic and Kinetic Stability of Refractory Materials at Ultra-High Temperatures.

AD-A235 490/0

100,246

HAYES, W.

Water Spray Suppression of Fully-Developed Wood Crib Fires in a Compartment

100,159

HAYNES, L. S.

Control of Flexible Robot Arm

PB91.174979

101,032

HAYNES, W. M.

Survey of Current Worldwide Research on the Thermophysical Properties of Alternative Refrigerants.

PB92-112366

101,198

HAYWARD, E.

Polarizability of the Photon

PB91-203166

101,686

E, $M$

Spinodal Decomposition in a Hydrogen-Bonded Polymer Blend.

100,489

HE, Y.

Calculation of Energy of Low-Angle Grain Boundaries. PB91-134601

101,464

HE, Y. Z

Single Pulse Shock Tube Study of the Reactions of Hydrogen Atoms with Aromatics. 4. Chlorotoluenes.

100,331

PEANEY, M. D.

Increased Facilitated Transport Related to Microstructura Changes in Heat-Treated lon-Exchange Membranes PB91-174805

100,323

HEARING, E. D.

Heat Capacities and Entropies of Organic Compounds in the Condensed

100,356

HEATH, J.

Difficulties in Parsing SGML.

PB91-189456

100,650

HEATLEY, S.

Validation of an OSI Transport Class 4 Simulator.

PB91-187724

100,568

HECKMANN, P. H.

Beam-Foil Study of Two-Electron Transitions in Cu-Like

PB91-23751

101,714

HEFNER, A. R.

Experimentally Verified IGBT Model Implemented in the Saber Circuit Simulator

PB92-116862

100,822

PEMANN, B. E.

Characterization of Tricresylphosphate Lubricating Films by Micro-Fourier Transform Infrared Spectroscopy.

101,179

HEHN, K. E.

Preparation and Analysis of a Marine Sediment Reference Material for the Determination of Trace Organic Constituents.

PB91-149237

101,377

HEILES, $\mathrm{C}$.

Detection of an Expanding $\mathrm{H}$ I Shell in the Old Supernova Remnant CTB 80.

PB91-174920

100,028

HEILWEIL, E. J.

Application of Ultrafast Broadband Infrared Spectroscopy to PB91-149955

100,308

Picosecond IR Studies of the Vibrational Dynamics of CO/

Pt(111).
PB91-161927

100,314

Population Lifetimes of $\mathrm{OH}(v=1)$ and $\mathrm{OD}(\mathrm{v}=1)$ Vibrations in Alcohols, Silanols and Crystalline Micas.

PB91-174813

100,324

HEIMANN, P. A.

Angle-Resolved Photoemission from the Ar $2 p$ Subshell.

PB91-194928

101,665

HEINKEL, J.

Gas Analysis Modeling System

PB91-175299

100,884

HEINRICH, B.

Magnetic Anisotropies in Ultrathin fcc Fe(001) Films Grown on $\mathrm{Cu}(001)$ Substrates.

101,565

HEINZEN, D. J.

303-MHz Frequency Standard Based on Trapped Be(+) lons.

101,722

Observation of Correlations in Finite, Strongly Coupled Ion

Plasmas.

101,678

Progress at NIST on Absolute Frequency Standards Using

Stored lons.

101,625

Reply to Comment on 'Quantum Zeno Effect'.

101,708 
HERMAN, M.

Analysis of Optical Flow Estimation Using Epipolar Plane Images.

Application of the PIPE Image Processing Machine to Scanning Microscopy

101,034

PB91-203190

Descrip-

Geometric Reasoni

PB91-237677

100,714

New Approach to Vision and Control for Road Following.

PB91-157156 101,743

Real-Time Hierarchical Planning for Multiple Mobile Robots.

PB91-158782 101,375

Three Dimensional Reconstruction from Optical Flow Using Temporal Integration.

PB92-112507

100,702

Video Compression for Remote Vehicle Driving

101,035

HERON, J. T.

Detection of Trace Disulfur Decafluoride in Sulfur Hexafluoride by Gas Chromatography/Mass Spectrometry.

PB91-189860

100, 197

HERRON, J. T.

Chemical Kinetic Data Base for Propellant, Combustion. 1. Reactions Involving NO, NO2, $\mathrm{HNO}, \mathrm{HNO} 2, \mathrm{HCN}$ and $\mathrm{N} 2 \mathrm{O}$ PB92-148071

Formation and Stability of SF5 and S2F 10

100,467

Letter to Editor of Chemical and Engineering News.

PB91-174847

100,327

Thermochemistry of Sulfoxides and Sulfones.

Zonal Model for Corona Discharge-Induced Oxidation of $\mathrm{SF} 6$ in $\mathrm{SF6} / \mathrm{O} 2 / \mathrm{H} 2 \mathrm{O}$ Gas Mixtures.

100,312

HERSMAN, F. W.

Electrofission in the Quasifree and Delta Regions.

PB91-147355

HESKESTAD, G.

Sprinkler/Hot Layer Interaction

PB91-206680

HESS, $\mathrm{B}$.

Photodissociation Dynamics of Water in the Second Absorption Band: Vibrational Exitation of $\mathrm{OH}$ (A (2)Sigma).

PB91-148726

100.291

HESTENES, M. $\mathbf{R}$

NBS-INA. The Institute for Numerical Analysis. UCLA 1947 1954

PB92-112408

101,264

HEUMANN, $B$.

Photodissociation Dynamics of Water in the Second Absorption Band: Vibrational Exitation of $\mathrm{OH}$ (A (2)Sigma).

PB91-148726

HEUMANN, K. G.

Atomic Weights of the Elements 1989

Isotopic Compositions of the Elements 1989 .

PB92-148220

HEYLIGER, P.

Capacitive Array Sensors for Nondestructive Evaluation

PB91-237313 100,947

Resonating-Orthotropic-Cube Method for Elastic Constants.

PB91-236794

101,586

HEYLIGER, P. R.

Characterization of Capacitive Array for NDE Applications.

Stress Intensity Factors by Enriched Mixed Finite Elements.
PB91-147371

PB91-147371

Charac.

terization of Dielectric Properties of Materials.

100,948

PB91-237610

HICHO, G. E.

Crack Arrest Fracture Toughness Measurements of Normalized and Inclusion Shape Controlled AAR TC128 Grade $B$ Steel, and Micro-Alloyed, Control-Rolled, and Inclusion Shape Controlled A BXX Grade B Steel.

PB91-167346

101,150

Effects of Varying Austenitizing Temperature and Cooling Rate on the Ability of HSLA-80 Steel to Achieve a Yield Strength Comparable to HSLA-100.

PB91-189464

101,156

Examination of the Excessive Retained Austenite on the Surface of a Section of 17-7 Precipitation Hardening Stain less Steel.

PB91-167171

101,149

Mechanical Properties and Fracture Toughness of AAR TC128 Grade B Steel in the Normalized, and Normalized and Stress Relieved Conditions.

PB92-108901

101,169

HIGGINS, P. D.

Mass Energy-Transfer and Mass Energy-Absorption Coefficients, Including In-Flight Positron Annihilation for Photon Energies $1 \mathrm{keV}$ to $100 \mathrm{MeV}$.
PB92-126473

101,445

D. A.

Diffraction by a Half-Plane in a Lossy Medium.

Generalization of the Cornu Spiral for Lossy Media.

101,437

PB91-203216

101,687

Near-Field and Far-Field Excitation of a Long Conductor in a Lossy Medium.

NIST Calibration Procedure for Vertically Polarized Monopole Antennas $30 \mathrm{kHz}$ to $300 \mathrm{MHz}$.

100,726

Quasi-Static Analysis of a Two-Wire Transmission Line Located at an Interface.
PB91-148734

100,564

HILL, M.

Comparison of Flux Dynamics in Two Samples of YBa2Cu3O7 with Different Pinning.

PB91.149419

101,069

Strength and Fracture Behavior of Ba-Y-Cu-O Superconducting Ceramics.

PB91-148205

101,474

Processing Bi-Pb-Sr-Ca-Cu-O Superconductors from Amorphous State. phous State.
PB91-187369

101,504

HILL, P. G.

Sixteen Thousand Evaluated Experimental Thermodynamic Property Data for Water and Steam.

100,475

Unified Fundamental Equation for the Thermodynamic Unified Fundamental Equation for the Thermodynamic
Properties of $\mathrm{H2O}$.
PB91-192534
100,364

100,364

HILL, W. T.

Laser-Driven lonization and Photoabsorption Spectroscopy of Atomic Ions.

PB91-174854

101,415

HILLS, M. M.

Chemical Effects of $\mathrm{Ne}+$ Bombardment on the MoS2(0001) Surface Studied by High-Resolution Photoelectron Spectroscopy.

HILLS, R. G.

Model Validation at the Las Cruces Trench Site. NUREG/CR.5716

100,399

HIRONAKA, $\mathbf{N}$.

New Inexpensive Frequency Calibration Service from NIST. PB91-200790

HIRSCH, J.

Al: Does It Have a Place in Building Simulation PB91-159293

100,063

HOCKEY, B. J.

Amorphization and Conductivity of Silicon and Germanium Induced by Indentation.

101,482

PB91-150045

Glass-

Application of Analytical Electron Microscopy PB91-147389

101,060

\section{HOKMABADI, M. S.}

High-Temperature High-Pressure Raman Spectra from PBg1-195735

HOLEMANS, $W$.

Low-Cost Low-Volume Carrier (Minilab) for Biotechnology and Fluids Experiments in Low Gravity.

PB91-150029

100,041

HOLLANDER, W. J.

Gyroscope-Weighing Experiment Revisited -- with a Null

$1-174722$

101,630

HOLLBERG, L

CW Dye Lasers.

Diode Lasers and

PB92-116904

Modulatable Narrow-Linewidth Semiconductor Lasers.

PB91-134106

101,404

HOLT, D. R.

Determination of Scattering Parameters with Respect to the Characteristic Impedance of Precision Coaxial Air-Line Standards.

PB91-134114

HOLUBEC, A.

100,770

Asymptotic Shooting Method for the Solution of Differential Equations.

HONG, T. H.

101,255

Analysis of Optical Flow Estimation Using Epipolar Plane

PB92-112564

100,703

Real-Time Algorithms and Data Structures for Underwater Mapping.

101,360

Three Dimensional Reconstruction from Optical Flow Using Temporal Integration.

PB92-112507

100,702

Video Compression for Remote Vehicle Driving. PB91-203208

101,035

\section{HOPP, T. H.}

Review of Current Geometric Tolerancing Theories and Inspection Data Analysis Algorithms.

PB91-167221

101,016

\section{HORLICK, J}

Directory of Accredited Laboratories, 1991. National Voluntary Laboratory

100,934

HORN, R. G.

Analytic Solution for the Three-Layer Multiple Beam Inter-

PB91-162107

101,007

HORST, J. A.

Assembly Code to Compute Sine and Cosine Using the Cordic Algorithm

100,638

Task Decomposition and Algorithm Development for RealTime Motion Control of a Continuous Mining Machine.

PB91-231597

HORVATH, C.

Method to Enhance Porosity of Micro-particles.

PB91-187328

101,357

HORVATH, J.

Temperature Measurements in Flames Using Thermally Assisted Laser-Induced Fluorescence of $\mathrm{Ga}$.

PB91-236851

HORVATH, $V$.

Liposome Flow Injection Immunoassay: Implications fo Sensitivity, Dynamic Range, and Antibody Regeneration.
PB91-147629

HOSTETTER, $\mathrm{B}$

Study of the Linear Viscoelastic Properties of Cyclic Polystyrenes Using Creep and Recovery Measurements.

PB91-195107

Report on 1989 Actions by International Institute of Welding.

HOUSTON, J.

Electronic and Radiometric Characteristics of Near Infrared Photodiodes.

PB91-174706

HOWARD, C. J.

High-Resolution Infrared Spectrum of the nu1 Band of

Spectroscopic Constants for the nu9 Infrared Band of PB91-189415

HOWARD, J.

Dynamics of Molecular Hydrogen Adsorbed in CoNa-A ZeoPBite. 195255

HOWARTH, I. D.

Terminal Velocities for a Large Sample of O Stars, B Supergiants, and Wolf-Rayet Stars.

PB91-149021

100,023

HOWE, S. E.

Guide to Available Mathematical Software Problem Classification System

HOWELL, B.

Morphology of Polymer Films and Single Molecules.

PB91-203224

HRUSZKEWYCZ, A. M.

8-Hydroxyguanine Content of Isolated Mitochondria Increases with Lipid Peroxidation. 


\section{PERSONAL AUTHOR INDEX}

Heats of Immersion, Friction, and Wear of Base Oil Frac-

tions

101,173

Institute for Materials Science and Engineering, Ceramics Technical Activities 1990

PB91-132233

101,057

Mechanisms of Additive Effectiveness.

101,172

Mechanisms of Additive Effectiveness.

101,174

Mechano-Chemical Descriptive Model for Wear Under Mixed Lubrication Conditions.

Oxidation Kinetics of Silicon Carbide Whiskers Studied by

$X$-ray Photoelectron Spectroscopy.

100,436

Study of Additive Response in a Series of Rerefined Base Qils Typical of Current Commercial Practice.

101,175

Study on the Nature of Boundary Lubricating Film: Analytical Method Development

101,176

Surface Oxidation Kinetics of $\mathrm{Si3N} 4-4 \%$ Y2O3 Powders

Studied by Bremsstrahlung-Excited Auger Spectroscopy.
PB91-23770 100,435

HSU, S. T.

Mechanisms of Additive Effectiveness.

PB91-158808

Mechanisms of Additive Effectiveness.

PB91-174862

101,172

UANG, $C$.

101,174

Magnetic Hysteresis of High-Temperature YBa2Cu3Ox-AgO Superconductors: Explanation of Magnetic Suspension. PB91-18950

HUANG, D. $X$.

RF-DC Differences of Thermal Voltage Converters Arising from Input Connectors.

PB91-236802

100,735

HUANG, H. M.

Task Decomposition and Algorithm Development for RealTime Motion Control of a Continuous Mining Machine.

PB91-231597

101,357

\section{HUANG, P. H.}

Assessment of Uncertainties in Measurement of pH in Hostile Environments Characteristic of Nuclear Repositories.
NUREG/CR-5711 NIST Calibration Services for Humidity Measuremen

101,367

High Humidity Sensing.

100,843

HUANG, Z. S.

Structure of Carbon Dioxide Dimer from Near Infrared Spectroscopy.

HUBBARD, C. R.

Quantitative Determination of Amorphous Content in Ceramic Materiais Using X-ray Powder Diffraction.

PB91-147553

101,061

PB91-147397

HUBBARD, J. B.

Mobility Fluctuations and Electrophoretic Light Scattering

from Macromolecular Solutions.
PB91-189514 100,345

Semiempirical Theory of Relaxation: Concentrated Polymer Solution Dynamics.
PB91-236638

HUBBELL, J, $\mathrm{H}$.

100,511

Certain Theorems on Bilateral Generating Functions Involving Hermite, Laguerre, and Gegenbauer Polynomials.
PB91-148759

Mass Energy-Transfer and Mass Energy-Absorption Coefficients, Including In-Flight Positron Annihilation for Photon Energies $1 \mathrm{keV}$ to $100 \mathrm{MeV}$

PB92-126473

101,445

HUBER, J. R.

Photodissociation of C(I)NO in the S(I) State: A OuantumMechanical ab initio Study.

PB91-14926

HUBER, M. E.

100,239

Correlation of Flux States Generated by Optical Switching of a Superconducting Circuit.

101,682

Excess Low-Frequency Flux Noise in dc Squids Incorporating Nb/Al-Oxide/Nb Josephson Junctions.
PB91-236810
100,736 HUBER, M. L.

00,736

Extension of an Improved One-Fluid Conformal Solution Theory to Real Fluid Mixtures with Large Size Differences.

Predictive Extended Corresponding States Model for Pure and Mixed Refrigerants

PB92.116748

HUDGENS, J. W.

101,199

Electronic Spectrum of the GeH3 Radical
PB91-189548

100,346

Resonance Enhanced Multiphoton Ionization Spectra of the $\mathrm{GeF}$ and $\mathrm{GeCl}$ Radicals from $400-500 \mathrm{~nm}$.

100,396

Resonance Enhanced Multiphoton Ionization Spectroscopy of $\mathrm{CHCl} 2$ and $\mathrm{CDCl} 2$

100,282

Resonance Enhanced Multiphoton Ionization Spectroscopy of $\mathrm{CHCl} 2$ and $\mathrm{CDCl} 2$.

PB91-174870

100,328

HUE, R. E.

Rate Constants for Reactions of Peroxyl Radicals in Fluid Solutions.
PB90-24464

HUGGINS, M.

100,261

Abstracts of Daylighting Research.

100,076

HUGHES, R. J.

New Test of Quantum Mechanics: Is Planck's Constant PB91-174755

101,632

HUIE, R. E.

Rate Constants for Hydrogen Abstraction Reactions of the Sulfate Radical, SO4- Alkanes and Ethers

PB91-203232

100,394

Rate Constants for Reduction of Substituted Methylperoxy Radicals by Ascorbate lons and $\mathrm{N}, \mathrm{N}, \mathrm{N}^{\prime}, \mathrm{N}^{\prime}$-TetramethylPara-Phenylenediamine.

PBg1-195230

100,379

Asymptotic Expansions of the Kernel Functions for Line Formation with Continuous Absorption.

101,688

Photospheres of Hot Stars. 4. Spectral Type 04

100,025

HUMPHREYS, J. C.

Effects of Absorbed Dose Rate, Irradiation Temperature and Post-Irradiation Temperature on the Gamma Ray Response of Red Perspex Dosimeters.

PB92-116441

101,363

NIST High-Dose Calibration Services.

PB91-203257

101,689

Temperature Dependence of Radiochromic Film Dosi-

PB92-117134

101,728

HUNSTON, D.

Assessment of the State-of-the-Art for Process Monitoring Sensors for Polymer Composites.

PB91-222612

100,510

Polymer Composite Processing. Industry Workshop (2nd). Held at Gaithersburg, Maryland on May 18, 1990

PB91-132191

101,115

HUNTER, J. S.

Design and Analysis of Experiments.

PB91-15882

101,274

TER,

Document Architecture for Open Systems.

HUNTER, W. G.

Design and Analysis of Experiments

PB91-158824

100,961

HURLE, D. T. J.

Effect of an Electric Field on the Morphological Stability of the Crystal-Melt Interface of a Binary Alloy. 3. Weakly Nonlinear Theory.

PB91-195743

HURLEY, J. A.

101,536

Automated High-Temperature Guarded-Hot-Plate Apparatus for Measuring Apparent Thermal Conductivity of Insulations

between 300 and

100,111

Ignition Characteristics of Selected SSME Alloys. PB91-174474

100,544

Unsteady Laminar Flow in a Circular Tube: A Test of the HERCOL (Hermitian Collocation) Computer Code. PB91-222679

101,395

HURST, W. S.

Dispersion of the Electronic Contribution to the Thrid-Order Nonlinear Susceptibility of $\mathrm{H} 2$.

PB91.149138

101,411

HUST, J. G.

Automated High-Temperature Guarded-Hot-Plate Apparatus for Measuring Apparent Thermal Conductivity of Insulations between 300 and $750 \mathrm{~K}$

PB91-162115

100,111

Round Robin on Apparent Thermal Conductivity of Several Loose-Fill Insulations.

PB91-149948

101,143

HUTCHINS, C. F.

Publications of the Manufacturing Engineering Laboratory Covering the Period January 1989-September 1991.

PB92-123041

101,053

HUTCHINSON, J. M. R.

Observation of Anomalous Isotope Ratios in SIRIS Measurements of Molydenum.

PB92-116789

101,361

Preparation and Properties of Monomolecular Films for Use as Radioactive Source Mounts.

101,608

HWANG, N. M.

Processing Bi-Pb-Sr-Ca-Cu-Q Superconductors from Amorphous State.

HYER, C. W.

101,504

Directory of State and Local Government Laboratory ACcreditation/Designation Programs.

PB92-108968

100,174

HYLAND, R. W.

NIST Calibration Services for Humidity Measurement

PB92-112499

100,520

HYSLOP, P. A.

Damage to the Bases in DNA Induced by Stimulated Human Neutrophils.

PB92-116912

101,312

Cross Sections for Collisions of Electrons and Photons with Atomic Oxygen.
PB90-244682

100,264

INGEL, R. P.

Physical and Thermo-Mechanical Properties of Monoclinic Single Crystals.
PB91-148767

101,065

INGHAM, $\mathrm{H}$.

Thermophysical Properties of Ethane.

PB92-110188

INGUSCIO, $M$

Accurate Frequency of the $119 \mathrm{mu} \mathrm{m}$ Methanol Laser from Tunable Far-Infrared Absorption Spectroscopy.

101,405

INN, K. G. W.

Interlaboratory Comparison of Actinides in Human Tissue (239) $\mathrm{Pu}+(240) \mathrm{Pu}$

IP, U. S.

Investigation of Simulated Qil-Well Blowout Fires. PB91-144337

101,323

IRACANE, D.

Model for the Non-Perturbative OCD (Ouantum Chromodynamics) Vacuum

IRWIN, R. B.

Round-Robin Study of Implants in $\mathrm{Si}$ and $\mathrm{SiO} 2$ by SIMS, RBS, and NAA.

ISHIDA, T.

Fundamental and Harmonic Susceptibilities of $\mathrm{YBa} 2 \mathrm{Cu} 3 \mathrm{O} 7$. PB91-134148

101,458

ISHII, K.

Spectral Data and Grotrian Diagrams for Highly Ionized Copper, Cu X-Cu XXIX

PB92-110121

100,441

TANO, W. M.

303-MHz Frequency Standard Based on Trapped $\mathrm{Be}(+)$

$\begin{array}{ll}\text { lons. } & 101,722\end{array}$

Observation of Correlations in Finite, Strongly Coupled Ion Plasmas.

101,678

Progress at NIST on Absolute Frequency Standards Using Stored lons.

Ouantum Optics of Single, Trapped lons

101,625

PB91-162123 


\section{PERSONAL AUTHOR INDEX}

JOLIE, J.

Tribological Characteristics of Synthesized Diamond Films on Silicon Carbide.
PB91-189530

IYENGAR, G. V.

101,186

Dietary Collection Procedures.

PB91-147413

Practical Constraints in Monitoring Micronutrient Intake.

PB91-147421

101,317

IYER, $\mathrm{H}$.

Eddy Current Probe Sensitivity as a Function of Coil Construction Parameters.
PB91-236562

IZUMITANI, T.

100,945

Small-Angle Neutron Scattering and Light Scattering Study on the Miscibility of Poly(styrene-ran-butadiene)/Polybutadiene Blends.
PB92-117274

100,519

JACH, T.

Molecular-Orbital Studies via Satellite-Free $X$-ray Fluorescence: $\mathrm{Cl} \mathrm{K}$ Absorption and K-Valence-Level Emission Spectra of Chlorofluoromethanes. PBectra of 237164

100,427

Polarized X-ray Emission Studies of Methyl Chloride and the Chlorofluoromethanes.

100,329

JACH, T. J.

Instrumentation of X-ray Beam Lines with PIN Diode Detectors.

PB91-174888

101,633

JACKSON, J. H.

Damage to the Bases in DNA Induced by Stimulated Human Neutrophils.

Human Neutrophi
PB92-116912

101,312

JACKSON, R. H. F.

Second Order Sensitivity Analysis in Factorable Programming: Theory and Applications

101,266

State-of-the-Art of Computational Testing of Mathematical Programming Algorithms.

PB91-17489

101,268

JACOB, I.

Structural Study of the Epitaxial Growth of fcc-Fe Films, Sandwiches, and Superlattices on Cu(100).

PB91-237420

101,555

JACOBS, J. A.

National Educators Workshop: Update '90. Standard Ex-

periments in Engineering Materials Science and Technolo-

gy.

101,253

JACOBSEN, R. T.

Thermodynamic Properties of Oxygen from the Triple Point to $300 \mathrm{~K}$ with Pressures to $80 \mathrm{MPa}$.

PB92-148147

100,474

ACoX, M. E.

Production and Spectroscopy of Small Polyatomic Molecular lons Isolated in

100,245

Production and Spectroscopy of Small Polyatomic Molecular lons Isolated in Solid Neon

PB91-203273

100,395

Vibrational and Electronic Energy Levels of Polyatomic Transient Molect

100,367

Vibrational Spectra of Molecular Ions Isolated in Solid

Neon. III. N4(+)
AD-A228 082/4

100,243

Vibrational Spectra of Molecular lons Isolated in Solid Neon. IV.NO(+) , NO(-), ONNO(+), aNd ONNO(-). AD-A230 957/3

100,244

Vibrational Spectra of Molecular lons Isolated in Solid Neon. 4. NO $(1+)$, NO(1-), ONNO(1 + ), and ONNO(1-).
PB91-149914

Vibrational Spectra of Molecular lons Isolated in Solid

Neon. 5. $\mathrm{N2O}(1+)$ and $\mathrm{NNO} 2(1-)$.

100,306

Vibrational Spectra of Molecular Ions Isolated in Solid Neon. 6. CO4(-).
AD-A238 $415 / 4$

100,249

Vibrational Spectra of Molecular lons Isolated in Solid Neon. 6. CO4(1-).

PB91-236836

100,419

Vibrational Spectra of Molecular Ions Isolated in Solid

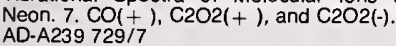

100,250

JAFAR, S. A.

Solubilities of Solids and Liquids of Low Volatility in Supercritical Carbon Dioxide.

100,470

JAHANMIR, $\mathbf{s}$.

ACTIS: Towards a Comprehensive Tribology Data Base.

PB91-174904 101,184 Numeric Database for Tribology: Format and Application

Issues.

101,045

Tribological Characteristics of Synthesized Diamond Films on Silicon Carbide.
PB91.189530

101,186

JAKUS, $K$.

Role of Glassy Interfaces in High Temperature Crack Growth in SiC Fiber Reinforced Alumina.

PB91-20368

101,127

AMES, C. $R$.

Numerical Modeling of Silicon Photodiodes for High-Accuracy Applications. Part 1. Simulation Programs.

100,763

Numerical Modeling of Silicon Photodiodes for High-Accuracy Applications. Part 2. Interpreting Oxide-bias Experiments.

Numerical Modeling of Silicon Photodiodes for High-Accuracy Applications. Part 3. Interpolating and Extrapolating In-

ternal Quantum-Efficiency Calibrations.
PB92-110311

100,765

Testing the Accuracy of Calculated Equilibrium Carrier Concentrations in the Presence of Surface Fields.

PB91-236729

101,549

JANEZIC, M. D.

Improved Technique for Measuring Permittıvity of Thin Die lectrics with a Cylindrical Resonant Cavity.

PB91-23684

100,858

\section{SON, N. H.}

Building and Fire Research Laboratory Publications, 1990.

PB91-187807

FIREDOC Users Manual, 2nd Edition.

PB91-178830

100,122

International Fire Detection Bibliography, 1975-1990 PB92-109156

100,547

Joint Panel Meeting of the UJNR Panel on Fire Research and Safety (11th). Held in Berkeley, CA. on October 19-24, 1989.

PB92-109164

100,166

Summaries of BFRL Fire Research In.House Projects and Grants, 1991

PB92-112218
JEANNERET, B.

100,168

Properties of YBa2Cu3O7-delta Thin Films Grown on OffAxis-Cut MgO Substrates.

PB91-203950

101,547

JECKELMANN, B. M

Investigating the Use of Multimeters to Measure Quantized Hall Resistance Standards.

P891-236554

100,734

JEFFERIES, $\mathbf{s}$.

Eddy Current Reflection Probe: Theory and Experiment

PB91-187120

JEFFERSON, D. $K$.

Data Management Standards in Computer-Aided Acquisition and Logistic Support (CALS)

101,339

Use of the IRDS Standard in CALS (Revised)

PB91-132209

100,684

JEFFERTS, S. R.

Nonresonant Charge Transfer in the Threshold Region for (3) $\mathrm{He}(+)+(4) \mathrm{He}($ sub $<-$ sup- $>)(3) \mathrm{He}+(4) \mathrm{He}(+)$.

\section{JEMIAN, P. R.}

High-Resolution Small-Angle $\mathrm{X}$-ray Scattering Camera for Anomalous Scattering

Small-Angle-Scattering Determination of the Microstructure of Porous Silica Precursor Bodies.

JENNINGS, D. A.

101,077

Accurate Frequency of the $119 \mathrm{mu} \mathrm{m}$ Methanol Laser from Tunable Far-Infrared Absorption Spectroscopy.

PB91-134130

101,405

Heterodyne Frequency Measurements of $\mathrm{CO}$ and OCS Beyond $2100 \mathrm{~cm}(-1)$.

PB91-236984

100,423

JENNINGS, $\mathrm{H}$.

Techniques to Observe the Fracture Zone in Mortar and Concrete.

PB91-134189

100,521

JENNINGS, $H . M$

Computer Simulated Hydration of a Cement Model.
PB91-162149 Design of High Strength Cement-Based Materials. Part 2. Microstructure.

100,099

Towards Computer-Based Microstructure Models for Cement-Based Systems.

PB91-158840

101,074

Stability of Aqueous Inorganic Lead Solutions in Polycarbonate Containers.

100,211

Studies on the Degradation Products of Paper with and without Pollutants in a Closed Environment. 1. Preliminary Results.

P891-143313

101,181

Study on the Nature of Boundary Lubricating Film: Analytical Method Development.

PB91-189407

101,176

JIANG, M.

Spinodal Decomposition in a Hydrogen-Bonded Polymer Blend.

PB91-162099

100,489

JIN, G. X.

Thermodynamic Properties of $1,1,1,2,-$ Tetrafluoroethane (R134a) in the Critical Region.

PB91-237578

100,233

JIN, M. F.

Kinetics of Copper Extraction Using (anti)-2-Hydroxy-5-Nonlybenzophenone Oxime.

PB91-148783

100,292

JINNAI, H.

Microstructure Effects on the Lower Critical Solution Temperature Phase Behavior of Deuterated Polybutadiene and Protonated Polyisoprene Blends Studied by Small-Angle Neutron Scattering

PB92-117266

100,518

Time-Resolved Small.Angle Neutron Scattering in Intermediate- and Late-Stage Spinodal Decomposition of (DPB) HPI) Blends.
PB91-16213

JIRSA, J. 0.

Proceedings of a Workshop on Evaluation, Repair, and Retrofit of Structures. U.S.-Japan Panel on Wind and Seismic Effects, UJNR. Held in Gaithersburg, MD., USA, on May 12 $14,1990$.

PB91-184846

100,145

JOHANSSON, M. E.

YBa2Cu3O7-delta/Insulator Multi-Layers for Crossover Fab rication.

PB91-194662

101,518

JOHNSON, B. C.

NIST-NRL Free-Electron Laser.

AD-A227 $310 \%$

101,399

JOHNSON, C.

Polymer Composite Processing. Industry Workshop (2nd) Held at Gaithersburg, Maryland on May 18, 1990. PB91-132191

101,115

JOHNSON, K. B.

Small Scale Demand Type Neon Liquefaction Plant

PB91-162008

100,224

JOHNSON, L. A.

Quantitative Determination of Amorphous Content in $\mathrm{Ce}$ ramic Materials Using X-ray Powder Diffraction. P891-147553

101,061

JOHNSON, R. D.

Electronic Spectrum of the GeH3 Radical.

B91-189548

100,346

Resonance Enhanced Multiphoton Ionization Spectra of the $\mathrm{GeF}$ and $\mathrm{GeCl}$ Radicals from $400-500 \mathrm{~nm}$

PB91-203281

100,396

JOHNSON, R. G.

Annual Report to the Strategic Defense Initiative Organization on the Free-Electron Laser Driven by the NIST CW Mi- 


\section{PERSONAL AUTHOR INDEX}

PB91-189753

101,656

JONES, $A$.

Issues in the Design and Implementation of a System Architecture for Computer Integrated Manufacturing.

JONES, J. A.

Saran Carbon Cryogenic Regenerator for Liquid Helium. PB91-175430

101,640

JONES, M. A.

Test Chip for the Evaluation of Surface-Diffusion Phenom-

ena in Sputtered Aluminum Planarization Processes.

JONES, M. C.

100,823

Fiber-Optic Fluorescence Array to Study Free Convection in Porous Media.

101,407

JONES, S. A.

Microstructural Studies of Ti-Al Alloys in the Vicinity of the 'Eutectoid' Reaction

PB92-116938

101,241

\section{JONES, $W . W$.}

Programmer's Reference Manual for CFAST, the Unified Model of Fire Growth and Smoke Transport.

100,096

Refinement of a Model for Fire Growth and Smoke Transport.

100,104

PB91-148510

\section{JORDAN, T. H.}

Digital Image Analysis Assisted Microradiography Measure ment of Mineral Content of Caries Lesions in Teeth PB91-187666

101,314

JOSEPH, J. P.

Opportunities for Innovation: Chemical and Biological Sen-

PB92-127315

100,214

JOSHI, A. A.

Users' Guide to BREAK1, the Berkeley Algorithm for Breaking Window Glass in a Compartment Fire.

PB92-112556

100,136

JOSHI, J. B.

Hydrodynamics and Mass Transfer in Two-Phase Aqueous Extraction Using Spray Columns.

101,300

Mass Transier Coefficients in Two-Phase Aqueous Extraction.

100,426

OSHI, Y. N.

$5 \mathrm{~s}(2) 5 \mathrm{p}(2) \cdot(5 \mathrm{~s}(2) 5 \mathrm{p} 5 \mathrm{~d}+5 \mathrm{~s} 5 \mathrm{p}(3)+5 \mathrm{~s}(2) 5 \mathrm{p} 6 \mathrm{~s}+$ $5 s(2) 5 p 7 s)$ Transitions in Sb II and $5 s(2) 5 p-(5 s 5 p(2)+$ $5 \mathrm{~s}(2)$ nl) Transitions in $\mathrm{Sb}$

PB91-200816

101,676

JOVANOVIC, s. v.

Antioxidation Mechanisms of Uric Acid.

PB91-237347 100,222

Mechanism of $\mathrm{OH}$ Radical Reactions with Thymine and Uracil Derivatives.
PB91-147447

100,216

JOYCE, S. A. Electron Attenuation Lengths at SiO2/Si Interfaces.
PB91.175612
101,499

JuCKS, K. W.

Structure of Carbon Dioxide Dimer from Near Infrared Spectroscopy.

100,279

JUDGE, P. G.

Radio-Continuum Observations of a Variety of Cool Stars.
PB91-174664 JULNME, P. S.

Associative Ionization of Ultra-Cold Laser Trapped Sodium Atoms.

PB91-236745

101,707

Notes and Comments on Roundtable Discussion on LaserAssisted Collisions and Collision-Induced Spectra. PB91-203307

101,690

JUNG, D.

Horizontal Flow Boiling Heat Transfer Experiments with a Mixture of R22/R114.

PB92-116946

101,200

JUNG, D. S.

Study of Flow Boiling Heat Transfer with Refrigerant Mixtures.

PB91-189563

101,196

JUNTTILA, M. L.

Molecular-Beam Optothermal Spectroscopy of the 9.6 mum nu14.

PB91-236869

100,421

JURRENS, K. K.

Test Plan for Validating a Context Driven Integrated Model (CDIM) for Sheet Metal Die Design. National PDES Testbed Report Series
PB92-112523

100,970

KACKER, R. N.

Taguchi's Orthogonal Arrays Are Classical Designs of Experiments.
PB92-126671

101,280

ETZEL, L. J.

Guide to the Use of the Cement and Concrete Research Remote Bulletin Board System (RBBS) Computer.
PB91-148528

100,524
PB91-148528 Guidelines for the Development of Computer Based Models
in a Cementitious Materials Modeling Laboratory.
PB91-240804

KAFAFI, S. A

Carbon Acidities of Aromatic Compounds. 2. Ionic Probes of Aromaticity in Annelated Rings.

PB91-195065

100,375

Dissociation of Substituted Anisoles: Substituent Effects on Bond Strengths.

KAHANER, D.

Algorithm and Computer Program for the Calculation of Envelope Curves.
PB91-158972

KAHANER, D. K

DEOSOL and ELLPACK: Problem-Solving Environments for Partial Differential Equations.

PB91-174441

100,645

Guide to Available Mathematical Software Problem Classifi-

cation System

101,282

VolksGrapher: a FORTRAN Plotting Package User's Guide, Version 3.0.

PB91-185108

100,647

KAHN, A. H.

Advanced Sensing of Materials Processing.

PB92-11742

100,949

KAHORA, P.

Secondary Ion Yield Changes in Silicon and Gallium Arsenide Due to Topography Changes during Oxygen or Cesium Ion Bombardment.

PB91-148064

101,471

KAIJSER, $P$.

Document Architecture for Open Systems. PB91-189522

100,961

KAILEY, J. B.

Programming Languages and Database Language SOL. Validated Processor List Including GOSIP Conformance
Testing Registers.
PB91-187716
100,649

\section{KAISER, D. L.}

Low-Field Flux Pinning in Twinned and Detwinned Single Crystals of $\mathrm{YBa} 2 \mathrm{Cu} 3 \mathrm{O} 7-x$

1-237552

101,559

KAMITAKAHARA, W. A.

Neutron Measurements of Intramolecular Vibrational Modes in $\mathrm{C} 60$.

KAMMER, R. G.

101.055

Advanced Technology Program: A New Role for NIST in Accelerating the Development of Commercially Important Technologies.

100,013

KANDA, M.

Estimation of Electromagnetic Fields in Complex Environments.

PB91-203315

101,691

Lattice Approach to Environments Irradiated by Unknown Sources.

100,850

Microstrip Patch Antenna as a Standard Transmitting and Receiving Antenna.

PB91-162156

100,591

Photonic Probes for the Measurement of Electromagnetic Fields Over Broad Bandwidths.

PB91-162206

100,750

KANEI, A.

Effect of Therma! Expansion Mismatch on Fiber Pull-Out in Glass Matrix Composites.

PB91-202952

101,125

KANIA, D. R.

Transitions and Energy Levels for Cu-Like $\mathrm{Yb}(41+)$ ) $\mathrm{Ta}(44+)$, and $\mathrm{U}(63+)$.

101,610

\section{KAO, J. Y.}

Direct Digital Control Based Building Automation System Design Criteria.

100,091

Guide Specification for Direct Digital Control Based Building Automation System.

PB91-216697

Variable Air Volume System Design Guide.

PB91-216655

100,092

KARAM, L. R.

Biomarkers of $\mathrm{OH}$ Radical Damage In vivo.

100,077

Formation of ortho-Tyrosine by Radiation and Organic Solvents in Chicken Tissue.

PB92-116953

101,290

KAREN, V. L

NIST*LATTICE: A Program to Analyze Lattice Relationships. Spring 1991 Version.

101,561

KARNI, $M$.

Ab-Initio Calculations and Ideal Gas Thermodynamic Functions of Cyclopentadiene and Cyclopentadiene Derivatives. PB92-148089

100,468

KARPAS, $z$.

Alkyl Transfer Reactions between Protonated Alcohols and Ethers. The Gas-Phase Alkylation of Formaldehyde.
PB91-147470

KASHANI, A.

SHOOT Flowmeter and Pressure Transducers.

PB91-147488

100,923

KATHREN, R. L.

Interlaboratory Comparison of Actinides in Human Tissue (239) Pu $+(240) \mathrm{Pu}$

PB91-20326

101,323

KATZ, J. L.

Microstructure and Elastic Properties of Dental Resin and Resin-Based Glass-Reinforced Composites: XRD, SEM and Ultrasonic Methods.

PB91-204081

100,050

KATZ, S.

STEP On-Line Information Service User's Guide. National PDES Testbed Report Series.

100,950

KATZ, S. B.

Configuration Management Concepts Document. National

PDES Testbed Report Series.
PB91-194480

100,984

Configuration Management of the STEP Documents: Procedures and System Requirements. National PDES Testbed Report Series

100,968

\section{KAUFMAN, M. J.}

Cleavage Step Formation and Resistance to Transgranular Stress Corrosion Cracking.

PB91-149047

101,133

Microstructural Studies of Ti-Al Alloys in the Vicinity of the 'Eutectoid' Reaction.

tectoid' R

101,241

KAUFMAN, $v$.

$5 s(2) 5 p(2)-(5 s(2) 5 p 5 d+5 s 5 p(3)+5 s(2) 5 p 6 s+$ $5 s(2) 5 p 7 s)$ Transitions in Sb II and $5 s(2) 5 p-(5 s 5 p(2)+$ $5 s(2) n l)$ Transition
PB91-200816

101,676

Extreme-Ultraviolet Spectra of $\mathrm{Pd}, \mathrm{Ag}, \mathrm{Cd}$, and $\mathrm{Sn}$ lons $\mathrm{Ob}$ served in a 1.3-keV Tokamak Plasma.

PB91-237495

101,712

Ouantum Electrodynamic Contributions to Spin Orbit Splitting in the Ground State of Aluminum-Like lons. PB91-189597

01,653

Spectra of the P I Isoelectronic Sequence from Co XIII to

$\begin{array}{ll}\text { Mo XXVIII. } & 101,713\end{array}$

Spectrum and Energy Levels of Seven-Times-Ionized Krypton (Kr Vill) and Resonance Lines of Eight-Times-lonized Krypton $(\mathrm{Kr} \mid \mathrm{X})$

Krypton (Kr IX)

101,673

Wavelengths and Energy Level Classifications for the Spectra of Aluminum (AI I through AI XIII).

100,472 
KAZMERSKI, L.

Analysis of the $\mathrm{YBa} 2 \mathrm{Cu} 3 \mathrm{O} 7 / \mathrm{SrTiO} 3$ Interface as a Function of Post-Deposition Annealing Temperature.

101,480

KEANE, C. J.

Transitions and Energy Levels for Cu-Like $\mathrm{Yb}(41+$ ), $\mathrm{Ta}(44+)$, and $\mathrm{U}(63+)$

101,610

KEARSLEY, A. J.

Merit Function for Inequality Constrained Nonlinear Programming Pro

101,271

KEDZIERSKI, M. A.

Visualization of Nucleate Flow Boiling for an R22/R114 Mixture and Its Components.

PB91-162164

100,225

KEENAN, J.

Round-Robin Study of Implants in $\mathrm{Si}$ and $\mathrm{SiO} 2$ by SIMS, RBS, and NAA.

PB92-11724

100,830

KEEAY, W. J.

Application of Transmission Electron Detection to X-ray Mask Calibrations and Inspection.

PB92-117217

100,828

KELLEHER, D. E.

Overview of the Ion Dynamic Effect in Line Broadening. and a Generalization of the Unified Theory.

101,442

KELLEY, E. F.

Effect of High Pressure on Prebreakdown Phenomena in $n$ Hexane.

PB91-135020

100,839

Electrical Breakdown and Streamer Statistics in N-hexane under Uniform Field Conditions.

PB91-134684

100,837

KELLEY, M. H.

Uses of Spin-Polarised Electrons in Fundamental ElectronAtom Collision Processes and the Analysis of Magnetic Mi-

crostructures.

101,490

KELLEY, R. D.

Influence of Sulfur on Methanation over Tungsten(110).

Influence of Sulfur on Methanation over Tungsten(110),
PB91-149369

Summary Abstract: The Influence ot Sulfur on Methanation

over $W(110)$

100,301

KELLY, E.

Proceedings of Cable ' 89 Workshop

PB91-161943

100,773

KELLY, G. E.

Rule-Based Diagnostic Method for HVAC Fault Detection.
PB91-194936

Testing and Rating of an Atmospheric, Gas-Fired Furnace Equipped with a Burner Air Inlet Damper.

100,894

KELLY, J. F.

Collisional Energy Transfer between Excited Sr Atoms.

PB91-204164 101,703

In situ Measurements of Bridged Crack Interfaces in the Scanning Electron Microscope.

101,068

KELLY, R. M.

Biological Sulfur Oxidation and Reduction for Coal Sulfur Speciation and Desulfurization.

PB91-203323

100,886

KELLY, W. $\mathbf{R}$.

Development of a New Tracer Technology Using Enriched Rare-Earth Isotopes.

PB91-195347

100,908

KEMMERER, $\mathbf{S}$. J.

NIST Support of the CALS Program: 1990 Synopsis.

PB91-19382

101,347

KENDELEWICZ, T.

Morphology and Barrier-Height Development of $\mathrm{Bi} / \mathrm{InP}(110)$ Interlaces.

PB91-147736 101,468

Surface Extended-X-ray-Absorption Fine Structure and

Scanning Tunneling Microscopy of Si(001)2×1-Sb.
PB91-237263
101,552

PB91-237263

Building-in Reliability: Making It Work

PB91-203992

100,812

KENNEDY, T. A.

Optically Detected Magnetic Resonance Study of Antisiteto-Acceptor and Related Recombination Processes in asGrown InP-Zn.

101,532

KESHAVARZNIA, N. R.

Fe Implantation in In0.53Ga0.47As/InP

PB91-189969

101,516

KESSLER, E. G.

Determination of the Neutron Mass and Implications for Fundamental Constants.
PB91-148692

101,615

KESTIN, J.

Equilibrium and Transport Properties of Gas Mixtures at Low Density: Eleven Polyatomic Gases and Five Noble Gases.

100,363

Thermal Conductivity of Nine Polyatomic Gases at Low Density.

PB91-192492

100,360

KEYSER, P. T.

Search for a Composition Dependent Force Using a Liquid Supported Torsion Balance.

101,639

Test of Newton's Inverse Square Law of Gravity Using the $300 \mathrm{~m}$ Tower at Erie, Colorado: Newton Vindicated on the Plains of Colorado.

PB91-175273

101,637

Test of the Inverse-Square Law of Gravitation Using the 300-m Tower at Erie, Colorado.

PB91-204115

101,702

KHAN, H. M.

Radiochromic Film Dosimeter for Gamma Radiation in the Absorbed-Dose Range 0.1 - $10 \mathrm{kGY}$.

101,364

Temperature Dependence of Radiochromic Film Dosi-

meters.
PB92-117134
101,728

KHERA, D.

Knowledge Extraction Techniques for Expert System Assisted Wafer Screening.

100,824

Knowledge Verification of Machine-Learning Procedures Based on Test Structure Measurements.

PB91-189571

100,709

KIKUCHf, R.

Grain Boundaries in a 2-D Superlattice.

$1-202853$

100,388

Phase Diagrams of Hexagonal Binary Ordering Alloys with Anisotropic Interactions

PB91-147512

101,211

KILMER, $R$.

Control System Architecture for Unmanned Ground VehiPB91-134726

KIM, B. F.

101,379

Superconducting Thin Films of Bi-Sr-Ca-Cu-O Obtained by Laser Ablation Processing.

PB91-189589

101,508

KIM, C. I.

Heat Flux, Mass Loss Rate and Upward Flame Spread for Burning Vertical Walls (1990).

PB91-143297

100,094

Turbulent Upward Flame Spread for Burning Vertical Walls Made ot Finite Thickness.

PB92-11253

100,170

KIM, $\mathrm{H}$.

Temperature Dependence of Probe Diffusion in Bulk Polymer Matrices.

PB91-236885

100,422

KIM, $O$.

Validation of an OSI Transport Class 4 Simulator.

PB91-187724

100,568

$\mathrm{K}$ iM, $\mathrm{S}$.

Elastic Constants, Debye Temperatures, and Electron Phonon Parameters of Superconducting Cuprates and RePB91-134262

KIM, S. A.

101,460

Elastic Constants of Polycrystalline Bi-Pb-Sr-Ca-Cu-O Superconductor.

101,461

KIM, W.

Electrofission in the Ouasifree and Delta Regions

PB91-147355

101,609

KIM, Y. K.

Electric Ouadrupole and Magnetic Dipole Transition Probabilities in Potassium Isoelectronic Sequence.

PB91-187088

101,646

Energy Levels and Transition Probabilities in the Ground State Configuration of Sulfur-Like lons.

101,658

Ouantum Electrodynamic Contributions to Spin Orbit Splitting in the Ground State of Aluminum-Like lons.
PB91-189597

Relativistic and Correlation Effects in the $2 \mathrm{~s} 3 p$ Configuration of Beryllium-Like Ions.

PB91-189605

101,654

Relativistic Distorted-Wave Cross Sections for ElectronImpact Excitations of Berylliumlike Ions

PB91-195503

101,672

KINARD, J. R.

AC-DC Difference Relationships for Current Shunt and Thermal Converter Combinations.

100,737

100,737
RF-DC Differences of Thermal Voltage Converters Arising RF-DC Differences of
from Input Connectors

PB91-236802

100,735

G, D. S.

Laser Induced Desorption of Molecules trom Surfaces.

PB91-147066

100,235

Molecular Desorption from Solid Surfaces: Laser Diagnosics and Chemical Dynamics.

BB91-203331

100,397

Photodissociation Dynamics of $\mathrm{Mo}(\mathrm{CO}) 6$ at $266 \mathrm{~nm}$ and

$355 \mathrm{~nm}$ : CO Photofragment Kinetic-Energy and InternalState Distributions.

PB91.236547

100,413

Surface-State-Mediated Photochemistry: Laser-Induced Desorption of NO trom Si (111).

100,320

Time- and State-Resolved Measurements of Nitric Oxide

Time- and State-Resolved Measurements of Nitric Oxide
Dimer Infrared Photodissociation.
PB91-202846

KINGSTON, M. L.

NIST Serial Holdings, 1991

PB91-171330

100,960

KINNEY, P. D.

Use of the Electrostatic Classification Method to Size 0.1 micrometer SRM

100,932

KIRCHNER, P. D.

Amorphization and Conductivity of Silicon and Germanium Induced by Indentation.

PB91-150045

101,482

KIRCHNER, $\mathbf{S}$.

Preliminary Results of the Environmental Evaluation of the Federal Records Center in Overland Missouri.

PB91-240747

100,082

KIRKLIN, D. R.

Energy of Combustion of Triphenylphosphate. PB91-189613

100,347

KISSICK, W. A

Improved Technique for Determining Complex Permittivity with the Transmission/Retlection Method.

PB91-161919

100,844

KITCHING, I. D.

Frequency and Time Stability of GPS and GLONASS

PB91-202937

100,582

KLAUS, E. E.

Mechano-Chemical Descriptive Model for Wear Under Mixed Lubrication Conditions.

101,177

Study of Additive Response in a Series of Rerefined Base Oils Typical ot Current Commercial Practice.

PB91-174953

101,175

KLAVINS, $P$

Crystal-Field Splittings in the Cubic Heusler Alloys HoPd2Sn and ErPd2Sn.

PB91-194902

101,525

KLEIN, $\mathbf{R}$.

Laser Photolysis of Trimethylgallium at $193 \mathrm{~nm}$ : Ouantum Yields for Methyl Radical and Ethane Production

AD-A227 868/7

100,234

Photomultiplier Housing for Vacuum Operation of Side-on 1P28-Type Tubes. 


\section{PERSONAL AUTHOR INDEX}

$\mathrm{KOCH}$, W. F.

Evaluation of Liquid Junction Potentials and Determination of $\mathrm{pH}$ Values of Strong Acids at Moderate Ionic Strengths. PB91-237859

NIST Standards for Sodium and Potassium.

100,191

Proposed New Electrolytic Conductivity Primary Standards

$\begin{array}{ll}\text { for } \mathrm{KCl} \text { Solutions. } & 100,339 \\ \mathrm{~PB} 91-187658 & \end{array}$

Research at NBS in Direct Potentiometric Measurements in Blood.

PB91-148874

100,188

Second Dissociation Constant and $\mathrm{pH}$ of N-2-hydroxyethylpiperazine-N'-2-ethanesulfonic Acid (HEPES) from 0 to PB91-189282

100,196

KOCK, M.

Unified Set of Atomic Transition Probabilities for Neutral Argon.

101,717

KOEHLER, $R$.

Numerical Modeling of Silicon Photodiodes for High-AccuraCy Applications. Part 2. Interpreting Oxide-bias Experiments. KOEHLER, T

Design and Analysis of Experiments.
PB91-158824

101,274

KOENIG, J. A.

Checking the Net Contents of Packaged Goods. Third Edition, Supplement 2.

100,171

Uniforms Laws and Regulations as Adopted by the National Conference on Weights and Measures (76th), 1991

PB92-112416

101,022

KOEPKE, G. H.

Calibration of Antenna Factor at a Ground Screen Field Site Using an Automatic Network Analyzer.

PB91-134239

100,724

KOHLER, $R$.

Generalized Photodiode Self-Calibration Formula.

PB91-236901

100,760

KOHN, G.

Dynamic Arc-Power Source Response in GMA Welding. PBg 1

Pozzolan Programs of the Cement and Concrete Reference

Laboratory.
PB91-149922

100,525

KONJEVIC, $N$.

Experimental Stark Widths and Shifts for Spectral Lines of Neutral and lonized Atoms: A Critical Review of Selected Data for the Period 1983 through 198

PB91-1925

101,659

KOO, B. C.

Detection of an Expanding H I Shell in the Old Supernova Remnant CTB 80.

PB91-174920

100,028

KOPANSKI, J. J.

Assessment of Reliability Concerns for Wide-Temperature Operation of Semiconductor Devices and Circuits.

PB92-116987

100,825

High Spatial Resolution Mapping of Semiconductor Resis-

tivity. 194829

100,809

Permittivity Measurements on Molecular-Sized Samples.

PB91-159244 100,800

Verification of the Relation between Two-Probe and Four.

Probe Resistances as Measured on Silicon Wafers.
PB91-147520

KORDE, $\mathbf{R}$.

Stable, High Ouantum Efficiency Silicon Photodiodes for Vacuum-UV Applications

PB91-203349

100,757

KORSZUN, Z. R.

Application of Osmotic Dewatering to the Controlled Crystallization of Biological Macromolecules and Organic Com-

PB91-195719

100,230

KORZUN, Z. R.

Low-Cost Low-Volume Carrier (Minilab) for Biotechnology and Fluids Experiments in Low Gravity.

100,041

KOSMAS, M. K.

Flexible Polymer with Excluded Volume at an Interacting Penetrable Surface of Variable Dimension: A Multiple eps Ion Perturbation Theory.

PB92-116698

100,517

KOSTER, B. J.

Alaska Marine Mammal Tissue Archival Project: Revised Collection Protocol

101,374

Preparation and Analysis of a Marine Sediment Reference Material for the Determination of Trace Organic Constitu. Material for the Determination of Trace Organic Constitu-
ents.
PB91-149237

101,377
KOSTKOWSKI, H. J.

Roughened Ouartz Surfaces and Teilon as Small-Angle Diffusers and Depolarizers between 200 and $400 \mathrm{~nm}$. 101,425 PB91-195644

KOTHARI, K. M.

Elfect of Pipe Surface Finish on Gas Flow Measurement with an Orifice Meter

101,387

Effect of Tube Bundle Flow Conditioners on Orifice Meter Discharge Coefficients.

PB91-14879

100,881

KOVAL, C. A.

Overview of Facilitated Transport Membrane Systems.

100,228

KRAKA, E.

Van der Waals Complexes in 1,3-Dipolar Cycloaddition Reactions: Ozone-Ethylene.

PB91-236737

KRAMER, T. R.

Error Prevention and Detection in Data Preparation for a Numerically Controlled Milling Machine.

100,980

Extracting STEP Geometry and Topology from a Solid Modeler: Parasolid-to-STEP.

100,983

Parser That Converts a Boundary Representation into a

Features Representation.

Features Repr
PB91-203356

KRANBUEHL, D.

Simulation Studies of Excluded Volume Effects on Polymer

Chain Dynamics in Several Nonlattice Models.
PB91-203364

100,507

\section{KAUSE, R. F.}

Effect of Slag Penetration on the Performance of Magnesia Chrome Refractories in Slagging Gasifiers.
PB91-134940

100,875

Test Method for Tensile Creep of Structural Ceramics Using Flexure Beams.
PB92-116995

101,099

KRAUSE, S.

Residual Defects in SIMOX: Threading Dislocations and Pipes.

PB92-117233 100,829

Sensitivity of Ellipsometric Modeling to the 'Islands' of Silicon Precipitates at the Bottom of the Buried Oxide Layer in Annealed SIMOX

PB92-116607

100,820

KRAUSS, $M$.

Hydrogen Bonding in Pt Ammıne Complexes.

PB91-189639 PB91-194837

100,370

KRAUSS, R.

Transport Properties of Fluid Oxygen.

PB91-192484

100,359

Assessment of Uncertainties in Measurement of $\mathrm{pH}$ in Hos-

tile Environments Characteristic of Nuclear Repositories.

101,367

Comparison of Amorphous and Ouasicrystalline Films of Sputtered Al0.72MnO.22Si0.06.

PB91-195552

101,530

Instability in $\mathrm{pH}$ Measurements of Sputtered IrO2 Films. PB91.147538

Sputtered Thin Film YBa2Cu3On.

PB91-189647

100,281

Transparent Thin Film Thermocouple.

PB91-190116

101,509

KREINER, W. A Optothermal-Detected Microwave-Sideband CO2-Laser
Spectroscopy of Ar-NH3.

PB91-236679

100,416

R, D. P.

Alignment Fixture for Millimeter Waveguide

PB91-2369

100,727

Effect of a Free Surface on Stress Distribution in a Bimaterial Composite

101,121

Improved Optical Diffraction Strain Measurement System. PB91-174433

101,581

Mechanical Properties of Alumina-Peek Unidirectional Composite: Compression, Shear, and Tension.

101,118

Numerical Simulation and Visualization Models of Stress Numerical Simulation and Visualization Mode Wave Propagatio
PB91-174946

101,120 Stress Intensity Factors by Enriched Mixed Finite Elements.
PB91-147371

KROCKEL, $\mathrm{H}$.

Factual Materials Databanks - The Need for Standards. PB91.147546

101,249

KROLL, C. $M$.

Amorphization and Conductivity of Silicon and Germanium Induced by Indentation.

PB91-150045

101,482

KRUEGER, S.

Characterization of the Densification of Alumina by Multiple Small-Angle Neutron Scattering.

101,100

Effect of Green Density and the Role of Magnesium Oxide

Effect of Green Density and the Role of Magnesium Oxide
Additive on the Densification of Alumina Measured by Small-Angle Neutron Scattering

PB92-117043

101,101

Small-Angle-Scattering Determination of the Microstructure of Porous Silica Precursor Bodies.

101,077

RUEGER, S. T.

Small Angle Neutron Scattering Characterization of Microporous Silica.
PB91-203489

101,089

KRUGER, J.

Structure of Surface Films on Magnesium and on Magnesium Alloys.

PB91.147637

101,212

Surface-Extended X-ray-Absorption Fine-Structure Experiments at Atmospheric Pressure by Means of a Photocathode Proportional Counter with Monolayer Sensitivity.

PB91-194951

100,372

Surface X-ray Absorption Spectroscopy, EXAFS and NEXAFS, for the In situ and Ex situ Study of Electrodes.
PB92-117035

KU, C. S.

Characterization of Tricresylphosphate Lubricating Films by Micro-Fourier Transform Infrared Spectroscopy.

1-203810

Mechanisms of Additive Effectiveness.

PB91-158808

101,179

Mechanisms of Additive Effectiveness.

101,172

101,174

Study of Additive Response in a Series of Rerefined Base Oils Typical of Current Commercial Practice.

101,175

KU, H, H.

Experimentation and Measurement.

PB91-203372

101,692

Uncertainty and Accuracy in Physical Measurements. PB91-167205

101,629

KUBOTA, T.

Species Produced in Fires Burning in Two-Layered and Homogeneous Vitiated Environments.

PB91-157180

100.542

KUCHINSKI, M. A.

Ouantitative Determination of Amorphous Content in $\mathrm{Ce}$ ramic Materials

101,061

KUENTZLER, $R$

Structural Instabilities and Superconductivity in Ouasi-Binary Mn5Si3-Type Compounds.

PB91-149427

101,215

KUHL, K.

Photodissociation Dynamics of Water in the Second $A b-$ sorption Band: Vibrational Exitation of $\mathrm{OH}$ (A (2)Sigma)
PB91-148726

KUHN, D. R.

Proceedings of the Workshop on High Integrity Software 
KURIYAMA, M.

Dynamical Diffraction Imaging (Topography) with X-ray Synchrotron Radiation.

101,510

High Resolution Diffraction Imaging of Crystals Grown in Microgravity and Closely Related Terrestrial Crystals.

High Resolution Synchrotron X-Radiation Diffraction Imag-
ing of Crystals Grown in Microgravity and Closely Related ing of Crystals Grown in Microgravity and Closely Related Terrestrial Crystals.

PB91-216747

101,548

Streaking Images That Appear Only in the Plane of Diffraction in Unoped GaAs Single Crystals: Diffraction Imaging (Topography) by Monochromatic Synchrotron Radiation. Structural Anomalies in Undoped Gallium Arsenide Observed in High Resolution Diffraction Imaging with Monochromatic Synchrotron Radiation.

PB91-237438

101,556

Electronic Structure of High-Tc Superconductors Studied

Using Photoelectron Spectroscopy.

Initial Stages of Degradation of Superconductor Surfaces: $\mathrm{O} 2, \mathrm{H} 2 \mathrm{O}, \mathrm{CO} 2$ and CO Chemisorption on $\mathrm{La2}-\mathrm{xSr} \times \mathrm{CuO} 4$. PB91-189670

KURYIO, M. J.

Group Reactivities in the Gas Phase Reactions of Hydroxyl Radicals with Ethers

KUSUDA, T.

100,330

Design Heat Loss Factors for Basement and Slab Floors

PB91-147561 100,059

Method for Measuring Heat Loss from Underground Heat Distribution Systems.

PB91-134221

100,888

KUYATT, C. E.

Center for Radiation Research. 1990 Technical Activities. BB91.178855

KWOK, s.

Energy Distributions of Symbiotic Novae.

PB91-200873

100,032

Evolution of Infrared Carbon Stars.

PB91-202861

100,033

LADBURY, J. M.

Mode-Stirred Chamber for Measuring Shielding Effectiveness of Cables and Connectors: Assessing MIL-STD-1344A Method 3008

PB92-116656

LAESECKE, A.

Transport Properties of Fluid Oxygen.

PB91-19248

100,776

LAFFERTY, W. J.

100,359

High-Pesolution Infrared Spectrum of the nu1 Band of

100,463

Rovibrational Analysis of the nu7(1) Intermolecular Hydrogen Bond Bending Vibration in HCN-HF Using Far Infrared Fourier Transform Spectroscopy.

PB91-237784

100,437

Structure of Carbon Dioxide Dimer from Near Infrared Spectroscopy.

LAGERGREN, E.S.

100,279

Taguchi's Orthogonal Arrays Are Classical Designs of Experiments.

101,280

LAHER, R. $R$.

Improved Fits for the Vibrational and Rotational Constants of Many States of Nitrogen and Oxygen. $\quad 100,469$
PB92-148097 100,469
PB92-148097 tron Impact on Atomic Oxygen.

PB90-244591

100,256

LAKDAWALA, V. K.

Transition from Trichel-Pulse Corona to Dielectric Barrier Discharge.
PB91-134213

100,835

LAL, $R$.

High Resolution Diffraction Imaging of Crystals Grown in Microgravity and Closely Related Terrestrial Crystals. PB92-109008

LAMAZE, G.P.

Cross-Section Measurements in the Intermediate-Energy Standard Neutron Field.

PB91-203398

101,693

Spectrum-Averaged Cross-Section Measurement of $(103) \mathrm{Rh}(\mathrm{n}, \mathrm{n})(103 \mathrm{~m}) \mathrm{Rh}$ in the $(252) \mathrm{Cf}$ Fission Neutron Spec-

PB91-203406

101,694

LANGLAND, J. $K$.

Low-Background Gamma-Ray Assay Laboralory for Activation Analysis.

100,192

LANNIN, J. S.

Neutron Measurements of Intramolecular Vibrational Modes in $\mathrm{C} 60$
PB92-116540

101,055

LAOR, U.

Streaking Images That Appear Only in the Plane of Diffraction in Unoped GaAs Single Crystals: Diffraction Imaging Topography by Monochromatic Synchrotron Radiation. (Topography) by Monochromatic Synchrotron Radiation, 101,511 Structural Anomalies in Undoped Gallium Arsenide Observed in High Resolution Diffraction Imaging with Monochromatic Synchrotron Radiation.

PB91-237438

101,556

LARRABEE, R. D.

Application of Transmission Electron Detection to X-ray

Mask Calibrations and Inspection.
PB92-117217

100,828

\section{ARSEN, E. B.}

Calibration of Antenna Factor at a Ground Screen Field Site Using an Automatic Network Analyzer.

100,724

NIST Calibration Frocedure for Vertically Polarized Monopole Antennas $30 \mathrm{kHz}$ to $300 \mathrm{MHz}$.

PB91-185124

100,726

LARSON,

Structural Anomalies in Undoped Gallium Arsenide Observed in High Resolution Diffraction Imaging with Monoch romatic Synchrotron Radiation.

PB91.237438

101,556

\section{LARSON, D.}

Streaking Images That Appear Only in the Plane of Diffraction in Unoped GaAs Single Crystals: Diffraction Imaging (Topography) by Monochromatic Synchrotron Radiation.
PB91-189662

LARSON, D. R.

Hydrogenated Amorphous Germanium Detectors Deposited onto Channel Waveguides.

PB91-194845

100,756

Integrated-Optic Waveguide Glass Lasers.

100,758

Semiconductor Claddings on Glass Waveguides for Polariz ers and Detectors.

101,416

LARSON, T.

Prospects for High Temperature Superconductor - Semiconductor Contacts.

PB91-203646

101,545

LASCOLA, $R$.

Structural Dependence of HF Vibrational Red Shifts in ArnHF, $n=1.4$, via High-Resolution Slit Jet Infrared Spectroscopy.

PB91-203562

100,401

LASHMORE, D.

Surface Properties and Coatings of Materials Microtailored

Alloys.

101,232

LATHABAI, $\mathbf{S}$.

Cyclic Fatigue from Frictional Degradation at Bridging Grains in Alumina.

101,092

Fracture Mechanics Model for Subthreshold Identation Flaws. Part 1. Equilibrium Fracture. 101,093

01,093

\section{LAUFER, A. H.}

Ultraviolet Absorption of the Vinyl Radical and Reaction with Oxygen.

\section{LAVILLA, R. E.}

100,391

Anomalous Lgamma2,3 X-ray Emission Spectrum of $\mathrm{Xe}_{101,670}$
PB91-195289

Anomalous Th M3O1 X-ray Emission Spectrum of ThO2

PB91-195297 100,381

Many Electron Effects in Lgamma2,3 X-ray Emission Spectroscopy Spectrum of $\mathrm{BaO}$

PB91-195305

100,382

Many-Electron Effects in Lgamma2,3 XES Spectra from

Rare Earth Elemen

100,383

Molecular-Orbital Studies via Satellite-Free $X$-ray Fluorescence: $\mathrm{Cl} \mathrm{K}$ Absorption and $\mathrm{K}$-Valence-Level Emission Spectra of Chlorofluoromethanes.

100,427

Polarized X-ray Emission Studies of Methyl Chloride and the Chlorofluoromethanes.

the Chlorofluor
PB91-174995

100,329

LAWN, B. R.

Cyclic Fatigue from Frictional Degradation at Bridging Grains in Alumina.

101,092

Fracture Mechanics Model for Subthreshold Identation Flaws. Part 1. Equilibrium Fracture.

101,093

Fundamental Condition for Existence of Microcrack Clouds in Monophase Ceramics.

PB91-189704

101,082

In situ Measurements of Bridged Crack Interfaces in the Scanning Electron Microscope.

101,068

Strength and Microstructure of Ceramics.

AD-A243 049/4

101,056

LAWRENCE, $\mathbf{S}$.

Phonon Density of States of Superconducting $\mathrm{YBa} 2 \mathrm{Cu} 3 \mathrm{O} 7$ and the Nonsuperconducting Analog YBa2Cu3O6.

PB91-159129

\section{LE-TRAN, T. T.}

Magnetic Anisotropies in Ultrathin tcc $\mathrm{Fe}(001)$ Films Grown on Cu(001) Substrates.

PB92-116631

101,565

LEA, J. S.

Co-Trial on ESR Identification and Estimates of gamma-Ray and Electron Absorbed Doses Given to Meat and Bones. PB91.194720

LEAKE, $S$.

Robot Sensor Language

PB91-174961

100,017

LECHNER, J. A.

Estimators for Censored (Log)Normal Samples

PB91-203414

\section{LEDBETTER, $\boldsymbol{H}$.}

Elastic Constants, Debye Temperatures, and ElectronPhonon Parameters of Superconducting Cuprates and Related Oxides.

Elastic Constants of a Tungsten-Particle Copper-Matrix

PB91-194852

101,234

Elastic Constants of Fiber-Reinforced Composites: A Fresh

Measurement Approach

101,123

Group and Phase Sound Velocities in an Eu1Ba2Cu3O7 Superconductor and Related Perovskite Oxides.

PB91-194860

101,523

Madelung Potentials and Valences in the $\mathrm{Y} 1 \mathrm{Ba} 2 \mathrm{Cu} 3 \mathrm{O} 7 \mathrm{Su}$ perconductor.

Mechanical Loss in a Glass-Epoxy Composite.
PB91-149435

101,459

101,070

Oxides and Oxide Superconductors: Elastic and Related Properties.

Resonating-Orthotropic-Cube Method for Elastic Constant PB91-236794

LEDBETTER, H. M.

Elastic Constants of Polycrystalline Bi-Pb-Sr-Ca-Cu-O Superconductor

\section{LEDFORD, A. E.}

Monitoring the Fate of Chlorine from MSW Sampling Ihrough Combustion.

PB91-174631

MSW Calorimetry

PB91-133942

100,917

LEE, $A$.

Effect of Crosslink Density on Physical Aging of Epoxy NetPB91-189712

101,244

Physical Aging and the Viscoelastic Response of Network PB91-189829

101,246

Viscoelastic Response of Epoxy Glasses Subjected to Different Thermal Treatments.

PB91-189720

101,245

LEE, $C$ 
LEI, M. Elastic Constants, Debye Temperatures, and Electron-
Phonon Parameters of Superconducting Cuprates and Related Oxides.

101,460

Elastic Constants of Fiber-Fieinforced Composites: A Fresh Measurement Approach.

PB91-194878

101,123

Madelung Potentials and Valences in the $\mathrm{Y}_{1} \mathrm{Ba} 2 \mathrm{Cu} 3 \mathrm{O} 7 \mathrm{Su}$ -

perconductor.

101,459

Oxides and Oxide Superconductors: Elastic and Related Properties.

PB92-112333

101,563

LENAERS, $G$

Silicon Reference Materials Certified for Isotope Abundances.
PB92-126713

100,213

LENNON, $E$.

Computer Security: Selected Articles.

PB91-187740

100,706

National Computer Systems Laboratory Annual Report, 1990.

PB91-201822

100,603

LEONE, S. R.

Diode Laser Probing of $1^{*}((2) \mathrm{P} 1 / 2)$ Doppler Profiles: Time Evolution of a Fast, Anisotropic Velocity Distribution in a Thermal Bath

100,310

General Method for Doppler Determination of Cylindrically Symmetric Velocity Distributions: An Application of Fourier Transform Doppler Spectroscopy.

PB91-149385

100,302

Laser-Induced Fluorescence Measurements of Drift-Velocity Distributions for $\mathrm{Ba}(+)$ in $\mathrm{Ar}$ : Moment Analysis and a Direct Measure of Skewness.

PB91-149005

100,298

Multireference-Configuration Interaction (MR-Cl) Caiculations on $\mathrm{HS}(2+)$ and Experimental Observation via Electron Impact lonization of $\mathrm{H} 2 \mathrm{~S}$

100,295

Novel Laser Gain and Time-Resolved FTIR Studies of Photochemistry.

100,237

PB91-148809

Study of the GaAs-Si(100) Interface Using Laser Probing of
Thermal Desorption Kinetics.
PB91-149294
101,477 PB91-14929

LEOPOLD, K. R.

Rotational Spectrum and Structure of the Complex ArCh3CN

100,415

LEPEK, A.

Israel's New Synchronized Time Scale, UTC(INPL).
100,577

LETT, P. D.

Associative Ionization of Ultra-Cold Laser Trapped Sodium

PB9 $1-236745$

101,707

Effects of Colored Pump Noise on Intensity Correlations of a Single Mode Dye Laser.

PB91-189738

101,423

Observation of Atoms Laser-Cooled Below the Doppler Limit.

101,655

Observation of Atoms Laser Cooled Below the Doppler Limit.

101,671

Prospects for Electromagnetic Manipulation and Trapping of Antihydrogen.

PB91-194886

101,663

Calibration of the NBS 10 mum Space Beads.

PB91-2034:

101,695

Dimensional Calibration of an NBS 0.3-mum-Diameter Particle-Sizing Standard.

101,696

Light-Scattering Measurement of the RMS Slopes of Rough

Surfaces.

101,436

NBS Submicron Particle Standards for Microcontamination Measurement

PB91-158873

Optical Levitation of Single Particles.

100,798

PB91-17498

101,634

LEUBNER, C Explicit Mapping of the Set of Dynamical Symmetries onto
the Set of Equivalent Lagrangians for Systems with One Degree of Freedom.
PB91.158881

LEUNG, H. $O$.

101,622

Microwave Spectrum and Molecular Structure of the N2. H20 Complex
PB91-203448

LEUSCHNER, $M$.

Electrofission in the Quasifree and Delta Regions. PB91-147355
LEVAN, S. L.

Kinetic Properties of the Components of Douglas-Fir and the Heat of Combustion of Their Volatile Pyrolysis Products.
PB91-203794

LEVELT SENGERS, J. M. $\boldsymbol{H}$.

Limiting versus Apparant Critical Behavior of Henry's Constants and K Factors.

100,277

Modeling the Thermodynamic Properties of Sodium Chloride in Steam Through Extended Corresponding States.

Phase Transitions.

PB91-162073

100,318

Refractive Index of Water and Steam as Function of Wavelength, Temperature and Density.

101,402

Thermodynamic Properties of Aqueous Solutions at High Temperatures; Needs, Methods, and Challenges. PB91-204065

100,410

Thermodynamics of Solutions Near the Solvent's Critical PB91-237297

100,232

Unified Description of Infinite-Dilution Thermodynamic Properties for Aqueous Solutes.

PB91-162081

100,319

LEVIN, $B$.

Role of Bench-Scale Test Data in Assessing Real-Scale Fire Toxicity.
PB91-167270

\section{LEVIN, B. C.}

100,156

Data for Fire Hazard Assessment of Selected Non-Halogenated and Halogenated Fire Retardants; Report of Test FR 3983.

PB92-112473

100,557

Large-Scale PB92-116458 Tests.
100,139

Reduction of Hydrogen Cyanide Concentrations and Acute Inhalation Toxicity from Flexible Polyurethane Foam Com. bustion Products by the Addition of Copper Compounds. Part 3. The Effect of Copper Additives on the Flammability Characteristics of Flexible Polyurethane Foam.

PB91-132167

101,140

LEVINE, J.

New Inexpensive Frequency Calibration Service from NIST.
PB91-200790

Rubidium Frequency Standard and a GPS Receiver: A Remotely Steered Clock System with Good Short-Term and Lotely Serm Stability.

Long-Term Stabil
PB91-200808

100,581

Synchronizing Computer Clocks Using a Local Area Network.

100,596

\section{LEVINE, R. S.}

Navy Safety Center Data on the Effects of Fire Protection Systems on Electrical Equipment.

LEW, H. S.

101,376

Performance of 1/3-Scale Model Precast Concrete BeamColumn Connections Subjected to Cyclic Inelastic Loads. Report No. 2

LEWANDOWSKI, W.

100,149

Precise Ephemerides for GPS Time Transfer.

PB91-236950

100,586

ANDOWSKI, W. W.

GPS Time Transfer with Implementation of Selective AvailNo1-25766/7

LEWIS, D. F.

Sensitometry of the Response of a New Radiochromic Film Dosimeter to Gamma Radiation and Electron Beams. PB91-237008

101,326

LEWIS, M. A.

Choosing Flow Conditioners and Their Location for Orifice Flow Measurement.

PB92-116490

101,397

Experimental Evaluation of Selected Orifice Flowmeter Upstream Installations.

LEWIS, R. L.

100,880

General Technique to Correct Probe Position Errors in Planar Near-Field Measurements to Arbitrary Accuracy.
PB91-237073 Personal Computer Codes for Analysis of Planar Near Fields.
PB92-112283

100,730

LI, F.

Neutron Measurements of Intramolecular Vibrational Modes

in C60.

101,055

LI, W. H.

Crystal-Field Splittings in the Cubic Heusler Alloys HoPd2Sn and ErPd2Sn.

101,525 PB91-194902

Oxygen

Deficient RBa2Cu3O6 + $x$.

PB91-194894

101,524

LI, $\mathbf{X}$

Limited International Intercomparison of Responsivity Scales at Fiber Optic Wavelengths.

100,567

LI, Y.

Possible 'Proximity Matrix' Route to High Current ConducPB91-148932

101,476

Ultrasonic Measurement of Sheet Steel Texture and Forma bility: Comparison with Neutron Diffraction and Mechanical Measurements.

\section{LI, Z. Y.}

Antiferromagnetic Order of $\mathrm{Cu}$ in $\mathrm{Sm} 2 \mathrm{CuO} 4$

PB92.117340 101,569

Phonon Density of States and Superconductivity in Nd1.85Ce0.15CuO4.

PB91-189787

101,514

LIANG, J. H.

2-Deoxy-D-Ribose Aqueous Solution as a Gamma-Ray DosB92-117126

101,366

\section{LIAS, S. G.}

Concerning the Formation and the Kinetics of Phenylium PB91-187138

100,336

Reactions of $\mathrm{C} 5 \mathrm{H} 3(+)$ and $\mathrm{C} 5 \mathrm{H} 5(+)$ lons with Acetylene and Diacetylene.

LIBES, D.

Choosing a Name for Your Computer

PB91-158899

100,384

Clock for the Manufacturing Systems Integration Testbed.

UNIX in the Government's Automated Manufacturing Research Facility (AMRF)

PB91-147579

100,963

LICITRA, B. A.

Calibration at $24 \mathrm{C}$ of a Heat-Flow-Meter Apparatus Having $610 \mathrm{~mm}$ Square Plates.

100,933

Comparison of Two Test Methods for Determining Transfe Function Coefficients for a Wall Using a Calibrated Ho

PB91-187278

100,121

LIDE, D. L.

Journal of Physical and Chemical Reference Data, Volume 20, Number 6, November/December 1991.

100,476

LIDE, D. R.

Critical Data in Physics and Chemistry.

PB91-203455

101,697

Journal of Physical and Chemical Reference Data, Volume 19, Number 2, 1990.

PB90-244609

100,257

Journal of Physical and Chemical Reference Data, Volume 19, Number 3, 1990

100,262

Journal of Physical and Chemical Reference Data, Volume 19, Number 4, July-August 1990.

PB91-192435

100,354

Journal of Physical and Chemical Reference Data, Volume

19 , Number 5 , September-October 1990.

100,358

Journal of Physical and Chemical Reference Data, Volume 19. Number 6, November-December 1990.

PB91-192542

100,365

Journal of Physical and Chemical Reference Data, Volume 
LIEBMAN, J. F.

Carbon Acidities of Aromatic Compounds. 2. Ionic Probes of Aromaticity in Annelated Rings.

PB91-195065

100,375

LIEN, S. J.

Fundamental studies of black liquor combustion. Repor No. 4, Phases, 2, 3, and 4: Final report, December 1987-December 1989

DE91018580

LIGGETT, $w$.

New Yorker in Japan

100,915

PB91-158907

101,275

LIGGETT, $W$. S.

HgCdTe Detector Reliability Study for the GOES Program. PB92-112382

Interlaboratory Comparison of Actinides in Human Tissue $(239) \mathrm{Pu}+(240) \mathrm{Pu}$.

101,323

Replicate Measurements in the Interpretation of Environmental Monitoring

100,919

LIGHTBODY, J. W.

Electrofission in the Quasiiree and Delta Regions.

101,609

Possible $\left(e, e^{\prime} 2 N\right)$ Studies at CEBAF

PB91-147587

101,612

LIM, D. S.

Ceramic Wear Maps

PB91-189498

100,561

LIN, R. S.

Mechanisms of Additive Effectiveness.

PB91-158808

101,172

PB91.174862

101,174

LIN, $\mathbf{S}$.

Group and Phase Sound Velocities in an Eu1Ba2Cu3O7 Superconductor and Related Perovskite Oxides. PB91-194860

101,523

LINCE, J. $R$.

Chemical Effects of $\mathrm{Ne}+$ Bombardment on the MoS2(0001) Surface Studied by High-Resolution Photoelectron Spectroscopy

PB91-203471

100,399

LINDAU, I.

Morphology and Barrier-Height Development of $\mathrm{Bi} / \operatorname{InP}(110)$ Interfaces.

101,468

Surface Extended-X-ray-Absorption Fine Structure and Scanning Tunneling Microscopy of $\mathrm{Si}(001) 2 \times 1-\mathrm{Sb} . \quad 101,552$ PB91-237263

LINDLE, D. W.

Angle-Resolved Photoemission from the Ar $2 p$ Subshell.

PB91-194928 101,665

Angular Distribution of Ejected Electrons in Resonant Auger Processes of $A r, \mathrm{Kr}$, and $\mathrm{Xe}$.

101,648

Direct Determination of Molecular Orbital Symmetry of H2S Using Polarized X-ray Emission.

100,335

Molecular-Orbital Studies via Satellite-Free $X$-ray Fluores-

cence: $\mathrm{Cl} \mathrm{K}$ Absorption and K-Valence-Level Emission

$\begin{array}{ll}\text { Spectra of Chlorofluoromethanes. } & \\ \text { PB91-237164 } & 100,427\end{array}$

100,427

Parity-Unfavored Transitions in Resonant Photoemission from Ar, Kr, and $\mathrm{Xe}$ : Experimental and Theoretical Results. PB91-133900 101,599

Photoionization Studies of Atoms and Molecules Using Synchrotron Radiation

101,664

Polarized X-ray Emission Studies of Methyl Chloride and the Chlorofluoromethanes.

100,329

Unusual Degree of Angular Anisotropy in the Resonant Auger Spectrum of $\mathrm{Kr}$.

PB91-187310

101,649

LINDSTROM, D. J.

Low-Background Gamma-Ray Assay Laboratory for Activation Analysis.

100,192

LINDSTROM, R. M.

Characterization of the Mineral Fraction in Botanical Reference Materials and Its Influence on Homogeneity and Analytical Results.

100,186

Low-Background Gamma-Ray Assay Laboratory for Activation Analysis.

100,192

Neutron Activation Analysis in Electronic Technology.

PB91-189779 100,807

Round-Robin Study of Implants in $\mathrm{Si}$ and $\mathrm{SiO} 2$ by SIMS,

RBS, and NAA.
PB92-117241

100,830

LINHOLM, L. W.

Characterizing VLSI Processes Using Test Structures.
PB91-175000

100,804

Design, Fabrication, and Testing of an Interconnect Test Structure for Evaluating VLSI Processes.

PB91.174581

100,803

Evaluation and Improvement of E-Beam Exposure Routines by Use of Microelectronic Test Structures.

100,805

Knowledge Extraction Techniques for Expert System Assisted Wafer Screening.

PB92-116979

100,824

Knowledge Verification of Machine-Learning Procedures $\begin{array}{ll}\text { Based on Test Structure Measurements. } \\ \text { PB91-189571 } & 100,709\end{array}$

Modified Sliding Wire Potentiometer Test Structure for Mapping Nanometer-Level Distances.

PB9 2-116664

100,821

LINN, R. J.

Application of Formal Description Techniques to the Specification of Distributed Test Systems.

100,566

Application of OSI Protocols for Plant Information Networks.

works.
PB91-147595

Features and Facilities of Estelle.

PB91-158915

100,974

LINSKY, J. L.

Extension of the Class of Magnetic B Star Nonthermal Radio Sources.

100,031

Ground-Truth Observations of Stellar Surface Structure from the Lunar Surface.

PB91-175026

100,029

Modelling the Coronae and Chromospheres of RS CVn Systems by the Analysis of Ultraviolet, X-ray and Radio Observations.

100,019

New Radio Detectors of Early-Type Pre-Main Sequence Stars.

PB91-149286

100,024

Radio-Continuum Observations of a Variety of Cool Stars.

PB9.174664 100,027

Some Concluding Thoughts for Cool Stars V!

PB91-175034

100,030

Spatially Resolved Flares in RS CVn Systems

PB91-148965

100,022

Studies of $\mathrm{HI}$ and D I in the Local Interstellar Medium

PB91-148957

100,021

Ultraviolet, Optical, Infrared, and Microwave Observations of HR 5110.

LINTON, R. W.

100,020

Use of Single Particle Standards for LAMMA Calibration PB91-147777

100,183

LIPE, T. E.

AC-DC Difference Relationships for Current Shunt and Thermal Converter Combinations.

100,737

$91-236893$

PIATT, B. C.

Energy Prices and Discount Factors for Life-Cycle Cost Analysis 1992. Annual Supplement to NIST Handbook 135 and NBS Special Publication 709

PB92-112515

100,899

TMAN, W.

Israel's New Synchronized Time Scale, UTC(INPL)

100,577

ITTLE-MARENIN, I. R.

Ultraviolet, Optical, Infrared, and Microwave Observations of HR 5110.

LITTLE, S. J.

Ultraviolet, Optical, Infrared, and Microwave Observations of HA 5110 .

PB91-148833

100,020

LITTLER, C. L.

Intrinsic Carrier Concentrations in Long Wavelength $\mathrm{HgCdTe}$ Based on the New, Nonlinear Temperature Dependence of $\mathrm{Eg}(\mathrm{x}, \mathrm{T})$

PB92-117316 101,568

Narrow-Gap Semiconductors and Related Materials.
PB91-162313

Phonon-Assisted Magneto-Donor Optical Transitions in $\mathrm{n}$ -

$\begin{array}{ll}\text { InSb. } & 101,467\end{array}$

LIU, L. $X$. Device for Audio-Frequency Power Measurement.
PB91-149401

LIU, R.

Group Reactivities in the Gas Phase Reactions of Hydroxyl Radicals with Ethers.

PB91-175059

100,330

LIU, S. H.

Angle-Resolved Photoemission from the Ar $2 p$ Subshell. PB91-194928

101,665

LIU, S. T.

Rule-Based Diagnostic Method for HVAC Fault Detection.

PB91-194936

100,072

Temperature Measurement of Glass Subjected to Solar Radiation.

Testing and Rating of an Atmospheric, Gas-Fired Furnace Equipped with a Burner Air Inlet Damper.

PB92-126440

100,894

LIU, Y.

Spinodal Decomposition in a Hydrogen-Bonded Polymer

PB91-162099

100,489

LIVINGTON, H. D.

Interlaboratory Comparison of Actinides in Human Tissue $(239) \mathrm{Pu}+(240) \mathrm{Pu}$.
PB91-203265

101,323

LLOYD, F. L.

High Speed Superconducting A/D Converter.

PB91-148700

100,731

OCASCIO-BROWN, L.

Immobilization of Binding Proteins on Nonporous Supports

Comparison of Protein Loading, Activity, and Stability
PB91-237198

101,308

Liposome Flow Injection Immunoassay: Implications for Sensitivity, Dynamic Range, and Antibody Regeneration.

PB91-147629

Planar Waveguide Optical Immunosensors.

PB91-174565

101,291

OCKWOOD, F. E.

Heats of Immersion, Friction, and Wear of Base Oil Fractions.

PB91-158923

101,173

LOEVINGER, $R$.

Absorbed Dose to Water: Comparison of Several Methods Using a Liquid lonization Chamber.

101,322

OLOEE, M. R.

Phonon-Assisted Magneto-Donor Optical Transitions in $\mathrm{n}$ InSb.

101,467

LOMBARDI, M. A.

Extending the Range and Accuracy of Phase Noise Measurements.
PB91-134874

NIST Time and Frequency Services.

PB91-231605

100,588

LONG, G. G.

Characterization of the Densification of Alumina by Multiple Small-Angle Neutron Scattering.

PB92-117001

101,100

Effect of Green Density and the Role of Magnesium Oxide Additive on the Densification of Alumina Measured by Small-Angle Neutron Scattering.

PB92-117043

101,101

High-Resolution Small-Angle $X$-ray Scattering Camera for Anomalous Scattering.

PB91-175067

100,929

Reflection-Extended-X-ray-Absorption-Fine-Structure Spectroscopy at the Carbon K-Edge.

100,371

Small Angle Neutron Scattering Characterization of Microporous Silica.
PB91-203489

101,089

Small-Angle-Scattering Determination of the Microstructure of Porous Silica Precursor Bodies. 


\section{PERSONAL AUTHOR INDEX}

LOUIE, B.

Transient Hydrogen Heat Transfer. Final Report for April 1986-April 1989.

PB92-116326

100,454

LOVAS, F. J.

Fluoromethanol: Synthesis, Microwave Spectrum, and Dipole Momen

PB91-148080

100,288

Hydration of Carbon Dioxide: The Structure of $\mathrm{H} 2 \mathrm{O}-\mathrm{H} 2 \mathrm{O}$

$\mathrm{CO} 2$ from Microwave Spectroscopy.

PB91-237172

100,428

Microwave Spectra and Electric Dipole Moments of $\mathrm{X}(4)$ Sigma(1-) $1 / 2$ VO and $\mathrm{NbO}$.

100,429

Microwave Spectrum and Molecular Structure of the N2. H20 Complex

100,398

Microwave Spectrum of Ar-H2O: Dipole Moment, Isotopic Studies, and (17)O Ouadrupole Coupling Constants.
PB91-148650

100,290

Ch3CN

PB91-236661 100,415

Rotational-Tunneling Spectrum of the Ar-SO2 van der Waals Complex

100,457

Structures of the $\mathrm{NH} 3-\mathrm{HCCCCH}$ and $\mathrm{H} 2 \mathrm{O}-\mathrm{HCCCCH}$ Complexes by Fourier-Transform Microwave Spectroscopy. 100,294 Van der Waals Complexes in 1,3-Dipolar Cycloaddition Re-

actions: Ozone-Ethylene.

100,417

\section{LOVEJOY, C. M.}

Mode Specific Internal and Direct Rotational Predissociation in HeHF, HeDF, and HeHCL: van der Waals Complex-

es in the Weak Binding Limit.

PB91-148841

Multiple Intermolecular Bend Vibrational Excitation of a $\mathrm{Hy}$ drogen Bond: An Extended Infrared Study of OCOHF.
PB91-203703 PB91-203703 100,404 Rotational Predissociation, Vibrational Mixing, and van de
Waals Intermolecular Potentials of NeDF. PB91.175505

Structural Dependence of HF Vibrational Red Shifts in ArnHF, $n=1.4$, via High-Resolution Slit Jet Infrared Spectroscopy.

100,401

LOW, S. R.

Static and Dynamic Strength Tests on Electrical Conductor Cables Specified for Airport Landing Structures.

PB92-112226

101,739

LOWE, D.

Reduction of Hydrogen Cyanide Concentrations and Acute Inhalation Toxicity from Flexible Polyurethane Foam Combustion Products by the Addition of Copper Compounds. Part 3. The Effect of Copper Additives on the Flammability Characteristics of Flexible Polyurethane Foam.

PB91-132167

101,140

LOWNEY, J. R.

Band-Gap Narrowing and III-V Heterostructure FETs.

PB92-117159

100,827

Comparison of High-Resistivity Measurements of Silicon by AC Impedance, DC Resistance, van der Pauw, and FourProbe Methads.

100,792

$\mathrm{HgCdTe}$ Detector Reliability Study for the GOES Program.

PB92-112382 100,766

High Spatial Resolution Mapping of Semiconductor Resis-

PBivity. 194829

100,809

Intrinsic Carrier Concentrations in Long Wavelength $\mathrm{HgCdTe}$ Based on the New, Nonlinear Temperature Dependence of $E g(x, T)$

PB92-117316 101,568

Lattice Relaxation in Silicon Doped with $4 d-$ and $5 d-T r a n s i-$

tion Metals.

101.541

Majority and Minority Electron and Hole Mobilities in Heavily Doped GaAs.

101,550

Persistent Photoconductivity in SIMOX Films.

100,826

Testing the Accuracy of Calculated Equilibrium Carrier ConTesting the Accuracy of Calculated Equilibrium Carrier Con
centrations in the Presence of Surface Fields.

101,549

\section{LOWRY, R.}

Fluorescence Monitoring of Polymer Processing: Mixing and Zero Shear Viscosity.

PB91-174482

100,492

LOZIER, D. W.

Closure and Precision in Level-Index Arithmetic. PB91-147652

100,597

LUCATORTO, T. B.

Giant Resonances in the Transition Regions of the Periodic Table.

PB91-147116

101,605
Of What Use Is Basic Research

PB91-175513

101,642

Manufacturing Systems Integration: Initial Architecture Doc-
ument. ument. 12242

100,994

LUCK, $\mathbf{R}$.

Analysis of Optical Flow Estimation Using Epipolar Plane mages.

100,703

Three Dimensional Reconstruction from Optical Flow Using Temporai Integration

PB92-112507

100,702

Neutron Powder Diffraction and Inelastic Scattering Study
of the Structures of Zr2Pd, Zr2PdD1.70, and Zr2PdD 1.96.

of the Structures of $\mathrm{Zr} 2 \mathrm{Pd}, \mathrm{Zr} 2 \mathrm{PdD} 1.70$, and $\mathrm{Zr2PdD} 1.96$.

LUMIA, R.

NASREM Robot Control System and Testbed.

PB91-194969

101,033

Short-Term Evolution for the Flight Telerobotic Servicer PB91-144352

101,731

LUNDY, $D$.

Studies of Magnetic Flux Penetration in a Chemically Syn-

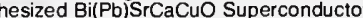

PB91.194977

101,526

LUO, J. C.

PLOTMD: An Interactive Program to Modify Molecular Plots on a Graphics Terminal

UX, G. E.

Round-Robin Study of Implants in $\mathrm{Si}$ and $\mathrm{SiO} 2$ by SIMS,

RBS, and NAA
PB92.117241

100,830

LWIN, T.

Calibration with Working Standards

PB91.158931

101,276

LYNN, J. W.

Antiferromagnetic Order of $\mathrm{Cu}$ in $\mathrm{Sm} 2 \mathrm{CuO} 4$

Poin Structures of $\mathrm{Nd} 2 \mathrm{CuO} 4_{4}$

Crystal and Spin in the Cubic Heusler Alloys HoPd2Sn and

101,525

Long-Pange Antiferromagnetic Order of the $\mathrm{Cu}$ in Oxygen Deficient RBBa2Cu3O6 $+x$

101,524

Low Temperature Magnetization and Magnetic Excitations in Amorphous Fe78B13Sig.

PB92-117464

101,573

Magnetic Ordering of $\mathrm{Er}$ in Powder and Single Crystals of ErBa2Cu3O7

PB91-187377 101,505

Phonon Density of States and Superconductivity in Nd1.85Ce0.15CuO4

PB91-189787

101,514

LYON, G.

Book Review: 'The Art of Computer Systems Performance Analysis' by A. Jain

100,719

Workloads, Observables, Benchmarks and Instrumentation.
100,598

LYONS, J. W.

Process Quality Enhancement for Machine Tools: Measurements and Standards.

PB91.194993

101,019

LYONS, R. M.

Bibliography of the NIST Electromagnetic Fields Division Publications.

PB91-13224

100,832

Bibliography of the NIST Electromagnetic Fields Division Publications.
PB92-116367

100,866

MA, L.

Cryogenic Fatigue of High-Strength Aluminum Alloys and Correlations with Tensile Properties.

PB91-236976

101,709

$M A, 1, M$.

Effect of Processing on 4-K Mechanical Properties of a Microalloyed Austenitic Stainless Steel.

PB91-237206

101,167

MACCREHAN, W. A.

Laser Enhanced Ionization as a Selective Detector for Liquid Chromatographic Determination of Alkyltins in SediLiquid

PB92-116755

100,210

Reverse-Phase HPLC Separation and Electrochemical Detection of Retinol and Its Isomers.

100,187

MACEK, A.

Advanced sensor development program for the pulp and paper industry. Final report.

101,248

MACGOWAN, B. J.

Transitions and Energy Levels for Cu-Like $\mathrm{Yb}(41+)$. a $(44+)$, and $\mathrm{U}(63+)$

PB91-147462

101,610

MACKAY, D. R.

Standardizing Standards.

100,953

MACREYNOLDS, $K$.

Evaluation of Dual-Port Circularly Polarized Probes for Planar Near-Field Measurements.

PB91-162040

100,590

\section{MADEY, T. E.}

Adsorption of Water and Oxygen on $\mathrm{Ag}(110)$ : A Study of the Interactions among Water Molecules, Hydroxyl Groups, and Oxygen Atoms. Influence of Sulfur on Methanation over Tungsten(110). PB91-149369 100,300 Initial Stages of Degradation of Superconductor Surfaces: O2, $\mathrm{H} 2 \mathrm{O}, \mathrm{CO} 2$ and $\mathrm{CO}$ Chemisorption on La2-xSrxCuO 4. PB91.189670 101,512 Summary Abstract: The Influence of Sulfur on Methanation over W(110).
PB91-149377

100,301

MADOU, M.

Opportunities for Innovation: Chemical and Biological Sen-

PBrs.

100,214

MADRZYKOWSKI, D.

Reduction in Fire Hazard in Corridors and Areas Adjoining Corridors Provided by Sprinklers.

100,162

MAEGLEY, $K$.

Chitin-Chitosan Membranes: Separations of Amino Acids and Polypeptides.

101,283

MAELAND, A. J.

Neutron Powder Diffraction and Inelastic Scattering Study of the Structures of Zr2Pd, Zr2PdD1.70, and Zr2PdD1.96 PB91-148866

101,213

Relationship of Hydrogen Site Occupancy to Diffusion Behavior in Crystalline and Amorphous $\mathrm{Zr} 2 \mathrm{PdHx}$.

PB91-187252

101,230

MAGEE, C. W.

Round-Robin Study of Implants in $\mathrm{Si}$ and $\mathrm{SiO} 2$ by SIMS, RBS, and NAA

PB92-117241

100,830

Rapid Low-Temperature Hopping of Hydrogen in a Pure Metal: The ScHx System.

Metal: The ScHx

101.217

MAGLEBY, S. P.

Conceptual Architecture for a Mechanical Parts Production System Based on STEP. National PDES Testbed Rept PB92-112234

100,993

MAJER, V.

Heat Capacities of Organic Compounds in Liquid State. 2 C1 to C18 n-Alkanes.

Heat Capacities of Organic Compounds in the Liquid State I. C1 to C18 1-Alkanols.

100,265

MAJKRZAK, C. F.

Fe-W Supermirrors for Polarizing Neutrons.

PB91-175521

101,643

MAJOR, J. R.

Dual Six-Port Reflectometer Systems Using Waveguide in 
MAKINEN, J. Test of Newton's Inverse Square Law of Gravity Using the
$300 \mathrm{~m}$ Tower at Erie, Colorado: Newton Vindicated on the Plains of Colorado.

PB91-175273

101,637

Test of the Inverse-Square Law of Gravitation Using the 300-m Tower at Erie, Colorado.

PB91-204115

MALDONADO, L.

Sputtered Thin Film YBa2Cu3On.

PB91-189647

101,702

MALGHAN, S. G.

Surface Oxidation Kinetics of $\mathrm{Si3N} 4 \cdot 4 \%$ Y2O3 Powders Studied by Bremsstrahlung-Excited Auger Spectroscopy. PB91-237701

100,435

MALLARD, W. G.

Single Pulse Shock Tube Study of the Reactions of Hydrogen Atoms with Aromatics. 4. Chlorotoluenes.

PB91-175133

100,331

MALONDA, A. G.

Calculation of Beta-Particle Counting Efficiency for Liquid Scintillation Systems with Three Phototubes.

PB91-175547

101,644

MALONE, K. J.

Integrated-Optic Waveguide Glass Lasers.

PB91-203976

100,758

MANDAK, J.

Slovak Metrological Society.

PB92-126721

100,938

Non-Linear Contour-to-Grid Digital Interpolation.

PB91-147678

101,351

MANDEL, J.

Nature of Collinearity.

PB91-203497

101,261

MANGANARO, $\mathrm{W} . \mathrm{M}$.

Cryogenic Properties of Copper (Wall Chart). B $91-148189$

MANGUM, B. W.

SRM 1970 - Succinonitrile Triple-Point Standard - And Its Use in Calibration of Thermistors.

PB91-134338

Stability of Thermistors.

PB91-134320

100,779

MANHEIMER, K. L.

Depot: A Framework for Sharing Software Installation Across Organizational and Unix Platform Boundaries. PB91-240754

100,659

MANN, W. B.

Preparation and Properties of Monomolecular Films for Use as Radioactive Source Mounts.

PB91-147249

101,608

MANNEY, C. $\mathrm{H}$.

Progress at NIST on Absolute Frequency Standards Using Stored lons.

MANNING, J. $R$

Atomistic Diffusion Equations and Kinetic Forces. PB91-195008

01,625

Rapid Solidification Alloys: Crystals, Ouasicrystals, and Metallic Glass.

MANSON, S. T.

101,220

Angle-Resolved Photoemission from the Ar 2p Subshell.
PB91-194928

MAOOI, $\mathrm{H}$.

Low-Pressure Symmetric Discharge Heat Pipe and DROGS

of Eu.
PB91-195016

101,666

MARANS, R. W.

Second-Level Post-Occupancy Evaluation Analysis.

PB91-147140

100,058

MARCHIANDO, J. F.

Ellipsometry SRM's for Use in Thin Film Measurements.

PB92-116599 100,819

Sensitivity of Ellipsometric Modeling to the 'Islands' of Silicon Precipitates at the Bottom of the Buried Oxide Layer in Annealed SIMOX.

PB92-116607

100,820

MARGRAVE, J. L.

Levitation Calorimetry.

PB91-158519

100,927

MARINENKO, G.

Research at NBS in Direct Potentiometric Measurements in Blood.

PB91-148874

100,188

MARINENKO, R. B.

Characteristics of Aerosols Produced by the Spark Discharge.

PB91-237727

100,209

Strategies for Background Subtraction in Electron Probe Microanalysis X-ray Compositional Mapping.
PB91-195180

100,202

MARINER-VOLPE, $B$.

Gas Analysis Modeling System.

PB91-175299

100,884

MARJENHOFF, W. A.

Development of an Adhesive System for Bonding to Hard Tooth Tissues.

100,052

Glass-Ceramic Inserts Anticipated for 'Megafilled' Composite Restorations.

MARKEN, K. R.

AC Loss Measurements of Two Multifilamentary NbTi Com. posite Strands.

PB91-133892

MARKOVITZ, P. Electronic Data Interchange in Message Handling Systems.

Guidelines for the Evaluation of File Transfer, Access and
Management Implementations. PB92-126580

100,699

MARKS, $R$

Characteristic Impedance Determination Using Propagation Constant Measurement.

100,775

Microwave Monolithic Integrated Circuit-Related Metrology at the National Institute of Standards and Technology. at the National Institute of Standards and Technology. 100,816
PB91-237255 MMIC Related Metrology at the National Institute of Standards and Technology.

PB91-149070

100,794

Translate LRL and LRM Calibrations.

PB91-237776

100,862

MARLER, F. E.

Dual Six-Port Reflectometer Systems Using Waveguide in the Frequency Range $18-50 \mathrm{GHz}$.

PB91-134916

100,838

MARSH, K. N.

Thermodynamic and Thermophysical Properties of Organic Nitrogen Compounds. Part 1. Methanamine, Ethanamine, 1. and 2-Propanamine, Benzenamine, 2-, 3-, and 4-Methylben-

zenamine.
PB91-192583

100,368

MARSHALL, M. D.

Microwave Spectrum and Molecular Structure of the N2H2O Complex.
PB91-203448

MARSHALL, R. D.

100,398

Measurement of Structural Response Characteristics of Full-Scale Buildings: Selection of Structures.

PB91-167239

MARTE, M. A. M.

Explicit Mapping of the Set of Dynamical Symmetries onto the Set of Equivalent Lagrangians for Systems with One Degree of Freedom.

PB91-158881

MARTIN, J. W.

Characterization of Cylindrical Holes in Metallic Substrates via Their Infrared Emission Patterns.

PB91-187187

101,228

Detection and Ouantitative Characterization of Blistering and Corrosion of Coatings on Steel Using Infrared Thermography.

101,139

Long Term Performance of Rubber in Seismic and NonSeismic Bearings: A Literature Review.

100,148 PB91-206714

Roughness Measures of Blasted Steel Surfaces Remotely
Imaged with a Thermographic Camera.
PB91.147686
Par,

PB91-147686

100,589

Accuracy Model for Phase Noise Measurements. PB91-134882

100,589

MARTIN, W. C.

Atomic Spectroscopy in the 20th Century - A Tribute to Sitterly, Charlotte Moore, on the Occasion of Her 90th Birth. PB92-116714

101,724

Energy Levels of Sulfur, S I Through S XVI.

100,355

Relativistic and Correlation Effects in the 2s3p Configuration of Beryllium-Like lons.

101,654

Wavelengths and Energy Level Classifications for the Spectra of Aluminum (A)I through AI XIII).

Tra of Aluminum

100,472

Wavelengths and Energy Level Classifications of Magnesium Spectra for All Stages of lonization (Mg I through Mg XII).

PB92-110139

100,442

Working Group 1: Atomic Spectra and Wavelength Standards.

PB91-158956

100,026

MARTINEZ, R. I.

CID Spectra of Selected Target Molecules.

AD-A243 170/8

100,180

Instrument-Independent Tandem Mass Spectrometry Database for XOO Instruments: The Dynamical Prerequisites. base for $X O O$

MARTINIS, J. M.

Noise-Affected I-V Curves in Small Hysteretic Josephson Junctions.

MARTINSON, I.

Beam-Foil Study of Two-Electron Transitions in Cu-Like lons.

PB91-237511

101,714

MARTON, $D$.

Ion Bombardment Induced Diffusion: A Case Study on a Sputtered Ag/Ni Layered System.

101,527

Ion-Induced Radiation-Enhanced Diffusion of Silver in Nickel.

PB91-175554

101,498

MARTZLOFF, F. D.

Annotated Bibliography: Diagnostic Methods and Measurement Approaches to Detect Incipient Defects Due to Aging

PB91-222687

101,369

Incompatibility between the 100/1300 Surge Test and Varistor Failure Rates.

Need for Power Ouality Testing Standards.

PB92-117076

100,854

Partial Discharges in Low Voltage Cables. PB91-134650

100,741

Real Realistic

PB91-189811

100,771

MARUTHAMUTHU, $P$.

Rate Constants for Reduction of Substituted Methylperoxy Radicals by Ascorbate lons and N,N,N', N'-TetramethylPara-Phenylenediamine.

PB91-195230

100,379

MARX, E.

Application of Transmission Electron Detection to X-ray Mask Calibrations and Inspection.

PB92-117217

100,828

Calibration of the NBS 10 mum Space Beads. PB91-203422

101,695

Computed Fields Near the Edge of a Dielectric Wedge.
PB91-203505 Determination of Fields Near a Silver Strip on a Glass Substrate.

PB91-147702

100,841

Relativistic Probability Amplitudes and State Preparation PB91-195032 101,667

Scattering by an Arbitrary Cylinder at a Plane Interface. PB91-203513

101,447

\section{MASON, A. R.}

Analysis of the $\mathrm{YBa} 2 \mathrm{Cu} 3 \mathrm{O} 7 / \mathrm{SrTiO} 3$ Interface as a Function of Post-Deposition Annealing Temperature.

101,480

Single-Target Magnetron Sputter-Deposition of High-Tc Superconducting Bi-Sr-Ca-Cu-O Thin Films.

PB92-116680

101,566

MASON, E. A.

Equilibrium and Transport Properties of Gas Mixtures at 
MATLOCK, D. K.

Metallographic Study of the Crack-Tip Region from Fracture Mechanics Specimens of Austenitic and Ferritic Steels. PB91-189951 101,158

MATSUMURA, $\mathrm{K}$.

Microwave Spectrum of Ar-H2O: Dipole Moment, Isotopic Studies, and (17)O Ouadrupole Coupling Constants PB91-148650 100,290 Rotational-Tunneling Spectrum of the Ar-SO2 van der Waals Complex.

100,457

Structures of the NH3-HCCCCH and $\mathrm{H} 2 \mathrm{O}-\mathrm{HCCCCH}$ Complexes by Fourier-Transform Microwave Spectroscopy.
PB91-148882 294

MATSUSHITA, Y.

Chain Conformation of a Block Polymer in a MicrophaseSeparated Structure.

PB91-148890

100,485

MATTHEW, J. A. D.

Core Hole Screening for Intermediate Size Metal Particles.
PB91-203539

MATTHIAS, C. L.

Simultaneous Butyltin Determinations in the Microlayer, Water Column and Sediment of a Northern Chesapeake Bay Marina and Receiving System.

100,918

MATTIESEN, D.

High Resolution Diffraction Imaging of Crystals Grown in Microgravity and Closely Related Terrestrial Crystals. PB92-109008

101,562

MATTINGLY, G. E.

Flowmeter Installation Effects: A New Approach to an Old but Prevalent Problem.

100,920

Gas Flow Measurement: Calibration Facilities and Fluid Metering Traceability at the National Bureau of Standards. 100,879 Particle Size Measurement of Inert Gas Atomized Powder. PB91-187203

101,229

MATTSSON, $O$.

Absorbed Dose to Water: Comparison of Several Methods Using a Liquid lonization Chamber.

101,322

\section{MAUTNER, M.}

Alkyl Transfer Reactions between Protonated Alcohols and Ethers. The Gas-Phase Alkylation of Formaldehyde. Carbon Acidities of Aromatic Compounds. 2. Ionic Probes of Aromaticity in Annelated Rings.

100,375

PB91-195065 100,375 mplications, and Role in Ion Solvation.

100,400

Multicomponent Cluster Ions. 2. Comparative Stabilities of

Multicomponent Cluster lons. 2. Comparative Stabilities of Cationic and Anionic Hydrogen Bonded Networks: Mixed
Clusters of Water and Hydrogen Cyanide.
PB91-147710

100,283

Proton Affinities and $\mathrm{pKa}$ Values of Tetraalkylhydrazines.

PB91-195214 100,378

Proton Affinity of Cyanogen and Ion-Molecule Reactions of $\mathrm{C} 2 \mathrm{~N} 2(+)$
$\mathrm{PB} 91-189902$

100,352

Slow Electron Transfer Reactions between Alkylhydrazines.
PB91-147785

Thermal Decomposition of Ions. 3. Protonated Ethanol and

Diethyl Ether.
PB91-195073 100,376

MAYER, I.

Ion Incorporation into Octacalcium Phosphate Hydrolyzates. MAYER, $R$.

Direct Determination of Molecular Orbital Symmetry of H2S Using Polarized X-ray Emission.

PB91-175562

100,335

MAYO, S.

$\mathrm{HgCdTe}$ Detector Reliability Study for the GOES Program.

Lattice Relaxation in Silicon Doped with $4 d$ - and $5 d-T r a n s i-$ tion Metals.

PB91-203554

101,541

Persistent Photoconductivity in SIMOX Films. PB92-117084

100,826

MAZER, J. A.

Characterizing VLSI Processes Using Test Structures.
PB91-175000 MCALISTER, A.

Microstructural Studies of Ti-Al Alloys in the Vicinity of the 'Eutectoid' Reaction.

PB92-116938

101,241

Transformation of the Icosahedral Phase in Rapidly Quenched Al-rich Al-Mn Alloys.

PB91-158964

101,221

MCAULIFFE, P. S.

Reusable Cryogenlc Foam Insulation for Advanced Aerospace Vehicles.
PB91-175570

MCCARRON, E. M.

Refinement of the Structure of Beta'-MoO3

MCCARTY, R. D.

New Wide Range Equation of State for Helium.

PB91-162222

Speed of Sound of Natural Gas Mixtures

PB91-162214

MCCLAIN, M.

Algorithm and Computer Program for the Calculation of Envelope Curves

MCCLUNEY, $R$.

Abstracts of Daylighting Research

PB91-206755

CCOLSKEY, J. D.

Aluminum Alloys for ALS Cryogenic Tanks: Comparative Measurements of Cryogenic Mechanical Properties of Al-Li Alloys and Alloy 2219.

AD-A242 956/1

101,204

Aluminum Alloys for ALS Cryogenic Tanks: Oxygen Compatibility. Volume

101,203

Aluminum Alloys for Cryogenic Tanks: Oxygen Compatibil-

AD-A229 229/0

101,201

Mechanical Properties of Alumina-Peek Unidirectional Com

posite: Compression, Shear, and Tension.

(162180

101,118

MCCORMICK, G.P.

Second Order Sensitivity Analysis in Factorable Programming: Theory and Applications.

CCOUBREY, A. O.

101,266

Guide for the Use of the International System of Units: The Modernized Metric System.

PB92-116383

100,962

MCCOWAN, C. N.

Aluminum Alloys for ALS Cryogenic Tanks: Oxygen Com patibility. Volume 2.

101,203

Cryogenic Material Properties of Stainless Steel Tube-toFlange Welds

101,145

Fracture Toughness of $316 \mathrm{~L}$ Stainless Steel Welds with Varying Inclusion Contents at $4 \mathrm{~K}$.

101,148

Image Ouality Indicator Design for Radioscopy and Tomog-

raphy.

101,018

MCDERMOTT, K. M.

Fusion of Mercury. A New Certified Standard for Differential Scanning Calorimetry

100,309

MCDONOUGH, W.

Assessment of the State-of-the-Art for Process Monitoring Sensors for Polymer Composites.

PB91-222612

100,510

MCEACHRAN, R.P.

Optical Potential Approach to Electron and Positron Scat tering from Noble Gases. 2. Neon.

101,619

MCEWAN, M. J.

Proton Affinity of Cyanogen and Ion-Molecule Reactions of $\mathrm{C} 2 \mathrm{~N} 2(+)$.

\section{MCFADDEN, G. B.}

Convective and Morphological Instabilities during Directional Solidification

101,219

Effect of an Electric Field on the Morphological Stability of the Crystal-Melt Interface of a Binary Alloy. 3. Weakly Nonlinear Theory.

101,536

Effect of Gravity Modulation on Thermosolutal Convection in an Infinite Layer of Fluid.

PB92-112291

101,396

Phase-Field Model for Isothermal Phase Transitions in Binary Alloys.

MCFADDIN, S. E.

101,240

Effect of Pipe Surface Finish on Gas Flow Measurement with an Orifice Meter.

PB91-148031

101,387

Effect of Pipe Surface Finish on the Orifice Discharge Coefficient

101,389

PB91-162347

on Orifice

Flowmeter Performance.

PB91-134445

100,921

MCFEELY, F. R.

Electron Attenuation Lengths at SiO2/Si Interfaces. PB91-175612

101,499

MCGARRY, E. D.

Cross-Section Measurements in the Intermediate-Energy

PB91-203398

101,693

MCGUIRE, T. J.

Residual Stress Detection in Railroad Wheels: An Uitrasonic System Using EMATs (Electromagnetic-Acoustic Transducers). Report No. 23.

PB91-222653

101,741

MCHENRY, H. I.

Materials Reliability. Technical Activities, 1990. (NAS-NRC Assessment Panel, January 31-February 1, 1991). PB91-143339

101,008

Materials Reliability. Technical Activities, 1991. (NAS-NRC Assessment Panel, February 13-14, 1992)

101,252

B92-126424

MCHUGH, M. P.

Gyroscope-Weighing Experiment Revisited -- with a Null

PB91-174722

101,630

Search for a Composition Dependent Force Using a LiquidSupported Torsion Balance.

101,639

Test of Newton's Inverse Square Law of Gravity Using the $300 \mathrm{~m}$ Tower at Erie, Colorado: Newton Vindicated on the Plains of Colorado.

PB91-175273

101,637

Test of the Inverse-Square Law of Gravitation Using the 300-m Tower at Erie, Colorado.

PB91-204115

101,702

MCILROY, A.

Structural Dependence of HF Vibrational Red Shifts in ArnHF, $n=1-4$, via High-Resolution Slit Jet Infrared Specroscopy.

PB91-203562

100,401

MCINROY, J. F.

Interlaboratory Comparison of Actinides in Human Tissue (239)Pu $+(240) \mathrm{Pu}$.

MCKENNA, G. B.

Effect of Crosslink Density on Physical Aging of Epoxy Networks.

101,244

Mechanical and Swelling Behavior of Crosslinked Natural Rubber: Consequences of the Flory-Rehner Hypothesis.

PB91-195081 101,141

Physical Aging and the Viscoelastic Response of Network PBlasses.

101,246

Relaxation of Crosslinked Networks: Theoretical Models

and Apparent Power Law Behavior
PB91-195099

100,501

Study of the Linear Viscoelastic Properties of Cyclic Polystyrenes Using Creep and Recovery Measurements. 100,502

Tests of the Flory-Rehner Hypothesis: Comparison of the Elastic Free Energy Function for Crosslinked Rubber in the Dry and Swollen States.

100,508

Viscoelastic Response of Epoxy Glasses Subjected to Different Thermal Treatments.

PB91-189720

101,245

MCKEOWN, D. A.

XANES of Transition Metal Zinc-Blende Semiconductors. PB92-117092

100,462

MCKINLAY, A

Proceedings of the Forum on Standards for High Integrity Software (DOD, Government, Industry). Held in Gaithersburg. Maryland on June 28, 1991

100,668

MCKNIGHT, M. E.

Detection and Ouantitative Characterization of Blistering and Corrosion of Coatings on Steel Using Infrared Thermography. 
Mechanisms of the Reduction Reactions of $\mathrm{Cr}(\mathrm{VI})$ in the Radiolysis of Acidic Potassium and Silver Dichromate Solutions in the Presence or Absence of Acetic Acid.
PB91-236489
100,241 Metal-Polysiloxane Shields for Radiation Therapy of Maxillo-Facial Tumors.

PB91-194761

100,054

Novel Radiation Dosimetry Systems.

PB92-11711

101,727

Photon Energy Dependence of the Sensitivity of Radiochromic Film and Comparison with Silver Halide Film and LiF TLDs Used for Brachytherapy Dosimetry.

101,296

Radiochromic Film Dosimeter for Gamma Radiation in the Radiochromic Film Dosimeter for Gamma Radiation in the
Absorbed-Dose Range $0.1-10 \mathrm{kGY}$.
PB92-116961

101,364

Sensitometry of the Response of a New Radiochromic Film Dosimeter to Gamma Radiation and Electron Beams.

PB91-237008 101,326

Temperature Dependence of Radiochromic Film Dosi-

101,728

MCLAY, M.

Validation Testing Laboratory User's Guide. National PDES Testbed Report Series.

PB92-123090

100,971

MCLEAN, C. $R$.

Research Tackles Automation Issues.

PB91-162230

101,005

CLINDEN, M. 0.

Horizontal Flow Boiling Heat Transfer Experiments with a Mixture of R22/R114

101,200

Study of Flow Boiling Heat Transfer with Refrigerant Mix-

101,196

Survey of Current Worldwide Research on the Thermophysical Properties of Alternative Refrigerants. - 101,198

101,198

Thermodynamic Diagrams for Refrigerant Mixtures.
PB91-236695

Use of Computer Algebra to Locate Critical Loci in Fluid Mixtures.

PB91-195263

101,390

MCNIFF, E. J.

Magnetic Hysteresis of High-Temperature YBa2Cu3Ox-AgO Superconductors: Explanation of Magnetic Suspension.

PB91-189506

MCQUARRIE, D. A.

Mobility Fluctuations and Electrophoretic Light Scattering from Macromolecular Solutions.

PB91-18951

MEADE, W.P.

First Pass at Computing the Cost of Fire Safety in a Modern Society

MEDHURST, L. J.

Angle-Resolved Photoemission from the Ar $2 p$ Subshell. PB91-194928

MEIER, U.

Photodissociation Dynamics of Water in the Second Absorption Band: Vibrational Exitation of $\mathrm{OH}$ (A (2)Sigma).

PB91-148726

MEIGOONI, A. S.

Photon Energy Dependence of the Sensitivity of Radiochromic Film and Comparison with Silver Halide Film and LiF TLDs Used for Brachytherapy Dosimetry.

PB92-117142

MEIJER, P. H. E.

Permittivity Measurements on Molecular-Sized Samples.

MEISEL, D.

Mechanism of Cyanide Release in the Radiolysis of Acetonitrile.

PB91-203679

100,240

MELAMUD, $M$.

Wigner-Seitz Local-Environment Study of the High Tc Superconductors

101,528

MELE, A.

Laser Photolysis of Trimethylgallium at $193 \mathrm{~nm}$ : Quantum Yields for Methyl Radical and Ethane Production.

AD-A227 868/7

100,234

Photomultiplier Housing for Vacuum Operation of Side-on 1P28-Type Tubes.

100,744

Scattered Light and Other Corrections in Absorption Coefficient Measurements in the Vacuum Ultraviolet: A Systems Approach.

AD-A227 869/5

100,179

MELMED, A. J.

Atomic Fingerprint of YBa2Cu3O7-x-Type High-Temperature Superconductors Observed by Field lon Microscopy.
PB91-203612

101,544 Interpretation of Field Ion Microscopy (FIM) Images of
Asymmetrical Specimens of 1,2,3-Type High-Tc Superconductors.
PB92-116730

101,567

Method of FIM-FEEM Specimen Preparation of Superconducting and Other Oxides.

101,542

Progress in Field Ion Microscopy Imaging of High-Tc Superconducting Oxides.

PB91-203604

101,543

CHANT, J. L.

Feasibility of Using Knowledge-Based Systems for Aiding

Inventors.

MERDES, D. $W$.

Perpendicular Bands of Cyclopropane in the $3.5 \mathrm{mum}$

PB91-237016

100,424

MESHKOV, $\mathbf{S}$.

Glueballs and Hadron Spectroscopy.

PB91-195123

METCALF, H. J.

101,668

Observation of Atoms Laser Cooled Below the Doppler Limit.

B91-195453

101,671

MEYER, C. $W$.

Dipole Moments of Seven Partially Halogenated Ethane Refrigerants.

100,377

MEYER, J. L.

Effect of Pyrophosphate Concentrations on Calcium Phosphate Growth on Well-Crystallized Octacalcium Phosphate and Hydroxyapatite Seed Crystals.

PB91-162024

101,193

MICHALOSKI, J.

TROI User's Guide

ICHEL, F. C.

Kinetics of Copper Extraction Using (anti)-2-Hydroxy-5-Nonybenzophenone Oxime

PB91-148783

100,292

MIGDALL, A. L.

Linearity of a Silicon Photodiode at $30 \mathrm{MHz}$ and Its Effect on Heterodyne Measurements.

PB91-187625

100,755

Use of Heterodyne Detection to Measure Optical Transmittance over a Wide Range.

PB91-148908

101,410

MIGHELL, A. D.

NIST*LATTICE: A Program to Analyze Lattice Relationships. Spring 1991 Version.

PB92-108877

101,561

MIGNOGNA, R. B.

Ultrasonic Texture and Stress Measurements in Anisotropic Polycrystalline Aggregates.

101,237

Use of Neutron Pole Figures to Calibrate Ultrasonic Techniques for On-Line Texture Control of Aluminum Plates. PB91-159111

101,222

MIILLER, A. P.

Dynamic Technique for Measuring Surface Tension at High Temperatures in Microgravity Environment.

N91-21347/0

101,206

Theory of Specific Heat of Solids.

MILES, D. S.

101,475

High Spatial Resolution Mapping of Semiconductor Resistivity

100,809

MILES, K. A.

American National Standard for C. Category: Software Standard. Subcategory: Programming Language.
FIPS PUB 160

od Crib

HLKE, J.

Water Spray Suppression of Fully-Developed Wood Crib
Fires in a Compartment. PB91-203620

100,159

MILKE, J. A.

Development of a Technique to Assess the Adequacy of the Municipal Water Supply for a Residential Sprinkler

PB92-123116

101,358

MILLAT, J.

Thermal Conductivity of Methane and Tetrafluoromethane in the Limit of Zero Density.

PB91-192500

100,361

Transport Prop

100,266

Viscosity and Thermal Conductivity of Pure Monatomic Gases from Their Normal Boiling Point Up to $5000 \mathrm{~K}$ in the Limit of Zero Density and at $0.101325 \mathrm{MPa}$.

100,366

MILLER, A.

Sensitometry of the Response of a New Radiochromic Film Dosimeter to Gamma Radiation and Electron Beams.

PB91-237008

MILLER, J. H.

Partial Equilibrium in Laminar Hydrocarbon Diffusion Flames.

PB91-237024

100,553

MILLER, K. J.

Hydrogen Bonding in Pt Ammine Complexes.

PB91-189639

100,348

MILLER, P. J.

Effects of Pressure on the Thermal Decomposition Kinetics

and Chemical Reactivity of Nitromethane.
PB91-147884

100,286

Multireference-Configuration Interaction (MR-Cl) Calcula tions on $\mathrm{HS}(2+)$ and Experimental Observation via Electron Impact lonization of $\mathrm{H} 2 \mathrm{~S}$.

PB91-148924

100,295

MILLER, R. E.

Structure of Carbon Dioxide Dimer from Near Infrared SBectroscopy.

100,279

MILLER, S. L.

Thermodynamics of the Krebs Cycle and Related Com-

PBounds.

100,357

MINGUEZ, E.

Muon Sticking in Catalysed d-t Fusion: Nuclear Resonance

PB91-147959

101,613

MINK, A.

Hardware Instrumentation Approach for Performance Measurement of a Shared-Memory Multiprocessor

PB91-159319

100,601

MINTZ, M. H.

Direct Detection of Atom Vaporization by Laser Resonance Ionization as a Probe of Gas-Surface Chemisorption Mechanisms.

PB91-237032

100,425

MISAKIAN, M.

Evaluation of Instrumentation Used to Measure AC Power

System Magnetic Fields.
PB91-162248

100,872

Measurements of Power Frequency Magnetic Fields Away from Power Lines.

PB91-175398

100,851

MISRA, D. N.

Adsorption and Orientation of Tetracycline on Hydroxyapatite.

MITCHELL, G. D.

Accurate and Precise Coulometric Determination of Sulfur Dioxide in Compressed Gas Mixtures.

100,912

Development of a Coulometric Method for Assessing the Concentration of Ambient Levels of CO2/Air in Compressed Gas Mixtures.

PB92-12664

100,913

TCHELL, $M$.

Development Plan: Application Protocols for Mechanical

Parts Production. National PDES Testbed Report Series.
PB91-222596 PB91-222596

ITCHELL, M. J.

Proposed Testing Methodology for STEP Application Protocol Validation. National PDES Testbed Report Serie

MITCHELL, R. A.

Eccentric Load Sensitivity of Force Sensors

PB91-175406

100,931

MITLER, H. E.

Algorithm for the Mass-Loss Rate of a Burning Wall.

PB91-158998 
MIYANO, K. E.

Morphology and Barrier-Height Development of $\mathrm{Bi} / \operatorname{InP}(110)$ Intertaces.

101,468

Surface Extended-X-ray-Absorption Fine Structure and Scanning Tunneling Microscopy of Si(001)2x1-Sb. 101,55

101,552

MOGI, Y.

Chain Conformation of a Block Polymer in a MicrophaseSeparated Structure.

PB91-148890

100,485

MOINI, $M$.

Reactions of $\mathrm{C}_{5} \mathrm{H} 3(+)$ and $\mathrm{C}_{5} \mathrm{H}_{5}(+)$ lons with Acetylene and Diacetylene.

MOLDOVER, M. $\mathbf{R}$

Accurate Acoustic Measurements in Gases Under Difficult Conditions.

PB92-116847

101,382

Alternative Refrigerants R123a, R134, R141b, R142b, and R152a: Critical Temperature, Refractive Index, Surface Tension, and Estimates of Liquid, Vapor, and Critical Densities.

101,189

Apparatus for Acoustic Measurements of Gases with Applications for High Temperatures.

100,458

Critical Exponent for the Viscosity of Four Binary Liquids.

Liquids.

Critical Exponent for Viscosity.

PB91-147009

MOLINE, J.

Book Review: Hypertext Hands-On
PB91-237040

100,656

Interactive Courseware is Leading the Multimedia Movement.

100,036

Linking Information to Objects: A Hypertext Prototype for Numismatists

100,657

Multimedia Courseware in an Open Systems Environment: A Federal Strategy.

100,039

MOLINO, B. B.

Critical Data in Physics and Chemistry.

PB91-203455

101,697

MOLLER, G.

Beam-Foil Study of Two-Electron Transitions in Cu-Like lons. 237511

101,714

MOLLER, T.

Low-Cost Low-Volume Carrier (Minilab) for Biotechnology and Fluids Experiments in Low Gravity.

PB91-150029

100,041

MONCARZ, H. T.

Apparel STEP Translator

PBPOE,

100,967

Observation of the Cesium Clock Transition Using LaserCooled Atoms in a Vapor Cell.

PB91-203638

101,698

MONTGOMERY, R. L.

Levitation Calorimetry.

PB91-158519

100,927

MOODY, J. R.

High Purity Chemicals and Clean Rooms for Trace Metal Analysis.
PB91-195156

100,200

High Purity Fluoropolymer Materials: Trace Element Content and Leaching.

PB91-162255

100,491

Recommended Inorganic Chemicals for Calibration. PB91-195164

100,201

MOOJANI, $K$.

Superconducting Thin Films of Bi-Sr-Ca-Cu-O Obtained by Laser Ablation Processing.

PB91-189589

101,508

MOOK, H. A.

Long-Range Antiferromagnetic Order of the $\mathrm{Cu}$ in Oxygen Deficient $\mathrm{RBa2Cu} 3 \mathrm{O} 6+\mathrm{x}$.

101,524

MOORE, J. L

Round-Robin Study of Implants in $\mathrm{Si}$ and $\mathrm{SiO} 2$ by SIMS, RBS, and NAA.

100,830

MoOS, H. W.

Studies of $\mathrm{H} \mathrm{I}$ and D I in the Local Interstellar Medium.
PB91-148957

MOPSIK, $\mathbf{F}$.

Assessment of the State-of-the-Art for Process Monitoring Sensors for Polymer Composites.

PB91-222612

100,510

MOPSIK, F. I.

Measurement of Space Charge Fields in Polymers.
PB91-158626

100,486

ORAN, K. L.

Structures and Properties of New Zeolite X-Type Zincophosphate and Beryllophosphate Molecular Sieves.
101,081 MORDFIN, L.

Mechanical Testing Revitalized.

PB91-159004

100,928

MOREHART, J. H.

Species Produced in Fires Burning in Two-Layered and Homogeneous Vitiated Environments.

PB91-157180

\section{MORELAND, $J$}

Break Junction Tunneling Spectroscopy of Single-Crysta Bismuth-Based High-Temperature Superconductors.
PB91-134478 $\begin{array}{lr}\text { PB91-134478 } & 101,462 \\ \text { High-Resolution, Tunneling-Stabilized Magnetic Imaging and }\end{array}$

PB91-134486

101,600

Morphology of Silver on YBa2Cu3O7-delta Thin Films.

PB91-195594 101,534

New Look at the Bitter Method of Magnetic Imaging

PB91-203919

101,701

Possible 'Proximity Matrix' Route to High Current Conduc-

tors. 14932

101,476

Prospects for High Temperature Superconductor - Semiconductor Contacts.

PB91-203646

101,545

Switching in High-Tc Superconductor Current Transport Measurements.
PB91-203117

101,539

Tunneling Stabilized Magnetic Force Microscopy: Prospects for Low Temperature Applications to Superconductors.

PB91-203653

101,546

MORGAN, R. S.

Collection of Technical Studies Completed for the Computer-Aided Acquisition and Logistic Support (CALS) Program Fiscal Year 1988. Volume 1 of 3. Text, Security and Data Management.

101,342

Collection of Technical Studies Completed for the Computer-Aided Acquisition and Logistic Support (CALS) Program, Fiscal Year 1988. Volume 2 of 3. Graphics, CGM MIL-

PB91-178897

101,343

Collection of Technical Studies Completed for the Comput-

er-Aided Acquisition and Logistic Support (CALS) Program, Fiscal Year 1988. Volume 3 of 3. CGM Registration.

MORI, $\mathrm{K}$.

Chain Conformation of a Block Polymer in a MicrophaseSeparated Structure.

PB91-148890

100,485

Spectral Data and Grotrian Diagrams for Highly lonized Iron, Fe Vill-XX

101,595

\section{MORKOC, $\mathrm{H}$.}

Emitter Ballasting Resistor Design for, and Current Handling Capability of AIGaAs/GaAs Power Heterojunction Bipolar Transistors.

PB91-236703

100,815

Uniform Junction Temperature AIGaAs/GaAs Power Heterojunction Bipolar Transistors on Silicon Substrates.
PB91-189399 MORLEY, P. D.

Exploding Foil Photoionization X-ray Laser.

PB91-237065

MORRISON, G.

Dipole Moments of Seven Partially Halogenated Ethane PBigerants.

100,377

Modelling Aqueous Solutions Near the Critical Point of

Water.

PB91-189837

100,349

Thermodynamic Diagrams for Refrigerant Mixtures.

PB91-236695

101,197

Thermodynamic Properties of Two Alternative Refrigerants: 1.1-dichloro-2 2, 2-trifuoroethane (R123) and 1,1,1,2-tetra1,1-dichloro-2,2,2-trif

PB91-175414

101,195

Use of Computer Algebra to Locate Critical Loci in Fluid Mixtures.

PB91-195263

101,390

MORRISON, M. A.

Near-Threshold Vibrational Excitation of $\mathrm{H} 2$ by Electron Impact: Resolution of Discrepancies between Experiment and Theory.

101,679

Moss, s. C.

Comparison of Amorphous and Ouasicrystalline Films of Sputtered Al0.72Mno.22Si0.06.

PB91-195552

101,530

MOSSERI, S.

Mechanism of Cyanide Release in the Radiolysis of Acetonitrile.

PB91.203679

100,240

Polyelectrolyte Stabilized Metal Oxide Hydrosols as Cataysts for the Photooxidation of Water by Zinc Porphyrins. 189852

100,351

Radiolytic Studies of the Redox Reactions of Ruthenium Porphyrins.

PB91-203661

100,402

Rate Constants for Reduction of Substituted Methylperoxyl Radicals by Ascorbate Ions and N,N,N',N'-TetramethylPara-Phenylenediamine.

PB91-195230

100,379

Reactivities of Chlorine Atoms and Peroxyl Radicals Formed in the Radiolysis of Dichloromethane.

PB91-200782

Redox Reactions of Osmium Porphyrins.

100,386

PB91-159012

100,217

MOULDER, J. C.

Characterization of Capacitive Array for NDE Applications. PB91.237305

Eddy Current Reflection Probe: Theory and Experiment. PB91-187120

MOULDER, J. D.

Progress in Uniform Field Eddy Current Methods. PB91-14775

100,944

\section{MOUNTAIN, R. D.}

Applicability of Smoluchowski's Equation with a Constant Kernel to Coalescence.

Computer Simulation of Fluid-Fluid Phase Coexistence in Mixtures of Nonadditive Soft Disks.

PB91-162263

101,388

Ergodic Convergence Properties of Supercooled Liquids and Glasses.

100,289

Ergodic Measures for the Simulation of Dielectric Properties of Water.

PB91-189845

100,350

MOZER, B.

6-D Structural Model for the Icosahedral (AI, Si)-Mn Ouasi-

PBystal.

101,501

N-Dimensional Crystallographic Description of the Icosahedral Phases; the Example of the Al73Mn21Si6 Ouasiperiodic Structure.

PB91-174771

101,226

\section{MUELLER, D.}

Electronic Structure of High-Tc Superconductors Studied Using Photoelectron Spectroscopy.

101,513

Initial Stages of Degradation of Superconductor Surfaces: $\mathrm{O} 2, \mathrm{H} 2 \mathrm{O}, \mathrm{CO} 2$ and CO Chemisorption on La2-xSrxCuO4. PB91-18967

MUENCH, P. J.

Photon Energy Dependence of the Sensitivity of Radiochromic Film and Comparison with Silver Halide Film and LiF TLDs Used for Brachytherapy Dosimetry.

101,296

MUIR, W. B.

Magnetic Anisotropies in Ultrathin fcc Fe(001) Films Grown

on Cu(001) Substrates.
PB92-116631

101,565

MULHOLLAND, G. W.

Applicability of Smoluchowski's Equation with a Constant 
MUNRO, R. G.

ACTIS: Towards a Comprehensive Tribology Data Base. PB91.174904 101,184 Ceramic Wear Maps

PB91-189498

101,184

Isothermal Phase Behavior of Ag3SbS3, ZnGeP2, and ZnS.

PB91-187237

101,080

MUNUTLA, L. V.

Saran Carbon Cryogenic Regenerator for Liquid Helium PB91-175430

101,640

MURPHY, D. W.

Parent Structure of the Layered High-Temperature SuperConductors.
PB91-195677

MURPHY, K. N.

101,535

Automating Robot Programming in the Cleaning and Deburring Workstation of the AMRF

PB91-195495

100,986

CAD Directed Robotic Deburring

100,985

1-195172

MURRAY, B. T.

Effect of Gravity Modulation on Thermosolutal Convection in an Infinite Layer of Fluid.

PB92-112291

101,396

MURTHY, J.

Studies of $\mathrm{HI}$ and D I in the Local Interstellar Medium
PB91-148957

100,021

MUSGROVE, A.

Energy Levels of Copper, Cu I through Cu XXIX

Energy Levels of Krypton, Kr I through $\mathrm{Kr}$ XXXVI.

PB92-148139

101,596

Energy Levels of Sulfur, S I Through S XVI.

100,473

PB91-192443

100,355

MUSSELMAN, I. H.

Use of Single Particle Standards for LAMMA Calibration PB91.147777

MUTH, L. A.

General Analytic Correction for Probe-Position Errors in Spherical Near-Field Measurements.

100,729

General Technique to Correct Probe Position Errors in Planar Near-Field Measurements to Arbitrary Accuracy.
PB91-237073

Persona

PB92-112283

100,730

MYERS, D. R.

Application of the Joint Excitation Gear Adjustment Technique to Low Frequencies and Heavy Robot Joints.

PB91-158576

101,028

Band-Gap Narrowing and III-V Heterostructure FETs.

PB92-117159

100,827

Self-Adjustment of a Robot Joint Controller.

PB91-158568

101,027

MYKLEBUST, $R$. L.

Strategies for Background Subtraction in Electron Probe Microanalysis X-ray Compositional Mapping

PB91-195180

100,202

NABINGER, S. J.

Preliminary Results of the Environmental Evaluation of the Federal Records Center in Overland Missouri. PB91-240747

100,082

NACHT, G.

Hardware Instrumentation Approach for Performance Measurement of a Shared-Memory Multiprocessor.

100,601

NACKERDIEN, $Z$.

Chemical Nature of In vivo DNA Base Damage in Hydrogen Peroxide-Treated Mammalian Cells.

PB91-187526

101,335

Modification of DNA Bases in Mammalian Chromatin by Radiation-Generated Free Radicals.

101,338

NAGARAJAN, A.

Knowledge Extraction Techniques for Expert System Assisted Wafer Screening.

PB92.116979

100,824

NAGASAWA, $M$

Chain Conformation of a Block Polymer in a MicrophaseSeparated Structure

PB91-148890

100,485

NAHOR, G. S

Polyelectrolyte Stabilized Metal Oxide Hydrosols as Catalysts for the Photooxidation of Water by Zinc Porphyrins.

PB91-189852

100,351

NAIR, S. V.

Role of Glassy Interfaces in High Temperature Crack Growth in $\mathrm{SiC}$ Fiber Reinforced Alumina.

101,127

NAKAGAKI, T.

Spectral Data and Grotrian Diagrams for Highly Ionized

Copper, Cu X-Cu XXIX.
PB92-110121

100,441

AKAGAWA, N.

Progress in Uniform Field Eddy Current Methods.
PB91.147751

NAKAI, Y.

Spectral Data and Grotrian Diagrams for Highly Ionized

Copper, Cu X-Cu XXIX

PB92-110121

100,441

Spectral Data and Grotrian Diagrams for Highly Ionized Iron, $\mathrm{Fe}$ VIII-XXVI.

NAKAJIMA, $\boldsymbol{H}$.

Interlaboratory Tension and Fracture Toughness Test Results for CSUS-JN1 (Fe-25Cr-15Ni-0.3N) Austenitic Stainless Steel at $4 \mathrm{~K}$

PB91-237081

101,166

NANZETTA, $P$.

Publications of the Manufacturing Engineering Laboratory

Covering the Period January 1989-September 1991

PB92-123041

101,053

NASHMAN, M.

Real-Time Model-Based Tracking Combining Spatial and Temporal Features.

PB91-216630

100,700

PBideo Compress

101,035

NASREEN, G.

Angle-Resolved Photoemission from the Ar $2 p$ Subshel. PB91-194928

101,665

NASSIMBENE, R. D.

Unsteady Laminar Flow in a Circular Tube: A Test of the HERCOL (Hermitian Collocation) Computer Code.
PB91-222679

101,395

NATH, $R$.

Photon Energy Dependence of the Sensitivity of Radiochro. mic Film and Comparison with Silver Halide Film and LiF TLDs Used for Brachytherapy Dosimetry.

101,296

NATRELLA, M. G.

Design and Analysis of Experiments

PB91-158824

101,274

NAVON, E.

Intensifying Effect of Metallic Screens on the Sensitivity of X-ray Films at $662 \mathrm{keV}$

101,669

Intensifying Effect of Metallic Screens on the Sensitivity of $X$-ray Films for $400-k V$ Bremsstrahlung Photons.

101,293

Use of Storage Phosphor Imaging Plates in Portal Imaging and High-Energy Radiography: The Intensifying Effect of and High-Energy Radiography: The Intensifying Effect of
Metallic Screens on the Sensitivity. Metallic Scree
PB91-236497

101,324

NAZARIO, N.

Standard Security Label for GOSIP: An Invitational Workshop. Held on April 9-10, 1991.

PB91-216671

NECHVATAL, J.

Public-Key Cryptography. Computer Security.

PB91-187864

100,712

NEDZELNITSKY, $v$.

Response of Personal Noise Dosimeters to Continuous and Impulse-Like Signals.

PB92-116359

100,914

NEFF, J.E.

Spatially Resolved Flares in RS CVn Systems. PB91-148965

100,022

NELSEN, S. F

Proton Aftinities and $\mathrm{pKa}$ Values of Tetraalkylhydrazines.

PB91-195214 100,378

Slow Electron Transfer Reactions between Alkylhydrazines.
PB91-147785

NELSON, A.

Analysis of the $\mathrm{YBa} 2 \mathrm{Cu} 3 \mathrm{O} 7 / \mathrm{SrTiO}$ Interface as a Function of Post-Deposition Annealing Temperature.

101,480

Morphology of Silver on YBa2Cu3O7-delta Thin Films.
PB91-195594

NELSON, D.

Investigation of Internal Rotor Dynamics of $\mathrm{NeDCl}$ and ArDCl via Infrar

100,409

NELSON, D. D.

$\mathrm{H}+\mathrm{O} 3$ Fourier-Transform Infrared Emission and Laser Absorption Studies of $\mathrm{OH}$ (X(2) Pi) Radical: An Experimental Dipole Moment Function and State-to-State Einstein A Coefficients.

PB91-148973

100,297

High-Resolution Infrared Flash Kinetic Spectroscopy of $\mathrm{OH}$ Radicals.

NELSON, H. E.

100,408

Engineering Analysis of Fire Development in the Hospice of

Southern Michigan, December 15, 1985.

PB91-195222

100,158

Engineering Analysis of the Fire Development in the Hillhaven Nursing Home Fire, October 5, 1989.

PB92-112317

100,169

FETOOL User's Guide.

PB92-108919

100,134

NELSON, P. G.

Gyroscope-Weighing Experiment Revisited -- with a Null

PB91-174722

101,630

\section{NELSON, T. L.}

Influence of Nonsinusoidal Waveforms on Voltmeters, Ammeters, and Phase Meters.

PB92-117167

100,869

NENADOVIC, T.

Mechanical Sputtering of Structural Stainless Steels

PB91-175448

101,152

NESBITT, D. J.

$\mathrm{H}+\mathrm{O} 3$ Fourier-Transform Infrared Emission and Laser Absorption Studies of $\mathrm{OH}(X(2)$ Pi) Radical: An Experimental Dipole Moment Function and State-to-State Einstein A Coefficients.

PB91-148973

100,297

High-Resolution Infrared Flash Kinetic Spectroscopy of $\mathrm{OH}$ Radicals.

PB91-204008 100,408

Investigation of fnternal Rotor Dynamics of $\mathrm{NeDCl}$ and ArDCl via Infrared Absorption Spectroscopy.

100,409

Mode Specific Internal and Direct Rotational Predissociation in $\mathrm{HeHF}, \mathrm{HeDF}$, and $\mathrm{HeHCL}$ : van der Waals Complexes in the Weak Binding Limit.

PB91-148841

100,293

Multiple Intermolecular Bend Vibrational Excitation of a $\mathrm{Hy}$ drogen Bond: An

Progress on the Determination of Intermolecular Potential

Energy Surfaces from High Resolution Spectroscopy
PB91-203695

Rotational Predissociation, Vibrational Mixing, and van der Waals Intermolecular Potentials of NeDF.

PB91-175505

100,334

Structural Dependence of HF Vibrational Red Shifts in ArnHF, $n=1-4$, via High-Resolution Slit Jet Intrared Spectroscopy.
PB91-203562

100,401

NETA, P.

Mechanism of Cyanide Release in the Radiolysis of AcetoPBitrile. 003679

100,240

Polyelectrolyte Stabilized Metal Oxide Hydrosols as Catalysts for the Photooxidation of Water by Zinc Porphyrins.
PB91-189852

Pulse Radiolysis Studies of Organic Electron Transfer Reactions

PB91-147801

100,285

Radiation Chem
PB91-147793

100,236

Radiolytic Studies of the Redox Reactions of Ruthenium Porphyrins.

Rate Constants for Reactions of Peroxyl Radicals in Fluid

Solutions.
PB90-244641

100,261 
NEWBURY, D.

Modeling Electron Beam Interactions in Semiconductors. PB91-159020

NEWBURY, D. E.

Strategies for Background Subtraction in Electron Probe Microanalysis X-ray Compositional Mapping.

PB91-195180

100,202

NEWELL, A. C

Alignment Fixture for Millimeter Waveguide.

PB91-236919

100,727

NEWMAN, D. S.

Near-Threshold Vibrational Excitation of $\mathrm{H}_{2}$ by Electron Impact: Resolution of Discrepancies between Experiment and Theory.

NEWMAN, H. M.

BACnet Communication Protocol for Building Automation Systems.

PB91-187286

100,069

NEWNAM, B. E.

Laser Induced Damage in Optical Materials: 1989

B91-148478

101,409

NEWTON, J.

Designing Data Entity Naming Conventions.

100,646

\section{NGUYEN, T.}

Characterization of Epoxide Coatings on Steel by Reflection/Absorption Fourier Transform Infrared Spectroscopy: Quantitative St

PB91-203729

101,109

Fourier Transform Infrared Spectroscopic Studies of the Degradation of One-Component Polyether Polyurethane Protective Coatings on Steel.

101,108

PB91-195248

of Thin

Reflection/Absorption FTIR Spectral Characteristics of

and Thick Crosslinked Epoxy Films on Steel Substrates.

PBOL

Dynamics of Molecular Hydrogen Adsorbed in CoNa-A Zeo-

PB91-195255

100,380

Structures and Properties of New Zeolite X-Type Zincophosphate and Beryllophosphate Molecular Sieves. PB91-189449

\section{NIEBAUER, T. M.}

Test of Newton's Inverse Square Law of Gravity Using the $300 \mathrm{~m}$ Tower at Erie, Colorado: Newton Vindicated on the Plains of Colorado.

PB91-175273

101,637

Test of the Inverse-Square Law of Gravitation Using the 300-m Tower at Erie, Colorado.

PB91-204115

101,702

NIELSEN, $\boldsymbol{F}$.

Document Architecture for Open Systems.

PB91-189522

100,961

NIELSON, G. C.

Use of Computer Algebra to Locate Critical Loci in Fluid

Mixtures.

101,390

NIESEN, $V, G$.

Critical Locus, (Vapor + Liquid) Equilibria, and Coexisting Densities of (Carbon Dioxide + Propane) at Temperatures rom $311 \mathrm{~K}$ to 361

PB91-134494

100,267

NIETO DE CASTRO, C. A.

Benzene: A Further Liquid Thermal Conductivity Standard.

PB90-244567 100,255

High-Temperature Transient Hot-wire Thermal Conductivity Apparatus for Fluids.

PB91-216713

100,411

Thermal Conductivity and Heat Capacity of Gaseous Argon.

PB91-134577 101,602

Thermal Conductivity of a Moderately Dense Gas.

PB91-134502

101,601

NIGHTINGALE, J. $S$.

GOSIP Conformance and Interoperation Testing and Regisration.

PB92-1 10105

100,694

NILSSON, D.

Electrofission in the Quasifree and Delta Regions.

PB91-147355

101,609

NISHIMURA, $H$.

Cross Sections and Related Data for Electron Collisions with Hydrogen Molecules and Molecular Ions. PB90-244674

100,263

NOBLE, $R$.

Enhanced Transport and Liquid Membranes in Bioseparations.

PB91.189886

101,287

NOBLE, R. D.

Kinetics of Copper Extraction Using (anti)-2-Hydroxy-5-Nonlybenzophenone Oxime.

lybenzophenon

100,292

Overview of Facilitated Transport Membrane Systems.
PB91-175463

100,228

NODA, I.

Chain Conformation of a Block Polymer in a MicrophaseSeparated Structure.

100,485

OGAMI, J.

Surface Extended-X-ray-Absorption Fine Structure and Scanning Tunneling Microscopy of Si(001)2x1-Sb. B $1-237263$

101,552

NOID, D. W.

Molecular Dynamics of Initial Events in the Thermal Degra. dation of Polymers.

PB91-195271

100,503

NONELLA, $M$.

Photodissociation of C(I)NO in the S(I) State: A QuantumMechanical ab initio Study.

PB91-149260

100,239

\section{NORCROSS, D. W.}

Close-Coupling Calculation for the (2)(Sigma sub $u)(+$ ) State of $(\mathrm{H}$ sub 2)(-)

101,614

Near-Threshold Vibrational Excitation of $\mathrm{H} 2$ by Electron mpact: Resolution of Discrepancies between Experiment and Theory.

PB91-200881

101,679

Spin-Dependent Electron-Impact Excitation of Sodium.

PB91-175497

101,641

NORCROSS, R. J.

Automating Robot Programming in the Cleaning and Deburring Workstation of the AMRF.

191-195495

100,986

CAD Directed Robotic Deburring

PB91-195172

Control Structure for Multi-Tasking Workstations. PQ1-203737

100,985

ORFORD, L. K.

Measurement of Thermal Characteristics of Office Build-

PB91-175471

100,068

NORTON, S. J.

Mossbauer Imaging.

PB91-203745

101,294

\section{NORTON, T. S.}

Laser-Induced Fluorescence of $\mathrm{CH}$. in a Laminar $\mathrm{CH} 4 / \mathrm{Air}$ Diffusion Flame: Implications for Diagnostic Measurements and Analysis of Chemical Rates. PB91-237099

100,554

NOTARIANNI, K. A.

Five Small Flaming Fire Tests in a Simulated Hospital Patient Room Protected by Automatic Fire Sprinklers. PB91-159038

NOVAK, S. W

Round-Robin Study of Implants in $\mathrm{Si}$ and $\mathrm{SiO} 2$ by SIMS, RBS, and NAA
PB92-117241

NOVOTNY, D. B.

High Spatial Resolution Mapping of Semiconductor Resis-

tivity.

Persistent Photoconductivity in SIMOX Films. PB92-117084

100,809

100,826

NUNNERMACKER, L.

Reference NO2 Calibration System for Ground-Based Intercomparisons during NASA's GTE/CITE II Mission. PB91-189340

100,034

NYDEN, $M$.

Molecular Dynamics of Initial Events in the Thermal Degradation of Polymers.

PB91-19527

100,503

NYDEN, M. $R$.

Molecular Dynamics Study of the Depolymerization Reaction in Simple Polymers.

100,495

NYYSSONEN, D.

New Approach to Image Modeling and Edge Detection in the SEM.

O'CONNOR, C

NIST Reactor: Summary of Activities July 1989 through

PB91-159772

101,488

'GALLAGHER, A. Calculated from the Frequency Domain: An U'pdate.
PB91-162388

the Fre-

Time Domain Frequency Stability Calculated rom Soltware Package to Calculate Time Domain Frequency Stability from the Frequency Domain.

100,587

92-123132

O'HAVER, T. C.

Laser Enhanced Ionization as a Selective Detector for Liquid Chromatographic Determination of Alkyltins in Sedi-

100,210

O'KEEFFE, T. $w$.

Evaluation and Improvement of E-Beam Exposure Routines by Use of Microelectronic Test Structures.

100,805

O'LEARY, D. P.

Computing the Eigenvalues and Eigenvectors of Symmetric Arrowhead Matrices.

PB91-147850

101,254

Some Algorithms for Approximating Convolutions.

PB91.175281

101,258

O'NEILL, R. P.

Gas Analysis Modeling System.

OETTINGER, F. F.

Thermal Characterization of Integrated Circuits - A Tutorial.

PB91.147819 100,789

Thermal Measurements of VLSI Packages: A Critical

Review.

PB91-147827

100,790

HLEMILLER, T. J.

Aspects of Smoldering Combustion

100,535

Forced Smolder Propagation and the Transition to Flaming in Cellulosic Insulation.

PB91-147835

100,101

Material Flammability Test Assessment for Space Station

PB91-216606 101,735

Smoldering Combustion Propagation Through a Permeable Horizontal Fuel Layer.

PB91-147843

100,102

OHNO, M.

Anomalous Lgamma2,3 X-ray Emission Spectrum of Xe.

Anomalous Th M3O1 X-ray Emission Spectrum of ThO2

PB91-195297 100,381

Many Electron Effects in Lgamma2,3 X-ray Emission Spectroscopy Spectrum of $\mathrm{BaO}$.

PB91-195305

100,382

Many-Electron Effects in Lgamma2,3 XES Spectra from Rare Earth Elements.

PB91-195313

100,383

OHTSU, M.

Modulatable Narrow-Linewidth Semiconductor Lasers. PB91-134106

101,404

OKABE, $\mathrm{H}$.

Dissociation Energetics of Simple Diazirines by Photon and Electron Impact.

100,405

Laser Photolysis of Trimethylgallium at $193 \mathrm{~nm}$ : Quantum Yields for Methyl Radical and Ethane Production.
AD-A227 868/7

Scattered Light and Other Corrections in Absorption Coefficient Measurements in the Vacuum Ultraviolet: A System Approach.

AD-A227 869/5

100,179

OLCHOWY, G. A. 
OLSON, G. J.

Biological Sulfur Oxidation and Reduction for Coal Sulfur Speciation and Desulfurization.

100,886

Bioprocessing of Fossil Fuels Using Hyperthermophilic Archaebacteria

100,883

Characterization by High Performance Liquid Chromatogra phy (HPLC) of the Solubilization of Phosphorus in Iron Ore by a Fungus.

101,162

Methods for the Analysis of Organometallic Compounds in Wastes.

PB91-148981

100,189

Newest Approaches to Quantitative Assessment of Bioac tive Organotins.

PB91-162032

101,328

Rate of Pyrite Bioleaching by 'Thiobacillus ferrooxidans'

Results of an Interlaboratory Comparison.

PB91-195321

101,161

Recent Progress in Coal Bioprocessing Research in the United States: Scope and Areas Needing Further Resear PB91-195339

100,885

OLTHOFF, J. K.

Collisional Electron-Detachment and Ion-Conversion Proc-

esses in SF6.
PB91-134528

Detection of Trace Disulfur Decafluoride in Sulfur Hexafluoride by Gas Chromatography/Mass Spectrometry. Off-Axis Measurements of Ion Kinetic Energies in RF Plasmas.

PB92-117225

101,444

OLVER, F. W. J.

Closure and Precision in Level-Index Arithmetic.
PB91-147652

Simplified Error Bounds for Newton's Rule.

PB91.159046

101,257

ONDOV, J. M.

Development of a New Tracer Technology Using Enriched Rare-Earth Isotopes.

PB91-195347

100,908

ONEIL, S. V.

Multireference-Configuration Interaction (MR-Cl) Calculations on $\mathrm{HS}(2+)$ and Experimental Observation via Elec
tron Impact lonization of $\mathrm{H} 2 \mathrm{~S}$. PB91.148924

100,295

ONO, R. $\mathrm{H}$.

Analysis of the $\mathrm{YBa} 2 \mathrm{Cu} 3 \mathrm{O} 7 / \mathrm{Sr} \mathrm{TiO} 3$ Interface as a Function f Post-Deposition Annealing Temperature.

101,480

Morphology of Silver on YBa2Cu307-delta Thin Films.

Morphology of Silver on YBa2Cu3O7-delta Thin Films.
PB91-195594 Single-Target Magnetron Sputter-Deposition of High-Tc Superconducting Bi-Sr-Ca-Cu-O Thin Films.

101,566

YBa2Cu3O7-delta/Insulator Multi-Layers for Crossover Fab-

rication.

OOSTRA, D. J.

101,518

Study of the GaAs-Si(100) Interface Using Laser Probing of Thermal Desorption Kinetics.

Thermal Desorpt
PB91-149294

101,477

OPPERMANN, H. V.

Specifications, Tolerances, and Other Technical Requirements for Weighing and Measuring Devices as Adopted by the 76th National Conference on Weights and Measures, 1991.

PB92-112424

101,023

OREF, I

Ab-Initio Calculations and Ideal Gas Thermodynamic Functions of Cyclopentadiene and Cyclopentadiene Derivatives.

PB92-148089

ORLANDO, J. J.

$\mathrm{H}+\mathrm{O} 3$ Fourier-Transform Infrared Emission and Laser Absorption Studies of $\mathrm{OH}(X(2)$ Pi) Radical: An Experimen tal Dipole Moment Function and State-to-State Einstein A Coefficients.

OATIGOSO, J.

100,297

High-Resolution Infrared Spectrum of the nu1 Band of

PB92-117175

100,463

OSBORNE, W. M.

Developing Federal Software Standards: A New Direction.

PB91-134536

100,637

OSKARD, D. N.

Real-Time Algorithms and Data Structures for Underwate Mapping.

OSOFSKY, M.

101,360

Electronic Structure of High-Tc Superconductors Studied Using Photoelectron Spectroscopy.

101,513

Phonon Density of States of Superconducting YBa2Cu3O7 and the Nonsuperconducting Analog YBa2Cu3O6.
PB91-159129

101,487

ERTAG, C.

Differential Sintering

Role of Glassy Interfaces in High Temperature Crack

Growth in SiC Fiber Reinforced Alumina.

PB91-203687

101,127

OSTERTAG, C. P.

Cathodoluminescence Measurement of Strained Alumina Single Crystals

101,094

Chemical Modification of the Orthorhombic Superconductor Ba2YCu3O7-delta.

PB91-159053

01,486

Reduction in Sintering Damage of Fiber Reinforced Com posites.

PB91-19536

101,124

OSTROM, $\boldsymbol{R}$.

Scanning tunneling microscopy studies of the surfaces of a$\mathrm{Si} H$ and a-SiGe:H films. Annual report, 1 December 1989 31 January 1991.

DE91002169

100,785

\section{OUTCALT, S. L.}

Hydrogen Component Fugacity Coefficients in Binary Mixtures with Etane: Pressure Dependence.

100,455

OVER

Domestic Implementation.

PB91-159061

OVERMAN, J. R.

GATT Standards Code Activities of the National Institute of Standards and Technology 1990

PB91-187823

100,177

OZA, D. H.

Overview of the Ion Dynamic Effect in Line Broadening and a Generalization of the Unified Theory.

101,442

PB91-14750

OZTURK, F.

Reactions of $\mathrm{C} 5 \mathrm{H} 3(+)$ and $\mathrm{C} 5 \mathrm{H} 5(+)$ lons with Acetylene and Diacetylene.

PB91-195370

PAABO, M.

Data for Fire Hazard Assessment of Selected Non-Halogenated and Halogenated Fire Retardants; Report of Test FR

PB92-112473

100,557

arge-Scale Validation of Bench-Scale Fire Toxicity Tests.

PB92-116458

Role of Bench-Scale Test Data in Assessing Real-Scale Fire Toxicity.

100,156

PAGE, R. A.

Characterization of the Densification of Alumina by Multiple Small-Angle Neutron Scattering.

101,100

Effect of Green Density and the Role of Magnesium Oxide Additive on the Densification of Alumina Measured by Small-Angle Neutron Scattering.

PB92-117043

101,101

PAGNI, P. J.

Users' Guide to BREAK1, the Berkeley Algorithm for Breaking Window Glass in a Compartment Fire.

PB92-112556

100,136

PAKANEN, J.

Prediction and Fault Detection of Building Energy Consumption Using Multi-Input, Single-Output Dynamical Mode

PALLETT, D. S.

Phone-Mediated Word Alignment for Speech Recognition Evaluation.

100,592

Scoring Continuous Speech in the DARPA Speech Recog. nition Program: Part 2

PB91-195396

100,593

PALMIERI, M. D.

Introduction to Supercritical Fluid Chromatography. 1. Principles and Instrumentation.

PB91-189878

100,198

PAN, L.

High Harmonic Generation in Hydrogenic lons. PB91-203778

101,699

PARAMESWARAN, $M$.

Micromachined Thermal Radiation Emitter from a Commercial CMOS Process.
PB91-148999

100,748

PARDEE, $R$.

National Bureau of Standards Publications 1977-1987. Volume 1. Citations, Key Words, and Abstracts. PB91-136507

100,957

National Bureau of Standards Publications 1977-1987. Volume 2. Indexes.

PB91-136515

100,958

PARISE, J.

New Different Forms of Ammonium Loaded and Partly Deammoniated Zeolite Rho Studied by Neutron Powder.

PB91-158717

100,311

ARISE, J. 8.

Refinement of the Structure of Beta'-MoO3.

PB91-203786

101,090

PARK, G. S.

Correlation of Flux States Generated by Optical Switching of a Superconducting Circuit.

PB91-202929

101,682

PARKER, W. J.

Kinetic Properties of the Components of Douglas-Fir and the Heat of Combustion of Their Volatile Pyrolysis Products. PB91-203794

PARKS, E. J.

Biological Sulfur Oxidation and Reduction for Coal Sulfur Speciation and Desulfurization.

PB91-203323

100,886

Characterization by High Performance Liquid ChromatograChy (HPLC) of the Solubilization of Phosphorus in Iron Ore by a Fungus.

101,162

Studies on the Degradation Products of Paper with and without Pollutants in a Closed Environment. 1. Preliminary

PB91-143313

101,181

PARKS, J. E.

Observation of Anomalous Isotope Ratios in SIRIS Measurements of Molydenum.

PB92-116789

101,36

PARR, A. C.

LBIR Facility User Handbook

PB92-102185

Shape Resonances in Molecular Fields.

PB91-187435

101,435

PARRIS, R. $M$.

Monitoring the Fate of Chlorine from MSW Sampling

PB91-174631

100,917

PARTLOW, W. D.

Design, Fabrication, and Testing of an Interconnect Test Structure for Evaluating VLSI Processes.

PB91-174581

100,803

PATIL, T. A.

Mass Transfer Coefficients in Two-Phase Aqueous Extraction.

$-237149$

100,426

PATTON, C. E

Anomalous Low-Frequency Butterfly Curves for Subsidiary and Ferromag

PAULSEN, P. J.

High Purity Fluoropolymer Materials: Trace Element Content and Leaching.

PB91-162255

100,491

PAULTER, N. G.

New Triple Correlation Technique for Measuring Ultrashort PB91-237156

101,434

New Triple Correlator Design for the Measurement of Ultrashort Laser Pulses.

PB91-203802

101,429

PEACOCK, R. D. 


\section{PERSONAL AUTHOR INDEX}

PEISER, H. S.

Silicon Reference Materials Certified for Isotope Abundances.

100,213

PELLEGRINI, G.

Real, Realistic Ring Waves for Surge Testing

PELLEGRINO, J.

Enhanced Transport and Liquid Membranes in Biosepara-

tions.

101,287

PELLEGRINO, J. J.

Chitin-Chitosan Membranes: Separations of Amino Acids and Polypeptides.

101,283

Increased Facilitated Transport Related to Microstructural Changes in Heat-Treated lon-Exchange Membranes. PB91-174805 100,323

Overview of Facilitated Transport Membrane Systems.

PB91-175463

100,228

PELTON, A. D.

Coupled Phase Diagram-Thermodynamic Analysis of the 24 Binary Systems, A2CO3-AX and A2SO4-AX Where $A=L i$, $\mathrm{Na}, \mathrm{K}$ and $\mathrm{X}=\mathrm{Cl}, \mathrm{F}, \mathrm{NO} 3, \mathrm{OH}$.

PENA, J, L.

Sputtered Thin Film YBa2Cu3On

PENG, $\mathrm{H}$.

Conformance Tester for X.25 DTE Implementations. PB91-189894

PENG, J. L.

Antiferromagnetic Order of $\mathrm{Cu}$ in $\mathrm{Sm}_{2} \mathrm{CuO} 4$

PB92-117340

Density of States and Superconductivity in Nd1.85Ce0.15CuO4

PENN R. W.

101,514

Microstructure and Elastic Properties of Dental Resin and Resin-Based Glass-Reinforced Composites: XRD, SEM and Ultrasonic Methods

PB91-204081

100,050

PENN, S. M.

Laser-Induced Fluorescence Measurements of Drift-Velocity Distributions for $\mathrm{Ba}(+)$ in $\mathrm{Ar}$ : Moment Analysis and a Direct Measure of Skewness.

100,298

PEPPLER, T. K

Frequency and Time Stability of GPS and GLONASS Clocks.

PB91-202937

100,582

PERCIVAL, D. R.

Interlaboratory Comparison of Actinides in Human Tissue $(239) \mathrm{Pu}+(240) \mathrm{Pu}$

PERERA, R. C. C.

101,323

Molecular-Orbital Studies via Satellite-Free $X$-ray Fluorescence: $\mathrm{Cl} \mathrm{K}$ Absorption and $\mathrm{K}$-Valence-Level Emission Spectra of Chlorofluoromethanes.

PB91-237164

100,427

Polarized X-ray Emission Studies of Methyl Chloride and the Chlorofluoromethanes.

100,329

\section{PEREZ, J. M.}

Characterization of Tricresylphosphate Lubricating Films by Micro-Fourier Transform Infrared Spectroscopy.
PB91-203810 Diesel Deposit Forming Tendencies - Microanalysis Methods.

101,178

PERIS, J. P.

Design and Implementation of a Five-Axis Robotic Micromanipulator for Inserting Parts into Precision Collets.

PB91-159194

101,029

PERKINS, R. A.

Experimental Thermal Conductivity, Thermal Diffusivity, and Specific Heat Values for Mixtures of Nitrogen, Oxygen, and ABg

100,369

Fiber-Optic Fluorescence Array to Study Free Convection in Porous Media

101,407

High-Temperature Transient Hot-wire Thermal Conductivity Apparatus for Fluids.

100,411

Thermal Conductivity and Heat Capacity of Gaseous Argon.
PB91-134577

Thermal Conductivity of a Moderately Dense Gas.

PB91-134502

101,601

PERREY, A. G.

Annotated Bibliography: Diagnostic Methods and Measurement Approaches to Detect Incipient Defects Due to Aging

of Cables.
PB91-222687

101,369

PERSILY, A.

Alr Exchange and Pollutant Concentration Measurements in Two Office Buildings.
PB91-195420

PERSILY, A. K.

Airtightness of Office-Building Envelopes.

100,909

Diagnostic Techniques for Evaluating Office Building Envelopes.

Measurement of Thermal Characteristics of Office Build-

ings. 175471

100,068

Preliminary Results of the Environmental Evaluation of the Federal Records Center in Overland Missouri.
PB91-240747

$\begin{array}{ll}\text { PB91-240747 } & 100,082 \\ & \end{array}$

Prototype Expert System for Diagnosing Moisture Problems
in Houses.

PB91-159079

Ventilation System Performance Evaluation Using Tracer Gas Technique
PB91-147876

100,060

PETERKOFSKY, A

Crystal Structure of a cAMP-Independent Form of Catabolite Gene Activator Protein with Adenosine Substituted in One of 2 CAMP-Binding Sites.

PB91-190074

101,305

PETERS, $M$.

Summary of the Intercomparison of the Force Standard Machines of the National Institute of Standards and Technology, USA, and the Physikalisch-Technische Bundesanstalt, Germany
PB92-126622

PETERS, P. N.

Magnetic Hysteresis of High-Temperature YBa2Cu3Ox-AgO Superconductors: Explanation of Magnetic Suspension.
PB91-189506

PETERSEN, $\mathbf{S}$. $\mathbf{R}$.

DISCOUNT-A Program for Discounting Computations in Life-Cycle Cost Analyses. User's Guide and Reference

NIST 'Building Life-Cycle Cost' (BLCC) Program (Version 3.0). User's Guide and Reference Manual.

PB91-159764

100,154

ZIP: The ZIP-Code Insulation Program, Version 2.0. Economic Insulation Levels for New and Existing Houses by Three-Digit Zip Code. Users' Guide and Reference Manual (Revised Edition).

PETERSEN, T. $W$.

100,064

Effect of Mechanical Deformation on Nb-Ti Filament Proximity-Effect Coupling at the Edges of SSC Cables.

PB91-203828

101,700

PETERSON, K.

Hydration of Carbon Dioxide: The Structure of $\mathrm{H} 2 \mathrm{O}-\mathrm{H} 2 \mathrm{O}$ CO2 from Microwave Spectroscopy.

PB91-237172

PETERSON, M. B.

Literature Review of the Galling Process.

PB91-134551

100,428

PB91-15926

101,208

Study of the Galling of Two Steels Using Two Test Meth-

Ods.

101,147

PETERSON, R. L.

Critical-Current Diffraction Patterns of Grain-Boundary Josephson Weak Links.

PB91-237180

101,551

Magnetization of Anisotropic Superconducting Grains

PB91-134569

101,463

Sidelobe Suppression in Small Josephson Junctions

PB91-195446

100,784

PETERSONS, 0

NIST Measurement Services: Calibration Service for Current Transformers.

PB91-216770

100,873

PETRIE, S. A. H.

Proton Affinity of Cyanogen and Ion-Molecule Reactions of $\mathrm{C} 2 \mathrm{~N} 2(+)$
$\mathrm{PB} 91-189902$

100,352

PEYERIMHOFF, S. D.

Photodissociation Dynamics of Water in the Second $A b-$ sorption Band: Vibrational Exitation of $\mathrm{OH}$ (A (2)Sigma).

PB91-148726

PFENNING, D. B.

Radiant Heat Fluxes from 100-200 MW Natural Gas/Air Diffusion Flames.
PB91-147306

100,538

PHAN, L. T.

Measurement of Structural Response Characteristics of Full-Scale Buildings: Selection of Structures.

PB91.167239

100,144

PHELAN, $F$.

Assessment of the State-of-the-Art for Process Monitoring Sensors for Polymer Composites.

100,510

PHELAN, R. J.

Hydrogenated Amorphous Germanium Detectors Deposited Onto Channel Waveguides.

100,756

Low-Temperature, Electrically Calibrated SOS Bolometer for Power and Energy Measurements.

PB91-147181

100,787

Semiconductor Claddings on Glass Waveguides for Polarizers and Detectors.

PB91-17514

101,416

PHELPS, A. $V$. Cross Sections and Swarm Coefficients for $\mathrm{H}+, \mathrm{H} 2+$
$\mathrm{H} 3+, \mathrm{H}, \mathrm{H} 2$, and $\mathrm{H}$-in $\mathrm{H} 2$ for Energies from $0.1 \mathrm{eV}$ to 10 PB90-244690 101,597

Cross Sections and Swarm Coefficients for Nitrogen lons and Neutrals in N2 and Argon lons and Neutrals in N2 and Argon lons and Neutrals in Ar for Energies from $0.1 \mathrm{eV} 10$ PB92-110246

100,452 Detection of Excited States by Laser-Induced Fluorescence and Analysis of Energy Transfer.

AD-A227 296/1

100,242

PHILLIPS, $K$.

Translate LRL and LRM Calibrations.

PB91-237776

100,862

PHILLIPS, T. E.

Superconducting Thin Films of $\mathrm{Bi}-\mathrm{Sr}-\mathrm{Ca}-\mathrm{Cu}-\mathrm{O}$ Obtained by Laser Ablation Processing.

PB91-189589

101,508

PHILLIPS, W. D.

Associative lonization of Ultra-Cold Laser Trapped Sodium Atoms.

PB91-236745

101,707

Observation of Atoms Laser-Cooled Below the Doppler

Limit.
PB91-189746

Observation of Atoms Laser Cooled Below the Doppler

PB91-195453

101,671

Prospects for Electromagnetic Manipulation and Trapping of Antihydrogen.

101,663

PHILPOTTS, J.A.

Possible Use of Pattern Recognition for the Analysis of Mars-Rover $X$-ray-Fluorescence Spectra.

101,733

PIANCASTELLI, M. N.

Angle-Resolved Photoemission from the Ar $2 p$ Subshell. PB91-194928

101,665

PIANETTA, P.

Surface Extended-X-ray-Absorption Fine Structure and Scanning Tunneling Microscopy of Si(001)2x1-Sb.
PB91-237263

PICKETT, $\mathrm{H}$. $\mathrm{M}$.

Fluoromethanol: Synthesis, Microwave Spectrum, and Dipole Moment.

100,288

PICONE, J.

Phone-Mediated Word Alignment for Speech Recognition Evaluation.

PICQUE, J. L

Photoionization and Collisional Ionization of Excited Atoms Using Synchrotron and Laser Radiations.

PB91-15930

101,626

PIELERT, J. $\mathrm{H}$

Activities of the Construction Materials Reference Laboratories Related to Laboratory Accreditation.

PB91-203836

100,531

Application of CCRL Data in the Development of Cement Standards. 
PIERMARINI, G. J.

Effects of Pressure on the Thermal Decomposition Kinetics and Chemical Reactivity of Nitromethane.

100,286

High-Temperature High-Pressure Raman Spectra from Liquid Water.

100,385

Isothermal Phase Behavior of Ag3SbS3, ZnGeP2, and ZnS. PB91-187237

01,08

PINA, C.

Knowledge Verification of Machine-Learning Procedures Based on Test Structure Measurements.

100,709

PINE, A. S

Molecular-Beam Optothermal Spectroscopy of the 9.6 mum

PB91-236869

100,421

Optothermal-Detected Microwave-Sideband CO2-Laser Spectroscopy of Ar-NH3.

100,416

Perpendicular Bands of Cyclopropane in the $3.5 \mathrm{mum}$ Region.

100,424

Vibrational, Rotational, and Tunneling Dependence of $\mathrm{Vi}$ brational Predissociation in the HF Dimer.

PITTS, w. M.

100,406

Development of a Line Camera for Real-Time Measure-

ments of Concentration in Turbulent Flow Fields.
PB91-147892

Effects of Global Density Ratio on the Centerline Mixing Behavior of Axisymmetric Turbulent Jets.

PB92-117183

100,014

Large-Scale Turbulent Structures and the Stabilization of Lifted Turbulent Jet Diffusion Flames.

100,558

Reynolds Number Effects on the Mixing Behavior of Axisymmetric Turbulent Jets.

PB92-117209

100,015

Wind Effects on Fires.

PB91-203851

100,549

Real-Time Radiology Standards: Results of a Workshop.

PB91-147900 101,010

Use of Storage Phosphor Imaging Plates in Portal Imaging and High-Energy Radiography: The Intensitying Effect of Metallic Screens on the Sensitivity.

PB91-236497

101,324

PLANT, A. L.

Immobilization of Binding Proteins on Nonporous Supports Comparison of Protein Loading, Activity, and Stability

PB91-237198

101,308

Liposome Flow Injection Immunoassay: Implications for

Sensitivity, Dynamic Range, and Antibody Regeneration.
PB91-147629

Study of the Linear Viscoelastic Properties of Cyclic Polys tyrenes Using Creep and Recovery Measurements.

PB91.195107

100,502

PLIVA, J.

Perpendicular Bands of Cyclopropane in the $3.5 \mathrm{mum}$

PB91-237016

100,424

PODIO, F. L.

Monitoring and Reporting Techniques for Error Rate and Error Distribution in Optical Disk Systems. PB92-12312

100,606

Test Methods for Optical Disk Media Characteristics (for $356 \mathrm{~mm}$ Ruggedized Magneto-optic Media)

100,605

POLITCH, J.

Use of Storage Phosphor Imaging Plates in Portal Imaging and High-Energy Radiography: The Intensifying Effect of Metallic Screens on the Sensitivity.

PB91-236497

101,324

POLLACK-DINGELS, $P$.

Investigation of Photoconductive Picosecond Microstripline Switches on Self-Implanted Silicon on Sapphire (SOS).
PB91-147918 POPOVIC, N.

Mechanical Sputtering of Structural Stainless Steels.

PB91-175448

101,152

POPPLEWELL, D. S

Interlaboratory Comparison of Actinides in Human Tissue (239) Pu + (240)Pu.

PB91-203265

101,323

PORTER, G.

Research. Services. Facilities. (National Institute of Standards and Technology

POSTEK, M. T.

100,012

Application of Transmission Electron Detection to X-ray

Mask Calibrations and Inspection.

100,828

PB92.117217

Low Accelerating Voltage SEM Imaging and Metrology Using Backscattered Electrons.
PB91-147926 101,469

Microchannel-Plate Detection Systems for Low Accelerating Voltage SEM

PB91-189928

101,515

SEM Imaging and Metrology at Low Accelerating Voltages Using Backscattered Electrons.

101,529

Submicrometer SEM Magnification Standard.

PB91-159087

100,799

POTZICK, J. E.

Standard Reference Materials: Bright-Chromium Linewidth Standard, SRM 476, for Calibration of Optical Microscope inewidth Measuring Systems.

PB91-167163

100,802

POWELL, C. J.

Inner-Shell lonization Cross Sections.

100,203

Recent Developments in Applied Surface Science. PB91-189936

100,203

Technical Activities 1990 Surface Science Division

PB91-240820

100,353

POWELL, R. C.

Refractive Index Gratings in Rare-Earth-Doped Alkaline Earth Glasses.

101,422

POWELL, R. M.

Electronics and Electrical Engineering Laboratory. 1991 Strategic Plan. Supporting Technology for U.S. Competitiveness in Electronics.

PB92-123082

100,870

PRATT, K. W.

Proposed New Electrolytic Conductivity Primary Standards for $\mathrm{KCl}$ Solutions.

B91-18765

100,339

PB91-195164

100,201

PRESSER, C

Application of Laser Diagnostic Techniques for the Examination of Liquid Fuel Spray Structure.

PB91-195487

100,229

Effect of Atomization Air on Droplet Dynamics of Spray PBa1-203869

100,550

Laser Diagnostics for Characterization of Fuel Sprays

PB91-149013

100,541

Velocity and Droplet Size Measurments in a Fuel Spray.

PB91-14793

100,539

PRINCE, E.

Ab Initio Phase Determination for X-ray Diffraction Data from Crystals of a Native Protein.

(1) 237362

101,309

Refinement of the Structure of Beta'-MoO3

PB91-203786

101,090

Terminal Velocities for a Large Sample of O Stars, B Supergiants, and Wolf-Rayet Stars.

pergiants, and

100,023

\section{PROCTOR, F. M.}

Automating Robot Programming in the Cleaning and Deburring Workstation

100,986

CAD Directed Robotic Deburring

PB91-195172

100,985

LBIR Facility User Handbook.

PRUITT, J. S.

Absorbed-Dose Calibration of Ionization Chambers in a(60)Co Gamma-Ray Beam.

PB91-132290

01,598

Absorbed Dose to Water: Comparison of Several Methods Using a Liquid Ionization Chamber

1-19505

101,322

Status of Beta-P
PB91-175265

101,636

PUGH, E. N.

Cleavage Step Formation and Resistance to Transgranular Stress Corrosion Cracking

PB91-149047

101,133

Institute for Materials Science and Engineering: Metallurgy Division, Technical Activities 1990.

101,207

Materials Research at the National Bureau of Standards.

PB91-149039 101,214

Metallurgy Division, Technical Activities 1991. (NAS-NRC Assessment Panel February 13-14, 1992)

101,171

PB92-126515

Characteristics of the Breakdown Voltage of Power MOS FETs After Total Dose Irradiation.

PB91-149062

100,793

PUI, D. Y. H.

Use of the Electrostatic Classification Method to Size 0.1 micrometer SRM Particles: A Feasibility Study.

PB91-187633

100,932

PULLEN, B. P.

Unusual Degree of Angular Anisotropy in the Resonant Auger Spectrum of $\mathrm{Kr}$.

PB91-187310

101,649

PUMMER, W. J.

Synthesis of Non-Ionic Water-Dispersible Resins for Use in Intaglio Inks Curing by Electron Beam Radiation.

PB91-194456

100,499

PURTSCHER, P. T.

Aluminum Alloys for ALS Cryogenic Tanks: Comparative Measurements of Cryogenic Mechanical Properties of Al-Li Alloys and Alloy 2219 .

AD-A242 956/1

101,204

Effect of Processing on 4-K Mechanical Properties of a Mi-

croalloyed Austenitic Stainless Steel.
PB91-237206

101,167

Interstitial Content on Fracture Toughness.

101,15

Interlaboratory Tension and Fracture Toughness Test Results for CSUS-JN1 (Fe-25Cr-15Ni-0.3N) Austenitic Stainless Steel at $4 \mathrm{~K}$

PB91-237081

101,166

Jlc Data Analysis with a 'Negative Crack Growth' Correction Procedure

100,930

Metallographic Study of the Crack-Tip Region from Fracture Mechanics Specimens of Austenitic and Ferritic Steels. PB91-189951

101,158

QIAN, W. J.

Relativistic Distorted-Wave Cross Sections for Electron Impact Excitations of Berylliumlike lons.

PB91-195503

101,672

QIAN, Z. T.

Device for Audio-Frequency Power Measurement. PB91-14940

100,733

QUATE, C. F.

Surface Extended-X-ray-Absorption Fine Structure and Scanning Tunneling Microscopy of Si(001)2x1-Sb. PB91-237263

101,552

\section{UINONEZ, A.}

Rovibrational Analysis of the nu7(1) Intermolecular Hydrogen Bond Bending Vibration in HCN-HF Using Far Infrared Fourier Transform Spectroscopy.

QUINTERO, R.

Task Decomposition and Algorithm Development for RealTime Motion Control of a Continuous Mining Machine.

PB91-231597

101,357

QUINTIERE, J. G.

Canadian Mass Fire Experiment, 1989.

PB91-143305

101,352

Flammability Characterization of Foam Plastics.

PB92-123033

Framework for Utilizing Fire Property Tests.

PB91-240788

100,142 
Optimization of a Pulse Tube Refrigerator for a Fixed Compressor Swept Volume.

PB91-203877

101,051

Review of Pulse Tube Refrigeration.

PB91-147942

101,046

Saran Carbon

um.

RADERMACHER, $\mathbf{R}$. Horizontal Flow Boiling Heat Transfer Experiments with a
Mixture of $\mathrm{R} 22 / \mathrm{R} 114$.

PB92-116946

101,200

Study of Flow Boiling Heat Transfer with Refrigerant Mixtures.
PB91-189563

101,196

RADOVANOV, S. B.

Off-Axis Measurements of Ion Kinetic Energies in RF Plas-

mas.

101,444

Muon Sticking in Catalysed d-t Fusion: Nuclear Resonance Effect.

PB91-147959

101,613

RAFFI, J. J.

Co-Trial on ESR Identification and Estimates of gamma-Ray

and Electron Absorbed Doses Given to Meat and Bones.
PB91-194720 PB91-194720

RAGHAVA RAO, K. S. M. S.

Electrokinetic Demixing of Two-Phase Aqueous Polymer Systems. 1. Separation Rates of Polyethylene Glycol-Dextran Mixtures.

PB91-159095 100,487

Electrokinetic Demixing of Two-Phase Aqueous Polymer Systems. 2. Separation Rates of Polyethylene Glycol-Maltodextrin Mixtures.

PB91-237230

RAGHAVAN, N. V.

Neutron Crystallography of Proteins.
PB91-175349

100,231

RAINS, T. C.

Recommended Inorganic Chemicals for Calibration

PB91-195164 100,201

Trace Determination of $\mathrm{Cr}(\mathrm{VI})$ by LC/AAS with on-Line Preconcentration.

RAINWATER, J. C.

100,184

Critical Locus, (Vapor + Liquid) Equilibria, and Coexisting Derisities of (Carbon Dioxide + Propane) at Temperatures from $311 \mathrm{~K}$ to $361 \mathrm{~K}$

PB91-134494

100,267

Thermal Conductivity of a Moderately Dense Gas. PB91-134502

101,601

RAIZEN, M. G

Progress at NIST on Absolute Frequency Standards Using Stored lons.

\section{RAM, R. S.}

101,625

Rovibrational Analysis of the nu7(1) Intermolecular Hydrogen Bond Bending Vibration in HCN-HF Using Far Infrared Fourier Transform Spectroscopy.

PB91-237784

100,437

RAMAGE, D.

Neutron Measurements of Intramolecular Vibrational Modes in 660 .

PB92-116540

101.055

\section{RAMANATHAN, G.}

Knowledge Extraction Techniques for Expert System Assisted Wafer Screening

100,824

RAMBOZ, J. D.

NIST Measurement Services: Calibration Service for Current Transformers.

Precision Oualification of Watthour Meters.

PB91-237214

100,873

RAMIRES, M. L. V.

Benzene: A Further Liquid Thermal Conductivity Standard. PB90-244567

RANDA, J.

Estimation of Electromagnetic Fields in Complex Environ-

101,691

Lattice Approach to Environments Irradiated by Unknown Sources.

100,850

Simultaneous versus Independent Injection Testing of Nonlinear Multiport Systems.

PB91-203885

100,857

Theoretical Considerations for a Thermo-Optic Microwave Electric-Field-Strength Probe.

PB91-237222

100,860

RANDOLPH, D.

Thin and Composite High-Flux Membranes of Perfluorosulfonated lon-Exchange Polymer.

100,512
RANGACHAR, $R$.

Analysis of Optical Flow Estimation Using Epipolar Plane Images.

100,703

Three Dimensional Reconstruction from Optical Flow Using Temporal Integration.

PB92-112507

100,702

RAO, G.

Chemical Nature of In vivo DNA Base Damage in Hydrogen Peroxide-Treated Mammalian Cells.

101,335

Damage to the DNA Bases in Mammalian Chromatin by Hydrogen Peroxide in the Presence of Ferric and Cupric lons.
PB91-189217

RAO, M. V.

Fe Implantation in In0.53Ga0.47As/InP
PB91-189969

101516

AASBERRY, S. D.

Reference Materials for Analytical Chemistry

PB91-203893

101,516

Reference Materials: Their Role in Measurement Accuracy.
PB91-159103

Role of Reference Materials in Ouality Assurance. PB91-203901

RAU, G.

100,206

Modification of DNA Bases in Mammalian Chromatin by Radiation-Generated Free Radicals.

PB91-189373

101,338

RAUFASTE, N. J.

Building and Fire Research Project Summaries, 1991.
PB91-216788

Wind and Seismic Effects. Proceedings of the Joint Meeting of the U.S.-Japan Cooperative Program in Natural ReSources Panel on Wind and Seismic Effects (23rd). Held in
Tsukuba, Japan, on May 14-17, 1991 . Tsukuba, Japan, on May 14-17, 1991.
PB92-116425

100,152

RAVIV, D.

Integration-Based Method for Depth Estimation. PB92-112275

100,701

New Approach to Vision and Control for Road Following.

PB91-157156

RAWLINS, $W$.

Apparatus for the Measurement of Regenerator Performance in Pulse Tube Refrigerators.

PB91-147967

101,047

RAWN, C. J.

Phase Equilibria of the System Strontium Oxide-Calcium Oxide-Cupric Oxide.

PB91-195628

101,085

RAY, $\mathbf{S}$.

Manufacturing Systems Integration Control Entity Interface Document.

100,991

Manufacturıng Systems Integration: Initial Architecture Doc-

PB92-112242

100,994

RAYNES, A.

Design Diagrams for Heavy Metal Fluoride Glass Windows.

PAYNES, A. S.

Strength and Fracture Behavior of Ba-Y-Cu-O Superconducting Ceramics.

PB91-148205

101,474

REACH, W. T.

Detection of an Expanding $\mathrm{H}$ I Shell in the Old Supernova Remnant CTB 80.

PB91-174920

100,028

AD, D. T.

Experimental and Numerical Studies of the J-Integral for a Surface Flaw.
PB91-147256

READER, J.

101,576

Spectrum and Energy Levels of Seven-Times-Ionized Krypton $(\mathrm{Kr}$ VIII) and Resonance Lines of Eight-Times-Ionized Krypton (Kr |X)

PBg1-195511

Spectrum and Energy Levels of the Xenonlike Ion Ce

Sp91-175364

REBULDELA, G.

101,638

RF-DC Differences of Thermal Voltage Converters Arising

from Input Connectors.
PB91-236802

100,735

REDFORS, A.

Spectrum and Energy Levels of the Xenonlike Ion Ce $\mathrm{V}$.

PB91-175364

101,638

AEED, R. P.

Aluminum Alloys for ALS Cryogenıc Tanks: Comparative Measurements of Cryogenic Mechanical Properties of Al-Li Alloys and Alloy 2219.

101,204

Aluminum Alloys for ALS Cryogenic Tanks: Oxygen Compatibility. Volume 2

AD-A231 830/1

101,203

Aluminum Alloys for Cryogenic Tanks: Oxygen Compatibility. Volume 1.

AD-A229 229/0

101,201

Creep of Copper: 4 to $295 \mathrm{~K}$

101,233

Cryogenic Fatigue of High-Strength Aluminum Alloys and Correlations with

101,709

Discontinuous Yielding during Tensile Tests at Low Tem-

PB91-189977

101,159

Effect of Nitrogen and Carbon on FCC-HCP Stability in Austenitic Steels

101,151

Effect of Processing on 4-K Mechanical Properties of a Microalloyed Austenitic Stainless Steel.

PB91-237206

101,167

forluence of Interstitial Content on Fracture Toughness.

101,157

Interlaboratory Tension and Fracture Toughness Test Results for CSUS-JN1 (Fe-25Cr-15Ni-0.3N) Austenitic Stainless Steel at $4 \mathrm{~K}$.

PB91-237081

101,166

Load-Controlled Tensile Tests of Austenitic Steels at $4 \mathrm{~K}$.

101,165

Metallographic Study of the Crack-Tip Region from Fracture Mechanics Specimens of Austenitic and Ferritic Steels.

PB91-189951

101,158

Nitrogen Strengthening of Austenitic Stainless Steels at Low Temperatures.

101,163

Review of Cryogenic Mechanical and Thermal Properties of Al-Li Alloys and Alloy 2219.

101,202

Strain Rate Effect on Tensile Properties at $4 \mathrm{~K}$ of a VAMAS Round-Robin Austenitic Steel.

101,168

REED, W. P.

Reference Materials: Their Role in Measurement Accuracy.
PB91-159103 REEVE, C. $P$.

Eccentric Load Sensitivity of Force Sensors.

PB91-175406

100,931

REEVE, G. R.

Microwave Monolithic Integrated Circuit-Related Metrology at the National Institute of Standards and Technology.
PB91-237255 MMIC Related Metrology at the National Institute of Standards and Technology.

PB91-149070

100,794

REHM, R. G.

Boussinesq Algorithm for Enclosed Buoyant Convection in Two Dimensions.

\section{REIF, A. K}

Standard Chemical Thermodynamic Properties of Polycyclic Aromatic Hydrocarbons and Their Isomer Groups. III. Naphthocoronene Series, Ovalene Series, and First Members of Some Higher Series.

PB90-244625

100,259

REILLY, M. L.

MSW Calorimetry.

PB91-133942

100,878

REIMANN, C. W.

Measurement Ouality Assurance.

PB91-149930

101,013 
REYNARD, $K$.

Factual Materials Databanks - The Need for Standards.
PB91-147546

RHEE, W. H.

101,249

Coherency Strain Induced Instability of Solid-Liquid Inter-

faces in the Mo-Ni System.
PB91-195537

101,235

RHYNE, J. J.

Low Temperature Magnetization and Magnetic Excitations in Amorphous Fe78B $13 \mathrm{Sig}$

101,573

Phonon Density of States of Superconducting YBa2Cu3O7

and the Nonsuperconducting Analog YBa2Cu3O6.

101,487

Structural and Magnetic Properties of Er Thin Films and Er/ $Y$ Superlattices: Magnetoelastic Effects.

101,489

RICE, $P$.

High-Pesolution, Tunneling-Stabilized Magnetic Imaging and Recording.

101,600

New Look at the Bitter Method of Magnetic Imaging.
PB91-203919

Tunneling Stabilized Magnetic Force Microscapy: Prospects for Low Temperature Applications to Superconductors. 101,546 PB91-203653

Rapid Low-Temperature Hopping of Hydrogen in a Pure Metal: The ScHx System.

PB91-15848

101,217

Laser Induced Desorption of Molecules from Surlaces.

PB91-147066 100,235

Photodissociation Dynamics of $\mathrm{Mo}(\mathrm{CO}) 6$ at $266 \mathrm{~nm}$ and $355 \mathrm{~nm}$ : CO Photofragment Kinetic-Energy and InternalState Distributions.

PB91-236547

100,413

Surface-State-Mediated Photochemistry: Laser-Induced Desorption of $\mathrm{NO}$ from $\mathrm{Si}$ (111).

ICHTER,

100,320

Surface Extended-X-ray-Absorption Fine Structure and Scanning Tunneling Microscopy of Si(001)2 $\times 1-\mathrm{Sb}$.

101,552

PB91-237263

RICKER, R. E.

Influence of Moisture and Pressure on the Mechanical Properties of a Glass-Epoxy Matrix Composite and a Graphite-Epoxy Matrix Composite.

101,114

AD-A239 509/3

ies of a

Influence of Water on the $M$

101,113

AD-A231 778/2

Stress Corrosion Cracking of Al-Li Alloys: The Role of Grain Boundary Precipitates.

PB91-17518

101,137

Particle Size Measurement of Inert Gas Atomized Powder.
PB91-187203

RINN, $K$.

and Their Probe for Real-Time Images of Particle Beams and Their
Analyses in a Merged-Beams Apparatus.
PB91-203067
101,683

RISKO, D. G.

Capacitance versus Stylus Measurements of Surface Poughness.

101,020

RITTER, J.

Studies of Magnetic Flux Penetration in a Chemically Synthesized $\mathrm{Bi}(\mathrm{Pb}) \mathrm{SrCaCuO}$ Superconductor.

PB91-194977

101,526

RITTER, J. J.

Change in Magnetic State of $\mathrm{Fe}+$ Silica Gel Nanocomposites Due to Low Temperature Treatment in Ammonia.

PB91-237321 101,129

Hermetically Sealed Inert Atmosphere Cell for X-ray Powder Diffraction.

Low Temperature Chemical Process for Precursors to Boride and Carbide Ceramics Powders.

PB91-149096

101,066

Low Temperature Chemical Route to Precursors of Boride and Carbide Ceramic Powders.

101,078

Low Temperature Synthesis of Ceramic Powders for Structural and Electronic Applications.

PB91-149104

101,067

Magnetic Behavior of Nanocomposites Prepared in a Vitreous Alumina Ge

101,131

Magnetic Properties of Iron/Silica Gel Nanocomposites.

PB91-204073 101,110

Phase Equilibria of the System Strontium Oxide-Calcium

Oxide-Cupric Oxide

Spin Glass Magnetic Behavior of Iron/Silica Gel Nanocomposites.
PB91-159178

101,117

VERA, $R$.

Chitin-Chitosan Membranes: Separations of Amino Acids and Polypeptides.

RIZICKA, V.

101,283

Heat Capacities of Organic Compounds in Liquid State. 2 C1 to C18 n-Alkanes.

PB92-110204

100,448

ROACH, R.

National Computer Systems Laboratory Annual Report, PB91-201822

ROBACK, E.

100,603

Automated Information Systems Security Program Hand-

book. 22638

Glossary of Computer Security Terminology.

100,716

National Aeronautics and Space Administration's (NASA)

Automated Information Security Handbook.

PB91-187781

100,707

ROBB, $\mathrm{H}$.

Trends in Technological Innovation

PB91-195545

ROBERTS, J. A.

Test Chip for the Evaluation of Surface-Diffusion Phenom ena in Sputtered Aluminum Planarization Processes. PB92-116920

ROBERTS, J. R.

Relative Populations of Excited Levels within the Ground Configuration of Si-Like $\mathrm{Cu}, \mathrm{Zn}, \mathrm{Ge}$, and Se lons.

PB91-158618

ROBERTS, K. $M$.

ISDN Conformance Testing: Layer 1. Physical Layer. Part 1 Basic Rate S/T Interlace, User Side.

100,572

North American ISDN Users' Forum Agreements on Integrated Services Digital Network.

PB92-102219

100,573

ROBERTSON, $B$

Frequency Dependence of Catalyzed Reactions in a Weak Oscillating Field.
PB91-203927

ROBERTSON, J. L.

Comparison of Amorphous and Quasicrystalline Films of Sputtered Al0.72Mn0.22SiO.06

ROBEY, S. W

Electronic Structure of High-Tc Superconductors Studied Using Photoelectron Spectroscopy.

91-18966

101,513

NS, L.

Influence of Filament Geometry on Hot Filament Growth of Diamond Films.

ROBINS, L. H.

Cathodoluminescence Measurement of Strained Alumina Single Crystals.
PB91-237131

101,094

Chemical Vapor Deposited Diamond.
AD-A243 095/7

101,106

Critical Assessment of Optical Properties of CVD Diamond

Determination of the Optical Constants of Thin ChemicalVapor-Deposited Diamond Windows from 0.5 to $6.5 \mathrm{eV}$

AD-A243 094/0 100,215

Effects of Multiple Filament Geometry in the Hot Filament Deposition of Diamond Films.

101,142

High Resolution Electron Microscopy of Diamond Film Growth Defects and

101,449

Inverse Correlation between the Intensity of Luminescence Excited by Electrons and by Visible Light in Chemical$\checkmark$ apor-Deposited Diamond Films.

PB91-195560

101,531

Optically Detected Magnetic Resonance Study of Antisite to-Acceptor and Related Recombination Processes in as Grown InP-Zn.

Photoluminescence Excitation by Band-Gap Optical Ab sorption in Chemical Vapor Deposition Diamond Films.

PB91-195586

101,533

Spatially and Spectrally Resolved Cathodoluminescence Measurements of CVD-Grown Diamond Particles and Films.

Spatially and Spectrally Resolved Cathodoluminescence of Hot-Filament Chemical-Vapor-Deposited Diamond Particles. AD-A236 485/9 100,247

Spatially and Spectrally Resolved Cathodoluminescence of Hot-Filament Chemical-Vapor-Deposited Diamond Particles. AD-A237 128/4

ROBINSON, A, M.

Micromachined Thermal Radiation Emitter from a Commercial CMOS Process.

PB91.148999

100,748

Numerical Modeling of Silicon Photodiodes for High-Accuracy Applications. Part 1. Simulation Programs.

100,763

Numerical Modeling of Silicon Photodiodes for High-Accura-
cy Applications. Part 2. Interpreting Oxide-bias Experiments cy Applications. Part 2. Interpreting Oxide-bias Experiments.
PB92-110303

Numerical Modeling of Silicon Photodiodes for High-Accuracy Applications. Part 3. Interpolating and Extrapolating Internal Quantum-Efficiency Calibrations.

PB92-110311

100,765

Testing the Accuracy of Calculated Equilibrium Carrier Concentrations in the Presence of Surface Fields.

PB91-236729

101,549

AOBINSON, $\mathrm{H}$

Observation of the Cesium Clock Transition Using LaserCooled Atoms in a Vapor Cell.

PB91-203638

101,698

ROBINSON, M. S.

High-Resolution Infrared Flash Kinetic Spectroscopy of $\mathrm{OH}$ Radicals

PB91-204008

100,408

ROBINSON, S. J.

Doppler Shift Attenuation Lifetime Measurements in (54)Cr Following Thermal Neutron Capture.

PB91-189753

Using the Harvard/NIST Mark VI Fire Simulation.

101,656

RODEL, J.

Cyclic Fatigue from Frictional Degradation at Bridging Grains in Aluming.

101,092

Fracture Mechanics Model for Subthreshold Identation Flaws. Part 1. Equilibrium Fracture.

101,093

In situ Measurements of Bridged Crack Interlaces in the Scanning Electron Microscope.

101,068

RODER, $H$. M.

High-Temperature Transient Hot-wire Thermal Conductivity Apparatus for Fluids.

Thermal Conductivity and Heat Capacity of Gaseous Argon PB91-134577

101,602

RODGERS, A. $S$

Thermodynamic and Thermophysical Properties of Organic Nitrogen Compounds. Part 1. Methanamine, Ethanamine, 1 and 2-Propanamine, Benzenamine, 2-, 3-, and 4-Methylbenzenamine.

PB91-192583

100,368

ROEHRIG, $\mathrm{H}$

Use of Storage Phosphor Imaging Plates in Portal Imaging and High-Energy Radiography: The Intensitying Effect of Metallic Screens on the Sensitivity

PB91-236497

101,324

ROGERS, E. E.

Concerning the Formation and the Kinetics of Phenylium lons.

100,336

ROGERS, J. E.

Interior-Point Method for Linear and Quadratic Programming Problems.

ROGERS, S. A

101,269

Multireference-Configuration Interaction (MR-Cl) Calcula tions on $\mathrm{HS}(2+)$ and Experimental Observation via Elec tions on $\mathrm{HS}(2+)$ and Experim

tron Impact loniz

ROITBURD, A.

Comparison of Flux Dynamics in Two Samples of YBa2Cu3O7 with Different Pinning. 


\section{PERSONAL AUTHOR INDEX}

RONGGEN, L.

Low-Pressure Symmetric Discharge Heat Pipe and DROGS

OP EU.

101,666

\section{ROOKE, S.}

Refrigerant Charge Effects on Heat Pump Performance. ROOP, B.

Use of Heterodyne Detection to Measure Optical Transmittance over a Wide Range.

PB91-148908

101,410

ROSASCO, G. J.

Dispersion of the Electronic Contribution to the Thrid-Order Nonlinear Susceptibility of $\mathrm{H} 2$.

101,411

Semiclassical Calculation of Self-Broadening in O2, N2, and CO Raman Spectra.

PB92-117050

100,461

ROSE, A. H.

High Speed Magnetic Field Sensors Based on Iron Gar-

PB91-147223

100,840

square

Iron-Garnet Magnetic Field Sen

100,751

Optical Fiber Current Sensors with Temperature Stabilities Near the Material Limit.

100,753

PB91-175109

Performance and Limitations of Faraday Effect Sensors.
PB91-149146

Practical Considerations in the Design of Optical Fiber Current Sensors.
PB91-134742

100,746

ROSE, J.

Capacitance versus Stylus Measurements of Surface Roughness.

ROSEN, B. K

Guide to Schema and Schema Extensibility.
PB92-126556

101,020

100,955

ROSENTHAL, $Y$.

Computerization of a Thermomechanical Processing Research System
PB91-175208

101,583

ROSENTHAL, Y. A.

JIc Data Analysis with a 'Negative Crack Growth' Correction Procedure.

PB91-175216

100,930

ROSHKO, A.

Morphology of Silver on YBa2Cu3O7-delta Thin Films.

PB91-195594 101,534

Switching in High-Tc Superconductor Current Transport Measurements.

ROSMAN, K. J. R.

Isotopic Compositions of the Elements 1989 PB92-148220

101,539

Ross, A. B.

Rate Constants for Reactions of Peroxyl Radicals in Fluid Solutions.

PB90-244641

ROSSITER, W. J.

Field Study of the Performance of EPDM Roofing at Air Force Facilities.

101,340

Field Testing of Adhesive-Bonded Searns of Rubber Roofing Membranes.
PB91-189993

100,124

Further Investigation of the Effect of Application Parameters on Adhesive-Bonded Seams of Single-Ply Roof Membranes.

100,105

Pulse-Echo Uttrasonic Evaluation of the Integrity of Seams

Pulse-Echo Ultrasonic Evaluation of the Integrity of Seams
of Single-Ply Roofing Membranes: Laboratory Evaluation of of Single-Ply Roofing Membranes: Laboratory Evaluation o a Prototype Te
PB91-157172

100,106

Pulse-Echo Ultrasonic Evaluation of the Integrity of Seams of Single-Ply Roofing Membranes: Results of Field Investigations and Recommendations.

100.110

Use of Nondestructive Methods for Inspection of Single-Ply Use of Nondestructive Methods for Inspection of Single-Ply
Roofing Membranes.

PB91.203935
ROSZMAN, L. J.

100.128

Dielectronic Recombination Rate Coefficients for lons of the Fluorine Isoelectronic Sequence.

PB91-195602

101,674

Dielectronic Recombination Rate Coefficients of $\mathrm{O}(5+)$ and $O(2+)$.

PB91-190009 101,657

Influence of Metastable States and Thermal Equilibration upon Dielectronic Recombination in Low Density to Moderate Density.

101,675

ROTH, R. S.

Chemistry of Electronic Ceramic Materials.
PB91-178822

New Layered Cuprate Structure-Type, (A1. $\left.X A^{\prime} x\right) 14 C u 24041$.
$P B 91-237339$

Parent Structure of the Layered High-Temperature Superconductors.

101,535

Phase Equilibria of the System Strontium Oxide-Calcium Oxide-Cupric Oxide.

PB91-195628

101,085

\section{ROTHER, J. E.}

Analysis of Wet Deposition (Acid Rain): Determination of the Major Anionic Constituents by Ion Chromatography.
PB91-236646

ROTTMAN, $C$.

Calculation of Energy of Low-Angle Grain Boundaries.

PB91-134601

101,464

\section{ROWAN, W. L.}

Extreme-Ultraviolet Spectra of Pd, Ag, Cd, and Sn lons Observed in a 1.3-keV Tokamak Plasma.

101,712

Spectra of the P I Isoelectronic Sequence from Co XIII to Spectra of the P I Isoelectronic Sequence from Co XIII to
Mo XXVIII.

ROWE, W. P.

Depot: A Framework for Sharing Software Installation Across Organizational and Unix Platform Boundaries.

PB91-240754

ROWLANDS, c. c.

Co-Trial on ESR Identification and Estimates of gamma-Ray and Electron Absorbed Doses Given to Meat and Bones. PB91-194720

\section{ROWLEY, R. L.}

Non-Equilibrium Molecular Dynamics Simulations of Structured Molecules. Part 1. Isomeric Effects on the Viscosity of Butanes.

PB91-195636

101,391

\section{ROYSTER, $C$.}

Guidelines for the Evaluation of File Transfer, Access and Management Implementations.

PB92-126580

100,699

ROYTBUAD, A.

Low-Field Flux Pinning in Twinned and Detwinned Single Crystals of YBa2Cu3O7-x.

PB91-237552

101,559

RUBIN, A.

Daylight Research Requirements. Workshop Proceedings. Held in Baltimore, Maryland on August 1-2, $1990 . \quad 100,078$

Intelligent Building Technology in Japan. PB91-187757

100,071

AUBIN, A. I.

Abstracts of Daylighting Research.

PB91-206755

RUBINSTEIN, $M$.

Mossbauer Study of the Effect of Oxygen Stoichiometry on the High Tc Superconductor Y1Ba2(CuO.97Fe0.03)3O7-x. PB91-237545

RUDD, J. M.

Magnetic Anisotropies in Ultrathin fcc Fe(001) Films Grown on Cu(001) Substrates.

PB92-116631

101,565

RUDGE, S. R.

Applied Electric Fields for Downstream Processing

PB91-14916

100,299

RUDMAN, D. A

Properties of YBa2Cu3O7-delta Thin Films Grown on OffAxis-Cut $\mathrm{MgO}$ Substrates

RUEGG, R. T.

101,547

Economic Analysis for Military Construction (MILCON) Design. Concepts, Techniques, and Applications for the An alyst. Instructor's Manual.

PB92-112440

101,349

Economic Analysis for Military Construction (MILCON) Design. Concepts. Techniques, and Applications for the Analyst. Student's Manual.

101,350

RUFF, A. W.

Comparison of Standard Test Methods for Non-Lubricated Sliding Wear

101,187

Numeric Database for Tribology: Format and Application

$\begin{array}{ll}\text { Issues. } & 101,045 \\ \text { PB91-203943 }\end{array}$

Sliding Wear Studies of Nickel-Copper Composition-Modulated Coatings on Steel.

PB91-190025

101,188

RUHL, M. K.

Software Reengineering: A Case Study and Lessons Learned

PB92-116417

100,669

RULE, D. L.

Thermal Conductivity of a Polymide Film between 4.2 and $300 \mathrm{~K}$, with and without Alumina Particles as Filler.

PB91-144402

100,483

Thermal Conductivity of Alumina Fiber/Epoxy and Alumina Fiber/PEEK from 4.2 to $310 \mathrm{~K}$

101,130

RUMACK, D. T.

Proton Affinities and pKa Values of Tetraalkylhydrazines.

PB91-195214 100,378

Slow Electron Transfer Reactions between Alkylhydrazines. 91-147785

RUMBLE, J.

Factual Materials Databanks - The Need for Standards.
PB91-147546

Numeric Database for Tribology: Format and Application Issues.

PB91-203943

101,045

RUSH, J. J.

Neutron Powder Diffraction and Inelastic Scattering Study of the Structures of $\mathrm{Zr} 2 \mathrm{Pd}, \mathrm{Zr} 2 \mathrm{PdD} 1.70$, and $\mathrm{Zr} 2 \mathrm{PdD} 1.96$.

101,213

Neutron Spectroscopic Evidence for Adsorbed Hydroxyl Species on Platinum Black.

PB91-237636

100,432

Phonon Density of States and Superconductivity in Nd1.85Ce0.15CuO4.

PB91-189787

Properties of Amorphous Zirconium Rhodium Hydride (Zr3RhHx) Prepared from Glassy and Crystalline Alloys.
PB91-174466

101,225

Rapid Low-Temperature
Metal: The ScHx System.

PB91-158485

101,217

Relationship of Hydrogen Site Occupancy to Diffusion Behavior in Crystalline and Amorphous $\mathrm{Zr} 2 \mathrm{PdH}$

101,230

RUSSEK, S. E.

Properties of YBa2Cu3O7-delta Thin Films Grown on OffAxis-Cut MgO Substrates.

PB91-203950

101,547

RUSSELL, D. $\mathrm{H}$

Dual Six-Port Reflectometer Systems Using Waveguide in the Frequency Range 18-50 GHz.

PB91-134916

100,838

RUSSELL, T. J.

Measurement of Capacitance on Wafers

PB91-149120

100,795

RUST, B. W.

Dosimetry of a Nearly Monoenergetic 6- to 7-MeV Photon Source by $\mathrm{Nal}(\mathrm{TI})$ Scintillation Spectrometry. PB92-116706

101,723

RUZICKA, V.

Heat Capacities of Organic Compounds in the Liquid State I. C1 to C18 1-Alkanols.

PB90-244716

100,265

SABRY, D. Y.

Redox Reactions of Osmium Porphyrins.

PB91-159012

100,217

SAGUCHI, $R$.

Chain Conformation of a Block Polymer in a MicrophaseSeparated Structure.

PB91-148890

100,485

SAHA, B. C.

Near-Threshold Vibrational Excitation of $\mathrm{H} 2$ by Electron Impact: Resolution of Discrepancies between Experiment and Theory.

SAKURAI, S.

101,679 
SALOMAN, E. B.

Energy Levels and Transition Probabilities in the Ground State Configuration of Sulfur-Like lons.

PB91.190033

Resonance Ionization Spectroscopy/Resonance lonization Mass Spectrometry Data Service.

100,207

Resonance Ionization Spectroscopy/Resonance Ionization Mass Spectrometry Data Service. II. Data Sheets for Al, Ca, $\mathrm{Cs}, \mathrm{Cr}, \mathrm{Co}, \mathrm{Cu}, \mathrm{Kr}, \mathrm{Mg}, \mathrm{Hg}$, and $\mathrm{Ni}$.

PB91-162297

100,193

SALTMAN, R. G

Electronic Data Interchange (EDI). Category: Software Standard. Subcategory: Electronic Data Interchange. FIPS PUB 161

100,677

SAMS, $R$.

Reference NO2 Calibration System for Ground-Based Inter-

comparisons during NASA's GTE/CITE II Mission.
PB91-189340

100,034

SAMWER, $K$.

Properties of Amorphous Zirconium Rhodium Hydride

(Zr3RhHx) Prepared from Glassy and Crystalline Alloys.
PB91-174466 SANDELL, A.

Electrofission in the Ouasifree and Delta Regions.

PB91-147355

ANDER, L. C.

Determination of Bonded Phase Thickness in Liquid Chro-

matography by Small Angle Neutron Scattering.
PB91-149187

101,609

100,190

Evaluation of Column Performance in Liquid Chromatography. 190041

Method to Enhance Porosity of Micro-particles.

PB91-187328

100,199

SANDERS, A. A.

Laser Measurements.

PB91-149195

101,185

SANFORD, D. T.

STEP: Standard for the Exchange of Product Model Data Resource Integration: Semantic and Syntactic Rules.
PB91-184788

SANFORD, N. A.

Integrated-Optic Waveguide Glass Lasers

SANFORD, R. J.

Determining the Dynamic Stress Intensity Factor with Strain Gages Using a Crack Tip Locating Algorithm.

PB91-174268

101,580

SANGSTER, J.

Coupled Phase Diagram-Thermodynamic Analysis of the 24

Binary Systems, A2CO3-AX and A2SO4-AX Where A $=\mathrm{Li}$,

$\mathrm{Na}, \mathrm{K}$ and $\mathrm{X}=\mathrm{Cl}, \mathrm{F}, \mathrm{NO}, \mathrm{OH}$.

PB91-192518

100,362

SANI, A. P.

Prototyping the IRDS: An Airport Application.

PB92-112580

SANSALONE, $M$.

Assessment: U.S. Office Building of Moscow.

PB91-237834 100,15

Extended Abstract: A Finite Element Study of the Stress and Displacement Fields Produced by Point Impact.

PB91-175224

101,584

Impact-Echo: A New Method for Inspecting Construction

PB92-116557

100,140

SANSONETTI, C. J.

Precise Experimental Test of Calculated Two-Electron Lamb Shifts in Helium.

101,617

Precise Laser Wavelength Measurements: What Can We Learn from Classical Spectroscopy.

101,430

SANTORO, A.

Models for Oxygen Ordering and Diffusion in Ba2YCu3OX and $\mathrm{Ba} Y \mathrm{YCu}-\mathrm{yMyO} \times(\mathrm{M}=\mathrm{Fe}, \mathrm{Co}, \mathrm{Al}, \mathrm{Ga})$.

101,496

Neutron Powder Diffraction and Inelastic Scattering Study of the Structures of Zr2Pd, Zr2PdD1.70, and Zr2PdD1.96. PB91-148866

101,213

SANTORO, R. J.

Application of Laser Diagnostic Techniques for the Examination of Liquid Fuel Spray Structure.

PB91-195487

100,229

Laser Diagnostics for Characterization of Fuel Sprays.

PB91-149013 100,541

Velocity and Droplet Size Measurments in a Fuel Spray.

PB91-147934 100,539

SARAIJI, $R$.

Abstracts of Daylighting Research

SARGENT, C. L.

100,076

Data-Reduction and Analysis Procedures Used in NIST'S

Thermomechanical Processing Research.
PB91-132282

100,925

SASAKI, $\mathrm{H}$.

Monitoring the Mass Standard via the Comparison of Mechanical to Electrical Power

PB91-237115

101,710

SASS, J. $K$.

Adsorption of Water and Oxygen on $\mathrm{Ag}(110)$ : A Study of the Interactions among Water Molecules, Hydroxyl Groups, and Oxygen Atoms.

SATO, $H$.

100,270

Sixteen Thousand Evaluated Experimental Thermodynamic Property Data for Water and Steam.

PB92-148154

100,475

SAUERS, I.

Detection of Trace Disulfur Decafluoride in Sulfur Hexafluoride by Gas Chromatography/Mass Spectrometry PB91-189860

100,197

SAUERWEIN, J. C.

NIST Standard Reference Data Products 1991 Catalog.
PB91-167312

SAULSON, P. R.

Gravitational Radiation Observations on the Moon

PB91-175083

101,635

Thermal Noise

101,578

SAUNDERS, B. V.

Effect of Gravity Modulation on Thermosolutal Convection in an Infinite Layer of Fluid.

101,396

Portable Vectorized Software for Bessel Function Evalua$\begin{array}{ll}\text { tion. } & 100,652\end{array}$

100,652

SAUNDERS, P. $B$.

Agency Exploits Flexibility of Software in Moving Info. PB91-159160

SAUNDERS, R. D.

Roughened Quartz Surfaces and Teflon as Small-Angle Diffusers and Depolarizers between 200 and $400 \mathrm{~nm}$ PB91-195644

101,425

SAWANO, $K$.

Comparison of Flux Dynamics in Two Samples of YBa2Cu3O7 with Different Pinning.

PB91-149419

SAWANT, S. B.

Hydrodynamics and Mass Transfer in Two-Phase Aqueous Extraction Using Spray Columns.

101,300

Mass Transfer Coefficients in Two-Phase Aqueous Extrac-

tion.

100,426

SAWLA, A.

Summary of the Intercomparison of the Force Standard Machines of the National Institute of Standards and Technology, USA, and the Physikalisch-Technische Bundesanstalt, Germany
PB92-126622

SCACE, R. I.

Metrology for the Semiconductor Industry.

PB91-240739

101,590

SCHAEFER, R. J.

Acoustic Emission Monitoring of Laser Drilling

PB91-158527

Phase Selection in Non-Equilibrium Processing.

PB91-195651

Transformation of the lcosahedral Phase in Ouenched Al-rich Al-Mn Alloys.

Rapidly

PB91-158964

101,221

AENMAN, $P$.

Estimated Impact of the Center for Fire Research Program on the Costs of Fire.

SCHAFFT, H. A.

Building-in Reliability: Making It Work

PB91-203992

100,161

Current Density Dependence of Electromigration $150 \mathrm{En}$ hancement Due to Pulsed Operation

PB91-134692

PB92-117282

100,786

SCHANTZ, M. M.

Preparation and Analysis of a Marine Sediment Reference Material for the Determination of Trace Organic Constitu-

PB91-149237

101,377

SCHAPS, S. R.

Residual Stress Detection in Railroad Wheels: An Ultrasonic System Using EMATs (Electromagnetic-Acoustic Transducers). Report No. 23.

PB91-222653

101,741

Tread Crack Detection in Railroad Wheels: An Ultrasonic System Using EMATs (Electromagnetic-Acoustic Transducers). Report No. 22.

101,742

SCHAUER, M. M.

Nonresonant Charge Transfer in the Threshold Region for (3) $\mathrm{He}(+)+(4) \mathrm{He}($ sub $<-$ sup- $>$ ) (3) $\mathrm{He}+(4) \mathrm{He}(+)$.

SCHEINFEIN, M. R.

Micromagnetic Calculations of $180 \mathrm{deg}$ Surface Domain. Wall Magnetization Profiles with Comparison to Measurements.

101,483

Micromagnetic Calculations of $180 \mathrm{deg}$ Surface Domain Walls.

PB91-237271

101,553

Micromagnetics of Domain Walls at Surfaces.

101,491

Scanning Electron Microscopy with Polarization Analysis (SEMPA)-Studies of Domains, Domain Walls and Magnetic Singularities at Surfaces and in Thin Films.

PB91-237289

101,554

SCHEN, M. A.

Development of Test Methods to Determine the Compatibil-

ity of Liquid Hazardous Materials with Polyethylene Packag-

ings.

100,943

SCHENCK, P. K

Graphics Program for Binary and Ternary Ceramic Phase Diagrams.

PB91-147991

101,063

Laser Vaporization Mass Spectrometry of Refractory Materials: Graphite and $\mathrm{YBa} 2 \mathrm{Cu} 3 \mathrm{Ox}$

Lead Zirconate-Titanate Thin Films Prepared by the Laser Ablation Technique.

PB91-187344

101,502

Thermodynamic and Kinetic Stability of Refractory Materials at Ultra-High Temperatures.

AD-A235 490/0

100,246

Biological Sulfur Oxidation and Reduction for Coal Sulfur Speciation and Desulfurization.

PB91-203323

100,886

Bioprocessing of Fossil Fuels Using Hyperthermophilic Archaebacteria.
PB91.162271

\section{SCHIEBENER, $P$.}

Refractive Index of Water and Steam as Function of Wave. length, Temperature and Density.

PB90-244708

101,402

SCHIFFMAN, A.

$\mathrm{H}+\mathrm{O} 3$ Fourier-Transform Infrared Emission and Laser Absorption Studies of $\mathrm{OH}(\mathrm{X}(2) \mathrm{Pi})$ Radical: An Experimen-
tal Dipole Moment Function and State-to-State Einstein A Coefficients

PB91-148973

100,297

High-Resolution Intrared Flash Kinetic Spectroscopy of $\mathrm{OH}$ Radicals.

PB91-204008

100,408

SCHILLER, S. B.

Precision Oualification of Watthour Meters

PB91-237214

100,859

SCHIMA, F. J.

Cross-Section Measurements in the Intermediate-Energy Standard Neutron Field.

PB91-203398

101,693

Spectrum-Averaged Cross-Section Measurement of $(103) R h(n, n)(103 m) R h$ in the $(252)$ Cf Fission Neutron Spectrum.

PB91-203406

101,694 
SCHNEEMAN, R. D.

Interactive Video Software Portability: Migrating a DOS Application to POSIX

SCHNEEMEYER, L. F.

New Layered Cuprate Structure-Type, (A1. PB91-237339

SCHNEIDER, S. J.

Standardization of Advanced Ceramics.

PB91-195669

SCHNEIR, J.

Pattern Generation on Semiconductor Surfaces by a Scanning Tunneling Microscope Operating in Air.

PB91-194712

100,808

Selective-Area Epitaxial Growth of Gallium Arsenide on Silicon Substrates Patterned Using a Scanning Tunneling Microscope Operating in Alr.

PB91-149880

100,796

SCHOENWETTER, H. K.

Recent Developments in Digital Oscilloscopes.

PB91-175240

100,849

SCHONBERGER, E.

Reverse-Phase HPLC Separation and Electrochemical Detection of Retinol and Its Isomers.

PB91-148858

100,187

SCHOOLEY, J. F.

Differences between Thermodynamic Temperature and (IPTS-68) in the Range $230 \mathrm{C}$ to $660 \mathrm{C}$

101,725 PB92-116722

SCHRAMM, R. E.

Residual Stress Detection in Raitroad Wheels: An Ultrasonic System Using EMATs (Electromagnetic-Acoustic Transducers). Report No. 23

101,741

Tread Crack Detection in Railroad Wheels: An Ultrasonic System Using EMATs (Electromagnetic-Acoustic Transducers). Report No. 22

101,742

Ultrasonic Measurement of Sheet Steel Texture and Formability: Comparison with Neutron Diffraction and Mechanical bility: Comparison with Neutron Diffraction and Mechanica PB92-116615

101,170

SCHRAUFSTATTER, I. U.

Damage to the Bases in DNA Induced by Stimulated Human Neutrophils.

PB92-116912

101,312

SCHRODER, $B$.

Electrofission in the Ouasifree and Delta Regions
PB91-147355

101,609

SCHRODT, D. J.

Effect of Thermochemical Treatments on the Strength and Microstructure of SiC Fibers.

CHROEDER, J. A.

Hydrogen Component Fugacity Coefficients in Binary Mixtures with Etane: Pressure Dependence.

PB92-116508

100,455

SCHUDER, $M$.

Investigation of Internal Rotor Dynamics of $\mathrm{NeDCl}$ and ArDCl via Intrared Absorption Spectroscopy.
PB91-204024

SCHULL, P. J.

Tread Crack Detection in Railroad Wheels: An Ultrasonic System Using EMATs (Electromagnetic-Acoustic Transducers). Report No. 22

PB91-222661

101,742

SCHWARTZ, B. B.

Magnetic Hysteresis of High-Temperature YBa2Cu3Ox-AgO Superconductors: Explanation of Magnetic Suspension. PB91-189506

SCHWARTZ, L. $\mathrm{H}$.

Advanced Materials: Opportunities and Challenges.
PB91-204032 100,009 International Co-operation: The Versailles Project on Advanced Materials and Standards.

PB91-159137

101,191

International Collaboration in Prestandards Research on Advanced Materials within the Context of VAMAS.
PB91-204040 101,251
U.S Assessment of the New Diamond Technology in Japan.

101,107

167395

101,192

VAMAS Bulletin.
PB91-159145

SCHWARZACHER, W.

Magnetic Anisotropies in Ultrathin fcc Fe(001) Films Grown on Cu(001) Substrates.

PB92-116631

101,565

SCOTT, $\mathrm{H}$.

Control System Architecture for Unmanned Ground Vehicles.

SCOTT, J. L.

101,379

Choosing Flow Conditioners and Their Location for Orifice Flow Measurement.
PB92-116490

101,397

SCRIBNER, C. F.

Investigation of the Collapse of L'Ambiance Plaza. PB91-204057

100,146

SEABAUGH, A. C.

Investigation of Photoconductive Picosecond Microstripline Switches on Self-Implanted Silicon on Sapphire (SOS)

PB91-147918

100,791

SEEM, J.

Method for Characterizing the Thermal Performance of a Solar Storage Wall from Measured Data

100,901

SEIFARTH, R. L.

Eccentric Load Sensitivity of Force Sensors

SEILER, D. G.

$\mathrm{HgCdTe}$ Detector Reliability Study for the GOES Program.
PB92-112382

Intrinsic Carrier Concentrations in Long Wavelength

$\mathrm{HgCdTe}$ Based on the New, Nonlinear Temperature Dependence of $\operatorname{Eg}(x, T)$.

B92-117316

101,568

Narrow-Gap Semiconductors and Related Materials

PB91-162313

101,492

Phonon-Assisted Magneto-Donor Optical Transitions in $n$ InSb.

101,467

SEILER, J. F.

Field Study of the Performance of EPDM Roofing at Air FB91-167247

101,340

Field Testing of Adhesive-Bonded Seams of Rubber Roofing Membranes.
PB91-189993

SEKERKA, R. F.

100,124

Numerical Study of Two-Dimensional Crystal Growth Forms in the Presence of Anisotropic Growth Kinetics.

PB91-200865

SELTZER, S. M.

Dosimetry of a Nearly Monoenergetic 6- to 7-MeV Photon Source by $\mathrm{Nal}(\mathrm{TI})$ Scintillation Spectrometry

PB92-116706

101,723

Mass Energy-Transfer and Mass Energy-Absorption Coefficients, Including In-Flight Positron Annihilation for Photon nergies $1 \mathrm{keV}$ to $100 \mathrm{MeV}$.

-126473

101,445

Possible Use of Pattern Recognition for the Analysis of Mars-Rover X-ray-Fluorescence Spectra.

PB91-237875

101,733

SELVAMANICKAM, $v$.

Current Record in Superconductors.

PB91-194753

101,521

SEMANCIK, S.

Chemical and Electronic Properties of $\mathrm{Pd} / \mathrm{SnO} 2(110)$ Model Gas Sensors.
PB91-203075

Instability in $\mathrm{pH}$ Measurements of Sputtered IrO2 Films.

PB91-147538

100,393

SEMERJIAN, $H$.

Temperature Measurements in Flames Using Thermally As sisted Laser-Induced Fluorescence of Ga.
PB91-236851
100,420

SEMERJIAN, H. G.

Advanced sensor development program for the pulp and paper industry. Final report.

101,248

Application of Laser Diagnostic Techniques for the Examination of Liquid Fuel Spray Structure.

100,229

Effect of Atomization Air on Droplet Dynamics of Spray Flames.

100,550

ICLASS-91 Proceedings of the International Conference on Liquid Atomization and Spray Systems (5th). Held in Gaithersburg, MD. on July 15-18, 1991

PB91-216838

100,552

Laser Diagnostics for Characterization of Fuel Sprays.

PB91-149013 100,541

Velocity and Droplet Size Measurments in a Fuel Spray.

PB91-147934

SENEHI, M. K.

Manufacturing Systems Integration Control Entity Interface Document.

100,991

Manufacturing Systems Integration: Initial Architecture Doc

ument.

100,994

SENEKOWITSCH, J.

Multireference-Configuration Interaction (MR-Cl) Calculations on $\mathrm{HS}(2+)$ and Experimental Observation via ElecIron Impact lonization of $\mathrm{H} 2 \mathrm{~S}$.

PB91-148924

100,295

SENF, $F$.

Resonant Photoemission and PSD of Rare Earth Oxides.

PB91-148015

100,287

SENGERS, J. M. H. L.

Sixteen Thousand Evaluated Experimental Thermodynamic Property Data for Water and Steam.

100,475

SENGERS, J. $\mathrm{V}$.

Thermodynamic Behavior of Fluids in the Supercritical PB91-2041,

101,394

Thermodynamic Properties of 1,1,1,2,-Tetrafluoroethane (R134a) in the Critical Region.

100,233

Transport Properties of Carbon Dioxide.

100,266

ETZMANN, $U$.

New Equation of State and Tables of Thermodynamic Properties for Methane Covering the Range from the Melting Line to $625 \mathrm{~K}$ at Pressures up to $1000 \mathrm{MPa}$.

PB92-148170

100,477

SEYOUM, H. M.

Superconducting Properties of Bi2-x-yPbxSnySr2Ca2Cu3Oz.
PB91-162321
101,076

SHAFER, $C$.

Real-Time Algorithms and Data Structures for Underwater Mapping.

SHAFFER, S. A

101,360

Research. Services. Facilities. (National Institute of Standards and Technology)

SHANNON, $R$.

100,012 New Different Forms of Ammonium Loaded and Partly
Deammoniated Zeolite Rho Studied by Neutron Powder. PB91-158717

SHAPIAA, Y.

Magnetic Hysteresis of High-Temperature $\mathrm{YBa} 2 \mathrm{Cu} 3 \mathrm{Ox}-\mathrm{AgO}$ Superconductors: Explanation of Magnetic Suspension PB91-189506

101,507

SHAPIRO, A.

Sputtered Thin Film YBa2Cu3On

1-189647

101,509

SHAPIRO, A. J.

Magnetic Behavior of Nanocomposites Prepared in a Vitreous Alumina Gel.

Magnetic Properties of Iron/Silica Gel Nanocomposites.

PB91-204073 101,110

Processing Bi-Pb-Sr-Ca-Cu-O Superconductors from Amor-

phous State.
PB91-187369 101,504

Superconducting Properties of $\mathrm{Bi2}-\mathrm{x}-\mathrm{yPbxSnySr} 2 \mathrm{Ca} 2 \mathrm{Cu} 3 \mathrm{Oz}$

PB91-162321

SHARPE, L. H.

Tiled Raster Graphics and MIL-R-28002A: A Tutorial and Implementation Guide

SHASTRI, L. V.

101,346

Rate Constants for Reduction of Substituted Methylperoxy Radicals by Ascorbate lons and N,N,N',N'-TetramethylPara-Phenylenediamine.

PB91-195230

100,379

SHAVIV, R.

Thermodynamics of the Divalent Metal Fluorides. 3. Heat Capacity of the Fast lon Conductor Sr SnF4 from 6 to 344 PB91-149864

100,305

SHEAHEN, L. A

Co-Trial on ESR Identification and Estimates of gamma-Ray and Electron Absorbed Doses Given to Meat and Bones. PB91-194720

100,017

SHECHTMAN, D.

High Resolution Electron Microscopy of Diamond Film Growth Defects and their Interactions.

Transmission Electron Microscopy of the CVD Diamond 
SHEPPARD, C. 1

Guide to Design, Implementation and Management of Disributed Databases.

PB91-187567

100,648

SHER, A. H.

Agency Exploits Flexibility of Software in Moving Info.
PB91-159160

SHERWOOD, G. V.

Air Gage Size Measurement of Microwave Standards PB92-117324

100,777

SHI, A. C.

Calculation of Energy of Low-Angle Grain Boundaries.

PB91-134601 Energy of Low-Angle Grain Boundaries. 101,464 SHI, D.

Strontium-Induced Oxygen Defect Structure and Hole Doping in La2- $\times \mathrm{Sr} \times \mathrm{CuO}$

PB91-148155

101,472

SHIELDS, J. R.

Reduction of Hydrogen Cyanide Concentrations and Acute Inhalation Toxicity from Flexible Polyurethane Foam Combustion Products by the Addition of Copper Compounds. Part 3. The Effect of Copper Additives on the Flammability Characteristics of Flexible Polyurethane Foam.

PB91-132167

101,140

SHIH, A.

Electronic Structure of High-Tc Superconductors Studied Using Photoelectron Spectroscopy.

101,513

Initial Stages of Degradation of Superconductor Surtaces: $\mathrm{O} 2, \mathrm{H} 2 \mathrm{O}, \mathrm{CO} 2$ and $\mathrm{CO}$ Chemisorption on La2-xSixCuO4.

PB91-189670

101.512

SHILSTONE, G. F.

Solubilities of Solids and Liquids of Low Volatility in Supercritical Carbon Dioxide.

PB92-148105

100,470

SHIMAMOTO, $\mathbf{s}$.

Interlaboratory Tension and Fracture Toughness Test Re sults for CSUS-JN1 (Fe-25Cr-15Ni-0.3N) Austenitic Stainless Steel at $4 \mathrm{~K}$

PB91-23708

101,166

SHIRAI, T.

Spectral Data and Grotrian Diagrams for Highly Ionized

Copper, Cu X-Cu XXIX. 100,441
PB92-110121

Spectral Data and Grotrian Diagrams for Highly lonized Iron, Fe VIII-XXVI.

PB90.244583

101,595

SHIRLEY, D. A.

Angle-Resolved Photoemission from the Ar $2 p$ Subshell.

PB91-194928

101,665

SHIVES, T. R.

Examination of the Excessive Retained Austenite on the Surface of a Section of 17.7 Precipitation Hardening Stain-

$\begin{array}{ll}\text { less Steel. } & 101,149\end{array}$

Intercomparison Study of Rockwell Hardness Test Blocks. PB91-167338

101,224

SHUKER, $P$.

Direct Detection of Atom Vaporization by Laser Resonance Ionization as a Probe of Gas-Surface Chemisorption Mech-

PB91-237032

100,425

SHULL, J. M.

Detection of an Expanding $\mathrm{H}$ I Shell in the Old Supernova Remnant CTB 80

PB91-174920

100,028

Caracitive Array Sensors for Nondestructive Evaluation

Capacitive Array Sensors for Nondestructive Evaluation.
PB91-237313 Characterization of Capacitive Array for NDE Applications. PB91-237305 100,946 Eddy Current Reflection Probe: Theory and Experiment. PB91-187120 100,944 Progress in Uniform Field Eddy Current Methods. PB91-147751

00,944

SHULL, $\mathbf{R}$.

101,039

Studies of Magnetic Flux Penetration in a Chemically Synthesized Bi(Pb)SrCaCuO Superconductor.

101,526

SHULL, R. D.

Atomic Fingerprint of YBa2Cu3O7-x-Type High-Temperature Superconductors Observed by Field lon Microscopy.
PB91-203612

101,544

Change in Magnetic State of $\mathrm{Fe}+$ Silica Gel Nanocomposites Due to Low Temperature Treatment in Ammonia. PB91-237321

101,129

High Magnetic Field Facilities in Japan Related to Superconductivity Research (Japan Technology Program).
PB91-240762

101,718

High T(sub C) Superconductors: Are They Magnetic.

N91-20208/5

101,453

Kelvin Water-Drop Experiment.

N91-20219/2

100,900

Levitation of Superconducting Composites.
PB91-187351

101,503

Low Carbon Steel: Metallurgical Structure vs. Mechanical Properties.

101,144

Magnetic Behavior of Nanocomposites Prepared in a Vitreous Alumina Gel.

PB92-117332

101,13

Magnetic Hysteresis of High-Temperature $\mathrm{YBa} 2 \mathrm{Cu} 3 \mathrm{Ox}-\mathrm{AgO}$ Superconductors: Explanation of Magnetic Suspension. PB91-189506

101,507

Magnetic Properties of Iron/Silica Gel Nanocomposites.

PB91-204073 101, 110

Microstructural Studies of Ti-Al Alloys in the Vicinity of the 'Eutectoid' Reaction.

PB92-116938

101,241

Processing Bi-Pb-Sr-Ca-Cu-O Superconductors from Amor-

$\begin{array}{ll}\text { phous State. } & 101,504 \\ \text { PB91-187369 } & \end{array}$

Progress in Field Ion Microscopy Imaging of High-Tc Superconducting Oxides.

PB91-203604

101,543

Spin Glass Magnetic Behavior of Iron/Silica Gel Nanocom-

PB91-159178 101,117

Superconducting Thin Films of Bi-Sr-Ca-Cu-O Obtained by Laser Ablation Processing

PB91-189589

101,508

SIBATA, C. H.

Mass Energy-Transfer and Mass Energy-Absorption Coefficients, Including In-Flight Positron Annihilation for Photon Energies $1 \mathrm{keV}$ to $100 \mathrm{MeV}$.

PB92-126473

101,445

SIDOROV, Y. V.

Squeezed Ouantum States of Relic Gravitons and Primordial Density Fluctuations.

PB91.203125

101,685

SIECK, L. W.

Proton Affinities and pKa Values of Tetraalkylhydrazines.

PB91-195214 100,378

Thermal Decomposition of lons. 3. Protonated Ethanol and Diethyl Ether.

SIEGRIST, T.

New Layered Cuprate Structure-Type, (A1XA'x) 14Cu24041. 101,095
PB91-237339

Parent Structure of the Layered High-Temperature Superconductors.

SIEGWARTH, J. D.

Apparatus for Measurement of Coefficient of Friction B91-148049

101,535

SIES, R. F.

Government Document Processing Requirements Report PB91-187773

100,574

SIEW, C.

Comparative Study of Bovine Pericardium Mineralization: A Basic and Practical Approach.

PB92-117407

SIEWERT, T. A.

Analysis and Characterization of Commercial Welding PB91-162339

101,003

Cryogenic Material Properties of Stainless Steel Tube-toFlange Welds.
PB91-134627

101,145

Dynamic Arc-Power Source Response in GMA Welding.

101,002

Fracture Toughness of $316 \mathrm{~L}$ Stainless Steel Welds with Varying Inclusion Contents at $4 \mathrm{~K}$. PB91-158980

101,148

Image Ouality Indicator Design for Radioscopy and TomogPB91-190058

101,018

Microstructure, Composition, and Hardness of Rockwell C Hardness Blocks.

PB91-184747

101,153

Real-Time Radiology Standards: Results of a Workshop.

PB91-147900 101,010

Report on 1989 Actions by International Institute of Weld-

PBg 1-134619

101,001

Sensing of GMAW Droplet Transfer Modes Using an ER 100S-1 Electrode.

PB91-174250

101,004

SIKDAR, S. K.

Application of Osmotic Dewatering to the Controlled Crystallization of Biological Macromolecules and Organic Compounds.

PB91-195719

100,230

Biodegradable Plastics: An Idea Whose Time Has Come.

PB91-150086 101,243

Hydrodynamics and Mass Transfer in Two-Phase Aqueous Extraction Using Spray Columns.

PB91-149229

101,300

Mass Transfer Coefficients in Two-Phase Aqueous Extraction.

PB91-237149

100,426

Thin and Composite High-Flux Membranes of Perfluorosultonated Ion-Exchange Polymer.

PB91-236653

100,512

SILBERSTEIN, $S$

Proposed Standard Practice for Assessing the Performance of Gas-Phase Air Cleaning Equipment.

PB91-167353

100,905

SILVA, J. M.

Measurements of Power Frequency Magnetic Fields Away from Power Lines.

PB91-175398

100,851

SILVERNALE, $G$

Validation Testing Laboratory User's Guide. National PDES Testbed Report Series.

PB92-123090

100,971

SIMCHICK, R.

High Resolution Diffraction Imaging of Crystals Grown in Nijcrogravity and Closely Related Terrestrial Crystals. PB92-109008

101,562

SIMIC, M. G.

Antioxidation Mechanisms of Uric Acid.

PB91-237347

100,222

Biomarkers of $\mathrm{OH}$ Radical Damage In vivo.

PB91-236877

101,325

Formation of ortho-Tyrosine by Radiation and Organic Solvents in Chicken Tissue.

PB92-116953

101,290

Mechanism of $\mathrm{OH}$ Radical Reactions with Thymine and Uracil Derivatives.

PB91-147447

100,216

SIMIU, E.

Identification of Dynamic Green's Functions in Structural Networks.

Impulse Response Functions for Elastic Structures with Rigid Body Degrees of Freedom.

PB91-237354

101,587

SIMMONS, J. A.

Deconvolution of Acoustic Emission and Other Causal Time Series.

PB91-216762

101,263

Root Projection of One-Sided Time Series.

PB91.216754

101,262

SIMMONS, J. D.

Metric Conversion Card

NIST Calibration Services Users Guide, 1991

PB92-126416

100,941

SIMON, N. J.

Aluminum Alloys for ALS Cryogenic Tanks: Comparative Measurements of Cryogenic Mechanical Properties of Al-L Alloys and Alloy 2219.

AD-A242 956/1

101,204

Aluminum Alloys for ALS Cryogenic Tanks: Oxygen Compatibility. Volume 2 . 
SIMOViC, M.

Preparation and Properties of Monomolecular Films for Use as Radioactive Source Mounts.

PB91-147

101,608

SIMS, J. S.

Boussinesq Algorithm for Enclosed Buoyant Convection in Two Dimensions.

PB91-178848

100,157

SIMSON, B. G.

Conformity Assessment Workshop on Electromagnetic Compatibility.
PB91-192989

100,579

Conformity Assessment Workshop on Pressure Vessels.

PB91-192997 101,049

Meeting of the Standards Working Group of the Joint U.S. U.S.S.R. Commercial Commission (1st). Held in Moscow on March 11-13, 1991.
PB91-194498

100,178

SINDT, C. F.

Choosing Flow Conditioners and Their Location for Orifice

Flow Measurement.
PB92-116490 101,397

Effect of Pipe Surface Finish on Gas Flow Measurement

with an Orifice Meter.
PB91-148031

Effect of Pipe Surface Finish on the Orifice Discharge Coefficient

101,389

Elfect of the Location of an In-Line Tube Bundle on Orifice Flowmeter Performance.

100,921

Experimental Evaluation of Selected Orifice Flowmeter Upstream Installations

PB91-147025

100,880

SINGH, A. K.

Electronic Structure of High-Tc Superconductors Studied Using Photoelectron Spectroscopy.

PB91-189688

101,513

SINGH, S.

Microstructure and Elastic Properties of Dental Resin and Resin-Based Glass-Peinforced Composites: XRD, SEM and

Ultrasonic Methods.
PB91-204081

100,050

SINHA, K.

List of New Group Divisible Designs.

PB92-126705

101,281

SJOLIN, L.

$\mathrm{Ab}$ Initio Phase Determination for X-ray Diffraction Data from Crystals of a Native Protein

PB91-237362

101,309

Crystal Structure of Phosphate-Free Ribonuclease.

PB91-175604 101,301

Multiple Conformations of Amino Acid Residues in Pibonu-

clease $A$

SKANDERA, $M$.

101,298

Computer Security Bulletin Board System. User's Guide.

PB92-112390

100,717

SKANTHAKUMAR, $\mathbf{S}$.

Antiferromagnetic Order of $\mathrm{Cu}$ in $\mathrm{Sm} 2 \mathrm{CuO} 4$.

PB92-117340

101,569

Crystal and Spin Structures of Nd2CuO4

PB91-148023

101,470

SKINNER, S. L.

New Radio Detectors of Early-Type Pre-Main Sequence

Stars.
PB91-149286

100,024

SKINNER, S. M.

Investigation of Simulated Oil-Well Blowout Fires.

PB91-144337

101,356

SKLIZKOV, G. V

Effect of Slag Penetration on the Performance of Magnesia

$\begin{array}{ll}\text { Chrome Refractories in Slagging Gasifiers. } & \\ \text { PB91-134940 } & 100,875\end{array}$

PB91-134940

SLABACK, L. A.

Low-Background Gamma-Ray Assay Laboratory for Activation Analysis.

100,192

SLAFF, G. F.

Book Review: Partitioning in Aqueous Two-Phase Systems: Theory, Methods, Uses, and Applications to Biotechnology.
PB91-175257

SLEIGHT, A. W.

Refinement of the Structure of Beta'-MoO3.

PB91-203786

101,090

SLIFKA, A. J.

Apparatus for Measurement of Coefficient of Friction.
PB91-148049

Apparatus for Measurement of Thermal Conductivity of Insulation Systems Subjected to Extreme Temperature Differences.

101,738

PB91-162016

Small Scale Demand Type Neon Liquefaction Plant.
PB91-162008
Thermal Conductivity of Evacuated Perlite at Low Temperatures as a Function of Load and Load History.

100,227 $-174672$

SLOCUM, A. H.

Design and Implementation of a Five-Axis Robotic Micromanipulator for Inserting Parts into Precision Collets. PB91-159194

101,029

Kinematic Couplings for Precision Fixturing. Part 2. Experimental Determination of Repeatability and Stiffness.

101,041

SLUPSKY, J. D.

Solubility of Carbon Dioxide in Water at Low Pressure. PB92-148196

100,479

SMALL, J. A.

Characteristics of Aerosols Produced by the Spark Discharge.
PB91-237727

100,209

Use of Single Particle Standards for LAMMA Calibration.

PB91-147777 100,183

Visibility of Chrysotile Asbestos in the Scanning Electron Microscope.
PB91-204099

SMID, M. E.

Data Encryption Standard: Past and Future

101,320

SMILGYS, R. V.

Study of the GaAs-Si(100) Interface Using Laser Probing of Thermal Desorption Kinetics.

PB91-149294

101,477

SMITH, D. R.

Apparent Thermal Conductivity of Fumed-Silica Standard Reference Materials.

PB92-117357

101,102

Automated High-Temperature Guarded-Hot-Plate Apparatus for Measuring Apparent Thermal Conductivity of Insulations between 300 and $750 \mathrm{~K}$

PB91-162115

100,111

Thermal Conductivity of a Polymide Film between 4.2 and $300 \mathrm{~K}$, with and without Alumina Particles as Filler. PB91-144402

100,483

SMITH, D. T.

Analytic Solution for the Three-Layer Multiple Beam Interferometer.

PB91-162107

101,007

SMITH, J. F.

Use of Neutron Pole Figures to Calibrate UItrasonic Techniques for On-Line Texture Control of Aluminum Plates. niques for On-Li

101,222

SMITH, J. H.

Institute for Materials Science and Engineering: Metallurgy Division, Technical Activities 1990.

101,207

B91-132225

Intercomparison Study of Rockwell Hardness Test Blocks.
PB91-167338

SMITH, L. E.

Polymers: Technical Activities 1990

Polymers: Tech

100,488

Polymers: Technical Activities 1991. (NAS-NRC Assessment Panel, February 13-14, 1992).

100,516

SMITH-MAGOWAN, D.

Thermodynamics of the Krebs Cycle and Related Compounds.

100,357

SMITH, R. L.

Concept of the Calculus of Fire Safety.

PB91-132134

100,093

SMODIS, B.

Characterization of the Mineral Fraction in Botanical Reference Materials and Its Influence on Homogeneity and Analytical Results
PB91.148817

100,186

SMYTH, K. C.

Laser-Induced Fluorescence of $\mathrm{CH}$. in a Laminar $\mathrm{CH}_{4} /$ Air Diffusion Flame: Implications for Diagnostic Measurements and Analysis of Chemical Rates.

PB91-237099

100,554

Partial Equilibrium in Laminar Hydrocarbon Diffusion PB91-237024

100,553

Photoluminescence Excitation by Band-Gap Optical Absorption in Chemical Vapor Deposition Diamond Films.

101,533

Relative $\mathrm{H}$-Atom and O-Atom Concentration Measurement in a Laminar, Methane/Air Diffusion Flame.

100,559

Short-Duration Autoignition Temperature Measurements for Hydrocarbon Fuels.

100,877

Short-Duration Autoignition Temperature Measurements for Hydrocarbon Fuels.

100,887

Signal Detection Efficiency in Multiphoton Ionization Flame Measurements.

100,540

SNELICK, R. D.

Performance Evaluation of Hypercube Applications: Using a Global Clock and Time Dilation.

PB91-222588

100,653

Workloads, Observables, Benchmarks and Instrumentation. PB91-147660

100,598

SNELL, J. E.

Application of Fire Research.

PB91-149302

100,153

SNIEGOSKI, L. T.

Synthesis of 3-Ouinuclidinol-(18)O, Benzilic-d5 Acid, and 3Ouinuclidinyl-(18)O, Benzilate-d5.

PB91-237370

100,223

SNITCHLER, G.

Near-Threshold Vibrational Excitation of $\mathrm{H} 2$ by Electron Impact: Resolution of Discrepancies between Experimen and Theory.

PB91-200881

101,679

Spin-Dependent

101,641

SNYDER, R. L.

RIR - Measurement and Use in Ouantitative XRD.

PB91-147397

101,466

SOARES, C. G.

Calibration of Ophthalmic Applicators at NIST: A Revised Approach.

PB91-237388

101,327

Dosimetry of a Nearly Monoenergetic 6- to 7-MeV Photon Source by Nal(TI) Scintillation Spectrometry.

101,723

Sensitometry of the Response of a New Radiochromic Film Dosimeter to Gamma Padiation and Electron Beams.

PB91-237008

101,326

Status of Beta-Particle Calibration Studies at NBS.

PB91-175265

101,636

SOCOLOW, R. H.

Measurement of Thermal Characteristics of Office Build

ings.

SOILEAU, M. J.

Laser Induced Damage in Optical Materials: 1989.

PB91-148478

SOISSON, J. P.

Biological Sulfur Oxidation and Peduction for Coal Sulfur Speciation and Desulfurization.

$91-203323$

100,886

SOMAYAJULU, G. R

Thermodynamic and Thermophysical Properties of Organic Nitrogen Compounds. Part 1. Methanamine, Ethanamine, 1 and 2-Propanamine, Benzenamine, 2-, 3-, and 4-Methylben-

PB91-192583

100,368

SOMERS, T. A.

Building Technology Publications 1986-1989.

PB91-216820

100,160

Evaluation of Fumed-Silica Insulation for a Thermal Conductivity Standard Reference Material.

PB91-147975

100,103

Thermal Conductivity of Fumed Silica Board at Foom Temperature.

PB91-190124

100,125

SONG, J. F. 
SPARKS, L. L.

Apparatus for Measurement of Coefficient of Friction.
PB91.148049 Apparatus for Measurement of Thermal Conductivity of In sulation Systems Subjected to Extreme Temperature Differ-

PB91-162016

101,738

Reusable Cryogenic Foam Insulation for Advanced Aerospace Vehicles

100,560 Small Scale Demand Type Neon Liquefaction Plant.
PB91-162008

100,224

Thermal Conductivity of a Polymide Film between 4.2 and $300 \mathrm{~K}$, with and without Alumina Particles as Filler.

100,483

Thermal Conductivity of Alumina Fiber/Epoxy and Alumina Fiber/PEEK from 4.2 to $310 \mathrm{~K}$.

PB92-117258

101,130

Thermal Conductivity of Evacuated Perlite at Low Temperatures as a Function of Load and Load History.

100,227

Test of Newton's Inverse Square Law of Gravity Using the $300 \mathrm{~m}$ Tower at Erie, Colorado: Newton Vindicated on the Plains of Colorado.

PB91-175273

101,637

Test of the Inverse-Square Law of Gravitation Using the $300-m$ Tower at Erie, Colorado.

PB91-204115

101,702

SPELLER, $\mathrm{C} . \mathrm{V}$.

Multicomponent Cluster Ions. 2. Comparative Stabilities of Cationic and Anionic Hydrogen Bonded Networks: Mixed Clusters of Water and Hydrogen Cyanide.

PB91-147710

100,283

SPENCER, W. P.

Field Study of the Performance of EPDM Roofing at Air Force Facilities.

PB91-167247

SPICER, W. E.

Surface Extended-X-ray-Absorption Fine Structure and Scanning Tunneling Microscopy of Si(001)2x1-Sb. 101,552

SPIDALIERE, $P$.

Recommended Fine Positioning Test for the Development Test Flight (DTF-1) of the NASA Flight Teterobotic Servicer (FTS)

$1-185090$

101,732

SPIEGEL, $V$.

Spectrum-Averaged Cross-Section Measurement of
$(103) \mathrm{Rh}(\mathrm{n}, \mathrm{n})(103 \mathrm{~m}) \mathrm{Rh}$ in the $(252) \mathrm{Cf}$ Fission Neutron Spec-

PB91-203406

SPIEGELMAN, C. H.

Calibration with Working Standards

PB91-158931

SPIELMAN, F. E.

Information Systems Engineering Program.

Raster Graphics Conformance Testing.

PB91-167296

MIL-28002A: A Tutorial and Implementation Guide.

PB91-187708

101,346

SPINDT, C. J.

Morphology and Barrier.Height Development of $\mathrm{Bi} / \mathrm{InP}(110)$ Interfaces.

PB91-147736

101,468

SPIVAK, A. L.

SHOOT Flowmeter and Pressure Transducers.

PB91-147488

100,923

SPLETT, J.

Eddy Current Probe Sensitivity as a Function of Coil Con-

PB91-23656

100,945

SPRING, C.

Evaluation of Test Methods for Measuring the Bond Strength of Portland Cement-Based Repair Materials to Concrete.

PB91-189621

100,530

SPRING, C. B.

Application of CCRL Data in the Development of Cement Standards.

PB91-175315

100,119

SRINIVASAN, G.

Anomalous Low-Frequency Butterfly Curves for Subsidiary and Ferromagnetic Resonance Overlap at $3 \mathrm{GHz}$. $\quad 101,519$ SRIVASTAVA, H. M.

Certain Theorems on Bilateral Generating Functions Involv. ing Hermite, Laguerre, and Gegenbauer Polynomials.

PB91-148759

STAFFORD, G.

Electrodeposition of Aluminum from Molten Salts

PB91-159202

101,223

Structural Study of Electrodeposited Aluminum-Manganese Alloys.
PB91-189423

101,231

STAFFORD, G. R.

Phase Formation in Electrodeposited and Thermally Annealed Al-Mn Alloys.

PB91-147314

101,209

STAFFORD, T. Accelerator (14)C Dating of Late Pleistocene Megafauna.
PB91-237396

STAFFORD, T. $W$.

Accelerator Radiocarbon Dates on Charcoal, Shell, and Hone from the Del Mar Site, California.

PB91-237404

STANLEY, H. B.

Crystal-Field Splittings in the Cubic Heusler Alloys HoPd2Sn and ErPd2Sn.

PB91-194902

101,525

STANSBURY, J. W.

Difunctional and Multifunctional Monomers Capable of Cyclopolymerization.

STARK, C.

100,504

Development Plan: Application Protocols for Mechanical Parts Production. National PDES Testbed Report Series.
PB91-222596

STAUFFER, A. D.

Asymptotic Shooting Method for the Solution of Differential

Equations.
PB91-148742 101,255

Optical Potential Approach to Electron and Positron Scattering from Noble Gases. 2. Neon.

PB91.149971

101,619

STAUFFER, J. A.

Asymptotic Shooting Method for the Solution of Differential Equations.

PB91-148742

101,255

STEBBINS, R. T.

Gravitational Radiation Observations on the Moon.

PB91-175083

101,635

unar Gravitational Wave Antenna Using a Laser Interferometer.

101,603

STECKLER, K. D.

Experimental Study of the Pyrolysis Rate of a Poly(methy methacrylate) (PMMA) Wall Panel in a Reduced-Scale Enclosure.

PB91-237412

100,555

STEEL, E.

Accuracy of Transmission Electron Microscopy Analysis of Asbestos on Filters: Interlabortory Study.

100,204

Rate Constants for Reduction of Substituted Methylperoxyl Radicals by Ascorbate lons and N,N,N',N'-TetramethylPara-Phenylenediamine.

PB91-195230

100,379

STEIGERWALD, D. A

Epitaxial fcc Fe Films on $\mathrm{Cu}(100)$

101,478

Structural Study of the Epitaxial Growth of fcc-Fe Films, Sandwiches, and Superlattices on $\mathrm{Cu}(100)$

PB91-237420

101,555

STEIN, S. E.

Dissociation of Substituted Anisoles: Substituent Effects on

Bond Strengths.
PB91-237537

100,431

Mechanism of Soot Formation in Acetylene-Oxygen Mixtures.

100,537

Stabilities of Substituted Benzyl Radicals: Dissociation Rates of Aminoethylbenzenes, Hydroxyethylbenzenes, and Cyanoethylbenzenes.

PB91-237529

100,430

STEINER, B.

High Resolution Diffraction Imaging of Crystals Grown in Microgravity and Closely Related Terrestrial Crystals.

PB92-109008

101,562

High Resolution Synchrotron X-Radiation Diffraction Imaging of Crystals Grown in Microgravity and Closely Related Terrestrial Crystals.

101,548

Streaking Images That Appear Only in the Plane of Diffraction in Unoped GaAs Single Crystals: Diffraction Imaging (Topography) by Monochromatic Synchrotron Radiation.
PB91-189662

101,511

Structural Anomalies in Undoped Gallium Arsenide Observed in High Resolution Diffraction Imaging with Monoch romatic Synchrotron Radiation.

PB91-237438

101,556

STEINER, B. W.

Dynamical Diffraction Imaging (Topography) with X-ray Synchrotron Radiation.

101,510

International Co-operation: The Versailles Project on Advanced Materials and Standards.

vanced Materias

101,191

International Collaboration in Prestandards Research on Advanced Materials within the Context of VAMAS.

PB91-204040

VAMAS Bulletin.

PB91-159145

101,251

101,192

NER, J.P.

Partial Discharges in Low Voltage Cables.

PB91-134650

100,771

STEINER, R. L.

Improvements for Automating Voltage Calibrations Using a 10-V Josephson Array.

100,739

Investigating the Use of Multimeters to Measure Ouantized Hall Resistance Standards.

PB91-236554

100,734

STENBAKKEN, G. N.

Comprehensive Approach for Modeling and Testing Analog and Mixed-Signal Devices.

PB91-149310

100,732

Cutting the High Cost of Testing.

PB91-162362

100,846

TEPHAN, $K$

Transport Properties of Fluid Oxygen

PB91-192484

STEPHENSON, B.

Levitation Calorimetry.

PB91-158519

TTEPHENSON, J. C.

Application of Ultrafast Broadband Infrared Spectroscopy to Measurement of Metal-Carbonyl Dynamics.

PB91-149955

100,308

Picosecond IR Studies of the Vibrational Dynamics of $\mathrm{CO} /$ Pt(111)

PB91-161927

100,314

Population Lifetimes of $\mathrm{OH}(\mathrm{v}=1)$ and $\mathrm{OD}(\mathrm{v}=1)$ Vibrations in Alcohols, Silanols and Crystalline Micas.
PB91-174813

Oxide

Time- and State-Resolved Mea

PB91-202846

100,387

STEVENS, $\mathbf{W} . J$.

Theoretical Model of Metal Binding Sites in Proteins.

PB91-194837

100,370

STEVIE, F.

Secondary Ion Yield Changes in Silicon and Galtium Arsenide Due to Topography Changes during Oxygen or Cesium on Bombardment.

PB91-148064

101,471

STEVIE, F. A

Round-Robin Study of Implants in $\mathrm{Si}$ and $\mathrm{SiO} 2$ by SIMS, ABS, and NAA

\section{STEWARD, D.}

Chitin-Chitosan Membranes: Separations of Amino Acids and Polypeptides.

PB91-134544

101,283

STEWARD, W. G

Transient Hydrogen Heat Transfer. Final Report for April 1986-April 1989

PB92-116326 
STOCK, K. D.

Limited International Intercomparison of Responsivity Scales at Fiber Optic Wavelengths.

100,567

STOCKBAUER, $\mathrm{A}$.

Initial Stages of Degradation of Superconductor Surfaces: $\mathrm{O} 2, \mathrm{H} 2 \mathrm{O}, \mathrm{CO} 2$ and $\mathrm{CO}$ Chemisorption on $\mathrm{La} 2-\times \mathrm{Sr} \times \mathrm{CuO} 4$. PB91-189670

101,512

STOCKBAUER, R. L.

Electronic Structure of High-Tc Superconductors Studied Using Photoelectron Spectroscopy.

PB91-189688

101,513

STOLARSKY, H. K

Effect of Logarithmic Singularity on the Free Edge Stress Intensity Factor of Composite Laminates.
PB91-147090
101,116

STONE, C. A.

Structure of Odd-Odd (132)Sb.

PB91-237461

STONE, W. C.

Preliminary Dynamic Analyses of the Ministry of Agriculture Building.

STORCH, P. J.

Analytical Model for the Refrigeration Power of the Orifice Pulse Tube Refrigerator.

PB91-187534

101,048

STOTTS, P. D.

Functional Meta-Structure for Hypertext Models and Systems.

100,655

STOUDT, M. R.

Influence of Moisture and Pressure on the Mechanical Properties of a Glass-Epoxy Matrix Composite and a Graphite-Epoxy Matrix Composite.

101,114

AD-A239 509/3

ies a

Influence of Water on the

AD-A231 $778 / 2$

101,113

STRATY, G. C.

Neutron Diffraction from Sheared D-Glycerol: Preliminary

Studies.
PB91-134676

100,268

STRAUB, J.

Refractive Index of Water and Steam as Function of Wavelength, Temperature and Density.

PB90-244708

101,402

STRAYER, W. T.

Error Prevention and Detection in Data Preparation for a Numerically Controlled Milling Machine.

PB91-174938

100,980

STRICKLETT, K. L.

Effect of High Pressure on Prebreakdown Phenomena in $n$ Hexane.

100,839

Electrical Breakdown and Streamer Statistics in N-hexane under Uniform Field Conditions.

PB91-134684

100,837

STROSCIO, J. A.

Geometric and Electronic Properties of Cs Structures on III$\checkmark(110)$ Surfaces: From $1 D$ and $2 D$ insulators to $3 D$ Metals. 101,517

Scanning Tunneling Microscopy Study of Clean and CsCovered $\operatorname{InSb}(110)$.

101,537

System for the Study of Magnetic Materials and Magnetic Imaging with the Scanning Tunneling Microscope. Imaging with the
PB91-194779

STROUP, D. W.

Suppression of Post-Flashover Compartment Fires Using Manually Applied Water Sprays.

PB91-240770

100,164

STRUBLE, L.

Microstructural Aspects of the Fracture of Hardened Cement Paste

100,133

Microstructure and Fracture at the Cement Paste-Aggre-

gate Interface.

100,523

STRUBLE, L. J.

Guidelines for the Development of Computer Based Models in a Cementitious Materials Modeling Laboratory. $\quad 100,532$ STUBENRAUCH, C. F.

Spherical Near-Field Scanning: Experimental and Theoretical Studies.

PB91-132274

100,723

STUCKY, G. D.

Structures and Properties of New Zeolite X-Type Zincophosphate and Beryllophosphate Molecular Sieves.
PB91-189449

STUTZMAN, P.

Microstructural Aspects of the Fracture of Hardened Cement Paste.
PB91-237479

100,133

TUTZMAN, P. E.

Characterization of Field Concrete.

PB91-178863

100,528

Field Study of the Performance of EPDM Roofing at Air

Force Facilities.
PB91-167247

101,340

Field Testing of Adhesive-Bonded Seams of Rubber Roofing Membranes.

STUVE, E. M.

100,124

Adsorption of Water and Oxygen on $A g(110)$ : A Study of the Interactions among Water Molecules, Hydroxyl Groups, and Oxygen Atoms.

TWALLEY, W. C.

100,270

Spectroscopy and Structure of the Alkali Hydride Diatomic Molecules and Their lons.

PB92-110147

100,443

Conformance Tester for X.25 DTE Implementations. PB91-189894

SU, $w$.

Network Structure in Epoxies. 6. The Growth Process Investigated by Neutron Scattering.

SUEHLE, J.S.

100,514

Current Density Dependence of Electromigration 150 Enhancement Due to Pulsed Operation.

PB91-134692

Measurement of Capacitance on Wafers

100,786

$91-149120$

100,795

SUENRAM, R. D.

Fluoromethanol: Synthesis, Microwave Spectrum, and

PB91-148080

100,288

Hydration of Carbon Dioxide: The Structure of $\mathrm{H}_{2} \mathrm{O}-\mathrm{H} 2 \mathrm{O}$ -

$\mathrm{CO} 2$ from Microwave Spectroscopy.

PB91-237172

100,428

Microwave Spectra and Electric Dipole Moments of $X(4)$ Sigma(1-) $1 / 2$ VO and $\mathrm{NbO}$.
$\mathrm{PB} 91-237487$

100,429

Microwave Spectrum and Molecular Structure of the N2H20 Complex.
PB91-203448

100,398

Microwave Spectrurn of Ar-H2O: Dipole Moment, Isotopic Studies, and (17)O Ouadrupole Coupling Constants.
PB91-148650 Rotational Spectrum and Structure of the Complex $\mathrm{Ar}$ $\mathrm{Ch} 3 \mathrm{CN}$

PB91-236661

100,415

Structures of the NH3-HCCCCH and $\mathrm{H} 2 \mathrm{O}-\mathrm{HCCCCH}$ Complexes by Fourier-Transform Microwave Spectroscopy.
PB91-148882 294

Van der Waals Complexes in 1,3-Dipolar Cycloaddition Reactions: Ozone-Ethylene.
PB91-236737

100,417

SUGAR, J.

Beam-Foil Study of Two-Electron Transitions in Cu-Like lons.
PB91-237511

101,714

Energy Levels of Copper, Cu I through Cu XXIX

PB90-244666

Energy Levels of Krypton, $\mathrm{Kr} I$ through $\mathrm{Kr} X X X \mathrm{XV}$.

101,596

Exploding Foil Pholoionization X-ray Laser.

100,473

PB91-237065 101,433

Extreme-Ultraviolet Spectra of Pd, Ag, Cd, and Sn lons ObServed in a 1.3-keV Tokamak Plasma.

101,712

Ouantum Electrodynamic Contributions to Spin Orbit Splitting in the Ground State of Aluminum-Like lons. PB91-189597

101,653

Spectra of the P I Isoelectronic Sequence from Co XIII to Mo XXVIII.

PB91-237503

101,713

Spectral Data and Grotrian Diagrams for Highly lonized Copper, Cu X-Cu XXIX

100,441

Spectral Data and Grotrian Diagrams for Highly lonized Iron, Fe VIII-XXVI.

101,595

SULLIVAN, F.

Algorithm Design for Large-Scale Computations.

100,640

SUMARLIN, I. W.

Phonon Density of States and Superconductivity in Nd1.85Ce0.15CuO4.

PB91-189787

SUMPTION, M. D.

AC Loss Measurements of Two Multifilamentary NbTi Composite Strands

100,769

SUN, J.

Effect of Slag Penetration on the Performance of Magnesia Chrome Refractories in Slagging Gasifiers.

PB91-134940

100,875

SUN, Y. N.

Stoichiometry of Reactively Evaporated Films. 91-158709

101,413

SUNDARESWARAN, P. C.

Levitation Calorimetry.

PB91-158519

100,927

SUNG, $P$.

Corrosion, Repassivation and Corrosion Fatigue Behavior of the Surgical Implant Alloy, Co-Cr-Mo.

PB91.134700

SUNSHINE, S. A.

New Layered Cuprate Structure-Type, (A1. $\left.x A^{\prime} x\right) 14 \mathrm{Cu} 24 \mathrm{O} 41$

PB91-237339

Dissociation of Substituted Anisoles: Substituent Effects on Bond Strengths.

100,431

Stabilities of Substituted Benzyl Radicals: Dissociation Rates of Aminoethylbenzenes, Hydroxyethylbenzenes, and Cyanoethylbenzenes.

PB91-237529

100,430

SUTER, H. U.

Photodissociation of $\mathrm{C}(\mathrm{I}) \mathrm{NO}$ in the $\mathrm{S}(\mathrm{I})$ State: A OuantumMechanical ab initio Study.

PB91-149260

100,239

SVENSSON, $H$.

Absorbed Dose to Water: Comparison of Several Methods Using a Liquid Ionization Chamber.

SVENSSON, L. A

101,322

Crystal Structure of Phosphate-Free Ribonuclease.
PB91-175604

Multiple Conformations of Amino Acid Residues in Ribonuclease $A$

PB91-148098

101,298

SWAFFIELD, J. A.

Plumbing Test Lab Accreditation

PB91-190066

100,089

SWANSON, $M$

Computer Security Bulletin Board System. User's Guide.

Computer Security: Selected Articles.

PB91-187740

100,717

SWANSON, P. A.

Study of the Galling of Two Steels Using Two Test Meth.

PB91-148106

101,147

SWANSON, P. L.

Tensile-Fracture Resistance Mechanisms in Brittle Polycrystals: An Ultrasonics and In-situ Microscopy Investigation. PB91.149344

01,190

SWARTZENDRUBER, L.

Examination of the Excessive Retained Austenite on the Surface of a Section of 17-7 Precipitation Hardening Stainless Steel.

101,149

Studies of Magnetic Flux Penetration in a Chemically Synthesized Bi(Pb)SrCaCuO Superconductor.

101,526

SWARTZENDRUBER, L. J.

Break Junction Tunneling Spectroscopy of Single-Crystal Bismuth-Based High-Temperature Superconductors. PB91-134478 101,462

Change in Magnetic State of Fe+ Silica Gel NanocompoSites Due to Low

Comparison of Flux Dynamics in Two Samples of

YBa2Cu3O7 with Different Pinning
PB91-149419 


\section{PERSONAL AUTHOR INDEX}

THOMPSON, R. B.

SWARTZLANDER, A. B.

Analysis of the $\mathrm{YBa} 2 \mathrm{Cu} 3 \mathrm{O} 7 / \mathrm{Sr} \mathrm{TiO}$ Interface as a Function of Post-Deposition Annealing Temperature.

101,480 PB91-1499

SWEET, L.

Calculated from the Frequency Domain: An Update. 100,578 Time Domain Frequency Stability Calculated from the Frequency Domain Description: Use of the SIGINT Software Package to Calculate Time Domain Frequency Stability from the Frequency Domain

PB92-123132

100,587

SWEET, $R$.

Calculated from the Frequency Domain: An Update.
PB91-162388

Time Domain Frequency Stability Calculated from the Fre quency Domain Description: Use of the SIGINT Software Package to Calculate Time Domain Frequency Stability from the Frequency Domain.

PB92-123132

100,587

SWENSON, D. R.

Probe for Real-Time Images of Particle Beams and Their Analyses in a Merged-Beams Apparatus. PB91-203067

101,683

SWYT, D. A.

Agenda for Progress in Technology Education: A Personal PB91-148114

100,037

Al in Manufacturing: The NBS AMRF as an Intelligent $\mathrm{Ma}$

100,973

PB91-134718

CIM, Data and Standardization within the NBS AMRF.
PB91-148122 Innovation in Manufacturing Technology: A View from NBS.
PB91-148130

New Concepts of Precision Dimensional Measurement for Modern Manufacturing.

PB91-240812

101,006

Transferring NBS Technology to Small Manufacturers Through State and Local Centers.
PB91-149351 101,025 U.S. Manufacturing Systems: Factories Past, Present, and

100,981

PB91-175091

SYTY, A.

Trace Determination of $\mathrm{Cr}(\mathrm{VI})$ by LC/AAS with on-Line Preconcentration.

100,184

SZABO, S.

Control System Architecture for Unmanned Ground Vehi-

PB91-134726

101,379

SZLAG, D. C.

Low-Cost Aqueous Two-Phase System for Enzyme ExtracPB91-237560

101,310

Statistical Mechanical Model of Aqueous Two-Phase Systems.

100,493

SZUROMI, P. D.

Influence of Sulfur on Methanation over Tungsten(1 10).
PB91-149369

Summary Abstract: The Influence of Sulfur on Methanation Over W(110).

TAATJES, C. A.

100,301

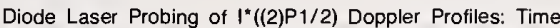
Evolution of a Fast, Anisotropic Velocity Distribution in Thermal Bath.

PB91-150052

100,310

General Method for Doppler Determination of Cylindrically Symmetric Velocity Distributions: An Application of Fourier

Transform Doppler Spectroscopy.
PB91-149385

100,302

TAKAGI, $\mathbf{S}$.

Digital Image Analysis Assisted Microradiography Measurement of Mineral Content of Caries Lesions in Teeth.

PB91-187666

Self-Setting Calcium Phosphate Cements

PB91-202879

101,314 Phosphate Cements.

PB91-148668

TAKENAKA, $M$.

ies on the Miscibility of Protonated Polyisoprene/Deuterat ed Polybutadiene Blends.

PB91-236786

PB91-148155

TANAKA, D. K.

Reflection-Extended-X-ray-Absorption-Fine-Structure Spectroscopy at the Carbon K-Edge

100,371

Structure of Surface Films on Magnesium and on Magnesium Alloys.

101,212

Surface-Extended X-ray-Absorption Fine-Structure Experiments at Atmospheric Pressure by Means of a Photocathode Proportional Counter with Monolayer Sensitivity.

PB91-194951

100,372

TANG, D.

Optical Fiber Current Sensors with Temperature Stabilities Near the Material Limit.

PB91-175109

100,753

Performance and Limitations of Faraday Effect Sensors.

$\begin{array}{ll}\text { PB91-149146 100,749 } & \end{array}$

Practical Considerations in the Design of Optical Fiber Current Sensors.

PB91-134742 100,746

Progress in the Development of Miniature Optical Fiber Current Sensors.

100,745

TANG, H. C.

Boussinesq Algorithm for Enclosed Buoyant Convection in Two Dimensions

TANG, $S$.

Thermodynamic Behavior of Fluids in the Supercritica

Region.
PB91-204123 101,394

Thermodynamic Properties of 1,1,1,2,-Tetrafluoroethane (R134a) in the Critical Region.

PB91-237578

100,233

TANGER, J. C.

Unified Description of Infinite-Dilution Thermodynamic Properties for Aqueous Solutes.

PB91-162081

100,319

TANK, R. C.

Rate Constant Functions for Strength Development of Concrete.

PB91-175117

100,527

TANNENBAUM, $D$.

Scanning tunneling microscopy studies of the surfaces of a$\mathrm{Si}: \mathrm{H}$ and a-SiGe:H films. Annual report, 1 December 1989 31 January 1991

TARLOV, M. J.

100,785 Assessment of Uncertainties in Measurement of $\mathrm{pH}$ in Hos
tile Environments Characteristic of Nuclear Repositories. tile Environments Characteristic of Nuclear Repositories.

TASSEY, G.

Industry and Government Strategies for Product Ouality. PB91-148163

101,011

Structural Change and Competitiveness: The U.S. Semiconductor Industry
PB91-195693

100,173

TAWARA, $\mathrm{H}$.

Cross Sections and Related Data for Electron Collisions with Hydrogen Molecules and Molecular Ions. PB90-244674

100,263

TAYLOR, A. H.

Reusable Cryogenic Foam Insulation for Advanced Aerospace Vehicles.

100,560

TAYLOR, B. N.

Adjustment of the Fundamental Physical Constants: A Report of the CODATA Task Group on Fundamental Constants, 1986

PB91-147124

101,606

Basic Standards and Fundamental Constants

PB91-237594

101,715

International System of Units (SI)--Translation

PB92-109032

101,719

Interpretation of the SI for the United States and Metric Conversion Policy for Federal Agencies.

101,729

New International Electrical Reference Standards Based on the Josephson and Ouantum Hall Effects.

100,740

PB91-237586

TAYLOR, J. E.

Theory of Orientation Textures Due to Surface Energy Anisotropies.

PB92-117381

101,571

TALEB-IBRAHIMI, A.

Chemical Effects of $\mathrm{Ne}+$ Bombardment on the MoS2(0001) Surface Studied by High-Resolution Photoelec tron Spectroscopy.

TAN, $\mathbf{Z}$.

100,399

Strontium-Induced Oxygen Defect Structure and Hole Doping in La2-xSrxCuO4.
Angular Distribution of Ejected Electrons in Resonant Auger Processes of $\mathrm{Ar}, \mathrm{Kr}$, and $\mathrm{Xe}$.

101,648

Parity-Unfavored Transitions in Resonant Photoemission from $\mathrm{Ar}, \mathrm{Kr}$, and Xe: Experimental and Theoretical Results.
PB91-133900

Unusual Degree of Angular Anisotropy in the Resonant Auger Spectrum of $\mathrm{Kr}$.

101,649

TAYLOR, K. T.

High Harmonic Generation in Hydrogenic lons. B91-203778

101,699

TAYLOR, P. C.

Optically Detected Magnetic Resonance Study of Antisiteto-Acceptor and Related Recombination Processes in as

Grown InP-Zn.

TEAGUE, E. C.

Determination of Fields Near a Silver Strip on a Glass Substrate.

PB91-147702

100,841

TEBBE, J.

Properties of Amorphous Zirconium Rhodium Hydride (Zr3RhHx) Prepared from Glassy and Crystalline Alloys. PB91-174466

TERLIZZI, C. P.

Testing and Rating of an Atmospheric, Gas-Fired Furnace Equipped with a Burner Air Inlet Damper.

PB92-126440

100,894

TESK, J. A.

Dental Biomaterials and Engineering Frontiers for the 90's.

Microstructure and Elastic Properties of Dental Resin and Resin-Based Glass-Reinforced Composites: XRD, SEM and Ultrasonic Methods.

PB91-204081

100,050

Visco-Elastic Deformation of Dental Porcelain and Porcelain-Metal Compatibility.

PB91-158493

100,042

TEW, $W$ L

Monitoring the Mass Standard via the Comparison of MeChanical to Electrical Power.

PB91-237115

101,710

TEWARI, Y. $B$.

Thermodynamics of Hydrolysis of Disaccharides: Lactulose, alpha-D - Melibiose, Palatinose, D - Trehalose, D - Turanose and 3-o-beta-D-Galactopyranosyl-D-arabinose.

PB91-195701

101,289

Thermodynamics of Hydrolysis of Oligosaccharides.

101,288

Thermodynamics of Industrially Important Enzyme Catalyzed Reactions

PB91-237602

101,311

Thermodynamics of the Disproportionation of Adenosine 5'diphosphate to Adenosine 5 'triphosphate and Adenosine 5'-monophosphate. 1. Equilibrium Model.

PB92-116854

100,459

Thermodynamics of the Disproportionation of Adenosine 5'. diphosphate to Adenosine $5^{\prime}$-triphosphate and Adenosine 5'-monophosphate. 2. Experimental Data.

PB92-117399

101,313

TEWARY, V. K.

Effect of a Free Surface on Stress Distribution in a Bimaterial Composite.

PB91-185116

101,121 perature for Cubic Solids.

PB91-149393 100,303

Theory of Capacitive Probe Method for Noncontact Characterization of Dielectric Properties of Materials.

PB91-237610

100,948

THIRUMALAI, D.

Ergodic Convergence Properties of Supercooled Liquids and Glasses.

Ergodic Measures for the Simulation of Dielectric Properties of Water.

PB91-189845

100,350

THOMAS, W. C.

Analysis of Moisture Accumulation in a Wood Frame Wall 
Use of Neutron Pole Figures to Calibrate Uitrasonic Tech niques for On-Line Texture Control of Aluminum Plates.
PB91-159111 THOMPSON, W. E.

Production and Spectroscopy of Small Polyatomic MolecuIar lons Isolated in Solid Neon.

AD-A234 043/8

100,245

Production and Spectroscopy of Small Polyatomic Molecular Ions Isolated in Solid Neon.

PB91-203273

100,395

Vibrational Spectra of Molecular Ions Isolated in Solid Neon. III. $\mathrm{N} 4(+)$

100,243

Vibrational Spectra of Molecular Ions Isolated in Solid Neon. 4. NO(1 + ), NO(1-), ONNO(1+), and ONNO(1-).

PB91-149914 100,307

Vibrational Spectra of Molecular Ions Isolated in Solid Neon. 6. $\mathrm{CO} 4(\cdot)$.
AD-A238 $415 / 4$

100,249

Vibrational Spectra of Molecular Ions Isolated in Solid

$\begin{array}{ll}\text { Neon. 6. CO4(1-). } & 100,419 \\ \text { PB91-236836 } & \end{array}$

Vibrational Spectra of Molecular lons Isolated in Solid $\mathrm{Neon} .7 . \mathrm{CO}(+), \mathrm{C} 2 \mathrm{O} 2(+)$, and $\mathrm{C} 2 \mathrm{O} 2(-)$.

100,250

THORSHEIM, H. R.

Associative Ionization of Ultra-Cold Laser Trapped Sodium Atoms.

PB91-236745

101,707

THURBER, W. R.

Comparison of High-Resistivity Measurements of Silicon by AC Impedance, DC Resistance, van der Pauw, and FourProbe Methods

TIBERIO, R. C.

100,792

Submicrometer SEM Magnification Standard.

PB91-159087

100,799

TIGGES, C. P.

Band-Gap Narrowing and III.V Heterostructure FETs.

PB92-117159

TILFORD, C. R.

Stabilization Techniques for Spinning Rotor Gage Residual Drag.

100,922

TIMMER, $\mathrm{C}$.

Probe for Real-Time Images of Particle Beams and Their Analyses in a Merged-Beams Apparatus.

PB91-203067

101,683

TISON, S. A.

Study of the Linearity of Transfer Leaks and a Helium Leak Detector.
PB91-147272

100,926

TJOSSEM, P. J. H.

Photoluminescence Excitation by Band-Gap Optical Absorption in Chemical Vapor Deposition Diamond Films.

sorption in Chemical Vapor Deposition Diamond Films.
PB91-195586

Relative $\mathrm{H}$-Atom and O-Atom Concentration Measurement in a Laminar, Methane/Air Diffusion Flame.

100,559

Signal Detection Efficiency in Multiphoton Ionization Flame Measurements.

PB91-148056

100,540

TOBLER, R. L

Cryogenic Fatigue of High-Strength Aluminum Alloys and Correlations with Tensile Properties.

PB91-236976

101,709

Interlaboratory Tension and Fracture Toughness Test Results for CSUS-JN1 (Fe-25Cr-15Ni-0.3N) Austenitic Stainless Steel at $4 \mathrm{~K}$

PB91-237081 101,166

Jlc Data Analysis with a 'Negative Crack Growth' Correc. tion Procedure.
PB91.175216

100,930

Strain Rate Effect on Tensile Properties at $4 \mathrm{~K}$ of a VAMAS Round-Robin Austenitic Steel.

Round-Robin
PB9 $1-237248$

101,168

TODD, D. R

Ultimate Strength of Masonry Shear Walls: Predictions versus Test Results.

100,138

TODD, J.

NBS-INA. The Institute for Numerical Analysis. UCLA 1947 1954

PB92-112408

101,264

TODD, $\mathbf{P}$.

Application of Osmotic Dewatering to the Controlled Crystallization of Biological Macromolecutes and Organic Compounds.

PB91-195719

100,230

Applied Electric

100,299

Electrokinetic Demixing of Two-Phase Aqueous Polymer Systems. 1. Separation Rates of Polyethylene Glycol-Dextran Mixtures.

100,487
Gravity-Dependent Phenomena at the Scale of the Singte PB91-134767

101,297

Low-Cost Low-Volume Carrier (Minilab) for Biotechnology and Fluids Experiments in Low Gravity.

100,041

ODD, P. W.

Electrokinetic Demixing of Two-Phase Aqueous Polyme Systems. 2. Separation Rates of Polyethylene Glycol-Maltodextrin Mixtures.

PB91-237230

100,231

TOGANO, $\mathrm{K}$.

Elastic Constants of Polycrystalline Bi-Pb-Sr-Ca-Cu-O Superconductor

101,461

TOLLE, J. W.

Convergence Properties of a Class of Rank-Two Updates. PB91-187799 101,260 Merit Function for Inequality Constrained Nonlinear Programming Problems.

PB92-116318

101,271

TOM, $\mathrm{H}$.

Congressional Districts of the United States. Category: Federal General Data Standard, Representations and Codes.
FIPS PUB 9-1

TOMAZIC, B. B.

Comparative Study of Bovine Pericardium Mineralization: A Basic and Practical Approach.

PB92-117407

100,056

Ion Incorporation into Octacalcium Phosphate Hydrolyzates. PB91-175125

100,219

TOMER, A.

Continuous-Cooling Transformation Characteristics and High-Temperature Flow Behavior of a Microalloyed SAE PB91-18785

101,155

Microstructure, Composition, and Hardness of Rockwell C Hardness Blocks
PB91-184747

101.153

TONG, G. Q.

Device for Audio-Frequency Power Measurement. PB91-14940

100,733

TORGESON, D. $\mathrm{R}$.

Relationship of Hydrogen Site Occupancy to Diffusion Behavior in Crystalline and Amorphous $\mathrm{Zr} 2 \mathrm{PdHx}$. PB91-187252

101,230

TOTH, $\mathrm{K}$.

Analysis of Wet Deposition (Acid Rain): Determination o the Major Anionic Constituents by lon Chromatography. PB91-236646

100,035

TOTH, L.

Electronic Structure of High-Tc Superconductors Studied Using Photoelectron Spectroscopy.

101,513 Initial Stages of Degradation of Superconductor Surfaces
$\mathrm{O} 2, \mathrm{H} 2 \mathrm{O}, \mathrm{CO} 2$ and $\mathrm{CO}$ Chemisorption on $\mathrm{La} 2-\times \mathrm{Sr} \times \mathrm{CuO} 4$. $\mathrm{O} 2, \mathrm{H} 2 \mathrm{O}, \mathrm{CO} 2$ and $\mathrm{CO}$ Chemisorption on La2-xSrxCuO4.
$\mathrm{PB} 91-189670$ Phonon Density of States of Superconducting YBa2Cu3O7 and the Nonsuperconducting Analog YBa2Cu3O6. PB91-159129

101,487

TOTH, R. B.

Standards Activities of Organizations in the United States. PB91-17777

TRABERT, E.

100,004

lons

PB91-237511

TRAHEY, N. M.

Directory of Accredited Laboratories, 1991. National Voluntary Laboratory Accreditation Program (NVLAP). PB91-222646

100,934

TRAIL, W. $K$

Near-Threshold Vibrational Excitation of $\mathrm{H} 2$ by Electron Impact: Resolution of Discrepancies between Experimen and Theory.

TRAN-CONG, $Q$.

Temperature Dependence of Probe Diffusion in Bulk Polymer Matrices.
PB91-236885

TRAPMANN, W. A.

Gas Analysis Modeling System.

PB91-175299

TREADO, S. J.

Measurement and Evaluation of Lighting/HVAC Interaction.

POMKA, J.

Possible Use of Pattern Recognition for the Analysis of Mars-Rover X-ray-Fluorescence Spectra.

101,733

TROUT, T. K.

Fourier Transform Infrared Analysis of Ceramic Powders: Quantitative Determination of Alpha, Beta, and Amorphous Phases of Silicon Nitride.

PB91-237628

101,096

TROY, E. F.

Thwarting the Hackers.

PB91-159228

100,705

TRUS, S.

Guidelines for the Evaluation of File Transter, Access and Management Implementations.

PB92-126580

100,699

TSAI, B. P.

Electronic Spectrum of the GeH3 Radical.

PB91-189548 100,346

Resonance Enhanced Multiphoton Ionization Spectra of the $\mathrm{GeF}$ and $\mathrm{GeCl}$ Radicals from $400-500 \mathrm{~nm}$.

PB91-203281

100,396

TSAI, C.

Critical Evaluation of Liquid Crystal Transition Temperatures I: 4,4'-Alkyl/alkoxyphenylbenzoates.

PB92-110154

100,444

TSAI, K. H.

Control of Flexible Robot Arm.

PB91-174979

101,032

TSAI, M.

Critical Evaluation of Liquid Crystal Transition Temperatures I: 4,4'-Alkyl/alkoxyphenylbenzoates.

PB92-110154

100,444

TSAI, T. M.

Performance Measures of a Robotic Micropositioner PB92-117019

101,037

TSANG, K. L.

Soft X-ray Absorption and Emission Spectra and the Electronic Structure of Some Exotic Materials.

PB91-194738

101,520

TSANG, $W$.

Chemical Kinetic Data Base for Combustion Chemistry Part 4. Isobutane.

100,252

Chemical Kinetic Data Base for Combustion Chemistry. Part 5. Propene.

Chemical Kinetic Data Base for Propellant, Combustion. 1. Reactions Involving NO, NO2, HNO, HNO2, $\mathrm{HCN}$ and N2O. PB92-148071

Formation and Stability of SF5 and S2F10.

PB91-174839

100,467

Letter to Editor of Chemical and Engineering News.
PB91-174847

100,326

Single Pulse Shock Tube Study of the Reactions of Hydrogen Atoms with

100,331

TSENG, $w$.

Pattern Generation on Semiconductor Surfaces by a Scanning Tunneling Microscope Operating in Air.

100,808

Selective-Area Epitaxial Growth of Gallium Arsenide on Silicon Substrates Patterned Using a Scanning Tunneling Microscope Operating in Air.

PB91-149880

100,796

TSUDAGAWA, $M$

Electronic and Radiometric Characteristics of Near Infrared Photodiodes.

PB91-174706

100,752

TU, K. M.

Engineering Analysis of the Fire Development in the Hillha. ven Nursing Home Fire, October 5, 1989

100,169

Experimental Study of Top Vented Compartment Fires. PB91-167254

100,155

TU, Y.

Analyzing Integrated Optical Waveguides: A Comparison of Two New Methods.

PB91-204131

100,759

TUNG, $\mathbf{W}$. 
TURNER, A. H.

Report of the National Conference on Weights and Meas Report of the National Conference on Weights and Measures (76th). Hel

101,024

TURNER, C.

Electrodeposition of Aluminum from Molten Salts.

PB91-159202

101,223

TURNER, $\mathbf{S}$.

Accuracy of Transmission Electron Microscopy Analysis of Asbestos on Filters: Interlabortory Study.

100,204

TYREE $Y$. C.

Knowledge Verification of Machine-Learning Procedures Best on Testructure Measurements.

PB91-189571

100,709

TYSON, R. A.

Accelerator Radiocarbon Dates on Charcoal, Shell, and Human Bone from the Del Mar Site, California.

101,355

PB91-23740

TZENG, L. S.

Theoretical Investigation of Piloted Ignition of Wood.

PB92-112432

100,556

UDOVIC, T.J.

Crystal-Field Splittings in the Cubic Heusler Alloys HoPd2Sn and ErPd2Sn.

PB91-194902

101,525

Neutron Spectroscopic Evidence for Adsorbed Hydroxyl Species on Platinum Black.

PB91-237636

100,432

Rapid Low-Temperature Hopping of Hydrogen in a Pure Metal: The $\mathrm{ScH}$ System.

PB91-158485

101,217

ULBIG, $\mathbf{S}$.

Doppler Shift Attenuation Lifetime Measurements in (54) Cr

Following Thermal Neutron Capture.

PB91-189753

101,656

UNGURIS, $J$.

Micromagnetics of Domain Walls at Surfaces.

Observation of Two Different Oscillation Periods in the Exchange Coupling of $\mathrm{Fe} / \mathrm{Cr} / \mathrm{Fe}(100)$.

PB92-117415

101,572

Scanning Electron Microscopy with Polarization Analysis (SEMPA)-Studies of Domains, Domain Walls and Magnetic Singularities at Surfaces and in Thin Films.

PB91-237289

101,554

UNLU, M. S.

Emitter Ballasting Resistor Design for, and Current Handling Capability of AlGaAs/GaAs Power Heterojunction Bipolar Transistors.

PB91-236703

100,815

Uniform Junction Temperature AIGaAs/GaAs Power Heter-

ojunction Bipolar Transistors on Silicon Substrates.

PB91-189399

URIANO, G. A.

Advanced Technology Program: A New Role for NIST in Accelerating the Development of Commercially Importan Technologies.

100,013

URIBE, F. J.

Equilibrium and Transport Properties of Gas Mixtures a Low Density: Eleven Polyatomic Gases and Five Noble

PBases.

100,363

Thermal Conductivity of Nine Polyatomic Gases at Low Density.

VALKIERS, $\mathbf{S}$.

100,360

Silicon Reference Materials Certified for Isotope Abun dances.

100,213

VAN BRUNT,

Collisional Electron-Detachment and Ion-Conversion Proc-

esses in SF6.
PB91-134528

Detection of Trace Disulfur Decafluoride in Sulfur Hexafluoride by Gas Chromatography/Mass Spectrometry. 100,197 Influence of Memory Propagation on Phase-Resolved Stochastic Behavior of AC-Generated Partial Discharges.
PB91-237644

Off-Axis Measurements of Ion Kinetic Energies in RF Plas-

PBa2-117225

101,444

Preface to Book Entitled 'Nonequilibrium Effects in Ion and Electron Transport'

PB91-159236 101,623

Transition from Trichel-Pulse Corona to Dielectric Barrier Discharge.
PB91-134213

100,835

Zonal Model for Corona Discharge-Induced Oxidation of $\mathrm{SF} 6$ in $\mathrm{SF} 6 / \mathrm{O} 2 / \mathrm{H} 2 \mathrm{O}$ Gas Mixtures.

PB91-158790

100,312

VAN DEN BERG, L.

High Resolution Diffraction Imaging of Crystals Grown in

Microgravity and Closely Related Terrestrial Crystals.
PB92-109008

101,562

AN DYK, G.

Sensitometry of the Response of a New Radiochromic Film Dosimeter to Gamma Radiation and Electron Beams.

PB9 1-237008

101,326

AN POOLEN, L. J.

Extended Phase Rule for Nonreactive, Multiphase, Multicomponent Chemical Systems.

PB91-237651

100,433

VAN ROGGEN, A.

Permittivity Measurements on Molecular-Sized Samples.

PB91-159244

100,800

VANDIVER, P. B.

Variations in the Practice of Ceramic Technology in Different Cultures: A Comparison of Korean and Chinese Celadon Glazes.

VANEK, M. D.

101,111

Extending the Range and Accuracy of Phase Noise Measurements.

PB91-134874

VANEY, M. C.

100,588

Crystal Structure of a CAMP-Independent Form of Catabolite Gene Activator Protein with Adenosine Substituted in One of 2 cAMP-Binding Sites.

PB91-190074

101,305

VANZURA, E. J.

Improved Technique for Determining Complex Permittivity with the Transmission/Reflection Method.

PB91-161919

100,844

VARMA-NAIR, $M$.

Heat Capacity and Other Thermodynamic Properties of Linear Macromolecules. 10. Update of the ATHAS 1980 Data Bank.

PB92-110196

100,515

VARNER, R. N.

Standard Reference Materials: Bright-Chromium Linewidth Standard, SRM 476, for Calibration of Optical Microscope Linewidth Measuring Systems

PB91-167163

100,802

VASQUEZ, I.

Ignition Characteristics of Selected SSME Alloys.

PB91-174474

100,544

VASUDEVAN, A. $K$.

Stress Corrosion Cracking of Al-Li Alloys: The Role of Grain Boundary Precipitates.

AUDIN, $M$.

Processing Bi-Pb-Sr-Ca-Cu-O Superconductors from Amor-

phous State.

VAZQUEZ, E.

Performance of OSI Transport over ACCUNET and IBERPAC.

PB91-134825

100,563

VEASEY, D. L.

Semiconductor Claddings on Glass Waveguides for Polariz ers and Detectors.

PB91-175141

101,416

VECCHIA, D. F.

Linear Calibration When the Coefficient of Variation is Constant.

PB91-149443

101,273

VEESER, L. R.

Faraday Effect Current Sensing Using a Sagnac Interferometer with a $3 \times 3$ Coupler.

PB91-175158

100,754

LARDE, G.

Muon Sticking in Catalysed d-t Fusion: Nuclear Resonance PBg1-14

VENTURINI, E. L.

101,613

Properties of Amorphous Zirconium Rhodium Hydride (Zr3RhHx) Prepared from Glassy and Crystalline Alloys.
101,225

101,225

VENZ, $S$.

Dental Composite Resins: An Update.

PB91-204149

100,051

Filler Systems Based on Calcium Metaphosphates.

PB91-194654

VERDIER, P. H.

Multiple Variable-Angle Light Scattering Detector for Gel Permeation Chromatography.

Simulation Studies of Excluded Volume Effects on Polyme Chain Dynamics in Several Nonlattice Models.

PB91-203364

100,507

VESOVIC, $V$

Thermal Conductivity of Methane and Tetrafluoromethane in the Limit of Zero Density.

PB91-192500

100,361

PB90-244724

100,266

VEZZETTI, C. F.

tandard Reference Materials: Bright-Chromium Linewidth Standard, SRM 476, for Calibration of Optical Microscope inewidth Measuring Systems.

PB91-167163

100,802

\section{VIDAL-MADJAR, A.}

Studies of $\mathrm{HI}$ and $\mathrm{DI}$ in the Local Interstellar Medium.

PB91-148957

VIGLIOTTI, D. P.

Cryogenic Material Properties of Stainless Steel Tube-toFlange Welds.

101,145

\section{VILLA, K. M.}

Cone Calorimeter Rate of Heat Release Measurements for Upholstered Composites of Polyurethane Foams.

PB92-108984

100,087

Material Flammability Test Assessment for Space Station Freedom.

PB91-216606

101,735

INCENT, M. A.

Orbit Determination and Gravitational Field Accuracy for a Mercury Transponder Satellite.

101,736

VINYES, J.

Performance of OSI Transport over ACCUNET and IBER-

B91-134825

100,563

VISITSERNGTRUKUL, $\mathbf{S}$.

Sensitivity of Ellipsometric Modeling to the 'Islands' of Silicon Precipitates at the Bottom of the Buried Oxide Layer in Annealed SIMOX

PB92-116607

100,820

Residual Defects in SIMOX: Threading Dislocations and Pipes.

PB92-117233

100,829

VOELS, S. A

Photospheres of Hot Stars. 4. Spectral Type O4 PB91-149849

100,025

VOGEL, E

Viscosity and Thermal Conductivity of Pure Monatomic Gases from Their Normal Boiling Point Up to $5000 \mathrm{~K}$ in the Limit of Zero Density and at $0.101325 \mathrm{MPa}$.

PB91-192559

100,366

VOLCHOK, H. L.

Interlaboratory Comparison of Actinides in Human Tissue (239) $\mathrm{Pu}+(240) \mathrm{Pu}$

PB91-203265

101,323

VOORHEES, P. W

Role of Elastic Energy in the Morphological Development of a Ni-Ti-Al Alloy.
PB91-187161

VORBURGER, T. V.

Gapacitance versus Stylus Measurements of Surface Roughness.

PB91-203463

101,020

Light-Scattering Measurement of the RMS Slopes of Rough

PB92-116532 101,436

Standard Reference Specimens in Quality Control of Engi- 
WAHLIN, E.

Probe for Real-Time Images of Particle Beams and Thei Analyses in a Merged-Beams Apparatus.

101,683

WAIT, D. F.

Intercomparison of NBS Noise Calibration Services. PB91-162370

WAKEHAM, W. A.

Benzene: A Further Liquid Thermal Conductivity Standard.
PB90-244567

Thermal Conductivity of Methane and Tetrafluoromethane in the Limit of Zero Density.

in the Limit of Zer
PB91-192500

100,361

Transport Properties of Carbon Dioxide.

PB90-244724

100,266

\section{WAKID, S. A.}

Conformance Tester for X.25 DTE Implementations.
PB91-189894

ISDN Conformance Testing: Layer 1. Physical Layer. Part 1

Basic Rate S/T Interface, User Side.

PB92-102201

100,572

North American ISDN Users' Forum Agreements on Integrated Services Digital Network.

\section{WALDOW, D.}

Temperature Dependence of Probe Diffusion in Bulk Polymer Matrices.
PB91-236885

WALKER, $\mathrm{C}$.

100,422

Application of Osmotic Dewatering to the Controlled Crystallization of Biological Macromolecules and Organic Com pounds.

PB91-195719

100,230

WALKER, E. L.

Geometric Reasoning for Constructing 3D Scene Descriptions for Images.

WALKER, H. N.

Techniques to Observe the Fracture Zone in Mortar and Concrete.

PB91-134189

100,521

WALLACE, D. $R$

Proceedings of the Forum on Standards for High Integrity Software (DOD, Government, Industry). Held in Gaithersburg, Maryland on June 28, 1991

PB92-112267

100,668

Proceedings of the Workshop on High Integrity Software. Held in Gaithersburg, MD. on January 22-23, 1991.
PB92-109040

100,667 Software Verification and Validation: An Overview.
PB91-237685 100,658

WALLACE, E. K.

Manufacturing Systems Integration Control Entity Interface

PB91-240796

100,991

Manufacturing Systems Integration: Initial Architecture Document.

PB92-112242

WALLACE, J S.

100,994

Effect of Thermochemical Treatments on the Strength and Microstructure of SiC Fibers.

PB91-194670

101,084

Strength and Fracture Behavior of Ba-Y-Cu-O Superconducting Ceramics.

PB91-148205

101,474

Superconducting Thin Films of $\mathrm{Bi}-\mathrm{Sr}-\mathrm{Ca}-\mathrm{Cu}-\mathrm{O}$ Obtained by Laser Ablation Processing.

WALLACE, S.

Manufacturing Systems Integration Control Entity Interface Document.

PB91-240796

100,991

Manufacturing Systems Integration: Initial Architecture Document.

WALLINGTON, T. J.

100,994

Group Reactivities in the Gas Phase Reactions of Hydroxyl Radicals with Ethers.

PB91-175059

100,330

WALLIS, $B$.

Stable, High Quantum Efficiency Silicon Photodiodes for Vacuum-UV Applications.

PB91-203349

100,757

\section{WALLS, F. L.}

Accuracy Model for Phase Noise Measurements.
PB91-134882

Calculated from the Frequency Domain: An Update.

PB91-162388

Environmental Effects on the Medium and Long Term Frequency Stability of Quartz Oscillators.

Environmental Sensitivities of Quartz Crystal Oscillators.

N91-25797/2 100,778

Extending the Range and Accuracy of Phase Noise Meas urements.
PB91-134874

100,588

Time Domain Frequency Stability Calculated from the Frequency Domain Description: Use of the SIGINT Software Package to Calculate Time Domain Frequency Stability from the Frequency Domain.

PB92-123132

100,587

WALRAFEN, G. E.

High-Temperature High-Pressure Raman Spectra from PB91-195735

WALSH, R. P.

100,385

Aluminum Alloys for ALS Cryogenic Tanks: Comparative Measurements of Cryogenic Mechanical Properties of Al-Li Alloys and Alloy 2219

AD-A242 956/1

101,204

Creep of Copper: 4 to $295 \mathrm{~K}$

101,233

Cryogenic Fatigue of High-Strength Aluminum Alloys and Correlations with Tensile Properties.

PB91-236976

101,709

Interlaboratory Tension and Fracture Toughness Test Results for CSUS-JN1 (Fe-25Cr-15Ni-0.3N) Austenitic Stainless Steel at $4 \mathrm{~K}$.

101,166

Strain Rate Effect on Tensile Properties at $4 \mathrm{~K}$ of a VAMAS

Strain Rate Effect on Tensile Properties at $4 \mathrm{~K}$ of a VAMAS
Round-Robin Austenitic Steel.
PB91-237248

WALTERS, W. B.

Structure of Odd-Odd (132)Sb

PB91-237461

101,711

WALTON, G. N.

Computer Program for Simulation of HVAC/Lighting Interactions: Initial Report

PB91-144386

100,889

WALTRIP, B. C.

Performance Evaluation of a New Audio-Frequency Power Bridge.

100,742

WANG, B. L.

Control of Flexible Robot Arm.

PB91-174979

101,032

WANG, $\mathbf{F}$.

Assessment of the State-of-the-Art for Process Monitoring Sensors for Polymer Composites.

100,510

Fluorescence Monitoring of Polymer Processing: Mixing and Zero Shear Viscosity.
PB91-174482

WANG, F. W.

100,492

Stability of Aqueous Inorganic Lead Solutions in Polycarbonate Containers.

WANG, $P$.

100,211

Database and Retrieval System for the NBS Tables of Chemical Thermodynamic Properties.

PB91-237693 100,434

Dielectric Constant of Water and Debye-Huiecke' Limiting Law Slopes.

WANG, P. S.

100,260

Oxidation Kinetics of Silicon Carbide Whiskers Studied by X-ray Photoelectron Spectroscopy.

100,436

Surface Oxidation Kinetics of Si3N4 - 4\% Y2O3 Powders Surface Oxidation Kinetics of Si3N4 - 4\% Y2O3 Powders
Studied by Bremsstrahlung-Excited Auger Spectroscopy.
PB91-237701

100,435

WANG, $Y$.

Collisional Electron-Detachment and Ion-Conversion Processes in SF6.

WANG, $z$. $x$.

Sliding Wear Studies of Nickel-Copper Composition-Modulated Coatings on Steel.

PB91-19002

101,188

WARD, D. B.

Study of the Linearity of Transfer Leaks and a Helium Leak PB91-147272

100,926

WARD, D. K.

Thermodynamic Properties of Two Alternative Refrigerants 1,1-dichloro-2,2,2-trifluoroethane (R123) and 1,1,1,2-tetraluoroethane (R134a)

PB91-175414

101,195

WARD, T. H.

Limited International Intercomparison of Responsivity Scales at Fiber Optic Wavelengths.

100,567

WARSAW, B.

TROI User's Guide.

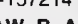

101,026

Depot: A Framework for Sharing Software Installation Across Organizational and Unix Platform Boundaries.

PB91-240754

WASZCZAK, J. V.

New Layered Cuprate Structure-Type, (A1.

PB91-237339

101,095

WATANABE, $H$.

Pulse-Echo Ultrasonic Evaluation of the Integrity of Seams of Single-Ply Roofing Membranes: Laboratory Evaluation of a Prototype Test Apparatus.

PB91-157172

100,106

Pulse-Echo Ultrasonic Evaluation of the Integrity of Seams of Single-Ply Roofing Membranes: Results of Field Investigations and Recommendations.

100,110

WATANABE, $K$.

Sixteen Thousand Evaluated Experimental Thermodynamic Property Data for Water and Steam.

100,475

Survey of Current Worldwide Research on the Thermophysical Properties of Alternative Refrigerants.

PB92-112366

101,198

\section{WATERSTRAT, R. $M$.}

Structural Instabilities and Superconductivity in Quasi-Binary Mn5Si3-Type Compounds.

101,215

WATSON, J. T. R.

Survey of Current Worldwide Research on the Thermophysical Properties of Alternative Refrigerants.

PB92-112366

101,198

Transport Properties of Carbon Dioxide.

PB90-244724

100,266

WATSON, P. R.

Critical Compilation of Surface Structures Determined by Ion Scattering Methods.

$-244559$

100,254

WATSON, R. E.

Crystalline and Glassy Phases of Transition-Metal - Metalloid Systems.

101,238

Wigner-Seitz Local-Environment Study of the High Tc Superconductors.

101,528

WATTERS, R. L.

Characteristics of Aerosols Produced by the Spark Dis-

PB91-237727

100,209

WATTS, R. N.

Associative Ionization of Ultra-Cold Laser Trapped Sodium

Atoms.

101,707

Observa

PBimit. 189746

Doppler

Observation of Atoms Laser Cooled Below the Doppler Limit.

PB91-195453

101,671

WAVERING, A.

NASREM Robot Control System and Testbed. PB91-194969

101,033

WAVERING, A. J

Recommended Fine Positioning Test for the Development Test Flight (DTF-1) of the NASA Flight Telerobotic Servicer (FTS).

101,732

WEBB, R. E.

Dentin-Bonding Molar Efficiency Using N-Phenylglycine, NPhenyl-beta-Alanine, or N-Methyl-N-Phenylglycine.

100,053

WEBER, A.

Molecular Spectroscopy Division of the National Bureau of 
WINELAND, D. J.

WEIL, C. M.

Dual Six-Port Reflectometer Systems Using Waveguide in the Frequency Range $18-50 \mathrm{GHz}$.

PB91-134916

WEIMER, C. $\mathbf{S}$.

Progress at NIST on Absolute Frequency Standards Using Stored lons.

WEINER, J.

101,625

Associative Ionization of Ultra-Cold Laser Trapped Sodium Atoms.

PB91-236745

101,707

WEIR, R. D.

Heat Capacity and Thermodynamic Properties of Deutrated Ammonium Hexafluorophosphate ND4PF 6 from $5.8 \mathrm{~K}$ to

PB91-147041

100,272

Heat Capacity of Deuterated Ammonium Tetrafluoroborate ND4BF4 from 7 to $348 \mathrm{~K}$

100,273

Thermodynamics of Ammonium Indates. 2. The Molar Heat Thermodynamics of Ammonium Indates. 2. The Molar Heat
Capacity of the Ammonium Pentabromoindate Monohydrate Capacity of the Ammonium Pentabromoindate Monohydrate
Salt (NH4)2 InBr5. H2O from 7.8 to $348 \mathrm{~K}$.
PB91-149831

100,304

Thermodynamics of the Divalent Metal Fluorides. 3. Heat Capacity of the Fast Ion Conductor Sr SnF4 from 6 to 344
K.
PB91-149864 100,305 100,305
4. Heat Thermodynamics of the Divalent-Metal Fluorides. 4. Heat Capacity of $\mathrm{MSnF}_{4}, \mathrm{M}=\mathrm{Pb}, \mathrm{Ba}$, or $\mathrm{Sr}$, at Temperatures from $300 \mathrm{~K}$ to $660 \mathrm{~K}$

WEISS, A. W.

100,456

Relativistic and Correlation Effects in the 2s3p Configuration of Beryllium-Like Ions

PB91-189605

101,654

WEISS, M. A.

New Inexpensive Frequency Calibration Service from NIST.

PB91-200790 100,580

Precise Ephemerides for GPS Time Transfer.

PB91-236950

100,586

WELCH, J. F.

Unsteady Laminar Flow in a Circular Tube: A Test of the HERCOL (Hermitian Collocation) Computer Code. PB91-222679

101,395

WELCH, M. J.

Stability of Uric Acid in Ammonium Hydroxide PB91-189266

101,292

WELLER, $M$.

Mechanical Loss in a Glass-Epoxy Composite.

101,070

Heterodyne Frequency Measurements of $\mathrm{CO}$ and OCS Beyond $2100 \mathrm{~cm}(-1)$

PB91-236984

100,423

WELSCH, L.

Difficulties in Parsing SGML.

PB91-189456

100,650

WELSCH, L. A.

Multimedia Courseware in an Open Systems Environment: A Federal Strategy.

WERIJ, H. G. C.

Collisional Energy Transfer between Excited Sr Atoms.

PB91-204164

WESSMAN, R. A.

Interlaboratory Comparison of Actinides in Human Tissue (239) Pu $+(240) \mathrm{Pu}$.

PB91-203265

PB91-149864

WEXLER, A.

NIST Calibration Services for Humidity Measurement PB92-112499

WHEELER, A. A

Effect of an Electric Field on the Morphological Stability of the Crystal-Melt Interface of a Binary Alloy. 3. Weakly Nonlinear Theory.

101,536

Effect of Gravity Modulation on Thermosolutal Convection in an Infinite Layer of Fluid.

101,396

Phase-Field Model for Isothermal Phase Transitions in Binary Alloys.
PB92-108992

101,240

WHETSTONE, J. $R$.

Advanced sensor development program for the pulp and paper industry. Final report.

DE91007688

101,248

WHITE, E.

Synthesis of 3-Ouinuclidinol-(18)O, Benzilic-d5 Acid, and 3Ouinuclidinyl-(18)O, Benzilate-d5.

100,223

WHITE, G. S.

Effects of Crystal Bonding on Brittle Fracture.

PB91-148221 101,064

Environmentally Enhanced Fracture of Gallium Arsenide.

PB91-237743 101,097

Indentation Determination of Crack Growth Parameters in Gallium Arsenide.

PB91-19008

101,083

WHITE, H. J.

Steam Tables.

WHITE, J. $W$.

Structure of a Stage-3 Cs - Graphite Intercalate.
PB91-134957

WHITE V, E.

Stability of Uric Acid in Ammonium Hydroxide. PB91-189266

WHITE, V. R.

Directory of Accredited Laboratories, 1991. National Voluntary Laboratory Accreditation Program (NVLAP)

NVLAP FY 90 Annual Report

PB91-206748

100,010

WHITENTON, E. P.

Method for Ouantitative Measurement of Galling Damage.
PB91-159269

Study of the Galling of Two Steels Using Two Test Methods.

WHITMAN, L. J.

Geometric and Electronic Properties of Cs Structures on III-

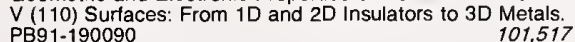
Scanning Tunneling Microscopy Study of Clean and CsCovered InSb(110).

PB91-195750

101,537

WHITTEN, B. L.

Spin-Dependent Electron-Impact Excitation of Sodium PB91-175497

WHITTENBERG, C. D.

Standard Reference Fibers for Calibration of the Optical Time Domain Reflectometer.

101,323

WESTBROOK, C. I.

Associative Ionization of Ultra-Cold Laser Trapped Sodium

101,707

Observation of Atoms Laser-Cooled Below the Doppler Limit

PB91-189746

101,655

Observation of Atoms Laser Cooled Below the Doppler Limit.

WESTRUM, E. F.

101,671

Fusion of Mercury. A New Certified Standard for Differentia Scanning Calorimetry.

PB91-150003

100,309

Heat Capacity and Thermodynamic Properties of Deutrated Ammonium Hexafluorophosphate ND4PF 6 from $5.8 \mathrm{~K}$ to $347 \mathrm{~K}$.

PB91-147041

100,272

Heat Capacity of Deuterated Ammonium Tetrafluoroborate ND4BF 4 from 7 to $348 \mathrm{~K}$

PB91-147058

100,273

Thermodynamics of Ammonium Indates. 2. The Molar Heat Capacity of the Ammonium Pentabromoindate Monohydrate Salt $(\mathrm{NH} 4) 2$ InBr5. H2O from 7.8 to $348 \mathrm{~K}$.

PB91-149831

100,304

Thermodynamics of the Divalent Metal Fluorides. 3. Heat Capacity of the Fast lon Conductor Sr SnF 4 from 6 to 344 $\mathrm{K}$.
PB91-147207

101,406

WICKMAN, G.

Absorbed Dose to Water: Comparison of Several Methods Using a Liquid Ionization Chamber.

101,322

WICKS, F. J.

Wawayandaite, a New Calcium Manganese Beryllium Boron Silicate from Franklin, New Jersey.

PB91-174680

101,359

WIEDERHORN, $\mathbf{S}$. $\mathrm{M}$.

Comparison of Creep Rupture Behavior in Tension and Bending.

101,087

Creep and Reliability of Ceramic Materials at Elevated Tem-

peratures.

101,091

Effect of Slag Penetration on the Performance of Magnesia Chrome Refract

100,875

Effect of Surface Forces on Subcritical Crack Growth in Glass.

PB92-117449

101,103

High Temperature Creep Testing of Ceramics.

B91-202838

101,088

Tensile Creep Behavior of Structural Ceramics. PB91-159277

101,075

WIELOPOLSKI, P. A

Structure of a Stage-3 Cs - Graphite Intercalate.

PB91-134957

100,269

WIEMAN, $\mathrm{C}$

Observation of the Cesium Clock Transition Using LaserCooled Atoms in a Vapor Cell.

PB91-203638

101,698

WIERENGA, P. J.

Model Validation at the Las Cruces Trench Site.

NUREG/CR-5716

101,368

WIESE, W. L.

Experimental Stark Widths and Shifts for Spectral Lines of

Neutral and lonized Atoms: A Critical Review of Selected Data for the Period 1983 through 1988.

PB91-192567

101,659

Spectral Data and Grotrian Diagrams for Highly Ionized Iron, Fe VIII-XXVI.

101,595

Spectroscopic Data Base for Carbon and Oxygen.
PB91-237750

ities for Neutral

Argon.
PB91-237768

Working Group 2: Atomic Transition Probabilities.
PB91-175588

101,717

WILCOX, R. A.

SHOOT Flowmeter and Pressure Transducers.

PB91-147488

WILHOIT, R. C.

Thermodynamic and Thermophysical Properties of Organic Nitrogen Compounds. Part 1. Methanamine, Ethanamine, 1. and 2-Propanamine, Benzenamine, 2-, 3-, and 4-Methylbenzenamine.

WILLGING, $P$. A

100,368

Physical and Thermo-Mechanical Properties of Monoclinic Single Crystals.

WILLIAMS, D.

101,065

Characteristic Impedance Determination Using Propagation Constant Measurement.

PB91-236992

Translate LRL and LRM Calibrations.
PB91-237776

100,775

WILLIAMS, E. R.

Monitoring the Mass Standard via the Comparison of Mechanical to Electrical Power

PB91-237115

101,710

WILLIAMSON, M. P.

3480 Type Tape Cartridge: Potential Data Storage Risks, and Care and Handling Procedures to Minimize Risks.

PB92-126549

101,381

Standard Reference Materials: Calibration of NIST Standard Reference Material 3201 for 0.5 Inch $(12.65 \mathrm{~mm})$ Serial Serpentine Magnetic Tape Cartridge.

PB91-187542

100,690

WILLMAN, N.

Development of an Optical Disk System for the Automated Retrieval of EASEAR Records.

PB92-108943

100,666

WILLMAN, N. E.

Standard Reference Materials: Calibration of NIST Standard Reference Material 3201 for 0.5 Inch $(12.65 \mathrm{~mm})$ Serial Serpentine Magnetic Tape Cartridge.

PB91-187542

100,690

WILSON, A. M.

Environmentally Enhanced Fracture of Gallium Arsenide.

101,097

Indentation Determination of Crack Growth Parameters in Gallium Arsenide. 
AD-A227 656/6

Liquid and Solid Ion Plasmas.

101,441

101,592

Observation of Correlations in Finite, Strongly Coupled Ion Plasmas.

PB91-200857

101,678

Progress at NIST on Absolute Frequency Standards Using PB91-159285

101,625

Quantum Optics of Single, Trapped lons

101,628

Reply to Comment on 'Quantum Zeno Effect'

101,708

Test of the Linearity of Quantum Mechanics by if Spectroscopy of the $(9) \mathrm{Be}(+)$ Ground State.

101,726

Trapped-Ion Frequency Standards.

N91-25760/0

100,575

WINNEWISSSER, $c$

Linearity of a Silicon Photodiode at $30 \mathrm{MHz}$ and Its Effect on Heterodyne Measurements.

PB91-187625

100,755

WINSTEAD, E.

Reference NO2 Calibration System for Ground-Based Intercomparisons during NASA's GTE/CITE II Mission.

PB91-189340

WINTER, C.

100,034

Doppler Shift Attenuation Lifetime Measurements in $(54) \mathrm{Cr}$ Following Thermal Neutron Capture.

PB91-189753

101,656

WINTERS, M. P.

Correlated Spontaneous Emission in a Zeeman Laser

PB91-175596

101,417

Correlated Spontaneous Emission in a Zeeman Laser.

PB91-204180

101,432

WISE, J. A

Procedure for the Effective Recalibration of Liquid-in-Glass Thermometers.

100,935

WISE, S.

Capillary Supercritical Fluid Chromatography of Explosives:

Investigations on the Interactions between the Analytes, the Mobile Phase and the Stationary Phase.

Mobile Phase

100,182

WISE, S. A.

Alaska Marine Mammal Tissue Archival Project: Revised Collection Protocol.

PB91-184796

101,374

Cryogenic-Temperature Fluorescence Spectra of Polynuclear Aromatic Hydrocarbons of Molecular Weight 302.
PB91-147157 100,274 Determination of Bonded Phase Thickness in Liquid Chromatography by Small Angle Neutron Scattering.

100,190

VITT, T. J.

New International Electrical Reference Standards Based on the Josephson and Quantum Hall Effects.

PB91-237586

WITTBERG, T. $N$.

100,740

Oxidation Kinetics

of Silicon Carbide Whiskers Studied by

PB91-237719 100,436

Surface Oxidation Kinetics of Si3N4 - 4\% Y2O3 Powders Studied by Bremsstrahlung-Excited Auger Spectroscopy.
PB91-237701
100,435

WITTMAN, R. C.

Spherical Near-Field Scanning: Determining the Incident Field Near a Rotatable Probe.

100,725

WITTMANN, R. C

Probe-Corrected Spherical Near-Field Scanning Theory in Acoustics.

101,383

Spherical Near-Field Scanning: Experimental and Theoretical Studies.

100,723

\section{WITZGALL, $C$.}

Convergence Behavior of Trajectories for Linear ProgramPB91-204198

101,270

Interior-Point Method for Linear and Quadratic Programming Problems.

WLODAWEA, A.

101,269

Crystal Structure of Phosphate-Free Ribonuclease. PB91-175604

101,301

Multiple Conformations of Amino Acid Residues in Ribonuclease A.

PB91-148098

101,298

Neutron Crystallography of Proteins.

PB91-175349

101,285

WOFFORD, B. A.

Rovibrational Analysis of the nu7(1) Intermolecular Hydrogen Bond Bending Vibration in HCN-HF Using Far Infrared Fourier Transform Spectroscopy.
PB91-237784

WOICIK, J. C.

Strontium-Induced Oxygen Defect Structure and Hole Doping in $\mathrm{La2}-x \mathrm{Sr} x \mathrm{CuO} 4$

Surface Extended-X-ray-Absorption Fine Structure and Scanning Tunneling Microscopy of Si(001)2x1-Sb. PB91-237263

WOLF, S.

Phonon Density of States of Superconducting $\mathrm{YBa} 2 \mathrm{Cu} 3 \mathrm{O} 7$ and the Nonsuperconducting Analog $\mathrm{YBa} 2 \mathrm{Cu} 3 \mathrm{O} 6$.

PB91-159129

101,487

\section{ONG-NG, W.}

Phase Equilibria in the System TI-Ca-Ba-Cu-O. 1. Stability of the 2122 Phase under Conditions of Oxygen Annealing. 101,465

Processing Bi-Pb-Sr-Ca-Cu-O Superconductors from Amorphous State.

101,504

Superconducting Properties of $\mathrm{Bi} 2-x-y \mathrm{~Pb} x \mathrm{SnyS} 2 \mathrm{Ca} 2 \mathrm{Cu} 3 \mathrm{Oz}$ PB91.162321

WOOD, L. L.

Publications of the Manufacturing Engineering Laboratory Covering the Period January 1989-September 1991.

PB92-123041

101,053

WOOD, M. L.

Mechanistic Studies of lonizing Radiation and Oxidative Mutagenesis: Genetic Effects of a Single 8-Hydroxyguanine (7Hydro-8-oxoguanine) Residue Inserted at a Unique Site in Viral Genome.

PB91-190108

WOODHOUSE, C. E.

SHOOT Flowmeter and Pressure Transducers.

PB91-147488

101,321

WORTHEY, J. A

Dealing with Obvious Issues in Lighting.

100,761

Effect of Veiling Relections on Vision of Colored Objects.

Light Source Area, Shading, and Glare.

PB91-237818

Role of Theory in Lighting Research and Design
PB91-237826

100,081

Newest Approaches to Quantitative Assessment of Bioactive Organotin

WRIGHT, R. N.

Al: Does It Have a Place in Building Simulation.

PB91-159293

101,328

100,063

PB91-237834

100,151

Research and Technology for Structural Engineering Productivity.

PB91-204206

100,147

WU, M. K.

Magnetic Hysteresis of High-Temperature $\mathrm{YBa} 2 \mathrm{Cu} 3 \mathrm{Ox}-\mathrm{AgO}$ PB91-189506

WU, S. T.

Computational Models Developed for the Corrosion of Pres tressing Steel.
PB91-167304

\section{WU, W. L.}

Examination of Mechanisms Responsible for Incoherent Scattering of Subthermal Neutrons by Polymers.

100,505 Network Structure in Epoxies. 6. The Growth Process In
vestigated by Neutron Scattering.
PB91-237842
100,514

Evaluation of Liquid Junction Potentials and Determination of pH Values of Strong Acids at Moderate Ionic Strengths.
PB91-237859 Proposed New Electrolytic Conductivity Primary Standards for $\mathrm{KCl}$ Solutions

PB91-187658

100,339

Second Dissociation Constant and $\mathrm{pH}$ of N-2-hydroxyethylpiperazine- $\mathrm{N}^{\prime}$-2-ethanesulfonic Acid (HEPES) from 0 to PB91-189282

100,196

WUILLEUMIER, F. J.

Photoionization and Collisional Ionization of Excited Atoms Using Synchrotron and Laser Radiations.

Using Synchrotro

101,626

WUNDERLICH, $\mathrm{B}$.

Heat Capacity and Other Thermodynamic Properties of Linear Macromolecules. 10. Update of the ATHAS 1980 Data Bank

PB92-110196

100,515

XIA, G. J.

Use of Heterodyne Detection to Measure Optical Transmittance over a Wide Range.

101,410

XIONG, L. $X$

Chloride Ion Diffusion in Low Water-to-Solid Cement Pastes.

101,138

$\mathrm{XU}, \mathrm{X} . \mathrm{Y}$.

Device for Audio-Frequency Power Measurement

PB91-149401

100,733

YAMAMOTO, $M$.

Temperature Dependence of Probe Diffusion in Bulk Polymer Matrices.
PB91-236885

YAMASHITA, $\mathrm{H}$.

Effect of High Pressure on Prebreakdown Phenomena in n-

PB91-135020

100,839

Electrical Breakdown and Streamer Statistics in $\mathrm{N}$-hexane under Uniform Field Conditions.

100,837

YANCEY, C. W. C.

Review of Research Literature on Masonry Shear Walls. PB91-167189

100,113

YANG, S. C.

Spectroscopy and Structure of the Alkali Hydride Diatomic Molecules and Their lons.

PB92-110147

100,443

YANG, W. H.

High-Temperature High-Pressure Raman Spectra from Liquid Water.
PB91-195735

100,385

YANG, Y.

STEP: Standard for the Exchange of Product Model Data Resource Integration: Semantic and Syntactic Rules.

YANIV, S. L.

100,966

Summary of the Intercomparison of the Force Standard Machines of the National Institute of Standards and Technology, USA, and the Physikalisch-Technische Bundesanstalt, Germany.

101,590

YAO, Y. C.

Linear Calibration When the Coefficient of Variation is Constant.

PB91-149443

YAP, W. T.

Electrochemical Behavior of Ferrocene in Polyethylene Oxide-400.

PB91-237867

100,439

YARMOFF, J. A.

Chemical Effects of $\mathrm{Ne}+$ Bombardment on the MoS2(0001) Surface Studied by High-Resolution Photoelectron Spectroscopy.

Electron Attenuation Lengths at $\mathrm{SiO} 2 / \mathrm{Si}$ Interfaces. PB91-175612

100,399

YATES, B. W.

Angular Distribution of Ejected Electrons in Resonant Auger Processes of $\mathrm{Ar}, \mathrm{Kr}$, and $\mathrm{Xe}$

PB91-187302

101,648

Parity-Unfavored Transitions in Resonant Photoemission from Ar, Kr, and $\mathrm{Xe}$ : Experimental and Theoretical Results.

101,599

Unusual Degree of Angular Anisotropy in the Resonant Auger Spectrum of Kr.

PB91-18731

101,649

YATES, $N$.

Reference NO2 Calibration System for Ground-Based Intercomparisons during NASA's GTE/CITE II Mission. PB91-189340 
PB91-187849

100,534

YOLKEN, H. T.

Intelligent Processing of Materials, Technical Activities 1991 (NAS-NRC Assessment Panel November 14-15, 1991).

PB92-112572

100,995

YOON, D. N.

Coherency Strain Induced Instability of Solid-Liquid Interfaces in the Mo-Ni System.

101,235

Effect of Coherency Strain on Alloy Formation: Migration of Liquid Films.

101,210 PB91-14732

inth

YOON, I. T.

Intrinsic Carrier Concentrations in Long Wavelength $\mathrm{HgCdTe}$ Based on the New, Nonlinear Temperature De-

pendence of $\operatorname{Eg}(x, T)$.

101,568

YOSHIDA, $K$

Interlaboratory Tension and Fracture Toughness Test Results for CSUS-JN1 (Fe-25Cr-15Ni-0.3N) Austenitic Stainless Steel at $4 \mathrm{~K}$

PB91-237081

101,166

YOSHINO, M.

Cross Sections and Related Data for Electron Collisions with Hydrogen Molecules and Molecular lons. PB90-244674

100,263

YU, D. Y.

Investigating the Use of Multimeters to Measure Quantized Hall Resistance Standards.

100,734

PB91-23655

(1)

Low Temperature Magnetization and Magnetic Excitations in Amorphous Fe78B13Sig.

101,573

PB92-11746

Mix

Mechanism of Soot Formation in Acetylene-Oxygen Mix-

tures.
PB91-147280

YUN-DONG, C.

Sensitometry of the Response of a New Radiochromic Film Dosimeter to Gamma Radiation and Electron Beams.

PB91-237008

YUST, $M$.

Transparent Thin Film Thermocouple.

PB91-190116

100,783

YUWONO, L.

Permittivity Measurements on Molecular-Sized Samples.
PB91-159244

ZABRANSKY, $M$.

Heat Capacities of Organic Compounds in Liquid State. 2. C1 to C18 n-Alkanes.
PB92-110204 100,448

Heat Capacities of Organic Compounds in the Liquid State . C1 to C18 1-Alkanols.

PB90-244716

100,265

ZAHURAK, S. M.

Parent Structure of the Layered High-Temperature Superconductors.

PB91-195677

Electronic and Radiometric Characteristics of Near Infrared Photodiodes

100,752

ZALUBAS, $R$.

Energy Levels of Sulfur, S I Through S XVI. PB91-192443

100,355

ZAPAS, L. J.

Multistep Stress-Relaxation Behavior in Uniaxial Extension of an Ethylene-Hexene Copolymer

PB91-148247

101,242

Phenomenological Theory of the Influence of Strain History on the Rate of Isothermal Stress Relaxation.

100,496

187385

Calibration at $24 \mathrm{C}$ of a Heat-Flow-Meter Apparatus Having $610 \mathrm{~mm}$ Square Plates.

100,933

Comparison of Two Test Methods for Determining Transfer Function Coefficients for a Wall Using a Calibrated Ho Fox Box. 187278

100,12

Evaluation of Fumed-Silica Insulation for a Thermal Conductivity Standard Reference Material.

PB91-147975

100,103

Evaluation of the Heat Flux Transducer Technique for Measuring the Thermal Performance of Walls.

100,126

Summary of Low-Density Glass-Fiber Reference Materials at NIST: $1980-1989$

PB91.175620

101,079

Thermal Conductivity of Fumed Silica Board at Room Temperature.

PB91-190124

100,125

ZAWADZKI, W.

Phonon-Assisted Magneto-Donor Optical Transitions in $n$ -

PB91-147603

101,467

ZEISLER, $R$.

Alaska Marine Mammal Tissue Archival Project: Revised Collection Protocol.

PB91-184796

101,374

ZELEZNIK, F. J.

Thermodynamic Properties of the Aqueous Sulfuric Acid System to $350 \mathrm{~K}$
PB92-148188

100,478

ZEMKE, W. T.

Spectroscopy and Structure of the Alkali Hydride Diatomic Molecules and Their lons.

PB92-110147

100,443

ZHANG, C. H.

Soft $X$-ray Absorption and Emission Spectra and the Electronic Structure of Some Exotic Materials.

tronic Structure

101,520

ZHANG, Y.

Diesel Deposit Forming Tendencies - Microanalysis Meth ods.

PB91-195412

101,178

ZHANG, $\mathbf{Z}$.

Structure of Surface Films on Magnesium and on Magnesium Alloys.

PB91-147637

101,212

ZHENG, Y. C.

Use of Heterodyne Detection to Measure Optical Transmittance over a Wide Range.

PB91-148908

101.410

ZHOU, H.

Permittivity Measurements on Molecular-Sized Samples. PB91-159244

100,800

ZHOU, H. L.

Spin-Dependent Electron-Impact Excitation of Sodium

PB91-175497

101,641

ZIMMERMAN, J.

Optimization of a Pulse Tube Refrigerator for a Fixed Com pressor Swept Volume.

PB91-203877

101,051

ZIMMERMAN, J. E.

Analytical Model for the Refrigeration Power of the Orifice Pulse Tube Refrigerator

PB91-187534

101,048

ZINK, L. $R$.

Accurate Frequency of the $119 \mathrm{mu}$ m Methanol Laser from Tunable Far-Infrared Absorption Spectroscopy. PB91-134130

101,405

ZOU, K.

Thermodynamic Functions and Properties of $\mathrm{MgO}$ at High Compression and High Temperature.

PB90-244542

100,253

ZUKOSKI, E. E.

Species Produced in Fires Burning in Two-Layered and Ho mogeneous Vitiated Environments.

100,542

ZUQIU, L.

Conformance Verification of FDDI Stations.

PB91-148254

100,747 



\section{SAMPLE ENTRY}

\section{Alr Pollution \& Control \\ Proposed Standard Practice for Assessing the Performance of Gas-Phase Air Cleaning Equipment \\ PB91-167353 \\ 100,905}

Keyword term
Titie

NTIS order number
Abstract number
2-METHYLPROPANE

Chemical Kinetic Data Base for Combustion Chemistry Part 4. Isobutan

PB90-244534

100,252

8-Hydroxyguanine Content of Isolated Mitochondria Increases with Lipid Peroxidation.

PB91.189472

101,286

Mechanistic Studies of lonizing Radiation and Oxidative Mutagenesis: Genetic Effects of a Single 8-Hydroxyguanine (7-Hydro-8-oxoguanine) Residue Inserted at a Unique Site in a Viral
PB91-190108

101,321

AB-INITIO CALCULATIONS

Ab-Initio Calculations and Ideal Gas Thermodynamic Functions of Cyclopentadiene and Cyclopentadiene Derivatives.

PB92-148089

100,468

ABRASIVE BLASTING

Roughness Measures of Blasted Steel Surfaces Remotely Imaged with a Thermographic Camera

101,146

ABSORPTION COEFFICIENTS

Scattered Light and Other Corrections in Absorption Coefficient Measurements in the Vacuum Ultraviolet: A Systems Approach.

AD-A227 869/5

100,179

ABSORPTIVITY

Letter to the Editor, Fire Technology, Comments on T.Z. Harmathy Viewpoint, May 1985. PB91.174797

100,545

ABSTRACTS

Abstract and Index Collection in the Research Information Center of the National Institute of Standards and Technology (Fourth Edition).

100,959

High-Temperature Superconductivity: Abstracts of NIST Publications, 1987-1991.

PB92-126564

101,105

AC LOSSES

AC Loss Measurements of Two Multifilamentary $\mathrm{NbTi}$ Composite Strands.

ACCESS

100,769

Configuration Management Concepts Document. National PDES Testbed Report Series.
PB91-194480

100,984

ACCESS CONTROL

Thwarting the Hackers.
PB91-159228

ACCESS METHODS

Issues in Transparent File Access.
PB91-187831

ACCIDENT INVESTIGATIONS

Investigation of the Collapse of L'Ambiance Plaza.
PB91-204057

ACCREDITATION

Plumbing Tést Lab Accreditation.
PB91-190066

Laboratory Accreditation in the United States. PB91-194415

ACCURACY

Uncertainty and Accuracy in Physical Measurements.

PB91-167205 101,629

Accuracy of Transmission Electron Microscopy Analysis of Asbestos on Filters: Interlabortory Study.
PB91-195727
100,204

ACETIC ACID

Studies on the Degradation Products of Paper with and without Pollutants in a Closed Environment. 1. Preliminary Results.

PB91-143313 101,181

Thermodynamics of the Krebs Cycle and Related Compounds.

PB91-192468

100,357

Mechanisms of the Reduction Reactions of $\mathrm{Cr}(\mathrm{VI})$ in the Radiolysis of Acidic Potassium and Silver Dichromate Solutions in the Presence or Absence of Acetic Acid PB91-236489

100,241

ACETONITRILE

Mechanism of Cyanide Release in the Radiolysis of Acetonitrile.

PB91-203679

100,240

Rotational Spectrum and Structure of the Complex Ar-

Ch3CN.

100,415

ACETYLENE

Mechanism of Soot Formation in Acetylene-Oxygen Mixtures.
PB91-147280

Reactions of $\mathrm{C} 5 \mathrm{H} 3(+)$ and $\mathrm{C} 5 \mathrm{H} 5(+)$ lons with Acety lene and Diacetylene.

PB91-195370

100,384

\section{ACID BONDED REACTION CEMENTS}

Setting Reactions and Compressive Strengths of Calcium Phosphate Cements.

PB91-148668

00,040

Effect of Catalyst Structure on the Synthesis of a Dental Restorative Monomer.

PB91-158691

100,043

Development of an Adhesive System for Bonding to Hard Tooth Tissues.

100,052

Dentin-Bonding Molar Efficiency Using N-Phenylglycine, $\mathrm{N}$-Phenyl-beta-Alanine, or N-Methyl-N-Phenylglycine. PB91-237735

100,053

ACID RAIN

Analysis of Wet Deposition (Acid Rain): Determination of the Major Anionic Constituents by lon Chromatography. PB91-236646

ACID RESISTANCE

Effects of $\mathrm{pH}$ and Calcium on Hydrolysis of Na2SiF6 and Na2SnF6: A Quasi-Constant Composition Titration Study. PB91-194746

ACOUSTIC DETECTORS

Material Dependency of Chip-Form Detection Using Acoustic Emission.

PB91-149450

ACOUSTIC EMISSION

Acoustic Emission Monitoring of Laser Drilling PB91-158527

101,216

COUSTIC EMISSIONS

Deconvolution of Acoustic Emission and Other Causal Time Series.

ACOUSTIC FIELDS

Probe-Corrected Spherical Near-Field Scanning Theory in Acoustics.

101,383

ACOUSTIC VELOCITY

Speed of Sound of Natural Gas Mixtures. 
PB91-162214

100,882

Group and Phase Sound Velocities in an Eu1Ba2Cu3O7 Superconductor and Related Perovskite Oxides.
PB91-194860 ACOUSTICS

Ultrasonic Texture and Stress Measurements in Anisotropic Polycrystalline Aggregates.

Accurate Acoustic Measurements in Gases Under Difficult Conditions
PB92-116847

101,382

ACOUSTICS \& SOUND

Device for Audio-Frequency Power Measurement.
PB91-149401 Extended Abstract: A Finite Element Study of the Stress and Displacement Fields Produced by Point Impact. PB91-175224 101,584 Acoustic Technique for Evaluation of Thermal Insulation. PB91-189324 100,123 Microstructure and Elastic Properties of Dental Resin and Resin-Based Glass-Reinforced Composites: XRD, SEM and Ultrasonic Methods.

100,050

Deconvolution of Acoustic Emission and Other Causal Time Series.

101,263

Apparatus for Acoustic Measurements of Gases with Applications for High Temperatures.

100,458

Accurate Acoustic Measurements in Gases Under Difficult Conditions
PB92-116847

101,382

Performance Evaluation of a New Audio-Frequency Power Bridge

100,742

\section{ACRYLATES}

Difunctional and Multifunctional Monomers Capable of Cyclopolymerization
PB91-195685

100,504

ACTINIDES

Interlaboratory Comparison of Actinides in Human Tissue (239)Pu $+(240) \mathrm{Pu}$.

101,323

ACTIS CODE

ACTIS: Towards a Comprehensive Tribology Data Base. PB.91-174904

101,184

ACTIVATION ANALYSIS

Low-Background Gamma-Ray Assay Laboratory for Activation Analysis
PB91-162198

100,192

ACTIVITY COEFFICIENTS

Research at NBS in Direct Potentiometric Measurements in Blood.

\section{DA PROGRAMMING LANGUAGE}

100,188

Ada Compiler Validation Summary Report. Certificate Number: 901109S1.11053, Digital Equipment Corporation VAX Ada, Version 2.2 VAX $8800=>$ VAX 8800

AD-A233 $961 / 2$

100,607

Ada Compiler Validation Summary Report: DDC International A/S, DACS VAX/VMS to 80186 Bare Ada Cross Compiler System, Version 4.6, VAX 8530 (Host) to Bare Board iSBC 186/93A (Host), 901129S1.11079.

AD-A234 083/4 Ada Compiler Validation Summary Report: Certificate Number: 901129S1.11112 DDC International A/S DACS 80386 DMS/OS Ada Compiler System, Version 4.6 IBM PS/2 Model 80-311 = > IBM PS/2 Model 80-311.
AD-A234 118/8 609 Ada Compiler Validation Summary Report: Certificate Number: 901129S1.11050 DDC International A/S DACS VAX/VMS Native Ada Compiler System, Version 4.6 VAX $8530=>$ VAX 8530

100,610

Ada Compiler Validation Summary Report: Digital Equipment Corporation, VAX Ada, Version 2.2, BAX 8800 (Host) $t$ VAX MicrovAX $\|$ Running VAXELN Version 4 (Target), 90110951.11054

Ada Compiler Validation Summary Report: Certificate Number: 901129S1.11051, DDC International A/S, DACS VAX/VMS to 68020 Bare Cross Compiler System, Version 4.6 MicroVAX 3100 Greater Than or Equal to Motorola MVME133.

AD-A234 380/4

100,612

Ada Compiler Validation Summary Report: Certificate Number: $901129 S 1.11077$ DDC International A/S DACS
VAX/VMS to 80186 Bare Ada Cross Compiler System with Rate Monotonic Scheduling, Version 4.6 VAX 8530 $=>$ Bare Board iSBC/03A.

AD-A234 438/0

100,613

Ada Compiler Validation Summary Report: Certificate Number: 901129S1.11075 DDC International A/S DACS 80386 UNIX $\vee$ Ada Compiler System, Version $4.6 \mathrm{ICL}$ DRS $300=>$ ICL DRS 300

AD-A234 439/8

100,614

Ada Compiler Validation Summary Report: Certificate Number: 901129S1.11074 DDC International A/S DACS
System, Version 4.6 VAX $8530=>$ Bare Board iSBC $386 / 21$.

AD-A234 529/6

100,615

Ada Compiler Validation Summary Report. Certificate Number: 910510S1.11161 UNISYS Corporation, UCS Ada, Version 1R1, 2200/600 (Host and Target). Revision.
AD-A239 715/6

100,616

Ada Compiler Validation Summary Report: Certificate (OPTIMIZE) VAX $11 / 785=>$ AN/AYK-14 (Bare

AD-A240 511/6

100,617

Ada Compiler Validation Summary Report: Certificate Number 910705S1.11192 InterAct Corporation InterAC Ada Mips Cross-Compiler System, Release 2.0 MicroVAX
3100 Cluster $=>$ Lockheed Sanders STAR MVP 3100 Cluster $=>$ Lockheed S

100,618

AD-A240 512/4

Ada Compiler Validation Summary Report: Cerlificate Number: $910502 S 1.11158$ DDC International A/S, DACS VAX/VMS to 80860 Bare Ada Cross Compiler System,
Version 4.6.1, VAX 8530 (Host) to Tadpole Technology Version 4.6.1', VAX 8530 (Host) to
PLC TP860M' (Bare Board) (Target).

PLC TP860M (B

100,619

Ada Compiler Validation Summary Report: Cerlificate Number: $910626 S 1.11176$ U.S. Navy, Ada/L, Version 4.0 (/Optimize), VAX 11/785 (Host) to AN/UYJ-43 (Single CPU) (Bare Board) (Target).

100,620 Ada Compiler Validation Summary Report: Certificate Number: 910626S1.11174 U.S. Navy, Ada/M, Version 4.0 (Optimize),
(Host) to AN/UYK-44 (EMR) (Bare Board) (Target). AD-A240 612/2

100,621

Ada Compiler Validation Summary Report: Certificate Number: 910626 S1.11175 U.S. Navy Ada/M, Version 4.0 (/Optimize), VAX 8550 (Host) to AN/AYK-14 (Bare

Board) (Target).
AD-A240 613/0
Ada

Ada Compiler Validation Summary Report. Certificate Number: 910705S1.11191, InterACT Corporation InterACT Ada 1750A Compiler System, Release 3.5, MicroVAX 3100 Cluster $=>$ InterACT MIL-STD-1750A InStruction Set Architecture Simulator, Release 2.3 (Bare
Machine).
AD-A240 762/5
100,623

Ada Compiler Validation Summary Report. Certificate Number: 910626S1.11178, U.S. Navy Ada/M, Version 4.0
(OPTIMIZE) VAX 11/785 $>$ AN/UYK-44 (EMR)

AD-A240 763/3

100,624

Ada Compiler Validation Summary Report: Certificate Number 910626S1.11173 U.S. Navy Ada/L, Version 4.0 (/Optimize) VAX $855=$ AN/UYK-43 (EMR) (Bare

Board).
AD-A240 783/1

100,625

Ada Compiler Validation Summary Report: Certificate Number Number $910626 S_{1} 11172$ U.S. Navy Ada/L, Version 4.0 (/Optimize) VAX $8550=$ AN/UYK-43 (Single CPU) (Bare Board)

100,626

Ada Compiler Validation Summary Report: Certificate Number: 910502S1.11159 DDC International A/S DACS Sun-3/SunOS to 68030 Bare Ada Cross Compiler
System, Version 4.6.4, MRI IEEE 695 (BASIC-MODE) System, Version 4.6.4, MRI IEEE 695 (BASIC-MODE)
Sun-3/50 = Motorola MVME143 Board (68030/68882). Sun-3/50 = Motorola MVME143 Board (68030/68882).
AD-A240 785/6
100,627 Ada Compiler Validation Summary Report. Certificate Number: 910502S1.11160 DDC International A/S DACS Sun-3/SunOS to 68030 Bare Ada Cross Compile System, Version 4.6.4, MRI IEEE 695 (SECURE MODE) Sun-3/50 $=>$ Motorola MVME143 Board (68030)

AD-A240 849/0

100,628

Ada Compiler Validation Summary Report. Certificate Number: 910626S1.11177 U.S. NAVY Ada/L, Version 4.0 (/OPTIMIZE) VAX $11 / 785=>$ AN/UYK-43 (EMR)
(Bare Board).
AD-A240 850/8
100,629

Ada Compiler Validation Summary Report: Certificate Number: 91051751.11163 U.S. Navy, AdaVAX, Version
5.0, (/NO OPTIMIZE) VAX 8350 (Host and Target). 5.0, (/NO OPTIMIZE) VAX 8350 (Host and Target).
AD-A242 268/1 Ada Compiler Validation Summary Report: Certificate Number: $910517 S 1.11162$ U.S. Navy, AdaVAX, Version 5.0, (/OPTIMIZE) VAX 8600 (Host and Target).
AD-A242 269/9 Ada Compiler Validation Summary Report: Certificate Number: 910517S1.11165 U.S. Navy, AdaVAX, Version 5.0 (/NO-OPTIMIZE), VAX 11/785 (Host and Target). Ada Compiler Validation Summary Report: Certificate
Number: 91051751.11164 U.S. Navy, AdaVAX, Version Number: 910517 S1.11164 U.S. Navy, AdaVAX, Version
5.0, (/OPTIMIZE), VAX11/785 (Host and Target).
AD-A242 273/1
100,633 Ada Compiler Validation Summary Report: Certificate Number 910918S1.11216, NEC Corporation, NEC Ada Compiler System for EWS-UX/V (Rel 4.0), Version Release 2.1(4.6) EWS $4800 / 220=$ EWS4800/220. AD-A242 609/6 100,634 Ada Compiler Validation Summary Report. Cerlificate Number: $911025 S 1.11226$, Digital Equipment Corporation DECstation 5000 Model 200

AD-A242 896/9

100,635

Validated Products List (Cobol, Fortran, ADA, Pascal, MUMPS, SQL)

100,671

ADAPTERS

Determining Adapter Efficiency by Envelope Averaging Swept Frequency Reflection Data

100,743

\section{ADENOSINE}

Crystal Structure of a cAMP-Independent Form of Catabolite Gene Activator Protein with

PB91-190074

101,305

ADENOSINE CYCLIC MONOPHOSPHATE

Crystallizing Catabolite Gene Activator Protein with CAMP

for Structural Analysis.
PB91-148213 101,299

Crystal Structure of a CAMP-Independent Form of Catabolite Gene Activator Protein with Adenosine Substituted in One of 2 CAMP-Binding Sites.

101,305

ADENOSINE PHOSPHATES

Thermodynamics of the Disproportionation of Adenosine 5 '-diphosphate to Adenosine 5 '-triphosphate and Adenosine 5 -monophosphate. 1. Equilibrium Model.

PB92-116854

100,459

Thermodynamics of the Disproportionation of Adenosine 5'-diphosphate to Adenosine 5'-triphosphate and Adenosine $5^{\prime}$-monophosphate. 2. Experimental Data.

PB92-117399

101,313

ADHESIVE BONDING

Further Investigation of the Effect of Application Parameters on Adhesive-Bonded Seams of Single-Ply Roof PB91-149153

100,105

Pulse-Echo Ultrasonic Evaluation of the Integrity of Seams of Single-Ply Roofing Membranes: Laboratory Evaluation of a Prototype Test Apparatus.

100,106

Pulse-Echo Ultrasonic Evaluation of the Integrity of Seams of Single-Ply Roofing Membranes: Results of Field Investigations and Recommendations.
PB91-159731 100,110

Field Testing of Adhesive-Bonded Seams of Rubber Roofing Membranes.

ADHESIVES

Development of an Adhesive System for Bonding to Hard PB91-236539

100,052

Dentin-Bonding Molar Efficiency Using N-Phenylglycine, N-Phenyl-beta-Alanine, or N-Methyl-N-Phenylglycine.
PB91-237735

ADP

Thermodynamics of the Disproportionation of Adenosine $5^{\prime}$-diphosphate to Adenosine $5^{\prime}$-triphosphate and Adenosine 5'-monophosphate. 1. Equilibrium Model. PB92-116854

100,459

\section{ADSORPTION}

Chitin-Chitosan Membranes: Separations of Amino Acids and Polypeptides.

How Does Surface Roughness Affect Polymer-Surface Interactions.

100,341

Dynamics of Molecular Hydrogen Adsorbed in CoNa-A Zeolite.

100,380

Neutron Spectroscopic Evidence for Adsorbed Hydroxyl Species on Platinum Black.

ADVANCED TECHNOLOGY PROGRAM

Advanced Technology Program: A New Role for NIST in Accelerating the Development of Commercially Important Technologies.

100,013

AEROSOLS

Characteristics of Aerosols Produced by the Spark Discharge.

AGE DETERMINATION

Accelerator (14)C Dating of Late Pleistocene Megafauna.
PB91-237396

Accelerator Radiocarbon Dates on Charcoal, Shell, and 
AIR CIRCULATION

Air Exchange and Pollutant Concentration Measurements in Two Office Buildings.

$$
\text { PB91-195420 }
$$

100,909

AIR CLEANERS

Proposed Standard Practice for Assessing the Performance of Gas-Phase Air Cleaning Equipment. PB91-167353

100,905

AIR CONDITIONING

Simulation of an Evaporator with Nonuniform One-Dimensional Air Distribution.

PB91-174649

100,066

Evaporator Performance Investigation for Residential AirConditioning Application Using Mixed Refrigerants. PB92-126432

100,893

AIR FLOW

Fire Propagation in Concurrent Flows.

PB91-157206

100,543

Compartment Fire-Generated Environment and Smoke

Filling.

100,107

Ceiling Jet in Fires

100,114

User Manual NBSAVIS CONTAM88. A User Interface for Air Movement and Contaminant Dispersal Analysis in Multizone Buildings.

PB91-206722

100,910

Variable Air Volume System Design Guide.

PB91-216655

100,077

AIR HANDLING UNITS

Rule-Based Diagnostic Method for HVAC Fault Detection.
PB91-194936

AIR INFILTRATION

General Routine for Analysis of Stack Effect.

General Routine

100,163

AIR POLLUTION CONTROL EQUIPMENT

Advanced sensor development program for the pulp and paper industry. Final report

101,248

Proposed Standard Practice for Assessing the Perform. Proposed Standard Practice for Assessing the Perform ance of Gas-P

100,905

\section{AIR POLLUTION DETECTION}

Accurate and Precise Coulometric Determination of Sulfur Dioxide in Compressed Gas Mixtures.

100,912

Development of a Coulometric Method for Assessing the

Development of a Coulometric Method for Assessing the
Concentration of Ambient Levels of $\mathrm{CO} 2 /$ Air in ComConcentration of Ambient Levels of CO2/Air in Compressed Gas

100,913

AIR POLLUTION MONITORING

Air Exchange and Pollutant Concentration Measurements in Two Office Buildings.

PB91-195420

100,909

AIRPORTS

Prototyping the IRDS: An Airport Application.

PB92-112580

101,740

ALANINES

Thermodynamics of the Krebs Cycle and Related Compounds.

100,357

ALASKA

Alaska Marine Mammal Tissue Archival Project: Revised Collection Protocol.

PB91-184796

101,374

ALBUMINS

Hydrodynamics and Mass Transfer in Two-Phase Aqueous Extraction Using Spray Columns.

101,300

ALCOHOLS

Heat Capacities of Organic Compounds in the Liquid State I. C1 to C18 1-Alkanols.

100,265

Alkyl Transfer Reactions between Protonated Alcohols and Ethers. The Gas-Phase Alkylation of Formaldehyde.
PB91-147470

Oxidation of Organic Compounds by Active Manganese Dioxide.

PB91-174730

100,218

Population Lifetimes of $\mathrm{OH}(v=1)$ and $\mathrm{OD}(v=1)$ Vibrations in Alcohols, Silanols and Crystalline Micas. 100,324

ALDEHYDES

Radiation Chemistry of Enones.
PB91-147793 100,236

ALGORITHM DESIGN Algorithm Design for Large-Scale Computations.
PB91-149336
100,640

Wall.

Algorithm for the Mass-Loss Rate of a Burning Wall.
100,108 Phone-Mediated Word Alignment for Speech Recognition

$\begin{array}{ll}\text { Evaluation. } & 100,592\end{array}$

Task Decomposition and Algorithm Development for Real-Time Motion Control of a Continuous Mining Machine.
PB91-231597

101,357

ALALINE EARTH METAL COMPOUNDS

New Layered Cuprate Structure-Type, (A1$\left.x A^{\prime} x\right) 14$ Cu24O4

101,095

ALKANES

Rate Constants for Hydrogen Abstraction Reactions of the Sulfate Radical, SO4- Alkanes and Ethers. PB91-203232

100,394

Heat Capacities of Organic Compounds in Liquid State. 2. $\mathrm{C} 1$ to $\mathrm{C} 18 \mathrm{n}$-Alkanes.

PB92-110204

100,448

ALKENE COMPOUNDS

Reactions of $\mathrm{C}_{5} \mathrm{H} 3(+)$ ) and $\mathrm{C} 5 \mathrm{H} 5(+)$ lons with Acetylene and Diacety

100,384

ALKOXY COMPOUNDS

Critical Evaluation of Liquid Crystal Transition Temperatures I: 4,4'-Alkyl/alkoxyphenylbenzoates.

PB92-110154

100,444

ALKYL COMPOUNDS

Critical Evaluation of Liquid Crystal Transition Temperatures I: 4,4'-Alkyl/alkoxyphenylbenzoates.

100,444

\section{ALKYL TRANSFER REACTIONS}

Alkyl Transfer Reactions between Protonated Alcohols and Ethers. The Gas-Phase Alkylation of Formaldehyde. PB91-147470

100,280

ALKYNE COMPOUNDS

Reactions of $\mathrm{C} 5 \mathrm{H} 3(+)$ and $\mathrm{C} 5 \mathrm{H} 5(+)$ lons with Acetyene and Diacetylene.

PB91-195370

100,384

ALLOYS

Effect of Coherency Strain on Alloy Formation: Migration of Liquid Films.

101,210

Neutron Powder Diffraction and Inelastic Scattering Study of the Structures of Zr2Pd, Zr2PdD1.70, and Zr2PdD1.96.
PB91-148866 Rapid Solidification Alloys: Crystals, Quasicrystals, and Metallic Glass.

101,220

Surface Properties and Coatings of Materials Microtailored Alloys.
PB91-189696

ALPHA PARTICLES

Evidence for Surface alpha Particle Clusters in (nat)AG(197)A
PB91-216812

101,705

\section{ALUMINUM}

Use of Neutron Pole Figures to Calibrate Ultrasonic Techniques for On-Line Texture Control of Aluminum Plates.

PB91-159111

101,222

Characterization of Cylindrical Holes in Metallic Substrates via Their Infrared Emission Patterns. PB91-187187

101,228

Wavelengths and Energy Level Classifications for the Spectra of Aluminum (AI I through AI XIII).

100,472

ALUMINUM ALLOYS

Aluminum Alloys for Cryogenic Tanks: Oxygen Compatibility. Volume 1

AD-A229 229/0

101,201

Review of Cryogenic Mechanical and Thermal Properties of Al-Li Alloys and Alloy 2219.

AD-A229 231/6 101,202

Aluminum Alloys for ALS Cryogenic Tanks: Oxygen Com-

patibility. Volume 2.
AD-A231 $830 / 1$

101,203

Aluminum Alloys for ALS Cryogenic Tanks: Comparative Measurements of Cryogenic

AD-A242 956/1

101,204

N-Dimensional Crystallographic Description of the Icosahedral Phases; the Example of the Al73Mn21Si6 Quasiperiodic Structure.

101,226

Stress Corrosion Cracking of Al-Li Alloys: The Role of Grain Boundary Precipitates.

101,137

Role of Elastic Energy in the Morphological Development of a Ni-Ti-Al Alloy.

101,227

Structural Study of Electrodeposited Aluminum-Manganese Alloys.

101,231

Phase Selection in Non-Equilibrium Processing.

PB91-195651

101,236

Cryogenic Fatigue of High-Strength Aluminum Alloys and Correlations with Tensile Properties.

PB91-236976

101,709

Microstructural Studies of Ti-Al Alloys in the Vicinity of the 'Eutectoid' Reaction. the 'Eutectoid'
PB92-116938

101,241

\section{ALUMINUM COATINGS}

Emissivity of Aluminized Mylar.

PB91-174656

101,119

ALUMINUM IONS

Energy Levels of Atomic Aluminum with Hyperfine Structure.

101,594

Wavelengths and Energy Level Classifications for the Spectra of Aluminum (AI I through AI XIII).

100,472

\section{UMINUM-LIKE IONS}

Ouantum Electrodynamic Contributions to Spin Orbit Splitting in the Ground State of Aluminum-Like Ions.

PB91-189597

\section{ALUMINUM MANGANESE ALLOYS}

Phase Formation in Electrodeposited and Thermally An-

nealed Al-Mn Alloys.
PB91-147314

101,209

Transformation of the Icosahedral Phase in Rapidly Ouenched Al-rich Al-Mn Alloys.

PB91-158964

Electrodeposition of Aluminum from Molten Salts.

PB91-159202

101,223

Comparison of Amorphous and Ouasicrystalline Films of Sputtered Al0.72Mn0.22Si0.06

PB91-195552

101,530

ALUMINUM OXIDE

Dihedral Angles in Magnesia and Alumina: Distributions from Surface Thermal Grooves.

PB91-147330

101,058

Application of Analytical Electron Microscopy to GlassBonded Aluminas.

Mechanical Properties of Alumina-Peek Unidirectional

Composite: Compression, Shear, and Tension.
PB91-162180

101,118

Cyclic Fatigue from Frictional Degradation at Bridging Grains in Alumina.
PB91-236927

101,092

Cathodoluminescence Measurement of Strained Alumina Single Crystals.
PB91-237131

\section{ALUMINUM OXIDES}

Characterization of the Densification of Alumina by Multiple Small-Angle Neutron Scattering

PB92-117001

101,100

Effect of Green Density and the Role of Magnesium Oxide Additive on the Densification of Alumina Measured by Small-Angle Neutron Scattering.

101,101

Magnetic Behavior of Nanocomposites Prepared in a Vitreous Alumina Gel.

PB92-117332

101,131

Oxidation of Organic Compounds by Active Manganese

PB91-174730

100,218

Thermodynamic and Thermophysical Properties of Or. ganic Nitrogen Compounds. Part 1. Methanamine, Ethan amine, 1- and 2-Propanamine, Benzenamine, 2-, 3-, and 4-Methylbenzenamine. 


\section{KEYWORD INDEX}

Heat Capacity of Deuterated Ammonium Tetrafluoroborate ND4BF4 from 7 to $348 \mathrm{~K}$

100,273

AMMONIUM PENTABROMOINDATE MONOHYDRATE

Thermodynamics of Ammonium Indates. 2. The Molar Heat Capacity of the Ammonium Pentabromoindate Monohydrate Salt $(\mathrm{NH} 4) 2 \mathrm{InBr5} . \mathrm{H} 2 \mathrm{O}$ from 7.8 to $348 \mathrm{~K}$. PB91-14983

AMORPHIZATION

Amorphization and Conductivity of Silicon and Germanium Induced by Indentation.

PB91-150045

101,482

\section{AMORPHOUS MATERIALS}

Ouantitative Determination of Amorphous Content in $\mathrm{Ce}$. ramic Materials Using $X$-ray Powder Diffraction. Properties of Amorphous Zirconium Rhodium Hydride (Zr3RhHx) Prepared from Glassy and Crystalline Alloys.
PB91-174466 Relationship of Hydrogen Site Occupancy to Diffusion Behavior in Crystalline and Amorphous $\mathrm{Zr} 2 \mathrm{PdHx}$. PB91-187252

ANALOG DEVICES

Comprehensive Approach for Modeling and Testing Analog and Mixed-Signal Devices.

100,732

NALOG TO DIGITAL CONVERTERS

High Speed Superconducting A/D Converter

100,731

\section{ANALYTICAL CHEMISTRY}

Scattered Light and Other Corrections in Absorption Coefficient Measurements in the Vacuum Ultraviolet: A SysAD-A227 869/5

100,179

CID Spectra of Selected Target Molecules.

AD-A243 170/8 100,180 Cryogenic-Temperature Fluorescence Spectra of PolynuPB91-147157

100,274

Dietary Collection Procedures.

101,317

Practical Constraints in Monitoring Micronutrient Intake. PB91-147421 101,318

Capillary Supercritical Fluid Chromatography of Explosives: Investigations on the Interactions between the Analytes, the Mobile Phase and the Stationary Phase.
PB91-147769 182 Use of Single Particle Standards for LAMMA Calibration. PB91-147777 100,183 Secondary Ion Yield Changes in Silicon and Gallium Arsenide Due to Topography Changes during Oxygen or Cesium Ion Bombardment.

101,471

Trace Determination of $\mathrm{Cr}(\mathrm{VI})$ by LC/AAS with on-Line Preconcentration. Preconcentration

Reverse-Phase HPLC Separation and Electrochemical Detection of Retinol and Its Isomers.

100,187

Research at NBS in Direct Potentiometric Measurements in Blood.

100,188
Methods for the Analysis of Organometallic Compounds in Wastes.

$\begin{array}{ll}\text { PB91-148981 100,189 } & \end{array}$

Determination of Bonded Phase Thickness in Liquid Chromatography by Small Angle Neutron Scattering. Low-Background Gamma-Ray Assay Laboratory for Activation Analysis.

100,192

High Purity Fluoropolymer Materials: Trace Element Con-

tent and Leaching.
PB91-162255

100,491

Robot Characterization Testing

101,030

Intrared Spectroscopy Applied to Atmospheric Chemistry PB91-175539 100,906

Alaska Marine Mammal Tissue Archival Project: Revised Collection Protocol.

101,374

Multiple Variable-Angle Light Scattering Detector for Gel Permeation Chromatography.

PB91-187641

100,195

Proposed New Electrolytic Conductivity Primary Standards for $\mathrm{KCl}$ Solutions.

PB91-187658

100,339

Stability of Uric Acid in Ammonium Hydroxide. PB91-189266

101,292

Second Dissociation Constant and $\mathrm{pH}$ of $\mathrm{N}$-2-hydroxyethylpiperazine-N'-2-ethanesulfonic Acid (HEPES) from 0 to PB91-189282

100,196

Reference NO2 Calibration System for Ground-Based Intercomparisons during NASA's GTE/CITE II Mission.
PB91-189340

Neutron Activation Analysis in Electronic Technology.
PB91-189779

100,807

etection of Trace Disulfur Decafluoride in Sulfur Hexafluoride by Gas Chromatography/Mass Spectrometry. 100,197
Evaluation of Column Pertormance in Liquid Chromatography.

100,199

High Purity Chemicals and Clean Rooms for Trace Metal Analysis.

100,200

Recommended Inorganic Chemicals for Calibration

100,201

Strategies for Background Subtraction in Electron Probe Microanalysis $X$-ray Compositional Mapping.

100,202

Development of a New Tracer Technology Using Enriched Rare-Earth Isotopes.
PB91-195347

100,908

Accuracy of Transmission Electron Microscopy Analysis of Asbestos on Filters: Interlabortory Study.
PB91-195727 100,204

from

High-Tempera

PB91-195735

100,385

Lanthanide Coordination Chemistry: Spectroscopic Properties of Terbium and Europium Poly(Pyrazol-1-YI) Borate nd Poly(Imidazol-1-YI) Borate.

3034

100,392

nterlaboratory Comparison of Actinides in Human Tissue (239)Pu $+(240) \mathrm{Pu}$.

PB91-203265

101,323

Mossbauer Imaging.

101,294

Visibility of Chrysotile Asbestos in the Scanning Electron Microscope.

101,320

Ouantitative Analysis of Impurities in SIMOX Samples Using Secondary lon Mass Spectrometry.

100,208

Analysis of Wet Deposition (Acid Rain): Determination of the Major Anionic Constituents by Ion Chromatography.
100,035

100,035

Accelerator (14)C Dating of Late Pleistocene Megafauna.
PB91-237396

Variations in the Practice of Ceramic Technology in Different Cultures: A Comparison of Korean and Chinese Celadon Glazes

101,111

Characteristics of Aerosols Produced by the Spark Discharge.
PB91-237727

100,209

Evaluation of Liquid Junction Potentials and Determina tion of $\mathrm{pH}$ Values of Strong Acids at Moderate Ionic Strengths

PB91-237859

100,438

Electrochemical Behavior of Ferrocene in Polyethylene

Oxide -400 .

100,439

Laser Enhanced Ionization as a Selective Detector for Liquid Chromatographic Determination of Alkyltins in PBO2-11675

New Way to Mount Particulate Material for Laser Microprobe Mass Analysis

PB92-116805

100,911

Laser Vaporization Mass Spectrometry of Refractory Materials: Graphite

100,464

Multi-Enterprise Concurrent Engineering through Interna-

tional Standards.
PB92-123058 100,997

Accurate and Precise Coulometric Determination of Sulfur Dioxide in Compressed Gas Mixtures.

100,912

Development of a Coulometric Method for Assessing the

Development of a Coulometric Method for Assessing the pressed Gas Mixtures.

ANIONS

Analysis of Wet Deposition (Acid Rain): Determination of the Major Anionic Constituents by lon Chromatography.
PB91-236646

ANISOLES

Dissociation of Substituted Anisoles: Substituent Effects on Bond Strengths

PB91-237537

100,431

ANISOTROPY

Numerical Study of Two-Dimensional Crystal Growth Forms in the Presence of Anisotropic Growth Kinetics.
PB91-200865

Ultrasonic Texture and Stress Measurements in Anisotropic Polycrystalline Aggregates.

PB91-202945
PBlaline Aggregates.

Monitoring of Anisotropic Material Elastic Properties Using Ultrasonic Receiving Rays.

101,126

ANNEALING

Ouantitative Analysis of Impurities in SIMOX Samples Using Secondary lon Mass Spectrometry.

PB91-236596

100,208

ANTENNA MEASUREMENTS

Spherical Near-Field Scanning: Experimental and Theo-

$\begin{array}{ll}\text { retical Studies. } & 100,723\end{array}$

Spherical Near-Field Scanning: Determining the Incident Rotatable Probe.

100,725

Evaluation of Dual-Port Circularly Polarized Probes for Planar Near-Field Measurements.

PB91-162040

100,590

ANTENNA RADIATION PATTERNS

General Analytic Correction for Probe-Position Errors in Spherical Near-Field Measurements.

100,729

Personal Computer Codes for Analysis of Planar Near Fields.

PB92-112283

100,730

ANTENNAS

Calibration of Antenna Factor at a Ground Screen Field Site Using an Automatic Network Analyzer

Alignment Fixture for Millimeter Waveguide.

100,724

PB91-236919

100,727

General Technique to Correct Probe Position Errors in Planar Near-Field Measurements to Arbitrary Accuracy.
PB91-237073

ANTIFERROMAGNETISM

Long-Range Antiferromagnetic Order of the $\mathrm{Cu}$ in Oxygen Deficient RBa2Cu3O6 $+x$

PB91-194894

101,524

ANTIHYDROGEN

Prospects for Electromagnetic Manipulation and Trapping of Antihydrogen.
PB91-194886

101,663

\section{ANTIMATTER}

Prospects for Electromagnetic Manipulation and Trapping of Antihydrogen.

101.663

\section{ANTIMISSILE DEFENSE SYSTEMS}

Annual Report to the Strategic Defense Initiative Organization on the Free-Electron Laser Driven by the NIST CW

AD-A207 806/1

101,398

ANTIMONIDES

Isothermal Phase Behavior of Ag3SbS3, ZnGeP2, and ZnS.

\section{ANTIMONY}

Surface Extended.X-ray-Absorption Fine Structure and Scanning Tunneling Microscopy of Si(001)2x1-Sb.

ANTIMONY 132

Structure of Odd-Odd (132)Sb

ANTIMONY IONS

$5 \mathrm{~s}(2) 5 \mathrm{p}(2)-(5 \mathrm{~s}(2) 5 \mathrm{p} 5 \mathrm{~d}+5 \mathrm{~s} 5 \mathrm{p}(3)+5 \mathrm{~s}(2) 5 \mathrm{p} 6 \mathrm{~s}+$ $5 s(2) 5 p 7 s)$ Transitions in Sb II and $5 s(2) 5 p \cdot(5 s 5 p(2)+$ $5 s(2) n l)$ Transitions in Sb III.

PB91.200816

101,676

ANTIPERSONNEL MINES

Summary of Experiments with the Separated Aperture AD-A230 438/4

100,721

ANTONOMOUS CONTROL

Control System Architecture for Unmanned Ground VehiPB91-134726

101,379

APATITES

Adsorption and Orientation of Tetracycline on Hydroxyapatite.

101,319

APP (APPLICATION PORTABILITY PROFILE)

Application Portability Profile (APP): The U.S. Government's Open System Environment Profile OSE/1 Version PB91-201004 
PB91-187658

Modelling Aqueous Solutions Near the Critical Point of Water.

PB91-189837 100,349

Phase Equilibria and Critical Lines in Model Water/Salt Mixtures.

PB91-236778

100,418

AQUEOUS SOLUTIONS

Rate Constants for Reactions of Peroxyl Radicals in Fluid

Solutions.
PB90-244641 100,261

Unified Description of Infinite-Dilution Thermodynamic Properties for Aqueous Solutes.

100,319

Thermodynamic Properties of Aqueous Solutions at High Temperatures; Needs, Methods, and Challenges.

PB91-204065

100,410

AQUEOUS SYSTEMS

Book Review: Partitioning in Aqueous Two-Phase Systems: Theory, Methods, Uses, and Applications to Biotechnology.

ARABINOSE

100,333

2-Deoxy-D-Ribose Aqueous Solution as a Gamma-Ray Dosimeter.
PB92-117126

101,366

ARCHAEOLOGICAL SPECIMENS Variations in the Practice of Ceramic Technology in Dif-
ferent Cultures: A Comparison of Korean and Chinese ferent Cultures: A Comparison of Korean and Chinese Celadon Glazes.
PB91-237669

101,111

ARGON

Parity-Unfavored Transitions in Resonant Photoemission from $\mathrm{Ar}, \mathrm{Kr}$, and $\mathrm{Xe}$ : Experimental and Theoretical Results.

101,599

Thermal Conductivity and Heat Capacity of Gaseous Argon.

PB91-134577 101,602
Effect of Noble Gas Mixtures on the Performance of Re-

Effect of Noble Gas Mixtures on the Performance of Re-
generative-Type Cryocoolers Analytical Estimate.
100,890

Unified Description of Intinite-Dilution Thermodynamic Properties for Aqueous Solutes.

PB91-162081

100,319

Angular Distribution of Ejected Electrons in Resonant Auger Processes of $\mathrm{Ar}, \mathrm{Kr}$, and $\mathrm{Xe}$

101,648

Viscosity and Thermal Conductivity of Pure Monatomic Viscosity and Thermal Conductivity of Pure Monatomic
Gases from Their Normal Boiling Point UP to $5000 \mathrm{~K}$ in Gases from Their Normal Boiling Point Up to $5000 \mathrm{~K}$ in
the Limit of Zero Density and at $0.101325 \mathrm{MPa}$. the Limit of Zero

100,366

Experimental Thermal Conductivity, Thermal Diffusivity, and Specific Heat Values for Mixtures of Nitrogen, Oxygen, and Argon.
PB91-194472

100,369

Angle-Resolved Photoemission from the Ar $2 p$ Subshell. PB91-194928 101,665 Unified Set of Atomic Transition Probabilities for Neutral

Argon.

101,717

ARGON COMPLEXES

Microwave Spectrum of Ar-H2O: Dipole Moment, Isotopic Studies, and (17)O Quadrupole Coupling Constants.
PB91-148650

100,290

Structural Dependence of HF Vibrational Red Shifts in
ArnHF, $n=1-4$, via Hign-Resolution Slit Jet Infrared Spectroscopy.
PB91-203562

100,401

Investigation of Internal Rotor Dynamics of $\mathrm{NeDCl}$ and ArDCl via Infrared Absorption Spectroscopy. 100,409 Rotational Spectrum and Structure of the Complex $\mathrm{Ar}$ $\mathrm{Ch} 3 \mathrm{CN}$.

PB91-236661

100,415

Optothermal-Detected Microwave-Sideband CO2-Laser Spectroscopy of $\mathrm{Ar}-\mathrm{NH} 3$

100,416

ARGON COMPOUNDS

Rotational-Tunneling Spectrum of the Ar-SO2 van der Waals Complex.

100,457

ARGON PLASMA

Off-Axis Measurements of lon Kinetic Energies in RF Plasmas.

PB92-117225

101,444

ARITHMETIC UNITS

Closure and Precision in Level-Index Arithmetic

ROMATIC COMPOUNDS

100,597

Carbon Acidities of Aromatic Compounds. 2. Ionic Probes of Aromaticity in Annelated Rings.

PB91-195065

100,375

AROMATIC POLYCYCLIC HYDROCARBONS

Standard Chemical Thermodynamic Properties of Polycyclic Aromatic Hydrocarbons and Their Isomer Groups. III

Naphthocoronene Series, Oval
Members of Some Higher Series.
PB90-244625

100,259

Cryogenic-Temperature Fluorescence Spectra of Polynuclear Aromatic Hydrocarbons of Molecular Weight 302. B91-147157

100,274

Preparation and Analysis of a Marine Sediment Reference Material for the Determination of Trace Organic Constituents.

PB91-149237

101,377

Carbon Acidities of Aromatic Compounds. 2. Ionic Probes of Aromaticity in Annelated Rings.

100,375

\section{ARRAYS}

Characterization of Capacitive Array for NDE Applications.

PB91-237305

100,946

Capacitive Array Sensors for Nondestructive Evaluation.

PB91-237313

100947

ARTIFICIAL INTELLIGENCE

Al in Manufacturing: The NBS AMRF as an Intelligent Machine.

PB91-134718

100,973

Toward Modular Semantic Application Layer Protocols (MS-ALPS): Communication between Heterogeneous Information Systems Using Artificial Intelligence (AI) Programming Techniques.

100,565

Al: Does It Have a Place in Building Simulation

PB91-159293

100,063

Intelligent Processing of Materials, Technical Activities 1991. (NAS-NRC Assessment Panel, November 14-15

100,995

Integrated Knowledge Systems for Concrete Science and Technology.
PB92-116821

100,141

ARTIFICIAL MEMBRANES

Enhanced Transport and Liquid Membranes in Bioseparations.

PB91-189886

101,287

ASBESTOS

Accuracy of Transmission Electron Microscopy Analysis of Asbestos on Filters: Interlabortory Study.

100,204

Visibility of Chrysotile Asbestos in the Scanning Electron Microscope.
PB91-204099

ASPARTIC ACID

Thermodynamics of the Krebs Cycle and Related Com-

pounds.

100,357

ASSESSMENTS

Development of a Technique to Assess the Adequacy of the Municipal Water Supply for a Residential Sprinkler System.

101,358

ASSOCIATIVE IONIZATION

Associative Ionization of Ultra-Cold Laser Trapped Sodium Atoms.

101,707

ATMOSPHERIC CHEMISTAY

Chemical Kinetic Data Sheets for High-Temperature Reactions. Part

PB92-148204

ATMOSPHERIC COMPOSITION

Infrared Spectroscopy Applied to Atmospheric Chemistry. PB91.175539

100,906

ATMOSPHERIC MOTION

Canadian Mass Fire Experiment, 1989.

PB91-143305

101,352

ATOM-MOLECULE COLLISIONS

Cross Sections and Swarm Coefficients for $\mathrm{H}+, \mathrm{H} 2+$ $\mathrm{H} 3+, \mathrm{H}, \mathrm{H} 2$, and $\mathrm{H}$-in $\mathrm{H}_{2}$ for Energies from 0.1 eV to 10 PB90-244690

101,597

\section{ATOM TRAPS}

Prospects for Electromagnetic Manipulation and Trapping of Antihydrogen
PB91-194886

ATOMIC CLOCKS

Progress at NIST on Absolute Frequency Standards Using Stored lons.

PB91-15928

101,625

Clocks, Atomic and Molecular.

101,677

Observation of the Cesium Clock Transition Using Laser-

Cooled Atoms in a Vapor Cell.

PB91-203638

101,698

303-MHz Frequency Standard Based on Trapped Be $(+)$ lons.

101,722

ATOMIC COLLISIONS

Measurements of Velocity-Changing Collision Kernels.

Measurements of Velocity-Changing Collision Kernels.
PB91-203083

Collisional Energy Transfer between Excited Sr Atoms

PB91-204164

101,703

ATOMIC ENERGY LEVELS

Energy Levels of Atomic Aluminum with Hyperfine Struc-

PB90-244575

101,594

Energy Levels of Copper, Cu I through Cu XXIX. PB90-244666

101,596

Transitions and Energy Levels for Cu-Like $\mathrm{Yb}(41+)$, $\mathrm{Ta}(44+)$, and $\mathrm{U}(63+)$.

91.147462

Journal of Physical and Chemical Reference Data,
Volume 19, Number 4, July-August 1990 .
PB91-192435 PB91-192435

Energy Levels of Sulfur, S I Through S XVI.

Spectroscopic Data Base for Carbon and Oxygen.

PB91-237750

100,355

Unified Set of Atomic Transition Probabilities for Neutral Argon.

Wavelengths and Energy Level Classifications of Magnesium Spectra for All Stages of Ionization (Mg I through $\mathrm{Mg}$ XII)

PB92.110139

100,442

ATOMIC FORCE MICROSCOPY

Stylus Profiling at High Resolution and Low Force. PB91-162354

101,015

ATOMIC IONS

Laser-Driven lonization and Photoabsorption Spectroscopy of Atomic lons.

PB91-174854

101,415

ATOMIC LIFETIMES

Unified Set of Atomic Transition Probabilities for Neutral Argon.
PB91-237768

101,717

ATOMIC \& MOLECULAR STUDIES

Cooled Ion Frequency Standard

AD-A227 806/7

101,591

Vibrational Spectra of Molecular Ions Isolated in Solid Neon. III. $\mathrm{N} 4(+)$.
AD-A228 082/4

100,243

Vibrational Spectra of Molecular lons Isolated in Solid Neon. IV.NO(+) $)$, NO(-), ONNO(+) , aNd ONNO(-).
AD-A230 $957 / 3$

Production and Spectroscopy of Small Polyatomic Molecular lons Isolated in Solid Neon.

AD-A234 043/8

100,245

Thermal Conductivity of a Moderately Dense Gas PB91-134502

101,601

Collisional Electron-Detachment and Ion-Conversion rocesses in SF6.

PB91-134528 100,836

Giant Resonances in the Transition Regions of the Periodic Table.

PB91-147116

101,605

Selective Excitation of $\mathrm{X}$-ray Emission Spectra.

PB91.147173

101,607

Structure of Carbon Dioxide Dimer from Near Infrared Spectroscopy
PB91-147454

100,279

Transitions and Energy Levels for Cu-Like $\mathrm{Yb}(41+)$, $\mathrm{Ta}(44+)$, and $\mathrm{U}(63+)$

101,610

Overview of the Ion Dynamic Effect in Line Broadening, 
Vibrational Spectra of Molecular lons Isolated in Solid Neon. 5. $\mathrm{N2O}(1+)$ and $\mathrm{NNO} 2(1-)$

100,306

Vibrational Spectra of Molecular lons Isolated in Solid Neon. 4. NO(1 +), $\mathrm{NO}(1-), \mathrm{ONNO}(1+)$, and ONNO(1-).
PB91-149914 Application of Ultrafast Broadband Infrared Spectroscopy to Measurement of Metal-Carbonyl Dynamics. 100,308

Optical Potential Approach to Electron and Positron Scattering from Noble Gases. 2. Neon.

PB91-149971

101,619

Emergence of Modern Nucleation Theory.

101,481

Nonlocal Phase Shifts Induced by Static Electric Fields in Neutron Interferometers When the Path-Enclosed Charge Vanishes.

PB91-150011

101,620

Diode Laser Probing of I*((2)P1/2) Doppler Profiles: Time

Evolution of a Fast, Anisotropic Velocity Distribution in a Thermal Bath.

100,310

Rapid Low-Temperature Hopping of Hydrogen in a Pure Metal: The ScHx System.

PB91.158485

101,217

Working Group 1: Atomic Spectra and Wavelength PB91.158956

100,026

Preface to Book Entitled 'Nonequilibrium Effects in Ion and Electron Transport'

PB91-159236

101,623

Permittivity Measurements on Molecular-Sized Samples.

PB91-159244 100,800

Photoionization and Collisional Ionization of Excited

Atoms Using Synchrotron and Laser Radiations.
PB91-159301

101,626

$\mathrm{H} 2 \mathrm{O} 2$ : Spectroscopy, Structure and Dynamics.

100,316

Resonant Tunneling with Electron-Phonon Interactions: An Exactly Solvable Model Applied to Desorption.
PB91-162065

Ouantum Optics of Single, Trapped lons.

101,628

Uses of Spin-Polarised Electrons in Fundamental Electron-Atom Collision Processes and the Analysis of Mag.

PB91-162172

101,490

Resonance lonization Spectroscopy/Resonance lonization Mass Spectrometry Data Service. II. Data Sheets for $\mathrm{Al}, \mathrm{Ca}, \mathrm{Cs}, \mathrm{Cr}, \mathrm{Co}, \mathrm{Cu}, \mathrm{Kr}, \mathrm{Mg}, \mathrm{Hg}$, and $\mathrm{Ni}$.

100,193

Oxidation of Organic Compounds by Active Manganese

Dioxide.
PB91-174730

100,218

Population Lifetimes of $\mathrm{OH}(\mathrm{v}=1)$ and $\mathrm{OD}(\mathrm{v}=1)$ Vibra tions in Alcohols, Silanols and Crystalline Micas.

PB91-174813

100,324

Laser-Driven Ionization and Photoabsorption Spectroscopy of Atomic lons.

Resonance Enhanced Multiphoton Ionization Spectroscopy of $\mathrm{CHCl} 2$ and $\mathrm{CDCl} 2$

100,328

Polarized X-ray Emission Studies of Methyl Chloride and the Chlorofluoromethanes.
PB91-174995 100,329 Molecular Spectroscopy Division of the National Bureau of Standards.
PB91.175174

100,332

Spectrum and Energy Levels of the Xenonlike Ion Ce V.

PB91-175364 101,638 Spin-Dependent Electron-Impact Excitation of Sodium. PB91-175497 101,641 Fe-W Supermirrors for Polarizing Neutrons.

PB91-175521 Direct Determination of Molecular Orbital Symmetry of $\mathrm{H} 2 \mathrm{~S}$ Using Polarized $X$-ray Emission. PB91-175562 100,335

Working Group 2: Atomic Transition Probabilities.

PB91.175588 101,645

Copper-lon-Dependent Damage to the Bases in DNA in the Presence of Hydrogen Peroxide.

101,330

Molecular Dynamics Study of the Depolymerization Reaction in Simple Polymers.

100,495

Angular Distribution of Ejected Electrons in Resonant Auger Processes of $\mathrm{Ar}, \mathrm{Kr}$, and $\mathrm{Xe}$.

01,648

Unusual Degree of Angular Anisotropy in the Resonan Auger Spectrum of $K$

101,649

Angular Distributions of Electrons in Resonant Auger Spectra.
PB91-187393

Shape Resonances in Molecular Fields
PB91-187435

100,338

Some Historical Remarks Concerning These Conferences: With Emphasis on the X-ray Component.

PB91-187443

101,652

Electronic Spectrum of the GeH3 Radical.

100,346

Ouantum Electrodynamic Contributions to Spin Orbit Splitting in the Ground State of Aluminum-Like Ions. PB91-189597 101,653 Relativistic and Correlation Effects in the 2s3p Configuration of Beryllium-Like lons.

PB91-189605

101,654

HBdrogen Bond

100,348

Observation of Atoms Laser-Cooled Below the Doppler PB91-189746

101,655

Fourier Transform Infrared Spectroscopy at the NIST: High Temperature Molecules and Atmospheric MolePB91-189803

101,424

Introduction to Supercritical Fluid Chromatography. 1. Principles and Instrumentation.

100,198

Energy Levels and Transition Probabilities in the Ground State Configuration of Sulfur-Like lons.

101,658

Prospects for Electromagnetic Manipulation and Trapping of Antihydrogen. 101,663

Photoionization Studies of Atoms and Molecules Using Synchrotron Radiation

PB91-194910

101,664

Angle-Resolved Photoemission from the Ar $2 p$ Subshell.

PB91-194928 101,665

Atomistic Diffusion Equations and Kinetic Forces.

PB91-195008 100,374

Dynamics of Molecular Hydrogen Adsorbed in CoNa-A

100,380

Many Electron Effects in Lgamma2,3 $X$-ray Emission Spectroscopy Spectrum of BaO.
PB91-195305

Many-Electron Effects in Lgamma2,3 XES Spectra from Rare Earth Elements.

PB91-195313 100,383

Observation of Atoms Laser Cooled Below the Doppler

PB91-195453

101,671

Non-Equilibrium Molecular Dynamics Simulations of Structured Molecules. Part 1. Isomeric Effects on the Viscosity of Butanes.

PB91-195636

101,391

Clocks, Atomic and Molecular.

PB91-200840 101,677

Observation of Correlations in Finite, Strongly Coupled lon Plasmas.

101,678

Production and Spectroscopy of Small Polyatomic Molecular Ions Isolated in Solid Neon.

ular lons Isolate

100,395

Resonance Enhanced Multiphoton Ionization Spectra of the GeF and GeCl Radicals from $400-500 \mathrm{~nm}$.

PB91-203281

100,396

Simulation Studies of Excluded Volume Effects on Poly-

mer Chain Dynamics in Several Nonlattice Models.
PB91-203364

Microwave Spectrum and Molecular Structure of the N2H20 Complex

PB91-203448

High Harmonic Generation in Hydrogenic lons.

100,398

101,699

Vibrational, Rotational, and Tunneling Dependence of $\mathrm{Vi}$ -

brational Predissociation in the HF Dimer.
PB91-203844

100,406

Problem of Detailed Balance and Model Lineshapes in Collision-Induced Rotovibrational Bands: $\mathrm{H}_{2}-\mathrm{H}_{2}$ and $\mathrm{H} 2-$

PB91-236521

100,412

Photodissociation Dynamics of $\mathrm{Mo}(\mathrm{CO}) 6$ at $266 \mathrm{~nm}$ and $355 \mathrm{~nm}$ : CO Photofragment Kinetic-Energy and InternalDistribution
1-236547

100,413

Rotational Spectrum and Structure of the Complex ArCh3CN

PB91-236661

100,415

Optothermal-Detected Microwave-Sideband CO2-Laser Spectroscopy of $\mathrm{Ar}-\mathrm{NH} 3$

100,416

Van der Waals Complexes in 1,3-Dipolar Cycloaddition Reactions: Ozone-Ethylene.

100,417

Associative Ionization of Ultra-Cold Laser Trapped

Sodium Atoms

Trapped

PB91.236745

Reply to Comment on 'Quantum Zeno Effect'.

PB91-236828

101,708

ibrational Spectra of Molecular Ions Isolated in Solid Neon. 6. $\mathrm{CO} 4(1-)$

100,419

Molecular-Beam Optothermal Spectroscopy of the 9.6 mum nu14.

PB91-236869

100,421

Heterodyne Frequency Measurements of $\mathrm{CO}$ and OCS Beyond $2100 \mathrm{~cm}(-1)$

Beyond $2100 \mathrm{~cm}$

100,423

Perpendicular Bands of Cyclopropane in the $3.5 \mathrm{mum}$

PB91-237016

100,424

Molecular-Orbital Studies via Satellite-Free $X$-ray Fluorescence: $\mathrm{Cl} \mathrm{K}$ Absorption and K-Valence-Level Emission Sectra of Chlorofluoromethanes.

PB91-237164

100,427

Hydration of Carbon Dioxide: The Structure of H2O-H2O CO2 from Microwave Spectroscopy.

100,428

Accelerator Radiocarbon Dates on Charcoal, Shell, and Accelerator Radiocarbon Dates on Charcoal, Shell, and
Human Bone from the Del Mar Site, California. B91-237404

Structure of Odd-Odd (132) Sb.

PB91-237461

101,355

101,711

Microwave Spectra and Electric Dipole Moments of (4)Sigma(1-)1/2 VO and $\mathrm{NbO}$

Extreme Ultraviolet Spectra of $\mathrm{Pd}, \mathrm{Ag}, \mathrm{Cd}$, and $\mathrm{Sn}$ lons 1.3-keV Tokamak Plasma.

101,712

Beam-Foil Study of Two-Electron Transitions in Cu-Like PB91-237511

101,714

Neutron Spectroscopic Evidence for Adsorbed Hydroxyl Species on Platinum Black.

PB91-237636

100,432

Spectroscopic Data Base for Carbon and Oxygen

PB91-237750

101,716

Rovibrational Analysis of the nu7(1) Intermolecular $\mathrm{Hy}$. drogen Bond Bending Vibration in HCN-HF Using Far Inrared Fourier Transform Spectroscopy.

100,437

303-MHz Frequency Standard Based on Trapped Be $(+)$

PB92-116474

101,722

Rotational-Tunneling Spectrum of the Ar-SO2 van der Waals Complex

100,457

Atomic Spectroscopy in the 20th Century - A Tribute to Sitterly, Charlotte Moore, on the Occasion of Her 90th Bime

101,724

Test of the Linearity of Ouantum Mechanics by $r$ Specroscopy of the (9)Be(+ ) Ground State.

101,726

Semiclassical Calculation of Self-Broadening in O2, N2, and CO Raman Spectra.

100,461

High-Resolution Infrared Spectrum of the nu1 Band of OC10.

PB92-117175

100,463

Off-Axis Measurements of lon Kinetic Energies in RF PB92-117225

101,444 


\section{KEYWORD INDEX}

AUTOMATION \& ROBOTICS

ATOMIZATION

Effect of Alomization Air on Droplet Dynamics of Spray

100,550

ICLASS-91 Proceedings of the International Conference on Liquid Atomization and Spray Systems (5th). Held in Gaithersburg, MD. on July 15-18, 1991.

PB91-216838

100,552

ATOMIZATION AIR

Effect of Atomization Air on Droplet Dynamics of Spray PB91-203869

100,550

ATOMIZERS

Particle Size Measurement of Inert Gas Atomized

PB91-187203

101,229

ATOMS

Single Pulse Shock Tube Study of the Reactions of $\mathrm{Hy}$ drogen Atoms with Aromatics. 4. Chlorotoluenes

PB91-175133 100,331

Reactivities of Chlorine Atoms and Peroxyl Radicals

Formed in the Radiolysis of Dichloromethane. 100,386

Partial Equilibrium in Laminar Hydrocarbon Diffusion Partial Equilibrium in Laminar Hydrocarbon Diffusion
Flames.
PB91-237024
100,553

ATP

Thermodynamics of the Disproportionation of Adenosine 5'-diphosphate to Adenosine 5'-triphosphate and Adenosine 5'-monophosphate. 1. Equilibrium Model.

PB92-116854

100,459

AUGER EFFECT

Angular Distribution of Ejected Electrons in Resonant Auger Processes of $\mathrm{Ar}, \mathrm{Kr}$, and $\mathrm{Xe}$.

101,648 PB91-187302 Unusual Degree of Angular Anisotropy in the Resonant
Auger Spectrum of Kr. PB91-187310

101,649

AUGER ELECTRON SPECTROSCOPY

Surface Oxidation Kinetics of Si3N $4-4 \%$ Y2O3 Powders Studied by Bremsstrahlung-Excited Auger Spectroscopy.
PB91-237701 100,435

\section{AUGER ELECTRONS}

Angular Distributions of Electrons in Resonant Auge Angutra.

PB91-187393

101,650

AUGERS Hydrogen Slush Production with a Large Auger.
PB91-147199
100,275

AUSTENITE

Examination of the Excessive Retained Austenite on the Surface of a Section of 17-7 Precipitation Hardening Stainless Steel.

PB91-167171

101,149

AUSTENITIC STAINLESS STEELS

Mechanical Sputtering of Structural Stainiess Steels.
PB91-175448 152

Nitrogen Strengthening of Austenitic Stainiess Steels at Low Temperatures.

PB91-195529 101,163

Load-Controlled Tensile Tests of Austenitic Steels at $4 \mathrm{~K}$. PB91-236943 101,165 Interlaboratory Tension and Fracture Toughness Test Results for CSUS-JN1 (Fe-25Cr-15Ni-0.3N) Austenitic Stainless Steel at $4 \mathrm{~K}$

PB91-237081 101,166

Effect of Processing on 4-K Mechanical Properties of a Microalloyed Austenitic Stainless Steel. 101,167

Strain Rate Effect on Tensile Properties at $4 \mathrm{~K}$ of a VAMAS Round-Robin Austenitic Steel.

101,168

PB91-237248

AUSTENITIC STEELS
Effect of Nitrogen and Carbon on FCC-HCP Stability in Effect of Nitrogen and Carbon on FCC-HCP Stability in
Austenitic Steels.
PB91-175372 101,151

101,151 Effects of Varying Austenitizing Temperature and Cooling
Rate on the Ability of HSLA-80 Steel to Achieve a Yield Strength Comparable to HSLA-100.

PB91-189464

101,156

Influence of Interstitial Content on Fracture Toughness

PB91-189944 101,157

Metallographic Study of the Crack-Tip Region from Fracture Mechanics Specimens of Austenitic and Ferritic

PB91-189951

101,158

Discontinuous Yielding during Tensile Tests at Low Tem-

$$
\text { peratures. }
$$

AUTOIGNITION

101,159

Short-Duration Autoignition Temperature Measurements

for Hydrocarbon Fue
AD-A242 816/7

100,877

Short-Duration Autoignition Temperature Measurements for Hydrocarbon Fuels.

PB92-108927 100,887

AUTOMATED MANUFACTURING RESEARCH FACILITY

Al in Manufacturing: The NBS AMRF as an Intelligent Machine.
PB91-134718

100973

UNIX in the Government's Automated Manufacturing Research Facility (AMRF)

PB91-147579

100,963

Research Tackles Automation Issues.

101,005

\section{AUTOMATIC CONTROL}

Low-Cost Low-Volume Carrier (Minilab) for Biotechnology and Fluids Experiments in Low Gravity.

100,041

Direct Digital Control Based Building Automation System Design Criteria.

PB91-216614 100,091

Guide Specification for Direct Digital Control Based Build ing Automation System.

PB91-216697

100,092

AUTOMATION

Research Tackles Automation Issues

PB91.162230

101,005

Automating Robot Programming in the Cleaning and Deburring Works

100,986

\section{AUTOMATION \& ROBOTICS}

Fed-X: The NIST Express Translator. Revised, November

PB91-132126

100,681

NIST PDES Toolkit: Technical Fundamentals. National PDES Testbed Report Series (Revised)

National

PB91.132159

Control System Architecture for Unmanned Ground Vehi-
cles.
PB91-134726 101,379

Short-Term Evolution for the Flight Telerobotic Servicer.

PB91-144352 101,731

UNIX in the Government's Automated Manufacturing Re. search Facility (AMRF)

PB91-147579

100,963

CIM, Data and Standardization within the NBS AMRF

PB91-148122 100,975

Innovation in Manufacturing Technology: A View from

PB91-148130

100,976

Transferring NBS Technology to Small Manufacturers Through State and Local Centers.

101,025

Material Dependency of Chip-Form Detection Using Acoustic Emission

PB91-149450

101,216

STEP On-Line Information Service User's Guide. Nationa PDES Testbed Report Series.

PB91-157123 100,950

New Approach to Vision and Control for Road Following.
PB91-157156

TROI User's Guide.

PB91-157214

101,026

Self-Adjustment of a Robot Joint Controiler.

101,027

Application of the Joint Excitation Gear Adjustment Technique to Low Frequencies and Heavy Robot Joints. nique to Low Frequencies and Heavy Robot Joints.
PB91-158576 Real-Time Hierarchical Planning for Multiple Mobile

PB91-158782

101,375

Kinematic Couplings for Precision Fixturing. Part 2. Experimental Determination of Repeatability and Stiffness.
PB91-159186

Design and Implementation of a Five-Axis Robotic Micromanipulator for inserting Parts into Precision Collets.

PB91.159194 101,029

National PDES Testbed Mail Server User's Guide. National PDES Testbed Report Series.

100,951

PB91-159756

101,005

Research Tackles Automation Issues.

ZIP: The ZIP.Code Insulation Program, Version 2.0. Economic Insulation Levels for New and Existing Houses by nomic Insulation Levels for New and Existing Houses by

PB91-167155

100,064

Review of Current Geometric Tolerancing Theories and Inspection Data Analysis Algorithms.

PB91-167221

101,016

Engineering Design Laboratory Guide.

100,977

High Precision Gaging with Computer Vision Systems.

PB91-174623 100,979

Error Prevention and Detection in Data Preparation for a

Numerically Controlled Milling Machine.

PB91-174938

100,980

Robot Sensor Language.

PB91-174961

101,031

Control of Flexib

101,032

U.S. Manufacturing Systems: Factories Past, Present, and Future.

PB91-175091

100,981

Computerization of a Thermomechanical Processing Research System
PB91-175208

101,583

Recommended Fine Positioning Test for the Development Test Flight (DTF-1) of the NASA Flight Telerobotic Servicer (FTS)

PB91-185090

101,732

AMRF Composites Fabrication Workstation: A Test MethAdology to Measure the Quality of Thermoplastic Composite Parts.

PB91-187765

101,017

Statistical Process Control Tactics for Coordinate Measuring Machines.

PB91-189225

101,042

Concurrent Engineering through Product Data Standards.

100,982

Extracting STEP Geometry and Topology from a Solid Modeler: Parasolid-to-STEP.

PB91-194449

100,983

NASREM Robot Control System and Testbed

PB91-194969

101,033

CAD Directed Robotic Deburring.

PB91-195172

100,985

Real-Time Algorithms and Data Structures for Underwater Mapping.

SEM Imaging and Metrology at Low Accelerating Voltages Using Backscattered Electrons.

PB91-195461

101,529

Automating Robot Programming in the Cleaning and De burring Workstation of the AMRF.

PB91-195495

100,986

Spectrum and Energy Levels of Seven-Times-lonized Krypton (Kr VIII) and Resonance Lines of Eight-Timeslonized Krypton (Kr IX)

PB91.195511

101,673

Computer Vision Based Station for Tool Setting and Tool Form Measurement

PB91-202978

101,043

Application of the PIPE Image Processing Machine to Scanning Microscopy

PB91-203190

101,034

Video Compression for Remote Vehicle Driving

PB91-203208

101,035

Control Structure for Multi-Tasking Workstations.

100,989

Real-Time Model-Based Tracking Combining Spatial and Temporal Features.

PB91-216630

100,700

Description of a Vibration Compensation System for the Small Scale Model Robot Crane Project.

PB91-21664

100,130

Standard Reference Specimens in Quality Control of En- 


\section{KEYWORD INDEX}

Mare Island Flexible Manufacturing Workstation. PB92-117027

100,996

Publications of the Manufacturing Engineering Laboratory Covering the Period January 1989-September 1991.
PB92-123041 Use of Solid Modeling in the Design of M3 Components. PB92-123066

\section{AUTOMOBILES}

Study of Additive Response in a Series of Rerefined Base Oils Typical of Current Commercial Practice.

\section{UTONOMOUS NAVIGATION}

Real-Time Algorithms and Data Structures for UnderwatPB91-195354

101,360

AWARDS

Interim Report to the President and to the Congress on the Malcolm Baldrige National Ouality Award.

PB91-167833

100,001

Feasibility of Using a Multiple Award Schedule for Specifying Paints in Government Painting Contracts. 100,002

B STARS

Extension of the Class of Magnetic B Star Nonthermal Radio Sources.
PB91-175042

BACILLUS SUBTILIS

Newest Approaches to Ouantitative Assessment of Bioactive Organotins.

\section{CKGROUND DISCRIMINATION}

Strategies for Background Subtraction in Electron Probe Microanalysis $X$-ray Compositional Mapping. PB91-195180

100,202

BACNET

BACnet Communication Protocol for Building Automation Systems.
PB91-187286

100,069

\section{BACNET PROTOCOL}

Draft Abstract Test Suite for Determining Conformance to the BACnet Protocol.

PB91-187732

100,070

BARIUM IONS

Laser-Induced Fluorescence Measurements of Drift-Velocity Distributions for $\mathrm{Ba}(+)$ in $\mathrm{Ar}$ : Moment Analysis and a Direct Measure of Skewnes.

PB91-149005

100,298

BARIUM OXIDES

Many Electron Effects in Lgamma2,3 X-ray Emission Spectroscopy Spectrum of BaO

100,382

BARRIER MATERIALS

Detection and Ouantitative Characterization of Blistering and Corrosion of Coatings on Steel Using Infrared Thermography.

101,139

BASEMENTS

Design Heat Loss Factors for Basement and Slab Floors. BSIC INTERACTIONS

100,059

Search for a Composition Dependent Force Using a Liquid-Supported Torsion Balance.

101,639 PB91-175380

BEAM STEERING

Of What Use Is Basic Research
PB91-175513

101,642

BEAMS (SUPPORTS)

Performance of 1/3-Scale Model Precast Concrete Beam-Column Connections Subjected to Cyclic Inelastic L.oads. Report No. 2

PB91-222570 100,149

Performance of Precast Concrete Beam-to-Column Connections Subject to Cyclic Loading.

PB91-236588

100,150

\section{BEARINGS}

Long Term Performance of Rubber in Seismic and NonSeismic Bearings: A Literature Review.

\section{ENEFICIATION}

Rate of Pyrite Bioleaching by 'Thiobacillus ferrooxidans' Results of an Interlaboratory Comparison.

100,148 PB91-195321

101,161

Characterization by High Performance Liquid Chromatography (HPLC) of the Solubilization of Phosphorus in Iron raphy (HPLC) of Ore by a Fungus.
PB91-195404

101,162

\section{BENEFIT COST ANALYSIS}

NIST 'Building Life-Cycle Cost' (BL.CC) Program (Version 3.0). User's Guide and Reference Manual. PB91-159764

100,154

BENZENE

Benzene: A Further Liquid Thermal Conductivity Standard.

100,255

Molecular-Beam Optothermal Spectroscopy of the 9.6 mum nu14.

100,421
BENZILIC ACID

Synthesis of 3-Ouinuclidinol-(18)O, Benzilic-d5 Acid, and 3-Ouinuclidinyl-(18)O, Benzilate-d5.
PB91-237370

100,223

BENZILIC ACID/ (QUINUCLIDINYL-ESTER)

Synthesis of 3-Ouinuclidinol-(18)O, Benzilic-d5 Acid, and 3-Ouinuclidinyl-(18)O, Benzilate-d5.

100,223

\section{BENZOIC ACID/ (PHENYL-ESTER)}

Critical Evaluation of Liquid Crystal Transition Temperatures I: 4,4'-Alkyl/alkoxyphenylbenzoates.
PB92-110154

100,444

BENZYL RADICALS

Stabilities of Substituted Benzyl Radicals: Dissociation Rates of Aminoethylbenzenes, Hydroxyethylbenzenes, and Cyanoethylbenzenes.

PB91-237529

100,430

BERYLLIUM 9

Test of the Linearity of Ouantum Mechanics by if Spectroscopy of the (9)Be(+) Ground State.

101,726

BERYLLIUM-LIKE IONS

Relativistic and Correlation Effects in the 2s3p Configuration of Beryllium-Like Ions.

PB91-189605

101,654

Relativistic Distorted-Wave Cross Sections for ElectronImpact Excitations of Berylliumlike Ions.

101,672

BERYLLIUM PHOSPHATES

Structures and Properties of New Zeolite X-Type Zincophosphate and Beryllophosphate Molecular Sieves. PB91-189449

BESSEL FUNCTIONS

Portable Vectorized Software for Bessel Function Evaluation. 216598

100,652

BETA DETECTION

Status of Beta-Particle Calibration Studies at NBS.
PB91-175265

Calculation of Beta-Particle Counting Efficiency for Liquid Scintillation Systems with Three Phototubes. 101,644

BETA DOSIMETRY

Calibration of Ophthalmic Applicators at NIST: A Revised Approach.
PB91-237388

BETA SOURCES

Status of Beta-Particle Calibration Studies at NBS.

BIBLIOGRAPHIES

Metrology for Electromagnetic Technology: A Bibliography of NIST Publications.
PB91-132266 100,833

National Bureau of Standards Publications 1977-1987. Volume 1. Citations, Key Words, and Abstracts. 100,957 National Bureau of Standards Publications 1977-1987. National Bureau of Standards Publications 1977-1987. Volume 2. Indexes.

100,958

Bibliography of Selected Computer Security Publications, January 1980-October 1989.

100,704

Bidirectional Scattering Distribution Function (BSDF): A Systematized Bibliography. Building and Fire Research Laboratory Publications,

100,122

PB91-187807

100,076

Abstracts of Dayt
PB91-206755

Publications of the National Institute of Standards and
Technology, 1990 Catalog. Technology, 1990 Catalog.
PB91-216531

100,011

Building Technology Publications 1986-1989.
PB91-216820 Annotated Bibliography: Diagnostic Methods and Measurement Approaches to Detect Incipient Defects Due to Aging of Cables.

International Fire Detection Bibliography, 1975-1990.

PB92-109156 100,088

Bibliography of the NIST Electromagnetic Fields Division Publications.
PB92-116367

100,866

Metrology for Electromagnetic Technology: A Bibliogra-

phy of NIST Publications.

100,867

Publications of the Manufacturing Engineering Laboratory

Publications of the Manufacturing Engineering Laboratory
Covering the Period January 1989-September 1991.

PB92-12304

101,053

NIST X-Ray Photoelectron Spectroscopy (XPS) Data-

PB92-123157

100,465

BICRYSTALS

Grain Boundaries in a 2-D Superlattice.

100,388

BIDIRECTIONAL SCATTERING DISTRIBUTION FUNCTION Bidirectional Scattering Distribution Function (BSDF): A Systematized Bibliography.

PB91-187674

101,421

INARY ALLOY SYSTEMS

Structural Instabilities and Superconductivity in OuasiBinary Mn5Si3-Type Compounds.

101,215

BINARY ALLOYS

Phase Diagrams of Hexagonal Binary Ordering Alloys with Anisotropic Interactions.

101,211

Convective and Morphological Instabilities during Directional Solidification.

PB91-158550

101,219

Coherency Strain Induced Instability of Solid-Liquid Interfaces in the Mo-Ni System.

101,235

Phase-Field Model for Isothermal Phase Transitions in Binary Alloys

PB92-108992 101,240

Microstructural Studies of Ti-Al Alloys in the Vicinity of the 'Eutectoid' Reaction.

PB92-116938

101,241

BINARY FLUIDS

Phase Equilibria from the One-Fluid Model.

PB91-134585

101,385

BINARY MIXTURES

Effect of Noble Gas Mixtures on the Performance of Regenerative-Type Cryocoolers Analytical Estimate.

100,890

Computer Simulation of Fluid-Fluid Phase Coexistence in Mixtures of Nonadditive Soft Disks.

PB91-162263

101,388

Viscosity Surface for Mixtures of Methane and Ethane.

PB91-174763 100,322

Critical Exponent for the Viscosity of Four Binary Liquids.
PB91-187195

Hydrogen Component Fugacity Coefficients in Binary Mixtures with Etane: Pressure Dependence.

PB92-116508

100,455

\section{BINARY STARS}

Ultraviolet, Optical, Infrared, and Microwave Observations of HR 5110 .

100,020

Spatially Resolved Flares in RS CVn Systems.

PB91-148965

100,022

\section{BINARY SYSTEM (MATERIALS)}

Statistical Mechanical Model of Aqueous Two-Phase SysPB91-17.

100,493

Small-Angle Neutron Scattering Studies of Compatible Blends of Linear Poly(Vinyl Methyl Ether) and Cross-

Linked Deuterated Polystyrene.
PB91-187153

100,494

BINDING ENERGY

Theoretical Model of Metal Binding Sites in Proteins.

PB91-194837

100,370

BINDING SITES

Theoretical Model of Metal Binding Sites in Proteins.
PB91-194837

Immobilization of Binding Proteins on Nonporous Supports Comparison of Protein Loading, Activity, and StabiliPB. $1-237198$

101,308

BIOASSAY

Biological Sulfur Oxidation and Reduction for Coal Sulfur Speciation and Desulfurization.

PB91-203323

100,886

BIOCHEMISTRY

Neutron Crystallography of Proteins.

PB91-175349 101,285 
BREAKDOWN (ELECTRONIC THRESHOLD)

BIOMONITORING

Practical Constraints in Monitoring Micronutrient Intake. PB91-147421

101,318

BIOPROCESSING

Bioprocessing of Fossil Fuels Using Hyperthermophilic Archaebacteria.

100,883

BIOSEPARATIONS

Enhanced Transport and Liquid Membranes in Biosepara-

tions.

101,287

BIOTECHNOLOGY

Liposome Flow Injection Immunoassay: Implications for Sensitivity, Dynamic Range, and Antibody Regeneration. PB91-147629

101,291

Radiation Chemistry of Enones.

100,236

Multiple Conformations of Amino Acid Residues in Ribonuclease $\mathrm{A}$.

101,298

Crystallizing Catabolite Gene Activator Protein with CAMP for Structural Analysis.

101,299

Low-Cost Low-Volume Carrier (Minilab) tor Biotechnology and Fluids Experiments in Low Gravity PB91-150029

100,041

Dental Biomaterials and Engineering Frontiers for the 90 's.

100,044

Newest Approaches to Quantitative Assessment of Bioactive Organotins

101,328

Bioprocessing of Fossil Fuels Using Hyperthermophilic Archaebacteria.

PB91-162271

100,883

Neutron Crystallography of Proteins.

101,285

Crystal Structure of Phosphate-Free Ribonuclease

PB91-175604 101,301

Base Modifications in Plasmid DNA Caused by Potassium

Permanganate

101,329

Damage to the Bases in DNA Induced by Hydrogen Peroxide and Ferric Ion Chelates.

101,331

Chemical Determination of Free Radical-Induced Damage

$\begin{array}{ll} & \\ \text { to DNA. } & 101,332\end{array}$

46Gas Chromatography-Mass Spectrometry of Free Rad-

ical-Induced Products of Pyrimidines and Purines in DNA.

PB91.187476 101,302

Measurement of DNA Base Damage and DNA-Protein

Cross-Links in Mammalian Chromatin.

101,303

Modification of Bases in DNA by Copper Ion-1,10-Phenanthroline Complexes.

101,333

Structure and Mechanism of Hydroxyl Radical-Induced
Formation of a DNA-Protein Cross-Link Involving Thy-

Formation of a DNA-Protein Cross-Link Involving Thy-
mine and Lysine in Nucleohistone.
PB91-187518

101,334

Chemical Nature of In vivo DNA Base Damage in Hydrogen Peroxide-Treated Mammalian Cells.

101,335 Damage to the DNA Bases in Mammalian Chromatin by
Hydrogen Peroxide in the Presence of Ferric and Cupric lons.

lons.
PB91-189217

101,336

Bleomycin-Dependent Damage to the Bases in DNA is a Minor Side Reaction.

101,337

Hydroxyl Radical Induced Cross-Linking of Cytosine and Tyrosine in Nucleohistone.

101,304

Modification of DNA Bases in Mammalian Chromatin by Radiation-Generated Free Radicals.

101,338

8-Hydroxyguanine Content of Isolated Mitochondria In. creases with Lipid Peroxidation. Crystal Structure of a CAMP-Independent Form of Cata. bolite Gene Activator Protein with Adenosine Substituted in One of 2 cAMP-Binding Sites.

PB91-190074

101,305

Mechanistic Studies of lonizing Radiation and Oxidative Mutagenesis: Genetic Effects of a Single 8-Hydroxyguanine (7-Hydro-8-oxoguanine) Residue Inserted at a Unique Site in a Viral Genome.

Site in a Viral
PB91.190108

101,321

Theoretical Model of Metal Binding Sites in Proteins.

PB91-194837

100,370

Rate of Pyrite Bioleaching by 'Thiobacillus ferrooxidans': Results of an Interlaboratory Comparison.

101,161

PB91-195321

Recent Progress in Coal Bioprocessing Research in the United States: Scope and Areas Needing Further Research.
PB91-195339

100,885

Characterization by High Performance Liquid Chromatography (HPLC) of the Solubilization of Phosphorus in Iron Ore by a Fungus.

PB91-195404

101,162

Iron Ion-Dependent Modification of Bases in DNA by the Superoxide Radical-Generating System Hypoxanthine/ Xanthine Oxidase.

101,306

Selected-Ion Mass Spectrometry: Assays of Oxidative

$\begin{array}{ll}\text { DNA Damage. } & 101,307 \\ \text { PB91-202960 } & \end{array}$

Biological Sulfur Oxidation and Reduction for Coal Sulfur Speciation and Desulfurization.

PB91-203323

100,886

Biomarkers ot $\mathrm{OH}$ Radical Damage In vivo.

101,325

Immobilization of Binding Proteins on Nonporous Supports Comparison of Protein Loading, Activity, and Stabili-

Pि.

101,308

Ab Initio Phase Determination for X-ray Diffraction Data from Crystals of a Native Protein.

101,309

Thermodynamics of the Disproportionation of Adenosine 5'-diphosphate to Adenosine 5'-triphosphate and Adenosine 5'-monophosphate. 1. Equilibrium Model.

100,459

Damage to the Bases in DNA Induced by Stimulated Human Neutrophils.

PB92-116912

101,312

Formation of ortho-Tyrosine by Radiation and Organic

Solvents in Chicken Tissue.

PB92-116953

101,290

Thermodynamics of the Disproportionation of Adenosine 5 -diphosphate to Adenosine 5'-triphosphate and Adenosine 5 '-monophosphate. 2 . Experimental

PB92-117399

101,313

Comparative Study of Bovine Pericardium Mineralization: A Basic and Practical Approach.

PB92-117407

100,056

Opportunıties for Innovation: Chemical and Biological Sensors.

100,214

BIPOLAR TRANSISTORS

Uniform Junction Temperature AIGaAs/GaAs Power Heterojunction Bipolar Transistors on Silicon Substrates.
PB91-189399

100,806

Emitter Ballasting Resistor Design for, and Current Handling Capability of AIGaAs/GaAs Power Heterojunction Bipolar Transistors.

PB91-236703

100,815

Experimentally Verified IGBT Model Implemented in the Saber Circuit Simulato

BIREFRINGENT COATINGS

Use of Birefringent Coatings in Fracture Mechanics.

BISMUTH

Morphology and Barrier-Height Development of Bi/ InP(110) Interiaces.

BISMUTH LEAD STRONTIUM CALCIUM CUPRATES

Studies of Magnetic Flux Penetration in a Chemically Synthesized Bi(Pb)SrCaCuO Superconductor.

101,526 1-194977

BISMUTH STRONTIUM CALCIUM CUPRATES

Processing $\mathrm{Bi}-\mathrm{Pb}-\mathrm{Sr}-\mathrm{Ca}-\mathrm{Cu}-\mathrm{O}$ Superconductors from

Amorphous State.
PB91-187369 101,504

Superconducting Thin Films of Bi-Sr-Ca-Cu-O Obtained by Laser Ablation Processing

101,508

Theoretical Model for the Tunneling-Gap Anisotropy Observed in Layered Copper-Oxide High-Temperature Su-

$\begin{array}{ll}\text { perconductors. } & 101,564 \\ \text { PB92-116565 } & \end{array}$

Single-Target Magnetron Sputter-Deposition of High-Tc Superconducting Bi-Sr-Ca-Cu-O Thin Films.

101,566

PBg -116680 New Look at the Bitter Method of Magnetic Imaging.
PB91-203919

BLACK LIQUIDS

Fundamental studies of black liquor combustion. Report No. 4, Phases, 2, 3, and 4: Final report, December 1987.December 1989.

\section{BLEOMYCIN}

Bleomycin-Dependent Damage to the Bases in DNA is a Minor Side Reaction

PB91-189357

101,337

BLOCK COPOLYMERS

Incorporation of Molecular Orientation into Systems of Lamellar Morphology. 1. Effects of Packing Entropy on the Lamellar Thickness of Block Copolymers. $\quad 100,484$
PB91-147231 Chain Conformation of a Block Polymer in a MicrophaseSeparated Structure.

PB91.148890

100,485

BLOCK DESIGN

List of New Group Divisible Designs.

PB92-126705

101,281

BLOOD

Research at NBS in Direct Potentiometric Measurements in Blood

PB91-148874

100,188

BLOOD CELLS

Thermodynamics of the Disproportionation of Adenosine 5 '-diphosphate to Adenosine $5^{\prime}$-triphosphate and Adeno-

sine 5'-monophosphate. 2. Experimental Data.
PB92-117399

101,313

BLOOD SERUM

NIST Standards for Sodium and Potassium. PB91-158865

100,191

BLOWOUTS

Investigation ot Simulated Oil-Well Blowout Fires.

BOILERS

Conformity Assessment Workshop on Pressure Vessels. PB91-192997

101,049

BOILING

Horizontal Flow Boiling Heat Transfer Experiments with a Mixture of R22/R114.

PB92-116946

101,200

BOLOMETERS

Low-Temperature, Electrically Calibrated SOS Bolometer for Power and Energy Measurements.

PB91-147181

100,787

BOND STRENGTH

Dissociation of Substituted Anisoles: Substituent Effects on Bond Strengths

PB91-237537

100,431

Determination of Bonded Phase Thickness in Liquid Chromatography by Small Angle Neutron Scaltering.

BONDING STRENGTH

Microstructure and Fracture at the Cement Paste-Aggregate Interface

100,523

Evaluation of Test Methods for Measuring the Bond Strength of Portland Cement-Based Repair Materials to Concrete.

PB91-189621

100,530

Hydrogen Bonding in Pt Ammine Complexes.

100,348

Dentin-Bonding Molar Efficiency Using N-Phenylglycine, N-Phenyl-beta-Alanine, or N-Methyl-N-Phenylglycine.
PB91-237735 Uniaxial Tensile Tests to Measure the Bond of In situ Concrete Overlays

BONES

Co-Trial on ESR Identification and Estimates of gamma-

Ray and Electron Absorbed Doses Given to Meat and

Bones.

PBo1-194720

100,017

BOOKS

Book Review: Hypertext Hands-On.

PB91-237040

100,656

BORATES

Lanthanide Coordination Chemistry: Spectroscopic Properties of Terbium and Europium Poly(Pyrazol-1-YI) Borate and Poly(Imidazol-1-YI) Borate.

PB91-203034

100,392

BORON CARBIDES

Low Temperature Chemical Process for Precursors to 


\section{KEYWORD INDEX}

BREAKING

Users' Guide to BREAK1, the Berkeley Algorithm for Breaking Window Glass in a Compartment Fire.

100,136

REWSTER ANGLE

Alternate Representation of Prism Refraction for Light In cident at the Brewster Angle.

PB91-203042

101,426

BRIDGE FOUNDATIONS

Proposed Design Criteria for Shallow Bridge Foundations PB91-187849

100,534

BRITTLE MATERIALS

Effects of Crystal Bonding on Brittle Fracture.

PB91-148221

101,064

Tensile-Fracture Resistance Mechanisms in Brittle Polycrystals: An Ultrasonics and In-situ Microscopy Investigation.

101,190

BROADCAST EPHEMERIDES Precise Ephemerides for GPS Time Transfer
PB91-236950

100,586

BSDF (BIDIRECTIONAL SCATTERING DISTRIBUTION

Bidirectional Scattering Distribution Function (BSDF): A Systematized Bibliography.

101,421

BUCKEYBALLS

Neutron Measurements of Intramolecular Vibrational Modes in $\mathrm{C} 60$

101,055

BUCKLING

Buckling and Post-Buckling Behavior of Elliptical Plates: Part 1. Analysis.
PB92-116573

101,588

Buckling and Post-Buckling Behavior of Elliptical Plates. Part 2. Results

101,589

BUCKMINSTERFULLERENCE

Neutron Measurements of Intramolecular Vibrational Modes in C60.
PB92-116540

BUFFERS (CHEMISTRY)

Second Dissociation Constant and $\mathrm{pH}$ of $\mathrm{N}$-2-hydroxyethylpiperazine- $N^{\prime}-2$-ethanesulfonic Acid (HEPES) from 0 to PB91-189282

100,196

BUILDING AND FIRE RESEARCH LABORATORY Building and Fire Research Project Summaries, 1991. PB91-216788 100,132

BUILDING AUTOMATION AND CONTROL NETWORK BACnet Communication Protocol for Building Automation

PB91-187286

100,069

BUILDING AUTOMATION CONTROL

Direct Digital Control Based Building Automation System Design Criteria.

100,091

BUILDING AUTOMATION SYSTEMS

Guide Specification for Direct Digital Control Based Building Automation System.

100,092

BUILDING CODES

Review of Research Literature on Masonry Shear Walls. PB91-167189 100,113 Improving the Fire Performance of Building Contents. PB91-187260

100,084

Plumbing Test Lab Accreditation. PB91-190066

100,089

BUILDING FIRES

Boussinesq Algorithm for Enclosed Buoyant Convection in Two Dimensions.

100,157

Data for Room Fire Model Comparisons PB92-110287

100,167

BUILDING TECHNOLOGY

Techniques to Observe the Fracture Zone in Mortar and Concrete.

100,521

Method for Measuring Heat Loss from Underground Heat Distribution Systems

PB91-134221

100,888

Preliminary Dynamic Analyses of the Ministry of Agriculture Building.

100,143

Outline of a National Plan on High-Performance Concrete: Report on the NIST/ACI Workshop. Held in Gaithersburg. MD on May 16-18, 1990.

PB91-143321

100,522

Computer Program for Simulation of HVAC/Lighting Interactions: Initial Report.

100,889

Calibration Procedures for Infrared Imaging Systems for Building Diagnostics.

100,057

Second-Level Post-Occupancy Evaluation Analysis.

PB91-147140
Design of High Strength Cement-Based Materials. Part 2. Microstructure.
PB91-147439

100,099

Design Heat Loss Factors for Basement and Slab Floors.

100,059

Temperature Measurement of Glass Subjected to Solar Radiation

PB91-147611

100,100

entilation System Performance Evaluation Using Tracer Gas Techniques.

PB91.147876

100,060

Microstructure and Fracture at the Cement Paste-Aggregate Interface

100,523

Guide to the Use of the Cement and Concrete Research Remote Bulletin Board System (RBBS) Computer.

PB91-148528 eters on Adhesive-Bonded Seams of Single-Ply Roof PB91-149153

Pozzolan Programs of the Cement and Concrete ReferPB91-149922

100,525

Pulse-Echo Ultrasonic Evaluation of the Integrity of Seams of Single-Ply Roofing Membranes: Laboratory Evaluation of a Prototype Test Apparatus. 100,10 Conference Coverage: Papers Presentations Shine. PB91-158535 100,061

Toward Modular Semantic Application Layer Protocols (MS-ALPs): Communication between Heterogeneous Inormation Systems Using Artificial Intelligence (Al) Programming Techniques.

151-158584

Rapporteur's Summary Materials Development for Coal

$\begin{array}{ll}\text { Gasification. } & 100,876\end{array}$

Towards Computer-Based Microstructure Models for Cement-Based Systems.

101,074

Prototype Expert System for Diagnosing Moisture Prob-

$\begin{array}{ll}\text { Pems in Houses. } & 100,062 \\ \text { PB91-159079 }\end{array}$

Al: Does It Have a Place in Building Simulation.

100,063 Pulse-Echo Ultrasonic Evaluation of the Integrity of
Seams of Single-Ply Roofing Membranes: Results of Field Investigations and Recommendations.
PB91-159731 NIST 'Building Life-Cycle Cost' (BLCC) Program (Version 3.0). User's Guide and Reference Manual.
PB91-159764

Computer Simulated Hydration of a Cement Model.
PB91-162149 100,112

Visualization of Nucleate Flow Boiling for an R22/R114 Mixture and Its Components.

PB91-162164

100,225

Review of Research Literature on Masonry Shear Walls. 100,113 Measurement of Structural Response Characteristics of Full-Scale Buildings: Selection of Structures.

PB91-167239

100,144

Field Study of the Performance of EPDM Roofing at Air Force Facilities

101,340

Computational Models Developed for the Corrosion of

$\begin{array}{ll}\text { Prestressing Steel. } & 100,115 \\ \text { PB91-167304 }\end{array}$

Experimentation, Analysis, and Correlation of Refrigerant22 Flow Through Short Tube Restrictors.

101,194

Research and Development of Heat Pumps Using Nonazeotropic Mixture Refrigerants.

100,891

Introduction to $\mathrm{ACl}$ 306.1-87: Specification for Cold Weather Concreting.

PB91-174524

100,526

Refrigerant Charge Effects on Heat Pump Performance.

100,065

Simulation of an Evaporator with Nonuniform One-Dimen-

sional Air Distribution.
PB91-174649 100,066

Rate Constant Functions for Strength Development of Concrete.

100,527

Airtightness of Office-Building Envelopes.

100,067

Application of CCRL Data in the Development of Cement Standards.

100,119

Measurement of Thermal Characteristics of Office Build

Measurement of Thermal Characteristics of Ofice Build

PB̈91-175471

100,068

Summary of Low-Density Glass-Fiber Reference Materials at NIST: 1980-1989.

101,079

Characterization of Field Concrete.

Proceedings of a Workshop on Evaluation, Repair, and Retrofit of Structures. U.S.-Japan Panel on Wind and Seismic Effects, UJNR. Held in Gaithersburg, MD., USA On May 12.14, 1990.

100,145

Multi-Zone Dispersal Analysis by Element Assembly.

PB91-187146

100,907

Percolation of Phases in a Three-Dimensional Cement Paste Microstructural Model.

100,529

Comparison of Two Test Methods for Determining Transfer Function Coefficients for a Wall Using a Calibrated Hot Box.

PB91-187278

100,121

BACnet Communication Protocol for Building Automation Systems.
PB91-187286

100,069

Chloride Ion Diffusion in Low Water-to-Solid Cemen

PB91-187690

101,138

Draft Abstract Test Suite for Determining Conformance to the BACnet Protocol.

PB91-187732

100,070

Intelligent Building Technology in Japan

100,071

Measured Performance of Residential Water Heaters Using Existing and Proposed Department of Energy Tes Procedures.
PB91.189274

100,085

Study of Flow Boiling Heat Transfer with Refrigerant Mix

PB91-189563

101,196

Evaluation of Test Methods for Measuring the Bond Strength of Portland Cement-Based Repair Materials to

PB91-189621

100,530

Field Testing of Adhesive-Bonded Seams of Rubbe

Roofing Membranes.
PB91-189993 100,124

Thermal Conductivity of Fumed Silica Board at Room

Temperature.

100,125

Evaluation of the Heat Flux Transducer Technique for Measuring the Thermal Performance of Walls.

PB91-194696

100,126

Rule-Based Diagnostic Method for HVAC Fault Detection. PB91-194936

Dipole Moments of Seven Partially Halogenated Ethane Refrigerants. Degradation of One-Component Polyether Polyurethane Protective Coatings on Steel.

Prediction and Fault Detection of Building Energy Consumption Using Multi-Input, Single-Output Dynamical

100,073

Air Exchange and Pollutant Concentration Measurements in Two Office Buildings.

PB91-195420

100,909

Diagnostic Techniques for Evaluating Office Building Envelopes.

100,074

Identification of Dynamic Green's Functions in Structural Networks.

PB91-202812 
Measurement and Evaluation of Lighting/HVAC Interaction.

100,892

PB91-206706

and Non-

Long Term Performance of Rubber in

100,148

PB91-206714

User Manual NBSAVIS CONTAM88. A User Interface for
Air Movement and Contaminant Dispersal Analysis in

Air Movement and Contaminant Dispersal Analysis in
Multizone Buildings.
PB91-206722
100,910 PB91-206722

Calibration at $24 \mathrm{C}$ of a Heat-Flow-Meter Apparatus Having $610 \mathrm{~mm}$ Square Plates.

PB91-206730

100,933

Abstracts of Daylighting Research

PB91-206755

100,076

Direct Digital Control Based Building Automation System Design Criteria.

100,091

Variable Air Volume System Design Guide.

PB91-216655

100,077

Guide Specification for Direct Digital Control Based Build-

ing Automation System.
PB91-216697

100,092

Building and Fire Research Project Summaries, 1991

PB91-216788

100,132

Building Technology Publications 1986-1989.

100,160

Pertormance of 1/3-Scale Model Precast Concrete Beam-Column Connections Subjected to Cyclic Inelastic Beam-Column Connections Subjected to Cyclic Inelastic

Loads. Report No. 2 .
PB91-222570

100,149

Standards for the Physical Protection of National Resources and Facilities
PB91-222695

100,006

Daylight Research Requirements. Workshop Proceedings. Held in Baltimore, Maryland on August 1-2, 1990.

Performance of Precast Concrete Beam-to-Column Connections Subject to Cyclic Loading.

PB91-236588

100,150

Impulse Response Functions for Elastic Structures with Rigid Body Degrees of Freedom.

PB91-237354

101,587

Microstructural Aspects of the Fracture of Hardened Cement Paste.

PB91-237479

100,133

Dealing with Obvious Issues in Lighting

100,761 Effect of Veiling Relections on Vision of Colored Objects.
PB91-237800

Light Source

100,080

Role of Theory in Lighting Research and Design.

PB91-237826

100,081

Assessment: U.S. Office Building of Moscow.

PB91.237834

100,151

Economic Analysis for Military Construction (MILCON) Design. Concepts, Techniques, and Applications for the Analyst. Instructor's Manual.

101,349

PB92-112440

Economic Analysis for Military Construction (MILCON) ( Concepts, Techniqu

PB92-112457

101,350

Uniaxial Tensile Tests to Measure the Bond of In situ Concrete Overlays.

100,533

Analysis of Moisture Accumulation in a Wood Frame Wal Subjected to Winter Climate.

PB92-116334

100,137

Ultimate Strength of Masonry Shear Walls: Predictions versus Test Results.

100,138

Wind and Seismic Effects. Proceedings of the Joint Meeting of the U.S.-Japan Cooperative Program in Natural Re sources Panel on Wind and Seismic Effects (23rd). Held in Tsukuba, Japan, on May 14-17, 1991

100,152

Impact-Echo: A New Method for Inspecting Construction Materials.

PB92-116557 100, 140
Integrated Knowledge Systems for Concrete Science and Technology.

100,141

Horizontal Flow Boiling Heat Transfer Experiments with a Mixture of R22/R114.

101,200

PB92-116946

Probe-Corrected Spherical Near-Field Scanning Theory in
Acoustics.
PB92-117456
101,383

PB92-117456

ential Air-

Conditioning Application Using Mixed Refrigerants.

Testing and Rating of an Atmospheric, Gas-Fired Furnace Equipped with a Burner Air Inlet Damper.

PB92-126440

100,894

Predicting the Remaining Service Life of Concrete.
PB92-126598

BUILDINGS

Concept of the Calculus of Fire Safety.

101,371 actions: Initial Report.

Using the Harvard/NIST Mark VI Fire Simulation.
PB91-144394

100,889

Programmer's Reference Manual for CFAST, the Unified Model of Fire Growth and Smoke Transport.

PB91-144436

100,096

Calibration Procedures for Infrared Imaging Systems for Building Diagnostics.

100,057

Refinement of a Model for Fire Growth and Smoke Transport.

PB91-148510

100,104

Compartment Fire-Generated Environment and Smoke Filling.

100,107

Evaluation of Building Systems Inventions.

PB91-158659

100,896

Al: Does It Have

100,063

NIST 'Building Life-Cycle Cost' (BLCC) Program (Version 3.0). User's Guide and Reference Manual.

PB91-159764

100,154

Measurement of Structural Response Characteristics of Full-Scale Buildings: Selection of Structures.

PB91-167239

100,144

Ceiling Jet-Driven Wall Flows in Compartment Fires.

PB91-174573

100,117

Draft Abstract Test Suite for Determining Conformance to the BACnet Protocol.

PB91-187732

100,070

Intelligent Building Technology in Japan.

PB91-187757

100,071

Building and Fire Research Laboratory Publications, 1990.

PB91-187807

100,122

Prediction and Fault Detection of Building Energy Consumption Using Multi-Input, Single-Output Dynamical

PB91-195388

100,073

Investigation of the Collapse of L'Ambiance Plaza.

PB91-204057

100,146

Applications of the Generalized Global Equivalence Ratio Model (GGERM) for Predicting the Generation Rate and Distribution of Products of Combustion in Two-Layer Fire Environments:
PB91-216689

100,131

Building and Fire Research Project Summaries, 1991

PB91-216788 100,132

Building Technology Publications 1986-1989.

100,160

Daylight Research Requirements. Workshop Proceedings. Held in Baltimore, Maryland on August 1-2, 1990. PB91-231563

General Routine for Analysis of Stack Effect. PB91-231589

100,163

Effect of Veiling Relections on Vision of Colored Objects. PB91-237800 100,079 Assessment: U.S. Office Building of Moscow.

100,151

Building Life Cycle Cost Computer Program, Version 3.2

$\begin{array}{ll}\text { (for Microcomputers). } & 100,898\end{array}$

Users' Guide to BREAK1, the Berkeley Algorithm for Breaking Window Glass in a Compartment Fire.

PB92-112556

100,136

BURNER BOX INLET DAMPERS

Testing and Rating of an Atmospheric, Gas-Fired Furnace Equipped with a Burner Air Inlet Damper.
PB92-126440

100,894

BURNING RATE

Algorithm for the Mass-Loss Rate of a Burning Wall. Technical Reference and User's Guide for FAST/FFM Version 3.

PBB91-206664

100,086

BUTANE

Non-Equilibrium Molecular Dynamics Simulations of Structured Molecules. Part 1. Isomeric Effects on the Viscosity of Butanes.

BUTANES

Chemical Kinetic Data Base for Combustion Chemistry Part 4. Isobutane.

PB90-244534

100,252

High-Resolution Infrared Flash Kinetic Spectroscopy of $\mathrm{OH}$ Radicals

100,408

BUTYNE

Reactions of $\mathrm{C} 5 \mathrm{H} 3(+)$ and $\mathrm{C} 5 \mathrm{H} 5(+)$ lons with Acety lene and Diacetylene.

PB91-195370

100,384

C PROGRAMMING LANGUAGE

American National Standard for C. Category: Software Standard. Subcategory: Programming Language.

\section{CABLES}

Annotated Bibliography: Diagnostic Methods and Meas urement Approaches to Detect Incipient Defects Due to Aging of Cables.

CALCIUM IONS

Research at NBS in Direct Potentiometric Measurements in Blood.

PB91-148874

100,188

Etfects of $\mathrm{pH}$ and Calcium on Hydrolysis of Na2SiF6 and Na2SnF6: A Ouasi-Constant Composition Titration Study. Theoretical Model of Metal Binding Sites in Proteins. PB91-194837

100,370

CALCIUM OXIDES

Phase Equilibria of the System Strontium Oxide-Calcium Oxide-Cupric Oxide.

CALCIUM PHOSPHATES

101.085

Setting Reactions and Compressive Strengths of Calcium Phosphate Cements.

PB91-148668

100,040

Effect of Pyrophosphate Concentrations on Calcium Phosphate Growth on Well-Crystallized Octacalcium Phosphate and Hydroxyapatite Seed Crystals.
PB91-162024

101,193

Ion Incorporation into Octacalcium Phosphate Hydrolyzates.

Filler Systems Based on Calcium Metaphosphates.

PB91-194654

Self-Setting Calcium Phosphate Cements

PB91-202879

100,048

CALCIUM STRONTIUM CUPRATES

Parent Structure of the Layered High-Temperature Superconductors.
PB91-195677

CALIBRATING

Linear Calibration When the Coefficient of Variation is PB91-149443

101,273

Calibration of Ophthalmic Applicators at NIST: A Revised Approach.
PB91-237388

101,327

CALIBRATION

Absorbed-Dose Calibration of Ionization Chambers in a(60)Co Gamma-Ray Beam.

PB91-132290

101,598

Eddy Current Probe Characterization Using an Impedance Plane Display Instrument.

100,834

Calibration of Antenna Factor at a Ground Screen Field Site Using an Automatic Network Analyzer.

PB91-134239

100,724 


\section{KEYWORD INDEX}

Translate LRL and LRM Calibrations.

PB91-237776

Procedure for the Effective Recalibration of Liquid-inGlass Thermometers.

100,935

Criteria for the Operation of Federally-Owned Secondary Calibration Laboratories (Ionizing Radiation).

PB92-112481

101,721

NIST Calibration Services for Humidity Measurement.

100,520

Voltage Calibration Systems Using Josephson Junction Arrays.

100,868

Application of Transmission Electron Detection to X-ray Mask Calibrations and Inspection.

100,828

NIST Calibration Services Users Guide, 1991 PB92-126416

100,936

ALORTFIC VALUE

MSW Calorimetry.

PB91-133942

100,878

CALORIMETERS

Temperature Gradient in Differential Scanning Calorimetry.

100,414

CALORIMETRY

Heat Capacity and Thermodynamic Properties of Deutrated Ammonium Hexafluorophosphate ND4PF6 from $5.8 \mathrm{~K}$ ed Ammonium Hexafluorophosphate ND4PF6 from $5.8 \mathrm{~K}$ o $347 \mathrm{~K}$.

100,272

Heat Capacity of Deuterated Ammonium Tetrafluoroborate ND4BF4 from 7 to $348 \mathrm{~K}$

PB91-147058

100,273

Levitation Calorimetry.

Combustion Calorimeters

PB91-189233

100,927

Thermodynamics of the Divalent-Metal Fluorides. 4. Heat Capacity of MSnF4, $\mathrm{M}=\mathrm{Pb}, \mathrm{Ba}$, or $\mathrm{Sr}$, at Temperatures from $300 \mathrm{~K}$ to $660 \mathrm{~K}$

CALS SQL3 Support for CALS Applications.

100,456

CALS (COMPUTER-AIDED ACQUISITTON AND LOGISTIC (

Collection of Technical Studies Completed for the Computer-Aided Acquisition and Logistic Support (CALS) Program. Fiscal Year 1988. Volume 1 of 3. Text, Security and Data Management.

101,342

Collection of Technical Studies Completed for the Computer-Aided Acquisition and Logistic Support (CALS) Program, Fiscal Year 1988. Volume 2 of 3. Graphics, CGM MIL-SPEC

PB91-178897

101,343

Collection of Technical Studies Completed for the Computer-Aided Acquisition and Logistic Support (CALS) Proputer-Aided Acquisition and Logistic Support (CALS) Pro-
gram. Fiscal Year 1988 . Volume 3 of 3 . CGM Registra-

101,344

PB91-178905

GISTICS

CALS (COM
SUPPORT)

Use of the IRDS Standard in CALS (Revised)

PB91-132209

100,684

NIST Support of the CALS Program: 1990 Synopsis.

101,347

CAMERAS

Development of a Line Camera for Real-Time Measurements of Concentration in Turbulent Flow Fields.

PB91-147892

101,386

High-Resolution Small-Angle X-ray Scattering Camera for Anomalous Scattering

APACITANCE

100,929

Measurement of Capacitance on Wafers.

PB91-149120

100,795

Characterization of Capacitive Array for NDE Applications.

100,946

Capacitive Array Sensors for Nondestructive Evaluation. PB91-237313 100,947

Theory of Capacitive Probe Method for Noncontact Characterization of Dielectric Properties of Materials. PB91-237610

100,948

CARBINOLS

Fluoromethanol: Synthesis, Microwave Spectrum, and Dipole Moment
PB91-148080

CARBON Spatially and Spectrally Resolved Cathodoluminescence
of Hot-Filament Chemical-Vapor-Deposited Diamond Particles.

AD-A236 485/9

100,247

Saran Carbon Cryogenic Regenerator for Liquid Helium. PB91-175430 101,640 Spectroscopic Data Base for Carbon and Oxygen.
PB91-237750

101,716

Neutron Measurements of Intramolecular Vibrational Modes in $\mathrm{C60}$.

101,055

New Way to Mount Particulate Material for Laser Microprobe Mass Analysis.

PB92-116805

100,911

CARBON ADDITIIONS

Effect of Nitrogen and Carbon on FCC-HCP Stability in Austenitic Steels.
PB91-175372

101,151

CARBON DIOXIDE

Transport Properties of Carbon Dioxide.

100,266

Critical Locus, (Vapor + Liquid) Equilibria, and Coexisting Densities of (Carbon Dioxide + Propane) at Temperatures from $311 \mathrm{~K}$ to $361 \mathrm{~K}$.

PB91-134494

100,267

Critical Exponent for Viscosity.

100,271

Air Exchange and Pollutant Concentration Measurements in Two Office Buildings.

100,909

Production and Spectroscopy of Small Polyatomic MolecUlar lons Isolated in Solid Neon.

PB91-203273 100,395

Heterodyne Frequency Measurements of $\mathrm{CO}$ and OCS Beyond $2100 \mathrm{~cm}(-1)$

100,423

Hydration of Carbon Dioxide: The Structure of $\mathrm{H} 2 \mathrm{O}-\mathrm{H} 2 \mathrm{O}$ $\mathrm{CO} 2$ from Microwave Spectroscopy.

100,428

Evaluation of Solubility Data of the System $\mathrm{CO}-\mathrm{H} 2 \mathrm{O}$ from Evaluation of Solubility Data of the System CO-H2O from
$273 \mathrm{~K}$ to the Critical Point of Water.
PB92-110253 100,453

100,453

Development of a Coulometric Method for Assessing the Concentration of Ambient Levels of CO2/Air in ComCons

100,913

Solubilities of Solids and Liquids of Low Volatility in Supercritical Carbon Dioxide. B92-148105

100,470

Solubility of Carbon Dioxide in Water at Low Pressure. PB92-148196

100,479

CARBON DIOXIDE DIMERS

Structure of Carbon Dioxide Dimer from Near Infrared Spectroscopy.

\section{CARBON MONOXIDE}

Vibrational Spectra of Molecular lons Isolated in Solid Neon. 7. $\mathrm{CO}(+), \mathrm{C} 2 \mathrm{O} 2(+)$, and $\mathrm{C} 2 \mathrm{O} 2(-)$.

100,279

AD-A239 729/7

100,250

Picosecond IR Studies of the Vibrational Dynamics of CO/Pt(111).
PB91-161927

100,314

Air Exchange and Pollutant Concentration Measurements in Two Office Buildings.

PB91-195420

100,909

Photodissociation Dynamics of $\mathrm{Mo}(\mathrm{CO}) 6$ at $266 \mathrm{~nm}$ and $355 \mathrm{~nm}$ : CO Photofragment Kinetic-Energy and InternalState Distributions.

100,413

1-23654

Semiclassical Calculation of Self-Broadening in O2, N2,
and CO Raman Spectra.
PB92-117050
$\begin{aligned} & 100,461\end{aligned}$ PB92-117050

OCs

Heterodyne Frequency Measurements of $\mathrm{CO}$ and OCS Beyond $2100 \mathrm{~cm}(-1)$

PB91-236984

100,423

CARBON STARS

Evolution of Infrared Carbon Stars.

PB91-202861

100,033

CARBON STEELS

Crack Arrest Fracture Toughness Measurements of Normalized and Inclusion Shape Controlled AAR TC128 Grade B Steel, and Micro-Alloyed, Control-Rolled, and in-
clusion Shape Controlled A 8XX Grade B Steel.
PB91-167346

101,150

Mechanical Properties and Fracture Toughness of AAR TC128 Grade B Steel in the Normalized, and Normalized and Stress Relieved Conditions.

PB92-108901

101,169

\section{CARBON TETRAFLUORTDE}

Thermal Conductivity of Methane and Tetrafluoromethane in the Limit of Zero Density.

100,361

\section{CARRIER DENSITY}

Testing the Accuracy of Calculated Equilibrium Carrier Concentrations in the Presence of Surface Fields. PB91-236729

101,549

CASTINGS

Thin and Composite High-Flux Membranes of Perfluorosulfonated Ion-Exchange Polymer

100,512

CATABOLITE GENE ACTIVATOR PROTEINS

Crystallizing Catabolite Gene Activator Protein with cAMP for Structural Analysis.

PB91-148213

101,299

Crystal Structure of a cAMP-Independent Form of Catabolite Gene Activator Protein with Adenosine Substituted in One of 2 cAMP-Binding Sites.

PB91-190074

101,305

CATALOGS (DOCUMENTATION)

NIST Serial Holdings, 1991.

PB91-171330

100,960

CATALOGS (PUBLICATIONS)

NIST Standard Reference Data Products 1991 Catalog.

PB91-167312

100,952

Publications of the National Institute of Standards and Technology, 1990 Catalog

CATALYSIS

100,011

Frequency Dependence of Catalyzed Reactions in a Weak Oscillating Field

PB91-203927

100,407

Thermodynamics of Industrially Important Enzyme Catalyzed Reactions
PB91-237602

101,311

CATALYSTS

Influence of Sulfur on Methanation over Tungsten(110). 149369

Summary Abstract: The Influence of Sulfur on Methanation over W(110)

PB91-149377

100,301

Polyelectrolyte Stabilized Metal Oxide Hydrosols as Catalysts for the Photooxidation of Water by Zinc Porphyrins.

Chemical and Electronic Properties of $\mathrm{Pd} / \mathrm{SnO} 2(110)$ Model Gas Sensors.

PB91-203075

100,393

Neutron Spectroscopic Evidence for Adsorbed Hydroxyl Species on Platinum Black.

PB91-237636

100,432

\section{CATHODOLUMTNESCENCE}

Spatially and Spectrally Resolved Cathodoluminescence of Hot-Filament Chemical-Vapor-Deposited Diamond Par-

AD-A237 128/4

100,248

Spatially and Spectrally Resolved Cathodoluminescence Measurements of CVD.Grown Diamond Particles and AD-A243 220/1

101,451

Inverse Correlation between the Intensity of Luminescence Excited by Electrons and by Visible Light in Chemical-Vapor-Deposited Diamond Films.

101,531

Cathodoluminescence Measurement of Strained Alumina Single Crystals.

101,094

CD ROM

Overview of the Product Data HyperStandard CD-ROM Prototype.
PB91-132258

101,380

CEILINGS (ARCHITECTURE)

Ceiling Jet in Fires.
PB91-167197 


\section{KEYWORD INDEX}

CHEMICAL DISSOCIATION

PB91-162149

100,112

Application of CCRL Data in the Development of Cement Standards.

100,119

Percolation of Phases in a Three-Dimensional Cement Paste Microstructural Model.

100,529

PB91-187179

100,049

Self-Setting Calcium Phosphate Cements.
PB91-202879

Microstructural Aspects of the Fracture of Hardened
Cement Paste. Cement Paste.

100,133

Guidelines for the Development of Computer Based Models in a Cementitious Materials Modeling Laboratory.
PB91-240804 Integrated Knowledge Systems for Concrete Science and Technology.

100,141

CENTER FOR FIRE RESEARCH

Application of Fire Research.
PB91-149302

100,153

CERAMIC FIBERS

Effect of Thermochemical Treatments on the Strength and Microstructure of SiC Fibers.

101,084

Effect of Thermal Expansion Mismatch on Fiber Pull-Out in Glass Matrix Composites.

101,125

Role of Glassy Interfaces in High Temperature Crack Growth in $\mathrm{SiC}$ Fiber Reinforced Alumina.

101,127

CERAMIC GLAZING

Variations in the Practice of Ceramic Technology in Different Cultures: A Comparison of Korean and Chinese Celadon Glazes.

PB91-237669

101,111

CERAMIC MATERIALS

Thermodynamic and Kinetic Stability of Refractory Materi-

als at Ultra-High Temperatures.
AD-A235 $490 \%$

100,246

CERAMIC MATRIX COMPOSITES

Mechanical Properties of Alumina-Peek Unidirectional Composite: Compression, Shear, and Tension. Effect of Thermochemical Treatments on the Strength

and Microstructure of SiC Fibers.
PB91-194670

101,084

Reduction in Sintering Damage of Fiber Reinforced Com-
posites.
PB91-195362
101,124

Role of Glassy Interfaces in High Temperature Crack

Growth in SiC Fiber Reinforced Alumina.

PB91-203687

101,127

Differential Sintering.

101,128

Magnetic Behavior of Nanocomposites Prepared in a Vitreous Alumina Gel.

PB92-117332

101,131

CERAMIC PRECURSORS

Small-Angle-Scattering Determination of the Microstructure of Porous Silica Precursor Bodies.

101,077

Low Temperature Chemical Route to Precursors of

Boride and Carbide Ceramic Powders.
PB91-175190 101,078

CERAMICS

Strength and Microstructure of Ceramics.
AD-A243 $049 / 4$

101,056

Institute for Materials Science and Engineering, Ceramics: Technical Activities 1990.

PB91-132233

101,057

Application of Analytical Electron Microscopy to Glass-

Bonded Aluminas.
PB91-147389 $\quad 101,060$

Quantitative Determination of Amorphous Content in Ceramic Materials Using X-ray Powder Diffraction. $\quad 101,061$ Graphics Program for Binary and Ternary Ceramic Phase

Diagrams.
PB91-147991 101,063

Effects of Crystal Bonding on Brittle Fracture.

PB91-14822

101,064

Physical and Thermo-Mechanical Properties of Monoclinic Single Crystals.

101,065

Low Temperature Chemical Process for Precursors to Boride and Carbide Ceramics Powders.
PB91-149096 Low Temperature Synthesis of Ceramic Powders for Structural and Electronic Applications

101,067

B91-149104

in the

In situ Measurements of Bridged

101,068

Comparison of Flux Dynamics in Two Samples of

YBa2Cu3O7 with Different Pinning.
PB91-149419

Improved Analysis for Flexural Creep with Application to Sialon Ceramics.

PB91-150037

101,071

Near Net Shape

101,072

National Bureau of Standards-American Ceramic Society Phase Diagram Data Program.

PB91-158774

101,073

Tensile Creep Behavior of Structural Ceramics. PB91-15927

101,075

Superconducting Properties of $\mathrm{Bi} 2-\mathrm{x}$ $\begin{array}{ll}\text { yPbxSnySr2Ca2Cu3Oz. } & 101,076\end{array}$

Small-Angle-Scattering Determination of the Microstructure of Porous Silica Precursor Bodies.

PB91-175075

101,077

Low Temperature Chemical Route to Precursors of Boride and Carbide Ceramic Powders.

PB91-175190

101,078

PB91-178822

101,500

Journal of Research of the National Institute of Standards and Technology. January-February 1991. Volume 96, Number 1 .

Method to Enhance Porosity of Micro-particles. PB91-187328

100,220

Ceramic Wear Maps.

PB91-189498

101,185

100,561 Films on Silicon Carbide.

101,186

Fundamental Condition for Existence of Microcrack Clouds in Monophase Ceramics.

Clouds in Mon

101,082

Indentation Determination of Crack Growth Parameters in Gallium Arsenide.

PB91-190082

101,083

Phase Equilibria of the System Strontium Oxide-Calcium Oxide-Cupric Oxide.

B91-195628

101,085

Standardization of Advanced Ceramics.

101,086

Comparison of Creep Rupture Behavior in Tension and

Comparison of Creep Rupture Behavior in Tension and
Bending.
PB91-202820 101,087

101,087

High Temperature Creep Testing of Ceramics.

PB91-202838

101,088

Chemical and Electronic Properties of $\mathrm{Pd} / \mathrm{SnO} 2(110)$ Model Gas Sensors.

100,393

Small Angle Neutron Scattering Characterization of Microporous Silica.
PB91-203489

101,089

Creep and Reliability of Ceramic Materials at Elevated Temperatures.

101,091

Effect of Selected Chemical Compounds on the Lubrication of Silicon Nitride.

PB91-236711

101,180

Microstructural Control Through Diffusion-Induced Grain Boundary Migration.

101,239

Cyclic Fatigue from Frictional Degradation at Bridging Grains in Alumina.

PB91-236927

101,092

Capacitive Array Sensors for Nondestructive Evaluation.

100,947

New Layered Cuprate Structure-Type, (A1. $\left.x A^{\prime} x\right) 14$ Cu24O41

PB91-237339

101,095

Fourier Transform Infrared Analysis of Ceramic Powders: Quantitative Determination of Alpha, Beta, and Amorphous Phases of Silicon Nitride.

PBous -237628

101,096

Surface Oxidation Kinetics of Si3N4 - 4\% Y2O3 Powders Studied by Bremsstrahlung-Excited Auger Spectroscopy.
PB91-237701 Oxidation Kinetics of Silicon Carbide Whiskers Studied by $X$-ray Photoelectron Spectroscopy.

100,436

PB91-237719 Gallium Arsenide Environmentally Enhanced Fracture of Gallium Arsenide.
PB91-237743 Heat Conductivity of Oxide Coatings by Photothermal Radiometry between 293 and $1173 \mathrm{~K}$

101,112

Test Method for Tensile Creep of Structural Ceramics Test Method for Tensile Creep of Structural Ceramics
Using Flexure Beams.
PB92-116995

101,099

Effect of Green Density and the Role of Magnesium Oxide Additive on the Densification of Alumina Measured by Small-Angle Neutron Scattering.

101,101

PB92-117043

Ceramics: Technical Activities 1991. (NAS-NRC Assessment Panel, February 13-14, 1992).

PB92-126507

101,104

High-Temperature Superconductivity: Abstracts of NIST Publications, 1987-1991.

PB92-126564

101,105

RIUM IONS

Spectrum and Energy Levels of the Xenonlike Ion Ce V.

PB91-175364

CERTIFICATION

Directory of Federal Government Laboratory Accreditation/Designation Programs.

B 91-167379

100,005

NVLAP FY 90 Annual Report.

PB91-206748

100,010

CERIFIED REFERENCE MATERIALS

Appendix: Standard Solutions and Certified Reference PB91-17423

100,194

CESIUM

Structure of a Stage-3 Cs - Graphite Intercalate.

PB91-134957

100,269

Geometric and Electronic Properties of Cs Structures on III-V (110) Surfaces: From $1 D$ and $2 D$ Insulators to $3 D$

PB91-190090

101,517

CGM (COMPUTER GRAPHICS METAFILE)

Collection of Technical Studies Completed for the Computer-Aided Acquisition and Logistic Support (CALS) Program, Fiscal Year 1988. Volume 3 of 3. CGM RegistraPB91-178905

101,344

CHAOS

Global Stability of the Chaotic State Near an Interior Crisis.

PB91-147496

101,611

CHARACTERISTIC IMPEDANCE

Characteristic Impedance Determination Using Propagation Constant Measurement

CHARACTERISTICS

Robot Characterization Testing

PB91-167361

100,775

CHARGE TRANSFER

Nonresonant Charge Transfer in the Threshold Region for (3) $\mathrm{He}(+)+$ (4) $\mathrm{He}($ sub $<$-sup- $>$ ) (3) $\mathrm{He}+$ PB91.149245

101,618

CHELATING AGENTS

Kinetics of Copper Extraction Using (anti)-2-Hydroxy-5-

Nonlybenzophenone Oxime.

100,292

Damage to the Bases in DNA Induced by Hydrogen Peroxide and Ferric lon Chelates.

PB91.187112

101,331

CHEMICAL

Determination of the Optical Constants of Thin Chemical Vapor-Deposited Diamond Windows from 0.5 to $6.5 \mathrm{eV}$. AD-A243 094/0

CHEMICAL ANALYSIS

Methods for the Analysis of Organometallic Compounds in Wastes. 


\section{KEYWORD INDEX}

PB91-202994

100,390

CHEMICAL EQUILIBRIUM

Second Dissociation Constant and $\mathrm{pH}$ of N-2-hydroxyethyjpiperazine- $\mathrm{N}^{\prime}$-2-ethanesulfonic Acid (HEPES) from 0 to PB91-189282

100,196

CHEMICAL INFORMATION SYSTEMS

Technical Activities 1990, Standard Reference Data Program.

100,321

CHEMICAL LABORATORIES

High Purity Chemicals and Clean Rooms for Trace Metal Analysis.

HEMICAL PROPERTIES

Critical Data in Physics and Chemistry
PB91-203455

100,200

101,697

Database and Retrieval System for the NBS Tables of Chemical Thermodynamic Properties.

100,434

CHEMICAL RADIATION EFFECTS

Biomarkers of $\mathrm{OH}$ Radical Damage In vivo.

PB91-236877

101,325

Dosimetry: New Approaches for New Challenges.

MEMICAL REACTION MECHANISMS

Kinetics and Mechanisms of the Gas-Phase Reactions of the NO3 Radical with Organic Compounds. PB92-110220

100,450

CHEMICAL REACTIONS

Enhanced Transport and Liquid Membranes in Biosepara. tions.

101,287

CHEMICAL STABILITY

Stabilities of Substituted Benzyl Radicals: Dissociation Rates of Aminoethylbenzenes, Hydroxyethylbenzenes, and Cyanoethylbenzenes.
PB91-237529

100,430

CHEMICAL YAPOR DEPOSITION

Determination of the Optical Constants of Thin ChemicalVapor-Deposited Diamond Windows from 0.5 to $6.5 \mathrm{eV}$. AD-A243 094/0

100,215

Chemical Vapor Deposited Diamond.

101,106

U.S. Assessment of the New Diamond Technology in Japan.

101,107

CHEMICALS

Opportunities for Innovation: Chemical and Biological Sensors.

100,214

CHEMISORPTION

Adsorption of Water and Oxygen on Ag(110): A Study of the Interactions among Water Molecules, Hydroxyl Groups, and Oxygen Atoms.

100,270

PB91-146985

Initial Stages of Degradation of Superconductor Surfaces $\mathrm{O} 2, \mathrm{H} 2 \mathrm{O}, \mathrm{CO} 2$ and $\mathrm{CO}$ Chemisorption on La2-xSrxCuO4. Geometric and Electronic Properties of Cs Structures on III-V (110) Surfaces: From $1 D$ and $2 D$ Insulators to $3 D$

PB91-190090

101,517

Direct Detection of Atom Vaporization by Laser Resonance Ionization as a Probe of Gas-Surface Chemisorption Mechanisms.

PB91-237032

100,425

CHESAPEAKE BAY

Simultaneous Butyltin Determınations in the Microlayer, Water Column and Sediment of a Northern Chesapeake Bay Marina and Receiving System.

CHIPS

100,918

Material Dependency of Chip-Form Detection Using

Acoustic Emission
PB91-149450

101,216

CHIPS (ELECTRONICS)

1987: The Year of the 386

PB91-158766

100,599

CHIRALITY

New Chemical and Stereochemical Applications of Organoiron Complexe

CHITINS

Chitin-Chitosan Membranes: Separations of Amino Acids and Polypeptides
PB91-134544

CHLORINE

Monitoring the Fate of Chlorine from MSW Sampling

through Combustion
PB91-174631

100,917

Reactivities of Chlorine Atoms and Peroxyl Radicals Formed in the Radiolysis of Dichloromethane. PB91-200782

100,386

CHLORINE IONS

Chloride Ion Diffusion in Low Water-to-Solid Cement Pastes.
PB91-187690

101,138

CHLORINE OXIDES

High-Resolution Infrared Spectrum of the nu1 Band of

PB92-117175

100,463

CHLOROALKANES

Standard Chemical Thermodynamic Properties of Isomer Groups of Monochloroalkanes.

100,258

\section{CHLOROMETHANES}

Polarızed X-ray Emission Studies of Methyl Chloride and the Chlorofluoromethanes.

PB91-174995

100,329

Molecular-Orbital Studies via Satellite-Free $X$-ray Fluores-

cence: $\mathrm{Cl} \mathrm{K}$ Absorption and $\mathrm{K}$-Valence-Level Emission Spectra of Chlorofluoromethanes.

CHLOROMETHYL RADICALS

100,427

Resonance Enhanced Multiphoton lonization Spectroscoy of $\mathrm{CHCl} 2$ and $\mathrm{CDCl} 2$.

100,328

CHLOROTOLUENES

Single Pulse Shock Tube Study of the Reactions of Hydrogen Atoms with Aromatics. 4. Chlorotoluenes. PB91-175133

CHLOROTRIFLUOROMETHANE

Polarized X-ray Emission Studies of Methyl Chloride and The Chlorofluoromethanes.

PB91-174995

100,329

Molecular-Orbital Studies via Satellite-Free $X$-ray Fluorescence: $\mathrm{Cl} \mathrm{K}$ Absorption and $\mathrm{K}$-Valence-Level Emission Spectra of Chlorofluoromethanes.

PB91-237164

100,427

CHROMATES

Trace Determination of $\mathrm{Cr}(\mathrm{VI})$ by LC/AAS with on-Line Preconcentration

100,184

Mechanisms of the Reduction Reactions of $\mathrm{Cr}(\mathrm{VI})$ in the Radiolysis of Acidic Potassium and Silver Dichromate So. utions in the Presence or Absence of Acetic Acid. PB91-236489

100,241

CHROMATIN

Measurement of DNA Base Damage and DNA-Protein Cross-Links in
PB91-187484

101,303

Damage to the DNA Bases in Mammalian Chromatin by Hydrogen Peroxide in the Presence of Ferric and Cupric

PB91-189217

101,336

Modification of DNA Bases in Mammalian Chromatin by Radiation-Generated Free Radicals.

101,338

CHROMATOGRAPHIC ANALYSIS

Capillary Supercritical Fluid Chromatography of Exploives: Investigations on the Interactions between the A alytes, the Mobile Phase and the Stationary Phase.

100,182

CHROMIUM

Trace Determination of $\mathrm{Cr}(\mathrm{VI})$ by LC/AAS with on-Line Preconcentration.

PB91-148148

CHROMIUM 54

Doppler Shift Attenuation Lifetime Measurements in 54) Cr Following Thermal Neutron Capture.

101,656

CHROMIUM ALLOYS

Corrosion, Repassivation and Corrosion Fatigue Behavior of the Surgical Implant Alloy, Co-Cr-Mo.

101,132

CHROMIUM NICKEL ALLOYS

Cryogenic Material Properties of Stainless Steel Tube-toPBange Welds

101,145

CHROMIUM-NICKEL STEELS

Examination of the Excessive Retained Austenite on the Surface of a Section of 17-7 Precipitation Hardening Stainless Steel
PB91-167171

CHRYSOTILE

Visibility of Chrysotile Asbestos in the Scanning Electron Microscope.

101,320

CIGARETTES

Aspects of Smoldering Combustion.

PB91-134510

CIRCUIT INTERCONNECTIONS

Prospects for High Temperature Superconductor - Semiconductor Contacts.

101,545

CIRCUIT SIMULATORS

Experimentally Verified IGBT Model Implemented in the Saber Circuit Simulator

100,822

CITRIC ACID

Thermodynamics of the Krebs Cycle and Related Compounds.

PB91-192468

100,357

CLASSIFICATIONS

Guide to Available Mathematical Software Problem Classification System.

PB91-132175

101,282

CLOCKS

Synchronizing Computer Clocks Using a Local Area Net-

N91-25793/1

100,596

Clock for the Manufacturing Systems Integration Testbed.

PB92-108885

CLOTHING INDUSTRY

Apparel STEP Translator.

100,967

CLUSTER IONS

Multicomponent Cluster lons. 2. Comparative Stabilities of Cationic and Anionic Hydrogen Bonded Networks: Mixed Clusters of Water and Hydrogen Cyanide. PB91-147710

CLUSTER MODEL

Evidence for Surface alpha Particle Clusters in (nat)AG(197)Au from the (e,alpha) Reaction.
PB91-216812

101,705

\section{CLUSTERING}

Ionic Hydrogen Bonds. Part 1. Thermochemistry, Structural Implications, and Role in Ion Solvation

100,400

CMS (CONFIGURATION MANAGEMENT SYSTEM)

Configuration Management Concepts Document. National PDES Testbed Report Series.

100,984

COAL

Bioprocessing of Fossil Fuels Using Hyperthermophilic Archaebacteria.

PB91-162271

100,883

Biological Sulfur Oxidation and Reduction for Coal Sulfur Speciation and
PB91-203323

100,886

COAL GASIFICATION

Effect of Slag Penetration on the Performance of Magnesia Chrome Refractories in Slagging Gasifiers.

100,875

Rapporteur's Summary Materials Development for Coal Gasification.
PB91-158592

100,876

COAL MINING

Task Decomposition and Algorithm Development for Real-Time Motion Control of a Continuous Mining MaPB91-231597

101,357

COAL PREPARATION

Recent Progress in Coal Bioprocessing Research in the United States: Scope and Areas Needing Further Re-

PB91-195339

100,885

COALESCENCE

Applicability of Smoluchowski's Equation with a Constant PB91-148940

100,296

COATINGS (MATERIALS)

Sliding Wear Studies of Nickel-Copper Composition-Modulated Coatings on Steet.

PB91-190025

101,188

Reflection/Absorption FTIR Spectral Characteristics of Thin and Thick Crosslinked Epoxy Films on Steel Sub-

PB91-203711

100,509

Characterization of Epoxide Coatings on Steel by Reflection/Absorption Fourier Transform Infrared Spectroscopy: Ouantitative Study. 
COLD WEATHER CONSTRUCTION

Introduction to $\mathrm{ACl}$ 306.1-87: Specification for Cold Weather Concreting

100,526

COLLAPSE

Investigation of the Collapse of L'Ambiance Plaza. PB91-204057

COLLECTION

NIST Serial Holdings, 1991

PB91-171330

100,960

COLLINEARITY

Nature of Collinearity.

PB91-203497

101,261

COLLISION CROSS SECTIONS

Cross Sections and Related Data for Electron Collisions with Hydrogen Molecules and Molecular Ions.

100,263

Cross Sections for Collisions of Electrons and Photons with Atomic Oxygen.
PB90-244682

100,264

Cross Sections and Swarm Coefficients for $\mathrm{H}+, \mathrm{H} 2+$
$\mathrm{H} 3+, \mathrm{H}, \mathrm{H} 2$, and $\mathrm{H}$-in $\mathrm{H} 2$ for Energies from $0.1 \mathrm{eV}$ to 10 PB90-244690

101,597

COLLISION INDUCED SPECTRA

Notes and Comments on Roundtable Discussion on

Laser-Assisted Collisions and Collision-Induced Spectra.
PB91-203307

COLLISION KERNALS

Measurements of Velocity-Changing Collision Kernels.

PB91-203083

COLORIMETERS

MSW Calorimetry

COLUMNS (PROCESS ENGINEERING)

Evaluation of Column Performance in Liquid Chromatography.

100,199

COLUMNS (SUPPORTS)

Performance of Precast Concrete Beam-to-Column Connections Subject to Cyclic Loading.

\section{PB91-236588}

100,150

Chemical Kinetic Data Base for Combustion Chemistry Part 4. Isobutane

PB90-244534

100,252

Aspects of Smo

100,535

Ignition Characteristics of the Nickel-Based Alloy UNS N07001 in Pressurized Oxygen

100,536

NBS Research Program in Municipal Solid Waste Combustion.

PB91-147264

100,916

Ignition Characteristics of Selected SSME Alloys.

100,544

Letter to Editor of Chemical and Engineering News.
PB91-174847

Molecular Dynamics Study of the Depolymerization Reac-

tion in Simple Polymers.

PB91-187229

100,495

Combustion Calorimeters.

100,340

High-Temperature Thermal Properties of UNS S44004 Using Multivariant Analysis.

101,160

Molecular Dynamics of Initial Events in the Thermal Degradation of Polymers.

PB91-19527

100,503

Applications of the Generalized Global Equivalence Ratio Model (GGERM) for Predicting the Generation Rate and Distribution of Products of Combustion in Two-Layer Fire Environments: Methane and Hexanes.

100,131

Chemical Kinetic Data Base for Combustion Chemistry Part 5. Propene.

PB92-110170 100,446

Theoretical Investigation of Piloted Ignition of Wood.

PB92-112432 100,556

Chemical Kinetic Data Base for Propellant, Combustion. 1. Reactions Involving NO, NO2, $\mathrm{HNO}, \mathrm{HNO} 2, \mathrm{HCN}$ and $\mathrm{N} 2 \mathrm{O}$.

PB92-148071 100,467
Chemical Kinetic Data Sheets for High-Temperature Reactions. Part 2.

100,480

COMBUSTION CONTROL

Advanced sensor development program for the pulp and paper industry. Final report.

\section{COMBUSTION KINETICS}

101,248

Laser-Induced Fluorescence of $\mathrm{CH}$. in a Laminar $\mathrm{CH}_{4} / \mathrm{Air}$ Diffusion Flame: Implications for Diagnostic Measurements and Analysis of Chemical Rates.

PB91-237099

100,554

COMBUSTION PRODUCTS

Reduction of Hydrogen Cyanide Concentrations and Acute Inhalation Toxicity from Flexible Polyurethane
Foam Combustion Products by the Addition of Copper Compounds. Part 3. The Effect of Copper Additives on the Flammability Characteristics of Flexible Polyurethane Foam

PB91-132167

101,140

Species Produced in Fires Burning in Two-Layered and Homogeneous Vitiated Environments

100,542 PB91-157180 Kinetic Properties of the Components of Douglas-Fir and ucts.

100,548

Technical Reference and User's Guide for FAST/FFM Version 3.

\section{COMETARY ATMOSPHERES}

Novel Laser Gain and Time-Resolved FTIR Studies of Photochemistry.

PB91-148809

100,237

COMFORT

Second-Level Post-Occupancy Evaluation Analysis

PB91-147140

100,058

COMMERCIAL BUILDINGS

Air Exchange and Pollutant Concentration Measurements in Two Office Buildings.

100,909

\section{COMMODITIES}

Checking the Net Contents of Packaged Goods. Third Edition, Supplement 2.

PB92-112549

100,171

COMMUNICATION NETWORKS

Interface between Data Terminal Equipment (DTE) and Data Circuit-Terminating Equipment (DCE) for Operation with Packet-Switched Data Networks (PSDN), or between Two DTEs, by Dedicated Circuit. Category: Hardware Standard. Subcategory: Data Transmission.
FIPS PUB 100-1 $\quad 100,672$ Application of OSI Protocols for Plant Information Net-

works.

100,974

Issues in Transparent File Access.

PB91-187831

100,569

Security in ISD

100,718

\section{COMPACT HEAT EXCHANGERS} Heat Transfer and Pressure Drop in a Compact Pin-Fin
Heat Exchanger with Pin Orientation at $18 \mathrm{deg}$ to the FBow Direction

101,050

COMPARTMENTS

Review of Four Compartment Fires with Four Compartment Fire Models.

Ceiling Jet-Driven Wall Flows in Compartment Fires.

PB91-174573

00,098

COMPATIBILITY

Aluminum Alloys for Cryogenic Tanks: Oxygen CompatADility. Volume 1 .

\section{COMPILERS}

Ada Compiler Validation Summary Report. Certificate Number: $901109 \mathrm{~S} 1.11053$, Digital Equipment Corporation VAX Ada, Version 2.2 VAX $8800=>$ VAX 8800.
AD-A233 $961 / 2$
100,607 Ada Compiler Validation Summary Report: DDC International A/S, DACS VAX/VMS to 80186 Bare Ada Cross Compiler System, Version 4.6, VAX 8530 (Host) to Bare Board isBC 186/93A (Host), 901129S1.11079. AD-A234 083/4

100,608

Ada Compiler Validation Summary Report: Certificate Number: 90112951.11112 DDC International A/S DACS PS $/ 2$ Model $80-311=>$ IBM PS $/ 2$ Model $80-311$. AD-A234 118/8

100,609

Ada Compiler Validation Summary Report: Certificate Number: 901129S1.11050 DDC International A/S DACS VAX/VMS Native Ada Compiler System, Version 4.6 VAX $8530=>$ VAX 8530

AD-A234 $323 / 4$

100,610

Ada Compiler Validation Summary Report: Digital Equipment Corporation, VAX Ada, Version 2.2, BAX 8800 (Host) \& VAX MicroVAX II Running VAXELN Version 4.1 (Target), $901109 \mathrm{~S} 1.11054$

100,611

Ada Compiler Validation Summary Report: Certificate Number: 901129S1.11051, DDC International A/S, DACS VAX/VMS to 68020 Bare Cross Compiler System, Version 4.6 MicroVAX 3100 Greater Than or Equal to MotorOla MVME133.

Ada Compiler Validation Summary Report: Cerificate Number: 901129S1.11077 DDC International A/S DACS VAX/VMS to 80186 Bare Ada Cross Compiler System with Rate Monotonic Scheduling, Version 4.6 VAX 8530 $=>$ Bare Board iSBC/03A.

100,613 Ada Compiler Validation Summary Report: Certificate Number: 90112951.11075 DDC International A/S DACS 80386 UNIX V Ada Compil
DRS $300=>$ ICL DRS 300

AD-A234 439/8

100,614

Ada Compiler Validation Summary Report: Certificate Number: 901129S1.11074 DDC International A/S DACS VAX/VMS 6080386 PM Bare Ada Cross Compiler System, Version 4.6 VAX $8530=>$ Bare Board iSBC $386 / 21$

AD-A234 529/6

100,615

Ada Compiler Validation Summary Report. Certificate Number: 910510S1.11161 UNISYS Corporation, UCS Ada, Version 1R1, 2200/600 (Host and Target). Revision. A239 715/

Ada Compiler Validation Summary Report: Certificate Number: 910626S1.11179 U.S. Navy Ada/M, Version 4.0 (OPTIMIZE) VAX $11 / 785=>$ AN/AYK-14 (Bare

AD-A240 $511 / 6$

100,617

Ada Compiler Validation Summary Report: Certificate Number 910705S1.11192 InterAct Corporation InterAct Ada Mips Cross-Compiler System, Release 2.0 MicroVAX 3100 Cluster $=>$ bockheed Sanders STAR MVP

AD-A240 512/4

100,618

Ada Compiler Validation Summary Report: Certificate Number: 910502S1.11158 DDC International A/S, DACS VAX/VMS to 80860 Bare Ada Cross Compiler System. Version 4.6.1 VAX 8530 (Host) to Tadpole Technology PLC TP860M (Bare Board) (Target)

100,619 AD-A240 610/6 Ada Compiler Validation Summary Report: Certificate Number: $910626 S 1.11176$ U.S. Navy, Ada/L, Version 4.0
(Optimize), VAX $11 / 785$ (Host) to AN/UYJ-43 (Single CPU) (Bare Board) (Target).

100,620

Ada Compiler Validation Summary Report: Certificate Number: 910626S1.11174 U.S. Navy, Ada/M, Version 4.0 (Optimize), VAX 8550, Running VAX/VMS Version 5.3 (Host) to AN/UYK-44 (EMR) (Bare Board) (Target) AD-A240 612/2

100,621

Ada Compiler Validation Summary Report: Certificate Number: $910626 S 1.11175$ U.S. Navy Ada/M, Version 4.0 (/Optimize), VAX 8550 (Host) to AN/AYK-14 (Bare Board) (Target).
AD-A240 $613 / 0$

100,622

Ada Compiler Validation Summary Report. Certificate Number: 910705S1.11191, InterACT Corporation InterACT Ada 1750A Compiler System, Release 3.5, MicroVAX 3100 Cluster $=>$ InterACT MIL-STD-1750A In-
struction Set Architecture Simulator, Release 2.3 (Bare Machine).

100,623

Ada Compiler Validation Summary Report. Certificate Number: 910626S1.11178, U.S. Navy Ada/M, Version 4.0 (/OPTIMIZE) VAX $11 / 785=>$ AN/UYK-44 (EMR) (Bare Board)

AD-A240 763/3

100,624

Ada Compiler Validation Summary Report: Certificate Number $910626 \$ 1.11173$ U.S. Navy Ada/L, Version 4.0 (/Optimize) VAX $855=$ AN/UYK-43 (EMR) (Bare

AD-A240 783/1

100,625

Ada Compiler Validation Summary Report: Certificate Number Number 910626S1.11172 U.S. Navy Ada/L, Version 4.0 (/Optimize) VAX $8550=$ AN/UYK-43 (Single CPU) (Bare Board)

100,626

Ada Compiler Validation Summary Report: Certificate Number: 910502S1.11159 DDC International A/S DACS 


\section{KEYWORD INDEX}

Compiler System for EWS-UX/V (Rel 4.0), Version Release $2.1(4.6)$ EWS4800/220 = EWS4800/220. 100,634
AD-A242 609/6 AD-A242609/6 100,634 Ada Compiler Validation Summan Rigital Equipment Corporation DEC Ada, Version 1.0 DECstation $5000 \mathrm{Model} 200=>$ DECstation 5000 Model 200.

AD-A242 896/9

100,635

Programming Languages and Database Language SOL Validated Processor List Including GOSIP Conformance Testing Registers.

PB91.187716

100649

FORTRAN Temporary Program Fixes, FCVS78 Version 2.0, Level 26.

100,651

MUMPS Validation Suite, Version 7.6 (Vax/VMS Backup),

1991.

PB91-507699

100,660

MUMPS Validation Suite, Version 7.6, 1991 (for Micro-

computers). MUMPS Validation Suite, Version 7.6 (ANSI), 1991.
PB91-507715 MUMPS Validation Suite, Version 7.6 (TAR), 1991. PB91-507723

100,663

COMPLEX COMPOUNDS

Microwave Spectrum and Molecular Structure of the N2. H20 Complex.
PB91-203448

100,398

\section{COMPLEXES}

Structures of the $\mathrm{NH} 3-\mathrm{HCCCCH}$ and $\mathrm{H} 2 \mathrm{O}-\mathrm{HCCCCH}$ Complexes by Fourier-Transform Microwave SpectroscoPB. $91-148882$

100,294

Progress on the Determination of Intermolecular Potential Energy Surfaces from High Resolution Spectroscopy.

00,403

Multiple Intermolecular Bend Vibrational Excitation of a Hydrogen Bond: An Extended Infrared Study of OCOHF.
PB91-203703

COMPOSITE FABRICATION

AMRF Composites Fabrication Workstation: A Test Methodology to Measure the Ouality of Thermoplastic Composite Parts.

101,017

COMPOSITE MATERIALS

Influence of Water on the Mechanical Properties of a Glass-Epoxy Matrix Composite.

101,113

Influence of Moisture and Pressure on the Mechanical Properties of a Glass-Epoxy Matrix Composite and a Graphite-Epoxy Matrix Composite.

101,114

Polymer Composite Processing. Industry Workshop (2nd) Held at Gaithersburg, Maryland on May 18, 1990.
PB91-132191 Thermal Conductivity of a Polymide Film between 4.2 and $300 \mathrm{~K}$, with and without Alumina Particles as Filler.
PB91-144402 100,483 PB91-144402
Spin Glass Magnetic Behavior of Iron/Silica Gel Nano

101,117 PB91-159178

Glass-Ceramic Inserts Anticipated for 'Megafilled' Com-
posite Restorations. posite Restoration
PB91-174458

Numerical Simulation and Visualization Models of Stress Wave Propagation Graphite/Epoxy Composites.
PB91-174946 120

Effect of a Free Surface on Stress Distribution in a Bima-

terial Composite
PB91-185116

101,121

Interlaminar Shear Fracture of Laminated Composites.
PB91-187336

Chloride Ion Diffusion in Low Water-to-Solid Cement

Pastes.

PB91-187690

101,138

Role of Glassy Interfaces in High Temperature Crack Growth in SiC Fiber Reinforced Alumina. Change in Magnetic State of $\mathrm{Fe}+$ Silica Gel Nanocomposites Due to Low Temperature Treatment in Ammonia.
PB91-237321 COMPOSITE STRUCTURES

Buckling and Post-Buckling Behavior of Elliptical Plates Part 1. Analysis.
PB92-116573

101,588

Buckling and Post-Buckling Behavior of Elliptical Plates. Part 2. Results.

101.589

COMPOSITION (PROPERTY)

Analysis and Characterization of Commercial Welding

Fluxes.
PB91-162339

101,003

COMPOSITIONAL MAPPING

Strategies for Background Subtraction in Electron Probe Microanalysis X-ray Compositional Mapping. COMPUTATIONAL FLUID DYNAMICS

COMPUTATIONAL FLUID DYNAMICS
Analysis of Laminar Vortex Shedding Behind a Circular Cylinder by Computer-Aided Flow Visualization.
PB91-203000

101,393

Unsteady Laminar Flow in a Circular Tube: A Test of the HERCOL (Hermitian Collocation) Computer Code. 101,395 COMPUTATIONAL GEOMETRY

Computing Delaunay Triangulations for Comet-Shaped Polygons.

101,265

COMPUTATIONAL TESTING

State-of-the-Art of Computational Testing of Mathematical Programming Algorithms.

101,268

COMPUTER-AIDED ACQUISITION AND LOGISTIC

Raster Graphics Conformance Testing.

PB91-167296

101,341

Standard Generalized Markup Language Encoding of the Office Document Architecture Document Application ProPB91-184812

101,345

COMPUTER AIDED DESIGN

NIST STEP Working Form Programmer's Reference. National PDES Testbed Report Series (Revised)

100,998

NIST Express Working Form Programmer's Reference (Revised November 1990). National PDES Testbed Report Series.
PB91-157164

100,964

Engıneering Design Laboratory Guide

CAD Directed Robotic Deburring.

PB91-195172

100,977

Apparel STEP Translator.

100,985

Ap91-216663

100,967

Test Plan for Validating a Context Driven Integrated Model (CDIM) for Sheet Metal Die Design. Nationa PDES Testbed Report Series.

PB92-112523

100,970

Validation Testing Laboratory User's Guide. National PDES Testbed Report Series.

100,971

COMPUTER AIDED MANUFACTURING

Software and Computer Integrated Manufacturing.
PB91-132142

PB91-132142 100,972 Report Series (Revised).

PB91-132183 100,683

Al in Manufacturing: The NBS AMRF as an Intelligent Machine.

100,973

NIST STEP Working Form Programmer's Reference. Na tional PDES Testbed Report Series (Revised)

100,998

Application of OSI Protocols for Plant Information Net-

works.

100,974

CIM, Data and Standardization within the NBS AMRF

PB91-148122 100,975

Innovation in Manufacturing Technology: A View from NBS.

100,976

NIST Express Working Form Programmer's Reference (Revised November 1990). National PDES Testbed Report Series.

SOL3 Support for CALS Applications.

PB91-167262

100,964

Engineering Design Laboratory Guide

100,965

100,977

Automan 2.0: Decision Support Software for Automated Manufacturing Investments. User Manual.

100,978

Error Prevention and Detection in Data Preparation for a

Numerically Controlled Milling Machine.

$\begin{array}{ll}100,980 \\ \text { UB } 91-174938 & \end{array}$ and Future.

PB91-175091

100,981

Concurrent Engineering through Product Data Standards.

PB91-193367

CAD Directed Robotic Deburring.
PB91-195172 100,985

PB91-195172 100,985

Automating Robot Programming in the Cleaning and De-
burring Workstation of the AMRF.
PB91-195495 100,986

tem $\mathrm{Ar}$

Issues in the Design and Implementation of a System Ar-
chitecture for Computer Integrated Manufacturing. chitecture for Computer Integrated Manufacturing.
PB91-203299

Parser That Converts a Boundary Representation into a Features Representation.

PB91-203356

100,988

Development Plan: Application Protocols for Mechanical Parts Production. National PDES Testbed Report Series. Parts Production. National PDES Testbed Report Series.
PB91-222596 Configuration Management of the STEP Documents: Procedures and System Requirements. National PDES Testbed Report Series.

PB91-222620

100,968

Manufacturing Systems Integration Control Entity Interface Document

100,991

Clock for the Manufacturing Systems Integration Testbed. PB92-108885 100,992 Conceptual Architecture for a Mechanical Parts Production System Based on STEP. National PDES Testbed Rept. Series.
PB92-112234

100,993

Manufacturing Systems Integration: Initial Architecture Document.

PB92-112242

100,994

Test Plan for Validating a Context Driven Integrated Model (CDIM) for Sheet Metal Die Design. National PDES Testbed Report Series.

100,970

Mare Island Flexible Manufacturing Workstation. PB92-117027

100,996

Multi-Enterprise Concurrent Engineering through International Standards.

100,997

Validation Testing Laboratory User's Guide. National PDES Testbed Report Series.

100,971

COMPUTER APPLICATIONS

Data Management Standards in Computer-Aided Acquisition and Logistic Support (CALS)

101,339

NIST 'Building Life-Cycle Cost' (BLCC) Program (Version 3.0). User's Guide and Reference Manual.

100,154

Users Guide for RAPID, Version 2.3

100,999 PB91-167403

an ER

Sensing of GMAW

100S-1 Electrod
PB91-174250

101,004

NIST Support for the Computer-Aided Acquisition and Logistic Support (CALS) Program in the Area of Graphics Standards, Calendar Year 1990

PB91-194506

101,348

Scoring Continuous Speech in the DARPA Speech Recognition Program: Part 2

PB91-195396

100,593

Application Portability Profile (APP): The U.S. Government's Open System Environment Profile OSE/1 Version 1.0 .

PB91-201004

$100,69 \dot{2}$

FPETOOL User's Guide.

PB92-108919

100,134

Users' Guide to BREAK1, the Berkeley Algorithm for Breaking Wind

COMPUTER ARCHITECTURE

Issues in the Design and Implementation of a System Architecture for Computer Integrated Manufacturing

OMPUTER ASSISTED INSTRUCTION

Multimedia Courseware in an Open Systems Environment: A Federal Strategy.

PB91-143362

100,039

Interactive Courseware is Leading the Multimedia Move. ment.

100,036

\section{COMPUTER CALCULATIONS}

Assembly Code to Compute Sine and Cosine Using the

PB91-143289 100,638

Development of a Three-Dimensional Finite Element Program for Large Strain Elastic-Plastic Solids.

101.494

Use of Computer Algebra to Locate Critical Loci in Fluid

Mixtures.

101,390

COMPUTER COMMUNICATIONS

Performance of OSI Transport over ACCUNET and IBERPBP

100,563 
Guidelines for the Evaluation of File Transfer, Access and Management Implementations.

100,699

$$
\text { PB92-126580 }
$$

COMPUTER GRAPHICS

Graphical Kernel System (GKS). Pascal Binding; Category: Soltware Standard; Subcategory: Graphics.
FIPS PUB 120-1A
100,636 Graphics Program for Binary and Ternary Ceramic Phase Diagrams.
PB91-147991 101,063

101,063

Raster Graphics Conformance Testing

101,341

Collection of Technical Studies Completed for the Computer-Aided Acquisition and Logistic Support (CALS) Pro-
gram, Fiscal Year 1988. Volume 2 of 3 . Graphics, CGM
MIL-SPEC.

PB91-178897

101,343

Tiled Raster Graphics and MIL-R-28002A: A Tutorial and Implementation Guide.

101,346

NIST Support for the Computer-Aided Acquisition and Logistic Support (CALS) Program in the Area of Graphics Standards, Calendar Year 1990

PB91-194506

101,348

COMPUTER INFORMATION SECURITY

Automated Information Systems Security Program Hand-

book.

100,713

Glossary of Computer Security Terminology.

100,716

Computer Security Bulletin Board System. User's Guide.
PB92-112390

Establishing a Computer Security Incident Response Capability (CSIRC).

COMPUTER NETWORK

Guidelines for the Evaluation of File Transfer, Access and Management Implementations.

PB92-126580

100,699

COMPUTER NETWORKS

Interface between Data Terminal Equipment (DTE) and Data Circuit-Terminating Equipment (DCE) for Operation Two DTEs, by Dedicated Circuit. Category: Hardware Standard. Subcategory: Data Transmission.

Standard. Subca
FIPS PUB 100-1

100,672

Government Open Systems Interconnection Profile

(GOSIP); Category: Hardware and Soltware Standards: Subcategory: Computer Network Protocols.
FIPS PUB 146-1

100.675

Synchronizing Computer Clocks Using a Local Area Net-

work.

100,596

Performance of OSI Transport over ACCUNET and IBER-

PAC

100,563

Stable Implementation Agreements for Open Systems Interconnection Protocols. Version 4, Edition 1, December 1990 .

PB91-171967

100,689

Validation of an OSI Transport Class 4 Simulator.

PB91-187724 100,568

GOSIP Conformance and Interoperation Testing and

Registration.

100,694

Stable Implementation Agreements for Open Systems Interconnection Protocols. Version 4, Edition 1, June 1991 Change Pages (Supplement)

PB92-112200

100,695

Security in ISDN

100,718

Government Open Systems Interconnection Profile Users' Guide, Version 2.

100,696

COMPUTER PERFORMANCE EVALUATION

Hardware Instrumentation Approach for Performance Measurement of a Shared-Memory Multiprocessor. Book Review: 'The Art of Computer Systems Performance Analysis' by R. Jain.

PB92-117068

100,719

COMPUTER PROGRAM PORTABILITY

Multimedia Courseware in an Open Systems Environment: A Federal Strategy.

100,039

Application Portability Profile (APP): The U.S. Government's Open System Environment Profile OSE/1 Version 1.0.
PB91-201004

100,692

Interactive Video Software Portability: Migrating a DOS Application to POSIX.

100,670

COMPUTER PROGRAM RELIABILITY

Proceedings of the Workshop on High Integrity Software. Held in Gaithersburg, MD. on January 22-23, 1991.
PB92-109040 100,667

COMPUTER PROGRAM VERIFICATION

Software Verification and Validation: An Overview.
PB91-237685

100,658

COMPUTER PROGRAMMING

Algorithm Design for Large-Scale Computations.

PB91-149336 100,640

DEOSOL and ELLPACK: Problem-Solving Environments for Partial Differential Equations.

100,645

Portable Vectorized Software for Bessel Function Evalua. tion. 216598

100,652

NIST "LATTICE: A Program to Analyze Lattice Relationships. Spring 1991 Version.

PB92-108877

101,561

COMPUTER PROGRAMS

PLOTMD: An Interactive Program to Modify Molecular Plots on a Graphics Terminal.

COMPUTER SCIENCE \& TECHNOLOGY

100,373

Ada Compiler Validation Summary Report. Certificate Number: 901109S1.11053, Digital Equipment Corporation VAX Ada, Version 2.2 VAX $8800=>$ VAX 8800 AD-A233 $961 / 2$

100,607

Ada Compiler Validation Summary Report: DDC International A/S, DACS VAX/VMS to 80186 Bare Ada Cross Compiler System, Version 4.6, VAX 8530 (Host) to Bare (Host), 901129 S 111079 .

AD-A234 083/4

100,608

Ada Compiler Validation Summary Report: Certificate Number: 901129S1.11112 DDC International A/S DACS 80386 DMS/OS Ada Compiler System, Version 4.6 IBM PS $/ 2$ Model 80-311 = > IBM PS/2 Model 80-311.

AD-A234 118/8

100,609

Ada Compiler Validation Summary Report: Certificate Number: 901129S1.11050 DDC International A/S DACS VAX/VMS Native Ada Compiler System, Version 4.6 VAX $8530=>$ VAX 8530

AD-A234 323/4

100,610

Ada Compiler Validation Summary Report: Digital Equipment Corporation, VAX Ada, Version 2.2, BAX 8800 (Host) t VAX MicroVAX II Running VAXELN Version 4.1 (Target), $901109 \mathrm{~S} 1.1105$

100,611

Ada Compiler Validation Summary Report: Certificate Number: 901129S1.11051, DDC International A/S, DACS VAX/VMS to 68020 Bare Cross Compiler System, Version 4.6 Micro VAX

AD-A234 380/4

100,612

Ada Compiler Validation Summary Report: Certificate Number: 901129S1.11077 DDC International A/S DACS VAX/VMS to 80186 Bare Ada Cross Compiler System with Rate Monotonic Scheduling. Version 4.6 VAX 8530 $\Rightarrow$ Bare Board iSBC/03A.

AD-A234 438 $/ 0$

100,613

Ada Compiler Validation Summary Report: Certificate Number: $90112951.11075 \mathrm{DDC}$ International A/S DACS DRS300 $=\underset{\text { AD-A234 }}{239 / 8}$ ICL DRS300.

100,614

Ada Compiler Validation Summary Report: Certificate Number: 901129S1.11074 DDC International A/S DACS VAX/VMS 60 80386 PM Bare Ada Cross Compiler System, Version 4.6 VAX $8530=>$ Bare Board iSBC $386 / 21$,

100,615

Ada Ada Compiler Validation Summary Report. Certificate Ada, Version 1R1, 2200/600 (Host and Target). Revision.
AD-A239 715/6

Ada Compiler Validation Summary Report: Certificate Number: 910626S1.11179 U.S. Navy Ada/M, Version 4.0 (OPTIMIZE) VAX $11 / 785=>$ AN/AYK-14 (Bare

AD-A240 511/6

100,617

Ada Compiler Validation Summary Report: Certificate Number 910705S1.11192 InterAct Corporation InterAct Ada Mips Cross-Compiler System, Release 2.0 MicroVAX R3000/R3010 board (Bare Machine).

$\mathrm{AD}-\mathrm{A} 240$ 512/4

100,618

Ada Compiler Validation Summary Report: Certificate Number: $910502 S 1.11158$ DDC International A/S, DACS VAX/VMS to 80860 Bare Ada Cross Compiler System,
Version 4.6.1, VAX 8530 (Host) to Tadpole Technology PLC TP860M (Bare Board) (Target)

100,619

Ada Compiler Validation Summary Report: Certificate Number: 910626S1.11176 U.S. Navy, Ada/L, Version 4.0 (/Optimize), VAX $11 / 785$ (Host) to AN/UYJ-43 (Single CPU) (Bare Board) (Target).

100,620

Ada Compiler Validation Summary Report: Certificate Ada Compiler Validation Summary Report: Certificate Number: 910626S1.11174 U.S. Navy, Ada/M, Version 4.0
(/Optimize), VAX 8550, Running VAX/VMS Version 5.3 ('Optimize), VAX 8550, Running VAX/NMS
(Host) to AN/UYK.44 (EMR) (Bare Board) (Target). (Host) to AN/UYK-44 (EMR) (Bare Board) (Target).
AD-A240 612/2 Ada Compiler Validation Summary Report: Certificate Number: $910626 \mathrm{~S} 1.11175$ U.S. Navy Ada/M, Version 4.0 (/Optimize), VAX 8550 (Host) to AN/AYK-14 (Bare Board) (Target).
AD-A240 613/0

100,622

Ada Compiler Validation Summary Report. Certificate
Number: $910705 S 1.11191$, InterACT Corporation Inter-

ACT Ada 1750A Compiler System, Release 3.5, Microstruction Set Architecture Simulator, Release 2.3 (Bare Machine)

100,623

Ada Compiler Validation Summary Report. Certificate Number: $910626 S 1.11178$, U.S. Navy Ada/M, Version 4.0 (/OPTIMIZE) VAX $11 / 785=>$ AN/UYK-44 (EMR) (Bare Board).
AD-A240 763/3

100,624

Ada Compiler Validation Summary Report: Certificate Number $910626 S 1.11173$ U.S. Navy Ada/L, Version 4.0
(/Optimize) VAX $855=$ AN/UYK-43 (EMR) (Bare

AD-A240 783/1

100,625

Ada Compiler Validation Summary Report: Certificate Number Number $910626 \mathrm{~S} 1.11172$ U.S. Navy Ada/L, Version 4.0 (/Optimize) VAX $8550=$ AN/UYK -43 (Single CPU) (Bare Board)

AD-A240 784/9

100,626

Ada Compiler Validation Summary Report: Certificate Number: 910502S1.11159 DDC International A/S DACS Sun-3/SunOS to 68030 Bare Ada Cross Compile System, Version 4.6.4, MRI IEEE 695 (BASIC-MODE) Sun-3/50 = Motorola MVME 143 Board $(68030 / 68882)$.
AD-A240 785/6 Ada Compiler Validation Summary Report. Certificate Number: 910502S1.11160 DDC International A/S DACS Sun-3/SunOS to 68030 Bare Ada Cross Compiler System, Version 4.6.4. MRI IEEE 695 (SECURE MODE)
Sun-3/50 $>>$ Motorola MVME143 Board (68030/

AD-A240 849/0

100,628

Ada Compiler Validation Summary Report. Certificate Number: 910626S1.11177 U.S. NAVY Ada/L, Version 4.0
(/OPTIMIZE) VAX $11 / 785=>$ AN/UYK-43 (EMR) (Bare Board).

AD-A240 850/8

100,629 Ada Compiler Validation Summary Report: Certificate
Number: $910517 S 1.11163$ U.S. Navy, AdaVAX, Version 5.0, (/NO OPTIMIZE) VAX 8350 (Host and Target).

AD-A242 268/1

100,630

Ada Compiler Validation Summary Report: Certificate Number: $910517 S 1.11162$ U.S. Navy, AdaVAX, Version 5.0, (/OPTIMIZE) VAX 8600 (Host and Target).

AD-A242 269/9

100,631

Ada Compiler Validation Summary Report: Certificate Number: 910517S1.11165 U.S. Navy, AdaVAX, Version 5.0 (/NO-OPTIMIZE), VAX 11/785 (Host and Target)

AD.A242 270/7

100,632

Ada Compiler Validation Summary Report: Certificate Number: 910517S1.11164 U.S. Navy, AdaVAX, Version 5.0, (/OPTIMIZE),
AD-A242 273/1

100,633

Ada Compiler Validation Summary Report: Certificate Number 910918S1.11216, NEC Corporation, NEC Ada Compiler System for EWS-UX/V (Rel 4.0), Version Release 2.1(4.6) EWS4800/220 = EWS4800/220 AD-A242 609/6

100,634

Ada Compiler Validation Summary Report. Certificate Number: 911025 S1.11226. Digital Equipment Corporation DEC Ada, Version 1.0 DECstation 5000 Model $200=$ DECstation 5000 Model 200.

AD-A242 896/9

100,635

Congressional Districts of the United States. Category: Federal General Data Standard, Representations and Codes.

100,679

Guideline: American National Dictionary for Information Systems. Category: Software; Subcategory: Documentation.

FIPS PUB 11-3 


\section{KEYWORD INDEX}

FIPS PUB 160

100,676

Electronic Data Interchange (EDI). Category: Software Standard. Subcategory: Electronic Data Interchange.
FIPS PUB 161 Federal Information Processing Standards Publication.

FIPS SET 1991 Synchronizing Computer Clocks Using a Local Area Net100,596

N91-25793/1 100,972

Software and

NIST Working Form for STEP: National PDES Testbed Report Series (Revised)

100,683

Use of the IRDS Standard in CALS (Revised).

PB91-132209

100,684

Overview of the Product Data HyperStandard CD-ROM

Prototype.

101,380

Developing Federal Soltware Standards: A New Direc-

PB91-134536

100,637

Information Systems Engineering Program.

100,685

Al in Manufacturing: The NBS AMRF as an Intelligent

Machin

100,973

Performance of OSI Transport over ACCUNET and IBER-

100,563

PB91-134825

sing the

Assembly Code

PB91.143289

100,638

Multimedia Courseware in an Open Systems Environ ment: A Federal Strategy.

100,039

NIST STEP Working Form Programmer's Reference. $\mathrm{Na}$ tional PDES Testbed Report Series (Revised).

PB91-144378

100,998

Working Implementation Agreements for Open Systems I

PB91-144444 Protols. 100,686

Application of OSI Protocols for Plant Information Net-

PB91-147595 100,974

Feasibility of Using Knowledge-Based Systems for Aiding inventors.

PB91-147728

100,895

Interactive Courseware is Leading the Multimedia Move-

100,036

Conformance Verification of FDDI Stations.

100,747

Bibliography of Selected Computer Security Publications, January 1980-October 1989.

100,704

UNIX Expert: A Prototype Knowledge-Based Software Development Workstation.

PB91-149179 100,639

NIST Express Working Form Programmer's Reference

(Revised November 1990). National PDES Testbed Report Series

PB91-157164

100,964

Proceedings of the Object-Oriented Database Task Group Workshop, Tuesday October 23, 1990, Chateau Laurier Hotel, Ottawa, Canada.

Specifications for a Federal Information Processing Standard Data Dictionary System.

PB91-158758

100,687

1987: The Year of the 386 .

PB91-158766

100,599

Data Management Standards in Computer-Aided Acquisi-

tion and Logistic Support (CALS).

PB91-158832

101,339

Choosing a Name for Your Computer

100,600

Features and Facilities of Estelle.

100,688

Algorithm and Computer Program for the Calculation of Algorithm and Computer Program for the Calculation of Envelope Curves.

101,414

Agency Exploits Flexibility of Software in Moving Info.
PB91-159160

Thwarting the Hackers.

100,705

Hardware Instrumentation Approach for Performance Measurement of a Shared-Memory Multiprocessor PB91-159319

100,601

Proceedings of the Object-Oriented Database Task Group Workshop. Held in Atlantic City, New Jersey on May 22, 1990

100,643

SOL3 Support for CALS Applications.

100,965

DISCOUNT-A Program for Discounting Computations in Life-Cycle Cost Analyses. User's Guide and Reference Manual.
PB91-167288

100,897

Raster Graphics

Functional Benchmarks for Fourth Generation Languages.

100,644

Automan 2.0: Decision Support Software for Automated Manufacturing Investments. User Manual.

100,978

Stable Implementation Agreements for Open Systems Interconnection Protocols. Version 4, Edition 1, December 1990.

PB91-171967

100,689

Designing Data Entity Naming Conventions.

100,646

Collection of Technical Studies Completed for the Computer-Aided Acquisition and Logistic Support (CALS) Program. Fiscal Year 1988. Volume 1 of 3. Text, Security and Data Management.

PB91-178889

101,342

Collection of Technical Studies Completed for the Computer-Aided Acquisition and Logistic Support (CALS) Program, Fiscal Year 1988. Volume 2 of 3. Graphics, CGM PBO1-178897

101,343

Collection of Technical Studies Completed for the Computer-Aided Acquisition and Logistic Support (CALS) Program, Fiscal Year 1988. Volume 3 of 3 . CGM Registra.

PB91-178905

101,344

Center for Electronics and Electrical Engineering Technical Publication Announcements Covering Center Programs, July to September 1990, with 1991 CEEE Events Calendar.

PB91-184754

100,852

Standard Generalized Markup Language Encoding of the Office Document Architecture Document Application Profile.

PB91-184812

101,345

VolksGrapher: a FORTRAN Plotting Package User's Guide, Version 3.0.

PB91-185108

100,647

Guide to Design, Implementation and Management of Distributed Databases.

PB91-187567 100,648

Tiled Raster Graphics and MIL-R-28002A: A Tutorial and mplementation Guide.

101,346

Programming Languages and Database Language SQL Validated Processor List Including GOSIP Conformance Testing Registers.

PB91-187716

100,649

Validation of an OSI Transport Class 4 Simulator.

PB91-187724 100,568

Computer Security: Selected Articles.

PB91-18774

100,706

Government Document Processing Requirements Report.

National Aeronautics and Space Administration's (NASA)

National Aeronautics and Space Administration's (NASA)
Automated Information Security Handbook.
PB91-187781

Issues in Transparent File Access.

PB91-187831

Public-Key Cryptography. Computer Security

187864

100,569

Difficulties in Parsing SGML.

PB91-189456

Document Architecture for Open Systems.

PB91-189522

100,650

Conformance Tester for X.25 DTE Implementations.

PB91-189894 100,602

Phone-Mediated Word Alignment for Speech Recognition Evaluation.

PB91-189910

NIST Support of the CALS Program: 1990 Synopsis.

PB91-19382

Configuration Management Concepts Document. National PDES Testbed Report Series.

100,984

NIST Support for the Computer-Aided Acquisition and Lo-

gistic Support (CALS) Program in the Area of Graphics Standards, Calendar Year 1990.

101,348

PLOTMD: An Interactive Program to Modify Molecular Plots on a Graphics Terminal.

100,373

Use of Computer Algebra to Locate Critical Loci in Fluid Use of Computer Algebra to Locate Critical Loci in Fluid
Mixtures.
PB91-195263

101,390

Scoring Continuous Speech in the DARPA Speech Recognition Program: Part 2

PB91-195396

100,593

Stable Implementation Agreements for Open Systems Interconnection Protocols. Version 4, Edition 1, March 1991. Change Pages (Supplement)

100.691

Application Portability Profile (APP): The U.S. Government's Open System Environment Profile OSE/1 Version 1.0 .

PB91-201004

100,692

TRAN Temporary Program Fixes, FCVS78 Version 2.0, Level 26.

100,651

National Computer Systems Laboratory Annual Report,

PB91-201822

100,603

How the Government Shapes UNIX Standards.

100,693

Issues in the Design and Implementation of a System Architecture for Computer Integrated Manufacturing. PB91-203299

100,987

Parser That Converts a Boundary Representation into a Features Representation.

PB91-203356

100,988

Static Analysis Tools for Software Security Certification. PB91-203380 100,710 Numeric Database for Tribology: Format and Application

PB91-203943

101,045

Data Encryption Standard: Past and Future.

PB91-204107 100,711 Electronic Data Interchange in Message Handling Sys-

PB91-216622

Apparel STEP Translator.

PB91-216663

100,571 Shop. Held on April 9-10, 1991.

Automated Information Systems Security Program Hand-

PB91-222638

100,713

Computer Implementation of a Discrete Set Algebra.

PB91-231571

100,654

Functional Meta-Structure for Hypertext Models and Sys-

PB91-236687

100,655

Linking Information to Objects: A Hypertext Prototype for Numismatists

100,657

Geometric Reasoning for Constructing 3D Scene Descriptions for Images.

100,714

Software Verification and Validation: An Overview.

PB91-237685

100,658

Database and Retrieval System for the NBS Tables of Chemical Thermodynamic Properties.

Depot: A Framework for Sharing Software Installation Across Organizational and Unix Platform Boundaries. PB91-240754

100,659

Guidelines for the Development of Computer Based 
Stable Implementation Agreements for Open Systems Interconnection Protocols. Version 4, Edition 1, June 1991 Change Pages (Supplement)

100,695

Glossary of Computer Security Terminology.

100,716

Proceedings of the Forum on Standards for High Integrity Software (DOD, Government, Industry). Held in Gaithersburg, Maryland on June 28, 1991.

100,668

Proposed Testing Methodology for STEP Application Protocol Validation. National PDES Testbed Report Series. PB92-112374. National PDES Testbed Report Series.
100,969 Computer Security Bulletin Board System. User's Guide. PB92-112390 100,717 Prototyping the IRDS: An Airport Application. PB92.112580

101,740

Security in ISDN.

PB92-116391 100,718

Test Methods for Optical Disk Media Characteristics (for

$356 \mathrm{~mm}$ Ruggedized Magnelo-optic Media).
PB92-116409

100,605

Software Reengineering: A Case Study and Lessons Learned.

100,669

Interactive Video Software Portability: Migrating a DOS Application to POSIX

PB92-117308

100,670

Government Open Systems Interconnection Profile

Users' Guide, Version 2.

Validation Testing Laboratory User's Guide. National PDES Testbed Report Series.

PB92-123090

100,971

Monitoring and Reporting Techniques for Error Rate and Error Distribution in Optical Disk Systems. Establishing a Computer Security Incident Response Capability (CSIRC).

100,720

Stable Implementation Agreements for Open Systems Interconnection Protocols. Version 4, Edition 1, Change Pages September 1991. Output from the September 1991 OSI Implementors Workshop. Held in Gaithersburg, Maryland.

PB92-126408 100,697

Working Implementation Agreements for Open Systems Interconnection Protocols.

PB92-126523
3480 Type Tape Cartridge: Potential Data Storage Risks

and Care and Handling Procedures to Minimize Risks.

PB92-126549

101,381

Guide to Schema and Schema Extensibility.

100,955

Guidelines for the Evaluation of File Transfer, Access and Management Implementations.

PB92-126580

100,699

Validated Products List (Cobol, Fortran, ADA, Pascal,

MUMPS, SQL).

100,671

COMPUTER SECURITY

Bibliography of Selected Computer Security Publications, January 1980 -October 1989

PB91-148486

100,704

Thwarting the Hackers.

100,705

Collection of Technical Studies Completed for the Computer-Aided Acquisition and Logistic Support (CALS) Program. Fiscal Year 1988. Volume 1 of 3 . Text, Security and Data Management

PB91.178889

101,342

Computer Security: Selected Articles.

100,706

National Aeronautics and Space Administration's (NASA) Automated Information Security Handbook.

PB91-187781

100,707

Public-Key Cryptography. Computer Security.

100,708

Static Analysis Tools for Software Security Certification.

PB91-203380 100,710

Standard Security Label for GOSIP: An Invitational Work-

shop. Held on April 9-10, 1991.

PB91-216671

100,712

Automated Information Systems Security Program Hand-

book

100,713

Proceedings of the Forum on Standards for High Integrity Software (DOD, Government, Industry). Held in Gaithers. burg, Maryland on June 28, 1991.

PB92-112267

100,668

Security in ISDN

100,718

COMPUTER SOFTWARE

Graphical Kernel System (GKS); Category: Software Standard; Subcategory: Graphics.

100,673

Graphical Kernel System (GKS). Ada Binding; Category:

Software Standard; Subcategory: Graphics.
FIPS PUB 120-18

100,674

Federal Information Processing Standards Publication

FIPS SET $1991 \quad 100,680$

Software and Computer Integrated Manufacturing.

PB91-132142 100,972

Guide to Available Mathematical Software Problem Classification System

101,282

Developing Federal Software Standards: A New Direc-

PB91-134536

100,637

Agency Exploits Flexibility of Software in Moving Info.

100,642

National Computer Systems Laboratory Annual Report,

1990.

PB91-201822

100,603

Static Analysis Tools for Software Security Certification.

Depot: A Framework for Sharing Software Installation Across Organizational and Unix Platform Boundaries.

PB91-240754

100,659

Proceedings of the Workshop on High Integrity Software. Held in Gaithersburg, MD. on January 22-23, 1991

PB92-109040

100,667

Proceedings of the Forum on Standards for High Integrity Software (DOD, Government, Industry). Held in Gaithersburg, Maryland on June 28, 1991

100,668

COMPUTER SOFTWARE MANAGEMENT

Software Verification and Validation: An Overview.

COMPUTER STORAGE DEVICES

Test Methods for Optical Disk Media Characteristics (for $356 \mathrm{~mm}$ Ruggedized Magneto-optic Media)

100,605

3480 Type Tape Cartridge: Polential Data Storage Risks, and Care and Handling Procedures to Minimize Risks.
PB92-126549

\section{COMPUTER SYSTEMS HARDWARE}

Closure and Precision in Level-Index Arithmetic PB91-147652

100,597

COMPUTER SYSTEMS PERFORMANCE

Workloads, Observables, Benchmarks and Instrumenta-

PB91-147660

100,598

Book Review: 'The Art of Computer Systems Performance Analysis' by R. Jain.

\section{COMPUTER SYSTEMS PROGRAMS}

100,719

Data-Reduction and Analysis Procedures Used in NIST's Thermomechanical Processing Research.

100,925

Clock for the Manufacturing Systems Integration Testbed.
PB92-108885

COMPUTER VISION

New Approach to Vision and Control for Road Following.
PB91-157156

High Precision Gaging with Computer Vision Systems.
PB91-174623

100,979 Form Measurement.

PB91-202978

101,043

Application of the PIPE Image Processing Machine to Scanning Microscopy.

101,034

Geometric Reasoning for Constructing 3D Scene Descriptions for Images
PB91-237677

\section{COMPUTERIZED SIMULATION}

Computer Program for Simulation of HVAC/Lighting Interactions: Initial Report

PB91-144386

100,889

Using the Harvard/NIST Mark VI Fire Simulation.

PB91-144394

100,095

Simulation of an Evaporator with Nonuniform One-Dimensional Air Distribution

Numerical Simulation and Visualization Models of Stress Wave Propagation Graphite/Epoxy Composites.
PB91.174946 Computerization of a Thermomechanical Processing Re. search System

101,583

Guidelines for the Development of Computer Based Models in a Cementitious Materials Modeling Laboratory.
PB91-240804 Journal of Research of the National Institute of Stand-

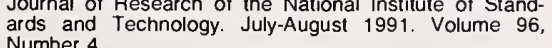

PB92-110261

100,762

Numerical Modeling of Silicon Photodiodes for High-Ac-

curacy Applications. Part 1. Simulation Programs
PB92-110295

100,763

Numerical Modeling of Silicon Photodiodes for High-Accuracy Applications. Part 2. Interpreting Oxide-bias Ex. periments.

PB92-110303

100,764

Numerical Modeling of Silicon Photodiodes for High-Ac. curacy Applications. Part 3 . Interpolating and Extrapolating Internal Quantum-Efficiency Calibrations.

PB92-110311

100,765

Three-Dimensional Simulations of High-Resolution Photoresist Processing.

PB92-116466

100,818

COMPUTERIZED TOMOGRAPHY

Mossbauer Imaging.

COMPUTERS

Choosing a Name for Your Computer.
PB91-158899

101,294

National Computer Systems Laboratory Annual Report, 1990.
PB91-201822

100,603

CONCENTRATION (COMPOSITION)

Effects of Global Density Ratio on the Centerline Mixing Behavior of Axisymmetric Turbulent Jets.

100,014

Reynolds Number Effects on the Mixing Behavior of Axisymmetric Turbulent Jets.

PB92-117209

100,015

Relative $\mathrm{H}$-Atom and O-Atom Concentration Measure. ment in a Laminar, Methane/Air Diffusion Flame.

100,559

CONCRETE CONSTRUCTION

Introduction to $\mathrm{ACl}$ 306.1-87: Specification for Cold Weather Concreting.

100,526

CONCAETE PAVEMENTS

Uniaxial Tensile Tests to Measure the Bond of In situ

Concrete Overlays.
PB92-116300

100,533

CONCRETE STRUCTURES

Preliminary Dynamic Analyses of the Ministry of Agriculture Building.
PB91-134668

\section{CONCRETES}

Techniques to Observe the Fracture Zone in Mortar and Concrete.

PB91-134189 100,521

Guide to the Use of the Cement and Concrete Research Remote Bulletin Board System (RBBS) Computer

100,524

Pozzolan Programs of the Cement and Concrete Refer-

$\begin{array}{ll}\text { ence Laboratory. } & 100,525 \\ \text { PB91-149922 }\end{array}$

Rate Constant Functions for Strength Development of Concrete.

PB91.175117

100,527

Characterization of Field Concrete.

PB91-178863

100,528

Impact-Echo: A New Method for Inspecting Construction Materials.

Integrated Knowledge Systems for Concrete Science and Technology.

100,141

Predicting the Remaining Service Life of Concrete.

PB92-126598

101,371

CONCURRENT ENGINEERING

Concurrent Engineering through Product Data Standards. PB91-193367 100,982

Multi-Enterprise Concurrent Engineering through International Standards

PB92-123058

100,997

CONDUCTION

Space Marching Difference Schemes in the Nonlinear Inverse Heat Conduction Problem 


\section{KEYWORD INDEX}

CONSTANTS

Improved Fits for the Vibrational and Rotational Constants of Many States of Nitrogen and Oxygen.

100,469

CONSTRUCTION JOINTS

Performance of 1/3-Scale Model Precast Concrete

Beam-Column Connections Subjected to Cyclic Inelastic Loads. Report No. 2.

PB91-222570

100,149

CONSTRUCTION MANAGEMENT

Economic Analysis for Military Construction (MILCON) Design. Concepts, Techniques, and Applications for the Analyst. Instructor's Manual.

AB92-112440

101,349

Economic Analysis for Military Construction (MILCON) Design. Concepts, Techniques, and Applications for the Analyst. Student's Manual.

PB92-112457

101,350

CONSTRUCTION MATERIALS

Meeting the Challenges of a Global Market - Construction Standards. Testing and Certification.

100,090

Activities of the Construction Materials Reference LabActivities of the Construction Materials Reference Lab-
oratories Related to Laboratory Accreditation.

100,531

Experimental Study of the Pyrolysis Rate of a Poly(methy methacrylate) (PMMA) Wall Panel in a Reduced-Scale Enclosure.

PB91-237412

100,555

Analysis of Moisture Accumulation in a Wood Frame Wall Subjected to Winter Climate.

100,137

Large-Scale Validation of Bench-Scale Fire Toxicity Tests.

$\begin{array}{lr}\text { PB92-116458 } & 100,139 \\ & \end{array}$

Impact-Echo: A New Method for Inspecting Construction

PB92-11655

100,140

Test Method for Tensile Creep of Structural Ceramics Using Flexure Beams.

NTAINERS

Stability of Aqueous Inorganic Lead Solutions in Polycarbonate Containers.

PB92-126499

101,099

CONTINUOUS MINING MACHINES

Task Decomposition and Algorithm Development for Real-Time Motion Control of a Continuous Mining Machine.

PB91-231597

101,357

CONTINUOUS WAVE LASERS

CW Dye Lasers.
PB92-116896

CONTOURS

Non-Linear Contour-to-Grid Digital Interpolation

PB91-147678

101,438

CONTRACTS

Feasibility of Using a Multiple Award Schedule for Specifying Paints in Government Painting Contracts. PB92-12648

100,211

CONTROL SYSTEMS

New Approach to Vision and Control for Road Following.

TROI User's Guide.

PB91-157214

101,026

Control of Flexible Robot Arm.

101,032

NASREM Robot Control System and Testbed.

101,033

Control Structure for Multi-Tasking Workstations.

PB91-203737

100,989

Generation of

101,036

Manufacturing Systems Integration Control Entity Interface Document

100,991

Performance Measures of a Robotic Micropositioner.

PB92-117019

101,037

CONTROL SYSTEMS DESIGN

Sensing of GMAW Droplet Transfer Modes Using an ER

100S-1 Electrode.

101,004

Task Decomposition and Algorithm Development for Real-Time Motion Control of a Continuous Mining Machine.

PB91-231597

101,357

CONVECTION

Fiber-Optic Fluorescence Array to Study Free Convection in Porous Media.

101,407

Boussinesq Algorithm for Enclosed Buoyant Convection in Two Dimensions

100,157

Effect of Gravity Modulation on Thermosolutal Convection in an Infinite Layer of Fluid.

101,396

CONVERGENCE

Convergence Behavior of Trajectories for Linear Programming.
PB91-204198

101,270

ONVERSION TABLES

Guide for the Use of the International System of Units: The Modernized Metric System.

100,962

CONVOLUTION

Some Algorithms for Approximating Convolutions. 101,258

COOL STARS

Radio-Continuum Observations of a Variety of Cool Stars.
100,027

Some Concluding Thoughts for Cool Stars VI.

PB91-175034

100,030

COOLING

Sprinkler/Hot Layer Interaction.

PB91-206680

100,129

COOLING LOAD

Measurement and Evaluation of Lighting/HVAC Interaction. 206706

COORDINATE MEASURING MACHINES

100,892

Review of Current Geometric Tolerancing Theories and Inspection Data Analysis Algorithms.

101,016

Statistical Process Control Tactics for Coordinate Measuring Machines.

uring Machines.

101,042

COPPER

Cryogenic Properties of Copper (Wall Chart)

PB91-148189

101,473

Measuring the

101,136

Copper-Ion-Dependent Damage to the Bases in DNA in the Presence of Hydrogen Peroxide.

PB91-187104

101,330

Damage to the DNA Bases in Mammalian Chromatin by Hydrogen Peroxide in the Presence of Ferric and Cupric PB91-189217

101,336

Creep of Copper: 4 to $295 \mathrm{~K}$

101,233

Elastic Constants of a Tungsten-Particle Copper-Matrix Composite.

101,234

COPPER ALLOYS

Cryogenic Properties of Copper (Wall Chart). $\quad 101,473$
PB91-148189

Sliding Wear Studies of Nickel-Copper Composition-Modulated Coatings on Steel.

PB91-190025

101,188

COPPER COMPLEXES

Kinetics of Copper Extraction Using (anti)-2-Hydroxy-5Nonlybenzophenone Oxime.

COPPER COMPOUNDS

Reduction of Hydrogen Cyanide Concentrations and Acute Inhalation Toxicity from Flexible Polyurethane Foam Combustion Products by the Addition of Coppe Compounds. Part 3. The Effect of Copper Additives on the Flammability Characteristics of Flexible Polyurethane

PB91-132167

101,140

COPPER IONS

Energy Levels of Copper, Cu I through Cu XXIX.
PB90-244666

Relative Populations of Excited Levels within the Ground Configuration of Si-Like Cu, Zn, Ge, and Se lons.

PB91-158618

101,443

Spectral Data and Grotrian Diagrams for Highly lonized Copper, Cu X-Cu XXIX

Antiferromagnetic Order of $\mathrm{Cu}$ in Sm2CuO4.

PB92-117340

100,441

COPPER-LIKE IONS

Exploding Foil Photoionization X-ray Laser. $\quad 101,433$
PB91-237065

Beam-Foil Study of Two-Electron Transitions in Cu-Like lons.

101,714

COPPER OXIDES

Phase Equilibria of the System Strontium Oxide-Calcium Oxide-Cupric Oxide.

101,085

CORDIC ALGORITHM

Assembly Code to Compute Sine and Cosine Using the Cordic Algorithm.

PB91-143289

100,638

CORONA DISCHARGES

Transition from Trichel-Pulse Corona to Dielectric Barrier Discharge.

00,835

Zonal Model for Corona Discharge-Induced Oxidation of $\mathrm{SF} 6$ in $\mathrm{SF} 6 / \mathrm{O} 2 / \mathrm{H} 2 \mathrm{O}$ Gas Mixtures.

PB91-158790

100,312

CORRELATORS

New Triple Correlator Design for the Measurement of UItrashort Laser Pulses.

PB91-203802

101,429

CORRIDORS

Reduction in Fire Hazard in Corridors and Areas Adjoining Corridors Provided by Sprinklers.

100,162

CORROSION

Rapporteur's Summary Materials Development for Coal Gasification.

PB91-158592

Corrosion Principles in Dental Implantology.

100,876

B91-194787

100,055

CORROSION ALLOYS

Corrosion, Repassivation and Corrosion Fatigue Behavior of the Surgical Implant Alloy, Co-Cr-Mo.

101,132

CORROSION MECHANISMS

Fourier Transform Infrared Spectroscopic Studies of the Degradation of One-Component Polyether Polyurethane Protective Coatings on Steel.

PB91-195248

101,108

\section{CORROSION PREVENTION}

Detection and Quantitative Characterization of Blistering and Corrosion

mography.

CORROSION TESTS

Corrosion Testing in Soil.

PB91-158667

Corrosion Behavior of Zirconium Alloy Nuclear Fuel Clad-

ding. 158733

101,372

Chloride Ion Diffusion in Low Water-to-Solid Cement

Pastes.

101,138

COST ANALYSIS

DISCOUNT-A Program for Discounting Computations in Life-Cycle Cost Analyses. User's Guide and Reference

100,897

Estimated Impact of the Center for Fire Research Pro. gram on the Costs of Fire.

100,161

Energy Prices and Discount Factors for Life-Cycle Cost Analysis 1992. Annual Supplement to NIST Handbook 135 and NBS Special Publication 709.

PB92-112515

COST ESTIMATES

First Pass at Computing the Cost of Fire Safety in a Modern Society.
PB91-216796

COUNTERMEASURES

Summary of Experiments with the Separated Aperture Technique of Dielectric Anomaly Detection. AD-A230 438/4

100,721

COUPLINGS

Kinematic Couplings for Precision Fixturing. Part 2. Experimental Determination of Repeatability and Stiffness. PB91.159186

CRACK ARREST

Plastic Zone Formation Around an Arresting Crack

PB91.189316

CRACK GROWTH

Indentation Determination of Crack Growth Parameters in Gallium Arsenide

CRACK INITIATION

Determining the Dynamic Stress Intensity Factor with Strain Gages Using a Crack Tip Locating Algorithr PB91-174268

CRACK OPENING DISPLACEMENT 
Effect of Surface Forces on Subcritical Crack Growth in Glass

PB92-117449

101,103

CRACKS

Tread Crack Detection in Railroad Wheels: An Ultrasonic System Using EMATs (Electromagnetic-Acoustic Transducers). Report No. 22

PB91-222661

101,742

CRANES (HOISTS)

Description of a Vibration Compensation System for the Small Scale Model Robot Crane Project.

PB91-216648

100,130

CREEP PROPERTIES

Creep of Copper: 4 to $295 \mathrm{~K}$.
PB91-189985

101,233

Comparison of Creep Rupture Behavior in Tension and Bending.

101,087

Creep and Reliability of Ceramic Materials at Elevated Temperatures.
PB91-204172

101,091

CREEP RUPTURE TESTS

Effect of Slag Penetration on the Performance of Magne-

sia Chrome Refractories in Slagging Gasifiers. 100875

100,875

\section{CREEP STRENGTH}

Improved Analysis for Flexural Creep with Application to Sialon Ceramics
PB91-150037

101,071

CREEP TESTS

Creep of Copper: 4 to $295 \mathrm{~K}$.

PB91-189985

101,233

Long Time Creep and Lifetime Behavior in Uniaxial Extension of a Linear Low Density Polyethylene.

PB91-236612

101,247

CRITICAL CURRENT

Guideline for $\mathrm{Nb}$ (sub 3) $\mathrm{Sn}$ critical current measurements

using fiberglass-epoxy composite sample mandrels.
DE9101,205

Current Supply for High-T(sub c) Superconductor Testing.

Transport Critical Current of Aligned Polycrystalline Y1Ba2Cu3O7-delta and Evidence for a Nonweak-Linked Component of Intergranular Current Conduction.

PB91-133983

101,456

High T(sub c) Superconductors and the Critical Current Measurement.

101,457

Integrity Tests for High-(T sub $c)$ and Conventional Critical-Current Measurement Systems.

PB91-148676

100,842

Current Record in Superconductors.

101,521

Sidelobe Suppression in Small Josephson Junctions.

Sidelobe Suppression in Small Josephson Junctions.
PB91-195446

Critical-Current Diffraction Patterns of Grain-Boundary Josephson Weak Links.

101,551

CRITICAL LINES

Phase Equilibria and Critical Lines in Model Water/Salt

Mixtures.
PB91-236778

100,418

CRITICAL POINT

Phase Equilibria and Critical Lines in Model Water/Salt Mixtures.
PB91-236778

100,418

Thermodynamics of Solutions Near the Solvent's Critical Point.

100,232

PB91-237297

oethane

Thermodynamic Properties
(R134a) in the Critical Region

100,233

CROSS SECTIONS

Cross Sections and Swarm Coefficients for Nitrogen. Ions and Argon lons and Neutrals in Ar for Energies from 0.1 eV $10 \mathrm{keV}$.

PB92-110246

100,452

CROSSLINKING

Etfect of Crosslink Density on Physical Aging of Epoxy Networks.
PB91-189712

101,244

Viscoelastic Response of Epoxy Glasses Subjected to Different Thermal Treatments.

101,245

Mechanical and Swelling Behavior of Crosslinked Natural

Mechanical and Swelling Behavior of Crosslinked Natural Rubber: Consequences of the Flory-Rehner Hypothesis.
PB91-195081

Models

Relaxation of Crosslinked Networks:

PB91-195099

100,501

CRYOGENIC COOLING

Review of Pulse Tube Refrigeration.
PB91-147942 101,046

$\begin{array}{ll}\text { PB91-147942 } & 101,046 \\ \text { Apparatus for the Measurement of Regenerator Perform. }\end{array}$ ance in Pulse Tube Refrigerators.

101,047
Analytical Model for the Refrigeration Power of the Orifice Pulse Tube Refrigerato

101,048

Optimization of a Pulse Tube Refrigerator for a Fixed Compressor Swept Volume.

101,051

\section{RYOGENIC FLUID STORAGE}

Reusable Cryogenic Foam Insulation for Advanced Aerospace Vehicles.

CRYOGENIC STORAGE DEVICES

Review of Cryogenic Mechanical and Thermal Properties

of Al-Li Alloys and Alloy 2219

AD-A229 231/6 101,202

Cryogenic Fatigue of High-Strength Aluminum Alloys and Correlations with Tensile Properties.

PB91-236976

101,709

CRYOGENICS

Aluminum Alloys for Cryogenic Tanks: Oxygen Compatibility. Volume 1 .

101,201

Aluminum Alloys for ALS Cryogenic Tanks: Comparative Measurements of Cryogenic Mechanical Properties of AlLi Alloys and Alloy 2219

AD-A242 956/1

101,204

Guideline for $\mathrm{Nb}$ (sub 3) Sn critical current measurements using fiberglass-epoxy composite sample mandrels.

DE91016274 101,205

Cryogenic Material Properties of Stainless Steel Tube-toFlange Welds

PB91-134627 $\quad 101,145$

Hydrogen Slush Production with a Large Auger.

Fracture Toughness of $316 \mathrm{~L}$ Stainless Steel Welds with Varying Inclusion Contents at $4 \mathrm{~K}$.

101,148

Influence of Interstitial Content on Fracture Toughness.

PB91-189944

101,157

CRYPTOGRAPHY

Public-Key Cryptography. Computer Security.

PB91-187864

100,708

CRYSTAL DEFECTS

Influence of Interstitial Content on Fracture Toughness.

PB91-189944 101,157

Atomic Fingerprint of YBa2Cu3O7-x-Type High-Tempera-

ture Superconductors Observed by Field Ion Microscopy.
PB91-203612

High Resolution Synchrotron X-Radiation Diffraction Imaging of Crystals Grown in Microgravity and Closely Related Terrestrial Crystals.

101,548

Structural Anomalies in Undoped Gallium Arsenide ObStructural Anomalies in Undoped Gallium Arsenide Ob-
served in High Resolution Diffraction Imaging with Monochromatic Synchrotron Radiation.

PB91-237438 101,556

Residual Defects in SIMOX: Threading Dislocations and

PB92-117233

100,829

CRYSTAL FIELD

Crystal-Field Splittings in the Cubic Heusler Alloys HoPd2Sn and ErPd2S

101,525

CRYSTAL GROWTH

Emergence of Modern Nucleation Theory.

PB91-149997

101,481

Numerical Study of Two-Dimensional Crystal Growth Forms in the Presence of Anisotropic Growth Kinetics.

PB91-200865

101,538

High Resolution Synchrotron X-Radiation Diffraction Imaging of Crystals Grown in Microgravity and Closely Related Terrestrial Crystals.

101,548

High Resolution Diffraction Imaging of Crystals Grown in Microgravity and Closely Related Terrestrial Crystals. PB92-109008

\section{CRYSTAL LATTICES}

NIST*LATTICE: A Program to Analyze Lattice Relationships. Spring 1991 Version.

MYSTAL-MELT INTERFACE

101,561

Effect of an Electric Field on the Morphological Stability of the Crystal-Melt Interface of a Binary Alloy. 3. Weakly Nonlinear Theory.

CRYSTAL MODELS

101,536

6-D Structural Model for the Icosahedral (AI, Si)-Mn QuaPBcrystal.

CRYSTAL OSCILLATORS

101,501 Environmental Sensitivities of Quartz Crystal Oscillators.
N91-25797/2

CRYSTAL STRUCTURE

Crystal and Spin Structures of $\mathrm{Nd}_{2} \mathrm{CuO} 4$

PB91-148023

101,470

Crystal Structure of Phosphate-Free Ribonuclease. PB91-175604 101,301 PLOTMD: An Interactive Program to Modify Molecular Plots on a Graphics Terminal.

PB91-194985

100,373

Phase Equilibria of the System Strontıum Oxide-Calcium Oxide-Cupric Oxide.

101,085

Parent Structure of the Layered High-Temperature Superconductors.

PB91-195677

101,535

Grain Boundaries in a 2-D Superlattice.

PB91-202853

Refinement of the Structure of Beta'-MoO3.

100,388

91-203786

101,090

New Layered Cuprate Structure-Type, (A1$\left.x A^{\prime} x\right) 14 C u 24 O 4$

101,095

Ab Initio Phase Determination for $\mathrm{X}$-ray Diffraction Data from Crystals of a Native Protein.

High Resolution Diffraction Imaging of Crystals Grown in Microgravity and Closely Related Terrestrial Crystals

PB92-109008

101,562

CRYSTALLIZATION

Crystal Structure of a cAMP-Independent Form of Cata-

bolite Gene Activator Protein with Adenosine Substituted in One of 2 CAMP-Binding Sites.

PB91-190074

101,305

Application of Osmotic Dewatering to the Controlled Crystallization of Biological Macromolecules and Organic PB91-195719

100,230

Theory of Orientation Textures Due to Surface Energy Anisotropies.

101,571

CRYSTALLOGRAPHY

PLOTMD: An Interactive Program to Modify Molecular

Plots on a Graphics Terminal.
PB91-194985

100,373

SIRC

Establishing a Computer Security Incident Response Capability (CSIRC)

100,720

CUPRATES

New Layered Cuprate Structure-Type, (A1XA'x) $14 \mathrm{Cu} 24 \mathrm{O} 41$

101,095

Antiferromagnetic Order of $\mathrm{Cu}$ in $\mathrm{Sm} 2 \mathrm{CuO} 4$

PB92-117340

101,569

CURING

Rate Constant Functions for Strength Development of Concrete

PB91-175117

100,527

CURRENT SOURCES

Current Supply for High-T(sub c) Superconductor Testing.

CURAENT TRANSFORMERS

NIST Measurement Services: Calibration Service for Current Transformers.

rent Transformer
PB91-216770

CURVE FITTING

Algorithm and Computer Program for the Calculation of Envelope Curves.

101,414

CYANIDE IONS

Multicomponent Cluster lons. 2. Comparative Stabilities of Cationic and Anionic Hydrogen Bonded Networks: Mixed Clusters of Water and Hydrogen Cyanide.

PB91-147710

100,283

CYANIDES

Mechanism of Cyanide Release in the Radiolysis of Ace- 


\section{KEYWORD INDEX}

CYCLOPROPANE

Perpendicular Bands of Cyclopropane in the $3.5 \mathrm{mum}$ Region.

PB91-237016

100,424

CYLINDERS

Analysis of Laminar Vortex Shedding Behind a Circular Cylinder by Computer-Aided Flow Visualization. PB91-203000

101,393

CVTOSINE

Hydroxyl Radical Induced Cross-Linking of Cytosine and Tyrosine in Nucleohistone.

Tyrosine in N
PB91-189365

101,304

DAMAGE ASSESSMENT

Assessment: U.S. Office Building of Moscow.

PB91-237834

100,151

DATA ACQUISITION

Computerization of a Thermomechanical Processing Research System

101,583

DATA ANALYSIS

OMNITAB 80 (ASCII): An Interactive System for Statistical and Numerical Data Analysis (Version 7.0).

101,278

OMNITAB 80 (UNIX): An Interactive System for Statistical and Numerical Data Analysis. Version 7.0. PB91-507962

101,279

DATA BASE MANAGEMENT SYSTEMS

Use of the IRDS Standard in CALS (Revised).

PB91-132209

100.684

SOL3 Support for CALS Applications.

100,965

Guide to Design, Implementation and Management of Distributed Databases.

PB91-187567

100,648

DATA BASES

Information Systems Engineering Program.

PB91-134635 100,685

Factual Materials Databanks - The Need for Standards.

PB91-147546 101,249

Instrument-Independent Tandem Mass Spectrometry Database for XOO Instruments: The Dynamical Prerequisites.

100,181

Guide to the Use of the Cement and Concrete Research Remote Bulletin Board System (RBBS) Computer. Remote Bulletin Board System (RBBS) Computer. 100,524
PB91-148528

Proceedings of the Object-Oriented Database Task Group Workshop, Tuesday, October 23, 1990, Chateau Laurier Hotel, Ottawa, Canada.
PB91-157198

100,641

Society

National Bureau of Standards-

101,073

PB91-158774

Agency Exploits Flexibility of Soltware in Moving Info.
PB91-159160

Proceedings of the Object-Oriented Database Task Group Workshop. Held in Atlantic City, New Jersey on May 22, 1990

100,643

NIST Standard Reference Data Products 1991 Catalog

NIST Standard Reference Data Products 1991 Catalog.
PB91-167312

ACTIS: Towards a Comprehensive Tribology Data Base

PB91-174904

101,184

Designing Data Entity Naming Conventions.

$\quad 100,646$

Taking the Measure of Fluid Properties Data Bases. PB91-200832 101,392 Numeric Database for Tribology: Format and Application Issues.

101,045

PB91-203943

Advanced Materials: Opportunities and Challenges.
PB91-204032

International Collaboration in Prestandards Research on Advanced Materials within the Context of VAMAS

PB91-204040

101,251

Data Bases Available in the Research Information Center of the National Institute of Standards and Technology.
PB92-109016

Integrated Knowledge Systems for Concrete Science and Technology.

PB92-116821

100,141

NIST X-Ray Photoelectron Spectroscopy (XPS) Data-

base.

100,465

DATA COMMUNICATIONS

Features and Facilities of Estelle.

PB91-158915

100,688

DATA DICTIONARIES

Specifications for a Federal Information Processing Standard Data Dictionary System.

PB91-158758

100,687

DATA ENCRYPTION

Data Encryption Standard: Past and Future. $\quad 100,711$
PB91-204107

DATA FILE

DARPA Air Travel Information System (ATISO) Speaker-

Dependent Training Data (on CD-ROM).
PB91-505370

100,594

Road Rally Conversational Speech Corpora (on CDPB91-509802

100,595

DATA MANAGEMENT

nformation Systems Engineering Program.

PB91-134635

CIM, Data and Standardization within the NBS AMRF.
PB91-148122

MRF.

Data Management Standards in Computer-Aided Acquisition and Logistic Support (CALS).

101,339

Designing Data Entity Naming Conventions.

100,646

PB91-175455 100,646 puter-Aided Acquisition and Logistic Support (CALS) Program. Fiscal Year 1988. Volume 1 of 3. Text, Security and Data Management.

PB91-178889

, 342

1-231571

Prototyping the IRDS: An Airport Application.

PB92-112580

100,654

Guide to Schema and Schema Extensibility.

101,740

B92.126556

100,955

\section{DATA PROCESSING}

Data-Reduction and Analysis Procedures Used in NIST's PB91-132282

100,925

Conceptual Architecture for a Mechanical Parts Production System Based on STEP. National PDES Testbed PB92-112234

100,993

DATA PROCESSING SECURITY

National Aeronautics and Space Administration's (NASA) Automated Information Security Handbook. Aus-187781

100,707

Glossary of Computer Security Terminology.

100,716

DATA PROCESSING SYSTEMS

Detail Specification for 62.5-mum Core Diameter/125mum Cladding Diameter Class la Multimode, GradedIndex Optical Waveguide Fibers.

FIPS PUB 159

100,562

DATA PROCESSING TERMINALS

Interface between Data Terminal Equipment (DTE) and Data Circuit-Terminating Equipment (DCE) for Operation with Packet-Switched Data Networks (PSDN), or between Two DTEs, by Dedicated Circuit. Category: Hardware Standard. Subcategory: Data Transmission.

100,672

ATA REDUCTION

Users Guide for RAPID, Version 2.3 PB91-167403

100,999

\section{DATA STORAGE DEVICES}

3480 Type Tape Cartridge: Potential Data Storage Risks, and Care and

101,381

DATA TERMINAL EQUIPMENT Conformance Tester for X.25 DTE Implementations.
PB91-189894 100,602

DATA TRANSMISSION

Electronic Data Interchange (EDI). Category: Software Standard. Subcategory: Electronic Data Interchange.

Electronic Data Interchange in Message Handling Sys-

PB91-216622

100,571

\section{AYLIGHTING}

Abstracts of Daylighting Research

100,076

Daylight Research Requirements. Workshop Proceedings. Held in Baltimore, Maryland on August 1-2, 1990. PB91-231563

Dealing with Obvious Issues in Lighting BB91-237792

100,761

DEBURRING

CAD Directed Robotic Deburring

PB91-195172

100,985

DEBYE-HUCKEL THEORY

Dielectric Constant of Water and Debye-Huieckel Limiting Law Slopes.

100,260

\section{DECISION SUPPORT SYSEMS}

Automan 2.0: Decision Support Software for Automated Manufacturing Investments. User Manual.

100,978

EFCTS

Annotated Bibliography: Diagnostic Methods and Measurement Approaches to Detect Incipient Defects Due to Aging of Cables
PB91-222687

DEGRADATION

101,369

Studies on the Degradation Products of Paper with and without Pollutants in a Closed Environment. 1. Preliminary Results.

PB91-143313

101,181

DEGREES OF FREEDOM

Impulse Response Functions for Elastic Structures with Rigid Body Degrees of Freedom.

DELAMINATING

Buckling and Post-Buckling Behavior of Elliptical Plates Part 2. Results.

PB92-116581

101,589

DELAUNEY TRIANGULATIONS

Computing Delaunay Triangulations for Comet-Shaped PB92-123108

101,265

DENSIFICATION

Effect of Green Density and the Role of Magnesium Oxide Additive on the Densification of Alumina Measured by Small-Angle Neutron Scaltering

PB92-117043

101,101

\section{DENSITY RATION}

Effects of Global Density Ratio on the Centerline Mixing PB92-117183

100,014

DENTAL CARIES

Digital Image Analysis Assisted Microradiography Meas urement of Mineral Content of Caries Lesions in Teeth.

PB91-187666

Protective Coatings for Tooth Crowns.

101,314

100.047

Filler Systems Based on Calcium Metaphosphates.

PB91-194654

100,048

Effects of $\mathrm{pH}$ and Calcium on Hydrolysis of Na2SiF6 and Na2SnF6: A Ouasi-Constant Composition Titration Study. 194746

101,315

Microstructure and Elastic Properties of Dental Resin and Resin-Based Glass-Reinforced Composites: XRD, SEM and Ultrasonic Methods.

PB91-204081

100,050

Dental Composite Resins: An Update.

100,051

Mathematical Model for Dental Caries: A Coupled Disso lution-Diffusion Process.

PB92-126689

101,316

DENTAL MATERIALS

Setting Reactions and Compressive Strengths of Calcium Phosphate Cements.

100,040

Visco-Elastic Deformation of Dental Porcelain and Porcelain-Metal Compatibility

100,042

Effect of Catalyst Structure on the Synthesis of a Dental Restorative Monomer

PB91-158691

100,043

Dental Biomaterials and Engineering Frontiers for the 90 's.

Glass-Ceramic Inserts Anticipated for 'Megafilled' Composite Restorations.

74458 


\section{KEYWORD INDEX}

DIFFUSION FLAMES

DEPOLARIZERS

Roughened Ouartz Suriaces and Teflon as Small-Angle Diffusers and Depolarizers between 200 and $400 \mathrm{~nm}$. PB91-195644

101,425

DEPOLYMERIZATION

Molecular Dynamics Study of the Depolymerization Reac tion in Simple Polymers.

PB91-187229

100,495

DEPOSITION

Analysis of Wet Deposition (Acid Rain): Determination of the Major Anionic Constituents by Ion Chromatography.

PB91-236646

100,035

DEPOSITS

Diesel Deposit Forming Tendencies - Microanalysis Meth-

PB91-195412

101,178

DEPTH FINDING

Integration-Based Method for Depth Estimation.
PB92-112275

DESIGN CRITERIA

Design Diagrams for Heavy Metal Fluoride Glass WinPB91-18

101,419

Proposed Design Criteria for Shallow Bridge Foundations. PB91-187849 100,534 Variable Air Volume System Design Guide. PB91-216655

100,077 DESORPTION

Study of the GaAs-Si(100) Interface Using Laser Probing of Thermal Desorption Kinetics.

PB91-149294

101,477

Resonant Tunneling with Electron-Phonon Interactions: An Exactly Solvable Model Applied to Desorption.

PB91-162065 100,317

Surface-State-Mediated Photochemistry: Laser-Induced Desorption of NO from Si (111).

PB91-162289

100,320

Molecular Desorption from Solid Surfaces: Laser Diagnostics and Chemical Dynamics.

100,397

DESULFURIZATION

Bioprocessing of Fossil Fuels Using Hyperthermophilic Archaebacteria.

100,883

Biological Sulfur Oxidation and Reduction for Coal Sulfur Speciation and Desulfurization.

PB91-203323

100,886

DETECTION

Summary of Experiments with the Separated Aperture Technique of Dielectric Anomaly Detection.

100,72

AD-A230 438/4

Assessment of the State-of-the-Art for Process Monitoring Sensors for Polymer Composites.

100,510

Capacitive Array Sensors for Nondestructive Evaluation PB91-237313

100,947

Laser Enhanced Ionization as a Selective Detector for Liquid Chromatographic Determination of Alkyltins in PB92-116755

100,210

Advanced Sensing of Materials Processing.

PB92-117423

100,949

Opportunities for Innovation: Chemical and Biological Sensors.
PB92-127315

100,214

DETONES

Radiation Chemistry of Enones.

PB91-147793

100,236

DEWATERING

Application of Osmotic Dewatering to the Controlled Crystallization of Biological Macromolecules and Organic Compounds.

100,230

DEXTRIN

Electrokinetic Demixing of Two-Phase Aqueous Polymer Systems. 2. Separation Rates of Polyethylene Glycol-Maltodextrin Mixtures.

PB91-237230

100,231

DIACETYLENE

Reactions of $\mathrm{C} 5 \mathrm{H} 3(+)$ and $\mathrm{C} 5 \mathrm{H} 5(+)$ lons with Acetylene and Diacetylene

PB91-195370

100,384

DIAGNOSTIC TECHNIQUES

Rule-Based Diagnostic Method for HVAC Fault Detection.

100,072

Diagnostic Techniques for Evaluating Office Building EnPB91-195438

100,074

DIAMOND

Spatially and Spectrally Resolved Cathodoluminescence of Hot-Filament Chemical-Vapor-Deposited Diamond Par-

AD-A236 485/9

100,247

Influence of Filament Geometry on Hot Filament Growth of Diamond Films.
AD-A231 818/6

101,448

Critical Assessment of Optical Properties of CVD DiaFilms.

AD-A236 746/4 101,400

High Resolution Electron Microscopy of Diamond Film Growth Defects and their Interactions.

AD-A243 093/2

101,449

Transmission Electron Microscopy of the CVD Diamond Film/Substrate Interface.

AD-A243 096/5

101,450

Spatially and Spectrally Resolved Cathodoluminescence Measurements of CVD-Grown Diamond Particles and

AD-A243 220/1

101,451

Inverse Correlation between the Intensity of Luminescence Excited by Electrons and by Visible Light in Chemical-Vapor-Deposited Diamond Films.

101,531

Photoluminescence Excitation by Band-Gap Optical Absorption in Chemical Vapor Deposition Diamond Films.
PB91-195586

\section{DIAMONDS}

Effects of Multiple Filament Geometry in the Hot Filament Deposition of Diamond Films.

AD-A236 708/4

101,142

Spatially and Spectrally Resolved Cathodoluminescence of Hot-Filament Chemical-Vapor-Deposited Diamond Par-

AD-A237 128/4

100,248

Determination of the Optical Constants of Thin Chemica Vapor-Deposited Diamond Windows from 0.5 to $6.5 \mathrm{eV}$. AD-A243 094/0

Chemical Vapor Deposited Diamond.

101,106

Use of Diamond as an Optical Material.

AD-A243 097/3

101,401

Spatially and Spectrally Resolved Cathodoluminescence Measurements of CVD-Grown Diamond Particles and Films.

AD-A243 220/1

101,451

Effects of Different $\mathrm{CH} 4-\mathrm{H} 2$ Gas Compositions on the Morphology and Growth of Diamond Grown by Hot FilaPB91-158683

U.S. Assessment of the New Diamond Technology in Japan.

PB91-167395

101,107

Tribological Characteristics of Synthesized Diamond Films on Silicon Carbide.

Films on Silic

101,186

DIATOMIC MOLECULES

Spectroscopy and Structure of the Alkali Hydride Diatomic Molecules and Their lons.

PB92-110147

100,443

DIAZIRINE

Dissociation Energetics of Simple Diazirines by Photon and Electron Impact.

DICHLORODIFLUOROMETHANE

Polarized X-ray Emission Studies of Methyl Chloride and the Chlorofluoromethanes.

PB91-174995

100.329

Molecular-Orbital Studies via Satellite-Free $X$-ray Fluorescence: $\mathrm{Cl} \mathrm{K}$ Absorption and K-Valence-Level Emission Spectra of Chlorofluoromethanes. PB91-237164

100,427

\section{DICHLOROMETHANE RADICALS}

Resonance Enhanced Multiphoton Ionization Spectroscopy of $\mathrm{CHCl} 2$ and $\mathrm{CDCl} 2$.

PB91-147645

Glossary of Computer Security Terminology.
PB92-112259

100,282

TIONARIES

Guideline: American National Dictionary for Information Systems. Category: Soitware; Subcategory: Documenta-

FIPS PUB 11-3

100,956

DIELECTRIC BREAKDOWN

Electrical Breakdown and Streamer Statistics in Nhexane under Uniform Field Conditions.

100,837

PB91-134684

Effect of High Pressure on Prebreakdown Phenomena in n-Hexane.

PB91-135020

100,839

DIELECTRIC FILMS

Measurement of Space Charge Fields in Polymers. 100,486

DIELECTRIC MATERIALS

Proceedings of Cable ' 89 Workshop.

PB91-161943

100,773

Chemistry of Electronic Ceramic Materials.

101,500

Theory of Capacitive Probe Method for Noncontact Characterization of Dielectric Properties of Materials.

PB91-237610

100,948

DIELECTRIC PROPERTIES

Dielectric Constant of Water and Debye-Huieckel Limiting Law Slopes.

PB90-244633

100,260

Transmission/Reflection and Short-Circuit Line Permittivity Measurements.

Formation and Stability of SF5 and S2F10.

100,848

PB91-174839

100,326

Core Hole Screening for Intermediate Size Metal Particles.

101,540

Optimization Techniques for Permittivity and Permeability Determination.

DIELECTRICS

Assessment of the State-of-the-Art for Process Monitoring Sensors for Polymer Composites.

PB91-222612

100,510

Improved Technique for Measuring Permittivity of Thin Dielectrics with a Cylindrical Resonant Cavity.

100,858

Characterization of Capacitive Array for NDE Applica-

Research for Electric Energy Systems: An Annual Report. PB92-112341

DIELECTRONIC RECOMBINATION

Dielectronic Recombination Rate Coefficients for lons of

the Fluorine Isoelectronic Sequence.

PB91-195602

101,674

DIES

Test Plan for Validating a Context Driven Integrated Model (CDIM) for Sheet Metal Die Design. National PDES Testbed Report Series.

PB92-112523

100,970

DIESEL ENGINES

Diesel Deposit Forming Tendencies - Microanalysis Meth-

PB91-195412

101,178

DIFFERENTIAL EQUATIONS

Asymptotic Shooting Method for the Solution of Differen tial Equations.

PB91-148742

101.255

DIFFERENTIAL THERMAL ANALYSIS

Fusion of Mercury. A New Certified Standard for Differential Scanning Calorimetry

DIFFRACTION

Improved Optical Diffraction Strain Measurement System.

Diffraction by a Half-Plane in a Lossy Medium PB92-116888

101,437

DIFFRACTION IMAGING

Dynamical Diffraction Imaging (Topography) with $X$-ray Synchrotron Radi
PB91-189654

101,510

Streaking Images That Appear Only in the Plane of Diffraction in Unoped GaAs Single Crystals: Diffraction Imaging (Topography) by Monochromatic Synchrotron Radi- 
Large-Scale Turbulent Structures and the Stabilization of Lilted Turbulent Jet Diffusion Flames.

100,558

Relative $\mathrm{H}$-Atom and O-Atom Concentration Measurement in a Laminar, Methane/Air Diffusion Flame.
PB92-117365
100,559

DIGITAL COMMAND SYSTEMS

Direct Digital Control Based Building Automation System Design Criteria.

100,091

Guide Specification for Direct Digital Control Based Building Automation System.

PB91-216697
IGITAL OSCILLOSCOPES

Recent Developments in Digital Oscilloscopes. PB91-175240

DIHEDRAL ANGLE

Dihedral Angles in Magnesia and Alumina: Distributions from Surface Thermal Grooves.

PB91-147330

101,058

Metal Reference Line Technique for Obtaining Dihedral Angles from Surface Thermal Grooves.

101,059

\section{DIMENSIONAL MEASUREMENT}

Determination of Fields Near a Silver Strip on a Glass Substrate.

100,841

Standard Reference Materials: Bright-Chromium Linewidth Standard, SRM 476, for Calibration of Optical Microscope Linewidth Measuring Systems.

100,802

Review of Current Geometric Tolerancing Theories and Inspection Data Analysis Algorithms.

101,016

Use of the Electrostatic Classification Method to Size 0.1 micrometer SAM Particles: A Feasibility Study. micrometer SR

100,932

Statistical Process Control Tactics for Coordinate Measuring Machines.
PB91-189225

101,042

Calibration of the NBS 10mum Space Beads.

PB91-203422

101,695

Dimensional Calibration of an NBS 0.3-mum-Diameter Particle-Sizing Standard.

101,696

New Concepts of Precision Dimensional Measurement for Modern Manufacturing.
PB91-240812 101,006

Ellipsometry SRM's for Use in Thin Film Measurements. PB92-116599 100,819

Modified Sliding Wire Potentiometer Test Structure Ior Mapping Nanometer-Level Distances.

100,821

Certification of NIST SRM 1961: 30 micrometers Diameter Polystyrene Spheres

PB92-126655

100,937

DIMENSIONAL MEASUREMENTS

Submicrometer SEM Magnification Standard. $\quad 100,799$

DIMERIZATION

$\mathrm{H} 2 \mathrm{O} 2$ : Spectroscopy, Structure and Dynamics.

PB

100,316

Fluoromethanol: Synthesis, Microwave Spectrum, and Dipole Momen

PB91-148080 100,288

Dipole Moments ol Seven Partially Halogenated Ethane Refrigerants.
PB91-195131

100,377

DIRECT DIGITAL CONTROL

Direct Digital Control Based Building Automation System Design Criteria

100,091

Guide Specilication for Direct Digital Control Based Building Automation System.

PB91-216697

100,092

DIRECTION SIGNS Visibility of Exit Signs and Directional Indicators.
PB91-161984

100,083

DIRECTIONAL SOLIDIFICATION (CRYSTALS)

Convective and Morphological Instabilities during Directional Solidification.

PB91-158550

101,219

DIRECTORIES

Directory of Federal Government Laboratory Accreditation/Designation Programs.

100,005

Standards Activities of Organizations in the United States.
PB91-177774 100,004

Directory of Accredited Laboratories, 1991. National Voluntary Laboratory Accreditation Program (NVLAP).
PB91-222646 Directory of State and Local Government Laboratory Accreditation/Designation Programs.

PB92-108968

100,174
DISACCHARIDES

Thermodynamics of Hydrolysis of Disaccharides: Lactuose, alpha-D - Melibiose, Palatinose, D - Trehalose, D Turanose and 3-o-beta-D-Galactopyranosyl-D-arabinose. DISCHARGE COEFFICIENTS

Effect of Tube Bundle Flow Conditioners on Orifice Meter Discharge Coelficients.

PB91-148791

100,881

DISK OPERATING SYSTEM (DOS)

Interactive Video Software Portability: Migrating a DOS Application to POSIX

100,670

DISPLACEMENT

Extended Abstract: A Finite Element Study of the Stress and Displacement Fields Produced by Point Impact.

PB91-175224

101,584

DISPROPORTIONATION

Thermodynamics of the Disproportionation of Adenosine 5 -diphosphate to Adenosine $5^{\prime}$-triphosphate and Adenosine 5'-monophosphate. 1. Equilibrium Model.

100,459

Thermodynamics of the Disproportionation ol Adenosine 5 -diphosphate to Adenosine 5'-triphosphate and Adenosine 5'-monophosphate. 2. Experimental Data. PB92-117399

101,313

DISSOCIATION

Second Dissociation Constant and $\mathrm{pH}$ of $\mathrm{N}$-2-hydroxyethylpiperazine-N'-2-ethanesulfonic Acid (HEPES) from 0 to

PB91-189282 100,196

Proton Alfinities and pKa Values ol Tetraalkylhydrazines.
PB91-195214 PB91-195214

Stabilities of Substituted Benzyl Radicals: Dissociation Rates of Aminoethylbenzenes, Hydroxyethylbenzenes, and Cyanoethylbenzenes.

100,430

Dissociation of Substituted Anisoles: Substituent Effects on Bond Strengths.

DISSOCIATION ENERGY

Dissociation Energetics of Simple Diazirines by Photon and Electron Impact

STRIBUTED COMPUTER SYSTEMS

Guide to Design, Implementation and Management of Distributed Databases.

PB91-187567

100,648

Depot: A Framework lor Sharing Soltware Installation

Across Organizational and Unix Platform Boundaries.
P891-240754 P891-240754

DISTRIBUTED PROCESSING

Application ol Formal Description Techniques to the Specification of Distributed Test Systems.

100.566

DNA-BINDING PROTEINS

Measurement of DNA Base Damage and DNA-Protein Cross-Links in Mammalian Chromatin.

PB91-187484

101,303

Structure and Mechanism of Hydroxyl Radical-Induced Formation of a DNA-Protein Cross-Link Involving Thymine and Lysine in Nucleohistone.

101,334

DNA DAMAGE

Base Modifications in Plasmid DNA Caused by Potassium Permanganate.

PB91-187070

101,329

Copper-Ion-Dependent Damage to the Bases in DNA in the Presence of Hydrogen Peroxide.

101,330

PB91-187104

Damage to the Bases in DNA Induced by Hydrogen Peroxide and Ferric Ion Chelates.

101,331

Chemical Determination of Free Radical-Induced Damage to DNA

PB91-187468

101,332

46Gas Chromatography-Mass Spectrometry ol Free Radical-Induced Products ol Pyrimidines and Purines in DNA.
PB91-187476

101,302

Measurement of DNA Base Damage and DNA-Protein Cross-Links in Mammalian Chromatin.

101,303

Modification of Bases in DNA by Copper Ion-1,10-Phenanthroline Complexes.

101,333

Structure and Mechanism of Hydroxyl Radical-Induced Formation ol a DNA-Protein Cross-Link Involving Thymine and Lysine in Nucleohistone.

101,334

Chemical Nature of In vivo DNA Base Damage in Hydro-

gen Peroxide-Treated Mammalian Cells.

Damage to the DNA Bases in Mammalian Chromatin by Hydrogen Peroxide in the Presence ol Ferric and Cupric

PB91-189217

101,336

Bleomycin-Dependent Damage to the Bases in DNA is a Mınor Side Reaction.

PB91-189357

101,337

Modification of DNA Bases in Mammalian Chromatin by Radiation-Generated Free Radicals.

PB91-189373

101,338

Selected-Ion Mass Spectrometry: Assays ol Oxidative PB91-202960

101,307

Damage to the Bases in DNA Induced by Stimulated Human Neutrophils.

PB92-116912

101,312

DOCUMENT PROCESSING

Document Architecture lor Open Systems.

PB91-18952

100,961

Standard Generalized Markup Language Encoding of the Office Document Architecture Document Application Pro-

PB91-184812

101,345

Document Architecture for Open Systems.

PB91-189522

100,961

DOMAIN NAME SYSTEM

Choosing a Name for Your Computer.

PB91-158899

100,600

DOMAIN WALLS

Micromagnetic Calculations of $180 \mathrm{deg}$ Surface DomainWall Magnetization Profiles with Comparison to Measure-

PB91-158501 101,483

Micromagnetics of Domain Walls at Surfaces.

101,491

Micromagnetic Calculations of 180 deg Surface Domain Walls

PB91-237271

101,553

Scanning Electron Microscopy with Polarization Analysis . ic Singularities at Surfaces and in Thin Films. PB91-237289

101,554

DOSEMETERS

Sensitometry of the Response of a New Radiochromic Film Dosimeter to Gamma Radiation and Electron

PB91-237008

101,326

DOSIMETERS

NIST High-Dose Calibration Services

PB91-203257 101,689

Criteria for the Operation of Federally-Owned Secondary Calibration Laboratories (Ionizing Radiation).

PB92-112481

101,721

Response ol Personal Noise Dosimeters to Continuous and Impulse-Like Signals.

100,914

Effects of Absorbed Dose Rate, Irradiation Temperature and Post-Irradiation Temperature on the Gamma Ray Response ol Red Perspex Dosimeters.

PB92-116441

101,363

Novel Radiation Dosimetry Systems.

101,727

2-Deoxy-D-Ribose Aqueous Solution as a Gamma-Ray

PB92-117126

101,366

Temperat

PB92-117134

101,728

DOSIMETRY

Use of Storage Phosphor Imaging Plates in Portal Imaging and High-Energy Radiography: The Intensifying Effect Ol Metallic Screens on the Sensitivity.

PB91-236497

101,324

Dosimetry: New Approaches for New Challenges.

PB92-117100 


\section{KEYWORD INDEX}

ELECTRICAL TRANSIENTS

CW Dye Lasers

PB92-116896

101,438

DYNAMIC RESPONSE

Dynamic Arc-Power Source Response in GMA Welding.

Measurement of Structural Response Characteristics of Full-Scale Buildings: Selection of Structures.

PB91-167239

100,144

Impulse Response Functions for Elastic Structures with Rigid Body Degrees of Freedom

101,587

DYNAMIC STRUCTURAL ANALYSIS

Preliminary Dynamic Analyses of the Ministry of Agricullure Building.

PB91-134668

100,143

dentification of Dynamic Green's Functions in Structural

Networks.

101,585

DYNAMICS

Effect of Atomization Air on Droplet Dynamics of Spray Flames.

PB91-203869

100,550

E2-TRANSITIONS

Electric Quadrupole and Magnetic Dipole Transition Prob. abilities in Potassium Isoelectronic Sequence. PB91-187088

101,646

EARLY STARS

Terminal Velocities for a Large Sample of O Stars, B Supergiants, and Wolf-Rayet Stars

New Radio Detectors of Early-Type Pre-Main Sequence

Stars.
PB91-149286

100,024

EARTHQUAKE ENGINEERING

Measurement of Structural Response Characteristics of Full-Scale Buildings: Selection of Structures.

PB91-167239

100,144

Proceedings of a Workshop on Evaluation, Repair, and Retrofit of Structures. U.S. Japan Panel on Wind and on May 12-14, 1990. PB91-184846

100,145

EARTHQUAKE RESISTANT STRUCTURES

Preliminary Dynamic Analyses of the Ministry of Agriculture Building.
PB91-134668

100,143

Proceedings of a Workshop on Evaluation, Repair, and Retrofit of Structures. U.S.-Japan Panel on Wind and Seismic Effects, UJNR. Held in Gaithersburg, MD., USA, on May 12-14, 1990

PB91.184846

100,145

Long Term Performance of Rubber in Seismic and NonSeismic Bearings: A Literature Review.

100,148

Performance of 1/3-Scale Model Precast Concrete Beam-Column Connections Subjected to Cyclic Inelastic Loads. Report No. 2

100,149

Wind and Seismic Effects. Proceedings of the Joint MeetWind and Seismic Effects. Proceedings of the Joint Meetsources Panel on Wind and Seismic Effects (23rd). Held in Tsukuba, Japan, on May 14-17, 1991.

100,152

ARTHOUAKES

Wind and Seismic Effects. Proceedings of the Joint Meeting of the U.S.-Japan Cooperative Program in Natural Resources Panel on Wind and Seismic Effects (23rd). Held in Tsukuba, Japan, on May 14-17, 1991. PB92-116425

100,152

ECCENTRICITY

Eccentric Load Sensitivity of Force Sensors.
PB91-175406

100,931

ECONOMIC ANALYSIS

NIST 'Building Life-Cycle Cost' (BLCC) Program (Version 3.0). User's Guide and Reference Manual. $\quad 100,154$
PB91-159764 PB91-159764

0. Eco. ZIP: The ZIP-Code Insulation Program, Version 200,154 Three-Digit Zip Code. Users' Guide and Reference Manual (Revised Edition).

PB91-167155

100,064

Building Life Cycle Cost Computer Program, Version 3.2 (for Microcomputers)

100,898

Economic Analysis for Military Construction (MILCON) Design. Concepts, Techniques, and Applications for the Analyst. Instructor's Manual.

PB92-112440

101,349

Economic Analysis for Military Construction (MILCON) Design. Concepts, Techniques, and Applications for the Analyst. Student's Manual.

PB92-112457

101,350

EDDY CURRENT TESTS

Eddy Current Probe Characterization Using an Imped ance Plane Display Instrument.

PB91-133868

100,834

Progress in Uniform Field Eddy Current Methods.

PB91-147751

101,039
Eddy Current Probe Sensitivity as a Function of Coil Construction Parameters.

100,945

EDDY CURRENTS

Eddy Current Reflection Probe: Theory and Experiment. PB91-187120

100,944

EDGE DETECTION

New Approach to Image Modeling and Edge Detection in PB91-203752

100,811

EDUCATION

Agenda for Progress in Technology Education: A Personal View.

PB91-148114

100,037

National Educators Workshop: Update ' 90 . Standard Experiments in Engineering Materials Science and TechnolPgy. 2 -126606

101,253

EFFECTIVENESS

Apparatus for the Measurement of Regenerator Performance in Pulse Tube Refrigerators.

PB91-147967

101,047

EICOSANE

Multistep Stress-Relaxation Study of a Single Crystal of $n$-Eicosane $(\mathrm{C} 2 \mathrm{OH} 42)$ in Torsion.

100,497

ASTIC ANALYSIS

mpulse Response Functions for Elastic Structures with Rigid Body Degrees of Freedom. PB91-237354

101,587

ELASTIC CONSTANTS

Resonating-Orthotropic-Cube Method for Elastic Con-

PB91-236794

101,586

ELASTIC PROPERTIES

Elastic Constants of a Tungsten-Particle Copper-Matrix Composite.

101,234

Elastic Constants of Fiber-Reinforced Composites: A Fresh Measurement Approach.

PB91-194878

101,123

Monitoring of Anisotropic Material Elastic Properties Using Ultrasonic Receiving Rays.

101,126

ELASTOMERS

Tests of the Flory-Rehner Hypothesis: Comparison of the Elastic Free Energy Function for Crosslinked Rubber in the Dry and Swollen States.

PB91-203570

100,508

ELECTRIC BRIDGES

Performance Evaluation of a New Audio-Frequency Power Bridge.

100,742

ELECTRIC CABLES

Partial Discharges in Low Voltage Cables.

PB91-134650

100,771

Proceedings of Cable ' 89 Workshop.

PB91-161943

100,773

Mode-Stirred Chamber for Measuring Shielding Effectiveness of Cables and Connectors: Assessing MIL-STD1344A Method 3008.

B92-116656

100,776

ELECTRIC COILS

Eddy Current Probe Sensitivity as a Function of Coil Construction Parameters

PB91-236562

100,945

ELECTRIC CONDUCTORS

Magnetoresistance of Multifilament Al/Al-Alloy ConducMors.

Near-Field and Far-Field Excitation of a Long Conductor in a Lossy Medium

PB91-143347

100,722

ELECTRIC CONNECTORS

Mode-Stirred Chamber for Measuring Shielding Effectiveness of Cables and Connectors: Assessing MIL-STD1344A Method 3008.

PB92-116656

100,776

ELECTRIC CONTACTS

Cratering.
PB91-203141

100,810

Prospects for High Temperature Superconductor - Semiconductor Contacts.

PB91-203646

101,545

\section{ELECTRIC CURRENT METERS}

Progress in the Development of Miniature Optical Fiber Current Sensors.

PB91-134734

100.745

Practical Considerations in the Design of Optical Fiber Current Sensors.

100,746

Performance and Limitations of Faraday Effect Sensors. PB91-149146 100,749

Optical Fiber Current Sensors with Temperature Stabilities Near the Material Limit.

PB91-175109

100,753

Faraday Effect Current Sensing Using a Sagnac Interferometer with a $3 \times 3$ Coupler.

PB91-175158

100,754

\section{ELECTRIC DIPOLE MOMENTS}

Microwave Spectra and Electric Dipole Moments of $\mathrm{X}(4)$ Sigma(1-) $1 / 2 \mathrm{VO}$ and $\mathrm{NbO}$.

100,429

ELECTRIC DISCHARGES

Partial Discharges in Low Voltage Cables.

PB91-134650

100,771

Electrical Breakdown and Streamer Statistics in $\mathrm{N}$. Exane under Uniform Field Conditions.

100,837

Effect of High Pressure on Prebreakdown Phenomena in n-Hexane.

PB91-135020

100,839

Influence of Memory Propagation on Phase-Resolved Stochastic Behavior of AC-Generated Partial Discharges. B91-237644

100,861

Characteristics of Aerosols Produced by the Spark Discharge.

100,209

ELECTRIC FIELD METERS

Photonic Probes for the Measurement of Electromagnetic Fields Over Broad Bandwidths.

PB91-162206

100,750

LECTRIC FIELDS

Applied Electric Fields for Downstream Processing.

Theoretical Considerations for a Thermo-Optic Microwave Electric-Field-Strength Probe.

100,860

Research for Electric Energy Systems: An Annual Report. PB92-112341

ELECTRIC POTENTIAL

Kelvin Water-Drop Experiment.

N91-20219/2

100,900

Evaluation of Liquid Junction Potentials and Determina tion of $\mathrm{pH}$ Values of Strong Acids at Moderate Ionic PB91-237859

100,438

ELECTRIC POWER PLANTS

Development of a New Tracer Technology Using Enriched Rare-E

100,908

ELECTRIC WIRE

Static and Dynamic Strength Tests on Electrical Conductor Cables Specified for Airport Landing Structures. PB92-112226

ELECTRICAL ENGINEERING

Center for Electronics and Electrical Engineering Technical Publication Announcements Covering Center Programs, October to December 1990, with 1991 CEEE Events Calendar. PB91-240838

100,863

PCTRICAL EQUIPMENT

Navy Safety Center Data on the Effects of Fire Protection 


\section{KEYWORD INDEX}

PB91-158626

100,486

ELECTROCHEMICAL COATING

Surface Properties and Coatings of Materials Microtailored Alloys.
PB91-189696

ELECTROCHEMICAL CORROSION

Concepts of Underground Corrosion

ECTROCHEMISTRY

Electrochemical Behavior of Ferrocene in Polyethylene Oxide- 400 .

PB91-237867

100,439

Surface X-ray Absorption Spectroscopy, EXAFS and NEXAFS, for the In situ and Ex situ Study of Electrodes. PB92-117035

100,460

ELECTRODEPOSITED COATINGS

Structural Study of Electrodeposited Aluminum-Manganese Alloys.
PB91-189423

101,231

ELECTRODEPOSITION

Electrodeposition of Aluminum from Molten Salts.
PB91-159202

Surface Properties and Coatings of Materials Microtai-

lored Alloys.
PB91-189696

101,232

ELECTRODES

Assessment of Uncertainties in Measurement of $\mathrm{pH}$ in Hostile Environments Characteristic of Nuclear Repositories.

101,367

Surface X-ray Absorption Spectroscopy, EXAFS and NEXAFS, for the In situ and EX situ Study of Electrodes.
PB92-117035

ELECTROFISSION

Electrofission in the Ouasifree and Delta Regions. PB91-147355

101,609

ELECTROLYTIC CONDUCTIVITY

Proposed New Electrolytic Conductivity Primary Stand. ards for $\mathrm{KCl}$ Solutions. PB91-187658

100,339

ELECTROMAGNETIC COMPATIBILITY

TEM/Reverberating Chamber Design/Concept Study: A Single Facility for Large System Radiated EMC Testing. $10 \mathrm{kHz}-40 \mathrm{Gh}$.

100,845

Conformity Assessment Workshop on Electromagnetic Compatibility.
PB91-192989

100,579

ELECTROMAGNETIC ENVIRONMENTS

Lattice Approach to Environments Irradiated by Unknown Sources.
PB91-175356

100,850

ELECTROMAGNETIC FIELDS

Bibliography of the NIST Electromagnetic Fields Division Publications.
PB91-132241

100,832

Calibration of Antenna Factor at a Ground Screen Field Site Using an Automatic Network Analyzer. Estimation of Electromagnetic Fields in Complex Environ-

101,691

PB91-203315

Division

Bibliography

PB92-116367

100,866

ELECTROMAGNETIC INTERFERENCE

Electronics and Electrical Engineering Laboratory Technical Publication Announcements Covering Laboratory Programs, January to March 1991, with 1991 EEEL Events PB92-112309

100,864

ELECTROMAGNETIC METROLOGY

Summary of Experiments with the Separated Aperture Technique of Dielectric Anomaly Detection.

AD-A230 438/4

100,721

Trapped-Ion Frequency Standards.

N91-25760/0

100,575

GPS Time Transfer with Implementation of Selective Availability.
N91-25766/7

100,576

Bibliography of the NIST Electromagnetic Fields Division Publications.

100,832

Metrology for Electromagnetic Technology: A Bibliography of NIST Publications.

100,833

Spherical Near-Field Scanning: Experimental and Theoretical Studies.
PB91-132274

100,723

Technical Digest-Symposium on Optical Fiber Measurements, 1990.

101,403

Determination of Scattering Parameters with Respect to the Characteristic Impedance of Precision Coaxial AirLine Standards.

100,770

Accurate Frequency of the $119 \mathrm{mu} \mathrm{m}$ Methanol Laser Accurate Frequency of the 119 mu m Methanol
from Tunable Far-Intrared Absorption Spectroscopy.
PB91-134130

101,405

Calibration of Antenna Factor at a Ground Screen Field Site Using an Automatic Network Analyzer.

100,724

Partial Discharges in Low Voltage Cables.

100,771

Progress in the Development of Miniature Optical Fibe

PB91-134734 100,745

Practical Considerations in the Design of Optical Fiber Current Sensors.

100,746

Accuracy Model for Phase Noise Measurements.
PB91-134882

100,589

Dual Six-Port Reflectometer Systems Using Waveguide in the Frequency Range 18-50 G

100,838

Near-Field and Far-Field Excitation of a Long Conductor in a Lossy Medium

PB91-143347

100,722

Verification of the Relation between Two-Probe and Fourrobe Resistances as Measured on Silicon Wafers.

PB91.147520 100,788

Determination of Fields Near a Silver Strip on a Glass

Substrate.
PB91-147702 100,841

Spherical Near-Field Scanning: Determining the Incident Field Near a Rotatable Probe.

100,725

Integrity Tests for High-(T sub c) and Conventional Critical-Current Measurement Systems.

100,842

Ouasi-Static Analysis of a Two-Wire Transmission Line Located at an Interface.

PB91-148734

100,564

MMIC Related Metrology at the National Institute of Standards and Technology.

100,794

Improved Technique for Determining Complex Permittivity with the Transmission/Reflection Method.

PB91-161919

100,844

TEM/Reverberating Chamber Design/Concept Study: A Single Facility for Large System Radiated EMC Testing $10 \mathrm{kHz}-40 \mathrm{Ghz}$

100,845

Evaluation of Dual-Port Circularly Polarized Probes for Planar Near-Field Measurements.

PB91-162040

100,590

Microstrip Patch Antenna as a Standard Transmitting and Receiving Antenna.

100,591

Photonic Probes for the Measurement of Electromagnetic Fields Over Broad Bandwidths.

PB91-162206

100,750

Evaluation of Instrumentation Used to Measure AC Power System Magnetic Fields.

PB91-162248

100,872

Intercomparison of NBS Noise Calibration Services.

PBo1-162370

100,847

Transmission/Reflection and Short-Circuit Line Permittivity Measurements.

PB91-171959

100,848

Lattice Approach to Environments Irradiated by Unknown Sources

100,850

Measurements of Power Frequency Magnetic Fields Away from Power Lines.

PB91-175398

100,851

NIST Calibration Procedure for Vertically Polarized Monopole Antennas $30 \mathrm{kHz}$ to $300 \mathrm{MHz}$.

Incompatibility between the $100 / 1300$ Surge Test and Varistor Failure Rates.

100,854

Conformity Assessment Workshop on Electromagnetic

$\begin{array}{ll}\text { Compatibility. } & 100,579\end{array}$

Rubidium Frequency Standard and a GPS Receiver: A

Remotely Steered Clock System with Good Short-Term and Long-Term Stability.

PB91-200808

100,581

TEM Driven Reverberating Chamber, a Single Facility for Radiated EMS/V Testing, $10 \mathrm{kHz}-18 \mathrm{GHz}$

100,856

Correlation of Flux States Generated by Optical Switching of a Superconducting Circuit.

PB91-202929

101,682

Established Micd 100,584

Generalization of the Cornu Spiral for Lossy Media. PB91-203216
Estimation of Electromagnetic Fields in Complex Environ-

101,691

Computed Fields Near the Edge of a Dielectric Wedge. PB91-203505

Scattering by an Arbitrary Cylinder at a Plane Interface. B91-203513 Simultaneous versus Independent Injection Testing of Nonlinear Multiport Systems.

PB91-203885

100,857

New Look at the Bitter Method of Magnetic Imaging.
PB91-203919 101,701
PB91-203919 rent Transformers.

PB91-216770

100,873

Eddy Current Probe Sensitivity as a Function of Coil Construction Parameters.

100,945

Determining Adapter Efficiency by Envelope Averaging Swept Frequency Reflection Data.

PB91-236620

100,743

RF-DC Differences of Thermal Voltage Converters Arising from Input Connectors.

$1-236802$

100,735

Excess Low-Frequency Flux Noise in dc Squids Incorporating $\mathrm{Nb} / \mathrm{Al}-\mathrm{Oxide} / \mathrm{Nb}$ Josephson Junctions.

PB91-236810

100,736

Improved Technique for Measuring Permittivity of Thin Dielectrics with a Cylindrical Resonant Cavity. $\quad 100,858$
PB91-236844

AC-DC Difference Relationships for Current Shunt and Thermal Converter Combinations.

PB91-236893

100,737

Alignment Fixture for Millimeter Waveguide. -236919

100,727

Precise Ephemerides for GPS Time Transfer.

PB91-236950

100,586

Characteristic Impedance Determination Using PropagaPB91-236992

100,775

General Technique to Correct Probe Position Errors in Planar Near-Field Measurements to Arbitrary Accuracy.
PB91-237073 New Low-Voltage Standards in the DC to $1-\mathrm{MHz}$ Frequency Range.

100,738

Monitoring the Mass Standard via the Comparison of Mechanical to Electrical Power.

PB91-237115

101,710

New Triple Correlation Technique for Measuring Ultrashort Laser Pulses.

PB91-237156

101,434

Critical-Current Diffraction Patterns of Grain-Boundary Josephson Weak Links.

PB91-237180

101,551

Precision Oualiti

100,859

Theoretical Considerations for a Thermo-Optic Microwave Electric-Field-Strength Probe.

PB91-237222

100,860

Microwave Monolithic Integrated Circuit-Related Metrology at the National Institute of Standards and Technology.
PB91-237255 Improvements for Automating Voltage Calibrations Using a $10-V$ Josephson Array.

PB91-237446

100,739

Influence of Mernory Propagation on Phase-Resolved Stochastic Behavior of AC-Generated Partial Discharges.

Translate LRL and LRM Calibrations.

PB91-237776

100,862

General Analytic Correction for Probe-Position Errors in Spherical Near-Field Measurements.

B92-110279

100,729 
ware Package to Calculate Time Domain Frequency Stability from the Frequency Domain.

100,587

Modified Airy Function and WKB Solutions to the Wave Equation.

PB92-126531

101,440

ELECTROMAGNETIC SCATTERING

Computed Fields Near the Edge of a Dielectric Wedge.

101,446

Scattering by an Arbitrary Cylinder at a Plane Interface.

PB91-203513 101,447
PBory

ELECTROMAGNETIC SHIELDING

Mode-Stirred Chamber for Measuring Shielding Effectiveness of Cables and Connectors: Assessing MIL-STD344A Method 3008

PB92-116656

100,776

ELECTROMAGNETIC SUSCEPTIBILITY

Simultaneous versus Independent Injection Testing of Nonlinear Multiport Systems.

PB91-203885

100,857

ELECTROMAGNETIC TESTING

TEM Driven Reverberating Chamber, a Single Facility for Radiated EMS $/ V$ Testing, $10 \mathrm{kHz}-18 \mathrm{GHz}$. PB91-202895

100,856

ELECTROMIGRATION

Current Density Dependence of Electromigration t50 Enhancement Due to Pulsed Operation.

100,786

\section{ELECTRON-ATOM COLLISIONS}

Updated Excitation and Ionization Cross Sections for Electron Impact on Atomic Oxygen.

100,256

Cross Sections for Collisions of Electrons and Photons with Atomic Oxygen

PB90-244682 100,264

Optical Potential Approach to Electron and Positron Scattering from Noble Gases. 2. Neon.

101,619

Uses of Spin-Polarised Electrons in Fundamental ElecUses of Spin-Polarised Electrons in Fundamental Elec-
tron-Atom Collision Processes and the Analysis of Magnetic Microstructures.

PBTRON BEAMS

101,490

Synthesis of Prototype Resins for Use as BEP Intaglio Ink Vehicles Curing by Electron Beam Radiation.

PB91-144345 101,182 Modeling Electron Beam Interactions in Semiconductors. PB91-159020 101,485 Sensitometry of the Response of a New Radiochromic Film Dosimeter to Gamma Radiation and Electron Beams.

PB91-237008

101,326

ELECTRON BOMBARDMENT

Intensifying Effect of Metallic Screens on the Sensitivity of X-ray Films for $400-\mathrm{kV}$ Bremsstrahlung Photons.

PB91-195198 101,293

Intensifying Effect of Metallic Screens on the Sensitivity of X-ray Films at $662 \mathrm{keV}$.

101,669

ELECTRON DETACHMENT

Collisional Electron-Detachment and Ion-Conversion Processes in SF6.

PB91-134528

100,836

ELECTRON-ION COLLISIONS

Cross Sections and Related Data for Electron Collisions with Hydrogen Molecules and Molecular lons.
PB90-244674 Relativistic Distorted-Wave Cross Sections for ElectronImpact Excitations of Berylliumlike Ions. PB91-195503

101,672

ELECTRON MICROSCOPY

Accuracy of Transmission Electron Microscopy Analysis of Asbestos on Filters: Interlabortory Study.

100,204

ELECTRON MOBILITY

Majority and Minority Electron and Hole Mobilities in Heavily Doped

101,550

ELECTRON-MOLECULE COLLISIONS

Cross Sections and Related Data for Electron Collisions with Hydrogen Molecules and Molecular lons.

Near-Threshold Vibrational Excitation of $\mathrm{H} 2$ by Electron Impact: Resolution of Discrepancies between Experiment and Theory.

101,679

ELECTRON PROBES

Strategies for Background Subtraction in Electron Probe Microanalysis $X$-ray Compositional Mapping. MB91-195180

100,202

ELECTRON REACTIONS

Evidence for Surface alpha Particle Clusters in (nat)AG(197)Au from the (e,alpha) Reaction PB91-216812

101,705

ELECTRON SCATTERING

Possible (e,e'2N) Studies at CEBAF

PB91-147587

101,612
Spin-Dependent Electron-Impact Excitation of Sodium. PB91-175497

101,641

ELECTRON SPECTRA

Resonance Enhanced Multiphoton Ionization Spectra of the $\mathrm{GeF}$ and $\mathrm{GeCl}$ Radicals from $400-500 \mathrm{~nm}$.

100,396

\section{ELECTRON SPIN POLARIZATION}

Uses of Spin-Polarised Electrons in Fundamental Electron-Atom Collision Processes and the Analysis of Mag-

PB91-162172

101,490

Book Review: Polarized Electrons at Surfaces by J. Kirschner.

PB91-174532

101,493

Book Review: 'Polarized Electrons at Surfaces' by J. Kirschner.
PB91-175323

101,497

\section{ELECTRON SPIN RESONANCE}

Vibrational Spectra of Molecular lons Isolated in Solid Neon. III. N4(+).

100,243

Electron Spin Resonance for Monitoring Radiation-Processed Meats Containing Bone.

PB92-116672

100,018

\section{ELECTRON TRANSFER}

Slow Electron Transfer Reactions between Alkylhydra-

100,284

Pulse Radiolysis Studies of Organic Electron Transfer Reactions.

PB91-147801

100,285

ELECTRON TRANSITIONS

Transitions and Energy Levels for Cu-Like Yb(41+), $\mathrm{Ta}(44+)$, and $\mathrm{U}(63+)$.

Working Group 2: Atomic Transition Probabilities PB91-175588

101,645

Resonance Enhanced Multiphoton Ionization Spectra of the GeF and $\mathrm{GeCl}$ Radicals from 400-500 nm. 100,396 XANES of Transition Metal Zinc-Blende Semiconductors. XANES of Transition Metal Zinc-Blende Semiconductors.
PB92-117092 100,462

100,462

\section{ELECTRON TRANSPORT}

Preface to Book Entitled 'Nonequilibrium Effects in Ion and Electron Transport'.

PB91-159236

101,623

Electron Attenuation Lengths at $\mathrm{SiO} 2 / \mathrm{Si}$ Interfaces PB91-175612

101,499

\section{ELECTRONIC DATA INTERCHANGE}

Electronic Data Interchange (EDI). Category: Software Standard. Subcategory: Electronic Data Interchange.

FIPS PUB 161

ELECTRONIC MAIL

National PDES Testbed Mail Server User's Guide. National PDES Testbed Report Series.

PB91-159756

100,951

Application of Formal Description Techniques to the Specification of Distributed Test Systems.

100,566

Electronic Data Interchange in Message Handling Systems.

100,571

ELECTRONIC PUBLISHING

Government Document Processing Requirements Report. PB91-187773

ELECTRONIC SPECTRA

Resonance Enhanced Multiphoton Ionization Spectroscopy of $\mathrm{CHCl} 2$ and $\mathrm{CDCl} 2$.

PB91-174870

100,328

ELECTRONIC STRUCTURE

Electronic Structure of High-Tc Superconductors Studied Using Photoelectron Spectroscopy.

101,513

Soft $X$-ray Absorption and Emission Spectra and the Electronic Structure of Some Exotic Materials.

PB91-194738

101,520

ELECTRONIC TECHNOLOGY

Scanning tunneling microscopy studies of the surfaces of a-Si:H and a-SiGe:H films. Annual report, 1 December 1989-31 January 199

DE91002169

100,785

Environmental Sensitivities of Quartz Crystal Oscillators.

N91-25797/2 100,778

Current Supply for High-T(sub c) Superconductor Testing.
PB91-133827

Electrical Breakdown and Streamer Statistics in $\mathrm{N}$ hexane under Uniform Field Conditions.

100,837

Current Density Dependence of Electromigration $150 \mathrm{En}$ Current Density Dependence of Electromigration
hancement Due to Pulsed Operation. PB91-134692 100,786 Effect of High Pressure on Prebreakdown Phenomena in
n-Hexane.
PB91-135020 100,839

Studies on the Degradation Products of Paper with and without Pollutants in a Closed Environment. 1. Preliminary

PB91-143313

101,181

Phonon-Assisted Magneto-Donor Optical Transitions in $n$ -

InSb.

101,467

Thermal Characterization of Integrated Circuits - A Tutori-

PB91-147819

100,789

Thermal Measurements of VLSI Packages: A Critical Review.

PB91-147827

100,790

Investigation of Photoconductive Picosecond Microstripline Switches on Self-Implanted Silicon on Sapphire (SOS).

100,791

Low Accelerating Voltage SEM Imaging and Metrology Using Backscattered Electrons.

101,469

Strontium-Induced Oxygen Defect Structure and Hole Doping in La2-xSrxCuO4.

Doping in La2-
PB91-148155

101,472

Comparison of High-Resistivity Measurements of Silicon by AC Impedance, DC Resistance, van der Pauw, and Four-Probe Methods.

PB91-148197

100,792

High Speed Superconducting A/D Converter

B91-148700

100,731

Micromachined Thermal Radiation Emitter from a Commercial CMOS Process.

PB91-148999

100,748

Characteristics of the Breakdown Voltage of Power MOSFETs After Total Dose Irradiation

B91-149062

100,793

Measurement of Capacitance on Wafers

PB91-149120

100,795

Selective-Area Epitaxial Growth of Gallium Arsenide on Silicon Substrates Patterned Using a Scanning Tunneling Microscope Operating in Air.

100,796

Modelling Gallium Arsenide Transistors.

100,797

NBS Submicron Particle Standards for Microcontamination Measurement.

PB91-158873

100,798

Modeling Electron Beam Interactions in Semiconductors.

PB91-159020

101,485

Center for Electronics and Electrical Engineering Technical Progress Bulletin Covering Center Programs, July to September 1990, with 1991 CEEE Events Calendar.
PB91-159749 801

Narrow-Gap Semiconductors and Related Materials. B91-162313

Cutting the High Cost of Testing

PB91-162362

101,492

Design, Fabrication, and Testing of an Interconnect Test Structure for Evaluating VLSI Processes.

1-174581 
Annotated Bibliography: Diagnostic Methods and Measurement Approaches to Detect Incipient Defects Due to Aging of Cables.

101369

Automated Reverse-Bias Second-Breakdown Transistor

PB91-236513 100,814

Emitter Ballasting Resistor Design for, and Current Handling Capability of AIGaAs/GaAs Power Heterojunction Bipolar Transistors.

PB91-236703

100,815

Testing the Accuracy of Calculated Equilibrium Carrier Concentrations in the Presence of Surface Fields.
PB91-236729

101,549

Generalized Photodiode Self-Calibration Formula

PB91-236901

100,760

Majority and Minority Electron and Hole Mobilities in Heavily Doped GaAs.

PB91-236968

101,550

Metrology for the Semiconductor Industry.

100,817

Center for Electronics and Electrical Engineering Technical Publication Announcements Covering Center Programs, October to December 1990, with 1991 CEEE Events Calendar.

PB91-240838

100,863

Video Processing with the Princeton Engine at NIST.

100,604

Numerical Modeling of Silicon Photodiodes for High-ACcuracy Applications. Part 1. Simulation Programs.

PB92-110295

100,763

Numerical Modeling of Silicon Photodiodes for High-ACcuracy Applications. Part 2. Interpreting Oxide-bias Experiments.

100,764

Numerical Modeling of Silicon Photodiodes for High-Accuracy Applications. Part 3. Interpolating and Extrapolating Internal Ouantum-Efficiency Calibrations.

PB92.110311

100,765

HgCdTe Detector Reliability Study for the GOES Pro.

gram.

100,766

Sensitivity of Ellipsometric Modeling to the 'Islands' of Silicon Precipitates at the Bottom of the Buried Oxide Layer in Annealed SIMOX.

LB92-116607

100,820

Modified Sliding Wire Potentiometer Test Structure for Mapping Nanometer-Level Distances.

100,821

Temperature Monitored/Controlled Silicon Photodiodes for Standardization.

100,767

PB92-116763

Experimentally Verified IGBT Model Implemented in the
Saber Circuit Simulator. Saber Circuit Simulator.

100,822

Knowledge Extraction Techniques for Expert System Assisted Wafer Screening.

PB92-116979

100,824

Assessment of Reliability Concerns for Wide-Temperature Operation of Semiconductor Devices and Circuits.
PB92-116987

100,825

Persistent Photoconductivity in SIMOX Films.

PB92-117084 100,826 Band-Gap Narrowing and III-V Heterostructure FET

PB92-117159 100,827

Influence of Nonsinusoidal Waveforms on Voltmeters, Ammeters, and Phase Meters.

PB92-117167 100,869

Residual Defects in SIMOX: Threading Dislocations and Pipes.

PB92-117233 100,829

Round-Robin Study of Implants in $\mathrm{Si}$ and $\mathrm{SiO} 2$ by SIMS, RBS, and NAA.

100,830

Measurement, Use, and Interpretation of TCR. $\quad 100,831$
PB92-117282 Intrinsic Carrier Concentrations in Long Wavelength $\mathrm{HgCdTe}$ Based on the New, Nonlinear Temperature Dependence of $\mathrm{Eg}(x, \mathrm{~T})$

PB92-117316

101,568

Electronics and Electrical Engineering Laboratory. 1991 Strategic Plan. Supporting Technology for U.S. Competitiveness in Electronics.

PB92-123082

100,870

ELECTRONICS

Center for Electronics and Electrical Engineering Technical Publication Announcements Covering Center Programs, October to December 1990, with 1991 CEEE Events Calend PB91-240838

100,863

ELECTROOPTICS

Electronics and Electrical Engineering Laboratory Technical Publication Announcements Covering Laboratory Programs, January to March 1991, with 1991 EEEL Events Calendar

PB92-112309

ELECTROPHORESIS

Electrokinetic Demixing of Two-Phase Aqueous Polymer Systems. 1. Separation Rates of Polyethylene GlycolDextran Mixtures.
PB91-159095

100,487

Electrokinetic Demixing of Two-Phase Aqueous Polymer Systems. 2. Separation Rates of Polyethylene Glycol-Mal-

PB91-237230

100,231

\section{ELLIPSOMETRY}

Ellipsometry SRM's for Use in Thin Film Measurements.

ELLIPTICAL CONFIGURATION

Buckling and Post-Buckling Behavior of Elliptical Plates: PB92-116573

EMERGENCY PLANNING

Visibility of Exit Directional Indicators.

PB91-184762

101,588

EMISSION SPECTROSCOPY

Advanced sensor development program for the pulp and paper industry. Final report.

DE91007688

101,248

ENCLOSURES

Mathematical Modeling of Enclosure Fires. PB92-108976

100,135

ENERGY ABSORPTION

Mass Energy-Transfer and Mass Energy-Absorption Coefficients, Including In-Flight Positron Annihilation for PB92-126473

101,445

\section{ENERGY CONSERVATION}

DISCOUNT-A Program for Discounting Computations in Life-Cycle Cost Analyses. User's Guide and Reference Manual

PB91-167288

100,897

Evaluation of the Heat Flux Transducer Technique for Measuring the Thermal Performance of Walls. PB91-194696

100,126

Diagnostic Techniques for Evaluating Office Building En-

PB91-195438

100,074

ENERGY CONSERVATION \& PRODUCTION

Short-Duration Autoignition Temperature Measurements ror Hydrocarbon Fuels

Round Robin on Apparent Thermal Conductivity of Several Loose-Fill Insulations.

PB91-149948

101,143

Evaluation of Building Systems Inventions.

100,896

Gas Analysis Modeling System.

PB91-175299

100,884

Energy Related Inventions Program: A Joint Program of the Department of Energy and the National Institute of Standards and Technology. Status Report for Recom. mendations 1 through 250 .

PB91-178871

100,902

Energy Related Inventions Program. A Joint Program of the Department of Energy and the National Institute of Standards and Technology. Status Report for Recommendations 251 through 523

100,903

Effect of Atomization Air on Droplet Dynamics of Spray Flames.

PB91-203869

100,550

Building Life Cycle Cost Computer Program, Version 3.2 (for Microcomputers)

100,898

Energy Prices and Discount Factors for Life-Cycle Cost Analysis 1992. Annual Supplement to NIST Handbook 135 and NBS Special Publication 709. PB92-112515

100,899

\section{ENERGY CONSUMPTION}

Prediction and Fault Detection of Building Energy Consumption Using Multi-Input, Single-Output Dynamical

100,073

PB91-195388

Interac-

PB91-206706

100,892

ENERGY GAP

Band-Gap Narrowing and III-V Heterostructure FETs. PB92-117159

100,827

\section{ENERGY LEVELS}

Spectrum and Energy Levels of the Xenonlike Ion Ce V. 101,638 Energy Levels and Transition Probabilities in the Ground State Configuration of Sulfur-Like Ions.

PB91-190033

101,658

Spectrum and Energy Levels of Seven-Times-Ionized Krypton (Kr VIII) and Resonance Lines of Eight-Timeslonized Krypton (Kr IX).

PB91-195511

101,673

Journal of Physical and Chemical Reference Data Volume 20, Number 5, September/October 1991. B92-148113

100,471

Wavelengths and Energy Level Classifications for the Spectra of Aluminum (AII through AI XIII).

100,472

Energy Levels of Krypton, $\mathrm{Kr} I$ through $\mathrm{Kr}$ XXXVI.

PB92-148139

100,473

ENERGY MANAGEMENT SYSTEMS

BACnet Communication Protocol for Building Automation Systems.

Draft Abstract Test Suite for Determining Conformance to the BACnet Protocol.

PB91-187732

100,070

Intelligent Building Technology in Japan.

00,071 Rule-Based Diagnostic Method for HVAC Fault Detection.
PB91-194936

ENERGY MODELS

Gas Analysis Modeling System.

ENERGY RELATED INVENTIONS PROGRAM

100,884

Energy Related Inventions Program. A Joint Program of the Department of Energy and the National Institute of Standards and Technology. Status Report for Recommendations 251 through 523.

100,903

ENERGY SOURCES

DISCOUNT-A Program for Discounting Computations in Life-Cycle Cost Analyses. User's Guide and Reference

PB91-167288

100,897

ENERGY TRANSFER

Mass Energy-Transfer and Mass Energy-Absorption Coefficients, Including In-Flight Positron Annihilation for Photon Energies $1 \mathrm{keV}$ to $100 \mathrm{Me}$

01,445

ENGINEERING DESIGN LABORATORY

Engineering Design Laboratory Guide.

ENGINEERING EDUCATION

Engineers' Statistical Literacy is Key to U.S. Competitive-

ENGINEERING/PRODUCT/INFORMATION STANDARDS

Domestic Implementation.

PB91-159061

100,176

International Co-operation: The Versailles Project on Advanced Materials and Standards.

101,191

Directory of Federal Government Laboratory Accredita

tion/Designation Programs.
PB91-167379

100,005

Standards Activities of Organizations in the United

States.

100,004

GATT Standards Code Activities of the National Institute of Standards and Technology 1990

PB91-187823

100,177

Standardizing Standards. 
Journal of Physical and Chemical Reference Data, Volume 19, Number 4, July-August 1990.

PB91-192435

100,354

Heat Capacities and Entropies of Organic Compounds in the Condensed Phase. Volume 2.

100,356

Thermodynamics of the Krebs Cycle and Related Com-

pounds.
PB91-192468

100,357

ENVELOPES

Airtightness of Office-Building Envelopes.
PB91-175307

100,067

ENVIRONMENTAL ENGINEERING

Preliminary Results of the Environmental Evaluation of the Federal Records Center in Overland Missouri. PB91-240747

100,082

ENVIRONMENTAL IMPACTS

Canadian Mass Fire Experiment, 1989 PB91-143305

101,352

ENVIRONMENTAL MONITORING

Replicate Measurements in the Interpretation of Environmental Monitoring.

PB91-189761

100,919

ENVIRONMENTAL STUDIES: POLLUTION MEASUREMENT Fundamental studies of black liquor combustion. Report No. 4, Phases, 2, 3, and 4: Final report, December 1987December 198

100,915

Thermodynamic Data for Modeling of Flue Gas Cleanup Systems (Abstract for Poster Session).

100,904

Proposed Standard Practice for Assessing the Performance of Gas-Phase Air Cleaning Equipment. 100,905
PB91-167353 Preliminary Results of the Environmental Evaluation of the Federal Records Center in Overland Missouri.
PB91-240747 100,082

ENVIRONMENTAL SURVEYS

Methods for the Analysis of Organometallic Compounds in Wastes.

100,189

ENZYMES

Low-Cost Aqueous Two-Phase System for Enzyme Extraction.

101,310

Thermodynamics of Industrially Important Enzyme Cata-

lyzed Reactions.
PB91-237602 101,311

Thermodynamics of the Disproportionation of Adenosine 5 '-diphosphate to Adenosine 5'-triphosphate and Adenosine 5'-monophosphate. 1. Equilibrium Model. PB92-116854

EPHEMERIS TIME

Precise Ephemerides for GPS Time Transfer.
PB91-236950

EPITAXIAL GROWTH

Epitaxial fcc Fe Films on $\mathrm{Cu}(100)$

PB91-149328

Selective-Area Epitaxial Growth of Gallium Arsenide on Silicon Substrates Patterned Using a Scanning Tunneling Microscope Operating in Air.

100,796

Structural Study of the Epitaxial Growth of fcc-Fe Films, Sandwiches, and Superlattices on $\mathrm{Cu}(100)$. PB91-237420

101,555

EPITAXY

XPS and Auger Forward Scattering in Epitaxial Films. PB91-174698

EPOXY COMPOSITES

Influence of Moisture and Pressure on the Mechanical Properties of a Glass-Epoxy Matrix Composite and a Graphite-Epoxy Matrix Composite.

AD-A239 509/3

101,114

EPOXY MATRIX COMPOSITES

Mechanical Loss in a Glass-Epoxy Composite. $\quad 101,070$
PB91-149435

Thermal Conductivity of Alumina Fiber/Epoxy and Alumina Fiber/PEEK from 4.2 to $310 \mathrm{~K}$.

PB92-117258

101,130

EPOXY RESINS

Effect of Crosslink Density on Physical Aging of Epoxy

PB91-189712 101,244

Viscoelastic Response of Epoxy Glasses Subjected to Different Thermal Treatments.

101,245

Physical Aging and the Viscoelastic Response of Network Glasses.

PB91-189829 101,246

Reflection/Absorption FTIR Spectral Characteristics of Thin and Thick Crosslinked Epoxy Films on Steel Sub-

PBg1-203711

100,509

Characterization of Epoxide Coatings on Steel by Reflection/Absorption Fourier Transform Infrared Spectroscopy: Quantitative Study.

PB91-203729

101,109
Network Structure in Epoxies. 6. The Growth Process Investigated by Neutron Scattering.

$1-237842$

100,514

EQUATIONS OF STATE

New Wide Range Equation of State for Helium.
PB91-162222 Thermodynamic Diagrams for Refrigerant Mixtures. PB91-236695 101,197 Thermodynamic Properties of 1,1,1,2,-Tetrafluoroethane (R134a) in the Critical Region.

100,233

New Equation of State and Tables of Thermodynamic New Equation of State and Tables of Thermodynamic
Properties for Methane Covering the Range from the Properties for Methane Covering the Range from the
Melting Line to $625 \mathrm{~K}$ at Pressures up to $1000 \mathrm{MPa}$.
PB92-148170
100,477

EQUILIBRIUM

Computer Simulation of Fluid-Fluid Phase Coexistence in Mixtures of Nonadditive Soft Disks.

PB91-162263

101,388

ERBIUM

Structural and Magnetic Properties of Er Thin Films and $\mathrm{Er} / \mathrm{Y}$ Superlattices: Magnetoelastic Effects.

101,489 PB91-161935

ERBIUM BARIUM CUPRATES

Magnetic Ordering of Er in Powder and Single Crystals of ErBa2Cu3O7.
PB91-187377

ERBIUM LASERS

101,505

Frequency Stabilization of an Erbium-Doped Fiber Laser A Potential Wavelength Standard for Optical Communica-

tions.
PB91-203091

100,570

Frequency Stabilization of a Tunable Erbium-Doped Fiber Laser.

PB91-203109

101,427

ERBIUM PALLADIUM STANNIDES

Crystal-Field Splittings in the Cubic Heusler Alloys PB91-194902

101,525

ERIP (ENERGY-RELATED INVENTIONS PROGRAM)

Feasibility of Using Knowledge-Based Systems for Aiding PB91-147728

100,895

EROSION

Mechanical Sputtering of Structural Stainless Steels. PB91-175448

101,152

ERROR ANALYSIS

Orthogonal Distance Regression.

ROR CORRECTION CODES

101,272

FORTRAN Temporary Program Fixes, FCVS78 Version 2.0, Level 26.

100,651

ERROR DETECTION CODES

Error Prevention and Detection in Data Preparation for a Numerically Controlled Milling Machine.

100,980

ESTELLE

Features and Facilities of Estelle.

PB91-158915

100,688

\section{ESTERS}

Difunctional and Multifunctional Monomers Capable of Cyclopolymerization

ESTIMATING

Estimation of Electromagnetic Fields in Complex Environ-

ments.
PB91-203315

101,691

ETHANE

Viscosity Surface for Mixtures of Methane and Ethane.
PB91-174763

Dipole Moments of Seven Partially Halogenated Ethane Refrigerants.

PB91-195131

100,377

High-Resolution Infrared Flash Kinetic Spectroscopy of OH Radicals

100,408

Thermophysical Properties of Ethane.

100,447

Hydrogen Component Fugacity Coefficients in Binary Mixtures with Etane: Pressure Dependence. PB92-116508

100,455

ETHANOL

Thermal Decomposition of lons. 3. Protonated Ethanol and Diethyl Ether

100,376

ETHERS

Alkyl Transfer Reactions between Protonated Alcohols and Ethers. The Gas.Phase Alkylation of Formaldehyde.
PB91-147470

100,280

Group Reactivities in the Gas Phase Reactions of Hydroxyl Radicals with Ethers.

100,330

Rate Constants for Hydrogen Abstraction Reactions of the Sulfate Radical, SO4- Alkanes and Ethers.
PB91-203232

\section{ETHYL ETHER}

Thermal Decomposition of lons. 3. Protonated Ethanol and Diethyl Ether.

THYLENE

100,376

Unified Description of Infinite-Dilution Thermodynamic Properties for Aqueous Solutes.

PB91-162081

100,319

Van der Waals Complexes in 1,3-Dipolar Cycloaddition Reactions: Ozone-Ethylene.

PB91-236737

100,417

\section{POENE COPOLYMERS}

Multistep Stress-Relaxation Behavior in Uniaxial Extension of an Ethylene-Hexene Copolymer.

101,242

\section{ETHYLENE PROPYLENE DIENE POLYMERS}

Further Investigation of the Effect of Application Parameters on Adhesive-Bonded Seams of Single-Ply Roof Membranes.

PB91-149153

100,105

EUROPE

Meeting the Challenges of a Global Market - Construc tion Standards, Testing and Certification.

100,090

UROPIUM

Low-Pressure Symmetric Discharge Heat Pipe and DROGS of Eu

PB91-195016

101,666

EUROPIUM BARIUM CUPRATES

Group and Phase Sound Velocities in an Eu1Ba2Cu3O7 Superconductor and Related Perovskite Oxides. PB91-194860

\section{EUROPIUM COMPLEXES}

Lanthanide Coordination Chemistry: Spectroscopic Properties of Terbium and Europium Poly(Pyrazol-1-YI) Borate

and Poly(Imidazol-1-YI) Borate.
PB91-203034

100,392

EUROPIUM OXIDES

Resonant Photoemission and PSD of Rare Earth Oxides. PB91-148015

100,287

EVACUATING (VACUUM)

Thermal Conductivity of Evacuated Perlite at Low Tem-

peratures as a Function of Load and Load History.
PB91-174672

EVAPORATORS

Simulation of an Evaporator with Nonuniform One-Dimensional Air Distribution.

PB91-174649

100,066

Evaporator Performance Investigation for Residential AirConditioning Application Using Mixed Refrigerants.
PB92-126432

EXCITED STATES

Detection of Excited States by Laser-Induced Fluorescence and Analysis of Energy Transfer.

AD-A227 296/1

100,242

Photoionization and Collisional Ionization of Excited Atoms Using Synchrotron and Laser Radiations. PB91-159301

EXCLUDED VOLUME

Flexible Polymer with Excluded Volume at an Interacting Penetrable Surface of Variable Dimension: A Multiple ep silon Perturbation Theory

EXITS

Visibility of Exit Signs and Directional Indicators. PB91-161984

100,517

XPERIMENTAL DESIGN 


\section{KEYWORD INDEX}

EXTRACTION

Low-Cost Aqueous Two-Phase System for Enzyme Extraction.

101,310

FACILITATED TRANSPORT MEMBRANE SYSTEMS Overview of Facilitated Transport Membrane Systems.

FACTORY AUTOMATION

Engineering Design Laboratory Guide.
PB91-167320
100,977

FAILURE ANALYSIS

Effects of Crystal Bonding on Brittle Fracture.
PB91-148221

101,06
101,002 Dynamic Arc-Power Source Response in GMA Welding.
PB91.149278

Spatially Overdetermined Analysis for Propagation Toughness Using Strain Gages.

101,164

FARADAY EFFECT

Performance and Limitations of Faraday Effect Sensors.
100,749

AST ION CONDUCTORS

Thermodynamics of the Divalent-Metal Fluorides. 4. Heat Capacity of MSnF4, M $=\mathrm{Pb}, \mathrm{Ba}$, or Sr, at Temperatures from $300 \mathrm{~K}$ to $660 \mathrm{~K}$

ATIGUE LIFE

Cyclic Fatigue from Frictional Degradation at Bridging Grains in Alumina.

AUJASITE

Structures and Properties of New Zeolite X-Type Zincophosphate and Beryllophosphate Molecular Sieves.
101,081

FDDI (FIBER DISTRIBUTED DATA INTERFACE)

Conformance Verification of FDDI Stations. PB91-148254

100,747

FEDERAL BUILDINGS

Direct Digital Control Based Building Automation System Design Criteria.

100,091

Guide Specification for Direct Digital Control Based Building Automation System.

PB91-216697

100,092

Standards for the Physical Protection of National Resources and Facilities.

100,006

Preliminary Results of the Environmental Evaluation of the Federal Records Center in Overland Missouri. PB91-240747

100,082

FEDERAL EMERGENCY MANAGEMENT AGENCY

Standards for the Physical Protection of National Resources and Facilities.

100,006

FEDERAL ENERGY MANAGEMENT PROGRAM

Energy Prices and Discount Factors for Life-Cycle Cost Analysis 1992. Annual Supplement to NIST Handbook 135 and NBS Special Publication 709. PB92-112515

100,899

FEDERAL GOVERNMENT CERTIFICATION

Directory of Federal Government Laboratory Accredita-

tion/Designation Programs.
PB91-167379

100,005

FEDERAL INFORMATION PROCESSING STANDARD

Graphical Kernel System (GKS). Ada Binding; Category: Software Standard; Subcategory: Graphics. $\quad 100,674$
FIPS PUB 120-1B American National Standard for C. Category: Software Standard. Subcategory: Programming Language.
FIPS PUB 160
100,676 $\begin{array}{ll} & 100,676 \\ & \text { FIPS PUB } 160\end{array}$ Standard. Subcategory: Electronic Data Interchange. FIPS PUB 161

FEDERAL INFORMATION PROCESSING STANDARDS Congressional Districts of the United States. Category:
Federal General Data Standard, Representations and Codes.

100,679

Guideline: American National Dictionary for Information Systems. Category: Software; Subcategory: DocumentaFIPS

100,956

Computer Output Microform (COM) Formats and Reduction Ratios, 16MM and 105MM. Category: Hardware Standard; Subcategory: Media. FIPS PUB 54-1

100,678

Interface between Data Terminal Equipment (DTE) and Data Circuit-Terminating Equipment (DCE) for Operation with Packet-Switched Data Networks (PSDN), or between Two DTEs, by Dedicated Circuit. Category: Hardware Standard. Subcategory: Data Transmission.

100,672

Graphical Kernel System (GKS); Category: Software Standard; Subcategory: Graphics. Government Open Systems Interconnection Profile Government Open Systems int Category: Hardware and Software Standards; Subcategory: Computer Network Protocols.
FIPS PUB 146.1 100,675

Federal Information 100.680

Developing Federal Software Standards: A New Direc-

PB91-134536 100,637

Specifications for a Federal Information Processing Standard Data Dictionary System.

PB91-158758

100,687

How the Government Shapes UNIX Standards. $\quad 100,693$
PB91-203133

GOSIP Conformance and Interoperation Testing and Registration

100,694

Validated Products List (Cobol, Fortran, ADA, Pascal, MUMPS, SOL).

FEES

NIST Calibration Services Users Guide, 1991. PB92-126416

100,671

FERRITIC STAINLESS STEELS

Metallographic Study of the Crack-Tip Region from Fracture Mechanics Specimens of Austenitic and Ferritic

189951

101,158

FERROCENE

Electrochemical Behavior of Ferrocene in Polyethylene PBide-400.

100,439

FERROMAGNETIC MATERIALS

System for the Study of Magnetic Materials and Magnetic Imaging with the Scanning Tunneling Microscope. PB91-194779

101,522

FIBER COMPOSITES

Polymer Composite Processing. Industry Workshop (2nd). Held at Gaithersburg, Maryland on May 18, 1990.

PB91-132191

101,115

Mechanical Loss in a Glass-Epoxy Composite.

PB91.149435

101,070

Mechanical Properties of Alumina-Peek Unidirectional Composite: Compression, Shear, and Tension.

PB91-162180

101,118

Elastic Constants of Fiber-Reinforced Composites: A Fresh Measurement Approach.

101,123

Reduction in Sintering Damage of Fiber Reinforced Composites.

PB91-195362

101,124

Effect of Thermal Expansion Mismatch on Fiber Pull-Out in Glass Matrix Composites.

PB91-202952

101,125

Microstructure and Elastic Properties of Dental Resin and Resin-Based Glass-Reinforced Composites: XRD, SEM and Ultrasonic Methods.

PB91-204081

100,050

Differential Sintering

101,128

Thermal Conductivity of Alumina Fiber/Epoxy and Alumina Fiber/PEEK from 4.2 to $310 \mathrm{~K}$

101,130

FIBER DISTRIBUTED DATA INTERFACE

Conformance Verification of FDDI Stations. $\quad 100,747$

FIBER OPTICS

Technical Digest-Symposium on Optical Fiber Measurements, 1990.

101,403

Progress in the Development of Miniature Optical Fiber Current Sensors.

Practical Considerations in the Design of Optical Fiber Current Sensors.
PB91-134742

100,746

Fiber-Optic Fluorescence Array to Study Free Convection in Porous Media.

101,407

Iron-Garnet Magnetic Field Sensors with $100 \mathrm{pT} / \mathrm{per}$ square root $\mathrm{Hz}$ Noise-Equivalent Field.

100,751

Faraday Effect Current Sensing Using a Sagnac Interferometer with a $3 \times 3$ Coupler.

ometer with a $3 x$

100,754

Methods of Analyzing Planar Optical Waveguides. PB91-236752

100,774

FIBERGLASS

Microstructure and Elastic Properties of Dental Resin and Resin-Based Glass-Reinforced Composites: XRD, SEM and Ultrasonic Methods.

PB91-204081

100,050

FIBERS

Accuracy of Transmission Electron Microscopy Analysis of Asbestos on Filters: Interlabortory Study. PB91-195727

100,204

FIELD EFFECT TRANSISTORS

Characteristics of the Breakdown Voltage of Power MOS. FETs After Total Dose Irradiation.

PB91-149062

100,793

and-Gap Narrowing and III-V Heterostructure FETs.

PBg2-117159

100,827

FIELD ELECTRON MICROSCOPY

Method of FIM-FEEM Specimen Preparation of SuperPB91-203596

101,542

FIELD ION MICROSCOPY

Method of FIM-FEEM Specimen Preparation of Superconducting and Other Oxides.

01,542

Progress in Field Ion Microscopy Imaging of High-Tc Superconducting Oxides.

101,543 Interpretation of Field Ion Microscopy (FIM) Images of
Asymmetrical Specimens of 1,2,3-Type High-Tc SuperPB92-116730

101,567

FIELD STRENGTH

Estimation of Electromagnetic Fields in Complex Environ-

PB91-203315

101,691

FIELD TESTS

Characterization of Field Concrete.

PB91-178863

100,528

FIFTH FORCE

Search for a Composition Dependent Force Using a Liquid-Supported Torsion Balance.

101,639

\section{FILAMENTS}

Effects of Multiple Filament Geometry in the Hot Filament Deposition of Diamond Films.

AD-A236 $708 / 4$

Issues in Transparent File Access.

PB91-187831

101,142 PDES Testbed Report Series.

100,984

FILLERS

Filler Systems Based on Calcium Metaphosphates.

PB91-194654

100,048

FILM DOSIMETRY

Sensitometry of the Response of a New Radiochromic Film Dosimeter to Gamma Radiation and Electron

PB91-237008

101,326

Calibration of Ophthalmic Applicators at NIST: A Revised Approach.

101,327

Radiochromic Film Dosimeter for Gamma Radiation in the Absorbed-Dose Range 0.1 - $10 \mathrm{kGY}$

PB92-11696

101,364

Temperature Dependence of Radiochromic Film Dosi-

PB92-117134

101,728

Photon Energy Dependence of the Sensitivity of Radiochromic Film and Comparison with Silver Halide Film and LiF TLDs Used for Brachytherapy Dosimetry. PB92-117142

101,296

FILM THICKNESS

Ellipsometry SRM's for Use in Thin Film Measurements. FILMS

Characterization of Tricresylphosphate Lubricating Films by Micro-Fourier Transform Infrared Spectroscopy

PB91-203810

FINITE DIFFERENCE METHOD

Numerical Simulation and Visualization Models of Stress Wave Propagation Graphite/Epoxy Composites. PB91-174946

101,120

FINITE ELEMENT METHOD

Extended Abstract: A Finite Element Study of the Stress and Displacement Fields Produced by Point Impact. PB91-175224 
PB91-146977

100,097

FPETOOL User's Guide.

PB92-108919

100,134

Data for Fire Hazard Assessment of Selected Non-Halogenated and Halogenated Fire Retardants; Report of PB92-112473.

100,557

FIRE MODELS

Mathematical Modeling of Enclosure Fires.

PB92-108976

100,135

FIRE PREVENTION

Summaries of BFRL Fire Research In-House Projects and Grants, 199

100,168

FIRE PROTECTION

Investigation of Simulated Oil-Well Blowout Fires.
PB91-144337

Five Small Flaming Fire Tests in a Simulated Hospital Patient Room Prolected by Automatic Fire Sprinklers.

PB91-159038

100,109

FPETOOL User's Guide.

PB92-108919

Navy Safety Center Data on the Effects of Fire Protection Systems on Electrical Equipment

PB92-108935

101,376

FIRE RESEARCH

Concept of the Calculus of Fire Satety.

100,093

Reduction of Hydrogen Cyanide Concentrations and Acute Inhalation Toxicity from Flexible Polyurethan Foam Combustion Products by the Addition of Copper Compounds. Part 3. The Effect of Copper Additives on the Flammability Characteristics of Flexible Polyurethane Foam.

PB91-132167

101,140

Aspects of Smoldering Combustion.

100,535

Heat Flux, Mass Loss Rate and Upward Flame Spread for Burning Vertical Walls (1990)

PB91-143297

100,094

Canadian Mass Fire Experiment, 1989

101,352

Investigation of Simulated Oil-Well Blowout Fires.

PB91-144337

01,356

Using the Harvard/NIST Mark VI Fire Simulation.
PB91-144394

Programmer's Reference Manual for CFAST, the Unified Model of Fire Growth and Smoke Transport.

PB91-144436

100,096

Heat Release Rate: The Single Most Important Variable in Fire Hazard.

100,097

Review of Four Compartment Fires with Four Compart ment Fire Models.

PB91-147215

100,098

Forced Smolder Propagation and the Transition to Flaming in Cellulosic Insulation.

100,101

Smoldering Combustion Propagation Through a Permeable Horizontal Fuel Layer.

PB91-147843

100,102

Development of a Line Camera for Real-Time Measurements of Concentration in Turbulent Flow Fields.

PB91-147892 101,386

Signal Detection Efficiency in Multiphoton Ionization Flame Measurements.

100,540

Refinement of a Model for Fire Growth and Smoke

Transport.

PB91-148510

100,104

Application of Fire Research

PB91-149302

100,153

Species Produced in Fires Burning in Two-Layered and Homogeneous Vitiated Environments.

PB91.157180

100,542

Fire Propagation in Concurrent Flows.

PB91-157206

00,543

Compartment Fire-Generated Environment and Smoke Filling.

100,107

Algorithm for the Mass-Loss Rate of a Burning Wall.

PB91-158998

100,108

Five Small Flaming Fire Tests in a Simulated Hospital Patient Room Protected by Automatic Fire Sprinklers.

PB91-159038

100,109

Ceiling Jet in Fires

PB91-167197

100,114

Experimental Study of Top Vented Compartment Fires.

Role of Bench-Scale Test Data in Assessing Real-Scale Fire Toxicity.

100,156

Users Guide for

100,999

Ceiling Jet-Driven Wall Flows in Compartment Fires.
PB91-174573

100,117

Letter to the Editor of Standardization News.

100,118

Letter to the Editor, Fire Technology, Comments on T.Z. Harmathy Viewpoint, May 1985.

PB91-174797

100,545

Surface Flame Spread.

BB91-175331

Smoke Production and Properties.

PB91-175422

100,546

FIREDOC Users Manual, 2nd Edition.

PB91-178830

100,120

Visibility of Exit Directional Indicators

100,547

PB91-184762

101,000

STEP: Standard for the Exchange of Product Model Data Resource Integration: Semantic and Syntactic Rules. PB91-184788 100,966 Improving the Fire Performance of Building Contents.

PB91-187260 100,084 Building and Fire Research Laboratory Publications,

PB91-187807

100,122

Engineering Analysis of Fire Development in the Hospice t Southern Michigan, December 15, 1985.

100,158

Molecular Dynamics of Initial Events in the Thermal Deg radation of Polymers.

PB91-195271

100,503

Water Spray Suppression of Fully-Developed Wood Crib Fies in a Compartment

PB91-203620

100,159

Kinetic Properties of the Components of Douglas-Fir and the Heat of Combustion of Their Volatile Pyrolysis Prod-

PB91-20379

100,548

Wind Effects on Fires.

100,549

Technical Reference and User's Guide for FAST/FFM Version 3.

PB91-206664

100,086

Sprinkler/Hot Layer Interaction.

BB91-206680

100,129

Material Flammability Test Assessment for Space Station

PB91-216606 101,735

Applications of the Generalized Global Equivalence Ratio Model (GGERM) for Predicting the Generation Rate an Distribution of Products of Combustion in Two-Layer Fire Environments: Methane and Hexanes.

PB91-216689

100,131

First Pass at Computing the Cost of Fire Safety in a Modern Society.

101,730

Interaction of an Isolated Sprinkler Spray and a TwoLayer Compartment Fire Environment. PB91-216804
100,551 Estimated Impact of the Center for Fire Research Program on the Costs of Fire.

PB91-217422

100,161

Reduction in Fire Hazard in Corridors and Areas Adjoining Corridors Provided by Sprinklers.

ing Corridors Pro

100,162

General Routine for Analysis of Stack Effect

PB91-231589

100,163

Partial

Flames.

100553

Laser-Induced Fluorescence of $\mathrm{CH}$. in a Laminar $\mathrm{CH} 4$ /Air Diffusion Flame: Implications for Diagnostic Measurements and Analysis of Chemical Rates.

PB91-237099

100,554

Experimental Study of the Pyrolysis Rate of a Poly(methy methacrylate) (PMMA) Wall Panel in a Reduced-Scale Enclosure.

100,555

Suppression of Post-Flashover Compartment Fires Using Manually Applied Water Sprays.

100,164

Framework for Utilizing Fire Property Tests.

PB91-240788

100,165

FPETOOL User's Guide.

PBg2-108919

Short-Duration Autoignition Temperature Measurements tor Hydrocarbon Fuels.

100,887

Navy Safety Center Data on the Effects of Fire Protection Systems on Electrical Equipment.

PB92-108935

101,376

Mathematical Modeling of Enclosure Fires.

Cone Calorimeter Rate of Heat Release Measurements for Upholstered Composites of Polyurethane Foams. PB92-108984

International Fire Detection Bibliography, 1975-1990.

PB92-10915

Joint Panel Meeting of the UJNR Panel on Fire Research and Safety (11th). Held in Berkeley, CA. on October 19 24, 1989.

PB92-109164

100,166

Data for Room Fire Model Comparisons.

PB92-110287

100,167

Summaries of BFRL Fire Research In-House Projects and Grants, 1991.

PB92-112218 100,168

Engineering Analysis of the Fire Development in the Hillhaven Nursing Home Fire, October 5, 1989.

PB92-112317

100,169

Theoretical Investigation of Piloted Ignition of Wood.

100,556

Data for Fire Hazard Assessment of Selected Non-Halogenated and Halogenated Fire Retardants; Report of Test FR 3983.

PB92-112473

100,557

Turbulent Upward Flame Spread for Burning Vertical Walls Made of Finite Thickness.

PB92-112531

100,170

Users' Guide to BREAK1, the Berkeley Algorithm for Breaking Window Glass in a Compartment Fire.

B92-112556

Large-Scale Validation of Bench-Scale Fire Toxicity

PB92-116458

100,139

Effects of Global Density Ratio on the Centerline Mixing Behavior of Axisymmetric Turbulent Jets.

100,014

Large-Scale Turbulent Structures and the Stabilization of Lifted Turbulent Jet Ditfusion Flames.

PB92-117191

100,558

Reynolds Number Effects on the Mixing Behavior of Axisymmetric Turbulent Jets.

PB92-117209

100,015

Relative $\mathrm{H}$-Atom and O-Atom Concentration Measurement in a Laminar, Methane/Air Diffusion Flame.

PB92-117365

Flammability Characterization of Foam Plastics.

PB92-123033

100,559

Generated Ceiling Jet Characteristics an Convective Heat Transfer to Ceiling and Wall Surfaces in a Two-Layer Zone-Type Fire Environment: Uniform Tem perature Ceiling and Walls.

PB92-123074

101,370

Development of a Technique to Assess the Adequacy of the Municipal Water Supply for a Residential Sprinkler System.

PB92-123116

101,358

FIRE RESEARCH INFORMATION SERVICES 


\section{KEYWORD INDEX}

PB91-216804

General Routin

100,163

Suppression of Post-Flashover Compartment Fires Using Manually Applied Water Sprays.

PB91-240770

100,164

Cone Calorimeter Rate of Heat Release Measurements for Upholstered Composites of Polyurethane Foams. 100,087 Summaries of BFRL Fire Research In-House Projects and Grants, 1991

PB92-112218

100,168

Turbulent Upward Flame Spread for Burning Vertical Walls Made of Finite Thickness.

100,170

Large-Scale Validation of Bench-Scale Fire Toxicity

Tests.

100,139

FIREDOC SYSTEMS

FiteDOC Users Manual, 2nd Edition.

PB91-178830

100,547

FIRES

Investigation of Simulated Oil-Well Blowout Fires. 101,356

PB91-144337

Using the Harvar

100,095

Programmer's Reference Manual for CFAST, the Unified

Model of Fire Growth and Smoke Transport

PB91-144436 100,096

Heat Release Rate: The Single Most Important Variable in Fire Hazard
PB91-146977

100,097

Review of Four Compartment Fires with Four Compartment Fire Models.

100,098

Refinement of a Model for Fire Growth and Smoke Transport

Transport.
PB91-148510

100,104

Compartment Fire-Generated Environment and Smoke

Filling.

100,107

Algorithm for the Mass-Loss Rate of a Burning Wall.

PBo1-158

100,108

Ceiling Jet in Fires.

PB91-167197

100,114

Experimental Study of Top Vented Compartment Fires.

$167254 \quad 100,155$

Role of Bench-Scale Test Data in Assessing Real-Scale Fire Toxicity.
PB91-167270

100,156

Ceiling Jet-Driven Wall Flows in Compartment Fires.

PB91-174573

100,117

Letter to the Editor, Fire Technology, Comments on T.Z. Harmathy Viewpoint, May 1985.

PB91-174797

100,545

Surface Flame Spread.

B91-175331

100,546

Smoke Production and Properties.

100,120

Engineering Analysis of Fire Development in the Hospice of Southern Michigan, December 15, 1985.

PB91-195222

100,158

Wind Effects on Fires.

PB91-203851

100,549

Technical Reference and User's Guide for FAST/FFM Version 3

PB91-206664

Sprinkler/Hot Layer Interaction.

100,086

100,129

Applications of the Generalized Global Equivalence Ratio Model (GGERM) for Predicting the Generation Rate and Distribution of Products of Combustion in Two-Layer Fire

Environments: Methane and Hexanes.
PB91-216689

100,131

Building and Fire

100,132

Framework for Utilizing Fire Property Tests.

PB91-240788

100,165

Mathematical Modeling of Enclosure Fires.

PB92-108976

100,135

Engineering Analysis of the Fire Development in the Hillhaven Nursing Home Fire, October 5, 1989.

100,169

Users' Guide to BREAK1, the Berkeley Algorithm for Breaking Window Glass in a Compartment Fire.
PB92-112556

100,136 Fire-Plume-Generated Ceiling Jet Characteristics and a Two-Layer Zone-Type Fire Environment: Uniform Temperature Ceiling and Walls.

PB92-123074

101,370

FISSION CROSS SECTIONS

Electrofission in the Quasifree and Delta Regions PB91-147355

101,609

FISSION PRODUCTS

Method to Enhance Porosity of Micro-particles.
PB91-187328

101,185

AME PROPAGATION

Forced Smolder Propagation and the Transition to Flaming in Cellulosic Insulation

100,101

91-147835

Permea-

Smoldering Combustion Pro

PB91-147843

100,102

Fire Propagation in Concurrent Flows.

PB91-157206

100,543

Surface Flame Spread.

PB91-175331

100,546

FLAME SPRAYING

Velocity and Droplet Size Measurments in a Fuel Spray.
100,539

FLAMES

Heat Flux, Mass Loss Rate and Upward Flame Spread for Burning Vertical Walls (1990).

PB91-143297

100,094

Effect of Atomization Air on Droplet Dynamics of Spray

PB91-203869

100,550

Temperature Measurements in Flames Using Thermally Assisted Laser-Induced Fluorescence of Ga.

100,420

FLAMMABIL

Improving the Fire Periormance of Building Contents.

PB91-187260

Plammability

100,084

AMMABILITY TESTING

Forced Smolder Propagation and the Transition to Flaming in Cellulosic Insulation

PB91-147835

100,10

Smoldering Combustion Propagation Through a Permeable Horizontal Fuel Layer.

100,102

Modern Test Methods for Flammability.

PB91-167676 100,116

Material Flammability Test Assessment for Space Station Freedom.

Framework for Utilizing Fire Property Tests. $\quad 100,165$

$\begin{array}{ll}\text { PB91-240788 } & 100,165\end{array}$

Data for Fire Hazard Assessment of Selected Non-Halo Test FR 3983

PB92-112473

100,557

FLEXURAL STRENGTH

Test Method for Tensile Creep of Structural Ceramics Using Flexure Beams.

101,099

FLIGHT TESTS

Recommended Fine Positioning Test for the Develop ment Test Flight (DTF-1) of the NASA Flight Telerobotic Servicer (FTS)

FLOOAS

Design Heat Loss Factors for Basement and Slab Floors.
PB90 100, 05967561

FLORY-HUGGINS PARAMETER

Mechanical and Swelling Behavior of Crosslinked Natura Rubber: Consequences of the Flory-Rehner Hypothesis. Rubber: Conseq

FLORY-AEHNER HYPOTHESIS

Mechanical and Swelling Behavior of Crosslinked Natura Rubber: Consequences of the Flory-Rehner Hypothesis. PB91-195081

101,141

FLOW CONDITIONERS

Choosing Flow Conditioners and Their Location for Orifice Flow Measur
PB92-116490

101,397

\section{FLOW INJECTION ANALYSIS}

Liposome Flow Injection Immunoassay: Implications for Sensitivity, Dynamic Range, and Antibody Regeneration.

FLOW MEASUREMENT

Gas Flow Measurement: Calibration Facilities and Fluid Metering Traceability at the National Bureau of Standards. 134403

100,879

Flowmeter Installation Effects: A New Approach to an Old but Prevalent Problem.

100,920

Effect of Pipe Surface Finish on Gas Flow Measurement

with an Orifice Meter.

Effect of Tube Bundle Flow Conditioners on Orifice Meter Discharge Coefficients.

100,881

FLOW MODELS

Multi-Zone Dispersal Analysis by Element Assembly. PB91-187146

100,907

\section{FLOW REGULATORS}

Effect of Tube Bundle Flow Conditioners on Orifice Meter Discharge Coefficients.

B 91-148791

100,881

FLOW VISUALIZATION

Flowmeter Installation Effects: A New Approach to an Old but Prevalent Problem.

PB91-134411

100,920

Development of a Line Camera for Real-Time Measurements of Concentration in Turbulent Flow Fields.

PB91-147892

101,386

Analysis of Laminar Vortex Shedding Behind a Circular Cylinder by Computer-Aided Flow Visualization. PB91-203000

101,393

\section{LOWMETERS}

Gas Flow Measurement: Calibration Facilities and Fluid Metering Traceability at the National Bureau of Stand-

PB91-134403

100,879

Effect of the Location of an In-Line Tube Bundle on Orifice Flowmeter Performance.

PB91-134445

100,921

SHOOT Flowmeter and Pressure Transducers. PB91-147488

100,923

\section{FLUE GASES}

Thermodynamic Data for Modeling of Flue Gas Cleanup Systems (Abstract for Poster Session).

100,904

Development of a New Tracer Technology Using Enriched Rare-Earth Isotopes.

100,908

FLUID-SOLID INTERACTIONS

How Does Surface Roughness Affect Polymer-Surface Interactions.

100,341

Study on the Nature of Boundary Lubricating Film: Analytical Method Development.

101,176

\section{FLUIDS}

Integrated theoretical and experimental study of the thermophysical properties of fluid mixtures. Progress report (February 1990-February 1991)

Extension of an Improved One-Fluid Conformal Solution Theory to Real Fluid Mixtures with Large Size Differ-

PB91-147405

100,278

Use of Computer Algebra to Locate Critical Loci in Fluid Mixtures.

101,390

Taking the Measure of Fluid Properties Data Bases. PB91-200832

101,392

Thermodynamic Behavior of Fluids in the Supercritical Region.

Thermodynamics of Solutions Near the Solvent's Critical

PBoint. 237297

100,232

FLUIDS: LIQUIDS/GASES/PLASMAS 


\section{KEYWORD INDEX}

FREE ENERGY

Analysis of Laminar Vortex Shedding Behind a Circular Cylinder by Computer-Aided Flow Visualization.

101,393

ICLASS-91 Proceedings of the International Conference on Liquid Atomization and Spray Systems (5th). Held in Gaithersburg, MD. on July 15-18, 1991.

100,552

Effect of Gravity Modulation on Thermosolutal Convection in an Infinite Layer of Fluid.

tion in an Infinit

101396

Hydrogen Component Fugacity Coefficients in Binary Mixtures with Etane: Pressure Dependence.

100,455

FLUOBORATES

Heat Capacity of Deuterated Ammonium Tetrafluoroborte ND4BF4 from 7 to $348 \mathrm{~K}$

PB91-147058

100,273

FLUORANTHENES

Cryogenic-Temperature Fluorescence Spectra of Polynuclear Aromatic Hydrocarbons of Molecular Weight 302. PB91-147157

FLUORESCENCE

Fiber-Optic Fluorescence Array to Study Free Convection in Porous Media.

101,407

Relative H-Atom and O-Atom Concentration Measurement in a Laminar, Methane/Air Diffusion Flame. PB92-117365

100,559

FLUORESCENCE SPECTROSCOPY

Cryogenic-Temperature Fluorescence Spectra of Polynuclear Aromatic Hydrocarbons of Molecular Weight 302. PB91-147157

100,274

Fluorescence Monitoring of Polymer Processing: Mixing and Zero Shear Viscosity.

100,492

FLUORIDES

Design Diagrams for Heavy Metal Fluoride Glass Windows.

101,419

FLUORINATED ALPHATIC HYDROCARBONS

Dipole Moments of Seven Partially Halogenated Ethane PBrigerants.

100,377

FLUORINE-LIKE IONS

Dielectronic Recombination Rate Coefficients for lons of the Fluorine Isoelectronic Sequence.

PB91-195602

101,674

FLUORINE ORGANIC COMPOUNDS

Fluoromethanol: Synthesis, Microwave Spectrum, and Dipole Moment

100,288

FLUOROHYDROCARBONS

Thermodynamic Properties of 1,1,1,2,-Tetrafluoroethane (R134a) in the Critical Region.

PB91-237578

100,233

FLUOROPOLYMERS

High Purity Fluoropolymer Materials: Trace Element Content and Leaching

100,491

FLUX PINNING

Low-Field Flux Pinning in Twinned and Detwinned Single Crystals of YBa2Cu3O7-x.

PB91-237552

101,559

FLY ASH

Development of a New Tracer Technology Using Enriched Rare-Earth Isotopes.

100,908

FOAM RUBBER

Reduction of Hydrogen Cyanide Concentrations and Acute Inhalation Toxicity from Flexible Polyurethane Foam Combustion Products by the Addition of Copper Compounds. Part 3. The Effect of Copper Additives on the Flammability Characteristics of Flexible Polyurethane Foam.

PB91-132167

101,140

FOOD ANALYSIS

Dietary Collection Procedures.

PB91-147413

101,317

amma-Iradiated Seafoods: Identification and Dosimetn by Electron Paramagnetic Resonance Spectroscopy. 100,016 Co-Trial on ESR Identification and Estimates of gammaRay and Electron Absorbed Doses Given to Meat and

PB91-194720

100,017

FOOD PROCESSING

gamma-Irradiated Seafoods: Identification and Dosimetr by Electron Paramagnetic Resonance Spectroscopy. Co-Trial on ESR Identification and Estimates of gammaRay and Electron Absorbed Doses Given to Meat and

PB91-194720 100,017

Electron Spin Resonance for Monitoring Radiation-Processed Meats Containing Bone.

PB92-116672

100,018

FORCE

Eccentric Load Sensitivity of Force Sensors
PB91-175406

100,931

\section{FORCE MEASUREMENT}

Summary of the Intercomparison of the Force Standard She National Institute of Standards and desanstalt, Germany.

PB92-126622

101,590

FORCE STANDARDS

Summary of the Intercomparison of the Force Standard Machines of the National Institute of Standards and Technology, USA, and the Physikalisch-Technische Bundesanstalt, Germany.

PB92-126622

101,590

FOREIGN TECHNOLOGY

Report on 1989 Actions by International Institute of Welding. 134619

101,001

Heat Capacity of Deuterated Ammonium Tetrafluoroborate ND4BF4 from 7 to $348 \mathrm{~K}$

100,273

Convergence Properties of a Class of Rank-Two Up-

dates.
PB91-187799

101,260

High Magnetic Field Facilities in Japan Related to Superconductivity Research (Japan Technology Program). PB91-240762

101,718 International Syst

101,719

Journal of Physical and Chemical Reference Data Volume 20, Number 6, November/December 1991 PB92-148162

100,476

FOREST FIRES

Canadian Mass Fire Experiment, 1989

\section{FORMALDEHYDE}

Air Exchange and Pollutant Concentration Measurements in Two Office Buildings.

ORMIC ACID

Thermodynamics of the Krebs Cycle and Related Compounds.

PB91-192468

100,357

FORTRAN PROGRAMMING LANGUAGE

FORTRAN Temporary Program Fixes, FCVS78 Version 2.0, Level 26

100,651

Validated Products List (Cobol, Fortran, ADA, Pascal MUMPS, SQL).
PB92-937300

100,671

FOSSILS

Accelerator (14)C Dating of Late Pleistocene Megafauna.

101,35
PB91-237396

Accelerator Radiocarbon Dates on Charcoal, Shell, and Human Bone
PB91-237404

101,355

FOURIER SPECTROSCOPY

Fourier Transform Infrared Spectroscopy at the NIST: High Temperature Molecules and Atmospheric Mole-

PB91-189803

101,424

FOURIER TRANSFORMATION

Fourier Transform Infrared Analysis of Ceramic Powders: Quantitative Determination of Alpha, Beta, and Amorphous Phases of Silicon Nitride.

PB91-237628

101,096

FOURTH GENERATION LANGUAGES

Functional Benchmarks for Fourth Generation Languages.

100,644

FPETOOL COMPUTER PROGRAM

FPETOOL User's Guide.

RACTALS

100,134

How Does Surface Roughness Affect Polymer-Surface Interactions.

FRACTIONATION

Study of the Linear Viscoelastic Properties of Cyclic Po-

lystyrenes Using Creep and Recovery Measurements.

FRACTURE (MECHANICS)

Strength and Microstructure of Ceramics.

AD-A243 049/4 101,056

Stress Intensity Factors by Enriched Mixed Finite Ele-

ments.

101,577

Effects of Crystal Bonding on Brittle Fracture.

PB91-148221

Cleavage Step Formation and Resistance to Transgranu-

lar Stress Corrosion Cracking.
PB91-149047

101,133

In situ Measurements of Bridged Crack Interfaces in the Scanning Electron Microscope.

101,068

Tensile-Fracture Resistance Mechanisms in Brittle Polycrystals: An Ultrasonics and In-situ Microscopy Investigation.

PB91-149344

101,190

Crack Arrest Fracture Toughness Measurements of Normalized and Inclusion Shape Controlled AAR TC128 Grade B Steel, and Micro-Alloyed, Control-Rolled, and Inclusion Shape Controlled A $8 \times \times$ Grade B Steel.

PB91-167346

101,150

Improved Optical Diffraction Strain Measurement System. PB91-174433 101,581

Use of Birefringent Coatings in Fracture Mechanics.

PB91-174599 101,582

Interlaminar Shear Fracture of Laminated Composites. PB91-187336 101,122

Plastic Zone Formation Around an Arresting Crack

PB91-189316

101,373

Fundamental Condition for Existence of Microcrack Clouds in Monophase Ceramics.

101,082

Influence of Interstitial Content on Fracture Toughness.

PB91-189944

101,157

Metallographic Study of the Crack-Tip Region from Fracture Mechanics Specimens of Austenitic and Ferritic

PB91-189951

101,158

Indentation Determination of Crack Growth Parameters in Gallium Arsenide.

101,083

Reduction in Sintering Damage of Fiber Reinforced Com-

PB91-195362

101,124

Role of Glassy Interfaces in High Temperature Crack

Growth in SiC Fiber Reinforced Alumina.
PB91-203687

101,127

Cyclic Fatigue from Frictional Degradation at Bridging rains in Alumina.

101,092

Microstructural Aspects of the Fracture of Hardened Cement Paste.

PB91-237479

100,133

Environmentally Enhanced Fracture of Gallium Arsenide.

PB91-237743

101,097

Mechanical Properties and Fracture Toughness of AAR TC128 Grade B Steel in the Normalized, and Normalized and Stress Relieved Conditions.

101,169 National Educators Workshop: Update '90. Standard Ex-
periments in Engineering Materials Science and Technolperiments in Engineering Materials Science and Technol-
ogy.
PBg2-126606

101,253

FRACTURE PROPERTIES

Experimental and Numerical Studies of the J-Integral for a Surface Flaw

101,576

Spatially Overdetermined Analysis for Propagation Toughness Using Strain Gages.

101,164

Interlaboratory Tension and Fracture Toughness Test ReInterlaboratory Tension and Fracture Toughness Test Re-
sults for CSUS-JN1 (Fe-25Cr-15Ni-0.3N) Austenitic Stainsults for CSUS-JN1

less Steel at 4
PB91-237081

101,166

Effect of Processing on 4-K Mechanical Properties of a Microalloyed Austenitic Stainless Steel.

PB91-237206

101,167

FRACTURE STRENGTH 


\section{KEYWORD INDEX}

FREE RADICALS

Chemical Determination of Free Radical-Induced Damage to DNA

101,332

46 Gas Chromatography-Mass Spectrometry of Free Radical-Induced Products of Pyrimidines and Purines in DNA. PB91-187476 101,302

Modification of DNA Bases in Mammalian Chromatin by Radiation-Generated Free Radicals.

PB91.189373

101,338

Rate Constants for Reduction of Substituted Methylperoxyl Radicals by Ascorbate lons and N,N,N', N'-Tetramethyl-Para-Phenylenediamin

PB91-195230

Biomarkers of $\mathrm{OH}$ Radical Damage In vivo.

100,379 PB91-236877

101,325

FREE SURFACES (CRYSTALLOGRAPHY)

Effect of a Free Surface on Stress Distribution in a Bimaterial Composite.
PB91.185116

FREQUENCY CALIBRATION

New Inexpensive Frequency Calibration Service from PB91-200790

100,580

FREQUENCY MEASUREMENT

Accurate Frequency of the $119 \mathrm{mu} \mathrm{m}$ Methanol Laser from Tunable Far-Infrared Absorption Spectroscopy. PB91-134130

101,405

FREQUENCY RESPONSE

Frequency Dependence of Catalyzed Reactions in a Weak Oscillating Field

FREQUENCY STABILITY

Environmental Effects on the Medium and Long Term Frequency Stability of Ouartz Oscillators.

100,407

PB91-134866

100,781

Calculated from the Frequency Domain: An Update.

PB91-162388

100,578

Rubidium Frequency Standard and a GPS Receiver: A Remotely Steered Clock System with Good Short-Term and Long-Term Stability.

100,581

Frequency and Time Stability of GPS and GLONASS

Clocks.

PB91-202937

100,582

Time Domain Frequency Stability Calculated from the Frequency Domain Description: Use of the SIGINT Software Package to Calculate Time Domain Frequency Stability from the Frequency Domain

100,587

FREQUENCY STANDARDS

Cooled Ion Frequency Standard.

Cooled Ion Frequency Standard.

AD-A242 030/5

101,591

Trapped-Ion Frequency Standards.

NG1-25760/0

101,593

Progress at NIST on Absolute Frequency Standards UBing Stored lons.

Clocks, Atomic and Molecular.

101,625

Clocks, Atomic

101,677

Advances in Science, Emerging Technologies and Global Competition: Opportunities and Challenge.

100,583

Established Microwave Frequency Standards.

PB91-203182

100,584

NIST Time and Frequency Services.

PB91-231605

100,585

PB92.116474

$\mathrm{Be}(+)$

FRESNEL DIFFRACTION

Generalization of the Cornu Spiral for Lossy Media.
PB91-203216

FRICTION

Effect of Selected Chemical Compounds on the Lubrica-

tion of Silicon Nitride.

FICTION FACTOR

Heat Transfer and Pressure Drop in a Compact Pin-Fin Heat Exchanger with Pin Orientation at 18 deg to the Flow Direction
PB91-194464

101,050

FRICTION MEASUREMENT

Apparatus for Measurement of Coefficient of Friction.
PB91-148049

FUEL SPRAYS

Laser Diagnostics for Characterization of Fuel Sprays. PB91-149013

100,541 FUELS

MSW Calorimetry.

PB91-133942

100,878

Short-Duration Autoignition Temperature Measurements lor Hydrocarbon Fuels.

100,887
FUGACITY

Hydrogen Component Fugacity Coefficients in Binary Mix tures with Etane: Pressure Dependence.

100,455

FUMARIC ACID

Thermodynamics of the Krebs Cycle and Related Com-

PB91-192468

100,357

FUMED SILICA

Apparent Thermal Conductivity of Fumed-Silica Standard Reference Materials.

101,102

\section{FUNCTIONAL BENCHMARKS}

Functional Benchmarks for Fourth Generation Languages.

100,644

FUNDAMENTAL CONSTANTS

Adjustment of the Fundamental Physical Constants: A Report of the CODATA Task Group on Fundamenta Constants, 1986

PB91-147124

101,606

Basic Standards and Fundamental Constants. B91-237594

101,715

FUNGI

Characterization by High Pertormance Liquid Chromatog raphy (HPLC) of the Solubilization of Phosphorus in Iron Ore by a Fungus.

PB91-19540

101,162

FURNITURE

Technical Reference and User's Guide for FAST/FFM

Version 3.
PB91-206664

100,086

FUSED SALTS

Electrodeposition of Aluminum from Molten Salts. PB91-159202

101,223

GAGING

High Precision Gaging with Computer Vision Systems.

GALLING

Literature Review of the Galling Process.

PB91-134551

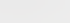

Study of the Galling of Two Steels Using Two Test MethPB91-148106

101,147

Method for Ouantitative Measurement of Galling Damage.
PB91-159269

101,183

GALLIUM ARSENIDES

Secondary Ion Yield Changes in Silicon and Gallium Ar. senide Due to Topography Changes during Oxygen or Cesium lon Bombardment.

101,471

Study of the GaAs-Si(100) Interface Using Laser Probing of Thermal Desorption Kinetics.

101,477

Selective-Area Epitaxial Growth of Gallium Arsenide on Selective-Area Epitaxial Growth of Gallium Arsenide on
Silicon Substrates Patterned Using a Scanning Tunneling Silicon Substrates Patterned Using a Scanning Tunneling
Microscope Operating in Air. PB91-149880

100,796

Modelling Gallium Arsenide Transistors

100,797

Indentation Determination of Crack Growth Parameters in Gallium Arsenide.

Gallium Arsenide

101,083

High Spatial Resolution Mapping of Semiconductor Re. sistivity.

PB91-194829

100,809

Majority and Minority Electron and Hole Mobilities in Heavily Doped GaAs.

101,550

Structural Anomalies in Undoped Gallium Arsenide ObStructural Anomalies in Undoped Gallium Arsenide Obochromatic Synchrotron Radiation.

PB91-237438

101,556

Environmentaliy Enhanced Fracture of Gallium Arsenide.
PB91-237743

High Resolution Diffraction Imaging of Crystals Grown in High Resolution Diffraction Imaging of Crystals Grown in
Microgravity and Closely Related Terrestrial Crystals.
PB92-109008

\section{GALLIUM INDIUM ARSENIDES} Fe Implantation in In0.53Ga0.47As/InP. PB91-189969

101,516

GALLIUM ORGANIC COMPOUNDS

Laser Photolysis of Trimethylgallium at $193 \mathrm{~nm}$ : Ouantum Yields for Methyl Radical and Ethane Production.
AD-A227 868/7 GAMMA COUNTERS

Low-Background Gamma-Ray Assay Laboratory for Activation Analysis.

100,192

GAMMA DOSIMETRY

Dosimetry of a Nearly Monoenergetic 6- to 7-MeV Photon Source by $\mathrm{Nal}(\mathrm{TI})$ Scintillation Spectrometry.

101,723

Radiochromic Film Dosimeter for Gamma Radiation in the Absorbed-Dose Range 0.1 - $10 \mathrm{kGY}$.

PB92-11696

101,364

GAMMA RAYS

Co-Trial on ESR Identification and Estimates of gammaRay and Electron Absorbed Doses Given to Meat and

100,017

Metal-Polysiloxane Shields for Radiation Therapy of Maxillo-Facial Tumors.

PB91-194761

100,054

Sensitometry of the Response of a New Radiochromic Film Dosimeter to Gamma Radiation and Electron

PB91-237008

101,326

2-Deoxy-D-Ribose Aqueous Solution as a Gamma-Ray Dosimeter.

GARBAGE

MSW Calorimetry

PB91-133942

101,366

GAS ANALYSIS MODELING SYSTEM

Gas Analysis Modeling System

PB91-175299

100,878

AS CHROMATOGRAPHY

Strategy for Chemical Analysis of Alternative RefrigerPB91-148502

100,185

Detection of Trace Disulfur Decafluoride in Sulfur Hexafluoride by Gas Chromatography/Mass Spectrometry.

AS DETECTORS

Chemical and Electronic Properties of $\mathrm{Pd} / \mathrm{SnO} 2(110)$ Model Gas Sensors.

PB91-203075

100,393

\section{AS DISCHARGES}

Preface to Book Entitled Nonequilibrium Effects in Ion and Electron Transport'.

101,623

Causes of $\mathrm{SiH} 4$ Dissociation in Silane dc discharges.

PB91-202986 100,389 Silane Dissociation Products in Deposition Discharges.
PB91-202994

GAS FURNACES

Testing and Rating of an Atmospheric, Gas-Fired Furnace Equipped

GAS METAL ARC WELDING

Dynamic Arc-Power Source Response in GMA Welding. PB91-149278

GAS METAL ORE WELDING

Sensing of GMAW Droplet Transfer Modes Using an ER 100S-1 Electrode.

PB91-174250

GAS METERS

Experimental Evaluation of Selected Orifice Flowmeter Upstream Installations.

PB91-147025

100,880

AS PIPELINES

Gas Analysis Modeling System.

PB91.175299

100,884

GAS-SOLID INTERFACES

Ouantitative Analysis of Impurities in SIMOX Samples Using Secondary lon Mass Spectrometry.

PB91-236596

100,208

GAS SUPPLY

Gas Analysis Modeling System.

PB91-175299

100,884

GASES 


\section{KEYWORD INDEX}

GENERAL THEORETICAL CHEMISTRY \& PHYSICS

GENERAL AGREEMENT ON TARIFFS AND TRADE

GATT Standards Code Activities of the National Institute of Standards and Technology 1990.

100,177

$$
\text { PB91-187823 }
$$

GENERAL INTEREST

Kelvin Water-Drop Experiment

N91-20219/2

100,900

Institute for Materials Science and Engineering: Metallurgy Division, Technical Activities 1990

101,207

Metrology for Electromagnetic Technology: A Bibliography of NIST Publications.

100,833

Gravity-Dependent Phenomena at the Scale of the Single

$\begin{array}{lr}\text { PB91-134767 } & 101,297 \\ \text { National Bureau of Standards Publications 1977-1987. }\end{array}$ Volume 1. Citations, Key Words, and Abstracts.

PB91-136507

100,957

National Bureau of Standards Publications 1977-1987. Volume 2. Indexes.

100,958

Materials Reliability. Technical Activities, 1990. (NAS NRC Assessment Panel, January 31-February 1, 1991). PB91-143339 101,008

Agenda for Progress in Technology Education: A Personal View.

100,037

Abstract and Index Collection in the Research Informa tion Center of the National Institute of Standards and Technology (Fourth Edition)

PB91-148494

100,959

Close-Coupling Calculation for the (2)(Sigma sub $u)(+)$ State of $(\mathrm{H}$ sub 2)(-).

101,614

Photodissociation Dynamics of Water in the Second $A b-$ sorption Band: Vibrational Exitation of $\mathrm{OH}$ (A (2)Sigma).
PB91-148726

Asymptotic Shooting Method for the Solution of Differen-

tial Equations.

101,255

Novel Laser Gain and Time-Resolved FTIR Studies of Photochemistry.

$\begin{array}{ll}\text { PB91-148809 } & 100,237\end{array}$

Modelling the Coronae and Chromospher Systems by the Analysis of Ultraviolet, $X$-ray and Radio Observations

100,019

Ultraviolet, Optical, Infrared, and Microwave Observations

$\begin{array}{ll}\text { of HR } 5110 . & 100,020\end{array}$

Mode Specific Internal and Direct Rotational Predissociation in HeHF HeDF, and HeHCL: van der Waals Complexes in the Weak Binding Limit.

100,293

Multireference-Configuration Interaction (MR-Cl) CalculaMultireference-Configuration Interaction (MR-Cl) Calcula-
tions on $\mathrm{HS}(2+)$ and Experimental Observation via Electron Impact lonization of

PB91-148924

100,295

Studies of $\mathrm{H} \mathrm{I}$ and $\mathrm{DI}$ in the Local Interstellar Medium.

B 14148957

100,021

Spatially Resolved Flares in RS CVn Systems. 100,022

PB91-148965 100,022 Absorption Studies of $\mathrm{OH}$ (X(2) Pi) Radical: An Experimental Dipole Moment Function and State-to-State Einstein A Coefficients.

PB91-148973

100,297

Laser-Induced Fluorescence Measurements of Drift-Ve locity Distributions for $\mathrm{Ba}(+)$ in Ar: Moment Analysis and

a Direct Measure of Skewness.

100,298

Terminal Velocities for a Large Sample of O Stars, B Supergiants, and Wolf-Rayet Stars.

PB91-149021

100,023

Materials Research at the National Bureau of Standards.

Thermal Noise in Mechanical Experiments.

101,214

PB91-149211

101,578

New Radio Detectors of Early-Type Pre-Main Sequence

100,024

General Method for Doppler Determination of Cylindrical-

ly Symmetric Velocity Distributions: An Application of

Fourier Transform Doppler Spectroscopy.

PB91-149385

100,302

Photospheres of Hot Stars. 4. Spectral Type O4.

PB91-149849

100,025

New Yorker in Japan

PB91-158907

101,275

Modern Test Methods for Flammability.

100,116

Interim Report to the President and to the Congress on

the Malcolm Baldrige National Quality Award.

PB91-167833

100,001

NIST Serial Holdings, 1991

100,960
Book Review: Polarized Electrons at Surfaces by J. Kirschner.

101,493

Padio-Continuum Observations of a Variety of Cool Stars.

Radio-Continuum Observations of a Variety of Cool Stars.
PB91-174664

Wawayandaite, a New Calcium Manganese Beryllium Boron Silicate from Franklin, New Jersey.

101,359

Gyroscope-Weighing Experiment Revisited -- with a Null

Result. 101,630

Detection of an Expanding $\mathrm{H}$ I Shell in the Old Supernova Remnant CTB 80.

100,028

Ground-Truth Observations of Stellar Surface Structure from the Lunar Surface.

PB91.175026

100,029

Some Concluding Thoughts for Cool Stars VI. 100,030

100,030
PB91-175034 Extension of the Class of Magnetic B Star Nontherma
Radio Sources.
PB91-175042
100,031

Orbit Determination and Gravitational Field Accuracy for a Mercury Transponder Satellite.

101,736

Book Review: Partitioning in Aqueous Two-Phase Systems: Theory, Methods, Uses, and Applications to Biotechnology.

100,333

Book Review: 'Polarized Electrons at Surfaces' by J.

101,497

Search for a Composition Dependent Force Using a Liquid-Supported Torsion Balance.

101,639

Rotational Predissociation, Vibrational Mixing, and van der Waals Intermolecular Potentials of NeDF.

PB91-175505

100,334

Of What Use Is Basic Research.

PB91-175513

101,642

Correlated Spontaneous Emission in a Zeeman Laser.

CB91.175596

101,417

NIST Research Reports, March 1991

PB91-187559

100,007

Proposed Design Criteria for Shallow Bridge Foundations. PB91-187849

100,534

Structural Change and Competitiveness: The U.S. Semiconductor Industry.

100,173

Energy Distributions of Symbiotic Novae

PB91-200873

100,032

Near-Threshold Vibrational Excitation of $\mathrm{H} 2$ by Electron Impact: Resolution of Discrepancies between Experiment and Theory.

Evolution of Infrared Carbon Stars

101,679

PB91-202861

100,033

Causes of Sit4

100,389

Silane Dissociation Products in Deposition Discharges.

PB91-202994

Lunar Laser Ranging

PB91-203026

100,390

Probe for Real-Time Images of Particle Beams and Their Analyses in a Merged-Beams Apparatus.

101,683

Measurements of Velocity-Changing Collision Kernels.

PB91-203083 101,684

Squeezed Quantum States of Relic Gravitons and Primordial Density Fluctuations.

101,685

Asymptotic Expansions of the Kernel Functions for Line Formation with Continuous Absorption.

PB91-203240

101,688

Structural Dependence of HF Vibrational Red Shifts in ArnHF, $n=1-4$, via High-Resolution Slit Jet Infrared Spectroscopy

100,401

Observation of the Cesium Clock Transition Using LaserCooled Atoms in a Vapor Cell.

PB91-203638

101,698

Progress on the Determination of Intermolecular Potential Energy Surfaces from High Resolution Spectroscopy.
PB91-203695 Multiple Intermolecular Bend Vibrational Excitation of a Hydrogen Bond: An Extended Infrared Study of OCOHF.

100,812

Building-in Reliability: Making It Work

High-Resolution Infrared Flash Kinetic Spectroscopy of $\mathrm{OH}$ Radicals.

100,408

Production and Diagnosis of a Highly Spin-Polarized $\mathrm{Na}$ PB91-204016

101,431

Investigation of Internal Rotor Dynamics of $\mathrm{NeDCl}$ and ArDCl via Infrared Absorption Spectroscopy.

PB91-204024

100,409

Test of the Inverse-Square Law of Gravitation Using the 300-m Tower at Erie, Colorado.

PB91-204115

101,702

Collisional Energy Transfer between Excited Sr Atoms.

PB91-204164

101,703

Correlated Spontaneous Emission in a Zeeman Laser.
PB91-204180

Publications of the National Institute of Standards and Pubchnology, 1990 Catalog.

PB91-216531

100,011

NIST Personnel Management Demonstration Project Design, Implementation and Accomplishments.

PB91-231555

Book Review: Hypertext Hands-On

100,003

Center B92-109016

Research. Services. Facilities. (National Institute of Standards and Technology)

100,012

Electronics and Electrical Engineering Laboratory Technical Publication Announcements Covering Laboratory Pro grams, January to March 1991, with 1991 EEEL Events PB92-112309

100,864

Book Review: 'The Art of Computer Systems Performance Analysis' by R. Jain.

100,719

Ouestions and Answers on Quality, the ISO 9000 Stand ard Series, Ouality System Registration, and Related

PB92-126465

100,175

Feasibility of Using a Multiple Award Schedule for Specifying Paints in Government Painting Contracts.

100,002

National Educators Workshop: Update '90. Standard Ex periments in Engineering Materials Science and Technol PBy.

101,253

List of New Group Divisible Designs.

PB92-12670

GENERAL THEORETICAL CHEMISTRY \& PHYSICS

Thermodynamic and Kinetic Stability of Refractory Materials at Uiltra-High Temperatures.

Spatially and Spectrally Resolved Cathodoluminescence of Hot-Filament Chemical-Vapor-Deposited Diamond Par-

AD-A236 485/9

100,247

Critical Assessment of Optical Properties of CVD Diamond Films.

AD-A236 746/4

101,400

Spatially and Spectrally Resolved Cathodoluminescence of Hot-Filament Chemical-Vapor-Deposited Diamond Par-

AD-A237 128/4

100,248 
PB91-222588

100,653

High Magnetic Field Facilities in Japan Related to Superconductivity Research (Japan Technology Program). PB91-240762

101,718

GENERATING FUNCTIONS

Certain Theorems on Bilateral Generating Functions Involving Hermite, Laguerre, and Gegenbauer Polynomials.

GEODESY

Lunar Laser Ranging

PB91-203026

101,256

EOGRAPHIC INFORMATION SYSTEMS

Prototyping the IRDS: An Airport Application.

EOLOGIC AGES

Accelerator (14)C Dating of Late Pleistocene Megafauna.
PB91-237396

Accelerator Radiocarbon Dates on Charcoal, Shell, and Human Bone from the Del Mar Site, California.
PB91-237404

GERMANIDES

Isothermal Phase Behavior of Ag3SbS3, ZnGeP2, and

PB91-187237

101,080

GERMANIUM

Amorphization and Conductivity of Silicon and Germanium Induced by Indentation.

101,482

Resonance Enhanced Multiphoton lonization Spectra of the $\mathrm{GeF}$ and $\mathrm{GeCl}$ Radicals from $400-500 \mathrm{~nm}$. PB91-203281

100,396

GERMANIUM CHLORIDE RADICALS

Resonance Enhanced Multiphoton Ionization Spectra of the $\mathrm{GeF}$ and $\mathrm{GeCl}$ Radicals from $400-500 \mathrm{~nm}$.

100,396

GERMANIUM COMPOUNDS

Scanning tunneling microscopy studies of the surfaces of a-Si:H and a-SiGe:H films. Annual report, 1 December 1989-31 January 1901 . DE91002169

100,785

GERMANIUM FLUORIDE RADICALS

Resonance Enhanced Multiphoton Ionization Spectra of the $\mathrm{GeF}$ and $\mathrm{GeCl}$ Radicals from $400-500 \mathrm{~nm}$. PB91-203281

100,396

GERMANIUM IONS

Relative Populations of Excited Levels within the Ground Configuration of Si-Like Cu, Zn, Ge, and Se lons.

101,443

GERMYL RADICALS

Electronic Spectrum of the GeH3 Radical. PB91-189548

100,346

GIBBS FREE ENERGY

Thermodynamics of the Krebs Cycle and Related Compounds.

100,357

GLASS

Influence of Moisture and Pressure on the Mechanical Properties of a Glass-Epoxy Matrix Composite and a

Graphite-Epoxy Matrix Composite.

101,114

Design Diagrams for Heavy Metal Fluoride Glass Windows.

101,419

Effect of Thermal Expansion Mismatch on Fiber Pull-Out in Glass Matrix Composites.

101,125

Semiempirical Theory of Relaxation: Concentrated Polymer Solution Dynamics.

100,511

Immobilization of Binding Proteins on Nonporous Sup

ports Comparison of Protein Loading, Activity, and Stabili-

PB91-237198

101,308

GLASS FIBER REINFORCED PLASTICS

Mechanical Loss in a Glass-Epoxy Composite.

101,070

GLASS FIBERS

Detail Specification for 62.5-mum Core Diameter/125mum Cladding Diameter Class la Multimode, GradedIndex Optical Waveguide Fibers.

Index Optical Waveguide Fibers.
FIPS PUB 159

Summary of Low-Density Glass-Fiber Reference Materials at NIST: 1980-1989.

PB91-175620

101,079

GLASSY CARBON

Reflection-Extended-X-ray-Absorption-Fine-Structure Spectroscopy at the Carbon K-Edge.

PB91-194944

100,371

GLAZED WARE

Variations in the Practice of Ceramic Technology in Different Cultures: A Comparison of Korean and Chinese Celadon Glazes.
PB91-237669

101,111

GLOBAL POSITIONING SYSTEM

GPS Time Transfer with Implementation of Selective Availability.

100,576 Rubidium Frequency Standard and a GPS Receiver: A
Remotely Steered Clock System with Good Short-Term and Long-Term Stability.

PB91-200808

100,581

Frequency and Time Stability of GPS and GLONASS PB91-202937

100,582

GLONASS SYSTEM

Frequency and Time Stability of GPS and GLONASS Clocks.
PB91-202937

100,582

GLUEBALLS

Glueballs and Hadron Spectroscopy

GLUTAMIC ACID

Thermodynamics of the Krebs Cycle and Related Compounds.

100,357

GLYCEROLS

Neutron Dittraction from Sheared D-Glycerol: Preliminary Studies.

PB91-134676

100,268

GLYCINE

Thermodynamics of the Krebs Cycle and Related Compounds

PB91-192468

100,357

GOES SATELLITES

$\mathrm{HgCdTe}$ Detector Reliability Study for the GOES Pro-

gram. 112382

100,766

GOLD 197 TARGET

Evidence for Surface alpha Particle Clusters in (nat)AG(197)Au from the (e,alpha) Reaction.

101,705

OSIP (GOVERNMENT OPEN SYSTEM

INTERCONNECTION)

Government Open Systems Interconnection Profile Users' Guide, Version

100,696

GOSIP (GOVERNMENT OPEN SYSTEMS

INTERCONNECTION PROFILE)

Standard Security Label for GOSIP: An Invitational Work-

Shop. Held on April 9-10, 1991.
PB91-216671

GOSIP Conformance and Interoperation Testing and Registration.
PB92-110105

100,694

GOVERNMENT PROCUREMENT

GOSIP Conformance and Interoperation Testing and PBgistration.

100,694

Feasibility of Using a Multiple Award Schedule for Specifying Paints in Government Painting Contracts.
PB92-126481

GRAIN BOUNDARIES

Calculation of Energy of Low-Angle Grain Boundaries. PB91-134601 101,464 Metal Reference Line Technique for Obtaining Dihedral Angles from Surface Thermal Grooves.

Grain Boundaries in a 2-D Superlattice.

101,059

PB91-202853

100,388

Microstructural Control Through Diffusion-Induced Grain Boundary Migration

101,239

GRAIN SIZE

Cyclic Fatigue from Frictional Degradation at Bridging Grains in Alumina.

GRAIN STRUCTURE

Transport Critical Current of Aligned Polycrystalline Y1Ba2Cu307-delta and Evidence for a Nonweak-Linked Component of Intergranular Current Conduction. PB91-133983

101,456

GRANTS

Evaluation of Building Systems Inventions. PB91-158659

100,896

GRAPHIC KERNEL SYSTEM

Graphical Kernel System (GKS); Category: Software Standard; Subcategory: Graphics.

FIPS PUB 120-1

100,673

Graphical Kernel System (GKS). Ada Binding; Category: Software Standard; Subcategory: Graphics.

100,674

GRAP

CHETHODS

Ceramic Wear
PB91-189498

100,561

PLOTMD: An Interactive Program to Modify Molecular Plots on a Graphics Terminal.

PB91-194985

100,373

GRAPHICAL KERNEL SYSTEM

Graphical Kernel System (GKS). Pascal Binding; Catego-

ry: Software Standard; Subcategory: Graphics.
FIPS PUB 120-1A

GRAPHITE

Structure ot a Stage-3 Cs - Graphite Intercalate.

100,269

Laser Vaporization Mass Spectrometry of Refractory Maerials: Graphite and $\mathrm{YBa} 2 \mathrm{Cu} 3 \mathrm{O}$

100,464

GRAPHITE COMPOSITES

Elastic Constants of Fiber-Reinforced Composites: A Fresh Measurement Approach.

PB91-194878

101,123

GRAPHITE EPOXY COMPOSITES

Influence of Moisture and Pressure on the Mechanical Properties of a Glass-Epoxy Matrix Composite and a Graphite-Epoxy Matrix Composite.

AD-A239 509/3

101,114

GRAPHS (CHARTS)

Cryogenic Properties ot Copper (Wall Chart)

PB91-1481

Refractive Index Gratings in Rare-Earth-Doped Alkaline Earth Glasses.

101,422

GRAVITATION

Test of Newton's Inverse Square Law of Gravity Using the $300 \mathrm{~m}$ Tower at Erie, Colorado: Newton Vindicated on the Plains of Colorado.

PB91-175273

101,637

Test of the Inverse-Square Law of Gravitation Using the 300-m Tower at Erie, Colorado.

101,702

GRAVITATIONAL FIELDS

Orbit Determination and Gravitational Field Accuracy for a Mercury Transponder Satellite.

PB91-175166

101,736

GRAVITATIONAL WAVE ANTENNAS

Lunar Gravitational Wave Antenna Using a Laser InterferPB91-134643

101,603

Gravitational Radiation Observations on the Moon.

PB91-175083

101,635

GRAVITONS

Squeezed Quantum States of Relic Gravitons and Primordial Density Fluctuations.

101,685

GRAVITY

Test of Newton's Inverse Square Law of Gravity Using the $300 \mathrm{~m}$ Tower at Erie, Colorado: Newton Vindicated the $300 \mathrm{~m}$ Tower at Erie,
on the Plains of Colorado.

PB91-175273

101,637

GREENS FUNCTION

Identification of Dynamic Green's Functions in Structural

PB91-202812

101,585

Impulse Response Functions for Elastic Structures with Rigid Body Degrees of Freedom. PB91-237354

101,587

GRIDS (COORDINATES)

Non-Linear Contour-to-Grid Digital Interpolation. PB91-147678

101,351

GROTRIAN DIAGRAMS

Spectral Data and Grotrian Diagrams for Highly lonized Copper, Cu X-Cu XXIX

100,441

GROUND VEHICLES

Control System Architecture for Unmanned Ground Vehicles. New Approach to Vision and Control for Road Following.
PB91-157156 Video Compression for Remote Vehicle Driving. PB91-203208

101,035

GUN BARRELS

Space Marching Difference Schemes in the Nonlinear Inverse Heat Conduction Problem.

PB91-144360

101,604

GYROSCOPES 
Specifications, Tolerances, and Other Technical Requirements for Weighing and Measuring Devices as Adopted by the 76 th National Conference on Weights and Measures, 1991.

Checking the Net Contents of Packaged Goods. Third Edition, Supplement 2.

PB92-112549

100,171

HARMONIC GENERATION

High Harmonic Generation in Hydrogenic lons.

HARVARD/NIST VI COMPUTER PROGRAM Using the Harvard/NIST Mark VI Fire Simulation. B 91-144394

100,095

HAZARDOUS MATERIALS

Development of Test Methods to Determine the Compatibility of Liquid Hazardous Materials with Polyethylene PB92-126457

100,943

HEALTH \& SAFETY

Corrosion, Repassivation and Corrosion Fatigue Behavior of the Surgical Implant Alloy, Co-Cr-Mo. Setting Reactions and Compressive Strengths of Calcium hosphate Cements.

PB91-148668 100,040

Visco-Elastic Deformation of Dental Porcelain and Porceain-Metal Compatibility.

PB91-158493 100,042

Effect of Catalyst Structure on the Synthesis of a Dental Restorative Monomer.

100,043

Dental Biomaterials and Engineering Frontiers for the

PB91-159210

100,044

Visibility of Exit Signs and Directional Indicators. 100,083

Glass-Ceramic Inserts Anticipated for 'Megafilled' Composite Restorations.

PB91-174458

100,045

Digital Image Analysis Assisted Microradiography Meas-

urement of Mineral Content of Caries Lesions in Teeth.

PB91-187666

101,314

Protective Coatings for Tooth Crowns.

PB91-189555

100,047

Filler Systems

100,048

Effects of $\mathrm{pH}$ and Calcium on Hydrolysis of Na2SiF6 and Na2SnF6: A Quasi-Constant Composition Titration Study. 101,315

Metal-Polysiloxane Shields for Radiation Therapy of Maxillo-Facial Tumors.

PB91-194761

100,054

Corrosion Principles in Dental Implantology.

100,055

Adsorption and Orientation of Tetracycline on Hydroxyaatite.

101,319

100,049

Self-Setting Calcium Phosphate Cements.

Development of an Adhesive System for Bonding to Hard Tooth Tissues.

100,052

Dentin-Bonding Molar Efficiency Using N-Phenylglycine,

N-Phenyl-beta-Alanine, or N-Methyl-N-Phenylglycine.
PB91-237735

Mathematical Model for Dental Caries: A Coupled Dissolution-Diffusion Process.

101,316

HEARING AIDS

Correction for Converting 2-CM(3)-Coupler Responses to Insertion Responses for Custom in-the-Ear Non-Direction-

al Hearing Aids.
PB91.174490

HEART VALVE PROSTHESIS

100,046

Comparative Study of Bovine Pericardium Mineralization: A Basic and Practical Approach.

100,056

EAT DISTRIBUTION SYSTEMS

Method for Measuring Heat Loss from Underground Heat Distribution Systems.

PBistribution Syst-134221

100,888

HEAT ENGINES

Ceramic Wear Maps

PB91-189498

100,561

Creep and Reliability of Ceramic Materials at Elevated Temperatures.

HEAT EXCHANGERS

101,091

Simulation of an Evaporator with Nonuniform One-Dimensional Air Distribution.

PB91-174649

100,066

Heat Transfer in a Compact Heat Exchanger Containing Rectangular Channels and Using Helium Gas. PB91-184804

101,734

HEAT FLOW METERS

Calibration at $24 \mathrm{C}$ of a Heat-Flow-Meter Apparatus Having $610 \mathrm{~mm}$ Square Plates.
PB91-206730

100,933

HEAT FLUX

Heat Flux, Mass Loss Rate and Upward Flame Spread or Burning Vertical Walls (1990)

PB91-143297

100,094

Radiant Heat Fluxes from 100-200 MW Natural Gas/Air

Diffusion Flames.
PB91-147306 100,538

Evaluation of the Heat Flux Transducer Technique for Measuring the Thermal Performance of Walis.

100,126

AT FLUX TRANSDUCERS

Evaluation of the Heat Flux Transducer Technique for Measuring the Thermal Pertormance of Walls. PB91-194696

100,126

HEAT LOSS

Method for Measuring Heat Loss from Underground Heat Distribution Systems.

100,888

Calibration Procedures for Infrared Imaging Systems for

Building Diagnostics.
PB91-147082 100,057

Design Heat Loss Factors for Basement and Slab Floors.

100,059

Measurement of Thermal Characteristics of Office Buildings.

100,068

\section{HEAT MEASUREMENT}

Method for Measuring Heat Loss from Underground Heat Distribution Systems.

Combustion Calorimeters.

PB91-189233

100,888

Calori-

PB91-236570

100,414

HEAT OF COMBUSTION

Heat Release Rate: The Single Most Important Variable in Fire Hazard.

PB91-146977

100,097

Energy of Combustion of Triphenylphosphate.

PB91-189613

100,347

Kinetic Properties of the Components of Douglas-Fir and the Heat of Combustion of Their Volatile Pyrolysis Prod-

PB91-203794

100,548

HEAT OF FORMATION

Energy of Combustion of Triphenylphosphate.
100,347

Thermodynamics of the Krebs Cycle and Related Com-

pounds.

100,357

HEAT OF FUSION

Fusion of Mercury. A New Certified Standard for Differential Scanning
PB91-150003

100,309

HEAT OF IMMERSION

Heats of Immersion, Friction, and Wear of Base Oil Frac-

PB91-158923

101,173

HEAT PIPES

Low-Pressure Symmetric Discharge Heat Pipe and DAOGS of Eu

101,666

HEAT PUMPS

Experimentation, Analysis, and Correlation of Refrigerant22 Flow Through Short Tube Restrictors.

101,194

B91-174243

Research and Development of Heat Pumps Using Nonazeotropic Mixture Refrigerants.

100,891

Refrigerant Charge Effects on Heat Pump Performance.

PB91-174607 100,065

Study of Flow Boiling Heat Transfer with Refrigerant Mix-

PB91-189563

101,196

HEAT RELEASE RATE

Heat Release Rate: The Single Most Important Variable PB91-146977

100,097

HEAT RESISTANT ALLOYS

PBition Characteristics of Selected SSME Alloys. 100,544

Continuous-Cooling Transformation Characteristics and High-Temperature Flow Behavior of a Microalloyed SAE 1141 Steel.

PB91-187856

101,155

HEAT RESISTANT MATERIALS

Creep and Reliability of Ceramic Materials at Elevated Temperatures
PB91-204172

101,091

HEAT TRANSFER

Effect of Noble Gas Mixtures on the Performance of Re. generative-Type Cryocoolers Analytical Estimate.

Method for Characterizing the Thermal Performance of a Solar Storage Wall from Measured Data.

PB91-159152

100,901

Letter to the Editor, Fire Technology, Comments on T.Z. Harmathy Viewpoint, May 1985.

PB91-174797

100,545

Heat Transfer in a Compact Heat Exchanger Containing Rectangular Channels and Using Helium Gas.

PB91-184804

101,734

Heat Transfer and Pressure Drop in a Compact Pin-Fin Heat Exchanger with Pin Orientation at $18 \mathrm{deg}$ to the Flow Direction.

PB91-194464

101,050

Transient Hydrogen Heat Transfer. Final Report for April 1986-April 1989

PB92-116326

100,454

Horizontal Flow Boiling Heat Transfer Experiments with a Mixture of R22/R114.

101,200

Fire-Plume-Generated Ceiling Jet Characteristics and Convective Heat Transfer to Ceiling and Wall Surfaces in a Two-Layer Zone-Type Fire Environment: Uniform Temperature Ceiling and Walls.

PB92-123074

101,370

HEAT TRANSFER COEFFICIENT

Comparison of Two Test Methods for Determining Transfer Function Coefficients for a Wall Using a Calibrated Hot Box

PB91-187278

100,121

\section{HEAT TREATMENT}

Effects of Varying Austenitizing Temperature and Cooling Aate on the Ability of HSLA-80 Steel to Achieve a Yield Strength Comparable to HSLA-100.

ELIUM

Effect of Noble Gas Mixtures on the Performance of Regenerative-Type Cryocoolers Analytical Estimate.

100,890

Precise Experimental Test of Calculated Two-Electron Lamb Shifts in Helium.

PB91-149203

101,617

Nonresonant Charge Transfer in the Threshold Region for $(3) \mathrm{He}(+)+(4) \mathrm{He}(\mathrm{sub}<-$ sup- $>)$ ) (3) $\mathrm{He}+$

PB91-149245

101,618

Viscosity and Thermal Conductivity of Pure Monatomic Gases from Their Normal Boiling Point Up to $5000 \mathrm{~K}$ in the Limit of Zero Density and at $0.101325 \mathrm{MPa}$. PB91-192559

100,366

\section{HELIUM COMPLEXES}

Mode Specific Internal and Direct Rotational Predissociaton in HeHF, HeDF, and HeHCL: van der Waals Complexes in the Weak Binding Limit.

PB91-148841

100,293

HELIUM NEON LASERS

Correlated Spontaneous Emission in a Zeeman Laser

PB91-175596

HENRY CONSTANT

Limiting versus Apparant Critical Behavior of Henry's Constants and $K$ Factors.

PB91-147363 
HIGH PRESSURE OXYGEN

Ignition Characteristics of the Nickel-Based Alloy UNS N07001 in Pressurized Oxygen.

PB91-144428

100,536

HIGH STRENGTH CONCRETES

Outline of a National Plan on High-Performance Concrete: Report on the NIST/ACI Workshop. Held in Gaithersburg, MD on May 16-18, 1990.

100,522

Design of High Strength Cement-Based Materiais. Part 2. Microstructure.

PB91-147439

100,099

HIGH STRENGTH STEELS

Load-Controlled Tensile Tests of Austenitic Steels at $4 \mathrm{~K}$

Strain Rate Effect on Tensile Properties at $4 \mathrm{~K}$ of a VAMAS Round-Robin Austenitic Steel.

PB91-237248

101,168

HIGH-TC SUPERCONDUCTORS

Superconducting Properties of Bi2-x yPbxSnySr2C

101,076

\section{HIGH TEMPERATURE}

Thermodynamic and Kinetic Stability of Refractory Materials at Ultra-High Temperatures.

100,246

HIGH TEMPERATURE SUPERCONDUCTERS

YBa2Cu3O7-delta/Insulator Multi-Layers for Crossover

PB91-194662 101,518

HIGH TEMPERATURE SUPERCONDUCTORS

High T(sub C) Superconductors: Are They Magnetic.

N91-20208/5 101,453

Current Supply for High-T(sub c) Superconductor Testing.
PB91-133827

Transport Critical Current of Aligned Polycrystalline Y1Ba2Cu3O7-delta and Evidence for a Nonweak-Linked Component of Intergranular Current Conduction.

PB91-133983 101,456

High $T$ (sub c) Superconductors and the Critical Current Measurement.

PB91.134056 101,457

Fundamental and Harmonic Susceptibilities of $\begin{array}{ll}\text { PB91-134148 } & 101,458\end{array}$

Madelung Potentials and Valences in the $\mathrm{Y} 1 \mathrm{Ba} 2 \mathrm{Cu} 3 \mathrm{O} 7$ Superconductor.

Elastic Constants, Debye Temperatures, and ElectronPhonon Parameters of Superconducting Cuprates and Related Oxides.

PB91-134262

101,460

Elastic Constants of Polycrystalline Bi-Pb-Sr-Ca-Cu-O Superconductor.

101,461

Break Junction Tunneling Spectroscopy of Single-Crysta Bismuth-Based High-Temperature Superconductors.
PB91-134478

Magnetization of Anisotropic Superconducting Grains

101,463

Phase Equilibria in the System TI-Ca-Ba-Cu-O. 1. Stability

of the 2122 Phase under Conditions of Oxygen Anneal-

ing. 147165

101,465

Strontium-Induced Oxygen Defect Structure and Hole Doping in La2-xSrxCuO4.

101,472

Strength and Fracture Behavior of Ba-Y.Cu-O Superconducting Ceramics.

PB91-148205 101,474

Possible 'Proximity Matrix' Route to High Current Conductors.

PB91-148932 101,476

Analysis of the YBa2Cu3O7/SrTiO3 Interface as a Function of Post-Deposition Annealing Temperature.

PB91-149963

101,480

Chemical Modification of the Orthorhombic Superconductor Ba2YCu3O7-delta.

PB91-159053

101,486

Phonon Density of States of Superconducting $\mathrm{YBa} 2 \mathrm{Cu} 3 \mathrm{O} 7$ and the Nonsuperconducting Analog YBa2Cu3O6.

101,487

Models for Oxygen Ordering and Diffusion in Ba2YCu3OX

and Ba2YCu3-yMyOx (M = Fe, Co, Al, Ga).

101,496

Levitation of Superconducting Composites.

101,503

Processing $\mathrm{Bi}-\mathrm{Pb}-\mathrm{Sr}-\mathrm{Ca}-\mathrm{Cu}-\mathrm{O}$ Superconductors from Amorphous State.

PB91-187369

101,504

Magnetic Ordering of $\mathrm{Er}$ in Powder and Single Crystals of ErBa2Cu3O7.

101,505

Magnetic Hysteresis of High-Temperature YBa2Cu3OX AgO Superconductors: Explanation of Magnetic Suspension.
PB91-189506

101,507

Superconducting Thin Films of Bi-Sr-Ca-Cu-O Obtained by Laser Ablation Processing

PB91.189589

101,508

Sputtered Thin Film YBa2Cu3On

101,509

nitial Stages of Degradation of Superconductor Surfaces: $\mathrm{O} 2, \mathrm{H} 2 \mathrm{O}, \mathrm{CO} 2$ and $\mathrm{CO}$ Chemisorption on $\mathrm{La} 2-\times \mathrm{Sr} \times \mathrm{CuO} 4$. PB91-189670

101,512

Electronic Structure of High-Tc Superconductors Studied Using Photoelectron Spectroscopy.

PB91-189688

101513

Phonon Density of States and Superconductivity in $\mathrm{Nd} 1.85 \mathrm{Ce} 0.15 \mathrm{CuO} 4$

PB91.189787

101,514

PB91-194753

101,521

Long-Range Antiferromagnetic Order of the $\mathrm{Cu}$ in Oxygen Deficient $\mathrm{ABa} 2 \mathrm{Cu} 3 \mathrm{O} 6+\mathrm{x}$

101,524

Studies of Magnetic Flux Penetration in a Chemically Synthesized $\mathrm{Bi}(\mathrm{Pb}) \mathrm{SrCaCuO}$ Superconductor.

PB91-194977

101,526

Wigner-Seitz Local-Environment Study of the High Tc Superconductors.

101,528

Morphology of Silver on YBa2Cu3O7-delta Thin Films.

101,534

Parent Structure of the Layered High-Temperature Super-

Conductors.
PB91-195677

101,535

Switching in High-Tc Superconductor Current Transport Measurements.

101,539

Progress in Field Ion Microscopy Imaging of High-Tc Superconducting Oxides.

101,543

Atomic Fingerprint of YBa2Cu3O7-X-Type High-Temperature Superconductors Observed by Field Ion Microscopy.
PB91-203612

Prospects for High Temperature Superconductor - Semiconductor Contacts.

PB91-203646

101,545

Properties of YBa2Cu3O7-delta Thin Films Grown on OffAxis-Cut MgO Substrates.

PB91-203950

101,547

Mossbauer Study of the Effect of Oxygen Stoichiometry on the High Tc Superconductor PB91-237545

101,558

Low-Field Flux Pinning in Twinned and Detwinned Single Crystals of YBa2Cu3O 7 -

101,559

Oxides and Oxide Superconductors: Elastic and Related Properties.

PB92-112333

101,563

Theoretical Model for the Tunneling-Gap Anisotropy ObTher in Layered Copper-Oxide High-Temperature Superconductors.

PB92-116565

101,564

Single-Target Magnetron Sputter-Deposition of High-Tc Superconducting Bi-Sr-Ca-Cu-O Thin Films. 101,566
PB92-116680 Interpretation of Field Ion Microscopy (FIM) Images of

PB92-116730

101,567

HIGH TEMPERATURE TESTS

High Temperature Creep Testing of Ceramics. 101,088

Chemical Kinetic Data Sheets for High-Temperature Reactions. Part 2.

PB92-148204

HOLE MOBILITY

Majority and Minority Electron and Hole Mobilities in

Heavily Doped GaAs.
PB91-236968

101,550

HOLES (MECHANICS)

Characterization of Cylindrical Holes in Metallic Substrates via Their Infrared Emission Patterns.

101,228

HOLMIUM PALLADIUM STANNIDES

Crystal-Field Splittings in the Cubic Heusler Alloys d2Sn and ErPd2Sn.

PB91-194902

101,525

HOSPITALs

Five Small Flaming Fire Tests in a Simulated Hospital Patient Room Protected by Automatic Fire Sprinklers. PB91-159038

HOT PLATES

Design and Fabrication of a Miniature High-Temperature Guarded-Hot-Plate Apparatus.

PB92-116797

101,098

HOT STARS

Photospheres of Hot Stars. 4. Spectral Type O4.

100,025

HOUSINGS

Photomultiplier Housing for Vacuum Operation of Side-on 1P28-Type Tubes.

AD-A227 836/4

100,744

HR 5110 STARS

Ultraviolet, Optical, Infrared, and Microwave Observations PB91-148833

100,020

HUMAN FACTORS ENGINEERING

Visibility of Exit Directional Indicators.

B 91-184762

101,000

HUMIDITY MEASUREMENT

Surface Acoustical Wave Spectroscopy for High Humidity PB91-158816 100,843

NIST Calibration Services for Humidity Measurement. PB92-112499

HVAC SYSTEMS

Computer Program for Simulation of HVAC/Lighting InterCetions: Initial Report

100,889

Prototype Expert System for Diagnosing Moisture Problems in Houses.

BACnet Communication Protocol for Building Automation Systems.

Rule-Based Diagnostic Method for HVAC Fault Detection. PB91-194936 100,072

Measurement and Evaluation of Lighting/HVAC InteracPB91-206706

100,892

\section{HYDRATION}

Computer Simulated Hydration of a Cement Model.

PB91-162149 100,112

Percolation of Phases in a Three-Dimensional Cement Paste Microstructural Model.

100,529

Hydration of Carbon Dioxide: The Structure of $\mathrm{H} 2 \mathrm{O}-\mathrm{H} 2 \mathrm{O}$ Hydration of Carbon Dioxide: The Structure of $\mathrm{H} 2 \mathrm{O}-\mathrm{H} 2 \mathrm{O}$ CO2 from Microwave Spectroscopy.

100,428

HYDRAZINE

Proton Affinities and pKa Values of Tetraalkylhydrazines. PB91-195214

00,378

HYDRAZINES

Slow Electron Transfer Reactions between Alkylhydra-

PB91-147785

100,284

Oxidation of Organic Compounds by Active Manganese

HYDROCARBON FUELS

100,218

Short-Duration Autoignition Temperature Measurements for Hydrocarbon Fuels.
AD-A242 816/7

100,877

HYDROCARBONS

Short-Duration Autoignition Temperature Measurements for Hydrocarbon Fuels

HYDRODYNAMICS

Hydrodynamics and Mass Transfer in Two-Phase Aque-

ous Extraction Using Spray Columns.
PB91-149229

101,300

HYDROGEN

Close-Coupling Calculation for the (2)(Sigma sub $u)(+)$ State of ( $\mathrm{H}$ sub 2)(-).

PB91.148684

101,614

Dispersion of the Electronic Contribution to the ThridOrder Nonlinear Susceptibility of $\mathrm{H} 2$.

PB9 1.149138 101,411

Rapid Low-Temperature Hopping of Hydrogen in a Pure Metal: The ScHx System.

PB91-158485

101,217 
P891-189639

100,348

Ionic Hydrogen Bonds. Part 1. Thermochemistry, Structural Implications, and Role in Ion Solvation.

PB91-203547

100,400

Multiple Intermolecular Bend Vibrational Excitation of a Hydrogen Bond: An Extended Infrared Study of OCOHF. $-203703$

100,404

Rovibrational Analysis of the nut(1) Intermolecular $\mathrm{Hy}$ drogen Bond Bending Vibration in HCN.HF Using Far Infrared Fourier Transform Spectroscopy.

100,437

HYDROGEN CYANIDE

Reduction of Hydrogen Cyanide Concentrations and Acute Inhalation Toxicity from Flexible Polyurethane Foam Combustion Products by the Addition of Copper Compounds. Part 3 . The Ellect of Copper Additives on the Flammabily Characteristics of Flexible Polyurethane

101,140

Rovibrational Analysis of the nu7(1) Intermolecular $\mathrm{Hy}$ drogen Bond Bending Vibration in HCN-HF Using Far Infrared Fourier Transform Spectroscopy

100,437 PB91.237784

HYDROGEN FLUORIDE

Vibrational, Rotational, and Tunneling Dependence of Vibrational Predissociation in the HF Dimer.

PB91-203844

100,406

Rovibrational Analysis of the nu7(1) Intermolecular Hydrogen Bond Bending Vibration in HCN-HF Using Far Infrared Fourier Transtorm Spectroscopy.

100,437

HYDROGEN IONS

Cross Sections and Related Data for Electron Collisions with Hydrogen Molecules and Molecular lons.

100,263

HYDROGEN IONS 1 MINUS

Cross Sections and Swarm Coefficients for $\mathrm{H}+, \mathrm{H} 2+$, $\mathrm{H} 3+, \mathrm{H}, \mathrm{H} 2$, and $\mathrm{H}$-in $\mathrm{H} 2$ for Energies from 0.1 eV to 10 PB90-244690

101,597

HYDROGEN IONS 1 PLUS

Cross Sections and Swarm Coefficients for $\mathrm{H}+, \mathrm{H} 2+$, (ros

PB90-244690

101,597

Carbon Acidities of Aromatic Compounds. 2. Ionic Probes of Aromaticity in Annelated Rings.

PB91-195065

100,375

HYDROGEN IONS 2 PLUS

Cross Sections and Swarm Coefficients for $\mathrm{H}+, \mathrm{H} 2+$ $\mathrm{H}_{3}+, \mathrm{H}, \mathrm{H} 2$, and $\mathrm{H}$-in $\mathrm{H} 2$ for Energies from $0.1 \mathrm{eV}$ to 10 kB

101,597

HYDROGEN IONS 3 PLUS

Cross Sections and Swarm Coefficients for $\mathrm{H}_{+}, \mathrm{H}_{2}+{ }^{\prime}$ keV.

PB90-244690

101,597

HYDROGEN-LIKE IONS

High Harmonic Generation in Hydrogenic Ions.
PB91-203778

PB91-203778
YDROGEN PEROXIDE

Copper-Ion-Dependent Damage to the Bases in DNA in the Presence of Hydrogen Peroxide.

101,330

Damage to the Bases in DNA Induced by Hydrogen Peroxide and Ferric lon Chelates.

PB91-187112

101,331

Chemical Nature of In vivo DNA Base Damage in Hydro-

gen Peroxide-Treated Mammalian Cells.

101,335

Damage to the DNA Bases in Mammalian Chromatin by Hydrogen Peroxide in the Presence of Ferric and Cupric lons.

PB91-189217

101,336

HYDROGEN SULFIDE

Direct Determination of Molecular Orbital Symmetry of 2S Using Polarized X-ray Emission.

PB91-175562

100,335

HYDROGEN SULFIDES

Multireference-Configuration Interaction (MR-Cl) Calculations on $\mathrm{HS}(2+)$ and Experimental Observation via Electron Impact lonization of H2S.

$$
\text { PB91-148924 }
$$

100,295

HYDROGEN TRASFER

Rate Constants for Hydrogen Abstraction Reactions of the Sulfate Radical, SO4- Alkanes and Ethers.

PB91-203232

100,394

HYDROGENIC IONS

High Harmonic Generation in Hydrogenic lons. PB91-203778

101,699

HYDROLYSIS

Effects of $\mathrm{pH}$ and Calcium on Hydrolysis of Na2SiF6 and Na2SnF6: A Quasi-Constant Composition Titration Study. YDROSOLS

Polyelectrolyte Stabilized Metal Oxide Hydrosols as Catalysts for the Photooxidation of Water by Zinc Porphyrins.
P891-189852

100,351

HYDROTHERMAL SYSTEMS

Thermodynamic Properties of Aqueous Solutions at High Temperatures; Needs, Methods, and Challenges
PB91-204065

100,410

HYDROXY COMPOUNDS

Adsorption and Orientation of Tetracycline on Hydroxya-

PB91-195149

101,319

HYDROXY RADICALS

Group Reactivities in the Gas Phase Reactions of Hydroxyl Radicals with Ethers.

PB91-175059

100,330

HYDROXYL COMPOUNDS

Neutron Spectroscopic Evidence for Adsorbed Hydroxyl Species on Platinum Black.

PB91-237636

100,432

HYDROXYL RADICALS

Mechanism of $\mathrm{OH}$ Radical Reactions with Thymine and Uracil Derivatives.

B91-147447

100,216

Multicomponent Cluster lons. 2. Comparative Stabilities of Cationic and Anionic Hydrogen Bonded Networks: Mixed Clusters of Water and Hydrogen Cyanide.

PB91-147710

100,283

$\mathrm{H}+\mathrm{O} 3$ Fourier-Transform Infrared Emission and Laser absorption Studies of $\mathrm{OH}(X(2) \mathrm{Pi})$ Radical: An Experimental Dipole Moment Function and State-to-State EinStein A Coefficients.

100,297

Structure and Mechanism of Hydroxyl Radical-Induced Formation of a DNA-Protein Cross-Link Involving Thymine and Lysine in Nucleohistone.

101,334

Hydroxyl Radical Induced Cross-Linking of Cytosine and Tyrosine in Nucleohiston.

PB91-189365

101,304

High-Resolution Infrared Flash Kinetic Spectroscopy of $\mathrm{OH}$ Radicals.

PB91-204008

100,408

Biomarkers of $\mathrm{OH}$ Radical Damage In vivo.

PB91-236877

101,325

HYGROMETERS

NIST Calibration Services for Humidity Measurement. PB92-112499

100,520

HYPERCUBE MULTIPROCESSORS

Performance Evaluation of Hypercube Applications: Using a Global Clock and Time Dilation.

PB91-222588

100,653

HYPERTEXT

Functional Meta-Structure for Hypertext Models and Sys-

tems.

$91-236687$

100,655

Book Review: Hypertext Hands-On.

PB91-237040

100,656

Linking Information to Objects: A Hypertext Prototype for Numismatists.

HYPOXANTHINES

100,657

ron Ion-Dependent Modification of Bases in DNA by the Superoxide Radical-Generating System Hypoxanthine/ Xanthine Oxidase

101,306

EDRONS

$\mathrm{N}$-Dimensional Crystallographic Description of the Icosa hedral Phases; the Example of the Al73Mn21Si6 Quasiperiodic Structure.

PB91-174771

101,226

IGNITION

Ignition Characteristics of the Nickel-Based Alloy UNS N07001 in Pressurized Oxygen.

PB91-144428

100,536

IGNITION TEMPERATURE

Ignition Characteristics of Selected SSME Alloys. PB91-174474

100,544

ILLUMINATING Conference Coverage: Papers Presentations Shine.
PB91-158535 0061

Dealing with Obvious Issues in Lighting.

Light Source Area, Shading, and Glare.

100,761

Role of Theory in Lighting Research and Design.

PB91-237826

100,080

LUNIMATING Effect of Veiling Relections on Vision of Colored Objects.
PB9 100,079

IMAGE ANALYSIS

Digital Image Analysis Assisted Microradiography Meas. urement of Mineral Content of Caries Lesions in Teeth. 101,314 Analysis of Optical Flow Estimation Using Epipolar Plane

Images.

100,703

IMAGE PROCESSING

Some Algorithms for Approximating Convolutions.

PB91.175281

101,258

Application of the PIPE Image Processing Machine to Scanning Microscopy.

101,034

Geometric Reasoning for Constructing 3D Scene Descriptions for Images.

Scriptions for
PB91-237677

100,714

Video Processing with the Princeton Engine at NIST

PB92-108950

100,604

IMAGE QUALITY INDICATORS

Image Quality Indicator Design for Radioscopy and Tomography.

101,018

\section{IMAGE RECONSTRUCTION}

Cross-Validation Procedure for Stopping the EM Algorithm and Deconvolution of Neutron Depth Protiling SpecPB91-161976

101,627

Integration-Based Method for Depth Estimation. PB92-112275

100,701

Three Dimensional Reconstruction from Optical Flow Using Temporal Integration.

100,702

Use of Cross-Validation as a Stopping Rule in Emission Tomography Image Reconstruction. PB92-116623

101,295

IMAGES

Tunneling Stabilized Magnetic Force Microscopy: Prospects for Low Temperature Applications to Superconducors.

PB91-203653

101,546

IMAGING TECHNIQUES

Mossbauer Imaging.

PB91-203745

101,294

New Approach to Image Modeling and Edge Detection in

PB91-203752

100,811

IMMUNOASSAY

Liposome Flow Injection Immunoassay: Implications for Sensitivity, Dynamic Range, and Antibody Regeneration. PB91-147629

IMMUNOSENSORS

Planar Waveguide Optical Immunosensors.

PB91-174565

101,291

MPACT ECHO TECHNIQUE

Impact-Echo: A New Method for Inspecting Construction PB92-116557

IMPACT-ECHO TESTS

100,140

Extended Abstract: A Finite Element Study of the Stress and Displacement Fields Produced by Point Impact.

PB91.175224

IMPLANTS

Corrosion Principles in Dental Implantology. 


\section{KEYWORD INDEX}

INDOOR AIR POLLUTION

Ventilation System Performance Evaluation Using Tracer

Gas Techniques.
PB91-147876

100,060

Proposed Standard Practice for Assessing the Performance of Gas-Phase Air Cleaning Equipment.

100,905

Air Exchange and Pollutant Concentration Measurements in Two Office Buildings.

PB91-195420

100,909

Indoor Ventilation Requirements for Manufactured Housing.

100,075

INDOOR AIR QUALITY

Multi-Zone Dispersal Analysis by Element Assembly.
PB91-187146

User Manual NBSAVIS CONTAM88. A User Interface for Air Movement and Contaminant Dispersal Analysis in Multizone Buildings.

ERT ATMOSPHERES

Hermetically Sealed Inert Atmosphere Cell for X-ray Powder Diffraction.

PB91-147983

101,062

NERTIA

Letter to the Editor, Fire Technology, Comments on T.Z. Harmathy Viewpoint, May 1985

100,545

INFILTRATION

Airtightness of Office-Building Envelopes.

PB91-175307

100,067

INFINITE DILUTION

Unified Description of Infinite-Dilution Thermodynamic Properties for Aqueous Solutes.

PB91-162081

100,319

INFORMATION CENTERS

NIST Serial Holdings, 1991

NFORMATION DISSEMINATION

FIREDOC Users Manual, 2nd Edition.

PB91-178830

100,960

FORMATION PROCESSING

Standard Generalized Markup Language Encoding of the Office Document Architecture Document Application ProPBile. 184812

101,345

INFORMATION RESOURCE DICTIONARY SYSTEM

Prototyping the IRDS: An Airport Application.

101,740

NFORMATION RETRIEVAL

Overview of the Product Data HyperStandard CD-ROM

Prototype.
PB91-132258

101,380

FIREDOC Users Manual, 2nd Edition.

100,547

Development of an Optical Disk System for the Automated Retrieval of EASEAR Records.

PB92-108943

100,666

INFORMATION SECURITY

National Aeronautics and Space Administration's (NASA) Automated Information Security Handbook.

100,707

INFORMATION SERVICES

STEP On-Line Information Service User's Guide. National PDES Testbed Report Series.

100,950

Data Bases Available in the Research Information Center Data Bases Available in the Research Information Center of the National Institute of Standards and Technology.
PB92-109016

INFORMATION SYSTEMS

Guideline: American National Dictionary for Information Systems. Category: Software; Subcategory: DocumentaFIPS PUB 11-3

100,956

Use of the IRDS Standard in CALS (Revised). $\quad 100,684$
PB91-132209 100,684
PB91-132209 Overview

PB91-132258

101,380

Information Systems Engineering Program.

100,685

Resonance Ionization Spectroscopy/Resonance Ionization Mass Spectrometry Data Service.

PB91-203968

100,207

Linking Information to Objects: A Hypertext Prototype for Numismatists.

100,657

Database and Retrieval System for the NBS Tables of Chemical Thermodynamic Properties.

PB91-237693

100,434

Guide to Schema and Scherna Extensibility. P892-126556

100,955

INFRARED DETECTORS

$\mathrm{HgCdTe}$ Detector Reliability Study for the GOES Pro-

gram. 292.112382

100,766
INFRARED EQUIPMENT

Calibration Procedures for Infrared Imaging Systems for Bullding Diagnostics.

PB91-147082

100,057

INFRARED MAPPING

Characterization of Cylindrical Holes in Metallic Substrates via Their Infrared Emission Patterns.

101,228

INFRARED SOURCES

Micromachined Thermal Radiation Emitter from a Commercial CMOS Process.

PB91-148999

100,748

INFRARED SPECTRA

Vibrational Spectra of Molecular lons Isolated in Solid Neon. 7. $\mathrm{CO}(+), \mathrm{C} 2 \mathrm{O} 2(+)$, and $\mathrm{C} 2 \mathrm{O} 2(-)$.
$\mathrm{AD}-\mathrm{A} 239729 / 7$

Production and Spectroscopy of Small Polyatomic Molecular Ions Isolated in Solid Neon.

Heterodyne Frequency Measurements of $\mathrm{CO}$ and OCS

Beyond $2100 \mathrm{~cm}(-1)$.
PB91-236984 100,423

Perpendicular Bands of Cyclopropane in the $3.5 \mathrm{mum}$ Region.

100,424

High-Resolution Infrared Spectrum of the nu1 Band of

PB92-117175

100,463

INFRARED SPECTROSCOPY

Strategy for Chemical Analysis of Alternative Refrigerants.

100,185

Infrared Spectroscopy Applied to Atmospheric Chemistry. 100,906 Spectroscopic Constants for the nug Infrared Band of HNO3.

PB91-189415

100,343

Fourier Transform Infrared Spectroscopy at the NIST: High Temperature Molecules and Atmospheric Molecules.

101,424

Fourier Transform Infrared Analysis of Ceramic Powders: Quantitative Determination of Alpha, Beta, and Amorhous Phases of Silicon Nitride.

PB91-237628

101,096

INFRARED THERMOGRAPHY

Fundamentals and Applications of Infrared Themography for Nondestructive Testing

101,009

\section{INJECTION TESTING}

Simultaneous versus Independent Injection Testing of Nonlinear Multiport Systems.

100,857

INKS

Synthesis of Prototype Resins for Use as BEP Intaglio nk Vehicles Curing by Electron Beam Radiation

PB91-144345 101,182

Synthesis of Non-lonic Water-Dispersible Resins for Use in Intaglio Inks Curing by Electron Beam Radiation. PB91-194456

100,499

INNER-SHELL IONIZATION

Angle-Resolved Photoemission from the Ar $2 p$ Subshell. PB91-194928

101,665

Inner-Shell lo

100,203

INORGANIC MATERIALS

Characterization of the Mineral Fraction in Botanical Reference Materials and Its Influence on Homogeneity and Analytical Results.

PB91-148817

100,186

INORGANIC PHOSPHATES

Heat Capacity and Thermodynamic Properties of Deutratd Ammonium Hexafluorophosphate ND4PF6 from $5.8 \mathrm{~K}$ to $347 \mathrm{~K}$.

PB91-147041

100,272

INORGANIC POLYMERS

Filler Systerns Based on Calcium Metaphosphates. PB91-194654

100,048

INORGANIC SILICATES

Wawayandaite, a New Calcium Manganese Beryllium Boron Silicate from Franklin, New Jersey.

101,359

INSPECTION

Field Study of the Performance of EPDM Roofing at Air Force Facilities.

PBD1-1672

101,340

STABILITY

Coherency Strain Induced Instability of Solid-Liquid Interaces in the Mo-Ni System.

PB91-195537

101,235

INSTALLING

Depot: A Framework for Sharing Software Installation Across Organizational and Unix Platform Boundaries. PB91-240754

100,659

INSTITUTE FOR NUMERICAL ANALYSIS

NBS-INA. The Institute for Numerical Analysis. UCLA 1947-1954.

PB92-112408

101,264

INSTRUCTORS

Economic Analysis for Military Construction (MILCON) Design. Concepts, Techniques, and Applications for the Analyst. Instructor's Manual.

PB92-112440

101,349

INSTRUMENTATION \& EXPERIMENTAL METHODS

Photomultiplier Housing for Vacuum Operation of Side-on 1P28-Type Tubes.

100,744

High Resolution Electron Microscopy of Diamond Film Dects and their Interactio

101,449

Transmission Electron Microscopy of the CVD Diamond Film/Substrate Interface.

$\begin{array}{ll}\text { AD-A243 096/5 } & 101,450 \\ \text { Spatially and Spectrally Resolved Cathodoluminescence }\end{array}$

Measurements of CVD-Grown Diamond Particles and

AD-A243 220/1

101,451

Advanced sensor development program for the pulp and paper industry. Final report.

101,248

Guideline for $\mathrm{Nb}$ (sub 3) $\mathrm{Sn}$ critical current measurements

using fiberglass-epoxy composite sample mandrels.
DE9101,205 Integrity tests for high-(Tc) and conventional critical-current measurements systems.

101,452

High-Speed Spatial (Linear) Scanning Pyrometer: A Too for Diagnostics, Temperature Mapping, and Property Determinations at High Temperatures.

N91-21361/1

100,924

Eddy Current Probe Characterization Using an Impedance Plane Display Instrument.

100,834

Flowmeter Installation Effects: A New Approach to an Old but Prevalent Problem.

PB91.134411

100,920

High-Resolution, Tunneling-Stabilized Magnetic Imaging and Recording

101,600

Chitin-Chitosan Membranes: Separations of Amino Acids and Polypeptides.

PB91-134544

101,283

Lunar Gravitational Wave Antenna Using a Laser Interfer-

PB91-134643

101,603

Synthesis of Prototype Resins for Use as BEP Intaglio Ink Vehicles Curing by Electron Bearn Radiation.

PB91-144345

101,182

Experimental Evaluation of Selected Orifice Flowmeter Upstream Installations.

Stabilization Techniques for Spinning Rotor Gage Residu-

PB91-147108

100,922

Fundamentals and Applications of Infrared Themography for Nondestructive Testing.

101,009

Low-Temperature, Electrically Calibrated SOS Bolometer for Power and Energy Measurements.

100,787

High Speed Magnetic Field Sensors Based on Iron Gar- 
Electrokinetic Demixing of Two-Phase Aqueous Polymer Systerns. 1. Separation Rates of Polyethylene GlycolDextran Mixtures.

100,487

Apparatus for Measurement of Thermal Conductivity of Insulation Systems Subjected to Extreme Temperature Differences.

101,738

Sensing of GMAW Droplet Transfer Modes Using an ER 100S-1 Electrode.

PB91-174250

101,004

Improved Optical Diffraction Strain Measurement System.

Correction for Converting 2-CM(3)-Coupler Responses to Insertion Responses for Custom in-the-Ear Non-Directional Hearing Aids.

Planar Waveguide Optical Immunosensors

100,046

PB91-174565

101,284

Use of Birefringent Coatings in Fracture Mechanics.

PB91-174599

101,582

Iron-Garnet Magnetic Field Sensors with $100 \mathrm{pT} / \mathrm{per}$ square root $\mathrm{Hz}$ Noise-Equivalent Field.

100,751

Instrumentation of X-ray Beam Lines with PIN Diode De-

Instrumentation of X-ray Beam Lines with PIN Diode De-

tectors.

101,633

High-Resolution Small-Angle X-ray Scattering Camera for Anomalous Scattering.

PB91-175067

100,929

Optical Fiber Current Sensors with Temperature Stabilities Near the Material Limit.

PB91-175109

100,753

Faraday Effect Current Sensing Using a Sagnac Interferometer with a $3 \times 3$ Coupler.

100,754

Overview of Facilitated Transport Membrane Systems.
PB91-175463

Reusable Cryogenic Foam Insulation for Advanced Aerospace Vehicles.

100,560

Heat Transfer in a Compact Heat Exchanger Containing Heat Transfer in a Compact Heat Exchanger Containing
Rectangular Channels and Using Helium Gas.
PB91-184804

101,734

Enhanced Transport and Liquid Membranes in Bioseparations.

101,287

Microchannel-Plate Detection Systems for Low AcceleratMicrochannel-Plate Detection Systems for Low Accelerat-
ing Voltage SEM.
PB91-189928
PO1,515

Comparison of Standard Test Methods for Non-Lubricated Sliding Wea

101,187

Image Quality Indicator Design for Radioscopy and Tomography.

101,018

Heat Transter and Pressure Drop in a Compact Pin-Fin Heat Exchanger with Pin Orientation at 18 deg to the Flow Direction.
PB91-194464

101,050

Application of Osmotic Dewatering to the Controlled Application of Osmotic Dewatering to the Controlled
Crystallization of Biological Macromolecules and Organic Crystallization of Biological Macromolecules and Organic
Compounds. Compounds.
PB91-195719

100,230

Frequency Stabilization of an Erbium-Doped Fiber Laser A Potential Wavelength Standard for Optical Communications.

PB91-203091

100,570

Note on the Precision Cementing of Small Optical Com-

ponents.
PB91-203158

101,428

Capacitance versus Stylus Measurements of Surface Poughness.

101,020

Integrated-Optic Waveguide Glass Lasers.

PB91-203976 100,758

High-Temperature Transient Hot-wire Thermal Conductivi-

ty Apparatus for Fluids.

PB91-216713

100,411

Automated Reverse-Bias Second-Breakdown Transistor

Tester.

PB91-216739 100,813

Investigating the Use of Multimeters to Measure Quan-

tized Hall Resistance Standards.

PB91-236554

100,734

Thin and Composite High-Flux Membranes of Perfluoro-

sulfonated Ion-Exchange Polymer.

100,512

Electrokinetic Demixing of Two-Phase Aqueous Polymer Systerns. 2. Separation Rates of Polyethylene Glycol-Maltodextrin Mixtures.

PB91-237230

100,231

Characterization of Capacitive Array for NDE Applica-

tions.

100,946

Theory of Capacitive Probe Method for Noncontact Char-

acterization of Dielectric Properties of Materials.
PB91-237610

100,948

Transient Hydrogen Heat Transfer. Final Report for April 1986-April 1989.

B92-116326

100,454

Response of Personal Noise Dosimeters to Continuous and Impulse-Like Signals.

100,914

Ultrasonic Measurement of Sheet Steel Texture and For mability: Comparison with Neutron Diffraction and $\mathrm{Me}$ chanical Measurements.

PB92-116615

101,170

Fourteen-Decade Photocurrent Measurements with Large-Area Silicon Photodiodes at Room Temperature. Large-Area 71 100,768
PB92-11671 Photodiodes at Room Temperature. Design and Fabrication of a Miniature High-Temperature Guarded-Hot-Plate Apparatus.

PB92-116797

101,098

Advanced Se

100,949

Development of Test Methods to Determine the Compatibility of Liquid Hazardous Materials with Polyethylene PBackagings.

100,943

INSULATING BOARDS

Apparent Thermal Conductivity of Fumed-Silica Standard Reference Materials.

PB92-117357

101,102

INTAGLIO

Synthesis of Non-lonic Water-Dispersible Resins for Use in Intaglio Inks Curing by Electron Beam Radiation. PB91-194456

INTAGLIO INK

Synthesis of Prototype Resins for Use as BEP Intaglio Ink Vehicles Curing by Electron Beam Radiation.

PB91-144345

101,182

NTEGRATED CIRCUITS

Current Density Dependence of Electromigration t50 Enhancement Due to Pulsed Operation.

PB91-134692

100,786

Thermal Characterization of Integrated Circuits - A Tutori-

PB91-147819

100,789

Thermal Measurements of VLSI Packages: A Critical Review.

100,790

MMIC Related Metrology at the National Institute of Standards and Technology.

PB91-149070

100,794

Measurement of Capacitance on Waters.

PB91-149120

100,795

Standard Reterence Materials: Bright-Chromium Linewidth Standard, SRM 476, for Calibration of Optica Microscope Linewidth Measuring Systems.

100,802

Design, Fabrication, and Testing of an Interconnect Test Design, Fabrication, and Testing of an Interconnect Test
Structure for Evaluating VLSI Processes.

Characterizing VLSI Processes Using Test Structures.

PB91-175000 100,804

Evaluation and Improvement of E-Beam Exposure Routines by Use of Microelectronic Test Structures.
PB91-175489

Microwave Monolithic Integrated Circuit-Related Metrology at the National Institute of Standards and Technology.
PB91-237255

Metrology fot the Semiconductor Industry.

100,817

Three-Dimensional Simulations of High-Resolution Pho-

Three-Dimensional Simulations of High-Resolution Photoresist Processing

Knowledge Extraction Techniques for Expert System Assisted Wafer Screening

PB92-116979

100,824

Application of Transmission Electron Detection to $\mathrm{X}$-ray

Mask Calibrations and Inspection.

PB92-117217

100,828

Measurement, Use, and Interpretation of TCR

PB92-117282

100,831

TEGRATED OPTICS

Integrated-Optic Waveguide Glass Lasers.

PB91-203976 100,758

Analyzing Integrated Optical Waveguides: A Comparison of Two New Methods.

PB91-204131

100,759

INTEGRATED SERVICES DIGITAL NETWORK

ISDN Conformance Testing: Layer 1. Physical Layer. Part 1. Basic Rate $S / T$ Interface, User Side.

PB92-102201

100,572

NTELLIGENT BUILDINGS

Intelligent Building Technology in Japan

PB91-187757

100,071

PRACTIVE GRAPHICS

Graphical Kernel System (GKS). Pascal Binding; Catego-

ry: Software Standard; Subcategory: Graphics.

FIPS PUB 120-1A

INTERACTIVE SYSTEMS

Multimedia Courseware in an Open Systems Environ-

ment: A Federal Strategy.

PB91-143362

100,039

INTERCALATION

Structure of a Stage-3 Cs - Graphite Intercalate.

PB91-134957

100,269

\section{INTERFACES}

Interface between Data Terminal Equipment (DTE) and Data Circuit-Terminating Equipment (DCE) for Operation with Packet-Switched Data Networks (PSDN), or between Two DTEs, by Dedicated Circuit. Category: Hardware Standard. Subcategory: Data Transmission.

100,672

Morphology and Barrier-Height Development of $\mathrm{Bi} /$ InP(110) Interfaces.

PB91-147736

101,468

Applied Electric Fields for Downstream Processing.

Apo,299

Study of the GaAs-Si(100) Interface Using Laser Probing of Thermal Desorption Kinetics.

PB91-149294

101,477

XPS and Auger Forward Scattering in Epitaxial Films.

PB91-174698 101,495

Surface Extended-X-ray-Absorption Fine Structure and Scanning Tunneling Microscopy of Si(001)2×1-Sb.

PB91-237263

101,552

Manufacturing Systems Integration Control Entity Interface Document

100,991

Kinematic Theory of Ballistic Electron Emission Spectros copy of Silicon-Silicide Interfaces.

PB92-117373

101,570

INTERFACIAL TENSION

Dynamic Technique for Measuring Surface Tension at

High Temperatures in Microgravity Environment.
N91-21347/0

101,206

Effect of Thermal Expansion Mismatch on Fiber Pull-Out in Glass Matrix Composites.

PB91-202952

101,125

\section{NTERFEROMETERS}

Analytic Solution for the Three-Layer Multiple Beam InterPB91-162107

101,007

INTERFEROMETRY

$X$-ray Interferometry and gamma-ray Wavelengths

PB91-158642

101,484

INTERLABORATORY COMPARISONS

Round Robin on Apparent Thermal Conductivity of Several Loose-Fill Insulations.

Comparison of Standard Test Methods for Non-Lubricated Sliding Wear

101,187

Interlaboratory Comparison of Actinides in Human Tissue (239) Pu $+(240) \mathrm{Pu}$.

PB91-203265

101,323

INTERNATIONAL COOPERATION

International Co-operation: The Versailles Project on Ad- 
Energy Related Inventions Program: A Joint Program of the Department of Energy and the National Institute of Standards and Technology. Status Report for Recom mendations 1 through 250 .

PB91-178871

100,902

Energy Related Inventions Program. A Joint Program of the Department of Energy and the National Institute of Standards and Technology. Status Report for Recom mendations 251 through 523 .

PB91-184770

100,903

INVESTMENTS

Automan 2.0: Decision Support Software for Automated Manufacturing Investments. User Manual.

100,978

IODINE ATOMS

General Method for Doppler Determination of Cylindrical-

ly Symmetric Velocity Distributions: An Application of Fourier Transform Doppler Spectroscopy.

100,302

Diode Laser Probing of I*((2)P1/2) Doppler Profiles: Time Evolution of a Fast, Anisotropic Velocity Distribution in a Thermal Bath.

ION BOMBARDMENT

Secondary Ion Yield Changes in Silicon and Gallium Arsenide Due to Topography Changes during Oxygen or Cesium Ion Bombardment

101,471

Chemical Effects of $\mathrm{Ne}+$ Bombardment on the MoS2(0001) Surface Studied by High-Resolution Photoelectron Spectroscopy.

PB91-203471

100,399

ION CHROMATOGRAPHY Analysis of Wet Deposition (Acid Rain): Determination of
the Major Anionic Constituents by Ion Chromatography. the Major Anionic Constituents by Ion Chromatography.
PB91-236646

\section{ION EXCHANGE MATERIALS}

Increased Facilitated Transport Related to Microstructural Changes in Heat-Treated Ion-Exchange Membranes. PB91-174805

100,323

ION EXCHANGE RESINS

New Different Forms of Ammonium Loaded and Partly Deammoniated Zeolite Rho Studied by Neutron Powder. PB91-158717

ION IMPLANTATION

Fe Implantation in In0 53Ga0 47As/lnP.

PB91-189969 101,516

Quantitative Analysis of Impurities in SIMOX Samples Using Secondary lon Mass Spectrometry.
PB91-236596 100,208 Round-Robin Study of Implants in $\mathrm{Si}$ and $\mathrm{SiO} 2$ by SIMS, RBS, and NAA.

PB92-117241

100,830

ION MICROSCOPY

Method of FIM-FEEM Specimen Preparation of Superconducting and Other Oxides.

PB91-203596

101,542

ION-MOLECULE COLLISIONS

Cross Sections and Swarm Coefficients for $\mathrm{H}+, \mathrm{H} 2+$ $\mathrm{H} 3+, \mathrm{H}, \mathrm{H} 2$, and $\mathrm{H}$-in $\mathrm{H} 2$ for Energies from $0.1 \mathrm{eV}$ to 10 PB90-244690

101,597

Collisional Electron-Detachment and Ion-Conversion Processes in SF6.

100,836

Cross Sections and Swarm Coefficients for Nitrogen Ions and Neutrals in N2 and Argon lons and Neutrals in N2 and Neutrals in N2 and Argon lons and Neutrals in N2 PB92-110246

100,452

ION MOLECULE INTERACTIONS

Vibrational Spectra of Molecular Ions Isolated in Solid Neon. 7. $\mathrm{CO}(+), \mathrm{C} 2 \mathrm{O} 2(+)$, and $\mathrm{C}_{2} \mathrm{O} 2(-)$

100,250

ON MOLECULE REACTIONS

Proton Affinity of Cyanogen and Ion-Molecule Reactions of C2N2 $(+)$.

100,352

ION PLASMAS

Liquid and Solid lon Plasmas.

AD-A242 029/7

101,592

Observation of Correlations in Finite, Strongly Coupled Ion Plasmas.
PB91-200857

101,678

ION SCATTERING

Critical Compilation of Surface Structures Determined by Ion Scattering Methods.

PB90-244559

100,254

ION STORAGE

Progress at NIST on Absolute Frequency Standards Using Stored lons.

PB91-159285

101,625

Quantum Optics of Single, Trapped lons.

PB91-162123

101,628

ION TRANSPORT

Preface to Book Entitled 'Nonequilibrium Effects in Ion and Electron Transport'.

PB91-159236

101,623
IONIC CONDUCTIVITY

Thermodynamics of the Divalent-Metal Fluorides. 4. Heat Capacity of MSnF4, M $=\mathrm{Pb}, \mathrm{Ba}$, or $\mathrm{Sr}$, at Temperatures from $300 \mathrm{~K}$ to $660 \mathrm{~K}$

PB92-116524

100,456

IONIC PARTITIONING

Applied Electric Fields for Downstream Processing. 100,299

\section{IONIZATION}

Resonance Enhanced Multiphoton lonization Spectroscopy of $\mathrm{CHCl} 2$ and $\mathrm{CDCl} 2$

PB91-174870

100,328

Spectroscopic Data Base for Carbon and Oxygen.
PB91-237750

101,716
Relative H.Atom and O-Atom Concentration Measurement in a Laminar, Methane/Air Diffusion Flame. PB92-117365

100,559

IONIZATION CHAMBERS

Absorbed-Dose Calibration of Ionization Chambers in a(60)Co Gamma-Ray Beam

101,598

Status of Beta-Particle Calibration Studies at NBS. PB91.175265

101,636

IONIZATION CROSS SECTIONS

Inner-Shell Ionization Cross Sections

PB91.195479

100,203

IONIZING RADIATION

Modification of DNA Bases in Mammalian Chromatin by Radiation-Generated Free Radicals.

PB91-189373

101,338

Mechanistic Studies of Ionizing Radiation and Oxidative Mutagenesis: Genetic Effects of a Single 8-Hydroxyguanine (7-Hydro-8-oxoguanine) Residue Inserted at a Unique Site in a Viral Genome

PB91-190108

101,321

Criteria for the Operation of Federally-Owned Secondary Calibration Laboratories (Ionizing Radiation).

101,721

Electron Spin Resonance for Monitoring Radiation-Processed Meats Containing Bone

IONS $92-116672$

100,018

Applied Electric Fields for Downstream Processing PB91-149161 100,299 Ionic Hydrogen Bonds. Part 1. Thermochemistry, Structural Implications, and Role in Ion Solvation. $\quad 100,400$
PB91-203547 PB91-203547 100,400 Spectroscopy and Structure of the Alkali Hydride Diato-
mic Molecules and Their lons. PB92-110147

RDS (INFORMATION RESOURCE DICTIONARY SYSTEM) Use of the IRDS Standard in CALS (Revised). PB91-132209

IRDS SYSTEM

Prototyping the IRDS: An Airport Application.

\section{PB92-112580}

Instability in $\mathrm{pH}$ Measurements of Sputtered IrO2 Films.

PB91-147538

Polyelectrolyte Stabilized Metal Oxide Hydrosols as Catalysts for the Photooxidation of Water by Zinc Porphyrins.
100,351

IRON

Epitaxial fcc Fe Films on $\mathrm{Cu}(100)$

PB91-149328

101,478

Copper-lon-Dependent Damage to the Bases in DNA in the Presence of Hydrogen Peroxide.

101,330

Damage to the Bases in DNA Induced by Hydrogen Peroxide and Ferric Ion Chelates.

PB91-187112

101,331

Damage to the DNA Bases in Mammalian Chromatin by Hydrogen Peroxide in the Presence of Ferric and Cupric

PB91-189217

101,336

Fe Implantation in In $0.53 \mathrm{Ga0} 0.47 \mathrm{As} / \mathrm{InP}$

PB91-189969

101,516

Characterization by High Performance Liquid Chromatography (HPLC) of the Solubilization of Phosphorus in Iron Ore by a Fungus.

101,162

Iron Ion-Dependent Modification of Bases in DNA by the Iron Ion-Dependent Modification of Bases in DNA by the
Superoxide Radical-Generating System Hypoxanthine/ Xanthine Oxidase.

PB91-200824

101,306

Magnetic Properties of Iron/Silica Gel Nanocomposites.

101,110

Structural Study of the Epitaxial Growth of fcc-Fe Films, Sandwiches, and Superlattices on Cu(100).

101,555

IRON ALLOYS

Crystalline and Glassy Phases of Transition-Metal - Metalloid System

101,238

Low Temperature Magnetization and Magnetic Excitations in Amorphous Fe78B13Sig.

PB92-117464

101,573

IRON COMPLEXES

Journal of Research of the National Institute of Standards and Technology. January-February 1991. Volume 96, Number 1

100,220

New Chemical and Stereochemical Applications of Organoiron Complexes.

PB91-184861

100,221

RON COMPOUNDS

Spin Glass Magnetic Behavior of Iron/Silica Gel NanoPB91-159178

101,117

Change in Magnetic State of $\mathrm{Fe}+$ Silica Gel Nanocomposites Due to Low Temperature Treatment in Ammonia.
101,129 Magnetic Behavior of Nanocomposites Prepared in a Vitreous Alumina Gel.

101,131

IRON IONS

Spectral Data and Grotrian Diagrams for Highly lonized Iron, $\mathrm{Fe} V \mathrm{VIII-XXV|}$.

PB90-244583

101,595

IRON ORGANIC COMPOUNDS

Journal of Research of the National Institute of Standards and Technology. January-February 1991. Volume

96, Number 1.
PB91-184853
100,220

New Chemical and Stereochemical Applications of Or. ganoiron Complexes.

100,221

IRON OXIDES

Surface-Extended X-ray-Absorption Fine-Structure Experiments at Atmospheric Pressure by Means of a Photocathode Proportional Counter with Monolayer Sensitivity.
PB91-194951

SDN (INTEGRATED SERVICES DIGITAL NETWORK)

North American ISDN Users' Forum Agreements on Integrated Services Digital Network.

PB92-102219

Security in ISDN

PB92-116391

100,573

ISOBUTYL RADICALS

Chemical Kinetic Data Base for Combustion Chemistry Part 4. Isobutan

100,252

ISOMERS

Standard Chemical Thermodynamic Properties of Isomer Groups of Monochloroalkanes.

100,258

Standard Chemical Thermodynamic Properties of PolycyStandard Chemical Thermodynamic Properties of Polycyclic Aromatic Hydrocarbons and Their Isomer Groups. III. Naphthocoronene Series, Ovalene Series, and First
Members of Some Higher Series.
PB90-244625
100,259

ISOTHERMAL TREATMENT

Isothermal Phase Behavior of Ag3SbS3, ZnGeP2, and Zns.

OTHERMS

Phenomenological Theory of the Influence of Strain History on the Rate of Isothermal Stress Relaxation. PB91-187385

100,496

ISOTOPE RATIO

Observation of Anomalous Isotope Ratios in SIRIS Measurements of Molydenum

101,361

ISOTOPE REFERENCE MATERIALS

Silicon Reference Materials Certified for Isotope Abundances. 
PB91-187757

100,071

JET FLOW

Ceiling Jet in Fires.

PB91-167197

100,114

Large-Scale Turbulent Structures and the Stabilization of Lifted Turbulent Jet Diffusion Flames.

PB92-117191

100,558

JET MIXING FLOW

Effects of Global Density Ratio on the Centerline Mixing Behavior of Axisymmetric Turbulent Jets.

100,014

Reynolds Number Effects on the Mixing Behavior of Axi-
symmetric Turbulent Jets.
PB92-117209 100,015

PB92-117209

01,027

Self-Adjustment of a Robot Joint Controller.

101,027

JOSEPHSON JUNCTIONS

Noise-Affected I-V Curves in Small Hysteretic Josephson Junctions.

PB91-174912

100,782

Sidelobe Suppression in Small Josephson Junctions.
PB91-195446

100,784
PB91-195446 sephson Weak Links.

101,551

Voltage Calibration Systems Using Josephson Junction Arrays.

PB92-116516

100,868

JUNCTION POTENTIAL

Evaluation of Liquid Junction Potentials and Determination of $\mathrm{pH}$ Values of Strong Acids at Moderate Ionic Strengths.

KEROSENE

Velocity and Droplet Size Measurments in a Fuel Spray.
PB91-147934

Application of Laser Diagnostic Techniques for the Examination of Liquid Fuel Spray Structure.

PB91.195487

100,229

KILOGRAM

Monitoring the Mass Standard via the Comparison of Mechanical to Electrical Power.

PB91.237115

101,710

KINETICS

Numerical Study of Two-Dimensional Crystal Growth Forms in the Presence of Anisotropic Growth Kinetics. PB91-200865

101,538

NOCK-OUT REACTIONS

Possible (e,e'2N) Studies at CEBAF.

101,612

KNOWLEDGE BASES (ARTIFICIAL INTELLIGENCE)

Knowledge Verification of Machine-Learning Procedures Based on Test Structure Measurements.

100,709

PBS

Journal of Physical and Chemical Reference Data, Volume 19, Number 4, July-August 1990

100,354

Thermodynamics of the Krebs Cycle and Related Com-

pounds.
PB91-192468

100,357

KRYPTON

Parity-Unfavored Transitions in Resonant Photoemission

from Ar, Kr, and Xe: Experimental and Theoretical Re-

sults.

101,599

Effect of Noble Gas Mixtures on the Performance of Re-

generative-Type Cryocoolers Analytical Estimate.

Angular Distribution of Ejected Electrons in Resonant Auger Processes of $\mathrm{Ar}, \mathrm{Kr}$, and $\mathrm{Xe}$.

101,648

Unusual Degree of Angular Anisotropy in the Resonant Auger Spectrum of $\mathrm{Kr}$.

101,649

Viscosity and Thermal Conductivity of Pure Monatomic Gases from Their Normal Boiling Point Up to $5000 \mathrm{~K}$ in the Limit of Zero Density and at $0.101325 \mathrm{MPa}$.

PB91-192559

100,366

Energy Levels of Krypton, $\mathrm{Kr} I$ through $\mathrm{Kr}$ XXXVI.

100,473

KRYPTON IONS

Spectrum and Energy Levels of Seven-Times-Ionized Krypton (Kr VIII) and Resonance Lines of Eight-Timeslonized Krypton (Kr IX).

101,673

Energy Levels of Krypton, $\mathrm{Kr} I$ through Kr XXXVI.
PB92-148139

LABELS

Checking the Net Contents of Packaged Goods. Third Edition, Supplement 2.

PB92-112549

100,171

LABORATORIES

Pozzolan Programs of the Cement and Concrete Reference Laboratory
PB91-149922

Raster Graphics Conformance Testing

100,525

PB91-167296

101,341

Directory of Federal Government Laboratory Accreditation/Designation Programs

100,005

Application of CCRL Data in the Development of Cement

Standards.
PB91-175315

100,119

NVLAP FY 90 Annual Report

100,010

Directory of Accredited Laboratories, 1991. National Voluntary Laboratory Accreditation Program (NVLAP).

PB91-222646

100,934

Criteria for the Operation of Federally-Owned Secondary Calibration Laboratories (lonizing Radiation).

101,721

LABORATORY ACCREDITATION

Laboratory Accreditation in the United States.

PB91-194415

100,172

Activities of the Construction Materials Reference Laboratories Related to Laboratory Accreditation PB91-203836

100,531

LAMB SHIFT

Precise Experimental Test of Calculated Two-Electron Lamb Shifts in Helium.

PB91-149203

101,617

LAMELLAR STRUCTURE

Incorporation of Molecular Orientation into Systems of Lamellar Morphology. 1. Effects of Packing Entropy on the Lamellar Thickness of Block Copolymers.

PB91-147231

100,484

LAMINAR FLOW

Unsteady Laminar Flow in a Circular Tube: A Test of the HERCOL (Hermitian Collocation) Computer Code. PB91-22267

101,395

LAMINATES

Effect of Logarithmic Singularity on the Free Edge Stress Intensity Factor of Composite Laminates.

101,116

PB91-147090

LANDING AIDS

Static and Dynamic Strength Tests on Electrical Conductor Cables Specified for Airport Landing Structures.

PB92-112226

101,739

LANGUAGE BINDING

Graphical Kernel System (GKS). Ada Binding; Category: Software Standard; Subcategory: Graphics.

100,674

\section{LANTHANUM COMPLEXES}

Lanthanide Coordination Chemistry: Spectroscopic Properties of Terbium and Europium Poly(Pyrazol-1-YI) Borate and Poly(Imidazol-1-YI) Borate.

PB91-203034

100,392

LANTHANUM STRONTIUM CUPRATES

Strontium-Induced Oxygen Defect Structure and Hole Doping in $\mathrm{La2}-x \mathrm{Sr} x \mathrm{CuO} 4$

101,472

Initial Stages of Degradation of Superconductor Surfaces:

$\mathrm{O} 2, \mathrm{H} 2 \mathrm{O}, \mathrm{CO} 2$ and $\mathrm{CO}$ Chemisorption on $\mathrm{La} 2-\mathrm{xS} \times \mathrm{CuO} 4$.

$\mathrm{O} 2, \mathrm{H} 2 \mathrm{O}, \mathrm{CO} 2$ and $\mathrm{CO}$ Chemisorption on La2-xSrxCuO4.
$\mathrm{PB91-189670}$

LARGE SPACE STRUCTURES

Probe Waveforms and the Reconstruction of Structural Dynamic Green's Functions.

PB91-161950

101,737

LASER APPLICATIONS

Improved Optical Diffraction Strain Measurement System.
PB91-174433

Relative $\mathrm{H}$-Atom and O-Atom Concentration Measurement in a Laminar, Methane/Air Diffusion Flame. PB92-117365

100,559

LASER ASSISTED COLLISIONS

Notes and Comments on Roundtable Discussion on Laser-Assisted Collisions and Collision-Induced Spectra.
PB91-203307

LASER COOLING

Cooled Ion Frequency Standard.

AD-A242 030/5

101,593

LASER DAMAGE

Laser Induced Damage in Optical Materials: 1989.

PB91-148478

101,409

LASER DRILLING

Acoustic Emission Monitoring of Laser Drilling PB91-158527

101,218

LASER ENHANCED IONIZATION

Laser Enhanced Ionization as a Selective Detector for Liquid Chromatographic Determination of Alkyltins in PB92-116755

100,210

\section{LASER FREQUENCIES}

Accurate Frequency of the $119 \mathrm{mu} \mathrm{m}$ Methanol Laser from Tunable Far-Intrared Absorption Spectroscopy.

LASER HEATING

Ignition Characteristics of the Nickel-Based Alloy UNS N07001 in Pressurized Oxygen.

100,536

LASER INDUCED DESORPTION

Laser Induced Desorption of Molecules from Surfaces

PB91-147066

100,235

LASER INDUCED FLUORESCENCE

Detection of Excited States by Laser-Induced Fluorescence and Analysis of Energy Transfer.

AD-A227 296/1

100,242

Laser-Induced Fluorescence of $\mathrm{CH}$. in a Laminar $\mathrm{CH} 4$ /Air

Diffusion Flame: Implications for Diagnostic Measurements and Analysis of Chemical Rates.

PB91-237099

100,554

LASER MICROPROBE MASS SPECTROSCOPY

Use of Single Particle Standards for LAMMA Calibration.

PB91-147777

LASER RADIATION

Laser Measurements.

PB91-149195

100,183

Precise Laser Wavelength Measurements: What Can We Learn from Classical Spectroscopy.

101,430

New Triple Correlation Technique for Measuring Ultrashort Laser Pulses.

LASER SPECTROSCOPY

Direct Detection of Atom Vaporization by Laser Reso. nance Ionization as a Probe of Gas-Surface Chemisorption Mechanisms.

tion Mechanism
PB91-237032

100,425

LASERS

Laser Measurements.

PB91-149195

101,412

LASERS \& THEIR APPLICATIONS

Annual Report to the Strategic Defense Initiative Organjzation on the Free-Electron Laser Driven by the NIST CW Microtron.

AD-A207 806/1

101,398

Detection of Excited States by Laser-Induced Fluorescence and Analysis of Energy Transfer.

AD-A227 296/1

100,242

NIST-NRL Free-Electron Laser.

101,399

Laser Photolysis of Trimethylgallium at $193 \mathrm{~nm}$ : Quantum Yields for Methyl Radical and Ethane Production.

AD-A227 868/7

100,234

Modulatable Narrow-Linewidth Semiconductor Lasers.

PB91-134106 101,404

Radiant Heat Fluxes from 100-200 MW Natural Gas/Air Diffusion Flames.

PB91-147306

100,538

Fiber-Optic Fluorescence Array to Study Free Convection

in Porous Media.
PB91-147868

101,407

Laser Induced Damage in Optical Materials: 1989.

101,409

Laser Diagnostics for Characterization of Fuel Sprays.
PB91-149013

Laser Measurements.

B91-149195

101,412

Effects of Colored Pump Noise on Intensity Correlations of a Single Mode Dye Laser.

PB91-189738 
LAW (JURISPRUDENCE)

Uniforms Laws and Regulations as Adopted by the $\mathrm{Na}$ tional Conference on Weights and Measures (76th), 199 PB92-112416

101,022

LBIR FACILITY

LBIR Facility User Handbook

PB92-102185

101,435

LEACHING

Rate of Pyrite Bioleaching by 'Thiobacillus ferrooxidans' Results of an Interlaboratory Comparison.

101,161

EAD ACETATE

Stability of Aqueous Inorganic Lead Solutions in Polycarbonate Containers.

AD INORGANIC COMPOUNDS

Stability of Aqueous Inorganic Lead Solutions in Polycarbonate Containers.

PB92-126499

100,211

LEAD TIN TELLURIDES

High Resolution Diffraction Imaging of Crystals Grown in

Microgravity and Closely Related Terrestrial Crystals.
PB92-109008

LEAD ZIRCONATE TITANATES

Lead Zirconate-Titanate Thin Films Prepared by the Laser Ablation Technique.

PB91-187344

101,502

LEAK DETECTORS

Study of the Linearity of Transfer Leaks and a Helium PB91-147272

100,926

LEAKAGE

Airtightness of Office-Building Envelopes.

PB91-175307

100,067

LEAVES (BOTANY)

Characterization of the Mineral Fraction in Botanical Reference Materials and Its Influence on Homogeneity and Analytical Results.

100,186

LENNARD-JONES POTENTIAL

Non-Equilibrium Molecular Dynamics Simulations of Structured Molecules. Part 1. Isomeric Effects on the Viscosity of Butanes

PB91-195636

101,391

LEVIATION MELTING

Levitation Calorimetry.

EVITATION

Optical Levitation of Single Particles
PB91-174987

100,927

101,634

LIFE CYCLE COSTS

DISCOUNT-A Program for Discounting Computations in Life-Cycle Cost Analyses. User's Guide and Reference PB91-16

100,897

Building Life Cycle Cost Computer Program, Version 3.2 (for Microcomputers).

PB91-507970

100,898

Energy Prices and Discount Factors for Life-Cycle Cost Analysis 1992. Annual Supplement to NIST Handbook 135 and NBS Special Publication 709.

100,899

FT SLAB CONSTRUCTION

Investigation of the Collapse of L'Ambiance Plaza.
PB91-204057 100,146

LIGHT PULSES

New Triple Correlation Technique for Measuring UltraShort Laser Pulses.

GHT SCATTERING

101,434

Determination of Fields Near a Silver Strip on a Glass PB91-147702

100,841

Multiple Variable-Angle Light Scattering Detector for Gel Permeation Chromatography.

100,195

PB91-187641

(BSDF): A

Bidirectional Scattering Dist
Systematized Bibliography.

Systematized

101,421

LIGHT SOURCES

Light Source Area, Shading, and Glare.

100,080

LIGHT TRANSMISSION

Algorithm and Computer Program for the Calculation of

Envelope Curves.
PB91-158972

101,414

LIGHTING SYSTEMS

Computer Program for Simulation of HVAC/Lighting Interactions: Initial Report

PB91-144386

100,889

Second-Level Post-Occupancy Evaluation Analysis.

PB91-147140 100,058

Measurement and Evaluation of Lighting/HVAC Interaction. 206706

LINE BROADENING

Overview of the Ion Dynamic Effect in Line Broadening,

and a Generalization of the Unified Theory.
PB91-147504

101,442

INE SPECTRA

Experimental Stark Widths and Shifts for Spectral Lines of Neutral and Ionized Atoms: A Critical Review of Selected Data for the Period 1983 through 1988 B91-192567

101,659

Spectrum and Energy Levels of Seven-Times-lonized Krypton ( $\mathrm{Kr}$ VIII) and Resonance Lines of Eight-Timeslonized Krypton ( $\mathrm{Kr} \mathrm{IX}$ )

101,673

Asymptotic Expansions of the Kernel Functions for Line Formation with Continuous Absorption.

101,688 PB91-203240

LINE WIDTH

Standard Reference Materials: Bright-Chromium Linewidth Standard, SRM 476, for Calibration of Optical Microscope Linewidth Measuring Systems.

100,802

INEAR PROGRAMMING

Interior-Point Method for Linear and Quadratic Programming Problems.

101,269

Convergence Behavior of Trajectories for Linear Programming.

101,270

LINEARITY

Study of the Linearity of Transfer Leaks and a Helium Leak Detector

100,926

LIPID PEROXIDATION

8-Hydroxyguanine Content of Isolated Mitochondria Increases with Lipid Peroxidation.

101,286

LIPOSOMES

Liposome Flow Injection Immunoassay: Implications for Sensitivity, Dynamic Range, and Antibody Regeneration.
PB91-147629

LIQUEFIED GASES

Small Scale Demand Type Neon Liquefaction Plant PB91-162008

100,224

LIQUID CHROMATOGRAPHY

Trace Determination of Cr(VI) by LC/AAS with on-Line Preconcentration.

100,184

Reverse-Phase HPLC Separation and Electrochemical Detection of Retinol and Its Isomers.

100,187

PB91-148858

matog.

raphy.

100,199

aser Enhanced Ionization as a Selective Detector for Liquid Chromatographic Determination of Alkyltins in PBdiment.

100,210

LIQUID COLUMN CHROMATOGRAPHY

Characterization by High Periormance Liquid Chromatography (HPLC) of the Solubilization of Phosphorus in Iron Ore by a Fungus.

101,162

\section{LOUID CRYSTALS}

Critical Evaluation of Liquid Crystal Transition Temperatures I: 4,4'-Alkyl/alkoxyphenylbenzoates.

100,444

\section{LIOUID FILMS}

Effect of Coherency Strain on Alloy Formation: Migration of Liquid Films.

101,210

LIQUID HELIUM

New Wide Range Equation of State for Helium. $\quad 100,226$ Saran Carbon Cryogenic Regenerator for Liquid Helium PB91-175430

101,640

LIOUID HYDROGEN

Transient Hydrogen Heat Transfer. Final Report for April 1586-April 1989

100,454

LIOUID METALS

Dynamic Technique for Measuring Surface Tension at High Temperatures in Microgravity Environment N91-21347/0

101,206

Levitation Calorimetry.

100,927

High-Temperature Thermal Properties of UNS S44004 Using Multivariant Analysis.

101,160

LIQUID OXYGEN

Aluminum Alloys for Cryogenic Tanks: Oxygen CompatAD-A229 229/0

101,201

Transport Properties of Fluid Oxygen.

100,359

LIOUID PHASES

Heat Capacities of Organic Compounds in Liquid State. 2. C1 to C18 n-Alkanes.

QUID SCINTILLATORS

Calculation of Beta-Particle Counting Efficiency for Liquid Scintillation Systems with Three Phototubes.

PB91-175547

101,644

LIQUID-SOLID INTERFACES

Coherency Strain Induced Instability of Solid-Liquid Interfaces in the Mo-Ni System.

PB91-195537

101,235

IOUID-VAPOR INTERFACES

Effect of Long Range Interfacial Forces on the Dynamics of Wetting Layers at a Liquid Vapor Interface.

100,344

LIOUIDS

Computer Simulation of Fluid-Fluid Phase Coexistence in Mixtures of Nonadditive Soft Disks.

PB91-162263 101,388

Critical Exponent for the Viscosity of Four Binary Liquids. PB91-187195 100,337 Journal of Physical and Chemical Reference Data Volume 19, Number 4, July-August 1990

100,354

Heat Capacities and Entropies of Organic Compounds in the Condensed Phase. Volume

PB91-192450

100,356

Diesel Deposit Forming Tendencies - Microanalysis Meth-

PB91-195412

101,178

ICLASS-91 Proceedings of the International Conference on Liquid Atomization and Spray Systems (5th). Held in Gaithersburg, MD. on July 15-18, 1991.

100,552

Stability of Aqueous Inorganic Lead Solutions in Polycarbonate Containers.

100,211

Solubilities of Solids and Liquids of Low Volatility in Supercritical Carbon Dioxide.

ITHIUM ALLOYS

100,470

Aluminum Alloys for Cryogenic Tanks: Oxygen Compatibility. Volume 1.

101,201

Review of Cryogenic Mechanical and Thermal Properties of Al-Li Alloys and Alloy 2219

AD-A229 231/6

101,202

Aluminum Alloys for ALS Cryogenic Tanks: Comparative Measurements of Cryogenic Mechanical Properties of AlLi Alloys and Alloy 2219

101,204

Stress Corrosion Cracking of Al-Li Alloys: The Role of Grain Boundary Precipitates.

PB91-175182

LOADS (FORCES)

Load-Controlled Tensile Tests of Austenitic Steels at $4 \mathrm{~K}$

LOCAL AREA NETWORKS

Synchronizing Computer Clocks Using a Local Area Net-

N91-25793/1

Conformance Verification of FODI Stations.

PB91-148254

100,596

LOC

Use of Computer Algebra to Locate Critical Loci in Fluid Mixtures.
PB91-195263

101,390

LOGISTICS SUPPORT

Data Management Standards in Computer-Aided Acquisition and Logistic Support (CALS).

PB91-158832

101,339

NIST Support for the Computer-Aided Acquisition and Logistic Support (CALS) Program in the Area of Graphics Standards, Calendar Year 1990.

PB91-194506

101,348 


\section{KEYWORD INDEX}

MAGNIFICATION STANDARDS

LOW TEMPERATURE SCIENCE \& ENGINEERING

Aluminum Alloys for Cryogenic Tanks: Oxygen CompatAD-A229 229/0

101,201

Review of Cryogenic Mechanical and Thermal Properties of Al-Li Alloys and Alloy 2219.

101,202

Aluminum Alloys for ALS Cryogenic Tanks: Oxygen Compatibility. Volume 2.

101,203

AD-A231 830/1 101,203

generative-Type Cryocoolers Analytical Estimate.

PB91-144410

100,890

Hydrogen Slush Production with a Large Auger.

PB91-147199

100,275

Review of Pulse Tube Refrigeration

101,046

Fracture Toughness of $316 \mathrm{~L}$ Stainless Steel Welds with

Varying Inclusion Contents at $4 \mathrm{~K}$.
PB91-158980

Small Scale Demand Type Neon Liquefaction Plant.

PB91-162008 100,224

Effect of Nitrogen and Carbon on FCC-HCP Stability in

$\begin{array}{ll}\text { Austenitic Steels. } & 101,151 \\ \text { PB91-175372 } & \end{array}$

Saran Carbon Cryogenic Regenerator for Liquid Helium.

PB9 1.175430

Discontinuous Yielding during Tensile Tests at Low Temperatures.

PB91-189977 101,159

Prospects for High Temperature Superconductor - Semiconductor Contacts.

101,545

Tunneling Stabilized Magnetic Force Microscopy: Prospects for Low Temperature Applications to Superconduc-

tors.

101,546

Optimization of a Pulse Tube Refrigerator for a Fixed Compressor Swept Volume.

101,051

Load-Controlled Tensile Tests of Austenitic Steels at $4 \mathrm{~K}$. PB91-236943 101,165

Cryogenic Fatigue of High-Strength Aluminum Alloys and Correlations with Tensile Properties.

PB91-236976

101,709

Interlaboratory Tension and Fracture Toughness Test Results for CSUS-JN1 (Fe-25Cr-15Ni-0.3N) Austenitic Stainless Steel at $4 \mathrm{~K}$

PB91-23708

101,166

Effect of Processing on 4-K Mechanical Properties of a Microalloyed Austenitic Stainless Steel.

PB91-237206

101,167

Strain Rate Effect on Tensile Properties at $4 \mathrm{~K}$ of a VAMAS Round-Robin Austenitic Steel.

101,168

PB91-237248

Related

Oxides and

PB92-112333

101,563

Measurement of Regenerator Performance in a Vuilleumier Refrigerato

PB92-116482

101,052

LOW TEMPERATURE TESTS

Discontinuous Yielding during Tensile Tests at Low Tem-

peratures.

101,159

Load-Controlled Tensile Tests of Austenitic Steels at $4 \mathrm{~K}$. PB91-236943 101,165 Interlaboratory Tension and Fracture Toughness Test Results for CSUS-JN1 (Fe-25Cr-15Ni-0.3N) Austenitic Stainless Steel at $4 \mathrm{~K}$.

PB91-237081

101,166

Strain Rate Effect on Tensile Properties at $4 \mathrm{~K}$ of a VAMAS Round-Robin Austenitic Steel.

101,168

UBRICANT ADDITIVES

Mechanisms of Additive Effectiveness

Mechanisms of Additive Effectiveness.

101,172

PB91-174862

101,174

Characterization of Tricresylphosphate Lubricating Films by Micro-Fourier Transform Infrared Spectroscopy.
PB91-203810 1019 Effect of Selected Chemical Compounds on the Lubrication of Silicon Nitride.

PB91-236711

101,180

LUBRICANTS

Diesel Deposit Forming Tendencies - Microanalysis Meth. Ods. 195412

101,178

LUBRICATING OILS

Heats of Immersion, Friction, and Wear of Base Oil Fractions.

101,173

Study of Additive Response in a Series of Rerefined Base Oils Typical of Current Commercial Practice.

PB91-174953

101,175
LUBRICATION

Study on the Nature of Boundary Lubricating Film: Analytcal Method Development.

101,176

Mechano-Chemical Descriptive Model for Wear Under Mixed Lubrication Conditions.

101,177

\section{UNAR OBSERVATORIES}

Ground-Truth Observations of Stellar Surface Structure from the Lunar Surface.

PB91-175026

100,029

Gravitational Radiation Observations on the Moon PB91-175083

101,635

LUNAR RANGEFINDING

Lunar Laser Ranging.

YSINE

Structure and Mechanism of Hydroxyl Radical-Induced Formation of a DNA.Protein Cross-Link Involving Thy mine and Lysine in Nucleohistone. PB91-187518

101,334

LYSOZYME

Application of Osmotic Dewatering to the Controlled Crystallization of Biological Macromolecules and Organic Compounds.

\section{M1-TRANSITIONS}

Electric Quadrupole and Magnetic Dipole Transition Probabilities in Potassium Isoelectronic Sequence.
PB91-187088

101,646

ACHINE LEARNING

Knowledge Verification of Machine-Learning Procedures Based on Test Structure Measurements.

100,709

ACHINE TOOLS

Using Surface Roughness as a Wear Criterion in HighPB91-158675

101,040

\section{MACHINING}

Material Dependency of Chip-Form Detection Using Acoustic Emission.

101,216

Parser That Converts a Boundary Representation into a Parser That Converts a Boundary Representation into a
Features Representation.
PB91-203356 100,988

100,988

MACROMOLECULES

Mobility Fluctuations and Electrophoretic Light Scattering rom Macromolecular Solutions.

PB91-189514

100,345

MAGNESITE REFRACTORIES

Effect of Slag Penetration on the Performance of Magnesia Chrome Refractories in Slagging Gasifiers. PB91-134940

100,875

MAGNESIUM

Structure of Surface Films on Magnesium and on Magnesium Alloys.

101,212

Wavelengths and Energy Level Classifications of Magnesium Spectra for All Stages of Ionization (Mg I through $\mathrm{Mg} X \mid 1)$

MAGNESIUM ALLOY AZ61

Structure of Surface Films on Magnesium and on Magnesium Alloys.
PB91-147637

MAGNESIUM IONS

Theoretical Model of Metal Binding Sites in Proteins.

Theoretical Model of Metal Binding Sites in Proteins.
PB91-194837

Thermodynamics of the Disproportionation of Adenosine 5'-diphosphate to Adenosine 5'-triphosphate and Adenosine 5'-monophosphate. 1. Equilibrium Model.

PB92-116854

100,459

Thermodynamics of the Disproportionation of Adenosine $5^{\prime}$-diphosphate to Adenosine 5'-triphosphate and Adenosine 5'-monophosphate. 2. Experimental Data. PB92-117399

101,313

\section{MAGNESIUM OXIDES}

Journal of Physical and Chemical Reference Data, Volume 19, Number 1, 1990.

PB90-244526

100,251

Thermodynamic Functions and Properties of $\mathrm{MgO}$ at High Thersion and High Temperature.

PB90-244542

100,253

Dihedral Angles in Magnesia and Alumina: Distributions from Surface Thermal Grooves.

PB91-147330

101,058

Structure of Surface Films on Magnesium and on Magnesium Alloys.

101,212

\section{MAGNETIC DETECTION}

Iron-Garnet Magnetic Field Sensors with $100 \mathrm{pT} / \mathrm{per}$ square root $\mathrm{H}$
PB91-174615

100,751

MAGNETIC DOMAINS

Scanning Electron Microscopy with Polarization Analysis (SEMPA)-Studies of Domains, Domain Walls and Magnetic Singularities at Surfaces and in Thin Films.

PB91-237289

101,554

MAGNETIC FIELDS

Near-Field and Far-Field Excitation of a Long Conductor in a Lossy Medium.

PB91-143347 100,722

High Speed Magnetic Field Sensors Based on Iron Gar-

PB91-147223

100,840

Performance and Limitations of Faraday Effect Sensors.

PB91-149146 100,749

Evaluation of Instrumentation Used to Measure AC Power System Magnetic Fields.

PB91-162248 100,872

Measurements of Power Frequency Magnetic Fields Away from Power Lines.

PB91-175398 100,851

High Magnetic Field Facilities in Japan Related to Superconductivity Research (Japan Technology Program).

Magnetic Anisotropies in Ultrathin fcc $\mathrm{Fe}(001)$ Films Grown on Cu(001) Substrates.

PB92-116631

101,565

MAGNETIC IMAGING New Look at the Bitter Method of Magnetic Imaging.
PB91-203919

MAGNETIC MATERIALS

Change in Magnetic State of $\mathrm{Fe}+$ Silica Gel Nanocomposites Due to Low Temperature Treatment in Ammonia.
PB91-237321

MAGNETIC MEASUREMENT

High Speed Magnetic Field Sensors Based on Iron Gar-

PB91-147223

100,840

Evaluation of Instrumentation Used to Measure AC Power System Magnetic Fields.

PB91-162248

100,872

MAGNETIC ORDERING

Magnetic Ordering of Er in Powder and Single Crystals of ErBa2Cu3O7.

PB91-187377

101,505

Long-Range Antiferromagnetic Order of the $\mathrm{Cu}$ in Oxygen PB91-194894

101,524

MAGNETIC PROPERTIES

Cryogenic Properties of Copper (Wall Chart)

Magnetic Properties of Iron/Silica Gel Nanocomposites. PB91-204073

101,110

MAGNETIC RECORDING

High-Resolution, Tunneling-Stabilized Magnetic Imaging and Recording.

MAGNETIC STARS

Extension of the Class of Magnetic B Star Nonthermal Radio Sources.

MAGNETIC SUSCEPTIBILITY

Fundamental and Harmonic Susceptibilities of YBa2Cu3O7-delta.

PB91-134148

MAGNETIC TAPES

Standard Reference Materials: Calibration of NIST Standard Reference Material 3201 for 0.5 Inch (12.65 mm) Serial Serpentine Magnetic Tape Cartridge. $\quad 100,690$ 3480 Type Tape Cartridge: Potential Data Storage Risks and Care and Handling Procedures to Minimize Risks.
PB92-126549

MAGNETIZATION

Observation of Two Different Oscillation Periods in the

Exchange Coupling of $\mathrm{Fe} / \mathrm{Cr} / \mathrm{Fe}(100)$. 


\section{KEYWORD INDEX}

MAGNONS

Magnetic Anisotropies in Ultrathin Icc $\mathrm{Fe}(001)$ Films Grown on Cu(001) Substrates.

PB92-116631

101,565

MAMMALS

Alaska Marine Mammal Tissue Archival Project: Revised Collection Protocol.

101,374

MAN MACHINE SYSTEMS

TROI User's Guide.

101,026

MANGANESE ADDITIONS

Structural Study of Electrodeposited Aluminum-Manganese Alloys.
PB91-189423

101,231

MANGANESE ALLOYS

Cryogenic Material Properties of Stainless Steel Tube-toFlange Welds.

101,145

Phase Selection in Non-Equilibrium Processing. PB91-195651

101,236

MANGANESE OXIDES

Oxidation of Organic Compounds by Active Manganese Dioxide

PB91-174730

100,218

MANIPULATORS

Design and Implementation of a Five-Axis Robotic Micromanipulator for Inserting Parts into Precision Collets.

PB91.159194

101,029

Generation of Smooth Trajectories without Planning PB91-2174

101,036

MANUFACTURED HOUSING

Indoor Ventilation Requirements for Manutactured Housing.

100,075

MANUFACTURING

Innovation in Manufacturing Technology: A View trom

PB91-148130

100,976

Research Tackles Automation Issues

101,005

Control Structure for Multi-Tasking Workstations

PB91-203737 100,989

New Concepts ot Precision Dimensional Measurement for Modern Manutacturing.

PB91-240812

101,006

MANUFACTURING ENGINEERING LABORATORY

Publications of the Manufacturing Engineering Laboratory

Covering the Period January 1989-September 1991

PB92-123041

101,053

MAPPING

Real-Time Algorithms and Data Structures Ior Underwater Mapping.
PB91-195354

101,360

MAPS

Non-Linear Contour-to-Grid Digital Interpolation

101,351

\section{MARINE ENVIRONMENTS}

Preparation and Analysis ot a Marine Sediment Reference Material for the Determination of Trace Organic Constituents.

101,377

MARS SAMPLE RETURN MISSIONS

Possible Use of Pattern Recognition for the Analysis of Mars-Rover X-ray-Fluorescence Spectra.

101,733

\section{MARS SURFACE SAMPLES}

Possible Use of Pattern Recognition tor the Analysis of Mars-Rover X-ray-Fluorescence Spectra.

101,733

$$
\text { PB91-237875 }
$$

Review of Research Literature on Masonry Shear Walls.
PB91-167189 PB91-167189

versuse Strength of PB92-116342

100,138

MASS

Monitoring the Mass Standard via the Comparison of Me chanical to Electrical Power.

101,710

MASS SPECTRA

CID Spectra of Selected Target Molecules.

AD-A243 170/8

100,180

MASS SPECTROMETRY

Selected-Ion Mass Spectrometry: Assays ol Oxidative DNA Damage.

101,307

MASS SPECTROSCOPY

Instrument-Independent Tandem Mass Spectrometry Database for XQO Instruments: The Dynamical Prerequisites.

100,181

Strategy for Chemical Analysis of Alternative Retrigerants.

100,185

PB91-148502

ur Hexa.

fluoride by Gas Chromatography/Mass Spectrometry.
PB91-189860

100,197

Resonance Ionization Spectroscopy/Resonance Ionization Mass Spectrometry Data Service.

100,207

\section{MASS TRANSFER}

Hydrodynamics and Mass Transler in Two-Phase Aqueous Extraction Using Spray Columns.

101,300

PB91-149229

100, 426

Mass T

PB91-237149

100,426

MATERIALS

Factual Materials Databanks - The Need for Standards.

PB91-147546 101,249

International Co-operation: The Versailles Project on Advanced Materials and Standards.

PB91-159137

101,191

VAMAS Bulletin

PB91-159145

101,192

PB91-204032

100,009

Intelligent Processing of Materials, Technical Activities 1991. (NAS-NRC Assessment Panel, November 14-15, PB92

100,995

Materials Reliability. Technical Activities, 1991. (NAS NRC Assessment Panel, February 13-14, 1992).
PB92-126424

MATERIALS TESTS Monitoring of Anisotropic Material Elastic Properties
Using Ultrasonic Receiving Rays.
PB91-203059
101,126 International Collaboration in Prestandards Research on Advanced Materials within the Context of VAMAS. PB91-204040

101,251

Guidelines for the Development of Computer Based Models in a Cementitious Materials Modeling Laboratory. Models in a Cementitious Materials Modeling Laboratory.
PB91-240804 National Educators Workshop: Update ' 90 . Standard Ex. periments in Engineering Materials Science and TechnolPBy. 126606

101,253

MATHEMATICAL MODELS

Refinement of a Model for Fire Growth and Smoke Transport.

PB91-148510

100,104

Modelling Gallium Arsenide Transistors.

100,797

Compartment Fire-Generated Environment and Smoke

PB91-158543

100,107

Zonal Model Ior Corona Discharge-Induced Oxidation of

$\mathrm{SF} 6$ in $\mathrm{SF} 6 / \mathrm{O} 2 / \mathrm{H} 2 \mathrm{O}$ Gas Mixtures.

100,312

Analytical Model lor the Refrigeration Power of the Orifice Pulse Tube Refrigerator.

PB91-187534

101,048

Frequency Dependence of Catalyzed Reactions in a Weak Oscillating Field.

PB91.203927

100,407

Phase Equilibria and Critical Lines in Model Water/Salt

Mixtures.
PB91-236778 100,418

Guidelines for the Development of Computer Based Models in a Cementitious Materials Modeling Laboratory.

Mathematical Modeling ol Enclosure Fires. PB92-108976

100,135

\section{MATHEMATICAL PROGRAMMING}

Second Order Sensitivity Analysis in Factorable Program-

ming: Theory and Applications. 101,266

State-of-the-Art of Computational Testing of Mathematical Programming Algorithms.

101,268

\section{MATHEMATICAL \& STATISTICAL METHODS}

Influence of Moisture and Pressure on the Mechanical Properties ol a Glass-Epoxy Matrix Composite and a Graphite-Epoxy Matrix Composite.

AD-A239 509/3

101,114

Guide to Available Mathematical Soltware Problem Clas. sification System.

101,282

Israel's New Synchronized Time Scale, UTC(INPL),
PB91-134593

Space Marching Difference Schemes in the Nonlinear In-

verse Heat Conduction Problem.

vB91-144360

101,604

Orthogonal Distance Regression

PB91-147017

101,272

Mechanism lor High Strain-Rate Shear Band Formation.

PB91-147033 101,575

Stress Intensity Factors by Enriched Mixed Finite Ele-

ments.

101,577

Global Stability of the Chaotic State Near an Interior Crisis.

PB91-147496

101,611

Closure and Precision in Level-Index Arithmetic PB91-147652

100,597

Non-Linear Contour-to-Grid Digital Interpolation PB91-147678

101,351

Computing the Eigenvalues and Eigenvectors of SymmetC Arrowhead Matrices.

101,254

Certain Theorems on Bilateral Generating Functions Involving Hermite, Laguerre, and Gegenbauer Polynomials.
PB91-148759 Second Order Sensitivity Analysis in Factorable Programming: Theory and Applications.

101,266

Comprehensive Approach Ior Modeling and Testing Analog and Mixed-Signal Devices.

PB91-149310

100,732

Algorithm Design for Large-Scale Computations.

100,640 Linear Calibration When the Coefficient of Variation is Constant.

PB91-149443

101,273

Engineers' Statistical Literacy is Key to U.S. Competitiveness.

PB91.158725

100,038

Design and Analysis of Experiments.

101,274

Explicit Mapping of the Set of Dynamical Symmetries oxto the Set of Equivalent Lagrangians lor Systems with Onto the Set of Equivalent

PB91-158881

101,622

Calibration with Working Standards.

PB91-158931

101,276

Simplilied Error Bounds tor Newton's Rule.

PB91-159046

101,257

Probe Wavetorms and the Reconstruction of Structural Dynamic Green's Functions.

101,737

Cross-Validation Procedure for Stopping the EM Algorithm and Deconvolution of Neutron Depth Profiling Spectra.

PB91-161976

101,627

Calculated from the Frequency Domain: An Update.

PB91-162388 100,578 DEOSOL and ELLPACK: Problem-Solving Environments for Partial Dilferential Equations.

PB91-174441

100,645

Development of a Three-Dimensional Finite Element Pro-

gram for Large Strain Elastic-Plastic Solids.
PB91-174557

101,494

State-ol-the-Art of Computational Testing of Mathematical Programming Algorithms.

101,268 
Root Projection of One-Sided Time Series. PB91-216754

101,262 Unsteady Laminar Flow in a Circular Tube: A Test of the HERCOL (Hermitian Collocation) Computer Code. ${ }_{101,395}$ Methods of Analyzing Planar Optical Waveguides. Methods of Analyzing Planar Optical Waveguides.
PB91-236752 OMNITAB 80 (ASCII): An Interactive System for Statistical and Numerical Data Analysis (Version 7.0). PB91-507954 101,278 OMNITAB 80 (UNIX): An Interactive System for Statistical and Numerical Data Analysis. Version 7.0.

PB91-507962

Phase-Field Model for Isothermal Phase Transitions in Binary Alloys.

PB92-108992

101,240

Optimization Techniques for Permittivity and Permeability

Determination

100,865

NBS-INA. The Institute for Numerical Analysis. UCLA 1947-1954.

PB92-112408 101,264

Merit Function for Inequality Constrained Nonlinear Programming Problems.

101,271

Three-Dimensional Simulations of High-Resolution Photoresist Processing

PB92-116466

100,818

Use of Cross-Validation as a Stopping Rule in Emission Tomography Image Reconstruction.

101,295

Computing Delaunay Triangulations for Comet-Shaped Polygons.

101,265

Optimization Techniques for Permittivity and Permeability Determination.
PB92-126663

100,871

Taguchi's Orthogonal Arrays Are Classical Designs of Experiments.

101,280

PB92.126

Computing the Eigenvalues and Eigenvectors of Symmetric Arrowhead Matrices.

PB91-147850

101,254

Algorithms for Special Tridiagonal Systems. PB91-187245

101,259

MATRIX ISOLATION

Production and Spectroscopy of Small Polyatomic Molecular lons Isolated in Solid Neon.

AD-A234 043/8

100,245

Vibrational Spectra of Molecular lons Isolated in Solid Neon. 6. CO4(1-)

PB91-236836

100,419

MATRIX MATERIALS

Effect of Thermal Expansion Mismatch on Fiber Pull-Out in Glass Matrix Composites.

PB91-202952

101,125

MAUV (MULTIPLE AUTONOMOUS UNDERWATER

EHICLES

Real-Time Algorithms and Data Structures for Underwater Mapping.

101,360

MEAN SPHERICAL APPROXIMATION

Phase Equilibria and Critical Lines in Model Water/Salt Mixtures.

PB91-236778

100,418

MEASUREMENT

Ventilation System Performance Evaluation Using Tracer Gas Techniques.

Measurement of Structural Response Characteristics of Full-Scale Buildings: Selection of Structures.

100,144

Replicate Measurements in the Interpretation of Environmental Monitoring

mental Monitorin

100,919

MEASUREMENT SCIENCE \& TECHNOLOGY

CALIBRATION

Study of the Linearity of Transfer Leaks and a Helium Leak Detecto

PB91.147272

100,926

NIST High-Dose Calibration Services.

101,689

101,689
Stable, High Quantum Efficiency Silicon Photodiodes for Vacuum-UV Applications.

PB91-203349

100,757

Procedure for the Effective Recalibration of Liquid-inGlass Thermometers.

100,935

Criteria for the Operation of Federally-Owned Secondary Calibration Laboratories (lonizing Radiation).

PB92-112481

101,721

NIST Calibration Services for Humidity Measurement.

PB92-112499

100,520

NIST Calibration Services Users Guide, 1991

PB92-126416
Silicon Reference Materials Certified for Isotope Abundances.
PB92-126713

100,213

列

STANDARDS \& FUNDAMENTAL CONSTANTS

Use of Diamond as an Optical Material.

AD-A243 097/3

101,401

SRM 1970 - Succinonitrile Triple-Point Standard - And Its Use in Calibration of Thermistors.

PB91-134338

100,780

tate-of-the-Art in Pressure Metrology.

101,038

Adjustment of the Fundamental Physical Constants: A Report of the CODATA Task Group on Fundamental Constants, 1986

PB91-147124

101,606

Standard Reference Fibers for Calibration of the Optical Time Domain Reflectometer.

PB91-147207

101,406

Velocity and Droplet Size Measurments in a Fuel Spray. PB91-147934 100,539

Determination of the Neutron Mass and Implications for Fundamental Constants.

PB91-148692

101,615

NBS Guality Assurance Support: Current and Planned

$\begin{array}{ll}\text { Services. } & 101,012 \\ \text { PB91-149088 }\end{array}$

Fusion of Mercury. A New Certified Standard for Differential Scanning Calorimetry

100,309

Progress at NIST on Absolute Frequency Standards Using Stored lons

PB91-159285 101,625

Uncertainty and Accuracy in Physical Measurements.

101,629

New Test of Ouantum Mechanics: Is Planck's Constant Unique

PB91-174755

101,632

Test of Newton's Inverse Square Law of Gravity Using the $300 \mathrm{~m}$ Tower at Erie, Colorado: Newton Vindicated on the Plains of Colorad

101,637

Limited International Intercomparison of Responsivity Scales at Fiber Optic Wavelengths.

100,567

PB91-187682

Diameter

Particle-Sizing Standard.

PB91-203430

101,696

NVLAP FY 90 Annual Report

PB91-206748

100,010

PB91-231605

100,585

Resonating-Orthotropic-Cube Method for Elastic Constants.

PB91-236794

101,586

Basic Standards and Fundamental Constants.

PB91-237594

101,715

Guide for the Use of the International System of Units: The Modernized Metric System.

PB92-116383

100,962

Voltage Calibration Systems Using Josephson Junction Arrays.

PB92-116516

100,868

Differences between Thermodynamic Temperature and t(IPTS-68) in the Range $230 \mathrm{C}$ to $660 \mathrm{C}$

101,725

Modernized Metric System (Chart)

100,939

Brief History of Measurement Systems with a Chart of the Modernized Metric System.

the Modernized

100,940

Metric Conversion Card.

PB92-163880

100,941

Metric Measures Up (Ruler)

PB92-163898

100,942

MEASUREMENT SCIENCE \& TECHNOLOGY: POLICY \&

STATE-OF-THE-ART SURVEYS

Measurement Ouality Assurance.

PB91-149930

101,013

Biodegradable Plastics: An Idea Whose Time Has Come

PB91-150086

101,243

Levitation Calorimetry.

PB91-158519

100,927

VAMAS Bulletin

PB91-159145

101,192

NIST Reactor: Summary of Activities July 1989 through June 1990

PB91-159772

101,488

Taking the Measure of Fluid Properties Data Bases. PB91-200832

101,392

Assessment of the State-of-the-Art for Process Monitoring Sensors for Polymer Composites.

PB91-222612

100,510

International System of Units (SI)--Translation. PB92-109032

101,719

Magnetic Measurements for High Energy Physics Applications.

PB92-112358

101,720

Interpretation of the SI for the United States and Metric Conversion Policy for Federal Agencies.

101,729

MEASURING INSTRUMENTS

Apparatus for the Measurement of Regenerator Performance in Pulse Tube Refrigerators.

PB91-147967

101,047

Specifications, Tolerances, and Other Technical Requirements for Weighing and Measuring Devices as Adopted by the 76 th National Conference on Weights and MeasPB92-112424

101,023

MEAT

Co-Trial on ESP Identification and Estimates of gammaRay and Electron Absorbed Doses Given to Meat and

PB91-194720

100,017

Electron Spin Resonance for Monitoring Radiation-Proc-

100,018

MECHANICAL DEVICES

Development Plan: Application Protocols for Mechanical Parts Production. National PDES Testbed Report Series. PB91-222596

100,990

Conceptual Architecture for a Mechanical Parts Production System Based on STEP. National PDES Testbed Rept. Series.
PB92-112234

MECHANICAL MEASUREMENT

Mechanical Loss in a Glass-Epoxy Composite. PB91-149435

100993

ECHANICAL OSCILLATORS

Thermal Noise in Mechanical Experiments.

PB91-149211

101,070

MECHANICAL PROPERTIES

Review of Cryogenic Mechanical and Thermal Properties of Al-Li Alloys and Alloy 2219.

AD-A229 231/6

101,202

Low Carbon Steel: Metallurgical Structure vs. Mechanical Properties.

Computerization of a Thermomechanical Processing Research System

MECHANICAL TESTS

Mechanical Testing Revitalized

PB91-159004

101,583 
MEETINGS

Polymer Composite Processing. Industry Workshop (2nd). Held at Gaithersburg, Maryland on May 18, 1990.

PB91-132191

101,115

Technical Digest-Symposium on Optical Fiber Measurements, 1990.

101,403

Outline of a National Plan on High-Performance Concrete: Report on the NIST/ACI Workshop. Held in Gaithersburg, MD on May 16-18, 1990

100,522

Laser Induced Damage in Optical Materials: 1989

PB91-148478

101,409

Proceedings of the Object-Oriented Database Task Group Workshop, Tuesday, October 23, 1990, Chateau Laurier Hotel, Ottawa, Canada.

PBa1-157198

100,641

Proceedings of the Object-Oriented Database Task Group Workshop. Held in Atlantic City, New Jersey on May 22, 1990

PB91-159723

100,643

Proceedings of Cable ' 89 Workshop.

PB91-161943

Narrow-Gap Semiconductors and Related Materials.

PB91-162313

101,492

Chemistry of
PB91-178822

101,500

Proceedings of a Workshop on Evaluation, Repair, and Retrofit of Structures. U.S.-Japan Panel on Wind and Seismic Effects, UJNR. Held in Gaithersburg, MD., USA on May $12-14,1990$.

100,145

Conformity Assessment Workshop on Electromagnetic Compatibility

100,579

Conformity Assessment Workshop on Pressure Vessels.

PB91-192997 101,049

Meeting of the Standards Working Group of the Joint U.S.-U.S.S.R. Commercial Commission (1st). Held in Moscow on March 11-13, 1991.

PB91-194498

100,178

Standard Security Label for GOSIP: An Invilational Workshop. Held on April 9-10, 1991.

PB91-216671

100,712

ICLASS-91 Proceedings of the International Conference on Liquid Atomization and Spray Systems $(5$ th). Held in Gaithersburg, MD. on July 15-18,1991.

PB91-216838

100,552

Daylight Research Requirements. Workshop Proceedings. Held in Baltimore, Maryland on August 1-2, 1990.
PB91-231563

Proceedings of the Workshop on High Integrity Software. Held in Gaithersburg, MD. on January 22-23, 1991.
PB92-109040 Joint Panel Meeting of the UJNR Panel on Fire Research and Safety (11 th). Held in Berkeley, CA. On October 19

PB'92-109164 100,166 Proceedings of the Forum on Standards for High Integrity
Software (DOD, Government, Industry). Held in Gaithersburg, Maryland on June 28, 1991.

PB92-112267

100,668

Report of the National Conference on Weights and Measures (76th). Held in Philadelphia, PA. on July 14-19 1991

101,024

Monitoring and Reporting Techniques for Error Rate and Error Distribution in Optical Disk Systems. PB92-123124

100,606

MELTING POINTS

Fusion of Mercury. A New Certified Standard for Differential Scanning Calorimetry.

MELTS

100,309

Semiempirical Theory of Relaxation: Concentrated Polymer Solution Dynamics.

PB91-236638

100,511

MEMBRANE TRANSPORT

Increased Facilitated Transport Related to Microstructura Changes in Heat-Treated lon-Exchange Membranes.

100,323 Overview of Facilitated Transport Membrane Systems.
PB91-175463 100,228
PB91-175463 Thin and Composite High-Flux Membranes of Perfluoro
sulfonated lon-Exchange Polymer. PB91-236653

100,512

\section{EMBRANES}

Chitin-Chitosan Membranes: Separations of Amino Acids and Polypeptides

101,283

Increased Facilitated Transport Related to Microstructural

Changes in Heat-Treated lon-Exchange Membranes.
PB91-174805

Use of Nondestructive Methods for Inspection of SinglePly Roofing Membranes

100,128

MERCURY

Fusion of Mercury. A New Certified Standard for Differential Scanning Calorimetry.
PB91-150003

100,309

ERCURY CADMIUM TELLURIDES

High Spatial Resolution Mapping of Semiconductor Re-

PBist-1948.

100,809

Intrinsic Carrier Concentrations in Long Wavelength $\mathrm{HgCdTe}$ Based on the New, Nonlinear Temperature Deendence of $\mathrm{Eg}(\mathbf{x}, \mathrm{T})$

PB92-117316

101,568

MERCURY IODIDES

High Resolution Diffraction Imaging of Crystals Grown in Microgravity and Closely Related Terrestrial Crystals. PB92-109008

101,562

MERCURY IONS

Ouantum Optics of Single, Trapped lons.

MERCURY (PLANET)

Orbit Determination and Gravitational Field Accuracy for Mercury Transponder Satellite.

101,736

\section{MERIT FUNCTIONS}

Merit Function for Inequality Constrained Nonlinear Programming Problems.

PB92-116318

101,271

MESSAGE PROCESSING

Electronic Data Interchange in Message Handling Systems.

100,571

\section{METABOLISM}

Bioprocessing of Fossil Fuels Using Hyperthermophilic PB91-162271

100,883

METAL ALKYL COMPOUNDS

Simultaneous Butyltin Determinations in the Microlayer, Water Column and Sediment of a Northern Chesapeake Bay Marina and Receiving System.

PB91-195040

100,918

METAL CARBONYLS

Application of Ultrafast Broadband Infrared Spectroscopy to Measurement of Metal-Carbonyl Dynamics.

100,308

HYDRIDES

Properties of Amorphous Zirconium Rhodium Hydride (Zr3RhHx) Prepared from Glassy and Crystalline Alloys.
PB91-174466

Spectroscopy and Structure of the Alkali Hydride Diatomic Molecules and Their lons.

100,443

METAL MATRIX COMPOSITES

Elastic Constants of a Tungsten-Particle Copper-Matrix

Composite.

101,234

Elastic Constants of Fiber-Reinforced Composites: A Fresh Measurement Approach.

101,123

1-194878

Heat Conductivity of Oxide Coatings by Photothermal Radiometry between 293 and $1173 \mathrm{~K}$

PB92-116813

101,112

METAL PARTICLES

Core Hole Screening for Intermediate Size Metal Particles.

101,540

METAL PLATES

Use of Neutron Pole Figures to Calibrate Ultrasonic Techniques for On-Line Texture Control of Aluminum

PB91-159111

101,222

Aniso-

tropic Polycrystalline Aggregates.

PB91-202945

METAL SHEETS

Test Plan for Validating a Context Driven Integrated Model (CDIM) for Sheet Metal Die Design. National PDES Testbed Report Series.

100,970

Ultrasonic Measurement of Sheet Steel Texture and Formability: Comparison with Neutron Diffraction and Mechanical Measurements.

PB92-116615

101,170

METAL SPINNING

Material Dependency of Chip-Form Detection Using Acoustic Emission

101,216

METALLIC GLASSES

Crystalline and Glassy Phases of Transition-Metal - Metalloid Systems.

101,238

METALLOID

Crystalline and Glassy Phases of Transition-Metal - Metalloid Systems.
PB91-204156

METALLURGY

101,238

Low Carbon Steel: Metallurgical Structure vs. Mechanical N91.20218

101,144

Institute for Materials Science and Engineering: Metallurgy Division, Technical Activities 1990.

PB91-132225

101,207

Metallurgy Division, Technical Activities 1991. (NAS-NRC Assessment Panel February 13-14, 1992).

101,171

METALS

Literature Review of the Galling Process.

PB91-134551

101,208

Materials Research at the National Bureau of Standards.

PB91.149039 101,214

Cleavage Step Formation and Resistance to Transgranular Stress Corrosion Cracking.

PB91-149047

101,133

Concepts of Underground Corrosion.

PB91-150078

101,134

Acoustic Emission Monitoring of Laser Drilling.

PB91-158527

101,218

Corrosion Testing in Soil.

PB91-158667

101,135

Rapid Solidification Alloys: Crystals, Quasicrystals, and Metallic Glass.

101,220

Method for Ouantitative Measurement of Galling Pamage.

101,183

Intercomparison Study of Rockwell Hardness Test Blocks.

PB91-167338

101,224

Ignition Characteristics of Selected SSME Alloys.

100,544

Characterization of Cylindrical Holes in Metallic Substrates via Their Infrared Emission Patterns.

PB91-187187

101,228

High Purity Chemicals and Clean Rooms for Trace Metal Analysis.
PB91-195156

100,200

Microstructural Control Through Diffusion-Induced Grain Boundary Migration.

PB91-236760

101,239

Direct Detection of Atom Vaporization by Laser Resonance Ionization as a Probe of Gas-Surface Chemisorption Mechanisms.

PB91-237032

100,425

Characteristics of Aerosols Produced by the Spark Dis-

charge.
PB91-237727

100,209

Advanced Sensing of Materials Processing PB92-117423

100.949

METHAN

Applications of the Generalized Global Equivalence Ratio Model (GGERM) for Predicting the Generation Rate and Distribution of Products of Combustion in Two-Layer Fire Environments: Methane and Hexanes.

100,131

(216689

Influence of Sulfur on Methanation over Tungsten(110). PB91-149369 00,300

Summary Abstract: The Influence of Sulfur on Methanation over $W(110)$

100,301

METHANE 
P892-116383

100,962

Interpretation of the SI for the United States and Metric Conversion Policy for Federal Agencies.

101,729

PB92-126572

100,939

Modernized Metric System (Chart).

Brief History of Measurement Systems with a Chart of
the Modernized Metric System. PB92-163872

100,940

METRICATION

Metric Conversion Card.

PB92-163880

100,941

METROLOGY

GPS Time Transfer with Implementation of Selective Availability.
N91-25766/7

100,576

Metrology for Electromagnetic Technology: A Bibliography of NIST Publications.

100,833

State-ot-the-Art in Pressure Metrology.

101,038

101,038
PB91-146993

Low Accelerating Voltage SEM

PB91-147926

101,469

MMIC Related Metrology at the National Institute of Standards and Technology.

100,794

Center for Electronics and Electrical Engineering Technical Progress Bulletin Covering Center Programs, July to September 1990, with 1991 CEEE Events Calendar.
PB91-159749

Center for Electronics and Electrical Engineering Technical Publication Announcements Covering Center Pro-
grams, July to September 1990, with 1991 CEEE Events

100,852

PB91-184754

and Tool

Computer Vision Ba

Form Measurem

101,043

Microwave Monolithic Integrated Circuit-Related Metrology at the National Institute of Standards and Technology.

100,816

Metronductor Industry.

100,817

Electronics and Electrical Engineering Laboratory Technical Publication Announcements Covering Laboratory ProCB92-112309

100,864

Metrology for Electromagnetic Technology: A Bibliography of NIST Publications.

100,867

NIST Calibration Services Users Guide, 1991

100,936

Journal of Research of the National Institute of StandJournal of Research of the National Institute of Stand96 , Number 5

PB92-126614

100,212

Slovak Metrological Society.

100,938

METROLOGY: PHYSICAL MEASUREMENTS

Dynamic Technique for Measuring Surface Tension at High Temperatures in Microgravity Environment.

N91-21347/0

101,206

Environmental Effects on the Medium and Long Term

Frequency Stability of Ouartz Oscillators.

100,781

PB91-134866

Extending the Range and Accuracy of Phase Noise
Measurements.
PB91-134874
100,588

Measurements.
PB91-134874

Submicrometer SEM Magnification Standard.

Gravitational Radiation Observations on the Moon.

PB91-175083

Status of Beta-Particle Calibration Studies at NBS.

Journal of Research of the National Institute of Standards and Technology. January-February 1991. Volume

$\begin{array}{ll}96, \text { Number } 1 . & 100,220 \\ \text { PB91-184853 } & \end{array}$

Particle Size Measurement of Inert Gas Atomized

PB91-18

101,229

Journal of Research of the National Institute of Standards and Technology. March-April 1991. Volume 96, Number 2.

101,420

Use of the Electrostatic Classification Method to Size 0.1 micrometer SRM Particles: A Feasibility Study.

PB91-187633

100,932

Journal of Research of the National Institute of Standards and Technology. May-June 1991. Volume 96 PB91-216705

101,704

New Concepts of Precision Dimensional Measurement for Modern Manufacturing.

101,006
Journal of Research of the National Institute of Standards and Technology. July-August 1991. Volume 96 Number 4.

100,762 Choosing Flow Conditioners and Their Location for Ori-

PB92-116490

101,397

Application of Transmission Electron Detection to $\mathrm{X}$-ray Mask Calibrations and Inspection

PB92-117217

100,828

Journal of Research of the National Institute of Standards and Technology, September-October 1991. Volume 96 , Number 5

100,212

Summary of the Intercomparison of the Force Standard Machines of the National Institute of Standards and Technology, USA, and the Physikalisch-Technische Bundesanslait, Germany.

PB92-126622

Slovak Metrological Society.

101,590 PB

Population Lifetimes of $\mathrm{OH}(\mathrm{v}=1)$ and $\mathrm{OD}(\mathrm{v}=1)$ Vibrations in Alcohols, Silanols and Crystalline Micas. PB91-174813

100,324

MICROALLOYED STEELS

Continuous-Cooling Transformation Characteristics and High-Temperature Flow Behavior of a Microalloyed SAE 1141 Steel.

MICROANALYSIS

Use of Single Particle Standards for LAMMA Calibration.
PB91.147777

Inner-Shell Ionization Cross Sections.

PB91-195479

100,203

MICROCHANNEL ELECTRON MULTIPLIERS

Microchannel-Plate Detection Systems for Low Accelerating Voltage SEM.

\section{MICAOCOMPUTERS}

Agency Exploits Flexibility of Software in Moving Info P91.159160 100,642 Guide to Expert System Building Tools for MicrocomputPB92-108893

100,715

\section{MICROELECTRONICS}

Cratering.
PB91-203141

100,810

Electronics and Electrical Engineering Laboratory Technical Publication Announcements Covering Laboratory Pro(o March 1991, with 1991 EEEL Events Calendar.

PB92-112309

100,864

MICROFICHE

Computer Output Microform (COM) Formats and Reduction Ratios, $16 \mathrm{MM}$ and $105 \mathrm{MM}$. Category: Hardware Standard; Subcategory: Media

FIPS PUB 54-1

100,678

MICROFILM

Computer Output Microform (COM) Formats and Reduction Ratios, 16MM and 105MM. Category: Hardware Standard; Subcategory: Media

FIPS PUB 54-1

100,678

MICROFORM

Computer Output Microform (COM) Formats and Reduction Ratios, 16MM and 105MM. Category: Hardware

Standard; Subcategory: Media.
FIPS PUB 54-1

100,678

MICROLITHOGRAPHY

Three-Dimensional Simulations of High-Resolution Photoresist Processing

MICRORADIOGRAPHY

100,818

Digital Image Analysis Assisted Microradiography Measurement of Mineral Content of Caries Lesions in Teeth. P891-187666

101,314

MICROSCOPY

Techniques to Observe the Fracture Zone in Mortar and

Poncrete.

MICROSPHERES

100,521

Use of the Electrostatic Classification Method to Size 0.1 micrometer SRM Particles: A Feasibility Study.

Calibration of the NBS 10 mum Space Beads.

PB91-203422

101,695

Dimensional Calibration of an NBS 0.3-mum-Diameter Particle-Sizing Standard.

PB91-203430

101,696

Certification of NIST SRM 1961: 30 micrometers Diameter Polystyrene Spheres.

100,937

MICROSTRIP ANTENNAS

Microstrip Patch Antenna as a Standard Transmitting and Receiving Anten
PB91-162156

100,591

MICROSTRUCTURE

Strength and Microstructure of Ceramics.

AD-A243 049/4

101,056

Design of High Strength Cement-Based Materials. Part 2. Microstructure.

100,099

Microstructure and Fracture at the Cement Paste-Aggre gate Interiace.

100,523

Computer Simulated Hydration of a Cement Model.

PB91.162149 100,112

Percolation of Phases in a Three-Dimensional Cement Paste Microstructural Model.

PB91-187179

100,529

Microstructural Control Through Diffusion-Induced Grain Boundary Migration

MICROWAVE REFLECTOMETERS

101,239

Dual Six-Port Reflectometer Systems Using Waveguide in the Frequency Range $18-50 \mathrm{GHz}$

100,838

MICROWAVE SPECTRA

Fluoromethanol: Synthesis, Microwave Spectrum, and Dipole Moment
PB91-148080

100,288

Microwave Spectrum and Molecular Structure of the N2 H20 Complex.

100,398

Rotational-Tunneling Spectrum of the Ar-SO2 van der Waals Complex.

MILITARY FACILITIES

Field Study of the Performance of EPDM Roofing at Air Force Facilities
PB91-167247

101,340

MILITARY SPECIFICATION MIL-R-28002A

Tiled Raster Graphics and MIL-R-28002A: A Tutorial and Implementation Guide.

PB91-187708

101,346

\section{MILITARY TRAINING}

Economic Analysis for Military Construction (MILCON) Design. Concepts, Techniques, and Applications for the Analyst. Instructor's Manual.

PB92.112440

101,349

Economic Analysis for Military Construction (MILCON) Design. Concepts, Techniques, and Applications for the Analyst. Student's Manual.

- 112457

101,350

MILITARY VEHICLES

Control System Architecture for Unmanned Ground Vehicles.

101,379

ILLING MACHINES

Error Prevention and Detection in Data Preparation for a Numerically Controlled Milling Machine.

PB91-174938

MINE DETECTORS

Summary of Experiments with the Separated Aperture

Technique of Dielectric Anomaly Detection.
AD-A230 438/4

100,721

Quantifying Standard Performance of ElectromagneticBased Mine Detectors

PB92-116292

101,378

MINERALIZATION

Comparative Study of Bovine Pericardium Mineralization A Basic and Practical Approach.

PB92.117407

100,056

MINING EQUIPMENT 
PB91-147215

100,098

Comprehensive Approach for Modeling and Testing Analog and Mixed-Signal Devices.

100,732

STEP: Standard for the Exchange of Product Model Data Resource Integration: Semantic and Syntactic Rules. PB91-184788 100,966 Functional Meta-Structure for Hypertext Models and Sys-

PB91-236687

100,655

Fracture Mechanics Model for Subthreshold Identation Flaws. Part 1. Equilibrium Fracture.

PB91-236935

101,093

Test Plan for Validating a Context Driven Integrated Model (CDIM) for Sheet Metal Die Design. National PDES Testbed Report Series.

100,970

MOISTURE CONTENT

Prototype Expert System for Diagnosing Moisture Problems in Houses.

100,062

Analysis of Moisture Accumulation in a Wood Frame Wall Subjected to Winter Climate.

PB92-116334

100,137

MOLAR VOLUME

Unified Description of Infinite-Dilution Thermodynamic Properties for Aqueous Solutes.

OLECULAR BEAM EPITAXY

100,319

Magnetic Anisotropies in Ultrathin fcc $\mathrm{Fe}(001)$ Films Grown on Cu(001) Substrates

PB92-116631

101,565

MOLECULAR COLLISIONS

Soluble Models in Surface Collision Dynamics.

PB91-147298

100,276

Problem of Detailed Balance and Model Lineshapes in Collision-Induced Rotovibrational Bands: $\mathrm{H} 2-\mathrm{H} 2$ and $\mathrm{H} 2$ PB.

100,412

MOLECULAR DYNAMICS

Molecular Dynamics Study of the Depolymerization Reaction in Simple Polymers.

PB91-187229

100,495

Dynamics of Molecular Hydrogen Adsorbed in CoNa-A

MB91-195255 100,380

100,380 radation of Polymers.

PB91-195271

100,503

MOLECULAR ELECTRONICS

Permittivity Measurements on Molecular-Sized Samples.
PB91-159244

MOLECULAR ENERGY LEVELS

Vibrational and Electronic Energy Levels of Polyatomic Transient Molecules. Supplement 1

PB91-192575

100,367

MOLECULAR GASES

Polarization and Anisotropy of X-ray Emission from MoleCules.

101,651

MOLECULAR IONS

Vibrational Spectra of Molecular Ions Isolated in Solid Neon. III. $\mathrm{N} 4(+)$

100,243

Production and Spectroscopy of Small Polyatomic Molec ular Ions Isolated in Solid Neon.

100,245

Vibrational Spectra of Molecular Ions Isolated in Solid Neon. 6. CO4(-).
AD-A238 415/4
100,249

Vibrational Spectra of Molecular Ions Isolated in Solid Neon. 7. $\mathrm{CO}(+), \mathrm{C} 2 \mathrm{O} 2(+)$, and C2O2(-).
AD-A239 $729 / 7$

Vibrational Spectra of Molecular Ions Isolated in Solid Neon. 5. N2O(1+) and NNO2(1-).

100,306

Vibrational Spectra of Molecular Ions Isolated in Solid Neon. 4. $\mathrm{NO}(1+)$, $\mathrm{NO}(1-), \mathrm{ONNO}(1+)$, and ONNO(1-).
PB91-149914

100,307

Production and Spectroscopy of Small Polyatomic Molec ular lons Isolated in Solid Neon.

100,395

Vibrational Spectra of Molecular Ions Isolated in Solid Neon. 6. $\mathrm{CO} 4(1-)$

\section{LECULAR MEASURING MACHINES}

Use of Solid Modeling in the Design of M3 Components. PB92-123066

101,044

MOLECULAR ORBITALS

Direct Determination of Molecular Orbital Symmetry of H2S Using Polarized X-ray Emission.
PB91-175562 PB91-175562

PB91-194837

100,370

Ab-Initio Calculations and Ideal Gas Thermodynamic Functions of Cyclopentadiene and Cyclopentadiene Derivatives.
PB92-148089

100,468

MOLECULAR RELAXATION

Core Hole Screening for Intermediate Size Metal PartiPB91-203539

101,540

MOLECULAR ROTATION

Improved Fits for the Vibrational and Rotational Constants of Many States of Nitrogen and Oxygen. PB92-148097

100,469

MOLECULAR SIEVES

Structures and Properties of New Zeolite X-Type Zincophosphate and Beryllophosphate Molecular Sieves. 101,081

MOLECULAR SPECTRA

Heterodyne Frequency Measurements of $\mathrm{CO}$ and OCS Beyond $2100 \mathrm{~cm}(-1)$

\section{OLECULAR SPECTROSCOPY}

100,423

Molecular Spectroscopy Division of the National Bureau of Standards.

100,332

Fourier Transform Infrared Spectroscopy at the NIST: High Temperature Molecules and Atmospheric MolePB91-189803

101,424

MOLECULAR STRUCTURE

Structure of Carbon Dioxide Dimer from Near Intrared Spectroscopy.

100,279

Structures of the $\mathrm{NH} 3-\mathrm{HCCCCH}$ and $\mathrm{H} 2 \mathrm{O}-\mathrm{HCCCCH}$ Complexes by Fourier-Transform Microwave SpectroscoPB91-148882

100,294

Microwave Spectrum and Molecular Structure of the N2. H20 Complex

100,398

Dissociation of Substituted Anisoles: Substituent Effects on Bond Strengths.

PB91-237537

100,431

Spectroscopy and Structure of the Alkali Hydride Diatomic Molecules and Their lons.

LECULAR VIBRATION

100,443

Vibrational Spectra of Molecular Ions Isolated in Solid Neon. 6. CO4(-).

Rovibrational Analysis of the nu7(1) Intermolecular Hydrogen Bond Bending Vibration in HCN-HF Using Far Infrared Fourier Transform Spectroscopy.

100,437 PB91-237784

Improved Fits for the Vibrational and Rotational Con-
stants of Many States of Nitrogen and Oxygen. PBants of Many

MOLECULAR WEIGHT

Multiple Variable-Angle Light Scattering Detector for Ge Permeation Chromatography.

PB91-187641

100,195

Study of the Linear Viscoelastic Properties of Cyclic Polystyrenes Using

100,502 PB91-195107

Cross Sections and Swarm Coefficients for $\mathrm{H}+, \mathrm{H} 2+$, $\mathrm{H} 3+, \mathrm{H}, \mathrm{H} 2$, and $\mathrm{H}$-in $\mathrm{H} 2$ for Energies from $0.1 \mathrm{eV}$ to 10 PB90-244690

101,597

Cross Sections and Swarm Coefficients for Nitrogen Ions and Neutrals in N2 and Argon lons and Neutrals in N2 and Argon lons and Neutrals in Ar for Energies from 0.1 eV $10 \mathrm{keV}$.

PB92-110246

100,452

MOLTEN SALTS

Coupled Phase Diagram-Thermodynamic Analysis of the 24 Binary Systems, A2CO3-AX and A2SO4-AX Where $\mathrm{A}=\mathrm{Li}, \mathrm{Na}, \mathrm{K}$ and $\mathrm{X}=\mathrm{Cl}, \mathrm{F}, \mathrm{NO} 3, \mathrm{OH}$

PB91-192518

100,362

\section{MOLYBDENUM ALLOYS}

Corrosion, Repassivation and Corrosion Fatigue Behavior of the Surgical Implant Alloy, Co-Cr-Mo.

101,132

Coherency Strain Induced Instability of Solid-Liquid Interfaces in the Mo-Ni System.

PB91-195537

101,235

MOLYBDENUM DISULFIDE

Chemical Effects of $\mathrm{Ne}+$ Bombardment on the MoS2(0001) Surface Studied by High-Resolution Photoelectron Spectros

100,399

\section{MOLYBDENUM ISOTOPES}

Observation of Anomalous Isotope Ratios in SIRIS Measurements of Molydenum.

PB92-116789

101,361

MOLYBDENUM OXIDES

Refinement of the Structure of Beta'-MoO3. $\quad 101,090$

\section{MONITORING}

Practical Constraints in Monitoring Micronutrient Intake.

ONOCLINIC LATTICES

Physical and Thermo-Mechanical Properties of Monoclinic Single Crystals.

PB91-148767

101,065

MONOMERS

Molecular Dynamics Study of the Depolymerization Reaction in Simple Polymers.

100,495

Difunctional and Multifunctional Monomers Capable of Cyclopolymerization.

MONOMOLECULAR FILMS

Preparation and Properties of Monomolecular Films for se as Radioactive Source Mounts.

391-147249

Morphology of Polymer Films and Single Molecules. PB91-203224

100,506

\section{MONOPOLE ANTENNAS}

NIST Calibration Procedure for Vertically Polarized Monopole Antennas $30 \mathrm{kHz}$ to $300 \mathrm{MHz}$

100,726

\section{MONTE CARLO METHOD}

Simulation Studies of Excluded Volume Effects on Polymer Chain Dynan
PB91.203364

100,507

MOAPHOLOGY

Role of Elastic Energy in the Morphological Development of a Ni-Ti-Al Alloy

PB91-187161

101,227

MOSFET

Characteristics of the Breakdown Voltage of Power MOS PB91-149062

100,793

MOSSBAUER EFFECT

Mossbauer Imaging

PB91-203745

101,294

Mossbauer Study of the Effect of Oxygen Stoichiometr on the High Tc Superconducto PB91-237545

101,558

\section{MOUNTINGS}

Preparation and Properties of Monomolecular Films for Use as Radioactive Source Mounts.

PB91-147249

101,608

MULTIMETERS

Investigating the Use of Multimeters to Measure Quanized Hall Resistance Standards.

PB91-236554

100,734

MULTIPHOTON IONIZATION

Signal Detection Efficiency in Multiphoton Ionization Flame Measuremen

100,540

Resonance Enhanced Multiphoton Ionization Spectra of the $\mathrm{GeF}$ and $\mathrm{GeCl}$ Radicals from $400-500 \mathrm{~nm}$. PB91-203281

100,396

MULTIPROCESSORS

Hardware Instrumentation Approach for Performance Measurement of a Shared-Memory Multiprocessor

MUMPS PROGRAMMING LANGUAGE

MUMPS Validation Suite, Version 7.6 (Vax/VMS Backup) PB91-5C

Validation Suite, Version 7.6, 1991 (for $N$ computers).

MUMPS Validation Suite, Version 7.6 (ANSI), 1991.

PB91-507715 100,662

MUMPS Validation Suite, Version 7.6 (TAR), 1991 


\section{KEYWORD INDEX}

NITROGEN

NANOCOMPOSITES

Spin Glass Magnetic Behavior of Iron/Silica Gel Nanocomposites.

101,117

Magnetic Properties of Iron/Silica Gel Nanocomposites. PB91-204073

101,110

NANOSTRUCTURES

Change in Magnetic State of $\mathrm{Fe}+$ Silica Gel Nanocomposites Due to Low Temperature Treatment in Ammonia.
PB91-237321

NARROW GAP SEMICONDUCTORS

Narrow-Gap Semiconductors and Related Materials. PB91-162313 101,492

NASREM

NASREM Robot Control System and Testbed. PB91-194969

101,033

NATIONAL BUREAU OF STANDARDS

National Bureau of Standards Publications 1977-1987. Volume 1. Citations, Key Words, and Abstracts. PB91-136507

100,957

National Bureau of Standards Publications 1977-1987. Volume 2. Indexes.

PB91-136515

100,958

NBS Quality Assurance Support: Current and Planned Services.

Measurement Quality Assurance.

101,012

PB91-149930

101,013

High Purity Chemicals and Clean Rooms for Trace Metal Analysis.

100,200

NATIONAL COMPUTER SYSTEMS LABORATORY

National Computer Systems Laboratory Annual Report,

PB91-201822

100,603

NATIONAL INSTITUTE OF STANDARDS AND

ADOLOGY

Abstract and Index Collection in the Research Information Center of the National Institute of Standards and Technology (Fourth Edition).

100,959 NIST Standard Reference Data Products 1991 Catalog.

Publications of the National Institute of Standards and Technology, 1990 Catalog.

100,011

NIST Personnel Management Demonstration Project: Design, Implementation and Accomplishments. 100,003
PB91-231555 Research. Services. Facilities. (National Institute of Standards and Technologil.

100,012

NIST Calibration Services Users Guide, 1991 PB92-126416

100,936

NATIONAL RESEARCH LABORATORY OF METROLOGY New Yorker in Japan.

PB91-158907

101,275

NATIONAL VOLUNTARY LABORATORY ACCREDITATION

PROGRAM

Directory of Accredited Laboratories, 1991. National Voluntary Laboratory Accreditation Program (NVLAP) PB91-222646

100,934

NATURAL GAS

Gas Flow Measurement: Calibration Facilities and Fluid Metering Traceability at the National Bureau of Standards.

100,879

PB91-134403

Gas/Air

Radiant Heat Fluxes

100,538

Speed of Sound of Natural Gas Mixtures.

100,882

PB91-162214

Gas Analysis

100,884

NATURAL RUBBER

Mechanical and Swelling Behavior of Crosslinked Natural Rubber: Consequences of the Flory-Rehner Hypothesis. PB91-195081

101,141

NAVIGATION SATELLITES

GPS Time Transfer with Implementation of Selective Availability.

100,576

NBSR REACTOR

NIST Reactor: Summary of Activities July 1989 through June 1990.

PB91-159772

101,488

NEBULAE

Detection of an Expanding $\mathrm{H}$ I Shell in the Old Supernova Remnant CTB 80 .

PB91-174920

100,028

NEODYMIUM 148

Development of a New Tracer Technology Using Enriched Rare-Earth Isotopes.

PB91-195347

100,908

NEODYMIUM BARIUM CUPRATES

Long-Range Antiferromagnetic Order of the $\mathrm{Cu}$ in Oxygen Deficient $\mathrm{RBa} 2 \mathrm{Cu} 3 \mathrm{O} 6+\mathrm{x}$.
PB91-194894

101,524

NEODYMIUM CERIUM CUPRATES

henon Density of States and Superconductivity in Nd1.85Ce0.15CuO4.

PB91-189787

101,514

NEODYMIUM CUPRATES

Crystal and Spin Structures of $\mathrm{Nd} 2 \mathrm{CuO} 4$

PB91-148023

101,470

NEODYMIUM OXIDES

Many-Electron Effects in Lgamma2,3 XES Spectra from are Earth Elements.

NEON

Vibrational Spectra of Molecular lons Isolated in Solid Neon. IV.NO( + ), NO(-), ONNO( +$)$, aNd ONNO(-).

Vibrational Spectra of Molecular lons Isolated in Solid

AD-A239 $729 / 7$, C2O2 $(+)$, and C2O2(-).

100,250

Optical Potential Approach to Electron and Positron Scat. from Noble Gases. 2. Neon.

B91-149971

101,619

Small Scale Demand Type Neon Liquefaction Plant

PB91-162008

100,224

Viscosity and Thermal Conductivity of Pure Monatomic Gases from Their Normal Boiling Point Up to $5000 \mathrm{~K}$ in the Limit of Zero Density and at $0.101325 \mathrm{MPa}$

100,366 1.192559 Vibrational Spectra of Molecular lons Isolated in Solid
Neon. 6. CO4(1-).
PB91-236836
PB PB91-236836

van Rotational Predissociation, Vibrational Mixing, and van
der Waals Intermolecular Potentials of NeDF.

PB91-175505

100,334

Investigation of Internal Rotor Dynamics of $\mathrm{NeDCl}$ and ArDCl via Infrared Absorption Spectroscopy.

100,409 PB91-204024

NEON MATRICES

Vibrational Spectra of Molecular Ions Isolated in Solid Neon. 6. CO4(-).

100,249

NET SHAPING

Near Net Shape Forming of Ceramics. PB91-158600

101,072

NETWORK ANALYZERS

Calibration of Antenna Factor at a Ground Screen Field Site Using an Automatic Network Analyzer

100,724

\section{NETWORKS}

Effect of Crosslink Density on Physical Aging of Epoxy PB91-189712

101,244

Viscoelastic Response of Epoxy Glasses Subjected to Different Thermal Treatments.

101,245

Physical Aging and the Viscoelastic Response of Network Glasses

NEUTRON

101,246

Small-Angle Neutron Scattering of Blends of CrossLinked and Linear Polystyrene. PB91-194688

100,500

NEUTRON ACTIVATION ANALYSIS

Neutron Activation Analysis in Electronic Technology.
PB91-189779

NEUTRON CHOPPERS

Transmission Properties of a Counter-Rotating Pair of Disk Choppers
PB91-236604

101,706

\section{NEUTRON CROSS SECTIONS}

Cross-Section Measurements in the Intermediate-Energy Standard Neutron Field.

101,693

Spectrum-Averaged Cross-Section Measurement of $(103) \mathrm{Rh}(n, n)(103 \mathrm{~m}) \mathrm{Rh}$ in the $(252) \mathrm{Cf}$ Fission Neutron PB91-203406

101,694

NEUTRON DIFFRACTION

Use of Neutron Pole Figures to Calibrate Ultrasonic Techniques for On-Line Texture Control of Aluminum

PB91-159111

101,222

Neutron Crystallography of Proteins.

PB91-175349

101,285

NEUTRON INTERFEROMETRY

Nonlocal Phase Shifts Induced by Static Electric Fields in Neutron Interferometers When the Path-Enclosed Charge Vanishes

EUTRON MASS

101,620

Determination of the Neutron Mass and Implications for Fundamental Constants.

PB91-148692

101,615

NEUTRON POLARIZERS

Fe-W Supermirrors for Polarizing Neutrons.

PB91-175521

101.643

NEUTRON SCATTERING

Microstructure Effects on the Lower Critical Solution Behavior of Deuterated Polybutadiene and Protonated Polyisoprene Blends Studied by Small-Angle Neutron Scattering

PB92-117266

100,518

NEUTRON SPECTRA

Cross-Validation Procedure for Stopping the EM Algorithm and Deconvolution of Neutron Depth Profiling Spec-

PB91-161976

101,627

NEUTROPHILS

Damage to the Bases in DNA Induced by Stimulated Human Neutrophils.

PB92-116912

101,312

NEWSPRINT

Studies on the Degradation Products of Paper with and without Pollutants in a Closed Environment. 1. Preliminary

PB91.143313

101,181

NEWTON METHOD

Simplified Error Bounds for Newton's Rule. PB91-159046

101,257

NICKEL ALLOYS

Role of Elastic Energy in the Morphological Developmen of a Ni-Ti-Al Alloy.

PB91-187161

101,227

Sliding Wear Studies of Nickel-Copper Composition-Modlated Coatings on Steel.

101,188

Coherency Strain Induced Instability of Solid-Liquid Interaces in the Mo-Ni System.

101,235

Crystalline and Glassy Phases of Transition-Metal - Metalloid Systems.

PB91-204156

101,238

Theory of Ballistic-Electron-Emission Spectroscopy of $\mathrm{NiSi} / \mathrm{Si}(111)$ Interfaces.

101,557

Kinematic Theory of Ballistic Electron Emission Spectroscopy of Silicon-Silicide Interfaces.

PB92-117373

101,570

NIOBIUM BASE ALLOYS

Guideline for $\mathrm{Nb}$ (sub 3) $\mathrm{Sn}$ critical current measurements using fiberglass-epoxy composite sample mandrels. DE91016274

NIOBIUM OXIDES

Microwave Spectra and Electric Dipole Moments of $X(4)$ Sigma(1-) $1 / 2$ VO and NbO.

PB91-237487

100,429

NIST

NIST Serial Holdings, 1991

PB91-171330

100,960

Data Bases Available in the Research Information Center 


\section{KEYWORD INDEX}

Improved Fits for the Vibrational and Rotational Constants of Many States of Nitrogen and Oxygen

100,469

\section{NITAOGEN ADDITIONS}

Effect of Nitrogen and Carbon on FCC-HCP Stability in Austenitic Steels.

NITROGEN DIOXIDE

101,151

Reference NO2 Calibration System for Ground-Based Intercomparisons during NASA's GTE/CITE II Mission. PB91-189340

100,034

NITROGEN IONS

Detection of Excited States by Laser-Induced Fluorescence and Analysis of Energy Transfer.

100,242

\section{NITROGEN OXIDES}

Thermodynamic Data for Modeling of Flue Gas Cleanup Systems (Abstract for Poster Session).

100,904

NITROMETHANE

Effects of Pressure on the Thermal Decomposition Kinetics and Chemical Reactivity of Nitromethane

100,286

NITROSYL CHLORIDE

Photodissociation of C(I)NO in the S(I) State: A OuantumMechanical ab initio Study.

PB91-149260

100,239

NITROUS OXIDE

Vibrational Spectra of Molecular Ions Isolated in Solid

Neon. 5. $\mathrm{N2O}(1+)$ and $\mathrm{NNO} 2(1-)$
PB91-149906

100,306

NITROXYL RADICALS

Kinetics and Mechanisms of the Gas-Phase Reactions of the NO3 Radical with Organic Compounds

100,450

OISE METERS

Response of Personal Noise Dosimeters to Continuous and Impulse-Like Signals.

PB92-116359

100,914

NOISE (SOUND)

Acoustic Technique for Evaluation of Thermal Insulation.
PB91-189324

NON-NEWTONIAN FLUIDS

Anisotropic Structure of a Simple Liquid.

PBg1-148718

101,616

\section{NONAZEOTROPIC MIXTURES}

Research and Development of Heat Pumps Using Nonazeotropic Mixture Refrigerants.

100,891

NONDESTRUCTIVE TESTS

Report on 1989 Actions by International Institute of Welding.

101,001

Fundamentals and Applicatıons of Infrared Themography for Nondestructive Testing.

101,009

PB91-147132

101,039

Progress in Uniform Field Eddy Current Methods.

PB91-14775

andards.

PB91-149039

Eddy Current Reflection Probe: Theory and Experiment.

B91-187120

AMRF Composites Fabrication Workstation: A Test Methodology to Measure the Ouality of Thermoplastic Composite Parts.

101,017

Use of Nondestructive Methods for Inspection of SinglePly Roofing Membranes

100,128

Automated Reverse-Bias Second-Breakdown Transistor Tester

100,813

PB91-216739

Annotated Bibliography: Diagnostic Methods and Measurement Approaches to Detect Incipient Defects Due to Aging of Cables.

101,369

Eddy Current Probe Sensitivity as a Function of Coil Construction Parameters.

PB91-236562

100,945

Characterization of Capacitive Array for NDE Applications.

100,946 Capacitive Array Sensors for Nondestructive Evaluation.
PB91-237313 Intelligent Processing of Materials, Technical Activities 1991. (NAS-NRC Assessment Panel, November 14-15, 1991)

100,995

Impact-Echo: A New Method for Inspecting Construction Materials.

PB92-116557

100,140

Advanced Sensing of Materials Processing

PB92-117423

100,949

NONELECTROLYTES

Modelling Aqueous Solutions Near the Critical Point of Water.
P91-189837

100,349

NONEQUILIBAIUM FLOW

Non-Equilibrium Molecular Dynamics Simulations of Structured Molecules. Part 1. Isomeric Effects on the Vis-

101,391

NONLINEAR PROGRAMMING

Merit Function for Inequality Constrained Nonlinear Proramming Problems.

101,271

NONMETALLIFEROUS MINERALS

Wawayandaite, a New Calcium Manganese Beryllium Boron Silicate from Franklin, New Jersey.

101,359

NOVAL

Energy Distributions of Symbiotic Novae.

B91-200873

100,032

NUCLEAR EXPLOSION EFFECTS

Wind Effects on Fires.

PB91-203851

100,549

NUCLEAR FUEL CLADDINGS

Corrosion Behavior of Zirconium Alloy Nuclear Fuel Cladding. 158733

101,372

NUCLEAR MEDICINE

Use of Storage Phosphor Imaging Plates in Portal Imag-

ing and High-Energy Radiography. The Intensifying Effect

of Metallic Screens on the Sensitivity. PB91-236497

101,324

NUCLEAR PHYSICS \& RADIATION TECHNOLOGY

Effects of Multiple Filament Geometry in the Hot Filament Deposition of Diamond Films.

AD-A236 708/4

101,142

Assessment of Uncertainties in Measurement of $\mathrm{pH}$ in Hostile Environments Characteristic of Nuclear Repositor-

NUREG/CR-5711

101,367

Model Validation at the Las Cruces Trench Site.

NUREG/CR-5716

101,368

Absorbed-Dose Calibration of Ionization Chambers in A

101,598 Parity-Unfavored Transitions in Resonant Photoemission
from $\mathrm{Ar}_{\mathrm{K}} \mathrm{Kr}$, and $\mathrm{Xe}$ : Experimental and Theoretical Refrom $A r, K r$, and $\mathrm{Xe}$ : Experimental and Theoretical Re-
sults.

PB91-133900

101,599

Preparation and Properties of Monomolecular Films for Use as Radioactive Source Mounts.

101,608

Electrofission in the Ouasifree and Delta Regions.

PB91-147355 101,609

Possible (e,e'2N) Studies at CEBAF

PB91-147587

101,612

PB91-147900

101,010

Muon Sticking in Catalysed d-t Fusion: Nuclear Resonance Effect.
PB91-147959

101,613

gamma-Irradiated Seafoods: Identification and Dosimetry by Electron Paramagnetic Resonance Spectroscopy

PB91-150060

100,016

Relative Populations of Excited Levels within the Ground Configuration of Si-Like $\mathrm{Cu}, \mathrm{Zn}, \mathrm{Ge}$, and Se Ions. PB91-158618

101,443

Accurate Spectroscopy in the $\mathrm{X}$-ray Region

PB91-158634

101,621

X-ray Interferom

101,484

XPS and Auger Forward Scattering in Epitaxial Films.
PB91-174698

101,495
Electronic and Radiometric Characteristics of Near InfraPB91-174706

100,752

Calculation of Beta-Particle Counting Efficiency for Liquid Scintillation Systems with Three Phototubes.

101,644

Center for Radiation Research. 1990 Technical Activities. PB91-178855 101,362

Electric Quadrupole and Magnetic Dipole Transition Probabilities in Potassium Isoelectronic Sequence.

101,646

Method to Enhance Porosity of Micro-particles

PB91-187328

101,185

Polarization and Anisotropy of X-ray Emission from Mole-

PB91-187401

101,651

Linearity of a Silicon Photodiode at $30 \mathrm{MHz}$ and Its Effect on Heterodyne Measurements.

100,755

Bidirectional Scattering Distribution Function (BSDF): A Systematized Bibliography.

PB91-187674

101,421

Spectroscopic Constants for the nu9 Infrared Band of

100,343

Dynamical Diffraction Imaging (Topography) with $X$-ray Dynamical Diffraction
Synchrotron Radiation.

PB91-189654

101,510

Doppler Shift Attenuation Lifetime Measurements in D4) Cr Following Thermal Neutron Capture.

101,656

Dielectronic Recombination Rate Coefficients of $\mathrm{O}(5+)$

$\begin{array}{ll}\text { and } \mathrm{O}(2+) \text {. } & 101,657\end{array}$

Stopping Power of Fast Charged Particles in Heavy Ele-

PB91-194399

Model for the Non-Perturbative OCD (Ouantum Chromodynamics) Vacuum

101,661

Co-Trial on ESR Identification and Estimates of gammaRay and Electron Absorbed Doses Given to Meat and Bones.

$\begin{array}{ll}\text { PB91-194720 100,017 } & \end{array}$

Soft $X$-ray Absorption and Emission Spectra and the Electronic Structure of Some Exotic Materials.

Reflection-Extended-X-ray-Absorption-Fine-Structure

Spectroscopy at the Carbon K-Edge.

PB91-194944

100,371

Low-Pressure Symmetric Discharge Heat Pipe and DROGS of Eu

101,666

Absorbed Dose to Water: Comparison of Several Methods Using a Liquid lonization Chamber.

101,322

Glueballs and Hadron Spectroscopy.

PB91-195123 101,668

Intensifying Effect of Metallic Screens on the Sensitivity of X-ray Films for $400-\mathrm{kV}$ Bremsstrahlung Photons.

PB91-195198

101,293

Intensifying Effect of Metallic Screens on the Sensitivity of X-ray Films at $662 \mathrm{keV}$.

101,669

Anomalous Lgamma2,3 $\mathrm{X}$-ray Emission Spectrum of $\mathrm{Xe}$ PB91-195289 101,670 Anomalous Th $\mathrm{M} 3 \mathrm{O} 1 \mathrm{X}$-ray Emission Spectrum of $\mathrm{ThO} 2$. Inner-Shell Ionization Cross Sections.

PB91-195479

100,381 Impact Excitations of Berylliumlike lons.

PB91-195503

101,672

Dielectronic Recombination Rate Coefficients for lons of the Fluorine Isoelectronic Sequence.

PB91-195602

101,674

Influence of Metastable States and Thermal Equilibration upon Dielectronic Recombination in Low Density to Moderate Density.

101,675

$5 s(2) 5 p(2)-(5 s(2) 5 p 5 d+5 s 5 p(3)+5 s(2) 5 p 6 s+$

$5 s(2) 5 p 7 s)$ Transitions in Sb II and $5 s(2) 5 p-(5 s 5 p(2)+$

$5 \mathrm{~s}(2) \mathrm{nl}$ ) Transitions in $\mathrm{Sb}$ III.

PB91-200816

101,676

Polarizability of the Photon

101,686

Cross-Section Measurements in the Intermediate-Energy 
PB92-102185

101,435

Effects of Absorbed Dose Rate, Irradiation Temperature and Post-Irradiation Temperature on the Gamma Ray Re. sponse of Red Perspex Dosimeters.

PB92-116441

101,363

Neutron Measurements of Intramolecular Vibrational Modes in $\mathrm{C} 60$

PB92-116540 $\quad 101,055$

Electron Spin Resonance for Monitoring Radiation-Processed Meats Containing Bone

100,018

PB92-116672

Dosimetry of a Nearly Monoenergetic 6- to 7-MeV Photon
Source by Nal(TI) Scintillation Spectrometry.

Source by Nal(TI) Scintillation Spectrometry.

101,723

Observation of Anomalous Isotope Ratios in SIRIS Meas. urements of Molydenum.

PB92-116799

101,361

Radiochromic Film Dosimeter for Gamma Radiation in the Absorbed-Dose Range 0.1 - $10 \mathrm{kGY}$. PB92-116961

101,364

Dosimetry: New Approaches for New Challenges.
PB92-117100

101,365

Novel Radiation Dosimetry Systems.

101,727

2-Deoxy-D-Ribose Aqueous Solution as a Gamma-Ray Dosimeter.

101,366

Temperature Dependence of Radiochromic Film Dosimeters

101,728

Photon Energy Dependence of the Sensitivity of Radiochromic Film and Comparison with Silver Halide Film and LiF TLDs Used for Brachytherapy Dosimetry.

PB92-117142

101,296

Mass Energy-Transfer and Mass Energy-Absorption Coefficients, Including In-Flight Positron Annihilation for Photon Energies $1 \mathrm{keV}$ to $100 \mathrm{MeV}$.

PB92-126473

101,445

NUCLEAR POWER PLANTS

Annotated Bibliography: Diagnostic Methods and Measurement Approaches to Detect Incipient Defects Due to Aging of Cables
PB91-222687

101,369

Predicting the Remaining Service Life of Concrete.
PB92-126598

NUCLEATE BOILING

Visualization of Nucleate Flow Boiling for an R22/R114 Mixture and Its Components.

PB91-162164

100,225

NUCLEATION

Emergence of Modern Nucleation Theory.

101,481

Theory of Orientation Textures Due to Surface Energy Anisotropies.

101,571

NUCLEOHISTONES

Structure and Mechanism of Hydroxyl Radical-Induced Formation of a DNA-Protein Cross-Link Involving Thymine and Lysine in Nucleohistone.

PB91-187518

101.334

Hydroxyl Radical Induced Cross-Linking of Cytosine and Tyrosine in Nucleohistone.

101,304

NUMERICAL ANALYSIS

Convergence Properties of a Class of Rank-Two Updates.
PB91-187799

101,260

OMNITAB 80 (ASCII): An Interactive System for Statistical and Numerical Data Analysis (Version 70 ).

101,278

OMNITAB 80 (UNIX): An Interactive System for Statistical OMNITAB 80 (UNIX): An Interactive System for Statistical and Numerical Data Analysis. Version 7.0.

101,279

NUMISMATICS

Linking Information to Objects: A Hypertext Prototype for Numismatists.

100,657

NURSING HOMES

Engineering Analysis of the Fire Development in the Hillhaven Nursing Home Fire, October 5, 1989. 100,169

VLAP (NATIONAL VOLUNTEER LABORATORY

ACCREDITATION PROGRAM)

NVLAP FY 90 Annual Report

100,010

NYLON

Immobilization of Binding Proteins on Nonporous Supports Comparison of Protein Loading, Activity, and StabiliPB91-237198

101,308

O STARS

Terminal Velocities for a Large Sample of O Stars, B Supergiants, and Wolf-Rayet Stars.

100,023

OBJECT-ORIENTED PROGRAMMING

Proceedings of the Object-Oriented Database Task Laurier Hotel, Ottawa, Canada.
PB91-157198

100,641

Proceedings of the Object-Oriented Database Task Group Workshop. Held in Atlantic City, New Jersey on

May 22, 1990

100,643

OCCUPATIONAL SAFETY AND HEALTH

Response of Personal NoIse Dosimeters to Continuous and Impulse-Like Signals

PB92-116359

100,914

A (OFFICE DOCUMENT ARCHITECTURE)

Document Architecture for Open Systems.

PB91-189522

100,961

OFFICE BUILDINGS

Second-Level Post-Occupancy Evaluation Analysis.
PB91-147140

Airtightness of Office-Building Envelopes.

PB91-175307

100,067

Measurement of Thermal Characteristics of Office Build-

ings. 175471

100,068

Diagnostic Techniques for Evaluating Office Building En-

PB91-195438

100,074

Variable Air Volume System Design Guide.

100,077

OIL WELLS

Investigation of Simulated Oil-Well Blowout Fires PB91-144337

101,356

OLIGOSACCHARIDES

Thermodynamics of Hydrolysis of Oligosaccharides.
101,288

ON LINE SYSTEMS

STEP On-Line Information Service User's Guide. National PDES Testbed Report Series.

PB91-157123

100,950

OPEN SYSTEM ENVIRONMENT

Application Portability Profile (APP): The U.S. Government's Open System Environment Profile OSE / 1 Version 1.0

-201004

100,692

OPEN SYSTEMS INTERCONNECTIONS Government Open Systems Interconnection Profile
(GOSIP); Category: Hardware and Software Standards; Subcategory: Computer Network Protocols

100,675 PUB 146-1

OPERATING SYSTEMS (COMPUTERS)

Programming Languages and Database Language SOL. Validated Processor List Including GOSIP Conformance Testing Registers.

100,649

OPERATIONS ANALYSIS \& APPLICATIONS

Applications of Operations Research Techniques to System Design and Evaluation.

PB91-174540

101,267

OPERATIONS RESEARCH

Applications of Operations Research Techniques to System Design and Evaluation.

101,267

OPHTHAMIC APPLICATORS

Calibration of Ophthalmic Applicators at NIST: A Revised Approach.

OPTHALMOLOGY

Calibration of Ophthalmic Applicators at NIST: A Revised Approach

PB91-237388

101,327

OPTICAL COMMUNICATION

Limited International Intercomparison of Responsivity Scales at Fiber Optic Wavelengths.

100,567

Frequency Stabilization of an Erbium-Doped Fiber Laser:
A Potential Wavelength Standard for Optical CommunicaA Potential Wavelength Standard for Optical Communica-
tions.
PB91-203091 100,570

100,570

OPTICAL CORRELATORS

New Triple Correlator Design for the Measurement of UItrashort Laser Pulses

PB91-203802

101,429

OPTICAL DETECTORS

Fourteen-Decade Photocurrent Measurements with Large-Area Silicon Photodiodes at Room Temperature.
PB92-116771 PB92-116771

OPTICAL DISKS

Development of an Optical Disk System for the Automated Retrieval of EASEAR Records.

100,666

Test Methods for Optical Disk Media Characteristics (for $356 \mathrm{~mm}$ Ruggedized Magneto-optic Media)

PB92-116409

100,605

Monitoring and Reporting Techniques for Error Rate and Error Distribution in Optical Disk Systems.
PB92-123124

PTICAL EQUIPMENT

Note on the Precision Cementing of Small Optical Components.

PB91-203158

101,428

OPTICAL FIBERS

Technical Digest-Symposium on Optical Fiber Measure-

PB91-132308

101,403

rogress in the Development of Miniature Optical Fiber Current Sensors.

Practical Considerations in the Design of Optical Fiber Current Sensors.

PB91-134742

100,746

Optical Fiber Current Sensors with Temperature Stabilities Near the Material Limil.

PB91-175109

100,753

Methods of Analyzing Planar Optical Waveguides PB91-236752

100,774

OPTICAL FILMS

Stoichiometry of Reactively Evaporated Films
PB91-158709

101,413

PTICAL FLOW

Analysis of Optical Flow Estimation Using Epipolar Plane Images.

2564

100,703

OPTICAL LEVITATION

Optical Levitation of Single Particles.

OPTICAL MATERIALS

Use of Diamond as an Optical Materia

AD-A243 097/3

101,634

Laser Induced Damage in Optical Materials: 1989 PB91.148478

Design Diagrams for Heavy Metal Fluoride Giass

dows.
PB91-187419

101.419

Alternate Representation of Prism Refraction for Light Incident at the Brewster Angle.

PB91-203042

101,426

OPTICAL MEASUREMENT

Technical Digest-Symposium on Optical Fiber Measurements, 1990.

101,403

Light-Scattering Measurement of the RMS Slopes of Rough Surfaces.

101,436

OPTICAL MEASURING INSTRUMENTS

Performance and Limitations of Faraday Effect Sensors.
PB91-149146

Faraday Effect Current Sensing Using a Sagnac Interferometer with a $3 \times 3$ Coupler.

PB91-175158

100,754

OPTICAL MICROSCOPES

Standard Reference Materials: Bright-Chromium Linewidth Standard, SRM 476, for Calibration of Optical Microscope Linewidth Measuring Systems. PB91-167163

100,802

OPTICAL PROPERTIES

Determination of the Optical Constants of Thin ChemicalVapor-Deposited Diamond Windows from 0.5 to $6.5 \mathrm{eV}$.

Use of Diamond as an Optical Material.

AD-A243 097/3

101,401

OPTICAL PUMPING 


\section{KEYWORD INDEX}

OPTOELECTRONIC DEVICES

Progress in the Development of Miniature Optical Fiber Current Sensors.

PB91-134734

100,745

Practical Considerations in the Design of Optical Fiber Current Sensors.

ORBIT CALCULATION

Orbit Determination and Gravitational Field Accuracy for a Mercury Transponder Satellite.

PB91-175166

ORGANIC ACIDS

Journal of Physical and Chemical Reference Data

Volume 19, Number 4, July-August 1990.

100,354
ed Com.

Thermodynamics of the Krebs Cycle and Related Com-
pounds.
PB91-192468
100,357

RGANIC COMPOUNDS

Journal of Physical and Chemical Reference Data

Volume 19, Number 4, July-August 1990.

Heat Capacities and Entropies of Organic Compounds in the Condensed Phase. Volume 2.

100,356

PB91-192450

Heat Capacities of Organic Compounds in Liquid State.
2. C1 to C18 n-Alkanes.
PB92-110204 100,448

Kinetics and Mechanisms of the Gas-Phase Reactions of the NO3 Radical with Organic Compounds.

100,450 PB92-110220

ORGANIC IONS

Alkyl Transier Reactions between Protonated Alcohols and Ethers. The Gas-Phase Alkylation of Formaldehyde.

100,280 Thermal Decomposition of Ions. 3. Protonated Ethanol and Diethyl Ether.

PB91-195073 100,376

Reactions of $\mathrm{C} 5 \mathrm{H} 3(+)$ and $\mathrm{C} 5 \mathrm{H} 5(+)$ lons with Acetylene and Diacetylene

100,384

ORGANIC NITROGEN COMPOUNDS

Thermodynamic and Thermophysical Properties of Organic Nitrogen Compounds. Part 1. Methanamine, Ethan amine, 1- and 2-Propanamine, Benzenamine, 2-, 3-, and 4-Melhybenzenamine.

PB91-192583

100,368

ORGANIC SEMICONDUCTORS

Permittivity Measurements on Molecular-Sized Samples. PB91-159244

mples.

ORGANIC SOLVENTS

Formation of ortho-Tyrosine by Radiation and Organic Solvents in Chicken Tissue.

PB92-116953

101,290

ORGANIZATIONS

Standards Activities of Organizations in the United States

11-177774

100,004

ORGANOMETALLIC COMPOUNDS

Methods for the Analysis of Organometallic Compounds PB91-14898

ORGANOTIN COMPOUNDS

Newest Approaches to Ouantitative Assessment of Bioactive Organotins.

Bioactive Organ
PB91-162032

100,189

\section{ORIFICE FLOW}

Effect of the Location of an In-Line Tube Bundle on Orifice Flowmeter Performance.

PB91-134445

100,921

Effect of Pipe Surface Finish on the Orifice Discharge Coefficient.

101,389

Choosing Flow Conditioners and Their Location for Orifice Flow Measurement.

PB92-116490

101,397

ORIFICE METERS

Effect of the Location of an In-Line Tube Bundle on Orifice Flowmeter Performance.

100,921

Experimental Evaluation of Selected Orifice Flowmeter

Upstream Installations.
PB91-147025
100,880

Effect of Pipe Surface Finish on Gas Flow Measurement with an Orifice Meter

101,387

Effect of Pipe Surface Finish on the Orifice Discharge

Coefficient.
PB91-162347 101,389

Choosing Flow Conditioners and Their Location for Orifice Flow Measurement.

PB92-116490

101,397

ORTHOGONAL ARRAYS

Taguchi's Orthogonal Arrays Are Classical Designs of Experiments.

ORTHOGONAL DISTANCE REGRESSION

101,280

Orthogonal Distance Regression.
PB91-147017

101,272

PILLATING FIELDS

Frequency Dependence of Catalyzed Reactions in a Weak Oscillating Field

100.407

\section{OSCILLOSCOPES} Recent Developments in Digital Oscilloscopes.
PB91-175240

100,849

OSI (OPEN SYSTEM INTERCONNECTION)

Stable Implementation Agreements for Open Systems Interconnection Protocols. Version 4, Edition 1, Change 1991 OSI Implementors Workshop. Held in Gaithersburg, Maryland.

OSI (OPEN SYSTEMS INTERCONNECTION)

100,697

Working Implementation Agreements for Open Systems Wrotocols.

100,686

Stable Implementation Agreements for Open Systems Interconnection Protocols. Version 4, Edition 1, DecemInterconnection Protocols. Version 4, Edition 1, Decem PB91-171967

Stable Implementation Agreements for Open Systems Interconnection Protocols. Version 4, Edition 1, March 1991. Change Pages (Supplement).

100,691 Stable Implementation Agreements for Open Systems
Interconnection Protocols. Version 4, Edition 1, June Interconnection Protocols. Version 4, Edition 1, June
1991 Change Pages (Supplement).

100695

Working Implementation Agreements for Open Systems Interconnection Protocols

PB92-126523

100,698

Guidelines for the Evaluation of File Transter, Access and Management Implementations.

100,699

OSMIUM

Redox Reactions of Osmium Porphyrins.

PB91-159012

100,217

OXIDATION

Stoichiometry of Reactively Evaporated Films.

PB91-158709

101,413

Zonal Model for Corona Discharge-Induced Oxidation of $\mathrm{SF} 6$ in $\mathrm{SF} 6 / \mathrm{O} 2 / \mathrm{H} 2 \mathrm{O}$ Gas Mixtures.

PB91-158790

100,312

Oxidation of Organic Compounds by Active Manganese Dioxide

PB91-174730

100,218

Selected-Ion Mass Spectrometry: Assays of Oxidative DNA Damage

101,307

Surface Oxidation Kinetics of Si3N4 - 4\% Y2O3 Powders Studied by Bremsstrahlung-Excited Auger Spectroscopy. PB91-237701

100,435

Oxidation Kinetics of Silicon Carbide Whiskers Studied by X-ray Photoelectron Spectroscopy.

100.436

\section{OXIDATION REDUCTION REACTIONS}

Mechanism of $\mathrm{OH}$ Radical Reactions with Thymine and Uracil Derivatives

PB91-147447

100,216

Redox Reactions of Osmium Porphyrins.

100.217

Radiolytic Studies of the Redox Reactions of Ruthenium Porphyrins.

B $91-203661$

100,402

Mechanisms of the Reduction Reactions of $\mathrm{Cr}(\mathrm{VI})$ in the Radiolysis of Acidic Potassium and Silver Dichromate Solutions in the Presence or Absence of Acetic Acid.

PB91-236489

Antioxidation Mechanisms of Uric Acid. PB91-237347

100,241

OXIMES

Kinetics of Copper Extraction Using (anti)-2-Hydroxy-5Nonlybenzophenone Oxime.

PB91-148783

100,292

OXYGEN

Adsorption of Water and Oxygen on $\mathrm{Ag}(110)$ : A Study of the Interactions among Water Molecules, Hydroxyl Groups, and Oxygen Atoms.

100,270

Mechanism of Soot Formation in Acetylene-Oxygen Mix-

PB91-147280

100,537

Transport Properties of Fluid Oxygen.

100,359

Experimental Thermal Conductivity, Thermal Diffusivity, and Specific Heat Values for Mixtures of Nitrogen, Oxygen, and Argon

100,369

Partial Equilibrium in Laminar Hydrocarbon Diffusion Flames.

100,553

(1-237024

Spectroscopic Data Base for Carbon and Oxygen.
PB91-237750 101,716 Semiclassical Calculation of Self-Broadening in O2, N2, and CO Raman Spectra.

PB92-117050

100,461

mproved Fits for the Vibrational and Rotational Conants of Many States of Nitrogen and Oxygen. 100,469 Thermodynamic Properties of Oxygen from the Triple Point to $300 \mathrm{~K}$ with Pressures to $80 \mathrm{MPa}$.

100,474

OXYGEN ATOMS

Updated Excitation and Ionization Cross Sections for Electron Impact on Atomic Oxygen.

B90-244591

100,256

Cross Sections for Colfisions of Electrons and Photons with Atomic Oxygen.

PB90-244682

100,264

OXYGEN COMPLEXES

Van der Waals Complexes in 1,3-Dipolar Cycloaddition Reactions: Ozone-Ethylene.

PB91-236737

100,417

\section{OXYGEN IONS}

Dielectronic Recombination Rate Coefficients of $\mathrm{O}(5+)$ and $\mathrm{O}(2+)$.

101,657

Production and Spectroscopy of Small Polyatomic Molecular lons Isolated in Solid Neon.

PB91-203273

100,395

OXYGEN-LIKE IONS

Influence of Metastable States and Thermal Equilibration upon Dielectronic Recombination in Low Density to Mod PB91-195610

101,675

OZONE

Van der Waals Complexes in 1,3-Dipolar Cycloaddition Reactions: Ozone-Ethylene.

PB91-236737

100.417

PACKAGING

Checking the Net Contents of Packaged Goods. Third Edition, Supplement 2.

PB92-112549

100,171

PACKAGING MATERIALS

Biodegradable Plastics: An Idea Whose Time Has Come BB91-150086

101,243

PALLADIUM

Neutron Powder Diffraction and Inelastic Scattering Study of the Structures of Zr2Pd, Zr2PdD1.70, and Zr2PdD1.96. Chemical and Electronic Properties of $\mathrm{Pd} / \mathrm{SnO} 2(110)$ Model Gas Sensors.

PB91-203075

100,393

PALLADIUM ALLOYS

Relationship of Hydrogen Site Occupancy to Diffusion Behavior in Crystalline and Amorphous $\mathrm{Zr} 2 \mathrm{PdHx}$ PB91-187252

101,230

PANCREATIC RIBONUCLEASE

Multiple Conformations of Amino Acid Residues in Ribonuclease A.

101,298

PANELS

Roughness Measures of Blasted Steel Surfaces Remote- 
PB91-174441

PARTIAL MOLAR ISOBARIC THERMAL EXPANSION

Modelling Aqueous Solutions Near the Critical Point of

PB91-189837

100,349

PARTIAL MOLAR VOLUME

Modelling Aqueous Solutions Near the Critical Point of

PB91-189837

100,349

PARTICLE BEAMS

Probe for Real-Time Images of Particle Beams and Their Analyses in a Merged-Beams Apparatus.

101,683

RTICLE SIZE

Use of the Electrostatic Classification Method to Size 0.1 micrometer SAM Particles: A Feasibility Study.

PB91-187633

100,932

Certification of NIST SRM 1961: 30 micrometers Diameter Polystyrene Spheres.

PB92-126655

100,937

ARTICLE SIZE DISTRIBUTION

Velocity and Droplet Size Measurments in a Fuel Spray.

Particle Size Measurement of Inert Gas Atomized PB91-187203

101,229

PARTICLE STANDARDS

NBS Submicron Particle Standards for Microcontamination Measurement

100,798

\section{PARTICULATE COMPOSITES}

Superconductor-Polymer Composites.

NT-4 954481

Elastic Constants of a Tungsten-Particle Copper-Matrix

Composite.
PB91-194852 101,234

Magnetic Properties of Iron/Silica Gel Nanocomposites.

PB91-204073 101,110

PARTICULATES

New Way to Mount Particulate Material for Laser Microprobe Mass Analysis.

PB92-116805

100,911

PARTITION

Book Review: Partitioning in Aqueous Two-Phase Systems: Theory, Methods, Uses, and Applications to Biotechnology.

PASCAL PROGRAMMING LANGUAGE

Validated Products List (Cobol, Fortran, ADA, Pascal, MUMPS, SQL).

PATTERN MAKING

Pattern Generation on Semiconductor Surfaces by a Scanning Tunneling Microscope Operating in Air.
PB91-194712

00,808

Possible Use of Pattern Recognition for the Analysis of Mars-Rover X-ray-Fluorescence Spectra. Mars-Rover $X$-ray
PB91-237875

101,733

PAVEMENT OVERLAYS

Uniaxial Tensile Tests to Measure the Bond of In situ Concrete Overlays.

\section{PAYMENT SYSTEMS}

NIST Personnel Management Demonstration Project: Design, Implementation and Accomplishments. $\quad 100,003$
PB91-231555

DES (PRODUCT DATA EXCHANGE SPECIFICATION)

Fed-X: The NIST Express Translator. Revised, November$$
1990 .
$$

PB91-132126

100,681

NIST PDES Toolkit: Technical Fundamentals. National PDES Testbed Report Series (Revised).

100,682

NIST Working Form for STEP: National PDES Testbed Report Series (Revised).

100,683

NIST STEP Working Form Programmer's Reference. NaNIST STEP Working Form Programmer's Reference. Na-
tional POES Testbed Report Series (Revised).
PB91-144378
100,998

PDES (PRODUCT DATA EXCHANGE SPECIFICATIONS) NIST Express Working Form Programmer's Reference
(Revised November 1990). National PDES Testbed
Report Series

PB91-157164

100,964

POES (PRODUCT DATA EXCHANGE USING STEP)

National PDES Testbed Mail Server User's Guide. National PDES Testbed Report Series.

100,951

PEEK

Thermal Conductivity of Alumina Fiber/Epoxy and Alumina Fiber/PEEK from 4.2 to $310 \mathrm{~K}$

101,130

PEEL TESTS

Further Investigation of the Effect of Application Parameters on Adhesive-Bonded Seams of Single-Ply Roof Membranes.
PB91-149153

100,105

PENLIT

Thermal Conductivity of Evacuated Perlite at Low Tem-

peratures as a Function of Load and Load History.

peratures as a Function of Load and Load History.
PB90,174672

\section{PEPTIDES}

Chitı-Chitosan Membranes: Separations of Amino Acids and Polypeptides.

PB91-134544

101,283

PERCARBONATE ION

Vibrational Spectra of Molecular Ions Isolated in Solid Neon. 6. CO4(1-)

PB91-236836

100,419

PERCOLATION

Percolation of Phases in a Three-Dimensional Cement

Paste Microstructural Model.

PB91-187179

100,529

\section{PERFLUORO COMPOUNDS}

Thin and Composite High-Flux Membranes of Perfluorosulfonated lon-Exchange Polymer.

PB91-236653

100,512

PERFORMANCE

Refrigerant Charge Effects on Heat Pump Performance. PB91-174607

100,065

PERFORMANCE EVALUATION

Workloads, Observables, Benchmarks and Instrumenta-

tion.

Robot Characterization Testing.

100,598

PB91-167361

101,030

Scoring Continuous Speech in the DARPA Speech Recognition Program: Part 2.

100.593

Measurement of Regenerator Performance in a Vuilleumier Refrigerato

PB92-116482

101,052

\section{PERICARDIUM}

Comparative Study of Bovine Pericardium Mineralization: A Basic and Practical Approach.

PERICLASE

Thermodynamic Functions and Properties of $\mathrm{MgO}$ at High Compression and High Temperature.

100,056 PB90-244542

100,253

PERIODICALS

NIST Serial Holdings, 1991

PB91-171330

100,960

PERMEABILITY

Optimization Techniques for Permittivity and Permeability

PB92-112325

100,865

Optimization Techniques for Permittivity and Permeability Determination
PB92-126663

100,871

\section{PERMITTIVITY}

Permittivity Measurements on Molecular-Sized Samples.

PB91-159244

100,800

Improved Technique for Determining Complex Permittivity with the Transmission/Reflection Method.

100,844

Improved Technique for Measuring Permittivity of Thin Improved Technique for Measuring Permittivity of Thin
Dielectrics with a Cylindrical Resonant Cavity.
PB91-236844

100,858

Optimization Techniques for Permittivity and Permeability PB92-112325

100,865

Optimization Techniques for Permittivity and Permeability Determination
PB92-126663

100,871

PERMITTIVITY MEASUREMENT

Transmission/Reflection and Short-Circuit Line Permittivity Measurements.

PEROXY RADICALS

Reactivities of Chlorine Atoms and Peroxyl Radicals Formed in the Radiolysis of Dichloromethane. PB91-200782

100,386

PEROXYL RADICALS

Rate Constants for Reactions of Peroxyl Radicals in Fluid Solutions.

PSONAL COMPUTERS

100,261

1987: The Year of the 386

PB91-158766

100,599

PERSONNEL DOSIMETRY

Response of Personal Noise Dosimeters to Continuous and Impulse-Like Signals. PB92-116359

100,914

PERSONNEL MANAGEMENT

NIST Personnel Management Demonstration Project: Design, Implementation and Accomplishments.

PERYLENES

Cryogenic-Temperature Fluorescence Spectra of Polynuclear Aromatic Hydrocarbons of Molecular Weight 302 PB91-147157

Assessment of Uncertainties in Measurement of $\mathrm{pH}$ in Hostile Environments Characteristic of Nuclear RepositorNUREG/CR-5711

101,367

Second Dissociation Constant and pH of N-2-hydroxyethylpıperaztne- $N^{\prime}$-2-ethanesulfonic Acid (HEPES) from 0 to

PB91-189282

100,196

Evaluation of Liquid Junction Potentials and Determination of $\mathrm{pH}$ Values of Strong Acids at Moderate lonic Strengths.

PB91-237859

100,438

PH METERS

Instability in pH Measurements of Sputtered IrO2 Films. PB91-147538

100,281

PHASE

Book Review: Partitioning in Aqueous Two-Phase Sys-

tems: Theory, Methods, Uses, and Applications to Biotechnology.

\section{PHASE DIAGRAMS}

100,333

Phase Diagrams of Hexagonal Binary Ordering Alloys with Anisotropic Interactions.

B91-147512

Graphics Program for Binary and Ternary Ceramic Phase

$\begin{array}{ll}\text { Diagrams. } & 101,063 \\ \text { PB91-147991 } & \end{array}$

N-Dimensional Crystallographic Description of the Icosahedral Phases; the Example of the Al73Mn21Si6 Ouasiperiodic Structure.

PB91-174771

101,226

Coupled Phase Diagram.Thermodynamic Analysis of the 24 Binary Systems, A2CO3-AX and A2SO4-AX Where $\mathrm{A}=\mathrm{Li}, \mathrm{Na}, \mathrm{K}$ and $\mathrm{X}=\mathrm{Cl}, \mathrm{F}, \mathrm{NO} 3, \mathrm{OH}$

PB91-192518

100,362

Phase Equilibria of the System Strontium Oxide-Calcium Oxide-Cupric Oxide.

101,085

Phase Selection in Non-Equilibrium Processing

PB91-195651

101,236

Phase Equilibria and Critical Lines in Model Water/Salt xtures.

100.418

Ab Initio Phase Determination for $\mathrm{X}$-ray Diffraction Data from Crystals of a Native Protein.

PB91-237362

101,309

HASE METERS

Influence of Nonsinusoidal Waveforms on Voltmeters, Ammeters, and Phase Meters.

100,869

\section{PHASE NOISE}

Extending the Range and Accuracy of Phase Noise Measurements

Accuracy Model for Phase Noise Measurements. PB91-134882

100,589

PHASE RULE

Extended Phase Rule for Nonreactive, Multiphase, Multicomponent Chemical Systems. 


\section{KEYWORD INDEX}

PHOTIONIZATION Angle-Resolved Photoemission from the Ar 2p Subshell.
PB91-194928 PHOTOABSORPTION

Laser-Driven lonization and Photoabsorption Spectrosco. py of Atomic lons
PB91-174854

101,415

PHOTOCHEMICAL REACTIONS

Novel Laser Gain and Time-Resolved FTIR Studies of Photochemistry
PB91-148809

100,237

PHOTOCONDUCTIVITY

Persistent Photoconductivity in SIMOX Films. PB92-117084

100,826

PHOTOCURRENTS

Fourteen-Decade Photocurrent Measurements with Large-Area Silicon Photodiodes at Room Temperature.
PB92-116771

PHOTODETECTORS

High-Resolution Small-Angle X-ray Scattering Camera for

Anomalous Scattering

100,929

Hydrogenated Amorphous Germanium Detectors Deposited onto Channel Waveguides.

PB91-194845

100,756

\section{PHOTODIODES}

Electronic and Radiometric Characteristics of Near Infra-

red Photodiodes.

PB91-174706 100,752

Temperature Dependence of High Accuracy-Photometer Heads.

101,418

Journal of Research of the National Institute of Stand-

Journal of Research of the National Institute of Stand-
ards and Technology. March-April 1991. Volume 96,

PB91-187617

101,420

Linearity of a Silicon Photodiode at $30 \mathrm{MHz}$ and Its Effect on Heterodyne Measurements.

100,755

Stable, High Ouantum Efficiency Silicon Photodiodes for

$\begin{array}{ll}\text { Vacuum-UV Applications. } & 100,757\end{array}$

Generalized Photodiode Self-Calibration Formula

PB91-236901

100,760

Journal of Research of the National Institute of Standards and Technology. July-August 1991. Volume 96, Number 4.

100,762

Numerical Modeling of Silicon Photodiodes for High-Accuracy Applications. Part 1. Simulation Programs.
PB92-110295 763

Numerical Modeling of Silicon Photodiodes for High-ACcuracy Applications. Part 2. Interpreting Oxide-bias Experiments.

periments.

100,764

Numerical Modeling of Silicon Photodiodes for High-Accuracy Applications. Part 3 . Interpolating and Extrapolating Internal Ouantum-Efficiency Calibrations.

PB92-110311

100,765

Fourteen-Decade Photocurrent Measurements with Large-Area Silicon Photodiodes at Room Temperature.

PHOTODISSOCIATION

Photodissociation Dynamics of Water in the Second $\mathrm{Ab}$ sorption Band: Vibrational Exitation of $\mathrm{OH}$ (A (2)Sigma). PB91-148726 100,291

Periodic Orbits and Diffuse Structures in the Photodissociation of Symmetric Triatomic Molecules.

100,238

Photodissociation of C(I)NO in the S(I) State: A OuantumMechanical ab initio Study.

PB91-149260

100,239

Time- and State-Resolved Measurements of Nitric Oxide Dimer Infrared Photodissociation.

100,387

Photodissociation Dynamics of Mo(CO) 6 at $266 \mathrm{~nm}$ and

Photodissociation Dynamics of $\mathrm{Mo}(\mathrm{CO}) 6$ at $266 \mathrm{~nm}$ and
$355 \mathrm{~nm}$ : CO Photofragment Kinetic-Energy and Internal$355 \mathrm{~nm}$ : CO Photofragment Kinetic-Energy and InternalState Distributions.
PB91-236547

100,413

PHOTOELECTRON SPECTROSCOPY

NIST X-Ray Photoelectron Spectroscopy (XPS) DataPB92-123157

100,465

PHOTOEMISSION

Parity-Unfavored Transitions in Resonant Photoemission from $\mathrm{Ar}, \mathrm{Kr}$, and $\mathrm{Xe}$ : Experimental and Theoretical Results.

101,599

Resonant Photoemission and PSD of Rare Earth Oxides. PB91-148015

100,287

PHOTOGRAPHIC FILM DOSEMETERS

Temperature Dependence of Radiochromic Film Dosi-

meters.
PB92-117134

101,728

PHOTOIONIZATION

Photoionization and Collisional Ionization of Excited Atoms Using Synchrotron and Laser Radiations.
PB91-159301

101,626

Photoionization Studies of Atoms and Molecules Using Synchrotron Radiation

PB91-194910

101,664

PHOTOLUMINESCENCE

nverse Correlation between the Intensity of Luminescence Excited by Electrons and by Visible Light in Chemical-Vapor-Deposited Diamond Films. Photoluminescence Excitation by Band-Gap Optical Absorption in Chemical Vapor Deposition Diamond Films. PB91-195586

101,533

\section{PHOTOLYSIS}

Laser Photolysis of Trimethylgallium at $193 \mathrm{~nm}$ : Ouantum Yields for Methyl Radical and Ethane Production. AD-A227 868/7

100,234

PHOTOMASKING

Standard Reference Materials: Bright-Chromium Linewidth Standard, SRM 476, for Calibration of Optical Microscope Linewidth Measuring Systems.

PB91-167163

100,802

PHOTOMETERS

Temperature Dependence of High Accuracy-Photometer

PB91-187096

101,418

Temperature Monitored/Controlled Silicon Photodiodes for Standardization.

PB92-116763

100,767

PHOTOMULTIPLIER TUBES

Photomultiplier Housing for Vacuum Operation of Side-on 1P28-Type Tubes.
AD-A227 $836 / 4$

100,744

\section{PHOTON-ATOM COLLISIONS}

Cross Sections for Collisions of Electrons and Photons with Atomic Oxygen

PHOTON CROSS SECTIONS

Mass Energy-Transfer and Mass Energy-Absorption Coefficients, Including In-Flight Positron Annihilation for Photon Energies $1 \mathrm{keV}$ to $100 \mathrm{MeV}$. PB92-126473

101,445

PHOTONICS

Photonic Probes for the Measurement of Electromagnetic Fields Over Broad Bandwidths.

PB91-162206

100,750

PHOTONS

Photon Energy Dependence of the Sensitivity of Radioch romic Film and Comparison with Silver Halide Film and LiF TLDs Used for Brachytherapy Dosimetry.

PB92-117142

101,296

\section{PHOTOOXIDATION}

Polyelectrolyte Stabilized Metal Oxide Hydrosols as Catalysts for the Photooxidation of Water by Zinc Porphyrins. PB91-189852

100,351

\section{PHYSICAL CHEMISTRY}

Journal of Physical and Chemical Reference Data Volume 19, Number 2, 1990

PB90-244609

100,257

Journal of Physical and Chemical Reference Data Volume 20, Number 2, March-April 1991.

100,445

Journal of Physical and Chemical Reference Data Volume 20, Number 3, May-June 1991.

100,449

PB92+110212

Journal of Physical and Chemical Reference Data,
Volume 20, Number 4, July/August 1991.

Volume 20, Number 4, July/August 1991

00,466

Journal of Physical and Chemical Reference Data Volume 20, Number 6, November/December 1991.
PB92-148162

PHYSICAL PROPERTIES

Critical Data in Physics and Chemistry.

PB91-203455 101,697

Journal of Physical and Chemical Reference Data. Volume 20, Number 1, 1991

PB92-110113

100,440

PIEZOELECTRIC GAGES

Monitoring of Anisotropic Material Elastic Properties Using Ultrasonic Receiving Rays.

101,126

\section{PILOT PLANTS}

Small Scale Demand Type Neon Liquefaction Plant. PB91-162008

100,224

PIN DIODES

Instrumentation of X-ray Beam Lines with PIN Diode Detectors.
PB91-174888

101.633

PINNING

Comparison of Flux Dynamics in Two Samples of YBa2Cu3O7 with
PB91-149419

101,069

PIPE FLOW

Flowmeter Installation Effects: A New Approach to an Old but Prevalent Problem.

PB91-13441

100,920

Effect of Pipe Surface Finish on the Orifice Discharge Coefficient.

PB91-162347

101,389

Experimentation, Analysis, and Correlation of Refrigerant22 Flow Through Short Tube Restrictors.

PB91-174243

101,194

Unsteady Laminar Flow in a Circular Tube: A Test of the HERCOL (Hermitian Collocation) Computer Code. PB91-222679

PIPES (TUBES)

Effect of Pipe Surface Finish on Gas Flow Measurement with an Orifice Meter.

PB91-148031

101,387

PLANAR WAVEGUIDES

Planar Waveguide Optical Immunosensors.

101,284

PLANCKS CONSTANT

New Test of Ouantum Mechanics: Is Planck's Constant Ung1-174

101,632

PLANETARY ATMOSPHERES

Novel Laser Gain and Time-Resolved FTIR Studies of Photochemistry
PB91-148809

100,237

\section{PLASMA SPECTRA}

Extreme-Ultraviolet Spectra of Pd, Ag, Cd, and Sn lons Obsed in a 1.3-keV Tokamak Plasma.

101,712 B91-237495 Spectra of the P I isoelectronic Sequence from Co XIII to
Mo XXVIII.
PB91-237503
101,713 PB91-237503

PLASMAS (PHYSICS)

Liquid and Solid lon Plasmas.

AD-A227 656/

101,441

PLASMIDS

Base Modifications in Plasmid DNA Caused by Potassium Permanganate.

101,329

PLASTIC PROPERTIES

Development of a Three-Dimensional Finite Element Program for Large Strain Elastic-Plastic Solids.
PB91-174557

101,494

\section{PLASTICITY}

Plastic Zone Formation Around an Arresting Crack. PB91-189316

101,373

PLASTICS

Biodegradable Plastics: An Idea Whose Time Has Come PB91-150086

101,243

Morphology of Polymer Films and Single Molecules. PB91-203224

100,506

Long Time Creep and Lifetime Behavior in Uniaxial Extension of a line

101,247

Experimental Study of the Pyrolysis Rate of a Poly(methyl methacrylate) (PMMA) Wall Panel in a Reduced-Scale Enclosure.

PB91-237412

100,555

Data for Fire Hazard Assessment of Selected Non-Halogenated and Halogenated Fire Retardants; Report of Test FA 3983

100,557 


\section{KEYWORD INDEX}

\section{POSITIONING}

Experimental Study of the Pyrolysis Rate of a Poly(methyl methacrylate) (PMMA) Wall Panel in a Reduced-Scale Enclosure.

PB91-237412

100,555

POLARIZERS

Semiconductor Claddings on Glass Waveguides for POlarizers and Detectors.

PB91-175141

101,416

Fe-W Supermirro

101,643

POLYATOMIC GASES

Thermal Conductivity of Nine Polyatomic Gases at Low Density.

PB91-192492

100,360

Equilibrium and Transport Properties of Gas Mixtures at Low Density: Eleven Polyatomic Gases and Five Noble

Gases.

100,363

POLYATOMIC MOLECULES

Vibrational and Electronic Energy Levels of Polyatomic Transient Molecules. Supplement 1

PB91-192575

100,367

POLYBUTADIENE

Small-Angle Neutron Scattering and Light Scattering Studies on the Miscibility of Protonated Polyisoprene/ Deuterated Polybutadiene Blends.

PB91-236786

100,513

Microstructure Effects on the Lower Critical Solution Temperature Phase Behavior of Deuterated Polybutadiene and Protonated Polyisoprene Blends Studied by Small-Angle Neutron Scattering.

PB92-117266

100,518

Small-Angle Neutron Scattering and Light Scattering Study on the Miscibility of Poly(styrene-ran-butadiene) Polybutadiene Blends.

POLYCHLOROBIPHENYL COMPOUNDS

100,519

Preparation and Analysis of a Marine Sediment Reference Material for the Determination of Trace Organic Constituents.

101,377

POLYCRYSTALS

Tensile-Fracture Resistance Mechanisms in Brittle Polycrystals: An Ultrasonics and In-situ Microscopy Investiga-

PB91-149344 101,190

Fundamental Condition for Existence of Microcrack Clouds in Monophase Ceramics

PB91-189704

101,082

POLYETHELENE

Development of Test Methods to Determine the Compatibility of Liquid Hazardous Materials with Polyethylene PB92-126457

100,943

POLYETHERS

Fourier Transform Infrared Spectroscopic Studies of the Degradation of One-Component Polyether Polyurethane Protective Coatings on Steel.

PB91-195248

101,108

POLYETHYLENE

Multistep Stress-Relaxation Behavior in Uniaxial Extension of an Ethylene-Hexene Copolyme

101,242

Molecular Dynamics of Initial Events in the Thermal Degradation of Polymers.

100,503

Long Time Creep and Lifetime Behavior in Uniaxial Ex tension of a Linear Low Density Polyethylene. PB91-236612

101,247

\section{OLYETHYLENE GLYCOLS}

Electrochemical Behavior of Ferrocene in Polyethylene Oxide-400.

OLYETHYLENE TEREPHTHALATE

Emissivity of Aluminized Mylar.

100,439

POLYIMIDE RESINS

Thermal Conductivity of a Polymide Film between 4.2 and $300 \mathrm{~K}$, with and without Alumina Particles as Filler PB91-144402

POLYISOPRENE

Small-Angle Neutron Scattering and Light Scattering Studies on the Miscibility of Protonated Polyisoprene/ Deuterated Polybutadiene Blends.

PB91-236786

100,513

Microstructure Effects on the Lower Critical Solution Temperature Phase Behavior of Deuterated Polybutadiene and Protonated Polyisoprene Blends Studied by

Small-Angle Neutron Scattering.
PB92-117266

100,518

POLYMER BLENDS

Spinodal Decomposition in a Hydrogen-Bonded Polymer

100,489

PB91-162099

Time-Resolved Small-Angle Neutron Scattering in Inter(DPB/HPI) Blends.

PB91-162131

100,490

Statistical Mechanical Model of Aqueous Two-Phase Systems.
PB91-174508

100,493

Small-Angle Neutron Scattering Studies of Compatible Blends of Linear Poly(Vinyl Methyl Ether) and CrossLinked Deuterated Polystyrene.

PB91-187153

100,494

Small-Angle Neutron Scattering of Blends of CrossLinked and Linear Polystyrene.

100,500

Polymers: Technical Activities 1991. (NAS-NRC Assessment Panel, February 13-14, 1992).

100,516

Microstructure Effects on the Lower Critical Solution Temperature Phase Behavior of Deuterated Polybutadiene and Protonated Polyisoprene Blends Studied by Small-Angle Neutron Scattering.

PB92-117266

100,518

Small-Angle Neutron Scattering and Light Scattering Study on the Miscibility of Poly(styrene-ran-butadiene) Polybutadiene Blends.

PB92-117274

100,519

\section{POLYMER MATRIX COMPOSITES}

Superconductor-Polymer Composites.

PATENT-4 954481

101,454

Polymer Composite Processing. Industry Workshop (2nd) Held at Gaithersburg, Maryland on May 18, 1990

101,115

Polymers: Technical Activities 1991. (NAS-NRC Assessment Panel, February 13-14, 1992).

100,516

Thermal Conductivity of Alumina Fiber/Epoxy and Alumina Fiber/PEEK from 4.2 to $310 \mathrm{~K}$

PB92-117258

101,130

\section{POLYMERIC FILMS}

Thermal Conductivity of a Polymide Film between 4.2 and $300 \mathrm{~K}$, with and without Alumina Particles as Filler.

PB91-144402

100,483

Measurement of Space Charge Fields in Polymers. 100,486

Study on the Nature of Boundary Lubricating Film: Analyt ical Method Developmen

PB91-189407

101,176

Protective Coatings for Tooth Crowns.

PB91-189555

100,047

Morphology of Polymer Films and Single Molecules.

PB91-203224

100,506

Reflection/Absorption FTIR Spectral Characteristics of Thin and Thick Crosslinked Epoxy Films on Steel Substrates.

100,509

Characterization of Epoxide Coatings on Steel by Reflection/Absorption Fourier Transform Infrared Spectroscopy: Quantitative Study

PB91-203729

101,109

Thin and Composite High-Flux Membranes of Perfluorosulfonated lon-Exchange Polymer.

100,512

POLYMERIZATION

Difunctional and Multifunctional Monomers Capable of Cyclopolymerization.

PB91-195685

100,504

Temperature Dependence of Probe Diffusion in Bulk Polymer Matrices.

100,422

Magnetic Behavior of Nanocomposites Prepared in a Vitreous Alumina Gel.

101,131

\section{POLYMERS}

Electrokinetic Demixing of Two-Phase Aqueous Polyme Systems. 1. Separation Rates of Polyethylene GlycolDextran Mixtures.

100,487

Spin Glass Magnetic Behavior of Iron/Silica Gel NanoComposites.

Polymers: Technical Activities 1990.

101,117

PB91-159715

100,488

Proceedings of Cable 89 Workshop.

100,773

Fluorescence Monitoring of Polymer Processing: Mixing and Zero Shear Viscosity

PB91-174482

100,492

Phenomenological Theory of the Influence of Strain History on the Rate of Isothermal Stress Relaxation. PB91-18738

100,496 Multistep Stress-Relaxation Study of a Single Crystal of n-Eicosane $(\mathrm{C} 2 \mathrm{OH} 42)$ in Torsion.

PB91-187427

100,497

Statistics of a Polymer Molecule in the Presence of Asymmetric Obstacles.

How Does Surface Roughness Affect Polymer-Surface Interactions.

100,341

Examination of Mechanisms Responsible for Incoherent Scattering of Subthermal Neutrons by Polymers.

PB91-202887

100,505

Simulation Studies of Excluded Volume Effects on Polymer Chain Dynamics in Several Nonlattice Models.

P891-203364

100,507

Tests of the Flory-Rehner Hypothesis: Comparison of the Elastic Free Energy Function for Crosslinked Rubber in

the Dry and Swollen States.
PB91-203570

100,508

Assessment of the State-of-the-Art for Process Monitoring Sensors for Polymer Composites.

100,510

Long Time Creep and Lifetime Behavior in Uniaxial Ex tension of a Linear Low Density Polyethylene.

PB91-236612

101,247

Semiempirical Theory of Relaxation: Concentrated Polymer Solution Dynamics.

PB91-236638

100,511

Heat Capacity and Other Thermodynamic Properties of Linear Macromolecules. 10. Update of the ATHAS 1980 Data Bank.

PB92-110196

100,515

Polymers: Technical Activities 1991. (NAS-NRC Assessment Panel, February 13-14, 1992).

100,516

Flexible Polymer with Excluded Volume at an Interacting Penetrable Surface of Variable Dimension: A Multiple epsilon Perturbation Theory.

PB92-116698

100,517

POLYMORPHISM

Crystalline and Glassy Phases of Transition-Metal - Metalloid System

PB91-204156

101,238

\section{OLYSTYRENE}

Chain Conformation of a Block Polymer in a Microphase Separated Structure.

Small-Angle Neutron Scattering of Blends of Cross Linked and Linear Polystyrene.

100,500

Study of the Linear Viscoelastic Properties of Cyclic Po lystyrenes Using Creep and Recovery Measurements.
PB91-195107 Morphology of Polymer Films and Single Molecules.

PB91-203224

100,506

Immobilization of Binding Proteins on Nonporous Sup ports Comparison of Protein Loading, Activity, and Stability.

101,308

POLYURETHANE FOAM 


\section{KEYWORD INDEX}

POSITRON-ATOM COLLISIONS

Optical Potential Approach to Electron and Positron Scattering from Noble Gases. 2. Neon.

PB91-149971

101,619

POSITRON EMISSION TOMOGRAPHY

Use of Cross-Validation as a Stopping Rule in Emission Tomography Image Reconstruction. PB92-116623

101,295

POSIX (PORTABLE OPERATING SYSTEM INTERFACE

FOR COMPUTER ENVIRONMENTS)

Interactive Video Software Portability: Migrating a DOS Application to POSIX.

PB92-117308

100,670

POTASSIUM

NIST Standards for Sodium and Potassium. PB91-158865

100,191

POTASSIUM CHLORIDE

Proposed New Electrolytic Conductivity Primary Stand ards for $\mathrm{KCl}$ Solutions.

PB91-187658

100,339

POTASSIUM IONS

Research at NBS in Direct Potentiometric Measurements in Blood.

PB91-148874

100,188

POTASSIUM PERMANGANATE

Base Modifications in Plasmid DNA Caused by Potassium Permanganate.

POTENTIAL ENERGY SURFACES

101329

Progress on the Determination of Intermolecular Potential Energy Surfaces from High Resolution Spectroscopy.
PB91-203695

100,403

POTENTIOMETERS (INSTRUMENTS)

Modified Sliding Wire Potentiometer Test Structure for Mapping Nanometer-Level Distances

100,821

POTENTIOMETRIC ANALYSIS

Research at NBS in Direct Potentiometric Measurements in Blood.

PB91-148874

100,138

POULTRY

Formation of ortho-Tyrosine by Radiation and Organic Solvents in Chicken Tissue.

PB92-116953

101,290

POWDER METALLURGY

Particle Size Measurement of Inert Gas Atomized Powder.

101,229

POWER MEASUREMENT

Device for Audio-Frequency Power Measurement PB91-149401

POWER SUPPLY CIRCUITS

Need for Power Ouality Testing Standards

POWER SYSTEMS

Evaluation of Instrumentation Used to Measure AC Power System Magnetic Fields.

PB91-162248

100,872

POWER TRANSMISSION LINES

Research for Electric Energy Systems: An Annual Report.
PB92-112341

POZZOLANS

Pozzolan Programs of the Cement and Concrete Reference Laboratory.

PRASEODYMIUM BARIUM CUPRATES

Mossbauer Study of the Effect of Oxygen Stoichiometry on the High Tc Superconductor Y1Ba2(CuO.97Fe0.03)3O7-x PB91-237545

101,558

PRE-MAIN SEQUENCE STARS

New Radio Detectors of Early-Type Pre-Main Sequence Stars.

100,024

PRECAST CONCRETE

Performance of $1 / 3$-Scale Model Precast Concrete Beam-Column Connections Subjected to Cyclic Inelastic Loads. Report No. 2

PB91-222570

100,149

Performance of Precast Concrete Beam-to-Column Connections Subject to Cyclic Loading.

100,150

PRECURSORS

Low Temperature Chemical Process for Precursors to .

101,066

Low Temperature Synthesis of Ceramic Powders for Structural and Electronic Applications.

101,067 PB91-149104

PREDISSOCIATION

Vibrational, Rotational, and Tunneling Dependence of Vibrational Predissociation in the HF Dimer.

PB91-203844

100,406

PRESSURE

Capillary Supercritical Fluid Chromatography of Exploalytes, the Mobile Phase and the Stationary Phase.
PB91-147769

100,182

RESSURE EFFECTS

Effect of High Pressure on Prebreakdown Phenomena in PB91-135020

100,839

PRESSURE GRADIENTS

Heat Transfer and Pressure Drop in a Compact Pin-Fin Heat Exchanger with Pin Orientation at $18 \mathrm{deg}$ to the Flow Direction.

PB91-194464

101,050

PRSURE MEASUREMENT

State-of-the-Art in Pressure Metrology.

101,038

$\mathrm{H} 2 \mathrm{O}(\mathrm{I})-\mathrm{H} 2 \mathrm{O}(\mathrm{III})-\mathrm{H} 2 \mathrm{O}(\mathrm{L})$ Triple Point of Water as a Fixed Pressure Point

PB91-187211

101,647

PRESSURE TRANSDUCERS

SHOOT Flowmeter and Pressure Transducers.

1.147488

100,923

PRESSURE VESSELS

Plastic Zone Formation Around an Arresting Crack.
PB91-189316

101,373

PB91-192997

101,049

PRESTRESSED CONCRETE

Computational Models Developed for the Corrosion of Prestressing Steel.

100,115

PRESTRESSING STEELS

Computational Models Developed for the Corrosion of Prestressing Steel.

100,115

PRICES

Energy Prices and Discount Factors for Life-Cycle Cost Analysis 1992. Annual Supplement to NIST Handbook and NBS Special Publication 709.

100,899

\section{RIMARY STANDARDS}

Proposed New Electrolytic Conductivity Primary Standards for $\mathrm{KCl}$ Solutions.

PB91-187658

100,339

PRINCETON ENGINE

Video Processing with the Princeton Engine at NIST.

PB92.108950

100,604

\section{PRISMATIC BODIES}

Alternate Representation of Prism Refraction for Light Incident at the Brewster Angle.

ROBABILITY THEORY

101,426

Engineers' Statistical Literacy is Key to U.S. Competitiveness.

100,038

PROBES

Temperature Dependence of Probe Diffusion in Bulk Polymer Matrices.

PB91-236885

100,422

PROBES (ELECTROMAGNETIC)

Evaluation of Dual-Port Circularly Polarized Probes for Planar Near-Field Measurements.

100,590

Probe for Real-Time Images of Particle Beams and Their Analyses in a Merged-Beams Apparatus.

101,683

Theoretical Considerations for a Thermo-Optic Microwave Electric-Field-Strength Probe.

100,860

PROCEDURES

Kelvin Water-Drop Experiment. N91-20219/2

100,900

POCESS COMPUTERS

Process Ouality Enhancement for Machine Tools: Measurements and

101,019

PROCESS CONTROL

Use of Neutron Pole Figures to Calibrate Ultrasonic Techniques for On-Line Texture Control of Aluminum

PB91-159111

101,222

Statistical Process Control Tactics for Coordinate Measuring Machines.

101,042

Surface Properties and Coatings of Materials Microtai-

lored Alloys.
PB91-189696 101,232

Process Ouality Enhancement for Machine Tools: Measurements and Standards.

its: Opportunilies and Challe Advanced Mate

100,009

Intelligent Processing of Materials, Technical Activities 1991. (NAS-NRC Assessment Panel, November 14-15, 1991).

100,995

Advanced Sensing of Materials Processing B 92.117423

100,949

\section{PROCESS MONITORING}

Assessment of the State-of-the-Art for Process Monitoring Sensors for Polymer Composites.

PB91-222612

100,510

PROCESSING \& PERFORMANCE OF MATERIALS

Polymer Composite Processing. Industry Workshop (2nd) Held at Gaithersburg, Maryland on May 18, 1990.
PB91-132191

101,115

Tew Temperature Chemical Process

PB91.149096

101,066

Low Temperature Synthesis of Ceramic Powders for Structural and Electronic Applications.

101,067

Concepts of Underground Corrosion.

PB91-150078

101,134

Convective and Morphological Instabilities during Directional Solidification.

Effects of Different $\mathrm{CH} 4-\mathrm{H}_{2}$ Gas Compositions on the Morphology and Growth of Diamond Grown by Hot FilaCVD.

Corrosion Behavior of Zirconium Alloy Nuclear Fuel Clad-

ding. 158733

101,372

Rapid Solidification Alloys: Crystals, Ouasicrystals, and Metallic Glass
PB91-158949

101,220

Chemical Modification of the Orthorhombic Superconductor $\mathrm{Ba} 2 \mathrm{YCu} 3 \mathrm{O} 7$-delta.

PB91-159053 101,486

Use of Neutron Pole Figures to Calibrate Ulirasonic Techniques for On-Line Texture Control of Aluminum

PB91-159111

101,222

Analysis and Characterization of Commercial Welding

PB91-162339

101,003

U.S. Assessment of the New Diamond Technology in

PB91.16739

01,107

Ignition Characte

Increased Facilitated Transport Related to Microstructural Changes in Heat-Treated lon-Exchange Membranes.

B91-174805

100,323

Study of Additive Response in a Series of Rerefined Base Oils Typical of Current Commercial Practice.

Low Temperature Chemical Route to Precursors of oride and Carbide Ceramic Powders.

101,078

Fe Implantation in In0.53Ga0.47As/InP.

101,516

Studies of Magnetic Flux Penetration in a Chemically ynthesized $\mathrm{Bi}(\mathrm{Pb}) \mathrm{SrCaCuO}$ Superconductor.

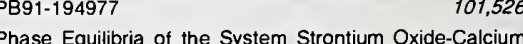

Phase Equilibria of

PB91-195628

101,085

Phase Selection in Non-Equilibrium Processing.

101,236

Low-Cost Aqueous Two-Phase System for Enzyme Ex- 


\section{KEYWORD INDEX}

PROPERTIES OF MATERIALS: STRUCTURAL/MECHANICAL

Proposed Testing Methodology for STEP Application Pro tocol Validation. National PDES Testbed Report Series.
PB92-112374

Multi-Enterprise Concurrent Engineering through International Standards.

$\begin{array}{ll}\text { PB92-123058 } & 100,997 \\ \text { Validation Testing Laboratory User's Guide. National }\end{array}$

$\begin{array}{ll}\text { Validation Testing Laboratory User's Guide. National } \\ \text { PDES Testbed Report Series. } & 100,971 \\ \text { PB92-123090 } & \end{array}$

Manufacturing Systems Integration: Initial Architecture Document.
PB92-112242

100,994

PRODUCTION PLANNING

Manufacturing Systems Integration: Initial Architecture Document.

100,994

PRODUCTIVITY

Research and Technology for Structural Engineering Productivity.
PB91-204206

100,147

PROFILOMETRY

Stylus Profiling at High Resolution and Low Force.

PB91-162354

101,015

PROGRAMMING LANGUAGES

American National Standard for C. Category: Software Standard. Subcategory: Programming Language.

FIPS PUB 160

Robot Sensor Language.

PB91-174961

100,676

PROJECT MANAGEMENT

NIST Support of the CALS Program: 1990 Synopsis. PB91-193821

101,031

PROPANE

Critical Locus, (Vapor + Liquid) Equilibria, and Coexisting Densities of (Carbon Dioxide + Propane) at Temperatures from $311 \mathrm{~K}$ to $361 \mathrm{~K}$

100,267

High-Resolution Infrared Flash Kinetic Spectroscopy of $\mathrm{OH}$ Radicals.

100,408

PROPELLANT TANKS

Reusable Cryogenic Foam Insulation for Advanced Aerospace Vehicles.

PB91-175570

100,560

PROPELLANTS

Fluorescence Monitoring of Polymer Processing: Mixing and Zero Shear Viscosity.

100,492

Chemical Kinetic Data Base for Propellant, Combustion 1. Reactions Involving NO, NO2, HNO, HNO2, HCN and
N2O

PB92-148071

100,467

PROPENES

Chemical Kinetic Data Base for Combustion Chemistry. Part 5. Propene.

100,446

PROPERTIES OF MATERIALS: ELECTRONIC/MAGNETIC/

PROPER
OPTICAL

High T(sub C) Superconductors: Are They Magnetic.

N91-20208/5 101,453

AC Loss Measurements of Two Multifilamentary $\mathrm{NbTi}$ Composite Strands.

100,769

Transport Critical Current of Aligned Polycrystalline Transport Critical Current of Aligned Polycrystalline
Y1Ba2Cu3O7-delta and Evidence for a Nonweak-Linked Y1Ba2Cu3O7-delta and Evidence for a Nonweak-Linked
Component of Intergranular Current Conduction.
PB91-133983 101,456

101,456 High T(sub c) Superconductors and the Critical Current
Measurement.
PB91-134056 Fundamental and Harmonic Susceptibilities of

YBa2Cu3O7-delta.
PB91-134148 101,458

Transition from Trichel-Pulse Corona to Dielectric Barrier Discharge.
PB91-134213

100,835

Break Junction Tunneling Spectroscopy of Single-Crystal Bismuth-Based High-Temperature Superconductors.
PB91-134478 PB91-134478 101,46

Magnetization of Anisotropic Superconducting Grains.
PB91-134569 Magnetoresistance of Multifilament Al/AI-Alloy Conduc-

tors.
PB91-134759 100,772

RIR - Measurement and Use in Ouantitative XRD.

PB91-147397 101,466

Hermetically Sealed Inert Atmosphere Cell for X-ray

Powder Diffraction.
PB91-147983

101,062

PBystal and Spin

101,470

Cryogenic Properties of Copper (Wall Chart).

PB91-148189

101,473

Use of Heterodyne Detection to Measure Optical Transmittance over a Wide Range.
PB91-148908

101,410

Possible 'Proximity Matrix' Route to High Current Conductors.

101,476

Comparison of Flux Dynamics in Two Sampies of YBa2Cu3O7 with Different Pinning.
PB91-149419 101,069 Hall Effect.

PB91-149898

101,479

Analysis of the $\mathrm{YBa} 2 \mathrm{Cu} 3 \mathrm{O} 7 / \mathrm{SrTiO} 3$ Interface as a Function of Post-Deposition Annealing Temperature.

PB91-149963

101,480

Amorphization and Conductivity of Silicon and Germanium Induced by Indentation.

101,482

Micromagnetic Calculations of 180 deg Surface Domain-

Micromagnetic Calculations of $180 \mathrm{deg}$ Surface Domain-
Wall Magnetization Profiles with Comparison to MeasureWall $M$

ments.
PB91-158501

101,483

Measurement of Space Charge Fields in Polymers

PB91-158626

100,486

National Bureau of Standards-American Ceramic Society

Phase Diagram Data Program

101,073

Spin Glass Magnetic Behavior of Iron/Silica Gel Nanocomposites.
PB91-159178

101,117

Electrodeposition of Aluminum from Molten Salts.
PB91-159202

Structural and Magnetic Properties of Er Thin Films and Er/Y Superlattices: Magnetoelastic Effects.

PB91-161935

101,489

Superconducting Properties of Bi2- $x$ -

yPbxSnySr2Ca

101,076

Anomalous Magnetoresistance in Al/Al-Alloy Composite

Conductors.
PB91-174748 101,631

Noise-Affected I-V Curves in Small Hysteretic Josephson Junctions.

Junctions.

100,782

Optical Levitation of Single Particles

PB91-174987

101,634

Semiconductor Claddings on Glass Waveguides for Poarizers and Detectors.

PB91-175141

101,416

Temperature Dependence of High Accuracy-Photometer

PB91-187096 101,418

Role of Elastic Energy in the Morphological Development of a Ni-Ti-Al Alloy.

101,227

Levitation of Superconducting Composites.

PB91-187351

101,503

Magnetic Ordering of Er in Powder and Single Crystals of ErBa2Cu307.

101,505

Refractive Index Gratings in Rare-Earth-Doped Alkaline Earth Glasses.

Superconducting Thin Films of Bi-Sr-Ca-Cu-O Obtained by Laser Ablation Processing.

101,508

Streaking Images That Appear Only in the Plane of Diffraction in Unoped GaAs Single Crystals: Diffraction Imfraction in Unoped GaAs Single Crystals: Diffraction Im-

101,511

PB91-189662

Subsidi-

Anomalous Low-Frequency Butterfly Curves for Subsidary and Ferromagnetic Resonance Overlap at $3 \mathrm{GHz}$.
PB91-194704

Current Record in Superconductors.

101,521

PB91-194753

System for the Study of Magnetic Materials and Magnetic Imaging with the Scanning Tunneling Microscope.
PB91-194779 Long-Range Antiferromagnetic Order of the $\mathrm{Cu}$ in Oxygen Deficient RBa2Cu3O6 + $x$

101,524

Crystal-Field Splittings in the Cubic Heusler Alloys HoPd2Sn and ErPd2Sn.

PB91-194902

101,525

Inverse Correlation between the Intensity of Luminescence Excited by Electrons and by Visible Light in Chemical-Vapor-Deposited Diamond Films.

PB91-195560

101,531

Optically Detected Magnetic Resonance Study of Antisite-to-Acceptor and Related Recombination Processes site-to-Acceptor and $R$

in as-Grown In

101,532

Photoluminescence Excitation by Band-Gap Optical Absorption in Chemical Vapor Deposition Diamond Films.

Morphology of Silver on YBa2Cu3O7-delta Thin Films.

PB91-195594 101,534

Enhanced Flux Creep in Nb-Ti Superconductors After an Increase in Temperature.

PB91-202903

101,680

Hall Probe Magnetometer for SSC Magnet Cables: Effect of Transport Current on Magnetization and Flux Creep.

Alternate Representation of Prism Refraction for Light Incident at the Brewster Angle.

PB91-203042

101,426

Switching in High-Tc Superconductor Current Transport Measurements

101,539

Progress in Field Ion Microscopy Imaging of High-Tc Superconducting Oxides.

PB91-203604

101,543

Atomic Fingerprint of YBa2Cu3O7-x-Type High-Temperature Superconductors Observed by Field Ion Microscopy.

Effect of Mechanical Deformation on $\mathrm{Nb}-\mathrm{Ti}$ Filament Proximity-Effect Coupling at the Edges of SSC Cables.
PB91-203828

Properties of YBa2Cu307-delta Thin Films Grown on Off-

Axis-Cut MgO Substrates

101,547

Magnetic Properties of Iron/Silica Gel Nanocomposites.
PB91-204073

High Resolution Synchrotron X-Radiation Diffraction Imaging of Crystals Grown in Microgravity and Closely Reined Terrestrial Crystals.

B91-216747

101,548

Small-Angle Neutron Scattering and Light Scattering Studies on the Miscibility of Protonated Polyisoprene/ Deuterated Polybutadiene Blends.

PB91-236786

100,513

Micromagnetic Calculations of $180 \mathrm{deg}$ Surface Domain

B $91-237271$

101,553

Change in Magnetic State of $\mathrm{Fe}+$ Silica Gel Nanocomposites Due to Low Temperature Treatment in Ammonia.
PB91-237321 New International Electrical Reference Standards Based on the Josephson and Ouantum Hall Effects. on the Joseph

100,740

Fourier Transform Infrared Analysis of Ceramic Powders: Quantitative Determination of Alpha, Beta, and Amorphous Phases of Silicon Nitride.

PB91-237628

101,096

Light-Scattering Measurement of the RMS Slopes of Rough Surfaces.

101,436

Theoretical Model for the Tunneling-Gap Anisotropy Observed in Layered Copper-Oxide High-Temperature Superconductors

B92-116565

Single-Target Magnetron Sputter-Deposition of High-Tc Superconducting Bi-Sr-Ca-Cu-O Thin Films. PB92-116680

101,566

Magnetic Behavior of Nanocomposites Prepared in a Vitreous Alumina Gel.

B92-117332

101,131

Antiferromagnetic Order of $\mathrm{Cu}$ in $\mathrm{Sm} 2 \mathrm{CuO} 4$.

PB92-117340

101,569

Low Temperature Magnetization and Magnetic Excitations in Amorphous Fe78B13Sig.

ROPERTIES OF MATERIALS: STRUCTURAL/

101,573

MECHANICAL

Influence of Water on the Mechanical Properties of a

Glass-Epoxy Matrix Composite.
AD-A231 778/2

101,113

Aluminum Alloys for ALS Cryogenic Tanks: Comparative Measurements of Cryogenic Mechanical Properties of Al- 


\section{KEYWORD INDEX}

PB91-134551

101,208

Calculation of

101.464

Report on 1989 Actions by International Institute of Weld-

ing. $91-134619$

101,001

Cryogenic Material Properties of Stainless Steel Tube-toFlange Welds
PB91-134627

101,145

Effect of Slag Penetration on the Performance of Magne-

sia Chrome Refractories in Slagging Gasifiers.

100,875

Structure of a Stage-3 Cs - Graphite Intercalate.

PB91-134957

100,269

Effect of Logarithmic Singularity on the Free Edge Stress Intensity Factor of Composite Laminates.

101,116

Phase Equilibria in the System TI-Ca-Ba-Cu-O 1 Stability Phase Equilibria in the System TI-Ca-Ba-Cu-O. 1. Stability
of the 2122 Phase under Conditions of Oxygen Annealing.

101,465

Incorporation of Molecular Orientation into Systems of Lamellar Morphology. 1. Effects of Packing Entropy on the Lamellar Thickness of Block Copolymers.

100,484 PB91-147231

ally An-

Phase Formation in

101,209

PB91-147314

Effect of Coherency Strain on Alloy Formation: Migration of Liquid Films

101,210

Dihedral Angles in Magnesia and Alumina: Distributions from Surface Thermal Grooves.

PB91-147330

101,058

Metal Reference Line Technique for Obtaining Dihedral Angies from Surface Thermal Grooves.

101,059

Application of Analytical Electron Microscopy to GlassBonded Aluminas.

PB91-147389 101,060

Ouantitative Determination of Amorphous Content in Ceramic Materials Using $X$-ray Powder Diffraction.

PB91-147553

101,061

Roughness Measures of Blasted Steel Surfaces Remotely Imaged with a Thermographic Camera.

101,146

Graphics Program for Binary and Ternary Ceramic Phase Diagrams.
PB91-147991

101,063

Study of the Galling of Two Steels Using Two Test Meth. ods.

101,147

Strength and Fracture Behavior of Ba-Y-Cu-O Superconducting Ceramics. ducting Ceramics.

101,474

Multistep Stress-Relaxation Behavior in Uniaxial Extension of an Ethylene-Hexene Copolymer.

PB91-148247

101,242

Neutron Powder Diffraction and Inelastic Scattering Study of the Structures of Zr2Pd, Zr2PdD1.70, and Zr2PdD1.96.
PB91-148866 Cleavage Step Formation and Resistance to Transgranular Stress Corrosion Cracking.

101,133

In situ Measurements of Bridged Crack Interfaces in the Scanning Electron Microscope.

PB91-149112

101,068

Tensile-Fracture Resistance Mechanisms in Brittle Polycrystals: An Ultrasonics and In-situ Microscopy Investiga-

tion.

101,190

Structural Instabilities and Superconductivity in OuasiBinary Mn5Si3-Type Compounds.

101,215

Mechanism for Shear Band Formation in the High StrainRate Torsion Test.

PB91-149872

101,579

Improved Analysis for Flexural Creep with Application to Sialon Ceramics.

Near Net Shape Forming of Ceramics.

101,071

PB91-158600

101,072

Corrosion Testing in Soil.

101,135

Using Surface Roughness as a Wear Criterion in HighSpeed Steel End-Milling.

101,040

New Different Forms of Ammonium Loaded and Partly Deammoniated Zeolite Rho Studied by Neutron Powder. PB91-158717 100,311

Heats of Immersion, Friction, and Wear of Base Oil Fractions.

101,173

Transformation of the Icosahedral Phase in Rapidly Transformation of the Icosahedral Phase in Rapidy
Ouenched Al-rich Al-Mn Alloys.
PB91-158964 101,221
PB91-158964 Phonon Density of States of Superconducting YBa2Cu3O6.
PB91-159129

101,487

Method for Ouantitative Measurement of Galling Dama1-15926

101,183

Tensile Creep Behavior of Structural Ceramics. PB91-159277

101,075

PB91-159715

100,488

Proceedings of Cable ' 89 Workshop.

100,773

Time-Resolved Small-Angle Neutron Scattering in Intermediate- and Late-Stage Spinodal Decomposition of (DPB/HPI) Blends.

PB91-162131

100,490

Mechanical Properties of Alumina-Peek Unidirectional Composite: Compression, Shear, and Tension.

101,118 Examination of the Excessive Retained Austenite on the
Surface of a Section of 17-7 Precipitation Hardening tainless Steel.

101,149

Intercomparison Study of Rockwell Hardness Test Blocks.

PB91-167338

101,224

Crack Arrest Fracture Toughness Measurements of Normalized and Inclusion Shape Controlled AAR TC128 Grade B Steel, and Micro-Alloyed, Control-Rolled, and Inclusion Shape Controlled A 8XX Grade B Stee:

B91-167346

101,150

Properties of Amorphous Zirconium Rhodium Hydride (Zr3RhHx) Prepared from Glassy and Crystalline Alloys.
101,225

Fluorescence Monitoring of Polymer Processing: Mixing and Zero Shear Viscosity.

PB91-174482

100,492

Measuring the Corrosion of Metals in Soil.

PB91-174714

101,136

$\mathrm{N}$-Dimensional Crystallographic Description of the Icosahedral Phases; the Example of the Al73Mn21Si6 Quasiperiodic Structure.

PB91-174771

101,226

ACTIS: Towards a Comprehensive Tribology Data Base.

ACT15: 174

101,184

Small-Angle-Scattering Determination of the Microstructure of Porous Silica Precursor Bodies.

101,077

Ion Incorporation into Octacalcium Phosphate Hydrolyzates

100,219

91-175125

Stress Corrosion Cracking of Al-Li Alloys: The Role of
Grain Boundary Precipitates. Grain Boundary Precipitates

Models for Oxygen Ordering and Diffusion in Ba2YCu3OX and $\mathrm{Ba} 2 \mathrm{YCu}_{\mathrm{C}}-\mathrm{yMyO}$ ( $\left.\mathrm{M}=\mathrm{Fe}, \mathrm{Co}, \mathrm{Al}, \mathrm{Ga}\right)$.

101,496

Chemistry of Electronic Ceramic Materials.

01500

Prediction of Elevated Temperature Deformation of Structural Steel under Anisothermal Conditions.

PB91-184838

101,154 terial Composite.

101,121

Small-Angle Neutron Scattering Studies of Compatible Blends of Linear Poly(Vinyl Methyl Ether) and CrossLinked Deuterated Polystyren

100,494

6-D Structural Model for the Icosahedral (Al, Si)-Mn Oua-

PB91-187294

101,501

Interlaminar Shear Fracture of Laminated Composites.

101,122

Processing Bi-Pb-Sr-Ca-Cu-O Superconductors from Amorphous State

PB91-187369

101,504

Phenomenological Theory of the Influence of Strain History on the Rate of Isothermal Stress Relaxation. 100,496 Design Diagrams for Heavy Metal Fluoride Glass Windows.

101,419

Multistep Stress-Relaxation Study of a Single Crystal of $n$-Eicosane $(\mathrm{C} 2 \mathrm{OH} 42)$ in Torsion.

100,497

Continuous-Cooling Transformation Characteristics and High-Temperature Flow Behavior of a Microalloyed SAE

PB91-187856

101,155

How Does Surface Roughness Affect Polymer-Surface Interactions.

PB91-189241

100,341

PBastic Zone

101,373

Study on the Nature of Boundary Lubricating Film: AnalytStid Method Development.

101,176

Structural Study of Electrodeposited Aluminum-Manganese Alloys.

PB91-189423

101,231

Structures and Properties of New Zeolite X-Type Zincophosphate and Beryllophosphate Molecular Sieves. 1-189449

101,081

Effects of Varying Austenitizing Temperature and Cooling Rate on the Ability of HSLA-80 Steel to Achieve a Yield Strength Comparable to HSLA-100.

PB91-189464

101,156

Ceramic Wear Maps.

100,561

Magnetic Hysteresis of High-Temperature YBa2Cu3OXAgO Superconductors: Explanation of Magnetic Suspen-

PB91-189506

101,507

Tribological Characteristics of Synthesized Diamond Films on Silicon Carbide.

101,186

Initial Stages of Degradation of Superconductor Surfaces: $\mathrm{O} 2, \mathrm{H} 2 \mathrm{O}, \mathrm{CO} 2$ and $\mathrm{CO}$ Chemisorption on La2-xSrxCuO4. PB91-189670 101,512

Electronic Structure of High-Tc Superconductors Studied Using Photoelectron Spectroscopy.

PB91-189688

101513

Fundamental Condition for Existence of Microcrack

$\begin{array}{ll}\text { Clouds in Monophase Ceramics. } & 101,082 \\ \text { PB91-189704 }\end{array}$

Effect of Crosslink Density on Physical Aging of Epoxy Networks.

Viscoelastic Response of Epoxy Glasses Subjected to Different Thermal Treatments.

PB91-189720

101,245

Phonon Density of States and Superconductivity in

991-189787

Physical Aging and the Viscoelastic Response of Network Glasses.
PB91-189829

Influence of In

Metallographic Study of the Crack-Tip Region from Fracture Mechanics Specimens of Austenitic and Ferritic

PB91-189951

101,158

Creep of Copper: 4 to $295 \mathrm{~K}$

PB91-189985

101,233

Sliding Wear Studies of Nickel-Copper Composition-Modulated Coatings on Steel.

PB91-190025

101,188

Indentation Determination of Crack Growth Parameters in

Synthesis of Non-lonic Water-Dispersible Resins for Use in Intaglio Inks Curing by Electron Beam Radiation. 194456

100,499 
Difunctional and Multifunctional Monomers Capable of Cyclopolymerization

100,504

Numerical Study of Two-Dimensional Crystal Growth Forms in the Presence of Anisotropic Growth Kinetics. Forms in the Presence of Anisotropic Growth Kinetics.
PB91-200865 Comparison of Creep Rupture Behavior in Tension and Bendin

PB91-202820

101,087

High Temperature Creep Testing of Ceramics.

Grain Boundaries in a 2-D Superlattice.

101,088

PB91-202853

100,388

Examination of Mechanisms Responsible for Incoherent Scattering of Subthermal Neutrons by Polymers. 100,505

Effect of Thermal Expansion Mismatch on Fiber Pull-Out in Glass Matrix Composites.

PB91-202952

101,125

Monitoring of Anisotropic Material Elastic Properties Using Ultrasonic Receiving Rays.

101,126

Morphology of Polymer Films and Single Molecules.
PB91-203224

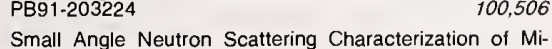

PB91-203489

101,089

Detection and Ouantitative Characterization of Blistering and Corrosion of Coatings on Steel Using Infrared Thermography.

101,139

Role of Glassy Interlaces in High Temperature Crack Growth in SiC Fiber Reinforced Alumina.

PB91-203687

101,127

Refinement of the Structure of Beta'-MoO3.

101,090 PB91-203786

Characterization of Tricresylphosphate Lubricating Films
by Micro-Fourier Transform Infrared Spectroscopy.
PB91-203810
101,179

PB91-203810

100,009

Advanced Materials: Opportunities and Challenges.

Dental Composite Resins: An Update.

PB91-204149

100,051

Crystalline and Glassy Phases of Transition-Metal - MePB91-204156

101,238

Creep and Reliability of Ceramic Materials at Elevated Temperatures
PB91-204172

101,091

Long Time Creep and Lifetime Behavior in Uniaxial Extension of a Linear Low Density Polyethylene.

Effect of Selected Chemical Compounds on the Lubrication of Silicon Nitride.

PB91-236711 101,180

Microstructural Control Through Diffusion-Induced Grain Boundary Migration.

101,239

Cyclic Fatigue from Frictional Degradation at Bridging Grains in Alumina.

101,092

Fracture Mechanics Model for Subthreshold Identation Flaws. Part 1. Equilibrium Fracture.

PB91-236935

101,093

Differential Sintering

101,128

Cathodoluminescence Measurement of Strained Alumina Single Crystals.
PB91-237131

101,094

New Layered Cuprate Structure-Type, (A1. XA'x) 14 Cu24O41.
PB91-237339 101,095

Structural Anomalies in Undoped Galtium Arsenide Observed in High Resolution Diffraction Imaging with Monochromatic Synchrotron Radiation.

PB91-237438

101,556

Mossbauer Study of the Effect of Oxygen Stoichiometry on the High Tc Superconducto Y1Ba2(CuO.97Fe0.03)3O7-x. Low-Field Flux Pinning in Twinned and Detwinned Single Crystals of YBa2Cu3O 7-x.

PB91-237552

101,559

Environmentally Enhanced Fracture of Gallium Arsenide.

PB91-237743 101.097 Network Structure in Epoxies. 6. The Growth Process In-

vestigated by Neutron Scattering
PB91-237842

100,514

NIST "LATTICE: A Program to Analyze Lattice Relation-

ships. Spring 1991 Version.

101,561

Mechanical Properties and Fracture Toughness of AAP TC128 Grade B Steel in the Normalized, and Normalized and Stress Relieved Conditions.

PB92-108901

101,169

High Resolution Diffraction Imaging of Crystals Grown in Microgravity and Closely Related Terrestrial Crystals.
PB92-109008

101,562

Static and Dynamic Strength Tests on Electrical Conductor Cables Specified for Airport Landing Structures. PB92-112226

Intelligent Processing of Materials, Technical Activities 1991. (NAS-NRC Assessment Panel, November 14-15,

PB92-112572

100,995

Polymers: Technical Activities 1991. (NAS-NRC Assessment Panel, February 13-14, 1992).

100,516

Thermodynamics of the Divalent-Metal Fluorides. 4. Heat Capacity of MSnF4, M $=\mathrm{Pb}, \mathrm{Ba}$, or Sr, at Temperatures from $300 \mathrm{~K}$ to $660 \mathrm{~K}$

PB92-116524

100,456

Buckling and Post-Buckling Behavior of Elliptical Plates: Part 1. Analysis.
PB92-116573

101,588

Buckling and Post-Buckling Behavior of Elliptical Plates. Part 2. Results

101,589

Flexible Polymer with Excluded Volume at an Interacting Penetrable Surface of Variable Dimension: A Multiple epsilon Perturbation Theory.

PB92-116698

100,517

interpretation of Field Ion Microscopy (FIM) Images of Asymmetrical Specimens of 1,2,3-Type High-Tc Superconductors.

101,567

Test Method for Tensile Creep of Structural Ceramics Using Flexure Beams.

PB92-116995

101,099

Characterization of the Densification of Alumina by Multiple Small-Angle Neutron Scattering

101,100

Surface X-ray Absorption Spectroscopy, EXAFS and NEXAFS, for the In situ and Ex situ Study of Electrodes. PB92-117035

100,460 Effect of Green Density and the Role of Magnesium
Oxide Additive on the Densification of Alumina Measured by Small-Angle Neutron Scattering.

101,101

XANES of Transition Metal Zinc-Blende Semiconductors.

XANES of Transition Metal Zinc-Blende Semiconductors.
PB92-117092

Microstructure Effects on the Lower Critical Solution Temperature Phase Behavior of Deuterated Polybutadiene and Protonated Polyisoprene Blends Studied by Small-Angle Neutron Scattering.

100,518

PB92-117266

Small-Angle Neutron Scattering and Light Scattering
Study on the Miscibility of Poly(styrene-ran-butadiene)/ Study on the Miscibiliy
Polybutadiene Blends.

Polybutadiene
PB92-117274

100,519

Effect of Surface Forces on Subcritical Crack Growth in

PBlass. 117449

101,103

Materials Reliability. Technical Activities, 1991. (NASNRC Assessment Panel, February 13-14, 1992). Ceramics: Technical Activities 1991. (NAS-NAC Assessment Panel, February 13-14, 1992)

PB92-126507

101, 104

High-Temperature Superconductivity: Abstracts of NIST Publications, 1987-1991.

PB92-126564

101,105

PROPERTIES OF MATERIALS: THERMODYNAMIC

TSPORT

Thermal Conductivity and Heat Capacity of Gaseous

PB91-134577 101,602

Thermal Conductivity of a Polymide Film between 4.2 and $300 \mathrm{~K}$, with and without Alumina Particles as Filler.

PB91-144402

100,483

Ignition Characteristics of the Nickel-Based Alloy UNS No7001 in Pressurized Oxygen.

100,536

Heat Capacity of Deuterated Ammonium Tetrafluoroborate ND4BF 4 from 7 to $348 \mathrm{~K}$

PB91-147058

100,273

Phase Diagrams of Hexagonal Binary Ordering Alloys with Anisotropic interactions.

101,211

Effects of Pressure on the Thermal Decomposition Kinetics and Chemical Reactivity of Nitromethane.

PB91-147884

100,286

Physical and Thermo-Mechanical Properties of Monoclinic Single Crystals.

101,065

Theory of Specific Heat of Solids.

101,475

Applicability of Smoluchowski's Equation with a Constant Kernel to Coalescence.

100,296

Relation between the Surface Energy and the Debye Relation between the Surface Energy and the Debye
Temperature for Cubic Solids.
PB91-149393 100,303

100,303

Thermodynamics of Ammonium Indates. 2. The Molar Heat Capacity of the Ammonium Pentabromoindate Monohydrate Salt (NH4)2 InBr5. H2O from 7.8 to $348 \mathrm{~K}$.

PB91-149831

100,304

Thermodynamics of the Divalent Metal Fluorides. 3. Heat Capacity of the Fast Ion Conductor Sr SnF4 from 6 to $344 \mathrm{~K}$

PB91-149864

100,305

Effect of Pyrophosphate Concentrations on Calcium Phosphate Growth on Well-Crystallized Octacalcium Phosphate and Hydroxyapatite Seed Crystals.

PB91-162024

101,193

Spinodal Decomposition in a Hydrogen-Bonded Polymer Blend.

PB91-162099

100,489

Speed of Sound of Natural Gas Mixtures.

PB91-162214

100,882

New Wide Range Equation of State for Helium PB91-162222

100,226

missivity of Aluminized Mylar.

Thermal Conductivity of Evacuated Perlite at Low Temperatures as a Function of Load and Load History.
PB91-174672 Critical Exponent for the Viscosity of Four Binary Liquids. Isothermal Phase Behavior of Ag3SbS3, ZnGeP2, and ZnS.

PB91-187237

101,080

Relationship of Hydrogen Site Occupancy to Diffusion Behavior in Crystalline and Amorphous Zr2PdHx.
PB91-187252

101,230

Mobility Fluctuations and Electrophoretic Light Scattering rom Macromolecular Solutions.

100,345

Experimental Thermal Conductivity, Thermal Diffusivity, and Specific Heat Values for Mixtures of Nitrogen, Oxygen, and Argon.

PB91-194472

100,369

Small-Angle Neutron Scattering of Blends of CrossLinked and Linear Polystyrene.

100,500

High-Temperature Thermal Properties of UNS $\$ 44004$ Using Multivariant Analysis.

PB91-194795

101,160

Simultaneous Butyltin Determinations in the Microlayer. Water Column and Sediment of a Northern Chesapeake Bay Marina and Receiving System.

PB97-195040

100,918

Semiempirical Theory of Relaxation: Concentrated Polymer Solution Dynamics.

100,511

Temperature Dependence of Probe Diffusion in Bulk Polymer Matrices.

PB91-236885

100,422

Mass Transfer Coefficients in Two-Phase Aqueous Extraction.

100,426

Survey of Current Worldwide Research on the Thermophysical Properties of Alternative Refrigerants. PB92-112366

101,198

Predictive Extended Corresponding States Model for

Pure and Mixed Refrigerants

101,199 


\section{KEYWORD INDEX}

Crystal Structure of a CAMP-Independent Form of Catabolite Gene Activator Protein with Adenosine Substituted in One of 2 CAMP-Binding Sites.

PB91-190074

101,305

PROTEINS

Neutron Crystallography of Proterns.

PB91-175349

101,285

Immobilization of Binding Proteins on Nonporous Supports Comparison of Protein Loading. Activity, and Stabili-

PB91-237198

101,308

Ab Initio Phase Determination for $\mathrm{X}$-ray Diffraction Data from Crystals of a Native Protein.

PB91-237362

101,309

PROTOCOL (COMPUTERS)

BACnet Communication Protocol for Building Automation Systems.

100,069

Draft Abstract Test Suite for Determining Conformance to the BACnet Protocol.

PB91-187732

100,070

PROTOCOLS

Performance of OSI Transport over ACCUNET and IBER-

100,563

Working Implementation Agreements for Qpen Systems interconnection Protocols

100,686

Application of QSI Protocols for Plant Information Net-

works.
PB91-147595

100,974

Toward Modular Semantic Application Layer Protocols (MS-ALPS): Communication between Heterogeneous Inormation Systems Using Artificial Intelligence (AI) Programming Techniques.

100,565

Stable Implementation Agreements for Qpen Systems Interconnection Protocols. Version 4, Edition 1, Decem. ber 1990.

PB91-171967

100,689

Validation of an QSI Transport Class 4 Simulator.
PB91-187724

Stable Implementation Agreements for Qpen Systems Interconnection Protocols. Version 4, Edition 1, March 1991. Change Pages (Supplement).

100,691

Development Plan: Application Protocols for Mechanical Parts Production. National PDES Testbed Report Series. Parts Production. National PDES Testbed Report Series.
PB91-222596 Stable Implementation Agreements for Qpen Systems Interconnection Protocols. Version 4, Edition 1, June 1991 Change Pages (Supplement)

100,695

Proposed Testing Methodology for STEP Application Protocol Validation. National PDES Testbed Report Series. PB92-112374
100,969 Government Qpen Systems Interconnection Profile Users' Guide, Version 2.

PB92-119676

100,696

Stable Implementation Agreements for Qpen Systems Interconnection Protocols. Version 4, Edition 1, Change 1991 OSI Implementors Workshop. Held in Gaithersburg, Maryland.

$\begin{array}{ll}\text { PB92-126408 100,697 } & \end{array}$

Working Implementation Agreements for Qpen Systems Interconnection Protocols.

100,698

PROTON AFFINITY

Proton Affinity of Cyanogen and Ion-Molecule Reactions of $\mathrm{C} 2 \mathrm{~N} 2(+)$.

100,352

Proton Affinities and pKa Values of Tetraalkylhydrazines. PB91-195214

100,378

PROTONS

Multicomponent Cluster lons. 2. Comparative Stabilities of Cationic and Anionic Hydrogen Bonded Networks: Mixed Clusters of Water and Hydrogen Cyanide. PB91-147710

Polarizability of the Photon.

PB91-203166

100,283

JBLIC BUILDINGS

Preliminary Dynamic Analyses of the Ministry of Agriculture Building.

UBLIC-KEY SYSTEMS

Public-Key Cryptography. Computer Security. B91-18786

PB91-147967

101,047

Analytical Model for the Refrigeration Power of the Qriice Pulse Tube Retrigerator.

PB91-187534

101,048

Qptimization of a Pulse Tube Refrigerator for a Fixed Compressor Swept Volume

101,051

PURINES

46Gas Chromatography-Mass Spectrometry of Free Radical-Induced Products of Pyrimidines and Purines in DNA.
PB91-187476

PYRENES

Cryogenic-Temperature Fluorescence Spectra of Polynuclear Aromatic Hydrocarbons of Molecular Weight 302

YRIDINES

Proton Affinities and pKa Values of Tetraalkylhydrazines.
PB91.195214

YRIMIDINES

46Gas Chromatography-Mass Spectrometry of Free Radical-Induced Products of Pyrimidines and Purines in DNA

PYRITE

Rate of Pyrite Bioleaching by 'Thiobacillus ferrooxidans' Results of an Interlaboratory Comparison.

PB91-195321

101,161

PYROCOCCUS FURIOSUS

Bıoprocessing of Fossil Fuels Using Hyperthermophilic Archaebacteria.

PYROLYSIS

Thermal Decomposition of Ions. 3. Protonated Ethano and Diethyl Ether.

PB91-195073

100,376

Kinetic Properties of the Components of Douglas-Fir and the Heat of Combustion of Their Volatile Pyrolysis Prod-

PB91-203794

100,548

Experimental Study of the Pyrolysis Rate of a Poly(methy methacrylate) (PMMA) Wall Panel in a Reduced-Scale

PB91-237412

100,555

Theoretical Investigation of Piloted Ignition of Wood. PB92-112432

100,556

PYROMETERS

High-Speed Spatial (Linear) Scanning Pyrometer: A Tool for Diagnostics, Temperature Mapping, and Properly Determinations at High Temperatures.

N91-21361/1

PYROPHOSPHATES

Effect of Pyrophosphate Concentrations on Calcium Phosphate Growth on Well-Crystallized Octacalcium Phosphate and Hydroxyapatite Seed Crystals. PB91-162024

101,193

PZT

Lead Zirconate-Titanate Thin Films Prepared by the Laser Ablation Technique.

PB91-187344

101,502

QUADRATIC PROGRAMMING

Interior-Point Method for Linear and Quadratic Programming Problems
PB91.187815

\section{QUALITATIVE ANALYSIS}

101,269

Biological Sulfur Qxidation and Reduction for Coal Sulfur Speciation and Desulfurization.

QUALITY

Industry and Government Strategies for Product Quality. PB91-148163

Need for Power Quality Testing Standards. PB92-117076

101,01

QUALITY ASSURANCE

NBS Quality Assurance Support: Current and Planned Services.

1.149088

Measurement Quality Assurance.

Laboratory Accreditation in the United States.

101,012

101,013

100.172

Process Quality Enhancement for Machine Tools: Measurements and Standards.

PB91-194993

101,019

Role of Reference Materials in Quality Assurance.

PB91-203901 100,206

Directory of State and Local Government Laboratory ACcreditation/Designation Programs.

100,174

PULSE COMPRESSION

Subpicosecond Pulse Compression and Raman Generation Using a Mode-Locked Erbium-Doped Fiber LaserAmplifier.

LSE TUBE REFRIGERATORS

Review of Pulse Tube Refrigeration.

PB91-147942

Apparatus for the Measurement of Regenerator Periormance in Pulse Tube Refrigerators.

PB91.216721

101,021

\section{QUALITY MANAGEMENT}

Interim Report to the President and to the Congress on PB91-167833

100,001

QUANTITATIVE ANALYSIS

Quantitative Analysis of Impurities in SIMQX Samples Using Secondary Ion Mass Spectrometry.

100,208

Fourier Transform Infrared Analysis of Ceramic Powders: Quantitative Determination of Alpha, Beta, and Amorhous Phases of Silicon Nitride.

PB91-237628

101,096

QUANTUM CHROMODYNAMICS

Model for the Non-Perturbative QCD (Quantum Chromodynamics) Vacuum

PB91-194407

101,661

QUANTUM ELECTRODYNAMICS

Relativistic Probability Amplitudes and State Preparation. PB91-195032

101,667

QUANTUM HALL EFFECT

Hall Effect.

101,479

Investigating the Use of Multimeters to Measure Quantized Hall Resistance Standards.

100,734

QUANTUM MECHANICS

New Test of Quantum Mechanics: Is Planck's Constant

101,632

Test of the Linearity of Quantum Mechanics by if Spectroscopy of the $(9) \mathrm{Be}(+)$ ) Ground State.

101,726

QUANTUM OPTICS

Quantum Qptics of Single, Trapped lons.

QUANTUM ZENO EFFECT

Reply to Comment on 'Quantum Zeno Effect'.

PB91-236828

101,628

QUARTZ RESONATORS

Environmental Sensitivities of Quartz Crystal Qscillators.
N91-25797/2

Environmental Effects on the Medium and Long Term Frequency Stability of Quartz Qscillators.

PB91-134866

100,781

QUASICRYSTALS

Rapid Solidification Alloys: Crystals, Quasicrystals, and

Metallic Glass.
PB91-158949

Transformation of the Icosahedral Phase in Rapidly Quenched Al-rich Al-Mn Alloys.

101,221

6-D Structural Model for the Icosahedral (Al, Si)-Mn Quasicrystal.

PB91-187294 101,501

Comparison of Amorphous and Quasicrystalline Films of Sputtered Al0.72Mno.22Sio.06.

UENCHING (COOLING)

Phase Selection in Non-Equilibrium Processing PB91-19565

101,530

OUINUCLIDINOL

Synthesis of 3-Quinuclidinol-(18)Q, Benzilic-d5 Acid, and 3-Quinuclidinyl-(18)Q, Benzilate-d5.

PB91-237370

100,223

RACETRACK MICROTRONS

Of What Use Is Basic Research.

PB91-175513

101,642

RADIATION

Formation of ortho-Tyrosine by Radiation and Organic Solvents in Chicken Tissue.

101,290

RADIATION CHEMISTRY

Radiation Chemistry of Enones.

PB91-147793

100,236

RADIATION CURING

Synthesis of Prototype Resins for Use as BEP Intaglio Ink Vehicles Curing by Electron Beam Radiation

PB91-144345

101,182

Effects of Absorbed Dose Rate, Irradiation Temperature and Post-Irradiation Temperature on the Gamma Ray Response of Red Perspex Dosimeters.

101,363 
NiST High-Dose Calibration Services

PB91-203257

101,689

Calibration of Ophthalmic Applicators at NIST: A Revised Approach.

RADIATION EFFECTS

Characteristics of the Breakdown Voltage of Power MOSFETs After Total Dose Irradiation.

PB91-149062

100,793

Intensifying Effect of Metallic Screens on the Sensitivity of X-ray Films for 400-kV Bremsstrahlung Photons.
PB91-195198

101,293

Intensifying Effect of Metallic Screens on the Sensitivity of X-ray Films at $662 \mathrm{keV}$.

PB91-195206

101,669

RADIATION ENHANCED DIFFUSION

Ion-Induced Radiation-Enhanced Diffusion of Silver in

PB91-175554

101,498

ADIATION MEASURING INSTRUMENTS

Center for Radiation Research. 1990 Technical Activities. PB91-178855

101,362

DIATION PRESSURE

Optical Levitation of Single Particles.

PB91.174987

101,634

RADIATION THERAPY

Metal-Polysiloxane Shields for Radiation Therapy of Maxillo-Facial Tumors.

100,054

RADIATIVE HEAT TRANSFER

Radiant Heat Fluxes from 100-200 MW Natural Gas/Air Diffusion Flames.

RADICALS

Rate Constants for Hydrogen Abstraction Reactions of the Sulfate Radical, SO4- Alkanes and Ethers

PB91-203232

100,394

RADIO BROADCASTING

NIST Time and Frequency Services.

ADIO SOURCES (ASTRONOMY)

Radio-Continuum Observations of a Variety of Cool Stars.

Extension of the Class of Magnetic B Star Nonthermal Extension of the Class of Magnetic B Star Nonthermal Radio Sources.

100,031

RADIOACTIVE WASTE FACILITIES

Model Validation at the Las Cruces Trench Site. NUREG/CR-5716

101,368

RADIOACTIVE WASTES

Assessment of Uncertainties in Measurement of $\mathrm{pH}$ in Hostile Environments Characteristic of Nuclear Repositor NUREG/CR-5711

101,367

RADIOACTIVITY

Preparation and Properties of Monomolecular Films for Use as Radioactive Source Mounts.

101,608

RADIOCARBON DATING

Accelerator (14)C Dating of Late Pleistocene Megafauna.
PB91-237396

Accelerator Radiocarbon Dates on Charcoal, Shell, and Human Bone from the Del Mar Site, California. PB91-237404

101,355

RADIOCHROMIC FILM

Radiochromic Film Dosimeter for Gamma Radiation in the Absorbed-Dose Range 0.1 - $10 \mathrm{kGY}$

PB92-116961

101,364

Photon Energy Dependence of the Sensitivity of Radiochromic Film and Comparison with Silver Halide Film and LiF TLDs Used for Brachytherapy Dosimetry.

101,296

ADIOGRAPHIC FILMS

Intensifying Effect of Metallic Screens on the Sensitivity of X-ray Films for $400-\mathrm{kV}$ Bremsstrahlung Photons. PB91-195198

101,293

RADIOGRAPHY

Real-Time Radiology Standards: Results of a Workshop.
PB91.147900

RADIOLOGY

Real-Time Radiology Standards: Results of a Workshop. PB91.147900

101,010

RADIOLYSIS

Mechanism of Cyanide Release in the Radiolysis of Ace-

tonitrile.
PB91-203679

100,240

RADIOMETERS

Temperature Monitored/Controlled Silicon Photodiodes for Standardization.

100,767

RADIOMETRIC ANALYSIS

Center for Radiation Research. 1990 Technical Activities. PB91-178855

101,362

RADIOMETRY

Electronic and Radiometric Characteristics of Near Infrared Photodiodes.
PB91-174706

100,752

ADIOSCOPY

Image Ouality Indicator Design for Radioscopy and ToPB91-190058

101,018

RADIOTHERAPY

Metal-Polysiloxane Shields for Radiation Therapy of Maxillo-Facial Tumors.

PB91-194761

100,054

RADIUS OF GYRATION

Chain Conformation of a Block Polymer in a MicrophaseSeparated Structur

100,485

RADON

Air Exchange and Pollutant Concentration Measurements

in Two Office Buildings.

100.909

RAILROAD CARS

Crack Arrest Fracture Toughness Measurements of Normalized and Inclusion Shape Controlled AAR TC128 Grade B Steel, and Micro-Alloyed, Control-Rolled, and in clusion Shape Controlled A 8XX Grade B Steel.

PB91-167346

101,150

Residual Stress Detection in Railroad Wheels: An Ultrasonic System Using EMATs (Electromagnetic-Acoustic Transducers). Report No. 23.

101,741

Tread Crack Detection in Railroad Wheels: An Ultrasonic

Tread Crack Detection in Railroad Wheels: An Ultrasonic System Using EMATs (Electromagnetic-Acoustic Transducers). Report No. 22

101,742

RAMAN SPECTRA

High-Temperature High-Pressure Raman Spectra from Liquid Water.

100,385

Semiclassical Calculation of Self-Broadening in O2, N2, and CO Raman Spectra.

PB92-11705

100,461

RAPID SOLIDIFICATION PROCESS

Rapid Solidification Alloys: Crystals, Ouasicrystals, and Metallic Glass

101,220

Role of Elastic Energy in the Morphological Development

of a Ni-Ti-Al Alloy.
PB91-187161

Structural Study of Electrodeposited Aluminum-Manga-

nese Alloys.

101,231

Phase Selection in Non-Equilibrium Processing.

PB91-195651

101,236

RARE EARTH ELEMENTS

Giant Resonances in the Transition Regions of the Periodic Table.

101,605

Development of a New Tracer Technology Using EnDevelopment of a New Tracer Technology Using En-
riched Rare-Earth Isotopes.
$\begin{array}{ll}\text { PB91-195347 } & 100,908\end{array}$

RARE GASES

Equilibrium and Transport Properties of Gas Mixtures at Low Density: Eleven Polyatomic Gases and Five Noble

$-192526$

100,363

RAYLEIGH SCATTERING

Temperature Dependence of Probe Diffusion in Bulk Polymer Matrices.

PB91-236885

100,422

REACTION KINETICS

Chemical Kinetic Data Base for Combustion Chemistry Part 4. Isobutane

100,252

Rate Constants for Reactions of Peroxyl Radicals in Fluid Solutions.

PB90-244641

100,261

Mechanism of Soot Formation in Acetylene-Oxygen Mix-

100,537

Alkyl Transfer Reactions between Protonated Alcohols and Ethers. The Gas-Phase Alkylation of Formaldehyde. PB91-147470 100,280

Slow Electron Transfer Reactions between Alkylhydrazines.

100.284

Effects of Pressure on the Thermal Decomposition Kinetics and Chemical Reactivity of Nitromethane.

100,286

Kinetics of Copper Extraction Using (anti)-2-Hydroxy-5Nonlybenzophenone Oxime.

PB91-148783

100,292

Influence of Sulfur on Methanation over Tungsten(110).

PB91-149369 100,300

Summary Abstract: The Influence of Sulfur on Methanation over $W(110)$

100,301

Zonal Model for Corona Discharge-Induced Oxidation of $\mathrm{SF} 6$ in $\mathrm{SF} 6 / \mathrm{O} 2 / \mathrm{H} 2 \mathrm{O}$ Gas Mixtures.

100,312

Group Reactivities in the Gas Phase Reactions of $\mathrm{Hy}$ droxyl Radicals with Ethers.

PB91-175059

100,330

Single Pulse Shock Tube Study of the Reactions of Hy drogen Atoms with Aromatics. 4. Chlorotoluenes.

PB91-175133

100,331

Concerning the Formation and the Kinetics of Phenylium

PB91-187138

100,336

Effects of $\mathrm{pH}$ and Calcium on Hydrolysis of Na2SiF6 and Na2SnF6: A Ouasi-Constant Composition Titration Study. PB91-194746

Atomistic Diffusion Equations and Kinetic Forces.

PB91.195008

100,374

Pate Constants for Reduction of Substituted Methylperoxyl Radicals by Ascorbate lons and N,N,N',N'-Tetramethyl-Para-Phenylenediamine.

100,379

Reactivities of Chlorine Atoms and Peroxyl Radicals Formed in the Radiolysis of Dichloromethane.

100,386

Rate Constants for Hydrogen Abstraction Reactions of the Sulfate Radical, $\mathrm{SO} 4$. Alkanes and Ethers.

PBg1-203232

100,394

Frequency Dependence of Catalyzed Reactions in a Weak Oscillating Field.

PB91-203927

100,407

High-Resolution Infrared Flash Kinetic Spectroscopy of $\mathrm{OH}$ Radicals.

100,408

Mechanisms of the Reduction Reactions of $\mathrm{Cr}(\mathrm{VI})$ in the Radiolysis of Acidic Potassium and Silver Dichromate Solutions in the Presence or Absence of Acetic Acid.

PB91-236489

Antioxidation Mechanisms of Uric Acid

PB91-237347

100,241

Stabilities of Substituted Benzyl Radicals: Dissociation PB91-23752

Surface Oxidation Kinetics of Si3N4 - 4\% Y2O3 Powders

Studied by Bremsstrahlung-Excited Auger Spectroscopy.

91-237701

100,435

Oxidation Kinetics of Silicon Carbide Whiskers Studied by $\mathrm{X}$-ray Photoelectron Spectroscopy.

100,436

Chemical Kinetic Data Base for Combustion Chemistry

Chemical Kinetic Data Base for Combustion Chemistry.
Part 5. Propene.

Kinetics and Mechanisms of the Gas-Phase Reactions of the NO3 Radical with Organic Compounds.

100,450

Chemical Kinetic Data Base for Propellant Combustion

Chemical Kinetic Data Base for Propellant, Combustion.
1. Reactions Involving NO, NO2, HNO, HNO2, HCN and 1. ReO

PB92-148071

100,467

Chemical Kinetic Data Sheets for High-Temperature Reactions. Part 2
PB92-14820

REACTIVITY

Reactivities of Chlorine Aloms and Peroxyl Radicals Formed in the Radiolysis of Dichloromethane.

100,386

REACTOR MATERIALS

Plastic Zone Formation Around an Arresting Crack

PB91-189316 
Dielectric Constant of Water and Debye-Huieckel Limiting Law Slopes.

100,260

Standard Reference Fibers for Calibration of the Optical Time Domain Reflectometer.

PB91-147207

101,406

Reference Materials: Their Role in Measurement Accura-

PB91-159103

101,014

Reference NO2 Calibration System for Ground-Based In tercomparisons during NASA's GTE/CITE II Mission. PB91-189340 00,034

Role of Reference Materials in Ouality Assurance. PB91-203901

100,206

Journal of Physical and Chemical Reference Data Volume 20, Number 1, 1991.

PB92-110113 100,440

Spectral Data and Grotrian Diagrams for Highly lonized Copper, Cu X-Cu XXIX.

100,441

Wavelengths and Energy Level Classifications of Magne sium Spectra for All Stages of Ionization ( $\mathrm{Mg}$ I through $\mathrm{Mg} X \mathrm{II})$.

PB92-110139

100,442

Spectroscopy and Structure of the Alkali Hydride Diatomic Molecules and Therr lons.

100,443

Journal of Physical and Chemical Reference Data

Volume 20, Number 2, March-April 1991.

100,445

Chemical Kinetic Data Base for Combustion Chemistry.

Part 5. Propene. Part 5. Propene.

Thermophysical Properties of Ethane

100,446

PB92-110188

100,447

Heat Capacity and Other Thermodynamic Properties of Linear Macromolecules. Data Bank

PB92-110196

100,515

Heat Capacities of Organic Compounds in Liquid State 2. C1 to $\mathrm{C} 18$ n-Alkanes.

PB92-110204

100,448

Journal of Physical and Chemical Reference Data, Volume 20, Number 3, May-June 1991

100,449

Kinetics and Mechanisms of the Gas-Phase Reactions of Kinetics and Mechanisms of the Gas-Phase Reactions of
the NO3 Radical with Organic Compounds.

PB92-110220 100,450

Thermodynamic Properties of the $\mathrm{NaBr}+\mathrm{H} 2 \mathrm{O}$ System.

PB92-110238 100,45

Silicon Reference Materials Certified for Isotope Abun-

PB92-126713

REFLECTOMETERS

Standard Reference Fibers for Calibration of the Optical Time Domain Reflectometer.

PB91-147207

100,213

REFRACTIVE INDEX

Refractive Index of Water and Steam as Function of

Wavelength Temperature and Density.

PB90-244708

101,402

REFRACTORIES

Rapporteur's Summary Materials Development for Coal Gasification.

PB91-158592

100,876

REFRACTORY MATERIALS

Thermodynamic and Kinetic Stability of Refractory Materials at Ultra-High Temperatures.

100,246

Elfect of Slag Penetration on the Performance of Magnesia Chrome Refractories in Slagging Gasifiers.

PB91-134940

100,875

REFRIGERANTS

Alternative Refrigerants R123a, R134, R141b, R142b and R152a: Critical Temperature, Refractive Index, Surface Tension, and Estimates of Liquid, Vapor, and Critical Densities.

101,189

Strategy for Chemical Analysis of Alternative Refriger-

ants.

100,185

Visualization of Nucleate Flow Boiling for an R22/R114
Mixture and lts Components.

PB91-162164

Experimentation, Analysis, and Correlation of Refrigerant22 Flow Through Short Tube Restrictors.

101,194

Research and Development of Heat Pumps Using Nonazeotropic Mixture Refrigerants

100,891

Pefrige Effects on Heat Pump Performance.

Refrigerant Charge Effects on Heat Pump Performance.
PB91-174607

Thermodynamic Properties of Two Alternative Refrigerants: 1,1-dichloro-2,2,2-trifluoroethane (R123) and 1,1,1,2-tetrafluoroethane $(\mathrm{R} 134 \mathrm{a})$

101,195

Study of Flow Boiling Heat Transfer with Refrigerant Mix Study
tures.
PB91-189563

101,196

Dipole Moments of Seven Partially Halogenated Ethane Refrigerants.

PB91-195131

100,377

PB91-236695

101,197

Survey of Current Worldwide Research on the Thermophysical Properties of Alternative Refrigerants.

101,198

Predictive Extended Corresponding States Model for Pure and Mixed Refrigerants

PB92-116748

101,199

Horizontal Flow Boiling Heat Transfer Experiments with a Mixture of R22/R114.

101,200

REFRIGERATION

Analytical Model for the Refrigeration Power of the Orifice Pulse Tube Refrigerator.

PB91.187534

101,048

REFRIGERATORS

Review of Pulse Tube Refrigeration

101,046

Apparatus for the Measurement of Regenerator Pertormance in Pulse Tube Refrigerators

101,047

Measurement of Regenerator Performance in a Vuilleumier Refrigerator.
PB92-116482

101,052

REGENERATIVE COOLING

Effect of Noble Gas Mixtures on the Periormance of Regenerative-Type Cryocoolers Analytical Estimate. PB91-144410

100,890

REGENERATORS

Apparatus for the Measurement of Regenerator Performance in Pulse Tube Refrigerators.

PB91-147967 101.047

Saran Carbon Cryogenic Regenerator for Liquid Helium. PB91-175430 101,640

Measurement of Regenerator Performance in a Vuilleumier Refrigerato

101,052

REGISTRATION

Ouestions and Answers on Ouality, the ISO 9000 Standard Series, Ouality System Registration, and Related

\section{REGULATIONS}

100,175

Uniforms Laws and Regulations as Adopted by the $\mathrm{Na}$ tional Conference on Weights and Measures (76th), 1991.

PB92-112416

101,022

Report of the National Conference on Weights and Measures (76th). Held in Philadelphia, PA. on July 14-19, PB92-112465

101,024

REINFORCED CONCRETE

Chloride Ion Diffusion in Low Water-to-Solid Cement Pastes

PB91-187690

101,138

REINFORCING STEELS

Chloride Ion Diffusion in Low Water-to-Solid Cement PB91-187690

101,138

\section{RELAXATION}

Relaxation of Crosslinked Networks: Theoretical Models and Apparent Power Law Behavior. PB91-195099

100,501

RELIABILITY

Materials Reliability. Technical Activities, 1990. (NASNRC Assessment Panel, January 31-February 1, 1991).
PB91-143339

101,008

Evaporator Performance Investigation for Residential AirConditioning Application Using Mixed Refrigerants. PB92-126432

100,893

RELIABILITY (ELECTRONICS)

Building-in Reliability: Making It Work.

100,812

Assessment of Reliability Concerns for Wide-Temperature Operation of Semiconductor Devices and Circuits.
PB92-116987

REMOTE BULLETIN BOARD SYSTEM

Guide to the Use of the Cement and Concrete Research Remote Bulletin Board System (RBBS) Computer. PB91-148528

REMOTE CONTROL

Low-Cost Low-Volume Carrier (Minilab) for Biotechnology and Fluids Experiments in Low Gravity. PB91-150029

100,041

REMOTE MANIPULATOR SYSTEM

Short-Term Evolution for the Flight Telerobotic Servicer. PB91-144352

101,731

REPLICATING

Replicate Measurements in the Interpretation of Environmental Monitoring

PB91-18976

100,919

RESEARCH

Journal of Physical and Chemical Reference Data,

Volume 19 , Number $3,1990$.

PB90-244658

100,262

NIST Research Reports, March 1991

PB91-187559

100,007

Journal of Physical and Chemical Reference Data olume 19, Number 6 November-December 1990. PB91-192542

100,365

Technical Activities 1990, Electron and Optical Physics Division.

Journal of Research of the National Institute of Standards and Technology. May-June 1991. Volume 96 (umber 3

PB91-216705

101,704

RESEARCH FACILITIES

BIR Facility User Handbook

PB92-102185

101,435

Research. Services. Facilities. (National Institute of Standards and Technology).

100,012

\section{RESEARCH MANAGEMENT}

Feasibility of Using Knowledge-Based Systems for Aiding Inventors.

Building Technology Publications 1986-1989

B91-216820

100,160

PB9 $1-237826$

100,081

RESEARCH PROGRAMS

Research. Services. Facilities. (National Institute of Standards and Technology)

PB92-109172

100,012

\section{RESEARCH PROJECTS}

Materials Reliability. Technical Activities, 1990. (NAS NRC Assessment Panel, January 31-February 1, 1991). 101,008 NBS Research Program in Municipal Solid Waste Com-

PB91-147264

100,916

UNIX in the Government's Automated Manufacturing Research Facility (AMRF)

100,963

Guide to the Use of the Cement and Concrete Research Remote Bulletin Board System (RBBS) Computer.

PB91-148528

00,524

Materials Research at the National Bureau of Standards.

101,214

Polymers: Technical Activities 1990

PB91-159715

100,488

Center for Radiation Research. 1990 Technical Activities. 101,362 Advanced Materials: Opportunities and Challenges. 
PB91-187336

101,122

Microstructure and Elastic Properties of Dental Resin and Resin-Based Glass-Reinforced Composites: XRD, SEM and Ultrasonic Methods.

PB91-204081

100,050

Dental Composite Resins: An Update.

100,051

Thermal Conductivity of Alumina Fiber/Epoxy and Alumina Fiber/PEEK from 4.2 to $310 \mathrm{~K}$.

PB92-117258

101,130

RESINS

Synthesis of Prototype Resins for Use as BEP Intaglio Ink Vehicles Curing by Electron Beam Radiation. PB91-144345

101,182

RESISTANCE STANDARDS

Investigating the Use of Multimeters to Measure Ouantized Hall Resistance Standards.

$\begin{array}{ll}\text { PB91-236554 100,734 } & \end{array}$

New International Electrical Reference Standards Based on the Josephson and Quantum Hall Effects.

PB91-237586

100,740

RESISTIVITY MAPPING

High Spatial Resolution Mapping of Semiconductor ReSistivity.

100,809

RESONANCE IONIZATION MASS SPECTROMETRY

Resonance Ionization Spectroscopy/Resonance Ionization Mass Spectrometry Data Service.

PB91-203968

100,207

RESONANCE IONIZATION MASS SPECTROSCOPY

Resonance Ionization Spectroscopy/Resonance Ionization Mass Spectrometry Data Service. II. Data Sheets for $\mathrm{Al}, \mathrm{Ca}, \mathrm{Cs}, \mathrm{Cr}, \mathrm{Co}, \mathrm{Cu}, \mathrm{Kr}, \mathrm{Mg}, \mathrm{Hg}$, and $\mathrm{Ni}$.

100,193 Observation of Anomalous Isotope Ratios in SIRIS Measurements of Molydenum.

PB92-116789

101,361

RESONANCE IONIZATION SPECTROSCOPY

Resonance Ionization Spectroscopy/Resonance Ionization Mass Spectrometry Data Service. II. Data Sheets for $\mathrm{Al}, \mathrm{Ca}, \mathrm{Cs}, \mathrm{Cr}, \mathrm{Co}, \mathrm{Cu}, \mathrm{Kr}, \mathrm{Mg}, \mathrm{Hg}$, and $\mathrm{N}$

100,193 PB91-162297

Resonance Ionization Spectroscopy/Resonance Ioniza-
tion Mass Spectrometry Data Service. PB91-203968

100,207

RESONANT TUNNELING

Resonant Tunneling with Electron-Phonon Interactions: An Exactly Solvable Model Applied to Desorption PB91-162065

100,317

RETINOL

Reverse-Phase HPLC Separation and Electrochemical Detection of Retinol and Its Isomers.

PB91-148858

100,187

REVERBERATION CHAMBERS

TEM/Reverberating Chamber Design/Concept Study: A Single Facility for Large System Radiated EMC Testing, $10 \mathrm{kHz}-40 \mathrm{Ghz}$

100,845

TEM Driven Reverberating Chamber, a Single Facility for

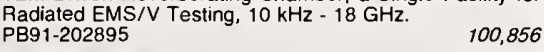

00,856

Application of Osmotic Dewatering to the Controlled Crystallization of Biological Macromolecules and Organic Compounds.

100,230

REVIEWS

Literature Review of the Galling Process.

PB91-134551

101,208

Book Review: Hypertext Hands-On

PB91-237040

100,656

REYNOLDS NUMBER

Reynolds Number Effects on the Mixing Behavior of Axisymmetric Turbulent Jets.

PB92-117209

100,015

RHEOLOGICAL PROPERTIES

Study of the Linear Viscoelastic Properties of Cyclic Polystyrenes Using Creep and Recovery Measurements.
PB91-195107

RHODIUM 103 TARGET

Spectrum-Averaged Cross-Section Measurement of $(103) R h(n, n)(103 m) R h$ in the $(252) C f$ Fission Neutron Spectrum.

PB91-203406

101,694

RHODIUM ALLOYS

Properties of Amorphous Zirconium Rhodium Hydride

(Zr3RhHx) Prepared from Glassy and Crystalline Alloys.
PB91-174466

PIBONUCLEASES

Crystal Structure of Phosphate-Free Ribonuclease PB91-175604

101,301

RISK ASSESSMENT

Concept of the Calculus of Fire Safety.

PB91-132134

100,093

ROBOT ARMS

Control of Flexible Robot Arm.

PB91.174979

101,032
ROBOT DYNAMICS

New Approach to Vision and Control for Road Following.
PB91-157156

Performance Measures of a Robotic Micropositioner. PB92-117019

101,037

ROBOT SENSORS

Robot Sensor Language.

PB91-174961

101,031

\section{OBOT VISION}

Real-Time Model-Based Tracking Combining Spatial and Temporal Features.

100,700

ROBOTICS

Short-Term Evolution for the Flight Telerobotic Servicer. PB91-144352 101,731

Real-Time Hierarchical Planning for Multiple Mobile

$\begin{array}{ll}\text { Robots. } & 101,375 \\ \text { PB91-158782 } & \end{array}$

Design and Implementation of a Five-Axis Robotic Micromanipulator for Inserting Parts into Precision Collets.

PB91-159194

101,029

Video Compression for Remote Vehicle Driving.

PB91-203208

101,035

Description of a Vibration Compensation System for the Small Scale Model Robot Crane Project.

PB91-216648

100,130

Performance Measures of a Robotic Micropositioner PB92-117019

101,037

ROBOTS

TROI User's Guide.

PB91-157214

101,026

Self-Adjustment of a Robot Joint Controller.

PB91-158568

101,027

Application of the Joint Excitation Gear Adjustment Technique to Low Frequencies and Heavy Robot Joints.

PB91-158576

101,028

Robot Characterization Testing.

PB91-16736

101,030

U.S. Manufacturing Systems: Factories Past, Present, and Future.

NASREM Robot Control System and Testbed.

PB91-194969

CAD Directed Robotic Deburring.

PB91-195172

101,033

Automating Robot Programming in the Cleaning and De-

burring Workstation of the AMRF
PB91-195495

100,986

Application of the PIPE Image Processing Machine to Scanning Microscopy.

ROCKWELL HARDNESS

Intercomparison Study of Rockwell Hardness Test

PB91-167338

101,224

Microstructure, Composition, and Hardness of Rockwell $C$ Hardness Blocks.

PB91-184747

101,153

ROD BUNDLES

Effect of the Location of an In-Line Tube Bundle on Orifice Flowmeter Performance.

PB91-134445

100,921

Choosing Flow Conditioners and Their Location for Orifice Flow Measurement.

PB92-116490

101,397

ROOFING

Further Investigation of the Effect of Application Parameters on Adhesive-Bonded Seams of Single-Ply Roo Membranes.

100,105

Pulse-Echo Ultrasonic Evaluation of the Integrity of Seams of Single-Ply Roofing Membranes: Laboratory Evaluation of a Prototype Test Apparatus.

100,106 PB91-157172

Pulse-Echo Ultrasonic Evaluation of the Integrity of
Seams of Single-Ply Roofing Membranes: Results of Seams of Single-Ply Roofing Membranes:

100,110 PB91-159731

Use of Nondestructive Methods for Inspection of Single-
Ply Roofing Membranes. Ply Roofing M

100,128

ROOFS

Field Study of the Performance of EPDM Roofing at Air Force Facilities.

101,340

Field Testing of Adhesive-Bonded Seams of Rubber Roofing Membranes.

100,124

ROOM FIRES

Data for Room Fire Model Comparisons.

PB92-110287

100,167

RUBBER

Tests of the Flory-Rehner Hypothesis: Comparison of the Elastic Free Energy Function for Crosslinked Rubber in the Dry and Swollen States.

100,508

Long Term Performance of Rubber in Seismic and Non Seismic Bearings: A Literature Review.

PB91-20671

100,148

RUBBER ADHESIVES

Field Testing of Adhesive-Bonded Seams of Rubber

Roofing Membranes.

PB91-189993

100,124

RUBIDIUM FREQUENCY STANDARDS

Rubidium Frequency Standard and a GPS Receiver: A Remotely Steered Clock System with Good Short-Term and Long-Term Stability.

PB91-200808

100,581

RUTHENIUM COMPOUNDS

Radiolytic Studies of the Redox Reactions of Ruthenium Porphyrins.

PB91-203661

100,402

RUTHENIUM OXIDES

Polyelectrolyte Stabilized Metal Oxide Hydrosols as Catalysts for the Photooxidation of Water by Zinc Porphyrins.
PB91-189852

RYDBERG STATES

Resonance Enhanced Multiphoton Ionization Spectra of the $\mathrm{GeF}$ and $\mathrm{GeCl}$ Radicals from $400-500 \mathrm{~nm}$

PB91-203281

100,396

SAMARIUM COMPOUNDS

Antiferromagnetic Order of $\mathrm{Cu}$ in $\mathrm{Sm} 2 \mathrm{CuO} 4$

PB92-117340

101,569

SAMARIUM OXIDES

Resonant Photoemission and PSD of Rare Earth Oxides. PB91-148015

100,287

Rare Earth Elements.

PB91-195313

100,383

SAMPLE PREPARATION

New Way to Mount Particulate Material for Laser Micro probe Mass Analysis.

PB92-116805

100,911

AW DEVICES

Surface Acoustical Wave Spectroscopy for High Humidity Sensing.

100,843

SCANDIUM

Rapid Low-Temperature Hopping of Hydrogen in a Pure Metal: The $\mathrm{ScH} x$ System.

PB91-158485

101,217

SCANNING ELECTRON MICROSCOPY

Low Accelerating Voltage SEM Imaging and Metrology Using Backscattered Electrons.

PB91.147926

101,469

Submicrometer SEM Magnification Standard.

100,799

Microchannel-Plate Detection Systems for Low Accelerating Voltage SEM

PB91-189928

101,515 


\section{KEYWORD INDEX}

PB91-236513

100,814

SECURITY

Standards for the Physical Protection of National Resources and Facilities.

SEDIMENTS

100,006

Preparation and Analysis of a Marine Sediment Reference Material for the Determination of Trace Organic Constituents.
PB91-149237

101,377

Laser Enhanced Ionization as a Selective Detector for Liquid Chromatographic Determination of Alkyltins in PB92-116755

100,210

SELENIUM IONS

Relative Populations of Excited Levels within the Ground Configuration of Si-Like Cu, Zn, Ge, and Se lons.
PB91-158618

ELF ADAPTIVE CONTROL SYSTEMS

Self-Adjustment of a Robot Joint Controller.

PB91-158568

101,443

\section{SEMICONDUCTOR DEVICES}

NBS Submicron Particle Standards for MicrocontaminaPB91-

Center for Electronics and Electrical Engineering Technical Progress Bulletin Covering Center Programs, July to September 1990, with 1991 CEEE Events Calendar.
PB91-159749

Center for Electronics and Electrical Engineering Technical Publication Announcements Covering Center Programs, July to September 1990, with 1991 CEEE Events Calendar.

PB91-184754

100,852

Pattern Generation on Semiconductor Surfaces by a Scanning Tunneling Microscope Operating in Air.

Prospects for High Temperature Superconductor - Semiconductor Contacts.

PB91-203646

101,545

Assessment of Reliability Concerns for Wide-Tempera

ture Operation of Semiconductor Devices and Circuits.
PB92-116987

SEMICONDUCTOR INDUSTRY

Structural Change and Competitiveness: The U.S. Semiconductor Industry.

PB91-195693

100,173

Metrology for

100,817

SEMICONDUCTOR LASERS

Modulatable Narrow-Linewidth Semiconductor Lasers.
PB91-134106

Diode Lasers and Their Application to Spectroscopy.
PB92-116904

SEMICONDUCTOR SWITCHES

Investigation of Photoconductive Picosecond Microstri-

pline Switches on Self-Implanted Silicon on Sapphire

(SOS)

PB91-147918

100,791

SEMICONDUCTORS

Low Accelerating Voltage SEM Imaging and Metrology $\begin{array}{ll}\text { Using Backscattered Electrons. } & 101,469\end{array}$ Modeling Electron Beam Interactions in Semiconductors. PB91-159020 101,485 Narrow-Gap Semiconductors and Related Materials.

PB91-162313 101,492

SEM Imaging and Metrology at Low Accelerating Voltages Using Backscattered Electrons.
PB91-195461

101,529

SEMICONDUCTORS (MATERIALS)

Effects of Crystal Bonding on Brittle Fracture.
PB91-148221

New Layered Cuprate Structure-Type, (A1$\begin{array}{ll}\left.X A^{\prime} X\right) 14 C u 24041 . & 101,095 \\ \text { PB91-237339 } & \end{array}$

SENSORS

Eccentric Load Sensitivity of Force Sensors

PARATION

Hydrodynamics and Mass Transfer in Two-Phase Aqueous Extraction Using Spray Columns.

PB91-149229

101,300

SEPARATION PROCESSES

Chitin-Chitosan Membranes: Separations of Amino Acids and Polypeptides.

101,283

Electrokinetic Demixing of Two-Phase Aqueous Polymer Systems. 1. Separation Rates of Polyethylene Glycol. Dextran Mixtures.

PB91-159095

100,487

Adsorption and Orientation of Tetracycline on Hydroxyapatite.

101,319

Electrokinetic Demixing of Two-Phase Aqueous Polyme Systems. 2. Separation Rates of Polyethylene Glycol-Maltodextrin Mixtures.
PB91-237230

100,231

SERVICE LIFE

Predicting the Remaining Service Life of Concrete.
PB92-126598

SET THEORY

Computer Implementation of a Discrete Set Algebra.

PB91-231571

100,654

SETTLEMENT (STRUCTURAL)

Proposed Design Criteria for Shallow Bridge Foundations.

SGML (STANDARD GENERALIZED MARKUP LANGUAGE) Standard Generalized Markup Language Encoding of the Office Document Architecture Document Application ProPB91-184812

101,345

Difficulties in Parsing SGML.

PB91-189456

100,650

SHAPE RESONANCES

Shape Resonances in Molecular Fields

PB91-187435

100,338

SHEAR PROPERTIES

Mechanism for Shear Band Formation in the High StrainRate Torsion Test

SHIELDING

Metal-Polysiloxane Shields for Radiation Therapy of Maxillo-Facial Tumors.

PB91-194761

100,054

SHIPBOARD FIRE CONTROL

Navy Safety Center Data on the Effects of Fire Protection Systems on Electrical Equipment.

101,376

SHIPPING CONTAINERS

Development of Test Methods to Determine the Compat ibility of Liquid Hazardous Materials with Polyethylene PB92-126457

100,943

SHPOL'SKII EFFECT

Cryogenic-Temperature Fluorescence Spectra of Polynuclear Aromatic Hydrocarbons of Molecular Weight 302. PB91-147157

SIALON

Improved Analysis for Flexural Creep with Application to Sialon Ceramics

PB91-150037

101,071

Visibility of Exit Signs and Directional Indicators. PB91-161984

100,083

SIGNAL PROCESSING

Root Projection of One-Sided Time Series.

101,262

Deconvolution of Acoustic Emission and Other Causa Time Series.

101,263

SILANE

Causes of SiH4 Dissociation in Silane dc discharges.

Silane Dissociation Products in Deposition Discharges

Silane Dissociation Products in Deposition Discharges.
PB91-202994

SILICA

Small-Angle-Scattering Determination of the Microstructure of Porous Silica Precursor Bodies.

101,077

Method to Enhance Porosity of Micro-particles.

PB91-187328

101,185

Small Angle Neutron Scattering Characterization of Microporous Silica.

101,089

Apparent Thermal Conductivity of Fumed-Silica Standard Reference Materials.

PB92-11735

101,102

SILICA GEL

Spin Glass Magnetic Behavior of Iron/Silica Gel NanoPB91-159178

101,117

Magnetic Properties of Iron/Silica Gel Nanocomposites.

Change in Magnetic State of Fe+ Silica Gel Nanocom

posites Due to Low Temperature Treatment in Ammonia.
PB91-237321

SILICA GLASS

Fracture Mechanics Model for Subthreshold Identation Flaws. Part 1. Equilibrium Fracture.

PB91-236935

101,093

Effect of Surface Forces on Subcritical Crack Growth in Glass

101,103

SILICON

Verification of the Relation between Two-Probe and FourProbe Resistances as Measured on Silicon Wafers.

PB91-147520

100,788

Secondary Ion Yield Changes in Silicon and Gallium Arsenide Due to Topography Changes during Oxygen or

101,471

Comparison of High-Resistivity Measurements of Silicon by AC Impedance, DC Resistance, van der Pauw, and Four-Probe Methods.

PB91-148197

100,792

Amorphization and Conductivity of Silicon and Germanium Induced by Indentation.

101,482

High Spatial Resolution Mapping of Semiconductor Re-

PB91-194829

100,809

Lattice Relaxation in Silicon Doped with 4d- and 5d-Transition Metals.

101,541

Quantitative Analysis of Impurities in SIMOX Samples Using Secondary Ion Mass Spectrometry.

100,208

Surface Extended-X-ray-Absorption Fine Structure and Scanning Tunneling Microscopy of Si(001)2x1-Sb.
PB91-237263 Theory of Ballistic-Electron-Emission Spectroscopy of NiSi2/Si(111) Interfaces.

PB91-237453

101,557

Round-Robin Study of Implants in SI and SiO2 by SIMS, RBS, and NAA.

100,830

Kinematic Theory of Ballistic Electron Emission Spectroscopy of Silicon-Silicide Interfaces.

PB92-117373

101,570

\section{SILON CARBIDES}

Thermodynamic and Kinetic Stability of Refractory Materials at Ultra-High Temperatures.

100,246

Low Temperature Chemical Process for Precursors to Boride and Carbide Ceramics Powders.

101,066

Low Temperature Synthesis of Ceramic Powders for Structural and Electronic Applications.

101,067

Tribological Characteristics of Synthesized Diamond Films on Silicon Carbide.

PB91-189530

101,186

Effect of Thermochemical Treatments on the Strength and Microstructure of SiC Fibers.

101,084

Comparison of Creep Rupture Behavior in Tension and Bending

PB91-202820

101,087

High Temperature Creep Testing of Ceramics.

101,088

Creep and Reliability of Ceramic Materials at Elevated Temperatures

101,091

Oxidation Kinetics of Silicon Carbide Whiskers Studied by X-ray Photoelectron Spectroscopy.

100,436

SILICON COMPOUNDS

Scanning tunneling microscopy studies of the surfaces of a-Si:H and a-SiGe:H films. Annual report, 1 December 1989-31 January 1991. DE91002169

100,785

SILICON DIOXIDE

Evaluation of Fumed-Silica Insulation for a Thermal Conductivity Standard Reference Material.

PB91-147975

100,103

Determination of Bonded Phase Thickness in Liquid Chromatography by Small Angle Neutron Scattering.
PB91-149187 190 Electron Attenuation Lengths at SiO2/Si Interfaces.

PB91-175612 101,499 Thermal Conductivity of Fumed Silica Board at Room

Temperature.
PB91-190124 100,125

Ellipsometry SRM's for Use in Thin Film Measurements.

Round-Robin Study of Implants in $\mathrm{Si}$ and $\mathrm{SiO} 2$ by SIMS, RBS, and NAA

SILICON ISOTOPES

Silicon Reference Materials Certified for Isotope Abundances.

100,213

ICON NITRIDES 


\section{KEYWORD INDEX}

SOLUTIONS

Evidence for Surface alpha Particle Clusters in (nat)AG(197)Au from the (e,alpha) Reaction

101,705

SILVER COATINGS

Possible 'Proximity Matrix' Route to High Current Conductors.

PB91-148932

101,476

SILVER INORGANIC COMPOUNDS

Isothermal Phase Behavior of Ag3SbS3, ZnGeP2, and

101,080

SILVER STRII

Determination of Fields Near a Silver Strip on a Glass Substrate.

SIMOX

Sensitivity of Ellipsometric Modeling to the 'Islands' of Silicon Precipitates at the Bottom of the Buried Oxide Layer in Annealed SIMOX.

PB92-116607 100,820

Persistent Photoconductivity in SIMOX Films.

PB92-117084

100,826

Residual Defects in SIMOX: Threading Dislocations and Pipes

100,829

SIMPLE CUBIC LATTICES

Relation between the Surface Energy and the Debye Temperature for Cubic Solids.

PB91-149393

100,303

SINGLE CRYSTALS

Physical and Thermo-Mechanical Properties of Monoclinic Single Crystals

Neutron Crystallography of Proteins.

101,065

PB91-175349

101,285

Isothermal Phase Behavior of Ag3SbS3, ZnGeP2, and

101,080

PB91-187237

Multistep Stress-Relaxation Study of a Single Crystal of $n$-Eicosane $(\mathrm{C} 2 \mathrm{OH} 42)$ in Torsion.

100,497

Cathodoluminescence Measurement of Strained Alumina Single Crystals
PB91-237131

101,094

SINGLE-PLY ROOFING MEMBRANES

Pulse-Echo Ultrasonic Evaluation of the Integrity of Seams of Single-Ply Roofing Membranes: Laboratory Evaluation of a Prototype Test Apparatus.

100,106

Pulse-Echo Uitrasonic Evaluation of the Integrity of Pulse-Echo Ultrasonic Evaluation of the Integrity of
Seams of Single-Ply Roofing Membranes: Results of Seams of Single-Ply Roofing Membranes: Results of
Field Investigations and Recommendations.

100,110

SINTERED MATERIALS

Differential Sintering.

PB91-237123

101,128

SINTERING

Reduction in Sintering Damage of Fiber Reinforced Com-

PBS1-195362

101,124

SITE SURVEYS

Accelerator Radiocarbon Dates on Charcoal, Shell, and Human Bone from the Del Mar Site, California PB91-237404

101,355

SLOVAK METROLOGICAL SOCIETY

Slovak Metrological Society

PB92-126721

100,938

SMALL ANGLE SCATTERING

High-Resolution Small-Angle X-ray Scattering Camera for Anomalous Scattering.

100,929

Small-Angle-Scattering Determination of the Microstructure of Porous Silica Precursor Bodies.

101,077

Small-Angle Neutron Scattering Studies of Compatible Small-Angle Neutron Scattering Studies of Compatible
Blends of Linear Poly(Vinyl Methyl Ether) and CrossLinked Deuterated Polystyrene.

PB91-187153

100,494

Small-Angle Neutron Scattering of Blends of CrossLinked and Linear Polystyrene.

100,500

Examination of Mechanisms Responsible for Incoherent Examination of Mechanisms Responsible for Incoherent
Scattering of Subthermal Neutrons by Polymers. Scattering of Subthermal Neutrons by Polymers.
PB91-202887 Small Angle Neutron Scattering Characterization of Microporous Silica.

101,089

SMALL BUSINESSES

Transferring NBS Technology to Small Manufacturers Through State and Local Centers.

PB91-149351

101,025

SMOKE

Programmer's Reference Manual for CFAST, the Unified Model of Fire Growth and Smoke Transport.

100,096

Refinement of a Model for Fire Growth and Smoke Transport.
PB91-148510

100,104

Compartment Fire-Generated Environment and Smoke Filling.

100,107

Smoke Production and Properties.

PB91-175422

100,120

Engineering Analysis of Fire Development in the Hospice of Southern Michigan, December 15, 1985.

100,158

SMOKE DET

Smoke Production and Properties.

PB91-175422

100,120

International Fire

100,088

SMOLUCHOWSKI EQUATION

Applicability of Smoluchowski's Equation with a Constant Kernel to Coalescence.

PB91-148940

100,296

OCIAL SECURITY ADMINISTRATION

Development of an Optical Disk System for the Automat

ed Retrieval of EASEAR Records.

100,666

SODIUM

Advanced sensor development program for the pulp and paper industry. Final report

DE91007688

101,248

NIST Standards for Sodium and Potassium

PB91-158865

100,191

Spin-Dependent Electron-Impact Excitation of Sodium

101,641

Production and Diagnosis of a Highly Spin-Polarized $\mathrm{Na}$ Beam

DOUM ATOMS

101,431

Associative Ionization of Ultra-Cold Laser Trapped Sodium Atoms.

SODIUM BROMIDES

Thermodynamic Properties of the $\mathrm{NaBr}+\mathrm{H} 2 \mathrm{O}$ System.

SODIUM CHLORIDE

Modeling the Thermodynamic Properties of Sodium Chloride in Steam Through Extended Corresponding States. PB91-189381

100,342

SODIUM IONS

Research at NBS in Direct Potentiometric Measurements

100,188

Theoretical Model of Metal Binding Sites in Proteins.

PB91-194837

100,370

SOFTWARE

MUMPS Validation Suite, Version 7.6 (Vax/VMS Backup),

PB91-507699

100,660

MUMPS Validation Suite, Version 7.6, 1991 (for Micro-

computers).

100,661

MUMPS Validation Suite, Version 7.6 (ANSI), 1991

PB91-507715 100,66

MUMPS Validation Suite, Version 7.6 (TAR), 1991

PB91-507723 100,663

OMNITAB 80 (ASCII): An Interactive System for StatistiCal and Numerical Data Analysis (Version 7.0).
PB91-507954,278

OMNITAB 80 (UNIX): An Interactive System for Statistical and Numerical Data Analysis. Version 7.0 .

101,279

PB91-507962

Building Life Cycle Cost Computer Program, Version 3.2
(for Microcomputers). (for Microcomput
PB91-507970

100,898

\section{SOFTWARE ENGINEERING}

Developing Federal Soltware Standards: A New DirecPB91-134536

100,637

Software Verification and Validation: An Overview.
PB91-237685 $\begin{array}{ll}\text { PB91-237685 } & 100,658 \\ \text { Software Reengineering: A Case Study and Lessons }\end{array}$ Learned.

PB92-116417

100,669

SOFTWARE REUSE

Software Reengineering: A Case Study and Lessons Learned.

PB92-116417

100,669

SOFTWARE TOOLS

NIST PDES Toolkit: Technical Fundamentals. National PDES Testbed
PB91-132159

100,682

NIST Express Working Form Programmer's Reference (Revised November 1990). National PDES Testbed Report Series.

PB91-157164

100,964

Static Analysis Tools for Software Security Certification.

PB91-203380 100,710

Guide to Expert System Building Tools for Microcomputers.

PB92-108893

100,715

SOIL PROPERTIES

Concepts of Underground Corrosion

SOILS

Corrosion Testing in Soil.

PB91-158667

Measuring the Corrosion of Metals in Soil

PB91.174714

Possible Use of Pattern Recognition for the Analysis of Mars-Rover X-ray-Fluorescence Spectra.

101,733

SOLAR

Method for Characterizing the Thermal Performance of a Solar Storage Wall from Measured Data.

PB91-159152

100,901

SOLAR RADIATION

Temperature Measurement of Glass Subjected to Solar PBadiation.

100,100

SOLID CRYOGENS

Hydrogen Slush Production with a Large Auger.

100,275

SOLID ELECTROLYTES

Electrochemical Behavior of Ferrocene in Polyethylene

PB91-237867

100,439

SOLID HYDROGEN

Hydrogen Slush Production with a Large Auger

91.147199

100,275

SOLID STATE PHYSICS

Core Hole Screening for Intermediate Size Metal Parti-

PBes. 203539

SOLID WASTES

NBS Research Program in Municipal Solid Waste Com-

PB91-147264

100,916

Monitoring the Fate of Chlorine from MSW Sampling through Combustion

100,917

SOLIDIFICATION

Phase Selection in Non-Equilibrium Processing

PB91-195651

101,236

SOLIDIFIED GASES

Hydrogen Slush Production with a Large Auger.

PB91-147199

100,275

SOLIDS

Relation between the Surface Energy and the Debye Temperature for Cubic Solids.

Journal of Physical and Chemical Reference Data, Volume 19, Number 4, July-August 1990.

PB91-192435

100,354

Heat Capacities and Entropies of Organic Compounds in the Condensed Phase. Volume

PB91-192450

100,356

Extracting STEP Geometry and Topology from a Solid Modeler: Parasolid-to-STEP.

PB91-194449

100,983

Molecular Desorption from Solid Surfaces: Laser Diag-

nostics and Chemical Dynamics. 
PB91-189837

100,349

Phase Equilibria and Critical Lines in Model Water/Salt Mixtures.

100,418

Thermodynamics of Solutions Near the Solvent's Critical Point.

SOLVATION

onic Hydrogen Bonds. Part 1. Thermochemistry, Struc tural Implications, and Role in Ion Solvation

100,232 $1-203547$

100,400

SOLVENT EXTRACTION

Electrokinetic Demixing of Two-Phase Aqueous Polymer Systems. 1. Separation Rates ot Polyethylene GlycolDextran Mixtures.

100,487

PB91-159095

lectrokinetic Demixing of Two-Phase Aqueous Polymer Systems. 2. Separatio

PB91-237230

100,231

SOLVENTS

Tests of the Flory-Rehner Hypothesis: Comparison of the Elastic Free Energy Function for Crosslinked Rubber in the Dry and Swollen States.

SOOT

100,508

Mechanism of Soot Formation in Acetylene-Oxygen MixPB91-147280

100,537

SOUND LEVEL METERS

Response of Personal Noise Dosimeters to Continuous and impulse-Like Signals.

PB92-116359 TRANSDUCERS

100,914

Apparatus for Acoustic Measurements of Gases with Applications for High Temperatures.

PB92-116839 100,458

Probe-Corrected Spherical Near-Field Scanning Theory in Acoustics.
PB92-117456

101,383

SOUND WAVES

Apparatus tor Acoustic Measurements of Gases with Applications for High Temperatures.

100,458

Accurate Acoustic Measurements in Gases Under Difficult Condition

101,382

SPACE LABORATORIES

Low-Cost Low-Volume Carrier (Minilab) for Biotechnology and Fluids Experiments in Low Gravity.

100,041

SPACE STATIONS

Material Flammability Test Assessment for Space Station Freedom.
PB91-216606

101,735

SPACECRAFT CONSTRUCTION MATERIALS

Material Flammability Test Assessment for Space Station PBeedom.

101,735

SPACECRAFT DESIGN

Apparatus for Measurement of Thermal Conductivity of Insulation Systems Subjected to Extreme Temperature DBences

101,738

SPACECRAFT MAINTENANCE

Recommended Fine Positioning Test for the Development Test Flight (DTF-1) of the NASA Flight Telerobotic Servicer (FTS)

PB91-185090

101,732

SPARSE MATRIX

Computing the Eigenvalues and Eigenvectors of Symmetic Arrowhead Matrices.

PB91-147850

101,254

SPECIFIC HEAT

Heat Capacities of Organic Compounds in the Liquid State I. C1 to C18 1.Alkanols.

100,265

Thermal Conductivity and Heat Capacity of Gaseous

101,602

PB91-134577

Heat Capacity and Thermodynamic Properties of Deutrated Ammonium Hexafluorophosphate ND4PF6 from $5.8 \mathrm{~K}$

100,272

Heat Capacity of Deuterated Ammonium Tetratluoroborate ND4BF4 from 7 to $348 \mathrm{~K}$

PB91-147058

100,273

Theory of Specific Heat of Solids.

PB91-148916

101,475

Relation between the Surface Energy and the Debye Temperature for Cubic Solids.

100,303

Thermodynamics of Ammonium Indates. 2. The Molar Heat Capacity of the Ammonium Pentabromoindate Mon ohydrate Salt (NH4)2 InBr5. H2O from 7.8 to $348 \mathrm{~K}$.

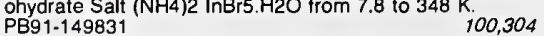

Thermodynamics of the Divalent Metal Fluorides. 3. Heat Capacity of the Fast lon Conductor Sr SnF4 from 6 to $344 \mathrm{~K}$.
PB91-149864

100,305

Unified Description of Infinite-Dilution Thermodynamic Properties for Aqueous Solutes.

100,319

1.162081

Journal of Physical and Chemical Reterence Data,
Volume 19, Number 4, July-August 1990.
PB91-192435 PB91-192435

ounds in

Heat Capacities and Entropies of

100,356
EB91-192450 Experimental Thermal Conductivity, Thermal Diffusivity, Oxygen, and Argon.

PB91-194472

100,369

Heat Capacity and Other Thermodynamic Properties of Linear Macromolecules. 10. Update of the ATHAS 1980 Data Bank.

100,515

Heat Capacities of Organic Compounds in Liquid State. 2. C1 to C18 n-Alkanes. 2. C1 to C18 n-Alkanes.

100,448

Thermodynamics of the Divalent-Metal Fluorides. 4. Hea Capacity of $\mathrm{MSnF} 4, \mathrm{M}=\mathrm{Pb}, \mathrm{Ba}$, or $\mathrm{Sr}$, at Temperatures trom $300 \mathrm{~K}$ to $660 \mathrm{~K}$

PB92-116524

100,456

SPECIFICATIONS

Congressional Districts of the United States. Category Federal General Data Standard, Representations and Codes.
FIPS PUB 9-1

100,679

Introduction to $\mathrm{ACl}$ 306.1-87: Specification for Cold Weather Concreting

PB91-174524

100,526

Application Portability Profile (APP): The U.S. Government's Open System Environment Profile OSE /1 Version 1.0.

100,692

B91-201004

sed Build-

ing Automation System.

PB91-216697

100,092

SPECTRORADIOMETERS

Roughened Quartz Surfaces and Teflon as Small-Angle Ditfusers and Depolarizers between 200 and $400 \mathrm{~nm}$.
PB91-195644

relding

OSCOPIC ANALYSIS

Analysis and Characterization of Commercial Welding

PBiuxes.

101,003

SPECTROSCOPIC DATA

Spectroscopic Data Base for Carbon and Oxygen. ${ }_{101,716}$
PB91-237750

SPECTROSCOPY

Mossbauer Imaging

Assessment of the State-of-the-Art for Process Monitoring Sensors for Polymer Composites

100,510

Diode Lasers and Their Application to Spectroscopy.

SPECTRUM ANALYSIS

Spectroscopic Constants tor the nu9 Infrared Band of HNO3.

PB91-189415

100,343

Strategies for Background Subtraction in Electron Probe Microanalysis X-ray Compositional Mapping

100,202

Lanthanide Coordination Chemistry: Spectroscopic Properties ot Terbium and Europium Poly (Pyrazol-1-YI) Borate and Poly(Imidazol-1-YI) Borate.

100,392

Spectral Data and Grotrian Diagrams tor Highly lonized Copper, Cu X-Cu XXIX.

100,441

Wavelengths and Energy Level Classifications of Magnesium Spectra tor All Stages of Ionization (Mg I through

PB92-110139

100,442

SPEECH RECOGNITION

Phone-Mediated Word Alignment for Speech Recognition Evaluation.

100,592

Scoring Continuous Speech in the DARPA Speech Rec-

Ognition Program: Part 2.
PB91-195396 100,593

DARPA Air Travel Information System (ATISO) SpeakerDependent Training Data (on CD-ROM).

100,594

Road Rally Conversational Speech Corpora (on CD Road Rally Conversational Speech Corpora (on CD-
ROM).
PB91-509802
100,595

SPIN GLASS

Spin Glass Magnetic Behavior ot Iron/Silica Gel Nanocomposites.

101,117

SPINODAL DECOMPOSITION

Spinodal Decomposition in a Hydrogen-Bonded Polymer Blend.

PB91-162099

100,489

Time-Resolved Small-Angle Neutron Scattering in Intermediate- and Late-Stage Spinodal Decomposition of DPB/HPI) Blends.

PB91-162131

100,490

SPRAYING

Suppression of Post-Flashover Compartment Fires Using Manually Applied Water Sprays.

PB91-240770

100,164

SPRAYS

Hydrodynamics and Mass Transier in Two-Phase Aqueous Extraction Using Spray Columns.

101,300

Application of Laser Diagnostic Techniques for the Exam. ination of Liquid Fuel Spray Structure.

100,229

Effect of Atomization Air on Droplet Dynamics ot Spray

PB91-203869

100,550

ICLASS-91 Proceedings of the International Conference on Liquid Atomization and Spray Systems (5th). Held in Gaithersburg, MD. on July 15-18, 1991

PB91-216838

100,552

SPRINKLER SYSTEMS

Interaction of an Isolated Sprinkler Spray and a TwoLayer Compartment Fire Environment.

100,551

SPRINKLERS

Five Small Flaming Fire Tests in a Simulated Hospital Patient Room Protected by Automatic Fire Sprinklers.

PB91-159038

Sprinkler/Hot Layer Interaction

PB91-206680

100,109

Reduction in Fire Hazard in Corridors and Areas Adjoining Corridors Provided by Sprinklers.

SPUTTERING

Mechanical Sputtering of Structural Stainless Steels PB91-175448

101,152

SOL3 DATABASE LANGUAGE

SOL3 Support for CALS Applications.

QUID DEVICES

Excess Low-Frequency Flux Noise in dc Squids Incorporating $\mathrm{Nb} / \mathrm{Al}-\mathrm{O} x \mathrm{ide} / \mathrm{Nb}$ Josephson Junctions

100,736

STABILITY

Stability of Uric Acid in Ammonium Hydroxide.
PB91-189266

Stability of Aqueous Inorganic Lead Solutions in Polycarbonate Containers.

PB92-126499

100,211

STAINLESS STEEL-304L

Cryogenic Material Properties of Stainless Steel Tube-toFlange Welds.
PB91.134627

101,145

STAINLESS STEEL-316L

Cryogenic Material Properties of Stainless Steel Tube-toFlange Welds

Fracture Toughness of $316 \mathrm{~L}$ Stainless Steel Welds with Varying Inclusion Contents at $4 \mathrm{~K}$

101,148

STAINLESS STEEL-440

High-Temperature Thermal Properties of UNS \$44004 Using Multivariant Analysis.

STAINLESS STEELS 
PB90-244583

101,595

Updated Excitation and Ionization Cross Sections for Electron Impact on Atomic Oxygen.

Journal of Physical and Chemical Reference Data, Volume 19, Number 2, 1990

PB90-244609

Standard Chemical Thermodynamic Properties of Isomer Groups of Monochloroalkanes.

100,258

Standard Chemical Thermodynamic Properties of Polycy clic Aromatic Hydrocarbons and Their Isomer Groups. III. Naphthocoronene Series, Ovalene Series, and First Members of Some Higher Series.

PB90-244625

100,259

Dielectric Constant of Water and Debye-Huieckel Limitıng Law Slopes.

100,260

Rate Constants for Reactions of Peroxyl Radicals in Fluid Solutions

PB90-244641

100,261

Journal of Physical and Chemical Reference Data Volume 19, Number 3, 1990

PB90-244658

100,262

Energy Levels of Copper, $\mathrm{Cu} I$ through $\mathrm{Cu} X \mathrm{XIX}$.

PB90-244666

101,596

Cross Sections and Related Data for Electron Collisions with Hydrogen Molecules and Molecular lons.
PB90-244674 Cross Sections for Collisions of Electrons and Photons with Atomic Oxygen

PB90-244682

100,264

Cross Sections and Swarm Coefficients for $\mathrm{H}+, \mathrm{H} 2+$ $\mathrm{H} 3+, \mathrm{H}, \mathrm{H} 2$, and $\mathrm{H}$-in $\mathrm{H} 2$ for Energies from $0.1 \mathrm{eV}$ to 10 PB90-244690

101.597

Refractive Index of Water and Steam as Function of Wavelength, Temperature and Density.

101,402

Heat Capacities of Organic Compounds in the Liquid State I. C1 to C18 1-Alkanols

100,265

Transport Properties of Carbon Dioxide.

PB90-244724

100,266

Factual Materials Databanks - The Need for Standards.

PB91-147546 101,249

Technical Activities 1990, Standard Reference Data Pro-

gram.

PB91-167213

100,321

NIST Standard

100,952

Journal of Physical and Chemical Reference Data

Journal of Physical and Chemical Reference Data,
Volume 19, Number 4, July-August 1990 .
PB91-192435
100,354

PB91-192435

Energy Levels of Sulfur, S I Through S XVI

100,355

Heat Capacities and Entropies of Organic Compounds in the Condensed Phase. Volume 2

PB91-192450

100,356

Thermodynamics of the Krebs Cycle and Related Compounds.

100,357

Journal of Physical and Chemical Reference Data,
Volume 19, Number 5, September-October 1990 .

Volume 19, Number 5, September-October 1990.
PB91.192476 100,358

Transport Properties of Fluid Oxygen.

PB91-192484

100,359

Thermal Conductivity of Nine Polyatomic Gases at Low

Density.

100,360

Thermal Conductivity of Methane and Tetrafluoromethane in the Limit of Zero Density.

PB91-192500

100,361

Coupled Phase Diagram-Thermodynamic Analysis of the 24 Binary Systems, A2CO3-AX and A2SO4-AX Where $\mathrm{A}=\mathrm{Li}, \mathrm{Na}, \mathrm{K}$ and $\mathrm{X}=\mathrm{Cl}, \mathrm{F}, \mathrm{NO}, \mathrm{OH}$

$\mathrm{A}=\mathrm{Li}, \mathrm{Na}, \mathrm{K}$
$\mathrm{PBg}$

100,362

Equilibrium and Transport Properties of Gas Mixtures at Low Density: Eleven Polyatomic Gases and Five Noble Gases.

PB91-192526

100,363

Unified Fundamental Equation for the Thermodynamic Properties of $\mathrm{H} 2 \mathrm{O}$

PB91-192534

100.364

Journal of Physical and Chemical Reference Data Volume 19, Number 6, November-December 1990.

PB91-192542

100,365

Viscosity and Thermal Conductivity of Pure Monatomic Gases from Their Normal Boiling Point Up to $5000 \mathrm{~K}$ in the Limit of Zero Density and at $0.101325 \mathrm{MPa}$.

PB91-192559

100,366

Experimental Stark Widths and Shifts for Spectral Lines of Neutral and lonized Atoms: A Critical Review of Selected Data for the Period 1983 through 1988

PB91-192567

101,659

Vibrational and Electronic Energy Levels of Polyatomic

Transient Molecules. Supplement 1.
PB91-192575

100,367

Thermodynamic and Thermophysical Properties of Organic Nitrogen Compounds. Part 1. Methanamine, Ethanamine, 1- and 2-Propanamine, Benzenamine, 2-, 3-, and -Methylbenzenamine

PB91-192583

100,368

Critical Data in Physics and Chemistry.

101,697

Journal of Physical and Chemical Reference Data, Volume 20, Number 1, 1991

PB92-110113

100,440

Spectral Data and Grotrian Diagrams for Highly lonized Copper, Cu X-Cu XXIX

PB92-110121

100,441

Wavelengths and Energy Level Classifications of Magnesium Spectra for All Stages of Ionization (Mg I through PBg2-1

100,442

Spectroscopy and Structure of the Alkali Hydride Diato-

mic Molecules and Their lons.
PB92-110147

100,443

Critical Evaluation of Liquid Crystal Transition Temperatures I: 4,4'-Alkyl/alkoxyphenylbenzoates.

100,444

Journal of Physical and Chemical Reference Data Volume 20 Number 2 March-April 1991 Volume 20, $\mathrm{Nun}$

100,445

Chemical Kinetic Data Base for Combustion Chemistry Part 5. Propene

PB92-110170

100,446

Thermophysical Properties of Ethane.

B92-110188

100,447

Heat Capacity and Other Thermodynamic Properties of Linear Macromolecules. 10. Update of the ATHAS 1980 Data Bank.

PB92-110196

100,515

Heat Capacities of Organic Compounds in Liquid State. 2. C1 to C18 n-Alkanes

100,448

B92-110204

Journal of Physical and Chemical
Volume 20 . Number 3, May-June 1991.
PB92-110212 PB92-110212

Kinetics and Mechanisms of the Gas-Phase Reactions of
the NO3 Radical with Organic Compounds. PB92-110220

Thermodynamic Properties of the $\mathrm{NaBr}+\mathrm{H} 2 \mathrm{O}$ System.
PB92-110238

Cross Sections and Swarm Coefficients for Nitrogen Ions and Neutrals in N2 and Argon lons and Neutrals in N2 and Argon lons and Neutrals in Ar for Energies from 0.1 eV $10 \mathrm{keV}$.

PB92-110246

100,452

Evaluation of Solubility Data of the System $\mathrm{CO} \cdot \mathrm{H} 2 \mathrm{O}$ from $273 \mathrm{~K}$ to the Critical Point of Water.

PB92-110253

100,453

NIST $X$-Ray Photoelectron Spectroscopy (XPS) Data-

PB92-123157

100,465

Journal of Physical and Chemical Reference Data, Volume 20, Number 4, July/August 1991.

PB92-148063

100,466

Chemical Kinetic Data Base for Propellant, Combustion. 1. Reactions Involving $\mathrm{NO}, \mathrm{NO} 2, \mathrm{HNO}, \mathrm{HNO} 2, \mathrm{HCN}$ and

PB92-148071

100,467

Ab-Initio Calculations and Ideal Gas Thermodynamic Functions of Cyclopentadiene and Cyclopentadiene De-

$\begin{array}{ll}\text { rivatives. } & 100,468\end{array}$

Improved Fits for the Vibrational and Rotational Constants of Many States of Nitrogen and Oxygen.

PB92-148097

100,469

Solubilities of Solids and Liquids of Low Volatility in Supercritical Carbon Dioxide

100,470

Journal of Physical and Chemical Reference Data, Volume 20, Number 5, September/October 1991

PB92-148113

100,471

Wavelengths and Energy Level Classifications for the Spectra of Aluminum (AI I through AI XIII).

Energy Levels of Krypton, $\mathrm{Kr} I$ through $\mathrm{Kr}$ XXXVI.
PB92-148139

100,473

Thermodynamic Properties of Oxygen from the Triple Point to 300

100,474

Sixteen Thousand Evaluated Experimental Thermodynamic Property Data for Water and Steam. PB92-148154

100,475

Journal of Physical and Chemical Reference Data, Volume 20, Number 6, November/December 1991. PB92-148162

New Equation of State and Tables of Thermodynamic Properties for Methane Covering the Range from Melting Line to $625 \mathrm{~K}$ at Pressures up to $1000 \mathrm{MPa}$.
PB92-148170 Thermodynamic Properties of the Aqueous Sulfuric Acid System to $350 \mathrm{~K}$.

PB92-148188

100,478

Solubility of Carbon Dioxide in Water at Low Pressure.

100,479

Chemical Kinetic Data Sheets for High-Temperature Re actions. Part 2.

100,480

Atomic Weights of the Elements 1989

PB92-148212

100,481

Isotopic Compositions of the Elements 1989

100,482

STANDARD REFERENCE FIBERS

Standard Reference Fibers for Calibration of the Optical Time Domain

101,406

\section{STANDARD REFERENCE MATERIALS}

SRM 1970 - Succinonitrile Triple-Point Standard - And Its Use in Calibration of Thermistors.

PB91-134338

100,780

Evaluation of Fumed-Silica Insulation for a Thermal Conductivity Standard Reference Material.

100,103

Characterization of the Mineral Fraction in Botanical Reference Materials and Its Influence on Homogeneity and Analytical Results

Analytical Result

100,186

Preparation and Analysis of a Marine Sediment Refer ence Material for the Determination of Trace Organic

Constituents.
PB91-149237

101,377

NIST Standards for Sodium and Potassium.

PB91-158865

100,191

Reference Materials: Their Role in Measurement Accura-

PB. 159103

101,014

Automated High-Temperature Guarded-Hot-Plate Apparalus for Measuring Apparent Thermal Conductivity of Insulatıons between 300 and $750 \mathrm{~K}$

100.111

Standard Reference Materials: Bright-Chromium Linewidth Standard, SRM 476 , for Calibration of Optical Microscope Linewidth Measuring Systems.

100,802

Appendix: Standard Solutions and Certified Reference Appendix. Slandard Solutions and Cerlified Reterence
Materials. PB91-174235

Summary of Low-Density Glass-Fiber Reference Materials at NIST: 1980-1989.

PB91-175620

101,079

Microstructure, Composition, and Hardness of Rockwell C Hardness Blocks 


\section{KEYWORD INDEX}

Proceedings of the Object-Oriented Database Task Group Workshop. Held in Atlantic City, New Jersey on May 22, 1990

100.643

Standards Activities of Organizations in the United States

100,004

PB91-177774 Meeting of the Standards Working Group of the Joint
U.S.U.S.S.R. Commercial Commission (1st). Held in Moscow on March 11-13, 1991.

PB91-194498

100,178

Standardization of Advanced Ceramics.

101,086

Validation Testıng Laboratory User's Guide. National PDES Testbed Report Series.

PB92-123090

100.971

\section{STANDARDS}

Computer Output Microform (COM) Formats and Reduction Ratios, I6MM and 105MM. Category: Hardware Standard; Subcategory: Media
FIPS PUB 54-1

100,678

Government Open Systems Interconnection Profile (GOSIP); Category: Hardware and Software Standards: Subcategory: Computer Network Protocols.

100,675

Detail Specification for 62.5-mum Core Diameter/125 mum Cladding Diameter Class la Multimode, GradedIndex Optical Waveguide Fibers. FIPS PUB 159

100,562

Benzene: A Further Liquid Thermal Conductivity Standard.

100,255

PB90-244567

NIST Working Form for STEP: National PDES Testbed
Report Series (Revised).

RB91-132183 (Revised)

Use of the IRDS Standard in CALS (Revised). 100,684
PB91-132209

Eddy Current Probe Characterization Using an Imped-

ance Plane Display Instrument.

Information Systems Engineering Program

100,834

PB91-134635 100,685

NIST STEP Working Form Programmer's Reference. National PDES Testbed Report Series (Revised).

PB91-144378

100,998

State-of-the-Art in Pressure Metrology

Experimental Evaluation of Selected Orifice Flowmeter Upstream Installations.

PB91-147025

100,880

Dietary Collection Procedures.

101,317

Factual Materials Databanks - The Need for Standards.
PB91-147546

Use of Single Particle Standards for LAMMA Calibration.

PB91-147777 100,183

Real-Time Radiology Standards: Results of a Workshop.

PB91-147900 101,010

$\mathrm{CIM}$, Data and Standardization within the NBS AMRF.

PB91-148122 100,975

Characterization of the Mineral Fraction in Botanical Reference Materials and Its Influence on Homogeneity and Analytical Results

PB91-148817

100,186

Preparation and Analysis of a Marine Sediment Reference Material for the Determination of Trace Organic Constituents.

101,377

Fusion of Mercury. A New Certified Standard for Differential Scanning Calorimetry.

100,309

NIST Express Working Form Programmer's Reterence (Revised November 1990). National PDES Testbed Report Series.

100,964

Data Management Standards in Computer-Aided Acquisition and Logistic Support (CALS)

101,339

NIST Standards for Sodium and Potassium.

100,191

NBS Submicron Particle Standards for Microcontamina$\begin{array}{ll} & \\ \text { tion Measurement. } & 100,798\end{array}$

100,688

Features and Facilities of Estelle

PB91-158915

100,176

Domestic Implementation.

Submicrometer SEM Magnification Standard.

100,799

International Co-operation: The Versailles Project on Advanced Materials and Standards.

PB91.159137

101,191

VAMAS Bulletin.

PB91.159145

101,192

Progress at NIST on Absolute Frequency Standards

Using Stored lons.
PB91-159285

101,625

Intercomparison of NBS Noise Calibration Services.

PB91-162370 100,847 Intercomparison Study of Rockwell Hardness Test

PB91-167338

101,224

Proposed Standard Practice for Assessing the Perform. ance of Gas-Phas

100,905

Appendix: Standard Solutions and Certified Reference Materials.

PB91-174235

100,194

etter to the Editor of Standardization News.

100,118

100,118
Application of CCRL Data in the Development of Cement Standards.

PB91-175315

100,119

Summary of Low-Density Glass-Fiber Reference Materials at NIST: $1980-1989$

101,079

Collection of Technical Studies Completed for the Computer-Aided Acquisition and Logistic Support (CALS) Program, Fiscal Year 1988. Volume 2 of 3. Graphics, CGM

PB91-178897

101,343

Microstructure, Composition, and Hardness of Rockwell C Hardness Blocks.

101,153

STEP: Standard for the Exchange of Product Model Data Resource Integration: Semantic and Syntactic Rules. PB91-184788 100,966

Standard Reference Materials: Calibration of NIST Standard Reference Material 3201 for 0.5 Inch (12.65 mm) Serial Serpentine Magnetic Tape Cartridge.

100,690

Proposed New Electrolytic Conductivity Primary Standards for $\mathrm{KCl}$ Solutions

100,339

Government Document Processing Requirements Report. PB91-187773 100,574

GATT Standards Code Activities of the National Institute of Standards and Technology 1990

100,177

Document Architecture for Open Systems.

100,961 $91 \cdot 189522$

Standardizing Standards

100,953

Comparison of Standard Test Methods for Non-Lubricated Sliding Wear
PB91-190017

101,187

Concurrent Engineering through Product Data Standards. 1.193367

100,982

NIST Support for the Computer-Aided Acquisition and Logistic Support (CALS) Program in the Area of Graphics PB91-194506

101,348

Meeting the Challenges of a Global Market - Construction Standards, Testing and Certification.

PB91-194811

100,090

Reference Materials for Analytical Chemistry. PB91-203893

100,205

International Collaboration in Prestandards Research on Advanced Materials within the Context of VAMAS

PB91-204040

101,251

Data Encryption Standard: Past and Future.

PB91-204107

100,711

Standard Security Label for GOSIP: An Invitational Workshop. Held on April 9-10, 1991.

.

Configuration Management of the STEP Documents: Procedures and System Requirements. National PDES Testbed Report Series.

B91.222620

100,968

Standards for the Physical Protection of National Resources and Facilities.

PB91-222695

100,006

Investigating the Use of Multimeters to Measure Ouanized Hall Resistance Standards.

100,734

New Low-Voltage Standards in the DC $101-\mathrm{MHz}$ Frequency Range.
PB91-237107

100,738

Monitoring the Mass Standard via the Comparison of Mechanical to Electrical Power.

101,710

Improvements for Automating Voltage Calibrations Using a $10-\mathrm{V}$ Josephson Array.

PB91-237446

100,739

New International Electrical Reference Standards Based on the Josephson and Ouantum Hall Effects. PB91-237586

100,740

Basic Standards and Fundamental Constants PB91-237594

101,715

North American ISDN Users' Forum Agreements on Integrated Services Digital Network.

100,573

Proceedings of the Workshop on High Integrity Software PB92-109040

00,667

Conceptual Architecture for a Mechanical Parts Produc tion System Based on STEP. National PDES Testbed Rept. Series.

PB92-112234

100,993

Proceedings of the Forum on Standards for High Integrity Software (DOD, Government, Industry). Held in Gaithersburg, Maryland on June 28, 1991.

PB92-112267

Proposed Testing Methodology for STEP Application Pro tocol Validation. National PDES Testbed Report Series. PB92-112374. 100,969

Large-Scale Validation of Bench-Scale Fire Toxicity PB92-116458

100,139

Voltage Calibration Systems Using Josephson Junction

Arrays.

100,868

Need for Power Ouality Testing Standards.

100,741

Air Gage Size Measurement of Microwave Standards

PB92-117324 100,777

Multi-Enterprise Concurrent Engineering through International Standards.

100,997

Ouestions and Answers on Ouality, the ISO 9000 Standard Series, Ouality System Registration, and Related

PB92-126465

100,175

Summary of the Intercomparison of the Force Standard Machines of the National Institute of Standards and Technology, USA, and the Physikalisch-Technische Bundesanstalt, Germany.

PB92-126622

101,590

Atomic Weights of the Elements 1989

PB92-148212

100,481

\section{TANNATES}

Thermodynamics of the Divalent-Metal Fluorides. 4. Heat Capacity of $\mathrm{MSnF} 4, \mathrm{M}=\mathrm{Pb}, \mathrm{Ba}$, or $\mathrm{Sr}$, at Temperatures from $300 \mathrm{~K}$ to $660 \mathrm{~K}$

STARK EFFECT

100,456 Experimental Stark Widths and Shifts for Spectral Lines lected Data for the Period 1983 through 1988 PB91.192567

101,659

STARS

Modelling the Coronae and Chromospheres of RS CVn Systems by the Analysis of Ultraviolet, $\mathrm{X}$-ray and Radio PB91-148825

\section{STATIC TESTS}

Static and Dynamic Strength Tests on Electrical Conductor Cables Specified for Airport Landing Structures

PB92-112226
ATISTICAL ANALYSIS

Statistics of a Polymer Molecule in the Presence of

$\begin{array}{ll}\text { Asymmetric Obstacles. } & 100,498 \\ \text { PB91-187450 }\end{array}$

OMNITAB 80 (ASCII): An Interactive System for Statistical and Numerical Data Analysis (Version 7.0). 
STEEL HSLA-100

Effects of Varying Austenitizing Temperature and Cooling Rate on the Ability of HSLA-80 Steel to Achieve a Yield Strength Comparable to HSLA-100.

PB91-189464

101,156

STEEL HSLA-80

Effects of Varying Austenitizing Temperature and Cooling Rate on the Ability of HSLA-80 Steel to Achieve a Yield Strength Comparable to HSLA-100

PB91-189464

101,156

STEEL-SAE-1141

Continuous-Cooling Transformation Characteristics and High-Temperature Flow Behavior of a Microalloyed SAE 1141 Steel.

PB91-187856

101,155

STEELS

Roughness Measures of Blasted Steel Surfaces Remotely Imaged with a Thermographic Camera.

101,146

PB91-147686

st Meth

ods.

PB91-148106

101,147

Concepts of Underground Corrosion.

PB91-150078

101,134

Using Surface Roughness as a Wear Criterion in HighSpeed Steel End-Milling.

101,040

Microstructure, Composition, and Hardness of Rockwell C Hardness Blocks. CHardness Blocks.
PB91-184747

101,153

Characterization of Cylindrical Holes in Metallic Substrates via Their Infrared Emission Patterns.

PB91-187187

101,228

Plastic Zone Formation Around an Arresting Crack

PB91-189316 101,373 Sliding Wear Studies of Nickel-Copper Composition-Modulated Coatings on Steel.

PB91-190025

101,188

Fourier Transform Infrared Spectroscopic Studies of the Degradation of One-Component Polyether Polyurethane Protective Coatings on Steel.

101,108

Reflection/Absorption FTIR Spectral Characteristics of Thin and Thick Crosslinked Epoxy Films on Steel SubThin and Thick Crosslinked Epoxy Films on Steel Sub Strates.

100,509

Characterization of Epoxide Coatings on Steel by Reflection/Absorption Fourier Transform Infrared Spectroscopy: Quantitative Study.

PB91-203729

101,109

Ultrasonic Measurement of Sheet Steel Texture and Formability: Comparison with Neutron Diffraction and $\mathrm{Me}$ chanical Measurements.

PB92-116615

101,170

STELLAR ACTIVITY

Ground-Truth Observations of Stellar Surface Structure from the Lunar Surface.

PB91.175026

100,029

TELLAR EVOLUTION

Evolution of Infrared Carbon Stars.

PB91-202861

STELLAR FLARES

Spatially Resolved Flares in RS CVn Systems.
PB91.148965

STELLAR WINDS

Terminal Velocities for a Large Sample of O Stars, B Supergiants, and Wolf-Rayet Stars.

pergiants, and

100,023

STEP (STANDARD FOR THE EXCHANGE OF PRODUCT

MDEL DATA)

NIST PDES Toolkit: Technical Fundamentals. National PDES Testbed Report Series (Revised).

100,682

NIST Working Form for STEP: National PDES Testbed Report Series (Revised)

PB91.132183

100,683

NIST STEP Working Form Programmer's Reference. National PDES Testbed Report Series (Revised).

PB91-144378

100,998

STEP: Standard for the Exchange of Product Model Data Resource Integration: Semantic and Syntactic Rules.

PB91-184788

100,966

Extracting STEP Geometry and Topology from a Solid Modeler: Parasolid

100,983

Configuration Management Concepts Document. National PDES Testbed Report Series.

PB91-194480

100,984

Apparel STEP Translator.

100,967

Development Plan: Application Protocols for Mechanical Parts Production. National PDES Testbed Report Series. PB91-222596 100,990

Configuration Management of the STEP Documents: Procedures and System Requirements. National PDES Testbed Report Series.

PB91-222620

100,968

Conceptual Architecture for a Mechanical Parts Production System Based on STEP. National PDES Testbed Rept. Series.
PB92-112234

100,993

Proposed Testing Methodology for STEP Application ProPocol Validation. National PDES Testbed Report Series. PB92-112374

STEREOCHEMISTRY

Statistics of a Polymer Molecule in the Presence of Asymmetric Obstacles.

PB91-187450

100,498

PLOTMD: An Interactive Program to Modify Molecular Plots on a Graphics Terminal.

PB91-194985

100,373

Species Produced in Fires Burning in Two-Layered and Species Produced in Fires Burning in Two-Layered and
Homogeneous Vitiated Environments.
PB91-157180

100,542

\section{STOPPING POWER}

Stopping Power of Fast Charged Particles in Heavy Elements.
PB91-194399

101,660

STRAIN ENERGY METHODS

Role of Elastic Energy in the Morphological Development of a Ni-Ti-Al Alloy.

PB91-18716

101,227

\section{AIN GAGES}

Mechanical Testing Revitalized.

Spatially Overdetermined Analysis for Propagation Toughness Using Strain Gages.

PB91-236505

101,164

STRAIN HARDENING

Metallographic Study of the Crack-Tip Region from Fracture Mechanics Specimens of Austenitic and Ferritic

PB91-189951

101,158

\section{STRAIN MEASUREMENT}

Study of Static and Dynamic Fracture Using Strain MeasPB91-13221

101,574

Determining the Dynamic Stress Intensity Factor with Strain Gages Using a Crack Tip Locating Algorithm.
PB91-174268 Improved Optical Diffraction Strain Measurement System. Use of Birefringent Coatings in Fracture Mechanics. PB91-174599

101,582

STRAIN RATE

Strain Rate Effect on Tensile Properties at $4 \mathrm{~K}$ of VAMAS Round-Robin Austenitic Steel.

PB91-237248

101,168

STRAIN TESTS

Phenomenological Theory of the Influence of Strain History on the Rate of Isothermal Stress Relaxation. PB91-187385

Plastic Zone Formation Around an Arresting Crack PB91-189316

\section{0,496}

STRATEGIC DEFENSE INITIATIVE Annual Report to the Strategic Defense Initiative Organi-
zation on the Free-Electron Laser Driven by the NIST CW Microtron.

AD-A207 806/1

101,398

STRENGTH

Rate Constant Functions for Strength Development of Concrete.

100,527

Static and Dynamic Strength Tests on Electrical Conductor Cables Specified for Airport Landing Structures. PB92-112226

STRENGTH (MECHANICS)

Strength and Microstructure of Ceramics.
AD-A243 049/4

101,739

RESS ANALYSIS

Effect of a Free Surface on Stress Distribution in a Bimaterial Composite
PB91-185116

STRESS CORROSION

Cleavage Step Formation and Resistance to Transgranular Stress Corrosion Cracking.

101,133

Computational Models Developed for the Corrosion of Prestressing Steel.

PB91-18

100,115

STRESS CORROSION CRACKING

Stress Corrosion Cracking of Al-Li Alloys: The Role of Grain Boundary Precipitates.

PB91-175182

101,137

STRESS INTENSITY FACTORS

Effect of Logarithmic Singularity on the Free Edge Stress Intensity Factor of Composite Laminates.

101,116

Stress Intensity Factors by Enriched Mixed Finite Elements.

PB91-147371

101,577

Determining the Dynamic Stress Intensity Factor with Strain Gages Using a Crack Tip Locating Algorithm.
PB91-174268 Use of Birefringent Coatings in Fracture Mechanics.

PB91-174599

101,582

\section{TRESS RELAXATION}

Multistep Stress-Rejaxation Behavior in Uniaxial Extension of an Ethylene-Hexene Copolymer.

PB91-148247

01,242

Phenomenological Theory of the Influence of Strain History on the Rate of Isothermal Stress Relaxation

PB91.187385

100,496

Multistep Stress-Relaxation Study of a Single Crystal of $n$-Eicosane $(\mathrm{C} 2 \mathrm{OH} 42)$ in Torsion.

100,497

Nitrogen Strengthening of Austenitic Stainless Steels at Low Temperatures.

PB91-195529

101,163

Semiempirical Theory of Relaxation: Concentrated Polymer Solution Dynamics.

PB91-236638

100,511

\section{TESTS}

Cryogenic Fatigue of High-Strength Aluminum Alloys and Correlations with Tensile Properties.

PB91-236976

101,709

STRESS WAVES

Numerical Simulation and Visualization Models of Stress Wave Propagation Graphite/Epoxy Composites.

PB91.174946

Extended Abstract: A Finite Element Study of the Stress and Displacement Fields Produced by Point Impact PB91-175224

101,584

TRONTIUM

Collisional Energy Transfer between Excited Sr Atoms.

STRONTIUM OXIDES

Phase Equilibria of the System Strontium Oxide-Calcium Oxide-Cupric Oxide.

PB91-195628

101,085

STRONTIUM TETRAFLUOROSTANNATES

Thermodynamics of the Divalent Metal Fluorides. 3. Hea Capacity of the Fast Ion Conductor Sr SnF4 from 6 to $344 \mathrm{~K}$

PB91-149864

100,305

STRONTIUM TIN FLUORIDES

Thermodynamics of the Divalent Metal Fluorides. 3. Hea Capacity of the Fast lon Conductor Sr SnF4 from 6 to

PB91-149864

100,305

STRUCTURAL ANALYSIS

Proceedings of a Workshop on Evaluation, Repair, and 作. Japan Panel on Wind and on May 12-14, 1990. 


\section{KEYWORD INDEX}

SUBSTITUENT EFFECTS

Dissociation of Substituted Anisoles: Substituent Effects on Bond Strengths.

PB Bond-237537

100,431

SUBSTITUTES

Strategy for Chemical Analysis of Alternative Refrigerants.

100,185

PB91-148502$$
100,370
$$

Theoretical Model of Metal Binding Sites in Protein

PB91-194837

00,370

SUCCINIC ACID

Thermodynamics of the Krebs Cycle and Related Com-

pounds.

100,357

SUCCINONITRILE

SRM 1970 - Succinonitrile Triple-Point Standard - And Its Use in Calibration of Thermistors.

100,780

SULFATES

Thermochemistry of Sulfoxides and Sulfones. PB91-17482

Rate Constants for Hydrogen Abstraction Reactions of the Sulfate Radical, SO4- Alkanes and Ethers.
PB91-203232

Thermochemistry of Sulfoxides and Sulfones. PB91-174821

100,325

SULFONES

Thermochemistry of Sulfoxides and Sulfones.

PB91-174821

100,325

SULFONIC ACIDS

Thin and Composite High-Flux Membranes of Perfluorosulfonated lon-Exchange Polymer.

100,512

SULFOXIDES

Thermochemistry of Sulfoxides and Sulfones.

PB91-174821

100,325

SULFUR

Influence of Sulfur on Methanation over Tungsten(110). P891-149369 100,300 Summary Abstract: The Influence of Sulfur on Methanation over $W(110)$.

100,301

Bioprocessing of Fossil Fuels Using Hyperthermophilic

Archaebacteria.
PB91-162271 100,883

Journal of Physical and Chemical Reference Data, Volume 19, Number 4, July-August 1990.

PB91-192435

100,354

Energy Levels of Sulfur, S I Through S XVI PB91-192443

100,355

SULFUR DIOXIDE

Studies on the Degradation Products of Paper with and without Pollutants in a Closed Environment. 1. Preliminary Results.

101,181

Thermodynamic Data for Modeling of Flue Gas Cleanup Thermodynamic Data for Modeling (Abstract for Poster Session). 100,904

PB91-158741 100,904 Rotational-Tunneling Spectrum of the Ar-SO2 van der
Waals Complex.
PB92-116649
Accurat

Accurate and Precise Coulometric Determination of Sulfur Dioxide in Compressed Gas Mixtures.

SULFUR FLUORIDES

Formation and Stability of SF5 and S2F10.

PB91-174839

100,912 fluoride by Gas Chromatography/Mass Spectrometry. PB91-189860

100,197

SULFUR HEXAFLUORIDE

Collisional Electron-Detachment and Ion-Conversion Processes in SF6.
PB91-134528

100,836

Zonal Model for Corona Discharge-Induced Oxidation of SF6 in SF6/O2/H2O Gas Mixtures.

PB91.158790

100,312

Formation and Stability of SF5 and S2F10.

100,326

Detection of Trace Disulfur Decafluoride in Sulfur Hexafluoride by Gas Chromatography/Mass Spectrometry.
PB91-189860 197

SULFUR IONS

Biological Sulfur Oxidation and Reduction for Coal Sulfur Speciation and Desulfurization.

PB91-203323

100,886

SULFUR-LIKE IONS

Energy Levels and Transition Probabilities in the Ground State Configuration of Sulfur-Like Ions.

PB91-190033

101,658

SULFURIC ACID

Thermodynamic Properties of the Aqueous Sulfuric Acid System to $350 \mathrm{~K}$

100,478
SUPERCOMPUTERS

Video Processing with the Princeton Engine at NIST. PB92-108950 100,604

SUPERCONDUCTING CABLES

Enhanced Flux Creep in Nb-Ti Superconductors After an Increase in Temperature.

101,680

Hall Probe Magnetometer for SSC Magnet Cables: Effect of Transport Current on Magnetization and Flux Creep. of Transport Current on Magnetization and Flux Creep.
PB91-202911

Effect of Mechanical Deformation on Nb-Ti Filament Proximity-Effect Coupling at the Edges of SSC Cables.
PB91-203828

Magnetic Measurements for High Energy Physics Appli-

cations.
PB92-112358

101,720

SUPERCONDUCTING COMPOSITES

Levitation of Superconducting Composites

PB91-187351

101,503

Magnetic Hysteresis of High-Temperature YBa2Cu3OXAgO Superconductors: Explanation of Magnetic Suspension. 189506

101,507

SUPERCONDUCTING DEVICES

High Speed Superconducting A/D Converter

SUPERCONDUCTING FILMS

Superconductor-Polymer Composites.

Possible 'Proximity Matrix' Route to High Current Con-

ductors.
PB91-148932

101,476

Analysis of the $\mathrm{YBa} 2 \mathrm{Cu} 3 \mathrm{O} 7 / \mathrm{SrTiO}_{3}$ Interface as a Function of Post-Deposition Annealing Temperature. B91.149963

101,480

Superconducting Thin Films of Bi-Sr-Ca-Cu-O Obtained by Laser Ablation Processing.

P891-189589

101,508

Sputtered Thin Film YBa2Cu3On.

YBa2Cu3O7-delta/Insulator Multi-Layers for Crossover Fabrication.

101,518

Morphology of Silver on YBa2Cu3O7-delta Thin Films.
PB91-195594

Properties of YBa2Cu3O7-delta Thin Films Grown on OffAxis-Cut MgO Substrates.

PB91-203950

101,547

Single-Target Magnetron Sputter-Deposition of High-Tc Superconducting Bi-Sr-Ca-Cu-O Thin Films.

101,566

SUPERCONDUCTING JUNCTIONS

Break Junction Tunneling Spectroscopy of Single-Crystal Bismuth-Based High-Temperature Superconductors.

PB91.134478

101,462

SUPERCONDUCTING MAGNETS

Magnetic Measurements for High Energy Physics Applications.
PB92-112358

101,720

SUPERCONDUCTING SUPER COLLIDER

Cryogenic Material Properties of Stainless Steel Tube-toFlange Welds.

101,145

Enhanced Flux Creep in Nb-Ti Superconductors After an Increase in Temperature.

101,680

Hall Probe Magnetometer for SSC Magnet Cables: Effect of Transport Current on Magnetization and Flux Creep. Effect of Mechanical Deformation on $\mathrm{Nb}-\mathrm{Ti}$ Filament Proximity-Effect Coupling at the Edges of SSC Cables. $\begin{aligned} & 101,700 \\ & P B 91-203828\end{aligned}$ Cryogenic Fatigue of High-Strength Aluminum Alloys and PB91-236976

101,709

SUPERCONDUCTING SWITCHES

Correlation of Flux States Generated by Optical Switching of a Superconducting Circuit.

101,682

\section{SUPERCONDUCTING WIRES}

AC Loss Measurements of Two Multifilamentary $\mathrm{NbTi}$ Composite Strands.

100,769

SUPERCONDUCTIVITY

Structural Instabilities and Superconductivity in OuasiBinary Mn5Si3-Type Compounds.

101,215

Oxides and Oxide Superconductors: Elastic and Related Properties.

101,563

High-Temperature Superconductivity: Abstracts of NIST Publications, 1987-1991.

101,105

SUPERCONDUCTORS

Guideline for $\mathrm{Nb}$ (sub 3) $\mathrm{Sn}$ critical current measurements using fiberglass-epoxy composite sample mandrels.

DE91016274

101,205

Integrity tests for high-(Tc) and conventional critical-current measurements systems.

DE91016275

101,452

Current Supply for High-T(sub c) Superconductor Testing.

Transport Critical Current of Aligned Polycrystalline Y1Ba2Cu3O7-delta and Evidence for a Nonweak-Linked Component of Intergranular Current Conduction.

PB91-133983

101,456

High $T$ (sub c) Superconductors and the Critical Current Measurement.

101,457

Fundamental and Harmonic Susceptibilities of YBa2Cu3O7-delta.

PB91-134148

101,458

Madelung Potentials and Valences in the $\mathrm{Y}_{1} \mathrm{Ba}_{2} \mathrm{Cu} 3 \mathrm{O} 7$ Superconductor

101,459

Elastic Constants, Debye Temperatures, and ElectronPhonon Parameters of Superconducting Cuprates and Related Oxides.

PB91-134262

101,460

Elastic Constants of Polycrystalline Bi-Pb-Sr-Ca-Cu-O Superconductor.

Magnetization of Anisotropic Superconducting Grains.

Phase Equilibria in the System TI-Ca-Ba-Cu-O. 1. Stability of the 2122 Phase under Conditions of Oxygen Annealing. 147165

101,465

Strontium-Induced Oxygen Defect Structure and Hole Doping in $\mathrm{La} 2-x \mathrm{SrxCuO} 4$

PB91-148155

101,472

Strength and Fracture Behavior of Ba-Y-Cu-O Supercon-

ducting Ceramics.
PB91-148205

Chemical Modification of the Orthorhombic Superconductor Ba2YCu3O7-delta.

PB91-159053

101,486

Phonon Density of States of Superconducting $\mathrm{YBa} 2 \mathrm{Cu} 3 \mathrm{O} 7$ and the Nonsuperconducting Analog YBa2Cu3O6.

PB91-159129

101,487

Models for Oxygen Ordering and Diffusion in Ba2YCu3OX and $\mathrm{Ba}$ YCu3-yMyOx (M = Fe, Co, Al, Ga).

B991-175232

Chemistry of Electronic Ceramic Materials.

101,496

Processing $\mathrm{Bi}-\mathrm{Pb}-\mathrm{Sr}-\mathrm{Ca}-\mathrm{Cu}-\mathrm{O}$ Superconductors from Amorphous State.

PB91-187369

101,504

Magnetic Ordering of $\mathrm{Er}$ in Powder and Single Crystals o $\mathrm{ErBa2} \mathrm{Cu} 3 \mathrm{O} 7$

PB91-187377

101,505

Initial Stages of Degradation of Superconductor Surfaces:

$\mathrm{O} 2, \mathrm{H} 2 \mathrm{O}, \mathrm{CO} 2$ and $\mathrm{CO}$ Chemisorption on La2-xSrxCuO4.

Electronic Structure of High-Tc Superconductors Studied Using Photoelectron Spectroscopy.

PB91-189688

101,513

Phonon Density of States and Superconductivity in Nd1.85Ce0.15CuO4.

PB91-189787

101,514

Current Record in Superconductors.

PB91-194753 
Low-Field Flux Pinning in Twinned and Detwinned Single Crystals of YBa2Cu3O7-x

101,559

High Magnetic Field Facilities in Japan Related to Superconductivity Research (Japan Technology Program) PB91-240762

101,718

Theoretical Model for the Tunneling-Gap Anisotropy Observed in Layered Copper-Oxide High-Temperature Superconductors

101,564

Interpretation of Field Ion Microscopy (FIM) Images of Asymmetrical Specimens of 1,2,3-Type High-Tc Superconductors.

PB92-116730

101,567

SUPERCOOLING

Ergodic Convergence Properties of Supercooled Liquids and Glasses.

100,289

SUPERCRITICAL FLUID CHROMATOGRAPHY

Capillary Supercritical Fluid Chromatography of Explosives: Investigations on the Interactions between the Analytes, the Mobile Phase and the Stationary Phase.

100,182

Introduction to Supercritical Fluid Chromatography. 1. Principles and Instrumentation.

100,198 PB91-189878

PB92-117381

101,571

SURFACE FORCES

Effect of Surface Forces on Subcritical Crack Growth in PB92-117449

101,103

SURFACE MAGNETISM

Scanning Electron Microscopy with Polarization Analysis (SEMPA)-Studies of Domains, Domain Walls and Magnetic Singularities at Surfaces and in Thin Films.

101,554

\section{SURFACE PROPERTIES}

Capillary Supercritical Fluid Chromatography of ExploShe Interactions between the Analytes, the Mobile Phase and the Stationary Phase. Flexible Polymer with Excluded Volume at an Interacting Penetrable Surface of Variable Dimension: A Multiple epsilon Perturbation Theory. PB92-116698

100,517

SURFACE REACTIONS

Laser Induced Desorption of Molecules from Surfaces PB91-147066

Soluble Models in Surface Collision Dynamics. PB91-147298

100,276

\section{SURFACE ROUGHNESS}

Roughness Measures of Blasted Steel Surfaces Remotely Imaged with a Thermographic Camera.

Introduction to Supercritical Fluid Chromatography. 1. Principles and
PB91-189878

100,198

SUPERCRITICAL STATE

Thermodynamic Behavior of Fluids in the Supercritical

Region.
PB91-204123

101,394

SUPERGIANT STARS

Terminal Velocities for a Large Sample of O Stars, B Su-

pergiants, and Wolf-Rayet Stars.
PB91-149021

100,023

SUPERLATTICES

Grain Boundaries in a 2-D Superlattice.

PB91-202853

100,388

SUPERMIRRORS

Fe-W Supermizrors for Polarizing Neutrons

PB91-175521

101,643

SUPERNOVA REMNANTS

Detection of an Expanding $\mathrm{H}$ I Shell in the Old Supernova Remnant CTB 80.

PB91-174920

100,028

SUPEROXIDE

Iron Ion-Dependent Modification of Bases in DNA by the Superoxide Radical-Generating System Hypoxanthine/ Xanthine Oxidas

PB91-200824

Particle Size Measurement of Inert Gas Atomized Powder.

PB91-187203

101,229

SUPERSONIC INERT GAS METAL ATOMIZER

Particle Size Measurement of Inert Gas Atomized Powder.

PB91-187203

101,229

SURFACE ACOUSTIC WAVE DEVICES

Surface Acoustical Wave Spectroscopy for High Humidity PB91-158816

100,843

SURFACE CHEMISTRY

Adsorption of Water and Oxygen on $\mathrm{Ag}(110)$ : A Study of the Interactions among Water Molecules, Hydroxyl Groups, and Oxygen Atoms.

PB91-146985

100,270

Influence of Sulfur on Methanation over Tungsten(110). PB91-149369 100,300 Summary Abstract: The Influence of Sulfur on Methanation over $W(110)$

100,301

Mechano-Chemical Descriptive Model for Wear Under Mixed Lubrication Condition

PB91-189480

101,177

Molecular Desorption from Solid Surfaces: Laser Diagnostics and Chemical Dynamics.

100,397

Direct Detection of Atom Vaporization by Laser Resonance lonization as a Probe of Gas-Surface Chemisorption Mechanism

100,425

PB91-237032

Hydroxyl

Neutron Spectroscopic Evide

100,432

Surface X-ray Absorption Spectroscopy, EXAFS and Surface X-ray Absorption Spectroscopy, EXAFS and
NEXAFS, for the In situ and Ex situ Study of Electrodes.
PB92-117035 PB92-117035

100,460

Relation between the Surface Energy and the Debye Temperature for Cubic Solids.

PB91-149393

100,303

Theory of Orientation Textures Due to Surface Energy Anisotropies.
Using Surface Roughness as a Wear Criterion in HighSpeed Steel End-Milling.

101,040

Effect of Pipe Surface Finish on the Orifice Discharge

Coelficient. 101,389 Interactions.

PB91-189241

100,341

Capacitance versus Stylus Measurements of Surface Roughness.
PB91-20346

101,020

Standard Reference Specimens in Ouality Control of En-

$\begin{array}{ll}\text { gineering Surfaces. } & 101,021\end{array}$

Light-Scattering Measurement of the RMS Slopes of Rough Surface

101,436

SURFACE SCIENCE

Technical Activities 1990 Surface Science Division

101,560

SURFACE STRUCTURE

Critical Compilation of Surface Structures Determined by on Scattering Methods.

PB90-244559

100,254

\section{SURFACE TENSION}

Dynamic Technique for Measuring Surface Tension at High Temperatures in Microgravity Environment.

N91-21347/0

101,206

SURFACES

Structure of Surface Films on Magnesium and on Magne sium Alloys.

101,212

Book Review: Polarized Electrons at Surfaces by $\mathrm{J}$ Kirschner.

PB91-174532 101,493

Book Review: 'Polarized Electrons at Surfaces' by J Kirschner.

101,497

$X$-ray Photoelectron Forward Scattering Studies of Surface Segregation in Epitaxial Cu-Ni-Ni(100) Sandwich Structures.

PB91-189258

101,506

Recent Developments in Applied Surface Science. PB91-189936

100,353

\section{SURFACES \& INTERFACES}

Influence of Filament Geometry on Hot Filament Growth of Diamond Films

101,448

Adsorption of Water and Oxygen on $\mathrm{Ag}(110)$ : A Study of Groups, and Oxygen Aloms.

PB91.146985

100,270

Laser Induced Desorption of Molecules from Surfaces. PB91-147066

Soluble Models in Surface Collision Dynamics.

PB91-147298 100,276

Instability in pH Measurements of Sputtered IrO2 Films.

PB91-147538 100,281

Structure of Surface Films on Magnesium and on Magne-

sium Alloys.

101,212

Morphology and Barrier-Height Development of $\mathrm{Bi}$ InP(110) Interfaces.

101,468

Resonant Photoemission and PSD of Rare Earth Oxides. PB91-148015 100,287 Kinetics of Copper Extraction Using (anti)-2-Hydroxy-5 Nonlybenzophenone Oxime.
How Does Surface Roughness Affect Polymer-Surface

PB91-240820 the Interactions among Water Molecules, Hydroxyl

PB91-148783

100,292

Epitaxial fcc Fe Films on Cu(100)

101,478

Influence of Sulfur on Methanation over Tungsten(110).
PB91-149369

Summary Abstract: The Influence of Sulfur on Methanation over $W(110)$

PB91-149377

100,301

Stoichiometry of Reactively Evaporated Films.

101,413

PB91-158808

101,172

Wetting Layers on Solid Substrates.

PB91-158857

100,313

Picosecond IR Studies of the Vibrational Dynamics of $\mathrm{CO} / \mathrm{Pt}(111)$.

PB91-161927

100,314

Analytic Solution for the Three-Layer Multiple Beam Intererometer.

PB91-162107

101,007

Surface-State-Mediated Photochemistry: Laser-Induced Desorption of NO from $\mathrm{Si}$ (111)

PB91-162289

100,320

Micromagnetics of Domain Walls at Surfaces.

PB91-162305

101,491

Stylus Profiling

Mechanisms of Additive Effectiveness.

PB91-174862

101.015

Mechanical Sputtering of Structural Stainless Steel

101,152

Ion-Induced Radiation-Enhanced Diffusion of Silver in

PB91-175554

101,498

Electron Atten

Lead Zirconate-Titanate Thin Films Prepared by the Laser Ablation Technique.

PB91-187344

101,502

$X$-ray Photoelectron Forward Scattering Studies of Surace Segregation in Epitaxial Cu-Ni-Ni(100) Sandwi Structures

101506

Mechano-Chemical Descriptive Model for Wear Under Mixed Lubrication Conditions

PB91-189480

101,177

Sputtered Thin Film YBa2Cu3On.

PB91-189647

Surface Properties and Coatings of Materials Microtailored Alloys.

PB91-189696

101,232

Recent Developments in Applied Surface Science.

100,353

Geometric and Electronic Properties of Cs Structures on III-V (110) Surfaces: From $1 D$ and $2 D$ Insulators to $3 D$

PB91-190090 
Method of FIM-FEEM Specimen Preparation of Superconducting and Other Oxides.

101,542

Direct Detection of Atom Vaporization by Laser Resonance lonization as a Probe of Gas-Surface Chemisorption Mechanisms.

PB91-237032

100,425

Surface Extended-X-ray-Absorption Fine Structure and Scanning Tunneling Microscopy of Si(001)2x1-Sb.
PB91-237263

101,552

(SEMPA)-Studies Microscopy with Polarization Analysis ic Singularities at Surfaces and in Thin Films.

101,554

Structural Study of the Epitaxial Growth of fcc-Fe Films, Sandwiches, and Superlattices on Cu(100).

101,555

Theory of Ballistic-Electron-Emission Spectroscopy of $\mathrm{NiSi2} / \mathrm{Si}(111)$ Interfaces.

101,557

Oxidation Kinetics of Silicon Carbide Whiskers Studied by X-ray Photoelectron Spectroscopy.

PB91-237719

100,436

Technical Activities 1990 Surface Science Division

PB91-240820 101,560

Magnetic Anisotropies in Ultrathin fcc $\mathrm{Fe}(001)$ Films Grown on Cu(001) Substrates.

PB92-116631

101,565

Heat Conductivity of Oxide Coatings by Photothermal Radiometry betw

101,112

Test Chip for the Evaluation of Surface-Diffusion Phenomena in Sputtered Aluminum Planarization Processes.

Kinematic Theory of Ballistic Electron Emission Spectroscopy of Silicon-Silicide Interfaces.

101,570

Observation of Two Different Oscillation Periods in the Exchange Coupling of $\mathrm{Fe} / \mathrm{Cr} / \mathrm{Fe}(100)$.

101,572

SURGES

Incompatibility between the 100/1300 Surge Test and Varistor Failure Rates.

Real, Realistic Ring Waves for Surge Testing.

PB91-189811

00,854

Research for Electric Energy Systems: An Annual Report

PB92-112341 100,874

Need for Power Ouality Testing Standards. PB92-117076

SURVEY MONITORS

Criteria for the Operation of Federally. Owned Secondary

Calibration Laboratories (Ionizing Radiation).
PB92-112481

101,721

SUSPENSIONS

Integrated theoretical and experimental study of the thermophysical properties of fluid mixtures. Progress report (February 1990-February 1991).

WEEP GENERATORS

Raster Graphics Conformance Testing.

101,384

PB91-167296

101,341

Tiled Raster Graphics and MIL-R-28002A: A Tutorial and Implementation Guide.

101,346

SWELLING

Mechanical and Swelling Behavior of Crosslinked Natural Rubber: Consequences of the Flory-Rehner Hypothesis.

Tests of the Flory-Rehner Hypothesis: Comparison of the

Tests of the Flory-Rehner Hypothesis: Comparison of the the Dry and Swollen States.

PB91-203570

SYMBIOTIC STARS

Energy Distributions of Symbiotic Novae.

PB91.200873

100,508

YNCHRONISM

Synchronizing Computer Clocks Using a Local Area NetWork.

100,596

SYNCHROTRON RADIATION SOURCES

Instrumentation of $X$-ray Beam Lines with PIN Diode De-

PB91-174888

101,633

SYNTHESIS (CHEMISTRY)

Molecular Dynamics Study of the Depolymerization Reaction in Simple Polymers.

Synthesis of 3-Ouinuclidinol-(18)O, Benzilic-d5 Acid, and 3-Ouinuclidinyl-(18)O, Benzilate-d5.

100,223

PNTHETIC ELASTOMERS

Relaxation of Crosslinked Networks: Theoretical Models and Apparent Power Law Behavior.

100,501

Small-Angle Neutron Scattering and Light Scattering Studies on the Miscibility of Protonated Polyisoprene/ Deuterated Polybutadiene Blends.
PB91-236786

100,513

Microstructure Effects on the Lower Critical Solution Temperature Phase Behavior of Deuterated Polybuta diene and Protonated Polyisoprene Blends Studied by Small-Angle Neutron Scattering.

100,518

Small-Angle Neutron Scattering and Light Scattering Study on the Miscibility of Poly(styrene-ran-butadiene)/ Polybutadiene Blends.

100,519

SYSTEMS ANALYSIS

Applications of Operations Research Techniques to System Design and Evaluation

101,267

SYSTEMS DESIGN

Applications of Operations Research Techniques to System Design and Evaluation.

PB91-174540

101,267

\section{SYSTEMS ENGINEERING}

Issues in the Design and Implementation of a System Architecture for Computer Integrated Manufacturing 100,987

Guide to Expert System Building Tools for MicrocomputPB92-108893

100,715

Manufacturing Systems Integration: Initial Architecture Document.

100,994

TANKS (CONTAINERS)

Aluminum Ailoys for Cryogenic Tanks: Oxygen Compatibility. Volume 1

101,201

Review of Cryogenic Mechanical and Thermal Properties of Al-Li Alloys and Alloy 2219

AD-A229 231/6

101,202

TANTALUM IONS

Transitions and Energy Levels for Cu-Like $\mathrm{Yb}(41+)$ $\mathrm{Ta}(44+)$, and $\mathrm{U}(63+)$

101,610

TCP

Characterization of Tricresyiphosphate Lubricating Films by Micro-Fourier Transform Infrared Spectroscopy.

\section{ECHNICAL INFORMATION CENTERS}

Abstract and Index Collection in the Research Information Center of the National Institute of Standards and Technology (Fourth Edition).

PB91-148494

100,959

TECHNOLOGY

Advanced Technology Program: A New Role for NIST in Accelerating the Development of Commercially Importan Technologies.

100,013

TECHNOLOGY ASSESSMENT

Innovation in Manufacturing Technology: A View from PB91-148130

100,976

Intelligent Building Technology in Japan

100,071

TECHNOLOGY INCENTIVES

Industry and Government Strategies for Product Ouality.

Trends in Technological Innovation.

PB91-195545

Advances in Science, Emerging Technologies and Global Competition: Opportunities and Challenge.

100,583

Advanced Technology Program: A New Role for NIST in Advanced Technology Program: A New Role for NIST in
Accelerating the Development of Commercially Important Technologies.

100,013

TECHNOLOGY INNOVATION

Trends in Technological Innovation.

PB91-195545

100,008

TECHNOLOGY TRANSFER

Agenda for Progress in Technology Education: A Personal View

PB91-148114

100,037

Transferring NBS Technology to Small Manufacturers PB91-149351

101,025

TECHNOLOGY UTILIZATION

Research Tackles Automation Issues.
PB91-162230

TEETH

Mathematical Model for Dental Caries: A Coupled Dissolution-Diffusion Process.

PB92-126689

101,316

TELECOMMUNICATIONS

Detail Specification for 62.5-mum Core Diameter/125-

mum Cladding Diameter Class la Multimode, Graded

Index Optical Waveguide Fibers.

FIPS PUB 159

100,562

TELEOPERATORS

Short-Term Evolution for the Flight Telerobotic Servicer.

PB91-144352

101,731

Video Compression for Remote Vehicle Driving.

PB91-203208

101,035

TELEROBOTICS

Recommended Fine Positioning Test for the Development Test Flight (DTF-1) of the NASA Flight Telerobotic Servicer (FTS)

PB91-185090

101,732

\section{MPERATURE}

Short-Duration Autoignition Temperature Measurements for Hydrocarbon Fuels.

100,877

Capillary Supercritical Fluid Chromatography of Explosives: Investigations on the Interactions between the Analytes, the Mobile Phase and the Stationary Phase.

PB91-147769

100,182

TEMPERATURE COEFFICIENT OF RESISTANCE

Measurement, Use, and Interpretation of TCR.

PB92-117282

100,831

TEMPERATURE GRADIENTS

Temperature Gradient in Differential Scanning Calori-

PB91-236570

100,414

\section{TEMPERATURE MEASUREMENT}

High-Speed Spatial (Linear) Scanning Pyrometer: A Tool for Diagnostics, Temperature Mapping, and Property Determinations at High Temperatures.

N91-21361/1

100,924

Temperature Measurement of Glass Subjected to Solar Radiation.

Temperature Measurements in Flames Using Thermally Assisted Laser-Induced Fluorescence of $\mathrm{Ga}$. PB91-236851

100,420

TEMPERATURE MEASURING INSTRUMENTS

Calibration Procedures for Infrared Imaging Systems for Building Diagnostics.

100,057

Comparison of Two Test Methods for Determining Transfer Function Coefficients for a Wall Using a Calibrated Hot Box

PB91-187278

100,121

Procedure for the Effective Recalibration of Liquid-in Glass Thermometers.

100,935

\section{TEMPERATURE SCALES}

Differences between Thermodynamic Temperature and $t($ IPTS-68) in the Range $230 \mathrm{C}$ to $660 \mathrm{C}$ PB92-116722

101,725

\section{TENSILE PROPERTIES}

Strain Rate Effect on Tensile Properties at $4 \mathrm{~K}$ of a VAMAS Round-Robin Austenitic Steel.

101,168

TENSILE STRENGTH

Effect of Thermochemical Treatments on the Strength and Microstructure of SiC Fibers.

101,084

TENSILE TESTS

Discontinuous Yielding during Tensile Tests at Low Temperatures.

101,159

TENSION TESTS

Cyclic Fatigue from Frictional Degradation at Bridging Grains in Alumina.

Load-Controlled Tensile Tests of Austenitic Steels at $4 \mathrm{~K}$. PB91-236943 101,165 Uniaxial Tensile Tests to Measure the Bond of In situ Concrete Overlays.

PB92-116300 
TEST FACILITIES

Calibration of Antenna Factor at a Ground Screen Field Site Using an Automatic Network Analyzer.

100,724

Pulse-Echo Ultrasonic Evaluation of the Integrity of Seams of Single-Ply Roofing Membranes: Laboratory Evaluation of a Prototype Test Apparatus.
PB91-157172 100,106 Apparatus for Measurement of Thermal Conductivity of Insulation Systems Subjected to Extreme Temperature Differences.

PB91-162016 101,738

Automated High-Temperature Guarded-Hot-Plate Apparatus for Measuring Apparent Thermal Conductivity of Insu-

lations between 300 and $750 \mathrm{~K}$.
PB91-162115

100,111

Status of Beta-Particle Calibration Studies at NBS

PB91-175265

101,636

Plumbing Test Lab Accreditation

PB91-190066

100,089

Laboratory Accreditation in the United States

100,172

Directory of State and Local Government Laboratory Accreditation/Designation Programs.

PB92-108968

100,174

Questions and Answers on Quality, the ISO 9000 Stand-

ard Series, Quality System Registration, and Related

PB92-126465

100,175

EST METHODS

Cutting the High Cost of Testing

ESTS

Comprehensive Approach for Modeling and Testing Analog and Mixed-Signal Devices.

100,732

Raster Graphics Conformance Testing

PB91-167296

101,341

Functional Benchmarks for Fourth Generation Lan-

guages.

100,644

Application of Formal Description Techniques to the Specification of Distributed Test Systems.

100,566

MUMPS Validation Suite, Version 7.6 (Vax/VMS Backup),

1991.

100,660

MUMPS Validation Suite, Version 7.6, 1991 (for Microcomputers).
PB91-507707

MUMPS Validation Suite Version 7.6 (ANSI), 1991 , 100,662

PB91-507715

100,662

MUMPS Validation Suite, Version 7.6 (TAR), 1991.
PB91-507723

Proposed Testing Methodology for STEP Application Pro-

tocol Validation. National PDES Testbed Report Series.

Test Methods for Optical Disk Media Characteristics (for

$356 \mathrm{~mm}$ Ruggedized Magneto-optic Media). 100,605

TETRACYCLINES

Adsorption and Orientation of Tetracycline on Hydroxya-

Patite.

101,319

TEXT PROCESSING

Collection of Technical Studies Completed for the Computer-Aided Acquisition and Logistic Support (CALS) Program. Fiscal Year 1988. Volume 1 of 3. Text, Security and Data Manag

101,342

THALLIUM BARIUM CALCIUM CUPRATES

Phase Equilibria in the System TI-Ca-Ba-Cu-O. 1. Stability of the 2122 Phase under Conditions of Oxygen AnnealPB91-147165

101,465

THEOPHYLLINE

Liposome Flow Injection Immunoassay: Implications for Sensitivity, Dynamic Range, and Antibody Regeneration. PB91-147629

101,291

Planar Waveg

101,284

THERMAL ANALYSIS

Diagnostic Techniques for Evaluating Office Building Envelopes.

PB91-195438 100,074

Temperature Gradient in Differential Scanning Calori-

metry.

100,414

THERMAL CONDUCTIVITY

Benzene: A Further Liquid Thermal Conductivity Standard

PB90-244567

100,255

Transport Properties of Carbon Dioxide.

100,266

Thermal Conductivity of a Moderately Dense Gas.

PB91-134502 101,601 Thermal Conductivity and Heat Capacity of Gaseous Argon.
PB91-134577

101,602

Thermal Conductivity of a Polymide Film between 4.2 and $300 \mathrm{~K}$, with and without Alumina Particles as Filler. 100,483

Evaluation of Fumed-Silica Insulation for a Thermal Conductivity Standard Reference Material.

100,103

Round Robin on Apparent Thermal Conductivity of Several Loose-Fill Insulations.

PB91-149948

101,143

Apparatus for Measurement of Thermal Conductivity of Insulation Systems Subjected to Extreme Temperature Differences.

101,738

Automated High-Temperature Guarded-Hot-Plate Apparatus for Measuring Apparent Thermal Conductivity of Insulations between 300 and $750 \mathrm{~K}$

100,111

Thermal Conductivity of Evacuated Perlite at Low Temperatures as a Function of Load and Load History.
100,227 Thermal Conductivity of Nine Polyatomic Gases at Low

PB91-192492

100,360

Thermal Conductivity of Methane and Tetrafluorometh. ane in the Limit of Zero Density.

100,361

Viscosity and Thermal Conductivity of Pure Monatomic Gases from Their Normal Boiling Point Up to $5000 \mathrm{~K}$ in the Limit of Zero Density and at $0.101325 \mathrm{MPa}$.

PB91-192559

100,366

Experimental Thermal Conductivity, Thermal Diffusivity, and Specific Heat Values for Mixtures of Nitrogen, Oxygen, and Argon.

100,369

High-Temperature Transient Hot-wire Thermal Conductivity Apparatus for Fluids.

100,411

Heat Conductivity of Oxide Coatings by Photothermal Ra. diometry between 293 and $1173 \mathrm{~K}$

diometry betwe

101,112

Thermal Conductivity of Alumina Fiber/Epoxy and Alumina Fiber/PEEK from 4.2 to $310 \mathrm{~K}$

PB92-117258

101,130

Apparent Thermal Conductivity of Fumed.Silica Standard Reference Materials.

PB92-117357

101,102

THERMAL CONVERTERS

AC-DC Difference Relationships for Current Shunt and

Thermal Converter Combinations.

PB91-236893

100,737

THERMAL DEGRADATION

Effects of Pressure on the Thermal Decomposition Kinet-

ics and Chemical Reactivity of Nitromethane.

PB91-147884

100,286

Molecular Dynamics of Initial Events in the Thermal Deg. radation of Polymers.

PB91-195271

100,503

THERMAL DIFFUSIVITY

Thermal Conductivity and Heat Capacity of Gaseous

101,602

PB91-134577

Experimental Thermal Conductivity, Thermal Diffusivity,
and Specific Heat Values for Mixtures of Nitrogen, Oxygen, and Argon.

100,369

High-Temperature Transient Hot-wire Thermal Conductivi-

High-Temperature Tran
ty Apparatus for Fluids.
PB91-216713

100,411

THERMAL EMISSION

Characterization of Cylindrical Holes in Metallic SubStrates via Their

101,228

THERMAL ENERGY STORAGE SYSTEMS

Method for Characterizing the Thermal Performance of a Solar Storage Wall from Measured Data

100,901

THERMAL EXPANSION

Effect of Thermal Expansion Mismatch on Fiber Pull-Out

in Glass Matrix Composites
PB91-202952

101,125

\section{THERMAL INSULATION}

Forced Smolder Propagation and the Transition to FlamPB91-147835ic Insulation. Smoldering Combustion Propagation Through a Permeable Horizontal Fuel Layer.
PB91-147843

100,102

Evaluation of Fumed-Silica Insulation for a Thermal Conductivity Standard Reference Material.

100,103

Round Robin on Apparent Thermal Conductivity of Sever. al Loose-Fill Insulations.

al Loose-Fill Insu

101,143

Apparatus for Measurement of Thermal Conductivity of Insulation Systems Subjected to Extreme Temperature Differences.
PB91-162016

101,738

Automated High-Temperature Guarded-Hot-Plate Apparatus for Measuring Apparent Thermal Conductivity of Insulations between 300 and $750 \mathrm{~K}$

162115

100,111

ZIP: The ZIP-Code Insulation Program, Version 2.0. Economic Insulation Levels for New and Existing Houses by Three-Digit Zip Code. Users' Guide and Reference Manual (Revised Edition).

PB91-167155

Emissivity of Aluminized Myla

PB91-174656

100,064

101,119

space Vehicles.

Summary of Low-Density Glass-Fiber Reference Materials at NIST: $1980-1989$

PB91-175620

101,079

Acoustic Technique for Evaluation of Thermal Insulation.

PB91-189324 100,123

Thermal Conductivity of Fumed Silica Board at Room Temperature.
PB91-190124

100,125

THERMAL MEASUREMENTS

Method for Measuring Heat Loss from Underground Heat Distribution Systems.

PB91-134221

100,888

Thermal

PB91-147827

100,790

Apparatus for Measurement of Thermal Conductivity of Insulation Systems Subjected to Extreme Temperature Differences.

PB91-162016

101,738

Measurement of Thermal Characteristics of Office Build-

ings.

100,068

Comparison of Two Test Methods for Determining Transfer Function Coefficients for a Wall Using a Calibrated Hot Box.

PB91-187278

100,121

Evaluation of the Heat Flux Transducer Technique for Measuring the Thermal Performance of Walls. PB91-194696

100,126

\section{THERMAL NEUTRONS}

Examination of Mechanisms Responsible for Incoherent Scattering of

THERMAL NOISE

Thermal Noise in Mechanical Experiments.

PB91-149211

Intercomparison of NBS Noise Calibration Services.

PB91-162370

\section{THERMAL PLASMAS}

Overview of the Ion Dynamic Effect in Line Broadening, and a Generaliza

THERMAL PROPERTIES

Review of Cryogenic Mechanical and Thermal Properties of Al-Li Alloys and Alloy 2219.

AD-A229 231/6

101,202

THERMAL RADIATION

Fadiant Heat Fluxes from 100-200 MW Natural Gas/Air

Diffusion Flames.
PB91-147306

100,538 


\section{KEYWORD INDEX}

THERMOCOUPLES

Automated High-Temperature Guarded-Hot-Plate Apparatus for Measuring Apparent Thermal Conductivity of Insulations between 300 and $750 \mathrm{~K}$

PB91-162115

100,111 Transparent

100,783

THERMODYNAMIC DIAGRAMS Thermodynamic Diagrams for Refrigerant Mixtures.
PB91-236695 107

THERMODYNAMIC EQUILIBRIUM

Critical Locus, (Vapor + Liquid) Equilibria, and Coexisting Densities of (Carbon Dioxide + Propane) at Temperatures from $311 \mathrm{~K}$ to $361 \mathrm{~K}$.

100,267

Phase Equilibria and Critical Lines in Model Water/Salt Mixtures.

100,418

PB91-236778

Thermodynamics of the Disproportionation of Adenosine sine 5'-monophosphate. 2. Experimental Data.

PB92-117399

101,313

\section{THERMODYNAMIC PROPERTIES}

Thermodynamic Functions and Properties of $\mathrm{MgO}$ at High Compression and High Temperature.

100,253

Standard Chemical Thermodynamic Properties of Isomer Groups of Monochloroalkanes.

100,258

Standard Chemical Thermodynamic Properties of Polycyclic Aromatic Hydrocarbons and Their Isomer Groups. III. Naphthocoronene Series, Ovalene Series, and First Members of Some Higher Series.

PB90-244625

100,259

Cryogenic Properties of Copper (Wall Chart).

PB91-148189

101,473

Thermodynamic Properties of Two Alternative Refrigerants: 1,1-dichloro-2,2,2-trifluoroethane (R123) and 1,1,1,2-tetrafluoroethane (R134a)

101,195

Modeling the Thermodynamic Properties of Sodium Chloride in Steam Through Extended Corresponding States.
PB91-189381 Modelling Aqueous Solutions Near the Critical Point of

Pater.

100,349

Journal of Physical and Chemical Reference Data, Volume 19, Number 5, September-October 1990. 192476

100,358

Unified Fundamental Equation for the Thermodynamic Properties of $\mathrm{H} 2 \mathrm{O}$.

100,364

Thermodynamic and Thermophysical Properties of Organic Nitrogen Compounds. Part 1. Methanamine, Ethanamine, 1 - and 2-Propanamine, Benzenamine, 2-, 3-, and
4-Methylbenzenamine.

PB91-192583 100,368

Thermodynamic Properties of Aqueous Solutions at High Temperatures; Needs, Methods, and Challenges. 100,410
PB91-204065 Thermodynamic Behavior of Fluids in the Supercritical

PB91-204123

101,394

Thermodynamic Diagrams for Refrigerant Mixtures.

101,197

Thermodynamic Properties of 1,1,1,2,-Tetrafluoroethane (R134a) in the Critical Region

PB91-237578

100,233

Database and Retrieval System for the NBS Tables of Chemical Thermodynamic Properties.

100,434

Heat Capacity and Other Thermodynamic Properties of Heat Capacity and Other Thermodynamic Properties of
Linear Macromolecules. 10. Update of the ATHAS 1980 Data Bank.

100,515

Thermodynamic Properties of the $\mathrm{NaBr}+\mathrm{H} 2 \mathrm{O}$ System.
PB92-110238

Horizontal Flow Boiling Heat Transfer Experiments with a Mixture of R22/R114. PB92-116946

101200

Ab-Initio Calculations and Ideal Gas Thermodynamic Functions of Cyclopentadiene and Cyclopentadiene De-

rivatives.
PB92-148089

100,468

Journal of Physical and Chemical Reference Data, Volume 20, Number 5, September/October 1991. Thermodynamic Properties of Oxygen from the Triple Thermodynamic Properties of Oxygen from the Triple Point to $300 \mathrm{~K}$ with Pressures to $80 \mathrm{MPa}$. $\quad 100,474$
PB92-148147 100,474
SBixteen Thousand Evaluated Experimental Thermodynamic Property Data for Water and Steam.

100,475

New Equation of State and Tables of Thermodynamic Properties for Methane Covering the Range from the Melting Line to $625 \mathrm{~K}$ at Pressures up to $1000 \mathrm{MPa}$. PB92.148170
100,477 Thermodynamic Properties of the Aqueous Sulfuric Acid System to $350 \mathrm{~K}$.
PB92-148188

100,478

THERMODYNAMICS

Thermodynamics of Hydrolysis of Oligosaccharides.
101,288

Thermodynamics of Hydrolysis of Disaccharides: Lactulose, alpha-D - Melibiose, Palatinose, D - Trehalose, D Turanose and 3-o-beta-D-Galactopyranosyl-D-arabinose,
PB91-195701

Thermodynamics of Industrially Important Enzyme Cata yzed Reactions

101,311

THERMODYNAMICS \& CHEMICAL KINETICS

MSW Calorimetry

Stability of Thermistors

100,878

PB91-134320

100,779

Critical Locus, (Vapor + Liquid) Equilibria, and Coexisting Densities of (Carbon Dioxide + Propane) at Temperatures from $311 \mathrm{~K}$ to $361 \mathrm{~K}$

PB91-134494

100,267

Critical Exponent for Viscosity.

B91.147009

100,271

Heat Capacity and Thermodynamic Properties of Deutrated Ammonium Hexafluorophosphate ND4PF6 from $5.8 \mathrm{~K}$ o $347 \mathrm{~K}$

PB91-147041

100,272

Alternative Refrigerants R123a, R134, R141b, R142b, and A152a: Critical Temperature, Refractive Index, Surface Tension, and Estimates of Liquid, Vapor, and Critical

101,189

NBS Research Program in Municipal Solid Waste Com-

PB91-147264

100,916

Mechanism of Soot Formation in Acetylene-Oxygen Mix-

PB91-147280

100,537

Limiting versus Apparant Critical Behavior of Henry's Constants and $K$ Factors.

PB91-147363 100,277

Mechanism of $\mathrm{OH}$ Radical Reactions with Thymine and Uracil Derivatives.

Alkyl Transfer Reactions between Protonated Alcohols and Ethers. The Gas-Phase Alkylation of Formaldehyde.

100,280

Instrument-Independent Tandem Mass Spectrometry Database for $X Q Q$ Instruments: The Dynamical Prerequi-

PB91-147694

100,181

Multicomponent Cluster Ions. 2. Comparative Stabilities of Cationic and Anionic Hydrogen Bonded Networks: Mixed Clusters of Water and Hydrogen Bonded PB91-147710

Pulse Radiolysis Studies of Organic Electron Transie Peactions.

100,285

Strategy for Chemical Analysis of Alternative Refriger-

PB91-148502

100,185

Anisotropic Structure of a Simple Liquid.

101,616

Zonal Model for Corona Discharge-Induced Oxidation of $\mathrm{SF} 6$ in $\mathrm{SF} 6 / \mathrm{O} 2 / \mathrm{H} 2 \mathrm{O}$ Gas Mixtures.

PB91-158790

100,312

Redox Reaction

100,217

Method for Characterizing the Thermal Performance of a Solar Storage Wall from Measured Data.

PB91-159152

Steam Tables

100,901

Research at NBS on Dynamic Measurements of Thermo physical Properties at High Temperature (Abstract).

Phase Transitions

Unified Description of Infinite-Dilution Thermodynamic Properties for Aqueous Solutes.

100,319

Computer Simulation of Fluid-Fluid Phase Coexistence in Mixtures of Nonadditive Soft Disks.

PB91-162263

101,388

Technical Activities 1990, Standard Reference Data Pro-

gram.

Monitoring the Fate of Chlorine from MSW Sampling through Combustion.

100,917

Viscosity Surface for Mixtures of Methane and Ethane.

PB91.174763

100,322

Thermochemistry of Sulfoxides and Sulfones.

100,325

Formation and Stability of SF5 and S2F10 PB91-174839

100,326

Letter to Editor of Chemical and Engineering News.

100,327

Group Reactivities in the Gas Phase Reactions of $\mathrm{Hy}$ droxyl Radicals with Ethers.

PB91-175059

100,330

Single Pulse Shock Tube Study of the Reactions of Hydrogen Atoms with Aromatics. 4. Chlorotoluenes.

PB91-175133

100,331

Concerning the Formation and the Kinetics of Phenylium

PB91-187138

100,336

$\mathrm{H}_{2} \mathrm{O}$ (I)- $\mathrm{H} 2 \mathrm{O}$ (III)- $\mathrm{H} 2 \mathrm{O}$ (L) Triple Point of Water as a Fixed PB91.187211

101,647

Combustion Calorimeters.

PB91.189233

100,340

Modeling the Thermodynamic Properties of Sodium Chlo-

ride in Steam Through Extended Corresponding States.

100,342

Elfect of Long Range Interfacial Forces on the Dynamics of Wetting Layers at a Liquid Vapor Interface.

PB91-189431

100,344

Energy of Combustion of Triphenylphosphate.

100,347

Modelling Aqueous Solutions Near the Critical Point of

PB91-189837

100,349

Ergodic Measures for the Simulation of Dielectric Proper-

ties of Water.

100,350

Polyelectrolyte Stabilized Metal Oxide Hydrosols as Cata lysts for the Photooxidation of Water by Zinc Porphyrins.
PB91-189852

Proton Affinity of Cyanogen and Ion-Molecule Reactions of $\mathrm{C} 2 \mathrm{~N} 2(+)$

100,352

Thermodynamics of Hydrolysis of Oligosaccharides

PB91-194803 101,288

Carbon Acidities of Aromatic Compounds. 2. Ionic Probes of Aromaticity in Annelated Rings.

PB91-195065

100,375

Thermal Decomposition of lons. 3. Protonated Ethanol and Diethyl Ether.

Proton Affinities

100,376

Rate Constants for Reduction of Substituted Methylper oxyl Radicals by Ascorbate Ions and N,N,N',N'-Tetramethyl-Para-Phenylenediamine.

PB91-195230

100,379

Reactions of $\mathrm{C} 5 \mathrm{H} 3(+)$ ) and $\mathrm{C} 5 \mathrm{H} 5(+)$ ) lons with Acetylene and Diacetylene.

PB91-195370

100,384

Thermodynamics of Hydrolysis of Disaccharides: Lactulose, alpha-D - Melibiose, Palatinose, D - Trehalose, D Turanose and 3-o-beta-D-Galactopyranosyl-D-arabinose. $-195701$

101,289

Reactivities of Chlorine Atoms and Peroxyl Radicals Formed in the Radiolysis of Dichloromethane.

PB91-200782 
PB91-236570

Thermodynamic Diagrams for Refrigerant Mixtures.

PB91-236695 101,197

Phase Equilibria and Critical Lines in Model Water/Salt Mixtures.

PB91-236778 100,418

Thermodynamics of Solutions Near the Solvent's Critical Point.

PB91-237297

100,232

Antioxidation Mechanisms of Uric Acid

100,222

PB91-237347

Synthesis of 3-Ouinuclidinol-(18)O, Benzilic-d5 Acid, and
3-Ouinuclidinyl-(18)O, Benzilate-d5.
PB91-237370 PB91-237370

Stabilities of Substituted Benzyl Radicals: Dissociation
Rates of Aminoethylbenzenes, Hydroxyethylbenzenes, Rates of Aminoethylbenzenes. Hydroxyethylbenzenes, and Cyanoethy
PB91-237529

100,430

Dissociation of Substituted Anisoles: Substituent Effects on Bond Strengths.

PB91-237537

100,431

Thermodynamic Properties of 1,1,1,2,-Tetrafluoroethane (R134a) in the Critical Region.

100,233

Thermodynamics of Industrially Important Enzyme Catalyzed Reactions.

PB91-237602

101,311

Extended Phase Rule for Nonreactive, Multiphase, Multicomponent Chemical Systems.

100,433

Surface Oxidation Kinetics of Si3N4 - 4\% Y2O3 Powders Studied by Bremsstrahlung-Excited Auger Spectroscopy.
PB91-237701

THERMOGRAPHY

Fundamentals and Applications of Infrared Themography for Nondestructive Testing.

PB91-147132
Roughness Measures of Blasted Steel Surfaces Remotely Imaged with a Thermographic Camera

101,146

THERMOLUMINESCENT DOSIMETRY

Photon Energy Dependence of the Sensitivity of Radiochromic Film and Comparison with Silver Halide Film and LiF TLDs Used for Brachytherapy Dosimetry.

101,296 PB92-117142

THERMOMECHANICAL TREATMENT

Low Carbon Steel: Metallurgical Structure vs. Mechanical Properties.

N9 1-20218/4

101,144

Continuous-Cooling Transformation Characteristics and High-Temperature Flow Behavior of a Microalloyed SAE 1141 Steel.

101,155

THERMOMECHANICAL TREATMENTS

Data-Reduction and Analysis Procedures Used in NIST'S Thermomechanical Processing Research.

100,925

HERMOMETERS

Procedure for the Effective Recalibration of Liquid-inGlass Thermometers

THERMOPHYSICAL PROPERTIES

Integrated theoretical and experimental study of the thermophysical properties of fluid mixtures. Progress report

(February 1990-February 1991).
DE91012660

101,384

Research at NBS on Dynamic Measurements of Thermophysical Properties at High Temperature (Abstract).
PB91.161968 Thermodynamic and Thermophysical Properties of Or. ganic Nitrogen Compounds. Part 1. Methanamine, Ethanamine, 1. and 2-Propanamine, Benzenamine, 2-, 3-, and 4-Methylbenzenamin

PB91-192583

100,368

Taking the Measure of Fluid Properties Data Bases.

PB91-200832 101,392

Thermodynamic Properties of Aqueous Solutions at High Temperatures; Needs, Methods, and Challenges

PB91-204065

100,410

Thermophysical Properties of Ethane.

100,447

Survey of Current Worldwide Research on the Thermophysical Properties of Alternative Refrigerants.
PB92-112366 Predictive Extended Corresponding States Model for Pure and Mixed Refrigerants.

PB92-116748

101,199

THERMOSETTING RESINS

Polymer Composite Processing. Industry Workshop (2nd).

Held at Gaithersburg, Maryland on May 18, 1990.
PB91-132191

101,115

THERMOSOLUTAL CONVECTION

Effect of Gravity Modulation on Thermosolutal Convec-

tion in an Infinite Layer of Fluid.

PB92-112291

101,396

THICKNESS

Determination of Bonded Phase Thickness in Liquid Chromatography by Small Angle Neutron Scattering.
PB91-149187

\section{THIN FILMS}

Assessment of Uncertainties in Measurement of $\mathrm{pH}$ in Hostile Environments Characteristic of Nuclear RepositorNUREG/CR-5711

101,367

Effects of Different CH4-H2 Gas Compositions on the Morphology and Growth of Diamond Grown by Hot Filament CVD.

PB91-158683

Transparent Thin Film Thermocouple.

101,054

B91.190116

100,783

Magnetic Anisotropies in Ultrathin fcc $\mathrm{Fe}(001)$ Films Grown on Cu(001) Substrates.

PB92-116631

101,565

THORIUM OXIDES

Anomalous Th M3O1 X-ray Emission Spectrum of ThO2 PB91-195297

100,381

THREE DIMENSIONAL MODELS

Development of a Three-Dimensional Finite Element Program for Large Strain Elastic-Plastic Solids

101,494

Extracting STEP Geometry and Topology from a Solid Modeler: Parasolid-to-STEP

100,983

Geometric Reasoning for Constructing 3D Scene Descriptions for Images.

PB91-237677

100,714

THYMINE

Mechanism of $\mathrm{OH}$ Radical Reactions with Thymine and Uracil Derivatives.

PB91-147447

100,216

Structure and Mechanism of Hydroxyl Radical-Induced Formation of a DNA-Protein Cross-Link Involving Thymine and Lysine
PB91-187518

101,334

\section{TIME MEASUREMENT}

GPS Time Transfer with Implementation of Selective N91-25766/7

100,576

Synchronizing Computer Clocks Using a Local Area Network.

Israel's New Synchronized Time Scale, UTC(INPL) PB91-134593

100,596

TIME SERIES ANALYSIS

Root Projection of One-Sided Time Series.

PB91-216754

100,577

Deconvolution

101,262

PB91-216762

101,263

TIME SIGNALS

NIST Time and Frequency Services.

PB91-231605

100,585

TIME STANDARDS

Advances in Science, Emerging Technologies and Global Competition: Opportunities and Challenge.

100,583

TIME TRANSFER

Precise Ephemerides for GPS Time Transfer. PB91-236950

100,586

TIN ALLOYS

Guideline for $\mathrm{Nb}$ (sub 3) Sn critical current measurements using fiberglass-epoxy composite sample mandrels. DE91016274

101,205

TIN ISOTOPES

Observation of Anomalous Isotope Ratios in SIRIS Measurements of Moly
PB92-116789

101,361

TIN ORGANIC COMPOUNDS

Laser Enhanced Ionization as a Selective Detector for Liquid Chromatographic Determination of Alkyltins in Sediment.

PB92-116755

100,210

TIN OXIDES

Transparent Thin Film Thermocouple.

PB91-190116

100,783

Chemical and Electronic Properties of $\mathrm{Pd} / \mathrm{SnO} 2(110)$ Model Gas Sensors.

Model Gas Sensc
PB91-203075

100,393

TISSUES (BIOLOGY)

Alaska Marine Mammal Tissue Archival Project: Revised Collection Protocol

PB91-184796

101,374

Interlaboratory Comparison of Actinides in Human Tissue (239) Pu $+(240) \mathrm{Pu}$.

101,323

\section{TITANIUM ALLOYS}

Role of Elastic Energy in the Morphological Development of a Ni-Ti-Al Alloy.
PB91-187161

Corrosion Principles in Dental Implantology.

101,227 PB91-194787

100,055

Microstructural Studies of Ti-Al Alloys in the Vicinity of the 'Eutectoid' Reaction

PB92-116938

101,241

\section{TITANIUM BORIDES}

Low Temperature Chemical Process for Precursors to Boride and Carbide Ceramics Powders.

PB91-149096

101,066

Low Temperature Synthesis of Ceramic Powders for Structural and Electronic Applications.

PB91-149104

101,067

TOKEN RING NETWORKS

Conformance Verification of FDDI Stations

PB91-148254

100,747

TANCES (MECHANICS)

Review of Current Geometric Tolerancing Theories and Inspection Data Analysis Algorithms.

PB91-167221

101,016

TOMOGRAPHY

Image Ouality Indicator Design for Radioscopy and Tomography.
PB91-190058

101,018

Use of Cross-Validation as a Stopping Rule in Emission Tomography Image Reconstruction.

TOOLS

Computer Vision Based Station for Tool Setting and Tool Form Measurement

PB91-202978

101,043

TORSION TESTS

Mechanism for High Strain-Rate Shear Band Formation.
PB91-147033

Mechanism for Shear Band Formation in the High StrainRate Torsion Test.

Rate Torsion Te
PB91-149872

101,579

\section{TOUGHNESS}

Spatially Overdetermined Analysis for Propagation

PB91-236505

101,164

Interlaboratory Tension and Fracture Toughness Test Results for CSUS-JN1 (Fe-25Cr-15Ni-0.3N) Austenitic Stainless Steel at $4 \mathrm{~K}$

PB91-237081

101,166

Effect of Processing on 4-K Mechanical Properties of a Microalloyed Aus
PB91-237206

101,167

Mechanical Properties and Fracture Toughness of AAR TC128 Grade B Steel in the Normalized, and Normalized and Stress Relieved Conditions.

PB92-108901

101,169

TOXIC SUBSTANCES

Newest Approaches to Quantitative Assessment of Bioactive Organotins.

TOXICITY

Newest Approaches to Ouantitative Assessment of Bioactive Organotins

PB91-162032

101,328

Base Modifications in Plasmid DNA Caused by Potassium Permanganate

101,329

Copper-lon-Dependent Damage to the Bases in DNA in the Presence of Hydrogen Peroxide.

PB91-187104 


\section{KEYWORD INDEX}

TRACE ANALYSIS

High Purity Chemicals and Clean Rooms for Trace Meta Analysis.
PB91-195156

TRACE ELEMENTS

Practical Constraints in Monitoring Micronutrient Intake. PB91-147421

101,318

Characterization of the Mineral Fraction in Botanical Reference Materials and Its Influence on Homogeneity and Analytical Results.

\section{TRACERS}

100,186

Ventilation System Performance Evaluation Using Tracer Gas Techniques

100,060

TRACKING (POSITION)

Real-Time Model-Based Tracking Combining Spatial and Temporal Features.

100,700

TRAINING

Multimedia Courseware in an Open Systems Environ. ment: A Federal Strategy.

PB91.143362

100,039

TRAINING DEVICES

Interactive Courseware is Leading the Multimedia Move-

PB91-147744

100,036

TRAJECTORIES

Convergence Behavior of Trajectories for Linear Programming.

101,270

TRAJECTORY CONTROL

Generation of Smooth Trajectories without Planning.

TANSDUC

Probe-Corrected Spherical Near-Field Scanning Theory in Acoustics.

PB92-117456

101,383

TRANSGRANULAR CORROSION

Cleavage Step Formation and Resistance to Transgranu. lar Stress Corrosion Cracking.

PB91-149047

101,133

TRANSIENT MOLECULES

Vibrational and Electronic Energy Levels of Polyatomic Transient Molecules. Supplement

100,367

$$
\text { PB91-192575 }
$$

100,797

Modelling Gallium Arsenide Transistors.

PB91-149989

Automated Reverse-Bias Second-Breakdown Transistor Tester.

100,813

Automated Reverse-Bias Second-Breakdown Transistor

Tester.
PB91-236513

100,814

TRANSITION METALS

Giant Resonances in the Transition Regions of the PeriOdic Table.

101,605

XANES of Transition Metal Zinc-Blende Semiconductors. PB92-117092

100,462

TRANSITION PROBABILITIES

Working Group 2: Atomic Transition Probabilities. 101,645

Spectroscopic Data Base for Carbon and Oxygen

PB91-237750 101,716

Unified Set of Atomic Transition Probabilities for Neutral

PB91-237768

101,717

TRANSITION TEMPERATURES

Critical Evaluation of Liquid Crystal Transition Temperatures I: 4,4'-Alkyl/alkoxyphenylbenzoates.
PB92-110154 100,444

TRANSLATORS

Fed-X: The NIST Express Translator. Revised, November 1990.

100,681

Extracting STEP Geometry and Topology from a Solid $\begin{array}{ll}\text { Modeler: Parasolid-to-STEP. } & 100,983 \\ \text { PB91-194449 }\end{array}$

RANSMISSION LINES

Ouasi-Static Analysis of a Two-Wire Transmission Line Located at an interface.

100,564

Characteristic Impedance Determination Usıng Propagation Constant Measurement.

100,775

Air Gage Size Measurement of Microwave Standards

PB92.117324 100,777

TRANSMITTANCE

Use of Heterodyne Detection to Measure Optical Transmittance over a Wide Range.

PB91-148908

101,410

TRANSPORT PROPERTIES

Multi-Zone Dispersal Analysis by Element Assembly.
PB91-187146
TRAPPED PARTICLES

Trapped-Ion Frequency Standards N91-25760/0

100,575

TRENDS

Trends in Technological Innovation. PB91-195545

100,008

TRIALKYLTINS

Laser Enhanced Ionization as a Selective Detector for Liquid Chromatographic Determination of Alkyltins in PB92-116755

100,210

TRIBOLOGY

Study of the Galling of Two Steels Using Two Test Meth-

PB91-148106

101,147

Mechanisms of Additive Effectiveness

101,172

Method for Ouantitative Measurement of Galling

PB91-159269

101,183

Mechanisms of Additive Effectiveness

101,174

ACTIS: Towards a Comprehensive Tribology Data Base.

PB91-174904 101,184

Study of Additive Response in a Series of Rerefined

Base Oits Typical of Current Commercial Practice.

101,175

Study on the Nature of Boundary Lubricating Film: Analytical Method Development.

101,176

Mechano-Chemical Descriptive Model for Wear Under Mechano-Chemical Descriptive Model for Wear Under Mixed Lubrication Condition.

101,177

Ceramic Wear Maps.

Tribological Characteristics of Synthesized Diamond Films on Silicon Carbide

Diamond

Comparison of Standard Test Methods for Non-Lubricated Sliding Wear.

PB91-190017

101,187

Sliding Wear Studies of Nickel-Copper Composition-Modulated Coatings on Steel.

PB91-190025

101,188

Diesel Deposit Forming Tendencies - Microanalysis Meth-

PB91-195412

101,178

Characterization of Tricresylphosphate Lubricating Films by Micro-Fourier Transform Infrared Spectroscopy. Numeric Database for Tribology: Format and Application

101,045

PB91-203943

101,180

tion of Silicon Nitride.

PB91-236711

TRIBUTYLTINS

Simultaneous Butyltin Determinations in the Microlayer, Water Column and Sediment of a Northern Chesapeake Bay Marina and Receiving System. PB91-195040

100,918

TRICHLOROFLUOROMETHANE

Polarized X-ray Emission Studies of Methyl Chloride and the Chlorofluoromethanes.

RIDIAGONAL MATRICES

Algorithms for Special Tridiagonal Systems. PB91-187245

100,329

IGLYCINE SULFATES

High Resolution Diffraction Imaging of Crystals Grown in Microgravity and Closely Related Terrestrial Crystals.
PB92-109008

101,562

TRIPHONYLPHOSPHATE

Energy of Combustion of Triphenylphosphate. PB91-189613

100,347

TROI (TELEROBOTIC OPERATOR INTERFACE) TROI User's Guide.

PB91-157214

101,026

UBE DISCHARGE FLOW CONDITIONERS

Effect of Tube Bundle Flow Conditioners on Orifice Meter DBscharge Co

100,881

TUNGSTEN

Influence of Sulfur on Methanation over Tungsten(110).
PB91-149369

Summary Abstract: The Influence of Sulfur on Methanation over $W(110)$

100,301

Elastic Constants of a Tungsten-Particle Copper-Matrix Composite.

101,234

TUNNEL EFFECT

Tunneling Stabilized Magnetic Force Microscopy: Prospects for Low Temperature Applications to Superconduc. pects for Low Temperature Applications to Superconductors. 203653

101,546

TUNNELING STABILIZED MAGNETIC FORCE

Tunneling Stabilized Magnetic Force Microscopy: Pros pects for Low Temperature Applications to Superconduc-

PB91-203653

101,546

TURBULENT FLOW

Development of a Line Camera for Real-Time Measurements of Concentration in Turbulent Flow Fields

PB91-147892 101,386

Effects of Global Density Ratio on the Centerline Mixing Behavior of Axisymmetric Turbulent Jets.

PB92-117183

100,014

Reynolds Number Effects on the Mixing Behavior of Axisymmetric Turbulent Jets.

PB92-117209

100,015

TWO-DIMENSIONAL CALCULATIONS

Numerical Study of Two-Dimensional Crystal Growth PB91-200865

TWO PHASE AQUEOUS EXTRACTION

Hydrodynamics and Mass Transfer in Two-Phase Aqueous Extraction Using Spray Columns

101,300

TYPE 2 SUPERCONDUCTORS

Magnetization of Anisotropic Superconducting Grains. PB91-134569

101,463

TYROSINE

Hydroxyl Radical Induced Cross-Linking of Cytosine and Tyrosine in Nucleohistone.

PB91-189365

101,304

Formation of ortho-Tyrosine by Radiation and Organic Solvents in Chicken Tissue.

101,290

ULTIMATE STRENGTH

Ultimate Strength of Masonry Shear Walls: Predictions versus Test Results.

PB92-116342

100,138

ULTRACOLD ATOMS

Observation of Atoms Laser-Cooled Below the Doppler Limit.

101,655

Observation of Atoms Laser Cooled Below the Doppler LBmit.

ULTRASONIC TESTS

Pulse-Echo Ultrasonic Evaluation of the Integrity of Seams of Single-Ply Roofing Membranes: Laboratory Evaluation of a Prototype Test Apparatus.

PB91-157172

100,106

Pulse-Echo Ultrasonic Evaluation of the Integrity of Peams of Single-Ply Roofing Membranes: Hesults of Field Investigations and Recommendations.

PB91-159731 100,110

Elastic Constants of Fiber-Reinforced Composites: A Fresh Measurement Approach

101,123

Ultrasonic Texture and Stress Measurements in Anisotropic Polycrystalline Aggregates.

101,237

Monitoring of Anisotropic Material Elastic Properties Using Uitrasonic Receiving Rays.

PB91-203059

101. 126

Residual Stress Detection in Railroad Wheels: An Ultrasonic System Using EMATs (Electromagnetic-Acoustic 
ULTRAVIOLET SPECTROMETERS

Scattered Light and Other Corrections in Absorption Co efficient Measurements in the Vacuum Ultraviolet: A Systems Approach.

100,179

UNCERTAINTY

Uncertainty and Accuracy in Physical Measurements.
PB91-167205

UNDERGROUND CORROSION

Measuring the Corrosion of Metals in Soil. DERGROUND MINING

Task Decomposition and Algorithm Development for Real-Time Motion Control of a Continuous Mining $\mathrm{Ma}$

PBine. 231597

101,357

UNDERWATER VEHICLES

Real-Time Hierarchical Planning for Multiple Mobile Robots.

101,375

UNITED STATES

Congressional Districts of the United States. Category: Federal General Data Standard, Representations and Codes.

FIPS PUB 9-1

100,679

Standards Activities of Organizations in the United States.

UNITS OF MEASUREMENT

100,004

International System of Units (SI)--Translation. PB92-109032

101,719

Metric Measures Up (Ruler).

PB92-163898

100,942

UNIX (OPERATING SYSTEM)

UNIX in the Government's Automated Manufacturing Research Facility (AMRF).

PB91-147579 100,963

UNIX Expert: A Prototype Knowledge-Based Software Development Workstation.

PB91-149179

100,639

How the Government Shapes UNIX Standards.

100,693

UPHOLSTERY

Cone Calorimeter Rate of Heat Release Measurements for Upholstered Composites of Polyurethane Foams. PB92-108984

100,087

UPWARD FLAME SPREAD

Turbulent Upward Flame Spread for Burning Vertical Walls Made of Finite Thickness.

100,170

URACILS

Mechanism of $\mathrm{OH}$ Radical Reactions with Thymine and Uracil Derivatives

URANIUM

100,216

Method to Enhance Porosity of Micro-particles.

PB91-187328

101,185

URANIUM 233

Electrofission in the Ouasifree and Delta Regions PB91-12

101,609

URANIUM 238

Electrofission in the Ouasifree and Delta Regions.

URANIUM IONS

Transitions and Energy Levels for Cu-Like $\mathrm{Yb}(41+)$, $\mathrm{Ta}(44+)$, and $\mathrm{U}(63+)$

PB91-147462

101,610

WBAN AREAS

Wind Effects on Fires.

URIC ACID

Stability of Uric Acid in Ammonium Hydroxide.
PB91-189266

Antioxidation Mechanisms of Uric Acid.

PB91-237347

100,549

US DOE

Energy Related Inventions Program: A Joint Program of the Department of Energy and the National Institute of PB91-178871

100,902

US EMBASSY (MOSCOW)

Assessment: U.S. Office Building of Moscow. PB91-237834

100,151

TECHNOLNAL

NIST Serial Holdings, 1991.

100,960

US NBS

National Bureau of Standards Publications 1977-1987. Volume 1. Citations, Key Words, and Abstracts.

PB91-136507

100,957

National Bureau of Standards Publications 1977-1987 Volume 2. Indexes.

100,958

Materials Research at the National Bureau of Standards.
PB91-149039

101,214

NBS Ouality Assurance Support: Current and Planned Services.

101,012

Measurement Ouality Assurance.

PB91-149930

101,013

Polymers: Technical Activities 1990

100,488

US NIST

Energy Related Inventions Program: A Joint Program of Energy and the National Institute of Status Report for Recom PB91-178871

100,902

\section{USER MANUALS (COMPUTER PROGRAMS)}

FIREDOC Users Manual, 2nd Edition.

PB91-178830

100,547

Technical Reference and User's Guide for FAST/FFM Version 3.

PB91-206664 100,086

User Manual NBSAVIS CONTAM88. A User Interface for Air Movement and Contaminant Dispersal Analysis in Multizone Buildings.

PB91-206722

100,910

VACUUM GAGES

Stabilization Techniques for Spinning Rotor Gage Residual Drag.

VACUUM STATES

Model for the Non-Perturbative OCD (Ouantum Chromodynamics) Vacuum.

PB91-194407

101,661

\section{VACUUM ULTRAVIOLET RADIATION}

Scattered Light and Other Corrections in Absorption Co efficient Measurements in the Vacuum Ultraviolet: A Systems Approach

VALIDATION SUMMARY REPORTS

Ada Compiler Validation Summary Report. Certificate Number: 901109S1.11053, Digital Equipment Corporation VAXAda, Version 2.2 VAX $8800=>$ VAX 8800 AD-A233 961/2

100,607

Ada Compiler Validation Summary Report: DDC International A/S, DACS VAX/VMS to 80186 Bare Ada Cross Compiler System, Version 4.6. VAX 8530 (Host) to Bare Board iSBC 186/93A (Host), 901129S1.11079. AD-A234 083/4

100,608

Ada Compiler Validation Summary Report: Certificate Number: 901129S1.11112 DDC International A/S DACS 80386 DMS/OS Ada Compiler System, Version 4.6 IBM PS/2 Model 80-311 = > IBM PS/2 Model 80-311 $\mathrm{AD}-\mathrm{A} 234118 / 8$

100,609

Ada Compiler Validation Summary Report: Certificate Number: 901129S1.11050 DDC International A/S DACS AXXVMS Native Ada Cor

AD-A234 323/4

100,610

Ada Compiler Validation Summary Report: Digital Equipment Corporation, VAX Ada, Version 2.2, BAX 8800 (Host) I VAX MicrovaX II Running VAXELN Version 4. (Target), 90110951.11054

AD-A234 350/7

100,611

Ada Compiler Validation Summary Report: Certificate Number: 901129S1.11051, DDC International A/S, DACS sion 4.6 MicroVAX 3100 Greater Than or Equal to Motorola MVME133.

AD-A234 380/4

100,612

Ada Compiler Validation Summary Report: Certificate Number: 901129S1.11077 DDC International A/S DACS VAX VuMS to 80186 Bare Ada Cross Compiler System with Rate Monotonic Scheduling, Version 4.6 VAX 8530 with Rate Monotonic Sched

AD-A234 $438 \%$

100,613

Ada Compiler Validation Summary Report: Certificate Number: 901129S1.11075 DDC International A/S DACS 80386 UNIX V Ada Compiler System, Version 4.6 ICL DRS $300=>$ ICL. DRS 300

AD-A234 439/8

100,614

Ada Compiler Validation Summary Report: Certificate Number: 90112951.11074 DDC International A/S DACS VAX/VMS 60 80386 PM Bare Ada Cross Compiler System, Version 4.6 VAX $8530=>$ Bare Board isBC $386 / 21$

AD-A234 529/6

100,615

Ada Compiler Validation Summary Report. Certificate Number: 910510S1.11161 UNISYS Corporation, UCS Ada, Version $1 R 1,2200 / 600$ (Host and Target). Revision AD-A239 715/6

100,616

Ada Compiler Validation Summary Report: Certificate Number: 910626S1.11179 U.S. Navy Ada/M, Version 4.0 (OPTIMIZE) VAX $11 / 785=>$ AN/AYK-14 (Bare Board).

Ada Compiler Validation Summary Report: Certificate Number 910705S1.11192 InterAct Corporation InterAct Ada Mips Cross-Compiler System, Release 2.0 MicroVAX 3100 Cluster $=>$ Lockheed Sanders STAR MVP R3000/R3010 board (Bare Machine)

100,618

Ada Compiler Validation Summary Report: Certificate Number: 910502S1.11158 DDC International A/S, DACS

VAX/VMS to 80860 Bare Ada Cross Compiler System, Version 4.6.1, VAX 8530 (Host) to Tadpole Technology PLC TP860M (Bare Board) (Target)

AD-A240 610/6

100,619

Ada Compiler Validation Summary Report: Certificate (/Optimize), VAX 11/785 (Host) to AN/UYJ-43 (Single CPU) (Bare Board) (Target).

AD-A240 $611 / 4$

100,620

Ada Compiler Validation Summary Report: Certificate Number: $910626 \mathrm{~S} 1.11174$ U.S. Navy, Ada/M, Version 4.0 (/Optimize), VAX 8550, Running VAX/VMS Version 5.3 (Host) to AN/UYK-44 (EMR) (Bare Board) (Target)

AD-A240 612/2 Number: 910626S1.11175 U.S. Navy Ada/M, Version 4.0 (/Optimize), VAX 8550 (Host) to AN/AYK-14 (Bare Board) (Target)

AD-A240 613/0

100,622

Ada Compiler Validation Summary Report. Certificate Number: 910705S1.11191, InterACT Corporation InterACT Ada 1750A Compiler System, Release 3.5, MicroVAX 3100 Cluster = Int struction Set Architecture Simulator, Release 2.3 (Bare

D-A240 762/ 5

100,623

Ada Compiler Validation Summary Report. Certificate Number: 910626S1.11178, U.S. Navy Ada/M, Version 4.0 (Bare Board)

AD-A240 $763 / 3$

100,624

Ada Compiler Validation Summary Report: Certificate Number 910626S1.11173 U.S. Navy Ada/L, Version 4.0 (/Optimize) VAX $855=$ AN/UYK-43 (EMR) (Bare

AD-A240 783/1

100,625

Ada Compiler Validation Summary Report: Certificate Number Number 910626S1.11172 U.S. Navy Ada/L, Version 4.0 (/Optimize) VAX $8550=$ AN/UYK-43 (Single AD-A240 784/9

100,626

Ada Compiler Validation Summary Report: Certificate Number: 910502S1.11159 DDC International A/S DACS Sun-3/SunOS to 68030 Bare Ada Cross Compiler
System, Version 4.6.4, MRI IEEE 695 (BASIC-MODE) Sun- $3 / 50=$ Motorola MVME143 Board $(68030 / 68882)$.
AD-A240 785/6

Ada Compiler Validation Summary Report. Certificate Number: 910502S1.11160 DDC International A/S DACS Sun-3/SunOS to 68030 Bare Ada Cross Compile 4. MRI IEEE 695 (SECURE MODE) Sun-3/50 = Motorola MVME143 Board (68030) AD-A240 849/0

100,628

Ada Compiler Validation Summary Report. Certificate Number: 910626S1.11177 U.S. NAVY Ada/L, Version 4.0 (/OPTIMIZE) VAX $11 / 785=>$ AN/UYK-43 (EMR) AD-A240 850/8

100,629

Ada Compiler Validation Summary Report: Certificate Number: 910517 S1.11163 U.S. Navy, AdaVAX, Version 5.0, (/NO OPTIMIZE) VAX 8350 (Host and Target) AD-A242 268/1

100,630

Ada Compiler Validation Summary Report: Certificate Number: 910517S1.11162 U.S. Navy, AdaVAX, Version 5.0, (/OPTIMIZE) VAX 8600 (Host and Target)
AD-A242 269/9

100,631 


\section{KEYWORD INDEX}

\section{VAMAS (VERSAILLES PROJECT ON ADVANCED}

\section{ERIALS AND STANDARDS}

International Collaboration in Prestandards Research on Advanced Materials within the Context of VAMAS PBg 1-204040

101,251

VANADIUM OXIDES

Microwave Spectra and Electric Dipole Moments of $X(4)$ Sigma(1-) $1 / 2$ VO and NbO
PB91-237487

100,429

VAPOR DEOSITION

Spatially and Spectrally Resolved Cathodoluminescence of Hot-Filament Chemical-Vapor-Deposited Diamond Par-

AD-A237 128/4

100,248

VAPOR DEPOSITED COATINGS

U.S. Assessment of the New Diamond Technology in Japan.

101,107

Tribological Characteristics of Synthesized Diamond Films on Silicon Carbide.

101,186

PB91-189530 Model Gas Sensors.

PB91-203075

100,393

VAPOR DEPOSITION

Chemical Vapor Deposited Diamond.

101,106

Apparent Thermal Conductivity of Fumed-Silica Standard Reference Materials.

PB92-117357

101,102

VAPORIZATION

Thermodynamic and Kinetic Stability of Refractory Materials at Uitra-High Temperatures.

100,246

VARISTORS

Incompatibility between the $100 / 1300$ Surge Test and Varistor Failure Rates.

PB91-189308

100,854

VELOCITY DISTRIBUTION

Unsteady Laminar Flow in a Circular Tube: A Test of the HERCOL (Hermitian Collocation) Computer Code.
PB91-222679 PB91-222679

VENTILATION

Ventilation System Performance Evaluation Using Tracer Gas Techniques.

PB91-147876

100,060

Airtightness of Office-Building Envelopes.
PB91-175307 $\quad 100,067$ $\begin{array}{ll}\text { PB91-175307 100,067 } & \\ \text { Indoor Ventilation Requirements for Manufactured Hous- }\end{array}$ ing. 100,075 Variable Air Volume System Design Guide. PB91-216655

100,077

VERY LARGE SCALE INTEGRATION

Thermal Measurements of VLSI Packages: A Critical Review.

100,790

Measurement of Capacitance on Wafers

100,795

Design, Fabrication, and Testing of an Interconnect Test Structure for Evaluating VLSI Processes.

PB91-174581

100,803

Characterizing VLSI Processes Using Test Structures.

Evaluation and Improvement of E-Beam Exposure Rou. tines by Use of Microelectronic Test Structures.
PB91-175489 New Approach to Image Modeling and Edge Detection in the SEM. PB91-203752

\section{VIBRATION DAMPING}

100,811

Description of a Vibration Compensation System for the Small Scale Model Robot Crane Project. PB91-216648

100,130

VIBRATION INSULATORS

Long Term Performance of Rubber in Seismic and NonSeismic Bearings: A Literature Review.

PB91-206714

100,148

VIBAATION TESTS

Resonating-Orthotropic-Cube Method for Elastic ConStants.

101,586

VIBRATIONAL SPECTRA

Vibrational Spectra of Molecular lons Isolated in Solid Neon. III. $\mathrm{N} 4(+)$

AD-A228 082/4 100,243

Vibrational Spectra of Molecular Ions Isolated in Solid Neon. IV.NO(+), NO(-), ONNO(+), aNd ONNO(-). Vibrational Spectra of Molecular Ions Isolated in Solid Vibrational Spectra of Molecular lons Isolated in Solid Neon. 6. CO4(-)

100,249

Vibrational Spectra of Molecular Ions Isolated in Solid $\mathrm{Neon}$. 7. $\mathrm{CO}(+), \mathrm{C} 2 \mathrm{O} 2(+)$, and $\mathrm{C} 2 \mathrm{O} 2(-)$.

100,250

Vibrational Spectra of Molecular Ions Isolated in Solid

Neon. 5. $\mathrm{N} 2 \mathrm{O}(1+)$ and NNO2(1-).
PB91-149906

100,306

Vibrational Spectra of Molecular Ions Isolated in Solid Neon. 4. NO(1 +), NO(1-), ONNO(1+), and ONNO(1-). 100,307

Vibrational Spectra of Molecular lons Isolated in Solid Neon. 6. $\mathrm{CO} 4(1-)$

100,419

Rovibrational Analysis of the nu7(1) Intermolecular Hy Rovibrational Analysis of the nut(1) Intermolecular $\mathrm{Hy}$ drogen Bond Bending Vibration in HCN-HF Using $\mathrm{Far}$ In-
frared Fourier Transform Spectroscopy.
PB91-237784

100,437

Neutron Measurements of Intramolecular Vibrational Modes in C60

101,055

VIBRATIONAL STATES

Picosecond IR Studies of the Vibrational Dynamics of PB91-161927

Population Lifetimes of $\mathrm{OH}(\mathrm{v}=1)$ and $\mathrm{OD}(\mathrm{v}=1)$ Vibrations in Alcohols, Silanols and Crystalline Micas

PB91-174813

100,324

Vibrational and Electronic Energy Levels of Polyatomic Transient Molecules. Supplement 1.

PB91-192575

100,367

VICKEAS HARDNESS

Cathodoluminescence Measurement of Strained Alumina Single Crystals.
PB91-237131

101,094

VINYL COPOL YMERS

Chain Conformation of a Block Polymer in a MicrophasePB91-148890

100,485

\section{VINYL ETHER RESINS}

Small-Angle Neutron Scattering Studies of Compatible Blends of Linear Poly(Vinyl Methyl Ether) and CrossLinked Deuterated Polystyrene.

PB91-187153

100,494

VINYL RADICALS

Ultraviolet Absorption of the Vinyl Radical and Reaction with Oxygen,

100,391

\section{VIRAL GENES}

Mechanistic Studies of lonizing Radiation and Oxidative Mutagenesis: Genetic Effects of a Single 8-Hydroxyguanine (7-Hydro-8-oxoguanine) Residue Inserted at a Unique Site in a Viral Genome.

PB91-190108

101,321

VISCOELASTICITY

Mechanism for Shear Band Formation in the High StrainRate Torsion Test

101,579

Viscoelastic Response of Epoxy Glasses Subjected to Different Thermal Treatments.

101,245

Relaxation of Crosslinked Networks: Theoretical Models and Apparent Power Law Behavior.

Study of the Linear Viscoelastic Properties of Cyclic Po lystyrenes Using Creep and Recovery Measurements.
PB91-195107 Semiempirical Theory of Pelaxation: Concentrated Polymer Solution Dynamics.

PB91-236638

100,511

VISCOSITY

Transport Properties of Carbon Dioxide.

PB90-244724

100,266

Critical Exponen

100,271

Viscosity Surface for Mixtures of Methane and Ethane.

891-174763 100,322

Critical Exponent for the Viscosity of Four Binary Liquids.
100,337

Viscosity and Thermal Conductivity of Pure Monatomic
Gases from Their Normal Boiling Point Up to $5000 \mathrm{~K}$ in the Limit of Zero Density and at $0.101325 \mathrm{MPa}$. PB91-192559

Non-Equilibrium Molecular Dynamics Simulations of
Structured Molecules. Part 1. Isomeric Effects on the Viscosity ol Butanes.

PB91-195636

101,391

VISIBILITY

Visibility of Chrysotile Asbestos in the Scanning Electron Microscope.

101,320

VITAMIN A

Reverse-Phase HPLC Separation and Electrochemical Detection of Retinol and Its Isomers. PB91-148858

100,187

VOLTAGE

New International Electrical Reference Standards Based on the Josephson and Ouantum Hall Effects. BB91-237586

100,740

\section{VOLTAGE STANDARDS}

New Low-Voltage Standards in the DC to $1-\mathrm{MHz}$ Frequency Range.
PB91-237107

100,738

Improvements for Automating Voltage Calibrations Using a 10-V Josephson Array.

PB91-237446

100,739

New International Electrical Reference Standards Based on the Josephson and Ouantum Hall Effects.

PB91-237586

100,740

Voltage Calibration Systems Using Josephson Junction

B92-116516

100,868

VOLTAMETRY

Accurate and Precise Coulometric Determination of Sulfur Dioxide in Compressed Gas Mixtures.

PB92-126630

100,912

Development of a Coulometric Method for Assessing the Concentration of Ambient Levels of CO2/Air in Compressed Gas Mixtures.

PB92-126648

100913

\section{VOLTMETERS}

Influence of Nonsinusoidal Waveforms on Voltmeters, Ammeters, and Phase Meters.

100,869

VORTEX SHEDDING

Analysis of Laminar Vortex Shedding Behind a Circular Cylinder by Computer-Aided Flow Visualization.

PB91-203000

101,393

VORTICES

Large-Scale Turbulent Structures and the Stabilization of Lifted Turbulent Jet Diffusion Flames.

100,558

\section{VUILLEUMIER REFAIGERATOR}

Measurement of Regenerator Performance in a Vuilleumier Refriger ator

101,052

WALLS

Heat Flux, Mass Loss Rate and Upward Flame Spread for Burning Vertical Walls (1990)

100,094

Algorithm for the Mass-Loss Rate of a Burning Wall.

PB91-158998

00,108

Method for Characterizing the Thermal Performance of a Solar Storage Wall from Measured Data

PB91-159152

100,901

Review of Research Literature on Masonry Shear Walls.

Ceiling Jet-Driven Wall Flows in Compartment Fires.

Ceiling Jet-Driven Wall Flows in Compartment Fires.
PB91-174573

Comparison of Two Test Methods for Determining Transfer Function Coefficients for a Wall Using a Calibrated Hot Box

PB91-187278

100,121

Evaluation of the Heat Flux Transducer Technique for Measuring the Thermal Performance of Walls. 1-194696

00,126

Turbulent Upward Flame Spread for Burning Vertical Walls Made of Finite Thickness.

PB92-112531

100,170

Analysis of Moisture Accumulation in a Wood Frame Wall Subjected to Winter Climate.

100,137

Ultimate Strength of Masonry Shear Walls: Predictions versus Test Results. 


\section{KEYWORD INDEX}

XENON

Polyelectrolyte Stabilized Metal Oxide Hydrosols as Cata lysts for the Photooxidation of Water by Zinc Porphyrins. Unified Fundamental Equation for the Thermodynamic Properties of $\mathrm{H} 2 \mathrm{O}$.

100,364

High-Temperature High-Pressure Raman Spectra from Liquid Water.

PB91-195735

100,385

Microwave Spectrum and Molecular Structure of the N2H20 Complex.

PB91-203448 100,398

Suppression of Post-Flashover Compartment Fires Using Manually Applied Water Sprays.

PB91-240770

100,164

Thermodynamic Properties of the $\mathrm{NaBr}+\mathrm{H} 2 \mathrm{O}$ System. PB92-110238 100,45 Evaluation of Solubility Data of the System $\mathrm{CO}-\mathrm{H} 2 \mathrm{O}$ from $273 \mathrm{~K}$ to the Critıcal Point of Water.

PB92-110253

100,453

Sixteen Thousand Evaluated Experimental Thermodynamic Property Data for Water and Steam.

100,475

\section{WATER COMPLEXES}

Structures of the NH3-HCCCCH and $\mathrm{H} 2 \mathrm{O}-\mathrm{HCCCCH}$ Complexes by Fourier-Transform Microwave Spectrosco-

Py. 148882

WATER HEATERS

100,294

Measured Performance of Residential Water Heaters Using Existing and Proposed Department of Energy Test Procedures.

WATER POLLUTION

Simultaneous Butyltin Determinations in the Microlayer,
Water Column and Sediment of a Northern Chesapeake Water Column and Sediment of a $\mathrm{N}$

Bay Marina and
PB91-195040

100,918

WATER SUPPLY

Development of a Technique to Assess the Adequacy of the Municipal Water Supply for a Residential Sprinkle

System.
PB92-123116

101,358

WATER VAPOR

Refractive Index of Water and Steam as Function of Wavelength, Temperature and Density.

101,402

WATT HOUR METERS

Precision Qualification of Watthour Meters.

PB91-237214

100,859

WAVE DIFFRACTION

Diffraction by a Half-Plane in a Lossy Medium. $\quad 101,437$
PB92-116888

WAVE PROPAGATION

Numerical Simulation and Visualization Models of Stress Wave Propagation Graphite/Epoxy Composites.
PB91-174946

Stress

Extended Abstract: A Finite Element Study of the Stress and Displacement Fields Produced by Point Impact

101,584

WAVEGUIDE FLANGES

Alignment Fixture for Millimeter Waveguide
PB91-236919

100,727

AVEGUIDE LASERS

Integrated-Optic Waveguide Glass Lasers PB91-203976

100,758

WAVEGUIDES

Accurate Acoustic Measurements in Gases Under Difficult Conditions.

PB92-116847

101,382

Air Gage Size Measurement of Microwave Standards. PB92-117324

\section{WAVELENGTH MEASUREMENT}

Precise Laser Wavelength Measurements: What Can We Learn from Classical Spectroscopy.

101,430

Wavelengths and Energy Level Classifications of Magnesium Spectra for All Stages of Ionization (Mg I through

PB92-110139 100,442

Wavelengths and Energy Level Classifications for the Spectra of Aluminum (Al I through Al XIII).

100,472

PBg -1

Precise Laser Wavelength Measurements: What Can We Learn from Classical Spectroscopy.

101,430

AWAYANDAITE

Wawayandaite, a New Calcium Manganese Beryllium Boron Silicate from Franklin, New Jersey.

PB91-174680

101,359

WEAR

Using Surface Roughness as a Wear Criterion in HighSpeed Steel End-Milling.

PB91-158675

101,040

Mechanisms of Additive Effectiveness.
PB91-158808

101,172

Mechano-Chemical Descriptive Model for Wear Under Mixed Lubrication Condition

PB91-189480

101,177

Ceramic Wear Maps.

PB91-189498

Tribological Characteristics of Synthesized Diamond Films on Silicon Carbide.

PB91-189530

101,186

WEAR TESTS

Comparison of Standard Test Methods for Non-Lubricated Sliding Wear.

Sliding Wear Studies of Nickel-Copper Composition-Mod ulated Coatings on Steel.

PB91-190025

101,188

Effect of Selected Chemical Compounds on the Lubrication of Silicon Nitride.

PB91-236711

101,180

\section{WEIGHT INDICATORS}

Specifications, Tolerances, and Other Technical Require ments for Weighing and Measuring Devices as Adopted by the 76th National Conference on Weights and Meas-

PB92-112424

101,023

\section{WEIGHT MEASUREMENT}

Uniforms Laws and Regulations as Adopted by the $\mathrm{Na}$ tional Conference on Weights and Measures (76th)

101,022

PB92-112416

Report of the National Conference on Weights and
Measures (76th). Held in Philadelphia, PA. on July $14-19$

PB92-112465

101,024

WEIGHT REDUCTION

Gyroscope-Weighing Experiment Revisited .. with a Null

PB91-174722

101,630

WEIGHTS AND MEASURES

Uniforms Laws and Regulations as Adopted by the $\mathrm{Na}$. tional Conference on Weights and Measures (76th),

PB92-112416

101,022

Report of the National Conference on Weights and Measures (76th). Held in Philadelphia, PA. on July 14-19,

PB92-112465

101,024

WELD DEFECTS

Fracture Toughness of $316 \mathrm{~L}$ Stainless Steel Welds with Varying Inclusion
PB91-158980

101,148

\section{WELDING}

Report on 1989 Actions by International Institute of Welding. 134619

101,001

Analysis and Characterization of Commercial Welding PB91-162339

101,003

WELDMENTS

Cryogenic Material Properties of Stainless Steel Tube-to Flange Welds.

101,145

WETTING

Wetting Layers on Solid Substrates.

PB91-158857

100,313

WHEELS

Residual Stress Detection in Railroad Wheels: An Ultra sonic System Using EMATs (Electromagnetic-Acoustic Transducers). Report No. 23.

PB91-222653

101,741

Tread Crack Detection in Railroad Wheels: An Ultrasonic System Using EMATs (Electromagnetic-Acoustic Transducers). Report No. 22

PB91-222661

101,742

\section{WIND PRESSURE}

Wind and Seismic Effects. Proceedings of the Joint Meeting of the U.S.-Japan Cooperative Program in Natural Resources Panel on Wind and Seismic Effects (23rd). Held PB92-116425

100,152

\section{WINDOW GLASS}

Design Diagrams for Heavy Metal Fluoride Glass Win. PB91-187419

101,419

Users' Guide to BREAK1, the Berkeley Algorithm for Breaking Window Glass in a Compartment Fire. $\quad 100,136$ INDOWS

Temperature Measurement of Glass Subjected to Solar Radiation.

PB91-147611

100,100

WIRE BONDS

Cratering.
PB91-203141

100,810

WOLF-RAYET STARS

Terminal Velocities for a Large Sample of O Stars, B Supergiants, and
PB91-149021

100,023

WOOD FUELS

Theoretical Investigation of Piloted Ignition of Wood

PB92-112432

100,556

\section{WOOD PRODUCTS}

Analysis of Moisture Accumulation in a Wood Frame Wall Subjected to Winter Climate.

PB92.116334

100,137

WORKSTATIONS

Automating Robot Programming in the Cleaning and Deburring Workstation of the AMRF.

PB91-195495

100,986

Control Structure for Multi-Tasking Workstations. PB91-203737

100,989

Mare Island Flexible Manufacturing Workstation PB92-117027

100,996

\section{-RAY ANALYSIS}

High-Resolution Small-Angle $X$-ray Scattering Camera for Anomalous Scattering.

100,929

Strategies for Background Subtraction in Electron Probe Microanalysis $X$-ray Compositional Mapping.

100,202

XANES of Transition Metal Zinc-Blende Semiconductors PB92-117092

$X$-RAY DETECTION

Instrumentation of X-ray Beam Lines with PIN Diode De-

PB91.174888

101,633

$X$ RAY DIFFRACTION

RIR - Measurement and Use in Quantitative XRD.

PB91-147397

101,466

Hermetically Sealed Inert Atmosphere Cell for X-ray Powder Diffraction

101,062

$X$ RAY FILM

Intensifying Effect of Metallic Screens on the Sensitivity of X-ray Films for 400-kV Bremsstrahlung Photons.

PB91-195198 101,293

Intensifying Effect of Metallic Screens on the Sensitivity of X-ray Films at $662 \mathrm{keV}$.

101,669

$X$-RAY FLUORESCENSE ANALYSIS

Possible Use of Pattern Recognition for the Analysis of Mars-Rover X-ray-Fluorescence Spectra.

PB91-237875

101,733

$X$ RAY LASERS

Exploding Foil Photoionization X-ray Lase

PB91-237065

101,433

$X$ RAY MASKS

Application of Transmission Electron Detection to X-ray

Mask Calibrations and Inspection 


\section{KEYWORD INDEX}

PB91-133900

101,599

Critical Exponent for Viscosity.

PB91-147009

100,271

Unified Description of Infinite-Dilution Thermodynamic Properties for Aqueous Solutes.

100,319

PB91-162081

Angular Distribution of Ejected Electrons in Resonan Auger Processes of $\mathrm{Ar}, \mathrm{Kr}$, and $\mathrm{Xe}$

PB91-187302

101,648

Viscosity and Thermal Conductivity of Pure Monatomic Gases from Their Normal Boiling Point Up to $5000 \mathrm{~K}$ in the Limit of Zero Density and at $0.101325 \mathrm{MPa}$.

PB91-192559

Anomalous Lgamma2,3 X-ray Emission Spectrum of $X e$.

PB91-195289

of $\mathrm{Xe}$.

YIELD STRENGTH

Effects of Varying Austenitizing Temperature and Cooling Rate on the Ability of HSLA-80 Steel to Achieve a Yield Strength Comparable to HSLA-100.

PB91-189464

101,156

Nitrogen Strengthening of Austenitic Stainless Steels at Low Temperatures.

101,163 PB91-195529

YTTERBIUM IONS

Transitions and Energy Levels for Cu-Like $\mathrm{Yb}(41+)$ $\mathrm{Ta}(44+)$, and $\mathrm{U}(63+$

PB91-147462

101,610

YTTERBIUM OXIDES

Resonant Photoemission and PSD of Rare Earth Oxides. TRIUM

Structural and Magnetic Properties of Er Thin Films and $\mathrm{Er} / \mathrm{Y}$ Superlattices: Magnetoelastic Effects.

101,489 PB91-161935

High T(sub C) Superconductors: Are They Magnetic.
N91-20208/5

Transport Critical Current of Aligned Polycrystalline Y1Ba2Cu3O7-delta and Evidence for a Nonweak-Linked Component of Intergranular Current Conduction.
PB91-133983 Fundamental and Harmonic Susceptibilities of YBa2Cu3O7-delta. Madelung Potentials and Vatences in the $\mathrm{Y} 1 \mathrm{Ba} 2 \mathrm{Cu} 3 \mathrm{O} 7$ Superconductor

101,459

Strength and Fracture Behavior of Ba-Y-Cu-O Superconducting Ceramics.

101,474

Possible 'Proximity Matrix' Route to High Current ConPossible 'Proximily Matrix' Route to High Current Con-
ductors. ductors.
PB91-148932

101,476

Comparison of Flux Dynamics in Two Samples of YBa2Cu3O7 with Different Pinning.
PB91-149419

101,069

Analysis of the YBa2Cu3O7/SrTiO3 Interface as a Function of Post-Deposition Annealing Temperature.

PB91-149963

101,480

Chemical Modification of the Orthorhombic Superconductor $\mathrm{Ba2YCu} 3 \mathrm{O} 7$-delta.

PB91-159053

101,486

Phonon Density of States of Superconducting YBa2Cu3O7 and the Nonsuperconducting Analog YBa2Cu3O6.

101,487

Models for Oxygen Ordering and Diffusion in Ba2YCu3OX and Ba2YCu3-yMyOx (M = Fe, Co, Al, Ga).
PB91-175232 Levitation of Superconducting Composites. PB91-187351

101,503

Magnetic Hysteresis of High-Temperature YBa2Cu3OXAgO Superconductors: Explanation of Magnetic Suspension.

101,507

Sputtered Thin Film YBa2Cu3On.

PB91-189647

101,509

YBa2Cu3O7-delta/Insulator Multi-Layers for Crossover Fabrication.

101,518

Current Record in Superconductors.

PB91-194753

101,521

Long-Pange Antiferromagnetic Order of the $\mathrm{Cu}$ in Oxygen Deficient $\mathrm{ABa2Cu} 3 \mathrm{O} 6+\mathrm{x}$

PB91-194894

101,524

Morphology of Silver on YBa2Cu3O7-delta Thin Films.

PB91-195594 101,534

Properties of YBa2Cu3O7-delta Thin Films Grown on OffAxis-Cut MgO Substrates.

PB91-203950

101,547

Mossbauer Study of the Effect of Oxygen Stoichiometry on the High Tc Superconductor On the High
Y1Ba2(CuO.97Fe0.03)3O7-x. TC

Low-Field Flux Pinning in Twinned and Detwinned Single Crystals of YBa2Cu3O7-x.

101,559

PB91-237552

Oxides and Oxide Superconductors: Elastic and Related
Properties.

PBoperties.

101,563

Laser Vaporization Mass Spectrometry of Refractory Materials: Graphite and YBa2Cu3Ox.

100,464

YTTRIUM IRON GARNETS

High Speed Magnetic Field Sensors Based on Iron Gar-

PBg1-14

100,840

Anomalous Low-Frequency Butterfly Curves for Subsidiary and Ferromagnetic Resonance Overlap at $3 \mathrm{GHz}$.
PB91-194704

101,519

ZEEMAN LASERS

Correlated Spontaneous Emission in a Zeeman Laser.

PB91-175596 101,417

Correlated Spontaneous Emission in a Zeeman Laser. PB91-204180

101,432

ZENO EFFECT

Reply to Comment on 'Quantum Zeno Effect'.

PB91-236828

101,708

ZEOLITES

New Different Forms of Ammonium Loaded and Partly Deammoniated Zeolite Rho Studied by Neutron Powder.

100,311

Structures and Properties of New Zeolite X-Type Zincophosphate and Beryllophosphate Molecular Sieves.

PB91-189449 101,081

Dynamics of Molecular Hydrogen Adsorbed in CoNa-A

Zeolite.

100,380

ZINC IONS

Relative Populations of Excited Levels within the Ground Configuration of Si-Like $\mathrm{Cu}, \mathrm{Zn}, \mathrm{Ge}$, and Se lons. PB91-158618

ZINC PHOSPHATES

Structures and Properties of New Zeolite X-Type Zincophosphate and Beryllophosphate Molecular Sieves.

PB91-189449

101,081

ZIRCALOYS

Corrosion Behavior of Zirconium Alloy Nuclear Fuel Clad-

PB91-158733

101,372

ZIRCONIUM ALLOYS

Structural Instabilities and Superconductivity in QuasiBinary Mn5Si3-Type Compounds.

PB91-149427

101,215

Properties of Amorphous Zirconium Rhodium Hydride (Zr3RhHx) Prepared from Glassy and Crystalline Alloys. PB91-174466 101,225 Relationship of Hydrogen Site Occupancy to Diffusion Behavior in Crystalline and Amorphous Zr2PdHx. PB91-187252

101,230

ZIRCONIUM HYDRIDES

Neutron Powder Diffraction and Inelastic Scattering Study of the Structures of $\mathrm{Zr} 2 \mathrm{Pd}, \mathrm{Zr} 2 \mathrm{PdD} 1.70$, and Zr2PdD1.96. PB91-148866

101,213

Relationship of Hydrogen Site Occupancy to Diffusion Behavior in Crystalline and Amorphous $\mathrm{Zr} 2 \mathrm{PdHx}$. PB91-187252

101,230

ZIRCONIUM OXIDES

Physical and Thermo-Mechanical Properties of Monoclinic Single Crystals.

101,065 


\section{SAMPLE ENTRY}

\author{
Proposed Standard Practice for Assessing the \\ Performance of Gas-Phase Air Cleaning Equipment \\ PB91-167353 \\ 100,905
}

Titie

NTIS order number Abstract number
Availability Price Code
2-Deoxy-D-Ribose Aqueous Solution as a Gamma-Ray Do-

101,366 Not available NTIS $5 s(2) 5 p(2)-(5 s(2) 5 p 5 d+5 s 5 p(3)+5 s(2) 5 p 6 s$
$5 s(2) 5 p 7 s)$ Transitions in $5 b \quad I$ and $5 s(2) 5 p-(5 s 5 p(2)+$ $5 \mathrm{~s}(2) 5 \mathrm{p} 7 \mathrm{~s})$ Transitions in $\mathrm{Sb} / \mathrm{l}$ and $5 \mathrm{~s}(2) 5 \mathrm{p}-(5 \mathrm{~s} 5 \mathrm{p}(2)+$
$\mathrm{s}(2) \mathrm{nl})$ Transitions in $\mathrm{Sb}$ III.
PB91-200816 101,676 Not available NTIS 6-D Structural Model for the Icosahedral (Al, Si)-Mn QuasiCrystal.

101,501 Not available NTIS

8-Hydroxyguanine Content of Isolated Mitochondria Increases with Lipid Peroxidation.

PB91-189472

101,286 Not available NTIS

46Gas Chromatography-Mass Spectrometry of Free Radical-Induced Products of Pyrimidines and Purines in DNA. PB91-187476 101,302 Not available NTIS

303-MHz Frequency Standard Based on Trapped Be(+) lons.

PB92-116474

101,722 Not available NTIS

1987: The Year of the 386 .

PB91-158766

100,599 Not available NTIS

3480 Type Tape Cartridge: Potential Data Storage Risks, and Care and Handling Procedures to Minimize Risks. PB92-126549 101,381 PC A04/MF A01

Ab-Initio Calculations and Ideal Gas Thermodynamic Functions of Cyclopentadiene and Cyclopentadiene Derivatives. PB92-148089 100,468 (Order as PB92-148063)

Ab Initio Phase Determination for $X$-ray Diffraction Data from Crystals of a Native Protein.

PB91-237362 101,309 Not available NTIS

Absorbed-Dose Calibration of Ionization Chambers in a(60)Co Gamma-Ray Beam.

PB91-132290

$101,598 \mathrm{PC} \mathrm{A04/MF} \mathrm{A01}$

Absorbed Dose to Water: Comparison of Several Methods Using a Liquid Ionization Chamber.

PB91-195057

101,322 Not available NTIS
Abstract and Index Collection in the Research Information Center of the National Institute of Standards and TechnoloCenter of the National Institute of Standards and Technolo-
Py (Fourth Edition).
PB90,148494 PC A03/MF A01 Abstracts of Daylighting Research.

PB91-206755 100,076 PC A06/MF A01

AC-DC Difference Relationships for Current Shunt and Thermal Converter Combinations.

PB91-236893

100,737 Not available NTIS

AC Loss Measurements of Two Multifilamentary NbTi Composite Strands.
PB91-133892

100,769 Not available NTIS

Accelerator Radiocarbon Dates on Charcoal, Shell, and Human Bone from the Del Mar Site, California.

PB91-237404 101,355 Not available NTIS

Accelerator (14)C Dating of Late Pleistocene Megafauna.
PB91-237396 101,354 Not available NTIS

Accuracy Model for Phase Noise Measurements.

PB91-134882 100,589 Not available NTIS

Accuracy of Transmission Electron Microscopy Analysis of

Asbestos on Filters: Interlabortory Study. 100,204 Not available NTIS

Accurate Acoustic Measurements in Gases Under Difficult Conditions.

PB92-116847

101,382 Not available NTIS

Accurate and Precise Coulometric Determination of Sulfur Dioxide in Compressed Gas Mixtures.

100,912

(Order as PB92-126614, PC A06/MF A02)

Accurate Frequency of the $119 \mathrm{mu} \mathrm{m}$ Methanol Laser from Tunable Far-Infrared Absorption Spectroscopy.

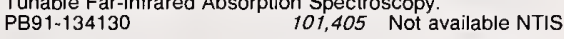

Accurate Spectroscopy in the X-ray Region.

PB91-158634 101,621 Not available NTIS

Acoustic Emission Monitoring of Laser Drilling.

Acoustic Emission Monitoring of Laser Drilling.
PB91-158527
Acoustic Technique for Evaluation of Thermal Insulation. Acoustic Technique for Evaluation of Thermal Insulation.
PB91-189324 100,123 Not available NTIS ACTIS: Towards a Comprehensive Tribology Data Base. PB91-174904 101,184 Not available NTIS

Activities of the Construction Materials Reference Laboratories Related to Laboratory Accreditation.

PB91-203836

100,531. Not available NTIS

Ada Compiler Validation Summary Report: Certificate Number Number 910626S1.11172 U.S. Navy Ada/L, Version 4.0 (/Optimize) VAX $8550=$ AN/UYK-43 (Single CPU) (Bare Board).

AD-A240 784/9

100,626 PC A05/MF A01

Ada Compiler Validation Summary Report. Certificate Number: 901109S1.11053, Digital Equipment Corporation VAX Ada, Version 2.2 VAX $8800=>$ VAX 8800 . AD-A233 961/2 100,607 PC A04/MF A01

Ada Compiler Validation Summary Report: Certificate Number: 901129S1.11050 DDC International A/S DACS VAX/VMS Native Ada Compiler System, Version 4.6 VAX $8530=>$ VAX 8530.

AD-A234 323/4

$100,610 \mathrm{PC}$ A04/MF A01

Ada Compiler Validation Summary Report: Certificate Number: 901129S1.11051, DDC International A/S, DACS VAX/VMS to 68020 Bare Cross Compiler System, Version 4.6 MicroVAX 3100 Greater Than or Equal to Motorola
MVME133. AD-A234 380/4

100,612 PC A03/MF A01

Ada Compiler Validation Summary Report: Certificate Number: 901129S1.11074 DDC International A/S DACS VAX/VMS 6080386 PM Bare Ada Cross Compiler System, Version 4.6 VAX $8530=>$ Bare Board iSBC $386 / 21$. AD-A234 529/6 100,615 PC A06/MF A01

Ada Compiler Validation Summary Report: Certificate Number: 901129S1.11075 DDC International A/S DACS 80386 UNIX V Ada Compiler System, Version 4.6 ICL

DRS300 $=\underset{439 / 8}{>}$ ICL DRS300.

100,614 PC A04/MF A01

Ada Compiler Validation Summary Report: Certificate Number: 901129S1.11077 DDC International A/S DACS 


\section{TITLE INDEX}

VAX/VMS to 80186 Bare Ada Cross Compiler System with Rate Monotonic Scheduling, Version 4.6 VAX $8530=>$ Bare Board iSBC/03A

100,613 PC A06/MF A01

Ada Compiler Validation Summary Report: Certificate Number: 901129S1.11112 DDC International A/S DACS B0386 DMS/OS Ada Compiler System, Version 4.6 IBM PS 12 M AD-A234 118/8 100,609 PC A06/MF A01

Ada Compiler Validation Summary Report: Certificate Number: $910502 S 1.11158$ DDC International A/S, DACS VAX/VMS to 80860 Bare Ada Cross Compiler System, Version 4.6.1, VAX 8530 (Host)

AD-A240 $610 / 6$

100,619 PC A03/MF A01

Ada Compiler Validation Summary Report: Certificate Number: 910502S1.11159 DDC Int Sun-3/SunOS to 88030 Bare Ada Cross Compiler System, Motorola MVME 143 Board (68030/68882)

$\begin{array}{lll}\text { AD-A240 785/6 } & 100,627 \text { PC A03/MF A01 }\end{array}$

Ada Compiler Validation Summary Report. Certificate Number: 910502S1.11160 DDC International A/S DACS Sun-3/SunOS to 68030 Bare Ada Cross Compiler System, Version 4.6.4, MRI IEEE 695 (SECURE MODE) SUn-3/50 AD-A240 $849 \%$

100,628 PC A03/MF A01

Ada Compiler Validation Summary Report. Certificate Number: 910510S1.11161 UNISYS Corporation, UCS Ada, Version $1 \mathrm{R} 1,2200 / 600$ (Host and Target). Revision.
AD-A239 715/6
100,616 PC A06/MF A02

Ada Compiler Validation Summary Report: Certificate Number: 910517 S1.11162 U.S. Navy, AdaVAX, Version 5.0, (/OPTIMIZE) VAX 8600 (Host and Target).
AD-A242 269/9 100,631 PC A05/MF A01 Ada Compiler Validation Summary Report: Certificate Number: 910517 S1.11163 U.S. Navy, AdaVAX, Version 5.0 (/NO OPTIMIZE) VAX 8350 (Host and Target)

AD-A242 268/1
100,630 PC A08/MF A02

Ada Compiler Validation Summary Report: Certificate Number: 91051751.11164 U.S. Navy, AdaVAX, Version 5.0

(/OPTIMIZE), VAX11/785 (Host and Target).
AD-A242 273/1 100,633 PC A04/MF A01

Ada Compiler Validation Summary Report: Certificate Number: 910517 S1.11165 U.S. Navy, AdaVAX, Version 5.0 (/NO-OPTIMIZE), VAX 11/785 (Host and Target)

AD-A242 270/7 100,632 PC A04/MF A01

Ada Compiler Validation Summary Report: Certificate Number 910626S1.11173 U.S. Navy Ada/L, Version 4.0 (/ Optimize) VAX $855=$ AN/UYK-43 (EMR) (Bare Board)
AD-A240 783/1
100,625 PC A05/MF A01

Ada Compiler Validation Summary Report: Certificate Ada Compiler Validation Summary Report: Certificate Number: 910626S1.11174 U.S. Navy, Ada/M, Version 4.0
(/Optimize). VAX 8550, Running VAX/VMS Version 5.3 (Host) to AN/UYK-44 (EMR) (Bare Board) (Target). (Host) to AN/UYK-44 (EMR) (Bare Board) (Target).
AD-A240 612/2

Ada Compiler Validation Summary Report: Certificate Number: 910626 S1.11175 U.S. Navy Ada/M, Version 4.0 (/ (Target).

Ada Compiler Validation Summary Report: Certificate Ada Compiler Validation Summary Report: Certificate Optimize), VAX 11/785 (Host) to AN/UYJ-43 (Single CPU) (Bare Board) (Target)

(Bare Board) (Targe
AD-A240 $611 / 4$

100,620 PC A05/MF A01

Ada Compiler Validation Summary Report. Certificate (OPTIMIZE) VAX $11 / 785=>$ AN/UYK-43 (EMR) (Bare Board)

AD-A240 850/8

100,629 PC A05/MF A01

Ada Compiler Validation Summary Report. Certificate (/OPTIMIZE) VAX $11 / 785=>$ AN/UYK-44 (EMR) (Bare Board)
AD-A240 $763 / 3$

100,624 PC A05/MF A01

Ada Compiler Validation Summary Report: Certificate Number: 910626S1.11179 U.S. Navy Ada/M, Version 4.0 (OPTIMIZE) VAX $11 / 785=>$ AN/AYK-14 (Bare Board).
AD-A240 511/6

Ada Compiler Validation Summary Report. Certificate Number: 910705S1.11191, InterACT Corporation InterAC MicroVAX 3100 Cluster $=>$ InterACT MIL-STD-1750A Instruction Set Architecture Simulator, Release 2.3 (Bare Machine)

AD-A240 762/5
100,623 PC A04/MF A01

Ada Compiler Validation Summary Report: Certificate Number 910705S1.11192 InterAct Corporation InterAct Ada Mips Cross-Compiler System, Release 2.0 MicroVAX 3100 Cluster $=>$ Lockheed Sanders STAR MVP R3000

R30 10 board (Bare Machine)
AD-A240 512/4

$100,618 \mathrm{PC} \mathrm{A04/MF} \mathrm{A01}$

Ada Compiler Validation Summary Report: Certificate Number 910918S1.11216, NEC Corporation, NEC Ada
Compiler System for EWS-UXIV (Rel 4.0), Version Release 2.1(4.6) EWS4800/220 = EWS4800/220. PC A05/MF A01
AD-A242 609/6 100,634 PC

Ada Compiler Validation Summary Report. Certificate Number: 911025 S1.11226, Digital Equipment Corporation Number: $911025 S 1.11226$, Digital Equipment Corporation
DEC Ada, Version 1.0 DECstation 5000 Model $200=>$ DECstation 5000 Model 200. AD-A242 896/9

100,635 PC A04/MF A01

Ada Compiler Validation Summary Report: DDC International A/S, DACS VAX/VMS to 80186 Bare Ada Cross Compiler System, Version 4.6, VAX 8530 (Host) to Bare Board iSBC $186 / 93 A$ (Host), 901129S1.11079.
AD-A234 083/4 100,608 PC A05/MF A01

100,608 PC A05/MF A01

Ada Compiler Validation Summary Report: Digital EquipVAX MicroVAX if Running VAXELN Version 4.1 (Target), $901109 \$ 1.1105$

100,611 PC A04/MF A01

Adjustment of the Fundamental Physical Constants: A Report of the CODATA Task Group on Fundamental ConStants, 1986.

101,606 Not available NTIS

Adsorption and Orientation of Tetracycline on HydroxyapaPB91-195149

101,319 Not available NTIS Adsorption of Water and Oxygen on $\mathrm{Ag}(110)$ : A Study of
the Interactions among Water Molecules, Hydroxyl Groups, and Oxygen Atoms.

100,270 Not available NTIS

Advanced Materials: Opportunities and Challenges.

PB91-204032 100,009 Not available NTIS

Advanced Sensing of Materials Processing.
100,949 NB

Advanced sensor development program for the pulp and paper industry. Final report.
DE91007688

101,248 PC A05/MF A01

Advanced Technology Program: A New Role for NIST in Accelerating the Development of Commercially Important Technologies
PB92-126697

100,013

(Order as PB92-126614, PC A06/MF A02)

Advances in Science, Emerging Technologies and Global Competition: Opportunities and Challenge.
PB91-203174 100,583 Not available NTIS

Agency Exploits Flexibility of Software in Moving Info.

PB91-159160 100,642 Not available NTIS

Agenda for Progress in Technology Education: A Personal PB91-148114

100,037 Not available NTIS

Al: Does It Have a Place in Building Simulation.

PB91-159293 100,063 Not available NTIS

Al in Manufacturing: The NBS AMRF as an Intelligent Ma. PBine.

100,973 Not available NTIS

Air Exchange and Pollutant Concentration Measurements in Two Office Buildings

100,909 Not available NTIS

Air Gage Size Measurement of Microwave Standards.

PB92-117324

100,777 Not available NTIS

Airtightness of Office-Building Envelopes.

PB91-175307

100,067 Not available NTIS

Alaska Marine Mammal Tissue Archival Project: Revised Collection Protocol

PB91.184796

101,374 PC A03/MF A01

Algorithm and Computer Program for the Calculation of Envelope Curves
PB91-158972

101,414 Not available NTIS

Algorithm Design for Large-Scale Computations.

PB91-149336 100,640 Not available NTIS

Algorithm for the Mass-Loss Rate of a Burning Wall.

PB91-158998 100,108 Not available NTIS

Algorithms for Special Tridiagonal Systems.

PB91-187245 101,259 Not available NTIS

Alignment Fixture for Millimeter Waveguide.

PB91-236919 100,727 Not available NTIS

Alkyl Transfer Reactions between Protonated Alcohols and Ethers. The Gas-Phase Alkylation of Formaldehyde.
PB91-147470 100,280 Not available NTIS

Alternate Representation of Prism Refraction for Light Incident at the Brewster Angle.

101,426 Not available NTIS

Alternative Refrigerants R123a, R134, R141b, R142b, and R152a: Critical Temperature, Refractive Index, Surlace Tension, and Estimates of Liquid, Vapor, and Critical Densities.
PB91.147074
Aluminum Alloys for ALS Cryogenic Tanks: Comparative Measurements of Cryogenic Mechanical Properties of Al-L Alloys and Alloy 2219 .

AD-A242 956/1

101,204 PC A07/MF A02

Aluminum Alloys for ALS Cryogenic Tanks: Oxygen Compatibility. Volume 2 .

101,203 PC A07/MF A01

Aluminum Alloys for Cryogenic Tanks: Oxygen Compatibility. Volume 1.

101,201 PC A10/MF A02

American National Standard for C Category: Sottware Standard. Subcategory: Programming Language.
FIPS PUB $160 \quad$ PC E15 Amorphization and Conductivity of Silicon and Germanium Induced by Indentation.

101,482 Not available NTIS

AMRF Composites Fabrication Workstation: A Test Methodology to Measure the Quality of Thermoplastic Compos

PB91-187765

101,017 PC A03/MF A01

Analysis and Characterization of Commercial Welding PBluxes.

101,003 Not available NTIS

Analysis of Laminar Vortex Shedding Behind a Circular Cylinder by Computer-Aided Flow Visualization.
PB91-203000 101,393 Not available NTIS

Analysis of Moisture Accumulation in a Wood Frame Wall Subjected to Winter Climate.

100,137 PC A04/MF A01

Analysis of Optical Flow Estimation Using Epipolar Plane Images.
PB92-112564

100,703 PC A03/MF A01

Analysis of the $\mathrm{YBa} 2 \mathrm{Cu} 3 \mathrm{O} 7 / \mathrm{SrTIO}$ Interface as a Function of Post-Deposition Annealing Temperature.

Analysis of Wet Deposition (Acid Rain): Determination of the Major Anionic Constituents by lon Chromatography. Analytic Solution for the Three-Layer Multiple Beam Interferometer.
PB91.162107

101,007 Not available NTIS

Analytical Model for the Refrigeration Power of the Orifice Pulse Tube Refrigerator.

101,048 PC A05/MF A01

PB91.187534

aveguides: A Comparison of Two New Methods.

PB91-204131

100, 759 Not available NTIS

Angle-Resolved Photoemission from the Ar $2 p$ Subshell.

PB91-194928

101,665 Not available NTIS

Angular Distribution of Ejected Electrons in Resonant Auger Processes of
PB91.187302

101,648 Not available NTIS

Angular Distributions of Electrons in Resonant Auger SpecPB91.187393

101,650 Not available NTIS

Anisotropic Structure of a Simple Liquid.

PB91-148718

101,616. Not available NTIS

Annotated Bibliography: Diagnostic Methods and Measurement Approaches to Detect Incipient Defects Due to Aging of Cables.

101,369 PC A10/MF A03

Annual Report to the Strategic Defense Initiative Organization on the Free-Electron Laser Driven by the NIST CW Mi-

AD-A207 806/

101,398 PC A06/MF A01

Anomalous Lgamma2,3 $X$-ray Emission Spectrum of $X e$. PB91-195289 101,670 Not available NTIS Anomalous Low-Frequency Butterfly Curves for Subsidiary

and Ferromagnetic Resonance Overlap at $3 \mathrm{GHz}$.
PB91-194704 Not

Anomalous Magnetoresistance in Al/AI-Alloy Composite Conductors.
PB91-174748

101,631 Not available NTIS

Anomalous Th $\mathrm{M} 3 \mathrm{O} 1 \mathrm{X}$-ray Emission Spectrum of ThO2.

100,381 Not available NTIS

Antiferromagnetic Order of $\mathrm{Cu}$ in $\mathrm{Sm}_{2} \mathrm{CuO} 4$

PB92-117340 Order of Cu in 101,569 Not available NTIS

Antioxidation Mechanisms of Uric Acid.

PB91-237347 100,222 Not available NTIS

Apparatus for Acoustic Measurements of Gases with Applications for High Temperatures.
PB92-116839 100,458 Not available NTIS

Apparatus for Measurement of Coefficient of Friction.

PB91-148049 101,250 Not available NTIS 


\section{TITLE INDEX}

Apparatus for Measurement of Thermal Conductivity of In sulation Systems Subjected to Extreme Temperature Differ-

PB91-162016

101,738 Not available NTIS

Apparatus for the Measurement of Regenerator Performance in Pulse Tube Refrigerators.

PB91-147967 101,047 Not available NTIS

Apparel STEP Translator

100,967 PC A03/MF A01

Apparent Thermal Conductivity of Fumed-Silica Standard Reference Materials

101,102 Not available NTIS

Appendix: Standard Solutions and Certified Reference Materials.

100,194 Not available NTIS

Applicability of Smoluchowski's Equation with a Constant

Kernel to Coalescence.
PB91-148940

100,296 Not available NTIS

Application of Analytical Electron Microscopy to GlassBonded Aluminas.

101,060 Not available NTIS

Application of CCRL Data in the Development of Cement Standards.

100,119 Not available NTIS

Application of Fire Research.

100,153 Not available NTIS

Application of Formal Description Techniques to the Speci-

fication of Distributed Test Systems.
PB91-175018 100,566 Not available NTIS

Application of Laser Diagnostic Techniques for the Examination of Liquid Fuel Spray Structure.
PB91-195487 Not available NTIS
PBS1-195487

100,229 Not available NTIS

Application of OSI Protocols for Plant Information Networks.
PB91-147595

100,974 Not available NTIS

Application of Osmotic Dewatering to the Controlled Crystallization of Biological Macromolecules and Organic Compounds.
PB91-195719

100,230 Not available NTIS

Application of the Joint Excitation Gear Adjustment Technique to Low Frequencies and Heavy Robot Joints.
PB91-158576 101,028 Not available NTIS

Application of the PIPE Image Processing Machine to Scanning Microscopy.
PB91-203190

101,034 Not available NTIS

Application of Transmission Electron Detection to X-ray

100,828 Not available NTIS

Application of Ultrafast Broadband Infrared Spectroscopy to easurement of Metal-Carbonyl Dynamics.

100,308 Not available NTIS

Application Portability Profile (APP): The U.S. Government's Open System Environment Profile OSE/1 Version 1.0.
PB91-201004 100,692 PC A05/MF A01

Applications of Operations Research Techniques to System Design and Evaluation.

PB9 $1-174540$

101,267 Not available NTIS

Applications of the Generalized Global Equivalence Ratio Model (GGERM) for Predicting the Generation Rate and Distribution of Products of Combustion in Two-Layer Fire Environments: Methane and Hexanes.

Applied Electric Fields for Downstream Processing

Applied Electric Fields for Downstream Processing.
PB91-149161 100,299 Not available NTIS

Aspects of Smoldering Combustion.

PB91-134510 100,535 Not available NTIS

Assembly Code to Compute Sine and Cosine Using the Cordic Algorithm

PB91-143289

100,638 PC A03/MF A01

Assessment of Reliability Concerns for Wide-Temperature Operation of Semiconductor Devices and Circuits.

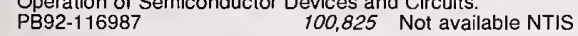

Assessment of the State-of-the-Art for Process Monitoring Sensors for Polymer Composites.

PB91-222612

100,510 PC A07/MF A02

Assessment of Uncertainties in Measurement of $\mathrm{pH}$ in Hostile Environments Characteristic of Nuclear Repositories.
NUREG/CR-5711 101,367 PC A06/MF A02 Assessment: U.S. Office Building of Moscow. PB91-237834 100,151 Not available NTIS Associative lonization of UItra-Cold Laser Trapped Sodium Atoms. PB91-236745 101,707 Not available NTIS Asymptotic Expansions of the Kernel Functions for Line Formation with Continuous Absorption.
PB91-203240

101,688 Not available NTIS

Asymptotic Shooting Method for the Solution of Differential Equations.

PB91-148742

101,255 Not available NTIS

Atomic Fingerprint of YBa2Cu3O7-x-Type High-Temperature Superconductors Observed by Field lon Microscopy.
PB91-203612 101,544 Not available NTIS

Atomic Spectroscopy in the 20th Century - A Tribute to Sitterly, Charlotte Moore, on the Occasion of Her 90th Birthday. 216714

101,724 Not available NTIS

Atomic Weights of the Elements 1989

PB92-148212 100,481 (Order as PB92-148162)

Atomistic Diffusion Equations and Kinetic Forces

PB91-195008 100,374 Not available NTIS

Automan 2.0: Decision Support Software for Automated

Manufacturing Investments. User Manual.
PB91.167700

Automated High-Temperature Guarded-Hot-Plate Apparatus for Measuring Apparent Thermal Conductivity of Insulations between 300 and $750 \mathrm{~K}$.

between 300 anc

100,111 Not available NTIS

Automated Information Systems Security Program Handbook.

100,713 PC A08/MF A02

Automated Reverse-Bias Second-Breakdown Transistor Tester

100,813

$1-216739$

(Order as PB91-216705, PC A07/MF A01)

PB91-236513 100,814 Not available NTIS

Automating Robot Programming in the Cleaning and Deburring Workstation of the AMRF.

ring Workstation

100,986 Not available NTIS

BACnet Communication Protocol for Building Automation Systems.
PB91-187286

100,069 Not available NTIS

Band-Gap Narrowing and III-V Heterostructure FETs.

PB92-117159 100,827 Not available NTIS
PBis

Base Modifications in Plasmid DNA Caused by Potassium Permanganate.

101,329 Not available NTIS

Basic Standards and Fundamental Constants.

PB91-237594 101,715 Not available NTIS

Beam-Foil Study of Two-Electron Transitions in Cu-Like lons.

101,714 Not available NTIS

Benzene: A Further Liquid Thermal Conductivity Standard. PB90-244567 100,255 (Order as PB90-244526)

Bibliography of Selected Computer Security Publications, January 1980-October 1989.

PB91-148486

100,704 PC A09/MF A02

Bibliography of the NIST Electromagnetic Fields Division Publications.

PB92- 116367

100,832 PC A06/MF A01

100,866 PC A06/MF A02

Bidirectional Scattering Distribution Function (BSDF): A Systematized
PB91-187674

101,421

(Order as PB91-187617, PC A06/MF A01)

Biodegradable Plastics: An Idea Whose Time Has Come.
PB91-150086

Biological Sulfur Oxidation and Reduction for Coal Sulfur Speciation and Desulfurization

PB91-203323

100,886 Not available NTIS

Biomarkers of $\mathrm{OH}$ Radical Damage In vivo

PB91-236877 101,325 Not available NTIS

Bioprocessing of Fossil Fuels Using Hyperthermophilic Archaebacteria.
PB91-162271

100,883 Not available NTIS

Bleomycin-Dependent Damage to the Bases in DNA is a Minor Side Reaction.

PB91-189357

101,337 Not available NTIS

Book Review: Hypertext Hands-On

PB91-237040 100,656 Not available NTIS

Book Review: Partitioning in Aqueous Two-Phase Systems: Theory, Methods, Uses, and Applications to Biotechnology.
PB91-175257

Book Review: Polarized Electrons at Surfaces by J. KirschPB91-174532

PB91-175323

101, 493 Not available NTIS

Book Review: 'The Art of Computer Systems Performance Analysis' by R. Jain.
PB92-117068

100,719 Not available NTIS

Boussinesq Algorithm for Enclosed Buoyant Convection in Two Dimensions.

PB91-178848

100,157 PC A03/MF A01

Break Junction Tunneling Spectroscopy of Single-Crystal Bismuth-Based High-Temperature Superconductors.

PB91-134478

101,462 Not available NTIS

Brief History of Measurement Systems with a Chart of the Modernized Metric System.

PB92-163872

100,940 Not available NTIS

Buckling and Post-Buckling Behavior of Elliptical Plates: Part 1. Analysis.

PB92-116573

101,588 Not available NTIS

Buckling and Post-Buckling Behavior of Elliptical Plates. Part 2. Results.

101,589 Not available NTIS

Building and Fire Research Laboratory Publications, 1990.

PB91-187807

100,122 PC A04/MF A01

Building and Fire Research Project Summaries, 1991

PB91-216788

100,132 PC A08/MF A02

Building-in Reliability: Making It Work

PB91-203992

100,812 Not available NTIS

Building Life Cycle Cost Computer Program, Version 3.2 (for Microcomputers).

PB91-507970

100,898 CP D02

Building Technology Publications 1986-1989.

PB91-216820

100,160 PC A08/MF A02

CAD Directed Robotic Deburring.

PB91-195172

100,985 Not available NTIS

Calculated from

Domain: An Update.

Calculation of Beta-Particle Counting Efficiency for Liquid Calculation of Beta-Particle Counting Efficiend
Scintillation Systems with Three Phototubes.

Scintillation Syst

101,644 Not available NTIS

Calculation of Energy of Low-Angle Grain Boundaries.

PB91-134601

101,464 Not available NTIS

Calibration at $24 \mathrm{C}$ of a Heat-Flow-Meter Apparatus Having $610 \mathrm{~mm}$ Square Plates.

PB91-206730

100,933 PC A03/MF A01

Calibration of Antenna Factor at a Ground Screen Field Site Using an Automatic Network Analyzer.

PB91-134239 100,724 Not available NTIS

Calibration of Ophthalmic Applicators at NIST: A Revised Approach.

PB91-237388

101,327 Not available NTIS

Calibration of the NBS 10 mum Space Beads.

PB91-203422

101,695 Not available NTIS

Calibration Procedures for Infrared Imaging Systems for Building Diagnostics.

PB91-147082

100,057 Not available NTIS

Calibration with Working Standards.

PB91-158931 101,276 Not available NTIS

Canadian Mass Fire Experiment, 1989

PB91-143305

101,352 PC A04/MF A01

Capacitance

Roughness.

Measurements of Surface

Capacitive Array Sensors for Nondestructive Evaluation

PB91-237313

100,947 Not available NTIS

Capillary Supercritical Fluid Chromatography of Explosives: Investigations on the Interactions between the Analytes, the Mebile Phase and the Stations between

PB91-147769

100,182 .

Carbon Acidities of Aromatic Compounds. 2. Ionic Probes of Aromaticity in Annelated Rings.

PB91-195065

100,375 Not available NTIS

Cathodoluminescence Measurement of Strained Alumina

Single Crystals.
PB91-237131

101,094 Not available NTIS

Causes of $\mathrm{SiH} 4$ Dissociation in Silane dc discharges.

PB91-202986

100,389 Not available NTIS

Ceiling Jet-Driven Wall Flows in Compartment Fires.

PB91-174573

100,117 Not available NTIS

Ceiling Jet in Fires.

PB91-167197

100,114 PC A04/MF A01

Center for Electronics and Electrical Engineering Technical Progress Bulletin Covering Center Programs, July to September 1990, with 1991 CEEE Events Calendar. PB91-159749

$100,801 \mathrm{PC}$ A03/MF A01 


\section{TITLE INDEX}

Center for Electronics and Electrical Engineering Technical Publication Announcements Covering Center Programs, July to September 1990, with 1991 CEEE Events Calendar.
PB91-184754 100,852 PC A03/MF A01

Center for Electronics and Electrical Engineering Technical Publication Announcements Covering Center Programs, October to December 1990 , with 1991 CEEE Events Calendar.

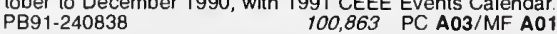

Center for Radiation Research. 1990 Technical Activities. PB91-178855 101,362 PC A08/MF A01

Ceramic Wear Maps

PB91-189498

100,561 Not available NTIS

Ceramics: Technical Activities 1991. (NAS-NRC Assessment Panel, February 13-14, 1992). 104 PC A09/MF A02

Certain Theorems on Bilateral Generating Functions Involving Hermite, Laguerre, and Gegenbauer Polynomials.

PB91-148759 101,256 Not available NTIS

Certification of NIST SRM 1961: 30 micrometers Diameter Polystyrene Spheres.

100,937

(Order as PB92-126614, PC A06/MF A02)

Chain Conformation of a Block Polymer in a MicrophaseSeparated Structure.

100,485 Not available NTIS

Change in Magnetic State of Fe+ Silica Gel Nanocomposites Due to Low Temperature Treatment in Ammonia.

PB91-237321 101,129 Not available NTIS

Characteristic Impedance Determination Using Propagation Constant Measurement.

100,775 Not available NTIS

Characteristics of Aerosols Produced by the Spark Discharge.
PB91-237727

100,209 Not available NTIS

Characteristics of the Breakdown Voltage of Power MOSFETs After Total Dose Irradiation.

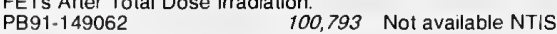

Characterization by High Performance Liquid Chromatography (HPLC) of the Solubilization of Phosphorus in Iron Ore by a Fungus.

Characterization of Capacitive Array for NDE Applications.

Characterization of Cylindrical Holes in Metallic Substrates

via Their Infrared Emission Patterns.
PB91-187187 PB91-187187

Characterization of Epoxide Coatings on Steel by Reflection/Absorption Fourier Transform Infrared Spectroscopy: Quantitative Stur

PB91-203729

101,109 Not available NTIS

Characterization of Field Concrete.

PB91-178863

100,528 PC A03/MF A01

Characterization of the Densification of Alumina by Multiple

Small-Angle Neutron Scattering, 101,100 Not available NTIS
PB92-117001

Characterization of the Mineral Fraction in Botanical Reference Materials and Its Influence on Homogeneity and Analytical Results

100,186 Not available NTIS

Characterization of Tricresylphosphate Lubricating Films by Micro-Fourier Transform Infrared Spectroscopy.
PB91-203810 101, Not available NTIS

Characterizing VLSI Processes Using Test Structures

PB91-175000 100,804 Not available NTIS

Checking the Net Contents of Packaged Goods. Third Edition, Supplement 2.

PB92-112549

100,171 PC A03/MF A01

Chemical and Electronic Properties of $\mathrm{Pd} / \mathrm{SnO} 2(110)$ Model Gas Sensors.
PB91-203075

100,393 Not available NTIS

Chemical Determination of Free Radical-Induced Damage to DNA.

PB91-187468

101,332 Not available NTIS

Chemical Effects of $\mathrm{Ne}+$ Bombardment on the MoS2(0001) Surface Studied by High-Resolution Photoelectron Spectroscopy

100,399 Not available NTIS

Chemical Kinetic Data Base for Combustion Chemistry Part 4. Isobutane.
PB90-244534

100,252 (Order as PB90-244526)

Chemical Kinetic Data Base for Combustion Chemistry. Part 5. Propene.
PB92-1 10170

100,446 (Order as PB92-110162)

Chemical Kinetic Data Base for Propellant, Combustion. 1 Reactions Involving NO, NO2, $\mathrm{HNO}, \mathrm{HNO} 2, \mathrm{HCN}$ and $\mathrm{N} 2 \mathrm{O}$.
PB92-148071

100,467 (Order as PB92-148063)

Chemical Kinetic Data Sheets for High-Temperature Reactions. Part 2.

100,480 (Order as PB92-148162)

Chemical Modification of the Orthorhombic Superconductor Ba2YCu307-delta.

PB91-159053

101,486 Not available NTIS

Chemical Nature of In vivo DNA Base Damage in Hydrogen Peroxide-Treated Mammalian Cells.
PB91-187526 Not available NTIS

Chemical Vapor Deposited Diamond.

AD-A243 095/7 101,106 PC A03/MF A01

Chemistry of Electronic Ceramic Materials.

PB91-178822

101,500 PC A23/MF A03

Chitin-Chitosan Membranes: Separations of Amino Acids and Polypeptides.

101,283 Not available NTIS

Chloride Ion Diffusion in Low Water-to-Solid Cement Pastes.

$101,138 \mathrm{PC} \mathrm{A03/MF} \mathrm{A01}$

Choosing a Name for Your Computer.

PB91-158899 100,600 Not available NTIS

Choosing Flow Conditioners and Their Location for Orifice Flow Measurement.

PB92-116490

101,397 Not available NTIS

CID Spectra of Selected Target Molecules

AD-A243 170/8 100,180 PC A04/MF A01

CIM, Data and Standardization within the NBS AMRF.

PB91-148122 100,975 Not available NTIS

Cleavage Step Formation and Resistance to Transgranular Stress Corrosion Cracking.

101, 133 Not available NTIS

Clock for the Manufacturing Systems Integration Testbed.

PB92-108885

100,992 PC A03/MF A01

Clocks, Atomic and Molecula

PB91-200840

101,677 Not available NTIS

Close-Coupling Calculation for the (2)(Sigma sub $u)(+$ ) State of $\left(\begin{array}{r}H \text { sub } 2)(-) \\ \text { PB91-148684 }\end{array}\right.$

101,614 Not available NTIS

Closure and Precision in Level-Index Arithmetic.
PB91-147652

Co-Trial on ESR Identification and Estimates of gamma-Ray and Electron Absorbed Doses Given to Meat and Bones. PB91-194720 100,017 Not available NTIS

Coherency Strain Induced Instability of Solid-Liquid Interfaces in the Mo-Ni System.

101,235 Not available NTIS

Collection of Technical Studies Completed for the Computer-Aided Acquisition and Logistic Support (CALS) Program. Fiscal Year 1988. Volume 1 of 3. Text, Security and Data Management.

101,342 PC A12/MF A02

Collection of Technical Studies Completed for the Computer-Aided Acquisition and Logistic Support (CALS) Program, Fiscal Year 1988. Volume 2 of 3 . Graphics, CGM MIL-

PB91-178897

101,343 PC A21/MF A03

Collection of Technical Studies Completed for the Computer-Aided Acquisition and Logistic Support (CALS) Program, Fiscal Year 1988. Volume 3 of 3. CGM Registration PB91-178905

101,344 PC A08/MF A01

Collisional Electron-Detachment and Ion-Conversion Processes in SF6.

100,836 Not available NTIS

Collisional Energy Transfer between Excited Sr Atoms.

PB91-204164

101,703 Not available NTIS

Combustion Calorimeters

PB91-189233

100,340 Not available NTIS

Comparative Study of Bovine Pericardium Mineralization: A Basic and Practical Approach. 100,056 Not available NTIS
PB92-117407

Comparison of Amorphous and Quasicrystalline Films of PB91-195552 101,530 Not available NTIS Comparison of Creep Rupture Behavior in Tension and Bending.

107,087 Not available NTIS

Comparison of Flux Dynamics in Two Samples of YBa2Cu3O7 with Different Pinning.
PB91-149419 No69 available NTIS Comparison of High-Resistivity Measurements of Silicon by AC Impedance, DC Resistance, van der Pauw, and FourProbe Methods.

100,792 Not avallable NTIS
Comparison of Standard Test Methods for Non-Lubricated Sliding Wear

101,187 Not available NTIS

Comparison of Two Test Methods for Determining Transfer Function Coefficients for a Wall Using a Calibrated Ho Box.
PB91-187278

100,121 Not available NTIS

Compartment Fire-Generated Environment and Smoke Filling.

100,107 Not available NTIS

Comprehensive Approach for Modeling and Testing Analog

and Mixed-Signal Devices
PB91-149310

100,732 Not available NTIS

Computational Models Developed for the Corrosion of Prestressing Steel.

$100,115 \mathrm{PC}$ A03/MF A01

Computed Fields Near the Edge of a Dielectric Wedge

PB91-203505 101,446 Not available NTIS

Computer Implementation of a Discrete Set Algebra.

PB91-231571

100,654 PC A03/MF A01

Computer Output Microform (COM) Formats and Reduction Ratios, $16 \mathrm{MM}$ and $105 \mathrm{MM}$. Category: Hardware Standard Subcategory: Media.

FIPS PUB 54-1

100,678 PC E18

Computer Program for Simulation of HVAC/Lighting Inter-

actions: Initial Report
PB91-144386

100,889 PC A05/MF A0

Computer Security Bulletin Board System. User's Guide. PB92-112390 100,717 PC A03/MF A01

Computer Security: Selected Articles.

PB91-187740 100,706 PC A03/MF A01

Computer Simulated Hydration of a Cement Model.

PB91-162149 100,112 Not available NTIS

Computer Simulation of Fluid-Fluid Phase Coexistence in Mixtures of Nonadditive Soft Disks.

PB91-162263

101,388 Not available NTIS

Computer Vision Based Station for Tool Setting and Tool Form Measurement

PB91-202978

101,043 Not available NTIS

Computerization of a Thermomechanical Processing Research System.
PB91-175208

101,583 Not available NTIS

Computing Delaunay Triangulations for Comet-Shaped Polygons.
PB92-123108

101,265 PC A03/MF A01

Computing the Eigenvalues and Eigenvectors of Symmetric

Arrowhead Matrices.
PB91-147850

101,254 Not available NTIS

Concept of the Calculus of Fire Safety.

PB91-132134

100,093 PC A03/MF A01

Concepts of Underground Corrosion.

PB91-150078 101,134 Not available NTIS

Conceptual Architecture for a Mechanical Parts Production System Based on STEP. National PDES Testbed Rept

PB92-112234

100,993 PC A03/MF A01

Concerning the Formation and the Kinetics of Phenylium lons.

PB91-187138

100,336 Not available NTIS

Concurrent Engineering through Product Data Standards.

PB91-193367 100,982 PC A05/MF A01

Cone Calorimeter Rate of Heat Release Measurements for Upholstered Composites of Polyurethane Foams.
PB92-108984 100,087 PC A03/MF A01

100,087 PC A03/M Conference Coverage: Papers Presentations Shine.
PB91-158535 100,061 Not available NTIS

Configuration Management Concepts Document. National PDES Testbed Report Series.

PB91-194480

$100,984 \mathrm{PC} \mathrm{A03/MF} \mathrm{A01}$

Configuration Management of the STEP Documents: Procedures and System Requirements. National PDES Testbed Report Series

100,968 PC A03/MF A01

Conformance Tester for X.25 DTE Implementations.

PB91-189894 100,602 Not available NTIS

Conformance Verification of FDDI Stations

PB91-148254 100,747 Not available NTIS

Conformity Assessment Workshop on Electromagnetic Compatibility.

100,579 PC A06/MF A02

Conformity Assessment Workshop on Pressure Vessels

PB91-192997

101,049 PC A06/MF A01 


\section{TITLE INDEX}

Congressional Districts of the United States. Category: Federal General Data Standard, Representations and Codes.

Continuous-Cooling Transformation Characteristics and High-Temperature Flow Behavior of a Microalloyed SAE 1141 Steel.

101,155 PC A03/MF A01

Control of Flexible Robot Arm. PB91-174979

101,032 Not available NTIS

Control Structure for Multi-Tasking Workstations.

PB91-203737 100,989 Not available NTIS

Control System Architecture for Unmanned Ground VehiPles. 134726

101,379 Not available NTIS

Convective and Morphological Instabilities during Directional Solidification.

101,219 Not available NTIS

Convergence Behavior of Trajectories for Linear Programming. 204198

101,270 Not available NTIS

Convergence Properties of a Class of Rank-Two Updates.
PB91-187799 101,260 PC A03/MF A0

Cooled Ion Frequency Standard.

AD-A227 806/7 101,591 PC A03/MF A01

AD-A242 030/5 101,593 PC A02/MF A01

Copper-Ion-Dependent Damage to the Bases in DNA in the Presence of Hydrogen Peroxide.
PB91-187104 330 Not available NTIS Core Hole Screening for Intermediate Size Metal Particles. PB91-203539 101,540 Not available NTis

Correction for Converting 2-CM(3)-Coupler Responses to Insertion Responses for Custom in-the-Ear Non-Directional Hearing Aids.

100,046 Not available NTIS

Correlated Spontaneous Emission in a Zeeman Laser

PB91-175596 101,417 Not available NTIS

PB91-204180 101,432 Not available NTIS

Correlation of Flux States Generated by Optical Switching of a Superconducting Circuit. 101,682 Not available NTIS
PB91-202929 Corrosion Benavior of Zirconium Alloy Nuclear Fuel CladPB91-158733 101,372 Not available NTIS Corrosion Principles in Dental Implantology.

PB91-194787 100,055 Not available NTIS

Corrosion, Repassivation and Corrosion Fatigue Behavior of the Surgical Implant Alloy, Co-Cr-Mo. Corrosion Testing in Soil.

1,132 Not available NTIS

PB91-158667

101, 135 Not available NTIS

Coupled Phase Diagram-Thermodynamic Analysis of the 24 Binary Systems, A2CO3-AX and A2SO4-AX Where $\mathrm{A}=\mathrm{Li}$,

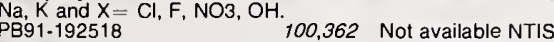

Crack Arrest Fracture Toughness Measurements of Normalized and Inclusion Shape Controlled AAR TC128 Grade $B$ Steel, and Micro-Alloyed, Control-Rolled, and Inclusion Shape Controlled A $8 \times X$ Grade B Steel.

PB91-167346

101,150 PC A03/MF A01

Cratering.

100,810 Not available NTIS

Creep and Reliability of Ceramic Materials at Elevated Temperatures.

101,091 Not available NTIS

Creep of Copper: 4 to $295 \mathrm{~K}$

PB91-189985

101,233 Not available NTIS

Criteria for the Operation of Federally-Owned Secondary

Calibration Laboratories (Ionizing Radiation).
PB92-112481

Critical Assessment of Optical Properties of CVD Diamond Films.

AD-A236 746/4

101,400 PC A02/MF A01

Critical Compilation of Surface Structures Determined by lon Scattering Methods.
PB90-244559 100,254 (Order as PB90-244526) Critical-Current Diffraction Patterns of Grain-Boundary Jo-

sephson Weak Links.
PB91-237180

101,551 Not available NTIS

Critical Data in Physics and Chemistry.

P891-203455 101,697 Not available NTIS

Critical Evaluation of Liquid Crystal Transition Temperatures l: $4,4^{\prime}$-Alkyl/alkoxyphenylbenzoates.
PB92-110154 100,444 (Order as PB92-110113) Critical Exponent for the Viscosity of Four Binary Liquids.
PB91-187195

100,337 Not available NTIS

Critical Exponent for Viscosity PB91-147009

100,271 Not available NTIS

Critical Locus, (Vapor + Liquid) Equilibria, and Coexisting Densities of (Carbon Dioxide + Propane) at Temperatures from $311 \mathrm{~K}$ to $361 \mathrm{~K}$

PB91-134494

100,267 Not available NTIS

Cross-Section Measurements in the Intermediate-Energy Standard Neutron Field.

101,693 Not available NTIS

Cross Sections and Related Data for Electron Collisions with Hydrogen Molecules and Molecular lons.

PB90-244674 100,263 (Order as PB90-244658)

Cross Sections and Swarm Coefficients for $\mathrm{H}_{+}, \mathrm{H} 2+$ $\mathrm{H} 3+, \mathrm{H}, \mathrm{H} 2$, and $\mathrm{H}$-in $\mathrm{H} 2$ for Energies from $0.1 \mathrm{eV}$ to 10 PB90-244690

101,597 (Order as PB90-244658)

Cross Sections and Swarm Coefficients for Nitrogen lons and Neutrals in N2 and Argon lons and Neutrals in N2 and Argon lons and Neutrals in Ar for Energies from $0.1 \mathrm{eV} 10$ PB92-110246

100,452 (Order as PB92-110212)

Cross Sections for Collisions of Electrons and Photons with Atomic Oxygen.
PB90-244682

100,264 (Order as PB90-244658)

Cross-Validation Procedure for Stopping the EM Algorithm

and Deconvolution of Neutron Depth Profiling Spectra.
PB91-161976 101,627 Not available NTIS

Cryogenic Fatigue of High-Strength Aluminum Alloys and Correlations with Tensile Properties.
PB91-236976 Not available NTIS

Cryogenic Material Properties of Stainless Steel Tube-toFlange Welds
PB91-134627

101, 145 Not available NTIS

Cryogenic Properties of Copper (Wall Chart)

PB91-148189 101,473 Not available NTIS

Cryogenic-Temperature Fluorescence Spectra of Polynuclear Aromatic Hydrocarbons of Molecular Weight 302.

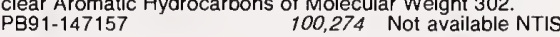

Crystal and Spin Structures of $\mathrm{Nd}_{2} \mathrm{CuO} 4$

PB91-148023 101,470 Not available NTIS

Crystal-Fieid Splittings in the Cubic Heusler Alloys HoPd2Sn and ErPd2Sn.

101,525 Not available NTIS

Crystal Structure of a CAMP-Independent Form of Catabolite Gene Activator Protein with Adenosine Substituted in One of 2 CAMP-Binding Sites.

PB91-190074

101,305 Not available NTIS

Crystal Structure of Phosphate-Free Ribonuclease

PB91-175604 101,301 Not available NTIS

Crystalline and Glassy Phases of Transition-Metal - Metalloid Systems.
PB91-204156

101,238 Not available NTIS

Crystallizing Catabolite Gene Activator Protein with CAMP for Structural Analysis.

101,299 Not available NTIS

Current Density Dependence of Electromigration t50 En-

hancement Due to Pulsed Operation.
PB91-134692 100,786 Not available NTIS

Current Record in Superconductors

PB91-194753 101,521 Not available NTIS

Current Supply for High-T(sub c) Superconductor Testing.

PB91-133827 101,455 Not available NTIS

Cutting the High Cost of Testing.

PB91-162362 100,846 Not available NTIS

CW Dye Lasers.

P892-116896

101,438 Not available NTIS

Cyclic Fatigue from Frictional Degradation at Bridging

Grains in Alumina.
PB91-236927 101,092 Not available NTIS

Damage to the Bases in DNA Induced by Hydrogen Peroxide and Ferric lon Chelates. 101,331 Not available NTIS
PB91-187112

Damage to the Bases in DNA Induced by Stimulated
Human Neutrophils.
PB92-116912 101,312 Not available NTIS PB92-116912

01,312 Not available NTIS

Damage to the DNA Bases in Mammalian Chromatin by Hydrogen Peroxide in the Presence of Ferric and Cupric lons.
PB91-189217

DARPA Air Travel Information System (ATISO) Speaker-Dependent Training Data (on CD ROM).

PB91-505370

100,594 CD-ROM $\$ 1200.00$

Data Bases Available in the Research Information Center of the National Institute of Standards and Technology.
PB92-109016

100,954 PC A07/MF A02

Data Encryption Standard: Past and Future.

PB91-204107 100,711 Not available NTIS

Data for Fire Hazard Assessment of Selected Non-Halogenated and Halogenated Fire Retardants; Report of Test FR

PB92-112473

100,557 PC A04/MF A01

Data for Room Fire Model Comparisons.

PBg -110287

100,167

(Order as PB92-110261, PC A05/MF A01)

Data Management Standards in Computer-Aided Acquisition and Logistic Support (CALS).

PB91-158832

101,339 Not available NTIS

Data-Reduction and Analysis Procedures Used in NIST'S Thermomechanical Processing Research.

PB91-132282

$100,925 \mathrm{PC} \mathrm{A04/MF} \mathrm{A01}$

Database and Retrieval System for the NBS Tables of Chemical Thermodynamic Properties.

PB91-237693

100,434 Not available NTIS

Daylight Research Requirements. Workshop Proceedings. Held in Baltimore, Maryland on August 1-2, 1990.

PB91-231563 100,078 PC A04/MF A01

Dealing with Obvious Issues in Lighting.

PB91-237792 100,761 Not available NTIS

Deconvolution of Acoustic Emission and Other Causal Time Series.

PB91-216762

101,263

(Order as PB91-216705, PC A07/MF A01)

Dental Biomaterials and Engineering Frontiers for the 90's. PB91-159210 100,044 Not available NTIS

Dental Composite Resins: An Update.

PB91-204149

100,051 Not available NTIS

Dentin-Bonding Molar Efficiency Using N-Phenylglycine, N-

Phenyl-beta-Alanine, or N-Methyl-N-Phenylglycine.
PB91-237735 100,053 Not available NTIS

Depot: A Framework for Sharing Software Installation

Across Organizational and Unix Platform Boundaries.
PB91-240754 100,659 PC A03/MF A01

DEQSOL and ELLPACK: Problem-Solving Environments for Partial Differential Equations.

PB91-174441

100,645 Not available NTIS

Description of a Vibration Compensation System for the Small Scale Model Robot Crane Project.

PB91-216648

100,130 PC A04/MF A01

Design and Analysis of Experiments.

PB91-158824 101,274 Not available NTIS

Design and Fabrication of a Miniature High-Temperature Guarded-Hot-Plate Apparatus.

PB92-116797

101,098 Not available NTIS

Design and Implementation of a Five-Axis Robotic Micromanipulator for Inserting Parts into Precision Collets.

PB91-159194

101,029 Not available NTIS

Design Diagrams for Heavy Metal Fluoride Glass Windows. PB91-187419

101,419 Not available NTIS

Design, Fabrication, and Testing of an Interconnect Test Structure for Evaluating VLSI Processes.

PB91-174581

100,803 Not available NTIS

Design Heat Loss Factors for Basement and Slab Floors.

PB91-147561

100,059 Not available NTIS

Design of High Strength Cement-Based Materials. Part 2. Microstructure.

100,099 Not available NTIS

Designing Data Entity Naming Conventions.

PB91-175455

100,646 Not available NTIS

Detail Specification for 62.5-mum Core Diameter/125-mum Cladding Diameter Class la Multimode, Graded-Index Optical Waveguide Fibers.

100,562 PC E07

Detection and Quantitative Characterization of Blistering and Corrosion of Coatings on Steel Using Infrared Thermography.

101,139 Not available NTIS

Detection of an Expanding $\mathrm{H}$ I Shell in the Old Supernova Remnant CTB 80.

PB91-174920

100,028 Not available NTIS

Detection of Excited States by Laser-Induced Fluorescence and Analysis of Energy Transfer. AD-A227 296/1

100,242 PC A04/MF A01

Detection of Trace Disulfur Decafluoride in Sulfur Hexafluoride by Gas Chromatography/Mass Spectrometry.

PB91-189860 100,197 Not available NTIS 


\section{TITLE INDEX}

Determination of Bonded Phase Thickness in Liquid Chromatography by Small Angle Neutron Scattering.
PB91-149187

Determination of Fields Near a Silver Strip on a Glass Substrate.

100,841 Not available NTIS

Determination of Scattering Parameters with Respect to the Characteristic Impedance of Precision Coaxial Air-Line Standards.

PB91-134114

100,770 Not available NTIS

Determination of the Neutron Mass and Implications for Fundamental Constants.

101,615 Not available NTIS

Determination of the Optical Constants of Thin Chemical Vapor-Deposited Diamond Windows from 0.5 to $6.5 \mathrm{eV}$.
$\mathrm{AD}-\mathrm{A} 243094 / 0$
100,215
$\mathrm{PC} \mathrm{A03/MF} \mathrm{A01}$

Determining Adapter Efficiency by Envelope Averaging Swept Frequency Reflection Data.
PB91-236620 100,743 Not available NTIS Determining the Dynamic Stress Intensity Factor with Strain Gages Using a Crack Tip Locating Algorithm.
PB91-174268 Developing Federal Software Standards: A New Direction. PB91-134536 100,637 Not available NTIS Development of a Coulometric Method for Assessing the Concentration of Ambient Levels of $\mathrm{CO} 2 /$ Air in Compressed Gas Mixtures.

100,913

(Order as PB92-126614, PC A06/MF A02)

Development of a Line Camera for Real-Time Measure-

ments of Concentration in Turbulent Flow Fields.
PB91-147892

Development of a New Tracer Technology Using Enriched Rare-Earth Isotopes.

PB91-195347

100,908 Not available NTIS

Development of a Technique to Assess the Adequacy of the Municipal Water Supply for a Residential Sprinkler System.
PB92-123116

101,358 PC A05/MF A01

Development of a Three-Dimensional Finite Element Pro-

gram for Large Strain Elastic-Plastic Solids.
PB91-174557 101,494 Not available NTIS

Development of an Adhesive System for Bonding to Hard Tooth Tissues.
PB91-236539

100,052 Not available NTIS

Development of an Optical Disk System for the Automated Retrieval of EASEAR Records.

PB92-108943

$100,666 \mathrm{PC} \mathrm{A07/MF} \mathrm{A02}$

Development of Test Methods to Determine the Compatibitity of Liquid Hazardous Materials with Polyethylene Packagings.
PB92-126457

100,943 PC A05/MF A01

Development Plan: Application Protocols for Mechanical Parts Production. National PDES Testbed Report Series.
PB91-222596 100,990 PC A03/MF A01

Device for Audio-Frequency Power Measurement.
PB91-149401 100,733 Not available NTIS

Diagnostic Techniques for Evaluating Office Building Envelopes.
PB91-195438

100,074 Not available NTIS

Dielectric Constant of Water and Debye-Huieckel Limiting Law Slopes.

100,260 (Order as PB90-244609)

Dielectronic Recombination Rate Coefficients for lons of the Fluorine Isoelectronic Sequence.

Dielectronic Recombination Rate Coefficients of $\mathrm{O}(5+)$ and $\mathrm{O}(2+)$.

101,657 Not available NTIS

Diesel Deposit Forming Tendencies - Microanalysis MethPB91-195412

101,178 Not available NTIS

Dietary Collection Procedures

PB91-147413

101,317 Not available NTIS

Differences between Thermodynamic Temperature and t(IPTS-68) in the Range $230 \mathrm{C}$ to $660 \mathrm{C}$.
PB92-116722 101,725 Not available NTIS Differential Sintering

PB91-237123

101,128 Not available NTIS

Difficulties in Parsing SGML. 100,650 Not available NTIS
PB91-189456

Diffraction by a Half-Plane in a Lossy Medium.

PB92-116888 101,437 Not available NTIS

Difunctional and Multifunctional Monomers Capable of Cyclopolymerization.
PB91-195685

100,504 Not available NTIS

Digital Image Analysis Assisted Microradiography Measurement of Mineral Content of Caries Lesions in Teeth. (Order as PB91-187617, PC A06/MF A01)

Dihedral Angles in Magnesia and Alumina: Distributions from Surface Thermal Grooves.

PB91-147330

101,058 Not available NTIS

Dimensional Calibration of an NBS 0.3-mum-Diameter Particle-Sizing Standard.

101,696 Not available NTIS Diode Laser Probing of $\mathrm{I}^{*}((2) \mathrm{P} 1 / 2)$ Doppler Profiles: Time
Evolution of a Fast, Anisotropic Velocity Distribution in a Thermal Bath

100,310 Not available NTIS

Diode Lasers and Their Application to Spectroscopy.
PB92-116904 Not available NTIS

Dipole Moments of Seven Partially Halogenated Ethane Refrigerants.

100,377 Not available NTIS

Direct Detection of Atom Vaporization by Laser Resonance lonization as a Probe of Gas-Surface Chemisorption Mechanisms. PB91-237032

100,425 Not available NTIS

Direct Determination of Molecular Orbital Symmetry of H2S Using Polarized X-ray Emission. 100,335 Not available NTIS
PB91-175562

Direct Digital Control Based Building Automation System Design Criteria.

100,091 PC A03/MF A01

PB91-216614

ories, 1991. National VolunDirectory of Accredited Labor PB91-222646 100,934 PC A08/MF A02

Directory of Federal Government Laboratory Accreditation/ Designation Programs.

100,005 PC A06/MF A0

PB91-167379

Directory of State and Local Government Laboratory AC
creditation/Designation Programs.
PB92-108968 100,174 PC A05/MF A01 PB92-108968

Discontinuous Yielding during Tensile Tests at Low Tem-
peratures.
PB91-189977
101,159 Not available NTIS

DISCOUNT-A Program for Discounting Computations in Life-Cycle Cost Analyses. User's Guide and Reference

Manual.

100,897 PC A03/MF A01

Dispersion of the Electronic Contribution to the Thrid-Order Nonlinear Susceptibility of $\mathrm{H} 2$.
PB91-149138 101,411 Not available NTIS Dissociation Energetics of Simple Diazirines by Photon and Electron Impact.

100,405 Not available NTIS

Dissociation of Substituted Anisoles: Substituent Effects on

Bond Strengths.
PB91-237537

100,431 Not available NTIS

Document Architecture for Open Systems.

PB91-189522 100,961 Not available NTIS

Domestic Implementation

PB91-159061

100,176 Not available NTIS

Doppler Shift Attenuation Lifetime Measurements in (54)Cr

Doppler Shift Attenuation Lifetime Measurements in (54)Cr
Following Thermal Neutron Capture.
PB91-189753 101,656 Not available NTIS

Dosimetry: New Approaches for New Challenges.

Dosimetry: New Approaches for New Challenges.
PB92-117100 Not available NTIS

Dosimetry of a Nearly Monoenergetic 6- to 7-MeV Photon

Source by Nal(TI) Scintillation Spectrometry.
PB92-116706

Draft Abstract Test Suite for Determining Conformance to the BACnet Protocol.

100,070 PC A06/MF A01

PB91-187732

ystems Using Waveguide in

Dual Six-Port Reflectometer Syste

PB91-134916

100,838 Not available NTIS

Dynamic Arc-Power Source Response in GMA Welding

PB91-149278

101,002 Not available NTIS

Dynamic Technique for Measuring Surface Tension at High Temperatures in Microgravity Environment.

101,206 N91-21347/0

(Order as N91-21331/4, PC A24/MF A03)

Dynamical Diffraction Imaging (Topography) with $X$-ray Syn-

chrotron Radiation.

101,510 Not available NTIS

Dynamics of Molecular Hydrogen Adsorbed in CoNa-A Zeolite.
PB91-195255

100,380 Not available NTIS

Eccentric Load Sensitivity of Force Sensors.

PB91-175406 100,931 Not available NTIS

Economic Analysis for Military Construction (MILCON) Design. Concepts, Techniques, and Applications for the Analyst. Instructor's Manual.

PB92-112440

101,349 PC A99/MF A06

Economic Analysis for Military Construction (MILCON) Design. Concepts, Techniques, and Applications for the Analyst. Student's Manual.

101,350 PC A21/MF A04

Eddy Current Probe Characterization Using an Impedance Plane Display Instrument.

PB91-133868

100,834 Not available NTIS

Eddy Current Probe Sensitivity as a Function of Coil Construction Parameters.

100,945 Not available NTIS

Eddy Current Reflection Probe: Theory and Experiment. PB91-187120 100,944 Not available NTIS

Effect of a Free Surface on Stress Distribution in a Bimaterial Composite.

$101,121 \mathrm{PC}$ A09/MF A01

Effect of an Electric Field on the Morphological Stability of the Crystal-Melt Interface of a Binary Alloy. 3. Weakly Nonlinear Theory.

101,536 Not available NTIS

Effect of Atomization Air on Droplet Dynamics of Spray

Flames.
PB91-203869

100,550 Not available NTIS

Effect of Catalyst Structure on the Synthesis of a Dental Restorative Monomer.

PB91-158691

100,043 Not available NTIS

Effect of Coherency Strain on Alloy Formation: Migration of Liquid Films.

101,210 Not available NTIS

Effect of Crosslink Density on Physical Aging of Epoxy Networks.

101,244 Not available NTIS

Effect of Gravity Modulation on Thermosolutal Convection in an Infinite Layer of Fluid.

101,396 PC A03/MF A01

Effect of Green Density and the Role of Magnesium Oxide Additive on the Densification of Alumina Measured by Small-A

PB92-117043

101,101 Not available NTIS

Effect of High Pressure on Prebreakdown Phenomena in $n$ Hexane.
PB91-135020

100,839 Not available NTIS

Effect of Logarithmic Singularity on the Free Edge Stress Intensity Factor of Composite Laminates.
PB91-147090 101,116 Not available NTIS

Effect of Long Range Interfacial Forces on the Dynamics of

Wetting Layers at a Liquid Vapor Interface.
PB91-189431 100,344 Not available NTIS

Effect of Mechanical Deformation on Nb-Ti Filament Proximity-Effect Coupling at the Edges of SSC Cables. PB91-203828 101,700 Not available NTIS

Effect of Nitrogen and Carbon on FCC-HCP Stability in Austenitic Steels.
PB91-175372

101,151 Not available NTIS

Effect of Noble Gas Mixtures on the Performance of Re-

generative-Type Cryocoolers Analytical Estimate.
100,890 PC A03/MF A01

Effect of Pipe Surface Finish on Gas Flow Measurement with an Orifice Meter.

101,387 Not available NTIS

Effect of Pipe Surface Finish on the Orifice Discharge Coefficient.

PB91-162347

101,389 Not available NTIS

Effect of Processing on 4-K Mechanical Properties of a MiCroalloyed Auste

101,167 Not available NTIS

Effect of Pyrophosphate Concentrations on Calcium Phosphate Growth on Well-Crystallized Octacalcium Phosphate .

and Hydroxyapat

101,193 Not available NTIS

Effect of Selected Chemical Compounds on the Lubrication of Silicon Nitride.

101,180 Not available NTIS

Effect of Slag Penetration on the Performance of Magnesia Chrome Retractories in Slagging Gasifiers.
PB91-134940 100,875 Not available NTIS

Effect of Surface Forces on Subcritical Crack Growth in Glass,

PB92-117449

101,103 Not available NTIS 


\section{TITLE INDEX}

Effect of the Location of an In-Line Tube Bundle on Orifice PB91-134445

100,921 Not available NTIS

Effect of Thermal Expansion Mismatch on Fiber Pull-Out in Glass Matrix Composites.

101,125 Not available NTIS

Effect of Thermochemical Treatments on the Strength arid Microstructure of SiC Fibers.

Microstructure
PB91-194670

101,084 Not available NTIS

Effect of Tube Bundle Flow Conditioners on Orifice Meter ischarge Coefficients.

PB91-148791

100,881 Not available NTIS

Effect of Veiling Relections on Vision of Colored Objects. PB91-237800 100,079 Not available NTIS

Effects of Absorbed Dose Rate, Irradiation Temperature and Post-Irradiation Temperature on the Gamma Ray Reonse of Red Perspex Dosim

PB92-116441 101,363 Not available NTIS

Effects of Colored Pump Noise on Intensity Correlations of a Single Mode Dye Laser.

PB91-189738

101,423 Not available NTIS

Effects of Crystal Bonding on Brittle Fracture

PB91-148221 101,064 Not available NTIS

Effects of Different $\mathrm{CH} 4-\mathrm{H} 2$ Gas Compositions on the Morphology and Growth of Diamond Grown by Hot Filament CVD.

101,054 Not available NTIS

Effects of Global Density Ratio on the Centerline Mixing Behavior of Axisymmetric Turbulent Jets

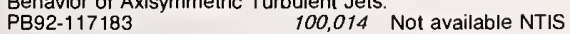

Effects of Multiple Filament Geometry in the Hot Filament Deposition of Diamond Films.

AD-A236 $708 / 4$

101,142 PC A02/MF A01

Effects of $\mathrm{pH}$ and Calcium on Hydrolysis of Na2SiF6 and Na2SnF6: A Quasi-Constant Composition Titration Study.

PB91-194746

101,315 Not available NTIS

Effects of Pressure on the Thermal Decomposition Kinetics and Chemical Reactivity of Nitromethane.

PB91-147884 100,286 Not available NTIS

Effects of Varying Austenitizing Temperature and Cooling Rate on the Ability of HSLA-80 Steel to Achieve a Yield Strength Comparable to HSLA-100.

PB91-189464 101,156 Not available NTIS

Elastic Constants, Debye Temperatures, and ElectronPhonon Parameters of Superconducting Cuprates and Related Oxides.

PB91-134262

101,460 Not available NTIS

Elastic Constants of a Tungsten-Particle Copper-Matrix Composite.
PB91-194852

101,234 Not available NTIS

Elastic Constants of Fiber-Reinforced Composites: A Fresh Measurement Approach.

PB91-194878

101,123 Not available NTIS

Elastic Constants of Polycrystalline Bi-Pb-Sr-Ca-Cu-O Superconductor.
PB91-134270

101,461 Not available NTIS

Electric Quadrupole and Magnetic Dipole Transition Probabilities in Potassium Isoelectronic Sequence.

PB91-187088 101,646 Not available NTIS

Electrical Breakdown and Streamer Statistics in N-hexane under Uniform Field Conditions.

PB91-134684 100,837 Not available NTIS

Electrochemical Behavior of Ferrocene in Polyethylene Oxide- 400 .

PB91-237867

100,439 Not available NTIS

Electrodeposition of Aluminum from Moiten Salts

PB91-159202 101,223 Not available NTIS

Electrofission in the Quasifree and Delta Regions.

PB91-147355 101,609 Not available NTIS

Electrokinetic Demixing of Two-Phase Aqueous Polymer Systems. 1. Separation Rates of Polyethylene Glycol-Dextran Mixtures

PB91-159095

100,487 Not available NTIS

Electrokinetic Demixing of Two-Phase Aqueous Polymer Systems. 2. Separation Rates of Polyethylene Glycol-Maltodextrin Mixtures.

PB91-237230

100,231 Not available NTIS

Electron Attenuation Lengths at $\mathrm{SiO} 2 / \mathrm{Si}$ Interfaces.

PB91-175612 101,499 Not available NTIS

Electron Spin Resonance for Monitoring Radiation-Processed Meats Containing Bone.
PB92-116672 100,018 Not available NTIS Electronic and Radiometric Characteristics of Near Infrared Photodiodes.

100,752 Not available NTIS
Electronic Data Interchange (EDI). Category: Software Standard. Subcategory: Electronic Data Interchange.
100,677 PC A02 Electronic Data Interchange in Message Handling Systems.
PB91-216622

Electronic Spectrum of the $\mathrm{GeH} 3$ Radical

PB91-189548 100,346 Not available NTIS

Electronic Structure of High-Tc Superconductors Studied Using Photoelectron Spectroscopy.
PB91-189688 Not available NTIS

Electronics and Electrical Engineering Laboratory Technical Publication Announcements Covering Laboratory Programs, January to March 1991, with 1991 EEEL Events Calendar.
PB92-112309 100,864 PC A03/MF A01

Electronics and Electrical Engineering Laboratory. 1991 Strategic Plan. Supporting Technology for U.S. Competitiveness in Electronics.

100,870 PC A03/MF A01

Ellipsometry SRM's for Use in Thin Film Measurements

PB92-116599

100,819 Not available NTIS

Emergence of Modern Nucleation Theory.

PB91-149997

101,481 Not available NTIS

Emissivity of Aluminized Mylar

PB91-174656

101,119 Not available NTIS

Emitter Ballasting Resistor Design for, and Current Handling Capability of AIGaAs/GaAs Power Heterojunction Bipolar Transistors.

100,815 Not available NTIS

Energy Distributions of Symbiotic Novae.

PB91-200873

100,032 Not available NTIS

Energy Levels and Transition Probabilities in the Ground

State Configuration of Sulfur-Like lons.
PB91-190033 Not available NTIS

Energy Levels of Atomic Aluminum with Hyperfine Struc-

PB90-244575

101,594 (Order as PB90-244526)

Energy Levels of Copper, Cu I through Cu XXIX

PB90-244666 101,596 (Order as PB90-244658)

Energy Levels of Krypton, $\mathrm{Kr} I$ through $\mathrm{Kr}$ XXXVI.

PB92-148139 100,473 (Order as PB92-148113)

Energy Levels of Sulfur, S I Through S XVI

PB91-192443 100,355 Not available NTIS

Energy of Combustion of Triphenylphosphate.

PB91-189613 100,347 Not available NTIS

Energy Prices and Discount Factors for Life-Cycle Cost Analysis 1992. Annual Supplement to NIST Handbook 135 and NBS Special Publication 709

PB92-112515

100,899 PC A04/MF A01

Energy Related Inventions Program: A Joint Program of the Department of Energy and the National Institute of Stand. ards and Technology. Status Report for Recommendations through 250

PB91-178871

100,902 PC A09/MF A01

Energy Related Inventions Program. A Joint Program of the Department of Energy and the National Institute of Standards and Technology. Status Report for Recommendations 251 through 523

100,903 PC A13/MF A02

Engineering Analysis of Fire Development in the Hospice of

Southern Michigan, December $15,1985$.
PB91-195222

Engineering Analysis of the Fire Development in the Hillhaven Nursing Home Fire, October 5, 1989.

100,169. PC A04/MF A01

Engineering Design Laboratory Guide

100977 PC A03/MF A01

Engineers' Statistical Literacy is Key to U.S. CompetitivePBS1-158725

100,038 Not available NTIS

Enhanced Flux Creep in Nb-Ti Superconductors After an Increase in Temperature.

101,680 Not available NTIS

Enhanced Transport and Liquid Membranes in Biosepara-

PB91-189886

101,287 Not available NTIS

Environmental Effects on the Medium and Long Term Frequency Stability of Quartz Oscillators.
PB91-134866 Not available NTIS

Environmental Sensitivities of Quartz Crystal Oscillators.

$\mathrm{N} 91-25797 / 2$

100,778

(Order as N91-25755/0, PC A99/MF A04)

Environmentally Enhanced Fracture of Gallium Arsenide. PB91-237743 101,097 Not available NTIS

Epitaxial fcc Fe Films on Cu(100).
PB91-149328

101,478 Not available NTIS

Equilibrium and Transport Properties of Gas Mixtures at Low Density: Eleven Polyatomic Gases and Five Noble

100,363 Not available NTIS

Ergodic Convergence Properties of Supercooled Liquids and Glasses.

100,289 Not available NTIS

Ergodic Measures for the Simulation of Dielectric Properties of Water.

100,350 Not available NTIS

Error Prevention and Detection in Data Preparation for a

Numerically Controlled Milling Machine.

ncy Standards. Established Microwave Frequency Standards.
PB91-203182 100,584 Not available NTIS Establishing a Computer Security Incident Response Capa-
bility (CSIRC).
PB92-123140 100,720 PC A03/MF A01

Estimated Impact of the Center for Fire Research Program on the Costs of Fire.

B91-217422

100,161 PC A05/MF A01

Estimation of Electromagnetic Fields in Complex Environ-

ments.
PB91-203315

101,691 Not available NTIS

Estimators for Censored (Log)Normal Samples.

PB91-203414 101,277 Not availabie NTIS

Evaluation and Improvement of E-Beam Exposure Routınes by Use of Microelectronic Test Structures.

PB91-175489 100,805 Not available NTIS

Evaluation of Building Systems Inventions.

PB91-158659 100,896 Not available NTIS

Evaluation of Column Performance in Liquid ChromatograPBy. 190041

100,199 Not available NTIS

Evaluation of Dual-Port Circularly Polarized Probes for Planar Near-Field Measurements.

PB91-162040

100,590 Not available NTIS

Evaluation of Fumed-Silica Insulation for a Thermal Conductivity Standard Reference Material.

PB91-147975 100,103 Not available NTIS

Evaluation of Instrumentation Used to Measure AC Power System Magnetic Fields.

100,872 Not available NTIS

Evaluation of Liquid Junction Potentials and Determination of pH Values of Strong Acids at Moderate lonic Strengths.
PB91-237859 100,438 Not available NTIS

Evaluation of Solubility Data of the System CO-H2O from

$273 \mathrm{~K}$ to the Critical Point of Water.
$\mathrm{PB92}-110253 \quad 100,453$ (Order as PB92-110212)

Evaluation of Test Methods for Measuring the Bond Strength of Portland Cement-Based Repair Materials to

PB91-189621

100,530 Not available NTIS

Evaluation of the Heat Flux Transducer Technique for

Measuring the Thermal Performance of Walls.
PB91-194696 100,126 Not available NTIS

Evaporator Performance Investigation for Residential Air-

Conditioning Application Using Mixed Refrigerants.
PB92-126432

Evidence for Surface alpha Particle Clusters in (nat)AG(197)Au from the (e,alpha) Reaction.

PB91.216812 101,705 PC A04/MF A01

Evolution of Infrared Carbon Stars.

PB91-202861

100,033 Not available NTIS

Examination of Mechanisms Responsible for Incoherent

Scattering of Subthermal Neutrons by Polymers.
PB91-202887 100,505 Not available NTIS

Examination of the Excessive Retained Austenite on the Surface of a Section of 17-7 Precipitation Hardening Stainless Steel.

PB91-167171

101,149 PC A03/MF A01

Excess Low-Frequency Flux Noise in dc Squids Incorporating $\mathrm{Nb} / \mathrm{Al}-\mathrm{Oxide} / \mathrm{Nb}$ Josephson Junctions.

PB91-236810 100,736 Not available NTIS

Experimental and Numerical Studies of the J-Integral for a Surface Flaw.
PB91-147256

101,576 Not available NTIS

Experimental Evaluation of Selected Orifice Flowmeter Upstream Installations

PB91-147025

100,880 Not available NTIS

Experimental Stark Widths and Shifts for Spectral Lines of Neutral and lonized Atoms: A Critical Review of Selected Data for the Period 1983 through 1988 


\section{TITLE INDEX}

PB91-192567

101,659 Not available NTIS

Experimental Study of the Pyrolysis Rate of a Poly(methyl methacrylate) (PMMA) Wall Panel in a Reduced-Scale En

100,555 Not available NTIS

Experimental Study of Top Vented Compartment Fires. PB91-167254 100,155 PC A03/MF A01

Experimental Thermal Conductivity, Thermal Diffusivity, and Specific Heat Values for Mixtures of Nitrogen, Oxygen, and

100,369 PC A04/MF A01

Experimentally Verified IGBT Model Implemented in the Saber Circuit Simulator

100,822 Not available NTIS

Experimentation, Analysis, and Correlation of Refrigerant-22 Flow Through Short Tube Restrictors.
PB91-174243

Experimentation and Measurement.
PB91-203372 No1,692 Not available NTIS

Explicit Mapping of the Set of Dynamical Symmetries onto the Set of Equivalent Lagrangians for Systems with One Degree of Freedom.

Degree of Freed
PB91-158881

101,622 Not available NTIS

Exploding Foil Photoionization $X$-ray Laser

PB91-237065 101,433 Not available NTIS

Extended Abstract: A Finite Element Study of the Stress

and Displacement Fields Produced by Point Impact.
PB91-175224

Extended Phase Rule for Nonreactive, Multiphase, Multi-

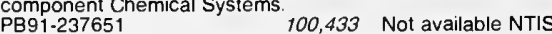

Extending the Range and Accuracy of Phase Noise Meas-

Urements.
PB91-134874

100,588 Not available NT1S

Extension of an Improved One-Fluid Conformal Solution Theory to Real Fluid Mixtures with Large Size Differences.
PB91-147405

Extension of the Class of Magnetic B Star Nonthermal Radio Sources.
PB91-175042

100,031 Not available NTIS

Extracting STEP Geometry and Topology from a Solid Modeler: Parasolid-to-STEP.

100,983 PC A04/MF A01

Extreme-Ultraviolet Spectra of $\mathrm{Pd}, \mathrm{Ag}, \mathrm{Cd}$, and $\mathrm{Sn}$ lons $\mathrm{Ob}$ served in a 1,3-keV Tokamak Plasma.
PB91-237495 Not available NTIS Factual Materials Databanks - The Need for Standards PB91-147546 101,249 Not available NTIS

Faraday Effect Current Sensing Using a Sagnac Interferometer with a $3 \times 3$ Coupler

100,754 Not available NTIS

Fe Implantation in In0.53Ga0.47As/InP

PB91-189969 101,516 Not available NTIS

Fe-W Supermirrors for Polarizing Neutrons

PB91-175521 101,643 Not available NTIS

Feasibility of Using a Multiple Award Schedule for Specifying Paints in Government Painting Contracts.
PB92-126481 100,002 PC A03/MF A01

Feasibility of Using Knowledge-Based Systems for Aiding Inventors. PB91-147728

100,895 Not available NTIS

Features and Facilities of Estelle.

PB91-158915 100,688 Not available NTIS

Fed-X: The NIST Express Translator. Revised, November 1990.

PB91-132126

100,681 PC A03/MF A0

Federal Information Processing Standards Publication. FIPS SET $1991 \quad 100,680$ PC\$2300.00

Fiber-Optic Fluorescence Array to Study Free Convection in Porous Media

101,407 Not available NTIS

Field Study of the Performance of EPDM Roofing at Air Force Facilities.

101,340 PC A05/MF A01

PB91-167247

Field Testing of Adhesive-Bonded Seams of Rubber Roofing Membranes

100,124 Not available NTIS

Filler Systems Based on Calcium Metaphosphates.

PB91-194654 100,048 Not available NTIS

Fire-Plume-Generated Ceiling Jet Characteristics and Convective Heat Transfer to Ceiling and Wall Surfaces in a Two-Layer Zone-Type Fire Environment: Uniform Temperature Ceiling and Walls.

PB92-123074

101,370 PC A04/MF A01
Fire Propagation in Concurrent Flows.

PB91-157206 100,543 PC A07/MF A01

FIREDOC Users Manual, 2nd Edition

PB91-178830 100,547 PC A03/MF A01

First Pass at Computing the Cost of Fire Safety in a Modern Society
PB91-216796

101,730 PC A04/MF A01

Five Small Flaming Fire Tests in a Simulated Hospital Patient Room Protected by Automatic Fire Sprinklers. PB91-159038 100, 109 Not available NTIS

Flammability Characterization of Foam Plastics.

PB92-123033

100, 142 PC A07/MF A02

Flexible Polymer with Excluded Volume at an Interacting Penetrable Surface of Variable Dimension: A Multiple epsiIon Perturbation Theory.

PB92-116698

100,517 Not available NTIS

Flowmeter Installation Effects: A New Approach to an Old but Prevalent Problem.

PB91-134411

100,920 Not available NTIS

Fluorescence Monitoring of Polymer Processing: Mixing and Zero Shear Viscosity.

PB91-174482

100,492 Not available NTIS

Fluoromethanol: Synthesis, Microwave Spectrum, and Dipole Moment

100,288 Not available NTIS

Forced Smolder Propagation and the Transition to Flaming in Cellulosic Insulation.

PB91-147835

100,101 Not available NTIS

Formation and Stability of SF5 and S2F10

PB91-174839 100,326 Not available NTIS

Formation of ortho-Tyrosine by Radiation and Organic Solvents in Chicken Tissue.

PB92-116953

101,290 Not available NTIS

FORTRAN Temporary Program Fixes, FCVS78 Version 2.0,

PB91-201772

100,651 PC A03/MF A01

Fourler Transform Infrared Analysis of Ceramic Powders Quantitative Determination of Alpha, Beta, and Amorphous Phases of Silicon Nitride.

PB91-237628

101,096 Not available NTIS

Fourier Transform Infrared Spectroscopic Studies of the Degradation of One-Component Polyether Polyurethane Protective Coatings on Steel.

PB91-195248

101,108 Not available NTIS

Fourier Transform Infrared Spectroscopy at the NIST: High Temperature Molecules and Atmospheric Molecules.

PB91.189803

101,424 Not available NTIS

Fourteen-Decade Photocurrent Measurements with LargeArea Silicon Photodiodes at Room Temperature.

PB92-116771

100,768 Not available NTIS

FPETOOL User's Guide

PB92-108919

100,134 PC A03/MF A01

Fracture Mechanics Model for Subthreshold Identation Flaws. Part 1. Equitibrium Fracture.

PB91-236935

101,093 Not available NTIS

Fracture Toughness of $316 \mathrm{~L}$ Stainless Steel Welds with Varying Inclusion Contents at $4 \mathrm{~K}$ PB91-158980

101,148 Not available NTIS

Framework for Utilizing Fire Property Tests.

PB91-240788

100, 165 PC A03/MF A01

Frequency and Time Stability of GPS and GLONASS Clocks.
PB91-202937

100,582 Not available NTIS

Frequency Dependence of Catalyzed Reactions in a Weak Oscillating Field.

PB91-203927

100,407 Not available NTIS

Frequency Stabilization of a Tunable Erbium-Doped Fiber Laser.

101,427 Not available NTIS

Frequency Stabilization of an Erbium-Doped Fiber Laser: A Potential Wavelength Standard for Optical Communications PB91-203091 100,570 Not available NTIS

Functional Benchmarks for Fourth Generation Languages.
PB91-167387 100,644 PC A04/MF A01

Functional Meta-Structure for Hypertext Models and Sys-

tems.
PB91-236687

100,655 Not available NTIS

Fundamental and Harmonic Susceptibilities of $\mathrm{YBa} 2 \mathrm{Cu} 3 \mathrm{O} 7$. delta.

PB91-134148

101,458 Not available NTIS

Fundamental Condition for Existence of Microcrack Clouds in Monophase Ceramics.

PB91-189704

101,082 Not available NTIS
Fundamental studies of black liquor combustion. Report No. 4, Phases, 2, 3, and 4: Final report, December 1987 . DE 91018580

100,915 PC A09/MF A02

Fundamentals and Applications of Infrared Themography for Nondestructive Testing. PB91-147132

101,009 Not available NTIS

Further Investigation of the Effect of Application Parameters on Adhesive-Bonded Seams of Single-Ply Roof Mem-

PB91-149153 100,105 Not available NTIS

Fusion of Mercury. A New Certified Standard for Differential Scanning Calorimetry.

PB91-150003

100,309 Not available NTIS

gamma-Irradiated Seafoods: Identification and Dosimetry by

Electron Paramagnetic Resonance Spectroscopy.
PB91-150060 100,016 Not available NTIS

Gas Analysis Modeling System

PB91-175299 100,884 Not available NTIS

Gas Flow Measurement: Calibration Facilities and Fluid Metering Traceability at the National Bureau of Standards.
PB91-134403 100,879 Not available NTIS

GATT Standards Code Activities of the National Institute of Standards and Technology 1990 PB 1-187823

100,177 PC A04/MF A01

General Analytic Correction for Probe-Position Errors in Spherical Near-Field Measurements.

(Order as PB92-110261, PC A05/MF A01)

General Method for Doppler Determination of Cylindrically Symmetric Velocity Distributions: An Application of Fourie Transform Doppler Spectroscopy.

PB91-149385 100,302 Not available NTIS General Routine for Analysis of Stack Effect PB91-231589

$100,163 \mathrm{PC} \mathrm{A03/MF} \mathrm{A01}$

General Technique to Correct Probe Position Errors in Planar Near-Field Measurements to Arbitrary Accuracy.
PB91-237073 No0,728 Not available NTIS Generalization of the Cornu Spiral for Lossy Media. PB91-203216 101,687 Not available NTIS Generalized Photodiode Self-Catibration Formula. PB91-236901 100,760 Not available NTIS Generation of Smooth Trajectories without Planning. PB91-217414 101,036 PC A03/MF A01
1000th Trajectories without Planning. Geometric and Electronic Properties of Cs Structures on IIIV (110) Surfaces: From $1 D$ and $2 \mathrm{D}$ Insulators to $3 \mathrm{D}$ Metals.
PB91-190090 101,517 Not available NTIS

Geometric Reasoning for Constructing 3D Scene Descriptions for Images.
PB91-237677

100,714 Not available NTIS

Giant Resonances in the Transition Regions of the Periodic Table.

PB91-147116

101,605 Not available NTIS

Glass-Ceramic Inserts Anticipated for 'Megafilled' Composite Restorations.

PB91-174458

100,045 Not available NTIS

Global Stability of the Chaotic State Near an Interior Crisis. PB91-147496 101,611 Not available NTIS

Glossary of Computer Security Terminology.

100,716 PC A09/MF A02

Glueballs and Hadron Spectroscopy.
PB91-195123 GOSIP Conformance and Interoperation Testing and Registration.

100,694 PC A05/MF A01

Government Document Processing Requirements Report. 100.574 PC A03/MF A01

Government Open Systems Interconnection Profile (GOSIP); Category: Hardware and Software Standards; Subcategory: Computer Network Protocols.
FIPS PUB 146.1 Government Open Systems Interconnection Profile Users' Guide, Version 2.

PB92-119676

100,696 PC A08/MF A02

GPS Time Transfer with Implementation of Selective Availability N91-25766/7 (Order as N91-25755/0, PC A99/MF A04) Grain Boundaries in a 2-D Superlattice.

PB91-202853 100,388 Not available NTIS Graphical Kernel System (GKS). Ada Binding; Category: Software Standard; Subcategory: Graphics.
FIPS PUB 120-1B 674 PC E12 


\section{TITLE INDEX}

Graphical Kernel System (GKS); Category. Software Standard; Subcategory: Graphics.

100,673 PC E15

Graphical Kernel System (GKS). Pascal Binding; Category Software Standard; Subcategory: Graphics

FIPS PUB 120-1A 100,636 PC E09

Graphics Program for Binary and Ternary Ceramic Phase Diagrams.

PB91-14799 101,063 Not available NTIS

Gravitational Radiation Observations on the Moon PB91-175083 101,635 Not available NTIS Gravity-Dependent Phenomena at the Scale of the Single
Cell. 101,297 Not available NTIS

Ground-Truth Observations of Stellar Surface Structure from the Lunar Surface.

100,029 Not available NTIS

Group and Phase Sound Velocities in an Eu1Ba2Cu3O7 Superconductor and Related Perovskite Oxides. PB91-194860 101,523 Not available NTIS

Group Reactivities in the Gas Phase Reactions of Hydroxy Radicals with Ethers

PB91-175059

100,330 Not available NTIS

Guide for the Use of the International System of Units: The Modernized Metric System.

PB92-116383

100,962 PC A03/MF A01

Guide Specification for Direct Digital Control Based Building Automation System.

PB91-216697

100,092 PC A04/MF A01

Guide to Available Mathematical Software Problem Classification System.

$101,282 \mathrm{PC} \mathrm{A03/MF} \mathrm{A01}$

Guide to Design, Implementation and Management of Dis tributed Databases.

PB91-187567

100,648 PC A04/MF A01

Guide to Expert System Building Tools for Microcomputers.
PB92-108893 100,715 PC A08/MF A02 PB92-108893

Guide to Schema and Schema Extensibility. PB92-126556

100,955 PC A03/MF A01

Guide to the Use of the Cement and Concrete Research Remote Bulletin Board System (RBBS) Computer

PB91-148528 100,524 PC A03/MF A0

Guideline: American National Dictionary for Information Systems. Category: Software; Subcategory: Documentation.
FIPS PUB 11-3

Guideline for $\mathrm{Nb}$ (sub 3) $\mathrm{Sn}$ critical current measurements

using fiberglass-epoxy composite sample mandrels.
DE91016274 101,205 PC A03/MF A01

Guidelines for the Development of Computer Based Models in a Cementitious Materials Modeling Laboratory.
PB91-240804 100,532 PC A03/MF A01

Guidelines for the Evaluation of File Transfer, Access and Management Implementations.

PB92-126580

100,699 PC A05/MF A01

Gyroscope-Weighing Experiment Revisited -- with a Null Result.

PB91- 174722

101,630 Not available NTIS

$\mathrm{H}+\mathrm{O} 3$ Fourier-Transform Infrared Emission and Lase Absorption Studies of $\mathrm{OH}(X(2)$ Pi) Radical: An Experimental Dipole Moment Function and State-10-State Einstein A Coefficients.

PB91-148973

100,297 Not available NTIS

$\mathrm{H} 2 \mathrm{O}(\mathrm{l})-\mathrm{H} 2 \mathrm{O}(\mathrm{III})-\mathrm{H} 2 \mathrm{O}(\mathrm{L})$ Triple Point of Water as a Fixed Pressure Point

101,647 Not available NTIS

$\mathrm{H} 2 \mathrm{O} 2:$ Spectroscopy, Structure and Dynamics.

PB91-162057 100,316 Not available NTIS

Hall Effect.

PB91-149898

101,479 Not available NTIS

Hall Probe Magnetometer for SSC Magnet Cables: Effect of Transport Current on Magnetization and Flux Creep.

PB91-202911 101,681 Not available NTIS

Hardware Instrumentation Approach for Performance Meas urement of a Shared-Memory Multiprocessor.

PB91.159319 100,601 Not available NTIS

Heat Capacities and Entropies of Organic Compounds in

the Condensed Phase. Volume 2.
PB91-192450 Not available NTIS

Heat Capacities of Organıc Compounds in Liquid State. 2. C1 to C18 n-Alkanes

100,448 (Order as PB92-110162)

Heat Capacities of Organic Compounds in the Liquid State I. C1 to C18 1-Alkanols.

PB90-244716

100,265 (Order as PB90-244658)
Heat Capacity and Other Thermodynamic Properties of Linear Macromolecules. 10. Update of the ATHAS 1980 Data Bank.

100,515 (Order as PB92-110162)

Heat Capacity and Thermodynamic Properties of Deutrated Ammonium Hexafluorophosphate ND4PF6 from $5.8 \mathrm{~K}$ to

100,272 Not available NTIS

Heat Capacity of Deuterated Ammonium Tetrafluoroborate ND4BF 4 from 7 to 348

100,273 Not available NTIS

Heat Conductivity of Oxide Coatings by Photothermal Radiometry between 293 and $1173 \mathrm{~K}$. 101,112 Not available NTIS
PB92-116813 Heat Flux, Mass Loss Rate and Upward Flame Spread for Burning Vertical Walls (1990)

100,094 PC A04/MF A01

Heat Release Rate: The Single Most Important Variable in Fire Hazard.

100,097 Not available NTIS

Heat Transfer and Pressure Drop in a Compact Pin-Fin Heat Exchanger with Pin Orientation at 18 deg to the Flow Direction.

PB91-194464

101,050 PC A05/MF A01

Heat Transfer in a Compact Heat Exchanger Containing Rectangular Channels and Using Helium Gas. PB91-184804

101,734 PC A05/MF A01

Heats of Immersion, Friction, and Wear of Base Oil Frac PB91-158923

101,173 Not available NTIS

Hermetically Sealed Inert Atmosphere Cell for X-ray Powder Diffraction.

101,062 Not available NTIS

Heterodyne Frequency Measurements of $\mathrm{CO}$ and OCS Beyond $2100 \mathrm{~cm}(-1)$

PB91-236984

100,423 Not available NTIS

$\mathrm{HgCdTe}$ Detector Reliability Study for the GOES Program.
PB92-112382 100,766 PC A05/MF A02

High Harmonic Generation in Hydrogenic lons.
PB91-203778 Not available NTIS

High Magnetic Field Facilities in Japan Related to Super conductivity Research (Japan Technology Program).
PB91-240762

High Precision Gaging with Computer Vision Systems. PB91-174623 100,979 Not available NTIS High Purity Chemicals and Clean Rooms for Trace Metal Analysis.
PB91-195156

100,200 Not available NTIS

High Purity Fluoropolymer Materials: Trace Element Con-

tent and Leaching
PB91-162255

100,491 Not available NTIS

High Resolution Diffraction Imaging of Crystals Grown in

Microgravity and Closely Related Terrestrial Crystals

101,562 PC A08/MF A02

High Resolution Electron Microscopy of Diamond Film Growth Defects and their Interactions.
AD-A243 093/2

High-Resolution Infrared Flash Kinetic Spectroscopy of $\mathrm{OH}$ Radicals.

100,408 Not available NTIS

High-Resolution Infrared Spectrum of the nu1 Band of OC10 10

100,463 Not available NTIS

$X$-ray Scattering Camera for

High-Resolution Small-A

PB91-175067

100,929 Not available NTIS

High Resolution Synchrotron X-Radiation Diffraction Imaging of Crystals Grown in Microgravity and Closely Related Terrestrial Crystals.

101,548

(Order as PB91-216705, PC A07/MF A01)

High-Resolution, Tunneling-Stabilized Magnetic Imaging and Recording.

101,600 Not available NTIS

High Spatial Resolution Mapping of Semiconductor Resis-

tivity. 194829

100,809 Not available NTIS

High Speed Magnetic Field Sensors Based on Iron Garnets. 147223

100,840 Not available NTIS

High-Speed Spatial (Linear) Scanning Pyrometer: A Tool for Diagnostics, Temperature Mapping, and Property Determinations at High Temperatures.

N91-21361/1

100,924

(Order as N91-21331/4, PC A24/MF A03)

High Speed Superconducting A/D Converter.
PB91-148700

100,731 Not available NTIS

High $T($ sub c) Superconductors and the Critical Current Measurement

PB91-134056

101,457 Not available NTIS

High T(sub C) Superconductors: Are They Magnetic. N91-20208/5

101,453

(Order as N91-20207/7, PC A05/MF A01)

High Temperature Creep Testing of Ceramics.

PB91-202838

101,088 Not available NTIS

High-Temperature High-Pressure Raman Spectra from Liquid Water

100,385 Not available NTIS

High-Temperature Superconductivity: Abstracts of NIST Publications, 1987-1991

PB92-126564

101,105 PC A05/MF A01

High-Temperature Thermal Properties of UNS $\$ 44004$ Using Multivariant Analysis.

PB91-194795

101,160 Not available NTIS

High-Temperature Transient Hot-wire Thermal Conductivity Apparatus for Fluids.

PB91-216713

100,41

(Order as PB91-216705, PC A07/MF A01)

Horizontal Flow Boiling Heat Transfer Experiments with a Mixture of R22/R114.

PB92-116946

101,200 Not available NTIS

How Does Surface Roughness Affect Polymer-Surface Interactions.

How the Government Shapes UNIX Standards.

PB91-203133

100,693 Not available NTIS

Hydration of Carbon Dioxide: The Structure of $\mathrm{H} 2 \mathrm{O}-\mathrm{H} 2 \mathrm{O}$ $\mathrm{CO} 2$ from Microwave Spectroscopy.

PB91-237172

100,428 Not available NTIS

Hydrodynamics and Mass Transfer in Two-Phase Aqueous Extraction Using Spray Columns

PB91-149229

101,300 Not available NTIS

Hydrogen Bonding in Pt Ammine Complexes.

PB91-189639 100,348 Not available NTIS

Hydrogen Component Fugacity Coefficients in Binary Mix tures with Etane: Pressure Dependence.

PB92-116508

100,455 Not available NTIS

Hydrogen Slush Production with a Large Auger.

PB91-147199

100,275 Not available NTIS

Hydrogenated Amorphous Germanium Detectors Deposited onto Channel Waveguides.

PB91-194845

100,756 Not available NTIS

Hydroxyl Radical Induced Cross-Linking of Cytosine and Tyrosine in Nucleohistone.

PB91-189365

101,304 Not available NTIS

ICLASS-91 Proceedings of the International Conference on Iquid Atomization and Spray Systems (5th). Held in Gaithersburg. MD. on July 15-18, 1991.

PB91-216838

100,552 PC A99/MF E08

Identification of Dynamic Green's Functions in Structural Networks.

PB91-202812

101,585 Not available NTIS

Ignition Characteristics of Selected SSME Alloys.

PB91-174474

100,544 Not available NTIS

Ignition Characteristics of the Nickel-Based Alloy UNS N07001 in Pressurized Oxygen

PB91-144428

100,536 PC A04/MF A01

Image Ouality Indicator Design for Radioscopy and Tomog. raphy.

101,018 Not available NTIS

Immobilization of Binding Proteins on Nonporous Supports Comparison of Protein Loading. Activity, and Stability. PB91-237198

101,308 Not available NTIS

Impact-Echo: A New Method for Inspecting Construction Materials.

Improved Analysis for Flexural Creep with Application Sialon Ceramics.

PB91.150037

100,140 Not available NTIS

Improved Fits for the Vibrational and Rotational Constants of Many States of Nitrogen and Oxygen.

PB92-148097 100,469 (Order as PB92-148063)

Improved Optical Diffraction Strain Measurement System

PB91-174433 101,581 Not available NTIS

Improved Technique for Determining Complex Permittivity with the Transmission/Reflection Method.

PB91-161919

100,844 Not available NTIS 


\section{TITLE INDEX}

Improved Technique for Measuring Permittivity of Thin Dielectrics with a Cylindrical Resonant Cavity.
PB9 1-236844 100,858 Not available NTIS Improvements for Automating Voltage Calibrations Using a $10-\mathrm{V}$ Josephson Array.

100,739 Not available NTIS

Improving the Fire Performance of Building Contents

100,084 Not available NTIS

Impulse Response Functions for Elastic Structures with Rigid Body Degrees of Freedom.
PB91-237354 101,587 Not available NTIS

In situ Measurements of Bridged Crack Interfaces in the Scanning Electron Microscope.
PB91-149112 Incompatibility between the 100/1300 Surge Test and Varistor Failure Rates.

100,854 Not available NTIS

Incorporation of Molecular Orientation into Systems of Lamellar Morphology. 1. Effects of Packing Entropy on the Lamellar Thickness of Block Copolymers.

Increased Facilitated Transport Related to Microstructural Changes in Heat-Treated lon-Exchange Membranes.
PB91-174805 Indentation Determination of Crack Growth Parameters in Gallium Arsenide

101,083 Not available NTIS Indoor Ventilation Requirements for Manufactured Housing
PB91-206698 Industry and Government Strategies for Product Quality
PB91-148163 101,011 Not available NTIS Influence of Filament Geometry on Hot Filament Growth of Diamond Films

101,448 PC A02/MF A01

Influence of Interstitial Content on Fracture Toughness

PB91-189944 101,157 Not available NTIS Influence of Memory Propagation on Phase-Resolved Stochastic Behavior of AC-Generated Partial Discharges.
PB91-237644 100,861 Not available NTIS

Influence of Metastable States and Thermal Equilibration upon Dielectronic Recombination in Low Density to Moderate Density. PB91-195610

101,675 Not available NTIS

Influence of Moisture and Pressure on the Mechanical Properties of a Glass-Epoxy Matrix Composite and a Graphite-Epoxy Matrix Composite. 101,114 PC A03/MF A01

Influence of Nonsinusoidal Waveforms on Voltmeters, $A m$

meters, and Phase Meters
PB92-117167

100,869 Not available NTIS

Influence of Sulfur on Methanation over Tungsten(110) PB91-149369

100,300 Not available NTIS

Influence of Water on the Mechanical Properties of a Glass-Epoxy Matrix Composite.
AD-A231 778/2

$101,113 \mathrm{PC} \mathrm{A03/MF} \mathrm{A01}$

Information Systems Engineering Program.

Information Systems Engineering Program.
PB91-134635 00,685 Not available NTIS Intrared Spectroscopy Applied to Atmospheric Chemistry. PB91-175539 100,906 Not available NTIS

Initial Stages of Degradation of Superconductor Surfaces: $\mathrm{Q} 2, \mathrm{H} 2 \mathrm{O}, \mathrm{CO} 2$ and $\mathrm{CO}$ Chemisorption on $\mathrm{La2}-\times \mathrm{Sr} \times \mathrm{CuO} 4$.
$\mathrm{PB} 91-189670$
101,512 Not available NTIS

Inner-Shell lonization Cross Sections.

PB91-195479 100,203 Not available NTIS

Innovation in Manufacturing Technology: A View from NBS.

PB91-148130 100,976 Not available NTIS

Instability in $\mathrm{pH}$ Measurements of Sputtered IrQ2 Films

PB91-147538 100,281 Not available NTIS

Institute for Materials Science and Engineering. Ceramics: Technical Activities 1990 . 101,057 PC A08/MF A01
PB91-132233

101,057 PC A08/MF A01

Institute for Materials Science and Engineering: Metallurgy Division, Technical Activities 1990.
PB91-132225

Instrument-Independent Tandem Mass Spectrometry Database for $X O O$ Instruments: The Dynamical Prerequisites.
PB91-147694
100,181 Not available NTIS

Instrumentation of $X$-ray Beam Lines with PIN Diode DetecPB91-174888

101,633 Not available NTIS

Integrated Knowledge Systems for Concrete Science and Technology.
PB92-116821 100,141 Not available NTIS

Integrated-Optic Waveguide Glass Lasers.
PB91-203976

100,758 Not available NTIS

Integrated theoretical and experimental study of the thermophysical properties of fluid mixtures. Progress report (February 1990-February 1991)

DE91012660

$101,384 \mathrm{PC} \mathrm{A03/MF} \mathrm{A01}$

Integration-Based Method for Depth Estimation

PB92-112275 100,701 PC A03/MF A01

Integrity Tests for High-(T sub $c)$ and Conventional CriticalCurrent Measurement Systems. PB91-14867

100,842 Not available NTIS

Integrity tests for high-(Tc) and conventional critical-current measurements systems

101,452 PC A03/MF A01

Intelligent Building Technology in Japan

PB91-18775

100,071 PC A04/MF A01

Intelligent Processing of Materials, Technical Activities 1991. (NAS-NRC Assessment Panel, November 14-15 PB92-112572

100,995 PC A05/MF A01

Intensifying Effect of Metallic Screens on the Sensitivity of $X$-ray Films at $662 \mathrm{ke}$

101,669 Not available NTIS

Intensifying Effect of Metallic Screens on the Sensitivity of X-ray Films for 400-kV Bremsstrahlung Photons.
PB91-195198 101,293 Not available NTIS

Interaction of an Isolated Sprinkler Spray and a Two-Layer Compartment Fire Environment.
PB91-216804

Interactive Courseware is Leading the Multimedia Movement. 100,036 Not available NTIS Interactive Video Software Portability: Migrating a DOS APplication to POSIX. $\quad 100,670$ Not available NTIS Intercomparison of NBS Noise Calibration Services. PB91-162370 100,847 Not available NTIS Intercomparison Study of Rockwell Hardness Test Blocks.
PB91-167338 Interface between Data Terminal Equipment (DTE) and Data Circuit-Terminating Equipment (DCE) for Operation with Packet-Switched Data Networks (PSDN), or between Two DTEs, by Dedicated Circuit. Category: Hardware Standard. Subcategory: Data Transmission

FIPS PUB 100-1

100,672 PC A03

Interim Report to the President and to the Congress on the

Malcolm Baldrige National Quality Award.
PB91-167833 PC A05/MF A01

Interior-Point Method for Linear and Quadratic Programming Problems.

PB91-187815

101,269 PC A03/MF A01

Interlaboratory Comparison of Actinides in Human Tissue (239) Pu $+(240) \mathrm{Pu}$

101,323 Not available NTIS

Interlaboratory Tension and Fracture Toughness Test Results for CSUS-JN1 (Fe-25Cr-15Ni-0.3N) Austenitic Stainless Steel at $4 \mathrm{~K}$

101,166 Not available NTIS

Interlaminar Shear Fracture of Laminated Composites.

PB91-187336 101,122 Not available NTIS

International Co-operation: The Versailles Project on Advanced Materials and Standards.
PB91-159137 101,191 Not available NTIS

International Collaboration in Prestandards Research on Advanced Materials within the Context of VAMAS.
PB91-204040 101,251 Not available NTIS

International Fire Detection Bibliography, 1975-1990.

PB92-109156

100,088 PC A09/MF A02

International System of Units (SI)--Translation.

PB92-109032

101,719 PC A04/MF A01

Interpretation of Field lon Microscopy (FIM) Images of Asymmetrical Specimens of 1,2,3-Type High-Tc Superconductors.

101,567 Not available NTIS

United States and Metric Conversion Policy for Federal Agencies PB92-126572

101,729 PC A03/MF A01
Intrinsic Carrier Concentrations in Long Wavelength
$\mathrm{HgCdTe}$ Based on the New, Nonlinear Temperature Dependence of $\operatorname{Eg}(x, T)$ 101,568 Not available NTIS

Introduction to $\mathrm{ACl}$ 306.1-87: Specification for Cold Weather Concreting
PB91-174524

100,526 Not available NTIS

Introduction to Supercritical Fluid Chromatography. 1. Principles and Instrumentation.
PB91-189878

100,198 Not available NTIS

nverse Correlation between the Intensity of Luminescence Excited by Electrons and by Visible Light in ChemicalVapor-Depositeo Diamond Film

PB91-195560

101,531 Not available NTIS

Investigating the Use of Multimeters to Measure Ouantized Hall Resistance Standards. PB91-236554

100,734 Not available NTIS

Investigation of Internal Rotor Dynamics of $\mathrm{NeDCl}$ and ArDCl via Infrared Absorption Spectroscopy. PB91-204024

100,409 Not available NTIS

Investigation of Photoconductive Picosecond Microstripline Switches on Self-Implanted Silicon on Sapphire (SOS).
PB91-147918 Investigation of Simulated Qil-Well Blowout Fires. PB91-144337

101,356 PC A05/MF A01

Investigation of the Collapse of L'Ambiance Plaza. PB91-204057

100,146 Not available NTIS

Ion Bombardment Induced Diffusion: A Case Study on a Sputtered Ag/Ni Layered System.

PB91-195024

101,527 Not available NTIS

Ion Incorporation into Octacalcium Phosphate Hydrolyzates.
PB91-175125

lon-Induced Radiation-Enhanced Diffusion of Silver in

Nickel.
PB91-175554

101,498 Not available NTIS

Ionic Hydrogen Bonds. Part 1. Thermochemistry, Structural Implications, and Role in Ion Solvation.

PB91-203547

100,400 Not available NTIS

Iron-Garnet Magnetic Field Sensors with $100 \mathrm{pT} /$ per square root $\mathrm{Hz}$ Noise-Equivalent Field.

PB91-174615

100,751 Not available NTIS

Iron Ion-Dependent Modification of Bases in DNA by the Superoxide Radical-Generating System Hypoxanthine/Xan-

thine Qxidase

101,306 Not available NTIS

ISDN Conformance Testing: Layer 1. Physical Layer. Part 1. Basic Rate S/T Interface, User Side

PB92-102201

100,572 PC A05/MF A01

Isothermal Phase Behavior of Ag3SbS3, ZnGeP2, and ZnS.
PB91-187237 101,080 Not available NTIS

Isotopic Compositions of the Elements 1989.

PB92-148220 100,482 (Order as PB92-148162)

Israel's New Synchronized Time Scale, UTC(INPL).

PB91-134593 100,577 Not available NTIS

Issues in the Design and Implementation of a System Ar-

chitecture for Computer Integrated Manufacturing.
PB91-203299 100,987 Not available NTIS

Issues in Transparent File Access.

PB91-187831

100,569 PC A05/MF A01

Jlc Data Analysis with a 'Negative Crack Growth' Correction Procedure.

100,930 Not available NTIS

Joint Panel Meeting of the UJNR Panel on Fire Research and Safety (11th). Held in Berkeley, CA. on October 19-24, PB92-109164

100,166 PC A16/MF A03

Journal of Physical and Chemical Reference Data, Volume 19, Number 1,1990

100,251 Not available NTIS

Journal of Physical and Chemical Feference Data, Volume 19, Number 2, 1990

100,257 Not available NTIS

Journal of Physical and Chemical Reference Data, Volume 19, Number 3, 1990

PB90-244658

100,262 Not available NTIS

Journal of Physical and Chemical Reference Data, Volume 19. Number 4, July-August 1990

PB91-192435

100,354 Not available NTIS

Journal of Physical and Chemical Reference Data, Volume

19, Number 5, September-October 1990.
PB91-192476

Journal of Physical and Chemical Reference Data, Volume 19, Number 6, November-December 1990

PB9 1-192542 100,365 Not available NTIS

Journal of Physical and Chemical Reference Data, Volume 20, Number 1, 1991.

PB92-110113

100,440 Not available NTIS

Journal of Physical and Chemical Reference Data, Volume 20, Number 2, March-April 1991.

PB92-110162

100,445 Not available NTIS

Journal of Physical and Chemical Reference Data, Volume 20, Number 3, May-June 1991. 
Journal of Physical and Chemical Reference Data, Volume 20, Number 4, July/August 199

PB'92-148063 100,466 Not available NTIS

Journal of Physical and Chemical Reference Data, Volume

20, Number 5. September/October 1991 . Not available NTIS
PB92-148113

Journal of Physical and Chemical Reference Data, Volume 20, Number 6, November/December 1991

PB92-148162 100,476 Not available NTIS

Journal of Research of the National Institute of Standards and Technology. January-February 1991. Volume 96, Number 1

PB91-184853

100,220 PC A07/MF A01

Journal of Research of the National Institute of Standards and Technology. March-April 1991. Volume 96, Number 2. PB91-187617 101,420 PC A06/MF A01 Journal of Research of the National Institute of Standards and Technology. May-June 1991. Volume 96, Number 3.
PB91-216705 101,704 PC A07/MF A02 Journal of Research of the National Institute of Standards and Technology, September-October 1991. Volume 96 , Number 5

PB92-126614

100,212 PC A06/MF A02

Journal of Research of the National Institute of Standards and Technology. July-August 1991. Volume 96, Number 4.

Kelvin Water-Drop Experiment.

N91-20219/2

100,900

(Order as N91-20207/7, PC A05/MF A01)

Kinematic Couplings for Precision Fixturıng. Part 2. Experimental Determination of Repeatability and Stiffness.
PB91-159186 101,041 Not available NTIS

Kinematic Theory of Ballistic Electron Emission Spectroscopy of Silicon-Silicide Interfaces.

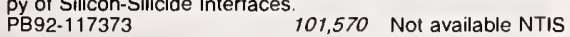

Kinetic Properties of the Components of Douglas-Fir and the Heat of Combustion of Their Volatile Pyrolysis Products. the Heat of Combustion of Their Volatile Pyrolysis Products.
PB91-203794 Not available NTIS Kinetics and Mechanisms of the Gas-Phase Reactions of

PB92-110220 100,450 (Order as PB92-110212)

Kinetics of Copper Extraction Using (anti)-2-Hydroxy-5-Nonlybenzophenone Oxime.
PB91-148783 100,292 Not available NTIS

Knowledge Extraction Techniques for Expert System Assisted Wafer Screening.
PB92-116979 100,824 Not available NTIS

Knowledge Verification of Machine-Learning Procedures Based on Test Structure Measurements.
PB91-189571 Not available NTIS

Laboratory Accreditation in the United States.

Laboratory Accreditation in the United States.
PB91-194415 100,172 PC A03/MF A01

Lanthanide Coordination Chemistry: Spectroscopic Properties of Terbium and Europium Poly(Pyrazol-1-Y1) Borate and

Poly(Imidazol-1-YI) Borate. 100,392 Not available NTIS
PB91-203034 PB91-203034

Large-Scale Turbulent Structures and the Stabilization of PBg2-117191 100,558 Not available NTIS
PBifted Turbulent Jet Diffun Flames.

Large-Scale Validation of Bench-Scale Fire Toxicity Tests. PB92-116458 100,139 Not available NTIS

Laser Diagnostics for Characterization of Fuel Sprays.
PB91-149013 100,541 Not available NTIS

Laser-Driven Ionization and Photoabsorption Spectroscopy of Atomic lons

101,415 Not available NTIS

Laser Enhanced Ionization as a Selective Detector for Liquid Chromatographic Determination of Alkyltins in Sediment.

100,210 Not available NTIS

PB92.116755

Materials: 1989

Laser Induced Damage in Optical Materials: 1989.
PB91-148478 101,409 PC A99/MF A04

Laser Induced Desorption of Molecules from Surfaces

PB91-147066

100,235 Not available NTIS

Laser-Induced Fluorescence Measurements of Drift-Velocity Distributions for $\mathrm{Ba}(+)$, in $\mathrm{Ar}$ : Moment Analysis and a Direct Measure of Skewness.
PB91-149005 100,298 Not available NTIS

Laser-Induced Fluorescence of $\mathrm{CH}$ in a Lammar $\mathrm{CH} 4 / \mathrm{Air}$ Diffusion Flame: Implications for Diagnostic Measurements and Analysis of Chemical Rates.

PB91-237099

100,554 Not available NTIS

Laser Measurements.
Laser Photolysis of Trimethylgallium at $193 \mathrm{~nm}$ : Ouantum Yields for Methyl Radical and Ethane Production AD-A227 868/7 100,234 PC A02/MF A01

Laser Vaporization Mass Spectrometry of Refractory Mate-

rials: Graphite and YBa2Cu3Ox.
PB92-117290 100,464 Not available NTIS

Lattice Approach to Environments Irradiated by Unknown

Sources.
PB91-175356

100,850 Not available NTIS

Lattice Relaxation in Silicon Doped with $4 \mathrm{~d}$ - and $5 \mathrm{~d}$-Transition Metals.

PB91-203554

101,541 Not available NTIS

LBIR Facility User Handbook.

PB92-102185

101,435 Not available NTIS

Lead Zirconate-Titanate Thin Films Prepared by the Laser Ablation Technique.

PB91-187344

101,502 Not available NTIS

Letter to Editor of Chemical and Engineering News.

PB91-174847 100,327 Not available NTIS

Letter to the Editor, Fire Technology, Comments on T.Z. Harmathy Viewpoint, May 1985 , 100,545 Not available NTIS Letter to the Editor of Standardization News.

PB91-174789 100,118 Not available NTIS

Levitation Calorimetry

PB91-158519

100,927 Not available NTIS

Levitation of Superconducting Composites.

PB91-187351 101,503 Not available NTIS

Light-Scattering Measurement of the RMS Slopes of Rough Surfaces.

101,436 Not available NTIS

Light Source Area, Shading, and Glare.

PB91-237818 100,080 Not available NTIS

Limited International Intercomparison of Responsivity Scales at Fiber Optic Wavelengths

100,567

(Order as PB91-187617, PC A06/MF A01)

Limiting versus Apparant Critical Behavior of Henry's ConStants and $K$ Factors

100,277 Not available NTIS

Linear Calibration When the Coefficient of Variation is Con-

PB91-149443

101,273 Not available NTIS

Linearity of a Silicon Photodiode at $30 \mathrm{MHz}$ and Its Effect on Heterodyine Measurements.

100,755

(Order as PB91-187617, PC A06/MF A01)

Linking Information to Objects: A Hypertext Prototype for Numismatists.
PB91-237057

100,657 Not available NTIS

Liposome Flow Injection Immunoassay: Implications for Sensitivity, Dynamic Range, and Antibody Regeneration.
PB91-147629 Not available NTIS

Liquid and Solid Ion Plasmas

AD-A227 656/6

101,441 PC A02/MF A01

AD-A242 029/7

101,592 PC A02/MF A01

List of New Group Divisible Designs

PB92-126705

101,281

(Order as PB92-126614, PC A06/MF A02)

Literature Review of the Galling Process.
PB91-134551

Load-Controlled Tensile Tests of Austenitic Steels at $4 \mathrm{~K}$

Load-Controlled
PB91-236943

101,165 Not available NTIS

Long-Range Antiferromagnetic Order of the $\mathrm{Cu}$ in Oxygen PB91-194894

101,524 Not available NTIS

Long Term Performance of Rubber in Seismic and NonSeismic Bearings: A Literature Review.

PB91-206714

100,148 PC A03/MF A01

Long Time Creep and Lifetime Behavior in Uniaxial Exten-

sion of a Linear Low Density Polyethylene.

101,247 Not available NTIS

Low Accelerating Voltage SEM Imaging and Metrology Using Backscattered Electrons. 101,469 Not available NTIS

Low-Background Gamma-Ray Assay Laboratory for Activation Analysis.

100,192 Not available NTIS

Low Carbon Steel: Metallurgical Structure vs. Mechanical

Properties.
N91-20218/4

101,144
Low-Cost Aqueous Two-Phase System for Enzyme Extraction.

101,310 Not available NTIS

Low-Cost Low-Volume Carrier (Mınilab) for Biotechnology and Fluids Experiments in Low Gravity.

PB91.150029

100,041 Not available NTIS

Low-Field Flux Pinning in Twinned and Detwinned Single Crystals of YBa2Cu3O7-x.

PB91-237552

101,559 Not available NTIS

Low-Pressure Symmetric Discharge Heat Pipe and DROGS PB91-195016

101,666 Not available NTIS

Low Temperature Chemical Process for Precursors to PB91-149096

101,066 Not available NTIS

Low Temperature Chemical Route to Precursors of Boride and Carbide Ceramic Powders

PB91-175190

101,078 Not available NTIS

Low-Temperature, Electrically Calibrated SOS Bolometer for Power and Energy Measurements.

PB91-147181

100,787 Not available NTIS

Low Temperature Magnetization and Magnetic Excitations in Amorphous Fe78B13Si9.

PB92.117464

101,573 Not available NTIS

Low Temperature Synthesis of Ceramic Powders for Struc tural and Electronic Applications.

PB91-149104

101,067 Not available NTIS

Lunar Gravitational Wave Antenna Using a Laser Interferometer

PB91-134643

101,603 Not available NTIS

Lunar Laser Ranging

(203026

101,353 Not available NTIS

Madelung Potentials and Valences in the $\mathrm{Y} 1 \mathrm{Ba} 2 \mathrm{Cu} 3 \mathrm{O} 7 \mathrm{Su}-$ perconductor.

101,459 Not available NTIS

Magnetic Anisotropies in Ultrathin fcc Fe(001) Films Grown on Cu(001) Substrates.

ous Alumina Gel
PB92-117332

101,565 Not available NTIS

Magnetic Hysteresis of High-Temperature YBa2Cu3Ox-AgO Superconductors
PB91-189506

101,507 Not available NTIS
10

Magnetic Measurements for High Energy Physics ApplicaPB92-112358

101,720 PC A04/MF A01

Magnetic Ordering of Er in Powder and Single Crystals of ErBa2Cu3O7.

101,505 Not available NTIS

Magnetic Properties of Iron/Silica Gel Nanocomposites

Magnetic Properties of Iron/Silica Gel Nanocomposites.
PB91-204073 101,110 Not available NTIS

Magnetization of Anisotropic Superconducting Grains.

Magnetization of Anisotropic Superconducting Grains.
PB91-134569 NTIS Not available NTIS

Magnetoresistance of Multifilament Al/Al-Alloy Conductors.

PB91-134759 100,772 Not available NTIS

Majority and Minority Electron and Hole Mobilities in Heavily Doped GaAs.

101,550 Not available NTIS

Manufacturing Systems Integration Control Entity Interface Document.

100,991 PC A03/MF A01

Manufacturing Systems Integration: Initial Architecture Document.

PB92-112242

100,994 PC A04/MF A01

Many Electron Effects in Lgamma2,3 X-ray Emission Spectroscopy Spectrum of $\mathrm{BaO}$.

PB91-195305

100,382 Not available NTIS

Many-Electron Effects in Lgamma2,3 XES Spectra from Rare Earth Elements.

100,383 Not available NTIS

Mare Island Flexible Manufacturing Workstation.

PB92-117027

100,996 Not available NTIS

Mass Energy-Transfer and Mass Energy-Absorption Coefficients, Including In-Flight Positron Annihilation for Photon Energies $1 \mathrm{keV}$ to $100 \mathrm{MeV}$.

PB92-126473

101,445 PC A04/MF A01

Mass Transfer Coefficients in Two-Phase Aqueous Extraction.

100,426 Not available NTIS

Material Dependency of Chip-Form Detection Using Acoustic Emission. 


\section{TITLE INDEX}

PB91-149450

101,216 Not available NTIS

Material Flammability Test Assessment for Space Station Freedom.

PB91-216606

101,735 PC A05/MF A01

Materials Reliability. Technical Activities, 1990. (NAS-NRC Assessment Panel, January 31-February 1, 1991).

PB91-143339 101,008 PC A05/MF A01

Materials Reliability. Technical Activities, 1991. (NAS-NRC

Assessment Panel, February $13-14,1992)$
PB92-126424
101,252 PC A05/MF A01

Materials Research at the National Bureau of Standards.

PB91-149039 101,214 Not available NTIS

Mathematical Model for Dental Caries: A Coupled Dissolution-Diffusion Process

101,316

(Order as PB92.126614, PC A06/MF A02)

Mathematical Modeling of Enclosure Fires

PB92-108976

100,135 PC A03/MF A01

Measured Performance of Residential Water Heaters Using Existing and Proposed Department of Energy Test Procedures.

100,085 Not available NTIS

Measurement and Evaluation of Lighting/HVAC Interaction. PB91-206706 100,892 PC A04/MF A01

Measurement of Capacitance on Wafers.

PB91-149120 100,795 Not available NTIS

Measurement of DNA Base Damage and DNA-Protein Cross-Links in Mammalian Chromatin.
PB91-187484 Not available NTIS Measurement of Regenerator Performance in a Vuilleumier Refrigerator.

101,052 Not available NTIS

Measurement of Space Charge Fields in Polymers.

PB91-158626 100,486 Not available NTIS

Measurement of Structural Response Characteristics of Full-Scale Buildings: Selection of Structures.
PB91-167239 100,144 PC A03/MF A01

100,144 PC A03/MF A01

Measurement of Thermal Characteristics of Office Build

ings.

100,068 Not available NTIS

Measurement Quality Assurance.

PB91-149930 101,013 Not available NTIS

Measurement Reliability: The Detection of Nonlinearities.

PB91-189290 100,853 Not available NTIS

Measurement, Use, and Interpretation of TCR

PB92-117282 100,831 Not available NTIS

Measurements of Power Frequency Magnetic Fields Away from Power Lines.

100,851 Not available NTIS

Measurements of Velocity-Changing Collision Kernels.

PB91-203083 of Velocity-Changing Collision Kernels.

Measuring the Corrosion of Metals in Soil.

PB91-174714 101,136 Not available NTIS

Mechanical and Swelling Behavior of Crosslinked Natura Rubber: Consequences of the Flory-Rehner Hypothesis.

PB91-195081 101,141 Not available NTIS

Mechanical Loss in a Glass-Epoxy Composite.

PB91-149435 101,070 Nol available NTIS
P

Mechanical Properties and Fracture Toughness of AAR TC128 Grade B Steel in the Normalized, and Normalized and Stress Relieved Conditions.

PB92-108901

101,169 PC A03/MF A01

Mechanical Properties of Alumina-Peek Unidirectional Composite: Compression, Shear, and Tension.
PB91-162180 10118 Not available NTIS

Mechanical Sputtering of Structural Stainless Steels

PB91-175448 101,152 Not available NTIS

Mechanical Testing Revitalized.

PB91-159004 100,928 Not available NTIS

Mechanism for High Strain-Rate Shear Band Formation

PB91.147033 101,575 Not available NTIS

Mechanism for Shear Band Formation in the High StrainRate Torsion Test

101,579 Not available NTIS

Mechanism of Cyanide Release in the Radiolysis of Acetonitrile

100,240 Not available NTIS

PB91-203679

Reactions with Thymine and Mechanism of $\mathrm{OH}$

100,216 Not available NTIS

PB91-147447

in Acetylene-Oxygen MixMech
PB91-147280

100,537 Not available NTIS

Mechanisms of Additive Effectiveness.

PB91-158808 101,172 Not available NTIS

PB91-174862

101,174 Not available NTIS

Mechanisms of the Reduction Reactions of Cr(VI) in the Radiolysis of Acidic Potassium and Silver Dichromate SoluRadiolysis of Acidic Potassium and Silver Dichromate Solu-
tions in the Presence or Absence of Acetic Acid. PB91-236489

100,241 Not available NTIS

Mechanistic Studies of lonizing Radiation and Oxidative $\mathrm{Mu}-$ tagenesis: Genetic Effects of a Single 8-Hydroxyguanine (7Hydro-8-oxoguanine) Residue Inserted at a Unique Site in a Viral Genome.

101,321 Not available NTIS

Mechano-Chemical Descriptive Model for Wear Under Mixed Lubrication Conditions.

PB91-189480

101,177 Not available NTIS

Meeting of the Standards Working Group of the Joint U.S. U.S.S.R. Commercial Commission (1st). Held in Moscow on March 11-13, 1991

PB91-194498

100,178 PC A11/MF A02

Meeting the Challenges of a Global Market - Construction Standards, Testing and Certification.

PB91-194811 100,090 Not available NTIS

Merit Function for Inequality Constrained Nonlinear Programming Problems.

101,271 PC A03/MF A01

Metal-Polysiloxane Shields for Radiation Therapy of Maxillo-Facial Tumors

PB91.194761

100,054 Not available NTIS

Metal Reference Line Technique for Obtaining Dihedra Angles from Surface Thermal Grooves.

PB91-147348 101,059 Not available NTIS

Metallographic Study of the Crack-Tip Region from Fracture Mechanics Specimens of Austenitic and Ferritic Steels. PB91-189951 101,158 Not available NTIS

Metallurgy Division, Technical Activities 1991. (NAS-NRC

Assessment Panel February 13-14, 1992).
PB92-126515

Method for Characterizing the Thermal Performance of a

Solar Storage Wall from Measured Data.
PB91-159152

Method for Measuring Heat Loss from Underground Heat Distribution Systems.

100,888 Not available NTIS

Method for Ouantitative Measurement of Galling Damage.
PB91-159269

Method of FIM-FEEM Specimen Preparation of Superconducting and Other Oxides.

101,542 Not available NTIS

Method to Enhance Porosity of Micro-particles

PB91-187328

101,185 Not available NTIS

Methods for the Analysis of Organometallic Compounds in Wastes.

PB91-148981

100,189 Not available NTIS

Methods of Analyzing Planar Optical Waveguides

PB91-236752

100,774 Not available NTIS

Metric Conversion Card

PB92-163880

100,941 Not available NTIS

Metric Measures Up (Ruler)

PB92-163898

100,942 Not available NTIS

Metrology for Electromagnetic Technology: A Bibliography of NIST Publications.

PB91-132266

100,833 PC A04/MF A01

PB92-116375

$100,867 \mathrm{PC}$ A04/MF A01

Metrology for the Semiconductor Industry.

PB91-240739

100,817. PC A03/MF A01

Microchannel-Plate Detection Systems for Low Accelerating Voltage SEM

101,515 Not available NTIS

Micromachined Thermal Radiation Emitter from a Commercial CMOS Process.

PB91-148999

100,748 Not available NTIS

Micromagnetic Calculations of $180 \mathrm{deg}$ Surface DomainWall Magnetization Profiles with Comparison to Measure-

ments.

101,483 Not available NTIS

Micromagnetic Calculations of $180 \mathrm{deg}$ Sirface Domain PB91-237271

101,553 Not available NTIS

Micromagnetics of Domain Walls at Surfaces.

PB91-162305

101,491 Not available NTIS

Microstrip Patch Antenna as a Standard Transmitting and Receiving Antenna.

PB91-162156

100,591 Not available NTIS
Microstructural Aspects of the Fracture of Hardened Cement Paste.

100,133 Not available NTIS

Microstructural Control Through Diffusion-Induced Grain Boundary Migration.

PB91-236760

101,239 Not available NTIS

Microstructural Studies of Ti-Al Alloys in the Vicinity of the 'Eutectoid' Reaction.

PB92-116938

101,241 Not available NTIS

Microstructure and Elastic Properties of Dental Resin and Resin-Based Glass-Reinforced Composites: XRD, SEM and Ultrasonic Methods.

PB91-204081

100,050 Not available NTIS

Microstructure and Fracture at the Cement Paste-Aggregate Interface

100,523 Not available NTIS

Microstructure, Composition, and Hardness of Rockwell C Hardness Blocks.

$101,153 \mathrm{PC} \mathrm{A03/MF} \mathrm{A01}$

Microstructure Effects on the Lower Critical Solution Temperature Phase Behavior of Deuterated Polybutadiene and Protonated Polyisoprene Blends Sludied by Small-Angle Neutron Scattering.

PB92-117266

100,518 Not available NTIS

Microwave Monolithic Integrated Circuit-Related Metrology at the National Institute of Standards and Technology PB91-237255

100,816 Not available NTIS

Microwave Spectra and Electric Dipole Moments of $X(4)$ Sigma(1-) 1/2 VO and $\mathrm{NbO}$
$\mathrm{PB9} 1-237487$

100,429 Not available NTIS

Microwave Spectrum and Molecular Structure of the N2. H20 Complex
PB91-203448

100,398 Not available NTIS

Microwave Spectrum of Ar-H2O: Dipole Moment, Isotopic Studies, and (17)O Ouadrupole Coupling Constants.
PB91-148650 100,290 Not available NTIS

MMIC Related Metrology at the National Institute of Standards and Technology.

PB91-149070

100,794 Not available NTIS

Mobility Fluctuations and Electrophoretic Light Scattering from Macromolecular Solutions.

PB91-189514

100,345 Not available NTIS

Mode Specific Internal and Direct Rotational Predissociation in HeHF, HeDF, and HeHCL: van der Waals Complexes in the Weak Binding Limit

PB91-148841

100,293 Not available NTIS

Mode-Stirred Chamber for Measuring Shielding Effectiveness of Cables and Connectors: Assessing MIL-STD-1344A

PB92-116656

100,776 Not available NTIS

Model for the Non-Perturbative OCD (Quantum Chromodynamics) Vacuum
PB91-194407

$101,661 \mathrm{PC} \mathrm{A03/MF} \mathrm{A01}$

Model Validation at the Las Cruces Trench Site

NUREG/CR-5716 101,368 PC A05/MF A01

Modeling Electron Beam Interactions in Semiconductors.

PB91-159020 101,485 Not available NTIS

Modeling the Thermodynamic Properties of Sodium Chloride in Sieam Through Extended Corresponding States. PB91-189381 100,342 Not available NTIS

Modelling Aqueous Solutions Near the Critical Point of Water.
PB91-189837 100,349 Not available NTIS

Modelling Gallium Arsenide Transistors.

PB91-149989 100,797 Not available NTIS

Modelling the Coronae and Chromospheres of RS CVn Systems by the Analysis of Ulitraviolet, X-ray and Radio Observations.

100,019 Not available NTIS

Models for Oxygen Ordering and Diffusion in $\mathrm{Ba} 2 \mathrm{YCu}_{3} \mathrm{O} \mathrm{x}$

and Ba2YCu3-yMyOx (M = Fe, Co, $\mathrm{Al}$, Ga).

Modern Test Methods for Flammability. PB91-167676

100,116 PC A03/MF A01

Modernized Metric System (Chart)

PB92-163864

100,939 Not available NTIS

Modification of Bases in DNA by Copper Ion-1,10-Phenanthroline Complexes.

101,333 Not available NTIS

Modification of DNA Bases in Mammalian Chromatin by Radiation-Generated Free Radicals.

PB91-189373

101,338 Not available NTIS

Modified Airy Function and WKB Solutions to the Wave

Equation.

$101,440 \quad \mathrm{PC}$ AOB/MF A02 


\section{TITLE INDEX}

Modified Sliding Wire Potentiometer Test Structure for Map ping Nanometer-Level Distances.
PB92-116664 100,821 Not available NTIS Modulatable Narrow-Linewidth Semiconductor Lasers. PB91-134106 101,404 Not available NTIS Molecular-Beam Optothermal Spectroscopy of the 9.6 mum nu14. 100,421 Not available NTIS Molecular Desorption from Solid Surfaces: Laser Diagnostics and Chemical Dynamics.
PB91-203331 100,397 Not available NTIS Molecular Dynamics of Initial Events in the Thermal Degra-
dation of Polymers.
PB91-195271 100,503 Not available NTIS PB91-195271 Molecular Dynamics Study of the Depolymerization Reac tion in Simple Polymers. $\quad 100,495$ Not available NTIS
PB91.187229 Molecular-Orbital Studies via Satellite-Free $\mathrm{X}$-ray Fluorescence: $\mathrm{Cl} \mathrm{K}$ Absorption and $\mathrm{K}$-Valence-Level Emission

PB91-237164 100,427 Not available NTIS

Molecular Spectroscopy Division of the National Bureau of Standards.

PB91-175174

100,332 Not available NTIS

Monitoring and Reporting Techniques for Error Rate and

Error Distribution in Optical Disk Systems.
PB92-123124 100,606 PC A05/MF A01

Monitoring of Anisotropic Material Elastic Properties Using Ultrasonic Receiving Rays.

PB91-203059

101,126 Not available NTIS

Monitoring the Fate of Chlorine from MSW Sampling through Combustion.

100,917 Not available NTIS

Monitoring the Mass Standard via the Comparison of Me-

chanical to Electrical Power.
PB91-237115

101,710 Not available NTIS

Morphology and Barrier-Height Development of $\mathrm{Bi} / \operatorname{lnP}(110)$ Interfaces.

101,468 Not available NTIS

Morphology of Polymer Films and Single Molecules.

PB91-203224 100,506 Not available NTIS

Morphology of Silver on YBa2Cu307-delta Thin Films.

PB91-195594 101,534 Not available NTIS

Mossbauer Imaging

PB91-203745

101,294 Not available NTIS

Mossbauer Study of the Effect of Qxygen Stoichiometry on the High Tc Superconductor $\mathrm{Y} 1 \mathrm{Ba2}(\mathrm{CuO} .97 \mathrm{Fe} 0.03) 307-\mathrm{x}$
$\mathrm{PB} 91-237545$ MSW Calorimetry.

PB91-133942

100,878 Not available NTIS

Multi-Enterprise Concurrent Engineering through International Standards

$100,997 \mathrm{PC} \mathrm{A03/MF} \mathrm{A01}$

Multi-Zone Dispersal Analysis by Element Assembly.

PB91-187146 100,907 Not available NTIS

Multicomponent Cluster lons. 2. Comparative Stabilities of Cationic and Anionic Hydrogen Bonded Networks: Mixed Clusters of Water and Hydrogen Cyanide.

PB91-147710 100,283 Not available NTIS

Multimedia Courseware in an Open Systems Environment: A Federal Strategy.

100,039 PC A04/MF A01

Multiple Conformations of Amino Acid Residues in Ribonuclease A.

101,298 Not available NTIS

Multiple Intermolecular Bend Vibrational Excitation of a Hydrogen Bond: An Extended Infrared Study of OCQHF.
PB91-203703 100,404 Not available NTIS

Multiple Variable-Angle Light Scattering Detector for Gel Permeation Chromatography.

100,195

(Order as PB91-187617, PC A06/MF A01)

Multireference-Configuration Interaction (MR-Cl) Calculations on $\mathrm{HS}(2+)$ and Experimental Observation via Electron Impact lonization of $\mathrm{H} 2 \mathrm{~S}$

PB91-148924

100,295 Not available NTIS

Multistep Stress-Relaxation Behavior in Uniaxial Extension

of an Ethylene-Hexene Copolymer.
PB91-148247 Not available NTIS

Multistep Stress-Relaxation Study of a Single Crystal of $n$ Eicosane $(\mathrm{C} 2 \mathrm{OH} 42)$ in Torsion.

PB91-187427

100,497 Not available NTIS

MUMPS Validation Suite, Version 7.6, 1991 (for Microcomputers).
P891-507707

100,661 CP D99

MUMPS Validation Suite, Version 7.6 (ANSI), 1991 PB91-507715 100,662 CP T99 MUMPS Validation Suite, Version 7.6 (TAR), 1991 PB91-507723 100,663 CP T99 MUMPS Validation Suite, Version 7.6 (Vax/VMS Backup) 1991

100,660 СР T99

Muon Sticking in Catalysed d-t Fusion: Nuclear Resonance Effect.
PB91-147959

101,613 Not available NTIS

N-Dimensional Crystallographic Description of the Icosahe dral Phases; the Example of the Al73Mn21Si6 Quasiperiodic Structure

101,226 Not available NTIS

Narrow-Gap Semiconductors and Related Materials.
PB91-162313

NASREM Robot Control System and Testbed

PB91-194969 101,033 Not available NTIS

National Aeronautics and Space Administration's (NASA)

Automated Information Security Handbook.
PB91-187781 100,707 PC A06/MF A01

National Bureau of Standards-American Ceramic Society Phase Diagram Data Program.

PB91-158774

101.073 Not available NTIS

National Bureau of Standards Publications 1977-1987. Volume 1. Citations, Key Words, and Abstracts. PB91-136507

100,957 PC A99/MF A99

National Bureau of Standards Publications 1977-1987. Volume 2. Indexes

100,958 PC A99/MF A99

PB91-136515

National Computer Systems Laboratory Annual Report,
1990.
PB91-201822
100,603 PC A05/MF A0

PB91-201822

Update ' 90 . Standard Ex-

National Educators Workshop: Update '90. Standard Ex-

gy. $92-126606$

101,253 PC A15/MF A03

National PDES Testbed Mail Server User's Guide. Nationa PDES Testbed Report Series.
PB91-159756

100,951 PC A02/MF A0

Nature of Collinearity.

PB91-203497

101,261 Not available NTIS

Navy Safety Center Data on the Effects of Fire Protection Systems on Electrical Equipmen

PB92-108935 101,376 PC A05/MF A01

NBS-INA. The Institute for Numerical Analysis. UCLA 1947 1954.

101,264 PC A09/MF A02

NBS Quality Assurance Support: Current and Planned Services. 149088

101,012 Not available NTIS

NBS Research on Protective Coatings for Buildings and Structures.
PB91-203521

100,127 Not available NTIS

NBS Research Program in Municipal Solid Waste Combustion. 147264

100,916 Not available NTIS

NBS Submicron Particle Standards for Microcontamination Measurement.

100,798 Not available NTIS

Near-Field and Far-Field Excitation of a Long Conductor in a Lossy Medium

100,722 PC A03/MF A01

Near Net Shape Forming of Ceramics.

PB91-158600

101,072 Not available NTIS

Near-Threshold Vibrational Excitation of $\mathrm{H} 2$ by Electron Impact: Resolution of Discrepancies between Experiment and Theory.

PB91-20088

101,679 Not available NTIS

Need for Power Quality Testing Standards.

PB92-117076 100,741 Not available NTIS

Network Structure in Epoxies. 6. The Growth Process Investigated by Neutron Scattering.
PB91.237842

Neutron Activation Analysis in Electronic Technology

PB91-189779 100,807 Not available NTIS

Neutron Crystallography of Protein

PB91-175349 101,285 Not available NTIS

Neutron Diffraction from Sheared D-Glycerol: Preliminary Studies

PB91-134676

100,268 Not available NTIS

Neutron Measurements of Intramolecular Vibrational Modes in $\mathrm{C} 60$.
PB92-116540

101,055 Not available NTIS

Neutron Powder Diffraction and Inelastic Scattering Study of the Structures of $\mathrm{Zr} 2 \mathrm{Pd}, \mathrm{Zr} 2 \mathrm{PdD} 1.70$, and $\mathrm{Zr} 2 \mathrm{PdD} 1.96$. PB91-148866 101,213 Not available NTIS

Neutron Spectroscopic Evidence for Adsorbed Hydroxy Species on Platinum Black

PB91-237636

100,432 Not available NTIS New Approach to Image Modeling and Edge Detection in
the SEM.

PB91-203752

100,811 Not available NTIS

New Approach to Vision and Control for Road Following

PB91-157156

$101,743 \mathrm{PC} \mathrm{A03/MF} \mathrm{A01}$

New Chemical and Stereochemical Applications of Organoiron Complexes

100,221

(Order as PB91-184853, PC A07/MF A01)

New Concepts of Precision Dimensional Measurement for

Modern Manufacturing

101,006 PC A04/MF A01

New Different Forms of Ammonium Loaded and Partly Deammoniated Zeolite Rho Studied by Neutron Powder PB91-158717 100,311 Not available NTIS

New Equation of State and Tables of Thermodynamic Properties for Methane Covering the Range from the Melting Line to $625 \mathrm{~K}$ at Pressures up to $1000 \mathrm{MP}$

PB92-148170 100,477 (Order as PB92-148162)

New Inexpensive Frequency Calibration Service from NIST. PB91-200790 100,580 Not available NTIS

New International Electrical Reference Standards Based on

the Josephson and Quantum Hall Effects.
PB91-237586 100,740 Not available NTIS

New Layered Cuprate Structure-Type, (A1 $\left.X A^{\prime} x\right) 14$ Cu24Q4

PB91-237339

101,095 Not available NTIS

New Look at the Bitter Method of Magnetic Imaging

PB91-203919 101,701 Not available NTIS

New Low-Voltage Standards in the DC to 1-MHz Frequency Range.

Range.
PB91-237107

100,738 Not available NTIS

New Radio Detectors of Early-Type Pre-Main Sequence Stars.

PB91-149286

100,024 Not available NTIS

New Test of Quantum Mechanics: Is Planck's Constan:

Unique.
PB91-174755

101,632 Not available NTIS

New Triple Correlation Technique for Measuring Ultrashor Laser Pulses.

PB91-237156

101,434 Not available NTIS

New Triple Correlator Design for the Measurement of Ultrashort Laser Pu'ses.

PB91-203802

101,429 Not available NTIS

New Way to Mount Particulate Material for Laser Microprobe Mass Analysis.

PB92-116805

100,911 Not available NTIS

New Wide Range Equation of State for Helium

PB91-162222

100,226 Not available NTIS

New Yorker in Japan.

PB91.158907

101,275 Not available NTIS

Newest Approaches to Quantitative Assessment of Bioac tive Organotins

101,328 Not available NTIS

NIST 'Building Life-Cycle Cost' (BLCC) Program (Version

3.0). User's Guide and Reference Manual.
PB91-159764 100,154 PC A04/MF A01

NIST Calibration Procedure for Vertically Polarized Monopole Antennas $30 \mathrm{kHz}$ to $300 \mathrm{MHz}$.

PB91-185124

$100,726 \mathrm{PC} \mathrm{A03/MF} \mathrm{A0}$

NIST Calibration Services for Humidity Measurement.

PB92-112499

$100,520 \mathrm{PC} \mathrm{A04/MF} \mathrm{A01}$

NIST Calibration Services Users Guide, 1991

PB92-126416

$100,936 \mathrm{PC} \mathrm{A10/MF} \mathrm{A03}$

NIST Express Working Form Programmer's Reference (Revised November 1990). National PDES Testbed Report

PB91-157164

100,964 PC A04/MF A01

NIST High-Dose Calibration Services.

PB91-203257

101,689 Not available NTIS

NIST "LATTICE: A Program to Analyze Lattice Relation ships. Spring 1991 Version.

$101.561 \mathrm{PC} \mathrm{A03/MF} \mathrm{A01}$

NIST Measurement Services: Calibration Service for Current Transformers. 


\section{TITLE INDEX}

PB91-216770

NIST-NRL Free-Electron Lase AD-A227 $310 / 0$

NIST PDES Toolkit: Technical Fundamentals. National PDES Testbed Report Series (Revised).

PB91-132159 100,682 PC A03/MF A01

NIST Personnel Management Demonstration Project: Design, Implementation and Accomplishments.
PB91-231555

NIST Reactor: Summary of Activities July 1989 through June 1990.

101,488 PC A05/MF A01

NIST Research Reports, March 1991

PB91-187559

100,007 PC A03/MF A01

NIST Serial Holdings, 1991.

PB91-171330

100,960 PC A12/MF A02

NIST Standard Reference Data Products 1991 Catalog

PB91-167312 100,952 PC A04/MF A01

NIST Standards for Sodium and Potassium.

PB91-158865 100,191 Not available NTIS NIST STEP Working Form Programmer's Reference. Na-

tional PDES Testbed Report Series (Revised).
PB91-144378
100,998 PC A03/MF A01

NIST Support for the Computer-Aided Acquisition and LOgistic Support (CALS) Program in the Area of Graphics

Standards, Calendar Year 1990
PB91-194506

101,348 PC A14/MF A02

NIST Support of the CALS Program: 1990 Synopsis.

NIST Time and Frequency Services.
PB91-231605 100,585 PC A03/MF A01

NIST Working Form for STEP: National PDES Testbed Report Series (Revised)

100,683 PC A03/MF A01

NIST X-Ray Photoelectron Spectroscopy (XPS) Database.

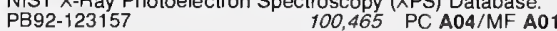

Nitrogen Strengthening of Austenitic Stainless Steels at Low Temperatures.

101,163 Not available NTIS

Noise-Affected I-V Curves in Small Hysteretic Josephson Junctions.

100,782 Not available NTIS

Non-Equilibrium Molecular Dynamics Simulations of Structured Molecules. Part 1. Isomeric Effects on the Viscosity of Butanes.
PB91-195636

101,391 Not available NTIS

Non-Linear Contour-to-Grid Digital Interpolation.

PB91-147678 101,351 Not available NTIS

Nonlocal Phase Shifts Induced by Static Electric Fields in Neutron Interferometers When the Path-Enclosed Charge Vanishes.

101,620 Not available NTIS

Nonresonant Charge Transfer in the Threshold Region fo

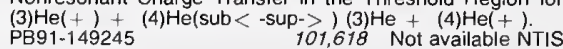

North American ISDN Users' Forum Agreements on Integrated Services Digital Network. 100,573 PC A08/MF A02 Note on the Precision Cementing of Small Optical Compo-

nents. 101,428 Not available NTIS

Notes and Comments on Roundtable Discussion on LaserAssisted Collisions and Collision-Induced Spectra

PB91-203307 101,690 Not available NTIS

Novel Laser Gain and Time-Resolved FTIR Studies of Photochemistry. 100,237 Not available NTIS

Novel Radiation Dosimetry Systems.

PB92-117118 101,727 Not available NTIS

Numeric Database for Tribology: Format and Application Issues.
PB91-203943 101,045 Not available NTIS

Numerical Modeling of Silicon Photodiodes for High-Accuracy Applications
PB92-110295

100,763

(Order as PB92-110261, PC A05/MF A01)

Numerical Modeling of Silicon Photodiodes for High-Accuracy Applications. Part 2. Interpreting Oxide-bias Experiments.
PB92-110303 (Order as PB92-110261, PC A05/MF 01)

Numerical Modeling of Silicon Photodiodes for High-Accuracy Applications. Part 3. Interpolating and Extrapolating Internal Quantum-Efficiency Calibrations.
PB92-110311

100,765

(Order as PB92-110261, PC A05/MF A01)

Numerical Simulation and Visualization Models of Stress Wave Propagation Graphite/Epoxy Composites.

PB91-174946 101,120 Not available NTIS

Numerical Study of Two-Dimensional Crystal Growth Forms in the Presence of Anisotropic Growth Kinetics.

PB91-200865 101,538 Not available NTIS

NVLAP FY 90 Annual Report

PB91-206748

100,010 PC A03/MF A01

Observation of Anomalous Isotope Ratios in SIRIS Measurements of Molydenum.

PB92-116789

101,361 Not available NTIS

Observation of Atoms Laser-Cooled Below the Doppler Limit.
PB91-189746

101,655 Not available NTIS PB91-195453

101,671 Not available NTIS

Observation of Correlations in Finite, Strongly Coupled Ion PB91-200857

101,678 Not available NTIS

Observation of the Cesium Clock Transition Using LaserCooled Atoms in a Vapor Cell. PB91-203638

101,698 Not available NTIS

Observation of Two Different Oscillation Periods in the Exchange Coupling of $\mathrm{Fe} / \mathrm{Cr} / \mathrm{Fe}(100)$.

PB92-117415 $\quad 101,572$ Not available NTIS

Of What Use Is Basic Research.

PB91-175513 101,642 Not available NTIS

Off-Axis Measurements of Ion Kinetic Energies in RF Plas-

PB92-117225 101,444 Not available NTIS

OMNITAB 80 (ASCII): An Interactive System for Statistical and Numerical Data Analysis (Version 7.0)

PB91-507954

101,278 CP T06

OMNITAB 80 (UNIX): An Interactive System for Statistical

and Numerical Data Analysis. Version 7.0. ${ }_{101,279 \text { CP T06 }}$

Opportunities for Innovation: Chemical and Biological SenPBS.

100,214 Not available NTIS

Optical Fiber Current Sensors with Temperature Stabilities Near the Material Limit.

PB91-175109

100,753 Not available NTIS

Optical Levitation of Single Particles.

PB91-174987

101,634 Not available NTIS

Optical Potential Approach to Electron and Positron Scat-

tering from Noble Gases. 2. Neon. 101,619 Not available NTIS
PB91-149971

Optically Detected Magnetic Resonance Study of Antisiteto-Acceptor and Related Recombination Processes in asGrown InP-Zn.
PB91-195578 101,532 Not available NTIS

Optimization of a Pulse Tube Refrigerator for a Fixed Compressor Swept Volume.
PB91-203877

101,051 Not available NTIS

Optimization Techniques for Permittivity and Permeability Determination.
PB92-112325

PB92-126663

100,865 PC A03/MF A01

100,871

(Order as PB92-126614, PC A06/MF A02)

Optothermal-Detected Microwave-Sideband CO2-Laser Spectroscopy of Ar-NH3.
PB91-236679
100,416 Not available NTIS

Orbit Determination and Gravitational Field Accuracy for a Mercury Transponder Satellite.

PB91-175166 101,736 Not available NTIS

Orthogonal Distance Regression.

PB91-147017

101,272 Not available NTIS

Outline of a National Plan on High-Performance Concrete: Report on the NIST/ACl Workshop. Held in Gaithersburg, MD on May $16-18,1990$

100,522 PC A04/MF A01

Overview of Facilitated Transport Membrane Systems. PB91-175463

100,228 Not available NTIS

Overview of the Ion Dynamic Effect in Line Broadening. and a Generalization of the Unified Theory.
PB91-147504 Not available NTIS Overview of the Product Data HyperStandard CD-ROM Pro$\begin{array}{lll}\text { totype. } & 101,380 & \text { PC A03/MF A01 }\end{array}$

$101,380 \mathrm{PC} \mathrm{A03} / \mathrm{MF} \mathrm{A0} 1$

Oxidation Kinetics of Silicon Carbide Whiskers Studied by
X-ray Photoelectron Spectroscopy. X-ray Photoelectron Spectroscopy.
PB91-237719 100,436 Not available NTIS
Oxidation of Organic Compounds by Active Manganese $\mathrm{Di}$ PB91-174730

100,218 Not available NTIS

Oxides and Oxide Superconductors: Elastic and Related Properties.

101,563 PC A05/MF A02

Parent Structure of the Layered High-Temperature Superconductors.

101,535 Not available NTIS

Parity-Unfavored Transitions in Resonant Photoemission from Ar, Kr, and Xe: Experimental and Theoretical Results.
PB91-133900 Not,599 available NTIS Parser That Converts a Boundary Representation into a Features Representation

100,988 Not available NTIS

Partial Discharges in Low Voltage Cables.

PB91-134650 771 Not available NTIS Partial Equilibrium in Laminar Hydrocarbon Diffusion PB91.237024

100,553 Not available NTIS

Particle Size Measurement of Inert Gas Atomized Powder. PB91-187203

101,229 Not available NTIS

Pattern Generation on Semiconductor Surfaces by a Scanning Tunneling Microscope Operating in Air.

100,808 Not available NTIS

Percolation of Phases in a Three-Dimensional Cement Paste Microstructural Model.

PB91-187179

100,529 Not available NTIS

Performance and Limitations of Faraday Effect Sensors.

PB91-149146 100,749 Not available NTIS

Performance Evaluation of a New Audio-Frequency Power Bridge.

100,742 Not available NTIS

Performance Evaluation of Hypercube Applications: Using a

Global Clock and Time Dilation
PB91-222588

$100,653 \mathrm{PC} \mathrm{A03/MF} \mathrm{A01}$

Performance Measures of a Robotic Micropositioner.

101,037 Not available NTIS

Performance of OSI Transport over ACCUNET and IBER-

PBC1-134825

100,563 Not availabie NTIS

Performance of Precast Concrete Beam-to-Column Connections Subject to Cyclic Loadin

100,150 Not available NTIS

Performance of 1/3-Scale Model Precast Concrete BeamColumn Connections Subjected to Cyclic Inelastic Loads. PB91-222570

100,149 PC A05/MF A01

Periodic Orbits and Diffuse Structures in the Photodissocia-

tion
PB91-149252 100,238 Not available NTIS

Permittivity Measurements on Molecular-Sized Samples.

PB91-159244

100,800 Not available NTIS

Perpendicular Bands of Cyclopropane in the $3.5 \mathrm{mum}$ Region.
PB91-237016 100,424 Not available NTIS

Persistent Photoconductivity in SIMOX Films.

PB92-117084 100,826 Not available NTIS

Personal Computer Codes for Analysis of Planar Nea

Fields.
PB92-112283

100,730 PC A04/MF A01

Phase Diagrams of Hexagonal Binary Ordering Alloys with

Anisotropic Interactions.
PB91-147512

101,211 Not available NTIS

Phase Equilibria and Critical Lines in Model Water/Salt Mix tures.

PB91-236778 100,418 Not available NTIS

Phase Equilibria from the One-Fluid Model.

PB91-134585 101,385 Not available NTIS

Phase Equilibria in the System TI-Ca-Ba-Cu-O. 1. Stability of the 2122 Phase under Conditions of Oxygen Annealing.
PB91-147165 101,465 Not available NTis

Phase Equilibria of the System Strontium Oxide-Calcium Oxide-Cupric Oxid

101,085 Not available NTIS

Phase-Field Model for Isothermal Phase Transitions in Binary Alloys.

101,240 PC A04/MF A01

Phase Formation in Electrodeposited and Thermally Annealed Al-Mn Alloys.

101,209 Not availabie NTIS

Phase Selection in Non-Equilibrium Processing.

PB91-195651 101,236 Not available NTIS 


\section{TITLE INDEX}

Phase Transitions

PB91-162073

100,318 Not available NTIS

Phenomenological Theory of the Influence of Strain History

on the Rate of Isothermal Stress Relaxation.
PB91-187385 100,496 Not available NTIS

Phone-Mediated Word Alignment for Speech Recognition Evaluation.
PB91-189910

100,592 Not available NTIS

Phonon-Assisted Magneto-Donor Optical Transitions in nInSb.

PB91-147603

101,467 Not available NTIS

Phonon Density of States and Superconductivity in $\begin{array}{ll}\mathrm{Nd} 1.85 \mathrm{Ce} 0.15 \mathrm{CuO} 4 . & 101,514 \text { Not available NTIS } \\ \text { PB91-189787 }\end{array}$ Phonon Density of States of Superconducting $\mathrm{YBa} 2 \mathrm{Cu} 3 \mathrm{O} 7$ and the Nonsuperconducting Analog YBa2Cu3O6.
PB91-159129 101,487 Not available NTIS

Photodissociation Dynamics of $\mathrm{Mo}(\mathrm{CO}) 6$ at $266 \mathrm{~nm}$ and $355 \mathrm{~nm}$ : CO Photofragment Kinetic-Energy and InternalState Distributions.

PB91-236547

100,413 Not available NTIS

Photodissociation Dynamics of Water in the Second AbSorption Band: Vibrational Exitation of $\mathrm{OH}$ (A (2)Sigma).
PB91-148726 100,291 Not available NTIS

Photodissociation of $\mathrm{C}(\mathrm{l}) \mathrm{NO}$ in the $\mathrm{S}(\mathrm{l})$ State: A OuantumMechanical ab initio Study.

100,239 Not available NTIS

Photoionization and Collisional Ionization of Excited Atoms

Using Synchrotron and Laser Radiations.
PB91-159301

Photoionization Studies of Atoms and Molecules Using Syn-

chrotron Radiation

101,664 Not available NTIS

Photoluminescence Excitation by Band-Gap Optical Absorption in Chemical Vapor Deposition Diamond Films.
PB91-195586

Photomultiplier Housıng for Vacuum Operation of Side-on 1P28-Type Tubes.

1P28-Type Tubes.

$100,744 \mathrm{PC}$ A01/MF A01

Photon Energy Dependence of the Sensitivity of Radiochromic Film and Comparison with Silver Halide Film and LiF TLDs Used for Brachytherapy Dosimetry.
PB92-117142 101,296 Not available NTIS

Photonic Probes for the Measurement of Electromagnetic Fields Over Broad Bandwidths.
PB91-162206 100,750 Not available NTIS

Photospheres of Hot Stars. 4. Spectral Type O4.
PB91-149849

Physical Aging and the Viscoelastic Response of Network Glasses

PB91-189829

101,246 Not available NTIS

Physical and Thermo-Mechanical Properties of Monoclinic Single Crystals
PBg1-148767

101,065 Not available NTIS

Picosecond IR Studies of the Vibrational Dynamics of $\mathrm{CO} /$

Pt(111).

100,314 Not available NTIS

Planar Waveguide Optical Immunosensors.

PB91-174565 101,284 Not available NTIS

Plastic Zone Formation Around an Arresting Crack

PB91-189316 101,373 Not available NTIS

PLOTMD: An Interactive Program to Modify Molecular Plots on a Graphics Terminal.

PB91-194985

100,373 Not available NTIS

Plumbing Test Lab Accreditation

PB91-190066 100,089 Not available NTIS

Polarizability of the Photon

PB91-203166

101,686 Not available NTIS

Polarization and Anisotropy of $X$-ray Emission from Mole-

PB91-187401

101,651 Not available NTIS

Polarized $\mathrm{X}$-ray Emission Studies of Methyl Chloride and the Chlorofluoromethanes.

PB91-174995

100,329 Not available NTIS

Polyelectrolyte Stabilized Metal Oxide Hydrosols as Catalysts for the Photooxidation of Water by Zinc Porphyrins.

Polymer Composite Processing. Industry Workshop (2nd) Held at Gaithersburg, Maryland on May 18, 1990 PB91-132191 101,115 PC A05/MF A01 Polymers: Technical Activities 1990

PB91-159715

$100,488 \mathrm{PC}$ A07/MF A01

Polymers: Technical Activities 1991. (NAS-NRC Assess ment Panel, February 13-14, 1992)
PB92-116284

100,516 PC A07/MF A02

Population Lifetimes of $\mathrm{OH}(v=1)$ and $O D(v=1)$ Vibra tions in Alcohols, Silanols and Crystalline Micas.
PB91-174813 100,324 Not available NTIS Portable Vectorized Software for Bessel Function Evalua PBon.

100,652 PC A03/MF A0

Possible (e,e'2N) Studies at CEBAF.

PB91-147587 101,612 Not available NTIS Possible 'Proximity Matrix' Route to High Current Conductors.

101,476 Not available NTIS

Possible Use of Pattern Recognition for the Analysis of Mars-Rover X-ray-Fluorescence Spectra.
PB91-237875 101,733 Not available NTIS

Pozzolan Programs of the Cement and Concrete Reference Laboratory.

100,525 Not available NTIS

Practical Considerations in the Design of Optical Fiber Current Sensors.

100,746 Not available NTIS

Practical Constraints in Monitoring Micronutrient Intake.

PB91-147421 101,318 Not available NTIS

Precise Ephemerides for GPS Time Transfer.

PB91-236950 100,586 Not available NTIS

Precise Experimental Test of Calculated Two-Electron Lamb Shifts in Helium.

PB91-149203

101,617 Not available NTIS

Precise Laser Wavelength Measurements: What Can We Learn from Classical Spectroscopy,
PB91-203984 Not available NTIS Precision Qualification of Watthour Meters PB91-237214 100,859 Not available NTIS Predicting the Remaining Service Life of Concrete PB92-126598 101,371 PC A05/MF A01 Prediction and Fault Detection of Building Energy Consumption Using Multi-Input, Single-Output Dynamical Model.
PB90 10,073 Not available NTIS

Prediction of Elevated Temperature Deformation of Struc

tural Steel under Anisothermal Conditions.
PB91-184838

Predictive Extended Corresponding States Model for Pure and Mixed Refrigerants.

PB92-116748

101,199 Not available NTIS

Preface to Book Entitled 'Nonequilibrium Effects in lon and

Electron Transport
PB91-159236

101,623 Not available NTIS

Preliminary Dynamic Analyses of the Ministry of Agriculture Building.
PB91-134668

100,143 Not available NTIS

Preliminary Results of the Environmental Evaluation of the Federal Records Center in Overland Missouri.

$100,082 \mathrm{PC} \mathrm{A04/MF} \mathrm{A01}$

Preparation and Analysis of a Marine Sediment Reference Material for the Determination of Trace Organic Constitu ents.

101,377 Not available NTIS

Preparation and Properties of Monomolecular Films for Use as Radioactive Source Mounts. 101,608 Not available NTIS

Probe-Corrected Spherical Near-Field Scanning Theory in Acoustics.

Acoustics.

101,383 Not available NTIS

Probe for Real-Time Images of Particle Beams and Their

Analyses in a Merged-Beams Apparatus.
PB91-203067

101,683 Not available NTIS

Probe Waveforms and the Reconstruction of Structural Dynamic Green's Functions.

PB91-161950

101,737 Not available NTIS

Problem of Detailed Balance and Model Lineshapes in Collision-Induced Rotovibrational Bands: $\mathrm{H} 2-\mathrm{H} 2$ and $\mathrm{H} 2-\mathrm{He}$. PB91-236521 100,412 Not available NTIS

Procedure for the Effective Recalibration of Liquid-in-Glass Thermometers

$100,935 \mathrm{PC} \mathrm{A03/MF} \mathrm{A0}$

Proceedings of a Workshop on Evaluation, Repair, and Retrofit of Structures. U.S. Japan Panel on Wind and Seismic Effects, UJNR. Held in Gaithersburg, MD., USA, on May 12. 14,1990

PBं91-184846

100,145 PC A14/MF A02

Proceedings of Cable ' 89 Workshop.

PB91-161943 100,773 Not available NTIS

Proceedings of the Forum on Standards for High Integrity Software (DOD, Government, Industry). Held in Gaithersburg, Maryland on June 28, 1991
PB92-112267

$100,668 \mathrm{PC}$ A04/MF A0

Proceedings of the Object-Oriented Database Task Group Workshop. Held in Atlantic City, New Jersey on May 22,

PB91.159723

100,643 PC A14/MF A02

Proceedings of the Object-Oriented Database Task Group Workshop, Tuesday, October 23, 1990, Chateau Laurie Hotel, Ottawa, Canada.

PB91-157198

100,641 PC A07/MF A01

Proceedings of the Workshop on High Integrity Software. Held in Gaithersburg, MD. on January 22-23, 1991.

PB92-109040

100,667 PC A03/MF A01

Process Ouality Enhancement for Machine Tools: Measure ments and Standards.

PB91-194993

101,019 Not available NTIS

Processing Bi-Pb-Sr-Ca-Cu-O Superconductors from Amorphous State.
PB91-187369

101,504 Not available NTIS

Production and Diagnosis of a Highly Spin-Polarized Na Beam.

PB91-204016

101,431 Not available NTIS

Production and Spectroscopy of Small Polyatomic Molecular lons Isolated in Solid Neon.

AD-A234 043/8

PB91-203273

100,245 PC A03/MF A0

Programmer's Reterence Manual for CFAST, the Unilied Model of Fire Growth and Smoke Transport PB91-144436

100,096 PC A06/MF A01

Programming Languages and Database Language SOL. Validated Processor List Including GOSIP Conformance Testing Register

PB91-187716

100,649 PC A04/MF A01

Progress at NIST on Absolute Frequency Standards Using Stored lons.

Progress in Field Ion Microscopy Imaging of High-Tc Super-

conducting Oxides.

101,543 Not available NTIS

Progress in the Development of Miniature Optical Fiber Current Sensors

Progress in Uniform Field Eddy Current Methods.

PBo $1-14775$

Progress on the Determination of Intermolecular Potential Energy Surfaces from High Resolution Spectroscopy 100,403 Not available NTIS

Properties of Amorphous Zirconium Rhodium Hydride (Zr3RhH H) Prepared from Glassy and Crystalline Alloys. PB91-17446 101,225 Not available NTIS

Properties of YBa2Cu3O7-delta Thin Films Grown on OffAxis-Cut MgO Substrates. PB91-203950

101,547 Not available NTIS

Proposed Design Criteria for Shallow Bridge Foundations PB91-187849 100 $534 \mathrm{PC} \mathrm{A04/MF} \mathrm{A01}$

Proposed New Electrolytic Conductivity Primary Standards for $\mathrm{KCl}$ Solution

100339

(Order as PB91-187617, PC A06/MF A01)

Proposed Standard Practice for Assessing the Performance of Gas-Phase Air Cleaning Equipment PB91-167353

100,905 PC A03/MF A0

Proposed Testing Methodology for STEP Application Protocol Validation. National PDES Testoe PB92-112374

$100,969 \mathrm{PC} \mathrm{A04/MF} \mathrm{A01}$

Prospects for Electromagnetic Manipulation and Trapping of Antihydrogen

101,663 Not available NTIS

Prospects for High Temperature Superconductor - Semiconductor Contacts.

PB91-203646

101,545 Not available NTIS

Protective Coatings for Tooth Crowns.

PB91-189555

100,047 Not available NTIS

Proton Affinities and pKa Values of Tetraalkylhydrazines. PB91-195214

100,378 Not available NTIS

Proton Affinity of Cyanogen and Ion-Molecule Reactions of $\mathrm{C} 2 \mathrm{~N} 2(+)$.

100,352 Not available NTIS

Prototype Expert System for Diagnosing Moisture Problems in Houses.

100,062 Not available NTIS

Prototyping the IRDS: An Airport Application

PB92-112580 101,740 PC A06/MF A02

Public-Key Cryptography. Computer Security. 


\section{TITLE INDEX}

PB91-187864

100,708 PC A08/MF A01

Publications of the Manufacturing Engineering Laboratory Covering the Period January 1989 -September 1991

PB92-123041 101,053 PC A05/MF A01

Publications of the National Institute of Standards and Technology, 1990 Catalog.
PB91-216531 100,011 PC A20/MF A04

Pulse-Echo Ultrasonic Evaluation of the Integrity of Seams of Single-Ply Roofing Membranes: Laboratory Evaluation of a Prototype Test Apparatus.

100,106 PC A03/MF A01

Pulse-Echo Ultrasonic Evaluation of the Integrity of Seams of Single-Ply Roofing Membranes: Results of Field Investigations and Recommendations.

100,110 PC A03/MF A0 1

Pulse Radiolysis Studies of Organic Electron Transfer Reactions.
PB91-147801

100,285 Not available NTIS

Ouantifying Standard Performance of ElectromagneticBased Mine Detectors.

101,378 PC A09/MF A02

Ouantitative Analysis of Impurities in SIMOX Samples Using Secondary lon Mass Spectrometry.
PB91-236596 Not available NTIS

Quantitative Determination of Amorphous Content in Ce-

ramic Materials Using X-ray Powder Diffraction.
PB91-147553

Ouantum Electrodynamic Contributions to Spin Orbit Split-

ting in the Ground State of Aluminum-Like lons.
PB91-189597 101,653 Not available NTIS

Quantum Optics of Single, Trapped lons.

PB91-162123 101,628 Not available NTIS

Ouasi-Static Analysis of a Two-Wire Transmission Line Located at an Interface.

100,564 Not available NTIS

Questions and Answers on Ouality, the ISO 9000 Standard Series, Quality System Registration, and Related Issues.
PB92-126465 100,175 PC A03/MF A01

Radiant Heat Fluxes from 100-200 MW Natural Gas/Air Diffusion Flames
PB91-147306

100,538 Not available NTIS

Radiation Chemistry of Enones.
PB91-147793 100,236 Not available NTIS

Radio-Continuum Observations of a Variety of Cool Stars.

PB91-174664 100,027 Not available NTIS

Radiochromic Film Dosimeter for Gamma Radiation in the

Absorbed-Dose Range 0.1 - 10 kGY.
PB92-116961

Radiolytic Studies of the Redox Reactions of Ruthenium PB91-203661

100,402 Not available NTIS

Rapid Low-Temperature Hopping of Hydrogen in a Pure Metal: The $\mathrm{ScH} \times$ System.

101,217 Not available NTIS

Rapid Solidification Alloys: Crystais, Ouasicrystals, and Metallic Glass.

101,220 Not available NTIS

Rapporteur's Summary Materials Development for Coal Gasification.

100,876 Not available NTIS

Raster Graphics Conformance Testing.
PB91-167296 101,341 PC A04/MF A01

PB91-167296 101,341 PC A04/MF A01
Rate Constant Functions for Strength Development of Con-

Rate Constant Functions for Strength Development of Con-
PB91-175117 100,527 Not available NTIS

100,527 Not available NTIS

Rate Constants for Hydrogen Abstraction Reactions of the Sulfate Radical, SO4- Alkanes and Ethers.
PB91-203232 100,394 Not available NTIS

Rate Constants for Reactions of Peroxyl Radicals in Fluid Solutions.

PB90-244641

100,261 (Order as PB90-244609)

Rate Constants for Reduction of Substituted Methylperoxyl Radicals by Ascorbate lons and N,N,N',N'-TetramethylPara-Phenylenediamine. $\quad 100379$ Not available NTIS

Rate of Pyrite Bioleaching by 'Thiobacillus ferrooxidans': Results of an Interlaboratory Comparison.
PB91-195321

Reactions of $\mathrm{C}_{5} \mathrm{H} 3(+)$ and $\mathrm{C}_{5} \mathrm{H} 5(+)$ lons with Acetylene and Diacetylene.
PB91-195370

100,384 Not available NTIS

Reactivities of Chlorine Atoms and Peroxyl Radicals

Formed in the Radiolysis of Dichloromethane.
PB91-200782 100,386 Not available NTIS

Real, Realistic Ring Waves for Surge Testing.
PB91-189811

100,855 Not available NTIS

Real-Time Algorithms and Data Structures for Underwate Mapping.
PB91-195354

101,360 Not available NTIS

Real-Time Hierarchical Planning for Multiple Mobile Robots.
PB91-158782 101,375 Not available NTIS

Real-Time Model-Based Tracking Combining Spatial and Temporal Features.

100,700 PC A03/MF A01

Real-Time Radiology Standards: Results of a Workshop PB91-147900 101,010 Not available NTIS Recent Developments in Applied Surface Science. PB91-189936 100,353 Not available NTIS Recent Developments in Digital Oscilloscopes.

PB91-175240 100,849 Not available NTIS

Recent Progress in Coal Bioprocessing Research in the United States: Scope and Areas Needing Further Research PB91-195339 100,885 Not available NTIS

Recommended Fine Positioning Test for the Development Test Flight (DTF-1) of the NASA Flight Telerobotic Service PB91-185090

101,732 PC A06/MF A01

Recommended Inorganic Chemicals for Calibration.

PB91-195164 100,201 Not available NTIS

Redox Reactions of Osmium Porphyrins.

PB91-159012 100,217 Not available NTIS

Reduction in Fire Hazard in Corridors and Areas Adjoining Corridors Provided by Sprinklers.

PB91-222604

100,162 PC A05/MF A01

Reduction in Sintering Damage of Fiber Reinforced Com-

posites.

101,124 Not available NTIS

Reduction of Hydrogen Cyanide Concentrations and Acute Inhalation Toxicity from Flexible Polyurethane Foam Combustion Products by the Addition of Copper Compounds. Part 3. The Effect of Copper Additives on the Flammability Characteristics of Flexible Polyurethane Foam.
PB91-132167

Reference Materials for Analytical Chemistry.

PB91-203893 100,205 Not available NTIS

Reference Materials: Their Role in Measurement Accuracy.
PB91-159103 101,014 Not available NTIS

Reference NO2 Calibration System for Ground-Based Intercomparisons during NASA's GTE/CITE II Mission. PB91-189340 100,034 Not available NTIS

Refinement of a Model for Fire Growth and Smoke Transport. 148510

100,104 PC A04/MF A01

Refinement of the Structure of Beta'-MoO3.

PB91-203786 101,090 Not available NTIS

Reflection/Absorption FTIR Spectral Characteristics of Thin and Thick Crosslinked Epoxy Films on Steel Substrates.
PB91-203711 100,509 Not available NTIS Reflection-Extended-X-ray-Absorption-Fine-Structure Spectroscopy at the Carbon K-Edge.
PB91-194944 100,371 Not available NTIS Refractive Index Gratings in Rare-Earth-Doped Alkaline Earth Glasses.
PB91-189332

101,422 Not available NTIS

Refractive Index of Water and Steam as Function of Wavelength, Temperature and Density.
PB90-244708 101,402 (Order as PB90-244658) Refrigerant Charge Effects on Heat Pump Performance.

Refrigerant Charge Effects on Heat Pump Performance.
PB91-174607 100,065 Not available NTIS

Relation between the Surface Energy and the Debye Tem. perature for Cubic Solids.

PB91-149393

100,303 Not available NTIS

Relationship of Hydrogen Site Occupancy to Diffusion Behavior in Crystalline and Amorphous $\mathrm{Zr} 2 \mathrm{PdHx}$.

havior in Crystalline and Amorphous $\mathrm{Zr} 2 \mathrm{PdHx}$.
PB91-187252 1030 Not available NTIS

Relative $\mathrm{H}$-Atom and O-Atom Concentration Measurement in a Laminar, Methane/Air Diffusion Flame.

PB92-117365

100,559 Not available NTIS

Relative Populations of Excited Levels within the Ground Configuration of Si-Like $\mathrm{Cu}, \mathrm{Zn}, \mathrm{Ge}$, and Se lons.

PB91-158618 101,443 Not available NTIS

Relativistic and Correlation Effects in the 2s3p Configuration of Beryllium-Like lons.

PB91-189605

101,654 Not available NTIS

Relativistic Distorted-Wave Cross Sections for ElectronImpact Excitations of Berylliumlike lons.

PB91-195503 101,672 Not available NTIS

Relativistic Probability Amplitudes and State Preparation.
PB91-195032

101,667 Not available NTIS

Relaxation of Crosslinked Networks: Theoretical Models and Apparent Power Law Behavior.

PB91-195099

100,501 Not available NTIS

Replicate Measurements in the Interpretation of Environmental Monitoring.

100,919 Not available NTIS

Reply to Comment on 'Ouantum Zeno Effect'.

PB91-236828

101,708 Not available NTIS

Report of the National Conference on Weights and Measures (76th). Held in Philadelphia, PA. on July 14-19, 1991.

101,024 PC A16/MF A03

Report on 1989 Actions by International Institute of WeldPB91-134619

101,001 Not available NTIS

Research and Development of Heat Pumps Using NonaPB91-174516

100,891 Not available NTIS

Research and Technology for Structural Engineering ProPB91-204206

100,147 Not available NTIS

Research at NBS in Direct Potentiometric Measurements in Blood.

PB91-148874

100,188 Not available NTIS

Research at NBS on Dynamic Measurements of Thermophysical Propertic

100,315 Not available NTIS

Research for Electric Energy Systems: An Annual Report. PB92-112341

100,874 PC A06/MF A02

Research. Services. Facilities. (National Institute of Standards and Technology)

100,012 PC A05/MF A01

Research Tackles Automation Issues.

PB91-162230 101,005 Not available NTIS

Residual Defects in SIMOX: Threading Dislocations and Pipes.

100,829 Not available NTiS

Residual Stress Detection in Railroad Wheels: An Ultrasonic System Using EMATs (Electromagnetic-Acoustic Transducers). Report No. 23

ducers). Report
PB91-222653

101,741 PC A03/MF A01

Resonance Enhanced Multiphoton lonization Spectra of the GeF and GeCl Radicals from $400-500 \mathrm{~nm}$.

100,396 Not available NTIS

Resonance Enhanced Multiphoton Ionization Spectroscopy of $\mathrm{CHCl} 2$ and $\mathrm{CDCl} 2$.

PB91-147645

100,282 Not available NTIS

PB91-174870

100,328 Not available NTIS

Resonance Ionization Spectroscopy/Resonance Ionization Mass Spectrometry Data Service.

PB91-203968

100,207 Not available NTIS

Resonance Ionization Spectroscopy/Resonance Ionization Mass Spectrometry Data Service. II. Data Sheets for $\mathrm{Al}, \mathrm{Ca}$, $\mathrm{Cs}, \mathrm{Cr}, \mathrm{Co}, \mathrm{Cu}, \mathrm{Kr}, \mathrm{Mg}, \mathrm{Hg}$, and $\mathrm{N}$.

PB91-162297

100,193 Not availabie NTIS

Resonant Photoemission and PSD of Rare Earth Oxides. PB91-148015

100,287 Not available NTIS

Resonant Tunneling with Electron-Phonon Interactions: An Exactly Solvable Model Applied to Desorption.

PB91-162065

100,317 Not available NTIS

Resonating-Orthotropic-Cube Method for Elastic Constants.
PB91-236794

Response of Personal Noise Dosimeters to Continuous and Impulse-Like Signals.

PB92-116359

100,914 PC A07/MF A02

Reusable Cryogenic Foam Insulation for Advanced Aerospace Vehicles.
PB91-175570

100,560 Not available NTIS

Reverse-Phase HPLC Separation and Electrochemical Detection of Retinol and Its Isomers.

PB91-148858

100,187 Not available NTIS

Review of Cryogenic Mechanical and Thermal Properties of Al-Li Alloys and Alloy 2219

AD-A229 231/6

101,202 PC A09/MF A02

Review of Current Geometric Tolerancing Theories and Inspection Data Analysis Algorithms.

PB91-167221

101,016 PC A03/MF A01

Review of Four Compartment Fires with Four Compartmen Fire Models.
PB91-147215

100,098 Not available NTIS

Review of Pulse Tube Refrigeration.

PB91-147942

101,046 Not available NTIS 
Review of Research Literature on Masonry Shear Walls. PB91-167189 100,113 PC A05/MF A01 Reynolds Number Effects on the Mixing Behavior of Axisymmetric Turbulent Jets.

100,015 Not available NTIS

RF-DC Differences of Thermal Voltage Converters Arising from Input Connectors.

100,735 Not available NTIS

RIR - Measurement and Use in Quantitative XRD

PB91-147397 101,466 Not available NTIS

Road Raliy Conversational Speech Corpora (on CD-ROM).

PB91-509802 100,595 CD-ROM\$500.00

Robot Characterization Testing

PB91-167361

$101,030 \mathrm{PC} \mathrm{A03/MF} \mathrm{A01}$

Robot Sensor Language.

101,031 Not available NTIS

Role of Bench-Scale Test Data in Assessing Real-Scale Fire Toxicity.

100,156 PC A06/MF A01

Role of Elastic Energy in the Morphological Development of a Ni-Ti-Al Alloy.

101,227 Not available NTIS

Role of Gíassy. Interfaces in High Temperature Crack Growth in SiC Fiber Reinforced Alumina.
PB91-203687

Role of Reference Materials in Quality Assurance.

PB91-203901 100,206 Not available NTIS

Role of Theory in Lighting Research and Design

PB91-237826 100,081 Not available NTIS

Root Projection of One-Sided Time Series.

PB91-¿16754

101,262

(Order as PB91-216705, PC A07/MF A01)

Rotational Predissociation, Vibrational Mixing, and van der

Waals Intermolecular Potentials of NeDF.
PB91-175505 100,334 Not available NTIS

Rotational Spectrum and Structure of the Complex ArCh3CN.

PB91-236661

100,415 Not available NTIS

Rotational-Tunneling Spectrum of the Ar-SO2 van der Waals Complex

100,457 Not available NTIS

Roughened Quartz Surfaces and Teflon as Small-Angle Diffusers and Depolarizers between 200 and $400 \mathrm{~nm}$.

PB91-195644 101,425 Not available NTIS

Roughness Measures of Blasted Steel Surfaces Remotely Imaged with a Thermographic Camera.
PB91-147686

Round Robin on Apparent Thermal Conductivity of Several Loose-Fill Insulations.

101,143 Not available NTIS

Round-Robin Study of Implants in $\mathrm{Si}$ and $\mathrm{SiO} 2$ by SIMS, RBS, and NAA

100,830 Not available NTIS

Rovibrational Analysis of the nu7(1) Intermolecular Hydrogen Bond Bending Vibration in HCN-HF Using Far Infrared

100,437 Not available NTIS

Rubidium Frequency Standard and a GPS Receiver: A Remotely Steered Clock System with Good Short-Term and Long-Term Stability.
PB91-200808 100,581 Not available NTIS

100,581 Not available NTIS

Rule-Based Diagnostic Method for HVAC Fault Detection. PB91-194936 100,072 Not available NTIS

Saran Carbon Cryogenic Regenerator for Liquid Helium.

PB91-175430 101,640 Not available NTIS

Scanning Electron Microscopy with Polarization Analysis (SEMPA)-Studies of Domains, Domain Walls and Magnetic

Singularities at Surfaces and in Thin Films.
PB91-237289

canning tunneling microscopy studies of the surfaces of a31 January 1991.

DE91002169

100,785 PC A03/MF A01

Scanning Tunneling Microscopy Study of Clean and Cs. Covered InSb(110)

101,537 Not available NTIS

Scattered Light and Other Corrections in Absorption Coefficient Measurements in the Vacuum Ultraviolet: A Systems

ADr-A227 869/5

100,179 PC A02/MF A01

Scattering by an Arbitrary Cylinder at a Plane Interface.

PB91-203513 101,447 Not available NTIS

Scoring Continuous Speech in the DARPA Speech Recognition Program: Part 2.
PB91-195396

100,593 Not available NTIS

Search for a Composition Dependent Force Using a LiquidSupported Torsion Balance. PB91-175380

101,639 Not available NTIS

Second Dissociation Constant and $\mathrm{pH}$ of $\mathrm{N}$-2-hydroxyethylpiperazine-N'-2-ethanesulfonic Acid (HEPES) trom 0 to

100,196 Not available NTIS

Second-Level Post-Occupancy Evaluation Analysis.

PB91-147140 100,058 Not available NTIS

Second Order Sensitivity Analysis in Factorable Programming: Theory and Applications. 101,266 Not available NTIS

Secondary lon Yield Changes in Silicon and Gallium Arsenide Due to Topography Changes during Oxygen or Cesium Ion Bombardment.

PB91-14806

101,471 Not available NTIS

Security in ISDN.

PB92-116391

100,718 PC A05/MF A01

Selected-lon Mass Spectrometry: Assays of Oxidative DNA Damage.

101,307 Not available NTIS Selective-Area Epitaxial Growth of Gallium Arsenide on Sili-
con Substrates Patterned Using a Scanning Tunneling Micon Substrates Patterned Using a Scanning Tunneling Mi-
croscope Operating in Air. croscope Operating in Ai

100,796 Not available NTIS

Selective Excitation of X-ray Emission Spectra.
PB91-147173

Self-Adjustment of a Robot Joint Controller

PB91-158568

101,027 Not available NTIS

Self-Setting Calcium Phosphate Cements.

PB91-202879

100,049 Not available NTIS

SEM Imaging and Metrology at Low Accelerating Voltages Using Backscattered Electrons.

101,529 Not available NTIS

Semiclassical Calculation of Self-Broadening in O2, N2, and

CO Raman Spectra.

100,461 Not available NTIS

Semiconductor Claddings on Glass Waveguides for Polarizers and Detectors.

101,416 Not available NTIS

PB91-175141

xation: Concentrated Polymer

Semiempirical Theory

PBo1-236638

100,511 Not available NTIS

Sensing of GMAW Droplet Transfer Modes Using an ER 100S-1 Electrode

PB91-174250

101,004 Not available NTIS

Sensitivity of Ellipsometric Modeling to the 'Islands' of Silicon Precipitates at the Bottom of the Buried Oxide Layer in Annealed SIMOX.

PB92-116607

100,820 Not available NTIS

Sensitometry of the Response of a New Radiochromic Film Dosimeter to Gamma Radiation and Electron Beams.

PB91-237008 101,326 Not available NTIS

Setting Reactions and Compressive Strengths of Calcium Phosphate Cements.

PB91-148668

100,040 Not available NTIS

Shape Resonanc
PB91-187435

100,338. Not available NTIS

SHOOT Flowmeter and Pressure Transducers.

PB91-147488

100,923 Not available NTIS

Short-Duration Autoignition Temperature Measurements for Hydrocarbon Fuels.

AD-A242 816/7

100,877 PC A03/MF A01

PB92-108927

100,887 PC A03/MF A01

Short-Term Evolution for the Flight Telerobotic Servicer.

PB91-144352

101,731 PC A04/MF A01

Sidelobe Suppression in Small Josephson Junctions.

PB91-195446

100,784 Not available NTIS

Signal Detection Efficiency in Multiphoton Ionization Flame

Measurements.

100,540 Not available NTIS

Silane Dissociation Products in Deposition Discharges.

PB91-202994

Deposition Discharges.
100,390 Not available NTIS

Silicon Reference Materials Certified for Isotope Abundances.

100,213

(Order as PB92-126614, PC A06/MF A02)

Simplified Error Bounds for Newton's Rule.

PB91-159046 101,257 Not available NTIS

Simulation of an Evaporator with Nonuniform One-Dimensional Air Distribution.

100,066 Not available NTIS
Simulation Studies of Excluded Volume Effects on Polyme Chain Dynamics in Several Nonlattice Models. PB91-203364 100,507 Not available NTIS

Simultaneous Butyltin Determinations in the Microlayer Water Column and Sediment of a Northern Chesapeake Bay Marina and Receiving System. PB91-195040

100,918 Not available NTIS

Simultaneous versus Independent Injection Testing of Nonlinear Multiport Systems.

PB91-203885

100,857 Not available NTIS

Single Pulse Shock Tube Study of the Reactions of Hydrogen Atoms with Aromatics. 4. Chlorotoluenes.

PB91-175133

100,331 Not available NTIS

Single-Target Magnetron Sputter-Deposition of High-Tc Superconducting Bi-Sr-Ca-Cu-O Thin Films.

PB92-116680

101,566 Not available NTIS

Sixteen Thousand Evaluated Experimental Thermodynamic Property Data for Water and Steam.

PB92-148154 $\quad 100,475$ (Order as PB92-148113)

Sliding Wear Studies of Nickel-Copper Composition-Modulated Coatings on Steel.

PB91-190025

101,188 Not available NTIS

Slovak Metrological Society.

PB92-126721

100938

(Order as PB92-126614, PC A06/MF A02)

Slow Electron Transfer Reactions between Alkylhydrazines.
PB91-147785 100,284 Not available NTIS

Small-Angle Neutron Scattering and Light Scattering Stud ies on the Miscibility of Protonated Polyisoprene/Deuterat ed Polybutadiene Blends.

PB91-236786

100,513 Not available NTIS

Small-Angle Neutron Scattering and Light Scattering Stud on the Miscibility of Poly(styrene-ran-butadiene)/Polybutadiene Blends.

100,519 Not available NTIS

Small Angle Neutron Scattering Characterization of Microporous Silica.

101,089 Not available NTIS

Small-Angle Neutron Scattering of Blends of Cross-Linked and Linear Polystyrene.

PB91-194688

100,500 Not available NTIS

Small-Angle Neutron Scattering Studies of Compatible Blends of Linear Poly(Vinyl Methyl Ether) and Cross-Linked Deuterated Polystyrene.

PB91-187153

100,494 Not available NTIS

Small-Angle-Scattering Determination of the Microstructure of Porous Silica Precursor Bodies.

PB91-175075 101,077 Not available NTIS

Small Scale Demand Type Neon Liquefaction Plant.

PB91-162008 100,224 Not available NTIS

Smoke Production and Properties.

PB91-175422

100,120 Not available NTIS

Smoldering Combustion Propagation Through a Permeable Horizontal Fuel Layer.

PB91-147843

100,102 Not available NTIS

Soft X-ray Absorption and Emission Spectra and the Electronic Structure of Some Exotic Materials.

tronic Structure of Some Exotic Materials.

Software and Computer Integrated Manufacturing

PB91-132142

100,972 PC A03/MF A01

Software Reengineering: A Case Study and Lessons Learned.

100,669 PC A03/MF A01

Software Verification and Validation: An Overview.

PB91-237685

100,658 Not available NTIS

Solubilities of Solids and Liquids of Low Volatility in Super. critical Carbon Dioxide.

PB92-148105

100,470 (Order as PB92-148063)

Solubility of Carbon Dioxide in Water at Low Pressure.

PB92-148196 100,479 (Order as PB92-148162)

Soluble Models in Surface Collision Dynamics.

PB91-147298 100,276 Not available NTIS

Some Algorithms for Approximating Convolutions.

PB91-175281 101,258 Not available NTIS

Some Concluding Thoughts for Cool Stars VI.

PB91-175034 100,030 Not available NTIS

Some Historical Remarks Concerning These Conferences: With Emphasis on the X-ray Component.

PB91-187443 101,652 Not available NTIS

Space Marching Difference Schemes in the Nonlinear In verse Heat Conduction Problem.

PB91-144360

101,604 PC A03/MF A0 


\section{TITLE INDEX}

Spatially and Spectrally Resolved Cathodoluminescence Measurements of CVD-Grown Diamond Particles and Films.
AD-A243 220/1 101,451 PC A03/MF A01

Spatially and Spectrally Resolved Cathodoluminescence of Hot-Filament Chemical-Vapor-Deposited Diamond Particles. AD-A236 485/9 100,247 PC A03/MF A01 AD-A237 128/4 100,248 PC A03/MF A01

Spatially Overdetermined Analysis for Propagation Toughness Using Strain Gages.

101,164 Not available NTIS

Spatially Resolved Flares in RS CVn Systems.

PB91-148965 100,022 Not available NTIS

Species Produced in Fires Burning in Two-Layered and Homogeneous Vitiated Environments.

PB91-157180

100,542 PC A12/MF A02

Specifications for a Federal Intormation Processing Standard Data Dictionary System.

PB91-158758

100,687 Not available NTIS

Specifications, Tolerances, and Other Technical Requirements for Weighing and Measuring Devices as Adopted by the 76th National Conference on Weights and Measures,

1991

101,023 PC A10/MF A03

Spectra of the P I Isoelectronic Sequence from Co XIII to
Mo XXVIII. Mo XXVIII.
PB91-23750

101,713 Not available NTIS

Spectral Data and Grotrian Diagrams for Highly lonized Copper, Cu X-Cu XXIX

PB92-110121 100,441 (Order as PB92-110113)

Spectral Data and Grotrian Diagrams tor Highly Ionized Iron, Fe VIII-XXV

PB90-244583

101,595 (Order as PB90-244526)

Spectroscopic Constants for the nu9 Infrared Band of $\mathrm{HNO} 3$.

100,343 Not available NTIS

Spectroscopic Data Base tor Carbon and Oxygen.

PB91-237750 101,716 Not available NTIS

Spectroscopy and Structure of the Alkali Hydride Diatomic

Molecules and Their lons.
PB92-110147 100,443 (Order as PB92-110113)

Spectrum and Energy Levels of Seven-Times-lonized Krypton (Kr VIII) and Resonance Lines of Eight-Times-Ionized Krypton (Kr IX)

PB91-195511

101,673 Not available NTIS

Spectrum and Energy Levels of the Xenonlike Ion Ce V

PB91-175364 101,638 Not available NTIS

Spectrum-Averaged Cross-Section Measurement of $(103) R h(n, n)(103 m) R h$ in the $(252) C f$ Fission Neutron SpecPB91-203406

101,694 Not available NTIS

Speed ot Sound of Natural Gas Mixtures

PB91-162214 100,882 Not available NTIS

Spherical Near-Field Scanning: Determining the Incident Field Near a Rotatable Probe.

PB91-148239

100,725 Not available NTIS

Spherical Near-Field Scannıng: Experimental and Theoretical Studies.

$100,723 \mathrm{PC} \mathrm{A07/MF} \mathrm{A01}$

Spin-Dependent Electron-Impact Excitation ot Sodium.
PB91-175497 NT1,641 Not available NTIS

Spin Glass Magnetic Behavior of Iron/Silica Gel Nanocomposites.

101,117 Not available NTIS

Spinodal Decomposition in a Hydrogen-Bonded Polymer Blend.
PB91-162099 100,489 Not avaiłable NTIS

Sprinkler/Hot Layer Interaction.

PB91-206680 100,129 PC A04/MF A01

Sputtered Thin Film YBa2Cu3O

PB91-189647 101,509 Not available NTIS

SOL3 Support for CALS Application

PB91-167262

100,965 PC A03/MF A01

Squeezed Ouantum States of Relic Gravitons and Primordial Density Fluctuations.

101,685 Not available NTIS

SRM 1970 - Succinonitrile Triple-Point Standard - And Its Use in Calibration of Thermistors.

PB91-134338

100,780 Not available NTIS

Stabilities of Substituted Benzyl Radicals: Dissociation Rates of Aminoethylbenzenes, Hydroxyethylbenzenes, and Cyanoethylbenzenes.

PB91-237529

100,430 Not available NTIS

Stability of Aqueous Inorganic Lead Solutions in Polycar bonate Containers.
PB92-126499

$100,211 \mathrm{PC} \mathrm{A03/MF} \mathrm{A01}$

Stability of Thermistors.

PB91-134320

100,779 Not available NTIS

Stability of Uric Acid in Ammonium Hydroxide.

PB91-189266 101,292 Not available NTIS

Stabilization Techniques tor Spinning Rotor Gage Residual Drag.

100,922 Not available NTIS

Stable, High Ouantum Efficiency Silicon Photodiodes for Vacuum-UV Applications.

100,757 Not available NTIS

Stable Implementation Agreements for Open Systems Interconnection Protocols. Version 4, Edition 1, Change Pages September 1991. Output from the September 1991 OSI Implementors Workshop. Held in Gaithersburg, Maryland.
PB92-126408

Stable Implementation Agreements for Open Systems Interconnection Protocols. Version 4, Edition 1. December 1990.
PB91-171967 100,689

Stable Implementation Agreements for Open Systems Interconnection Protocols Version 4, Edition 1 June 1991

Change Pages (Supplement). 100,695 PC A04/MF A01
PB92-112200

Stable Implementation Agreements for Open Systems Interconnection Protocols. Version 4, Edition 1, March 1991. Change Pages (Supplement).

100,691 PC A11/MF A02

Standard Chemical Thermodynamic Properties of Isomer

Groups ot Monochloroalkanes.
PB90-244617 100,258 (Order as PB90-244609)

Standard Chemical Thermodynamic Properties of Polycyclic Aromatic Hydrocarbons and Their Isomer Groups. III. Naphthocoronene Series, Ovalene Series, and First Members of Some Higher Series.
PB90-244625 100,259 (Order as PB90-244609)

Standard Generalized Markup Language Encoding of the Otfice Document Architecture Document Application Protile PB91-184812
101,345 PC A03/MF A01

Standard Reference Fibers for Calibration of the Optical Time Domain Reflectometer.

PB91-147207

101,406 Not available NTIS

Standard Reference Materials: Bright-Chromium Linewidth Standard, SRM 476, tor Calibration of Optical Microscope Linewidth Measuring Systems.

PB91-167163

100,802 PC A03/MF A01

Standard Reference Materials: Calibration of NIST Standard Reference Material 3201 for 0.5 Inch $(12.65 \mathrm{~mm})$ Serial Serpentine Magnetic Tape Cartridge.
PB91.187542 Standard Reference Specimens in Ouality Control of Engineering Surfaces

101,021

(Order as PB91-216705, PC A07/MF A01)

Standard Security Label for GOSIP: An Invitational Workshop. Held on April 9-10, 1991.

PB91-216671

100,712 PC A07/MF A02

Standardization of Advanced Ceramics.

PB91-195669 101,086 Not available NTIS

Standardizing Standards

PB91-189795 100,953 Not available NTIS

Standards Activities ot Organizations in the United States. PB91-177774 100,004 PC A99/MF A04

Standards for the Physical Protection of National Resources and Facilities.

100,006 PC A05/MF A01

State-ot-the-Art in Pressure Metrology.
PB91-146993 Not available NTIS

PB91-146993 101,038 Not available NTIS

State-ot-the-Art of Computational Testing of Mathematical Programming Algorithms. $\quad 101,268$ Not available NTIS
PB91-174896

Static Analysis Tools for Soltware Security Certification.
PB91-203380 100,710 Not available NTIS

Static and Dynamic Strength Tests on Electrical Conductor

Cables Specified for Airport Landing Structures.
PB92-112226

Statistical Mechanical Model ot Aqueous Two-Phase SysPB91-174508

100,493 Not available NTIS

Statistical Process Control Tactics for Coordinate Measuring Machines.

101,042 Not available NTIS

Statistics of a Polymer Molecule in the Presence of Asymmetric Obstacles
PB91-187450 100,498 Not available NTIS

Status of Beta-Particle Calibration Studies at NBS.
PB91-175265

101,636 Not available NTIS

Steam Tables

101,624 Not available NTIS

STEP On-Line Information Service User's Guide. National PDES Testbed Report Series.

100,950 PC A03/MF A01

STEP: Standard for the Exchange of Product Model Data Resource Integration: Semantic and Syntactic Rules. PB91-184788

100,966 PC A03/MF A01

Stoichiometry of Reactively Evaporated Films.
PB91-158709

Stopping Power of Fast Charged Particles in Heavy Elements.

101,660 PC A04/MF A01

Strain Rate Eftect on Tensile Properties at $4 \mathrm{~K}$ of a VAMAS Round-Robin Austenitic Steel.

PB91-237248

101,168 Not available NTIS

Strategies for Background Subtraction in Electron Probe Mi-

croanalysis X-ray Compositional Mapping.
PB91-195180 100,202 Not available NTIS

Strategy for Chemical Analysis of Alternative Retrigerants.
PB91-148502 100,185 PC A06/MF A01

Streaking Images That Appear Only in the Plane of Diffraction in Unoped GaAs Single Crystals: Diffraction Imaging (Topography) by Monochromatic Synchrotron Radiation.
PB91-189662

Strength and Fracture Behavior of Ba-Y-Cu-O Supercon-

ducting Ceramics.
PB91-148205

101,474 Not available NTIS

Strength and Microstructure of Ceramics.

AD-A243 049/4

101,056 PC A08/MF A02

Stress Corrosion Cracking of Al-Li Alloys: The Role of Grain Boundary Precipitates.

PB91-175182

101,137 Not available NTIS

Stress Intensity Factors by Enriched Mixed Finite Elements. PB91-147371 101,577 Not available NTIS

Strontium-Induced Oxygen Detect Structure and Hole Doping in La2-xSrxCuO4.

101,472 Not available NTIS

Structural and Magnetic Properties of Er Thin Films and $\mathrm{Er} /$

Y Superlattices: Magnetoelastic Effects.
PB91.161935 Not available NTIS

Structural Anomalies in Undoped Gallium Arsenide Observed in High Resolution Diffraction Imaging with Monochromatic Synchrotron Radiatio

PB91-237438

101,556 Not available NTIS

Structural Change and Competitiveness: The U.S. Semiconductor Industry
PB91-195693

100,173 Not available NTIS

Structural Dependence of HF Vibrational Red Shifts in ArnHF, $n=1-4$, via High-Resolution Slit Jet Infrared Spectroscopy.

100,401 Not available NTIS

Structural Instabilities and Superconductivity in Ouasi-Binary Mn5Si3-Type Compounds.

101,215 Not available NTIS

Structural Study of Electrodeposited Aluminum-Manganese Alloys.

101,231 Not available NTIS

Structural Study of the Epitaxial Growth of tcc-Fe Films, Sandwiches, and Superlattices on $\mathrm{Cu}(100)$.
PB91-237420 available NTIS

Structure and Mechanism of Hydroxyl Radical-Induced Formation of a DNA-Protein Cross-Link Involving Thymine and ysine in Nucleohistone.

PB91-187518

101,334 Not available NTIS

Structure ot a Stage-3 Cs - Graphite Intercalate.

PB91-134957 100,269 Not available NTIS

Structure of Carbon Dioxide Dimer from Near Infrared Spectroscopy

100,279 Not available NTIS

Structure of Odd-Odd (132)Sb.

PB91-237461

101,711 Not available NTIS

Structure ot Surface Films on Magnesium and on Magnesium Alloys.

101,212 Not available NTIS

Structures and Properties of New Zeolite X-Type Zinco phosphate and Beryllophosphate Molecular Sieves.

PB91-189449

101,081 Not available NTIS

Structures of the $\mathrm{NH} 3-\mathrm{HCCCCH}$ and $\mathrm{H} 2 \mathrm{O}-\mathrm{HCCCCH}$ Com plexes by Fourier-Transform Microwave Spectroscopy.
PB91-148882 100,294 Not available NTIS Studies of $\mathrm{HI}$ and $\mathrm{D} I$ in the Local Interstellar Medium. 


\section{TITLE INDEX}

PB91-148957

100,021 Not available NTIS

Studies of Magnetic Flux Penetration in a Chemically Synthesized Bi(Pb)SrCaCuO Superconductor. Not available NTIS

Studies on the Degradation Products of Paper with and without Pollutants in a Closed Environment. 1. Preliminary Results.

PB91.143313

101,181 PC A03/MF A01

Study of Additive Response in a Series of Rerefined Base Oils Typical of Current Commercial Practice.

PB91-174953 101,175 Not available NTIS

Study of Flow Boiling Heat Transfer with Refrigerant Mixtures.

101,196 Not available NTIS

Study of Static and Dynamic Fracture Using Strain Measurements.

101,574 PC A09/MF A01

Study of the GaAs-Si(100) Interface Using Laser Probing of Thermal Desorption Kinetics.

PB91-149294

101,477 Not available NTIS

Study of the Galling of Two Steels Using Two Test Meth Ods.

101,147 Not available NTIS

Study of the Linear Viscoelastic Properties of Cyclic Polys

tyrenes Using Creep and Recovery Measurements.
PB91-195107 N02 Not available NTIS

Study of the Linearity of Transfer Leaks and a Helium Leak Detector

PB91-147272

100,926 Not available NTIS

Study on the Nature of Boundary Lubricating Film: Analytical Method Development

PB91-189407

101,176 Not available NTIS

Stylus Profiling at High Resolution and Low Force.

PB91-162354 101,015 Not available NTIS

Submicrometer SEM Magnification Standard.

PB91-159087 100,799 Not available NTIS

Subpicosecond Pulse Compression and Raman Generation Using a Mode-Locked Erbium-Doped Fiber Laser-Amplifier.

Summaries of BFRL Fire Research In-House Projects and Grants, 1991.

100,168 PC A09/MF A03

Summary Abstract: The Influence of Sulfur on Methanation over W(110).
PB91-149377

100,301 Not available NTIS

Summary of Experiments with the Separated Aperture Technique of Dielectric Anomaly Detection

AD-A230 438/4

100,721 PC A02/MF A01

Summary of Low-Density Glass-Fiber Reference Materials at NIST: $1980-1989$

PB91-175620

101,079 Not available NTIS

Summary of the Intercomparison of the Force Standard Machines of the National Institute of Standards and Technology, USA, and the Physikalisch-Technische Bundesanstalt, Germany.
PB92-126622

101,590

(Order as PB92-126614, PC A06/MF A02)

Superconducting Properties of $\mathrm{Bi} 2-x-y \mathrm{PbxSnySr} 2 \mathrm{Ca} 2 \mathrm{Cu} 3 \mathrm{Oz}$. PB91-162321 101,076 Not available NTIS Superconducting Thin Films of $\mathrm{Bi}-\mathrm{Sr}-\mathrm{Ca}-\mathrm{Cu}-\mathrm{O}$ Obtained by Laser Ablation Processing.

101,508 Not available NTIS

Superconductor-Polymer Composites.
PATENT-4 $954481 \quad$ Not available NTIS

Suppression of Post-Flashover Compartment Fires Using Manually Applied Water Sprays. 100,164 PC A03/MF A01
PB91-240770 Surface Acoustical Wave Spectroscopy for High Humidity Sensing.
PB91-158816

100,843 Not available NTIS

Surface Extended-X-ray-Absorption Fine Structure and

Scanning Tunneling Microscopy of Si(001)2x1-Sb.
PB91-237263 101,552 Not available NTIS

Surface-Extended $X$-ray-Absorption Fine-Structure Experiments at Atmospheric Pressure by Means of a Photocathode Proportional Counter with Monolayer Sensitivity.
PB91-194951 100,372 Not available NTIS

Surface Flame Spread.

100,546 Not available NTIS

PB91-175331 100,546 Not available NTIS

Surface Oxidation Kinetics of Si3N4 - 4\% Y2O3 Powders Studied by Bremsstrahlung-Excited Auger Spectroscopy.
PB91-237701 100,435 Not available NTIS

Surface Properties and Coatings of Materials Microtailored Surface
Alloys.
PB91-189696

101,232 Not available NTIS

Surface-State-Mediated Photochemistry: Laser-Induced Desorption of NO from Si (111).

PB91-162289

100,320 Not available NTIS

Surface X-ray Absorption Spectroscopy, EXAFS and NEXAFS, for the In situ and Ex situ Study of Electrodes. PB92-117035

100,460 Not available NTIS

Survey of Current Worldwide Research on the Thermophy-
sical Properties of Alternative Refrigerants. PB92-112366

101, 198 PC A05/MF A01

Switching in High-Tc Superconductor Current Transport
Measurements.
PB91-203117
101,539 Not available NTIS PB91-203117

Synchronizing Computer Clocks Using a Local Area Net-

Nork. $25793 / 1$

100,596

(Order as N91-25755/0, PC A99/MF A04)

Synthesis of Non-Ionic Water-Dispersible Resins for Use in Intaglio Inks Curing by Electron Beam Radiation PB91-194456

100,499 PC A03/MF A01

Synthesis of Prototype Resins for Use as BEP Intaglio Ink Vehicles Curing by Electron Beam Radiation.
PB91-144345 01,182 PC A03/MF A01

Synthesis of 3-Quinuclidinol-(18)O, Benzilic-d5 Acid, and 3Quinuclidinyl-(18)O, Benzilate-d5.
100,223 Not available NTIS

System for the Study of Magnetic Materials and Magnetic System for the Study of Magnetic Materials and Magnetic
Imaging with the Scanning Tunneling Microscope. Imaging with the Scanning Tunneling Microscope.
PB91-194779 101,522 Not available NTIS

Taguchi's Orthogonal Arrays Are Classical Designs of Ex. periments.

101,280

(Order as PB92-126614, PC A06/MF A02)

Taking the Measure of Fluid Properties Data Bases

PB91-200832 101,392 Not available NTIS

Task Decomposition and Algorithm Development for RealTime Motion Control of a Continuous Mining Machin PB91-231597 101,357 PC A03/MF A01

Technical Activities 1990, Ëlectron and Optical Physics Division.

101,662 PC A04/MF A01

Technical Activities 1990, Standard Reference Data Program.

100,321 PC A05/MF A01

Technical Activities 1990 Surface Science Division.

PB91-240820 101,560 PC A04/MF A01

Technical Digest-Symposium on Optical Fiber Measurements, 1990.

PB91-132308

101,403 PC A10/MF A02

Technical Reference and User's Guide for FAST/FFM Version 3

PB91-206664

100,086 PC A08/MF A01

Techniques to Observe the Fracture Zone in Mortar and Concrete.
PB91-134189

100,521 Not available NTIS

TEM Driven Reverberating Chamber, a Single Facility for Radiated EMS $/ V$ Testing, $10 \mathrm{kHz}=18 \mathrm{GHz}$
PB91-202895. 100,856 Not available NTIS

TEM/Reverberating Chamber Design/Concept Study: A Single Facility for Large System Radiated EMC Testing, 10 $\mathrm{kHz}=40 \mathrm{Ghz}$.

100,845 Not available NTIS

Temperature Dependence of High Accuracy-Photometer Heads.
PB91-187096

101,418 Not available NTIS

Temperature Dependence of Probe Diffusion in Bulk Polymer Matrices.

100,422 Not available NTIS

Temperature Dependence of Radiochromic Film Dosimeters.
PB92-117134

101,728 Not available NTIS

Temperature Gradient in Differential Scanning Calorimetry. PB91-236570 100,414 Not available NTis

Temper ature Measurement of Glass Subjected to Solar Radiation.

100,100 Not available NTIS

Temperature Measurements in Flames Using Thermally Assisted Laser-Induced Fluorescence of Ga.
PB91-236851 100,420 Not available NTIS

Temperature Monitored/Controlled Silicon Photodiodes for Standardization

100,767 Not available NTIS

Tensile Creep Behavior of Structural Ceramics.

PB91-159277 101,075 Not available NTIS
Tensile-Fracture Resistance Mechanisms in Brittle Polycrystals: An Ultrasonics and In-situ Microscopy Investigation. PB91-149344

101,190 Not available NTIS

Terminal Velocities for a Large Sample of O Stars, B Supergiants, and Wolf-Rayet Stars.

pBgi-149021

100,023 Not available NTIS

Test Chip for the Evaluation of Surface-Diffusion Phenomena in Sputtered Aluminum Planarization Processes.

PB92-116920

100,823 Not available NTIS

Test Method for Tensile Creep of Structural Ceramics Using Flexure Beams.

PB92-116995

101,099 Not available NTIS

Test Methods for Optical Disk Media Characteristics (fo $356 \mathrm{~mm}$ Ruggedized Magneto-optic Media).

PB92-116409

100,605 PC A05/MF A0

Test of Newton's Inverse Square Law of Gravity Using the $300 \mathrm{~m}$ Tower at Erie, Colorado: Newton Vindicated on the Plains of Colorado.

PB91-175273

101,637 Not available NTIS

Test of the Inverse-Square Law of Gravitation Using the 300-m Tower at Erie, Couare

300-m Tower at
PB91-204115

101,702 Not available NTIS

Test of the Linearity of Quantum Mechanics by if Spectroscopy of the (9)Be( + ) Ground State.

Copy of the (9)
PB92-116870

101,726 Not available NTIS

Test Plan for Validating a Context Driven Integrated Model (CDIM) for Sheet Metal Die Design. National PDES Testbed Report Series

100,970 PC A04/MF A01

Testing and Rating of an Atmospheric, Gas-Fired Furnace Equipped with a Burner Air Inlet Damper.

PB92-126440

100,894 PC A04/MF A01

Testing the Accuracy of Calculated Equilibrium Carrier Con-

centrations in the Presence of Surface Fields.
PB91-236729

Tests of the Flory-Rehner Hypothesis: Comparison of the Elastic Free Energy Function for Crosslinked Rubber in the Dry and Swollen States.

PB91-203570

100,508 Not available NTIS

Theoretical Considerations for a Thermo-Optic Microwave Electric-Field-Strength Probe.

PB91-237222

100,860 Not available NTIS

Theoretical Investigation of Piloted Ignition of Wood.

PB92-112432

100,556 PC A11/MF A03

Theoretical Model for the Tunneling-Gap Anisotropy Observed in Layered Copper-Oxide High-Temperature Superconductors.

101,564 Not available NTIS

Theoretical Model of Metal Binding Sites in Proteins.

PBe 1-194837

100,370 Not available NTIS

Theory of Ballistic-Electron-Emission Spectroscopy of

NiSi2/Si(111) Interfaces.

101,557 Not available NTIS

Theory of Capacitive Probe Method for Noncontact Charac-

terization of Dielectric Properties of Materials.
PB91-237610 100,948 Not available NTIS

Theory of Orientation Textures Due to Surface Energy Anisotropies.
PB92-117381

101,571 Not available NTIS

Theory of Specific Heat of Solids.

101,475 Not available NTIS

Thermal Characterization of Integrated Circuits - A Tutorial

PB91-147819 100,789 Not available NTIS

Thermal Conductivity and Heat Capacity of Gaseous Argon. PB91-134577 101,602 Not available NTIS

Thermal Conductivity of a Moderately Dense Gas.

PB91-134502 101,601 Not available NTIS

Thermal Conductivity of a Polymide Film between 4.2 and

$300 \mathrm{~K}$, with and without Alumina Particles as Filler.
PB91-144402 100,483 PC A05/MF A01

Thermal Conductivity of Alumina Fiber/Epoxy and Alumina Fiber/PEEK from 4.2 to $310 \mathrm{~K}$

PB92-117258

101,130 Not available NTIS

Thermal Conductivity of Evacuated Perlite at Low Temperatures as a Function of Load and Load History.

100,227 Not available NTIS

Thermal Conductivity of Fumed Silica Board at Room Temperature.

100,125 Not available NTIS

Thermal Conductivity of Methane and Tetrafluoromethane in the Limit of Zero Density.

100,36? Not available NTIS

Thermal Conductivity of Nine Polyatomic Gases at Low Density. 


\section{TITLE INDEX}

PB91-192492

100,360 Not available NTIS

Thermal Decomposition of lons. 3. Protonated Ethanol and Diethyl Ether.

100,376 Not available NTIS

Thermal Measurements of VLSI Packages: A Critical Review.

100,790 Not available NTIS

PB91-147827

Thermal Noise in Mechanical Experiments.
PB91-149211

Thermochemistry of Sulfoxides and Sulfones.

PB91-174821 100,325 Not available NTIS

Thermodynamic and Kinetic Stability of Refractory Materials at Uitra-High Temperatures.

AD-A235 490/0

100,246 PC A03/MF A0

Thermodynamic and Thermophysical Properties of Organic Nitrogen Compounds. Part 1. Methanamine, Ethanamine, 1 and 2-Propanamine, Benzenamine, 2-, 3-, and 4-Methylbenzenamine.

100,368 Not available NTIS

Thermodynamic Behavior of Fluids in the Supercritical Region.

101,394 Not available NTIS

deling of Flue Gas Cleanup Systems (Abstract for Poster Session).

PB91-158741 100,904 Not available NTIS

Thermodynamic Diagrams for Refrigerant Mixtures.

PB91-236695 101,197 Not available NTIS

Thermodynamic Functions and Properties of $\mathrm{MgO}$ at High

Compression and High Temperature.
PB90-244542 100,253 (Order as PB90-244526)

Thermodynamic Properties of Aqueous Solutions at High

Temperatures; Needs, Methods, and Challenges.
PB91-204065

Thermodynamic Properties of Oxygen from the Triple Point

to $300 \mathrm{~K}$ with Pressures to $80 \mathrm{MPa}$.
$\mathrm{PB} 92-148147$
100,474
(Order as PB92-148113)

Thermodynamic Properties of the Aqueous Sulfuric Acid System to $350 \mathrm{~K}$

PB92-148188

100,478 (Order as PB92-148162)

Thermodynamic Properties of the $\mathrm{NaBr}+\mathrm{H} 2 \mathrm{O}$ System.
PB92-110238 100,45t (Order as PB92-110212)

Thermodynamic Properties of Two Alternative Refrigerants: 1,1-dichloro-2,2,2-trifluoroethane (R123) and 1,1,1,2-tetrafluoroethane (R134a).

PB91-175414

101,195 Not available NTIS

Thermodynamic Properties of 1,1,1,2,-Tetrafluoroethane (R134a) in the Critical Region. 100,233 Not available NTIS
PB91-237578 Thermodynamics of Ammonium Indates. 2. The Molar Heat Capacity of the Ammonium Pentabromoindate Monohydrat Salt (NH4) 2 InBr5. H2O from 7.8 to $348 \mathrm{~K}$.

PB91-149831 100,304 Not available NTIS

Thermodynamics of Hydrolysis of Disaccharides: Lactulose, alpha-D - Melibiose, Palatinose, D. Trehalose, D. Turanose and 3-o-beta-D-Galactopyranosyl-D-arabinose.

PB91-195701 101,289 Not available NTIS

Thermodynamics of Hydrolysis of Oligosaccharides.

PB91-194803 101,288 Not available NTIS

Thermodynamics of Industrially Important Enzyme Catalyzed Reactions

101,311 Not available NTIS

Thermodynamics of Solutions Near the Solvent's Critical Point.
PB91-237297 100,232 Not available NTIS

Thermodynamics of the Disproportionation of Adenosine 5'diphosphate to Adenosine 5'-triphosphate and Adenosine 5 '-monophosphate. 1. Equilibrium Model

PB92-116854 100,459 Not available NTIS

Thermodynamics of the Disproportionation of Adenosine 5' diphosphate to Adenosine 5 '-triphosphate and Adenosine $5^{\prime}$-monophosphate. 2. Experimental Data. Thermodynamics of the Divalent Metal Fluorides. 3. Heat Capacity of the Fast lon Conductor Sr SnF4 from 6 to 344 PB91-149864

100,305 Not available NTIS

Thermodynamics of the Divalent-Metal Fluorides. 4. Heat Capacity of MSnF4, M $=\mathrm{Pb}, \mathrm{Ba}$, or $\mathrm{Sr}$, at Temperatures

from $300 \mathrm{~K}$ to $660 \mathrm{~K}$. $\quad 100,456$ Not available NTIS
PB92-116524 (1) 116524

Thermodynamics of the Krebs Cycle and Related Compounds.

100,357 Not available NTIS

Thermophysical Properties of Ethane.
PB92-110188 100,447 (Order as PB92-110162)
Thin and Composite High-Flux Membranes of Perfluorosulfonated lon-Exchange Polyme PB91.236653

100,512 Not available NTIS

Three Dimensional Reconstruction from Optical Flow Using Temporal integration

100,702 PC A03/MF A01

Three-Dimensional Simulations of High-Resolution Photoresist Processing.

100,818 Not available NTIS

Thwarting the Hackers.

PB91.159228

100,705 Not available NTIS

Tiled Raster Graphics and MIL-R-28002A: A Tutorial and Implementation Guide.

PB91-187708

101,346 PC A06/MF A01

Time- and State-Resolved Measurements of Nitric Oxide Dimer Infrared Photodissociation.

PB91-202846

100,387 Not available NTIS

Time Domain Frequency Stability Calculated from the Fre quency Domain Description: Use of the SIGINT Software Package to Calculate Time Domain Frequency Stability from the Frequency Domain.

PB92-123132

100,587 PC A03/MF A01

Time-Resolved Small-Angle Neutron Scattering in Intermediate- and Late-Stage Spinodal Decomposition of (DPB) $\mathrm{HPI}$ ) Blends.

PB91-16213

100,490 Not available NTIS

Toward Modular Semantic Application Layer Protocols (MS. ALPS): Communication between Heterogeneous Information Systems Using Artificial Intelligence (Al) Programming Techniques.

PB91-158584

100,565 Not available NTIS

Towards Computer-Based Microstructure Models for Cement-Based Systems.

101,074 Not available NTIS

Trace Determination of $\mathrm{Cr}(\mathrm{VI})$ by LC/AAS with on-Line Preconcentration.
PB91-148148

100, 184 Not available NTIS

Transferring NBS Technology to Small Manufacturers Through State and Local Centers.
PB91-149351

Transformation of the Icosahedral Phase in Rapidly Ouenched Al-rich AI-Mn Alloys.
PB91-158964 101,221 Not available NTIS Transient Hydrogen Heat Transfer. Final Report for April
1986-April 1989.
PB92-116326 100,454 PC A03/MF A01 PB92-116326

$100,454 \mathrm{PC} \mathrm{A03/MF} \mathrm{A01}$

Transition from Trichel-Pulse Corona to Dielectric Barrier Discharge.
PB91-134213

100,835 Not available NTIS

Transitions and Energy Levels for Cu-Like $\mathrm{Yb}(41+)$ $\mathrm{Ta}(44+)$, and $\mathrm{U}(63+)$.
PB91-147462

Translate LRL and LRM Calibrations.
PB91-237776
100,862 Not available NTIS

Transmission Electron Microscopy of the CVD Diamond Film/Substrate Interface.

101,450 PC A03/MF A01

Transmission Properties of a Counter-Rotating Pair of Disk

Choppers.
PB91-236604

101,706 Not available NTIS

Transmission/Reflection and Short-Circuit Line Permittivity Measurements

100,848 PC A08/MF A01

PB91-171959

100,783 Not available NTIS

PB91-190116

Transport Critical Current of Aligned Polycrystalline Y1Ba2Cu3O7-delta and Evidence for a Nonweak-Linked
Component of Intergranular Current Conduction.
PB91-133983 101,456 Not available NTIS

101,456

Transport Properties of Carbon Dioxide.
PB90-244724 100,266 (Order as PB90-244658)

Transport Properties of Fluid Oxygen.

PB91-192484 100,359 Not available NTIS

Trapped-Ion Frequency Standards.

N91-25760/0

Tread Crack Detection in Railroad Wheels: An Ultrasonic Tread Crack Detection in Railroad Wheels: An Ultrasonic ers). Report No. 22

PB91-222661

101,742 PC A04/MF A01

Trends in Technological Innovation

PB91-195545 100,008 Not available NTIS

Tribological Characteristics of Synthesized Diamond Films on Silicon Carbide.

PB91-189530

101,186 Not available NTIS
TROI User's Guide.

PB91-157214

101,026 PC A03/MF A01

Tunneling Stabilized Magnetic Force Microscopy: Prospects for Low Temperature Applications to Superconductors.

PB91-203653

101,546 Not available NTIS

Turbulent Upward Flame Spread for Burning Vertical Walis Made of Finite Thickness.

PB92-112531

100,170 PC A05/MF A01

U.S. Assessment of the New Diamond Technology in PB91-167395

101,107 PC A05/MF A01

U.S. Manufacturing Systems: Factories Past, Present, and Future.
PB91-175091

100,981 Not available NTIS

Ultimate Strength of Masonry Shear Walls: Predictions versus Test Results.

$100,138 \mathrm{PC} \mathrm{A03/MF} \mathrm{A01}$

Ultrasonic Measurement of Sheet Steel Texture and Forma. bility: Comparison with Neutron Diffraction and Mechanical

Measurements.

101,170 Not available NTIS

Ultrasonic Texture and Stress Measurements in Anisotropic Polycrystalline Aggregates.

PB91-202945

101,237 Not available NTIS

Ultraviolet Absorption of the Vinyl Radical and Reaction with Oxygen.

100,391 Not available NTIS

Ultraviolet, Optical, Infrared, and Microwave Observations of HR 5110.

100,020 Not available NTIS

Uncertainty and Accuracy in Physical Measurements.

PB91-167205

101,629 PC A03/MF A01

Uniaxial Tensile Tests to Measure the Bond of In situ Concrete Overlays.

100,533 PC A03/MF A01

Unified Description of Infinite-Dilution Thermodynamic Properties for Aqueous Solutes.

PB91-162081

100,319 Not available NTIS

Unified Fundamental Equation for the Thermodynamic

Unified Set of Atomic Transition Probabilities for Neutral Argon.

101,717 Not available NTIS

Uniform Junction Temperature AIGaAs/GaAs Power Heterojunction Bipolar Transistors on Silicon Substrates.

PB91-189399 100,806 Not available NTIS

Uniforms Laws and Regulations as Adopted by the National Conference on Weights and Measures (76th), 1991.
PB92-112416 101,022 PC A08/MF A02

UNIX Expert: A Prototype Knowledge-Based Software Development Workstation.

PB91-149179

100,639 Not available NT1S

UNIX in the Government's Automated Manufacturing Research Facility (AMRF)

100,963 Not available NTIS

Unsteady Laminar Flow in a Circular Tube: A Test of the HERCOL (Hermitian Collocation) Computer Code.

PB91-222679 101,395 PC A03/MF A01

Unusual Degree of Angular Anisotropy in the Resonant Auger Spectrum of $\mathrm{Kr}$

101,649 Not available NTIS

Updated Excitation and Ionization Cross Sections for Electron Impact on Atomic Oxygen.
PB90-244591 100,256 (Order as PB90-244526)

Use of Birefringent Coatings in Fracture Mechanics.

PB91-174599 101,582 Not available NTIS

Use of Computer Algebra to Locate Critical Loci in Fluid Mixtures.
PB91-195263

101,390 Not available NTIS

Use of Cross-Validation as a Stopping Rule in Emission Tomography Image Reconstruction.
PB92-116623 101,295 Not available NTIS

Use of Diamond as an Optical Material.

AD-A243 097/3 101,401 PC A03/MF A01

Use of Heterodyne Detection to Measure Optical Transmittance over a Wide Range.

101,410 Not available NTIS

Use of Neutron Pole Figures to Calibrate Ultrasonic Techniques for On-Lin

101,222 Not available NTIS

Use of Nondestructive Methods for Inspection of Single-Ply Rooting Membranes.

PB91-203935

100,128 Not available NTIS 


\section{TITLE INDEX}

Use of Single Particle Standards for LAMMA Calibration.
PB91-147777 100,183 Not available NTIS Use of Solid Modeling in the Design of M3 Components. PB92-123066 101,044 PC A03/MF A0 Use of Storage Phosphor Imaging Plates in Portal Imaging and High-Energy Radiography: The Intensifying Effect of Metallic Screens on the Sensitivity.
PB91-236497 Not available NTIS Use of the Electrostatic Classification Method to Size 0.1 micrometer SRM Particles: A Feasibility Study. $\quad 100,932$ (Order as PB91-187617, PC A06/MF A01) Use of the IRDS Standard in CALS (Revised). PB91-132209 100,684 PC A03/MF A01 User Manual NBSAVIS CONTAM88. A User Interface for Air Movement and Contaminant Dispersal Analysis in Multizone Buildings.
PB91-206722

$100,910 \mathrm{PC}$ A07/MF A01

Users Guide for RAPID, Version 2.3

PB91-167403 100,999 PC A06/MF A01 Users' Guide to BREAK1, the Berkeley Algorithm for Break-

ing Window Glass in a Compartment Fire.
PB92-112556 100,136 PC A03/MF A01

Uses of Spin-Polarised Electrons in Fundamental ElectronAtom Collision Processes and the Analysis of Magnetic Microstructures.
PB91-162172

101,490 Not available NTIS

Using Surface Roughness as a Wear Criterion in High. Speed Steel End-Milling. $\quad 101,040 \quad$ Not available NTIS
PB91-158675

Using the Harvard/NIST Mark VI Fire Simulation.
PB91-144394 100,095 PC A03/MF A01 Validated Processor List (Cobol, Fortran, ADA, Pascal, SQL). July 1991

100,664 Not available NTIS

Validated Processor List (Cobol, Fortran, ADA, Pascal, SOL). October 1991

100,665 Not available NTIS

Validated Products List (Cobol, Fortran, ADA, Pascal, MUMPS, SQL)

100,671 Standing Order

Validation of an OSI Transport Class 4 Simulator.
PB91-187724
100,568 PC A03/MF A01

Validation Testing Laboratory User's Guide. National PDES Testbed Report Series.

PB92-123090

$100,971 \mathrm{PC}$ A06/MF A02

VAMAS Bulletin

101,192 Not available NTIS

Van der Waals Complexes in 1,3-Dipolar Cycloaddition Reactions: Ozone-Ethylene.

100,417 Not available NTIS

\section{sign Guide.}

Variable Air Vol
PB91-216655

100,077 PC A03/MF A01

Variations in the Practice of Ceramic Technology in Different Cultures: A Comparison of Korean and Chinese Celadon Glazes.

101,111 Not available NTIS

Velocity and Droplet Size Measurments in a Fuel Spray,
PB91-147934

Ventilation System Performance Evaluation Using Tracer Gas Techniques.
PB91-147876

100,060 Not available NTIS

Verification of the Relation between Two-Probe and FourProbe Resistances as Measured on Silicon Wafers. PB91-147520 100,788 Not available NTIS

Vibrational and Electronic Energy Levels of Polyatomic Transient Molecules. Supplement PB91-192575

100,367 Not available NTIS

Vibrational, Rotational, and Tunneling Dependence of Vi-

brational Predissociation in the HF Dimer.
PB91-203844 100,406 Not available NTIS

Vibrational Spectra of Molecular Ions Isolated in Solid Neon. III. $\mathrm{N} 4(+)$

100,243 PC A02/MF A01

Vibrational Spectra of Molecular Ions Isolated in Solid

Neon. IV.NO( + ), NO(-), ONNO( + ), aNd ONNO(-).
AD-A230 957/3
100,244 PC A03/MF A01

Vibrational Spectra of Molecular Ions Isolated in Solid Neon. 4. NO(1+), NO(1-), ONNO(1+), and ONNO(1-) PB91-149914 100,307 Not available NTIS

Vibrational Spectra of Molecular lons Isolated in Solid

Neon. 5. N2O $(1+)$ and NNO2(1-).
PB91-149906 100,306 Not available NTIS

Vibrational Spectra of Molecular Ions Isolated in Solid Neon. 6. CO4(-)

100,249 Not available NTIS

Vibrational Spectra of Molecular Ions Isolated in Solid Neon. 6. $\mathrm{CO} 4(1-)$

100,419 Not available NTIS

Vibrational Spectra of Molecular Ions Isolated in Solid

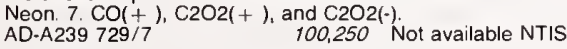
Video Compression for Remote Vehicle Driving PB91-203208 101,035 Not available NTIS Video Processing with the Princeton Engine at NIST. PB92-108950 100,604 PC A04/MF A01

Visco-Elastic Deformation of Dental Porcelaın and Porcelain-Metal Compatibility.

100,042 Not available NTIS

Viscoelastic Response of Epoxy Glasses Subjected to Dif. ferent Thermal Treatments.

PB91-189720

101,245 Not available NTIS

Viscosity and Thermal Conductivity of Pure Monatomic Gases from Their Normal Boiling Point Up to $5000 \mathrm{~K}$ in the Limit of Zero Density and at $0.101325 \mathrm{MPa}$.

PB91-192559 100,366 Not available NTIS

Viscosity Surface for Mixtures of Methane and Ethane,

PB91-174763 100,322 Not available NTIS

Visibility of Chrysotile Asbestos in the Scanning Electron Microscope.
PB91-204099

101,320 Not available NTIS

Visibility of Exit Directional Indicators

PB91-184762

$101,000 \mathrm{PC} \mathrm{A03/MF} \mathrm{A01}$

Visibility of Exit Signs and Directional Indicators.

PB91-161984 100,083 Not available NTIS

Visualization of Nucleate Flow Boiling for an R22/R114 Mixture and Its Components. PB91-162164

100,225 Not available NTIS

VolksGrapher: a FORTRAN Plotting Package User's Guide, Version 3.0 .

PB91-185108

100,647 PC A04/MF A01
Voltage Calibration Systems Using Josephson Junction Arrays

PB92-116516

100,868 Not available NTIS

Water Spray Suppression of Fully-Developed Wood Crib Fires in a Compartment.

PB91-203620

100,159 Not available NTIS

Wavelengths and Energy Level Classifications for the Spectra of Aluminum (AI I through AI XIII).

PB92-148121 100,472 (Order as PB92-148113)

Wavelengths and Energy Level Classifications of Magnesium Spectra for All Stages of lonization ( $\mathrm{Mg}$ I through $\mathrm{Mg}$

PB92-110139

100,442 (Order as PB92-110113)

Wawayandaite, a New Calcium Manganese Beryllium Boron Silicate from Franklin, New Jersey.

PB91-174680

101,359 Not available NTIS

Wetting Layers on Solid Substrates.

PB91-158857 100,313 Not available NTIS

Wigner-Seitz Local-Environment Study of the High Tc Superconductors

101,528 Not available NTIS

Wind and Seismic Effects. Proceedings of the Joint Meeting of the U.S.-Japan Cooperative Program in Natural Resources Panel on Wind and Seismic Effects (23rd). Held in Tsukuba, Japan, on May 14-17,

PB92-116425

100,152 PC A99/MF A06

Wind Effects on Fires.

PB91-203851

100,549 Not available NTIS

Working Group 1: Atomic Spectra and Wavelength Standards.

PB91-158956

100,026 Not available NTIS

Working Group 2: Atomic Transition Probabilities PB91-175588

101,645 Not available NTIS

Working Implementation Agreements for Open Systems Interconnection Protocols

PB91-144444

100686 PC A99/MF A04

PB92-126523

100,698 PC A17/MF A04

Workloads, Observables, Benchmarks and Instrumentation.

$X$-ray Interferometry and gamma-ray Wavelengths. PB91-158642

101,484 Not available NTIS

X-ray Photoelectron Forward Scatterıng Studies of Surface Segregation in Epitaxial Cu-Ni-Ni(100) Sandwich Structures. PB91-189258 101,506 Not available NTIS

XANES of Transition Metal Zinc-Blende Semiconductors PB92-117092 100,462 Not available NTIS

XPS and Auger Forward Scattering in Epitaxial Films.

PB91.174698

YBa2Cu3O7-delta/Insulator Multi-Layers for Crossover Fab-

PB91-194662

101,518 Not available NTIS

ZIP: The ZIP-Code Insulation Program, Version 2.0. Economic Insulation Levels for New and Existing Houses by Three-Digit Zip Code. Users' Guide and Reference Manual (Revised Edition).

PB91-167155

100,064 PC A03/MF A01

Zonal Model for Corona Discharge-Induced Oxidation of $\mathrm{SF} 6$ in $\mathrm{SF} 6 / \mathrm{O} 2 / \mathrm{H} 2 \mathrm{O}$ Gas Mixtures. PB91-158790

100,312 Not available NTIS 



\section{NTIS ORDER/REPORT NUMBER INDEX}

\section{SAMPLE ENTRY}

\section{NISTIR-4523}

Proposed Standard Practice for Assessing the Performance of Gas-Phase Air Cieaning Equipment

PB91-167353 100,905 PC A03/MF A01

\section{PB91-167353}

Proposed Standard Practice for Assessing the Performance of Gas-Phase Alr Cleaning Equipment PB91-167353
PC A03/MF A01

\section{Report or serles number}

Titie

NTIS order number

Abstract number

Availability Prlce Code

\section{Report or serles number}

Title

NTIS order number
Abstract number
Availability Prlce code
AD-A207 806/1

Annual Report to the Strategic Defense Initiative Organization on the Free-Electron Laser Driven by the NIST CW Microtron.

AD-A207 806/1

AD-A227 296/1

Detection of Excited States by Laser-Induced Fluorescence and Analysis of Energy Transfer.

AD-A227 296/1

101,398 PC A06/MF A01

AD-A227 310/0

NIST-NRL Free-Electron Laser.
AD-A227 $310 / 0$

AD-A227 656/6

Liquid and Solid lon Plasmas.

AD-A227 656/6

AD.A227 $806 / 7$

Cooled Ion Frequency Standard.

AD-A227 806/7

AD-A227 836/4

Photomultiplier Housing for Vacuum Operation of Side-on 1P28-Type Tubes.

AD-A227 868/7

Laser Photolysis of Trimethylgallium at $193 \mathrm{~nm}$ : Quantum Yields for Methyl Radical and Ethane Production.

AD-A227 868/7

100,234 PC A02/MF A01
AD-A227 869/5

Scattered Light and Other Corrections in Absorption Coefticient Measurements in the Vacuum Ultraviolet: A Systems Approach.

AD-A228 082/4

Vibrational Spectra of Molecular Ions Isolated in Solid Neon. III. $\mathrm{N} 4(+)$

AD-A228 082/4

AD-A229 229/0

100,243 PC A02/MF A01

Aluminum Alloys for Cryogenic Tanks: Oxygen Compatibility. Volume 1.

AD-Á229 231/6

101,201 PC A10/MF A02

Review of Cryogenic Mechanical and Thermal Properties of Al-Li Alloys and Alloy 2219. AD-A229 231/6

101,202 PC A09/MF A02 AD-A230 438/4

Summary of Experiments with the Separated Aperture Technique of Dielectric Anomaly Detection. AD-A230 957/3

Vibrational Spectra of Molecular lons Isolated in Solid Neon. IV.NO(+), NO(-), ONNO(+), aNd ONNO(-)

ADA230 957/3, NO(-), ONNO(+), aNd ON A03/MF A01 AD-A231 778/2

Influence of Water on the Mechanical Properties of a Glass-Epoxy Matrix Composite.
AD-A231 778/2

AD-A231 818/6

Influence of Filament Geometry on Hot Filament Growth of Diamond Films.

AD-A231 818/

AD-A231 830/1

101,448 PC A02/MF A01

Aluminum Alloys for ALS Cryogenic Tanks: Oxygen Com. patibility. Volume 2

A231 $830 / 1$

$101,203 \mathrm{PC} \mathrm{A07/MF} \mathrm{A01}$ AD-A233 961/2

Ada Compiler Validation Summary Report. Certificate Number: 901109S1.11053, Digital Equipment Corporation VAX Ada, Version 2.2 VAX $8800=>$ VAX 8800

AD-A233 961/2 100,607 PC A04/MF A01 AD-A234043/8

Production and Spectroscopy of Small Polyatomic Molecular lons Isolated in Solid Neon.

AD.A234 043/8

100,245 PC A03/MF A01 -A234083/4

Ada Compiler Validation Summary Report: DDC International A/S, DACS VAX/VMS to 80186 Bare Ada Cross Compital A System, Version 4.6 VAX 8530 (Host) to Bare Board iSBC 186/93A (Host), 901129S1 11079 .

AD-A234 118/8 Ada Compiler Validation Summary Report: Certificate
Number: 90112951.11112 DDC International A/S DACS 
80386 DMS/OS Ada Compiler System, Version 4.6 IBM PS/2 Model $80-311=>$ IBM PS/2 Model 80.311 AD-A234 $118 / 8$ 100,609 PC A06/MF A01 AD-A234 323/4

Ada Compiler Validation Summary Report: Certificate Number: 901129S1.11050 DDC International A/S DACS VAX/VMS Native Ada Compiler System, Version 4.6 VAX $8530=>\operatorname{VAX} 8530$

AD-A234 $323 / 4$

100,610 PC A04/MF A01

AD-A234 350/7

Ada Compiler Validation Summary Report: Digital Equipment Corporation, VAX Ada, Version 2.2, BAX 8800 (Host) $t$ 901109S1.11054.

AD-A234 380/4

100,611 PC A04/MF A01

Ada Compiler Validation Summary Report: Certificate Number: 901129S1.11051, DDC International A/S, DACS VAX/VMS to 68020 Bare Cross Compiler System, Version 4.6 MicrovAX 3100 Greater Than or Equal to Motorola MVME133.

AD-A234 $380 / 4$

AD-A234 438/0

100,612 PC A03/MF A01

Ada Compiler Validation Summary Report: Certificate NAX/Y VAX VMS to 80186 Bare Ada Cross Compiler System with Bare Board iSBC/03A.

AD-A234 $439 / 8$

100,613 PC A06/MF A01

Ada Compiler Validation Summary Report: Certificate Number: 901129S1.11075 DDC International A/S DACS 80386 UNIX V Ada Compiler System, Version 4.6 ICL DRS300 $=>$ ICL DRS 300 .

AD-A234 529/6

100,614 PC A04/MF A01

Ada Compiler Validation Summary Report: Certificate VAX VMS 6080386 PM Bare Ada Cross Compiler System

Version 4.6 VAX $8530=>$ Bare Board iSBC $386 / 21$.

100,615 PC A06/MF A01

AD-A235 490/0

Thermodynamic and Kinetic Stability of Refractory Materials at Ultra-High Temperatures.

AD-A235 490/0

100,246 PC A03/MF A01

AD-A236 485/9

Spatially and Spectrally Resolved Cathodoluminescence of Hot-Filament Chemical-Vapor-Deposited Diamond Particles.
AD-A236 485/9 100,247 PC A03/MF A01

AD-A236 708/4

Effects of Multiple Filament Geometry in the Hot Filament Deposition of Diamond Films.

AD-A236 74614

$101,142 \mathrm{PC} \mathrm{A02/MF} \mathrm{A01}$

Critical Assessment of Optical Properties of CVD Diamond Films

AD-A236 746/4

101,400 PC A02/MF A01

AD-A237 128/4

Spatially and Spectrally Resolved Cathodoluminescence of

Hot-Filament Chemical-Vapor-Deposited Diamond Particles.
AD-A237 128/4 100,248 PC A03/MF A01 AD-A237 $128 / 4$

Vibrational Spectra of Molecular lons Isolated in Solid Neon. 6. $\mathrm{CO} 4(-)$

AD-A238 415/

AD-A239 509/3

100,249 Not available NTIS

Influence of Moisture and Pressure on the Mechanical Properties of a Glass-Epoxy Matrix Composite and a Graphite-Epoxy Matrix Composite.

AD-A239 509/3 101,114 PC A03/MF A01 $A D-A 2397$

Ada Compiler Validation Summary Report. Certificate Number: 910510 S1.11161 UNiSYS Corporation, UCS Ada, Version 1R1, 2200/600 (Host and Target). Revision.
AD-A239 715/6 100,616 PC A06/MF A02 AD-A239 729/7

Vibrational Spectra of Molecular Ions Isolated in Solid Neon. 7. $\mathrm{CO}(+), \mathrm{C}_{2} \mathrm{O} 2(+)$, and $\mathrm{C} 2 \mathrm{O} 2(-)$. AD-A239 $729 / 7$

AD-A240 511/6

Ada Compiler Validation Summary Report: Certificate Number: $910626 S 1.11179$ U.S. Navy Ada/M, Version 4.0 (OPTIMIZE) VAX $11 / 785=>$ AN/AYK-14 (Bare Board).
AD-A240 511/6 DD-A240 512/4

Ada Compiler Validation Summary Report: Certificate Number 010705 1.11192 InterAct Corporation InterAct Ada Mips Cross-Compiler System, Release 2.0 MicroVAX 3100 Cluster $=>$ Lockheed Sanders STAR MVP R3000/ A3010 board (Bare Machine).

AD-A240 610/6

Ada Compiler Validation Summary Report: Certificate Number: 910502S1.11158 DDC International A/S, DACS VAX/VMS to 80860 Bare Ada Cross Compiler System, Version 4.6.1, VAX 8530 (Host) to Tadpole Technology PLC TP860M (Bare Board) (Target).

AD-A240 610/6

Ada Compiler Validation Summary Report: Certificate
Number: $910626 S 1.11176$ U.S. Navy, Ada/L, Version 4.0 (/
Optimize), VAX 11/785 (Host) to AN/UYJ-43 (Single CPU) (Bare Board) (Target)

AD-A240 612/2

Ada Compiler Validation Summary Report: Certificate Number: $910626 S 1.11174$ U.S. Navy, Ada/M, Version 4.0 (/Optimize), VAX 8550, Running VAX/VMS Version 5.3 Host) to AN/UYK-44 (EMR) (Bare Board) (Target).

AD-A240 612/2

AD-A240 613/0

Ada Compiler Validation Summary Report: Certificate Number: 910626 S1.11175 U.S. Navy Ada/M, Version 4.0 Optimize), VAX 8550 (Host) to AN/AYK-14 (Bare Board) (Target).

AD-A240 762/5

Ada Compiler Vałidation Summary Report. Certificate Number: 910705S1.11191, InterACT Corporation InterACT Ada 1750A Compiler System, Release 3.5, MicroVAX 3100 Cluster $=>$ InterACT MIL.STD.1750A Instruction Set Architecture Simulator, Release 23 (Bare Machine) AD-A240 $762 / 5$

AD-A240 763/3

Ada Compiler Validation Summary Report. Certificate Number: $910626 S 1.11178$, U.S. Navy Ada/M, Version 4. /OPTIMIZE) VAX $11 / 785=$ AN/UYK-44 (EMR) (Bare Board).

100,624 PC A05/MF A01

\section{AD-A240 783/1}

Ada Compiler Validation Summary Report: Certificate Optimize) VAX 55511173 U.S. Navy Ada/L, Version 4.0

Optimize) VAX $855=$ AN/UYK-43 (EMR) (Bare Board)
AD-A240 783/1
100,625 PC A05/MF A0 1

AD-A240 7841

Ada Compiler Validation Summary Report: Certificate Number Number 910626 S1.11172 U.S. Navy Ada/L, Version 4.0 (/Optimize) VAX $8550=$ AN/UYK-43 (Single CPU) (Bare Board)
AD-A2 $40784 / 9$

AD-A240 784/9
AD-A240 785/6

100,626 PC A05/MF A01

Ada Compiler Validation Summary Report: Certificate Number: 910502S1.11159 DDC International A/S DACS Sun-3/SunOS to 68030 Bare Ada Cross Compiler System, Version 4.6.4. MRI IEEE 695 (BASIC-MODE) Sun-3/50= Version 4.6.4. MRI IEEE 695 (BASIC-MODE)

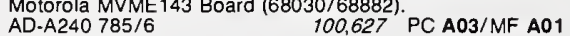
AD-A240 849/0

Ada Compiler Validation Summary Report. Certificate Number: 910502S1.11160 DDC International A/S DACS Sun-3/SunOS to 68030 Bare Ada Cross Compiler System, Version 4.6.4, MRI IEEE 695 (SECURE MODE) Sun-3/50 $=>$ Motorola MVME143 Board $(68030 / 68882)$. AD-A240 849/0

$100,628 \mathrm{PC} \mathrm{A03/MF} \mathrm{A01}$

Ada Compiler Validation Summary Report. Certificate Number: 910626S1.11177 U.S. NAVY Ada/L, Version 4.0
(/OPTIMIZE) VAX $11 / 785=>$ AN/UYK-43 (EMR) (Bare (/OPTIM

AD-A240 850/8

00,629 PC A05/MF A01

AD-A242 029/7

Liquid and Solid lon Plasmas.
AD-A242 029/7

AD-A242 030/5

Cooled Ion Frequency Standard.

AD-A242 030/5

AD-A242 268/1

Ada Compiler Validation Summary Report: Certificate Number: 91051751.11163 U.S. Navy, AdaVAX, Version 5.0, (NO OPTIMIZE) VAX 8350 (Host and Target) AD-A242 268/1

AD-A242 269/9

Ada Compiler Validation Summary Report: Certificat Number: 910517 S1.11162 U.S. Navy, AdaVAX, Version 5.0 /OPTIMIZE) VAX 8600 (Host and Target).

AD-A242 269/9

AD-A242 270/7

Ada Compiler Validation Summary Report: Certificate Number: 91051751.11165 U.S. Navy, AdaVAX, Version 5.0 (NO-OPTIMIZE), VAX 11/785 (Host and Target).

AD-A 4

Ada Compiler Validation Summary Report: Certificate Number: 910517 S1.11164 U.S. Navy, AdaVAX, Version 5.0 (/OPTIMIZE), VAX11/785 (Host and Target).

AD-A242 273/

AD-A242 609/6

Ada Compiler Validation Summary Report: Certificate Number 910918S1.11216, NEC Corporation, NEC Ada Compiler System for EWS-UX $N$ (Rel 4.0), Version Release 2.1 (4.6) EWS $4800 / 220=$ EWS4800/220 AD-A242 609/

100,634 PC A05/MF A01 AD-A242 816/7

Short-Duration Autoignition Temperature Measurements fo Hydrocarbon Fuels
AD-A242 $816 / 7$

\section{AD-A242 896/9}

100,877 PC A03/MF A01

Ada Compiler Validation Summary Report. Certificate Number: 911025S1.11226, Digital Equipment Corporation DEC Ada, Version 1.0 DECstation 5000 Model $200=>$ $5000 \mathrm{Model} 200$

$100,635 \mathrm{PC} \mathrm{A04/MF} \mathrm{A01}$

AD-A242 956/1

Aluminum Alioys for ALS Cryogenic Tanks: Comparative Measurements of Cryogenic Mechanical Properties of Al-Li Alloys and Alloy 2219.

AD-A242 956/

101,204 PC A07/MF A02

AD-A243 049/4

Strength and Microstructure of Ceramic

AD-A243 049/4

101,056 PC A08/MF A02

AD-A243 093/2

High Resolution Electron Microscopy of Diamond Film nd their interac

AD-A243 093/2

101,449 PC A03/MF A01

AD-A243 094/0

Determination of the Optical Constants of Thin Chemical. Vapor-Deposited Diamond Windows from 0.5 to $6.5 \mathrm{eV}$.
AD-A243 094/0 100,215 PC A03/MF A0

AD-A243 095/7

Chemical Vapor Deposited Diamond.

AD-A243 095/7

101,106 PC A03/MF A01

AD-A243 096/5

Transmission Electron Microscopy of the CVD Diamond Film/ Substrate Interface.

AD-A243 096/5

101,450 PC A03/MF A01

AD-A243 097/3

Use of Diamond as an Optical Material.

AD.A243 097/3

101,401 PC A03/MF A01

AD-A243 170/8

CID Spectra of Sele
AD-A243 $170 / 8$

AD-A243 220/1

Spatially and Spectrally Resolved Cathodoluminescence Measurements of CVD-Grown Diamond Particles and Films.

AD-A243 220/

$101,451 \mathrm{PC} \mathrm{A03/MF} \mathrm{A01}$

AFESC/ESL-TR-88-71

CID Spectra of Selected Target Molecules.

AD-A243 170/8

100,180 PC A04/MF A01

AFESC/ESL-TR-90-43

Short-Duration Autoignition Temperature Measurements for Hydrocarbon Fuels.
AD-A242 816/7

AFOSR-TR-91-0447

$100,877 \mathrm{PC} \mathrm{A03/MF} \mathrm{A01}$

Thermodynamic and Kinetic Stability of Refractory Materials at Ultra-High Temperatures.

AD-A235.

100,246 PC A03/MF A0

AFOSR-TR-91-0892

Strength and Microstructure of Ceramics.

AD-A243 049/4

101,056 PC A08/MF A02

A-TR-90-063-VOL-1

Aluminum Alloys for Cryogenic Tanks: Oxygen Compatibility. Volume 1.

AL-TR-90-064

101,201 PC A10/MF A02

Review of Cryogenic Mechanical and Thermal Properties of Al-Li Alloys and Alloy 2219

$101,202 \mathrm{PC} \mathrm{A09/MF} \mathrm{A02}$

AL-TR-90-0630-VOL-2

Aluminum Alloys for ALS Cryogenic Tanks: Oxygen Compatibility. Volume 2

ARO-25664.2

101,203 PC A07/MF A0

Production and Spectroscopy of Small Polyatomic Molecular lons Isolated in Solid Neon

AD-A234 043/8

100.245 PC A03/MF AO

ARO-25664.4-CH

Vibrational Spectra of Molecular Ions Isolated in Solid Neon. III. $\mathrm{N} 4(+)$
AD-A228 $082 / 4$

ARO-25664.7-CH

$100,243 \mathrm{PC} \mathrm{A02/MF} \mathrm{A0}$

Vibrational Spectra of Molecular lons Isolated in Solid Neon. IV.NO(+) $)$, NO(-), ONNO(+), aNd ONNO(-).
AD-A230 957/3
100,244 PC A03/MF A01

ARO-25664.9-CH

Vibrational Spectra of Molecular Ions Isolated in Solid Neon. 6. $\mathrm{CO} 4(-)$

ARO-25664.10-CH 
AD-A233 $961 / 2$

CONF-890701-30

Integrity tests for high-(Tc) and conventional critical-current measurements systems.

DE91016275

CONF-890701-31

101,452 PC A03/MF A01

Guideline for $\mathrm{Nb}$ (sub 3) $\mathrm{Sn}$ critical current measurements sing fiberglass-epoxy composite sample mandrels. DE91016274

101,205 PC A03/MF A01 DE91002169

Scanning tunneling microscopy studies of the surfaces of a$\mathrm{Si}: \mathrm{H}$ and a-SiGe:H films. Annual report, 1 December 1989 . 31 January 1991

$100,785 \mathrm{PC} \mathrm{A03/MF} \mathrm{A01}$

DE91007688

Advanced sensor development program for the pulp and paper industry. Final report

DE9100768

101,248 PC A05/MF A01

DE91012660

Integrated theoretical and experimental study of the thermophysical properties of fluid mixtures. Progress report (February 1990-February 1991)

DE91012660

DE9101627

$101,384 \mathrm{PC} \mathrm{A03/MF} \mathrm{A01}$

Guideline for $\mathrm{Nb}(\mathrm{sub} 3$ ) $\mathrm{Sn}$ critical current measurements using fiberglass-epoxy composite sample mandrels.

DE91016275

101,205 PC A03/MF A01

Integrity tests for high-(Tc) and conventional critical-current measurements systems.

DE91016275

101,452 PC A03/MF A01

DE91018580

Fundamental studies of black liquor combustion. Report No. 4, Phases, 2, 3, and 4: Final report, December 1987-December 1989.

DE91018580

DOE/CE/40637-T9

100,915 PC A09/MF A02

Fundamental studies of black liquor combustion. Report No. 4, Phases, 2, 3, and 4: Final report, December 1987-December 1989

91018580

$100,915 \mathrm{PC}$ A09/MF A02

DOE/CE/40748-T

Advanced sensor development program for the pulp and

paper industry. Final report.

101,248 PC A05/MF A01

DOE/ER/13992-T1

Integrated theoretical and experimental study of the thermophysical properties of fluid mixtures. Progress report (February 1990-February 1991)

DE9101266

101,384 PC A03/MF A01

FIPS PUB 9-1

Congressional Districts of the United States. Category: Federal General Data Standard, Representations and Codes.

FIPS PUB 9.1

FIPS PUB 11-3

Guideline: American National Dictionary for Information

Systems. Category: Software; Subcategory: Documentation.

FIPS PUB 11 -

FIPS PUB 54-1

Computer Output Microform (COM) Formats and Reduction

Ratios, $16 \mathrm{MM}$ and $105 \mathrm{MM}$. Category: Hardware Standard;

Subcategory: Media.

PUB 54-1

100,678 PC E18

Interface between Data Terminal Equipment (DTE) and Data Circuit-Terminating Equipment (DCE) for Operation with Packet-Switched Data Networks (PSDN), or between Two DTEs, by Dedicated Circuit. Category: Hardware Standard. Subcategory: Data Transmission

FIPS PUB 100-1

$100,672 \mathrm{PC} \mathrm{A03}$

IPS PUB 120-1

Graphical Kernel System (GKS); Category: Software Standard; Subcategory: Graphics.

FIPS PUB $120-$

100,673 PC E15

FIPS PUB 120-1A

Graphical Kernel System (GKS). Pascal Binding; Category: Soltware Standard; Subcategory: Graphics.

FIPS PUB 120

Graphical Kernel System (GKS). Ada Binding; Category: Software Standard; Subcategory: Graphics.

FIPS PUB 120-1B

100,674 PCE12

UB 146-1

Government Open Systems Interconnection Profile (GOSIP); Category: Hardware and Software Standards; Subcategory: Computer Network Protocols.

FIPS PUB 159

Detail Specification for 62.5-mum Core Diameter/125-mum Cladding Diameter Class la Multimode, Graded-Index Optical Waveguide Fibers.
FIPS PUB 159

FIPS PUB 160

100,562 PC E07

American National Standard for C. Category: Software Standard. Subcategory: Programming Language.
FIPS PUB 160 FIPS PUB 161

Electronic Data Interchange (EDI). Category: Software Standard. Subcategory: Electronic Data Interchange.
FIPS PUB 161

FIPS SET 1991

Federal Information Processing Standards Publication

FIPS SET 1991

N91-20208/5

High T(sub C) Superconductors: Are They Magnetic N91-20208/5

(Order as N91-20207/7, PC A05/MF A01)

N91-20218/4

Low Carbon Steel: Metallurgical Structure vs. Mechanical Properties.

N91-20218/4

(Order as N91-20207/7, PC A05/MF A01)

N91-20219/2

Kelvin Water-Drop Experiment.

N91-20219/2

(Order as N91-20207/7, PC A05/MF A01)

N91-21347/0

Dynamic Technique for Measuring Surface Tension at High Temperatures in Microgravity Environment.

N91-21347/0

101,206
(Order as N91-21331/4, PC A24/MF A03)

\section{N91-21361/1}

High-Speed Spatial (Linear) Scanning Pyrometer: A Tool for Diagnostics, Temperature Mappin

nations at High Temperatures.

(Order as N91-21331/4, PC A24/MF A03)

N91-25760/0

Trapped-lon Frequency Standards.

N91-25760/0

(Order as N91-25755/0, PC A99/MF A04)

N91-25766/7

GPS Time Transfer with Implementation of Selective Avail-

N91-25766/7

100,576

\section{N91-25793/1}

Synchronizing Computer Clocks Using a Local Area Net-

Nork. $25793 / 1$

(Order as N91-25755/0, PC 100,596

N91-25797/2

N91-25797/2 100, 778

NASA-CR-187115

Material Flammability Test Assessment for Space Station Freedom.

NIST/DF/VD-91/003

DARPA Air Travel Information System (ATISO) Speaker-Dependent Training Data (on CD-ROM).

PB91-505370

100,594 CD-ROM $\$ 1200.00$

NIST/DF/VD-91/015

Road Rally Conversational Speech Corpora (on CD-ROM). PB91.509802

NIST/GCR-90/581

Investigation of Simulated Oil-Well Blowout Fires.

PB91.144337

101,356 PC A05/MF A01

NIST/GCR-90/582

Ceiling Jet in Fires.

PB91-167197

NIST/GCR-90/584

Heat Flux, Mass Loss Rate and Upward Flame Spread for Burning Vertical Walls (1990)

NIST/GCR-90/585

Species Produced in Fires Burning in Two-Layered and Homogeneous Vitiated Environments.

PB91-157180

NIST/GCR-90/586

Fire Propagation in Concurrent Flows.

PB91-157206

NIST/GCR-91/589 sion 3

$91-206664$

IST/ĠCR-91/590

Sprinkler/Hot Layer Interactio

PB91-206680

NIST/GCR-91/591

Estimated Impact of the Center for Fire Research Program on the Costs of Fire.

PBT1-21

First Pass at Computing the Cost of Fire Safety in a Modern Society.

PB91-216796

NIST/GCR-91/593-1

101,730 PC A04/MF A01

Opportunities for Innovation: Chemical and Biological Sen-

sors.

100,214 Not available NTIS

NIST/GCR-91/600

Development of a Technique to Assess the Adequacy of the Municipal Water Supply for a Residential Sprinkter System

PB92-123116

101,358 PC A05/MF A01

NIST/GCR-91-595

Theoretical Investigation of Piloted Ignition of Wood

PB92-112432

100,556 PCA11/MF A03

NIST/GCR-91/596

Users' Guide to BREAK1, the Berkeley Algorithm for Breaking Window Glass in a Compartment Fire.

PB92-112556

$100,136 \mathrm{PC}$ A03/MF A01 NIST/GCR-91/597

Turbulent Upward Flame Spread for Burning Vertical Walls Made of Finite Thickness.

PB92-112531

100,170 PC A05/MF A01

NIST/HB-44-1992 ED

Specifications, Tolerances, and Other Technical Requirements for Weighing and Measuring Devices as Adopted by the 76th National Conference on Weights and Measures, 1991.

101,023 PC A 10/MF A03

NIST/HB-130-1992 ED

Uniforms Laws and Regulations as Adopted by the National Conference on Weights and Measures (76th) 1991

PB92-112416

101,022 PC A08/MF A02

NIST/HB-133-ED-3-SUPPL 2

Checking the Net Contents of Packaged Goods. Third Edition, Supplement 2

NIST/HB-147

LBIR Facility User Handbook

PB92-102185
NIST/MONO-176

100,171 PC A03/MF A01

101,435 Not available NTIS

Modified Airy Function and WKB Solutions to the Wave

Equation.

101,440 PC A08/MF A02

IST/SP-250/36

NIST Measurement
rent Transformers.

PB91-216770

NIST/SP-250/40

Absorbed-Dose Calibration of Ionization Chambers in a(60)Co Gamma-Ray Beam

PB91-132290

101,598 PC A04/MF A01

NTP-250-91-ED

NIST Calibration Services Users Guide, 1991

PB92-126416

100,936 PC A 10/MF A03

NIST/SP-260/114

Standard Reterence Materials: Bright-Chromium Linewidth

Standard, SRM 476, for Calibration of Optical Microscope Linewidth Measuring Systems.

PB91-167163

NIST/SP-260-115

Standard Reference Materials: Calibration of NIST Standard Reference Material 3201 for 0.5 Inch $(12.65 \mathrm{~mm})$ Serial Serpentine Magnetic Tape Cartridge.

PB91-187542

00,690 PC A03/MF A01

NIST/SP-304-91-ED

Modernized Metric System (Chart)

PB92-163864

100,939 Not available NTIS

NIST/SP-304A-91-ED

Brief History of Measurement Systems with a Chart of the Modernized Metric System.

92.163872

100,940 Not available NTIS

NIST/SP-305-SUPPL-22

Publications of the National Institute of Standards and Technology, 1990 Catalog.

PB91-216531

100,011 PC A20/MF A04

ST/SP-330

International System of Units (SI)--Translation

PB92-109032

101,719 PC A04/MF A01

NIST/SP-365-91-ED 


\section{NTIS ORDER/REPORT NUMBER INDEX}

NIST/SP-500/183SUPPL1

Stable Implementation Agreements for Open Systems Interconnection Protocols. Version 4, Edition 1, March 1991 Change Pages (Supplement)

100,691 PC A11/MF A02

NIST/SP-500/184

Functional Benchmarks for Fourth Generation Languages. PB91-167387

NIST/SP-500/185

Guide to Design, Implementation and Management of Distributed Database

PB91-187567

100,648 PC A04/MF A01

NIST/SP-500/186

Issues in Transparent File Access.

PB91-187831

100,569 PC A05/MF A01

NIST/SP-500/187

Application Portability Profile (APP): The U.S. Government's Open System Environment Profile OSE/1 Version 1.0

PB91-201004 100,692 PC A05/MF A01

NIST/SP-500/18

Guide to Expert System Building Tools for Microcomputers. PB92-108893 100,715 PC A08/MF A02

NIST/SP-500/189

Security in ISDN.

PB92-116391

NIST/SP-500/190

100,718 PC A05/MF A01

Proceedings of the Workshop on High Integrity Software. Held in Gauthersburg, MD. on January 22-23, 1991

PB92-109040

100.667 PC A03/MF A01

NIST/SP-500/191

Test Methods for Optical Disk Media Characteristics (for $356 \mathrm{~mm}$ Ruggedized Magneto-op tic Media)

PB92-116409

100,605 PC A05/MF A01 NIST/SP-500/192

Government Open Systems Interconnection Profile Users' Guide, Version 2 .

PB92-119676

NIST/SP.500/193

100,696 PC A08/MF A02

Software Reengıneering: A Case Study and Lessons Learned.

$100,669 \mathrm{PC} \mathrm{A03/MF} \mathrm{A01}$

ISDN Conformance Testing: Layer 1. Physical Layer. Part 1. Basic Rate S/T Interface, User Side.
PB92-102201

NIST/SP-500/195

North American ISDN Users' Forum Agreements on Inte-

grated Services Digital Network
PB92-102219

100,573 PC A08/MF A02

NIST/SP-500/196

Guidelines for the Evaluation of File Transfer, Access and Management Implementations.
PB92-126580 100,699 PC A05/MF A01 NIST/SP-500/197

Guide to Schema and Schema Extensibility.
PB92-126556 NIST/SP- 500

Monitoring and Reporting Techniques for Error Rate and Error Distribution in Optical Disk Systems.

PB92-123124 100,606 PC A05/MF A01
A

NIST/SP-500/199

3480 Type Tape Cartridge: Potential Data Storage Risks, and Care and Handling Procedures to Minimize Risks.

PB92-126549 101.381 PC A04/MF A01

NIST/SP-730

NBS-INA. The Institute for Numerical Analysis. UCLA 19471954

PB92-112408

101,264 PC A09/MF A02

NIST/SP-777

NIST Serial Holdings, 1991

PB91-171330

$100,960 \mathrm{PC} \mathrm{A12/MF} \mathrm{A02}$

NIST/SP-782

NIST Standard Reference Data Products 1991 Catalog

PB91-167312

100,952 PC A04/MF A01

NIST/SP-790-VOL-

National Bureau of Standards Publications 1977-1987. Volume 1. Citations, Key Words, and Abstracts.
PB91-136507

0.957 PC A99/MF A99

NIST/SP-790-VOL-2

National Bureau of Standards Publications 1977-1987. Volume 2. Indexes.

PB91-136515

100,958 PC A99/MF A99

NIST/SP-792

Technical Digest-Symposium on Optical Fiber Measurements, 1990.

NIST/SP-798

101,403 PC A10/MF A02

Users Guide for RAPID, Version 2.3.
PB91-167403 100,999 PC A06/MF A01

NIST/SP-800/1

Bibliography of Selected Computer Security Publications,
January 1980-October 1989

PB91-148486

100,704 PC A09/MF A02

NIST/SP-800/2

Public-Key Cryptography. Computer Security.
PB91-187864 100,708 PC A08/MF A01

NIST/SP-800/3

Establishing a Computer Security Incident Response Capability (CSIRC)

NIST/SP-801

100,720 PC A03/MF A01

Laser Induced Damage in Optical Materials: 1989 PB91-148478

NIST/SP-802

101,409 PC A99/MF A04

Effect of a Free Surface on Stress Distribution in a Bimaterial Composite.
PB91-185116

NIST/SP-803

101,121 PC A09/MF A01

Abstract and Index Collection in the Research Information Center of the National Institute of Standards and Technology (Fourth Edition)

NIST/SP-804

$100,959 \mathrm{PC} \mathrm{A03/MF} \mathrm{A01}$

Chemistry of Electronic Ceramic Materials.

PB91-178822

NIST/SP-805

Uncertainty and Accuracy in Physical Measurements PB91-167205

NIST/SP-806

Standards Activities of Organizations in the United States

PB91-177774

NIST/SP-807

U.S. Assessment of the New Diamond Technology in PB91-1

NIST/SP-808

101, 107 PC A05/MF A01

Directory of Federal Government Laboratory Accreditation/ Designation Programs.

100,005 PC A06/MF A01

NIST/SP-809

NIST Research Reports, March 1991

PB91-187559

$100,007 \mathrm{PC} \mathrm{A03/MF} \mathrm{A0}$

IST/SP-810

Directory of Accredited Laboratories, 1991. National Voluntary Laboratory Accreditation Program (NVLAP)

NIST/SP-811

100,934 PC A08/MF A02

Guide for the Use of the International System of Units: The Modernized Metric System.

PB92-116383

$100,962 \mathrm{PC} \mathrm{A03/MF} \mathrm{A01}$

NIST/SP-812

Criteria for the Operation of Federally-Owned Secondary Cailbration Laboratories (lonizing Radiation).

PB92-11248

NIST/SP-813

101,721 PC A04/MF A01

ICLASS-91 Proceedings of the International Conference on Liquid Atomization and Spray Systems (5th). Held in Gaithersburg, MD. on July 15-18, 1991 .

PB91-216838

100,552 PC A99/MF E08

NIST/SP-814

Interpretation of the SI for the United States and Metric Conversion Policy for Federal Agencies. PB92-126572

NIST/SP-815

Directory of State and Local Government Laboratory ACcreditation/Designation Programs.

PB92-108968

NIST/SP-816

Report of the National Conference on Weights and Measures (76th). Held in Philadelphia, PA. on July 14-19, 1991. PB92-112465

NIST/SP-817

Research. Services. Facilities. (National Institute of Stand ards and Technology).

NIST/SP,

100,012 PC A05/MF A01

Data Bases Available in the Research Information Center of the National Institute of Standards and Technology.
PB92-109016 100,954 PC A07/MF A02

NIST/SP-819

Procedure for the Effective Recalibration of Liquid-in-Glass Thermometers

NIST/SP-820

$100,935 \mathrm{PC} \mathrm{A03/MF} \mathrm{A01}$

Wind and Seismic Effects. Proceedings of the Joint Meeting the U.S.-Japan Cooperative Program in Natural Resources Panel on Wind and Seismic Effects (23rd). Held in

2-116425

NIST/SP-822

100,152 PC A99/MF A06

National Educators Workshop: Update '90. Standard Experiments in Engineering Materials Science and Technology. $92-126606$

101,253 PC A 15/MF A03

NIST/SP-826

High-Temperature Superconductivity: Abstracts of NIST

Publications, 1987-1991.

PB92-126564

$101,105 \mathrm{PC}$ A05/MF A01

NIST/SW/DK-91/007A

Automan 2.0: Decision Support Software for Automated Manufacturing Investments. User Manual.

IST/SW/DK-91/009A

Automan 2.0: Decision Support Software for Automated Manufacturing Investments. User Manual.

PB91-167700

100,978 PC A04/MF A01

NIST/SW/DK-91/012

MUMPS Validation Suite, Version 7.6, 1991 (for Microcomputers).

100,661 CP D99

NIST/SW/DK-91/013

Building Life Cycle Cost Computer Program, Version 3.2 (for Microcomputers)

100,898 CP D02

NIST/SW/MT-91/011

MUMPS Validation Suite, Version 7.6 (Vax/VMS Backup), 1991.

PB91-507699 100,660 CP T99

OMNITAB 80 (ASCII): An Interactive System for Statistical and Numerical Data Analysis (Version 7.0)

PB91-507954

101,278 CP T06

NIST/SW/MT-91/012

OMNITAB 80 (UNIX): An Interactive System for Statistical and Numerical Data Analysis. Version 7.0.

$-507962$

101.279 СР T06

NIST/SW/MT-91/013

MUMPS Validation Suite, Version 7.6 (ANSI), 1991

IST/SW/MT-91/014

MUMPS Validation Sute, Version 7.6 (TAR), 1991.

PB91-507723

NIST/TN-1282

Refinement of a Model for Fire Growth and Smoke Transport.

PB91-148510

100,104 PC A04/MF A01

NIST/TN-1283

Programmer's Reference Manual for CFAST, the Unified Model of Fire Growth and Smoke Transport

PB91-144436

100,096 PC A06/MF A01

NIST/TN-1284

Role of Bench-Scale Test Data in Assessing Real-Scale Fire Toxicity.

$100,156 \mathrm{PC}$ A06/MF A0

NIST/TN-1285

NIST Reactor: Summary of Activities July 1989 through June 1990.

PB91-159772

101.488 PC A05/MF A01

NIST/TN-1286

Response of Personal Noise Dosimeters to Continuous and Impulse-Like Signals

PB92-116359

100,914 PC A07/MF A02

NIST/TN-1287

High Resolution Diffraction Imaging of Crystals Grown in Microgravity and Closely Related Terrestrial Crystals.

PB92-109008

$101,562 \mathrm{PC}$ A08/MF A02

NIST/TN-1288

Video Processing with the Princeton Engine at NIST.

PB92-108950

100,604 PC A04/MF A0

NIST/TN-1289

NIST X-Ray Photoelectron Spectroscopy (XPS) Database.

PB92-123157

NIST/TN-1290

NIST*LATTICE: A Program to Analyze Lattice Relationships. Spring 1991 Version.

$-108877$

$101,561 \mathrm{PC}$ A03/MF A01

NIST/TN-1340

for Chemical Analysis of Alternative Refrigerants. PB91-148502 
NISTIR-85/3273-6

Energy Prices and Discount Factors for Life-Cycle Cost Analysis 1992. Annual Supplement to NIST Handbook 135 and NBS Special Publication 709

PB92-112515

NISTIR-88/3801-1

100,899 PC A04/MF A01

ZIP: The ZIP.Code Insulation Program, Version 2.0. Economic Insulation Levels for New and Existing Houses by Three-Digit Zip Code. Users' Guide and Reference Manual (Revised Edition)

PB91-167155

100,064 PC A03/MF A01 NISTIR-88-3884

Static and Dynamic Strength Tests on Electrical Conductor Cables Specified for Airport Landing Structures.

PB92-112226

101,739 PC A05/MF A01

NISTIR-89/3916-REV

Time Domain Frequency Stability Calculated from the Frequency Domain Description: Use of the SIGINT Software Package to Calculate Time Domain Frequency Stability Irom the Frequency Domain.

PB92-123132

100,587 PC A03/MF A01

NISTIR-89-4169-REV

Use of the IRDS Standard in CALS (Revised)

PB91.132209

100,684 PC A03/MF A01

Effect of Noble Gas Mixtures on the Performance of Re-

generative-Type Cryocoolers Analytical Estimate.
PB91-144410
100,890 NISTIR-90/4238

VolksGrapher: a FORTRAN Plotting Package User's Guide, Version 3.0 .

PB91-185108

NISTIR-90/4248

100,647 PC A04/MF A01

Proposed Design Criteria for Shallow Bridge Foundations

PB91-187849

100,534 PC A04/MiF A01
PC

NISTIR-90/4255

Economic Analysis for Military Construction (MILCON)

Design. Concepts, Techniques, and Applications for the Analyst. Student's Manual.

NISTIR-90/4256

101,350 PC A21/MF A04

Economic Analysis for Military Construction (MILCON) Design. Concepts. Techniques, and Applications for the Analyst. Instructor's Manual.

101,349 PC A99/MF A06

NISTIR-90-4294

Mathematical Modeling of Enclosure Fires

PB92-108976

100, 135 PC A03/MF A01

NISTIR-90/4395

Materials Reliability. Technical Activities, 1990. (NAS-NRC Assessment Panel, January 31-February 1, 1991)

NISTIR-3945

101,008 PC A05/MF A01

Bibliography of the NIST Electromagnetic Fields Division Publications.

NISTIR-3946

100,832 PC A06/MF A01

Metrology for Electromagnetic Technology: A Bibliography

PB91-132266

100,833 PC A04/MF A01

Ignition Characteristics of the Nickel-Based Alloy UNS No7001 in Pressurized Oxygen.

PB91-144428

NISTIR-3948

100,536 PC A04/MF A01

Thermal Conductivity of a Polymide Film between 4.2 and $300 \mathrm{~K}$, with and without Alumina Particles as Filler. PB91-144402

NISTIR-3950

100.483 PC A05/MF A01

Data-Reduction and Analysis Procedures Used in NIST's Thermomechanical Processing Research

PB91-132282

100,925 PC A04/MF A01

Transient Hydrogen Heat Transter. Final Report for April 1986-April 1989

PB92-116326

NISTIR-3952

100,454 PC A03/MF A.01

Study of

PB91-132217

101,574 PC A09/MF A01

Near-Field and Far-Field Excitation of a Long Conductor in a Lossy Medium

PB91-143347

100,722 PC A03/MF A01

Spherical Near-Field Scanning: Experimental and Theoretical Studies.

IISTIR-39:

100,723 PC A07/MF A01

NISTIR-3959

Heat Transfer in a Compact Heat Exchanger Containing Rectangular Channels and Using Helium Gas.

PB91-184804

NISTIR-3960

101,734 PC A05/MF A01

Microstructure, Com

PB91-184747

NISTIR-3961

101,153 PC A03/MF A01

Experimental Thermal Conductivity, Thermal Diffusivity, and Specific Heat Values for Mixtures of Nitrogen, Oxygen, and Argon.
PB91-194472

100,369 PC A04/MF A01

NISTIR-3963

Unsteady Laminar Flow in a Circular Tube: A Test of the HERCOL (Hermitian Collocation) Computer Code PB91-222679

101,395 PC A03/MF A01 NISTIR-3964

Continuous-Cooling Transformation Characteristics and High-Temperature Flow Behavior of a Microalloyed SAE 114 Steel.

PB91-18785

NISTIR-3965

101, 155 PC A03/MF A01

Heat Transfer and Pressure Drop in a Compact Pin-Fin Heat Exchanger with Pin Orientation at 18 deg to the Flow Direction.

PB91-194464
NISTIR-3967

101,050 PC A05/MF A01

Tread Crack Detection in Railroad Wheels: An Ultrasonic System Using EMATs (Electromagnetic-Acoustic Transducers.. Report No. 22

PB91-22266

101,742 PC A04/MF A01

Residual Stress Detection in Railroad Wheels: An Ultrasonic Systern. Using EMATs (Electromagnetic-Acoustic Transducers). Report No. 23

PB91-222653

101,741 PC A03/MF A01

NISTIR-3969

Survey of Current Worldwide Research on the Thermophysical Properties of Alternative Refrigerants

PB92-112366

101,198 PC A05/MF A01

ISTIR-3970

Personal Computer Codes for Analysis of Planar Near Fields

NISTIR-3972

100,730 PC A04/MF A01

Metrology for Electromagnetic Technology: A Bibliography of NIST Publications

NISTIR-3973

100,867 PC A04/MF A01

Bibliography of the NIST Electromagnetic Fields Division Publications.
PB92-116367

NISTIR-3975

100,866 PC A06/MF A02

Magnetic Measurements for High Energy Physics Applications

PB92.112358

101,720 PC A04/MF A01

NISTIR-3980

Oxides and Oxide Superconductors: Elastic and Related Properties.

PB92-112333

NISTIR-3982

Quantifying Standard Periormance of ElectromagneticBased Mine Detectors

101,378 PC A09/MF A02

\section{NISTIR-4315}

Collection of Technical Studies Completed for the Computer-Aided Acquisition and Logistic Support (CALS) Program. Management.

PB91-178889

NISTIR-4316

101,342 PC A12/MF A02

Collection of Technical Studies Completed for the Computer-Aided Acquisition and Logistic Support (CALS) Program, Fiscal Year 1988. Volume 2 of 3 . Graphics, CGM MIL-

PB91-178897

101,343 PC A21/MF A03

NISTIR-4317

Collection of Technical Studies Completed for the Computer-Aided Acquisition and Logistic Support (CALS) Program,

Fiscal Year 1988 Volume 3 of 3. CGM Registration.

PB91-178905 101,344 PC A08/MF A01 NISTIR-4326

Modern Test Methods for Flammability.
PB91-167676 100,116 PC A03/MF A01 NISTIR-4335/REV

NIST PDES Tookit: Technical Fundamentals. National PDES Testbed Report Series (Revised).

PDES Testbed

100.682 PC A03/MF A01 NISTIR-4351/REV

NIST Working Form for STEP: National PDES Testbed Report Series (Revised)

PB91-132183

$100,683 \mathrm{PC} \mathrm{A03/MF} \mathrm{A01}$

NISTIR-4353/REV

NIST STEP Working Form Programmer's Reference. National PDES Testbed Report Series (Revised).
PB91-144378
100,998 PC A03/MF A01 NISTIR-4371-REV

Fed-X: The NIST Express Translator. Revised, November

PB91-132126

100,681 PC A03/MF A01

\section{NISTIR-4394}

Institute for Materials Science and Engineering, Ceramics: Technical Activities 1990

101,057 PC A08/MF A01

NISTIR-4396

Polymers: Technical Activities 1990.

PB91-159715

100,488 PC A07/MF A01

NISTIR-4397

Institute for Materials Science and Engineering: Metallurgy Division, Technical Activities 1990

PB91-132225

$101,207 \mathrm{PC}$ A07/MF A01

NISTIR-4407

NIST Express Working Form Programmer's Reference (Revised November 1990). National PDES Testbed Report

PB91-157164

$100,964 \mathrm{PC}$ A04/MF A01

\section{NISTIR-4424}

Pulse-Echo Ultrasonic Evaluation of the Integrity of Seams Pingle-Ply Roofing Membranes: Laboratory Evaluation of a Prototype Test Apparatus.

PB91-157172

100,106 PC A03/MF A01

NISTIR-4425

Pulse-Echo Ultrasonic Evaluation of the Integrity of Seams of Single-Ply Roofing Membranes: Results of Field Investigations and Recommendations.

PB91-159731

$100,110 \mathrm{PC} \mathrm{A03/MF} \mathrm{A01}$

ISTIR-4429

Measurement and Evaluation of Lighting/HVAC Interaction.
PB91-206706 NISTIR-4436

Depot: A Framework for Sharing Software Installation Across Organizational and Unix Platform Boundaries.

PB91-240754

100,659 PC A03/MF A01

ISTIR-4439

FPETOOL User's Guide.

PB92.108919

$100,134 \mathrm{PC} \mathrm{A03/MF} \mathrm{A01}$

\section{NISTIR-4441}

Reduction of Hydrogen Cyanide Concentrations and Acute Inhalation Toxicity from Flexible Polyurethane Foam Combustion Products by the Addition of Copper Compounds. Characteristics of Flexible Polyurethane Foam. PB91-132167

101,140 PC A03/MF A01

NISTIR-4444

Canadian Mass Fire Experiment, 1989

PB91-143305

101,352 PC A04/MF A01

NISTIR-4448

Working Implementation Agreements for Open Systems Interconnection Protocols.

PB91-144444

100,686 PC A99/MF A04

\section{ISTIR-4449}

Joint Panel Meeting of the UJNR Panel on Fire Research and Safety (11th). Held in Berkeley, CA, on October 19-24,

PB92-109164

$100,166 \mathrm{PC} \mathrm{A16/MF} \mathrm{A03}$

NISTIR-4455

Computational Models Developed for the Corrosion of Prestressing Steel.

\section{NISTIR-4456}

$100,115 \mathrm{PC}$ A03/MF A01

Studies on the Degradation Products of Paper with and without

Results.

PB91-143313

$101,181 \mathrm{PC}$ A03/MF A01 


\section{NTIS ORDER/REPORT NUMBER INDEX}

NISTIR-4474

Synthesis of Prototype Resins for Use as BEP Intaglio Ink Vehicles Curing by Electron Beam Radiation.

PB91-144345

$101,182 \mathrm{PC} \mathrm{A03/MF} \mathrm{A01}$

NISTIR-4475

Guide to Available Mathematical Software Problem Classification System

NISTIR-4476

$101,282 \mathrm{PC} \mathrm{A03/MF} \mathrm{A01}$

New Approach to Vision and Control for Road Following PB91-157156

NISTIR-4478

101, $743 \mathrm{PC} \mathrm{A03/MF} \mathrm{A01}$

Recommended Fine Positioning Test for the Development Test Flight (DTF-1) of the NASA Flight Telerobotic Servicer (FTS)

101,732 PC A06/MF A01

NISTIR-4480

Assembly Code to Compute Sine and Cosine Using the Cordic Algorithm

100,638 PC A03/MF A01

NIST 'Building Life-Cycle Cost' (BLCC) Program (Version 3.0). User's Guide and Reference Manual.

PB91-159764

NISTIR-4482

100,154 PC A04/MF A0

Space Marching Difference Schemes in the Nonlinear Inverse Heat Conduction Problem

PB91-144360

101,604 PC A03/MF A01 NISTIR-4484

Multimedia Courseware in an Open Systems Environment: A Federal Strategy.

PB91-143362

100,039 PC A04/MF A01

NISTIR-4485

Annotated Bibliography: Diagnostic Methods and Measure ment Approaches to Detect Incipient Defects Due to Aging of Cables.

PB91-222687

101,369 PC A10/MF A03

NISTIR-4488

Proceedings of the Object-Oriented Database Task Group Workshop, Tuesday, October 23, 1990, Chateau Laurier Hotel, Ottawa, Canada.

PB91-157198

100,641 PC A07/MF A01

NISTIR-4491

STEP On-Line Information Service User's Guide. National PDES Testbed Report Series.

PB91-157123

$100,950 \mathrm{PC} \mathrm{A03/MF} \mathrm{A01}$

National Computer Systems Laboratory Annual Report, 1990.

PB91-201822

100,603 PC A05/MF A01

NISTIR-4494

SOL3 Support for CALS Applications.

PB91-167262

$100,965 \mathrm{PC} \mathrm{A03/MF} \mathrm{A01}$

NISTIR-4495

Evidence for Surface alpha Particle Clusters in (nat)AG(197)Au from the (e,alpha) Reaction.
PB91-216812 NISTIR-4496

Center for Electronics and Electrical Engineering Technical
Progress Bulletin Covering Center Programs, July to SepProgress Bulletin Covering Center Programs, July to Sep-
tember 1990 , with 1991 CEEE Events Calendar.

$100,801 \mathrm{PC} \mathrm{A03/MF} \mathrm{A01}$

NISTIR-4497

Prediction of Elevated Temperature Deformation of Structural Steel under Anisothermal Conditions.

PB91-184838

$101,154 \mathrm{PC} \mathrm{A03/MF} \mathrm{A01}$

NISTIR-4499

Experimental Study of Top Vented Compartment Fires

PB91-167254

100.155 PC A03/MF A01

NISTIR-4501

Crack Arrest Fracture Toughness Measurements of Normalized and Inclusion Shape Controlled AAR TC128 Grade $B$ Steel, and Micro-Alloyed, Control-Rolled, and Inclusion Shape Controlled A 8XX Grade B Steel. PB91-167346

$101,150 \mathrm{PC} \mathrm{A03/MF} \mathrm{A01}$ NISTIR-4502

Examination of the Excessive Retained Austenite on the Surface of a Section of 17-7 Precipitation Hardening Stainless Steel.

NISTIR-4503

101,149 PC A03/MF A01

Proceedings of the Object-Oriented Database Task Group Workshop. Held in Atlantic City, New Jersey on May 22, 1990.

PB91-159723

NISTIR-4504

Field Study of the Performance of EPDM Roofing at Air Force Facilities.

NISTIR-4505

101,340 PC A05/MF A01

Technical Activities 1990, Standard Reference Data Pro. gram.

100,321 PC A05/MF A01

NISTIR-4506

Center for Radiation Research. 1990 Technical Activities

PB91.178855

1990 Technical Activities.
101,362 PC A08/MF A01

NISTIR-4507

Working Implementation Agreements for Open Systems Interconnection Protocols.
PB92-126523

NISTIR-4508

National PDES Testbed Mail Server User's Guide. National

PDES Testbed Report Series.

PB91-159756

NISTIR-4509

Review of Current Geometric Tolerancing Theories and Inspection Data Analysis Algorithms.

PB91-167221 101,016 PC A03/MF A01 NISTIR-4510

Robot Characterization Testing

PB91-16736

$101,030 \mathrm{PC} \mathrm{A03/MF} \mathrm{A01}$

NISTIR-4511

Measurement of Structural Response Characteristics of Full-Scale Buildings: Selection of Structures.

100,144 PC A03/MF A01 NISTIR-4512

Review of Research Literature on Masonry Shear Walls.

PB91-167189

100,113 PC A05/MF A01
PC NISTIR-4513

DISCOUNT-A Program for Discounting Computations in Life-Cycle Cost Analyses. User's Guide and Reference Manual

PB91-167288

100,897 PC A03/MF A0

NISTIR-4514

Assessment of the State-of-the-Art for Process Monitoring

Sensors for Polymer Composites.

PB91-222612

NISTIR-4515

Proceedings of a Workshop on Evaluation, Repair, and Retrofit of Structures. U.S.-Japan Panel on Wind and Seismic Effects, UJNR. Held in Gaithersburg, MD., USA, on May 12$14,1990$.

PB'91-184846

NISTIR-4516

100,145 PC A14/MF A02

Char acterization of Field Concrete.

PB91-178863

100,528 PC A03/MF A01 NISTIR-4518

National Aeronautics and Space Admunistration's (NASA) Automated Information Security Handbook

100,707 PC A06/MF A0

NISTIR-4519

Engineering Design Laboratory Guide.

PB91-167320

100,977 PC A03/MF A01 NISTIR-4523

Proposed Standard Practice for Assessing the Performance of Gas-Phase Air Cleaning Equipment.

100,905 PC A03/MF A0 NISTIR-4524

Raster Graphics Conformance Testing. 101,341 PC A04/MF A01 NISTIR-4526

FIREDOC Users Manual, 2nd Edition.

PB91-178830

100,547 PC A03/MF AO

NISTIR-4528

STEP: Standard for the Exchange of Product Model Data Resource Integration: Semantic and Syntactic Rules.

Resource Integration: Semantic and Syntactic Rules.
PB91-184788 $100,966 \quad \mathrm{PC} \mathrm{A03/MF} \mathrm{A01}$ NISTIR-45

Alaska Marine Mammal Tissue Archival Project: Revised Collection Protocol.

PB91-184796

101,374 PC A03/MF A0

NISTIR-4530

Validation of an OSI Transport Class 4 Simulator. PB91-187724

100,568 PC A03/MF A01 NISTIR-453

Intercomparison Study of Rockwell Hardness Test Blocks. PB91-167338

101,224 PC A04/MF A0

NISTIR-4532

Visibility of Exit Directional Indicators.

PB91-184762

$101,000 \mathrm{PC} \mathrm{A03/MF} \mathrm{A01}$

Energy Related Inventions Program: A Joint Program of the Department of Energy and the National Institute of Stand ards and Technology. Status Report for Recommendation 1 through 250

NISTIR.4534

$100,902 \mathrm{PC}$ A09/MF A0

Energy Related Inventions Program. A Joint Program of the Department of Energy and the National Institute of Standards and Technology. Status Report for Recommendations 251 through 523.

PB91-184770

NISTIR-4537

$100,903 \mathrm{PC} \mathrm{A13/MF} \mathrm{A02}$

Center for Electronics and Electrical Engineering Technical Publication Announcements Covering Center Programs, July to September 1990, with 1991 CEEE Events Calendar.
100,852 PC A03/MF A01 NISTIR-4538

$100,852 \mathrm{PC} \mathrm{A03/MF} \mathrm{A0}$

Configuration Management Concepts Document. National PDES Testbed Report Series.

P891-194480

100,984 PC A03/MF A0

ISTIR-4539

Calibration at $24 \mathrm{C}$ of a Heat-Flow-Meter Apparatus Having $610 \mathrm{~mm}$ Square Plates.

NISTIR-4540

$100933 \mathrm{PC} \mathrm{A03/MF} \mathrm{A01}$

Boussinesq Algorithm for Enclosed Buoyant Convection in Two Dimensions.

PB91-178848

100,157 PC A03/MF A01

NISTIR-4542

Conformity Assessment Workshop on Pressure Vessels.

PB91-192997

101,049 PC A06/MF A01

NISTIR-4543

Automan 2.0: Decision Support Software for Automated Manufacturing Investments. User Manual.

Manufacturing Investments. User Manual. 100,978 PC A04/MF A01 NISTIR-4545

Computer Security: Selected Articles.

PB91-187740

100,706 PC A03/MF A01

NISTIR-4546

Intelligent Building Technology in Japan

P891-187757

NISTIR-4547

100,071 PC A04/MF A01

Standard Generalized Markup Language Encoding of the Office Document Architecture Document Application Profile.
101,345 PC A03/MF A01 NISTIR-4549

Chloride Ion Diffusion in Low Water-to-Solid Cement Pastes.

PB91-187690

$101,138 \mathrm{PC} \mathrm{A03/MF} \mathrm{A01}$

NISTIR-4550

Stopping Power of Fast Charged Particles in Heavy Elements.

$101,660 \mathrm{PC}$ A04/MF A01

NISTIR-4552

AMRF Composites Fabrication Workstation: A Test MethAMRF Com the Ouality of Thermoplastic Compt ite Parts.

PB91-187765

$101,017 \mathrm{PC} \mathrm{A03/MF} \mathrm{A01}$

NISTIR-4553

Technical Activities 1990, Electron and Optical Physics Divi-

Sion. 19423

101,662 PC A04/MF A01

NISTIR-4554

Convergence Properties of a Class of Rank-Two Updates.

PB91-187799

$101,260 \mathrm{PC} \mathrm{A03/MF} \mathrm{A01}$

NISTIR-4555

Model for the Non-Perturbative OCD (Ouantum Chromodynamics) Vacuum

NISTIR-4556

$101,661 \mathrm{PC} \mathrm{A03/MF} \mathrm{A01}$

Interior-Point Method for Linear and Quadratic Programming Problems.
PB91-187815

NISTIR-4557

$101,269 \mathrm{PC} \mathrm{A03/MF} \mathrm{A01}$

Programming Languages and Database Language SOL. Validated Processor List Including GOSIP Conformance Testing Registers.

NISTIR-4559

GATT Standards Code Activities of the National Institute of Standards and Technology 1990.

PB91-187823

100,177 PC A04/MF A01

NISTIR-4560

Government Document Processing Requirements Report.

PB9 1-187773 
PB91-206698

100,075 PC A03/MF A01

NISTIR-4576

Laboratory Accreditation in the United States.

PB91-194415

100,172 PC A03/MF A0

NISTIR-4577

Extracting STEP Geometry and Topology from a Solid Modeler: Parasolid-to-STEP.

100,983 PC A04/MF A0

NISTIR-4578

Abstracts of Daylighting Research.

PB91-206755

100,076 PC A06/MF A01

NISTIR-4579

NIST Support for the Computer-Aided Acquisition and Logistic Support (CALS) Program in the Area of Graphics Standards, Calendar Year 1990

PB91-194506

101,348 PC A14/MF A02

NISTIR-4582

Building and Fire Research Project Summaries, 1991

PB91-216788

100, 132 PC A08/MF A02

NISTIR-4585

User Manual NBSAVIS CONTAM88 A User Interface for Air Movement and Contaminant Dispersal Analysis in Multizone Buildings

PB91-206722

$100,910 \mathrm{PC} \mathrm{A07/MF} \mathrm{A01}$

NISTIR-4587

Interaction of an Isolated Sprinkler Spray and a Two-Layer Compartment Fire Environment.

PB91-216804

NISTIR-4588

General Routine for Analysis of Stack Effect.

PB91-231589

$100,163 \mathrm{PC} \mathrm{A03/MF} \mathrm{A01}$

NISTIR-4589

Performance of 1/3-Scale Model Precast Concrete Beam Column Connections Subjected to Cyclic Inelastic Loads. Report No. 2.

PB91-222570

100,149 PC A05/MF A0

NISTIR-4590 Applications of the Generalized Global Equivalence Ratio
Model (GGERM) for Predicting the Generation Rate and Distribution of Products of Combustion in Two-Layer Fire Environments: Methane and Hexanes.

PB91-216689

$100,131 \mathrm{PC}$ A05/MF A0

NISTIR-4591

Material Flammability Test Assessment for Space Station Freedom.
PB91-216606

PB91-216606
NISTIR-4593

101,735 PC A05/MF A0

High Magnetic Field Facilities in Japan Related to Superconductivity Research (Japan Technology Program)

PB91-240762

101,718 PC A04/MF A0

NISTIR-4594

GOSIP Conformance and Interoperation Testing and Registration

PB92.110105

$100,694 \mathrm{PC} \mathrm{A05/MF} \mathrm{A0}$

ISTIR-4595

Description of a Vibration Compensation System for the Small Scale Model Robot Crane Project.

PB91-216648

$100,130 \mathrm{PC} \mathrm{A04/MF} \mathrm{A01}$

NISTIR-4596

Task Decomposition and Algorithm Development for RealTime Motion Control of a Continuous Mining Machine

PB91-231597

101,357 PC A03/MF A0

NISTIR-4599

NVLAP FY 90 Annual Report.

PB91-206748

100,010 PC A03/MF A0

ISTIR-4604

Direct Digital Control Based Building Automation System Design Criteria
PB91-216614

NISTIR-4605

100,091 PC A03/MF A01

Variable Air Volume System Design Guide.

PB91-216655

NISTIR-4606

Guide Specification for Direct Digital Control Based Building Automation System.

PB91-216697

100,092 PC A04/MF A0

NISTIR-4608

Electronic Data Interchange in Message Handling Systems

NISTIR-4609

NIST Support of the CALS Program: 1990 Synopsis

PB91.193821

NISTIR-4610

Real-Time Model-Based Tracking Combining Spatial and Temporal Features.

PB91-216630

100,700 PC A03/MF A01

NISTIR-461

Conformity Assessment Workshop on Electromagnetic Compatibility.

NISTIR-4612

Apparel STEP Translator

PB91-216663

NISTIR-4613

Long Term Performance of Rubber in Seismic and NonSeismic Bearings: A Literature Review.
PB91-206714

$100,148 \mathrm{PC} \mathrm{A03/MF} \mathrm{A01}$

NISTIR-4614

Standard Security Label for GOSIP: An Invitational Workshop. Held on April 9-10, 1991.

PB91-216671

NISTIR-4615

100,712 PC A07/MF A02

Portable Vectorized Software for Bessel Function Evalua tion.

PB91-216598

NISTIR-4616

Building Technology Publications 1986-1989.

PB91-216820

$100,160 \mathrm{PC}$ A08/MF A02

NISTIR-4618

Standards for the Physical Protection of National Resources and Facilities

100,006 PC A05/MF A0

NISTIR-4619

Framework for Utilizing Fire Property Tests.

PB91-240788

100,165 PC A03/MF A01 NISTIR-4620

Navy Safety Center Data on the Effects of Fire Protection Systems on Electrical Equipment

PB92-108935

101,376 PC A05/MF A0

NISTIR-4622

Generation of Smooth Trajectories without Planning

PB91-217414

101036 PC A03/MF A01

NISTIR-4623

Validated Processor List (Cobol, Fortran, ADA, Pascal SQL). July 199

NISTIR-4625

Suppression of Post-Flashover Compartment Fires Using

Manually Applied Water Sprays

NISTIR-4626

Manufacturing Systems Integration Control Entity Interface Document

PB91-240796

NISTIR-4628

$100,991 \mathrm{PC}$ A03/MF A0

Development Plan: Application Protocols for Mechanical Parts Production. National PDES Testbed Report Series

PB91-222596

NISTIR-4629

$100,990 \mathrm{PC} \mathrm{A03/MF} \mathrm{A0}$

Configuration Management of the STEP Documents: Procedures and System Requirements. National PDES Testbed Report Series

NISTIR-4630

$100,968 \mathrm{PC} \mathrm{A03/MF} \mathrm{A0}$

Performance Evaluation of Hypercube Applications: Using a Global Clock and Time Dilation.

PB91-222588

NISTIR-4631

100,653 $\mathrm{PC} \mathrm{A03/MF} \mathrm{A0}$

Reduction in Fire Hazard in Corridors and Areas Adjoining Corridors Provided by Sprinklers.

PB91-222604

NISTIR-4633

Ultimate Strength of Masonry Shear Walls: Predictions versus Test Result

PB92-116342

NISTIR-4634

$100,138 \mathrm{PC} \mathrm{A03/MF} \mathrm{A0}$

Preliminary Results of the Environmental Evaluation of the Federal Records Center in Overland Missouri.

100,082 PC A04/MF A01

NISTIR-4636

Automated Information Systems Security Program HandPB91.

NISTIR-4637

100,713 PC A08/MF A02

Computer Implementation of a Discrete Set Algebra PB91-23157

PC A03/MF AO

NISTIR-4639

Daylight Research Requirements. Workshop Proceedings Held in Baltimore, Maryland on August 1-2, 1990.

PB91-231563

100,078 PC A04/MF A01

NISTIR-4640

NIST Personnel Management Demonstration Project Design, Implementation and Accomplishments.

PB91-231555 100,003 PC A05/MF A01

NISTIR-4643

Technical Activities 1990 Surface Science Division PB91-240820

101,560 PC A04/MF A01 NISTIR-4644

New Concepts of Precision Dimensional Measurement for Modern Manufacturing.

PB91-240812

101,006 PC A04/MF A0

NISTIR-4645

Center for Electronics and Electrical Engineering Technical Publication Announcements Covering Center Programs, October to December 1990, with 1991 CEEE Events Calenda 1.240838

NISTIR-4648

100,863 PC A03/MF A01

Uniaxial Tensile Tests to Measure the Bond of In situ Concrete Overlays.

NISTIR-4649

$100,533 \mathrm{PC} \mathrm{A03/MF} \mathrm{A01}$

Data for Fire Hazard Assessment of Selected Non-Halogenated and Halogenated Fire Retardants; Report of Test FA 3983

PB92-112473

$100,557 \mathrm{PC} \mathrm{A04/MF} \mathrm{A01}$

NISTIR-4650

Guidelines for the Development of Computer Based Models

in a Cementitious Materials Modeling Laboratory

PB91-240804 100,532 PC A03/MF A01

NISTIR-4652

Cone Calorimeter Rate of Heat Release Measurements for

Upholstered Composites of Polyurethane Foams.
PB92-108984 100,087 PC A03/MF A01

NISTIR-4653

Metrology for the Semiconductor Industry

PB91-240739

100,817 PC A03/MF A0

NISTIR-4654

Development of an Optical Disk System for the Automated Retrieval of EASEAR Records.

PB92-108943

100,666 PC A07/MF A02

NISTIR-4656

Proceedings of the Forum on Standards for High Integrity Soltware (DOD, Government, Industry). Held in Gaithersburg, Maryland on June 28, 1991.

PB92-112267

100,668 PC A04/MF A01

NISTIR-4659

Glossary of Computer Security Terminology.

PB92-112259

100,716 PC A09/MF A02

NISTIR-4660

Mechanical Properties and Fracture Toughness of AAR

TC128 Grade B Steel in the Normalized, and Normalized and Stress Relieved Conditions.

PB92-108901

$101,169 \mathrm{PC}$ A03/MF A01

NISTIR-4661

International Fire Detection Bibliography, 1975-1990

PB92-109156

100,088 PC A09/MF A02

NISTIR-4662

Phase-Field Model for Isothermal Phase Transitions in Binary Alloys.

NISTIR-4664

Flammability Characterization of Foam Plastıcs.

PB92-123033

101,240 PC A04/MF A01

NISTIR-4665

Engineering Analysis of the Fire Development in the Hillha

ven Nursing Home Fire, October 5, 1989.
PB92-112317

NISTIR-4666

Clock for the Manufacturing Systems Integration Testbed

PB92-108885

NISTIR-4667

Computer Security Bulletin Board System. User's Guide.

PB92-112390

NISTIR-4669

Integration-Based Method for Depth Estimation

PB92-112275

$100,701 \mathrm{PC} \mathrm{A03/MF} \mathrm{A01}$

NISTIR-4674

Analysis of Moisture Accumulation in a Wood Frame Wall Subjected to Winter Climate.

PB92-116334

100,137 PC A04/MF A01

NISTIR-4675

Electronics and Electrical Engineering Laboratory Technical Publication Announcements Covering Laboratory Programs, January to March 1991, with 1991 EEEL Events Calendar. 


\section{NISTIR-4687}

HgCdTe Detector Reliability Study for the GOES Program. NISTIR-4688

Prototyping the IRDS: An Airport Application

PB92-112580

101,740 PC A06/MF A02

NISTIR 4690

Validated Processor List (Cobol, Fortran, ADA, Pascal, SOL). October 1991

PB91-937302

100,665 Not available NTIS

NISTIR.4691

Research for Electric Energy Systems: An Annual Report.
PB92-112341

NISTIR-4693 Inte!ligent Processing of Materials, Technical Activities
1991. (NAS-NRC Assessment Panel, November 14-15, 1991).

100,995 PC A05/MF A01

NISTIR-4694

Ceramics: Technical Activities 1991. (NAS-NRC Assessment Panel, February 13-14, 1992). 101,104 PC A09/MF A02
PB92-126507

Materials Reliability. Technical Activities, 1991. (NAS-NRC Assessment Panel, February 13-14, 1992)

PB92-12642

NISTIR-4696

Polymers: Technical Activities 1991. (NAS-NRC Assessment Panel, February 13.14, 1992)

PB92-116284

NISTIR-4697

Metallurgy Division, Technical Activities 1991. (NAS-NRC Assessment Panel February 13-14, 1992)

PB92-126515

$101,171 \mathrm{PC}$ A07/MF A02

NISTIR-4699

Test Plan for Validating a Context Driven Integrated Model (CDIM) for Sheet Metal Die Design. National PDES Testbed Report Series.

PB92-112523

$100.970 \mathrm{PC} \mathrm{A04/MF} \mathrm{A01}$

NISTIR-4702

Merit Function for Inequality Constrained Nonlinear Programming Problems.

$101,271 \mathrm{PC}$ A03/MF A01

NISTIR-4705

Fire-Plume-Generated Ceiling Jet Characteristics and Convective Heat Transfer to Ceiling and Wall Surfaces in a Two-Layer Zone-Type Fire Environment: Uniform Temperaure Ceiling and Walls.

PB92-12307

101,370 PC A04/MF A01

NISTIR-4706

Feasibility of Using a Multiple Award Schedule for Specify-

ing Paints in Government Painting Contracts.
PB92-126481 100,002 PC A03/MF A01

NISTIR-4708

Multi-Enterprise Concurrent Engineering through International Standards

PB92-123058

NISTIR-47 10

Use of Solid Modeling in the Design of M3 Components

PB92-123066

NISTIR-4712

Predicting the Remaining Service Life of Concrete.

101,371 PC A05/MF A01

NISTIR•4713

Publications of the Manufacturing Engineerıng Laboratory Covering the Period January 1989-September 199

PB92-123041 101,053 PC A05/MF A01

NISTIR-4714

Electronics and Electrical Engineering Laboratory. 1991 Strategic Plan. Supporting Technology for U.S. Competitiveness in Electronics.

$100,870 \mathrm{PC}$ A03/MF A01

NISTIR-47 16

Computing Delaunay Triangulations for Comet-Shaped Polygons.
PB92-123108

NISTIR-4717

101,265 PC A03/MF A0

Testing and Rating of an Atmospheric, Gas-Fired Furnace

Equipped with a Burner Air Inlet Damper.
PB92-126440 $100,894 \quad$ PC A04/MF A01

NISTIR-4721

Ouestions and Answers on Ouality, the ISO 9000 Standard Series, Ouality System Registration, and Related Issues.
PB92-126465 100,175 PC A03/MF A01

NISTIR-4723

Evaporator Performance Investigation for Residential AirConditioning Application Using Mixed Refrigerants.

100,893 PC A07/MF A02

NISTIR-4725

Stability of Aqueous Inorganic Lead Solutions in Polycarbonate Containers.

NISTIR-4729

$100,211 \mathrm{PC} \mathrm{A03/MF} \mathrm{A01}$

Development of Test Methods to Determine the Compatibility of Liquid Hazardous Materials with Polyethylene PackagPB92-126457 100,943 PC A05/MF A01
NUREG/CR-5711

Assessment of Uncertainties in Measurement of $\mathrm{pH}$ in Hostile Environments Characteristic of Nuclear Repositories.

Model Validation at the Las Cruces Trench Site

NUREG/CR-5716 101,368 PC A05/MF A01

PAT-APPL-7.292 601

Superconductor-Polymer Composites.

PATENT-4 954481

Superconductor-Polymer Composites.
PATENT-4 $954481 \quad$ Not, 454 available NTIS PB90-244526

Journal of Physical and Chemical Reference Data, Volume 19, Number 1,1990 . 100,251 Not available NTIS

\section{PB90-244534}

Chemical Kinetic Data Base for Combustion Chemistry Part 4. Isobutane.

PB90-244542

100,252 (Order as PB90-244526)

Thermodynamic Functions and Properties of $\mathrm{MgO}$ at High

Compression and High Temperature.
PB90-244542 100,253 (Order as PB90-244526)

PB90-244559

Critical Compilation of Surface Structures Determined by Ion Scattering Methods.

PB90-244567

Benzene: A Further Liquid Thermal Conductivity Standard. PB90-244567 100,255 (Order as PB90-244526)

PB90-244575

Energy Levels of Atomic Aluminum with Hyperfine Structure.

PB90-244583

Spectral Data and Grotrian Diagrams for Highly lonized ron, Fe VIII-XXVI.

PB90-24458

101,595 (Order as PB90-244526)

PB90-244591

Updated Excitation and Ionization Cross Sections for Electron Impact on Atomic Oxygen.

PB90-244609

Journal of Physical and Chemical Reference Data, Volume 19. Number 2,1990

PB90-244609

PB90-244617

100,257 Not available NTIS

Standard Chemical Thermodynamic Properties of Isomer Groups of Monochloroalkanes

PB90-244617 100,258 (Order as PB90-244609) PB90-24462

Standard Chemical Thermodynamic Properties of Polycyclic Aromatic Hydrocarbons and Their Isomer Groups. III. Naphthocoronene Series, Ovalene Series, and First Mem-

bers of Some Higher Series.
PB90-244625 100,259 (Order as PB90-244609)

PB90-244633

Dielectric Constant of Water and Debye-Huieckel Limiting Law Slopes.

PB90-244641

100,260 (Order as PB90-244609)

Rate Constants for Reactions of Peroxyl Radicals in Fluid Solutions.

PB90-244641

100,261 (Order as PB90-244609)

\section{PB90-244658}

Journal of Physical and Chemical Reference Data, Volume 19. Number 3,1990

PB90-244658
PB90-244666

Energy Levels of Copper, Cu I through Cu XXIX.

PB90-244666

100,262 Not available NTIS PB90-244666

101,596 (Order as PB90-244658)

P90-244674

Cross Sections and Related Data for Electron Collisions with Hydrogen Molecules and Molecular lons

PB90-244674 100,263 (Order as PB90-244658)

PB90-244682

Cross Sections for Collisions of Electrons and Photons with Atomic Oxygen

PB90-244690

100,264 (Order as PB90-244658)

Cross Sections and Swarm Coefficients for $\mathrm{H}+, \mathrm{H} 2+$ $\mathrm{H} 3+, \mathrm{H}, \mathrm{H}_{2}$, and $\mathrm{H}$-in $\mathrm{H} 2$ for Energies from $0.1 \mathrm{eV}$ to 10 keV.

PB90-244690

101,597. (Order as PB90-244658)

Refractive Index of Water and Steam as Function of Wavelength, Temperature and Density

PB90-244708

101,402 (Order as PB90-244658)

PB90-244716

Heat Capacities of Organic Compounds in the Liquid State I. C1 to C18 1-Alkanols.

B $90-244716$

100,265 (Order as PB90-244658)

Transport Properties of Carbon Dioxide.

PB90-244724

100,266 (Order as PB90-244658)

PB91-132126

Fed-X: The NIST Express Translator. Revised, November

PB91-132126

$100,681 \mathrm{PC}$ A03/MF A01

PB91-132134

Concept of the Calculus of Fire Safety.

PB91-132134

100.093 PC A03/MF A01

PB91-132142

Software and Computer Integrated Manufacturing.

PB91-132142

100,972 PC A03/MF A01

PB91-132159

NIST PDES Toolkit: Technical Fundamentals. National

PDES Testbed Report Series (Revised).

100,682 PC A03/MF A01

PB91-132167

Reduction of Hydrogen Cyanide Concentrations and Acute Inhalation Toxicity from Flexible Polyurethane Foam Com Part 3. The Effect of Copper Additives on the Flammability Characteristics of Flexible Polyurethane Foam.

PB91-132167

101,140 PC A03/MF A01

PB91-132175

Guide to Available Mathematical Software Problem Classification System.
PB91-132175

PB91-132183

101,282 PC A03/MF A01

NIST Working Form for STEP: Nationa! PDES Testbed

Report Series (Revised)

PB91-132183

100,683 PC A03/MF A01

PB91-132191

Polymer Composite Processing. Industry Workshop (2nd)

Held at Gaithersburg, Maryland on May 18, 1990.

PB91-132191

101,115 PC A05/MF A01

PB91.132209

Use of the IRDS Standard in CALS (Revised)

PB91-132209

100,684 PC A03/MF A01

PB91-132217

Study of Static and Dynamic Fracture Using Strain Measurements.
PB91-132217

101,574 PC A09/MF A01

PB91-132225

Institute for Materials Science and Engineering: Metallurgy Division, Technical Activities 1990.

PB91-132225

PB91-132233

Institute for Materials Science and Engineering, Ceramics Technical Activities 1990

PB91-132233

101,057 PC A08/MF A01

PB91.132241

Bibliography of the NIST Electromagnetic Fields Division PBblications.

100,832 PC A06/MF A0 1

PB91-132258

Overview of the Product Data HyperStandard CD-ROM Prototype.

$101,380 \mathrm{PC} \mathrm{A03/MF} \mathrm{A01}$

B91-132266

Metrology for Electromagnetic Technology: A Bibliography of NIST Publications.

PB91-132266

100,833 PC A04/MF A0

PB91-132274

Spherical Near-Field Scanning: Experimental and Theoretical Studies.
PB91-132274

PB91-132282

100,723 PC A07/MF A01

Data-Reduction and Analysis Procedures Used in NIST's PB91-132282

PB91-132290

Absorbed-Dose Calibration of Ionization Chambers in a(60)Co Gamma-Ray Beam

PB91-132290

PB91-132308

Technical Digest-Symposium on Optical Fiber Measurements, 1990

PB91-133827

Current Supply for High-T(sub c) Superconductor Testing.

PB91-133868

Eddy Current Probe Characterization Using an Impedance 
PB91-133983

Transport Critical Current of Aligned Polycrystalline Y1Ba2Cu307-delta and Evidence for a Nonweak-Linked Component of Intergranular Current Conduction.

PB91-133983

101,456 Not available NTIS

PB91-134056

High T(sub c) Superconductors and the Critical Current PB91-134056

101,457 Not available NTIS

PB91-134106

Modulatable Narrow-Linewidth Semiconductor Lasers

PB91-134106

101,404 Not available NTIS

B91-134114

Determination of Scattering Parameters with Respect to the Characteristic Impedance of Precision Coaxial Air-Line Standards.

B91-134130

100,770 Not available NTIS

Accurate Frequency of the $119 \mathrm{mu} \mathrm{m}$ Methanol Laser from Tunable Far-Infrared Absorption Spectroscopy.

PB91-134130

101,405 Not available NTIS

PB91-134148

Fundamental and Harmonic Susceptibilities of $\mathrm{YBa} 2 \mathrm{Cu} 3 \mathrm{O} 7$ delta.

PB91-134148

101,458 Not available NTIS

PB91-134189

Techniques to Observe the Fracture Zone in Mortar and Concrete.

PB91-134189

100,521 Not available NTIS

Transition from Trichel-Pulse Corona to Dielectric Barrier Discharge.

PB91-134221

100,835 Not available NTIS

Method for Measuring Heat Loss from Underground Heat Distribution Systems.

PB91-13422

100,888 Not available NTIS

PB91-134239

Calibration of Antenna Factor at a Ground Screen Field Site Using an Automatic Network Analyze

PB91-134239 100,724 Not available NTIS PB91-134254

Madelung Potentials and Valences in the $\mathrm{Y} 1 \mathrm{Ba} 2 \mathrm{Cu} 3 \mathrm{O} 7 \mathrm{Su}$ perconductor

PB91-13425
391-134262

101,459 Not available NTIS

Elastic Constants, Debye Temperatures, and ElectronPhonon Parameters of Superconducting Cuprates and Related Oxides

PB91-134262

Elastic Constants of Polycrystalline Bi-Pb-Sr-Ca-Cu-O Superconductor

PB91-134270

PB91-134320

Stability of Thermistors.

PB91-134320

01,461 Not available NTIS

PB91-134338

00,779 Not available NTIS

SRM 1970 - Succinonitrile Triple-Point Standard - And Its Use in Calibration of Thermistors.

PB91-134338

100,780 Not available NTIS

B91-134403

Gas Flow Measurement: Calibration Facilities and Fluid Metering Traceability at the National Bureau of Standards

PB91-134403

100,879 Not available NTIS

B91-134411

Flowmeter Installation Effects: A New Approach to an Old but Prevalent Problem

$1-134411$

100,920 Not available NTIS

Effect of the Location of an In-Line Tube Bundle on Orifice Flowmeter Performance. PB91-134445

PB91-134478

100,921 Not available NTIS

Break Junction Tunneling Spectroscopy of Single-Crysta Bismuth-Based High-Temperature Superconductors.

101,462 Not available NTIS

B91-134486

High-Resolution, Tunneling-Stabilized Magnetic Imaging and Recording.

PB91-134486

101,600 Not available NTIS

Critical Locus, (Vapor + Liquid) Equilibria, and Coexisting Critical Locus, (Vapor + Liquid) Equilibria, and Coexisting
Densities of (Carbon Dioxide + Propane) at Temperatures from $311 \mathrm{~K}$ to $361 \mathrm{~K}$

PB91-134494

100,267 Not available NTIS

PB91-134502

Thermal Conductivity of a Moderately Dense Gas.

PB91-134502 101,601 Not available NTIS

PB91-134510

Aspects of Smoldering Combustion

PB91-134510

100,535 Not available NTIS

B9 1-134528

Collisional Electron-Detachment and Ion-Conversion Processes in SF6.

B91-134536

100,836 Not available NTIS

Developing Federal Software Standards: A New Direction
PB91.134536

100,637 Not available NTIS

B91-134544

Chitin-Chitosan Membranes: Separations of Amino Acids and Polypeptides.

PB 1-134551

101,283 Not available NTIS

Literature Review of the Galling Process
PB91-134551

PB91-134569

Magnetization of Anisotropic Superconducting Grains

PB91-134569

PB91-134577

Thermal Conductivity and Heat Capacity of Gaseous Argon.

PB91-134577

101,602 Not available NTIS

PB91-134585

Phase Equilibria from the One-Fluid Model

PB91-134585

101,385 Not available NTIS

$91-134593$

Israel's New Synchronized Time Scale, UTC(INPL)

PB91-134593

PB91-134601

Calculation of Energy of Low-Angle Grain Boundaries.

PB91-134601

101,464 Not available NTIS

PB91-134619

Report on 1989 Actions by International Institute of Weld-

ing.

101,001 Not available NTIS

PB91-134627

Cryogenic Material Properties of Stainless Steel Tube-toFlange Welds

PB91-134635

101,145 Not available NTIS

Information Systems Engineerıng Program

PB91-134635

100,685 Not available NTIS

PB91-134643

Lunar Gravitational Wave Antenna Using a Laser InterferLmeter.

PB91-134643

PB91-134650

Partial Discharges in Low Voltage Cables

PB91-134650

101,603 Not available NTIS

PB91-134650

Preliminary Dynamic Analyses of the Ministry of Agriculture Building.

PB91-134668

100,143 Not available NTIS

PB91-134676

Neutron Diffraction from Sheared D-Glycerol: Preliminary Studies.

PB91-134676

100,268 Not available NTIS

PB91-134684

Electrical Breakdown and Streamer Statistics in $\mathrm{N}$-hexane under Uniform Field Conditions.

PB91-134684

100,837 Not available NTIS

PB91-134692

Current Density Dependence of Electromigration t50 Enhancement D

PB91-134700

Corrosion, Repassivation and Corrosion Fatigue Behavior of the Surgical Implant Alloy, Co-Cr-Mo.

PB91-134700 101,132 Not available NTIS

PB91-134718

Al in Manufacturing: The NBS AMRF as an Intelligent Ma-

100,973 Not available NTIS

PB91-134726

Control System Architecture for Unmanned Ground Vehicles.

$1 \cdot 13472$

101,379 Not available NTIS

PB91-134734

Progress in the Development of Miniature Optical Fiber Cur rent Sensors.
PB91-134734

PB91-134742

100,745 Not available NTIS

Practical Considerations in the Design of Optical Fiber Current Sensors

PB91-134759

100.746 Not available NTIS

Magnetoresistance of Multifilament Al/Al-Alloy Conductors.

PB91-134759

PB91-134767

Gravity-Dependent Phenomena at the Scale of the Single

PB91-134767

101,297 Not available NTIS

PB91-134825

Performance of OSI Transport over ACCUNET and IBER-

PB91-134825

100,563 Not available NTIS

PB91-134866

Environmental Effects on the Medium and Long Term Frequency Stability of Quartz Oscillators.

PB91-134866

PB91-134874

Extending the Range and Accuracy of Phase Noise Measurements.

PB91-134874

100,588 Not available NTIS

PB91-134882

Accuracy Model for Phase Noise Measurements.

PB91-134882

100,589 Not available NTIS

PB91-134916

Dual Six-Port Reflectometer Systems Using Waveguide in the Frequency Range $18-50 \mathrm{GHz}$

PB91-134916

100,838 Not available NTIS

PB91-134940

Effect of Slag Penetration on the Performance of Magnesia Chrome Refractories in Slagging Gasifiers.

PB91-134940 100,875 Not available NTIS

PB91-134957

Structure of a Stage-3 Cs - Graphite Intercalate.

PB91-134957

100,269 Not available NTIS

B91-135020

Effect of High Pressure on Prebreakdown Phenomena in $n$

PB91-135020

100,839 Not available NTIS

PB91-136507

National Bureau of Standards Publications 1977-1987 Volume 1. Citations, Key Words, and Abstracts

PB91-136507

100,957 PC A99/MF A99

PB91-136515

National Bureau of Standards Publications 1977-1987.

Volume 2. Indexes.

PB91-136515

100,958 PC A99/MF A99

PB91-143289

Assembly Code to Compute Sine and Cosine Using the Cordic Algorithm.

PB91-143289

PB91-143297

$100,638 \mathrm{PC} \mathrm{A03/MF} \mathrm{A01}$

Heat Flux, Mass Loss Rate and Upward Flame Spread for Burning Vertical Walls (1990)

PB91-143297

100,094 PC A04/MF A01

PB91-143305

Canadian Mass Fire Experiment, 1989

PB91-143305

101,352 PC A04/MF A01

PB91.143313

Studies on the Degradation Products of Paper with and without Pollutants in a Closed Environment. 1 . Preliminary without

PB91-143313

101,181 PC A03/MF A01

PB91-143321

Outline of a National Plan on High-Performance Concrete: On the NIST/ACI Workshop. Held in Gaithersburg, MD on May $16 \cdot 18,1990$

PB91-143321

100,522 PC A04/MF A01

PB91-143339

Materials Reliability. Technical Activities, 1990. (NAS-NRC Assessment Panel, January 31.February 1, 1991)

PB91.143339

PB91.143347

Near-Field and Far-Field Excitation of a Long Conductor in a Lossy Medium

PB91-143347

PB91-143362

Multımedia Courseware in an Open Systems Environment A Federal Strategy.

PB91.14336

100,039 PC A04/MF A01

PB91-144337 
PB91-144428

Ignition Characteristics of the Nickel-Based Alloy UNS No7001 in Pressurized Oxygen.

PB91-144428

$100,536 \mathrm{PC} \mathrm{A04/MF} \mathrm{A01}$

\section{PB91-144436}

Programmer's Reference Manual for CFAST, the Unified Model of Fire Growth and Smoke Transport

PB91-144436

100,096 PC A06/MF A01

PB91-144444

Working Implementation Agreements for Open Systems Interconnection Protocols.

PB91-144444

100,686 PC A99/MF A04

PB91-146977

Heat Release Rate: The Single Most Important Variable in Fire Hazard.
PB91-146977

PB91-146985

100,097 Not available NTIS

Adsorption of Water and Oxygen on $A g(110)$ : A Study of the Interactions among Water Molecules. Hydroxyl Groups, and Oxygen Atoms.

PB91-14698

100,270 Not available NTIS

PB91-146993

State-of-the-Art in Pressure Metrology

PB91-146993

PB91-147009

Critical Exponent for Viscosity

PB91-147009

101,038 Not available NTIS

PB91-147017

100,271 Not available NTIS

Orthogonal Distance Regression

PB91-147017

101,272 Not available NTIS

PB91-147025

Experimental Evaluation of Selected Orifice Flowmeter Upstream Installations
PB91-147025

PB91-147033

100,880 Not available NTIS

Mechanism for High Strain-Rate Shear Band Formation

PB91-147033

PB91-147041

101575 Not available NTIS

Heat Capacity and Thermodynamic Properties of Deutrated Ammonium Hexafluorophosphate ND4PF6 from $5.8 \mathrm{~K}$ to $347 \mathrm{~K}$

PB91-147041

100,272 Not available NTIS

PB91-147058

Heat Capacity of Deuterated Ammonium Tetrafluoroborate ND4BF4 from 7 to $348 \mathrm{~K}$

PB91-147058

PB91-147066

100,273 Not available NTIS

Laser Induced Desorption of Molecules from Surfaces

PB91-147066 100,235 Not available NTIS

PB91-147074

Alternative Refrigerants R123a, R134, R141b, R142b, and R152a: Critical Temperature, Refractive Index, Surface Tension, and Estimates of Liquid, Vapor, and Critical Densities. PB 1-1470

Calibration Procedures for Infrared Imaging Systems for Building Diagnostics.

PB91-147082

100,057 Not available NTIS

PB91-147090

Effect of Logarithmic Singularity on the Free Edge Stress Intensity Factor of Composite Laminates.

Intensity Factor of Composite Laminates.

PB91-147

Stabilization Techniques for Spinning Rotor Gage Residual

PBag. 147108

100,922 Not available NTIS

PB91-147116

Giant Resonances in the Transition Regions of the Periodic Table.

101,605 Not available NTIS

PB91-147124

Adjustment of the Fundamental Physical Constants: A Report of the CODATA Task Group on Fundamental ConStants, 1986.

PB91-147132

101,606 Not available NTIS

Fundamentals and Applications of Infrared Themography for Nondestructive Testing

PB91-147132

101,009 Not available NTIS

PB91-147140

Second-Level Post-Occupancy Evaluation Analysis.

PB91-147140

100,058 Not available NTIS

PB91-147157

Cryogenic-Temperature Fluorescence Spectra of Polynuclear Aromatic Hydrocarbons of Molecular Weight 302.
PB91-147157 100,274 Not available NTIS PB91-147165

Phase Equilibria in the System TI-Ca-Ba-Cu-O. 1. Stability of the 2122 Phase under Conditions of Oxygen Annealing. PB91-147165

PB91-147173

Selective Excitation of X-ray Emission Spectra PB91-147173

101,607 Not available NTIS PB91-147181

Low-Temperature, Electrically Calibrated SOS Bolometer lor Power and Energy Measurements.
PB91-147181

PB91-147199

Hydrogen Slush Production with a Large Auger.
PB91-147199 100,275 Not available NTIS

PB91-147207

Standard Reference Fibers for Calibration of the Optical Time Domain Reflectometer

PB91-147207

101,406 Not available NTIS

PB91-147215

Review of Four Compartment Fires with Four Compartment Fire Models.

PB91-147223

100,098 Not available NTIS

High Speed Magnetic Field Sensors Based on Iron Garnets.

PB91-147223

PB91-147231

100,840 Not available NTIS

Incorporation of Molecular Orientation into Systems of Lamellar Morphology. 1. Effects of Packing Entropy on the La-

mellar Thickness of Block Copolymers.
PB91-147231 No0,484 available NTIS

PB91-14

Preparation and Properties of Monomolecular Films for Use as Radioactive Source Mounts

PB91-147249

PB91-147256

Experimental and Numerical Studies of the J-Integral for a Surface Flaw

PB91-147264

NBS Research Program in Municipal Solid Waste Combus tion.

PB91.14726

PB91-147272

100,916 Not available NTIS

Study of the Linearity of Transfer Leaks and a Helium Leak

Detector.

100,926 Not available NTIS

PB91-147280

Mechanism of Soot Formation in Acetylene-Oxygen Mixtures.

PB91-147280

PB91-147298

100,537 Not available NTIS

Soluble Models in Surface Collision Dynamics

PB91-147298

100,276 Not available NTIS

PB91-147306

Radiant Heat Fluxes from 100-200 MW Natural Gas/Air Diffusion Flames.

PB91-147314

100,538 Not available NTIS

Phase Formation in Electrodeposited and Thermally Annealed Al-Mn Alloys.

PB 1-147322

101,209 Not available NTIS

Effect of Coherency Strain on Alloy Formation: Migration of Liquid Films.
PB91-147322

PB91-147330

101,210 Not available NTIS

Dihedral Angles in Magnesia and Alumina: Distributions

from Surface Thermal Grooves

101,058 Not available NTIS

PB91-147348

Metal Reference Line Technique for Obtaining Dihedral Angles from Surface Thermal Grooves

PB91-147348

PB91-147355

Electrofission in the Ouasifree and Delta Regions.

PB91-147355

101,609 Not available NTIS

PB91-147363

Limiting versus Apparant Critical Behavior of Henry's Constants and K Factors

PB91-147363

100,277 Not available NTIS

PB91-147371

Stress Intensity Factors by Enriched Mixed Finite Elements. PB91-147371

101.577 Not available NTIS

PB91-147389

Application of Analytical Electron Microscopy to GlassBonded Aluminas.

PB91-147389

PB91-147397

101,060 Not available NTIS

RIR - Measurement and Use in Ouantitative XRD

PB91-147397

101,466 Not available NTIS

PB91-147405

Extension of an Improved One-Fluid Conformal Solution

Theory to Real Fluid Mixtures with Large Size Differences.
PB91-147405 100,278 Not available NTIS

Dietary Collection Procedures.

PB91-147413

PB91-147421

101,317 Not available NTIS

Practical Constraints in Monitoring Micronutrient Intake.

PB91-14742

PB91-147439

101,318 Not available NTIS

Design of High

Microstructure

PB91-147447

Mechanism of $\mathrm{OH}$ Radical Reactions with Thymine and Uracil Derivatives.

PB91-147447

100,216 Not available NTIS

PB91-147454

Structure of Carbon Dioxide Dimer from Near Infrared Spectroscopy
PB91-147454

PB91-147462

100,279 Not available NTIS

Transitions and Energy Levels for Cu-Like $\mathrm{Yb}(41+)$ ), $\mathrm{Ta}(44+)$, and $\mathrm{U}(63+)$.

PB91-147462

PB91-147470

Alkyl Transfer Reactions between Protonated Alcohols and Ethers. The Gas-Phase Alkylation of Formaldehyde

PB91-147470

100,280 Not available NTIS

PB91-147488

SHOOT Flowmeter and Pressure Transducers.

PB91-147488

100,923 Not available NTIS

PB91-147496

Global Stability of the Chaotic State Near an Interior Crisis.
PB91-147496

PB91-147504

Overview of the Ion Dynamic Effect in Line Broadening. and a Generalization of the Unified Theory.
PB91-147504 PB91-147512

Phase Diagrams of Hexagonal Binary Orderıng Alloys with Anisotropic Interactions.

PB91-147512

101,211 Not available NTIS

PB91-147520

Verification of the Relation between Two-Probe and FourProbe Resistances as Measured on Silicon Wafers.

PB91-147520

PB91-147538

Instability in $\mathrm{pH}$ Measurements of Sputtered $\mathrm{IrO} 2$ Films.

PB91-147538

100,281 Not available NTIS

PB91-147546

Factual Materials Databanks - The Need for Standards.

PB91-147546

101,249 Not available NTIS

1-147553

Quantitative Determination of Amorphous Content in $\mathrm{Ce}$ ramic Materials Using X-ray Powder Diffraction

PB91-14755

101,061 Not available NTIS

PB91-147561

Design Heat Loss Factors for Basement and Slab Floors.
PB91-147561

PB91-147561

PB91-147579

UNIX in the Government's Automated Manufacturing Research Facility (AMRF)

PB91-147579

100,963 Not available NTIS

PB91-147587

Possible $\left(e, e^{\prime} 2 N\right)$ Studies at CEBAF.

PB91.147587

101,612 Not available NTIS

PB91-147595

Application of OSI Protocols for Plant Information Net-

PB91-147595

100,974 Not available NTIS

PB91-147603

Phonon-Assisted Magneto-Donor Optical Transitions in nInSb.

PB91-147603

101,467 Not available NTIS

PB91-147611

Temperature Measurement of Glass Subjected to Solar Ra- 
PB91-147702

100,841 Not available NTIS

PB91-147710

Multicomponent Cluster Ions. 2. Comparative Stabilities of Cationic and Anionic Hydrogen Bonded Networks: Mixed

Clusters of Water and Hydrogen Cyanide.
PB91-147710 100,283 Not available NTIS PB91-147728

Feasibility of Using Knowledge-Based Systems for Aiding Inventors.

PB91-147736

100,895 Not available NTIS

Morphology and Barrier-Height Development of $\mathrm{Bi} / \mathrm{InP}(110)$ Interfaces.

PB91-147736

101,468 Not available NTIS

PB91-147744

Interactive Courseware is Leading the Multimedia Movement.

100,036 Not available NTIS

PB91-147744

Progress in Uniform Field Eddy Current Methods.

PB91.147751 101,039 Not available NTIS

PB91-147769

Capillary Supercritical Fluid Chromatography of Explosives: Investigations on the Interactions between the Analytes, the Mobile Phase and the Stationary Phase.

PB91-147769

100,182 Not available NTIS

PB91-147777

Use of Single Particle Standards for LAMMA Calibration

PB91-147777

100,183 Not available NTIS

PB91-147785

Slow Electron Transfer Reactions between Alkylhydrazines.

PB91-147785

100,284 Not available NTIS

B91-147793

Radiation Chemistry of Enones.
PB91.147793 100,236 Not available NTIS

PBg1

P91-147801

Pulse Radiolysis Studies of Organic Electron Transfer Re-

actions.

100,285 Not available NTIS

PB91-147819

Thermal Characterization of Integrated Circuits - A Tutorial. PB91-147819

PB91-147827 100,789 Not available NTIS

Thermal Measurements of VLSI Packages: A Critical

PB91-147827

Forced Smolder Propagation and the Transition to Flaming in Cellulosic Insulation.

PB91-147835

B91-147843

100,101 Not available NTIS

Smoldering Combustion Propagation Through a Permeable Horizontal Fuel Layer.

PB91-147843

100,102 Not available NTIS

PB91-147850

Computing the Eigenvalues and Eigenvectors of Symmetric Arrowhead Matrices.

PB91-147850

101,254 Not available NTIS

B91-147868

Fiber-Optic Fluorescence Array to Study Free Convection in Porous Media.

PB91-147876

101,407 Not available NTIS

Ventilation System Performance Evaluation Using Tracer Gas Techniques.

PB91-14787

100,060 Not available NTIS

PB91-147884

Effects of Pressure on the Thermal Decomposition Kinetics and Chemical Reactivity of Nitromethane.

100,286 Not available NTIS

PB91-147892

Development of a Line Camera for Real-Time Measure ments of Concentration in Turbulent Flow Fields.

PB91-147892

PB91-147900

101,386 Not available NTIS

Real-Time Radiology Standards: Results of a Workshop.

PB91-147900

S: Results of a Workshop.
101,010 Not available NTIS

PB91-147918

Investigation of Photoconductive Picosecond Microstrıpline Switches on Self-Implanted Silicon on Sapphire (SOS).

PB91-147918

100,791 Not available NTIS

B91-147926

Low Accelerating Voltage SEM Imaging and Metrology Using Backscattered Electrons.
PB91-147926 469 Not available NTIS PB91-147934

Velocity and Droplet Size Measurments in a Fuel Spray. PB91-147934

100,539 Not available NTIS

PB91-147942

Review of Pulse Tube Refrigeration.

PB91-147959

Muon Sticking in Catalysed d-t Fusion: Nuclear Resonance Effect.

PB91-147959

101,613 Not available NTIS

PB91-147967

Apparatus for the Measurement of Regenerator Performance in Pulse Tube Retrigerators.
PB91-147967

PB91-147975

Evaluation of Fumed-Silica Insulation for a Thermal Conductivity Standard Reference Material.

PB91-147975

PB91-147983

Hermetically Sealed Inert Atmosphere Cell for X-ray Powder Diffraction.

PB91-147983

101,062 Not available NTIS

PB91-147991

Graphics Program for Binary and Ternary Ceramic Phase PB91.147991

PB91-148007

101,063 Not available NTIS

Subpicosecond Pulse Compression and Raman Generation Using a Mode-Locked Erbium-Doped Fiber Laser-Amplifier.

PB1-148015

Resonant Photoemission and PSD of Rare Earth Oxides PB91-148015

PB91-148023

Crystal and Spin Structures of $\mathrm{Nd} 2 \mathrm{CuO} 4$

PB91-148023

101,470 Not available NTIS

PB91-148031

Effect of Pipe Surface Finish on Gas Flow Measurement with an Orifice Meter.

PB91-148031

101,387 Not available NTIS

91-148049

Apparatus for Measurement of Coefficient of Friction.

PB91-148049

PB91-148056

101,250 Not available NTIS

Signai Detection

MB91.148056

PB91-148064

100,540 Not available NTIS

Secondary Ion Yield Changes in Silicon and Gallium Arsenide Due to Topography Changes during Oxygen or Cesium Ion Bombardment.

PB91-148064

101,471 Not available NTIS

\section{PB91-148072}

Microstructure and Fracture at the Cement Paste-Aggregate Interface.
PB91-148072

PB91-148080

100,523 Not available NTIS

Fluoromethanol: Synthesis, Microwave Spectrum, and Dipole Moment

PB91-148098

100,288 Not available NTIS

Multiple Conformations of Amino Acid Residues in Ribonuclease A.

PB91-148098

101,298 Not available NTIS

PB91-148106

Study of the Galking of Two Steels Using Two Test MethOds.

101,147 Not available NTIS

PB91-148114

Agenda for Progress in Technology Education: A Personal VBew.

91-148114

100,037 Not available NTIS

PB91-148122

CIM, Data and Standardization within the NBS AMRF.

PB91-148122

100,975 Not available NTIS

\section{1-148130}

Innovation in Manufacturing Technology: A View from NBS. PB91-148130

PB91-148148

100,976 Not available NTIS

Trace Determination of $\mathrm{Cr}(\mathrm{VI})$ by LC/AAS with on-Line Preconcentration.

PB91-148155

Strontium-Induced Oxygen Defect Structure and Hole Doping in $\mathrm{La} 2-x \mathrm{Sr} \times \mathrm{CuO} 4$

PB91-148155

PB91-148163

Industry and Government Strategies for Product Ouality.

PB91-148163

PB91-148171

Ergodic Convergence Properties of Supercooled Liquids and Glasses.

PB91-148189

Cryogenic Properties of Copper (Wall Chart).

PB91-14818

PB91-148197

Comparison of High-Resistivity Measurements of Silicon by AC Impedance, DC Resistance, van der Pauw, and FourProbe Methods.

PB91-148197

100,792 Not avallable NTIS

PB91-148205

Strength and Fracture Behavior of $\mathrm{Ba}-\mathrm{Y}-\mathrm{Cu}-\mathrm{O}$ Supercon. ducting Ceramics.

PB91-148205

PB91-148213

101,474 Not available NTIS

Crystallizıng Catabolite Gene Activator Protein with CAMP for Structural Analysis.

PB91-148213

PB91-148221

Effects of Crystal Bonding on Brittle Fracture.
PB91-148221

PB91-148239

Spherical Near-Field Scanning: Determining the Incident Field Near a Rotatable Probe.

PB91-148239

PB91-148247

Multistep Stress-Relaxation Behavior in Uniaxial Extension

of an Ethylene-Hexene Copolymer.

PB91-148247 101,242 Not available NTIS

PB91-148254

Conformance Verification of FDDI Stations.

PB91-148254

100,747 Not available NTIS

B91-148478

Laser Induced Damage in Optical Materials: 1989

PB91-148478

PB91-148486

Bibliography of Selected Computer Security Publications, January 1980 -October 1989

PB91-148494

Abstract and Index Collection in the Research Information Center of the National Institute of Standards and Technology (Fourth Edition)

PB91-148502

Strategy for Chemical Analysis of Alternative Refrigerants. PB91-148502

PB91-148510

Refinement of a Model for Fire Growth and Smoke Transport.

48510

100,104 PC A04/MF A01

PB91-148528

Guide to the Use of the Cement and Concrete Research Remote Bulletin

PB91-14852

PB91-148650

Microwave Spectrum of Ar-H2O: Dipole Moment, Isotopic Studies, and (17)O Ouadrupole Coupling Constants.

PB91-148650

PB1-148668

Setting Reactions and Compressive Strengths of Calcium Phosphate Cements.

PB91-148668

100,040 Not available NTIS

PB9 1-148676

Integrity Tests for High-(T sub c) and Conventional CriticalCurrent Measurement Systems.

PB91-148684

Close-Coupling Calculation for the (2)(Sigma sub $\mathrm{u})(+$ ) State of ( $\mathrm{H}$ sub 2)(-).

PB91-148684

101,614 Not available NTIS

PB91-148692

Determination of the Neutron Mass and Implications for Fundamental Constants.

PB91-148692

101,615 Not available NTIS

PB91-148700

High Speed Superconducting A/D Converter

PB91-148700

PB91-148718

Anisotropic Structure of a Simple Liquid.

PB91-14871

101,616 Not available NTIS

PB91-148726

Photodissociation Dynamics of Water in the Second $\mathrm{Ab}$

sorption Band: Vibrational Exitation of $\mathrm{OH}$ (A (2)Sigma).
PB91-148726 100,291 Not available NTIS 
PB91-148809

Novel Laser Gain and Time-Resolved FTIR Studies of Photochemistry.
PB91-148809

PB91-148817

100,237 Not available NTIS

Characterization of the Mineral Fraction in Botanical Reference Materials and Its Influence on Homogeneity and Analytical Results

PB91-148825

100,186 Not available NTIS

Modelling the Coronae and Chromospheres of RS CVn Systems by the Analysis of Ultraviolet, $\mathrm{X}$-ray and Radio Observations.

PB91-148825

PB91-148833

100,019 Not available NTIS

Ultraviolet, Optical, Infrared, and Microwave Observations of HR 5110 .

PB91-148841

100,020 Not available NTIS

Mode Specific Internal and Direct Rotational Predissociation in HeHF, HeDF, and HeHCL: van der Waals Complexes in the Weak Binding Limit.

PB91-148841

PB91-148858

Reverse-Phase HPLC Separation and Electrochemical Detection of Retinol and Its Isomers.
PB91-148858 100, 187 Not available NTIS

PB91-148866

Neutron Powder Diffraction and Inelastic Scattering Study of the Structures of $\mathrm{Zr} 2 \mathrm{Pd}, \mathrm{Zr} 2 \mathrm{PdD} 1.70$, and $\mathrm{Zr} 2 \mathrm{PdD} 1.96$. of the Structures of Zr2Pd, Zr2PdD1.70, and Zr2PdD1.96.
PB91-148866 101,213 Not available NTIS PB91-148874

Research at NBS in Direct Potentiometric Measurements in Blood.

PB91-148874

100,188 Not available NTIS

PB91.148882

Structures of the NH3-HCCCCH and $\mathrm{H} 2 \mathrm{O}-\mathrm{HCCCCH}$ Complexes by Fourier-Transform Microwave Spectroscopy.
PB91-148882 100,294 Not available NTIS

PB91-148890

Chain Conformation of a Block Polymer in a MicrophaseSeparated Structure

PB91-148890

100,485 Not available NTIS

PB91-148908

Use of Heterodyne Detection to Measure Optical Transmittance over a Wide Range. 101,410 Not available NTIS
PB91-148908

PB91-148916

Theory of Specific Heat of Solids.
PB91-148916

PB91-148924

Multireference-Configuration Interaction (MR-Cl) Calculations on $\mathrm{HS}(2+)$ and Experimental Observation via Electron Impact lonization of H2S.
PB91-148924 100,295 Not available NTIS PB91-148932

Possible 'Proximity Matrix' Route to High Current Conductors.
PB91-148932

PB91-148940

Applicability of Smoluchowski's Equation with a Constant Kernel to Coalescence.

PB91-148940

PB91-148957

Studies of $\mathrm{HI}$ and D I in the Local Interstellar Medium

PB91-148957 100,021 Not available NTIS

PB91-148965

Spatially Resolved Flares in RS CVn Systems.

PB91-148965

100,022 Not available NTIS

PB91-148973

$\mathrm{H}+\mathrm{O} 3$ Fourier-Transform Infrared Emission and Laser tal Dipole Moment Function and State-to-State Einstein A Coefficients

PB91-148973

PB91-148981

100,297 Not available NTIS

Methods for the Analysis of Organometallic Compounds in Wastes

PB91-148999

100,189 Not available NTIS

Micromachined Thermal Radiation Emitter from a Commer cial CMOS Process.
PB91-148999

100,748 Not available NTIS

PB91-149005

Laser-Induced Fluorescence Measurements of Drift-Velocity Distributions for $\mathrm{Ba}(+)$ in $\mathrm{Ar}$ : Moment Analysis and a Direct Measure of Skewness.

PB91-149005

100,298 Not available NTIS

PB91-149013

Laser Diagnostics for Characterization of Fuel Sprays.

PB91-149013 100,541 Not available NTIS PB91-149021

Terminal Velocities for a Large Sample of O Stars, B Supergiants, and Wolf-Rayet Stars.

PB91-149021

100,023 Not available NTIS

PB91-149039

Materials Research at the National Bureau of Standards.
PB91-149039

101,214 Not available NTIS

PB91-149047

Cleavage Step Formation and Resistance to Transgranula Stress Corrosion Cracking

PB91-14904

101,133 Not available NTIS

\section{$1-149062$}

Characteristics of the Breakdown Voltage of Power MOSFETs After Total Dose Irradiation
PB91-149062 100,793 Not available NTIS

PB91-149070

MMIC Related Metrology at the National Institute of Standards and Technology

PB91-14907

100,794 Not available NTIS

NBS Ouality Assurance Support: Current and Planned Serv PB91-149088

PB91-149096

101,012 Not available NTIS

Low Temperature Chemical Process for Precursors to Boride and Carbide Ceramics Powders. Not available NTIS
PB91-149096 101,066 N PB91-149104

Low Temperature Synthesis of Ceramic Powders for Structural and Electronic Applications.

PB91-149104

\section{PB91-149112}

In situ Measurements of Bridged Crack Interfaces in the Scanning Electron Microscope.
PB91-149112 101,068 Not available NTIS

PB91-149120

Measurement of Capacitance on Wafers

PB91-149120

100,795 Not available NTIS

PB91-149138

Dispersion of the Electronic Contribution to the Thrid-Orde Nonlinear Susceptibility of $\mathrm{H}_{2}$.

PB91-149138

101,411 Not available NTIS

PB91-149146

Performance and Limitations of Faraday Effect Sensors.

PB91-149146

PB91-149153

Further Investigation of the Effect of Application Parameters on Adhesive-Bonded Seams of Single-Ply Roof Mem branes.

PB91-149153

100,105 Not available NTIS

PB91-149161

Applied Electric Fields for Downstream Processing PB91-149161

PB91-149179

100,299 Not available NTIS

UNIX Expert: A Prototype Knowledge-Based Software Development Workstation.

PB91-149179

100,639 Not available NTIS

PB91-149187

Determination of Bonded Phase Thickness in Liquid Chro-

matography by Small Angle Neutron Scattering

PB91-149187 100,190 Not available NTIS

PB91-149195

Laser Measurements

PB91-149203 Precise Experimental Test of Calculated Two-Electron
Lamb Shifts in Helium.

PB91-149203

PB91-149211

Thermal Noise in Mechanical Experiments.

PB91-149211

PB91-149229

Hydrodynamics and Mass Transfer in Two-Phase Aqueous Extraction Using Spray Columns.

PBtraction Usi

PB91-149237

Preparation and Analysis of a Marine Sediment Reference

Material for the Determination of Trace Organic Constituents.
PB91-149237

PB9 1-149245

101,377 Not available NTIS

Nonresonant Charge Transfer in the Threshold Region for

(3) $\mathrm{He}(+)+(4) \mathrm{He}(\mathrm{sub}<-$ sup- $>)$ ) (3) $\mathrm{He}+(4) \mathrm{He}(+)$. PB91-149245

PB91-149252

Periodic Orbits and Diffuse Structures in the Photodissocia-

tion of Symmetric Triatomic Molecules.
PB91-149252 100,238 Not available NTIS

\section{PB91-149260}

Photodissociation of C(I)NO in the S(I) State: A Quantum Mechanical ab initio Study.

PB91-149260

PB91-149278

Dynamic Arc-Power Source Response in GMA Welding PB91-149278

100,239 Not available NTIS PB91.149286

New Radio Detectors of Early-Type Pre-Main Sequence Stars

PB91-149286

PB91-149294

Study of the GaAs-Si(100) Interface Using Laser Probing of Thermal Desorption Kinetics.

PB91-149294

101,477 Not available NTIS

PB91-149302

Application of Fire Research.

PB91-149302

100,153 Not available NTIS

PB91-149310

Comprehensive Approach for Modeling and Testing Analog and Mixed-Signal Devices.

PB91.149310

100,732 Not available NTIS

PB91-149328

Epitaxial fcc Fe Films on $\mathrm{Cu}(100)$

PB91-149328 101,478 Not available NTIS

PB91-149336

Algorithm Design for Large-Scale Computations

PB91-149336

100,640 Not available NTIS

PB91-149344

Tensile-Fracture Resistance Mechanisms in Brittle Polycrystals: An Ultrasonics and In-situ Microscopy Investigation.

101,190 Not available NTIS

PB91-14935

Transferring NBS Technology to Small Manufacturers Through State and Local Centers. PB1-149369

Influence of Sulfur on Methanation over Tungsten(110). PB91-149369

100,300 Not available NTIS

PB91-149377

Summary Abstract: The Influence of Sulfur on Methanation over W(110).

PB91-149385

100,301 Not available NTIS

General Method for Doppler Determination of Cylindrically Symmetric Velocity Distributions: An Application of Fourier Transform Doppler Spectroscopy. An Application of Fourier PB91-149385

PB91-149393

Relation between the Surface Energy and the Debye Temperature for Cubic Solids.

PB91-149393

PB91-149401

Device for Audio-Frequency Power Measurement

PB91-149401

100,733 Not available NTIS

PB91-149419

Comparison of Flux Dynamics in Two Samples of YBa2Cu3O7 with Different Pinning.
PB91-149419 Not available NTIS

PB91-149427

Structural Instabilities and Superconductivity in Ouasi-Binary

Mn5Si3-Type Compounds
PB91-149427

PB91-149435

Mechanical Loss in a Glass-Epoxy Composite.

PB91-149435

101,215 Not available NTIS

PB91-149443

Linear Calibration When the Coefficient of Variation is Con-

Stant. 14943

101,273 Not available NTIS

PB91-149450

Material Dependency of Chip-Form Detection Using Acoustic Emission.

101,216 Not available NTIS

PB91-149831

Thermodynamics of Ammonium Indates. 2. The Molar Heat Capacity of the Ammonium Pentabromoindate Monohydrate Salt (NH4)2 InBr5. H2O from 7.8 to $348 \mathrm{~K}$.

PB91-149831

100,304 Not available NTIS

PB91-149849

Photospheres of Hot Stars. 4. Spectral Type O4.

PB91-149849

100.025 Not available NTIS

PB91-149864

Thermodynamics of the Divalent Metal Fluorides. 3. Heat Capacity of the Fast lon Conductor Sr SnF4 from 6 to 344 PB91-149864

100,305 Not available NTIS

PB91-149872

Mechanism for Shear Band Formation in the High StrainRate Torsion Test.

PB91-149872

101,579 Not available NTIS 
PB91-149922

Pozzolan Programs of the Cement and Concrete Reference Laboratory.

PB91-149930

Measurement Ouality Assurance.

PB91-149930

100,525 Not available NTIS

PB91-149948

Round Robin on Apparent Thermal Conductivity of Several Loose-Fill Insulations.

PB91-149948

101,143 Not available NTIS

PB91-149955

Application of Ultrafast Broadband Infrared Spectroscopy to Measurement of Metal-Carbonyl Dynamics. PB91-149955

PB91-149963

Analysis of the $\mathrm{Y} 8 \mathrm{a} 2 \mathrm{Cu} 3 \mathrm{O} 7 / \mathrm{SrTiO}$ Interface as a Function of Post-Deposition Annealing Temperature.

PB91-149963

101,480 Not available NTIS

PB91-149971

Optical Potential Approach to Electron and Positron Scattering from Noble Gases. 2. Neon.

tering from Noble Gases. 2. Neon. 101,619 Not available NTIS

PB91-149989

Modelling Gallium Arsenide Transistors.
P891-149989 Not available NTIS

PB91-149997

Emergence of Modern Nucleation Theory

P891-149997

101,481 Not available NTIS

PB91-150003

Fusion of Mercury. A New Certified Standard for Differential Scanning Calorimetry.

P891-150003

100,309 Not available NTIS

PB91-150011

Nonlocal Phase Shifts Induced by Static Electric Fields in Neutron Interferometers When the Path-Enclosed Charge Vanishes.

PB91-150011

101,620 Not available NTIS

PB91-150029

Low-Cost Low-Volume Carrier (Minilab) for Biotechnology and Fluids Experiments in Low Gravity

PB91-150029

100,041 Not available NTIS

B91-150037

Improved Analysis for Flexural Creep with Application to Sialon Ceramics.

PB91-150045

101,071 Not available NTIS

Amorphization and Conductivity of Silicon and Germanium Induced by Indentation.

PB91-150045

101,482 Not available NTIS

\section{PB91-150052}

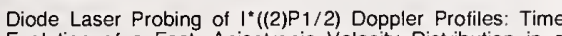
Evolution of a Fast, Anisotropic Velocity Distribution in a Thermal Bath.

PB91-15005
PB91-150060

100,310 Not available NTIS

gamma-Irradiated Seafoods: Identification and Dosimetry by gamma-Irradiated Seafoods: Identification and Dosir

PB91-150060 100,016 Not available NTIS

PB91-150078

Concepts of Underground Corrosion,
PB91-150078 101,134 Not available NTIS

PB91-150086

Biodegradable Plastics: An Idea Whose Time Has Come

PB91-150086

01,243 Not available NTIS

PB91-157123

STEP On-Line Information Service User's Guide. National PDES Testbed Report Series.

P891-157123

$100,950 \mathrm{PC} \mathrm{A03/MF} \mathrm{A01}$

PB91-157156

New Approach to Vision and Control for Road Following PB91-157156

101,743 PC A03/MF A01

PB91-157164

NIST Express Working Form Programmer's Reference (Revised November 1990). National PDES Testbed Report Series.

PB91-157164

100,964 PC A04/MF A01

PB91-157172

Pulse-Echo Ultrasonic Evaluation of the Integrity of Seams of Single-Ply Roofing Membranes: Laboratory Evaluation of a Prototype Test Apparatus.

P891-157172

100,106 PC A03/MF A01

PB91-157180

Species Produced in Fires Burning in Two-Layered and Homogeneous Vitiated Environments.

P891-157180

100,542 PC A12/MF A02

PB91-157198

Proceedings of the Object-Oriented Database Task Group Workshop, Tuesday, October 23, 1990, Chateau Laurier Hotel, Ottawa, Canada.

PB91-157198

$100,641 \mathrm{PC} \mathrm{A07/MF} \mathrm{A01}$

PB91-157206

Fire Propagation in Concurrent Flows.

P891-157206

100,543 PC A07/MF A01

PB91-157214

TROI User's Guide.
P891-157214

$101,026 \mathrm{PC} \mathrm{A03/MF} \mathrm{A01}$

PB91-158485

Rapid Low-Temperature Hopping of Hydrogen in a Pure Metal: The ScHx System.

PB91-158485

101,217 Not available NTIS

PB91-158493

Visco-Elastic Deformation of Dental Porcelain and Porcelain-Metal Compatibility.

PB91-158493

100,042 Not available NTIS

PB91-158501

Micromagnetic Calculations of $180 \mathrm{deg}$ Surface DomainWall Magnetization Profiles with Comparison to Measure-

ments.

101,483 Not available NTIS

PB91-158519

Levitation Calorimetry.

P891.158519

PB91-158527

Acoustic Emission Monitoring of Laser Drilling.

P891-158527

PB91-158535

Conference Coverage: Papers Presentations Shine.

PB91-158535

PB91-158543

Compartment Fire-Generated Environment and Smoke Fill-

ing. $151-158543$

PB91-158550

100,107 Not available NTIS

Convective and Morphological Instabilities during Directional Solidification

PB91-158568

101,219 Not available NTIS

Self-Adjustment of a Robot Joint Controller

PB91-158568

101,027 Not available NTIS

PB91-158576

Application of the Joint Excitation Gear Adjustment Technique to Low Frequencies and Heavy Robot Joints.

P891-158576

PB91-158584

101,028 Not available NTIS

Toward Modular Semantic Application Layer Protocols (MSALPs): Communication between Heterogeneous Information Systems Using Artificial Intelligence (Al) Programming Techniques.

P891-158584

PB91-158592

Rapporteur's Summary Materials Development for Coal Gasification.

PB91-158600

100,876 Not available NTIS

Near Net Shape Forming of Ceramics.

PB91-158600

101,072 Not avaitable NTIS

PB91-158618

Relative Populations of Excited Levels within the Ground Configuration of Si-Like Cu, Zn, Ge, and Se lons.

P891-158618 101,443 Not available NTIS

PB91-158626

Measurement of Space Charge Fields in Polymers.

PB91-158626

100,486 Not available NTIS

PB91-158634

Accurate Spectroscopy in the $X$-ray Region

PB91-158634

101,621 Not available NTIS

B91-158642

$\mathrm{X}$-ray Interferometry and gamma-ray Wavelengths.

PB91-158659

Evaluation of Building Systems Inventions.

PB91-158659

01.484 Not available NTIS

PB91-158659.

100,896 Not available NTIS

Corrosion Testing in Soil.

PB91-158667

PB91-158675

Using Surface Roughness as a Wear Criterion in HighSpeed Steel End-Milling.

PB91-158675

101,040 Not available NTIS

PB91-158683

Effects of Different CH4-H2 Gas Compositions on the Morphology and Growth of Diamond Grown by Hot Filament

P891-158683

101,054 Not available NTIS

PB91-158691

Effect of Catalyst Structure on the Synthesis of a Dental Restorative Monomer

391-158691

PB91-158709

100,043 Not available NTIS

Stoichiometry

PB91-158717

New Different Forms of Ammonium Loaded and Partly Deammoniated Zeolite Rho Studied by Neutron Powder PB91-158717 00,311 Not available NTIS

PB91-158725

Engineers' Statistical Literacy is Key to U.S. Competitiveness.

100,038 Not available NTIS

PB91-158733

Corrosion Behavior of Zirconium Alloy Nuclear Fuel Cladding.

P891-158733

101,372 Not available NTIS

PB91-15874

Thermodynamic Data for Modeling of Flue Gas Cleanup Systems (Abstract for Poster Session).

PB91-158741

PB91-158758

Specifications for a Federal Information Processing Stand-

ard Data Dictionary System.

P891-158758

PB91-158766

1987: The Year of the 386

P891-158766

100,687 Not available NTIS

PB91-158774

National Bureau of Standards-American Ceramic Society Phase Diagram Data Program.

PB91-158774

PB91-158782

Real-Time Hierarchical Planning for Multiple Mobile Robots PB91-158782

PB91-158790

Zonal Model for Corona Discharge-Induced Oxidation of $\mathrm{SF} 6$ in $\mathrm{SF} 6 / \mathrm{O} 2 / \mathrm{H} 2 \mathrm{O}$ Gas Mixtures.

PB91-158790

PB91-158808

Mechanisms of Additive Effectiveness.

PB91-158808

172 Not available NTIS

PB 1-158816

Surface Acoustical Wave Spectroscopy for High Humidity

Sensing

P891-158816

100,843 Not available NTIS

91-158824

Design and Analysis of Experiments

P891-158824

PB91-158832

Data Management Standards in Computer-Aided Acquisition and Logistic Support (CALS).

PB91-158832

101,339 Not available NTIS

PB91-158840

Towards Computer-Based Microstructure Models for Cement-Based Systems.

PB91-158840

101,074 Not available NTIS

PB91-158857

Wetting Layers on Solid Substrates.

PB91-158857

100,313 Not availabie NTIS

PB91-158865

NIST Standards for Sodium and Potassium.

P891-158865

100,191 Not available NTIS

PB9 1-158873

NBS Submicron Particle Standards for Microcontamination Measurement

P891-158873

PB91-158881

100,798 Not available NTIS

Explicit Mapping of the Set of Dynamical Symmetries onto the Set of Equivalent Lagrangians for Systems with One Degree of Freedom

158881

101,622 Not available NTIS

PB91-158899

Choosing a Name for Your Computer.

PB91-158899 
PB91-159004

Mechanical Testing Revitalized.

PB91-159004

PB91-159012

Redox Reactions of Osmium Porphyrins

PB91-159012

PB91-159020

Modeling Electron Beam Interactions in Semiconductors.

PB91-159020

PB91-159038

Five Small Flaming Fire Tests in a Simulated Hospital Patient Room Protected by Automatic Fire Sprinklers.

100,109 Not available NTIS

PB91-159038

Simplified Error Bounds for Newton's Rule.

PB91-159046

PB91-159053

101,257 Not available NTIS

Chemical Modification of the Orthorhombic Superconductor Ba2YCu3O7-delta.

PB91-159061

101,486 Not avaitable NTIS

Domestic Implementation

PB91-159061

PB91-159079

100,176 Not available NTIS

Prototype Expert System for Diagnosing Moisture Problems in Houses.

PB91-159087

100,062 Not available NTIS

Submicrometer SEM Magnification Standard

PB91-159087

PB91-159095

Electrokinetic Demixing of Two-Phase Aqueous Polyme Systems. 1. Separation Rates of Polyethylene Glycol-Dex PB91-159095

PB91-159103

100,487 Not available NTIS

Reference Materials: Their Role in Measurement Accuracy

PB91-159103

PB91-159111

101,014 Not available NTIS

Use of Neutron Pole Figures to Calibrate Ultrasonic Techniques for On-Line Texture Control of Aluminum Plates.

PB91-159111 101,222 Not available NTIS

PB91-159129

Phonon Density of States of Superconducting YBa2Cu3O7 and the Nonsuperconducting Analog $\mathrm{YBa} 2 \mathrm{Cu} 3 \mathrm{O} 6$.

PB91-159129 101,487 Not available NTIS

PB91-159137

International Co-operation: The Versailles Project on Advanced Materials and Standards

PB91-159137

PB91-159145

VAMAS Bulletin.

PB91-159145

101,191 Not available NTIS

PB91-159152

101,192 Not available NTIS

Method for Characterizıng the Thermal Performance of a Solar Storage Wall from Measured Data.

PB91-159152 100,901 Not available NTIS

PB91-159160

Agency Exploits Flexibility of Software in Moving Info.

PB91-159178

100,642 Not available NTiS
Software in Moving Info.

Spin Glass Magnetic Behavior of Iron/Silica Gel Nanocom-

posites.
PB91-159178

PB91-159186

101,117 Not available NTIS

Kinematic Couplings for Precision Fixturing. Part 2. Experimental Determination of Pepeatability and

PB91-15918

101,041 Not available NTIS

PB91-159194

Design and Implementation of a Five-Axis Robotic Micromanipulator for Inserting Parts into Precision Collets

PB91-159194 101,029 Not available NTIS

PB91-159202

Electrodeposition of Alumınum from Molten Salts

PB91-159202 101,223 Not available NTIS

PB91-159210

Dental Biomaterials and Engineering Frontiers for the 90 's.

PB91-159210

100,044 Not available NTIS
10ering Frontiers for the 90 's.

PB91-159228

Thwarting the Hackers

PB91-159228

100,705 Not available NTIS

Preface to Book Entitled 'Nonequilibrium Effects in lon and

Electron Transport'

101,623 Not available NTIS

PB91-159244

Permittivity Measurements on Molecular-Sized Samples.

PB91-159244

PB91-159251

Steam Tables

Steam Tables

PB91-159269

Method for Ouantitative Measurement of Galling Damage.

PB91-159269

PB91-159277

Tensile Creep Behavior of Structural Ceramics.
PB91.159277

101,075 Not available NTIS

PB91-159285

Progress at NIST on Absolute Frequency Standards Using PB91-159285

PB91-159293

Al: Does It Have a Place in Building Simulation.

PB91-159293

PB91-159301

Photoionization and Collisional Ionization of Excited Atoms Using Synchrotron and Laser Padiation

PB91-159301

101,626 Not available NTIS

PB91-159319

Hardware Instrumentation Approach for Performance Meas urement of a Shared-Memory Multiprocessor

PB91-159319

100,601 Not available NTIS

PB91-159715

Polymers: Technical Activities 1990

PB91-159715

$100,488 \mathrm{PC} \mathrm{A07/MF} \mathrm{A01}$

PB91-159723

Proceedings of the Object-Oriented Database Task Group Workshop. Held in Atlantic City, New Jersey on May 22,

PB91-159723

100,643 PC A14/MF A02

PB91-159731

Pulse-Echo Ultrasonic Evaluation of the Integrity of Seams of Single-Ply Roofing Membranes: Results of Field Investigations and Recommendations.

100,110 PC A03/MF A01

PB91-159749

Center for Electronics and Electrical Engineering Technical Progress Bulletin Covering Center Programs, July to September 1990, with 1991 CEEE Events Calendar PB91-159749

100,801 PC A03/MF A01

\section{$1-15975$}

National PDES Testbed Mail Server User's Guide. Nationa PDES Testbed Report Series.

PB91-15975

$100,951 \mathrm{PC}$ A02/MF A0

PB91-15976

NIST 'Building Life-Cycle Cost' (BLCC) Program (Version

3.0). User's Guide and Reference Manual.
PB91-159764 100,154 PC A04/MF A01

PB91-159772

NIST Reactor: Summary of Activities July 1989 through June 1990.

PB91-159772

101,488 PC A05/MF A01

PB91-161919

Improved Technique for Determining Complex Permittivity with the Transmission/Reflect

PB91-161927

Picosecond IR Studies of the Vibrational Dynamics of $\mathrm{CO}$ Pt(111).

PB91-161927

100,314 Not available NTIS

PB91-161935

Structural and Magnetic Properties of Er Thin Films and $\mathrm{Er}$ / $Y$ Superlattices: Magnetoelastic Effects.

PB91-161935

PB91-161943

Proceedings of Cable ' 89 Workshop.

PB91-161943

100,773 Not available NTIS

PB91-161950

Probe Waveforms and the Reconstruction of Structural Dynamic Green's Functions.

PB91-161950

101,737 Not available NTIS

PB91-161968

Research at NBS on Dynamic Measurements of Thermo physical Properties at High Temperature (Abstract).

PB91-161968

100,315 Not a

PB91-161976

Cross-Validation Procedure for Stopping the EM Algorithm and Deconvolution of Neutron Depth Profiling Spectra.

PB91-161976 101,627 Not available NTIS

PB91-161984

Visibility of Exit Signs and Directional Indıcators.

PB91-161984

100,083 Not available NTIS

PB91-161992

TEM/Reverberating Chamber Design/Concept Study: A Single Facility for Large System Radiated EMC Testing, 10 $\mathrm{kHz}=40 \mathrm{Ghz}$

PB91-162008

Small Scale Demand Type Neon Liquefaction Plant PB91-162008

PB91-162016

100,224 Not available NTIS

Apparatus for Measurement of Thermal Conductivity of in sulation Systems Subjected to Extreme Temperature Differnces.

PB91-162016

101,738 Not available NTIS

PB91-162024

Effect of Pyrophosphate Concentrations on Calcium Phosphate Growth on Well-Crystallized Octacalcium Phosphate and Hydroxyapatite Seed Crystals.

PB91-162024

101, 193 Not available NTIS

PB91-162032

Newest Approaches to Ouantitative Assessment of Bioac-

tive Organotins.

PB91-162032

101,328 Not available NTIS

PB91-162040

Evaluation of Dual-Port Circularly Polarized Probes for Elanar Near-Field Measurements.

PB91-162040 100,590 Not available NTIS

PB91-162057

$\mathrm{H} 2 \mathrm{O} 2$ : Spectroscopy, Structure and Dynamics.

PB91-162057 100,316 Not available NTIS

PB91-162065

Resonant Tunneling with Electron-Phonon Interactions: An Exactly Solvable Model Applied to Desorption.

PB91-162065

PB91.162073

Phase Transitions.

PB91-162073

100,317 Not available NTIS

Unified Description of Infinite-Dilution Thermodynamic Properties for Aqueous Solutes.

PB91-162081

PB91-162099

Spinodal Decomposition in a Hydrogen-Bonded Polymer

PB91-162099

100,489 Not available NTIS

PB91-162107

Analytic Solution for the Three-Layer Multiple Beam Intererometer.

PB91-162107

101,007 Not available NTIS

PB91.162115

Automated High-Temperature Guarded-Hot-Plate Apparatus for Measuring Apparent Thermal Conductivity of Insulations between 300 and $750 \mathrm{~K}$

PB91-162115

100,111 Not available NTIS

PB91-162123

Ouantum Optics of Single, Trapped lons.

PB91-162123

101,628 Not available NTIS

PB91-162131

Time-Resolved Small-Angle Neutron Scattering in Intermediate- and Late-Stage Spinodal Decomposition of (DPB/ PB91-162131

PB91-162149

Computer Simulated Hydration of a Cement Model.

PB91-162149

100,112 Not available NTIS

91-162156

Microstrip Patch Antenna as a Standard Transmitting and Receiving Antenn

100,591 Not available NTIS

PB91-162164

Visualization of Nucleate Flow Boiling for an R22/R114 Mixture and Its Components.

PB91-162164

100,225 Not available NTIS

PB91-162172

Uses of Spın-Polarised Electrons in Fundamental ElectronAtom Collision Processes and the Analysis of Magnetic Microstructures.

101,490 Not available NTIS

PB91-162180

Mechanical Properties of Alumina-Peek Unidirectional Com-

posite: Compression, Shear, and Tension.
PB91-162180

PB91-162198

Low-Background Gamma-Ray Assay Laboratory for Activation Analysis.

100,192 Not available NTIS

PB91-162206

Photonic Probes for the Measurement of Electromagnetic Fields Over Broad Bandwidths.

PB91-162206 
PB91-162289

Surface-State-Mediated Photochemistry: Laser-Induced Desorption of NO from Si (111)

PB91-162289

PB91-162297

Resonance Ionization Spectroscopy/Resonance Ionization Mass Spectrometry Data Service. II. Data Sheets for Al, Ca, $\mathrm{Cs}, \mathrm{Cr}, \mathrm{Co}, \mathrm{Cu}, \mathrm{Kr}, \mathrm{Mg}, \mathrm{Hg}$, and $\mathrm{Ni}$

PB91-162297

100,193 Not available NTIS

PB91-162305

Micromagnetics of Domain Walls at Surfaces.

PB91-162305

101,491 Not available NTIS

PB91-162313

Narrow-Gap Semiconductors and Related Materials.

PB91-162313

101,492 Not available NTIS

PB91-162321

Superconducting Properties of $\mathrm{Bi} 2-\mathrm{x}-\mathrm{yPb}$ P SnySr2CazCu3Oz
101,076 Not available NTIS
PB91-162321

PB91-162339

Analysis and Characterization of Commercial Welding

PB91.162339

101,003 Not available NTIS

PB91-162347

Effect of Pipe Surface Finish on the Orifice Discharge Coefficient.

PB91-162347

101,389 Not available NTIS

PB91-162354

Stylus Profiling at High Resolution and Low Force.

PB91-162354 101.015 Not available NTIS

PB91-162362

Cutting the High Cost of Testing.
PB91-162362

Intercomparison of NBS Noise Calibration Services.

PB91-162370 100,847 Not available NTIS

PB91-162

Calculated from the Frequency Domain: An Update

PB91-162388 100,578 Not available NTIS

PB91-167155

ZIP: The ZIP-Code Insulation Program, Version 2.0. Economic Insulation Levels for New and Existing Houses by Three-Digit Zip Code Users' Guide and Reference Manual Revised Edition

$100,064 \mathrm{PC} \mathrm{A03/MF} \mathrm{A01}$

PB91-167163

Standard Reference Materials: Bright-Chromium Linewidth Standard, SRM 476, for Calibration of Optical Microscope Linewidth Measuring Systems.

PB91-167163

PB91-167171

Examination of the Excessive Retained Austenite on the Surface of a Section of 17-7 Precipitation Hardening Stain less Steel.

PB91-16717

$101,149 \mathrm{PC}$ A03/MF A01

PB91-167189

Review of Research Literature on Masonry Shear Walls.

PB91-167189

100,113 PC A05/MF A0
Pasonry Shear Walls.

PB91-167197

Ceiling Jet in Fires.
PB91-167197

PB91-167205

Uncertainty and Accuracy in Physical Measurements.

PB91-167205

100,114 PC A04/MF A01

PB91-167213

Technical Activities 1990, Standard Reference Data Program.

$91-167213$

$100,321 \mathrm{PC}$ A05/MF A0

PB91-16722

Review of Current Geometric Tolerancing Theories and In spection Data Analysis Algorithms.

PB91-167221

PB91-167239

Measurement of Structural Response Characteristics of Full-Scale Buildings: Selection of Structures.

PB91-167239

100,144 PC A03/MF A01

PB91-167247

Field Study of the Performance of EPDM Roofing at Air Force Facilities

PB91-167254

Experimental Study of Top Vented Compartment Fires

PB91-167254

PB91-167262

SOL3 Support for CALS Applications.

PB91-167262

PB91-167270

Role of Bench-Scale Test Data in Assessing Real-Scale PB91-167270

PB91-167288

100,156 PC A06/MF A0

DISCOUNT-A Program for Discounting Computations in Life-Cycle Cost Analyses. User's Guide and Reference

PB91-167288

$100,897 \mathrm{PC} \mathrm{A03/MF} \mathrm{A0}$

PB91-167296

Raster Graphics Conformance Testing
PB91-167296

101,341 PC A04/MF A01

B91-167304

Computational Models Developed for the Corrosion of Prestressing Steel.

PB91-167312

$100,115 \mathrm{PC} \mathrm{A03/MF} \mathrm{A0}$

NIST Standard Reference Data Products 1991 Catalog.

PB91-167312 100,952 PC A04/MF A01

PB91-167

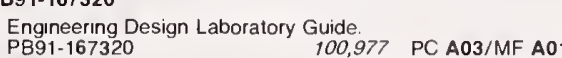

PB91-167338

Intercomparıson Study of Rockwell Hardness Test Blocks.

PB91-167338

PB91-167346

Crack Arrest Fracture Toughness Measurements of Nor malized and Inclusion Shape Controlled AAR TC128 Grade $B$ Steel, and Micro-Alloyed, Control-Rolled, and Inclusion Shape Controlled A $8 \times \times$ Grade B Steel.

PB91-167346

PB91.167353

Proposed Standard Practice for Assessing the Performance of Gas-Phase Air Cleaning Equipment

PB91-167353 100,905 PC A03/MF A01

PB91-167361

Robot Characterization Testing

PB91-16736

PB91-167379

Directory of Federal Government Laboratory Accreditation Designation Programs.

PB91-167379

PB91-167387

Functional Benchmarks for Fourth Generation Languages

PB91-167387

PB91-167395

U.S. Assessment of the New Diamond Technology in Japan.

PB91-167395

101,107 PC A05/MF A0

PB91-167403

Users Guide for RAPID, Version 2.3

PB91-167403

100,999 PC A06/MF A0

91-167676

Modern Test Methods for Flammability.

PB91-167676

$100,116 \mathrm{PC} \mathrm{A03/MF} \mathrm{A01}$

PB91-167700

Automan 2.0: Decision Support Software for Automated Manufacturing Investments. User Manual

PB91.167700

100,978 PC A04/MF A01

PB91-167833

Interim Report to the President and to the Congress on the Malcolm Baldrige National Ouality Award.

PB91-167833

100,001 PC A05/MF A0

PB91-171330

NIST Serial Holdings, 199

PB91-171330

100,960 PC A12/MF A02

PB91-171959

Transmission/Reflection and Short-Circuit Line Permittivity Measurements

PB91-171967

100,848 PC A08/MF A01

Stable Implementation Agreements for Open Systems Interconnection Protocols. Version 4, Edition 1, December 1990 PB91-17196

100,689 PC A99/MF E06

PB91-174235

Appendix: Standard Solutions and Certified Reference Materials.

PB91-174235

100,194 Not available NTIS

PB91-174243

Experimentation, Analysis, and Correlation of Refrigerant-2 Flow Through Short Tube Restrictors.

PB91-174243

PB91-174250

Sensing of GMAW Droplet Transfer Modes Using an ER 100S-1 Electrode.

B91-174268

Determining the Dynamic Stress Intensity Factor with Strain Gages Using a Crack Tip Locating Algorithm.

PB91.174268 101,580 Not available NTIS PB91-174433

Improved Optical Diffraction Strain Measurement System PB91-174433

101,581 Not available NTIS

PB91-174441

DEQSOL and ELLPACK: Problem-Solving Environments for Partial Differential Equations.

PB91-17444

PB91-174458

Glass-Ceramic Inserts Anticipated for 'Megafilled' Composite Restorations.
PB91-174458

PB91-174466

100,045 Not available NTIS

Properties of Amorphous Zirconium Rhodium Hydride (Zr3RhHx) Prepared from Glassy and Crystalline Alloys.

PB91-174466

PB91-174474

Ignition Characteristics of Selected SSME Alloys.

P891-174474

100,544 Not available NTIS

PB91-174482

Fluorescence Monitoring of Polymer Processing: Mixing and Zero Shear Viscosity.

PB91-174482

100,492 Not available NTIS

PB91-174490

Correction for Converting 2-CM(3)-Coupler Responses to Insertion Responses for Custom in-the-Ear Non-Directional Hearing Aids.

PB91-174490

100,046 Not available NTIS

Statistical Mechanical Model of Aqueous Two-Phase Systems.

PB91-174508

PB91-174516

Research and Development of Heat Pumps Using Nonazeotropic Mixture Refrigerants.

PB91-174516

100,891 Not available NTIS

PB91-17452

Introduction to $\mathrm{ACI}$ 306.1-87: Specification for Cold Weather Concreting.

PB91-174532

Book Review: Polarized Electrons at Surfaces by J. KirschPB91-174532

101,493 Not available NTIS

PB91-174540

Applications of Operations Research Techniques to System Design and Evaluation.

PB91-17

101,267 Not available NTIS

PB91-174557

Development of a Three-Dimensional Finite Element Program for Large Strain Elastic-Plastic Solids

PB91-174557

101,494 Not available NTIS

PB91-174565

Planar Waveguide Optical Immunosensors.

PB91-174565

101,284 Not available NTIS

PB91-174573

Ceiling Jet-Driven Wall Flows in Compartment Fires

PB91-174573

100,117 Not available NTIS

PB91-174581

Design, Fabrication, and Testing of an Interconnect Tes Structure for Evaluating VLSI Processes.

PB91-17458

PB91-174599

Use of Birefringent Coatings in Fracture Mechanics.

PB91-174599

PB91-174607

Refrigerant Charge Elfects on Heat Pump Performance

PB91-174607

PB91.174615

Iron-Garnet Magnetic Field Sensors with $100 \mathrm{pT} /$ per square root $\mathrm{Hz}$ Noise-Equivalent Field.

PB91-174615

PB91-174623

High Precision Gaging with Computer Vision Systems.

PB91-174623

PB91-174631

Monitoring the Fate of Chlorine from MSW Sampling through Combustion

-174631

100,917 Not available NTIS

PB91-174649

Simulation of an Evaporator with Nonuniform One-Dimen sional Air Distribution.

PB91-174649

100,066 Not available NTIS

PB91-174656

Emissivity of Aluminized Mylar

PB91-174656

101,119 Not available NTIS

PB91-174664

Radio-Continuum Observations of a Variety of Cool Stars. PB91-174664

PB91-174672 
PB91-174730

Oxidation of Organic Compounds by Active Manganese Di-

PB91-174730

PB91-174748

Anomalous Magnetoresistance in Al/Al-Alloy Composite Conductors.

B 1-174755

New Test of Quantum Mechanics: Is Planck's Constant Unique.

PB91-174755

PB91-174763

Viscosity Surface for Mixtures of Methane and Ethane

PB91-174763

PB91-174771

$\mathrm{N}$-Dimensional Crystallographic Description of the Icosahedral Phases; the Example of the Al73Mn21Si6 Quasiperiodic Structure.

PB91-174771

PB91-174789

101,226 Not available NTIS

Letter to the Editor of Standardization News.

PB91-174789 100,118 Not available NTIS

PB91-174797

Letter to the Editor, Fire Technology, Comments on T.Z. Harmathy Viewpoint, May 1985.

PB91-174797

100,545 Not available NTIS

PB91-174805

Increased Facilitated Transport Related to Microstructural Changes in Heat-Treated lon-Exchange Membranes.

PB91-174805 100,323 Not available NTIS

PB91-174813

Population Lifetimes of $\mathrm{OH}(\mathrm{v}=1)$ and $\mathrm{OD}(\mathrm{v}=1)$ Vibrations in Alcohols, Silanols and Crystalline Micas.

PB91-174813 100,324 Not available NTIS

PB91-174821

Thermochemistry of Sulfoxides and Sulfones.

PB91-174821 100,325 Not available NTIS

PB91-174839

Formation and Stability of SF5 and S2F10

PB91-174839 100,326 Not available NTIS

PB91-174847

Letter to Editor of Chemical and Engineering News.

PB91-174847 100,327 Not available NTIS

PB91-174854

Laser-Driven Ionization and Photoabsorption Spectroscopy of Atomic lons.

PB91-174862

101,415 Not available NTIS

Mechanisms of Additive Effectiveness.

PB91.174862

101,174 Not available NTIS

PB91-174870

Resonance Enhanced Multiphoton Ionization Spectroscopy

of $\mathrm{CHCl} 2$ and $\mathrm{CDCl} 2$
$\mathrm{~PB} 91-174870$

PB91-174888

100,328 Not available NTIS

Instrumentation of X-ray Beam Lines with PIN Diode Detectors.

PB91-174888

101,633 Not available NTIS

PB91-174896

State-of-the-Art of Computational Testing of Mathematical Programming Algorithms.

PB91-174904

101,268 Not available NTIS

ACTIS: Towards a Comprehensive Tribology Data Base.

PB91-174904

101,184 Not available NTIS

PB91-174912

Noise-Affected I-V Curves in Small Hysteretic Josephson Junctions.

PB91-174912

PB91-174920

100,782 Not available NTIS

Detection of an Expanding $\mathrm{H}$ I Shell in the Old Supernova Remnant CTB 80

100,028 Not available NTIS B91-174938

Error Prevention and Detection in Data Preparation for a

Numerically Controlled Milling Machine.
PB91-174938 PB91-174946

Numerical Simulation and Visualization Models of Stress Wave Propagation Graphite/Epoxy Composites.

PB91-1749

101,120 Not available NTIS B91-174953

Study of Additive Response in a Series of Rerefined Base Oils Typical of Current Commercial Practice.

PB91-174953 101,175 Not available NTIS

PB91-174961

Robot Sensor Language

101,031 Not available NTIS

PB91-174979

Control of Flexible Robot Arm.
PB91-174979 101,032 Not available NTIS PB91-174987

Optical Levitation of Single Particles.

101,634 Not available NTIS PB91-174995

Polarızed X-ray Emission Studies of Methyl Chloride and the Chlorofluoromethanes.
PB91-174995

PB91-175000

Characterizing VLSI Processes Using Test Structures.

PB91-175000 100,804 Not available NTIS

PB91-175018

Application of Formal Description Techniques to the Specification of Distributed Test Syste

100,566 Not available NTIS PB91-1750

Ground-Truth Observations of Stellar Surface Structure from the Lunar Surface.

PB91-175026

100,029 Not available NTIS

PB91-175034

Some Concluding Thoughts for Cool Stars $\mathrm{VI}$

PB91-175034

100,030 Not available NTIS

PB91-175042

Extension of the Class of Magnetic B Star Nonthermal Radio Sources

PB91.175059

100,031 Not available NTIS

Group Reactivities in the Gas Phase Reactions of Hydroxyl Radicals with Ethers.

PB91-175059

100,330 Not available NTIS

PB91-175067

High-Resolution Small-Angle X-ray Scattering Camera for Anomalous Scattering

PB91-175067

100,929 Not available NTIS PB91-175075

Small-Angle-Scattering Determination of the Microstructure of Porous Silica Precursor Bodies.

PB91-175075

101,077 Not available NTIS

PB91-175083

Gravitational Radiation Observations on the Moon.

P891-175083

101,635 Not available NTIS

PB91-175091

U.S. Manufacturing Systems: Factories Past, Present, and PB91-175091

100,981 Not available NTIS

PB91-175109

Optical Fiber Current Sensors with Temperature Stabilities Near the Material Limit.

PB91-175109

PB91-175117

Rate Constant Functions for Strength Development of ConPB9 1-175117

100,753 Not available NTIS

100,527 Not available NTIS

B91-175125

Ion Incorporation into Octacalcium Phosphate Hydrolyzates.
PB91-175125 100,219 Not available NTIS

B91-175133

Single Pulse Shock Tube Study of the Reactions of Hydrogen Atoms with Aromatics. 4. Chlorotoluenes.

PB91-175133

100,331 Not available NTIS

PB91-175141

Semiconductor Claddings on Glass Waveguides for Polarizers and Detectors.

PB91-175141

PB91-175158

101,416 Not available NTIS

Faraday Effect Current Sensing Using a Sagnac Interferometer with a $3 \times 3$ Coupler

100,754 Not available NTIS

PB91-175166

Orbit Determination and Gravitational Field Accuracy for a Mercury Transponder Satellite.

PB91-175166

PB91-175174

Molecular Spectroscopy Division of the National Bureau of Standards.

PB91-175174

PB91-175182

Stress Corrosion Cracking of Al-Li Alloys: The Role of Grain Boundary Precipitates.

1-175182

101,137 Not available NTIS PB91-175190

Low Temperature Chemical Route to Precursors of Boride and Carbide Ceramic Powders.

PB91-175190

PB91-175208

Computerization of a Thermomechanical Processing Research System

PB91-175216

101,583 Not available NTIS

Jlc Data Analysis with a 'Negative Crack Growth' Correction Procedure.

PB91-175224

Extended Abstract: A Finite Element Study of the Stress

and Displacement Fields Produced by Point Impact.
PB91-175224 No1,584 Not available NTIS

PB91-175232

Models for Oxygen Ordering and Diffusion in $\mathrm{Ba} 2 \mathrm{YCu} 3 \mathrm{O}$ and $\mathrm{Ba} 2 \mathrm{YCu} 3-\mathrm{yMyOx}(\mathrm{M}=\mathrm{Fe}, \mathrm{Co}, \mathrm{Al}, \mathrm{Ga}$ ).

and $\mathrm{Ba2YCu3-yMyOx} \mathrm{(M} \mathrm{=} \mathrm{Fe,} \mathrm{Co}, \mathrm{Al}$, Ga). PB91-175240

Recent Developments in Digital Oscilloscopes.

PB91-175240

100,849 Not available NTIS

PB91-175257

Book Review: Partitioning in Aqueous Two-Phase Systems: Theory, Methods, Uses, and Applications to Biotechnology.
PB91-175257 PB91-175265

Status of Beta-Particle Calibration Studies at NBS.

PB91-175265 101,636 Not available NTIS

PB91-175273

Test of Newton's Inverse Square Law of Gravity Using the $300 \mathrm{~m}$ Tower at Erie, Colorado: Newton Vindicated on the Plains of Colorado.

PB91-175273

PB91-175281

101,637 Not available NTIS

Some Algorithms for Approximating Convolutions.

PB91-175281 101,258 Not available NTIS

PB91-175299

Gas Analysis Modeling System.

PB91-175299

PB91-175307

Airtightness of Office-Building Envelopes

PB91-175307

PB91-175315

100,067 Not available NTIS

Application

Standards.

PB91-175323

Book Review: 'Polarized Electrons at Surfaces' by J.

Kirschner.

PB91-175323

1,497 Not available NTIS

.175331

Surface Flame Spread.

PB91-17533

PB91-175349

Neutron Crystallography of Proteins.

PB91-175349

PB91-175356

Lattice Approach to Environments Irradiated by Unknown

PB91-175356

PB91-175364

Spectrum and Energy Levels of the Xenonlike Ion Ce V. PB91-175364

PB91-175372

Effect of Nitrogen and Carbon on FCC-HCP Stability in Austenitic Steels.

PB91-175380

101,151 Not available NTIS

Search for a Composition Dependent Force Using a LiquidSupported Torsion Balance.

PB91-175380

101,639 Not available NTIS

PB91-175398

Measurements of Power Frequency Magnetic Fields Away from Power Lines.

PB91-175406

Eccentric Load Sensitivity of Force Sensors. PB91-175406

100,851 Not available NTIS

PB91-1754

Thermodynamic Properties of Two Alternative Refrigerants 1,1-dichloro-2,2,2-trifluoroethane (R123) and 1,1,1,2-tetrafluoroethane (R134a)

PB91-175414

101,195 Not available NTIS

PB91-175422

Smoke Production and Properties.

PB91-175422

PB91-175430

Saran Carbon Cryogenic Regenerator for Liquid Helium

PB91-175430

PB91-175448

Mechanical Sputtering of Structural Stainless Steels.

PB91-17544

PB91-175455

Designing Data Entity Naming Conventions.

PB91-175455

100,646 Not available NTIS

PB91.175463 
PB91-175513

101,642 Not available NTIS

PB91-175521

Fe-W Supermirrors for Polarizing Neutrons

PB91-175521

101,643 Not available NTIS

PB91-175539

Intrared Spectroscopy Applied to Atmospheric Chemistry.

PB91-175539 100,906 Not available NTIS

PB91-175547

Calculation of Beta-Particle Counting Effıciency for Liquid Scintillation Systems with Three Phototubes.

PB91-175547 101,644 Not available NTIS PB91-175554

Ion-Induced Radiation-Enhanced Diffusion of Silver in Nickel.

PB91-175554

101,498 Not available NT1S

PB91-175562

Direct Determination of Molecular Orbital Symmetry of H2S Using Polarized X-ray Emission. PB91-17556

PB91-175570

Reusable Cryogenic Foam Insulation for Advanced AeroSpace Vehicles
PB91-175570

PB91.175588

Working Group 2: Atomic Transition Probabilities

PB91-175588 101,645 Not available NTIS

PB91-175596

Correlated Spontaneous Emission in a Zeeman Laser

PB91.175596

101,417 Not available NTIS

PB91-175604

Crystal Structure of Phosphate-Free Ribonuclease.

PB91-175604 101,301 Not available NTIS

PB91-175612

Electron Attenuation Lengths at $\mathrm{SiO} 2 / \mathrm{Si}$ Interfaces

PB91-175612

101,499 Not available NTIS

B91-175620

Summary of Low-Density Glass-Fiber Reference Materials at NIST: 1980-1989.

PB91-175620

101,079 Not available NTIS

PB91-176032

101.454 Not available NTIS
Nosites.

Superconductor-Poly
PATENT-4 954481

PB91-177774

Standards Activities of Organizations in the United States

PB91-177774

100,004 PC A99/MF A0

B91-178822

Chemistry of Electronic Ceramic Materials.

PB91.178822

101,500 PC A23/MF A03

PB91-178830

FIREDOC Users Manual, 2nd Edition

PB91-178830

100,547 PC A03/MF A01

B91-178848

Boussinesq Algorithm for Enclosed Buoyant Convection in Two Dimensions.

PB91-178855

Center for Radiation Research. 1990 Technical Activities

PB91-178855

100,157 PC A03/MF A01

PB91-178863

Characterization of Field Concrete

PB91-178863

101,362 PC A08/MF A01

PB91-178871

Energy Related Inventions Program: A Joint Program of the Thent of Energy and the National Institute of Standards and Technology. Status Report for Recommendations 1 through 250

PB91-178889

100902 PC A09/MF A01

Collection of Technical Studies Completed for the Computer-Aided Acquisition and Logistic Support (CALS) Program. Fiscal Year 1988. Volume 1 of 3. Text, Security and Data Management.

PB91-178897

101,342 PC A12/MF A02

Colfection of Technical Studies Completed for the Computer-Aided Acquisition and Logistic Support (CALS) Program, Fiscal Year 1988 . Volume 2 of 3 . Graphics, CGM MIL: SPEC

PB91-178897

101,343 PC A21/MF A03

B91-178905

Collection of Technical Studies Completed for the Computer-Aided Acquisition and Logistic Support (CALS) Program, Fiscal Year 1988. Volume 3 of 3 . CGM Registration

PB91-178905

101,344 PC A08/MF A01

PB 1-184747

Microstructure, Composition, and Hardness of Rockwell C Hardness Blocks
PB91-184747

P91-184754

$101,153 \mathrm{PC} \mathrm{A03/MF} \mathrm{A01}$

Center for Electronics and Electrical Engineering Technical Publication Announcements Covering Center Programs, July to September 1990, with 1991 CEEE Events Calendar PB91-184754

100,852 PC A03/MF A01

184762

Visibility of Exit Directional Indicators.

PB91-184762

$101,000 \quad \mathrm{PC} \mathrm{A03/MF} \mathrm{A01}$
PB91-184770

Energy Related Inventions Program. A Joint Program of the Department of Energy and the National Institute of Standards and Technology. Status Report for Recommendations 251 through 523

PB91-184788

100,903 PC A13/MF A02

STEP: Standard for the Exchange of Product Model Data Resource Integration: Semantic and Syntactic Rules. PB91-184788

100,966 PC A03/MF A01

\section{B91-184796}

Alaska Marine Mammal Tissue Archival Project: Revised Collection Protocol

PB91.184796

101,374 PC A03/MF A01

PB91-184804

Heat Transfer in a Compact Heat Exchanger Containing Rectangular Channels and Using Helium Gas.

PB91-184804 101,734 PC A05/MF A01

PB91-184812

101,734 PC A05/MF A01

Standard Generalized Markup Language Encoding of the

Office Document Architecture Document Application Profile.
PB91-184812 101,345 PC A03/MF A01

Prediction of Elevated Temperature Deformation of Struc. tural Steel under Anisothermal Condition

PB91-18483

101,154 PC A03/MF A01

PB91-184846

Proceedings of a Workshop on Evaluation, Repair, and Retrofit of Structures. U.S.-Japan Panel on Wind and Seismic Effects, UJNR. Held in Gaithersburg, MD., USA, on May 1214,1990 .

PB 91.184846

100,145 PC A14/MF A02

PB91-184853

Journal of Research of the National Institute of Standards and Technology. January-February 1991. Volume 96,

NB 1 184853

100,220 PC A07/MF A01

PB91-184861

New Chemical and Stereochemical Applications of Organoiron Complex
PB91-184861

100,221
(Order as PB91-184853, PC A07/MF A01)

\section{PB91-185090}

Recommended Fine Positioning Test for the Development Test Flight (DTF-1) of the NASA Flight Telerobotic Servicer (FTS

101,732 PC A06/MF A01

PB91-18

tting Package User's Guide,

VolksGraph

Version 3.0.

PB91-185108

100,647 PC A04/MF A01

991-185116

Effect of a Free Surface on Stress Distribution in a Bimaterial Composite.

PB91-185124

$101,121 \mathrm{PC} \mathrm{A09/MF} \mathrm{A01}$

NIST Calibration Procedure for Vertically Polarized Monopole Antennas $30 \mathrm{kHz}$ to $300 \mathrm{MHz}$.

pB A1-185124

100,726 PC A03/MF A01

PB91-187070

Base Modifications in Plasmid DNA Caused by Potassium Permanganate
PB91-187070

PB91-187088

101,329 Not available NTIS

Electric Quadrupole and Magnetic Dipole Transition Prob-

abilities in Potassium Isoelectronic Sequence.
PB91-187088 101,646 Not available NTIS

PB91-187096

Temperature Dependence of High Accuracy-Photometer Heads.

PB91-187096

101,418 Not available NTIS

PB1.187104

Copper-Ion-Dependent Damage to the Bases in DNA in the Presence of Hydrogen Peroxide

PB91-187104

101,330 Not available NTIS

PB91-187112

Damage to the Bases in DNA Induced by Hydrogen Peroxide and Ferric Ion Chelates.

PB91-187112

101,331 Not available NTIS

B91-187120

Eddy Current Reflection Probe: Theory and Experiment.

PB91-187120

PB91-187138

100,944 Not available NTIS

Concerning the Formation and the Kinetics of Phenylium lons.

PB91-187146

100,336 Not available NTIS

Multi-Zone Dispersal Analysis by Element Assembly

PB91-187146

100,907 Not available NTIS

B91-187153

Small-Angle Neutron Scattering Studies of Compatible Blends of Linear Poly(Vinyl Methyl Ether) and Cross-Linked Deuterated Polystyrene.

PB91-187153

100,494 Not available NTIS

PB91-187161

Role of Elastic Energy in the Morphological Development of a Ni-Ti-Al Alloy

101,227 Not available NTIS

PB91-187179

Percolation of Phases in a Three-Dimensional Cement Paste Microstructural Model.

100,529 Not available NTIS

PB91-187187

Characterization of Cylindrical Holes in Metallic Substrates via Their Infrared Emission Patterns.

PB91.187187 101,228 Not available NTIS PB91-187195

Critical Exponent for the Viscosity of Four Binary Liquids.

PB91-187195 100,337 Not available NTIS

PB91-187203

Particle Size Measurement of Inert Gas Atomized Powder.

PB91-187203

PB91-187211

$\mathrm{H}_{2} \mathrm{O}(\mathrm{I})-\mathrm{H} 2 \mathrm{O}(\mathrm{III})-\mathrm{H} 2 \mathrm{O}(\mathrm{L})$ Triple Point of Water as a Fixed

PB91-187211

PB91-187229

Molecular Dynamics Study of the Depolymerization Reaction in Simple Polymers.

PB91-187229

PB91-187237

Isothermal Phase Behavior of Ag3SbS3, ZnGeP2, and ZnS. PB91-187237

PB91-187245

Algorithms for Special Tridiagonal Systems.

PB91-187245

101,259 Not available NTIS

PB91-187252

Relationship of Hydrogen Site Occupancy to Diffusion Behavior in Crystalline and Amorphous $\mathrm{Zr} 2 \mathrm{PdH}$

PB91-187252

101,230 Not available NTIS

PB91-187260

Improving the Fire Performance of Building Contents.

PB91-187260

PB91-187278

00,084 Not available NTIS

Comparison of Two Test Methods for Determining Transfer Function Coefficients for a Wall Using a Calibrated Hot Box.

100,121 Not available NTIS

PB91-187286

BACnet Communication Protocol for Building Automation

PB91-187286

PB91-187294

6-D Structural Model for the Icosahedra! (Al, Si)-Mn Quasicrystal.

PB91-187294

101,501 Not available NTIS

PB91-187302

Angular Distribution of Ejected Electrons in Resonant Auger Processes of $\mathrm{Ar}, \mathrm{Kr}$, and $\mathrm{Xe}$

PB91-187310

Unusual Degree of Angular Anisotropy in the Resonant Auger Spectrum of $\mathrm{Kr}$.

187310

Method to Enhance Porosity of Micro-particles.

PB91-187328

PB91-187336

Interlaminar Shear Fracture of Laminated Composites

PB91-187336

PB91-187344 


\section{NTIS ORDER/REPORT NUMBER INDEX}

PB91-187427

100,497 Not available NTIS

PB91.187435

Shape Resonances in Molecular Fields.

100,338. Not available NTIS

PB91.187443

Some Historical Remarks Concerning These Conferences With Emphasis on the $X$-ray Componen

PB91-187443 101,652 Not available NTIS

PB91-187450

Statistics of a Polymer Molecule in the Presence of Asymmetric Obstacles.

PB91-187450

100,498 Not available NTIS

Chemical Determination of Free Radical-Induced Damage 10 DNA.

PB91-187468

PB91-187476

101,332 Not available NTIS

46Gas Chromatography-Mass Spectrometry of Free Radical-Induced Products of Pyrimidines and Purines in DNA.

PB91-187476 101,302 Not available NTIS

PB91-18747
PB91-187484

Measurement of DNA Base Damage and DNA-Protein Cross-Links in Mammalian Chromatin

PB91-187484 101,303 Not available NTIS

PB91-187492

Modification of Bases in DNA by Copper Ion-1,10-Phenanthroline Complexes.

PB91-187492

101,333 Not available NTIS

PB91-187518

Structure and Mechanism of Hydroxyl Radical-Induced Formation of a DNA-Protein Cross-Link Involving Thymine and Lysine in Nucleohistone

PB91-187518

101,334 Not available NTIS

PB91-187526

Chemical Nature of In vivo DNA Base Damage in Hydrogen Peroxide-Treated Mammalian Cells.

PB91-187526 101,335 Not available NTIS

PB91-187534

Analytical Model for the Refrigeration Power of the Orifice Pulse Tube Refrigerator

PB91-18753

101,048 PC A05/MF A01

PB91-187542

Standard Reference Materiałs: Calibration of NIST Standard Reference Material 3201 for 0.5 Inch $(12.65 \mathrm{~mm})$ Serial Serpentine Magnetic Tape Cartridge. 690 PC A03/MF A01 PB91.187559

NIST Research Reports, March 1991

PB91-187559

100,007 PC A03/MF A01

PB91-187567

Guide to Design, Implementation and Management of Distributed Databases.

PB91-187567

PB91-187617

100,648 PC A04/MF A01

Journal of Research of the National Institute of Standards and Technology. March-April 1991. Volume 96, Number 2. PB91-187617 101,420 PC A06/MF A01 PB91-187625

Linearity of a Silicon Photodiode at $30 \mathrm{MHz}$ and Its Effect on Heterodyne Measurements.

PB91-187625

Order as PB91-187617, PC A06/MF 100,755

PB91-187633

Use of the Electrostatic Classification Method to Size 0.1 micrometer SRM Particles: A Feasibility Study.

PB91-187633

100,932
(Order as PB91-187617, PC A06/MF A01)

PB91-187641

Multiple Variable-Angle Light Scattering Detector for Gel PB91-187641

(Order as PB91-187617, PC A06/MF 195

PB91-187658

Proposed New Electrolytic Conductivity Primary Standards for $\mathrm{KCl}$ Solutions.

PB91-187658

(Order as PB91-187617, PC A06/MF A01)

PB91-187666

Digital Image Analysis Assisted Microradiography Measurement of Mineral Content of Caries Lesions in Teeth.

PB91-187666 Content or Caries Lesions in Feeth. 101314

PB91-187674

(Order as PB91-187617, PC A06/MF A01)

Bidirectional Scattering Distribution Function (BSDF): A Systematized Bibliography.

PB91-187674

(Order as PB91-187617, PC A06/MF A01)

PB91-187682

Limited International Intercomparison of Responsivity Scales at Fiber Optic Wavelengths.

100,567
(Order as PB91-187617, PC A06/MF A01)

PB91.187682

PB91-187690

Chloride Ion Diffusion in Low Water-to-Solid Cement Pastes.

PB91-187690

$101,138 \mathrm{PC} \mathrm{A03/MF} \mathrm{A01}$

PB91-187708

Tiled Raster Graphics and MIL-R-28002A: A Tutorial and Implementation Guide.
PB91-187708

PB91-187716

101,346 PC A06/MF A01

Programming Languages and Database Language SOL. Validated Processor List Including GOSIP Conformance Testing Registers

100,649 PC A04/MF A01

PB91-187724

Validation of an OSI Transport Class 4 Simulator PB91-187724

100,568 PC A03/MF A01

Draft Abstract Test Suite for Determining Conformance to the BACnet Protocol.

100,070 PC A06/MF A01

PB91.187740

Computer Security: Selected Articles.

PB91-187740

100,706 PC A03/MF A01

PB91-187757

Intelligent Building Technology in Japan

PB91-187757

100,071 PC A04/MF A01

PB9 1-187765

AMRF Composites Fabrication Workstation: A Test Methodology to Measure the Ouality of Thermoplastic Composite Parts.

PB91-187765

$101,017 \mathrm{PC}$ A03/MF A01

PB91-187773

Government Document Processing Requirements Report.

PB91-187773

100,574 PC A03/MF A01

PB91-187781

National Aeronautics and Space Administration's (NASA) Automated Information Security Handbook

Automated Information Security Handbook

PB:1-18,

100,707 PC A06/MF A01

Convergence Properties of a Class of Rank-Two Updates.

PB91-187799

PB91-187807

Buildıng and Fire Research Laboratory Publications, 1990

PB91-187807

ratory Publications, 1990 .
100,122 PC A04/MF A01

PB91-187815

Interior-Point Method for Linear and Ouadratic Programming Problems

PB91-187815

PB91.187823

101,269 PC A03/MF A01

GATT Standards Code Activities of the National Institute of Standards and Technology 1990.

87823

100,177 PC A04/MF A01

PB91-187831

Issues in Transparent File Access.

PB91-187831

100,569 PC A05/MF A01

B 91-187849

Proposed Design Criteria for Shallow Bridge Foundations.

PB91-187849

PB91-187856

Continuous-Cooling Transformation Characteristics and High-Temperature Flow Behavior of a Microalloyed SAE 1141 Steel.

PB91-187856

PB91-187864

Public-Key Cryptography. Computer Security.

PB91-187864

PB91-189217

100,708 PC A08/MF A01

Damage to the DNA Bases in Mammalian Chromatin by Hydrogen Peroxide in the Presence of Ferric and Cupric lons.
PB91-189217

PB91-189225

Statistical Process Control Tactics for Coordinate Measuring Machines.
PB91-189225

PB91-189233

Combustion Calorimeters

PB91-189233

101,042 Not available NTIS

PB91-1892

100,340 Not available NTIS

How Does Surface Roughness Affect Polymer-Surface Interactions.
PB91-189241

PB91-189258

100,341 Not available NTIS

$X$-ray Photoelectron Forward Scattering Studies of Surtace Segregation in Epitaxial Cu-Ni-Ni(100) Sandwich Structures Segregation in Epitaxial Cu-Ni-Ni(100) Sandwich Structures.
PB91-189258 PB91-189266

Stability of Uric Acid in Ammonium Hydroxide.

PB91-189266

101,292 Not available NTIS
.

PB91-189274

Measured Performance of Residential Water Heaters Using Existing and Proposed Department of Energy Test Procedures.

PB91-189274

100,085 Not available NTIS PB91-189282 Second Dissociation Constant and $\mathrm{pH}$ of $\mathrm{N}$-2-hydroxyethyl-
piperazine-N'-2-ethanesulfonic Acid (HEPES) from 0 to

$50 \mathrm{C}$.
$\mathrm{PB} 91-189282$

100,196 Not available NTIS

Measurement Reliability: The Detection of Nonlinearities.
PB91-189290

PB91-189308

Incompatibility between the $100 / 1300$ Surge Test and Varistor Failure Rates.

PB91-189308

100,854 Not available NTIS

B91-189316

Plastic Zone Formation Around an Arresting Crack

PB91-189316 101,373 Not available NTIS

PB91-189324

Acoustic Technique for Evaluation of Thermal Insulation

PB91-189324

PB91-189332

Refractive Index Gratings in Rare-Earth-Doped Alkaline

Earth Glasses.

PB91-189340

Reference NO2 Calibration System for Ground-Based Intercomparisons during NASA's GTE/CITE II Mission

PB91-189340

100,034 Not available NTIS

PB91-189357

Bleomycin-Dependent Damage to the Bases in DNA is a Minor Side Reaction.

PB91-189365

Hydroxyl Radical Induced Cross-Linking of Cytosine and Tyrosine in Nucleohistone.

B91-189365

101,304 Not available NTIS

Modification of DNA Bases in Mammalian Chromatin by Radiation-Generated Free Radicals.

PB91-189373

101,338 Not available NTIS

PB91-189381

Modeling the Thermodynamic Properties of Sodium Chlo-

ride in Steam Through Extended Corresponding States.

PB91-189399

Uniform Junction Temperature AIGaAs/GaAs Power Heter-

Ojunction Bipolar Transistors on Silicon Substrates.
PB91-189399 100,806 Not available NTIS

PB91-189407

Study on the Nature of Boundary Lubricating Film: Analytical Method Development

PBg1-189407

101,176 Not available NTIS

PB91-189415

Spectroscopic Constants for the nu9 Infrared Band of PBO1-18

B91-189423

100,343 Not available NTIS

Structural Study of Electrodeposited Aluminum-Manganese Alloys.
PB91-189423

PB91-189431

101,231 Not available NTIS

Effect of Long Range Interfacial Forces on the Dynamics of Wetting Layers at a Liquid Vapor Interface.

PB91-189431

100,344 Not available NTIS

PB91-189449

Structures and Properties of New Zeolite X-Type Zinco-

phosphate and

PB91-189456

Difficulties in Parsing SGML.
PB91-189456

PB91-189464

Effects of Varying Austenitizing Temperature and Cooling

Rate on the Ability of HSLA-80 Steel to Achieve a Yield

Strength Comparable to HSLA-100

PB91-189464

PB91-189472

8-Hydroxyguanine Content of Isolated Mitochondria Increases with Lipid Peroxidation.

PB91-189472

PB91-189480 
PB91-189563

Study of Flow Boiling Heat Transfer with Refrigerant Mix-

PB91-189563

PB91.189571

101,196 Not available NTIS

Knowledge Verification of Machine-Learning Procedures Based on Test Structure Measurements.

PB91-189571 100,709 Not available NTIS

PB91-189589

Superconducting Thin Films of Bi-Sr-Ca-Cu-O Obtained by Laser Ablation Processing.

PB91-18958

101,508 Not available NTIS

PB91-189597

Ouantum Electrodynamic Contributions to Spin Orbit Splitting in the Ground State of Aluminum-Like lons.

ting in the Grour

101,653 Not available NTIS

PB91.189605

Relativistic and Correlation Effects in the 2s3p Configuration of Beryllium-Like lons.

PB91-189605

101,654 Not available NTIS

B91-189613

Energy of Combustion of Triphenytphosphate.

PB91-189613

100,347 Not available NTIS

PB91-189621

Evaluation of Test Methods for Measuring the Bond Strength of Portland Cement-Based Repair Materials to Concrete.

PB91-189621

100,530 Not available NTIS

PB91-189639

Hydrogen Bonding in Pt Ammine Complexes.

PB91-189639 100,348 Not available NTIS

PB91-189647

Sputtered Thin Film YBa2Cu3O

PB91-189647

101,509 Not available NTIS

PB91-189654

Dynamical Diffraction Imaging (Topography) with X-ray Synchrotron Radiation.

Chrotron Radiati

101,510 Not available NTIS

PB91-189662

Streaking Images That Appear Only in the Plane of Diffraction in Unoped GaAs Single Crystals: Diffraction Imaging (Topography) by Monochromatic Synchrotron Radiation. PB91-189662 101,511 Not available NTIS

PB91-189670

Initial Stages of Degradation of Superconductor Surfaces: $\mathrm{O} 2, \mathrm{H} 2 \mathrm{O}, \mathrm{CO} 2$ and $\mathrm{CO}$ Chemisorption on La2-xSrxCuO4

PB91-18967

101,512 Not available NTIS

PB91-189688

Electronic Structure of High-Tc Superconductors Studied

Using Photoelectron Spectroscopy.
PB91-189688 101,513 Not available NTIS

PB91-18968

Surface Properties and Coatings of Materials Microtailored

Alloys.

PB91.189696

101,232 Not available NTIS

PB91-189704

Fundamental Condition for Existence of Microcrack Clouds in Monophase Ceramics.

PB91-189704

101,082 Not available NTIS

PB91-189712

Effect of Crosslink Density on Physical Aging of Epoxy Networks.

PB91-189712

101,244 Not available NTIS

PB91-189720

Viscoelastic Response of Epoxy Glasses Subjected to Ditferent Thermal Treatments.

PB91-189720

PB91-189738

Effects of Colored Pump Noise on Intensity Correlations of a Single Mode Dye Laser

PB9 1-189746

Observation of Atoms Laser-Cooled Below the Doppler

Limit.

$91-189746$

101,655 Not available NTIS

PB91-189753

Doppler Shift Attenuation Lifetime Measurements in (54) Cr

Following Thermal Neutron Capture.

PB91-189753

101,656 Not available NTIS

PQ91-189761

Replicate Measurements in the Interpretation of Environmental Monitoring.

PB91-189779

100,919 Not available NTIS

Neutron Activation Analysis in Electronic Technology

PB91-189779

PB91-189787

100,807 Not available NTIS

Phonon Density of States and Superconductivity in $\mathrm{Nd1} .85 \mathrm{Ce} 0.15 \mathrm{CuO} 4$.

PB91-189787 101,514 Not available NTIS

B91-189795

Standardizing Standards

PB91-189803

100,953 Not available NTIS

Fourier Transform Infrared Spectroscopy at the NIST: High Temperature Molecules and Atmospheric Molecules.
PB91-189803

101,424 Not available NTIS

91-189811

Real, Realistic Ring Waves for Surge Testing

100855 Not available NTIS

PB91-189829

Physical Aging and the Viscoelastic Response of Network

Glasses.

PB91-189829

PB91-189837

Modelling Aqueous Solutions Near the Critical Point of Water.

PB91-189837

PB91-189845

100,349 Not available NTIS

Ergodic M

of Water.

PB91-189852

Polyelectrolyte Stabilized Metal Oxide Hydrosols as Cata lysts for the Photooxidation of Water by Zinc Porphyrins.

PB91-18985

PB91-189860

Detection of Trace Disulfur Decafluoride in Sulfur Hexa-

fluoride by Gas Chromatography/Mass Spectrometry.
PB91-189860 100,197 Not available NTIS

PB91-189878

Introduction to Supercritical Fluid Chromatography. 1. Principles and Instrumentation.

PB91-189878

PB91-189886

100,198 Not available NTIS

Enhanced Transport and Liquid Membranes in Biosepara. tions.

$-18988$

PB91-189894

Conformance Tester for X.25 DTE Implementations

PB91-189894

PB91-189902

Proton Affinity of Cyanogen and lon-Molecule Reactions of $\mathrm{C} 2 \mathrm{~N} 2(+)$.

PB91-189902

PB91-189910

Phone-Mediated Word Alignment for Speech Recognition Evaluation.

189910

100,592 Not available NTIS

PB1.189928

Microchannel-Plate Detection Systems for Low Accelerating Voltage SEM.
PB91-189928

PB91-189936

Recent Developments in Applied Surface Science

PB91-189936

101,515 Not available NTIS

PB91.189944

Influence of Interstitial Content on Fracture Toughness. PB91-189944

100,353 Not available NTIS

P91.189951

101,157 Not available NTIS

Metallographic Study of the Crack-Tip Region from Fracture

Mechanics Specimens of Austenitic and Ferritic Steels.
PB91-189951 101,158 Not available NTIS

PB91-189969

Fe Implantation in In0.53Ga0.47As/InP.

PB91-189969

101,516 Not available NTIS

PB91-189977

Discontinuous Yielding during Tensile Tests at Low Temperatures.

PB91-189977

PB91-189985

101,159 Not available NTIS

Creep of Copper: 4 to $295 \mathrm{~K}$.

101,233 Not available NTIS

PB91-189993

Field Testing of Adhesive-Bonded Seams of Rubber Roofing Membranes

PB91-190009

Dielectronic Recombination Rate Coefficients of $\mathrm{O}(5+)$ and $\mathrm{O}(2+)$.

PB91-190017

Comparison of Standard Test Methods for Non-Lubricated Sliding Wear.

$01-190025$

101,187 Not available NTIS

Sliding Wear Studies of Nickel-Copper Composition-Modulated Coatings on Steel.

PB91-190025

101,188 Not available NTIS

PB91-190033

Energy Levels and Transition Probabilities in the Ground State Configuration of Sulfur-Like lons.

PB91-190033

101,658 Not available NTIS

PB91-190041

Evaluation of Column Performance in Liquid Chromatogra-

Phy.

100,199 Not available NTIS

PB91-190058

Image Quality Indicator Design for Radioscopy and Tomography.

PB91-190066

101,018 Not available NTIS

Plumbing Test Lab Accreditation.

PB91-190066

100,089 Not available NTIS

\section{PB91-190074}

Crystal Structure of a CAMP-Independent Form of Catabo-

lite Gene Activator Protein with Adenosine Substituted in

One of 2 CAMP-Binding Sites
PB91-190074

PB91-190082

Indentation Determination of Crack Growth Parameters in Gallium Arsenide.

PB91-190090

101,083 Not available NTIS

Geometric and Electronic Properties of Cs Structures on III$V(110)$ Surfaces: From $1 D$ and $2 D$ Insulators to $3 D$ Metals.

PB91-190090

PB91-190108

Mechanistic Studies of lonizing Radiation and Oxidative Mutagenesis: Genetic Effects of a Single 8-Hydroxyguanine (7Hydro-8-oxoguanine) Residue Inserted at a Unique Site in a Viral Genome.

PB91-190108

PB91-190116

Transparent Thin Film Thermocouple.

PB91-190116

100,783 Not available NTIS

PB91-190124

Thermal Conductivity of Fumed Silica Board at Room Tem-

perature.

100,125 Not available NTIS

B91-192435

Journal of Physical and Chemical Reference Data, Volume

19. Number 4, July-August 1990

PB91-192435

PB91-192443

Energy Levels of Sulfur, S I Through S XVI.

PB91-192443

PB91-192450

Heat Capacities and Entropies of Organic Compounds in the Condensed Phase. Volume 2.

PB91-192450

100,356 Not available NTIS

PB91-192468

Thermodynamics of the Krebs Cycle and Related Com

pounds

PB91-192468

100,357 Not available NTIS

B91-192476

Journal of Physical and Chemical Rererence Data, Volume

19, Number 5, September-October 1990.

PB91-19247

B91-192484

Transport Properties of Fluid Oxygen

PB91-192484

100.359 Not available NTIS

PE91-192492

Thermal

Density.

PB91.192500

Thermal Conductivity of Methane and Tetrafluoromethane in the Limit of Zero Density.

PB $1-1$

100,361 Not available NTIS

19251

Coupled Phase Diagram-Thermodynamic Analysis of the 24 Binary Systems, A2CO3-AX and A2SO4-AX Where A= Li, $\mathrm{Na}, \mathrm{K}$ and $\mathrm{X}=\mathrm{Cl}, \mathrm{F}, \mathrm{NO} 3, \mathrm{OH}$.

PB91-192518

PB91-192526

Equilibrium and Transport Properties of Gas Mixtures at 
PB91-192989

Conformity Assessment Workshop on Electromagnetic Compatibility.

PB91-1929

100,579 PC A06/MF A02

Conformity Assessment Workshop on Pressure Vessels

PB91-192997 101,049 PC A06/MF A01

PB91-193367

Concurrent Engineering through Product Data Standards.

100,982 PC A05/MF A01

PB91-193821

NIST Support of the CALS Program: 1990 Synopsis

PB91-193821 101,347 PC A03/MF A01

PB91-194399

Stopping Power of Fast Charged Particles in Heavy Elements.

101,660 PC A04/MF A01

PB91-194407

Model for the Non-Perturbative QCD (Quantum Chromodynamics) Vacuum.

PB91-194415

$101,661 \mathrm{PC} \mathrm{A03/MF} \mathrm{A01}$

Laboratory Accreditation in the United States.

PB91-194415

100,172 PC A03/MF A01

PB91-194423

Technical Activities 1990, Electron and Optical Physics DiviPB91-194423

$101,662 \mathrm{PC} \mathrm{A04/MF} \mathrm{A01}$

\section{PB91-194449}

Extracting STEP Geometry and Topology from a Solid Mod$\begin{array}{ll}\text { eler: Parasolid-to-STEP. } & 100,983 \text { PC A04/MF A01 } \\ \text { PB91-194449 }\end{array}$

Synthesis of Non-lonic Water-Dispersible Resins for Use in Intaglio Inks Curing by Electron Beam Radiation.

PB91-194456 100,499 PC A03/MF A01

PB91-194464

Heat Transfer and Pressure Drop in a Compact Pin-Fin Heat Exchanger with Pin Orientation at $18 \mathrm{deg}$ to the Flow

Direction.
PB91-194464

$101,050 \mathrm{PC}$ A05/MF A01

PB91-194472

Experimental Thermal Conductivity, Thermal Diffusivity, and Specific Heat Values for Mixtures of Nitrogen, Oxygen, and Argon.

$91-194472$

100,369 PC A04/MF A01

PB91-194480

Configuration Management Concepts Document. National PDES Testbed Report Series. $100,984 \quad$ PC A03/MF A01
PB91-194480

PB91-194498 Meeting of the Standards Working Group of the Joint U.S.-
U.S.S.R. Commercial Commission (1st). Held in Moscow on March 11-13, 1991.

PB91-194498

100,178 PC A11/MF A02

PB91-194506

NIST Support for the Computer-Aided Acquisition and Logistic Support (CALS) Program in the Area of Graphics Standards, Calendar Year 1990

PB91-194506

101,348 PC A14/MF A02

PB91-194654

Filler Systems Based on Calcium Metaphosphates.
PB91-194654 100,048 Not available NTIS

PB91-194662

YBa2Cu3O7-delta/Insulator Multi-Layers for Crossover Fabrication.

PB91.194662

PB91.194670

101,518 Not available NTIS

Effect of Thermochemical Treatments on the Strength and Microstructure of SiC Fibers.

PB91-194670

101,084 Not available NTIS

PB91-194688

Small-Angle Neutron Scattering of Blends of Cross-Linked and Linear Polystyrene.

PB91-194688

PB91-194696

100,500 Not available NTIS

Evaluation of the Heat Flux Transducer Technique for

Measuring the Thermal Performance of Walls.
PB91-194696 100,126 Not available NTIS

PB91-194704

Anomalous Low-Frequency Butterfly Curves for Subsidiary and Ferromagnetic Resonance Overlap at $3 \mathrm{GHz}$.

PB91-194704 101,519 Not available NTIS

PB91-194712

Pattern Generation on Semiconductor Surfaces by a Scanning Tunneling Microscope Operating in Air.

PB91.194712

100,808 Not available NTIS

PB91-194720

Co-Trial on ESR Identification and Estimates of gamma-Ray and Electron Absorbed Doses Given to Meat and Bones.

PB91-194720 100,017 Not available NTIS

PB91-194738

Soft X-ray Absorption and Emission Spectra and the Elec. tronic Structure of Some Exotic Materials.

PB91-194738

101,520 Not available NTIS

PB91-194746

Effects of $\mathrm{pH}$ and Calcium on Hydrolysis of Na2SiF6 and
PB91-194746

101,315 Not available NTIS

1.194753

Current Record in Superconductors.
PB91-194753 Not available NTIS PB91-194761

Metal-Polysiloxane Shields for Radiation Therapy of Maxillo-Facial Tumors.

PB91-19476

100,054 Not available NTIS

B91-194779

System for the Study of Magnetic Materials and Magnetic Imaging with the Scanning Tunneling Microscope

PB91-194779

101,522 Not available NTIS

PB91-194787

Corrosion Principles in Dental Implantology.

PB91-194787

100,055 Not available NTIS

PB91-194795

High-Temperature Thermal Properties of UNS 544004 Using Multivariant Analysis. PB91-194795

101,160 Not available NTIS

PB91-194803

Thermodynamics of Hydrolysis of Oligosaccharides. PB91-194803

101,288 Not available NTIS

PB91-194811

Meeting the Challenges of a Global Market - Construction tandards, Testing and Certification.

PB91-194811

100,090 Not available NTIS

PB91-194829

High Spatial Resolution Mapping of Semiconductor Resistivity.

100,809 Not available NTIS

PB91-194837

Theoretical Model of Metal Binding Sites in Proteins.

PB91-194837

PB91-194845

100,370 Not available NTIS

Hydrogenated Amorphous Germanium Detectors Deposited onto Channel Waveguides.

100,756 Not available NTIS

PB91-194852

Elastic Constants of a Tungsten-Particle Copper-Matrix

Composite.
PB91-194852

PB91-194860

101,234 Not available NTIS

Group and Phase Sound Velocities in an Eu1Ba2Cu3O7 Superconductor and Related Perovskite Oxides.

PB91.194860

101,523 Not available NTIS

PB91-194878

Elastic Constants of Fiber-Reinforced Composites: A Fresh Measurement Approach.

PB91-194878

101,123 Not available NTIS

PB91-194886

Prospects for Electromagnetic Manipulation and Trapping of Antihydrogen

PB91-194894

101,663 Not available NTIS

Long-Range Antiferromagnetic Order of the $\mathrm{Cu}$ in Oxygen Deficient $\mathrm{RBa} 2 \mathrm{Cu} 3 \mathrm{O} 6+x$

PB91-194894

101,524 Not available NTIS

PB91-194902

Crystal-Field Splittings in the Cubic Heusler Alloys HoPd2Sn and ErPd2S

101,525 Not available NTIS

PB91-194910

Photoionization Studies of Atoms and Molecules Using Synchrotron Radiation

PB91-194910

101,664 Not available NTIS

PB91-194928

Angle-Resolved Photoemission from the Ar $2 p$ Subshell.

PB91-194928

01,665 Not available NTIS

Rule-Based Diagnostic Method for HVAC Fault Detection. PB91-194936

100,072 Not available NTIS PB91-194944

Reflection-Extended-X-ray-Absorption-Fine-Structure Spectroscopy at the Carbon K-Edge.

PB91.194944

100,371 Not available NTIS

PB91-194951

Surface-Extended X-ray-Absorption Fine-Structure Experiments at Atmospheric Pressure by Means of a Photocathode Proportional Counter with Monolayer Sensitivity.

PB91-194951 100,372 Not available NTIS PB91-194969

NASREM Robot Control Systern and Testbed.

PB91-194969 101,033 Not available NTIS

PB91-194977

Studies of Magnetic Flux Penetration in a Chemically Synthesized $\mathrm{Bi}(\mathrm{Pb}) \mathrm{SrCaCuO}$ Superconductor.

PB91-194977 PB91-19498

101,526 Not available NTIS

PLOTMD: An Interactive Program to Modify Molecular Plots on a Graphics Terminal.

PB91-194985

100,373 Not available NTIS

PB9 1-194993

Process Quality Enhancement for Machine Tools: Measurements and Standards.

101,019 Not available NTIS PB91-195008

Atomistic Diffusion Equations and Kinetic Forces.

PB91-195008

100,374 Not available NTIS

PB91-195016

Low-Pressure Symmetric Discharge Heat Pipe and DROGS of Eu.

PB91-195016

PB91-195024

101,666 Not available NTIS

Ion Bombardment Induced Diffusion: A Case Study on a Sputtered Ag/Ni Layered System.

PB91-195024

101,527 Not available NTIS

PB91-195032

Relativistic Probability Amplitudes and State Preparation.

PB91-195032

101,667 Not available NTIS

PB91-195040

Simultaneous Butyltin Determinations in the Microlayer,

Water Column and Sediment of a Northern Chesapeake

Bay Marina and Receiving System

PB91-195040

100,918 Not available NTIS

PB9 1-195057

Absorbed Dose to Water: Comparison of Several Methods Using a Liquid lonization Chamber.

PB91-195065

Carbon Acidities of Aromatic Compounds. 2. Ionic Probes of Aromaticity in Annelated Rings.

PB91-195065

PB91-195073

Thermal Decomposition of lons. 3. Protonated Ethanol and

Diethyl Ether.

PB91-195073

PB91-195081

100,376 Not available NTIS

Mechanical and Swelling Behavior of Crosslinked Natural Rubber: Consequences of the Flory-Rehner Hypothesis.

PB91-195081

101,141 Not available NTIS

PB91-195099

Relaxation of Crosslinked Networks: Theoretical Models and Apparent Power Law Behavior.

PB91-195099

100,501 Not available NTIS

PB91-195107

Study of the Linear Viscoelastic Properties of Cyclic Polystyrenes Using Creep and Recovery Measurements.

100,502 Not available NTIS

PB91-195115

Wigner-Seitz Local-Environment Study of the High Tc Superconductors.

PB91-195123

101,528 Not available NTIS

Glueballs and Hadron Spectroscopy.

PB91-195123

101,668 Not available NTIS

PB91-195131

Dipole Moments of Seven Partially Halogenated Ethane Refrigerants.
PB91-195131

PB91-195149

100,377 Not available NTIS

Adsorption and Orientation of Tetracycline on Hydroxyapa-

tite.

PB91-195156

High Purity Chemicals and Clean Rooms for Trace Metal

PB91-195156

PB91-195164 
PB91-195255

Dynamics of Molecular Hydrogen Adsorbed in CoNa-A ZeoPB91-195255

100,380 Not available NTIS

PB91-195263

Use of Computer Algebra to Locate Critical Loci in Fluid Mixtures.

PB91-195263

101,390 Not available NTIS

PB91-19527

Molecular Dynamics of initial Events in the Thermal Degra dation of Polymers

PB91-195271

PB91-195289

Anomalous Lgamma2,3 X-ray Emission Spectrum of $X e$.

PB91-195289
PB91-195297

100,503 Not available NTIS

Anomalous Th M3O1 X-ray Emission Spectrum of ThO2

PB91-195297

101,670 Not available NTIS

PB91-195305

100,381 Not available NTIS

Many Electron Effects in Lgamma2,3 X-ray Emission Spec troscopy Spectrum of $\mathrm{BaO}$.

PB91-195305

100,382 Not available NTIS

PB91-195313

Many-Electron Effects in Lgamma2,3 XES Spectra from Rare Earth Elements.

PB91-195321

100,383 Not available NTIS

Rate of Pyrite Bioleaching by 'Thiobacillus ferrooxidans': Results of an Interiaboratory Comparison.

PB91-195321

101,161 Not availabie NTIS

PB91-195339

Recent Progress in Coal Bioprocessing Research in the United States: Scope and Areas Needing Further Research PB91-195339

PB91-195347

Development of a New Tracer Technology Using Enriched Rare-Earth isotopes.

PB91-195347

100,908 Not available NTIS

Real-Time Algorithms and Data Structures for Underwater Mapping.

MBapping.

101,360 Not available NTIS

PB91-195362

Reduction in Sintering Damage of Fiber Reinforced Com

posites.

PB91-195370

101,124 Not available NTIS

Reactions of $\mathrm{C} 5 \mathrm{H} 3(+)$ and $\mathrm{C} 5 \mathrm{H} 5(+)$ lons with Acetylene and Diacetylene.

PB91-195388

Prediction and Fault Detection of Building Energy Consumption Using Multi-Input, Single-Output Dynamical Model. PB91-195388 100,073 Not available NTIS

PB91-195396

Scoring Continuous Speech in the DARPA Speech Recognition Program: Part 2

PB91-195396

PB91-195404

100,593 Not available NTIS

Characterization by High Performance Liquid Chromatography (HPLC) of the Solubilization of Phosphorus in Iron Ore by a Fungus.

PB91-195412

101,162 Not available NTIS

Diesel Deposit Forming Tendencies - Microanalysis Methods.

101,178 Not available NTIS

PB91-195420

Air Exchange and Pollutant Concentration Measurements in Two Otfice Buildings.

PB91-195420

100,909 Not available NTIS

PB91-195438

Diagnostic Techniques for Evaluating Office Building EnvePBg $1-19$

PB91-195446

Sidelobe Suppression in Small Josephson Junctions.

PB91-195446

100,074 Not available NTIS

PB91-195453

Observation of Atoms Laser Cooled Below the Dopple

PB91-195453

PB91-195461

101,671 Not available NTIS

SEM imaging and Metrology at Low Accelerating Voltages Using Backscattered Electrons. 101,529 Not available NTIS PB91-19

PB91-195479

inner-Shell lonization Cross Sections.

PB91-195479

100,203 Not available NTIS

PB91-195487

Application of Laser Diagnostic Techniques for the Examination of Liquid Fuel Spray Structure.

PB91-19548

PB91-195495

Automatıng Robot Programming in the Cleaning and Deburring Workstation of the AMRF.
PB91-195495

100,986 Not available NTIS

91-195503

Relativistic Distorted-Wave Cross Sections for ElectronImpact Excitations of Beryiliumlike Ions

PB91-195503

101672 Not available NTIS

PB91.195511

Spectrum and Energy Levels of Seven-Times-Ionized Krypton ( $\mathrm{Kr}$ VIil) and Resonance Lines of Eight-Times-Ionized Krypton (Kr IX)

PB91-1955

101,673 Not available NTIS

Nitrogen Strengthening of Austenitic Stainless Steels at Low Temperatures.

PB91-195529

PB91-195537

101,163 Not available NTIS

Coherency Strain Induced Instability of Solid-Liquid Interfaces in the Mo-Ni System.

PB91-195537

101,235 Not available NTIS

PB1-195545

Trends in Technological Innovation.

PB91-195545

100,008 Not available NTIS

91-195552

Comparison of Amorphous and Quasicrystalline Films of Sputtered Al0.72Mn0.22Si0.06.

PB91-195552

PB91-195560

Inverse Correlation between the Intensity of Luminescence Excited by Electrons and by Visible Light in ChemicalVapor-Deposited Diamond Fiims.

PB91-195560

101,531 Not available NTIS

PB91-195578

Optically Detected Magnetic Resonance Study of Antisiteto-Acceptor and Related Recombination Processes in as Grown InP-Zn.

PB91-195586

101,532 Not available NTIS

Photoluminescence Excitation by Band-Gap Optical Absorption in Chemical Vapor Deposition Diamond Films. PB91-195586 101,533 Not available NTiS

PB91-195594

Morphology of Silver on YBa2Cu3O7-delta Thin Films.

PB91-19559

PB91-195602

vailable NTIS

Dielectronic Recombination Rate Coefficients for Ions of the Fluorine Isoelectronic Sequence.

PB91-195602

101.674 Not available NTIS

PB91-195610

Influence of Metastable States and Thermal Equilibration upon Dielectronic Recombination in Low Density to Moderate Density.

PB91-195610

PB91-195628

101,675 Not available NTIS

Phase Equilibria of the System Strontium Oxide-Calcium Oxide-Cupric Oxide

PB91-195628

101.085 Not available NTIS

PB91-195636

Non-Equilibrium Molecular Dynamics Simulations of Structured Molecules. Part 1. isomeric Effects on the Viscosity of

PB91-195636

PB91-195644

101,391 Not available NTIS

Roughened Ouartz Surfaces and Teflon as Small-Angle Diffusers and Depolarizers between 200 and 400 nm.

101,425 Not available NTIS

PB91-195651

Phase Selection in Non-Equilibrium Processing.

PB91-195651

PB91-195669

101,236 Not available NTIS

Standardization of Advanced Ceramics.

PB91-195669

101.086 Not available NTIS

PB91-195677

Parent Structure of the Layered High-Temperature SuperPB91-195677

PB91-195685

101,535 Not available NTIS

Difunctional and Multifunctionai Monomers Capable of $\mathrm{Cy}$ clopolymerization

PB91-195685

100,504 Not available NTIS

PB91-195693

Structural Change and Competitiveness: The U.S. Semiconductor Industry.

PB91-195701

100,173 Not available NTIS

Thermodynamics of Hydrolysis of Disaccharides: Lactulose alpha-D - Melibiose, Palatinose, D - Trehalose, D - Turanose and 3-o-beta-D-Galactopyranosyl-D-arabinos

PB91-19570

PB91-195719

Application of Osmotic Dewatering to the Controlled Crystallization of Biological Macromolecules and Organic Compounds.

PB91-195719

100,230 Not available NTIS

PB91-195727

tron Microscopy Analysis of Accuracy of Transmission Electron Micros

Asbestos on Filters: Interlabortory Study.
PB91-195727 100,204 Not available NTIS

PB91-195735

High-Temperature High-Pressure Raman Spectra from Liquid Water.

PB91-195735

100,385 Not available NTIS

PB91-195743

Effect of an Electric Field on the Morphological Stability of

the Crystal-Melt Interface of a Binary Alloy. 3. Weakly Non-

PB91-195743

PB91-195750

101,536 Not available NTIS

Scanning Tunneling Microscopy Study of Clean and Cs Covered InSb(110)

PB91-195750

101,537 Not available NTIS

PB91-197004

Stable implementation Agreements for Open Systems interconnection Protocols. Version 4, Edition 1, March 1991 Change Pages (Supplement).

PB91-197004

PB91-200782

Reactivities of Chlorine Atoms and Peroxyl Radicals Formed in the Radiolysis of Dichloromethane.

PB91-200782

100,386 Not avallable NTIS

PB91-200790

New Inexpensive Frequency Calibration Service from NIST. PB91-200790

PB91-200808

Rubidium Frequency Standard and a GPS Receiver: A Remotely Steered Clock System with Good Short-Term and Long-Term Stability.

PB91-200808

100,581 Not available NTIS

PB91-200816

$5 s(2) 5 p(2)-(5 s(2) 5 p 5 d+5 s 5 p(3)+5 s(2) 5 p 6 s$

$5 s(2) 5 p 7 s)$ Transitions in $\mathrm{sb} I I$ and $5 s(2) 5 p$

$5 \mathrm{~s}(2) \mathrm{nl})$ Transitions in Sb III.

1-200816

101,676 Not available NTIS

PB91-200824

iron Ion-Dependent Modification of Bases in DNA by the Superoxide Radical-Generating System Hypoxanthine/Xan thine Oxidase.

PB91-200824

PB91-200832

Taking the Measure of Fluid Properties Data Bases.

PB91-200832

B91-200840

Clocks, Atomic and Molecular.

PB91-200840

PB91-200857

Observation of Correlations in Finite, Strongly Coupled Ion Piasmas.

PB91-200857

101.678 Not available NTIS

PB91-200865

Numerical Study of Two-Dimensional Crystal Growth Forms in the Presence of Anisotropic Growth Kinetics.

PB91-200865

PB91-200873

Energy Distributions of Symbiotic Novae.

PB91-200873

PB91-200881

Near-Threshold Vibrational Excitation of $\mathrm{H}_{2}$ by Electron Impact: Resolution of Discrepancies between Experiment and Theory.

PB91-200881 
PB91-202887

Examination of Mechanisms Responsible for Incoheren Scattering of Subthermal Neutrons by Polymers.

PB91-202887

100,505 Not available NTIS

PB91-202895

TEM Driven Reverberating Chamber, a Single Facility for Radiated EMS/V Testing, $10 \mathrm{kHz}-18 \mathrm{GHz}$

PB91-202895 100,856 Not available NTIS PB91-202903

Enhanced Flux Creep in Nb-Ti Superconductors After an In crease in Temperature.

PB91-202903

101,680 Not available NTIS

PB91-202911

Hall Probe Magnetometer for SSC Magnet Cables: Effect of Transport Current on Magnetization and Flux Creep.
PB91-202911 101,681 Not available NTIS PB91-202929

Correlation of Flux States Generated by Optical Switching of a Superconducting Circuit.

101,682 Not available NTIS

391-202937

Frequency and Time Stability of GPS and GLONASS Clocks.

PB91-202945

100,582 Not available NTIS

Ultrasonic Texture and Stress Measurements in Anisotropic Polycrystalline Aggregates.

101,237 Not available NTIS

PB91-202952

Effect of Thermal Expansion Mismatch on Fiber Pull-Out in Glass Matrix Composites.

PB91-202952

PB91-202960

Selected-Ion Mass Spectrometry: Assays of Oxidative DNA Damage.

PB91-202960

PB91-202978

101,307 Not available NTIS

Computer Vision Based Station for Tool Setting and Tool

Form Measurement
PB91-202978

101,043 Not available NTIS

PB91-202986

Causes of $\mathrm{SiH} 4$ Dissociation in Silane dc discharges

PB91-202986 100,389 Not available NTIS

PB91-202994

Silane Dissociation Products in Deposition Discharges.

100,390 Not available NTIS

PB91-203000

Analysis of Laminar Vortex Shedding Behind a Circular Cylinder by Computer-Aided Flow Visualization.

PB91-203000 101,393 Not available NTIS

PB91-203018

Ultraviolet Absorption of the Vinyl Radical and Reaction with Oxygen.
PB91-203018

PB91-203026

Lunar Laser Ranging

PB91-203026

100,391 Not available NTIS

PB91-203034

101,353 Not available NTIS

Lanthanide Coordination Chemistry: Spectroscopic Properties of Terbium and Europium Poly(Pyrazol-1-YI) Borate and Poly(Imidazol-1-Yl) Borate.

PB91-203034

PB91-203042

100,392 Not available NTIS

Alternate Representation of Prism Refraction for Light Incident at the Brewster Angle.

101,426 Not available NTIS

PB91-203059

Monitoring of Anisotropic Material Elastic Properties Using Ultrasonic Receiving Rays.

101,126 Not available NTIS

B9 1-203067

Probe for Real-Time Images of Particle Beams and Their Analyses in a Merged-Beams Apparatus.

101,683 Not available NTIS

PB91-203075

Chemical and Electronic Properties of Pd/SnO2(110) Model Gas Sensors.

PB91-203083

Measurements of Velocity-Changing Collision Kernels.

PB91-203083

PB91-203091

Frequency Stabilization of an Erbium-Doped Fiber Laser: A Potential Wavelength Standard for Optical Communications. PB91-203091 100,570 Not available NTIS

PB91-203109

Frequency Stabilization of a Tunable Erbium-Doped Fiber Laser.

101,427 Not available NTIS

PB $1-203$

Switching in High-Tc Superconductor Current Transport Measurements
PB91-203117

PB91-203125

101,539 Not available NTIS

Squeezed Ouantum States of Relic Gravitons and Primordial Density Fluctuations.
PB91-203125

101,685 Not available NTIS

PB91-203133

How the Government Shapes UNIX Standards

PB91-203133

100,693 Not available NTIS

PB91-203141

Cratering.

PB91-203141

100,810 Not available NTIS

PB91-203158

Note on the Precision Cementing of Small Optical Components.

B91-203158

101,428 Not available NTIS

PB91-203166

Polarizability of the Photon

PB91-203166

101,686 Not available NTIS

PB91-203174

Advances in Science, Emerging Technologies and Global Competition: Opportunities and Challenge.
PB91-203174 100,583 Not available NTIS

PB91-203182

Established Microwave Frequency Standards.

PB91-203182

100,584 Not available NTIS

PB91-203190

Application of the PIPE Image Processing Machine to Scanning Microscopy. 101,034 Not available NTIS
PB91-203190

PB91-203208

Video Compression for Remote Vehicle Driving.

PB91-203208 101,035 Not available NTIS

PB91-203216

Generalization of the Cornu Spiral for Lossy Media.
PB91-203216

PB91-203224

Morphology of Polymer Films and Single Molecules.

PB91-203224

PB91-203232

Rate Constants for Hydrogen Abstraction Reactions of the Sulfate Radical, SO4- Alkanes and Ethers.

PB91-203232

100,394 Not available NTIS

PB91-203240

Asymptotic Expansions of the Kernel Functions for Line Formation with Continuous Absorption.

PB91-203240

PB91-203257

NIST High-Dose Calibration Services.

PB91-203257

101,689 Not available NTIS

PB91-203265

Interlaboratory Comparison of Actinides in Human Tissue (239) $\mathrm{Pu}+(240) \mathrm{Pu}$

PB91-203265

PB91-203273

Production and Spectroscopy of Small Polyatomic Molecular Ions Isolated in Solid Neon

PB91-20327

100,395 Not available NTIS

PB91-203281

Resonance Enhanced Multiphoton lonization Spectra of the $\mathrm{GeF}$ and $\mathrm{GeCl}$ Radicals from $400-500 \mathrm{~nm}$

GB91-203281 Radicals from $400-500 \mathrm{~nm}$.

PB91-203299

Issues in the Design and Implementation of a System Ar-

chitecture for Computer Integrated Manufacturing.
PB91-203299 100,987 Not available NTIS

PB91-203307

Notes and Comments on Roundtable Discussion on LaserAssisted Collisions and Collision-Induced Spectra.

PB91-203307

101,690 Not available NTIS

PB91-203315

Estimation of Electromagnetic Fields in Complex Environ-

ments.

101,691 Not available NTIS

PB91-203323

Biological Sulfur Oxidation and Reduction for Coal Sulfur Speciation and Desulfurization

PB91-203323

100,886 Not available NTIS

PB91-203331

Molecular Desorption from Solid Surfaces: Laser Diagnostics and Chemical Dynamics.

PB91-203331

PB91-203349

Stable, High Ouantum Efficiency Silicon Photodiodes for Vacuum-UV Applications.

PB91-203349

PB91-203356

Parser That Converts a Boundary Representation into a Features Representation.

100,988 Not available NTIS

PB91-203364

Simulation Studies of Excluded Volume Effects on Polymer Chain Dynamics in Several Nonlattice Models.

PB91-203364

100,507 Not available NTIS

PB91-203372

Experimentation and Measurement.

PB91-203372

101,692 Not available NTIS

PB91-203380

Static Analysis Tools for Software Security Certification

PB91-203380

PB91-203398

Cross-Section Measurements in the Intermediate-Energy Standard Neutron Field.

PB91-203398

101,693 Not available NTIS

PB91-203406

Spectrum-Averaged Cross-Section Measurement of $(103) \operatorname{Rh}(n, n)(103 \mathrm{~m}) \mathrm{Rh}$ in the (252)Cf Fission Neutron SpecPB91-203406

101,694 Not available NTIS

PB91-203414

Estimators for Censored (Log)Normal Samples. PB91-203414

101,277 Not available NTIS

PB91-203422

Calibration of the NBS 10 mum Space Beads.

PB91-203422

101,695 Not available NTIS

Dimensional Calibration of an NBS 0.3-mum-Diameter Particle-Sizing Standard.

101,696 Not available NTIS

PB91-203448

Microwave Spectrum and Molecular Structure of the N2-

H20 Complex
PB91-203448

PB91-203455

100,398 Not available NTIS

Critical Data in

PB91-203463

Capacitance versus Stylus Measurements of Surface Roughness.
PB91-203463

101,020 Not available NTIS

PB91-203471

Chemical Effects of $\mathrm{Ne}+$ Bombardment on the MoS2(0001) Surface Studied by High-Resolution Photoelectron Spectroscopy.

PB91-203489

100,399 Not available NTIS

Small Angle Neutron Scattering Characterization of Microporous Silica.

PB91-203497

Nature of Collinearity.

PB91-203497

101,089 Not available NTIS

PB91-203505

Computed Fields Near the Edge of a Dielectric Wedge.

PB91-203505

PB91-203513

Scattering by an Arbitrary Cylinder at a Plane Interface.

PB91-203513

101,447 Not available NTIS

PB91-203521

NBS Research on Protective Coatings for Buildings and Structures.

PB91-203539

100,127 Not available NTIS

Core Hole Screening for Intermediate Size Metal Particles.

PB91-203539

101,540 Not available NTIS

PB1-203547

Ionic Hydrogen Bonds. Part 1. Thermochemistry, Structural Implications, and Role in lon Solvation.
PB91-203547

Lattice Relaxation in Silicon Doped with $4 \mathrm{~d}$ - and $5 \mathrm{~d}$-Transition Metals.

PB91-203554

PB91-203562

101,541 Not available NTIS

Structural Dependence of HF Vibrational Red Shifts in ArnHF, $n=1-4$, via High-Resolution Slit Jet Infrared SpecPB91-203562

100,401 Not available NTIS

PB91-203570

Tests of the Flory-Rehner Hypothesis: Comparison of the Elastic Free Energy Function for Crosslinked Rubber in the Dry and Swollen States.

PB91-203570 
PB91-203638

Observation of the Cesium Clock Transition Using LaserCooled Atoms in a Vapor Cell. 101,698 Not available NTIS

Prospects for High Temperature Superconductor - Semiconductor Contacts.

PB91.203646

101,545 Not available NTIS

PB91-203653

Tunneling Stabilized Magnetic Force Microscopy: Prospects for Low Temperature Applications to Superconductors.

PB91-203653 101,546 Not available NTIS

PB91-203661

Radiolytic Studies of the Redox Reactions of Ruthenium Porphyrins.

PB91-20366

100,402 Not available NTIS

Mechanism of Cyanide Release in the Radiolysis of Aceto-

nitrile.

100,240 Not available NTIS

PB91-203687

Role of Glassy Interlaces in High Temperature Crack

Growth in SiC Fiber Reinforced Alumina.

PB91-203687 101,127 Not available NTIS

PB91-203695

Progress on the Determination of Intermolecular Potential

Energy Surfaces from High Resolution Spectroscopy.
PB91-203695

PB91-203703

Multiple Intermolecular Bend Vibrational Excitation of a $\mathrm{Hy}$ drogen Bond: An Extended Infrared Study of OCOHF.

drogen Bond: An Extended Infrared Study of OCOHF.

PB91-20370

100,404 Not available NTIS

Reflection/Absorption FTIIR Spectral Characteristics of Thin and Thick Crosslinked Epoxy Films on Steel Substrates.

PB91-203711 100,509 Not available NTIS

PB91-203729

Characterization of Epoxide Coatings on Steel by Reflec tion/Absorption Fourier Transform Infrared Spectroscopy: Quantitative Study.

PB91-203729

101,109 Not available NTIS

Control Structure for Multi-Tasking Workstations

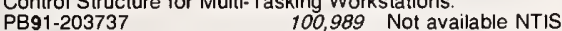

PBg1-

Mossbauer Imaging

PB91-203745

101,294 Not available NTIS

PB91-203752

New Approach to Image Modeling and Edge Detection in

PB91-203752

100,811 Not available NTIS

PB91-203760

Dissociation Energetics of Simple Diazirines by Photon and Electron Impact.

PB91-203760

100,405 Not available NTIS

PB91-203778

High Harmonic Generation in Hydrogenic lons.

PB91-203778 101,699 Not available NTIS

PB91-203786

Refinement of the Structure of Beta'-MoO3.

PB91-203786

PB91-203794

101,090 Not available NTIS

Kinetic Properties of the Components of Douglas-Fir and the Heat of Combustion of Their Volatile Pyrolysis Products.

PB91-203794

100,548 Not available NTIS

PB91-203802

New Triple Correlator Design for the Measurement of Ultrashort Laser Pulses.

PB91-203802

PB91-203810

101,429 Not available NTIS

Characterization of Tricresylphosphate Lubricating Films by

Micro-Fourier Transform Infrared Spectroscopy.
PB91-203810 101,179 Not available NTIS

PB91-203828

Effect of Mechanical Deformation on Nb-Ti Filament Proximity-Effect Coupling at the Edges of SSC Cables.

PB91-203828

101,700 Not available NTIS

PB91-203836

Activities of the Construction Materials Reference Laborato ries Related to Laboratory Accreditation.

PB91-203836 100,531 Not available NTIS

PB91-203844

Vibrational, Rotational, and Tunneling Dependence of Vi-

brational Predissociation in the HF Dimer.
PB91-203844 100,406 Not available NTIS

PB91-203844

B91-203851

Wind Effects on Fires

PB91-203851

100,549 Not available NTIS

PB91-203869

Effect of Atomization Air on Droplet Dynamics of Spray

Fiames.

100,550 Not available NTIS

PB91-203877

Optimization of a Pulse Tube Refrigerator for a Fixed Compressor Swept Volume.

101,051 Not available NTIS
PB91-203885

Simultaneous versus Independent Injection Testing of Nonlinear Multiport Systems.

PB91-203885

100,857 Not available NTIS

PB91-203893

Reference Materials for Analytical Chemistry

PB91-203893

100,205 Not available NTIS

PB91-203901

Role of Reference Materials in Quality Assurance.

PB91-203901

100,206 Not available NTIS

PB91-203919

New Look at the Bitter Method of Magnetic Imaging

PB91-203919

101,701 Not available NTIS

PB91-203927

Frequency Dependence of Catalyzed Reactions in a Weak Oscillating Field
PB91-203927

PB91-2039

100,407 Not available NTIS

Use of Nondestruclive Methods for Inspection of Single-Ply Roofing Membranes.

PB91-20393

100,128 Not available NTIS

PB91-203943

Numeric Database for Tribology: Format and Application (1)

101,045 Not available NTIS

PB91-203950

Properties of YBa2Cu3O7-delta Thin Films Grown on OffAxis-Cut MgO Substrates.

PB91-203950

101,547 Not available NTIS

PB91-203968

Resonance Ionization Spectroscopy/Resonance Ionization

Mass Spectrometry Data Service.
PB91-203968 100,207 Not available NTIS PB91-203968

PB91-203976

Integrated-Optic Waveguide Glass Lasers.

PB91-203976

100,758 Not available NTIS

PB91-203984

Precise Laser Wavelength Measurements: What Can We Learn from Classical Spectroscopy.
PB91-203984 Not available NTIS

PB91-203992

Building-in Reliability: Making It Work

PB91-203992

PB91-204008

100.812 Not available NTIS

High-Resolution Infrared Flash Kinetic Spectroscopy of $\mathrm{OH}$ Radicals.

PB91.204008

100,408 Not available NTIS

PB91-204016

Production and Diagnosis of a Highly Spin-Polarized $\mathrm{N}$ Beam.

PB91-204016

101,431 Not available NTIS

PB91-204024

Investigation of Internal Rotor Dynamics of $\mathrm{NeDCl}$ and ArDCl via Infrared Absorption Spectroscopy

PB91-204024

100,409 Not available NTIS

PB91-204032

Advanced Materials: Opportunities and Challenges

PB91-204032

100,009 Not available NTIS

PB91-204040

International Collaboration in Prestandards Research on Advanced Materials within the Context of VAMAS

PB91-204040

101,251 Not available NTIS

PB91-204057

Investigation of the Collapse of L'Ambiance Plaza

PB91-204057

100,146 Not available NTIS

PB91-204065

Thermodynamic Properties of Aqueous Solutions at High Temperatures; Needs, Methods, and Challenges.

PB91-204065 100,410 Not available NTIS

PB91-204073

Magnetic Properties of Iron/Silica Gel Nanocomposites.

PB91-204073

PB91-204081

Microstructure and Elastic Properties of Dental Resin and Resin-Based Glass-Reinforced Composites: XRD, SEM and Ultrasonic Methods.

PB91-204081

PB9 1-204099

Visibility of Chrysotile Asbestos in the Scanning Electron Microscope.

PB91-204107

Data Encryption Standard: Past and Future.

P'B91-204107

PB91-204115

Test of the Inverse-Square Law of Gravitation Using the 300-m Tower at Erie, Colorado.

PB91-204115

101,702 Not available NTIS

PB91.204123

Thermodynamic Behavior of Fluids in the Supercritica

PBgion.

PB91-204131

101,394 Not available NTIS

Analyzing Integrated Optical Waveguides: A Comparison of Two New Methods.

100,759 Not available NTIS

PB91-204149

Dental Composite Resins: An Update.

PB91.204149

100,051 Not available NTIS

PB91-204156

Crystalline and Glassy Phases of Transition-Metal - Metalloid Systems.

PB91-204164

Collisional Energy Transfer between Excited Sr Atoms.

PB91-204164

PB91-204172

Creep and Reliability of Ceramic Materials at Elevated Tem peratures.

PB91-204172

101,091 Not available NTIS

PB91-204180

Correlated Spontaneous Emission in a Zeeman Lase

PB91-204180

101,432 Not available NTIS

PB91-204198

Convergence Behavior of Trajectories for Linear Program-

ming. 204198

PB91-204206

101,270 Not available NTIS

Research and Technology for Structural Engineering Pro-

ductivity.

100,147 Not available NTIS

PB91-206664

Technical Reference and User's Guide for FAST/FFM Version 3

PB91-206664

100,086 PC A08/MF A0

PB91-206680

Sprinkler/Hot Layer Interaction

PB91-206680

$100,129 \mathrm{PC}$ A04/MF A01

PB91-206698

Indoor Ventilation Requirements for Manufactured Housing.
PB91-206698

PB91-206706

Measurement and Evaluation of Lighting/HVAC Interaction PB91-206706

PB91-206714

Long Term Performance of Rubber in Seismic and NonSeismic Bearıngs: A Literature Review. PB91-206714

PB91-206722

User Manual NBSAVIS CONTAM88. A User Interface for Air Movement and Contaminant Dispersal Analysis in Multizone Buildings.

PB91-206730

Calibration at $24 \mathrm{C}$ of a Heat-Flow-Meter Apparatus Having $610 \mathrm{~mm}$ Square Plates.

PB91-206730

PB91-206748

NVLAP FY 90 Annual Report.

PB91-206748

100,933 PC A03/MF A0

PB91-206755

Abstracts of Daylighting Research

PB91-206755

$100,010 \mathrm{PC} \mathrm{A03/MF} \mathrm{A01}$

PB91-216531

Publications of the National Institute of Standards and Technology, 1990 Catalog.

PB91-216531

PB91-216598

Portable Vectorized Software for Bessel Function Evaluation

PB91-216598 
PB91-216689

Applications of the Generalized Global Equivalence Ratio Model (GGERM) for Predicting the Generation Rate and Distribution of Products of Combustion in Two-Layer Fire Environments: Methane and Hexanes.

PB91-21668

100,131 PC A05/MF A01

PB91-216697

Guide Specification for Direct Digital Control Based Building Automation System.

PB91-216697

100,092 PC A04/MF A01

PB91-216705

Journal of Research of the National Institute of Standards and Technology. May-June 1991. Volume 96, Number 3.

PB91-216705

101,704 PC A07/MF A02

PB91-216713

High-Temperature Transient Hot-wire Thermal Conductivity Apparatus for Fluids.

100,411

B91-216721

Order as PB91-216705, PC A07/MF

Standard Reference Specimens in Ouality Control of Engineering Surfaces.

(Order as PB91-216705, PC A07/MF A01)

PB91-216739

Automated Reverse-Bias Second-Breakdown Transisto

Tester.

100,813

B91-216747

(Order as PB91-216705, PC A07/MF A01)

High Resolution Synchrotron X-Radiation Diffraction Imaging of Crystals Grown in Microgravity and Closely Related Terrestrial Crystals

(Order as PB91-216705, PC A07/MF

PB91-216754

Root Projection of One-Sided Time Series.

PB91-216754

101,262
(Order as PB91-216705, PC A07/MF A01)

PB91-216762

Deconvolution of Acoustic Emission and Other Causal Time Series.

PB91-216762

101,263
(Order as PB91-216705, PC A07/MF A01)

\section{PB91-216770}

NIST Measurement Services: Calibration Service for Current Transforme

PB91-216788

100,873 PC A04/MF A0

Building and Fire Research Project Summaries, 1991. PB91-216788

PB91-216796

$100,132 \mathrm{PC} \mathrm{A08/MF} \mathrm{A02}$

First Pass at Computing the Cost of Fire Safety in a Modern Societ

PB91-216804

101.730 PC A04/MF A0

Interaction of an Isolated Sprinkler Spray and a Two-Layer Compartment Fire Environment.

PB91-216804

$100,551 \mathrm{PC} \mathrm{A03/MF} \mathrm{A01}$

PB91-216812

Evidence for Surface alpha Particle Clusters in (nat)AG(197)Au from the (e,alpha) Reaction.
PB91-216812 101,705 PC A04/MF A01 PB91-216820

Building Technology Publications 1986-1989.

PB91-216820

100,160 PC A08/MF A02

PB91-216838

ICLASS-91 Proceedings of the International Conference on Liquid Atomization and Spray Systems (5th). Held in Gaith ersburg, MD. on July 15-18, 1991 .
PB91-216838 100,552 PC A99/MF E08 PB91-217414

Generation of Smooth Trajectories without Planning.
PB91-217414 101,036 PC A03/MF A01

PB91-217422

Estimated Impact of the Center for Fire Research Program
on the Costs of Fire.
PB91-217422 100,161 PC A05/MF A0 On the Costs of Fire.
P891-217422

PB91-222570

Performance of 1/3-Scale Model Precast Concrete BeamColumn Connections Subjected to Cyclic Inelastic Loads. Report No. 2

B 91-222588

100,149 PC A05/MF A0

Performance Evaluation of Hypercube Applications: Using a Global Clock and Time Dilation.

PB91-222588

PB91-222596

100,653 PC A03/MF A0

Development Plan: Application Protocols for Mechanical Parts Production. National PDES Testbed Report Series. B9 1-222596

PB91-222604

Reduction in Fire Hazard in Corridors and Areas Adjoining Corridors Provided by Sprinklers.

PB91-222604

PB91-222612

Assessment of the State-of-the-Art for Process Monitoring Sensors for Polymer Composites.
PB91-222612

$100,510 \mathrm{PC}$ A07/MF A02

\section{PB91-222620}

Configuration Management of the STEP Documents: Procedures and System Requirements. National PDES Testbed Report Series.

PB91-222638

Automated Information Systems Security Program Handbook.

PB91-222638

100,713 PC A08/MF A02

PB91-222646

Directory of Accredited Laboratories, 1991. National Voluntary Laboratory Accreditation Program (NVLAP)

tary Laboratory Accreditation Program (NVLAP). PB91-222653

Residual Stress Detection in Railroad Wheels: An Uitrasonic System Using EMATs (Electromagnetic-Acoustic Transducers). Report No. 23

PB91-222653

101,741 PC A03/MF A01

\section{1-222661}

Tread Crack Detection in Railroad Wheels: An Ultrasonic System Using EMATs (Electromagnetic-Acoustic Transducers). Report No. 22

PB91-222661

101,742 PC A04/MF A01

\section{PB91-222679}

Unsteady Laminar Flow in a Circular Tube: A Test of the HERCOL (Hermitian Collocation) Computer Code.

PB91-222679

101,395 PC A03/MF A01

PB91-222687

Annotated Bibliography: Diagnostic Methods and Measurement Approaches to Detect Incipient Defects Due to Aging of Cables.

PB91-222695

$101,369 \mathrm{PC} \mathrm{A10/MF} \mathrm{A03}$

Standards for the Physical Protection of National Resources and Facilities.

100,006 PC A05/MF A01

PB91-231555

NIST Personnel Management Demonstration Project Design, Implementation and Accomplishments.

PB91-231555

100,003 PC A05/MF A01

PB91-231563

Daylight Research Requirements. Workshop Proceedings Held in Baltimore, Maryland on August 1-2, 1990.

PB91-231563

00078 PC A04/MF A01

PB91-231571

Computer Implementation of a Discrete Set Algebra.

PB91-231571

100,654 PC A03/MF A01

PB91-231589

General Routine for Analysis of Stack Effect

PB91-231589

$100,163 \mathrm{PC}$ A03/MF A0

PB91-231597

Task Decomposition and Algorithm Development for RealTime Motion Control of a Continuous Mining Machine

101357 PC A03/MF A01

PB91-231605

NIST Time and Frequency Services.

PB91-231605

$100,585 \mathrm{PC}$ A03/MF A01

PB91-236489

Mechanisms of the Reduction Reactions of $\mathrm{Cr}(\mathrm{VI})$ in the Radiolysis of Acidic Potassium and Silver Dichromate Solutions in the Presence or Absence of Acetic Acid.

PB91-236489

PB91-236497

Use of Storage Phosphor Imaging Plates in Portal Imaging and High-Energy Radiography: The Intensifying Effect of the Sensitivity.

PB91-236497

101,324 Not available NTIS

PB91-23650

Spatially Overdetermined Analysis for Propagation Toughness Using Strain Gages
PB91-236505

PB91-236513

101,164 Not available NTIS

Automated Reverse-Bias Second-Breakdown Transistor Tester

PB91-236513

100,814 Not available NTIS

PB91-23652

Problem of Detailed Balance and Model Lineshapes in Collision-Induced Rotovibrational Bands: $\mathrm{H} 2-\mathrm{H} 2$ and $\mathrm{H} 2-\mathrm{He}$. PB91-236521

PB91-236539

Development of an Adhesive System for Bonding to Hard Tooth Tissues.
PB91-236539

PB91-236547

100,052 Not available NTIS

Photodissociation Dynamics of $\mathrm{Mo}(\mathrm{CO}) 6$ at $266 \mathrm{~nm}$ and $355 \mathrm{~nm}$ : CO Photofragment Kinetic-Energy and InternalState Distributions.

P891-236547

100,413 Not available NTIS

PB91-236554

Investigating the Use of Multimeters to Measure Ouantized Hall Resistance Standards.

P891-236554

PB91-236562

100,734 Not available NTIS

Eddy Current Probe Sensitivity as a Function of Coil Construction Parameters.

1.236562

PB91-236570

Temperature Gradient in Differential Scanning Calorimetry.
PB91-236570 100,414 Not available NTIS B91-236588

Performance of Precast Concrete Beam-to-Column Connections Subject to Cyclic Loading. PB91-23658

PB91-236596

Ouantitative Analysis of Impurities in SIMOX Samples Using Secondary lon Mass Spectrometry.

PB91-236596

100,208 Not available NTIS

PB91-236604

Transmission Properties of a Counter-Rotating Pair of Disk PB91-236604

PB91-236612

Long Time Creep and Lifetime Behavior in Uniaxial Extension of a Linear Low Density Polyethylene.

PB91-236612

PB91-236620

Determining Adapter Efficiency by Envelope Averaging Swept Frequency Reflection Data.
PB91-236620 100,743 Not available NTIS PB91-236638

Semiempirical Theory of Relaxation: Concentrated Polymer Solution Dynamics.

PB91.236638

PB91-236646

100,511 Not available NTIS

Analysis of Wet Deposition (Acid Rain): Determination of the Major Anionic Constituents by lon Chromatography.
PB91-236646 100,035 Not available NTIS

PB91-236653

Thin and Composite High-Flux Membranes of Perfluorosultonated lon-Exchange Polymer

PB91-23665

100,512 Not available NTIS

PB91-236661

Rotational Spectrum and Structure of the Complex Ar$\mathrm{Ch} 3 \mathrm{CN}$

PB91-23666

100,415 Not available NTIS

PB91-236679

Optothermal-Detected Microwave-Sideband CO2-Laser Spectroscopy of Ar-NH3.

PB91-236679

100,416 Not available NTIS

PB91-236687

Functional Meta-Structure for Hypertext Models and Sys-

PB91-236687

100,655 Not available NTIS

PB91-236695

Thermodynamic Diagrams for Refrigerant Mixtures.

PB91-236695

101,197 Not available NTIS

PB91-236703

Emitter Ballasting Resistor Design for, and Current Handling Capability of AIGaAs/GaAs Power Heterojunction Bipolar Transistors. PB91-236703

PB91-236711

Effect of Selected Chemical Compounds on the Lubrication of Silicon Nitride.
PB91-236711

Testing the Accuracy of Calculated Equilibrium Carrier ConTentrations in the Presence of Surface Fields.

PB91-236729

PB91-236737

Van der Waals Complexes in 1,3-Dipolar Cycloaddition Reactions: Ozone-Ethylene.

PB91-236737

100,417 Not available NTIS

PB91-236745

Associative Ionization of Ultra-Cold Laser Trapped Sodium

PB91-236745

101,707 Not available NTIS 
PB91-236810

Excess Low-Frequency Flux Noise in dc Squids Incorporating $\mathrm{Nb} / \mathrm{Al}$-Oxide/ $\mathrm{Nb}$ Josephson Junctions.

ing $\mathrm{Nb} / \mathrm{Al}$-Oxide/ $\mathrm{Nb}$ Josephson Junctions. PB91-236810

Reply to Comment on 'Ouantum Zeno Effect'.

PB91-236828 101,708 Not available NTIS

PB91-236836

Vibrational Spectra of Molecular Ions Isolated in Solid Neon. 6. $\mathrm{CO} 4(1-)$

PB91-236836
PB91-236844

100,419 Not available NTIS

Improved Technique for Measuring Permittivity of Thin Die-

lectrics with a Cylindrical Resonant Cavity.
PB91-236844
100,858 Not available NTIS

PB91-236844

Temperature Measurements in Flames Using Thermally Assisted Laser-Induced Fluorescence of $\mathrm{Ga}$.

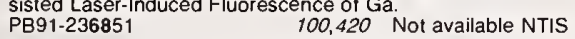

PB91-236869

Molecular-Beam Optothermal Spectroscopy of the 9.6 mum nu14.

PB91-236869

100,421 Not available NTIS

PB91-236877

Biomarkers of $\mathrm{OH}$ Radical Damage In vivo

PB91-236877

101,325 Not available NTIS

PB91-236885

Temperature Dependence of Probe Diffusion in Bulk Polymer Matrices.
PB91-236885

PB91-236893

100,422 Not available NTIS

AC-DC Difference Relationships for Current Shunt and Thermal Converter Combinations.
PB91-236893 Not available NTIS

PB91-236901

Generalizeo Photodiode Self-Calibration Formula

PB91-236901 100,760 Not available NTIS

PB91-236919

Alignment Fixture for Millimeter Waveguide.

PB91-236919 100,727 Not available NTIS

PB91-236927

Cyclic Fatigue from Frictional Degradation at Bridging Grains in Alumina.

PB91-236935

101,092 Not available NTIS

Fracture Mechanics Model for Subthreshold Identation Flaws. Part 1. Equilibrium Fracture.
PB91-236935

PB91-236943

Load-Controlled Tensile Tests of Austenitic Steels at $4 \mathrm{~K}$

PB91-236943

PB91-236950

Precise Ephemerides for GPS Time Transfer.

PB91-236950 100,586 Not available NTIS

PB91-236968

Majority and Minority Electron and Hole Mobilities in Heavily Doped GaAs.

PB91-236968

PB91-236976

101,550 Not available NTIS

Cryogenic Fatigue of High-Strength Aluminum Alloys and Correlations with Tensile Properties.
PB91-236976 101,709 Not available NTIS

PB91-236984

Heterodyne Frequency Measurements of CO and OCS Beyond $2100 \mathrm{~cm}(-1)$.

B91-236992

100,423 Not available NTIS

Characteristic Impedance Determination Using Propagation Constant Measurement.

PB91-236992

PB91-237008

100,775 Not available NTIS

Sensitometry of the Response of a New Radiochromic Film Dosimeter to Gamma Radiation and Electron Beams.
PB91.237008 101,326 Not available NTIS

PB91-237016

Perpendicular Bands of Cyclopropane in the 3.5 mum Region.

PB91-237016

PB91-237024

Partial Equilibrium in Laminar Hydrocarbon Diffusion Flames.

PB91-23702

100,553 Not available NTIS

PB91-237032

Direct Detection of Atom Vaporization by Laser Resonance Ionization as a Probe of Gas-Surface Chemisorption Mechanisms.

PB91-237032

100,425 Not available NTIS

PB91-237040

Book Review: Hypertext Hands-On

PB91-237040

100,656 Not available NTIS

PB91-23705

Linking Information to Objects: A Hypertext Prototype for Numismatists.
PB91-237057

PB91-237065

100,657 Not available NTIS

Exploding Foil Photoionization X-ray Laser.
PB91-237065

101,433 Not available NTIS 37073

General Technique to Correct Probe Position Errors in Planar Near-Field Measurements to Arbitrary Accuracy.
PB91-237073 100,728 Not available NTIS

PB91-237081

Interlaboratory Tension and Fracture Toughness Test $\mathrm{Re}$ sults for CSUS-JN1 (Fe-25Cr-15Ni-0.3N) Austenitic Stain

less Steel at $4 \mathrm{~K}$.

PB91-237099

Laser-Induced Fluorescence of $\mathrm{CH}$. in a Laminar $\mathrm{CH} 4 /$ Air Diffusion Flame: Implications for Diagnostic Measurements and Analysis of Chemical Rates.

PB91-237099

PB91-237107

New Low-Voltage Standards in the DC to $1-\mathrm{MHz}$ Frequency

Range.

100,738 Not available NTIS

PB9 1-237115

Monitoring the Mass Standard via the Comparison of $\mathrm{Me}$ chanical to Electrical Power.

PB91-237115

101,710 Not available NTIS

PB91-237123

Differential Sintering

PB 1-237131

101,128 Not available NTIS

Cathodoluminescence Measurement of Strained Alumina Single Crystals.

PB91-237

101,094 Not available NTIS

Mass Transfer Coefficients in Two-Phase Aqueous Extraction.

PB91-237156

100,426 Not available NTIS

New Triple Cor

Laser Pulses.

PB91-237164

Molecular-Orbital Studies via Satellite-Free $X$-ray Fluorescence: $\mathrm{Cl} \mathrm{K}$ Absorption and $\mathrm{K}$-Valence-Level Emission Spectra of Chloroluorometha

PB91-237164

100,427 Not available NTIS

PB91-237172

Hydration of Carbon Dioxide: The Structure of $\mathrm{H} 2 \mathrm{O}-\mathrm{H} 2 \mathrm{O}$ $\mathrm{CO} 2$ from Microwave Spectroscopy.
$\mathrm{PB} 91-237172$
100,428 Not available NTIS

PB91-237180

Critical-Current Diffraction Patterns of Grain-Boundary Josephson Weak Links.

PB91-237

101,551 Not available NTIS

Immobilization of Binding Proteins on Nonporous Supports Comparison of Protein Loading, Activity, and Stability.

PB91-237198 101,308 Not available NTIS

PB9 1-237206

Effect of Processing on 4-K Mechanical Properties of a Mi-

croalloyed Austenitic Stainless Steel.
PB91-237206

PB91-237214

Precision Oualification of Watthour Meters.

PB91-237214

100,859 Not available NTIS

PB91-237222

Theoretical Considerations for a Thermo-Optic Microwave Electric-Field-Strength Probe.

PB91-237222

PB9 1-237230

Electrokinetic Demixing of Two-Phase Aqueous Polymer Systems. 2. Separation Rates of Polyethylene Glycol-Maltodextrin Mixtures.

100,231 Not available NTIS

1-237248

Strain Rate Effect on Tensile Properties at $4 \mathrm{~K}$ of a VAMAS Round-Robin Austenitic Steel.

PB91-23724

PB91-237255

101, 168 Not available NTIS

Microwave Monolithic Integrated Circuit-Related Metrology

at the National Institute of Standards and Technology.
PB91-237255

PB91-237263

Surface Extended-X-ray-Absorption Fine Structure and

Scanning Tunneling Microscopy of Si(001)2 $2 \times 1-$ Sb.
PB91-237263

PB91-237271

Micromagnetic Calculations of $180 \mathrm{deg}$ Surface Domain WBalls

101,553 Not available NTIS

PB91-237289

Scanning Electron Microscopy with Polarization Analysis (SEMPA)-Studies of Domains, Domain Walls and Magnetic Singularities at Surfaces and in Thin Films.

PB91-237289

PB91-237297

101,554 Not available NTIS

Thermodynamics of Solutions Near the Solvent's Critical PBg1-

$1-237297$

100,232 Not available NTIS

PB91-237305

Characterization of Capacitive Array for NDE Applications.

PB91-237305

100,946 Not available NTIS

891-237313

Capacitive Array Sensors for Nondestructive Evaluation

PB91-237313

PB91-237321

Change in Magnetic State of $\mathrm{Fe}+$ Silica Gel Nanocomposiles Due to Low Temperature Treatment in Ammonia.

PB91-237321

PB91-237339

New Layered Cuprate Structure-Type, (A1 $\left.x A^{\prime} x\right) 14$ Cu24O 41

PB91-237339

PB91-237347

Antioxidation Mechanisms of Uric Acid

PB91-237347

PB91-237354

Impulse Response Functions for Elastic Structures with Rigid Body Degrees of Freedom.

PB91-237354

PB91-237362

Ab Initio Phase Determination for X-ray Diffraction Data from Crystals of a Native Protein.

PB91-237362

PB91-237370

Synthesis of 3-Ouinuclidinol-(18)O, Benzilic-d5 Acid, and 3 Ouinuclidinyl-(18)O, Benzilate-d5.

PB91-237370

PB91-237388

Calibration of Ophthalmic Applicators at NIST: A Revised Approach.

PB91-237396

Accelerator (14)C Dating of Late Pleistocene Megafauna.

PB91-237396

PB91-237404

Accelerator Radiocarbon Dates on Charcoal, Shell, and Human Bone from the Del Mar Site, California.

PB91-237404

101,355 Not available NTIS

PB91-237412

Experimental Study of the Pyrolysis Rate of a Poly(methy methacrylate) (PMMA) Wall Panel in a Reduced-Scale EnPB91-237412

100,555 Not available NTIS

PB91-237420

Structural Study of the Epitaxial Growth of fcc-Fe Films, Sandwiches, and Superlattices on Cu(100).

PB91-237420

101,555 Not available NTIS

PB91-237438

Structural Anomalies in Undoped Gallium Arsenide Observed in High Resolution Diffraction Imaging with Monochromatic Synchrotron Radiation.

PB91-237438

PB91-237446

Improvements for Automating Voltage Calibrations Using a 10-V Josephson Array.

PB91-237

PB91-237453

Theory of Ballistic-Electron-Emission Spectroscopy of $\mathrm{NiSi} / \mathrm{Si}(111)$ Interfaces

PB91-237453

101,557 Not availabje NTIS

PB91-23746

Structure of Odd-Odd (132)Sb.

PB91-237461

101,711 Not available NTIS

\subsection{9}

Microstructural Aspects of the Fracture of Hardened Cement Paste
PB91-237479

PB91-237487

100,133 Not available NTIS

Microwave Spectra and Electric Dipole Moments of $\mathrm{X}(4)$ Sigma(1-)1/2 $\mathrm{VO}$ and $\mathrm{NbO}$

PB91-237487

PB91-237495

Extreme-Ultraviolet Spectra of Pd, Ag, Cd, and Sn lons Observed in a 1.3-keV Tokamak Plasma.

PB91-237495

PB91-237503

Spectra of the P I Isoelectronic Sequence from Co XIII to Mo XXV11. 
PB91-237552

Low-Field Flux Pinning in Twinned and Detwinned Single

Crystals of YBa2Cu3O7-x
PBg1-237552

101,559 Not available NTIS

PB91-237560

Low-Cost Aqueous Two-Phase System for Enzyme Extrac-

PB91-237560

101,310 Not available NTIS

PB91-237578

Thermodynamic Properties of 1,1,1,2,-Tetrafluoroethane (R134a) in the Critical Region

PB91-237578

100,233 Not available NTIS

PB91-237586

New International Electrical Reference Standards Based on

the Josephson and Quantum Hall Effects.

PB91-237586

Basic Standards and Fundamental Constants.

PBg1-237594 101,715 Not available NTIS PB91-237602

Thermodynamics of Industrially Important Enzyme Catalyzed Reaction

PBg1-237602
P91-237610

101,311 Not available NTIS

Theory of Capacitive Probe Method for Noncontact Characterization of Dielectric Properties of Materials.

PB91-237610 100,948 Not available NTIS PB91-237628

Fourier Transform Infrared Analysis of Ceramic Powders: Ouantitative Determination of Alpha, Beta, and Amorphous Phases of Silicon Nitride.

PB91-237628

101,096 Not available NTIS

PB91-237636

Neutron Spectroscopic Evidence for Adsorbed Hydroxyl Species on Platinum Black. 100,432 Not available NTIS
PBg1-237636

PB91-237644

Influence of Memory Propagation on Phase-Resolved Stochastic Behavior of AC-Generated Partial Discharges.

PB91-237644 100,861 Not available NTIS

PB91-23765

Extended Phase Rule for Nonreactive, Multiphase, MultiComponent Chemical Systems.
PB91-237651 100,433 Not available NTIS PB91-237669

Variations in the Practice of Ceramic Technology in Different Cultures: A Comparison of Korean and Chinese Celadon Glazes

don Glazes.

PB91-237677

101,111 Not available NTIS

Geometric Reasoning for Constructing 3D Scene Descriptions for Images
PBg $_{1-237677}$

PB91-237685

Software Verification and Validation: An Overview.
PBg1-237685

PB91-237693

Database and Retrieval System for the NBS Tables of

Chemical Thermodynamic Properties.
PBg1-237693 PB91-23770

Surface Oxidation Kinetics of Si3N4 - 4\% Y2O3 Powders Studied by Bremsstrahlung-Excited Auger Spectroscopy.
PBg 1-237701 100,435 Not available NTIS PB91-237719

Oxidation Kinetics of Silicon Carbide Whiskers Studied by X-ray Photoelectron Spectroscopy.
PBg1-237719 100,436 Not available NTIS

PB9 1-237727

Characteristics of Aerosols Produced by the Spark Dis-

PBg $1-237727$

100,209 Not available NTIS

PB91-237735

Dentin-Bonding Molar Efficiency Using N-Phenylglycine, NPhenyl-beta-Alanine, or N-Methyl-N-Phenylglycine.
PB91-237735 100,053 Not available NTIS

PB91-237743

Environmentally Enhanced Fracture of Gallium Arsenide.

PBg1-237743

101,097 Not available NTIS

PB91-237750

Spectroscopic Data Base for Carbon and Oxygen

PBg 1-237750 101,716 Not available NTIS

PB91-237768

Unified Set of Atomic Transition Probabilities for Neutral Argon.
PBg $1-237768$

101,717 Not available NTIS

PB91-237776

Translate LRL and LRM Calibrations.

PB91-237776

100,862 Not available NTIS

PB91-237784

Rovibrational Analysis of the nu7(1) Intermolecular Hydrogen Bond Bending Vibration in HCN-HF Using Far Infrared Fourier Transform Spectroscopy

PBg1-237784

PB91-237792

Dealing with Obvious Issues in Lighting.
PB91-237792

100,761 Not available NTIS

B91-237800

Effect of Veiling Relections on Vision of Colored Objects

PBg 1-237800 100,079 Not available NTIS

PB91-237818

Light Source Area, Shading, and Glare.

PBg 1.237818

100,080 Not available NTIS

PB91-237826

Role of Theory in Lighting Research and Design.

PBg1-237826 100,081 Not available NTIS

PB91-237834

Assessment: U.S. Office Building of Moscow.

PBg1-237834

100,151 Not available NTIS

\section{PB91-237842}

Network Structure in Epoxies. 6. The Growth Process Investigated by
PBg $1-237842$

\section{PB91-237859}

Evaluation of Liquid Junction Potentials and Determination of $\mathrm{pH}$ Values of Strong Acids at Moderate lonic Strengths. PB9 1-237859

PB91-237867

Electrochemical Behavior of Ferrocene in Polyethylene Oxide-400.

PB91-237867

100.439 Not available NTIS

Possible Use of Pattern Recognition for the Analysis of Mars-Rover X-ray-Fluorescence Spectra.

PB91-237875 101,733 Not available NTIS

PB91-240739

PB91-240739

100,817 PC A03/MF A01

PB91-240747

Preliminary Results of the Environmental Evaluation of the Federal Records Center in Overland Missouri.

PB91-240747

100,082 PC A04/MF A01

\section{1-240754}

Depot: A Framework for Sharing Software Installation Across Organizational and Unix Platform Boundarie

PB91-240762

High Magnetic Field Facilities in Japan Related to Superconductivity Research (Japan Technology Program).

PB91-240762

PB91-240770

Suppression of Post-Flashover Compartment Fires Using Manually Applied Water Sprays.

PB91-240770

PB91-240788

Framework for Utilizing Fire Property Tests.

Pramework for

PB91-240796

Manufacturing Systems Integration Control Entity Interface Document.
PB91.240796

PB91-240804

100,991 PC A03/MF A01

Guidelines for the Development of Computer Based Models in a Cementitious Materials Modeling Laboratory.
100,532 PC A03/MF A01

\section{PB91-240812}

New Concepts of Precision Dimensional Measurement for

PBg1-240812

101,006 PC A04/MF A01

\section{PB91-240820}

Technical Activities 1990 Surface Science Division PBg 1-240820

\section{PB91-240838}

101,560 PC A04/MF A01

Center for Electronics and Electrical Engineering Technical Publication Announcements Covering Center Programs, October to December 1990, with $199_{1}$ CEEE Events Calendar. PB91-505370

DARPA Air Travel Information System (ATISO) Speaker-Dependent Training Data (on CD-ROM).

PBg $1-505370$

PB91-507699

100,594 CD-ROM\$1200.00

MUMPS Validation Suite, Version 7.6 (Vax/VMS Backup), 1991

PBg1-50769g

100,660 CP T99

PB91-507707

MUMPS Validation Suite, Version 7.6, 1991 (for Microcomputers)

PB91-507707

100,661 CP D99

1-507715

MUMPS Validation Suite, Version 7.6 (ANSI), 1991.

PB91-507715

100,662 CP T99

PB91-507723

MUMPS Validation Suite, Version 7.6 (TAR), 1991

PBg 1-507723

100,663 CP T99

PB91-507954

OMNITAB 80 (ASCII): An Interactive System for Statistical and Numerical Data Analysis (Version 7.0)

PB91-507962

OMNITAB 80 (UNIX): An Interactive System for Statistical and Numerical Data Analysis. Version 7.0.

PB91.507962

101,279 CP T06

PB91-507970

Building Life Cycle Cost Computer Program, Version 3.2 (for Microcomputers)

PBg $1-507970$

PB91-509802

Road Rally Conversational Speech Corpora (on CD-ROM). PB91-509802

PB91-937301

Validated Processor List (Cobol, Fortran, ADA, Pascal, $\mathrm{SOL})$. July 1991.

PB1-937302

Validated Processor List (Cobol, Fortran, ADA, Pascal, $\mathrm{SOL})$. October 1991.

PBg1-937302

PB92-102185

LBIR Facility User Handbook.

PBg2-102185

100,665 Not available NTIS

PB92-102201

ISDN Conformance Testing: Layer 1. Physical Layer. Part 1. Basic Rate S/T Interface, User Side.

PB92-10220

PB92-102219

North American ISDN Users' Forum Agreements on Integrated Services Digital Network

-10221

100,573 PC A08/MF A02

892-108877

NIST*LATTICE: A Program to Analyze Lattice Relationships. Spring 1991 Version.

PB92-10887

101,561 PC A03/MF A01

PB92-108885

Clock for the Manufacturing Systems Integration Testbed.

PB92-108885

100,992 PC A03/MF A01

PB92-108893

Guide to Expert System Building Tools for Microcomputers.

B92-108901

Mechanical Properties and Fracture Toughness of AAR TC128 Grade B Steel in the Normalized, and Normalized and Stress Relieved Conditions.

PB92-10890

101,169 PC A03/MF A01

B92-108919

FPETOOL User's Guide.

PB92-108927

$100,134 \mathrm{PC} \mathrm{A03/MF} \mathrm{A01}$

Short-Duration Autoignition Temperature Measurements for Hydrocarbon Fuels.

PBg2-108927

$100,887 \mathrm{PC} \mathrm{A03/MF} \mathrm{A01}$

B92-108935

Navy Safety Center Data on the Effects of Fire Protection Systems on Electrical Equipment.

PBg2-108935

101,376 PC A05/MF A01

PB92-108943

Development of an Optical Disk System for the Automated Retrieval of EASEAR Records.

100,666 PC A07/MF A02

PB92-108950

Video Processing with the Princeton Engine at NIST PBg2-108950

100,604 PC A04/MF A01

PB92-108968

Directory of State and Local Government Laboratory ACcreditation/Designation Programs.

PB92-108968

PB92-108976

Mathematical Modeling of Enclosure Fires.

PB92-108976

\section{PB92-108984}

Cone Calorimeter Rate of Heat Reiease Measurements for Upholstered Con 
PB92- 109156

International Fire Detection Bibliography, 1975-1990 PB92-109156

100.088 PC A09/MF A02

PB92-10916

Joint Panel Meeting of the UJNR Panel on Fire Research and Safety (11th). Held in Berkeley, CA. on October 19-24,

PB92-109164

$100,166 \mathrm{PC} \mathrm{A16/MF} \mathrm{A03}$

PB92-109172

Research. Services. Facilities. (National Institute of Standards and Technology)

PB92-109172

100,012 PC A05/MF A0

PB92-110105

GOSIP Conformance and Interoperation Testing and Regis-

tration.

100,694 PC A05/MF AO

PB92-110113

Journal of Physical and Chemical Reference Data, Volume 20, Number 1, 1991

PB92-110113

100,440 Not available NTIS

PB92-110121

Spectral Data and Grotrian Diagrams for Highly lonized Copper, Cu X-Cu XXIX.

PB92-110121

100,441 (Order as PB92-110113)

PB92-110139

Wavelengths and Energy Level Classifications of Magnesium Spectra for All Stages of Ionization (Mg / through Mg

PB92-110139

100,442 (Order as PB92-110113)

PB92-110147

Spectroscopy and Structure of the Alkali Hydride Diatomic Molecules and Their lons.

PB92-110147

100,443 (Order as PB92-110113)

PB92-110154

Critical Evaluation of Liquid Crystal Transition Temperatures : 4,4'-Alkyl/alkoxyphenylbenzoates.

PB92-11015

100,444 (Order as PB92-110113)

PB92-110162

Journal of Physical and Chemical Reference Data, Volume 20, Number 2, March-April 1991

PB92-110162

100,445 Not available NTIS

PB92-110170

Chemical Kinetic Data Base for Combustion Chemistry. Part 5. Propene.

PB92-110170

100,446 (Order as PB92-110162)

PB92-110188

Thermophysical Properties of Ethane

PB92-110188

100,447 (Order as PB92-110162)

PB92-110196

Heat Capacity and Other Thermodynamic Properties of Linear Macromolecules. 10. Update of the ATHAS 1980 Data Bank.

PB92-110196

PB92-110204

100,515 (Order as PB92-110162)

Heat Capacities of Organic Compounds in Liquid State. 2 C1 to C18 n-Alkanes.

PB92-110204

100,448 (Order as PB92-110162)

PB92-110212

Journal of Physical and Chemical Reference Data, Volume

20, Number 3, May-June 1991

PB92-110220

Kinetics and Mechanisms of the Gas-Phase Reactions of the NO3 Radical with Organic Compounds.

the NO3 Radical with Organic Componds.

PB92-110220

100,450 (Order as PB92-110212)

Thermodynamic Properties of the $\mathrm{NaBr}+\mathrm{H}_{2} \mathrm{O}$ System.

PB92-110238 100,451 (Order as PB92-110212)

PB92-110246

Cross Sections and Swarm Coefficients for Nitrogen lons and Neutrals in N2 and Argon lons and Neutrals in N2 an Argon lons and Neutrals in $\mathrm{Ar}$ for Energies from $0.1 \mathrm{eV} 10$

100,452 (Order as PB92-110212)

PB92-110253

Evaluation of Solubility Data of the System $\mathrm{CO}-\mathrm{H} 2 \mathrm{O}$ from $273 \mathrm{~K}$ to the Critical Point of Water.

PB92-110253

100,453 (Order as PB92-110212)

PB92-110261

Journal of Research of the National Institute of Standards and Technology. July-August 1991. Volume 96, Number 4.

PB92-110279

General Analytic Correction for Probe-Position Errors in Spherical Near-Field Measurements.

PB92-110279

(Order as PB92-110261, PC A05/MF A01)

PB92-110287

Data for Room Fire Model Comparisons.

PB92-110287

100, 167
(Order as PB92-110261, PC A05/MF A01)

PB92-110295

Numerical Modeling of Silicon Photodiodes for High-Accuracy Applications. Part 1. Simulation Programs.

Cy Applications.

Oorder as PB92-110261, PC A05/MF, 103

PB92-110303

Numerical Modeling of Silicon Photodiodes for High-Accura-

cy Applications. Part 2. Interpreting Oxide-bias Experiments.
PB92-110303

100,764
(Order as PB92-110261, PC A05/MF 01)

PB92-110311

Numerical Modeling of Silicon Photodiodes for High-Accuracy Applications. Part 3 . Interpolating and Extrapolating In

PB92-110311

100,765
(Order as PB92-110261, PC A05/MF A01)

PB92-112200

Stable Implementation Agreements for Open Systems Interconnection Protocols. Version 4, Edition 1, June 1991 Change Pages (Supplement)

PB92-112200

PB92-112218

Summaries of BFRL Fire Research In-House Projects and Grants, 1991

PB92-11222

$100,168 \mathrm{PC}$ A09/MF A03

Static and Dynamic Strength Tests on Electrical Conductor Cables Specified for Airport Landing Structures.

PB92-112226

101739 PC A05/MF A01

PB92-112234

Conceptual Architecture for a Mechanical Parts Production System Based on STEP. National PDES Testbed Rept.

PB92-112234

100,993 PC A03/MF A01

PB92-112242

Manufacturing Systems Integration: Initial Architecture Document.

PB92-112242

PB92-112259

Glossary of Computer Security Terminology.

PB92-112259

$100,994 \mathrm{PC} \mathrm{A04/MF} \mathrm{A0}$

100,716 PC A09/MF A02

B92-112267

Proceedings of the Forum on Standards for High Integrity Software (DOD, Government, Industry). Held in Gaithersburg, Maryland on June 28, 1991.

PB92-112267

$100,668 \mathrm{PC}$ A04/MF A0

PB92-112275

Integration-Based Method for Depth Estimation

PB92-112275

100,701 PC A03/MF A01

PB92-112283

Personal Computer Codes for Analysis of Planar Near Fields.

$100,730 \mathrm{PC} \mathrm{A04/MF} \mathrm{A01}$

B92-112283

Thermosolutal Convection

892-112291

Effect of Gravity Modulation on Thermosolutal Convection
in an Infinite Layer of Fluid.
PB92-112291 101,396 PC A03/MF A0

PB92-112309

Electronics and Electrical Engineering Laboratory Technical Publication Announcements Covering Laboratory Programs, January to March 1991, with 1991 EEEL Events Calendar.

PB92-112309

Engineering Analysis of the Fire Development in the Hillhaven Nursing Home Fire, October 5, 1989. PB92-112317

100,169 PC A04/MF AO

PB92-112325

Optimization Techniques for Permittivity and Permeability Determination.

PB92-112333

$100,865 \mathrm{PC} \mathrm{A03/MF} \mathrm{A0}$

Oxides and Oxide Superconductors: Elastic and Related Properties.

PB92-112

101,563 PC A05/MF A02

Research for Electric Energy Systems: An Annual Report

PB92-112341

PB92-112358

Magnetic Measurements for High Energy Physics Applications.

PB92-112358

101,720 PC A04/MF A0

PB92-112366

Survey of Current Worldwide Research on the Thermophy sical Properties of Alternative Refrigerants.

PB92-11236

101, 198 PC A05/MF A01

PB92-112374

Proposed Testing Methodology for STEP Application Protocol Validation. National PDES Testbed Report Series.

PB92-112374

100969 PC A04/MF A01

PB92-112382

$\mathrm{HgCdTe}$ Detector Reliability Study for the GOES Program.

PB92-112382

PB92-112390

Computer Security Bulletin Board System. User's Guide.

PB92-112390

PB92-112408

NBS-INA. The Institute for Numerical Analysis. UCLA 19471954.

PB92-112408

PB92-112416

101,264 PC A09/MF A02

Uniforms Laws and Regulations as Adopted by the National Conference on Weights and Measures (76th), 1991.

101,022 PC A08/MF A02

PB92-112424

Specifications, Tolerances, and Other Technical Requirements for Weighing and Measuring Devices as Adopted by

the 76th National Conference on Weights and Measures,

PB92-112424

101,023 PC A10/MF A03

PB92-112432

Theoretical Investigation of Piloted Ignition of Wood.

PB92-112432

PB92-112440

100556 PC A11/MF A03

Economic Analysis for Military Construction (MILCON) (t) An alyst. Instructor's Manual.

PB92-112440

101,349 PC A99/MF A06

PB92-112457

Economic Analysis for Military Construction (MILCON) Design. Concepts, Techniques, and Applications for the Analyst. Student's Manual.

PB92-112457

PB92-112465

101,350 PC A21/MF A04

Report of the National Conference on Weights and Measures (76th). Held in Philadelphia, PA on July 14-19, 1991.

PB92-112465

101,024 PC A16/MF A03

PB92-112473

Data for Fire Hazard Assessment of Selected Non-Halogenated and Halogenated Fire Retardants; Report of Test FA

PB92-112473

100,557 PC A04/MF A01

PB92-112481

Criteria for the Operation of Federally-Owned Secondary Calibration Laboratories (Ionizing Radiation). PB92.112481

PB92-112499

NIST Calibration Services for Humidity Measurement

PB92-112499

PB92-112507

Three Dimensional Reconstruction from Optical Flow Using Temporal Integration

PB92-112507

$100,702 \mathrm{PC} \mathrm{A03/MF} \mathrm{A0}$

PB92-112515

Energy Prices and Discount Factors for Life-Cycle Cos Analysis 1992. Annual Supplement to NIST Handbook 135 and NBS Special Publication 709.

PB92-112515

PB92-112523

Test Plan for Validating a Context Driven Integrated Mode (CDIM) for Sheet Metal Die Design. National PDES Testbed Repon Series.

PB92-112531

$100,970 \mathrm{PC}$ A04/MF A0

Turbulent Upward Flame Spread for Burning Vertical Walls Made of Finite Thickness.

PB92-11253

100,170 PC A05/MF A01

PB92-112549

Checking the Net Contents of Packaged Goods. Third Edition, Supplement 2

PB92-112549

PB92-112556

$100,171 \mathrm{PC} \mathrm{A03/MF} \mathrm{A01}$

Users' Guide to BREAK1, the Berkeley Algorithm for Break- 
PB92-116342

PB92-116359

Response of Personal Noise Dosimeters to Continuous and Impulse-Like Signals.

PB92-11635

100,914 PC A07/MF A02

PB92-11636

Bibliography of the NIST Electromagnetic Fields Division Publications.
PB92-116367

PB92-116375

Metrology for Electromagnetic Technology: A Bibliography of NIST Publications.

100,867 PC A04/MF A01

Q92-116383

Guide for the Use of the International System of Units: The Modernized Metric System.

PB92-116383

PB92-116391

Security in ISDN.

100,962 PC A03/MF A01

B92-116409

100,718 PC A05/MF A01

Test Methods for Optical Disk Media Characteristics (for

$356 \mathrm{~mm}$ Ruggedized Magneto-optic Media).
PB92-116409

PB92-116417

Software Reengineering: A Case Study and Lessons Learned.

100,669 PC A03/MF A01

PB92-116425

Wind and Seismic Effects. Proceedings of the Joint Meeting of the U.S.Japan Cooperative Program in Natural Resources Panel on Wind and Seismic Effects (23rd). Held in Tsukuba, Japan, on May 14-17, 1991. PB92-116425

100,152 PC A99/MF A06

PB92-116

Effects of Absorbed Dose Rate, Irradiation Temperature and Post-Irradiation Temperature on the Gamma Ray Response of Red Perspex Dosimeters.

PB92-11644

101,363 Not available NTIS

Large-Scale Validation of Bench-Scale Fire Toxicity Tests.

PB92-116458

100,139 Not available NTIS

PB92-116466

Three-Dimensional Simulations of High-Resolution Photoresist Processing
PB92-116466

PB92-116474

100,818 Not available NTIS

303-MHz Frequency Standard Based on Trapped $\mathrm{Be}(+)$ lons.

101,722 Not available NTIS

PB92-116482

Measurement of Regenerator Performance in a Vuilleumier Refrigerator.

PB92-116490

101,052 Not available NTIS

Choosing Flow Conditioners and Their Location for Orifice Flow Measurement

101,397 Not available NTIS

B92-116508

Hydrogen Component Fugacity Coefficients in Binary Mix-

tures with Etane: Pressure Dependence.
PB92-116508 100,455 Not available NTIS

PB92-116516

Voltage Calibration Systems Using Josephson Junction Arrays.

PB92-116524

100,868 Not available NTIS

Thermodynamics of the Divalent-Metal Fluorides. 4. Heat Capacity of $\mathrm{MSnF} 4, \mathrm{M}=\mathrm{Pb}, \mathrm{Ba}$, or $\mathrm{Sr}$, at Temperatures from $300 \mathrm{~K}$ to $660 \mathrm{~K}$

PB92-116524

PB92-116532

100,456 Not available NTIS

Light-Scattering Measurement of the RMS Slopes of Rough Surfaces.

PB92-116540

101,436 Not available NTIS

Neutron Measurements of Intramolecular Vibrational Modes

in C60. 116540

PB92-116557

101,055 Not available NTIS

Impact-Echo: A New Method for Inspecting Construction Materials.

PB92-11655

PB92-116565

100,140 Not available NTIS

Theoretical Model for the Tunneling-Gap Anisotropy Observed in Layered Copper-Oxide High-Temperature Superconductors.

PB92-116573

Buckling and Post-Buckling Behavior of Elliptical Plates: Part 1. Analysis.

PB92-116573
PB92-116581

101,588 Not available NTIS

Buckling and Post-Buckling Behavior of Elliptical Plates Part 2. Results.

PB92-116599

Ellipsometry SRM's for Use in Thin Film Measurements
PB92-116599

PB92-116607

100,819 Not available NTIS

Sensitivity of Ellipsometric Modeling to the 'Islands' of Silicon Precipitates at the Bottom of the Buried Oxide Layer in Annealed SIMOX

PB92-116607

PB92-116607

100,820 Not available NTIS

Uitrasonic Measurement of Sheet Steel Texture and Formability: Comparison with Neutron Diffraction and Mechanical Measurements.

PB92-116623

101,170 Not available NTIS

Use of Cross-Validation as a Stopping Rule in Emission To-

mography Image Reconstruction. PB92-116631

Magnetic Anisotropies in Ultrathin fcc Fe(001) Films Grown on Cu(001) Substrates.

PB92-11663

101,565 Not available NTIS

PB92-116649

Rotational-Tunneling Spectrum of the Ar-SO2 van der Waals Complex

PB92-1166

100,457 Not available NTIS

Mode-Stirred Chamber for Measuring Shielding Effectiveness of Cables and Connectors: Assessing MIL-STD-1344A Method 3008

PB92-116656

PB92-116664

100,776 Not available NTIS

Modified Sliding Wire Potentiometer Test Structure for Mapping Nanometer-Level Distances.

ping Nanometer

100,821 Not available NTIS

PB92-116672

Electron Spin Resonance for Monitoring Radiation-Processed Meats Containing Bone.

PB92-116672

100,018 Not available NTIS

PB92-116680

Single-Target Magnetron Sputter-Deposition of High-TC Superconducting Bi-Sr-Ca-Cu-O Thin Films

2-116680

101,566 Not available NTIS

PB92-116698

Flexible Polymer with Excluded Volume at an Interacting Penetrable Surface of Variable Dimension: A Multiple epsilon Perturbation Theory.

PB92- $\uparrow 16698$

100,517 Not available NTIS

PB92-116706

Dosimetry of a Nearly Monoenergetic 6- to 7-MeV Photon Source by $\mathrm{Nal}(\mathrm{TI})$ Scintillation Spectrometry.
101,723 Not available NTIS

PB92-116714

101,723 Not available NTIS

Atomic Spectroscopy in the 20th Century - A Tribute to Sitterly, Charlotte Moore, on the Occasion of Her 90th Birthday.

101,724 Not available NTIS

PB92-11672

Differences between Thermodynamic Temperature and t(IPTS-68) in the Range $230 \mathrm{C}$ to $660 \mathrm{C}$.
PB92-116722 Not available NTIS

PB92-116730

Interpretation of Field Ion Microscopy (FIM) Images of Asymmetrical Specimens of 1,2,3-Type High-Tc Superconductors.

PB92-116730

101,567 Not available NTIS

PB92-116748

Predictive Extended Corresponding States Model for Pure and Mixed Refrigerants.

PB92-116755

101,199 Not available NTIS

Laser Enhanced Ionization as a Selective Detector for Liquid Chromatographic Determination of Alkyltins in Sedi-

PB92-116755

100,210 Not available NTIS

PB92-116763

Temperature Monitored/Controlled Silicon Photodiodes for Standardization

PB92-116771

100,767 Not available NTIS

Fourteen-Decade Photocurrent Measurements with LargeArea Silicon Photodiodes at Room Temperature.

PB92-116771

PB92-116789

100,768 Not available NTIS

Observation of Anomalous Isotope Ratios in SIRIS Measurements of Molydenum.

PB92-116789

PB92-116797

101,361 Not available NTIS

Design and Fabrication of a Miniature High-Temperature Guarded-Hot-Plate Apparatus.

PB92-116797

PB92-116805

New Way to Mount Particulate Material for Laser Microprobe Mass Analysis.

PB92-116805

100,911 Not available NTIS

PB92-116813

Heat Conductivity of Oxide Coatings by Photothermal Radiometry betweer

PB92-116821

Integrated Knowledge Systems for Concrete Science and Technology.

PB92-11682

100,141 Not available NTIS

PB92-116839

Apparatus for Acoustic Measurements of Gases with Applications for High Temperatures. 100,458 Not available NTIS

PB92-116847

Accurate Acoustic Measurements in Gases Under Difficult candations.

PB92-116847

PB92-116854

101,382 Not available NTIS

Thermodynamics of the Disproportionation of Adenosine 5'diphosphate to Adenosine $5^{\prime}$-triphosphate and Adenosine

5'-monophosphate. 1. Equilibrium Model.
PB92-116854 Not available NTIS

\section{PB92-116862}

Experimentally Verified IGBT Model Implemented in the Saber Circuit Simulator.

PB92-116862

100,822 Not available NTIS

PB92-116870

Test of the Linearity of Quantum Mechanics by if Spectros-

copy of the $(9) \mathrm{Be}(+)$ Ground State. PB92-116888

Diffraction by a Half-Plane in a Lossy Medium.

PB92-116888

PB92-116896

CW Dye Lasers.

PB92-116896

Diode Lasers and Their Application to Spectroscopy.

Diode Lasers and Their Application to Spectroscopy.
101,439 Not available NTIS

PB92-116912

Damage to the Bases in DNA Induced by Stimulated Human Neutrophils.

101,312 Not available NTIS

Test Chip for the Evaluation of Surface-Diffusion Phenom ena in Sputtered Aluminum Planarization Processes.

PB92-116920 100,823 Not available NTIS

PB92-116938

Microstructural Studies of Ti-Al Alloys in the Vicinity of the 'Eutectoid' Reaction

PB92-116938

101,241 Not available NTIS

PB92-116946

Horizontal Flow Boiling Heat Transfer Experiments with a Mixture of R22/R114.

PB92-116946

101,200 Not available NTIS

PB92-116953

Formation of ortho-Tyrosine by Radiation and Organic Solvents in Chicken Tissue.

PB92-116953

101,290 Not available NTIS

PB92-116961

Radiochromic Film Dosimeter for Gamma Radiation in the Absorbed-Dose Range 0.1 - $10 \mathrm{kGY}$

PB92.116961

101,364 Not available NTIS

PB92-116979

Knowledge Extraction Techniques for Expert System Assisted Wafer Screening

100,824 Not available NTIS

PB92-116987

Assessment of Reliability Concerns for Wide-Temperature Operation of

PB92-11698

PB92-116995

Test Method for Tensile Creep of Structural Ceramics Using Flexure Beams.

PB92-116995

101,099 Not available NTIS 
PB92-117076

Need for Power Quality Testing Standards.
PB92-117076 100,741 Not available NTIS

PB92-117084

Persistent Photoconductivity in SIMOX Films.

PB92-117084

PB92-117092

XANES of Transition Metal Zinc-Blende Semiconductors

PB92-117092

PB92-117100

Dosimetry: New Approaches for New Challenges.
PB92-117100 101,365 Not available NTIS

PB92-117118

Novel Radiation Dosimetry Systems.

PB92-117118 101,727 Not available NTIS

PB92-117126

2-Deoxy-D-Ribose Aqueous Solution as a Gamma-Ray Dosimeter.

PB92-117126

101,366 Not available NTIS

PB92-117134

Temperature Dependence of Radiochromic Film Dosi-

meters.

101,728 Not available NTIS

PB92-117142

Photon Energy Dependence of the Sensitivity of Radiochromic Film and Comparison with Silver Halide Film and LiF TLDs Used for Brachytherapy Dosimetry

PB92-117142

101,296 Not available NTIS

PB92-117159

Band-Gap Narrowing and III-V Heterostructure FETs

PB92-117159

100,827 Not available NTIS

PB92-117167

Influence of Nonsinusoidal Waveforms on Voltmeters, Ammeters, and Phase Meters

PB92-117167

100,869 Not available NTIS

PB92-117175

High-Resolution Infrared Spectrum of the nu1 Band of OC10.

PB92- 117175

100,463 Not available NTIS

PB92-117183

Effects of Global Density Ratio on the Centerline Mixing Behavior of Axisymmetric Turbulent Jets.

PB92-117183 100,014 Not available NTIS

PB92-117191

Large-Scale Turbulent Structures and the Stabilization of Lifted Turbulent Jet Diffusion Flames.

PB92-117191 100,558 Not available NTIS

PB92-117209

Reynolds Number Effects on the Mixing Behavior of Axisymmetric Turbulent Jets.

PB92-117209

100,015 Not available NTIS

PB92-117217

Application of Transmission Electron Detection to $X$-ray Mask Calibrations and Inspection.

PB92-117217

100,828 Not available NTIS

PB92-117225

Off-Axis Measurements of lon Kinetic Energies in RF Plas-

mas.

PB92-117233

101,444 Not available NTIS

Residual Defects in SIMOX: Threading Dislocations and

Pipes.

100,829 Not available NTIS

PB92-117241

Round-Robin Study of Implants in $\mathrm{Si}$ and $\mathrm{SiO} 2$ by SIMS, RBS, and NAA

PB92-117258

Thermal Conductivity of Alumina Fiber/Epoxy and Alumina Fiber/PEEK from 4.2 to $310 \mathrm{~K}$. 101,130 Not available NTIS
PB92-117258

PB92-117266

Microstructure Effects on the Lower Critical Solution Temperature Phase Behavior of Deuterated Polybutadiene and Protonated Polyisoprene Blends Studied by Small-Angle Neutron Scattering

PB92-117266

100,518 Not available NTIS

PB92-117274

Small-Angle Neutron Scattering and Light Scattering Study on the Miscibility of Poly(styrene-ran-butadiene)/Polybutadiene Blends.
PB92- 117274

PB92-117282

Measurement, Use, and Interpretation of TCR

PB92-117282

100,519 Not available NTIS

PB92-117290

Laser Vaporization Mass Spectrometry of Refractory Materials: Graphite and YBa2Cu3OX

PB92-117290

100,831 Not available NTIS

PBg2-117308

Interactive Video Software Portability: Migrating a DOS APplication to POSIX.

PB92-11730

100,670 Not available NTIS

PB92-117316

Intrinsic Carrier Concentrations in Long Wavelength HgCdTe Based on the New, Nonlinear Temperature De pendence of $E g(x, T)$.
PB92-117316

101,568 Not available NTIS

92-11732

Air Gage Size Measurement of Microwave Standards.

PB92-117324

PB92-117332

Magnetic Behavior of Nanocomposites Prepared in a Vitreous Alumina Gel

PB92-117340

101,131 Not available NTIS

Antiferromagnetic Order of $\mathrm{Cu}$ in $\mathrm{Sm} 2 \mathrm{CuO} 4$

PB92-117340

PB92-117357

101,569 Not available NTIS

Apparent Thermal Conductivity of Fumed-Silica Standard Reference Materials.

PB92-117357

PB92-117365

101,102 Not available NTIS

Relative H-Atom and O-Atom Concentration Measurement in a Laminar, Methane/Air Diffusion Flame.

PB92-117365

100,559 Not available NTIS

PB92-117373

Kinematic Theory of Ballistic Electron Emission Spectrosco yy of Silicon-Silicide Intertaces.

PB92-117373

PB92-117381

Theory of Orientation Textures Due to Surface Energy AniSotropies.

PB92-117399

101,571 Not available NTIS

Thermodynamics of the Disproportionation of Adenosine 5'diphosphate to Adenosine 5 '-triphosphate and Adenosine -monophosphate. 2. Experimental Data.

PB92-117399

101,313 Not available NTIS

2-117407

Comparative Study of Bovine Pericardium Mineralization: A Basic and Practical Approach.

100,056 Not available NTIS

PB92-117415

Observation of Two Different Oscillation Periods in the Ex hange Coupling of $\mathrm{Fe} / \mathrm{Cr} / \mathrm{Fe}(100)$.

PB92-117415

PB92-117423

Advanced Sensing of Materials Processing

PB92-117423

100,949 Not available NTIS

PB92-11743

Performance Evaluation of a New Audio-Frequency Power

PBridge. 11743

100,742 Not available NTIS

982-117449

Effect of Surface Forces on Subcritical Crack Growth in Glass.

PB92-117456

101,103 Not available NTIS

Probe-Corrected Spherical Near-Field Scanning Theory in Acoustics.

PB92-117456

PB92-117464

101,383 Not available NTIS

Low Temperature Magnetization and Magnetic Excitations in Amorphous Fe78B13Sig.

PB92.11746

PB92-119676

Government Open Systems Interconnection Profile Users' Guide, Version 2.

PB92-123033

Flammability Characterization of Foam Plastics.

PB92-123033

100,142 PC A07/MF A02

PB92-12304

Publications of the Manufacturing Engineering Laboratory Covering the Period January 1989-September PB92-12304

PB92-123058

101,053 PC A05/MF A0

Multi-Enterprise Concurrent Engineering through International Standards

PB92-12306

100,997 PC A03/MF A0

Use of Solid Modeling in the Design of M3 Components.
PB92-123066

PB92-123074

Fire-Plume-Generated Ceiling Jet Characteristics and Convective Heat Transfer to Ceiling and Wall Surfaces in Two-Layer Zone-Type Fire Environment: Uniform Temperature Ceiling and Walls.

PB92.123074

$101,370 \mathrm{PC} \mathrm{A04/MF} \mathrm{A01}$

PB92-123082

Electronics and Electrical Engineering Laboratory. 1991 Strategic Plan. Supporting Technology for U.S. Competitiveness in Electronics.

PB92-123082

100,870 PC A03/MF A0

PB92-123090

Validation Testing Laboratory User's Guide. National PDES Testbed Report Series.

PB92-123090

100,971 PC A06/MF A02

PB92-123108

Computing Delaunay Triangulations for Comet-Shaped Polygons.
PB92.123108

PB92-123116

$101,265 \mathrm{PC} \mathrm{A03/MF} \mathrm{A01}$

Development of a Technique to Assess the Adequacy of the Municipal Water Supply for a Residential Sprinkler System.

PB92-123116

$101,358 \mathrm{PC}$ A05/MF A0

PB92-123124

Monitoring and Reporting Techniques for Error Rate and Error Distribution in Optical Disk Systems

PB92.123124

PB92-123132

Time Domain Frequency Stability Calculated from the Frequency Domain Description: Package to Calculate Time Domain Frequency Stability from the Frequency Domain

PB92-12313

100,587 PC A03/MF A0

$92-123140$

Establishing a Computer Security Incident Response Capability (CSIRC)

PB92-123157

NIST X-Ray Photoelectron Spectroscopy (XPS) Database.
PB92-123157 100,465 PC A04/MF A01

92-126408

Stable Implementation Agreements for Open Systems Interconnection Protocols. Version 4, Edition 1, Change Page September 1991. Output from the September 1991 OSI Im-

plementors Workshop. Held in Gaithersburg, Maryland
PB92-126408
100,697 PC A11/MF

100,697 PC A11/MF A03

PB92-126416

NIST Calibration Services Users Guide, 1991

PB92-126416

100 936 PC A 10/MF A03

PB92-126424

Materials Reliability. Technical Activities, 1991. (NAS-NRC Assessment Panel, February 13-14, 1992)

PB92-126424

101,252 PC A05/MF A01

PB92-126432

Evaporator Performance Investigation for Residential AirConditioning Application Using Mixed Refrigerants

PB92-126432

100,893 PC A07/MF A02

PB92-126440

Testing and Rating of an Atmospheric, Gas-Fired Furnace Equipped with a Burner Air Inlet Damper.

PB92-126440

100,894 PC A04/MF A01

PB92-126457

Development of Test Methods to Determine the Compatibility of Liquid Hazardous Materials with Polyethylene Packag ings.

100943 PC A05/MF A01

PB92-126465

Questions and Answers on Quality, the ISO 9000 Standard Series, Quality System Registration, and Related Issues. PB92-126465

PB92-126473

Mass Energy-Transfer and Mass Energy-Absorption Coefficients, Including In-Flight Positron Annihilation for Photon Energies $1 \mathrm{keV}$ to $100 \mathrm{MeV}$

PB92-126473

$101,445 \mathrm{PC} \mathrm{A04/MF} \mathrm{A0}$

PB92-126481

Feasibility of Using a Multiple Award Schedule for Specifying Paints in Government Painting Contracts

PB92-126499

Stability of Aqueous Inorganic Lead Solutions in Polycarbonate Containers.

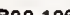


PB92-126598

Predicting the Remaining Service Life of Concrete.
PB92-126598
101,371 PC A05/MF A01

PB92-126606

National Educators Workshop: Update '90. Standard Experiments in Engineering Materials Science and Technolo

gy. 2 2-126606

101,253 PC A15/MF A03

PB92-126614

Journal of Research of the National Institute of Standards and Technology, September-October 1991. Volume 96 Number 5.

PB92-126614

PB92-126622

100,212 PC A06/MF A02

Summary of the Intercomparison of the Force Standard Machines of the National Institute of Standards and Technology, USA, and the Physikalisch-Technische BundesanPB92-126622

101,590

PB92-126630

(Order as PB92-126614, PC A06/MF A02)

Accurate and Precise Coulometric Determination of Sulfur Dioxide in Compressed Gas Mixture

PB92-126630

(Order as PB92-126614, PC A06/MF A02)

PB92-126648

Development of a Coulometric Method for Assessing the
Concentration of Ambient Levels of $\mathrm{CO}_{2} /$ Air in Com.

pressed Gas Mixtures.

100,913

PB92-126648

(Order as PB92-126614, PC A06/MF AD2)

PB92-126655

Certification of NIST SAM 1961: 30 micrometers Diameter Polystyrene Spheres.

100,937

PB92-12665

(Order as PB92-126614, PC A06/MF A02)

PB92-126663

Optimization Techniques for Permittivity and Permeability Determination

100871

PB92-126671

(Order as PB92-126614, PC A06/MF A02)

Taguchi's Orthogonal Arrays Are Classical Designs of Ex-

PB92-126671

101,280

PB92-126689

Mathematical Model for Dental Caries: A Coupled Dissolution-Diffusion Process.

PB92-126689

101,316
(Order as PB92-126614, PC A06/MF A02)

PB92-126697

Advanced Technology Program: A New Role for NIST in Accelerating the Development of Commercially Important Technologies.

(Order as PB92-126614, PC A06/MF A02) PB92-126697

PB92-126705

List of New Group Divisible Designs
PB92-126705

(Order as PB92-126614, PC A06/MF A02)

PB92-126713

Silicon Reference Materials Certified for Isotope Abundances.
PB92-126713

100,213
(Order as PB92-126614, PC A06/MF A02)

PB92-126721

Slovak Metrological Society.

PB92-126721 100938

\section{PB92-127315}

Opportunities for Innovation: Chemical and Biological Sensors.

(Order as PB92-126614, PC A06/MF A02)

PB92-127315

100,214 Not available NTIS

PB92-148063

Journal of Physical and Chemical Reference Data, Volume 20, Number 4, July/August 1991. PB92-148063

\section{PB92-148071}

Chemical Kinetic Data Base for Propellant, Combustion. 1 Reactions Involving NO, NO2. $\mathrm{HNO}, \mathrm{HNO} 2, \mathrm{HCN}$ and $\mathrm{N} 2 \mathrm{O}$. 00,467 (Order as PB92-148063)

\section{PB92-148089}

Ab-Initio Calculations and Ideal Gas Thermodynamic Func tions of Cyclopentadiene and Cyclopentadiene Derivatives. PB92-148089 100.468 (Order as PB92-148063)

PB92-148097

Improved Fits for the Vibrational and Rotational Constants of Many States of Nitrogen and Oxygen

$\begin{array}{ll}\mathrm{PB} 92-148097 \quad 100,469 & \text { (Order as PB92-148063) }\end{array}$

PB92-148105

Solubilities of Solids and Liquids of Low Volatility in Supercritical Carbon Dioxide.

B92-148113

Journal of Physical and Chemical Reference Data, Volume 20, Number 5, September/October 1991 .

PB92-148113 $\quad 100,471$ Not available NTIS

\section{PB92-148121}

Wavelengths and Energy Level Classifications for the Spectra of Aluminum (AI I through AI XIII).

100,472 (Order as PB92-148113)

PB92-148139

Energy Levels of Krypton, $\mathrm{Kr}$ I through $\mathrm{Kr}$ XXXVI.

PB92-148139 100,473 (Order as PB92-148113) PB92-148147

Thermodynamic Properties of Oxygen from the Triple Poin to $300 \mathrm{~K}$ with Pressures to $80 \mathrm{MPa}$.

PB92-148147 100,474 (Order as PB92-148113)

\section{PB92-148154}

Sixteen Thousand Evaluated Experimental Thermodynamic Property Data for Water and Steam.
PB92-148154 100,475 (Order as PB92-148113) PB92-148162

Journal of Physical and Chemical Reference Data, Volume 20, Number 6, November/December 1991.

PB92-148162 100.476 Not available NTIS PB92-148170

New Equation of State and Tables of Thermodynamic Properties for Methane Covering the Range from the Melting Line to $625 \mathrm{~K}$ at Pressures up to $1000 \mathrm{MP}$

PB92-148170 100,477 (Order as PB92-148162) PB92-148188

Thermodynamic Properties of the Aqueous Sulfuric Acid System to $350 \mathrm{~K}$
PB92-148188

100,478 (Order as PB92-148162)

PB92-148196

Solubility of Carbon Dioxide in Water at Low Pressure.

PB92-148196 100,479 (Order as PB92-148162)

PB92-148204

Chemical Kinetic Data Sheets for High-Temperature Reactions. Part 2.

14820

PB92-148212

Atomic Weights of the Elements 1989

PB92-148212

100,480 (Order as PB92-148162)

PB92-148220

Isotopic Compositions of the Elements 1989

PB92-148220 100,482 (Order as PB92-148162)

PB92-163864

Modernized Metric System (Char).

PB92-163864

100,939 Not available NTIS

PB92-163872

Brief History of Measurement Systems with a Chart of the Modernized Metric System.

100,940 Not available NTIS

PB92-163880

Metric Conversion Card

PB92-163880

100,941 Not available NTIS

PB92-163898

Metric Measures Up (Ruler).

PB92-163898

100,942 Not available NTIS

PB92-937300

Validated Products List (Cobol, Fortran, ADA, Pascal, MUMPS, SQL)

100,671 Standing Order

REPT-21

Crack Arrest Fracture Toughness Measurements of Nor. malized and Inclusion Shape Controlied AAR TC128 Grade $B$ Steel, and Micro-Alloyed, Control-Rolled, and Inclusion Shape Controlled A 8XX Grade B Steel

01,150 PC A03/MF A01

REPT-24

Mechanical Properties and Fracture Toughness of AAR TC128 Grade B Steel in the Normalized, and Normalized and Stress Relieved Conditions.

PB92-108901

$101,169 \mathrm{PC} \mathrm{A03/MF} \mathrm{A01}$

SERI/TP-2 14-4409

Scanning tunneling microscopy studies of the surfaces of aSi:H and a-SiGe:H films. Annual report, 1 December 1989 31 January 1991

DE91002169

100,785 PC A03/MF A01

SR-723-29-89

Summary of Experiments with the Separated Aperture Technique of Dielectric Anomaly Detection.

AD-A230 438/4 100,721 PC A02/MF A01

TR-1

Influence of Filament Geometry on Hot Filament Growth of Diamond Films

WRDC-TR-90-2081

$101,448 \mathrm{PC} \mathrm{A02/MF} \mathrm{A01}$

Detection of Excited States by Laser-Induced Fluorescence and Analysis of Energy Transfer.

AD.A227 296/1

100,242 PC A04/MF A01 


\section{APPENDIX A \\ List of Depository Libraries In the United States}

\section{ALABAMA}

\section{Auburn}

Auburn University Ralph Brown Draughon Library (1873)

\section{Blrmingham}

Birmingham Public Library (1895)

Birmingham-Southem College Library (1932)

Jefferson State Community College James B. Allen Library (1970)

Samford University Library Hawwell G. Davis Library (1884)

\section{Enterprlse}

Enterprise State Junior College Leaming Resources Center (1967)

\section{Fayette}

Brewer State Junior College Leaming Resources Center Library (1979)

\section{Florence}

University of North Alabama Collier Library (1932)

\section{Gadsden}

Gadsden Public Library (1963)

\section{Huntsville}

University of Alabama in Huntsville Library (1964)

\section{Jacksonvllle}

Jacksonville State University Houston Cole Library (1929)

\section{Moblle}

Mobile Public Library (1963)

Spring Hill College Thomas Byme Memorial Library (1937)

University of South Alabama Library (1968)

\section{Montgomery}

Alabana Public Library Service (1984)

Alabama Supreme Court and State Law Library (1884)

Aubum University at Montgomery Library (1971) REGIONAL

Air University Library Maxwell Air Force Base (1963)

\section{Normal}

Alabama Agricultural and Mechanical University J. F. Drake Mernorial Leaming Resources Center (1963)

\section{Troy}

Troy State University Library (1963)

\section{Tuscaloosa}

University of Alabarna Arnelia Gayle Gorgas Library (1860) REGIONAL University of Alabama School of Law Library (1967)

\section{Tuskegee}

Tuskegee University Hollis Burke Frissell Library (1907)

\section{ALASKA}

\section{Anchorage}

Anchorage Law Library (1973)

Anchorage Municipal Libraries Z. J. Loussac Public Library (1978)

University of Alaska at Anchorage Library (1961)

U.S. Alaska Resources Library (1981)

U.S. District Court Law Library (1983)

\section{Falrbanks}

University of Alaska Elmer E. Rasmuson Library (1922)

\section{Juneau}

Alaska State Library (1900)

University of Alaska-Juneau Library (1981)

\section{Kotchlkan}

Ketchikan College Library (1970)

\section{AMERICAN SAMOA}

\section{Pago Pago}

American Samoa Community College Learning Resources Center (1985)

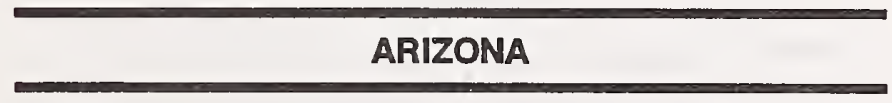

\section{Coolldge}

Central Arizona College Instructional Materials Center (1973)

\section{Flagstaff}

Northem Arizona University Cline Library (1937) 


\section{Glendale}

Glendale Public LIbrary (1986)

\section{Holbrook}

Northland Pioneer College Leaming Resources Center (1985)

\section{Mesa}

Mesa Public Library (1983)

\section{Phoenlx}

Department of Library Archives, and Public Records (unknown) REGIONAL

Grand Canyon University Fleming Library (1978)

Phoenix Public Library (1917)

U.S. Court of Appeals 9th Circuit Library (1984)

\section{Prescott}

Yavapai College Library (1976)

\section{Tempe}

Arizona State University College of Law Library (1977)

Arizona State University Hayden Library (1944)

\section{Tucson}

Tucson Public Library (1970)

University of Arizona Library (1907) REGIONAL

\section{Yuma}

Yuma County Library (1963)

\section{ARKANSAS}

\section{Arkadelphla}

Ouachita Baptist University Riley Hickingbotham Library (1963)

\section{Batesville}

Arkansas College Library (1963)

\section{Clarksville}

University of the Ozarks Dobson Memorial Library (1925)

\section{Conway}

Hendrix College Olin C. Balley Library (1903)

\section{Fayetteville}

University of Arkansas Mullins Library (1907)

University of Arkansas School of Law Library Robert A. Leflar (1978)

\section{Little Rock}

Arkansas State Library (1978) REGIONAL

Arkansas Supreme Court Library (1962)

Central Arkansas Library System Main Llbrary (1953)

University of Arkansas at Little Rock Library Ottenheimer Library (1973)

University of Arkansas at Little Rock Pulaski County Law Library (1979)

\section{Magnolla}

Southem Arkansas University Magale Library (1956)

\section{Monticello}

University of Arkansas at Monticello Library (1956)

\section{PIne Bluff}

University of Arkansas at Pine Bluff Watson Memorial Library (1976)

\section{Russellville}

Arkansas Technical University Tomlinson Library (1925)

\section{Searcy}

Harding University Brackett Library (1963)

\section{State Unlversity}

Arkansas State University Dean B. Ellis Library (1913)

\section{Walnut RIdge}

Southem Baptist College Felix Goodson Library (1967)

\section{CALIFORNIA}

\section{Anahelm}

Anaheim Public Library (1963)

\section{Arcadla}

Arcadia Public Library (1975)

\section{Arcata}

Humboldt State University Library (1963)

\section{Bakersfleld}

Califomia State University Bakersfield Library (1974) Kem County, Beale Memorial Library (1943)

\section{Berkeley}

University of Califomia General Library (1907) University of Califomia Boalt Law Library (1963) 


\section{Carson}

California State University Dominguez Hills Library (1973)

Carson Regional Library (1973)

\section{Chilco}

California State University, Chico Merriam Library (1962)

\section{Claremont}

Government Publications and Microforms Department Honnold Library, Claremont College (1913)

\section{Compton}

Compton Public Library (1972)

\section{Culver Clty}

Culver City Public Library (1966)

\section{Davls}

University of California Shields Library (1953)

University of Califomia at Davis Law Library (1972)

\section{Downoy}

Downey City Library (1963)

\section{Fresno}

Califomia State University, Fresno, Henry Madden Library (1962)

Government Documents Department Library (1920)

\section{Fullerton}

California State University at Fullerton Library (1963)

\section{Garden Grove}

Orange County Public Library (1963)

\section{Gardena}

County of Los Angeles Public Library (1966)

\section{Hayward}

Califomia State University, Hayward Library (1963)

\section{Huntlngton Park}

Huntington Park Library (1970)

\section{Inglowood}

Inglewood Public Library (1963)

\section{Irvlne}

University of Callfomia at Invine Main Library (1963)

\section{La Jolla}

University of California at San Diego Central University Library (1963)
Angelo lacoboni Public Library (1970)

\section{Lancaster}

Lancaster Library (1967)

\section{La Verne}

University of La Verne College of Law Library (1979)

\section{Long Boach}

California State University at Long Beach Library (1962) Long Beach Public Library (1933)

\section{Los Angeles}

California State University, Los Angeles University Library (1956) Los Angeles County Law Library (1963)

Los Angeles Public Library (1891)

Loyola Marymount University Charles Von der Ahe Library (1933) Loyola Law School William M. Rains Law Library (1979) Occidental College Library (1941)

Southwestern University School of Law Library (1975)

University of California, University Research Library (1932)

University of California, Los Angeles Law Library (1958)

University of Southem Califomia Doheny Memorial Library (1933)

University of Southern Califomia Law Library (1978)

U.S. Court of Appeals Ninth Circuit Library (1981)

Whittier College School of Law Library (1978)

\section{Mallbu}

Pepperdine University Payson Library (1963)

\section{Menlo Park}

U.S. Geological Survey Library (1962)

\section{Montebello}

Montebello Regional Library (1966)

\section{Monterey}

U.S. Naval Postgraduate School Dudley Knox Library (1963)

$$
\text { Monterey Park }
$$

Bruggemeyer Memorial Library (1964)

\section{Northrldge}

California State University at Northridge Oviatt Library (1958)

\section{Norwalk}

Norwalk Regional Library (1973)

\section{Oakland}

Mills College Library (1966)

Oakland Public Library (1923) 


\section{Ontarlo}

Ontario City Library (1974)

\section{Palm Springs}

Palm Springs Public Library (1980)

\section{Pasadena}

California Institute of Technology Millikan Memorial Library (1933) Pasadena Public Library (1963)

\section{Pleasant HIII}

Contra Costa County Library (1964)

\section{Reddling}

Shasta County Library (1956)

\section{Redlands}

University of Redlands Armacost Library (1933)

\section{Redwood Clty}

Redwood City Public Library (1966)

\section{Reseda}

West Valley Regional Branch Library (1966)

\section{Richmond}

Richmond Public Library (1943)

\section{Rlverside}

Riverside City and County Public Library (1947)

University of Califomia at Riverside Library (1963)

\section{Sacramento}

Califomia State Library (1895) REGIONAL

Califomia State University Sacramento Library (1963)

Sacramento County Law Library (1963)

Sacramento Public Library (1880)

University of the Pacific McGeorge School of Law Library (1978)

\section{San Bernardino}

Don A. Tumer County Law Library (1984)

San Bernardino County Library (1964)

\section{San Dlego}

National University Law Library (1989)

San Diego County Law Library (1973)

San Diego County Library (1973)

San Diego Public Library (1895)

San Diego State University Library (1962)

University of San Diego Legal Research Center (1967)

\section{San Franclsco}

Golden Gate University School of Law Library (1979)
University of California Hastings College of Law Library (1972) San Francisco Public Library (1889)

San Francisco State University J. Paul Leonard Library (1955)

Supreme Court of California Library (1979)

U.S. Court of Appeals Ninth Circuit Library (1971)

University of San Francisco Richard A. Gleeson Library (1963)

\section{San Jose}

San Jose State University Clark Library (1962)

\section{San Leandro}

San Leandro Community Library Center (1961)

\section{San Luls Oblspo}

California Polytechnic State University Robert E. Kennedy Library (1969)

\section{San Mateo}

College of San Mateo Library (1987)

\section{San Rafael}

Marin County Free Library (1975)

\section{Santa Ana}

Orange County Law Library (1975)

Santa Ana Public Library (1959)

\section{Santa Barbara}

University of California at Santa Barbara Library (1960)

\section{Santa Clara}

Santa Clara University Orradre Library (1963)

\section{Santa Cruz}

University of Califomia at Santa Cnz McHenry Library (1963)

\section{Santa Rosa}

Sonoma County Library (1896)

\section{Stanford}

Stanford University Libraries (1895)

Stanford University Robert Crown Law Library (1978)

\section{Stockton}

Public Library of Stockton and San Joaquin County (1884)

Thousand Oaks

Califomia Lutheran University Pearson Library (1964)

\section{Torrance}




\section{Turlock}

California State University, Stanislaus Library (1964)

\section{Vallejo}

Solano County Library John F. Kennedy Library (1982)

\section{Valencla}

Valencia Regional Library (1972)

\section{Ventura}

Ventura County Library Services Agency (1975)

\section{Visalla}

Tulare County Free Library (1967)

\section{Walnut}

Mount San Antonio College Library (1966)

\section{West Covina}

West Covina Regional Library (1966)

\section{Whittler}

Whittier College Wardman Library (1963)

\section{COLORADO}

\section{Alamosa}

Adams State College Llbrary (1963)

\section{Aurora}

Aurora Public Library (1984)

\section{Boulder}

University of Colorado at Boulder Norlin Library (1879) REGIONAL University of Colorado School of Law Library (1988)

\section{Colorado Springs}

Colorado College Tutt Library (1880)

University of Colorado at Colorado Springs Library (1974)

U.S. Air Force Academy Library (1956)

\section{Denver}

Auraria Library (1978)

Colorado Supreme Court Library (1978)

Denver Public Library (1884) REGIONAL

Department of the Interior Library (1962)

Regis College Dayton Memorial Library (1915)

U.S. Courts Library (1973)
University of Denver Penrose Library (1909)

University of Denver College of Law Westminster Law Library (1978)

\section{Fort Collins}

Colorado State University Libraries (1907)

\section{Golden}

Colorado School of Mines Arthur Lakes Library (1939)

\section{Grand Junctlon}

Mesa State College Tomlinson Library (1978) Mesa County Public Library (1975)

\section{Greeley}

University of Northem Colorado James A. Michener Library (1966)

\section{Gunnlson}

Western State College Leslie J. Savage Library (1932)

\section{La Junta}

Otero Junior College Wheeler Library (1963)

\section{Lakewood}

Jefferson County Public Library Lakewood Library (1968)

\section{Puoblo}

Pueblo Library District (1893)

University of Southem Colorado Library (1965)

CONNECTICUT

\section{Brldgeport}

Bridgeport Public Library (1884)

University of Bridgeport School of Law Library Wahlstrom Library (1979)

\section{Danbury}

Westem Connecticut State University Ruth A. Haas Library (1967)

\section{Danlelson}

Quinebaug Valley Community College Audrey P. Beck Library (1968)

\section{Enfleld}

Enfield Contral Library (1967)

\section{Hartford}

Connecticut State Library (unknown) REGIONAL Hartford Public Library (1945) 
Trinity College Library (1895)

University of Connecticur School of Law Library (1978)

\section{Middletown}

Wesleyan University Olin Library (1906)

\section{Mystlc}

Mystic Seaport Museum, Inc., G. W. Blunt White Library (1964)

\section{Now Brltaln}

Central Connecticut State University Elihu Burritt Library (1973)

\section{New Haven}

Southerm Connecticut State University Hilton C. Buley Library (1968) Yale Law Library (1981)

Yale University Seeley G. Mudd Library (1859)

\section{Now London}

Connecticut College C. E. Shain Library (1926)

U.S. Coast Guard Academy Library (1939)

\section{Stamford}

Ferguson Library (1973)

\section{Storrs}

University of Connecticut Homer Babbidge Library (1907)

\section{Waterbury}

Post College Traurig Library and Learning Resources Center (1977) Silas Bronson Public Library (1869)

\section{West Haven}

University of New Haven Marvin K. Peterson Library (1971)

\section{DELAWARE}

\section{Dover}

State Law Library in Kent County (unknown)

\section{Georgetown}

Delaware Technical and Community College Southern Campus Library (1968)

\section{Newark}

University of Delaware Library (1907)

\section{Wilmington}

Wideme University School of Law Library (1976)
DISTRICT OF COLUMBIA

\section{Washlngton}

Administrative Conference of the United States Library (1972) Advisory Commission on Intergovernmental Relations Library (1977) American University Washington College of Law Library (1983)

Catholic University of America Robert J. White Law Library (1979)

Comptroller of the Currency Library (1986)

Pentagon Library (1969)

Department of Commerce Library (1955)

Department of Education (1988)

Department of Health and Human Services Library and Information Center (1954)

Department of Housing and Urban Development Library (1969)

Department of the Interior Natural Resources Library (1895)

Department of Justice Main Library (1895)

Department of Labor Library (1976)

Department of the Navy Library (1895)

Department of State Library (1895)

Department of State Law Library (1966)

Department of Transportation Main Library (1982)

Department of Transportation, U.S. Coast Guard Law Library (1982)

Department of Veteran's Affairs Librany (1967)

Department of the Treasury Library (1895)

District of Columbia Court of Appeals Library (1981)

District of Columbia Public Library (1943)

Equal Employment Opportunity Commission Library (1984)

Executive Office of the President, Library \& Information Service Division (1965)

Federal Deposit Insurance Corporation Library (1972)

Federal Election Commission Law Library (1975)

Federal Energy Regulatory Commission Library (1983)

Federal Mine Safety \& Health Review Commission Library (1979)

Federal Reserve System Board of Govemors Research Library (1978)

Federal Reserve System Law Library (1976)

General Accounting Office Office of Library Services (1974)

General Servlces Administration Library (1975)

Georgetown University Library (1969)

Georgetown University Law Center Edward Bennett Williams Library (1978)

George Washington University Melvin Gelman Library (1983)

George Washington University National Law Center Jacob Burns Law Library (1978)

Library of Congress Congressional Research Service (1978)

Library of Congress Serial and Government Publications (1977)

Merit Systems Protection Board Library (1979)

National Defense University Library (1895)

Pension Benefit Guaranty Corporation Legal Dept. Library (1984)

U.S. Court of Appeals Judges' Library (1975)

U.S. Court of Appeals for the Federal Circuit Library (1986)

U.S. Information Agency Library (1984)

U.S. Otfice of Personnel Management Library (1963)

U.S. Postal Service Library (1895)

U.S. Senate Library (1979)

U.S. Supreme Court Library (1978)

University of the District of Columbia Library Leaming Resources Division (1970)

Veterans' Administration Central Office Library (1967)

\section{FLORIDA}

\section{Boca Raton}

Forida Atlantic University S. E. Wimberly Library (1963) 
Casselberry

Seminole County Public Library (1989)

Coral Gables

University of Miami Otto G. Richter Library (1939)

\section{Daytona Beach}

Volusia County Library Center (1963)

\section{De Land}

Stetson University duPont-Ball Library (1887)

\section{Fort Lauderdale}

Broward County Main Library (1967)

Nova University Law Library (1967)

\section{Fort Plerce}

Indian River Community College Library (1975)

\section{Galnesville}

University of Florida College of Law Library (1978)

University of Florida Libraries (1907) REGIONAL

\section{Jacksonvllle}

Haydon Bums Public Library (1914)

Jacksonville University Swisher Library (1962)

University of North Florida Thomas G. Carpenter Library (1972)

\section{Key West}

Florida Keys Community College Key West Campus Library (1989)

\section{Lakeland}

Lakeland Public Library (1928)

\section{Leesburg}

Lake-Sumter Community College Library (1963)

\section{Melbourne}

Florida Institute of Technology Library (1963)

\section{Mlaml}

Florida International University Library Tamiarni Trail (1970) Miami-Dade Public Library (1952)

\section{North Mlaml}

Florida International University Bay Vista Campus Library (1977)

\section{Opa Locka}

St. Thomas University Library (1977)
Orlando

University of Central Florida Library (1966)

\section{Palatka}

Saint Johns River Community College Library (1963)

\section{Panama Clty}

Bay County Public Library (1983)

\section{Pensacola}

University of West Florida John C. Pace Library (1966)

\section{Port Charlotte}

Charlotte-Glades Library System (1973)

\section{Salnt Petersburg}

Saint Petersburg Public Library (1965)

Stetson University College of Law Charles A. Dana Law Library (1975)

\section{Sarasota}

Selby Public Library (1970)

\section{Tallahassee}

Florida Agricultural and Mechanical University Coleman Memonial Library (1936)

Florida State University Law Library (1978)

Florida State University Strozier Library (1941)

Florida Supreme Court Library (1974)

State Library of Florida (1929)

\section{Tampa}

Tampa-Hillsborough County Public Library (1965)

University of South Florida Library (1962)

University of Tampa Ment Kelce Librery (1953)

\section{Winter Park}

Rollins College Olin Library (1909)

\section{GEORGIA}

\section{Albany}

Dougherty County Public Library (1964)

\section{Amerlcus}

Georgia Southwestem College Jarnes Eart Carter Library (1966)

\section{Athens}

University of Georgia Libraries (1907) REGIONAL

University of Georgia School of Law Library (1979) 


\section{Atlanta}

Atlanta-Fulton Public Library (1880)

Atlanta University Center Robert W. Woodruff Library (1962)

Emory University Law School Library (1968)

Emory University Woodruff Library (1928)

Georgia Institute of Technology Price Gilbert Memorial Library (1963)

Georgia State Law Library (unknown)

Georgia State University William Russell Pullen Library (1970)

Georgia State University College of Law Library (1983)

U.S. Court of Appeals 11th Circuit Library (1980)

\section{Augusta}

Augusta College Reese Library (1962)

Medical College of Georgia Library (1986)

\section{Brunswlck}

Brunswick-Glynn County Regional Library (1965)

\section{Carroliton}

West Georgia College Irvine Sullivan Ingram Library (1962)

\section{Columbus}

Columbus College Simon Schwob Memorial Library (1975)

\section{Dahlonega}

North Georgia College Stewart Library (1939)

\section{Dalton}

Dalton College Library (1978)

\section{Macon}

Mercer University Stetson Main Library (1964)

Mercer University Walter F. George School of Law Library (1978)

\section{Marlotta}

Kennesaw State College Horace W. Sturgis Library (1968)

\section{Milledgeville}

Georgia College Ina Dillard Russell Library (1950)

\section{Rome}

Berry College Memorial Library (1970)

\section{Savannah}

Chatham-Effingham Liberty Regional Library (1857)

\section{Statesboro}

Georgia Southem University Zach S. Henderson Library (1939)

\section{Valdosta}

Valdosta State College Library (1956)

\section{GUAM}

\section{Agana}

Nieves M. Flores Memorial Library (1962)

\section{Mangllao}

University of Guam Robert F. Kennedy Memorial Library (1978)

\section{HAWAII}

\section{HIlo}

University of Hawaii at Hilo Edwin H. Mookini Library (1962)

\section{Honolulu}

Hawaii Medical Library Incorporated (1968)

Hawaii State Libraiy (1929)

Municipal Reference \& Records Center (1965)

Supreme Court Law Library (1973)

University of Hawaii Hamilton Library (1907) REGIONAL

University of Hawaii William S. Richardson School of Law Library (1978)

\section{Lale}

Joseph F. Smith Library Brigham Young University Haviaii Campus (1964)

\section{Lhue}

Lihue Public Library (1967)

\section{Pearl Clty}

Leeward Community College Library (1967)

Walluku

Maui Public Library (1962)

IDAHO

\section{Bolse}

Boise Public Library and Information Center (1929)

Boise State University Library (1966)

Idaho State Law Library (unknown)

Idaho State Library (unknown)

\section{Caldwell}

College of Idaho Terteling Library (1930)

\section{Crawfordsville}

Wabash College Lily Library 


\section{Moscow}

University of Idaho College of Law Library (1978)

University of Idaho Library (1907) REGIONAL

\section{Nampa}

Northwest Nazarene College John E. Riley Library (1984)

\section{Pocatello}

Idaho State University Eli Oboler Library (1908)

\section{Rexburg}

Davis O. McKay Library (1946)

Twin Falls

College of Southern Idaho Library (1970)

\section{ILLINOIS}

\section{Bloomington}

Illinois Wesleyan University, Sheөan Library (1964)

\section{Carbondale}

Southem Illinois University at Carbondale Morris Library (1932) Southern Illinois University School of Law Library (1978)

\section{Carllnville}

Blackbum College Lumpkin Llbrary (1954)

\section{Carterville}

Shawne日 Library System (1971)

\section{Champalgn}

University of Illinois Law Library (1965)

\section{Charleston}

Eastern Illinois University Booth Library (1962)

\section{Chlcago}

Chicago Public Library (1876)

Chicago State University Paul and Emily Douglas Library (1954)

DePaul University Law Library (1979)

Field Museum of Natural History Library (1963)

Illinois Institute of Technology Chicago-Kent College of Law Library (1978)

Illinois Institute of Technology Paul V. Galvln Library (1982)

John Marshall Law School Library (1981)

Loyola University of Chicago E. M. Cudahy Memorial Llbrary (1966)

Loyola University School of Law Library (1979)

Northeastern Illinois University Ronald Williams Library (1961)

Northwestem University School of Law Library (1978)

University of Chicago Law Library (1964)

University of Chicago Library (1897)
University of Illinois at Chicago Library (1957)

William J. Campbell Library of the U.S. Courts (1979)

\section{Decatur}

Decatur Public Library (1954)

De Kalb

Northern Illinois University Founders' Memorial Library (1960) Northern Illinois University College of Law Library (1978)

\section{Des Plalnes}

Government Information Center and The Northwest Municipal Conference Oakton Community College Library (1976)

\section{Edwardsvllle}

Southern Illinois University at Edwardsville Lovejoy Memorial Library (1959)

\section{Elsah}

Principia College Marshall Brooks Library (1957)

\section{Evanston}

Northwestem University Library (1876)

\section{Freoport}

Freeport Public Library (1905)

\section{Galesburg}

Gajesburg Public Library (1896)

\section{Jacksonvllle}

MacMurray College Henry Pfeiffer Library (1929)

\section{Kankakeө}

Olivet Nazarene University Bonner Library and Learning Center (1946)

\section{Lake Forest}

Lake Forest College Donnelley Library (1962)

\section{Lebanon}

McKendree College Holman Library (1968)

\section{LIsle}

Illinois Benedictine College Theodore F. Lownik Library (1911)

\section{Macomb}

Western Illinois University Govemment Publications \& Legal Reference Library (1962)

\section{Mollne}

Black Hawk College Leaming Resources Center (1970) 
Monmouth

Monmouth College Hewes Llbrary (1860)

\section{Mount Carmel}

Wabash Valley College Bauer Media Center (1975)

\section{Mount Prospoct}

Mount Prospect Public Library (1977)

\section{Normal}

Illinois State University Milner Library (1877)

\section{Oak Park}

Oak Park Public Library (1963)

Oglesby

Illinois Valley Community College Jacobs Memorial Library (1976)

\section{Palos Hills}

Moraine Valley Community College Leaming Resources Center (1972)

\section{Peorla}

Cullom-Davis Library (1963)

Peoria Public Library (1883)

\section{Rlver Forest}

Rosary College Rebecca Crown Llbrary (1966)

\section{Rockford}

Rockford Public Library (1895)

\section{Romeoville}

Lewis University Library (1952)

\section{South Holland}

South Suburban College Learning Resources Center

\section{Springfleid}

Illinois State Library (unknown) REGIONAL

\section{Stroamwood}

Poplar Creok Public Library (1980)

\section{Universlty Park}

Govemors' State University Library (1974)

\section{Urbana}

University of Illinois Documents Library (1907)

\section{Wheaton}

Wheaton College Buswell Memorial Llbrary (1964)
Woodstock

Woodstock Public Library (1963)

INDIANA

\section{Anderson}

Anderson College Charles E. Wilson Library (1959) Anderson Public Library (1983)

\section{Bloomington}

Indiana University Library (1881)

Indiana University Law Library (1978)

\section{Crawfordsville}

Wabash College Lilly Library (1906)

\section{Evansville}

Evansville and Vanderburgh County Public Library (1928) University of Southem Indiana Library Services (1969)

\section{Fort Wayne}

Allen County Public Library (1896) Indiana University-Purdue University at Fort Wayne (1965)

\section{Franklln}

Franklin College Llbrary (1976)

\section{Gary}

Gary Public Library (1943)

Indiana University Northwest Library (1966)

\section{Greencastle}

De Pauw University Roy O. West Library (1879)

\section{Hammond}

Hammond Public Library (1964)

\section{Hanover}

Hanover College Duggan Library (1892)

\section{Huntlngton}

Huntington College Richlyn Library (1964)

\section{Indlanapolls}

Butter University Irwin Library (1965) Indianapolis-Marion County Public Library (1906) Indiana State Library (unknown) REGIONAL Indiana Supreme Court Law Library (1975) Indiana University School of Law Library (1967) Indiana University-Purdue University Library (1979) 


\section{Kokomo}

Indiana University at Kokomo Learning Resource Center (1969)

\section{Muncle}

Ball State University Alexander M. Bracken Library (1959) Muncie Public Library (1906)

\section{Now Albany}

Indiana University Southeast Library (1965)

\section{Notre Dame}

Notre Dame Law School Kresge Law Library (1985)

University of Notre Dame Hesburgh Library (1883)

\section{Rensselaer}

Saint Joseph's College Library (1964)

\section{Richmond}

Earham College Lilly Library (1964)

Morrison-Reeves Library (1906)

\section{South Bend}

Indiana University at South Bend Franklin D. Schurz Library (1965)

\section{Terre Haute}

Indiana State University Cunningham Memorial Library (1906)

\section{Valparalso}

Valparaiso University Moellering Memorial Library (1930)

Valparaiso University Law Library (1978)

\section{West Lafayette}

Purdue University Libraries (1907)

\section{IOWA}

\section{Ames}

lowa State University Parks Library (1907)

\section{Codar Falls}

University of Northem lowa Donald O. Rod Library (1946)

\section{Codar Raplds}

Cedar Rapids Public Library (1986)

\section{Councll Blutfs}

Free Public Library (1885)

lowa Westem Community College Herbert Hoover Library (1972)

\section{Davenport}

Davenport Public Library (1973)

\section{Des Molnes}

Drake University Cowles Library (1966)

Drake University Law Library (1972)

Public Library of Des Moines (1888)

State Library of lowa (unknown)

\section{Dubuque}

Carnegie-Stout Public Library (unknown)

Loras College Wahlert Memorial Library (1967)

\section{Fayette}

Upper lowa University Henderson-Wilder Library (1974)

\section{GrInnell}

Grinnell College Burling Library (1874)

\section{lowa Clty}

University of lowa College of Law Library (1968)

University of lowa Libraries (1884) REGIONAL.

\section{Lamonl}

Graceland College Frederick Madison Smith Library (1927)

\section{Mason Clty}

North lowa Area Community College Library (1976)

\section{Mount Vernon}

Comell College Russell D. Cole Library (1896)

\section{Orange Clty}

Northwestem College Ramaker Library (1970)

\section{Sloux Clity}

Sioux City Public Library (1894)

\section{KANSAS}

\section{Atchlson}

Benedictine College Library (1965)

\section{Baldwin Clty}

Baker University Collins Library (1908)

\section{Colby}

Colby Community College H. F. Davis Memorial Library (1968) 
Emporla

Emporia State University William Allen White Library (1909)

Hays

Fort Hays State University Forsyth Library (1926)

\section{Hutchlnson}

Hutchinson Public Library (1963)

\section{Lawrence}

University of Kansas Law Library (1971)

University of Kansas Map Library (1869) REGIONAL

\section{Manhattan}

Kansas State University Farrell Library (1907)

\section{Plttsburg}

Pittsburg State University Leonard H. Axe Library (1952)

\section{Sallna}

Kansas Wesleyan University Memorial Library (1930)

\section{Shawnee Mlsslon}

Johnson County Library (1979)

\section{Topoka}

Kansas State Historical Society Library (1877)

Kansas State Library (unknown)

Kansas Supreme Court Law Library (1975)

Washbum University of Topeka Law Library (1971)

\section{Wichlta}

Wichita State University Ablah Library (1901)

\section{KENTUCKY}

\section{Ashland}

Ashland Community College Library (1946)

\section{Barbourville}

Unlon College Abigail E. Weeks Memorial Library (1958)

\section{Bowllng Green}

Westem Kentucky University Helm-Cravens Library (1934)

\section{Columbla}

LIndsey Wilson College Katie Murrell Library (1987)

\section{Crestvlew HIlls}

Thomas More College Library (1970)

\section{Danville}

Centre College Grace Doherty Library (1884)

\section{Frankfort}

Kentucky Department of Libraries and Archives (1967)

Kentucky State Law Library (unknown)

Kentucky State University Blazer Library (1972)

\section{Hazard}

Hazard Community College Library (1988)

\section{Highland Helghts}

Northern Kentucky University W. Frank Steely Library (1973)

\section{Lexington}

University of Kentucky Law Library (1968)

University of Kentucky Libraries (1907) REGIONAL

\section{Loulsville}

Louisville Free Public Library (1904)

University of Louisville Ekstrom Library (1925)

University of Louisville Law Library (1975)

\section{Morehead}

Morehead State University Camden-Carroll Library (1955)

\section{Murray}

Murray State University Waterfield Library (1924)

\section{Owensboro}

Kentucky-Wesleyan College Library Learning Center (1966)

\section{Richmond}

Eastem Kentucky University John Grant Crabbe Library (1966)

\section{WIIllamsburg}

Cumberland College Norma Perkins Hagan (1988)

\section{LOUISIANA}

\section{Baton Rouge}

Louisiana State Library (1976)

Louisiana State University Middleton Library (1907) REGIONAL Louisiana State University Paul M. Hebert Law Center Libray (1929) Southem University John B. Cade Library (1952)

Southem University Law School Library (1979) 


\section{Eunlce}

Louisiana State University at Eunice LeDoux Library (1969)

\section{Hammond}

Southeastem Louisiana University Sims Memorial Library (1966)

\section{Lafayotte}

University of Southwestem Louisiana Dupre Library (1938)

\section{Lake Charles}

McNeese State University Lether E. Frazar Memorial Library (1941)

\section{Monroe}

Northeast Louisiana University Sandel Library (1963)

\section{Natchltoches}

Northwestem State University Watson Memorial Library

(1887)

\section{Now Orleans}

Law Library of Louisiana (unknown)

Loyola University Government Documents Library (1942)

Loyola University Law Library (1978)

New Orleans Public Library (1883)

Our Lady of Holy Cross College Library (1968)

Southem University in New Orleans Leonard S. Washington Memorial Library (1962)

Tulane University Law Library (1976)

Tulane University Howard-Tilton Memorial Library (1942)

U.S. Court of Appeals 5th Circuit Library (1973)

University of New Orleans Earl K. Long Library (1963)

\section{PIneville}

Louisiana College Righthard W. Norton Memorial Library (1969)

\section{Ruston}

Lovisiana Technical University Prescott Memorial Library (1896) REGIONAL

\section{Shroveport}

Louisiana State University at Shreveport Library (1967)

Shreve Memorial Library (1923)

\section{Thlbodaux}

Nicholls State University Ellender Memorial Library (1962)

\section{MAINE}

\section{Augusta}

Maine Law and Legislative Reference Library (1973)

Maine State Library (unknown)

Bangor

Bangor Public Library (1884)

\section{Brunswlck}

Bowdoin College Library (1884)

\section{Castlne}

Maine Maritime Academy Nutting Memonal Library (1969)

\section{Lewiston}

Bates College George and Helen Ladd Library (1883)

\section{Orono}

University of Maine Raymond H. Fogler Library (1907) REGIONAL

\section{Portland}

Portland Public Library (1884)

University of Maine School of Law Garbrecht Law Library (1964)

\section{Presque Isle}

University of Maine at Presque Isle Library/Leaming Resources Center (1979)

\section{Sanford}

Louis B. Goodall Memorial Library (1984)

\section{Watervilile}

Colby College Miller Library (1884)

\section{MARYLAND}

\section{Annapolls}

Maryland State Law Library (unknown)

U.S. Naval Academy Nimitz Library (1895)

\section{Baitlmore}

Enoch Pratt Free Library (1887)

Johns Hopkins University Milton S. Eisenhower Library (1882)

Morgan State University Soper Library (1940)

University of Baltimore Langsdale Library (1973)

University of Baltimore Law Library (1980)

University of Maryland School of Law Marshall Law Library (1969)

U.S. Court of Appeals 4th Circuit Library (1982)

\section{Bel Alr}

Harford Community College Library (1967)

\section{Beltsville}

Department of Agriculture National Agricultural Library (1895)

\section{Bothesda}

Department of Health and Human Services National Library of Medicine (1978)

Uniformed Services University of Health Sciences Leaming Resource Center (1983) 
Catonsville

University of Maryland, Baltimore County Albin O. Kuhn Library \& Gallery (1971)

\section{Chestertown}

Washington College Clifton M. Miller Library (1891)

\section{College Park}

University of Maryland Hombake Library (1925) REGIONAL

\section{Cumberland}

Allegany Community College Library (1974)

\section{Frostburg}

Frostburg State University Library (1967)

\section{Patuxent Rlver}

Patuxent River Central Library (1968)

\section{Rockvllle}

Montgomery County Department of Public Libraries (1951)

\section{Sallsbury}

Salisbury State College Blackwell Library (1965)

\section{Towson}

Goucher College Julia Rogers Library (1966)

Towson State University Cook Library (1979)

\section{WestmInster}

Westem Maryland College Hoover Library (1886)

\section{MASSACHUSETTS}

\section{Amherst}

Amherst College Library (1884)

University of Massachusetts University Library (1907)

\section{Boston}

Boston Athenaeum Library (unknown)

Boston Public Library (1859) REGIONAL

Boston University School of Law (1979)

Northeastem University Snell Library (1962)

State Library of Massachusetts (unknown)

Suffolk University Law Library (1979)

Supreme Judicial Court Social Law Library (1979)

U.S. Court of Appeals First Circuit Library (1978)

\section{Brookllne}

Publlc Library of Brookline (1925)

\section{Cambrldge}

Harvard College Library (1860)

Harvard Law School Library (1981)

Massachusetts Institute of Technology Library (1946)

\section{Chestnut HIII}

Boston College Thomas P. O'Neill Jr., Library (1963)

\section{Chlcopee}

College of Our Lady of the Elms Alumnae Library (1969)

\section{Lowell}

University of Lowell O'Leary Library (1952)

\section{Medford}

Tufts University Wessel Library (1899)

Militon

Curry College Levin Library (1972)

\section{New Bedford}

New Bedford Free Public Library (1858)

\section{Newton Centre}

Boston College Law School Library (1979)

\section{North Dartmouth}

Southeastem Massachusetts University Library (1965)

\section{North Easton}

Stonehill College Cushing-Martin Library (1962)

\section{Springfleld}

Springfield City Library (1966)

Western New England College School of Law Library (1978)

\section{Waltham}

Brandeis University Library (1965)

Waltham Public Library (1982)

\section{Wellesley}

Wellesley College Margaret Clapp Library (1943)

\section{Wenham}

Gordon College Jenks Learning Resource Center (1963)

\section{WIIllamstown}

Williams College Sawyer Library (unknown) 


\section{Worcester}

American Antiquarian Society Library (1814)

University of Massachusetts Medical Center Library (1972)

Worcester Public Library (1859)

\section{MICHIGAN}

\section{Alblon}

Albion College Stockwell-Mudd Library (1966)

\section{Allendale}

Grand Valley State College Zumberge Library (1963)

Alma

Alma College Library (1963)

\section{Ann Arbor}

University of Michigan Harlan Hatcher Graduate Library (1884)

University of Michigan Law Library (1978)

\section{Benton Harbor}

Benton Harbor Public Library (1907)

\section{Bloomfleld Hills}

Cranbrook Institute of Science Library (1940)

\section{Dearborn}

Henry Ford Centennial Library (1969)

Henry Ford Community College Library (1957)

\section{Detrolt}

Detroit College of Law Library (1979)

Detroit Public Library (1868) REGIONAL

Marygrove College Library (1965)

Mercy College of Detroit Library (1965)

University of Detroit Library (1884)

University of Detroit School of Law Library (1978)

Wayne State University Purdy/Kresge Library (1937)

Wayne State University Arthur Neef Law Librany (1971)

\section{Dowaglac}

Southwestern Michigan College Matthews Library (1971)

\section{East Lansing}

Michigan State University Government Documents Llbrary (1907)

\section{Farmington HIlls}

Oakland Community College Martin L. King Leaming Resources Center (1968)
Fllnt

Filnt Public Library (1967)

University of Michigan-Flint Library (1977)

\section{Grand Raplds}

Calvin College \& Seminary Library (1967)

Grand Rapids Public Library (1876)

\section{Houghton}

Michigan Technological University J. Robert Van Pelt Library (1876)

Jackson

Jackson District Library (1965)

\section{Kalamazoo}

Kalamazoo Public Library (1907)

Western Michigan University Dwight B. Waldo Library (1963)

\section{Lansing}

Library of Michigan (unknown) REGIONAL

Thomas M. Cooley Law School Library (1978)

\section{Llvonla}

Livonia Public Library (1987)

Schoolcraft College Library (1962)

\section{Madison Helghts}

Madison Heights Public Library (1982)

\section{Marquette}

Northem Michigan University Lydia M. Olson Library (1963)

\section{Monroe}

Monroe County Library System (1974)

\section{Mount Clemens}

Macomb County Library (1968)

\section{Mount Pleasant}

Central Michigan University Charles V. Park Library (1958)

\section{Muskegon}

Hackley Public Library (1894)

\section{Petoskey}

North Central Michigan College Library (1962)

\section{Port Huron}

Saint Clalr County Library (1876) 
Rochester

Oakland University Kresge Library (1964)

\section{Royal Oak}

Royal Oak Public Library (1984)

\section{Saglnaw}

Hoyt Public Librany (1890)

\section{Sault Ste. Marle}

Lake Superior State University Kenneth Shouldice Library (1982)

\section{Traverse Clty}

Northwestem Michigan College Mark and Helen Osterlin Library (1964)

\section{Unlverslty Center}

Delta College Library (1963)

\section{Warren}

Warren Public Library Arthur J. Miller Branch (1973)

Ypsllantl

Eastem Michigan University Library (1965)

\section{MICRONESIA}

\section{East Carollne Islands}

Community College of Micronesia Library (1982)

\section{MINNESOTA}

\section{Bemld]I}

Bernidji State University A.C. Clark Library (1963)

\section{Blalne}

Anoka County Library (1971)

\section{Collegeville}

Saint John's University Alcuin Library (1954)

\section{Cottage Grove}

Washington County Library-Park Grove Branch (1983)
Duluth Public Library (1909)

Duluth

University of Minnesota Duluth Library (1984)

Eagan

Dakota County Library-Westcott Branch (1983)

\section{Edlna}

Southdale-Hennepin Area Library (1971)

\section{Mankato}

Mankato State University Memorial Library (1962)

\section{Marshall}

Southwest State University Library (1986)

\section{Minneapolls}

Minneapolis Public Library (1893)

University of Minnesota Law School Library (1978)

University of Minnesota Wilson Library (1907) REGIONAL

\section{Moorhead}

Moorhead State University Livingston Lord Library (1956)

\section{Morrls}

University of Minnesota, Morris, Rodney A. Briggs Library (1963)

\section{Northfleld}

Carleton College Library (1930)

Saint Olaf College Rolvaag Mernorial Library (1930)

\section{Salnt Cloud}

Saint Cloud State University, Leaming Rescources Center (1962)

\section{Salnt Paul}

Harnline University School of Law Library (1978)

Minnesota Historical Society Library (1867)

Minnesota State Law Library (unknown)

Saint Paul Public Library (1914)

University of Minnesota Saint Paul Campus Library (1974)

William Mitchell College of Law Library (1979)

\section{Salnt Peter}

Gustavus Adolphus College Fake Bernadette Memorial Library (1941)

\section{Winona}

Winona State University Maxwell Library (1969) 


\section{MISSISSIPPI}

\section{Cleveland}

Delta State University W. B. Roberts Library (1975)

\section{Columbus}

Mississippi University for Women John Clayton Fant Memorial Library (1929)

\section{Hattlesburg}

University of Southem Mississippi Joseph A. Cook Memorial Library (1935)

\section{Jackson}

Jackson State University Henry Thomas Sampson Library (1968)

Millsaps College Millsaps-Wilson Library (1963)

Mississippi College School of Law Library (1977)

Mississippi Library Commission (1947)

Mississippi State Law Library (unknown)

\section{Lorman}

Alcom State University J. D. Boyd Library (1970)

\section{MIssissippl State}

Mississippi State University Mitchell Memorial Library (1907)

\section{Unlversity}

University of Mississippi J. D. Williams Library (1883) REGIONAL University of Mississippi James O. Eastland Law Library (1967)

\section{MISSOURI}

\section{Cape Glrardeau}

Southeast Missouri State University Kent Library (1916)

\section{Columbla}

University of Missouri at Columbia Ellis Library (1862) REGIONAL University of Missouri-Columbia Law Library (1978)

\section{Fulton}

Westminster College Reeves Library (1875)

\section{HIllsboro}

Jefferson College Library (1984)

\section{Jefferson Clity}

Lincoln University Inman E. Page Library (1944)

Missouri State Library (1963)

Missouri Supreme Court Library (unknown)

$$
\text { Joplln }
$$

Missouri Southern State College George A. Spiva Library (1966)

\section{Kansas Clty}

Kansas City Missouri Public Library (1881)

Rockhurst College Greenlease Library (1917)

University of Missouri at Kansas City General Library (1938)

University of Missouri Kansas City Leon E. Bloch Law Library (1978)

\section{Klrksville}

Northeast Missouri State University Pickler Memorial Library (1966)

\section{Llberty}

William Jewell College Charles F. Cury Library (1900)

\section{Maryville}

Northwest Missouri State University B. D. Owens Library (1982)

\section{Rolla}

University of Missouri-Rolla Curtis Laws Wilson Library (1907)

\section{Salnt Charles}

Kisker Road Branch Library

Lindenwood College Margaret Leggat Butler Library (1973)

\section{Salnt Joseph}

River Bluffs Regional Public Library (1891)

\section{Salnt Louls}

Maryville College Library (1976)

Saint Louis County Library (1970)

Saint Louis Public Library (1866)

Saint Louis University Law Library (1967)

Saint Louis University Pius XII Mernorial Library (1866)

U.S. Court of Appeals Eighth Circuit Library (1972)

University of Missouri at Saint Louis Thomas Jefferson Library (1966)

Washington University John M. Olin Library (1906)

Washington University Law Library (1978)

\section{Sprlngfleld}

Drury College, Walker Library (1874)

Southwest Missouri State University Duane G. Meyer Library (1963)

\section{Warrensburg}

Central Missouri State University Ward Edwards Library (1914) 


\section{MONTANA}

\section{Bllilings}

Eastern Montana College Library (1958)

\section{Bozeman}

Montana State University Libraries (1907)

\section{Butto}

Montana College of Mineral Science and Technology Library (1901)

\section{Havre}

Northem Montana College Vande Bogart Library (1980)

\section{Helena}

Carroll College Corrette Library (1974)

Montana State Library (1966)

State Law Library of Montana (1977)

\section{Missoula}

University of Montana Maurene \& Mike Mansfield Library (1909) REGIONAL

\section{NEBRASKA}

\section{Blalr}

C. A. Dana College Dana-LIFE Library (1924)

\section{Crete}

Doane College Perkins Library (1944)

\section{Fremont}

Midland Lutheran College Luther Library (1924)

\section{Kearney}

Keamey State College Calvin T. Ayan Library (1962)

\section{Lincoln}

Nebraska Library Commission (1972)

Nebraska State Library (unknown)

University of Nebraska-Lincoln College of Law Library (1981)

University of Nebraska-Lincoln D. L. Love Memorial Library (1907) REGIONAL

\section{Omaha}

Creighton University Reinert/Alumni Library (1964)

Creighton University School of Law Library (1979)

Omaha Public Library W. Dale Clark Library (1880)

University of Nebraska at Omaha University Library (1939)
Scottsbluff Public Library (1925)

\section{Wayne}

Wayne State College U.S. Conn Library (1970)

\section{NEVADA}

\section{Carson Clty}

Nevada State Library and Archives (unknown) Nevada Supreme Court Library (1973)

\section{Las Vegas}

Clark County Law Library (1988)

Las Vegas-Clark County Library (1974)

University of Nevada at Las Vegas James Dickinson Library (1959)

\section{Reno}

National Judicial College Law Library (1979)

Nevada Historical Society Library (1974)

University of Nevada-Reno Library (1907) REGIONAL

Washoe County Library (1980)

\section{NEW HAMPSHIRE}

\section{Concord}

Franklin Pierce Law Center Library (1973)

New Hampshire State Library (unknown)

\section{Durham}

University of New Hampshire Library (1907)

\section{Hanover}

Dartmouth College Library (1884)

\section{Hennlker}

New England College Danforth Library (1966)

\section{Manchester}

Manchester City Library (1884)

New Hampshire College H. A. B. Shapiro Memorial Library (1976)

Saint Anselm College Geisel Library (1963)

\section{Nashua}

Nashua Public Library (1971) 
NEW JERSEY

Bayonne

Bayonne Free Public Library (1909)

\section{Bloomfleid}

Bloomfield Public Library (1965)

\section{Bridgeton}

Cumberland County Library (1966)

\section{Camden}

Rutgers University Camden Library (1966)

Rutgers University School of Law Library (1979)

\section{Convent Station}

College of Saint Elizabeth Mahoney Library (1938)

\section{East Brunswlck}

East Brunswick Public Library (1977)

\section{East Orange}

East Orange Public Library (1966)

\section{Elizabeth}

Free Public Library of Elizabeth (1895)

\section{Glassboro}

Glassboro State College Savitz Library (1963)

\section{Hackensack}

Johnson Free Public Library (1966)

\section{Irvington}

Irvington Public Library (1966)

\section{Jersey Clty}

Jersey City Public Library (1879)

Jersey City State College Forrest A. Inwin Library (1963)

\section{Lawrenceville}

Rider College Franklin F. Moore Library (1975)

\section{Madison}

Drew University Library (1939)
Ramapo College Library (1971)

Mount Holly

Burlington County Library (1966)

\section{Now Brunswick}

Rutgers University Alexander Library (1907)

\section{Nowark}

Newark Public Library (1906) REGIONAL

Rutgers University John Cotton Dana Library (1966)

Rutgers University Law Library (1979)

Seton Hall University Law Library (1979)

\section{Newton}

Sussex County Library (1986)

\section{Passalc}

Passaic Public Library (1964)

\section{Philllpsburg}

Phillipsburg Free Public Library (1976)

\section{Plainfleid}

Plainfield Public Library (1971)

\section{Pomona}

Stockton State College Library (1972)

\section{Princeton}

Princeton University Firestone Library (1884)

\section{Randolph}

County College of Morris Sherman H. Masten Learning Resource Center (1975)

\section{Rutherford}

Fairleigh Dickinson University Messler Library (1953)

\section{Shrowsbury}

Monmouth County Library (1968)

\section{South Orange}

Seton Hall University Library (1947)

\section{Teaneck}

Fairleigh Dickinson University Weiner Library (1963) 


\section{Toms Rlver}

Ocean County College Learning Resources Center (1966)

\section{Trenton}

New Jersey State Library (unknown)

Trenton Free Public Library (1902)

\section{Unlon}

Kean College of New Jersey Nancy Thompson Library (1971)

\section{Upper Montclalr}

Montclair State College Harry A. Sprague Library (1967)

Wayne

Wayne Public Library (1972)

\section{West Long Branch}

Monmouth College Guggenheim Memorial Library (1963)

\section{Woodbrldge}

Woodbridge Public Library (1965)

\section{NEW MEXICO}

\section{Albuquerque}

University of New Mexico Medical Center Library (1973)

University of New Mexico School of Law Library (1973)

University of New Mexico General Library (1896) REGIONAL

\section{Hobbs}

New Mexico Junior College Pannell Library (1969)

\section{Las Cruces}

New Mexico State University Library (1907)

\section{Las Vegas}

New Mexico Highlands University Donnelly Library (1913)

\section{Portales}

Eastern New Mexico University Golden Library (1962)

\section{Santa $\mathrm{Fe}$}

New Mexico State Library (1960) REGIONAL New Mexico Supreme Court Law Library (unknown)

\section{Sllver Clty}

Western New Mexico University Miller Library (1972)

\section{Socorro}

New Mexico Institute of Mining \& Technology Martin Speare Memorial Library (1984)

\section{NEW YORK}

\section{Albany}

Albany Law School Schaffer Law Library (1979)

New York State Library (unknown) REGIONAL

State University of New York at Albany University Library (1964)

\section{Auburn}

Seymour Library (1972)

\section{Bellport}

East Islip Public Library (1973)

\section{Binghamton}

State University of New York at Binghamton Glenn G. Bartle Library (1962)

\section{Brockport}

State University of New York at Brockport Drake Memorial Library (1967)

\section{Bronx}

Fordham University Library (1937)

Lehman College Library (1967)

New York Public Library (1973)

State University of New York Maritime College Stephen B. Luce Library (1947)

\section{Bronxvllle}

Sarah Lawrence College Esther Raushenbush Library (1969)

\section{Brooklyn}

Brooklyn College Library (1936)

Brooklyn Law School Library (1974)

Brooklyn Public Library Business Library (1984)

Brooklyn Public Library (1908)

Pratt Institute Library (1891)

State University of New York Health Center at Brooklyn Library (1958)

\section{Buffalo}

Buffalo and Erie County Public Library (1895)

State University of New York at Buffalo Charles B. Sears Law Library (1978)

State University of New York at Buffalo Lockwood Memorial Library (1963)

\section{Canton}

Saint Lawrence University Owen D. Young Library (1920) 


\section{Corning}

Corning Community College Arthur A. Houghton Jr. Library (1963)

\section{Cortland}

State University of New York at Cortland Memorial Library (1964)

\section{Delhi}

State University College of Technology Resnick Library (1970)

\section{Elmira}

Elmira College Gannett Tripp Learning Center (1956)

\section{Farmingdale}

State University of New York at Farmingdale Greenley Library (1917)

\section{Flushlng}

CUNY Law School at Queens College CUNY Law Library (1983) Queens College Benjamin S. Rosenthal Library (1939)

\section{Garden Clty}

Adelphi University Swirbul Library (1966)

\section{Geneseo}

State University of New York at Geneseo Milne Library (1967)

\section{Greenvale}

Long Island University B. Davis Schwartz Memonial Library (1964)

\section{Hamilton}

Colgate University, Everett Needham Case Library (1902)

\section{Hempstead}

Hofstra University Library (1964)

Hofstra University School of Law Library (1979)

\section{Huntington}

Touro College Jacob D. Fuchsberg Law Center Library (1985)

\section{Ithaca}

Comell University Library (1907)

Cornell Law Library (1978)

Comell University Albert R. Mann Library (1943)

\section{Jamalca}

Queens Borough Public Library (1926)

Saint John's University Library (1956)

Saint John's University School of Law Library (1978)

\section{KIngs Polnt}

U.S. Merchant Marine Acaderny Schuyler Otis Bland Library (1962)

\section{Long Island Clty}

Fiorello H. LaGuardia Community College Library (1981)

\section{Middletown}

Thrall Library (1986)

\section{Mount Vernon}

Mount Vemon Public Library (1962)

\section{New Paltz}

State University College at New Paltz Sojoumer Truth Library (1965)

\section{Now York Clty}

City College of City University of New York Cohen Library (1884)

College of Insurance Library (1965)

Columbia University Libraries (1882)

Columbia University School of Law Library (1981)

Cooper Union for the Advancement of Science and Arts Library (1930)

Fordham Law School Library (1987)

Medical Library Center of New York (1976)

New York Law Institute Library (1909)

New York Law School Library (1979)

New York Public Library Astor Branch (1907)

New York Public Library Lenox Branch (1884)

New York University Law Library (1974)

New York University Elmer Holmes Bobst Library (1967)

U.S. Court of Appeals Second Circuit Library (1976)

Yeshiva University Chutick Law Library Cardozo School of Law (1979)

Yeshiva University Pollack Library (1979)

\section{Nowburgh}

Newburgh Free Library (1909)

\section{Nlagara Falls}

Niagara Falls Public Library (1976)

\section{Oakdale}

Dowling College Library (1965)

\section{Oneonta}

State University College at Onenonta James M. Miine Library (1966)

\section{Oswego}

State University of New York at Oswego Penfield Library (1966)

\section{Plattsburgh}

State University College at Plattsburgh Benjamin F. Feinberg Library (1967) 


\section{Potsdam}

Clarkson University Harriet Call Bumap Memorial Library (1938)

State University College of New York at Potsdam Frederick W. Crumb Memorial Library (1964)

\section{Poughkeopsle}

Vassar College Library (1943)

\section{Purchase}

State University of New York at Purchase Library (1969)

\section{Rochester}

Rochester Public Library (1963)

University of Rochester Rush Rhees Library (1880)

\section{Salnt Bonaventure}

Saint Bonaventure University Friedsam Memorial Library (1938)

\section{Saratoga Sprlngs}

Skidmore College Library (1964)

\section{Schenectady}

Union College Schaffer Library (1901)

\section{Southampton}

Long Island University Southhampton Campus Library (1973)

\section{SparkIII}

St. Thomas Aquinas College Loughe日d Library (1984)

\section{Staten Island}

Wagner College Hormann Library (1953)

\section{Stony Brook}

State University of New York at Stony Brook Main Library (1963)

\section{Syracuse}

Onondaga County Public Library (1978)

Syracuse University Library (1878)

Syracuse University College of Law H. Douglas Barclay Law Library (1978)

\section{Troy}

Troy Public Library (1869)

\section{Unlondale}

Nassau Library System (1965)

\section{Utica}

Utica Public Library (1885)

SUNY Institute of Technology Library (1977)

\section{West Polnt}

U.S. Military Academy Library (unknown)

\section{White Plalns}

Pace University Law Library (1978)

\section{Yonkers}

Yonkers Public Library Getty Square Branch (1910)

\section{Yorktown Helghts}

Mercy College Library (1976)

\section{NORTH CAROLINA}

\section{Ashovllle}

University of North Carolina at Asheville D. Hiden Ramsey Library (1965)

\section{Bolling Springs}

Gardner-Webb College Dover Memorial Library (1974)

\section{Boone}

Appalachian State University Carol Grotnes Belk Library (1963)

\section{Bules Crook}

Campbell University Carrie Rich Memorial Library (1965)

\section{Chapel HIII}

University of North Carolina at Chapel Hill Davis Library (1884) REGIONAL

University of North Carolina at Chapel Hill Law Library (1978)

\section{Charlotte}

Public Library of Charlotte and Mecklenburg County (1964) Queens College Everett Library (1927)

University of North Carolina at Charlotte Atkins Library (1964)

\section{Cullowhee}

Westem Carolina University Hunter Library (1953)

\section{Davldson}

Davidson College Library (1893) 


\section{Durham}

Duke University School of Law Library (1978)

Duke University William R. Perkins Library (1890)

North Carolina Central University Law School Library (1979)

North Carolina Central University James E. Shepard Memonal Library (1973)

\section{Elon College}

Elon College Ins Holt McEwen Library (1971)

\section{Fayetteville}

Fayetteville State University Charles W. Chesnutt Library (1971)

\section{Greensboro}

North Carolina Agricultural and Technical State University F. D. Bluford Library (1937)

University of North Carolina at Greensboro Walter Clinton Jackson Library (1963)

\section{Greenville}

East Carolina University J. Y. Joyner Library (1951)

\section{Laurlnburg}

Saint Andrews Presbyterian College DeTamble Library (1969)

\section{Lexlngton}

Davidson County Public Library (1971)

\section{Mount Ollve}

Mount Olive College Moye Library (1971)

\section{Pembroke}

Pembroke State University Mary H. Livermore Library (1956)

\section{Ralelgh}

Department of Cultural Resources Division of State Library (unknown) North Carolina State University D. H. Hill Library (1923)

North Carolina Supreme Court Library (1972)

\section{Rocky Mount}

North Carolina Wesleyan College Library (1969)

\section{Sallsbury}

Catawba College Library (1925)

\section{Wilmington}

University of North Carollna at Wilmington William M. Randall Library (1965)
Forsyth County Public Library (1954)

Wake Forest University School of Law Library (1902)

\section{NORTH DAKOTA}

\section{Blsmarck}

North Dakota State Library (1971)

North Dakota Supreme Court Law Library (unknown)

State Historical Society of North Dakota State Archives \& Historical Research Library (1907)

Veterans' Memorial Public Library (1967)

\section{Dicklnson}

Dickinson State University Stoxen Library (1968)

\section{Fargo}

Fargo Public Library (1964)

North Dakota State University Library (1907) REGIONAL

\section{Grand Forks}

University of North Dakota Chester Fritz Library (1890)

Minot

Minot State University Memorial Library (1925)

\section{Valley Clty}

Valley City State University Allen Memorial Library (1913)

\section{NORTHERN MARIANA ISLANDS}

\section{Salpan}

Northem Marianas College Olympio T. Borja Memorial Library (1988)

\section{OHIO}

\section{Ada}

Ohio Northem University J. P. Taggart Law Library (1965)

\section{Akron}

Akron-Summit County Public Library (1952)

University of Akron Bierce Library (1963)

University of Akron School of Law Library (1978)

\section{Allance}

Mount Union Colloge Library (1888) 


\section{Ashland}

Ashland University Library (1938)

\section{Athens}

Ohio University Alden Library (1886)

\section{Batavla}

University of Cincinnati Clermont College Library (1973)

\section{Bluffton}

Bluffton College Musselman Library (1951)

\section{Bowling Green}

Bowling Green State University Jerome Library (1933)

\section{Canton}

Malone College Everett L. Cattel Library (1970)

\section{Chardon}

Chardon Public Library (1971)

\section{CIncInnatl}

Public Library of Cincinnati and Hamilton County (1884)

University of Cincinnati Langsam Library (1929)

University of Cincinnati College of Law Marx Law Library (1978)

U.S. Court of Appeals 6th Circuit Library (1986)

\section{Cleveland}

Case Westem Reserve University Freiberger Library (1913)

Case Westem Reserve University School of Law Library (1979)

Cleveland Public Library (1886)

Cleveland State University Cleveland-Marshall College of Law, Joseph W. Bartunek III Law Library (1978)

Cleveland State University Library (1966)

Municipal Reference Library (1970)

\section{Cleveland Helghts}

Cleveland Heights-University Heights Public Library (1970)

\section{Columbus}

Capital University Law School Library (1980)

Capital University Library (1968)

Columbus Metropolitan Library (1885)

Ohio State University College of Law Library (1984)

Ohio State University Libraries (1907)

Ohio Supreme Court Law Library (1973)

State Library of Ohio (unknown) REGIONAL

\section{Dayton}

Dayton and Montgomery County Public Library (1909)

University of Dayton Roesch Library (1969)

Wright State University Library (1965)
Delaware

Ohio Wesleyan University L. A. Beeghly Library (1845)

Elyrla

Elyria Public Library (1966)

FIndlay

Findlay University of Shafer Library (1969)

\section{Gambler}

Kenyon College Library (1873)

\section{Granvllle}

Denison University Libraries William H. Doane Library (1884)

Hiram

Hiram College Teachout-Price Memorial Library (1874)

\section{Kent}

Kent State University Libraries (1962)

Marletta

Marietta College Dawes Memorial Library (1884)

Marlon

Marion Public Library (1979)

\section{Middletown}

Miami University Middletown Gardner-Harvey Library (1970)

\section{New Concord}

Muskingum College Library (1966)

\section{Oberlln}

Oberlin College Library (1858)

\section{Oxford}

Miami University Libraries King Library (1909)

\section{Portsmouth}

Shawnee State University Library (1987)

\section{Rlo Grande}

University of Rio Grande Jeanette Albiez Davis Library (1966)

\section{Springfleld}

Clark County Public Library (1884) 


\section{Steubenville}

Franciscan University of Steubenville John Paul II Library (1971)

Public Library of Steubenville and Jefferson County (1950)

\section{Tiffin}

Heidelberg College Beeghly Library (1964)

\section{Toledo}

Toledo-Lucus County Public Library (1884)

University of Toledo College of Law Library (1981)

University of Toledo Library (1963)

\section{Unlverslty Helghts}

John Carroll University Grasselli Library (1963)

\section{Westerville}

Otterbein College Courtright Memorial Library (1967)

\section{Wilmington}

Wilmington College S. Arthur Watson Library (1986)

\section{Wooster}

College of Wooster Andrews Library (1966)

\section{Worthlngton}

Worthington Public Library (1984)

\section{Youngstown}

Public Library of Youngstown and Mahoning County (1923)

Youngstown State University William F. Maag Library (1971)

\section{OKLAHOMA}

\section{Ada}

East Central Oklahoma State University Linscheid Library (1914)

\section{Alva}

Northwestem OKlahoma State University J. W. Martin Library (1907)

\section{Bethany}

Southem Nazarene University R. T. Williams Leaming Resources Center (1971)

\section{Durant}

Southeastem OKlahoma State University Henry G. Bennett Memorial Library (1929)

\section{Edmond}

Central State University Library (1934)

\section{Enld}

Public Library of Enid and Garfield County (1908)

\section{Langston}

Langston University G. Lamar Harrison Library (1941)

\section{Lawton}

Lawton Public Library (1987)

\section{Norman}

University of Oklahoma Libraries Bizzell Mermorial Library (1893) University of Oklahoma Law Library (1978)

\section{Oklahoma Clty}

Metropolitan Library System Main Library (1974)

Oklahoma City University Dulaney Browne Library (1963)

Oklahoma Department of Libraries (1893) REGIONAL

\section{Shawnee}

Oklahoma Baptist University Library (1933)

\section{Stlllwator}

Oklahoma State University Edmon Low Library (1907) REGIONAL

\section{Tahlequah}

Northeastem Oklahoma State University John Vaughan Library (1923)

\section{Tulsa}

Tu'sa City-County Library System (1963)

University of Tulsa College of Law Library (1979)

University of Tulsa McFarlin Library (1929)

\section{Weatherford}

Southwestern OKlahoma State University Al Harris Library (1958)

OREGON

\section{Ashland}

Southem Oregon State College Library (1953)

\section{Bend}

Central Oregon Community College Library/Media Service (1985) 
Corvallis

Oregon State University Library (1907)

\section{Eugene}

University of Oregon Law Library (1979)

University of Oregon Library (1883)

\section{Forest Grove}

Pacific University Harvey W. Scott Memorial Library (1897)

\section{Klamath Falls}

Oregon Institute of Technology Library (1982)

\section{La Grande}

Eastem Oregon State College Walter M. Pierce Library (1954)

\section{McMinnville}

Linfield College Northup Library (1965)

\section{Monmouth}

Westem Oregon State College Library (1967)

\section{Pendleton}

Blue Mountain Community College Library (1983)

\section{Portland}

Lewis and Clark College Aubrey R. Watzek Library (1967)

Multnomah County Portland (1884)

Northwestern School of Law Lewis and Clark College Paul L. Boley Law Library (1979)

Portland State University Millar Library (1963) REGIONAL

Reed College Library (1912)

U.S Department of Energy Bonneville Power Administration Library (1962)

\section{Salem}

Oregon State Library (unknown)

Oregon Supreme Court Law Library (1974)

Willamette University College of Law Library (1979)

Willamette University Main Library (1969)

\section{PANAMA}

\section{Balboa Helghts}

Panama Canal Commission Technical Resources Center

\section{PENNSYLVANIA}

Altoona Area Public Library (1969)

\section{Bethel Park}

Bethel Park Public Library (1980)

\section{Bethlehem}

Lehigh University Libraries Linderman Library (1876)

\section{Blue Bell}

Montgomery County Community College Leaming Resources Center (1975)

\section{Bradford}

University of Pittsburgh at Bradford T. Edwards \& Tullah Hanley Library (1979)

\section{Broomall}

Marple Public Library (1988)

\section{Callfornla}

California University of Pennsylvania Louis L. Manderino Librany (1986)

\section{Carllsle}

Dickinson College Boyd Lee Spahr Library (1947)

Dickinson School of Law Sheeley-Lee Law Library (1978)

\section{Cheyney}

Cheyney University Leslie Pinckney Hill Library (1967)

$$
\text { Collegeville }
$$

Ursinus College Myrin Library (1963)

\section{Coraopolls}

Robert Morris College Library (1978)

\section{Doylestown}

Bucks County Free Library (1970)

\section{East Stroudsburg}

East Stroudsburg University Kemp Library (1966)

$$
\text { Erle }
$$

Erie County Library System (1897)

\section{Greenvllle}

Thiel College Langenheim Memorial Library (1963) 


\section{Harrisburg}

State Library of Pennsylvania (unknown) REGIONAL

Widener University Harrisburg Campus School of Law Library

\section{Haverford}

Haverford College Magill Library (1897)

\section{Hazleton}

Hazleton Area Public Library (1964)

\section{Indlana}

Indiana University of Pennsylvania Stapleton Library (1962)

\section{Johnstown}

Cambria County Library System Glosser Memorial Library Building (1965)

\section{Lancaster}

Bucknell University Ellen Clarke Bertrand Library

Franklin and Marshall College Shadek-Fackenthal Library (1895)

\section{Lewlsburg}

Bucknell University Ellen Clarke Bertrand Library (1963)

\section{Mansfleld}

Mansfield University Library (1968)

\section{Meadville}

Allegheny College Lawrence Lee Pelletier Library (1907)

\section{MIllersville}

Millersville University Helen A. Ganser Library (1966)

\section{Monessen}

Monessen Public Library (1969)

\section{Now Castle}

New Castle Public Library (1963)

\section{Newton}

Bucks County Community College Library (1968)

\section{Norrlstown}

Montgomery County-Nomistown Public Library (1969)

\section{Phlladelphla}

Drexel University W. W. Hagerty Library (1963)

Free Library of Philadelphia (1897)
Saint Joseph's University Drexel Library (1974)

Temple University Paley Library (1947)

Temple University Law Library (1979)

U.S. Court of Appeals Third Circuit Library (1973)

University of Pennsylvania Biddle Law Library (1974)

University of Pennsylvania Library (1886)

\section{Plttsburgh}

Allegheny County Law Library (1977)

Camegie Library of Pittsburgh Allegheny Regional Branch (1924)

Carnegie Library of Pittsburgh (1895)

Duquesne University Law Library (1978)

La Roche College John J. Wright Library (1974)

U.S. Bureau of Mines Library (1962)

University of Pittsburgh Hillman Library (1910)

University of Pittsburgh Barco Law Library (1979)

\section{Pottsville}

Pottsville Free Public Library (1967)

\section{Readling}

Reading Public Library (1901)

\section{Scranton}

Scranton Public Library (1895)

\section{Shlppensburg}

Shippensburg University Ezra Lehman Memorial Library (1973)

\section{Sllppery Rock}

Bailey Library (1965)

\section{Swarthmore}

Swarthmore College McCabe Library (1923)

\section{Unlverslty Park}

Pennsylvania State University Libraries Pattee Library (1907)

\section{VIllanova}

Villanova University Law School Pulling Law Library (1964)

\section{Warren}

Warren Library Association Warren Public Library (1885)

\section{Waynesburg}

Waynesburg College Library (1964)

\section{West Chester}

West Chester University Francis Harvey Green Library (1967)

\section{Wilkes-Barre}

King's College D. Leonard Corgan Library (1949) 


\section{WIIllamsport}

Lycoming College Librery (1970)

\section{York}

York College of Pennsylvania Schmidt Library (1963)

\section{Youngwood}

Westmoreland County Community College Leaming Resources Center (1972)

\section{PUERTO RICO}

\section{Mayaguez}

University of Puerto Rico Mayaguez Campus Library (1928)

\section{Ponce}

Catholic University of Puerto Rico Encamacion Valdes Library (1966) Catholic University of Puerto Rico School of Law Library (1978)

\section{Rlo Pledras}

University of Puerto Rico J. M. Lazaro Library (1928)

\section{RHODE ISLAND}

\section{Barrlngton}

Barrington Public Library (1986)

\section{KIngston}

University of Rhode Island Library (1907)

Newport

U.S. Naval War College Library (1963)

\section{Provldence}

Brown University John D. Rockefeller Jr. Library (unknown) Providence College Phillips Memorial Library (1969)

Providence Public Library (1884)

Rhode Island College James P. Adams Library (1965)

Rhode Island State Law Library (1979)

Rhode Island State Library (1895)

\section{Warwlck}

Warwick Public Library (1966)

\section{Westerly}

Westerly Public Library (1909)
Woonsocket

Woonsocket Harris Public Library (1977)

\section{SOUTH CAROLINA}

\section{Alken}

University of South Carolina-Aiken Gregg-Graniteville Library (1989)

\section{Charleston}

Baptist College at Charleston L. Mendel Rivers Library (1967)

The Citadel Military College Daniel Library (1962)

College of Charleston Robert Scott Small Library (1869)

\section{Clemson}

Clemson University Cooper Library (1893)

\section{Columbla}

Benedict College Library Payton Leaming Resources Center (1969) South Carolina State Library (1895)

University of South Carolina Coleman Karesh Law Library (1983)

University of South Carolina Thomas Cooper Library (1884)

\section{Conway}

University of South Carolina Coastal Carolina College Kimbel Library (1974)

\section{Due West}

Erskine College McCain Library (1968)

\section{Florence}

Florence County Library (1967)

Francis Marion College James A. Rogers Library (1970)

\section{Greenville}

Furman University Library (1962)

Greenville County Library (1966)

\section{Greenwood}

Lander College Jackson Library (1967)

\section{Lancaster}

University of South Carolina-Lancaster Medford Library

\section{Orangeburg}

South Carolina State College Miller F. Whittaker Library (1953)

\section{Rock HIII}

Winthrop College Dacus Library (1896) 


\section{Spartanburg}

Spartanburg County Public Library (1967)

\section{SOUTH DAKOTA}

\section{Aberdeen}

Northem State College Beulah Williams Library (1963)

\section{Brooklngs}

South Dakota State University H. M. Briggs Library (1889)

\section{Plerre}

South Dakota State Library (1973)

South Dakota Supreme Court Library (1978)

\section{Rapld Clty}

Rapid City Public Library (1963)

South Dakota School of Mines and Technology Devereaux Library (1963)

\section{Sloux Falls}

Augustana College Mikkelsen Library (1969)

Sioux Falls Public Library (1903)

\section{Spearflsh}

Black Hills State University Library E. Y. Berry (1942)

\section{Vermillion}

University of South Dakota I. D. Weeks Library (1889)

\section{TENNESSEE}

\section{Brlstol}

King College E. W. King Library (1970)

\section{Chattanooga}

Chattanooga-Hamilton County Bicentennial Librany (1908)

U.S. Tennessee Valley Authority Technical Library (1976)

\section{Clarksvllle}

Austin Peay State University Felix G. Woodward Library (1945)

\section{Cleveland}

Cleveland State Community College Library (1973)

\section{Columbla}

Columbia State Community College John W. Finney Memorial Library (1973)

\section{Cookevllle}

Tennessee Technological University Library (1969)

\section{Jackson}

Lambuth College Luther L. Gobbel Library (1967)

\section{Jefferson Clty}

Carson-Newman College Stephens-Bumett Library (1964)

\section{Johnson Clty}

East Tennessee State University Sherrod Library (1942)

\section{Knoxvllle}

Knoxville County Public Library System Lawson McGhee Library (1973)

University of Tennessee at Knoxville John C. Hodges Library (1907) University of Tennessee Law Library (1971)

\section{MartIn}

University of Tennessee at Martin Paul Meek Library (1957)

\section{Memphls}

Memphis-Shelby County Public Library and Information Center (1896) Memphis State University Cecil C. Humphreys School of Law Library (1979)

Memphis State University Libraries (1966)

\section{Murfreesboro}

Middle Tennessee State University Todd Library (1912)

\section{Nashvllle}

Fisk University Library (1965)

Public Library of Nashville and Davidson County (1884)

Tennessee State Library and Archives (unknown)

Tennessee State University Brown-Daniel Library (1972)

Vanderbilt University Alyne Queener Massey Law Library (1976)

Vanderbilt University Library (1884)

\section{Sewanee}

University of the South Jessie Ball duPont Library (1873)

\section{TEXAS}

Abllene
Abilene Christian University Margaret and Herman Brown Library (1978)
Hardin-Simmons University Rupert and Pauline Richardson Library
(1940)

\section{Arllngton}

Arlington Public Library (1970)

University of Texas at Arlington Library (1963) 


\section{Austln}

Texas State Law Library (1972)

Texas State Library (unknown) REGIONAL

University of Texas at Austin Perry-Castaneda Library (1884)

University of Texas at Austin Edie and Lew Wasserman Public Affairs Librany (1966)

University of Texas at Austin Tartton Law Library (1965)

Baytown

Lee College Library (1970)

\section{Beaumont}

Lamar University Mary and John Gray Library (1957)

\section{Brownwood}

Howard Payne University Walker Memorial Library (1964)

\section{Canyon}

West Texas State University Comette Library (1928)

\section{College Station}

Texas Agricultural and Mechanical University Sterling G. Evans Library (1907)

\section{Commerce}

East Texas State University James Gilliarn Gee Library (1937)

\section{Corpus Christl}

Corpus Christi State University Library (1976)

\section{Corslcana}

Navarro College Learning Resource Center (1965)

\section{Dallas}

Dallas Baptist University Vance Memorial Library (1967) Dallas Public Library (1900)

Southem Methodist University Fondren Library (1925)

University of Texas Southwestern Medical Center Library (1975)

\section{Denton}

North Texas State University Library (1948)

\section{Edlnburg}

University of Texax-Pan American Library (1959)

\section{El Paso}

El Paso Public Library (1906)

University of Texas at El Paso Library (1966)

\section{Fort Worth}

Fort Worth Public Library (1905)

Texas Christian University Mary Couts Bumett Library (1916)
Rosenberg Library (1909)

\section{Garland}

Nicholson Memorial Library System (1990)

\section{Houston}

Houston Public Library (1884)

North Harris County College (1974)

Rice University Fondren Library (1967)

South Texas College of Law Library (1981)

Texas Southem University Thurgood Marshall School of Law Library (1982)

University of Houston-Clear Lake Alfred R. Neumann Library (1980)

University of Houston M. D. Anderson Library (1957)

University of Houston School of Law Library (1979)

\section{Huntsvllle}

Sam Houston State University Newton Gresham Library (1949)

\section{Irvlng}

Irving Public Library System (1974)

\section{KIngsvllle}

Texas Arts and Industries University Jemigan Library (1944)

\section{Laredo}

Laredo Junior College Harold R. Yeary Library (1970)

\section{Longvlew}

Longview Public Library (1961)

\section{Lubbock}

Texas Technical University Library (1935) REGIONAL Texas Technical University School of Law Library (1978)

\section{Nacogdoches}

Stephen F. Austin State University Steen Library (1965)

\section{Rlchardson}

University of Texas at Dallas McDermott Library (1972)

\section{San Angelo}

Angelo State University Port Henderson Library (1964)

\section{San Antonlo}

Palo Alto College Learning Resources Center Saint Mary's University Academic Library (1964)

Saint Mary's University Sarita Kenedy East Law Library (1982)

San Antonio College Library (1972)

San Antonio Public Library (1899)

Trinity University Elizabeth Coates Maddux Library (1964)

University of Texas at San Antonio Library (1973) 


\section{San Marcos}

Southwest Texas State University Library (1955)

\section{Seguln}

Texas Lutheran College Blumberg Memorial Library (1970)

\section{Sherman}

Austin College Abell Library (1963)

\section{Texarkana}

Texarkana College Palmer Memorial Library (1963)

\section{Vlctorla}

Victoria College/University of Houston Victoria Library (1973)

\section{Waco}

Baylor University Caston Law Library (1982)

Baylor University Moody Memorial Library (1905)

\section{Wichlta Falls}

Midwestern State University Moffett Library (1963)

\section{UTAH}

\section{Codar Clty}

Southem Utah State College Library (1964)

\section{Ephralm}

Snow College Lucy A. Phillips Library (1963)

\section{Logan}

Utah State University Merrill Library and Learning Resources Center (1907) REGIONAL

\section{Ogden}

Weber State College Stewart Library (1962)

\section{Provo}

Brigham Young University Harold B. Lee Library (1908)

Brigham Young University Law Library (1972)

\section{Salt Lake Clty}

University of Utah Eccles Health Sciences Library (1970)

University of Utah Law Library (1966)

University of Utah Marriott Library (1893)

Utah State Library (unknown)

Utah State Supreme Court Law Llbrary (1975)

\section{VERMONT}

\section{Burllngton}

University of Vermont Bailey/Howe Library (1907)

\section{Castleton}

Castleton State College Calvin Coolidge Library (1969)

\section{Johnson}

Johnson State College John Dewey Library (1955)

\section{Lyndonvilie}

Lyndon State College Samuel Reed Hall Library (1969)

$$
\text { Middlebury }
$$

Middlebury College Egbert Starr Library (1884)

$$
\text { Montpeller }
$$

Vermont Department of Libraries (1845)

\section{Northfleld}

Norwich University Chaplin Library (1908)

\section{South Royalton}

Vermont Law School Library (1978)

\section{VIRGIN ISLANDS}

\section{Salnt Crolx}

Florence Williarns Public Library (1968)

\section{Salnt Thomas}

University of the Virgin Islands Ralph M. Paiewonsky Library (1973)

\section{VIRGINA}

\section{Alexandrla}

Dept. of the Navy Office of Judge Advocate General Law Library (1963)

\section{Arllngton}

George Mason University School of Law Library (1981)

U.S. Patent \& Trademark Office Scientific and Technical Information Center (1986) 


\section{Blacksburg}

Virginia Polytechnic Institute and State University Carol M. Newman Library (1907)

\section{Brldgewater}

Bridgewater College Alexander Mack Memorial Library (1902)

\section{Charlottesville}

University of Virginia Alderman Library (1910) REGIONAL

University of Virginia Arthur J. Morris Law Library (1964)

\section{Chesapeake}

Chesapeake Public Library (1970)

\section{Danville}

Danville Community College Leaming Resources Center (1969)

\section{Emory}

Emory and Henry College Kelly Library (1884)

\section{Falrfax}

George Mason University Fenwick Library (1960)

\section{Frederlcksburg}

Mary Washington College Library (1940)

\section{Hampden-Sydney}

Hampden-Sydney College Eggleston Library (1891)

\section{Hampton}

Hampton University Huntington Mermorial Library (1977)

\section{Harrlsonburg}

James Madison University Carrier Library (1973)

\section{Lexlngton}

Virginia Military Institute Preston Library (1874)

Washington and Lee University University Library (1910)

Washington and Lee University Wilbur C. Hall Law Library (1978)

\section{Martinsville}

Patrick Henry Community College Learning Resource Center (1971)

\section{Norfolk}

Norfolk Publlc Library (1895)

Old Dominion University Library (1963)

U.S. Armed Forces Staff College Library (1963)

\section{Petersburg}

Virginla State University Johnston Memorial Llbrary (1907)

\section{Quantlco}

Federal Bureau of Investigation Academy Library (1970)

Marine Corps Education Center MCCDC James Carson Breckinridge Library (1967)

\section{Reston}

Department of the Interior Geological Survey Library (1963)

\section{Rlchmond}

U.S. Court of Appeals Fourth Circuit Library (1973)

University of Richmond Boatwright Memorial Library (1900)

University of Richmond Law School Library (1982)

Virginia Commonwealth University Library Services (1971)

Virginia State Law Library (1973)

Virginia State Library \& Archives (unknown)

\section{Roanoke}

Hollins College Fisburn Library (1967)

\section{Salom}

Roanoke College Library (1886)

\section{WIIllamsburg}

College of William and Mary Marshall-Wythe Law Library (1978) College of William and Mary Swem Library (1936)

\section{WIse}

Clinch Valley College John Cook Wyllie Library (1971)

\section{WASHINGTON}

\section{Bellevue}

Bellevue Public Library (1990)

\section{Bellingham}

Westem Washington University Mable Zoe Wilson Library (1963)

\section{Cheney}

Eastern Washington University JFK Library (1966)

\section{Des Molnes}

Highline Community College Library (1983)

\section{Ellensburg}

Central Washington University Llbrary (1962)

\section{Everett}

Everett Publlc Llbrary (1914) 


\section{Olympla}

Evergreen State College Daniel J. Evans Library (1972)

Washington State Law Library (1979)

Washington State Library (unknown) REGIONAL

\section{Port Angeles}

North Olympic Library System (1965)

\section{Pullman}

Washington State University Holland Library (unknown)

\section{Seattle}

Seattle Public Library (1908)

University of Washington Suzzallo Library (1890)

University of Washington Marian Gould Gallagher Law Library (1969)

U.S. Court of Appeals 9th Circuit Library (1981)

\section{Spokane}

Gonzaga University School of Law Library (1979)

Spokane Public Library (1910)

\section{Tacoma}

Tacoma Public Librany (1894)

University of Puget Sound Collins Mernorial Librany (1938)

University of Puget Sound School of Law Library (1978)

\section{Vancouver}

Fort Vancouver Regional Library (1962)

\section{Walla Walla}

Whitman College Penrose Mernorial Library (1890)

\section{WEST VIRGINIA}

\section{Athens}

Concord College J. Frank Marsh Library (1924)

\section{Bluefleld}

Bluefield State College Hardway Library (1972)

\section{Charleston}

Kanawha County Public Library (1952)

West Virginia Library Commission (1975)

West Virglnia Supreme Court Law Library (1977)

\section{Elkins}

Davls and Elkins College Library (1913)

\section{Falrmont}

Fairmont State College Llbrary (1884)

\section{Huntington}

Marshall University James E. Morrow Library (1925)

\section{Instltute}

West Virginia State College Drain-Jordon Library (1907)

\section{Montgomery}

West Virginia Institute of Technology Vining Library (1985)

\section{Morgantown}

West Virginia University Library (1907) REGIONAL

\section{Salem}

Salem-Tolkyo University Benedum Library (1921)

\section{Shepherdstown}

Shepherd College Scarborough Library (1971)

\section{Welrton}

Mary H. Weir Public Library (1963)

\section{WISCONSIN}

\section{Appleton}

Lawrence University Seeley G. Mudd Library (1869)

\section{Belolt}

Beloit College Col. Robert H. Morse Library (1888)

\section{Eau Clalre}

University of Wisconsin-Eau Claire William D. Mclntyre Library (1951)

\section{Fond du Lac}

Fond du Lac Public Library (1966)

\section{Green Bay}

University of Wisconsin-Green Bay Library Leaming Center (1968)

\section{La Crosse}

La Crosse Public Library (1883)

University of Wisconsin-La Crosse Murphy Library (1965)

\section{Madlson}

Madison Publlc Library (1965)

State Historical Society of Wisconsin Library (1870) REGIONAL University of Wisconsin-Madison Mernorial Library (1939)

University of Wisconsin-Madison Law Library (1981)

Wisconsin State Law Librany (unknown) 


\section{Milwaukee}

Alverno College Library/Media Center (1971)

Marquette University Law Library (1987)

Medical College of Wisconsin, Inc. Todd Wehr Library (1980)

Milwaukee County Law and Reference Library (1934)

Milwaukee Public Library (1861) REGIONAL

Mount Mary College Haggerty Library (1964)

University of Wisconsin-Milwaukee Golda Meir Library (1960)

\section{Oshkosh}

University of Wisconsin-Oshkosh Forrest R. Polk Library (1956)

\section{Plattevllie}

University of Wisconsin-Platteville Karmann Library (1964)

\section{Raclne}

Racine Public Library (1898)

\section{RIpon}

Ripon College Library (1982)

\section{Rlver Falls}

University of Wisconsin-River Falls Chalmer Davee Library (1962)

\section{Sheboygan}

Mead Public Library (1983)

\section{Stevens Polnt}

University of Wisconsin-Stevens Point Library (1951)

\section{Superlor}

Superior Public Library (1908)

University of Wisconsin-Superior Jim Dan Hill Library (1935)

\section{Waukesha}

Waukesha Public Library (1966)

\section{Wausau}

Marathon County Public Library (1971)

\section{Whitewater}

University of Wisconsin-Whitewater Library and Learning Resources (1963)

\section{WYOMING}

\section{Casper}

Natrona County Public Library (1929)
Wyoming State Law Library (1977)

Wyoming State Library (unknown) REGIONAL

\section{GIllette}

Campbell County Public Library (1980)

\section{Laramle}

University of Wyoming, Coe Library (1907)

University of Wyoming Law Library (1978)

\section{Powell}

Northwest Community College John Taggart Hinckley Library (1967)

\section{Rlverton}

Central Wyoming College Learning Resources Center (1969)

\section{Rock Sprlngs}

Westem Wyoming Community College Library (1969)

\section{Sherldan}

Sheridan College Griffith Memorial Library (1963) 


\section{Alabama}

Blrmingham-2015 2nd Avenue North, 3rd Floor, Berry Building, 35203, Area Code 205 Tel 731-1331, FAX 205-731-0076

\section{ALASKA}

Anchorage-4201 Tudor Center Drive, Suite 319, World Trade Center, Tel. 907-271-6237, FAX 907-271-6242

\section{ARIZONA}

Phoenlx-Federal Building \& U.S. Courthouse, 230 N. 1st Avenue, Room 3412, 85025, Area Code 602 Tel 261-3285, FAX 602-379-4324

\section{ARKANSAS}

Little Rock-Suite 811, 320 W. Capitol Avenue, 72201, Area Code 501 Tel 378-5794, FAX 501-324-7380

\section{CALIFORNIA}

Los Angeles-Room 9200, 11000 Willshire Blvd., 90024, Area Code 310 Tel 575-7105, FAX 575-7220

-San Dlego-6363 Greenwich Drive, Suite 230, 92122, Area Code 619 Tel 557-5395, FAX 619-557-6176

- San Franclsco-Federal Building, Box 6013, 450 Golden Gate Avenue, 94102, Area Code 415 Tel 705-2300, FAX 415-705-2299

- Santa Ana-116 W. 4th Street, Suite \#1, 92701, Area Code 714 Tel 836-2461, FAX 714-836-2332

\section{COLORADO}

Denver-1625 Broadway, Suite 68D, 80202, Area Code 303 Tel 8443246, FAX 303-844-5651

\section{CONNECTICUT}

Hartford-Room 610-B, Federal Office Building, 450 Main Street, 06103, Area Code 203 Tel 240-3530, FAX 203-240-3470

\section{FLORIDA}

- Clearwater-128 North Osceola Avenue, 34615, Area Code 813 Tel 461-0011, FAX 813-449-2889

Mlaml-Suite 224, Federal Building, 51 S.W. First Avenue, 33130, Area Code 305 Tel 536-5267, FAX 305-536-4765

- Orlando-Room 346, University of Central Florida, 32802, Area Code 407 Tel 648-1608

- Denotes regional office with management and administrative oversight responsibilities (no direct business counseling)

- Denotes trade specialist at post or duty station
-Tallahassee-Collins Building, Room 401, 107 W. Gaines Street, 32304, Area Code 904 Tel 488-6469, FAX 904-487-1407

\section{GEORGIA}

Atlanta-Suite 310,4380 Chamblee-Dunwoody Road, 30341, Area Code 404 Tel 452-9102, FAX 404-452-9105

Savannah-120 Barnard Street, A-107, 31401, Area Code 912 Tel 652-4204, FAX 912-652-4241

\section{HAWAII}

Honolulu-4106 Federal Building, P.O. Box 50026, 300 Ala Moana Blvd., 96850, Area Code 808 Tel 541-1782, FAX 808-541-3435

\section{IDAHO}

Bolse (Denver, Colorado District)-700 W. State Street, 83720, Area Code 208 Tel 334-3857, FAX 208-334-2787

\section{ILLINOIS}

Chlcago-1406 Mid Continental Plaza Building, 55 E. Monroe Street, 60603, Area Code 312 Tel 353-4450, FTS 8 353-4450

-Palatlne-W. R. Harper College, Algonquin \& Rodelle Road, 60067, Area Code 312 Tel 397-3000, ×2532

-Rockiord-515 N. Court Street, P.O. Box 1747, 61110-0247, Area Code 815 Tel $987-8123$, FTS 8 363-4347

- Wheaton-Illinois Institute \& Technology, 201 E. Loop Road, 60187, Area Code 312 Tel 353-4332, FAX 312-353-4336

\section{INDIANA}

Indlanapolls-One N. Capitol, Suite 520, 46204, Area Code 317 Tel 269-6214, FAX 317-226-6139

IOWA

Des Molnes-817 Federal Building, 210 Walnut Street, 50309, Area Code 515 Tel 284-4222, FAX 515-284-4021

\section{KANSAS}

- Wlchlta (Kansas Clty, Mlssourl Distrlct)-151 North Volutsia, 672144695, Area Code 316 Tel 269-6160, FAX 316-683-7326

\section{KENTUCKY}

Loulsville-Room 6368, 601 W. Broadway, 40202, Area Code 502 Tel 582-5066, FAX 502-582-6573

\section{LOUISIANA}

New Orleans-432 World Trade Center, No. 2 Canal Street, 70130 , Area Code 504 Tel 589-6546, FAX 207-626-9156 


\section{MAINE}

-Augusta (Boston, Distrlct Offlce)-77 Sewell Street, 04330, Area Code 207 Tel 622-8249, FAX 207-626-9156

\section{MARYLAND}

Baltimore-415 U.S. Customhouse, 40 South Gay Streets, 21202, Area Code $301 \mathrm{Tel}$ 962-3560, FAX 410-962-7813

- Galthersburg-c/0 National Institute of Standards \& Technology, Bldg. A11, 20899 Area Code 301 Tel 975-3904, FAX 301-948-4360

\section{MASSACHUSETTS}

Boston-World Trade Center, Suite 307, Commonwealth Pier Area, 02210, Area Code 617 Tel 565-8563, FAX 617-565-8530

\section{MICHIGAN}

Detrolt-1140 McNamara Building, 477 Michigan Avenue, 48226, Area Code 313 Tel 226-3650, FAX 313-226-3657

- Grand Rapids-300 Monroe N.W., Room 409, 49503, Area Code 616 Tel 456-2411, FAX 616-456-2695

\section{MINNESOTA}

Minneapolls-108 Federal Building, 110 S. 4th St., 55401, Area Code 612 Tel 349-1638, FAX 612-349-1650

\section{MISSISSIPPI}

Jackson-328 Jackson Mall Office Center, 300 Woodrow Wilson Blvd., 39213, Area Code 601 Tel 965-4388, FAX 601-965-5386

\section{MISSOURI}

Kansas Clty-Room 635, 601 E. 12th Street, 64106, Area Code 816 Tel 426-3141, FAX 816-426-3140

-St. Louls-7911 Forsyth Blvd., Suite 610, 63105, Area Code 314 Tel 425-3302-4, FAX 314-425-3381

\section{NEBRASKA}

Omaha-11133 O Street, 68137, Area Code 402 Tel 221-3664, FAX 402-221-3668

\section{NEVADA}

Reno-1755 E. Plumb Lane, Room 152, 89502, Area Code 702 Tel 784-5203, FAX 702-784-5343

\section{NEW JERSEY}

Trenton-3131 Princeton Pike Building, Suite 100, 08648, Area Code 609 Tel 989-2100, FAX 609-989-2395

\section{NEW MEXICO}

Albuquerque-625 Silver S.W. Suite 320, 87102, Area Code 505 Tel 706-2070, FAX 505-766-1057

\section{NEW YORK}

Buffalo-1312 Federal Building, $111 \mathrm{~W}$. Huron Street, 14202, Area Code 716 Tel 846-4191, FAX 716-846-5290
New York-Federal Office Building, 26 Federal Plaza, Foley Square, 10278, Area Code 212 Tel 264-0634, FAX 264-1350

-Rochester-111 E. Avenue, Suite 220, 14604, Area Code 716 Tel 263-6480, FAX 716-325-6505

\section{NORTH CAROLINA}

Greensboro-400 W. Market Street, P.O. Box 1950, 27402, Area Code 919 Tel 333-5345, FAX 919-333-5158

\section{OHIO}

CIncInnatl-9504 Federal Building, 550 Main Street, 45202, Area Code 513 Tel 684-2944, FAX 513-684-3200

Cleveland-Room 600, 668 Euclid Avenue, 44114, Area Code 216 Tel 522-4750, FAX 216-522-2235

\section{OKLAHOMA}

Oklahoma Clty-5 Broadway Executive Park, Suite 200, 6601 Broadway Extension, 73116, Area Code 405 Tel 231-5302, FAX 405-8415245

- Tulsa-440 S. Houston Steet, 74127, Area Code 918 Tel 581-7650, FAX 918-581-2844

\section{OREGON}

Portland-Suite 242 One World Trade Center, 97204, Area Code 503 Tel 326-3001, FAX 503-326-6351

\section{PENNSYLVANIA}

Phlladelphla-475 Allendale Road, King of Prussia, 19106, Area Code 215 Tel 951.7954 , FAX 215-951-7959

Plttsburgh-2002 Federal Building, 1000 Liberty Avenue, 15222, Area Code 412 Tel 644-2850, FAX 412-644-4875

\section{PUERTO RICO}

San Juan (Hato Rey)-Room G-55, Federal Building, Chardon Avenue, 00918, Area Code 809 Tel 786-5555, Ext. 555, FAX 809-786-5692

\section{RHODE ISLAND}

-Provldence (Hartford DIstrlct Offlce)-7 Jackson Walkway, 02903, Area Code 401 Tel 528-5104, FAX 401-528-5067

\section{SOUTH CAROLINA}

- Charleston-Room 128, 9 Library Street, 29424, Area Code $803 \mathrm{Tel}$ $727-4361$

Columbla-Strom Thurmond Federal Building, Suite 172, 1835 Assembly Street, 29201, Area Code 803 Tel 765-5345, FAX 803-253-3614

\section{TENNESSEE}

Knoxville-301 E. Church Avenue, 37915, Area Code 615 Tel 549-9265

-Memphls-22 N. Front Street, Suite 200, 38103, Area Code 901 Tel 544-4137, FAX $901575-3510$

Nashville-Suite 1114, Parkway Towers, 404 James Robertson Parkway, 37219-1505, Area Code 615 Tel 736-5161, FAX 615-736-2454 


\section{TEXAS}

- AustIn-P.O. Box 12728, Suite 1200, 816 Congress Avenue, 78701, Area Code 512 Tel 482-5939, FAX 512-320-9674

Dallas-Room 7A5, 1100 Commerce Street, 75242, Area Code 214 Tel 767-0542, FAX 214-767-8240

Houston-2625 Federal Courthouse Building, 615 Rusk Street, 77002, Area Code 713 Tel 229-2578, FAX 713-229-2203

\section{UTAH}

Salt Lake Clty-324 S. State Street, Suite 105, 84111, Area Code 801 Tel 524-5116, FAX 801-524-5880

\section{VIRGINIA}

Rlchmond-8010 Federal Building, 400 North 8 th Street, 23240, Area Code 804 Tel 771-2246, FAX 804-771-2390

\section{WASHINGTON}

Seattle-3131 Elliott Avenue, Suite 290, 98121, Area Code 206 Tel 553-5616, FAX 206-563-7253

\section{WEST VIRGINIA}

Charleston-405 Capitol Street, 26301, Area Code 304 Tel 347-5123, FAX 304-347-5408

\section{WISCONSIN}

Mllwaukee-605 Federal Building, 517 E. Wisconsin Avenue, 53202, Area Code 414 Tel 297-3473, FAX 414-292-3470 


\begin{tabular}{|c|c|c|}
\hline \multirow[t]{3}{*}{$\begin{array}{l}\text { NIST-114A } \\
\text { (REV. 3-90) }\end{array}$} & \multirow[t]{2}{*}{$\begin{array}{l}\text { U.S. DEPARTMENT OF COMMERCE } \\
\text { NATIONAL INSTITUTE OF STANDARDS AND TECHNOLOGY }\end{array}$} & $\begin{array}{l}\text { 1. PUBLICATION OR AEPORT NUMBER } \\
\text { NIST/SP }-305 / 23\end{array}$ \\
\hline & & 2. PERFORMING ORGANIZATION REPORT NUMBER \\
\hline & BIBLIOGRAPHIC DATA SHEET & $\begin{array}{l}\text { 3. PUBLCATION DATE } \\
\text { June } 1992\end{array}$ \\
\hline
\end{tabular}

4. TITLE AND SUBTITLE

Publications of the National Institute of Standards and Technology, 1991 Catalog

5. AUTHOR(S)

Ernestine T. Gladden, Editor

6. PERFORMING ORGANIZATION (IF JOINT OR OTHER THAN NIST, SEE INSTRUCTIONS)

U.S. DEPARTMENT OF COMMERCE

NATIONAL INSTITUTE OF STANDARDS AND TECHNOLOGY（TS）

GAITHERSBURG, MO 20899

7. CONTRACT/GRANT NUMBER

8. TYPE OF REPORT AND PERIOD COVERED January-December 1991

9. SPONSORING ORGANIZATION NAME AND COMPLETE ADDRESS (STREET, CITY, STATE, ZIP)

Same as item 6.

10. SUPPLEMENTARY NOTES

11. ABSTRACT (A 200-WORD OR LESS FACTUAL SUMMARY OF MOST SIGNIFICANT INFORMATION. IF DOCUMENT INCLUDES A SIGNIFICANT BIBLOGRAPHY OR LTERATURE SURVEY, MENTION IT HERE.)

The 23rd Supplement to Special Publication 305 contains full bibliographic citations including keywords and abstracts for National Institute of Standards and Technology (NIST) 1991 papers published and entered into the National Technical Information Service (NTIS) collection. (Also included are NBS/NIST papers published prior to 1991, but not reported in previous supplements of this annual catalog.) Four indexes are included to allow the user to identify NBS/NIST papers by personal author, keywords, title, or NTIS/order report number.

12. KEY WORDS (6 TO 12 ENTRIES; ALPHABETICAL ORDER; CAPITALIZE ONLY PROPER NAMES; AND SEPARATE KEY WORDS BY SEMICOLONS) abstract, NBS/NIST publications; catalog, NBS/NIST publications; NBS/NIST publications; publications, NBS/NIST

13. AVAILABILITY

\section{$\mathrm{X}$ UNLIMITED}

FOR OfFICIAL DISTRIBUTION. DO NOT RELEASE TO NATIONAL TECHNICAL INFORMATION SERVICE (NTIS).

ORDER FAOM SUPERINTENDENT OF DOCUMENTS, U.S. GOVERNMENT PRINTING OFFICE, WASHINGTON, DC 20402.

OAOER FROM NATIONAL TECHWIGALINFORMATION SERYICE (NTIS) SPRIMGFIELD, VA 22161.

14. NUMBER OF PRINTED PAGES

\section{3}

15. PRICE 


\section{ANNOUNCEMENT OF NEW PUBLICATIONS OF THE NATIONAL INSTITUTE OF STANDARDS AND TECHNOLOGY}

Superintendent of Documents

Government Printing Office

Washington, DC 20402

Dear Sir:

Please add my name to the announcement list of new publications as issued by the National Institute of Standards and Technology.

Name

Company

Address

City

State

Zip Code

(Notifleation key N519) 

Please Type or Print (Form is aligned for typewriter use.)

\begin{tabular}{|c|c|c|c|c|}
\hline Qty. & Stock Number & Title & $\begin{array}{c}\text { Price } \\
\text { Each }\end{array}$ & $\begin{array}{c}\text { Total } \\
\text { Price }\end{array}$ \\
\hline & & & & \\
\hline & & & & \\
\hline & & & & \\
\hline & & & & \\
\hline & & \multicolumn{2}{|c|}{ Total for Publications } & \\
\hline
\end{tabular}

Subscriptions

\begin{tabular}{|c|c|c|c|c|}
\hline Qty. & (List ID) & Title & $\begin{array}{l}\text { Price } \\
\text { Each }\end{array}$ & $\begin{array}{l}\text { Total } \\
\text { Price }\end{array}$ \\
\hline & & & & \\
\hline & & & & \\
\hline & & & 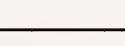 & \\
\hline & & & & \\
\hline $\begin{array}{l}\text { NOTE: } P \\
\text { Internatio }\end{array}$ & omers pl & & criptions & \\
\hline
\end{tabular}

(Company or Personal Name)

(Please type or print)

(Additional address/attention line)

\section{(Street address)}

(City, State. ZIP Code)

(Daytime phone including area code)

$$
\text { (Purchase Order No.) }
$$

May we make your name/address available to other mailers?

\section{Please Choose Method of Payment:}

$\square$ Check Payable to the Superintendent of Documents

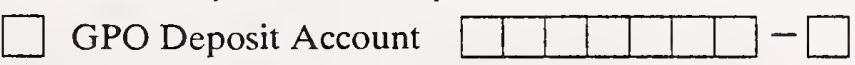

$\square$ VISA or MasterCard Account

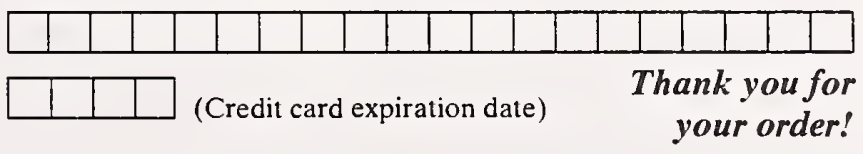

\section{(Authorizing Signature)}

Mail To: New Orders, Superintendent of Documents P.O. Box 371954, Pittsburgh, PA 15250-7954 

9S TELEPHONE ORDERS Call (703) 487.4650
TELEX 89-9405 Telecopier (703) 321-8547 Subscriptions: (703) 487-4630

(See reverse side for RUSH and EXPRESS ordering options)

- HANDLING FEE: A handling fee is required for each order except for Express, Rush, Subscription, QuikORDER, or Pickup orders.

- SHIPPING: U.S.: Printed reports and microfiche copies are shipped First Class Mail or equivalent.

FOREIGN: Regular service: Printed reports and microfiche copies are shipped surface mail.

Air Mail service to Canada and Mexico: add $\$ 3$ per printed report; $75 \notin$ per microfiche copy.

Air Mail service to all other addresses: add $\$ 6$ per printed report; $75 \&$ per microfiche copy.

SUBSCRIPTIONS and standing orders are sent surface mail; contact NTIS for air mail rates.

\section{Address Information}

PURCHASER: DATE:

\begin{tabular}{l}
\hline Last Name \\
\hline Title \\
\hline Company/Organization Initial \\
\hline Address \\
\hline City/State/ZIP \\
\hline Attention \\
\hline Telephone number
\end{tabular}

DTiC Usere Code: Contract No. SHIP TO (Enter ONLY if different from purchaser):

\begin{tabular}{l} 
Last Name \\
\hline Title \\
\hline Company/Organization \\
\hline Address \\
\hline City/Stale/ZIP \\
Attention
\end{tabular}

Telephone number

\section{Method of Payment}

$\square$ Charge my NTIS Deposit Account _ _ _ - - -

Charge my $\square$ Amer. Express $\square$ VISA $\square$ MasterCard

Account No. Exp.

Signature:

(Required to valldate all orders)
Check/Money order enclosed for \$

Please bill ADD $\$ 7.50$ per order (See below for restrictions)t

\section{Order Selection}

Enter NTIS order number(s)

(Ordering by title only will delay your order)

(For computer products, see reverse)

1

2.

3.

4.

5.

6.

7.

OVER - Order continued on reverse

t Billing Service: This service is restricted to customers in the United States, Canada, and Mexico for an additional $\$ 7.50$ per order. A late payment charge will be applied to all billings more than 30 days overdue.

t† Customer Routing Code: NTIS can label each item for routing within your organization. If you want this service, put your routing code in this box.
Purchase Order No.

\section{Rev. 5/20/88}




\section{Order Selection (Cont.)}

\section{Enter the NTIS order number(s) \\ (Ordering by tite only will delay your order)}

8

9.

10.

11.

12.

13.

14.

15.

16.

17.

18.

19.

\begin{tabular}{|c|c|c|c|c|c|}
\hline & \multicolumn{2}{|c|}{ QUANTITY } & \multirow[b]{2}{*}{$\begin{array}{l}\text { UNIT } \\
\text { PAICE }\end{array}$} & \multirow[b]{2}{*}{$\begin{array}{l}\text { Foreign } \\
\text { Air Mail }\end{array}$} & \multirow[b]{2}{*}{$\begin{array}{l}\text { TOTAL } \\
\text { PAICE }\end{array}$} \\
\hline $\begin{array}{l}\text { Customer } \\
\text { Routing }\end{array}$ & $\begin{array}{c}\text { Printed } \\
\text { Copy }\end{array}$ & $\begin{array}{l}\text { Micro- } \\
\text { fiche }\end{array}$ & & & \\
\hline & & & & & \\
\hline & & & & & \\
\hline & & & & & \\
\hline & & & & & \\
\hline & & & & & \\
\hline & & & & & \\
\hline & & & & & \\
\hline & & & & & \\
\hline & & & & & \\
\hline & & & & & \\
\hline & & & & & \\
\hline & & & & & \\
\hline & & & & Subtotal & \\
\hline
\end{tabular}

ENTER this amount on the other side of this form.

\section{Computer Products}

If you have questions about a particular computer product, please call our Federal Computer Products Center at (703) 487-4763.
Enter the NTIS order number(s)

(Ordering by titte only will delay your order)

20.

21.

22.

23.

All magnetic tapes are sent air mail or equivalent service to both U.S. and foreign addresses.

\begin{tabular}{|c|c|c|c|}
\hline \multirow[b]{2}{*}{$\begin{array}{l}\text { Customer } \\
\text { Routing }\end{array}$} & \multicolumn{2}{|c|}{$\begin{array}{c}\text { TAPE DENSITY } \\
(9 \text { track })\end{array}$} & \multirow[b]{2}{*}{$\begin{array}{l}\text { TOTAL } \\
\text { PRICE }\end{array}$} \\
\hline & $1600 \mathrm{bpi}$ & $6250 \mathrm{bpi}$ & \\
\hline & & & \\
\hline & & & \\
\hline & & & \\
\hline & \multicolumn{2}{|c|}{ Subtotal } & \\
\hline
\end{tabular}

ENTER this amount on the other side of this form.
SPECIAL RUSH and EXPRESS ORDERING OPTIONS

Telephone: (800) 336-4700

in Virginia call

(703) $487-4700$
RUSH SERVICE-Add \$10 per item: Orders are processed within 24 hours and sent First Class or equivalent. Available to U.S. addresses.

EXPRESS SERVICE-Add $\$ 20$ per item: Orders are processed within 24 hours AND delivered by overnight courier. Available to U.S. addresses only. 
Journal of Research of the National Institute of Standards and Technology-Reports NIST research and development in those disciplines of the physical and engineering sciences in which the Institute is active. These include physics, chemistry, engineering, mathematics, and computer sciences. Papers cover a broad range of subjects, with major emphasis on measurement methodology and the basic technology underlying standardization. Also included from time to time are survey articles on topics closely related to. the Institute's technical and scientific programs. Issued six times a year.

\section{Nonperiodicals}

Monographs - Major contributions to the technical literature on various subjects related to the Institute's scientific and technical activities.

Handbooks - Recommended codes of engineering and industrial practice (including safety codes) developed in cooperation with interested industries, professional organizations, and regulatory bodies.

Special Publications - Include proceedings of conferences sponsored by NIST, NIST annual reports, and other special publications appropriate to this grouping such as wall charts, pocket cards, and bibliographies.

Applied Mathematics Series - Mathematical tables, manuals, and studies of special interest to physicists, engineers, chemists, biologists, mathematicians, computer programmers, and others engaged in scientific and technical work.

National Standard Reference Data Series - Provides quantitative data on the physical and chemical properties of materials, compiled from the world's literature and critically evaluated. Developed under a worldwide program coordinated by NIST under the authority of the National Standard Data Act (Public Law 90-396). NOTE: The Journal of Physical and Chemical Reference Data (JPCRD) is published bi-monthly for NIST by the American Chemical Society (ACS) and the American Institute of Physics (AIP). Subscriptions, reprints, and supplements are available from ACS, 1155 Sixteenth St., NW., Washington, DC 20056.

Building Science Series-Disseminates technical information developed at the Institute on building materials, components, systems, and whole structures. The series presents research results, test methods, and performance criteria related to the structural and environmental functions and the durability and safety characteristics of building elements and systems.

Technical Notes - Studies or reports which are complete in themselves but restrictive in their treatment of a subject. Analogous to monographs but not so comprehensive in scope or definitive in treatment of the subject area. Often serve as a vehicle for final reports of work performed at NIST under the sponsorship of other government agencies.

Voluntary Product Standards - Developed under procedures published by the Department of Commerce in Part 10, Title 15, of the Code of Federal Regulations. The standards establish nationally recognized requirements for products, and provide all concerned interests with a basis for common understanding of the characteristics of the products. NIST administers this program as a supplement to the activities of the private sector standardizing organizations.

Consumer Information Series - Practical information, based on NIST research and experience, covering areas of interest to the consumer. Easily understandable language and illustrations provide useful background knowledge for shopping in today's technological marketplace. Order the above NIST publications from: Superintendent of Documents, Government Printing Office, Washington, DC 20402.

Order the following NIST publications-FIPS and NISTIRs-from the National Technical Information Service, Springfield, VA 22161.

Federal Information Processing Standards Publications (FIPS PUB) - Publications in this series collectively constitute the Federal Information Processing Standards Register. The Register serves as the official source of information in the Federal Government regarding standards issued by NIST pursuant to the Federal Property and Administrative Services Act of 1949 as amended, Public Law 89-306 (79 Stat. 1127), and as implemented by Executive Order 11717 (38 FR 12315, dated May 11, 1973) and Part 6 of Title 15 CFR (Code of Federal Regulations).

NIST Interagency Reports (NISTIR) - A special series of interim or final reports on work performed by NIST for outside sponsors (both government and non-government). In general, initial distribution is handled by the sponsor; public distribution is by the National Technical Information Service, Springfield, VA 22161, in paper copy or microfiche form. 
ADMINISTRATION \& MANAGEMENT

AERONAUTICS \& AERODYNAMICS

AGRICULTURE \& FOOD

ASTRONOMY \& ASTROPHYSICS

ATMOSPHERIC SCIENCES

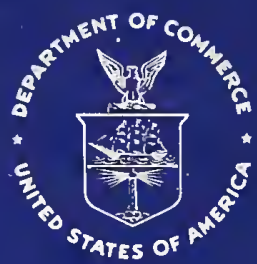

BEHAVIOR \& SOCIETY

BIOMEDICAL TECḢNOLOGY \& HUMAN FACTORS ENGINEERING

BUILDING INDUSTRY TECHNOLOGY

BUSINESS \& ECONOMICS

CHEMISTRY

CIVIL ENGINEERING

COMBUSTION, ENGINES \& PROPELLANTS

COMMUNICATION

COMPUTERS, CONTROL \& INFORMATION THEORY

DETECTION \& COUNTERMEASURES

ELECTROTECHNOLOGY

ENERGY

ENVIRONMENTAL POLLUTION \& CONTROL

HEALTH CARE

INDUSTRIAL \& MECHANICAL ENGINEERING

LIBRARY \& INFORMATION SCIENCES

MANUFACTURING TECHNOLOGY

MATERIALS SCIENCES

MATHEMATICAL SCIENCES

MEDICINE \& BIOLOGY

MILITARY SCIENCES

MISSILE TECHNOLOGY

NATURAL RESOURCES \& EARTH SCIENCES

NAVIGATION, GUIDANCE \& CONTROL

NUCLEAR SCIENCE \& TECHNOLOGY

OCEAN TECHNOLOGY \& ENGINEERING

ORDNANCE

PHOTOGRAPHY \& RECORDING DEVICES

PHYSICS

PROBLEM-SOLVING INFORMATION FOR STATE \& LOCAL GOVERNMENTS

SPACE TECHNOLOGY

TRANSPORTATION

URBAN \& REGIONAL TECHNOLOGY \& DEVELOPMENT 